\title{
Arterial Thromboembolism
}

\section{ACUTE CORONARY SYNDROMES}

LPB0001 | Impact of Heparin Induced Thrombocytopenia on the Outcomes of Hospitalizations for Acute Myocardial Infarction: Analysis of National Inpatient Sample

M.U. Almani ${ }^{1}$; M.J. Tariq ${ }^{1}$; M. Usman ${ }^{1} ;$ M. Qudrat Ullah ${ }^{2}$; M. Yousuf ${ }^{3}$; N. Fatima ${ }^{4}$; E. Edigin ${ }^{1}$; A. Vij ${ }^{1}$; J. Gomez Valencia ${ }^{1}$

${ }^{1}$ John H. Stroger Jr. Hospital of Cook County, Chicago, United States;

${ }^{2}$ Texas Tech University Health Sciences at Permian Basin, Odessa, United States; ${ }^{3}$ Quaid-e-Azam Medical College, Bahawalpur, Pakistan; ${ }^{4}$ Nishtar University Multan, Multan, Pakistan

Background: Heparin induced thrombocytopenia (HIT) can be a lifethreatening complication in patients with acute myocardial infarction (AMI) who receive heparin products. Impact of HIT on morbidity and mortality in AMI patients is not well studied.

Aims: We sought to determine the impact of HIT on outcomes of and treatment strategies for patients admitted with AMI.

Methods: Data was extracted from the National Inpatient Sample (NIS) 2016-2018 Database. The NIS was searched for hospitalizations for adult patients with AMI as principal discharge diagnosis with and without HIT as secondary diagnosis using ICD10 codes. The primary outcome was inpatient mortality. Secondary outcomes are shown in Table 1. Multivariate logistic and linear regression analysis was used accordingly to adjust for confounders. Data was analyzed using STATA.

Results: A total of $1,960,949$ patients were admitted for AMI, of which 2560 (0.13\%) developed HIT. Mean age of patients with HIT was 67.7 years with $37.7 \%$ females. Whereas, mean age of patients without HIT was 66.9 years, with $37.9 \%$ of them being females. The adjusted odds ratio (aOR) for inpatient mortality for AMI with HIT compared to those without HIT was $1.68(95 \% \mathrm{Cl} 1.226-2.303$, $P=0.001)$. Patients with co-morbid HIT had 10 days higher mean difference in length of stay $(95 \% \mathrm{Cl}: 8.9-11.2, P<0.001)$ and $\$ 238,831$ higher mean difference in hospital charges $(95 \% \mathrm{Cl}$ : $189,781$ - 287,881, $P<0.001)$ compared to the patients without HIT. AMI patients with HIT were more likely to get coronary artery bypass graft (aOR: 6.05, 95\% Cl: $5.00-7.31, P<0.001$ ) and less likely to have percutaneous coronary intervention (aOR: $0.48,95 \%$ Cl: $0.39-0.60, P<0.001)$ compared to their counterparts. Detailed outcomes are listed in Table 1.

TABLE 1 Clinical outcomes of patients admitted for acute myocardial infarction with and without co-existing heparin induced thrombocytopenia (HIT) in the U.S from 2016 through 2018, analysis of inpatient sample

\begin{tabular}{|c|c|c|c|c|}
\hline Outcome & Without HIT, \% & With HIT, \% & aOR $(95 \% \mathrm{Cl})$ & $P$-value ${ }^{*}$ \\
\hline \multicolumn{5}{|l|}{ Primary outcome } \\
\hline In hospital mortality & 4.6 & 13.7 & $1.68(1.226-2.303)$ & $0.001^{*}$ \\
\hline \multicolumn{5}{|l|}{ Secondary outcomes } \\
\hline Length of stay (days), mean & 4.4 & 15.8 & $10.07(8.921-11.217)^{\#}$ & $<0.001^{*}$ \\
\hline Total hospital charges (US\$), mean & 94899 & 354,149 & $238,831(189,781-287,881)^{\#}$ & $<0.001^{*}$ \\
\hline Percutaneous coronary intervention & 47.9 & 28.5 & $0.48(0.392-0.604)$ & $<0.001^{*}$ \\
\hline Coronary artery bypass graft & 8.7 & 37.9 & $6.05(5.000-7.312)$ & $<0.001^{*}$ \\
\hline $\begin{array}{l}\text { Intra-operative and postoperative complications of } \\
\text { circulatory system }\end{array}$ & 2.3 & 9.0 & $3.16(2.289-4.354)$ & $<0.001^{*}$ \\
\hline Post-procedural cardiogenic shock & 0.2 & 2.2 & $5.61(2.999-10.502)$ & $<0.001^{*}$ \\
\hline Cardiac arrest & 2.9 & 8.0 & $1.67(1.175-2.383)$ & $0.004^{*}$ \\
\hline Cardiogenic shock & 6.2 & 28.1 & $3.55(2.796-4.504)$ & $<0.001^{*}$ \\
\hline IABP placement & 4.0 & 19.7 & $4.29(3.328-5.536)$ & $<0.001^{*}$ \\
\hline
\end{tabular}

Abbreviations: *; statistically significant, \#; adjusted mean difference, aOR: adjusted odds ratio, Cl: confidence interval, IABP: Intra-aortic balloon pump. Adjusting factors: age, sex, race, charlson comorbidity index, hospital location and teaching status, hospital bed size, hospital region, dyslipidemia, hypertension, obesity, chronic kidney disease, liver disease, smoking, presence of pacemaker, chronic obstructive pulmonary disease, cerebral infarction and peripheral vascular disease.

This is an open access article under the terms of the Creative Commons Attribution-NonCommercial-NoDerivs License, which permits use and distribution in any medium, provided the original work is properly cited, the use is non-commercial and no modifications or adaptations are made. (c) 2020 The Authors. Research and Practice in Thrombosis and Haemostasis published by Wiley Periodicals LLC on behalf of International Society on Thrombosis and Haemostasis (ISTH). 
Conclusions: Development of HIT in patients with AMI is associated with increased mortality, hospital stay, hospital charges, periprocedural complications, cardiac arrest and cardiogenic shock.

PB0001 | High Concentration of Symmetric Dimethylarginine is Associated with Low Platelet Reactivity and Increased Bleeding Risk in Patients with Acute Coronary Syndrome

C. Eyileten ${ }^{1}$; J. Jarosz-Popek ${ }^{1,2}$; D. Jakubik ${ }^{1}$; A. Gąsecka ${ }^{3}$;

M. Wolska ${ }^{1}$; A. Fitas ${ }^{1}$; P. Czajka ${ }^{1}$; A. Nowak ${ }^{1}$; M. Ufnal ${ }^{4}$;

M. Postuła ${ }^{1}$; J.M. Siller-Matuła ${ }^{5,1}$

${ }^{1}$ Center for Preclinical Research and Technology CEPT, Department of Experimental and Clinical Pharmacology, Medical University of Warsaw, Warsaw, Poland; ${ }^{2}$ Doctoral School, Medical University of Warsaw, Warsaw, Poland; ${ }^{3} 1$ st Chair and Department of Cardiology, Medical University of Warsaw, Warsaw, Poland; ${ }^{4}$ Department of Experimental Physiology and Pathophysiology, Laboratory of Centre for Preclinical Research, Medical University of Warsaw, Warsaw, Poland;

${ }^{5}$ Department of Internal Medicine II, Division of Cardiology, Medical University of Vienna, Vienna, Austria

Background: One of the promising biomarkers in CVD are asymmetric dimethylarginine (ADMA) and symmetric dimethylarginine (SDMA), which are products of L-arginine methylation and are both involved in endothelial dysfunction. ADMA, SDMA and Lhomoarginine, have emerged as biomarkers linked to cardiovascular outcomes.

Aims: To investigate the association of SDMA with platelet reactivity and bleeding risk in patients with acute coronary syndrome (ACS) treated with potent $\mathrm{P} 2 \mathrm{Y} 12$ inhibitors prasugrel and ticagrelor.

Methods: Our prospective observational study enrolled 292 patients. Plasma concentrations of SDMA were measured during the hospitalization for ACS. Impedance aggregometry was used. The primary study endpoint was the concentration of metabolites and platelet reactivity.

Results: There was an inverse correlation between SDMA serum levels and platelet reactivity $(r=-0.25 ; P<0.000)$. The ADP+PGE1induced platelet reactivity was $33 \%$ lower among patients with the highest SDMA quartile (4th) as compared to those with the 1-3rd SDMA quartile (8 [0-29] vs 12 [0-126] U; $P<0.001)$. The AA-induced platelet reactivity was $56 \%$ lower among patients with the highest SDMA quartile (4th) as compared to those with the 1-3rd SDMA quartile (4 [0-48] vs 9 [0-133]; $P<0.001$ ). In a multivariate model, the highest SDMA (4th) quartile was found to be an independent predictor of the lowest ADP+PGE1 and AA induced platelet aggregation (OR: 2.666, 95\% Cl [1.184-5.999], $P=0.018$ ).
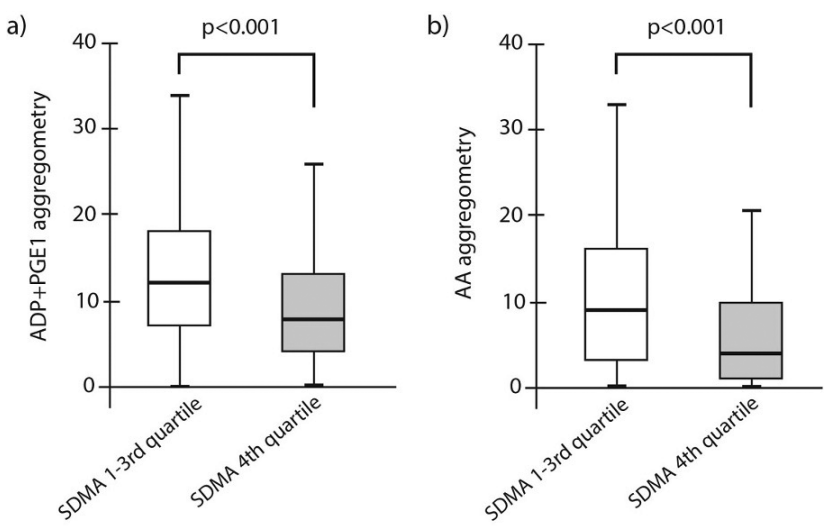

c)

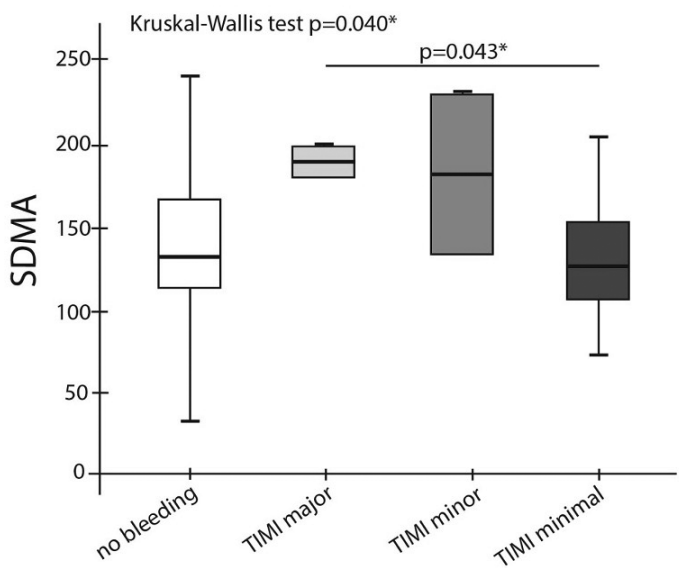

TIMI bleeding during 12-month of DAPT treatment

FIGURE 1 Platelet reactivity as assessed with the Multiple Aggregometry (MEA) for comparison between plasma SDMA quartiles. a) ADP+PGE1-induced platelet aggregation, b) Arachidonic acid (AA)-induced platelet aggregation. c) Distribution of plasma SDMA concentration across patients with and without TIMI bleeding events during 12 months of DAPT treatment. SDMA concentration differed among the four groups ( $P=0.040$, Kruskal - Wallis' test). SDMA concentration in the TIMI major group was higher than that in the TIMI minimal group significantly ( $P=0.043$, Mann-Whitney's $U$ test) 


\begin{tabular}{|c|c|c|c|c|}
\hline \multirow[t]{2}{*}{ Variable } & \multirow[t]{2}{*}{ OR } & \multicolumn{2}{|c|}{$95 \% \mathrm{CI}$} & \multirow[t]{2}{*}{ p-value } \\
\hline & & Lower & Upper & \\
\hline SDMA quartile 4th vs 1 to 3 & 2.666 & 1.065 & 5.758 & 0.018 \\
\hline Age & 1.002 & 0.968 & 1.038 & 0.890 \\
\hline Male (gender) & 2.158 & 0.956 & 4.874 & 0.064 \\
\hline Smoking & 1.014 & 0.449 & 2.290 & 0.972 \\
\hline Diabetes & 0.929 & 0.385 & 2.242 & 0.870 \\
\hline Dyslipidemia & 1.220 & 0.587 & 2.540 & 0.593 \\
\hline $\begin{array}{l}\text { eGFR (assessed with Cockcroft-Gault } \\
\text { formula) }\end{array}$ & 0.995 & 0.976 & 1.014 & 0.585 \\
\hline History of MI & 0.841 & 0.280 & 2.531 & 0.759 \\
\hline History of PCI & 1.994 & 0.508 & 7.833 & 0.323 \\
\hline CRP & 0.986 & 0.832 & 1.168 & 0.870 \\
\hline Hemoglobin & 0.972 & 0.801 & 1.180 & 0.777 \\
\hline $\begin{array}{l}\text { OR, odd ratio; } \mathrm{MI} \text {, myocardial infarction; } \mathrm{PCl} \\
95 \% \text { confidence interval }\end{array}$ & , percut & eous coro & ry interv & ion; $95 \% \mathrm{C}$ \\
\hline
\end{tabular}

FIGURE 2 Multivariate logistic regression analysis of factors which correlates with lowest platelet reactivity (lowest quartile of $A A$ and ADP+PGE1- induced platelet aggregation)

Conclusions: Our study shows that high plasma concentration of SDMA, but not ADMA, is independently associated with low platelet reactivity to ADP and $A A$ and is associated with major and minor bleeding events in patients with ACS on potent antiplatelet therapies. Therefore, SDMA might have a potential to be further evaluated as a blood biomarker for individualization of duration and potency of antiplatelet therapies in an ACS population at high risk of bleeding complications.
PB0002 | A Machine-learning-Based Bio-psycho-Social Model for the Prediction of Non-obstructive and Obstructive Coronary Artery Disease

V. Raparelli ${ }^{1}$; M. Proietti ${ }^{2}$; G.F. Romiti ${ }^{3}$; R. Seccia ${ }^{4}$; G. Di Teodoro ${ }^{4}$; G. Tanzilli ${ }^{5}$; R. Marrapodi ${ }^{3}$; B. Corica ${ }^{3}$; D. Flego ${ }^{3}$; R. Cangemi ${ }^{3}$; L. Palagi ${ }^{4}$; S. Basili ${ }^{3}$; L. Stefanini ${ }^{3}$

${ }^{1}$ Dept. Translational Medicine, University of Ferrara, Ferrara, Italy; ${ }^{2}$ IRCCS Istituti Clinici Scientifici Maugeri, Milan, Italy; ${ }^{3}$ Dept. Translational and Precision Medicine, Sapienza University of Rome, Rome, Italy; ${ }^{4}$ Dept. Computer Control and Management Engineering, Sapienza University of Rome, Rome, Italy; ${ }^{5}$ Dept. Clinical, Internal, Anesthesiology and Cardiovascular Sciences, Sapienza University of Rome, Rome, Italy

Background: Although cardiovascular disease is the leading cause of mortality in both females and males, women are more likely to have non-obstructive ischemic heart disease (IHD) than men. However, the underlying sex- and gender-specific mechanisms and differences in IHD manifestations are still not fully understood.

Aims: To develop an interpretable machine learning (ML) model to gain insight on the clinical, functional, biological and psychosocial features playing a major role in the supervised prediction of nonobstructive versus obstructive coronary artery disease (CAD).

Methods: From the EVA study, we analysed a consecutive unselected cohort of adults hospitalised for IHD undergoing coronary angiography. Non-obstructive CAD was defined by a coronary stenosis at the angiogram $<50 \%$. Baseline clinical and psycho-socio-cultural characteristics were used for computing a frailty index based on Rockwood and Mitnitsky model, and gender score according to GENESIS-PRAXY methodology. The serum concentration of inflammatory cytokines was measured with a multiplex flow cytometry assay. An XGBoost classifier combined to an explainable artificial intelligence tool (SHAP) was employed to identify the most influential features in discriminating obstructive versus non-obstructive CAD.

Results: Among the overall EVA cohort $(n=509), 311$ individuals (mean age $67 \pm 11$ years, 38\% females; $67 \%$ obstructive CAD) with complete data were analysed. The ML-based model (83\% accuracy and $87 \%$ precision) revealed that while obstructive CAD associated with a lower frailty index (i.e., lower physiological reserve), older age and a cytokine signature characterised by IL-1 $\beta$, IL-12p70 and IL-33, non-obstructive CAD is more likely associated with higher gender score (i.e., social characteristics traditionally ascribed to women, regardless of biological sex) and with a cytokine signature characterised by IL-18, IL-8, IL-23.

Conclusions: Integrating clinical, biological and psychosocial features, we have optimised a sex- and gender-unbiased model that discriminates obstructive and non-obstructive CAD. Further mechanistic studies will shed light on the biological plausibility of the observed associations. 
PB0003 | A Coupled 1D-3D Hybrid Model for Patient-specific Coronary Flow Simulations: Bridging the Gap between Macroscale Hemodynamics and Microscale Thrombosis Models

N. Grande Gutierrez; T. Sinno; S. Diamond

University of Pennsylvania, Philadelphia, United States

Background: Thrombosis is the main complication associated with cardiovascular disease, resulting in myocardial infarction and stroke, the leading causes of death globally. Adverse cardiovascular events in coronary artery disease patients are the result of plaque rupture or erosion in combination with occlusive thrombosis-promoting factors. Highly resolved 3D hemodynamic data at the stenosis is essential to model shear-sensitive thrombotic events in coronary artery disease.

Aims: This study aims to develop a computational framework for patient-specific, multi-scale, multi-physics simulations to investigate the process of clot formation from initial platelet deposition to total occlusion in atherothrombosis.

Methods: We developed a hybrid 1D-3D simulation framework to compute patient-specific coronary hemodynamics efficiently. A 1D model of the coronary flow is coupled to an image-based 3D model of the region of interest. This framework provides the advantages of reduced-order modeling, decreasing the global computational cost allowing us to investigate fluid structures and transport phenomena at a very high resolution.

Results: We validated our 1D-3D model against full 3D coronary simulations in healthy and diseased conditions. Our results showed good agreement between the 3D and the 1D-3D models while reducing the computational cost by 40 -fold compared to the $3 \mathrm{D}$ simulation. The 1D-3D model predicted left/right coronary flow distribution within $3 \%$ and provided an accurate estimation of fractional flow reserve and wall shear stress distribution at the stenosis comparable to the 3D simulation.
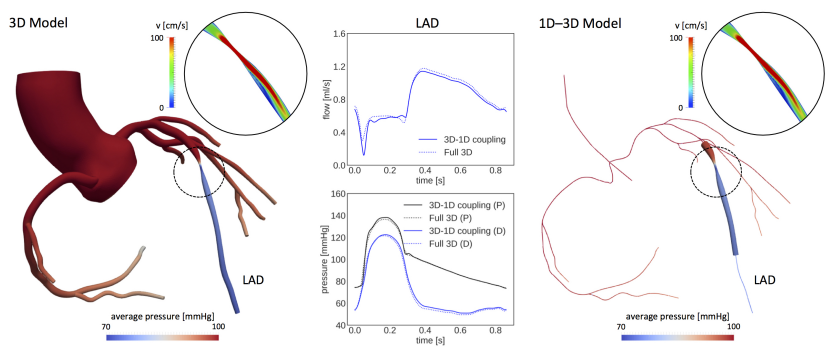

FIGURE 1 Comparison of full 3D and hybrid 1D-3D coronary flow simulation. Pressure distribution and velocity field (longitudinal slice at the location of the stenosis)

Conclusions: Significant savings in computational cost may allow modeling situations with changing geometry, such as growing thrombosis. Also, this approach would allow quantifying the timedependent effect of thrombotic growth and occlusion on the global coronary circulation. We expect this modeling framework provides not only a tool to investigate adverse cardiovascular outcomes such as coronary thrombosis, but also a test-bed for anticoagulation drug testing.

\section{PB0004 | Comparison of Platelet Function Tests during P2Y} Inhibition

P.P. Wadowski ${ }^{1}$; J. Pultar ${ }^{1}$; C. Weikert ${ }^{1}$; B. Eichelberger ${ }^{2}$; I.M. Lang ${ }^{3}$; R. Koppensteiner ${ }^{1}$; S. Panzer ${ }^{2}$; T. Gremmel ${ }^{1,4}$

${ }^{1}$ Medical University of Vienna, Department of Internal Medicine II, Division of Angiology, Vienna, Austria; ${ }^{2}$ Medical University of Vienna, Department of Blood Group Serology and Transfusion Medicine, Vienna, Austria; ${ }^{3}$ Medical University of Vienna, Department of Internal Medicine II, Division of Cardiology, Vienna, Austria; ${ }^{4}$ Landesklinikum Mistelbach-Gänserndorf, Department of Internal Medicine I,

Mistelbach, Mistelbach, Austria

Background: Data on the agreement between light transmission aggregometry (LTA) and multiple electrode aggregometry (MEA) in patients on the more potent $\mathrm{P}_{2} \mathrm{Y}_{12}$ inhibitors are missing so far.

Aims: Therefore, we investigated if the evaluation of the responsiveness to therapy by LTA can be replaced by MEA in 160 acute coronary syndrome (ACS) patients on dual antiplatelet therapy with aspirin and prasugrel or ticagrelor ( $n=80$ each).

Methods: Platelet aggregation was measured using adenosine diphosphate (ADP) or arachidonic acid (AA) as agonists. Cut-off values for high on-treatment residual platelet reactivity (HRPR) to ADP and AA were defined according to previous studies showing an association of HRPR with the occurrence of adverse ischemic outcomes.

Results: ADP-inducible platelet reactivity by MEA correlated significantly with LTA ADP in prasugrel-treated patients $(r=0.4$, $P<0.001)$, but not in those receiving ticagrelor $(r=0.09 ; P=0.45)$. AA-inducible platelet aggregation by LTA and MEA did not correlate in prasugrel- and ticagrelor-treated patients. Sensitivity/specificity of HRPR by MEA to detect HRPR by LTA were $25 \% / 99 \%$ for MEA ADP and $100 \% / 79 \%$ for MEA AA in prasugrel-treated patients, and $0 \% / 100 \%$ for MEA ADP and $70 \% / 83 \%$ for MEA AA in ticagrelortreated patients.

Conclusions: On-treatment residual ADP-inducible platelet reactivity by LTA and MEA shows a significant correlation in prasugrel- but not ticagrelor-treated patients. However, in both groups LTA and MEA revealed heterogeneous results regarding the classification of patients as responders or non-responders to $\mathrm{P}_{2} \mathrm{Y}_{12}$ inhibition. Accordingly, these tests are not interchangeable in the assessment of the response to antiplatelet therapy in ACS patients undergoing $\mathrm{PCI}$. 


\section{PB0005 | A Rare Presentation of ST-segment Elevation}

Myocardial Infarction Associated with Ovarian Stimulation in the Absence of Ovarian Hyperstimulation Syndrome

K.Y. Kim ${ }^{1}$; Y. Gerais ${ }^{1}$; A. Prasad ${ }^{1}$; N.H. Kim ${ }^{1}$; C. Sun ${ }^{1}$; S. Hussein ${ }^{1}$; P. Thapa ${ }^{1}$; R. Ramadurai ${ }^{2}$

${ }^{1}$ Amita Health Saint Joseph Hospital, Chicago, United States; ${ }^{2}$ Amita Health Saint Mary of Nazareth Hospital, Chicago, United States

Background: Follitropin Beta (Follistim) is a recombinant human follicle stimulating hormone (rhFSH) used to stimulate ovarian follicular growth in women. Both venous and arterial thrombosis have been reported in association with ovarian stimulation with and without ovarian hyperstimulation syndrome (OHSS).

Aims: By sharing the presentation of a rare yet serious condition of ST-segment elevation myocardial infarction (STEMI) associated with ovarian stimulation, we aim to emphasize the possible risk of major thromboembolic events associated with exogenous gonadotropin administration for ovarian induction.

Methods: We present a rare association between ovarian stimulation with the use of rhFSH and STEMI, which to our knowledge, is the first case of STEMI in the absence of OHSS associated with ovarian stimulation to be reported.

Results:

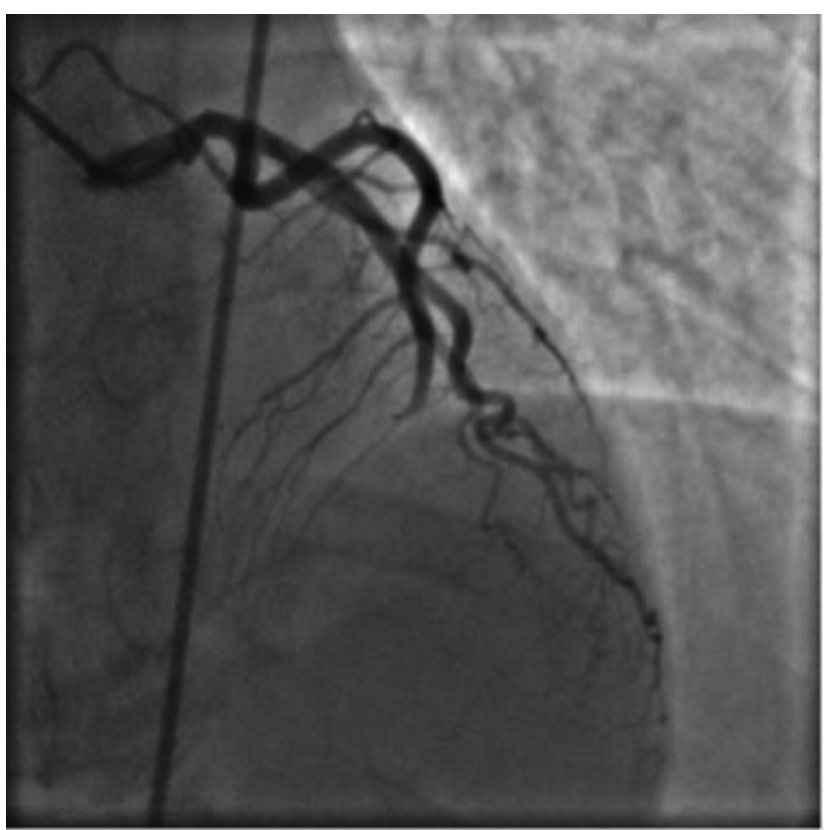

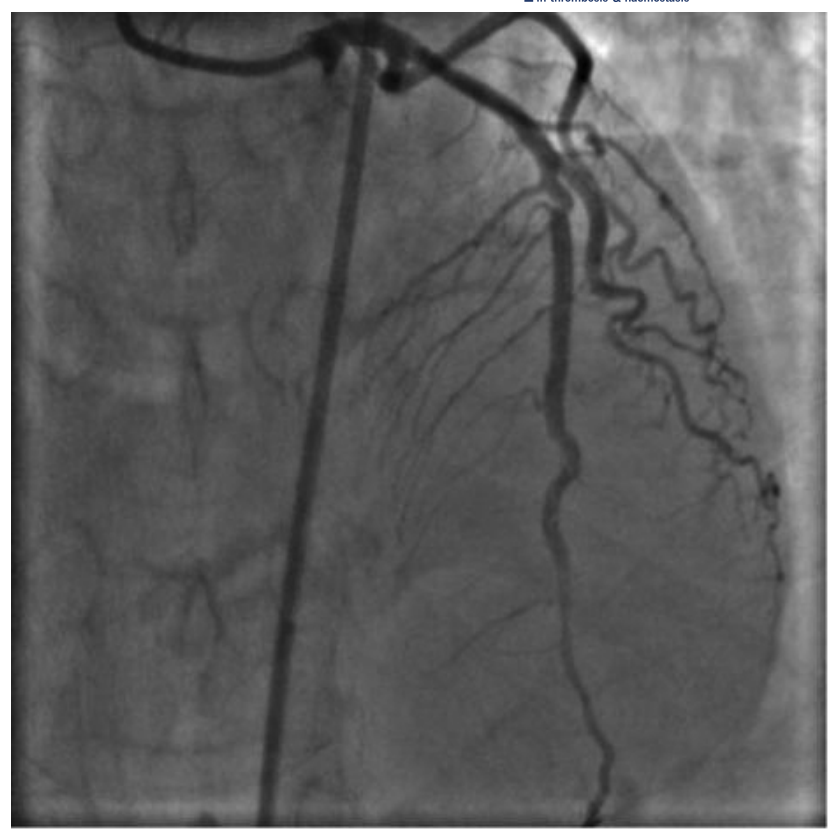

FIGURE 2 Successful recanalization of the LAD after stent placement

A 47-year-old athletic female without any risk factors for cardiovascular disease or predisposing conditions for hypercoagulable state, presented to the emergency room with new onset chest pain. 12lead ECG showed ST-segment elevation in the anterolateral leads with elevated serum troponin levels. Percutaneous coronary intervention $(\mathrm{PCl})$ showed $100 \%$ occlusion of the mid left anterior descending artery (LAD). Two stents were successfully deployed to the LAD.

Conclusions: As our patient with no symptoms of OHSS, cardiovascular risk, or underlying hypercoagulable conditions presented with ACS associated with rhFSH use, the risk of major thromboembolic events associated with exogenous gonadotropin administration for ovarian induction is emphasized. Clinicians should be prompted to evaluate for cardiovascular risk and discuss the risk of thromboembolic events with patients when considering ovarian stimulation. Further analysis of serum estrogen levels in patients developing thromboembolic events with ovarian stimulation may provide guidance into risk stratification of thromboembolic events and the need for prophylactic anticoagulation accordingly. As there are no current guidelines on prophylactic anticoagulation during ovarian stimulation, further studies are needed to provide guidance in anticoagulation therapies and risk stratification for thromboembolic events in these patients.

FIGURE 1 Percutaneous coronary angiography revealing complete occlusion in mid-LAD 
PB0006 | Flowcytometric Assay of Platelet-derived Microparticles and the Dual Anti-platelet Therapy in Egyptian Patients with Ischemic Heart Disease, a Promising Indicator

N. Farouqe ${ }^{1}$; M. Wahba ${ }^{1} ;$ A. Saad ${ }^{1} ;$ R. El-Gamal ${ }^{1} ;$ Y. Elsakhawy $^{1}$; M. Farouk ${ }^{2}$

${ }^{1}$ Ain Shams University Hospitals, Clinical Pathology Department, Cairo, Egypt; ${ }^{2}$ Ain Shams University Hospitals, Cardiology Department, Cairo, Egypt

Background: Platelet-derived microparticles (PMPs) measured by flowcytometry (FCM) have proved recently to be an important pro coagulant element along the pathogenesis and the therapeutic challenge of ischemic heart disease (IHD), especially the acute coronary syndrome (ACS). Therapeutic Program of which includes either a single or a more effective dual anti-platelet therapy (DAPT), with wide range of variability among patients' response, whereas an old-modality partially-subjective platelet function assay, namely light transmission aggregometry (LTA) were used to monitor such response.

Aims: To evaluate the FCM-PMPs role in monitoring the response of DAPT therapy of ACS, in comparison to single anti platelet therapy among Egyptian patients of ischemic heart disease.

Methods: One hundred and thirty individuals, divided as 100 Egyptian patients with proven IHD presented to Ain Shams university hospital- CCU (of which included 68 ACS patients), and 30 age- and sex- matched controls. None had active inflammatory condition causing platelet activation. They all had consent, history taking, especially for their anti-platelet drug intake (single/ DAPT). They also had physical examination and Flowcytometric assay for PMPs in their 3.2\% citrated platelet-free plasma samples, standardized with Magamix beads, expressed in both terms of $\%$ of activation and number/microlitre.

Results: On comparing the citrated-plasma PMPs' measurements, as regards the \% of activation and number/microlitre, among patients treated with single anti-platelet and those with DAPT, using a MannWhitney test of significance, all results were statistically significant.

Conclusions: Flowcytometric PMPs analysis in citrated platelet-free plasma is a promising indicator assay in monitoring IHD (especially ACS) therapeutic response, and aiding in dose escalation, especially when DAPT is adopted. Moreover, they could efficiently replace LTA.
P0117 | A Case of Recurrent Myocardial Infarction and Heterozygous Factor V Leiden

E. Grinsztejn ${ }^{1}$; J.P. Dugan ${ }^{1}$; E.X. Stavrou ${ }^{2,3}$

${ }^{1}$ Case Western Reserve University/University Hospitals Cleveland Medical Center, Cleveland, United States; ${ }^{2}$ Case Western Reserve University School of Medicine, Cleveland, United States; ${ }^{3}$ Louis Stokes Cleveland Veterans Administration Medical Center, VA Northeast Ohio Healthcare System, Cleveland, United States

Background: Factor $\mathrm{V}$ Leiden (FVL) or resistance to activated Protein $\mathrm{C}(\mathrm{aPC})$ results from a single-point mutation in the Factor $\mathrm{V}$ gene, which renders activated Factor $V(F V a)$ enzymatically active but resistant to deactivation by activated protein $\mathrm{C}$ (aPC), leading to a hypercoagulable state. FVL confers a 4 to 5 -fold risk for venous thromboembolism, however its contribution to arterial thrombosis remains controversial.

Aims: Discuss the risk of arterial atherothrombotic events in heterozygous FVL individuals.

Methods: We report a case of a 71-year-old heterozygous FVL man who presented with recurrent acute myocardial infarction (AMI) prompted by interruptions in antiplatelet and anticoagulation therapy.

Results: This patient was diagnosed with heterozygous FVL at 58 years of age when he presented with post-operative pulmonary embolism following a biceps repair. He had no other comorbidities and never smoked. However, within 12 months from his hypercoagulable workup, patient was diagnosed with intermediate grade prostate cancer and was treated with surgery and chemoradiation, achieving complete remission. At 66 years old, he had his first AMI resulting from complete right coronary artery (RCA) occlusion, for which he was placed on dual-antiplatelet therapy and warfarin. After six months of treatment, warfarin was switched to rivaroxaban. The following year, he had another AMI secondary to acute RCA thrombosis, approximately one month after discontinuing rivaroxaban. He had two subsequent AMIs in the following four years, both soon after stopping therapeutic anticoagulation (Tables 1 and 2). Ultimate management was determined to be a combination of low-dose rivaroxaban and clopidogrel.

TABLE 1 Acute Myocardial InH6pfarction Data

Date

$06 / 2014$

$\mathrm{AMI} /$ Coronary artery

Angiographic Findings STEMI/RCA

$100 \%$ occlusion of RCA
Treatment
Thrombectomy + DESx1 to RCA; DAPT + warfarin. Warfarin switched to rivaroxaban after 6 months
$10 / 2015$

STEMI/RCA

Thrombosis of RCA

$03 / 2018$

STEMI/RCA

Acute thrombus; severe in-stent-restenosis at the previous distal stent at bifurcation of rPDA \& rPLV

Thrombectomy + DESx3 to RCA: DAPT + warfarin
Angioplasty to rPLV + DESx1; DAPT + therapeutic enoxaparin

\section{8/2019}

STEMI/RCA

Acute thrombus of RCA

Intravascular aspiration; clopidogrel + rivaroxaban 


\section{Date}

$06 / 2014$

Right common femoral vein DVT

Preceding None

Anticoagulation
$10 / 2015$

Left gastrocnemius vein DVT

Rivaroxaban stopped due to financial burden 1 month prior

Bone metastatic prostate cancer
$03 / 2018$

08/2019

None

Warfarin stopped 1 week prior for colonoscopy

None (PSA undetectable, not on active treatment)
None

Therapeutic enoxaparin stopped 10 days prior due to expanding gluteal hematoma with lower extremity paresthesia and acute anemia

None (PSA undetectable, not on active treatment) cancer

$\mathrm{AMI}=$ acute myocardial infarction; STEMI = ST-elevation myocardial infarction; RCA = right coronary artery

rPDA = right posterior descending artery; $r P L V=$ right posterior-lateral ventricular branch

DES = drug-eluting stent; DAPT $=$ dual-antiplatelet therapy; VTE = venous thromboembolism

DVT = deep vein thrombosis; PSA = prostate-specific antigen

TABLE 2 Cardiovascular Risk Profile

\begin{tabular}{|c|c|c|c|c|}
\hline Date & $06 / 2014$ & $10 / 2015$ & $03 / 2018$ & $08 / 2019$ \\
\hline Total Cholesterol & 145 & 186 & 259 & 142 \\
\hline HDL & 36 & 55 & 74 & 48 \\
\hline LDL & 82 & 113 & 164 & 84 \\
\hline Triglycerides & 134 & 89 & 126 & 48 \\
\hline Hemoglobin A1c (\%) & 5.1 & 5.5 & 5.4 & 5.6 \\
\hline Blood Pressure (mmHg) & $100 / 65$ & $110 / 80$ & $107 / 70$ & $94 / 59$ \\
\hline
\end{tabular}

Conclusions: This case illustrates that FVL may interact with certain genetic and environmental risk factors to promote arterial thrombosis. Therefore, in select patient groups with established cardiovascular disease, a concomitant diagnosis of FVL carrier state may warrant aggressive escalation of therapy for secondary prevention of arterial thrombotic events.

\section{ATHEROSCLEROSIS}

LPB0048 | Association between Plaque Vulnerability and Neutrophil Extracellular Traps (NETs) Levels: The Plaque at RISK Study

J.J. de Vries ${ }^{1}$; A.S. Autar ${ }^{1}$; D.H. van Dam-Nolen ${ }^{1}$; S.J. Donkel ${ }^{1}$; M. Kassem ${ }^{2}$; A.G. van der Kolk ${ }^{3,4}$; T.J. van Velzen ${ }^{5}$; ME. Kooi ${ }^{2}$; J. Hendrikse ${ }^{4}$; P.J. Nederkoorn ${ }^{5}$; D. Bos ${ }^{1}$; A. van der Lugt $^{1}$; M.P. de Maat ${ }^{1}$; H.M. van Beusekom ${ }^{1}$

${ }^{1}$ Erasmus Medical Center, Rotterdam, Netherlands; ${ }^{2}$ Maastricht University Medical Center, Maastricht, Netherlands; ${ }^{3}$ Antoni van Leeuwenhoek Hospital, Amsterdam, Netherlands; ${ }^{4}$ University Medical Center Utrecht, Utrecht, Netherlands; ${ }^{5}$ Academic Medical Center, Amsterdam, Netherlands

Background: Carotid atherosclerotic plaque rupture and its sequelae are among the leading causes of acute ischemic stroke. The risk of rupture and subsequent thrombosis is, among others, determined by vulnerable plaque characteristics and linked to activation of the immune system, in which neutrophil extracellular traps (NETs) potentially play a role.

Aims: To investigate how plaque vulnerability is associated with plasma levels of NETs.

Methods: In this study, we included 182 patients from the Plaque At RISK (PARISK) study in whom carotid imaging was performed to determine the presence and size of plaque ulceration, the presence of a thin or ruptured fibrous cap, and the presence and volume of intraplaque hemorrhage (IPH), lipid-rich necrotic core (LRNC) and calcifications. The PARISK study was approved by the medical ethics committee and informed consent was obtained from patients. Principal component analysis generated a 'vulnerability index' comprising all measured vulnerable plaque characteristics. Plasma levels of the highly specific NETs marker myeloperoxidase-DNA complex were measured using ELISA and patients were divided in two groups based on these levels. The association between the vulnerability index (independent variable) and low or high plasma levels of NETs (dependent variable) was assessed by logistic regression, adjusted for age, sex and time between event and blood sampling.

Results: No significant association between the vulnerability index and NETs levels was detected in the total population (OR 1.28, 95\% $\mathrm{Cl}$ 0.90-1.83). However, in the subgroup of patients naive to statins or antithrombotic medication before the index event, this association was statistically significant (OR 2.08, 95\% Cl 1.04-4.17). Further analyses revealed that this positive association was mainly driven by IPH, LRNC and ulceration (Table). 
TABLE 1 Association between plaque characteristics and myeloperoxidase-DNA levels in subgroups stratified by medication use before the index event

\begin{tabular}{|c|c|c|c|c|}
\hline \multirow[b]{2}{*}{ Plaque characteristic } & \multicolumn{2}{|c|}{$\begin{array}{l}\text { Patients without statins or antithrombotic } \\
\text { medication }(n=72)\end{array}$} & \multicolumn{2}{|c|}{$\begin{array}{l}\text { Patients with statins or antithrombotic } \\
\text { medication }(n=109)\end{array}$} \\
\hline & OR $[95 \% \mathrm{Cl}]$ & $P$-value & OR $[95 \% \mathrm{Cl}]$ & $P$-value \\
\hline Relative IPH volume (\%) & $1.19[1.04-1.35]$ & $0.01^{*}$ & $1.00[0.94-1.06]$ & 0.91 \\
\hline LRNC presence & $2.45[0.78-7.69]$ & 0.13 & $0.61[0.26-1.47]$ & 0.27 \\
\hline Ulceration presence & $5.93[1.38-25.37]$ & $0.02^{*}$ & $2.04[0.81-5.13]$ & 0.13 \\
\hline Ulceration size & $1.70[0.97-3.00]$ & 0.07 & 1.14 [0.77-1.69] & 0.52 \\
\hline Thin or ruptured fibrous cap & $1.51[0.55-4.13]$ & 0.42 & $0.75[0.31-1.80]$ & 0.51 \\
\hline Calcification presence & $0.85[0.18-3.95]$ & 0.84 & - & 0.99 \\
\hline
\end{tabular}

Conclusions: In patients with symptomatic carotid atherosclerotic plaques, plaque vulnerability is positively associated with NETs levels, but only in patients naive to statins or antithrombotic medication before the index event.

\section{LPB0096 | Development of a 3D Tissue-engineered} Neointimal Model as an Alternative to Animal Models of Human Atherosclerosis

$\underline{\text { J. Echrish }}^{1,2}$; Y. Yang ${ }^{3}$; A. Harper ${ }^{1,3}$

${ }^{1}$ School of Medicine/Keele University, Staffordshire, United Kingdom;

${ }^{2}$ School of Pharmacy \&Bioengineering, stoke-on-Trent, United Kingdom;

${ }^{3}$ School of Pharmacy \& Bioengineering/Keele University, Stoke-on-

Trent, United Kingdom

Background: Ischemic heart disease is the leading cause of death worldwide. Most cases are caused by atherosclerosis. Erosion of this plaque triggers unwanted blood clotting which blocks the blood supply to the heart, triggering heart attacks. This process is currently principally studied in animal models.

Aims: To create a tissue-engineered neointima as an alternative to current animal models and assess its effect on primary and secondary haemostasis.

Methods: THP-1 derived foam cells were cultured within collagen hydrogels and triggered to differentiate into foam cells by treatment with lipopolysaccharide, IFN- $\gamma$, and oxidised low-density lipoprotein. Prothrombin times were measured to assess the procoagulant activity of the tissue-engineered neointimal constructs using platelet-poor plasma prepared from blood of healthy medication-free volunteers. Platelet aggregation was assessed using light transmission aggregometry. Tissue factor activity was measured using the fluorogenic substrate SN-17.

Results: This novel in vitro neo-intima tissue can trigger rapid coagulation of human plasma due to the presence of significant tissue factor activity. Prothrombin times were $87.5 \pm 8.4 \mathrm{~s}, 158.3 \pm 12.7 \mathrm{~s}$ and 376.6 $\pm 28.1 \mathrm{~s}$ for gels containing foams cell, M1 cells or no additional cells respectively ( $n=6 ; P<0.05)$. Collagen hydrogels containing THP-1 derived foam cells samples had significantly greater tissue factor activity compared to cell-free hydrogels or M1 macrophage-containing hydrogels. The cell-free collagen hydrogel was found not to trigger platelet aggregation, whilst both the M1- and foam cell-containing hydrogels were found to initiate a slow, but reproducible, platelet activation.

Conclusions: Our tissue-engineered neointimal model can recreate the pro-thrombotic potential of human atherosclerotic plaques. This could form the basis for a novel in vitro 3D model of human atherosclerosis to replace current animal models.

LPB0097 | Role of Low-dose Aspirin on the Release of Circulating Microvesicles in Patients at Cardiovascular Risk with and without Type 2 Diabetes

P. Simeone; R. Liani; R. Tripaldi; S. Ciotti; P. Lanuti; M. Marchisio;

S. Miscia; F. Santilli

Department of Medicine and Aging, Center for Advanced Studies and Technology (CAST), University "G. d'Annunzio" of Chieti-Pescara, Chieti, Italy

Background: Microvesicles(MVs) are small vesicles deriving from all cell type. MVs seems to be one of the procoagulant determinants in type 2 diabetes(T2DM).

Aims: To assess the effects of chronic low dose aspirin(ASA) on levels of total MVs and specific subtypes, such as platelet(PMV), endothelial(EMV), leucocytes(LMV) in DM and non-DM patients in the 24-hour interval between 2 witnessed ASA administrations.

Methods: We enrolled 59 patients with and 41 without T2DM, at cardiovascular risk, on chronic low-dose ASA treatment. The kinetics of platelet cyclooxygenase-1 recovery(COX-1) was characterized by measuring serum $\mathrm{TXB}_{2}$ after 10 and $24 \mathrm{~h}$ after a witnessed ASA administration. Nine healthy subjects were enrolled to verify the number of MVs on circadian measurements. Each subject signed written informed consent. Protocol was approved. 
Results: Total MVs levels and the number of CD45+ LMV were reduced at T10 as compared to T24 within both $\mathrm{DM}(P<0.001$ and $P=0.009$, respectively $)$ and noDM patients $(P<0.001$ and $P=0.034)$ and in the two groups $(P<0.001$ both).The CD41a+PMVs and CD31+EMV did not show differences between T10 and T24 in DM and in non-DM patients, whereas reduced levels at T10 vs. T24 were observed for Annex-positive CD41a+ PMVs. In healthy subjects, nodifference in the levels of total MV, PMV and EMV was observed between $\mathrm{T} 10$ and T24, over $24 \mathrm{~h}$ between two ASA administrations.

A
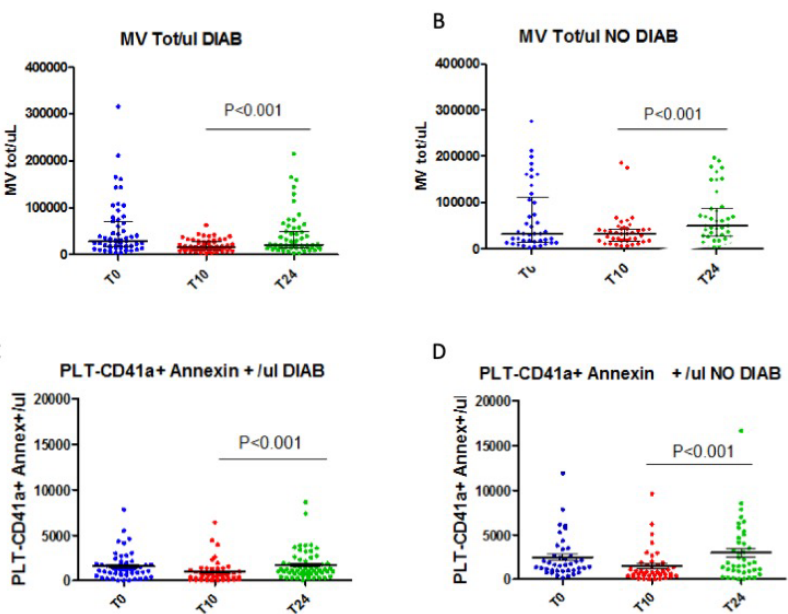

FIGURE 1 Levels of Total Microvescicles, and Platelet derived Microvescicles Annex positive in patients with and without diabetes after 10 and $24 \mathrm{~h}$ after a witnessed aspirin administration

Conclusions: In ASA-treated subjects, daily administration may inhibit the release of total $M V$ and annexin-positive, phosphatydilserine -exposing, PMVduring the 24-hour dosing interval. After excluding a circadian variation in MV levels, mirrored by the stability of the phenotype in healthy subjects, the inhibition after $10 \mathrm{~h}$ since a witnessed ASA administration suggests that COX-1 dependent mechanisms may be involved in this inhibition. With the limits of a cross-sectional study, our findings suggest a previously unappreciated effect of ASA.

PB0007 | The Role of TEC Family Kinases in oxLDL-mediated Platelet Activity in vitro and Platelet and Endothelial Dysfunction in vivo

T. Kohs ${ }^{1}$; T. Zheng ${ }^{1}$; I. Parra-Izquierdoㅎ․ S. Olson ${ }^{1} ; \mathrm{A} . \mathrm{Xie}^{1}$; J.

Hodovan $^{1}$; M. Muller ${ }^{1}$; C. McArthur ${ }^{1}$; J. Johnson ${ }^{1}$; M. Wallisch ${ }^{1}$;

C. Lorentz ${ }^{1}$; N. Verbout ${ }^{1}$; P. Kievit ${ }^{1}$; M. Larson ${ }^{2}$; J. Aslan ${ }^{1}$; C. Puy ${ }^{1}$;

J. Lindner ${ }^{1}$; O. McCarty ${ }^{1}$; J. Shatzel ${ }^{1}$

${ }^{1}$ Oregon Health \& Science University, Portland, United States;

${ }^{2}$ Augustana University, Sioux Falls, United States

Background: Atherosclerosis is characterized by endothelial dysfunction, increased expression of cellular adhesion molecules, and accumulation of oxidized low-density lipoprotein (oxLDL). While it has been shown that oxLDL signals through the Src and TEC-family kinase pathway to potentiate platelet activation in vitro, whether TEC-family kinases regulate platelet and endothelial cell (EC) activity during the development and progression of atherosclerosis in vivo is unclear.

Aims: Investigate the role of the TEC-family kinase pathway in oxLDL-mediated platelet activity in vitro, and platelet and EC dysfunction in vivo.

Methods: Platelet aggregation in response to platelet agonist, oxLDL, was measured using light aggregometry. Platelet integrin activation and granule secretion in response to glycoprotein VI-agonist, crosslinked collagen-related peptide (CRP-XL), were measured using flow cytometry. In a nonhuman primate model (NHP) of early atherosclerosis, two obese rhesus macaques were administered the TEC-family kinase inhibitor, ibrutinib, for one week. Targeted contrast-enhanced ultrasound molecular imaging was used to measure vascular cell adhesion molecule-1 (VCAM-1) as a marker of EC activity and platelet glycoprotein-Iba (GPIba) at the carotid bifurcation before and after treatment with a TEC-family kinase inhibitor.

Results: Platelet aggregation in response to oxLDL was inhibited following pre-incubation with two pharmacologically distinct TECfamily kinase inhibitors, including the Bruton's tyrosine kinase inhibitor, ibrutinib. We observed a reduction in platelet integrin activation and granule secretion in response to CRP-XL following preincubation with ibrutinib. In a NHP model of early atherosclerosis, in vivo molecular imaging showed that treatment with ibrutinib decreased signal for both EC VCAM-1 and platelet GPIba.

Conclusions: oxLDL potentiates platelet activity in vitro through TEC-family kinase pathway, while the TEC-family kinase inhibitor, ibrutinib, decreased the markers of EC dysfunction and platelet activity in vivo. These results suggest that TEC family kinases contribute to the pathogenesis of atherosclerosis and could represent a novel therapeutic target.

PB0008 | Tissue Inhibitor of Metalloproteinases-1 (TIMP1) is Related to Residual Thromboxane Dependent Platelet Activation in Patients with Type 2 Diabetes Mellitus Receiving Low Dose Aspirin

P. Simeone ${ }^{1}$; R. Tripaldi ${ }^{1}$; R. Liani ${ }^{1} ;$ S. Ciotti ${ }^{1}$; V. Cavalca ${ }^{2}$; M. Camera ${ }^{2}$; E. Tremoli ${ }^{2}$; F. Santilli ${ }^{1}$

${ }^{1}$ Department of Medicine and Aging, Center for Advanced Studies and Technology (CAST), University 'G. d'Annunzio' of Chieti-Pescara, Chieti, Italy; ${ }^{2}$ Monzino Cardiology Center, IRCCS, Milan, Italy

Background: Thromboxane(TX)-dependent platelet activation, plays a key role in atherothrombosis. Incomplete suppression by ASA of urinary 11-dehydro- $\mathrm{TXB}_{2}$ excretion is predictive of cardiovascular events. TIMP1 is secreted by platelets. However, no data exist on the relationship between TIMP1 and TX dependent platelet activation. 
Aims: To evaluate intraplatelet(i) and circulating(c)TIMP1 levels in diabetic(T2DM) and noT2DM patients receiving low dose ASA, and whether they may be associated with residual TX dependent platelet activation.

Methods: Thirty-nine patients (20 with and 19 without T2DM) were evaluated. All patients were treated with ASA(100 mg). Twenty-four had metabolic syndrome(MS) and twenty had nonalcoholic fatty liver disease(NAFLD). Plasma and iTIMP1 was measured by ELISA Urinary 11-dehydro- $\mathrm{TXB}_{2}$ was measured by mass-spectrometry. Each subject signed written informed consent, Protocol was approved (GR-2011-02350450).

Results: In the whole group of patients and in noT2DM patients, cTIMP1 and iTIMP1 correlated directly $(P=0.039$ and $P<0.001)$ according with the hypothesis of platelets as major source of circulating TIMP1. cTIMP1 levels were comparable between T2DM and no T2DM patients $(P=0.513)$. iTIMP1 were higher in T2DM( $P=0.027)$. Patients with MS had higher levels of ITIMP1 in the whole group and in patients with and without $\operatorname{T2DM}(P<0.001 ; P=0.032$ and $P=0.009$ respectively). Higher levels of iTIMP1 were found in patients with NAFLD both in the whole group and in patients with $\operatorname{T2DM}(P=0.030$ and $P=0.043)$. cTIMP1 was directly related to 11-dehydro-TXB ${ }_{2}$ in the whole group and in T2DM patients $(P=0.006$ and $P=0.027$ ) (figure) suggesting a role for TIMP-1 in residual TXdependent platelet activation.

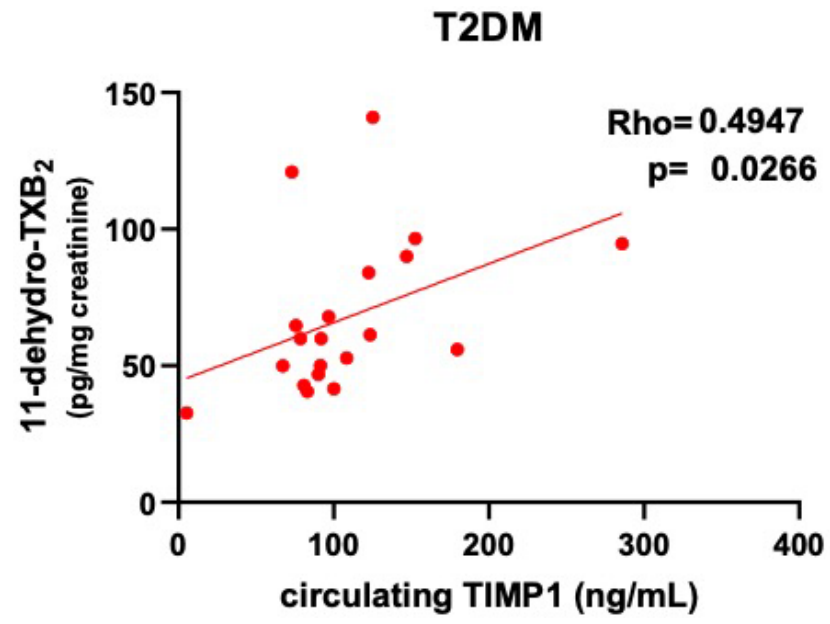

FIGURE 1 Correlation between circulating TIMP1 and urinary 11-dehydro-TXB2 in the patients with type 2 diabetes mellitus

Conclusions: In patients in treatment with ASA (I) higher iTIMP1 levels are associated with metabolic diseases (T2DM, MS and NAFLD); (II) iTIMP1 may be a major source of CTIMP1 and (III) CTIMP1 is directly related to urinary 11-dehydro- $\mathrm{TXB}_{2}$, suggesting a contribution of residual TX to TIMP1 release, which may further amplify platelet activation, or vice versa a role for TIMP1 as a determinant of TXdependent platelet activation.
PB0009 | Combined Focused Ultrasound and Electrohydraulic Shock Wave Thrombolytic Therapy Accompanied by Tissue Plasminogen Activator Administration Reduce Thrombus Content in the Arterial Atherothrombotic Stenosis

$\underline{\text { H. Mehrad }}^{1,2} ;$ A. Foletti ${ }^{3,2}$

${ }^{1}$ Islamic Azad University, Basic Sciences Faculty, Department of Physics, Tabriz Branch, Tabriz, Iran, Islamic Republic of; ${ }^{2}$ Mehrad Research Lab, Division of Translational Development of Noninvasive Treatments, Tabriz, Iran, Islamic Republic of; ${ }^{3}$ Italian National Research Council, Institute of Translational Pharmacology IFT, Rome, Italy

Background: Vascular disease arises through the complications of atherosclerosis, a complex chronic inflammatory condition affecting the arterial circulation. It leads to the development of vascular lesions or soft atherosclerotic plaques, which manifest as asymmetrical thickenings of the intima of medium to large sized elastic and muscular arteries. A soft plaque may rupture with high risk of subsequent thrombus mediated acute clinical events such as myocardial infarction and stroke.

Aims: The aim of this study was to generate a hamster model of common carotid artery atherothrombotic stenosis and the subsequent investigating the feasibility of combined focused ultrasound and electrohydraulic shock wave - mediated thrombolytic therapy accompanied by tissue plasminogen activator (tPA) administration in this model.

Methods: Briefly, golden Syrian hamsters were submitted to common carotid artery atherothrombotic stenosis by primary balloon injury followed $2 \%$ cholesterol- rich diet injury for eight weeks and finally perivascular liquid nitrogen- mediated severe cold injury. Then treatment group underwent combined focused ultrasound $\left(I=4 \mathrm{~W} / \mathrm{cm}^{2}, F=1.2 \mathrm{MHz}, \mathrm{PD}=120 \mathrm{~ms}\right)$ and electrohydraulic shock wave $(V=15 \mathrm{Kv}, F=0.3 \mathrm{~Hz}$, Impulses = 100) - mediated thrombolytic therapy accompanied by tPA $(0.6 \mathrm{mg} / \mathrm{kg})$ administration.

Results: Results from histopathology, B-mode and color Doppler ultrasonography, showed a significant reduction in the mean value for thrombus content, blood mean velocity and a significant increase in the mean value for blood volume flow at the stenotic region in the treatment group compared with the other groups $(P<0.05)$.

Conclusions: Focal thrombus disruption mechanism was partly clarified as originated from microjet formation upon bubble collapse. Enhanced anti- thrombotic effect of tPA, due to enhanced microjets, induced by combined focused ultrasound and electrohydraulic shock wave therapy, can cause to reduce the thrombus content and significantly dilate the luminal cross-sectional area of stenosis and lower treatment time in comparison with conventional tPA thrombolytic therapy. 
PB0010 | Investigation of Red Blood Cells Influence on Atherosclerotic Plaque by Scanning Flow Cytometry

E. Yastrebova $^{1,2}$; A. Karpenko ${ }^{2}$

${ }^{1}$ Voevodsky Institute of Chemical Kinetics and Combustion, Novosibirsk, Russian Federation; ${ }^{2}$ Meshalkin National Medical Research Center, Novosibirsk, Russian Federation

Background: Erythrocytes gas transport function controls the diffusion of chloride and bicarbonate through erythrocyte membrane and limits the rate of $\mathrm{CO} 2$ transfer from tissues to lungs. Detailed characterization of morpho-functional erythrocyte parameters is necessary to control risk of hypoxic stage in organism. This is especially important in case of cardiovascular diseases. It can lead to formation of unstable atheroma in places with increased oxygen demand.

Aims: This study aims to verify the hypothesis that deviations in erythrocyte parameters (anion permeability) correlate with atheroma types (stable or unstable).

Methods: Carotid artery plaques were obtained by endarterectomy and measured by AxioCam MRc5. Whole blood was taken by venopuncture. Then cells were used in experiments at room temperature $\left(22^{\circ} \mathrm{C}\right)$. All donors were divided in three groups: patients with stable and unstable plaques and healthy donors. Experiments were carried out on scanning flow cytometer (fabricated by CytoNova Ltd., Novosibirsk, Russia, http://cyto.kinetics.nsc.ru) which permit to determine characteristics of individual blood cells.

Results: We obtained for each measured RBC in samples the $\mathrm{Hb}$ content simultaneously with morphological characteristics. We define the anion permeability in terms of mean number of 'effective' (parameter derived from erythrocyte isotonic lysis in ammonium chloride) and 'activated' (parameter derived from addition of nifedipine solution before isotonic hemolysis) erythrocyte Band 3 protein. Total 45 patients and 45 donors were included in this study. It turns out that patients with stable atherosclerotic plaque have increased ratio between 'activated' and 'effective' anion exchangers compare to even healthy group. Patients with unstable plaque have significant nonactive fraction of anion carriers (up to 80\%).

Conclusions: Deviations in gas transport function can be explained as the protection mechanism from hypoxic conditions in places with increased oxygen demand, such as atherosclerotic plaque. And, consequently, decreased anion exchange can be a predictor of atheroma destabilization.
P0100 | Early Stage Atherosclerosis Regression, Using Highdose Atorvastatin Administration Accompanied by B-mode Ultrasound-guided 5-aminolevulinic Acid-mediated Catheterbased Low Level Blue Laser Photodynamic Therapy

H. Mehrad $^{1,2}$; A. sultan-Qurraie ${ }^{3}$

${ }^{1}$ Islamic Azad University, Basic Sciences Faculty, Department of Physics, Tabriz Branch, Tabriz, Iran, Islamic Republic of; ${ }^{2}$ Division of Translational Development of Noninvasive Treatments, Mehrad Research Lab, Tabriz, Iran, Islamic Republic of; ${ }^{3}$ University of Washington, Valley Medical Center. Neuroscience Institute, Renton, Washington, United States

Background: Atherosclerosis is the leading cause of stroke and cardiovascular disease. As is well recognized, the majority of atherosclerosis start with an inflammatory process. Excessive lipid accumulation by macrophages plays a crucial role in atherosclerosis. Foam cells are generated by uncontrolled uptake of modified LDL, especially oxidized LDL (oxLDL). Photodynamic therapy is a treatment that uses photosensitizing agents, along with light to kill cancerous and hyperplasia cells.

Aims: The aim of this study was to evaluate the effect of high-dose atorvastatin administration accompanied by 5 - Aminolevulinic Acidmediated catheter- based low level blue laser photodynamic therapy on early stage atherosclerotic regression, wherein diagnostic B- mode ultrasound is combined with laser system, with a goal of increased safety.

Methods: Golden Syrian Hamsters underwent primary balloon dilatation injury at the right common carotid artery followed by a $1.5 \%$ cholesterol-rich diet injury for three weeks. Histopathology results showed development of macrophages- derived foam cells in intimal layer and early stage atherosclerosis formation in all of the hamsters' arteries. Then common carotid arteries of the treatment group $(n=10)$ at the injured segment, treated using B- mode ultrasound- guided 5-Aminolevulinic Acid- mediated catheter-based low level blue laser $\left(\lambda=450 \mathrm{~nm}, E / A=15 \mathrm{~J} / \mathrm{cm}^{2}\right)$ photodynamic therapy accompanied by high-dose atorvastatin ( $5 \mathrm{mg} / \mathrm{kg} / \mathrm{day}$ ) administration. Foam cells density were evaluated in the treatment group compared with the control group using B-mode ultrasonography and histopathology.

Results: Ultrasonography and histopathology results showed a significant reduction in the mean value for foam cells density within the atherosclerotic lesion in the treatment group compared with the control group $(P<0.05)$.

Conclusions: Apoptotic effect of 5-Aminolevulinic Acid, induced by low level blue laser accompanied by enhanced pleiotropic and lipophilic effects of atorvastatin-induced by high-dose administration, can cause to early stage atherosclerosis regression and significantly reduce the foam cells-mediated inflammation. 
P0101 | Lipid-based Encapsulated Methotrexate Nanoparticlesmediated Low Level Focused Ultrasound Sonoporation Therapy Decrease Inflammation and Restenosis after ArF Excimer Laser Angioplasty of Fibrocalcific Atherosclerosis

\section{H. Mehrad ${ }^{1,2}$; A. Foletti ${ }^{3,2}$}

${ }^{1}$ Islamic Azad University, Basic Sciences Faculty, Department of Physics, Tabriz Branch, Tabriz, Iran, Islamic Republic of; ${ }^{2}$ Mehrad Research Lab, Division of Translational Development of Noninvasive Treatments, Tabriz, Iran, Islamic Republic of; ${ }^{3}$ Italian National Research Council, Institute of Translational Pharmacology IFT, Rome, Italy

Background: The management of advanced atherosclerotic stenosis reduces the risk of stroke and myocardial infarction and their related deaths. The laser atherectomy methods that are currently in use, cause to inflammation and subsequent restenosis.

Aims: The aim of this study was to evaluate the effect of Lipid- based encapsulated methotrexate nanoparticles - mediated ultrasound sonoporation therapy on inflammation and intimal hyperplasia reduction after laser angioplasty in the hamster abdominal aorta model of fibrocalcific atherosclerosis (fibrotic plaque with a large calcified content), wherein diagnostic B- mode ultrasound is combined with sonoporation therapy system, with a goal of increased safety.

Methods: Golden Syrian Hamsters were submitted to the fibrocalcific atherosclerosis at the abdominal aorta (approximately $0.5 \mathrm{~cm}$ superior to the iliac bifurcation). Histopathology results showed the formation of advanced atherosclerosis with fibrous- derived extracellular matrix- rich plaque and calcification, resulted to severe stenosis (>80\%) in all of the hamsters' arteries. Then treatment group underwent ArF excimer laser (193 nm) angioplasty followed by lowlevel focused ultrasound ( $F=1.2 \mathrm{MHz}, I=10 \mathrm{~J} / \mathrm{cm}^{2}, \mathrm{PD}=150 \mathrm{~ms}$ )mediated sonoporation therapy in combination with lipid- based encapsulated methotrexate nanoparticles $(10 \mathrm{mg} / \mathrm{kg})$ administration. Results: Results from histopathology showed a significant reduction in the mean value for macrophages and smooth muscle hyperplasia cells density after laser thermal angioplasty in the sonoporation therapy group compared with the other groups $(P<0.05)$.

Conclusions: Enhanced apoptotic and anti- proliferative effect of methotrexate - induced by enhanced sonoporation effect of focused ultrasound, due to inertial cavitation- mediated collapsed capsules, can cause to reduce inflammation and restenosis after ArF excimer laser angioplasty of fibrocalcific atherosclerosis.
P0102 | Ultrasound-guided Catheter-based ${ }^{192}$ Ir -mediated $\beta^{-}$brachytherapy of Neointimal Hyperplasia Accompanied by Protoporphyrin IX-loaded Microbubbles-mediated Pulsed Low Level Focused Ultrasound Sonodynamic Therapy

$\underline{\text { H. Mehrad }}^{1,2} ;$ H. Ahmadi Noubari ${ }^{3,2}$

${ }^{1}$ Islamic Azad University, Basic Sciences Faculty, Department of Physics, Tabriz Branch, Tabriz, Iran, Islamic Republic of; ${ }^{2}$ Mehrad Research Lab, Division of Translational Development of Noninvasive Treatments, Tabriz, Iran, Islamic Republic of; ${ }^{3}$ University of British Columbia, Electrical and Computer Engineering Faculty, Vancouver, Canada

Background: Neointimal hyperplasia is usually defined in an artery as thickening of the intimal layer after an injury such as angioplasty, stenting or surgical repair.

Aims: In this study, we developed an experimental combined $\beta^{-}$ brachytherapy and protoporphyrin IX- loaded microbubbles- mediated ultrasound sonodynamic therapy protocol, and investigated its effectiveness on neointimal hyperplasia reduction, wherein diagnostic ultrasound system is adjuncted with treatment system, with a goal of increased safety.

Methods: Briefly, rats underwent perivascular severe cold injury using liquid nitrogen at the abdominal aorta (approximately $0.5 \mathrm{~cm}$ superior to the iliac bifurcation). After eight weeks, the histopathology results showed progressive inflammation and smooth muscle cells proliferation in intimal layer, resulting in vessel wall thickening. Then treatment group underwent catheter- based $\beta^{-}$brachytherapy ( $\left.{ }^{192} \mathrm{Ir}, 15 \mathrm{~Gy}\right)$ in combination with pulsed low level focused ultrasound ( $P=15 \mathrm{~W}, \mathrm{PD}=250 \mathrm{~ms}$ )- mediated sonodynamic therapy accompanied by sonosensitizer protoporphyrin IX - loaded PESDA (Perfluorocarbon- Exposed Sonicated Dextrose Albumin) microbubbles $\left(100 \mathrm{ml} / \mathrm{kg}, 2-5 \times 10^{5}\right.$ bubbles $\left./ \mathrm{ml}\right)$ administration and simultaneously B- mode ultrasound imaging.

Results: Results from ultrasonography and histopathology, showed a significant reduction in the mean value for wall mean thickness and percentage of luminal cross-sectional area of stenosis at the neointimal hyperplasia region in the treatment group compared with the other groups $(P<0.05)$. Moreover, cell morphology with electron microscopy showed the apoptosis of smooth muscle cells in intimal layer after combination therapy.

Conclusions: Apoptotic effect of $\beta^{-}$brachytherapy in combination with enhanced anti- inflammatory effect of protoporphyrin IX, induced by enhanced sonodynamic therapy effect of ultrasound, due to collapsed microbubbles, can cause to reduce the smooth muscle hyperplasia cells in intimal layer and significantly dilate the luminal cross-sectional area of stenosis. These findings provide the basis for developing of combined $\beta^{-}$brachytherapy and sonodynamic therapy for a successful clinical application in the treatment of hyperplastic conditions such as restenosis. 
P0106 | Stable Fibroatheromatic Plaque Regression Using High-dose Atorvastatin-loaded PESDA Microbubbles-mediated Extracorporeal Shock Wave Sonoporation Therapy

\section{$\underline{\text { H. Mehrad }}^{1,2}$; M. Farhoudi ${ }^{3}$}

${ }^{1}$ Islamic Azad University, Basic Sciences Faculty, Department of Physics, Tabriz Branch, Tabriz, Iran, Islamic Republic of; ${ }^{2}$ Division of Translational Development of Noninvasive Treatments, Mehrad Research Lab, Tabriz, Iran, Islamic Republic of; ${ }^{3}$ Neurosciences Research Center (NSRC), Tabriz University of Medical Sciences, Tabriz, Iran, Islamic Republic of

Background: Large mature or immature lipid core (atheroma) within a thick connective tissue containing of macrophages, smooth muscle cells, lipid laden- cells and a lot of fibrous- derived extracellular matrix with a low probability of calcification, is called stable fibroatheromatic plaque. The management of stable fibroatheromatic plaque reduces the risk of ischemic stroke and its related deaths. Management options are invasive and include carotid endarterectomy and carotid artery stenting.

Aims: In this study, we developed an experimental electrohydraulic shock wave generator (0-20 kv), and investigated its effectiveness on stable fibroatheromatic plaque regression in the golden Syrian hamster common carotid artery.

Methods: Stable fibroatheromatic plaque with severe stenosis (>80\%) was induced at the right common carotid artery of golden Syrian hamsters. The animals treated by repeated electrohydraulic focused shock waves ( $V=20 \mathrm{Kv}, F=0.3 \mathrm{~Hz}$, Impulses = 120) accompanied by PESDA microbubbles $\left(100 \mu \mathrm{l} / \mathrm{kg}, 2-5 \times 10^{5}\right.$ bubbles $/ \mathrm{ml}$ ) and high- dose atorvastatin ( $5 \mathrm{mg} / \mathrm{kg} /$ day) administration. Blood volume flow and blood mean velocity were measured by color Doppler ultrasonography at the stenotic region. Moreover, wall mean thickness and percentage of luminal cross-sectional area of stenosis were measured by B-mode ultrasound and histology.

Results: Results from histopathology, color Doppler and B-mode ultrasonography showed a significant reduction in the mean value for blood mean velocity, wall mean thickness and the percentage of luminal cross-sectional area of stenosis and a significant increase in the mean value for blood volume flow in the treatment group compared with the other groups $(P<0.05)$.

Conclusions: Enhanced inertial cavitation- mediated sonoporation effect of shock waves, induced by collapsed microbubbles accompanied by enhanced lipophilic and pleiotropic effects of atorvastatin- induced by high-dose administration, can cause to reduce the lipid- laden cells, macrophages in the plaque lipid core and significantly dilate the luminal cross-sectional area of stenosis and regress the thick- cap fibroatheromatic plaque.
P0107 | Ultrasound-guided Laser-mediated Thermal Angioplasty of Occluded Artery Accompanied by Combined 5-Aminolevulinic Acid-loaded Microbubbles-mediated Photodynamic Therapy and Catheter-based ${ }^{192}$ Ir-mediated $\beta^{-}$brachytherapy

H. Mehrad $^{1,2} ;$ H. Ahmadi Noubari ${ }^{3,2}$

${ }^{1}$ Islamic Azad University, Basic Sciences Faculty, Department of Physics, Tabriz Branch, Tabriz, Iran, Islamic Republic of; ${ }^{2}$ Mehrad Research Lab, Division of Translational Development of Noninvasive Treatments, Tabriz, Iran, Islamic Republic of; ${ }^{3}$ University of British Columbia, Electrical and Computer Engineering Faculty, Vancouver, Canada

Background: The laser atherectomy methods that are currently in use, cause to inflammation and subsequent restenosis.

Aims: The aim of this study was to evaluate the effect of combined 5- Aminolevulinic Acid- loaded microbubbles- mediated photodynamic therapy and catheter- based ${ }^{192} \mathrm{Ir}$ - mediated $\beta^{-}$brachytherapy on inflammation and intimal hyperplasia reduction after laser angioplasty of the animal occluded femoral artery, wherein diagnostic ultrasound is adjuncted with angioplasty and combination therapy system, with a goal of increased safety.

Methods: Briefly, New Zealand white rabbits were submitted to femoral artery advanced atherosclerotic occlusion by primary perivascular severe cold injury followed by a $2 \%$ cholesterol- rich diet for fourteen weeks. Histopathology results showed the formation of stable advanced atherosclerosis with lipid and neovessel rich plaque, resulted in occlusion in all of the rabbits' arteries. Then treatment group underwent B- mode ultrasound- guided argon laser $\left(488 \mathrm{~nm}\right.$ ) angioplasty followed by catheter- based $\beta^{-}$brachytherapy $\left({ }^{192} \mathrm{Ir}, 15 \mathrm{~Gy}\right)$ in combination with photodynamic therapy with red diode laser $\left(\mathrm{WL}=635 \mathrm{~nm}, \mathrm{E} / \mathrm{A}=120 \mathrm{~J} / \mathrm{cm}^{2}\right)$ accompanied by photosensitizer 5- Aminolevulinic Acid- loaded PESDA (PerfluorocarbonExposed Sonicated Dextrose Albumin) microbubbles $(100 \mathrm{ml} / \mathrm{kg}$, $2-5 \times 10^{5}$ bubbles $/ \mathrm{ml}$ ) administration and simultaneously ultrasound imaging.

Results: Results from ultrasonography and histopathology showed a significant reduction in the mean value for immune cells and smooth muscle hyperplasia cells density after angioplasty in the treatment group compared with the other groups $(P<0.05)$.

Conclusions: Apoptotic effect of $\beta^{-}$brachytherapy in combination with anti- inflammatory effect of 5- Aminolevulinic Acid, induced by photodynamic therapy effect of red diode laser, can cause to reduce the density of macrophage cells and smooth muscle hyperplasia cells in the intimal layer. These findings provide the basis for developing of combined $\beta^{-}$brachytherapy and photodynamic therapy for a successful clinical application in the treatment of neointimal hyperplasia after laser angioplasty. 
P0108 | Improving of the of Biomechanical Parameters in an Experimental Animal Abdominal Aorta Model of Thick- cap Fibroatheromatic Plaque Using Ultrasound-guided High-dose Atorvastatin-loaded PESDA Microbubbles and Low Level Focused Shock Waves

$\underline{\text { H. Mehrad }}^{1,2} ;$ H. Ahmadi Noubari ${ }^{3,2}$

${ }^{1}$ Islamic Azad University, Basic Sciences Faculty, Department of Physics, Tabriz Branch, Tabriz, Iran, Islamic Republic of; ${ }^{2}$ Mehrad Research Lab, Division of Translational Development of Noninvasive Treatments, Tabriz, Iran, Islamic Republic of; ${ }^{3}$ University of British Columbia, Electrical and Computer Engineering Faculty, Vancouver, Canada

Background: It is known that high-fat diet, induce hypercholesterolemia, deterioration of arterial wall, both morphologically and mechanically. Mechanical changes include thickening of arterial wall, alteration of arterial elasticity, contraction of smooth muscle, increase in sensitivity to pharmacological stimulation and increase in arterial viscoelasticity, i.e., arteriosclerosis. The management of stable fibroatheromatic plaque reduces the risk of ischemic diseases and its related deaths. Customary management options are invasive and include arterial atherectomy and stenting.

Aims: In this study, we developed an experimental electrohydraulic shock wave generator system (0-20 kv), and investigated its effectiveness on stable fibroatheromatic plaque regression in the hamster abdominal aorta, wherein diagnostic B-mode ultrasound is combined with shock wave therapy system, with a goal of increased safety.

Methods: Thick- cap fibroatheromatic plaque with severe stenosis (>80\%) was induced at the right common carotid artery of Golden Syrian Hamsters. The animals treated by repeated electrohydraulic focused shock waves ( $V=15 \mathrm{Kv}, F=0.5 \mathrm{~Hz}$, Impulses = 100) accompanied by PESDA microbubbles ( $100 \mu \mathrm{l} / \mathrm{kg}, 2-5 \times 10^{5}$ bubbles $/ \mathrm{ml}$ ) and high-dose atorvastatin ( $5 \mathrm{mg} / \mathrm{kg} /$ day) administration. Arterial biomechanical parameters were evaluated in the different groups using B- mode ultrasound images.

Results: Results showed a significant reduction in the mean value for shear elastic modulus, resistive index and a significant increase in the mean value for radial strain, compliance and distensibility index in the treatment group compared with the other groups $(P<0.05)$

Conclusions: Enhanced inertial cavitation- mediated sonoporation effect of shock waves, induced by collapsed microbubbles accompanied by enhanced lipophilic and pleiotropic effects of atorvastatin- induced by high-dose administration, accompanied by anti- inflammatory effect of shock waves, can cause to reduce the lipid- laden cells in the plaque lipid core and significantly dilate the luminal cross-sectional area of stenosis and regress the thick- cap fibroatheromatic plaque. These features can cause to improve the arterial biomechanical parameters.
P0109 | Development of an Experimental Animal Carotid Artery Model of Thick-cap Fibroatheromatic Plaque with Severe Stenosis (>80\%) Using High Power Blue Laser-mediated Thermal Injury and High-cholesterol Diet Injury

$\underline{\text { H. Mehrad }}^{1,2}$; A. Sultan- Qurraie ${ }^{3}$

${ }^{1}$ Islamic Azad University, Basic Sciences Faculty, Department of Physics, Tabriz Branch, Tabriz, Iran, Islamic Republic of; ${ }^{2}$ Division of Translational Development of Noninvasive Treatments, Mehrad Research Lab, Tabriz, Iran, Islamic Republic of; ${ }^{3}$ University of Washington, Valley Medical Center, Neuroscience Institute, Renton, United States

Background: The use of animal models in ischemic stroke has improved our understanding of stroke pathophysiology. An ideal animal model for atherosclerosis-mediated stroke would mimic the pathophysiology in humans.

Aims: We sought to develop an easily reproducible and inexpensive experimental hamster carotid artery model to study severe stenosis (>80\%) with thick-cap fibroatheromatic plaque.

Methods: Thick- cap fibroatheromatic plaque was induced via perivascularly using high power blue laser $(\lambda=450 \mathrm{~nm}, P=20 \mathrm{~W})$ thermal injury at the right common carotid artery in Golden Syrian Hamsters. Hamsters were then fed a $2 \%$ cholesterol-rich diet for six weeks. Histopathology was evaluated after six weeks.

Results: Histopathologic evaluation revealed progressive smooth muscle cell and extracellular lipid droplet proliferation in the intimal layer, resulting in vessel wall thickening, increased inflammation and a large immature lipid core formation with a thick- fibrous cap. In addition, increased collagen-derived extracellular matrix was found in the intimal layer compared with the other groups. These conditions resulted in the formation of a thick-cap fibroatheromatic plaque. Percentage of luminal-cross sectional area of stenosis was 81.42[ASQ1] $\pm 6.31(P<0.05)$ compared with the other groups. Results from color Doppler ultrasonography showed a significant increase in the mean value for blood Peak Systolic Velocity (PSV), End Diastolic Velocity (EDV), Mean Velocity (MV), Resistance Index (RI), Pulsatility Index (PI) and significant reduction in the mean value for blood Volume Flow (VF) at the stenotic region in the atherosclerotic group compared with the control group $(P<0.05)$.

Conclusions: We successfully produced an easily reproducible and inexpensive [ASQ1] experimental hamster cerebrovascular model of atherosclerotic thick-cap plaque using high power blue laser- mediated thermal injury, similar to the condition seen in human subjects. This condition in hamsters can be properly assessed by color Doppler ultrasonography. 
P0110 | Ultrasound-guided Balloon Angioplasty of Occluded Artery Accompanied by Combined Electrohydraulic Low Level Shock Wave Therapy and 125I- Mediated Gamma-intravascular Brachytherapy

\section{H. Mehrad $^{1,2} ;$ H. Ahmadi Noubari ${ }^{3,2}$}

${ }^{1}$ Islamic Azad University, Basic Sciences Faculty, Department of Physics, Tabriz Branch, Tabriz, Iran, Islamic Republic of; ${ }^{2}$ Mehrad Research Lab, Division of Translational Development of Noninvasive Treatments, Tabriz, Iran, Islamic Republic of; ${ }^{3}$ University of British Columbia, Electrical and Computer Engineering Faculty, Vancouver, Canada

Background: Three mechanisms are responsible for the development of restenosis: elastic recoil, intimal hyperplasia and late vascular constriction, all grouped under the catch phrase "negative remodeling". Neointimal hyperplasia is usually defined in an artery as thickening of the intimal layer after an injury such as different angioplasty methods, mechanical atherectomy or surgical repair The balloon angioplasty methods that are currently in use, cause to inflammation and subsequent restenosis.

Aims: The aim of this study was to evaluate the effect of combined electrohydraulic shock wave therapy and ${ }^{125}$ - mediated gammaintravascular brachytherapy on inflammation and restenosis reduction after balloon angioplasty of the animal occluded common carotid artery model, wherein diagnostic ultrasound is adjuncted with angioplasty and combination therapy system, with a goal of in creased safety.

Methods: Briefly, Golden Syrian Hamsters were submitted to common carotid artery advanced atherosclerotic occlusion by primary perivascular $\mathrm{Co}_{2}$ thermal laser injury followed by a $2 \%$ cholesterolrich diet for six weeks. Histopathology results showed the formation of a thick- cap fibroatheromatic plaque, resulted to occlusion in all of the hamsters' arteries. Then treatment group $(n=10)$ underwent B- mode ultrasound- guided balloon angioplasty followed by combined shock wave ( $V=12 \mathrm{Kv}, F=0.5 \mathrm{~Hz}$, Impulses = 120) therapy and gamma-intravascular brachytherapy $\left({ }^{125} \mathrm{I}-, 18 \mathrm{~Gy}\right)$ and simultaneously ultrasound imaging.

Results: Results from histopathology showed a significant reduction in the mean value for macrophages and smooth muscle hyperplasia cells density after balloon angioplasty in the treated group compared with the other groups $(P<0.05)$.

Conclusions: Anti- inflammatory effect of shock waves accompanied by apoptotic effect of gamma brachytherapy, can cause to reduce the inflammation and smooth muscle hyperplasia cells in the intimal layer. These findings provide the basis for developing of combined low level electrohydraulic focused shock wave therapy and ${ }^{125} \mathrm{I}$ - mediated gamma-intravascular brachytherapy for a successful clinical application in the treatment of restenosis after balloon angioplasty.
P0111 | Intermediate Stage Atherosclerosis Regression Using Lipid-based Encapsulated Methotrexate Nanoparticles-mediated B-mode Ultrasound-guided Pulsed Low Level Focused Ultrasound Sonoporation Therapy

H. Mehrad $^{1,2}$; M. Kasiri ${ }^{2}$

${ }^{1}$ Islamic Azad University, Basic Sciences Faculty, Department of Physics, Tabriz Branch, Tabriz, Iran, Islamic Republic of; ${ }^{2}$ Mehrad Research Lab, Division of Translational Development of Noninvasive Treatments, Tabriz, Iran, Islamic Republic of

Background: Atherosclerosis is a slow, progressive disease that may begin as early as childhood. Although the exact cause is unknown, atherosclerosis may start with damage or injury to the inner layer of an artery. Atherosclerosis is the main cause of stroke and myocardial infarction. In atherosclerosis, local inflammation and associated macrophage activity can lead to foam cell- rich lesion formation, making inflammation an important therapeutic target in cerebrovascular and cardiovascular diseases.

Aims: In this study, we developed an experimental pulsed low level focused ultrasound system, and investigated its effectiveness on intermediate stage atherosclerosis regression accompanied by intravenous lipid- based encapsulated methotrexate nanoparticles administration in the Golden Syrian Hamster common carotid artery, wherein diagnostic B- mode ultrasound is combined with ultrasound therapy system, with a goal of increased safety.

Methods: In this study, common carotid arteries of hamsters submitted to intermediate stage atherosclerosis using intravascular balloon injury and high- cholesterol diet injury. Then common carotid arteries of the treatment group $(n=10)$ at the injured region, underwent pulsed low level focused ultrasound $(F=1.3 \mathrm{MHz}, P=24$ $\mathrm{W}, \mathrm{PD}=100 \mathrm{~ms}$ ) - mediated sonoporation therapy accompanied by intravenous lipid- based encapsulated methotrexate nanoparticles (10 ml/kg) administration and simultaneously B- mode ultrasound imaging. Wall mean thickness and percentage of luminal crosssectional area of stenosis were measured by ultrasonography and histopathology.

Results: Results from ultrasonography and histopathology showed a significant reduction in the mean value for wall mean thickness, percentage of luminal cross-sectional area of stenosis, lipid- laden cells and lipid droplets density at the stenotic region in the treatment group compared with the other groups $(P<0.05)$.

Conclusions: Enhanced apoptotic and anti- proliferative effect of methotrexate, induced by enhanced inertial cavitation- based sonoporation effect of focused ultrasound, due to collapsed capsules, can cause to regress of the intermediate stage atherosclerosis and significantly dilate the luminal cross-sectional area of stenosis. 
P0112 | Treatment of Advanced Atherosclerotic Plaque with Neovascularization Using B-mode Ultrasound-guided HDL-loaded Microbubbles-mediated Low Level Focused Electrohydraulic Shock Wave Sonoporation Therapy and Cholesterol Diet Withdrawal

$\underline{\text { H. Mehrad }}^{1,2}$; A. Foletti ${ }^{3,2}$

${ }^{1}$ Islamic Azad University, Basic Sciences Faculty, Department of Physics, Tabriz Branch, Tabriz, Iran, Islamic Republic of; ${ }^{2}$ Mehrad Research Lab, Division of Translational Development of Noninvasive Treatments, Tabriz, Iran, Islamic Republic of; ${ }^{3}$ Italian National Research Council, Institute of Translational Pharmacology IFT, Rome, Italy

Background: Atherosclerosis is the leading cause of cerebrovascular and cardiovascular diseases. The management of advanced atherosclerosis with neovascularization reduces the risk of stroke and myocardial infarction and their related deaths.

Aims: In this study, we developed an experimental low level focused shock wave therapy protocol, and investigated its effectiveness on intraplaque neovessels destruction and advanced atherosclerotic plaque regression, wherein diagnostic B-mode ultrasound is combined with focused-electrohydraulic shock wave generator, with a goal of increased safety.

Methods: Briefly, golden Syrian hamsters underwent primary perivascular severe cold injury at the right common carotid artery followed by a $1.5 \%$ cholesterol-rich diet injury for 12 weeks. Histopathology and ultrasonography results showed the formation of advanced atherosclerosis with neovessel - rich plaque and severe stenosis (>70\%) in all of the hamsters' arteries. The animals treated by low level focused electrohydraulic shock wave $(V=8$ $\mathrm{Kv}, \mathrm{F}=5 \mathrm{~Hz}$, Impulses = 100) accompanied by HDL- loaded PESDA (Perfluorocarbon- Exposed Sonicated Dextrose Albumin) microbubbles administration and cholesterol diet withdrawal.

Results: Results from histopathology, B-mode and color Doppler ultrasonography showed a significant reduction in the mean value for blood mean velocity, intraplaque neovessels density, wall mean thickness and percentage of luminal cross- sectional area of stenosis and significant increase in the mean value for blood volume flow in the treatment group compared with the other groups $(P<0.05)$.

Conclusions: Enhanced destruction effect of shock wave- mediated microjets, induced by collapsed microbubbles within the neovessels, concomitant with the enhanced reverse cholesterol transport effect of HDL, induced by sonoporation effect of shock wave and collapsed microbubbles, accompanied by macrophages egress and foam cells efferocytosis effects of cholesterol diet withdrawal and HDL administration, can cause to destroy the intraplaque neovessels, reduce the lesion lipid content and significantly dilate the luminal cross- sectional area of stenosis.

\section{CARDIOVASCULAR RISK FACTORS}

LPB0049 | Cardiovascular Outcomes in Patients with Essential Thrombocythemia: Analysis of the National Inpatient Sample (NIS)

G. Tafur $^{1}$; H. Saadaldin ${ }^{2,3}$; F. Velazquez ${ }^{4}$; E. Marinescu ${ }^{5}$; A. Tafur ${ }^{6,7}$

${ }^{1}$ Weinberg College of Arts and Sciences, Northwestern University, Evanston, United States; ${ }^{2}$ October 6 University, 6th of October City, Egypt; ${ }^{3}$ American Venous Forum, East Dundee, United States;

${ }^{4}$ Advocate Aurora Health Care Saint Luke's South Shire Medical Center, Department of Internal Medicine, Cudahy, United States; ${ }^{5}$ Miami University, Oxford, United States; ${ }^{6}$ NorthShore University HealthSystem, Skokie, United States; ${ }^{7}$ The University of Chicago Pritzker School of Medicine, Chicago, United States

Background: Essential Thrombocythemia (ET) is a myeloproliferative neoplasm characterized by an overproduction of platelets by the bone marrow. Furthermore, it can cause severe complications such as stroke or venous thromboembolism. Despite the intrinsic connection between ET and cardiovascular events, there is a paucity of data on hospitalization risk for cardiovascular events in patients with ET. Aims: Our objective was to identify prevalent comorbidities among patients with ET who were hospitalized for cardiovascular disease.

Methods: We performed a retrospective analysis of the National Inpatient Sample (NIS) database from 2016 to 2017. Patients included were at least 18 years of age and had an ICD-10 code for ET. To identify cardiovascular outcomes, an ICD-10 code for Venous Thromboembolism, Stroke, Acute Coronary Syndrome, or Major Adverse Limb Events must be present in the top three discharge diagnoses. We used descriptive statistics to find common chronic comorbidities and performed binary logistic regression to identify prevalent risk factors for hospitalizations due to cardiovascular disease. All statistical analyses were performed in SPSS.

Results: We identified a total of 234,225 (weighted) hospitalizations of patients with ET. Patients had a mean age of 59.72 years of age (SD $=18.679)$, and they were predominantly female $(57 \%)$. Cardiovascular outcomes were present in 18,955 hospitalizations (8.10\%). prevalence for cardiovascular outcomes among patients with ET were: valvular disease (odds ratio, $1.33[95 \% \mathrm{Cl}, 1.23-$ 1.43]; $P<0.0001$ ), peripheral vascular disease (odds ratio, 1.97 [95\% Cl, 1.87-2.07]; $P<0.0001$ ), paralysis (odds ratio, 1.63 [95\% $\mathrm{Cl}, 1.54-1.73] ; P<0.0001$ ), renal failure (odds ratio, $1.06[95 \% \mathrm{Cl}$, 1.01-1.11]; $P=0.013$ ), hypertension (odds ratio, $1.36[95 \% \mathrm{Cl}, 1.32$ 1.41]; $P<0.0001$ ), and cancer (odds ratio, 1.06 [95\% Cl, 1.01-1.12]; $P=0.020)$. 
TABLE 1 Patient Characteristics

\begin{tabular}{|c|c|c|c|c|}
\hline \multirow{3}{*}{ Characteristic } & \multicolumn{2}{|c|}{ No CV outcome } & \multicolumn{2}{|c|}{ CV outcome } \\
\hline & $\mathbf{N}$ & $\%$ & $\mathbf{N}$ & $\%$ \\
\hline & 215,270 & $91.90 \%$ & 18,955 & $8.10 \%$ \\
\hline Age (Mean, SD) & 59.33 & 18.752 & 64.22 & 17.205 \\
\hline Female sex & 122,830 & $57.10 \%$ & 10,520 & $55.50 \%$ \\
\hline \multicolumn{5}{|c|}{ Comorbidities } \\
\hline Heart Failure & 21,710 & $10.09 \%$ & 2,195 & $11.58 \%$ \\
\hline Valvular Disease & 6,600 & $3.07 \%$ & 810 & $4.27 \%$ \\
\hline Peripheral Vascular Disease & 11,815 & $5.49 \%$ & 2,030 & $10.71 \%$ \\
\hline Paralysis & 11,110 & $5.16 \%$ & 1,510 & $7.97 \%$ \\
\hline Renal Failure & 25,235 & $11.72 \%$ & 2,555 & $13.48 \%$ \\
\hline Hypertension & 101,465 & $47.13 \%$ & 10,565 & $55.74 \%$ \\
\hline Metastatic Cancer & 11,240 & $5.22 \%$ & 915 & $4.83 \%$ \\
\hline Solid Tumor w/out metastasis & 7.790 & $3.62 \%$ & 775 & $4.09 \%$ \\
\hline Hypothyroidism & 22,455 & $10.43 \%$ & 1,880 & $9.92 \%$ \\
\hline Liver Disease & 9.130 & $4.24 \%$ & 485 & $2.56 \%$ \\
\hline Diabetes w/o Chronic Complications & 19,115 & $8.88 \%$ & 1,855 & $9.79 \%$ \\
\hline Hadoeres & 25.230 & $11.72 \%$ & 2.385 & \\
\hline
\end{tabular}

Conclusions: The most prevalent risk factors of hospitalizations for cardiovascular events among patients with ET include valvular disease, peripheral vascular disease, and paralysis. Additional evaluation should be done to consider optimization of prevention strategies for cardiovascular outcomes.

\section{LPB0050 | History of Cancer is Associated with Increased} Platelet Activity and Atherothrombosis in Patients with Peripheral Artery Disease

L. Cofer ${ }^{1}$; T. Barrett ${ }^{2}$; Y. Xia ${ }^{3}$; E. Luttrell-Williams ${ }^{4} ;$ K. Myndzar $^{4}$; K.-K. Wong ${ }^{5,6}$; D. Wise ${ }^{7}$ J. Berger ${ }^{2,8}$

${ }^{1}$ NYU Grossman School of Medicine, New York, United States; ${ }^{2}$ Leon

H. Charney Division of Cardiology, Department of Medicine, NYU Grossman School of Medicine, New York, United States; ${ }^{3}$ Division of Biostatistics, Department of Population Health, NYU Grossman School of Medicine, New York, United States; ${ }^{4}$ NYU Langone Health, New York, United States; ${ }^{5}$ Division of Hematology and Medical Oncology, NYU Grossman School of Medicine, New York, United States; ${ }^{6}$ Laura and Isaac Perlmutter Cancer Center, NYU Langone Health, New York, United States; ${ }^{7}$ Department of Medicine, NYU Grossman School of Medicine, New York, United States; ${ }^{8}$ Center for the Prevention of Cardiovascular Disease, NYU Langone Health, New York, United States

Background: Cancer and peripheral artery disease (PAD) share common risk factors and are frequently coprevalent. Platelets are culprits in the pathogenesis of PAD and mediators of arterial cardiovascular events. The association between platelet activity and cardiovascular events in patients with versus without cancer is uncertain. Aims: To investigate if cancer history is associated with platelet activity and incident cardiovascular events in a cohort of patients with PAD.

Methods: 289 patients with PAD undergoing lower extremity revascularization enrolled in the Platelet Activity and Cardiovascular Events (PACE) study were followed longitudinally for a median of 18 months. Prior to revascularization, patients had platelet activity measured via light transmission aggregometry in response to ADP, collagen, epinephrine, and serotonin. The primary clinical outcome was myocardial infarction (MI). Other endpoints were $\mathrm{Ml} /$ stroke and major adverse cardiovascular event (MACE; MI/stroke/death).

Results: 64 patients (22.1\%) reported a cancer history, 10 (15.6\%) with metastatic and 10 (15.6\%) with active cancer. Patients with (versus without) cancer history were older, less often Hispanic, and less frequently current smokers $(P<0.05$ for each). There was no difference in prevalent diabetes, coronary artery disease, hypertension, or antiplatelet therapy between groups. Platelet aggregation in response to submaximal $\operatorname{ADP}(0.4 \mu \mathrm{M}, 1.0 \mu \mathrm{M})$, collagen $(0.2 \mu \mathrm{g} / \mathrm{ml}$, $1.0 \mathrm{ug} / \mathrm{ml})$, and serotonin $(10 \mu \mathrm{M})$ was higher in patients with versus without cancer history. Consistently, patients with cancer history experienced more incident $\mathrm{MI}(18.8 \%$ vs. $7.6 \%, P=0.02), \mathrm{Ml} /$ stroke (25.0\% vs. 9.3\%, $P=0.002$ ), and MACE (35.9\% vs. $22.2 \%, P=0.04$ ). After adjustment for age, sex, race/ethnicity, smoking, diabetes, prior stroke, CAD, revascularization procedure, and antiplatelet therapy, patients with cancer history were at higher hazard for MI, $\mathrm{MI} /$ stroke, and MACE (Figure). The association between cancer and thrombotic events was most apparent in patients with metastatic and active cancer (Figure).

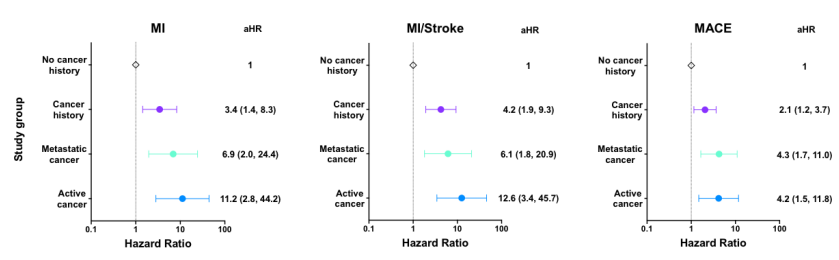

FIGURE 1 Adjusted hazard ratios for cardiovascular events by study group

Conclusions: In patients with PAD, cancer history was associated with increased platelet aggregation and risk for arterial thrombotic events.

LPB0098 | A Simple Score to Evaluate Bleeding Risk in Medical Patients Taking Antiplatelets for Cardiovascular Prevention

S. Barbar ${ }^{1}$; A. Vecchiato ${ }^{2}$; F. Moro ${ }^{2}$; F. Boscaro ${ }^{2}$; F. Fabris ${ }^{2}$

${ }^{1}$ Medicina - Ospedale di Cittadella - Azienda Ulss6 Euganea, Cittadella, Italy; ${ }^{2}$ Università degli Studi di Padova, Padova, Italy

Background: Antiplatelets are widely prescribed in medical patients, both for primary and secondary prevention of cardiovascular diseases and they may increase the patient's bleeding risk. At present, no appropriate tools are available for hemorrhagic risk assessment in this population.

Aims: In a large cohort of medical patients, we aimed to define the incidence of antiplatelets-related hemorrhage, identify clinical features associated with bleeding and develop a simple score able to predict them. 
Methods: Medical patients were considered eligible for this cohort study, provided they were taking antiplatelets and weren't on anticoagulant treatment. In a 12-months follow-up, we calculated the incidence of bleeding and identified factors independently associated with bleeding. We then developed a bedside risk score including the most predictive risk factors, weighted according to their strength of association with the bleeding complication.

Results: We enrolled 1275 patients; mean age was $75.3 \pm 13.6$ years. In a 1-year follow-up, 164 (12.9\%) major/non-major clinically relevant (NMCR) bleeds occurred, while the incidence of minor bleeding was $14.6 \%$. Gastrointestinal tract was the most frequent site of bleeding (87\% composite of

major/NMCR and minor bleeding). Clinical features associated with bleeding at the univariate analysis $(P>0.020)$ were tested in a logistic regression model. Female sex, history of anemia/previous bleeding, gastrointestinal tract disease, frequent falls/loss of consciousness and severe renal impairment have been found as independent risk factors for bleeding in antiplatelets-treated patients. These features have been associated in a five-item score that reached specificity $71 \%$, sensibility $91 \%$ and AUC $0.80(95 \% \mathrm{Cl} 0.71-0.94)$ in the derivation cohort (Fig. 1). Compared with low-risk participants, high-risk participants had an 8-fold greater risk of bleeding (HR 7.91; 95\%Cl 5.63-11.1).

\begin{tabular}{lccc}
\hline Risk factor & $\boldsymbol{R} \boldsymbol{R}$ & $\boldsymbol{C I 9 5 \%}$ & Score \\
\hline History of anemia/bleeding & 69,1 & $\mathbf{4 6 , 4 - 1 2 6 , 6}$ & $\mathbf{5}$ \\
Knoun gastrointestinal disease & 10,2 & $5,7-17,0$ & $\mathbf{3}$ \\
Recurrent fall/loss of consciousness & 5,6 & $1,8-22,4$ & $\mathbf{2}$ \\
Severe renal impairment & 3,3 & $1,5-7,4$ & $\mathbf{1}$ \\
Female sex & 3,7 & $1,99-6,22$ & $\mathbf{1}$ \\
\hline & & & Cut-off $\geq 6$ \\
\hline
\end{tabular}

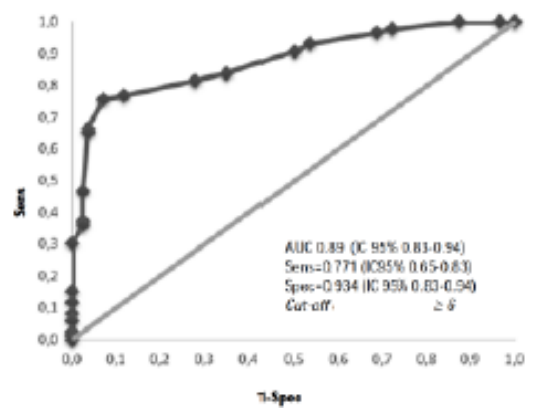

FIGURE 1 The newly derived score and its ROC curve in the derivation cohort

Conclusions: Despite their bleeding risk, antiplatelets are broadly prescribed in medical patients. A new simple score may be helpful in predicting individual hemorrhagic risk in antiplatelets-treated patients. Further studies are needed to confirm these findings.
PB0011 | Platelet-derived Let-7e is an Independent Predictor of Long-term All-cause Mortality in Patients with Type 2 Diabetes Mellitus

C. Eyileten ${ }^{1}$; J. Pordzik ${ }^{1}$; D. Jakubik ${ }^{1}$; P. Czajka ${ }^{1}$; M. Wolska ${ }^{1}$; J. Jarosz-Popek ${ }^{1,2}$; A. Fitas ${ }^{1}$; A. Nowak ${ }^{1}$; S. De Rosa ${ }^{3}$; A. Gąsecka ${ }^{4}$; A. Kapłon-Cieślicka ${ }^{4}$; J.M. Siller-Matula ${ }^{5}$; M. Postuła ${ }^{1}$ ${ }^{1}$ Center for Preclinical Research and Technology CEPT, Department of Experimental and Clinical Pharmacology, Medical University of Warsaw, Warsaw, Poland; ${ }^{2}$ Doctoral School, Medical University of Warsaw, Warsaw, Poland; ${ }^{3}$ Division of Cardiology, Department of Medical and Surgical Sciences, 'Magna Graecia' University, Catanzaro, Italy; ${ }^{4} 1$ st Chair and Department of Cardiology, Medical University of Warsaw, Warsaw, Poland; ${ }^{5}$ Department of Internal Medicine II, Division of Cardiology, Medical University of Vienna, Vienna, Austria

Background: In the light of growing prevalence of type 2 diabetes mellitus (T2DM), efforts are made to discover novel biomarkers. MicroRNAs (miRNAs-miR) are non-coding RNAs used in various processes involved in regulating gene expression which play a role in platelet function.

Aims: To analyze the ability of platelet-derived miRNAs in prediction of mortality and response to antiplatelet treatment among T2DM-patients.

Methods: 252 diabetic subjects were enrolled and were receiving either acetylsalicylic acid (ASA) $75 \mathrm{mg}$ (65\%) or $150 \mathrm{mg}$ (15\%) or clopidogrel (19\%). Plasma miR-126, miR-223, miR-125a-3p and Let-7e expressions were assessed by qRT-PCR and compared between the patients who survived and those who died. Median observation time was 5.9 years. Adjusted Cox-regression analysis was used for prediction of mortality. Differential miRNAs expression due to different antiplatelet treatment was analyzed.

Results: ROC curve analysis revealed increasing concentrations of miR-126, Let-7e and miR-125a-3p levels had a diagnostic ability for prediction of long-term all-cause mortality (c-index $=0.75, P<0.001$; $0.72, P<0.001 ; 0.72, P=0.001$, respectively). Multivariate Cox regression model revealed high miR-126 and Let-7e expressions which were strong and independent predictors of all-cause long-term mortality $(\mathrm{HR}=5.08,95 \% \mathrm{Cl}: 1.92-13.43 ; \mathrm{P}=0.001 ; \mathrm{HR}=5.94,95 \%$ Cl:1.98-17.79; $P=0.001$, respectively). After including all miRNAs into one multivariate Cox regression model, only Let-7e was predictive of future occurrence of long-term all-cause death $(H R=7.83$, 95\% Cl: 1.2-51.1; $P=0.032$ ). MiR-126, Let-7e and miR-223 expressions in the clopidogrel group were significantly higher than in the ASA group ( $P=0.014 ; P=0.013 ; P=0.028$, respectively). 
a)

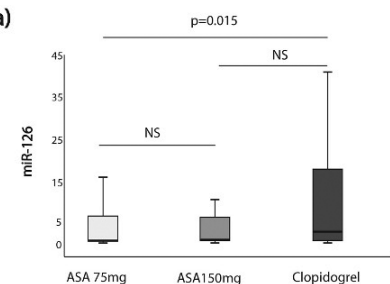

c)

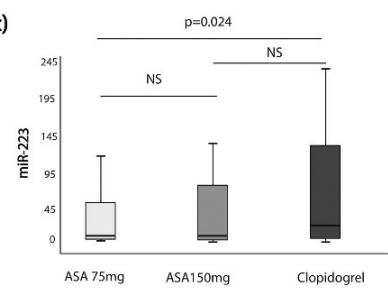

b)

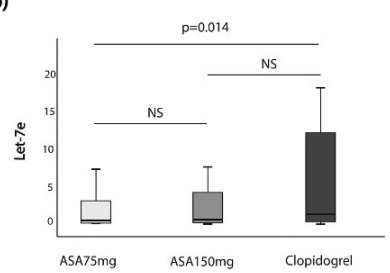

d)

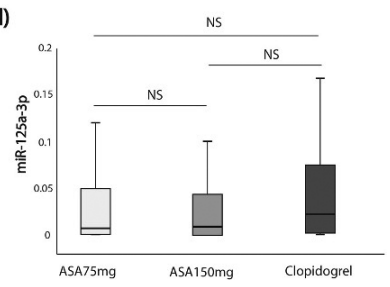

FIGURE 1 MiRNAs expressions in regard to the type of antiplatelet treatment based on randomization [ASA 75 mg vs ASA 150 mg vs Clopidogrel]; a) miR-126; b) Let-7e; c) miR-223; d) miR-125a-3p

\begin{tabular}{lllll}
\hline Variable & HR & $\mathbf{9 5 \% C I}$ & & p-value \\
\hline & & Lower & Upper & \\
\hline High miR-223 & 0.326 & 0.088 & 1.207 & 0.093 \\
High miR-126 & 3.754 & 0.943 & 14.941 & 0.061 \\
High Let-7e & 7.829 & 1.200 & 51.095 & $\mathbf{0 . 0 3 2}$ \\
High miR-125a-3p & 0.657 & 0.224 & 1.928 & 0.445 \\
Hypertension & 2.828 & 0.558 & 14.344 & 0.210 \\
Dyslipidemia & 1.022 & 0.344 & 3.034 & 0.968 \\
Age & 1.099 & 1.031 & 1.171 & $\mathbf{0 . 0 0 4}$ \\
Gender (male) & 5.968 & 1.977 & 18.016 & $\mathbf{0 . 0 0 2}$ \\
History of MI & 1.753 & $\mathbf{0 . 6 4 8}$ & 4.737 & 0.269 \\
Current smoking & 3.034 & 0.589 & 15.621 & 0.184 \\
Clopidogrel & 2.471 & 0.894 & 6.828 & 0.081 \\
HR, hazard ratio; MI, myocardial infarction, $95 \%$ CI, $95 \%$ confidence interval; & \\
\hline & & & & \\
\hline
\end{tabular}

FIGURE 2 Multivariate Cox regression model including high levels of miRNAs and clinical data

Conclusions: Let-7e expression is a strong and independent predictor of long-term all-cause mortality among patients with T2DM. MiR-223, miR-126 and Let-7e present significant interactions with antiplatelet treatment and clinical outcomes.

PB0012 | Prognostic Significance of von Willebrand Factor in Flow-mediated Dilatation Test at Patients with Arterial Hypertension and Diabetes Type 2

\section{E. Shorikov; D. Shorikova; P. Shorikov}

Bukovinian State Medical University, Chernivtsi, Ukraine

Background: A poor prognosis of concomitant courses of arterial hypertension and diabetes mellitus type 2 on the population morbidity

especially deals with the thrombotic complications which are situated in locally injured vessels.

Aims: To set von Willebrand factor (vWF) level at the patients with arterial hypertension (AH) and type 2 diabetes mellitus (DM) and analyze its prognostic relationship due to vasodilation and assessment of endothelial function.

Methods: The activity of VWF was estimated in plasma of 146 patients with $\mathrm{AH}$ and $\mathrm{DM}$ and in 50 healthy persons using specific standardized VWF-reagent. The research of endothelial dysfunction was conducted in flow-mediated dilatation. Prognostic level of VWF was analyzed in the true-positive rate test and, then, with plotting a function of receiver operating characteristic.

Results: Initially, it was set, that basal level of vWF at patients with $\mathrm{AH}$ and type $2 \mathrm{DM}$ was reliably higher $(113.3 \pm 13.9 \%)$ than in the control group (85.2 $\pm 8.9 \% ; P<0.01)$. It was determined the negative reliable association in VWF activity and index of absolute diameter increasing ( $r=-0.49, P=0.02$ ). Then we have measured sensitivity, specificity and, set the predictive value of vWF level in relation to disorders of endothelial function. The prognostic value of the model was sufficiently high, area under the curve (AUC) obtains $64.6 \% \pm 6.74 \%(P=0.015)$, that is a difference to $50.0 \%$ of AUC level. It was set the prognostic points for vWF activity between 81.5 $\%$ and $131.0 \%$.

Conclusions: The results undertaken in the study testify that elevation of vWF activity is associated with the decline of absolute increase of brachial diameters in flow-mediated dilatation test with prognostic level of vWF between $81.5 \%$ and $131.0 \%$.

PB0013 | The Coagulation and Fibrinolisys Changes Undergoing the Ramipril and Losartan Treatment of Concomitant Arterial Hypertension and Diabetes Mellitus Type 2

\section{E. Shorikov; D. Shorikova; P. Shorikov; O. Khukhlina}

Bukovinian State Medical University, Chernivtsi, Ukraine

Background: ESC 2018 Guidelines of the management of arterial hypertension yet have set ACE-inhibitors and angiotensin-receptor blockers (BRA) as drugs of choice in treatment of associated pathology. We have interested in some specific pleiotropic features in the activity of them such as the influence on the state coagulation and fibrinolisys.

Aims: To learn potential activity of ramopril versus losartan on some factors of coagulation and plasma fibrinolisys during their use in patients with arterial hypertension and diabetes mellitus of type 2 .

Methods: Monotherapy of ramipril (from 5 to $10 \mathrm{mg})(\mathrm{N}=48)$ or losartan (from 50 to $100 \mathrm{mg}$ ) ( $N=41)$ per day were prescribed for 89 patients with arterial hypertension and diabetes mellitus type 2 on the basic use of metformin and statins for 36 weeks. The activities of protein C, antithrombin III (AT III), and factor XIII, plasma fibrinolitic activity (PFA) were measured. The estimation of efficacy was conducted by calculation of "before-after" changes, the therapeutical benefit (changes of attributable risk - CAR and odds ratio (OR). 
Results: It has been established the same direction of anticoagulative activity changes under the influence of both drugs. We set the reliable increase of protein C activity (ramipril group - from $0.82 \pm 0.25$ u. to $1.09 \pm 0.14$ u. $P<0.001$ and losartan group $-0.86 \pm 0.22 \mathrm{u}$. to $1.12 \pm 0.37$ u. $P<0.001$ ), but the changes of AT III and factor XIII were unreliable. As to state of fibrinolisys, we have found the incrimination of the activity after this period of treatment. Administration of ramipril forwards more considerable raise of PFA, the absolute advantage ramipril against losartan was set at CAR $=16.4 \%, \mathrm{OR}=$ $1.92(0.74-9.84)$

Conclusions: Both drugs (ramipril and losartan) have a certain equal level of the influence on coagulation; however ramipril have been shown greater ability on the changes of fibrinolitic activity.

P0113 | Is There an Association of Diffuse Large B-cell Lymphoma with Coronary Artery Disease?

H. Holik $^{1,2} ;$ B. Coha ${ }^{1}$

${ }^{1}$ General Hospital dr. Josip Benčević, Slavonski Brod, Croatia;

${ }^{2}$ University of Osijek Josip Juraj Strossmayer, Osijek, Croatia

Background: The association of malignancies with venous thromboembolism is known but the association with arterial thromboembolism is not so clear. There is also insufficient data on the association of certain lymphoma entities with coronary artery disease (CAD).

Aims: To determine the prevalence of CAD in different types of lymphoma.

Methods: We collected data retrospectively from patients with Hodgkin's and non-Hodgkin's lymphoma at the General Hospital Dr. Josip Benčević from the beginning of 2011. by August 2020.

Results: 121 patients were included in this study, 67 (55\%) female. 11 (9\%) patients had CAD, 8 male and 3 female. Out of a 121 patients with lymphoma, 51 (42\%) had diffuse large B-cell lymphoma (DLBCL), 13 (11\%) had follicular lymphoma (FL), other indolent lymphomas had 20 (17\%), 13 (11\%) had Hodgkin's lymphoma, T lymphomas 6 (5\%) and 18 (15\%) patients had other types of lymphoma. The time from the first manifestation of CAD to the diagnosis of lymphoma ranged from 3 months to 20 years. Of the 11 patients with CAD, 7 (64\%) had DLBCL, 2 (18\%) small cell lymphoma, 1 (9\%) hairy cell leukemia, and 1 (9\%) FL. No one had CAD after lymphoma diagnosis.

Conclusions: This study suggests a higher presence of CAD in patients with DLBCL than in other types of lymphoma. The prevalence of CAD in DLBCL is almost twice as high as in the general population (6.7\% for the general population older than 20 years) while for other types of lymphoma it is similar to prevalence in the general population. Interestingly in our study was higher prevalence of female with lymphoma while CAD was almost 3 times more common in male. Further studies with more patients are needed to confirm this observations and eventually to find a link between DLBCL and CAD.
P0114 | Endothelin and General Oxidative Ability as Markers of Deadaptation in Sailors in the conditions of the Arctic Flight

$\underline{\text { N. Vorobyeva }}^{1,2} ;$ A. Vorobyeva ${ }^{2}$

${ }^{1}$ National Medical Research Center for Hematology (Northern Branch) Ministry of Health of Russia, Arckhangelsk, Russian Federation;

${ }^{2}$ Northern State Medical University Ministry of Health of Russia, Arckhangelsk, Russian Federation

Background: One of the areas of clinical medicine is the study of the mechanisms of adaptation of the vascular endothelium as a risk factor for thrombosis.

Aims: The study aimed to determine the possible relationship between the development of endothelial dysfunction and the antioxidant system with modifiable risk factors in a trans-latitudinal flight in the Arctic.

Methods: The prospective study included 32 crewmembers of the research vessel "Mikhail Somov» during the "TransArctic-2019» integrated marine scientific expedition. Venous blood sampling was carried out before the went on a voyage (zero point) to the city of Arkhangelsk ( $64^{\circ} 33^{\prime}$ north latitude $40^{\circ} 32^{\prime}$ east longitude) and at the highest point of the expedition - Hayes Island $\left(80^{\circ} 34^{\prime}\right.$ north latitude $57^{\circ} 41^{\prime}$ east longitude). The determination of the concentration of endothelin-1 and the total antioxidant ability of serum (AOS) by enzyme-linked immunosorbent assay. The study was supported by a grant from the Russian Foundation for Basic Research - project No. 18-00-00814-COMFI (18-00-00478).

Results: Statistically differences were obtained in the concentration of endothelin at zero $(M=4.79 \pm 2.1) \mathrm{pg} / \mathrm{ml}$ and high points $(M=7.02$ $\pm 2.42) \mathrm{pg} / \mathrm{ml}, t=-3.6532, \mathrm{df}=31, P<0.001$, the concentration of endothelin-1 significantly increases, which indicates the first signs of the formation of maladaptation of the vascular endothelium in the form of vasoconstriction. $84.4 \%$ of crewmembers at a high point showed a high overall antioxidant ability, which may indicate compensation for antioxidant defence mechanisms. It was shown that AEC in the study group among smokers was statistically significantly higher than in non-smokers.

Conclusions: Moreover, the predominance of high AOC among the crew of the vessel indicates an increased oxidative load on the human body to neutralize the excess amount of reactive oxygen species.

\section{P0115 | Seasonal Changes of Platelet Aggregation Indexes at} the Patients with Stable Angina

D. Shorikova; E. Shorikov; P. Shorikov

Bukovinian State Medical University, Chernivtsi, Ukraine

Background: Seasonal variations of cardiovascular complications is signed by different authors (Turin T. C. et al., 2008; Khan F. A. et al., 2005). The activation of clotting is rapid protective reaction to vessel damage. At the same time, the studies which present correlations 
of platelets aggregation with seasonality, and, also, describe clinical risk of vascular complications are limited.

Aims: To analyze the seasonal changes of platelet aggregation indexes, and, also, risk of thrombotic complications at patients with stable angina in emergency care clinic.

Methods: The study included 939 people (male - 507, female - 432, average age $-42.1 \pm 8.9$ years). The analysis of platelet aggregation was carried out on the analyzer of platelet aggregation "BIOLA" 230LA by J. Born method.

Results: It is set that the highest risk of thrombotic complications has been on February and March, where contribution of patients with thromboembolism are $20.9 \%(P=0.001)$. Probability of thromboembolism in the $1^{\text {st }}$ quarter of the year among surgical patients in the postoperative period is set as $29.9 \%(P<0.005)$. At the patients with thrombotic complications are estimated the higher level of spontaneous $(P<0.05)$, ADP-induced aggregation $(P<0.05)$, and the speed of induced aggregation in compare with healthy volunteers $(P<0.05)$. Due to seasonal changes the $63.0 \%$ patients have higher level of ADP-induced aggregation than grouping median in the 1-st quarter of year, and the lowest risk due to the aggregation parameters are set at the $4^{\text {th }}$ quarter.

Conclusions: In patients with stable angina were proved seasonal variations of thrombotic complications: the highest risk of thrombosis occurs in February and March due to increased level of spontaneous and ADP-induced platelet aggregation.

\section{P0118 | Homocysteine Exchange in Athletes in the Arctic}

\section{Region}

N. Vorobyeva $^{1,2} ;$ N. Malyshkina ${ }^{2}$

"National Medical Research Center for Hematology" (Northern branch) Ministry of Health of Russia, Arckhangelsk, Russian Federation; ${ }^{2}$ Northern State Medical University Ministry of Health of Russia,

Arckhangelsk, Russian Federation

Background: The impact of lifestyle on blood homocysteine levels is undeniable. The role of hyperhomocysteinemia in the development of vascular pathology is also well known. The climatic and geographical features of human life in the Arctic region also leave their mark. Aims: Determination of changes in blood homocysteine levels in athletes living in the Arctic region of the Russian.

Methods: The study included a survey of 98 athletes involved in various sports living in the Arctic region of the RF. The median age was $19.5(16 ; 25)$ years. Determination of blood serum homocysteine level was done by enzyme immunoassay. Polymorphisms of three folate cycle genes were studied: MTHFR $677 \mathrm{C}>\mathrm{T}$ polymorphism, MTHFR polymorphism (glu429ala); MTRR A66G polymorphism.

Results: Homocysteine levels were from 3.33 to $50.0(\mathrm{Me}=15.84)$ $\mu \mathrm{mol} / \mathrm{L}$. A weak correlation was found between the level of homocysteine and the gender of the athlete $(r=0.037, P=0.774)$, the age of the athlete $(r=0.222, P=0.081)$, the level of sportsmanship $(r=0.079, P=0.540$ ). The polymorphism MTHFR $677 \mathrm{C}>\mathrm{T}$ was found in $45 \%$ of cases ( $\mathrm{Cl}$ : 34-57\%), homozygotes - 5\% (Cl: 1.5-12\%) of alleles and heterozygotes - 40\% (Cl: 30-52\%). MTHFR (glu429ala) polymorphism was found in $48 \%$ ( $\mathrm{Cl}: 36-59 \%)$, the homozygous allele $\mathrm{A} 1298 \mathrm{C}$ was in 3.6\% (Cl: 0.8-10.3\%). Heterozygotes - in 44\% of cases ( $\mathrm{Cl}$ : 33-55\%). The genetic marker MTRR A66G had a frequency of $76 \%$ (Cl: $65-84 \%)$; homozygous variant in $23 \%$ (Cl: $15-34 \%)$, heterozygous in $52 \%$ (Cl: $41-64 \%)$.

Conclusions: Demonstrated a mild to moderate degree of hyperhomocysteinemia. There were no differences in the level of homocysteine depending on the allelic variants of the folate cycle enzyme genes $(P=0.395, P=0.987, P=0.852)$. Differences in blood homocysteine concentration in athletes with different alleles of the same gene of the folate cycle are insignificant. Which may indicate the influence of external factors on the high level of homocysteine in the blood.

\section{CEREBROVASCULAR DISORDERS}

\section{LPB0002 | Safety and Pharmacokinetics of Direct Oral} Anticoagulants after Bariatric Surgery: A Systematic Review

R. Leong ${ }^{1}$; D.K Chu ${ }^{2,3}$; M.A Crowther ${ }^{2}$; S. Mithoowani ${ }^{2}$

${ }^{1}$ McMaster University, Faculty of Health Sciences, Hamilton, Canada;

${ }^{2}$ McMaster University, Department of Medicine, Hamilton, Canada;

${ }^{3}$ McMaster University, Department of Health Research Methods, Evidence and Impact, Hamilton, Canada

Background: Bariatric surgery likely impairs the absorption of direct oral anticoagulants (DOACs). The optimal DOAC after bariatric surgery is unclear.

Aims: To report on the safety and pharmacokinetics of DOACs after bariatric surgery in adults.

Methods: We systematically searched MEDLINE, EMBASE, Cochrane Library, CINAHL and ClinicalTrials.gov from Jan 2000-Jul 2020 for randomized and non-randomized studies. Two reviewers independently screened titles, abstracts and full-text articles. We used the Newcastle-Ottawa scale to assess risk of bias in nonrandomized studies excluding case reports. This review was registered with PROSPERO (CRD42020202636).

Results: We screened 2,086 titles and abstracts and included 19 records ( $n=2,233$ patients): no randomized trials, seven cohort studies, two case series, and ten case reports. Patients were taking DOACs for a variety of indications. Most studies had a moderate-high risk of bias. Of 2,123 patients taking apixaban, two (0.1\%) developed venous thromboembolism (VTE) and six (0.3\%) had major bleeding. Peak apixaban levels were measured in 13 patients (mean $182 \mathrm{ng} / \mathrm{mL}$, 95\% confidence interval [Cl] 142-223 $\mathrm{ng} / \mathrm{mL}$ ); none were outside the therapeutic range seen in phase II studies. Of 94 patients taking rivaroxaban, two developed VTE (2.1\%) and five (5.3\%) had major bleeding. Peak rivaroxaban levels were measured in 17 patients (mean $249 \mathrm{ng} / \mathrm{mL}, 95 \% \mathrm{Cl} 162-336$ $\mathrm{ng} / \mathrm{mL}$ ); eight (47\%) were below and one (5.9\%) was above the 
therapeutic range. Of 16 patients taking dabigatran, one patient (6.3\%) each developed VTE, stroke and major bleeding. Peak dabigatran levels were measured in 12 patients (mean $57 \mathrm{ng} / \mathrm{mL}, 95 \%$ $\mathrm{Cl} 22-91 \mathrm{ng} / \mathrm{mL}$ ); nine (75\%) were below and none were above the therapeutic range.

Conclusions: The preferred DOAC after bariatric surgery is uncertain. Additional cohort and registry studies are warranted given how frequently such patients require anticoagulant therapy.

LPB0003 | Persistence Pattern to Oral Anticoagulants among Non-valvular Atrial Fibrillation Patients who Switched from Vitamin K Antagonists to Direct Oral Anticoagulants: A Cohort Study

M.M. Toorop ${ }^{1}$; Q. Chen ${ }^{1}$; M.J. Kruip ${ }^{2,3}$; F.J. van der Meer ${ }^{1}$; M. Nierman ${ }^{4}$; L. Faber ${ }^{5}$; L. Goede ${ }^{6}$; S.C. Cannegieter ${ }^{1}$; W.M. Lijfering ${ }^{1}$

${ }^{1}$ Leiden University Medical Center, Leiden, Netherlands; ${ }^{2}$ Erasmus University Medical Center, Rotterdam, Netherlands; ${ }^{3}$ Thrombosis Service Star-shl, Rotterdam, Netherlands; ${ }^{4}$ Thrombosis Service Atalmedial, Amsterdam, Netherlands; ${ }^{5}$ Red Cross Hospital, Beverwijk, Netherlands; ${ }^{6}$ Thrombosis Service Saltro, Utrecht, Netherlands

Background: Patients with non-valvular atrial fibrillation (NVAF) who use vitamin $\mathrm{K}$ antagonist (VKA) and have low Time in Therapeutic
Range (TTR) should switch to direct oral anticoagulants (DOAC) according to international guidelines.

Aims: To determine if patients on VKA with a low TTR who are switched to DOAC have a same persistence rate to oral anticoagulants (OAC) as patients on VKA with a high TTR who are also switched to DOAC.

Methods: Individual data was obtained from anticoagulation clinics and Statistic Netherlands. Adult NVAF patients who were switched from VKA to DOAC between April-2013 and September-2018 were stratified by baseline TTR and followed from the date of switch. OAC prescription records were examined to determine non-persistence, based on a 100-day gap. Cumulative incidences of non-persistence to OAC (allowing a switch back to VKA) and DOAC (not allowing a switch back to VKA) after 6 months, 1 and 4 years of follow-up were estimated and multivariable Cox regression models were employed to evaluate the associations of baseline TTR with OAC non-persistence.

Results: 4100 patients were included of which $28 \%$ had a baseline TTR $\leq 45 \% .8 .5 \%(95 \% \mathrm{Cl} 6.8-10.4)$ of patients with a baseline TTR $\leq 45 \%$ were non-persistent to OAC at 1 year after the switch compared to $4.7 \%(95 \% \mathrm{Cl} 3.9-5.5)$ of patients with a baseline TTR $>45 \%$ (Table 1). After adjustments for age, sex, anticoagulation clinics, and comorbidities, a baseline TTR $\leq 45 \%$ was associated with a higher risk of non-persistence to OAC (Hazard ratio $1.63,95 \% \mathrm{Cl}$ 1.35-2.03) when compared to TTR > 45\% (Table 2). This trend was similar when using other cut-off points for baseline TTR (60\%;70\%).

TABLE 1 Cumulative incidences of non-persistence to OAC and DOAC, stratified by baseline TTR

\begin{tabular}{|c|c|c|c|c|c|c|c|c|}
\hline \multirow{2}{*}{$\begin{array}{l}\text { Time since } \\
\text { switch to } \\
\text { DOAC }\end{array}$} & \multicolumn{4}{|c|}{ Non-persistent to all OAC (allowing a switch back to VKA) } & \multicolumn{4}{|c|}{ Non-persistent to DOAC (not allowing a switch back to VKA) } \\
\hline & $\begin{array}{l}\text { Follow-up } \\
\text { (years) }\end{array}$ & No. at risk & $\begin{array}{l}\text { No. } \\
\text { event }\end{array}$ & $\begin{array}{l}\text { Cumulative incidence } \\
(95 \% \mathrm{Cl})(\%)^{*}\end{array}$ & $\begin{array}{l}\text { Follow-up } \\
\text { (years) }\end{array}$ & $\begin{array}{l}\text { No. at } \\
\text { risk }\end{array}$ & $\begin{array}{l}\text { No. } \\
\text { event }\end{array}$ & $\begin{array}{l}\text { Cumulative incidence } \\
(95 \% \mathrm{Cl})(\%)^{*}\end{array}$ \\
\hline \multicolumn{9}{|l|}{$\mathrm{TTR} \leq 45 \%$} \\
\hline $0-6$ months & 483.83 & 1132 & 43 & $4.12(3.03-5.45)$ & 466.82 & 1132 & 91 & $8.59(7-10.38)$ \\
\hline 0-1 year & 857.31 & 1132 & 80 & $8.52(6.83-10.43)$ & 819.23 & 1132 & 133 & $13.58(11.49-15.83)$ \\
\hline $0-4$ year & 1697.32 & 1132 & 141 & $24.06(19.63-28.75)$ & 1584.05 & 1132 & 195 & $29.69(24.91-34.62)$ \\
\hline \multicolumn{9}{|l|}{ TTR $>45 \%$} \\
\hline $0-6$ months & 1335.69 & 2968 & 81 & $2.87(2.3-3.54)$ & 1291.54 & 2968 & 195 & $6.83(5.94-7.8)$ \\
\hline 0-1 year & 2434.01 & 2968 & 124 & $4.69(3.92-5.54)$ & 2332.42 & 2968 & 256 & $9.42(8.35-10.56)$ \\
\hline $0-4$ year & 5518.46 & 2968 & 267 & $16.16(14.13-18.31)$ & 5185.26 & 2968 & 389 & $20.26(18.15-22.45)$ \\
\hline
\end{tabular}

"estimated by cumulative incidence competing risk (CICR) method.

Abbreviations: OAC, oral anticoagulants; DOAC, direct oral anticoagulants; VKA, Vitamin $\mathrm{K}$ antagonists; $\mathrm{Cl}$, confidence interval; TTR, time in therapeutic range. 
TABLE 2 Risk of non-persistence to OAC between different levels of TTR during VKA treatment

\begin{tabular}{|c|c|c|c|c|c|c|c|}
\hline & $\begin{array}{l}\text { Observation } \\
\text { time } \\
\text { (person-year) }\end{array}$ & No. event & $\begin{array}{l}\text { Incidence rate } \\
(95 \% \mathrm{Cl}) \text { (per } \\
100 \text {-person } \\
\text { year) }\end{array}$ & HR $(95 \% \mathrm{Cl})$ & $\mathrm{HR}^{*}(95 \% \mathrm{Cl})$ & $\mathrm{HR}^{\dagger}(95 \% \mathrm{Cl})$ & $\mathrm{HR}^{\dagger \ddagger}(95 \% \mathrm{Cl})$ \\
\hline TTR > 45\% & 5735.75 & 290 & $5.06(4.49-5.67)$ & 1 (Reference) & 1 (Reference) & 1 (Reference) & 1 (Reference) \\
\hline TTR $\leq 45 \%$ & 1736.54 & 144 & $8.29(6.99-9.76)$ & $1.64(1.34-2.01)$ & $1.66(1.35-2.03)$ & $1.63(1.32-2)$ & $1.49(1.17-1.9)$ \\
\hline TTR > 60\% & 4564.35 & 213 & $4.67(4.06-5.34)$ & 1 (Reference) & 1 (Reference) & 1 (Reference) & 1 (Reference) \\
\hline TTR > 70\% & 3656.69 & 169 & $4.62(3.95-5.37)$ & 1 (Reference) & 1 (Reference) & 1 (Reference) & 1 (Reference) \\
\hline TTR $\leq 70 \%$ & 3815.61 & 265 & $6.95(6.13-7.83)$ & $1.52(1.21-1.92)$ & $1.51(1.19-1.91)$ & $1.47(1.16-1.87)$ & $1.47(1.16-1.87)$ \\
\hline
\end{tabular}

*Adjusted for age, sex, and anticoagulation clinics.

${ }^{\dagger}$ Adjusted for age, sex, anticoagulation clinics, and comorbidities (chronic obstructive pulmonary disease, asthma, other chronic lung diseases, congestive heart failure, hypertension, myocardial infarction history, abnormal liver function, gastroesophageal reflux disease, peptic ulcer disease, abnormal renal function, anemia, coagulopathy, diabetes mellitus, thyroid disease, autoimmune disease, systemic connective tissue disorders, stroke/ TIA history, Alzheimer's disease, Parkinson's disease, peripheral artery disease, deep vein thrombosis, pulmonary embolism, arterial embolism and thrombosis, major bleeding history, malignant tumor).

${ }^{\ddagger}$ Restricted to patients who had a baseline CHA2DS2-VASc $\geq 2$.

Abbreviations: DOAC, direct oral anticoagulants; OAC, oral anticoagulants; TTR, time in therapeutic range; VKA, Vitamin K antagonists; HR, hazard ratio; $\mathrm{Cl}$, confidence interval.

Conclusions: For NVAF patients who switched from VKA to DOAC, a low baseline TTR was associated with an increased risk of being non-persistent to OAC. Clinicians should consider this risk before switching a patient from VKA to DOAC.

\section{LPB0004 | Safety and Efficacy of Using Direct Oral}

Anticoagulants for Stroke Prevention in Atrial Fibrillation Patients with Pre-obesity and Obesity

Q. Chen; M.M. Toorop; S.C. Cannegieter; W.M. Lijfering

Leiden University Medical Center, Leiden, Netherlands

Background: Evidence of direct oral anticoagulants (DOACs) use in obese atrial fibrillation (AF) patients is limited.

Aims: To investigate the safety and efficacy of DOACs for ischemic stroke prevention among AF patients with different baseline body mass index (BMI).

Methods: Prevalent adult AF patients using DOACs for stroke prevention on 1/1/2017 in the Netherlands were screened and those participated in the Dutch Health Monitor, a survey conducted in participants randomly sampled from the adult Dutch population in (September-December) 2016, were included. BMI from the survey was used to categorize the patients as normal weight $\left(18.5-25 \mathrm{~kg} / \mathrm{m}^{2}\right)$, pre-obesity $\left(25-30 \mathrm{~kg} / \mathrm{m}^{2}\right)$, and obesity $\left(\geq 30 \mathrm{~kg} / \mathrm{m}^{2}\right)$. Underweight patients $\left(<18.5 \mathrm{~kg} / \mathrm{m}^{2}\right)$ or those with extreme or missing value of weight ( $\leq 25$ or $\geq 275 \mathrm{~kg}$ ), height ( $\leq 75$ or $\geq 230 \mathrm{~cm}$ ), or BMI $\left(<14\right.$ or $\geq 50 \mathrm{~kg} / \mathrm{m}^{2}$ ) were excluded. The patients were followed from $1 / 1 / 2017$ to death or 31/12/2018, whichever came first. Major bleeding and ischemic stroke were the study outcomes. Cumulative incidences of the outcomes were estimated by Kaplan-Meier methods, and associations between baseline BMI categories and the outcomes were evaluated by Cox regression models, adjusting for age, sex, HAS-BLED score, $\mathrm{CHA}_{2} \mathrm{DS}_{2}$-VASc score, and household income.
Results: A total of 3,033 patients were included (mean age $73.94 \pm 7.83$ years, $59.3 \%$ male), of which 1003 had normal weight (mean BMI $22.93 \pm 1.57 \mathrm{~kg} / \mathrm{m}^{2}$ ), 1379 had pre-obesity (mean BMI $27.24 \pm 1.40 \mathrm{~kg} / \mathrm{m}^{2}$ ), and 651 had obesity (mean BMI $33.43 \pm 3.28 \mathrm{~kg} / \mathrm{m}^{2}$ ). Cumulative incidences of major bleeding within 1 year were $1.20 \%(95 \% \mathrm{Cl} 0.52 \%-1.87 \%), 1.53 \%(95 \% \mathrm{Cl}$ $0.87 \%-2.17 \%)$, and $1.39 \%(95 \% \mathrm{Cl} 0.48 \%-2.28 \%)$ respectively; and were $0.70 \%$ (95\% Cl 0.18\%-1.21\%), 0.95\% (95\% Cl 0.43\%-1.45\%), and $0.31 \%$ (95\% Cl 0.00\%-0.73\%) for ischemic stroke. No statistically significant associations were found between baseline BMI categories and the outcomes.
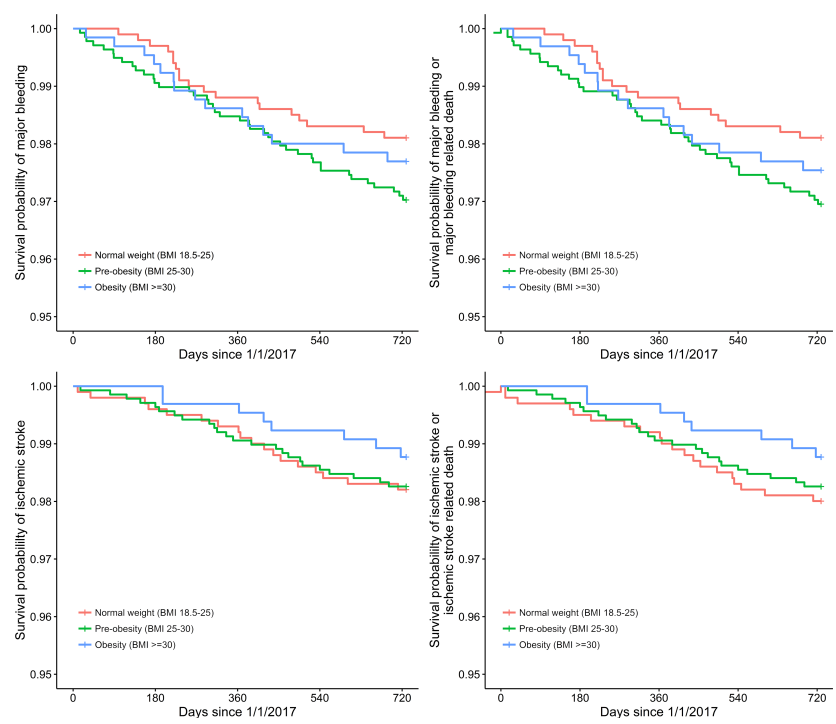

FIGURE 1 Kaplan-Meier curves for the studied clinical outcomes according to categories of baseline body mass index (BMI) 
TABLE 1 Associations between baseline body mass index (BMI) and prognosis

\begin{tabular}{|c|c|c|c|}
\hline Clinical outcomes & Crude HR $(95 \% \mathrm{Cl})$ & $\begin{array}{l}\mathrm{HR}(95 \% \mathrm{Cl}) \text {, } \\
\text { adjusting for age, } \\
\text { sex }\end{array}$ & $\begin{array}{l}\mathrm{HR}(95 \% \mathrm{Cl}) \text {, adjusting for } \\
\text { age, sex, } \mathrm{HAS} \text { - BLED score, } \\
\mathrm{CHA}_{2} \mathrm{DS}_{2} \text {-VASc score, } \\
\text { household income }\end{array}$ \\
\hline $\begin{array}{l}\text { Major bleeding: Pre-obesity }\left(25-30 \mathrm{~kg} / \mathrm{m}^{2}\right) \\
\left.\text { (VS normal weight, } 18.5-25 \mathrm{~kg} / \mathrm{m}^{2}\right)\end{array}$ & $1.58(0.92-2.72)$ & $1.70(0.98-2.93)$ & $1.64(0.95-2.83)$ \\
\hline $\begin{array}{l}\text { Major bleeding: Obesity }\left(\geq 30 \mathrm{~kg} / \mathrm{m}^{2} \text { ) }\right. \\
\text { (VS normal weight, } 18.5-25 \mathrm{~kg} / \mathrm{m}^{2} \text { ) }\end{array}$ & $1.22(0.62-2.40)$ & $1.43(0.72-2.83)$ & $1.26(0.63-2.53)$ \\
\hline $\begin{array}{l}\text { Major bleeding or major bleeding related death: Pre-obesity }\left(25-30 \mathrm{~kg} / \mathrm{m}^{2}\right) \\
\left.\text { (VS normal weight, } 18.5-25 \mathrm{~kg} / \mathrm{m}^{2}\right)\end{array}$ & $1.58(0.92-2.72)$ & $1.70(0.99-2.94)$ & $1.65(0.95-2.85)$ \\
\hline $\begin{array}{l}\text { Major bleeding or major bleeding related death: Obesity }\left(\geq 30 \mathrm{~kg} / \mathrm{m}^{2}\right) \\
\text { (VS normal weight, } 18.5-25 \mathrm{~kg} / \mathrm{m}^{2} \text { ) }\end{array}$ & $1.30(0.67-2.53)$ & $1.54(0.79-3.02)$ & $1.37(0.69-2.70)$ \\
\hline $\begin{array}{l}\text { Ischemic stroke: Pre-obesity }\left(25-30 \mathrm{~kg} / \mathrm{m}^{2}\right) \\
\left.\text { (VS normal weight, } 18.5-25 \mathrm{~kg} / \mathrm{m}^{2}\right)\end{array}$ & $0.97(0.53-1.79)$ & $1.06(0.57-1.95)$ & $1.04(0.56-1.94)$ \\
\hline $\begin{array}{l}\text { Ischemic stroke or ischemic stroke related death: Pre-obesity }\left(25-30 \mathrm{~kg} / \mathrm{m}^{2}\right) \\
\left.\text { (VS normal weight, } 18.5-25 \mathrm{~kg} / \mathrm{m}^{2}\right)\end{array}$ & $0.92(0.50-1.68)$ & $1.00(0.55-1.83)$ & $0.99(0.54-1.82)$ \\
\hline $\begin{array}{l}\text { Ischemic stroke or ischemic stroke related death: Obesity }\left(\geq 30 \mathrm{~kg} / \mathrm{m}^{2}\right) \\
\left.\text { (VS normal weight, } 18.5-25 \mathrm{~kg} / \mathrm{m}^{2}\right)\end{array}$ & $0.65(0.28-1.47)$ & $0.78(0.34-1.79)$ & $0.60(0.26-1.40)$ \\
\hline
\end{tabular}

Conclusions: DOACs appear to provide consistent safety and efficacy in patients with high BMI compared to patients with normal weight, but findings need to be confirmed in a larger cohort.

LPB0051 | ACTIMIS Trial: Safety Interim Analysis Data of Glenzocimab, a Novel Antiplatelet Agent on Top of Acute Ischemic Stroke Standard of Care

M. Mazighi ${ }^{1}$; A. Peeters ${ }^{2}$; S. Richard ${ }^{3}$; C. Molina ${ }^{4}$; R. Lemmens ${ }^{5}$; D. Toni $^{6}$; Y. Plétan ${ }^{7}$; M. Jandrot-Perrus ${ }^{8}$; A. Comenducci ${ }^{7}$; G. Avenard ${ }^{7}$; P. Lyrer ${ }^{9}$; M. Kohrmann ${ }^{10}$; ACTIMIS Study Group

${ }^{1}$ AP-HP, Lariboisière Hospital, University of Paris, Paris, France; ${ }^{2}$ Saint Luc University Hospital, Brussels, Belgium; ${ }^{3} \mathrm{CHRU}$ de Nancy - Hôpital Central, Nancy, France; ${ }^{4}$ Hospital Vall d'Hebron, Barcelona, Spain; ${ }^{5}$ UZ Leuven, Leuven, Belgium; ${ }^{6}$ University Rome Sapienza, Roma, Italy; ${ }^{7}$ Acticor Biotech, Paris, France; ${ }^{8}$ UMR_S 1148 Inserm University of Paris, Paris, France; ${ }^{9}$ Universitätsspital Basel, Basel, Switzerland;

${ }^{10}$ Universitätsklinikum Essen (AöR), Essen, Germany

Background: Standard of care (SoC) for acute ischemic stroke (AIS), (intravenous tPA and mechanical thrombectomy) showed great benefit. However, still many patients suffer from an unfavourable outcome, highlighting the importance for developing new reperfusion strategies. Glenzocimab, a novel antibody fragment targeting platelet GPVI, is evaluated to safely improve reperfusion therapy.

Aims: The goal of the ACTIMIS trial is to address the safety of glenzocimab on top of the SoC in AIS patients. The first study milestone was to complete the escalating dose phase lb and to choose a safe dose to be administered during phase Ila.
Methods: ACTIMIS is an exploratory, multinational, multicenter, randomized, double blind, placebo-controlled, single parallel escalating dose study. Patients eligible to tPA with stroke symptoms onset $\leq 4.5 \mathrm{hr}$ and some of them to mechanical thrombectomy were randomly assigned to receive either placebo $(n=12)$ or glenzocimab (6-hour infusion) following a sequential escalating dose scheme, $125 \mathrm{mg}, 250 \mathrm{mg}, 500 \mathrm{mg}$ and $1000 \mathrm{mg}$ ( $n=12$ per dose). The primary endpoint is the occurrence of symptomatic and non-symptomatic intracranial hemorrhages ( $\mathrm{s}$ - and $\mathrm{ns}-\mathrm{ICHs}$ ), serious adverse events (SAEs), bleeding-related events (BREs) and deaths.

Results: Sixty patients, treated with IV tPA, including 50\% with an additional MT, were included. Among the 40 SAEs reported, 17 of them were reported as ICHs ( 1 symptomatic, 16 non-symptomatic including 15 hemorrhagic transformations). Of the 23 other reported SAEs, none was related to the study drug, the majority of them recovered and 5 had a fatal outcome within 90 days. Among non-ICH bleeding-related events, none was related to study drug. There was no dose-effect for any of the safety related parameters. Conclusions: Glenzocimab showed a favorable safety profile with a $1.7 \% \mathrm{~s}-\mathrm{ICH}$ rate, lower than the reported percentage in IPA RCTs, representing a promising achievement. The highest dose of $1000 \mathrm{mg}$ was selected for phase Ila. 
PB0014 | Natural Changes in CHA2DS2-VASc Scores in Newly Diagnosed Non-valvular Atrial Fibrillation Patients with Low Stroke Risk

Q. Chen; M.M. Toorop; S.C. Cannegieter; W.M. Lijfering Leiden University Medical Center, Leiden, Netherlands

Background: Non-valvular atrial fibrillation (NVAF) patients with low stroke risk require regular re-evaluation of this risk given its dynamic nature. The 2020 ESC guideline for NVAF suggests the first re-evaluation should be made 4-6 months after the index evaluation based on limited evidence.

Aims: To investigate natural changes in $\mathrm{CHA}_{2} \mathrm{DS}_{2}-\mathrm{VASc}$ scores in newly diagnosed NVAF patients with low stroke risk.

Methods: The study used nationwide register data provided by Statistic Netherlands and included newly diagnosed NVAF patients (2013-2018) in the Netherlands with a baseline $\mathrm{CHA}_{2} \mathrm{DS}_{2}$-VASc score $<2$, who were considered to be at low risk of stroke according to current guidelines. Patients were followed from first NVAF diagnosis until the first oral anticoagulant (OAC) prescription, 31/12/2018, or death, whichever came first. $\mathrm{CHA}_{2} \mathrm{DS}_{2}$-VASc scores were daily re-evaluated, and the study outcome was a score reaching $\geq 2$. We investigated cumulative incidences and predictors of the outcome by Kaplan-Meier methods and Cox regression analysis.

Results: A total of 50,811 patients were included. The cumulative incidence of developing a $\mathrm{CHA}_{2} \mathrm{DS}_{2}$-VASc score $\geq 2$ within 3 months was $1.95 \%(95 \% \mathrm{Cl} 1.71 \%-2.16 \%)$ in male patients with a baseline score of $0,13.79 \%(95 \% \mathrm{Cl} 12.30 \%-13.48 \%)$ in male patients with a baseline score of 1 , and $5.19 \%(95 \% \mathrm{Cl} 4.54 \%-5.58 \%)$ in female patients with a baseline score of 1 .

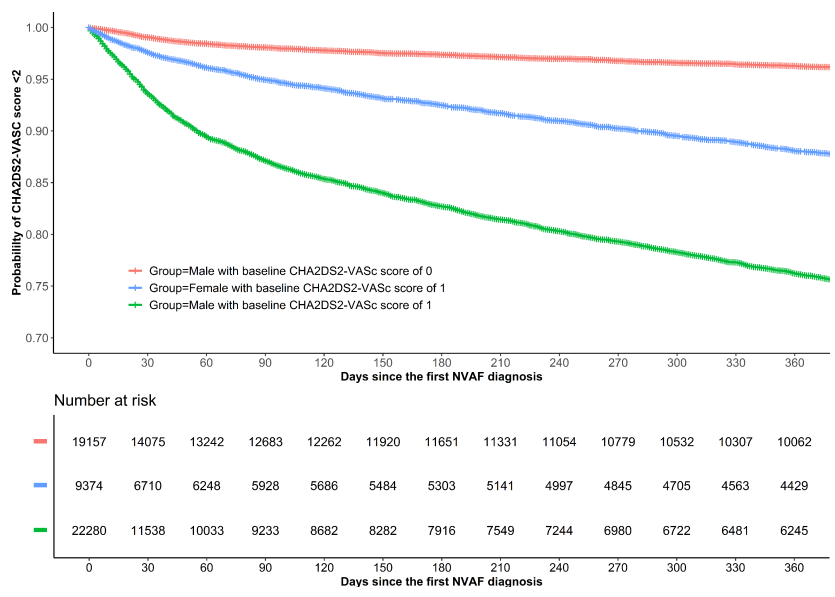

FIGURE 1 Kaplan-Meier curves for developing a CHA2DS2-VASc score $\geq 2$ in newly diagnosed non-valvular atrial fibrillation patients with low stroke risk

Older age, a baseline HAS-BLED score $\geq 3$ (for female patients), and having a medical history of congestive heart failure or diabetes were associated with an increased risk of developing a $\mathrm{CHA}_{2} \mathrm{DS}_{2}-\mathrm{VASc}$ score $\geq 2$ within 3 months.

TABLE 1 Baseline characteristics associated with developing a CHA2DS2-VASc score $\geq 2$ in newly diagnosed non-valvular atrial fibrillation patients with low stroke risk

\begin{tabular}{|c|c|c|c|c|c|c|c|c|}
\hline & \multicolumn{4}{|c|}{ Developing a $\mathrm{CHA}_{2} \mathrm{DS}_{2}$-VASc score $\geq 2$ within 3 months } & \multicolumn{4}{|c|}{ Developing a $\mathrm{CHA}_{2} \mathrm{DS}_{2}$-VASc score $\geq 2$ within 12 months } \\
\hline & $\begin{array}{l}\text { Male } \\
\text { baseline } \\
\text { score = } 0\end{array}$ & $\begin{array}{l}\text { Male } \\
\text { baseline } \\
\text { score = } 1\end{array}$ & $\begin{array}{l}\text { Male } \\
\text { baseline } \\
\text { score = } 1\end{array}$ & $\begin{array}{l}\text { Female } \\
\text { baseline } \\
\text { score = } 1\end{array}$ & $\begin{array}{l}\text { Male } \\
\text { baseline } \\
\text { score = } 0\end{array}$ & $\begin{array}{l}\text { Male } \\
\text { baseline } \\
\text { score = } 1\end{array}$ & $\begin{array}{l}\text { Male } \\
\text { baseline } \\
\text { score = } 1\end{array}$ & $\begin{array}{l}\text { Female } \\
\text { baseline } \\
\text { score = } 1\end{array}$ \\
\hline & $<65$ years & $<65$ years & $\geq 65$ years & $<65$ years & $<65$ years & $<65$ years & $\geq 65$ years & $<65$ years \\
\hline $\begin{array}{l}\text { Age (per year } \\
\text { increase) }\end{array}$ & $\begin{array}{c}1.11(1.09- \\
1.13)\end{array}$ & $\begin{array}{c}1.11(1.09- \\
1.13)\end{array}$ & $\begin{array}{c}1.10(1.08- \\
1.13)\end{array}$ & $\begin{array}{c}1.10(1.08- \\
1.12)\end{array}$ & $\begin{array}{c}1.11(1.09- \\
1.12)\end{array}$ & $\begin{array}{c}1.16(1.14- \\
1.18)\end{array}$ & $\begin{array}{c}1.18(1.16- \\
1.20)\end{array}$ & $\begin{array}{c}1.20(1.17- \\
1.22)\end{array}$ \\
\hline $\begin{array}{l}\text { HAS-BLED score } \\
\quad=1 \\
\text { (vs HAS-BLED } \\
\text { score }=0 \text { ) }\end{array}$ & $\begin{array}{c}1.86(1.45- \\
2.39)\end{array}$ & $\begin{array}{c}1.23 \text { (1.03- } \\
1.48)\end{array}$ & $\begin{array}{c}0.61(0.51- \\
0.72)\end{array}$ & $\begin{array}{c}1.22(0.98- \\
1.52)\end{array}$ & $\begin{array}{c}1.94(1.61- \\
2.35)\end{array}$ & $\begin{array}{c}1.32(1.15- \\
1.52)\end{array}$ & $\begin{array}{c}0.63(0.54- \\
0.72)\end{array}$ & $\begin{array}{c}1.36(1.17- \\
1.58)\end{array}$ \\
\hline
\end{tabular}




\begin{tabular}{|c|c|c|c|c|c|c|c|c|}
\hline & \multicolumn{4}{|c|}{ Developing a $\mathrm{CHA}_{2} \mathrm{DS}_{2}-\mathrm{VASc}$ score $\geq 2$ within 3 months } & \multicolumn{4}{|c|}{ Developing a $\mathrm{CHA}_{2} \mathrm{DS}_{2}-\mathrm{VASc}$ score $\geq 2$ within 12 months } \\
\hline & $\begin{array}{l}\text { Male } \\
\text { baseline } \\
\text { score = } 0 \\
<65 \text { years }\end{array}$ & $\begin{array}{l}\text { Male } \\
\text { baseline } \\
\text { score = } 1 \\
<65 \text { years }\end{array}$ & $\begin{array}{l}\text { Male } \\
\text { baseline } \\
\text { score = } 1 \\
\geq 65 \text { years }\end{array}$ & $\begin{array}{l}\text { Female } \\
\text { baseline } \\
\text { score = } 1 \\
<65 \text { years }\end{array}$ & $\begin{array}{l}\text { Male } \\
\text { baseline } \\
\text { score }=0 \\
<65 \text { years }\end{array}$ & $\begin{array}{l}\text { Male } \\
\text { baseline } \\
\text { score = } 1 \\
<65 \text { years }\end{array}$ & $\begin{array}{l}\text { Male } \\
\text { baseline } \\
\text { score = } 1 \\
\geq 65 \text { years }\end{array}$ & $\begin{array}{l}\text { Female } \\
\text { baseline } \\
\text { score = } 1 \\
<65 \text { years }\end{array}$ \\
\hline $\begin{array}{l}\text { HAS-BLED score } \\
\quad=2 \\
\text { (vs HAS-BLED } \\
\text { score }=0 \text { ) }\end{array}$ & $\begin{array}{c}3.66(2.46- \\
5.43)\end{array}$ & $\begin{array}{c}1.36(1.04- \\
1.78)\end{array}$ & $\begin{array}{c}0.76(0.64- \\
0.90)\end{array}$ & $\begin{array}{c}2.28(1.56- \\
3.32)\end{array}$ & $\begin{array}{c}3.98(2.96- \\
5.36)\end{array}$ & $\begin{array}{c}1.70(1.4- \\
2.07)\end{array}$ & $\begin{array}{c}0.81(0.70- \\
0.93)\end{array}$ & $\begin{array}{c}2.39(1.83- \\
3.11)\end{array}$ \\
\hline $\begin{array}{l}\text { HAS-BLED score } \\
\quad \geq 3 \\
\text { (vs HAS-BLED } \\
\text { score }=0 \text { ) } \\
\text { Contribution to } \\
\text { the baseline } \\
\mathrm{CHA}_{2} \mathrm{DS}_{2}- \\
\text { VASc score }\end{array}$ & $\begin{array}{c}0.99(0.14- \\
7.05)\end{array}$ & $\begin{array}{c}1.01(0.52- \\
1.97)\end{array}$ & - & $\begin{array}{c}3.06(1.26- \\
7.45)\end{array}$ & $\begin{array}{c}4.52(2.13- \\
9.61)\end{array}$ & $\begin{array}{c}1.62(1.04- \\
2.52)\end{array}$ & - & $\begin{array}{c}2.21(0.99- \\
4.95)\end{array}$ \\
\hline $\begin{array}{l}\text { Congestive heart } \\
\text { failure }\end{array}$ & - & $\begin{array}{c}1.52(1.16- \\
1.99)\end{array}$ & - & - & - & $\begin{array}{c}1.46(1.17- \\
1.82)\end{array}$ & - & - \\
\hline Hypertension & - & $\begin{array}{c}1.02(0.84- \\
1.24)\end{array}$ & - & - & - & $\begin{array}{c}0.97(0.84- \\
1.13)\end{array}$ & - & - \\
\hline Diabetes & - & $\begin{array}{c}1.31(1.01- \\
1.71)\end{array}$ & - & - & - & $\begin{array}{c}1.31(1.07- \\
1.60)\end{array}$ & - & - \\
\hline
\end{tabular}

Conclusions: More frequent re-evaluation of stroke risk in lowstroke-risk NVAF patients should be considered in male patients with a baseline score of 1 and those with a high baseline HAS-BLED score. Investigations on whether delayed re-evaluation of stroke risk and delayed use of OAC increase risk of ischemic stroke are warranted in future studies.

PB0015 | Is Microvessel Perfusion Associated with Platelets in Acute Cerebrovascular Disorders?

P.P. Wadowski ${ }^{1}$; C. Schörgenhofer ${ }^{2}$; T. Rieder ${ }^{2}$; S. Ertl ${ }^{1}$; J. Pultar ${ }^{1}$; W. Serles ${ }^{3}$; T. Sycha ${ }^{3}$; F. Mayer ${ }^{3}$; R. Koppensteiner ${ }^{1}$; T. Gremmel ${ }^{1}$; B. Jilma ${ }^{2}$

${ }^{1}$ Medical University of Vienna, Department of Internal Medicine II, Division of Angiology, Vienna, Austria; ${ }^{2}$ Medical University of Vienna, Department of Clinical Pharmacology, Vienna, Austria; ${ }^{3}$ Medical University of Vienna, Department of Neurology, Vienna, Austria

Background: Capillary density rarefaction and endothelial dysfunction contribute to chronic hypoperfusion and cerebral small vessel disease. Prior animal experiments revealed spatiotemporal microvascular remodelling directing post-stroke brain reorganization.

Aims: We hypothesized that microvessel perfusion changes during acute cerebrovascular events could be reflected systemically and might be associated with platelet function.
Methods: In a prospective observational trial in vivo sublingual Sidestream Darkfield videomicroscopy was performed in twentyone patients with acute ischemic/haemorrhagic stroke or transitory ischemic attacks within twenty-four hours after hospital admission and compared to an age- and sex-matched control group. Platelet function was assessed using multiple platelet aggregometry.

Results: Functional (3060 vs $3717 / \mathrm{mm}^{2}, P=0.001$ ) and perfused total (5263 vs $6550 / \mathrm{mm}^{2}, P=0.002$ ) capillary density was rarefied in patients when compared to healthy controls. There were significant associations between platelet count and functional $(r=0.57$, $P=0.007)$ or perfused total capillary density $(r=0.52, P=0.015)$ as well as platelet aggregation and perfused total capillary density (thrombin receptor-activating peptide $6: r=0.57, P=0.007$ ) or capillary density ratio (arachidonic acid: $r=-0.46, P=0.037$; collagen: $r=-0.55, P=0.012$ ).

Conclusions: Cerebrovascular events are associated with altered systemic microvascular perfusion. Further, microvessel perfusion correlates with platelet count and function in our patient group. 
PB0016 | "Red Cardiac versus White Atherosclerotic Thromboemboli in Acute Ischemic Stroke": Challenging the Concept via a Large-scale, Detailed Multi-parametric Histological Analysis

S. Staessens $^{1}$; O. François ${ }^{2}$; E. Boulleaux ${ }^{3}$; D. Corseaux ${ }^{3}$; L. Desender $^{1}$; A. Vandenbulcke ${ }^{1}$; J. de Boer ${ }^{4}$; C. Vens ${ }^{4}$; A.-S. De Sloovere $^{2}$; T. Dewaele ${ }^{2}$; P. Vanacker ${ }^{5,6,7}$; S. Susen ${ }^{3,8} ; \mathrm{K}$. Vanhoorelbeke $^{1}$; C. Cordonnier ${ }^{9}$; T. Andersson ${ }^{2,10}$; S.F. De Meyer ${ }^{1}$ ${ }^{1}$ Laboratory for Thrombosis Research, KU Leuven Campus Kulak Kortrijk, Kortrijk, Belgium; ${ }^{2}$ Department of Medical Imaging, AZ Groeninge, Kortrijk, Belgium; ${ }^{3}$ University of Lille, Inserm, CHU Lille, Institut Pasteur de Lille, U1011-EGID, Lille, France; ${ }^{4}$ Department of Public Health and Primary Care, KU Leuven Campus Kulak Kortrijk, Kortrijk, Belgium; ${ }^{5}$ Department of Neurology, AZ Groeninge, Kortrijk, Belgium; ${ }^{6}$ Department of Neurology, University Hospitals Antwerp, Antwerp, Belgium; ${ }^{7}$ Department of Translational Neuroscience, University of Antwerp, Antwerp, Belgium; ${ }^{8}$ Department of Hematology Transfusion, Centre Hospitalier Universitaire de Lille, Lille, France; ${ }^{9}$ INSERM U1172 - Lille NeuroScience \& Cognition (LiINCog), University of Lille, Centre Hospitalier Universitaire de Lille, Lille, France;

${ }^{10}$ Departments of Neuroradiology, Karolinska University Hospital, and Clinical Neuroscience, Karolinska Institutet, Stockholm, Sweden

Background: In ischemic stroke, thromboemboli are often described as white (platelet-rich) or red (red blood cell (RBC)-rich) when having an atherosclerotic or cardiac origin respectively. Secondary stroke prevention is mainly based on antiplatelet therapy for atherosclerotic etiologies and anticoagulation for cardioembolic etiologies. Yet, despite prevention, up to $25 \%$ of patients experience a recurrent stroke within the first 5 years after the initial event. Better understanding of thromboembolus composition can improve our understanding of underlying pathophysiologies and help in designing improved prevention strategies.

Aims: To perform a large-sample-study including multi-parameter quantitative histological analysis to investigate differences in composition of thromboemboli of different etiologies.

Methods: Thromboemboli ( $n=501)$ were collected from thrombectomy-treated ischemic stroke patients at Groeninge Hospital (Kortrijk, Belgium) and CHU Lille (Lille, France). Stroke etiology was categorized according to the TOAST classification. Quantitative histological analysis was performed for the amount RBCs, platelets, fibrin, von Willebrand factor (VWF), leukocytes, citrullinated histone $\mathrm{H} 3$ (H3Cit) and extracellular (ex)DNA.

Results: Compared to large artery atherosclerotic (LAA) thromboemboli (Figure 1), cardioembolic (CE) thromboemboli contained significantly less RBCs $(51.6 \% \pm 19.0 \%$ vs. $37.9 \% \pm 19.0 \%)$ and significantly more platelets $(29.0 \% \pm 14.0 \%$ vs. $36.9 \% \pm 18.2 \%)$, fibrin $(24.7 \% \pm 13.0 \%$ vs. $34.6 \% \pm 16.9 \%)$, leukocytes $(14.8 \% \pm 10.6 \%$ vs. $19.7 \% \pm 14.1 \%$ ) and exDNA (74\% vs. $88 \%$ scoring moderate/high). VWF $(35.5 \% \pm 16.6 \%$ vs. $33.4 \% \pm 15.4 \%)$ and $\mathrm{H} 3 \mathrm{Cit}(6.6 \% \pm 7.6 \%$ vs. $5.4 \pm 6.8 \%$ ) content were not different in CE/LAA thromboemboli respectively. Intriguingly, cryptogenic thromboemboli contained relatively low amounts of RBCs and high amounts of platelets (Figure 1), similar to CE thromboemboli. Notwithstanding these population differences, a typical CE or LAA thromboembolus cannot be defined given the observed heterogeneity in RBC/platelets.

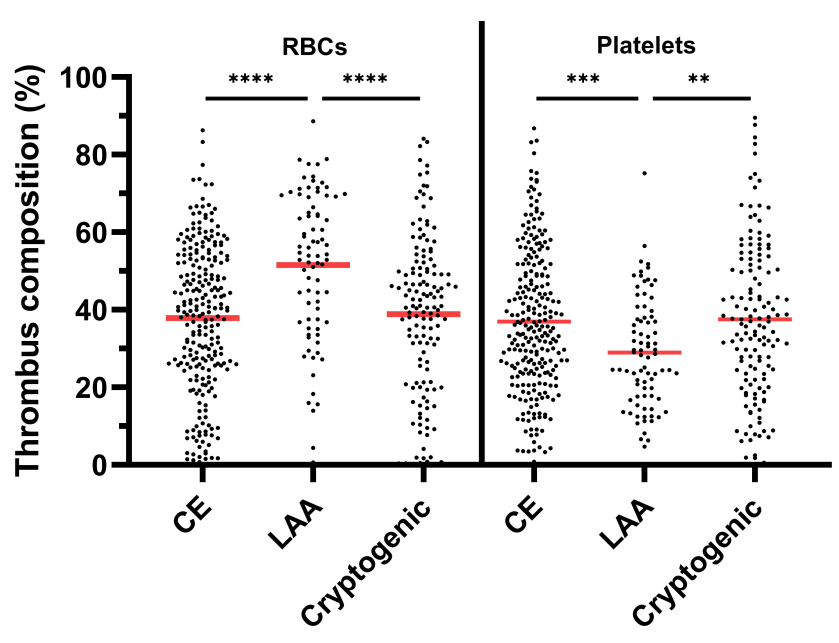

FIGURE 1 Histological comparison (RBCs and platelets) of CE, LAA and cryptogenic thromboemboli

Conclusions: In contrast to current concepts of 'white' atherosclerotic and 'red' cardiac thrombi, CE thromboemboli are more platelet-rich compared to RBC-rich LAA thromboemboli, with a heterogenous $\mathrm{RBC}$ /platelet thrombus profile. Such data could hint at combining antiplatelet with anticoagulation therapy in prevention of stroke, as was recently shown to be beneficial in the COMPASS-trial.

PB0017 | Soluble P-selectin is Associated with Increased Risk of Ischemic Stroke in Patients with Atrial Fibrillation on Hemodialysis: Results from the VIVALDI Study

O. Koenigsbruegge ${ }^{1}$; S. Schmaldienst ${ }^{2} ;$ M. Lorenz ${ }^{3}$; R. Klauser-Braun ${ }^{4}$; I. Pabinger ${ }^{1}$; M. Säemann ${ }^{5}$; C. Ay ${ }^{1}$

${ }^{1}$ Medical University of Vienna, Vienna, Austria; ${ }^{2}$ Clinic Favoriten, Vienna, Austria; ${ }^{3}$ Vienna Dialysis Center, Vienna, Austria; ${ }^{4}$ Clinic Donaustadt, Vienna, Austria; ${ }^{5}$ Clinic Ottakring, Vienna, Austria

Background: Atrial fibrillation in patients on hemodialysis (HD) is associated with an increased risk of stroke, transient ischemic attack (TIA) and systemic embolism (SE). Identification of patients at high risk would be of clinical relevance in order to personalize anticoagulation. Soluble Pselectin (sP-selectin) is a marker of platelet activation and is associated with a prothrombotic state in AF patients in the general population.

Aims: We aimed to investigate the association between sP-selectin and the occurrence of thromboembolic outcomes.

Methods: In a cohort study of end-stage renal failure patients on HD, we measured plasma levels of sP-selectin using an enzyme-linked 
immunoassay (R\&D Systems, Minneapolis, USA). Occurrence of ischemic stroke, TIA, SE, and major bleeding were recorded over a maximum observation period of 45 months (median 29 months). Risk of outcome occurrence was calculated with risk regression considering for the competing risk of death and $\mathrm{AF}$ as a time-dependent variable.

Results: Of 595 patients, 28 patients had stroke, 7 TIA and 5 SE. In AF patients (224 patients, 76 women [33.9\%], median age 71 years) levels of sP-selectin were not significantly increased compared to non-AF patients (39.9 vs $39.2 \mathrm{ng} / \mathrm{ml}, P=0.64$ ), and sP-selectin levels were not significantly lower in patients treated with anticoagulants (38.0 vs $39.8 \mathrm{ng} / \mathrm{ml}, P=0.30$ ) or anti-platelet agents (39.2 vs 39.7 $\mathrm{ng} / \mathrm{ml}, P=0.72$ ) compared to those without anti-thrombotic agents. In AF patients, sP-selectin was associated with a 1.8-fold increased risk for occurrence of the composite thromboembolic endpoint (stroke, TIA, SE) (SHR 1.81 per double increase, $95 \% \mathrm{Cl} 1.01-3.25$, $P=0.045)$ adjusted for age, sex, diabetes, prior stroke, prior cardiovascular disease and antiplatelet comedication, but not with occurrence of major bleeding (SHR 0.70 per double increase, $95 \% \mathrm{Cl} 0.34$ $-1.43, P=0.325)$.

Conclusions: Increasing sP-selectin levels predict the risk of thromboembolic complications such as stroke, TIA, and SE in hemodialysis patients with AF.

PB0018 | Evaluation of the Need for Cytoreduction and its Potential Carcinogenicity in Children and Young Adults with Myeloproliferative Neoplasms

$\underline{\text { H.S. Choi }}^{1}$; J. Hong ${ }^{2}$; S.M. Hwang ${ }^{3}$; J.H. Lee ${ }^{4}$; Y. Ma ${ }^{1}$; S.-A Kim ${ }^{4}$; J.Y. Lee ${ }^{4}$; J.-O. Lee ${ }^{4}$; S.-M. Bang ${ }^{4}$

${ }^{1}$ Department of Pediatrics, Seoul National University Bundang Hospital, Seongnam, Korea, Republic of; ${ }^{2}$ Department of Internal Medicine, Seoul National University Hospital, Seoul, Korea, Republic of; ${ }^{3}$ Department of Laboratory Medicine, Seoul National University Bundang Hospital, Seongnam, Korea, Republic of; ${ }^{4}$ Department of Internal Medicine, Seoul National University Bundang Hospital, Seongnam, Korea, Republic of

Background: Myeloproliferative neoplasms are rare at a young age and few reports have described the disease characteristics and outcomes in this group.

Aims: This study aimed to elucidate the clinical course of essential thrombocythemia (ET) and polycythemia vera (PV) in children and young adults aged $<39$ years focusing on thromboembolic events (TE) and second primary malignancies (SPMs).

Methods: We conducted an epidemiological study to elucidate the clinical course of ET and PV in children and young adults under the age of 39 years, focusing on thromboembolic events (TE), and SPMs by analyzing the Health Insurance Review and Assessment Service (HIRA) database, which includes almost all hospital-based patient information in Korea.
Results: A total of 990 patients who were diagnosed from 2008 to 2017 were included. The incidence was 2.53 per $1,000,000$ for ET (643 patients; 276 male patients; median 31 years) and 1.37 per 1,000,000 for PV (347 patients; 309 male patients; median 32 years). Three ET patients developed secondary acute myelogenous leukemia and three developed secondary myelofibrosis. The 5-year cumulative incidence of TE was $14.2 \%$ in ET and $21.3 \%$ in PV. Thus, the incidence was higher in PV; in particular, arterial TE (ATE) was evidently higher in PV than in ET. The 5-year cumulative incidence of SPMs was $2.5 \%$ in ET and $2.6 \%$ in PV. While the use of both aspirin and hydroxyurea reduced the incidence of ATE, hydroxyurea significantly increased the incidence of SPMs.

Conclusions: The incidence of ET and PV was very low, and ET was more common than PV in children and young adults. The high incidence of TE in young patients suggests the importance of thrombosis prevention. However, hydroxyurea appears to increase the incidence of SPMs; therefore, the risks and benefits should be considered.

\section{TABLE 1}

Increased occurrence of SPMs in hydroxyurea-exposed MPN patients

\begin{tabular}{|c|c|c|c|c|c|c|c|c|c|c|}
\hline \multirow[t]{2}{*}{ Medications } & & \multicolumn{2}{|c|}{ TE (\%) } & \multirow[t]{2}{*}{$P$} & \multicolumn{2}{|c|}{ ATE (\%) } & \multirow[t]{2}{*}{$P$} & \multicolumn{2}{|c|}{ SPMs (\%) } & \multirow[t]{2}{*}{$P$} \\
\hline & & Yes & No & & Yes & No & & Yes & No & \\
\hline \multirow{2}{*}{ Aspirin } & Yes & $58(11.2)$ & $461(88.8)$ & $<0.0001$ & $51(9.8)$ & $467(90.2)$ & $<0.0001$ & $16(2.7)$ & $570(97.3)$ & 0.2821 \\
\hline & No & $107(22.7)$ & $364(77.3)$ & & $95(20.1)$ & $377(79.9)$ & & $16(4.0)$ & $388(96.0)$ & \\
\hline \multirow{2}{*}{ Hydroxyurea } & Yes & $36(13.4)$ & $233(86.6)$ & 0.0904 & $29(10.8)$ & $240(89.2)$ & 0.0315 & $15(5.0)$ & $285(95.0)$ & 0.0381 \\
\hline & No & $129(17.9)$ & $592(82.1)$ & & $117(16.2)$ & $604(83.8)$ & & $17(2.5)$ & $673(97.5)$ & \\
\hline \multirow{2}{*}{ Anagrelide } & Yes & $31(12.4)$ & $218(87.6)$ & 0.039 & $29(11.6)$ & $221(88.4)$ & 0.1045 & $9(3.3)$ & $262(96.7)$ & 0.9228 \\
\hline & No & $134(18.1)$ & $607(81.9)$ & & $117(15.8)$ & $623(84.2)$ & & $23(3.2)$ & $696(96.8)$ & \\
\hline
\end{tabular}

PB0019 | Long-term Outcome of Percutaneous or Surgical revascularization with and without Prior Stroke in patients with Three-vessel Disease

N. Xu; L. Jiang; C. Zhang; J. Xu; R. Liu; Y. Zhang; X. Zhao; R. Gao; L. Song; J. Yuan

Fuwai Hospital, Chinese Academy of Medical Sciences, Beijing, China

Background: Presently, there are no established consensus and adequate data for optimal coronary revascularization strategy for complex CAD patients with prior stroke.

Aims: The study aims to determine whether high-risk patients with three-vessel disease (TVD) with and without prior stroke preferentially benefit from three strategies [percutaneous coronary intervention ( $\mathrm{PCl}$ ), coronary artery bypass grafting (CABG), and medical therapy (MT)].

Methods: A total of 8,943 patients with TVD were included. Patients were stratified into 2 categories according to presence or absence of prior stroke. The primary endpoint was all-cause death. Secondary endpoints included stroke and major adverse cardiac and cerebrovascular events (MACCE), a composite of death, myocardial infarction, unplanned revascularization and stroke. 
Results: Prior stroke was present in 888 of 8,943 patients (9.9\%). During a median follow-up of 7.5 years, patients with prior stroke were strongly associated with increased risks of all-cause death, cardiac death, stroke and MACCE (all adjusted $P<0.01$ ). Patients with prior stroke was strongly associated with increased MACCE compared with no prior stroke driven by higher rates of all-cause mortality and stroke, prior stroke was the independent risk factor of all-cause death, stroke and MACCE after adjusting for confounding variables. Revascularization strategy (PCI or CABG) was associated with a lower incidence of all-cause death and MACCE compared with MT alone, CABG showed lower risk of MACCE and similar risk of all-cause death compared with $\mathrm{PCl}$ irrespective of prior stroke. The prevalence of stroke was significantly higher after CABG when compared with $\mathrm{PCl}$ or MT among no prior stroke patients (HR 1.429, $95 \% \mathrm{Cl} 1.132-1.805$ for CABG vs.MT, HR 1.703, 95\% Cl 1.371-2.116 for CABG vs. PCI).

Conclusions: Patients with TVD and prior stroke have poor longterm outcomes. It is essential to balance benefit and risk when determining optimum revascularization strategy for TVD with and without prior stroke.

PB0020 | Single Centre Retrospective Chart Review to Examine the Management of Timing of Anticoagulation and Outcomes in Patients with Cerebral Venous Sinus Thrombosis

G. Mak; N. Chan; K. Perera

McMaster University, Hamilton, Canada

Background: Cerebral venous sinus thrombosis (CVST) accounts for $<1 \%$ of all strokes. Prior studies have demonstrated that anticoagulation in CVST is safe and reduces adverse events.

Aims: The objective of our study was to characterize the clinical features, identify the timing and duration of anticoagulation therapy, and establish the outcomes of patients diagnosed with CVST.

Methods: Using ICD-9 and ICD-10 codes, we conducted a retrospective chart review of patients admitted to Hamilton Health Sciences (HHS) from 2015 to 2020 with CVST confirmed on imaging.

Results: We included 96 patients ( 57 females; 39 males), mean age of 47.9 (SD 18.1). The most common clinical presentation was headache (43.8\%). Trauma was the most common identified risk factor (15.6\%), followed by oral contraceptive use (8.3\%) and infection (8.3\%). A cause was not identified in $27 \%$ of individuals. Most patients $(57.3 \%)$ received full dose anticoagulation within $24 \mathrm{~h}$ of identified CVST diagnosis. Reasons for delay in anticoagulation included the presence of venous infarct or haemorrhage (27.1\%), traumatic brain injury (31.8\%), post-op from neurosurgery (9.1\%), but in $31.8 \%$ of patients there was no clear rationale. Moreover, there was a greater proportion of patients disabled (modified Rankin Scale, mRS, score > 2) and dead (mRS 6) at discharge with a delay in initiating anticoagulation (72\%) compared to those who received anticoagulation within $24 \mathrm{~h}$ (40\%) of identified CVST. The majority of patients (55\%) had greater than 12 months to ongoing follow-up. The recurrence rate for CVST was $2.5 \%$, while bleeding complications during anticoagulation therapy was $6.3 \%$, all of which did not require hospitalization.

Conclusions: Our findings suggest that unjustified delay in anticoagulation may result in poorer clinical outcomes, however further studies are needed to confirm our findings and investigate whether prompt start of anticoagulation improves outcomes in CVST.

PB0021 | Platelet-derived microRNAs Are Increased after Transcatheter Aortic Valve Implantation and May Predict Adverse Outcomes in Patients with Heart Failure

C. Eyileten ${ }^{1}$; A. Fitas ${ }^{1}$; J. Jarosz-Popek ${ }^{1,2}$; T. Adem ${ }^{1}$; D. Jakubik ${ }^{1}$; M. Wolska ${ }^{1}$; A. Nowak ${ }^{1}$; P. Czajka ${ }^{1}$; M. Postuła ${ }^{1}$; A. Gąsecka ${ }^{3}$ ${ }^{1}$ Center for Preclinical Research and Technology CEPT, Department of Experimental and Clinical Pharmacology, Medical University of Warsaw, Warsaw, Poland; ${ }^{2}$ Doctoral School, Medical University of Warsaw, Warsaw, Poland; ${ }^{3} 1$ st Chair and Department of Cardiology, Medical University of Warsaw, Warsaw, Poland

Background: Transcatheter aortic valve implantation (TAVI) is an emerging invasive therapeutic strategy for patients with severe aortic stenosis (AS) significantly enhancing not only quality-of-life measures but also improving short-and long-term survival rates.

Aims: We aimed to analyze correlation between expression levels of platelet-derived microRNAs in patients with heart failure (HF) due to AS who underwent TAVI procedure and assess their association with primary MACE (major adverse cardiac events) defined by all cause mortality and secondary MACE defined as cardiovascular mortality, ischemic stroke, and non-fatal myocardial infarction.

Methods: 61 patients before and after the TAVI procedure were included. PlasmaRNA was extracted by mirVANA PARIS Kit and quality of RNA was assessed by fluorometric assay. GEP analysis was performed using the Clariom D pico chips, analyzed on the Affymetrix platform. RT-PCR was performed in order to validate the miRNAs in 61 patients by using the Taqman advanced protocol. MiRNA related to platelet function/antiplatelet treatment were chosen among those with the most relevant modulation between the groups. Wilcoxon test was performed for miRNAs comparison before and after TAVI. Calculations were performed using SPSS version 22.0, $P<0.05$ (IBM Corporation, Chicago, USA).

Results: We have found that miR-223, miR-125b and miR-125a were significantly increased in patients after the TAVI procedure. ROC analysis showed that increased miR-223 expression after the TAVI procedure might slightly have protective value against MACE outcome. 
a)
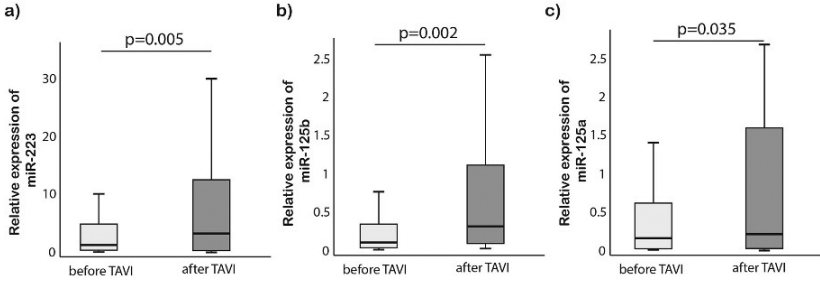

FIGURE 1 Comparison of plasma miRNA expression before and after transcatheter aortic valve implantation. A: miR-233, B: miR-125b, C: miR-125a

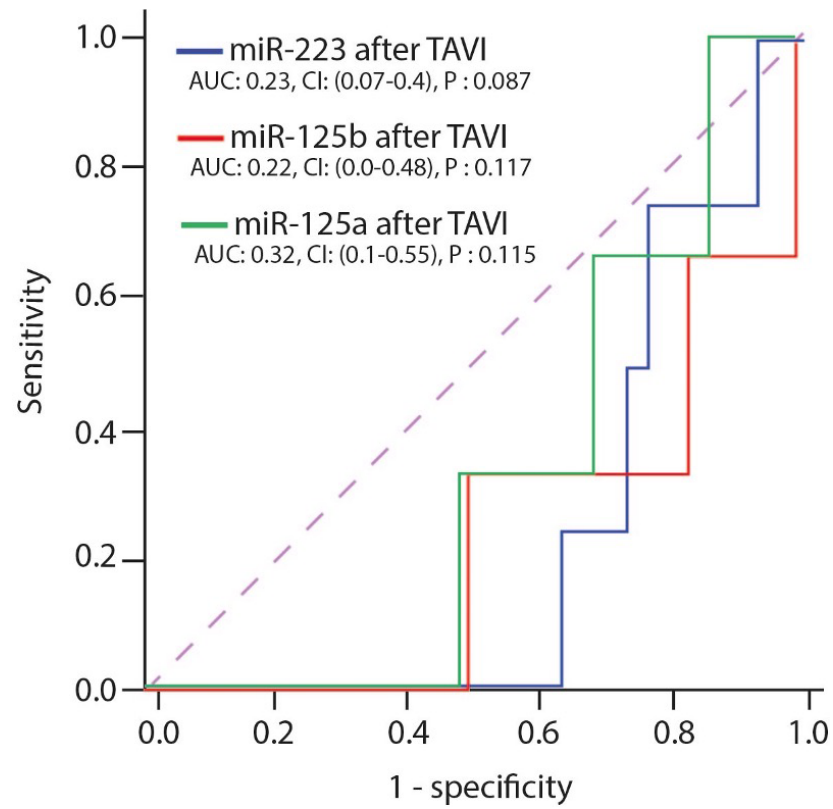

FIGURE 2 Receiver operating characteristic (ROC) curves of miRNAs relative expression after TAVI procedure. ROC curves of different miRNAs for prediction MACE

We have found that miR-223, miR-125b and miR-125a were significantly increased in patients after the TAVI procedure. ROC analysis showed that increased miR-223 expression after the TAVI procedure might slightly have protective value against MACE outcome.

Conclusions: Our analysis showed alteration of circulating miRNAs after the TAVI procedure and miR-223 might have a predictive value for MACE prognosis in patients with HF due to AS who underwent TAVI procedure.
PB0022 | Role of the Interaction between Thrombophilia and Other Risk Factors in the Occurring of Ischemic Stroke in Young Patients: Algerian Restrospective Study about 75 Cases

M. El Horri ${ }^{1}$; I. Khachaa ${ }^{1}$; A. Chikh Khelifa ${ }^{1}$; A. Berrah ${ }^{1}$; M. Chekkal ${ }^{2}$; F. Bemmoussat ${ }^{1}$; L. Benmahdi ${ }^{1}$; S.E. Belakehal ${ }^{1}$

${ }^{1}$ Hospital University Center of Oran, Oran, Algeria; ${ }^{2}$ University Hospital Establissement of Oran, Oran, Algeria

Background: Thrombophilia is a multifactorial disease which is mono- or plurigenic and its clinical expression is associated with a heterogeneous expression. The possible association between thrombophilia and ischemic stroke remains controversial. but it is true that its prevalence increases in young patients compared to the oldest.

Aims: We aimed to study inherited and acquired thrombophilias as risk factors for ischemic stroke in young patients, and the interaction with the other thromboembolic risk factors.

Methods: A total of 75 patients aged 18 to 50 years with a confirmed ischemic stroke were included in the study. Investigation for acquired and inherited thrombophilia was done in laboratory hematology unit at the Military University Hospital of Oran, Algeria from 1 January 2017 to 31 December 2019. The same assessment was carried out in 80 healthy subjects considered as controls. Clinical informations were recolted from medical records of neurology unit. Results: A positive thrombophilia test was identified in 15 (20\%) patients. One patient presented with protein $C$ deficiency, 5 patients with protein $\mathrm{S}$ deficiency. Activated protein $\mathrm{C}$ resistance in two patients. Hyperhomocysteinemia was observed in one patient. Antiphospholipid antibodies were found in 6 patients. The thrombophilia results came back negative in all subjects of the control population. In the presence of thrombophilia the patients had an OR of 1.25 [Cl: $1.12-1.40]$ of having an ischemic stroke compared to uninjured subjects.

In 03 patients Thrombophilia has been identified as the only risk factor (02 protein $\mathrm{S}$ deficiencies and 01 case of activated protein C resistance), while for the rest of patients (12), it was associated with other risk factors, like high blood pressure, diabetes, smoking, contraception and dyslipidemia. 
TABLE 1 Baseline Characteristics and prevalence of thrombophilia in the study patients

Epidemiological and clinical characteristics

Age: 37 years [IQR : 31 - 45]

Risk factors

Sexe: Male: 32 (42.7\%); Female: 43

(57.3\%)

Number of episodes: 01 episode: 65

(86.7\%)

More than 02 episodes: 10 (13.3\%)

Cryptogenic: 60 (80\%)

History of DVT or ischemic stroke: 09

(12\%)

History of foetal loss: 05 (6.7\%)
Prevalence of thrombophilia in the study population

Inherited thrombophilia: 08 (12\%)

Protein C deficiency: 01 (1.30\%)

Protein S deficiency: 05 (6.80\%)

Activated protein $\mathrm{C}$ resistance: 02 (2.70\%)

Antiphospholipid syndrome: 06 (08\%)

Lupus anticoagulant: 04 (05.30\%)

Anti-ß2GPI: 02 (2.70\%)

Anticardiolipin: 03 (04\%)
Conclusions: Thrombophilia is associated with ishemic stroke in young patients. This association may be stronger in the presence of traditional risk factors.

PB0023 | Photodynamic Therapy with 5- Aminolevulinic Acid and B-mode Ultrasound-guided Catheter-based Low-level Red Laser Results in Cerebrovascular Neointimal Hyperplasia Regression

H. Mehrad ${ }^{1,2}$; A. Sultan- Qurraie ${ }^{3}$

${ }^{1}$ Islamic Azad University, Basic Sciences Faculty, Department of Physics, Tabriz Branch, Tabriz, Iran, Islamic Republic of; ${ }^{2}$ Division of Translational Development of Noninvasive Treatments, Mehrad Research Lab, Tabriz, Iran, Islamic Republic of; ${ }^{3}$ University of Washington, Valley Medical Center, Neuroscience Institute, Renton, Washington, United States

Background: Intimal hyperplasia refers to proliferation and migration of vascular smooth muscle cells primarily in the tunica intima, resulting in arterial wall thickening and decreased arterial lumen size. Neointimal hyperplasia is the major cause of restenosis after percutaneous carotid interventions such as stenting or angioplasty. Photodynamic therapy is a two-stage treatment that combines light energy with a drug (photosensitizer) designed to destroy cancerous, precancerous and hyperplasia cells after light activation. Photosensitizers are activated by a specific wavelength of light energy. 5-Aminolevulinic Acid is an endogenous precursor of the natural photosensitizer protoporphyrin IX.

Aims: To investigate the effect of 5-Aminolevulinic Acid activated by low-level red laser on cerebrovascular neointimal hyperplasia in a prospective animal cohort study, wherein diagnostic B- mode ultrasound is combined with laser system, with a goal of increased safety. Methods: Endothelial balloon catheter denudation of the right common carotid artery of Golden Syrian Hamsters $(n=10)$ was performed. Histopathologic evaluation confirmed neointimal hyperplasia formation in all of the hamsters' arteries. The treatment group underwent intravenous 5- Aminolevulinic Acid administration accompanied by catheter- based low-level red laser $(\lambda=650 \mathrm{~nm}$, $E / A=10 \mathrm{~J} / \mathrm{cm}^{2}$ ) photodynamic therapy guided by simultaneous $B$ mode ultrasound imaging.

Results: B- mode ultrasound guided photodynamic therapy was feasible and appeared safe for the targeting of stenosis in the carotid artery. Furthermore, histopathologic results showed a significant reduction in the mean value for smooth muscle cells density, lumen wall thickness and percentage of luminal cross- sectional area of stenosis in the treatment group $(P<0.05)$.

Conclusions: 5-Aminolevulinic Acid in conjunction with low-level red laser is significantly associated with reduced carotid artery stenosis in hamsters. The mechanism may relate to reduced smooth muscle hyperplasia in the tunica intima.

PB0024 | miR-19a-3p Predicts Stroke Severity and miR-186-5p Might Be a Diagnostic Biomarker in Ischemic Stroke Patients with Hyper Platelet Reactivity

C. Eyileten ${ }^{1}$; D. Jakubik ${ }^{1}$; J. Jarosz-Popek ${ }^{1,2}$; P. Czajka ${ }^{1}$;

M. Wolska ${ }^{1}$; A. Fitas ${ }^{1}$; A. Nowak ${ }^{1}$; Z. Wicik ${ }^{3}$; D. Mirowska-Guzel ${ }^{1}$;

A. Członkowska ${ }^{4}$; M. Postuła ${ }^{1}$

${ }^{1}$ Center for Preclinical Research and Technology CEPT, Department of Experimental and Clinical Pharmacology, Medical University of Warsaw, Warsaw, Poland; ${ }^{2}$ Doctoral School, Medical University of Warsaw, Warsaw, Poland; ${ }^{3}$ Centro de Matemática, Computação e Cognição, Universidade Federal do ABC, São Paulo, Brazil; ${ }^{4}$ 2nd Department of Neurology, Institute of Psychiatry and Neurology,

Warsaw, Poland

Background: Ischemic stroke (IS) is one of the most frequent causes of death. In 2018, for every 6 deaths from cardiovascular disease, one was caused by stroke. Although there are well established treatment strategies diminishing post-stroke cell death, there are no available long-term diagnostic and prognostic methods to predict stroke severity. MicroRNAs (miRNAs) are a class of endogenous, non-coding RNA molecules. Since miRNAs have been illustrated to 
play an important role in various processes through regulation of multiple genes, and platelet function.

Aims: Analysis of plasma platelet-derived miR-19a-3p and miR186-5p expression levels $24-\mathrm{h}$ and 7-days after IS as novel diagnostic and prognostic biomarkers.

Methods: Blood samples of 28 patients diagnosed with acute IS with hyper platelet reactivity were collected 24 -h and 7-days after stroke and 26 age- and gender-matched individuals free of stroke with multiple risk factors for cardiovascular disease. PlasmaRNA was extracted: mirVANAPARISKit; quality RNA was assessed: fluorometric assay; microarray: Affymetrix platform; RT-PCR for validation the miRNAs with Taqman advanced. $P<0.05$ (SPSS, IBM Corporation, Chicago, USA).

Results: MiR-186-5p levels were significantly lower in the study group 7 days post-stroke vs. in controls. MiR-19a-3p levels were significantly lower 7 days post-stroke vs. at 1 day of stroke and significantly higher among patients with moderate vs. minor stroke at 1 day of IS.
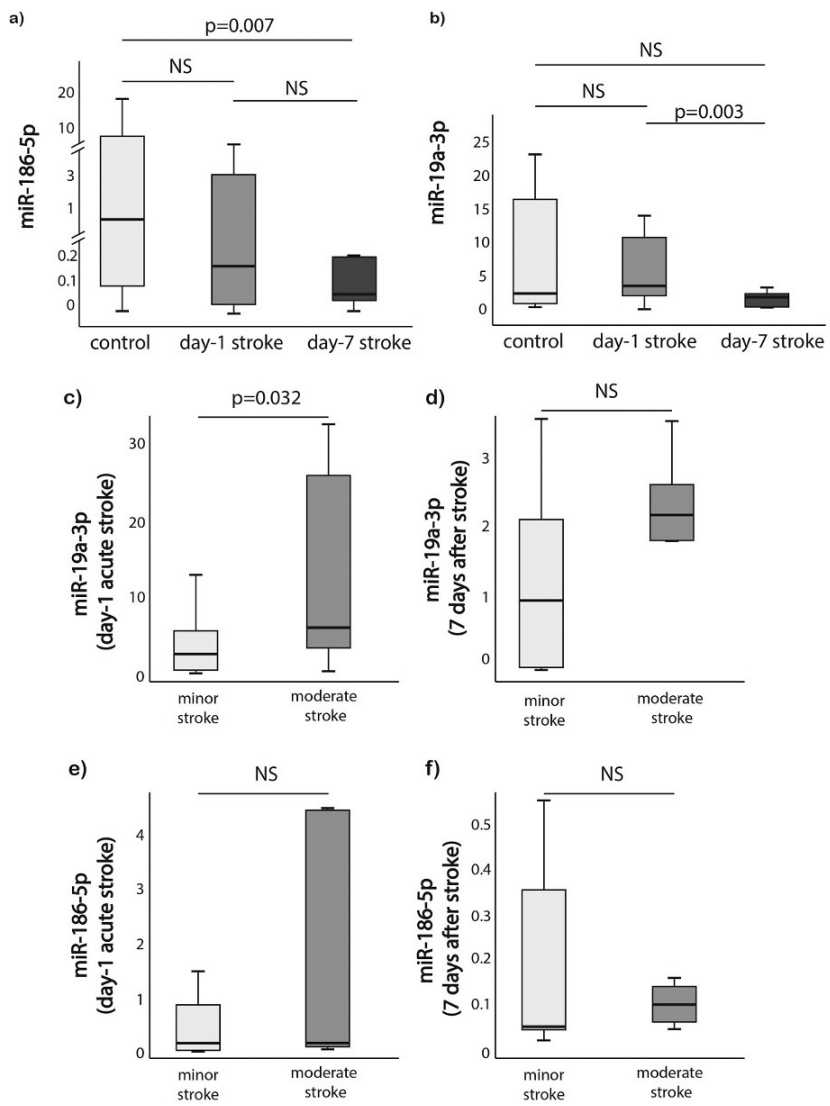

FIGURE 1 MiRNAs expression difference between groups and miRNAs expression difference based on stroke severity. a) miR-186-5p b) miR-19a-3p c) miR-19a-3p acute stroke; d) miR-19a-3p 7 days after stroke; e) miR-186-5p acute stroke; f) miR-186-5p 7 days after stroke. The initial stroke severity as measured by the NIHSS at admission. Minor stroke: 1-4 and moderate stroke: 5-15; $p$
Stroke severity

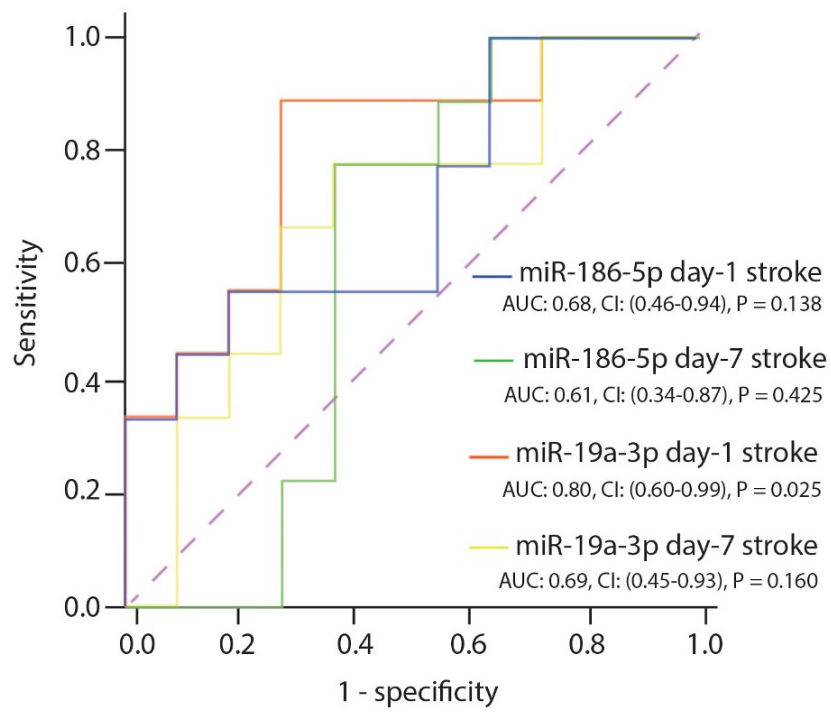

FIGURE 2 Receiver operating characteristic (ROC) curves of miR$19 a-3 p$ and miR-186-5p at the day-1 and day-7 stroke for prediction of stroke severity

Conclusions: Our analysis showed alteration of circulating miRNAs after IS. MiR-19a-3p might have a predictive value for stroke severity prognosis and miR-186-5p might be a novel diagnostic biomarker for patients IS with hyper platelet reactivity.

P0103 | B-mode Ultrasound-guided Combined Catheter-based Q-switched-Nd: YAG Laser and Pulsed Low Level Dual Frequency Focused Ultrasound Thrombolytic Therapy Reduce Thrombus Content in the Arterial Atherothrombotic Stenosis

H. Mehrad $^{1,2} ;$ M. Farhoudi ${ }^{3}$

${ }^{1}$ Islamic Azad University, Basic Sciences Faculty, Department of Physics, Tabriz Branch, Tabriz, Iran, Islamic Republic of; ${ }^{2}$ Mehrad Research Lab, Division of Translational Development of Noninvasive Treatments, Tabriz, Iran, Islamic Republic of; ${ }^{3}$ Tabriz University of Medical Sciences, Neurosciences Research Center (NSRC), Tabriz, Iran, Islamic Republic of

Background: A plaque may rupture with high risk of subsequent thrombus mediated acute clinical events such as stroke. The efficacy of intravenous anti-anticoagulant drugs is limited owing to a relatively poor recanalization rate and incomplete function recovery in the majority of treated patients. Therefore, other treatment modalities should be explored.

Aims: The aim of this study was to generate a hamster model of common carotid artery atherothrombotic stenosis and the subsequent investigating the feasibility of combined catheter-based QSwitched-Nd:YAG laser plus pulsed low level dual frequency focused ultrasound-mediated thrombolytic therapy in this model, wherein diagnostic B-mode ultrasound is combined with thrombolytic therapy system, with a goal of increased safety. 
Methods: Briefly, golden Syrian hamsters were submitted to common carotid artery atherothrombotic stenosis by primary balloon injury followed $1.5 \%$ cholesterol- rich diet injury for 8 weeks and finally perivascular severe liquid nitrogen-based cold injury. Then treatment group underwent combined B-mode ultrasound-guided Q-switched Nd:YAG laser ( $\lambda=532 \mathrm{~nm}, P=10 \mathrm{~W}, \mathrm{PD}=5 \mathrm{~ns}$ ) and pulsed low level dual frequency focused ultrasound $(F=750 \mathrm{KHz}$, $\mathrm{I}=10 \mathrm{~W} / \mathrm{cm}^{2}$, Duty Factor $=20 \%$ and $F=1.2 \mathrm{MHz}, \mathrm{I}=24 \mathrm{~W} / \mathrm{cm}^{2}$, Duty Factor $=50 \%$ )- mediated thrombolytic therapy.

Results: Results from histopathology, B-mode and color Doppler ultrasonography, showed a significant reduction in the mean value for thrombus content, percentage of luminal cross-sectional area of stenosis, blood mean velocity and a significant increase in the mean value for blood volume flow at the stenotic region in the treatment group compared with the other groups $(P<0.05)$.

Conclusions: Enhanced anti- thrombotic effect of mechanical inertial cavitation bubbles, induced by combined Q- Switched- Nd:YAG laser and pulsed low level dual frequency focused ultrasound, can cause to reduce the thrombus content and significantly dilate the luminal cross-sectional area of stenosis and lower treatment time.

P0104 | Extracorporeally Thrombolytic Therapy of Embolic Common Carotid Artery Using Q-switched Nd:YAG Laser and Confocal Dual Pulse Electrohydraulic Shock Waves Accompanied by PESDA Microbubbles Administration

\section{H. Mehrad $^{1,2} ;$ M. Farhoudi ${ }^{3}$}

${ }^{1}$ Islamic Azad University, Basic Sciences Faculty, Department of Physics, Tabriz Branch, Tabriz, Iran, Islamic Republic of; ${ }^{2}$ Mehrad Research Lab, Division of Translational Development of Noninvasive Treatments, Tabriz, Iran, Islamic Republic of; ${ }^{3}$ Tabriz University of Medical Sciences, Neurosciences Research Center (NSRC), Tabriz, Iran, Islamic Republic of

Background: An embolism is the lodging of an embolus, a blockagecausing piece of material, inside a blood vessel. The embolus may be a blood clot (thrombus), a fat globule (fat embolism), a bubble of air or other gas (gas embolism), amniotic fluid (amniotic fluid embolism), or foreign material. A thin- cap atheromatous plaque may rupture with high risk of subsequent thromboembolism- mediated acute clinical events such as acute stroke.

Aims: The aim of this study was to investigating the feasibility of extracorporeally and simultaneously application of Q-switched Nd:YAG laser and electrohydraulic shock waves accompanied by PESDA microbubbles administration on clot embolism reduction in the hamster carotid artery.

Methods: Briefly, golden Syrian hamsters were submitted to common carotid artery thromboembolism. Then treatment group underwent extracorporeally and simultaneously Q-switched Nd:YAG laser ( $\lambda=532 \mathrm{~nm}, P=10 \mathrm{w}, \mathrm{PD}=5 \mathrm{~ns}$ ) therapy and confocal dual pulse electrohydraulic shock wave $(V=10 \mathrm{kv}, F=0.2 \mathrm{~Hz}$, Impulses $=100$ and $V=15 \mathrm{kv}, F=0.5 \mathrm{~Hz}$, Impulses = 150) therapy accompanied by PESDA microbubbles $\left(100 \mathrm{ml} / \mathrm{kg}, 2-5 \times 10^{5}\right.$ bubbles $\left./ \mathrm{ml}\right)$ administration. Blood volume flow and blood mean velocity were measured by color Doppler ultrasonography. Moreover, percentage of luminal cross-sectional area of stenosis was measured by B-mode ultrasound at the occluded region. Moreover, occluded region was evaluated by histopathology.

Results: Quantitative ultrasonography and histopathological results showed a significant reduction in the mean value for blood mean velocity and the percentage of luminal cross-sectional area of stenosis and a significant increase in the mean value for blood volume flow in the treatment group compared with the other groups $(P<0.05)$. Conclusions: Enhanced thrombolytic effect of mechanical inertial cavitation effect of Q-switched Nd:YAG laser- induced by collapsed PESDA microbubbles and confocal dual pulse electrohydraulic shock waves, can cause to reduce the thrombosis and significantly dilate the luminal cross-sectional area of stenosis.

\section{PERIPHERAL ARTERY DISEASE}

PB0025 | Characterisation of Patients with Peripheral Artery Disease at Increased Risk for Cardiovascular Events and Mortality

B. Kremers $^{1}$; J.-W. Daemen ${ }^{2}$; H. ten Cate ${ }^{1,2}$; H. Spronk ${ }^{1}$; B. Mees ${ }^{2}$; A. ten Cate-Hoek ${ }^{1,2}$

${ }^{1}$ Maastricht University, Maastricht, Netherlands; ${ }^{2}$ Maastricht University Medical Center, Maastricht, Netherlands

Background: Peripheral artery disease (PAD) is characterised by atherosclerotic narrowing of peripheral arteries. Despite current medical treatment strategies, the incidence of cardiovascular events and mortality remains high in PAD populations.

Aims: To characterise PAD patients at increased risk for cardiovascular events and mortality.

Methods: Between 2018 and 2020, 250 PAD outpatients (17 newly diagnosed, 233 with known PAD) were enrolled in this observational cohort study. The study was approved by the ethical review board of MUMC+ and all participants gave written informed consent. Patient data and blood samples were collected upon inclusion and the composite endpoint (myocardial infarction, elective coronary revascularisation, stroke, acute limb ischemia, mortality) was evaluated after one year. Platelet reactivity was assessed using citrated platelet-poor plasma in the VerifyNow assay and medication adherence was assessed by the licensed Morisky Medication Adherence Scale-8 (MMAS-8) developed by Morisky et al, categorised as high (8 points), medium (6-7) and low (<6).

Results: The cohort comprised 211 claudicants and 39 chronic limb ischemia patients. Twenty-six patients reached the composite endpoint (Table 1). Prior myocardial infarction (OR 3.2 (1.4-7.3)), stroke (OR 4.4 (1.8-10.6)), aortic plaque formation (OR 3.2 (1-10.8)) and higher creatinine (OR 5.1 (2.1-12.1)), but lower high-density lipoprotein (OR 4.1 (1.5-11.4)) and haemoglobin levels (OR 3 (1.3-6.8)) were associated with events. Overall medication adherence was 
sufficient (75.6\% highly adherent), although high adherence was associated with events (OR 4.3 (1-18.7)) due to increased awareness.
(HTPR) on aspirin (OR 6.1 (1-3-28.3)) and clopidogrel (OR 4.5 (120.7))) (Figure 1).

Patients with events had more high on-treatment platelet reactivity

TABLE 1 Baseline characteristics for the whole cohort and distribution between patients with and without cardiovascular events and mortality during follow-up

\begin{tabular}{lllll} 
& Whole cohort & Event group & No event group \\
& Mean \pm SD/n (\%) & Mean \pm SD/n (\%) & Mean \pm SD/n (\%) & P-value \\
Age & $68.7 \pm 9.1$ & $71.5 \pm 7.9$ & $68.3 \pm 9.2$ & 0.094 \\
Male gender & $144(57.6)$ & $17(65.4)$ & $127(56.7)$ & 0.396 \\
Current smoking & $95(38)$ & $11(42.3)$ & $84(37.5)$ & 0.633 \\
Body Mass Index & $26.5 \pm 4.4$ & $27.4 \pm 5.9$ & $26.4 \pm 4.2$ & 0.403 \\
Diabetes Mellitus II & $69(27.6)$ & $10(38.5)$ & $59(26.3)$ & 0.191 \\
Lipid-lowering agents & $225(90)$ & $23(88.5)$ & $202(90.2)$ & 0.388 \\
Antihypertensive agents & $183(73.2)$ & $19(73.1)$ & $164(73.2)$ & 0.832 \\
Antiplatelet agents & $250(100)$ & $26(100)$ & $224(100)$ & 1.000 \\
\hline
\end{tabular}
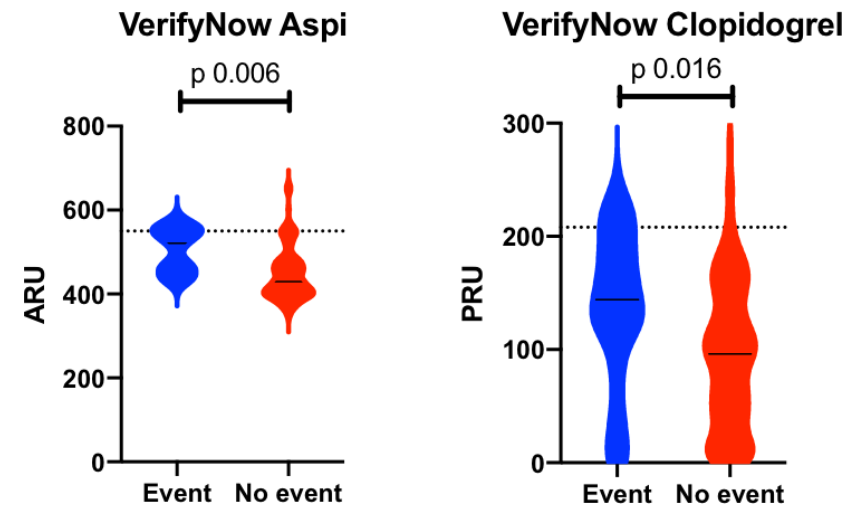

FIGURE 1 Platelet reactivity measured by the use of the VerifyNow assay in Aspirin Reactions Units (ARU) for aspirin users and P2Y12 Reaction Units (PRU) for clopidogrel users. Dotted lines represent HTPR which is an ARU > 550 for aspirin and a PRU > 208 for clopidogrel

Conclusions: PAD patients at increased risk for events had more often a prior myocardial infarction or stroke, extensive atherosclerosis with aortic plaque formation and renal insufficiency. Moreover, cardiovascular medication appeared insufficiently protective as lower levels of high-density lipoprotein and more HTPR were observed in patients with events despite adequate medication adherence.

\section{PB0026 | Leo Buerger's Disease: About 13 Cases}

I. Chabchoub; R. Ben Salah; N. Regaieg; F. Frikha; Z. Bahloul Internal Medicine Departement, Hedi Chaker Hospital, Sfax, Tunisia

Background: Leo Buerger's disease is a non-atherosclerotic arteriopathy, characterised by segmental and inflammatory obliteration affecting the small and medium-sized arteries and veins of four limbs. This pathology mainly affects young men who smoke.

Aims: To specify the clinical characteristics and the evolutionary profile of patients suffering from this condition.

Methods: A retrospective study including 13cases of Leo Buerger's disease diagnosed over a period of 22 years. The diagnosis was based on Mozes' criteria.

Results: There were 12 men and one woman, all smokers. The average age at diagnosis was 39.9 years. The disease is revealed by acute ischaemia of the limb in 6 cases, intermittent claudication of the limb in 4 cases, dry digital gangrene in 2 cases and venous thrombosis in 2 cases. 12 patients had distal arterial ischaemia. The ischaemia unilaterally affected the lower limb in 10 patients and the upper limb in4 patients. A decrease in peripheral pulses was noted in 4 cases and abolition in 8 cases. Trophic complications were found in 12 patients, involving digital ulcerations ( 3 cases), soft tissue infections ( 5 cases), osteitis (3 cases), distal necrosis (3 cases), gangrene ( 1 case) and sensory disturbances ( 2 cases). Raynaud's phenomenon was only observed in 2 patients, as was superficial thrombophlebitis. Arterial echodopler, performed on 9 patients, confirmed the distal location in all cases and confirmed bilateral damage in 6 patients. The arteriography carried out on 2 patients revealed left tibiofibulopedious stenosis in one case and distal arteritis in one case. An angioscan of the lower limbs carried out in 7 patients showed distal arterial occlusions. Therapeutically, smoking cessation and local care were offered in all patients. All patients received medical treatment 
including one to two vasodilators and a platelet anti-aggregant. Heparin therapy was prescribed for 4 patients.Surgery was necessary in 8 patients. The evolution was favourable in 6 cases. A recurrence of digital ischaemia was observed in 2 cases.

Conclusions: Leo Buerger's disease is a rare condition with difficult diagnosis to confirm due to the lack of specific markers.

\section{PB0027 | Changes in Vascular Physiology in Patients with End Stage Renal Disease Compared to Patients with ESRD and Concomitant Cardiovascular Disease}

\section{J. Goldstein; R. Dieter; K. Wieschhaus; J. Fareed}

Loyola University of Chicago Stritch School of Medicine, Maywood, United States

Background: Dialysis-dependent patients with end-stage renal disease (ESRD) commonly present with a variety of comorbidities, including an increased risk of cardiovascular disease. Biomarkers such as, tPA, D-dimer, and nitrotyrosine can serve as predictive factors for the estimation of vascular condition in patients with ESRD. Atrial fibrillation (AFIB) and peripheral arterial disease (PAD), can alter these biomarkers adversely affecting the health of the patients.

Aims: The objective of this study was to firstly establish a profile of the aforementioned biomarkers in patients with ESRD, ESRD and atrial fibrillation, as well as ESRD and PAD; and secondly, compare how these biomarkers and lab parameters have changed in patients who have cardiovascular comorbidities in addition to ESRD.

Methods: Blood samples were drawn from 95 patients with ESRD enrolled from Loyola Medical Center under the approved IRB protocol (\#LU107346). The biomarker levels were measured from citrated plasma levels of these samples using commercially available enzyme linked immune-absorbent assays (ELISAs). Lab parameters and patient comorbidities were obtained through a review of the patient medical records. The comorbidities were determined through provider notes, and evidence of applicable testing.

Results: Of the 94 patients with ESRD, $14.89 \%$ of patients were found to have atrial fibrillation ( $n=14$ ), $30.85 \%$ of patients were found to have peripheral arterial disease $(n=29)$, and $6.38 \%$ of patients were found to have both peripheral arterial disease and atrial fibrillation $(n=6)$. When compared to patients with only ESRD, patients with ESRD and PAD showed elevated levels of D-Dimer $(P=.0314)$ and nitrotyrosine $(P=.0330)$. When compared to patients with only ESRD, patients with atrial fibrillation showed elevated levels of D-Dimer $(P=.0372)$, nitrotyrosine $(P=.0322)$, and tPA $(P=.0198)$.

Conclusions: Atrial fibrillation and PAD is frequently encountered in patients with ESRD. Nitrotyrosine and d-dimer levels are elevated in patients with ESRD and concomitant PAD or atrial fibrillation.
P0116 | Clinical Outcomes and Natural History of Critical Limb Ischemia: A Real World Experience

D. Ceccato; S. Ragazzo; F. Boscaro; F. Ceccato; G. Camporese;

G. Avruscio

Azienda Ospedaliera di Padova, Padova, Italy

Background: Peripheral artery disease (PAD) is a chronic condition determining a compromised blood flow to extremities and the most severe form is the critical limb ischemia (CLI). The cornerstone therapy for CLI patients is endovascular revascularization. However, up to one-third of CLI patients are not suitable for surgery and amputation or conservative treatment is most commonly performed.

Aims: We investigate the comorbidities, survival rates and the amputation free-survival (AFL) of patients with CLI.

Methods: From Jan 2015 to Sept 2020 we selected consecutive patients with CLI after being taken to our Angiology Units. Baseline information were collected. The cohort was followed at least 6 months in order to assess primary outcome as amputation free-survive (AFS) and all causerelated death. Data were analysed using chi-squared test or Fisher's exact test. Survival time were determined by Kaplan-Meier analysis.

Results: We evaluated 103 patients with a CLI: Table 1 summarized the baseline characteristics. Median age was 76 year and 51 was male; most frequent risk factors were hypertension (91.3\%), dyslipidaemia (68\%) and history of cardiovascular events (49\%). During 5 years follow up, 70 (68\%) underwent surgical treatment and 101 (98.7\%) infusional treatment. Amputation were performed in $30(28.9 \%)$ patients, 9 above and 21 under the knee respectively. Complete recovered was reached in 26 (25.2\%) patients, while 57 (55.3\%) have had a wound stabilization. The overall AFS (above or under knee) was 78.7\% (SD \pm 4.7 ) (Figure 1). 14 (13.5\%) patients died during the period: the survival rate for all cause-related death was $88.4 \%$ (SD \pm 3.7 ). No differences were found in overall survival comparing amputation vs no amputation subject.

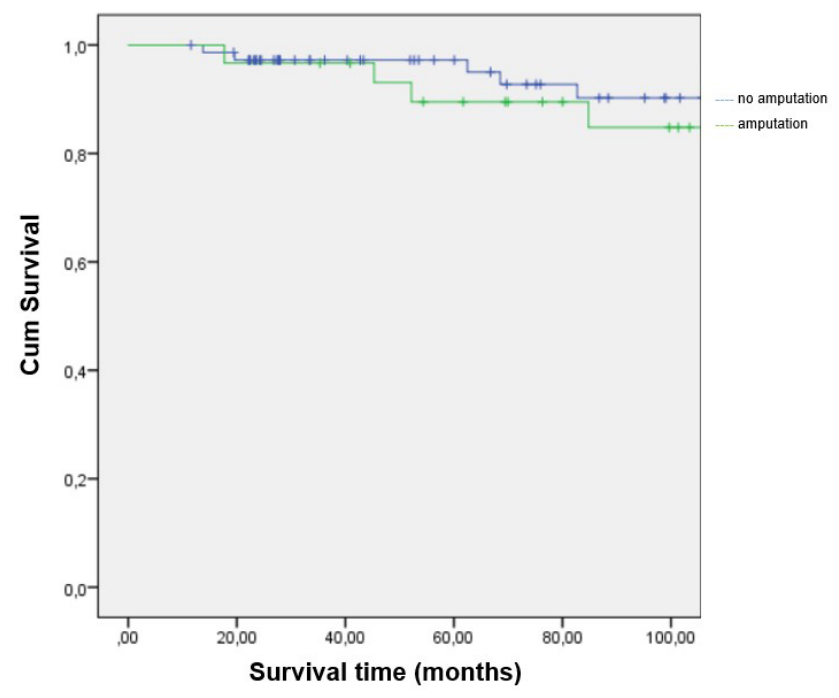




\section{TABLE 1}

\begin{tabular}{|c|c|c|c|c|}
\hline & Overall (N 103) & Amputation (30) & No amputation (73) & $p$-value \\
\hline Hypertension & $94(91.2 \%)$ & $28(93.3 \%)$ & $66(90.4 \%)$ & 0,61 \\
\hline Diabetes & $31(30.1 \%)$ & $11(36.1 \%)$ & $20(27.4 \%)$ & 0,35 \\
\hline Dyslipidaemia & $71(68.9 \%)$ & $21(70 \%)$ & $50(68.5 \%)$ & 0,8 \\
\hline $\mathrm{BMI}>30 \mathrm{Kg} / \mathrm{m}^{2}$ & $30(29.1 \%)$ & $8(26.7 \%)$ & $22(30.1 \%)$ & 0,75 \\
\hline COPD & $42(40.8 \%)$ & $11(36.7 \%)$ & $31(42.5 \%)$ & 0,58 \\
\hline Cardiovascular diseases & $49(47.6 \%)$ & $15(50 \%)$ & $34(46.6)$ & 0,72 \\
\hline Personal history for VTE & $19(18.4 \%)$ & $7(23.3 \%)$ & $12(16.4 \%)$ & 0,41 \\
\hline Autoimmune disease & $25(24.3 \%)$ & $10(33.3 \%)$ & $15(20.5 \%)$ & 0,1 \\
\hline Personal history of cancer & $38(36,9 \%)$ & $7(23.3 \%)$ & $31(42,5 \%)$ & 0,06 \\
\hline
\end{tabular}

Conclusions: Patients with CLI in charge to a high qualify angiology unit, mortality and amputations are still common treatment, however surgical revascularization and infusional therapy improve the prognosis of these patients.

\section{COAGULATION AND NATURAL ANTICOAGULANTS}

\section{ANIMAL MODELS IN THROMBOSIS AND HEMOSTASIS}

LPB0005 | Tissue-engineered Human Arteries Replicate

Primary and Secondary Haemostatic Functions Seen in vivo: A Replacement for Mouse Arterial Thrombosis Models?

J. Ranjbar; Y. Yang; A. Harper

Keele University, Stoke-on-Trent, United Kingdom

Background: Intravital microscopy in mice is widely used to study in vivo thrombus formation. Previously we have developed a 3D, tissue-engineered human arterial construct (TEAC) that replicates the primary haemostatic properties of the native artery [1].

Aims: To assess whether our TEAC can trigger the activation of the extrinsic coagulation cascade, and whether this can be used to generate a human arterial thrombosis model to replace current in vivo studies.

Methods: Medial and adventitial arterial layers were replicated by seeding human coronary artery smooth muscle cells and adventitial fibroblasts into a collagen hydrogel. A complete TEAC was created by co-culturing the medial layer with HUVEC-coated nanofibers. Prothrombin times were measured by exposing these constructs to Platelet-poor plasma (PPP) from healthy human volunteers. A fluorogenic tissue factor assay was performed to assess tissue factor activity by incubation with factor VII and SN17a. Platelet activation was assessed using physiological flow conditions in a custom-made 3D printed flow chamber.
Results: Both the medial and adventitial layer constructs are able to trigger rapid coagulation when exposed to PPP, and have measurable tissue factor activity. Tissue factor activity in the medial layer construct was enhanced by supplementation of ascorbic acid in the culture media. Ascorbic acid supplementation of the medial construct also significantly increased platelet adhesion and aggregation on this construct under flow conditions. Incorporation of an endothelial lining atop of the media layer construct can allow us to replicate commonly used thrombosis models.

Conclusions: Our data demonstrates that our TEACs can replicate all of the major haemostatic processes of the native blood vessel, and is a viable replacement to current murine thrombosis models.

This work is supported by an NC3R- and British Heart Foundation co-funded PhD studentship

Refs: [1] Musa et al. (2016) Tissue Eng Part C Methods 22, 691-699.

LPB0099 | Absence of CCR5/CCL5 Axis Inhibits Thrombus Resolution through Reduced uPA, tPA and VEGF Expressions in DVT Model

M. Nosaka ${ }^{1}$; Y. Ishida ${ }^{1}$; A. Ishigami ${ }^{1}$; Y. Kuninaka ${ }^{1}$; H. Yamamoto ${ }^{1}$; E. Shimada ${ }^{1}$; Y. Hashizume ${ }^{1}$; M. Kawaguchi ${ }^{1}$; A. Kimura ${ }^{1}$; N. Mukaida ${ }^{2}$; T. Kondo ${ }^{1}$.

${ }^{1}$ Department of Forensic Medicine, Wakayama Medical University, Wakayama, Japan; ${ }^{2}$ Division of Molecular Bioregulation, Cancer Research Institute, Kanazawa University, Kanazawa, Japan

Background: Deep vein thrombosis (DVT) is multifactorial and often results from a combination of risk factors such as genetic conditions, obesity, drugs, pregnancy, aging, trauma and malignancy. And DVT is a complex biological event, with endothelial injury, venous stasis and blood hypercoagulability.

Aims: In this study, we examined the pathophysiological roles of CCR5-mediated signals in the formation and resolution of DVT using C57BL/6 (WT) and Ccr5-/- (KO) mice.

Methods: Inferior vena cava (IVC) was ligated with silk sutures and kept alive for 1 to 21 days, and then thrombi were collected for immunohistochemical staining and gene expression analysis of factors involved in thrombolysis.

Results: After IVC ligation, Ccr5 gene expression was up-regulated in the thrombi of WT mice and intrathrombotic $\mathrm{CCR}^{+}$cells were detected (Figure 1). When KO mice were treated with the same manner, thrombus size was much larger, compared with WT ones (Figure 2). Moreover, recovery of the IVC blood flow was more impaired in KO mice than in WT ones. Intrathrombotic Plat, Plau and Vegf mRNA expressions were significantly reduced in $\mathrm{KO}$ mice, compared with WT ones. We explored the contribution of bone marrow (BM)-derived $\mathrm{CCR}^{+}$cells to thrombolysis by using BM chimeric mice generated from WT and KO mice. Both mice transplanted with WT-derived BM cells exhibited a higher thrombolytic activity than those transplanted with KO-derived BM cells. Supportingly, recombinant murine CCL5 treatment enhanced gene expression of 
Plau, Plat and Vegf in WT-derived but not KO-derived macrophages. CCR5/CCL5 axis-deficiency impaired fibrinolytic activity and collagen production.

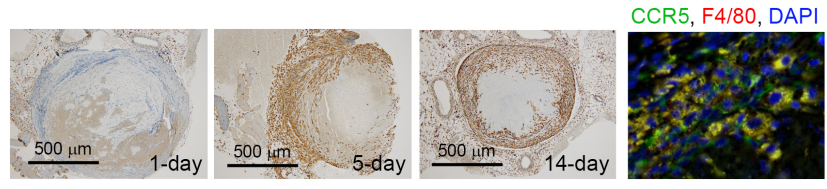

FIGURE 1 Immunohistochemical staining for CCR5 and double-color immunofluorescence staining images for CCR5 and F4/80 in thrombi of WT mice
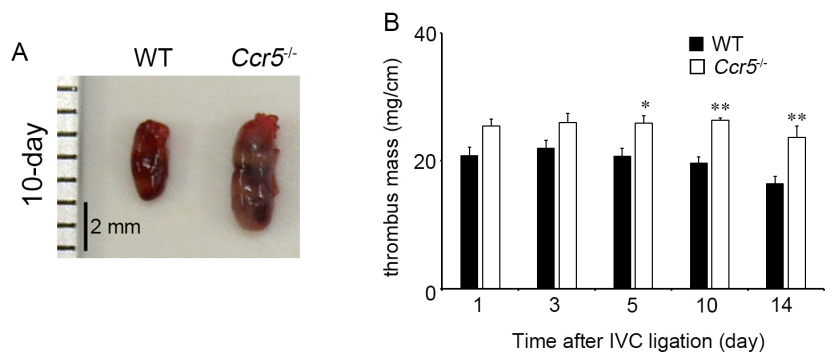

FIGURE 2 Overview of thrombi in the mouse model of DVT. A: Macroscopic observation of thrombi. B: Thrombus size of WT and KO mice. The thrombus size of KO mice were mach larger than WT ones

Conclusions: Collectively, the lack of CCR5-mediated signal pathways would have detrimental roles in the thrombus resolution by suppressing UPA, tPA and VEGF expression respectively. The CCR5/ CCL5 axis can be a good molecular target for the DVT treatment.

PB0029 | A Novel Mouse Model for Cerebral Venous Thrombosis

M.-C. Bourrienne ${ }^{1}$; S. Loyau ${ }^{1}$; S. Benichi' ${ }^{2}$ J. Gay ${ }^{1}$; M. SoloNomenjanahary $^{1}$; D. Faille ${ }^{1,3}$; C. Journé ${ }^{4}$; L. Di Meglio' ${ }^{1}$; A. Freiherr von Seckendorff ${ }^{1}$; J.-P. Desilles ${ }^{1,5}$; B. Ho-Tin-Noé ${ }^{1}$; M. Mazighi ${ }^{1,5,6}$; N. Ajzenberg ${ }^{1,3}$

${ }^{1}$ Université de Paris, Laboratory for Vascular Translational Science (LVTS), INSERM UMR_1148, Paris Cedex 18, France; ${ }^{2}$ Pediatric Neurosurgery Department, AP-HP, Necker Children Hospital, Paris, France; ${ }^{3}$ Laboratory of Hematology, AP-HP, Bichat Hospital, Paris Cedex 18, France; ${ }^{4}$ Université de Paris, INSERM UMS 34, Fédération de Recherche en Imagerie Multimodalités (FRIM), Faculté de Médecine X. Bichat, Paris, France; ${ }^{5}$ Department of Interventional Neuroradiology, Rothschild Foundation Hospital, Paris, France; ${ }^{6}$ Department of Neurology, AP-HP, Lariboisière Hospital, Paris, France

Background: Cerebral venous sinus thrombosis (CVST) is an uncommon cause of stroke resulting in parenchymal injuries associated with heterogeneous clinical symptoms and prognosis. Therefore, experimental animal model is required to further study underlying mechanisms involved in CVST.

Aims: This study aims to develop a novel murine model suitable and relevant for evaluating injury patterns during CVST and studying its clinically aspects.

Methods: CVST was achieved in C57BL/6J mice by autologous clot injection into the superior sagittal sinus (SSS) combined with bilateral ligation of external jugular veins. Clot was prepared ex vivo using thrombin before injection. On day 1 and 7 after CVST, SSS occlusion and associated-parenchymal lesions were monitored using different modalities: in vivo real-time intravital microscopy, magnetic resonance imaging (MRI), and immuno-histology. In addition, mice were subjected to a neurological sensory-motor evaluation.

Results: Thrombin-induced clot provided fibrin- and erythrocytesrich thrombus that lead to reproducible SSS occlusion at day 1 after CVST induction. On day 7 post-CVST, venous occlusion monitoring (MRI, intravital microscopy) showed that initial injected-thrombus size did not significantly change demonstrating no early spontaneous recanalization.

Microscopic histological and immunofluorescence analysis revealed that SSS occlusion resulted in brain edema, extensive fibrin-rich venular thrombotic occlusion, ischemic and hemorrhagic lesions. Mice with CVST showed a significant lower neurological score on post-operative day 1 and 7, compared to sham-operated group.

Conclusions: Here, we established a novel clinically CVST relevant model with a persistent and reproducible SSS occlusion responsible for symptomatic ischemic and hemorrhagic lesions. We showed that SSS thrombosis also lead to thrombosis extension in cortical veins that contributes to thrombotic occlusion of parts of the microcirculatory bed. This method provides a reliable model to study CVST physiopathology and evaluation of therapeutic new regimens.

PB0030 | High Shear Conditions Takes Place at the Edge of the Wound after Human and Mouse Vessel Injury

A. Yakusheva ${ }^{1,2,3}$; K. Butov ${ }^{2,3,4}$; F. Ataullakhanov ${ }^{2,3,5}$; C. Gachet ${ }^{1}$; M. Panteleev ${ }^{2,3,5}$; P. Mangin ${ }^{1}$

${ }^{1}$ Université de Strasbourg, INSERM, EFS Grand-Est, BPPS UMR-S 1255, FMTS, Strasbourg, France; ${ }^{2}$ Center for Theoretical Problems of Physicochemical Pharmacology, Moscow, Russian Federation; ${ }^{3} \mathrm{Federal}$ Research and Clinical Centre of Pediatric Hematology, Oncology and Immunology, Moscow, Russian Federation; ${ }^{4}$ Histology Department, Pirogov Russian National Research Medical University, Ministry of Healthcare of the Russian Federation, Moscow, Russian Federation; ${ }^{5}$ Faculty of Physics, Moscow State University, Moscow, Russian Federation

Background: The importance of blood flow in regulating hemostasis is well known. The current view is that low flow $\left(100 \mathrm{~s}^{-1}\right.$ up to $\left.2,000 \mathrm{~s}^{-1}\right)$ occurs in healthy vessels, while high shear $\left(>2,000 \mathrm{~s}^{-1}\right)$ is relevant to arterial thrombosis, notably around stenosed vessels. 
Targeting high shear has been proposed as an innovative strategy to selectively block thrombosis with a minor impact on hemostasis. However, the rheology occurring after vessel wall injury has never been measured experimentally.

Aims: To evaluate the shear rates at the edges of different wounds in mice and humans.

Methods: Intravital and electron microscopy was used to image thrombus formation in several novel models of hemostasis following needle-induced puncture or scissors-induced disruption of various vessels. Two laser doppler probes on both sides of the injury measured blood flow in the vessels throughout the hemostatic process, and ComSol software allowed to recalculate shear rates in the wound. In human the shear rate was calculated by applying Poiseuille's equation with volumetric rates of blood loss after catheter placement.

Results: The shear rate at the edge of the wound induced with a needle was 80-, 10-, 33- and 100-times higher in mouse artery, aorta, vein and vena cava than the values in the same uninjured vessels (Table1). In the scissors-disruption model of the spermatic artery, a 3-fold increase at the lesion site was obtained when compared to homeostatic conditions. In humans, the shear rate upon the wound of cubital vein was $14,500 \pm 5,000 \mathrm{~s}^{-1}$ and $3,700 \pm 800 \mathrm{~s}^{-1}$ for 310 and $410 \mu \mathrm{m}$ of injury diameter (Table1), indicating that the shear reaches high levels following vessel damage.

TABLE 1 The shear rates calculated for different scenarios

\begin{tabular}{|c|c|c|c|c|c|c|}
\hline Vessel & Species & $\begin{array}{l}\text { Type of vessel } \\
\text { injury }\end{array}$ & $\begin{array}{l}\text { Diameter of the } \\
\text { injury, } \mu \mathrm{m}\end{array}$ & \multicolumn{2}{|c|}{ Shear rate at the wound, $\mathrm{s}^{-1}$} & $\begin{array}{l}\text { Shear rate in the } \\
\text { intact vessel, } \mathrm{s}^{-1}\end{array}$ \\
\hline Carotid artery & mouse & puncture & $125-140$ & 11,600 & $6,700-30,400$ & 950 \\
\hline Aorta & mouse & puncture & $40-300$ & 29,000 & $4,700-680,000$ & 900 \\
\hline Femoral vein & mouse & puncture & $80-165$ & 22,200 & $7,100-50,000$ & 1,000 \\
\hline Vena cava & mouse & puncture & $70-280$ & 40,500 & $24,100-2.7 * 10^{6}$ & 200 \\
\hline \multirow[t]{2}{*}{ Median cubital vein } & human & puncture & 310 & 7,400 & $5,400-27,300$ & 250 \\
\hline & & & 410 & 3,500 & $2,300-5,400$ & 250 \\
\hline
\end{tabular}

Conclusions: Our results indicate that various types of injuries in small and large mouse and human vessels result in high shear rates. Elevated shear rate is not specific to arterial thrombosis, but also relevant to hemostasis.

PB0031 | Mass Cytometry Analysis of Venous Thrombi Demonstrates Key Roles for VWF Interactions in Inflammationassociated DVT

$\underline{\text { S.J. Choi }}^{1}$; M. Cormier ${ }^{1}$; L. Rapkin ${ }^{2}$; C. Hindmarch ${ }^{3}$; D. Lillicrap ${ }^{1}$

${ }^{1}$ Department of Pathology and Molecular Medicine, Queen's University, Kingston, Canada; ${ }^{2}$ Fluidigm Corporation, Markham, Canada; ${ }^{3}$ Queen's Cardiopulmonary Unit (QCPU), Translational Institute of Medicine (TIME), Department of Medicine, Queen's University, Kingston, Canada

Background: We have previously shown that lipopolysaccharide (LPS)-induced inflammation increased deep vein thrombosis (DVT) incidence associated with increased levels of von Willebrand factor (VWF). Venous thrombus architecture has been explored previously with conventional histology methods.

Aims: To simultaneously and quantitatively analyze the localization and interaction of ten venous thrombus constituents in control and endotoxemic mice.

Methods: The inferior vena cava stenosis model was used to induce DVT in wildtype mice, followed by intraperitoneal injections of $0.5 \mathrm{mg} / \mathrm{kg}$ LPS or vehicle control. We employed Hyperion mass cytometry imaging to simultaneously analyze ten thrombus targets in these mice. Data was analyzed using Ilastik, CellProfiler, HistoCAT, and RStudio software programs.

Results: Regardless of LPS-induced endotoxemia; platelets, Pselectin, high-mobility group box 1 (HMGB1), leukocytes, VWF and fibrin localized significantly $(P<0.05)$ in the white thrombus. Red blood cells (RBCs) were predominant in the red thrombus and neutrophil extracellular traps (NETs) were present throughout the whole thrombus. While endotoxemia did not significantly affect the overall VWF content, in those thrombi, VWF expression in the white thrombus positively correlated with HMGB1 and platelets and negatively with RBCs. VWF significantly correlated with P-selectin and fibrin in both the control and LPS cohorts. Although not statistically significant, NET-DNA correlations with VWF and fibrin were negative in the controls but positive in the LPS cohort. Correlations are shown in Figure 1. Clustering analysis identified the second and top highest VWF expression clusters among the 2 most common clusters in the control and LPS cohorts, respectively. 


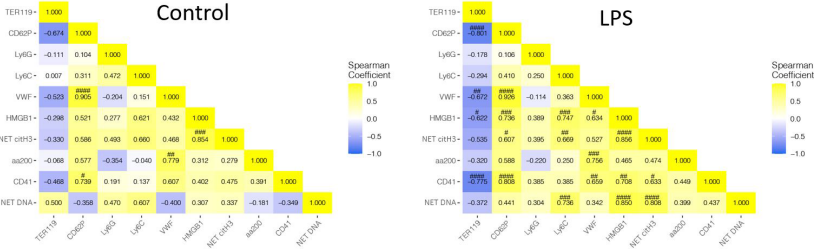

FIGURE 1 Correlational heatmaps of the average intensity of expression of TER119 (RBCs), CD62P (P-selectin), Ly6G (neutrophils), Ly6C (monocytes), VWF, HMGB1, NET citH3 (NET-associated citrullinated histones), aa200 (fibrin), CD41 (platelets), and NET DNA (NET-associated DNA) in Phenograph clusters in control (left) and LPS cohorts (right). Bonferroni-corrected P-values: \# $\mathrm{p}$

Conclusions: The majority of venous thrombus interactions, except for RBCs and NETs, occur in the white thrombus. Endotoxemia enhances VWF interactions with platelets and potentially plateletderived HMGB1. While the VWF-fibrin thrombus scaffold remains constant, endotoxemia promotes NET interactions with VWF and fibrin. The importance of VWF's role in venous thrombi is highlighted by the high representation of high-VWF expression clusters.

PB0032 | Absolute Measurement of Bovine and Porcine Heparin Red Assay Utilizing the Heparin Red Assay. Applications in the Study of Pharmacokinetics and Pharmacodynamics

W. Jeske ${ }^{1}$; A. Kouta ${ }^{1}$; D. Hoppensteadt ${ }^{1}$; L. Cera ${ }^{1}$; R. Duff ${ }^{1}$;

J. Fareed ${ }^{1}$; R. Kraemer ${ }^{2}$

${ }^{1}$ Loyola University Medical Center, Maywood, United States;

${ }^{2}$ Heidelberg University, Heidelberg, Germany

Background: Recent primate studies have suggested that when bovine and porcine heparins are dosed on the basis of equivalent antiXa units, equivalent anti-Ila and anti-Xa responses are observed. The pharmacokinetic behavior of heparins has traditionally been based on antithrombin-dependent anti-Xa and anti-Ila activities. Heparin Red is a polycationic substance whose intrinsic fluorescence is quenched upon binding to heparin. As such, assays utilizing Heparin Red detect all heparin oligosaccharides in a sample: those that bind to AT and produce anti-Xa and anti-Ila activities, and those that do not.

Aims: Thus, the pharmacokinetic behavior of heparin (half-life, etc.) as determined using a Heparin Red assay may more closely reflect the absolute levels.

Methods: Primates were administered PMH (Medefil, Glendale Heights, IL) or BMH (KinMaster, Passo Fundo, Brazil) at a dose of 100 anti-Xa U/kg IV. Blood samples were collected prior to and at 15 30, 60 and 120 min post-heparin administration. Heparin levels were assessed using a chromogenic anti-Xa assay and a Heparin Red assay relative to product-specific calibration curves. Pharmacokinetic parameters were assessed using a non-compartmental model.

Results: Peak levels of $1.45 \pm 0.11$ and $1.48 \pm 0.08 \mathrm{U} / \mathrm{ml}$ were observed in $\mathrm{PMH}$ and $\mathrm{BMH}$-treated primates, respectively. Using drug levels determined by anti-Xa assay, AUCs for bovine and porcine heparin treated animals were calculated to be $111.5 \pm 11.0$ and $108.8 \pm 26.7 \mathrm{U}^{*} \mathrm{~min}^{*} \mathrm{ml}^{-1}$, respectively. By Heparin Red assay, peak circulating heparin levels were higher following $\mathrm{BMH}$ administration. Conclusions: $\mathrm{BMH}$, when administered at equivalent anti- $\mathrm{Xa}$ unit doses, produces comparable pharmacodynamic effects as $\mathrm{PMH}$. Heparin Red assay may be useful for identifying the appropriate dose of protamine to completely neutralize $\mathrm{BMH}$. Furthermore absolute quantitation of GAGs by Red Probe method in these studies provides a true pharmacodynamic effect of heparins accounting for the cumulative actions of both AT dependent and independent actions.

\section{PB0033 | Short Peptides in the Regulation Blood Cells} Aggregation in Thrombogenesis

\section{Golubeva}

Lomonosov Moscow State University, Moscow, Russian Federation

Background: It has been shown that hormones of neurohypophysis and hypothalamus can have effects on blood clotting when interacting with specific receptors of blood cells and endothelium. Along with the neuropeptides themselves, their fragments formed during enzymatic degradation can have independent biological activity.

Aims: In this study was to compare the effect of small regulatory peptides, which are fragments of neurohormones, in vitro experiments on the aggregation of red blood cells and platelets under the action of epinephrine.

Methods: C - terminal fragments of vasopressin, oxytocin, luliberin and the hormone thyroliberin was used Pro-Arg-Gly-NH2. - vasopressin fragment (P1) Pro-Leu-Gly-NH2-oxytocin fragment, (P2) Arg-Pro-Gly-NH2-lyuliberin fragment (P3) Glu-His - Pro - NH2-tyroliberin (P4) Peptides are added in a concentration range of 10-1 - 10-10 M or an equal volume of saline added, or to a pool of washed platelets, or erythrocytes and the measured change in aggregation by the action of epinephrine $0.02 \mathrm{mmol} / \mathrm{L}$ on a aggregometer. The results were treated statistically.

Results: It has been shown that small regulatory peptides, which are products of the proteolysis of neuropeptides, have a different action on the aggregative state of blood cells stimulated by adrenaline. It was found that PI causes a significant increase in the aggregation of both red blood cells and platelets, $(P<0.001)$, whereas P2 inhibited only erythrocyte aggregation, without changing platelet aggregation. P3 had virtually no effect on platelet aggregation. P4 increased both platelet aggregation and erythrocyte aggregation $(P<0.001)$. Conclusions: The effect of the studied tripeptides on hemostasis can be carried out through specific receptors on both blood cells and endothelium. Given their high efficiency, a differentiated approach to the therapy of thrombotic complication is required. 
PB0034 | USP Potency Adjusted Bovine Mucosal Heparins Are Comparable to Porcine Mucosal Heparin and may be interchangeable for anticoagulation

A. Kouta; N. Baig; W. Jeske; D. Hoppensteadt; M. lacobelli; O. Iqbal; M. Bakhos; J. Fareed

Loyola University Medical Center, Maywood, United States

Background: Most of the heparins used clinically are derived from porcine intestinal mucosa. In contrast to the porcine mucosal heparin (PMH), the active pharmaceutical ingredient (API) of bovine mucosal heparin (BMH) exhibit a somewhat weaker USP potency as cross-referenced against $\mathrm{PMH}$.

Aims: We hypothesized that at equivalent potencies as adjusted by using the USP reference, the BMH may exhibit comparable effects. Methods: Molecular weight profile and in vitro anticoagulant and antiprotease assays were used to compare the $\mathrm{BMH}$ with $\mathrm{PMH}$. USP potency was measured in the amidolytic assays. Potency adjustments in reference to USP standard were made by weight basis. Potency adjusted $\mathrm{BMH}$ and $\mathrm{PMH}$ were injected at identical dosages $(50 \mathrm{U} / \mathrm{kg}$ and $100 \mathrm{U} / \mathrm{kg}$ ) to groups of human primates $(n=4)$ via intravenous route and blood samples were collected for a $6 \mathrm{~h}$ period of time.

Results: BMH exhibited higher molecular weight profiles compared to $\mathrm{PMH}$ as determined by size exclusion chromatography $\mathrm{BMH}$ $(\mathrm{Mw}) 18.6 \pm 0.5 \mathrm{kDa}$ and $\mathrm{PMH} 15.4 \pm 0.4 \mathrm{kDa}$. BMH showed a potency of $130 \mathrm{U} / \mathrm{mg}$ whereas PMH showed a potency of $190 \mathrm{U} / \mathrm{mg}$. When the $\mathrm{BMH}$ was compared at a potency adjusted concentration with $\mathrm{PMH}$, it showed identical calibration curves in the APTT and anti-protease assays. Mean t1/2 ranged from $54 \pm 11 \mathrm{~min} \&$ to 71 \pm 18 min for $\mathrm{PMH} \& \mathrm{BMH}$ respectively $(P=>0.05)$. Mean AUC values based on anti-Xa or anti-Ila activities were comparable for both heparins. Mean $\mathrm{Vd}(\sim 60 \mathrm{ml} / \mathrm{kg})$ and $\mathrm{Cl}(\sim 0.75 \mathrm{ml} / \mathrm{kg} / \mathrm{min})$ were also comparable for both heparins.

Conclusions: Potency adjusted based dosing results in comparable anticoagulants and pharmacokinetic profiles for $\mathrm{BMH}$ and $\mathrm{PMH}$ Therefore, such dosing may provide uniform levels of anticoagulation for the parenteral indications for heparins. These observations

TABLE 1 Thrombin generation and atrial histology analysis suggest that potency equated $\mathrm{BMH}$ and $\mathrm{PMH}$ produced comparable anticoagulant effects and may be interchangeable.

\section{PB0035 | Coagulation Potential, Electrophysiological} Characteristics and Structural Remodeling due to Atrial Fibrillation in Young and Aged Goats

E. D'Alessandro ${ }^{1}$; B. Scaf ${ }^{2}$; V. Sobota ${ }^{2}$; A. van Hunnik²; M. Kuiper²; J. Winters ${ }^{2}$; R. van Oerle ${ }^{1}$; H. Spronk ${ }^{1}$; F. van Nieuwenhoven ${ }^{2}$; H. ten Cate ${ }^{1}$; S. Verheule ${ }^{2}$; U. Schotten ${ }^{2}$

${ }^{1}$ Departments of Biochemistry and Internal Medicine, Maastricht University, Maastricht, Netherlands; ${ }^{2}$ Department of Physiology, Maastricht University, Maastricht, Netherlands

Background: Age is a risk factor for atrial fibrillation (AF) as well as for stroke in patients with AF. The effect of ageing on AF mechanisms and on coagulation activity is not well understood.

Aims: To evaluate the effect of age and AF on coagulation activity, AF characteristics, and structural remodeling.

Methods: Four groups of goats were investigated: Young sham ( $Y$ Sh: sinus rhythm, $<3$ years old, $n=9$ ), Young AF (Y-AF: 4 weeks of $A F,<3$ years old, $n=7$ ), Old sham (O-Sh: sinus rhythm, $>8$ years old, $n=6$ ), and Old AF (O-AF, 4 weeks of $A F$, $>8$ years old, $n=8$ ). Groups were matched for body weight. AF was maintained using implantable pacemakers in both AF groups. Clotting potential was measured using tissue factor-induced thrombin generation (TG) assays at baseline and 4 weeks (final). A terminal experiment was performed with atrial contact mapping to study electrophysiological AF characteristics and to collect atrial tissue for histological analysis.

Results: The clotting potential, expressed as the ability of activated plasma to generate thrombin, did not differ among groups. However, AF in old goats caused an increase in coagulation potential (thrombin $\mathrm{nM}$ ) while this was not the case in young goats. Complexity of fibrillatory conduction (no. of waves) was higher in AF groups after 4 weeks as compared to Sham, but there was no effect of age. AFrelated atrial myocyte hypertrophy occurred in old but not in young goats. Endomysial fibrosis was similar in all groups.

\begin{tabular}{|c|c|c|c|c|c|c|c|c|c|}
\hline \multirow[b]{2}{*}{ Thrombin generation ${ }^{*}$} & \multirow[b]{2}{*}{ Y-Sh } & \multirow[b]{2}{*}{ Y-AF } & \multirow[b]{2}{*}{ O-Sh } & \multirow[b]{2}{*}{ O-AF } & \multicolumn{3}{|c|}{ Tukey's post hoc } & \multirow[b]{2}{*}{$\begin{array}{l}\text { O-Sh } \\
\text { vs. } \\
\text { O-AF }\end{array}$} & \multirow[b]{2}{*}{$\begin{array}{l}\text { Y-AF } \\
\text { vs. } \\
\text { O-AF }\end{array}$} \\
\hline & & & & & $\begin{array}{l}\text { ANOVA } \\
\text { (all groups) }\end{array}$ & Y-Sh vs. Y-AF & $\begin{array}{l}\text { Y-Sh } \\
\text { vs. } \\
\text { O-Sh }\end{array}$ & & \\
\hline Peak height (nM) - baseline & $157 \pm 50$ & $175 \pm 43$ & $155 \pm 54$ & $175 \pm 52$ & ns & ns & ns & ns & ns \\
\hline $\begin{array}{l}\text { Peak height (nM) - final } \\
\text { Atrial histology }\end{array}$ & $179 \pm 55$ & $199 \pm 45$ & $145 \pm 17$ & $220 \pm 52^{* *}$ & ns & ns & ns & ns & ns \\
\hline LA myocyte size $(\mu \mathrm{m})$ & $12.1 \pm 1.5$ & $11.7 \pm 0.9$ & $12.3 \pm 1.0$ & $13.4 \pm 0.8$ & $P<0.05$ & ns & ns & ${ }^{*}$ & ns \\
\hline RA myocyte size $(\mu \mathrm{m})$ & $11.2 \pm 1.1$ & $11.2 \pm 0.8$ & $11.9 \pm 0.8$ & $12.6 \pm 1.0$ & $P<0.05$ & ns & ns & * & ns \\
\hline LA endomysial fibrosis ( $\mu \mathrm{m})$ & $5.8 \pm 0.3$ & $5.8 \pm 0.6$ & $5.6 \pm 0.3$ & $6.0 \pm 0.4$ & ns & ns & ns & ns & ns \\
\hline RA endomysial fibrosis ( $\mu \mathrm{m})$ & $6.2 \pm 0.3$ & $6.0 \pm 0.3$ & $6.0 \pm 0.6$ & $6.2 \pm 0.5$ & ns & ns & ns & ns & Ns \\
\hline
\end{tabular}

*Thrombin generation; Peak height baseline vs. final (paired $t$-test): $O-A F P<0.01^{* *}(n=7)$. All values mean $\pm S D$. 
Conclusions: Four weeks of AF caused an increase in clotting potential and myocyte size in old but not in young goats. Age did not cause fibrosis or increased AF complexity.

PB0036 | New Thromboplastin-based Modification of Thromboembolic Ischemic Stroke Model in Rats

I. Ostrova ${ }^{1}$; Z. Tsokolaeva ${ }^{1,2}$; S. Kalabushev ${ }^{1,3}$; I. Ryzhkov ${ }^{1}$

${ }^{1}$ V. A. Negovsky Research Institute of General Reanimatology,

Federal Research and Clinical Center of Intensive Care Medicine and

Rehabilitology, Moscow, Russian Federation; ${ }^{2}$ FSBI MSRC of Cardiology,

Moscow, Russian Federation; ${ }^{3}$ Research Institute of functional genomics, Lomonosov MSU, Moscow, Russian Federation

Background: Clinical relevance is an essential requirement for animal models of acute cerebrovascular disease. Middle cerebral artery embolic occlusion (eMCAo) in rats closely resembles human ischemic stroke. But this model is technically intricate and/or require more than a day of preparation before surgery.

Aims: Our aim was to elaborate straightforward thrombotic focal cerebral ischemia rat model, which would reproduce key components of human thromboembolic stroke.

Methods: Male Wistar rats (300-400 g) were subjected to eMCAo by administration of an autologous blood clot in the following way. Under anesthesia (6\% chloral hydrate, $300 \mathrm{mg} / \mathrm{kg}$ i.p.) left common carotid, external carotid and pterygopalatine arteries were ligated. The thrombus formed within $20 \mathrm{~min}$ in a syringe $(100 \mu \mathrm{l}$ blood mixed with thromboplastin and $\mathrm{CaCl}_{2}$ ) was introduced into the left internal carotid artery. Control animals $(n=5)$ were injected with saline. The incision was sutured $10 \mathrm{~min}$ after catheter removal. Neurological status was assessed two hours before euthanasia using 20-point scale. After $6 \mathrm{~h}(n=4), 1(n=3), 3(n=6)$ and $6(n=5)$ days, rat's brain was removed, fixed and embedded in paraffin. Histological evaluation of neuronal damage was performed on hematoxylin-eosin stained coronal sections of brain.

Results: The overall mortality rate was $22 \%$ (during surgery - $5 \%$, first day $-16 \%)$. Motor dysfunctions were found in rats after 1,3 , and 6 days $(P<0.05)$ (Figure 1). Histological analysis $6 \mathrm{~h}$ after surgery revealed foci of necrosis in striatum and cortex of ipsilateral hemisphere (ILH) in one rat, 3 days after - in four. On days 1 and 6, all rats had necrotic zones in ILH cortex, hippocampus, and subcortical regions. Quantitative analysis revealed a decrease in hippocampal pyramidal neurons density $(P<0.05)$ (Table 2$)$.

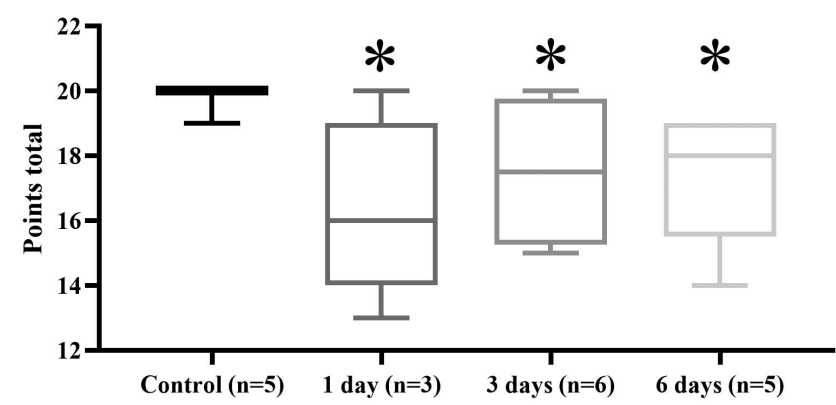

FIGURE 1 Neurological status assessment of eMCAo surged rats (20-point scale; ${ }^{*}-P$

TABLE 1 Pyramidal neurons density in hippocampus of eMCAo surged rats (number of neurons per 1 mm of laier length, ob. 20x; ${ }^{*} P$

\begin{tabular}{|c|c|c|c|c|c|}
\hline $\begin{array}{l}\text { Hippocampus } \\
\text { zone }\end{array}$ & $\begin{array}{l}\text { Group } \\
\text { Control }\end{array}$ & $6 \mathrm{~h}$ & 1 day & 3 days & 6 days \\
\hline CA1 & $\begin{array}{l}206.5 \\
{[199.1 ; 209.5]}\end{array}$ & $\begin{array}{l}202.5 \\
{[97.3 ; 216.8]}\end{array}$ & $\begin{array}{l}178.3^{*} \\
{[137.4 ; 188.5]}\end{array}$ & $\begin{array}{l}152.5^{*} \\
{[64.9 ; 178.7]}\end{array}$ & $\begin{array}{l}81.5^{*} \\
{[13.0 ; 124.6]}\end{array}$ \\
\hline CA4 & $\begin{array}{l}118.4 \\
{[118.4 ; 119.5]}\end{array}$ & $\begin{array}{l}103.5^{*} \\
{[87.5 ; 110.5]}\end{array}$ & $\begin{array}{l}77.7^{*} \\
{[71.7 ; 98.4]}\end{array}$ & $\begin{array}{l}107.4^{*} \\
{[63.4 ; 123.2]}\end{array}$ & $\begin{array}{l}94.9^{*} \\
{[84.8 ; 98.9]}\end{array}$ \\
\hline
\end{tabular}

Conclusions: Presented thromboembolic stroke model doesn't require microsurgery, long-term preparation, one surgery takes approximately $45 \mathrm{~min}$ and leads to reproducible manifested morphofunctional brain alterations. 
PB0037 | Rat Model of Metabolic Disorders: Dynamics of Hemostasis and Endothelial Function Changes

T. Obergan; L. Lyapina; M. Grigorjeva; T. Shubina

Lomonosov Moscow State University, Moscow, Russian Federation

Background: Metabolic disorders resulting from the consumption of rich-fat and rich-carbohydrates foods are accompanied by hypercoagulation, endothelial dysfunction and, as a result, the occurrence of thrombotic complications. Rodent models are used to improve the understanding of the causes and progression of these diseases, as well as to create therapeutic methods for their correction.

Aims: To evaluate changes of the hemostatic system and endothelial function in rats with metabolic disorders induced by a high-calorie diet, both with and without additional administration of L-NAME (endothelial synthase blocker).

Methods: Metabolic disorders were induced in adult male Wistar rats by a high-calorie diet (HCD, $3840 \mathrm{kcal} / \mathrm{kg}, 130 \%$ of standard feed) and $10 \%$ glucose solution as a drink. At $43^{\text {rd }}$ day of experiment, to enhance endothelial dysfunction, HCD-rats (group 3) were performed intragastric administration of L-NAME $(10 \mathrm{mg} / \mathrm{kg}$, once daily, 5 days). Normal animals (group 1, control) and HCD-rats without LNAME (group 2) received $0.85 \%$ saline. At $48^{\text {th }}$ and $55^{\text {th }}$ day of $H C D$ blood samples were collected to assess platelet aggregation (PA), anticoagulant activity (APTT test), coagulation factor XIIIa (FXIIIa) and tissue plasminogen activator (t-PA) activities.

Results: As shown in Table 1 , at $48^{\text {th }}$ day HCD-rats had hypercoagulation (APTT decreased to $88 \%$ vs. control), increased values of PA and FXIIla (115\% and $120 \%$ vs. control, respectively) and impaired endothelial function (reduced t-PA activity to $72 \%$ vs. control), which persisted until the $55^{\text {th }}$ day of experiment. The L-NAME introduction further enhanced the blood clotting potential (Group 3): PA, APTT and FXIIla were 136,80 and $113 \%$ vs. HCD-rats, respectively. This was accompanied by a more pronounced decrease in endothelial function (t-PA - 68\% vs. control), which increased 7 days after discontinuation of L-NAME treatment (75\% vs. HCD- rats).
Conclusions: Thus, the proposed experimental model can be useful in the study of the hemostasis disorders that occur in metabolic diseases.

P0119 | The Role of Thromboelastography in Search for Drugcandidates

D. Lipatov; V. Korunas; K. Enikeeva; R. Kamilova; L. Bashirova;

I. Bashirov; A. Samorodov

Bashkir State Medical University, Ufa, Russian Federation

Background: To date, there are no strict recommendations for realization screening preclinical researches to estimate effects of new drug candidates on the hemostasis system.

Aims: In this work has demonstrated opportunities of thromboelastography as a screening method for remedies, which affecting the hemostasis system , for example $2 \times 10^{-3} \mathrm{M}$ acetylsalicylic acid (Shandong Xinghua Pharmaceutical Co., LTD, China).

Methods: The research was approved by the ethics committee of Bashkir State Medical University (№2 от 17.10.2012). Informed consent was obtained from all study participants before blood sampling. Modeling hyperactivity of hemostasis system was carried out in vitro by the method of activated thromboelastography on the blood of healthy male donors $(n=100)$ aged $18-24$ years. Thromboelastography was carried out on a TEG 5000 (Haemonetics Corp., Braintree, MA). As a TEG activator were used $0.2 \mathrm{M} \mathrm{CaCl}$, recombinant tissue factor (Innovin ${ }^{\circledR}$, Dade Behring, Germany) and thrombin ("Technologiya-Standart», Russia). In the analysis of thromboelastograms was determined the general tendency of coagulation $(R)$, the functional activity of platelets and fibrinogen (MA, Angle), the activity of fibrinolysis (CLT), and the physicomechanical properties of the formed clots $(\mathrm{G})$

TABLE 1 Hemostasis parameters in rat blood plasma on the 48th and 55th days of the experiment (M \pm SD)

\begin{tabular}{|c|c|c|c|c|c|}
\hline Parameters & Day of experiment & $\begin{array}{l}\text { Group } 1 \\
\text { Normal rats (control) } \\
(n=10)\end{array}$ & $\begin{array}{l}\text { Group } 2 \\
\text { HCD } \\
(n=10)\end{array}$ & $\begin{array}{l}\text { Group } 3 \\
\text { HCD + L-NAME } \\
(n=10)\end{array}$ & $P_{2-3}$-value \\
\hline \multirow[t]{2}{*}{ PA (\%) } & Day 48 & $100 \pm 4.8$ & $115 \pm 12^{*}$ & $157 \pm 18^{*}$ & $<0.05$ \\
\hline & Day 55 & $100 \pm 9.4$ & $124 \pm 14^{*}$ & $131 \pm 12^{*}$ & $<0.05$ \\
\hline $\operatorname{APTT}(\mathrm{s})$ & Day 48 & $31.5 \pm 6.9$ & $27.7 \pm 4.2^{*}$ & $22.3 \pm 2.2^{*}$ & $<0.05$ \\
\hline FXIIla (U/ml) & Day 55 & $65.0 \pm 10.8$ & $86.5 \pm 5.3^{*}$ & $112.4 \pm 8.0^{*}$ & $<0.05$ \\
\hline \multirow[t]{2}{*}{$\mathrm{t}-\mathrm{PA}\left(\mathrm{mm}^{2}\right)$} & Day 48 & $42.0 \pm 10.6$ & $30.2 \pm 4.0^{*}$ & $28.4 \pm 0.8^{*}$ & NS \\
\hline & Day 55 & $55.8 \pm 5.3$ & $33.4 \pm 4.2^{*}$ & $25.0 \pm 2.4^{*}$ & $<0.05$ \\
\hline
\end{tabular}

${ }^{*} P_{1}<0.05$ - significance of differences from Control (Group 1);

$\mathrm{p}_{2-3}$ - significance of differences between groups 2 and 3 , NS - not significant 
Results: The effect of aspirin is characterized by a significant decrease in the MA indicator. It is worth noting that the antiaggregatory activity of aspirin depends on the blood coagulation activator and is more effective when coagulation is activated by $0.2 \mathrm{M} \mathrm{CaCl}_{2}$ compared with thrombin and tissue factor.

Conclusions: Thus, the possibilities of thromboelastography as a screening method of affecting new pharmacological agents on the hemostasis system at the preclinical research stage with the possibility of modeling diseases in which there is an excess of thrombin or tissue factor.

\section{COAGULATION FACTORS AND INHIBITORS}

\section{LPB0006 | Recombinant Heparin: An Old Drug for the Modern World}

B.E. Thacker ${ }^{1} ;$ K. Thorne ${ }^{1}$; C. Cartwright ${ }^{1}$; J. Park ${ }^{1} ;$ K. Glass ${ }^{1}$; A. Chea ${ }^{1}$; J.D. Esko ${ }^{2}$; W. Jeske ${ }^{3}$; J.M. Walenga ${ }^{3}$; C.A. Glass ${ }^{1}$ ${ }^{1}$ TEGA Therapeutics, Inc., San Diego, United States; ${ }^{2}$ University of California, San Diego, La Jolla, United States; ${ }^{3}$ Loyola University Medical Center, Maywood, United States

Background: Although most biologics are produced using recombinant technologies, heparin persists as a product purified from animal tissues. A cell based system for production of heparin would eliminate risk of supply shortage and contamination. While heparin is produced uniquely by mast cells, all mammalian cells produce heparan sulfate, a closely related and non-anticoagulant polysaccharide, in a shared biosynthetic pathway. Production of recombinant heparin in mammalian cells requires engineering the heparan sulfate biosynthetic pathway, which consists of more than 20 biosynthetic enzymes, to produce material with anticoagulant activity.

Aims: This work is focused on engineering mammalian cell lines and bioprocess methods to produce recombinant heparin.

Methods: The heparan sulfate biosynthetic pathway of mastocytoma cells was genetically engineered to alter the expression of heparan sulfate sulfotransferases. The resulting cell lines were screened for production of anti-Xa activity. Heparan sulfate production from a candidate cell line in chemically defined medium was tested. The recombinant product was characterized structurally and in clotting and anti-protease assays.

Results: Overexpression of sulfotransferases in engineered mastocytoma cells produced heparan sulfate with anti-Xa $(297 \mathrm{U} / \mathrm{mg})$ and anti-Ila (311 U/mg) activity exceeding the USP requirement for unfractionated heparin despite having lower sulfate content. APTT activity was similar to unfractionated heparin. Chain length was longer than unfractionated heparin.

Conclusions: Genetic manipulation of the biosynthetic pathway yielded heparan sulfate with anticoagulant potency. These results demonstrate the feasibility of producing a substitute for unfractionated heparin from recombinant cell culture.
LPB0100 | Mapping Prothrombin Binding on Pseutarin C by Site-directed PEGylation

F.I. Ustok; J.A. Huntington

University of Cambridge, Cambridge Institute of Medical Research, Department of Haematology, Cambridge, United Kingdom

Background: Prothrombinase (Ptase) is the enzyme complex that converts prothrombin to thrombin. It is composed of factor ( $f$ ) $\mathrm{Va}$, and $\mathrm{fXa}$, assembled on the surface of negatively charged membranes in the presence of calcium ions. Pseutarin $\mathrm{C}$ is an orthologue of human (h) Ptase and processes prothrombin in the same way as hPtase, predominantly through initial cleavage at R320 (meizothrombin intermediate), even in the absence of membranes. The molecular basis of Ptase assembly and function is of central importance to understanding haemostasis.

Aims: To investigate the interaction between $\mathrm{fVa}$ and prothrombin by mutating residues within and outside the proposed binding site on Pseutarin $\mathrm{C}$ and assessing the effects on prothrombin processing. Methods: Residues on the $\mathrm{A} 1$ and $\mathrm{A} 2$ domains and a1-loop were mutated to Cys and modified with a polyethylene glycol (PEG) moiety. The complete a1-loop was also swapped with that of $h f \mathrm{~V}$ and $h \mathrm{VVIII}$ to assess its role in prothrombin recognition.

Results: As a control, Ser578, located in the fXa interface in Pseutarin C, was mutated to Cys and PEGylated. The Cys mutation had no effect on prothrombin processing, but PEGylation completely abolished activity. As predicted by our model, PEGylation of residues 134, 168 and 540 had no effect on thrombin generation, whereas 276, 302, 441 and 455 slowed thrombin generation by 7-, 12.6-, 408- and 12.4-fold, respectively. PEGylation of individual a1loop residues reduced the rate of thrombin generation to a similar extent. Swapping the a1-loop for that of $h$ fVIII reduced the rate by 6.6-fold. Swapping the a1-loop for that of $h f V$ increased the rate by 3.6-fold, and channeled processing exclusively through the meizothrombin intermediate, with no evidence of prethrombin-2.

Conclusions: These results support our proposed prothrombin binding site on Pseutarin C. The composition of the a1-loop plays a key role in prothrombin binding and in presentation of the R320 site for cleavage. 
LPB0101 | Nucleotides Inhibit Serine Proteases Involved in Blood Clotting and Other Cellular Processes

F. Birkle $^{1}$; Y. Wang ${ }^{2}$; N. Collins ${ }^{3} ;$ J.H. Morrissey ${ }^{1}$

${ }^{1}$ University of Michigan Medical School, Ann Arbor, United States;

${ }^{2}$ Bayer Pharmaceuticals (formerly at University of Illinois), San

Francisco, United States; ${ }^{3}$ University of Michigan, Ann Arbor, United

States

Background: Recent studies have shown that physiological cytosolic ATP concentrations ( 1-10 mM) enhance the stability of many intracellular proteins and help keep them soluble. Similar nucleotide concentrations are present in the endoplasmic reticulum (ER) and Golgi. We hypothesize that low mM concentrations of ATP and other nucleotides in the ER and Golgi lumen could stabilize nascent serine proteases (especially, any prematurely activated zymogens) by inhibiting their enzymatic activity prior to secretion.

Aims: Investigate how physiologic concentrations of nucleotides effect serine protease activity.

Methods: We screened 12 serine proteases involved in different cellular processes including blood clotting (tissue factor-factor VIla, factor Xa, factor Xla, factor Xlla, kallikrein, thrombin), fibrinolysis (plasmin, urokinase, tissue plasminogen activator) and digestion (trypsin, chymotrypsin). Enzymatic activities were measured in the presence of nucleotides (including ADP, ATP, UTP, GTP, CTP). Surface Plasmon Resonance (SPR) with active site-inhibited serine proteases was used to determine $K_{D}$ values for ATP binding.

Results: Low mM concentrations of ATP or ADP strongly inhibited the activity of most serine proteases in our screen. UTP, GTP and CTP showed protease inhibition comparable to ATP. IC ${ }_{50}$ for inhibition of serine proteases by ATP ranged from 2-11 mM. In-depth kinetic studies for one of the proteases (tissue factor-factor VIla) revealed that ADP is an uncompetitive inhibitor, with $K_{i}=0.7-2.5$ $\mathrm{mM}$. SPR experiments showed cooperative binding of ATP to serine proteases with $\mathrm{K}_{\mathrm{D}}=16-23 \mathrm{mM}$ and Hill coefficients $=1.5-1.8$.

Conclusions: Our kinetic studies indicate that physiologic concentrations of nucleotides strongly inhibit multiple secreted serine proteases. SPR studies suggest a cooperative binding mode, with more than one ATP binding the protease. Inhibition of serine proteases by nucleotides could serve as a cellular safety mechanism to keep any prematurely activated proteases in the lumen of the ER and Golgi quiescent prior to secretion.
PB0038 | Generation of Anticoagulant RNA Aptamers Targeting Cofactors that Inhibit Clotting in Normal and COVID-19 Patient Samples

H. Yu ${ }^{1}$; E. Soule ${ }^{1}$; L. Olson ${ }^{1}$; I. Naqvi ${ }^{1}$; S. Kumar ${ }^{2}$; S. Krishnaswamy ${ }^{2}$; B. Sullenger ${ }^{1}$

${ }^{1}$ Duke University, Pharmacology and Cancer Biology, Durham, United States; ${ }^{2}$ Children's Hospital of Philadelphia, University of Pennsylvania, Philadelphia, United States

Background: Coagulation cascade cofactors are essential proteins that control homeostasis and are appealing targets for anticoagulants. However, targeting such proteins has been challenging due to their lack of an active site.

Aims: Development of FV/FVa and FVIII-inhibiting aptamers as potent and reversible anticoagulants that can be used to aid (or replace) heparin for therapeutic anticoagulation. Reveal new mechanisms of cofactor inhibition.

Methods: 2'F modified RNA Aptamers were identified via in vitro systematic evolution of ligand by exponential enrichment (SELEX) and post-SELEX truncation. Aptamer affinity was characterized by nitrocellulose filter binding assays and surface plasmon resonance. Aptamer bioactivity was analyzed using clotting assays and biochemical assays. Aptamers' binding mechanism was determined using fluorescent anisotropy, fluorescence resonance energy transfer, dynamic light scattering, and molecular modeling.

Results: An aptamer termed T18.3 binds to both human FV/FVa with $\mathrm{K}_{\mathrm{D}} \sim 10 \mathrm{nM}$ and prolongs aPTT in normal human plasma by 3.5 -fold at a concentration of $500 \mathrm{nM}$. The aptamer inhibits membrane docking of FVa by binding to FV/FVa light chain, thereby reduces prothrombinase assembly and thrombin generation. Notably, the aptamer showed similar anticoagulant potency in normal, FV Leiden, and COVID-19 patient plasma. The aptamer also synergistically inhibits clotting with enoxaparin and can be rapidly reversed by protamine in in vitro assays. Another aptamer termed F8-3.1 binds to human FVIII with a $\mathrm{K}_{\mathrm{D}}$ of $0.67 \mathrm{nM}$ and prolongs aPTT in normal human plasma by 1.9-fold at a concentration of $500 \mathrm{nM}$. F8-3.1 also has full cross-reactivity to canine FVIII $\left(K_{D}=0.68 \mathrm{nM}\right)$ and significantly prolongs the aPTT in canine and porcine plasma.

Conclusions: The work describes the generation of aptamers targeting FV/FVa and FVIII that can achieve clinically relevant anticoagulant activity. These results not only demonstrate the feasibility of using cofactor-binding aptamers as therapeutic anticoagulants but also reveal a novel mechanism of aptamer-mediated protein inhibition by interrupting protein-membrane interactions. 
PB0039 | The FV East Texas Variant Causes Bleeding in a Three Generation Family from Indiana

J. Peterson ${ }^{1}$; S. Gupta ${ }^{2}$; N. Martinez ${ }^{1}$; A. Mast ${ }^{1}$

${ }^{1}$ Versiti Blood Research Institute, Milwaukee, United States; ${ }^{2}$ Indiana

Hemophilia and Thrombosis Center, Indianapolis, United States

Background: The FV east Texas (FVET) bleeding disorder produces a 5- to 10-fold increase in the Tissue Factor Pathway Inhibitor-alpha (TFPla) plasma concentration. It is caused by a FV B-domain mutation producing FVshort. The TFPla C-terminus has homology with the FV B-domain basic region and tightly binds the FVshort $\mathrm{B}$-domain acidic region in FVET patients. The rapid inhibition of prothrombinase by TFPla bound to FVshort may be the primary anticoagulant mechanism causing bleeding in FVET patients.

Aims: Characterize three generations of an extended family who presented with variable bleeding and laboratory phenotypes.

Methods: Plasma TFPla was measured by ELISA, Western blot, and calibrated automated thrombography. The FV B-domain was sequenced.

Results: Over 13 years, a proband (male) presented with undiagnosed mild mucocutaneous bleeding symptoms, and significant post-surgical and post-traumatic hemorrhage, treated with rFVIla due to failure of universal hemostatic agents including DDAVP and antifibrinolytics. Similar bleeding was present in eight family members across three generations. The only abnormality in the hemostatic evaluation of the proband and some symptomatic family members was variably prolonged PT and aPTT tests. The proband and all symptomatic family members carried the FVET mutation, indicating complete penetrance of the mutation, and had 5- to 10-fold elevated plasma TFPIa. Thrombin generation in patient plasma was undetectable in reactions initiated with 1 pM TF but was restored to normal upon addition of a monoclonal antibody specific for the $\mathrm{K} 2$ domain of TFPI.

Conclusions: The FVET bleeding disorder was diagnosed in an extended Indiana family, genetically unlinked to the original east Texas pedigree. Evaluation of rare bleeding disorders should include measurement of plasma TFPla and DNA sequencing of FV B-domain. In vitro thrombin generation assays suggest that antibodies directed against the TFPI K2 domain, which are in development for hemophilia prophylaxis, may be therapeutically effective in FVET patients or others with mutations producing FVshort.
PB0040 | Reversal of Oral Anticoagulants: A Survey of Contemporary Practice Trends (ReACT)

H. Deng $^{1}$; R. DiDomenico ${ }^{2,3}$; E. Nutescu ${ }^{2,3}$

${ }^{1}$ Department of Pharmacy Systems, Outcomes and Policy, University of Illinois at Chicago, Chicago, United States; ${ }^{2}$ Department of Pharmacy

Practice, University of Illinois at Chicago, Chicago, United States;

${ }^{3}$ Center for Pharmacoeconomic \& Pharmacoepidemiology Research, University of Illinois at Chicago, Chicago, United States

Background: Inconsistencies exist in current guideline recommendations of oral anticoagulant reversal.

Aims: This study aims to evaluate current practice patterns and factors influencing treatment decisions when reversing oral anticoagulants.

Methods: A three-section survey with 27 closed and open-ended questions was electronically distributed to anticoagulation specialists of the Anticoagulation Forum. Questions were designed to capture practice trends in urgent or emergent reversals of warfarin, factor-Xa inhibitors, and factor-lla inhibitors. Continuous and categorical data were analyzed to generate descriptive statistics and compared with current guideline recommendations. Data for openended questions were summarized by thematic categories.

Results: 173 responses were collected, $77.8 \%$ were pharmacists, 76.1\% were direct patient care providers, and $84.3 \%$ were based in the US. Among various reversal agents, vitamin $\mathrm{K}(91.3 \%)$, activated charcoal (80.4\%), and plasma (72.8\%) were mostly reported as on formulary without restrictions; $87.0 \%$ reported four-factor prothrombin complex concentrate (4F-PCC) on formulary either with (52.2\%) or without (34.8\%) restrictions. Although 4F-PCC and andexanet alfa are suggested by current guidelines as first-line agents to reverse warfarin and factor-Xa inhibitors, respectively, vitamin $\mathrm{K}$ (98.8\%) was the most commonly used reversal agent for warfarin and 4F-PCC (71.6\%) for factor-Xa inhibitors. Weight-based dosing for 4F-PCC, as recommended by guidelines, is utilized only in $59.4 \%$, $41.2 \%$, and $44.4 \%$ of warfarin, factor-Xa inhibitor, and factor-lla inhibitor reversals, respectively. Cost, efficacy, and safety of 4F-PCC relative to other reversal agents were identified as both top facilitators and barriers for 4F-PCC adoption in practice. The majority of the respondents' institutions (90.2\%) utilized a guideline or protocol for urgent or emergent reversal of oral anticoagulants. 
TABLE 1 First-line Agents and 4F-PCC Dosages used in Oral Anticoagulant Reversal*

Reversal of warfarin

$(N=83)$

First-line agents

4F-PCC dosages

Vitamin K (98.8\%)
4F-PCC (61.5\%)
Plasma (12.2\%)
Activated charcoal (6.0\%)
Recombinant factor-VIIa (2.4\%)
PCC3 (2.4\%)

Weight-based dosage according to INR (59.4\%)

Other $^{* *}(26.6 \%)$

Fixed dose: 1,500 units for all (15.6\%)

Fixed dose: 1,500 units for $\mathrm{ICH}$, 1,000 units for all other (10.9\%) Fixed dose: 1,000 units for all (3.1\%)

Fixed dose: 500 units for all (1.6\%)
Reversal of factor-Xa inhibitors Reversal of factor-lla inhibitors

$(N=81) \quad(N=80)$

4F-PCC (71.6\%)
Activated Charcoal (28.4\%)
Andexanet Alfa (19.75\%)
Plasma (6.2\%)
PCC3 (2.5\%)

Idarucizumab (82.5\%)

Activated Charcoal (23.8\%)

4F-PCC (7.5\%)

Plasma (6.25\%)

PCC3 (3.8\%)

FEIBA (1.2\%)

Weight-based dosage 50 units/kg (41.2\%)

Other $^{* * *}(25.0 \%)$

Fixed dosing: 2000 units for all (20.6\%)

Weight-based dosage 25 units/kg (8.8\%)

Fixed dose: 1,500 units for $\mathrm{ICH}, 1,000$ units for all other $(7.4 \%)$

Fixed dose: 500 units for all (2.9\%)

Fixed dose: 1,500 units for

all (1.5\%)

Fixed dose: 1,000 units for

all $1.5 \%)$

"More than one option could be selected by the respondents

** Other dosages for warfarin reversal: weight-based doses for ICH and fixed doses for other indications, other weight-based dosages according to INR, or fixed dosages with a different dose

*** Other dosages for factor-Xa reversal: weight-based doses for ICH and fixed doses for others, or other weight-based dosages according to INR

**** Other dosages for factor-lla reversal: weight-based dosage 25 units $/ \mathrm{kg}$, or 2000 units for all

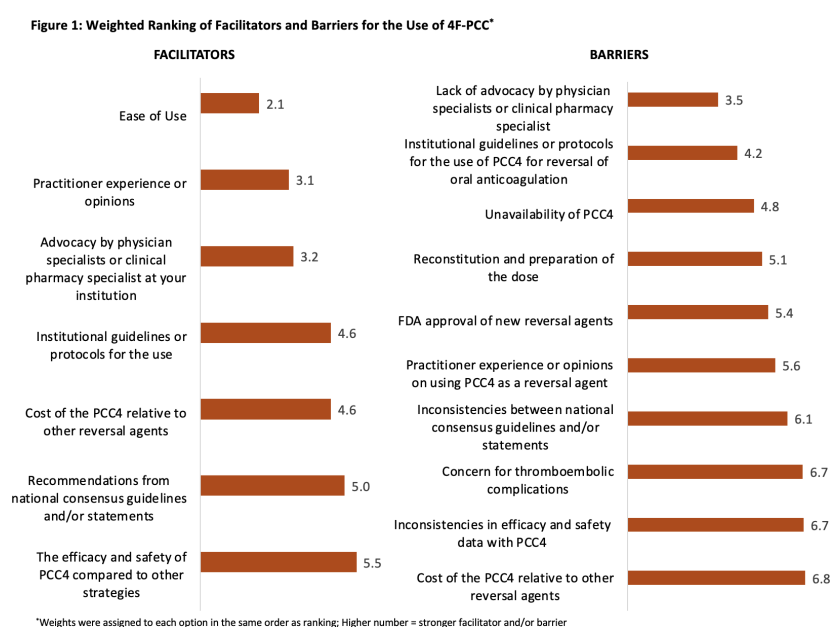

FIGURE 1 Weighted Ranking of Facilitators and Barriers for the Use of $4 \mathrm{~F}-\mathrm{PCC}$
Conclusions: Our findings revealed that guideline recommendations for reversal of warfarin and factor-Xa inhibitors are not followed by a sizable proportion of institutions. Additional studies with a larger sample size are needed to confirm evolving reversal practice patterns.

PB0041 | Reversal of Antithrombotic Effects of FXla Inhibitor Milvexian (BMS-986177/JNJ-70033093) by Non-specific Agents in a Rabbit AV-shunt Model of Thrombosis

X. Wang; Q. Li; F. Du; J. Caruso; A. Nawrocki; M. Chintala Janssen Pharmaceuticals of JNJ, Spring House, United States

Background: Factor Xia (FXla) inhibition is a promising therapeutic approach with the potential for an improved therapeutic index over currently approved oral anticoagulants. However, reversal of anticoagulation will be required in situations of unexpected major bleeding or for emergency surgery. 
Aims: Milvexian is a novel small molecule inhibitor of FXla currently in Phase II clinical trials. This study evaluated the feasibility of nonspecific reversal agents and recombinant activated factor VII to reverse the antithrombotic effects of milvexian in a rabbit model of arterial-venous (AV)- shunt thrombosis.

Methods: The AV-shunt model was conducted in anesthetized rabbits. Based on a prior efficacy dose-response study, the milvexian dose of $4.0 \mathrm{mg} / \mathrm{kg}$ bolus $+2.68 \mathrm{mg} / \mathrm{kg} / \mathrm{h}$ infusion was selected and various doses of FEIBA (0, 5, 25, 50 and 200 Units/kg), Kcentra (0, $5,15,50$ units $/ \mathrm{kg})$ or NovoSeven $(10,30,90 \mu \mathrm{g} / \mathrm{kg})$, iv, provided 5 min prior to initiation of the AV-shunt were evaluated. Thrombus weights served as the primary efficacy end-point. Blood was collected to measure activated partial thromboplastin time, prothrombin time, thrombin time, and plasma concentration of milvexian.

Results: Milvexian significantly reduced thrombus weight in the rabbit AV-shunt model, with $71.3 \pm 5.2 \%(P<0.001, n=8)$ inhibition vs. vehicle. The antithrombotic effect of milvexian in this model was completely abolished by 25 units/kg FEIBA (222\% increase vs. vehicle in thrombus weight) or $30 \mathrm{\mu g} / \mathrm{kg}$ NovoSeven (64.3\% increase), but only partially reversed by Kcentra (at the highest dose of 50 units $/ \mathrm{kg}$, with $58.6 \%$ inhibition). Plasma levels of milvexian were not altered in the presence of these non-specific reversal agents.

Conclusions: Our studies demonstrated that either FEIBA or NovoSeven but not Kcentra could effectively reverse the antithrombotic effects of BMS-177/JNJ-3093 in the rabbit AV-shunt model.

PB0042 | Polycationic Molecules Towards the Inhibition of Procoagulant and Antifibrinolytic Properties of Nucleic Acids

S. Vappala $^{1,2,3}$; M.T. Kalathottukaren ${ }^{1,2,3}$; C. La ${ }^{1,2,4} ;$ S. Abbina ${ }^{1,2,3}$; L. Abraham ${ }^{1,5}$; I. Constantinescu ${ }^{1,2,3}$; E. M. Conway ${ }^{1,2,6}$; C. A Haynes $^{1,2,7}$; J. N. Kizhakkedathu ${ }^{1,2,3,8}$

${ }^{1}$ University of British Columbia, Vancouver, Canada; ${ }^{2}$ Centre for Blood Research, Vancouver, Canada; ${ }^{3}$ Department of Pathology and Laboratory Medicine, Vancouver, Canada; ${ }^{4}$ Department of Chemistry, Vancouver, Canada; ${ }^{5}$ Microbiology and Immunology, Vancouver, Canada; ${ }^{6}$ Department of Medicine, Vancouver, Canada; ${ }^{7}$ Chemical and Biological Engineering, Vancouver, Canada; ${ }^{8}$ School of Biomedical Engineering, Vancouver, Canada

Background: Nucleic acids (polyanionic molecules) including, cellfree DNA, RNA, and the assembly of Neutrophil Extracellular Traps (NETs) are indicated as mediators of thrombosis. They are shown to promote thrombosis post-chemotherapy treatment and in sepsis. Besides, these polyanionic molecules are also found to inhibit clot lysis and alter clot structure. Targeting these mediators instead of key coagulation enzymes could potentially inhibit thrombosis and may lead to safer antithrombotics. Furthermore, this strategy could improve the outcomes of thrombolysis where nucleic acids are involved.
Aims: Screen a library of polycationic molecules (PNBIs) and identify safe and effective candidates that target polyanionic nucleic acids and reverse their accelerated blood clotting potential in vitro.

Methods: Thrombin generation was measured using Stago Thrombinoscope. FITC labeled nucleic acid was used to study its binding to the Alexa-546 labeled fibrin clot. Plasma clot lysis assay was performed in the presence of thrombin $\mathrm{CaCl}_{2}$ and tPA.

Results: We identified lead candidates with optimal neutralization potential for nucleic acids using plasma clotting and thrombin generation assays. PNBI inhibited thrombin generation triggered by nucleic acids (Fig. 1). We also found PNBIs inhibit nucleic acid-mediated FXII activation in plasma. PNBI's also inhibited the binding of FITC labeled poly I: $\mathrm{C}$ to the fibrin clot and normalize the clot structure. From the clot lysis studies, we found that PNBIs reverse the antifibrinolytic potential of nucleic acids (Fig. 2). Fluorescently labeled PNBls are seen to bind NETs and potentially inhibit thrombin generation.

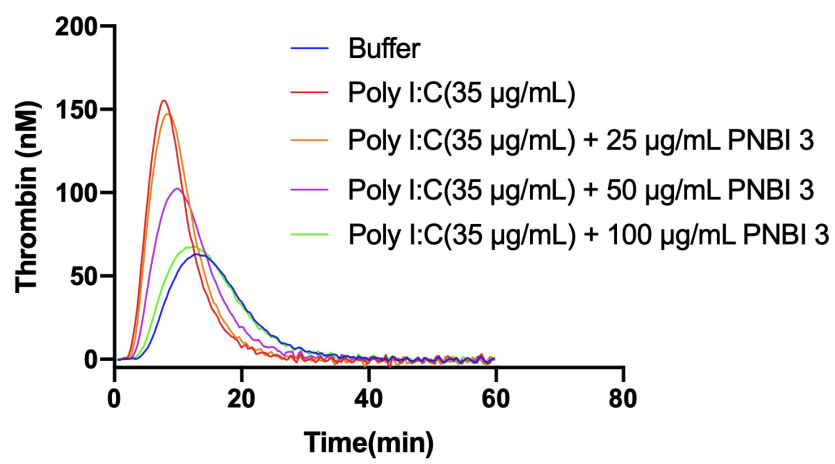

FIGURE 1 Inhibition of thrombin generation triggered by Poly I: C by PNBI 3 in plasma

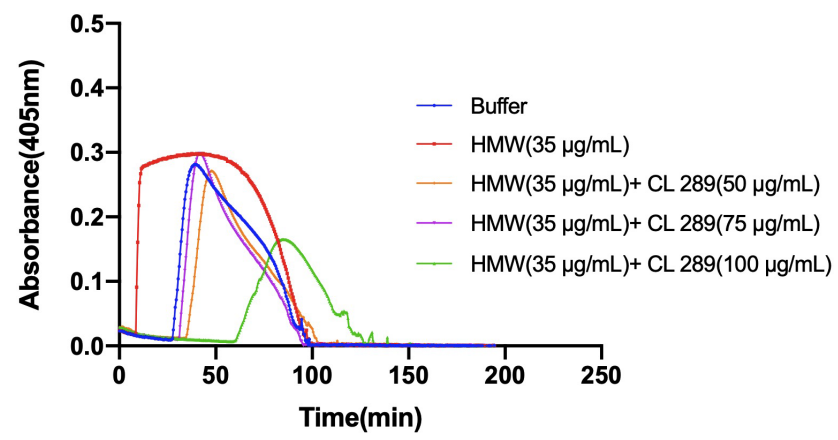

FIGURE 2 Improvement of Plasma clot lysis by PNBI 3 in plasma clot lysis assay (Representative data)

Conclusions: Our results demonstrate that PNBIs can reverse the procoagulant potential of nucleic acids- making them a potential antithrombotic agent. The strategy of targeting accelerators rather than coagulation enzymes offers a pathway to develop safer antithrombotic agents. PNBIs also enhance the effectiveness of tPAmediated clot lysis attributed to nucleic acids. Studies are ongoing to 
determine the efficacy of PNBIs in inhibiting thrombosis in relevant animal models.

PB0043 | Management of Intentional Low Molecular Weight Heparin Overdose: The McMaster University Experience

C. Lu $^{1}$; M.A Crowther ${ }^{1}$; P.L Gross ${ }^{1,2}$; S.M Bates ${ }^{1,2}$; S. Mithoowani ${ }^{1}$

${ }^{1}$ McMaster University, Hamilton, Canada; ${ }^{2}$ Thrombosis and

Atherosclerosis Research Institute, Hamilton, Canada

Background: Low molecular weight heparin (LMWH) overdose is a rare entity that may result in serious bleeding. There is no consensus on optimal management.

Aims: We explore management strategies for LMWH overdose.

Methods: We report four cases of intentional dalteparin overdose involving two patients and review the literature on $\mathrm{LMWH}$ overdose in adults. Results: The first patient was a 42-year-old man who self-injected 225,000 units of dalteparin with intent to self-harm. Eighteen hours later, he developed left arm anterior compartment syndrome attributed to traumatic venipuncture and required urgent fasciotomy. Peak LMWH anti-Xa level was $8.94 \mathrm{U} / \mathrm{mL}$ with activated partial thromboplastin time (aPTT) $>150 \mathrm{~s}$. He was treated with five doses of protamine sulfate $50 \mathrm{mg}$ intravenously. Serial LMWH anti-Xa levels demonstrated a transient biochemical response to protamine sulfate. Anti-Xa levels remained elevated despite normalization of aPTT (Figure 1). After recovery, he was discharged on a direct oral anticoagulant with Thrombosis and Psychiatry follow-up.

The second patient was a 39-year-old woman who on three separate occasions spanning 10 months, self-injected between 225,000-360,000 units of dalteparin with intent to self-harm. Peak LMWH anti-Xa levels were 7.33$10.0 \mathrm{U} / \mathrm{mL}$ with initial aPPTs $>150 \mathrm{~s}$. On two occasions, she was treated with empiric protamine sulfate with no bleeding complications and subsequent normalization of coagulation parameters (Figure 2, third episode). We identified 10 case reports describing 16 cases of LMWH overdose. One patient died and five had major bleeding. Protamine sulfate, tranexamic acid and/or recombinant factor VIla were used to treat most overdoses.

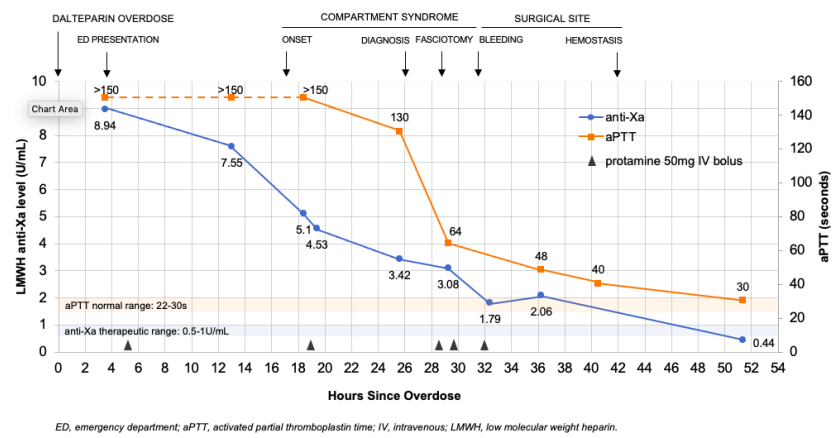

FIGURE 1 Clinical and biochemical timeline for patient 1

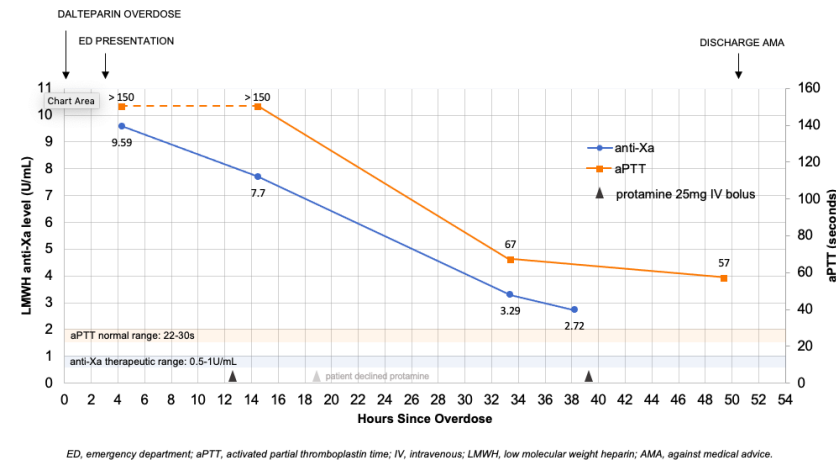

FIGURE 2 Clinical and biochemical timeline for patient 2, third episode

Conclusions: Pharmacokinetics and bleeding complications in LMWH overdose can be unpredictable. Our experience supports the use of empiric protamine sulfate to both prevent and treat bleeding. Serial LMWH anti-Xa activity measurements can help guide therapy. Patients often have complex medical and psychiatric comorbidities complicating the decision to resume anticoagulation following LMWH overdose.

PB0044 | Factor Xla Inhibitor Milvexian (BMS-986177/JNJ70033093) Suppresses Plasma Clot Formation and Reduces Resistance to Fibrinolysis in vitro

L.A. Holle ${ }^{1}$; J.M. Luettgen ${ }^{2}$; A.S. Wolberg ${ }^{1}$

${ }^{1}$ University of North Carolina, Department of Pathology and

Laboratory Medicine, Chapel Hill, United States; ${ }^{2}$ Bristol Myers Squibb, Lawrenceville, United States

Background: Venous thromboembolism is a leading cause of death and disability worldwide. Targeting factor XI (FXI) may prevent thrombosis without increasing bleeding risk. Milvexian (formerly referred to as BMS-986177/JNJ-70033093) is an orally-bioavailable, small molecule that inhibits FXla with high affinity and selectivity. In preclinical models, milvexian has shown antithrombotic activity while preserving hemostasis, and in healthy humans appears safe and well-tolerated.

Aims: Characterize the pharmacodynamic effect of milvexian on plasma clot formation and stability in vitro.

Methods: Clot formation in normal pooled plasma and in plasmas from 5 healthy individuals was initiated in the presence of tissue factor, phospholipids, $\mathrm{CaCl}_{2}$, and varying concentrations of milvexian or buffer. Fibrinolysis experiments included tissue plasminogen activator. Reactions were monitored by turbidity. Descriptive parameters were calculated and analyzed to yield concentrations exhibiting half-maximal effects.

Results: Milvexian delayed the onset of clot formation and time to the turbidity plateau or peak, and reduced the rate of clot formation in a dose-dependent manner. Milvexian also reduced the final clot turbidity in clot formation assays and the area under the curve in fibrinolysis assays, suggesting reduced fibrin incorporation into the clots. Milvexian also shortened the clot lysis time, indicating decreased resistance of 
clots to fibrinolysis. Effects were seen within dose ranges achieved following oral administration in humans, demonstrating pharmacodynamic effects at pharmacologically-relevant concentrations. For most parameters, half-maximal effects on clot formation and stability were achieved between 3-4 $\mathrm{\mu g} / \mathrm{mL}$ milvexian. Anticoagulant effects were observed at levels below functional thresholds previously associated with bleeding risk in FXI-deficient patients.

Conclusions: Milvexian delays the time to clot formation, decreases fibrin incorporation into the clot, and reduces the resistance of clots to fibrinolysis. These findings reveal the mechanism of action for a FXla active-site inhibitor and generate hypotheses for how to achieve antithrombotic activity without increasing bleeding risk.

PB0045 | D-dimer and the Risk of Diabetes: The REasons for Geographic and Racial Differences in Stroke (REGARDS)

D. Kamin Mukaz ${ }^{1}$; D.L. Long ${ }^{2}$; T.B. Plante ${ }^{1}$; S.E. Judd ${ }^{2}$; L.A. McClure ; A.S. Wolberg ${ }^{4}$; A.P. Carson ${ }^{2}$; B. Guo ${ }^{2}$; M. Cushman ${ }^{1}$

${ }^{1}$ University of Vermont, Burlington, United States; ${ }^{2}$ University of Alabama at Birmingham, Birmingham, United States; ${ }^{3}$ Drexel

University, Philadelphia, United States; ${ }^{4}$ University of North Carolina at Chapel Hill, Chapel Hill, United States

Background: Diabetes is the seventh leading cause of death in the U.S, yet much remains unknown about its pathogenesis. D-dimer, a fibrin degradation product, is higher in Black individuals and women. Higher concentrations are also associated with risk of several chronic diseases. We hypothesized that higher D-dimer is associated with increased diabetes risk.

Aims: We aimed to determine the association of baseline D-dimer with incident diabetes in a biracial cohort study, and to identify differences in the association by race or gender.

Methods: The study included 3235 participants of the REasons for Geographic And Racial Differences in Stroke (REGARDS) cohort. All were diabetes-free at baseline, and they were followed for a median of 9.5 years for diabetes. Risk ratios of diabetes by baseline D-dimer were estimated, and interactions between D-dimer and race, and between D-dimer and gender were tested.

Results: About half of participants were women and $44 \%$ were Black; 434 developed diabetes over follow up (205 women, 229 men, 266 Black participants and 168 White participants). The Table shows for modeling results adjusting for age, gender, race, education and income, higher D-dimer was not associated with diabetes incidence; however, in the fully adjusted model, the risk was lower in the highest compared to the lowest quartile. The association differed by gender $(P$-interaction $\leq 0.05)$ but not race $(P$-interaction $>0.10)$. In women, higher D-dimer was modestly associated with increased incidence of diabetes, but this was attenuated after full adjustment for risk factors (model 2). In men, D-dimer in the highest compared to the lowest quartile was associated with a $43 \%$ lower risk of diabetes in the fully adjusted model.

TABLE 1 Association of D-dimer and incident diabetes across quartiles of D-dimer. Gender-stratified models shown due to a significant interaction of D-dimer*gender

$\begin{array}{lllll}\text { Q1 } & \text { Q2 } & \text { Q3 } & \text { Q4 } & \\ 0.01-0.27 \mu \mathrm{g} / \mathrm{ml} & 0.27-0.39 \mu \mathrm{g} / \mathrm{ml} & 0.39-0.61 \mu \mathrm{g} / \mathrm{ml} & 0.61-20.1 \mu \mathrm{g} / \mathrm{ml} & P \text {-value for trend }\end{array}$

\begin{tabular}{|c|c|c|}
\hline \multicolumn{3}{|l|}{ All } \\
\hline \multicolumn{3}{|c|}{434 cases/3235 at risk } \\
\hline Model 1 & 1.0 & $1.06(0.82$ \\
\hline Model 2 & 1.0 & $0.96(0.74$ \\
\hline \multicolumn{3}{|l|}{ Women } \\
\hline \multicolumn{3}{|c|}{205 cases/1632 at risk } \\
\hline Model 1 & 1.0 & $1.11(0.74$ \\
\hline Model 2 & 1.0 & $0.98(0.65$ \\
\hline \multicolumn{3}{|l|}{ Men } \\
\hline \multicolumn{3}{|c|}{229 cases/1603 at risk } \\
\hline Model 1 & 1.0 & $1.04(0.74$ \\
\hline Model 2 & 1.0 & $0.94(0.67$ \\
\hline
\end{tabular}


Conclusions: Among men but not women, higher D-dimer was associated with lower risk of incident diabetes. Future studies are needed to understand biological mechanisms of this unexpected finding.

PB0046 | Induced Forms of $a_{2}$-Macroglobulin Neutralize Heparin and Direct Oral AntiCoagulant Effects

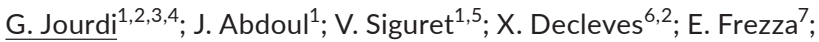
C. Pailleret ${ }^{1,8}$; I. Gouin-Thibault ${ }^{9,10} ;$ S. Gandrille ${ }^{1,11}$; N. Neveux ${ }^{2}$; C.-M. Samama ${ }^{1,2}$; S. Pasquali ${ }^{7}$; P. Gaussem ${ }^{1,11}$

${ }^{1}$ Université de Paris, Innovative Therapies in Haemostasis, INSERM UMR_S 1140, Paris, France; ${ }^{2}$ AP-HP, Centre-Université de Paris, Hôpital Cochin, Paris, France; ${ }^{3}$ Montreal Heart Institute, Montreal, Canada; ${ }^{4}$ Montreal University, Montreal, Canada; ${ }^{5}$ AP-HP, Nord-Université de Paris, Hôpital Lariboisière, Paris, France; ${ }^{6}$ Université de Paris, Variabilité de Réponse aux Psychotropes, INSERM UMR_S 1144, Paris, France; ' Laboratoire CiTCoM, Université de Paris, CNRS, Paris, France; ${ }^{8}$ Clinique du Mont Louis, Paris, France; ${ }^{9}$ Laboratoire d'Hématologie, CHU Pontchaillou, Rennes, France; ${ }^{10}$ Université de Rennes 1, CICInserm 1414, Rennes, France; ${ }^{11}$ AP-HP, Centre-Université de Paris, Hôpital Européen Georges Pompidou, Paris, France

Background: Specific antidotes are already commercialized or in development for some anticoagulant compounds while they are still lacking for others. As such, no universal antidote to direct oral anticoagulants (DOAC) and heparins is yet available. Such antidote would make it easier for healthcare professionals to manage anticoagulant compounds in case of major bleeding or urgent procedures. Aims: We investigated the mechanism and ability of Gla-domainless factor Xa (GDFXa)- and methylamine (MA)-induced $a_{2}$-Macroglobulin $\left(a_{2} M\right)$, to restore coagulation in presence of anticoagulants in vitro, ex vivo and in vivo.

Methods: Induced forms of $a_{2} M$ were prepared and characterized by chromogenic assays, electrophoresis, coagulation assays and immunonephelometry. Samples from healthy volunteers and anticoagulated patients with atrial fibrillation or venous thromboembolism who gave their written informed consent, were included. In vivo neutralization of anticoagulants was evaluated in C57BI/6JRj mouse bleeding model. Clot waveform assay and rotational thromboelastometry were triggered in plasma and whole blood, respectively. Binding sites of anticoagulants on induced $a_{2} M$ were depicted by computer-aided energy minimization modeling.

Results: GDFXa-induced $\mathrm{a}_{2} \mathrm{M}$ neutralized anticoagulants in plasma and whole blood samples spiked with DOAC (up to $600 \mathrm{ng} / \mathrm{mL}$ ) or heparins (up to $1.5 \mathrm{IU} / \mathrm{mL}$ ). In mouse, a single IV dose of GDFXainduced $a_{2} \mathrm{M}$ given following anticoagulant administration significantly reduced blood loss and bleeding time. Being easier to prepare and less expensive than GDFXa-induced $a_{2} M$, we investigated the efficacy of MA-induced $a_{2} M$. Molecular docking analysis evidenced that MA-induced $\mathrm{a}_{2} \mathrm{M}$ binds non-covalently (hydrogen bonds) to DOAC and heparins via some deeply buried binding sites. MAinduced $\mathrm{a}_{2} \mathrm{M}$ neutralized anticoagulants in spiked plasma samples in a concentration-dependent manner and in samples from 57 patients receiving DOAC or heparins.

Conclusions: Induced forms of $\mathrm{a}_{2} \mathrm{M}$ have the potential to neutralize a wide range of anticoagulant drugs and might be developed as a universal antidote in case of major bleeding or urgent surgery in anticoagulated patients.

PB0047 | Comparing the Real-world and Clinical Trial Bleeding Rates Associated with Anticoagulation Treatment for Atrial Fibrillation

R. Buderi' ${ }^{1}$; A. Yiं ${ }^{2}$; P. Allen ${ }^{1}$; T. Bebi ${ }^{1}$; C. Vigna ${ }^{1}$; R. Jain ${ }^{1}$;

A. Majumdar ${ }^{1}$; D. Bloomfield ${ }^{2}$; J. Salter ${ }^{2}$; D. Freedholm²;

A. Galaznik ${ }^{1}$

${ }^{1}$ Medidata Solutions, a Dassault Systèmes Company, Boston, United States; ${ }^{2}$ Anthos Therapeutics, Cambridge, United States

Background: In clinical trials, DOACs were as effective as warfarin in preventing stroke with improved safety profiles in patients with atrial fibrillation (AF).

Aims: The main objective of the study was to compare the bleeding rates among patients that receive DOACs in the clinical trial (CT) setting relative to the real world (RW) setting.

Methods: CT data from five recent open-label industry sponsored AF trials was compared with RW data from HealthVerity PS20 commercial and Medicare insurance claims. AF patients with DOAC use in both the databases were assessed for baseline demographic and clinical characteristics. Data on all bleeding events were collected and classified as 'major' or 'minor' based on RW criteria from published literature. The bleeding incidence rates within 12 months after treatment initiation were calculated and time-to-first bleeding event was stratified by subgroups.

Results: Relative to the patients in the CT $(n=3,207)$ database, patients in the RW $(n=61,214)$ were older (RW vs CT: $72.2 \pm 11.9$ vs $65.3 \pm 10.7$ ), with a higher percentage male ( $71 \%$ vs $53 \%$ ) and a higher baseline risk of bleeding (HAS-BLED scores: $2.13 \pm 1.02$ vs $1.04 \pm 0.93)$ and stroke $\left(\mathrm{CHA}_{2} \mathrm{DS}_{2}\right.$-VASc score: $3.98 \pm 1.9$ vs $2.87 \pm$ 1.7). In the RW setting, patients had higher 12-month post treatment major bleeding (18.97 vs 10.69 per 100 person-years), minor bleeding ( 51.55 vs 30.58 per 100 person-years) and any bleeding (59.30 vs 40.32 per 100 person-years) incidence rates. Similar patterns were observed when stratified by age and relevant medical histories.

Conclusions: AF patients in a RW setting have a higher risk profile with respect to age, gender, comorbidities, and the baseline risk of stroke and bleeding than AF patients studied in a CT setting. This correlates with a significantly higher 12-month rates of bleeding events. CT data may underestimate the burden of bleeding in RW patients. 
PB0048 | Sensitivity Analysis of a Reduced Model of

Thrombosis under Flow: Roles of Factor IX, Factor XI, and $\gamma$ '-Fibrin

J. Chen; S.L. Diamond

University of Pennsylvania, Philadelphia, United States

Background: By including thrombin feedback activation of FXI and the antithrombin-I activities of fibrin, a previously developed reduced model of flowing whole blood clotting on collagen/tissue factor was able to predict the rate of fibrin generation and the thrombin flux from a growing clot in the presence and absence of fibrin.

Aims: To explore the sensitivity of a reduced extrinsic pathway model for clotting in a 15-micron thick platelet deposit.

Methods: We performed the local sensitivity analysis where each of 5 plasma zymogens and 2 fibrin binding sites for thrombin were varied $\pm 50 \%$ from their published value. We conducted 10,000 Monte Carlo simulations where 7 variables were changed simultaneously and independently exploring variations in the main zymogens and thrombin binding sites of fibrin.

Results: A sensitivity analysis of zymogen concentrations indicated that FIX most influenced thrombin generation, a result expected from hemophilia A and B. Averaging all MC simulations confirmed both the mean and standard deviation of measured fibrin generation on 1 tissue factor (TF) molecule per $\mu \mathrm{m}^{2}$. Across all simulations, free diffusible thrombin in the layer ranged from 20 to $300 \mathrm{nM}$ (mean: $50 \mathrm{nM}$ ). The top $2 \%$ of simulations that produced maximal fibrin were dominated by conditions with low fibrin antithrombin-I activity (decreased weak and strong sites) and high FIX concentration. In contrast, the bottom $2 \%$ of simulations that produced minimal fibrin were dominated by low FIX and FX. The percent reduction of fibrin by an ideal FXla inhibitor $(F X I=0)$ ranged from $71 \%$ fibrin reduction in the top $2 \%$ of $M C$ simulations to only $34 \%$ fibrin reduction in the bottom $2 \%$ of $\mathrm{MC}$ simulations. Thus, the antithrombotic potency of FXla inhibitors may vary depending on normal ranges of zymogen concentrations.

A.
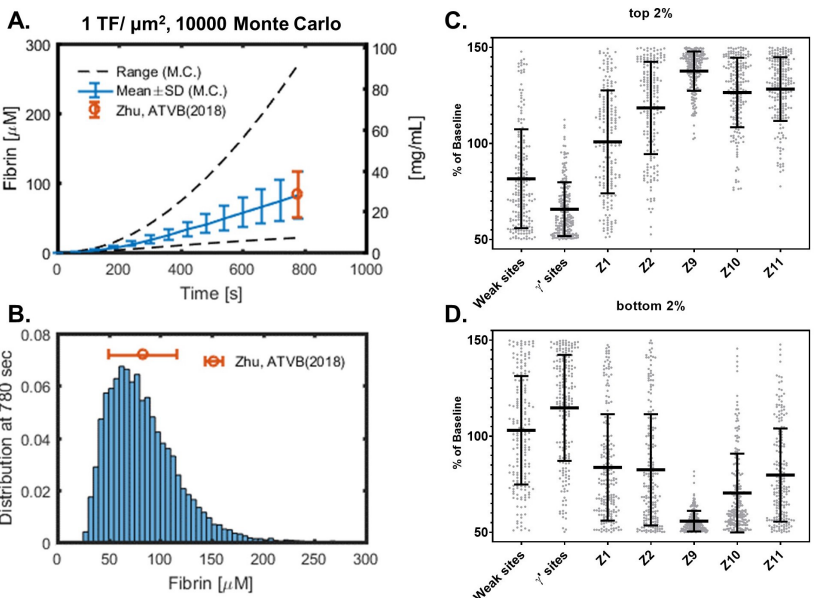

to experimental data of blood clotting over collagen/ TF under venous flow rate. Plasma protein levels and thrombin binding sites distribution of the subsets of top (C) and bottom $2 \%$ (D) of fibrin concentration.

Conclusions: This approach identified key zymogens variations that enhanced or reduced fibrin production during coagulation under flow conditions.

PB0049 | Rapid Detection of DOACs in DVT Patients Using a Single-use Microfluidic Chip under Whole Blood Flow

J.M. Rossi ${ }^{1}$; K.A. Panckeri' ${ }^{2}$; A.C. Cuker ${ }^{2}$; S.L. Diamond ${ }^{1}$

${ }^{1}$ University of Pennsylvania, Chemical and Biomolecular Engineering, Philadelphia, United States; ${ }^{2}$ University of Pennsylvania, Perelman School of Medicine, Philadelphia, United States

Background: Rapid detection of DOAC levels and effective reversal in patients would aid clinical decisions. Point-of-Care (POC) storagestable microfluidic chips offer rapid whole blood testing.

Aims: Evaluate an injection-molded 8-channel microfluidic chip for POC detection of DOACs in DVT patients utilizing the response of whole blood to ex vivo dosing of DOAC reversal agents.

Methods: Using a 1" $\times 3$ " device with 8-parallel flow paths crossing a bio-printed surface of collagen/tissue factor (200 s ${ }^{-1}$ wall shear rate), we measured platelets and fibrin accumulation with time using CTItreated blood from DVT patients $(N=7)$. Platelet and fibrin clotting was imaged for blood that was unmodified or treated ex vivo with the reversal agent Andexxa at two different doses or high-dose FXa inhibitor.

Results: For whole blood clotting at a wall shear rate of $200 \mathrm{~s}^{-1}$, a statistically significant difference was observed between channels with $300 \mathrm{nM}$ of reversal agent Andexanet Alfa present when compared with unaltered blood for 7 DVT patients on apixaban or rivaroxaban. Fluorescence intensity in the fibrin channel was increased in a dose dependent manner by the presence of the reversal agent. By evaluating the ratio of a patient's untreated and anticoagulantreversed blood and comparing with a known dose response curve for DOACs in this assay, patient drug concentrations can be predicted quantitatively

FIGURE 1 The mean, standard deviation, range (A), and the distribution (B) of fibrin concentration of 10,000 simulations compared 

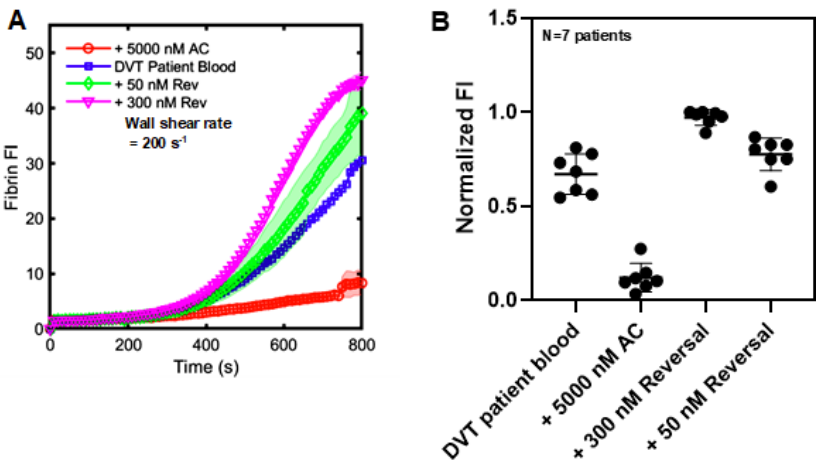

FIGURE 1 (A) Fibrin accumulation with time for DVT patient on apixaban (blue) or treated with Low (green) or high (purple) dose reversal agent or high dose Factor Xa inhibitor (red). (B) Fibrin fluorescence results evaluated at the time point where the high reversal condition intensity is $80 \%$ of the 15 min max for each patient, then normalized by the maximum fluorescence intensity for that patient across the whole assay at that time point

Conclusions: We demonstrate the ability to detect the presence of DOACs in whole blood samples drawn from DVT patients on oral Xa inhibitors.

PB0050 | The Relationship of 4 Factor PCC or Plasma to INR and Factor Levels: An Exploratory Analysis of 2 Clinical Trials

C. Hood ${ }^{1}$; P. Bajcic ${ }^{1}$; B. Goldstein ${ }^{1}$; J. Goldstein ${ }^{2}$; T. Milling ${ }^{3}$;

M. Refaai ${ }^{4} ;$ R. Sarode $^{5}$

${ }^{1}$ CSL Behring, King of Prussia, United States; ${ }^{2}$ Massachusetts General Hospital, Boston, United States; ${ }^{3}$ Dell Medical School at the University of Texas, Austin, United States; ${ }^{4}$ University of Rochester, Rochester, United States; ${ }^{5}$ UT Southwestern, Austin, United States

Background: Two Phase III prospective randomized clinical trials (RCTs) illustrated that non-activated 4 factor prothrombin complex concentrate (4F-PCC) was successful in decreasing INR to $\leq 1.3$ at 30 min after infusion and achieving hemostatic efficacy for vitamin $\mathrm{K}$ antagonist (VKA) reversal. However, many experts consider INR $\leq 1.5$ as a "successful" post-reversal INR. These data are clinically relevant and are not included in the trial results.

Aims: Explore the impact of 4F-PCC vs. plasma on INR reduction at different thresholds and vitamin $k$ dependent factor (VKDF) levels using the full dataset.

Methods: A post-hoc analysis of data from two RCTs comparing 4F-PCC to plasma for VKA reversal was performed $(n=369)$. Weight- and INR-based dosing of 4F-PCC and plasma was used. All post-infusion INR values were analyzed.

Results: Post-infusion, 85.9\% (159/185) of 4F-PCC patients achieved INR at $\leq 1.5$ vs. $39.1 \%(72 / 184)$ of plasma patients (Table 1 ).

Mean levels of FII and FX returned to $\geq$ LLN within 30 min after 4FPCC in $72.3 \%$ and $76.7 \%$ of patients, respectively, compared to $9.2 \%$ and $7.4 \%$ after plasma (Table 2 ).
In patients with a post-reversal INR $\leq 1.5$, VKDF were significantly higher after infusion of 4F-PCC compared to plasma. Even in 4FPCC patients with a post-reversal INR $>1.5$, mean VKDF levels were $>50 \%$ at $30 \mathrm{~min}$.

TABLE 1 Cumulative Frequency Distribution 30 min $^{*}$ Post-Reversal INR in 4F-PCC vs Plasma Patients

\begin{tabular}{llll} 
INR & 4F-PCC (\%) & Plasma (\%) & P-value \\
$\leq 3.5$ & 100 & 98.9 & 0.25 \\
$\leq 3.0$ & 99.5 & 95.7 & 0.02 \\
$\leq 2.5$ & 98.9 & 91.8 & 0.001 \\
$\leq 2.0$ & 97.8 & 82.6 & $<0.0001$ \\
$\leq 1.8$ & 92.4 & 70.7 & $<0.0001$ \\
$\leq 1.5$ & 85.9 & 39.1 & $<0.0001$ \\
$\leq 1.3$ & 65.4 & 12.5 & $<0.0001$ \\
\hline
\end{tabular}

"All patients who had an initial post-reversal INR were assessed, irrespective of timing of lab draw.

TABLE 2 Percentage of patients with factor levels $\geq$ LLN

\begin{tabular}{llll}
$\begin{array}{l}\text { Factor (normal } \\
\text { range \%) }\end{array}$ & $\begin{array}{l}\text { 4F-PCC } \% \\
\text { patients) }\end{array}$ & $\begin{array}{l}\text { Plasma (\% } \\
\text { patients) }\end{array}$ & P-value \\
II (70-146) & & & \\
30 min $^{* *}$ & 72.3 & 9.2 & $<0.0001$ \\
$24 \mathrm{~h}$ & 67.6 & 25.4 & $<0.0001$ \\
VII (67-143) & & & \\
30 min & & & \\
$24 \mathrm{~h}$ & 26.0 & 10.4 & 0.0003 \\
IX (55-163) & 66.5 & 74.0 & 0.13 \\
& & & \\
30 min & & & $<0.0001$ \\
$24 \mathrm{~h}$ & 76.1 & 44.6 & 0.01 \\
$X(70-152)$ & 82.1 & 91.3 & \\
$30 \mathrm{~min}$ & & & $<0.0001$ \\
$24 \mathrm{~h}$ & & & $<0.0001$ \\
\hline
\end{tabular}

"Physiologic range for VKDFs

${ }^{* *}$ Time after start of infusion

Conclusions: 4F-PCC rapidly reduced the INR to $\leq 1.5$ in $~ 86 \%$ of patients and $\leq 1.8$ in $92 \%$ of patients after infusion compared to $39 \%$ and $71 \%$ of patients receiving plasma, respectively. In addition, $4 \mathrm{~F}-$ PCC restored VKDF's to normal levels at a significantly higher rate than plasma. All VKDF levels were $>50 \%$ after administration of $4 \mathrm{~F}$ PCC at all time points assessed, regardless of INR achieved. When the full range of data is analyzed, 4F-PCC is highly successful at factor restoration and INR correction as compared to plasma. 
PB0051 | Combining Heparin with a FX/FXa Aptamer to Reduce Thrombin Generation in Models of Cardiopulmonary Bypass and COVID-19 Associated Coagulopathy

C.V. Chabata ${ }^{1,2}$; J.W. Frederiksen ${ }^{1}$; L.B. Olson ${ }^{2,3}$; I.A. Naqvi ${ }^{4}$; B.A. Sullenger ${ }^{1}$

${ }^{1}$ Department of Surgery, Duke University Medical Centre, Durham, United States; ${ }^{2}$ Department of Pharmacology and Cancer Biology, Duke University, Durham, United States; ${ }^{3}$ Medical Scientist Training Program, Duke University, Durham, United States; ${ }^{4}$ Department of Anaesthesiology, Duke University Medical Centre, Durham, United States

Background: Unfractionated heparin (UFH) is the primary anticoagulant for highly procoagulant indications including cardiopulmonary bypass (CPB) and severe COVID-19. UFH is unable to fully inhibit thrombin generation increasing the risk for thrombo-inflammatory adverse events (AEs).

We attempt to address this limitation by evaluating the FX/Xa aptamer, 11F7t. We hypothesized that combinations of UFH and $11 \mathrm{~F} 7 \mathrm{t}$ would achieve enhanced anticoagulation while minimizing thrombin generation in models of CPB and COVID-19 associated coagulopathy (CoAC).

Aims: We determined optimal combinations of UFH and $11 \mathrm{~F} 7 \mathrm{t}$ that produced CPB-sufficient anticoagulation and minimized thrombin generation in whole blood in vitro and ex vivo models of $\mathrm{CPB}$. Furthermore, we aimed to determine the utility of this anticoagulation strategy in addressing CoAC.

Methods: We employed whole blood coagulation assays to determine the optimal doses of UFH and 11F7t. Fresh human blood was treated with $\mathrm{UFH}+11 \mathrm{~F} 7 \mathrm{t}$ combinations and continuously circulated in an ex vivo oxygenator circuit for $2 \mathrm{~h}$ to model CPB. Thereafter, thrombin generation was assessed using ELISAs. For CoAC studies, thrombin generation from severely ill COVID-19 patient plasma was determined using the Calibrated Automated Thrombogram (CAT) assay.

Results: In in vitro and ex vivo models of CPB, a combined dose of $2 \mathrm{U} / \mathrm{mL} \mathrm{UFH}$ with $2 \mu \mathrm{M}$ 11F7t was able to achieve anticoagulation similar to the therapeutic dose of UFH $(5 \mathrm{U} / \mathrm{mL})$. Further investigation into thrombin generation with ex vivo experiments showed that the combinations of UFH and $11 F 7 t$ are more effective than UFH alone at limiting thrombin generation (Figure 1). Similar anticoagulation and reduced thrombin generation was observed when blood from severely ill COVID-19 patients on heparin therapy was treated with $2 \mu \mathrm{M}$ 11F7t (Figure 2).

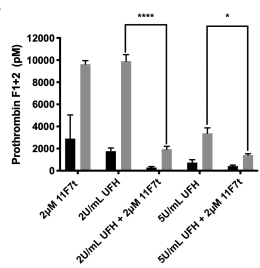

FIGURE 1 Thrombin generation during extracorporeal circulation of human blood. a) Levels of prothrombin fragment F1+2 in plasma prepared from blood that went through circulation for $120 \mathrm{~min}$ and that which did not were measured. b) Levels of thrombin-antithrombin (TAT) in plasma prepared from blood that went through circulation for 120 min and that which did not were measured. In both $A$ and $\mathrm{B},(n=3)$ for $5 \mathrm{U} / \mathrm{mL} U \mathrm{FH}, 2 \mu \mathrm{M}$ 11F7t, $2 \mathrm{U} / \mathrm{mL} U \mathrm{FH}+2 \mu \mathrm{M}$ 11F7t, and $5 \mathrm{U} / \mathrm{mL} U F H+2 \mu M$ 11F7t. $(n=4)$ for $2 \mathrm{U} / \mathrm{mL} U F H$. Statistical analysis was performed by one way ANOVA followed by Sidak's multiple comparisons test. For panel A, (prothrombin F1+2) comparison between $2 \mathrm{U} / \mathrm{mL}$ UFH versus $2 \mathrm{U} / \mathrm{mL} \mathrm{UFH}+2 \mu \mathrm{M} 11 \mathrm{~F} 7 \mathrm{t}, \mathrm{P}$
A

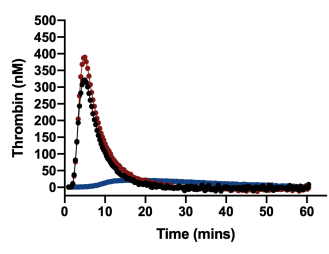

B

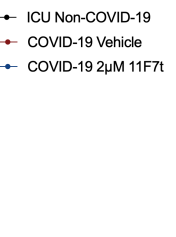

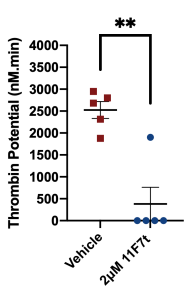

FIGURE 2 Effect of 11F7t on thrombin generation in heparin-treated COVID-19 patient blood. Calibrated Automated Thrombogram assays were performed to measure thrombin generation in platelet poor plasma from severely ill COVID-19 patients. All COVID-19 patients were treated with heparin the day before a) Thrombograms of plasma from a severely ill COVID-19 negative patient $(n=1)$, COVID-19 positive patients $(n=5)$ treated with either Vehicle or $2 \mu \mathrm{M}$ 11F7t. b) Plotted is the area under the curve or ETP for each treatment group. Statistical analysis was performed by paired $t$-test with $P=0.0025$

Conclusions: This study demonstrates that combining UFH with a $\mathrm{FX} / \mathrm{Xa}$ aptamer produces potent anticoagulation with reduced thrombin generation and has the potential to address CPBassociated AEs and has potential utility in other procoagulant settings such as CoAC. 
PB0052 | Evaluation of a New Thromboplastin Reagent Containing Human Recombinant Tissue Factor for the Determination of Prothrombin Time Reagent: the (h)PT-Phen ${ }^{\mathrm{TM}}$ LRT

I. Martin-Toutain ${ }^{1}$; M. Gaaloul ${ }^{1}$; J.-P. Delorme ${ }^{2}$; Y. Bourti' ${ }^{2}$; C. Frere ${ }^{1,3}$

${ }^{1}$ Assistance Publique Hôpitaux de Paris, Paris, France; ${ }^{2}$ Sysmex France, Villepinte, France; ${ }^{3}$ Sorbonne Université, INSERM UMRS_1166, Institute of Cardiometabolism and Nutrition, Paris, France

Background: The prothrombin time (PT) is widely used to screen single or combined deficiencies of the extrinsic coagulation pathway and to monitor vitamin $\mathrm{K}$ antagonist (VKA) anticoagulant therapy. The (h)PT-Phen ${ }^{\text {TM }}$ LRT reagent (HYPHEN BioMed, Neuville-sur-Oise, France) is a new liquid, ready to use thromboplastin reagent containing human recombinant tissue factor for the determination of PT on citrated human plasma. However, data on both its analytical performance and its use in current practice are scarce.

Aims: To assess the analytical performances of the (h)PT-Phen ${ }^{\mathrm{TM}}$ LRT and to compare this reagent with a widely used routine PT reagent (Neoplastine ${ }^{\circledR} \mathrm{Cl}$ Plus, Stago, France).

Methods: Analytical performances of the (h)PT-Phen ${ }^{\text {TM }}$ LRT reagent were assessed on a CN-6000 analyzer (Sysmex Corp., Japan). Blood samples from 120 healthy volunteers were collected to generate reference-values. PT was further determined in plasma samples from 84 patients treated with VKA, 28 patients suffering from hepatic failure, and 35 patients with low levels of factor $V$ with the $(h)$ PT-Phen ${ }^{\text {TM }}$ LRT and with the Neoplastine ${ }^{\circledR} \mathrm{Cl}$ Plus reagent on $\mathrm{CN}$ 6000 and STA-R Max $2^{\circledR}$ analyzers, respectively.
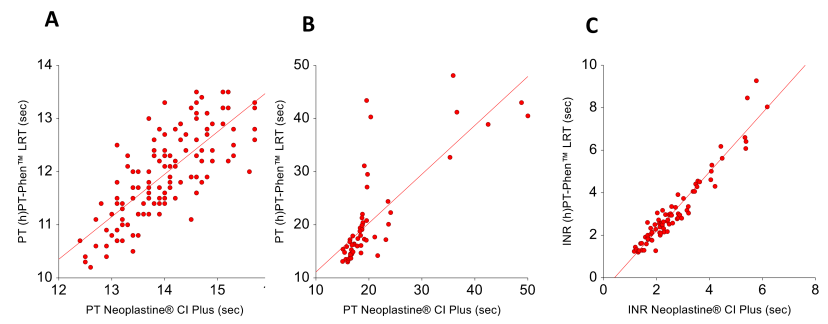

FIGURE 1 PT or INR values in healthy volunteers (A), in patients with hepatic failure and known factors $V$ low levels (B), and in patients treated with vitamin $\mathrm{K}$ antagonist (C)

Results: A good open-vial on-board stability over 14 days was observed. Excellent repeatability and intermediate precision measurements were obtained ( $C V<1.6 \%)$. Significant correlations were observed between the 2 reagents in normal subjects $(r=0.76$, $P<0.0001$, Figure $1 \mathrm{~A}$ ) and in those with hepatic failure and known factors $\vee$ low levels $(r=0.69, P<0.0001$, Figure $1 B)$. There was a good agreement between methods in samples from patients receiving VKA and no significant differences between methods with increasing INR values (Figure $1 \mathrm{C}$ ).
Conclusions: In light of its excellent analytical performance and its good agreement with a relevant comparator reagent, the (h)PTPhen $^{\text {TM }}$ LRT appears as suitable for routine use to detect factor II, V, VII and X deficiency and to monitor VKA in laboratories with high sample throughput.

PB0053 | The Effect of Lower-leg Trauma and Knee Arthroscopy on Thrombin Generation

C.E. Touw ${ }^{1}$; B. Nemeth ${ }^{1}$; R. van Adrichem ${ }^{1}$; W. Lijfering ${ }^{1}$; A. van Hylckama Vlieg ${ }^{1}$; R. Nelissen ${ }^{1}$; T. Lisman ${ }^{2}$; S. Cannegieter ${ }^{1}$ ${ }^{1}$ Leiden University Medical Center, Leiden, Netherlands; ${ }^{2}$ University Medical Center Groningen, Groningen, Netherlands

Background: Patients with lower-leg cast immobilization and knee arthroscopy patients are at high risk of venous thromboembolism (VTE), despite low-molecular-weight-heparin as prophylaxis. Improved prophylactic strategies are needed for which knowledge on the underlying mechanism of VTE in these patients is essential. For this it is of interest to what extent thrombin generation is affected by lower-leg trauma and knee arthroscopy.

Aims: To measure the effect of (1) lower-leg trauma and (2) knee arthroscopy on thrombin generation.

Methods: Blood samples were collected from POT-CAST (Prevention Of Thrombosis after Lower Leg Plaster Cast) and POTKAST (Prevention Of Thrombosis after Knee Arthroscopy) trials. Blood draw took place shortly after lower-leg trauma and within four hours before/after knee arthroscopy. A selection of patients who did not develop VTE were selected for current analyses: 89 patients with lower-leg injury and 88 knee arthroscopy patients. Thrombin generation was measured using thrombomodulin-modified CAT (Calibrated Automated Thrombography). Furthermore, plasma levels of thrombin-antithrombin complexes, prothrombin fragment 1+2 and fibrinopeptide A were measured in a smaller subset of patients by commercially available enzyme-linked immunosorbent assays.

Aim 1: lower-leg injured patients were compared with preoperative measurements of arthroscopy patients (controls), resulting in mean differences (or mean ratios if In-retransformed).

Aim 2: pre- and postoperative measurements in arthroscopy patients were compared, resulting in paired mean changes.

Results: Ex vivo thrombin generating potential and in vivo activation of coagulation were elevated after lower leg trauma (Table 1). Ex vivo thrombin generating potential and in vivo activation of coagulation did not change after knee arthroscopy (Table 2). 
TABLE 1 Aim 1: Thrombin generation parameters and plasma levels of associated parameters in patients with lower-leg injury compared to patients without lower-leg injury

\begin{tabular}{|c|c|c|c|c|}
\hline & $\begin{array}{l}\text { Lower-leg injury } \\
(N=89) \text {, mean (SD) }\end{array}$ & $\begin{array}{l}\text { No lower-leg injury } \\
(N=88) \text {, mean (SD) }\end{array}$ & $\begin{array}{l}\text { Mean difference/ratio } \\
(95 \% \mathrm{Cl})^{\dagger}\end{array}$ & $\begin{array}{l}\text { Adj. mean difference/ } \\
\text { ratio }(95 \% \mathrm{Cl})^{\dagger^{*}}\end{array}$ \\
\hline $\operatorname{ETP}\left(\mathrm{nM} I 1 a^{*} \min \right)$ & $566.0(202.2)$ & $451.4(239.4)$ & 114.6 (48.8 to 180.3$)$ & $120.0(55.0$ to 185.1$)$ \\
\hline Lag time (min) & $2.0(0.5)$ & $2.1(0.6)$ & $-0.1(-0.3$ to 0.0$)$ & $-0.1(-0.3$ to 0.0$)$ \\
\hline Velocity index (nM Ila/min) & $72.8(33.8)$ & $55.7(37.5)$ & 17.0 (6.4 to 27.6$)$ & $18.0(7.4$ to 28.6$)$ \\
\hline Prothrombin fragment $1+2(\mathrm{pmol} / \mathrm{l})^{\ddagger}$ & $\begin{array}{l}260.7(231.2 \text { to } \\
294.0)\end{array}$ & 242.7 (218.6 to 269.3 ) & $1.1(0.9 \text { to } 1.3)^{\dagger}$ & $1.0(0.8 \text { to } 1.2)^{\dagger}$ \\
\hline Fibrinopeptide A (ng/ml) & $143.9(91.5)$ & $95.1(64.3)$ & 48.8 (11.0 to 86.7 ) & 43.8 (5.9 to 81.8$)$ \\
\hline
\end{tabular}

ETP, endogenous thrombin potential; TAT, thrombin-antithrombin complexes.

*Adjusted for age, sex, Body Mass Index (BMI) and comorbidities.

${ }^{\dagger}$ Mean ratios are denoted in the table with this symbol and in italics.

‡TAT and F1+2 were not normally distributed and therefore In-retransformed, resulting in geometric means with $95 \% \mathrm{Cls}$ and mean ratios.

TABLE 2 Aim 2: Pre- and postoperative plasma levels of thrombin generation parameters and plasma levels of associated parameters in patients who underwent knee arthroscopy

\begin{tabular}{|c|c|c|c|}
\hline & Preoperative ( $N=88)$, mean $(S D)$ & Postoperative $(N=85)^{*}$, mean $(S D)$ & Paired mean change $(95 \% \mathrm{Cl})$ \\
\hline Thrombin peak (nM Ila) & $117.3(67.4)$ & $121.0(72.0)$ & $5.0(-4.0$ to 13.9$)$ \\
\hline Velocity index (nM Ila/min) & $55.7(37.5)$ & $58.7(42.1)$ & $3.2(-2.0$ to 8.3$)$ \\
\hline $\begin{array}{l}\text { In vivo markers of coagulation } \\
\text { activation }\end{array}$ & $(N=28)$ & $(N=28)$ & \\
\hline TAT complexes $(\mu \mathrm{g} / \mathrm{l})^{\dagger}$ & 2.3 (2.1 to 2.5$)$ & 2.1 (1.9 to 2.3 ) & $-0.2(-0.5$ to 0.2$)$ \\
\hline $\begin{array}{l}\text { Prothrombin fragment } 1+2 \\
\quad(\mathrm{pmol} / \mathrm{l})^{\dagger}\end{array}$ & 242.7 (218.6 to 269.3 ) & 226.6 (201.4 to 255.1$)$ & $-14.9(-38.4$ to 8.5$)$ \\
\hline Fibrinopeptide A (ng/ml) & $95.1(64.3)$ & $101.2(64.0)$ & $6.1(-20.8$ to 33.1$)$ \\
\hline
\end{tabular}

ETP, endogenous thrombin potential; TAT, thrombin-antithrombin complexes.

"Thrombin generation was not measured in the postoperative samples of three patients due to lack of plasma $(N=2)$ or inadequate sample quality $(N=1)$.

${ }^{\dagger}$ TAT and F1+2 were not normally distributed and therefore In-retransformed, resulting in geometric means with $95 \% \mathrm{Cls}$.

Conclusions: Lower-leg injury is associated with an enhanced ex vivo thrombin generating potential and in vivo thrombin generation, while knee arthroscopy has no effect on ex vivo thrombin generation, and surprisingly does not result in in vivo activation of coagulation. The pathogenesis of VTE therefore may be different for these patient groups.

PB0055 | HemosIL Liquid Anti-Xa Apixaban Measurement in Patients with High Risk of Major Bleeding

J. Hammelburger; Z. Cao; C. Kung

Research and Development, Instrumentation Laboratory, Orangeburg, United States

Background: The HemosIL Liquid Anti-Xa Assay, when used with HemosIL Apixaban Calibrators and Controls, is an automated, one-stage anti-Xa chromogenic assay for in vitro diagnostic use by laboratory professionals in clinical laboratories for the quantitative determination of apixaban in human citrated plasma on ACL TOP Family and ACL TOP Family 50 Series models.

Aims: The method comparison study was conducted to demonstrate the performance of the HemosIL Liquid Anti-Xa Assay for apixaban measurement on ACL TOP Family analyzers in a clinical setting, with liquid chromatography tandem mass spectrometry (LC-MS/MS) used as the reference method. 
Methods: 367 samples, including 301 from patients requiring emergency surgery or at high risk for major bleeding, were tested at 3 clinical sites with HemosIL Liquid Anti-Xa assay on ACL TOP Family analyzers and at an external site for LC-MS/MS measurement. Weighted Deming regression analysis was performed for the method comparison study using LC-MS/MS as the reference method.

Results: As shown in Figure 1 and Table 1 the regression analysis for the pooled sites showed a correlation coefficient of 0.995 , a slope of 1.10 and a mean bias of $6.8 \%$. The bias at medical decision levels was $1.9 \%$ (at $30 \mathrm{ng} / \mathrm{mL}$ apixaban) and $9.4 \%$ (at $350 \mathrm{ng} / \mathrm{mL}$ apixaban), respectively.
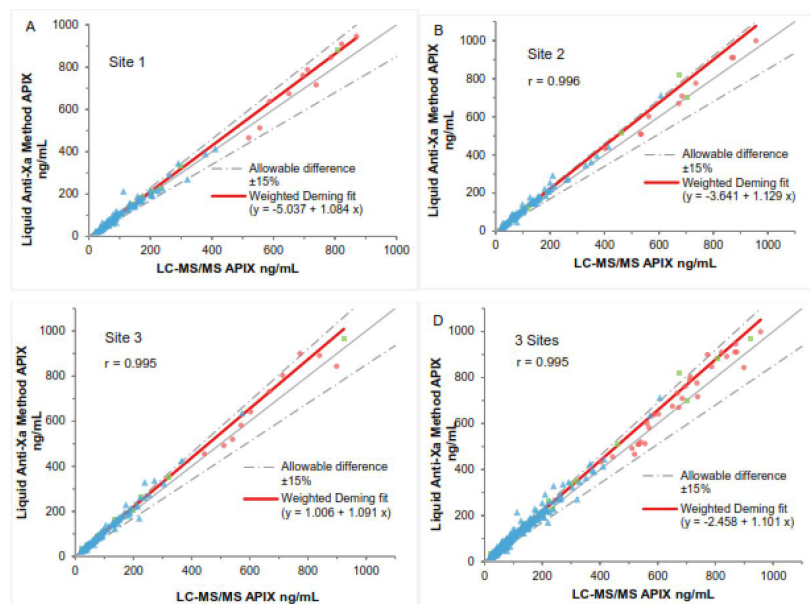

FIGURE 1 HemosIL Liquid Anti-Xa vs. LC-MS/MS Weighted Deming Regression Plots of Individual (Panels A through C) and Pooled (Panel D) Data Sets

TABLE 1 HemosIL Liquid Anti-Xa vs. LC-MS/MS Method Comparison Results

\begin{tabular}{|c|c|c|c|c|c|c|c|c|c|}
\hline Site & $\begin{array}{l}\text { Sample } \\
\text { Count } \\
\text { (N) }\end{array}$ & $\begin{array}{c}\underset{\operatorname{Min}}{\text { APIX }} \\
\text { (ng/mL) }\end{array}$ & $\begin{array}{c}\text { Max } \\
\text { APIX } \\
\text { (ng/mL) }\end{array}$ & $\begin{array}{c}R \\
\geq 0.950\end{array}$ & $\begin{array}{c}\text { Slope } \\
0.85-1.15\end{array}$ & Intercept & $\begin{array}{c}\text { Mean Bias } \\
\text { (Bland } \\
\text { Altman) } \\
\leq 15 \%\end{array}$ & $\begin{array}{c}\text { Bias at } \\
30 \mathrm{ng} / \mathrm{mL} \\
\leq 15 \%\end{array}$ & $\begin{array}{c}\text { Bias at } \\
350 \mathrm{ng} / \mathrm{mL} \\
\leq 15 \%\end{array}$ \\
\hline Site 1 & 120 & 21.9 & 945.4 & 0.995 & 1.084 & -5.0 & $1.6 \%$ & $-8.4 \%$ & $7.0 \%$ \\
\hline Site 2 & 121 & 21.3 & & 0.996 & 1.129 & -3.6 & $7.6 \%$ & $0.8 \%$ & $11.8 \%$ \\
\hline Site 3 & 126 & 21.7 & 967.4 & 0.995 & 1.091 & 1.006 & $10.8 \%$ & $12.5 \%$ & $9.4 \%$ \\
\hline Pooled & 367 & 21.3 & 998.2 & 0.995 & 1.101 & -2.458 & $6.8 \%$ & $1.9 \%$ & $9.4 \%$ \\
\hline
\end{tabular}

Conclusions: Method comparison demonstrates performance equivalency of the HemosIL Liquid Anti-Xa Assay, when used with
HemosIL Apixaban Calibrators and Controls, to the LC-MS/MS method in a patient population requiring emergency surgery or at high risk for major bleeding.

PB0056 | The Impact of Lower-leg Injury and Knee Arthroscopy on Procoagulant Factor Levels

C.E. Touw; B. Nemeth; I. Schipper; R. Nelissen; S. Cannegieter Leiden University Medical Center, Leiden, Netherlands

Background: Despite treatment with low-molecular-weight-heparin as thromboprophylaxis, patients with lower-leg cast immobilization and patients who undergo knee arthroscopy, are at high risk for developing Venous Thromboembolism (VTE). Establishing the mechanism of VTE in these patients could reveal new targets for thromboprophylactic strategies. It is currently unknown whether levels of procoagulant factors are influenced by lower-leg trauma and knee arthroscopy.

Aims: To measure the impact of

(1) lower-leg trauma and

(2) knee arthroscopy on plasma levels of procoagulant factors.

Methods: Blood samples of POT-CAST (Prevention Of Thrombosis following CAST immobilization) and POT-KAST (Prevention Of Thrombosis after Knee Arthroscopy) trial participants were analysed. Blood was collected directly after lower-leg trauma, and $\leq 4 \mathrm{~h}$ before and after knee arthroscopy. Factor (F)VIII, IX, XI, fibrinogen (FG), von Willebrand Factor (VWF) and D-dimer were measured. For aim 1, we compared measurements in lower-leg injury patients with those in preoperative blood samples of knee arthroscopy patients (controls). Linear regression was used to obtain mean differences, adjusted for age, sex, Body Mass Index (BMI), comorbidities, malignancy, and oral contraceptive use. Data were In-retransformed in case of skewedness, resulting in mean ratios. For aim 2, we compared pre- and postoperative factor levels of knee arthroscopy patients, using paired $t$-tests which resulted in mean changes.

Results: In patients with lower-leg injury all procoagulant factor levels were clearly higher than in controls, especially FVIII and VWF (Table 1). After knee arthroscopy, all procoagulant factor levels decreased (Table 2).

TABLE 1 Aim 1: Mean differences in procoagulant factor levels between patients with lower-leg injury and controls

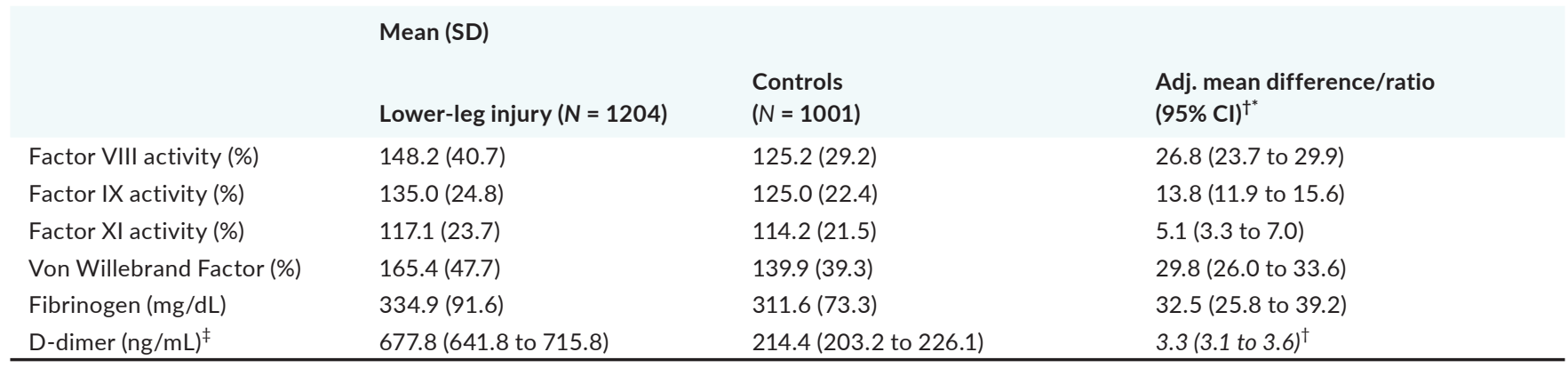

Adjusted for sex, age, BMI, comorbidity, presence of malignancy, use of oral contraceptives.

${ }^{\dagger}$ Mean ratios are denoted in the table with this symbol and in italics.

${ }^{\ddagger}$ D-dimer not normally distributed and therefore In-retransformed, resulting in geometric means with $95 \% \mathrm{Cls}$ and mean ratios. 
TABLE 2 Aim 2: Change of procoagulant factor levels after knee arthroscopy

Mean (SD)

Preoperative $(N=715)$

Factor VIII activity (\%)

$123.0(27.9)$

$123.1(22.0)$

$112.4(20.8)$

$139.9(38.9)$

308.9 (72.3)

215.6 (201.5 to 230.7)
Postoperative $(N=715)$

113.8 (35.7)

$110.1(21.8)$

$101.0(21.1)$

$132.7(40.4)$

$276.9(67.3)$

204.7 (193.1 to 216.9 )
Mean change

$(95 \% \mathrm{Cl}) *$

$-9.1(-11.1$ to -7.1$)$

$-12.9(-14.0$ to -11.9$)$

$-11.5(-12.6$ to -10.3$)$

$-7.2(-8.6$ to -5.7$)$

$-32.1(-34.7$ to -29.5$)$

$-53.5(-88.9$ to -18.0$)$

${ }^{*}$ Mean of difference between preoperative and postoperative paired per patient.

${ }^{\dagger}$ Ln-transformed data because of skewedness, resulting in geometric means.

Conclusions: In contrast to knee arthroscopy, lower-leg injury was associated with increased plasma levels of procoagulant factors, especially FVIII and VWF, suggesting a different underlying pathophysiology of VTE in these patient groups.

PB0057 | Ex vivo Evaluation of the Effect of Plasma-derived Factor VIII/von Willebrand Factor in Patients with Severe Hemophilia A on Prophylaxis with Emicizumab by ROTEM

E. Monzón Manzano ${ }^{1}$; E. Arias-Salgado ${ }^{1}$; P. Acuña ${ }^{1}$; M.-I. Bravo² ${ }^{2}$ A. Raventos ${ }^{2} ;$ A. Perez ${ }^{2}$; M.T. Álvarez Román ${ }^{1} ;$ N. Butta ${ }^{1}$; M. Costa ${ }^{2} ;$ T. Willis ${ }^{3}$; V. Jimenez-Yuste ${ }^{1,4}$

${ }^{1}$ Hematology Unit, Hospital Universitario La Paz-Idipaz, Madrid, Spain; ${ }^{2}$ Discovery Research, Bioscience Research Group, Grifols, Barcelona, Spain; ${ }^{3}$ Discovery Research, Bioscience Research Group, Grifols, Raleigh, United States; ${ }^{4}$ Universidad Autónoma de Madrid, Madrid, Spain

Background: Hemophilia A (HA) patients under emicizumab prophylaxis treatment may require the concomitant use of procoagulant factors for breakthrough bleedings or immune tolerance induction. Previous in vitro studies demonstrated that the combination of emicizumab and plasma-derived Factor VIII/Von Willebrand Factor (pdFVIII/VWF) has a non-additive effect on thrombin generation (TG). Aims: The aim of this study was to evaluate the ex vivo combination of emicizumab with a pdFVIII/VWF concentrate by rotational thromboelastometry (ROTEM).

Methods: Blood samples obtained from 12 adult patients with severe HA without inhibitors on prophylaxis with emicizumab were drawn in citrate plus corn trypsin inhibitor (CTI) tubes. Modified Tissue Factor (TF) activated ROTEM assay (EXTEM) was performed by using reagent diluted 1:100,000 (low concentration of TF). Clotting time (CT) and time to maximum clot formation velocity (MAXV-t) parameters were registered. In vitro spiking was performed with increasing concentrations of pdFVIII/VWF (10 to 400 $\mathrm{IU} / \mathrm{dL}$ ), recombinant activated factor VII (rFVIla, $0.9 \mathrm{mg} / \mathrm{mL}$ ) or activated prothrombin complex concentrate (aPCC, $0.5 \mathrm{U} / \mathrm{mL})$.
Results: ROTEM showed that whole blood CT (Figure 1) and MAXV-t (Figure 2) of patients treated with emicizumab were longer than those from healthy controls, and addition of pdFVIII/VWF at $50 \mathrm{IU} /$ $\mathrm{dL}$ ( $\equiv 25 \mathrm{IU} / \mathrm{kg}$ ) normalized these parameters. Increasing ex vivo concentrations of pdFVIII/VWF shortened CT and MAXV-t, with high concentrations of pdFVIII/VWF producing an enhanced procoagulant effect of emicizumab, similar to rFVIla. The addition of aPCC resulted in a higher synergistic procoagulant profile, which was not reached with the highest concentration of pdFVIII/VWF (400 IU/ $\mathrm{dL})$.

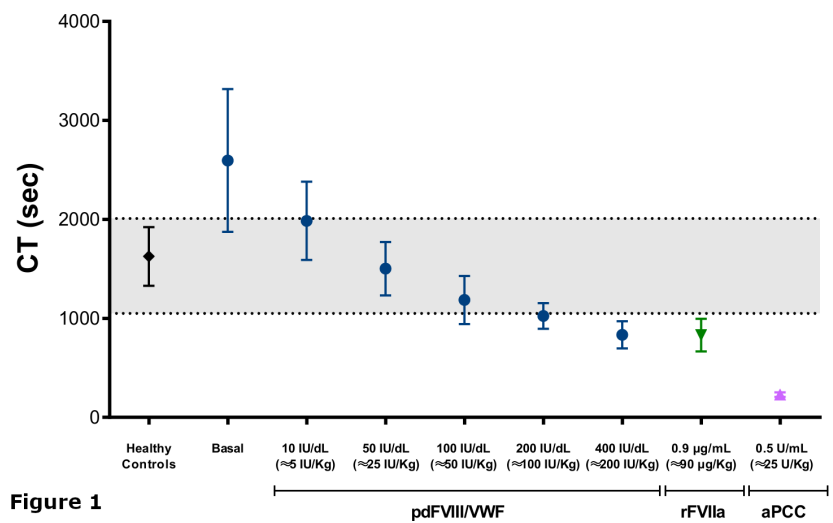

FIGURE 1 Clotting time (CT) in whole blood samples obtained from hemophilia $A$ patients treated with emicizumab and spiked with plasma-derived Factor VIII/Von Willebrand Factor (pdFVIII/VWF), recombinant activated factor VII (rFVIla) or activated prothrombin complex concentrate (aPCC). Bars represent mean \pm SD. Greyshaded bands correspond to maximum and minimum range of $\mathrm{CT}$ normal values 


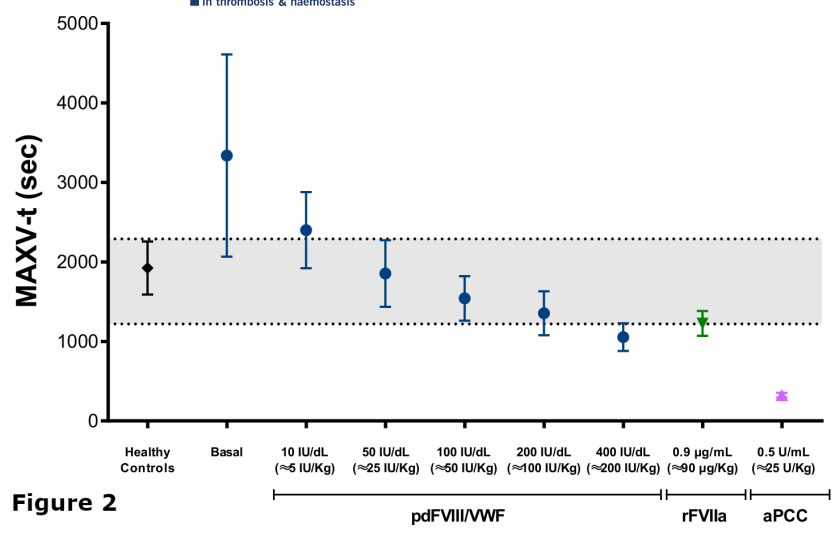

FIGURE 2 Time to maximum clot formation velocity (MAXV-t) in whole blood samples obtained from hemophilia $A$ patients treated with emicizumab and spiked with plasma-derived Factor VIII/Von Willebrand Factor (pdFVIII/VWF), recombinant activated factor VII ( $r F V I l a)$ or activated prothrombin complex concentrate (aPCC). Bars represent mean $\pm \mathrm{SD}$. Grey-shaded bands correspond to maximum and minimum range of MAXV-t normal values

Conclusions: ROTEM with CTI samples triggered by a low concentration of TF was a suitable method to evaluate the concomitant use of pdFVIII/VWF in patients on prophylaxis with emicizumab. These results showed that emicizumab plus pdFVIII/VWF combination did not trigger a multiplying effect on clot formation and were aligned with previous in vitro data using TG assay.
PB0058 | Are Direct Oral Anti-Xa Inhibitors Absorbed in Cancer Patients after Gastrectomy? A Case Series

H.C. Puhr; A. Ilhan-Mutlu; P. Quehenberger; P.A. Kyrle; S. Eichinger; L. Eischer

Medical University of Vienna, Vienna, Austria

Background: Direct oral anticoagulants (DOAC) including the anti$X a$ inhibitors apixaban, edoxaban and rivaroxaban are effective and safe for the treatment of venous thromboembolism (VTE) also in cancer patients. DOAC are absorbed predominantly in the upper gastrointestinal tract, which raises concern in cancer patients after gastrectomy regarding achievement of adequate DOAC plasma levels.

Aims: We aimed to evaluate the absorption of oral anti-Xa inhibitors in cancer patients after gastrectomy.

Methods: Using chromogenic anti-Xa assays calibrated for apixaban, edoxaban or rivaroxaban (STA Liquid Anti-Xa, Diagnostica Stago), we measured anti-Xa levels in plasma of eight cancer patients after partial or total gastrectomy who required oral anticoagulation because of VTE or atrial fibrillation. Levels were determined immediately before (trough) and 2-3 h after drug intake (peak). Rivaroxaban was administered with food.

Results: Patient characteristics including anti-Xa levels and coagulation parameters of eight patient with upper gastrointestinal cancer who underwent total or partial gastrectomy before and after administration of an anti-Xa inhibitor are shown in Table 1 and Table 2. All patients had anti-Xa levels well above the detection limit 2-3 $\mathrm{h}$ after drug intake. In two patients edoxaban trough levels were below the detection limit. One Patient was switched to rivaroxaban with trough levels slightly above the detection limit. One patient discontinued edoxaban and refused further anticoagulation. None of the patients had a thromboembolic event during treatment with DOAC, one patient had major bleeding after brachial artery puncture.

TABLE 1 Patient characteristics and medical history. Abbreviations: PE - pulmonary embolism, DVT - deep vein thrombosis , A-fib - atrial fibrillation; * time from first intake of DOAC until end of observation period

\begin{tabular}{|c|c|c|c|c|c|c|}
\hline Patient number & Sex & Age & Weight in kg & Surgery & Indication & $\begin{array}{l}\text { Duration of } \\
\text { anticoagulation in } \\
\text { months }^{*}\end{array}$ \\
\hline 2 & w & 37 & 43 & total gastrectomy & PE, DVT & 8 \\
\hline 3 & $\mathrm{~m}$ & 76 & 65 & partial gastrectomy & DVT & 50 \\
\hline 5 & $\mathrm{~m}$ & 76 & 84 & partial gastrectomy & $\mathrm{PE}$ & 11 \\
\hline 6 & $\mathrm{~m}$ & 76 & 92 & total gastrectomy & DVT, PE & 4 \\
\hline 7 & $\mathrm{~m}$ & 80 & 88 & total gastrectomy & A-fib & 14 \\
\hline 8 & w & 81 & 39 & total gastrectomy & A-fib & 0.1 \\
\hline
\end{tabular}


TABLE 2 Trough and peak anti-Xa levels and coagulation parameters. Abbreviations: DOAC - direct oral anticoagulant, PT - prothrombin time, aPTT - activated partial thromboplastin time, n.a. - not available; Creatinine clearance estimated with the Cockcroft-Gault equation

\begin{tabular}{|c|c|c|c|c|c|c|c|c|c|}
\hline $\begin{array}{l}\text { Patient } \\
\text { number }\end{array}$ & DOAC & Dosage & $\begin{array}{l}\text { Creatinine } \\
\text { Clearance } \\
\text { (ml/min) }\end{array}$ & $\begin{array}{l}\text { trough level } \\
\text { anti-Xa (ng/ } \\
\mathrm{ml} \text { ) }\end{array}$ & PT (\%) & aPTT (s) & $\begin{array}{l}\text { peak leve } \\
\text { anti-Xa } \\
\text { (ng/ml) }\end{array}$ & PT (\%) & aPTT (s) \\
\hline 2 & Edoxaban & $1 \times 30 \mathrm{mg}$ & 67.9 & $<20$ & 71 & 28.8 & 83 & 60 & 33.6 \\
\hline 2 & Rivaroxaban & $1 \times 20 \mathrm{mg}$ & 75.8 & 22 & 65 & 29.9 & 39 & 65 & 31.2 \\
\hline 4 & Rivaroxaban & $1 \times 20 \mathrm{mg}$ & 93.7 & 27 & 93 & 30.4 & 437 & 72 & 39.4 \\
\hline 5 & Rivaroxaban & $1 \times 20 \mathrm{mg}$ & 67.9 & 65 & 58 & 34.5 & 257 & 47 & 43.0 \\
\hline 6 & Rivaroxaban & $1 \times 20 \mathrm{mg}$ & 54.5 & 40 & 80 & 32.8 & 228 & 67 & 40.8 \\
\hline 7 & Edoxaban & $1 \times 60 \mathrm{mg}$ & n.a. & 51 & 73 & 39.5 & 195 & 66 & 47.7 \\
\hline 8 & Edoxaban & $1 \times 30 \mathrm{mg}$ & 39.9 & $<20$ & 62 & 40.4 & 171 & 56 & 49.9 \\
\hline
\end{tabular}

Conclusions: Direct oral anti-Xa inhibitors are well absorbed in cancer patients after partial or total gastrectomy, but trough and peak levels vary considerably. Studies investigating clinical endpoints are warranted.

PB0059 | Synthesis and Binding Study of Two Small Heterocyclic Compounds with factor Xa Inhibitory Activity

T. Sengupta $^{1}$; S. Karthikeyan ${ }^{2}$; R. Vasanthan ${ }^{3}$; C Suganthi ${ }^{1}$

${ }^{1}$ Sri Sivasubramaniya Nadar College of Engineering, Chennai, India; ${ }^{2} \mathrm{G}$. $S$ Gill Research Institute, Guru Nanak College, Chennai, India; ${ }^{3}$ Indian Institute of Technology Bombay, Mumbai, India

Background: Inhibition of clotting factor $\mathrm{Xa}(\mathrm{fXa})$ activity has been emerged as an effective therapeutic option for treating various thrombotic complications. Unlike warfarin, the widely used medication for thrombosis, $\mathrm{fXa}$ inhbitors do not require routine monitoring as they have predictable anticoagulant effect. Therefore, there is a constant search for novel small molecules fXa inhibitors with improved pharmacological and clinical profile.

Aims: Synthesis, activity and binding study of two small heterocyclic compounds 5-hydroxymethyl imidazolidine -2,4, dione, and indole-2 -carboxylic acid, having fXa inhibitory activity.
Methods: The imidazolidine compound was prepared by the reaction between serine and KCNO in acidic condition. The fXa, and fVIla structures were obtained from protein data bank (PDB ID: 2P16, 3TH2) and further structurally optimized using protein preparation wizard Schrodinger. Grid based ligand docking with energetics (GLIDE) was used to find favorable interactions between ligand and the receptors with flexible conformations of ligand. DFT calculations were carried out using Schrödinger inbuilt Jaguar FXa activity was measured in a Microtitre plate reader (using substrate S2765) following the normal procedure.

Results: Molecular docking simulation is a major tool to understand the binding information of protein - drug interaction mechanism at atomic level. For carboxylic acid, the best binding energy score was shown to be $-29.18 \mathrm{kcal} / \mathrm{mol},-35.03 \mathrm{kcal} / \mathrm{mol}$ in complex with factor $\mathrm{Xa}$, and fVIla respectively. On the other hand, the imidazolidine compound showed binding energy of $-24.68 \mathrm{kcal} / \mathrm{mol},-32.14 \mathrm{kcal} / \mathrm{mol}$, in complex with $\mathrm{fXa}$ and fVIla respectively. Further, to understand intermolecular stability, DFT was performed for free carboxylic acid and 5-hydroxymethyl imidazolidine compound and the DFT parameter as shown in Table 1. Both compounds inhibit fXa in a concentration dependent manner ( $\left.k_{d} \sim 200-300 n M\right)$.

TABLE 1 DFT parameters for Indole-2 -carboxylic acid and 5-hydroxymethyl imidazolidine -2,4, dione compound

\begin{tabular}{lll} 
Surface Name & Indole -2- carboxylic acid & 5-hydroxymethyl imidazolidine \\
HOMO (Unit: Hatree) & $\mathrm{E}=-0.215566$ occ $=1.00$ & $\mathrm{E}=-0.265629$ occ $=1.00$ \\
LUMO (Unit: Hatree) & $\mathrm{E}=-0.049872$ occ $=0.00$ & $\mathrm{E}=-0.012673$ occ $=0.00$ \\
average_local_ionization_energy units: $\mathrm{kcal} / \mathrm{mol}$ & 205.576 & 228.703 \\
electron_density units: electrons/bohr**3 & 0.001 & 0.001 \\
Density laplacian & 0 & 0 \\
electrostatic_potential units: $\mathrm{kcal} / \mathrm{mol}$ & -32.1462 & -33.4682 \\
Reduced density gradient units: none & 0.3 & 0.3 \\
Interaction strength units: electrons/bohr**3 & -0.05 & -0.05 \\
\hline
\end{tabular}




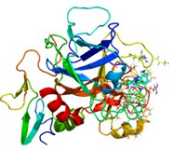

B

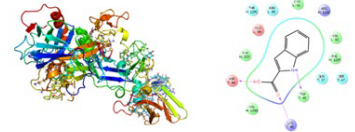

c

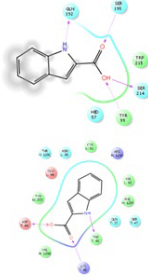

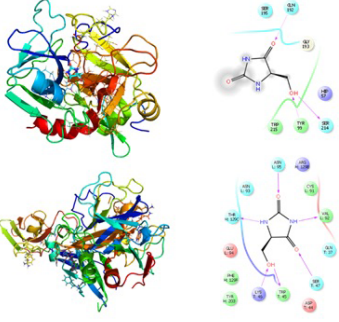

FIGURE 1 Best binding pose of Indole-2-carboxylic acid in complex with (A) fXa (B) fVIla, and of 5-hydroxymethyl imidazolidine -2,4, dione in complex with (C) fXa(D) fVIla

Conclusions: Initial computational and experimental studies show that both compounds bind well and inhibit fXa, Further characterization of the compounds are underway.

PB0060 | The Effect of Dextran Sulfate in Heparin Monitoring

\section{Reagents}

\section{E. Dean}

The Royal College of Pathologists of Australasia Quality Assurance Program (RCPAQAP), Sydney, Australia

Background: Heparins are a class of anticoagulant drugs that are used to prevent and treat thrombosis. The most effective way of monitoring heparin therapy is through anti-Xa assays; these are important to ensure clinical effectiveness and reduce the risk of overanticoagulation. ${ }^{1}$ Inclusion or exclusion of dextran sulfate (DS) in heparin assay reagents impacts measured unfractionated heparin (UFH) levels. The purpose of including DS in reagents is to increase stability and prevent false-low heparin results due to the binding of non-specific proteins to heparin in vivo. ${ }^{2}$ However, reagents that include DS may overestimate the in vivo heparin level. ${ }^{3}$

Aims: To investigate the impact of dextran sulfate in heparin monitoring reagents on external quality assessment (EQA) results.

Methods: Data from RCPAQAP's heparin monitoring surveys was analysed, including comparing the median of all participants to the medians of our 3 largest reagent groups: Siemens INNOVANCE heparin, HemosIL Liquid Anti-Xa, and Stago STA-Liquid Anti-Xa.

Results: Siemens INNOVANCE heparin and HemosIL Liquid Anti-Xa include dextran sulfate, while Stago STA-Liquid Anti-Xa does not. ${ }^{2}$ Inclusion of DS promotes stability and releases heparin from Platelet Factor 4 complexes, establishing free (and active) heparin. However, not all protein-bound heparin is active in vitro, therefore addition of DS may overestimate functional heparin. ${ }^{3}$

As seen in Figure 1, Siemens INNOVANCE heparin and HemosIL Liquid Anti-Xa, the reagents that include DS have increased median UFH results compared to Stago STA-Liquid Anti-Xa which does not include DS.

The differences between DS including and excluding reagents may be reduced in EQA reports such as RCPAQAP's since dextran is added as a stabilising excipient, and the process of lyophilising can have an impact. ${ }^{1}$

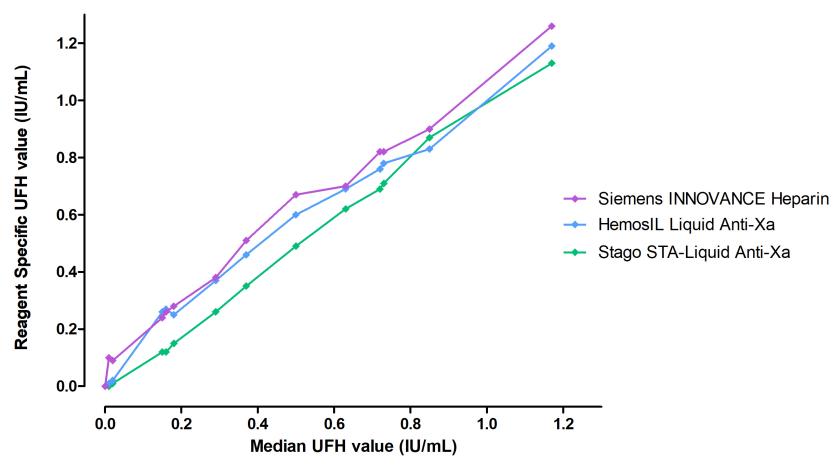

FIGURE 1 Bias of reagent groups due to inclusion or exclusion of dextran sulfate in RCPAQAP surveys

Conclusions: RCPAQAP's heparin monitoring survey data aligns with manufacturers data, where it has been found that heparin reagents that include dextran sulfate have increased anti-Xa results compared to reagents that exclude it.

PB0061 | Influence of Low-generation Polyamidoamine (PAMAM) Dendrimers on Structure and Function of Fibrinogen, Generation, Amidolytic and Coagulating Activity of Thrombin in vitro

L. Mukhametova ${ }^{1,2} ;$ R. Aisina ${ }^{1} ;$ E. Ivanova $^{1}$

${ }^{1}$ Lomonosov Moscow State University, Moscow, Russian Federation;

${ }^{2}$ Bauman Thechnical University, Moscow, Russian Federation

Background: PAMAM dendrimers are promising nanocarriers for drug delivery. They can interact with plasma proteins and affect the activity of enzymes. It is known that high-generation cationic dendrimers can cause platelet aggregation, inhibit thrombin generation, and cause the formation of fibrinogen aggregates. The mechanism of this coagulopathy is currently unclear.

Aims: Comparing of effect of low-generation anionic and cationic PAMAM on function of fibrinogen and activity of key enzyme of coagulation system of thrombin (Tb).

Methods: Effect of cationic (G1-G3) and anionic (G1.5-G3.5) PAMAM dendrimers on amidolytic and coagulating activities of $\mathrm{Tb}$, thrombin time (TT), prothrombin time (PT), thrombin generation in human plasma; fluorescent-spectrum, zeta-potential, and fibrinogen polymerization by Tb were studied.

Results: Thrombin activity was increased with increasing concentration and generation of cationic dendrimers, anionic inhibited Tb-activity. The cationic dendrimers suppressed Tb-generation in plasma at lower concentrations than anionic. Comparison of cationic and anionic dendrimers with the same number of surface-charged groups showed that cationic dendrimers cause greater changes in generation parameters of thrombogram. G3-dendrimer at concentration of $7 \mathrm{mkM}$ completely suppressed Tb-generation. PT increased with increasing concentration of cationic dendrimers and was independent of anionic dendrimers concentration. Cationic dendrimers elongated PT to greater extent than TT. Studying effect 
of dendrimers on fluorescent-spectrum, zeta-potential, and fibrinogen polymerization by thrombin showed that cationic dendrimers bind to fibrinogen strongly than anionic dendrimers, and change its conformation and coagulability. Anionic dendrimers didn't affect on kinetics of fibrinogen polymerization unlike cationic, both type of dendrimers reduced plasma clotting with thrombin.

Conclusions: Compared to anionic (G1.5-G3.5), cationic dendrimers (G1-G3) more strongly suppress the generation of thrombin, alter conformation of fibrinogen and its clotting rate by thrombin in a concentration-dependent manner. The observed effects of cationic PAMAM dendrimers are probably caused by strong electrostatic interactions between positively charged surface of dendrimers and negatively charged sites of thrombin and fibrinogen.

\section{PB0062 | Influence of Tissue-factor Pathway Inhibitor on} Thrombin Generation Assay

M. Didembourg ${ }^{1}$; J. Douxfils ${ }^{1,2}$; A. Carlo ${ }^{3}$; L. Morimont ${ }^{1,2}$

${ }^{1}$ University of Namur, Faculty of Medicine, Department of Pharmacy, Namur, Belgium; ${ }^{2}$ Qualiblood sa, Namur, Belgium; ${ }^{3}$ Diagnostica Stago, Asnières-sur-Seine, France

Background: Tissue-factor pathway inhibitor (TFPI) is an anticoagulant protein that exerts its activity by forming an inactive FXa-TFPITF-FVIla quaternary complex. In plasma, TFPI is normally found at a concentration of about $70 \mathrm{ng} / \mathrm{mL}$ but many pathologies impact its level, modifying the hemostatic function of individuals. In COVID-19 patients, in spite of a hypercoagulability state, increased levels of TFPI up to $400 \mathrm{ng} / \mathrm{mL}$ were observed.

Aims: This study aimed to assess the impact of TFPI on thrombin generation assay (TGA), when performed on the Calibrated Automated Thrombogram (CAT) and on the ST-Genesia system.

Methods: Normal pooled plasma (NPP) constituted of 50 healthy individuals was used as matrix. Recombinant TFPI was spiked in NPP at five relevant plasma concentrations (0 [=Phosphate buffer saline], 50, 100, 200 and $400 \mathrm{ng} / \mathrm{mL}$ ). TGA was assessed on both platforms by 3 independent runs and the triggering reagent was the STGThromboScreen-TM (Stago, France). The percentages of change from baseline were calculated for each TGA parameter.

Results: On both platforms, based on mean values, statistically significant differences were observed between the five tested concentrations ( $P$-value $<0.05)$. Though not statistically significant, percentages of change from baseline were more pronounced on the ST-Genesia compared to the CAT system, especially for the endogenous thrombin potential (ETP) (decrease of $25 \%$ and $17 \%$, respectively), for the lag time (LT) (prolongation of $17 \%$ and $10 \%$, respectively) and for the peak height $(\mathrm{PH})$ (decrease of $47 \%$ and $35 \%$, respectively).

Conclusions: Regarding the physiological role of TFPI, impact on the LT parameter was expected. On the other hand, the important inhibitory effect of TFPI on the ETP and PH is a novel observation. This study showed that TFPI levels impacted in a dose-dependent manner TGA performed either on the CAT or on the ST-Genesia system. Any acute or mechanistic situations in which TFPI levels may be impacted will thus interfere with TGA.

\section{PB0063 | Monitoring Heparin: Stability of Two Different Anti-Xa} Assays in Citrated vs CTAD Tubes

T. Sinegre; M. Gremillet; V. Fourneyron; M. Tillier; A. Trayaud;

L. Talon; A. Lebreton

CHU Clermont-Ferrand, Clermont-Ferrand, France

Background: Unfractionated heparin (UFH) and low molecular weight heparin (LMWH) are the main injectable anticoagulant. The monitoring by anti-Xa activity is mainly used for UFH and occasionally for LMWH but data on stability before analysis remains limited. According to medical societies anti-Xa activity should be done within $2 \mathrm{~h}$ for UFH and $6 \mathrm{~h}$ for LMWH. However, discrepancies exist depending on the reagents used and the type of tubes.

Aims: The aim of this study is to determine the stability of anti-Xa UFH and LMWH activities on Stago and Siemens analyzers, with citrated tubes with and without theophylline, adenosine dipyridamole (CTAD).

Methods: The first 14 patients from the Clermont-Ferrand University hospital anticoagulated with UFH or LMWH, whose blood was drawn on citrated tubes or CTAD were included (total of 56 patients). The samples were centrifuged at $2200 \mathrm{~g}$ for $15 \mathrm{~min}$ within an hour (T1) and the anti-Xa activity was determined immediately then $4 \mathrm{~h}$ and $6 \mathrm{~h}(\mathrm{~T} 6)$ after the blood sample. Anti-Xa activities were determined on StaR with STALiquid anti-Xa reagent (Stago) and on CS2100 with Innovance Heparin reagent (Siemens). Results (U/ $\mathrm{mL}$ ) are expressed as the median (min-max).

Results: UFH anticoagulated patients show stable anti-Xa activities for up to $6 \mathrm{~h}$ after collection on CTAD tube. Anti-Xa activities on citrated tube are significantly reduced at $6 \mathrm{~h}$ with respectively 0.25 (T1) (0.12-0.63) and $0.20(0.10-0.55)(P<0.01)$ for Stago and 0.28 (T1) $(0.18-0.50)$ and $0.26(0.16-0.47)(P<0.001)$ for Siemens (Figure 1). 

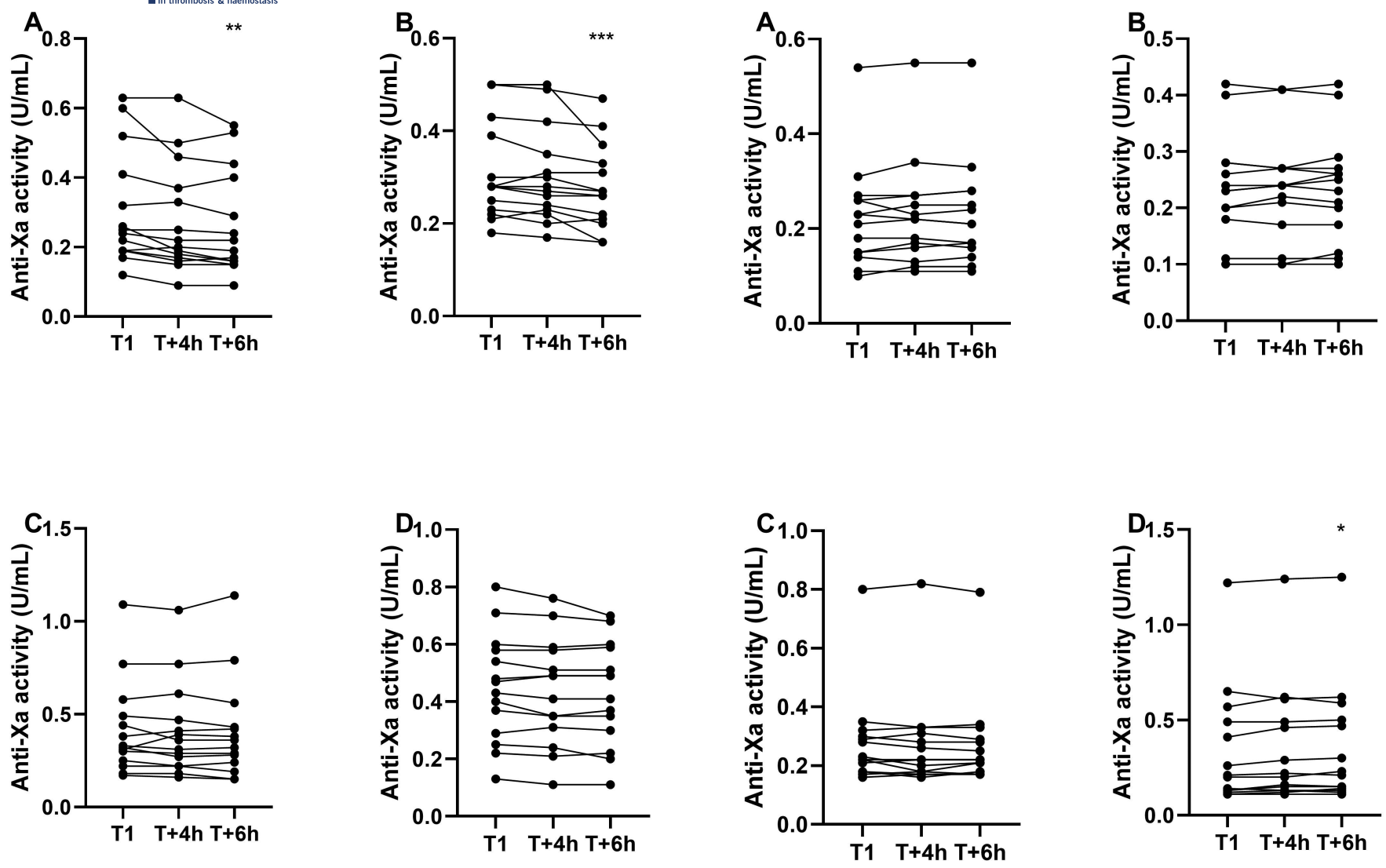

FIGURE 1 Stability of anti-Xa UFH activity. A. Sampling on citrated

FIGURE 2 Stability of anti-Xa LMWH activity. A. Sampling on cittube analyzed on Stago. B. Sampling on citrated tube analyzed on Siemens. C. Sampling on CTAD tube analyzed on Stago. D. Sampling on CTAD tube analyzed on Siemens

Patients anticoagulated with LMWH show stable anti-Xa activities for up to $6 \mathrm{~h}$. Anti-Xa activities on CTAD tube are stable at $6 \mathrm{~h}$ for Stago and $4 \mathrm{~h}$ for Siemens. Anti-Xa activities are increased at $6 \mathrm{~h}$ for Siemens with respectively 0.20 (T1) (0.11-1.22) and 0.22 (0.11-1.25) $(P<0.05)$ (Figure 2). rated tube analyzed on Stago. B. Sampling on citrated tube analyzed on Siemens. C. Sampling on CTAD tube analyzed on Stago. D. Sampling on CTAD tube analyzed on Siemens

Conclusions: Anti-Xa UFH activity on citrated tube is reduced at $6 \mathrm{~h}$ without clinical impact whatever the reagent.

PB0064 | Performance Evaluation of the INNOVANCE Anti-Xa Assay for the Quantitative Determination of Rivaroxaban

D. Pahren ${ }^{1}$; M. Wilkens ${ }^{1}$; A. Doimo ${ }^{1}$; A. Wilhelm¹; I. Birschmann²; E. Hod ${ }^{3}$; K. Madlener ${ }^{4}$; M. Merz ${ }^{1}$; D. Bussfeld ${ }^{1}$; U.-P. Schobel ${ }^{1}$ ${ }^{1}$ Siemens Healthcare Diagnostics Products GmbH, Marburg, Germany; ${ }^{2}$ Herz- und Diabeteszentrum Nordrhein-Westfalen, Universitätsklinik der Ruhr-Universität Bochum, Institut für Laboratoriums- und Transfusionsmedizin, Bad Oeynhausen, Germany; ${ }^{3}$ New York Presbyterian Hospital Columbia University, New York, United States; ${ }^{4}$ Kerckhoff-Klinik GmbH, Bad Nauheim, Germany

Background: Rivaroxaban (Xarelto) is a direct factor Xa inhibitor used in the prevention of thrombotic diseases. Due to its predictable pharmacokinetics and wide therapeutic window, no therapeutic range in which the clinical outcome is optimized has been established within rivaroxaban market approval. Since hemostasis is a balance between the tendency to clot and the risk of bleeding, anticoagulants have an inherent risk of causing bleeding, which is the 
most common side effect of rivaroxaban. Although, according to the manufacturer of rivaroxaban regular monitoring is not needed, quantitative assays should be available to aid in the assessment of a patient's anticoagulant status.

Aims: Performance evaluation of the INNOVANCE Anti-Xa assay for the quantitative determination of rivaroxaban on the Sysmex ${ }^{\circledR}$ CS2500 / CS-5100 System, Atellica ${ }^{\circledR}$ COAG 360 System, and BCS ${ }^{\circledR}$ XP System.

Methods: The following studies were conducted: limit of quantitation (LoQ), linearity, precision, dilution recovery, and method comparison.

Results: Based on the results from studies for each application mentioned above, the analytical measuring interval $(20-350 \mathrm{ng} / \mathrm{mL}$ ) was defined. An extended measuring interval (1:2 sample dilution) of $350-700 \mathrm{ng} / \mathrm{mL}$ was demonstrated. Good comparability of the INNOVANCE Anti-Xa assay on the Sysmex CS-2500 System versus the STA-Liquid Anti-Xa assay on the STA COMPACT MAX system could be shown., The INNOVANCE Anti-Xa assay compares well on the Sysmex CS-5100 System, BCS XP System, and Atellica COAG 360 System versus the Sysmex CS-2500 System.

Conclusions: The INNOVANCE Anti-Xa assay demonstrates good performance on the Sysmex CS-2500 / CS-5100 System, BCS XP System, and Atellica COAG 360 System and can be used for the quantitative determination of rivaroxaban in human citrated plasma. Disclaimer: Atellica COAG 360 System is not available in the US INNOVACE Anti-Xa is under development and not commercially available. Its future availability cannot be ensured.

PB0065 | The Coagulation Balance: Intracranial Hemorrhage in a Patient with Antithrombin Deficiency. A Case Report

I. Machado; S. Teixeira; D. Cibele Gonçalves; C. Monteiro;

M. Carvalho; L. Ricca Gonçalves; C. Neves; M.d.C. Koch

Centro Hospitalar Universitário São João, Porto, Portugal

Background: Deficiency of Antithrombin (AT) represents a risk factor for thromboembolic disease. However, there is considerable variation in its expression and the management of anticoagulant therapy can be challenging. In contrast, cerebral arteriovenous malformations (AVMs) are relatively uncommon but an important cause of intracranial hemorrhage (ICH).

Aims: To present a case report of a patient with AT deficiency, under anticoagulation, with an ICH due to AVM.

Methods: We report a case of a 40-year-old male with deficiency of AT and history of recurrent deep vein thrombosis under rivaroxaban $20 \mathrm{mg} /$ day. The patient was admitted to the emergency department due to an ICH associated with an AVM.

Results: When indicated, we use prothrombin complex concentrates (PCC) to reverse anticoagulation in patients under rivaroxaban, as andexanet alfa is not available. As rivaroxaban concentration was < $25 \mathrm{ng} / \mathrm{mL}$, it was decided not to use PCC. After a hemorrhagic event, the approach involves managing the thrombotic and bleeding recurrence risk. This patient, after embolization, developed hemorrhagic coagulopathy needing transfusion support (fresh frozen plasma and fibrinogen concentrate), as he had a large femoral hematoma when the catheter was removed. This coagulopathy was assumed to be related to contrast extravasation as he had an iatrogenic femoral pseudoaneurysm, whereby deferring the anticoagulation resumption. When resuming anticoagulation in this patient, unfractionated heparin will be used and AT replacement may be necessary to enhance heparin anticoagulant efficacy. Alternatively, a parenteral direct thrombin inhibitor such as argatroban, which does not require AT function, may be used.

Conclusions: Deficiency of AT, after a hemorrhagic event, may represent a huge challenge to restart anticoagulation. The balance of coagulation is not linear and decisions regarding therapy are individualized depending on the specific status of the patient. Depending on the circumstances, the hemorrhagic or the thrombotic risk prevails.

PB0066 | Non-thrombogenic Effect Observed with Simulated Concomitant Administration of Anti-hemophilic Factor, Emicizumab and rFVIla on Thrombin Generation

M.-I. Bravo ${ }^{1}$; A. Raventos ${ }^{1}$; A. Perez ${ }^{1} ;$ M. Costa ${ }^{1}$; T. Willis ${ }^{2}$

${ }^{1}$ Discovery Research, Bioscience Research Group, Grifols, Barcelona, Spain; ${ }^{2}$ Discovery Research, Bioscience Research Group, Grifols, Raleigh, United States

Background: Emicizumab, a non-factor therapy for hemophilia A (HA) treatment, may require concomitant pro-hemostatic therapies to fully protect patients against breakthrough bleedings. Further, HA patients with inhibitors treated with emicizumab may also require FVIII for immune tolerance induction (ITI). Previously, we demonstrated in vitro that the combination of emicizumab, plasma-derived factor VIII concentrate containing von Willebrand factor (pdFVIII/ VWF), and rFVIla did not trigger a multiplying effect on thrombin generation (TG). Since several pdFVIII/VWF concentrates with different purification and production processes are available, further studies are needed.

Aims: To assess the TG profiles of another pdFVIII/VWF concentrate, Anti-Hemophilic Factor (AHF) (Koate, Grifols) in combination with emicizumab and rFVIla, in a simulated in vitro model of ITI with bleeds.

Methods: Pooled HA plasma with inhibitors (HAp-i, Technoclone) (19 BU), containing emicizumab (50 $\mathrm{gg} / \mathrm{mL}$ ) plus AHF (1 to $4.5 \mathrm{IU} /$ $\mathrm{mL}$ ) was combined with rFVIla (0.9 and $7 \mu \mathrm{g} / \mathrm{mL}$ ). Samples were analyzed using a Calibrated Automated Thrombogram (CAT) assay and PPP Reagent Low (Stago). TG reaction parameters were calculated using CAT software.

Results: Thrombin peak (TP) range of normal plasma was established based on 13 healthy individual plasma samples (TP: 47-104 nM). In HAp-i with emicizumab $50 \mathrm{mg} / \mathrm{mL}$, TP levels were below normal ranges. Addition of high concentration of AHF (4.5 IU/mL) to HAp-i with emicizumab slightly increased TP compared to HAp-i without emicizumab (TP:47 and 34 nM, respectively), reaching the lowest 
level of TP normal range. When rFVIla was added, the triple combination did not trigger a multiplying effect on TP but an additive increase comparable to normal plasma TP ranges, even at high concentrations of AHF (TP: 74 and $138 \mathrm{nM}$, for 0.9 and $7 \mu \mathrm{g} / \mathrm{mL} \mathrm{rFVIla}$, respectively).

Conclusions: In agreement with previous data with a similar pdFVIII/ VWF, the combination of emicizumab plus AHF and rFVIla did not trigger a multiplying, but an additive effect on TG.

PB0067 | Evaluation and Performance Characteristics of the Automated Sysmex CN-6000 Haemostasis Analyzer

I. Martin-Toutain ${ }^{1}$; M. Gaaloul ${ }^{1}$; M. Berber ${ }^{1}$; E. Bailly ${ }^{1}$;

J.-P. Delorme ${ }^{2}$; Y. Bourti ${ }^{2}$; C. Frere ${ }^{1,3}$

${ }^{1}$ Assistance Publique Hôpitaux de Paris, Paris, France; ${ }^{2}$ Sysmex France, Villepinte, France; ${ }^{3}$ Sorbonne Université, INSERM UMRS_1166,

Institute of Cardiometabolism and Nutrition, Paris, France

Background: Centralization of laboratory diagnostics with nonstandard samples and urgent requests raise the need for highthroughput analyzers with short turn-around times for coagulation tests. The newly launched CN-6000 is a fully automated haemostasis random-access multiparameter analyzer, designed to perform blood coagulation assays.

Aims: To evaluate the performance of the $\mathrm{CN}-6000$ analyzer against a predicate device.

Methods: We evaluated the $\mathrm{CN}-6000$ analyzer (Sysmex Corp., Japan) with a unique portfolio of liquid and ready to use haemostasis reagents in comparison to the STA R Max $2^{\circledR}$ (Stago, France) analyzer with dedicated reagents for 10 different tests including determination of prothrombin time (PT), activated partial prothrombin time (aPTT), fibrinogen, anti-Xa activity, D-Dimers and antithrombin levels. We used 343 samples from normal subjects, anticoagulated patients, critically ill patients, plasmas with high or low fibrinogen content and plasmas with abnormal levels of factor II, V, VII and X. We evaluated imprecision, throughput and sample turn-around time (STAT) function.

Results: Total precision (evaluated as the coefficient of variation) was below $6 \%$ for all parameters both in normal and in pathological ranges. Test results obtained on the $\mathrm{CN}-6000$ analyzer were well correlated with those obtained on the STA R Max $2{ }^{\circledR}$ analyzer for all parameters (Pearson correlation coefficients between 0.908 and 0.987). Agreement between both analyzers was excellent for all assays. CN-6000 throughput was 30\% higher than STA R Max $2^{\circledR}$ throughput (258 vs 185 tests per hour for PT, aPTT, fibrinogen, DDimer, anti-Xa and factor V). STAT for critical care samples testing was $<7 \mathrm{~min}$.

Conclusions: The $\mathrm{CN}-6000$ performs equivalently or better than the reference methods with a significant improvement in throughput. This new hemostasis analyzer is therefore particularly well suited for coagulation laboratories managing a high number of nonstandard and critical care samples and requiring a high sample throughput.
PB0068 | Performance Evaluation of a New VWF:GPIbM VWF Activity Assay in a Multicenter Study

M. Kahl ${ }^{1} ;$ M. Boehm-Weigert ${ }^{1}$; C. Flickinger ${ }^{2}$; N. DeSimone ${ }^{3}$; D. Dietzen ${ }^{4}$; C. Eby ${ }^{4}$; K. Friedman ${ }^{5}$; K. Kottke-Marchant ${ }^{6}$; T. Ortel ${ }^{7}$; J. Patzke ${ }^{1}$; S. Pipe ${ }^{8}$; R. Sarode ${ }^{3} ;$ M. Merz ${ }^{1}$

${ }^{1}$ Siemens Healthcare Diagnostics Products $\mathrm{GmbH}$, Marburg, Germany; ${ }^{2}$ Siemens Healthcare Diagnostics, Inc., Newark, United States; ${ }^{3}$ UTSW Clinical Laboratory Services, Division of Transfusion Medicine and Hemostasis, UT Southwestern Medical Center, Dallas, United States; ${ }^{4}$ Washington University School of Medicine, St. Louis, United States; ${ }^{5}$ Versiti Wisconsin, Inc, Milwaukee, United States; ${ }^{6}$ Cleveland Clinic Foundation, Cleveland, United States; ${ }^{7}$ Duke Health, Duke Hematology Clinic, Durham, United States; ${ }^{8}$ University of Michigan, Ann Arbor, United States

Background: Deficiency or dysfunction of VWF may lead to von Willebrand disease. Initial evaluation of patients with suspected VWD usually includes coagulation factor VIII, VWF antigen, and VWF activity (initial test profile). The new INNOVANCE ${ }^{\circledR}$ VWF Ac Activity Assay, utilizing a glycoprotein lb (GPIb) fragment with two gain-of-function mutations, measures binding of VWF to GPIb in the absence of ristocetin (VWF:GPIbM).

Aims: In this study the analytical and clinical performance of the INNOVANCE VWF Ac Assay on the BCS ${ }^{\circledR}$ XP System was evaluated. Methods: Analytical performance data includes linearity, limit of quantitation (LoQ), precision, reproducibility, and reference intervals. Diagnostic accuracy was evaluated by comparison of diagnostic assessments when using the INNOVANCE VWF Ac Assay vs. a variety of VWF activity assays (commercially available assays, Laboratory Developed Tests, different analyzers).

Results: The assay measuring interval is 4 to $300 \%$ of norm as demonstrated by LoQ and linearity studies. Studies of internal precision and external reproducibility at three clinical sites demonstrated that the highest observed CV was $7.2 \%$. The reference intervals established are comparable to those for other VWF activity assays. The diagnostic accuracy study showed that the use of INNOVANCE VWF Ac (instead of currently used VWF activity assays) did not change the diagnostic assessment of the initial VWF profile results for most patients. For patients with discordant assessments, the benefit/risk ratio was higher compared to current VWF activity methods.

Conclusions: The INNOVANCE VWF Ac Assay on the BCS XP System is very precise can be used to aid in the diagnosis of VWD. Disclaimer: BCS, INNOVANCE, and all associated marks are trademarks of Siemens Healthcare Diagnostics Products $\mathrm{GmbH}$ or its affiliates. Under FDA review (DE Novo classification request). Not available for sale in the USA. Product availability may vary from country to country and is subject to varying regulatory requirements. 
PB0069 | Influence of Para-sulfonato-calixarenes on Components of Coagulation System

E. Ivanova $^{1}$; R. Aisina ${ }^{1}$; K. Gershkovich ${ }^{2}$

${ }^{1}$ Moscow State University, Moscow, Russian Federation; ${ }^{2}$ Emanuel Institute of Biochemical Physics of RAS, Moscow, Russian Federation

Background: Para-sulfonato-calix[n]arenes $(\mathrm{CnS})$ are capable of complexing biologically active molecules. The $\mathrm{CnS}$ hydrophobic core and anionic rim can facilitate protein recognition by capturing the arginine or lysine side chains. The potential bioactivity of $\mathrm{CnS}$ derivatives in relation to some components of the hemostatic system has been shown.

Aims: In this work, the influence of C4S (rigid cone structure) and C6S (flexible double partial cone structure) on the coagulation system components was studied.

Methods: C4S and C6S effect on thrombin and Xa-factor, plasma overall haemostatic potential (OHP), prothrombin time, APTT, Zpotential and fluorescent spectrum of fibrinogen (Fg), and Fg coagulation by thrombin were studied.

Results: C6S was a stronger noncompetitive inhibitor of thrombin $(\mathrm{Ki}=0.27 \mathrm{mM})$ than $\mathrm{C} 4 \mathrm{~S}(\mathrm{Ki}=1.1 \mathrm{mM}) . \mathrm{C} 4 \mathrm{~S}$ and $\mathrm{C} 6 \mathrm{~S}$ decreased $\mathrm{Xa}$ activity. C4S had little effect on plasma OHP, C6S increased the lag phase and reduced the amount of fibrin formed. C4S had no effect, C6S significantly increased prothrombin time and APTT. C4S and C6S form complexes with Fg and change its conformation: the fluorescence quenching constants are 1.3*104 and 1.1*104 M-1, respectively. Analysis of the Z-potential showed C6S increasing the negative charge of Fg stronglier than $\mathrm{C} 4 \mathrm{~S}$. C4S reduced the lag phase and increased the rate of Fg clotting by thrombin, while C6S $(>100 \mu \mathrm{M})$ increased the lag phase and decreased the amount of fibrin until the complete suppression of Fg clotting.

Conclusions: The differences between $\mathrm{C} 4 \mathrm{~S}$ and $\mathrm{C} 6 \mathrm{~S}$ are related to their structure, flexibility and the number of negative charges. C4S stimulates the clotting of fibrinogen, mainly by a change in the protein conformation upon complexation with a rigid $\mathrm{C} 4 \mathrm{~S}$ structure. C6S inhibits Fg coagulation due to strong inhibition of thrombin activity and influence on the coagulation factors cascade. Innocuous C4S may be promising for stopping local bleeding.

(Funds of the State Registration topicno.AAA-A16-116052010081-5)
PB0071 | Performance Evaluation of the INNOVANCE Anti-Xa Assay for the Quantitative Determination of Apixaban

M. Wilkens ${ }^{1}$; A. Doimo ${ }^{1}$; A. Wilhelm ${ }^{1}$; I. Birschmann 2 ; E. Hod $^{3}$; K. Madlener ${ }^{4}$; M. Merz ${ }^{1}$; D. Bussfeld ${ }^{1}$; U.-P. Schobel ${ }^{1}$

${ }^{1}$ Siemens Healthcare Diagnostics Products $\mathrm{GmbH}$, Marburg, Germany;

${ }^{2}$ Herz- und Diabeteszentrum Nordrhein-Westfalen, Universitätsklinik der Ruhr-Universität Bochum, Institut für Laboratoriums- und Transfusionsmedizin, Bad Oeynhausen, Germany; ${ }^{3}$ New York Presbyterian Hospital Columbia University, New York, United States; ${ }^{4}$ Kerckhoff-Klinik GmbH, Bad Nauheim, Germany

Background: Apixaban (Eliquis) is a direct factor Xa inhibitor used in the prevention of thrombotic diseases. Due to its predictable pharmacokinetics and wide therapeutic window, no therapeutic range in which the clinical outcome is optimized has been established within market approval. Since hemostasis is a balance between the tendency to clot and the risk of bleeding, anticoagulants have an inherent risk of causing bleeding, which is the most common side effect of apixaban. Although, according to the manufacturer of apixaban regular monitoring is not needed, quantitative assays should be available to aid in the assessment of a patient's anticoagulant status. Aims: Performance evaluation of the INNOVANCE ${ }^{\circledR}$ Anti-Xa assay for the quantitative determination of apixaban on the Sysmex ${ }^{\circledR}$ CS2500 / CS-5100 System, Atellica ${ }^{\circledR}$ COAG 360 System*, and BCS ${ }^{\circledR}$ XP System.

Methods: The following studies were conducted: limit of quantitation (LoQ), linearity, precision, dilution recovery, and method comparison.

Results: Based on the results from studies for each application mentioned above, the analytical measuring interval $(20-350 \mathrm{ng} / \mathrm{mL})$ was defined. An extended measuring interval (1:2 sample dilution) of $350-700 \mathrm{ng} / \mathrm{mL}$ was demonstrated. Good comparability of the INNOVANCE Anti-Xa assay on the Sysmex CS-2500 System versus the STA- Liquid Anti-Xa assay on the STA COMPACT MAX system could be shown. The INNOVANCE Anti-Xa assay compares well on the Sysmex CS-5100 System, BCS XP System, and Atellica COAG 360 System versus the Sysmex CS-2500 System.

Conclusions: The INNOVANCE Anti-Xa assay demonstrates good performance on the Sysmex CS-2500 / CS-5100 System, BCS XP System, and Atellica COAG 360 System and can be used for the quantitative determination of apixaban in human citrated plasma. Disclaimer: Atellica COAG 360 System is not available in the US. INNOVACE Anti-Xa is under development and not commercially available. Its future availability cannot be ensured. 


\section{PB0072 | Analysis of Coagulation Factors in Fresh-frozen Plasma}

M. Pikta ${ }^{1,2}$; T. Szanto ${ }^{3} ;$ R. Reimal ${ }^{4}$; P. Muliin ${ }^{4} ;$ H. König ${ }^{5} ;$ G. Blinova ${ }^{5}$; V. Banys ${ }^{6}$

${ }^{1}$ Department of Laboratory Medicine, North Estonia Medical Centre, Tallinn, Estonia; ${ }^{2}$ Department of Health Technologies, Tallinn University of Technology, Tallinn, Estonia; ${ }^{3}$ Research Program Unit of Systems Oncology, Helsinki University Hospital, Unit of Coagulation Disorders, Helsinki, Finland; ${ }^{4}$ Blood Centre, Foundation Pärnu Hospital, Pärnu, Estonia; ${ }^{5}$ Blood Service, Ida-Viru Central Hospital, Kohtla-Järve, Estonia; ${ }^{6}$ Faculty of Medicine, Institute of Biomedical Sciences, Department of Physiology, Biochemistry, Microbiology and Laboratory Medicine, Vilnius University, Vilnius, Lithuania

Background: Fresh frozen plasma (FFP) is used to correct deficiencies of coagulation factors. The levels of these factors in FFP vary with donor demographics, ex-vivo processing of plasma. Quality control monitoring of FFP plays an important role.

Aims: The aim of study was to analyze the levels of coagulation factors, the amount and structure of von Willebrand factor (VWF) in FFP units.

Methods: In total 30 FFP units were evaluated (20 from Foundation Pärnu Hospital Blood Centre and 10 from Ida-Viru Central Hospital Blood service). Blood of donors was collected with citrate, separated from blood erythrocytes, rapidly frozen within $24 \mathrm{~h}$ after phlebotomy at $-25^{\circ} \mathrm{C}$. FFP was thawed at $+37^{\circ} \mathrm{C}$ and immediately tested for fibrinogen, FII, FV, FVII, FVIII, FIX, FX, FXI, FXII, VWF:Ag levels, VWF activity (VWF:Ac) and VWF multimers (VWF:MM).

Results: All 30 FFP samples had normal coagulation factors activities, normal fibrinogen levels and the levels were within the routinely applied reference intervals. There was no significant difference in VWF:Ag, VWF:Ac and FVIII levels between blood group $O(n=11)$ and non-O blood group $(n=19)$ samples $(P=0.182$, $P=0.271, P=0.401$ ). The mean value of FVIII (after freezing and thawing) was not less than $70 \mathrm{IU}$ of Factor VIII per $100 \mathrm{~mL}$ (mean $115 \%$, SD \pm 35.7). The VWF:MM evaluation by densitometer demonstrated patterns comparable to internal reference plasma, which is used as internal quality control. No significant differences in high molecular weight multimers (HMWM) between blood group $\mathrm{O}$ and non-O samples were found $(P=0.491)$.

Conclusions: Our results demonstrate that retention of all coagulation factors in FFP produced from whole blood within $24 \mathrm{~h}$ of collection is satisfactory. The quantity and quality of VWF as well as VWF:MM patterns represent healthy population. To our best knowledge, this report on FFP quality is the first study in Estonia.
P0121 | Hemostatic Defects Associated with IgA Monoclonal Gammopathy

M.C. Shen ${ }^{1,2}$; C.-W. Huang ${ }^{1}$; G.-M. Lai ${ }^{1}$; Y.-H. Shih ${ }^{1}$; C.-Y.L. Lin ${ }^{1}$; H.-Y. Lin ${ }^{1}$; S.-F. Kuo ${ }^{1}$; J.-S. Lin ${ }^{1}$

${ }^{1}$ Changhua Christian Hospital, Changhua City, Taiwan, Province of China; ${ }^{2}$ National Taiwan University Hospital, Taipei, Taiwan, Province of China

Background: Acquired hemostatic defects are frequent manifestations of dysproteinemia. Hemostatic abnormalities are often varied. The main pathogenesis involve inhibition of hemostasis and accelerated clearance of coagulation proteins.

Aims: Hemostatic defects associated with IgA monoclonal gammopathy in 3 patients were studied for their abnormalities and pathogenesis.

Methods: Coagulation and platelet functional tests were done routinely. Mixing coagulation studies of patient's plasma 1:1 mixed with normal pool plasma (NPP) or normal platelet rich plasma (PRP) 1:1 mixed with patient's plasma were performed to detect any coagulation protein inhibitors or impaired platelet functions, respectively.

Results: In patients 1, 2 and 3, aged/sex were 44/F (female), 71/M (male) and 82/M, affected with lymphoproliferative disorder, lymphoplasmacytoid lymphoma and multiple myeloma, respectively; IgA levels were $2760 \mathrm{mg} / \mathrm{dl}, 3310 \mathrm{mg} / \mathrm{dl}$ and $8347.7 \mathrm{mg} / \mathrm{dl}$, respectively, and experienced hypermenorrhea and easy gun bleeding, prolonged postextraction bleeding and hematoma-like muscle swelling in the back, and free of abnormal bleeding, respectively; platelet count was $153,000 / \mu \mathrm{l}, 114,000 / \mu \mathrm{l}$ and $37,000 / \mu \mathrm{l}$, prothrombin time (s)/partial thromboplastin time $(\mathrm{s}) /$ thrombin time $(\mathrm{s})$ were 13.3/46.0/21.6, 15.7/49/28.4 and >160/>240/26.5, respectively; fibrinogen (mg/dl)/ VIII:C (IU/dl)/VWF:Ag (IU/dl)/VWF:Activity (IU/dl)/factor II, V, VII, IX and $X$ coagulant activity levels were 132.5/16.3/21.9/22.9/normal levels, 880/33.4/19.6/20.6/normal levels and 62/114/240/229/ coagulant activities decrease to 20-30 IU/dl, respectively. Platelet aggregation tests in patient 1 showed very poor response to agonists and it was difficult to isolate PRP for test in patients 2 and 3 . Mixing studies with NPP of VWF and factor VIII:C in patients 1 and 2 and other clotting actors in patient 3 all failed to detect inhibitors, and platelet aggregation test of NPRP mixed with PPP all showed normal results.

Conclusions: Similar IgA monoclonal gammopathy affected coagulation differently. Patient 1 and 2 manifested acquired von Willebrand disease, patient 3 manifested multiple coagulation factors-deficiencies, high possibly caused by accelerated clearance of coagulation proteins. However all showed platelet dysfunctions. 


\section{P0123 | Hemostatic Function in Patients with Factor XI}

\section{Deficiency}

L. Montero Martín ${ }^{1}$; N. Butta ${ }^{2}$; M.C Vázquez Rodriguez ${ }^{1}$; E. Monzón Manzano $^{3}$; P. Auña ${ }^{3}$; E.G. Arias Salgado ${ }^{3}$

${ }^{1}$ Haematology and Hemotherapy Department, Mostoles University Hospital, Móstoles, Spain; ${ }^{2}$ Head of Coagulopathies and Haemostasis Disorders Research Laboratory (Hematology Unit, La Paz University Hospital-IDIPaz), Madrid, Spain; ${ }^{3}$ Coagulopathies and Haemostasis Disorders Research Laboratory (Hematology Unit, La Paz University Hospital-IDIPaz), Madrid, Spain

Background: Factor (F) XI deficiency is a rare genetic disorder. The bleeding tendency is poorly correlated with its plasma levels, but seems to be affected by von Willebrand factor (VWF).

Aims: We aimed to evaluate haemostasis in patients with partial FXI deficiency, its dependency on VWF levels and the possible therapeutic effect of emicizumab in these patients.

Methods: Project was approved by the Ethics Committee for Clinical Research (CEIC) of the Mostoles University Hospital (CEIC 2020/037). Fourteen patients with partial FXI deficit (levels 16-60\%) were included. Its hemorrhagic phenotype was determined using the bleeding score (BS) proposed by Tosseto (2008) for Von Willebrand disease. Thirteen healthy individuals (controls) were also recruited. Blood was drawn with citrate as anticoagulant and platelets poor plasma was obtained. Activated partial thromboplastin time (APTT) with kaolin and Synthasil, plasma activity of FXI, antigenic dosage of VWF (WVF:Ag) and of the ristocetin cofactor of VWF (VWF:RCo) were determined. Calibrated automated thrombinography (CAT) was performed with PPP-low reagent (1 pM tissue factor and $4 \mu \mathrm{M}$ phospholipids). Fifty $\mu \mathrm{g} / \mathrm{ml}$ of emicizumab as added in vitro to test this drug on APTT and on CAT. Differences between groups were analyzed with Student's $t$ test and correlations were determined by Pearson test.

Results: Features of patients are shown in Table 1. FXI levels correlated with APTT results determined with both kaolin $(r=0.661$, $P<0.05)$ and Synthasil $(r=0.560, P<0.05)$, but did not correlate with CAT parameters. Moreover, WVF:Ag and VWF:RCo did not modify APTT and CAT results. Emicizumab reduced APTT value (Figure 1), but its effect was not detected in CAT experiments (Table 1).

Conclusions: Emicizumab surge como una posible alternativa para el tratamiento de pacientes con deficiencia o inhibidores del FXI. La CAT, al menos en las condiciones experimentales utilizadas, no fue útil para evaluar la hemostasia en pacientes con deficiencia de FXI.

\section{P0124 | Recurrent Thrombosis and Antithrombin Deficiency}

L. Stanciakova ${ }^{1}$; P. Holly ${ }^{1}$; J. Palova ${ }^{1}$; M. Brunclikova ${ }^{1}$;

M. Dobrotova ${ }^{1}$; T. Bolek ${ }^{2}$; M. Samos ${ }^{2}$; P. Kubisz ${ }^{1}$; J. Stasko

${ }^{1}$ National Center of Hemostasis and Thrombosis, Department of

Hematology and Transfusion Medicine, Comenius University in

Bratislava, Jessenius Faculty of Medicine in Martin, Martin University

Hospital, Martin, Slovakia; ${ }^{2}$ Department of the Internal Medicine I,

Comenius University in Bratislava, Jessenius Faculty of Medicine in

Martin, Martin University Hospital, Martin, Slovakia

Background: Antithrombin as thrombin inhibitor inhibits also activated coagulation factors IX, X, XI, XII, kalikrein and plasmin. Deficiency of antithrombin confers 3-7-fold increased risk of the venous thromboembolism (VTE) in the comparison with other thrombophilic states. Antithrombin deficiency may be inherited or acquired. Inherited form is rare thrombophilic state inherited in an autosomal dominant manner.

Aims: To present a case of patient with demanding management of repeated thrombotic complications and antithrombin deficiency.

Methods: The authors present the study of the patient with mild antithrombin deficiency. Despite this non-severe form of antithrombin activity, repeated thrombotic events were developed. Therefore, the authors discuss risk factors, diagnosis and treatment possibilities of this thrombophilia.

Results: During the last year, antithrombotic management improved the quality of life of the patient who has been the last year without further thrombotic complications.

Conclusions: Antithrombin deficiency is a high-risk and quite rare thrombophilia. Despite adequate anticoagulation, repeated thrombosis can occur and has severe clinical consequences. Thus, the close monitoring of the patient is inevitable.

Acknowledgements: Authors thank the support of the projects of the Scientific Grant Agency (Vega) 1/0549/19 and Agency for the Support of Research and Development APVV-16-0020.

\section{CONTACT PATHWAY}

\section{PB0073 | Selective Small Molecule Factor XIla Inhibitor KV998083 Protects against FeCl3 Induced Arterial Thrombosis in} Mice

S. Whitney ${ }^{1}$; A. Clermont ${ }^{1}$; N. Murugesan ${ }^{1} ;$ H. Edwards ${ }^{2}$; E. Duckworth ${ }^{2}$; S. Pethen ${ }^{2}$; S. Hampton ${ }^{2}$; E. Feener ${ }^{1}$

${ }^{1}$ KalVista Pharmaceuticals, Cambridge, United States; ${ }^{2}$ KalVista Pharmaceuticals, Salisbury, United Kingdom

Background: FXIla has been implicated as a target for anticoagulants that may prevent thrombosis without affecting hemostasis.

Aims: This study evaluates a selective small molecule FXIla inhibitor, KV998083, on thrombosis in mice. 
Methods: Right carotid artery thrombosis in male mice was initiated by $3.5 \% \mathrm{FeCl}_{3}$ patch for $3 \mathrm{~min}$. A Doppler flow probe (Transonic TS420) was used to monitor blood flow for up to $20 \mathrm{~min}$. Time to occlusion (TTO) was determined in WT mice administered vehicle or 14.4 mg/kg/day KV998083 by micro-osmotic pump (Alzet, 1003D) implanted subcutaneously at $24 \mathrm{~h}$ prior to $\mathrm{FeCl}_{3}$ injury and in $\mathrm{FXII}$ knockout mice. Tail bleeding time (TBT) was assessed following a 5 $\mathrm{mm}$ tail tip transection.

Results: The $\mathrm{IC}_{50}$ of KV998083 for human FXIla is $36.9 \mathrm{nM}$ and $>40 \mu \mathrm{M}$ for FXIa, FXa and thrombin. KV998083 prolongs APTT in both human and mouse plasma and has no effect on PT at the highest dose tested $(33 \mu \mathrm{M})$. The $\mathrm{FeCl}_{3}$ induced TTO in WT mice $(n=6)$ was $6.6 \pm 1.1 \mathrm{~min}$, while all FXII knockout mice $(n=6)$ were completely protected against occlusion. In mice treated with KV998083, 4 out of 6 animals showed no evidence of occlusion, with 2 mice showing TTOs of 10.7 and $12.1 \mathrm{~min}$. Vehicle treated animals had a TTO of $9.9 \pm 0.9$ min. At 10 mins post $\mathrm{FeCl}_{3}$ injury, \% of baseline flow was significantly higher in the KV998083 treated animals at $87.9 \%$ compared to vehicle at $4.8 \%(P<0.05)$. TBT for WT and FXII knockout mice were similar, $1.6 \pm 0.6$ and $1.6 \pm 0.3$ min, respectively. Conclusions: KV998083 is a selective FXIla inhibitor that protects against $\mathrm{FeCl}_{3}$ induced thrombosis in mice. Selective small molecule FXIla inhibitors may provide a novel class of antithrombotic that do not increase bleeding risk.

PB0074 | Extra-terrestrial Soils Activate Coagulation Factor XII and Manage Bleeding in a Porcine Model of Liver Laceration: Implications for Hemorrhage Control during Space Exploration

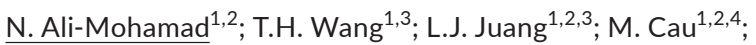
K. Cannon ${ }^{5}$; C. Kastrup ${ }^{1,2,3}$

${ }^{1}$ Michael Smith Laboratories, The University of British Columbia, Vancouver, Canada; ${ }^{2}$ Centre for Blood Research, The University of British Columbia, Vancouver, Canada; ${ }^{3}$ Department of Biochemistry and Molecular Biology, The University of British Columbia, Vancouver, Canada; ${ }^{4}$ School of Biomedical Engineering, The University of British Columbia, Vancouver, Canada; ${ }^{5}$ Geology and Geological Engineering, Colorado School of Mines, Golden, United States

Background: Bleeding is a leading cause of death in humans during trauma and surgery. Coagulation factor XII (FXII) is activated by silicates, including in hemostatic materials such as gauze used for hemorrhage control during trauma. Soils with a high silicon content accelerate clotting in humans and mice in a FXII-dependent manner. Aims: To determine whether extra-terrestrial soil simulants (ETSS) and true meteorite samples are effective hemostatic agents due to their high silicon concentrations like terrestrial soils.

Methods: Several ETSS, their component minerals and crushed chondrite meteorites were assessed for hemostatic potential. A plasma turbidity-based clotting assay was first used to compare clot times of normal control plasma and plasma deficient in FXII when ETSS and true meteorites were added. Thrombin generation was measured by comparing plasma exposed to ETSS treated with or without corn trypsin inhibitor to inhibit FXII activity. Two ETSS and chondrite meteorites were loosely place on gauze and administered to a deep porcine liver bleed to assess clotting time and blood loss compared to plain gauze.

Results: ETSS significantly accelerated in vitro clot formation $(P<0.01)$ and induced more thrombin generation in normal plasma, but not in FXII deficient plasma. Gauze loaded with ETSS, including chondrite meteorite, reduced blood loss in pigs following administration to deep liver lacerations: $25.52 \mathrm{~mL} \pm 5.34 \mathrm{~mL}$ (mean \pm SEM, $n=18)$ compared to $69.47 \mathrm{~mL} \pm 25.45 \mathrm{~mL}(n=7)$ in normal gauze. Conclusions: ETSS may be useful as an in situ material to promote hemostasis in future space missions such as on lunar and martian bases.

PB0075 | Titanium Compounds Bind and Activate Factor XII and Prekallikrein

M. Litvak ${ }^{1}$; A. Matafonov ${ }^{1}$; A. Shamanaev ${ }^{1}$; A. Kobrin ${ }^{1}$; A. Gruber ${ }^{2,3}$; O.J. McCarty ${ }^{2}$; D. Gailani ${ }^{1}$

${ }^{1}$ Department of Pathology, Microbiology and Immunology, Vanderbilt University Medical Center, Nashville, United States; ${ }^{2}$ Department of Biomedical Engineering, Division of Hematology / Medical Oncology, School of Medicine, Oregon Health \& Science University, Portland, United States; ${ }^{3}$ Aronora Inc., Portland, United States

Background: Titanium (Ti) is incorporated into a variety of medical implants because of its strength, resistance to corrosion, capacity for osseo-integration and presumed inertness. However, it is now clear that Ti-containing metals are not completely bio-inert. $\mathrm{Ti}$ is thrombogenic, complicating its use in devices that contact blood such as Ventricular Assist Devices (VAD).

Aims: investigate the effects of Ti-containing compounds and VAD components on the plasma contact zymogen/proteases factor XII(a) [fXII(a)] and prekallikrein/kallikrein.

Methods: Normal plasma (NP) was incubated on surfaces of Ti or titanium nitride (TiN) components of a HeartWare VAD (Framingham, MA) for $10 \mathrm{~min}$, followed by determination of the recalcification clotting time (RCT). Suspensions of $\mathrm{Ti}$ and TiN powders were incubated with normal plasma in the absence or presence of fXIla or kallikrein inhibiting antibodies for $10 \mathrm{~min}$, followed by determination of RCTs. Results: RCTs of normal plasma were substantially reduced after incubation on Ti (207 $\pm 28 \mathrm{~s}$ ) or TiN ( $226 \pm 51 \mathrm{~s})$ VAD surfaces, compared to polypropylene $(607.7 \pm 46.5 \mathrm{~s})$. RCTs of normal plasma were also reduced after incubation with $\mathrm{Ti}(308 \pm 83 \mathrm{~s})$ or $\mathrm{TiN}(184 \pm 58 \mathrm{~s})$ powders compared with control (611 $\pm 126 \mathrm{~s})$. RCTs of fXII-deficient plasma were not shortened by incubation with Ti or TiN. An antifXIla IgG prolonged RCTs with $\mathrm{Ti}$ and TiN, while anti-kallikrein IgG prolonged clotting only with $\mathrm{TiN}$. Both $\mathrm{Ti}$ and $\mathrm{TiN}$ bind fXII and prekallikrein, and deplete plasma of both proteins (figure). 

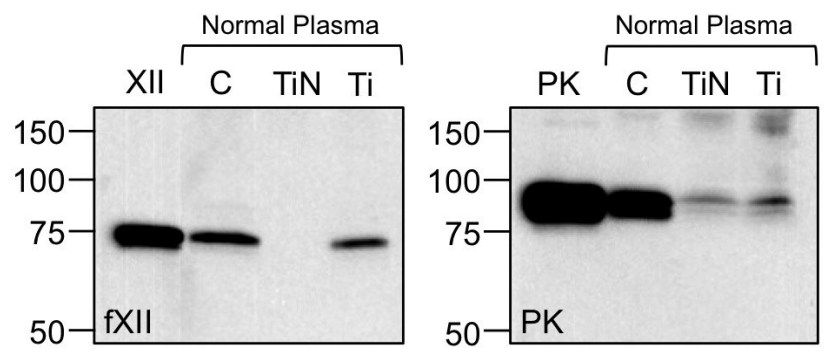

FIGURE 1 Western blots. Normal plasma was incubated with Ti or TiN microspheres or without microspheres (C - control) for $15 \mathrm{~min}$. Microspheres were pelleted and supernatant plasma was subjected to western blotting using HRP-conjugated antibodies to factor XII (fXII) or prekallikrein (PK). Left hand lanes contain 50 ng of pure fXII (XII) or PK

Conclusions: Ti-containing compounds used in biomedical devices bind the plasma contact factors fXII and prekallikrein, facilitating contact activation. The importance of prekallikrein to contact activation may vary depending on the type of Ti-containing compound. Ti surfaces likely contribute to the thrombotic potential of VADs and related intravascular devices. Pharmaceuticals targeting of fXIla and/or kallikrein may help alleviate this effect without exacerbating bleeding.

PB0076 | FXI-independent Pathways of Contact Activation: Analysis of Activated Partial Thromboplastin Time (aPTT) Data Using Mathematical Modelling

A. Rezvani-Sharif ${ }^{1}$; S. Rauth ${ }^{1}$; J. Arthur ${ }^{1}$; P. Niebl' ${ }^{2}$; S. Dower ${ }^{1}$; C. Panousis $^{1}$; I. Muir ${ }^{1}$

${ }^{1}$ CSL Innovation, Bio21 Institute, Parkville, Australia; ${ }^{2}$ CSL Behring, Marburg, Germany

Background: Despite a few studies showing the potential for FXIla and kallikrein to contribute to clot formation in a FXI-independent manner, thrombin generation via the intrinsic pathway is often considered FXI-dependent.

Aims: Investigate FXI bypass pathways of coagulation via selective inhibition of the contact activation system and mathematical modelling.

Methods: Various aPTT data sets were generated using different concentrations of functional antibodies against FXII/XIla (termed 3F7), FXI/Xla, and kallikrein. The aPTT data sets were simulated in three stages to mimic the in vitro assay:

1) binding of antibodies to their targets,

2) incubation of plasma with phospholipids and negatively charged FXII activator,

3) addition of calcium ions to trigger coagulation.

Results: Four distinct reaction pathways need to be included in the mathematical model to accurately reproduce the full range of aPTT data sets: rapid activation of FXI by FXIla, direct activation of FIX by kallikrein, and slow activation of prothrombin by both kallikrein and FXIla (Figure 1). High concentrations of 3F7 block all four downstream pathways of thrombin generation. Blocking the activation of FIX with a FXI/Xla antibody has little effect on the clotting time due to upstream reactions circumventing this reaction. Inhibition of kallikrein prolongs the clotting time only slightly, as the activation of FXII is the dominant trigger in an APTT assay. Blocking both kallikrein and FXla prolonged the clotting time more than threefold, highlighting the role of kallikrein in the absence of FXI and the presence of a thrombin generation pathway independent of FXI and kallikrein (Figure 2).

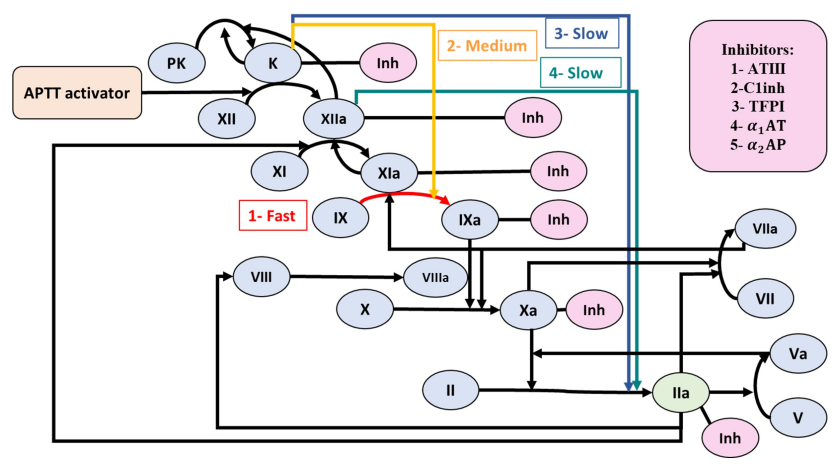

FIGURE 1 Modified reaction scheme of the contact activation system

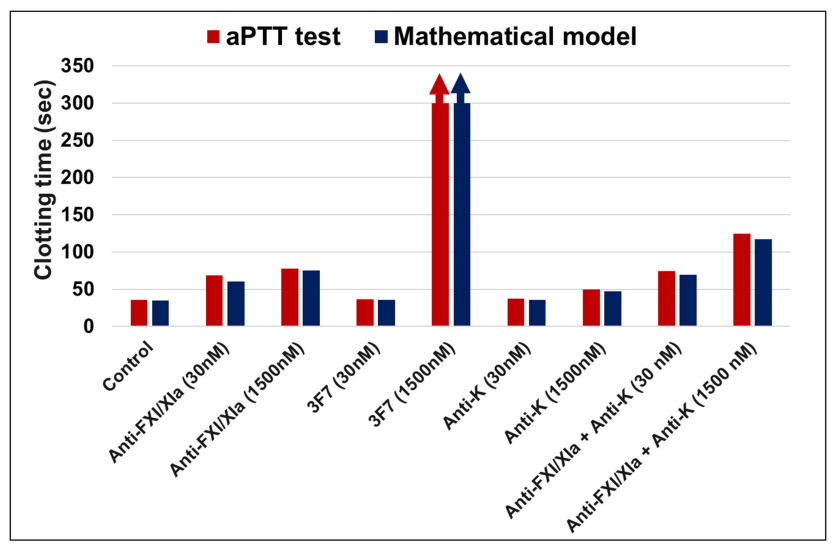

FIGURE 2 Comparison of the model output with experimental data. The maximum measurable clotting time is $300 \mathrm{~s}$

Conclusions: Our results suggest that in the absence of $\mathrm{FXI}$, both kallikrein and FXIla are able to generate thrombin via the contact activation pathway of coagulation. 
PB0077 | Pharmacokinetics and Pharmacodynamics of Abelacimab (MAA868), a Novel Dual Inhibitor of Factor XI and Factor Xla

B.A. Yi ${ }^{1}$; D. Freedholm ${ }^{1}$; N. Widener ${ }^{1} ;$ X. Wang ${ }^{2}$; E. Simard ${ }^{2}$; L. Galitz ${ }^{3}$; N.M. Al-Saady ${ }^{4}$; N.E. Lepor ${ }^{5}$; M. Lovern ${ }^{2}$; D. Bloomfield ${ }^{1}$

${ }^{1}$ Anthos Therapeutics, Cambridge, United States; ${ }^{2}$ Certara, Princeton, United States; ${ }^{3}$ Covance Clinical Research, Daytona Beach, United States; ${ }^{4}$ Covance by LabCorp, Maidenhead, United Kingdom; ${ }^{5}$ Westside Medical Associates of Los Angeles, Beverly Hills, United States

Background: Factor XI (FXI) inhibition offers the promise of hemostasis-sparing anticoagulation for the prevention and treatment of arterial and venous thromboembolic events. Abelacimab is a novel fully human monoclonal antibody that targets the catalytic domain and has dual activity against the inactive zymogen Factor X and the activated FXI.

Aims: To investigate the safety, pharmacokinetic (PK), and pharmacodynamic (PD) properties of single intravenous infusion and monthly subcutaneous administration of abelacimab in healthy volunteers and patients with atrial fibrillation, respectively.

Methods: In study ANT-003, healthy volunteers were administered single ascending doses of intravenous abelacimab (30 to $150 \mathrm{mg}$ ) or placebo. The ANT-003 study also included a cohort of obese but otherwise healthy subjects to evaluate the effect of body weight on PK properties. In study ANT-004, patients with atrial fibrillation were administered monthly subcutaneous doses of abelacimab (120 $\mathrm{mg}$ and $180 \mathrm{mg}$ ), or placebo, for 3 months. Key PK and PD parameters, including activated partial thromboplastin time (aPTT) and free FXI levels were assessed. These studies were approved by the local ethics committee and all volunteers provided written informed consent.

Results: Following intravenous administration of abelacimab, PK increased in an approximately dose-proportional manner. The mean terminal elimination half-life ranged from 25 to 30 days similar to that observed with subcutaneous dosing. Rapid dose- and timedependent reductions from baseline in free FXI levels $>99 \%$ were seen within an hour after intravenous infusion. Similarly, profound reductions in free FXI levels were maintained with subcutaneous monthly administration. Single intravenous infusion and multiple subcutaneous administration of abelacimab demonstrated a favorable safety profile with no major or clinically relevant non-major bleeding events.

Conclusions: The results provide a detailed profile of the PK and PD properties of abelacimab and support future clinical development of abelacimab as a once monthly anticoagulant.

\section{CRITICAL CARE AND PERIOPERATIVE}

\section{LPB0052 | Characterization of Andexanet Alfa-associated} Heparin Resistance: Implications for Management

N. Thalji ${ }^{1}$; C. Chabata ${ }^{2}$; P. Patel ${ }^{3}$; B. Sullenger ${ }^{2}$; R. Camire

${ }^{1}$ Hospital of the University of Pennsylvania, Philadelphia, United States; ${ }^{2}$ Duke University, Durham, United States; ${ }^{3}$ Yale University School of Medicine, New Haven, United States; ${ }^{4}$ Children's Hospital of Philadelphia, Philadelphia, United States

Background: Andexanet alfa ( $\mathrm{AnXa}$ ) is an inactive variant of factor $\mathrm{Xa}$ ( $\mathrm{FXa}$ ) that functions as a stoichiometric drug-sequestering antidote for direct FXa inhibitors. Case reports suggest that AnXa administration prior to administration of unfractionated heparin (UFH) for cardiopulmonary bypass (CPB) may result in inadequate anticoagulation.

Aims: We set out to determine whether AnXa interferes with the effects of UFH in point-of-care assays, and, if so, whether this effect resulted in inadequate anticoagulation on artificial surfaces. Further, we attempted to elucidate the mechanism of AnXa-associated heparin resistance and identify clinical countermeasures should it occur. Methods: We measured the activated clotting time (ACT) in human whole blood supplemented with varying amounts of AnXa and UFH. Further, we flowed AnXa and UFH-supplemented human whole blood in an extracorporeal oxygenator membrane model to assess whether administration of UFH following AnXa provided adequate anticoagulation. Finally, we supplemented plasma with AT in thrombin generation (TG) studies to determine if additional AT could overcome AnXa-associated heparin resistance.

Results: Both low and high dose AnXa prevented the expected ACT prolongation after usual cardiopulmonary bypass (CPB) doses of $\mathrm{UFH}(4 \mathrm{U} / \mathrm{mL})$. AnXa also prevented high doses $(16 \mathrm{U} / \mathrm{mL})$ of $\mathrm{UFH}$ from prolonging the ACT beyond $400 \mathrm{~s}$. In an extracorporeal model, concomitant administration of $\mathrm{AnXa}$ and $4 \mathrm{U} / \mathrm{mL} \mathrm{UFH}$ resulted rapid circuit thrombosis not seen with UFH alone (Figure 1), suggesting that AnXa-associated heparin resistance is not simply an in vitro effect. In TG studies, excess UFH restored anticoagulation when lowdose AnXa was used, but at high AnXa doses, AT supplementation in addition to excess UFH was needed to restore anticoagulation.
A

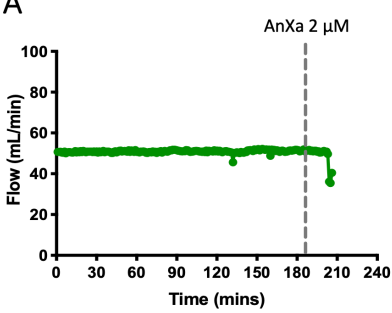

B

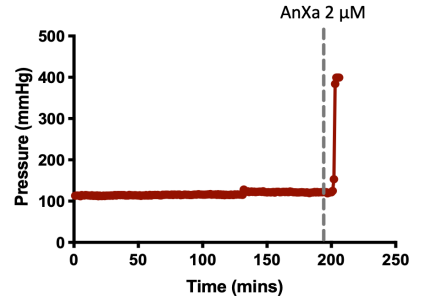

FIGURE 1 Administration of Andexanet alfa to an adequately heparinized $(4 \mathrm{U} / \mathrm{mL})$ extracorporeal circuit results in rapid circuit thrombosis as evidenced by an abrupt decrease in circuit flow (A) and increase in circuit pressure (B) 
Conclusions: AnXa produces heparin resistance that results in insufficient anticoagulation for cardiopulmonary bypass due to binding to UFH-AT complexes. Depending on the dose of AnXa used, extreme doses of UFH and sometimes AT supplementation may be necessary to achieve adequate anticoagulation with UFH.

PB0078 | Big-data and Machine Learning Prediction of Early and Late Mortality in ICU Patients with Venous Thromboembolism and Comparison with Classic Scoring Systems

V. Danilatou ${ }^{1}$; D. Mavroidis ${ }^{2}$; D. Antonakaki ${ }^{2}$; A. Kanterakis ${ }^{2}$; C. Tzagkarakis $^{2}$; T. Kostoulas ${ }^{3}$; S. loannidis ${ }^{4,2}$

${ }^{1}$ SPHYNX Technology Solutions, Nicosia, Cyprus; ${ }^{2}$ FORTH, Heraklion, Greece; ${ }^{3}$ Aegean University, Samos, Greece; ${ }^{4}$ Technical University of Crete, Chania, Greece

Background: Patients with venous thromboembolism (VTE) occasionally need intensive care unit (ICU) hospitalisation, suffering from high mortality. Several scoring systems predict early ICU mortality but only PESI is specific for VTE early mortality and no score exists for late mortality after ICU hospitalization. Huge amount of information stored in ICUs moves medical research towards "machinelearning" (ML) predictive modelling.

Aims: ML-based prediction of early and late mortality in ICU-VTE patients using a high-dimensional feature space. Comparison with known ICU scores. Identification of clinically-meaningful features to improve existing scores.

Methods: 2,468 ICU-VTE patients, from MIMIC-III database stratified in three groups based on their outcome: (1) died during first admission, (2) died after discharge (3) alive, months after discharge. 1,471 features selected from $>20,000$ were studied (demographic, clinical, laboratory, prescriptions, procedures, well-established scores and medical notes) and predictive performance was compared with 9 scores (Tables 1 and 2). We examine two ML classification tasks, early and late mortality prediction. Ridge logistic regression, random forest (RF) and support vector machines were used for ML classification,statistically equivalent signature and LASSO for feature selection.

TABLE 1 Description of clinical and laboratory features selected from MIMIC III database. The first column describes the corresponding table from the MIMIC III database

$\begin{array}{llllll}\text { Group } & \text { Description } & \begin{array}{l}\text { Overall } \\ \text { features }\end{array} & \begin{array}{l}\text { Selected } \\ \text { features }\end{array} & \text { Average } & \text { Median } \\ \text { ChartEvents } & \begin{array}{l}\text { Vital signs, labs, clinical } \\ \text { information }\end{array} & 7,212 & 235 & 433 & 77 \\ \text { LabEvents } & \text { Laboratory indices } & 753 & 45 & 1,237 & 1,157\end{array}$

\begin{tabular}{|c|c|c|c|c|c|c|}
\hline Procedures & $\begin{array}{l}\text { Several procedures, } \\
\text { including } \\
\text { transfusion } \\
\text { and mechanical } \\
\text { ventilation }\end{array}$ & 3,882 & 526 & 24.3 & 6 & $\begin{array}{l}\text { Venous cathetirization, enteral } \\
\text { nutrition, endotracheal } \\
\text { intubation, mechanical } \\
\text { ventilation, for more than } \\
96 \mathrm{~h}\end{array}$ \\
\hline InputEvents & $\begin{array}{l}\text { Transfusion and } \\
\text { parenteral nutrition }\end{array}$ & $\begin{array}{l}422 \\
2,938\end{array}$ & $\begin{array}{l}12(\mathrm{MV}) \\
10(\mathrm{CV})\end{array}$ & & & $\begin{array}{l}\text { RBC transfusions, PLT } \\
\text { transfusions, plasma } \\
\text { transfusions }\end{array}$ \\
\hline Prescriptions & Medications & 4,525 & 91 & 132 & 14 & $\begin{array}{l}\text { Heparin, insulin, warfarin, } \\
\text { aspirin, enoxaparin, } \\
\text { norepinephrine, } \\
\text { phytonadione, atorvastatin }\end{array}$ \\
\hline NoteEvents & $\begin{array}{l}\text { Unstructured medical } \\
\text { notes }\end{array}$ & & 50 & $\begin{array}{l}48 \text { entries } \\
2,408 \\
\quad \text { characters }\end{array}$ & 1,382 characters & N/A \\
\hline Concepts & $\begin{array}{l}\text { Scores, first day labs, } \\
\text { first day vitals } \\
\text { doses and durations } \\
\text { of medications }\end{array}$ & & 493 & & & $\begin{array}{l}\text { Comorbidity indices, severity } \\
\text { illness scores, organ failure } \\
\text { scores, sepsis scores, GCS, } \\
\text { first day labs, first day vital } \\
\text { signs, transfusions }\end{array}$ \\
\hline
\end{tabular}

\section{Most common features}

Common labs, blood gases, blood pressure

Hematocrit, hemoglobin, white blood cells, platelets, red blood cells, renal and liver function tests, hemostasis screening tests, sepsis indices

enous cathetirization, enteral ventilation, for more than

$\mathrm{BC}$ transfusions, PLT transfusions, plasma parin, insulin, warfarin aspirin, enoxaparin norepinephrine, phytonadione, atorvastatin signs, transfusions 
TABLE 2 Performance of various medical scores in predicting early and late mortality in ICU patients with VTE and most important clinical features selected

\begin{tabular}{|c|c|c|c|c|c|}
\hline Score & $\begin{array}{l}\text { Number of } \\
\text { features }\end{array}$ & $\begin{array}{l}\text { Early } \\
\text { mortality } \\
\text { AUC }\end{array}$ & Important features & $\begin{array}{l}\text { Late mortality } \\
\text { AUC }\end{array}$ & Important features \\
\hline APS II & 14 & $\begin{array}{l}0.81 \\
{[0.76,0.85]}\end{array}$ & GCS, BUN & $\begin{array}{l}0.65 \\
{[0.59,0.70]}\end{array}$ & $\begin{array}{l}\text { Urine output, BUN, } \\
\text { hematocrit }\end{array}$ \\
\hline SAPS & 13 & $\begin{array}{l}0.8 \\
{[0.76 .0 .84]}\end{array}$ & $\begin{array}{l}\text { BUN, age, WBC, } \\
\text { bicarbonate }\end{array}$ & $\begin{array}{l}0.67 \\
{[0.63,0.72]}\end{array}$ & Age, BUN \\
\hline SAPS II & 175 & $\begin{array}{l}0.85 \\
{[0.810,0.884]}\end{array}$ & Comorbidity, age, bilirubin & $\begin{array}{l}0.76 \\
{[0.72,0.81]}\end{array}$ & $\begin{array}{l}\text { Age, BUN,comorbidity, } \\
\text { urine output }\end{array}$ \\
\hline SOFA & 9 & $\begin{array}{l}0.764 \\
{[0.719,0.807]}\end{array}$ & - & $\begin{array}{l}0.58 \\
{[0.52,0.63]}\end{array}$ & Renal \\
\hline SIRS & 4 & $\begin{array}{l}0.64 \\
{[0.587,0.685]}\end{array}$ & WBC, temperature & 0.5 & - \\
\hline OASIS & 10 & $\begin{array}{l}0.81 \\
{[0.763,0.846]}\end{array}$ & $\begin{array}{l}\text { Age, GCS, respiratory } \\
\text { rate,elective surgery, } \\
\text { urine output, mean BP }\end{array}$ & $\begin{array}{l}0.68 \\
{[0.63,0.73]}\end{array}$ & $\begin{array}{l}\text { Age, } \\
\text { urine output, elective } \\
\text { surgery }\end{array}$ \\
\hline LODS & 6 & $\begin{array}{l}0.77 \\
{[0.723,0.810]}\end{array}$ & $\begin{array}{l}\text { Renal, cardiovascular, } \\
\text { neurologic, pulmonary, } \\
\text { hepatic, haematologic }\end{array}$ & $\begin{array}{l}0.61 \\
{[0.55,0.65]}\end{array}$ & $\begin{array}{l}\text { Renal, pulmonary, } \\
\text { hematologic }\end{array}$ \\
\hline Comorbidity & 126 & $\begin{array}{l}0.78 \\
{[0.74,0.82]}\end{array}$ & $\begin{array}{c}\text { Metastatic cancer, } \\
\text { depression }\end{array}$ & $\begin{array}{l}0.74 \\
{[0.69,0.79]}\end{array}$ & $\begin{array}{l}\text { Metastatic cancer, obesity, } \\
\text { congestive heart } \\
\text { failure, } \\
\text { solid tumor, chronic } \\
\text { pulmonary }\end{array}$ \\
\hline MLODS & 6 & $\begin{array}{l}0.768 \\
{[0.72,0.81]}\end{array}$ & $\begin{array}{l}\text { Hepatic, cardiovascular, } \\
\text { renal }\end{array}$ & $\begin{array}{l}0.57 \\
{[0.51,0.62]}\end{array}$ & $\begin{array}{c}\text { Pulmonary, renal, } \\
\text { hematologic }\end{array}$ \\
\hline All scores & 91 & $\begin{array}{l}0.86 \\
{[0.82,0.89]}\end{array}$ & $\begin{array}{l}\text { SAPS II: comorbidity, } \\
\text { paO2, age, bilirubin, } \\
\text { bicarbonate } \\
\text { LODS: hepatic } \\
\text { SOFA: coagulation, OASIS: } \\
\text { respiratory rate }\end{array}$ & $\begin{array}{l}0.76 \\
{[0.71,0.81]}\end{array}$ & SAPS II (comorbidity, age) \\
\hline $\begin{array}{l}\text { All features } \\
\text { (Big-data approach) }\end{array}$ & 1471 & $\begin{array}{l}0.93 \\
{[0.91,0.95]}\end{array}$ & $\begin{array}{l}\text { Sepsis Martin score } \\
\text { (respiratory, warfarin, } \\
\text { diastolic blood pressure } \\
\text { average, SAPSII, PT } \\
\text { average, Arterial pH } \\
\text { average, vasopressor } \\
\text { duration, RDW average }\end{array}$ & $\begin{array}{l}0.82 \\
{[0.79,0.84]}\end{array}$ & $\begin{array}{l}\text { Age, vasopressor } \\
\text { (phenylephrine), RDW, } \\
\text { metastatic cancer, ALP } \\
\text { average, Creatinine } \\
\text { average, CPK average, } \\
\text { warfarin, cancer, } \\
\text { comorbidities }\end{array}$ \\
\hline
\end{tabular}

Results: RF was the best $M L$ algorithm to predict early ( $A U C=0.93$ ) and late (AUC $=0.82$ ) mortality in ICU-VTE patients, using all features. In subgroup analysis, Concepts and ChartEvents showed the best performance in early ( $A \cup C=0.92$ ), and late (AUC $=0.77$ ) mortality, followed by SAPSII and combination of all scores. Significant predictive features of early and late mortality were cancer, sepsis, age,warfarin, GCS, vasopressors and RDW.

Conclusions: Predicting late mortality in ICU-VTE patients is a difficult task, and medical scores are still lacking. $M L$ and use of bigdata could outperform classic statistical approaches. Identification of clinically meaningful features allows the discovering of new biomarkers. RDW could be a candidate rediscovered biomarker, since it is known that it correlates with early mortality from VTE. Further validation is needed in prospective population study.
PB0079 | Elucidating Thrombus Formation in ECMO Circuits: A Detailed Histological Study

S. Staessens ${ }^{1,2} ;$ M. Moussa ${ }^{2,3} ;$ A. Pierarche ${ }^{4}$; E. Boulleaux ${ }^{2}$; A. Ung ${ }^{2}$; L. Desender ${ }^{1}$; B. Pradines ${ }^{2,5}$; N. Rousse ${ }^{2,6} ;$ A. Duhamel ${ }^{4}$; A. Rauch ${ }^{2,5}$; K. Vanhoorelbeke ${ }^{1}$; D. Corseaux ${ }^{2}$; S.F. De Meyer ${ }^{1}$; S. Susen ${ }^{2,5}$

${ }^{1}$ Laboratory for Thrombosis Research, KU Leuven Campus Kulak Kortrijk, Kortrijk, Belgium; ${ }^{2}$ University of Lille, Inserm, $\mathrm{CHU}$, Institut Pasteur de Lille, U 1011-EGID, Lille, France; ${ }^{3}$ Department of Anesthesiology and Critical Care, Centre Hospitalier Universitaire de Lille, Lille, France; ${ }^{4}$ ULR 2694-METRICS (Evaluation des Technologies de Santé et des Pratiques Médicales), University of Lille, Centre Hospitalier Universitaire Lille, Lille, France; ${ }^{5}$ Department of Hematology 
Transfusion, Centre Hospitalier Universitaire de Lille, Lille, France;

${ }^{6}$ Department of Cardiac Surgery, Centre Hospitalier Universitaire de Lille, Lille, France

Background: Intra-device thrombosis remains one of the most common complications during extracorporeal membrane oxygenation (ECMO) support. Despite monitored-heparin anticoagulation, $\pm 35 \%$ of patients develop acute thrombosis in the membrane oxygenator or blood pumps. This often results in equipment malfunctioning which necessitates hazardous elective/full system exchanges. Yet, surprisingly little is known about the composition of thrombi formed in the ECMO circuit. Aims: To investigate thrombus formation at different site in the ECMO circuit via histological analysis of ECMO thrombi.

Methods: Thrombi $(n=51)$ were collected immediately after venoarterial ECMO circuit removal from 15 patients at the $\mathrm{CHU}$ Lille (Lille, France). Of those thrombi, 13 originated from the pump, 15 from the oxygenator and 23 from the tubing. Quantitative histological analysis was performed for the amount red blood cells (RBCs), platelets, fibrin, von Willebrand factor (VWF), leukocytes and citrullinated histone $\mathrm{H} 3$ (H3Cit).

Results: ECMO thrombi consist of a heterogenous composition with fibrin $(40.9 \pm 17.2 \%)$ and VWF $(34.7 \pm 15.6 \%)$ being the major thrombus components, followed by RBCs $(25.4 \pm 22.8 \%)$, platelets $(24.0 \pm 17.1 \%)$ and smaller amounts of H3Cit (5.4 $\pm 6.3 \%)$. Hierarchal clustering analysis of the four major histological parameters identified two typical thrombus types: RBC-rich and fibrin-rich thrombi with no significant differences in VWF/platelet content. Thrombus location (pump, oxygenator or tubing) was not significantly associated with individual histological parameters or thrombus types. Our data, yet preliminary, indicate that the pump/oxygenator type might influence thrombus composition as Quadrox oxygenators induce the formation of fibrin-rich thrombi $(50.1 \% \pm 20.0 \%)$ compared to A.LONE oxygenators $(27.9 \% \pm 6.4 \%)$. In contrast to Revolution pumps $(26.7 \% \pm 28.6 \%$ RBCs, $15.0 \% \pm 12.5 \%$ platelets $)$, Rotaflow pumps (38.4\% $\pm 26.9 \% \mathrm{RBCs}, 39.2 \% \pm 25.0 \%$ platelets) seem to induce the formation of RBC-rich and platelet-rich thrombi.

Conclusions: Despite monitored-heparin anticoagulation, overall ECMO thrombus composition is heterogenous, consisting mainly of fibrin/VWF but regardless of thrombus location. Such data are important to further improve current anticoagulation strategies in ECMO patients and to reduce ECMO circuit thrombogenicity.

PB0080 | A Comparative Study of SEER Sonorheometry versus Thromboelastography during Cardiac Surgery with Cardiopulmonary Bypass

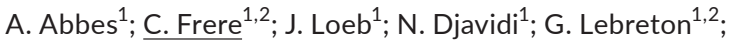
A. Bougle $\mathrm{e}^{1,3}$

${ }^{1}$ Assistance Publique Hôpitaux de Paris, Paris, France; ${ }^{2}$ Sorbonne Université, INSERM UMRS_1166, Institute of Cardiometabolism and Nutrition, Paris, France; ${ }^{3}$ Sorbonne Université, GRC 29, Paris, France
Background: Coagulation point-of-care (POC) tests are commonly used for hemostasis monitoring during cardiac surgery. The Quantra ${ }^{\circledR}$ system is a new coagulation POC device based on Sonic Estimation of Elasticity via Resonance (SEER) Sonorheometry equipped with a disposable QPlus ${ }^{\circledR}$ 4-channels cartridge. QPlus ${ }^{\circledR}$ parameters have been shown to well correlate with conventional coagulation tests and rotational thromboelastometry parameters, but no prior study compared them to those obtained with the $\mathrm{TEG}^{\circledR} 6 \mathrm{~s}$ citrated and platelet mapping (PM) cartridges.

Aims: To compare the Quantra ${ }^{\circledR}$ QPlus ${ }^{\circledR}$ and the TEG ${ }^{\circledR}$ 6s cartridges parameters for POC monitoring of hemostasis during cardiac surgery with cardiopulmonary bypass.

Methods: 21 patients were included in this cohort study. Blood was sampled before surgery, intraoperatively and $10 \mathrm{~min}$ after protamine administration. SEER Sonorheometry was performed on a Quantra ${ }^{\circledR}$ Hemostasis analyzer with the QPlus Cartridge (HemoSonics LLC) and thromboelastography on a TEG ${ }^{\circledR}$ 6s Hemostasis Analyzer with the citrated and PM cartridges (Haemonetics).

Results: The median age was 60 (53-71) years, with a median BMI of $25(23-27) \mathrm{Kg} / \mathrm{m}^{2}$, and 15 (71\%) patients were men. The median Euroscore was 2.9 (1.8-4.1). Quantra ${ }^{\circledR}$ parameters assessing clot stiffness (CS) significantly dropped over the course of cardiac surgery [CS $(P<0.0001)$, fibrinogen contribution to CS (FCS, $P=0.0001)$ and platelet contribution to CS (PCS, $P<0.0001)$ ]. Quantra ${ }^{\circledR}$ clot time significantly correlated with TEG ${ }^{\circledR}$ 6-CK R-time $(r=0.51)$, and Quantra ${ }^{\circledR}$ clot time with heparinase with TEG ${ }^{\circledR} 6 \mathrm{~s}-\mathrm{CKH}$ R-time $(r=0.73)$. Quantra ${ }^{\circledR} \mathrm{CS}$ showed a strong correlation with TEG ${ }^{\circledR}$ 6s CRT MA $(r=0.88)$ and Quantra ${ }^{\circledR}$ FCS with both TEG ${ }^{\circledR} 6 \mathrm{~s}$ CFF-MA $(r=0.86)$ and fibrinogen levels $(r=0.74)$. Finally, Quantra ${ }^{\circledR}$ PCS weakly correlated with TEG ${ }^{\circledR}$ 6s PM-AA $(r=0.61)$, TEG ${ }^{\circledR} 6$ s PM$\operatorname{ADP}(r=0.45)$ and platelet count $(r=0.57)$. However, Bland-Altman plots revealed a poor agreement between Quantra ${ }^{\circledR}$ QPlus and TEG ${ }^{\circledR}$ 6s parameters.
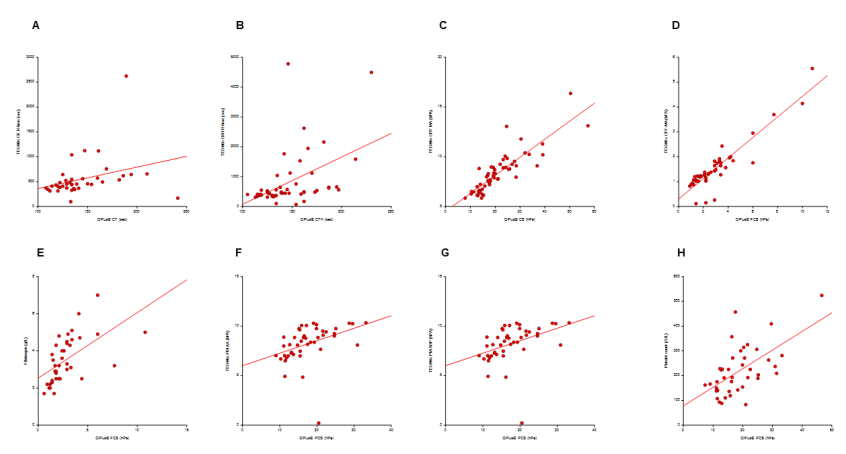

FIGURE 1 Spearman rank correlations between Quantra ${ }^{\circledR}$ and TEG ${ }^{\circledR}$ 6s parameters. A: QPlus CT (s) vs TEG ${ }^{\circledR}$ 6s CK R-time (s); B: QPlus CTH (s) vs TEG ${ }^{\circledR}$ 6s CKH R-time (s); C: QPlus CS (hPa) vs TEG ${ }^{\circledR}$ 6s CRT MA (hPA); D: QPlus FCS (hPa) vs TEG ${ }^{\circledR}$ 6s CFF-MA (hPA); E: QPlus FCS (hPa) vs Fibrinogen (g/L); F: QPlus PCS (hPa) vs TEG ${ }^{\circledR}$ 6s PM-AA (hPA) ; G: QPlus PCS (hPa) vs TEG ${ }^{\circledR}$ 6s PM-ADP (hPA) ; H: QPlus PCS $(\mathrm{hPa})$ vs platelet count $(\mathrm{G} / \mathrm{L})$ 

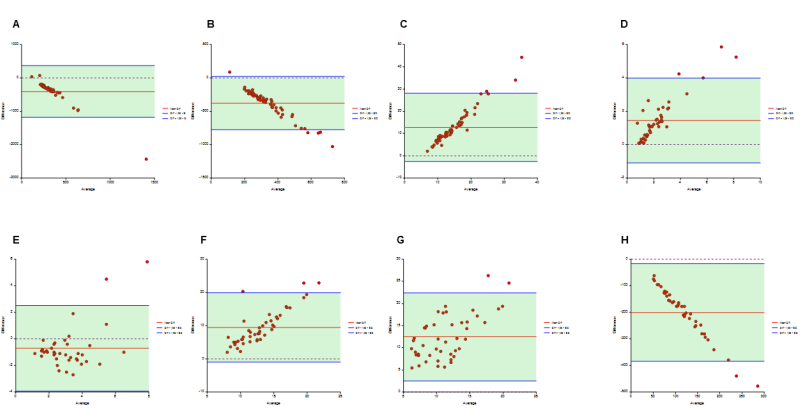

FIGURE 2 Bland-Altman plots to evaluate the agreement between Quantra ${ }^{\circledR}$ and TEG ${ }^{\circledR}$ 6s parameters. A: QPlus CT (s) vs TEG ${ }^{\circledR}$ 6s CK R-time (s); B: QPlus CTH (s) vs TEG ${ }^{\circledR}$ 6s CKH R-time (s); C: QPlus CS (hPa) vs TEG ${ }^{\circledR}$ 6s CRT MA (hPA); D: QPlus FCS (hPa) vs TEG ${ }^{\circledR}$ 6s CFF-MA (hPA) ; E: QPlus FCS (hPa) vs Fibrinogen (g/L); F: QPlus PCS (hPa) vs TEG ${ }^{\circledR}$ 6s PM-AA (hPA) ; G: QPlus PCS (hPa) vs TEG ${ }^{\circledR}$ 6s PMADP (hPA) ; H: QPlus PCS (hPa) vs platelet count (G/L)

Conclusions: Despite acceptable correlations between the Quantra ${ }^{\circledR}$ and TEG $^{\circledR} 6$ s parameters, these measurements are not interchangeable.

PB0081 | Management of Bleeding and Urgent Invasive Procedures in Patients Treated with Direct Oral Anticoagulants: An Observational Study over a Five-year Perio

A. Pontis $^{1,2}$; A. Mansour ${ }^{2,3} ;$ A. Maurice ${ }^{3}$; C. Rousseau ${ }^{4}$;

F. Nédelec-Gac ${ }^{1}$; P. Guéret ${ }^{1}$; L. Soulat ${ }^{5}$; P. Jégo ${ }^{6}$; C. Ecoffey ${ }^{3}$; I. Gouin-Thibault ${ }^{1,2,7}$

${ }^{1}$ University Hospital of Rennes, Department of Hematology and Hemostasis, Rennes, France; ${ }^{2}$ CIC INSERM 1414, Rennes, France;
${ }^{3}$ University Hospital of Rennes, Department of Anesthesia and Critical Care, Rennes, France; ${ }^{4}$ University Hospital of Rennes, Department of Clinical Pharmacology, Rennes, France; ${ }^{5}$ University Hospital of Rennes, Department of Emergency Medicine, Rennes, France; ${ }^{6}$ University Hospital of Rennes, Department of Internal Medicine and Clinical Immunology, Rennes, France; ${ }^{7}$ IRSET - Inserm UMR_S 1085, Rennes, France

Background: Management of bleeding and emergency invasive procedures remains challenging in patients on Direct Oral AntiCoagulants (DOACs). To manage these patients, the French working Group on Perioperative Hemostasis (GIHP) published guidelines ${ }^{1}$, based on DOAC level.

Aims: Evaluation of the management of bleeding and emergency invasive procedures in patients on DOAC according to the GIHP guidelines $^{1}$; and identification of risk factors associated with poor outcomes, over a five-year period.

Methods: We retrospectively included all patients with a DOAC measurement admitted for the management of an emergency invasive procedure or bleeding in the University Hospital of Rennes between 2014 and 2018. Poor outcomes were bleeding (ISTH criteria) or thrombotic complications, and death.

Results: We included 595 patients with 1006 DOAC measurements, corresponding to 592 situations: 432 emergency invasive procedures and 160 bleedings.The characteristics of patients in the invasive procedure and bleeding situations are presented in Table 1. Emergency procedures were mainly orthopedics (34\%) and neurosurgery (12\%). Bleeding were mainly critical (39\%) or severe $(29 \%)$, with $34 \%$ intracranial and $20 \%$ gastrointestinal. Adherence to the guidelines was $85 \%$, of which $91 \%$ of urgent procedures and $67 \%$ of bleedings.

TABLE 1 General characteristics of the study patients. SD: standard deviation, AF: atrial fibrillation, VTE: venous thrombo-embolism, DOAC: direct oral anticoagulant * bleedings versus invasive procedures

\begin{tabular}{|c|c|c|c|}
\hline & $\begin{array}{l}\text { Invasive procedures group } \\
n=432\end{array}$ & $\begin{array}{l}\text { Bleeding group } \\
n=160\end{array}$ & $P^{*}$ \\
\hline $\begin{array}{l}\text { Age (years) } \\
\text { Mean } \pm \text { SD }\end{array}$ & $76.19 \pm 12.56$ & $73.94 \pm 12.91$ & 0.0561 \\
\hline $\begin{array}{l}\text { Indication } n(\%) \\
\text { AF } \\
\text { VTE } \\
\text { Others/Unknown }\end{array}$ & $\begin{array}{l}347(80.3 \%) \\
55(12.7 \%) \\
30(6.9 \%)\end{array}$ & $\begin{array}{l}131(81.9 \%) \\
20(12.5 \%) \\
9(5.6 \%)\end{array}$ & 0.9056 \\
\hline $\begin{array}{l}\text { DOAC n (\%) } \\
\text { Apixaban } \\
\text { Rivaroxaban } \\
\text { Dabigatran }\end{array}$ & $\begin{array}{l}205(47.5 \%) \\
168(38.9 \%) \\
59(13.7 \%)\end{array}$ & $\begin{array}{l}65(40.6 \%) \\
75(46.9 \%) \\
20(12.5 \%)\end{array}$ & 0.2096 \\
\hline $\begin{array}{l}\text { Apixaban } n(\%) \\
<50 \mathrm{ng} / \mathrm{mL} \\
{[50-200[\mathrm{ng} / \mathrm{mL}} \\
\geq 200 \mathrm{ng} / \mathrm{mL}\end{array}$ & $\begin{array}{l}68(33.2 \%) \\
108(52.7 \%) \\
29(14.1 \%)\end{array}$ & $\begin{array}{l}12(18.5 \%) \\
44(64.6 \%) \\
11(16.9 \%)\end{array}$ & 0.049 \\
\hline $\begin{array}{l}\text { Rivaroxaban } n \text { (\%) } \\
<50 \mathrm{ng} / \mathrm{mL} \\
{[50-200[\mathrm{ng} / \mathrm{mL}}\end{array}$ & $\begin{array}{l}101(60.1 \%) \\
46(27.4 \%) \\
21(12.5 \%)\end{array}$ & $\begin{array}{l}19(25.3 \%) \\
31(41.3 \%) \\
25(33.3 \%)\end{array}$ & $<0.0001$ \\
\hline
\end{tabular}

$\geq 200 \mathrm{ng} / \mathrm{mL}$ 


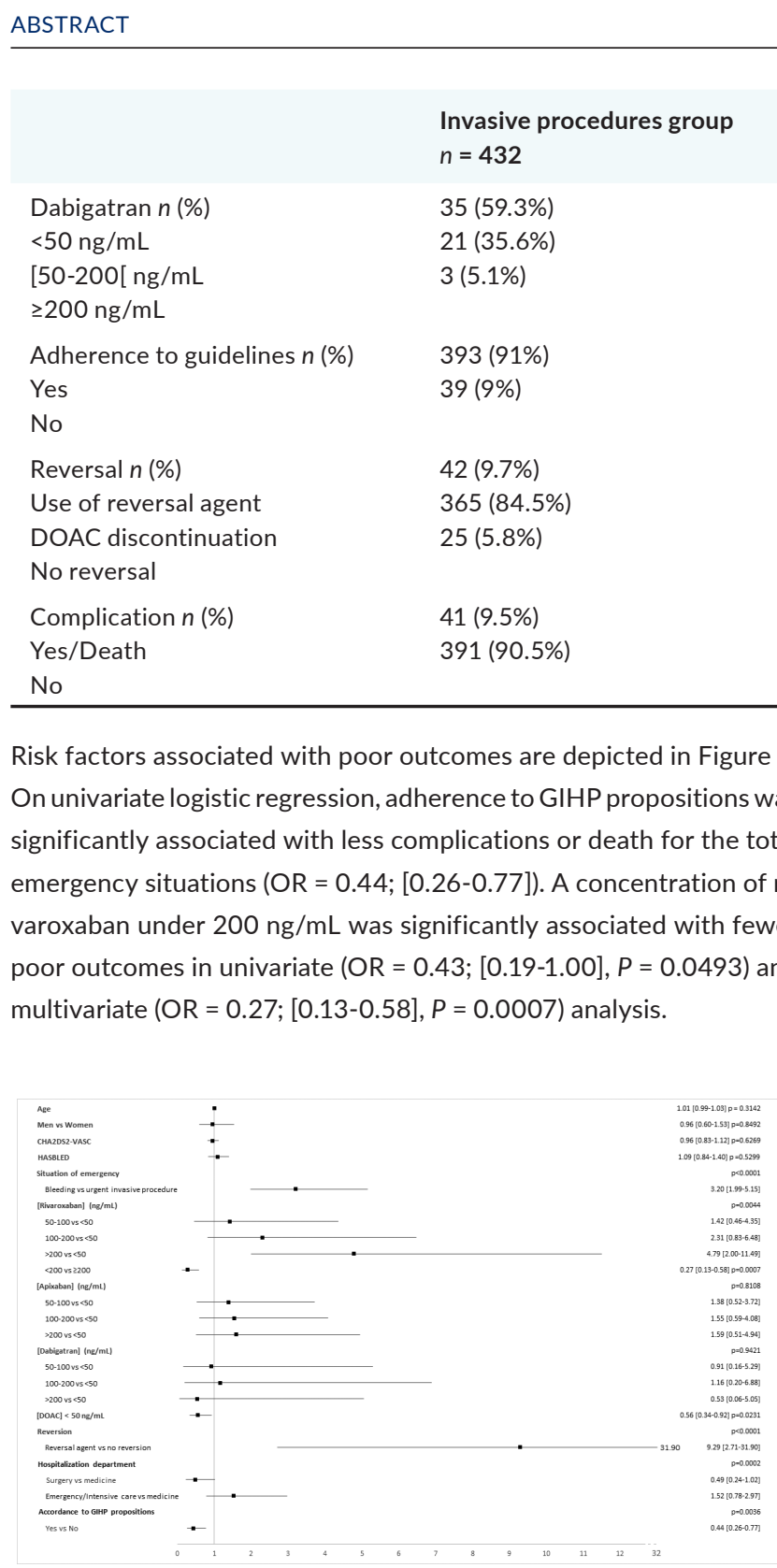

FIGURE 1 Forest plot of factors associated with poor outcomes (bleeding or thrombotic complications and death) in the total population (urgent invasive procedures and bleeding). Odds ratio (OR), 95\% confidence interval and $P$ are presented for each factor. DOAC: Direct oral anticoagulant, GIHP: French working Group on Perioperative Hemostasis

Conclusions: Adherence to the GIHP propositions was associated with fewer complications or death in emergency situations. We although found an association between higher plasmatic levels of rivaroxaban and the occurrence of poor outcomes that has not been described so far, and may deserve further investigations.
1. Albaladejo $P$ et al, Anaesthesia Critical Care \& Pain Medicine. 2018;37(4):391-399.

\section{PB0082 | Altered Hemostasis and Complement in Pediatric} Patients Treated with Cardiopulmonary Bypass

K. Todd Jr.; J. Tweddell; S. Jodele; E. Mullins; J. Palumbo Cincinnati Children's Hospital Medical Center and University of Cincinnati College of Medicine, Cincinnati, United States

Background: Cardiopulmonary bypass (CPB) causes significant thrombotic complications despite aggressive anticoagulation, and postoperative hemorrhage remains a problematic morbidity. Growing evidence also suggests that pathologic complement activation predisposes to catastrophic microangiopathies in CPB. The effects of CPB on the hemostatic and complement systems in children have not been adequately studied, representing a critical knowledge gap.

Aims: Identify the changes in hemostasis and complement activation in children undergoing CPB.

Methods: IRB approval and informed consent were obtained prior to enrollment. Samples were prospectively collected at baseline, $5 \mathrm{~min}$ and $1 \mathrm{~h}$ on $\mathrm{CPB}$, at the end of CPB, and $24 \mathrm{~h}$ postoperatively.

Results: 16 patients with mean age of 49.7 months (range 8 days to 17 years) were analyzed. Average CPB time was $160 \mathrm{~min}$ (range 59-407 min). We observed a statistically significant increase in VWF antigen and factor VIII shortly after bypass initiation (Fig. 1). We also observed statistically significant but modest diminutions in fibrinogen and factors II, VII, XI, XII and XIII after bypass initiation. We saw a major 10 -fold diminution in factor $V$ that far exceeded the changes we saw in other soluble clotting factors (Fig. 1). We also observed rapid and significant evidence of complement activation with $\mathrm{CPB}$ (increased $\mathrm{C} 3 \mathrm{a}$ and $\mathrm{s}$ C5b-9) that resolved $24 \mathrm{~h}$ following CPB (Fig. 2). 

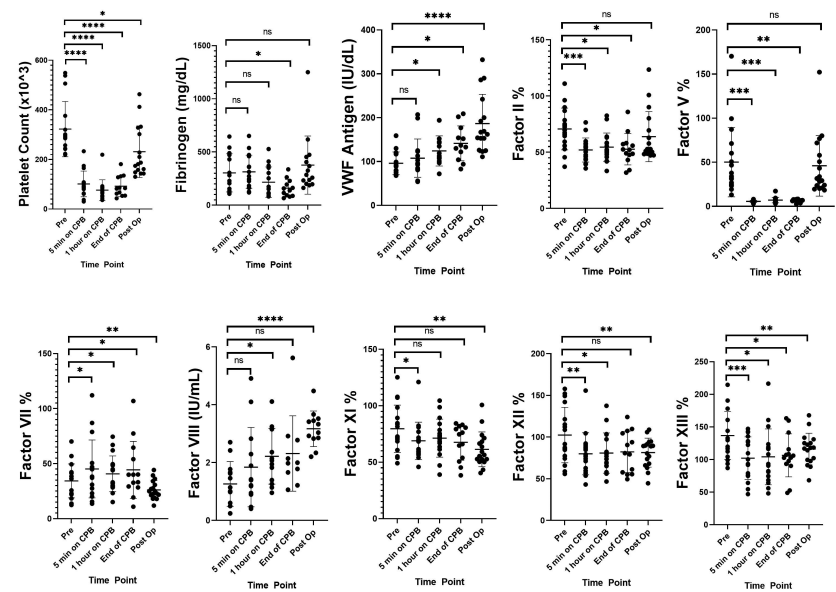

FIGURE 1 Alterations in hemostasis during CPB. KEY: ns $=P>0.05$ ${ }^{*}=P \leq 0.05,{ }^{* *}=P \leq 0.01,{ }^{* * *}=P \leq 0.001,{ }^{* * * *}=P \leq 0.0001$

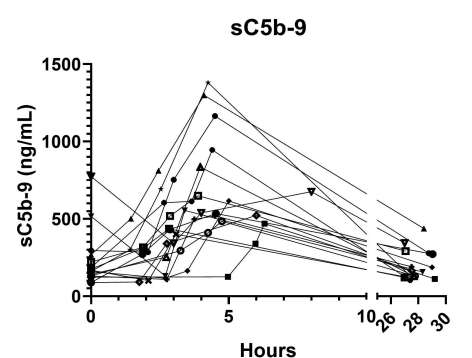

C3a

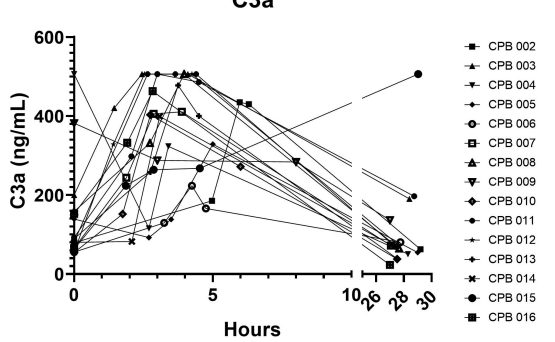

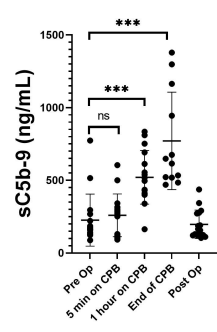

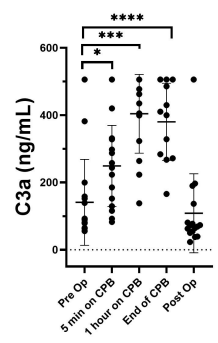

FIGURE 2 Alterations in complement during CPB. KEY: ns $=P>0.05$, ${ }^{*}=P \leq 0.05,{ }^{* *}=P \leq 0.01,{ }^{* * *}=P \leq 0.001,{ }^{* * * *}=P \leq 0.0001$

Conclusions: Pediatric patients undergoing CPB experienced multiple modifiable alterations in hemostasis and complement that may negatively impact outcomes. Specific correction of the major diminution in factor $V$ may represent a novel way to prevent postoperative hemorrhage as factor $V$ is diminished out of proportion to other factors in $\mathrm{CPB}$. Elevations in VWF may contribute to postoperative thrombotic risk. Complement activation may predispose to microangiopathy and multisystem organ damage. As multiple agents targeting C5 and C3 exist or are in development, studies targeting complement activation in pediatric CPB would be of major clinical interest.
PB0083 | Changes in the Global Hemostatic Profile after Subarachnoid Aneurismatic Hemorrhage

$\underline{\text { K. Nanwani }^{1} \text {; I. Fernandez }}{ }^{2}$; E. Monzón ${ }^{2}$; R. Justo ${ }^{2}$; N. Butta ${ }^{2}$; M. Quintana ${ }^{1}$

${ }^{1}$ Intensive Medical Department, La Paz Universitary Hospital, Madrid, Spain; ${ }^{2}$ Hematology Department, La Paz Universitary Hospital, Madrid, Spain

Background: Subarachnoid Aneurysmal Hemorrhage (SAH) evolves towards prothrombotic state that might influence development of $\mathrm{SAH}$ complications. Analysis of global thrombin generation (TG) by Calibrated Automatic Thrombinography (CAT) and clot-formation kinetics by thromboelastometry (ROTEM) could offer new insights on SAH.

Aims: Assessment of changes in patients' hemostatic profile after SAH.

Methods: A pilot, prospective, single-center and observational study was performed to assess changes in the global hemostatic profile on days (D) 1, 3 and 7 after SAH by CAT (TG peak) and ROTEM (maximun clot firmness by fibTEM test [MCF]). Platelet count, fibrinogen (FIB) and C-reactive protein (CRP) were also determined.

Results: Data of 15 patients $\geq 18$ years of age (60\% women) were analysed. Vasospasm, late neurological damage (LND), and death were observed in $28 \%, 20 \%$, and $13 \%$ of patients, respectively (Table 1). CRP was out of range after SAH, increasing along D1-D7, indicating an stepped development of a proinflammatory state. In D1 and D3, prothrombotic state due to an increment in TG was observed (Table 2). On the contrary, ROTEM showed a later fibrinogendependent prothrombotic state on D3 (MFC vs FIB; $r=0.703$; $P=0.035$ ) and D7 (MCF vs FIB; $r=0.900 ; P<0.001$ ). No patient was treated with anticoagulants during the study. We could not find any relationship between global hemostatic status and incidence of death, vasospasm or LND.

TABLE 1 Clinical and demographic data. LN = Late neurological damage; IHM = In-hospital mortality

\begin{tabular}{|c|c|c|c|}
\hline Parameter & $\begin{array}{l}\text { Patients } \\
(N=15)\end{array}$ & $\begin{array}{l}\text { Controls } \\
(N=29)\end{array}$ & $P$-value \\
\hline Age (year) (mean $\pm S D)$ & $56 \pm 12$ & $51 \pm 8$ & 0.070 \\
\hline Sex female (\%) & 60 & 59 & 1.000 \\
\hline Vasospasm (\%) & 28 & - & - \\
\hline LND (\%) & 20 & - & - \\
\hline IHM (\%) & 13 & - & - \\
\hline
\end{tabular}


TABLE 2 Thrombin generation, thromboelastometry and proinflammatory markers; median [25-75 percentile]; "statistically significant; $N=$ Normal range; $¥$ = median value out of normal range

\begin{tabular}{|c|c|c|c|c|c|}
\hline Parameter & $N$ & Day & Patients & Controls & $P$-value \\
\hline \multirow[t]{3}{*}{ Thrombin Peak (nM) } & 15 & 1 & 344 [274-377] & \multirow[t]{3}{*}{229 [136-338] } & $0.006^{*}$ \\
\hline & 14 & 3 & $296[235-434]$ & & $0.021^{*}$ \\
\hline & 10 & 7 & 219 [177-271] & & 0.700 \\
\hline \multirow[t]{3}{*}{ MCF-fibTEM (mm) } & 13 & 1 & $16[14-21]$ & \multirow[t]{3}{*}{$9-25^{N}$} & \multirow[t]{3}{*}{-} \\
\hline & 13 & 3 & $26[24-40]^{*}$ & & \\
\hline & 13 & 7 & $28[21-80]^{*}$ & & \\
\hline \multirow[t]{3}{*}{ Fibrinogen (mg/dL) } & 15 & 1 & 318 [286-407] & \multirow[t]{3}{*}{$200-400^{N}$} & \multirow[t]{3}{*}{-} \\
\hline & 14 & 3 & $544[408-683] ¥$ & & \\
\hline & 14 & 7 & $473[402-643]^{*}$ & & \\
\hline
\end{tabular}

Conclusions: After SAH, early hypercoagulable state develops due to increments in TG. In D3 and D7, though normalization of TG was observed, the prothrombotic status continues at the expense of fibrinogen-dependent increment of the clot MCF probably linked to the development of an acute proinflammatory state. Wider studies are required to determine mechanisms involved in the origin of the $\mathrm{SAH}$-related prothrombotic state and their relationship with the development of vasospasm, LND and death in SAH.

PB0084 | Coagulation Management Strategies in Cardiac Surgery: Viscoelastic tests

I. Rodriguez Martin; Isabel Rodriguez Martin

Hospital Universitario Virgen Macarena, Sevilla, Spain

Background: Viscoelastic tests (rotational thromboelastometry, ROTEM ), together with the implementation of a specific algorithm for coagulation management in cardiac surgery, enable perioperative coagulopathy to be better controlled.

Aims: Demonstrate that the implementation of viscoelastic tests, such as the case of ROTEM ${ }^{\circledR}$, allows a more selective and efficient transfusion therapy, achieving an improvement in health outcomes.

Methods: Retrospective cohort study including 675 patients who underwent cardiac surgery with cardiopulmonary bypass. The incidence of allogeneic blood transfusions and clinical postoperative complications were analyzed before and after ROTEM implementation.

Results: Following viscoelastic testing and the implementation of a specific algorithm for coagulation management, the incidence of any allogeneic blood transfusion decreased ( $41.4 \%$ vs $31.9 \%, P=0.026)$ during the perioperative period. In the group monitored with ROTEM , decreased incidence of transfusion was observed for packed red blood cells ( $31.3 \%$ vs $19.8 \%, P=0.002)$, fresh frozen plasma $(9.8 \%$ vs $3.8 \%, P=0.008)$, prothrombin complex concentrate administration (0.9\% vs $0.3 \%, P=0.599)$ and activated recombinant factor VII $(0.3 \%$ vs $0.0 \%, P=0.603$ ). Increased incidence was observed for platelet transfusion $(4.8 \%$ vs $6.8 \%, P=0.530)$ and fibrinogen concentrate (0.9\% vs $3.5 \%, P=0.066)$, tranexamic acid $(0.0 \%$ vs $0.6 \%, P=0.370)$ and protamine administration ( $0.6 \%$ vs $0.9 \%, P=0.908)$. Similar results were observed in the postoperative period, but with a decreased incidence of platelet transfusion (4.8\% vs $3.8 \%, P=0.813$ ). In addition, statistically significant reductions were detected in the incidence of postoperative bleeding ( $9.5 \%$ vs $5.3 \%, P=0.037$ ), surgical reexploration (6.0\% vs $2.9 \%, P=0.035)$, and length of Intensive Care Unit (ICU) stay (6.0 days vs 5.3 days, $P=0.026$ ).

Conclusions: The monitoring of hemostasis by ROTEM in cardiac surgery, was associated with decreased incidence of allogeneic blood transfusion, clinical hematologic postoperative complications and lengths of ICU stay.

\section{PB0085 | Evolution of Thrombin Generation in Pediatric Liver Transplantation}

K. Regling ${ }^{1}$; W. Hollon ${ }^{2}$; K. Collins ${ }^{2}$; N. Zoubi ${ }^{2}$; M. Rajpurkar ${ }^{1}$; M. Chitlur ${ }^{1}$

${ }^{1}$ Central Michigan University / Detroit Medical Center / Children's Hospital of Michigan, Detroit, United States; ${ }^{2}$ Detroit Medical Center / Children's Hospital of Michigan, Detroit, United States

Background: Orthotopic liver transplantation (OLT) is standard of care for patients with end-stage liver disease who are at increased risk of hemostatic complications. Previous adult studies demonstrated decreased thrombin generation intraoperatively with rebound post-operatively and resistance to thrombomodulin, compared to healthy controls. Assessment of coagulation in children in the peri-operative period remains challenging and global coagulation assays allow for better assessment of clot formation and lysis.

Aims: The specific aims of this project are to evaluate thrombin generation pre-OLT, intraoperatively and post-OLT using thrombin generation assay (TGA)and thromboelastography (TEG) and determine if there is correlation.

Methods: A retrospective study in 14 patients who underwent OLT at Children's Hospital of Michigan from 2014 - 2019 was performed with institutional review board approval and was funded by the Ashok and Ingrid Sarnaik Endowment Grant. Patient demographics, routine laboratory data and TEG parameters were collected. 11/14 
patients had TEG data and 9/14 patients had extra frozen platelet poor plasma sample for TGA. Each patient acted as their own control. Results: Patient demographics are listed in Table 1. TGA data showed a decrease in endogenous thrombin potential (ETP) and velocity index (VI) after liver reperfusion and rebounded to near pre-operative levels in the post-OLT period (Table 2). The underlying etiology of liver disease did not change the thrombin generation pattern. Thrombin generation as evaluated by maximum rate of thrombin generation (MRTG) on TEG also demonstrated intraoperative decrease, however no recovery was seen postoperatively. No bleeding or thrombotic complications occurred.

TABLE 1 Patient Demographics

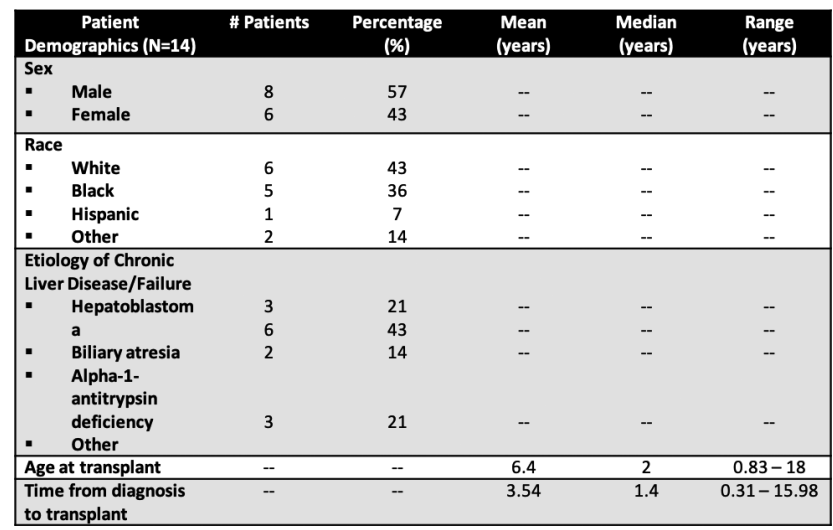

TABLE 2 Patient Data: Thrombin Generation Assay and Thromboelastography

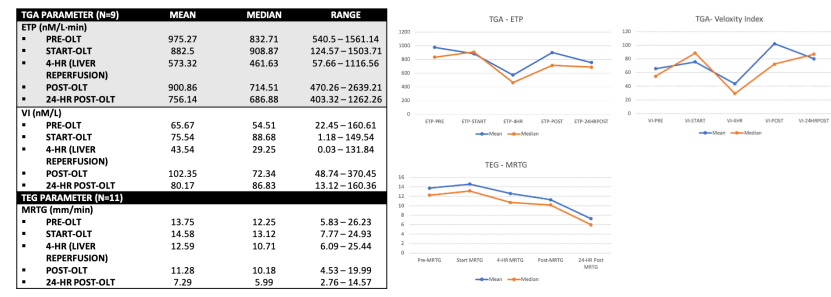

Conclusions: Like adults, children undergoing OLT show decreased thrombin generation peri-operatively, demonstrated by the decrease in ETP and VI, and show rebound increased thrombin generation post-OLT. We hypothesize that the TEG only partially reflects this finding due to the dominant reflection of fibrinogen and platelet function, which may not have normalized in the immediate postoperative period.
PB0086 | Anticoagulation and Antiplatelet Management during the Perioperative Period: A Daily Practice Cohort Prospective Study

O. Duranteau; A. Hamriti; T. Tuna; B. Ickx; L. Van Obbergh

Hopital Erasme, Bruxelles, Belgium

Background: For some patients, the performance of a planned surgery depends on the adjustment of their daily antithrombotic medication.

Aims: The aim of this study was to evaluate the consequences of the non-strict compliance with the published recommendations on the morbidity and mortality of patients.

Methods: We conducted a prospective cohort observational monocentric study in an academic hospital over 6 months in 2019.

All patients on antithrombotic agents scheduled for an elective surgery were included.

The endpoints were morbidity and mortality at 1 month, compliance with local and French-speaking guidelines, blood loss during the surgery, and length of stay.

Results: Among the 679 patients included in the study, 77 complications were recorded (11.3\%). 19 patients had a haemorrhage event and 6 died of it, 30 patients had an ischemic event and 4 died of it, 28 died of another cause. Forty percent anaesthesia consultations complied with the french-guidelines, $75.8 \%$ with local recommendations. The association was not was not statistically significant regarding morbidity and adherence to local guidelines $(P$-value $=0.12)$ but statistically significant with French speaking guidelines ( $P$-value $=0.027)$. Regarding guidelines respect and survival at 1 month, the association was not statistically significant with local guidelines $(P$ value $=0.80)$ and with french-speaking guidelines $(P$-value $=0.23$. No statistical relation can be made regarding compliance with recommendations and the volume of intraoperative bleeding, nor with the length of stay.

Conclusions: No association was found between adherence to local guidelines and morbi-mortality, but association was found with french-speaking ones. 
PB0087 | Off-label Use of Prothrombin Complex Concentrate in the Clinical Practice of a Portuguese Tertiary Hospital

D. Cibele; S. Teixeira; I. Machado; L. Gonçalves; C. Monteiro;

M. Carvalho; M. Koch

Centro Hospitalar Universitário São João, Porto, Portugal

Background: Prothrombin complex concentrates (PCCs) contain lyophilized purified vitamin $\mathrm{K}$-dependent clotting actors (II, VII, IX, X), and natural anticoagulants (protein $C$ and protein S). They are approved for urgent reversal of oral anticoagulants (OAC) but are often used in other clinical settings, namely coagulopathy associated to trauma, shock, perioperative bleeding or liver disease. Nonetheless, PCCs are associated to a potential increased risk of thromboembolic events.
Aims: Evaluate our hospital's experience with the use of PCC in patients not taking OAC, in a 3-year period, and analyze associated transfusional needs and adverse outcomes.

Methods: We performed a retrospective analysis of PCC use in 212 patients not treated with OAC, in different clinical settings, from January 2018 to December 2020, in Hospital de São João, Porto. A P value of 0.05 indicates statistical significance. Values are presented as appropriate. Analyses were performed with SPSS software.

Results: Over this 3-year period, CCP was administered in 692 occasions, 212 (30.64 \%) of which to patients who were not under OAC but showed laboratorial parameters compatible with coagulopathy and presented major bleeding or required urgent invasive procedures. Table 1 represents our population. Table 2 portrays the clinical context presented when PCC was used and associated outcomes.

TABLE 1 Demographics of the study population (IQR - Interquartile range)

\begin{tabular}{|c|c|c|c|c|}
\hline Characteristic & Urgent invasive procedure & Major bleeding & Total & $P$ value \\
\hline $\mathrm{N}(\%)$ & $117(54.9)$ & $95(44.6)$ & 212 & \\
\hline Age, mean (SD) & $57.6(12.73)$ & $57.15(10.52)$ & $57.36(11.74)$ & $P=0.798$ \\
\hline Male sex, $n(\%)$ & $79(67.6)$ & $75(78.9)$ & $154(72.6)$ & $P=0.063$ \\
\hline Liver disease, $n(\%)$ & $85(72.6)$ & $69(72.6)$ & $154(72.6)$ & $P=0.998$ \\
\hline
\end{tabular}

TABLE 2 Off-label PCC use: Clinical context and outcomes (IU - International Units, FFP - Fresh Frozen Plasma, y - years, d - days)

\begin{tabular}{|c|c|c|c|c|}
\hline Characteristic & $\begin{array}{l}\text { Urgent invasive } \\
\text { procedure }\end{array}$ & Major bleeding & Total & $P$ value \\
\hline Emergency department, $n$ (\%) & $24(20.5)$ & $24(25.3)$ & $48(22.6)$ & $P=0.411$ \\
\hline $\begin{array}{l}\text { Simultaneous administration of blood products, } n \\
(\%)\end{array}$ & $53(45.3)$ & $84(88.4)$ & $154(72.6)$ & $P<0.001$ \\
\hline FFP x 200 ml, median (IQR) & $0(0)$ & $2(6)$ & $0(3)$ & $P<0.001$ \\
\hline Fibrinogen g, median (IQR) & $0(0)$ & $1(2)$ & $0(2)$ & $P<0.001$ \\
\hline 3-y mortality (\%) & $73(62.4)$ & $68(71.6)$ & $142(67)$ & $P=0.159$ \\
\hline 90-d thrombotic event (\%) & $3(2.6)$ & $5(5.3)$ & $8(3.8)$ & $P=0.305$ \\
\hline
\end{tabular}

Conclusions: In patients with an acquired deficiency of the vitamin $\mathrm{K}$ dependent coagulation factors - due to severe hepatic dysfunction, trauma, major bleeding, sepsis - PCC is a useful tool for its rapid correction. Despite the high mortality observed in these patients, the low incidence of thromboembolism is noticeable - despite their acute hemostatic frailty, they might not present an hypercoagulable state associated with PCC administration. 
PB0088 | Adaptation of fixed prothrombin complex concentrate dosing for vitamin $\mathrm{K}$ antagonist-related bleeding in the Netherlands

$\underline{\text { R. Abdoellakhan; K. Meijer }}{ }^{1}$; E. Roelofsen ${ }^{2}$; V. van de Craats $^{3}$; N. Khorsand ${ }^{4}$

${ }^{1}$ University Medical Center Groningen, Groningen, Netherlands;

${ }^{2}$ Haaglanden Medical Center, The Hague, Netherlands;

${ }^{3}$ HagaZiekenhuis, The Hague, Netherlands; ${ }^{4}$ OLVG, Amsterdam, Netherlands

Background: The recommended reversal agent for vitamin $\mathrm{K}$ antagonists (VKA) related bleeding is four-factor prothrombin complex concentrate (4F-PCC). Despite over 40 years of clinical experience with 4F-PCC, literature remains ambiguous about the optimal dosing strategy, with current guidelines advising fixed doses or variable doses based on INR and bodyweight.

Aims: To investigate the proportion of hospitals using a fixed dosing strategy for 4F-PCC in treatment of VKA related bleeding in the Netherlands.

Methods: A survey was conducted among hospital pharmacists. The hospital pharmacist responsible for local anticoagulation policy of each hospital in the Netherlands was first identified by phone and then e-mailed access to an online survey. The main questions regarded the 4F-PCC dosing strategies in VKA-related extracranial and intracranial bleeding.

Results: Of the 106 identified hospitals, 74 responded $69.8 \%$ response rate). A total of 39 hospitals (52.7\%) reported the use of a fixed dose in VKA-related extracranial or intracranial bleeding emergencies, 30 hospitals used only the variable dosing strategy (40.5\%). Most fixed strategies were implemented for extracranial bleeds (51.4\%), either 1000 IU (84.2\%) or 500 IU (15.8\%). For intracranial bleeds 23 hospitals (31.1\%) used a fixed dose, mostly 1000 IU (65.2\%), however larger doses up to 2000 IU were seen. The implementation of fixed dosing in academic hospitals seemed to be not as widespread as in non-academic hospitals (20\% versus 55.1\%). Furthermore smaller hospitals had implemented the fixed dose more frequently, possibly for pragmatic reasons and costs. $95.9 \%$ of hospitals reported to have a local treatment protocol and $83.8 \%$ had 4F-PCC stored in the emergency department, minimizing door-to-needle time.

Conclusions: A fixed dose was implemented in $52.7 \%$ of hospitals in the Netherlands. The uptake of fixed dosing is considerable, given the scarcity of data, especially in intracranial bleeding. Future research should provide a decisive answer on the positioning of dosing strategies.

PB0089 | Persistently Low Levels of Antithrombin in Trauma Patients Associated with Disseminated Intravascular Coagulation

S. Gando ${ }^{1,2} ;$ T. Wada ${ }^{2}$

${ }^{1}$ Sapporo Tokushukai Hospital, Sapporo, Japan; ${ }^{2}$ Hokkaido University Faculty of Medicine, Sapporo, Japan

Background: Antithrombin is a major anticoagulant controlling inflammation as well as coagulation. Low antithrombin level has been considered one of causes of organ dysfunction and poor outcome in disseminated intravascular coagulation (DIC).

Aims: We retrospectively analyzed serial changes in the levels of antithrombin to clarify its roles in trauma patients associated with DIC. Methods: The 259 trauma patients were included and subdivided into 153 DIC and 106 non-DIC patients. Serial changes in platelet counts and coagulofibrinolytic parameters as well as antithrombin levels were evaluated from immediately after arrival at the emergency department (day 0), day 1 and days 2 to 6 (Late stage). During late stage, lowest antithrombin levels were used for analysis. Organ dysfunction was evaluated by Sequential Organ Failure Assessment (SOFA) socre and SOFA>12 was defined as multiple organ dysfunction syndome (MODS). Outcome measure was all-cause hospital mortality.

Results: DIC patients showed peresistently lower antithrombin levels at day 0 (63.8\%), day 1 (60.4\%), and Late stage (60.9\%) thant non-DIC patients $(79.0,77.0,74.5 \%)(P<0.001)$ and was associated significantly higher rates of MODS (24.8 vs. $18.9 \%)$ and hospital mortarity (28.1 vs. $7.5 \%$ ) than non-DIC patients. In addition, antithrombin levels at day 0 in died DIC patients (56.8\%) were lower than that of survivors (66.5\%) $(P<0.001)$. Antithrombin levels at day 0 were independent predictor of death (Odds, 0.97; $95 \% \mathrm{Cl} 0.95$ $0.98, P<0.001)$.

Conclusions: DIC, especially died DIC patients, showed persistently low antithrombin levels immediate to late stage of trauma. Low antithrombin levels may have role in poor prognosis of trauma patients. 
PB0090 | Global Coagulation Assay in a Patient Affected by AL Amyloidosis

S. Arcudi ${ }^{1}$; A. Artoni ${ }^{2}$; E. Scalambrino ${ }^{2} ;$ M. Clerici ${ }^{2} ;$ F. Valsecchi $^{3}$; M. Capecchi ${ }^{4}$; A. Tripodi ${ }^{2}$; F. Peyvandi ${ }^{1,2}$

${ }^{1}$ Fondazione IRCCS Ca' Granda Ospedale Maggiore Policlinico, Angelo Bianchi Bonomi Hemophilia and Thrombosis Center, Università degli Studi di Milano, Department of Pathophysiology and Transplantation, Milan, Italy; ${ }^{2}$ Fondazione IRCCS Ca' Granda Ospedale Maggiore Policlinico, Angelo Bianchi Bonomi Hemophilia and Thrombosis Center, Milan, Italy; ${ }^{3}$ Università degli Studi di Milano, Milan, Italy; ${ }^{4}$ Fondazione IRCCS Ca' Granda Ospedale Maggiore Policlinico, Angelo Bianchi Bonomi Hemophilia and Thrombosis Center, Università degli Studi di Milano, Department of Biomedical Sciences for Health, Milan, Italy

Background: Systemic Immunoglobulin Light Chain Amyloidosis (AL-Amyloidosis) is known to cause an acquired Factor $X(F X)$ deficiency, but FX activity does not predict the hemorrhagic risk of these patients.

Aims: To determine if the Thrombin Generation Assay (TGA) could be a useful haemostatic parameter in the management of a patient affected with acquired FX deficiency, going through an invasive procedure.

Methods: A 74-year-old female with a recent diagnosis of nephrotic syndrome had prolonged PT and aPTT (patient-to-normal) ratios corrected by mixing tests. A first coagulation assessment revealed a FX activity of $6 \%$ (baseline blood tests are reported in Table 1). The patients had to go through a kidney biopsy to confirm the clinical suspicion of AL-Amyloidosis. She was treated with $30 \mathrm{U} /$ $\mathrm{Kg}$ of Prothrombin Complex Concentrate (PCC), which increased FX activity to $14 \%$ in 30 min. Coagulation testing was performed at baseline and $30 \mathrm{~min}$ after $30 \mathrm{U} / \mathrm{Kg}$ of PCC treatment. TGA was assessed in platelet-poor-plasma with $1 \mathrm{pM}$ Tissue Factor (TF) and $1 \mu \mathrm{M}$ synthetic phospholipids, and then in platelet-rich-plasma after addition of TF (1 pM).

TABLE 1 Basal blood tests examination

\begin{tabular}{lll} 
Coagulation tests & Patient values & Normal values \\
PT (patient-to-normal) ratio & 1.82 & $0.84-1.20$ \\
aPTT ratio & 1.47 & $0.86-1.20$ \\
Protein C (\%) & 260 & $72-160$ \\
Protein S (\%) & 126 & $58-114$ \\
FII (\%) & 115 & $73-113$ \\
FVII (\%) & 135 & $62-138$ \\
FX (\%) & 6 & $66-126$ \\
\hline
\end{tabular}

Results: As shown in Table 2, patient baseline TGA showed a very prolonged lag-time (>20 $\mathrm{min}$ ) with the other parameters within the $97.5^{\circ}$ percentiles. After PCC treatment, the prolonged lag-time was only partially corrected but ETP increased over measurable limits. We repeated the analysis after mixing with $50 \%$ of normal plasma as a source of FX, with a partial correction of the lag-time. The patient underwent renal biopsy without bleeding complications and the diagnosis of AL-amyloidosis was confirmed.

TABLE 2 Thrombin Generation Assay (TGA) parameters. TGA was assessed according to Hemker with a homemade method, performing the test in a platelet-poor-plasma (PPP) with $1 \mathrm{pM}$ of Tissue Factor (TF) and $1 \mu \mathrm{M}$ of synthetic phospholipids (PL), and then in platelets-rich-plasma (PRP) by addition of TF (1 pM). "PCC" = Prothrombin Complex Concentrate.

\begin{tabular}{|c|c|c|c|c|}
\hline & PLASMA/triggers & LAG-TIME (min) & $\operatorname{ETP}\left(\mathrm{nM}^{*} \min \right)$ & PEAK HEIGHT (nM) \\
\hline Patient (pre PCC) & $\mathrm{PPP} / 1 \mathrm{pM} T F+1 \mu \mathrm{M} \mathrm{PL}$ & 20 (>99ºct) & $2685\left(97.5^{\circ} \mathrm{pct}\right)$ & 441 (97.5pct) \\
\hline Patient (pre PCC) & PRP/1 pM TF & 21 (>99ºct) & 2570 (95pct) & 141 (40pct) \\
\hline Patient (post PCC) & $\mathrm{PPP} / 1 \mathrm{pM} \mathrm{TF}+1 \mu \mathrm{M} \mathrm{PL}$ & 18 (>99ºct) & Not measurable & 675 (<99 $\mathrm{pct})$ \\
\hline Patient (post PCC) & PRP/1 pM TF & 18 (>99 $\left.{ }^{\circ} \mathrm{pct}\right)$ & Not measurable & 360 (<99 $\left.{ }^{\circ} \mathrm{pct}\right)$ \\
\hline Healthy donor & $\mathrm{PPP} / 1 \mathrm{pM} \mathrm{TF}+1 \mu \mathrm{M} \mathrm{PL}$ & $5\left(20^{\circ} \mathrm{pct}\right)$ & 1987 (45ำct) & $310\left(50^{\circ} \mathrm{pct}\right)$ \\
\hline Healthy donor & PRP/1 pM TF & $7\left(30^{\circ} \mathrm{pct}\right)$ & 2039 (60pct) & $137\left(25^{\circ} \mathrm{pct}\right)$ \\
\hline $\begin{array}{r}\text { Patient prePCC + } \\
\text { healthy donor }\end{array}$ & $\mathrm{PPP} / 1 \mathrm{pM} T F+1 \mu \mathrm{M}$ PL & 9 (>99 $\left.{ }^{\circ} \mathrm{pct}\right)$ & 2437 (90pct) & $443\left(97.5^{\circ} \mathrm{pct}\right)$ \\
\hline $\begin{array}{c}\text { Patient postPCC }+ \\
\text { healthy donor }\end{array}$ & PPP/1 pM TF+1 $\mu \mathrm{M}$ PL & 9 (>99 $\left.{ }^{\circ} \mathrm{pct}\right)$ & 3522 (>99 $\left.{ }^{\circ} \mathrm{pct}\right)$ & 526 (>99 $\left.{ }^{\circ} \mathrm{pct}\right)$ \\
\hline
\end{tabular}

Conclusions: AL-Amyloidosis is known to provoke an acquired FX deficiency, but the consequences on the haemostasis are still not predictable. TGA could provide a better assessment of haemostasis in these patients in order to avoid unnecessary bleeding or thrombotic risks. 
PB0091 | Severe Coagulopathy Induced by Therapeutic Plasma Exchange - A Case Report

$\underline{\text { S. Lopes }}^{1}$; R. Pombal ${ }^{1}$; L. Vieira ${ }^{1}$; R. Neto ${ }^{1}$; M.A. Canelas ${ }^{2}$;

T. Fonseca ${ }^{2}$; H. Cruz Gomes ${ }^{1}$; D. Ferreira ${ }^{1}$; P. Castelões ${ }^{2}$

${ }^{1}$ Serviço Imunohemoterapia, Centro Hospitalar Vila Nova de Gaia/

Espinho, Vila Nova de Gaia, Portugal; ${ }^{2}$ Serviço de Medicina Intensiva

Polivalente, Centro Hospitalar Vila Nova de Gaia/Espinho, Vila Nova de Gaia, Portugal

Background: Therapeutic plasma exchange (TPE) is increasingly used for treatment of acute pancreatitis with hypertriglyceridemia. There is a concern that TPE may increase risk of bleeding, although data is limited.

Aims: Case-report of coagulopathy induced by TPE.

Methods: Collection of clinical data in SClínico ${ }^{\circledR}$ application.

Results: 41-year old man, history of alcohol abuse and possible chronic liver disease, without other relevant background. Admitted to the Intermediate Medical Care Unit with acute pancreatitis associated with severe hypertriglyceridemia and acute kidney injury, with indication for TPE. After two sessions, on consecutive days, of TPE with albumin replacement, there was a hypertriglyceridemia improvement (decrease from 6930 to 497 mg/dL). After TPEtreatment, patient presented coagulopathy: aPTT $77.7 \mathrm{~s}$ (previous 40.5 s; normal 24-34), INR 1.31 (previous 0.92). Mixing studies confirmed deficit of intrinsic coagulation pathway factor. Within $24 \mathrm{~h}$, presented severe abdominal pain, fever, tachycardia and psychomotor agitation. CT-scan was suggestive of intestinal ischemia and compartment syndrome, with indication for emergent laparotomy. Transferred to Intensive Care Unit with hemodynamic instability and multiorgan dysfunction. Pre-surgically, had to be transfused with 6 units of fresh frozen plasma (FFP) and 1 pool of platelets, postponing surgical timing. Temporary correction of coagulopathy was achieved. Exploratory laparotomy revealed retroperitoneal necrosis, without evidence of active bleeding. Procedure progressed without major haemorrhagic complications. The patient remained refractory to the instituted therapies and died within $24 \mathrm{~h}$ after surgery.

Conclusions: In critically ill patients, TPE should be carefully considered and FFP should be generally preferred over albumin as replacement fluid to avoid depletion of coagulation factor and perioperative bleeding. TPE replaced with albumin induces significant changes in global haemostasis parameters thus potentially increasing bleeding risk. In this case, the replacement of coagulation factors preoperatively with FFP was crucial to avoid hemorrhagic complications, despite having postponed the surgery.
PB0092 | Viscoelastic Haemostatic Assay Comparison for Perioperative Coagulation Assessment

J. Fanning ${ }^{1,2,3} ;$ S. Roberts ${ }^{4,1}$; C. Anstey ${ }^{5}$; K. Poon ${ }^{3,6} ;$ A. Incani $i^{3,1}$; J. Fraser ${ }^{2,1,3}$

${ }^{1}$ University of Queensland, Herston, Australia; ${ }^{2}$ Critical Care

Research Group, Chermside, Australia; ${ }^{3}$ St. Andrew's War Memorial Hospital, Spring Hill, Australia; ${ }^{4}$ The Princess Alexandra Hospital, Woolloongabba, Australia; ${ }^{5}$ Griffith University, Southport, Australia;

${ }^{6}$ The Prince Charles Hospital, Chermside, Australia

Background: Viscoelastic haemostatic assays (VHA) now supersede laboratory assessments for the perioperative assessment and management of coagulation. Despite being used interchangeably in clinical practice, the devices in current use have rarely been directly compared.

Aims: This study directly compared standard laboratory coagulation tests and the two most commonly used VHA devices-TEG6s and ROTEMSigma-in a setting where large fluctuations in coagulation status are typical, to determine associations and interchangeability between key coagulation measures.

Methods: Patients undergoing cardiac surgery (requiring cardiopulmonary bypass) or interventional (transcatheter aortic valve implantation or percutaneous coronary intervention) were recruited prospectively. Blood samples ( $n=164$ ) were obtained at four timepoints (see Figure1). Each sample was assessed concurrently for standard laboratory tests (PT/INR, aPTT, thrombin clotting time, platelet count, direct fibrinogen), complete+hep(ROTEMSigma), and citrated(TEG6s) cartridges.

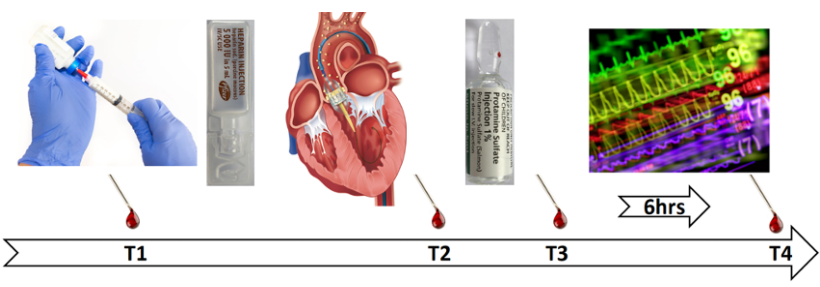

FIGURE 1 Time points for sample collection: T1, baseline; T2, postheparin administration; T3, post-protamine reversal; and, T4, sixhours postoperatively

Results: TEG6s required significantly less blood per test than ROTEMSigma ( $300 \mu \mathrm{L}$ vs. $1.8 \mathrm{~mL}$ ). Conversely, TEG6s is an open system requiring pipetting, whereas ROTEM is entirely enclosed. For intrinsically-activated assays, strong correlations existed for both 
'time to initiation of clot formation' (CT-INTEM vs. R-CK $=0.81$; CT-INTEM vs. R-CRT $=0.89$ ) and clot strength (MCF-INTEM vs. MA-CK, $r=0.81$; MCF-INTEM vs. MA-CRT, $r=0.87)$. For extrinsically-activated assays, a strong correlation existed for clot strength (MCF-EXTEM vs. MA-CRT, $r=0.89$ ), but not 'time to initiation of clot formation' (CT-EXTEM vs. R-CRT, $r=0.34$ ). Particularly important for both cardiac surgery and trauma settings is that measurements of the fibrinogen contribution to clot strength were highly correlated (MA-CFF vs. MCF-FIBTEM, $r=0.94$ ) and correlated with standard laboratory fibrinogen levels (fibrinogen vs. MA-CFF, $r=0.85$; fibrinogen vs. MCF-FIBTEM, $r=0.86$ ). Poor correlation was evident assessing time to clot formation between the two systems' heparinase channels (CT-HEPTEM vs. R-CKH, $r=0.53$ ) and clot strength (MCF-HEPTEM vs. MA-CKH, $r=0.53$ ).

Conclusions: Though ROTEMSigma and TEG6s are strongly correlated in some measurements, they are poorly correlated in others, suggesting that their results, and therefore clinical use, are not interchangeable.

\section{PB0093 | Surveillance of Anticoagulation in Patients with Extracorporal Oxygenation Membrane (ECMO)}

L.H. Gonzalez-Figueroa; F.A. Grimaldo-Gómez; X. García-Jiménez; V. Anguiano-Alvarez; E. Cortina-de la Rosa; M.O. Romero-Arroyo; G.A. Altamirano-Solorzano; G. Rojas-Velazco; R.A. Izaguirre-Avila National Institute of Cardiology Ignacio Chavez, Mexico City, Mexico

Background: Bleeding and thrombotic complications are the main contributors to morbidity and mortality during ECMO. Anticoagulation in patients with ECMO is challenging. There is no gold standard for anticoagulant surveillance and it is recommended to establish protocols in each institution according to the resources available.

Aims: Patients from the Intensive Care Unit treated with ECMO at the National Institute of Cardiology, assessed by Hematology, with a report of Activated Clotting Time (ACT), Activated Partial Thromboplastin Time (aPTT), Anti Xa, Antithrombin III (ATII), Fibrinogen (Fg) during ECMO use.

Methods: Observational, descriptive, retrospective, cross-sectional study. Patients from the Intensive Care Unit of the National Institute of Cardiology Dr. Ignacio Chávez treated with ECMO in the period 2019-2020 were included.

Results: Thirteen patients anticoagulated with unfractionated heparin during ECMO therapy were included. The laboratory tests for surveillance were: aPTT, Fibrinogen, platelet count, D-Dimer, Ant $\mathrm{Xa}, \mathrm{ACT}$. The ACT test had a very low correlation with heparinemia. In complications, a direct relationship was found between the report of ischemic CVD and subtherapeutic anticoagulant therapy. In the outcome, 8 patients were discharged home and 5 patients died (38.4\%)

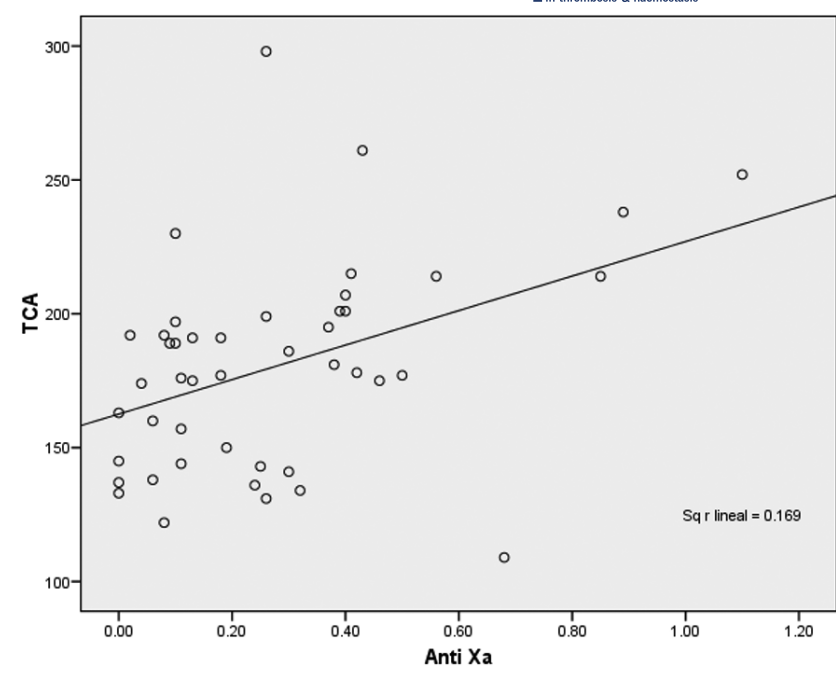

FIGURE 1 Dispersion of heparinemia values by Anti Xa vs TCA. The $\mathrm{ACT}$ test has a very low correlation with heparinemia and should not be used to monitor anticoagulation status in ECMO

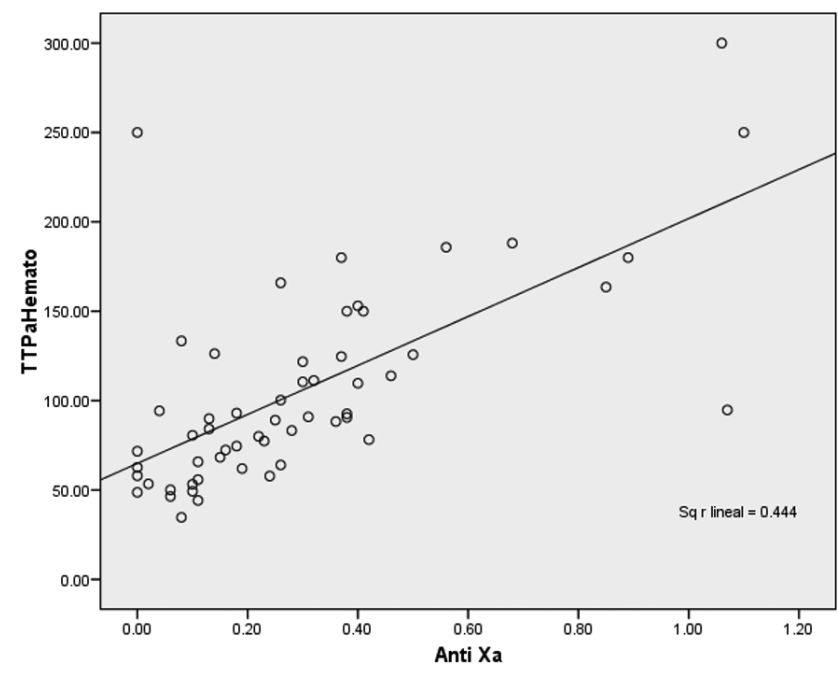

FIGURE 2 Dispersion of heparinemia values by Anti Xa vs aPTT. Spearman's correlation coefficient to determine the correlation between aPTT results and Anti Xa heparinemia levels

Conclusions: To achieve optimal anticoagulation of the patient on ECMO, close clinical surveillance and reliable measurements of the anticoagulation level are necessary, such as surveillance with measurement of antiXa levels. Calibration of aPTT with anti-Xa heparinemia is always recommended to be able to use aPTT in monitoring treatment with unfractionated heparin. Prospective multicenter trials are needed to investigate the optimal anticoagulation management strategy during ECMO support. 
PB0094 | Control of Anti-coagulant Treatment in Patients with Acute Stroke by the Results of the «Thrombodynamics» Test

E. Klychnikova; G. Ramazanov; E. Tazina; S. Abudeev; A. Vyshlova N.V. Sklifosovsky Research Institute for Emergency Medicine, Public Healthcare Institution of Moscow Healthcare Department, Moscow, Russian Federation

Background: In practice of preventing secondary thrombotic complications, unfractionated heparins are used.

Aims: To assess the effectiveness of laboratory control over anticoagulant therapy in patients with acute stroke.

Methods: 27 patients (mean age $65.6 \pm 14.4$ ) were examined. By the structure of neurological pathology: stroke was identified in 21 cases; cerebral venous sinus thrombosis was diagnosed in 2 cases, intracerebral hematoma was found in 2 cases; subarachnoid hemorrhage (SAH) was observed in 2 cases. Head computed tomography (CT), as well as CT - angiography, was made to all admitted patients. Ultrasonography of the brachiocephalic arteries, transcranial duplex scanning, echocardiography were also performed. Disaggregant therapy was not prescribed for patients with hemorrhagic stroke and $\mathrm{SAH}$, anticoagulants in such cases were prescribed $72 \mathrm{~h}$ after the development of a cerebral catastrophe, drug dosages were selected under the control of a coagulogram and Thrombodynamics (TD) test.

Results: Upon admission to ICU, the severity of the patient's condition according to APACHE II scale was from 12 to 25 points, the median was 21 points. The severity of stroke was assessed by NIHSS scale and ranged from 0 to 19 points, the median was 9 points. On admission, the hemostasiogram indices were within the normal range. However, there was a significant increase of D-dimer level - $1.49(1.90-5.68) \mathrm{mg} / \mathrm{I}(P<0.001)$ relative to the norm, as well as an increase of fibrinogen's level -4.26 (1.44-4.90) $\mathrm{g} / \mathrm{l}$. According to TD test, at the moment of admission the hemostasis system in patients was heterogeneous in terms of the dynamic characteristics Vi and Vst, as well as the size of the clot. $48 \%$ of patients were in the area of hypercoagulation of varying severity and $41 \%$ - in hypocoagulation state.

Conclusions: The global test "Thrombodynamics" is an effective too for assessing the state of the plasma hemostasis and monitoring anticoagulant therapy during the treatment of patients.

PB0095 | Ability of Fibrin Monomers and Fibrinogen Levels to Predict In-hospital Mortality in Patients with Severe Trauma

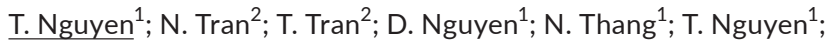
T. $\operatorname{Tran}^{1}$

${ }^{1}$ Cho Ray Hospital, Ho Chi Minh City, Vietnam; ${ }^{2}$ University of Medicine and Pharmacy, Ho Chi Minh City, Vietnam

Background: In trauma patients, trauma-induced coagulopathy (TIC) is a leading cause of mortality. Fibrin monomers (FM) and fibrinogen are markers of coagulation activation. Therefore, we speculate that these markers can be potential predictors of mortality in trauma patients.

Aims: Evaluate the in-hospital mortality predictive value of early fibrin monomers and fibrinogen levels in severe trauma.

Methods: Trauma patients with Injury Severity Scores (ISS) > 15 were recruited. FM and fibrinogen levels were checked at admission and again after $24 \mathrm{~h}$ (if the patient survived the first $24 \mathrm{~h}$.) The lowest FM and the highest fibrinogen values were defined as nadir FM (minFM) and peak fibrinogen levels (maxFib.) The patients were observed until being discharged from the hospital or dying during treatment.

Results: 19 patients were recruited in this preliminary study. 8 patients survived and 11 died in-hospital, Mean minFM and maxFib of the non-survivors were $122.92 \pm 81.59 \mu \mathrm{g} / \mathrm{ml}$ and $2.74 \pm 1.89$ $\mathrm{g} / \mathrm{L}$, respectively. Mean minFM and maxFib of the survivors were $9.06 \pm 4.98 \mu \mathrm{g} / \mathrm{ml}$ and $4.93 \pm 1.43 \mathrm{~g} / \mathrm{L}$, respectively. Both minFM and maxFib were significantly different between survivors and nonsurvivors $(P<0.05$.) A minFM of $>36.97 \mu \mathrm{g} / \mathrm{ml}$ had a sensitivity of $81.8 \%$ and specificity of $100 \%$ for in-hospital mortality prediction. A maxFib of $<4.56 \mathrm{~g} / \mathrm{L}$ had a sensitivity of $72.7 \%$ and specificity of $87.5 \%$ for in-hospital mortality prediction.

Conclusions: Our results highlight the role of coagulopathy in trauma. More specifically, since both FM and fibrinogen are very sensitive markers of fibrinolysis, we hypothesize that hyperfibrinolysis is the main etiology of TIC. Therefore, close monitoring of hyperfibrinolysis with assays like FM or fibrinogen levels may be crucial in trauma management. Further studies are warranted to clarify the roles of these assay in the clinical setting.

\section{PB0096 | Preoperative Hemorrhagic Risk Assessment: Knowledge, Attitude and Practice among Surgeons and Anesthesiologists}

E. Hammami; M. Cheikhrouhou; M.Y. Kaabar; S. Guermazi Department of Hematology, Charles Nicolle Hospital, Tunis, Tunisia

Background: Among the various challenges facing the coagulation system, surgery seems to be one of the most important. Thus, assessing the hemorrhagic risk preoperatively is paramount. Since the development of coagulation tests (CT) in the early 90's, considerable progress had been made in the understanding of the patient hemostatic status. Hence, medical societies issued recommendations regarding preoperative ordering of CT. This was done in an attempt to reduce inappropriate $\mathrm{CT}$. However, this practice is still widespread among physicians.

Aims: Our study aimed to evaluate the knowledge, attitude, and practices of surgeons and anesthesiologists regarding preoperative hemorrhagic risk assessment.

Methods: We conducted a prospective study in a Tunisian teaching hospital: Charles Nicole. We targeted residents and attendees in seven surgical departments and in the anesthesiology department. 
Data were collected via a 21-questions, self-administrated questionnaire prior to and after a training session. During this session, we have raised awareness among surgeons regarding coagulation testing's preoperative indications and interpretation.

Results: Fifty-seven physicians were included, 33 of whom frequently ordered CT. Fifteen participants $(26 \%)$ did not consider that they were ordering excessive CT. No anesthesiologist stated that they routinely ordered CT preoperatively versus $55 \%$ of surgeons $(P<0.01)$ and no anesthesiologist was unaware of the French society of anesthesiology and reanimation recommendations versus $38 \%$ of surgeons $(P<0.01)$. The top reason physicians said they order unnecessary CT is concern about malpractice issues. The median score for the knowledge evaluation questions was 5/8 [2.5-8] and 7/8 [48] after the training session.

Conclusions: Continuing medical education is an essential tool to ensure quality care and to limit resource waste in the healthcare system. Our action to streamline the prescription of CT through education seemed to be efficient. Nevertheless, it requires remote re-evaluation and long-term reinforcement.

PB0097 | First Case of Wunderlich Syndrome under Apixaban - Case Report

T. Quaresma $^{1}$; S. Maltês ${ }^{2}$; C. Carvalho Gouveia ${ }^{3}$; D.J. Canudo ${ }^{4}$; M. Rebelo ${ }^{5}$; F. Serrazina ${ }^{6}$; M.M. Deveza ${ }^{1}$; M. Galvão ${ }^{1}$

${ }^{1}$ Centro Hospitalar Universitário Lisboa Norte - Imunohemoterapia, Lisbon, Portugal; ${ }^{2}$ Centro Hospitalar Lisboa Ocidental - Hosp. Santa Cruz - Cardiologia, Lisbon, Portugal; ${ }^{3}$ Centro Hospitalar Lisboa Ocidental - Hosp. São Francisco Xavier - Medicina Interna, Lisbon, Portugal; ${ }^{4}$ Hospital Espírito Santo - Évora - Medicina Interna, Évora, Portugal; ${ }^{5}$ Centro Hospitalar Lisboa Ocidental - Hosp. Egas Moniz - Unidade de Cuidados Intensivos Polivalente - ICU, Lisbon, Portugal; ${ }^{6}$ Centro Hospitalar Lisboa Ocidental - Hosp. Egas Moniz - Neurologia, Lisbon, Portugal

Background: Wunderlich syndrome (WS) is a rare and life-threatening syndrome presenting as spontaneous nontraumatic renal rupture with subcapsular and perinephric hemorrhage. Apixaban, a direct oral anticoagulant (DOAC) recommended in patients with atrial fibrillation (AF), has previously been associated with atraumatic solid organ rupture but, to date, no case of apixaban-related WS has been reported.

Aims: To discuss the first case of Wunderlich syndrome under Apixaban.

Methods: We report a case of a 79-year-old female with known atrial fibrillation under apixaban. She was admitted with sudden left flank pain and hypotension, blood tests revealed a hemoglobin of $9.2 \mathrm{~g} / \mathrm{dL}$, platelets count of $188 \times 10^{9} / \mathrm{L}$, creatinine of $2.05 \mathrm{mg} / \mathrm{dL}$ (baseline of $0.85 \mathrm{mg} / \mathrm{dL}$ ). A computed tomography (CT) scan revealed an upper left renal rupture with extensive perirenal hemorrhage. Thus, a diagnosis of Wunderlich syndrome was made. She later developed obnubilation and hypotension. Blood analysis revealed hemoglobin of
$6.4 \mathrm{~g} / \mathrm{dL}$, mixed acidemia with a pH of 6.9 and lactate of $13.0 \mathrm{mmol} / \mathrm{L}$ (reference value $<2.0 \mathrm{mmol} / \mathrm{L}$ ). A diagnosis of hemorrhagic shock was assumed and the patient was transferred to the Intensive Care Unit for initiation of blood transfusion, vasopressor support and mechanical ventilation. Anticoagulation was suspended.

Results: Hemodynamic stability was achieved, however, at day seven of admission, she developed multiple 'de novo' cerebral embolic infarctions. She was deemed unsuitable for anticoagulation resumption given the recent major bleeding. At day 47 of admission, after excluding new bleeding events, anticoagulation with apixaban was resumed and she was discharged with ongoing improvement of neurological deficits and normal renal function.

Conclusions: We present the first-ever report of WS associated with DOAC. A high-suspicion index must be maintained in patients presenting with flank pain and features of hemorrhagic shock, especially in those under anticoagulation. Management of patients with active bleeding while on DOAC is complex, particularly when simultaneous thromboembolic events occur.

P0122 | Liver Resection and Perioperative Coagulation Management in Patient with Cirrhosis and HCC - A Case Report

M. Skoko ${ }^{1}$; I. Mihic Lasan $^{1} ;$ K. Bilic ${ }^{1}$; A. Stefic ${ }^{1}$; A. Jularic ${ }^{1}$; J. Dodig ${ }^{1}$; T. Bosnjak ${ }^{2}$; S. Misir Situm²; G. Brozovic ${ }^{2}$; D.V. Vrdoljak²; M. Mihaljevic ${ }^{3}$; T. Vucemilo ${ }^{1}$; T. Marinkovic Strumberger ${ }^{1}$

${ }^{1}$ University Hospital Center Sestre Milosrdnice, Department for Transfusion and Regenerative Medicine, Zagreb, Croatia; ${ }^{2}$ University Hospital Center Sestre Milosrdnice, University Hospital for Tumors, Zagreb, Croatia; ${ }^{3}$ University Hospital Center Zagreb, Zagreb, Croatia

Background: In the past, chirrotic patients were usually considered as patients with high risk of bleeding and hipocoagulability. The common practice was to correct coagulation parameters with plasma products and platelet. Cirrhosis is characterized by an impaired synthesis of all clotting factors and thrombocytopenia. FVIII and $v W f$ activity is usually increased.

Aims: A case report of coagulation managament in a patient with hepatocellular carcinoma after hepatitis B active disease.

Methods: 69 year old patient was admitted to our hospital for the liver operation. Preoperative routine blood tests were done.

PT (prothrombin time) was prolonged (58\%, INR 1.28) and fVII activity was slightly under normal range (54\%).

Results: Very large piece of the liver was resected and the bleeding during the operation was severe so they decided to administer recombinant FVII.

On second postoperative day PT was $22 \%$, INR 2.68. The third postoperative day patient developed oedema of the right leg. D-dimers were very high $>34.4 \mathrm{mg} / \mathrm{L}$ (normal range $<0.5 \mathrm{mg} / \mathrm{L}$ ).

Ultrasound showed deep vein thrombosis of femoral and popliteal vein.

On 10th day patient was infected with the SARS Cov-2 virus. We continued therapeutic doses of $\mathrm{LMWH}$. The coagulation status was 
normal (PV 76\%) and D-dimer were $6.54 \mathrm{mg} / \mathrm{L}$. She did not develop any major COVID - 19 symptoms.

Conclusions: Our case report shows the poor prognosis of routine coagulation tests in patients with cirrhosis. The theory that includes two cascade ways is not enough in this case. In 2001 a new cell based model was proposed by Hoffman and Monroe. This model includes three phases. All phases of coagulation leads to thrombin generation. In the end thrombin initiates the conversion of fibrin resulting in a fibrin burst. Thrombin generation in these patients is normal or even enlarged and the risk for venous thromboembolisam is enlarged.

\section{FVIII/IX}

\section{LPB0053 | Activated FVIII Inactivation Occurs Outside of the Intrinsic Xase Complex}

A. Wilhelm $^{1}$; L. George ${ }^{1,2,3}$

${ }^{1}$ The Children's Hospital of Philadelphia, Philadelphia, United States;

${ }^{2}$ The Raymond G. Perelman Center for Cellular and Molecular

Therapeutics, Philadelphia, United States; ${ }^{3}$ Perelman School of Medicine, University of Pennsylvania, Philadelphia, United States

Background: The FVIIla heterotrimer is stabilized by weak, electrostatic interactions. Inactivation of FVIIIa is due to either spontaneous A2-domain dissociation or activated protein C (APC) cleavage. Previous work demonstrated FIXa and lipids stabilize the A2-domain. Additionally, FIXa and FX have been shown to protect FVIIla from APC cleavage. Importantly, many of these studies used FVIIla concentrations above the $K_{d}$ of A2-domain dissociation (200-300 nM) and FIXa concentrations where FVIIIa/FIXa is $100 \%$ bound. Collectively this suggests FVIIla inactivation would be marginal when bound within the intrinsic Xase; however, given that the plasma concentration of FVIII $(1 \mathrm{nM})$ is below the $\mathrm{K}_{d}$ of FVIIla-A2domain dissociation and FIXa binding, the physiologic significance of these observations is unclear.

Aims: We sought to evaluate the stability and inactivation of FVIIla within the intrinsic Xase.

Methods: FVIII was activated with thrombin and quenched with hirudin. FVIIla was then incubated with active-site-blocked FIXa (FIXa-PPACK) at varying concentrations (0-5 nM), FX, and lipids in the presence or absence of APC and Protein S (PS). Inactivation of FVIIla was then determined using a FXa generation assay. Samples were evaluated by western blot to identify APC cleavage fragments. FIXa-PPACK concentrations were chosen to approximate $0-100 \%$ bound FVIIla according to the reported $K_{d}$ of $2 \mathrm{nM}$ (Table 1).
TABLE 1 Approximate fraction of FVIIla bound within the intrinsic Xase enzyme complex assuming a Kd of 2 nM for FVIIla and FIXa/lipids

$\begin{array}{lll}\text { FVIIIa (nM) } & \text { FIXa-PPACK (nM) } & \text { FVIIla bound (\%) } \\ 2 & 0 & 0 \\ 2 & 0.5 & 12 \\ 2 & 5 & 90\end{array}$

Results: When 90\% FVIIla was bound within the Xase, no loss in activity was observed with or without APC/PS present (Figure 1). In the absence of FIXa-PPACK, FVIIla was sensitive to inactivation by A2-domain dissociation and APC cleavage (confirmed by western blot). When only $15 \%$ of FVIIla was bound within the Xase, intermediate FVIIla inactivation and Xase stabilizing effect were observed.

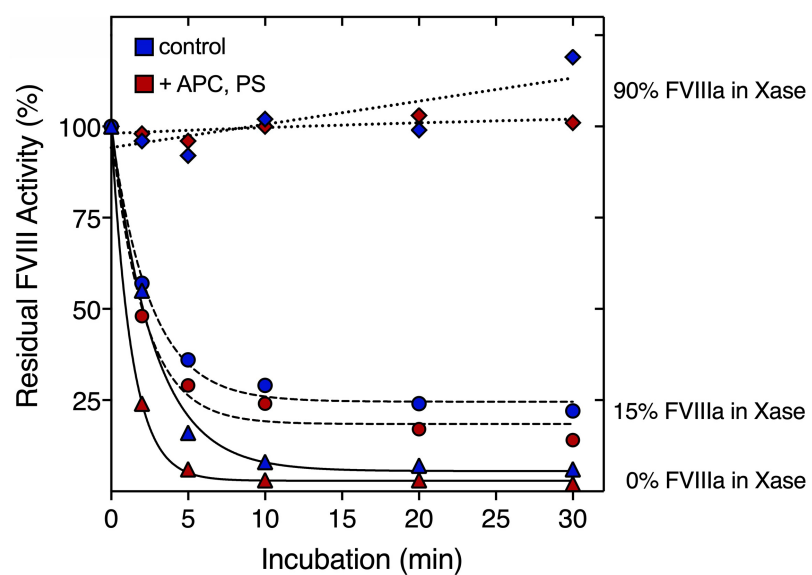

First, FVIII (16 nM) was incubated with Ila (100 nM) and quenched with hirudin $(300 \mathrm{nM})$. Then, FVIIla $(2 \mathrm{nM})$ was incubated with FIXa-PPACK (0-5 nM), FX (20 nM), and lipids $(20 \mu \mathrm{M})$ with or without APC $(6 \mathrm{nM})$ and PS $(50 \mathrm{nM})$. Next, samples were measured by FXa generation assay to determine residual FVIIla activity at timepoints indicated on the x-axis. Final concentrations within the FXa generation assay were $0.25 \mathrm{nM} \mathrm{FVIIla,}$

$20 \mathrm{nM}$ FIXa, $200 \mathrm{nM}$ FX, and $20 \mu \mathrm{M}$ lipids.

FIGURE 1 Residual FVIIla inactivation over time in the presence or absence of APC and PS evaluated by FXa generation

Conclusions: Irrespective of the mechanism of inactivation, FVIIla is remarkably stable when bound to FIXa-PPACK, FX, and lipids. These data support FVIIla inactivation occurs largely or completely outside the intrinsic Xase enzyme complex.

PB0098 | Development of a Cell-based Assay to Test Factor VIII Internalization Mediated by the Low-density Lipoprotein Receptor (LDLR) and LDLR-related Protein 1 (LRP1)

H. Chun; A.G Sarafanov

U. S. Food and Drug Administration, Silver Spring, United States

Background: Extension of the plasma half-life of therapeutic factor VIII (FVIII) products is important for improving their efficacy in 
treatment of Hemophilia A. This requires understanding mechanisms of FVIII plasma clearance mediated by hepatic low-density lipoprotein receptor (LDLR) and LDLR-related protein 1 (LRP1), which were shown to significantly contribute to FVIII clearance in mice model (Bovenschen, 2005). Therefore, it is of interest to establish also a cellculture based model to study these interactions.

Aims: To develop a method to test FVIII internalization mediated by LDLR/LRP1 in cell culture.

Methods: We measured the expression level of LDLR and LRP1 in four human hepatic established cell lines and evaluated relative contribution of both receptors on FVIII internalization by RNAi silencing. FVIII internalization in the presence and absence of LRP1 fragments and FVIII inhibitors were measured using flow cytometry. Results: Huh-7 human hepatic cells showed the highest expression of LDLR and LRP1 compared to HepG2, Hep3B, and SK-HEP1 cells. Using Huh-7 cells, we observed dose- and time-dependent FVIII uptake that allowed to select the optimal assay conditions. Inhibition of LRP1 expression by RNAi or use of competitive ligands, specific to either LDLR/LRP1 or FVIII, resulted in $60 \sim 70 \%$ decrease of FVIII internalization, indicating a more prominent role of LRP1 than LDLR in FVIII uptake.

Conclusions: We developed a novel cell-based assay to measure LDLR/LRP1-mediated uptake of FVIII using Huh-7 cells. This model system can be used to study mechanisms of LDLR/LRP1-mediated clearance of FVIII. In particular, this system can be used for testing FVIII variants with extended plasma half-life to facilitate generation of more efficient therapeutic FVIII products.

Disclaimer: This is an informal communication and it represents the authors' own best judgment. These comments do not bind or obligate FDA.

\section{PB0099 | Real-world Effectiveness of BAY 94-9027 to Treat Hemophilia A in the ATHNdataset}

C. He ${ }^{1}$; M. Chandler ${ }^{1} ;$ M. Recht ${ }^{1}$; J. Charlet ${ }^{2}$; T. Moulton $^{2}$; P. Vashi ${ }^{2}$

${ }^{1}$ American Thrombosis \& Hemostasis Network, Rochester, United States; ${ }^{2}$ Bayer US LLC, Whippany, United States

Background: The ATHNdataset is a HIPAA-compliant, de-identified database sponsored by the American Thrombosis and Hemostasis Network. Over 40,000 people with a bleeding or clotting disorder followed at a U.S. hemophilia treatment center (HTC) have submitted health information, including 15,747 hemophilia A patients.

Aims: Evaluate the effectiveness of BAY 94-9027 (Jivi $\left.{ }^{\circledR}\right)$ to treat hemophilia A in a real-world setting.

Methods: The ATHNdataset was queried for patients being treated with BAY 94-9027 prophylactically or episodically. Collected data included baseline demographic data, treatment history, comorbidities, and bleed rates. The data were captured via patient charts between January 1, 2010 and October 31, 2020.

Results: At data cutoff, 134 patients were being treated with BAY 94-9027. In this group, 99 (74\%) have severe hemophilia A and 35 (26\%) have mild/moderate disease. There are 131 (98\%) male patients and 3 (2\%) female patients. Of the total, 19 (14\%) patients had a history of inhibitors. The mean age of patients was 31.5 years old (median $=29.2$ ), with 6 patients $(4 \%)$ under the age of 12 at data cutoff. Patients had an average of 1.3 years of therapy and most (75/134, 56\%) had over 12 months of treatment.

There were 124 (92.5\%) patients with documented bleed rates for patients treated both prophylactically and episodically (Table 1). Mean overall annualized bleed rate (ABR) was 0.27 . Mean spontaneous $A B R$ was 0.17 . In patients with over 12 months of treatment experience, the mean overall $A B R$ was 0.20 and the mean spontaneous $A B R$ was 0.07 . Proportion of patients with zero bleeds during the entire observational period was $114 / 124$ (92\%).

TABLE 1 Evaluation of bleeding rates between all patients who were treated with BAY 94-9027 (stratified based on duration of therapy and prophylactic treatment regimens

\section{All Patients}

Full Analysis Group

$(n=124)$

Overall $A B R$

Mean \pm SD

Median (Q1-Q3)

$0.27 \pm 1.12$

$0(0-0)$

Spontaneous $A B R$

Mean \pm SD

$0.17 \pm 0.87$

Median (Q1-Q3)

$0(0-0)$

Proportion of patients with 0 bleeds

N

$\%$
114

$91.9 \%$
Prophylactic Patients ( $n=97)$

$0.28 \pm 1.20$

$0(0-0)$

$0.21 \pm 0.98$

$0(0-0)$

90

$92.8 \%$

>12 months treatment

Full Analysis Group $(n=72)$

$0.20 \pm 0.81$

$0(0-0)$

$0.07 \pm 0.32$

$0(0-0)$

69

$95.8 \%$
Prophylactic Patients $(n=60)$

$0.14 \pm 0.77$

$0(0-0)$

$0.07 \pm 0.34$

$0(0-0)$ 
Conclusions: The data show that BAY 94-9027 provides protection from bleeding events in the real world with $92 \%$ of patients with ABR data in this cohort reporting zero bleeds. These data should be interpreted with caution due to limitations in real world studies.

\section{PB0100 | Validation of a New Kit for the Quantitative Determination of Chromogenic Factor IX Activity}

T. Quinton; R. Clarke; J. Fraser; K.M Black; A. Sadeghi-Khomami

Precision BioLogic Inc, Dartmouth, Canada

Background: Factor IX assay methodology falls into two categories: one-stage clot-based (OSC) assays based on activated partial thromboplastin time (APTT) and two-stage chromogenic substrate (CS) assays. Unlike APTT-based assays, the CS assay offers the advantage of being less prone to impact from common interferents in samples. Aims: The aim of the study was to characterize a new chromogenic assay for the quantitative determination of factor IX (FIX) activity in citrated plasma samples by evaluating the reproducibility, reference interval, and on-board stability of the reagents.

Methods: All studies were performed according to applicable CLSI guidelines on IL ACL TOP series instruments. Reproducibility was determined through a 5 day $\times 2$ run $\times 3$ replicate study using three reagent lots at a single site by testing six plasma samples containing FIX spanning the assay range. The reference interval was measured by testing 120 frozen plasma samples (venipuncture, 3.2\% citrate) from ostensibly healthy individuals with three lots of reagents on two analyzers and calculated using a non-parametric method. On-board stability was assessed by repeated testing of five freshly-thawed plasma samples using the same set of reagents over the course of $49 \mathrm{~h}$.

Results: The between-lot and within-site assay precision was $<10 \%$ CV for four plasma samples with high FIX ( $\geq 10 \%)$ activity and SD of $<0.8 \%$ for two low FIX $(<10 \%)$ plasma samples. The reference interval was 74 to $151 \%$ FIX activity. Reagents demonstrated a robust onboard stability over nine hours with highly consistent results across the time course.

Conclusions: We observed excellent performance and lot-to-lot consistency of our factor IX chromogenic assay when measuring reproducibility, reference interval, and on-board stability. The reagents are stable throughout a typical working day and the convenient frozen format and size expedite preparation time and minimize reagent wastage.

PB0101 | Comparison of a Chromogenic vs. APTT-based Factor VIII Activity Assay in the Recovery of a Pegylated Factor VIII Replacement Therapy in Plasma Samples

T. Quinton; J. Fraser; K.M Black; A. Sadeghi-Khomami

Precision BioLogic Inc, Dartmouth, Canada

Background: The standard treatment for hemophilia A patients is FVIII replacement therapy with recombinant FVIII (rFVIII) or plasma-derived FVIII concentrates. Discrepancies in the measurements of extended half-life FVIII products have been observed depending on the assay and reagents used.

Aims: The study aim was to evaluate the recovery of a pegylated rFVIII product by a chromogenic FVIII (CS) and one-stage clotting (OSC) assay.

Methods: Recovery was investigated in a BAY94-9027 ECAT evaluation set containing five levels of FVIII activity $(0.05,0.10,0.25$, $0.50,1.50 \mathrm{IU} / \mathrm{mL}$ ) in a lyophilized format. After reconstitution, each level was measured in triplicate at three dilutions (neat, 1:2, and 1:4). BAY94-9027 was also diluted in congenital FVIII deficient and FVIII immunodepleted plasma to create five levels analogous to the ECAT sample set. Two pre-dilutions (1:2 and 1:4) of the neat plasma were prepared and measured in triplicate as fresh and frozen samples on an IL ACL TOP instrument using Chromogenic Factor VIII (Precision BioLogic) and HemosIL SynthAsil assays.

Results: The mean percent recoveries across all levels in the ECAT sample set were $73.7 \%$ and $96.7 \%$ for the CS and OSC assay, respectively. The recovery was improved by directly spiking drug product into congenital FVIII deficient plasma with mean recoveries of $85.3 \%$ and $101.4 \%$ for fresh and $87.6 \%$ and $101.1 \%$ for frozen samples by the CS and OSC assays, respectively. For the CS assay the recovery was dose-dependent with increased recovery $(\geq 96 \%)$ in the normal range (0.5-1.5 IU/mL) than at lower doses. Switching the matrix to FVIII immunodepleted plasma showed a significant dose-dependent impact on the recovery across all levels for the OSC assay but not the CS assay. Conclusions: The CS and OSC assays demonstrated acceptable recovery at clinically relevant concentrations when BAY94-9027 was directly spiked into congenital FVIII deficient plasma. The CS assay was less susceptible to matrix effects.

PB0102 | Optimizing Variant Curation Guidelines to Improve Clinical Genetic Testing for Hemophilia A and B

$\underline{\text { K. Lee }}{ }^{1} ;$ X. Chen ${ }^{2}$; Q. Ding ${ }^{3}$; K. Downes ${ }^{4}$; B. Doshi ${ }^{5}$; E.P. Ewen ${ }^{6}$; A. Frantz ${ }^{1}$; J. McCreery ${ }^{7}$; S. Mohan ${ }^{1}$; A. Payne ${ }^{8}$; X. Tian'; V. Trapp-Stamborski ${ }^{7}$; N.S. Key ${ }^{10}$; B.A. Konkle ${ }^{11}$; K. Gomez ${ }^{12}$ ${ }^{1}$ Department of Genetics, University of North Carolina at Chapel Hill, Chapel Hill, United States; ${ }^{2}$ Geneseeq Technology, Inc., Toronto, Canada; ${ }^{3}$ Department of Molecular Biology and Genetics, Cornell University, Ithaca, United States; ${ }^{4}$ Cambridge University Hospitals, Cambridge, United Kingdom; ${ }^{5}$ Division of Hematology, Children's Hospital of Philadelphia, Philadelphia, United States; ${ }^{6}$ Agilent Technologies, Santa Clara, United States; ${ }^{7}$ Versiti Blood Center of Wisconsin, Milwaukee, United States; ${ }^{8}$ National Center on Birth Defects and Developmental Disabilities, Centers for Disease Control and Prevention, Atlanta, United States; ${ }^{9}$ Human Genome Sequencing Center, Baylor College of Medicine, Houston, United States;

${ }^{10}$ Department of Medicine, University of North Carolina at Chapel Hill, Chapel Hill, United States; ${ }^{11}$ Department of Medicine, University of Washington, Seattle, United States; ${ }^{12}$ University College \& Royal Free Hospital, London, United Kingdom 
Background: Genetic testing for hemophilia A/B aids in risk stratification and family planning and is a prerequisite for delivering precision medicine. As tailoring treatment will require knowledge of the disease-causing variant, limiting the number of variants of uncertain significance (VUS) and increasing the number of accurately curated variants in public databases will be critically important.

Aims: In 2015, the American College of Medical Genetics and Association for Molecular Pathology (ACMG/AMP) developed a framework to assess variant pathogenicity; however, the guidelines were written broadly. The Clinical Genome Resource (ClinGen), funded by the National Human Genome Research Institute, convened the Coagulation Factor Deficiency Variant Curation Expert Panel (CFD-VCEP) in December 2018 to develop rule specifications for curating variants in the F8/F9 genes through their FDA-approved process.

Methods: The CFD-VCEP membership meet monthly to specify ACMG-AMP rules for F8/F9 genes and discuss resulting variant assertions. Each rule was assessed for its appropriateness for hemophilia and whether there would be any benefit to adding detailed criteria for its use and/or modifying its strength based on availability of evidence. Results: Variant curation specifications were applied to 19 ACMGAMP rule criteria and 9 rules were deemed not applicable to these genes. Criteria modifications included setting allele frequencies in normal controls, defining key functional domains and residues and conditions for adjusting strength. A pilot study of 80 F9 and F8 variants, covering all bleeding severities, is currently underway to test the rule specifications. The goal is to curate 15 pathogenic/likely pathogenic, 10 VUS and 15 benign/likely benign variants for each gene. Rule specifications and variant classifications to be presented. Conclusions: Results of this effort will aid clinical variant interpretation for the hemophilia genes and help standardize variant curations across laboratories. Expert level variant curation from this VCEP wil be deposited in the ClinVar database for public access.

PB0103 | Interaction of the Factor IXa with the Subpopulations of Activated Platelets

$\underline{\text { P.A. Soloveva }}^{1,2}$; N.A. Podoplelova ${ }^{1,3}$; F.I. Ataullakhanov ${ }^{1,2,3,4}$; M.A. Panteleev ${ }^{1,2,3,4}$

${ }^{1}$ Center for Theoretical Problems of Physicochemical Pharmacology, Moscow, Russian Federation; ${ }^{2}$ Moscow Institute of Physics and Technology, Dolgoprudny, Russian Federation; ${ }^{3}$ Dmitriy Rogachev National Medical Research Center of Pediatric Hematology, Onkology, Immunology Ministry of Healthcare of Russian Federation, Moscow, Russian Federation; ${ }^{4}$ Moscow State University, Moscow, Russian Federation

Background: One of the major membrane-dependent reactions of blood coagulation is activation factor $\mathrm{X}$ by intrinsic tenase complex. However, it is not clear how these coagulation factors interact with each other and with the platelet membrane of different subpopulations.
Aims: To investigate the equilibrium and kinetics binding of factors IXa and $X$ with subpopulations of activated platelets or phospholipid vesicles, as well as the effect of factor VIIla on this binding.

Methods: Platelets were obtained from donor blood by gel filtration purification, and phospholipid vesicles were formed by extrusion. Native fIXa and fX were labeling with Alexa Fluor 647, commercial FITC-labeled antibodies were used for observation of recombinant fVIIla. A flow cytometer BD FACSCanto II (BD Bioscience, San Jose, CA, USA) was used to assess the binding of factors to platelets and vesicles.

Results: The binding of factor IXa to the membrane of procoagulant platelets occurs through one low-affinity binding site, which is a negatively charged phosphatidylserine. In this case, the presence of factor VIII strongly increases the binding of factors IXa and X. At the same time, this binding was calcium-dependent and reversible. The association of intrinsic tenase factors with the PS- subpopulation was insignificant and linear in the studied range. There were obtained the equilibrium constants of binding of intrinsic tenase factors to the membrane of activated platelets PS+ subpopulation: fIXa $0.7 \pm 0.1 \mu \mathrm{M}, \mathrm{flXa}$ in the presence of fVIIla $67 \pm 16 \mathrm{nM}, \mathrm{fX}$ in the presence of fVIIla $109 \pm 10 \mathrm{nM}$; and to phospholipid vesicles: fIXa $1.0 \pm 0.1 \mu \mathrm{M}$.

Conclusions: Here, we quantitatively characterized the interaction of fIXa in the presence and absence of fVIIla with the two subpopulations of activated platelets.

The study was supported by the Russian Foundation for Basic Research grants 19-04-00615.

\section{HEMOSTASIS AND ORGAN DYSFUNCTION}

\section{LPB0054 | Experimental Acetaminophen-induced Acute Liver} Failure Is Associated with a Profound Consumptive Coagulopathy

D. Groeneveld ${ }^{1}$; E.G. Bouck ${ }^{2}$; L.G. Poole ${ }^{1}$; H. Cline-Fedewa ${ }^{1}$; K.J. Williams ${ }^{1}$; A.S. Wolberg ${ }^{2}$; J.P. Luyendyk ${ }^{1}$

${ }^{1}$ Michigan State University, East Lansing, United States; ${ }^{2}$ UNC Blood Research Center, University of North Carolina, Chapel Hill, United States

Background: Marked changes in the hemostatic system are observed in patients with acute liver failure (ALF) induced by acetaminophen (APAP) overdose. The mechanisms driving these changes are poorly understood, in part, because standard experimental settings of APAP hepatotoxicity (i.e., $300 \mathrm{mg} / \mathrm{kg}$ APAP) do not adequately model acute liver failure.

Aims: To define the hemostatic state in experimental settings of liver injury/repair and liver failure. 
Methods: Wild-type mice were challenged with APAP (300, 450 or $600 \mathrm{mg} / \mathrm{kg}$, i.p.) or vehicle (saline) and blood and liver samples were collected $24 \mathrm{~h}$ after challenge.

Results: There was no obvious difference in centrilobular necrosis across each dose of APAP. However, mice challenged with $600 \mathrm{mg} /$ kg APAP showed clear signs of hepatic dysfunction as direct bilirubin and albumin levels were respectively increased and decreased. Histological changes such as hemorrhage/congestion, hepatocyte vacuolization and sinusoidal perturbation distinguished mice challenged with $600 \mathrm{mg} / \mathrm{kg}$ APAP from lower APAP doses. Thrombin generation was reduced in APAP-challenged mice, with only subtle differences observed across doses. Despite this subtle difference, mice challenged with $600 \mathrm{mg} / \mathrm{kg}$ APAP displayed profound changes consistent with a consumptive coagulopathy as evidenced by a substantially prolonged prothrombin time, near undetectable levels of plasma fibrinogen, and dramatic elevation in markers of coagulation activation (thrombin anti-thrombin complexes) and fibrinolysis (d-dimer). Ex vivo addition of mouse fibrinogen to restore plasma fibrinogen levels in mice challenged with $600 \mathrm{mg} / \mathrm{kg}$ APAP only partially corrected the prolonged prothrombin time. Interestingly, hepatic fibrin(ogen) deposition did not display any connection to APAP dose, whereas hepatic VWF deposition was dramatically increased in mice challenged with $600 \mathrm{mg} / \mathrm{kg}$ compared to lower APAP doses. Conclusions: APAP-induced ALF in mice is associated with profound coagulopathy not evident in standard APAP hepatotoxicity. These results suggest experimental APAP dose-response studies could uncover the mechanistic basis of hemostatic changes observed in ALF patients.

PB0104 | Risk Factors for Non-variceal Hemorrhage in Patients with Chronic Liver Disease

P. Kesavan ${ }^{1}$; A. Afzal ${ }^{1}$; S. Luo ${ }^{1,2}$; B. Gage ${ }^{1}$; M. Schoen ${ }^{2}$;

K. Sanfilippo ${ }^{1,2}$

${ }^{1}$ Washington University School of Medicine, St. Louis, United States;

${ }^{2}$ St. Louis Veterans Health Administration Medical Center, St. Louis, United States

Background: Patients with chronic liver disease (CLD) frequently experience non-variceal hemorrhage, which results in considerable morbidity, mortality, and healthcare costs. Available literature focuses on the prevention of variceal hemorrhage; however, studies identifying risk factors for non-variceal hemorrhage have been limited.

Aims: We assessed the role of various clinical and laboratory parameters in predicting the risk of non-variceal hemorrhage among CLD patients.
Methods: This is a large, retrospective cohort study of U.S. veterans diagnosed with CLD between October 1, 2002 and September 30,2016 . We excluded patients with a history of malignancy, artificial heart valves, atrial fibrillation, prior venous thromboembolism, and anticoagulation. We selected candidate predictors from the HEMORR ${ }_{2}$ HAGES, HAS-BLED, and Child-Pugh risk prediction models. When appropriate, variables were analyzed as time-varying. We used competing risk analysis by methods of Fine and Grey to identify predictors of non-variceal hemorrhage within one year of CLD diagnosis.

Results: A total of 14,281 CLD patients were included in the study, and 576 hemorrhagic events were identified within one year of followup (Table 1). Most of the events occurred in the upper gastrointestinal (GI) tract (381), followed by lower GI tract (104), intracranial (35), and nasal/respiratory (31) hemorrhages. In competing risk analysis (Table 2), the following predictors were independently associated with non-variceal hemorrhage: elevated INR between 1.5 and 3, aspirin use, high bilirubin, low albumin, low glomerular filtration rate, prior hemorrhage, anemia, alcohol abuse, hemi- or paraplegia, and dementia.

TABLE 1 Demographic and Clinical Characteristics Comparison for CLD Patients Diagnosed from 2002 to 2016

\begin{tabular}{|c|c|c|c|}
\hline \multirow[b]{2}{*}{$\begin{array}{l}\text { Demographic clinical } \\
\text { characteristics }\end{array}$} & \multicolumn{2}{|c|}{ Total $(\mathrm{N}=\mathbf{1 4}, \mathbf{2 8 1})$} & \multirow[b]{2}{*}{ P-value } \\
\hline & $\begin{array}{c}\text { Hemorrhage } \\
n=576\end{array}$ & $\begin{array}{c}\text { No Hemorrhage } \\
n=13,705\end{array}$ & \\
\hline Age (mean years) & 58.0 & 57.2 & $0.03+$ \\
\hline Male (\%) & 96.7 & 96.8 & $0.88^{*}$ \\
\hline Race (\%) & & & $0.01^{*}$ \\
\hline White & 81.1 & 85.6 & \\
\hline Black & 15.6 & 11.8 & \\
\hline Other & 3.3 & 2.5 & \\
\hline Prior hemorrhage & 3.7 & 1.3 & $<0.001^{*}$ \\
\hline Cardiovascular disease (\%) & 9.4 & 7.5 & $0.09 *$ \\
\hline Congestive heart failure (\%) & 6.9 & 4.8 & $0.02^{*}$ \\
\hline Dementia (\%) & 1.2 & 0.5 & $0.01 *$ \\
\hline Chronic lung disease (\%) & 11.8 & 11.8 & $0.98^{*}$ \\
\hline Connective tissue disease (\%) & 0.9 & 0.7 & $0.67^{*}$ \\
\hline Peptic ulcer (\%) & 1.9 & 1.4 & $0.35^{*}$ \\
\hline Diabetes (\%) & 22.1 & 23.1 & $0.56^{*}$ \\
\hline Hemi- or paraplegia (\%) & 1.0 & 0.3 & $<0.001^{*}$ \\
\hline HIV (\%) & 0.2 & 1.0 & $0.05^{*}$ \\
\hline Anemia (\%) & 29.5 & 18.3 & $<0.001^{*}$ \\
\hline Hypertension (\%) & 42.7 & 40.7 & $0.34^{*}$ \\
\hline Alcohol abuse (\%) & 38.4 & 29.7 & $<0.001 *$ \\
\hline Ascites (\%) & 4.9 & 4.2 & $0.44^{*}$ \\
\hline Antiplatelet use (\%) & 3.4 & 2.7 & $0.07^{*}$ \\
\hline Aspirin use (\%) & 15.6 & 12.7 & $0.04^{*}$ \\
\hline Platelet count (median) & 119,371 & 118,804 & $0.21 \neq$ \\
\hline INR (median) & 1.34 & 1.30 & $<0.001 \ddagger$ \\
\hline Albumin (Median) & 2.9 & 3.1 & $<0.001 \neq$ \\
\hline Bilirubin (Median) & 1.8 & 1.6 & $<0.001 \ddagger$ \\
\hline eGFR (Median) & 91.1 & 88.4 & $<0.001 \neq$ \\
\hline
\end{tabular}


TABLE 2 Multivariate Analysis of Risk Factors for Non-Variceal Hemorrhage

\begin{tabular}{|c|c|c|}
\hline & $\begin{array}{l}\text { Hazard } \\
\text { Ratio }\end{array}$ & $\begin{array}{l}95 \% \\
\text { Confidence } \\
\text { Interval }\end{array}$ \\
\hline$A g e^{1}$ & 0.99 & $0.98-1.00$ \\
\hline Sex $(\text { male })^{1}$ & 1.07 & $0.67-1.70$ \\
\hline Platelet count $(<50,000)^{4}$ & 0.86 & $0.59-1.25$ \\
\hline INR 1.5 to $<2$ & 1.79 & $1.44-2.22$ \\
\hline INR 2 to 3 & 1.99 & $1.42-2.79$ \\
\hline INR $>3$ to 4 & 2.05 & $0.75-5.67$ \\
\hline$I N R \geq 4^{4}$ & 1.77 & $0.54-5.84$ \\
\hline Total bilirubin ( $\geq 2 \mathrm{mg} / \mathrm{dL})^{4}$ & 1.26 & $1.02-1.56$ \\
\hline Albumin $(<3.5 \mathrm{mg} / \mathrm{dL})^{4}$ & 3.16 & 2.44-4.10 \\
\hline Kidney disease $(e G F R<30 \mathrm{~mL} / \mathrm{min})^{4}$ & 2.12 & $1.57-2.85$ \\
\hline Antiplatelet (excluding aspirin) ${ }^{4}$ & 1.31 & $0.75-2.30$ \\
\hline Aspirin use ${ }^{4}$ & 1.63 & $1.21-2.18$ \\
\hline Prior hemorrhage $^{3}$ & 2.04 & $1.28-3.23$ \\
\hline Anemia $^{2}$ & 1.66 & $1.37-2.02$ \\
\hline Hypertension ${ }^{2}$ & 0.98 & $0.81-1.20$ \\
\hline Alcohol abuse ${ }^{2}$ & 1.29 & $1.08-1.55$ \\
\hline Ascites $^{2}$ & 0.93 & $0.63-1.38$ \\
\hline Congestive heart failure ${ }^{2}$ & 1.19 & $0.84-1.70$ \\
\hline Dementia $^{2}$ & 2.99 & $1.35-6.61$ \\
\hline Cardiovascular disease $^{2}$ & 1.03 & $0.75-1.41$ \\
\hline Chronic lung disease $^{2}$ & 0.90 & $0.69-1.19$ \\
\hline Connective tissue disease ${ }^{2}$ & 1.07 & $0.43-2.66$ \\
\hline Peptic Ulcer ${ }^{2}$ & 0.98 & $0.52-1.86$ \\
\hline Diabetes $^{2}$ & 0.92 & $0.73-1.16$ \\
\hline Hemi- or paraplegia ${ }^{2}$ & 2.47 & $1.09-5.59$ \\
\hline$H I V^{2}$ & 0.17 & $0.02-1.25$ \\
\hline
\end{tabular}

Candidate predictors with a significant association with non-variceal hemorrhage are bolded. ${ }^{1}$ Assessed at time 0 (CLD diagnosis). ${ }^{2}$ Assessed within 12 months prior to time $0 .{ }^{3}$ Assessed within 5 years prior to time $0 .{ }^{4}$ Time-varying variable.

Conclusions: Our study of over 14,000 veterans shows that severity of liver disease at presentation, aspirin use, concurrent renal failure, alcohol abuse, prior hemorrhage, and compromised self-care by either dementia or paraplegia predict a higher risk of hemorrhage within the first year after CLD diagnosis. This study was supported by the American Society of Hematology.

\section{PB0105 | Thrombin Generation Assay in Crohn Disease}

H. Ben Youssef; H. Baccouche; S. Meriem; C. Aya; F. Monia;

J. Boubaker; N. Ben Romdhane

Faculty of Medecine Tunis, Tunis, Tunisia

Background: Thrombosis risk is increased in Crohn disease (CD). thrombin generation assay (TGA) have been evaluated during CD in several studies but the results remain controversial.

Aims: Evaluate the hypercoagulable state in $C D$ patients through TGA and correlate it with the clinical and biological status of the disease.

Methods: This was a comparative study, including 50 patients with $\mathrm{CD}$ and 50 matched controls according to age and sex. Data related to CD were collected. TGA parameters: Lag time, time to peak, peak, endogenous thrombin potential (ETP) and velocity index were analyzed. C reactive protein (CRP), fibrinogen and factor VIII (FVIII) were also investigated.

Results: Median age was 44 years-old (29men and 21women). 66\% of patients were in remission. The mean level of FVIII ( $285 \pm 175$ vs $133 \pm 41 ; P=0.001$ ) was significantly higher in patients with $C D$ vs controls. Regarding to TGA parameters; index velocity was higher in patients than controls ( $163 \pm 41$ vs $138 \pm 67 \mathrm{nM} / \mathrm{min} ; P=0.04$ ) and in patients with fistulizing or stenosing phenotype compared to those with inflammatory phenotype. ETP was more increased in hospitalized patients vs outpatients (1515 \pm 406 vs $1248 \pm 380$ nM.min; $P=0.05)$. High values of ETP were also associated to corticotherapy $\left(\mathrm{ETP}=1718.7 \pm 126.9 \mathrm{nM}{ }^{*} \min ; P=0.03\right)$. Thrombin generation was higher in patients with CRP $>6 \mathrm{mg} / \mathrm{L}$ vs those with normal CRP (ETP:1515 \pm 384 vs $1026 \pm 199$ nM.min; $P=0.007$ ); in those with fibrinogen level $>4 \mathrm{~g} / \mathrm{l}$ vs those with normal fibrinogen (Peak:351 \pm 74 vs $295 \mathrm{nM} \pm 64 ; P=0.03$ ) and in those with elevated FVIII vs those with normal FVIII (ETP:1473.3 \pm 397 vs $1245 \pm 348$ nM.min; $P=0.02)$. A significant positive correlation was noticed between the parameters of TGT; CRP; fibrinogen and FVIII. Thus; two biological scores correlating ETP to CRP (ETP $=1384+2.9 \times C R P)$; as well as to fibrinogen (ETP = 1060.7+130.5xFibrinogen).

Conclusions: Thrombin generation is more accelerated in CD patients than controls. This hypercoagulability is associated with hospitalization, stenosing and fistulizing phenotypes, corticosteroid therapy, elevated values of CRP, fibrinogen and FVIII. ETP determination from CRP and fibrinogen would be a valuable tool for assessing the state of coagulability in these patients.

PB0106 | Delivery of a Resuscitation Fluid Cocktail Containing Fibrinogen and Vasopressin as an Infusion Rather than Boluses Improves Hemorrhage in Swine Polytrauma Model

A. St. John; X. Wang; K. Ringgold; S. Stern; N. White University of Washington School of Medicine, Seattle, United States

Background: Hemorrhage is a major contributor to the massive burden caused by trauma, and treatment of trauma victims in austere settings can be challenging. We have previously shown that a low-volume resuscitation fluid cocktail containing vasopressin and fibrinogen improves vital organ perfusion while minimizing hemorrhage when delivered in two boluses $30 \mathrm{~min}$ apart in a swine model of polytrauma. However, each bolus caused a spike in mean arterial pressure, possibly leading to thrombus failure and contributing to hemorrhage. If the vasoactive cocktail were administered in a slower fashion, it could blunt these blood pressure spikes and further mitigate blood loss.

Aims: Determine whether administration of this multifunctional resuscitation fluid cocktail as a continuous infusion rather than as two boluses can improve outcomes in a swine model of polytrauma.

Methods: Eighteen immature, anesthetized, splenectomized Yorkshire swine were subjected to fluid percussion brain injury, femur fracture, catheter hemorrhage, and aortic tear. After a shock period, animals received $14 \mathrm{~mL} / \mathrm{kg}$ of the previously described fluid resuscitation cocktail (hydroxyethyl starch solution with $0.8 \mathrm{U} /$ $\mathrm{kg}$ vasopressin and $100 \mathrm{mg} / \mathrm{kg}$ fibrinogen). They were randomized to receive this either divided into two boluses delivered at 0 and 40 min after resuscitation began $(n=9)$ or as a continuous infusion over $60 \mathrm{~min}(n=9)$. Animals were monitored for six hours or until 
time of death, after which intraperitoneal blood loss was measured. Hemorrhage volume was compared by $t$-test, and survival was compared by Kaplan-Meier log rank test.

Results: Baseline characteristics were similar between groups. Hemorrhage volume was lower with slow infusion compared to boluses (15.0 \pm 11.2 vs. $26.1 \pm 13.4 \mathrm{~mL} / \mathrm{kg}, P=0.04)$. Survival was not significantly changed with infusion compared to boluses (78 vs $44 \%$ at six hours, $P=0.16$ ).

Conclusions: Delivery of a previously identified multifunctional resuscitation fluid cocktail as a continuous infusion rather than as boluses improved blood loss though not survival after injury.

\section{PB0107 | Atrial Flutter and Intracardiac Thrombus after Cardiac} Surgery

S. Karaman ${ }^{1}$; D. Gokceer Karabulut ${ }^{2}$; A. Unuvar ${ }^{1}$; S. Sahin ${ }^{1}$; K. Nisli ${ }^{2}$; D. Tugcu ${ }^{1}$; G. Tanyildiz ${ }^{1}$; R. Tuna ${ }^{1}$; M. Bilici ${ }^{1}$; Z. Karakaş ${ }^{1}$

${ }^{1}$ Istanbul University Faculty of Medicine/Pediatric HematologyOncology, Istanbul, Turkey; ${ }^{2}$ Istanbul University Faculty of Medicine/ Pediatric Cardiology, Istanbul, Turkey

Background: Myocarditis,pericarditis,acute infections and atrial surgery are risk factors for the development of atrial flutter.

Aims: Here,a 15-year-old male patient who underwent cardiac surgery at newborn period and developed atrial flutter and intraatrial thrombus in the follow-up is presented together with the diagnostic and therapeutic approaches.

Methods: A fifteen-year-old male patient was admitted to the emergency room due to palpitations. It was learned that he had undergone a total correction operation with the diagnosis of "Total pulmonary venous return anomaly and atrial septal defect"at newborn period, and received metoprolol treatment for ventricular extrasystoles. All blood tests were found to be normal.Physical examination revealed bradycardia and sinus node dysfunction.During the treadmill exercise test,atrial flutter developed in the patient (Figure 1).Oral antithrombotic therapy was started because of the high risk of thromboembolism.In transesophageal ECHO(TEE),thrombus was detected at the apex of the right atrium (Figure 2)and LMWH treatment was started.Hyperhomocysteinemia and heterozygous FV Leiden mutations were detected in the thrombosis panel

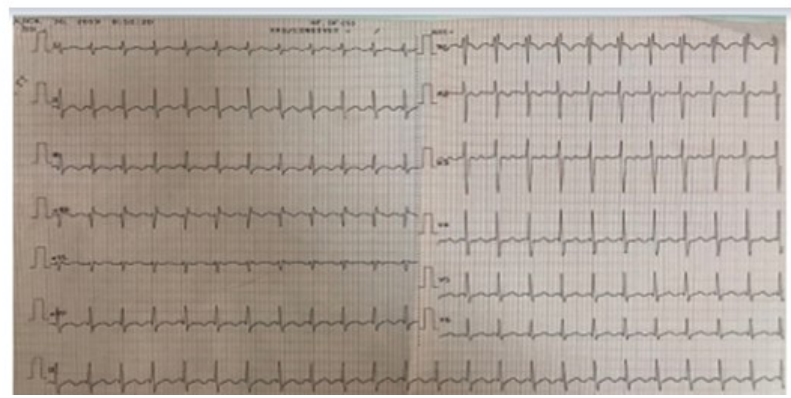

FIGURE 1 Atriyal flutter in EKG

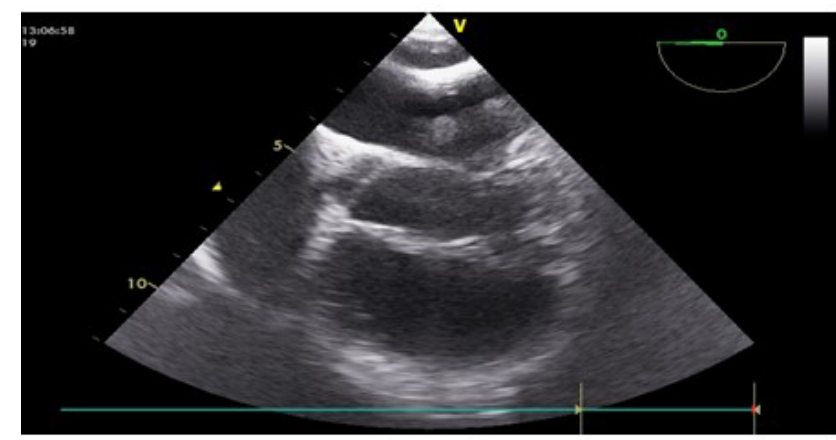

FIGURE 2 Presence of thrombus in the apex of the right atrium

Results: It was observed that the thrombus had shrunk 3 weeks after anticoagulant treatment.For medical cardioversion,amiodarone was started.Electrical cardioversion was applied to the patient who did not respond to medical cardioversion. It was observed that the atrial flutter rhythm was improved.In the TEE performed for control,the thrombus in the atrium was in an organized appearance. Due to the development of sinus node dysfunction secondary to the operation,the patient was implanted with a pacemaker.No thrombus was detected in $\mathrm{ECHO}$ at the last examination, and the patient continues to take oral anticoagulants, digoxin,beloc,cordarone and folbiol.

Conclusions: There may be a tendency to thrombosis in congenital/ acquired heart diseases,surgical conditions or rhythm disturbances. Atrial flutter is an important risk factor for the development of atrial thrombosis.The diameter of the clot and its motility play a role in treatment.Systemic anticoagulant therapy is recommended in high risk situations.Thrombectomy or thrombolytic therapy is controversial and should be evaluated individually according to the patient.

PB0108 | Coagulation Abnormalities in Patients without Congenital Bleeding Disorders: Analysis of Secondary Causes of Low Clotting Factors Levels

A. Lorenzo Jambrina; M. Mosquera Tapia; A. Torres Tienza; E. Zato Hernández; V. Cardos Gómez; P. Galán Álvarez; C. Olivier Cornacchia; J.A. Queizán Herández Hospital General de Segovia, Segovia, Spain

Background: Coagulation factor assays usually are performed in patients with congenital bleeding disorders, Assays in the special coagulation laboratory are affected by numerous situations. However, when we review all the factor assays performed in our center in last 12 years, we found many assays had been performed in hospitalized patients or with pathologies affect hemostasis.

Aims: Analyze the secondary causes of low clotting factors leves in patients in our center.

Methods: We retrospectively reviewed all factor assays (II, V, VII, VIII, IX, X, XI) performed in our center from 02/09/2009 to 08/31/2020. 3911 assays performed, in 594 cases level was below the reference range and 100 of these test, were performed on patients with multiple pathologies. 
Results: Table 2 shows the data of our series. Secondary cause that that most frequently produced low factors levels was liver disease (viral hepatitis, autoimmune hepatitis and liver metastases), followed by the presence of a lupus anticoagulant and vitamin $\mathrm{K}$ deficiency. In FII series, $100 \%$ of low levels corresponded to secondary causes, $55 \%$ in $\mathrm{FV}, 47 \%$ in $\mathrm{FX}$ and $37 \%$ in $\mathrm{FVII}$ series (27\% of patients had a severe infection). 14 low factor levels results corresponded to 7 patients with hematological diseases (plasma cell dyscrasias, chronic myeloproliferative syndromes and a lymphoma), all patients had a normal coagulation test prior to the diagnosis of hematological disease. In 10 cases the analysis was performed in hospitalized patients with active Bleeding and in another 10 cases in patients with severe active infection.

TABLE 1 Secondary causes of low clotting factor levels

\begin{tabular}{|c|c|c|c|c|c|c|c|c|c|c|c|c|}
\hline & & II & & V & & VII & VIII & IX & $x$ & & $\mathrm{XI}$ & Total \\
\hline \multicolumn{2}{|l|}{ Vitamina K deficiency } & 5 & & 0 & & 6 & 0 & 2 & 4 & & 0 & 17 \\
\hline \multicolumn{2}{|l|}{ Infection } & 0 & & 0 & & 9 & 0 & 1 & 0 & & 0 & 10 \\
\hline \multicolumn{2}{|c|}{$\begin{array}{l}\text { Hematological diseases } \\
\text { - Myeloproliferative } \\
\text { síndromes }\end{array}$} & & & & & & & & & & & 5 \\
\hline \multicolumn{2}{|l|}{ Polycythemia vera } & 0 & & 2 & & 0 & 0 & 0 & 1 & & 1 & \\
\hline \multicolumn{2}{|l|}{ Myelofibrosis } & 0 & & 0 & & 0 & 0 & 0 & 0 & & 1 & \\
\hline \multicolumn{2}{|c|}{ - Plasma cell dyscrasias } & & & & & & & & & & & 8 \\
\hline \multicolumn{2}{|l|}{ Multiple myeloma } & 0 & & 3 & & 2 & 0 & 0 & 1 & & 0 & \\
\hline \multicolumn{2}{|c|}{ Inflammatory bowel disease } & 0 & & 0 & & 1 & 0 & 0 & 1 & & 0 & 2 \\
\hline \multicolumn{2}{|l|}{ Others } & 0 & & 0 & & 1 & 0 & 2 & 0 & & 1 & 4 \\
\hline \multicolumn{2}{|l|}{ Total } & 10 & & 20 & & 33 & 2 & 8 & 16 & & 11 & 100 \\
\hline \multicolumn{13}{|l|}{2 Factor ass } \\
\hline FACTOR & II & & V & & VII & & VIII & IX & & $x$ & & XI \\
\hline Total factor assays & 113 & & 144 & & 365 & & 1330 & 723 & & 173 & & 1063 \\
\hline Total patients & 107 & & 104 & & 104 & & 895 & 558 & & 156 & & 558 \\
\hline Low levels assays & 12 & & 54 & & 156 & & 104 & 78 & & 42 & & 148 \\
\hline
\end{tabular}

Conclusions: Multiple pathologies and clinical situations may affect coagulation tests and factors assays. liver diseases, elderly patients with vitamin $\mathrm{K}$ deficiency, and patients with infections.

We recommend not performed clotting factor assays in hospitalized patients or with pathologies that affect hemostasis, to avoid unnecessary tests and falsely altered results.
In our series, many factor assays were performed in patients with 
P0120 | Risk Factors for Venous Thromboembolism at the University College Hospital, Ibadan

W. Shokunbi; P. Ogundeji; O. Aworanti; C. Akunwata

University College Hospital and College of Medicine, University of Ibadan, Ibadan, Nigeria

Background: Venous thromboembolism (VTE) poses significant public health challenges as it is responsible for a high morbidity and mortality worldwide. Risk factors (modifiable and non-modifiable) can be identified and play a major role in its pathogenesis. Considering the cost of managing VTE in poor resource settings such as found in Nigeria, VTE risk factor identification and thromboprophylaxis will reduce the burden of disease and its treatment cost.

Aims: The study aimed to review the pattern of presentation, and risk factors, in patients diagnosed with VTE at the University College Hospital (UCH), Ibadan.

Methods: This is a retrospective study of the hospital records of 65 confirmed VTE patients (doppler ultrasound scan for DVT and CT-angiography for PE) diagnosed and managed at $\mathrm{UCH}$, Ibadan between July 2016 and December 2017. Demographic data and possible risk factors documented in the case note were retrieved. The patients were treated for 3 to 6 months based on the type of VTE. Microsoft excel version 2013 was employed for carrying out the statistical analysis.

Results: 65 patients were studied of which 27 were males and $38 \mathrm{fe}$ males $(M: F=1: 1.4)$. Their age ranged between 15 and 87 years with a mean age of $51 \pm 17$ years. Only 6 patients $(9.4 \%)$ were $<30$ years; $44.6 \%$ were between $30-50$ years, and $46 \%$ were $>50$ years. 53 patients (81.5\%) had DVT of which $65 \%$ were unilateral and $16.9 \%$ bilateral DVT. Immobilization, found in $32.3 \%$ of the patients, was the most frequently identified risk factor, followed by a prior diagnosis of malignancy (15.4\%) and the use of femoral cannulation for haemodialysis (12.3\%).

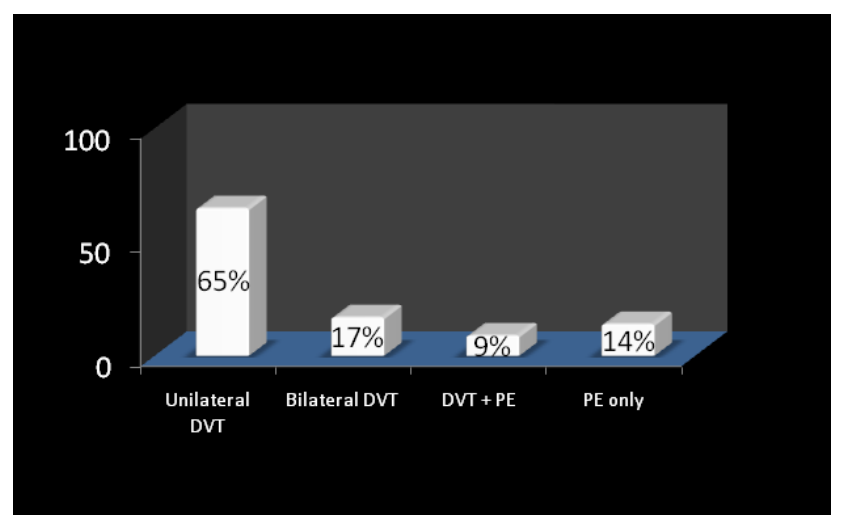

FIGURE 1 Common Types of Venous Thromboembolism

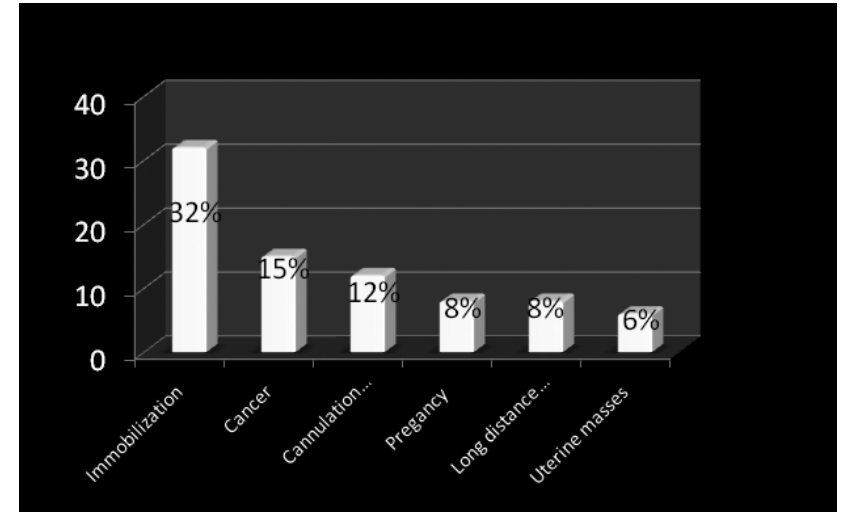

FIGURE 2 Risk Factors for Venous Thromboembolism

Conclusions: All cases of DVT (bilateral for example) should be fully screened to exclude malignancy. Prolonged immobilization should be avoided and prevented using physiotherapy and prophylactic anticoagulation. A high index of suspicion for DVT is required in cases of neurotrauma, pelvic surgery and fractures.

\section{MICROPARTICLES}

PB0109 | Peridontal Injury Prompts Remote Spleen Activation and the Generation of Spleen Procoagulant Microvesicles in a Periodontitis Murine Model

H. EL Itawi ${ }^{1}$; S. Amissi ${ }^{1}$; F. Batool ${ }^{2}$; C. Stutz ${ }^{1}$; F. El Ghazouani ${ }^{1}$;

A.B. Chaker ${ }^{1}$; A.W. Qureshi ${ }^{1}$; F. Moschovaki-Filippidou ${ }^{1}$; C. Auger ${ }^{1}$; E. Anglès-Cano ${ }^{3}$; O. Huck ${ }^{2}$; F. Toti ${ }^{4}$

${ }^{1}$ INSERM UMR 1260, CRBS, Université de Strasbourg, Strasbourg, France; ${ }^{2}$ INSERM UMR 1260, CRBS, Faculté d'Odontologie, Université de Strasbourg, Strasbourg, France; ${ }^{3}$ Inserm UMR_S 1140 Innovative Therapies in Haemostasis, Faculté de Pharmacie de Paris, Paris, France; ${ }^{4}$ INSERM UMR 1260, CRBS, Faculté de Pharmacie, Université de Strasbourg, Strasbourg, France

Background: We previously reported that porphyromonas gingivalis ( $P$. gingivalis), a periodontal pathogen, activates HUVEC endothelial cells and the shedding of microvesicles (MVs) that act as autocrine pro-inflammatory and apoptotic effectors [Bugueno et al., Sci Rep. 2020;10(1):1778].

Aims: To assess the effects of mechanical jaw injury, eventually combined to local $P$. gingivalis infection, and of intraperitoneal $P$. gingivalis infection alone on the spleen response in a C57BL 6 murine model of periondontitis.

Methods: A 2-weeks jaw injury was performed by silk (LIG) or $P$. gingivalis soaked ligatures (LIG-PG) and compared to $P$. gingivalis intraperitoneal injection (IP). Inhibition of Netosis was performed in IP-treated mice by simultaneous injection of $\mathrm{Cl}$-amidine, a PAD4-4 
inhibitor. Spleen MVs (SMVs) were extracted and characterized by protein and prothrombinase assays, and their diameter measured by TRPS. Cellular composition of the spleen was determined by flow cytometry, reactive oxygen species (ROS) measured on sections by DHE fluorescent probing, markers of inflammation and activation by western blot.

Results: In spleen-treated mice, a 2-fold spleen weight was associated with elevated SMV concentration (IP: $210 \pm 38$, LIG-PG: $175 \pm 5$, LIG:150 \pm 4 vs. CTL:100 \pm 6 nM PhtdSer eq., $P<0.05$ ) having a 188$200 \mathrm{~nm}$ diameter range. ROS accumulation followed the same pattern (IP: $77.27 \pm 0.5$, LIG-PG: $59.5 \pm 13$, LIG: $31 \pm$ 12, CTL:6.7 \pm 1.3 , A.U./ $P<0.05)$. In LIG and LIG-PG, tissue factor and VCAM expression was doubled. In IP-treated mice, spleen neutrophils (15\% vs $0.1 \%$ ), granulocytes ( $10 \%$ vs. $0.5 \%$ ) and monocytes ( $4.5 \%$ vs $0.5 \%$ ) increased drastically while T4, T8 and B lymphocytes were reduced by half $(P<0.05)$. Cl-amidine inhibited $79 \%$ of IP-induced ROS accumulation, pointing at Netosis contribution.

Conclusions: Data suggest that $P$. gingivalis-associated periodontitis induces remote spleen activation, Netosis and the shedding of procoagulant SMVs. Measurement of plasma Nets fragments and further characterization of SMV effects on coronary endothelial cells are needed to decipher the link between cardiovascular risk and periodontitis.

PB0110 | Coagulation Activity of Circulating Microparticles in Patients with Cardiovascular Diseases

A. Mazurov; I. Zyuryaev; A. Komarov; T. Martynyuk; N. Golubeva; O. Antonova

National Medical Research Center of Cardiology, Moscow, Russian

Federation

Background: Microparticles (MP) released by activated/apoptotic cells exhibit coagulation activity since they express phosphatidylserine (PS) and some of them tissue factor (TF).

Aims: Coagulation activity of circulating MP from patients with myocardial infarction ( $\mathrm{MI}, n=32$ ), polyvascular atherosclerotic disease (PAD, $n=23$ ) and idiopathic pulmonary arterial hypertension (IPAH $n=15)$ in comparison with healthy volunteers $(n=34)$ was evaluated. Methods: MP were sedimented from the blood plasma (20000 g, 30 min). Coagulation activity of MP from $100 \mu$ l plasma was evaluated by recalcification assay [Khaspekova et al. Biochemistry (Moscow) 2016;81:114-121]. Substrate plasma containing MP was recalcified, clotting was followed by changes of absorbance (A450), the lag phase (min) and $V \max (\triangle A 450 / 1 \mathrm{~min}$ ) were registered. MP were counted in a flow cytometer as PS+ (lactadherin-FITC stained) particles $<1 \mu \mathrm{m}$ (10 $/ \mu$ l plasma). Data are presented as median [25\%-75\%].

Results: Total MP from all groups of patients accelerated clotting effectively than MP from HV. The lag phase in comparison with HV (11.8 [11.0-13.1]) was shorter in MI (9.5 [7.3-12.1], P 0.030), PAD
(8.0 [6.5-10.0], P 0.001), IPAH (8.8 [5.0-12.0], P 0.007) patients, and Vmax in comparison with HV (13.1 [11.7-13.8]) was higher in PAD (24.4 [19.9-27.7], P 0.001), IPAH (18.6 [15.1-30.5], P 0.001), but not MI (12.6 [11.1-15.7], $P>0.05)$ patients. MP count in comparison with HV (0.57 [0.34-0.82]) was lower in MI (0.28 [0.23-0.54], P 0.001), tended to be lower in IPAH (0.40 [0.24-0.62], P 0.111) and was higher in PAD (0.90 [0.58-1.42], P 0.003) patients. Anti-TF antibody did not change clotting parameters in the presence of MP from all groups. Conclusions: Coagulation activity of total MP in MI, PAD and IPAH patients was higher than in HV. This increase was not associated with TF expression, and in MI and IPAH patients was achieved despite the decrease of MP count.

\section{PB0111 | Extracellular Microparticles as Aortic Valves Calcification Vectors, Proof of Concepts}

M. Flores Garcia ${ }^{1}$; A. De la Peña Diaz ${ }^{2,1}$; E. Soria Castro ${ }^{1}$; M. Peña

Duque $^{1}$; J.R. Hernández López ${ }^{2}$; D. Reyes Munguía ${ }^{1}$; M. López

Granados $^{1}$; J.C. Villegas Hernández ${ }^{1}$

${ }^{1}$ Instituto Nacional de Cardiología, CDMX, Mexico; ${ }^{2}$ Universidad

Nacional Autonoma de Mexico, CDMX, Mexico

Background: Tissue's calcification, particularly in the cardiovascular system, decreases the elasticity of the blood vessels and favors valvular disease, mainly the aorta.

It increases with age, chronic kidney or inflammatory disease.

There are many possible mechanisms in the calcification process, including vascular smooth muscle cell (VSMC) instability, degradation of calcium, phosphate and elastin, differentiation of osteoclasts, and apoptosis.

The Mps are structures whose size ranges between 50 and 1000 nm and are horizontal vectors of different molecules including proteins, nucleic acids. They are involved in several pathological processes and the evidence is increasing in the participation of the Mps in the calcification of aortic valve.

Aims: To identify the presence of minerals in the Mps from patients undergoing primary aortic valve replacement.

Methods: We studied the presence of calcium in the Mps of 3 individuals undergoing primary replacement of the aortic valve at the National Institute of Cardiology. The protocol was approved by the Research and Ethics Committee with No. 18-1043 and the individuals signed an informed consent. Mps were obtained by the ultracentrifugation method, the concentration was obtained by determining the proteins and was expressed as $\mathrm{mg} / \mathrm{mL}$. The Mps were analyzed in a scanning microscope Jeol JSM-7401F (Field Emission Scanning Electron Microscope), with an excitation potential of $10 \mathrm{KeV}$, for microanalysis during $300 \mathrm{~s}$ in an area of $200 \mathrm{~nm}$. 
Results:
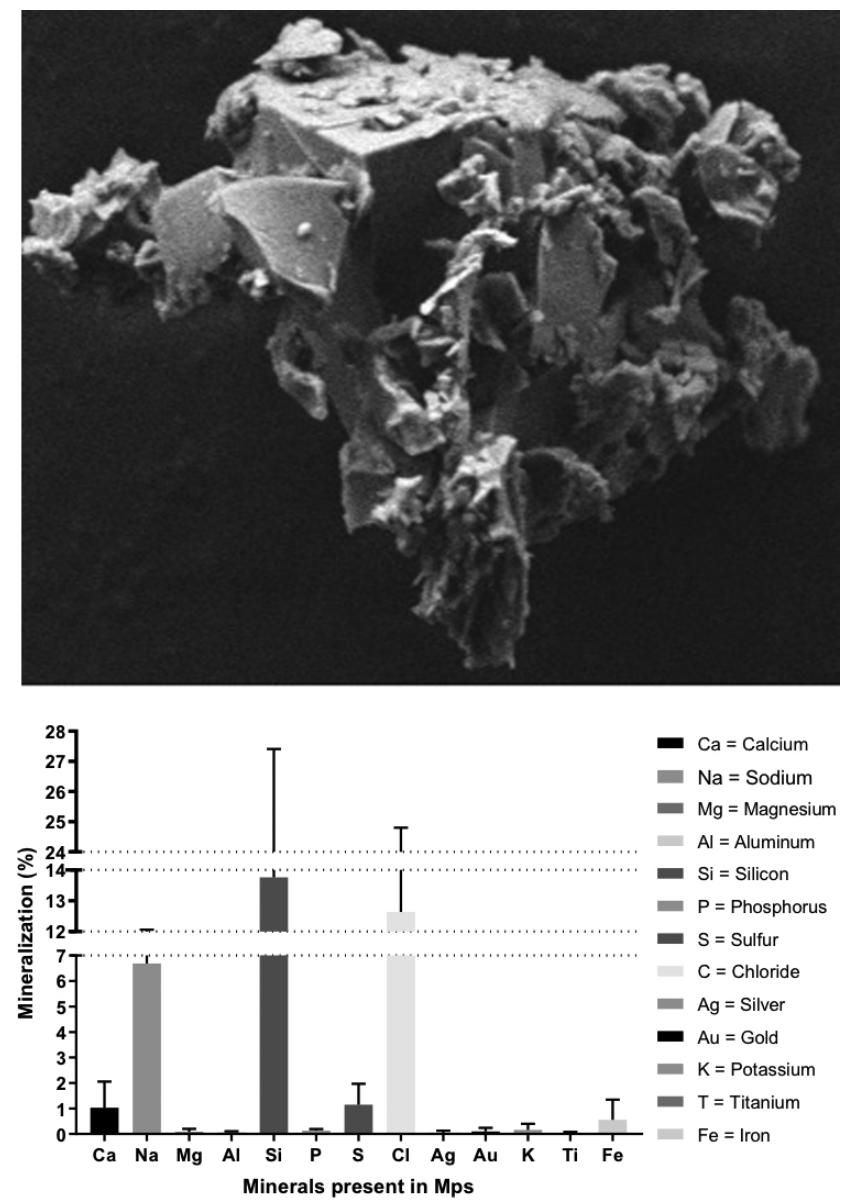

FIGURE 1 (A) As a proof of concept result, it shows the image obtained from a sample that corresponded to a 56-year-old male adult. (B) Presence of various minerals is appreciated, such as $\mathrm{Ca}, \mathrm{Na}, \mathrm{Si}, \mathrm{S}$, $\mathrm{Cl}$, Fe including trazes of $\mathrm{Mg}, \mathrm{Al}, \mathrm{P}, \mathrm{Ag}, \mathrm{Au}, \mathrm{K}$ and $\mathrm{Ti}$.

Conclusions: Our results show the presence of minerals in Mps of patients with aortic valve stenosis.

\section{PROTEIN C PATHWAY}

LPB0007 | Activated Protein C (APC) Resistance in FV-W1920R (FV-NARA) Was Caused by the Defective Association between FV-W1920R and Protein S (PS) in the APC/PS/FVa Assembly

N. Shimonishi; K. Ogiwara; J. Yoshida; K. Horie; K. Nogami Nara Medical University, Kashihara, Japan

Background: Coagulation factor $\mathrm{V}(\mathrm{FV})$ has pro-coagulant and anticoagulant functions. We reported that the FV-W1920R mutation (FV-NARA) in a patient with deep vein thrombosis and were resistant to APC (Nogami et al. Blood 2014). However, this mutation was present in the $\mathrm{C} 1$ domain, which is remote from the cleavage sites of APC, and the mechanism(s) in which FV-W1920R caused APC resistance remains unknown.
Aims: To elucidate the mechanism(s) of APC resistance in FV-W1920R Methods: We constructed HEK293 cells that express full-length FV stably using PiggyBac transposon. We evaluated the direct binding of FV to active-site blocked EGR-APC and PS by a surface plasmon resonance (SPR)-based assay. And we compared the cleavage and inactivation rates by APC using FV-WT and FV-W1920R in the presence and absence of PS. To investigate the effect of APC, PS, and phospholipids, APC-mediated inactivation of FV was compared using various concentrations of them.

Results: SPR-based assay revealed that FV-W1920R (Kd 12.8 nM) showed similar binding affinity to FV-WT (Kd $10.7 \mathrm{nM}$ ) for EGR-APC. And FV-W1920R (Kd $15.3 \mathrm{nM}$ ) and FV-WT (Kd $7.56 \mathrm{nM}$ ) showed little differences in binding affinity for PS. In FV-WT, cleavage of Arg306 was confirmed only in the presence of PS, but in FV-W1920R, cleavage of Arg306 was not observed, irrespective of the presence of protein S. In FV-WT, the inactivation rate by APC was enhanced by the presence of PS, whilst in FV-Nara, the enhancement effect by PS was not observed, even in high concentration of PS.

Conclusions: The APC resistance in FV-W1920R was caused by the defective association between mutated FV molecule and PS in the PS/APC/FVa assembly

PB0112 | Identification of a Novel PROC Mutation in a Newborn Causing Severe Protein C Deficiency Successfully Treated with Liver Transplantation

A. Le Moigne ${ }^{1}$; D. Habes ${ }^{2}$; G. Hery ${ }^{3}$; G. Staiti ${ }^{4}$; V. Fouquet ${ }^{3}$;

C. Baujard ${ }^{4}$; J. Miatello ${ }^{4}$; P. Durand ${ }^{4}$; M. Alhenc-Gelas ${ }^{5}$; O. Ackermann²; D. Mitanchez ${ }^{6}$; G. Leverger ${ }^{1}$; R. Favier ${ }^{7}$ V. Proulle ${ }^{8}$; M. Simonin ${ }^{1}$ ${ }^{1}$ Department of Pediatric Hematology and Oncology, AP-HP, Armand Trousseau Hospital, Sorbonne Université, Paris, France; ${ }^{2}$ Hôpital Bicêtre, Hépatologie et Transplantation Hépatique Pédiatriques, Université Paris Saclay, AP-HP, Le Kremlin-Bicêtre, France; ${ }^{3}$ Department of Pediatric Surgery, Hôpitaux Universitaires Paris-Sud (AP-HP), Hôpital Bicêtre, Le Kremlin-Bicêtre, France; ${ }^{4}$ Département d'Anesthésie-Réanimation, Hôpital de Bicêtre, AP-HP, Paris, France; ${ }^{5}$ Service d'Hématologie Biologique, Hôpital Européen Georges Pompidou, AP-HP, Paris, France; ${ }^{6}$ Department of Perinatality, APHP, GHUEP, Armand Trousseau Hospital, Medicine Sorbonne University, Paris, France; ${ }^{7}$ Laboratory of Hematology, Hôpital Trousseau, AP-HP, Paris, France; ${ }^{8}$ Service d'Hématologie Biologique, Institut Cochin, AP-HP, Paris, France

Background: nherited severe Protein $\mathrm{C}$ deficiency (SPCD) is a rare disease resulting from mutations in the $P R O C$ gene. It is responsible for neonatal purpura fulminans and severe thrombotic complications. Therapeutic options are often limited to life-long PC supplementation and anticoagulation. Liver transplantation (LT) remains an exceptional curative treatment. Aims: We present the case of a patient with a novel PROC mutation causing SPCD successfully treated with LT after multimodal therapy. Methods: At H30 of life, she developed skin necrosis and coagulopathy consistent with disseminated intravascular coagulation and SPCD. She was blind and lost the right index. PC supplementation was initiated 
intravenously on the $4^{\text {th }}$ day of life, in association with anticoagulation (LMWH). Due to large PC supplementation requirements, intolerance to subcutaneous administration, recurrent infectious and thrombotic complications over the next 18 months, a deceased-donor LT was considered. Results: Genetic sequencing of the PROC gene revealed an unreported homozygous mutation (p.Leu338Hisfs*36) in the proposita. Her asymptomatic non-consanguineous parents were heterozygous for the same mutation. A left lateral segment graft was successfully implanted $(250 \mathrm{~g})$ during a 12 -hours long surgery at the age of $19^{\text {th }}$ months. LT was uneventful under usual procedures, with post-surgery thromboprophylaxis (LMWH: $100 \mathrm{UI} / \mathrm{Kg} / \mathrm{d}$, aspirin: $3 \mathrm{mg} / \mathrm{Kg} / \mathrm{d}$ ) and immunosuppression (Tacrolimus). PC supplementation included an initial bolus (200 UI/Kg) followed by continuous infusion (100 UI/ $\mathrm{Kg} / \mathrm{h}$ ) aiming to maintain $\mathrm{PC}>75 \%$ and D-Dimers $<500 \mathrm{ng} / \mathrm{mL}$ during surgery. Replacement therapy was stopped after 4 more boluses (100 Ul/Kg/12 h) and PC stabilize spontaneously (50-60\%). In January 2021, at the age of 4, Tacrolimus ( $0.4 \mathrm{mg}$ bid) being is only long-term treatment; she had no other physical deficit than her blindness.

Conclusions: We describe a novel PROC gene mutation responsible of SPCD. Our case highlights the benefice of early LT in outweighing the complex and costly management of children with SPCD and resulting in a better quality of life for such patients.

\section{PB0113 | HIV-1 and SARS-CoV-2 Both Cause Protein S, but through Different Mechanisms}

M.M. Sim ${ }^{1}$; H.R. Alfar ${ }^{1}$; M. Hollifield ${ }^{1}$; D.W. Chung ${ }^{2}$; X. Fu' ${ }^{2}$; M. Banerjee ${ }^{1}$; X. Li ${ }^{1}$; A.C. Thornton ${ }^{1}$; J.Z. Porterfield ${ }^{1}$; J.L. Sturgill' ${ }^{1}$; G.A. Sievert ${ }^{1}$; M. Barton-Baxter ${ }^{1}$; K.S. Campbell ${ }^{1}$; J.G. Woodward ${ }^{1}$; J.A. Lopez ${ }^{2}$; S.W. Whiteheart ${ }^{1}$; B.A. Garvy ${ }^{1}$; J.P. Wood ${ }^{1}$

${ }^{1}$ University of Kentucky, Lexington, United States; ${ }^{2}$ Bloodworks
Northwest, Seattle, United States

Background: The anticoagulant protein S (PS) circulates in two plasma pools: free (functional) or bound to complement factor 4-binding protein (c4bp). PS deficiency commonly occurs in Human Immunodeficiency Virus-1 (HIV-1)+ patients and is associated with thrombosis. We hypothesized a similar process contributes to thrombosis in COVID-19.

Aims: To assess the regulation of PS in viral coagulopathies.

Methods: This study was approved by the Institutional Review Board. Citrated plasma was collected from consenting HIV-1+ (19 naïve, 11 on antiretroviral therapy, ART) or SARS-CoV-2+ (28 inpatients, 49 outpatients) and healthy controls for both populations (10, 31 , respectively).

Results: HIV-1+ patients had lower total PS than controls (94.12 $\pm 8.71 \%$ vs $133.77 \pm 10.45 \%, P=0.008)$, in both naïve $(42 \%)$ and ART-treated (27\%) patients. Total PS negatively correlated with endogenous thrombin potential $(P=0.01)$, suggesting PS deficiency contributes to increased thrombin generation in these patients.

Total PS was not reduced in SARS-CoV-2+ patients, but free PS was (Table). To determine the cause of free PS deficiency, we measured known PS-binding proteins C4bp, protein C (PC), and Mer, and found no differences between patients and controls. By native gel, we identified PS bound to C4bp, Mer, PC, tissue factor pathway inhibitor (TFPI), and von Willebrand Factor (VWF). VWF was markedly elevated in inpatients. Purified VWF dose-dependently decreased free, but not total, PS when added to control plasma, and blocked the TFPI cofactor activity of PS. PS was also identified as a plasma binding partner of VWF by mass spectrometry, and this interaction increased 10-million-fold with shearing. Finally, despite anticoagulation, plasma thrombin generation in inpatient samples was comparable to controls, suggesting a profound hypercoagulability, possibly exacerbated by PS deficiency.

TABLE 1 Coagulation-related parameters in COVID-19 patients

\begin{tabular}{|c|c|c|c|c|c|c|c|}
\hline Parameters & $\begin{array}{l}\text { Controls } \\
(n=31)\end{array}$ & $\begin{array}{l}\text { Inpatients } \\
(n=28)\end{array}$ & $P$-value & $\begin{array}{l}\text { Outpatients } \\
(n=48)\end{array}$ & $P$-value & $\begin{array}{l}\text { All COVID-19 } \\
(n=76)\end{array}$ & $P$-value \\
\hline Free PS (\%) & $142 \pm 50.4$ & $104.6 \pm 54.2$ & $0.02^{*}$ & $103 \pm 50.5$ & $0.01^{*}$ & $103.7 \pm 51.7$ & $0.01^{*}$ \\
\hline Total PS (\%) & $109.9 \pm 30.1$ & $153.9 \pm 138.2$ & 0.11 & $118 \pm 47.2$ & 0.43 & $133.2 \pm 97.5$ & 0.09 \\
\hline Free PS/Total PS & $1.4 \pm 0.7$ & $0.9 \pm 0.6$ & $0.02^{*}$ & $1 \pm 0.5$ & $0.02^{*}$ & $0.9 \pm 0.5$ & $0.01^{*}$ \\
\hline Protein C (\%) & $106 \pm 36$ & $155.3 \pm 107.3$ & $0.03^{*}$ & $117.1 \pm 46.4$ & 0.32 & $133 \pm 79.8$ & $0.04^{*}$ \\
\hline VWF (\% of average control) & $103.6 \pm 38.9$ & $378.1 \pm 176.7$ & $<0.0001^{*}$ & $117.1 \pm 37.4$ & 0.14 & $234.8 \pm 178$ & $<0.0001^{*}$ \\
\hline C4BP- $\beta$ (pg/mg total protein) & $22.5 \pm 11.6$ & $25.2 \pm 22$ & 0.49 & $43.5 \pm 49.3$ & 0.69 & $35.3 \pm 40.1$ & 0.95 \\
\hline Soluble Mer (pg/mg total protein) & $101.9 \pm 39.3$ & $133.7 \pm 81.9$ & 0.11 & $102.6 \pm 75.5$ & 0.95 & $114.7 \pm 78.9$ & 0.38 \\
\hline Lag time (s) & $22.5 \pm 11.6$ & $20.2 \pm 14.1$ & 0.47 & $17.7 \pm 10.3$ & 0.05 & $18.5 \pm 11.5$ & 0.09 \\
\hline $\begin{array}{l}\text { Endogenous thrombin potential } \\
\text { (nmol.min) }\end{array}$ & $952.2 \pm 326.8$ & $926 \pm 450.8$ & 0.78 & $1032.6 \pm 294$ & 0.25 & $997.8 \pm 353.9$ & 0.51 \\
\hline
\end{tabular}

Conclusions: In HIV-1, PS consumption leads to total PS deficiency. In SARS-CoV-2, VWF increases and binds PS, reducing the free pool. Thus, viruses can cause PS deficiency through multiple mechanisms, promoting thrombosis by shifting the procoagulant-anticoagulant balance. 
PB0114 | Various Receptors Are Related with the APC-induced Suppression of Osteoclast Differentiation

N. Akita $^{1}$; K. Yoshida ${ }^{2}$; T. Okamoto ${ }^{3} ;$ K. Asanuma ${ }^{4}$; K. Suzuki ${ }^{1}$;

T. Hayashi ${ }^{5}$

${ }^{1}$ Suzuka University of Medical Science, Suzuka, Japan; ${ }^{2}$ Ise Municipal

General Hospital, Ise, Japan; ${ }^{3}$ Faculty of Medicine, Shimane University, Izumo, Japan; ${ }^{4}$ Mie University Graduate School of Medicine, Tsu, Japan; ${ }^{5}$ Mie Prefectural College of Nursing, Tsu, Japan

Background: Protein C (PC) is activated by thrombin complexed with thrombomodulin (TM), which is present on vascular endothelial cells. Activated PC (APC) regulates blood coagulation by inactivating the factors Va and VIIla. We have so far demonstrated the inhibitory effect of APC on the differentiation of osteoclast precursor cells into osteoclasts via endothelial cell protein C receptor (EPCR) and PAR-1. Aims: In the present study, to further elucidate the relationship between bone remodeling and blood coagulation, we investigated the detailed signal transduction mechanism of APC-induced suppression of osteoclast differentiation.

Methods: Normal human osteoclast precursor cells were cultured in a growth medium including soluble RANKL, M-CSF, and FBS, and on days 4 and 7 , the culture medium was replaced with the same medium containing various concentrations of APC, FTY720, or APC + various substances without FBS. On day 8, TRAP-positive multinucleated cells were counted manually using a light microscope. The NF-kB and NFATc1 activation in the presence or absence of APC were evaluated using specific ELISA.

Results: APC inhibited the osteoclast differentiation, and FTY720, an agonist to the S1P receptor, had no effect on the osteoclast differentiation. However, FTY720 inhibited the APC-induced suppression of osteoclast differentiation. Similarly to anti-EPCR antibody and anti-PAR-1 antibody, anti-apoER2 antibody and recombinant apoER2 also inhibited the APC-induced suppression of osteoclast differentiation. Further, APC had no effect on NF-kB and NFATc1 activation.

Conclusions: APC suppresses human osteoclast differentiation mainly by inhibiting the formation of multinucleated cells via EPCR PAR-1, S1P receptor and apoER2.

\section{REGULATION OF COAGULATION}

LPB0055 | Exploration of TFPI-mediated Inhibitory Mechanisms in Coagulation Flow Model

K. Miyazawa ${ }^{1}$; A. Fogelson ${ }^{2}$; K. Leiderman ${ }^{1}$

${ }^{1}$ Colorado School of Mines, Golden, United States; ${ }^{2}$ The University of Utah, Salt Lake City, United States

Background: Our previous mathematical study suggested that lownormal levels of $\mathrm{FV}$ can rescue thrombin generation in hemophilia $\mathrm{A}$.
Recent experiments suggest TFPI can inhibit partially activated FV ( $F a^{\text {short }}$ ) and affect thrombin generation.

Aims: The objective was to embed TFPI-FVa ${ }^{\text {short }}$ interactions into a mathematical model of flow-mediated coagulation, and use it to determine the effect of platelet-surface-mediated TFPI inhibition on thrombin generation for both normal and hemophilia A blood.

Methods: We extended a mathematical model of coagulation kinetics to include production of $\mathrm{FVa}^{\text {short }}$ via platelet secretion and activation by FXa, TFPI binding to FVa ${ }^{\text {short }}$ and platelet-bound FXa, and all the possible interactions leading to the formation of a ternary complex between FXa, FVa ${ }^{\text {short }}$, and TFPI.

Results: Addition of the new TFPI-mediated inhibitory mechanisms increased the critical TF level necessary to produce a strong thrombin response. For physiologic levels of TFPI and fixed flow rate and TF density, the maximum thrombin generated is similar under all types of TFPI inhibition but occurs after different lag times, particularly at low TF levels. Previous version of this model only included TFPI inhibition of plasma-FXa and subendothelial TF:VIla, and showed little sensitivity to different TFPI levels under the flow. With the new TFPI inhibitory mechanisms, the model displays remarkably different thrombin dynamics under variation of TFPI levels. Under a hemophilic condition (1\% FVIII) and low TF, where little to no thrombin generation would occur, reducing TFPI to $50 \%$ enables substantial thrombin generation albeit with a long lag. A reduction of TFPI to $10 \%$ leads to a substantial reduction in this lag time.

Conclusions: Platelet-surface-mediated TFPI inhibition may play an important inhibitory role in thrombin generation under normal and hemophilic conditions. Such results suggest that blocking or reducing these inhibitory mechanisms may be beneficial to enhance thrombin generation.

PB0115 | Refining and Validating a Model of FXI Activation and Activity in Thrombin Generation and Blood Coagulation

H.H.S. Lakshmanan ${ }^{1}$; A. Estonilo ${ }^{2}$; C. Puy ${ }^{1}$; T. J. Zheng ${ }^{1}$; J. Maddala ${ }^{3}$; D. Gailani ${ }^{4}$; J. J. Shatzel ${ }^{1}$; A. Gruber ${ }^{1}$; P. L. Jurney ${ }^{2}$;

O. J.T. McCarty ${ }^{1}$

${ }^{1}$ Oregon Health \& Science University, Portland, United States; ${ }^{2}$ San José State University, San José, United States; ${ }^{3}$ West Virginia University, Morgantown, United States; ${ }^{4}$ Vanderbilt University, Tennessee, United States

Background: Factor $\mathrm{XI}(\mathrm{FXI})$ is a procoagulant enzyme that interacts with multiple substrates to promote thrombin generation. This is achieved directly through activation of coagulation factors and indirectly through inactivation of anticoagulants, including tissue factor pathway inhibitor (TFPI). Current computational models of coagulation do not incorporate functions of FXla other than FXla activation of factor IX.

Aims: To study the effect of TFPI inactivation by FXIa on thrombin generation using a mathematical model of thrombin generation. 
Methods: We expanded the ordinary differential equations model of thrombin generation in plasma to include TFPI inactivation by FXla. In simulations, we recorded time to generate $2 \mathrm{nM}$ thrombin, maximum thrombin concentration (peak thrombin) and time to reach peak thrombin concentration. Simulations were initiated with tissue factor (TF, 1-10 pM). In select simulations, TFPI inactivation by FXla was included by adjusting this reaction rate constant to $\geq 0$, while the rate of feedback activation of FXI by thrombin was accelerated by the presence of polyphosphate.

Results: Including inactivation of TFPI by FXIa increased maximum thrombin concentration and decreased time to reach maximum thrombin concentration. However, our model predicted that time to initially generate $2 \mathrm{nM}$ of thrombin by TF was insensitive to TFPI inactivation by FXIa. Inactivation of TFPI by FXIa led to a decrease in time to generate $2 \mathrm{nM}$ of thrombin and time to reach peak thrombin as a function of the rate of FXla generation by thrombin, while peak thrombin generation was unaffected. In all scenarios, the kinetics of thrombin generation was sensitive to the initial TF concentration.

Conclusions: Numerical simulations suggest that inactivation of TFPI by FXIa increases the initial rate and extent of thrombin generation. The rate of thrombin generation is amplified and is sensitive to inactivation of TFPI by FXla when feedback activation of FXI by thrombin is accelerated by polyphosphate.

PB0117 | Differences in Thrombin and Plasmin Generation Potential between East African and Western European Adults: The Role of Genetic and Non-genetic Factors

G.S. Temba ${ }^{1}$; N. Vadaq ${ }^{1}$; J. Wan²; V. Kullaya ${ }^{1}$; D. Huskens ${ }^{2}$; T. Pecht ${ }^{3}$; M. Jaeger ${ }^{1}$; C.K. Boahen ${ }^{1}$; V. Matzaraki ${ }^{1}$; W. Broeders ${ }^{1}$; L. Joosten ${ }^{1}$; P.G. de Groot ${ }^{1}$; J. Schultze ${ }^{3}$; A.J. van der Ven ${ }^{1}$; V. Kumar ${ }^{1}$; B.T. Mmbaga ${ }^{4}$; B. de Laat ${ }^{2}$; M.G. Netea ${ }^{1}$; M. Roest ${ }^{2}$; Q. de Mast ${ }^{1}$ ${ }^{1}$ Department of General Internal Medicine and Radboudumc Center for Infectious Diseases, Radboud University Medical Center, Nijmegen, Netherlands; ${ }^{2}$ Synapse Research Institute, Cardiovascular Research Institute Maastricht, Maastricht University Medical Center, Maastricht, Netherlands; ${ }^{3}$ Department for Genomics and Immunoregulation, Life \& Medical Sciences (LIMES) Institute, University of Bonn, Bonn, Germany; ${ }^{4}$ Kilimanjaro Clinical Research Institute, Kilimanjaro Christian Medical Center, Moshi, Tanzania, United Republic of

Background: Geographic variability in thrombosis incidence and coagulation and fibrinolysis potential are poorly understood.

Aims: The aim of the present study was to evaluate thrombin (TG) and plasmin generation (PG) potential in healthy Tanzanians compared with healthy individuals of Western-European ancestry and to assess the effects of environmental, genetic and non-genetic host factors.

Methods: We used data from the 300-Tanzania-FG $(n=313)$ and the Dutch 500FG cohort $(n=392)$, in which TG and PG were measured using modified calibrated automated thrombography. We determined associations of TG and PG with cohort characteristics and inflammatory mediators, and the impact of genetic variations through a genome-wide association study in both cohorts. Seasonal dietary influence on TG and PG was assessed using food metabolome data in Tanzanians.

Results: Tanzanians exhibited enhanced TG, PG, and TG/PG ratio compared with Dutch participants, and an increased normalized thrombomodulin sensitivity ratio, suggesting reduced sensitivity to the anticoagulant actions of protein C (Figure 1A). Female sex, BMI and age were associated with TG and PG in both cohorts. TG and PG in Tanzanians were also associated with food-derived plasma metabolites. The Tanzanians had higher concentrations of proinflammatory cytokines, which correlated strongly with TG and PG (Figure 1B). There was limited overlap in genetic variation associated with TG and PG between both cohorts (Figure 2A). Pathway analysis in the Tanzanian cohorts revealed multiple immune pathways that were enriched with TG and PG traits (Figure 2B), confirming the importance of coregulation of coagulation and inflammation.

\section{Figure 1.}
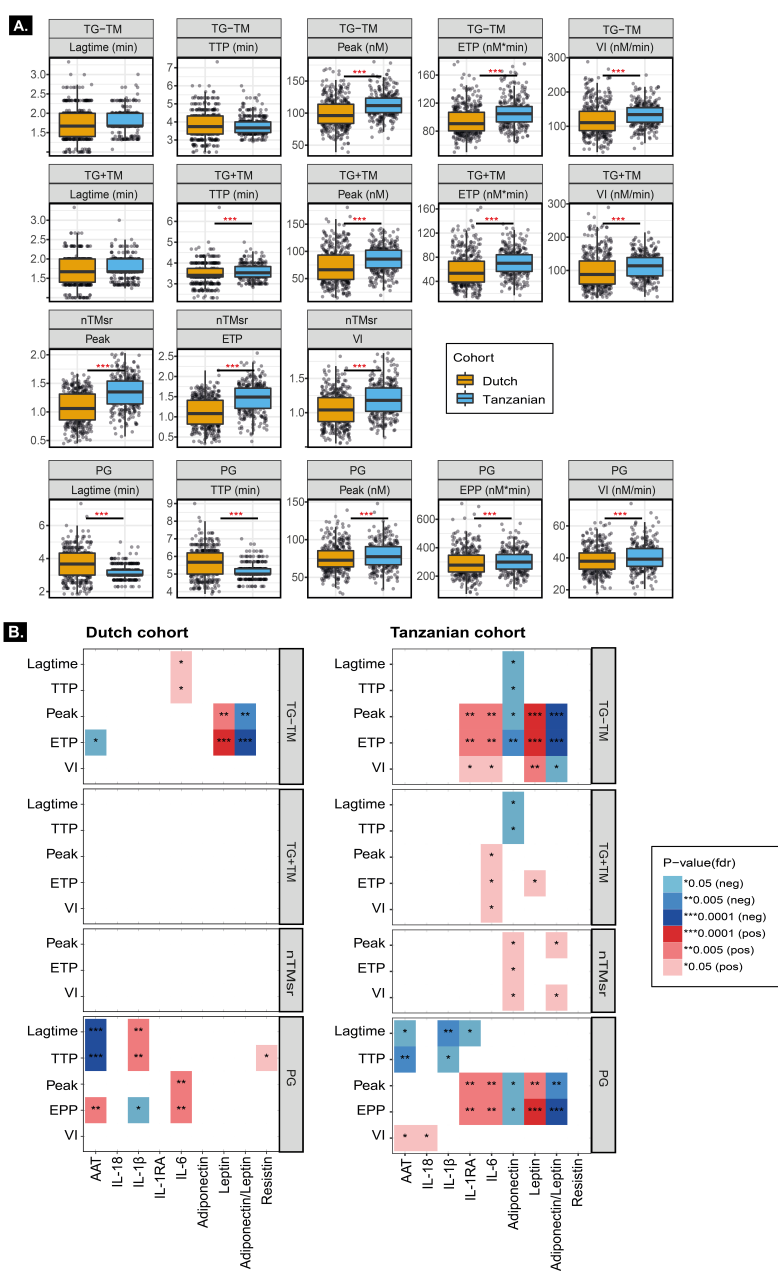

FIGURE $1 \mathrm{~A}$. Boxplots depict comparison between TG in the absence and presence of thrombomodulin (TM) and PG in the Tanzanian (blue) and Dutch cohort (yellow). In all box plots, the in-box line defines the median value, hinges depict 25 th and 75 th percentiles and whiskers extend to \pm 1.5 interquartile ranges; each dot indicates individual participant. Significance level was set by FDR P-value 
Figure 2.

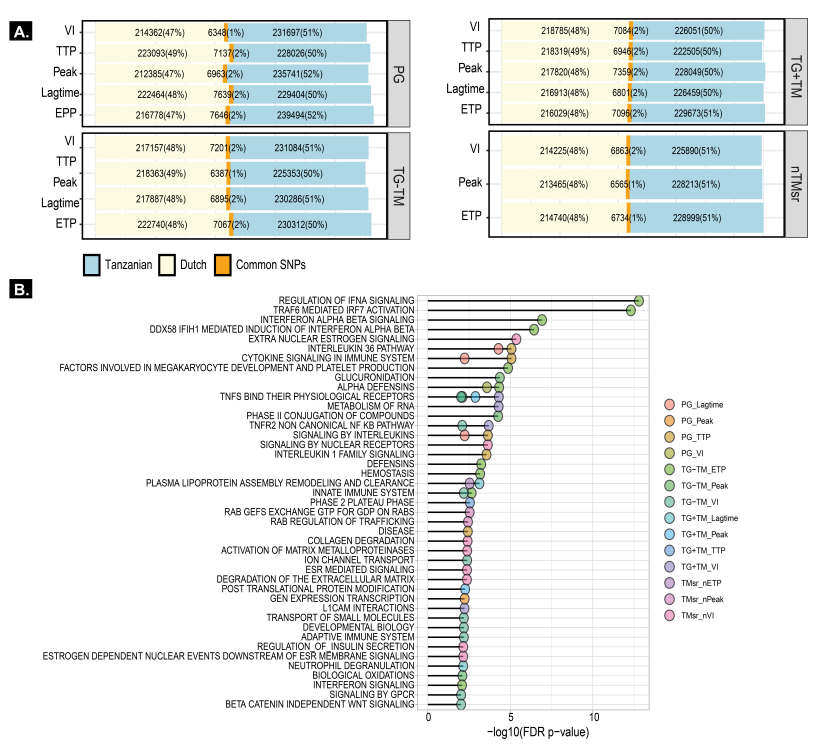

FIGURE 2 A. Frequency of common SNPs ( $P$-value < 0.05$)$ between the Tanzanian and Dutch cohort for each TG and PG parameters. B. Significant pathway in the Tanzanian and Dutch cohorts (FDR $P$ value < 0.01). Abbreviations: TG-TM = thrombin generation in the absence of thrombomodulin; TG+TM = thrombin generation in the presence of thrombomodulin; nTMsr = normalized thrombomodulin sensitivity ratio; TTP = time to peak; $\mathrm{VI}$ = velocity index; $\mathrm{ETP} / \mathrm{EPP}=$ endogenous thrombin/plasmin potential

Conclusions: Collectively, we show that Tanzanians have enhanced TG and PG potential relative to people of Western-European, which relates to a pro-inflammatory status and different genetic regulation. Also, increased TG/PG ratio as well as reduced sensitivity to protein $C$ activation indicates a hypercoagulable tendency. These findings are important in view of geographic variability in thrombosis and the rapid increase in non-communicable diseases in sub-Saharan Africa.

PB0118 | Neutrophils Can Promote Clotting by Secreting Proteins that Activate FXI while NETs Promote Clotting Independently of FXI in vitro

Y. Shi ${ }^{1}$; J.S. Gauer ${ }^{1}$; S.R. Baker ${ }^{2,1}$; H. Philippou ${ }^{1}$; S.D. Connell ${ }^{3}$; R.A.S. Ariëns ${ }^{1}$

${ }^{1}$ University of Leeds, Leeds Institute of Cardiovascular and Metabolic Medicine, Discovery and Translational Science Department, Leeds, United Kingdom; ${ }^{2}$ Wake Forest University, Department of Physics, Winston Salem, United States; ${ }^{3}$ University of Leeds, The Astbury Centre for Structural Molecular Biology, Molecular \& Nanoscale Physics, Leeds, United Kingdom

Background: Neutrophils release mediators (e.g. matrix metalloproteinases and serine proteases) which can influence thrombus formation. Neutrophil Extracellular Traps (NETs), which are extruded from neutrophils, have been shown to provide a "scaffold" for thrombosis and to increase the resistance of clots to fibrinolysis and thrombolysis. However, the interactions between neutrophils or NETs and fibrin(ogen) in clots, as well as the mechanisms behind these interactions are not yet fully understood.

Aims: To investigate the role of neutrophils/NETs in blood coagulation, fibrin formation, clot stability and clot porosity, in order to decipher how neutrophils/NETs interact with the fibrin network.

Methods: Human neutrophils were isolated from whole blood by the standard density gradient centrifugation method. Neutrophils were stimulated by Phorbol 12-myristate 13-acetate to generate NETs. Turbidity measurements were used to measure the kinetics of clot formation. Confocal microscopy was used to investigate the effects of neutrophils/NETs on overall clot network structure. Permeation experiments were carried out to investigate clot porosity.

Results: Neutrophils and NETs promote clotting in plasma without the addition of other coagulation triggers, but not in purified fibrinogen, indicating that other factors mediate the interaction. Both neutrophils and NETs also delayed clot lysis in plasma. The procoagulant effects of neutrophils and NETs were also observed in FXII- and FVII-deficient plasma. In FXI-deficient plasma, only the procoagulant effects of NETs were observed, but not of neutrophils. NETs increased the density of clots, particularly in the vicinity of the NETs, while neutrophils-induced clots were less stable and more porous.

Conclusions: NETs accelerate clotting and contribute to the formation of a denser clot architecture that is more resistant to lysis. Neutrophils, or their released mediators, may induce clotting in a different manner to NETs, mediated by FXI.

\section{PB0119 | Neutrophils Promote Tissue Factor-dependent Thrombin Generation in the Absence of Platelets}

I.I. Ivanov; M.T. Harper

University of Cambridge, Cambridge, United Kingdom

Background: Thrombin generation is central to haemostasis. Although tissue factor (TF) initiates coagulation, thrombin generation is amplified and propagated on cell membranes, particularly phosphatidylserine-exposing platelets. Decreasing platelet counts in thrombocytopenia are associated with reduced thrombin generation. Recently, some studies have suggested that neutrophils also enhance thrombin generation.

Aims: To investigate the contribution of neutrophils to thrombin generation in the presence and absence of platelets.

Methods: Blood from healthy drug-free donors was drawn into sodium citrate with informed, written consent. Platelets and plateletpoor plasma (PPP) were isolated by differential centrifugation. PPP was filtered through a $0.22 \mu \mathrm{m}$ filter to remove the majority of circulating microparticles. Neutrophils were isolated using a Ficoll gradient. Neutrophils and platelets were added to PPP to achieve different cell counts. Thrombin generation following recalification 
of plasma was monitored using a fluorogenic thrombin substrate (zGGR-AMC) in a microplate reader. TF (Dade, Innovin) was added to some samples.

Results: TF ( 1 or $5 \mathrm{pM}$ ) initiated thrombin generation in PPP. This was significantly enhanced when neutrophils were also present. In contrast, no thrombin generation was detected with neutrophils in the absence of TF. Neutrophils also enhanced thrombin generation in the presence of platelets up to $1 \times 10^{8}$ platelets $/ \mathrm{ml}$, although at $5 \times 10^{8}$ platelets $/ \mathrm{ml}$, neutrophils had little additional effect. Neutrophils had no effect on thrombin generation if they were fixed with paraformaldehyde prior to being added to PPP, indicating that neutrophil activation is required. In a screen of potential activators, stimulation of PAR2 by the peptide agonist, LIGRLO, also enhanced TF-initiated thrombin generation in PPP in the absence of TF. In contrast, SFLLRN (agonist of PAR1), AYPGKF (PAR4), lipopolysaccharide (TLR4) or fMLP (FPR1) had no effect.

Conclusions: Neutrophils enhance TF-initiated thrombin generation in plasma in the absence of platelets or at low platelet counts. This effect might contribute to thrombin generation during thrombocytopenia.

\section{PB0120 | Identifying Vulnerable Patients with Direct Oral} Anticoagulant (DOAC) Levels Outside the Expected Range

D. Braeken ${ }^{1}$; R. Bavalia ${ }^{2}$; Y. Henskens ${ }^{3}$; H. ten Cate ${ }^{1,4}$;

J. Veenhuizen ${ }^{2}$; R. Hengeveld ${ }^{5}$; B. Hutten ${ }^{6}$; S. Middeldorp ${ }^{2,7}$; M. Coppens ${ }^{2} ;$ A. Stroobants ${ }^{2}$

${ }^{1}$ Maastricht UMC, Thrombosis Expertise Centre, Maastricht, Netherlands; ${ }^{2}$ Amsterdam UMC, University of Amsterdam, Department of Vascular Medicine, Amsterdam, Netherlands; ${ }^{3}$ Maastricht UMC, Department of Clinical Chemistry, Maastricht, Netherlands; ${ }^{4}$ Maastricht UMC, Department of Internal Medicine, Maastricht, Netherlands; ${ }^{5}$ Amsterdam UMC, University of Amsterdam, Department of Clinical Chemistry, Amsterdam, Netherlands; ${ }^{6}$ Amsterdam UMC, University of Amsterdam, Department of Epidemiology and Data Science, Amsterdam, Netherlands; ${ }^{7}$ Radboud UMC, Department of Internal Medicine \& Radboud Institute of Health Sciences (RIHS), Nijmegen, Netherlands

Background: Monitoring direct oral anticoagulants (DOAC) is not recommended, although DOAC levels are frequently measured in clinical practice. Interpretation of extreme levels and appropriate changes for anticoagulant management have not been established yet.

Aims: Identify vulnerable patients with extreme DOAC levels and describe consequences for anticoagulant management.

Methods: In Amsterdam UMC and Maastricht UMC all patients with DOAC level measurement between 2013-2019 were included. DOAC levels below lower limit trough and above upper limit peak ranges were assigned as extreme(Douxfils 2018). One way ANOVA and Chi-square test were performed to assess differences between patients with levels outside and within the expected range.

Results: Of the 604 patients with a DOAC level measurement, 108 (17.9\%) had extreme levels. Patients with extremely high levels $(n=64)$ were significantly older (71.1 \pm 12.1 years), had more often creatinine clearance levels $<50 \mathrm{ml} / \mathrm{min}$. (32.8\%) and were more often using antithrombotic co-medication (25.0\%) than patients with levels within expected ranges $(60.6 \pm 17.0$ years, $P \leq 0.001 ; 13.9 \%, P \leq 0.001$; $13.1 \%, P=0.011$ ) and patients with extremely low levels ( $n=44$; $59.8 \pm 15.4$ years, $P=0.002 ; 11.4 \%, P=0.003 ; 6.8 \%, P=0.015)$. Atrial fibrillation (AF) as indication was associated with extreme levels (62.5\% vs. $61.4 \%$ vs. $39.1 \%, P \leq 0.001)$. In 33 (51.6\%) patients with extremely high and 19 (43.2\%) with extremely low levels, anticoagulation management was adjusted after DOAC level measurement. Of patients with a follow-up measurement $(n=31), 46.7 \%$ and $81.3 \%$ respectively, now had levels within expected ranges.

Conclusions: Despite broad ranges, almost one-fifth of patients had extreme DOAC levels. Patients with extremely high levels were older, had worse creatinine clearance levels, more antithrombotic co-medication and AF as DOAC indication. About half of the patients with extreme levels underwent adjustments in anticoagulant management. Larger, prospective studies are essential to investigate whether identification of patients with extreme DOAC levels is necessary and if they lead to justified adjustments in anticoagulant management.

PB0121 | Fixed-dose 1500-unit 4F-PCC for Factor Xa inhibitor Reversal in Spontaneous or Traumatic Intracranial Hemorrhage

S. Lujan; C. Rodriguez; M. Mason; L. Anselmo; T. Goot; A. Burnett University of New Mexico, Albuquerque, United States

Background: Andexanet alfa is FDA-approved for apixaban and rivaroxaban reversal. Due to concerns with cost, safety, logistics, and lack of comparative data, many hospitals use four factor prothrombin concentrate (4F-PCC), an off-label prohemostatic agent, as the standard of care.

Aims: Assess hemostatic efficacy of fixed-dose 1500 units of 4FPCC for Factor Xa inhibitor reversal in adult patients with intracranial hemorrhage (ICH).

Methods: IRB-approved, retrospective, single-center study of adult patients on apixaban or rivaroxaban receiving 4F-PCC 1500 units for ICH from August 2017 to September 2021 at an academic, level-1 trauma hospital. Exclusions were anticoagulants other than apixaban or rivaroxaban, 4F-PCC dose other than a fixed 1500 units or receipt of reversal outside our hospital. The primary outcome was evidence of any hematoma expansion on repeat computed tomography (CT) of the head (Table 1). Secondary outcomes included time from order to administration of $4 \mathrm{~F}-\mathrm{PCC}$, documented plan to resume anticoagulation, concomitant antiplatelet use, 30-day thromboembolism, and 30-day all-cause mortality. 
TABLE 1 Criteria for hemostatic efficacy

$\begin{array}{ll}\text { Hemostasis } & \text { Definition } \\ \text { Excellent/Good } & \text { No hematoma expansion } \\ \text { Poor/None } & \text { Hematoma expansion }\end{array}$

Results: Forty-one patients were included. Nine patients presented with $\mathrm{ICH}$ considered non-survivable and did not undergo repeat head computed tomography (CT). One patient had CT completed at an outside facility. Average time from order to administration was $43 \mathrm{~min}$. Thirty-one patients underwent repeat head CT within $24 \mathrm{~h}$. Twenty-two (71\%) had no hematoma expansion. Twenty-four of 28 patients (86\%) discharged alive had documented plans to resume anticoagulation. Eleven discharged patients (40\%) were lost to follow up. A minimum 16 patients (13 prior to discharge, 3 after discharge) and maximum 27 patients (assuming all 11 patients lost to follow- up died) expired, for 30-day mortality of 39-66\%. No thromboembolic events occurred in 28 discharged patients up to day 30 , and possibly all 11 patients lost to follow-up, for an incidence of $0-39 \%$.

TABLE 2 Demographic information

\begin{tabular}{ll} 
Demographic & $N=41$ \\
Age in yrs, avg (range) & $78(47-94)$ \\
Male, $n$ (\%) & $26(63)$ \\
Weight in kg, avg (range) & $78.3(42.9-148)$ \\
FXa inhibitor, $n$ (\%) & $25(61)$ apixaban \\
& $16(39)$ rivaroxaban \\
Concomitant antiplatelet, $n$ (\%) & $18(43)$ \\
Type of ICH, $n$ (\%) & \\
& Spontaneous 16 (39) \\
ICH score, avg (range) & Traumatic 25 (61) \\
Glasgow Coma Score, avg (range) & $12.5(3-15)$ \\
\hline
\end{tabular}

Conclusions: Fixed-dose 1500 unit 4F-PCC, administered within one hour, resulted in hemostasis in $71 \%$ of all $\mathrm{ICH}$.

Results:

TABLE 1 Patients short characteristics and laboratory findings
PB0122 | Interaction of Phenyramidol $\mathrm{HCl}$ and Warfarin: A Case Series Report

B. Murat ${ }^{1}$; E. Kivanc ${ }^{1}$; R. Dizman ${ }^{1}$; S. Murat ${ }^{2}$

${ }^{1}$ Eskisehir City Hospital, Eskisehir, Turkey; ${ }^{2}$ Eskisehir Osmnagazi University, Medical Faculty, Eskisehir, Turkey

Background: Phenyramidol is a 2-(beta-hydroxyphenethylamino) -pyridine hydrochloride and was first introduced into practice in 1960 as a muscle relaxant and analgesic. Warfarin acts by reducing the amount of active vitamin $\mathrm{K}$ required for the activation of coagulation factors. It is metabolized in the liver by cytochrome p450 enzymes. There are very rare cases of interaction between phenyramidol and warfarin in the literature

Aims: We present here the largest case series of the interaction of phenyramidol and warfarin.

Methods: It is aretrospective case series report.

Case reports: Seven patients admitted to hospital ( 3 patients to emergency department with haematuria and 4 patients to cardiology outpatient clinic with haemoptysis, ecchymosis). All patients were using warfarin due to atrial fibrillation (AF). The physical examination of the patients were normal. The other blood test were completely normal except PT (prothrombin time) and INR (international normalized ratio) (Table 1). When the patients' anamnesis was questioned in terms of food and drugs that might affect warfarin metabolism we learned that pheniramidol was started to patients (Table 1). All patients hospitalized in cardiology ward and fresh frozen plasma (FFP) were administered as intravenous infusion and one ampoule of vitamin $\mathrm{K}$ was administered intramuscularly. The control INR value taken from the patients on the $3^{\text {rd }}$ day is given in the Table 1. Three patients were switched to Novel oral anticoagulants (NOAC) during the hospital stay. While no overdose was observed in the follow-up in 4 cases where phenyramidol treatments were discontinued and warfarin was continued with the same dose, in one case, NOAC was switched to because the INR was below the therapeutic range.

Conclusions: Although the relationship between warfarin activity and phenyramidol is not definitively proven, it is important for physicians to be careful for the use of phenyramidol in patients using warfarin, to prevent bleeding complications.

\begin{tabular}{|c|c|c|c|c|c|}
\hline Age/sex & Diagnosis & $\begin{array}{l}\text { INR before } \\
\text { Phenyramidol }\end{array}$ & $\begin{array}{l}\text { Phenyramidol initiation } \\
\text { time (day) }\end{array}$ & $\begin{array}{l}\text { INR after } \\
\text { Phenyramidol }\end{array}$ & $\begin{array}{l}\text { INR after } \\
\text { Phenyramidol } \\
\text { discontinuation }\end{array}$ \\
\hline 61 years/female & Nonvalvular AF & 3.5 & 15 & 10 & 2.7 \\
\hline 73 years/female & Nonvalvular AF & 2.21 & 10 & 14.53 & 1.43 \\
\hline 70 years/females & Nonvalvular AF & 2.92 & 8 & 14.13 & 1.98 \\
\hline 93 years/male & Valvular AF & 2.65 & 5 & 17.1 & 2.31 \\
\hline
\end{tabular}




\section{PB0123 | On the Diagnosis of Thrombinemia in the Arctic}

N. Vorobyeva ${ }^{1,2} ;$ A. Vorobyeva ${ }^{2}$

"National Medical Research Center for Hematology" (Northern

Branch) Ministry of Health of Russia, Arckhangelsk, Russian Federation;

${ }^{2}$ Northern State Medical University Ministry of Health of Russia, Arckhangelsk, Russian Federation

Background: There is evidence of activation of the hemostasis system in the form of hypercoagulation formation during rotational work in circumpolar territories. A new promising method capable of assessing both hyper- and hypocoagulable states is the thrombin generation test.

Aims: The aim of the study is to analyze the dynamics of the parameters of the thrombin generation test (TGT) in the conditions of a trans-latitudinal Arctic sea voyage.

Methods: A prospective clinical and laboratory study was carried out on 52 subjects during the trans-latitudinal expedition "TransArktika-2019." Plasma samples for the determination of the thrombin generation test were taken at the zero point (Arkhangelsk $\left(64^{\circ} 33^{\prime} \mathrm{N} 40^{\circ} 32^{\prime} \mathrm{E}\right)$ prior to the ship's voyage; and at the highest point of the expedition (Heiss Island of the Archipelago Frans - Joseph ( $\left.80^{\circ} 34^{\prime} \mathrm{N} 57^{\circ} 41^{\prime} \mathrm{E}\right)$ on $18-20$ days of the Arctic voyage. The determination of the parameters of thrombin kinetics was carried out on a Ceveron-alpha analyzer with a TGA-module using Ceveron TGA High reagents. Analyzed: lag phase time (tLag), time of thrombin peak formation (tPeak), thrombin peak (Peak), endogenous thrombin potential (AUC).

Results: At the high point of the tran-latitudinal voyage, the time tLag ( $\mathrm{min}$ ) and the time to reach the peak of thrombin - tPeak (min) were statistically less than at the initial zero point, which indicated a state of hypercoagulability. The AUC (nM) of the expedition members was statistically significantly higher at the high point.

Conclusions: Being in high latitudes is possibly associated with the formation of a state of hypercoagulability. The study was supported by a grant from the Russian Foundation for Basic Research - project no. 18-00-00814-KOMFI (18-00-00478).

\section{PB0124 | Thrombin Generation is Affected by Freezing} Independent of Storage Temperature

M. Ninivaggi ${ }^{1}$; A. Carlo ${ }^{2}$; R. de Laat - Kremers ${ }^{1}$; B. de Laat $^{1}$

${ }^{1}$ Synapse Research Institute, Maastricht, Netherlands; ${ }^{2}$ Diagnostica

Stago S.A.S., Asnières sur Seine, France

Background: Thrombin generation (TG) is used to measure the overall coagulation potential of a person and to detect hypocoagulability and hypercoagulability. In practice, the assay is also used on an individual level to monitor the effect of anticoagulant treatment or to predict the effect of a drug on haemostasis.

Aims: The goal of this study was to investigate a possible effect of freezing of plasma on TG.
Methods: Platelet poor plasma was obtained from 25 healthy donors after double centrifugation of their citrated blood tubes. TG was measured either in fresh plasma, plasma stored at $-20^{\circ} \mathrm{C}$ or stored at $-80^{\circ} \mathrm{C}$. We measured TG on the ST Genesia with three different reagents: STG-BleedScreen (low tissue factor (TF)), STG-ThromboScreen \pm thrombomodulin (medium TF) and STGDrugScreen (high TF). Samples were tested fresh and after storage at $-20^{\circ} \mathrm{C}$ and $-80^{\circ} \mathrm{C}$ for 1 day. Wilcoxon matched-pairs signed rank test was used for statistical analysis.

Results: Overall, freezing of plasma samples caused an increased TG displayed in a significantly higher peak height (mean increase \pm SD; $28.7 \% \pm 18.3 \%$ ) and endogenous thrombin potential (ETP; $36.1 \% \pm 25.2 \%)$, and a significantly shorter lagtime $(-10.5 \% \pm 3.4$; $P$-values were all $<0.05)$. This observation was independent of the trigger used and the storage temperature. ETP inhibition by thrombomodulin decreased significantly after freezing, independently of the storage temperature $(-31.4 \% \pm 3.5 \%)$. The procoagulant effect on velocity index and time-to-peak differed in significance between storage temperatures.

Conclusions: Freezing plasma samples either at $-20^{\circ} \mathrm{C}$ or $-80^{\circ} \mathrm{C}$ affects TG significantly compared to fresh samples. Therefore results can only be compared when TG is measured in samples that are equally stored.

\section{PB0126 | Validation of the Bioequivalence of USP Potency} Adjusted Porcine, Ovine and Bovine Heparins

N. Baig; M. Jaradeh; A. Kouta; W. Jeske; D. Hoppensteadt;

J. Walenga; O. Iqbal; F. Siddiqui; M. Bakhos; J. Fareed

Loyola University Chicago Stritch School of Medicine, Maywood, United States

Background: The current shortage of porcine heparin has warranted the development of alternate source heparins from bovine and ovine mucosa. On a mass basis, porcine and ovine heparins exhibit similar potencies (200 units/mg) which are higher than their bovine counterparts (130-150 units/mg).

Aims: We compared porcine, ovine, and bovine heparins at USP potency-equated levels in standardized assays used for measuring and monitoring heparins.

Methods: Porcine (200 units/mg), ovine (200 units/mg), and bovine (140 units/mg) heparins were obtained from Medefil Inc. (Glendale Heights, IL), Ronnsi Pharmaceutical (Jiangsu, China), and Kin Master Pharmaceuticals (Posso Fundo, Brazil), respectively. The anticoagulant effect of all heparins was evaluated using whole blood clotting assays such as ACT and thromboelastographic methods and clot based assays such as aPTT, TT, and prothrombinase induced clotting time ( $\mathrm{PiCT}$ ). Thrombin generation inhibition assay was carried out using a kinetic assay. Protamine and heparinase neutralization of these agents were also investigated in both the mass and potency adjusted concentrations. All results were tabulated and compared, and applicable statistical methods were applied. 
Results: The USP potency adjusted heparin exhibited comparable anticoagulant effects in both the ACT and TEG assays. At equigravimetric levels porcine and ovine heparins produced comparable anticoagulant effects and bovine heparin produced weaker anticoagulant effects. All heparins produced similar anticoagulant effects at potency adjusted dosages. In the anti-Xa, anti-Ila, and thrombin generation assays, all agents were comparable. Potency adjusted bovine heparin required higher amount of protamine for the complete neutralization in comparison to ovine and porcine heparin.

Conclusions: These results show that, at potency adjusted concentrations, porcine, ovine, and bovine heparins exhibit comparable biochemical and anticoagulant responses in the plasma-based systems. Therefore, it appears that potency-equated porcine, ovine, and bovine heparins exhibit comparable biochemical and anticoagulant activities.

PB0127 | Heparin-binding Copolymer Completely Reverses the Anticoagulant Effect of Subcutaneously Administered Enoxaparin in Mice

J. Swieton $^{1}$; B. Kalaska ${ }^{1}$; J. Miklosz ${ }^{1}$; S.-I. Yusa ${ }^{2}$; K. Szczubialka ${ }^{3}$; D. Pawlak ${ }^{1}$; A. Mogielnicki ${ }^{1}$

${ }^{1}$ Medical University of Bialystok, Bialystok, Poland; ${ }^{2}$ University of Hyogo, Himeji, Japan; ${ }^{3}$ Jagiellonian University, Krakow, Poland

Background: Predictable pharmacokinetics allows for subcutaneous injection of enoxaparin once daily. Uncontrolled bleeding after enoxaparin is rare but may occur. The only registered antidote - protamine sulfate has only $60 \%$ efficacy and can cause severe complications. We developed heparin-binding copolymer (HBC), a diblock copolymer that reverses intravenously administered heparins and fondaparinux (Kalaska et al., Trans Res, 2016, Kalaska et al., J Pharmacol Exp Ther, 2020).

Aims: We focused on the optimal dosage regimen of HBC for reversing the anticoagulant activity of subcutaneously-administered enoxaparin in healthy mice.

Methods: Male BALB/c mice were subcutaneously injected with enoxaparin ( $5 \mathrm{mg} / \mathrm{kg}$ ). After $110 \mathrm{~min}$, vehicle (PBS), HBC (6.25 mg/kg or $12.5 \mathrm{mg} / \mathrm{kg}$ ) or protamine $(5 \mathrm{mg} / \mathrm{kg}$ or $10 \mathrm{mg} / \mathrm{kg}$ ) were administered into the tail vein. The blood was collected after 3, 10, 60, 180, 360 , and 600 min after their administration. Then, the activity of anti-factor $\mathrm{Xa}$ and Ila, and blood parameters were measured. All procedures involving animals were approved (Permit Numbers 6/2021) and conducted according to Directive 2010/63/EU. The data were analyzed with GraphPad Prism 6 software using the Mann-Whitney test. $P$ values less than 0.05 were considered significant.

Results: HBC at a lower dose reversed the effect of enoxaparin on anti-factor Xa activity after 3 and $10 \mathrm{~min}$, whereas higher dose reversed anti-factor Xa activity, whenever it was increased by enoxaparin (Figure 1). Both doses of HBC completely reversed the effect of enoxaparin on anti-factor Ila activity. Protamine did not reverse anti-factor Xa activity, and only partially reversed anti-factor Ila activity increased by enoxaparin. Safety studies revealed decreased number of blood platelets, and elevated liver enzymes in group treated with $\mathrm{HBC}$ at higher dose.
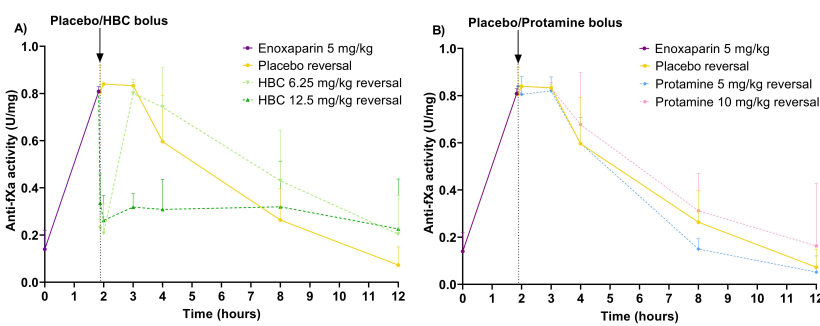

FIGURE 1 The neutralization of enoxaparin by HBC $(6.25 \mathrm{mg} / \mathrm{kg}$ and $12.5 \mathrm{mg} / \mathrm{kg}$ ) or protamine sulfate ( $5 \mathrm{mg} / \mathrm{and} 10 \mathrm{mg} / \mathrm{kg}$ ) in mice 3,10 , $60,180,360$, and 600 min after enoxaparin administration, measured by anti-factor Xa activity

Conclusions: HBC might be an efficient substitute for protamine sulfate to stop major bleeding that may occur in patients receiving subcutaneously enoxaparin.

Funding: National Science Centre, Poland (2016/21/B/ST5/00837).

PB0128 | The Thickness of Fibrin Fibers is Associated with High Fibrin Clot Visco-elasticity in Carcinomatosis Patients

M. Ullah ${ }^{1}$; S. Mirshahi ${ }^{2,1}$; I. Aldybiat ${ }^{1}$; G. Contant ${ }^{2}$; M. Pocard ${ }^{1}$; J. Soria ${ }^{1}$ M. Mirshahi ${ }^{1}$

${ }^{1}$ Université de Paris, CAP-Paris Tech., INSERM U 1275, Hôpital Lariboisière, Paris, France; ${ }^{2}$ Prospective Research, Diagnostica Stago, Gennevilliers, France

Background: Fibrin structure plays a crucial role in pathological events (Ząbczyk M, Undas A. Pol Arch Intern Med. 2017.).

Aims: To analyse the rheological properties of Fibrin clots formed in vitro in plasma from patients with carcinomatosis.

Methods: Fibrin clots formed in blood from peritoneal carcinomatosis patients $(n=44)$ of different origins (colic:9, pseudomysoma:7, gastric:6, rectal:5, ovarian:6, others:11), from controls without cancer $(n=31[C P G 1])$ and from healthy volunteers $(n=21)$ were analyzed. The viscoelastic parameters: clot elasticity or stiffness ( $G$ ') and clot viscosity (G") were determined using an Anton-Paar Rheometer (oscillation, frequency $1 \mathrm{~Hz}$, Strain ( $\gamma$ ) 1\%, time 1500 s). G' and G" determination was done using whole blood or fresh plasma. Fibrin clot degradation was performed using tissue plasminogen activator (tPA) incorporated into the clots and fibrin degradation products were evaluated using level of D-Dimers. The nucleotide materials in the clots were identified by confocal microscopy using DAPI and picogreen. Electron microscopy (SEM) analysis was performed using a S260 CAMBRIDGE apparatus to compare the structural shape and thickness of fibrin fibers in the clots. Results: Our results indicated that:

(i) In carcinomatosis patients, G' is increased in $57 \%$ of patients, 
(ii) Ratio of G'/fibrinogen level is higher in cancer patients, as compared to controls,

(iii) The plasma clot from cancer patients with high G' value has low fibrin degradability ( $R=-0.55$ ) compared to controls,

(iv) Nucleotide material was detected at higher concentration in the clots from cancer patients compared to controls,

(v) SEM analysis indicate a significant difference of morphological aspect as well as the thickness of fibrin fibers between Fibrin clots with low and those with high G'.

Conclusions: In cancer patients, the increase in visco-elasticity[CPG1] associated to an increased thickness[CPG2] of Fibrin fibers could explain the residual fibrin deposit on the peritoneal surface, probably due to a decreased fibrin degradation of clots.

PB0129 | Determination of DNA and Histone 3 Plasma Levels for Evaluation of Leukocyte Activation in Plasma of Patients with Peritoneal Carcinomatosis

S. Mirshahi $^{1,2}$; J. Soria ${ }^{2}$; I. Aldybiat ${ }^{2} ;$ M. Pocard ${ }^{2}$; M. Mirshahi ${ }^{2}$

${ }^{1}$ Prospective Research, Diagnostica Stago, Gennevilliers, France;

${ }^{2}$ Université de Paris, CAP-Paris Tech., INSERM U 1275, Hôpital

Lariboisière, Paris, France

Background: Circulating DNA or Neutrophil extracellular traps (NETs) modify fibrin structure (Longstaff $C$ et al. The journal of biological chemistry 2013).

Aims: Interpretation of the results of plasma DNA and Histones, in peritoneal cancer patients.

Methods: 77 patients with peritoneal carcinomatosis of several origins (ovarian19, colon 22, pseudomyxoma 9, gastric 9, appendix 4 others origins 13) were tested. Nets in plasma were evaluated by measuring DNA by fluorimetry using Green fluorescence protein, Histone 3 and Histone 4 were determined by ELISA.

Results: (i) The levels of circulating DNA and of Histone-3 in the 77 plasmas of cancer patients were found to be higher than in controls (mean levels of DNA and of $\mathrm{H} 3$ were respectively $0.84 \pm 0.97$ and $2.71 \pm 4.28$ in cancer patients versus $0.27 \pm 0.07$ and $0.56 \pm 0.06$ in controls),

(ii) DNA and $\mathrm{H} 3$ as well as $\mathrm{H} 4$ levels varied according to the origin of cancer: Patients with gastric cancer, pseudomyxoma and cancer of appendix expressed respectively 8, 5 and 8 times more Histone-3 than controls,

(iii) 40 patients with cancer (about 50\%) have an increased level of DNA but only 20 of them presented an increased level of Histone 3 (iv) Of the 37 other patients for whom plasma level of DNA was normal, 11 presented increased levels of Histone $\mathrm{H} 3$,

(v) The levels of Histone 4 were found to be higher in patients mainly with gastric cancer and pseudomyxoma (2.4 and 3.4 times more respectively) than in controls.
Consequently, the increased levels of DNA and of histone $\mathrm{H} 3$ in the 51 patients of the 77 with cancer testify an activation of polynuclear cells. Therefore, it was concluded that $66 \%$ of cancer patients present leukocyte activation.

Conclusions: Circulating DNA and Histone-3 were increased mainly in plasma from patients with gastric cancer, pseudomyxoma and cancer of appendix, and are associated with leukocyte activation in these patients.

PB0130 | Impact of DNA on the Fibrin Clot Structure in Peritoneal Carcinomatosis Patients

S. Mirshahi ${ }^{1,2}$; I. Aldybiat ${ }^{2}$; G. Contant ${ }^{1}$; M. Pocard ${ }^{2}$; J. Soria ${ }^{2}$; M. Mirshahi ${ }^{2}$

${ }^{1}$ Prospective Research, Diagnostica Stago,, Gennevilliers, France;

${ }^{2}$ Université de Paris, CAP-Paris Tech., INSERM U 1275, Hôpital

Lariboisière, Paris, France

Background: Circulating DNA modify fibrin degradability (Longstaff C et al. The journal of biological chemistry 2013)

Aims: To analyse the degradability of t-PA induced plasma clots formed in vitro in patients with peritoneal carcinomatosis.

Methods: i) Clot degradation: $200 \mathrm{~mL}$ of citrated plasma from normal pool plasma (NPP) or from patients with peritoneal carcinomatosis of several origins (7 ovarian, 3 pseudomyxoma, 3 mesothelioma, 2 colon) were clotted in absence or in presence of $10 \mathrm{ng}$ of t-PA, by 0.04 units of calcic thrombin. After 25 min incubation at $37^{\circ} \mathrm{C}$, degradation was stopped by adding $20 \mathrm{~mL}$ of aprotinin. The supernatants were kept for $\mathrm{D}$ dimer determination. The residual clots were transferred in a 24 well plate containing $400 \mathrm{~mL}$ of $\mathrm{NaCl} 0.15 \mathrm{M}$ in each well, that was placed above a light source, to be photographed. ii) Purified DNA was added before clotting in some experiments in NPP.

iii) Clot structure was determined by confocal microscopy, the fibrin fibers being detected in green after perfusion with glutaraldehyde and DNA being detected in blue using DAPI.

Results: i) After action of t-PA on the fibrin clot formed from plasma controls, the residual clot is very small, while in all patients suffering from peritoneal cancer - except one - the residual clot is large associated to a lower D dimer concentration[CPG1] .

ii) Clot structure of patient's plasmas was compared to the one of normal plasmas to which purified DNA was added before clotting. We observed that in both cases, DNA stained in blue was incorporated into Fibrin fibres that appear in green.

Conclusions: In peritoneal carcinomatosis patients[CPG1] , clot degradation by $\mathrm{t}-\mathrm{PA}$ is defective. This phenomenon is due to the impact of DNA on the Fibrin clot structure. We observed that DNA was incorporated into Fibrin fibres, down regulating clot degradation by fibrinolytic enzymes. 
PB0131 | Management of a Non-vitamin K Antagonist Oral Anticoagulant Associated Bleeding - A Case Report

R. Pombal; S. Lopes; L. Vieira; R. Neto; H. Gomes; M. Figueiredo Centre of Thrombosis and Hemostasis and Department of Transfusion Medicine, Centro Hospitalar Vila Nova de Gaia/Espinho, E.P.E., Vila Nova de Gaia, Portugal

Background: Prescription of Non-vitamin K antagonist oral anticoagulants (NOACs) has been increasing. This brings new challenges in the management of bleeding events, which may be triggered or potentiated by excessive NOACs plasma concentration.

Aims: Description of an acute bleeding in a patient treated with dabigatran.

Methods: Collection of clinical data in SClínico ${ }^{\circledR}$ application.

Results: Woman, 87 years old, was found prostrate on the ground and brought to Emergency Room. Medical history: arterial hypertension and atrial fibrillation anticoagulated with dabigatran. At the admission she had hypotension (70/30 mmHg), Glasgow Coma Scale E4V4M6 and a frontal acute bleeding incised wound. Acute cerebral haemorrhage was excluded by computed tomography (CT). Analytical study revealed acute renal failure, haemoglobin of $8.8 \mathrm{~g} /$ $\mathrm{dL}$ (previous $10 \mathrm{~g} / \mathrm{dL}$ ) and an altered coagulation study: aPTT $73.6 \mathrm{~s}$ (24.0-34.0 s), PT Rate 20\% (>70\%), INR 3.05, with dabigatran concentration $>460 \mathrm{ng} / \mathrm{ml}(52-275 \mathrm{ng} / \mathrm{ml})$. After fluid administration, tension profile has improved (109/52 $\mathrm{mmHg}$ ). As she was hemodynamically stable, the attending physician decide not to administer idarucizumab and to keep the patient under surveillance. The next day, brain CT scan was repeated, without further changes, but two red blood cell (RBC) units were transfused due to a decrease in haemoglobin $(7 \mathrm{~g} / \mathrm{dL})$. On the $3^{\text {rd }}$ and $4^{\text {th }}$ days of hospitalization, she had isolated self-limited episodes of hematuria and melena, respectively. Dabigatran levels, without antidote administration, returned to nontherapeutic levels on the $6^{\text {th }}$ day of hospitalization. The patient remained hospitalized for 15 days, without anticoagulation therapy, with progressive improvement of renal function and haemoglobin $(9.2 \mathrm{~g} / \mathrm{dL})$. No further bleeding occurred until discharge.

Conclusions: NOACs associated bleeding can be difficult to manage. In this case, the option of no administration of idarucizumab, its impact on patient bleeding and need of RBC transfusion, encourages clinical reflection on the topic. Thereby, it is important to create and work with interdisciplinary bleeding management teams.

\section{PB0132 | Natural Anticoagulants from Peonies}

\section{Lyapina; M. Kalugina}

Lomonosov Moscow State University, Moscow, Russian Federation

Background: In many plants, anticoagulants of different nature (heparin-like, peptides) have been found.

Aims: To study the possibility of displaying synergistic effects on the anticoagulant activity of the extract from the roots of the peony
"Ivan Gorozhankin", which was created by crossing the peobies "Lactiflora" and "Medicinal", in a comparative aspect with peony "Lactiflora".

Methods: A method for obtaining extracts from the roots of peonies "Ivan Gorozhankin" and "Lactiflora" has been developed. The anticoagulant activity of the extracts was determined in comparison with the standard low-molecular-heparin (LMH) of the Celsus company (USA) according to tests: the APTT, prothrombin time (PT) and thrombin time (TT).

Results: It was shown that extracts from both peony preparations had anticoagulant activity, but to different degrees. The anticoagulant activity of the extract from the peony "Lactoflora" corresponded to the same activity of the standard LMH. In extracts from the peony "Ivan Gorozhankin", synergistic effects were noted, namely, an excess of APTT by $20-30 \%$, PT - by $5-7 \%$ and TT-by $18-20 \%$ compared to those observed in extracts from the peony "Lactiflora" at concentrations from $10^{-1}-10^{-4} \mathrm{mg} / \mathrm{ml}$., Possible mechanisms of the activating effect of the extract from peony "Ivan Gorozhankin" on the anticoagulant properties of plasma were considered. This is due to the blockade of the activity of the thrombin and factors of the internal mechanism of blood clotting. According to the biochemical method, the presence of a heparin-like substance in the extract from the peony "Ivan Gorozhankin" was first detected.

Conclusions: For the first time, the ability of the extract from the peony "Ivan Gorozhankina" to show synergistic anticoagulant effects exceeding those of the extract from the peony "Lactiflore" was established. On the basis of the obtained data, there is a need to study the peony "Ivan Gorozhankin" as an antithrombotic, and possibly antiatherosclerotic agent

P0125 | Associated Factors of Adherence to the Use of Coumarin Derivatives or Direct Oral Anticoagulants: Protocol of a Systematic Review

W. Sousa ${ }^{1,2}$; N. Guimarães ${ }^{1}$; C. Viana ${ }^{1}$; P. Machado ${ }^{1}$; A. Medeiros ${ }^{1,3}$; M. Vianna ${ }^{1}$; M.A. Martins ${ }^{1,2,3}$

${ }^{1}$ Universidade Federal de Minas Gerais, Belo Horizonte, Brazil; ${ }^{2}$ Hospital das Clínicas da Universidade Federal de Minas Gerais, Belo Horizonte, Brazil; ${ }^{3}$ Hospital Risoleta Tolentino Neves, Belo Horizonte, Brazil

Background: Thromboembolic events are a public health problem worldwide. Oral anticoagulants are widely used for primary and secondary thromboprophylaxis. Adherence to oral anticoagulation is essential to achieve therapeutic goals.

Aims: This systematic review will assess the factors associated with adherence to treatment with coumarin derivatives or direct oral anticoagulants (DOACs).

Methods: Search strategies will be based on indexed terms from Medical Subject Heading (MeSH) and applied on MEDLINE/PubMed, EMBASE, CINAHL, and Latin American and Caribbean Health Sciences Information System (LILACS). Additional articles will be searched from gray literature and by manual search in the reference 
lists of selected studies. There will be no date limit or language restriction. Inclusion criteria: participants' age $>18$ years, both sexes, on chronic anticoagulation with coumarin derivatives or DOACs, observational or experimental studies using any method to assess adherence to oral anticoagulation. Exclusion criteria: duplicates, experimental studies involving animals, narrative or systematic reviews and case reports/series. The titles and abstracts will be evaluated and the selected studies will be fully read. The data of interest will be extracted and presented in tables, including sociodemographic, behavioral, clinical, drug therapy data and analytical methods. Two independent reviewers will participate of the selection of articles and data extraction. The discrepancies will be resolved by a third reviewer, if necessary. Validated instruments will be used to assess the quality of studies according to the study design. The systematic review was approved by PROSPERO (code CRD42020223555) and will be conducted in accordance with the PRISMA 2020 statement. Results: A total of 830 articles were retrieved in the initial search on MEDLINE (639), EMBASE (9), CINAHL (160) and LILACS (22).

Conclusions: Our findings are expected to contribute to the understanding of adherence to oral anticoagulation, bringing perspectives for the design of effective interventions in clinical practice.

\section{TISSUE FACTOR PATHWAY}

LPB0008 | ADP-induced Platelet-associated Tissue Factor Expression: Unique Involvement of the P2Y12 Receptor and Modulation by Clopidogrel Treatment

M. Brambilla ${ }^{1}$; P. Canzano ${ }^{1}$; G. Rovati ${ }^{2}$; N. Cosentino ${ }^{1}$; A. Becchetti ${ }^{1}$; J. Campodonico ${ }^{1}$; M. Cattaneo ${ }^{3}$; D. Trabattoni ${ }^{1}$; C. Pinna ${ }^{2}$; E. Tremoli ${ }^{1}$; M. Camera ${ }^{2}$

${ }^{1}$ Centro Cardiologico Monzino IRCCS, Milan, Italy; ${ }^{2}$ Dept of Pharmaceutical Sciences, Università degli Studi di Milano, Milan, Italy; ${ }^{3}$ Unità di Medicina II, ASST Santi Paolo e Carlo, Dept of Scienze della Salute, Università degli Studi di Milano, Milan, Italy

Background: Clopidogrel treatment is effective, besides inhibiting platelet aggregation, also in impairing platelet procoagulant activity which is supported by both phosphatidylserine and Tissue Factor (TF) exposure. Platelet-ADP stimulation results in TF expression. However, the role of the $2 \mathrm{ADP}$ purinergic receptors in platelet-TF modulation is still unknown.

Aims: To assess:

1) whether $P 2 Y_{1}$ and $P 2 Y_{12}$ receptors equally contribute to ADPinduced TF expression;

2) whether clopidogrel treatment modulate TF exposure;

3) TF intracellular localization through a pharmacological approach.

Methods: Platelets from healthy subjects were treated with the $\mathrm{P}_{2} \mathrm{Y}_{1}$ antagonist MRS-2250 or the P2Y ${ }_{12}$ antagonist AR-C69931MX (1pM-100nM). Expression of platelet-TF and P-selectin, as marker of alpha-granule secretion, were analyzed by flow cytometry upon ADP stimulation $(10 \mu \mathrm{M})$ and $\mathrm{pA} 2$ of the two P2Y-antagonists to inhibit their expression was calculated. The relationship between TF expression and the clopidogrel antiplatelet effect, assessed by the VASP-assay, was investigated in 106 coronary artery disease (CAD) patients.

Results: $\mathrm{P}_{2} \mathrm{Y}_{12}$-antagonist, but not $\mathrm{P}_{2} \mathrm{Y}_{1}$-antagonist, concentrationdependently prevented ADP-induced TF exposure. TF-activity, measured by thrombin generation assay, behaved similarly, being completely inhibited at $100 \mathrm{nM}$ AR-C69931MX. Platelet-TF expression in CAD patients with poor clopidogrel response (VASP > 50\%) was significantly higher compared to good-responders. Conversely, the extent of P-selectin inhibition was comparable between the two groups indicating that clopidogrel concentration able to inhibit P-selectin are less effective in reducing TF exposure. Indeed, AR-C69931MX pA2 to inhibit TF is 150-times lower than that of P-selectin. Inhibition of open canalicular system (OCS) by cytochalasin D impaired ADP-induced TF expression but it did not affect P-selectin, suggesting a different intracellular localization of the two proteins.

Conclusions: Data indicate that 1) $\mathrm{P}_{2} \mathrm{Y}_{12}$ only regulates surface expression of OCS-stored TF; 2) the therapeutic benefits of clopidogrel may also rely on inhibition of platelet-TF expression, although at higher concentrations than those required to regulate alphagranule secretion.

PB0133 | Mutagenesis Study Reveals Factor X Gla-domain Residues Critical for Initiation of the Clotting Cascade

D. Paul; S.M. Medfisch; R.C. Bailey; J.H. Morrissey

University of Michigan-Ann Arbor, Ann Arbor, United States

Background: Factor $X(F X)$ is proteolytically activated by the Tissue Factor (TF) and Factor VIla (FVIla) complex on membrane surfaces to initiate the clotting cascade. The $\mathrm{N}$-terminal $\gamma$-carboxyglutamaterich domain (Gla domain) of FX enables it to bind membranes. Besides membrane interactions, several studies have shown that the FX Gla-domain is also required for protein-protein interactions with the TF/FVIla complex. However, the exact residues in FX-Gla domain engaging with (TF/FVIIa) remain unclear.

Aims: To elucidate the molecular interactions between FX Gladomain residues and TF/FVIla.

Methods: 12 FX Gla-domain predicted by molecular dynamics simulation studies to interact with phosphatidylserine (PS) headgroups were mutated and expressed in HEK293 cells. Influence of the FX Gla-domain mutations were tested on rates of FX activation by TF/ FVIla in solution or on PS-containing membrane surfaces. Further, membrane-specific interactions were evaluated by quantifying membrane binding affinities using Nanodiscs immobilized on microring resonators.

Results: Mutating Lys-9, Arg-15, Gla-25, Arg-28 or Gla-32 had little effect on the rates of FX activation by soluble TF/FVIla complex, while mutating Gla-7, Gla-20 or Gla-29 reduced the rates by 50-80\%. For membrane-bound TF/FVIla, mutating Gla-7, Gla-14, 
Gla-19, Gla-20, Gla-25, Gla-29 or Gla-32 reduced FX activation rates by $90 \%$ whereas mutating Lys-9, Lys-10, Arg-15 or Arg-28 reduced it by $40 \%$. Membrane binding affinity of FX was impaired upon mutating Lys-9, Lys-10, Gla-19, Arg-28 or Gla-32, while membrane binding was undetectable upon mutating Gla-7, Gla-14, Gla-20, Gla-25 or Gla-29. We found a positive correlation $\left(R^{2}=0.685\right)$ between $\mathrm{FX}$ membrane binding affinity and $F X$ activation rates by membranebound TF/FVIla.

Conclusions: Our study shows that Lys-9, Lys-10, Arg-15, Gla-19, Gla-25, Arg-28 and Gla-32 are most critical for the membrane binding affinity of the FX Gla-domain. However, Gla-7, Gla-14, Gla-20 and Gla-29 are crucial residues in FX Gla-domain that participate in both protein-membrane and protein-protein interactions with the TF/FVIIa.

PB0134 | Genome-wide Knockdown of Chromatin Binding and Regulatory Protein Genes and Regulation of Zebrafish tfpia Gene Expression

R. Raman; W. Fallatah; A. Al Qaryoute; P. Jagadeeswaran University of North Texas, Denton, United States

Background: Tissue Factor Pathway Inhibitor (TFPI) is an anticoagulant protein that inhibits factor VIIa and Xa. It has been shown that FOXP3 is a TFPI repressor at the transcriptional level. At present, there are limited studies on the control of TFPI expression.

Aims: To identify the Chromatin Binding and Regulatory Proteins (CBRPs) involved in tfpia gene expression using the zebrafish model. Methods: The RNAs encoding CBRPs were selected from zebrafish thrombocyte Single-cell RNASeq data. Piggyback knockdowns of these CBRPs using ASOs and the quantitative Real Time PCR (qRT$P C R)$ were performed in adult zebrafish. Knockdowns and laserinduced thrombosis to measure TTO were performed on larvae.

Results: To identify the CBRPs involved in tfpia gene expression, we selected 103 CBRP mRNAs. We eliminated 34 mRNAs encoding histone-modifying enzymes. Thus, we targeted 69 mRNAs and performed 3-mRNA knockdowns using 3 ASOs each in 23 sets. After $48 \mathrm{~h}, \mathrm{qRT}$-PCR results showed knockdown of brd7, brd9, ccdc61, ing2, ing3, ing 4 , supt16h, suz12a, and suz12b increased tfpia gene expression. In the secondary screen, we found knockdown of 5 of these genes, brd7, ing2, ing3, ing4, and suz12b showed an increase in tfpia mRNA levels. We confirmed these 5 genes by a tertiary knockdown. We then examined the effects of simultaneous knockdown of all these 5 genes and found increased tfpia gene expression but was not greater than that shown by individual gene knockdowns. We also confirmed the above adult knockdown results of 5 genes in larvae by TTO studies.

Conclusions: Our results revealed that proteins encoded by brd7, ing2, ing3, ing 4 , and suz12b are negatively regulating tfpia gene transcription and the reduction in their gene expression led to increased tfpia mRNA levels. This information should be useful in designing novel drugs that inhibit these proteins such that Tfpia levels could be increased to treat patients with thrombotic risk.

PB0135 | Anti TFPI for Hemostasis Induction in Patients with Rare Bleeding Disorders, an ex vivo Thrombin Generation (TG) Guided Study

T. Brutman-Barazani ${ }^{1}$; E. Avishai ${ }^{1}$; I. Budnik²; A.A. Barg ${ }^{1}$; S. LeviMendelovich $^{1}$; T. Livnat ${ }^{1}$; G. Kenet ${ }^{1}$

${ }^{1}$ Sheba Medical Center, Tel Hashomer, Israel; ${ }^{2}$ Sechenov University, Moscow, Russian Federation

Background: Rare bleeding disorders (RBD) are inherited coagulopathies with variable bleeding phenotype, whose hemostatic control is based upon replacement therapy. Marstacimab (PF-06741086) is a fully human monoclonal IgG that targets the Kunitz2 domain of tissue factor pathway inhibitor [TFPI]. PF-06741086 is currently in development for bleeding prophylaxis in patients with hemophilia.

Aims: To assess the potential impact of PF-06741086 upon thrombin generation (TG) in RBD patients' plasma samples.

Methods: Citrated samples from RBD patients were collected and processed into platelet poor plasma (PPP) or platelet rich plasma (PRP). Samples were spiked with $150 \mu \mathrm{g} / \mathrm{ml}$ of PF-06741086 and incubated for $30 \mathrm{~min}$. TG was measured by calibrated automated thrombogram and compared to baseline samples and normal controls.

Results: Our study cohort included 20 RBD patients, with severe deficiencies: 5 VWD type 3, 4 FVII, 3 FXI, 2 FXIII deficiency and 1 patient with each of the following: FX, FV+FVIII, Fibrinogen, combined vitamin K dependent factors' deficiency, Glanzman thrombasthenia and Bernard Soulier. Figure 1 shows representative TG curves before and after spiking with PF-06741086. Among all patients a significantly reduced baseline TG was observed as compared to controls. Improvement of TG (lag time, peak and ETP) was observed in PF-06741086 spiked samples from $8 \mathrm{~min}, 99 \mathrm{nM}, 1116 \mathrm{nM} / \mathrm{min}$ to 5.5 min, 194 nM,1614 nM/min, respectively- see Figure 2. Notably, when PF-06741086 was added to normal controls' PPP, no significant TG increase was noted. None of the values measured among RBD patients exceeded normal controls. 
Figure 1.

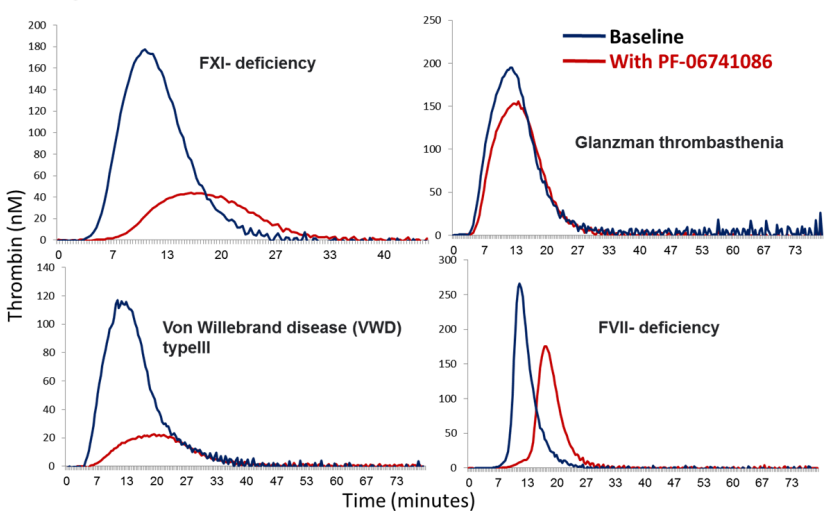

FIGURE 1 TG curves before and after spiking with PF-06741086

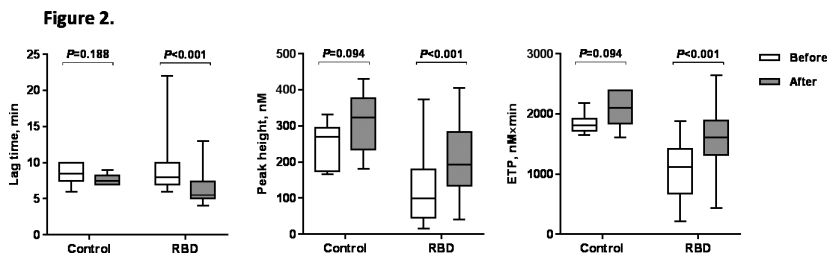

FIGURE 2 PF-06741086 induced TG improvement in RBD patients' plasma

Conclusions: These in vitro data suggest that PF-06741086 may serve as a promising approach for restoring the hemostatic balance and correction of TG in various RBD.

\section{COVID AND COAGULATION}

\section{COVID AND COAGULATION, BASIC SCIENCE}

LPB0102 | Blockade Of COVID-19 Antiphospholipid AntibodyInduced Thrombosis by a Specific Inhibitor of the TF Initiation Complex

D. Pedrosa $^{1}$; N. Müller-Calleja ${ }^{2,1}$; A. Hollerbach ${ }^{2}$; K. Lackner ${ }^{2}$; W. Ruf ${ }^{1,3}$

${ }^{1}$ Center for Thrombosis and Hemostasis (CTH) / Johannes Gutenberg University Medical Center, Mainz, Germany; ${ }^{2}$ Institute for Clinical Chemistry and Laboratory Medicine / Johannes Gutenberg University Medical Center, Mainz, Germany; ${ }^{3}$ Department of Immunology and Microbiology / Scripps Research, La Jolla, United States

Background: Antiphospholipid antibodies (aPL) cause the antiphospholipid syndrome (APS) and promote rapid complement- and protein disulfide isomerase (PDI)-dependent tissue factor (TF) decryption on monocytes. In this context, aPL induce procoagulant phosphatidylserine exposure by a rapid thrombin-dependent translocation of acidic sphingomyelinase (ASM) to the cell surface, where ASM is specifically activated by the endosomal lysobisphosphatidic acid (LBPA) presented by the endothelial protein C receptor (EPCR). aPL are also found in COVID-19 patients and effective therapeutic approaches to prevent TF-dependent thrombosis in APS associated with viral infections are needed.

Aims: Analyze the effect of rNAPc2, a hookworm-derived inhibitor of the TF initiation complex, in COVID-19 aPL immunoglobulin (Ig)induced thrombosis in mice.

Methods: Isolated Ig from hospitalized COVID-19 patients or healthy controls were injected into $\mathrm{C} 57 \mathrm{BI} / 6 \mathrm{~J}$ wild type mice to induce aPLamplified thrombosis in a flow restricted inferior vena cava (IVC) model. Labeled platelets and leukocytes were tracked over $3 \mathrm{~h}$ and thrombus size was quantified by intravital microscopy.

Results: COVID-19 Ig accelerated thrombus formation significantly in comparison to isolated Ig from healthy controls (31528 vs 302 $\mu \mathrm{m} 2 ; P<0.0001$ ). This increase was not impaired by non-inhibitory aEPCR injected prior to COVID-19 Ig but blocked by aEPCR which specifically inhibits EPCR-LBPA induction of ASM activity. These data showed that COVID-19 Ig triggered the same prothrombotic pathway elucidated for aPL from APS patients. Administration of the TF complex inhibitor rNAPc2 30 min prior to COVID-19 Ig-induced thrombosis markedly reduced thrombus size to levels comparable to control Ig injected mice (31527 vs 1399 um2; $P<0.0001$ ), demonstrating that thrombosis is induced by TF activation.

Conclusions: Blockade of TF with rNAPc2 is effective to prevent COVID-19 Ig-induced thrombosis in a preclinical model of APS.

\section{LPB0103 | Circulating Cellular Clusters Are Correlated with} Thrombotic Complications and Clinical Outcomes in COVID-19

A. Dorken Gallastegi ${ }^{1}$; L. Naar ${ }^{1}$; E.M. Van Cott $^{1}$; R.P. Rosovsky ${ }^{1}$; D.J. Gregory ${ }^{1}$; D. Annamalai ${ }^{2}$; J. Lee ${ }^{1}$; H.M.A. Kaafarani ${ }^{1}$; G. Velmahos ${ }^{1}$; R.G. Tompkins ${ }^{1}$; G.H. Frydman ${ }^{1,2}$

${ }^{1}$ Massachusetts General Hospital, Boston, United States;

${ }^{2}$ Massachusetts Institute of Technology, Cambridge, United States

Background: COVID-19 triggers a pro-inflammatory and prothrombotic state. The role of circulating cellular clusters in the setting of COVID-19 remains unclear.

Aims: This study explores the phenotype of circulating clusters and their potential relationship with clinical outcomes in COVID-19 patients.

Methods: Blood samples were collected between July - August 2020 from patients with a positive SARS-CoV2 PCR treated at a large academic medical center. Imaging flow cytometry was used to detect various circulating cellular clusters, including: platelet (plt)leukocyte aggregates (PLAs: 1 leukocyte + plt), leukocyte clusters (LC: $\geq 2$ leukocytes \pm any other cell) and platelet-erythrocyte aggregates (PEAs: $\geq 1$ erythrocyte + plt) (Figure 1). Cluster phenotypes were compared in patients with and without COVID-19 and were retrospectively correlated with clinical outcomes. 
Figure 1. Circulating cellular clusters in imaging flowcytometry.

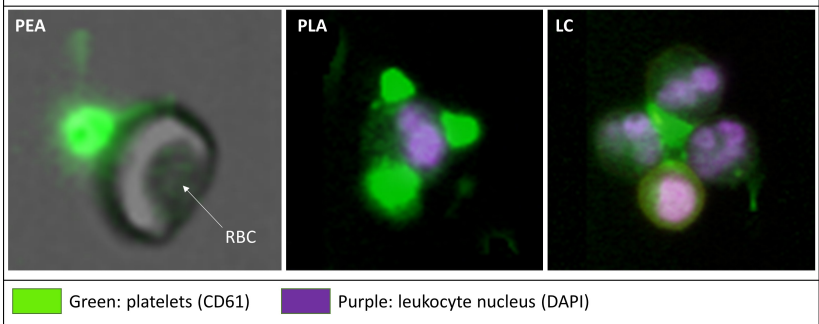

FIGURE 1 Circulating Cellular Clusters

Results: Forty-six COVID and 12 non-COVID samples were analyzed. Patients with COVID-19 had higher circulating PEAs $(2.58 \% \pm 0.12 \%$ vs $1.41 \% \pm 0.72 \%, P=.001)$ and manually-counted PLAs $(0.15 \% \pm 0.11 \%$ vs. $0.06 \% \pm 0.03 \% P=.007$ ) compared to healthy controls. Table 1 shows the relationship between specific populations of clusters and clinical outcomes in patients with COVID-19. The presence of LCs, in particular, significantly correlated with thrombotic events $(P=0.006)$, whereas PLAs and PEAs did not ( $P=0.73$ and $P=0.9$, respectively). Blood type was also correlated to LCs $(P=0.021)$, with Type $O$ having the least LCs, followed by Types A, AB and B.
Background: Coronavirus disease 2019 (COVID-19) is associated with a significant risk of thrombosis. A resistance to fibrinolysis has been highlighted in COVID-19 patients but its clinical relevance has not been established yet. In experimental stroke models, $\mathrm{N}$ acetylcysteine (NAC) promoted lysis of tPA-resistant thrombi by reducing large von Willebrand factor (VWF) multimers.

Aims: To better characterize the clinical relevance of fibrinolysis resistance in COVID-19 patients and to evaluate the capacity of NAC to restore fibrinolysis in vitro.

Methods: 28 critically ill patients with COVID-19 and 28 healthy controls were included in this single-center observational study. A modified thromboelastography assay (ROTEM ${ }^{\circledR}$ Delta) using EXTEM $^{\circledR}$ reagent and $150 \mathrm{ng} \mathrm{mL}^{-1}$ t-PA (Actilyse ${ }^{\circledR}$ ) was performed in the presence of $10 \mathrm{mM}$ NAC or buffer. Residual percentage of clot firmness 30 min after coagulation (LI30) and time required to complete lysis (LT) assessed the efficiency of t-PA-triggered fibrinolysis. Results: Blood clots from COVID-19 patients were resistant to fibrinolysis as indicated by increased LI30 (median 94\% [35-100] versus $1 \%$ [0-45], $P=0.0007$ ) and prolonged LT (2609 s [1855-3043] versus $1560 \mathrm{~s}$ [1376-1994], $P=0.0003$ ) compared to healthy controls. LI30 and LT were positively correlated with vWF levels $(r=0.6$,

TABLE 1 Correlations between circulating cell clusters and clinical outcomes in patients with COVID-19

\begin{tabular}{lllllll} 
& Mortality & Admitted to ICU & $\begin{array}{l}\text { Mechanical } \\
\text { Ventilation }\end{array}$ & $\begin{array}{l}\text { Acute Kidney } \\
\text { Injury }\end{array}$ & $\begin{array}{l}\text { Thrombotic } \\
\text { Complication }\end{array}$ & $\begin{array}{l}\text { Infectious } \\
\text { Complication }\end{array}$ \\
PEAs & $P=0.31$ & $P=0.13$ & $P=0.04$ & $P=0.11$ & $P=0.90$ & $P=0.045$ \\
PLAs & $P=0.41$ & $P=0.28$ & $P=0.47$ & $P=0.73$ & $P=0.73$ & $P=0.54$ \\
LCS & $P=0.49$ & $P=0.67$ & $P=0.91$ & $P=0.07$ & $P=0.006$ & $P=0.36$ \\
\hline
\end{tabular}

Thrombotic complication: Deep vein thrombosis, pulmonary embolism, clotting in lines, myocardial infarction, stroke or acute bowel ischemia. Infectious complications: Pneumonia, CLABSI, surgical site infection or UTI.

Conclusions: Circulating cellular clusters are correlated with significant clinical outcomes and cluster phenotypes appear to be associated with specific outcomes, including thrombotic events. These immuno-thrombotic complexes may play a significant role in the development of thrombosis and resultant end-organ damage. Further study of the role of the cellular component in COVID-19 may lead to the development of prediction models and help identify novel drug targets for inflammation-related thrombosis.

LPB0104 | Resistance to Fibrinolysis in Critically III Patients with COVID-19 Can Be Overcome by $\mathrm{N}$-acetylcysteine in vitro

D. Faille ${ }^{1}$; M.-C. Bourrienne ${ }^{2}$; J. Gay ${ }^{1}$; B. Ho-Tin-Noé2 ; S. Loyau ${ }^{2}$;

V. Andrieu ${ }^{1}$; C. Achard ${ }^{1}$; C. Cavalié ${ }^{1} ;$ M. Jandrot-Perrus ${ }^{2}$;

C.V Denis ${ }^{3}$; S. Ruckly ${ }^{1}$; J. Patrier ${ }^{1}$; P.-H. Wicky ${ }^{1}$; E. de Raucourt ${ }^{4}$;

R. Sonneville ${ }^{1}$; J.-F. Timsit ${ }^{1}$; N. Ajzenberg ${ }^{1}$

${ }^{1}$ University of Paris, Bichat Hospital, PARIS, France; ${ }^{2}$ LVTS INSERM

U1148, PARIS, France; ${ }^{3}$ HIT INSERM UMR_S 1176, Paris, France;

${ }^{4}$ Beaujon Hospital, PARIS, France
$P=0.009$ and $r=0.5, P=0.04$, respectively), suggesting a role for VWF in fibrinolysis resistance. Resistance to tPA-induced fibrinolysis was delayed in patients with thrombosis $(n=10)$ compared to patients without thrombosis, as indicated by higher LI30 (100\% [97100] versus $82.5 \%$ [0.5-97]; $P=0.002$ ) and prolonged LT (3189 $\mathrm{s}$ [2706-3772] versus $2217 \mathrm{~s}$ [1583 2840]; $P=0.008)$. Both LI30 and LT were correlated with SOFA $(r=0.7, P=0.0003$ and $r=0.5, P=0.01$, respectively) and Simplified Acute Physiology Score (SAPS) II scores ( $r=0.7, P=0.0004$ and $r=0.7, P=0.0009$, respectively). In vitro, NAC efficiently restored fibrinolysis in COVID-19 patient blood.

Conclusions: Resistance to fibrinolysis in critically ill COVID-19 patients is associated with thrombosis and clinical severity. NAC could represent a new adjunct therapy to promote endogenous fibrinolysis in severe COVID-19 patients. 
PB0136 | SARS-CoV-2 Derived Peptides Potentiate Proinflammatory and Pro-coagulant Responses to the TLR3 Ligand Poly(I:C) in Human Endothelial Cells and Mice

$\underline{\text { V. Bharathi }}^{1}$; Y. Zhang ${ }^{2}$; G. Wong ${ }^{2}$; N. Mackman ${ }^{1}$; S. Antoniak ${ }^{1}$

${ }^{1}$ University of North Carolina, Chapel Hill, United States; ${ }^{2}$ University of California, Los Angeles, United States

Background: COVID-19 disease, caused by SARS-CoV-2 infection, is associated with cytokine storm and thrombosis. The doublestranded RNA (dsRNA) mimetic poly(l:C) induces cytokines and Tissue Factor (TF) expression in endothelial cells. We demonstrated that host-encoded antimicrobial peptides (AMP) can organize dsRNA into pro-inflammatory nanocrystals with optimal spacing to stimulate toll-like receptor-mediated immune responses.

Aims: We determined if pro-inflammatory sequences in the SARSCoV-2 proteome enhanced host inflammatory and pro-coagulant responses to poly(l:C) in-vitro and in-vivo.

Methods: We identified pro-inflammatory sequences from the SARS-CoV-2 proteome using a machine learning-based classifier trained on innate immune peptide sequences. We identified multiple peptide sequences encoded by ORF1ab, ORF3, S, and N genes. We tested these peptides for their ability to enhance poly $(\mathrm{I}: \mathrm{C})$ induction of TF activity in Human umbilical vein endothelial cells (HUVECs) by stimulating with poly $(\mathrm{l}: \mathrm{C})(5 \mu \mathrm{g} / \mathrm{ml})$ and/or the peptides. TF activity in cell lysates was determined using two-stage FXa generation assay $6 \mathrm{~h}$ after stimulation. Peptide $6(2.2 \mu \mathrm{M})$, derived from ORF1ab showed the highest potency. Mice were administered poly $(\mathrm{l}: \mathrm{C})(100 \mu \mathrm{g})$ or poly $(\mathrm{l}: \mathrm{C})$ and peptide $6(344 \mu \mathrm{g})$ intravenously. Cytokine levels in HUVEC supernatants (IL-8) or mouse plasma (IL-6 and CXCL1) and plasma Thrombin-antithrombin complex (TAT) levels were analyzed by ELISA.

Results: Co-stimulation of HUVECs with poly(I:C) and peptide 6 significantly increased IL-8 release $(P<0.0001)$ and TF activity $(P<0.0001)$ compared to poly $(I: C)$ alone. Mice co-treated with poly $(\mathrm{I}: \mathrm{C}) /$ peptide 6 had significantly increased plasma levels of CXCL1 (mouse homolog of IL-8) $(P=0.005)$ and IL-6 $(P=0.015)$ at $2 \mathrm{~h}$ compared to poly(l:C)-treated control mice. We also observed an increase in TAT complexes, a marker of activation of coagulation in mice receiving poly $(\mathrm{l}: \mathrm{C}) /$ peptide 6 compared to poly $(\mathrm{l}: \mathrm{C})$ alone, although this increase did not reach statistical significance.

Conclusions: Our data demonstrate that SARS-CoV-2 derived AMPlike peptides, such as peptide 6 (encoded in ORF1ab), enhance proinflammatory and pro-coagulant responses to dsRNA in human endothelial cells and inflammation in mice.
PB0137 | Impact of COVID-19 on Platelet Activity and the Platelet Releasate

S. Comer $^{1,2}$; S. Cullivann ${ }^{1,3}$; P. Szklanna ${ }^{1,2} ;$ L. Weiss ${ }^{1,2} ;$ S. Cullen ${ }^{4}$; S. Kelliher ${ }^{5}$; A. Smolenski ${ }^{6}$; C. Murphy ${ }^{1,7}$; H. Altaie ${ }^{8}$; J. Curran ${ }^{9}$; K. O'Reilly ${ }^{3,6}$; A. Cotter ${ }^{6,10,11}$; B. Marsh ${ }^{6,12}$; S. Gaine ${ }^{3,6}$; P. Mallon ${ }^{6,13}$; B. McCullagh ${ }^{3,6}$; N. Moran ${ }^{4}$; F. Ní Áinle ${ }^{1,5,6,14}$; B. Kevane ${ }^{1,5,6}$; P. Maguire $\mathrm{e}^{1,2,15}$

${ }^{1}$ Conway SPHERE Research Group, Conway Institute, University College, Dublin, Ireland; ${ }^{2}$ School of Biomolecular and Biomedical Science, University College, Dublin, Ireland; ${ }^{3}$ Department of Respiratory Medicine, Mater Misericordiae University Hospital, Dublin, Ireland; ${ }^{4}$ School of Pharmacy and Biomolecular Sciences, Royal College of Surgeons in Ireland, Dublin, Ireland; ${ }^{5}$ Department of Haematology, Mater Misericordiae University Hospital, Dublin, Ireland; ${ }^{6}$ School of Medicine, University College, Dublin, Ireland; ${ }^{7}$ Department of Paediatrics, Royal College of Surgeons in Ireland, Dublin, Ireland; ${ }^{8}$ SAS UK Headquarters, Marlow, United Kingdom; ${ }^{9}$ SAS Institute Ltd, Dublin, Ireland; ${ }^{10}$ UCD Centre for Experimental Pathogen and Host Research (CEPHR), Dublin, Ireland; ${ }^{11}$ Department of Infectious Diseases, Mater Misericordiae University Hospital, Dublin, Ireland; ${ }^{12}$ Department of Critical Care Medicine, Mater Misericordiae University Hospital, Dublin, Ireland; ${ }^{13}$ Department of Infectious Diseases, St Vincent's University Hospital, Dublin, Ireland; ${ }^{14}$ Department of Haematology, Rotunda Hospital, Dublin, Ireland; ${ }^{15}$ UCD Institute for Discovery, University College, Dublin, Ireland

Background: Coronavirus disease 2019 (COVID-19), caused by severe acute respiratory syndrome coronavirus 2 (SARS-CoV-2), has affected over 100 million globally to date. Although high rates of venous thromboembolism and evidence of COVID-19-induced endothelial dysfunction have been reported, the precise aetiology of the increased thrombotic risk associated with COVID-19 infection remains to be fully elucidated. Several studies to date suggest a role for platelets in COVID-19-associated thrombosis.

Aims: To assess the impact of COVID-19 on platelet activity and to characterise the proteome of the platelet releasate from COVID-19 patients, compared with healthy controls.

Methods: Ethical approval was granted by the Institutional Review Board of the Mater Misericordiae University Hospital. Haematologic parameters of patients with severe COVID-19 disease (requiring intensive care; $n=34$ ), with non-severe disease (not requiring intensive care; $n=20$ ) and in general medical in-patients without COVID-19 $(n=20)$ were assessed. Platelet function and activity were evaluated by secretion and platelet marker analysis ( $n=6$ each cohort). The proteome of the platelet releasate was assessed using label-free mass spectrometry.

Results: We demonstrated agonist-induced ADP release was 30to-90 fold higher in COVID-19 patients compared with hospitalised 
controls (Fig. 1) and circulating levels of platelet-factor 4 (PF4), soluble P-selectin (sP-selectin) and thrombopoietin (TPO) were also significantly elevated in COVID-19. This study shows that COVID-19 patients possess hyperactive circulating platelets combined with a decreased activation threshold. Mass spectrometry analysis identified over 400 proteins from the releasate of COVID-19 patients and controls, including a multitude of inflammatory, vasoactive and vesicular proteins. The release of a subset of highly-relevant platelet proteins was modified based on the severity of COVID-19 infection.
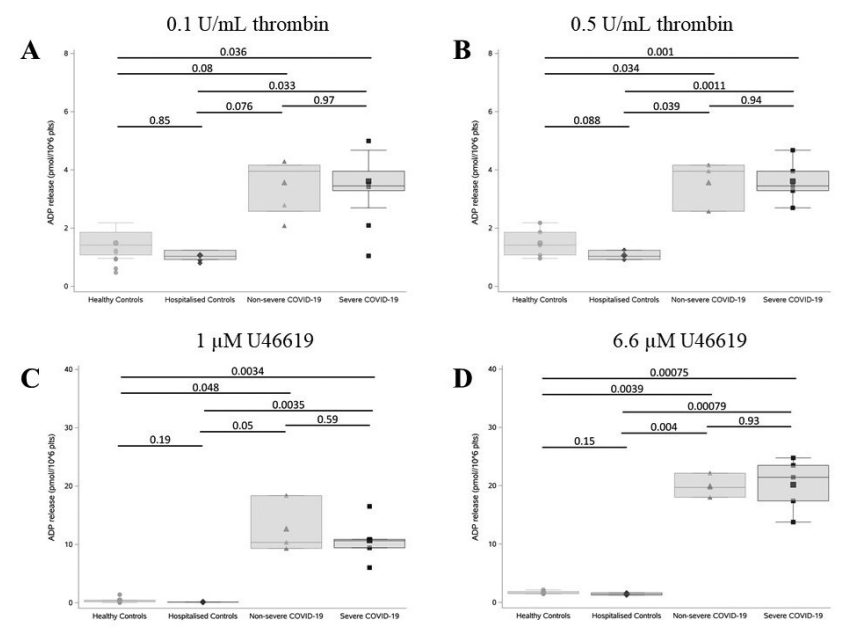

FIGURE 1 Agonist-induced ADP release was dramatically higher in COVID-19 patients compared with non-COVID-19 hospitalized patients

Conclusions: Our data suggest abnormal platelet reactivity may contribute to hypercoagulability in COVID- 19. Platelet releasate analysis confirmed this finding, enabling novel insights into the pathology of COVID-19.

PB0138 | Immunothrombosis Biomarkers for Distinguishing COVID-19 Patients from Non-COVID Septic Pneumonia Patients and for Predicting ICU Mortality

E. Cani ${ }^{1}$; D. Dwivedi ${ }^{1}$; K.L. Liaw ${ }^{2}$; D. Fraser ${ }^{3}$; C. Yeh ${ }^{4}$; C. Martin ${ }^{3}$; M. Slessarev ${ }^{3}$; S. Cerroni ${ }^{1}$; A. Fox-Robichaud ${ }^{1}$; J. Weitz ${ }^{1}$; P. Kim ${ }^{1}$; P. Liaw ${ }^{1}$; DYNAMICS and COVID-BEACONS Investigators

${ }^{1}$ McMaster University, Thrombosis \& Atherosclerosis Research Institute (TaARI), Hamilton, Canada; ${ }^{2}$ McMaster University, Hamilton, Canada; ${ }^{3}$ Western University, London, Canada; ${ }^{4}$ University of Toronto, Toronto, Canada

Background: COVID-19 infection is characterized by immunothrombosis that likely reflects hypercoagulation, endothelial dysfunction, and increased formation of neutrophil extracellular traps.

Aims: In this study, we investigated the utility of immunothrombosis biomarkers to distinguish between COVID-19 patients and
non-COVID septic pneumonia patients. We also investigated the prognostic utility of the biomarkers in predicting ICU mortality in the two patients groups.

Methods: The participants in this study were ICU COVID-19 patients ( $n=14$ ), ICU non-COVID septic pneumonia patients ( $n=19$ ), and age- and sex-matched healthy controls $(n=14)$. Blood samples were collected on Days 4, 7, 10, and/or 14. We measured plasma levels of the following biomarkers: thrombin-antithrombin (TAT) complexes, protein $C$, antithrombin, soluble TM, soluble EPCR, fibrinogen, D-dimer, cell-free DNA (cfDNA), and citrullinated histones (H3-Cit). Data analysis was based on binomial logit models and receiver operating characteristic curve analyses.

Results: We identified 8 biomarkers that distinguish COVID-19 patients from healthy individuals: cfDNA, D-dimer, sEPCR, PC, sTM, fibrinogen, H3-Cit, and TAT complexes. In comparison, 4 biomarkers distinguish COVID-19 from non-COVID septic pneumonia patients: fibrinogen, sEPCR, antithrombin, and cfDNA. With respect to prognosis, the main predictors of ICU mortality differ between the two patient groups. In COVID-19 patients, non-survivors have higher STM and H3-Cit compared with survivors. In septic pneumonia patients, non-survivor patients have lower levels of protein $C$ and higher cfDNA levels compared with survivors. In addition, the most recent values of the biomarkers have stronger prognostic value compared to their Day 1 values.

Conclusions: Our results suggest that fibrinogen, sEPCR, antithrombin, and cfDNA have utility for distinguishing COVID-19 patients from non-COVID septic pneumonia patients. Our data also suggest that the predictors of ICU mortality differ between the two patient groups: STM and H3-Cit for COVID-19 patients, and protein $\mathrm{C}$ and cfDNA for non-COVID septic pneumonia patients. These findings suggests that there are pathophysiological differences between the two patients groups.

PB0139 | Fibrinogen Adsorption on Hemofilter Membranes Associates with Severe COVID-19 and Renal Replacement Therapy Circuit Failure

D. Choi ${ }^{1}$; F. Liu' ${ }^{2}$; A. Borczuk ${ }^{3}$

${ }^{1}$ Division of Hematology and Oncology, New York-Presbyterian Hospital/Weill Cornell Medicine, New York, United States; ${ }^{2}$ Division of Nephrology and Hypertension, New York-Presbyterian Hospital/Weill Cornell Medicine, New York, United States; ${ }^{3}$ Department of Pathology and Laboratory Medicine, New York-Presbyterian Hospital/Weill Cornell Medicine, New York, United States

Background: Continuous renal replacement therapy (CRRT) is an important modality in critical care that utilizes a closed blood circuit with a hollow fiber membrane hemofilter (Figure 1A). CRRT circuit failure is a costly complication with an unclear mechanism, prominently observed during the coronavirus disease 2019 (COVID-19) pandemic. 
Aims: In this retrospective case series, we study the basis of circuit failure, particularly in the setting of COVID-19, via histopathologic examination of hemofilters after use in CRRT circuits.

Methods: Study patients were identified from critically ill patients, admitted to Weill Cornell Medical Center between April and June 2020, who had acute kidney injury requiring CRRT. Circuit failure was defined as the inability of the circuit to provide the prescribed continuous blood flow rate to the patient, necessitating a hemofilter change in advance of its 72-hour lifespan. Hemofilters from failed and functional circuits were formalin-fixed, sectioned and evaluated with histopathologic stains and immunohistochemistry (IHC). Chart review was completed in accordance with IRB-approved protocols.
Results: Circuit failure occurred in 4 out of 6 study patients. Two patients with severe COVID-19 experienced circuit failure despite anticoagulation with argatroban or regional citrate (Table 1). Al hemofilters displayed the accumulation of dense, platelet-rich aggregates at the arterial ends (Figure 1B-C), with a greater volume found in failed hemofilters. The hollow fiber membranes in hemofilters used for patients with severe COVID-19 displayed fibrinogen localized along the blood-facing lumens by IHC (Figure 1D). While hemofilters used for patients with COVID-19 exhibited such luminal fibrinogen staining regardless of circuit function, for patients without COVID-19 this was seen only in failed hemofilters.

TABLE 1 Demographic and laboratory data for study patients. Laboratory values are peak values measured in the $72 \mathrm{~h}$ prior to hemofilter collection. Abbreviations: SARS-CoV-2: severe acute respiratory syndrome coronavirus 2 (testing via nasopharyngeal swab), ESRD: end stage renal disease, DM2: type 2 diabetes mellitus, HTN: hypertension, CAD: coronary artery disease, CKD: chronic kidney disease, CABG: coronary artery bypass grafting

\begin{tabular}{|c|c|c|c|c|c|c|c|c|c|}
\hline $\begin{array}{l}\text { Case } \\
\#\end{array}$ & $\begin{array}{l}\text { Medical } \\
\text { History }\end{array}$ & $\begin{array}{l}\text { Reason for } \\
\text { Admission }\end{array}$ & $\begin{array}{l}\text { SARS- } \\
\text { CoV-2 } \\
\text { RT-PCR }\end{array}$ & $\begin{array}{l}\text { Circuit } \\
\text { Function at } \\
\text { Hemofilter } \\
\text { Collection }\end{array}$ & $\begin{array}{l}\text { Anticoagulation } \\
\text { at Hemofilter } \\
\text { Collection }\end{array}$ & $\begin{array}{l}\text { Neutrophil } \\
\text { Count } \\
\left(10^{3} / \mu \mathrm{l}\right)\end{array}$ & $\begin{array}{l}\text { Platelet } \\
\text { Count } \\
\left(10^{3} / \mu \mathrm{l}\right)\end{array}$ & $\begin{array}{l}\text { D- } \\
\text { dimer } \\
\text { (ng/ } \\
\mathrm{ml} \text { ) }\end{array}$ & $\begin{array}{l}\text { Fibrinogen } \\
\text { (mg/dl) }\end{array}$ \\
\hline 1 & $\begin{array}{l}\text { 67-year-old } \\
\text { woman } \\
\text { with ESRD, } \\
\text { vasculitis }\end{array}$ & $\begin{array}{l}\text { COVID-19 } \\
\text { respiratory } \\
\text { failure }\end{array}$ & Positive & Functional & $\begin{array}{l}\text { Unfractionated } \\
\text { heparin } \\
\text { Regional citrate }\end{array}$ & 20.8 & 333 & 2838 & 473 \\
\hline 3 & $\begin{array}{l}\text { 81-year-old- } \\
\text { man with } \\
\text { ESRD, } \\
\text { HTN, DM2 }\end{array}$ & $\begin{array}{l}\text { COVID-19 } \\
\text { respiratory } \\
\text { failure }\end{array}$ & Positive & Failed & Regional citrate & 14.9 & 39 & 5162 & 283 \\
\hline 5 & $\begin{array}{l}\text { 63-year-old } \\
\text { man with } \\
\text { heart } \\
\text { failure, } \\
\text { CKD }\end{array}$ & $\begin{array}{l}\text { Acute on } \\
\text { chronic } \\
\text { heart failure }\end{array}$ & Negative & Failed & None & 6.8 & 157 & 2813 & 164 \\
\hline 6 & $\begin{array}{l}\text { 61-year-old } \\
\text { man with } \\
\text { alcohol- } \\
\text { related } \\
\text { cirrhosis }\end{array}$ & $\begin{array}{c}\text { Orthotopic liver } \\
\text { transplant }\end{array}$ & Negative & Failed & None & 8.7 & 23 & 9939 & 122 \\
\hline
\end{tabular}


A

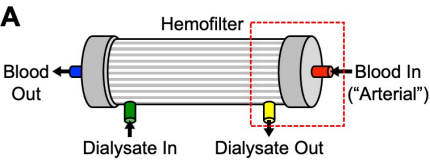

B
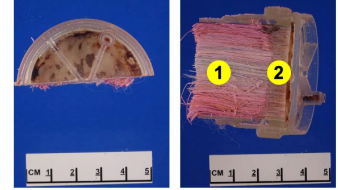

$\begin{array}{cc}\text { Hemofilter } & \begin{array}{c}\text { Hemofilter } \\ \text { Fibers }\end{array} \\ \text { Arterial Side }\end{array}$
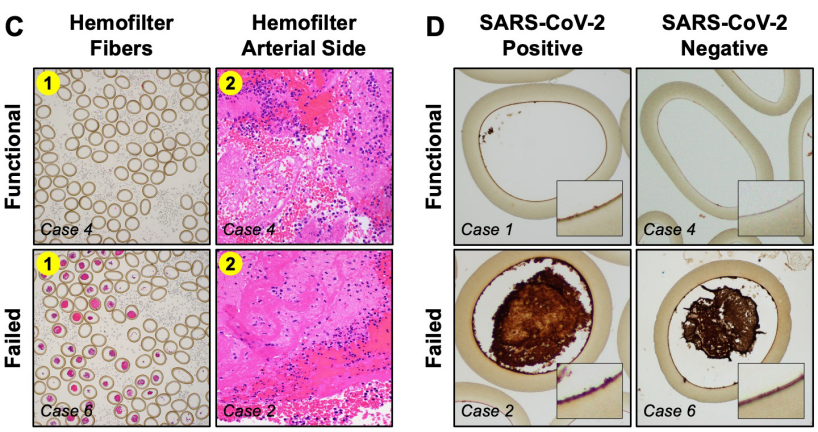

FIGURE 1(A) Schematic representation of a hemofilter, with proximal end prepared for histopathologic analysis boxed in red. (B) Representative gross appearance of the proximal end of a failed hemofilter. Hemofilter fibers (1) and arterial side (2) were examined. (C) Representative hematoxylin and eosin staining of hemofilter cross sections (1) and cellular material collected from the arterial sides (2) of functional and failed hemofilters. (D) IHC for fibrinogen is positive along blood-facing fiber surfaces of hemofilters used for SARS-CoV-2 positive patients and in failed hemofilters, but not in a functional hemofilter used for a SARS-CoV-2 negative patient. Insets show detail under high power

Conclusions: Fibrinogen adsorption on biocompatible hemofilter surfaces exposed to patient blood appears to be an initial event preceding CRRT circuit failure. With COVID-19, this adsorption occurs despite intensified anticoagulation. Further mechanistic study of hemofilter failure may facilitate direct therapeutic strategies to prevent this complication.

PB0141 | Contact System and Intrinsic Pathway Are Activated and Associated with Clinical and Laboratory Outcomes in COVID-19 Patients

M.W Henderson ${ }^{1,2}$; F. Lima ${ }^{3}$; C.R.P. Moraes ${ }^{3}$; A. Ilich ${ }^{2,4}$; S. Huber ${ }^{5}$; S.A.L. Montalvao ${ }^{5}$; M.S. Barbosa ${ }^{3}$; A.C Palma ${ }^{3}$; R.G Ulaf ${ }^{3}$; L.C. Ribeiro ${ }^{3}$; A.F. Bernardes ${ }^{3}$; M.L. Moretti ${ }^{3}$; S. Strickland ${ }^{6}$; J.M Annichino-Bizzacchi ${ }^{3,5}$; F.A. Orsi ${ }^{3}$; E. Mansour ${ }^{3}$; L.A Velloso ${ }^{3,7}$; N.S Key ${ }^{1,2,4}$; E.V De Paula ${ }^{3,5}$

${ }^{1}$ Department of Pathology and Laboratory Medicine University of North Carolina at Chapel Hill, Chapel Hill, United States; ${ }^{2}$ UNC Blood Research Center University of North Carolina at Chapel Hill, Chapel Hill, United States; ${ }^{3}$ University of Campinas, School of Medical Sciences, Campinas, Brazil; ${ }^{4}$ Department of Medicine Division of Hematology University of North Carolina at Chapel Hill, Chapel Hill, United States; ${ }^{5}$ University of Campinas, Hematology and Hemotherapy Center, Campinas, Brazil; ${ }^{6}$ The Rockefeller University, New York, United States; ${ }^{7}$ University of Campinas, Obesity and Comorbidities Research Center, Campinas, Brazil
Background: Hemostasis activation is considered a key pathogenic element of COVID-19, as evidenced by the frequency of lung microvascular thrombosis and of venous thromboembolism. As shown in other conditions, pathways that mediate hemostasis activation during inflammation are not necessarily the same as those that drive hemostasis in non-inflammatory states. In particular, contact system and intrinsic pathway activation have been increasingly explored as potential mediators of hemostasis activation and prothrombotic states observed in both sterile and infectious inflammatory conditions.

Aims: To assess the activation of contact system and intrinsic pathway activation in COVID-19.

Methods: Protease:serpin complexes were measured in plasma from inpatients with COVID-19 and healthy individuals (HI). All samples were collected within $24 \mathrm{~h}$ of COVID-19 diagnosis. Associations with laboratory and clinical markers of hemostasis activation and disease severity were explored. The study was approved by the IRB and all participants provided written informed consent.

Results: In total, 30 patients with COVID-19 and 30 age and sex-matched $\mathrm{HI}$ were enrolled. Main characteristics of the study population are shown in Table 1. As expected, higher levels of classical coagulation activation parameters were observed in COVID-19 patients. Contact system and intrinsic pathway activation in COVID-19 was demonstrated by increased levels of several protease:serpin complexes (Table 2). Interestingly, a consistent and significant association was observed between FIXa:antithrombin complexes with both clinical endpoints (need of ICU admission, length of ICU stay, total length of stay and extent of lung CT alterations), and with laboratory markers of immunothrombosis (neutrophil:lymphocyte ratio, C-reactive protein, D-dimer, PAP, VWF:Ag), as well as with several other activation markers of contact system and intrinsic pathway.

Center, Campinas, Brazil


TABLE 1 Demographic and clinical characteristics of the study population

\begin{tabular}{llll} 
& Patients & Healthy individuals & $P^{* *}$ \\
& $(n=30)$ & $(n=30)$ & - \\
Time from symptom onset, days ${ }^{\pi}$ & $8.1 \pm 2.3$ & - \\
Length of hospital stay, days & $12.9 \pm 9.8$ & - \\
Need for intensive care, yes (\%) & $12 / 30(40 \%)$ & - & $<0.001$ \\
D-dimer, $\mathrm{ng} / \mathrm{mL}^{\pi}$ & $3,609 \pm 14,440$ & $325.0(283.4-343.4)$ & $<0.001$ \\
Fibrinogen, $\mathrm{mg} / \mathrm{dL}^{*}$ & $773.7(696.8-913.1)$ & $132.1 \pm 31.3$ & $<0.001$ \\
FVIII activity, \% & $219.9 \pm 93.3$ & $162.3(95.3-189.5)$ & $<0.001$ \\
VWF:Ag, IU/dL & $248.0(238.2-475.8)$ & $0.35(0.30-0.46)$ & \\
PAP, $\mu \mathrm{gg} / \mathrm{mL}^{*}$ & $1.24(0.99-2.10)$ & & \\
\hline
\end{tabular}

VWF:Ag: Von Willebrand Factor antigen; PAP: plasmin-antiplasmin complexes; ${ }^{\top}$ mean \pm SD; ${ }^{*}$ median (interquartile range); ${ }^{* *}$ Mann-Whitney test or $t$-test for non-parametric and parametric data respectively.

TABLE 2 Contact system and intrinsic pathway protease:serpin complexes in COVID-19

\begin{tabular}{|c|c|c|c|}
\hline & $\begin{array}{l}\text { Patients } \\
(n=30)\end{array}$ & $\begin{array}{l}\text { Healthy individuals } \\
(n=30)\end{array}$ & $P^{* *}$ \\
\hline HMWK (intact), nM" & $80.0 \pm 40.9$ & $43.5 \pm 14.3$ & $<0.001$ \\
\hline Kallikrein-C1 esterase, $\mathrm{nm}^{\pi}$ & $2.57 \pm 1.11$ & $1.64 \pm 0.58$ & $<0.001$ \\
\hline FXIla-C1 esterase, $\mathrm{nM}^{*}$ & $2.60(2.01-3.45)$ & $0.86(0.53-1.52)$ & $<0.001$ \\
\hline FXla-antithrombin, $\mathrm{nM}^{*}$ & $0.54(0.52-0.57)$ & $0.53(0.48-0.58)$ & 0.63 \\
\hline FIXa-antithrombin, nM ${ }^{*}$ & $0.53(0.44-0.69)$ & $0.39(0.32-0.45)$ & $<0.001$ \\
\hline
\end{tabular}

HMWK: high molecular weigth kininogen; ${ }^{\top}$ mean $\pm \mathrm{SD} ;{ }^{*}$ median (interquartile range); ${ }^{* *}$ Mann-Whitney test or $t$-test for non-parametric and parametric data respectively.

Conclusions: Contact system and intrinsic pathway activation contribute to hemostasis activation in COVID-19. The association of FIXa:antithrombin complexes with disease severity suggests that these pathways contribute to the pathogenesis of the prothrombotic state of COVID-19.

\section{PB0142 | Time Course of the Development of} Immunothrombosis during COVID-19 Hospitalization

J. Oliveira $^{1}$; G. Vieira-Damiani ${ }^{1,2}$; G. Locachevic ${ }^{3}$; J. Mariolano ${ }^{3}$;

K. Soares ${ }^{3}$; A. Santos ${ }^{1}$; B. Jacintho ${ }^{1}$; C. Vaz ${ }^{1}$; G. Mesquita ${ }^{1}$;

E. Paula ${ }^{4,5} ;$ F. Orsi $^{4,3}$

${ }^{1}$ School of Medical Sciences, Department of Clinical Medicine, University of Campinas, Campinas, Brazil; ${ }^{2}$ Federal Institute of Education Science and Technology of São Paulo, Capivari-SP. Department of Biology, Campinas, Brazil; ${ }^{3}$ School of Medical Sciences, Department of Clinical Pathology, University of Campinas, Campinas, Brazil; ${ }^{4}$ Hematology and Hemotherapy Center, University of Campinas, Campinas, Brazil; ${ }^{5}$ School of Medical Sciences, University of Campinas, Campinas, Brazil

Background: Hypercoagulability in COVID-19 has been attributed to immunothrombosis, a process that involves the formation of neutrophils extracellular traps (NETs). The moment of the COVID-19 evolution in which immunothrombosis mechanisms are triggered is not established. Aims: To describe the association of the kinetics of NETs release during COVID-19 hospitalization with death and thrombosis.

Methods: We quantified markers of NETs (citrullinated H3) and inflammatory cytokines (TNF- $a$, IL-6) on 4 time points during COVID-19 hospitalization (admission, day 4, day 8 and last day) between May and July 2020. The association between changes in these markers levels and clinical outcomes was determined.

Results: Table 1 summarizes the patients characteristics. 101 patients were included, $59 \%$ were critically ill, $11 \%$ had a thrombotic event and 21\% died. Figure 1 illustrates the changes in citH3, IL-6 and TNF- a levels during hospitalization. IL-6 levels were high on admission in survivors (median 25.32, IQR 24.19-28.15) and nonsurvivors (median 24.19, IQR 12.51-27.19), but gradually decreased after day 4 in survivors. TNF-a levels remained 2 times higher in non-survivors than in survivors during the entire hospitalization period. CitH3 levels were similar between non-survivors and survivors until day 4. On day 8, citH3 increased by 3-fold (median 3.80, IQR 1.98-10.15) in non-survivors and 2-fold (median 2.60, IQR 1.22-5.01) in survivors. While IL-6 and TNF-a levels were similar between patients with and without thrombosis, citH3 levels increased shortly before the occurrence of a thrombotic event. 
TABLE 1 Clinical characteristics at baseline and main outcomes during hospitalization of 101 COVID-19 patients hospitalized between May and July 2020 at the UNICAMP University Hospital in Campinas, Brazil

Patients $(n=101)$

Age in years, median (SD)

$58(13.72)$

Men, $n(\%)$

$63(62.4)$

Days in-hospital, median (SD)

$15(25.68)$

Days in ICU, median (SD)

$20(17.47)$

Obesity (BMI > $\left.30 \mathrm{~kg} / \mathrm{m}^{2}\right), n(\%)$

$27(26.7 \%)$

Diabetes, $n(\%)$

$44(43.6)$

Hypertension, $n(\%)$

$54(53.5)$

Pacients with a thrombotic event $11(10.9)$

during hospitalization, $n$ (\%)

Death during hospitalization, $n(\%) \quad 21(20.9)$
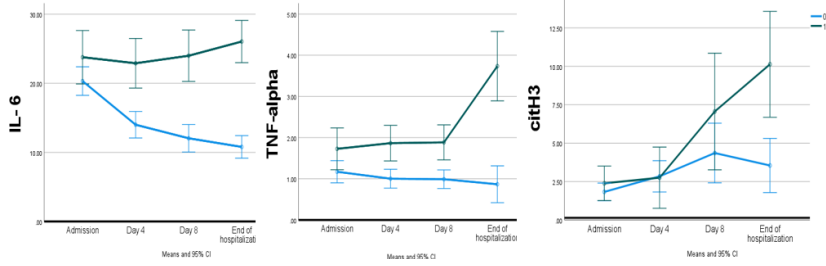

FIGURE 1 Change in citH3, IL- 6 and TNF- a levels during hospitalization by clinical outcomes of 101 COVID-19 patients hospitalized between May and July 2020 at the UNICAMP University Hospital in Campinas, Brazil. Patients are groups as survivors (indicated in blue and "0") and non-survivors (indicated in green and as "1")

Conclusions: Markers of inflammation and immunothrombosis were associated with poor outcomes in COVID-19; however, these disorders were detected in different moments during COVID-19 course. While an increased inflammatory response was observed since the beginning of hospitalization, markers of immunothrombosis arose latter during the course of the disease. Acknowledgment of the timecourse of immunothrombosis development in COVID-19 is important for planning therapeutic strategies against this pathological process.

PB0143 | Whole Blood Assays Demonstrate Reduced Platelet Function in Early SARS-CoV-2 Infection

K. Krauel $^{1,2} ;$ M. Rieder ${ }^{1,2} ;$ N. Baldus ${ }^{1,2} ;$ D. Stallmann ${ }^{1,2}$;

M. Jeserich ${ }^{1,2}$; I. Goller ${ }^{1,2}$; L. Wirth ${ }^{3}$; L. Pollmeier ${ }^{3}$; M. Hofmann ${ }^{4}$;

C. Bode ${ }^{1,2}$; H.-J. Busch ${ }^{5}$; B. Schmid ${ }^{5}$; N. Gauchel ${ }^{1,2}$; A. Lother ${ }^{1,2,3}$;

D. Duerschmied ${ }^{1,2}$

${ }^{1}$ Department of Cardiology and Angiology I, Heart Center, University

of Freiburg, Freiburg, Germany; ${ }^{2}$ Department of Medicine III

(Interdisciplinary Medical Intensive Care), Medical Center, Faculty

of Medicine, University of Freiburg, Freiburg, Germany; ${ }^{3}$ Institute of

Experimental and Clinical Pharmacology and Toxicology, Faculty of
Medicine, University of Freiburg, Freiburg, Germany; ${ }^{4}$ Department of Medicine II, University Hospital Freiburg, Faculty of Medicine, University of Freiburg, Freiburg, Germany; ${ }^{5}$ Department of Emergency Medicine, University Hospital of Freiburg, Faculty of Medicine, University of Freiburg, Freiburg, Germany

Background: Patients with COVID-19 frequently present with hypercoagulability. It has been shown that an increased reactivity of platelets might contribute to this prothrombotic disposition. However, previous studies were mostly performed with purified platelets and focused on severe COVID-19 patients.

Aims: To assess platelet function in early SARS-CoV-2 infection using whole blood assays.

Methods: We performed whole blood impedance aggregometry in 20 SARS-CoV-2 positive patients and 58 SARS-CoV-2 negative patients from an all-comers cohort presenting with suspected or confirmed COVID-19 at our emergency department. 15 healthy volunteers were included as controls. Thrombin receptor activating peptide 6 (TRAP-6), adenosine diphosphate (ADP) and arachidonic acid (AA) were used as agonists. In a subset of SARS-CoV-2 positive patients $(n=14)$, SARS-CoV-2 negative patients $(n=7)$ and healthy controls $(n=7)$, we investigated the expression of typical surface platelet activation markers (P-selectin, active GPIIb/IIla) using flow cytometry. One-way ANOVA and Bonferroni's multiple comparisons test were used for statistical analyses. The study complies with the Declaration of Helsinki and was approved by the ethical committee of the University of Freiburg. Written informed consent was obtained from all participants.

Results: Surprisingly, platelet aggregation was significantly reduced in SARS-CoV-2 positive patients in response to all agonists tested compared to SARS-CoV-2 negative patients (TRAP: $P<0.01$; ADP: $P<0.05 ; A A: P<0.01)$. The difference was even more pronounced when comparing with healthy controls (TRAP: $P<0.0001$; ADP: $P<0.001 ;$ AA: $P<0.001)$. P-selectin surface expression was not different between groups. However, GPIIb/IIla activation was decreased in SARS-CoV-2 positive patients compared to healthy controls (TRAP: $P<0.01$; ADP: $P<0.05$ ).

Conclusions: In contrast to previous findings, our study revealed decreased platelet reactivity in patients with COVID-19. This discrepancy could be due to the use of different methodological approaches for testing platelet function and different severity levels of COVID-19 patients. We conclude that anti-platelet medication is not required in early SARS-CoV-2 infection. 
PB0144 | Association of the Angiopoietin/Tie2 and VEGF-A Pathways with clinical and Laboratory Markers of Disease Severity in COVID-19

C.R.P. Moraes $^{1}$; F. Lima ${ }^{1}$; I.T. Borba Junior ${ }^{1}$; M.S. Barbosa ${ }^{1}$;

L.C Ribeiro ${ }^{1}$; A.C Palma ${ }^{1}$; R.G Ulaf ${ }^{1}$; A.F. Bernardes ${ }^{1}$; E. Mansour ${ }^{1}$;

F.T.M. Costa ${ }^{2}$; F.A. Orsi ${ }^{1}$; J.M Annichino-Bizzacchi ${ }^{1,3}$; M.L. Moretti ${ }^{1}$; L.A Velloso ${ }^{1,4}$; E.V. De Paula ${ }^{1,3}$

${ }^{1}$ University of Campinas, School of Medical Sciences, Campinas, Brazil; ${ }^{2}$ University of Campinas, Institute of Biology, Campinas, Brazil;

${ }^{3}$ University of Campinas, Hematology and Hemotherapy Center, Campinas, Brazil; ${ }^{4}$ University of Campinas, Obesity and Comorbidities Research Center, Campinas, Brazil

Background: Endothelial barrier (EB) disruption is an important part of immunothrombosis, allowing the access of leukocytes to in flamed tissues. Pathways involving angiopoietin (Ang) 1 and 2 and their receptor Tie2, and VEGF-A/VE-cadherin (VEC) are key regulators of EB integrity. While the association of these mediators with sepsis severity have been known for more than 15 years, it was only recently that their role in coagulation activation was described. Moreover, these proteins also mediate angiogenesis, which has been shown to be upregulated in COVID-19.

Aims: To measure circulating levels of key mediators of Ang/Tie2 and VEGF-A pathways in COVID-19 patients, and to explore their association with disease severity and hemostatic activation.

Methods: Samples were obtained from patients admitted to a COVID-19 ward, within $24 \mathrm{~h}$ from COVID-19 confirmation. EB mediator levels were measured by immunological methods (Elisa or multiplex assays). The study was approved by the IRB and all participants provided written informed consent.

Results: Data were obtained from 30 patients and 30 age and sexmatched healthy individuals. Mean length of hospital stay (LOS) was $12.9 \pm 9.8$ days respectively, twelve patients $(40 \%)$ required intensive care (ICU), and 28/30 patients survived. Mean D-dimer was $3,609 \pm 14,440 \mathrm{ng} / \mathrm{mL}$. Levels of EB mediators are shown in Table 1. Associations between these parameters with relevant clinical and laboratory markers of disease severity are shown in Table 2.

TABLE 1 Circulating levels of endothelial barrier integrity mediators in COVID-19

\begin{tabular}{llll} 
& Patients & Healthy individuals & $\boldsymbol{P}^{* *}$ \\
& $(n=30)$ & $(n=30)$ & $<0.0001$ \\
Angiopoietin-1, pg/mL & $463.2 \pm 194.6$ & $237.4 \pm 104.9$ & $<0.0001$ \\
Angiopoietin-2, pg/mL & $1,926(1,275-3,134)$ & $1,215(9-1,444)$ & $<0.0001$ \\
Tie2, pg/mL & $10,753 \pm 2,377$ & $8,603 \pm 1,851$ & 0.001 \\
VE-cadherin, $\mathrm{ng} / \mathrm{mL}$ & $1,234 \pm 318$ & $1,539 \pm 363$ & $<0.0001$ \\
VEGF-A, pg/mL & $94.7(73.4-116.0)$ & $45.9(39.7-57.0)$ & \\
\hline
\end{tabular}

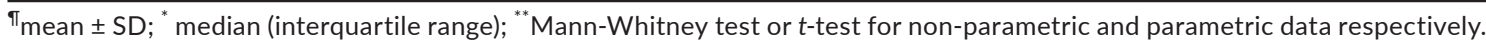

TABLE 2 Correlation of mediators of EB integrity with clinical and laboratory markers of coagulation activation and disease severity

\begin{tabular}{|c|c|c|c|}
\hline & Marker of coagulation, fibrinolysis or EC activation & Correlation coefficient ${ }^{*}$ & $P$ \\
\hline \multirow[t]{8}{*}{ Angiopoietin-1 } & FVIII:C & 0.329 & 0.01 \\
\hline & FIX: & 0.371 & 0.004 \\
\hline & FXII:C & 0.301 & 0.02 \\
\hline & Fibrinogen & 0.568 & $<0.0001$ \\
\hline & VWF:Ag & 0.478 & $<0.0001$ \\
\hline & UPAR & 0.725 & $<0.0001$ \\
\hline & P-selectin & 0.383 & $<0.0001$ \\
\hline & PAI-1 & 0.373 & 0.003 \\
\hline \multirow[t]{8}{*}{ Angiopoietin-2 } & ICU stay, days & 0.409 & 0.03 \\
\hline & Extension of lung disease (CT score) & 0.404 & 0.02 \\
\hline & aPTT & 0.331 & 0.01 \\
\hline & Fibrinogen & 0.371 & 0.004 \\
\hline & VWF:Ag & 0.514 & $<0.0001$ \\
\hline & UPAR & 0.560 & $<0.0001$ \\
\hline & P-selectin & 0.414 & 0.001 \\
\hline & PAI-1 & 0.446 & $<0.0001$ \\
\hline \multirow[t]{6}{*}{ Soluble Tie2 } & Platelet count & -0.336 & 001 \\
\hline & D-dimer & 0.467 & $<0.0001$ \\
\hline & VWF:Ag & 0.466 & $<0.0001$ \\
\hline & UPAR & 0.315 & 0.01 \\
\hline & P-selectin & 0.315 & 0.01 \\
\hline & PAI-1 & 0.323 & 0.01 \\
\hline
\end{tabular}


Marker of coagulation, fibrinolysis or EC activation

Soluble

VE-cadherin

Fibrinogen

FVIII:C

FIX:C

VEGF-A

Extension of lung disease (CT score)
Hemoglobin
FVIII:C
FIX:C
Fibrinogen
VWF:Ag
UPAR
P-selectin
PAI-1

$\begin{array}{ll}\text { Correlation coefficient }^{*} & P \\ -0.458 & 0.02 \\ -0.305 & 0.02 \\ -0.485 & <0.0001 \\ 0.418 & 0.02 \\ -0.545 & 0.002 \\ 0.515 & <0.0001 \\ 0.455 & <0.0001 \\ 0.675 & <0.0001 \\ 0.579 & <0.0001 \\ 0.852 & <0.0001 \\ 0.666 & <0.0001 \\ 0.600 & <0.0001\end{array}$

FVIII:C: factor VIII activity measured by one-stage coagulometric assay; VWF:Ag: Von Willebrand Factor antigen; uPAR: urokinase-type plasminogen activator receptor; PAI-1: plasminogen activator inhibitor-1; *correlation coefficients were Pearson or Spearman for parametric and non-parametric distributed data respectively.

Conclusions: All mediators of EB disruption were significantly elevated in COVID-19 patients. In addition, these mediators were consistently associated with proteins involved in immunothrombosis, in particular with fibrinogen, VWF:Ag, uPAR, PAI-1 and P-selectin. Clinically significant associations were observed between Ang-2 and VEGF-A and extension of lung disease (for both) and ICU stay (for VEGF-A). Additional studies are warranted to explore the crosstalk between Ang/Tie2 and VEGF-A pathways with hemostasis in COVID-19.

PB0145 | Fibrin Clot Characteristics and Anticoagulant Response in a SARS-CoV-2 Infected Endothelial Cell Model

C. McCafferty ${ }^{1}$; L. Lee ${ }^{2}$; T. Cai ${ }^{1}$; S. Praporski ${ }^{1}$; V. Karlaftis ${ }^{1}$; C. Attard ${ }^{1}$; S. Van Den Helm ${ }^{1}$; N. Letunica ${ }^{1}$; J. Stolper ${ }^{1}$; D. Elliott ${ }^{1}$; K. Subbarao ${ }^{3,2}$; P. Monagle ${ }^{4,5,1}$; V. Ignjatovic ${ }^{1,4}$

${ }^{1}$ Murdoch Children's Research Institute, Melbourne, Australia;

${ }^{2}$ Department of Microbiology, University of Melbourne at the Peter Doherty Institute for Infection and Immunity, Melbourne, Australia; ${ }^{3}$ WHO Collaborating Centre for Reference and Research on Influenza, University of Melbourne at The Peter Doherty Institute for Infection and Immunity, Melbourne, Australia; ${ }^{4}$ Department of Paediatrics, The University of Melbourne, Melbourne, Australia; ${ }^{5}$ Department of Clinical Haematology, The Royal Children's Hospital, Melbourne, Australia

Background: SARS-CoV-2 infection is associated with risk of thrombosis however not all SARS-CoV-2 positive patients have equal risk of developing thrombi. Evidence of COVID-19 associated coagulation has not been demonstrated in children, whilst adults with a known vasculopathy, have been identified as high-risk. Currently the mechanism through which SARS-Cov-2 infection promotes thrombosis is unknown. Aims: We hypothesise that plasma factors interact with SARS-CoV-2 infected endothelium and play a significant role in this process.

Methods: We developed a novel tool for modelling coagulation in vitro. Human Umbilical Vein Endothelial Cells (HUVECs) were infected with SARS-CoV-2; Uninfected cells and cells infected with Influenza A/Singapore/6/86 were used as controls. Wells were incubated with recalcified plasma from healthy children, healthy adults or vasculopathic adults (previous stroke) for $2 \mathrm{~h}$. This experiment was repeated in the presence of anticoagulants: Bivalirudin $(0.005$ $\mu \mathrm{g} / \mu \mathrm{L})$, Defibrotide $(0.02 \mu \mathrm{g} / \mu \mathrm{L})$ and therapeutic and prophylactic doses of Low Molecular Weight Heparin (1 U/mL, $0.5 \mathrm{U} / \mathrm{mL}$ ) and Unfractionated Heparin $(0.7 \mathrm{U} / \mathrm{mL}, 0.4 \mathrm{U} / \mathrm{mL})$.

Fibrin clots were studied using scanning electron microscopy and fibrin characteristics were quantified manually using ImageJ software by two blinded reviewers.

Results: Fibrin strands from child plasma had the largest thickness $(0.32 \mu \mathrm{m})$, followed by adult plasma $(0.30 \mu \mathrm{m})$ and finally vasculopath plasma $(0.27 \mu \mathrm{m})$.

Defibrotide and Bivalirudin had no effect, whilst Low Molecular Weight Heparin and Unfractionated Heparin completely eliminated clot formation at therapeutic and prophylactic doses.

Conclusions: We demonstrate that SARS-CoV-2 infection of endothelial cells promotes thrombosis differently in plasma samples from children, adults and vasculopaths. Thinner fibrin strands in vasculopathy suggests formation of clots that are more resistant to lysis. Our results support that children are resistant to COVID-19 associated coagulation, and that adults with vasculopathies are an at-risk population. This novel cell-culture model for visualising coagulation is a valuable step towards incorporating the endothelium into haemostatic research.

\section{PB0146 | Increased Thrombin Generation in COVID-19 is} Mediated by Platelet-dependent and -Independent Mechanisms

A.S. Eustes $^{1}$; J. Swamy ${ }^{1}$; S. Kudchadkar ${ }^{1}$; M. Jensen ${ }^{1}$; K.M. Wilson ${ }^{1}$; U. Perepu ${ }^{1} ;$ F. Miller ${ }^{2}$; S.R. Lentz ${ }^{1}$; S. Dayal ${ }^{1}$

${ }^{1}$ University of lowa, lowa City, United States; ${ }^{2}$ Duke University, Durham, United States

Background: Severe COVID-19 leads to inflammation and coagulopathy with progression to multiple organ failure. Several prothrombotic mechanisms have been proposed, including platelet hyperactivation, elevation in procoagulant microvesicles and tissue factor (TF), and NETosis with release of citrullinated histones. The 
contribution of these factors to enhanced thrombin generation in COVID-19 remains elusive.

Aims: We hypothesized that plasma from COVID-19 patients enhances thrombin generation through both platelet-dependent and -independent mechanisms.

Methods: Platelet poor plasma (PPP) was isolated from 136 COVID-19 patients enrolled in a multicenter randomized clinical trial comparing standard prophylactic dose to intermediate dose enoxaparin in hospitalized patients with severe COVID-19 (NCT04360824). Samples were also collected from healthy control subjects $(n=33)$. Thrombin generation was triggered by either tissue factor (TF), phospholipids, or both, and was measured in a calibrated automated thrombogram. Citrullinated histone $\mathrm{H} 3$ ( $\mathrm{H} 3 \mathrm{Cit}$ ) and tissue factor pathway inhibitor (TFPI) were measured by immunoassay.

Results: When thrombin generation in PPP was triggered with TF and phospholipids, samples from COVID-19 patients exhibited higher en dogenous thrombin potential (ETP), peak thrombin, and velocity index, and a delayed lag time compared to controls. The delayed lag time correlated with increased levels of TFPI in COVID-19 samples $(P=0.01)$. Significant increases in thrombin generation in COVID-19 samples also occurred in response to phospholipids or TF alone, suggesting the presence of endogenous TF-positive microvesicles in COVID-19 plasma. To examine platelet-dependent thrombin generation, control platelets were incubated with PPP prior to measurement of thrombin generation triggered by TF. Significantly higher peak thrombin was observed with platelets incubated with plasma from COVID-19 patients; this effect was decreased by the histone aptamer KU7, suggesting histone-mediated platelet-dependent thrombin generation.

Conclusions: These findings suggest that in COVID-19, histones mechanistically drive increased thrombin generation via platelets; and microvesicles and TF contributes to thrombin generation in a platelet-independent manner.

PB0147 | Thrombotic Events in COVID-19 Are Associated with a Lower Use of Prophylactic Anticoagulation Before Hospitalization and Followed by Decreases in Platelet Reactivity

C. Clark ${ }^{1}$; B. Jukema ${ }^{1}$; A. Barendrecht ${ }^{1} ;$ J. Spanjaard ${ }^{2}$; N. Jorritsma ${ }^{2}$; S. Smits ${ }^{1}$; S. de Maat ${ }^{1}$; C. Seinen ${ }^{1}$; S. Sebastian ${ }^{1}$; A. Koekman ${ }^{1}$; A. van Wesel ${ }^{1}$; H. van Goor ${ }^{3}$; R. Spijkerman ${ }^{2,4}$; S. Bongers ${ }^{2,5}$; E. van der Vries $^{1}$; S. Nierkens ${ }^{2}$; M. Boes ${ }^{2}$; L. Koenderman ${ }^{2,4}$; K. Kaasjager ${ }^{3}$; C. Maas ${ }^{1}$

${ }^{1}$ Department of CDL research, University Medical Center Utrecht, Utrecht University, Utrecht, Netherlands; ${ }^{2}$ Center for Translational Immunology (CTI), University Medical Center Utrecht, Utrecht University, Utrecht, Netherlands; ${ }^{3}$ Department of Internal Medicine, University Medical Center Utrecht, Utrecht University, Utrecht, Netherlands; ${ }^{4}$ Department of Respiratory Medicine, University Medical Center Utrecht, Utrecht University, Utrecht, Netherlands; ${ }^{5}$ Department of Trauma Surgery, University Medical Center Utrecht, Utrecht University, Utrecht, Netherlands

Background: Coronavirus disease of 2019 (COVID-19) is associated with a prothrombotic state and high incidence of thrombotic events
(TE). Platelet hyperreactivity has been reported in COVID-19 patients and might contribute to TE development.

Aims: To study platelet reactivity in hospitalized COVID-19 patients and to determine a possible association with the clinical outcomes thrombosis and all-cause mortality.

Methods: 79 hospitalized COVID-19 patients were enrolled in this retrospective cohort study and provided blood samples in which platelet reactivity in response to stimulation with ADP and TRAP-6 was determined using flow cytometry. Clinical outcomes included thrombotic events, and all-cause mortality.

Results: The incidence of TE in this study was $28 \%$ and all-cause mortality $16 \%$. Patients that developed a TE were younger than patients that did not (median age of 55 versus 70 years; adjusted odds ratio (AOR), 0.96 per 1 year of age [95\% $\mathrm{Cl}, 0.92-1.00$ ]; $P=0.042$ ). Furthermore, patients using preexisting thromboprophylaxis were less likely to develop a TE than patients that were not (18\% versus 54\%; adjusted odds ratio, 0.18 [95\% $\mathrm{Cl}, 0.04-0.82]$; $P=0.026$ ). Conversely, having asthma strongly increased the risk on TE development (adjusted odds ratio, 6.4 [95\% Cl, 1.17-35.4]; $P=0.032$ ). No significant differences in baseline P-selectin expression or platelet reactivity were observed between the COVID-19 positive patients $(n=79)$ and COVID-19 negative hospitalized control patients $(n=24)$, nor between COVID-19 survivors or non-survivors. However, patients showed decreased platelet reactivity in response TRAP-6 following TE development compared to patients without TE.

Conclusions: We observed an association between the use of preexisting thromboprophylaxis and a decreased risk of TE during COVID-19. This suggests that these therapies are beneficial for coping with COVID-19 associated hypercoagulability. This highlights the importance of patient therapy adherence. We observed lowered platelet reactivity after the development of TE, which might be attributed to platelet desensitization during thromboinflammation.

\section{PB0148 | Targeting Thymidine Phosphorylase May Be a Novel} Therapy for COVID-19

H. Yue; K. Denning; W. Li

Marshall University/Joan C. Edwards School of Medicine, Huntington, United States

Background: Coronavirus disease 2019 (COVID-19), caused by the severe acute respiratory syndrome coronavirus 2 (SARS-COV-2), is a human respiratory disease. Hitherto, there is no effective treatment has been established. Patients with cardiovascular or diabetes comorbidities are a high-risk cohort. COVID-19 is accompanied by excessive systemic thrombotic events, but the mechanism is not yet known. Our recent studies found that thymidine phosphorylase (TYMP) plays an important role in platelet activation, thrombosis, and TYMP expression is increased in obese and diabetic patients.

Aims: To test the hypothesis that TYMP participates in the host response to SARS-CoV-2.

Methods: By co-transfection of SARS-CoV-2 spike protein (SP) and human ACE2 into Cos-7 cells, we examined how SP 
regulates ACE2 and TYMP expression. By using data provided by the MGH (Massachusetts General Hospital) Emergency Department COVID-19 Cohort with Olink Proteomics we analyzed the correlation between plasma TYMP and the severity of COVID-19.

Results: Overexpression of $\mathrm{S}$ protein or its receptor-binding domain led to ACE2 shedding into a different size. S protein also increased TYMP expression in Cos-7 cells. In comparing to COVID-19 negative patients, plasma TYMP in COVID-19 patients was significantly increased in a severity-dependent manner. The increase of plasma TYMP was earlier than the increase of $C$ creative protein, a predictive factor for inflammation and future risk of cardiovascular events. The increase of TYMP is positively associated with plasma D-dimer and lactate dehydrogenase, the presence of pulmonary symptoms, as well as IFN- $\gamma$ and IFN- $\lambda$, especially IFN- $\lambda$. We also found that TYMP is highly expressed in mouse asthmatic lungs and human type II alveolar epithelial cells and bronchial epithelium, which mediate SARS-CoV-2 entry.

Conclusions: TYMP is positively correlated with COVID-19 acuity and COVID-19-associated thrombotic event, inflammation, and organ damages. TYMP could be an acuity marker for COVID-19 diagnosis. Targeting TYMP could be a novel effective medicine for COVID-19.

PB0149 | Distinctive Biomarker Features in the Endotheliopathy of Covid-19 and Septic Syndromes

A.B. Moreno-Castaño ${ }^{1,2,3} ;$ S. Fernandez ${ }^{4}$; M. Palomo ${ }^{5,2,3}$; J. Martinez-Sanchez ${ }^{5,2,3}$; S. Torramade-Moix ${ }^{2,3}$; A. Tellez ${ }^{4}$; H. Ventosa ${ }^{4}$; F. Segui ${ }^{4}$; G. Escolar ${ }^{1,2,3}$; E. Carreras ${ }^{5,3}$; J.M. Nicolas ${ }^{4,6}$; E. Richardson ${ }^{7}$; D. Garcia-Bernal ${ }^{8}$; C. Carlo-Stella ${ }^{9}$; J.M. Moraleda ${ }^{8}$; P. Richardson ${ }^{10}$; M. Diaz-Ricart ${ }^{1,2,3}$; P. Castro ${ }^{4,2,6}$ ${ }^{1}$ Hematopathology, Pathology Department, CDB, Hospital Clinic, Barcelona, Spain; ${ }^{2}$ Institut d'Investigacions Biomèdiques August Pi i Sunyer (IDIBAPS), University of Barcelona, Barcelona, Spain; ${ }^{3}$ Barcelona Endothelium Team, Barcelona, Spain; ${ }^{4}$ Medical Intensive Care Unit, Hospital Clinic, Barcelona, Spain; ${ }^{5}$ Josep Carreras Leukaemia Research Institute, Hospital Clinic, University of Barcelona, Barcelona, Spain; ${ }^{6}$ School of Medicine, University of Barcelona, Barcelona, Spain; ${ }^{7}$ Frank H. Netter M.D. School of Medicine at Quinnipiac University, New Haven, United States; ${ }^{8}$ Hematopoietic Transplant and Cellular Therapy Unit, Instituto Murciano de Investigación Biosanitaria IMIB-Arrixaca, Virgen de la Arrixaca University Hospital, University of Murcia, Murcia, Spain; ${ }^{9}$ Humanitas Clinical and Research CenterIRCCS, Rozzano-Milano,Italy, Milano, Italy; ${ }^{10}$ Dana-Farber Cancer Institute, Jerome Lipper Multiple Myeloma Center, Harvard Medical School, Boston, United States
Background: Endotheliopathy is a key element in COVID-19 pathophysiology, contributing to both morbidity and mortality. Biomarkers distinguishing different COVID-19 phenotypes from sepsis syndromes remain poorly understood.

Aims: We aimed to characterize circulating biomarkers of endothelial damage in different COVID-19 clinical disease stages in comparison to sepsis syndromes and normal volunteers.

Methods:
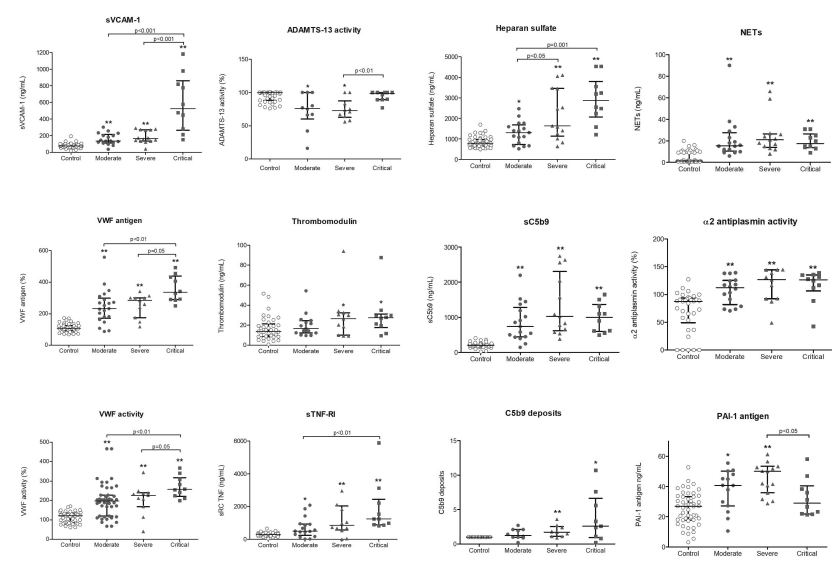

Patients with COVID-19 pneumonia $(n=49)$ were included and classified into moderate, severe or critical (life-threatening). Patients with other septic syndromes: sepsis (S, $n=7$ ), septic shock (SS, $n=14$ ) and patients with non-infectious systemic inflammatory response syndrome (NI-SIRS, $n=7$ ) were also included. Plasma samples were collected within 48-72 $\mathrm{h}$ of hospitalization to analyze endothelial activation markers, including sVCAM-1, VWF, ADAMTS-13 activity, thrombomodulin (TM) and soluble TNF receptor I (sTNFRI); heparan sulfate (HS) for endothelial glycocalyx degradation; C5b9 deposits on endothelial cells in culture and soluble C5b9 for complement activation; circulating dsDNA for neutrophil extracellular traps (NETs) presence, and a2-antiplasmin and PAI-1 as parameters of fibrinolysis. Results were compared to healthy donors as controls $(n=45)$; all three COVID-19 groups and septic shock patients.

Results: All analyzed biomarkers were increased in COVID-19 patients vs. controls $(P<0.001)$, except for ADAMTS-13 activity which was normal in both groups. Correlation with disease severity was observed for sVCAM-1, VWF, sTNFRI and HS $(P<0.05)$. SS patients showed significantly higher levels of VWF, TM, sTNFRI and NETS, with reduced ADAMTS-13 activity $(P<0.05)$. Importantly, a2-antiplasmin activity was higher in critical COVID-19 $(P<0.001)$ vs. SS. 

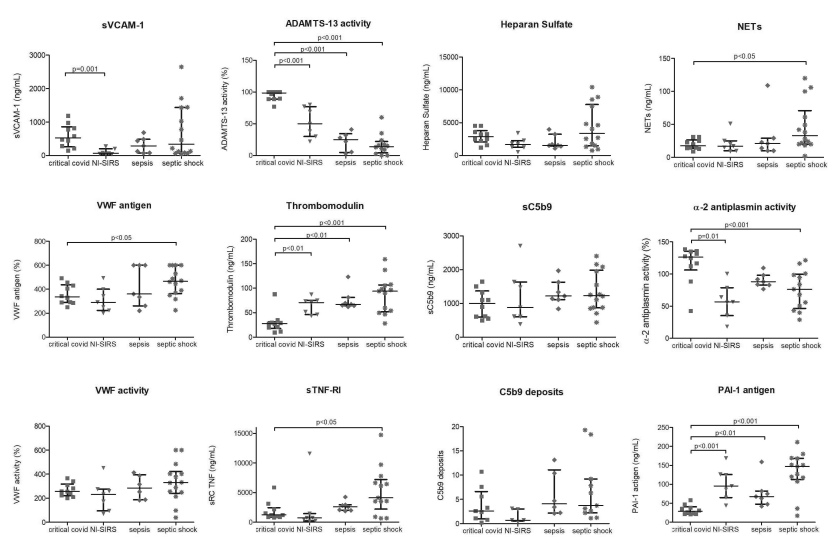

\section{FIGURE 1}

Conclusions: COVID-19 patients present increased circulating endothelial stress products, complement activation and fibrinolytic dysregulation, associated with disease severity. COVID-19 endotheliopathy differs from SS, in which endothelial damage is also a critical feature of pathobiology. These biomarker profiles may inform therapeutic intervention in COVID-19.

This study was supported by Fundació Clínic, Barcelona (HCB/2020/0401) and Jazz Pharmaceuticals (IST-16-10355).

PB0150 | Multi-organ Vascular Defects and General Morphological Changes in a Murine Model of SARS CoV-2 Infection

M. Paidas ${ }^{1}$; A. Mohamed ${ }^{1}$; A. Saad ${ }^{2} ;$ A. Jayakumar ${ }^{1}$

${ }^{1}$ Department of Obstetrics, Gynecology and Reproductive Sciences, University of Miami, Miami, United States; ${ }^{2}$ Department of Pathology, University of Miami, Miami, United States

Background: Infection with SARS-CoV-2, the virus responsible for the Covid-19 global pandemic, causes a respiratory illness and can severely impact other organ systems, possibly precipitated by cytokine storm, septic shock, thrombosis and oxidative/nitrative stress. SARS CoV-2 infected individuals may be asymptomatic or may experience mild, moderate, or severe symptoms with or without pneumonia.

Aims: The mechanisms by which SARS-CoV-2 infect humans is largely unknown.

Methods: Murine Hepatitis Virus 1 infusion in mice was used in this study which is a highly relevant and surrogate animal model for SARS-CoV-2.

Results: We found that MHV-1-induced animal death (60\%), as well as weight loss. MHV-1-infected mice at day 7-8 showed thrombi of pulmonary vessels, arterial endothelial swelling, inflammation and granular degeneration of vascular endothelial cells, and margination of leukocytes into the interstitium. Furthermore, peribronchiolar interstitial infiltration, bronchiolar epithelial cell necrosis and intra-alveolar necrotic debris, alveolar exudation (surrounding alveolar walls have capillaries that are dilated and filled with RBCs), mononuclear cell infiltration, hyaline membrane formation and interstitial edema were also observed in these mice. When compared to those uninfected, infected mice showed severe liver vascular congestion, luminal thrombosis of portal and sinusoidal vessels, hepatocytes degeneration, cells necrosis and hemorrhagic changes. Acute tubular necrosis and thrombosis were observed in the kidney. The heart also showed thrombosis, severe interstitial edema, vascular congestion and dilation, and red blood cells extravasation into interstitium.

Conclusions: Our findings suggest that the widespread thrombosis seen in highly relevant and surrogate animal model for SARS-CoV-2 mimics the reported findings in SARS-CoV-2-infected humans and represents an attractive, safer, animal model to study SARS-CoV-2 infection, pathophysiologic mechanisms and potential therapeutic interventions.

PB0152 | Identifying Tools to Track Hypercoagulability in COVID-19 Patients. Exploring Global Haemostasis (ROTEM) and Neutrophil Extracellular Traps (NETs) Immunoassays

S. Stanford ${ }^{1}$; C. Rea ${ }^{2}$; A. Roy ${ }^{3}$; B. Harris ${ }^{3}$; A. Ashton ${ }^{3}$; S. Mangles ${ }^{3}$; T. Everington ${ }^{3}$; K. Chandrakumaran ${ }^{3}$; E. Arbuthnot ${ }^{3}$; T. Cecil ${ }^{3}$ ${ }^{1}$ Hampshire Hospitals NHS Foundation Trust, Basingstoke, United Kingdom; ${ }^{2}$ Kings College Hospital NHS Foundation Trust, London, United Kingdom; ${ }^{3}$ Hampshire Hospitals NHS Foundation Trust, Hampshire, United Kingdom

Background: Synergy between coagulation and inflammation is clear in SARS-CoV-2 infection making patients susceptible to thrombosis. Numerous articles describe the prognostic value of individual or combined laboratory biomarkers. However, there is no single test that can be used to provide a composite assessment to easily identify and track emerging hypercoagulability. We present two methods via which a single assessment could be used as a global measure of inflammation (NETs) and coagulation (ROTEM).

Aims: Investigate whether patients with COVID-19 demonstrate ROTEM parameters and or elevated NETs markers (H3.1nucleosomes), indicating hypercoagulability, early in admission. (ii) Whether patients admitted to ITU have a significantly higher Maximum Clot Firmness (MCF) and H3.1-nucleosomes compared to those on the ward.

Methods: Consenting patients were tested for ROTEM on admission and daily for 10 days and weekly thereafter until discharge from hospital. H3.1-nucleosomes were measured at all time points in 3 patients ( 1 admitted directly to ITU, 1 to ITU during their hospital stay and 1 remaining on the ward) using Nu.Q ${ }^{\mathrm{TM}}$ H3.1-nucleosome ELISA (Belgian Volition SRL, Isnes, Belgium) according to the manufacturer's instruction.

Results: ROTEM demonstrated a hypercoagulable state compared to controls on admission for FIBTEM CT $(P<0.001)$ and MCF $(P<0.001)$, EXTEM CT, CFT, MCF and $M L(P<0.002)$ and INTEM 
CT $(P<0.028)$, MCF $(P<0.004)$ and $M L(P<0.001)$. In our cohort MCF ( $P=0.849)$ was not significantly higher in those admitted to ITU compared to those on the ward however moderate significance was observed in the EXTEM CFT $(P=0.051)$ and EXTEM ML $(P=0.056)$. In the 3 assessed with serial $\mathrm{H} 3.1$, the values closely track their clinical course (Fig 1).

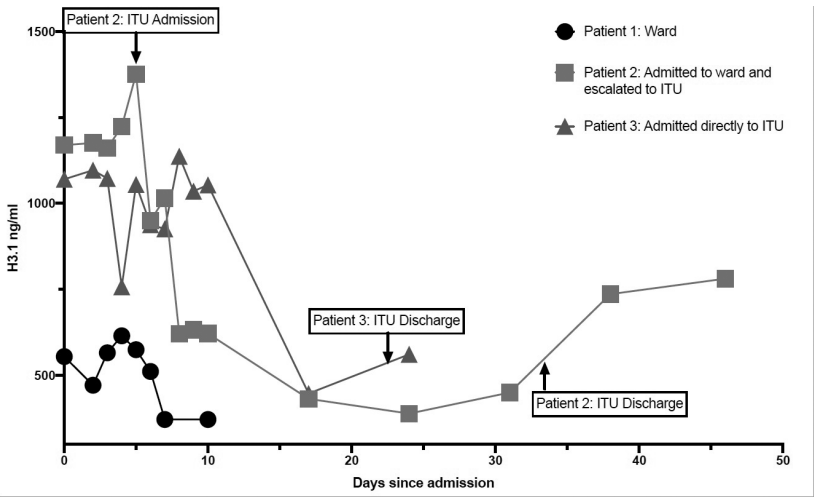

FIGURE 1 Serial H3.1-nucleosomes measurements in 3 patients: One admitted to directly to ITU, 1 admitted to ITU during hospital stay and 1 on the ward

Conclusions: ROTEM measures a hypercoagulable state compared to controls with increased MCF and hypofibrinolysis. Although this is a small, exploratory study, the H3.1 nucleosome findings suggest that it may be able to risk stratify on admission and track clinical course.

PB0153 | Tissue Factor-stimulated Plasma Thrombin Generation Is Enhanced in Hospitalised Patients with COVID-19 Despite Pharmacological Thromboprophylaxis

S. Kelliher $^{1,2}$; L. Weiss ${ }^{2,3}$; S. Cullivan ${ }^{4,2}$; E. O'Rouke ${ }^{1}$;

C. Murphy ${ }^{2,5}$; S. Toolan'; A. Lennon"; P.B Szklanna ${ }^{2,3}$; S. Comer ${ }^{2,3}$;

H. Macleod ${ }^{2}$; S. Gaine ${ }^{4,6}$; K. O'Reilly,6; B. McCullagh ${ }^{4,6}$;

J. Stack ${ }^{7,6}$; P.WG Mallon ${ }^{6,8,9}$; A. Cotter ${ }^{6,9,10}$; P.B Maguire ${ }^{2,3,11}$;

F. Ní Áinle ${ }^{1,2,6,12,13}$; B. Kevane ${ }^{1,2,6,13}$

${ }^{1}$ Department of Haematology, Mater Misericordiae University Hospital, Dublin, Ireland; ${ }^{2}$ UCD Conway SPHERE Research Group, University College Dublin, Dublin, Ireland; ${ }^{3}$ School of Biomolecular and Biomedical Science, University College Dublin, Dublin, Ireland; ${ }^{4}$ Department of Respiratory Medicine, Mater Misericordiae University Hospital, Dublin, Ireland; ${ }^{5}$ Department of Neonatology, Rotunda Hospital, Dublin, Ireland; ${ }^{6}$ School of Medicine, University College Dublin, Dublin, Ireland; ${ }^{7}$ Department of Rheumatology, Mater Misericordiae University Hospital, Dublin, Ireland; ${ }^{8}$ Department of Infectious Diseases, St Vincent's University Hospital, Dublin, Ireland; ${ }^{9}$ UCD Centre for Experimental Pathogen and Host Research, Dublin, Ireland; ${ }^{10}$ Department of Infectious Diseases, Mater Misericordiae University Hospital, Dublin, Ireland; ${ }^{11}$ UCD Institute for Discovery, University College Dublin, Dublin, Ireland; ${ }^{12}$ Department of Haematology, Rotunda Hospital, Dublin,
Ireland; ${ }^{13}$ Irish Network for VTE Research (INViTE), Dublin, Ireland

Background: COVID-19 confers an increased risk of thrombosis however the mechanisms underlying this coagulopathy and the optimal approach to thromboprophylaxis are unknown. Thrombotic risk is likely greatest among patients with severe COVID-19 requiring critical organ support however patients with moderate disease may be at risk and might also benefit from intensified thromboprophylaxis. Aims: To characterise plasma thrombin generation (TG) in patients with COVID-19 of moderate severity, treated with pharmacological thromboprophylaxis.

Methods: Blood was collected from individuals admitted to hospital with COVID-19 of moderate severity (not requiring critical care support) and a group of age-matched patients admitted with infective/ inflammatory illness (negative for COVID-19). All subjects received standard dose low molecular weight heparin (LMWH) thromboprophylaxis with samples taken at time of predicted trough levels (confirmed by measuring anti-FXa activity). TG in platelet-poor plasma was determined by calibrated automated thrombography in the presence/absence of tissue factor (TF) (ppp-LOW reagent, $1 \mathrm{pM}$ TF \& $4 \mu \mathrm{M}$ phospholipid; MP-reagent, $4 \mu \mathrm{M}$ phospholipid; Thrombinoscope BV ${ }^{\mathrm{TM}}$ ).

Results: Fourteen COVID-19 positive subjects and 11 hospitalised COVID-19 negative controls were recruited. Mean trough plasma anti-Xa activity was similar in both groups $(0.06 \mathrm{vs} 0.04 \mathrm{IU} / \mathrm{mL}$; $P=0.2$ ). In the presence of TF, mean endogenous thrombin potential was significantly higher in the COVID group in comparison to controls $\left(1929 \pm 119.7\right.$ vs $1528 \pm 138.9 \mathrm{nM}^{*}$ min; $\left.P=0.02\right)$. Peak thrombin was also higher in COVID-19 (267.3 \pm 22.2 vs $208.6 \pm 17.8 \mathrm{nM}$; $P=0.06)$. Despite increased TG overall, lagtime to TG was significantly prolonged in COVID-19 (8.1 \pm 0.5 vs $6.2 \pm 0.5$ mins; $P=0.02$ ). No difference in any parameter of TG was observed between groups in the absence of TF.

Conclusions: Despite pharmacological thromboprophylaxis plasma TG is enhanced in COVID-19. The underlying mechanisms remain to be elucidated. Specific clinical implications of increased TG despite pharmacological thromboprophylaxis have yet to be determined although clinical trials evaluating intensified anticoagulant regimens in a similar population are ongoing.

PB0154 | Haematological, Coagulation and Biochemical Abnormalities in Covid-19 Patients in India: Do We Behave Differently?

N. Awasthi; V. Tiwari; S. Naqvi; P. Das; S. Aswal; N. Husain; A. Tripathi RMLIMS, Lucknow, India

Background: COVID-19 infection which is usually a mild viral illness, may become life threatening. Progression and mortality is associated with wide range of laboratory abnormalities. Coagulopathy is frequently reported. After a year of pandemic, Indian data remains to be scarce. 
Aims: To explore haematological, coagulation and biochemical parameters of COVID-19 infection in Indian patients.

Methods: Prospective exploratory study included 52 patients of RTPCR confirmed COVID-19 admitted to this tertiary care dedicated Covid-19 facility.

Cases were categorised as mild (23) or severe (29) as per WHO interim guidance document. Following parameters were assessed at admission and after a mean of 7 days of hospital stay: Complete blood count, Prothrombin time (PT), activated partial thromboplastin time (APTT), D-dimer, Factors V,VII,VIII, Fibrinogen, AntithrombinIII, hs-CRP, Procalcitonin(PCT),IL-6, S.ferritin, LDH, Triglyceride. There were 40 survivors and 12 non-survivors. Parameters were statistically analysed among mild and severe disease and in survivors and non-survivors for both day 1 and day7.

Results: Day1 parameters significantly higher in severe group were TLC, Neutrophil\%,ANC, NLR, PT, D-dimer, HsCRP and lower were PLR, Lymphocyte\%, Monocyte\%, ALC. APTT on day7 was prolonged $\sim 9 \mathrm{~s}$ in severe but with no change in fibrinogen as expected (Table 1). None of the Coagulation factor assays (FV,VII,VIII,Fibrinogen,ATIII) revealed significant difference in mild/severe or survivors/non survivors on Day 1 and 7, however mean values of FVIII, Fibrinogen and D-dimer were higher than normal. Non-survivors revealed significant fall in $\mathrm{Hb}$, Lymphocyte\%, Monocyte\%, ALC,AMC, Platelet and increase in Neutrophil\%, APTT, D-dimer, NLR on Day7 in nonsurvivors (Table 2). HsCRP, LDH, IL-6, Ferritin, PCT were altered with disease severity but were not significant in our patient cohort.

TABLE 1 Unpaired $t$-test for parameters showing significantly different means in mild and severe groups on Day 1 or subsequent testing (mean Day7) ( $n=$ Mild 23, Severe 29)

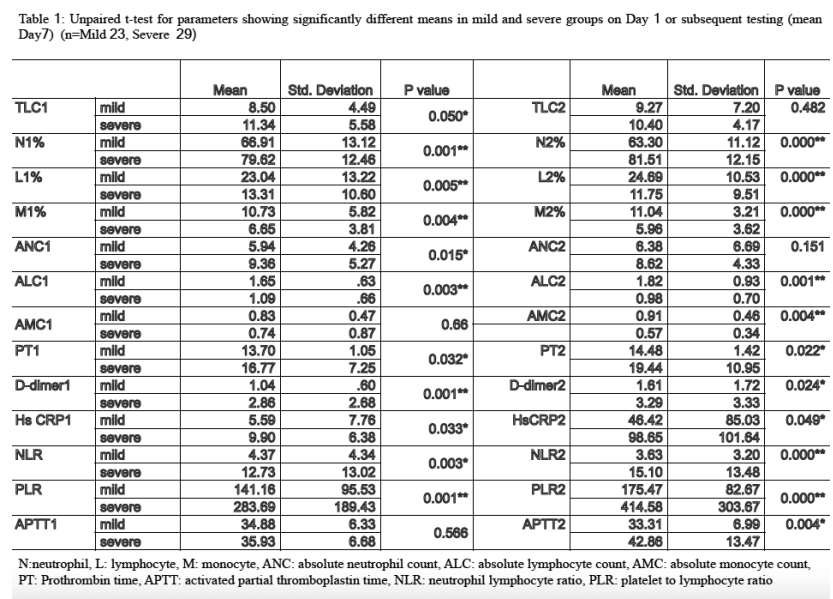

Conclusions: Dynamics of Leucocyte parameters, platelets and prolongation in PT, APTT with high D-dimer are most informative markers for disease progression and mortality. High levels of Fibrinogen and Factor VIII need further evaluation. Biochemical parameters appear to have no prognostic relevance.
TABLE 2 Mean change in parameters on day 1 and day 7 that were significant $(p$

Table 2: Mean change in parameters on day 1 and day 7 that were significant $(p<0.05)$ in ANCOVA with non-survival as covariate ( $n=$ Survivors: 40 , non survivors: 12)

\begin{tabular}{|l|c|c|c|c|}
\hline & $\begin{array}{l}\text { Paired } \\
\text { Differences } \\
\text { Mean }\end{array}$ & Std. Deviation & $\begin{array}{l}\text { Paired } \\
\text { Differences } \\
\text { Mean }\end{array}$ & Std. Deviation \\
\hline & \multicolumn{2}{|c|}{ Non survivors } & \multicolumn{2}{c|}{ Survivors } \\
\hline Hb2 - Hb 1 (g/d) & -0.65 & 0.68 & 0.15 & 0.96 \\
\hline N2- N1 (\%) & 10.75 & 9.90 & -3.92 & 13.62 \\
\hline L2-L1(\%) & -7.50 & 9.62 & 2.07 & 11.84 \\
\hline M2-M1(\%) & -3.50 & 5.43 & 0.72 & 5.33 \\
\hline $\begin{array}{l}\text { PLT2- } \\
\text { PLT1(x10 } 3 / \mathrm{ul})\end{array}$ & -19.68 & 80.96 & 105.40 & 138.57 \\
\hline $\begin{array}{l}\text { ALC2- } \\
\text { ALC1(x103/ul) }\end{array}$ & -0.48 & 0.43 & 0.16 & 0.90 \\
\hline $\begin{array}{l}\text { AMC2- } \\
\text { AMC1(x103/ul) }\end{array}$ & -0.51 & 1.45 & 0.07 & 0.42 \\
\hline $\begin{array}{l}\text { APTT2- } \\
\text { APTT1(sec) }\end{array}$ & 9.70 & 16.20 & 1.35 & 9.86 \\
\hline $\begin{array}{l}\text { D-dimer2-D- } \\
\text { dimer 1(ug/ml) }\end{array}$ & 2.08 & 4.08 & -0.01 & 2.29 \\
\hline NLR2- NLR1 & 10.41 & 13.20 & -1.86 & 9.02 \\
\hline
\end{tabular}

1: value at admission, 2: subsequent testing (mean day7) $\mathrm{Hb}$ : haemoglobin, N:neutrophil, L: lymphocyte, M: monocyte, PLT: platelet, ALC: absolute lymphocyte count, AMC: absolute monocyte count, APTT: activated partial thromboplastin time, NLR: neutrophil to lymphocyte ratio, ANCOVA: analysis of covariance. ${ }^{*}$ Values in negative show a decrease at the time of subsequent testing, positive values show an increase at the time of subsequent testing |

PB0155 | Platelets of COVID-19 Patients Are Refractory due to Thrombin Receptor Desensitizing in the Bloodstream

A. Martyanov $^{1,2,3}$; A.E. Boldova²; M.G. Stepanyan ${ }^{4,2}$ O.I. $\mathrm{An}^{5,2}$; S.V. Tsarenko ${ }^{6}$; S.A. Rumyantsev ${ }^{7}$; S.S. Karamzin ${ }^{2}$; M.A. Panteleev ${ }^{2,1,4}$; A.G. Rumyantsev ${ }^{1}$; F.I. Ataullakhanov ${ }^{2,1,4}$; A.N. Sveshnikova ${ }^{4,1,2,5}$

${ }^{1}$ NMRC PHOI named after Dmitry Rogachev, Moscow, Russian Federation; ${ }^{2}$ CTP PCP RAS, Moscow, Russian Federation; ${ }^{3}$ Emmanuel Institute for Biochemical Physics, Moscow, Russian Federation; ${ }^{4}$ Lomonosov Moscow State University, Physics Faculty, Moscow, Russian Federation; ${ }^{5}$ I.M. Sechenov First Moscow Medical University (Sechenov University), Moscow, Russian Federation; ${ }^{6}$ Moscow State Hospital 52, Moscow, Russian Federation; ${ }^{7}$ Federal State Hospital of the Russian Academy of Sciences, Troitsk, Russian Federation

Background: Coagulopathy is among the most alarming drivers of COVID-19-induced pathology. COVID-19 can result in blockade of the microvasculature in the lungs with microthrombi. While most of the studies concern COVID-19 impact on plasma coagulation, platelet dysfunction has been reported as well. However, the mechanism of platelet malfunctioning in COVID-19 has not been described yet.

Aims: To determine the mechanism of COVID-19 induced platelet disfunction.

Methods: 46 patients with confirmed COVID-19 (11 non-ICU, 26 ICU, and $9 \mathrm{ECMO}$ ) and 26 healthy volunteers were studied (independent ethics committee of NMRC PHOI No 3/2020). Citrated whole blood samples were diluted in Tyrode's buffer and activated by collagen and TRAP-6. Platelets of healthy donors were additionally washed and pre-treated by $0.5 \mathrm{nM}$ of thrombin. Samples were analyzed using BC Navios flow cytometer. Additionally, light transmission aggregometry (AP-2110 SOLAR) of citrated platelet-rich plasma (PRP) with TRAP- 6 and fucoidan as activators was conducted. 
Results: Platelet forward scattering parameter (FSC-A) for COVID-19 patients was significantly increased compared to healthy donors. This parameter reversibly correlated with mild thrombocytopenia observed in some patients. The amount of Annexin- $V$ positive platelets was increased in all patients as well. Relative CD42b and CD62p binding upon activation were decreased, being statistically different in non-ICU and ICU patients. Both of the parameters also correlated to CRP concentration in patient blood plasma. Platelet aggregation was reduced as well. Altogether, platelets demonstrated refractoriness resembling desensitization of receptors. To prove this hypothesis, we performed thrombin pre-treatment of healthy donor platelets, which resulted in a phenotype resembling the COVID-19. Conclusions: Platelets of COVID-19 patients demonstrate refractoriness to activation through PAR1 receptor, probably because of a previous activation with thrombin in circulation. Together with their increased size and fraction of necrotic platelets, this suggests that in COVID-19 platelets encounter thrombin in circulation. The study was supported by Russian Science Foundation (Grant 21-45-00012).

PB0156 | Thrombin Generation Kinetics, Neutrophil Extracellular Traps, and von Willebrand Factor in COVID-19 Associated Coagulopathy: A Pilot Study

T. MacArthur ${ }^{1}$; J. Goswami ${ }^{1}$; M. Sridharan ${ }^{2}$; J. Tange ${ }^{2}$; A. Kirmse ${ }^{1}$; K. Lundell ${ }^{1}$; M. Auton ${ }^{2}$; T. Chon ${ }^{3}$; R. Hurt ${ }^{3}$; B. Salonen ${ }^{3}$; R. Ganesh ${ }^{3}$; J.-F. Dong ${ }^{4}$; R. Kozar ${ }^{5}$; S. Heller ${ }^{1}$; E. Loomis ${ }^{1}$; A. Johnstone ${ }^{6}$; K. Bailey ${ }^{7}$; G. Spears ${ }^{7}$; M. Park ${ }^{1}$

${ }^{1}$ Mayo Clinic, Department of Surgery, Rochester, United States; ${ }^{2}$ Mayo Clinic, Department of Hematology, Rochester, United States; ${ }^{3}$ Mayo Clinic, Department of Internal Medicine, Rochester, United States; ${ }^{4}$ University of Washington School of Medicine, Bloodworks Research Institute, Seattle, United States; ${ }^{5}$ Shock Trauma Center, University of
Maryland School of Medicine, Baltimore, United States; ${ }^{6}$ EpiCypher Inc., Durham, United States; ${ }^{7}$ Mayo Clinic, Department of Health Sciences Research, Rochester, United States

Background: SARS-CoV-2 virus infection (COVID-19) is associated with high rates of venous thromboembolism (VTE). Immune mediated thrombosis has been implicated as a driving factor.

Aims: To characterize coagulopathy by assessing thrombin generation kinetics and Von Willebrand Factor (VWF) and to characterize thromboinflammation through neutrophil extracellular trap (NET) formation in COVID-19 patients (pts).

Methods: We collected plasma samples from 67 COVID-19 pts in the Emergency Department (ED) and 38 healthy volunteers (HV). Plasma thrombin generation kinetics were assessed using Calibrated Automated Thrombogram (CAT) and expressed as lag time (LT), peak height $(\mathrm{PH})$, time to peak (ttPeak), and endogenous thrombin potential (ETP-total thrombin generated). NET formation was quantified through citrullinated nucleosome ELISA (H3NUC). VWF antigen/activity levels were determined by latex immunoassay. Factor VIII levels were performed using one-stage optical clot detection. VTE events were tracked through electronic medical records. This study was approved by the Institutional Review Board and patients were consented. MannWhitney test was performed, $P<0.05$ considered significant.

Results: Subject characteristics are described in Table 1. Of the 67 pts, $50.7 \%$ required admission and $31.3 \%$ supplemental oxygen. COVID-19 pts had longer LT and ttPeak, greater H3NUC, VWF antigen and activity level, and Factor VIII levels as compared to HV (Table 2). Admitted pts had longer LT (4.33 min [3.67, 5.67]; $3.33 \mathrm{~min}$ [3.00, 4.00], $P<0.001)$, ttPeak (7.73 $\min [6.84,8.50] ; 7.15 \min [6.05$, 7.75], $P=0.011$ ), greater VWF antigen (247 IU/dL [208, 298]; $168 \mathrm{IU} /$ $\mathrm{dL}[103,222], P<0.001)$, activity (196 IU/dL [164, 238]; $130 \mathrm{IU} / \mathrm{dL}$ [94, 172], $P<0.001)$, and Factor VIII (159 IU/dL [129, 194]; $119 \mathrm{IU} / \mathrm{dL}$ $[88,169], P=0.023)$ as compared to those not admitted.

TABLE 1 Clinical and Demographic Information for COVID-19 Patients and Healthy Volunteers Enrolled in this Study. Age, days since COVID-19 (+) PCR test, and days of symptoms prior to sample collection are expressed as median and interquartile range [IQR]. "Indicates percentage of only those patients who were admitted. ICU = intensive care unit, VTE = venous thromboembolism, DVT = deep vein thrombosis, PE = pulmonary embolism.

$\begin{array}{ll}\text { COVID-19 } & \text { Healthy Volunteers } \\ (n=67) & (n=38) \\ 58[33-70] & 41[30-51] \\ 30(44.8 \%) & 18(50 \%) \\ 8[4.25-10] & - \\ 5[1-9] & - \\ 66(98.5 \%) & - \\ 21(31.3 \%) & - \\ 34(50.7 \%) & - \\ 3(8.8 \%) & \\ 33(97.1 \%) & \end{array}$

Age (years)

Sex (\% male)

Days of symptoms prior to sample collection

Days since COVID-19 PCR test

Symptomatic (\%)

Supplemental Oxygen Requirement (\%)

Admitted (\%)

ICU (\%)

VTE prophylaxis within $24 \mathrm{~h}^{*}$ 
COVID-19

$(n=67)$

5 (7.5\%)

VTE (\%)

DVT (\%)

PE (\%)

Mortality (\%)
Healthy Volunteers

$(n=38)$

TABLE 2 Thrombin generation kinetics, NETosis, and VWF in COVID-19 Patients vs. Healthy Volunteers. Plasma thrombin generation kinetics performed for COVID-19 patients and healthy volunteers using calibrated automated thrombogram (CAT) and expressed as lag time (LT), peak height (PH), time to peak (ttPeak), and endogenous thrombin potential (ETP). Neutrophil extracellular trap (NET) formation measured by quantification of citrullinated nucleosomes (H3NUC). VWF antigen levels, activity, and factor VIII levels all in IU/dL and calibrated to international standard. All values are expressed as median and interquartile range [IQR], $P<0.05$ considered significant. ${ }^{*}$ ETP and H3NUC unavailable for one COVID-19 patient. ${ }^{* *}$ VWF and Factor VIII unavailable for one healthy volunteer.

COVID-19 Healthy Volunteers

$(n=67)$

4.00

[3.26-4.67]

233.9

[210.2-288.8]

ttPeak (min)

\subsection{3}

[6.33-8.04]

$\operatorname{ETP}\left(\mathrm{nM}^{*} \min \right)^{*}$

1475

[1217-1779]

H3NUC (ng/mL)

79.5

[66.8-215.0]

VWF: Ag (IU/dL)

212

[158-275]

VWF Activity (IU/dL)

$$
165
$$

[128-221]

Factor VIII (IU/dL)

$$
148
$$

[106-190] $(n=38)$

$P$-value

2.95

[2.67-3.10]

206.2

[167.0-249.2]

6.45

[5.99-7.53]

1318

[1163-1557]

66.8

[66.8-114.4]

110

[88-128]

89

[70-109]

106

[85-129]
Conclusions: Symptomatic COVID-19 pts have prolonged initiation of thrombin generation, especially those requiring admission. Greater VWF activity and NETosis suggest systemic thromboinflammation in COVID-19 associated coagulopathy.

\section{PB0157 | SARS-CoV2 Spike Protein Can Activate Platelets} through Integrin Alpha Ilb Beta 3

C. Williams; J. Khalil; T. Walsh; K. Gupta; E. Aitken; S. Mundell; I. Berger; A. Poole; I. Hers

University of Bristol, Bristol, United Kingdom

Background: Patients with severe coronavirus disease 19 (COVID-19) are at increased risk of thrombosis, which can be a challenge to manage and is associated with elevated mortality. The virus responsible for COVID19, the severe acute respiratory syndrome coronavirus 2 
(SARS-CoV2), is unique amongst coronaviruses that infect humans in that its envelope spike protein includes an arginine-glutamateaspartate (RGD) peptide sequence in its receptor-binding domain (RBD). In platelets, RGD sequences are recognised by the main platelet activation and adhesion integrin, $a_{11 b} \beta_{3}$, triggering integrin activation, outside-in signalling, and platelet activation.

Aims: To investigate whether the RGD sequence in the spike protein is able to activate platelets through integrin $a_{\| l} b_{3}$.

Methods: Fibrinogen, collagen, purified spike and RBD protein were coated on glass slides and platelets were allowed to adhere under static or flow conditions. Slides were washed, stained platelets with ActinGreen and imaged by confocal microscopy.

Results: Purified SARS-CoV2 spike protein and RBD protein triggered platelet spreading and this was blocked by incubating the platelets with the clinically used non-peptide RGD mimetic $a_{11} \beta_{3}$ integrin blocker, tirofiban. In an in vitro thrombosis model using healthy donor blood, we find surprisingly that this ability to activate platelet integrins does not translate into an enhancement in thrombus formation on collagen, and platelets cannot form thrombi on the spike protein under arterial or venous conditions.

Conclusions: We conclude therefore that although SARS-CoV2 spike contains an RGD sequence that can activate platelet integrins, whether this contributes to enhanced thrombosis under pathological conditions in Covid-19 patients is not known. Structural analysis of the RGD site suggests a buried location in the spike, which may be revealed by other activatory mechanisms and receptors, and which will require further study.

PB0159 | Altered Protein S Activity Contributes Significantly to COVID-19 Pro-thrombotic State

T. Nguyen ${ }^{1}$; D. Melancon ${ }^{1}$; M. Dean ${ }^{2}$; A. Zea ${ }^{3}$; S. Chatterjee ${ }^{1}$;

J. Harman ${ }^{1}$; V. Pilli ${ }^{1}$; R. Nossaman ${ }^{4}$; J. Ochoa ${ }^{4}$; A. Ochoa ${ }^{2}$;

R. Majumder ${ }^{1}$

${ }^{1}$ LSUHSC, New Orleans, United States; ${ }^{2}$ Louisiana State University

Cancer Center, New Orleans, United States; ${ }^{3}$ Louisiana State University

Health Sciences Center, New Orleans, United States; ${ }^{4}$ Ochsner Medical

Center, New Orleans, United States

Background: Since January 2020, the SARS-CoV-2 pandemic has caused $\sim 500,000$ deaths in the United States alone. Many fatalities result from thrombotic events such as stroke, pulmonary embolism, or heart attack secondary to disseminated intravascular coagulation. Clinicians use anticoagulant and antiplatelet therapies to mitigate the risk of clotting, but these therapies increase the likelihood of bleeding. We propose that a decrease in activity of the anticoagulant Protein S (PS) significantly increases thrombotic risk in individuals with COVID-19.

Aims: Determine whether deficiency or modification of PS in hospitalized COVID-19 patients increases the risk of venous thromboembolism.
Methods: The Ochsner Clinical Foundation and Louisiana State University Health Sciences Center Biorepository provided plasma samples from hospitalized patients who tested positive for SARSCoV-2. To measure the amounts of bound and free PS, thrombomodulin, and PA1, we performed ELISA, immunoblot, and thrombin generation assays.

Results: ELISA data showed that free PS was deficient in severe COVID-19 patients (Figure 1A); bound PS was in the normal range (data not shown). We observed a significant rise in peak thrombin with most hospitalized patient samples, and thrombin generation reverted to control level by addition of free PS. However, addition of thrombomodulin did not have a significant similar effect (Figure 1B). Immunoblots (probed with PS antibodies) of hospitalized samples (low free PS; Figure 2) and controls (average is shown) revealed a significant decrease in PS amount (determined by densitometry) of the hospitalized samples compared with control samples. We immunoprecipitated PS from hospitalized and control samples to assess the presence of post translational modifications in Protein S that might cause a decrease in free PS. These samples are being analyzed by mass spectrometry.

\section{Figure 1}
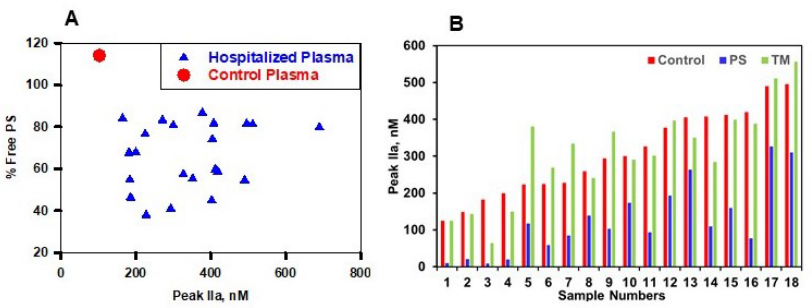

\section{FIGURE 1}

\section{Figure 2}

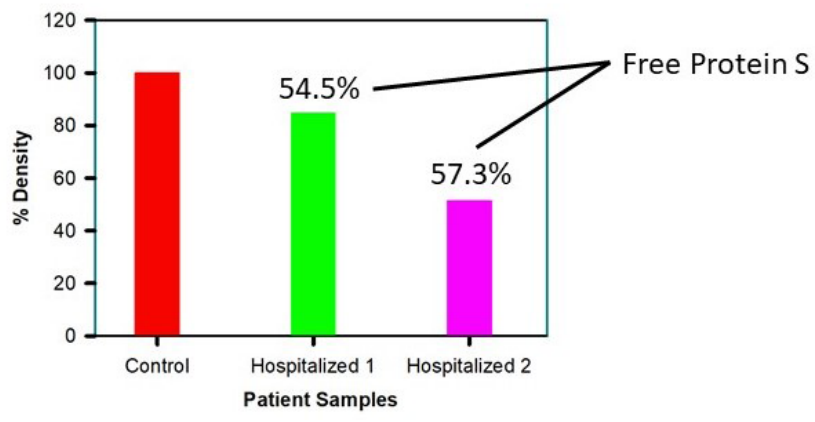

FIGURE 2

Conclusions: Our study indicates that PS is a significant contributor for the hypercoagulable states of hospitalized COVID-19 patients. 
PB0160 | Lymphocyte-Platelet Adhesion and Lymphocyteplatelet Clusters in Patients with Coronavirus Infection SARSCOV-2 (COVID-19)

A. Emelyanov; A. Emelyanova; E. Zaytseva; V. Kuchinskaya;

S. Igumnov; J. Igumnova; Y. Vitkovsky Chita State Medical Academy, Chita, Russian Federation

Background: Lymphocyte-platelet adhesion (LPA) is the ability of lymphocytes with $\mathrm{CD}^{+}, \mathrm{CD}^{+}, \mathrm{CD} 16^{+}$cell surface markers to form coaggregates with platelets using adhesion molecules, that allows lymphocytes to adhere to the damaged endothelium and migrate into the damaged surface of the vascular wall.

Aims: Investigation of the amount of lymphocyte-platelet aggregates and lymphocyte-platelet clusters in patients with COVID-19. Methods: The research involved 168 patients with SARS-CoV-2 (COVID-19) and 100 healthy people of the same age and gender. Patient's blood samples were taken on the 1st-2d, 10th-12th, 21st24th days. The participants of the study were Caucasian race and lived in the Trans-Baikal Territory. The examine of LPA was carried out by the method of Yuri Vitkovsky et al. (1999). The number of lymphocyte-platelet clusters (LPC) was estimated and expressed in rel. units per 100 free-standing cells. The results were expressed in averages and the standard deviation $(M \pm S D)$ was calculated Significant differences were considered for $P<0.05$.

Results: The authors revealed that on the 1 st-2d day of disease among patients the number of LPA increased to $28.9 \pm 3.2 \%$, LPC - to $5.0 \pm 2.3 \%$ as compared with the control group $(P<0.001)$. The average platelets volume LPA-rasio also increased by 2.9 times $(P<0.05)$. Among patients during 10-12 days of the disease there was a notable increase of LPA and LPA-rasio up to $41.3 \pm 1.9 \%$ and $5.8 \pm 0.48$ respectively (higher by 3 times as compared to the control group). On the 21-24th days of the disease with patients being in the hospital, there were no significant differences in the studied parameters among the patients and the control group.

Conclusions: There is an increase in the ability of lymphocytes to adhere platelets to their surface and contact with other lymphocytes while forming clusters in the acute phase of COVID-19. Probably the process of clustering is mediated by platelet and leukocyte adhesive molecules.

PB0161 | Is Viscoelastometric Testing a Good Tool to Assess Hemostasis of COVID-19 Patients?

M. Bareille ${ }^{1}$; M. Hardy ${ }^{2}$; T. Lecompte ${ }^{3}$; F. Mullier ${ }^{1}$

${ }^{1}$ Université Catholique de Louvain, Hematology laboratory, Namur Thrombosis and Hemostasis Center (NTHC), CHU UCL Namur, Yvoir, Belgium; ${ }^{2}$ Université Catholique de Louvain, Anesthesiology Department, Namur Thrombosis and Hemostasis Center (NTHC), CHU UCL Namur, Yvoir, Belgium; ${ }^{3}$ Departments of Medicine, Division of Angiology and Haemostasis, Geneva University Hospitals, and Geneva Platelet Group (GpG), Faculty of Medicine, University of Geneva, Geneva, Switzerland
Background: Infection by SARS-CoV-2 is associated with a high risk of thrombosis. The laboratory documentation of hypercoagulability and impaired fibrinolysis remains a challenge.

Aims: Our main aim was to assess the potential usefulness of viscoelastometric testing (VET) to predict thrombotic events in COVID-19 patients according to the literature. Our secondary aims were: (i) to analyze the impact of anticoagulation and the methods used to neutralize heparin, (ii) to see whether maximal clot mechanical strength brings more information than Clauss fibrinogen, and (iii) to point out results from studies with enhanced fibrinolysis modified tests.

Methods: We performed a systematic search in PubMed and Scopus databases, until December 31st, 2020 (Figure 1). VETs methods and parameters, as well as patients'features and outcomes were extracted.

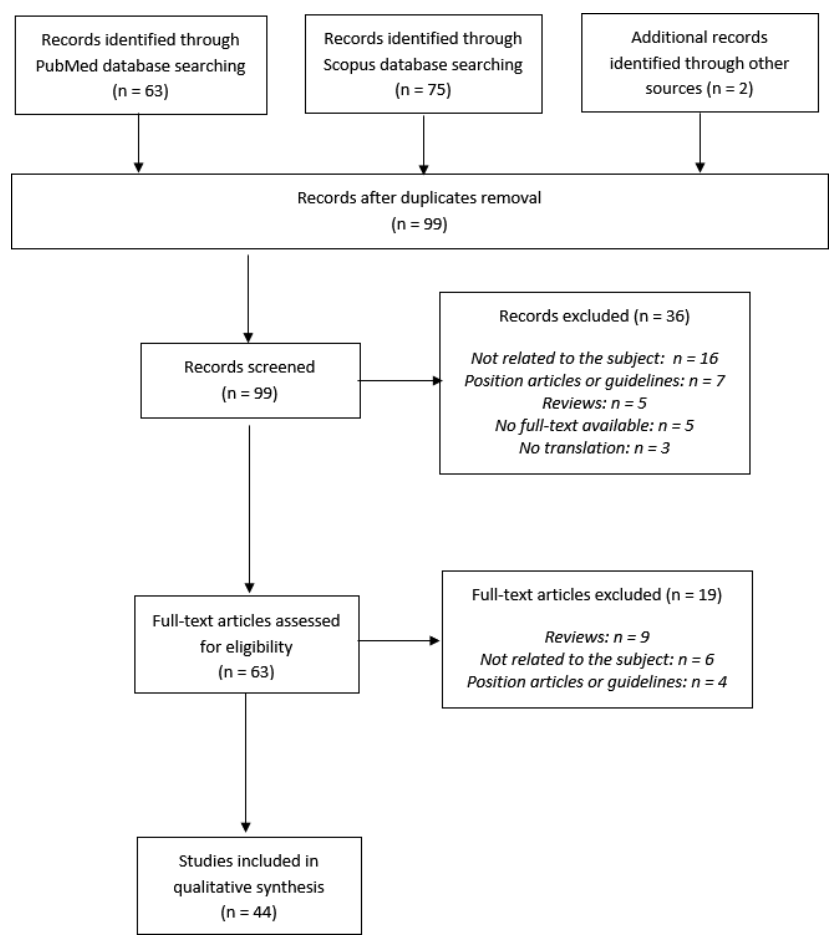

FIGURE 1 Literature search and selection flow chart according to PRISMA statement

Results: VET was performed for 1063 patients (893 ICU and 170 non-ICU, $n=44$ studies). There was a huge heterogeneity concerning study design, the VET device (ROTEM, TEG, Quantra and ClotPro) and reagents (with non-systematic use of heparin neutralization by heparinase and/or polybrene), timing of assay, and definition of hypercoagulable state.

The common findings were an increased clot mechanical strength mainly due to an excessive fibrinogen component with impaired to absent fibrinolysis, more conspicuous in the presence of an added plasminogen activator. This profile was associated with an uncontrolled thrombin generation despite a standard thromboprophylaxis. However, only 4 studies out of 16 that addressed this point found an association of VETs with thrombotic events. 
Functional fibrinogen assessed by VET showed a variable correlation with Clauss fibrinogen.

Abnormal VET pattern tended to normalization after an enhancement in thromboprophylaxis.

Notably, only 4 studies out of 25 using ROTEM reported data where heparin is neutralized by heparinase (HEPTEM).

Conclusions: The heterogeneity among VET studies and small sample sizes do not permit to point out an association between the illdefined hypercoagulable state and thrombotic events.

PB0162 | Hemostasiological Blood Properties in Patients with COVID-19 Associated Viral Pneumonia

M. Presnyakova; E. Galova; O. Kostina

Federal State Budgetary Educational Institution of Higher Education 'Privolzhsky Research Medical University' of the Ministry of Health of the Russian Federation, Nizny Novgorod, Russian Federation

Background: Impaired hemostasis is one of the main elements of COVID-19 associated pneumonia pathogenesis. Early detection and adequate correction of hemostasis impairments determine the pneumonia course and outcome.

Aims: The investigation objective was to study the changes of standard hemostasiological tests and thromboelastography indices in COVID-19 associated pneumonia patients.

Methods: We determined APTT, prothrombin time (PT), fibrinogen (F), D-dimers (D-d), antitrombin III (AT III), C-reactive protein (CRP), platelet count, and studied viscosity and elasticity of blood clots using kaolin-activated thromboelastography (TEG) in 49 patients aged 40-69 with clinical presentation of viral infection and bilatera multi-segmental pneumonia. According to CT, the severity of $100 \%$ cases corresponded to CT2-CT3. Acute respiratory failure (ARF) was diagnosed in 16 patients. A control group included 25 apparently healthy subjects.

Results: There were revealed a marked acute-phase reaction characterized by increased fibrinogen (5.44 [4.34; 7.0]) and CRP (34.5 [16.0; 108]) concentrations, high D-d concentration (819 [472; 1769]) indicating intravascular homeostasis activation, and PT prolongation, which are descriptive of protein synthesis hepatic function (12.8 [12.5; 13.5]). D-d concentration correlated with patient's age $(r=0.61 ; P<0.05)$ and comorbidity $(r=0.58 ; P<0.05)$. We found neither the alterations in TEG findings, nor the association between TEG parameters characterizing blood clot density (MA and G) and a fibrinogen level; yet there was revealed the relationship of the characteristics and platelet count $(r=0.61 ; P<0.05)$.

Conclusions: Patients with COVID-19 associated pneumonia present impaired hemostatic system of thrombogenic nature. Moreover kaolin-activated thromboelastography appeared to be less informative, so further investigations are required to make an objective assessment of blood viscosity and elasticity.
PB0163 | Thrombotic Microangiopathy in Two Patient with Covid 19 Disease

P. Bradacova ${ }^{1}$; L. Slavik ${ }^{2}$; J. Ullrychova ${ }^{1}$; J. Ulehlova ${ }^{2}$;

J. Prochazkova²; A. Hlusi ${ }^{2}$

${ }^{1}$ Masaryk Hospital, Department Clinical Hematology, Usti nad Labem, Czech Republic; ${ }^{2}$ Faculty of Medicine and Dentistry, Palacky University Olomouc, University Hospital Olomouc, Department of Hematooncology, Olomouc, Czech Republic

Background: The new, pandemic disease of Severe Acute Respiratory Syndrome Coronairus 2 (SARS-CoV-2) causes a variety of symptoms in infected individuals, from very mild flu-like symptoms to fatal severe pneumonia and organ failure.

However, it is very interesting that we are seeing an increase in thrombotic microagiopathies (TMA) in connection with the disease caused by the new coronavirus (Covid 19). The persistent action of coronavir activates the complement system, including activation of coagulation. This is accompanied by the production of antibodies against ADAMTS13, which leads to a reduction in ADAMTS13 activity and the development of TMA with microthrombotizations in blood vessels, tissues and organ failure.

Aims: Description of two case reports of hospitalized patients in the intensive care unit with Covid 19 and subsequently developed thrombotic microangiopathy.

Methods: To diagnose TMA, two patients with Covid 19 infections had the following tests: blood count, platelet count (PLT), schistocyte count, lactate dehydrogenase, urea, creatinine, ADAMTS13 activity, and ADAMTS13 inhibitor.

Results: Case report 1 - male 68 years old, Covid 19 positive, on the 5 day TMA is developed, PLT is $49 \times 109 /$, schistocytes $68 / 1000$ erythrocytes, ADAMTS13 activity 43\%, inhibitor $12 \mathrm{lU} / \mathrm{ml}$. After exchange plasmapheresis patient's condition improves. Case report 2 male 64 years old, Covid 19 positive, on the 8 day TMA is developed, PLT is $16 \times 109 /$, schistocytes 64/1000 erythrocytes, ADAMTS13 activity $29 \%$, inhibitor $68 \mathrm{lU} / \mathrm{ml}$. After exchange plasmapheresis patient's laboratory finding improves, but condition is very serious and patient die.

Conclusions: Covid 19 infection can damage the endothelium, activate coagulation and complement, and subsequently develop thrombotic microangiopathy. Covid 19 brings a wide range of overlapping signs and symptoms, making it difficult to diagnose severe TMA. For this reason, treatment is often started late and some patients die. Suported by grant: LF-2021-001 a MH CZ - DRO (FNOI, 00098892) 


\section{COVID AND COAGULATION, CLINICAL}

LPB0009 | Systematic Screening for Pulmonary Embolism in Patients with Suspected Covid-19 in the Emergency Department: A Prospective Cohort Study

I. Luu; F. Kroon; J. Buijs; J. Krdzalic; M. de Kruif; M. Leers;

G. Mostard; R. Martens; R. Mostard; D. van Twist

Zuyderland Medical Centre, Sittard/Heerlen, Netherlands

Background: Pulmonary embolism (PE) seems to be a prevalent complication in patients with Coronavirus disease 2019 (COVID-19). However, all studies thus far were retrospective analyses without systematic PE-screening, thus highly susceptible to selection bias. Therefore, the true prevalence of PE and optimal D-dimer cut-off cannot be derived from such studies.

Aims: We aimed to evaluate the true prevalence of PE in patients with suspected COVID-19 and the diagnostic yield of systematic PE screening. Methods: We evaluated all patients with suspected COVID-19 who were admitted to our hospital via the Emergency Department between April 7th and May 31st 2020. Patients were assessed according to a prespecified clinical protocol and were evaluated for PE using the YEARS-algorithm. CT-pulmonary angiography (CTPA) was performed in case D-dimer levels were $\geq 1000 \mathrm{ng} / \mathrm{mL}$, or $\geq 500 \mathrm{ng}$ / $\mathrm{mL}$ if patients had $\geq 1$ YEARS item (hemoptysis, clinical signs for deep vein thrombosis, and/or PE as the most likely diagnosis). Patients using anticoagulant drugs were excluded.

Results: 666 patients with suspected COVID-19 were included in the present analyses (Figure 1). CTPA was performed in 393 patients, revealing PE in 51 patients. Thus, 1 out of 7.7 CTPAs was positive for PE. Interestingly, the PE-prevalence did not differ between patients with COVID-19 $(n=190)$ and those without $(13.5 \%$ vs. $12.8 \%$, $P=0.87$ ). D-dimer levels were higher in patients with PE (Figure 2). The lowest D-dimer value ruling out PE was 1258 Mg/L. In multivariate regression analysis, only $\mathrm{D}$-dimer was associated with PE (OR 1.07 for every $1000 \mu \mathrm{g} / \mathrm{L}$ increase, $95 \% \mathrm{Cl} 1.02-1.13, P=0.007$ ).

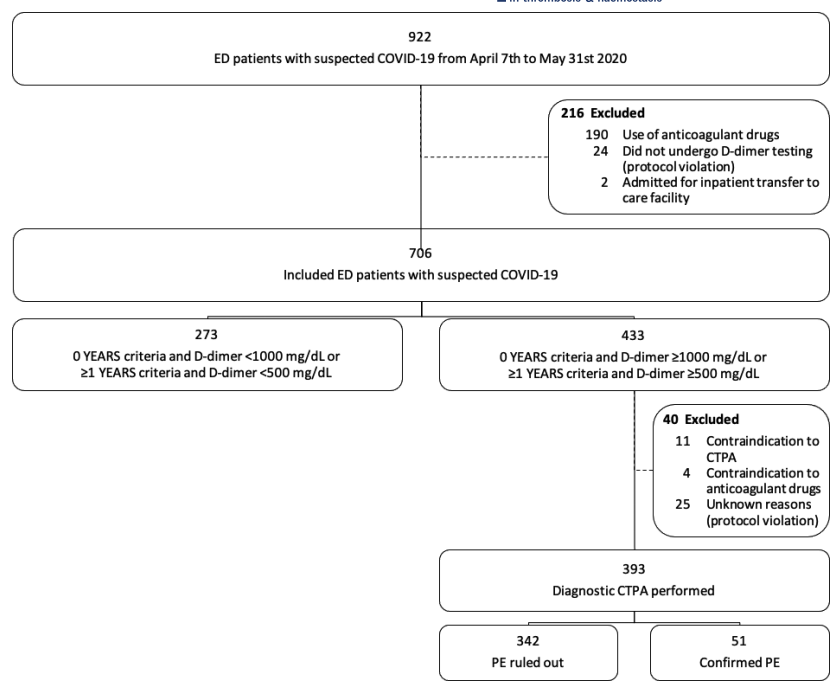

FIGURE 1 Flowchart of study population

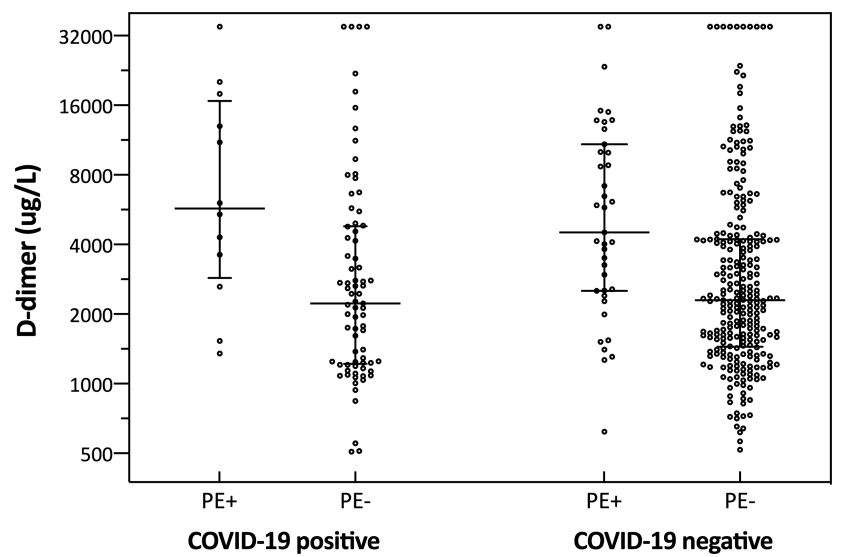

FIGURE 2 Scatter plot of the D-dimer levels in COVID-19 positive and COVID-19 negative patients with and without PE on a base-2 log scale. Each dot represents one patient. Horizontal lines show the median, error bars show the interquartile range

Conclusions: In the first study on systematic PE screening in ED patients with suspected COVID-19, we found an overall PE prevalence of $7.7 \%$. We believe this is a feasible approach for early PE-detection. Our analysis does not support the use of higher D-dimer thresholds to rule out $\mathrm{PE}$ in this population.

LPB0010 | Prevalence of Venous Thromboembolism and Pulmonary Embolism among Patients Hospitalized with a COVID-19 Diagnosis in France

A. Gabet ${ }^{1}$; C. Grave ${ }^{1}$; P. Tuppin ${ }^{2}$; J. Emmerich ${ }^{3}$; V. Olié ${ }^{1}$

${ }^{1}$ Santé Publique France, Saint Maurice, France; ${ }^{2}$ Caisse Nationale d'Assurance maladie, Paris, France; ${ }^{3}$ University of Paris, Groupe Hospitalier Paris Saint-Joseph and INSERM CRESS 1153, Paris, France

Background: Infection to SARS-CoV-2 has been found to be directly associated with an increased risk of venous thromboembolism (VTE). 
Thromboprophylaxy has been early implemented to reduce the risk of pulmonary embolism (PE) in COVID-19 patients.

Aims: The aims were to analyze time-trends in the prevalence of VTE among patients hospitalized with a SARS-CoV-2 infection and to describe the related in-hospital mortality rates.

Methods: We used the French national healthcare database. All patients hospitalized with COVID-19 diagnosis based on either biological sample or CT scan in France between January and September of 2020 were included. During the Covid19 hospitalization we identified concomitant VTE and specifically Pulmonary embolism (PE) using all diagnosis available during hospitalization.

Results: During the 2020 study period, 151,908 Covid-19 patients were identified. Among them, the prevalence of overall VTE was $5.1 \%$ and $3.4 \%$ for PE. The prevalence increased up to $5.1 \%$ for $\mathrm{PE}$ and $6.3 \%$ for VTE in the middle of May 2020. Higher prevalence of PE and VTE were found in COVID-19 patients admitted to a resuscitation unit (9.5\% and 15.6\%) as compared to COVID-19 patients not admitted to those units (2.5\% and 3.5\%). In-hospital mortality rate was higher in patients with PE (20.9\%) than in patients without PE (15.1\%). Same results were observed in resuscitation units $(31.9 \%$ vs $27.6 \%, P<0.0001)$. Between the first week of March and June 2020, in-hospital mortality rates were divided by five for COVID-19 patients with PE (from $70.0 \%$ to $11.3 \%$ ), and by four among those without PE (from $40.3 \%$ to $7.7 \%$ ).

Conclusions: Prevalence of hospitalized VTE decreased over the COVID-19 pandemic, as well as the related-in-hospital mortality rates, but seemed to increase again at the end of the study period, i.e. at the beginning of the second wave of the pandemic in France.

\section{LPB0011 | Immature Platelets as a Biomarker for Disease Severity and Mortality in COVID-19 Patients}

D. Welder; H. Jeon-Slaughter; B. Ashraf; S.-H. Choi; W. Chen; I. Ibrahim; T. Bat

University of Texas Southwestern Medical Center, Dallas, United States

Background: COVID-19, caused by SARS-CoV-2, is a contagious life-threatening viral disease that has killed more than $2.13 \mathrm{M}$ people worldwide. Attempts have been made to identify a biomarker to stratify disease severity to improve resource allocation. Patients with SARS-COV-2 infection manifest with higher inflammatory response and platelet hyperactivity; this raises the question of the role of thrombopoiesis in COVID-19. Immature platelet fraction (IPF\%) can be used to assess bone marrow activity, thrombopoiesis.

Aims: This study investigates whether the level of thrombopoiesis associates with COVID-19 severity defined by ICU stay, ventilator use, and mortality.

Methods: A large cohort of 678 (658, 97\% hospitalized) wellcharacterized COVID-19 patients was analyzed. This included $52.4 \%$ males, 38.9\% White, 26.8\% Black, 25.2\% Hispanic, 2.5\% Asian/Al/ $\mathrm{PI}$, and $6.5 \%$ Other. Overall age was $61.5 \pm 16.7$ years. Other characteristics reviewed included smoking status, comorbidities, and steroid use at the time of admission. Additionally, 5.6\% $(n=38)$ had thrombosis during admission. Group differences in continuous and categorical variables were tested using Two-sided Wilcoxon rank sum test and Mantel Chi-Square statistics, respectively.

Results: Elevated IPF\% at presentation was predictive of length of hospitalization $(P<0.01)$. Additionally, peak values of IPF\% were significantly higher among deceased patients compared to recovered patients $(7.9 \pm 6.3$ vs $5.4 \pm 7.8, P<0.01)$. Approximately $20 \%$ of hospitalized patients were admitted to the intensive care unit (ICU) with an average length of stay $11 \pm 12$ days; these had significantly higher IPF\% at presentation compared to those who did not require ICU care $(5.8 \pm 4.6$ vs $4.7 \pm 2.6, P<0.01)$.

\begin{tabular}{|c|c|c|c|c|c|c|c|c|c|}
\hline Characteristics & & Deceased? & & Admitted to IC & $\bar{c} \mathrm{C}$ among hoss & italized & Ventilator u & se among hospi & alized \\
\hline & Yes $(n=56)$ & No $(n=622)$ & $\mathrm{P}^{*}$ & Yes $(n=139)$ & No $(\mathrm{n}=519)$ & $\mathrm{P}^{*}$ & Yes $(n=79)$ & No $(\mathrm{n}=579)$ & $\mathrm{P}^{*}$ \\
\hline 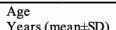 & $70.2 \pm 12.9$ & $60.8 \pm 16.7$ & $<0.01$ & $63.8 \pm 15.7$ & $60.7 \pm 16.8$ & 0.06 & $62.8 \pm 13.9$ & $61.4 \pm 17.0$ & 0.57 \\
\hline Males $(\mathrm{n}, \%)$ & $38(67.9 \%)$ & $317(51.0 \%)$ & 0.02 & $81(58.3 \%)$ & $266(51.3 \%)$ & 0.14 & $50(63.3 \%)$ & $305(50.9 \%)$ & 0.04 \\
\hline $\begin{array}{l}\text { Ondexamethasone } \\
(\mathrm{n} \%)\end{array}$ & $53(94.6 \%)$ & $509(81.8 \%)$ & 0.01 & $126(90.1 \%)$ & $431(83.0 \%)$ & 0.03 & $77(97.5 \%)$ & $485(81.0 \%)$ & $<0.01$ \\
\hline 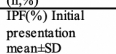 & $6.4+5.7$ & $4.8+2.8$ & 0.09 & $5.8 \pm 4.6$ & $4.7 \pm 2.6$ & 0.03 & $5.8 \pm 4.3$ & $4.8 \pm 3.0$ & 0.06 \\
\hline $\begin{array}{l}\text { IPF(\%) Peak } \\
\text { meantSD }\end{array}$ & $7.9 \pm 6.3$ & $5.4 \pm 7.8$ & $<0.01$ & $6.9 \pm 5.1$ & $5.3 \pm 8.4$ & $<0.01$ & $7.5 \pm 5.0$ & $5.4 \pm 8.1$ & $<0.01$ \\
\hline $\begin{array}{l}\text { Platelet count } \\
(\mathrm{x} 10(9) / /) \text { at } \\
\text { initial presentation } \\
\text { mean } \leq \mathrm{SD}\end{array}$ & $180.9 \pm 97.4$ & $226.0 \pm 98.3$ & $<0.01$ & $219.7 \pm 104.5$ & $224.0 \pm 98.5$ & 0.60 & $221.5 \pm 111.9$ & $223.3 \pm 98.0$ & 0.07 \\
\hline $\begin{array}{l}\text { Platelet count } \\
\text { (x10(9)//) at peak } \\
\text { meantSD }\end{array}$ & $223.1 \pm 119.2$ & $273.3 \pm 127.3$ & 0.01 & $278.9 \pm 136.1$ & $268.8 \pm 126.0$ & 0.30 & $292.4 \pm 137.6$ & $268.0 \pm 126.7$ & 0.80 \\
\hline 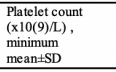 & $158.4 \pm 107.1$ & $\begin{array}{l}218.8 \pm 96.7 \\
\end{array}$ & $<0.01$ & $\begin{array}{l}193.4 \pm 101.8 \\
\end{array}$ & $220.0 \pm 98.2$ & $<0.01$ & $185.6 \pm 110.4$ & $218.3 \pm 97.4$ & $<0.01$ \\
\hline
\end{tabular}

FIGURE 1 Baseline characteristics, IPF (\%), and platelet counts of the study cohort $(n=678)$

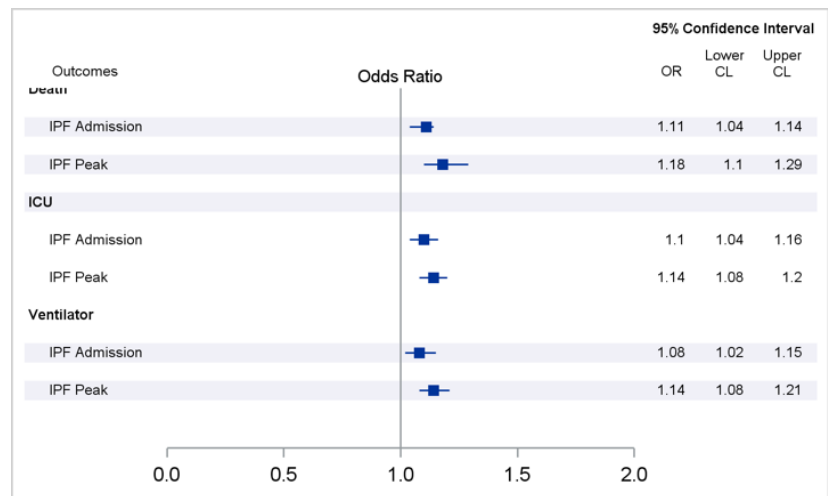

FIGURE 2 Logistic regression results of IPF\% on death, ICU, and Ventilator outcomes Note: 95\% confidence interval (lower CL-upper $\mathrm{CL}$ ) of Odds Ratios (OR) does not include ' 1 ' is statistically significant under $\alpha=0.05$.

Conclusions: Higher IPF\%, as a biomarker of thrombopoiesis, can predict disease severity, length of stay, and mortality risk among hospitalized COVID-19 patients. 
LPB0056 | Pre-admission Anticoagulant Therapy and Mortality in Hospitalized COVID-19 Patients: A Covid Predict Retrospective Cohort Study

T.F. van Haaps $^{1}$; D. Collard ${ }^{1}$; S. Middeldorp ${ }^{1}$; M. Coppens ${ }^{1}$; M.D. de Kruif ${ }^{2}$; H. ten Cate $^{3}$; P.R. Tuinman ${ }^{4}$; R.A. Douma ${ }^{5}$; M.C. Muller ${ }^{6}$; A.C. Reidinga ; M.A. Heuvelmans ${ }^{8}$; M. Oudkerk ${ }^{8}$; F.H. van Osch ${ }^{9}$; J.P. van den Bergh ${ }^{9}$; A. Maas ${ }^{9}$; N. Gritters ${ }^{10}$; M. Ten Wolde ${ }^{5}$; N.P. Juffermans ${ }^{11} ;$ N. van $\mathrm{Es}^{1}$; on behalf of the Dutch COVID \& Thrombosis Coalition

${ }^{1}$ Department of Vascular Medicine, Amsterdam Cardiovascular Sciences, Amsterdam University Medical Center, Amsterdam, Netherlands; ${ }^{2}$ Department of Pulmonary Medicine, Zuyderland Medical Centre, Heerlen, Netherlands; ${ }^{3}$ Department of Internal Medicine, Cardiovascular Research Institute Maastricht, Maastricht, Netherlands; ${ }^{4}$ Department of Intensive Care Medicine, Amsterdam University Medical Centers, Location VUmc, Amsterdam, Netherlands; ${ }^{5}$ Department of Internal Medicine, Flevo Hospital, Almere, Netherlands; ${ }^{6}$ Department of Intensive Care Medicine, Amsterdam University Medical Center, Amsterdam, Netherlands; ${ }^{7}$ Department of Anaesthesiology, Martini Hospital, Groningen, Netherlands; ${ }^{8}$ Institute of Diagnostic Accuracy (iDNA), Groningen, Netherlands; ${ }^{9}$ Department of Internal Medicine, VieCuri Medical Center, Venlo, Netherlands; ${ }^{10}$ Department of Intensive Care Medicine, Treant Zorggroep, Emmen, Netherlands; ${ }^{11}$ Department of Intensive Care Medicine, Onze Lieve Vrouwe Gasthuis, Amsterdam, Netherlands

Background: A high incidence of venous thromboembolism (VTE) is observed in patients with COVID-19. Furthermore, several studies show that hypercoagulability is associated with mortality.

Aims: To investigate whether pre-admission anticoagulant therapy is associated with a lower risk of all-cause mortality in hospitalized COVID-19 patients.

Methods: Retrospective data from 1,851 consecutive patients with PCR-confirmed SARS-CoV-2 infection hospitalized in eight Dutch centres between February $27^{\text {th }}$ and August $1^{\text {st }} 2020$ were used. During this period, Dutch guidelines recommended routine thromboprophylaxis for all hospitalized COVID-19 patients.

After 1:1 propensity score nearest-neighbour matching based on age, sex, and 17 comorbidities, the association between pre-admission anticoagulant therapy for VTE, atrial fibrillation, or other indications (i.e. direct oral anticoagulants or vitamin $\mathrm{K}$ antagonists) and all-cause mortality and intensive care unit (ICU) admission was evaluated.
A secondary analysis was performed with a broader definition of antithrombotic therapy including anticoagulants and antiplatelet drugs. Results: Mean age was 66.4 years (SD, 14.8) and 39\% were women. Pre-admission, 678 patients (37\%) were using anticoagulant and/or antiplatelet therapy of whom 287 (16\%) used anticoagulant therapy only, 408 (22\%) antiplatelet therapy only, and 17 both anticoagulant and antiplatelet therapy. 253 anticoagulant users and 253 patients not using therapeutic anticoagulation were matched.

During a median follow-up of 21 days [IQR: 9.8-21.0], anticoagulant therapy was neither associated with all-cause mortality (hazard ratio [HR], 0.95; 95\%-Cl, 0.70-1.27; Figure 1) nor with ICU admission (HR, $1.0 ; 95 \%-\mathrm{Cl}, 0.59-1.70)$. Results did not materially change in the secondary analysis of anticoagulant and/or antiplatelet therapy (HR for mortality, 1.18 [95\%-Cl, 0.87-1.59] and HR for ICU admission, 2.98 [95\%-Cl, 0.60-1.39]).
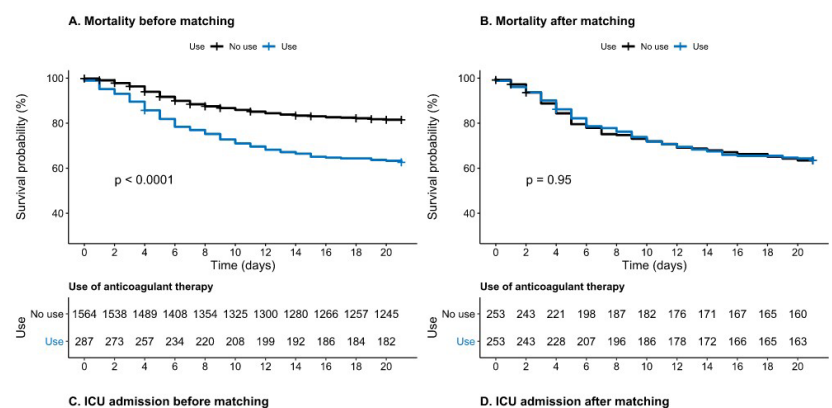

use + Nouse + usa

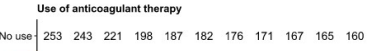

\begin{tabular}{l|lllllllllll} 
No use & 253 & 243 & 221 & 198 & 187 & 182 & 176 & 171 & 167 & 165 & 160
\end{tabular}

\begin{tabular}{cccccccccccc} 
Use- 253 & 243 & 228 & 207 & 196 & 186 & 178 & 172 & 166 & 165 & 163 \\
\hline 0 & $\dot{1}$ & 4 & $\dot{6}$ & 8 & 10 & 12 & 14 & 16 & 18 & 20
\end{tabular} D. ICU admission after matching

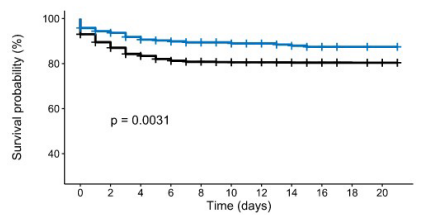

Use of anticoagulant therap

go No use 15641382126111621104108410661056104910441042

Use $287 \begin{array}{lllllllllll}260 & 239 & 215 & 200 & 190 & 182 & 174 & 167 & 165 & 163\end{array}$

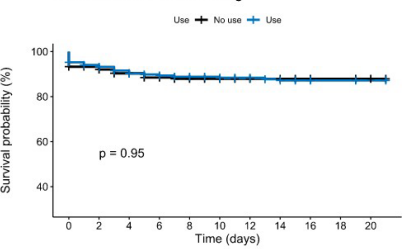

Use of anticoagulant therapy

No use - $253 \quad 228 \begin{array}{lllllllll}202 & 176 & 165 & 160 & 154 & 151 & 148 & 146 & 145\end{array}$

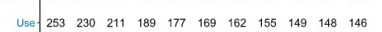
$\begin{array}{lllllllllll}0 & 2 & 4 & \dot{6} & 8 & 10 & 12 & 14 & 16 & 18 & 20\end{array}$

FIGURE 1 All-cause mortality (A-B) and ICU admission (C-D) in patients with and without anticoagulant therapy. Time from hospital admission to death and ICU admission overall (A-C) and in the matched groups (B-D). 
TABLE 1 Patient characteristics overall and in matched groups with asterisk is also used in propensity matched model

\begin{tabular}{|c|c|c|c|c|c|c|c|c|}
\hline $\begin{array}{l}\text { Total population } n= \\
1851\end{array}$ & $\begin{array}{l}\text { Anticoagulant } \\
\text { use } \\
(n=287)\end{array}$ & $\begin{array}{l}\text { No } \\
\text { anticoagulant } \\
\text { use } \\
(n=1564)\end{array}$ & $P$-value & $\begin{array}{l}\text { Standardized } \\
\text { mean } \\
\text { difference }\end{array}$ & $\begin{array}{l}\text { Anticoagulant } \\
\text { use } \\
(n=253)\end{array}$ & $\begin{array}{l}\text { No } \\
\text { anticoagulant } \\
\text { use } \\
(n=253)\end{array}$ & $P$-value & $\begin{array}{l}\text { Standardized } \\
\text { mean } \\
\text { difference }\end{array}$ \\
\hline $\mathrm{Age}^{*}$, years mean $(\mathrm{SD})$ & $76.75(10.36)$ & $64.54(14.75)$ & $<0.001$ & 0.957 & $76.47(16.00)$ & $75.89(10.10)$ & 0.523 & 0.012 \\
\hline $\begin{array}{c}\text { Time in pandemic in } \\
\text { days, mean (SD) }\end{array}$ & $45.69(15.56)$ & $46.88(16.37)$ & 0.253 & 0.075 & $45.55(15.28)$ & $46.96(16.21)$ & 0.419 & 0.071 \\
\hline Women", n (\%) & $98(34)$ & $615(39)$ & 0.112 & 0.108 & $89(35)$ & $91(36)$ & 1.000 & 0.008 \\
\hline Diabetes $^{*}, n(\%)$ & $100(35)$ & $382(25)$ & $<0.001$ & 0.228 & $89(35)$ & $100(39)$ & 0.359 & 0.008 \\
\hline Asthma ${ }^{*}, n(\%)$ & $21(7)$ & $171(11)$ & 0.077 & 0.128 & $20(8)$ & $23(9)$ & 0.750 & 0.045 \\
\hline Obesity ${ }^{*}, n(\%)$ & $85(32)$ & $439(30)$ & 0.726 & 0.028 & $81(32)$ & $87(34)$ & 0.571 & 0.043 \\
\hline Hypertension, n (\%) & $168(59)$ & $684(44)$ & $<0.001$ & 0.296 & $152(60)$ & $156(62)$ & 0.785 & 0.032 \\
\hline $\begin{array}{l}\text { Temperature at } \\
\text { admission in }{ }^{\circ} \mathrm{C} \text {, } \\
\text { mean (SD) }\end{array}$ & $37.68(1.28)$ & $37.82(1.16)$ & 0.062 & 0.116 & $37.70(1.31)$ & $37.81(1.24)$ & 0.308 & 0.091 \\
\hline $\begin{array}{c}\text { Oxygen saturation in } \\
\%, \text { mean (SD) }\end{array}$ & $93.2(6.38)$ & $93.1(5.85)$ & 0.737 & 0.021 & $93.3(6.44)$ & $92.9(5.85)$ & 0.453 & 0.067 \\
\hline
\end{tabular}

Conclusions: In this retrospective cohort study, pre-admission anticoagulant use was not associated with a lower risk of mortality or ICU admission in hospitalized COVID-19 patients. Further data from randomized controlled trials are needed to determine the riskbenefit ratio of initiating anticoagulant therapy during admission for COVID-19.

LPB0057 | Diagnostic Management of Suspected Pulmonary Embolism in Patients with COVID-19: A Prospective Cohort Study

M.A.M. Stals ${ }^{1}$; F.HJ Kaptein ${ }^{1}$; RHH Bemelmans ${ }^{2}$; T van Bemmel ${ }^{3}$; DCW Braeken ${ }^{4}$; DD Deenstra ${ }^{5}$; YPA van Dooren ${ }^{6}$; LM Faber; MJJH Grootenboers ${ }^{8}$; LR de Haan ${ }^{9}$; C Haazer $^{10}$; A Iglesias del Sol ${ }^{11}$; S Kelliher ${ }^{12}$; RI Meijer ${ }^{13}$; PE Westerweel ${ }^{14}$; FA Klok ${ }^{1}$; MV Huisman ${ }^{1}$; on behalf of the Dutch COVID \& Thrombosis Coalition (DCTC) ${ }^{1}$ Department of Thrombosis and Hemostasis, Leiden University Medical Center, Leiden, Netherlands; ${ }^{2}$ Department of Internal Medicine, Gelderse Vallei Hospital, Ede, Netherlands; ${ }^{3}$ Department of Internal Medicine, Gelre Hospitals, Apeldoorn, Netherlands; ${ }^{4}$ Thrombosis Expertise Center, Maastricht University Medical Center, Maastricht, Netherlands; ${ }^{5}$ Department of Pulmonology, Catharina Hospital, Eindhoven, Netherlands; ${ }^{6}$ Department of Pulmonology, Groene Hart Hospital, Gouda, Netherlands; ${ }^{7}$ Department of Haematology, Red Cross Hospital, Beverwijk, Netherlands; ${ }^{8}$ Department of Pulmonology, Amphia Hospital, Breda, Netherlands; ${ }^{9}$ Department of Internal Medicine, Flevo Hospital, Almere, Netherlands; ${ }^{10}$ Department of Internal Medicine, Reinier de Graaf Hospital, Delft, Netherlands; ${ }^{11}$ Department of Internal Medicine, Alrijne Hospital Leiderdorp, Leiderdorp, Netherlands; ${ }^{12}$ Department of Haematology, Mater Misericordiae University Hospital, Ireland and Irish Network for VTE Research, Dublin, Ireland; ${ }^{13}$ Department of Internal Medicine, Amsterdam University Medical Center, location VUmc, Amsterdam, Netherlands; ${ }^{14}$ Department of Haematology, Albert Schweitzer
Hospital, Dordrecht, Netherlands

Background: Diagnostic strategies for suspected pulmonary embolism (PE) have not been prospectively evaluated in COVID-19 patients.

Aims: Evaluate diagnostic strategies for suspected PE in patients with COVID-19.

Methods: Prospective, multicenter, cohort study in 708 patients with suspected PE and (suspected) COVID-19, without anticoagulant therapy, from 14 hospitals (March 1 - October 29, 2020). The study was approved by Institutional Review Boards of these hospitals and informed consent was obtained by opt-out approach. Different management protocols for suspected PE were used in the participating hospitals: YEARS algorithm, computed tomography pulmonary angiography (CTPA) only, or Wells rule with variable D-dimer threshold. We evaluated the safety (3-month failure rate) and efficiency (number of CTPAs avoided) of the three strategies.

Results: Baseline characteristics are presented in Table 1; PE prevalence was $28 \%$. YEARS was applied in $36 \%$, Wells rule in $4.2 \%$ and CTPA only in 52\%; $7.3 \%$ was not tested because of hemodynamic instability. Within YEARS, PE was considered excluded without CTPA in $29 \%$, of which one patient developed non-fatal PE during followup (failure rate $1.4 \% ; 95 \% \mathrm{Cl} 0.03-7.4$; Table 2). 117 patients had a negative CTPA within YEARS (46\%), of whom 10 were diagnosed with non-fatal venous thromboembolism (VTE) during follow-up (failure rate $8.5 \%$; $95 \% \mathrm{Cl} 4.2-15$; Table 2 ). In patients imaged with CTPA only, $65 \%$ had an initial negative CTPA, of whom 10 patients were diagnosed with non-fatal VTE during follow-up (failure rate 4.1\%; $95 \% \mathrm{Cl} 2.0-7.5$ ). CTPA was avoided in 2 patients (6.7\%) managed with Wells rule. 
TABLE 1 Baseline characteristics

\section{Baseline characteristics}

Age (mean, SD)

Male sex (number, \%)

Body Mass Index (median, IQR)

Active cancer (number, \%)

Prior history VTE (number, \%)

Admitted to the ICU at time of suspected PE event (number, \%)

Confirmed COVID-19 status* at time of suspected PE event (number, \%)
$N=708$

$62(15)$

399 (56)

$27(24-30)$

$73(10)$

45 (6.4)

$152(22)$

$425(60)$

Note: SD: standard deviation; IQR: interquartile range; VTE: venous thromboembolism; ICU: Intensive Care Unit; PE: pulmonary embolism. *COVID-19 status was confirmed in patients with a positive polymerase chain reaction (PCR) test or considered positive in patients with a negative PCR but highly suggestive symptoms and typical COVID-19 abnormalities on CT-scan of the chest (CO-RADS 4 or 5 following Dutch Radiology Society) with no alternative diagnosis.

TABLE 2 Details of the diagnostic strategies for suspected PE in 708 study patients

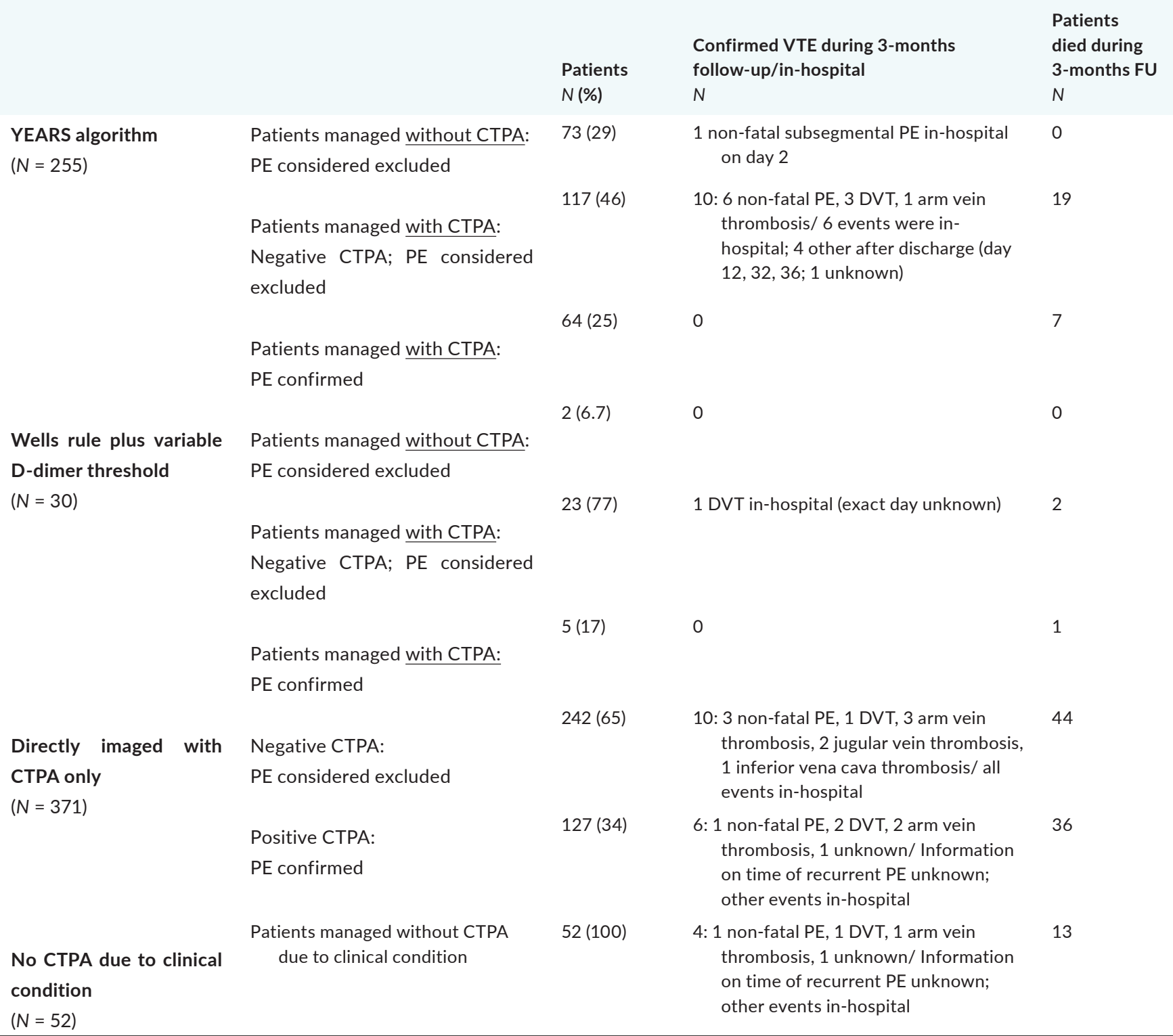

Note: PE: pulmonary embolism, CTPA: computed tomography pulmonary angiography; N: number; VTE: venous thromboembolism; FU: follow-up; DVT: deep vein thrombosis 
Conclusions: CTPA could be avoided in $29 \%$ of patients managed by YEARS, with a low failure rate (1.4\%). The failure rate of a negative CTPA, used as a sole test or within YEARS, was higher than in a non-COVID-19 population and warrants ongoing suspicion in these patients. Our results underline the applicability of the YEARS algorithm in COVID-19 patients with suspected PE.

LPB0058 | D-dimer Elevation at Time of Admission Is Associated with Need for Ventilator Support among Pediatric Patients with COVID-19 Infection

N. Wilken ${ }^{1}$; V. Kostousov ${ }^{1,2}$; K. Bruzdoski ${ }^{2}$; S. Sartain ${ }^{3}$; K. Krum ${ }^{1,2}$; L. Hensch ${ }^{1,2}$; J. Teruya ${ }^{1,2}$; S.-K.R. Hui ${ }^{1,2}$

${ }^{1}$ Department of Pathology \& Immunology, Baylor College of Medicine, Houston, United States, ${ }^{2}$ Department of Pathology, Texas Children's Hospital, Houston, United States, ${ }^{3}$ Department of Pediatrics, Section of Hematology-Oncology, Baylor College of Medicine, Texas Children's Hospital, Houston, United States

Background: One of the biggest challenges in the care of COVID-19 infected patients is predicting the severity of disease course and the need for ICU care and/or ventilator support. Published studies have suggested that $\mathrm{D}$-dimer on admission can predict in-hospital mortality and prognosis. However, these data are mostly limited to adult populations with limited studies in pediatric populations.

Aims: To determine if coagulation parameters at admission are associated with clinical severity of COVID-19 infection among pediatric patients.

Methods: We retrospectively reviewed admission coagulation studies [Diagnostica Stago, Inc] (D-dimer, Prothrombin Time, PTTHepzyme, Fibrinogen and Platelet (PLT) count) in children with a
COVID-19 diagnosis at a tertiary care pediatric hospital from April 2020 through February 2021. Disease severity was determined by ICU admission, length of stay (LOS), and need for ventilator support. Statistical analysis, including Mann Whitney $U$ test and Pearson correlation was performed with data presented as mean \pm SD with significance of $P<0.05$.

Results: There were 110 pediatric patients (57 females) ranging from 0.5 months to 18 years who had coagulation studies collected within $24 \mathrm{~h}$ of admission. Patients were divided into three groups based on ICU admission and ventilation support (see Table). Patients who required ICU admission and ventilation support had significantly higher D-dimer $(P=0.0006)$ and PT $(P=0.0083)$ values compared to patients who required neither. In addition, $D$-dimer was higher in this group when compared to those in the ICU only group $(P=0.0099)$. Only D-dimer showed moderate correlation with LOS in the total cohort of patients $(r=0.46, P<0.0001)$ (Fig. 1).

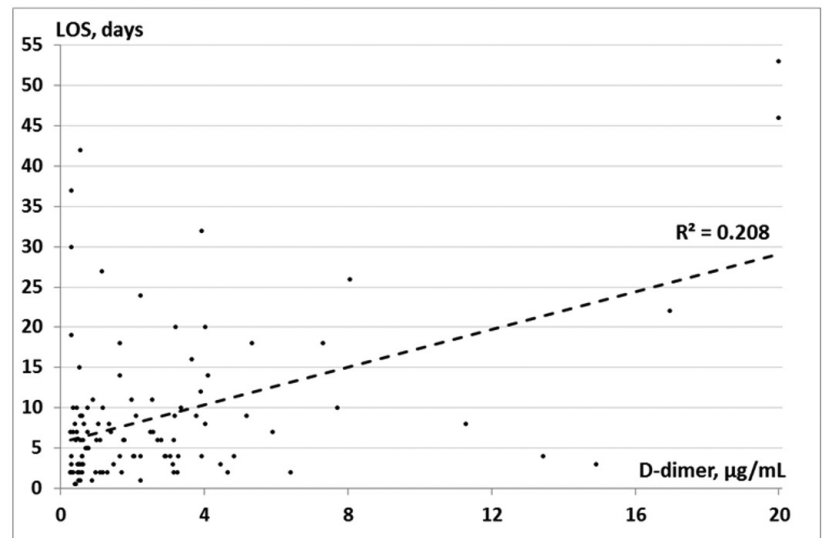

FIGURE 1 Correlation Between Length of Stay (LOS) and D-dimer

TABLE 1 Coagulation Studies on Admission and Length of Stay (LOS) for Patients without ICU Admission, with ICU Admission and with Ventilator Support

\begin{tabular}{llll} 
& & & \multicolumn{1}{c}{$\begin{array}{l}\text { ICU Admission and Ventilator } \\
\text { Support }\end{array}$} \\
Patients & No ICU Admission & ICU Admission & 19 \\
Age (years) & 54 & 37 & $11.8 \pm 3.5$ \\
Fibrinogen $(220-440 \mathrm{mg} / \mathrm{dL})$ & $9.4 \pm 6.4$ & $10.7 \pm 5.3$ & $463 \pm 158$ \\
D-dimer $(<0.4 \mu \mathrm{g} / \mathrm{mL})$ & $423 \pm 166$ & $446 \pm 146$ & $5.7 \pm 6.3$ \\
PTT-Hepzyme $(25.5-33.2 \mathrm{~s})$ & $2.0 \pm 2.8$ & $2.2 \pm 2.3$ & $32.4 \pm 6.5$ \\
PT (10.5-15.7 s) & $32.5 \pm 7.0$ & $31.9 \pm 5.5$ & $15.8 \pm 1.6$ \\
Platelet $\left(150-450 \times 10^{3} / \mu \mathrm{L}\right)$ & $14.8 \pm 1.6$ & $15.2 \pm 1.4$ & $222 \pm 160$ \\
LOS (days) & $258 \pm 122$ & $204 \pm 118$ & $21 \pm 14$ \\
\hline
\end{tabular}

Conclusions: Elevated D-dimer significantly correlated with severity of disease and LOS, while elevated PT only correlated with disease severity. Our data suggest that D-dimer at admission may predict a pediatric patient's need for ICU care or ventilator support. 
LPB0059 | Population Pharmacokinetics of Anti-Xa Levels in COVID-19 ICU Patients Receiving Prophylaxis for Thromboembolic Events Using Nadroparin

L.G.R. Romano ${ }^{1}$; H. Endeman ${ }^{2}$; N.G.M. Hunfeld ${ }^{2,3}$; T. Preijers ${ }^{3}$

${ }^{1}$ Department of Hematology, Erasmus MC, Erasmus University Medical Center, Rotterdam, Netherlands; ${ }^{2}$ Department of Adult Intensive Care, Erasmus MC, Erasmus University Medical Center, Rotterdam, Netherlands; ${ }^{3}$ Department of Hospital Pharmacy - Clinical Pharmacology, Erasmus MC, Erasmus University Medical Center,

Rotterdam, Netherlands

Background: An increased incidence of thrombo-embolic events has been described in Coronavirus Disease 2019 (COVID-19) patients, especially in critically-ill patients. In response, ISTH guidelines consider use of intermediate-dose low-molecular-weight heparins (LMWH) in critically-ill COVID-19 patients. Therefore, twice daily nadroparin dosing can been applied to prevent thrombo-embolic events. Although population pharmacokinetic (PK) parameters of nadroparin are unknown in ICU COVID-19 patients, these parameters would allow evaluation of the proposed dosing schemes from current guidelines.

Aims: To construct a population PK model describing anti-Xa levels subsequent to nadroparin administration, allowing assessment of population PK parameters and their associated interpatient variability (IIV) in COVID-19 Intensive Care Unit (ICU)-patients.

Methods: Retrospective data of 65 ICU patients with at least one positive SARS-CoV-2 RT-PCR test were included (Table 1/Results). At start of ICU admission, patients received twice daily 5700 IU nadroparin according to local protocol. Anti-Xa sampling was applied routinely daily. Population PK parameters were estimated using nonlinear mixed-effect modelling (NONMEM v7.4.1). Data collection was approved by the local ethics committee.
Results: Anti-Xa level versus time profiles were adequately described using a population PK model with one elimination and one absorption compartment (Figure 2). Including a transit-compartment did not result in significantly better data description. Population PK parameter estimates and IIV (\%) were: absorption rate: $0.245 \mathrm{~h}^{-1}$, clearance (CL): $2010 \mathrm{~mL} / \mathrm{h}$ (33.9\%), V1: $10.0 \mathrm{~L}$. CL increased exponentially with rising CRP, D-dimer and renal function (CKD-EPI eGFR). When applying corticosteroids or vasopressors, CL was decreased by $22.4 \%$ and $20.3 \%$ respectively.

TABLE 1 General characteristics of the study population

\begin{tabular}{|c|c|c|c|}
\hline & $\begin{array}{l}\text { Total population } \\
\text { No. (\%) or median [interquartile } \\
\text { range] }\end{array}$ & $\begin{array}{l}\text { Male } \\
\text { No. (\%) or median [interquartile } \\
\text { range] }\end{array}$ & $\begin{array}{l}\text { Female } \\
\text { No. (\%) or median [interquartile } \\
\text { range] }\end{array}$ \\
\hline Age (years) & $62[53-68.5]$ & $66[53-71]$ & $60.5[54.2-65]$ \\
\hline Body weight (kg) & 89 [78 - 100] & 90.5 [83.1 - 105] & 85 [72 - 95] \\
\hline D-dimer (mg/l) & $1.32[0.74-2.24]$ & 1.27 [0.73 - 2.28] & $1.32[1.02-2.22]$ \\
\hline $\mathrm{CRP}(\mathrm{mg} / \mathrm{l})$ & 119 [74.7 - 195] & $126[74.7-205]$ & 114 [78.7 - 165] \\
\hline $\begin{array}{l}\text { Vasopression use at } \\
\text { start }\end{array}$ & $57(88)$ & $35(88)$ & $22(88)$ \\
\hline
\end{tabular}




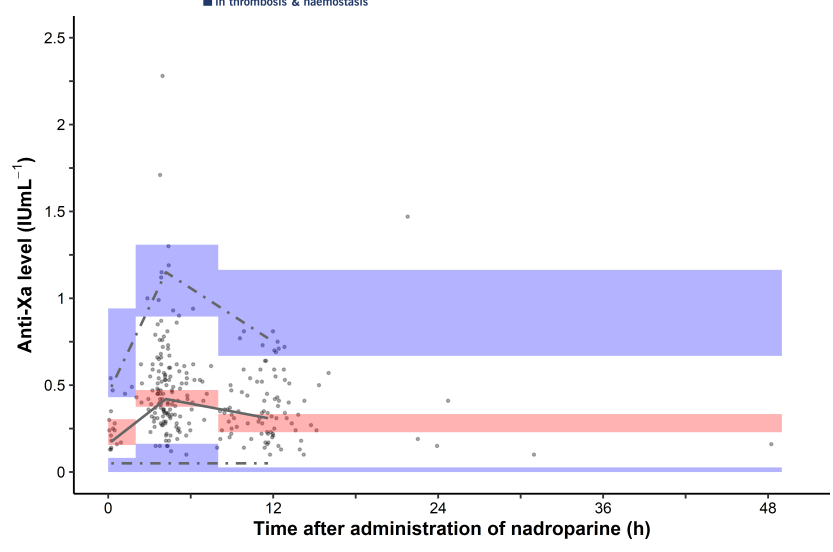

FIGURE 2 Prediction-corrected Visual Predictive Check of the final model. Black dots represent the measured anti-Xa levels. Solid grey line represents the median and the dashed grey lines represent the 2.5 th and 97.5th quantiles of the measured anti-Xa levels. Red and blueshaded areas show the $95 \%$ confidence intervals for the predicted individual anti-Xa levels, as obtained by 2000 Monte Carlo simulations using the final model.

Conclusions: In critically-ill COVID-19 patients, measured anti-Xa levels were adequately described by the established population PK model. Our model shows that nadroparin clearance, reported as anti-Xa levels, is influenced by inflammation. This model will be used to evaluate limited sampling schemes for anti-Xa to facilitate blood sampling in clinical practice.

LPB0105 | External Validation of IMPROVE-DD Risk Assessment Model for Venous Thromboembolism among Inpatients with COVID-19

S.K. Lin ${ }^{1}$; M. Goldin ${ }^{1,2,3}$; N. Kohn²; M. Qiu ${ }^{4}$; S.L. Cohen ${ }^{1,2} ;$ M.A. Barish $^{3}$; E. Gianos ${ }^{1,5}$; D. Giannis ${ }^{2}$; S. Chatterjee ${ }^{1,3}$; K. Coppa ${ }^{4}$; A. Diaz $^{1}$; S. Richardson ${ }^{1,2}$; J.S. Hirsch ${ }^{1,2,4}$; S. Ngu' ${ }^{6}$ S. Firoozan ${ }^{6} ;$ T. McGinn $^{1,2}$; A.C. Spyropoulos ${ }^{1,2}$

${ }^{1}$ Donald \& Barbara Zucker School of Medicine at Hofstra/Northwell, Hempstead, United States; ${ }^{2}$ Feinstein Institutes for Medical Research,
Northwell Health, Manhasset, United States; ${ }^{3}$ North Shore University Hospital, Northwell Health, Manhasset, United States; ${ }^{4}$ Department of Information Services, Northwell Health, New Hyde Park, United States; ${ }^{5}$ Division of Cardiology, Lenox Hill Hospital, Northwell Health, New York, United States; ${ }^{6}$ Department of Medicine, Northwell Health, Manhasset, United States

Background: There is a need to move towards an individualized, risk-adapted approach in order to discriminate higher risk groups for venous thromboembolism (VTE) in hospitalized COVID-19 patients and inform thromboprophylaxis strategies. The IMPROVE-DD VTE risk assessment model (RAM) has previously demonstrated good discrimination in non-COVID populations.

Aims: This study aimed to externally validate the IMRPOVE-DD RAM for VTE in a hospitalized, COVID-19 medical population in a large, integrated academic health center.

Methods: In this retrospective cohort study, the IMPROVE-DD VTE RAM was evaluated on adult patients with COVID-19 admitted to one of thirteen Northwell Health hospitals in the New York metropolitan area between March 1, 2020 and April 27, 2020. VTE was defined as a new onset deep venous thrombosis or pulmonary embolism, as identified on Radiology Department imaging or pointof-care ultrasound and manually adjudicated by two attending radiologists. To assess the predictive value of the RAM, the receiver operating characteristic (ROC) curve was plotted, and area under the curve (AUC) was calculated. Sensitivity, specificity, positive predictive value (PPV), and negative predictive value (NPV) were calculated using standard methods.

Results: Of the 9,407 patients who met the study criteria, 274 patients developed VTE with a prevalence of $2.91 \%$. The VTE rate was $0.41 \%$ for IMPROVE-DD score 0-1 (low risk), $1.21 \%$ for score 2-3 (moderate risk), and 5.30\% for score $\geq 4$ (high risk) (Table 1). Approximately $45.7 \%$ of patients were classified as high VTE risk, $33.3 \%$ moderate risk, and $21.0 \%$ low risk. The RAM discrimination of low versus moderate-high VTE risk demonstrated sensitivity 0.971 , specificity 0.215 , PPV 0.036, and NPV 0.996. ROC AUC was 0.703 (Figure 1).

TABLE 1 Observed VTE Events of IMPROVE-DD VTE RAM among COVID-19 Inpatients, based on Score Thresholds

\begin{tabular}{llll} 
& VTE Events & & Total \\
IMPROVE -DD & VTE & No VTE & $1975(21.00 \%)$ \\
0-1, Low Risk & $8(0.41 \%)$ & $1967(99.59 \%)$ & $3129(33.26 \%)$ \\
2-3, Moderate Risk & $38(1.21 \%)$ & $3091(98.79 \%)$ & $4303(45.74 \%)$ \\
3-12, High Risk & $228(5.30 \%)$ & $4075(94.70 \%)$ & $9407(100.0 \%)$ \\
Total & $274(2.91 \%)$ & $9133(97.09 \%)$ & \\
\hline
\end{tabular}




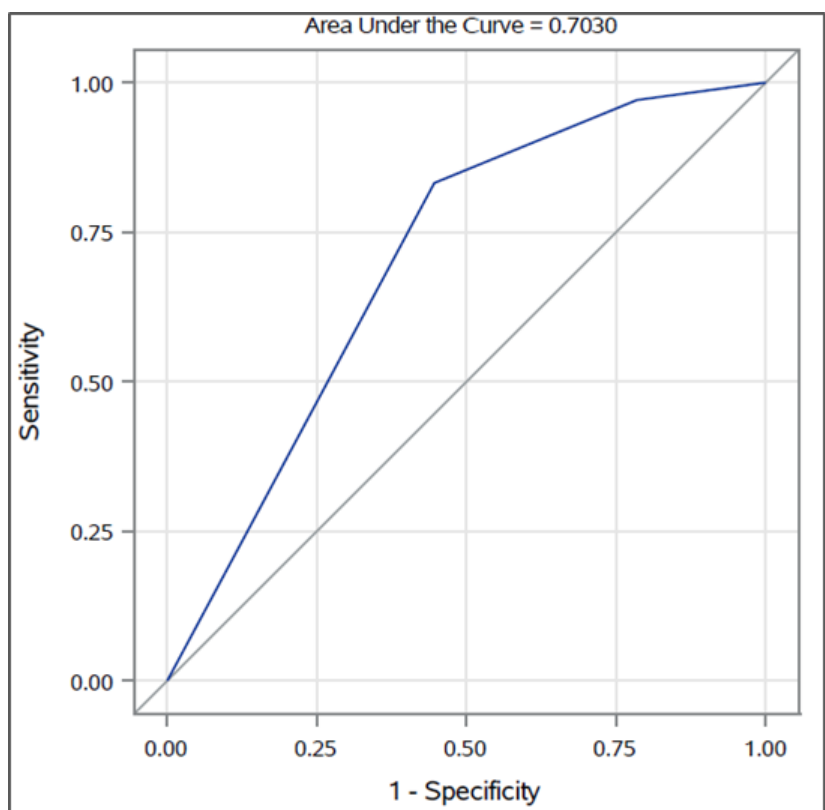

FIGURE 1 Logistic Regression with Receiver Operating Characteristic Curve for Inpatient VTE for IMPROVE-DD Score

Conclusions: In this external validation study, the IMPROVE-DD VTE RAM demonstrated very good degree of discrimination to identify hospitalized COVID-19 patients at low, moderate, and high VTE risk.

\section{PB0164 | Circulating Histones Are Associated with Disease} Severity and Coagulopathy in COVID-19

R.J Shaw ${ }^{1}$; J. Austin ${ }^{1}$; J. Taylor ${ }^{2}$; T. Dutt ${ }^{2}$; G. Wang ${ }^{1}$; S.T Abrams ${ }^{1}$; C.-H. Toh $^{1}$

${ }^{1}$ University of Liverpool, Liverpool, United Kingdom; ${ }^{2}$ Liverpool

University Hospitals NHS Foundation Trust, Liverpool, United Kingdom

Background: COVID-19 has highlighted lethal consequences of cross-talk between coagulation, inflammation and innate immune processes. Hospitalised COVID-19 patients have evidence of immune cell death, which would result in the release of nuclear material, such as histones. Extracellular histones are associated with adverse clinical outcomes and our work has shown they are procoagulant, pro-inflammatory and can cause pulmonary thrombosis. We hypothesize that circulating histones play a central role in poor outcomes in COVID-19.

Aims: We aim to determine the pathological role of circulating histones in disease severity, coagulation activation, inflammation and organ injury in COVID-19.

Methods: One hundred and three COVID-19 patients were recruited at the Royal Liverpool University Hospital, in accordance with ISARIC/WHO Clinical Characterisation Protocol for Severe Emerging Infections in the UK. Inclusion criteria: (1) swab positive/ high likelihood of infection OR (2) fever $\geq 38^{\circ} \mathrm{C}$, cough, dyspnoea/ tachypnoea. Circulating histones were quantified in patient plasma and patients were categorised into three groups based on severity of infection: mild (minimal symptoms/incidental finding), severe (dyspnoea/hypoxia) and critical (respiratory failure/multi-organ failure).

Results: Admission histone levels were significantly $(P<0.001)$ elevated in patients with increasing severity of COVID-19 infection (Mild; $2.00 \mu \mathrm{g} / \mathrm{ml}$ [0.68-6.62], Severe; $9.75 \mu \mathrm{g} / \mathrm{ml}$ [3.61-21.88], Critical; $23.37 \mu \mathrm{g} / \mathrm{ml}$ [11.35-30.02]). Histones were associated with a pro-coagulant (histones vs d-dimer; $R=0.596, P<0.001$ ) and proinflammatory phenotype (histones vs CRP; $R=0.730, P<0.001$, histones vs fibrinogen; $R=0.677, P<0.001$ ). Increased circulating histones were associated with organ dysfunction including hypoxia (oxygen saturations $\leq 93 \%$; $P=0.008$ ), raised bilirubin $(R=0.568$, $P=0.002)$ and elevated serum creatinine $(R=0.508, P=0.009)$. Patients with elevated histones required critical care admission $(P<0.001)$, increased duration of mechanical ventilation $(R=0.778$, $P=0.022)$ and overall length of hospital stay $(R=0.618, P<0.001)$.

Conclusions: Admission histone levels are associated with disease severity, coagulation activation, inflammation and organ dysfunction in COVID-19. This study indicates that elevated circulating histones might play a key role in the immuno-thrombotic pathogenesis of COVID-19.

PB0165 | COVID-19-Related Thrombosis in Japan: Results of a Questionnaire-based Survey in 2020

H. Horiuchi $^{1}$; E. Morishita ${ }^{2}$; T. Urano ${ }^{3}$; K. Yokoyama ${ }^{4}$; - The Questionnaire-Survey Joint Team on the COVID-19-Related Thrombosis $5,6,7$

${ }^{1}$ Tohoku University, Sendai, Japan; ${ }^{2}$ Kanazawa University, Kanazawa, Japan; ${ }^{3}$ Hamamatsu University School of Medicine, Hamamatsu, Japan; ${ }^{4}$ Tokai University Hachioji Hospital, Tokyo, Japan; ${ }^{5}$ The Japanese Society of Thrombosis and Hemostasis, Tokyo, Japan; ${ }^{6}$ The Japan Atherosclerosis Society, Tokyo, Japan; ${ }^{7}$ The Research Study Team for Intractable Disease (Blood Coagulation Abnormalities) Supported by the Ministry of Health, Labour and Welfare of Japan, Kanazawa, Japan

Background: COVID-19 became pandemic. Many reportssuggest that thrombosis is intimately involved in clinical deterioration of COVID-19 patients. However, data on COVID-19-related thrombosis in Japan were limited.

Aims: A questionnaire-based survey was conducted in order to characterize COVID-19-related thrombosis in Japan by the joint survey team including members of JSTH.

Methods: A questionnaire was sent to 399 hospitals throughout Japan.

Results: Responses were received from 111 (27.8\%) with information on 6,202 COVID-19 patients hospitalized before Aug 31, 2020. Of these, 333 and 56 required ventilation or extracorporeal membrane oxygenation (ECMO), respectively, and 212 died (3.4\%). D-dimer levels were measured in $75.0 \%$ of the patients, revealing 
that $9.2 \%$ and $7.6 \%$ exhibited D-dimer increases of 3-8-fold and $\geq 8$ fold the reference value, respectively. Thrombotic events occurred in 108 patients ( $1.86 \%$ of the 5,807 patients with available data) including symptomatic cerebral infarction in 24 , myocardial infarction in 7, deep vein thrombosis in 41, pulmonary thromboembolism in 30 , and other thrombotic events in 22 . Some patients developed multiple thrombotic events. Thrombosis occurred in 32 patients with mild or moderate COVID-19 severity $0.59 \%$ of those with data available) and in 52 patients on ventilation or ECMO (13.5\% of severe patients for whom data were available). Thrombosis occurred in 67 patients during worsening clinical condition and in 26 during recovery. Anticoagulant therapy was provided to 893 patients (14.6\% of the 6,119 patients with available data), the main reasons being provided as elevated D-dimer levels and worsening clinical condition.

Conclusions: Thus, the incidence of thrombotic events in mild/moderate COVID-19 patients in Japan might be much lower than that in the Western countries, while that in severe COVID-19 patients seems comparable/or slightly lower. Of the thromboses, although deep vein thrombosis and pulmonary thromboembolism were most frequent, symptomatic cerebral infarction was also frequent, occurring as $22 \%$ of thromboses, which might be a feature specific to Japanese.

\section{PB0166 | Von Willebrand Factor and ADAMTS13 Activity as Endothelial Injury Markers and their Prognostic Value in COVID-19}

A. Marco ${ }^{1,2}$; P. Marco ${ }^{1,2,3}$

${ }^{1}$ University General Hospital in Alicante, Alicante, Spain; ${ }^{2}$ Biomedical Research Institute, Alicante, Spain; ${ }^{3}$ Clinical Medicine Department, Miguel Hernandez University, Alicante, Spain

Background: The coronavirus disease 2019 (COVID-19) increases thrombotic risk. The mechanisms that lead to this prothrombotic state are unclear.

Aims: The main aim was to evaluate the von Willebrand factor (VWF) antigen and plasma ADAMTS13 activity as endothelial injury markers in COVID-19 and their prognostic value in COVID-19 evolution. Methods: We present a prospective study in COVID-19 patients recruited in our institution. The patients were divided into 2 groups depending on whether hospitalization was needed. Inpatients were subclassified into ward patients and those requiring intensive care. Thirty non-COVID-19 inpatients and 30 non-COVID-19 healthy individuals were recruited. VWF antigen, ADAMTS13 activity, D-dimer, and fibrinogen were measured during the first week once COVID-19 was diagnosed. Quantitative data were expressed as median ( $\mathrm{p} 25$ p75) and qualitative data as percentage.

Results: Fifty COVID-19 inpatients (44\% in the intensive care unit [ICU]) and 102 COVID-19 outpatients were enrolled. Inpatients were older and had a higher incidence of hypertension and diabetes. The COVID-19 inpatients had higher D-dimer, fibrinogen, and VWF antigen levels and a lower ADAMTS13 activity compared with the COVID-19 outpatients $(P<0.05)$. ICU patients had higher D-dimer and VWF antigen levels compared with the ward patients and the lowest ADAMTS13 activity $(P<0.05)$. An imbalance in VWF antigen/ ADAMTS13 activity ratio was observed in COVID-19, reaching the highest in ICU patients. In contrast to other acute inflammatory diseases, a significative reduction in ADAMTS13 activity was observed in all COVID-19 patients.

TABLE 1 Hemostatic parameters: inpatients versus outpatients

Inpatients $(n=50)$

D-Dimer $(\mu \mathrm{g} / \mathrm{mL})$

$2.48(0.88-6.86)$

Fibrinogen $(\mathrm{mg} / \mathrm{dL})$

ADAMTS13 activity (\%)

$44.4(32.5-60.8)$

VWF antigen (\%)
Outpatients $(n=102)$

$0.4(0.27-0.56)$

$P<0.05$

$346.5(291-374)$

$P<0.05$

59.9 (43.4-78.75)

$P<0.05$

121.6 (95.75-151.95)

$P<0.05$ 
TABLE 2 Hemostatic parameters: ward patients versus ICU patients

Ward patients $(n=28)$

$0.89(0.73-2.31)$

D-Dimer $(\mu \mathrm{g} / \mathrm{mL})$

Fibrinogen (mg/dL)

ADAMTS13 activity(\%)

$511(383-561)$

$46.5(40.4-60.9)$

VWF antigen (\%)
ICU patients $(n=22)$

$4.64(2.66-11.04)$

$P<0.05$

$505(400-576)$

$P<0.05$

$38.85(26-60)$

$P<0.05$

$368.6(336.3-400)$

$P<0.05$
Conclusions: There is an increase in VWF antigen and an ADAMTS13 activity reduction in COVID-19 related to disease severity and could predict poor clinical outcomes. The ADAMTS13 activity reduction could be a marker associated with COVID-19 in contrast to other inflammatory conditions.

PB0167 | The Kallikrein-kinin System in Bronchoalveolar Lavage Fluid from Patients with COVID-19

C.P. Martens ${ }^{1,2}$; P. Van $\mathrm{Mol}^{3,4}$; J. Wauters ${ }^{5,6}$; E. Wauters ${ }^{7}$;

T. Gangnus ${ }^{8}$; B. Noppen ${ }^{9}$; L. Liesenborghs ${ }^{1,10}$; E. Heylen ${ }^{10}$;

S. Jansen ${ }^{10}$; L.C. Velásquez Pereira ${ }^{1}$; M.M Engelen ${ }^{1,2}$; A. Van Herck ${ }^{7}$;

R. Vos ${ }^{7,4}$; C. Vandenbriele ${ }^{1,2}$; K. Martinod ${ }^{1}$; B.B Burckhardt ${ }^{8}$;

M. Vanhove ${ }^{9}$; P. Verhamme ${ }^{1,2}$; J. Neyts ${ }^{10} ;$ T. Vanassche ${ }^{1,2}$

${ }^{1}$ Center for Molecular and Vascular Biology, Department of Cardiovascular Sciences, KU Leuven, Leuven, Belgium; ${ }^{2}$ Department of Cardiovascular Diseases, University Hospitals Leuven, Leuven, Belgium; ${ }^{3}$ Laboratory of Translational Genetics, VIB - KU Leuven, Leuven, Belgium; ${ }^{4}$ Department of Respiratory Diseases, University Hospitals Leuven, Leuven, Belgium; ${ }^{5}$ Medical Intensive Care Unit, Department of General Internal Medicine, University Hospitals Leuven, Leuven, Belgium; ${ }^{6}$ Laboratory for Clinical Infectious and Inflammatory Diseases, Department of Microbiology and Immunity, KU Leuven, Leuven, Belgium; ${ }^{7}$ Department of Chronic Diseases and Metabolism (CHROMETA), Laboratory of Respiratory Diseases and Thoracic Surgery (BREATHE), KU Leuven, Leuven, Belgium; ${ }^{8}$ Institute of Clinical Pharmacy and Pharmacotherapy, Heinrich Heine University, Düsseldorf, Germany; ${ }^{9}$ Oxurion NV, Leuven, Belgium; ${ }^{10}$ Laboratory of Virology and Chemotherapy, Department of Microbiology, Immunology and Transplantation, Rega Institute, KU Leuven, Leuven, Belgium

Background: Markers of both inflammation and coagulation are linked to clinical outcome in coronavirus disease 2019 (COVID-19).
Binding of the severe acute respiratory syndrome coronavirus 2 (SARS-CoV-2) to the angiotensin-converting enzyme 2 receptor, which is involved in kinin breakdown, interferes with the kallikreinkinin pathway. This could result in increased vascular permeability, fluid excess in the lungs and pulmonary edema. Furthermore, the kallikrein-kinin pathway links coagulation and inflammation through its interactions with the contact activation pathway of coagulation via factor XII and with neutrophil extracellular traps (NETs). These insights could help to explain the clinical presentation of COVID-19 pneumonia with pulmonary coagulopathy and the high incidence of thromboembolic complications in COVID-19.

Aims: Given the lack of clinical evidence to support this hypothesis, we studied the kallikrein-kinin system in bronchoalveolar lavage (BAL) fluid.

Methods: In BAL fluid samples from patients with or without COVID-19, we performed in-depth analyses of kinin peptides (bradykinin, Lys-bradykinin, Lys-bradykinin-(1-8), bradykinin-(1-8), bradykinin-(1-7), and bradykinin-(1-5)) using a liquid chromatography with tandem mass spectrometry assay, along with measurements of plasma and tissue kallikrein hydrolytic activity and myeloperoxidase (MPO)-DNA complexes as a biomarker for NETs. Informed consent and ethical approval were obtained.

Results: We observed higher levels of the most downstream kinin peptide bradykinin-(1-5) (Figure 1), higher tissue kallikrein activity (Figure 2), and higher levels of MPO-DNA complexes $(699.0 \mathrm{ng} / \mathrm{mL}$ [66.0-142621.0], median [range], $n=21$ vs 70.5 [9.9-960.0], $n=19$; $P<0.001)$ in BAL fluid from patients with COVID-19 compared to those in BAL fluid from patients without COVID-19. 


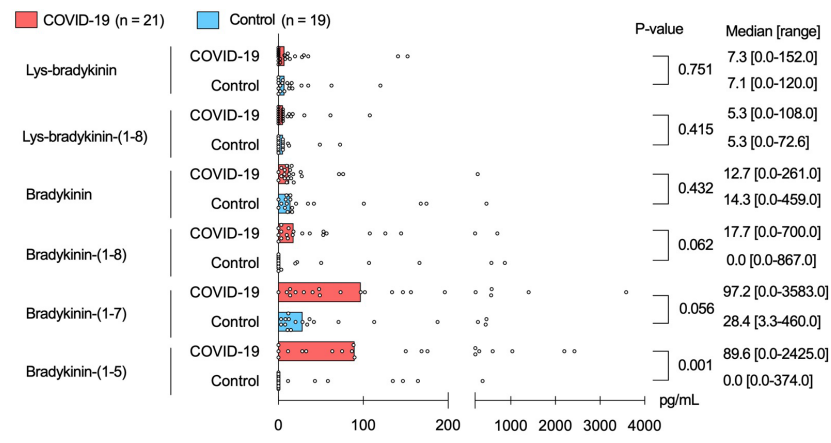

FIGURE 1 Levels of kinin peptides in bronchoalveolar lavage fluid samples from patients with COVID-19 compared to patients without COVID-19. Graphs show individual dots representing patient data and bar representing median of each patient group. Pairwise comparisons were made between patients with COVID-19 $(n=21)$ and patients without COVID-19 $(n=19)$. Median [min-max] and $P$-value are shown

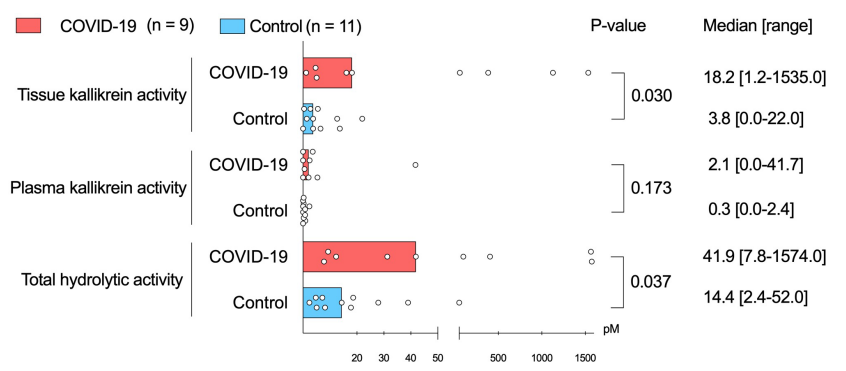

FIGURE 2 Kallikrein hydrolytic activity in bronchoalveolar lavage fluid samples from patients with COVID-19 compared to patients without COVID-19. Graphs show individual dots representing patient data and bar representing median of each patient group. Pairwise comparisons were made between patients with COVID-19 $(n=9)$ and patients without COVID-19 $(n=11)$. Median [min-max] and $P$-value are shown

Conclusions: Our data support the hypothesis that SARS-CoV-2 induces dysregulation of the kallikrein-kinin system, which contributes to thromboinflammation in COVID-19. These findings encourage the investigation of drugs that target the kallikrein-kinin system as a potential treatment option for patients with COVID-19.

Funding: Research Foundation-Flanders (G0G4720N, 1843418N), KU Leuven COVID research fund.

\section{PB0168 | Adjusting D-dimer to Lung Disease Extent to Exclude Pulmonary Embolism in COVID-19 Patients (Co-LEAD)}

L. Khider ${ }^{1}$; B. Planquette ${ }^{1}$; A. Le Berre ${ }^{2}$; S. Soudet ${ }^{3}$; G. Pernod ${ }^{4}$; R. Le Mao ${ }^{5}$; M. Besutti ${ }^{6}$; N. Gendron ${ }^{1}$; A. Yannoutsos ${ }^{2}$; D.M. Smadja $^{1}$; G. Goudot ${ }^{1}$; T. Mirault ${ }^{1}$; E. Messas ${ }^{1}$; J. Emmerich ${ }^{2}$; F. Couturaud ${ }^{5}$; G. Ferretti ${ }^{4}$; M.-A. Sevestre ${ }^{3}$; N. Meneveau ${ }^{6}$; G. Chatellier ${ }^{1}$; O. Sanchez ${ }^{1}$ ${ }^{1}$ Hopital Europeen Georges Pompidou, Paris, France; ${ }^{2}$ Groupe Hospitalier Paris Saint Joseph, Paris, France; ${ }^{3} \mathrm{CHU}$ Amiens, Amiens, France; ${ }^{4} \mathrm{CHU}$ Grenoble, Grenoble, France; ${ }^{5} \mathrm{CHU}$ Brest, Brest, France; ${ }^{6} \mathrm{CHU}$ Besançon, Bensançon, France

Background: D-dimer is a safe tool to exclude pulmonary embolism (PE) but its specificity is decreased in COVID-19.

Aims: Our aim was to derive a new algorithm with $\mathrm{D}$-dimer threshold adjusted to $\mathrm{CT}$ extent of lung damage.

Methods: We conducted a multicenter, retrospective cohort study among 774 COVID-19 patients with suspected PE. D-dimer threshold adjusted to CT extent of lung damage was derived in a patient set $(n=337)$, and its safety assessed in an independent validation set $(n=337)$.

Results: According to ROC curves, D-dimer safely excluded PE, with one false negative when using a $900 \mathrm{ng} / \mathrm{mL}$ threshold when lung damage extent was $<50 \%$ and $1700 \mathrm{ng} / \mathrm{mL}$ when lung damage extent was $\geq 50 \%$. In the derivation set, the algorithm's sensitivity was 98.2\% (95\% Cl: 94.7-100.0), and its specificity $28.4 \%$ (95\% Cl: $24.1-$ 32.3). The negative likelihood ratio (NLR) was 0.06 (95\% Cl: $0.01-$ $0.44)$ and the AUC was 0.63 (95\% Cl: $0.60-0.67)$. In the validation set, sensitivity and specificity were $96.7 \%(95 \% \mathrm{Cl}: 88.7-99.6)$ and $39.2 \%$ (95\% Cl: 32.2-46.1), respectively. The NLR was 0.08 (95\% Cl: $0.02-0.33)$, and the AUC did not differ from that of the derivation set $(0.68$ (95\% Cl: 0.64-0.72), $P=0.097)$. Using the Co-LEAD algorithm, 76/250 (30.4\%) COVID-19 patients with suspected PE could have been managed without CT pulmonary angiography (CTPA).

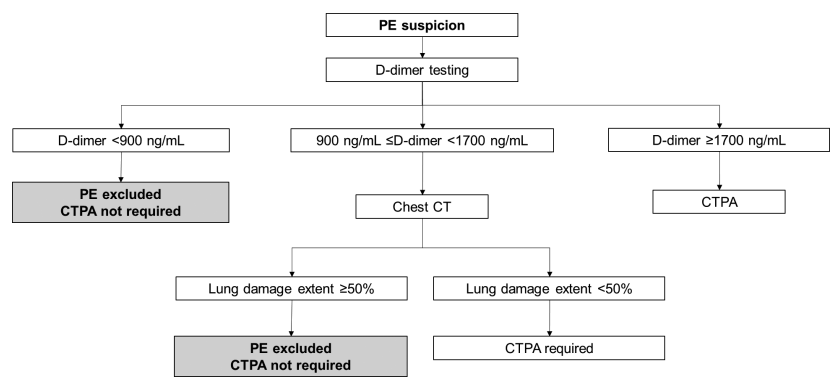

FIGURE 1 Combined strategy for PE suspicion in COVID-19 patients: Co-LEAD algorithm

Conclusions: The Co-LEAD algorithm safely excludes PE, and allows reducing the use of CTPA in COVID-19 patients. Prospective management studies are necessary to validate this strategy. 
PB0169 | ITP Secondary to COVID-19: A Comprehensive Review of Reported Cases

\section{B. Berger ${ }^{1}$; G. Rodgers ${ }^{2}$}

${ }^{1}$ University of Utah School of Medicine, Salt Lake City, United States;

${ }^{2}$ Huntsman Cancer Institute, Salt Lake City, United States

Background: The COVID-19 pandemic has infected 116 million people to date. Hematologic complications of COVID-19 are common, and associated thrombocytopenia correlates with disease severity. Immune thrombocytopenia (ITP) secondary to COVID-19 has been reported, but the characteristics of this novel entity remain unknown.

Aims: To elucidate the demographics, clinical presentations, COVID-19 symptoms, management strategies, complications, and outcomes of patients with ITP secondary to COVID-19. While case series and reviews of ITP secondary to COVID-19 have been published (PMID: 32677007, 32984764), this comprehensive review provides a greater sample size and details regarding management and outcomes.

Methods: A comprehensive literature review was performed through 2/28/21 in PubMed and Embase using keywords related to ITP and COVID-19. Articles were excluded based on diagnostic uncertainty, duplication, or irrelevance. Statistical analyses were performed using IBM SPSS Version 27.0. Means and SD reported.

Results: Among 106 unique articles identified, 38 were reviewed. Patient demographics and clinical presentations are presented in Table 1. Management and outcomes are described in Table 2.

TABLE 1 Demographics and Presentation

\begin{tabular}{|l|c|}
\hline \multicolumn{2}{|c|}{ Demographics } \\
\hline Cases & $\mathbf{n}(\%$ of cohort) \\
\hline Adult, new diagnosis & $51(81 \%)$ \\
\hline Adult, relapse & $7(11.1 \%)$ \\
\hline Pediatric, new diagnosis & $5(7.9 \%)$ \\
\hline Pediatric, relapse & $0(0.0 \%)$ \\
\hline Total & $63(100 \%)$ \\
\hline Age at diagnosis (years) & $53.5 \pm 22$ \\
\hline & \\
\hline Sex & $\mathbf{n}(\%$ of cohort) \\
\hline Male & $29(46 \%)$ \\
\hline Female & $30(47.6 \%)$ \\
\hline Not reported & $4(6.3 \%)$ \\
\hline & \\
\hline Comborbid conditions at & \\
time of diagnosis & $n(\%$ of cohort) \\
\hline Autoimmune disease & $8(12.7 \%)$ \\
\hline Cancer & $5(7.9 \%)$ \\
\hline Thrombosis & $4(6.3 \%)$ \\
\hline Pregnancy & $2(3.2 \%)$ \\
\hline
\end{tabular}

\begin{tabular}{|c|c|}
\hline \multicolumn{2}{|l|}{ Presentation } \\
\hline Bleeding symptoms & $n$ (\% of cohort) \\
\hline Petechiae & $20(31.7 \%)$ \\
\hline Purpura & $15(23.8 \%)$ \\
\hline Epistaxis & $14(22.2 \%)$ \\
\hline Gingival bleeding & $12(19 \%)$ \\
\hline Ecchymoses & $7(11.1 \%)$ \\
\hline Oral hemorrhagic bullae & $7(11.1 \%)$ \\
\hline Hematuria & $4(6.3 \%)$ \\
\hline Intracranial hemorrhage & $3(4.8 \%)$ \\
\hline Venipuncture site bleeding & $2(3.2 \%)$ \\
\hline Platelet count nadir (cells $/ \mu L$ ) & $12,600 \pm 20,200$ \\
\hline COVID-19 symptoms & $n$ (\% of cohort) \\
\hline Experienced symptoms & $56(88.9 \%)$ \\
\hline $\begin{array}{l}\text { Symptomatic at time of } \\
\text { diagnosis }\end{array}$ & $16(28.6 \%)$ \\
\hline $\begin{array}{l}\text { Post-symptomatic at time } \\
\text { of diagnosis }\end{array}$ & $8(14.2 \%)$ \\
\hline $\begin{array}{l}\text { Unclear timing of } \\
\text { symptoms and diagnosis }\end{array}$ & 32 (57.1\%) \\
\hline Never symptomatic & $5(7.9 \%)$ \\
\hline Not reported & $2(3.2 \%)$ \\
\hline $\begin{array}{l}\text { Time from symptom onset to } \\
\text { diagnosis (days) }\end{array}$ & $12.8 \pm 8.1$ \\
\hline
\end{tabular}

Table 1. Patient demographics and clinical presentations.
TABLE 2 Management and Outcomes

\begin{tabular}{|l|c|c|}
\hline \multicolumn{3}{|c|}{ Management } \\
\hline Treatment & $\mathbf{n}$ & $\begin{array}{c}\text { Rate of Complete } \\
\text { Response }\end{array}$ \\
\hline Steroids, IVIG, TPO-RA & 4 & $100.0 \%$ \\
\hline IVIG, TPO-RA & 6 & $83.3 \%$ \\
\hline Steroids & 13 & $77.0 \%$ \\
\hline Steroids, IVIG & 20 & $56.0 \%$ \\
\hline IVIG & 13 & $53.8 \%$ \\
\hline Regimen including TPO-RA & 10 & $90.0 \%$ \\
\hline Regimen including steroids & 37 & $70.2 \%$ \\
\hline Regimen including IVIG & 44 & $63.6 \%$ \\
\hline & & \\
\hline Complications of therapy & $\mathbf{n}$ & $\%$ of cohort \\
\hline Thrombocytosis & 1 & $1.6 \%$ \\
\hline $\begin{array}{l}\text { Acute heart failure secondary } \\
\text { to volume overload }\end{array}$ & 1 & $1.6 \%$ \\
\hline Hyperglycemia & 1 & $1.6 \%$ \\
\hline Constitutional symptoms & 1 & $1.6 \%$ \\
\hline
\end{tabular}

\begin{tabular}{|c|c|}
\hline \multicolumn{2}{|c|}{ Outcomes } \\
\hline Outcomes & $\mathrm{n}$ (\% of cohort) \\
\hline \multicolumn{2}{|l|}{ Response } \\
\hline Complete & $41(65 \%)$ \\
\hline Partial & $20(32.7 \%)$ \\
\hline Major bleeding & $7(11.1 \%)$ \\
\hline \multicolumn{2}{|l|}{ Mortality } \\
\hline $\begin{array}{l}\text { Due to complications } \\
\text { of ITP }\end{array}$ & $1(1.6 \%)$ \\
\hline $\begin{array}{l}\text { Due to complications } \\
\text { of COVID-19 }\end{array}$ & $1(1.6 \%)$ \\
\hline Level of care & $n$ (\% of cohort) \\
\hline Inpatient & 39 (61.9\%) \\
\hline ICU & $8(12.7 \%)$ \\
\hline Outpatient & $2(3.2 \%)$ \\
\hline Not specified & $17(27 \%)$ \\
\hline
\end{tabular}

Table 2. Management and outcomes. Complete response is defined as platelet count $>100,000$ cells $/ \mu \mathrm{L}$ at most recent follow-up and partial response is defined as platelet count $>30,000$ cells $/ \mu \mathrm{L}$ or double the platelet baseline at most recent follow-up. Major bleeding is defined in accordance with ISTH guidelines. Abbreviations: Intravenous immunoglobulins (IVIG), thrombopoietin receptor agonists (TPO-RA), intensive care unit (ICU).

Conclusions: SARS-CoV-2 is a virologic trigger of ITP. Middle-aged adults are most commonly affected, with no gender predilection. Autoimmune disease and cancer are common comorbidities. Mucocutaneous bleeding is the most common presenting symptom. While most patients had COVID-19 symptoms at the time of diagnosis, $12.6 \%$ were post-symptomatic and $7.9 \%$ never experienced symptoms. Treatment regimens including TPO-RAs were most effective in obtaining a complete response, and steroids may have been more effective than IVIG in the reported population. Treatment complications were rare. Patients with chronic and newly diagnosed ITP had similar presentations and outcomes. Prognostic data were reassuring: the vast majority of patients had complete or partial responses to therapy, and only 1 death was attributable to ITP. Rates of major bleeding were similar to typical ITP. Steroids may be a favorable treatment option given their apparent efficacy for both ITP and COVID-19.

PB0170 | Extended Outpatient Thromboprophylaxis for Individuals with COVID-19

A. Szuman ${ }^{1}$; Z. Kirkham ${ }^{1,2}$; R. Price ${ }^{1}$; S. Ahmed ${ }^{3}$; C. Macaulay ${ }^{4}$; K. Talks ${ }^{5}$; B. Greystoke ${ }^{5}$; K. Musgrave 2,5

${ }^{1}$ The Newcastle upon Tyne Hospitals NHS Foundation Trust, Newcastle upon Tyne, United Kingdom; ${ }^{2}$ Translational and Clinical Research Institute, Newcastle University, Newcastle upon Tyne, United Kingdom; ${ }^{3}$ Queen Elizabeth Hospital, Gateshead Health NHS Foundation Trust, Gateshead, United Kingdom; ${ }^{4}$ Leeds Teaching Hospitals NHS Trust, Leeds, United Kingdom; ${ }^{5}$ Haematology Department, The Newcastle upon Tyne Hospitals NHS Foundation Trust, Newcastle upon Tyne, United Kingdom

Background: Coronavirus disease 19 (COVID-19) is associated with an increased risk of venous thromboembolism (VTE).

Aims: To assess the effectiveness of outpatient thromboprophylaxis in patients with COVID-19. 
Methods: Consecutive patients hospitalized for SARS from March to May, 2020 were selected. Descriptive statistics, chi-square and ttests were used to compare COVID-19 and non-COVID-19 patients. Results: The Newcastle upon Tyne Hospital NHS Foundation Trust is a large UK-based teaching hospital. All individuals admitted with COVID-19 were offered outpatient thromboprophylaxis (rivaroxaban $10 \mathrm{mg}$ daily for 28 days) on discharge. Electronic medical records were reviewed for all admissions between 1st April and 15th May 2020, and for up to six months following discharge. Thromboprophylaxis was offered to those not at increased bleeding risk (see Figure 1). The study compares two groups, those individuals who received outpatient thromboprophylaxis (thromboprophylaxis) and those who did not (no thromboprophylaxis). Statistical analysis was performed using Fisher's exact test for categorical data and unpaired $t$ test for continuous data.

Data were collected from 179 medical inpatients with COVID-19, of these, 98 were eligible for thromboprophylaxis (Figure 1). The thromboprophylaxis group included 57 (58\%) individuals and the no thromboprophylaxis 41 (42\%).

All medical records were reviewed at 3 and 6 months but all VTE and bleeding events occurred within the 3 months following discharge (see Figure 2). At 3-month follow up there was no difference in the rate of VTE events between the groups (thromboprophylaxis, 1; no thromboprophylaxis, $1 ; P=1.00$ ). All VTE events occurred between stopping thromboprophylaxis (at 28 days) and before the 3-month point.

TABLE 1 Recorded VTE and bleeding events

\section{Thromboprophylaxis group}

Number of patients

Median age (years)

Number of males

VTE events at 3 months

VTE events at 6 months

(no new events in months 3-6)

Bleeding events at 3 months Intracranial bleed after fall 2 months after completing VTE prophylaxis

Bleeding events at 6 months 57

73

$28(49 \%)$

1

DVT \& PE in the same patient at 8 weeks post-discharge

(no new events in months 3-6)
The use of thromboprophylaxis was not associated with an increased risk of major bleeding (thromboprophylaxis, 1; no thromboprophylaxis, $0 ; P=1.00)$. The single bleeding event in the thromboprophylaxis group was an intracranial bleed following trauma and occurred two months after stopping the thromboprophylaxis.

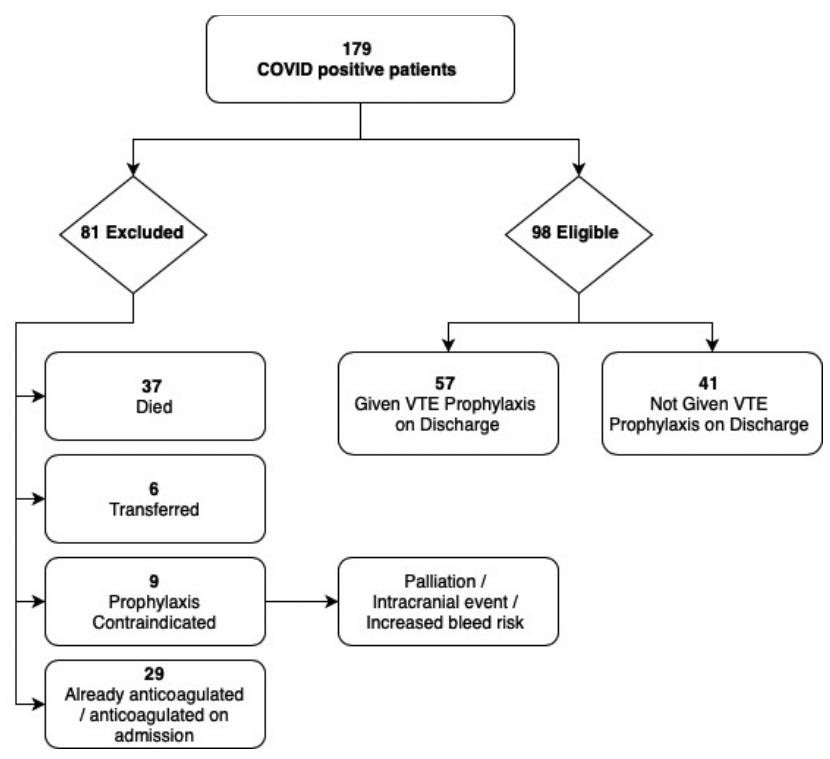

FIGURE 1 Flowchart of eligibility for thromboprophylaxis
No thromboprophylaxis group

$P$-value

41

70

0.43

$26(63 \%)$

0.21
DVT at 11 weeks post-discharge

1

(no new events in months 3-6)

0

1.00

0

Conclusions: Extended thromboprophylaxis was not associated with an increased risk of major bleeding. No VTE events occurred whilst an individual was taking thromboprophylaxis. Further work is needed to investigate the optimum duration of thromboprophylaxis. 
PB0171 | Venous Thromboembolism in Hospitalized Critical and Non-critical COVID-19 Patients: A Systematic Review and Metaanalysis

E. Mansory $^{1,2}$; S. Srigunapalan ${ }^{1} ;$ A. Lazolangner ${ }^{1}$

${ }^{1}$ Western University, London, Canada; ${ }^{2}$ King Abdulaziz University, Jeddah, Saudi Arabia

Background: Venous Thromboembolism has been observed as a frequent complication in patients with severe COVID-19 infection requiring hospital admission.

Aims: To evaluate the epidemiology of venous thromboembolisms in hospitalized intensive care (ICU) and non-ICU patients.

Methods: PubMed was searched up to $13^{\text {th }}$ of Nov 2020 and updated in December $12^{\text {th }}, 2020$. We included studies that evaluated the epidemiology of VTE, including PE and/or DVT, in patients with COVID-19. See Figure 1.

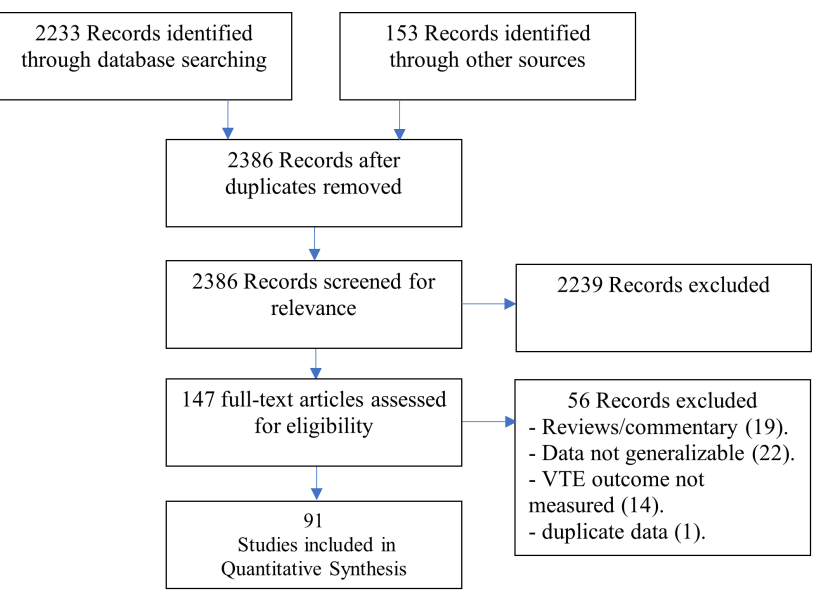

FIGURE 1 Preferred Reporting Items for Systematic Reviews and Meta- Analyses (PRISMA) flow diagram for study selection

Results: A total of 91 studies reporting on 35,017 patients with COVID were included. The overall frequency of VTE in all patients, ICU and non-ICU was $12.8 \%$ (95\% CI 11.103 to 14.605), 24.1\% (95\% $\mathrm{Cl} 20.070$ to 28.280 ), and $7.7 \%$ (95\% Cl 5.956 to 9.700 ), respectively. PE occurred in $8.5 \%$ (95\% $\mathrm{Cl} 6.911$ to 10.208$)$, and proximal DVT occurred in $8.2 \%$ (95\% Cl 6.675 to 9.874 ) of all hospitalized patients. The relative risk for VTE associated with ICU admission was 2.99 (95\% $\mathrm{Cl} 2.301$ to $3.887, P$ value $<0.001$ ). DVT and PE estimates in studies that adopted some form of systematic screening were higher compared to studies with symptom-triggered screening. Analysis restricted to studies in the 5 th quintile of sample size reported significantly lower VTE estimates.
TABLE 1 Overall proportion of COVID-19 patients with venous thromboembolism in different health care settings

\begin{tabular}{llll} 
& $\begin{array}{l}\text { patient } \\
\text { number of } \\
\text { patients }\end{array}$ & $\begin{array}{l}\text { with } \\
\mathrm{VTE}(\%)\end{array}$ & $\begin{array}{l}\text { 95\% Confidence } \\
\text { Interval }\end{array}$ \\
All Patients & 35017 & 12.827 & $\begin{array}{c}11.117 \text { to } \\
14.641\end{array}$ \\
ICU Patient & 8719 & 24.055 & $\begin{array}{c}20.070 \text { to } \\
28.280\end{array}$ \\
& & & 5.956 to 9.700 \\
\hline
\end{tabular}

Conclusions: This study confirmed a high risk of VTE in hospitalized COVID-19 patients specially those admitted to the ICU. Nevertheless, sensitivity analysis suggests that previously reported frequencies of VTE in COVID-19 might have been overestimated.

PB0172 | Does Hydroxychloroquine Prevent Symptomatic Venous Thromboembolism in Patients with Mild to Moderate COVID-19?

P.-M. Roy ${ }^{1,2,3} ;$ V. Dubée ${ }^{4,5} ;$ B. Vielle ${ }^{6}$ O. Blanchet ${ }^{7}$; J.-M. Lemée ${ }^{8}$; R. Dhersin ${ }^{4}$; S. Boucher ${ }^{9,1}$; C. Belizna ${ }^{10,1}$; G. Urbanski ${ }^{11,1}$; O. Sanchez ${ }^{12,13,3}$; P. Girard ${ }^{14,3}$; A. Elias ${ }^{15,3}$; D. Douillet ${ }^{16,1,3}$; I. Pellier ${ }^{17,5}$; HYCOVID Study Group ${ }^{1}$ UNIV Angers, Institut MitoVasc, UMR CNRS 6215 INSERM 1083, Angers, France; ${ }^{2} \mathrm{CHU}$ Angers, Direction de la Recherche et de I'Innovation, Département de Médecine d'Urgence, Angers, France; ${ }^{3}$ F-CRIN InnoVTE, Saint-Etienne, France; ${ }^{4} \mathrm{CHU}$ Angers, Service des Maladies Infectieuses, Angers, France; ${ }^{5}$ UNIV Angers et Université de Nantes, CRCINA, Inserm, Nantes, France; ${ }^{6} \mathrm{CHU}$ Angers, Direction de la Recherche et de I'Innovation, Département de Biostatistique et Méthodologie, Angers, France; ${ }^{7} \mathrm{CHU}$ Angers, Centre de Ressources Biologiques, BB-0033-00038, Angers, France; ${ }^{8} \mathrm{CHU}$ Angers, Service de Neurochirurgie, Angers, France; ${ }^{9} \mathrm{CHU}$ Angers, Service d'ORL et Chirurgie Cervico-faciale, Angers, France; ${ }^{10} \mathrm{CHU}$ Angers, Service de Médecine Vasculaire, Angers, France; ${ }^{11} \mathrm{CHU}$ Angers, Service de Médecine Interne, Angers, France; ${ }^{12}$ APHP, Hôpital Européen Georges Pompidou, Service de Pneumologie et Soins Intensifs, Paris, France;

${ }^{13}$ University of Paris; INSERM UMR-S 1140 Innovaties Therapies in Haemostasis, Paris, France; ${ }^{14}$ Institut Montsouris, Service de Pneumologie, Paris, France; ${ }^{15} \mathrm{CH}$ Sainte Musse, Département de Cardiologie et Médecine Vasculaire, Toulon, France; ${ }^{16} \mathrm{CHU}$ Angers, Département de Médecine d'Urgence, Angers, France; ${ }^{17} \mathrm{CHU}$ Angers, Immnologie, Hématologie et Oncologie Pédiatrique, Angers, France

Background: SARS-CoV-2 infection can trigger an important immune reaction, induce antiphospholipid antibodies, and is associated with a high-risk of venous thromboembolism (VTE), especially in patients with severe or critical disease. Hydroxychloroquine is an immunomodulator that had proven some efficacy in preventing thrombosis in antiphospholipid syndrome.

Aims: To investigate if hydroxychloroquine prevents symptomatic VTE in patients with mild to moderate COVID-19. 
Methods: Ancillary study of HYCOVID trial, a prospective, multicenter, randomized, double-blind trial aiming to assess the efficacy of hydroxychloroquine with regard to the 14-day rate of death or invasive ventilation (https://clinicaltrials.gov/ct2/show/NCT04 325893). COVID-19 patients were included, with their informed consent, if they had at least one of the following risk factors for worsening: need for supplemental oxygen, age $\geq 75$ years, age between 60 - 74 years and presence of at least one comorbidity. Eligible patients were randomized to receive either $800 \mathrm{mg}$ hydroxychloroquine on Day 0 followed by $400 \mathrm{mg}$ per day for 8 days, or a placebo. In the present study, we assessed the 28-day cumulative rate of symptomatic VTE. All suspected events were adjudicated by an independent committee, blinded to treatment allocation.

Results: Two hundred and fifty patients were enrolled; 124 and 123 patients received hydroxychloroquine or placebo and were included in the modified intention-to-treat analysis, respectively. Eightyseven percent of the patients received an anticoagulant treatment during hospitalization (104 [83.9\%] and 111 [91.2\%] in the hydroxychloroquine and the placebo group, respectively) (Table). Two patients in each group experienced VTE within the 28 days following inclusion. All events were pulmonary embolism (PE), including one fatal PE in the placebo group. The VTE rate was $1.61 \%(95 \% \mathrm{Cl}: 0.20$ to 5.70 ) in the hydroxychloroquine group and $1.63 \%$ (95\% confidence interval: 0.20 to 5.75$)$ in the placebo group $(P=1.00)$.
INNOVTE, Angers, France; ${ }^{3}$ UNIV Angers, Micro et Nano Médecines Translationnelles, MINT, UMR INSERM 1066, UMR CNRS 6021, Angers, France; ${ }^{4}$ Methodology and Biostatistics Department, Delegation to Clinical Research and Innovation, Angers University Hospital, Angers, France; ${ }^{5}$ Emergency Department, Cliniques Universitaires Saint-Luc, Université Catholique de Louvain, Bruxelles, Belgium; ${ }^{6} \mathrm{CH}$ Cholet, Cholet, France; ${ }^{7}$ EHESP, Irset, Inserm, UMR S1085, CAPTV CDC, University of Rennes, Angers, France; ${ }^{8}$ Department of Infectious Diseases, Angers University Hospital, Angers, France; ${ }^{9}$ CRCINA, Inserm, University of Nantes, UNIV Angers, Angers, France

Background: Severely ill patients with SARS-CoV-2 have at increased risk of venous thromboembolism (VTE). However, the VTE risk in patients with mild to moderate COVID-19 remains uncertain. Aims: To assess the risk of VTE in patients with mild to moderate COVID-19 patients versus similar patients without COVID-19 and to define VTE risk factors in COVID-19 patients.

Methods: A prospective cohort of patients presented to ED for acute dyspnea or chest pain and having confirmed or probable mild to moderate COVID-19 was retrospectively compared to a prospective cohort of similar ED patients using propensity score matching. Patients having diagnostic testing for suspected PE and for whom PE was initially ruled-out were included. Univariate and multivariate analysis of possible VTE risk factors were performed in the

TABLE 1 Characteristics of the patients

\begin{tabular}{|c|c|c|}
\hline Patients' characteristics & Hydroxychloroquine $(N=124)$ & Placebo $(N=123)$ \\
\hline Median age - year (IQR) & $76.5(59.5-85.0)$ & $78.0(57.0-87.0)$ \\
\hline Ambulatory treatment - no. (\%) & $2(1.6)$ & $1(0.8)$ \\
\hline Hospitalization, no oxygen therapy - no. (\%) & $45(36.3)$ & $50(40.7)$ \\
\hline Hospitalization, oxygen therapy ( $\leq 3 \mathrm{~L} / \mathrm{min}$ ) - no. (\%) & $77(62.1)$ & $72(58.5)$ \\
\hline \multicolumn{3}{|c|}{$\begin{array}{l}\text { Anticoagulant treatment during hospitalization ( } 1 \text { missing data } \\
\text { in placebo group) }\end{array}$} \\
\hline No treatment - no. (\%) & $20(16.1)$ & $11(9.0)$ \\
\hline Preventive dose - no. (\%) & $72(58.1)$ & $68(55.7)$ \\
\hline Intermediate dose - no. (\%) & $12(9.7)$ & $22(18.0)$ \\
\hline Curative dose - no. (\%) & $20(16.1)$ & $21(17.2)$ \\
\hline
\end{tabular}

Conclusions: In patients hospitalized for mild to moderate COVID-19, the rate of symptomatic VTE was low with no evidence of benefit of hydroxychloroquine.

PB0173 | Are Mild or Moderate COVID-19 Patients at Higher Risk of VTE? A Comparison of Two Prospective European Cohorts in Emergency Departments

D. Douillet ${ }^{1,2} ;$ J. Riou ${ }^{3,4}$; A. Penaloza ${ }^{5} ;$ T. Moumneh ${ }^{1,2}$; C. Soulié6; D. Savary ${ }^{1,7}$; F. Morin ${ }^{1}$; R. Mahieu ${ }^{8,9}$; P.-M. Roy ${ }^{1,2}$

${ }^{1}$ Emergency Department, Angers University Hospital, Angers, France;

${ }^{2}$ UNIV Angers, UMR MitoVasc CNRS 6015 - INSERM 1083. FCRIN,
COVID-19 patients. The main outcome was the rate of symptomatic VTE, deep venous thrombosis or pulmonary embolism, within the 28 days after ED presentation.

Results: A total of 2292 patients were included in the COVID-19 cohort and 1539 in the comparison cohort. After adjustment, the 28 -day rate of symptomatic VTE was $1.87 \%$ (95\% Cl: 0.95 to 3.24$)$ in the COVID-19 cohort and $0.18 \%(95 \% \mathrm{Cl}: 0.01$ to 1.63$)$ in the comparison cohort. The absolute difference was $+1.69 \%(95 \% \mathrm{Cl}: 0.88$ to $2.51, P<0.001),+3.45 \%$ (95\% Cl: 1.80 to 5.11 ) in hospitalized patients and $+0.50 \%$ ( $95 \% \mathrm{Cl}: 0.22$ to 1.23 ) in patients treated at home. In the COVID-19 cohort, hospitalization and age > 65 years were 
independent risk factors of symptomatic VTE but not the lack of thromboprophylaxis.

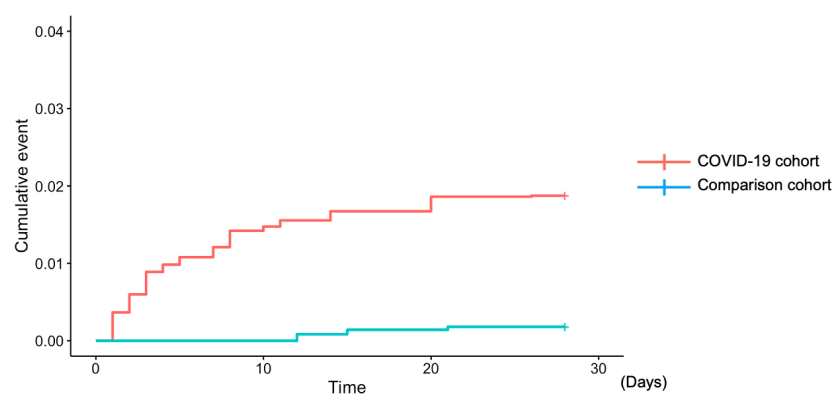

FIGURE 1 Kaplan-Meier cumulative VTE rate for the primary outcome

Conclusions: Patients with mild-to-moderate COVID-19 presenting to ED had a higher risk of subsequent VTE than similar patients, especially if they are older than 65 years and required hospitalization.

PB0174 | High Incidence and Early Presence of arterial thrombotic events in a COVID-19 hospitalized population: an overlooked problem?

M. de Oliveira ${ }^{1}$; F. Cubal ${ }^{2}$; J. Oliveira ${ }^{3}$; E. Cruz ${ }^{1}$; M. Coutinho ${ }^{1}$; M. Pereira ${ }^{1}$; R. Matos ${ }^{1}$; N. Pinho ${ }^{1}$; L. Moreira ${ }^{1}$; N. Seidi ${ }^{1}$; S. Morais ${ }^{1}$ ${ }^{1}$ Centro Hospitalar Universitário do Porto, Porto, Portugal; ${ }^{2}$ Centro Hospitalar de Trás-os-Montes e Alto Douro, Vila Real, Portugal; ${ }^{3}$ Hospital do Divino Espírito Santo de Ponta Delgada, Ponta Delgada, Portugal
Background: COVID-19 patients appear to be at increased risk for venous thromboembolism (VTE), however data on arterial thromboembolism (ATE) is still limited.

Aims: We report a case series of thromboembolic events (TE) in COVID-19 patients admitted to a Portuguese hospital.

Methods: In 290 patients with confirmed COVID-19 admitted between October and December 2020, clinical records were searched for TE. Admission levels of fibrinogen, D-dimers, C-reactive protein (CRP), ferritin, lactate dehydrogenase (LDH) and complete blood counts were evaluated. Statistical analysis was conducted using IBM SPSS $^{\circledR}$.

Results: Of the 290 patients (M/F 179/111; median age 70-years-old (interquartile range: 58-78); 79 in Intensive Care Units (ICU)), 8.6\% had TE $(n=25)$. Of these, $64 \%(n=16)$ had ATE $(44 \%$ ischaemic strokes and $20 \%$ ST-elevation myocardial infarctions); $28 \%(n=7)$ had VTE ( $16 \%$ pulmonary embolisms and $12 \%$ other) and $8 \%$ had multiple events. The overall incidence of ATE was $6.2 \%$ and VTE $3.1 \%$.

Most TE ( $n=17,68 \%$ ), of which $76 \%$ ATE, were detected upon admission to hospital, despite prior antiplatelet therapy in six patients (35.3\%). Among TE in hospitalized patients, 87.5\% occurred despite anticoagulant therapy.

Admissions to ICU and mortality were higher in patients with TE than in patients without thrombosis (non-TE) (44\% vs $27.2 \%$; $P=0.003$ and $28 \%$ vs $20.76 \% ; P=0.402$, respectively). Patients with TE presented significantly lower levels of fibrinogen and CRP and higher D-dimers, when compared with matched non-TE patients (Table 1). Further statistically significant differences were found were found between ATE and VTE groups, as presented in Table 1.

TABLE 1 Median values of relevant laboratorial parameters upon admission and comparison between groups. Interquartile range shown in brackets. Statistical significance calculated using a Kruskal-Wallis test. Non-TE patients were matched with TE patients by age, sex and severity of disease (defined by length of stay and highest level of care). Patients with multiple events were not included in the ATE/VTE subgroups analysis. Lack of statistical significance indicated as n.s.

\begin{tabular}{|c|c|c|c|c|c|c|c|c|}
\hline Admission Levels & $\begin{array}{l}\text { Non-TE } \\
(n=24)\end{array}$ & $\begin{array}{l}\text { TE } \\
(n=25)\end{array}$ & $\begin{array}{l}\text { ATE } \\
(n=16)\end{array}$ & $\begin{array}{l}\text { VTE } \\
(n=7)\end{array}$ & TE/non-TE & ATE/non-TE & VTE/non-TE & ATE/VTE \\
\hline Fibrinogen $(\mathrm{g} / \mathrm{L})$ & $\begin{array}{l}6.20 \\
(4.56-7.22)\end{array}$ & $\begin{array}{l}4.63 \\
(3.51-5.00)\end{array}$ & $\begin{array}{l}4.41 \\
(3.32-4.94)\end{array}$ & $\begin{array}{l}4.35 \\
(3.73-7.30)\end{array}$ & $P=0.014$ & $P=0.010$ & n.s. & n.s. \\
\hline $\mathrm{CRP}(\mathrm{mg} / \mathrm{L})$ & $\begin{array}{l}126.4 \\
(100.6-181)\end{array}$ & $\begin{array}{l}77.0 \\
(13.5-169.5)\end{array}$ & $\begin{array}{l}41.6 \\
(7.8-136.7)\end{array}$ & $\begin{array}{l}78.4 \\
(41.2-215)\end{array}$ & $P=0.021$ & $P=0.007$ & n.s. & n.s. \\
\hline D-dimer (ng/mL) & $\begin{array}{l}753.5 \\
(363-1112)\end{array}$ & $\begin{array}{l}1128 \\
(451-5250)\end{array}$ & $\begin{array}{l}666 \\
(420-1428)\end{array}$ & $\begin{array}{l}2645 \\
(872-5250)\end{array}$ & $P=0.049$ & n.s. & $P=0.014$ & n.s. \\
\hline $\mathrm{LDH}(\mathrm{U} / \mathrm{L})$ & $\begin{array}{l}361 \\
(270-492)\end{array}$ & $\begin{array}{l}357 \\
(204-554)\end{array}$ & $\begin{array}{l}237 \\
(189-371)\end{array}$ & $\begin{array}{l}478 \\
(392-561)\end{array}$ & n.s. & $P=0.037$ & n.s. & $P=0.018$ \\
\hline
\end{tabular}




\begin{tabular}{|c|c|c|c|c|c|c|c|c|}
\hline 146 of 972 & & & & & & & & ABSTRACT \\
\hline Admission Levels & $\begin{array}{l}\text { Non-TE } \\
(n=24)\end{array}$ & $\begin{array}{l}\text { TE } \\
(n=25)\end{array}$ & $\begin{array}{l}\text { ATE } \\
(n=16)\end{array}$ & $\begin{array}{l}\text { VTE } \\
(n=7)\end{array}$ & TE/non-TE & ATE/non-TE & VTE/non-TE & ATE/VTE \\
\hline Ferritin (ng/mL) & $\begin{array}{l}1555 \\
(887-2158)\end{array}$ & $\begin{array}{l}1220 \\
(325-1978)\end{array}$ & $\begin{array}{l}730 \\
(300-1483)\end{array}$ & $\begin{array}{l}1707 \\
(808-2318)\end{array}$ & n.s. & $P=0.045$ & n.s. & n.s. \\
\hline
\end{tabular}

Conclusions: Data from these patients point to an early occurrence of TE and an increased incidence of ATE over VTE. The significantly diminished inflammation markers, together with the early presence of TE (68\% at admission) in patients otherwise without reason for hospitalization, may suggest, at least for ATE events, a direct role of SARS-CoV-2 in thrombotic process.

PB0175 | Prior Use of Therapeutic Anticoagulation Does Not Protect against COVID-19 Related Clinical Outcomes in Hospitalized Patients: A Propensity Score-matched Cohort Study

J. Spiegelenberg ${ }^{1}$; M. van Gelder ${ }^{1}$; M. Maas ${ }^{2}$; M. Hovens ${ }^{3}$; A. Esselink ${ }^{4}$; A. Kerckhoffs ${ }^{5}$; J. Hoogerwerf ${ }^{1}$; N. Janssen ${ }^{1}$; M. van Apeldoorn $^{5}$; K. Veerman ${ }^{6}$; M. Blaauw ${ }^{1}$; R. Janssen ${ }^{4}$; R.J. Hassing ${ }^{3}$; T. Dofferhoff ${ }^{4} ;$ J. van de Maat ${ }^{1} ; K^{\text {K. Kramers }}{ }^{1}$; J. Leentjens ${ }^{1}$

${ }^{1}$ Radboudumc, Nijmegen, Netherlands; ${ }^{2}$ Bernhoven Hospital, Uden, Netherlands; ${ }^{3}$ Rijnstate Hospital, Arnhem, Netherlands; ${ }^{4}$ Canisius Wilhelmina Hospital, Nijmegen, Netherlands; ${ }^{5}$ Jeroen Bosch Hospital, Den Bosch, Netherlands; 'Sint Maartenskliniek, Nijmegen, Netherlands

Background: Patients with COVID-19 have a hypercoagulable state with increased risk of thrombotic venous events (VTE). These thrombotic complications could be responsible for a significant part of the morbidity and mortality seen in COVID-19 patients. The high incidence of VTE is seen even despite the use of apparently adequate thrombosis prophylaxis. Therefore, it is suggested that in COVID-19 patients increased intensity thromboprophylaxis or therapeutic anticoagulation should be considered.

Aims: We investigated whether the use of therapeutic anticoagulation prior to infection has a beneficial effect on morbidity and mortality in hospitalized COVID-19 patients.

Methods: In this multicenter retrospective cohort study, all $\geq 18$ years old COVID-19 patients admitted to 6 hospitals in the Netherlands between March and May 2020 were included. We applied 1:3 propensity score matching to evaluate the association between prior therapeutic anticoagulation use and clinical outcome, with in hospital mortality as primary endpoint. Relevant secondary outcomes included admission to the intensive care unit (ICU), need for invasive mechanical ventilation, pulmonary embolism and length of hospital stay

Results: A total of 1154 patients were included, of whom 190 (16\%) patients used therapeutic anticoagulation prior to admission. In the propensity score matched analyses, we observed no association between prior use of therapeutic anticoagulation and in hospital mortality compared to no prior use of anticoagulation (RR 1.02 (95\% $\mathrm{Cl}$; 0.80-1.30). We also found no significant differences in secondary outcomes apart from a lower risk of pulmonary embolism in patients using therapeutic anticoagulation prior to infection (RR 0.19 (95\% $\mathrm{Cl}$; 0.05-0.80).

Conclusions: Although prior therapeutic anticoagulation use is associated with reduced PE occurrence, it is not associated with better outcome parameters in hospitalized COVID-19 patients in terms of all-cause mortality, ICU admittance, need for mechanical ventilation, and length of hospital stay.

PB0176 | Pulmonary Thromboembolism (PTE) in Hospitalised Patients with COVID-19: A National Study of Patients Managed in Critical Care and Ward Environments

M. McGettrick ${ }^{1}$; A. MacLellan ${ }^{1}$; P. McCaughey ${ }^{1}$; C. Bagot $^{2}$;

M. Brewis ${ }^{1}$; N.N Lang ${ }^{3}$; M. Johnson ${ }^{1}$; A.C. Church ${ }^{1}$

${ }^{1}$ Scottish Pulmonary Vascular Unit, Golden Jubilee National Hospital, Glasgow, United Kingdom; ${ }^{2}$ Department of Haematology, Glasgow Royal Infirmary, Glasgow, United Kingdom; ${ }^{3}$ College of Medical, Veterinary and Life Sciences, University of Glasgow, Glasgow, United Kingdom

Background: Since the emergence of Severe Acute Respiratory Syndrome Coronavirus 2(SARS-CoV-2) in late December 2019, there has been increasing recognition of the pro-thrombotic risk this virus can cause as part of Coronavirus disease 2019

(Covid-19).

Aims: To assess if the rate of PTE was increased in those with COVID-19 in both critical care and ward patients.

To assess the effect of right heart strain or requirement for critical care on mortality.

Methods: We reviewed all computed tomography pulmonary angiograms (CTPA) performed in Scotland between $23^{\text {rd }}$ March and $31^{\text {st }}$ May 2020 (1st wave) and identified those inpatients with COVID-19 using either classical radiological appearances, or positive COVID-19 polymerase chain reaction swab. 
Results:

\section{Right Heart Strain Survival to 90 days}

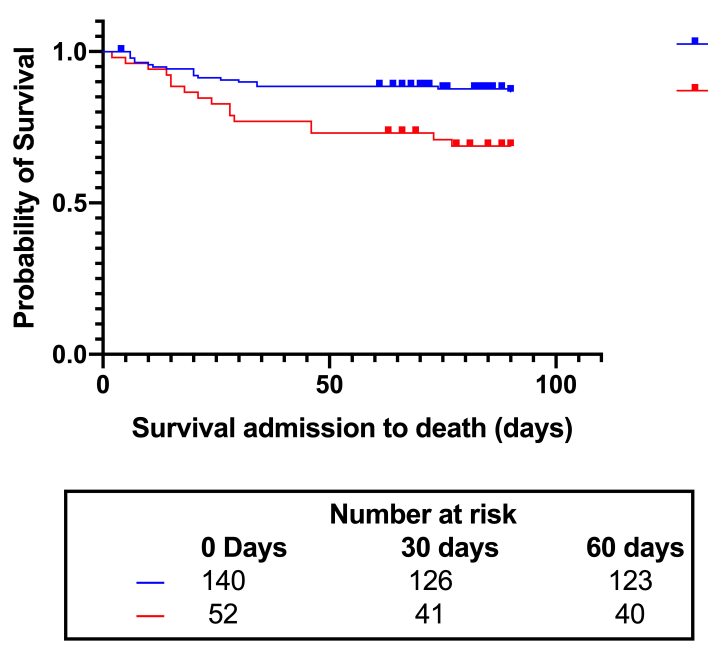

FIGURE 1 Right heart strain survival to 90 days

3401 CTPAs were reviewed. 192 were positive for PTE in patients with evidence of COVID-19 either real-time polymerase chain reaction (RT-PCR) swab positive for SARS-CoV-2 [ $n=104]$ or having radiological changes consistent with COVID-19 $[n=88]$ ). The total number of hospital admissions in Scotland between $23^{\text {rd }}$ March 2020 and $31^{\text {st }}$ May 2020 with COVID-19 was 5195. The incidence of PTE during this time was $3.7 \%$ in all patients admitted to all hospitals in Scotland with COVID-19 during this period. 475 hospitalised patients were managed in critical care (both level 2 and leve 3 care), in whom the incidence of PTE was 6\% ( $n=29) .4720$ patients required ward based care alone and the incidence of PTE was $3.5 \%(n=163)$. This compares to the national pre-Covid rate of $1 \%$. There was increased risk of death with right heart strain $(25 / 52$ vs $128 / 140[P<0.01])$ and in critical care $(15 / 29$ vs $146 / 163[P<0.01])$. Conclusions: In this national study, we have demonstrated an increased risk of pulmonary thromboembolism in both critical care and ward based environments.
PB0177 | COVID-19 Vaccination and Anticoagulation: Potential Effects on Anticoagulation Control in Patients Using Vitamin K Antagonists

C. Visser ${ }^{1}$; J.S. Biedermann ${ }^{1}$; M.C. Nierman ${ }^{2}$; F.J.M. van der Meer ${ }^{3}$; S.C. Cannegieter ${ }^{3,4}$; W.M. Lijfering ${ }^{4}$; M.J.H.A. Kruip ${ }^{1}$

${ }^{1}$ Department of Haematology, Erasmus MC, Erasmus University Medical Center, Rotterdam, Netherlands; ${ }^{2}$ Department of Thrombosis and Anticoagulation, Atalmedial Medical Diagnostics Centers,

Amsterdam, Netherlands; ${ }^{3}$ Department of Internal Medicine, section of Thrombosis and Hemostasis, Leiden University Medical Center, Leiden, Netherlands; ${ }^{4}$ Department of Clinical Epidemiology, Leiden University Medical Center, Leiden, Netherlands

Background: In January 2021, the Dutch vaccination programme against SARS-CoV-2 was started. Clinical studies have shown that systemic reactions including fever and chills occur in up to $50 \%$ of vaccine recipients. It is unclear whether these systematic reactions have an effect on anticoagulation control, potentially leading to an increased risk of thrombotic events and bleeding complications.

Aims: To investigate whether COVID-19 vaccination with the Pfizer vaccine is associated with suboptimal anticoagulation control in patients using Vitamin $\mathrm{K}$ antagonists (VKAs).

Methods: A case-crossover study was performed in a cohort of outpatients from three Dutch anticoagulation clinics who received a COVID-19 vaccination. All patients had their international normalized ratio (INR) measured 0-6 weeks before and 1-2 weeks after vaccination. INR results and VKA dosages before the first COVID-19 vaccination, the reference period, were compared with those after the first vaccination. Data extraction after the second vaccination will be performed in the near future, after which these analyses will be repeated and included in the final presentation at the congress.

Results: A total of 2197 outpatient VKA-users were included, with a mean age of 86 years, of whom $38.5 \%$ were male and $71.7 \%$ used acenocoumarol (Table 1). There was no difference in mean INR level before and after vaccination ( 2.51 vs 2.54 , mean difference 0.033 (95\% Cl, -0.071 to 0.0051 ). The mean dosage of phenprocoumon did not differ before and after vaccination (0.47 tablets/day (0.25)). Similarly, the mean dosage of acenocoumarol was comparable before and after vaccination (1.72 tablets/day (0.81) versus 1.71 tablets/ day (0.82). Most vaccine recipients remained in therapeutic range and INR > 5 was as likely to be reported after vaccination $(1.0 \%$ and $2.6 \%$ ) as it was before vaccination (1.0\% and $1.6 \%$ ) (Table 2 ). 
TABLE 1 Clinical characteristics of outpatients Vitamin K Antagonist users

\begin{tabular}{lll} 
& Phenprocoumon & Acenocoumarol \\
Patients $(n, \%)$ & $621(28.3 \%)$ & $1576(71.7 \%)$ \\
Age (mean, SD) & $85.12(10.95)$ & $86.34(10.07)$ \\
Men $(n, \%)$ & $247(39.8 \%)$ & $599(38.0 \%)$ \\
Days between INR measurement and vaccination & & \\
$\quad$ Before vaccination (median, IQR) & $3.00(6.00)$ & $3.00(6.00)$ \\
After vaccination (median, IQR) & $8.00(5.00)$ & $7.00(7.00)$ \\
Target INR & & $1437(91.2 \%)$ \\
{$[2.0-3.0](n, \%)$} & $571(91.9 \%)$ & $139(8.8 \%)$ \\
{$[2.5-3.5](n, \%)$} & $50(8.1 \%)$ & \\
\hline
\end{tabular}

TABLE 2 Anticoagulation control before and after vaccination

\begin{tabular}{|c|c|c|}
\hline & $\begin{array}{l}0-6 \text { weeks prior } \\
\text { vaccination }\end{array}$ & $\begin{array}{l}5-21 \text { days after } \\
\text { vaccination }\end{array}$ \\
\hline Mean INR level (SD) & $2.51(0.72)$ & $2.54(0.74)$ \\
\hline $\begin{array}{l}\text { Mean } \\
\text { phenprocoumon } \\
\text { dosage (SD) }\end{array}$ & $0.47(0.25)$ & $0.47(0.25)$ \\
\hline $\begin{array}{l}\text { Mean acenocoumarol } \\
\text { dosage (SD) }\end{array}$ & $1.72(0.81)$ & $1.71(0.82)$ \\
\hline INR in range 2-3 & 1364 (67.9\%) & 1354 (67.4\%) \\
\hline INR $>5$ & 20 (1.0\%) & 20 (1.0\%) \\
\hline INR in range $2.5-3.5$ & $108(57.1 \%)$ & $113(59.8 \%)$ \\
\hline INR $>5$ & $5(2.6 \%)$ & $3(1.6 \%)$ \\
\hline
\end{tabular}

Conclusions: COVID-19 vaccination did not influence anticoagulation control in patients using VKAs.

PB0178 | COVID-19 Diagnosis and its Impact on Thrombotic Risk in a Cohort of Consecutive Patients Hospitalized due to Severe Acute Respiratory Syndrome

A. Coy Canguçu $^{1}$; G. Aparecida Locachevic ${ }^{2}$; J.C. Silva Mariolano²; K.H. de Oliveira Soares²; J.D. Oliveira²; C. de Oliveira Vaz; G. Vieira Damiani²; B. Mazetto²; J. Annichino-Bizzacchi ${ }^{2}$;

E. V. de Paula ${ }^{2}$; F. Andrade Orsi ${ }^{2}$

${ }^{1}$ Pontifícia Universidade Católica de Campinas (PUCC), Campinas,

Brazil; ${ }^{2}$ Universidade Estadual de Campinas (UNICAMP), Campinas,

Brazil

Background: Previous evidence suggest that the thromboembolic risk is greater among patients with COVID-19 than those affected by other types of severe acute respiratory syndrome (SARS). However, such comparison has been mainly evaluated in historical cohorts.

Aims: To evaluate thromboembolic events in patients with COVID-19 and other SARS hospitalized in the same time period.

Methods: Consecutive patients hospitalized for SARS from March to May, 2020 were selected. Descriptive statistics, chi-square and $t$ tests were used to compare COVID-19 and non-COVID-19 patients.
Results: In all, 377 patients were admitted during this period. 100 COVID-19 and 100 non-COVID-19 patients were included. Table 1 demonstrates their baseline features. Both groups showed similar baseline risk of hospital-associated thrombosis, such as previous thromboembolism event, recognized "thrombophilia", infarction or stroke within the past 4 weeks, and trauma or surgery within the past 4 weeks. Reduced mobility, however, was more frequent among non-COVID-19 than COVID-19 patients. Oxygen saturation was lower in COVID-19 (92\% IQR 89\% to 96\%) than in non-COVID-19 patients (95\% IQR 90\% to 97\%, $P=0.03$ ); accordingly, the need for invasive oxygen support was greater ( $44 \%$ vs. $33 \%, P=0.05)$ and longer $(16.00$ days IQR 8.50 to 22.50 vs. 12.50 days IQR 4.75 to $21.25, P=0.002$ ) in COVID-19 than in non-COVID-19 patients. Coagulation markers such as activated partial thromboplastin time, prothrombin time, platelet count, and D-dimer levels $(1,700.00 \mathrm{ng} / \mathrm{mL}$ IQR 752.00 to $3,417.00$ non-COVID-19; $1,426.50$ ng/mL IQR 744.25 to $3,461.00$ COVID-19, $P=0.17)$ were similar between groups. Although thromboprophylaxis was more frequently administered to COVID-19 (76\%) than nonCOVID-19 patients (42\%, $P<0.0001)$, thrombotic events were more recurrent in the former group ( $22 \%$ vs. $12 \%, P=0.06)$. Table 2 demonstrates the thrombotic manifestations' characteristics. 
TABLE 1 Baseline demographic and clinical features of patients hospitalized due to SARS at the UNICAMP Clinical Hospital between March and May, 2020. Abbreviations: IQR interquartile range; CKD chronic kidney disease. * Gout, arthrosis, systemic lupus erythematosus, rheumatoid arthritis, systemic sclerosis, myasthenia gravis; ${ }^{* *}$ Tuberculosis, Hansen's disease, syphilis, gonorrhea, HIV, chronic hepatitis C and B.

\begin{tabular}{llll} 
& Non-COVID-19 $(n=100)$ & COVID-19 $(n=100)$ & $P$ value \\
Male, $n(\%)$ & $55(55.00 \%)$ & $67(67.00 \%)$ & 0.08 \\
Age in years, median (IQR) & $53.87(43.94$ to 68.91) & $57.36(45.84$ to 65.83) & 0.30 \\
Arterial hypertension, $n(\%)$ & $47(47.00 \%)$ & $47(47.00 \%)$ & 1.00 \\
Diabetes, $n$ (\%) & $30(30.00 \%)$ & $35(35.00 \%)$ & 0.45 \\
Obesity, $n(\%)$ & $14(14.00 \%)$ & $23(23.00 \%)$ & 0.10 \\
Active cancer, $n(\%)$ & $20(20.00 \%)$ & $10(10.00 \%)$ & 0.05 \\
Systemic autoimmune disease ${ }^{*} n(\%)$ & $8(8.00 \%)$ & $4(4.00 \%)$ & 0.23 \\
Chronic infectious disease,$n(\%)$ & $8(8.00 \%)$ & $4(4.00 \%)$ & 0.23 \\
End-stage CKD, $n(\%)$ & $6(6.00 \%)$ & $8(8.00 \%)$ & 0.58 \\
\hline
\end{tabular}

TABLE 2 Thrombotic complications during hospitalization due to SARS at the UNICAMP Clinical Hospital between March and May, 2020. Abbreviations: ICU intensive care unit; IQR interquartile range. "Upper limb (1 non-COVID-19; 2 COVID-19), neck (0 non-COVID-19; 2 COVID-19), renal artery (0 non-COVID-19; 1 COVID-19), iliac artery (0 non-COVID-19; 1 COVID-19), hepatic artery (0 non-COVID-19; 1 COVID-19).

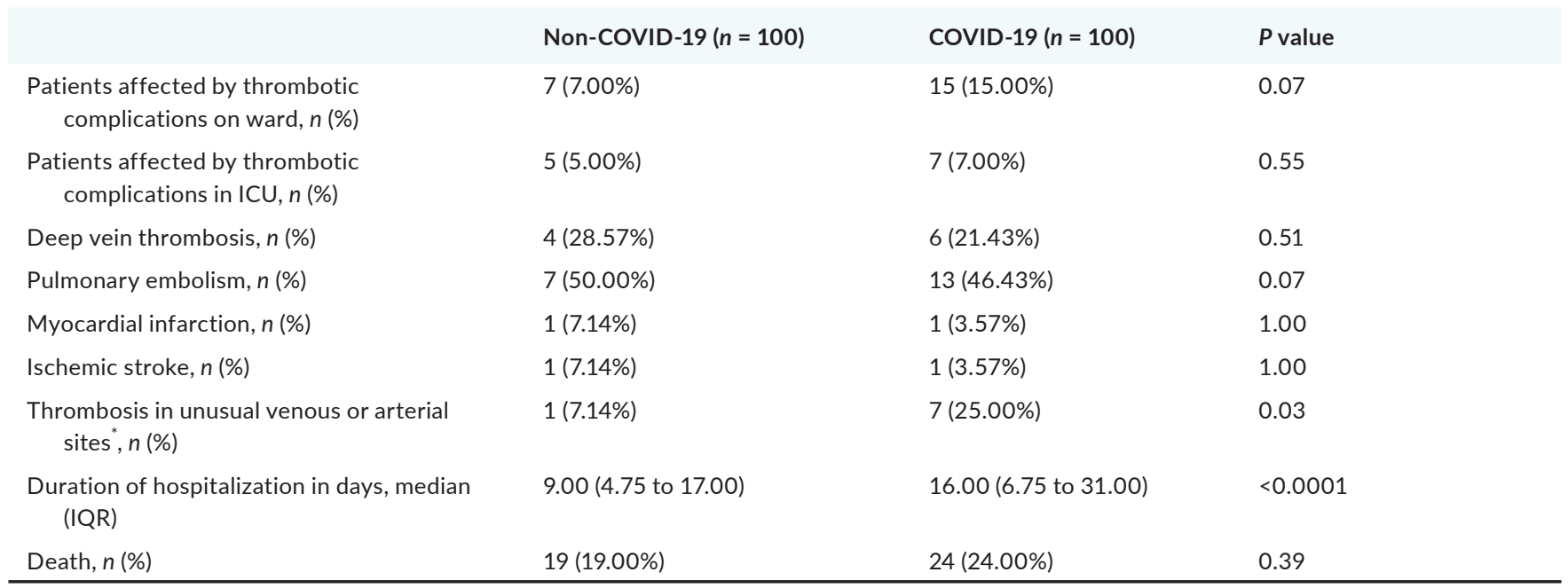

Conclusions: In this study, we provide clinical evidence that the risk of thrombosis is more pronounced in COVID-19 than in other SARS. D-dimer analysis was not capable of differentiating the thrombotic risk between these conditions. 
PB0179 | COVID-19 Infection is Associated with Antibodymediated Procoagulant Platelets: Data from an Observational Study

$\underline{\text { K. Althaus }}^{1,2}$; I. Marini ${ }^{1}$; J. Zlamal ${ }^{1}$; H. Häberle ${ }^{3}$; M. Mehrländer ${ }^{3}$; L. Pelzl ${ }^{1}$; A. Singh ${ }^{1}$; S. Hammer²; M. Bitzer ${ }^{4}$; N. Malek ${ }^{4}$; D. Rath ${ }^{5}$; H. Bösmüller ${ }^{6}$; M. Gawaz ${ }^{5}$; P. Rosenberger ${ }^{3}$; T. Bakchoul ${ }^{1,2}$ ${ }^{1}$ Transfusion Medicine, Medical Faculty of Tuebingen, University Hospital of Tuebingen, Tuebingen, Germany; ${ }^{2}$ Centre for Clinical Transfusion Medicine, University Hospital of Tuebingen, Tuebingen, Germany; ${ }^{3}$ Department of Anesthesiology and Intensive Care Medicine, University Hospital of Tuebingen, Tuebingen, Germany; ${ }^{4}$ Department of Internal Medicine I, University Hospital of Tuebingen, Tuebingen, Germany; ${ }^{5}$ Department of Medicine III, University Hospital of Tuebingen, Tuebingen, Germany; ${ }^{6}$ Institute for Pathology, University Hospital of Tuebingen, Tuebingen, Germany

Background: The pathophysiology of COVID-19-associated coagulopathy is to be complex and multifactorial. In this study, we hypothesized that the coagulopathy in COVID-19 is accompanied by immune mediated procoagulant platelets with subsequent alteration of the coagulation system.

Aims: We aimed to further analyze the role of platelets (PLTs) leading to increased thromboembolic events in patients with severe COVID-19.

Methods: Blood samples from COVID-19 intensive care unit (ICU) patients were analyzed for procoagulant markers. Flow cytometry was used to investigate depolarization of mitochondrial inner transmembrane potential $\left(\Delta \Psi_{\mathrm{m}}\right)$, intracellular $\mathrm{Ca} 2+$ concentration, and phosphatidylserine (PS) externalization.

Results: PLTs from COVID-19 patients $(n=21)$ showed significantly higher $\Delta \Psi \mathrm{m}$ depolarization (1.39 \pm 0.07 vs. $0.99 \pm 0.06, P=0.0005$ ), Ca2+ concentration $(2.73 \pm 0.31$ vs. $1.00 \pm 0.05, P<0.0001)$ and PS externalization ( $2.05 \pm 0.48$ vs. $0.86 \pm 0.11, P=0.0236)$, compared to healthy control $(\mathrm{HC})$, respectively. Most importantly, PS exposure was associated with SOFA score (sequential organ failure assessment, $r=0.5635, P=0.0078$ ) and plasma levels of $D$-Dimer $(r=0.4473, P=0.0420)$. Finally, patients with thromboembolic events had higher PS externalization compared to those without thrombosis ( $2.85 \pm 0.75$ vs. $0.99 \pm 0.20, P=0.0340)$. Sera from COVID-19 patients also induced significant increase in procoagulant markers $\left(\Delta \Psi_{\mathrm{m}}\right.$ depolarization (1.52 \pm 0.117 vs. $\left.0.958 \pm 0.082, P=0.0086\right)$, $\mathrm{Ca} 2+$ concentration (1.372 \pm 0.074 vs. $0.984 \pm 0.055, P=0.0036)$ and PS externalization (1.624 \pm 0.126 vs. $0.969 \pm 0.100, P=0.0051)$ ) compared to sera from HC. Interestingly, immunoglobulin G (IgG) fractions from COVID-19 patients induced an Fc gamma receptor IIA dependent platelet apoptosis $\left(\Delta \Psi_{\mathrm{m}}\right.$ depolarization $(2.23 \pm 0.25$ vs. $1.22 \pm 0.12, P=0.0216), \mathrm{Ca} 2+$ concentration $(1.80 \pm 0.15$ vs. $0.58 \pm 0.04)$ and PS externalization (9.59 \pm 1.52 vs. $2.12 \pm 0.20$, $P=0.0371)$ ).

Conclusions: Our study shows that COVID-19 patients had increased IgG-mediated procoagulant platelets. The strong correlations between procoagulant PLTs and increased D-Dimer levels as well as the incidence of thromboembolic complications may indicate that procoagulant platelets potentially contribute to sustained increased thromboembolic risk in COVID-19 ICU patients.

\section{PB0180 | Weight Adjusted Prophylactic to Intermediate Dosed Thromboprophylaxis in COVID-19}

M.M Engelen ${ }^{1}$; C.P Martens ${ }^{1}$; V. Spalart ${ }^{1}$; K. Peerlinck ${ }^{1}$; R. Willems ${ }^{1}$; B. Vandenberk ${ }^{1}$; P. De Munter ${ }^{1}$; N. Lorent ${ }^{1}$; I. Guler ${ }^{2}$; G. Pieters ${ }^{1}$; P. Sinonquel ${ }^{1}$; J. Vermeulen ${ }^{1}$; K. Martinod ${ }^{2}$; P. Verhamme ${ }^{1}$;

C. Vandenbriele ${ }^{1} ;$ T. Vanassche ${ }^{1}$

${ }^{1}$ University Hospitals Leuven, Leuven, Belgium; ${ }^{2}$ KU Leuven, Leuven, Belgium

Background: COVID-19 is frequently associated with venous thromboembolism (VTE), and the use of thromboprophylaxis has been suggested to improve hospitalized patients' outcomes. We, therefore, intensified our thromboprophylactic protocol starting March 31st.

Aims: We aimed to validate the implementation of an intensified thromboprophylactic protocol by reporting VTE incidence and safety while awaiting randomized controlled trials.

Methods: On March 31st, 2020, we implemented an intensified thromboprophylactic protocol based on weight and disease severity (50 IU anti-Xa LMWH/kg, once daily at the ward, twice daily at the intensive care unit (ICU)). ICU patients were monitored daily with anti-Xa serum levels. Full therapeutic doses were restricted to patients with a prior indication for therapeutic anticoagulation or confirmed VTE. As early reports demonstrated high VTE incidence, screening with duplex ultrasound became standard of care in our center as soon as logistically possible. We excluded patients with a prior indication for therapeutic anticoagulation and incidental findings of COVID-19 for analysis. The ethical committee has approved this observational study.

Results: We analyzed 412 symptomatic and confirmed Covid-19 cases, of which 116 were admitted to the ICU. All symptomatic VTE cases were reported, and $20 \%$ of all patients (38\% of ICU patients) received screening with venous ultrasound. In 219 patients who received the standard dose of LMWH, 16 patients (7.3\%) had VTE, 10 of which were symptomatic (4.6\%) (Figure 1). In 193 patients who 
received intensified thromboprophylaxis, there were no symptomatic VTE cases, three incidental DVT cases (1.6\%), and one incidental pulmonary embolism (0.5\%). Interestingly, rates of major bleeding were low (Figure 2).

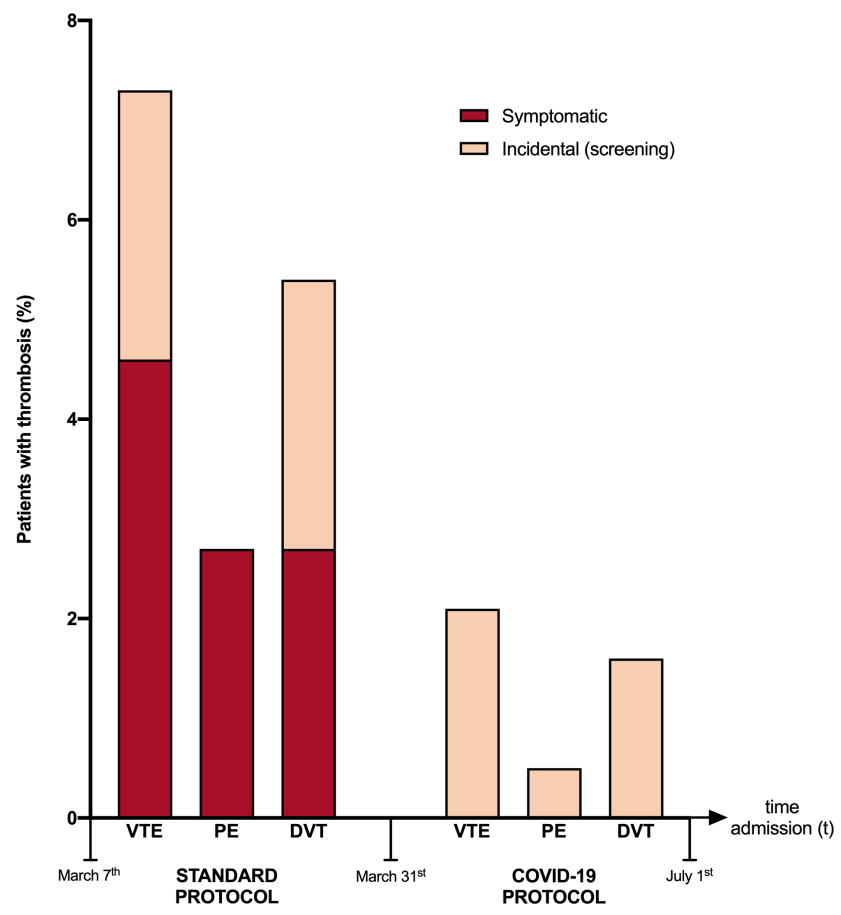

Figure 1. Incidence of venous thromboembolism.

Venous thromboembolism, VTE; pulmonary embolism, PE; deep vein thrombosis, DVT.

FIGURE 1 Incidence of venous thromboembolism

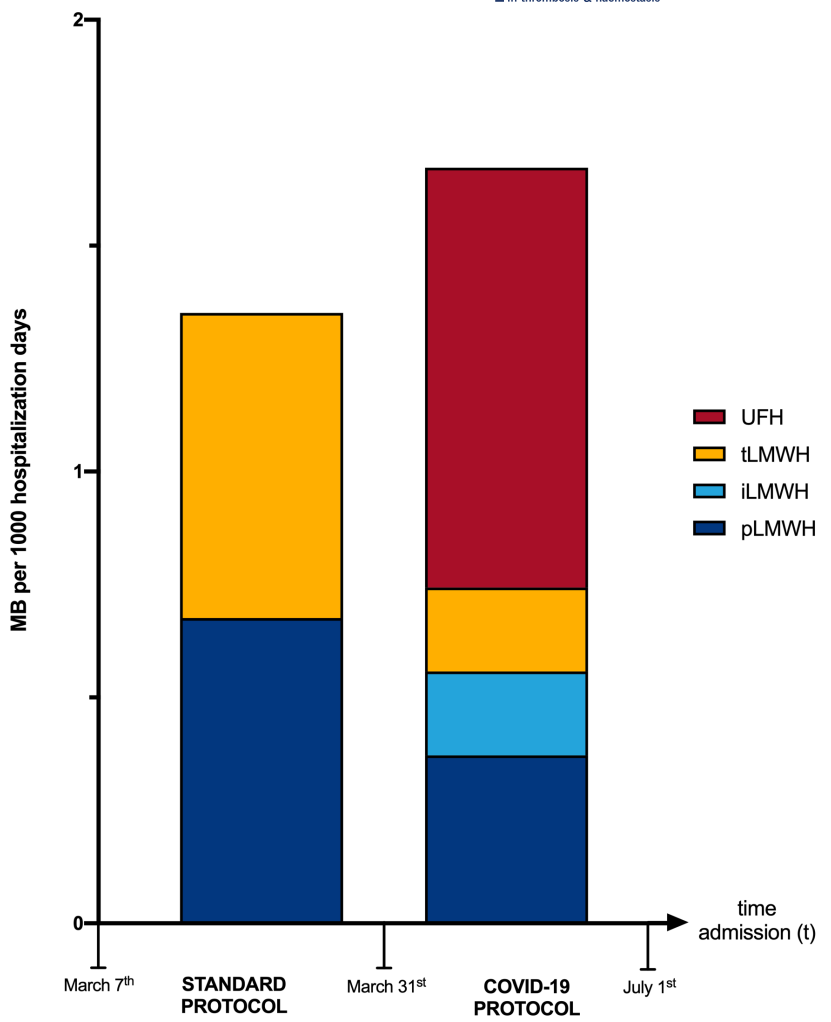

Figure 2. Major bleeding per 1000 hospitalization days.

Major bleeding as defined by the International Society on Thrombosis and Haemostasis, MB; unfractionated heparin, UFH, low molecular weight heparin, LMWH; therapeutic dosed LMWH tLMWH; intermediated dosed LMWH, iLMWH; prophylactic dosed LMWH, pLMWH.

FIGURE 2 Major bleeding per 1000 hospitalization days 
Conclusions: In a large cohort of hospitalized patients with COVID-19, we report no symptomatic VTE after implementing systematic thromboprophylaxis with weight-adjusted prophylactic (ward) to intermediate (ICU), but not therapeutic doses of LMWH. This strategy was associated with a low risk of major bleeding.

\section{PB0181 | Hemostatic Disorders Are Associated with the Course} and Outcomes of COVID-19

$\underline{\text { R.I. Litvinov }}^{1,2}$; N.G. Evtugina ${ }^{2}$; A.D. Peshkova ${ }^{2}$; S.I. Safiullina ${ }^{2,3}$; I.A. Andrianova ${ }^{2}$; R.R. Khismatullin²; S.S. Sannikova ${ }^{4}$;

S.M. Abdullaeva ${ }^{2}$; A.I. Khabirova ${ }^{2}$; J.W. Weisel ${ }^{1}$

${ }^{1}$ University of Pennsylvania School of Medicine, Philadelphia, United States; ${ }^{2}$ Kazan (Volga Region) Federal University, Kazan, Russian Federation; ${ }^{3}$ Medical Center "Aibolit", Kazan, Russian Federation; ${ }^{4}$ City Hospital No. 16, Kazan, Russian Federation

Background: Thrombotic events are common in COVID-19 but little understood.

Aims: To reveal if coagulopathies relate to the course and outcomes of COVID-19.

Methods: We examined 235 patients with moderate and severe COVID-19, receiving anticoagulants and immunosuppressive drugs Informed consent was obtained and the study was approved by the Ethics Committee of K(V)FU. Hemostasis was assessed using a thrombodynamics assay, thromboelastography, fibrinogen and Ddimer levels, prothrombin time, and soluble fibrin complexes (ethanol gelation test). Blood clot contraction (retraction) kinetics was performed along with electron microscopy of platelets and clots and platelet flow cytometry. The hemostatic parameters were correlated with biochemical and hematological tests.

Results: Coagulopathy manifested predominantly as hypercoagulability that correlated directly with systemic inflammation, disease severity, comorbidities, and mortality risk. The prolonged clotting tests in about $1 / 4$ cases were associated with a high level of C-reactive protein, which may impede coagulation in vitro and mask the inflammatory thrombophilia in vivo. Contraction of blood clots was hindered and incomplete in about $1 / 2$ patients, especially in severe and fatal cases. Suppression of clot contraction correlated directly with prothrombotic parameters, including a high D-dimer level. A decrease in the platelet contractility was mainly due to moderate thrombocytopenia in combination with chronic platelet activation and secondary platelet dysfunction. Clots with impaired contraction were porous, had a low content of compressed polyhedral erythrocytes (polyhedrocytes) and an even distribution of fibrin, suggesting that the uncompact intravital clots are more obstructive but could also be prone to bleeding. Persistent hyperfibrinogenemia and high D-dimer in the absence of consumption coagulopathy suggest the predominance of local and/or regional microthrombosis over disseminated intravascular coagulation.
Conclusions: The results obtained i) confirm the importance of hemostatic disorders in COVID-19; ii) justify monitoring of hemostasis, including the kinetics of blood clot contraction; iii) substantiate the active prophylaxis of thrombotic complications in COVID-19.

PB0183 | COVID-19 Related Abnormalities in the Hematologic Characteristics among Inpatient Children. Results of the PICNIC Registry

S. Tehseen $^{1}$; J. Robinson ${ }^{2}$; S. Williams ${ }^{3}$; L. Lieberman ${ }^{4}$; PICNIC Paediatric Investigator Collaborative Network on Infections in Canada

${ }^{1}$ Saskatchewan Health Authority, Saskatoon, Canada; ${ }^{2}$ Department of Pediatrics. University of Alberta, Edmonton, Canada; ${ }^{3}$ Division of Hematology Oncology, Hospital for Sick Children, Toronto, Canada; ${ }^{4}$ University of Toronto, UHN and Affiliated Hospitals, Toronto, Canada

Background: Hematologic complications, including thrombosis, are well described in adults with COVID-19. Information regarding pediatric hematologic complications is limited.

Aims: Describe the hematologic complications of pediatric inpatients with COVID-19

Methods: An international, multi-center retrospective registry was created in June 2020 describing the clinical spectrum of COVID-19 hospitalized children. Information collected included: demographics, comorbidities, presentation, multi-system inflammatory syndrome (MIS-C) diagnosis, investigations, management and outcomes. Hematologic variables: were bleeding, signs of disseminated intravascular coagulation (DIC) and thrombosis or bleeding. Bleeding and MIS-C were defined per WHO criteria. DIC diagnosis was based on clinical judgement. Information was collected in Redcap and data analyzed using SAS.

Categorical data was reported as frequencies and proportions, continuous variables as means with standard deviations, or medians with interquartile range (IQRs) if data were skewed.

Results: 295 children were included and 104/295 (35\%) had MISC. 107 (36.3\%) children were admitted for alternate diagnosis with associated COVID-19 infection. 2 children (0.6\%) developed thrombosis. 1 had an internal jugular vein thrombus and the other had extensive thromboembolism including pulmonary embolus (PE). 1 patient developed both PE and gastrointestinal (GI) hemorrhage. 5 children (1.7\%) experienced bleeding where 4 had epistaxis and/or $\mathrm{Gl}$ hemorrhage and 1 had pulmonary hemorrhage. 2 patients had DIC with bleeding. 4 children required blood products for management of hemorrhage. Rates of thrombosis and bleeding were similar between MIS-C and other COVID-19 patients. 117 (40\%) children received prophylactic anti-coagulation (22 [7.4\%] MIS-C patients) based on hospital specific policies. 5 (1.7\%) deaths occurred in the cohort; all unrelated to COVID-19 associated hematologic complications. 
TABLE 1 Demographics, clinical and laboratory features of inpatient children with COVID-19 and MISC

\begin{tabular}{|c|c|c|c|}
\hline & All $(N=295)$ & MIS-C (N = 104) & Covid-19 infection $(N=191)$ \\
\hline Demographics & 4 (IQR: 1-9) & 5 (IQR: 2-8) & 3 (IQR: 0-10) \\
\hline Age (years): Median (IQR) & $128(43 \%)$ & 51 (49\%) & 77 (40\%) \\
\hline \multicolumn{4}{|l|}{ Female gender $N(\%) F$} \\
\hline Co-morbid disorders & $109(37 \%)$ & 0 & $12(6.2 \%)$ \\
\hline Cancer & $12(4 \%)$ & $1(0.9 \%)$ & $1(0.6 \%)$ \\
\hline Thrombophilia & $2(0.6 \%)$ & 0 & $2(1 \%)$ \\
\hline Bleeding disorder & $2(0.6 \%)$ & 0 & $10(5 \%)$ \\
\hline Immunodeficiency & $10(3 \%)$ & 0 & $6(3 \%)$ \\
\hline CHD & $6(2 \%)$ & 0 & $4(2 \%)$ \\
\hline Chronic lung disease & $4(1.2 \%)$ & 0 & $1(0.6 \%)$ \\
\hline Sickle cell & $1(0.3 \%)$ & 0 & $2(1 \%)$ \\
\hline End stage renal Disease & $2(0.6 \%)$ & 0 & $5(2.6 \%)$ \\
\hline Hypertension & $5(1.7 \%)$ & $3(3 \%)$ & $12(6.2 \%)$ \\
\hline Obesity & $15(5 \%)$ & & \\
\hline Clinical hematologic complications $\mathrm{N}$ & $8(2.7 \%)$ & 1 (APS: RLE DVT, PE) & 1 (CVL: IJV clot) \\
\hline (Risk Factors: anatomic site) & 2 & 0 & 3 (thrombocytopenia, MAS, CHD: \\
\hline Thrombosis only & 3 & 1 (Sepsis: GI bleed) & Epistaxis, lung and GI bleed) \\
\hline Bleeding only & 2 & 1 (CVL, Obesity: PE and GI & 1 (AML, sepsis: Epistaxis) \\
\hline DIC with bleeding & 1 & bleed) & 0 \\
\hline \multicolumn{4}{|l|}{ Thrombosis and bleeding } \\
\hline$\underline{\text { Hematologic parameters }}$ & 103 (IQR: 90-120) & 97 (IQR: 88-107) & 112 (IQR: 92-127) \\
\hline Median (IQR) & 241 (IQR: 154-342) & 463 (IQR: 345-655) & 340 (IQR: 230-433) ${ }^{*}$ \\
\hline Hemoglobin g/dl & 43 (IQR: 38-46) & 42.5 (IQR: 39-45) & 44 (IQR: 32-47) \\
\hline Platelets $\times 10^{9} / \mathrm{L}$ & 6 (IQR: 4.9-6.8) & 6.1(IQR: 5-7) & 5.1 (IQR: 4.5-6.4) \\
\hline $\operatorname{PTT}(s)$ & 2660 (IQR: 1200-4001) & 2710 (IQR: 1120-4001) & 1530 (IQR: 1220-3535) \\
\hline \multicolumn{4}{|l|}{ Fibrinogen $\mathrm{g} / \mathrm{dl}$} \\
\hline \multicolumn{4}{|l|}{ D-Dimer ug/I } \\
\hline Patients with significant hematologic & $19(6.4 \%)$ & $4(4 \%)$ & $15(8 \%)$ \\
\hline lab abnormalities N (\%) & $15(5 \%)$ & $2(2 \%)$ & $13(7 \%)$ \\
\hline Hemoglobin $\leq 70 \mathrm{~g} / \mathrm{dl}$ & $2(0.6 \%)$ & 0 & $2(1 \%)$ \\
\hline Platelets $\leq 50 \times 10^{9} / \mathrm{L}$ & $2(0.6 \%)$ & 0 & $2(1 \%)$ \\
\hline Platelets $\leq 10 \times 10^{9} / \mathrm{L}$ & $1(0.3 \%)$ & 0 & $1(0.5 \%)$ \\
\hline Fibrinogen $\leq 1 \mathrm{~g} / \mathrm{dl}$ & 92 (31\%) & 53 (51\%) & $39(20 \%)$ \\
\hline$P T T \geq 55$ secs & $42(14 \%)$ & $36(35 \%)$ & $6(3 \%)$ \\
\hline Platelets $\geq 450 \times 10^{9} / \mathrm{L}$ & 99 (33\%) & 74 (71\%) & $25(13 \%)$ \\
\hline \multicolumn{4}{|l|}{ Fibrinogen $\geq 5 \mathrm{~g} / \mathrm{dl}$} \\
\hline \multicolumn{4}{|l|}{ Abnormal D-Dimer } \\
\hline Prophylactic anti-coagulation & $117(40 \%)$ & $22(21 \%)$ & $50(26 \%)$ \\
\hline
\end{tabular}




$\begin{array}{ll}\text { Cause of Death N (Risk Factors) } & 5 \text { (1.7\%) } \\ \text { COVID related respiratory } & 4 \text { (developmental delay, } \\ \text { failure } \pm \text { Sepsis } & \text { CLD, malignancy) } \\ \text { COVID related cardiac and respiratory } & 1 \text { (Renal failure with re- } \\ \text { dysfunction } & \text { flux disease of kidney) }\end{array}$

MIS-C: Multi system inflammatory syndrome in children. DIC: Disseminated intravascular coagulation. PE: Pulmonary Embolism, RLE: Right lower extremity DVT: Deep venous thrombosis, APS: Anti-phospholipid antibody syndrome, IJV: Internal jugular vein. Sepsis: Bacterial or fungal infection with DIC, MAS: Macrophage activation syndrome, CHD: hemodynamically significant congenital heart disease, CVL: Central venous line, ARDS: Acute respiratory distress syndrome, CLD: Chronic Lung Disease, AML: Acute Myeloid leukemia.

*assumes significance at $P$-value $>0.05$. Patients with MIS-C had a higher median age and platelet count with a lower hemoglobin compared to children without MIS-C.

Conclusions: This registry describes COVID-19 related hematologic complications in a large m8ulti-center pediatric cohort. The frequency of these complications is low and related to underlying comorbidities in children. Ongoing research is crucial to understand covid-associated hematological challenges in children and to determine the indications of prophylactic anti-coagulation.

PB0184 | Anti-factor Xa Activity after Intermediate Dose Nadroparin for Thrombosis Prophylaxis in Critically III COVID-19 Patients with and without Acute Kidney Injury

R. Eck ${ }^{1}$; J. van Leur ${ }^{2} ;$ M. Lukens ${ }^{1} ;$ W. Bult ${ }^{1} ;$ R. Gans ${ }^{1} ;$ K. Meijer ${ }^{1}$;

F. Keus ${ }^{1}$

${ }^{1}$ University Medical Center Groningen, University of Groningen, Groningen, Netherlands; ${ }^{2}$ Isala Zwolle, Zwolle, Netherlands

Background: The optimal prophylaxis for prevention of venous thromboembolism (VTE) in critically ill COVID-19 patients with acute kidney injury (AKI) is uncertain. Low-molecular-weight heparins are mainly excreted by the kidney and AKI may predispose to bioaccumulation with increased risk of bleeding. Dose adjustment or switch to unfractionated heparin is generally recommended, based on low quality evidence. Aims: To describe trough anti-Xa activity in critically ill patients with and without $A K I$ receiving prophylaxis with intermediate dose nadroparin.

Methods: This was a prospective cohort study including critically il COVID-19 patients receiving intermediate dose nadroparin (5700 IU once daily). Trough anti-Xa levels were measured twice weekly 20-25 h after the previous dose. AKI was defined according to guideline criteria using serum creatinine and renal replacement therapy. The main outcome was the proportion of patients with bioaccumulation, defined as a trough anti-Xa level $>0.2 \mathrm{IU} / \mathrm{mL}$ at any time, in which case the dose had to be reduced. In patients with $\mathrm{AKI}$ and repeated measurements we calculated the range of trough anti-Xa values as a proxy for within-patient variation. Results: There were 234 trough anti-Xa measurements in 108 patients. In twenty-four patients 36 measurements were made during AKI. Two patients had evidence of bioaccumulation on a single measurement, 1 with and 1 without AKI (Figure 1). In 20 patients who had $\mathrm{AKI}$ and repeated measurements, the median range of
anti-Xa values was $0.05 \mathrm{IU} / \mathrm{mL}$ (IQR $<0.04 \mathrm{IU} / \mathrm{mL}-0.08 \mathrm{IU} / \mathrm{mL}$; lowest range $<0.04 \mathrm{IU} / \mathrm{mL}$; highest range $0.20 \mathrm{IU} / \mathrm{mL}$, Figure 2). Four patients had major bleeding events during hospital stay $(2.4 \%$; $95 \% \mathrm{Cl}$ $0.04 \%-8.9 \%$ ) but none while on intermediate dose nadroparin.

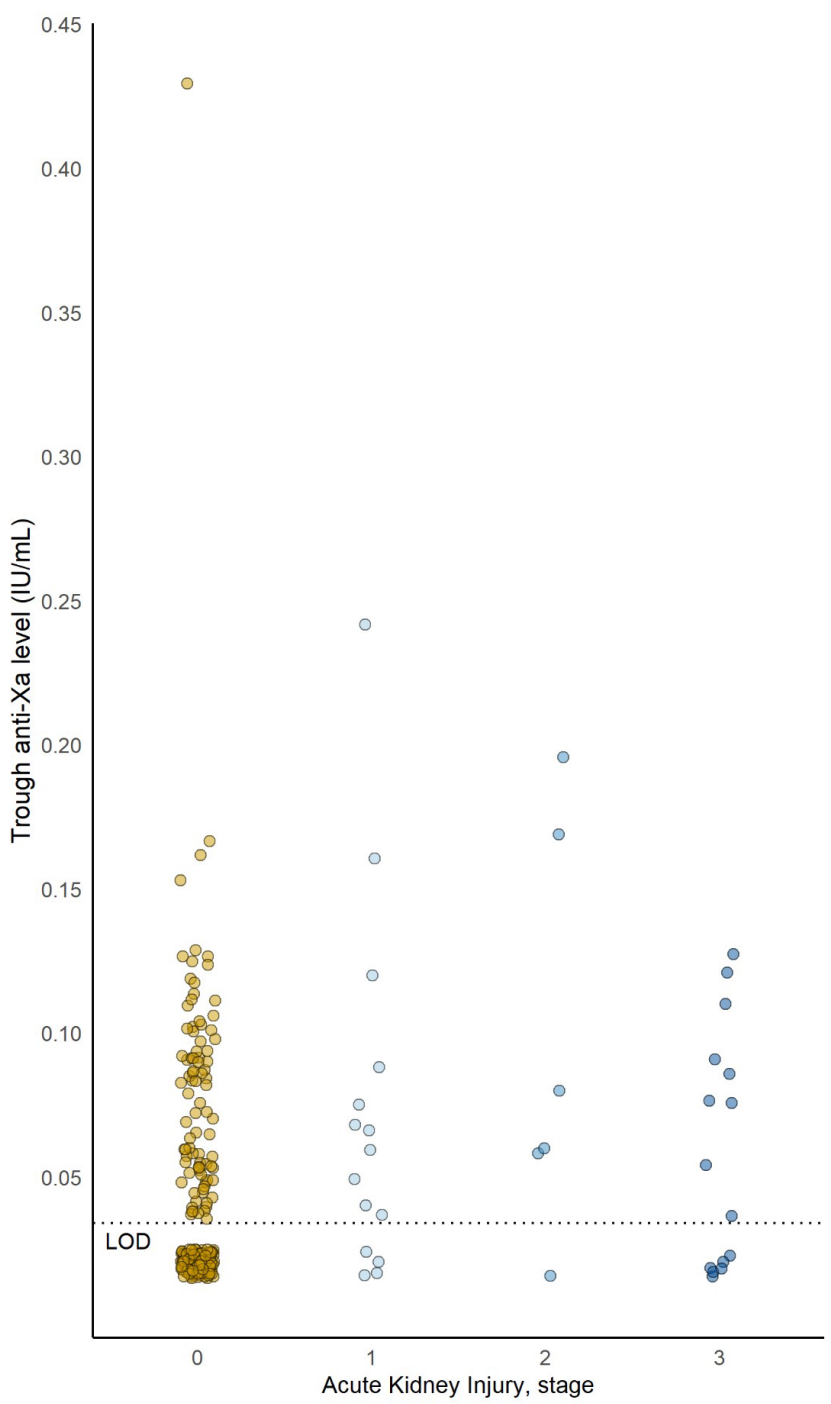

FIGURE 1 Trough anti-Xa level according to acute kidney injury stage 


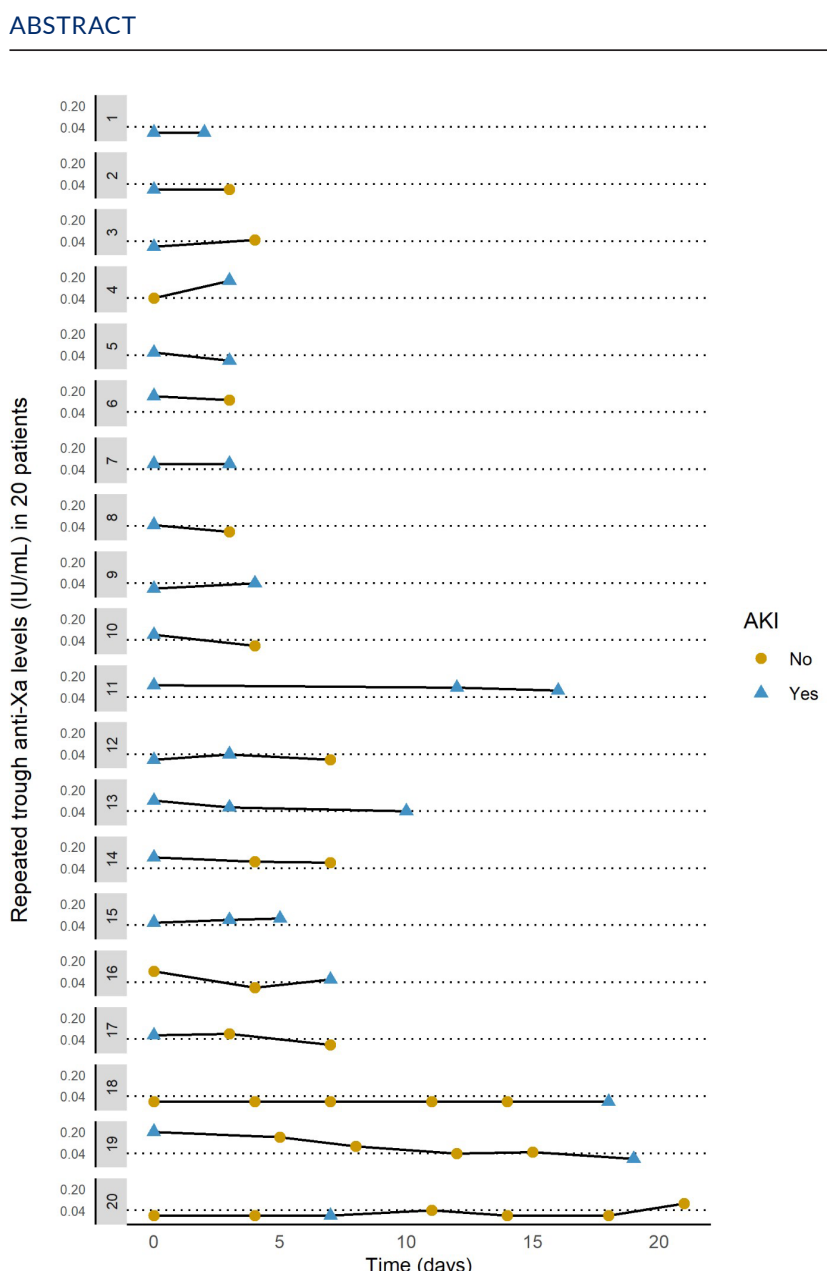

FIGURE 2 Repeated trough anti-Xa levels in twenty patients with acute kidney injury

Conclusions: Bioaccumulation was rare in critically ill patients with AKI who received intermediate dose nadroparin for thrombosis prophylaxis. These data suggest there is no need for dose adjustment nor monitoring of nadroparin prophylaxis in patients with COVID-19 and AKI. The selected population may limit generalization to other patients.

\section{PB0185 | Post Hospital Discharge Thrombosis in Patients with} COVID-19

$\underline{\text { P. Li }}^{1}$; S. Kaatz ${ }^{2}$; W. Zhao ${ }^{3}$; K. Latack ${ }^{1}$; L. Schultz ${ }^{1}$; L. Poisson ${ }^{1}$

${ }^{1}$ Public Health Sciences, Henry Ford Health System, Detroit, United States; ${ }^{2}$ Division of Hospital Medicine, Henry Ford Health System, Detroit, United States; ${ }^{3}$ Department of Internal Medicine, Ascension St. John Hospital, Detroit, United States

Background: Coronavirus disease 2019 (COVID-19) is associated with a high incidence of thrombotic events during hospitalization, however, the need for postdischarge thromboprophylaxis remains unclear.

Aims: To quantify the 90-day post hospital discharge rates of venous and arterial thromboembolism in COVID-19.
Methods: A retrospective single institution observational study of adult patients hospitalized with COVID-19 confirmed by positive SARS-CoV-2 testing from 3/1/2020 to $8 / 31 / 2020$. Patients were excluded if they: remained hospitalized at time of analysis, died during hospitalization or were discharged to hospice. For patients with multiple admissions related to COVID-19, the first admission was included. Thromboembolism was identified with ICD-10 codes up to 90 days of discharge: pulmonary embolism (PE: I26), deep vein thrombosis (DVT: 182), portal vein thrombosis (I81), intracranial venous thrombosis (167),transient ischemia attack (TIA: G45), stroke (I63), acute coronary syndrome (ACS: I20, 121, I22, I24), intracardiac thrombosis (I23, I51.3), and acute limb ischemia (174).

Results: Of 1653 hospitalized patients, 26 (1.6\%) developed post discharge venous thrombosis events (12 PE, 13 DVT and 1 portal vein thrombosis). Eleven (0.7\%) post discharge arterial thrombosis events were observed(1 TIA and 10 ACS). The risk of VTE decreases with time (Mann-Kendall trend test $P$-value $<0.001$ ) with median event time 15.5 days (IQR: 6-27). The risk of arterial thrombosis is constant with time (Mann-Kendall trend test $P$-value $=0.86$ ) with median event time 54 days (IQR: 24-65).

Conclusions: The rate thromboembolism is relatively low among COVID-19 patients after they leave the hospital. Results of ongoing randomized trials of the efficacy of post-discharge anticoagulation prophylaxis are eagerly awaited.

PB0186 | A Weight-based Approach to Thromboprophylaxis Dosing for Medical Inpatients with COVID-19

$\underline{\text { R. Price }}^{1}$; Z. Kirkham ${ }^{1,2}$; A. Szuman ${ }^{1}$; S. Ahmed ${ }^{3}$; C. Macaulay ${ }^{4}$; K. Talks ${ }^{5}$; B. Greystoke ${ }^{5}$; K. Musgrave ${ }^{2,5}$

${ }^{1}$ The Newcastle upon Tyne Hospitals NHS Foundation Trust, Newcastle upon Tyne, United Kingdom; ${ }^{2}$ Translational and Clinical Research Institute, Newcastle University, Newcastle upon Tyne, United Kingdom; ${ }^{3}$ Gateshead Health NHS Foundation Trust, Gateshead, United Kingdom; ${ }^{4}$ Leeds Teaching Hospitals NHS Trust, Leeds, United Kingdom; ${ }^{5}$ Haematology Department, The Newcastle upon Tyne Hospitals NHS Foundation Trust, Newcastle upon Tyne, United Kingdom

Background: COVID-19 is associated with a higher risk of venous thromboembolism (VTE). There is no consensus on the optimum dose of thromboprophylaxis.

Aims: To assess the effectiveness of weight-based thromboprophylaxis dosing.

Methods: This is a retrospective cohort study of all medical patients at a large UK-based teaching hospital, between April 1st and 15th May 2020. Electronic medical records were reviewed. People who were PCR positive for SARS-CoV-2 (COVID-19 positive) received a weight-based thromboprophylactic dose of enoxaparin (Table 1). Those PCR negative for SARS-CoV-2 (COVID-19 negative) received a standard dose (enoxaparin $40 \mathrm{mg}$ daily). Chi square and Fisher's exact test were used for categorical variables, unpaired $t$ test for continuous. 
TABLE 1 Weight-based dosing of thromboprophylaxis with enoxaparin for COVID-19 positive patients. (kg- kilogram, CrCL - creatinine clearance, OD - once daily)

$\begin{array}{lll}\text { Weight }(\mathrm{kg}) & \text { Crcl } \geq 30 & \text { Crcl 15-29 } \\ \leq 49 & 20 \mathrm{mg} \text { OD } & \text { Consider the use of unfractionated heparin } \\ 50-99 & 40 \mathrm{mg} \text { OD } & 20 \mathrm{mg} \text { OD } \\ 100-149 & 40 \mathrm{mg} \text { OD } & 40 \mathrm{mg} \text { OD } \\ \geq 150 & 60 \mathrm{mg} \text { OD } & 40 \mathrm{mg} \text { OD }\end{array}$

Results: 569 cases (179 COVID-19 positive, 390 COVID-19 negative) were identified. The COVID-19 positive group had a significantly higher average age, a similar proportion of males and a greater average weight (Table 2). $86 \%$ of the COVID-19 positive group received the correct thromboprophylaxis dose for their weight. Most receiving an incorrect dose weighed $>100 \mathrm{~kg}(54 \%)$ and received a lower dose than recommended. The incidence of new VTE was similar in the COVID-19 positive group (12,7\%) compared to those in the COVID-19 negative (16, 4\%, $P=0.18)$. Most VTE events were proven radiologically apart from two COVID-19 positive patients diagnosed by clinical suspicion alone. Eight cases (67\%) of VTE in patients with COVID-19 were pulmonary thrombosis, compared to twelve $(75 \%)$ in patients without $(P=0.69)$. Two cases of VTE occurred in the COVID-19 positive group whilst they were therapeutically anticoagulated compared to none in the COVID-19 negative group. Two major bleeding episodes occurred in the COVID-19 positive group and one in the COVID-19 negative $(P=0.23)$.
PB0187 | Physicians' Knowledge and Management Practices of COVID-19 Coagulopathies in Pregnancy

S. Jevtic ${ }^{1}$; A.K. Malinowski ${ }^{2,3}$; M. Othman ${ }^{4,5}$; R. Abdul Kadir ${ }^{6}$

${ }^{1}$ McMaster University, Hamilton, Canada; ${ }^{2}$ University of Toronto, Toronto, Canada; ${ }^{3}$ Lunenfeld-Tanenbaum Research Institute, Toronto, Canada; ${ }^{4}$ Queen's University, Kingston, Canada; ${ }^{5}$ Lawrence College, School of Baccalaureate Nursing, Kingston, Canada; ${ }^{6}$ University College London, London, United Kingdom

Background: Coronavirus disease 2019 (COVID-19) occurs following infection with the highly transmissible, and potentially fatal, severeacute-respiratory-syndrome-coronavirus-2 (SARS-CoV-2) virus. Infection can be complicated by coagulopathy featuring abnormal coagulation parameters, thrombo-inflammation, and thrombocytopenia, collectively termed COVID-19 associated coagulopathy (CAC). Data concerning CAC prevalence and management in pregnancy is

TABLE 2 Comparison of the COVID-19 positive and COVID-19 negative groups. (GI - gastrointestinal, \%- percentage)

\begin{tabular}{llll} 
& COVID-19 positive & COVID-19 negative & P value \\
Number & 179 & 390 & $<0.00001$ \\
Median age, years (range) & $74(19-101)$ & $66(17-99)$ & 0.947 \\
Male & $96(54 \%)$ & $208(53 \%)$ & 0.602 \\
Median weight, kg (range) & $74(38-155)$ & $70(33-241)$ & 0.18 \\
VTE & $12(7 \%)$ & $16(4 \%)$ & 0.69 \\
Pulmonary thrombosis & $8(67 \%)$ & $12(75 \%)$ & 0.23 \\
Major bleeding & $2(1 \%)$ & $1(1 \%)$ & $($ upper GI bleed, 1) \\
& (upper Gl bleed, 1; upper and lower GI & \\
\hline
\end{tabular}

Conclusions: There was a similar incidence of VTE in medical patients with COVID-19 compared to those without. Weight-based thromboprophylaxis was not associated with an increased rate of bleeding. limited. Evaluation of physician experiences in assessing and managing CAC is essential to identify current practice patterns and knowledge gaps.

Aims: To determine physician experiences and practice patterns relevant to CAC prevention and management in pregnancy.

Methods: A cross-sectional study with self-administered online questionnaire using the RedCap online platform; supported by the ISTH Subcommittee on Women's Health Issues in Thrombosis and Hemostasis.

Results: Of 115 physicians who opened the survey, 74 (38\% Maternal-Fetal Medicine, 31\% Haematology/Thrombosis) provided 
responses conducive to analysis. There were 1503 reported cases of COVID-19, from which 1288 contained data regarding disease severity. Sixty-five percent of COVID-19 infections were mild while only $4 \%$ were severe. Of all reported cases, $1 \%$ developed CAC, of which $63 \%$ were in the severe spectrum of COVID-19 infection. The most frequently identified abnormalities included thrombocytopenia, elevated CRP, D-dimer, and lymphopenia. Low-molecularweight heparin was the anticoagulant of choice, provided by $67 \%$ of respondents, with $56 \%$ using standard-prophylactic dosing for CAC. Thrombosis occurred in 6 patients on anticoagulation; 50\% were receiving standard prophylactic dosing, while the remaining $50 \%$ were unspecified. Anticoagulation duration depended on disease severity and additional thrombosis risk factors.

Conclusions: CAC is uncommon in pregnancy with a predilection for higher disease severity. Anticoagulation practices vary and may not reflect current guideline recommendations. Development of thrombosis has been observed in CAC on standard prophylactic anticoagulation, thus emphasizing the need for re-evaluation of anticoagulant regime in severe cases. Urgent research is required to determine appropriate anticoagulant dosing and duration in these patients.

PB0188 | COVID-19 Pneumonia Worse Prognosis Related to Lupus Anticoagulant Transient Positive Test

J.M. Ceresetto ${ }^{1}$; S. Rivarola ${ }^{2}$; C. Duboscq ${ }^{1}$; M. Carreras ${ }^{1}$;

A. Cantillo ${ }^{1}$; L. Zapata ${ }^{1}$; K. Oliveros ${ }^{1}$; A. Avila ${ }^{1}$; C. Sernaque ${ }^{1}$;

M. Quarchioni ${ }^{1}$; N. Flegler ${ }^{1}$; S. Palmer ${ }^{1}$; G. Stemmelin ${ }^{1}$

${ }^{1}$ Hospital Britanico de Buenos Aires, Buenos Aires, Argentina; ${ }^{2}$ Hospital

Britanico de Buenos Aires, Buenoas Aires, Argentina

Background: Lupus anticoagulant (LAC) has been reported in almost $50 \%$ of patients with COVID-19 disease, but its significance is not clear yet.

Aims: To evaluate prospectively the presence of LAC test in hospitalized symptomatic COVID-19 pneumonia patients and to report clinical evolution, thromboembolism (VTE), bleeding events, and persistence of the LAC positive (LAC+) 1-3 months after discharge.

Methods: 278 patients hospitalized with COVID-19 symptomatic disease (August-November 2020). LAC was performed according to ISTH guidelines. Heparin must be $<0.35 a n t i X a U / m L$. D-dimer (immunoturbidimetic) was performed with initial laboratory. Patients were followed for any thrombotic/hemorrhagic events for three months. Chi-square test and multivariate logistic regression (statistix 7.0) were used for the statistical analysis.

Results: From 278 patients with COVID-19 admitted at the hospital, 149 (54\%) had LAC+ in the first $48 \mathrm{~h}$. Most of them were dRVVT positive, only $12 / 149$ had both dRVVT/SCT positive. Average age 59 (range18-92) 103 males and this wasn't different from LAC(-). $46 / 278$ (17\%) patients required intensive care admission for ventilatory support, 9 (3\%) had clinical VTE, 5 (2\%) had major bleeding and $16(6 \%)$ died. LAC+ patients had significantly more severe COVID-19 pneumonia as they required more intensive care support (34 vs 12 patients $P=0.002$ ) and died more (13 vs 3 deaths $P=0.003$ ) They also had more VTE (7vs2). Five patients had major bleeding (three LAC+, two LAC(-) but 4/5 were under full dose anticoagulation (three $A F$, one VTE). D-dimer was nor different between LAC+/LAC(-) patients (average 1104 ng/FEU LAC+, 1249 ng/FEU LAC(-). A new LAC was performed 1-3 months after discharge in 63/137 (46\%) patients. No one had thrombosis and 43/63 (68\%) had LAC(-) when COVID-19 resolved.

Conclusions: LAC+ seems to be related to worse prognosis as they required more intensive care support and died more in patients with COVID-19 disease, although this pro thrombotic condition is transitory in most of them.

\section{PB0189 | Does Thromboelastography Predict Clinical Outcomes} in COVID-19?

M. Gaddam ${ }^{1}$; A. Wahab ${ }^{2}$; A. Borgert ${ }^{1}$; S. Frankki ${ }^{1}$; U. Perepu ${ }^{2}$;

S. Lentz ${ }^{2}$; L. Rosenstein ${ }^{1}$

${ }^{1}$ Gundersen Health System, Lacrosse, United States; ${ }^{2}$ University of lowa, lowa, United States

Background: COVID-19 infection is associated with thrombosis and death, but the role of global coagulation assays such as thromboelastography (TEG) in predicting clinical outcomes is unclear.

Aims: To assess TEG patterns in patients with COVID-19 and to determine association with mortality and thrombosis.

Methods: TEGs were performed prospectively as part of a multicenter randomized controlled trial comparing standard vs intermediate dosing of prophylactic anticoagulation in hospitalized patients with severe COVID-19 (NCT04360824). All enrolled patients had a modified ISTH DIC score>3 and/or were admitted to an intensive care unit. TEG and baseline coagulation labs were obtained at study entry. TEG parameters and baseline labs and patient demographics were analyzed and correlated with the primary outcome (30-day mortality) and secondary outcomes (arterial and venous thrombosis, acute kidney injury, and major and minor bleeding).

Results: The baseline characteristics for 131 patients for which TEG results were available are shown in Table 1. Thrombotic events occurred in $9.9 \%$ of patients ( $N=13$ ), acute kidney injury in $13 \%$ $(N=18)$ and the 30 -day mortality rate was $16 \%(N=21)$. No significant associations were noted between baseline TEG results and patient outcomes based on standard reference ranges. When comparing the continuous TEG data against relevant outcomes, only LY30 was associated with any outcome (30-day mortality). White blood cell count, D-dimer, and creatinine were better predictors of major patient outcomes. A multivariable model including categorical representations of age, LY30, and D-dimer values showed good performance in predicting 30-day mortality (Table 2). 


\begin{tabular}{|c|c|}
\hline Variable & Data \\
\hline \multicolumn{2}{|l|}{ Demographics } \\
\hline Patients, $\mathrm{n}$ & 131 \\
\hline Age, y, median (range) & $64(24-86)$ \\
\hline $\mathrm{BMI}, \mathrm{kg} / \mathrm{m}^{2}$, median (range) & $30.8(16.6-56.4)$ \\
\hline Sex, male, $n(\%)$ & $78(59.5)$ \\
\hline Labs & Median (range) \\
\hline White blood cell count, $\mathrm{k} / \mathrm{mm}^{3}$ & $9.4(2.8-114.7)$ \\
\hline Hemoglobin, g/dl & $12.4(5.9-16.9)$ \\
\hline Platelet count, $10^{9} / \mathrm{L}$ & $293(70-854)$ \\
\hline Prothrombin time, s & $11(10-15)$ \\
\hline Partial thromboplastin time, $s$ & $27(20-51)$ \\
\hline D-dimer, $\mathrm{mg} / \mathrm{mL}$ & $1.4(0.2-35.2)$ \\
\hline Fibrinogen, $\mathrm{mg} / \mathrm{dL}$ & $549(225-1000)$ \\
\hline Creatinine, $\mathrm{mg} / \mathrm{dL}$ & $0.8(0.4-5.2)$ \\
\hline TEG parameters (normal range) & Median (range) \\
\hline R-time, $\min (5.0-10.0)$ & $4.1(1.8-7.5)$ \\
\hline Kinetics, $\min (1.0-3.0)$ & $1.1(0.8-2.3)$ \\
\hline Angle, deg (50.0-72.0) & $74.1(51.6-83.4)$ \\
\hline $\begin{array}{l}\text { Maximum amplitude, } \mathrm{mm} \text { (50.0- } \\
70.0)\end{array}$ & $71.8(57.1-85.9)$ \\
\hline Ly30 (0.0-8.0) & $0.2(0.0-8.9)$ \\
\hline Coagulation index $(-3.0-3.0)$ & $3.4(-0.7-6.4)$ \\
\hline Clot strength (4.5-11.0) & $12.7(6.6-30.4)$ \\
\hline \multicolumn{2}{|l|}{ Outcomes, $n(\%)$} \\
\hline Deceased by day 30 & $21(16)$ \\
\hline Acute Kidney Injury & $18(13.7)$ \\
\hline Arterial Thrombosis & $6(4.6)$ \\
\hline Venous Thrombosis & $8(6.1)$ \\
\hline Arterial or Venous Thrombosis & $13(9.9)$ \\
\hline Major Bleeding & $4(3.1)$ \\
\hline Minor Bleeding & $10(7.6)$ \\
\hline
\end{tabular}

TABLE 2 Multivariable analysis

\begin{tabular}{|l|l|l|}
\hline Variable & Odds Ratio (95\% Confidence Interval) & P-value \\
\hline Age $\geq 60$ vs. Age $<60$ & $4.90(1.03,23.27)$ & 0.045 \\
\hline 30 minute lysis $<0.5 \%$ vs. $\geq 0.5 \%$ & $3.20(0.83,12.30)$ & 0.091 \\
\hline D-dimer $\geq 2.6 \mathrm{mg} / \mathrm{mL}$ vs. $<2.6 \mathrm{mg} / \mathrm{mL}$ & $4.46(1.54,12.93)$ & 0.006 \\
\hline & & \\
\hline C-statistic & 0.80 & \\
\hline
\end{tabular}

Conclusions: Hypercoagulability and thrombotic events occur frequently in hospitalized COVID-19 patients and are associated with adverse outcomes. TEG data independently was not predictive of major outcomes, but the addition of TEG results to a multivariate model improved prognostic implications for mortality.
A. Lundström $^{1}$; L. Ziegler ${ }^{1}$; S. Havervall ${ }^{1}$; A.-S. Rudberg ${ }^{1}$; F. Von Meijenfeldt ${ }^{2}$; T. Lisman ${ }^{2}$; N. Mackman ${ }^{3}$; P. Sandén ${ }^{1}$; C. Thålin ${ }^{1}$ ${ }^{1}$ Karolinska Institutet, Dept of Clinical Sciences Danderyd Hospital, Stockholm, Sweden; ${ }^{2}$ University of Groningen, Dept of Surgery, Groningen, Netherlands; ${ }^{3}$ University of North Carolina at Chapel Hill, Div of Hematology, Dept of Medicine, Chapel Hill, United States

Background: Angiotensin-converting enzyme 2 (ACE2) is the main entry receptor for SARS-CoV-2, but how virus-receptor interactions affect the RAS-balance and COVID-19 pathology is largely unknown. Aims: To measure soluble ACE2 (SACE2) and SACE during and after COVID-19 and investigate associations with risk factors for severe COVID-19, outcome and markers of thromboinflammation.

Methods: Plasma sACE2 and sACE were measured by ELISA in 114 hospital-treated patients with COVID-19 and 10 healthy controls. Follow-up samples after four months were available for 58/114 patients. Markers of inflammation and endothelial dysfunction during COVID-19 were available from routine testing or had been previously determined (VWF, factor VIII, D-dimer, IL-6, TNFa and PAI-1). Results: Levels of SACE2 were higher in COVID-19 patients than in healthy controls, median 5.0 (interquartile range $2.8-11.8$ ) $\mathrm{ng} / \mathrm{ml}$ versus 1.4 (1.1-1.6) ng/ml, $P<0.0001$. sACE2 was higher in men than women, but was not affected by other risk factors for severe COVID-19. sACE2 decreased to 2.3 (1.6-3.9) ng/ml at follow-up, $P<0.0001$, but remained higher than in healthy controls, $P=0.012$. Follow-up sACE2 was associated with several risk factors for severe COVID-19 and treatment with RAS-inhibition. SACE was marginally lower during COVID-19 compared with four months later $57(45-70) \mathrm{ng} / \mathrm{ml}$ versus 72 (52-87) ng/ $\mathrm{ml}, P=0.008$. Levels of sACE2 and sACE were not associated with survival or disease severity (care level, respiratory support). SACE2 during COVID-19 correlated positively with monocyte count and platelet count, while SACE was negatively correlated with both. SACE2 correlated with VWF, fVIII and D-dimer, while sACE correlated with IL-6, TNFa and PAI-1.

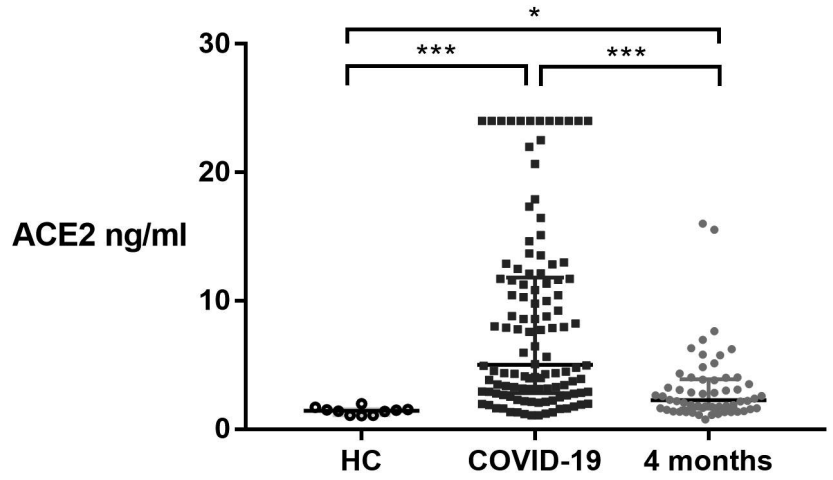

FIGURE 1 sACE2 concentrations for 10 healthy controls (HC, left), 114 patients with COVID-19 (middle) and 58 patients at follow-up after four months (right). The differences between COVID-19 patients and $\mathrm{HC}$ were analyzed by Mann-Whitney U-test. The difference between acute COVID-19 and follow-up four months later was analyzed by Wilcoxon signed rank tests. ${ }^{* * *} P<0.0001,{ }^{*} P<0.05$ 
TABLE 1 Spearman correlation coefficients for significant correlations between acute SACE2 respectively SACE and markers of inflammation and endothelial dysfunction. Correlation coefficients with $|r|>0.25$ are reported. N: number of patients analyzed. WBC: white blood cell count; VWF: von Willebrand factor; PAI-1: plasminogen activator inhibitor 1; IL-6: interleukin 6; TNFa: tumor necrosis factor a. ${ }^{*} P 0.01-0.049,{ }^{* *} P 0.001-0.0099,{ }^{* * *} P<0.001$.

\begin{tabular}{lll} 
& SACE2 & SACE \\
White blood cell count & $0.26^{*}$ & NS \\
Monocyte count & $0.39^{* * *}$ & $-0.26^{*}$ \\
Platelet count & $0.32^{* *}$ & $-0.34^{* *}$ \\
VWF & $0.36^{* * *}$ & NS \\
Factor VIII & $0.55^{* * *}$ & NS \\
D-dimer & $0.30^{* *}$ & NS \\
IL-6 & NS & $0.43^{* * *}$ \\
TNFa & NS & $0.43^{* * *}$ \\
PAI-1 & NS & $0.44^{* * *}$ \\
\hline
\end{tabular}

Conclusions: Shedding of ACE2 is increased and shedding of ACE is marginally decreased in COVID-19. SACE2 and SACE differ distinctly in correlations with markers of thromboinflammation, which may suggest different types of underlying endothelial injury, alternatively release from different cell types or vascular beds.

PB0191 | Differences of Venous Thromboembolism (VTE) Events in COVID-19 and Non-COVID-19 Hospitalized Patients

$\underline{\text { M.C. Chindamo }}^{1,2}$; M.B. Manhaes ${ }^{2}$; L.D. Carvalho²; G.G. Sardinha ${ }^{2}$; A.M. Marques ${ }^{2}$; H.C. Goudar ${ }^{2}$; A.B. Nunes ${ }^{2}$; P.R. Carmo Jr ${ }^{1,2}$; D.R. Salgado ${ }^{1,2}$

${ }^{1}$ Federal University of Rio de Janeiro, Rio de Janeiro, Brazil; ${ }^{2} \mathrm{Hospital}$ Barra D'Or, Rio de Janeiro, Brazil

Background: Although the novel coronavirus disease 2019 (COVID-19) has been associated with an increased risk of venous thromboembolism (VTE), different VTE incidences are reported according to the population profile.

Aims: To compare risk factors, prophylaxis regimens, laboratory data, incidence and mortality rates of VTE associated with COVID-19 and non-COVID-19 patients, hospitalized in an institution with best practices in thromboprophylaxis.

Methods: We retrospectively analyzed all confirmed cases of VTE (pulmonary embolism [PE] and/or deep vein thrombosis [DVT]) reported in electronic medical records diagnosed at admission, during hospitalization or readmitted to the hospital within 90 days after discharge, between January/2020 and February/2021. Characteristics of VTE events associated with COVID-19 and VTE associated with other diseases (non-COVID patients), were compared.

Results: Over the study period, 177 patients presented VTE events (63.8\% male, mean age $63.8 \pm 18.9$ years, $38.4 \%$ confirmed COVID-19; $85 \%$ critically ill patients). Clinical characteristics were summarized in Table 1. In contrast to non-COVID group, COVID-19 patients were predominantly male (78\% vs. $55 \% ; P=0.002)$ and older (66 vs. 61 years old ; $P=0.034$ ), had less clinical risk factors for VTE, and developed VTE more frequently during hospital stay, despite using higher doses of enoxaparin (Tables 1 and 2). Mortality rate was higher in the COVID group (38.2\% vs. 5.5\%; $P<0.0001$ ). Additionally, COVID group presented higher D-Dimer levels (17481 $\pm 26278 \mathrm{UI} /$ $\mathrm{ml}$ vs. $7291 \pm 11708 \mathrm{UI} / \mathrm{ml} ; P=0.004)$, but similar C-reactive protein levels $(12.5 \pm 11.8$ vs.13.8 $\pm 79.2 ; P=0.892)$. Regarding critically ill patients, COVID-19 was associated with an increased risk of VTE compared to non-COVID group (5.6\% [34/602] vs. 0.5\% [20/3690], unadjusted $\mathrm{OR}=10.98$ [95\% Cl 6.3-19.2; $\mathrm{P}<0.00001]$ ) (Table 2).

Conclusions: Despite of having less clinical risk factors for VTE and using greater doses of enoxaparin, COVID-19 patients had higher incidence of In-hospital VTE and higher mortality rate. These findings suggest that a hypercoagulability state could be induced by the coronavirus itself.

TABLE 1 Baseline characteristics of patients with VTE according to COVID-19 infection Clinical characteristics and VTE presentation ( $n$, \%) All patients ( $N=177$ ) COVID-19 $(n=68)$ non-COVID-19 $(n=109)$ $P$ value Age, years 62.9 (16-103) 66.4 (36-103) 60.7 (16-95) 0.034 Obesity 63 (35.6) 27 (39.7) 36 (33.0) 0.367 Malignant neoplasm 18 (10.2) 4 (5.9) 14 (12.8) 0.201 Previous VTE 18 (10.2) 2 (2.9) 16 (14.7) 0.011 Trauma 9 (5.1) 09 (8.3\%) 0.013 Previous surgery < 30 days 16 (9.1) 3 (4.5) 13 (11.9) 0.114 VTE diagnosis at hospital admission 105 (59.3) 28 (41.2) 77 (70.6) < 0.0001 VTE diagnosis during hospital stay 54 (30.5) 34 (50.0) 20 (18.3) Postdischarge VTE 18 (10.2) 6 (8.8) 12 (11.0)

TABLE 2 Analysis of subgroup of patients with VTE in-hospital events VTE anticoagulation regimes All patients $(n=54)$ COVID-19 $(n=34)$ NON- COVID-19 $(n=20) P$ value Standard prophylaxis (enoxaparin $40 \mathrm{mg} /$ day) 35 (64.8) 16 (47.1) 19 (95.0) 0.003 Intermediate prophylaxis (enoxaparin $40 \mathrm{mg}$ bid or $0.5 \mathrm{mg} / \mathrm{kg}$ bid) 13 (24.1) 13 (38.2) 0 Full anticoagulation (enoxaparin $1 \mathrm{mg} / \mathrm{kg}$ bid) ou I.V. unfractionated heparin 3 (5.6) 3 (8.8) 0 Mechanical methods 3 (5.6) 2 (5.9) 1 (5.0) VTE incidence and mortality Patients at intensive care unit 4292 $6023690<0.0001$ Mortality rates 24 (44.4) 21 (61.8) 3 (15.0) 0.001 
PB0192 | Lupus Anticoagulant Positivity is Not Associated with Venous Thromboembolism or In-hospital Mortality In COVID-19

N. Gendron ${ }^{1}$; M.-A. Dragon-Durey ${ }^{1}$; R. Chocron ${ }^{1}$; L. Darnige ${ }^{1}$;

G. Jourdi ${ }^{2}$; A. Philippe ${ }^{1}$; J. Hadjadj ${ }^{2}$; J. Duchemin ${ }^{2}$; L. Khider ${ }^{1}$;

N. Yatim²; L. Mauge ${ }^{1}$; P. Gaussem ${ }^{1}$; C.M. Samama ${ }^{2}$; B. Planquette ${ }^{1}$;

O. Sanchez ${ }^{1}$; J.L. Diehl ${ }^{1}$; T. Mirault ${ }^{1}$; M. Fontenay ${ }^{2}$; B. Terrier ${ }^{2}$;

D.M Smadja ${ }^{1}$

${ }^{1}$ Hôpital Européen Georges Pompidou, Paris, France; ${ }^{2}$ Hôpital Cochin,

Paris, France

Background: Antiphospholipid antibodies (APA) clinical relevance in COVID-19 is controversial.

Aims: We aimed to investigate the prevalence and prognostic value of conventional and non-conventional APA in COVID-19 patients.

Methods: This study was a multi-centric, prospective observational French cohort of patients hospitalized for COVID-19 suspicion.

Results: 249 patients were hospitalized for suspected COVID-19, including 154 (61.8\%) with confirmed COVID-19 and 95 (38.2\%) not confirmed. We found a significant increase in lupus anticoagulant (LA) positivity among COVID-19 positive patients (60.9\% versus $23.7 \%$ in non-COVID19 patients, $P<0.001$ ), while prevalence of conventional (LA, IgG, IgM and IgA isotypes) and non-conventional APA (anti-phosphatidylserine/prothrombin IgG and IgM) were low in both groups. COVID-19 patients with LA positivity had higher levels of fibrinogen (6.0 g/L, IQR 5.0-7.0 versus 5.3 IQR 4.3-6.4, $P=0.028$ ) and C-reactive protein (CRP, 115.5 IQR 66.0-204.8 versus 91.8 $\mathrm{mg} / \mathrm{L}$, IQR 27.0-155.1, $\mathrm{P}=0$ 0.019). Univariate analysis did not show any association between LA positivity and higher risk of venous thromboembolism (VTE, OR 1.02, 95\% Cl 0.44-2.43, $P=0.95$ ) or inhospital mortality (OR 1.80, 95\% Cl 0.70-5.05, $P=0.24)$. Unadjusted and adjusted (to CRP, age and sex) Kaplan-Meier survival curves according to LA positivity confirmed the absence of association with VTE or in-hospital mortality (unadjusted: $P=0.64$ and $P=0.26$, respectively; adjusted: hazard ratio $=1.1395 \% \mathrm{Cl} 0.48-2.60$ and 1.80 95\% Cl 0.67-5.01).

Conclusions: COVID-19 patients have an increased prevalence of LA positivity associated with biological inflammation markers. However, positive LA at admission is not associated with VTE risk and/or inhospital mortality.
PB0193 | Atherothrombotic Events in Hospitalized COVID-19 Patients Are Associated with Diabetes mellitus

D. Morozova $^{1}$; K. Nezhdanov ${ }^{1}$; O. Eliseeva ${ }^{2}$; L. Minushkina ${ }^{3}$;

D. Zateyshchikov ${ }^{3}$

${ }^{1}$ Lomonosov Moscow State University, Moscow, Russian Federation;

${ }^{2}$ Moscow Institut of Physics and Technology, Moscow, Russian Federation; ${ }^{3}$ City Clinical Hospital 51, Moscow, Russian Federation

Background: The significant number of hospitalized patients with COVID-19 suffer from type 2 diabetes mellitus (DM), a strong risk factor of atherothrombotic events.

Aims: To evaluate the association of atherothrombotic events with DM in hospitalized patients with COVID-19 infection.

Methods: 138 patients with DM and 452 patients without DM were followed during hospitalization for COVID-19. Data on atherothrombotic endpoints (myocardial infarction (MI) and ischemic stroke (IS) and mesenteric thrombosis (MT)), clinical and laboratory features was collected. Considering substantial group differences, propensity score matching (PSM) was conducted to equalize patients with and without DM in Stata/MP v.14.2 StataCorp. Sex, age and body mass index (BMI) were chosen as potential covariates-confounders. Further analysis was implemented by Kernel weighting approach with the help of psmatch2 command.

Results: 8 Mls, 8 ISs and 3 MTs occurred during hospitalization, including 9 (6.5\%) atherothrombotic events in DM group and 10 $(2.2 \%)$ in non-DM group $(P=0.022)$. The percentage of women in DM group was $63.4 \%$ and in non-DM - 46.9\% ( $P=0.07)$. Median age in DM-patients was 72 years old and 67 years old for non-DM group ( $P<0.001$ ). $50 \%$ of patients with $D M$ and $1 / 3$ of non-DM had obesity ( $\mathrm{BMI}>=30 \mathrm{~kg} / \mathrm{m} 2)$. After the PSM procedure the prevalence of atherothrombotic events in DM group remained significant. Thus, diabetics had 7 times higher chances of these complications ( $P=0.005$, OR 6.95, Cl: 1.78-27.07).

Conclusions: The combination of DM and COVID-19 is extremely dangerous for the development of an atherothrombotic event.

PB0194 | COVID-19 and Patients with Hemorrhagic Congenital Disease: Experience from Regional Heamophilia Treatment Center of Strasbourg (France)

M. Wald; O. Feugeas; D. Desprez

Regional Hemophilia Treatment Center, University Hospital, Strasbourg, France

Background: Severe acute respiratory syndrome coronavirus 2 (SARS-CoV 2) infection is a systemic disease with a pulmonary prognosis. Alsace, in northern France was one of the first hotspot for Covid-19.

Aims: Among the patients with hemorrhagic congenital disease (HCD), data about its incidence are scarce. 
Methods: We retrospectively report cases of Covid-19 in the Regional heamophilia treatment Center of Strasbourg in France since the beginning of the SARS-CoV 2 outbreak.

Results: Among the 620 patients followed, we retrieved 26 biologically confirmed cases of Covid-19: 18 diagnosed with a positive PCR-based viral RNA detection from nasopharyngeal swabs and 8 with serological testing.

We found 14 hemophilia's patients, 1 factor Xl's severe deficiency, 6 patients with thrombopathy and 5 Willebrand diseases. Demographic characteristics are listed in Table 1. Half of the infection occurred in the spring 2020. Nine patients were asymptomatic. No specific antiviral treatment were used. Two patients were hospitalized (10 and 90 days) including one in intensive care. The last one exhibited an acute respiratory distress syndrome and needed for mechanical ventilation. Despite his congenital thrombopathy, he required curative anticoagulation because of a pulmonary embolism. A significant activation of coagulation was also found in a young woman with Glanzmann's thrombasthenia without clinically proven thromboembolism disease. The two patients featured a major hemorrhagic syndrome (menorrhagia and hematuria). This condition increased complexity in their management, with the need for platelet transfusion. The outcome was favorable for all cases.

TABLE 1 Demographic characteristics and hemorrhagic congenital disease associted with Covid-19

\begin{tabular}{|l|c|}
\hline & 26 documented cases \\
\hline Hemophilias & 14 \\
\hline Willebrand disease & 5 \\
\hline Factor XI deficiency & 1 \\
\hline Thrombopahties & 6 \\
\hline & Characteristics of cases \\
\hline Age (mean, years) & 40 (range 12-66) \\
\hline Sex ratio & 0,9 \\
\hline BMI (mean, $\mathrm{kg} / \mathrm{m} 2)$ & 23 (range $19-49)$ \\
\hline Comorbidities & 4 obese patients with BMI > 30 kg/m2 \\
\hline & 4 patients with arterial hypertension \\
\hline & 2 other cardiac comorbidities \\
\hline
\end{tabular}

Conclusions: Coagulation abnormalities and the reported high frequency of venous thromboembolism during SARS CoV 2 infection makes the management of patients with HCD trickier. It would be interesting to collect cases of Covid-19 in patients with HCD on a national and international level.
PB0195 | Clinical Outcomes of Prophylactic vs. Therapeutic Doses of Anticoagulation In COVID-19 Patients: A Systematic Review and Meta-analysis

M.A. Elsebaie $^{1}$; B. Baral ${ }^{1}$; T. Shrivastava ${ }^{1}$; M.J. Tariq ${ }^{1}$; D. Kumi ${ }^{1}$; M. Elsebaie ${ }^{2}$

${ }^{1}$ John. H Stroger Hospital of Cook County, Chicago, United States;

${ }^{2}$ Faculty of Medicine, Ain Shams University, Cairo, Egypt

Background: Thromboprophylaxis is currently recommended for all COVID-19 patients requiring hospitalization. Whether therapeutic anticoagulation should be considered is highly debated.

Aims: Compare mortality, thromboembolism, and bleeding rates between prophylactic and therapeutic anticoagulation.

Methods: We performed a systematic search on PubMed and the Cochrane COVID-19 Study Register for studies comparing prophylactic and therapeutic anticoagulation, up to January 31, 2021. Search terms were: ("anticoagulant" OR "anticoagulation" OR "heparin" OR "thromboprophylaxis") AND ("COVID-19" OR "SARSCoV-2"). No language restriction was applied. Filters were applied to display comparative studies, clinical trials, and observational cohort studies. The Newcastle-Ottawa scale was used to assess the quality of included studies.

Results: We identified 472 records and screened 412 for eligibility. Thirty-eight studies were found to be eligible, including thirty-four retrospective cohorts and four prospective studies. Twenty-three, twenty-two, and twenty-three studies were included in the metaanalysis of mortality, thromboembolism, and bleeding outcomes respectively. Patients receiving prophylactic anticoagulation had better survival compared to those treated with therapeutic anticoagulation (OR, 0.65, 95\% Cl, 0.46-0.91). Therapeutic anticoagulation did not decrease risk of thromboembolism and was associated with significantly higher risk of bleeding (Figures 1 and 2). Severe COVID-19 patients admitted to intensive care unit (ICU) showed a non-significant trend towards improved mortality (OR, 1.85, 95\% Cl 0.90-3.81), and lower rates of thromboembolism (OR, 1.46, 95\% Cl, $0.83-2.57)$ with therapeutic anticoagulation.

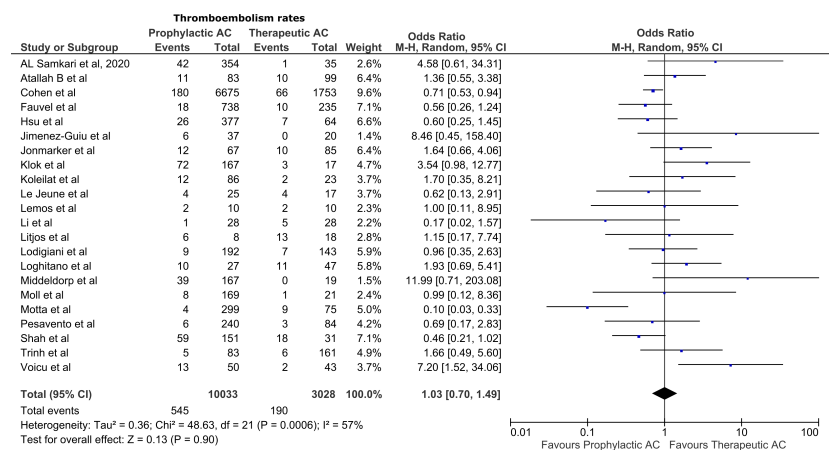

FIGURE 1 Forest plot of thromboembolism rates in patients receiving prophylactic vs. therapeutic anticoagulation (AC) 


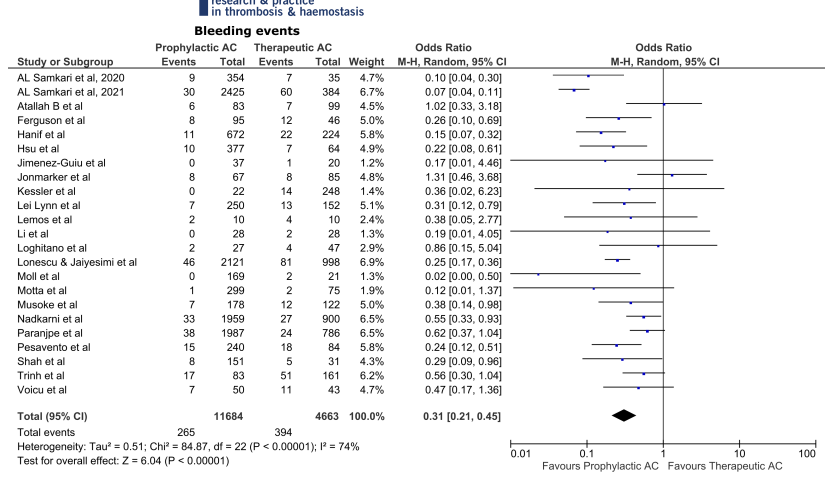

FIGURE 2 Forest plot of bleeding rates in patients receiving prophylactic vs. therapeutic anticoagulation (AC)

Conclusions: To our knowledge, this is the first in-depth review of the outcomes of prophylactic versus therapeutic anticoagulation in COVID-19. Our findings argue against the empiric use of therapeutic anticoagulation in COVID-19 patients. Compared to prophylactic anticoagulation, therapeutic anticoagulation did not improve survival or reduce risk of thromboembolism, and was associated with significantly higher risk of bleeding. Prospective trials are encouraged to explore the benefit of therapeutic anticoagulation in critically ill COVID-19 patients admitted to ICU.

\section{PB0196 | Bleeding in Critically III Patients with COVID-19}

T. Tassava; C. Callahan; M. Mayo; R. Bzeih

Saint Joseph Mercy Hospital, Ann Arbor, United States

Background: COVID-19 is a prothrombotic infection but there is limited data on bleeding risks.

Aims: This study aims to identify bleeding and thrombosis rates in relationship to anticoagulants in ventilated COVID-19 patients at a community hospital from March 1, 2020 until Feb 1, 2021.

Methods: This study was approved by the Institutional Review Board. All ventilated COVID-19 patients were included. Data was gathered by electronic medical record review. Bleed events were classified by ISTH criteria. Thrombosis events included venous thrombosis/arterial thrombosis/MI/stroke. Anticoagulant/antiplatelet agents at the time of the bleed/thrombosis event, and percent of hospitalization spent on full anticoagulation were recorded. Bleeding/thrombosis rates according to duration/degree/type of anticoagulant were calculated.

Results: Of 145 pts analyzed, bleeds occurred in 40/145 (28\%), (25/145 (17\%) major, 15/145 (10\%) minor) with 10/40 (25\%) bleeds contributing to cause of death. Of 73 patients who received full dose anticoagulation at some point during their hospitalization, 31/73 (44\%) had a bleeding event. Of patients who received full dose anticoagulants for $75-100 \%$ of their hospitalization: $8 / 21$ (38\%) bled, 50 74\%: 7/15 (46\%) bled, 25-49\%: 8/14 (53\%) bled, 1-24\%: 9/23 (39\%) bled. All patients who bled were on full anticoagulation at the time of the bleed. Of the 72 patients who never received full dose anticoagulation, 9/72 (12\%) bled. Multiple anticoagulants were involved in bleeding events (Figure 1). Of patients who bled, 14/40 (35\%) were also on antiplatelet agents. Site of bleed was predominantly pulmonary/tracheal (Figure 2). Indications for full anticoagulation were predominantly atrial fibrillation (14/31 $(45 \%)$ and pulmonary emboli (6/31 (19\%)). Thrombosis rates were 15\%, with 16/21 (76\%) on prophylactic and $5 / 21$ (24\%) on full anticoagulation.

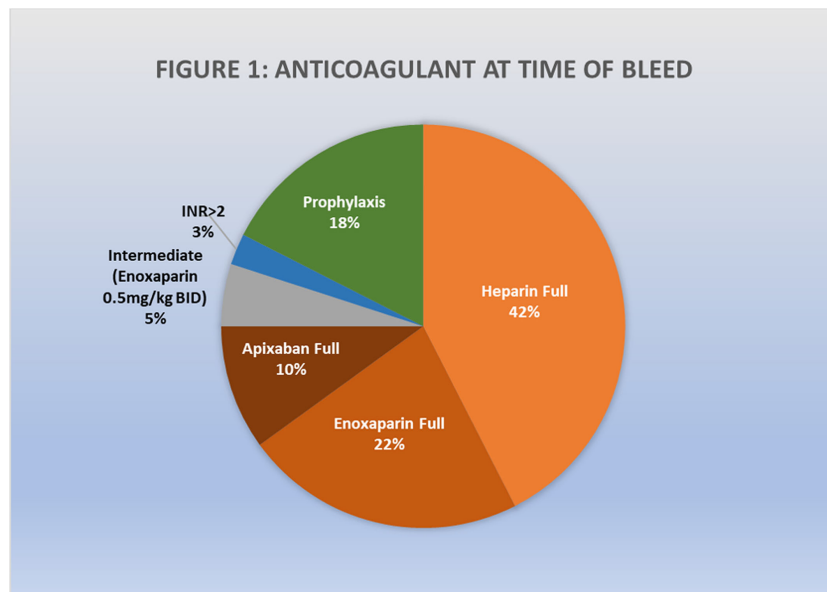

FIGURE 1 Anticoagulant at Time of Bleed

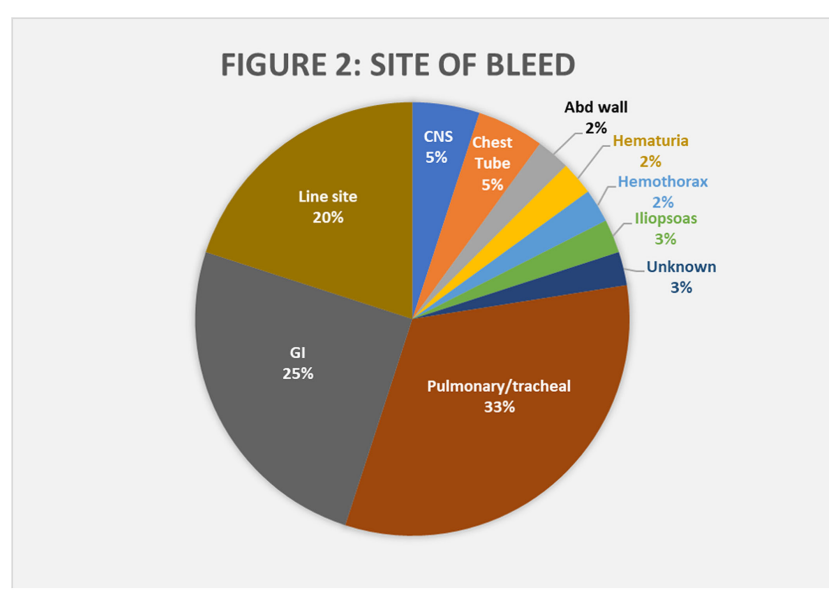

FIGURE 2 Site of Bleed

Conclusions: Ventilated COVID-19 patients had rates of thromboses consistent with other critically ill patients, but bleed rates were extremely high with significant mortality. Pulmonary bleeds predominated. Bleeds occurred regardless of type of full anticoagulant. Bleeding was highly correlated with use but not duration of full dose anticoagulation. Anticoagulant decisions for COVID-19 patients remain challenging. 
PB0197 | Stability of Oral Anticoagulant Treatment with Vitamin K Antagonists in COVID-19 Patients

E. Camilleri ${ }^{1}$; N. van Rein ${ }^{1,2}$; F.J.M. van der Meer ${ }^{3,4}$; M.C. Nierman ${ }^{5}$; W.M. Lijfering ${ }^{1,4}$; S.C. Cannegieter ${ }^{1,4}$

${ }^{1}$ Department of Clinical Epidemiology, Leiden University Medical Center, Leiden, Netherlands; ${ }^{2}$ Department of Pharmacy, Amsterdam University Medical Centers - Location AMC, University of Amsterdam, Amsterdam, Netherlands; ${ }^{3}$ Anticoagulation Clinic Leiden, Leiden, Netherlands; ${ }^{4}$ Department of Internal Medicine, Division of Thrombosis and Hemostasis, Leiden University Medical Center, Leiden, Netherlands; ${ }^{5}$ Department of Thrombosis and Anticoagulation, Atalmedial Medical Diagnostics Centers, Amsterdam, Netherlands

Background: Coagulopathy has been reported in severely ill patients with COVID-19, but data are lacking in outpatient settings. In patients treated with vitamin $\mathrm{K}$ antagonists (VKAs), whose anticoagulant effect is monitored through international normalized ratios (INRs), such coagulation abnormalities might lead to unstable control of anticoagulation. This could influence their thrombosis and bleeding risk.
Aims: To assess stability of VKA therapy in COVID-19 patients through a case-crossover study.

Methods: Between February-July 2020, we included patients with a positive COVID-19 test from two anticoagulant clinics in the Netherlands. We collected INRs between 26 weeks prior to diagnosis up to 12 weeks after. Time in Therapeutic Range (TTR), stability between INRs expressed as the Variance Growth Rate (VGR), and proportion of INR $\geq 5.0$ were calculated and compared within patients with paired sample $t$-test (in the 26 weeks before infection, in the first 6 weeks after and between 6 and 12 weeks after diagnosis). Results: 51 COVID-19 patients (mean age 84) were included, of whom 15 (29\%) were men. Mean TTR in the 26 weeks prior to COVID-19 infection was $80 \%(95 \% \mathrm{Cl} 75-85)$ compared to $59 \%(95 \% \mathrm{Cl} 51-68)$ in the 6 weeks after (Table 1). Mean TTR difference was $-23 \%(95 \% \mathrm{Cl}$ -32 to -14$)$ with a time above therapeutic range of $38 \%(95 \% \mathrm{Cl} 30$ $47)$ in the 6 weeks after diagnosis. The TTR rose again to $79 \%(95 \% \mathrm{Cl}$ 69-89) between 6 and 12 weeks after diagnosis (Figure 2). Also, VGR increased, with a mean increase of $4.8(95 \% \mathrm{Cl} 2.1-7.5)$ in the first 6 weeks. The risk of INRs $\geq 5.0$ was $4.4(95 \% \mathrm{Cl} 2.7-7.3)$ times higher in the 6 weeks after diagnosis compared with the 26 weeks before.

TABLE 1 Stability of anticoagulation before and after COVID-19 diagnosis in positive patients

\begin{tabular}{|c|c|c|c|c|c|c|c|c|}
\hline & $\begin{array}{l}\text { Mean } \\
\text { TTR, \% } \\
(95 \% \mathrm{Cl})\end{array}$ & $\begin{array}{l}\text { Mean } \\
\text { difference } \\
\text { TTR, \% } \\
(95 \% \mathrm{Cl})\end{array}$ & $\begin{array}{l}\text { Mean time } \\
\text { above range, } \\
\% \\
(95 \% \mathrm{Cl})\end{array}$ & $\begin{array}{l}\text { Mean } \\
\text { difference } \\
\text { time above } \\
\text { range,\% } \\
(95 \% \mathrm{Cl})\end{array}$ & $\begin{array}{l}\text { Mean } \\
\text { VGR } \\
(95 \% \mathrm{Cl})\end{array}$ & $\begin{array}{l}\text { Mean } \\
\text { difference } \\
\text { VGR } \\
(95 \% \mathrm{Cl})\end{array}$ & $\begin{array}{l}\text { INR } \geq 5.0 \\
\text { /all INR } \\
(\%)\end{array}$ & $\begin{array}{l}\text { RR of } \\
\text { INR } \geq 5.0 \\
(95 \% \mathrm{Cl})\end{array}$ \\
\hline $\begin{array}{l}26 \text { weeks before } \\
\text { COVID-19 }\end{array}$ & $\begin{array}{l}80 \\
(75 \text { to } 85)\end{array}$ & reference & $\begin{array}{l}17 \\
\text { (13 to } 22)\end{array}$ & reference & $\begin{array}{l}1.4 \\
(0.8 \text { to } \\
2.0)\end{array}$ & reference & $\begin{array}{l}19 / 641 \\
(3 \%)\end{array}$ & reference \\
\hline \multicolumn{9}{|l|}{6 weeks after } \\
\hline COVID-19 & $\begin{array}{l}59 \\
\text { (51 to } 68)\end{array}$ & $\begin{array}{l}-23 \\
(-32 \text { to }-14)\end{array}$ & $\begin{array}{l}38 \\
(30 \text { to } 47)\end{array}$ & $\begin{array}{l}24 \\
(14 \text { to } 33)\end{array}$ & $\begin{array}{l}5.7 \\
(3.0 \text { to } \\
8.5)\end{array}$ & $\begin{array}{l}4.8 \\
(2.1 \text { to } 7.5)\end{array}$ & $\begin{array}{l}35 / 247 \\
(14 \%)\end{array}$ & $\begin{array}{l}4.8 \\
\text { (2.8 to } 8.2 \text { ) }\end{array}$ \\
\hline
\end{tabular}

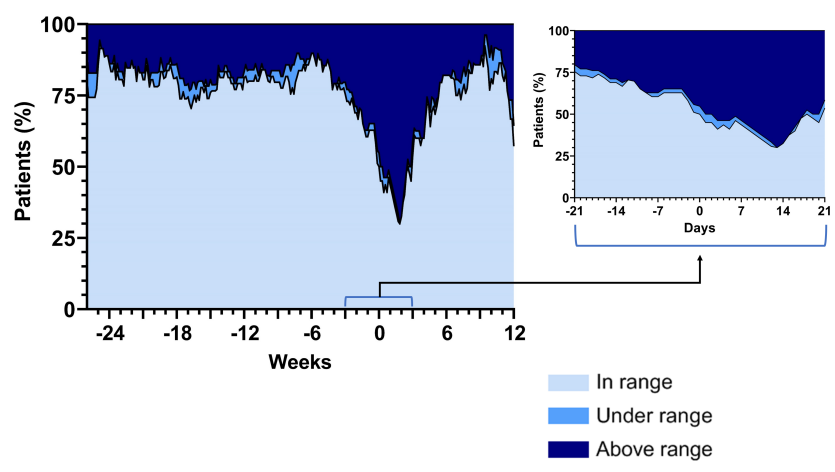

Conclusions: COVID-19 infection was associated with a strong decrease in TTR and INR stability in VKA users compared to their values before infection. Additional monitoring is advised to maintain therapeutic stability, particularly to prevent supratherapeutic INRs.

FIGURE 1 Percentage of patients in, above and under therapeutic range over time - Day 0 is the date of positive COVID-19 test 
PB0198 | Increased von Willebrand Factor (VWF) and Factor VIII (FVIII) Levels in Severe Acute Respiratory Syndrome Sars-Cov2positive Pneumonia Compared with Severe Acute Respiratory Syndrome Sars-Cov2-negative: A Case-control Study

M. Sacco ${ }^{1}$; R. De Cristofaro ${ }^{2,1}$; G. Liuzzo ${ }^{3}$; S. Lancellotti ${ }^{2}$;

D. Pedicino ${ }^{3}$ F. Andreotti ${ }^{3}$

${ }^{1}$ Catholic University School of Medicine, Translational Medicine and Surgery Department, Rome, Italy; ${ }^{2}$ Fondazione Policlinico Universitario A. Gemelli, Istituto di Ricovero e Cura a Carattere Scientifico (IRCCS), Center for Hemorrhagic and Thrombotic Diseases and Haemophilia Center, Rome, Italy; ${ }^{3}$ Fondazione Policlinico Universitario A. Gemelli, Istituto di Ricovero e Cura a Carattere Scientifico (IRCCS), Department of Cardiovascular Science, Rome, Italy

Background: The SARS-COV-2 direct interaction with AngiotensinConverting-Enzyme-2 (ACE2) membrane receptor on pulmonary epithelial and endothelial cells, impairs angiotensin 1-7 production and its vasculoprotective role, promoting an upregulation of the ACE/angiotensinll/angiotensinll receptor type-1 pathway. The consequent endothelial activation and increasing secretion of VWF and FVIII levels contributes to complement activation and leukocyte extravasation, leading to local microcirculation damage.

Aims: Our case-control study is aimed to investigate the characteristics of thrombotic microangiopathies (TMA)-like syndromes in COVID-19 evaluating VWF, FVIII and ADAMTS-13 levels, because the complement-mediated TMA and multiorgan microvascular lesions, observed in COVID-19 patients, seem not attributable to typical TMA.

Methods: Ten COVID-19 cases presenting fever, cough and sore throat, admitted to the Fondazione Policlinico Gemelli IRCCS, Rome, and diagnosed by RT-PCR, were compared to ten control patients with non-SARS-CoV-2 interstitial pneumonia, diagnosed by chest Xray/computerized tomography; notably no patient needed mechanical ventilation.

ADAMTS-13 activity was measured by a FRET-based assay; FVIII levels by a two-stage clotting assay, while VWF:antigen and VWF:activity were evaluated by chemiluminescence assays. Platelet count, schistocytes, D-dimer, C-reactive protein were centrally measured.

Results: COVID-19 pneumonia patients compared with controls showed a marked elevation of both VWF:Ag (median values: 324.1 Vs $139.5 \%, P<0.0001$ ) and VWF:act levels (median values: 341.5 Vs $133 \%, P<0.001$ ), as well as FVIII levels (median values: 202.5 Vs $123 \%, P<0.0001$ ), consistent with the presence of a thrombophilic condition; without significant differences in ADAMTS-13 activity (median values: $69 \mathrm{Vs} 76 \%, P=0.473$ ). In both groups, $\mathrm{D}$-dimer and C-reactive protein levels were elevated, but not statistically different, platelet counts were normal, and schistocytes, a typical TMAs hallmark, were not observed

Conclusions: The increased VWF/FVIII levels in COVID-19 pneumonia cases compared with non-COVID-19 controls cannot be fully explained by the inflammatory state alone and was likely caused by
SARS-CoV-2-mediated downregulation of the ACE2 axis, resulting in damage to the local microcirculation and increased leukocyte extravasation.

PB0199 | TEG/ROTEM: A Tool to Aid in the Diagnosis and Management of COVID 19 Coagulopathies - A Systematic Review

S. Kazi ${ }^{1}$; A. Noureldin ${ }^{2}$; K. Fainchtein ${ }^{2}$; Y. Deng ${ }^{2}$; M. Othman ${ }^{2,3}$

${ }^{1}$ Newcastle NHS Foundation Trust, Newcastle upon Tyne, United Kingdom; ${ }^{2}$ Queen's University, Kingston, Canada; ${ }^{3}$ School of Baccalaureate Nursing, St. Lawrence College, Kingston, Canada

Background: Venous thromboembolism (VTE) remains a significant complication of severe COVID-19 infection in spite of pharmacological thromboprophylaxis. Various coaguopathies including thrombocytopenia, thrombocytosis, DIC and fibrinolytic shutdown have been reported. Interpretation of isolated coagulation and fibrinolysis biomarkers can be complicated by septic states, disease severity and thrombo-inflammation. Thromboelastography (TEG) and Rotational Thromboelastography (ROTEM) are whole blood viscoelastic (VE) tests that provide rapid and full assessment of haemostasis process. Aims: A systematic review and meta-analysis, to evaluate if TEG/ ROTEM can help detect hypercoagulability and/or predict VTE risk in COVID-19 patients.

Methods: We searched MEDLINE, EMBASE, and EPUB Ahead of Print \& Other Non-Indexed Citations (from inception to $18^{\text {th }}$ October 2020). Studies included were observational or RCTs evaluating VE tests with COVID-19 infection. Review was registered with PROSPERO (CRD42021229814). 2 reviewers (SK, MO) reviewed all full-text versions of all eligible studies. Data abstracted on study design, demographic characteristics and clinical outcomes-VTE, mortality and laboratory tests and VE parameters. The effect estimates were expressed as odds ratio (OR) or standardized mean difference (SMD) with 95\% confidence interval.

Results: We identified 153 articles (PRISMA in Figure 1). A total of 841 patients from 20 selected studies (3 prospective,17 retrospective) were identified. 841 COVID-19 patients with 589 (70\%)who had viscoelastic tests (10 ROTEM,10 TEG). 431 (73\%) had elevated D-dimer. 293 (50\%) patients were hypercoagulable on TEG/ROTEM and 245 (41\%) normal. 3 studies reported fibrinolytic shutdown. 241 (29\%) patients had VTE events. ROTEM showed a higher sensitivity (93\%) and specificity (87\%) compared to TEG with a sensitivity of $51 \%$ and specificity of $57 \%$. The OR was 1.77 (95\% Cl: 0.62-to 5.04 , $\mathrm{I}^{2}=57 \%, P=0.29$ ). 


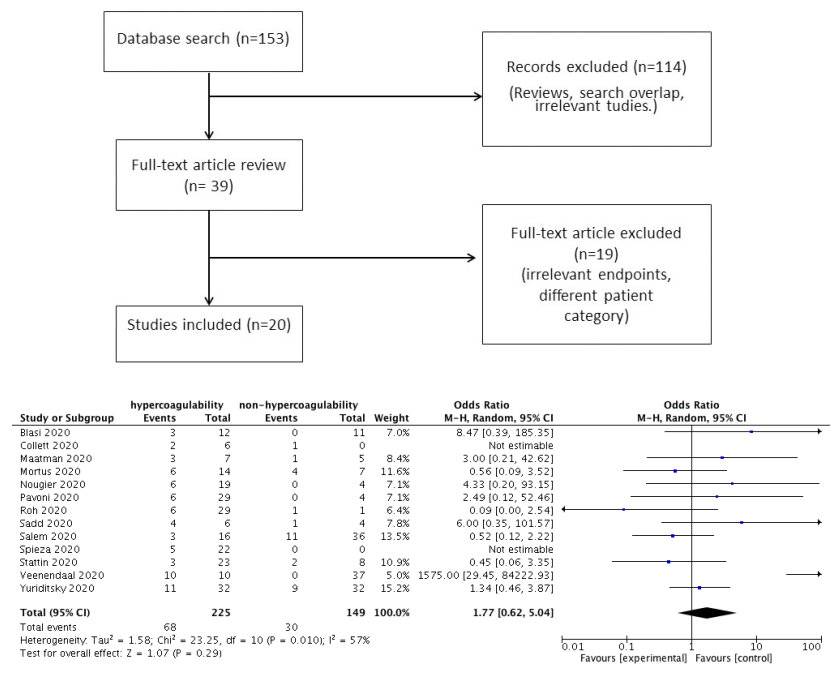

FIGURE 1 A, PRISMA flow diagram. B, Forest plot of VTE in COVID-19 patients undergoing VE testing with hypercoagulable vs normal VE result

Conclusions: TEG $^{\circledR} /$ ROTEM $^{\circledR}$ can detect hypercoagulability in association with COVID19 coagulopathy but may not be sufficient to predict VTE. Clot mass and viscoelasticity (MCF in ROTEM or MA in TEG) appear to be the most critical parameters.

PB0200 | Does a National Lockdown Affect the Characterises of Deep Vein Thromboses and Patients in the Community?

A. Shahid $^{1}$; N. Aden ${ }^{2}$; M. Howard ${ }^{1}$; S. Narayanamoorthi ${ }^{1} ;$ T. Khan $^{1}$

${ }^{1}$ Northern Care Alliance NHS Group, Oldham, United Kingdom;

${ }^{2}$ University of Manchester, Manchester, United Kingdom

Background: There is growing evidence identifying COVID-19 as a significant risk factor for thrombosis in in-patients. However it remains uncertain if patients in the community have been influenced during the COVID-19 pandemic and national lockdown. This study, across four centres in the UK, reviewed out-patients with a Deep Vein Thrombosis (DVT).

Aims: Has lockdown and COVID-19 led to a change in to the characteristics of DVT's and patients who have them alongside a review of the DVT service.

Methods: Data was collected retrospectively from electronic patient records system for the following periods: $1^{\text {st }}$ April until $30^{\text {th }}$ June 2019 and $1^{\text {st }}$ April until $30^{\text {th }}$ June 2020. These were the key months during the first United Kingdom national lockdown. Data was analysed for patient demographics, risk factors, characteristics of the DVT, management and DVT reoccurrence. Statistical analyses were preformed using GraphPad Prism 8.

Results: 227 patients sustained community DVT's in 2019 and 211 patients in 2020 during the study period. 13 of these patients in 2020 were COVID-19 positive. There was a difference in gender distribution $(P=0.0128)$ with 128 males and 99 females in 2019, 93 males and 118 females in 2020. No significant difference was noted for the incidence of thrombophilia with 9 in 2019 and 3 in 2020 ( $P=0.1437)$. Fewer long-haul journeys were made $(P=0.012)$ with 16 in 2019 and only 2 in 2020 . Fewer patients had immobility as a risk factor with 79 in 2019 and 55 in 2020 ( $P=0.0494)$. However, there were more patients using the contraceptive pill $(P=0.0086)$ with 1 in 2019 and 9 in 2020.

Conclusions: There is no significant difference in the characteristics, extent and management of DVT's prior to and during Lockdown during COVID-19. National Lockdowns do not affect community DVT's however it is important to highlight the surrounding inpatient numbers.

\section{PB0201 | Platelets from Donors of Convalescent Plasma after Mild COVID-19 Do Not Express Procoagulant Phenotype}

G. Uzun ${ }^{1}$; L. Pelzl ${ }^{2}$; W. Abou-Khalel ${ }^{2}$; A. Singh ${ }^{2}$; S. Nowak-Harnau ${ }^{1}$; K. Althaus ${ }^{1,2} ;$ T. Bakchoul ${ }^{1,2}$

${ }^{1}$ Center for Clinical Transfusion Medicine, Tübingen, Germany;

${ }^{2}$ Institute for Clinical and Experimental Transfusion Medicine (IKET),

University Hospital of Tübingen, Tübingen, Germany

Background: Coronavirus diesease-2019 (COVID-19) is associated with increased thromboembolic complications. COVID-19 related coagulotpathy (CAC) involves derangements in both cellular and plasma elements of the coagulation system. We found that COVID19 infection is accompanied with the development of procoagulant platelet phenotype and an increased resistance to clot lysis. As more people recover from COVID-19 infection but still develop thrombotic complications, discussion has begun over the possibility of persistent CAC even after the acute infection period.

Aims: To investigate the platelet activation after remission of COVID-19 infection.

Methods: We included plasma donors who had a PCR confirmed mild COVID-19 infection within the last 6 months (exCOVID-19 Group) in the study. A control group consisted of donors who did not have COVID-19. We collected citrate blood from study participant and investigated CD62p expression, Annexin V and GPIV shedding with flow cytometry. All participants gave informed consent and institutional ethics committee approved the study protocol. We compared the data with Mann-Whitney test using GraphPad Prism.

Results: Twenty-four convalescent plasma donors (11 Male, 13 Female) with a history of COVID-19 infection were included. Mean age (SD) was $39 \pm 13$ years. Median duration (interquartile range) after acute COVID-19 infection was 82 days (73-106). Baseline GPIV shedding rate was higher in exCOVID-19 group ( $1 \pm 0$ vs. $1.13 \pm$ 0.06). However, platelet response to collagen-related peptide (CRP) did not differ between both groups. Baseline CD62p expression and CD62 $p$ expression after activation with thrombin receptor associated protein (TRAP) was similar between exCOVID-19 group and control group. The percentage of CD62p and Annexin $V$ double positive platelets was similar between groups.

Conclusions: Our findings suggest that procoagulant platelet phenotype is not present after mild COVID-19. Further studies should 
investigate the alterations in plasma components of coagulation and fibrinolytic system after COVID-19.

PB0202 | Anticoagulation Strategies and Risk Of Bleeding Events in Critically ill COVID-19 Patients

C. Gabara ${ }^{1}$; B. Solarat ${ }^{2}$; P. Castro ${ }^{1,3}$; S. Fernández ${ }^{1,3}$; J.R. Badia ${ }^{2}$; D. Toapanta ${ }^{4}$; S. Schulman ${ }^{5}$; J.C. Reverter ${ }^{6}$; A. Soriano; J. Moises²; J. Aibar ${ }^{1}$

${ }^{1}$ Internal Medicine Department, Hospital Clínic, IDIBAPS - University of Barcelona, Barcelona, Spain; ${ }^{2}$ Pneumology Department, Hospital Clínic, IDIBAPS - University of Barcelona., Barcelona, Spain; ${ }^{3}$ Medical Intensive Care Unit, Hospital Clínic, IDIBAPS - University of Barcelona, Barcelona, Spain; ${ }^{4}$ Liver ICU, Hospital Clínic, IDIBAPS - University of Barcelona, Barcelona, Spain; ${ }^{5}$ Department of Medicine, Thrombosis and Atherosclerosis Research Institute, McMaster University, Hamilton, Hamilton, Canada; ${ }^{6}$ Hematology Department, Hospital Clínic, IDIBAPS - University of Barcelona, Barcelona, Spain; ${ }^{7}$ Infectious disease Department, Hospital Clínic, IDIBAPS - University of Barcelona, Barcelona, Spain

Background: Previous reports describe high rates of venous thromboembolism (VTE) in critically ill COVID-19 patients. Consequently, intermediate and therapeutic doses anticoagulation have been used in clinical practice, potentially exposing patients to a higher risk of bleeding.

Aims: To evaluate the rate of thrombosis, bleeding and mortality comparing prophylactic, intermediate or therapeutic doses in critically ill COVID-19 patients.

Methods: All COVID-19 patients admitted to the intensive care unit of a tertiary hospital between March and April 2020 were included. Patients were categorized into three groups according to the highest anticoagulant dose received: prophylactic, intermediate and therapeutic. Incidence of VTE, bleeding and mortality were compared between groups. We performed two logistic multivariable regressions to test the association between VTE and bleeding with clinical characteristics and the anticoagulant regimen.

Results: 201 patients were included. 78 (39\%) received prophylactic, 94 (47\%) intermediate and 29 (14\%) therapeutic doses. There were no differences in VTE and mortality between groups. In contrast, bleeding events were more frequent in patients receiving therapeutic (31\%) and intermediate (15\%) doses than in those receiving prophylactic doses $(5 \%)(P<0.001$ and $P<0.05$ respectively). Major bleedings were also more frequent in patients with anticoagulant doses $(P<0.01)$. The anticoagulant dose was the strongest determinant for bleeding (odds ratio [OR] 2.4, 95\% confidence interval (CI) $1.26-4.58, P=0.008)$ but had no impact on VTE (OR $0.97,95 \% \mathrm{Cl}$ $0.58-1.68, P=0.92$ ).

Conclusions: Critically ill COVID-19 patients receiving intermediate or therapeutic doses of heparin appear to have a higher risk of bleeding without a decrease of VTE events and mortality.
PB0203 | Management of COVID-19 Related Venous Thromboembolism - Data from Ljubljana Registry

G. Tratar; A. Mavri; T. Vižintin Cuderman; N. Vene; M. Žitko;

A. Rehberger Likozar

University Medical Centre, Department of Vascular Diseases, Ljubljana, Slovenia

Background: COVID-19 is a well recognised risk factor for thromboembolic events. So far, there is little data about efficacy of conventional treatment of venous thromboembolism (VTE) in COVID-19 patients.

Aims: To establish efficacy and safety of conventional VTE treatment in COVID-19 related VTE.

Methods: We analysed data of outpatients with COVID-19 associated VTE from the Ljubljana registry. Basic demographic and clinical data, selection of anticoagulants and complications of anticoagulant treatment were recorded.

Results: Between October 2020 and February 2021, 69 COVID-19 associated VTE were recorded. These patients represented $23 \%$ of all VTE patients treated in our institution. Compared to the same time frame one year before, the number of VTE patients increased by $25 \%$. Mean age was $63 \pm 16$ years, $65 \%$ of patients were male. In 22 (38\%) patients, COVID-19 severity required hospitalization. In 10 (46\%) of these patients, VTE occurred only after discharge from the hospital. Clinical presentation, anticoagulant treatment and treatment related complications are shown in Table 1.

TABLE 1 Clinical presentation, anticoagulant treatment and treatment complications in patients with COVID-19 related venous thromboembolism

$\begin{array}{ll}\text { Clinical presentation } & \\ \text { Pulmonary Embolism N (\%) } & 35 \text { (50.7\%) } \\ \text { Proximal Deep Vein Thrombosis N (\%) } & 26 \text { (37.7\%) } \\ \text { Calf Deep Vein Thrombosis N (\%) } & 4(5.8 \%) \\ \text { Upper Extremity Deep Vein Thrombosis N (\%) } & 2(2.9 \%) \\ \text { Superficial Vein Thrombosis N (\%) } & 2(2.9 \%) \\ \text { Anticoagulant Treatment } & \\ \text { Apixaban N (\%) } & 33(49.3 \%) \\ \text { Rivaroxaban N (\%) } & 17(24.6 \%) \\ \text { Warfarin N (\%) } & 7(10.1 \%) \\ \text { Low Molecular Weight Heparin N (\%) } & 7(10.1 \%) \\ \text { Dabigatran N (\%) } & 4(5.8 \%) \\ \text { Complications of Anticoagulant Treatment } & \\ \text { Recurrent Venous Thromboembolism } & 0 \\ \text { Major Bleeding } & 0 \\ \text { Death } & 0\end{array}$

Conclusions: In our cohort of outpatients with COVID-19 related VTE, treatment with apixaban and rivaroxaban was effective and safe. Larger scale observational trials are needed to confirm these findings. 
PB0204 | Anemia and Enhancement of Coagulation Are Associated during Severe COVID-19 Infection

$\underline{\text { T. Helin }^{1}}$; M. Lemponen ${ }^{1} ;$ T. Lahtiharju ${ }^{1} ;$ R. Lassila ${ }^{2}$;

L. Joutsi-Korhonen ${ }^{1}$

${ }^{1}$ Coagulation Disorders Unit, HUS Diagnostics Center, HUSLAB, Helsinki University Hospital and University of Helsinki, Helsinki, Finland; ${ }^{2}$ Coagulation Disorders Unit, Helsinki University Hospital, Research Program Unit in Systems Oncology, University of Helsinki, Helsinki, Finland

Background: Coagulation disturbances are common during severe COVID-19 infection. While classic disseminated intravascular coagulation (DIC) is rare, inflammation markedly enhances coagulation activity in certain COVID-19 patients.

Aims: We examined laboratory markers in hospitalized patients during the first wave of the pandemic in Finland to differentiate between patients of severe phenotype (ICU-admitted vs.ward patients) using inflammatory and coagulation markers, and blood cell counts.
Methods: We analyzed a wide panel of routine coagulation tests from anonymously collected samples of 78 hospitalized COVID-19 patients treated in specific wards, either in $\operatorname{ICU}(n=34)$ or medical wards $(n=44)$, at Helsinki University hospital in April-May 2020. These data were supplemented with the laboratory information system results, including complete blood count, creatinine and $\mathrm{C}$ reactive protein (CRP).

Results: Inflammatory and coagulation markers were elevated in most patients: CRP in 94\%, fibrinogen 77\%, D-dimer 74\%, FVIII 52\% and platelet count in $37 \%$. Anemia and decreased erythrocyte count were common, especially in men ( $73 \%$ vs, $44 \%$ in women; Table). Men admitted to the ICU during their hospital stay presented with higher platelet count, leukocytes, and FVIII but lower hemoglobin. Also, both men and women in ICU had higher fibrinogen and Ddimer $(P<0.01)$. Not a single patient exhibited DIC criteria, but $31 \%$ had D-dimer level of at least $1.5 \mathrm{mg} / \mathrm{L}$. Antithrombin was reduced in $47 \%$ of the patients but did not distinguish severity. In contrast, high $\mathrm{D}$ dimer and the presence of anemia clearly associated with severity. Patient age and FVIII levels were also higher in anemic patients (Figure), and among ICU-admitted patients.

TABLE 1 Laboratory findings in hospitalized COVID-19 patients

\begin{tabular}{|c|c|c|c|c|c|c|c|c|c|}
\hline & $N$ & Mean & Median & SD & Min & Max & Ref interval & $\begin{array}{l}\text { Below ref } \\
\text { interval, } N \\
\text { (\%) }\end{array}$ & $\begin{array}{l}\text { Above ref } \\
\text { interval. } N \\
\text { (\%) }\end{array}$ \\
\hline APTT (s) & 67 & 40 & 34 & 17 & 18 & 103 & $28-37 \mathrm{~s}$ & 2 (3.0\%) & 19 (28.4\%) \\
\hline D dimer (mg/l) & 78 & 2.2 & .9 & 6.5 & 0.2 & 56.7 & $<0.5 \mathrm{mg} / \mathrm{L}$ & - & $58(74.4 \%)$ \\
\hline $\begin{array}{l}\text { Antithrombin (U/ } \\
\mathrm{mL})\end{array}$ & 66 & 0.82 & 0.88 & 0.33 & 0.07 & 1.49 & $0.85-1.25 \mathrm{U} / \mathrm{mL}$ & 31 (47\%) & $4(6.1 \%)$ \\
\hline $\mathrm{CRP}(\mathrm{mg} / \mathrm{L})$ & 76 & 81 & 56 & 84 & 4 & 398 & $<4 \mathrm{mg} / \mathrm{L}$ & - & 71 (94.4\%) \\
\hline
\end{tabular}



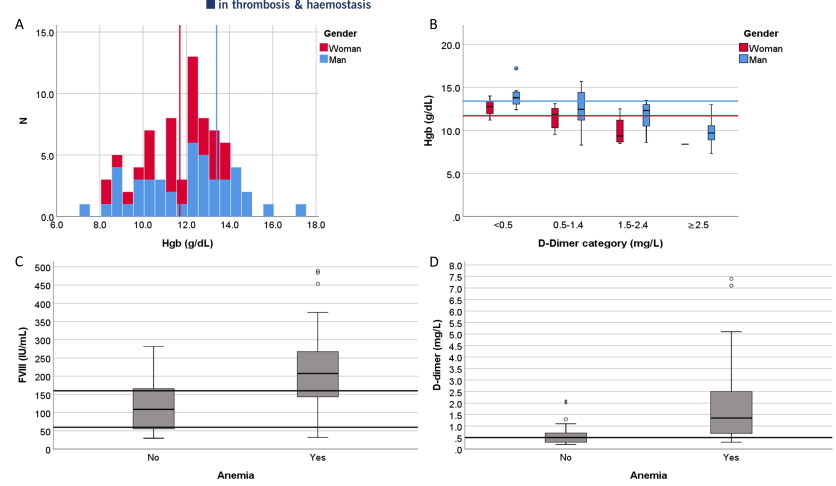

FIGURE 1 Stacked histogram of hemoglobin distribution among patients (A), hemoglobin across different D dimer levels (B), FVIII (C) and (D) D-Dimer according to their anemia status. Anemia was defined as $\mathrm{Hgb}$ below reference interval. The highest D-dimer result of $57 \mathrm{mg} / \mathrm{L}$ is not shown. Variables presented were selected according to medians with significant difference (Mann Whitney $U$ test). The number of patients with anemia was 48/78 (62\%) in total, men 32/44 (72\%), women 16/34 (47\%), ICU-patients 27/34 (79\%), non-ICU patients 7/44 (16\%). Lines, reference intervals; Thick line in box, median; box, first and third quartiles; whiskers, range; outlier $\geq 1.5$ box lengths from median, circle; extreme outlier $\geq 3.0$ box lengths from median, asterisk.

Conclusions: High inflammatory response, translated to elevated FVIII, fibrinogen and D-dimer and characterized hospitalized COVID-19 patients. In our patients, contrary to earlier reports, anemia was common, especially in men, and associated with coagulation activity, suggesting the role of red cells on COVID-19 pathology. We aim to further detect cell-derived microvesicles in these patients.

\section{PB0205 | Impact of COVID-19 Pandemic on the Quality and} Performance of Haemostasis Laboratories

$\underline{\text { R.A. Arisz }}{ }^{1}$; P. Meijer ${ }^{2}$; N.CV Péquériaux ${ }^{3}$; S.J van de Leur ${ }^{4}$; M.V Lukens ${ }^{5}$; M.PM de Maat ${ }^{1}$; M.J Hollestelle ${ }^{2}$

${ }^{1}$ Department of Hematology, Erasmus MC, University Medical Center Rotterdam, Rotterdam, Netherlands; ${ }^{2}$ ECAT Foundation (External quality Control for Assays and Tests), Voorschoten, Netherlands; ${ }^{3}$ Department of Clinical Chemistry and Hematology, Jeroen Bosch Hospital, 's Hertogenbosch, Netherlands; ${ }^{4}$ Department of Laboratory Medicine, Isala Hospital, Zwolle, Netherlands; ${ }^{5}$ Department of Laboratory Medicine, University Medical Center Groningen, University of Groningen, Groningen, Netherlands

Background: The high incidence of thrombotic events in patients with COVID-19 affects healthcare worldwide and results in an increased workload in haemostasis laboratories. Especially D-dimer, haemostatic parameters and anti-Xa tests are expected to be analysed more frequently. However, the impact of this increase in assay requests on the quality of performance in haemostasis laboratories remains unclear.
Aims: To evaluate the impact of the COVID-19 pandemic on the quality performance and management of haemostasis laboratories.

Methods: The impact on the quality of performance was studied using external quality assessment (EQA) data from 2019 and 2020 derived from ECAT surveys. A questionnaire was sent to Dutch haemostasis laboratories to identify challenges and management strategies during the COVID-19 pandemic with focus on D-dimer, anti-Xa, factor VIII and fibrinogen. Furthermore, the number of assays performed in 2019 and 2020 was collected from four Dutch hospitals, located in regions with different disease incidence.

Results: No differences in response rate nor the quality of the measurements were observed between the EQA surveys in 2019 and 2020 (Table 1). Fifty-five percent of the laboratories observed a more than $25 \%$ increase in test requests for anti-Xa and D-dimer assays in 2020 compared to 2019. Twenty-seven percent also experienced this increase in requested fibrinogen assays. Additionally, $84 \%$ of the respondents indicated to experience increased work pressure and increased absenteeism in $71 \%$ of the participating laboratories was observed. Evaluation of the number of assays performed over the year showed extreme peaks in anti-Xa assays (144 - 772\%) in all four hospitals and D-dimer assays (368\%, 670\%) in the two hospitals that are located in areas with high disease incidence.

TABLE 1 Comparison of international external quality data of haemostasis variables

\begin{tabular}{llll} 
& CV (\%) & & \\
& Pre-COVID19 & COVID19 & \\
& Mean \pm SD & Mean \pm SD & $P$ value \\
& $6.8 \pm 1.3$ & $8.1 \pm 1.4$ & n.s. \\
Fibrinogen (g/L) & $10.0 \pm 3.0$ & $9.4 \pm 2.3$ & n.s. \\
D-dimer (mg/L) & $8.1 \pm 1.4$ & $10.5 \pm 4.3$ & n.s. \\
Factor VIII (\%) & $6.5 \pm 1.5$ & $7.3 \pm 3.9$ & n.s. \\
Anti-Xa (UFH) (IU/mL) & $10.2 \pm 4.2$ & $10.0 \pm 3.9$ & n.s. \\
Anti-Xa (LMWH) (IU/ & & & \\
\hline mL) & & & \\
\hline
\end{tabular}

$\mathrm{CV}$, between participants coefficient of variation of two similar samples derived from two different surveys

n.s., not significant difference between pre-COVID19 and COVID19

Conclusions: The enormous increase in work pressure and test requests, especially for D-dimer assays and anti-Xa activity, did not affect the quality of performance within haemostatic laboratories during the COVID-19 pandemic. 
PB0206 | Hypercoagulability Detected by Seer Sonorheometry in Covid-19 Patients at Hospital Admission

F.J. López Jaime ${ }^{1}$; S. Martín-Téllez ${ }^{1} ;$ A. Doblas-Márquez ${ }^{1}$;

I. Márquez-Gómez ${ }^{2}$; J. Alemany ${ }^{1}$; M. Pérez-Raya ${ }^{1}$; A.M. Mena-

Santano $^{1}$; D.F. Muñoz-López ${ }^{1}$; M.I. Muñoz-Pérez ${ }^{1}$; I. Fernández-

Bello $^{3}$; A. Montaño ${ }^{1}$

${ }^{1}$ Unidad de Hemostasia y Trombosis, Hospital Universitario Regional de Málaga, IBIMA., Málaga, Spain; ${ }^{2}$ Servicio de Enfermedades Infecciosas, Hospital Universitario Regional de Málaga, Málaga, Spain; ${ }^{3}$ Biopharm, Novo Nordisk, Madrid, Spain

Background: Hospitalized COVID-19 patients often show a hypercoagulable profile, mainly elevated fibrinogen and D-dimer, associated with worse outcomes in terms of ICU admission, thrombotic events and mortality. Increased clot stiffness on whole blood viscoelastic testing (VET) is another hypercoagulable profile observed in COVID-19 patients. Sonic Estimation of Elasticity by Resonance (SEER) sonorheometry (Quantra ${ }^{\circledR}$ system) is a novel ultrasound based VET technology.

Aims: To characterize the hemostatic status of COVID-19 patients with standard and viscoelastic testings and its association with disease severity.

Methods: Blood samples from adult COVID-19 patients at hospital admission were analyzed with a panel of tests including markers of inflammation, coagulation and Quantra VET parameters. Fisher's exact test was used to statistically compare the moderate and severe/critical disease groups.

Results: The cohort of 37 patients showed a mean age (SD) of 59.6 (16) years, with 23 of them in the moderate disease group and 14 in the severe/critical disease group. Markers of inflammation were elevated in $44-84 \%$ of patients, while of coagulation tests, only fibrinogen (79\%) and D-dimer (60\%) levels were elevated. Clot stiffness (CS), as well as the contribution of platelets (PCS) and fibrinogen (FCS) measured by Quantra, were also above normal values at $64.3,50$ and $78.6 \%$, respectively. These parameters were the only ones significantly associated with the severe/critical disease group
Conclusions: The study of global hemostatic status using Quantra can be a powerful tool for the analysis of COVID-19 patients at admission, helping in risk stratification and triage decisions of patients. This profile, characterized by incresased clot stiffness from both platelet and fibrinogen contribution, was most prevalent in patients with severe/critical disease, and could therefore be established as a new prognostic marker.

\section{PB0207 | Effects of Dalteparin on Anti-Xa Levels Cannot Be Predicted in Critically III COVID-19 Patients}

C. van der Heijden; E. Kooistra; R. ter Heine; R. Brüggemann;

J. Walburgh Schmidt; E. de Grouw; T. Frenzel; P. Pickkers;

J. Leentjens

Radboud University Medical Center, Nijmegen, Netherlands

Background: Critically ill patients with COVID-19 are at high risk of thromboembolic events, despite thromboprophylaxis with lowmolecular weight heparins (LWMH), while increased-intensity thromboprophylaxis in this patient population is associated with bleeding. This raises the question whether pharmacokinetic (PK) effects of LMWHs are predictable in these patients.

Aims: To investigate whether a dosing algorithm for dalteparin administration could be designed based on clinical parameters, using PK modeling with anti-Xa levels as readout.

Methods: In this explorative, observational study, we prospectively included 15 adult COVID-19 patients admitted to the intensive care unit receiving dalteparin in prophylactic-intensity (5000 IU dalteparin once daily (OD) for those $<100 \mathrm{~kg}, 5000 \mathrm{IU}$ dalteparin bidaily (BD) for those $\geq 100 \mathrm{~kg}$ ) and therapeutic-intensity (100 IU/kg BD). A minimum of 4 anti-Xa samples per day were collected on regular timepoints over 1-3 days. PK analysis of dalteparin was performed by nonlinear mixed-effect modeling (NONMEM v7.4). The final model was used to perform Monte Carlo simulations to predict anti-Xa levels with different dalteparin regimens. The study was approved by the local medical ethics committee.

(Table 1), where most of the adverse outcome were observed. According to this result, the median of these parameters was significantly different between the two groups $(P=0.011 / 0.029 / 0.008)$.

TABLE 1 Parameters of coagulation in COVID-19 patients at hospital admission: proportion (\%) above the upper limit of normal (ULN).

\begin{tabular}{llllll} 
Test & ULN & All (\%) & Moderate (\%) & Severe/Critical (\%) & $P$ value \\
Platelet count $\left(\times 10^{3} / \mu \mathrm{L}\right)$ & 450 & $1 / 37(2.7)$ & $0 / 23(0.0)$ & $1 / 14(7.1)$ & 0.378 \\
Fibrinogen $(\mathrm{mg} / \mathrm{dL})$ & 400 & $27 / 34(79.4)$ & $14 / 20(70.0)$ & $13 / 14(92.9)$ & 0.198 \\
D-dimer $(\mu \mathrm{g} / \mathrm{L})$ & 500 & $22 / 37(59.5)$ & $12 / 23(52.2)$ & $10 / 14(71.4)$ & 0.314 \\
CT $(\mathrm{s})$ & 164 & $1 / 37(2.7)$ & $1 / 23(4.3)$ & $0 / 14(0.0)$ & 1.000 \\
CSL $(\%)$ & 99 & $8 / 26(30.8)$ & $2 / 13(15.4)$ & $6 / 13(46.2)$ & 0.202 \\
CS $(\mathrm{hPa})$ & 33.2 & $12 / 37(32.4)$ & $3 / 23(13.0)$ & $9 / 14(64.3)$ & 0.003 \\
PCS $(\mathrm{hPa})$ & 29.8 & $9 / 36(25.0)$ & $2 / 22(9.1)$ & $7 / 14(50.0)$ & 0.014 \\
FCS $(\mathrm{hPa})$ & 3.7 & $21 / 37(56.8)$ & $10 / 23(43.5)$ & $11 / 14(78.6)$ & 0.048 \\
\hline
\end{tabular}


Results: The data were well-fitted to a linear one compartment model. Wide interindividual variation in the parameters absorption (78\%) and clearance (34\%) of dalteparin was observed, not explained by clinical covariates such as creatinine clearance for elimination rate. Simulations show that prophylactic dosing in individuals $<100 \mathrm{~kg}$ result in anti-Xa levels within generally used prophylactic targets, while increased-prophylactic dosing in those $\geq 100 \mathrm{~kg}$ result in supraprophylactic levels in $40 \%$ of patients. With therapeuticintensity dosing in secondary thromboprophylaxis, $22 \%$ of patients would be subtherapeutically, and $19 \%$ patients supratherapeutically dosed.

Conclusions: Anti-Xa levels during dalteparin treatment in the critically ill COVID-19 patient are difficult to predict and often off-target. Until data from randomized clinical trials conclude on the best dosing, this suggests that anti-Xa measurements are needed to guide high-intensity dosing in the individual patient.

PB0208 | Platelet Dynamics in Hospitalized Patients with SARS CoV-2 Infection

V.E. Delgado Pinos ${ }^{1}$; R.M. Martín Rojas ${ }^{1}$; C. Pascual Izquierdo ${ }^{1,2}$ J.L. Díez Martín ${ }^{1,2,3}$; G. Perez Rus ${ }^{1,3}$

${ }^{1}$ Department of Hematology, Hospital General Universitario Gregorio Marañón, Madrid, Spain; ${ }^{2}$ Gregorio Marañón Health Research Institute, Madrid, Spain; ${ }^{3}$ Department of Medicine, Universidad Complutense de Madrid, Madrid, Spain
Background: A state of hypercoagulability and thrombotic complications have been reported as one of the main pathological events in SARS CoV2 infection. Platelets play an important role in pathological thrombosis, systemic inflammatory response and coordinate vascular function through complex interactions with endothelial cells and leukocytes. The platelet count and its dynamics are of interest for the understanding of these processes.

Aims: To describe the platelet dynamics in patients with SARS CoV-2 infection and its differences based on mortality and severity of the disease.

Methods: This is an observational and retrospective study that includes 221 patients with confirmed SARS CoV-2 infection, admitted to a tertiary Spanish hospital between March and April 2020. Patients with a history of known thrombocytopenia were excluded. Statistics were performed using SPSS v25 software.

Results: Mean age of the patients was $63.8 \pm 13.6$ years and the average hospital stay was $26.25 \pm 25.32$ days. Platelets count throughout hospitalization was lower in patients admitted to a critical care unit than in those in general wards. Mean platelet count nadir in the first group was $90.93 \pm 65 \times 10^{3} / \mu \mathrm{L}$ and it was reached on average at day $+19 \pm 9$ of hospitalization, while the platelet count of non-critical patients was $194.34 \pm 89 \times 10^{3} / \mu \mathrm{L}$ and was reached at day $+7 \pm 9$ $(P<0.001)$.

Non-survivors had significantly lower platelet counts during their hospitalization on days +7 and +15 compared to survivors. Platelets nadir of non-survivors was $115.29 \pm 116 \times 10^{3} / \mu \mathrm{L}$ vs $183.30 \pm$ $88 \times 10^{3} / \mu \mathrm{L}$ in the survivors group $(P<0.001)$. The platelet count peak was also lower in the first group. (Table and Figure 1).

TABLE 1 Platelet count, nadir and peak throughout hospitalized in patients with SARS CoV-2 infection

\begin{tabular}{|c|c|c|c|c|c|c|}
\hline Parameters & Critical $(n=42)$ & $\begin{array}{l}\text { Non-critical } \\
(n=179)\end{array}$ & $P$-value & Survivors $(n=193$ ) & $\begin{array}{l}\text { Non-survivors } \\
(n=28)\end{array}$ & $P$-value \\
\hline $\begin{array}{c}\text { Platelets }\left(\times 10^{3} / \mu \mathrm{L}\right) \\
\text { on admission }\end{array}$ & $195.97 \pm 73$ & $231.35 \pm 110$ & $0.01^{*}$ & $229.57 \pm 105$ & $190.54 \pm 93$ & 0.06 \\
\hline $\begin{array}{l}\text { Platelets }\left(\times 10^{3} / \mu \mathrm{L}\right) \\
\quad \text { day }+7\end{array}$ & $249.09 \pm 107$ & $330.22 \pm 144$ & $0.001^{*}$ & $322.48 \pm 139$ & $249.36 \pm 143$ & $0.01^{*}$ \\
\hline $\begin{array}{l}\text { Platelets }\left(\times 10^{3} / \mu \mathrm{L}\right) \\
\quad \text { day }+15\end{array}$ & $204.38 \pm 144$ & $292.49 \pm 124$ & $<0.001^{*}$ & $284.65 \pm 129$ & $134.77 \pm 111$ & $<0.001^{*}$ \\
\hline $\begin{array}{l}\text { Platelets nadir }\left(\times 10^{3} /\right. \\
\quad \mu \mathrm{L})\end{array}$ & $90.93 \pm 65$ & $194.34 \pm 89$ & $<0.001^{*}$ & $183.30 \pm 88$ & $115.29 \pm 116$ & $<0.001^{*}$ \\
\hline $\begin{array}{l}\text { Platelets peak }\left(\times 10^{3} /\right. \\
\quad \mu \mathrm{L})\end{array}$ & $382.69 \pm 152$ & $380.98 \pm 147$ & 0.946 & $390.76 \pm 144$ & $316.18 \pm 161$ & $0.012^{*}$ \\
\hline $\begin{array}{l}\text { Days from admission } \\
\text { at nadir }\end{array}$ & $19.38 \pm 9$ & $7.08 \pm 9$ & $<0.001^{*}$ & $9.22 \pm 10$ & $11.14 \pm 10$ & 0.393 \\
\hline $\begin{array}{l}\text { Days from admission } \\
\text { at peak }\end{array}$ & $20.74 \pm 15$ & $8.27 \pm 6$ & $<0.001^{*}$ & $10.65 \pm 10$ & $10.57 \pm 13$ & 0.191 \\
\hline
\end{tabular}




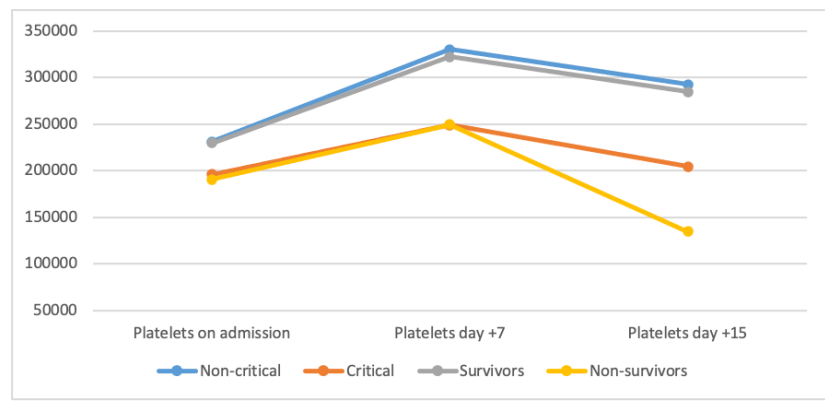

FIGURE 1 Platelet dynamics in admitted patients with SARS CoV-2 infection

Conclusions: Platelet dynamics in the first 15 days of hospitalization is similar in all groups. However, patients in critical care units and those who die, have lower platelet counts both on admission and throughout their hospitalization compared to non-critical and survivors patients.

\section{PB0209 | Hemoreologic Disorders in COVID-19 Patients}

O. Kostina; E. Galova; A. Popovicheva; L. Sosnina; M. Presnyakova Federal State Budgetary Educational Institution of Higher Education 'Privolzhsky Research Medical University' of the Ministry of Health of the Russian Federation, Nizhny Novgorod, Russian Federation

Background: Hemoreologic disorders are of great importance in acute inflammatory response pathogenesis, and complicate the course of different infectious diseases contributing to hypoxia, acidosis and micro-thrombosing. However, hemorheology change characteristics in COVID-19 are not understood that determined the urgency of the present study.

Aims: To study the hemorheological changes in COVID-19.

Methods: We studied erythrocytes aggregation, disaggregation and deformation properties, and determined fibrinogen concentration in 50 patients with COVID-19. 23 patients appeared to have acute respiratory failure (ARF).

Results: Regardless ARF, the patients were found to have erythrocyte deformability deterioration: 2.6 times as much compared to healthy people $(P=0.01)$. Maximum deformability was revealed in $11 \%$ erythrocytes; the reference value being $72.9 \%$, and all patients had $20 \%$ and $31 \%$ increase, respectively, in red blood cell aggregation degree and rate. At a low shearing flow rate (5s-1 and $10 s-1)$ the aggregate stability exceeded the parameters in healthy individuals by $30 \%$ and $18 \%$, respectively. There was found the continuing formation of erythrocyte aggregates at a low flow rate in $24 \%$ patients, and in an increased shearing rate there was the most expressed aggregate dispersion compared to other patients. At higher shear flow rates, $15 \mathrm{~s}-1$ and $20 \mathrm{~s}-1$, violation of the disaggregational properties of red blood cells was observed in $40 \%$ of patients - the strength of aggregates was increased by 1.2 times.
Impaired red blood cell functional activity was combined with hyperfibrinogenemia increasing blood plasma viscosity.

Conclusions: Newly diagnosed critical changes of erythrocyte deformability, impaired red blood cell aggregation and disaggregation in COVID-19 patients result in erythrocyte decreased passage through a capillary blood flow, enhancing an impaired blood function of oxygen transportation in micro- and macro-circulatory bloodstream.

PB0210 | Do Thrombotic and Bleeding Phenotypes Differ in Patients with Severe COVID-19 Compared to Other Viral Pneumonias in the Context of Extracorporeal Membrane Oxygenation?

A. Weatherill ${ }^{1}$; M. Gaspar ${ }^{2}$; M. Passariello ${ }^{3}$; M. Laffan ${ }^{4}$; S. Ledot ${ }^{3}$; D.R. Arachchillage ${ }^{2}$

${ }^{1}$ Imperial College Healthcare NHS Trust, London, United Kingdom;

${ }^{2}$ Department of Haematology, Royal Brompton Hospital, London, United Kingdom; ${ }^{3}$ Department of Intensive Care Medicine, Royal Brompton Hospital, London, United Kingdom; ${ }^{4}$ Department of Haematology, Imperial College London, London, United Kingdom

Background: Respiratory failure is a major complication in patients with COVID-19. Some patients rapidly develop severe acute respiratory distress syndrome (ARDS) with profound hypoxaemia despite mechanical ventilation and rescue therapies such as nitric oxide and require veno-venous extracorporeal membrane oxygenation (VVECMO). Bleeding and thrombosis are major complications in patients treated with ECMO irrespective of the cause for underlying respiratory failure. Thrombosis, mainly pulmonary thrombosis or pulmonary embolism, is frequently seen in patients with severe COVID-19. Aims: To compare thrombosis, bleeding and mortality rates in a cohort of patients with severe COVID-19 pneumonia to a matched cohort of patients with other viral pneumonia, supported with VV-ECMO.

Methods: A retrospective single-centre observational study in a tertiary ECMO referral centre. Fifty-four COVID-19 patients admitted for VV-ECMO from 17/3/20 to 26/5/20 were compared with a matched sample of 44 patients received VV-ECMO for nonCOVID-19 viral pneumonia from 5/4/18 to $18 / 1 / 19$. Thrombosis, bleeding and mortality rates were compared.

Results: Baseline characteristics were comparable between cohorts. COVID-19 patients had higher admission platelet count and fibrinogen level.

Sixty-nine thrombotic events occurred in 43/54 (80\%) COVID-19 patients, compared to 34 events in 26/44 (59\%) non-COVID-19 patients. Unlike the non-COVID-19 cohort, the majority of thromboses were pulmonary (57\%), diagnosed early in the course of VV-ECMO. Twenty-four major bleeding (MB) events occurred in 19/54 (35\%) COVID-19 patients and 11 in 9/44 (20\%) non-COVID-19 patients. All 21 major haemorrhages during VV-ECMO in the COVID-19 cohort were preceded by thrombosis. 
Mortality rate was comparable: 9/54 (17\%) versus 7/44 (16\%). 5/9 COVID-19 fatalities were caused by MB; haemorrhagic transformation of ischemic stroke, versus none in the non-COVID-19 cohort. Conclusions: COVID-19 patients receiving VV-ECMO are at higher risk of thrombosis and fatal $\mathrm{MB}$ than patients receiving $\mathrm{VV}$-ECMO for non-COVID-19 viral pneumonia. Further work is required to characterise the COVID-19/VV-ECMO coagulopathies and optimise anticoagulation strategy.

PB0211 | Serum ACE2 Activity for the Assessment of Disease Prognosis in COVID-19

B. Nagy Jr. ${ }^{1}$; Z. Fejes ${ }^{1}$; Z. Nagy ${ }^{2}$; R. Sütő ${ }^{3}$; E. Bíró ${ }^{4}$; G. Bekő ${ }^{4}$; Z. Szentkereszty ${ }^{3}$; I. Várkonyi ${ }^{2}$; J. Kappelmayer ${ }^{1}$; Z. Papp ${ }^{5}$; A. Tóth ${ }^{5}$; M. Fagyas ${ }^{5}$

${ }^{1}$ University of Debrecen, Faculty of Medicine, Department of Laboratory Medicine, Debrecen, Hungary; ${ }^{2}$ University of Debrecen, Faculty of Medicine, Department of Infectious Diseases, Debrecen, Hungary; ${ }^{3}$ University of Debrecen, Gyula Kenézy County Hospital, Intensive Care Unit, Debrecen, Hungary; ${ }^{4}$ South-Pest Central Hospital, National Institute of Hematology and Infectology, Budapest, Hungary; ${ }^{5}$ University of Debrecen, Faculty of Medicine, Department of Cardiology, Division of Clinical Physiology, Debrecen, Hungary

Background: Coronavirus disease 2019 (COVID-19) is associated with significant morbidity and mortality worldwide. Angiotensinconverting enzyme 2 (ACE2) represents the main receptor for SARSCoV-2 to enter endothelial cells. Interestingly, controversial data have been reported for soluble ACE2 levels in COVID-19 patients.

Aims: Here we analyzed circulating ACE2 activity to correlate with routinely measured laboratory parameters as well as the severity and outcome of COVID-19 disease.

Methods: In this retrospective, dual-center study, we determined serum ACE2 activity from 57 subjects with moderate COVID-19 and 119 critically ill patients. ACE2 levels were correlated with IL-6 and ferritin concentrations and clinical outcome. In addition, ACE2 levels were also measured in follow-up samples $(n=106)$.

Results: ACE2 activity was significantly higher in patients with severe COVID-19 (127.9 [83.1-209.7] vs. 73.8 [54.6-89.6] mU/L in nonsevere patients; $P<0.0001)$. Nevertheless, all COVID-19 patients had higher ACE2 than formerly measured serum ACE2 levels of healthy individuals $(16.2 \pm 0.8 \mathrm{mU} / \mathrm{L})$ and of hypertensives $(24.8 \pm$ $0.8 \mathrm{mU} / \mathrm{L}$ ) (Úri K et al., 2016). Circulating ACE2 showed a significant $(P<0.0001)$ but moderate correlation with IL-6 $(r=0.345)$ and ferritin $(r=0.277)$. Substantial area under the receiver operating characteristic curve (ROC-AUC) value was determined for baseline ACE2 to indicate disease severity $(0.774$ [95\% $\mathrm{Cl} 0.702-0.846] ; P<0.0001)$ Furthermore, significantly higher serum ACE2 was measured in nonsurviving vs. surviving COVID-19 patients (131.2 [84.8-217.1] vs. 77.8 [56.5-123.0] mU/L; $P<0.0001$ ), and a high ACE2 level before treatment predicted poor outcome with a ROC-AUC value of 0.709 [95\% Cl 0.632-0.787] $(P<0.0001)$. Finally, a larger elevation in ACE2 activity was observed compared to baseline values during monitoring in non-survivors vs. survivors $(P<0.0001$ vs. $P=0.076)$.

Conclusions: Elevated serum ACE2 activity strongly correlates with both COVID-19 severity and prognosis, thus represents a promising laboratory biomarker.

\section{PB0212 | Thrombotic Risk of Hospitalized Patients with COVID-19 and a Co-infection}

I. Akpan ${ }^{1}$; L. Van Doren ${ }^{1}$; J. Travers ${ }^{2} ;$ K. Runcie ${ }^{1} ;$ A. Wei ${ }^{1}$; J. Cogan ${ }^{1}$; Y. Pumpalova ${ }^{1}$; D. Diuguid ${ }^{1}$; A. Eisenberger ${ }^{1}$; R. Francis ${ }^{1}$

${ }^{1}$ Columbia University Irving Medical Center/NewYork Presbyterian Hospital, New York, United States; ${ }^{2}$ New York University Rory Meyers College of Nursing, New York, United States

Background: Coronavirus disease 2019 (COVID-19) has emerged as a worldwide health crisis characterized by high rates of thrombotic events and increased inflammatory markers. It is likely that co-infections alongside COVID-19 increase the likelihood of the occurrence of a thrombotic event, however this information is unknown.

Aims: To assess the association between co-infections and thrombotic events among hospitalized COVID-19 patients who received a non-malignant hematology consult.

Methods: This was a retrospective observational chart review that evaluated 80 hospitalized patients with COVID-19 infection at a single institution who received a non-malignant hematology consult between 3/15/2020-5/30/2020. Multivariable logistic regression analyses were performed to examine the association between a coinfected patient and thrombotic event. Patients with a co-infection were only included in study analyses if their co-infection was diagnosed no later than $48 \mathrm{~h}$ post-thrombotic event.

Results: Of the 80 patients, 69 met eligibility criteria and were on average, 56 years of age and majority Hispanic. Fifty-nine percent ( $n=41)$ had a co-infection and $36.59 \%(n=15)$ experienced a thrombotic event compared to $35.71 \%(n=10)$ without a co-infection $(P=0.941$, Table 1). The likelihood of a co-infected patient experiencing a thrombotic event was 2.91 times that of a patient without a co-infection ( $P=0.135$, Table 2). Eighty-five percent $(n=59)$ of all patients received anticoagulation on admission with no difference between patients who did and did not have a co-infection $(P=0.968)$. 
TABLE 1 Hospitalized COVID-19 Positive Patients with a CoInfection Who Developed a Thrombotic Event and Received a Nonmalignant Hematology Consult

\begin{tabular}{lrrrr}
\hline & $\begin{array}{r}\text { Total } \\
(\mathbf{n = 6 9 )} \\
\mathbf{n}(\%)\end{array}$ & $\begin{array}{r}\text { No Co-Infection } \\
(\mathbf{n = 2 8}) \\
\mathbf{n}(\%)\end{array}$ & $\begin{array}{r}\text { Co-Infection } \\
\mathbf{( n = 4 1 )} \\
\mathbf{n}(\%)\end{array}$ & P-value \\
\hline Age (mean, SD) & $56.94 \pm 13.27$ & $54.86 \pm 16.10$ & $58.37 \pm 10.92$ & 0.281 \\
\hline Gender (Male) & $44(63.77 \%)$ & $15(34.10 \%)$ & $29(70.73 \%)$ & 0.145 \\
\hline Ethnicity (Hispanic) & $38(55.07 \%)$ & $15(53.57 \%)$ & $23(56.10 \%)$ & 0.836 \\
\hline Anticoagulation on & $59(85.81 \%)$ & $24(85.71 \%)$ & $35(85.37 \%)$ & 0.968 \\
Admission (yes) & & & & \\
\hline D-dimer (mean, SD) & $5.99 \pm 7.04$ & $5.50 \pm 5.78$ & $6.33 \pm 7.85$ & 0.636 \\
\hline IL-6 (mean, SD) & $100.45 \pm 110.51$ & $112.68 \pm 123.73$ & $91.28 \pm 100.28$ & 0.447 \\
\hline Co-morbidities & & & & 0.609 \\
\hline $0-1$ & $32(46.38 \%)$ & $15(53.57 \%)$ & $17(41.46 \%)$ & \\
$2-3$ & $26(37.68 \%)$ & $9(32.14 \%)$ & $17(41.46 \%)$ & \\
$4-6$ & $11(15.94 \%)$ & $4(14.29 \%)$ & $7(17.07 \%)$ & \\
\hline Thrombotic Event (yes) & $25(36.23 \%)$ & $10(35.71 \%)$ & $15(36.59 \%)$ & 0.941 \\
\hline *Thrombotic events - venous, arterial, superficial, and deep thrombotic events &
\end{tabular}

TABLE 2 Logistic Regression Analysis Assessing the Association between Co-infections and Thrombotic Event Among Hospitalized COVID-19-infected Patients with a Non-malignant Hematology Consult

\begin{tabular}{lccc}
\hline & Odds Ratio & Confidence Interval & P-value \\
\hline Thrombotic Event & & & \\
\hline Co-infection & 2.91 & $.72-11.79$ & 0.135
\end{tabular}

*Adjusted for age, gender, ethnicity, anticoagulation on admission, d-dimer, fibrinogen (not shown), ㄴ-6, number of comorbidities

Conclusions: We did not find a significant increase of thrombotic events in patients who had a co-infection compared to those without. Limitations include, small sample size, single-institution study, and sample restricted to a sub-specialized service. Patients with presumed thrombotic events (i.e., without objective documentation of thrombosis) were also not included. Future research with larger sample sizes is needed to clarify the association between co-infection and thrombotic events in COVID-19 infected patients.
PB0213 | Excess Mortality in a Population of Vitamin K Antagonist Users during the First Wave of the COVID-19 Pandemic in the Netherlands Compared with Excess Mortality in the General Dutch Population

Q. Chen ${ }^{1}$; M.M.A. Toorop ${ }^{1}$; C. Visser ${ }^{2}$; F.J.M. van der Meer ${ }^{1}$; M.C. Nierman ${ }^{3}$; L. Goede ${ }^{4}$; H. ten Cate ${ }^{5}$; M.J.H.A. Kruip ${ }^{2,6}$; W.M. Lijfering ${ }^{1}$; S.C. Cannegieter ${ }^{1}$; Dutch COVID \& Thrombosis Coalition

${ }^{1}$ Leiden University Medical Center, Leiden, Netherlands; ${ }^{2}$ Erasmus University Medical Center, Rotterdam, Netherlands; ${ }^{3}$ Atalmedial Medical Diagnostics Centers, Amsterdam, Netherlands; ${ }^{4}$ Saltro, Utrecht, Netherlands; ${ }^{5}$ Cardiovascular Research Institute Maastricht, Maastricht, Netherlands; ${ }^{6}$ Thrombosis Service Star-shl, Rotterdam, Netherlands

Background: Excess mortality has been observed in the general population during the COVID-19 pandemic, but it is unknown whether preexisting anticoagulant treatment affects survival, given that COVID-19 associated hypercoagulability adversely impacts prognosis.

Aims: To investigate whether preexisting vitamin K antagonist (VKA) treatment is associated with lower excess mortality during the first wave of the COVID-19 pandemic in the Netherlands when compared with excess mortality in the general population.

Methods: All atrial fibrillation (AF) patients ( $\geq 60$ years) receiving long-term VKA therapy before week 11, 2020 were included from three Dutch anticoagulation clinics. The corresponding patient population managed by the same clinics in 2019 (i.e., all AF patients ( $\geq 60$ years) receiving long-term VKA therapy before week 11, 2019) was enrolled as a control cohort. Difference in survival within 9 weeks (i.e., week 11 to 19) between the two cohorts was evaluated by Cox regression analysis. This was compared with the difference in survival during the same time frame of the general elderly ( $\geq 60$ years) Dutch populations in 2020 versus 2019.

Results: The study included 22,730 VKA users for the cohort in 2019 and 19,476 for the cohort in 2020, of which baseline characteristics were comparable. The cumulative incidences for all-cause mortality of the VKA users and the general population are presented in Table 1. 
TABLE 1 Cumulative incidences of all-cause mortality during the study period of the cohorts of Vitamin K antagonist users and general population, all aged $\geq 60$ years, estimated by Kaplan-Meier method

\section{Vitamin $\mathrm{K}$ antagonist users}

Cohort 2019

$(N=22,730)$

\begin{tabular}{|c|c|c|c|c|c|c|c|c|}
\hline $\begin{array}{l}\text { Follow up } \\
\text { time (days) }\end{array}$ & No. death & $\begin{array}{l}\text { Cumulative } \\
\text { incidence (\%) } \\
(95 \% \mathrm{Cl})\end{array}$ & No. death & $\begin{array}{l}\text { Cumulative } \\
\text { incidence (\%) } \\
(95 \% \mathrm{Cl})\end{array}$ & No. death & $\begin{array}{l}\text { Cumulative } \\
\text { incidence (\%) } \\
(95 \% \mathrm{Cl})\end{array}$ & No. death & $\begin{array}{l}\text { Cumulative } \\
\text { incidence (\%) } \\
(95 \% \mathrm{Cl})\end{array}$ \\
\hline $0-10$ & 22 & $\begin{array}{l}0.10 \\
\quad(0.06-0.14)\end{array}$ & 19 & $\begin{array}{l}0.10 \\
\quad(0.05-0.14)\end{array}$ & 4,591 & $\begin{array}{l}0.09 \\
\quad(0.09-0.09)\end{array}$ & 4,781 & $\begin{array}{l}0.09 \\
\quad(0.09-0.09)\end{array}$ \\
\hline $0-20$ & 74 & $\begin{array}{l}0.33 \\
\quad(0.25-0.40)\end{array}$ & 67 & $\begin{array}{l}0.34 \\
\quad(0.26-0.43)\end{array}$ & 8,919 & $\begin{array}{l}0.17 \\
\quad(0.17-0.17)\end{array}$ & 10,868 & $\begin{array}{l}0.20 \\
\quad(0.20-0.21)\end{array}$ \\
\hline $0-30$ & 129 & $\begin{array}{l}0.57 \\
\quad(0.47-0.67)\end{array}$ & 132 & $\begin{array}{l}0.68 \\
\quad(0.56-0.79)\end{array}$ & 13,084 & $\begin{array}{l}0.25 \\
\quad(0.25-0.26)\end{array}$ & 18,245 & $\begin{array}{l}0.34 \\
\quad(0.34-0.35)\end{array}$ \\
\hline $0-40$ & 192 & $\begin{array}{l}0.84 \\
\quad(0.73-0.96)\end{array}$ & 209 & $\begin{array}{l}1.07 \\
\quad(0.93-1.22)\end{array}$ & 17,351 & $\begin{array}{l}0.33 \\
\quad(0.33-0.34)\end{array}$ & 24,794 & $\begin{array}{l}0.46 \\
\quad(0.46-0.47)\end{array}$ \\
\hline $0-50$ & 263 & $\begin{array}{l}1.16 \\
\quad(1.02-1.30)\end{array}$ & 268 & $\begin{array}{l}1.38 \\
\quad(1.21-1.54)\end{array}$ & 21,533 & $\begin{array}{l}0.41 \\
\quad(0.41-0.42)\end{array}$ & 30,273 & $\begin{array}{l}0.57 \\
\quad(0.56-0.57)\end{array}$ \\
\hline $0-60$ & 308 & $\begin{array}{l}1.36 \\
\quad(1.20-1.51)\end{array}$ & 312 & $\begin{array}{l}1.60 \\
\quad(1.43-1.78)\end{array}$ & 25,592 & $\begin{array}{l}0.49 \\
\quad(0.49-0.50)\end{array}$ & 34,827 & $\begin{array}{l}0.65 \\
\quad(0.64-0.66)\end{array}$ \\
\hline $0-62$ & 319 & $\begin{array}{l}1.40 \\
\quad(1.25-1.56)\end{array}$ & 321 & $\begin{array}{l}1.65 \\
\quad(1.47-1.83)\end{array}$ & 26,350 & $\begin{array}{l}0.51 \\
\quad(0.50-0.51)\end{array}$ & 35,609 & $\begin{array}{l}0.67 \\
\quad(0.66-0.67)\end{array}$ \\
\hline
\end{tabular}

After adjusting for age, sex, and clinics, all-cause mortality risk of the VKA users in 2020 was $16 \%$ higher than in 2019 (95\% Cl -1\% to 35\%).

During the same time frame, the contemporaneous general elderly

Dutch population saw a $30 \%$ increase in all-cause mortality risk $(95 \%$

Cl 28\%-32\%, adjusting for age, sex) (Table 2).

TABLE 2 Incidence rates and hazard ratios of all-cause mortality during the study period of the cohorts of Vitamin $\mathrm{K}$ antagonist users and general population, all aged $\geq 60$ years

\begin{tabular}{|c|c|c|c|c|}
\hline & \multicolumn{2}{|c|}{ Vitamin $\mathrm{K}$ antagonist users } & \multicolumn{2}{|c|}{ General population } \\
\hline & Cohort 2019 & Cohort 2020 & Cohort 2019 & Cohort 2020 \\
\hline $\begin{array}{l}\text { Incidence rate }(95 \% \mathrm{Cl}) \text {, per } 100 \\
\text { person-years }\end{array}$ & $8.32(7.43-9.28)$ & $9.78(8.74-10.91)$ & $2.99(2.95-3.02)$ & $3.94(3.90-3.98)$ \\
\hline $\begin{array}{l}\text { Incidence rate difference }(95 \% \mathrm{Cl}) \\
\text { per } 100 \text { person-years, Crude }\end{array}$ & 0 (Reference) & $1.46(0.06-2.87)$ & 0 (Reference) & $0.95(0.90-1.01)$ \\
\hline $\begin{array}{l}\text { Incidence rate difference ( } 95 \% \\
\text { CI), per } 100 \text { person-years, } \\
\text { Age-adjusted }\end{array}$ & 0 (Reference) & $1.46(0.06-2.87)$ & 0 (Reference) & $2.25(2.10-2.39)$ \\
\hline Incidence rate ratio, Crude & 1 (Reference) & $1.18(1.01-1.37)$ & 1 (Reference) & $1.32(1.30-1.34)$ \\
\hline Incidence rate ratio, Age-adjusted & 1 (Reference) & $1.18(1.01-1.37)$ & 1 (Reference) & $1.33(1.31-1.36)$ \\
\hline Hazard ratio, Crude & 1 (Reference) & $1.18(1.01-1.37)$ & 1 (Reference) & $1.32(1.30-1.34)$ \\
\hline $\begin{array}{l}\text { Hazard ratio, Model } 1 \text { (adjusting } \\
\quad \text { for age, sex) }\end{array}$ & 1 (Reference) & $1.16(0.99-1.35)$ & 1 (Reference) & $1.30(1.28-1.32)$ \\
\hline $\begin{array}{l}\text { Hazard ratio, Model } 2 \text { (Model } 1+ \\
\text { anticoagulation clinics) }\end{array}$ & 1 (Reference) & $1.16(0.99-1.35)$ & - & - \\
\hline
\end{tabular}




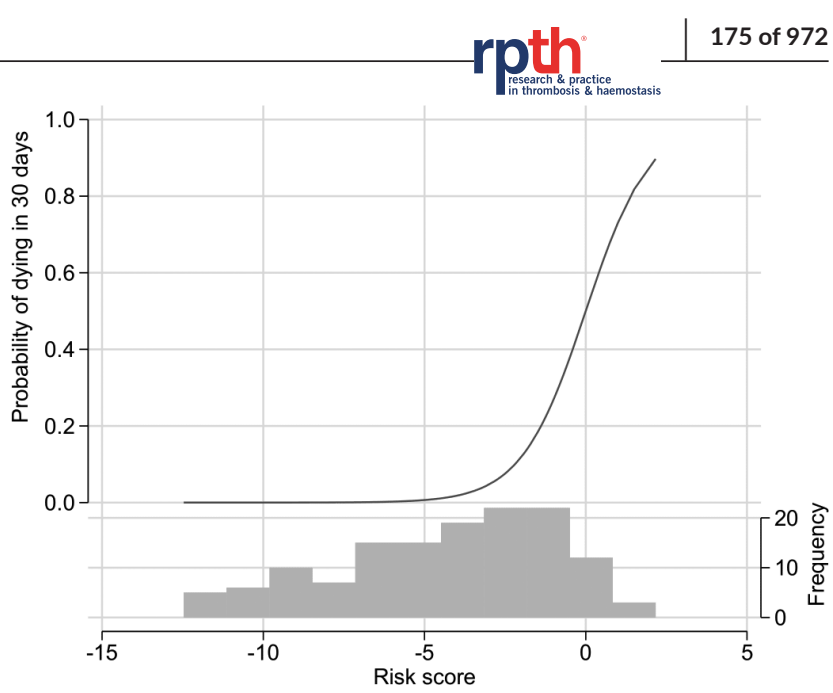

Conclusions: Elderly patients with AF receiving long-term VKA therapy in the Netherlands appeared to have a lower excess mortality during the first wave of the COVID-19 pandemic when compared to the general elderly population.

PB0215 | Hypercoagulation by Rotational Thromboelastometry (ROTEM) Predicts Mortality in COVID-19: A Prospective Observational Study

L. Almskog ${ }^{1}$; A. Wikman ${ }^{2}$; J. Svensson ${ }^{3}$; C.M. Wahlgren ${ }^{1}$; M. Wanecek ${ }^{4}$; M. Bottai ${ }^{5}$; J. van der Linden ${ }^{1}$; A. Ågren ${ }^{1,6}$ ${ }^{1}$ Department of Molecular Medicine and Surgery, Karolinska Institutet, Stockholm, Sweden; ${ }^{2}$ Department of Clinical Immunology and Transfusion Medicine, Karolinska University Hospital and Department of CLINTEC, Karolinska Institutet, Huddinge, Sweden; ${ }^{3}$ Department of Clinical Neuroscience, Karolinska Institutet, Stockholm, Sweden; ${ }^{4}$ Department of Physiology and Pharmacology, Karolinska Institutet, Solna, Sweden; ${ }^{5}$ Division of Biostatistics, Institute of Environmental Medicine, Karolinska Institutet, Stockholm, Sweden; ${ }^{6}$ Department of Clinical Sciences, Danderyd Hospital, Stockholm, Sweden

Background: Severe disease due to COVID-19 has been shown to be associated with coagulopathy with a high rate of micro- and macrothrombosis, and early identification of prothrombotic patients may guide treatment. Rotational Thromboelastometry (ROTEM) is an established point-of-care (POC) blood test, used for detecting and monitoring hypo- and hypercoagulation.

Aims: The primary aim of this study was to assess whether analysis by ROTEM alone or in combination with other risk markers can be a predictor of mortality in COVID-19.

Methods: This was a single center, prospective, observational study where COVID-19 positive patients over 18 years in need of hospitalization were eligible for inclusion. Conventional coagulation tests and ROTEM were taken day 1-3 after hospital admission and patients were followed for 30 days. A logistic regression approach was applied to the ROTEM data and possible important covariates.

Results: 141 patients were included, $62 \%$ men, median age 63 years. 18 patients (13\%) died within 30 days. Comorbidities were common, and most patients had laboratory markers indicating hypercoagulation (e.g. d-dimer, P-fibrinogen and ROTEM). The logistic model presented that the risk of death within 30 days for a patient hospitalized due to COVID-19 was increased with increased age, respiratory frequency and the ROTEM variable EXTEM-MCF (all predictors $P<0.05)$. When the model is applied to the data, the ROC curve $A U C$ is 0.91 .

\section{PB0216 | Extracorporeal Membrane Oxygenation Induces Early Alterations in Coagulation and Fibrinolysis Profiles in COVID-19 Patients with Acute Respiratory Distress Syndrome}

G. Hékimian ${ }^{1,2}$; P. Masi ${ }^{2}$; M. Lejeune ${ }^{1,2}$; G. Lebreton ${ }^{1,2}$; J. Chommeloux ${ }^{2}$; C. Desnos ${ }^{2}$; M. Pineton De Chambrun²; I. Martin-Toutain ${ }^{2}$; A. Nieszkowska ${ }^{2}$; N. Bréchot ${ }^{2}$; M. Schmidt ${ }^{1,2}$; P. Leprince ${ }^{1,2}$; C.-E. Luyt ${ }^{1,2} ;$ A. Combes ${ }^{1,2} ;{\text { C. } \text { Frere }^{1,2}}^{2}$

${ }^{1}$ Sorbonne Université, INSERM UMRS_1166, Institute of Cardiometabolism and Nutrition, Paris, France; ${ }^{2}$ Assistance Publique Hôpitaux de Paris, Paris, France

Background: Extracorporeal membrane oxygenation (ECMO) support induces complex hemostatic changes that have been yet poorly described, particularly in COVID-19 patients.

Aims: To comprehensively analyze changes in coagulation and fibrinolysis profiles occurring during ECMO support in COVID-19 and non-COVID-19 patients with severe acute respiratory distress syndrome (ARDS).

Methods: All consecutive patients with ARDS undergoing ECMO were eligible to participate in this prospective monocentric study. Clinical characteristics were recorded on admission. Blood was sampled before and then 24 h, 7 and 14 days after ECMO implantation for longitudinal measurement of coagulation and fibrinolysis markers. Clinical outcomes were prospectively assessed until discharge from the ICU or death. 
Results: We included 20 COVID-19 and 10 non-COVID-19 participants. The median age was $47(35-56)$ years, with a median body mass index of 30 (27-35) kg/m², and a SOFA score of 12 (8-16). Baseline levels of von Willebrand factor, fibrinogen, factor VIII, prothrombin F1+2, thrombin-antithrombin, D-Dimers and PAI-1 were elevated in both groups, indicating that endothelial activation, endogenous thrombin generation and fibrinolysis shut-down occur in all ARDS patients before ECMO implantation. From baseline to day 7 , platelet count $(P<0.0001)$ and fibrinogen level $(P<0.001)$ significantly decreased, resulting from increases in thrombin generation (prothrombin $\mathrm{F} 1+2, P<0.01$ ) and fibrin formation (fibrin monomers, $P<0.001)$. PAl-1 levels significantly decreased from baseline to day $7(P<0.0001)$ in all ARDS patients. These changes were more marked in COVID-19 patients, resulting in 3 fatal bleeding.

Conclusions: ECMO circuit triggers early coagulation activation, resulting in significant fibrinogen and platelets consumption, with subsequent hypofibrinogenemia and thrombocytopenia, which may have contributed to the high prevalence of bleeding complications observed in COVID-19 ARDS patients rescued by ECMO. Additional studies are warranted to determine whether individualized anticoagulation might help to reduce bleeding complications during ECMO support. For now, daily monitoring of platelet count and fibrinogen should be part of ECMO management.

PB0217 | Impact of the COVID-19 Pandemic and a National Lockdown on Hospitalizations for Venous Thromboembolism and Pulmonary Embolism in France

A. Gabet ${ }^{1}$; C. Grave ${ }^{1}$; P. Tuppin ${ }^{2}$; J. Emmerich ${ }^{3}$; V. Olié ${ }^{1}$

${ }^{1}$ Santé Publique France, Saint Maurice, France; ${ }^{2}$ Caisse Nationale d'Assurance Maladie, Paris, France; ${ }^{3}$ University of Paris, Groupe Hospitalier Paris Saint-Joseph and INSERM CRESS 1153, Paris, France

Background: The COVID-19 pandemic and the national lockdown implemented in France have disturbed populations behavior and overwhelmed hospitals. Furthermore, infection to SARS-CoV-2 has been found to be directly associated with an increased risk of pulmonary embolism (PE).

Aims: The aims of this study were to analyze time-trends in patients hospitalized for PE in France, to describe the prevalence of a SARSCoV-2 infection over time among these patients and the related inhospital mortality rates.

Methods: We used the French national healthcare database. All patients hospitalized with a diagnosis of PE in France between January and September (Weeks 1 - 40) of each year from 2017 to 2020 were selected. Weekly incidence rates ratios (IRR) were computed to compare the rates of patients hospitalized between 2020 and 2017 2019.The COVID-19 diagnosis was based on biologic sample or CT scan.

Results: During 2020 study period, 35,446 patients were hospitalized with a primary diagnosis of PE and 23,759 with an associated diagnosis of PE. The prevalence of COVID-19 diagnosis was respectively $3.1 \%$ and $15.9 \%$, and reached $9.5 \%$ and $45.1 \%$ during the lockdown (weeks 12-19). As compared with same 2017-2019 weeks, rates of patients hospitalized with a primary diagnosis of PE in 2020 decreased significantly during the first week of the lockdown $(-42 \%)$. By contrast, rates of patients hospitalized with an associated diagnosis of PE doubled during the lockdown. Adjusted in-hospital death probability was multiplied by 3 among patients hospitalized with an associated diagnosis of $\mathrm{PE}(\mathrm{OR}=3.1[2.0-4.8]$ for week 13 of 2020 vs 2017-2019) but remained steady among those hospitalized with a primary diagnosis of $\mathrm{PE}$.

Conclusions: Substantial changes in the epidemiology of PE have been found and were partly related to the global massive decrease in hospitalizations, and also directly linked to the increased number of PE with SARS-CoV-2 infections and their higher severity.

PB0218 | Systemic Platelet Exhaustion in Critically-ill Covid-19 Patients

G. Manukjan ${ }^{1}$; J. Herrmann² ; J. Kleiß ${ }^{1}$; M. Zeitlhöfler ${ }^{1}$; P. Burkard ${ }^{3}$; P. Kranke ${ }^{2}$; T. Schlesinger ${ }^{2}$; T. Rose ${ }^{1}$; F. Simon ${ }^{1}$; L. Weiß ${ }^{1}$; D. Weismann ${ }^{4}$; C. Lotz ${ }^{2}$; B. Nieswandt ${ }^{1}$; P. Meybohm²; $\underline{\text { H. Schulze }}^{1}$ ${ }^{1}$ University Hospital of Würzburg; Institute of Experimental Biomedicine, Würzburg, Germany; ${ }^{2}$ University Hospital of Würzburg; Department of Anaesthesiology and Intensive Care Medicine, Würzburg, Germany; ${ }^{3}$ University Hospital of Würzburg; Institute of Experimental Biomedicine, Würzbrug, Germany; ${ }^{4}$ University Hospital of Würzburg; Department of Internal Medicine I, Würzburg, Germany

Background: Infection with SARS-CoV-2 leads to an altered hemostatic system and Covid-19 associated coagulopathy (CAC). Platelet counts remain overall unaltered, but thromboembolic events are frequently reported. Studies on the contribution of platelets to CAC are emerging but still lacking precise cohort comparison and broad analyses of platelet markers.

Aims: We aimed to analyze platelet receptor expression and function on platelets and biomarkers in platelet-poor plasma to investigate the role of platelets in the onset of critical progression of CAC. Methods: Extensive platelet function analyses were performed on 34 critically-ill patients with Covid-19 and data was compared to sepsis patients $(n=24)$ and non-SARS-CoV-2 acute infection $(n=18)$. Tests included PFA-200, aggregometry, flow cytometry and whole mount TEM. Plasma levels of TPO, sCD62P and sGPVI were determined by ELISA. For all patients, relatives, and for healthy controls $(n=10)$ informed consent was obtained.

Results: While platelet counts in patients of our Covid-19 cohort were expectably unaltered, platelet function was severely impaired in multiple assays. Platelets failed to aggregate in response to ADP or TRAP- 6 and could not activate integrin response or release agranules. The amount of platelet-leukocyte aggregates was markedly elevated, indicating previous platelet activation in line with higher levels of sCD62P and sGPVI. Remarkably, we observed platelet exhaustion in Covid-19 patients using whole mount TEM by 
means of a lack of dense granules corroborating with impaired uptake of mepacrine.

Conclusions: Our data imply that SARS-CoV-2 infection leads to a sub-threshold activation of platelets in a way that they become activated already before critical disease progression, without being cleared from the circulation, which is in striking contrast to sepsis. The platelet pool appears to be exhausted with detrimental consequences for thrombus stability and the risk of thromboembolic events. The mere platelet count in Covid-19 does thus not reflect progression to CAC, whereas platelet function is of high prognostic relevance.

PB0219 | Role of Endothelium Activation Biomarkers in Prediction COVID-19 Related Intra-hospital Mortality

M. Marchetti ${ }^{1}$; P. Gomez-Rosas ${ }^{2,1}$; L. Russo ${ }^{1}$; E. Sanga ${ }^{1}$; S. Gamba ${ }^{1}$; C. Verzeroli ${ }^{1}$; F. Restuccia ${ }^{1}$; F. Schieppati ${ }^{1}$; E. Bonanomi ${ }^{1}$; M. Rizzi ${ }^{1}$; S. Fagiuoli ${ }^{1}$; A. D'Alessio ${ }^{3}$; L. Lorini ${ }^{1}$; A. Falanga ${ }^{1,4}$

${ }^{1}$ ASST Papa Giovanni XXIII, Bergamo, Italy; ${ }^{2}$ Hospital General Regional Tecamac IMSS, Mexico, Mexico; ${ }^{3}$ Policlinico San Marco, Bergamo, Italy; ${ }^{4}$ University of Milano Bicocca, Milano, Italy

Background: Endothelial damage and hypercoagulability are major players behind the hemostatic derangement in SARS-CoV-2 infection.

Aims: In this prospective cohort study of COVID-19 patients, we aimed to assess the role of circulating endothelial activation/damage biomarkers in predicting in-hospital mortality.

Methods: Clinical data of COVID-19 patients hospitalized in intensive care (ICU) and non-ICU units at 2 Bergamo (Italy) hospitals from March 23 to May 30, 2020, were analyzed. Markers of endothelium activation including von-Willebrand factor (vWF), soluble thrombomodulin (sTM), and fibrinolytic proteins (t-PA and PAI-1) were measured. Additionally, D-dimer, Fibrinogen, FVIII, nucleosomes, CRP and procalcitonin were assessed.

Results: Sixty-three (45 ICU, and 18 non-ICU) patients, with a median age of 62 years were analyzed. Increased plasma levels of Ddimer, FVIII, fibrinogen, nucleosomes, CRP, and procalcitonin were observed in the whole cohort. Extremely elevated vWF levels characterized all patients (highest values in ICU-subjects). Patients with a moderate and severe ARDS (i.e. $\mathrm{PaO} 2 / \mathrm{FiO} 2 \leq 200 \%$ ) have considerably higher vWF and sTM levels, and lower t-PA/PAI-1 values compared to patients in the mild ARDS group (i.e. $\mathrm{PaO} 2 / \mathrm{FiO} 2>200 \%$ ). After a median time of 30 days, death occurred in 13 (21\%) patients. By multivariable analysis, vWF-activity, neutrophil-count and $\mathrm{PaO} 2 /$ $\mathrm{FiO} 2$ were significantly associated with death. Using these variables, we generated a linear score with 3-risk groups (AUC 0.903) that provided a cumulative incidence of death of $0 \%$ in the low-, $32 \%$ in the intermediate-, and $78 \%$ in the high-risk group $(P<0.001)$.

Conclusions: In conclusion, our study provides an extensive overview of the endothelial damage induced by SARSCoV-2 infection in hospitalized patients with virus-induced pneumonia and different degrees of disease severity. In addition, despite the small sample size and the need for the external validation, we could generate an accurate score based on circulating VWF to predicting mortality in severe COVID-19 patients.

PB0220 | Extracellular Vesicles and Citrullinated Histone H3 in Coronavirus Disease 2019 (COVID-19) Patients

L. Traby; M. Kollars; M. Kussmann; M. Karer; H. Sinkovec;

E. Lobmeyr; A. Hermann; T. Staudinger; P. Schellongowski;

B. Rössler; H. Burgmann; P.A. Kyrle; S. Eichinger

Medical University of Vienna, Vienna, Austria

Background: Pulmonary thrombus formation is a hallmark of COVID-19. A dysregulated immune response culminating in thromboinflammation has been described, but the pathomechanisms remain unclear.

Aims: To evaluate the role of extracellular vesicles (EV) and citrullinated histone $\mathrm{H} 3$ (citH3) as markers of coagulopathy and inflammation.

Methods: We studied 41 adult COVID-19 patients with a positive result on a reverse-transcriptase polymerase-chain-reaction assay and 37 sex-and age-matched healthy controls. Number and surface characteristics of $\mathrm{EV}$ and citH3 levels were determined in plasma upon admission by flowcytometry and immunoassay, respectively. Values are given as median $\left(25^{\text {th }}, 75^{\text {th }}\right.$ percentile) and differences by geometric mean ratios (GMR $[95 \% \mathrm{Cl}]$ ) or mean differences ( $\triangle \mathrm{s}$ Mean [95\% CI]).

Results: 20 patients had severe and 21 mild disease. Patients exhibited significantly higher numbers of total $\mathrm{EV}$, and of $\mathrm{EV}$ derived from platelets, endothelial cells, leukocytes, or neutrophils than controls (Table 1). EV from alveolar-macrophages and alveolar-epithelial-cells were detectable in plasma and were significantly higher in patients. ICAM-1 positive EV levels were higher in patients while no difference between TF-positive and ACE-positive EV was seen between the two groups. Levels of EV did not differ between patients with severe and mild COVID-19. citH3 levels were higher in patients than in controls [1.42 $(0.6,3.37)$ vs $0.31(0.14,0.6)$, GMR $4.44(2.57,7.66)$; $P<0.001]$, and were significantly lower in patients with mild disease compared to those with severe disease. 
TABLE 1 Extracellular vesicles in COVID-19 patients and in healthy controls

\begin{tabular}{|c|c|c|c|}
\hline & Patients & Healthy controls & \\
\hline $\begin{array}{l}\mathrm{EV} \\
\left(\times 10^{3} \mathrm{~mL}^{-1}\right)\end{array}$ & $\begin{array}{l}658.8 \\
(353.24,876.85)\end{array}$ & $\begin{array}{l}435.5 \\
(332.5,585.25)\end{array}$ & $\begin{array}{l}\text { GMR: } 2.6 \\
(1.88,3.59) ; P<0.001\end{array}$ \\
\hline $\begin{array}{l}\text { PLT+EV (CD61+) } \\
\left(\times 10^{3} \mathrm{~mL}^{-1}\right)\end{array}$ & $\begin{array}{l}343.52 \\
(189.35,641.67)\end{array}$ & $\begin{array}{l}68.06 \\
(39.05,138.08)\end{array}$ & $\begin{array}{l}\text { GMR: } 4.67 \\
(2.63,8.29) ; P<0.001\end{array}$ \\
\hline $\begin{array}{l}\mathrm{EC}+\mathrm{EV}(\mathrm{CD} 144+) \\
\left(\times 10^{3} \mathrm{~mL}^{-1}\right)\end{array}$ & $\begin{array}{l}38.89 \\
(30.09,62.96)\end{array}$ & $\begin{array}{l}0.46 \\
(0.46,0.93)\end{array}$ & $\begin{array}{l}\text { GMR: } 1.97 \\
(1.39,2.79) ; P<0.001\end{array}$ \\
\hline $\begin{array}{l}\text { AlvMac+EV (CD11c+) } \\
\left(\times 10^{3} \mathrm{~mL}^{-1}\right)\end{array}$ & $\begin{array}{l}0.93 \\
(0.46,2.31)\end{array}$ & $\begin{array}{l}0.46 \\
(0.46,0.93)\end{array}$ & $\begin{array}{l}\Delta \text { sMean: } 0.39 \\
(0.13,0.66) ; P=0.005\end{array}$ \\
\hline $\begin{array}{l}\text { AlvEpC+EV (CD326+) } \\
\left(\times 10^{3} \mathrm{~mL}^{-1}\right)\end{array}$ & $\begin{array}{l}1.39 \\
(0.46,2.31)\end{array}$ & $\begin{array}{l}0.46 \\
(0.46,0.93)\end{array}$ & $\begin{array}{l}\Delta \text { sMean: } 0.48 \\
(0.17,0.8) ; P=0.004\end{array}$ \\
\hline $\begin{array}{l}\text { Leu+EV (CD45+) } \\
\left(\times 10^{3} \mathrm{~mL}^{-1}\right)\end{array}$ & $\begin{array}{l}183.1 \\
(147.8,252.66)\end{array}$ & $\begin{array}{l}114.35 \\
(100.23,136.34)\end{array}$ & $\begin{array}{l}\text { GMR: } 1.72 \\
(1.31,2.25) ; P<0.001\end{array}$ \\
\hline $\begin{array}{l}\mathrm{Neu}+\mathrm{EV}(\mathrm{CD} 11 \mathrm{~b}+) \\
\left(\times 10^{3} \mathrm{~mL}^{-1}\right)\end{array}$ & $\begin{array}{l}73.38 \\
(59.84,94.44)\end{array}$ & $\begin{array}{l}51.39 \\
(37.73,62.04)\end{array}$ & $\begin{array}{l}\text { GMR: } 1.53 \\
(1.24,1.89) ; P<0.001\end{array}$ \\
\hline $\begin{array}{l}\text { ICAM1+EV (CD54+) } \\
\left(\times 10^{3} \mathrm{~mL}^{-1}\right)\end{array}$ & $\begin{array}{l}65.51 \\
(55.09,74.31)\end{array}$ & $\begin{array}{l}54.63 \\
(47.22,60.88)\end{array}$ & $\begin{array}{l}\text { GMR: } 1.21 \\
(1.08,1.37) ; P=0.002\end{array}$ \\
\hline $\begin{array}{l}\text { TF+EV (CD142+) } \\
\left(\times 10^{3} \mathrm{~mL}^{-1}\right)\end{array}$ & $\begin{array}{l}10.19 \\
(7.41,12.96)\end{array}$ & $\begin{array}{l}10.19 \\
(6.94,12.04)\end{array}$ & $\begin{array}{l}\Delta \text { sMean: } 0 \\
(-0.39,0.39) ; P=0.994\end{array}$ \\
\hline $\begin{array}{l}\mathrm{ACE}+\mathrm{EV}(\mathrm{CD} 143+) \\
\left(\times 10^{3} \mathrm{~mL}^{-1}\right)\end{array}$ & $\begin{array}{l}101.39 \\
(87.04,121.3)\end{array}$ & $\begin{array}{l}96.06 \\
(82.41,111.11)\end{array}$ & $\begin{array}{l}\Delta \text { sMean: } 0.22 \\
(-0.65,1.08) ; P=0.624\end{array}$ \\
\hline
\end{tabular}

Conclusions: EV and citH3 are associated with COVID-19. They provide information regarding pathophysiology and could be explored as markers of the disease.

\section{PB0221 | D-dimer as a Predictive Marker for Mortality in Hospitalized COVID-19 Patients}

S. Hassan ${ }^{1,2}$; B. Ferrari ${ }^{3}$; R. Rossio ${ }^{4}$; V. la Mura ${ }^{5}$; A. Artoni ${ }^{5}$; F. R. Rosendaal ${ }^{2}$; R. Gualtierotti ${ }^{1,3}$; I. Martinelli ${ }^{3}$; F. Peyvandi ${ }^{1,3}$; COVID-19 hospital network

${ }^{1}$ Department of Pathophysiology and Transplantation, University of Milan, Milan, Italy; ${ }^{2}$ Department of Clinical Epidemiology, Leiden University Medical Center, Leiden, Netherlands; ${ }^{3}$ Angelo Bianchi Bonomi Haemophilia and Thrombosis Centre and Luigi Villa Foundation, Fondazione IRCCS Ca' Granda Ospedale Maggiore Policlinico, Milan, Italy; ${ }^{4}$ Department of Pathophysiology and Transplantation, Fondazione IRCCS Ca' Granda Ospedale Maggiore Policlinico, Milan, Italy; ${ }^{5}$ U.O.C. Medicina Generale Emostasi e Trombosi, Fondazione IRCCS Ca' Granda Ospedale Maggiore Policlinico, University of Milan, Milan, Italy

Background: The novel coronavirus disease 2019 (COVID-19) presents an important and urgent threat to global health. Identifying strong predictors of mortality could assist medical staff in treating patients and allocating limited healthcare resources. 
Aims: The primary aim of this paper was to study the effect of $d$ dimer levels at admission as a predictive marker for in-hospital mortality.

Methods: This was a retrospective cohort study evaluating hospitalized patients (age > 18 years), who were positive for COVID-19 based on real-time PCR at one of nine COVID-19 units during the period of the first COVID-19 wave in Lombardy, Italy. The primary endpoint was in-hospital mortality. Information was obtained from patient records. Statistical analyses were performed using a Fine-Gray competing risk survival model. Predictive power was assessed using Harrell's C-index. Results: Out of 1049 patients that were admitted to the emergency department and subsequently hospitalized, 501 patients had evaluable data for d-dimer. Of these 501 patients, 96 did not survive. Cumulative incidence of in-hospital mortality within 30 days was $20 \%$, and the majority of deaths occurred within the first 10 days. (Figure 1)

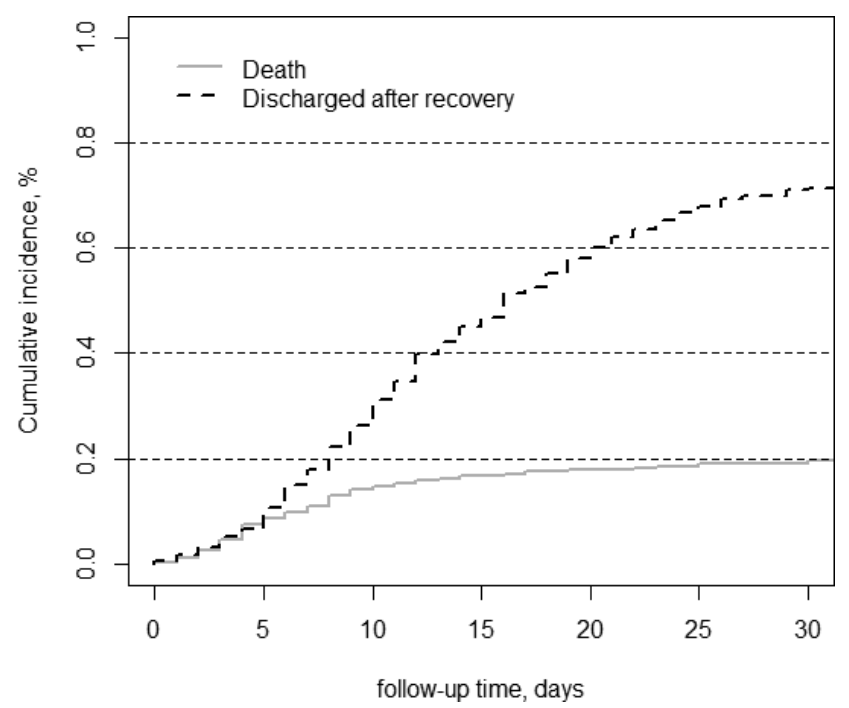

FIGURE 1 Cumulative incidence function of the cohort.

When compared to patients in the lowest quartile of $d$-dimer blood concentration, the hazard ratio of in-hospital mortality for patients in the $2^{\text {nd }}, 3^{\text {rd }}$ and $4^{\text {th }}$ quartile was $3.9(95 \mathrm{Cl}: 1.5-10.0), 5.8$ ( $95 \mathrm{Cl}: 2.3-$ 14.7), and 4.6 ( $95 \mathrm{Cl}: 1.8-11.5)$ respectively, after multivariable adjustment for age, sex and number of comorbidities. The $\mathrm{C}$-statistic of d-dimer for in-hospital mortality was 0.67 (95Cl: 0.62-0.71). (Table 2)
Conclusions: Higher d-dimer levels were strongly associated with inhospital mortality. However, the predictive power of d-dimer alone was not high enough to be useful as a risk prediction score. Future research should focus on the added value of $d$-dimer as part of a larger risk prediction score.

PB0222 | Dynamic Monitoring of Hemostasis and Correction of Heparin Therapy Can Halve the Number of Thrombotic Complications in Patients with Severe COVID-19

F. Ataullakhanov $^{1,2,3,4,5} ;$ S. Shakhidzhanov ${ }^{1}$; D. Nechipurenko ${ }^{4,1,3}$; I. Serebrisky ${ }^{6}$; S. Karamzin ${ }^{1}$; I. Spiridonov ${ }^{1}$; D. Zateyshchikov ${ }^{7}$; M. Panteleev 1,3,4,5; V. Mustyatsa ${ }^{3,1}$; D. Morozova ${ }^{8}$; E. Koltsova ${ }^{1,3}$; E. Seregina ${ }^{3,1}$; S. Ratchina ${ }^{9}$; A. Gubkin ${ }^{10}$; A. Martyanov ${ }^{3,1}$; E. Grishchuk ${ }^{2}$; D. Protsenko ${ }^{11}$; C. Tsarenko ${ }^{12}$; H. Vasilieva ${ }^{13}$; A. Rumyantsev ${ }^{3}$ ${ }^{1}$ Center for Theoretical Problems of Physicochemical Pharmacology, RAS, Moscow, Russian Federation; ${ }^{2}$ Department of Physiology, Perelman School of Medicine, University of Pennsylvania, Philadelphia, United States; ${ }^{3}$ Dmitry Rogachev National Research Center of Pediatric Hematology, Oncology and Immunology, Moscow, Russian Federation; ${ }^{4}$ Faculty of Physics of Lomonosov Moscow State University, Moscow, Russian Federation; ${ }^{5}$ Moscow Institute of of Physics and Technology, Moscow, Russian Federation; ${ }^{6}$ Moscow hospital \#40, Moscow, Russian Federation; ${ }^{7}$ Moscow hospital \#51, Moscow, Russian Federation; ${ }^{8}$ Faculty of Medicine of Lomonosov Moscow State University, Moscow, Russian Federation; ${ }^{9}$ Sechenov First Moscow State Medical University, Moscow, Russian Federation; ${ }^{10}$ Semashko Railroad Clinical Hospital, Moscow, Russian Federation; ${ }^{11}$ Moscow Hospital \#40, Moscow, Russian Federation; ${ }^{12}$ Moscow Hospital \#52, Moscow, Russian Federation;

${ }^{13}$ Moscow Hospital \#23, Moscow, Russian Federation

Background: Anticoagulant thromboprophylaxis is recommended for all hospitalized COVID-19 patients. However, the dosage regimen is debated, as thrombogenicity may vary in different patients and during the course of infection.

TABLE 1 Association between d-dimer values and in-hospital mortality.

\begin{tabular}{|c|c|c|c|c|c|}
\hline \multicolumn{6}{|l|}{ D-dimer } \\
\hline$<538 \mathrm{ng} / \mathrm{mL}$ & 126 & 5 & 0.04 & ref & ref \\
\hline $538-957 \mathrm{ng} / \mathrm{mL}$ & 124 & 20 & 0.16 & $4.2(1.6-11.1)$ & $3.9(1.5-10.0)$ \\
\hline $957-1764 \mathrm{ng} / \mathrm{mL}$ & 124 & 36 & 0.30 & $8.6(3.4-21.8)$ & $5.8(2.3-14.7)$ \\
\hline C-statistic $(95 \% \mathrm{Cl}$ & & & & $0.67(0.62-0.71)$ & - \\
\hline
\end{tabular}


Aims: 1. Conduct observational study of various hemostatic tests to achieve accurate dynamic monitoring in COVID-19 patients. 2. Evaluate effectiveness of heparin therapy correction in response to the ongoing changes in hemostasis system.

Methods: 1. Hemostasis was monitored in 1,859 patients with severe COVID-19 in seven hospitals in Moscow. Blood was taken at hospital admission before the start of thromboprophylaxis and every 1-3 days throughout the treatment. To minimize the presence of heparin in blood sample, blood was collected $12 \mathrm{~h}$ after heparin injection. Hemostasis was investigated using APTT, INR, D-dimer test, Thromboelastography and Thrombodynamics.

2. Correction of heparin therapy was carried out in 218 COVID-19 patients monitored using Thrombodynamics in three hospitals.

Results: 1. APTT and INR parameters did not change after the start of heparin therapy and indicated hypocoagulation during entire thromboprophylaxis. D-dimer was strongly elevated in most patients but was also unchanged during the therapy. Thromboelastography and Thrombodynamics showed hypercoagulability in $60-70 \%$ of patients prior to therapy. Subsequently, these tests revealed normal coagulation in $70 \%$ of the patients. From all examined hemostatic tests, the most sensitive indicator of the state of hemostasis was the rate of clot growth determined with Thrombodynamics.

2. Based on the results of the observational study, heparin therapy was corrected in ICU patients to maintain the rate of clot growth at 12-20 $\mu \mathrm{m} / \mathrm{min}$, as determined with Thrombodynamics. Thrombotic complications were observed in $19 \%$ of patients, compared with $32 \%$ in the control group with 151 patients, who received standard heparin therapy with no correction.

Conclusions: Dynamic monitoring of hemostasis via Thrombodynamics in patients with severe COVID-19 enables effective correction of heparin therapy, significantly reducing thrombotic complications.
PB0223 | Use of D-Dimer as a Predictor of Venous Thromboembolism Events (VTE) in Covid-19 Patients

M.C. Chindamo $^{1,2}$; M.B. Manhães ${ }^{1}$; L.D. Carvalho ${ }^{1}$; G.G. Sardinha ${ }^{1}$; A.M. Marques ${ }^{1}$; H.C. Goudar ${ }^{1}$; A.B. Nunes ${ }^{1}$; P.R. Carmo Jr ${ }^{1,2}$; D.R. Salgado ${ }^{1,2}$

${ }^{1}$ Hospital Barra D'Or, Rio de Janeiro, Brazil; ${ }^{2}$ Federal University of Rio de Janeiro, Rio de Janeiro, Brazil

Background: D-Dimer (DD) values are often elevated in coronavirus disease 2019 (COVID-19), but its role as a diagnostic test of VTE is controversial.

Aims: To analyze the performance of DD in the VTE events diagnosis in a cohort of COVID-19 patients.

Methods: We retrospectively analyzed, from March/2020 to February/2021, all cases of proven COVID-19 submitted to a compression ultrasound (CUS) or CT pulmonary angiogram (CTPA) to investigate deep venous thrombosis [DVT] and/or pulmonary embolism [PE] DD levels were recorded at hospital admission and at the day ( $\pm 24 \mathrm{~h}$ ) of each imaging studies. DD levels were categorized into 3 groups according to terciles, in order to determine VTE risk. ROC curve analysis was used to determine optimal DD cut-off to predict VTE.

Results: From 717 COVID-19 admissions, we identified 169 patients (median age 65 years [20-103], 66\% male) submitted to a $208 \mathrm{im}$ aging studies (74 CTPA, 134 CUS). In the overall cohort, 54 (32\%) patients had VTE diagnosis according to 62 positive exams (39 CUS and 23 CTPA). Confirmed VTE patients had higher median levels of DD at admission (2364 vs. $831 \mathrm{ng} / \mathrm{mL} ; P=0.004$ ) and at the day of exam (7013 vs. $1378 \mathrm{ng} / \mathrm{mL} ; P<0.0001$ ). The AUROC for DD and VTE at admission and at the day of imaging study was 0.72 (95\% $\mathrm{Cl}, 0.63-0.81)$ and $0.80(95 \% \mathrm{Cl}, 0.73-0.87)$, respectively. The best DD cut-off point to predict VTE was $2000 \mathrm{ng} / \mathrm{mL}$ ( $86 \%$ sensitivity, $63 \%$ specificity). Greater values of DD were associated with an increased rate of VTE diagnosis: group I $\leq 1200 \mathrm{ng} / \mathrm{mL}$ : 7.6\%; group II-1201-5000 ng/mL: $35.8 \%$ and group III>5000 ng/mL: $60.3 \%$ $(P<0.001)$. The OR of group II and III vs. group I was $6.8(95 \% \mathrm{Cl} 2.4-$ 19.3; $P<0.0001)$ and 18.5 (95\%Cl6.6-52.0; $P<0.0001)$, respectively. Conclusions: In a cohort of hospitalized COVID-19 patients, DD test was a good discriminator of VTE events. Serial measurements of DD could help physicians to initiate anticoagulation therapy in COVID-19 patients suspected of VTE diagnosis.

PB0224 | Significance of D-Dimers in Patients with COVID-19 Having Venous Thromboembolism

B. Moiz; H. Alam

Aga Khan University, Karachi, Pakistan

Background: Coagulopathy is a recognized feature of severe COVID-19. While high d-dimers are associated with the disease severity and poor outcome, its role in venous thromboembolism (VTE) is evolving. 
Aims: This study was done to evaluate the d-dimer levels in patients having COVID-19 with and without venous thromboembolic events. Methods: Patients admitted with COVID-19 during March to December 2020 were identified through ICD10 and data was collected through in-house computerized system. Frequency of myocardial infarction (MI), stroke, deep venous thrombosis (DVT), pulmonary embolism (PE) and cerebral venous sinus thrombosis (CVST) was determined. D-dimer was assayed on CS2500 (Sysmex, Kobe, Japan) and was compared in patients with and without DVT/ PE during their hospital stay using non-parametric methods (SPSS version 19).

Results: The thromboembolic events included stroke ( $n=41)$, MI (169), DVT $(n=4), \operatorname{PE}(n=21)$ and CVST $(n=3)$ in a total of 2454 patients. Patients having COVID-19 (76 in ICU and 25 with VTE) including 22 females and 79 males with a median (IQR) of 59 years (49.5-65.5) were evaluated for $d$-dimer levels. There was no statistically significant difference in median (IQR) d-dimers ( $\mathrm{mg} / \mathrm{L}$ ) in patients with and without VTE at the time of presentation [1.5 (0.6-3.2) vs. 1.6 (0.7-5.9); P 0.943] and at the last follow-up [2.4 (1.0-4.6) vs. 3.3 (1.3-7.1); $P$ 0.345]. However, there were significantly higher median (IQR) d-dimers of 8.1 (2.7-15.2) in patients having VTE events during hospital stay compared to those who did not have any VTE [3.4 (1.4-10.9), P 0.047]. Moreover, significantly higher median (IQR) d-dimers ( $\mathrm{mg} / \mathrm{L}$ ) i.e. 5.0 (2.5-12.0) was observed at the last follow-up in the patients who expired $(n=48)$ compared to those $(n=53)$ who were discharged alive from the hospital [1.7 (0.7-3.7); $P$ 0.022].

Conclusions: Patients with COVID-19 having VTE had higher level of d-dimers during hospital stay compared to ICU-patients admitted with severe disease.

PB0225 | Venous Thromboembolism in Cancer Patients with SARS-CoV-2 Infection

D. Attia ${ }^{1} ;$ W. Wei ${ }^{2}$; M. Wilks ${ }^{1}$; B. Tripp ${ }^{1} ;$ C. D'Andrea ${ }^{1}$;

D.E. Angelini ${ }^{1}$; A.A. Khorana ${ }^{1}$

${ }^{1}$ Taussig Cancer Institute, Cleveland Clinic Foundation, Cleveland, United States; ${ }^{2}$ Department of Quantitative Health Sciences, Cleveland Clinic Foundation, Cleveland, United States

Background: Venous thromboembolism (VTE) is a major cause of morbidity and mortality in cancer patients. Cancer patients with COVID-19, especially those who are hospitalized, may be at higher risk to develop VTE but this is incompletely understood.

Aims: To investigate the rate of confirmed VTE in cancer patients with COVID-19.

Methods: A retrospective cohort study of consecutive cancer patients with a diagnosis of COVID-19 at the Cleveland Clinic-Taussig Cancer Institute from 3/14/20 to 5/26/20. VTE (deep venous thrombosis (DVT) and pulmonary embolism (PE) was included only if confirmed by imaging.Clinical and laboratory features associated with VTE within 2-months post-test date were analyzed using Wilcoxon rank sum and Fisher's exact test.Time-to-VTE was estimated by Kaplan-Meier method and compared using log rank test.

Results: A total of 305 patients, of whom $47.2 \%$ were male.Median age was 69 (range23-96) years.Median follow-up was 0.7 months (range: 0.1-2.3) after the positive test date.169 patients (55.4\%) were hospitalized,185 patients $(60.7 \%)$ received prophylactic and $16(5.2 \%)$ received therapeutic anticoagulation. Baseline characteristics of the study population are shown in Table 1.16 patients (5.2\%) developed VTE (8 DVT, 4 PE, 4 both DVT/PE) who were hospitalized. Hospitalization was significantly associated with VTE within 2 month $(P=0.04)$. VTE patients were found to have significantly higher CRP levels (Median 12.7, range [2.3-39.9] vs. 8.7[0.2-48.3] $P=0.03$ ) and higher D-Dimer levels (Median 5750, range [1300$35200]$ vs. $1035[0.95-35200], P<0.0001)$. Overall survival was negatively impacted by hypertension $(2-\mathrm{mo}$ OS rate $=0.65,95 \% \mathrm{Cl}$ $0.54-0.77$ vs. $0.94[0.88-1], P=0.0005$. females had significantly better OS (2-mo OS rate $=0.86,95 \% \mathrm{Cl}(0.79-0.93)$ vs. 0.62 [0.48-0.78], $P=0.009$ ). Hospitalized patients had worse time-to-VTE (1-mo VTEfree rate $=0.89,95 \% \mathrm{Cl}[0.83-0.96]$ vs. 0.97[0.93-1], $P=0.03$ )and a significantly worse OS (1-mo OS rate $=0.69,95 \% \mathrm{Cl}(0.61-0.78)$ vs $0.94[0.89-0.99], P<0.0001)$. There was no significant difference in OS between VTE groups $(P=0.44)$.

TABLE 1 Baseline Characteristics of Cancer Patients with COVID-19

With VTE within 2 months $\mathrm{N}$ (\%)

Median age

$74.5(16-91)$

Sex (Males)

$10(6.49 \%)$

Females

$6(3.73 \%)$

Cancer types Hematological
Without VTE within 2 months $\mathrm{N}(\%)$

$69(23-96)$ 0.25

$134(93.06 \%)$

0.3

155 (96.27\%)

$22(84.62)$ 


\begin{tabular}{lll} 
& With VTE within 2 months $\mathbf{N}(\%)$ & Without VTE within 2 months $\mathbf{N}(\%)$ \\
Thoracic & $1(5.88 \%)$ & $16(94.12)$ \\
GI & $1(3.85 \%)$ & $25(96.15)$ \\
Others & $10(4.27 \%)$ & $224(95.73)$ \\
Mortality & $4(8.51)$ & $43(91.49)$ \\
\hline
\end{tabular}

Conclusions: VTE patients were found to have higher CRP and DDimer level. Hypertension and hospitalization status are independent risk factors for VTE and females had better OS.

PB0226 | Hypercoagulable Rotational Thromboelastometry NaHEPTEM Profile is Related to Mechanical Ventilation Requirement among Critically ill COVID-19 Patients

I. Carboni Bisso ${ }^{1}$; I. Huespe ${ }^{2,3}$; M. Las Heras ${ }^{1}$; E. Prado ${ }^{1}$; M. Garbarini ${ }^{4}$; M.S. López ${ }^{5,6}$; L. Barrera ${ }^{5,6}$; J. Sinner ${ }^{1}$; M. Martinuzzo ${ }^{5,6}$

${ }^{1}$ Hospital Italiano de Buenos Aires, Unidad de Terapia Intensiva de Adultos, CABA, Argentina; ${ }^{2}$ Hospital Italiano de Buenos Aires, Unidad de Terapia Intensiva de Adultos, Area de Investigación en Medicina Interna, CABA, Argentina; ${ }^{3}$ Instituto Universitario del Hospital Italiano de Buenos Aires, Instituto de Medicina Traslacional e Ingeniería Biomédica, CONICET, CABA, Argentina; ${ }^{4}$ Hospital Italiano de Buenos Aires, Unidad de Terapia Intensiva de Adultos, Hematología, CABA, Argentina; ${ }^{5}$ Hospital Italiano de Buenos Aires, Laboratorio Central, CABA, Argentina; ${ }^{6}$ Instituto Universitario del Hospital Italiano de Buenos Aires, CABA, Argentina

Background: Hypercoagulability and pulmonary microvascular thrombosis has been related to COVID-19 hypoxemia. Rotational thromboelastometry (RT) could identify the procoagulant state.
Aims: - To evaluate maximum clot firmness(MCF) and other RT parameters among COVID-19 patients in intensive care unit(ICU) compared to healthy controls $(\mathrm{HC})$

- To compare them according to mechanical ventilation (MV) requirement

Methods: Prospective observational cohort study (AugustNovember 2020). ICU cohort: all adult patients admitted due to COVID-19. HC cohort: healthy volunteers.

Coagulation profile was evaluated by RT NaHEPTEM assay in ROTEM Delta at day 1 (T1), 5 (T5) and 10 (T10) from ICU admission. $\mathrm{D}$-Dimer and Fibrinogen were also evaluated.

Results: Twenty three COVID-19 patients (under prophylactic dose enoxaparin) and $19 \mathrm{HC}$ were included. MCF was statistically higher in ICU patients vs HC at admission (T1) and further increasing at T5. (Table 1)

Distribution of NaHEPTEM parameters, DD and Fibrinogen in samples from ICU patients under MV or not are shown in Figure 1. ICU patients under MV compared to non-MV presented higher levels of fibrinogen from T1 to T10, DD and MCF at T5,and shorter clotting formation time (CFT), higher maximum velocity (MaxV) and 5 min Amplitude (A5) at T1. Maximum Lysis (ML) was significantly lower at T5 and T10 compared to T1, $P=0.003$ and $P=0.008$, respectively, but not associated with MV. (TABLE1).

COVID-19 patients discharged from hospital before T10 $(n=5)$ presented at T5 significant lower values of DD, Fibrinogen and RT MaxV compared to patients with longer $\mathrm{UCl}$ stay.

TABLE 1 Laboratory findings on different time points. Abbreviations: MV: mechanical ventilation; CT: Clotting time; CFT: Clotting formation time; A5: Amplitude at 5 min; MCF: Maximum clot firmness; ML: Maximum lysis; MaxV: maximum velocity of clot formation. ${ }^{a} P=0.051$; ${ }^{\mathrm{b}} P=0.058 ;{ }^{\mathrm{c}} P=0.019 ;{ }^{\mathrm{d}} P=0.022 ;{ }^{\mathrm{e}} P<0.001 ;{ }^{\mathrm{f}} P=0.042$

\begin{tabular}{|c|c|c|c|c|c|c|c|}
\hline & $\begin{array}{l}\mathrm{HC} \\
(n=19)\end{array}$ & $\begin{array}{l}\text { T1 } \\
\text { non-MV } \\
(n=12)\end{array}$ & $\begin{array}{l}\text { T1 } \\
\text { MV } \\
(n=11)\end{array}$ & $\begin{array}{l}\text { T5 } \\
\text { non-MV } \\
(n=8)\end{array}$ & $\begin{array}{l}\text { T5 } \\
\text { MV } \\
(n=15)\end{array}$ & $\begin{array}{l}\text { T10 non-MV } \\
(n=4)\end{array}$ & $\begin{array}{l}\text { T10 MV } \\
(n=13)\end{array}$ \\
\hline $\mathrm{CT}$, seg & $\begin{array}{l}568 \\
\quad(473-613)\end{array}$ & $577(493-647)$ & $\begin{array}{l}582 \\
\quad(493-619)\end{array}$ & $462(423-508)$ & $540(439-593)$ & $\begin{array}{l}480 \\
\quad(404-510)\end{array}$ & $509(444-548)$ \\
\hline CFT, seg & $\begin{array}{l}154 \\
\quad(134-207)\end{array}$ & $\begin{array}{l}173 \\
\quad(153-223)^{a}\end{array}$ & $\begin{array}{l}113 \\
(95-174)^{a}\end{array}$ & $139(114-151)$ & 125 (93-149) & $122(98-148)$ & $126(102-139)$ \\
\hline $\mathrm{A} 5, \mathrm{~mm}$ & $33(30-39)$ & $34(29-38)^{b}$ & $45(34-51)^{b}$ & $41(39-43)$ & $45(40-53)$ & $36(33-39)$ & $50(40-58)$ \\
\hline MCF, mm & $56(52-60)$ & $64(56-66)$ & $64(61-71)$ & $65(64-67)^{c}$ & $70(68-71)^{c}$ & $66(61-69)$ & 70 (67-74) \\
\hline ML, \% & $8(5-10)$ & $9(6-14)$ & $10(7-14)$ & $9(7-10)$ & $6(4-10)$ & $6(4-10)$ & $6(3-11)$ \\
\hline $\begin{array}{l}\mathrm{MaxV}, \mathrm{mm} / \\
\min \end{array}$ & $8(7-10)$ & $8(7-9)^{d}$ & $12(9-16)^{d}$ & $11(9-12)$ & $13(10-15)$ & $11(9-15)$ & $12(10-15)$ \\
\hline $\begin{array}{c}\text { Platelets X } \\
109 / \mathrm{L}\end{array}$ & $249(222-265)$ & $261(173-317)$ & $\begin{array}{l}231 \\
\quad(194-269)\end{array}$ & $356(307-444)$ & $279(243-319)^{*}$ & $\begin{array}{l}296 \\
\quad(286-313)\end{array}$ & $273(208-354)$ \\
\hline
\end{tabular}




\begin{tabular}{|c|c|c|c|c|c|c|c|}
\hline & & $\mathrm{T} 1$ & $\mathrm{~T} 1$ & T5 & T5 & & \\
\hline & $\begin{array}{l}\mathrm{HC} \\
(n=19)\end{array}$ & $\begin{array}{l}\text { non-MV } \\
(n=12)\end{array}$ & $\begin{array}{l}\text { MV } \\
(n=11)\end{array}$ & $\begin{array}{l}\text { non-MV } \\
(n=8)\end{array}$ & $\begin{array}{l}\text { MV } \\
(n=15)\end{array}$ & $\begin{array}{l}\text { T10 non-MV } \\
(n=4)\end{array}$ & $\begin{array}{l}\text { T10 MV } \\
(n=13)\end{array}$ \\
\hline $\begin{array}{c}\text { D-Dimer, } \\
\mathrm{ng} / \mathrm{ml} \\
\mathrm{FEU}\end{array}$ & - & $\begin{array}{l}750 \\
\quad(562-1286)\end{array}$ & $\begin{array}{c}1032(540- \\
1395)\end{array}$ & $\begin{array}{l}421 \\
\quad(273-776)^{\mathrm{e}}\end{array}$ & $2340(1419-4079)^{\mathrm{e}}$ & $\begin{array}{c}1691(1302- \\
2008)\end{array}$ & $\begin{array}{c}1874(1529- \\
1980)\end{array}$ \\
\hline $\begin{array}{c}\text { Fibrinogen, } \\
\text { mg/dL }\end{array}$ & $\begin{array}{l}311 \\
\quad(280-354)\end{array}$ & $\begin{array}{l}456 \\
\quad(423-542)^{f}\end{array}$ & $\begin{array}{c}579(487- \\
682)^{f}\end{array}$ & $\begin{array}{l}354 \\
\quad(315-373)^{\mathrm{e}}\end{array}$ & $568(510-647)^{e}$ & $437(351-502)$ & $605(547-696)$ \\
\hline
\end{tabular}

a)
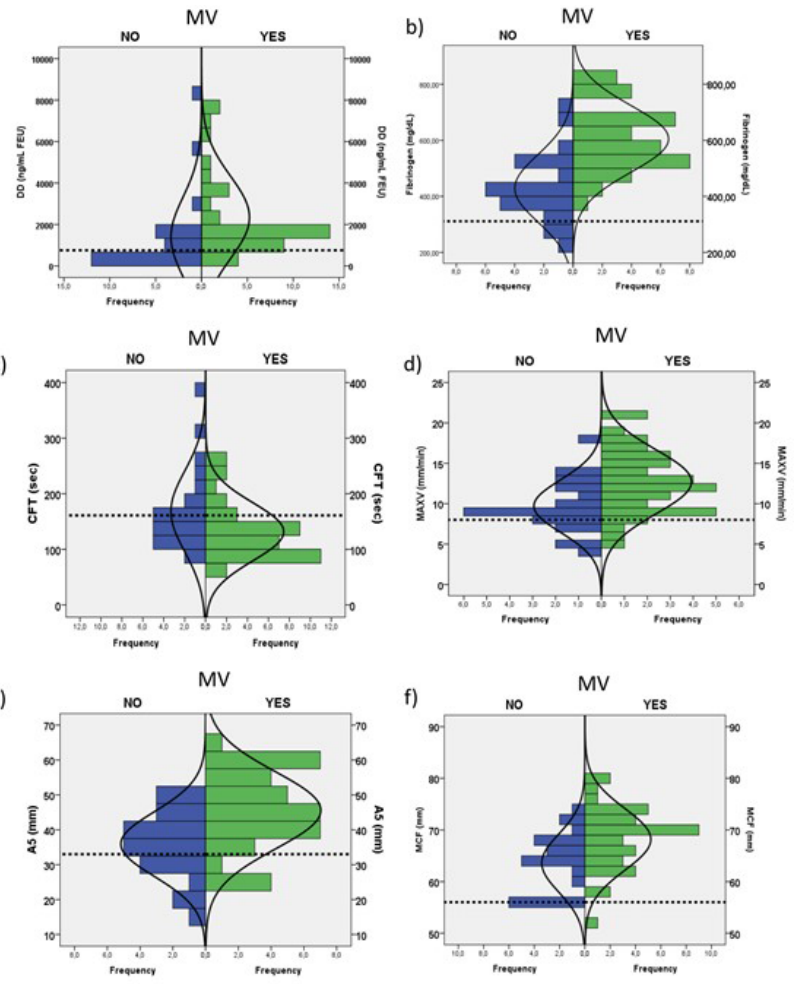

FIGURE 1 Distribution of hemostatic parameters: D-Dimer (a) and fibrinogen (b), as well as NaHEPTEM parameters CFT(c), MaxV(d), $\mathrm{A} 5(\mathrm{e})$ and MCF (f) in all blood samples performed from ICU patients with COVID-19, according to the need of mechanical ventilation treatment. Dashed lines indicated the parameter median obtained in the healthy control group

Conclusions: NaHEPTEM assay could detect hypercoagulability among COVID-19 critically ill patients. Velocity parameters(CFT, MaxV) and $A 5$ seem to be further altered in patients that required MV at early stages after ICU admission, probably reflecting increased thrombin generation. MCF and DD were higher at T5 post ICU admission in patients under MV. ML decreased along to study period without association to MV and no difference to HC. Further studies are needed to evaluate its clinical usefulness.

PB0228 | Suspicion of Heparin-induced Thrombocytopenia in COVID-19: Results from a Multi-centric Retrospective Study

N. Gendron ${ }^{1}$; A. Lillo-Le Louet ${ }^{1}$; W. Amara ${ }^{1}$; C. Le Beller ${ }^{1}$;

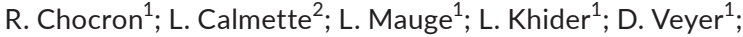

B. Planquette ${ }^{1}$; B. Rance ${ }^{1}$; B. Huynh ${ }^{3}$; P. Gaussem ${ }^{1}$; O. Sanchez ${ }^{1}$; J.L. Diehl' ${ }^{1}$; T. Mirault ${ }^{1}$; D.M Smadja ${ }^{1}$; D. Helley ${ }^{1}$

${ }^{1}$ Hôpital Européen Georges Pompidou, Paris, France; ${ }^{2}$ Hôpital

Ambroise-Paré, Boulogne-Billancourt, France; ${ }^{3}$ Institut Mutualiste

Montsouris, Paris, France

Background: High rates of venous thromboembolic events associated to $\mathrm{LMWH}$ /heparin therapy lead to numerous heparin-induced thrombocytopenia (HIT) suspicion during COVID-19 outbreak.

Aims: We aim to describe HIT-suspected patient's characteristics and prevalence between March 15 and April 15 of 2020.

Methods: This is a multi-centric retrospective cohort study of HITsuspected patients referred to our center. 4T score has been realized by experienced hematologist and/or pharmacologist and allowed us to trigger specific HIT assays if score was $>3$ (IgG anti-PF4/H and 14C-serotonine release assay, SRA). We included all consecutive HIT-suspected patients during COVID-19 outbreak compared to the same period in 2019.

Results: During 2019 and 2020-study periods we identified, respectively, 17 and 41 consecutive HIT-suspected patients. Among the 2020-group, 23 were COVID-19 and 18 were non-COVID-19 patients. Clinical and biological characteristics were not significantly different between the 2019, 2020 non-COVID-19 and COVID-19 HIT-suspected patients. During 2019-period study, 11 (64.7\%) patients had a 4T score $>3,4$ (36.3\%) of them had positive anti-PF4/H antibodies and only one had a positive SRA assay. During 2020-period study, 8 (44.4\%) non-COVID-19 and 10 (43.5\%) COVID-19 patients had a 4 T score $>3$. Among them, respectively, 3 (37.5\%) and 3 (30.\%) had positive anti-PF4/H antibodies. SRA assay was positive in 3 non-COVID-19 patients tested and in the only one COVID-19 tested patient. The 4T score was able to exclude HIT in 67\% of COVID-19 patients suspected. In 2020-study period, when comparing COVID-19 and non-COVID-19 patients, the only significantly difference in term of HIT suspicion criteria was the mean duration of heparin exposition before suspicion: 9.9 days \pm 6.3 for non-COVID-19 patients versus 15.2 days \pm 8.8 for COVID-19 patients, $P=0.043$ ).

Conclusions: HIT suspicion in COVID-19 occurs after longer anticoagulation time than non-COVID-19. We did not observe more confirmed HIT in COVID-19 in contrast our two non-COVID-19 control groups. 
PB0229 | The Characteristics and Outcomes of Critically III Patients with COVID-19 who Received Systemic Thrombolysis for Presumed Pulmonary Embolism: An Observational Study

M. So ${ }^{1}$; D. Steiger ${ }^{1}$; M. Takahashi ${ }^{1}$; N. Egorova ${ }^{2}$; T. Kuno ${ }^{1}$.

${ }^{1}$ Mount Sinai Beth Israel Medical Center, New York, United States;

${ }^{2}$ Ichan School of Medicine at Mount Sinai, New York, United States

Background: Coronavirus disease 2019 (COVID-19) is associated with abnormal hemostasis, autopsy evidence of systemic microthrombosis, and a high prevalence of venous thromboembolic disease (VTE). Tissue plasminogen activator (tPA) has been used in COVID-19 patients with severe hypoxia with high clinical suspicion of pulmonary embolism (PE).

Aims: We aimed to describe the clinical outcomes of critically ill COVID-19 patients who received tPA.

Methods: A retrospective cohort study was conducted on 6,095 hospitalized COVID-19 patients in the Mount Sinai Health System at 5 hospitals in New York. 57 patients with COVID-19, who were admitted from $3 / 10$ to 4/27, 2020 and received tPA for presumed PE were included in the analysis. Baseline demographic and clinical characteristics, indication for tPA, and overall mortality were reported.

Results: Among the 57 patients who received tPA, the mean age was $60.8 \pm 10.8$ years, and $71.9 \%$ (41/57) were male. PE was suspected among $75.4 \%$ (43/57) of patients with supporting findings who had rapidly worsening hypoxia or hypotension. Right ventricular (RV) strain was present in $15.8 \%$ (9/57), deep venous thrombosis (DVT) in $7.0 \%$ (4/57), increased dead space ventilation $\left(V_{d}\right)$ in $31.6 \%(18 / 57)$ of patients. RV strain and RV thrombus were present in 3.5\% (2/57), RV strain and DVT in 5.3\% (3/57), RV strain and increased $\mathrm{V}_{d}$ in $8.8 \%$ (5/57), and DVT and increased $V_{d}$ in $3.5 \%(2 / 57)$ of patients. No chest CT angiography was performed for any patients due to clinical instability from critical illness. Following tPA infusion, 49.1\% (28/57) of patients demonstrated improvement in either of $\mathrm{PaO}_{2} / \mathrm{FiO}_{2}$ ratio, blood pressure or partial arterial carbon dioxide. Bleeding complication was seen in 1 patient. Six patients (10.5\%) survived to hospital discharge. Overall mortality was $89.5 \%(51 / 57)$.

TABLE 1 Tissue plasminogen activator indications for patients with presumptive diagnosis of pulmonary embolism in deteriorating hypoxia and shock

$\begin{array}{ll}\text { tPA indications, } n(\%) & \begin{array}{l}\text { Total number of patients } \\ (n=57)\end{array} \\ \text { Sole RV strain } & 9(21.1 \%) \\ \text { RV strain and RV thrombus } & 2(3.5 \%) \\ \text { RV strain and DVT } & 3(5.3 \%) \\ \text { RV strain and increased } \mathrm{V}_{d} & 5(8.8 \%) \\ \text { Sole DVT on US } & 4(7.0 \%) \\ \text { Sole increased } \mathrm{V}_{d} & 18(31.6 \%) \\ \text { DVT and increased } \mathrm{V}_{d} & 2(3.5 \%) \\ \text { Hypoxia and shock without clear } & 14(24.6 \%) \\ \quad \text { evidence of PE } & \end{array}$

TABLE 2 Improvement with successful weaning of ventilator settings and vasopressors, outcomes and complications

\begin{tabular}{ll}
$\begin{array}{l}\text { Outcomes and complications, } \\
n(\%)\end{array}$ & $\begin{array}{l}\text { Total number of patients } \\
(n=57)\end{array}$ \\
Improvement after tPA infusion & $28(49.1 \%)$ \\
$\begin{array}{l}\text { Bleeding complications within } \\
\quad 36 \text { h of tPA infusion }\end{array}$ & $1(1.8 \%)$ \\
Death & $51(89.5 \%)$ \\
\hline
\end{tabular}

Conclusions: The overall mortality of critically ill COVID-19 patients who received tPA for presumed PE was $89.5 \%$. The utility of tPA for this indicaition warrants further studies.

PB0230 | Anticoagulation with Tinzaparin in COVID-19 Patients in ICU: Are Low Anti-Xa Levels Associated with the Development of Thrombo-embolic Events?

$\underline{\text { R. Van Lancker }}^{1}$; S. Demeester ${ }^{2}$; K. Jochmans ${ }^{2}$; M. Mekeirele ${ }^{1}$

${ }^{1}$ Vrije Universiteit Brussel (VUB), Universitair Ziekenhuis Brussel, Departement of Intensive Care Medicine, Brussels, Belgium; ${ }^{2}$ Vrije Universiteit Brussel (VUB), Universitair Ziekenhuis Brussel, Departement of Haematology, Brussels, Belgium

Background: During the first wave of COVID-19 infections, a significantly higher incidence of thrombo-embolic events (TE) was reported. This led to some international guidelines favouring the administration of a higher dose of low molecular weight heparin $(\mathrm{LMWH})$ as a prophylactic anticoagulation strategy. Despite switching to a dose of $100 \mathrm{IU} / \mathrm{kg}$ tinzaparin in COVID-19 infected patients in our intensive care unit (ICU), we still experienced several ischemic events.

Aims: To establish if anti-Xa plasma levels can determine the development of TE in COVID-19 infected ICU patients.

Methods: After obtaining ethical approval, we retrospectively collected data on anti-Xa levels from all COVID-19 infected ICU patients, treated once daily with tinzaparin, on 2 random dates in November 2020 in UZ Brussel. Samples were obtained $4 \mathrm{~h}$ after injection, as required to measure reliable peak levels. We investigated whether these patients developed a TE within 14 days following sample collection.

Results: We analysed 32 anti-Xa plasma levels from 24 patients, 19 from patients receiving a high prophylactic dose of tinzaparin (median dose = $103 \mathrm{IU} / \mathrm{kg}$ ) and 13 from patients receiving a therapeutic dose (median dose $=177 \mathrm{IU} / \mathrm{kg}$ ). None of the patients in the therapeutic group developed a TE within 14 days, in contrast with 6 patients in the high prophylactic group (Figure 1). All patients with anti-Xa levels below $0.23 \mathrm{IU} / \mathrm{mL}(n=4)$ developed a TE, as opposed to only 2 out of 15 with higher levels. However, due to a wide range in anti-Xa levels and a small sample size, there was no statistically significant difference in anti-Xa levels in patients with and without TE ( $P$-value $=0.123)$. 


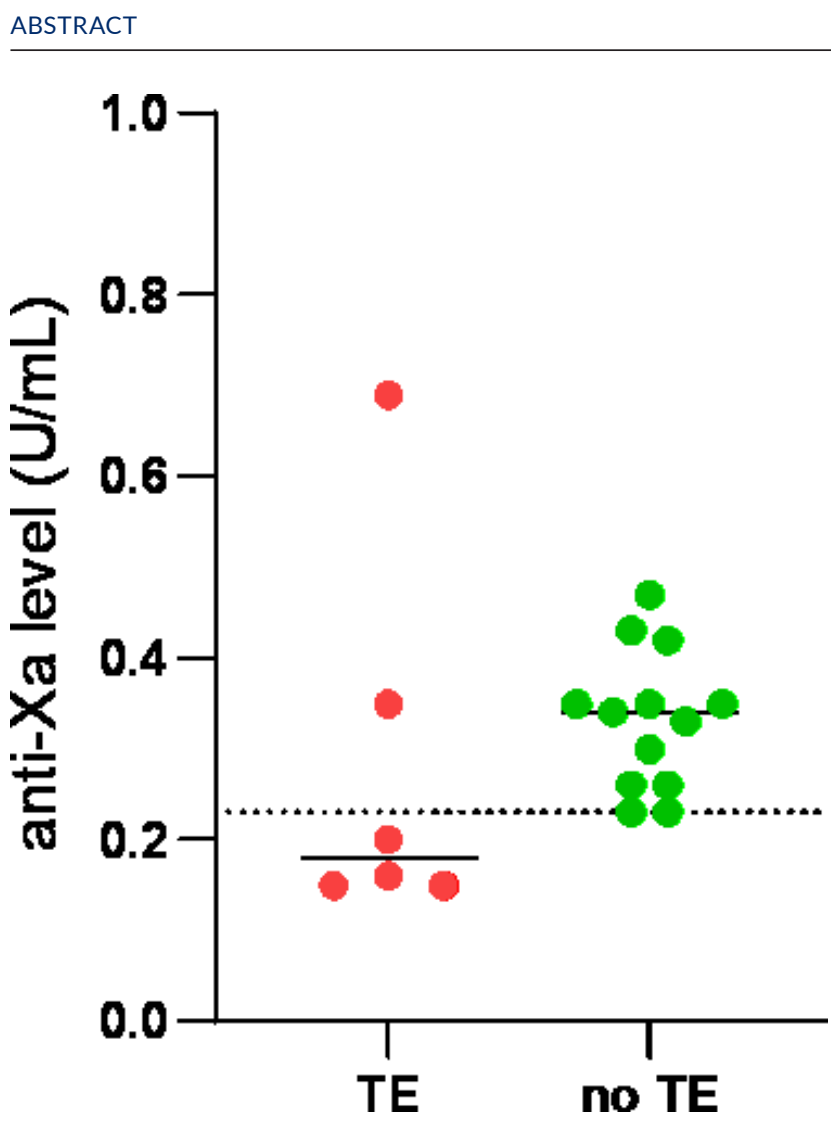

FIGURE 1 Anti-Xa peak levels in COVID-19 infected ICU patients receiving high prophylactic dose of tinzaparin with and without $T E$

Conclusions: Anti-Xa peak levels below $0.23 \mathrm{IU} / \mathrm{mL}$ might predict TE within 14 days in COVID-19 infected ICU patients receiving a high prophylactic dose of LMWH. After validation in larger patient groups, this could lead to integration of systematic anti-Xa level monitoring in future COVID-19 guidelines.
PB0231 | Effect of Dexamethasone on Direct Oral Anticoagulant Plasma Levels in Patients with COVID-19

F. Bosch $^{1,2}$; M. Candeloro ${ }^{3}$; N. Potere ${ }^{3}$; E. Porreca ${ }^{3}$; M. Di Nisio ${ }^{3}$; P.W. Kamphuisen ${ }^{1,2}$

${ }^{1}$ Tergooi Hospitals, Hilversum, Netherlands; ${ }^{2}$ Amsterdam UMC, Location AMC, Amsterdam, Netherlands; ${ }^{3}$ Gabriele D'Annunzio

University, Chieti, Italy

Background: Dexamethasone is part of the standard treatment of COVID-19 patients who need oxygen support. COVID-19 patients have a high risk of venous and arterial thrombosis, therefore adequate anticoagulation is of vital importance. Direct oral anticoagulants (DOACs) are generally not recommended in patients with dexamethasone due to possible drug-drug interactions which may decrease DOACs plasma levels. Therefore, data on the interaction between dexamethasone and DOACs is urgently needed.

Aims: To assess DOAC plasma levels in patients with simoultaneous use of dexamethasone.

Methods: Trough and peak DOAC plasma levels, by means of antiactivated factor $X$ (anti-Xa) were prospectively collected in hospitalized COVID-19 patients treated with dexamethasone and DOACs (apixaban, rivaroxaban and edoxaban) and in hospitalized COVID-19 patients treated with DOACs only, to assess whether these values were within reference range.

Results: Data were collected across two centres in Italy and the Netherlands. A total of 20 patients, 16 with DOACs and dexamethasone and 4 with DOACs only were enrolled. Twelve patients were on anticoagulant treatment for atrial fibrillation, seven for venous thromboembolism, and one for myocardial infarction. In 15 patients DOACs were started during the hospitalization. None of the patients had trough DOAC plasma levels below reference range. Only one patient (6.3\%) treated with rivaroxaban had peak levels below reference range. Six patients (37.5\%) in the dexamethasone group and two control patients (50\%) had peak or trough DOAC plasma levels above reference range. 
TABLE 1 DOAC plasma levels and characteristics per patient

\begin{tabular}{|c|c|c|c|c|c|c|c|c|c|}
\hline $\begin{array}{l}\text { Dexamethasone } \\
\text { use }\end{array}$ & DOAC dose & Indication & $\begin{array}{l}\text { New/ } \\
\text { chronic } \\
\text { users }\end{array}$ & Age & Sex & $\begin{array}{l}\text { Weight } \\
\text { (kg) }\end{array}$ & $\begin{array}{l}\text { eGFR } \\
\text { (mL/min) }\end{array}$ & $\begin{array}{l}\text { DOAC } \\
\text { trough level } \\
(\mathrm{ng} / \mathrm{mL})\end{array}$ & $\begin{array}{l}\text { DOAC } \\
\text { peak level } \\
(\mathrm{ng} / \mathrm{mL})\end{array}$ \\
\hline \multicolumn{10}{|l|}{ Yes } \\
\hline & Apixaban & $\mathrm{AF}$ & New & 88 & $M$ & 78 & 35 & 179 (high) & 227 (high) \\
\hline & $2.5 \mathrm{mg}$ bid & $\mathrm{AF}$ & New & 95 & $M$ & 66 & 19 & 36 & 69 \\
\hline & & $\mathrm{AF}$ & New & 80 & $M$ & 79 & 56 & 150 & 170 \\
\hline & Apixaban & $\mathrm{AF}$ & Chronic & 84 & $\mathrm{~F}$ & 109 & 80 & 122 & 182 \\
\hline & $5 \mathrm{mg}$ bid & VTE & New & 66 & $M$ & 87 & 132 & 32 & 110 \\
\hline & & VTE & New & 82 & $M$ & 69 & 41 & 192 & 308 \\
\hline & Apixaban & & & & & & & & \\
\hline & $10 \mathrm{mg}$ bid & & & & & & & & \\
\hline & & Post AMI & Chronic & 78 & $M$ & 78 & 82 & 41 (high) & 125 \\
\hline & Rivaroxaban & & & & & & & & \\
\hline & $10 \mathrm{mg}$ od & & & & & & & & \\
\hline & & $\mathrm{AF}$ & New & 86 & $\mathrm{~F}$ & 67 & 43 & 52 & 283 \\
\hline & Rivaroxaban & & & & & & & & \\
\hline & $15 \mathrm{mg}$ od & & & & & & & & \\
\hline & Rivaroxaban & $\mathrm{AF}$ & Chronic & 86 & $M$ & 68 & 66 & 81 & 545 (high) \\
\hline & $20 \mathrm{mg}$ od & $\mathrm{AF}$ & New & 83 & $M$ & 90 & 89 & 64 & 306 \\
\hline & & $\mathrm{AF}$ & New & 74 & $M$ & 110 & 75 & 76 & 263 \\
\hline & & $\mathrm{AF}$ & New & 71 & $M$ & 116 & 90 & 26 & 158 (low) \\
\hline & & VTE & New & 77 & $\mathrm{~F}$ & 62 & 88 & 79 & 256 \\
\hline & Rivaroxaban & & & & & & & & \\
\hline & $15 \mathrm{mg}$ bid & & & & & & & & \\
\hline & Edoxaban & VTE & Chronic & 79 & $M$ & 94 & 61 & 64 (high) & 347 (high) \\
\hline & $60 \mathrm{mg}$ od & VTE & New & 84 & $\mathrm{~F}$ & 76 & 61 & 35 & 388 (high) \\
\hline & & VTE & New & 72 & $M$ & 80 & 90 & 28 & 223 \\
\hline \multicolumn{10}{|l|}{ No } \\
\hline & Apixaban & $\mathrm{AF}$ & New & 85 & $\mathrm{~F}$ & 62 & 23 & 99 & 121 \\
\hline & $2.5 \mathrm{mg}$ bid & $\mathrm{AF}$ & Chronic & 90 & $M$ & 73 & 43 & 85 & 91 \\
\hline & & $\mathrm{AF}$ & New & 73 & $M$ & 96 & 28 & 107 & 156 \\
\hline & & VTE & New & 60 & $M$ & 88 & 82 & 61 & 286 \\
\hline & Apixaban & & & & & & & & \\
\hline & $10 \mathrm{mg}$ bid & & & & & & & & \\
\hline
\end{tabular}

Conclusions: In COVID-19 patients, the effect of dexamethasone use on DOAC plasma levels seems limited. This suggests that DOACs can be safely started or continued in COVID-19 patients treated with dexamethasone.
PB0232 | Coagulopathy in Patients with Severe COVID-19

$\underline{\text { R. Cacciola }}^{1}$; E. Gentilini Cacciola ${ }^{2}$; V. Vecchio ${ }^{3}$; E. Cacciola ${ }^{1}$

${ }^{1}$ Haemostasis Unit University of Catania, Catania, Italy; ${ }^{2}$ Infectious Diseases Unit Sapienza University of Rome, Rome, Italy; ${ }^{3}$ University of Catania, Catania, Italy

Background: Coagulopathy has been reported in severe coronavirus disease 2019 (COVID-19) but its pathogenesis remains elusive.

Aims: We assessed the inflammation, endothelial and haemostatic markers including IL-6 and Tumor Necrosis Factor-a (TNFa), Tissue Factor (TF), von Willebrand Factor (VWF), and Tissue Factor Pathway Inhibitor (TFPI) as a natural anticoagulant known to 
attenuate inflammation and vascular thrombosis, and DD, thrombin antithrombin complex (TAT), Fibrinogen (Fib), and Platelet Factor 4 (PF4) and b-Thromboglobulin (b-TG).

Methods: We prospectively enrolled 100 severe $(60$ men, 40 women; mean age 51 years, 32-60) and 60 mild/asymptomatic (40 men, 20 women; mean age 50 years, 31-59) patients COVID-19 reverse transcription polymerase chain reaction (RT-PCR)-confirmed cases. Severe COVID-19 patients had fever, respiratory symptoms and viral pneumonia on computed tomography scan (CT) receiving mechanical ventilation. Our management protocol included antithrombotic prophylaxis with 40 to $60 \mathrm{mg}$ of enoxaparin per day and empirical antimicrobial treatment and antiviral therapy and corticosteroid during the course of hospitalization. IL-6 and TNF-a were measured using multiplex bead array, TF, VWF and TFPI and PF4 and $\beta$-TG by ELISA.

Results: Severe COVID-19 had elevated IL- 6 and TNF-a, and TF VWF and TFPI $(50 \pm 10 \mathrm{pg} / \mathrm{ml}$ and $40 \pm 5 \mathrm{pg} / \mathrm{ml}$ and $2000 \pm 400$ $\mathrm{pg} / \mathrm{ml}, 390 \pm 50 \%$ and $176 \pm 60 \mathrm{ng} / \mathrm{ml}$ ) compared with mild/asymptomatic COVID-19 $(4 \pm 1 \mathrm{pg} / \mathrm{ml}$ and $8 \pm 2 \mathrm{pg} / \mathrm{ml}$ and $19 \pm 2 \mathrm{pg} / \mathrm{ml}$, $51 \pm 8 \%$ and $74 \pm 10 \mathrm{ng} / \mathrm{ml})$ as well as DD, TAT and Fib (540 \pm 100 $\mathrm{mg} / \mathrm{l}, 72 \pm 10 \mathrm{mg} / \mathrm{l}$ and $610 \pm 20 \mathrm{mg} / \mathrm{dl}$ ) compared with mild/asymptomatic COVID-19 (59 $\pm 5 \mathrm{mg} / \mathrm{l}, 2 \pm 1 \mathrm{mg} / \mathrm{l}$ and $175 \pm 10 \mathrm{mg} / \mathrm{l}$ ) and PF4 and b-TG $(160 \pm 63 \mathrm{IU} / \mathrm{ml}$ and $250 \pm 16 \mathrm{IU} / \mathrm{ml})$ compared with mild/asymptomatic COVID-19 ( $3 \pm 2 \mathrm{UI} / \mathrm{ml}$ and $9 \pm 5 \mathrm{IU} / \mathrm{ml})$.

A positive correlation there was between studied parameters $(P<.05)$.

Conclusions: These findings demonstrate that severe COVID-19 has profound inflammation associated to severe endotheliopathy leading increased uncontrolled TFPI haemostatic activity.

\section{PB0233 | Rotational Thromboelastometry (ROTEM) Profiling of} COVID-19 Patients

M. Mitrovic ${ }^{1,2} ;$ N. Sabljic ${ }^{1} ;$ Z. Svetkovic ${ }^{3,2} ;$ N. Pantic ${ }^{1} ;$ Z. Bukumiric ${ }^{4}$; N. Savic ${ }^{5}$ B. Milenkovic ${ }^{6,2}$; M. Virijevic ${ }^{1,2}$; Z. Pravdic ${ }^{1}$; N. Suvajdzic $^{1,2}$; J. Fareed ${ }^{7}$; D. Antic ${ }^{1,2}$

${ }^{1}$ Clinic of Hematology, Clinical Center of Serbia, Belgrade, Serbia;

${ }^{2}$ Faculty of Medicine, University of Belgrade, Belgrade, Serbia; ${ }^{3}$ Clinical Hospital Center Zemun, Belgrade, Serbia; ${ }^{4}$ Institute for Medical Statistics and Informatics, Faculty of Medicine, University of Belgrade, Belgrade, Serbia; ${ }^{5}$ Clinic of Vascular Surgery, Clinical Center of Serbia, Belgrade, Serbia; ${ }^{6}$ Clinic of Pulmology, Clinical Center of Serbia, Belgrade, Serbia; ${ }^{7}$ Departments of Pathology and Pharmacology, Loyola University Center, Maywood, United States

Background: Administration of a standard-dose thromboprophylaxis in all hospitalized Coronavirus disease (COVID-19) patients is recommended. However, despite thromboprophylaxis, frequent thrombotic complications are diagnosed. Rotational thromboelastometry (ROTEM) is a method to access hypercoagulable state in whole blood from these patients.
Aims: To analyze ROTEM parameters during the entire clinical COVID-19 stages including mild, moderate and critical phases and to assess the extent of hypercoagulablity by profiling ROTEM patterns. Methods: We evaluated coagulation abnormalities via traditional tests and ROTEM profile in a group of 94 patients with confirmed SARS-CoV-2 infection with different severity of pneumonia (34 moderate, 25 severe, 35 critical). Shorter than normal clotting time (CT) and higher than normal maximum clot firmness (MCF) in extrinsic rotational thromboelastometry (EXTEM) and fibrinogen rotational thromboelastometry (FIBTEM), shorter than normal EXTEM clot formation time (CFT), and higher than normal a-angle were identified as markers of hypercoagulable state.

Results: At least one hypercoagulable ROTEM parameter had 62 (66\%) patients. Increment in the number of patients with $\geq 2$ hypercoagulable parameters, higher $\operatorname{EXTEM~}(P=.0001)$, FIBTEM MCF $(P=0.0001)$ and maximum lysis decrement $(P=0.002)$ with increment in disease severity was observed $(P=0.0001)$. Significant positive correlations between IL6 and CT EXTEM $(P=0.003)$, MCF $\operatorname{EXTEM}(P=0.033), \operatorname{MCF}$ FIBTEM $(P=0.01)$, and negative with $\mathrm{ML}$ $\operatorname{EXTEM}(P=0.006)$ were seen.

Conclusions: These findings confirm that a hypercoagulable ROTEM profile characterized by clot formation acceleration, high clot strength, and reduced fibrinolysis was more frequent in advanced disease groups and patients with elevated IL6. These results underscore the need for different thromboprophylactic approaches for different severity groups.

\section{PB0234 | Severe SARS-CoV-2 Infection is Associated with Impaired Fibrinolysis: A Single Center Study of the Viscoelastic Properties of Blood Clot}

S. Hammer $^{1}$; H. Häberle ${ }^{2}$; K. Althaus ${ }^{1,3}$; T. Bakchoul ${ }^{1,3}$

${ }^{1}$ Center for Clinical Transfusion Medicine, University Hospital of Tuebingen, Tuebingen, Germany; ${ }^{2}$ Department of Anaesthesiology and Intensive Care, University Hospital of Tuebingen, Tuebingen, Germany;

${ }^{3}$ Transfusion Medicine, Medical Faculty of Tuebingen, Tuebingen, Germany

Background: Growing evidence indicates an association between SARS-CoV-2 infection and dysregulation of the coagulation system. Most reports on COVID-19-associated coagulopathy focused on the increased activation of the plasmatic coagulation system. Recent studies described hypofibrinolysis and the resistance to clot lysis by thromboelastography to be associated with increased risk for thromboembolic complications.

Aims: In this study we systematically analyze the viscoelastic changes in clotting system with a focus on plasmin resistance.

Methods: Consecutive 29 COVID-19 patients admitted to normal wards or to the intensive care unit (ICU) were included in this study. Whole blood samples were investigated by using a new viscoelastic test system to assess coagulation and fibrinolysis. Additionally, standard routine coagulation testing and immunoassays for 
factors of fibrinolysis as plasminogen activator inhibitor-1 (PAI-1), tissue plasminogen activator (tPA), plasminogen activity and alpha 2-antiplasmin (A2AP) were performed.

Results: Significantly elevated resistance to clot lysis and longer time of lysis (LT) after adding tissue plasminogen activator were observed in blood samples from ICU COVID-19 patients compared to healthy controls (LT: $365.7 \mathrm{~s} \pm 44.6$ vs. 193.2s $\pm 16.3, P=0.0014)$. PAI-1 activity was significantly higher in plasma samples of ICU COVID-19 patients. Most importantly, a positive correlation was found between the activity of PAI-1 and the lysis time of the formed clot $(r=0.70$, $P=0.0006)$.

Conclusions: Our data support that severe SARS-CoV-2 infection is associated with impaired fibrinolysis in blood, where fibrinolytic inhibitors are elevated leading to an increased resistance to clot lysis. Thromboelastography could be useful to investigate the contribution of the fibrinolytic status to the procoagulatory condition in COVID-19.

PB0235 | Fibrinogen and D-dimer Are Significantly Associated with Severity of COVID-19 Disease

M. El Horri; S.N.E. Touati; I. Khachaa; A. Chikh Khelifa; A. Berrah; L. Benmahdi; S.E. Belakehal

Military University Hospital of Oran, Oran, Algeria

Background: Since the emergence of COVID-19 pandemic, a growing evidence showed that this disease is associated with disturbances in hemostatic components, defining a COVID-19 associated coagulopathy. This is reflected in the lab tests with an increase in D-dimer and Fibrinogen, which correlate with severity and outcomes of disease.

Aims: To assess the association between elevation of fibrinogen and d-dimer with worsening of COVID-19 disease, as well as the prognostic value of these two markers.

Methods: We conducted a prospective single-center study, including patients with confirmed COVID-19 consecutively admitted over a period of 05 months. Fibrinogen and D-Dimer were tested and the correlation with clinical stages, and prognosis were studied.

Results: 131 patients with confirmed COVID-19 (Mean age 47 years; $13 \%$ with severe and critical cases; $73.3 \%$ male) were included in this study. The most frequent comorbidities were : high blood pressure $27.9 \%$ and diabetes $23.5 \%$.

Levels of D-Dimer and Fibrinogen increased considerably in severe and critical cases compared to mild, moderate and asymptomatic cases. For Fibrinogen: 4.81 gr/L [Cl 4.40 - 5.22] Vs 3.53 gr/L [Cl 3.28

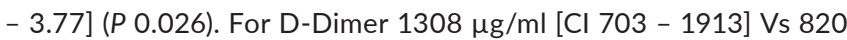
$\mu \mathrm{g} / \mathrm{ml}[\mathrm{Cl} 338$ - 1302] (P 0.0001).

The results from ROC analyses for predicting severe and critical cases showed that the AUC of Fibrinogen the AUC was at 0.75 also with two cut-off values : $4.46 \mathrm{gr} / \mathrm{L}$ (Se : $67.9 \%$, Sp : 78.2\%) and 5.49 gr/L (Se : 34\%, Sp : 97.4\% ). For D-Dimer AUC was 0.7, with two cutoff values : $750 \mu \mathrm{g} / \mathrm{ml}$ (Se : $56.8 \%$, Sp : $78.72 \%$ ) and $1790 \mu \mathrm{g} / \mathrm{ml}$ (Se : $24.32 \%, \mathrm{Sp}:$ : $95.7 \%)$.

TABLE 1 Association between increased levels of Fibrinogen, D-Dimer and COVID-19 severity

\begin{tabular}{|c|c|c|}
\hline & Severe and critical cases & Asymptomatic, mild and moderate cases \\
\hline $\mathrm{N}$ & $17(13 \%)$ & $114(87 \%)$ \\
\hline Fibrinogen mean (gr/L) & $4.81 \mathrm{gr} / \mathrm{L}[\mathrm{Cl} 4.40-5.22]$ & $3.53 \mathrm{gr} / \mathrm{L}[\mathrm{Cl} 3.28-3.77]$ \\
\hline$P$ & \multicolumn{2}{|l|}{0.026} \\
\hline \multirow[t]{3}{*}{ Fibrinogen Cut-off values } & \multicolumn{2}{|l|}{$A \cup C=0.756$} \\
\hline & \multicolumn{2}{|c|}{ 1st Cut-off value : $4.46 \mathrm{gr} / \mathrm{L}$ (Se 67.9\% Sp 78.2\% VPP 31.8\% VPN 94.2\%). } \\
\hline & \multicolumn{2}{|c|}{ 2nd cut-off value : 5.49 gr/L (Se 34\% Sp 97.4\% VPP 66.4\% VPN 90.8\%). } \\
\hline D-Dimer mean $(\mu \mathrm{g} / \mathrm{ml})$ & $1308 \mu \mathrm{g} / \mathrm{ml}[\mathrm{Cl} 95 \%: 703-1913]$ & $820 \mu \mathrm{g} / \mathrm{ml}[\mathrm{Cl} 95 \%: 338-1302]$ \\
\hline$P$ & \multicolumn{2}{|l|}{0.0001} \\
\hline \multicolumn{3}{|l|}{ D-Dimer Cut-off values } \\
\hline & \multicolumn{2}{|l|}{$A \cup C=0.700$} \\
\hline & \multicolumn{2}{|c|}{ 1st Cut-off value : 750 g/ml (Se 56.8\% Sp 78.7\% VPP 28.5\% VPN 92.4\%). } \\
\hline & \multicolumn{2}{|c|}{ 2nd cut-off value : 1790 g/ml (Se 24.32\% Sp 95.74\% VPP 46.1\% VPN 89.4\%). } \\
\hline
\end{tabular}




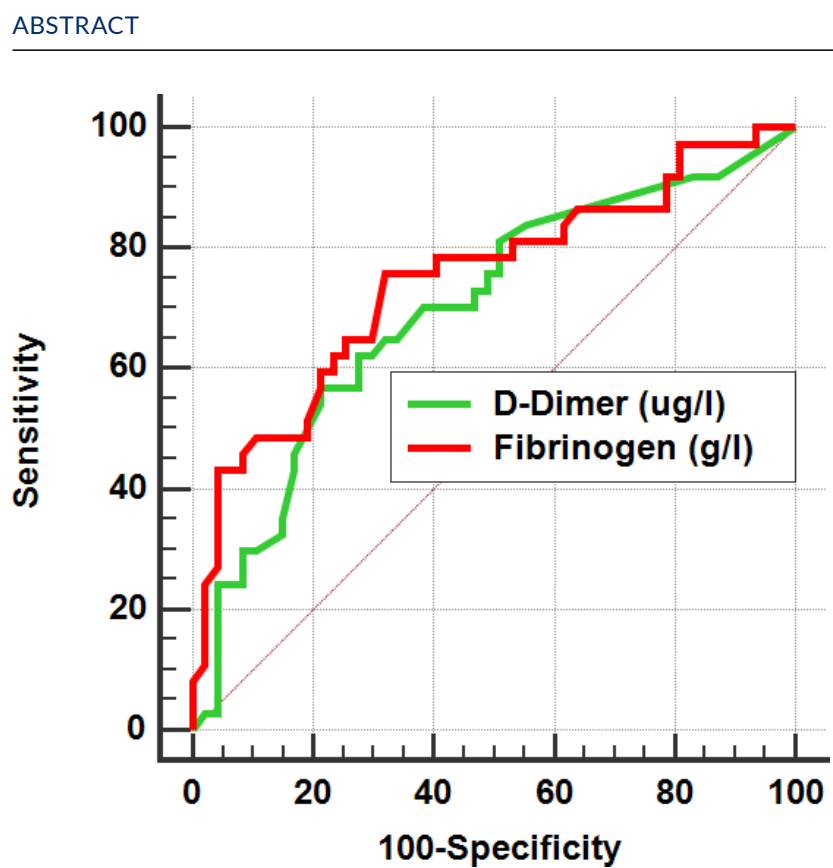

FIGURE 1 The relationship between Fibrinogen, D-Dimer and COVID-19 severity. ROC curve of Fibrinogen and D-Dimer in predicting COVID-19 severity

Conclusions: Fibrinogen and D-dimers in patients with COVID-19 should be used as prognostic markers for warning of severe cases.

PB0236 | Prognostic Coagulation Parameters in COVID-19 Patients at the ICU

S. van de Leur; S. Spanjersberg; E. ter Haar

Isala, Zwolle, Netherlands

Background: Studies have shown that thrombotic complications and coagulopathy occur more frequently in ARDS caused by COViD-19 than with other pathogens. This coagulopathy is associated with a higher morbidity and mortality at the ICU.

Aims: In this study we aimed to find the most prognostic coagulation factors for the incidence of thrombotic complications, duration of admittance to the ICU and mortality.

Methods: In a retrospective cohort study blood samples of 55 COVID-19 patients at our ICU were frozen for further research. In our pilot study von Willebrand activity (vWFAc) and antigen (vWFAg) , Factor VIII activity (FVIII), the D-dimer and ADAMTS-13 showed the most promising parameters. Medical data were extracted from the patient files.

Results: The VWFAc, the VWFAg, the FVIII and the D-dimer were more than fivefold higher than our reference values. High values of VWFAc, vWFAg and FVIII midway and the end of the ICU admittance were significant predictors of mortality (Figure 1). Higher values of these coagulation parameters correlated with a longer stay on the ICU and gave a high risk at thrombotic compications.

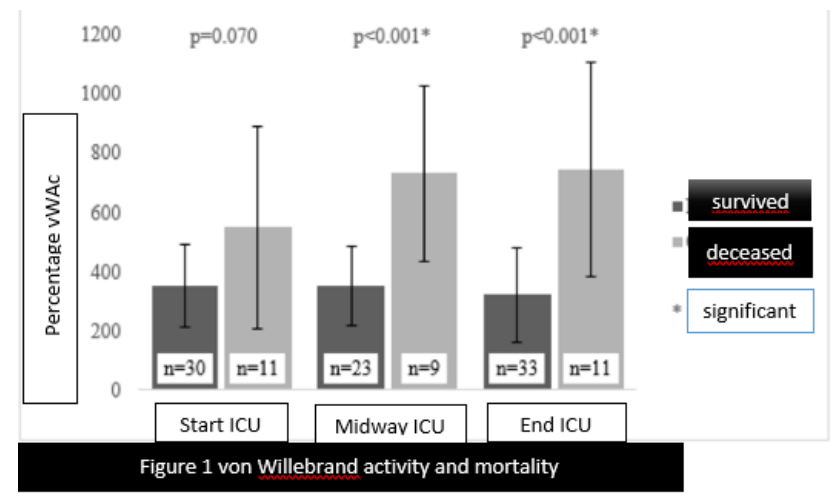

FIGURE 1 von Willebrand activity and mortality at the ICU

Conclusions: vWFAc,.vWFAg and FVIII are significant predictors of mortality for patients with COVID-19 infections at the ICU. There is also a higher risk for thrombotic complications and a longer hospitalization.

PB0237 | Thrombosis and Bleeding in Critically III COVID-19 Patients at Higher than Prophylactic Dose of Heparins

A. Mavri ${ }^{1}$; M. Lukić ${ }^{2}$; M. Mavrič ${ }^{2}$; N. Šoštarič ${ }^{2}$; D. Zupančič ${ }^{2}$

${ }^{1}$ Department of Vascular Diseases, University Medical Centre Ljubljana, Ljubljana, Slovenia; ${ }^{2}$ Department of Infectious Diseases, University

Medical Centre Ljubljana, Ljubljana, Slovenia

Background: COVID-19 pneumonia is associated with high rates of thromboembolic complications particularly in patients on intensive care unit (ICU). Optimal intensity of pharmacological thromboprophylaxis remains unclear.

Aims: To evaluate incidence of venous thromboembolism (VTE) and major bleeding in critically ill COVID-19 patients.

Methods: We included 92 consecutive patients admitted to one ICU of our hospital between October 2020 and March 2021. All patients required invasive mechanical ventilation. In 5 patients pulmonary embolism (PE) was confirmed on admission and they received a therapeutic dose of dalteparine. Therapeutic dalteparin or unfractionated heparin was used in some patients with a history of thromboembolism or renal failure. The majority of other patients were treated with a half-therapeutic dalteparin dose. Only 8 patients received prophylactic dalteparin in one patient thromboprophylaxis was omitted due to severe thrombocytopenia (Table 1). Compression ultrasonography of femoral, axillar and jugular veins was performed weekly until ICU discharge. CT angiography of the thorax was performed only if clinically indicated.

Results: We diagnosed 30 thromboembolic events; 1 arterial embolism and 29 VTE: 4 PE (4\%), 24 (26\%) catheter-related and 1 spontaneous venous thrombosis (VT). Two patients with catheter-related VT suffered segmental PE. The rates of major bleeding and mortality are shown in Table 1. Among patients with PE on admission no one suffered recurrent VTE or major bleeding, 1 died. 
TABLE 1 Characteristics of patients with venous thrombembolism (VTE) and without VTE during ICU stay. Results are shown as mean and standard deviation or as percentage

\begin{tabular}{|c|c|c|c|}
\hline & $\begin{array}{l}\text { All admitted patients } \\
N=92\end{array}$ & $\begin{array}{l}\text { Patients with VTE during } \\
\text { hospitalization } \\
N=29\end{array}$ & $\begin{array}{l}\text { Patients without VTE during } \\
\text { hospitalization } \\
N=58\end{array}$ \\
\hline Age (years) & $67 \pm 13$ & $67 \pm 11$ & $68 \pm 12$ \\
\hline Male (\%) & $67(73)$ & $22(76)$ & $41(71)$ \\
\hline VTE on admission & 5 & 0 & - \\
\hline \multicolumn{4}{|l|}{ Heparins: } \\
\hline Dalteparin: half-therapeutic (\%) & $68(73.9)$ & $21(72.4)$ & $47(81.0)$ \\
\hline Dalteparin/UH: therapeutic & $14(15.2)$ & $3(11.4)$ & $7(12.1)$ \\
\hline Major bleeding & $9(9.8)$ & $5(17.2)$ & $4(6.9)$ \\
\hline In-hospital mortality & $27(29.3)$ & $10(34.4)$ & $16(27.5)$ \\
\hline ICU length of stay (weeks) & $4 \pm 2$ & $5 \pm 3$ & $3 \pm 2$ \\
\hline
\end{tabular}

$\mathrm{BMI}$ - body mass index, UH-unfractionated heparin

Conclusions: Almost one-third of critically ill COVID-19 patients suffered VTE, despite using a half-therapeutic dose of dalteparin. Most of the events were catheter-related VT and in those patients risk of major bleeding increases after therapeutic anticoagulation with dalteparin. The mortality rate was similar between those who developed VTE and those who did not.

PB0239 | Hemostastatic Alterations with Disease Severity in the Early Symptomatic Phase of COVID-19

S. Huber ${ }^{1}$; S. Montalvão ${ }^{1}$; S. Martins ${ }^{1}$; L. da Silva ${ }^{1}$;

C. Bacchin ${ }^{1}$; N. Santos ${ }^{1}$; T. Diaz ${ }^{2}$; C. Wroclawsk ${ }^{3}$; C. Filho ${ }^{3}$;

E. de Paula ${ }^{1} ; \underline{\text { J. Annichino-Bizzacchi }}{ }^{1}$

${ }^{1}$ State University of Campinas - UNICAMP, Campinas, Brazil; ${ }^{2}$ Anhembi Field Municipal Hospital, São Paulo, Brazil; ${ }^{3}$ Sirio Libanes Hospital, São Paulo, Brazil

Background: The main factors associated with disease severity in COVID-19 are age, sex, body weight, hypertension, and diabetes. Biomarkers of hemostatic activation have been shown to be independent predictors of disease severity in different populations of inpatients.

Aims: To evaluate whether biomarkers of hemostatic activation were associated with clinical outcomes in patients admitted to a field hospital, set up to provide initial care to patients in the early symptomatic phase of COVID-19.

Methods: Data and samples were obtained from June to September 2020. Laboratory evaluation included complete blood counts, PT, aPTT, fibrinogen, D-dimer, factor VIII activity, Von Willebrand
Factor (VWF) (activity and antigen), $\mathrm{C}$ reactive protein (CRP) and $\mathrm{P}$ selectin (ELISA). Patients were segregated by outcome, with clinical deterioration defined as need for ICU, mechanical ventilation, pulmonary embolism, deep vein thrombosis or death.

Results: In total 209 were enrolled in the study, of which 24 presented clinical deterioration (11.5\%). Clinical data are described in Table 1. No differences could be observed between patient subgroups regarding the presence of fever (63.2\% vs. 62.5\%), dry cough (75.1\% vs. $87.5 \%)$ and dyspnea (65.9\% vs. $54.2 \%)$ at admission. As main comorbidities, the groups presented chronic obstructive pulmonary disease $(2.2 \%$ vs $8.3 \%$ ), asthma ( $3.2 \%$ vs $4.2 \%)$, chronic heart failure ( $1.1 \%$ vs $8.3 \%$ ), arterial hypertension ( $46 \%$ vs $41.7 \%$ ) and diabetes ( $28.1 \%$ vs $33.3 \%$ ) in comparing improved with clinical deterioration patients. Laboratory markers of hemostatic activation are shown in Table 2. In general, it was verified a significant decrease in platelet number, and an increase in the parameters of aPTT, CRP, vWF antigen and ristocetin cofactor. 
TABLE 1 Clinical data of improved and clinical deterioration patients

$\begin{array}{lll} & \text { Improved } & \text { Clinical derioration } \\ & N=185 & N=24 \\ \text { Sex F/M } & 89(48.1 \%) / 96(51.9 \%) & 10(41.7 \%) / 14(58.3 \%) \\ \text { Mean Age } \pm \text { SD } & 53.6 \pm 14.0 & 58.1 \pm 13.6 \\ \text { BMI } & 30.3 \pm 5.6 & 30.0 \pm 5.2 \\ \text { Smoking } & 6(3.2 \%) & 0 \\ \text { Alveolar Infiltrate } & 76(41.1 \%) & 12(50 \%) \\ \text { Mean time of initial symptoms and RT-PCR exam (days) } & 5.7 \pm 4 & 4.6 \pm 2.8 \\ \text { Mean time symptoms to Hospitalization (days) } & 12.1 \pm 5.7 & 11.0 \pm 5.0\end{array}$

TABLE 2 Laboratory markers of hemostatic activation and inflammation in COVID-19 patients

\begin{tabular}{llll} 
& Improved & Clinical derioration & \\
& $N=185$ & $N=24$ & $P^{* *}$ \\
Platelet Number $\left(\times 10^{3}\right.$ cells/uL) & $256 \pm 108.1$ & $203.8 \pm 92.4$ & 0.0426 \\
CRP $(\mathrm{mg} / \mathrm{L})$ & $64.2 \pm 65.6$ & $98.3 \pm 90.9$ & 0.0450 \\
aPTT $(\mathrm{s})$ & $29 \pm 10.0$ & $33.7 \pm 5.8$ & 0.0084 \\
VWF antigen $(\mathrm{UI} / \mathrm{dL})$ & $333.2 \pm 139.2$ & $411.4 \pm 106.4$ & 0.0022 \\
vWF ristocetin cofactor $(\mathrm{UI} / \mathrm{dL})$ & $270 \pm 111$ & $324.4 \pm 95.2$ & 0.0032 \\
\hline
\end{tabular}

Conclusions: Our results demonstrate that hemostasis activation is associated with clinical deterioration even at the early phases of COVID-19.

Supported by FAPESP number 2016/1417-2. Approved by Ethics Research Committee of Unicamp.

PB0240 | The Comparison of Different Coagulation Markers in Thrombosis Prediction in COVID-19 Pneumonia

M. Krykhtina; K. Bielosludtseva; T. Pertseva; L. Konopkina

Dnipropetrovsk Medical Academy, Dnipro, Ukraine

Background: Coagulation disturbances in COVID-19 pneumonia patients is already a well-known fact, but what marker can really reflect the disease progression is still unknown.
Aims: The aim was to estimate the diagnostic and prognostic role of different coagulation markers in hospitalized patients with confirmed COVID-19 pneumonia.

Methods: The main group was 53 patients (age - 59 (52; 65), men - 22 (41.5\%)) with COVID-19 pneumonia, divided into 3 subgroups: subgroup 1 - 36 patients with moderate COVID-19 pneumonia, subgroup 2 - 12 patients with severe COVID-19 pneumonia, subgroup 3 - 5 patients with critical COVID-19 pneumonia. General clinical analysis, blood coagulation test, determination of D-dimer and fibrinogen was performed at admission before starting of antibacterial and anticoagulant treatment.

Results: Individual analysis showed that 28 patients from group 2 and 3 had progression of COVID-19 pneumonia in dynamic, which was manifested with increased respiratory failure and connected with lung vessel thrombosis.

The ROC-analysis showed that only the level of D-dimer at admission can really reflects the severity of COVID-19 pneumonia and predicts the prognosis of the disease.

TABLE 1 Levels of coagulation parameters in hospitalized patients with COVID-19 pneumonia

\begin{tabular}{|c|c|c|c|c|c|}
\hline & Subgroups & & & & \\
\hline Parameter & $\begin{array}{l}1 \\
(n=36)\end{array}$ & $\begin{array}{l}2 \\
(n=12)\end{array}$ & $\begin{array}{l}3 \\
(n=5)\end{array}$ & References & $P$ \\
\hline INR & $\begin{array}{l}1.06 \\
(0.95 ; 1.2)\end{array}$ & $\begin{array}{l}1.05 \\
(1.0 ; 1.1)\end{array}$ & $\begin{array}{l}1.1 \\
(1.01 ; 1.2)\end{array}$ & $0.8-1.2$ & $\begin{array}{l}P_{1-2}=0.910 \\
P_{1-3}=0.480 \\
P_{2-3}=0.386\end{array}$ \\
\hline
\end{tabular}


Subgroups

1

Parameter

prothrombin, \%

prothrombine time, $s$

13.5

$(12.2 ; 14.5)$

3.7

$(3.0 ; 4.7)$

D-dimer, $\mathrm{ng} / \mathrm{ml}$

679.9

(118.1;

170.0)
2

$(n=12)$

94.5

(76.2; 103.1)

13.6

$(12.1 ; 14.1)$

4.2

$(3.7 ; 4.9)$

1103.1

(188.9;

1803.5)
3

$(n=5)$

81.4

(72.0; 90.0)

15.2

(93.0;

7.0

$(6.5 ; 7.5)$

5720

$0-285$

(4800; 7500)

\section{References $P$}

80-120

$P_{1-2}=0.900$

$P_{1-3}=0.197$

$P_{2-3}=0.102$

$P_{1-2}=0.830$

$P_{1-3}=0.183$

$P_{2-3}=0.342$

$P_{1-2}=0.044$

$P_{1-3}=0.033$

$P_{2-3}=0.015$

$P_{1-2}=0.149$

$P_{1-3}=0.000$

$P_{2-3}=0.009$

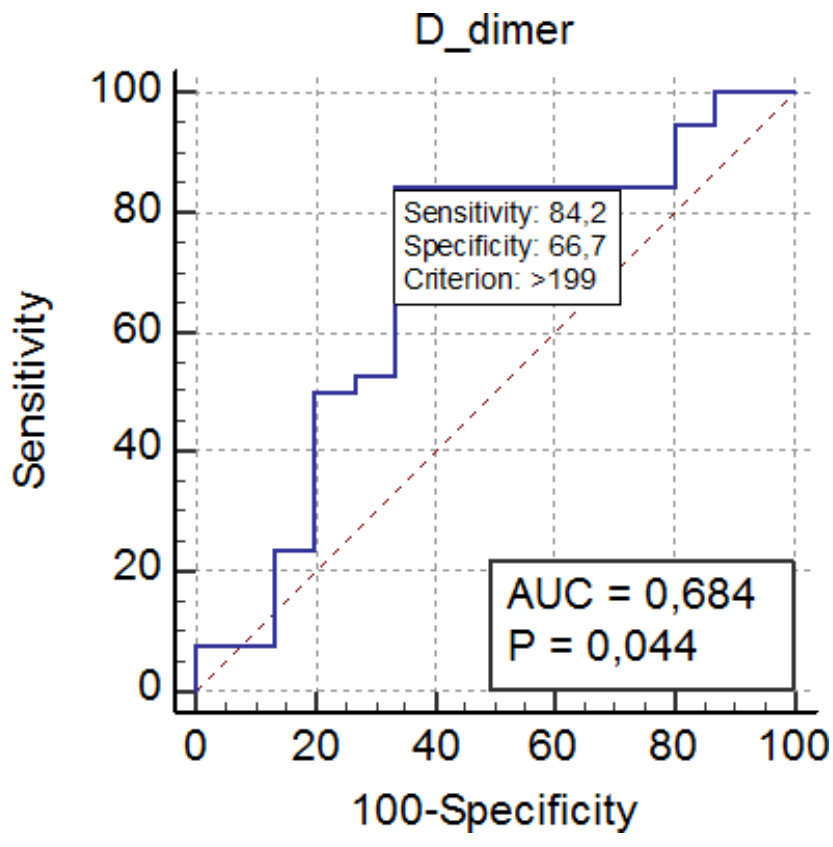

FIGURE 1 ROC-curve of D-dimer

Conclusions: (1) common coagulation parameters neither reflect severity no predict thrombosis in hospitalized patients with COVID-19; (2) Increasing of D-dimer on level $200 \mathrm{ng} / \mathrm{ml}$ at admission not only reflects the severe or critical course of in hospitalized patients with COVID-19, but also associated with lung vessel thrombosis and predicts the progression of disease.
PB0241 | Efficiency of Anticoagulant Protein C System in Patients with Coronavirus Infection Caused by the SARS-CoV-2

O. Matvienkoํㅜ N. Korsakova ${ }^{1}$; A. Lerner ${ }^{2}$; T. Shvedova ${ }^{2}$; L. Papayan ${ }^{1}$; O. Golovina ${ }^{1}$

${ }^{1}$ Russian Research Institute of Hematology and Transfusiology, Saint Petersburg, Russian Federation; ${ }^{2}$ Vsevolozhsk Clinical Interdistrict Hospital, Vsevolozsk, Russian Federation

Background: The concept of immunothrombosis has established as a central pathogenic factor leading to thrombosis complications, respiratory failure, and a multiple organ failure in patents with COVID-19. Studying of the hypercoagulability in patients with COVID-19 could be useful for improving of disease's outcomes. Estimation of anticoagulants efficiency can be informative for thrombotic risk assessment. The highest interest presents protein $C$ system, because, as we know, infection and inflammation lead to disfunction of this anticoagulant system.

Aims: To estimate the efficiency of protein $C$ anticoagulant system in COVID-19 patients.

Methods: The study included 60 patients with COVID-19 and 21 healthy controls. Thrombin generation was assessed by CAT according to Hemker et al. Measure was conducted in platelet poor plasma with or without presence of thrombomodulin. The following parameters were determined: endogenous thrombin potential (ETP, $\mathrm{nM}^{*}$ min), peak thrombin (Peak, nM). Sensitivity ETP and Peak for thrombomodulin were calculated as percent of decreasing of these parameters after adding thrombomodulin to the assay. Reduction of sensitivity for thrombomodulin indicates dysfunction of anticoagulant protein $\mathrm{C}$ system. Protein $\mathrm{C}$ activity and free protein $\mathrm{S}$ level were measured by " $\mathrm{ACL}$ ELIT PRO", Instrumentation Laboratory, USA. STATISTICA 12.0 package was used. Results are presented as median with 95\% confidence intervals, $P<0.05$ was considered statistically significant $\left({ }^{*}\right)$.

Results: Sensitivity ETP and Peak for thrombomodulin, protein C activity and free protein $\mathrm{S}$ level are presented in the table. 
TABLE 1 Sensitivity ETP and Peak for thrombomodulin, protein C activity and free protein $\mathrm{S}$ level

\begin{tabular}{lll} 
Parameters & Patients $(\boldsymbol{n}=60)$ & Controls $(\boldsymbol{n}=\mathbf{2 1})$ \\
Sensitivity ETP $(\%)$ & $11.0(0.0-61.3)^{*}$ & $51.5(22.5-65.5)$ \\
Sensitivity Peak (\%) & $4.0(0.0-39.0)^{*}$ & $35.5(14.6-53.8)$ \\
Protein C activity (\%) & $99.0(62.5-132.6)$ & $102.0(76.6-145.0)$ \\
Free protein S level (\%) & $64.2(34.1-106.5)^{*}$ & $79.0(58.3-112.0)$ \\
\hline
\end{tabular}

Sensitivity ETP and Peak for thrombomodulin were vastly lower in patients with COVID-19. Free protein S level was significantly reduced in patient's group, protein $C$ activity did not differ in the groups.

Conclusions: The efficiency of protein $\mathrm{C}$ anticoagulant system is depressed in COVID-19 patients. It may be associate with protein $\mathrm{S}$ level decreasing. The disability of anticoagulant protein $\mathrm{C}$ system can lead to hypercoagulability and it may be cause of thrombotic complication.

PB0242 | Indices of the Hemostasis System in Patients with COVID-19 Depending on the Outcome

E. Klychnikova; S. Petrikov; K. Popugaev; E. Tazina; S. Badygov N.V. Sklifosovsky Research Institute for Emergency Medicine, Public Healthcare Institution of Moscow Healthcare DepartmentN.V. Sklifosovsky Research Institute for Emergency Medicine, Public Healthcare Institution of Moscow Healthcare Department, Moscow, Russian Federation
Background: According to the literature, patients with COVID-19 who has been observed severe disorders in the hemostasis system more often had an unfavorable outcome.

Aims: To compare the indices of the hemostasis system in patients who had COVID-19 with favorable outcome and unfavorable outcome.

Methods: A prospective study of 122 patients (men was 53\%, women was $47 \%$ ) with a severe course of COVID-19 was carried out. The age was $59 \pm 12$ years. The patients' condition, assessed by the NEWS and SOFA severity score, was $4.5 \pm 1.4$ and $6.1 \pm 1.3$ points, respectively. The patients were separated in 2 groups:

1 group (61 people) - "survivors",

2 group (61 people) - "dead persons".

The control group (norm) consisted of 20 apparently healthy donors. The research was performed on day 1 and 7 from the moment of admission to the hospital.

Results: The differences in APTT between two groups were no significant. The difference between two groups in PT's values was significant on day 1 and on day 7. The difference between two groups in FG's level was significant on day 7. The difference between two groups in D-dimer's level was significant both on day 1 and day 7 of the research. The difference between two groups of patients in the activity of AT III was significant on day 7. The difference between two groups of patients in the activity of $\operatorname{Pr} C$ was significant on day 1. The difference between two groups in platelet count was significant on day 7 (Table 1).

TABLE 1 Indices of the hemostasis system in patients with COVID-19 depending on the outcome

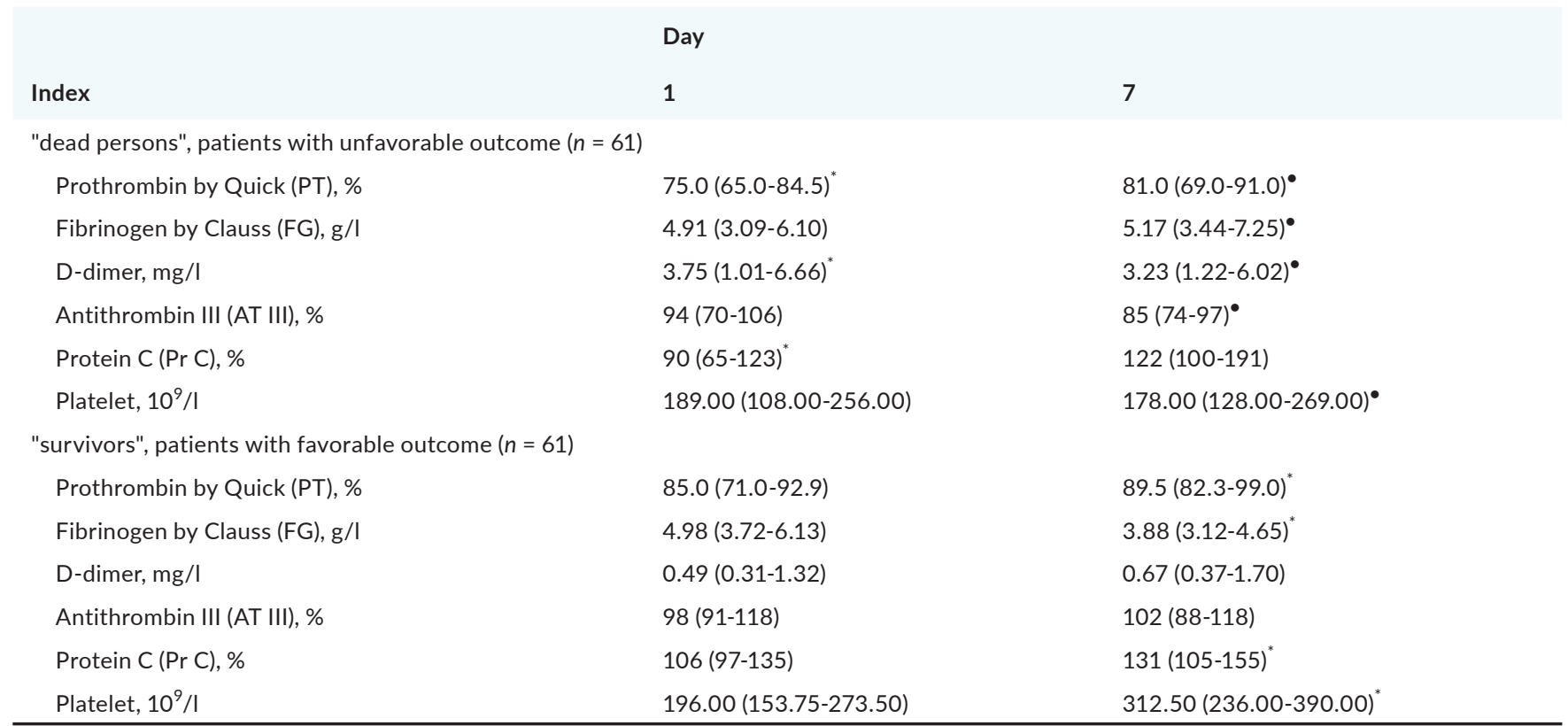

Note: ${ }^{*} P<0.05$ relative to the group of "Survivors, day 1 "

- $P<0.05$ relative to the group of "Survivors, day 7" 
Conclusions: In patients with a severe course of COVID-19 unfavorable factors in the development of the outcome were:

1. High level of D-dimer, decrease of PT and protein C on day 1 of the study.

2. Decrease of platelet count and antithrombin III, increase of Ddimer and fibrinogen on day 7 after admission.

\section{PB0243 | Influence of C-reactive Protein on Thrombin}

\section{Generation Assay}

M. Didembourg ${ }^{1}$; J. Douxfils ${ }^{1,2} ;$ F. Mullier $^{3}$; M. Hardy ${ }^{4}$;

J. Favresse ${ }^{1,5}$; L. Morimont ${ }^{1,2}$

${ }^{1}$ University of Namur, Faculty of Medicine, Department of Pharmacy, Namur, Belgium; ${ }^{2}$ Qualiblood sa, Namur, Belgium; ${ }^{3}$ Université Catholique de Louvain, CHU UCL Namur, Laboratory Hematology, Yvoir, Belgium; ${ }^{4}$ Université Catholique de Louvain, CHU UCL Namur, Department of Anesthesiology, Yvoir, Belgium; ${ }^{5}$ Clinique Saint-LuC Bouge A.S.B.L 'Santé et Prévoyance', Bouge, Belgium

Background: C-reactive protein (CRP) is an acute phase reactant plasma protein considered as biomarker representative of the burden of inflammation also linked to the development of prothrombotic states. In moderate to severe COVID-19 patients, persistent elevated levels of CRP were observed contributing to the risk of venous thromboembolic events. CRP is known to artefactually interfere with certain coagulation assays. As the use of global coagulation test like thrombin generation assay (TGA) may be relevant to characterize the evolution of the disease or identify its severity, it is interesting to investigate the impact of CRP on TGA.

Aims: This study aimed to assess how CRP impacts TGA on the automated ST Genesia system, using the STG-ThromboScreen- TM as triggering reagent. A comparison with the Calibrated Automated Thrombogram (CAT) system was performed.

Methods: Normal pooled plasma (NPP) constituted of 50 healthy individuals was used as the matrix. Human CRP (Merk, Germany) was spiked in NPP at five relevant plasma concentrations (0 [=Phosphate buffer saline], 50, 100, 200 and $350 \mathrm{mg} / \mathrm{L}$ ). Both TGA methods were performed in duplicate and assessed by 3 independent runs.

Results: Based on mean values, no statistically significant difference was observed between the five tested concentrations ( $P$-value $>0.05$ ), regardless of the platform used. On the other hand, the comparison between both analyzers showed significant differences for lag-time and time-to-peak, which were significantly higher when TGA was performed on the CAT system. These differences are possibly associated with the specific algorithm of each platform.

Conclusions: This study demonstrated that CRP levels up to $350 \mathrm{mg} / \mathrm{L}$ did not impact significantly thrombin generation performed either on CAT or on ST Genesia system. Therefore, TGA could be an efficient test to assess the hemostatic function of patients with elevated CRP, like those in sepsis and suffering from chronic or acute inflammatory conditions, such as COVID-19.
PB0244 | Global Haemostatic Tests Demonstrate the Absence of Hypercoagulability in Non-supplemental Oxygen Dependent COVID-19 Patients

B.E. Fan $^{1,2,3,4}$; C.L.L. Sum ${ }^{5}$; R.M. Lee ${ }^{6,3,4}$; J.Y. Lim ${ }^{6,3,4}$; C.F. Bok ${ }^{7,8}$; B.E. Young, ${ }^{7,8,3,4}$; C.B. Lye ${ }^{7,8,3,4}$; Y.W. Chia ${ }^{9,3,3,4}$; K.H. Ong ${ }^{1,2,3,4}$ ${ }^{1}$ Department of Haematology, Tan Tock Seng Hospital, Singapore, Singapore; ${ }^{2}$ Department of Laboratory Medicine, Khoo Teck Puat Hospital, Singapore, Singapore; ${ }^{3}$ Lee Kong Chian School of Medicine, Nanyang Technological University, Singapore, Singapore; ${ }^{4}$ Yong Loo Lin School of Medicine, National University of Singapore, Singapore, Singapore; ${ }^{5}$ Department of Laboratory Medicine, Tan Tock Seng Hospital, Singapore, Singapore; ${ }^{6}$ Department of Anaesthesiology, Intensive Care and Pain Medicine, Tan Tock Seng Hospital, Singapore, Singapore; ${ }^{7}$ Department of Infectious Diseases, Tan Tock Seng Hospital, Singapore, Singapore; ${ }^{8}$ National Centre for Infectious Diseases, Singapore, Singapore; ${ }^{9}$ Department of Cardiology, Tan Tock Seng Hospital, Singapore, Singapore

Background: Critically-ill COVID-19 patients demonstrate a hypercoagulable state, hence necessitating thromboprophylaxis. However, in non-critically ill COVID-19 patients, the haemostatic profile is unknown.

Aims: A prospective, observational study was performed to evaluate coagulation parameters, and thrombotic outcomes in critically and non-critically ill COVID-19 patients.

Methods: Informed consent was obtained from 10 critically ill (oxygen dependent, $\mathrm{PaO} 2 / \mathrm{FiO} 2$ ratio<300) PCR positive COVID-19 patients matched for age and gender with 10 non-critically ill patients (nonoxygen dependent). On recruitment, laboratory (FBC/LDH/CRP/ procalcitonin) and coagulation tests (PT/APTT/D-Dimer/Fibrinogen/ TCT/Factors II,V,VII,VIII,IX,X,XI/vWF/anti-thrombinIII/ProteinC/ ProteinS/antiphospholipid antibodies), Thromboelastography(TEG), Clot Waveform Analysis(CWA) were performed, with repeat TEG/ CWA every 3 days, till 21 days of admission or discharge. This study was DSRB approved and supported by an NHG-NCID grant.

Results: The median age was 60 years $(49.5,64.5)$ with 16 males and 4 females. Median Padua score of critically ill patients was 5 with $\mathrm{PaO} 2 / \mathrm{FiO} 2$ ratio 194.5 (174, 241). Hypercoagulability was present in critically ill patients with elevated median levels of Fibrinogen $5.6 \mathrm{~g} / \mathrm{L}(4.9,6.6)$, D-dimer $1.0 \mu \mathrm{g} / \mathrm{ml}(0.6,1.4)$, Factor VIII 206\%(171, $230)$, von Willebrand Factor $265 \%(206,321)$ as compared with lower levels in non-critically ill patients. Hypercoagulability was shown in TEG with increased CRT Angle $78.9^{\circ}(78.3,80.0)$, CFF MA $34.6 \mathrm{~mm}(27.4,38.6)$ and CFF A10 $30.9 \mathrm{~s}$ (25.5, 34.0); and CWA had increased clot velocity, aPTT Min1 7.7\%/s(6.4, 8.3). CK K, CK Angle, CK MA, CRT MA were higher in critically ill patients (Table 1). In noncritically ill patients, D-dimer levels were normal, $0.3 \mu \mathrm{g} / \mathrm{mL}(0.3$, $0.4)$ while Factor VIII levels of $176 \%(157,192)$ and vWF levels of $225 \%(158,237)$ were mildly elevated, with TEG and CWA demonstrating no hypercoagulability. 2 critically-ill patients developed thromboembolism(stroke, DVT) while no non-critically ill patients (not on thromboprophylaxis) had thrombosis. 
TABLE 1 Comparison of statistically significant results (Thromboelastography and Clot Waveform Analysis) between critically ill and noncritically ill COVID-19 patients

Tests (Reference Range)

Thromoboelastography (TEG ${ }^{\dagger}$ )

CK K (TEG) (0.8-2.1 min)

Baseline

Day 3

Day 6

Day 9

Day 12

Day 15

Day $18^{*}$

Day $21^{*}$

CK Angle (TEG) (63-78 degrees)

Baseline

Day 3

Day 6

Day 9

Day 12

Day 15

Day $18^{*}$

Day $21^{*}$

CK MA (TEG) (52-69 mm)

Baseline

Day 3

Day 6

Day 9

Day 12

Day 15

Day $18^{*}$

Day $21^{*}$

CRT Angle(TEG) (60-78 degrees)

Baseline

Day 3

Day 6

Day 9

Day 12

Day 15

Day $18^{*}$

Day $21^{*}$
Non critically ill patients

$(n=10)$

$1.6(1.3,2.1)$

$1.3(0.9,1.6)$

$0.8(0.8,1.3)$

$0.8(0.8,1.1)$

$1.0(0.8,1.2)$

$1.1(1.0,1.3)$

$1.2(1.0,1.8)$

$1.1(1.1,1.1)$

$0.8(0.8,0.8)$

$73.9(69.1,76.0)$

$69.1(64.6,74.5)$

$76.8(71.5,78.2)$

$77.6(74.6,80.5)$

$75.7(73.8,78.1)$

$73.0(64.5,74.6)$

$74.4(68.6,77.8)$

$74.8(74.8,74.8)$

$78.8(78.8,78.8)$

$62.4(58.7,67.8)$

$58.8(57.2,59.9)$

$68.2(65.6,69.3)$

$67.8(65.2,70.5)$

$68.5(65.5,69.9)$

$67.2(64.6,71.3)$

$64.6(63.3,67.8)$

$69.3(69.3,69.3)$

$70.2(70.2,70.2)$

77.7 (73.2, 79.2)

$74.1(72.0,75.2)$

$78.4(77.6,81.0)$

$79.5(77.9,81.6)$

79.8 (77.7, 81.2)

$79.7(78.1,80.0)$

$77.5(76.8,79.6)$

$81.0(81.0,81.0)$

$80.5(80.5,80.5)$
Critically ill patients ( $n=10)$

P-value

$1.1(0.9,1.2)$
$0.8(0.8,1.3)$
$0.8(0.8,1.1)$
$1.0(0.8,1.2)$
$1.1(1.0,1.3)$
$1.2(1.0,1.8)$
$1.1(1.1,1.1)$
$0.8(0.8,0.8)$

$75.3(73.4,77.2)$

0.01

$76.8(71.5,78.2)$

$77.6(74.6,80.5)$

$75.7(73.8,78.1)$

$73.0(64.5,74.6)$

$74.4(68.6,77.8)$

$74.8(74.8,74.8)$

$78.8(78.8,78.8)$

$66.6(64.2,69.6)$

0.008

$68.2(65.6,69.3)$

$67.8(65.2,70.5)$

$68.5(65.5,69.9)$

$67.2(64.6,71.3)$

$64.6(63.3,67.8)$

$69.3(69.3,69.3)$

$70.2(70.2,70.2)$

$78.9(78.3,80.0)$

$78.4(77.6,81.0)$

$79.5(77.9,81.6)$

$79.8(77.7,81.2)$

79.7 (78.1, 80.0)

$77.5(76.8,79.6)$

$81.0(81.0,81.0)$

$80.5(80.5,80.5)$
0.002

0.009

$-$

-

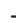

-

$-$

-

-$$
\text { - }
$$ 


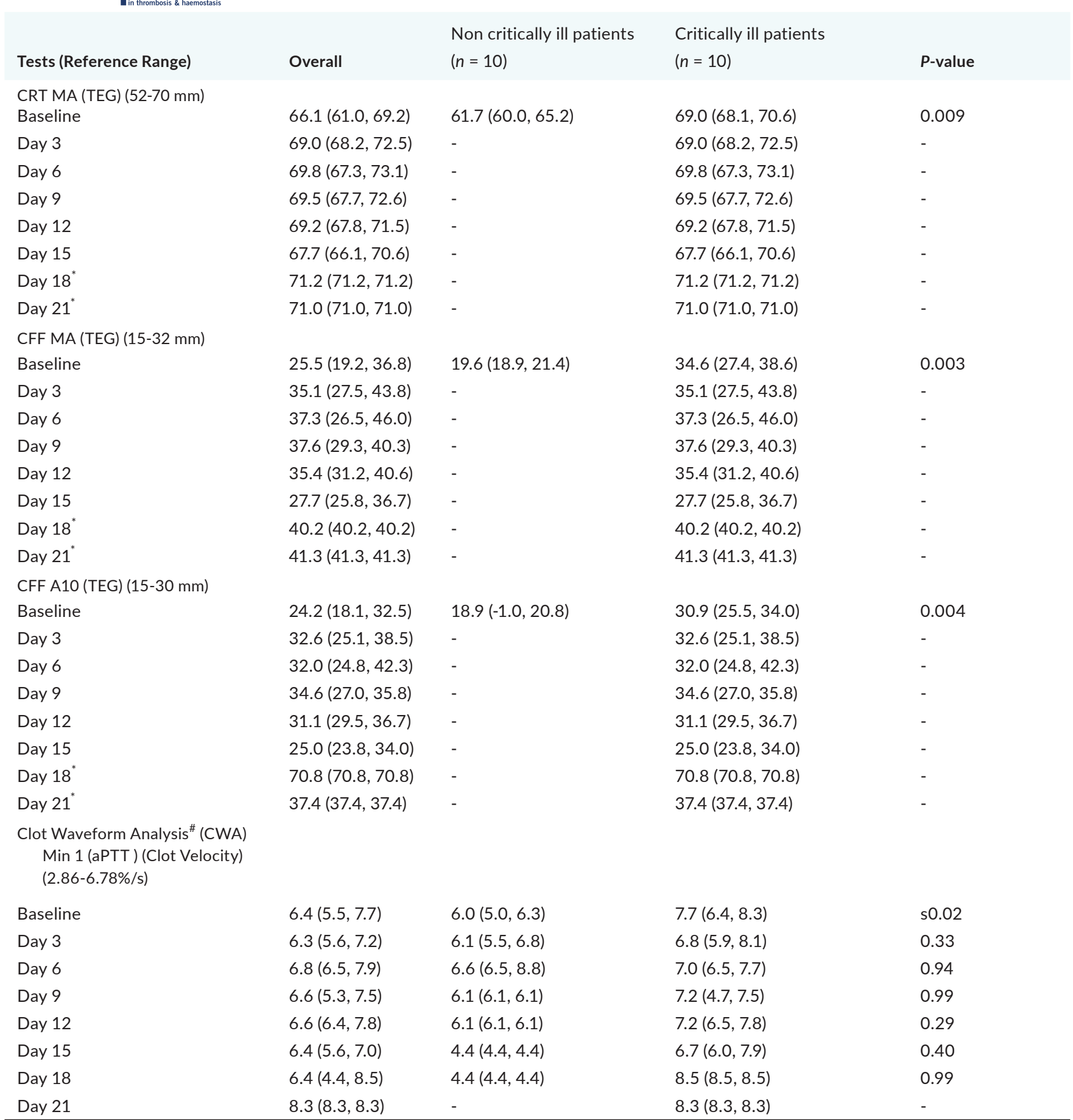

*Day 18 and Day 21 had only 2 critically ill patients remaining on study, these had stroke and lower limb deep vein thrombosis, this hypercoagulable state was still persistent in parameters on TEG and CWA.

${ }^{\dagger}$ TEG 6s Hemostasis Analyzer, Haemonetics, USA

${ }^{\#} \mathrm{CN}-6000$ Coagulation Analyser, Sysmex Corporation, Japan

Conclusions: Critically ill COVID-19 patients demonstrate a hypercoagulable state with raised fibrinogen and Factor VIII levels correlating with raised CK, CRT, CFF maximal amplitude and increased CWA clot velocity(min1), while non-critical patients showed an absence of hypercoagulability in global tests of haemostasis. 
PB0245 | Sex Differences in Thromboinflammatory Biomarkers in Patients with COVID-19: Preliminary Findings from a Retrospective, Single Center Study

G.F. Romiti ${ }^{1}$; D. Flego ${ }^{1}$; R. Marrapodi ${ }^{1}$; B. Corica ${ }^{1}$; P. Pasculli ${ }^{2}$; S.M. Carli ${ }^{2}$; M.A. Zingaropoli ${ }^{2}$; R. Cangemi ${ }^{1}$; V. Raparelli ${ }^{3}$; M.R. Ciardi ${ }^{2}$; C. Mastroianni ${ }^{2}$; S. Basilii ${ }^{1}$. L. Stefanini ${ }^{1}$ ${ }^{1}$ Dept. Translational and Precision Medicine, Sapienza University of Rome, Rome, Italy; ${ }^{2}$ Dept. Public Health and Infectious Disease, Sapienza University of Rome, Rome, Italy; ${ }^{3}$ Dept. Translational Medicine, University of Ferrara, Ferrara, Italy

Background: COVID-19 imposes a high burden of morbidity and mortality worldwide; since the beginning of this pandemic, several sex-related differences have been described in the pathogenesis and clinical course of the disease. Although the identification of biomarkers to stratify the severity of the disease is recognized as pivotal to identify patients with a higher risk of clinical deterioration, few studies investigated sex-related differences in the performance of these biomarkers.

Aims: To evaluate sex differences in the serum concentrations of biomarkers of platelet activation and vascular inflammation in patients with COVID-19.

Methods: We performed a retrospective, preliminary analysis of a single center study which is recruiting patients with COVID-19 at different stages of severity. Vascular inflammation and platelet activation markers were measured in batch in serum samples collected from the venous circulation by multiplex bead-based flow cytometry assay.

Results: Among 74 COVID-19 patients (median age: 69 years; $65 \%$ males; $55 \%$ with clinically-diagnosed severe disease), data on individual biomarkers were available for 69 individuals (36\% females). No significant differences were observed for age between males and females. Compared to SARS-Cov2-negative subjects, the serum concentration of soluble CD40L (sCD40L), Plasminogen Activator Inhibitor 1, Matrix Metalloproteinase-9, Myeloid-Related Protein $8 / 14$, Myeloperoxidase, Osteopontin in SARS-Cov2-positive subjects increased significantly in relation to disease severity. Among these, men, compared to women, showed higher levels of SCD40L, an X-linked marker of platelet activation, (Median: $17892 \mathrm{pg} / \mathrm{ml}$ [Interquartile Range, IQR: 11197-34520] vs. 11225 pg/ml [IQR: 5625-22644], $P=0.019$ ) and osteopontin, a sialoprotein implicated in estrogen-stimulated endothelial repair (Median: $151184 \mathrm{pg} / \mathrm{ml}$ [IQR: 82739-200141] vs. 74219 pg/ml [31602-144440], $P=0.011$ ).

Conclusions: In this preliminary analysis, we found that potentially relevant sex-based differences may exists in the levels of thromboinflammatory biomarkers which are currently investigated for the prognostic stratification of COVID-19 patients. Pre-specified analysis disaggregated by sex are needed to take into account differences in the performance of these biomarkers.
PB0246 | Enoxaparin in COVID-19 Moderate to Severe Hospitalized Patients (INHIXACOV19)

B. Cosmi ${ }^{1}$; M. Giannella ${ }^{2}$; C.M. Re ${ }^{3}$; F. Cristini ${ }^{4}$; E.G. Bignami ${ }^{5}$; A. Montineri ${ }^{6}$; S. Testa ${ }^{7}$; S. Casari ${ }^{8}$; E. Grandone ${ }^{9}$; R. Rozzini ${ }^{10}$; E. Tacconelli ${ }^{11}$; A. Castagna ${ }^{12}$; G. Di Perri ${ }^{13}$; M. Salvetti ${ }^{14}$; A. Stella ${ }^{15} ;$ P. Viale ${ }^{2}$

${ }^{1}$ Dept Angiology and Blood Coagulation, Sant'Orsola Malpighi University Hospital IRCSS, University of Bologna, Bologna, Italy;

${ }^{2}$ Infectious Diseases Unit, Department of Medical and Surgical Sciences, Policlinico Sant'Orsola IRCSS, University of Bologna, Bologna, Italy; ${ }^{3}$ Clinical Microbiology Unit, Department of Specialty Diagnostics and Experimental Medicine Policlinico Sant'Orsola IRCSS, University of Bologna, Bologna, Italy; ${ }^{4}$ Morgagni-Pierantoni Hospital, Forli, Italy; ${ }^{5}$ AOU Parma, Parma, Italy; ${ }^{6}$ San Marco Hospital, Catania, Italy; ${ }^{7}$ ASST Cremona, Cremona, Italy; ${ }^{8}$ Carlo Poma Hospital, Mantova, Italy; ${ }^{9}$ Padre Pio Hospital - San Giovanni Rotondo, Foggia, Italy; ${ }^{10}$ Poliambulanza Hospital, Brescia, Italy; ${ }^{11}$ AOU Verona, Verona, Italy; ${ }^{12}$ San Raffaele Hospital, Milano, Italy; ${ }^{13}$ Amedeo di Savoia Hospital, Torino, Italy; ${ }^{14}$ ASST Spedali Civili, Brescia, Italy; ${ }^{15}$ Alma Mater Studiorum, University of Bologna, Bologna, Italy

Background: COVID-19 (Coronavirus Disease 2019) is associated with High rates of thrombosis in hospitalized patients leading to varying pharmacologic thromboprophylaxis use based on rapidly changing societal guidance,institutional protocols from local expertise, and geographic patterns of practice.

Aims: To assess the efficacy and safety of enoxaparin in hospitalized patients with moderate to severe COVID-19 infection.

Methods: Phase II single-arm interventional prospective study including all patients treated with the study drug and an observational prospective cohort study including all patients screened for receiving the study drug but not included in the phase II study. Each patient was followed-up for a minimum of 90 days after COVID19 diagnosis. Patients included in the interventional study received subcutaneous enoxaparin in a single daily dose of:60 mg once daily in case of body weight of 45 to $60 \mathrm{~kg} 80 \mathrm{mg}$ per day in case of weight from 61 to 100 $\mathrm{kg}$ or $100 \mathrm{mg}$ once daily in case of bodyweight $>100 \mathrm{~kg}$ for 14 days, with dose adjustments on the basis of anti-factor Xa activity monitoring. Patients included in the observational cohort received standard thrombo-prophylaxis with subcutaneous enoxaparin $40 \mathrm{mg} / \mathrm{die}$. Primary outcomes were all-cause in-hospital 30-day and 90 mortality rates. Secondary outcomes were the proportion of patients in the severe or critical stage of disease at the end of treatment, proportion of patients who developed major and non-major bleeding events and thromboembolic complications, time to first negative RT-PCR on nasofaringeal swab, reduction of viral load in blood.

Results: Recruitment of 100 patients enrolled phase II single-arm interventional prospective study has been completed, while the recruitment of 200 patients in the observational prospective cohort study is ongoing.

Conclusions: Full results will be available by June 2021 
PB0247 | Apparent Different of Lupus Anticoagulant Disappearance in Novel Coronavirus 2019 Patients as a Distinct Clinical Feature

H. Kim ${ }^{1}$; M.-S. Kim² $;$ S. Jang ${ }^{1}$

${ }^{1}$ University of Ulsan College of Medicine and Asan Medical Center, Seoul, Korea, Republic of; ${ }^{2}$ Soon Chun Hyang University Medical Center, Cheonan-si, Korea, Republic of

Background: SARS-CoV-2 was reported to affect hemostasis, and thrombosis laboratory indices but its effect on lupus anticoagulant remains controversial.

Aims: The purpose of this study is to learn more about the properties of lupus anticoagulant in patients with COVID-19 in Korea.

Methods: We retrospectively searched for COVID-19 confirmed patients at the Asan Medical Center from March 2020 to February 2021. Medical chart review was conducted to collect their clinical information and hematology laboratory data. Among 142 confirmed patients, 67 patients with lupus anticoagulant testing were included to determine the differences in laboratory indices, especially LA.

Results: Most of the patients in our study had mild clinical courses. Lupus anticoagulant was identified in $67.8 \%$ of survivors and $25.0 \%$ of deaths. In the LA confirmatory test, 97.5\% DRVVT and 10.0\% SCT positivity were found. Among 40 LA-positive patients, 13 patients were lost for follow up, and 12 patients were unable to determine time-lapse until negative transition of LA. Of the 15 patients who had serial follow-up LA test data, 10 patients (66.7\%) were found with rapid disappearance within 6 weeks and 4 case $(26.7 \%)$ of negative conversion within 6 to 12 weeks. Most of cases (93.3\%) disappearance of LA less than 12 weeks. There were no other antiphospholipid syndrome-related antibodies, such as anti-cardiolipin antibodies or anti-beta-2-gylcoprotein, except for 2 of the 40 patients who were LA positive.

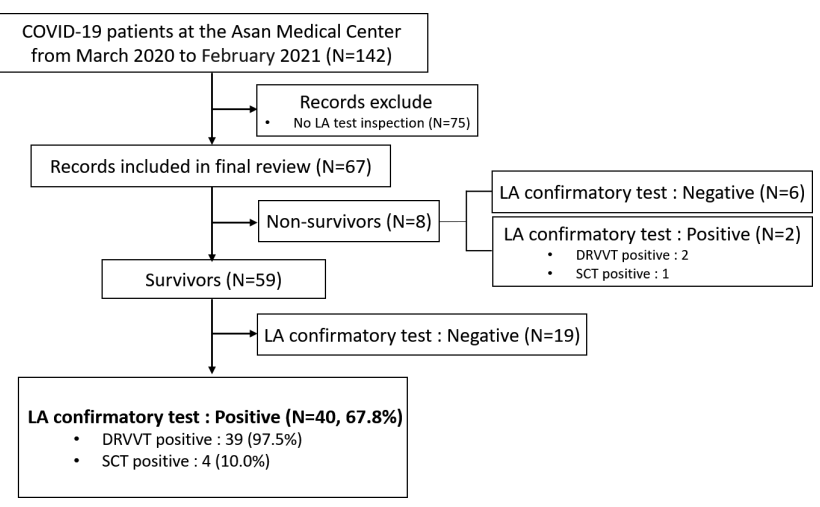

FIGURE 1 Classification of COVID-19 patients based on Lupus anticoagulant test
TABLE 1 Hematology laboratory parameters of COVID-19 patients based on Lupus anticoagulant test

\begin{tabular}{|c|c|c|c|}
\hline \multirow[t]{2}{*}{ Parameter } & \multicolumn{2}{|c|}{ Survivors $(n=59)$} & \multirow[t]{2}{*}{$P$ value } \\
\hline & LA negative $(n=19)$ & LA positive $(n=40)$ & \\
\hline Age (years) & $55.8 \pm 20.0$ & $60.3 \pm 17.4$ & 0.351 \\
\hline $\operatorname{Sex}(M / F)$ & $(12 / 7)$ & $(21 / 19)$ & \\
\hline WBC $\left(X 10^{9} / L\right)$ & $6.1(1.4-12.5)$ & $6.9(0.0-21.8)$ & 0.455 \\
\hline $\mathrm{Hb}(\mathrm{g} / \mathrm{L})$ & $119.7(83.0-158.0)$ & $121.7(78.0-166.0)$ & 0.632 \\
\hline $\mathrm{Plt}\left(\mathrm{X} 10^{\circ} / \mathrm{L}\right)$ & $233.2(33.0-403.0)$ & $205.8(36.0-736.0)$ & 0.153 \\
\hline NLR & $3.8(0.3-12.0)$ & $5.6(0.8-26.0)$ & 0.253 \\
\hline PT (sec) & $12.4(10.5-15.3)$ & $13.0(11.1-16.8)$ & 0.038 \\
\hline aPTT (sec) & $28.6(22.8-38.0)$ & $31.8(24.6-39.9)$ & 0.007 \\
\hline CRP (mg/L) & $0.27(0.01-2.36)$ & $0.66(0.01-2.23)$ & 0.000 \\
\hline LD (IU/L) & $262.5(81.0-469.0)$ & $302.9(117.0-612.0)$ & 0.215 \\
\hline Fibrinogen $(\mathbf{g} / \mathrm{L})$ & $4.0(2.5-8.7)$ & $5.2(1.1-8.7)$ & 0.009 \\
\hline D-dimer $(\mu \mathrm{g} / \mathrm{ml} \mathrm{FEU})$ & $0.8(0.2-2.5)$ & $2.2(0.2-23.1)$ & 0.073 \\
\hline $\mathrm{FDP}(\mu \mathrm{g} / \mathrm{ml})$ & $8.8(2.5-82.1)$ & $9.9(2.5-99.4)$ & 0.008 \\
\hline Antithrombin III (\%) & $94.7(64.0-111.0)$ & $87.6(25.0-110.0)$ & 0.054 \\
\hline \multicolumn{4}{|c|}{$\begin{array}{l}\text { Abbreviations: } L A \text {, Lupus anticoagulant; } M \text {, male; } F \text { female; } W B C \text {, white blood cells; } \mathrm{Hb} \text {, hemoglobin; } \\
\text { Plt, platelet; NLR, neutrophil to lymphocyte ratio; } P T \text {, prothrombin time; aPTT, activated partial } \\
\text { thromboplastin time; CRP, C-reactive protein; LD, lactate dehydrogenase; FDP, fibrin degradation } \\
\text { product. }\end{array}$} \\
\hline
\end{tabular}

Conclusions: As previously reported, LA was found in a high proportion of COVID-19 patients. This is the first study to confirm that the proportion of LA positivity (67.8\%) is high in Korean COVID patients. Most LA positivity disappeared within 12 weeks (93.3\%). The clinical significance of high LA positive rates and rapid negative transitions in COVID-19 patients is currently unknown, but needs confirmation.

PB0248 | Low Incidence of Thrombotic Events in Patients with Covid19: A Cohort Study

C. Vazquez; M. Vallone; F. Chuliber; V. Privitera; A. Ferraris;

R. Cantarella; M.F. Indo; V. Peuchot; F.J. Vazquez

Hospital Italiano de Buenos Aires, Buenos Aires, Argentina

Background: Since the beginning of the SARS-CoV 2 pandemic, there have been many reports of increased incidence of VTE (venous thromboembolism) and arterial events as a complication, which were the basis for the hypothesis that patients should receive higher doses of thromboprophylaxis (TP) or full anticoagulation.

Aims: To determine the incidence of thrombotic events (TE) in patients hospitalized for moderate or severe SARS-CoV2 disease (Covid-19).

Methods: Retrospective single center cohort study with adult patients with a positive rt-PCR for SARS-CoV2, included as from the date of hospital admission due to Covid-19 and followed for 90 days or until death.

Results: A total of 1621 patients were included. The mean age was 68.7 years (SD 21.1) and 57\% (913) were female, of which 6.5\% (59) were pregnant. Overall mortality was $21.6 \%$ (348). The overall incidence of symptomatic thrombotics events within 90 days was $1.72 \%$ (28/1621, 95\%Cl $1.19-2.49 \%)$, whereas the incidence of pulmonary embolism (PE) was $0.98 \%(15,95 \% \mathrm{Cl} 0.60-0.16 \%)$, deep venous thrombosis (DVT) $0.74 \%(11,95 \% \mathrm{Cl} 0.42-0.12 \%)$, ischemic stroke $0.24 \%$ 
$(4,95 \% \mathrm{Cl} 0.09-0.65 \%)$ and ischemic arterial events $0.06 \%(1,95 \% \mathrm{Cl}$ $0.008-0.43 \%)$. No acute coronary syndrome events were recorded. Median hospital stay length percentage under adequate TP was $78 \%$.

The median time since positive rt-PCR for the thrombotic event to develop was 22 days (IQR 25th-75th 19-43 days).

Median hospital stay length percentage under adequate thromboprophylaxis was $78 \%$ (IQR 25th-75th 50-89\%).

Conclusions: Unlike previous reports, the incidence of thrombotic events was low among our patients with moderate or severe Covid-19.

PB0249 | Artificial Neural Networks to Predict Covid-19 Progression of Moderate Hospitalized Patients using Early Clinical Parameters and Blood Tests

T.D. Martins $^{1}$; S.D. Martins ${ }^{2}$; S.A.L. Montalvão²; G.Y. Ottaiano ${ }^{1}$; M. Al Bannoud ${ }^{1}$; L.Q. Silva² ; S.C. Huber²; T.S. Diaz ${ }^{3}$;

C.K. Wroclawski ${ }^{4}$; C.C. Filho ${ }^{4}$; R.M. Filho ${ }^{5}$; J.M. Annichino-Bizzacchi ${ }^{2}$

${ }^{1}$ Universidade Federal de São Paulo, Diadema, Brazil; ${ }^{2}$ Hemostasis and Thrombosis Laboratory, Hematology and Hemotherapy Center, University of Campinas, Campinas, Brazil; ${ }^{3}$ Anhembi Field Municipal Hospital, São Paulo, Brazil; ${ }^{4}$ Thrombosis and Hemostasis Center, Sirio Libanes Hospital, São Paulo, Brazil; ${ }^{5}$ School of Chemical Engineering,

University of Campinas, Campinas, Brazil

TABLE 1 Summary of the proposed ANNs

\begin{tabular}{llll} 
ANN model & Outcome & Number of patients & Inclusion criteria \\
ANN 1 & Intubation (yes) & 80 & Confirmed Covid-19 patients \\
& Discharge (no) & 380 & \\
ANN 2 & Death (yes) & 27 & Patients intubated during Covid-19 treatment \\
& Discharge (no) & 53 & \\
\hline
\end{tabular}

Results: The main results are shown at Table 2 . The best models were obtained with different ANN's structures, which show the influence of the different outcome. The models presented high ACC,
Background: The early prediction of Covid-19 progression could improve patient's treatment. It is important to develop mathematical models to perform this task using simple blood tests.

Aims: To obtain a neural network (ANN) to predict the progression (death vs discharge and intubation vs discharge) of Covid-19 in patients with confirmed diagnosis.

Methods: The patients included in this work were diagnosed with Covid-19 by RT-PCR. All data were collected from hospitalized patients admitted to Anhembi Field Municipal Hospital (São Paulo-Brazil), a hospital set up for initial care to patients with moderate symptoms during the pandemic, between June/2020 and October/2020. Blood was collected at the patient's admission. The inputs considered were: sex, age, ethinicity, body mass index, tabagism, ex-tabagism, alveolar infiltrate, arterial hypertension, diabetes, heart rate, respiration rate, body temperature, oxygen saturation, D-dimer, activated partial thromboplastin time, prothrombin time, levels of: hemoglobin, platelet, leukocytes, lymphocytes, monocytes, neutrophils, lactate dehydrogenase, C-reactive protein, and creatinine. Two ANNs were proposed, as shown at Table 1. The best ANN was defined by a 5-fold cross-validation scheme. Finally, a test step was performed to verify the ANN performance. ANNs with one and two hidden layers were tested. The number of neurons ranged from 5 to 35 .
AUC, PPV, NPV, and TNR. The ANN 2 presented better performance than ANN 1. We believe that this may be due the data homogeneity that rises from the inclusion criteria adopted in the study.

TABLE 2 Summary results obtained for the test dataset for the ANNs proposed in this study

\begin{tabular}{lll} 
& \\
& ANN 1 & ANN 2 \\
Performance Metric & structure 25-15-15-1 & structure 25-15-20-1 \\
Accuracy (ACC) & $87.5 \%$ & $93.7 \%$ \\
AUC & 0.823 & 1.000 \\
Positive Predicted Value (PPV) & 0.600 & 1.000 \\
Negative Predicted Value (NPV) & 0.938 & 0.917 \\
True Positive Rate (TPR) & 0.693 & 0.800 \\
True Negative Rate (TNR) & 0.910 & 1.000 \\
False Negative Rate (FNR) & 0.090 & 0.200 \\
Positive Likelihood Ratio (LR+) & 7.731 & infinity \\
Negative Likelihood Ratio (LR-) & 0.338 & 0.200 \\
\hline
\end{tabular}


Conclusions: The results showed that the ANNs could be used to predict the progression of Covid-19 based on simple blood tests. The models could be used in the future after an external validation with high number of patients.

PB0250 | The Role of Thromboelastography and Follow-up of Fibrinolysis in Evaluating the Course of the Disease, Treatment Response, and Outcome of Pediatric Patients with COVID-19

T. Hilkay Karapınar ${ }^{1}$; E. Böncüoğlu ${ }^{1}$; Y. Oymak ${ }^{1}$; E. Kıymet ${ }^{1}$; Ö. Tüfekçi' ; A. Akaslan Kara ${ }^{1}$; K. Ötiken Arıkan ${ }^{1}$; Ş. Şahinkaya ${ }^{1}$; T. Çelik ${ }^{1}$; S.N. Bayram¹; I. Devrim ${ }^{1}$; $\underline{\text { H. Ören }}{ }^{2}$

${ }^{1}$ Dr. Behçet Uz Children's Hospital, İzmir, Turkey; ${ }^{2}$ Dokuz Eylül University Faculty of Medicine, İmir, Turkey

Background: Thromboelastography (TEG) may estimate the dynamics of blood coagulation from the activation of clotting factors to fibrin formation, clot stabilization, and clot lysis. Therefore, TEG may be helpful to predict thromboembolic events and estimate clinical outcome in patients with COVID-19.

Aims: In this study, we wanted to evaluate the coagulation abnormalities including TEG analyses in children that are hospitalized for COVID-19 and aimed to assess the utility of TEG in clinical practice in terms of course of the disease, treatment response, and outcome. Methods: A total of 66 patients who were $\leq 18$ years old and hospitalized in infection ward for COVID-19 were evaluated prospectively. Clinical and laboratory findings at admission, course of the disease, treatment modalities, development of MIS-C, and outcome of patients were noted. Haemoscope TEG analyzer (Haemoscope, USA) was used.

Results: Five patients (7.6\%) had thrombocytopenia. PT was prolonged in 5 patients (7.6\%) and APTT in 3 patients (4.5\%). Eight patients (12.1\%) had elevated levels of D-dimer. A total of 16 patients (24.2\%) had at least one abnormality at in TEG analysis. Eleven patients (16.6\%) had an abnormality related to hypercoagulation such as decreased $\mathrm{R} / \mathrm{K}$, increased MA/Angle or increased $\mathrm{Cl}$. MIS-C developed in 3 patients (4.5\%) during clinical follow-up; all of them had a normal graphic for TEG, even though they had increased D-dimer levels (>3000 $\mathrm{ng} / \mathrm{mL}$ ) at admission. There was no difference in TEG abnormalities between treated and untreated patients $(P=0.76)$. Thromboembolic complications or bleeding events were not observed. None of the patients needed mechanical ventilation. None of them died.

Conclusions: Abnormal TEG features may be the most common finding regarding coagulopathy at admission in these patients, followed by elevated D-dimer levels. Abnormal TEG results at admission is not able to predict the course of the disease, treatment results, and outcome in pediatric patients who did not need mechanical ventilation.

\section{PB0251 | Potential Markers to Predict Mortality in COVID-19}

C. Klapheke; H. McRae; T. Love; M. Refaai

University of Rochester Medical Center, Rochester, United States

Background: COVID-19 has a wide clinical spectrum, but hallmarks include acute kidney injury, fever, cough, dyspnea, lymphopenia, pneumonia, myalgia, and arterial and venous thromboembolism. A strong association with disseminated coagulopathy (DIC) has also been documented in acute COVID-19 cases. However, unlike the DIC usual clinical scenario, COVID-19 cases seem to be significantly hypercoagulable. Laboratory screening tests, such as PT, PTT, D-Dimer, fibrinogen, and platelet count are commonly used for thrombosis risk assessment. Studies also showed that mortality in acute COVID-19 patients is correlated with elevated D-Dimer levels. However, the association of other laboratory markers on in-hospital mortality is not well understood

Aims: We performed a retrospective data analysis of COVID-19 hospitalized patients in order to determine whether changes in laboratory values correlated with differences in in-hospital mortality.

Methods: Data were collected from March 2020 through January 2021 from adults who were admitted to our tertiary medical center with suspected diagnosis of Sars-CoV-2 and who had more than two D-dimer values recorded during the course of their admissions. 69 patients were included in this study, and changes in coagulation and metabolic laboratory values were compared between patients who survived ( $n=59$; median age 63.5 years) and expired ( $n=10$; median age 69.4 years) during the study period (Table). The Wilcoxon ranksum test was used to evaluate differences between cohorts.

Results: Using classification trees, the percent change in $\mathrm{pH}(<-$ $0.335 \%$ ) from baseline was the best prognostic indicator (Table). The maximum daily percentage change in D-dimer (>478.1\%) was a similar predictor of in-hospital mortality (Figure).

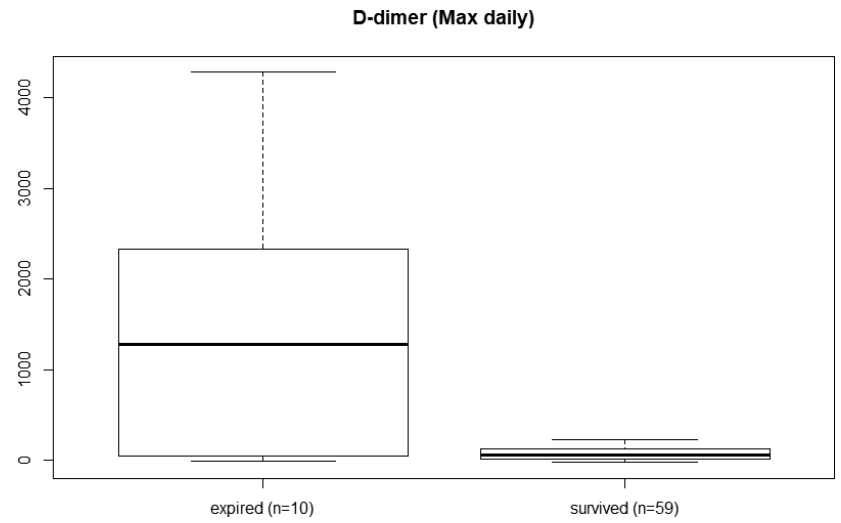

FIGURE 1 Maximum daily D-dimer percentage change during hospitalization for expired and survived COVID-19 patients 
TABLE 1 Laboratory value differences between expired versus survived hospitalized COVID-19 patients. Data shown in percentage change over the hospitalization period except for $\mathrm{D}$-dimer, which also is shown as daily percentage change and peak value

\begin{tabular}{|c|c|c|c|c|c|c|c|c|c|c|}
\hline Percent Change & Unit & \multicolumn{4}{|c|}{ Expired $(n=10)$} & \multicolumn{4}{|c|}{ Survived $(n=59)$} & $P$-value \\
\hline $\mathrm{pH}$ & - & 8 & -0.67 & -3.86 & 1.35 & 41 & 0.67 & -1.74 & 5.61 & 0.001 \\
\hline Hemoglobin & $\mathrm{g} / \mathrm{dL}$ & 10 & -31.23 & -58.11 & 27.54 & 58 & -15.19 & -35.14 & 13.90 & 0.073 \\
\hline Sodium ( $\mathrm{Na}$ ) & $\mathrm{mmol} / \mathrm{L}$ & 10 & 0.71 & -1.45 & 3.55 & 58 & 2.20 & -8.33 & 888.36 & 0.435 \\
\hline Potassium (K) & $\mathrm{mmol} / \mathrm{L}$ & 10 & 1.26 & -36.36 & 58.82 & 58 & 0.00 & -31.82 & 32.35 & 0.709 \\
\hline Lactate & $\mathrm{mmol} / \mathrm{L}$ & 8 & -6.00 & -50.00 & 842.86 & 31 & 0.00 & -65.71 & 188.89 & 1.000 \\
\hline $\begin{array}{l}\text { Prothrombin Time } \\
\quad(\mathrm{PT})\end{array}$ & seconds & 9 & 10.27 & -53.45 & 232.30 & 42 & 1.70 & -44.20 & 40.15 & 0.186 \\
\hline $\begin{array}{l}\text { Partial } \\
\text { Thromboplastin } \\
\text { Time (PTT) }\end{array}$ & seconds & 5 & 30.70 & -14.70 & 43.30 & 32 & 2.95 & -26.20 & 28.10 & 0.095 \\
\hline $\begin{array}{l}\text { D-dimer (Minimum } \\
\text { Daily) }\end{array}$ & $\mu \mathrm{g} / \mathrm{mL}$ & 10 & -64.55 & -8970 & -3.18 & 59 & -39.93 & -97.86 & 121.99 & 0.043 \\
\hline D-dimer (Peak Value) & $\mu \mathrm{g} / \mathrm{mL}$ & 10 & 49.72 & 3.54 & 128.00 & 59 & 2.25 & 0.40 & 128.00 & 0.001 \\
\hline
\end{tabular}

Conclusions: Our data confirm the previously reported association between D-dimer levels and mortality risk in COVID-19 hospitalized patients. The daily percentage change in D-dimer along with decreased arterial $\mathrm{pH}$ levels may jointly serve as a stronger prognostic marker for mortality in this population.

\section{PB0252 | IMPACT of COVID-19 Associated Coagulopathy in} Disease Severity

F. Santos; R. Marinho; M. Carneiro; R. Matos; N. Pinho; L. Moreira; N. Seidi; M. Coutinho; S. Morais; E. Cruz

Centro Hospitalar Universitário do Porto, Porto, Portugal

Background: Severe COVID-19 infection is associated with a significant coagulopathy, characterized predominantly by D-dimers elevation and correlated with poor prognosis. Thromboembolic events (TE) have been identified in many patients, mostly in severe cases.

Aims: The aim of this study was to characterize COVID-19 associatedcoagulopathy and to study correlations with disease-severity (DS) and outcomes (thrombosis and mortality).

Methods: Seventy-nine COVID-19 patients (45M/34F; mean $70 \pm 15$ years-old) admitted between March and May 2020, were divided in 4 subgroups according to DS: mild ( $n=17,21.5 \%$ ), pneumonia without hypoxemia ( $n=9,11.4 \%$ ), pneumonia with hypoxemia ( $n=34,43.0 \%$ ) and multi-organic dysfunction disease ( $n=19,24.1 \%$ ). Several samples from each patient were collected and characterized according to DS, in a total of 292 samples. D-dimers, fibrinogen, PT, aPTT and blood counts were analyzed at admission and during hospitalization. Co-morbidities, TE and mortality were recorded in patients.
Results: There were no significant differences in gender, age and co-morbidities between DS subgroups. D-dimers and fibrinogen at admission were significantly associated with DS $(P<0.05$ and $P<0.01$, respectively). During hospitalization, there was a positive and strong correlation of DS with D-dimers, fibrinogen and platelets, and a negative correlation with hemoglobin $(P<0.001)$. Venous TE (VTE) was observed in 6 patients (7.6\%) and arterial TE in $3(3.8 \%)$, which represents $17.0 \%(9 / 53)$ of patients in the severe stages, compared with TE incidence of $0 \%(0 / 26)$ in the lower stages $(P<0.05)$. TE occurred in $78 \%$ of patients, despite anticoagulation prophylaxis. Overall mortality was $24.1 \%$ ( $n=19$ ), significantly associated with DS $(P<0.01)$ and with admission values of $D$-dimers and fibrinogen (both $P<0.05$ ). See Table1 for results. 
TABLE 1 Median values (minimum - maximum) of relevant laboratorial parameters at admission and during hospitalization, according to disease severity. Mortality is show in percentage according to disease severity. Statistical significance was calculated using Kruskal-Wallis or Chi-square tests as appropriate

\begin{tabular}{|c|c|c|c|c|c|c|}
\hline Disease severity & & $\begin{array}{l}\text { Mild } \\
\text { symptoms }\end{array}$ & $\begin{array}{l}\text { Pneumonia } \\
\text { without } \\
\text { hypoxemia }\end{array}$ & $\begin{array}{l}\text { Pneumonia } \\
\text { with hypoxemia }\end{array}$ & $\begin{array}{l}\text { Multi-organic } \\
\text { disfunction }\end{array}$ & $P$ values \\
\hline Results at admission & $\begin{array}{l}\text { D-dimers } \\
\text { (ng/mL) }\end{array}$ & $\begin{array}{l}869 \\
\quad(135-5250)\end{array}$ & $628(124-4012)$ & $776(153-5250)$ & $1380(377-5250)$ & $<0.05$ \\
\hline Nr. of samples $(n=292)$ & & 26 & 15 & 84 & 157 & \\
\hline \multirow[t]{3}{*}{$\begin{array}{l}\text { Results during } \\
\text { hospitalization }\end{array}$} & $\begin{array}{l}\text { D-dimers } \\
(\mathrm{ng} / \mathrm{mL})\end{array}$ & $\begin{array}{l}849 \\
\quad(135-5250)\end{array}$ & $375(124-4012)$ & $760(153-5250)$ & 2041 (312-5250) & $<0.001$ \\
\hline & $\begin{array}{l}\text { Platelets } \\
\left(\times 10^{9} / \mathrm{L}\right)\end{array}$ & $193(74-486)$ & $145(91-343)$ & $254(98-645)$ & $217(32-842)$ & $<0.001$ \\
\hline & $\begin{array}{l}\text { Hemoblobin } \\
\text { (g/dL) }\end{array}$ & $\begin{array}{l}11.9 \\
(8.6-15.5)\end{array}$ & $13.7(8.5-16.4)$ & $12.6(7.7-17.0)$ & $10.5(7.1-17.3)$ & $<0.001$ \\
\hline Mortality $(n=19,24.1 \%)$ & & $1(5.9 \%)$ & $3(33.3 \%)$ & $7(20.6 \%)$ & $8(42.1 \%)$ & $<0.01$ \\
\hline
\end{tabular}

Conclusions: These findings validate fibrinogen as another prognostic marker of COVID-19 severity and confirm the importance of coagulation changes, namely elevation of D-dimers and fibrinogen, for disease progression monitoring and prognosis prediction. In addition, TE appear to be a consequence of DS and independent of anticoagulation prophylaxis.

PB0253 | Platelets and Neutrophil Extracellular Traps (NETs) in Immunothrombosis Progression at COVID-19

I. Vasilenko ${ }^{1,2}$; A. Gur'ev ${ }^{1}$; D. Kassina ${ }^{1}$; V. Metelin ${ }^{3,2}$; V. Schelkova ${ }^{1}$

${ }^{1}$ M.F. Vladimirsky Moscow Regional Clinical and Research Institute (MONIKI), Moscow, Russian Federation; ${ }^{2}$ A.N. Kosygin Russian State University, Moscow, Russian Federation; ${ }^{3}$ Moscow Regional Research Institute of Obstetrics and Gynecology (MONIIAG), Moscow, Russian Federation

Background: Virus infection COVID-19 caused by beta-coronavirus SARS-CoV-2 spread over the majority of countries and continents within several months. Pathophysiology of the disease is directly connected to uncontrollable progression of system inflammation, resulting in developed immunothrombosis of microvasculature and multiple organ dysfunction.
Aims: In the present investigation, the effort was made to to clarify several pathophysiological mechanism of COVID-19 development, to determine diagnostic and prognostic criteria of disease severity and outcome.

Methods: The blood of 87 patients with COVID-19 was examined using routine hemostasiological tests. In parallel, morphological and functional features of living functioning platelets were evaluated using quantitative phase imaging (QPI) technology at the level of superresolution and 3D visualization of cells and subcellular structures. According to the author's technique, the formation of neutrophil extracellular traps during netosis (NETosis) of neutrophils was analyzed in whole blood smears.

Results: It was found that patients are characterized by the development of COVID-associated coagulopathy, which correlates with the severity of the disease. We found that, compared with control, the patients had a elevated plasma D-dimer, fibrinogen and FDPs, as well as shortened TT. We identified the increasing by $27 \%$ platelets with low and high level of activation (64 $\pm 6.9 \%$ vs $37 \%$ in norm) in $83 \%$ of patients. The level of NETs was statistically significantly $(53.1 \pm 7.2 \%, P<0.05)$ higher than healthy volunteers $(9.3 \pm 3.5 \%)$ and progressively increased with the development of acute respiratory distress syndrome.

Conclusions: Hemostatic disorders during SARS-CoV-2 infection can be caused by hyperactivity of platelets, hypercoagulation, 
hyperfibrinolysis and an inadequate immune response due to the formation NETs, finally causing coronavirus-induced coagulopathy. Platelets and neutrophils not only serve as markers of acute infection, but also, as a source of neutrophilic extracellular traps, play a key role in the development of immunothrombosis, which ultimately leads to acute respiratory failure with COVID-19.

PB0254 | Oral Anticoagulation with Rivaroxaban as Thromboprophylaxis in Patients Recovered from COVID-19 Pneumonia in Veracruz, Mexico

L. Del Carpio-Orantes; S. García-Méndez; J.S. Sánchez-Diaz; K.G. Peniche-Moguel; E.E. Aparicio-Sánchez; O.I. SeguraRodríguez; A. Aguilar-Silva; O. García-Hernández; A. EscobarHuerta; A. Ahumada-Zamudio; A.R. Velasco-Caicero; O. González-Segovia; E.J. Pacheco-Pérez; A.E. Munguia-Sereno; E.R. Contreras-Sánchez; S.N. Hernández-Hernández; L.J. MedranoRios; A.L. Rosas-Lozano Instituto Mexicano del Seguro Social, Veracruz, Mexico

Background: Patients affected by COVID-19 are at risk of various venous and arterial thrombotic events, as well as embolic risks, the risk can vary from $17 \%$ to $78 \%$ according to the different published series. therefore, thromboprophylaxis must be imperative.

Aims: To assess the use of rivaroxaban as thromboprophylaxis in recovered COVID-19 patients who are at thrombotic risk

Methods: Descriptive and analytical study in patients who presented pneumonia due to COVID-19 in April and May 2020, who received LMWH during their hospital stay and at discharge with rivaroxaban $10 \mathrm{mg} /$ day for 2 months. D-dimer was measured at the beginning of the study and 1 month after discharge. Thrombotic or hemorrhagic episodes are controlled after 1 and 2 months of treatment (June - July 2020).

Results: 50 patients, women $40 \%$ and men $60 \%$, with a median age of 42.9 years. $64 \%$ had mild pneumonia and $36 \%$ had severe pneumonia, mean initial D-dimer $556.5 \mathrm{ng} / \mathrm{ml}, 56 \%$ had D-dimer $\geq 500 \mathrm{ng} /$ $\mathrm{ml}$ at the time of hospital admission. Baseline D-dimer values were significantly higher in severe pneumonia. In the follow-up at one and two months, we found that D-dimer values were significantly higher among patients with severe pneumonia and also, in this group of patients, the percentage of patients with D-dimer levels $\geq 500 \mathrm{ng} / \mathrm{mL}$ in the first month of follow-up, was significantly higher in the group of patients who were hospitalized for severe pneumonia. During the first month of follow-up, there was a thrombotic event and a hemorrhagic event in the group of patients with severe pneumonia; By the second month of follow-up, there was a hemorrhagic event in the group with mild pneumonia, but this difference in frequencies was not statistically significant.
TABLE 1 Variables stratified by type of pneumonia

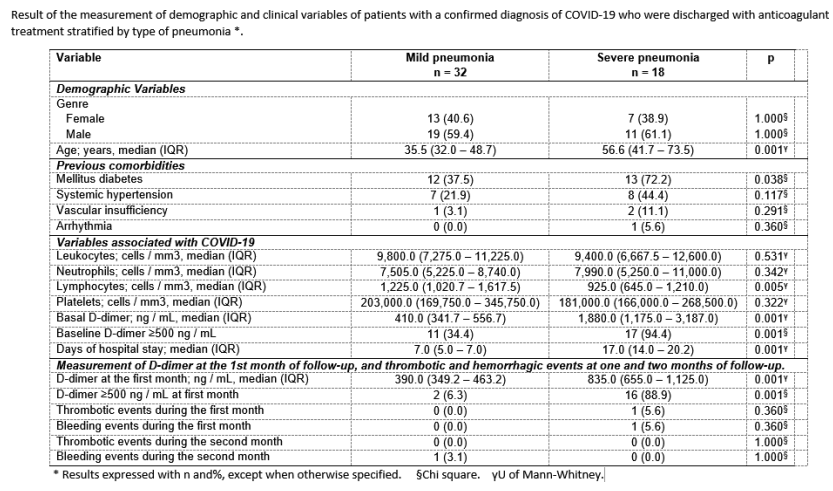

Conclusions: The incidence of thrombotic and hemorrhagic events was low, so the thromboprophylaxis scheme used in patients with recovered pneumonia due to COVID-19 is recommended. Rivaroxaban is safe to use like thromboprophylaxis.

PB0255 | Cross-talk between the Inflammatory Response and Coagulopathy: Single-center Report

Y.B. Tor ${ }^{1}$; S. Kalayoglu Besisik ${ }^{2}$; M. Ozbalak ${ }^{2}$; M. Kose ${ }^{1}$;

A. Medetalibeyoglu ${ }^{1}$; N. Senkal ${ }^{1}$; A. Cagatay ${ }^{3}$; S. Simsek Yavuz ${ }^{3}$; A. Gul ${ }^{4} ;$ M. Erelel ${ }^{5} ;$ F. Esen ${ }^{6} ;$ T. Tukek ${ }^{1}$

${ }^{1}$ Istanbul University Istanbul Medical Faculty, Department of Internal Medicine, Istanbul, Turkey; ${ }^{2}$ Istanbul University Istanbul Medical Faculty, Department of Internal Medicine, Division of Hematology, Istanbul, Turkey; ${ }^{3}$ Istanbul University Istanbul Medical Faculty, Department of Infectious Diseases and Clinical Microbiology, Istanbul, Turkey; ${ }^{4}$ Istanbul University Istanbul Medical Faculty, Department of Internal Medicine, Division of Rheumatology, Istanbul, Turkey; ${ }^{5}$ Istanbul University Istanbul Medical Faculty, Department of Pulmonary Diseases, Istanbul, Turkey; ${ }^{6}$ Istanbul University Istanbul Medical Faculty, Department of Anesthesiology and Reanimation, Istanbul, Turkey

Background: Covid-19 appeared quaint with evolving hyperinflammation phase, vasculoendothelial dysfunction, and a distinct coagulopathy.

Aims: We present our experience regarding coagulopathy predictive factors in hospitalized Covid-19 patients just after pandemic declaration.

Methods: The data were obtained retrospectively by screening the institution's electronic data system between March and May 2020. The treatment protocol based on Health Ministry guidelines, includes hydroxychloroquine, azithromycin, favipiravir, low-molecular-weight heparin, dipyridamole, and anti-cytokine agents on the hyperinflammation phase. We stratified 3 groups, patients with proven coagulopathy, highly suspected coagulopathy, and patients without 
coagulopathy. Highly suspected coagulopathy encompasses clinical deterioration with sudden and inconsistent $\mathrm{D}$-dimer elevation.

Results: A total of 511 patients were screened. Forty-nine of them were excluded due to accompanying conditions resulting in high D-dimer levels. The median age of the remaining patients was 56 years with a male/female ratio of $284 / 178$. Proven coagulopathy as documented thrombosis developed in $3.2 \%$ with a male predominance (60\%). Highly suspected coagulopathy was decided in $10.1 \%$ of patients.

Among predictive factors for coagulopathy, the risk factors at admission were being over 65-year-old, having coronary artery disease, dyspnea, severe lymphopenia $(<500 / \mu \mathrm{l})$, monocytopenia $(<300 /$ $\mu \mathrm{l})$, and elevated LDH. For highly suspected coagulopathy, in addition to these having more than 3 comorbidities, high initial ferritin (>1000 $\mathrm{ng} / \mathrm{ml}$ ) and d-dimer levels as greater than $3600 \mathrm{U} / \mathrm{ml}$ were also predictive.

The clinical pictures in the proven coagulopathy group included 5 myocardial infarctions, 4 disseminated intravascular coagulation (DIC), 2 deep vein thrombosis, 1 catheter-related venous thrombosis, 1 catheter-related venous thrombosis, and pulmonary embolism, 1 lower extremity arterial thrombosis, 1 stroke. All DIC cases had gram-negative bacterial sepsis.

TABLE 1 Association of demographic features, comorbidities, disease characteristics, and initial serum biomarkers with coagulopathy and highly suspected coagulopathy

\begin{tabular}{|c|c|c|c|c|}
\hline \multirow[b]{2}{*}{ Factor } & \multicolumn{2}{|c|}{$\begin{array}{l}\text { Coagulopathy } \\
(n=15)\end{array}$} & \multicolumn{2}{|c|}{$\begin{array}{l}\text { Highly suspected coagulopathy } \\
(\mathrm{n}=47)\end{array}$} \\
\hline & positive / total & $\mathbf{p}$ & positive / total & $\mathbf{p}$ \\
\hline $\begin{array}{l}\text { Age } \\
\text { age }>=65 \\
\text { Age }<65\end{array}$ & $\begin{array}{l}9 / 138 \\
6 / 324\end{array}$ & 0.01 & $\begin{array}{l}27 / 138 \\
20 / 324\end{array}$ & $<0.001$ \\
\hline $\begin{array}{l}\text { CAD } \\
\text { present } \\
\text { absent }\end{array}$ & $\begin{array}{c}5 / 51 \\
10 / 409\end{array}$ & 0.005 & $\begin{array}{l}12 / 51 \\
35 / 409\end{array}$ & 0.001 \\
\hline $\begin{array}{l}\text { CHF } \\
\text { present } \\
\text { absent }\end{array}$ & $\begin{array}{l}2 / 30 \\
13 / 430\end{array}$ & 0.277 & $\begin{array}{l}8 / 30 \\
39 / 430\end{array}$ & 0.002 \\
\hline $\begin{aligned} \begin{array}{l}\text { Number of } \\
\text { comorbidities }\end{array} & \\
& >=3 \\
<3 & \end{aligned}$ & $\begin{array}{c}4 / 69 \\
11 / 389\end{array}$ & 0.202 & $\begin{array}{c}12 / 69 \\
35 / 389\end{array}$ & 0.034 \\
\hline $\begin{array}{l}\text { Dyspnea } \\
\text { present } \\
\text { absent }\end{array}$ & $\begin{array}{c}12 / 198 \\
3 / 263\end{array}$ & 0.003 & $\begin{array}{l}34 / 198 \\
13 / 263\end{array}$ & $<0.001$ \\
\hline $\begin{array}{l}\text { Initial disease } \\
\text { Severity } \\
\text { severe } \\
\text { moderate }\end{array}$ & $\begin{array}{l}8 / 137 \\
7 / 325\end{array}$ & 0.041 & $\begin{array}{l}28 / 137 \\
19 / 325\end{array}$ & $<0.001$ \\
\hline 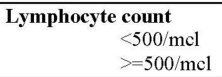 & $\begin{array}{c}5 / 32 \\
10 / 430\end{array}$ & $<0.001$ & $\begin{array}{c}8 / 32 \\
39 / 430\end{array}$ & 0.004 \\
\hline $\begin{array}{l}\text { Monocyte count } \\
\qquad \begin{array}{l}<00 / \mathrm{mcl} \\
>=300 / \mathrm{mcl}\end{array}\end{array}$ & $\begin{array}{c}5 / 70 \\
10 / 392\end{array}$ & 0.046 & $\begin{array}{l}12 / 70 \\
35 / 392\end{array}$ & 0.036 \\
\hline $\begin{array}{l}\text { Serum LDH } \\
\text { concentration } \\
\qquad \begin{aligned}>480 \mathrm{IU} / \mathrm{L} \\
<=480 \mathrm{IU} / \mathrm{L}\end{aligned}\end{array}$ & $\begin{array}{c}4 / 23 \\
10 / 435\end{array}$ & $<0.001$ & $\begin{array}{c}8 / 23 \\
36 / 435\end{array}$ & $<0.001$ \\
\hline $\begin{array}{l}\text { Serum ferritin } \\
\text { concentration } \\
>1000 \mathrm{ng} / \mathrm{ml} \\
<=1000 \mathrm{ng} / \mathrm{ml}\end{array}$ & $\begin{array}{c}1 / 68 \\
13 / 387\end{array}$ & 0.406 & $\begin{array}{l}14 / 68 \\
31 / 387\end{array}$ & 0.001 \\
\hline $\begin{array}{l}\text { Serum D-dimer } \\
\text { concentration } \\
>3600 \mathrm{U} / \mathrm{ml} \\
<=3600 \mathrm{U} / \mathrm{ml}\end{array}$ & $\begin{array}{c}2 / 3 \\
12 / 427\end{array}$ & 0.255 & $\begin{array}{c}8 / 31 \\
38 / 427\end{array}$ & 0.002 \\
\hline
\end{tabular}

Conclusions: Our data suggest coagulopathy is not directly correlated with inflammation severity but patients in hyperinflamation phase should be pursued for possible proven coagulopathy.

\section{PB0256 | Association of Elevated D-dimers with Severity and Outcome of Covid-19 Infection in Children}

N. Saqlain; N. Mazher; A. Bari; A. Ch; S. Farhan; N. Ahmed

The Children's Hospital \& ICH, Lahore, Pakistan

Background: COVID-19 caused by novel coronavirus (SARS-CoV-2) resulted in global pandemic. In Pakistan, the first case was reported on 26th February 2020. Out of total confirmed cases, children and adolescents $\leq 20$ years of age represented $10.6 \%$. Hypercoagulability has been reported in COVID-19 infection. Multiple adult studies have highlighted relation of elevated $D$ - dimers with disease severity. Aims: To determine association of $D$-dimers with the disease severity and outcome of children with COVID-19 and MIS-C/KD presenting to The Children's hospital, Lahore, Pakistan.

Methods: This cross-sectional study was done at The Children's Hospital, Lahore, from March 15 to December 31, 2020. We analyzed data of all laboratory-confirmed pediatric cases of severe acute respiratory syndrome coronavirus infection and post-COVID MIS-C/KD admitted to the Corona ward.

Results: Of 110 children, $73.6 \%$ were COVID and $26.4 \%$ postCOVID MIS-C/KD. $63.6 \%$ had mild-moderate illness followed by severe disease $21.8 \%$. In $41 \%$ there was a pre-existing comorbidity, the most common of which was congenital heart and chronic kidney disease. The mean age was $7.03 \pm 4.42$ years with a male preponderance $58.2 \%$. Fever (74.5\%), respiratory (46.4\%), and gastrointestinal symptoms (14.5\%) were the predominant symptoms. Ddimers were done in 67 patients with the mean of $3.68 \pm 2.08 \mu \mathrm{g} / \mathrm{ml}$ and a higher $D$-dimer value was significantly associated with severe and critical disease $(P=0.001)$. $D$-dimer levels were high in MIS-C $(4.61 \pm 4.0 \mu \mathrm{g} / \mathrm{ml})$ as compared to COVID $(3.05 \pm 4.6)(P=0.030)$. There were six deaths, comorbid conditions were associated with a fatal outcome in five cases and one death was due to MIS-C. A high $D$-dimer value was significantly associated with poor outcome $(P=0.009)$.

Conclusions: COVID-19 in children is generally mild with good outcome. A significant proportion of children presented with postCOVID MIS-C/KD. A high D-dimer value is significantly associated with severe disease and poor outcome. The presence of a co-morbid condition was a risk factor for poor outcome. 
PB0257 | Value of Laboratory Parameters in the Clinical Monitoring of Patients with SARS-COV-2 Infection (COVID-19)

M. Moreno Carbonell ${ }^{1}$; A. Gómez Martínez ${ }^{1}$; C. Hernández Mata ${ }^{1}$; E. González Gómez ${ }^{1}$; S. Martín-Consuegra Ramos ${ }^{1}$; M. Civeira Marín ${ }^{1}$; A. López Peña ${ }^{1}$; C. Rodríguez Lefler ${ }^{1}$; A.J. García Ortego; S.F. Pinzón Mariño ${ }^{2}$ N N. Fernández Mosteirin ${ }^{1}$; J.M. Calvo Villas ${ }^{1}$ ${ }^{1}$ Hospital Universitario Miguel Servet, Zaragoza, Spain; ${ }^{2}$ Hospital Del Mar, Barcelona, Spain

Background: Association between some analytical parameters and clinical evolution of patients infected by SARS-COV-2 is a controversial issue.

Aims: Review the relationship between laboratory parameters and severity and/or lethality of the COVID-19.

Methods: Observational, cross-sectional and retrospective study. Data obtained from electronic health record of patients over 18 years of age admitted in our hospital, between March and May 2020 with suspected SARS-COV-2 infection. After descriptive statistical study, we did an inferential analysis using Mann-Whitney $U$ test for independent samples, in order to verify association between the results of analytical parameters and the clinical severity (defined by hospitalization in conventional ward vs. intensive care units (ICU)) and/or lethality (alive vs. death at the end of the study).

Results: 668 patients were included, 345 men (52\%) and 323 women (48\%), with a median age of 70 years. $80 \%$ of patients had previous comorbidities, $10 \%$ were admitted to the ICU, and $17 \%$ died.Differences were found in hematimetric parameter between the different groups: leukocytes $\left(10^{3} / \mu \mathrm{l}\right): 8.3$ in ICU patients vs. 6.5 in those admitted to the ward; platelet count $\left(10^{3} / \mu \mathrm{l}\right)$ of $182 \mathrm{l}$ in death patients vs. 223 in survivors; hemostasis parameters: D-dimer (DD) ( $\mu \mathrm{g} / \mathrm{L})$ of 1379 and 1236 in deceased and admitted to the ICU respectively, compared to 734 and 786 in survivors and not admitted to ICU; and biochemical figures (increase in C-reactive protein (CRP), lactate dehydrogenase (LDH) or cardiac enzymes, the latter, especially, in patients who were deceased, with a median ultrasensitive troponin I (ng/L) of 34.75 vs. 7.1 in survivors. The differences were statistically significant ( $P$ value $<0.01$ ).

TABLE 1 ICU vs. Ward admitted/Defeated vs survival. Inferential statistics

\begin{tabular}{|c|c|c|c|c|c|c|c|c|c|}
\hline & a.ve & $w$ & Total & xal & $\begin{array}{l}\text { Ward } \\
\text { watituted }\end{array}$ & 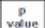 & Defonated & Survivat & po \\
\hline mood count & & & & & & & & & \\
\hline Hoesenogobin & (12-16.5) & $g / 2$ & $13.1(1.77)$ & $12.55(2.27)$ & $13.4(1.78)$ & 0.211 & $12.4(2.22)$ & $13.1(1.73)$ & 2014 \\
\hline Leukomes & $(3,6-10.5)$ & $1003 / 4$ & $68(6.97)$ & $8.3(4.72)$ & $6.5(7.2)$ & 0 & $8.35(7.29)$ & $6.5(6.86)$ & 。 \\
\hline Neutrophtils & (2.5.7.7) & $\operatorname{ton} x / \mu$ & $4.7(5.04)$ & $7.25(4.4)$ & $4.5(5.06)$ & 。 & $7.2(4.55)$ & $4 A 5(5.05)$ & 。 \\
\hline Linfocytes & (1.1.4.5) & $100 x / / \mu t$ & $1(2.24)$ & $0.7(0.4)$ & $1.1(2.46)$ & 。 & $0.7(5.25)$ & $1.1(0.87)$ & 。 \\
\hline Pistestints & $(140-770)=$ & $20 \times 3 / \mu$ & 217 (340.9) & $229(120.9)$ & $214(143.1)$ & 0.507 & $102(97.25)$ & 223 (1947.31) & 。 \\
\hline \begin{tabular}{|l|} 
Hoemostasia \\
\end{tabular} & & & & & & & & & \\
\hline$D D^{3}$ & $(0.500)$ & $\mu g / 2$ & $021(7015.58)$ & $3230(4701.19)$ & $7 n 0(72001.18)$ & 0 & $1279(4420.09)$ & $734(7420.4)$ & 。 \\
\hline $\mathrm{PT}^{\mathrm{4}}$ & $(0,5,-24,3)$ & $\mathrm{seg}$ & $129(0.05)$ & $13.1(2,04)$ & $12.9\langle 8,47\rangle$ & 0,121 & $13.4(15.72)$ & $32.9(4 . .55)$ & 0.013 \\
\hline $10 \pi^{5}$ & $(25 \cdot 37,9)$ & $\mathrm{seg}$ & $30\langle 5,7\rangle$ & $20.35(6.5)$ & 30 $(5,73)$ & 0.197 & $31.25(0.75)$ & $29.7(4.54)$ & 。 \\
\hline \begin{tabular}{|l} 
Biochemistry \\
\end{tabular} & & & & & & & & & \\
\hline wHo & $(0.248)$ & $u x$ & $279(147.03)$ & 400.5 (181.02) & $270(127,11)$ & 。 & $292(195.04)$ & $200(119.4)$ & 。 \\
\hline Fernen & (15-300) & nos/met & $617(6100.7\rangle$ & 1579.8(20071) & $600(6372.04\}$ & 0 & $766.9(1444.17)$ & $\begin{array}{l}509.6 \\
(60579,9\}\end{array}$ & 0.006 \\
\hline n- $-6^{2}$ & $(0-7)$ & se/mst & 29.10950 .927 & 78.15 (465.34) & $26.77(900.9)$ & 0.05 & $65.23(2243.64)$ & $\begin{array}{l}24,43 \\
(1173.07)\end{array}$ & 0.09 \\
\hline CnDos & $(0.0 .5)$ & $m g / d t$ & $7 A(0.42)$ & $15.24(12.29)$ & $7.2(0.66)$ & 0 & 11.87 (12..32) & $0.75(2.45)$ & 。 \\
\hline $\mathrm{PCT}$ & $(0.0 .5)$ & $\mathrm{mg} / \mathrm{mt}$ & $0.11(12.25)$ & $0.725(155.54)$ & $0.1(11.79)$ & 。 & 0.2 (20.5) & $0.09(2.13)$ & 。 \\
\hline Tn! $]^{\infty}$ & $(0-19.8)$ & $n g / 2$ & $8.3(404.43)\}$ & $14.1(51.4)$ & $8.1(420)$ & 0.002 & $34.75(732.46)$ & $7.1\{306.1\}$ & 。 \\
\hline
\end{tabular}

Af analytical data expressed as median (standard deviation). 1. Reference values in our iaborotary 2. Units of measurement. 3. D-dimer.

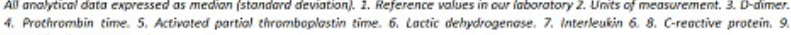
4. Prothrombin time. 5. Actioter.
Conclusions: Correlation exist between severity of infection and the increase in leukocytes, neutrophils, DD, CRP and LDH, and lymphopenia, in our sample. Decrease in the platelet count and the increase in cardiac enzymes were associated with an increased risk of mortality.

PB0258 | D-dimer Increase during Hospitalization is Associated with Negative Outcomes in Patients with COVID-19 Infection

E. Panchenko; E. Kropacheva; A. Komarov; A. Suvorov; F. Paveev;

N. Pogosova; S. Boytson

National Medical Research Center of Cardiology Ministry of Health of the Russian Federation, Moscow, Russian Federation

Background: Abnormal markers of coagulation including elevated D-dimers (DD) are associated with a poor prognosis in COVID-19 infection. However, the dynamics of DD have not been used consistently to identify patients at high risk of negative outcomes.

Aims: To identify the relationship between the changing of DD level and adverse outcomes in hospitalized patients with COVID-19 infection.

Methods: We retrospectively analyzed the data of 424 patients with PCR-confirmed SARS-CoV-2 infection from 759 pts, who were hospitalized at the National Medical Research Center of Cardiology between 15 April and 11 June, 2020. The median age was 61 years (IQR, 51-72 years); $55.7 \%$ were male. The median hospitalization period was 15 days (IQR 12.0; 19.0). During hospitalization, the majority of patients $(92.9 \%)$ received parenteral anticoagulants. The therapeutically dose of parenteral anticoagulants at any period of hospitalization received 174 pts (41,0\%). The delta of DD was calculated using initial and peak DD levels. End points were deaths from any cause, critical illness (sum of acute respiratory distress syndrome, multiple organ failure, and the need for any artificial ventilation), venous and arterial thrombotic events (TE) and major/clinical relevant bleedings BARC 2-5 during hospitalization.

Results: Frequency of deaths was $10.4 \%$, critical illness - $16.5 \%$, TE - $3.8 \%$, BARC 2-5 bleedings - 9.2\%. Significant correlations were found between the peak of DD level and the peak of C-reactive protein $(R 0.524 P=0.000)$, and the minimum of platelet count $(R-0.301$ $P=0.000$ ). Significant DD rising was found in severe COVID-19 patients with negative outcomes: death, bleeding, critical illness. 
TABLE 1 Initial, peak level and delta of D-dimer and outcomes in hospitalized patients with COVID-19 infection

\begin{tabular}{|c|c|c|c|c|c|c|}
\hline Outcomes & $\begin{array}{l}\text { D-dimer, initial level } \\
\text { (ng/ml) }\end{array}$ & $P$ & $\begin{array}{l}\text { D-dimer, peak level } \\
\text { (ng/ml) }\end{array}$ & $P$ & $\begin{array}{l}\text { Delta of D-dimer } \\
\text { (ng/ml) }\end{array}$ & $P$ \\
\hline $\begin{array}{l}\text { No death, Me [IQR } \\
25 ; 75 \%]\end{array}$ & $240[160 ; 410]$ & 0.001 & $320[195 ; 624]$ & 0.000 & $0[0 ; 128]$ & 0.005 \\
\hline $\begin{array}{l}\text { Death, Me [IQR } \\
\text { 25;75\%] }\end{array}$ & $560[315 ; 1145]$ & & 2160 [854; 3008] & & $535[0 ; 2222]$ & \\
\hline $\begin{array}{l}\text { Bleedings BARC } \\
\text { 2-5 (-), Me [IQR } \\
25 ; 75 \%]\end{array}$ & $245[165 ; 440]$ & 0.334 & $345[197 ; 688]$ & 0.000 & $0[0 ; 130]$ & 0.006 \\
\hline $\begin{array}{l}\text { Bleedings BARC (+), } \\
\quad \text { Me [IQR 25;75\%] }\end{array}$ & $370[217 ; 690]$ & & $1118[639 ; 24110]$ & & 512 [12; 1513] & \\
\hline $\begin{array}{c}\text { Thrombotic events (-), } \\
\text { Me [IQR 25;75\%] }\end{array}$ & $249[166 ; 444]$ & 0.059 & $360[209 ; 780]$ & 0.03 & $0[0 ; 164]$ & 0.471 \\
\hline $\begin{array}{l}\text { Critical illness (+), Me } \\
\text { [IQR 25;75\%] }\end{array}$ & 385 [219; 909] & & $1165[400 ; 2626]$ & & $381[0 ; 1476]$ & \\
\hline
\end{tabular}

Conclusions: D-dimer level and its increase during hospitalization associated with negative prognosis-determined outcomes in patients with COVID-19 infection.

PB0259 | Heparin-induced Thrombocytopenia (HIT) during Extracorporeal Membrane Oxygenation (ECMO) in COVID-19: A Case Report

G.A. Altamirano-Solorzano ${ }^{1}$; E. Cortina-de la Rosa ${ }^{1}$; F.A. GrimaldoGomez $^{1}$; V.M. Anguiano-Alvarez ${ }^{1}$; K.G. Cortes-Cortes ${ }^{1}$; M. PorresAguilar $^{2,3}$; G. Rojas-Velasco ${ }^{1}$; R. Izaguirre-Avila ${ }^{1}$

${ }^{1}$ National Institute of Cardiology Ignacio Chavez, Mexico City, Mexico;

${ }^{2}$ Department of Medicine, Division of Hospital Medicine, Northcentral Baptist Medical Center, San Antonio, Texas; USA and Department of Medicine, Division of Hospital Medicine, Sierra Providence Healthcare Network and Hospitals, El Paso, United States; ${ }^{3}$ Department of Medicine, Division of Cardiovascular Diseases; Texas Tech University Health Sciences Center and Paul L. Foster School of Medicine, El Paso, United States

Background: Heparin-induced thrombocytopenia (HIT) is an antibody-mediated reaction against the heparin-platelet factor 4 complex (H-PF4). Incidence in patients with extracorporeal circulation membrane (ECMO) is unknown.

Aims: We describe a case of HIT confirmed during ECMO support in a COVID-19 patient.

Methods: N/A
Results:

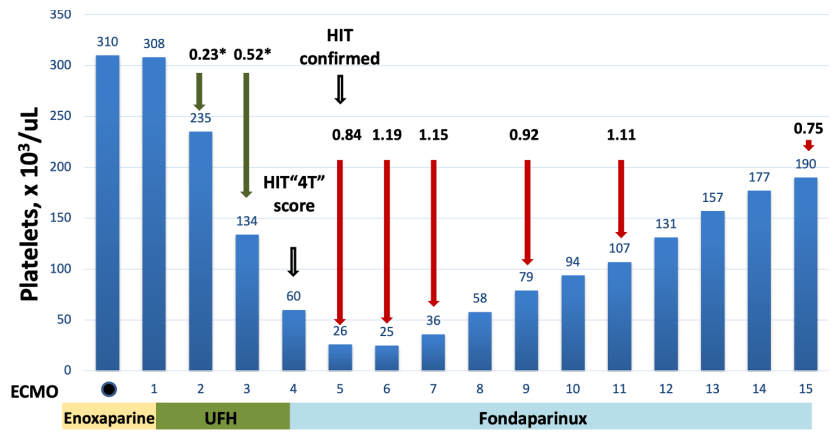

FIGURE 1 Platelet count in relation to time and response to the treatment. Blue bars: platelet count. Yellow bar: Enoxaparine $80 \mathrm{mg}$ BID, Green bar: infusion time with UFH (unfrationated heparine) 700 $\mathrm{UI} / \mathrm{h}$. Light blue bar: treatment time with fondaparinux $7.5 \mathrm{mg} \mathrm{SC}$. QD. Green arrows: anti-Xa levels for UFH expressed in IU/mL. Red arrows: serum levels of fondaparinux (micrograms $/ \mathrm{mL}$ )

A 48 year-old man with severe COVID-19 confirmed by RT-PCR for SARS-CoV2, was admitted to intensive care unit (ICU). After 21 days of the diagnosis he required mechanical ventilation support and V-V ECMO. Since his admission he received anticoagulation with enoxaparin $80 \mathrm{mg} \mathrm{SC}$ every $12 \mathrm{~h}$, when ECMO started, he continued anticoagulation with intravenous infusion of $700 \mathrm{U} /$ hour of unfractionated heparin (UFH). On the third day of infusion, there was a decrease in platelets $>50 \%$ (nadir $25,000 / \mathrm{mm}^{3}$ ), it was documented 6 points in a 4 T HIT score having a probability $64 \%$ for HIT. It was confirmed by functional test with platelet aggregometry induced by UFH using the Born method. UFH was suspended and fondaparinux $7.5 \mathrm{mg}$ SC every $24 \mathrm{~h}$. We made serial measurements of anti-Xa $\left(\operatorname{Stago}^{\circledR}\right)$. Therapeutic response to HIT was documented at day 9 from the start of fondaparinux, without requiring an ECMO membrane change. 
Conclusions: The diagnosis of HIT was made by clinical suspicion, using the 4T HIT score and later confirmation platelet aggregometryUFH induced. The usual pharmacological treatment is based on argatroban, bivalirudin, and lepirudin. To our knowledge, there is only one case reported with fondaparinux as treatment in ECMO. Determination of plasma levels by antiXa activity was used to guide dosing because previous studies have reported bleeding rates between 10 to $22 \%$ with fondaparinux. This is a success case of fondaparinux as treatment for HIT in an ICU patient with ECMO support, and the first one in the clinical context of severe COVID -19 infection.

PB0260 | Clinical Course of COVID-19 in Patients with Inherited Bleeding Disorders: An Interim Analysis of 28 Cases

A. Sikorska ${ }^{1}$; E. Stefanska-Windyga ${ }^{2} ;$ J. Windyga $^{1}$

${ }^{1}$ Department of Hemostasis Disorders and Internal Medicine, Institute of Hematology and Transfusion Medicine, Warsaw, Poland; ${ }^{2}$ Outpatient Clinic for People with Disorders of Hemostasis, Institute of Hematology and Transfusion Medicine, Warsaw, Poland

Background: The clinical course of severe respiratory syndrome Coronavirus 2 (SARS-CoV-2) in persons with haemophilia (PWH) and other congenital bleeding disorders (CBD) is not fully recognized despite worldwide spread of the disease.

Aims: The study aim was to describe the clinical course of COVID-19 in PWH and other CBD.

Methods: Between October 2020 and February 2021 we identified 28 cases of Coronavirus Disease 2019 (COVID-19) among PWH and other CBD treated in our Centre. COVID-19 was PCR confirmed in all.

Results: Of 28 patients aged 19-76 (median 46) enrolled in the study, 17 (61\%) had severe haemophilia A [5 with factor (F) VIII inhibitor], 3 - non-severe haemophilia A, 1 - severe haemophilia B, 5 - von Willebrand Disease, 1 - FVII deficiency, 1 - hypofibrinogenemia. At COVID-19 diagnosis the underlying bleeding disorder was treated with long-term prophylaxis in 18/28 (64\%) patients (the remaining patients were treated on-demand). Conditions/comorbidities that put our study patients at higher risk of severe COVID-19 were arterial hypertension $(11 / 28,39 \%)$, diabetes mellitus $(3 / 28,11 \%)$ obesity (5/28, 18\%). COVID-19 was mild in 27/28 (96\%) patients who required no hospitalization. For those patients no changes in therapy of underlying bleeding disorder were introduced with 5 exceptions:prophylaxis was intensified $(n=2)$, reduced $(n=2)$ or temporarily discontinued $(n=1)$. One severe haemophilia A patient without FVIII inhibitor (obese, arterial hypertension) required hospitalization in ICU for respiratory failure. He received low-molecular weight heparin for thromboprophylaxis (half therapeutic dose) with daily FVIII (35 IU/kg) replacement with no bleeding/thrombotic complications. All patients recovered with no bleeding/thrombotic complications. Several weeks after recovery from COVID-19 cardiac arrhythmia was identified In 2 patients.

Conclusions: The course of COVID-19 in most patients with haemophilia and allied disorders was mild with no significant impact on the therapy of the underlying bleeding disorder.

\section{PB0261 | Venous Thromboembolic Disease in Patients Wwith} SARS-CoV2 Infection: In Search of a Predictive Biomarker

P. Silva de Tena ${ }^{1}$; R. Gómez de Antonio ${ }^{1}$; R.M. Martín Rojas ${ }^{1}$; G. Perez Rus ${ }^{1,2,3}$; J.L. Díez-Martín ${ }^{1,2,3}$; C. Pascual Izquierdo ${ }^{1,2,3}$

${ }^{1}$ Hospital General Universitario Gregorio Marañón, Madrid, Spain;

${ }^{2}$ Gregorio Marañón Health Research Institute, Madrid, Spain;

${ }^{3}$ Universidad Complutense de Madrid, Madrid, Spain

Background: It is widely reported that patients infected with severe acute respiratory syndrome coronavirus 2 (Sars-CoV-2) are at increased risk of developing venous thromboembolic disease (VTD) requiring prophylactic heparin.

Aims: To describe predictive biomarkers at the time of emergency room (ER) admission that could predict the development of VTD in these patients.

Methods: From March 2020 to April 2020, 105 patients with COVID-19 were included in this retrospective study. Demographic characteristics, clinical history and blood test data at time of ER admission were collected. Data were compared using Mann-Whitney $U$ test for continuous variables and Chi-squared test for categorical variables. Univariate and multivariate logistic regression analysis were performed to identify independent impact on VTD risk.

Results: Forty-seven patients (44\%) presented thromboembolic event (group 1). Thirty-four (32\%) of them developed VTD during hospitalization while 13 patients (12\%) presented it at the time of ER admission. Blood test results at the time of ER admission are shown in Table 1.

TABLE 1 Baseline characteristics of the patients. Group 1: patients with VTD. Group 2: patients without VTD

$\begin{array}{llll} & \text { Group 1 (N: 47) } & \text { Group 2 (N: 58) } & \text { P-value } \\ \text { D-dimer (ng/dl) } & 3613(96-32967) & 2448(95-50354) & 0.007 \\ \text { Platelet count }(\mathrm{cell} / \mu \mathrm{L}) & 214000(46000-554000) & 216000(21000-519000) & 0.97 \\ \text { Lymphocyte count }(\mathrm{cell} / \mu \mathrm{L}) & 859(200-1800) & 998(200-3100) & 0.47 \\ \text { International Normalized Ratio } & 1.1(0.3-1.62) & 1.27(0.95-5.57) & 0.38 \\ \text { Fibrinogen }(\mathrm{mg} / \mathrm{dl}) & 716(440-949) & 741(232-1000) & 0.44\end{array}$




\begin{tabular}{|c|c|c|c|}
\hline & Group 1 (N: 47) & Group 2 (N: 58) & $P$-value \\
\hline Creatinine (mg/dl) & $1.03(0.52-2.07)$ & $0.92(0.49-1.48)$ & 0.072 \\
\hline Oxygen saturation level (\%) & $86 \%(55-98)$ & $92 \%(76-99)$ & 0.002 \\
\hline Length of stay (days) & $32(5-119)$ & $16(2-71)$ & 0.001 \\
\hline Exitus (\%) & 19.1 & 19 & 0.98 \\
\hline
\end{tabular}

Statistical differences between both groups were not significant in terms of personal background data collected: age, gender, cardiovascular risk factors, VTD risk factors (obesity, smoking, active cancer, previous immobilization, recent surgical intervention, former VTD or hormone therapy) and previous anticoagulant treatment. In group 1, 17 patients (36\%) required ICU admission vs 11 patients (19\%) in group $2(P=0.047)$. Representative univariate and multivariate regression analysis are shown in Table 2 . High D-dimer levels and low oxygen saturation levels were discovered to be independent predictors of VTD. In ROC curve analysis, D-dimer levels above 500 ng/dl detects VTD development with a sensitivity of $71.9 \%$ and a specificity of $58.6 \%$ (AUC: 0.672 ).

TABLE 2 Univariate and multivariate regression analysis. ICU: intensive care unit. OR: odds ratio

\begin{tabular}{|c|c|c|c|c|}
\hline & \multicolumn{2}{|c|}{ Univariate } & \multicolumn{2}{|c|}{ Multivariate } \\
\hline D-dimer > $500 \mathrm{ng} / \mathrm{dl}$ & 3.620 & 0.007 & 6.734 & 0.013 \\
\hline D-dimer > 1000 ng/dl & 5.444 & 0.001 & & \\
\hline Oxygen saturation level < 95\% & 6.289 & 0.002 & 11.650 & 0.002 \\
\hline Oxygen saturation level < $90 \%$ & 2,262 & 0.078 & & \\
\hline High flow oxygen therapy & 3.258 & 0.005 & 2.831 & 0.137 \\
\hline
\end{tabular}

Conclusions: In our study, higher D-dimer values and lower oxygen saturation levels at the time of ER admission seems to be predictors of development of VTD during hospitalization in patients with SarsCov2 infection.

\section{PB0262 | Anticoagulant Response to Tailored Low Molecular} Weight Heparin Regimens in Patients with COVID-19 Infection

A. Artoni ${ }^{1}$; V. De Zan ${ }^{1}$; M. Boscarino ${ }^{1}$; C. Novembrino ${ }^{1}$;

E. Scalambrino ${ }^{1}$; S. Aliberti ${ }^{2}$; M. Panigada ${ }^{3}$; I. Martinelli ${ }^{1}$; A. Tripodi ${ }^{1}$; F. Peyvandi ${ }^{1,4}$

${ }^{1}$ A Bianchi Bonomi Hemophilia and Thrombosis Center, Fondazione IRCCS Cà Granda Ospedale Maggiore Policlinico, Milan, Italy;

${ }^{2}$ Fondazione IRCCS Ca' Granda Ospedale Maggiore Policlinico, UOC Pneumologia, Milan, Italy; ${ }^{3}$ Fondazione IRCCS Ca' Granda Ospedale Maggiore Policlinico, UOC Anestesia e Terapia Intensiva Adulti, Milan, Italy; ${ }^{4}$ Department of Pathophysiology and Transplantation, Università degli Studi di Milano, Milan, Italy

Background: Covid-19 infection is associated with a widespread global activation of coagulation and affected patients are at an increased risk of thrombosis.

Aims: Heparin therapy is effective in various setting in preventing thromboembolic complications and aim of this study was to assess heparin response in COVID-19 patients through anti-FXa test.
Methods: In 52 patients, M:F ratio 59:41, median age 59 years old, admitted in different intensity of care units of our hospital, treated with different regimens of heparin $(100 \mathrm{U} / \mathrm{kg}$ every $24 \mathrm{~h}$ in low intensity care, $70 \mathrm{U} / \mathrm{kg}$ every $12 \mathrm{~h}$ in intermediate intensity care and $100 \mathrm{U} / \mathrm{kg}$ every $12 \mathrm{~h}$ in intensive care unit), anti-FXa levels were measured immediately before and $3 \mathrm{~h}$ after subcutaneous enoxaparin administration. On the same samples thrombin generation tests were performed.

Results: Patients treated with $100 \mathrm{U} / \mathrm{kg}$ every $24 \mathrm{~h}$ and $70 \mathrm{U} /$ $\mathrm{kg}$ every $12 \mathrm{~h}$ had median anti-FXa basal levels in the prophylactic range, respectively 0.18 and $0.22 \mathrm{U} / \mathrm{ml}$, while patients treated with $100 \mathrm{U} / \mathrm{kg}$ every $12 \mathrm{~h}$ were in the anticoagulant range (0.37 U/ $\mathrm{ml}$ ). Despite heparin therapy thrombin generation was elevated in COVID-19 patients, indicating a high level of coagulation activation. Conclusions: In conclusion we demonstrated that the biological response to enoxaparin in COVID-19 patients is in the expected range using anti-FXa assay and patients are not resistant to heparin therapy. 
PB0263 | Longitudinal Measurement of Whole Blood Viscoelastic Properties throughout the Course of Extracorporeal Membrane Oxygenation in COVID-19 Patients with Severe Acute Respiratory Distress Syndrome

M. Lejeune ${ }^{1,2}$; G. Hekimian ${ }^{1,2}$; P. Masi ${ }^{1}$; G. Lebreton ${ }^{1,2}$;

J. Chommeloux ${ }^{1}$; C. Desnos ${ }^{1}$; M. Pineton De Chambrun ${ }^{1}$; I. MartinToutain $^{1}$; A. Nieszkowska ${ }^{1}$; N. Bréchot ${ }^{1}$; M. Schmidt ${ }^{1,2}$; C.-E. Luyt ${ }^{1,2}$; P. Leprince ${ }^{1,2} ;$ A. Combes ${ }^{1,2} ;$. Frere $^{1,2}$

${ }^{1}$ Assistance Publique Hôpitaux de Paris, Paris, France; ${ }^{2}$ Sorbonne

Université, INSERM UMRS_1166, Institute of Cardiometabolism and

Nutrition, Paris, France

Background: Venovenous (vv)-extracorporeal membrane oxygenation (ECMO) support is used as rescue therapy in COVID-19 patients with severe acute respiratory distress syndrome (ARDS). However COVID-19 is associated with a hypercoagulable state with high rates of thrombosis. Whether ECMO implantation exacerbates COVID19-associated coagulopathy is unknown.

Aims: To perform a longitudinal evaluation of whole blood viscoelastic properties throughout the course of vv-ECMO in COVID-19 ARDS patients.

Methods: 20 COVID-19 patients undergoing vv-ECMO were included in this prospective study. Blood was sampled before ECMO implantation and then $24 \mathrm{~h}$ and 7 days after ECMO implantation. SEER Sonorheometry was performed on a Quantra ${ }^{\circledR}$ hemostasis analyzer with the QPlus ${ }^{\circledR}$ Cartridge (HemoSonics LLC). All patients received UFH to a target anti-Xa activity of $0.3-0.5 \mathrm{IU} / \mathrm{mL}$.

Results: The median age was 48 (42-58) years, with a median body mass index of $30.5(28.2-38.5) \mathrm{Kg} / \mathrm{m}^{2}$, and 15 (71\%) patients were men. The median SAPSII and SOFA scores on admission were 52.5 (44.3-65.5) and 12.0 (8.5-15.8), respectively. Baseline clot times (CT) and baseline clot times with heparinase (CTH) were within the normal range [median 150 (127-178) s and 129 (118-151) s, respectively] and did not vary throughout the course of ECMO. COVID-19 patients exhibited markedly increased baseline values of clot stiffness [CS, median 49.9 (35.5-69.2 hPA)], fibrinogen contribution to CS [FCS, median $12.80(6.20-20.10)$ hPA] and platelet contribution to CS [PCS, median 38.5 (28.7-52.4) hPA]. CS, FCS and PCS decreased from baseline to day 7. CT significantly correlated with aPTT $(r=0.75, P<0.0001)$, FCS with fibrinogen levels $(r=0.81$, $P<0.0001)$ and PCS with platelet count $(r=0.85, P<0.0001)$ but result delivery was much faster with the Quantra ${ }^{\circledR}$ analyzer compared to conventional tests ( 15 versus $\sim 60 \mathrm{~min}$ ).
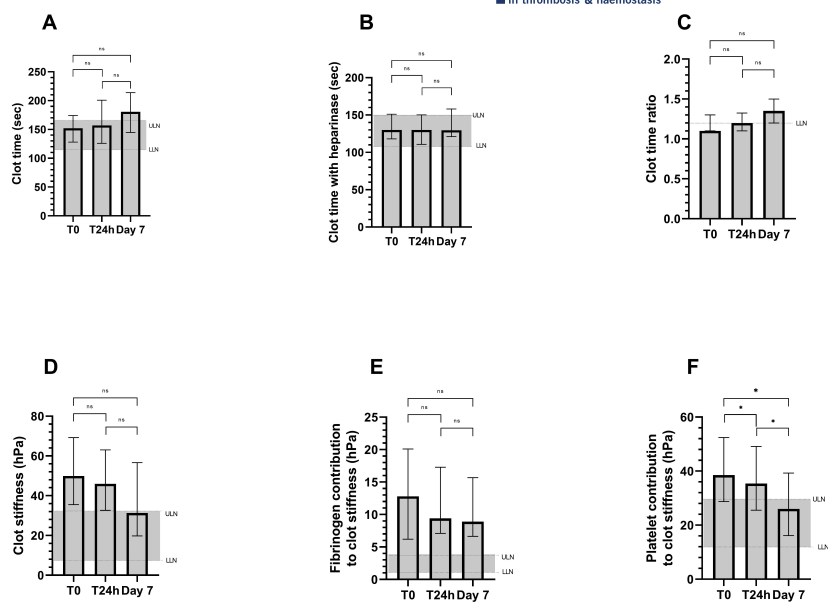

FIGURE 1 Viscoelastic properties of whole blood measured before (baseline), and $24 \mathrm{~h}$, and 7 days after vv-ECMO implantation.: clot time (A), clot time with heparinase (B), clot time ratio (C), clot stiffness (D), fibrinogen contribution to clot stiffness (E) and platelet contribution of clot stiffness (F); ns, non-significant; ${ }^{*} P<0.05$

Conclusions: COVID-19 patients with ARDS exhibited a pronounced baseline procoagulant state that partially resolved over the first 7 days of ECMO support.

PB0264 | Correlation of Coagulation Parameters with Prognosis of COVID-19

O. Smirnova ${ }^{1}$; O. Matvienko우 N. Korsakova ${ }^{1}$; A. Lerner ${ }^{2}$;

T. Shvedova ${ }^{2}$; O. Golovina ${ }^{1}$; L. Papayan ${ }^{1}$

${ }^{1}$ Russian Research Institute of Hematology and Transfusiology, SaintPetersburg, Russian Federation; ${ }^{2}$ Vsevolozhsk Clinical Interdistrict Hospital, Vsevolozhsk, Russian Federation

Background: COVID-19 demonstrates a high mortality because of rapidly progress to severe and critical cases with respiratory distress syndrome, coagulation dysfunction, multiple organ failure, etc. Therefore, early identification of the disease expansion is very important to the clinical diagnosis and treatment of COVID-19.

Aims: The aim of our study was to investigate the value of some coagulation parameters - prothrombin test (PT), activated partial thromboplastin time (APTT), fibrinogen (Fg), D-dimer (DD), factor VIII activity (FVIII), ristocetin-cofactor von Willebrand's factor activity (VWF:RCo), von Willebrand's factor antigen (vWF:Ag), antithrombin (AT) and homocysteine (HCY) - in predicting of prognosis of COVID-19.

Methods: These laboratory data were collected at hospitalization in 104 patients with COVID-19. Outcomes were divided into two types: hospital discharge $(n=81)$ and death $(n=23)$. Statistical analysis was performed by non-parametric methods (median (Me), 95\% confidence interval $(95 \% \mathrm{Cl})$ and Mann-Whitney $U$ test, Statistica 12.0), $P<0.05$ was considered statistically significant.

Results: Coagulation profiles observed in both groups of patients reflect a hypercoagulability, but PT and AT were significantly lower, 
while DD and HCY levels - significantly higher in patients with poor prognosis, and no difference in the other parameters was observed (data shown in the table).

\section{TABLE 1}

\begin{tabular}{lll}
$\begin{array}{l}\text { Parameter } \\
\text { (Me, 95\% CI) }\end{array}$ & $\begin{array}{l}\text { Recovery } \\
N=81\end{array}$ & $\begin{array}{l}\text { Death } \\
N=23\end{array}$ \\
PT, \% & $82.8(54.0-120.0)$ & $66.8^{*}(37.0-110.0)$ \\
APTT, s & $26.3(17.4-38.6)$ & $24.5(20.3-83.2)$ \\
Fg,g/I & $5.1(2.3-10.4)$ & $5.1(1.4-11.4)$ \\
DD,ng/ml & $342.0(63.0-3580.0)$ & $842.0^{*}$ \\
& & $(155.0-1336.0)$ \\
FVIII, \% & $136.2(49.3-307.7)$ & $130.5(53.6-330.9)$ \\
vWF:RCo, \% & $295.0(120.0-470.0)$ & $290.0(235.0-660.0)$ \\
vWF-Ag, \% & $216.2(131.3-790.7)$ & $238.2(149.9-1000.0)$ \\
AT, \% & $99.2(58.5-136.0)$ & $87.9^{*}(54.1-122.0)$ \\
HCY, $\mu \mathrm{mol} / \mathrm{I}$ & $15.6(6.9-39.0)$ & $21.2^{*}(12.1-31.1)$ \\
\hline
\end{tabular}

${ }^{*} \mathrm{P}<0.005$ with recovery patients.

Conclusions: The results of this study showed that hypercoagulation was present in patients with COVID-19. Severe coagulation dysfunction is more likely to occur in critically ill patients. PT, AT, DD and $\mathrm{HCY}$ could serve as diagnostic indicators for disease outcome.

PB0265 | Pulmonary Embolism in COVID-19-Patients: Diagnostic Accuracy of D-dimer

F. Laouan Brem ${ }^{1}$; C. Miri' ${ }^{1}$; H. Rasras ${ }^{1}$; A. Boudouh ${ }^{2}$; Y. Amane ${ }^{3}$; M.-A. Bouazzaoui ${ }^{4}$; S. Nasri ${ }^{3}$; I. Skiker ${ }^{3}$; N. Abda ${ }^{4}$; H. Kouismi ${ }^{2}$; Z. Bazid ${ }^{1} ;$ N. Ismailii, ${ }^{1,4}$; N. El Ouafi ${ }^{1,4}$

${ }^{1}$ Department of Cardiology, Mohammed VI University Hospital, Faculty of Medicine and Pharmacy of Oujda, Mohammed First University, Oujda, Morocco; ${ }^{2}$ Department of Pneumology, Mohammed VI University Hospital, Faculty of Medicine and Pharmacy of Oujda, Mohammed First University, Oujda, MAR, OUJDA, Morocco;
${ }^{3}$ Department of Radiology, Mohammed VI University Hospital, Faculty of Medicine and Pharmacy of Oujda, Mohammed First University, Oujda, MAR, Oujda, Morocco; ${ }^{4}$ Epidemiological Laboratory of Clinical Research and Public Health, Faculty of Medicine and Pharmacy of Oujda, Mohammed First University, Oujda, MAR, OUJDA, Morocco

Background: Since the spread of the novel Coronavirus 2(Sarscov2) lately in December 2019, proinflammatory and hypercoagulable states with marked elevation seen in D-Dimer levels have been widely described in COVID-19-patients even in the absence of pulmonary embolism (PE).

Aims: We sought to compare D-dimer levels in COVID-19-patients with and without pulmonary embolism and to establish an optimal D-dimer threshold for predicting the incidence of pulmonary embolism which guides the pulmonary computed tomography angiography (CTPA) indications.

Methods: We carried out a retrospective analysis of all COVID19-patients admitted between October 1st and November 22th, 2020, at the University Hospital Center of Mohammed VI, Oujda (Morocco) who were suspected to have PE and underwent a CTPA. Demographic, clinical, characteristics as well as blood test results at the time of PE suspicion, were compared between patients with and without PE. D-dimer levels were compared using the non-parametric test of U-man Whitney. The optimal D-Dimer' threshold to predict the occurrence of PE was established based on the receiver operating characteristics curve constructed.

Results: 84 confirmed-RT-PCR COVID-19-patients with a mean age of 64.93 years $(S D 14,19)$ were included in the final analysis. PE was diagnosed on CTPA in 31 (36.9\%) patients. Clinical symptoms and inhospital outcomes were similar in both groups except that more men had PE $(P=0.025)$. The median value of $D$-dimer was significantly higher in the PE-positive group compared to the PE-negative group (14680 [IQR 33620-3450] ng/mL vs 2980 [IQR 6870-1600] ng/mL respectively, $(P<0.001)$ ). (Table1) A D-dimer at $2600 \mathrm{ng} / \mathrm{mL}$ was the optimal threshold for predicting PE with a sensitivity of $90.3 \%$ and AUC was 0.773 [Cl 95\%,0.667-0.876).

TABLE 1 Demographic, clinical and biological finding of the study population

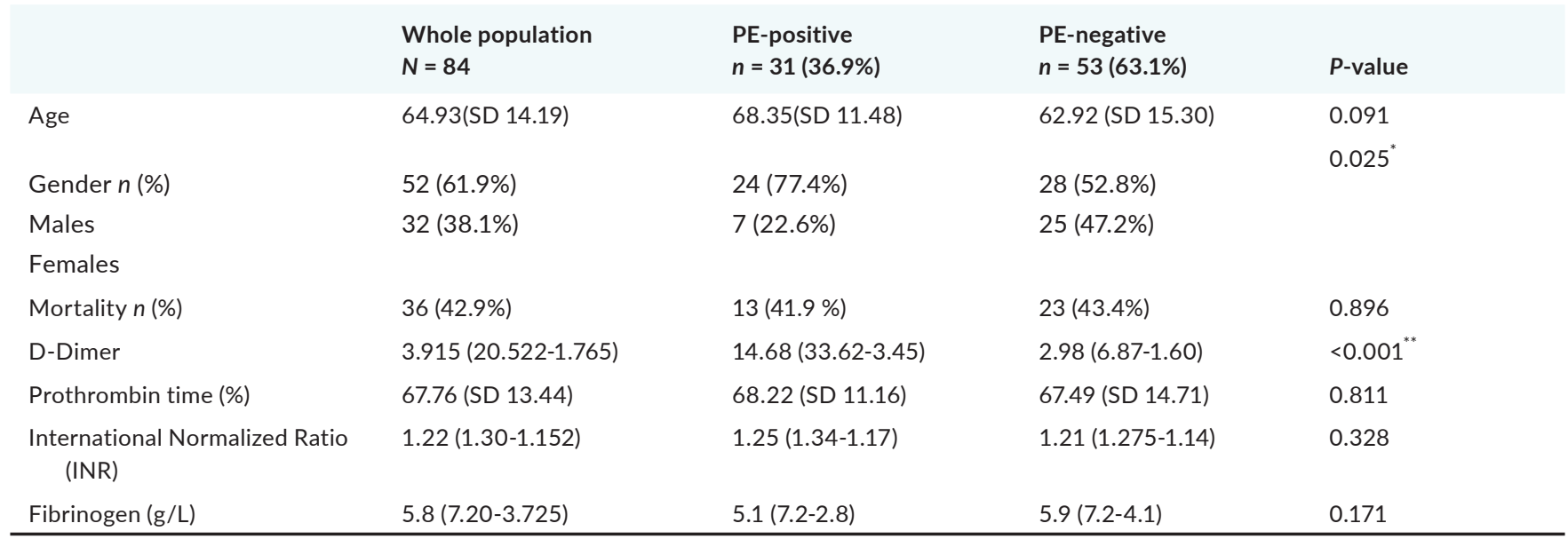


Conclusions: A D-dimer cut-off value of $2600 \mathrm{ng} / \mathrm{mL}$ is a significant predictor of PE in COVID-19-patients with a sensitivity of $90.3 \%$ and a higher threshold at $3285 \mathrm{ng} / \mathrm{mL}$ a with a sensitivity of $83.9 \%$.

PB0266 | Hemostasis Disorder in COVID-19 Patients: Disseminated Intravascular Clotting (DIC)?

E. Klychnikova; K. Popugaev; E. Tazina; S. Petrikov; S. Badygov;

\section{A. Bulanov}

N.V. Sklifosovsky Research Institute for Emergency Medicine, Public Healthcare Institution of Moscow Healthcare Department, Moscow, Russian Federation

Background: Hemostasis disorders in COVID-19 have specific clinical and laboratory features that differ from the "classic" form of DIC. Aims: To investigate the change in hemostasis indices in patients with severe course of COVID-19.

Methods: A prospective study of 122 patients (men 53\%, women $47 \%)$ with a severe course of COVID-19 was carried out. The age was $59 \pm 12$ years. The patients' condition, assessed by the NEWS and SOFA severity score, was $4.5 \pm 1.4$ and $6.1 \pm 1.3$ points. The control group was consisted of 20 apparently healthy donors. The research was performed on the 1st and 7th days from the moment of admission to the hospital.

Results: There was a significant increase of D-dimer's level in patients on the first day $(1.17(0.45-4.09) \mathrm{mg} / \mathrm{l}, \mathrm{p}<0.001)$ and on the 7th day (1.28 (0.46-3.23) mg/l, $\mathrm{p}<0.001)$. FG level was also significantly high in patients on the 1st day of the study (4.93 (3.49-6.11) $\mathrm{g} / \mathrm{l}, \mathrm{p}<0.001)$ and on day 7 (4.23 (3.15-5.68) g/l, p<0.001). PT was significantly lower in patients with COVID-19 than in the control group on the 1st day (78.7 (68.0-91.0) \%, $P=0.0003$ ) and remained within normal values on the 7th day. Platelet count was significantly lower in patients than in the control group on the 1st day (190 (127266) $10^{9} / \mathrm{I}, P=0.006$ ) and remained within normal values on the 7 th day. We calculated the index according to the ISTH's scoring scale and found that most patients with COVID-19 didn't score points to diagnose DIC-syndrome (Fig. 1).

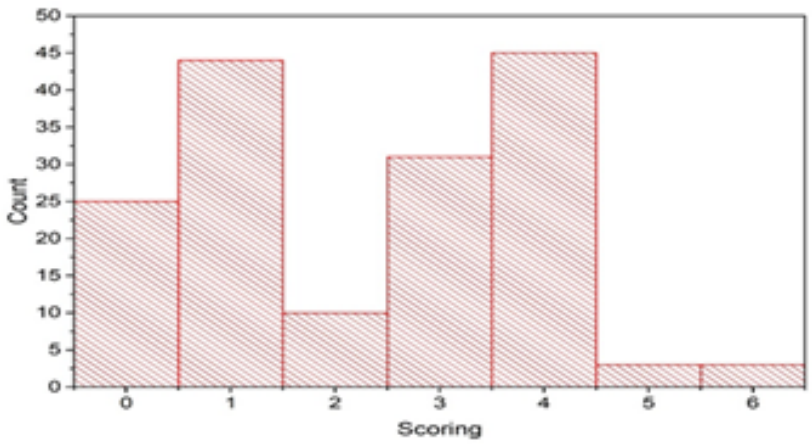

FIGURE 1 Scoring scale to confirm DIC in patients with COVID-19 who were being treated in N.V. Sklifosovsky Research Institute for Emergency Medicine
Conclusions: According to our data, prolongation of prothrombin time, thrombocytopenia and hypofibrinogenemia, decrease of antithrombin III in patients with COVID-19 on the 1st day and 7th were rare. Most patients showed a significant increase of $\mathrm{D}$-dimer, the rest of the hemostasis indices didn't correspond to the DIC-syndrome.

\section{PB0267 | Incidence of Disseminated Intravascular Coagulation} (DIC) in Critical Patients with Covid-19

C.F. Hernandez Mata; N. Fernández Mosteirín; I. Sancho Val;

M.d.M. Herrero Gutierrez; A. Esteban Molina; M. Peiro Chamarro;

A. Gómez Martínez; S. Martín-Consuegra Rámos; M. Moreno Carbonell; M. Civeira Marín; E. González Gómez; C. Rodríguez Lefler; A. López Peña; M.S. Ordas Miguelez; P.E. Lopez Gomez; J.M. Calvo Villas

Hospital Universitario Miguel Servet, Zaragoza, Spain

Background: A first publication by Tang et in March 2020 reported a high incidence of Disseminated Intravascular Coagulation (DIC) in patients who died from SARS-COV-2 (COVID-19) infection. Since then, some studies have questioned this high incidence.

Aims: To investigate the incidence of DIC in patients with confirmed diagnosis of severe COVID-19, who required admission to the ICU.

Methods: Observational, descriptive, and retrospective study that includes patients older than 18 years with severe COVID-19 infection admitted to the ICU of the "Miguel Servet" University Hospital, between March to November 2020. All clinical and laboratory parameters, as well as the clinical conduct, were requested and carried out according to the usual clinical practice.

Results: We included 130 patients $60 \%$ men, median age of 67 years (24-79), mortality of $40.8 \%, 9$ cases (6.9\%) of established DIC were documented, with impact in survival (Figure 1).

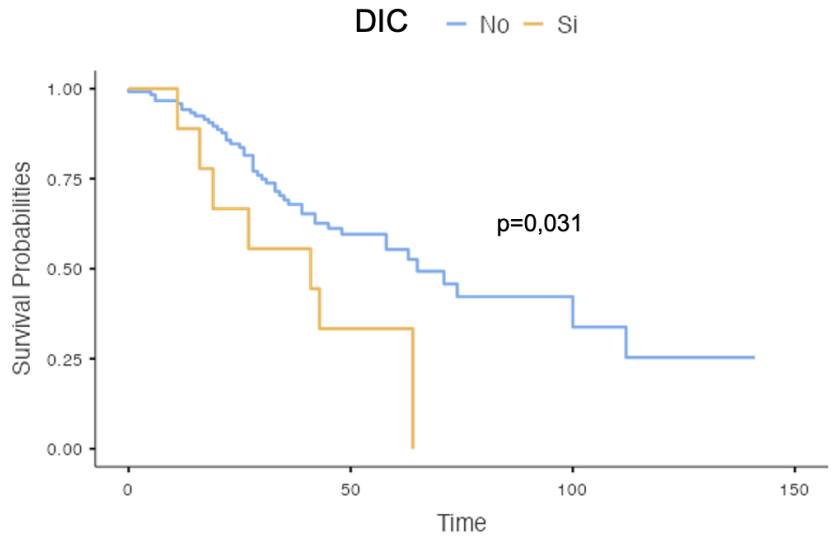

FIGURE 1 Survival in patients with and without DIC

No one with DIC had hypofibrinogenemia. More thrombosis and bleeding were found in patients with DIC (Table 1). 
TABLE 1 Overall results

Age, Median

(IQR) [range]

Years

Platelets me-

dian (IQR)

$[N=130]$

cell $\times 10^{3} / \mu \mathrm{L}$

PT, me-

dian (IQR)

$[N=130]$

$\mathrm{S}$

Fibrinogen, median (IQR)

[ $N=69]$

$\mathrm{g} / \mathrm{L}$

D-dimer, me-

dian (IQR)

$[N=130]$

$\mu \mathrm{g} / \mathrm{L}$

Thrombosis

None

Yes

Bleeding

None

Yes

APACHE,

Median

(IQR)

[range],

SOFA, Median

(IQR)

[range],
276 (195-386)

204 (125-355)

0.019

266

(181-376)

7 (5.7-7)

$7(6.4-7)$

0.13

7 (6.1-7)

$5.7(2.37-7)$

0.07
1960

(957-5215)
5447 (1736- 8330)

0.004

$2356(995-$
$6238)$

7933 (594125175)

0.107

$46(86.8)$

73 (94.8)

7 (13.2)

4 (5.2)

$113(93.4)$

$6(66.7)$

$8(6.6)$

3 (33.3)

0.055

40 (75.5)

68 (88.3)

$13(24.5)$

13 (9-18)

9 (11.7)

14 (10-19)

[7-38]

[4-38]

$11(8-15)$

[4-31]

$3(2-4)[1-14]$

$3(2-4)[1-10]$

$4(3-5)[1-14]$

$$
\begin{array}{ll}
103(85.1) & 5(55.6) \\
18(14.9) & 4(44.4) \\
12(9-18) & 16(10-24) \\
{[4-34]} & {[7-38]}
\end{array}
$$

$0.012 \quad 12(9-18) \quad 16(10-24)$

0.005

0.022

0.12

Conclusions: Mortality in our series is similar to that reported (40\%), the incidence in our series is higher than a recently published metaanalysis (6.9 VS 3\%).

The low incidence of DIC is interesting, because the high frequency of coagulopathy and elevation of D-dimer in the published series so, this raises the question, if there really is a classic DIC in patients with COVID-19. In our series, none of the patients with DIC presented hypofibrinogenemia. These findings support the theory that coagulopathy in COVID-19 patients is different from classic DIC and requires different management and classification. 
The published data suggest that patients with COVID-19 the coagulopathy is different from DIC, although, the ISTH score can identify a group of patients with a higher risk of complications and a worse outcome. This score must consider with other risk predictors, to increase surveillance or modify therapeutic measures.

PB0268 | Circulating Nucleosome Immunoassay: Evaluating a Clinically-applicable Test to Risk Stratify COVID-19 and Target Anticoagulation

C. Rea; S. Napoli; S. Mujib; F. Trovato; A. Zamalloa; J. Smith;

D. Nagra; K. O'Gallagher; M. McPhail

Kings College NHS Foundation Trust, London, United Kingdom

Background: Early therapeutic anticoagulation may decrease thrombotic and non-thrombotic complications in infection with SARSCoV-2 (COVID-19), improving outcomes. Accurately targeting only high risk patients would improve safety of this approach; bloodbased biomarkers to accurately risk-stratify would be of value. Dysregulated neutrophil extracellular traps (NETs) drive pulmonary inflammation, thrombosis and mortality and may therefore represent a useful biomarker. NETs determined via free DNA quantification are elevated in severe COVID-19 and correlate with outcome, but the technical requirements of these tests preclude their clinical use.

Aims: We assessed the potential of a clinically-applicable immunoassay for the quantification of cell free H3.1-nucleosome as a NETs biomarker and predictor of thrombosis and mortality.

Methods: Plasma samples on admission, day 3, 7 and 10 were evaluated from 20 patients with severe COVID-19 requiring organ support (severe cohort) and compared with 28 samples from COVID-19 patients requiring hospitalization, but not organ support (non-severe cohort). Circulating H3.1-nucleosomes were measured using Nu.Q ${ }^{\text {TM }}$ H3.1-nucleosome ELISA (Belgian Volition SRL, Isnes, Belgium) as per manufacturer's instruction.

Results: Patient demographics: Severe cohort: Age (median/ range) $=63$ (43-84). Gender distribution: $F=5, M=15$. Non-severe cohort: Age $=51.5(28-80) . F=11, M=17$. H3.1 nucleosome levels were significantly elevated in the severe versus non-severe cohort (Figure 1a). H3.1 nucleosome levels could not be used to predict thrombotic outcome, but there was an association with 28 day mortality, with significantly higher admission values recorded in ITU patients who died (Mann-whitney $P=0.014, n=6$ ) and comparatively higher H3.1-nucleosome values maintained during day 1-7 of ITU admission (Figure 1b).

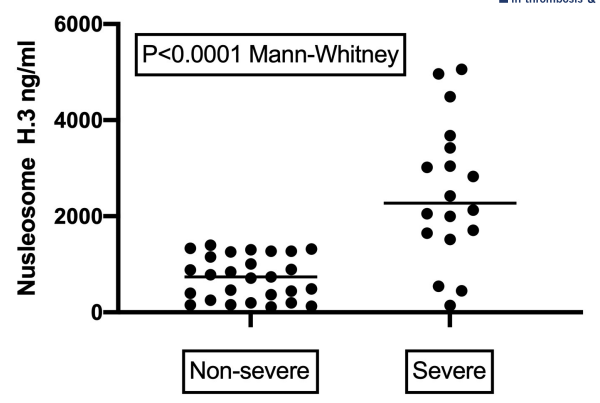

Figure 1a

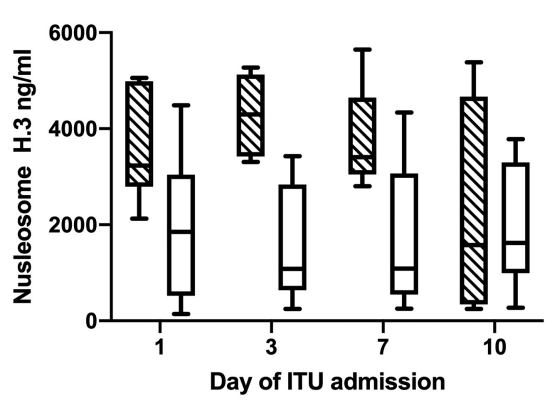

Death 28 days

$\square$ Survival 28 days

FIGURE 1 Panel 1a: Illustrates H3.1-nucleosome levels (ng/ml) in non-severe versus severe COVID-19 patients and the group median. Panel 1b: Charts the median and range of values for H3.1nucleosome levels $(\mathrm{ng} / \mathrm{ml}$ ) over the first 10 days of ITU admission in severe patients who survived versus those who died upto 28 days

Conclusions: The immunoassay NETs biomarker replicated findings of free DNA quantification studies; NETs correlate with disease severity. Although of no value in predicting thrombosis in this study, there is an indication that elevated values predict poor outcomes in patient admitted to ITU and may be of value in risk stratifying to treatments such as therapeutic anticoagulation and tracking response to treatment.

PB0269 | Immune Thrombocytopenia during Covid-19

Pandemic: An Italian Monocentric Experience

G. Lapietra; A. Ferretti; E. Baldacci; M.L. De Luca; S.M. Aprile;

F. Barone; S. Olivieri; A. Chistolini; C. Santoro

Unit of Hematology, Department of Translational and Precision

Medicine, Sapienza-University of Rome, Roma, Italy

Background: Since the pandemic caused by severe acute respiratory syndrome coronavirus-2(SARS-CoV2), known as COVID-19, has started in February 2020, different cases of immune thrombocytopenia (ITP) in patients (pts) affected by SARS-CoV2 have been reported. The management of COVID-19 in pts with simultaneous/previous ITP is challenging for the involvement of the haemostatic system.

Aims: To describe the management and outcome of pts with ITP and COVID-19.

Methods: Data were collected from clinical charts. All pts expressed their agreement. 
Results: 17 pts had RT-PCR confirmed SARS-CoV2 infection on a nasopharyngeal swab (October 2020-January 2021). Six pts were male (35.3\%), 11 female (64.7\%); median age 57 years (30-90). At the time of the infection, as regards ITP, pts were grouped as follows: 3 had simultaneous newly-diagnosed (ND) ITP (17.6\%) and 1 experienced a relapse (5.8\%) [median platelet count $5.5 \times 10^{9} / \mathrm{L}$ $\left.\left(2-30 \times 10^{9} / \mathrm{L}\right)\right] ; 7$ had chronic ITP on treatment (41.2\%) (eltrombopag, $n=5$; romiplostim, $n=1$; prednisone, $n=1$ ) and 2 had stable chronic ITP off-therapy (11.8\%) [(median platelet count $63 \times 10^{9} / \mathrm{L}$ $\left.\left(30-100 \times 10^{9} / \mathrm{L}\right)\right]$; $4 \mathrm{pts}$ had a previous ITP (complete responders, CR) on follow-up (23.6\%) (platelet count $>100 \times 10^{9} / \mathrm{L}$ ). Only 2 pts had no COVID-related symptoms (11.8\%). The most common symptoms were fever, anosmia, articular pain, mild-to-moderate respiratory distress. Three pts required hospitalization for acute platelet decrease and mucocutaneous bleeding (ND-ITP, $n=2$; relapse, $n=1)$ and were responsive to intravenous dexamethasone (40 mg/ day, days 1-4) and immunoglobulins (1 g/kg) (17.6\%). Three pts were hospitalized for pneumonia (ND-ITP, $n=1$; chronic ITP, $n=1$; CR, $n=1$ ) and required antibiotics and oral corticosteroid (17.6\%). Eleven pts recovered at home without bleeding; their platelet count did not show any change at the evaluation after quarantine (64.7\%). All pts had seroconversion; no death occurred.

Conclusions: ND-ITP triggered by COVID-19 is responsive to immunoglobulins and steroids. Outcome is favourable also for COVID-19 pts with off-therapy or on treatment ITP.

PB0270 | After the Storm; 6-month Follow up Outcomes and Management of COVID-19 Related Venous Thromboembolism

M. Ahmed; R. Khan; E. Xenou; M. Fowler; S. Sinha; A. Lokare;

C. Kartsios

Haematology Department, Heartlands Hospital, University Hospitals

Birmingham, Birmingham, United Kingdom

Background: Thrombotic events that frequently occur in COVID-19 are predominantly venous thromboemboli (VTE) and are associated with increasing disease severity and worse clinical outcomes.

Aims: In this audit we sought to distinguish COVID-19 hospitalised patients with a VTE diagnosis over a period of 5 months (01/02/2020-30/06/2020). We recorded their intermediate-term management and clinical outcomes.

Methods: We identified 54 patients with a diagnosis of COVID and VTE (M/F:29/25, median age:68 (29-94) years. VTE was the presenting symptom on admission in $71 \%$ of patients. Pulmonary embolism was diagnosed in $92 \%, 15 \%$ required intensive care and $5.5 \%$ underwent thrombolysis.

Results: Following VTE diagnosis, 37 patients were treated with unfractionated heparin/enoxaparin while 16 (apixaban: 12, rivaroxaban: 4) were treated with direct anticoagulants (DOACs). 70\% (38/54) patients recovered successfully; Upon discharge, $92 \%$ of patients were on DOACs (apixaban: 29, rivaroxaban: 5, edoxaban: 2) while only 2 continued enoxaparin. After a median follow up of 241 (0-350) days,
36 (66\%) patients were alive. Most deaths (12/18) occurred within a week following admission, another 4 patients died within a month and they were 2 late deaths (on days +114 and +198 ). 14 patients had their anticoagulants stopped at 3 months while 9 patients remained on anticoagulation between 3 and up to 6 months. 12 patients received anticoagulation for $>6$ months with a clinical decision to offer lifelong anticoagulation cover in 8/12 patients. We recorded one VTE recurrence $(2 \%)$ following anticoagulant discontinuation, one episode of major and one episode of minor bleeding. One patient developed pulmonary hypertension while post-COVID interstitial lung disease was diagnosed in 4 patients and 4 patients developed long COVID.

Conclusions: DOACs are safe and effective in COVID-related VTE. COVID is a complex clinical entity; In our patient cohort, $15 \%$ required lifelong anticoagulation following VTE.

PB0271 | Association between Race and the Incidence of Thrombosis in Hospitalized COVID-19 Patients Across a New York City Hospital System

P.P. Theprungsirikul; S.E. Saith

NYU Langone Health/NYU Grossman School of Medicine, New York, United States

Background: The 2019 novel coronavirus disease (COVID-19) is associated with significant thrombotic complications, contributing to the excess mortality observed with severe disease. Currently, there is insufficient evidence to support therapeutic dose anticoagulation in hospitalized COVID-19 patients outside of clinical trials. There is a greater need to understand the epidemiological and clinical factors associated with thrombotic complications.

Aims: To investigate the clinical characteristics in self-reported Asian race, who were hospitalized with COVID-19 early in the pandemic and the incidence of thrombosis.

Methods: We conducted a retrospective observational study of index hospitalizations for all patients, age $\geq 18$, with confirmed SARS-CoV-2 infection between March 13 and April 4, 2020 across two hospitals in New York City. Clinical demographics including selfreported race and admission labs were collected. Confirmed arterial and/or venous thrombosis were confirmed with imaging during the encounter with chart review.

Results: There were 118 patients, who self-identified as Asian, hospitalized with COVID-19 during the study period. During their hospitalization, there were 24 patients (20.3\%) who experienced thrombotic event during their encounter. There were 14 events described as venous thrombosis (7 deep venous thrombosis, 6 pulmonary embolisms, 1 superficial vein thrombosis) and 10 who experienced an arterial thrombosis (5 cerebrovascular accidents, 4 myocardial infarctions and 1 with splenic infarction). Body mass index $(P=0.027)$ and a history of cancer $(P=0.026)$ were significant demographic factors associated with thrombosis. $D$-dimer $(P$ $=0.045)$, $C$-reactive protein $(P<0.001)$, ferritin $(P=0.024)$ and troponin I $(P=0.036)$ were significantly elevated on admission. 
TABLE 1 Demographic and Clinical Characteristics of Hospitalized COVID-19 Patients with Asian Race by Incidence of Thrombotic Events

\begin{tabular}{|c|c|c|c|c|}
\hline & Total $(n=118)$ & $\begin{array}{c}\text { Overall } \\
\text { Thrombosis* } \\
\text { (n=24) }\end{array}$ & $\begin{array}{l}\text { No Thrombosis } \\
\quad(n=94)\end{array}$ & $p$-value \\
\hline Age, Years, Median (IQR) & $62(48-71)$ & $62(51-68)$ & $62(47-72)$ & 0.988 \\
\hline Female Gender, $\mathrm{n}(\%)$ & $50(42.4)$ & $8(33.3)$ & $42(44.7)$ & 0.315 \\
\hline BMI, kg/m², Median (IQR) & $25.8(22.3-29.5)$ & $27.5(24.4-32.3)$ & $25.5(21.9-28.8)$ & 0.027 \\
\hline \multicolumn{4}{|l|}{ Smoking Status, n (\%) } & 0.954 \\
\hline Current & $4(3.4)$ & $0(0.0)$ & $4(4.3)$ & \\
\hline Former & $17(14.4)$ & $3(12.5)$ & $14(14.9)$ & \\
\hline Never & $72(61.0)$ & $16(66.7)$ & $56(59.6)$ & \\
\hline Unknown & $25(21.2)$ & $5(20.8)$ & $20(21.3)$ & \\
\hline \multicolumn{5}{|l|}{ Admission Labs, Median (IQR) } \\
\hline $\mathrm{D}$-dimer $(\mathrm{ng} / \mathrm{mL}$ ) & $361(215-622)$ & $498(331-796)$ & $314(196-619)$ & 0.045 \\
\hline ESR $(\mathrm{mm} / \mathrm{hr})$ & $97(66-117)$ & $106(81-116)$ & $91(53-117)$ & 0.167 \\
\hline $\operatorname{CRP}(\mathrm{mg} / \mathrm{L})$ & $86(40-140)$ & $139(72-238)$ & $68(32-135)$ & $<0.001$ \\
\hline Ferritin $(\mathrm{ng} / \mathrm{mL})$ & 841 (294-1811) & $1456(659-3022)$ & $795(203-1678)$ & 0.024 \\
\hline Platelets $(1,000 / \mu \mathrm{L})$ & $190(139-244)$ & $203(131-281)$ & $189(142-243)$ & 0.919 \\
\hline Troponin I (ng/mL) & $0.032(0.017-0.11)$ & $0.095(0.0-0.955)$ & $0.03(0.017-0.085)$ & 0.036 \\
\hline \multicolumn{5}{|l|}{ Comorbidities, $n(\%)$} \\
\hline Cancer & $14(11.9)$ & $6(25)$ & $8(8.5)$ & 0.026 \\
\hline Coronary Artery Disease & $11(9.3)$ & $4(16.7)$ & $7(7.5)$ & 0.231 \\
\hline Atrial Fibrillation & $2(1.7)$ & $1(4.2)$ & $11(1.1)$ & 0.367 \\
\hline COPD & $4(3.4)$ & $0(0.0)$ & $4(4.3)$ & 0.581 \\
\hline Asthma & $16(13.6)$ & $5(20.8)$ & $11(11.7)$ & 0.314 \\
\hline CVA & $1(0.9)$ & $0(0.0)$ & $1(1.1)$ & 1.000 \\
\hline Seizure & $1(0.9)$ & $0(0.0)$ & $1(1.1)$ & 1.000 \\
\hline Hypertension & $57(48.3)$ & $15(62.5)$ & $42(44.7)$ & 0.119 \\
\hline Diabetes & \begin{tabular}{|l|l|}
$39(33.1)$ \\
\end{tabular} & $9(37.5)$ & $30(31.9)$ & 0.604 \\
\hline \multicolumn{4}{|c|}{ Anticoagulation Prior to Admission, $\mathbf{n}(\%)$} & 0.001 \\
\hline Warfarin & $1(0.9)$ & $1(4.2)$ & $0(0.0)$ & \\
\hline DOAC & \begin{tabular}{|l|}
$7(5.9)$ \\
\end{tabular} & $5(20.8)$ & $2(2.1)$ & \\
\hline Mortality, $n(\%)$ & $36(30.5)$ & $12(50)$ & $24(25.5)$ & \\
\hline
\end{tabular}

Abbreviations - BMI: Body Mass Index; COPD - Chronic Obstructive Pulmonary Disease; CVA - Cerebrovascular Accident; ESR - Erythrocyte Sedimentation Rate; CRP - C-reactive Protein; DOAC - Direct Oral Anticoagulant; IQR -

Interquartile Range

*Overall Thrombosis includes venous and/or arterial thrombotic events: pulmonary embolism (PE), deep vein

thrombosis (DVT), CVA, myocardial infarction (MI), acute limb ischemia, and splenic infarct, among others

Conclusions: There are differences in clinical characteristics on admission in self-identified Asians, associated with thrombosis in COVID-19. Understanding these associated factors is helpful to delineate both the spectrum and pathophysiology of COVID-19.

\section{PB0272 | Hemostasis Abnormalities in Post-COVID-19}

\section{Syndrome}

M. Noniashvili; T. Saralidze; T. Svanidze; T. Kandashvili; I.

Mamatsashvili

Tbilisi State Medical University, Tbilisi, Georgia

Background: Coronavirus disease 2019 (COVID-19) causes a spectrum of disease; some patients develop a severe proinflammatory state which can be associated with a unique coagulopathy. Initially, COVID-19 infection produces a prominent elevation of fibrinogen and D-dimer/fibrin(ogen) degradation products. This is associated with systemic hypercoagulability and frequent venous thromboembolic events. The degree of D-dimer elevation positively correlates with mortality in COVID-19 patients. Here, we review the current state of knowledge of COVID-19 and hemostasis.

Aims: Aim of our study was investigation of hemostasis in patients with post-COVID-19 syndrome (PCS).

Methods: Materials and methods: We studied 136 patients with PCS, 78 were men, 58 - women. Average age was $49.9 \pm 4.7$ years. Control group included 20 healthy people. Patients were divided into 3 groups. In the 1st group were united 20 patients with chronic respiratory diseases (CRD) and chronic cardiovascular diseases
(CCVD), in second group - 56 patients with different CRD, in third - 60 patients with CCVD. Fibrinogen concentration (F), D-dimer, activated partial thromboplastin time (APTT), and prothrombin index (P) with INR were studied in all patients and control group by standard methods.

Results: For the $1^{\text {st }}$ and $3^{\mathrm{d}}$ groups was characteristic increased level of F, d-dimer and APTT, compared with healthy persons. In the $2^{d}$ group $\mathrm{F}$ level was also increased comparing with control group. $\mathrm{P}$ and INR was similar to control group in all 3 groups, though in $1^{\text {st }}$ and $2^{\text {d }}$ groups tendency of $\mathrm{P}$ diminishing was revealed. $\mathrm{F}$ and $\mathrm{d}$-dimer level was high, but different in all groups. In the $1^{\text {st }}$ group $\mathrm{F}$ and d-dimer level was higher on $18.8 \%$, than in the $3^{\mathrm{d}}$ group and higher on $14.5 \%$, than in $2^{\mathrm{d}}$ one.

Conclusions: Hemostasis abnormalities revealed in the patients with comorbid diseases points to high risk of thromboembolic complications and underlines the necessity of monitoring coagulation tests for appropriate treatment.

\section{PB0273 | Is Procalcitonin Involved on the Severity of COVID-19?}

A. Zakeri ${ }^{1}$; S. Matin ${ }^{1}$; E. Rezaei Ghalechi ${ }^{2}$

${ }^{1}$ Department of Internal Medicine, School of Medicine, Ardabil

University of Medical Sciences, Ardabil, Iran, Islamic Republic of; ${ }^{2}$ Duke Medical Center Department of Surgery, Durham, United States

Background: Inflammatory response plays a key role in Coronavirus Disease 2019 (COVID-19) as it has been shown that the resulting cytokine storm increases its severity.

Aims: This study aimed to investigate the role of procalcitonin (PCT) on patients afflicted with severe forms of COVID-19.

Methods: This study was a retrospective cohort study. For the purpose of this research, 150 severe cases of COVID-19 who were hospitalized in ICU were investigated. The required data about patients' demographic information, clinical and laboratory findings, and their PCT level were collected upon their admission to the hospital and from the disease outcome data. Then the needed statistical analyses were performed on them.

Results: From the 150 patients who entered the study, 77 cases were discharged from the hospital alive. The mean age of the patients was $60.92 \pm 16.33$ years and $51.3 \%$ of them were male. The mean PCT level of the deceased cases was $2.43 \pm 3.40$ while that of the surviving cases was $0.73 \pm 1.28$, which represented a statistically significant difference between the two groups in this regard. Results indicated that PCT can be used as one of the independent factors for predicting mortality.

Conclusions: Serum PCT level can predict mortality and disease outcome in patients suffering from severe forms of COVID-19. 
PB0274 | National Survey about Management of Venous Thromboembolism (VTE) Prevention in COVID-19 Patients in Uruguay

A. Pizzarossa; M.N. Spangenberg; F. Munka; B. Borges; M. Sosa;

F. Passarella; M. Aguirre; E. Falero; C. Guillermo

Unidad de Hemostasis y Trombosis. Hospital de Clínicas. Facultad de Medicina, UdelaR, Montevideo, Uruguay

Background: Coagulopathy is one of the serious manifestations of COVID-19, however this infection is also related to an increased incidence of VTE. VTE prevention in these patients was not standardized, especially at the beginning of the pandemic.

Aims: To evaluate the VTE prevention strategies in patients with COVID-19 in Uruguay.

Methods: Descriptive observational study, based on an online survey, whose target population includes physicians from Uruguay who assisted COVID-19 patients. It was available online from 4/9 to $5 / 10 / 2020$. However, given the increase in cases in Uruguay, the survey was relaunched in March 2021.

Results: 42 physicians completed the survey. 29 assisted patients with moderate and 17 assisted patients with severe COVID-19 (4 assisted both groups).

Moderate COVID (required hospitalization)

23 used pharmacological and 2 used mechanical thromboprophylaxis. 12 used a LMWH dose of $40 \mathrm{mg} /$ day, 7 used $1 \mathrm{mg} / \mathrm{kg} /$ day, 4 used $60 \mathrm{mg} /$ day. 13 had doubts about whether to indicate thromboprophylaxis.10 requested D-dimers and in 9 cases they were augmented.

Severe COVID (required admission to ICU)

All used pharmacological thromboprophylaxis. 5 used a LMWH dose of $1 \mathrm{mg} / \mathrm{kg}$ every $12 \mathrm{~h}, 4$ used $40 \mathrm{mg} /$ day, 4 used $1 \mathrm{mg} / \mathrm{kg} /$ day, 2 used $60 \mathrm{mg} /$ day and 1 used $1.5 \mathrm{mg} / \mathrm{kg} /$ day.

16 requested $\mathrm{D}$ dimers, in all cases they were increased.

Regarding the preliminary results of the second phase. 17 doctors completed the survey, all of them cared for patients with moderate COVID. 14 used pharmacological thromboprophylaxis, 12 with doses of LMWH of $40 \mathrm{mg} /$ day and 2 with doses of $1 \mathrm{mg} / \mathrm{kg} /$ day. 5 had doubts whether to indicate thromboprophylaxis. 11 cared for patients with severe COVID, 10 used pharmacological thromboprophylaxis (5 used LMWH $1 \mathrm{mg} / \mathrm{kg}$ every 12 h, 2 used $1 \mathrm{mg} / \mathrm{kg} / \mathrm{day}$ and 3 used $40 \mathrm{mg} /$ day).

Conclusions: A heterogeneous approach to VTE prevention has been observed among physicians who cared for COVID-19 patients.
PB0275 | The Role of D-dimer in Prognosis of Patients with Pulmonary Thrombembolism: Some Aspects for COVID-19

M. Bosevski ${ }^{1}$; G. Krstevski ${ }^{1}$; E. Dodic ${ }^{2}$; K. Kapsarov ${ }^{3}$; N. Petkovic ${ }^{4}$; National Registry on Venous Thrombembolism ${ }^{1}$ St. Cyril and Methodius Faculty of Medicine, University Cardiology Clinic, Skopje, North Macedonia, The Republic of: ${ }^{2}$ General Hospital Kumanovo, Kumanovo, North Macedonia, The Republic of; ${ }^{3}$ University Clinic for Infective Diseases, Skopje, North Macedonia, The Republic of; ${ }^{4}$ General Hospital 8th September, Skopje, North Macedonia, The Republic of

Background: Despite the diagnostic role of D-dimer in the identification of pulmonary thrombembolism (PE), many questions still remain regarding its potential role in the clinical prognosis of these conditions. In recent times, PE has taken on new importance, as a common clinical exacerbation following disease state of COVID-19.

Aims: Paper aims to assess the predictive value of $D$-dimer when combined with other parameters (prothrombin time, platelet count, ultrasound parameters and risk factors) in the long term prognosis of patients with PE. Additionally, a group of patients with COVID-19 were tested for D-dimer to establish a prognostic role for the biomarker.

Methods: Here we present, stable, clear data from a national registry taken from multiple centers. 29 participants (1st group on age $58.7 \pm 9$ ) were recruited on hospitalization for moderate to severe COVID-19 and followed up during hospitalization. 84 patients with PE (2nd group, on age $60.3+12.5$ ), were selected and followed up for 6 months.

Results: Significant differences in D-dimer levels at admission were noted in COVID-19 patients who would require artificial ventilation $(1250 \pm 210 \mathrm{ng} / \mathrm{ml})$ compared to those who did not (650 $\pm 175 \mathrm{ng} /$ $\mathrm{ml}$ respectively; $P<0.05$ ). However multivariate analysis did not show independent value of $\mathrm{D}$-dimer in a relation to clinical course of these pts. Number of comorbidities was presented as a risk factor for poorer COVID-19 outcomes.

In PE pts regression analysis revealed that $\mathrm{D}$-dimer level (mean value $2654.5 \pm 420.3 \mathrm{ng} / \mathrm{ml}$ ) is a predictor for length of hospitalization ( $\beta$ $0.25, P 0.05)$, together with right ventricular diameter as a factor for duration of anticoagulation.

Conclusions: Our results confirmed the predictive value of D-dimer when added on top of echocardiographic parameters in relation to the prognosis of patients with pulmonary embolism. This biomarker might be superior to other thrombotic biomarkers in COVID-19 pts in a relation to their clinical course. 
PB0276 | Optimization of Operational Flow of D-dimer Test in a Core Laboratory in a Brazilian Tertiary Hospital to Ensure Operational Efficiency during the COVID-19 Pandemic

J. Guerra; V. Aranda; C. Mendes; A. Villarinho; C. Monteiro;

\section{R. Penteado}

Albert Einstein Hospital, São Paulo, Brazil

Background: D-dimer tests are very important tests in follow-up and treatment of patients affected by infection by SARS-COV-2. These results are directly related to morbidity and mortality. Due to high and sudden demand, our institution faced to an unacceptable risk of lack of reagents, concomitantly to an increase of employees sick pay in the laboratory's team.

Aims: The present study aims to demonstrate the need to modify the flows of processing the laboratory tests in a critical situation, and with it offer to our clients a quick and reliable result.

Methods: Before the pandemic, D-dimer tests were performed in two different methodologies. First, by immunoturbidimetry and for confirmation, just for results above $500 \mathrm{ng} / \mathrm{ml}$, a immunoenzymatically test used to realized, both by automated equipment. This check was necessary for decreasing the risk for false-positive in patients affected by thrombosis. In the face of a risk scenario, we performed a fishbone diagram to identify possible causes for an effect or problem and a PDCA (plan-do-check-act) cycle to minimize the risks (Figure 1). In the first phase (PLAN), we planned a many actions involving doctors and laboratory professionals with the aim of reduce by $80 \%$ of repetitions of this test in 6 months. In the second phase (DO), we started a new flow operational, performed the test with just one methodology with close monitoring by medical team.

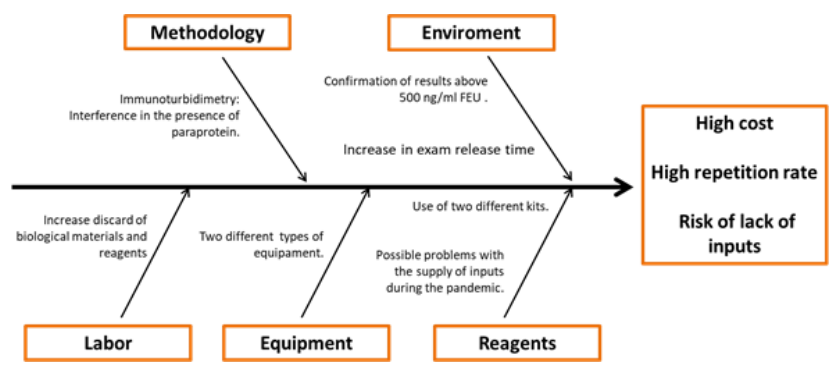

FIGURE 1 Fishbone diagram (Ishikawa diagram)

Results: Checking our operational results (third phase), was observed $96 \%$ decrease in repetitions in 6 months (April to September) with an important cost reduction from around $27 \%$ (Figure).

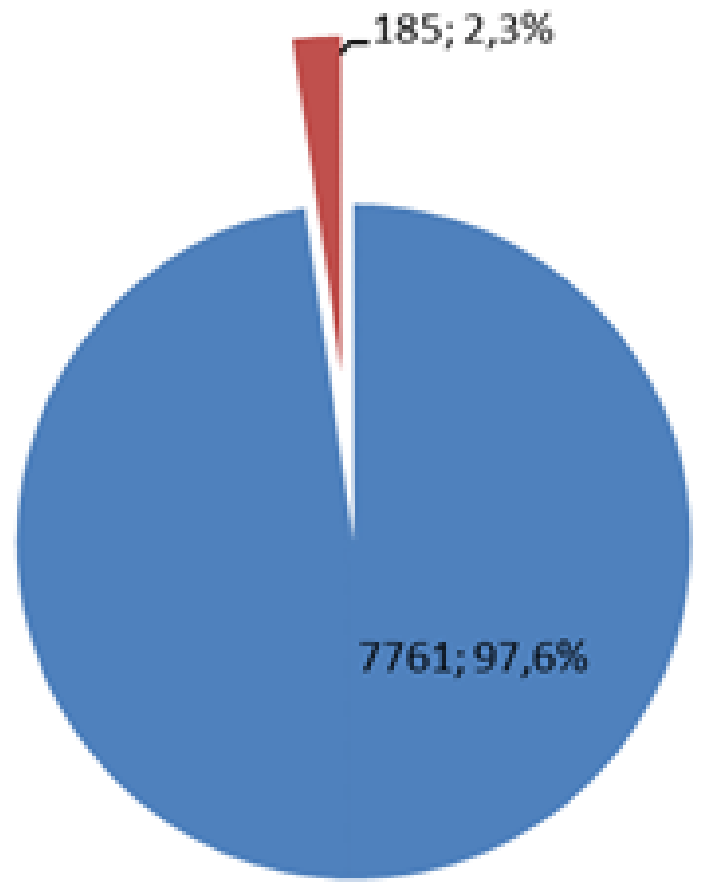

Results > $500 \mathrm{ng} / \mathrm{ml} \mathrm{FEU} \quad$ Repetitions

FIGURE 2 Repetition rate of the d-dimer test after modifying the operational flow

Conclusions: This joint work enabled a optimization of the equipments and reagents, wilth a improvement of our produtivity without losses of quality and security. Now we are in the last phase, when we are consolidating our new flow, with quarterly evaluations and continuous monitoring of technical and economical results.

PB0277 | The Effect of Covid-19 Lockdown on Patients Taking Warfarin

\section{Roberts}

University of Glamorgan, Pontyprydd, United Kingdom

Background: Warfarin is one of the most widely used anticoagulants in the United Kingdom. Patients taking Warfarin need regular International Normalised Ratio (INR) blood tests to ensure that their levels remain within a set therapeutic range. When data showed a drop in patient TTR during lockdown, and an increase in high range INR results, a nursing strategy was developed to improve these issues in the event of further lockdown restrictions. Patient education was reassessed, amendments were made to clinic attendance and steps were taking to evaluate if patients could be switched from Warfarin to a Direct Oral Anticoagulant (DOAC).

Aims: The aims of this study were to determine if the covid-19 induced lockdown of 2020 had an impact on patient INR results and patient time in range.

Methods: Following discussions with patients, during lockdown, it was found that many were reporting lifestyle changes. Many had 
taken up exercise, stopped drinking alcohol, and subsequently lost weight. Other patients had performed less exercise and stated they felt they gained weight. It was decided to study patient TTR trends to determine if patients had found that Covid-19, specifically the period of lockdown (March 232020 to 4th July 2020), had impacted their TTR. DAWN AC Software, was used to study data 14 weeks before the first case of Covid-19 in the UK, 14 weeks pre lockdown, 14 weeks duration of lockdown and the subsequent 14 weeks post lockdown, to monitor any general changes in patient time in range. Results: Results showed patient TTR was impacted. Patients TTR was worse during the period of lockdown and more likely to report high INRs.

Conclusions: Discussions were held with patients to increase options regarding testing, switching to DOACs and re-education was made a priority. Letters were sent to GPs and all patients reminding them of warfarin interactions and the study results.

PB0278 | Glenzocimab, a Novel Antiplatelet Therapy in COVID-19 Related Acute Respiratory Distress Syndrome (ARDS): The GARDEN Study, a Phase 2 Clinical Trial

A. Comenducci' ${ }^{1}$; S. Lebel-Binay ${ }^{1}$; G. Avenard ${ }^{1}$; J. Pottecher ${ }^{2}$; V. Sato ${ }^{3}$; M. Jandrot-Perrus ${ }^{4}$; Y. Plétan ${ }^{1}$

${ }^{1}$ Acticor Biotech, Paris, France; ${ }^{2}$ Strasbourg University Hospital, Strasbourg, France; ${ }^{3}$ Internacional Research Center, Hospital Alemão Oswaldo Cruz, Sau Paulo, Brazil; ${ }^{4}$ UMR_S 1148 Inserm University of Paris, Paris, France

Background: Glenzocimab is a fully-humanized fragment of monoclonal antibody directed against the human platelet glycoprotein $\mathrm{VI}$ (GPVI), which is involved in the formation, growth and stability of thrombi. Thrombosis mechanism in COVID-19 has not been completely elucidated. In severe COVID-19 disease, platelets may contribute to both micro and macro-thrombosis (particularly involving lung vasculature) and to the inflammatory syndrome. Furthermore, evidence involving platelets in lung fibrosis, via GPVI, have been described. In COVID-19-induced ARDS, developing new treatments aiming at reducing the contribution of platelets to uncontrolled lung inflammation and at preventing pro-thrombotic downstream complications is an unmet medical need. Such treatments should also be safe, avoiding undesirable bleeding.

Aims: The primary objective of GARDEN, a phase 2 clinical trial, is to offer an additional treatment option to SARS-CoV-2 infected patients presenting with an ARDS, and beyond, preventing downstream complications due to pro-thrombotic and pro-inflammatory conditions.

Methods: GARDEN (NCT04659109) is a randomized, double blind, multicenter, placebo-controlled, parallel group, fixed dose phase $2 b$ clinical trial. Sixty evaluable patients will be randomized in a $1 / 1$ ratio to glenzocimab (1000 mg/day for 3 days, 6 h IV infusion) or to a matching placebo. Main inclusion criteria are: a symptomatic and biologically-confirmed SARS-CoV-2 infection with signs of moderate but progressive pulmonary disease; a prothrombotic status and elevated inflammation markers. Patients requiring invasive mechanical ventilation or with a disseminated intravascular coagulation are excluded. The primary objective is to evaluate the effect of glenzocimab in preventing clinical progression of disease when added to standard-of-care. Secondary safety endpoints include mortality, chest CT scan, SAEs and bleeding-related events.

Results: GARDEN study has been approved in France and Brazil. To date, 16 patients have been recruited, no safety signal requiring a DSMB evaluation occurred.

Conclusions: The study is expected to be completed by June 2021 .

PB0279 | Change in Platelet Indices in Patients with Coronavirus Infection Disease (COVID-19): A Reflection of Platelet Activation and Contribution to Immunothrombosis?

M. Mezgebe; B.F. Jacobson; S. Louw

University of the Witwatersrand (WITS), National Health Laboratory

Service (NHLS), Johannesburg, South Africa

Background: Activation of the coagulation system, as reflected by raised D-dimers, is prevalent in patients with COVID-19 resulting in coagulopathies as well as venous and arterial macro- and microthromboses. Laboratory tests in patients admitted with COVID-19 should include prothrombin time (PT) and D-dimers. The role of platelets in COVID-19 related immunothrombosis has not been extensively documented.

Aims: Investigate platelet indices in patients with COVID-19 with raised D-dimer levels and compare the results to COVID-19 infected patients with normal D-dimer levels as well as to COVID-19 uninfected patients with raised D-dimers.

Methods: This retrospective observational study was approved by the Human Research Ethics Committee of the University of the Witwatersrand (M201010). The laboratory results of patients admitted to a quaternary referral centre in Johannesburg, South Africa from June to December 2020 were reviewed. Platelet indices including platelet large cell ratio (P-LCR), mean platelet volume (MPV) and platelet distribution width (PDW) were recorded and analysed in the following 3 cohorts: 281 COVID-19 patients with raised D-dimers, 72 COVID-19 patients without raised D-dimers and 51 COVID-19 negative patients with raised $\mathrm{D}$-dimers and disseminated intravascular coagulation (DIC).

Results: The platelet indices P-LCR, MPV and PDW in COVID-19 positive patients were statistically different from patients without COVID-19 ( $P<0.001)$ (Table 1). No statistically significant difference in the platelet indices was however observed between COVID-19 patients with and without raised D-dimers. 
TABLE 1 Platelet parameters in COVID-19 positive patients with and without raised D-dimers vs. COVID-19 negative patients with raised D-dimers

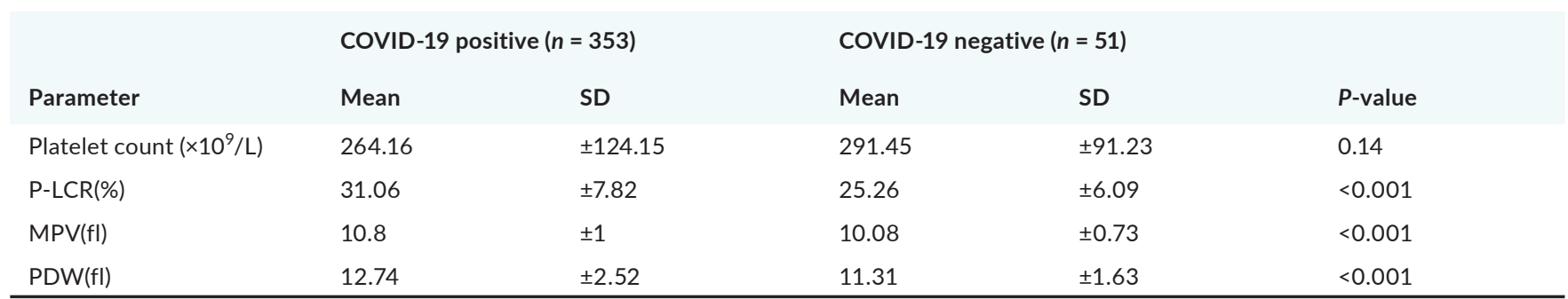

COVID-19, Corona virus disease-19; n, number of patients; SD, standard deviation; P-LCR, platelet large cell ratio; MPV, mean platelet volume; PDW, platelet distribution width.

Conclusions: Platelet laboratory indices are statistically different between COVID-19 positive and COVID-19 negative patients but were not predictive of the presence of an underlying coagulopathy in COVID-19 as reflected by elevated D-dimer levels. The alterations in platelet indices in COVID-19 patients probably reflect activation of platelets and contribution to immunothrombosis.

PB0280 | Anti-coagulant Drugs among Moderate, Severe and Critically III COVID 19 Patients in the Second Referral Hospital in Surabaya

P.Z. Romadhon; S.D. Suryantoro; C. Windradi; B.A. Mahdi;

E.D. Agustin; D. Novendrianto; K.N. Widiastuti; M. Savitri;

P.N.A. Amrita; M Noordiansyah; S.U.Y. Bintoro; A. Ashariati

University of Airlangga, Faculty of Medicine, Surabaya, Indonesia

Background: The establishment of definitive treatment against COVID-19 infection has not yet reached the finish line. Thrombosis and hypercoagulation in advanced stages are other vital issues. Further study on how to use anticoagulants is still being analyzed in order to embody the best prognosis.

Aims: Evaluating the use of anticoagulants in moderate to critically ill COVID-19 patients.

Methods: We conducted a retrospective cohort study involving 218 patients with moderate to critically ill conditions, in which 42 of them received anticoagulants. All cause-mortality, length of stay, and the average day of death were investigated.

Results: Of the 42 patients who received anticoagulants, 18 cases of moderate COVID were given anticoagulants and there was no death with a length of stay of 16.1 days vs 12.58 days without anticoagulants $(P=0.054)$. Five severe cases of COVID were given anticoagulants and no deaths occurred in severe cases with a length of treatment of 13.2 days vs 16.57 days without anticoagulants $(P=$ 0.315). In cases of critical illness, death occurred in 9 cases of 19 who were given anticoagulants compared to 9 deaths that occurred in 33 cases without anticoagulants with a comparison of length of treatment of 15.8 vs 15 days (without anticoagulants) $(P=0.72)$. The mean days of death on anticoagulant administration in critically ill cases were 20.7 days and 25.3 days in patients without anticoagulants $(P=0.274)$.

Conclusions: The administration of anticoagulants to moderate to critically-ill COVID-19 patients did not ameliorate the length of treatment and all-cause mortality.

PB0281 | Prevalence, Clinical Profile and Bleeding Risk of Hospitalized COVID-19 Patients Receiving Anticoagulation: A Retrospective Cross-sectional Study

\section{Imperial}

University of Santo Tomas Hospital, Manila, Philippines

Background: A major complication of COVID-19 is arterial and venous thromboembolism. It complicates hospitalized patients and contributes to adverse outcome. The use of anticoagulation to prevent thrombosis in COVID-19 is recommended. However, with use of anticoagulants there is always the risk of bleeding.

Aims: Determine the prevalence of bleeding in hospitalized COVID-19 patients on anticoagulation. And determine association of bleeding with their clinical profile.

Methods: Retrospective, cross-sectional study. Included all hospitalized confirmed COVID-19 patients $>18 \mathrm{y} / \mathrm{o}$ via RT-PCR on anticoagulation. Mean and standard deviation were used for continuous data, while frequency and percentage for categorical variable. Bleeding prevalence is presented with a $95 \%$ confidence interval. Univariate and multivariate logistic regression to determine the factors that affect the occurrence of bleeding was done in final analysis with level of significance at $5 \%$.

Results: A total of 83 patients where included in the study. The mean age at diagnosis is 61 years old (SD \pm 17.3 ) with predominance of males, $53 \%(n=44)$. The most common comorbid disease is Hypertension (72.3\%). The prevalent severity of COVID-19 that patients had was moderate disease $(n=39,47.6 \%)$ followed by critically ill ( $n=27,32.9 \%$ ). The prevalence of bleeding is $36.1 \%$, with the most frequent source of major bleeding is gastrointestinal bleeding at $36.7 \%$. No association was found in the level of inflammatory markers, Platelet count, PT, aPTT, Fibrinogen and D-dimer with the risk of bleeding. The is increased risk of bleeding in patients on anticoagulation with severe to critical COVID-19 with an OR $3.82(P-$ value 0.0175 ) and increased risk of death in patients who received anticoagulation and had bleeding with an OR 8.40 ( $P$-value 0.0004). 
Conclusions: The risk of major bleeding is increased with anticoagulation. Bleeding is associated with high rate of mortality in patients with COVID-19. Comprehensive evaluation of patients with COVID-19 who will receive anticoagulation is imperative to prevent morbidity and mortality.

\section{PB0282 | Hematologic Parameters of Ghanaian COVID-19 Patients at Diagnosis}

D. Fiawoo $^{1}$; B. Opoku-Asare ${ }^{2}$; E. Olayemi ${ }^{1}$

${ }^{1}$ Department of Haematology, Korle Bu Teaching Hospital, Accra, Ghana; ${ }^{2}$ Department of Internal Medicine,Korle Bu Teaching Hospital, Accra, Ghana

Background: The Corona virus disease 2019 (COVID -19) is caused by an RNA virus that belongs to the betacorona (betacov2) genus. The first confirmed cases in Ghana were found March 13, 2020.The clinical spectrum noted in Covid-19 patients range from asymptomatic to critical. Mild disease is defined by symptoms such as fever, loss of taste and smell etc. without dyspnoea or abnormal imaging. Moderate cases have evidence of lower respiratory disease with $\mathrm{SpO}_{2} \geq 94 \%$ on room air. Severe cases have $\mathrm{SpO}_{2}<94 \%$ on room air, respiratory rate of $>30$ cycles/min, lung infiltrates $>50 \%$. Abnormal laboratory findings in Covid-19 infection include elevated d-dimers, thrombocytopenia and lymphopenia and these findings vary among countries.

Aims: To evaluate baseline haematologic parameters in Covid-19 patients and determine any association between them and severity of the disease.

Methods: A retrospective study of Covid-19 positive patients seen at the Fevers and Medical unit of the Korle-bu Teaching Hospital (KBTH), Accra Ghana between April - November 2020 was conducted. A total of 109 out of 320 Covid-19 positive patients with complete laboratory parameters were included in this study and divided into 2 groups: Symptomatic and Asymptomatic. Symptomatic patients had moderate or severe disease and Asymptomatic cases had mild disease or no symptoms. Data extracted include the baseline d-dimer and platelet count. The study was approved by the ethics committee, KBTH.

Results: $60.6 \%$ (66/109) were Symptomatic and 39.4\% (43/109) were Asymptomatic. There was no significant difference between platelet count in both groups (Fishers exact $=1$ ). There was an association between high D-dimer concentration and symptomatic infection $(P=0.00001)$.

TABLE 1 Difference in Platelet levels

\begin{tabular}{lll} 
PLATELET COUNT & & \\
$\left(\times 10^{9} / \mathrm{L}\right)$ & SYMPTOMATIC & ASYMPTOMATIC \\
$\leq 150$ & 6 & 3 \\
$>150$ & 60 & 40 \\
\hline
\end{tabular}

Fishers exact test $=1$ Not significant at $P<0.05$
TABLE 2 Difference in D-dimer levels

\begin{tabular}{lll} 
D-DIMER (mg/l) & SYMPTOMATIC & ASYMPTOMATIC \\
$\leq 1$ & 19 & 31 \\
$>1$ & 47 & 12 \\
\hline
\end{tabular}

Chi square analysis : $P$-value $=0.00001$

Conclusions: Our study shows an association between elevated d-dimer concentration and symptomatic (moderate or severe) Covid-19 infection, which agrees with previous studies. Monitoring of the d-dimer concentration may help identify patients at increased risk of venous thromboembolism and poor outcomes.

\section{PB0283 | Hematologic Abnormalities in Moderate Forms of Covid-19}

M. Ben Ayed; M. Chelli; H. Baccouche; S. Saffar; A. Chakroun;

S. Mahjoub; N. Ben Romdhan

La Rabta Hospital, Tunis, Tunisia

Background: The number of COVID-19 infected patients is dramatically increasing worldwide. Although this infection is primarily manifested as a respiratory tract infection, emerging data indicate that it may be also a systemic disease involving multiple systems including hematopoietic system and hemostasis.

Aims: This study aimed to describe the main hematologic abnormalities in patients with moderate forms of covid-19 and their course during hospitalization.

Methods: We conducted a prospective study including 87 patients with coronavirus infectious disease hospitalized in departments other than Intensive Care Unit between November $16^{\text {th }}$ and December $30^{\text {th }}$ 2020. Hematologic investigations were performed on admission and during hospitalization including Blood count, Ddimers and fibrinogen.

Results: Among 87 patients, 52 were males and 35 were females. The mean age was 65 years old [26-100]. The average length of hospitalization was 11 days [1-39]. Of the 87 COVID-19 infected patients, 58 (67\%) had neutrophilia during hospitalization, 7 (8\%) had leucopenia, 63 (72\%) had lymphopenia, 13 (15\%) had thrombocytopenia, 12 (13.8\%) had thrombocytosis and 19 patients had myelemia $>5 \%$. Regarding D-dimers, 82 (94\%) patients had d-dimers $>500 \mathrm{ng} /$ $\mathrm{ml}$ and 68 (78\%) had d-dimers $>1000 \mathrm{ng} / \mathrm{ml}$. Concerning fibrinogen, 34 (39\%) patients had a rate of fibrinogen $>4 \mathrm{~g} / \mathrm{L}$ and 21 (17.9\%) patients had a rate of fibrinogen $>6 \mathrm{~g} / \mathrm{L}$.

The results of hematologic investigations performed on admission, day 5 and day 10 are summarized in the table below (Table 1). 
TABLE 1 Changes in hematologic investigations during hospitalization

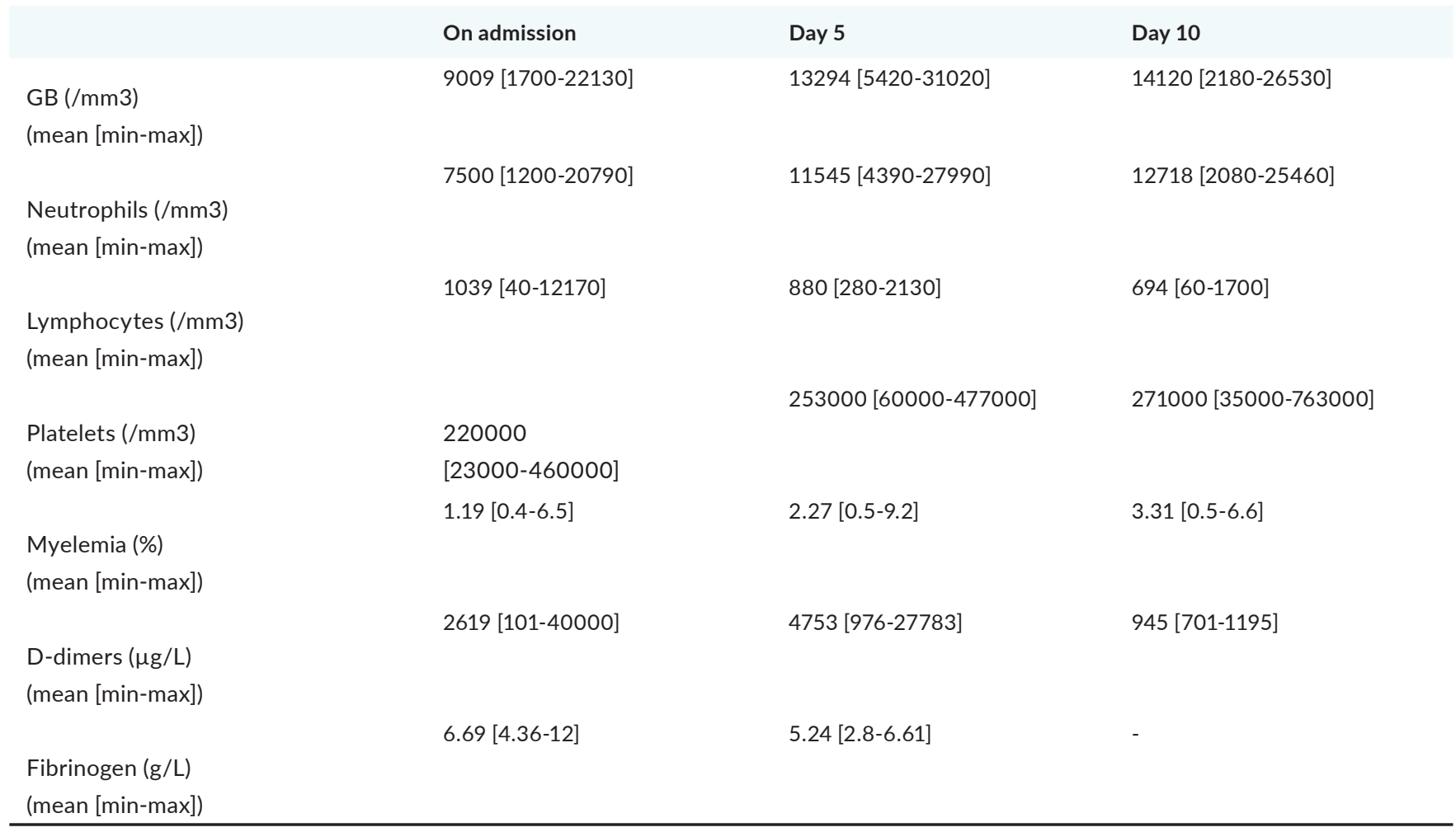

Conclusions: Increased values of $D$ dimers were found in almost all patients with moderate forms of COVID-19. These values are higher during hospitalization than on admission revealing a hypercoagulable state. The other most common haematological abnormalities were neutrophilia, lymphopenia and high values of fibrinogen.

\section{PB0284 | Lupus Anticoagulant and Outcome of COVID-19}

\section{Patients}

O. Oyedeji; R. Laforest; S. Philip; Z. Otrock

Henry Ford Hospital, Detroit, United States

Background: Patients with coronavirus disease 2019 (COVID-19) are at increased risk of thromboembolic events, partly due to the procoagulant state and excessive inflammatory response to the infection. An increased prevalence of lupus anticoagulant (LAC) among Covid-19 patients has been reported, which emphasized the acquired prothrombotic state during COVID-19. However, most published studies were conducted on patients receiving anticoagulants or expressing high C-reactive protein (CRP) levels, both of which are known to interfere with LAC testing.

Aims: To study LAC and outcome of COVID-19 patients.
Methods: Retrospective review of COVID-19 patients evaluated for LAC between March 2020 and December 2020 at a tertiary-care center. The study was approved by the Institutional Review Board. We included cases tested for LAC within 30 days from positive COVID-19 testing. Patients were not on anticoagulation at the time of LAC testing. Our special coagulation laboratory follows a strict triaging process for $L A C$ requests, and specimens containing anticoagulants are cancelled. LAC testing follows an algorithm utilizing two parallel methods: dilute Russell's viper venom time and PTTlupus anticoagulant, including their respective confirmatory testing. Eligible patients were stratified by the LAC testing results. Analyses were performed using SPSS; a $P$ value of $<0.05$ was considered significant.

Results: 19 patients were included; 10 were LAC-positive. The median age was 46 years (Range 23-83); 13 (68\%) were females. There was no difference between LAC-positive and LAC-negative patients in age, gender, race, comorbid conditions (results not shown), CRP levels, thrombotic events, need for ICU care, or survival 
TABLE 1 Characteristics and outcome of Covid-19 positive patients stratified by lupus anticoagulant testing results. LAC, lupus anticoagulant; AA, African American; SD, standard deviation; CRP, C-reactive protein, ICU, intensive care unit

\begin{tabular}{|c|c|c|c|}
\hline Variable & LAC-positive patients $(N=10)$ & LAC-negative patients $(N=9)$ & $P$ value \\
\hline Age (median, range) & $52(23-83)$ years & $41(23-59)$ years & 0.112 \\
\hline Gender & 3 & 3 & 1.0 \\
\hline Male & 7 & 6 & \\
\hline \multicolumn{4}{|l|}{ Female } \\
\hline & & & 0.65 \\
\hline Race & 5 & 6 & \\
\hline White & 5 & 3 & \\
\hline \multicolumn{4}{|l|}{ AA } \\
\hline Platelet count (mean \pm SD) & $276.8 \times 10^{9} / \mathrm{L} \pm 129.7 \times 10^{9} / \mathrm{L}$ & $261.1 \times 10^{9} / \mathrm{L} \pm 66.3 \times 10^{9} / \mathrm{L}$ & 0.761 \\
\hline $\mathrm{CRP}($ mean $\pm \mathrm{SD})$ & $12.3 \mathrm{mg} / \mathrm{L} \pm 7.0 \mathrm{mg} / \mathrm{L}$ & $9.6 \mathrm{mg} / \mathrm{L} \pm 11.7 \mathrm{mg} / \mathrm{L}$ & 0.628 \\
\hline & & & 1.0 \\
\hline Thrombosis & 5 & 4 & \\
\hline Yes & 5 & 5 & \\
\hline \multicolumn{4}{|l|}{ No } \\
\hline & & & 0.141 \\
\hline ICU care & 5 & 1 & \\
\hline Yes & 5 & 8 & \\
\hline \multicolumn{4}{|l|}{ No } \\
\hline & & & $\underline{0.02}$ \\
\hline Hospital stay & 3 & 8 & \\
\hline$<12$ days & 7 & 1 & \\
\hline \multicolumn{4}{|l|}{$\geq 12$ days } \\
\hline & & & 0.582 \\
\hline Survival & 7 & 8 & \\
\hline Alive & 3 & 1 & \\
\hline Dead & & & \\
\hline
\end{tabular}

LAC-positive patients had a longer hospital stay ( $\geq 12$ days) compared to LAC-negative patients $(P=0.02)$. Anticardiolipin and beta-2-glycoprotein antibodies did not differ between the two groups.

Conclusions: Covid-19 patients with positive LAC had longer hospital stay than patients with negative LAC. We have eliminated anticoagulation interference and false-positive testing. Our results warrant further testing in larger cohorts.

PB0285 | The Comparison of ISTH Criteria for Disseminated Intravascular Coagulation (DIC) Scale, Padua Scale, D-dimer and Fibrinogen Like the Best Predictor of Thrombosis in COVID-19 Pneumonia

K. Bielosludtseva; T. Pertseva; L. Konopkina; M. Krykhtina

Dnipropetrovsk Medical Academy, Dnipro, Ukraine

Background: Coagulation disturbances in COVID-19 pneumonia patients is already a well-known fact, but what marker can really reflect the disease progression is still unknown.

Aims: The aim was to estimate the diagnostic and prognostic role of different coagulation markers and scales in hospitalized patients with confirmed COVID-19 pneumonia.
Methods: The main group was 53 patients (age - 59 (52; 65), men - 22 (41.5\%)) with COVID-19 pneumonia, divided into 3 subgroups: subgroup 1 - 36 patients with moderate COVID-19 pneumonia, subgroup 2 - 12 patients with severe COVID-19 pneumonia, subgroup 3 - 5 patients with critical COVID-19 pneumonia. General clinical analysis, blood coagulation test, determination of D-dimer and fibrinogen, ISTH Criteria for DIC, Padua Scale count were performed at admission before starting of antibacterial and anticoagulant treatment.

Results: Individual analysis showed that 28 patients from group 2 and 3 had progression of COVID-19 pneumonia in dynamic due to thrombosis, which was manifested with increased respiratory failure. 


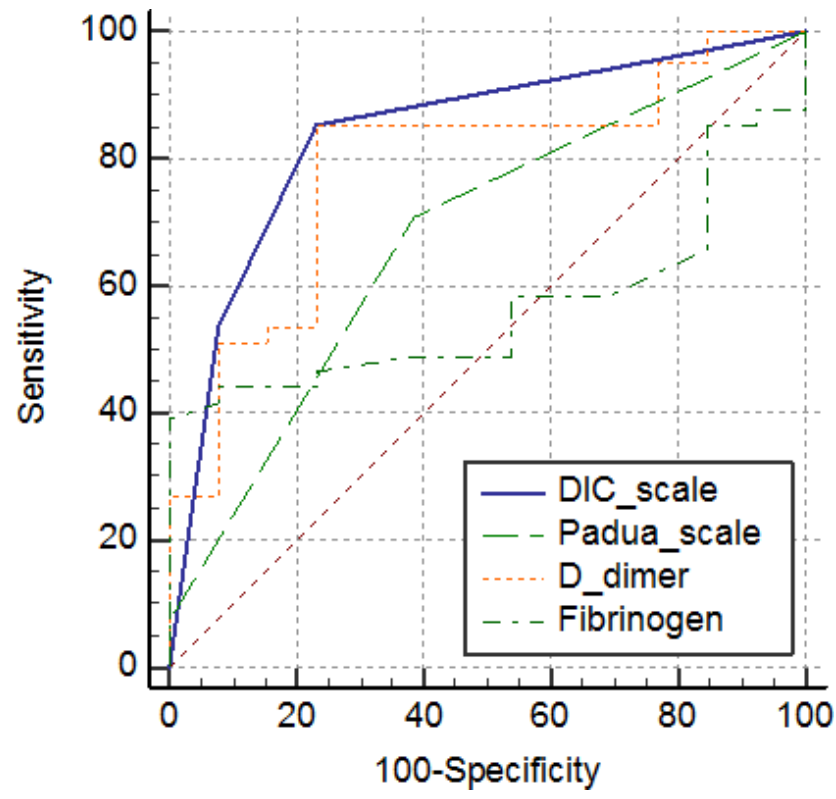

FIGURE 1 100-Specificy

Conclusions: The ROC-analysis showed that ISTH Criteria DIC, Padua scale and D-dimer can really reflect the severity of COVID-19 pneumonia and predict the prognosis of the disease.

1) the best parameter for thrombosis prediction in hospitalized patients with COVID-19 is ISTH Criteria DIC;

2) Increasing of ISTH Criteria DIC score at admission can reflect the risk of severe systemic coagulation disturbances which play a great role in the progression of disease.

\section{PB0286 | Head-to-Head Comparison of Cytokines Storm-} induced Coagulopathy in Septic Shock and Covid-19

$\underline{\text { J. De Poortere }}{ }^{1}$; M. Dechamps ${ }^{1,2}$; P.-F. Laterre ${ }^{3}$;

M. Octave ${ }^{1}$; A. Gigion ${ }^{1}$; V. Robaux ${ }^{1}$; L. Pirotton ${ }^{1}$; J. Bodart ${ }^{1}$;

L. Gerard ${ }^{1,3}$; D. Gruson ${ }^{4}$; M.-A. van Dievoet ${ }^{4}$; J. Douxfils ${ }^{5}$;

M. Derive ${ }^{6}$; L. Gatto ${ }^{7}$; C. Bouzin ${ }^{1}$; D. Castanares-Zapatero ${ }^{1,3}$;

L. Bertrand ${ }^{1}$; S. Horman ${ }^{1}$; C. Beauloye $e^{1,8}$

${ }^{1}$ Université catholique de Louvain, Institut de Recherche Expérimentale et Clinique (IREC), Brussels, Belgium; ${ }^{2}$ Cliniques Universitaires St Luc, Cardiovascular Intensive Care, Brussels, Belgium; ${ }^{3}$ Cliniques Universitaires St Luc, Intensive Care, Brussels, Belgium; ${ }^{4}$ Cliniques Universitaires St Luc, Clinical Biology, Brussels, Belgium; ${ }^{5}$ University of Namur, Namur Research Institute for Life Sciences (Narilis), Department of Pharmacy, Namur, Belgium; ${ }^{\circ}$ Inotrem SA, Vandoeuvreles-Nancy, France; ${ }^{7}$ Université catholique de Louvain, Institut de Duve (DDUV), Brussels, Belgium; ${ }^{8}$ Cliniques Universitaires St Luc, Cardiology, Brussels, Belgium

Background: Since December 2019, the coronavirus disease 2019 (Covid-19) is the main health concern around the world. Host immune response to the virus is variable and can induce a dysregulated inflammatory response associated with venous and arterial thrombosis called Covid-19 associated coagulopathy (CAC). During septic shock, inflammatory reaction generates endothelial activation and procoagulant state with microvascular thrombi inducing disseminated intravascular coagulation (DIC). Although CAC and DIC induce altered coagulation and fibrinolytic responses, their clinical outcomes are different.

Aims: We investigated and compared coagulopathy between septic shock and critical Covid-19 patients.

Methods: Septic shock patients were diagnosed following the Survival Sepsis Campaign guidelines. They were admitted in intensive care unit (ICU) and included in the study within 2 days after admission. Covid-19 patients were admitted in ICU for severe Acute Respiratory Distress Syndrome (ARDS) due to SARS-Cov2 infection and included within 2 days after admission. Patient's plasma was isolated and used to measure circulating biomarkers by ELISA.

Results: We observed an increase in VWF and TFPI in both septic and Covid-19 patients compared to controls, highlighting endothelial damage. Interestingly, circulating TF was only elevated in Covid-19 patients. Platelet activation differed between the two cohorts of patients. P-selectin and Trem-like transcript 1 were specifically heightened in septic shock whereas CD40L was only augmented in Covid-19. Coagulation markers were increased in a diseasedependent way, with PAI-1, tPA and D-Dimers higher in septic shock and fibrinogen level, higher in Covid-19.

Conclusions: Covid-19 patients had longer length-of-stay with more pronounced respiratory failure. This strong lung disruption overtime induced plasmatic tissue factor release with sustained inflammatory response characterized by $\mathrm{SCD} 40 \mathrm{~L}$ and fibrinogen secretion. Given the similarities between Covid-19 and septic shock regarding fibrinolysis and coagulation, but not platelet activation, endothelium seems to play a central role in Covid-19 and might explain the differences between CAC and DIC.

PB0287 | Clinical Impact of Immune Thrombocytopenic Purpura in the Context of SARS-CoV-2 Infection

${\text { C. } \text { Tarniceriu }^{1,2} \text {; L.L. Hurjui }}^{3}$; L. Iozneanu ${ }^{2}$; C. Delianu ${ }^{4}$; I. Hurjui ${ }^{5}$ ${ }^{1}$ St. Spiridon Hospital, Iasi, Iaşi, Romania; " “Grigore T. Popa” University of Medicine and Pharmacy, "St. Spiridon" Emergency County Hospital, Iasi, Romania; "'Sf. Spiridon" County Clinical Emergency Hospital, "Grigore T. Popa" University of Medicine and Pharmacy, Iasi, Romania;

4"St. Spiridon" County Clinical Emergency Hospital, Iasi, Romania;

5"Grigore T. Popa" University of Medicine and Pharmacy, Iasi, Romania

Background: Immune thrombocytopenic purpura (ITP) is a bleeding disorder that is characterized by transient or persistent thrombocytopenia. ITP may be classified as primary or secondary; but in many cases, patients with primary or secondary immune thrombocytopenic purpura are treated similarly. ITP can also be classified as: newly diagnosed (early ITP), persistent and chronic ITP. Diagnosing ITP secondary to COVID-19 can be a major challenge for clinicians due to various concomitant factors associated with SARS-CoV-2 infection. 
Aims: To present the challenges of ITP diagnosis and treatment in actual pandemic context.

Methods: We present a series of four cases of ITP and their challenges in context of SARS Cov-2 infection and a short review of literature data.

Results: Case 1: a patient with a history of ITP and splenectomy presents a relapse of ITP associated with a mild form COVID-19 disease. Case 2: a newly diagnosed ITP in a pregnant patient associated with asymptomatic form of COVID-19 disease. Case 3: a chronic ITP with splenectomy in treatment with romiplostim and stable platelet count presents a severe thrombocytopenia and later became refractory to romiplostim in the mild form COVID-19 disease. Case 4: a newly diagnosed ITP secondary to a moderate form of SARS CoV- 2 infection that evolved with severe thrombocytopenia and bleeding diathesis. We note that in all four cases, severe thrombocytopenia was found even if a severe acute respiratory syndrome was not discussed.

Conclusions: Although thrombotic events are most commonly described as being associated with COVID-19 disease, immune thrombocytopenia with severe hemorrhagic manifestations is a clinical reality in the current pandemic context. We highlighted that viral infection with SARS-CoV-2 could be a critical pathogenic factor for ITP.

\section{PB0288 | Prophylactic Anticoagulation in Hospitalized Patients} with COVID-19: A Survey of Practices in Latin America

F. de Andrade Orsi ${ }^{1}$; P. Casais ${ }^{2}$; G. Cesarman-Maus ${ }^{3}$; C. Guillermo ${ }^{4}$; R. Martinez ${ }^{4}$; S. Rezende ${ }^{5}$

${ }^{1}$ School of Medical Sciences, University of campinas, Brazil, Campinas, Brazil; ${ }^{2}$ Instituto de Investigación en Salud Pública, Buenos Aires, Argentina; ${ }^{3}$ Instituto Nacional de Cancerología, Mexico, Mexico; ${ }^{4}$ Hospital de Clínicas, Facultad de Medicina UDELAR, Montevideo, Uruguay; ${ }^{5}$ Hospital Universitario, Universidad Federal de Minas Gerais de Belo Horizonte, Belo Horizonte, Brazil

Background: Prophylactic anticoagulation is now considered standard-of-care for hospitalized COVID-19 patients. Since evidence-based guidelines for thrombo-prophylaxis are lacking, clinical practice has been based evidence showing a high risk of venous thromboembolism (VTE) among these patients.

Aims: To evaluate the change in anticoagulation practice patterns in hospitalized patients with COVID-19 among Hemostasis and Thrombosis (H\&T) experts in Latin America.

Methods: An invitation was sent by email and website to CLAHT members. The same questionnaire was issued twice: May-June and August-September 2020. with five multiple-choice questions regarding the use of standard or weight-adjusted dosing (SWAD), intermediate or full-dose (IFD) of low molecular weight heparin (LMWH), or no prophylaxis in the following scenarios: i.Hospitalized patients without comorbidities or ii. with comorbidities and D-dimer (DD) levels three-fold above reference values; iii. Patients on mechanical ventilation (MV), iv. Patients on MV with suspected VTE;
iv.LMWH management based solely on DD. Differences between surveys were analyzed using chi-square test.

Results: The first and second surveys were answered by 200 and 174 individuals respectively, representing themselves or institutional practices. Distribution of participants by country is shown in Table 1. Clinical practice was heterogeneous. When comparing surveys, we found that preference for IDF for non-ICU patients without comorbidities increased (6.4\%-14\%) while SWAD decrease from $88.6 \%$ to $72 \%$. IFD preference also increased from (40.6\%-49\%) for patients with comorbidities and high DD. Half (49\%) suggested using IFD for ICU patients without VTE, while 90\% preferred IFD for established or suspected VTE. More aggressive prophylaxis was favored initially in patients based on DD levels 6-fold above baseline, decreasing by $20 \%$ (from $60.6 \%$ to $49.1 \%$ ) in the second survey.

\begin{tabular}{|c|c|c|c|c|c|c|c|c|c|}
\hline Country & \multicolumn{2}{|c|}{$\begin{array}{c}1^{\circ} \text { Survey } \\
n(\%)\end{array}$} & \multicolumn{2}{|c|}{$\begin{array}{c}\text { 2 }^{\circ} \text { Survey } \\
\text { n (\%) }\end{array}$} & \multirow{2}{*}{$\begin{array}{l}\text { Country } \\
\text { Guatemala }\end{array}$} & \multicolumn{2}{|c|}{$\begin{array}{c}1^{\circ} \text { Survey } \\
n(\%)\end{array}$} & \multicolumn{2}{|c|}{$\begin{array}{c}2^{\circ} \text { Survey } \\
n(\%)\end{array}$} \\
\hline Argentina & & $(7.5)$ & & (10.9) & & 3 & (1.5) & 3 & (1.7) \\
\hline Bolivia & 2 & $(1.0)$ & 5 & (2.9) & Mexico & 74 & $(37.0)$ & 79 & (45.4) \\
\hline Brazil & & (23.0) & & (8.6) & Nicaragua & 1 & $(0.5)$ & 2 & (1.1) \\
\hline Canada & 1 & $(0 . .5)$ & & 0 & Panama & 4 & $(2.0)$ & & 0 \\
\hline Colombia & 8 & $(4 . .0)$ & 8 & $(4 . .6)$ & Paraguay & 1 & $(0.5)$ & 7 & (4.0) \\
\hline Costa Rica & 3 & (1..5) & & 0 & Peru & 9 & (4.5) & 12 & (6.9) \\
\hline $\begin{array}{l}\text { Dominican } \\
\text { Republic }\end{array}$ & 2 & (1.0) & 3 & (1.7) & United States & & (11.5) & 1 & $(0.6)$ \\
\hline Ecuador & & $(1.5)$ & & (3.4) & Uruguay & 3 & (1.5) & 5 & (2.9) \\
\hline El Salvador & 1 & $(0.5)$ & & $(0.6)$ & Venezuela & 1 & $(0.5)$ & 8 & (4.6) \\
\hline
\end{tabular}

\section{FIGURE 1}

Conclusions: Prophylactic anticoagulation for COVID-19 varied, with a trend towards a decrease in preference for IFD, likely reflecting reported bleeding incidents. Awaited final publication of results from prospective studies will likely homogenize clinical practice worldwide.

\section{PB0289 | Haemostatic Dysfunctions in COVID-19 Infection: A} Systematic Review

\author{
S. Abegunde $^{1} ;$ A. Adedeji ${ }^{2}$ \\ ${ }^{1}$ University of New Brunswick, Saint John, Canada; ${ }^{2}$ WAEC Staff \\ Medical Centre, Lagos, Nigeria
}

Background: Coronavirus disease-2019 (COVID-19) is a viral respiratory infection caused by the severe acute respiratory syndromecoronavirus-2(SARS-CoV-2) that may be associated with serious thrombotic complications. These complications may contribute to morbidity and mortality in COVID-19 patients.

Aims: This review aimed at synthesizing evidence that point to haemostatic dysfunctions as essential pathologic components 
of SARS-CoV-2 infection, their prognostic relevance and the antithrombotic management of symptomatic COVID-19 infection.

Methods: The PUBMED, MEDLINE and Cochrane Database of systematic reviews were searched for relevant English-based studies for current best evidence regarding haemostatic findings and complications in symptomatic and asymptomatic COVID-19 patients. Studies were eligible for inclusion if they were meta-analyses, systematic reviews, observational studies and case-series. Articles were screened and the references were searched to identify additional studies.

Results: Our search yielded 112 potentially eligible studies including two meta-analysis studies $(N=2120)$ and nine systematic reviews. Remainder being observational studies and case series. Thrombocytopenia and elevated D-dimer levels were the most common haemostatic abnormalities seen in moderate to severely symptomatic patients. High D-dimer levels were significantly associated with increased requirement for mechanical ventilation, ICU admission and mortality $(P<0.05)$. There were variations in individual studies regarding association between disease severity and other haemostatic findings including prolonged prothrombin-time, INR, thrombin-time and shortened APTT. Vascular platelet-fibrin clots were commonly reported abnormal haemostatic findings in COVID19-related-autopsies. There were variations in individual studies with respect to antithrombotic treatment protocols, dosing, and additional interventions. Parenteral anticoagulation was recommended in most cases with known thrombotic disease. Anticoagulation was frequently needed to maintain circuit patency in patients having extracorporeal membrane oxygenation.

Conclusions: Thrombotic episodes may be precedent factors or incident complications in COVID-19 patients. There is weak recommendation but moderate quality evidence regarding preventive and therapeutic use of antithrombotic-agents in COVID-19, hence need for high-quality studies evaluating patient-important clinical outcomes is hereby emphasized
PB0290 | Native Rotational Thromboelastometry Observations in Critically III COVID-19 Patients

A.-M. Hulshof ${ }^{1}$; M. Nagy²; F. Tjahjadi ${ }^{2}$; F. Heubel-Moenen ${ }^{1}$; T. Hackeng ${ }^{2}$; H. ten Cate ${ }^{2}$; B. van Bussel ${ }^{1}$; H. Spronk ${ }^{2}$; Y. Henskens ${ }^{1}$ ${ }^{1}$ Maastricht University Medical Center+, Maastricht, Netherlands;

${ }^{2}$ Maastricht University, Maastricht, Netherlands

Background: COVID-19 patients show profound hemostatic abnormalities and are at increased risk for thrombosis. Native rotational thromboelastometry (NATEM-ROTEM) is a viscoelastic assay in which the patient sample is solely re-calcified in absence of an activator of hemostasis and hence the activation originates from the sample itself. NATEM might better reflect in vivo hemostatic abnormalities in COVID-19 patients.

Aims: We aimed to evaluate the applicability of NATEM to detect hemostatic abnormalities in critically ill COVID-19 patients and the association with contact activation biomarkers.

Methods: NATEM was performed in whole blood(WB) and platelet poor plasma(PPP) with and without a thermostable inhibitor of contact activation(TICA) in 13 COVID-19 patients. All patients were admitted to the ICU and received prophylactic $(n=6)$ or therapeutic $(n=7)$ LMWH. Prolonged clotting time(CT), in WB and PPP, was expected due to LMWH administration[Scharbert, 2012]. Healthy volunteers served as NATEM controls. Activated coagulation factor inhibitor complexes (thrombin:antithrombin(T:AT), FXla:AT, FXlaa1AT, FXla:C1Inh, and FIXa:AT) were measured by ELISA to evaluate in vivo coagulation status in COVID-19 patients.

Results: CT did not differ in WB between COVID-19 patients and controls, but was prolonged in PPP NATEM(Table 1). In contrast, Maximum clot firmness(MCF) was increased in WB in COVID-19 patients compared to controls, but PPP did not differ between the groups. Addition of TICA resulted in prolonged CT values. Contact activation biomarkers(FXla:inhibitor complexes, FIXa:AT) were generally within the normal range and did not correlate with NATEM parameters or TICA-mediated changes; T:AT levels showed a negative correlation with $\mathrm{CT}(\rho=-0.76, P<0.01)$.

TABLE 1 NATEM-ROTEM clotting time and maximum clot firmness results in whole blood and platelet poor plasma of COVID-19 patients and healthy volunteers. 'WB MCF was unmeasurable for 1 COVID-19 patient. ${ }^{* *}$ PPP MCF was unmeasurable for 3 COVID-19 patients

\begin{tabular}{|c|c|c|c|c|}
\hline Parameter & $\begin{array}{l}\text { Manufacturer reference } \\
\text { values }\end{array}$ & COVID-19 patients & Healthy volunteers & $P$-value (Mann-Whitney U) \\
\hline Whole blood & $N=13$ & $N=22$ & & \\
\hline Clotting time (in s) & $300-1000$ & 808 [651-1106] & 817 [661-868] & 0.66 \\
\hline $\begin{array}{l}\text { Maximum clot firmness } \\
\quad \text { (in } \mathrm{mm})\end{array}$ & $40-65$ & $73[59-75]^{*}$ & $52[48-55]$ & $<0.001$ \\
\hline Platelet poor plasma & $N=13$ & $N=17$ & & \\
\hline Clotting time (in s) & - & 2141 [1378-3873] & 966 [831-1273] & $<0.001$ \\
\hline $\begin{array}{l}\text { Maximum clot firmness } \\
\quad \text { (in } \mathrm{mm} \text { ) }\end{array}$ & - & $30[21-35]^{* *}$ & 24 [19-27] & 0.096 \\
\hline
\end{tabular}


Conclusions: NATEM shows a hypercoagulable phenotype in WB samples from COVID-19 patients, illustrated by increased MCF and a lack of LMWH-mediated CT prolongation, that is absent in PPP. Contact activation biomarkers did not explain the observed results, suggesting other factors are important for NATEM clot development. This might suggest a role for endogenous tissue factor expression, leukocytes or platelets in COVID-19-associated hypercoagulability.

PB0291 | CATS-MICA Score in Predicting Venous Thromboembolism in SARS-CoV-2-infected Cancer Patients

D.S. Juliao Caamaño $^{1}$; C.L. Jimenez ${ }^{1}$; C. Blanco Abad ${ }^{2}$; J.L. Catoya Villa ${ }^{2}$; A. Gutierrez ORtiz de la Tabla ${ }^{3}$; I. Aparicio Salcedo ${ }^{3}$;

L. Ortega Moran ${ }^{3}$; M. Martin Jimenez ${ }^{3}$; A. Muñoz Martin ${ }^{3}$

${ }^{1}$ Hospital General Universitario Gregorio Marañon, Madrid, Spain;

${ }^{2}$ Hospital Universitario de Burgos, Burgos, Spain; ${ }^{3}$ Hospital General

Universitario Gregorio Marañón, Madrid, Spain

Background: A recent meta-analysis found a strong relationship between COVID-19 and venous thromboembolism (VTE) in hospitalized patients. Findings on cancer patients and VTE risk remain controversial, mostly in outpatient setting. CATS-MICA score (CMS) was developed as a simple tool (normogram), with only two variables (tumor-site and D-dimer concentration), to calculate predicted 6 month risk of venous thromboembolism (P6RV).

Aims: Assessing the prediction capability of VTE risk in SARS-CoV2-infected cancer patients (inpatients and outpatients) using CMS normogram.

Methods: We performed an observational, retrospective, singlecenter study. Consecutive SARS-CoV-2-infected cancer patients attended in Hospital General Universitario Gregorio Marañón Madrid Spain, in the first wave from March 2020 to June 2020, were included in the analysis. According to tumor location and D-Dimer value, CMS was individually calculated based on the online risk calculator (available at http://catscore.meduniwien.ac.at/) at COVID-19 diagnosis. Data were collected from electronic medical records.

Results: 80 patients were included (Table 1). Median follow-up was 117 days. There was no statistically significant association between CMS and VTE (median CMS for VTE patients was 6.18\% and for non VTE patients was $4.93 \%, P=0.6$ ). There was a statistical significant difference between the CMS of death cases and survivors. Survival analysis (Figure 1) showed a significant difference between low $(<5 \%)$ and high $(\geq 5 \%)$ risk groups $(P 0.02)$.

Conclusions: Limitations such as sample size and heterogeneity, plus use of anticoagulation on most patients at the moment of COVID-19 diagnosis blur the results. Further studies are required to demonstrate the usefulness of the CMS for prediction of VTE in this specific setting. Regardless, there were significant differences in mortality between high and low risk groups of P6RV values at survival analysis, suggesting a valuable relationship between this tool and mortality, that could be more thoroughly investigated.
PB0292 | Thromboembolism and Coagulopathy in COVID-19: A Review of Quality Indicators at Sunnybrook Health Sciences Centre

A. Suleman ${ }^{1}$; S. Carlin ${ }^{2}$; W. Geerts ${ }^{2}$

${ }^{1}$ University of Toronto, Toronto, Canada; ${ }^{2}$ Sunnybrook Health Sciences Centre, Toronto, Canada

Background: The incidence of thromboembolic events (TE) in COVID-19 ranges from $1.1 \%$ to $69 \%$. An elevated D-Dimer may indicate a high risk for TE, and is also an independent prognostic factor for development of severe disease.

Aims: We aimed to document our experience with TE in a cohort of COVID-19 patients admitted to a large Canadian teaching centre and to assess quality of care indicators including the use of D-Dimer and thromboprophylaxis.

Methods: A retrospective review of patients admitted with COVID-19 from March 12 to June 282020 at Sunnybrook Health Sciences Centre was performed. The frequencies and values of key laboratory tests, intensity of thromboprophylaxis, TE events and mortality were collected.

Results: A total of 105 patients were included. Twenty-five patients (24\%) had a D-Dimer on admission, and 36 patients (34\%) had a DDimer at any point during their hospital stay. Only five patients (5\%) had a fibrinogen during admission. VTE prophylaxis was initiated at any point during admission in 81 patients previously not on anticoagulation (88\%) and within two days of admission in 69 of these patients (75\%). High-dose prophylaxis use increased from $16 \%$ in the first two months to $78 \%$ in the last two months. Outcomes are shown in Table 1. Nineteen patients (18\%) died with 14 (74\%) attributable to COVID-19 or COVID-19-related complications.

TABLE 1 Arterial and venous thromboembolic outcomes of patients admitted to Sunnybrook Health Sciences Centre with COVID-19.

\begin{tabular}{lll} 
& $N$ (out of 105) & Percentage \\
$\begin{array}{l}\text { Investigation for Deep Vein } \\
\quad \text { Thrombosis }\end{array}$ & 8 & $7.6 \%$ \\
$\begin{array}{l}\text { Investigation for Pulmonary } \\
\quad \text { Embolism }\end{array}$ & 23 & $21.9 \%$ \\
DVT & 1 & $1.0 \%$ \\
Pulmonary Embolism & 3 & $2.9 \%$ \\
Line Thrombosis & 1 & $1.0 \%$ \\
Central Venous Sinus & 1 & $1.0 \%$ \\
$\quad$ Thrombosis & 3 & $2.9 \%$ \\
Stroke & 4 & $3.8 \%$ \\
Arterial TE Event (non-stroke) & 2 & $1.9 \%$ \\
Small Vessel Vasculopathy & & \\
\hline
\end{tabular}


Conclusions: The rate of symptomatic venous or arterial TE in patients who were subjected to investigation was only $12 \%$, low compared to other studies. VTE prophylaxis was used in a majority of cases, often at high dose. The routine use of D-Dimer and fibrinogen was low and did not appear to be used for risk stratification. Use of COVID-specific order sets may help to increase compliance with best practices and uptake of prognostic laboratory indices at the time of hospital and ICU admission.

PB0293 | The Assessment of Thrombotic Complication in Patients with COVID-19

V.M. Popov; M. ANDREESCU; M. OMER; M. Popescu; C. Despan; L. Iliescu; C. Pirvu; A. Grigorie; M. Niculae; O. Constantin; A. Rus;

A. Badea; I. Simion; M.I. Balea

Colentina Clinical Hospital, Bucharest, Romania

Background: COVID-19 frequently associated thrombotic complication that could determine severe evolution. Inflammation was proved as important pathogenic mechanism of thrombosis.

Aims: The main objective was to evaluate the role of inflammation in increased risk of thrombosis in COVID 19 patients.

Methods: Our study was prospective and included all patients diagnosed with COVID 19 between April-September 2020 in Hematology, Pneumology and Intensive Care Unit from Colentina Clinical Hospital (285 patients). The diagnosis was established using molecular test for SARS-Cov2.

Results: Thrombotic complication was presented in 56 COVID-19 patients $(19,65 \%)$, The higher incidence of thrombosis was observed in severe form of COVID-19: stage 3 (66\%) and stage 2 (26.3\%), Comorbidities: diabetes mellitus, obesity and arterial hypertension were presented in majority of COVID 19 patients with thrombosis. Acute thrombosis (stroke, myocardial infarction or pulmonary embolism) was diagnosed in 14 patients; all of them were admitted in Intensive care unit due severe form of COVID-19. Inflammatory markers including $C$ reactive protein (CRP), procalcitonin, ferritin are significantly increased in COVID-19 group with acute thrombosis compared with COVID -19 patients with thrombosis in medical history CRP $148.86 \mathrm{mg} / \mathrm{L}(2.96-386.5)$ vs. $58.24 \mathrm{mg} / \mathrm{L}(\min 0.25$, max 212.98) $P=0.005$; procalcitonin $0.93 \mathrm{ng} / \mathrm{ml}(0.04-784)$ vs $0.18(\mathrm{~min}$ 0.02 , $\max 14.1) P=0.02$; ferritin $702 \mathrm{ng} / \mathrm{ml}(\min 102$, $\max 4070)$ vs. $1195 \mathrm{ng} / \mathrm{ml}(\min 358, \max 12800) P=0.03$. There is no significant difference between haematological parameters in COVID-19 patients with acute thrombosis or in their medical history. D Dimers are significant increased in patients with acute thrombosis $4.79 \mathrm{ug} / \mathrm{ml}(0.51-20)$ vs patients with medical history of thrombosis $2.12(0.31-20), P=0.02$ The level of protein C, protein S and antitrombine III, antiphospholipid antibodies are not significant modified in the both groups.

Conclusions: The assessment of inflammation parameters are very important in COVID-19 patients especially those with a history of thrombosis or who have significant comorbidities (diabetes mellitus, arterial hypertension or obesity).
PB0294 | Circulating Biologic Markers of Endothelial Dysfunction in COVID-19 Patients

O. Matvienko ${ }^{1}$; N. Korsakova ${ }^{1}$; O. Smirnova ${ }^{1}$; A. Lerner ${ }^{2}$; T. Shwedova ${ }^{2}$; O. Golovina ${ }^{1}$; L. Papayan ${ }^{1}$

${ }^{1}$ Russian Research Institute of Hematology and Transfusiology, Saint Petersburg, Russian Federation; ${ }^{2}$ Vsevolozhsk Clinical Interdistrict Hospital, Vsevolozsk, Russian Federation

Background: COVID-19 course is characterized of thrombosis complications, respiratory failure, and a multiple organ failure development. One of the central pathogenic mechanism of COVID-19 is endothelial dysfunction which can lead to the hypercoagulability. Studying of the endothelial dysfunction markers in patients with COVID-19 could be useful for improving of disease's outcomes.

Aims: To estimate potential endothelial damage in COVID-19 patients by measuring biomarkers of endothelial disfunction.

Methods: The study included 221 patients with COVID-19 and 68 healthy controls. We studied ristocetin-cofactor von Willebrand's factor activity (vWF:RCo, \%), antigen von Willebrand's factor (vWF:Ag,\%), and homocysteine (HCY, $\mu \mathrm{mol} / \mathrm{l})$. STATISTICA 12.0 package was used. Statistical analysis was performed by non-parametric methods (median (Me), 95\% confidence interval (95\% Cl), Mann-Whitney $U$ test), $P<0.05$ was considered statistically significant.

Results:

\section{TABLE 1}

$\begin{array}{lll}\begin{array}{l}\text { Parameter (Me, } \\ \text { 95\% Cl) }\end{array} & \begin{array}{l}\text { Patients with COVID-19 } \\ \mathrm{N}=221\end{array} & \begin{array}{l}\text { Controls } \\ \mathrm{N}=68\end{array} \\ \text { vWF:RCo, \% } & 290.0^{*} & 97.0 \\ & (109.7-724.5) & (63.0-44.3) \\ \text { vWF: Ag, \% } & & \\ & 225.5^{*} & 107.0 \\ & (136.1-880.4) & (74.6-161.7) \\ \mathrm{HCY}, \mu \mathrm{mol} / \mathrm{L} & & \\ & 16.1^{*} & 10.1 \\ & (7.2-36.6) & (5.1-14.8)\end{array}$

${ }^{*} P=0.00001$ with controls.

Data are presented in the table. The vWF:RCo activity, vWF:Ag and $\mathrm{HCY}$ were significantly higher in patients with COVID-19 than in controls. Higher vWF:Ag was found in patients with fatal outcomes compared recovered patients

(Me-244.0, Cl: 216.5-930.0 vs. Me-219 Cl: 142.9-829.6, $P=0.01$ ).

Conclusions: The increase of vWF:RCo activity, vWF:Ag and HCY may indicate endothelial damage in COVID-19 patients that can lead to hypercoagulability and predispose to thromboembolic complications. High vWF:Ag could be predictive marker of poor outcomes in COVID-19 patients. 
PB0295 | The Effects of COVID-19 Pandemic on Children with Haemophilia

F.B. Kurtipek; D. Gürlek Gökçebay; V. Çulha; N.Y. Özbek Ankara City Hospital, Ankara, Turkey

Background: Hemophilia is a rare inherited bleeding disorder and patients need regular clotting-factor replacement (prophylaxis) or on-demand treatment. Recommended preventive measures by the World Foundation of Hemophilia (WFH) to reduce COVID-19 infections in patients with bleeding disorders are to limit routine clinical visits which may lead to limited access to medical care and drugs.

Aims: In this study we aimed to investigate the health problems of patients with hemophilia during COVID-19 pandemic between March and November 2020.

Methods: A telephone survey was used to collect data on 49 patients with hemophilia who are followed-up at the Ministry of Health Ankara City Hospital Pediatric Hematology outpatient clinic. The parents of patients filled a questionnaire with 10 open-ended questions.

Results: The mean age of the patients was 13 (1-28) years. All of patients had moderate or severe hemophilia, and four of them had inhibitors. Thirty seven of the patients were on prophylaxis (31 recombinant, 6 plasma derived products), and 34 of them received their prophylaxis regularly. Twenty patients had at least one bleeding episode (median 3 times) during the study period, and 16 of them required to hospital admission for treatment. Thirteen of this patients admitted to the hospital. However, 3 of 20 patients who had bleeding did not admit to the hospital and treated by himself or parents. Four of the patients had contact with SARS-CoV-2 positive people and 3 of them recovered of COVID 19 infection without need for hospitalization. None of our patients with prophylaxis had difficulty in accessing their drugs except 3 who are obtained them with a few days delay.

Conclusions: In conclusion, during COVID-19 pandemic limitation of routine visits may harmful for physical and social health of patients with hemophilia. Widespread use of telemedicine and proactive approach of physicians can help reducing the impact of the pandemic.

PB0296 | Management of Clot in Transit in a Postpartum COVID-19 Patient

A. Peruri; A. Kadl; C. Ennen; L. Wilkins; R. Ramcharitar; N. Teman;

A. Sharma; A. Mihalek; L. Man

University of Virginia, Charlottesville, United States

Background: The prothrombotic state of COVID-19 infection is well documented, but recommendations for management of clot in transit in the post-partum state are lacking.

Aims: We describe a unique case of a 39-year-old female, 34-week gravida with pregnancy complicated by COVID-19 and clot in transit.
Methods: Our patient presented to our hospital for a week of worsening dyspnea, cough, and fevers. Given her respiratory decline, she underwent urgent cesarean delivery of a healthy male infant. Initial chest $\mathrm{x}$-ray was consistent with severe COVID-19 pneumonia. Noninvasive methods of oxygenation were exhausted, and she was eventually intubated. Troponin was $<0.02 \mathrm{ng} / \mathrm{mL}$, BNP was $71 \mathrm{pg} / \mathrm{mL}$, and D-Dimer increased from 601 to $13.000 \mathrm{ng} / \mathrm{mL}$. Transthoracic echocardiogram showed preserved cardiac function, but imaging was sub-optimal for the right ventricle (RV). On post-cesarean day 6, CT Pulmonary Angiogram (CTPA) showed bilateral segmental and subsegmental Pulmonary Embolism (PE) with filling defect in the RV consistent with clot in transit (CIT). No RV strain was noted on Echo or CTPA. Lower extremity venous duplex showed only acute calf thrombus. She was anticoagulated with enoxaparin at $1 \mathrm{mg} /$ $\mathrm{kg}$, but her oxygen requirements worsened requiring $100 \% \mathrm{FiO} 2$ on the ventilator. Emergent multidisciplinary discussion was held by PE Response Team (PERT) regarding use of catheter directed lysis, systemic lysis, ECMO or surgical embolectomy.

Results: PERT team ultimately recommended $50 \mathrm{mg}$ alteplase be given systemically with family's consent. After alteplase was given, her oxygen requirements quickly improved to $45 \% \mathrm{FiO} 2$ and her vitals remained normal. CT Head reported no hemorrhage, and repeat CTPA showed near-complete resolution of RV thrombus.

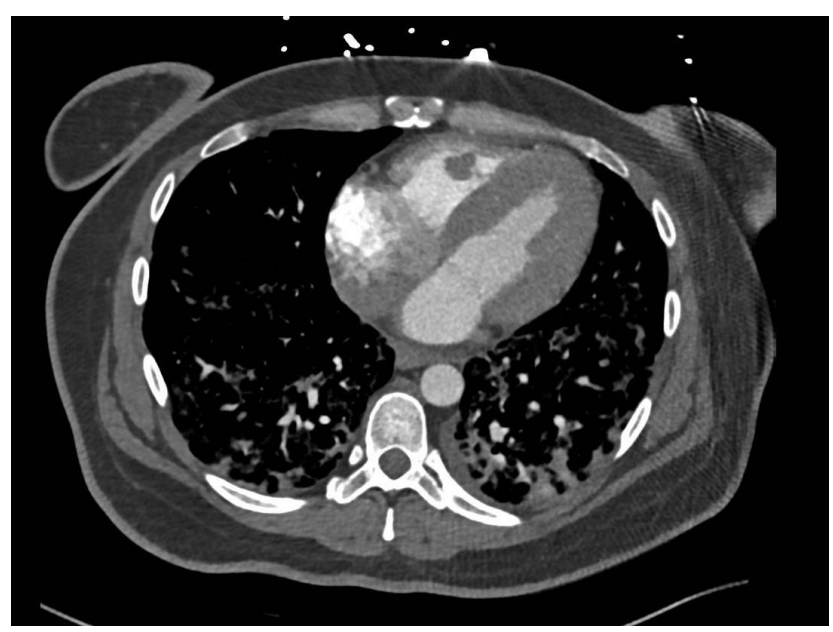

FIGURE 1 CTPA axial view of thrombus in right ventricle

Conclusions: This case exemplifies the role of both emergent multidisciplinary decision-making in complex case scenarios, and systemic thrombolysis for the management of COVID-19 related CIT in patients who recently underwent cesarean delivery. 
PB0297 | The Effect of Chronic Anticoagulation and Antiplatelets on COVID-19 Infection

J. Kaur; A. Abdou; M. Mohammed; Y. Nadeem; M. Kulkarni; P. Dixit;

Q. Jehangir; A. Morani; Y. Lee; I. Mohamed; M. Qamar; P. Pulapati;

J. Duong

Saint Joseph Mercy Oakland Hospital, Pontiac, United States

Background: Severe acute respiratory syndrome coronavirus -2 caused by the novel coronavirus 2019 (Covid-19) has resulted in a global pandemic. Covid-19 disease is associated with a hypercoagulable state, leading to microvascular and/or macrovascular thrombosis. The role of anticoagulation in Covid-19 is debatable.

Aims: To compare outcomes of chronic anticoagulation and/or antiplatelets versus no chronic anticoagulation or antiplatelets in Covid-19 patients.

Methods: This is a retrospective cohort study of hospitalized patients with polymerase chain reaction confirmed Covid-19 and over the age of 18 years who presented to the Trinity Health hospitals from March 8, 2020, to May 15, 2020. The exposed group was defined as patients who received chronic anticoagulation (warfarin, direct oral anticoagulant) or antiplatelet therapy or both(for more than one-month duration) for reasons other than Covid-19 disease while control group patients were defined as those who did not receive these therapies prior to admission. The primary outcome of the study is a composite outcome to compare mortality, length of hospital stay, readmission rate, rate of intubation, and length of Intensive-Care-Unit admission. Baseline characteristics and Covid-19 related treatment were compared in both groups (Table 1). The chi-square test and the student's $t$-test were used to compare the outcome in both groups. Statistical analysis was performed using SPSS version 25 .
Results: There were 3180 patients who were Covid-19 positive during the study period; 452 patients met the inclusion criteria. There were 183 patients in the exposed group and 269 patients in the control group. In the exposed group there was a significantly higher three- months mortality rate $(30.6 \%$ vs. $16 \%$; $P$ < 0.0005$)$ compared to the control group. There were no significant associations between readmission rate, rate of intubation, length of hospital stay, and length of ICU stay by the group.

Conclusions: Our results showed higher mortality in patients on chronic anticoagulation or antiplatelet therapy.

\section{PB0298 | Relationship between von Willebrand Factor and Clinical Outcome in Patients with Severe COVID-19}

F. Rivera-Buendia; L.E. Garcia-Chavez; E. Cortina-de la Rosa;

F.A. Grimaldo-Gomez; G.A. Altamirano-Solorzano; V.M. AnguianoAlvarez; M.O. Romero-Arroyo; R. Izaguirre-Avila

National Institute of Cardiology Ignacio Chavez, Mexico City, Mexico

Background: COVID-19 is associated with an intense inflammatory response and an increased risk of thrombosis. High levels of D-dimer (DD), fibrinogen (Fg), von Willebrand factor (VWF) and factor VIII (FVIII) was reported among patients with severe disease.

Aims: We aimed to characterize the relationship between vWF and other coagulation factors and clinical outcome among inpatients with severe COVID-19.

TABLE 1 Comparing baseline characteristics between exposed and control group.

\begin{tabular}{|c|c|c|c|}
\hline Baseline Charecteristich & Exposed group $(n=183)$ & Control group $(n=269)$ & $P$-value \\
\hline Age (Mean \pm SD) & $76 \pm 14$ & $61 \pm 20$ & $<0.0005$ \\
\hline Race & $164(89.6 \%)$ & 209 (77.7\%) & $<.0005$ \\
\hline Black (\%) & $3(1.6 \%)$ & 40 (15\%) & \\
\hline \multirow[t]{2}{*}{ White (\%) } & $16(8.7 \%)$ & $20(7.4 \%)$ & \\
\hline & & & 0.628 \\
\hline Sex & $81(44.3 \%)$ & $112(41.6 \%)$ & \\
\hline Male (\%) & $102(55.7 \%)$ & $157(58.4 \%)$ & \\
\hline \multicolumn{4}{|l|}{ Female (\%) } \\
\hline Obesity (\%) & $71(38.8 \%)$ & $134(50 \%)$ & 0.048 \\
\hline Hypertension (Yes, \%) & $157(86 \%)$ & $160(60 \%)$ & $<0.0005$ \\
\hline Diabetes Mellitus (yes, \%) & $76(41.5 \%)$ & $90(33.5 \%)$ & 0.091 \\
\hline Cerebrovascular accident (yes,\%) & $47(26 \%)$ & $15(5.6 \%)$ & $<0.0005$ \\
\hline Chronic obstructive lung disease (yes,\%) & $38(21 \%)$ & $36(13.4 \%)$ & 0.039 \\
\hline Smoking & $67(36.6 \%)$ & $123(46 \%)$ & $<0.0005$ \\
\hline Former smoker (yes, \%) & $58(31.7 \%)$ & 40 (15\%) & \\
\hline Current smoker (yes,\%) & $22(12 \%)$ & 40 (15\%) & \\
\hline Never smoked (yes, \%) & $36(19.7 \%)$ & $66(24 \%)$ & \\
\hline Unknown (yes, \%) & & & \\
\hline
\end{tabular}


Methods: We conducted a cross-sectional study of COVID-19 inpatients admitted in Mexico National Institute of Cardiology between April and December 2020. Patients with severe COVID-19 diagnosis who were admitted to the Intensive Care Unit were included. Blood samples were collected and processed according to the standardized institutional procedures. For patients who had anticoagulation therapy with heparin, anti-Xa level was determined. Patient's demographic and clinical characteristics, inflammatory and coagulation markers, and clinical outcome (thrombosis, mechanical ventilation, and death) were retrieved from the electronic medical records. Descriptive statistics and multivariate models were performed to assess associations between coagulation factors and clinical outcome.
Results: A total of 58 patients were included during the study period. The mean age was $53.7+14$ years and $75.9 \%$ were men. Overall, $84.2 \%$ required mechanical ventilation, $25 \%$ had thrombosis (pulmonary embolism and/or deep vein thrombosis).

Both vWFAg and vWFRCo were increased; the median value was $362.2 \%$ and $297.3 \%$ respectively. Among patients who died, a significantly increase in VWF measured by both methods was found. Of the 58 patients, 38 (65.5\%) had mechanical ventilation and 22 (57.9\%) died. Mortality was significantly higher among patients with mechanical ventilation $(57.9 \%$ vs $42.1 \%$; $P<0.001$.

TABLE 1 Clinical characteristics and factors associated with death among COVID-19 patients. * The measure of central tendency and dispersion was chosen based on the distribution of the data according to the Kolmogorov-Smirnov test.

\begin{tabular}{|c|c|c|c|c|c|}
\hline & $\begin{array}{l}\text { Total } \\
58(\%)\end{array}$ & $\begin{array}{l}\text { Alive } \\
36(\%)\end{array}$ & $\begin{array}{l}\text { Death } \\
22(\%)\end{array}$ & OR (Cl 95\%) & $P$-value \\
\hline Male & 44 (75.9) & $24(66.7)$ & 20 (90.9) & $5(0.99-25.02)$ & 0.057 \\
\hline Age $\geq 60$ years & $18(31)$ & $11(30.6)$ & $7(31.8)$ & $1.06(0.34-3.33)$ & 0.920 \\
\hline \multicolumn{6}{|c|}{ Biochemical markers", mean (SD) or median (RIQ) } \\
\hline C-reactive protein & $124.4(53.2-201.9)$ & $100.7(40-140.6)$ & $151.1(95-359.8)$ & - & 0.011 \\
\hline Fibrinogen & $5.9(2.1)$ & $5.9(2)$ & $6.2(2.5)$ & - & 0.513 \\
\hline D-dimer & $1.3(0.63-2)$ & $1.3(0.6-2.5)$ & $1.4(0.8-1.9)$ & - & 0.694 \\
\hline Factor VIII (\%) & $296.5(113.1)$ & $262.6(115.3)$ & $305.8(112)$ & - & 0.853 \\
\hline $\begin{array}{l}\text { Factor von Wilebrand } \\
\qquad \mathrm{Ag}(\%)\end{array}$ & $362.2(261.4-481.6)$ & $299.5(235.1-400.5)$ & $421.8(299.6-521.6)$ & - & 0.023 \\
\hline $\begin{array}{l}\text { Factor von Wilebrand } \\
\text { CoRi (\%) }\end{array}$ & $297.3(203.3-487.1)$ & $247(191.9-383)$ & 364.9 (247.3-539) & - & 0.023 \\
\hline
\end{tabular}

TABLE 2 Clinical characteristics and factors associated with mechanical ventilation among COVID-19 patients. "The measure of central tendency and dispersion was chosen based on the distribution of the data according to the Kolmogorov-Smirnov test.

Without mechanical

ventilation

(\%)

Biochemical markers ${ }^{*}$, mean (SD) or median (RIQ)

Factor von Wilebrand $\mathrm{Ag}(\%)$
$\begin{aligned} & \text { Factor von Wilebrand CoRi } \\ & \quad 202.5(220.5-299.5) \\ & \quad(\%)\end{aligned}$

(\%)

Outcomes, $n(\%)$

Days from admission to discharge or death, median (IQR)

\section{Thrombosis}

Deep vein thrombosis

Pulmonary embolism
$11(7-15)$

$44(22-55)$

7 (18.4)

6 (15.8)

$1(2.6)$

$\begin{array}{ll}5(25) & 7(18.4) \\ 3(15) & 6(15.8) \\ 3(15) & 1(2.6)\end{array}$

\section{OR (CI 95\%)}

P-value

421.8 (334.4-517.5)

$-\quad<0.001$

$<0.001$
$0.68(0.18-2.49)$

1.06 (0.24-4.79)

$0.15(0.02-1.58)$
$<0.001$

0.734

1

0.114 
Conclusions: In this study, we determined the levels of antigenic and RCo vWF in 58 patients with moderate-severe COVID-19. The vWF was markedly increased among patients with severe clinical presentation. Both antigenic and functional vWF were significantly associated with mechanical ventilation and death.

\section{PB0299 | Acute Liver Necrosis in a Sars-Cov-2 Positive Patient with Triple Positive Antiphospholipid Syndrome}

V. La Mura $^{1,2}$; R. Gualtierotti ${ }^{1,3}$; I. Martinelli ${ }^{1}$; B. Ferrari ${ }^{1}$; A.M. Ierardi ${ }^{4}$; N. Bitto ${ }^{1}$; G. Ghigliazza ${ }^{1}$; A. Giachi ${ }^{1}$; M. Maggioni ${ }^{5}$; M. Cugno ${ }^{1,3}$; A.L. Fracanzani ${ }^{3,6}$; G. Carrafiello ${ }^{4,7}$; F. Peyvandi ${ }^{1,3}$ ${ }^{1}$ IRCCS Fondazione Ca' Granda - Ospedale Maggiore Policlinico - UOC Medicina Generale Emostasi e Trombosi, Milan, Italy; ${ }^{2}$ Università degli Studi di Milano - Dipartimento di Scienze Biomediche per la Salute, Mllan, Italy; ${ }^{3}$ Università degli Studi di Milano - Dipartimento di Fisiopatologia Medico-Chirurgica e dei Trapianti, Milan, Italy; ${ }^{4}$ IRCCS Fondazione Ca' Granda - Ospedale Maggiore Policlinico - UO Radiologia, Milan, Italy; ${ }^{5}$ IRCCS Fondazione Ca' Granda - Ospedale Maggiore Policlinico - UO Anatomia Patologica, Milan, Italy; ${ }^{6} I R C C S$ Fondazione C $\mathrm{Ca}^{\prime}$ Granda - Ospedale Maggiore Policlinico - UOC Medicina Generale a Indirizzo Metabolico, Milan, Italy; ${ }^{7}$ Università degli Studi di Milano - Dipartimento di Scienze della Salute, Milan, Italy

Background: Several thrombotic manifestations have been reported with SARS-Cov-2 infection including liver vascular involvement.

Aims: We present a dramatic case of acute liver necrosis in a 36-year-old SARS-Cov-2 positive Italian woman with no respiratory symptoms and triple positive antiphospholipid syndrome (APS).

Methods: The patient was referred to our University Hospital for acute hypertransaminasemia and liver failure (Figure). She had systemic lupus erythematosus (positivity for: ANA, anti-dsDNA, complement activation, Coombs; thrombocytopenia, previous arthritis). Anti-phopspholipid antibodies (aPL) were detected for the first time in 2015 during routine pregnancy screening and chronically treated with aspirin. Apparently, no venous/arterial nor obstetric events were recorded up to the recent hospitalization.
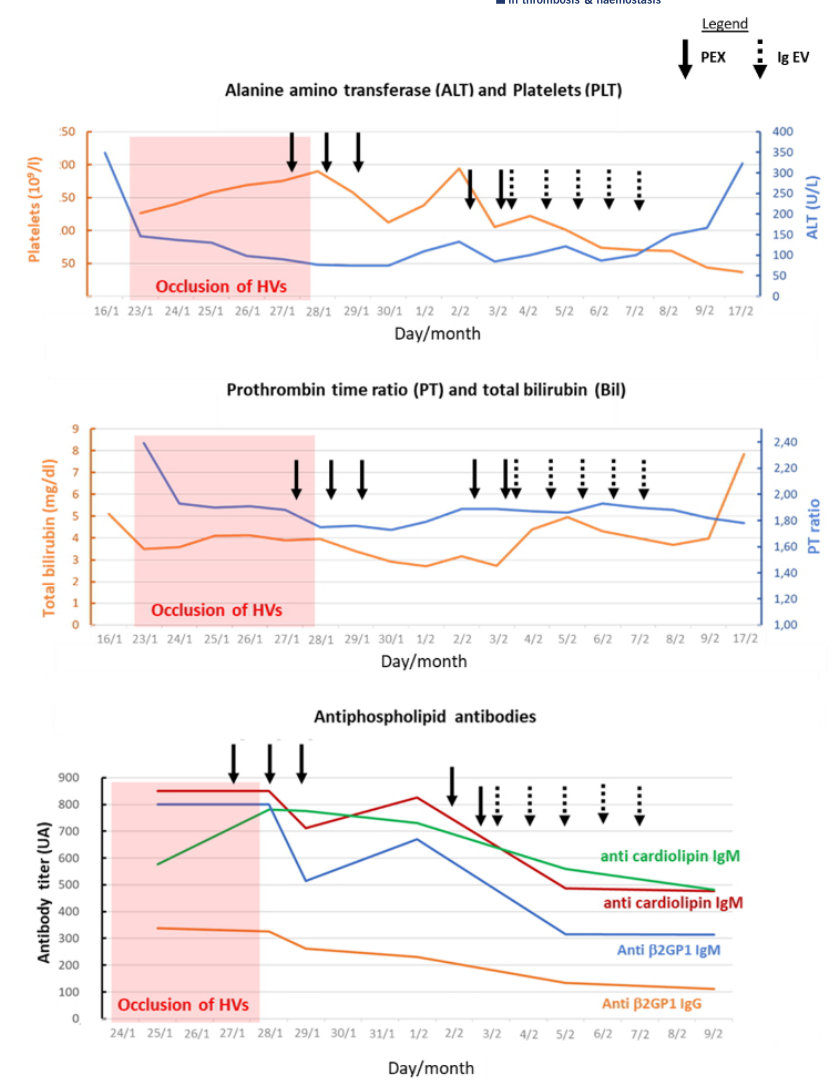

FIGURE 1

Results: At arrival, US-Doppler and CT-scan were consistent with signs of chronic liver disease and occlusion of the three hepatic veins defining a Budd-Chiari syndrome. We opted for a stepwise approach considering anticoagulation (clexane $100 \mathrm{UI} / \mathrm{Kg}$ b.i.d) the first line of therapy before any invasive intervention. Dexamethasone $6 \mathrm{mg} /$ day b.i.d., 6 sessions of plasma-exchange, i.v.-immunoglobulin were sequentially planned to revert the liver damage sustained by aPL. After 5-days, two hepatic-veins resulted recanalized in association with amelioration of liver-enzyme/function and aPL quantification. Then we performed hepatic vein catheterization and transjugular liver biopsy. The histology showed multiple areas of necrosis associated with liver cirrhosis. Unexpectedly, no signs of acute Budd-Chiari were observed (e.g. intraparenchymal hemorrhages, centrilobular congestion, sinusoidal dilation). Other etiologies were also excluded and we hypothesized the involvement of small arteries of the liver in a triple positive APS in a patients with SLE. We finally addressed the patient to a liver transplant program and a tight multispecialistic follow-up.

Conclusions: Thrombosis of arterial/venous vessels or microcirculation causes liver damage in some patients with aPL. Our report suggests that SARS-Cov-2 can exacerbate this prothrombotic condition determining a life-threatening complication such as acute liver failure. 
PB0300 | Analysing Risk Factors for Thrombosis in Covid-19 and Cancer Patients

B. Obispo Portero; J. Rogado; G. Serrano; C. Pangua; A. Lopez;

A. Martin; M. Perez; M.A. Lara

Hospital Universitario Infanta Leonor, Madrid, Spain

Background: Cancer patients are at increased risk of thrombosis due to multiple factors. Millions of cases of SARS-CoV-2 infection have been detected worldwide and there is multiple evidence of its association with arterial and venous thrombosis. At present, we do not know if Covid-19 adds an increased thrombotic risk to cancer patients.

Aims: The aim of our study is to analyze the incidence and risk factors of thrombosis in oncological patients with Covid-19.

Methods: We retrospectively reviewed 86 patients with active cancer and Covid-19 admitted to the general ward oh the Hospital Infanta Leonor (Madrid) between March 5th, 2020 to May 3th, 2020. Study data were collected and managed using REDCap electronic data capture tool.

Results: We diagnosed 10 thrombotic events in 8 oncological patients with a cumulative incidence of $9.3 \%$. A statistically significant association were found regarding thrombosis and history of obesity $(P=0.009)$. Atrend towards significance were detected regarding a previous history of chronic kidney disease $i$ were we detected 2 patients in the thrombosis cohort (25\%) versus only 6 patients of the $78(7.6 \%)$ without thrombosis $(P=0.108)$. No statistically significant differences were found in tumor stage, history of hypertension, acute coronary sindrome, heart disease, history of chronic obstructive pulmonary disease, diabetes, dyslipemia and smoking.

Conclusions: In patients with covid-19 and cancer, a statistically significant difference was found for the history of obesity being a classic factor predictor of the development of thrombotic events in our patients.The prothrombotic effect of Covid-19 infection does not seem to be as evident in cancer patients, probably due to the high mortality in this population group before developing thrombosis. More studies are needed to assess the impact of covid 19 in this population.

PB0301 | Combining Artificial Neural Networks and Blood Tests to Diagnose Covid-19 Infection

T.D. Martins ${ }^{1}$; S.D. Martins ${ }^{2}$; S.A.L. Montalvão ${ }^{2}$; M. Al Bannoud ${ }^{1}$; G.Y. Ottaiano ${ }^{1}$; L.Q. Silva ${ }^{2}$; S.C. Huber ${ }^{2}$; T.S.P. Diaz ${ }^{3}$; C.K. Wroclawski ${ }^{4}$; C.C. Filho ${ }^{4}$; R.M. Filho ${ }^{5}$; J.M. Annichino-Bizzacchi ${ }^{2}$ ${ }^{1}$ Universidade Federal de São Paulo, Diadema, Brazil; ${ }^{2}$ Hemostasis and Thrombosis Laboratory, Hematology and Hemotherapy Center, University of Campinas, Campinas, Brazil; ${ }^{3}$ Anhembi Field Municipal Hospital, São Paulo, Brazil; ${ }^{4}$ Thrombosis and Hemostasis Center, Sirio Libanes Hospital, São Paulo, Brazil; ${ }^{5}$ School of Chemical Engineering, University of Campinas, Campinas, Brazil

Background: Fast and accurate diagnosis of COVID-19 is important to prevent dissemination and disease progression. Artificial
Intelligence is known as a universal fitting tool and can be used on the formulation of predictive models for the disease's diagnosis.

Aims: Obtain a neural network (ANN) to diagnose patients as positive or negative COVID-19 based on patient data and blood tests.

Methods: Data from 678 patients with moderate symptoms from the Anhembi Field Municipal Hospital (São Paulo-Brazil), followed between June/2020 and October/2020 were used. Covid-19 by RTPCR was confirmed in 460 patients. The inputs considered were: sex, age, ethinicity, body mass index, tabagism, ex-tabagism, alveolar infiltrate, arterial hypertension, diabetes, heart rate, respiration rate, body temperature, oxygen saturation, D-dimer, activated partial thromboplastin time, prothrombin time, levels of: hemoglobin, platelet, leukocytes, lymphocytes, monocytes, neutrophils, lactate dehydrogenase, C-reactive protein, and creatinine. Blood was collected at the patient's admission. The ANNs had 25 inputs and the output was the Covid-19 diagnosis. The best ANN was defined by a 5 -fold cross-validation scheme. Then, a test step was performed to assess the model's performance. ANNs with one and two hidden layers were proposed. The number of neurons ranged from 5 to 35 . Results: The best result was obtained with an ANN containing 25 and 30 neurons in the first and second hidden layers, respectively. All the statistical parameters found for the best model are shown at Table 1. The model presented accuracy of $83.3 \%$, high capacity for the prediction of true positives (PPV $=0.917$ and $L R+=5.188$ ), and moderate probability to indicate false negatives $(L R-=0.202)$.

TABLE 1 Summary results obtained for the test dataset

$\begin{array}{ll}\text { Performance Metric } & \text { Value } \\ \text { Accuracy (ACC) } & 83.3 \% \\ \text { AUC } & 0.810 \\ \text { Positive Predicted Value (PPV) } & 0.917 \\ \text { Negative Predicted Value (NPV) } & 0.700 \\ \text { True Positive Rate (TPR) } & 0.830 \\ \text { False Positive Rate (FPR) } & 0.160 \\ \text { True Negative Rate (TNR) } & 0.840 \\ \text { Positive Likelihood Ratio (LR+) } & 5.188 \\ \text { Negative Likelihood Ratio (LR-) } & 0.202\end{array}$

Conclusions: The results showed that the ANNs are promising to diagnose Covid-19 based on clinical parameters and blood tests. After future refinements and proper validation, this model could be used to diagnose Covid-19 on daily basis.

\section{PB0302 | Incidence of Spontaneous Iliopsoas Hematoma in} COVID-19 Patients

G. Sottilotta ${ }^{1}$; C. Mangano ${ }^{2}$; R. Basile ${ }^{2}$; C. Falcone ${ }^{3}$; F. Luise $^{4}$; D. Megalizzi ${ }^{1}$; G.M. Nicolò ${ }^{5}$; V. Oriana ${ }^{1}$; A. Piromalli ${ }^{4}$ ${ }^{1}$ Hemophilia Centre - Thrombosis and Hemostasis Service, Great Metropolitan Hospital, Reggio Calabria, Italy; ${ }^{2}$ Unit of Infectious Disease, Great Metropolitan Hospital, Reggio Calabria, Italy; ${ }^{3}$ Unit of Radiology, 
Great Metropolitan Hospital, Reggio Calabria, Italy; ${ }^{4}$ Analysis Laboratory, Great Metropolitan Hospital, Reggio Calabria, Italy; ${ }^{5}$ Clinical Pathology and Clinical Biochemistry, University of Catania, Catania, Italy

Background: Low molecular weight heparin (LMWH) is recommended in hospitalized patients with coronavirus disease 2019 (COVID-19) due to high risk of thromboembolism. However, LMWH must be carefully dosed for the bleeding risk related to the hemostatic changes in COVID-9 patients.

Aims: To investigate the correlation between COVID-19 related hemorrhagic alterations and the onset of spontaneous lliopsoas hematoma (IPH).

Methods: We analyzed data from our patient's medical records and the reports found in PUBMED.

Results: There are currently very few reports about unprovoked IPS in COVID-19 patients: a 69-year-old male, on aspirin, who was started on daily 4000 I.U. LWMH at hospitalization, and who developed severe IPH after 20 days; a 74-year-old man treated with LMWH 4000 IU twice daily (b.i.d.) for atrial fibrillation (AF),with a worsening prolongation of PT and PTT during hospitalization, and a 56-year-old man, on LMWH 4000 b.i.d. for AF, with normal coagulation pattern at the admission, but with a moderately increased PT and a thrombocytopenia during the hospitalization, who developed IPH after 27 days. A bout our experience, we reported two cases (patients' features summarized in Table 1): a 75-year-old male on clopidogrel due to an ischemic stroke, which was substituted by LMWH 4000 I.U. b.i.d. and who had a transient ischemic attack after 7 days: for this reason, the LMWH dosage was increased to 6000 I.U. b.i.d. After ten days he developed IPS: a moderate and transient thrombocytopenia, disappeared spontaneously after some days, was observed without coagulation alterations. The second case was a 79-year-old obese male, who started increased LMWH prophylaxis with enoxaparin 6000 I.U. b.i.d. Two weeks after admission, the patient manifested a spontaneous IPS. No changes were observed in coagulation parameters, nor a reduction in the platelet count.
Conclusions: Differently from other reports, in our experience, IPH in COVID-19 patients cannot be certainly or directly related to depletion of coagulation factors or consuming of platelets. Further studies are needed to assess the hemorrhagic risk of antithrombotic therapy, as well as investigation of virus-related acquired coagulation disorders predisposing to bleeding in COVID-19.

\section{PB0303 | Immune Thrombocytopenia Purpura Associated with} Novel Coronavirus Infection

$\underline{\text { R.M. Camelo }}^{1,2}$; P.C. Gontijo ${ }^{2,3,4}$

${ }^{1}$ Faculty of Medicine of the Universidade Federal de Minas Gerais, Belo Horizonte, Brazil; ${ }^{2}$ Unimed-BH, Hospital Unimed Betim, Betim, Brazil;

${ }^{3}$ Unimed-BH, Unidade Belo Horizonte, Belo Horizonte, Brazil; ${ }^{4}$ CETUS Oncologia, Belo Horizonte, Brazil

Background: During the current COVID-19 pandemic, thrombocytopenia was noted in up to one third of hospitalized patients, being lower in those with very severe disease. In most COVID-19related thrombocytopenia, the platelet count does not fall below $100 \times 10^{9} / \mathrm{L}$. Severe degree of thrombocytopenia $\left(<20 \times 10^{9} / \mathrm{L}\right.$, or a sudden drop $>50 \%$ over $24-48 \mathrm{~h}$ ) is likely to indicate an immune aetiology.

Aims: We described a rare case of immune thrombocytopenic purpura (ITP) associated with COVID-19.

TABLE 1 Main features of patients with Iliopsoas hematoma admitted to our hospital

$\begin{array}{lll}\text { Basal Features } & \text { Patient } 1 & \text { Patient } 2 \\ \text { Age, Sex } & 75, \text { Male } & 79, \text { Male } \\ \text { Personal History } & \begin{array}{l}\text { Chronic obstructive pulmonary disease, carotid } \\ \text { atheromatous plaques, ischemic stroke }\end{array} & \text { Hypertension } \\ \text { Body mass index } & 26.4 & 30.4 \\ \text { Iliopsoas Hematoma Features } & \text { Bilateral } & \text { Monolateral } \\ \text { Max Dimension } & \text { Right } 52.6 \mathrm{~mm} \text {; Left } 40.5 \mathrm{~mm} & 91.6 \mathrm{~mm} \\ \text { Onset after admission } & 10 \text { days } & 14 \text { days } \\ \text { Enoxaparin Dosage before onset } & 6000 \text { I.U. twice daily } & 6000 \text { I.U. twice daily } \\ \text { Enoxaparin Dosage after onset } & 4000 \text { I.U. twice daily } & 5000 \text { I.U. twice daily } \\ \text { Time until remission } & 27 \text { days } & 21 \text { days }\end{array}$


Methods: Patient medical files were retrospectively reviewed.

Results: A previously healthy 23-year-old woman visited the Emergency Department after 2 days of sneezing, cough, sorethroat, shivers, and fever. She also complained petechiae and spontaneous mild gingival hemorrhage, with a normal blood count. Three days later, she was admitted due to worsening of the petechiae and a drop in the platelet $\left(231.9 \times 10^{9} / \mathrm{L}\right.$ to $\left.5.8 \times 10^{9} / \mathrm{L}\right)$. Nasopharynxswab RT-qPCR detected SARS-CoV-2. Reticulocyte count was normal and no schistocyte was observed. Clotting assays and fibrinogen were normal, and D-dimers were slightly increased. She had microscopic hematuria with normal renal function tests. Total/fractionated bilirubin, and transaminases were normal, although lactic dehydrogenase was slightly elevated. Thyroid function was normal. Protein electrophoresis was normal. Folate and vitamin B12 levels were normal. Anti-nuclear antibodies, syphilis, human immunodeficiency virus, and hepatitis $C$ virus tests were non-reagent. She was immunized against hepatitis B virus. Anticardiolipin antibodies were negative, but direct Coombs and lupus anticoagulant tests were positive. Thoracic computed tomography was normal. Prednisone was administered and she was discharged home with a platelet count of $191.9 \times 10^{9} / \mathrm{L}$. Two months after prednisone withdrawal, direct Coombs and lupus anticoagulant tests were negative, while platelets were $354.0 \times 10^{9} / \mathrm{L}$

TABLE 1 Blood count profiles in different moments. MCV, mean corpuscular volume; $\mathrm{MCH}$, mean corpuscular hemoglobin; $\mathrm{MCHC}$ mean corpuscular hemoglobin concentration; RDW, red cell distribution width.

\begin{tabular}{|c|c|c|c|c|c|c|c|c|c|}
\hline Blood count & $\begin{array}{l}\text { Basedine } \\
\text { Seper/1/2019 }\end{array}$ & Sun/2z/2020 & Ju/ $01 / 2020$ & 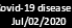 & 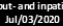 & tit]/04/2020 & $J u / 05 / 2020$ & | on & tit:5 \\
\hline blood cells $\left(x \times 10^{-1 / 4}\right.$ & 4.45 & 5.16 & 4.68 & 4.27 & 4.16 & 4.38 & 4.40 & 4.47 & 452 \\
\hline $\begin{array}{l}\text { Hemoglabin }(g / d L) \\
\text { Hemattort }\end{array}$ & 13.6 & 149 & 13.7 & 12.5 & 12.1 & 12.8 & 13.1 & 14.1 & 14.0 \\
\hline McV (ft) & $\begin{array}{l}38.9 \\
87.4\end{array}$ & $\begin{array}{l}83.2 \\
83.7\end{array}$ & 83.1 & $\begin{array}{l}35.9 \\
82.9\end{array}$ & 83.4 & $\begin{array}{l}86.4 \\
83.1\end{array}$ & $\begin{array}{l}36.0 \\
83.0\end{array}$ & $\begin{array}{l}389.8 \\
89.1\end{array}$ & $\begin{array}{l}80.6 \\
90.6\end{array}$ \\
\hline & 30.6 & 289 & 29.3 & 29.3 & 29.1 & 29.2 .2 & 29.8 & 31.5 & 31.0 \\
\hline $\operatorname{RDW}(\%)$ & 作 & & 势, & 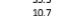 & 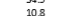 & 势, & & 作 & \\
\hline White blood cells $\times 100 / 10$ & 9.84 & 9.65 & 7.2 & 9.39 & 5.98 & 9.09 & 9.24 & 8.10 & 9.30 \\
\hline Segmented neutrophis (x) & 65.6 & 640 & 77.0 & 880 & 89.0 & 80.9 & 87.8 & 69.5 & 75.8 \\
\hline 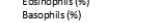 & $\begin{array}{l}10 \\
0.3 \\
10\end{array}$ & 0.9 & 0.0 & $\begin{array}{l}0.0 \\
0.0\end{array}$ & 0.6 & $\begin{array}{l}0.2 \\
0.8\end{array}$ & $\begin{array}{l}0.1 \\
0.4\end{array}$ & 0.7 & $\begin{array}{l}0.7 \\
0.7\end{array}$ \\
\hline ooreses $(x)$ & $\begin{array}{l}24.6 \\
8.5\end{array}$ & 25.9 & 9.00 & $\begin{array}{l}9.0 \\
30\end{array}$ & 8.1 & 58 & 8.7 & 24.1 & $\begin{array}{ll}16.1 \\
67\end{array}$ \\
\hline 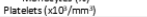 & 3080 & 231.9 & 5.8 & 10.0 & 572 & & $\begin{array}{l}3.0 \\
191.9\end{array}$ & 77.0 & \\
\hline
\end{tabular}

Conclusions: Although thrombocytopenia may be a predictor of worse prognosis in COVID-19, no relationship between ITP and outcome was described yet. Currently, after excluding other causes of (severe) thrombocytopenia, the treatment of COVID-19-associated ITP should be similar the treatment of non-COVID-19 individuals.
PB0304 | Transient Lupus Anticoagulant Presence in COVID-19 Pneumonia, a Probably Epiphenomenon

M. Martinuzzo $^{1,2}$; M.S. López ${ }^{1,2}$; I. Carboni Bisso ${ }^{3}$; I. Huespe ${ }^{4,5}$; M. Las Heras ${ }^{3}$; E. Prado ${ }^{3}$; V. Privitera ${ }^{6}$; M. Garbarini ${ }^{3,6}$; L. Barrera ${ }^{7,2}$; J. Sinner ${ }^{3}$

${ }^{1}$ Hospital Italiano de Buenos Aires. Laboratorio Central, Caba, Argentina; ${ }^{2}$ Instituto Universitario del Hospital Italiano de Buenos Aires, Caba, Argentina; ${ }^{3}$ Hospital Italiano de Buenos Aires. Unidad de Terapia Intensiva de Adultos, Caba, Argentina; ${ }^{4}$ Hospital Italiano de Buenos Aires. Unidad de Terapia Intensiva de Adultos, Area de Investigación en Medicina Interna, Caba, Argentina; ${ }^{5}$ Instituto Universitario del Hospital Italiano de Buenos Aires, Instituto de Medicina Traslacional e Ingeniería Biomédica. CONICET, Caba, Argentina; ${ }^{6} \mathrm{Hospital}$ Italiano de Buenos Aires. Hematología, Caba, Argentina; ${ }^{7}$ Hospital Italiano de Buenos Aires. Laboratorio Central, Ciudad Autonoma de Buenos Aires, Argentina

Background: High prevalence of Lupus anticoagulant (LA) has been reported in COVID-19 infection.

Aims: To determine the presence and the evolution of LA in COVID-19 pneumonia en the first 10 days at Intensive Care Unit (UCI).

Methods: Prospective observational cohort study: Consecutive adult COVID-19 patients admitted to ICU. Exclusion criteria: age > 80 years, anticoagulation, tocilizumab, convalescent plasma transfusion, thrombophilia, pregnancy and cancer.Blood samples on day 1, 5 and 10 from $\mathrm{UCl}$ admission. Studies: PT, APTT, silica clotting time [HemosILSCT, Instrumentation laboratory(IL)], diluted Russell viper venom time HemosILDRVVT(IL) and STADRVVT(STAGO Diagnostic). Screening. Mixing with normal pooled plasma (NP) and confirmatory tests should be above their cur off points to be consider LA+. Biomarkers: D Dimer(DD), Reactive Protein C high sentitivity(cRP-H), Ferritina, LDH and interleukin 6(IL 6).

Results: Patients included: 23, age 57 y (IQR52-71), 70\% male, 15 required mechanical ventilation(MV).Twelve(52.1\%) had LA+ by HemosILDRVVT in at least one time point, 3 in 3, 1 in 2(T5,10) and 8 in one(7/8 T1, 1/8 T5); 4/5 patients with hospital discharge before T10 presented LA+ only at T1. LA prevalence was lower with STADRVVT(Table 1). SCT was negative in all samples.

TABLE 1 Results of coagulation tests used for Lupus Anticoagulant diagnosis.

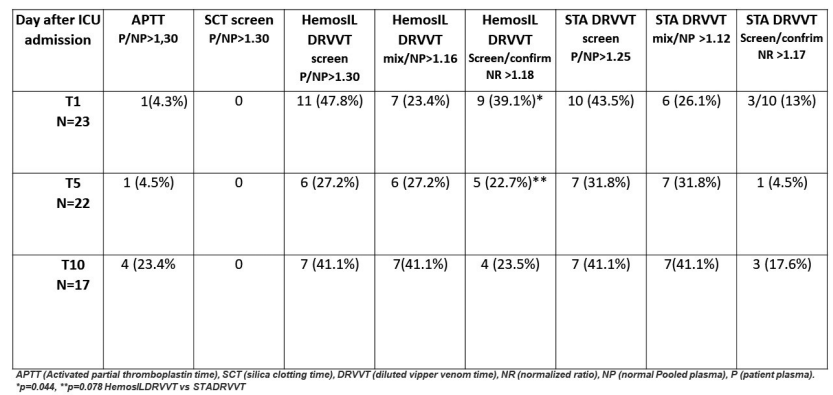


CRP-H, IL6 and Ferritin were higher in LA+, particularly at T5 and T10(Table 2). We cannot exclude CRP interference in DRVVT many samples had CRP > 126 (maximum concentration tested in vitro on NP).

TABLE 2 Inflammatory biomarkers levels and mechanical ventilation requirement in LA Pos and Neg patients at each time point.

\begin{tabular}{|c|c|c|c|c|c|c|}
\hline & $\begin{array}{c}\text { T1 } \\
\text { LAPOS } \\
n=10\end{array}$ & $\begin{array}{c}\text { T1 } \\
\text { LANEG } \\
n=13\end{array}$ & $\begin{array}{c}\text { T5 } \\
\text { LAPOS } \\
\text { n=5 }\end{array}$ & $\begin{array}{c}\text { T5 } \\
\text { LANEG } \\
n=17\end{array}$ & $\begin{array}{c}\text { T10 } \\
\text { LAPOS } \\
n=4\end{array}$ & $\begin{array}{c}\text { T10 } \\
\text { LANEG } \\
n=13\end{array}$ \\
\hline DD & 583 & 1033 & 2340 & 1592 & 1941 & 1664 \\
\hline $\begin{array}{l}\text { (nd/mL FEU) } \\
\text { Median (IQR) }\end{array}$ & $(476-1123)$ & $(580-3240)$ & (1125-5551) & $(420-3038]$ & (1384-6262) & (1511-2389) \\
\hline CRP-H $(\mathbb{Z} / \mathrm{L} / \mathrm{L})$ & 89 & 85 & $251^{*}$ & 46 & 150 & 76 \\
\hline Median (IQR) & (61-268) & $(37-147)$ & (134-292) & (6-100) & (91-233) & (42-249) \\
\hline $\operatorname{LOH}(\mathrm{U} / \mathrm{L})$ & 315 & 340 & 275 & 242 & 291 & 240 \\
\hline Median (IIR) & $(262-401)$ & {$[275-387]$} & {$[216-344]$} & (1144-314] & $(267-305)$ & $(184-316)$ \\
\hline Ferritin (mg/dal) & 1554 & 753 & 1781 & 734 & $2116^{5}$ & 700 \\
\hline Median (IQR) & (540-1787) & (543-1164) & (745-2053) & (479-1447) & (1549-2618) & $(626-1430)$ \\
\hline $166(\mathrm{pg} / \mathrm{mL})$ & 22 & 19 & $180^{4}$ & 43 & 89 & 61 \\
\hline Median (IIR) & (9.84) & $(10-114)$ & (69-299) & (498) & (39-487) & 20-129) \\
\hline vM & $4(40)$ & $7(53.8)$ & $5(100) \S$ & $9(52.9)$ & $4(100) \xi$ & $9(69.2)$ \\
\hline
\end{tabular}

Patients received prophylactic enoxaparin, samples were taken at through, antiXa $=0.08(0.04-0.12) \mathrm{U} / \mathrm{mL}$, ruling out interference. $\mathrm{LA}+$ was not associated with death $(n=4)$ or VM requirement. Only one LA- patient developed pulmonary thromboembolism after leaving ICU.

Conclusions: LA presence was demonstrated in this cohort of COVID-19 Pneumonia patients. Its presence was transient during the short period evaluated, LA was diagnosed through DRVVT with differences between regents. LA presence was associated with inflammatory biomarkers but not with MV requirement or death. These results confirm that LA is probably an epiphenomena.

\section{PB0305 | Role of Hematological Parameters in Stratification of Covid-19 Disease Severity}

S. Taj; A. Kashif; S. Arzinda Fatima; S. Imran; A. Lone; Q. Ahmed FMH College of Medicine and Dentistry, Lahore, Pakistan

Background: Covid-19 disease involves respiratory as well as other body systems including cardiovascular, gastrointestinal, neurological, immunological and hematopoietic system. Patient of covid-19 pneumonia presents with wide range of hemostatic abnormalities. These hemostatic abnormalities in COVID-19 are related with disease progression, severity and mortality.

Aims: The aim of our study is to evaluate the role of hematological parameters in determination of COVID-19 disease severity.

Methods: This was a retrospective study, conducted in Department of Pathology and Department of medicine, FMH college of Medicine and Dentistry from May 2020 to July 2020. Total of 101, confirmed cases of covid-19 disease, both genders between 17 and 75-year age were included. Hematological parameters were compared in mild, moderate, severe and critical disease group. Continuous variables were analyzed by using non parametric, Kruskal Wallis test while categorical variables were analyzed by chi-square test.

Results: Out of 101 patients, 20.8\%, 51.8\%,19.8\% and 7.9\% were in mild, moderate, severe and critical group respectively. Median (IQR) values of WBCs ( $P$-value 0.004$)$, ANC ( $P$-value 0.002$)$, NLR ( $P$-value $0.001)$, D-dimer level ( $P$-value 0.001), ferritin (0.0001), LDH (0.0001) were significantly increased in patients with critical disease. Median (IQR) values of APTT ( $P$-value 0.003 ) and CRP ( $P$-value 0.0001$)$ were suggestively higher in patients with severe disease. Other parameters like Hemoglobin, MCV, HCT, ALC, Platelet count, prothrombin time did not show statistically significant association with severity of disease.

Conclusions: The study concluded that Leukocytosis, neutrophilia, elevated Neutrophil to lymphocyte ratio, APTT, D-dimer, LDH, serum ferritin and CRP are associated with severity of covid-19 disease.

PB0306 | Ddimer and the Correlation with the Mortality Rate in Covid Patients: A Single Centre Report in Medan Indonesia

S.S. Widjaja ${ }^{1}$; L. Mutiara²; S. Tan²; F. Fenny ${ }^{2}$

${ }^{1}$ Universitas Sumatera Utara, Medan, Indonesia; ${ }^{2}$ Murni Teguh Memorial Hospital, Medan, Indonesia

Background: Covid 19 was first reported in the early of march 2020, increasingly rapidly and today reaching $1,386,556$ cases. Meanwhile the worldwide cases reach 117,727,822 cases as reported at 9 of march 2021. Clotting plays a big role in mortality and morbidity of Covid-19 infection, Ddimer a fibrin degradation product was used as a marker of hypercoagulable state were studied among patients admitted in Murni Teguh Memorial Hospital (MTMH) between March 2020 until February 2021.

Aims: This study aims to study the relationship between the increase of Ddimer levels and the number of deaths among Covid 19 patients. Methods: This is a cross-sectional study to investigate the increase of Ddimer levels among the death cases in Covid 19 patients.

Results: A total of 981 covid 19 cases admitted at MTMH with the number of deaths 156 cases (15.8\%), The ddimer level was elevated in $80.7 \%$ death cases. There was no statistical difference within the survive and death cases. $(P=0.085)$. The mortality rate of the renal failure ongoing dialysis with covid 19 was $100 \%$ with ddimer level above 2 (cut off level 0.5). Prothrombin time and Activated partial thromboplastin time levels were elevated in $57 \%$ of death cases and there was a statistical difference between the survival and death cases $(P=0.035)$.

Conclusions: Ddimer levels were elevated in 80.7 of death cases in Covid 19 patients, with $57 \%$ cases elevated in prothrombin time and activated partial thromboplastin time. 
PB0307 | Thrombin Generation (TG) Reveals Underlying Hypercoagulable Process in COVID-19 Patients Despite Low Molecular Weight Heparin (LMWH) Therapy

A. Scazziota $^{1,2}$; S. Pons ${ }^{1}$; M.d.L. Herrera ${ }^{1}$; S. Aguirre $^{1}$; V. Monserrat ${ }^{1}$

${ }^{1}$ Universidad de Buenos Aires, Buenos Aires, Argentina; ${ }^{2}$ Hospital de Clinicas, Buenos Aires, Argentina

Background: Infection with SARS-CoV-2 is responsible for systemic inflammation and consequent coagulation activation. Therapeutic or prophylactic LMWH choices might influence the procoagulant state. Aims: To determine the coagulation and inflammation parameters and to investigate the hypercoagulable process of COVID-19 patients receiving LMWH, employing TG assay.

Methods: All hemostasis measurements were performed on the Sta Compact (Stago). C - Reactive Protein (CRP) by immunoturbidimetric assay. TG was determined by CAT (Thrombinoscope BV) triggered with 5 pM TF. The parameters evaluated were: lag time (LT), peak time (ttP), endogenous thrombin potential (ETP) and thrombin peak (peak height). Patients with moderate COVID-19 admitted to the General ward (GW) or in the intensive care unit (ICU), were included. A group of patients at GW were receiving enoxaparin $40 \mathrm{mg}$ sc once daily (group B) and the patients at ICU received $0.5 \mathrm{mg} / \mathrm{kg}$ sc BID (group C). No Covid-19 patients at GW were used as control group (group D). Results: At the GW, there weren't difference in fjbrinogen and CRP values between group $A$ and group $B$, but both were higher than group D. Group A showed lower D-dimer values compared with group $B(P$ 0.01) and $C(P$ 0.037).Group $C$ had increased fibrinogen levels compared with Covid-19 patients admitted to the GW (Group $A, B$ ) .No significant differences were found in TG (Peak, ETP and ttP) between Group A and B but Group C had a lower ETP and longer LT than those in the GW, despite that, Group $C$, had similar thrombin generation than control group (ETP C vs D P 0.89, Peak CvsD P 0.57).

TABLE 1 Comparisons of TG parameters, fibrinogen and D dimer values in COVID-19 and no Covid-19 patients receiving or not LMWH, admitted to either general ward or intensive care unit. $P$-value $<0.05$ Data are presented as mean \pm SD [min-max]

\begin{tabular}{|c|c|c|c|c|c|c|c|}
\hline \multirow{2}{*}{$\begin{array}{l}\text { Patients at } \\
\text { admission }\end{array}$} & \multicolumn{2}{|c|}{$\begin{array}{c}\text { COVID-19 } \\
\text { General ward }\end{array}$} & \multirow{2}{*}{$\begin{array}{c}\begin{array}{c}\text { COVID-19 } \\
\text { ICU }\end{array} \\
\text { C } \\
\text { with LMWH } \\
\text { n } 22\end{array}$} & \multirow{2}{*}{$\begin{array}{c}\begin{array}{c}\text { General ward } \\
\text { control }\end{array} \\
\begin{array}{c}D \\
\text { without } L M W H \\
\mathrm{n} 28\end{array} \\
\end{array}$} & \multicolumn{3}{|c|}{$p$ values } \\
\hline & $\begin{array}{c}\text { A } \\
\text { without LMWH } \\
\text { n } 14\end{array}$ & $\begin{array}{c}\text { B } \\
\text { with LMWH } \\
\text { n } 16\end{array}$ & & & A vs D & $A$ vs $B$ & B vs C \\
\hline Age (years) & $\begin{array}{l}59 \pm 14 \\
{[46-93]}\end{array}$ & $\begin{array}{l}65 \pm 20 \\
{[26-87]}\end{array}$ & $\begin{array}{l}73 \pm 15 \\
(33-89)\end{array}$ & $\begin{array}{l}40 \pm 13 \\
{[26-62]}\end{array}$ & 0,00 & 0,42 & 0,17 \\
\hline $\begin{array}{l}\text { Peak height } \\
\mathrm{nM}\end{array}$ & $\begin{array}{c}364 \pm 66 \\
{[227-424]}\end{array}$ & $\begin{array}{c}328 \pm 148 \\
{[14-548]}\end{array}$ & $\begin{array}{c}253 \pm 149 \\
{[6-495]}\end{array}$ & $\begin{array}{c}272 \pm 84 \\
{[103-440]}\end{array}$ & \begin{tabular}{|l|l|}
0,003 \\
\end{tabular} & 0,48 & 0,13 \\
\hline$\underset{\substack{\text { ETP } \\
n M^{*} \min }}{ }$ & $\begin{array}{c}1600 \pm 377 \\
{[1187-2266]}\end{array}$ & $\begin{array}{l}1820 \pm 851 \\
{[180-3393]}\end{array}$ & $\begin{array}{l}1248 \pm 604 \\
{[130-257]}\end{array}$ & $\begin{array}{l}1229 \pm 448 \\
{[732-2525]}\end{array}$ & \begin{tabular}{|l|}
0,025 \\
\end{tabular} & 0,45 & 0,02 \\
\hline$\underset{\min }{\text { Lag time }}$ & $\begin{array}{c}3.40 \pm 0.71 \\
{[2.5-4.67]}\end{array}$ & $\begin{array}{l}5.72 \pm 2.27 \\
{[3.33-10.33]}\end{array}$ & $\left.\begin{array}{c}8,2 \pm 4,3 \\
{[3,33-16,83}\end{array}\right]$ & $\begin{array}{c}3.25 \pm 0.89 \\
{[1.5-5]}\end{array}$ & 0,6 & 0,005 & 0,04 \\
\hline $\begin{array}{l}\text { tte } \\
\text { min }\end{array}$ & $\begin{array}{c}8.81 \pm 9.86 \\
{[4-33.2]}\end{array}$ & $\begin{array}{l}8.83 \pm 3.95 \\
{[5.83-20.5]}\end{array}$ & $\begin{array}{c}12.3 \pm 7.7 \\
{[5-32.5]}\end{array}$ & $\begin{array}{c}6,27 \pm 1,35 \\
{[3,5-10,3]}\end{array}$ & 0,16 & 0,99 & 0,11 \\
\hline $\begin{array}{l}\text { Fibrinogen } \\
\mathrm{mg} / \mathrm{dL}\end{array}$ & $\begin{array}{l}453 \pm 92 \\
{[320-598]}\end{array}$ & $\begin{array}{l}484 \pm 119 \\
{[310-719]}\end{array}$ & $\begin{array}{l}581 \pm 176 \\
{[300-975]}\end{array}$ & $\begin{array}{c}330 \pm 44 \\
{[254-418]}\end{array}$ & 0.00 & 0,49 & 0,035 \\
\hline $\begin{array}{l}\text { D dimer } \\
\text { Hg/mL FEU }\end{array}$ & $\begin{array}{c}0.38 \pm 0.53 \\
{[0.1-1.83]}\end{array}$ & $\begin{array}{c}1.96 \pm 1.30 \\
{[0.1-4.54]}\end{array}$ & $\begin{array}{c}3.60 \pm 4,6 \\
{[0.42-21.50]}\end{array}$ & $\begin{array}{l}0,27 \pm 0,14 \\
{[0,23-0,32]}\end{array}$ & 0,31 & 0,001 & 0,176 \\
\hline $\begin{array}{l}\text { CRP } \\
\mathrm{mg} / \mathrm{L}\end{array}$ & $\begin{array}{c}31 \pm 366 \\
{[2-84]}\end{array}$ & $\begin{array}{c}32 \pm 60 \\
{[0.25-188]}\end{array}$ & $\begin{array}{c}34+39 \\
{[0,56-115]}\end{array}$ & $\begin{array}{c}2,8 \pm 1,4 \\
{[0-5]}\end{array}$ & 0.00 & 0,96 & 0,90 \\
\hline
\end{tabular}

Conclusions: Our results show that TG is a useful test to demostrate the hypercoagulable state in patients with COVID-19. In adittion TG assay revels that standard LMWH doses could be inefficient in critical patients.

P0126 | D Dimer and other Biomarkers in Dengue Patients within a Doble Viral Circulation Scenery (Dengue/COVID-19)

F. Chuliber ${ }^{1}$; R. vanden Ryn ${ }^{2}$; N. Schutz ${ }^{1,3} ;$ L. Barrera ${ }^{2,3}$; M.S. López ${ }^{2,3}$; C. Raff ${ }^{2}$; V. Privitera ${ }^{1}$; D. Mezzarobba ${ }^{1}$; M. Villagra Iturre $^{1}$; V. Estela $\mathrm{S}^{1,3}$; D. Penchasky ${ }^{1,3}$; G. Jimenez ${ }^{2,3}$; M. Rolan ${ }^{2}$; D. Arrigo ${ }^{2,3}$; J. Arbelbide ${ }^{1,3}$; F. Seoane ${ }^{2}$; M. Martinuzzo ${ }^{2,3}$ ${ }^{1}$ Hospital Italiano de Buenos Aires. Hematología, CABA, Argentina; ${ }^{2}$ Hospital Italiano de Buenos Aires. Laboratorio Central, CABA, Argentina; ${ }^{3}$ Instituto Universitario del Hospital Italiano de Buenos Aires, CABA, Argentina

Background: SARs-CoV-2 and DENGUE share clinical symptoms triggering inflammatory processes with high biomarkers levels that can hinder their differentiation in periods of double viral circulation. Aims: To compare levels of D-dimer(DD), High Sentivity C-reactive protein(CRP-H), Ferritin, Lactic-Dehydrogenase(LDH) and haematological parameters among patients with COVID-19 or DENGUE at first hospital consultation in emergency department(FCED).

Methods: Retrospective cohort study: consecutive patients (Age > 18y.o.) at FCED on suspicion of symptomatic COVID-19 infection (March-June 2020), in which DD (VIDAS-Exclusion II, Biomerieux) was measured. Only dengue or COVID-19 positive patients were included and classified as Dengue, Mild upper airway infection (MUAI) by COVID-19 and COVID-19 pneumonia. DD(ng/ $\mathrm{mL}$ FEU), CRP-H, ferritin and LDH(Beckman-Coulter), PT and APTT(ACLTOP,Instrumentation Laboratory) and haematological parameters(DxH800, Beckman Coulter), were compared.

Statistics: SPSS-23 Software

Results: A total of 229 patients were included. Non severe Dengue patients presented significantly higher DD levels than MUAI, but not different to COVID-19-positive pneumonia at FCED (Table 1).

The leukocytes, neutrophils, lymphocytes, and platelets count(both considering FCED and nadir) were lower in DENGUE, compared to COVID-19 patients (Table 2). 
TABLE 1 Coagulation tests, DD, and other biomarkers in Dengue and COVID-19 patients evaluated in the First consultation at emergency department. (MUAI Mild upper airways intection, CRP C-Reactive Protein high sensitivity. LDH Lactic dehydrogenase, DD D-Dimer, NS: Not significant)

$N(\%)$

$\begin{array}{lllll} & \text { COVID-19+ MUAI } & & \text { COVID-19 + Pneumonia } \\ \text { DENGUE } n=67 & n=85 & \text { P vs. DENGUE } & n=78 & \text { P vs DENGUE } \\ 31(47.7) & 40(47.1) & \text { NS } & 42(50.3) & \text { NS }\end{array}$

Female Sex

31 (47.7)

$40(47.1)$

NS

NS

$<0.001$

\begin{tabular}{|c|c|c|c|c|c|}
\hline Age, Years & 45 & 38 & & 38 & \\
\hline \multirow[t]{2}{*}{ Median (IQR) } & $(32-61)$ & $(30-51)$ & & $(30-51)$ & \\
\hline & & & NS & & NS \\
\hline PT (\% act) & 88 & 83 & & 82 & \\
\hline \multirow[t]{2}{*}{ Median (IQR) } & $(76-99)$ & $(74-92)$ & & $(74-91)$ & \\
\hline & & & NS & & 0.024 \\
\hline $\operatorname{APTT}(\mathrm{s})$ & 34 & 33 & & 31 & \\
\hline \multirow[t]{2}{*}{ Median (IQR) } & $(30-37)$ & $(30-36)$ & & $(28-35)$ & \\
\hline & & & $<0.001$ & & NS \\
\hline DD & 795 & 301 & & 593 & \\
\hline (ng/mL FEU) & $(502-1239)$ & (191-472) & & (331-1470) & \\
\hline \multicolumn{6}{|l|}{ Median (IQR) } \\
\hline & & & 0.039 & & $<0.001$ \\
\hline CRP-H mg/L) & 5.3 & 3.8 & & 16.9 & \\
\hline \multirow[t]{2}{*}{ Median (IQR) } & $(3.3-15.1)$ & $(2.1-9.9)$ & & $(7.3-49.2)$ & \\
\hline & & & 0.002 & & 0.091 \\
\hline LDH (U/L) & 190 & 165 & & 227 & \\
\hline \multirow[t]{2}{*}{ Median (IQR) } & $(167-233)$ & (136-193) & & $(174-256)$ & \\
\hline & & & $<0.001$ & & NS \\
\hline Ferritin (mg/dL) & 304.0 & 104 & & 290 & \\
\hline Median (IQR) & $(219-601)$ & $(58-300)$ & & $(140-827)$ & \\
\hline
\end{tabular}

TABLE 2 Hematological parameters in Dengue and COVID-19 patients in the first consultation at emergency department. MUAI Mild upper airways intection, HTC (Hematocrit), HB (Hemoglobin), NS (not significant)

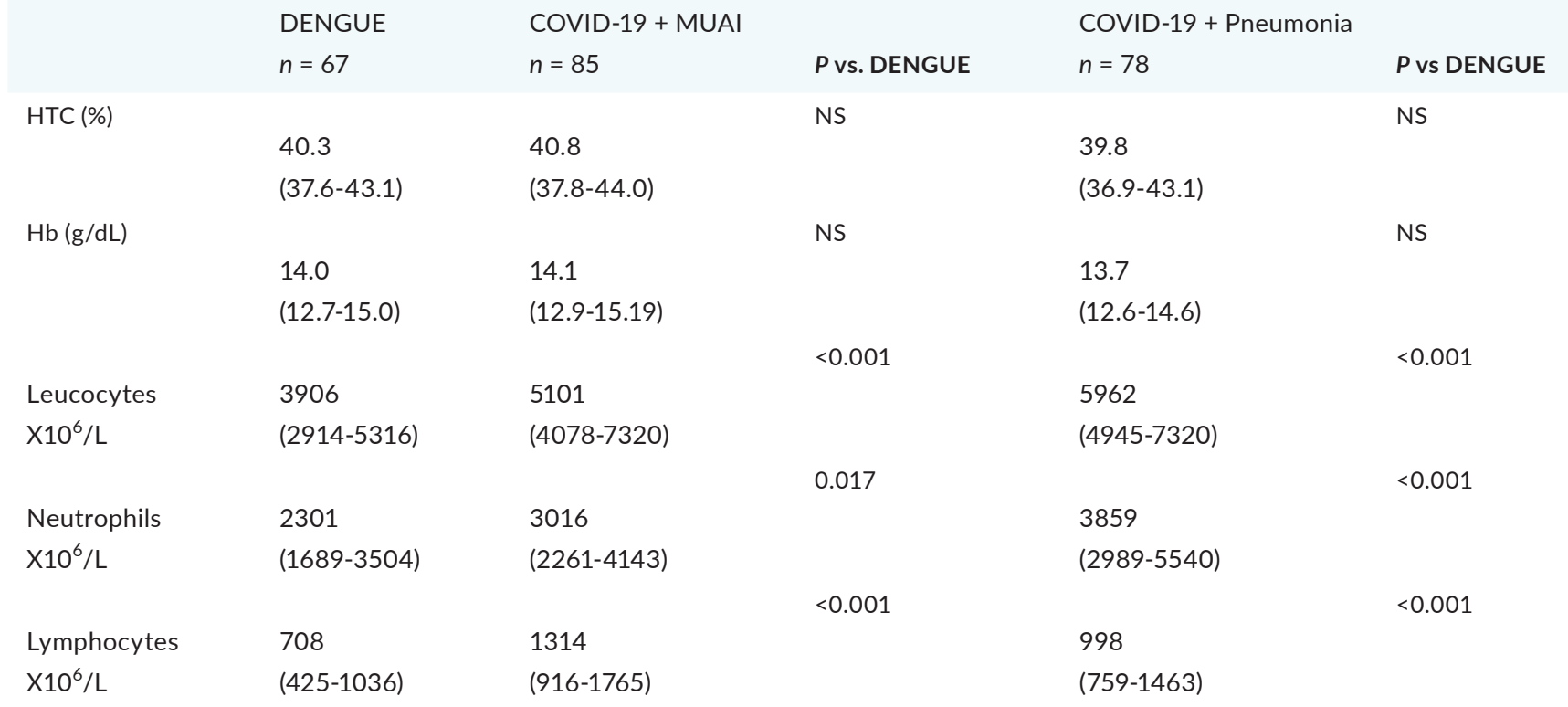




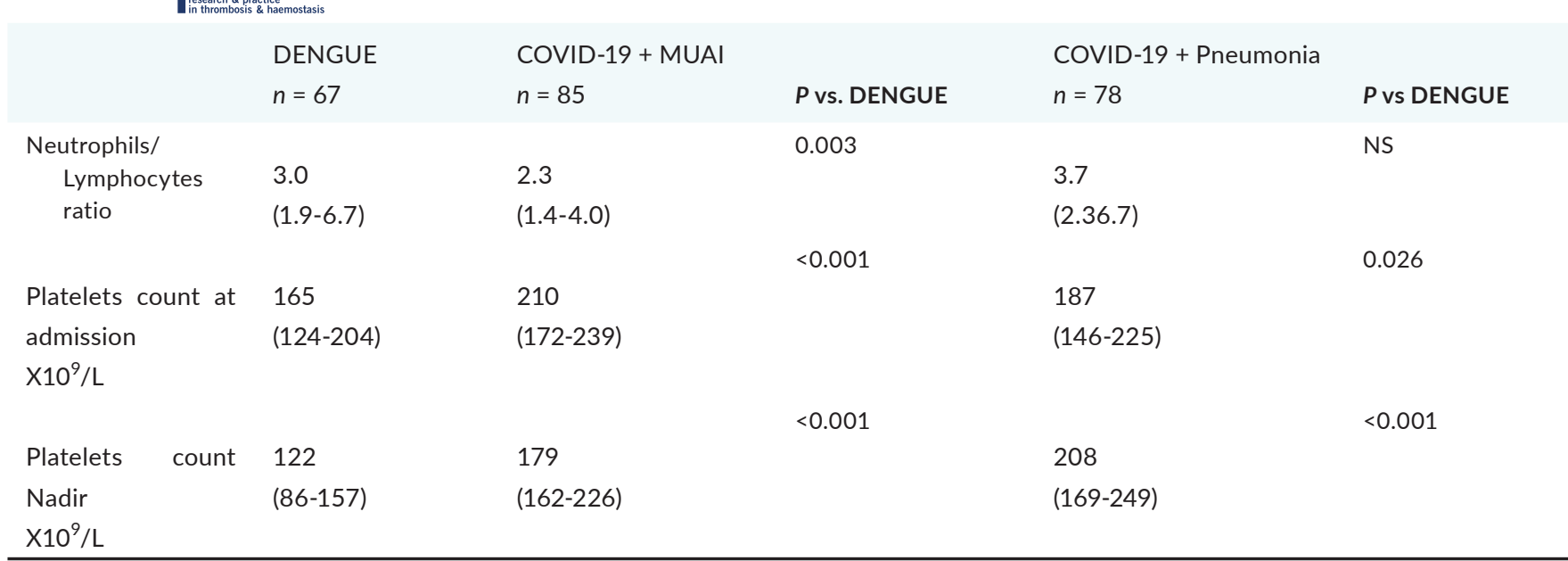

CRP-H levels were higher in DENGUE than in Covid-19 MUAI, but lower than COVID-19 pneumonia. DENGUE LDH and ferritin levels were higher than those of MUAI but comparable to those of Covid-19 pneumonia (Table 1). Ferritin and LDH show a statistically correlation to DD in DENGUE and COVID-19-positive patients, but DD and CRP-H only correlated in COVID-19 patients(r:0.567). Platelets correlated negatively(r:-0.437) with DD only in dengue. Dengue patients with platelets nadir $<100 \times 109 / \mathrm{L}$ and elevated liver transaminases have higher DD levels than those with higher platelels count and not liver inflamation.

Conclusions: Dengue and COVID-19 infections can trigger inflammatory processes with increased biomarkers, including DD. We showed that in non severe dengue the magnitude of response may be greater than in MUAI and similar to pneumonia COVID-19 positive. Studies are needed to define and compare the prognostic role of biomarkers in these entities.

P0127 | Association between FGB - 455 G/A and PAI-1 - 675 5G/4G Gene Polymorphisms and Covid-19 Severity

B. Vatseba; N. Virstyuk

Ivano-Frankivsk National Medical University, Ivano-Frankivsk, Ukraine

Background: Coronavirus infection (COVID-19) is characterized by an increased inflammatory response that can lead to serious manifestations, including coagulopathy. Limited data indicate a high incidence of DVT and pulmonary embolism in more than $40 \%$ of patients. Identifying specific genetic factors can help to determine the prognosis and management of such patients.

Aims: To determine the effect of PAI-1-675 and FGB - 455 G/A gene polymorphisms on the severity of Covid-19 disease course.

Methods: We examined 47 patients (ps) with laboratory-confirmed Covid-19 (26 females/21 males; age, $44.8 \pm 5.6$ year). Plasminogen activator inhibitor (PAI)-1-675 and beta-fibrinogen (FGB ) 455 G/A gene polymorphism were analysed by PCR. Interleukin 6 (IL-6), Ddimer, fibrinogen, $\mathrm{C}$-reactive protein (CRP) and PAI-1 were measured by the immuno-assay method.
Results: Patients were divided into three groups: 9 (19.1\%) ps with mild Covid-19 who were treated on an outpatient basis (I), 17 (36.2\%) ps with moderate of Covid-19 who were hospitalized for bilateral pneumonia (II) and 21 (44.7\%) ps with severe Covid-19 who were treated in intensive care for ARDS and required oxygen therapy (III). The level of D-dimer in group III was 4.7 times higher than in group I $(P<0.001)$. Fibrinogen level in group III was 2.5 times higher than in group I $(P<0.05)$. In patients of group I PAI-1-675 5G/5G (100.0\%), FGB-455 G/A (33.3\%); in patients of group II - PAI-1-675 5G/4G (82.4\%), FGB-455 G/A (47.1\%); in patients of group III - PAI 1-675 5G/4G (38.1\%) and PAI-1-675 4G/4G (61.9\%), FGB-455 G/A (19.1\%) and FGB-455 A/A (23.8\%) were detected. The median RAI-1 levels for the $5 \mathrm{G} / 5 \mathrm{G}, 5 \mathrm{G} / 4 \mathrm{G}$, and $4 \mathrm{G} / 4 \mathrm{G}$ genotypes of this gene were 34.6 , 79.8 and $172.6 \mu \mathrm{mol} / \mathrm{L},(P=0.002)$. The direct correlations between PAI-1 and IL-6, CRP ( $r=0.6209 ; r=0.6905$; accordantly; $P<0.001$ ) were detected.

Conclusions: FGB-455 G/A, PAI-1-675 5G/4G, and especially 4G/4G gene polymorphism may be a predictors of severe Covid-19 disease. 
P0128 | Immune Thrombocytopenia Refractory to Corticoid Therapy Secondary to Covid-19. A Single-centre Experience

B.L. Diaz Jordan; V.B. Yepez Espinales; A. Valverde Templado Valdepenas General Hospital, Valdepenas, Spain

Background: Although immune thrombocytopenia (IT) is uncommon, it has been described as one of the complications secondary to infection by SARS-CoV-2, probably secondary, amongst other factors, to the underlying immune dysregulation. The very few cases reported in the literature show excellent responses to corticoid therapy.

Aims: The objective of this study is to show our experience with 2 patients who had severe symptomatic corticoid-refractory IT as a complication of COVID-19.

Methods: A descriptive, single-centre study that shows our experience with 2 patients diagnosed with severe COVID-19 and a long-term stay at the Intensive Care Unit (ICU), who had severe symptomatic corticoid-refractory IT, an uncommon manifestation according to the very few series reported to date.

Results: Two patients (women aged 65-67 years) with no relevant medical history, diagnosed in 03-2020 and 04-2020, respectively, with bibasal pneumonia secondary to severe COVID-19, needing stay at the ICU and assisted ventilation and long-term stay. On discharge they had severe IT associated with skin bleeding events, initially treated with oral prednisone $\mathrm{mg} / \mathrm{kg} /$ day plus unspecific IVIg $\mathrm{g} / \mathrm{kg} /$ day, initially obtaining a good response, but followed by early loss of response in both cases. It was decided to switch protocol to IV dexamethasone $40 \mathrm{mg}$ for 4 days plus unspecific IVIg g/ $\mathrm{kg} /$ day every 15 days, of which they received 3 cycles, without response and with increased skin stigmata of bleeding. Second-line treatment was started with TPO analogues (oral eltrombopag $50 \mathrm{mg} / \mathrm{day}$, with dose escalation to $75 \mathrm{mg} /$ day), obtaining haematological response on day +7 of start and complete remission on day +21 of treatment. Currently complete response persists, with no documented adverse events.

Conclusions: Although uncommon, corticoid-refractory IT is a documented bleeding diathesis in severe COVID-19. Our experience shows that the early start of TPO analogues (in the absence of response to corticoid therapy) is an effective, well-tolerated option in patients with this complication.

P0129 | Alterations of the Coagulation Profile in Peruvians Admitted for Pneumonia due to SARS-COV2: Cross-sectional Study

R. Pichardo Rodriguez ${ }^{1,2}$; W. Peña-Oscuvilca ${ }^{1}$; L. Cordova-Cueva ${ }^{3}$; O. Ruiz-Franco ${ }^{2}$; F. Garmendia-Lorena ${ }^{2}$

${ }^{1}$ Hematology Service, National Hospital 'Dos de Mayo', Lima, Peru;

${ }^{2}$ Institute of Clinical Research 'Dr. Fausto Garmendia Lorena', Faculty of Medicine, National University of San Marcos, Lima, Peru; ${ }^{3}$ Nephrology Service, National Hospital 'Dos de Mayo', Lima, Peru
Background: In SARS-COV2 infection, little is known about the changes in the coagulation profile that occur at admission that could predict future events such as bleeding or thrombosis.

Aims: To establish the alterations in the coagulation profile of Peruvians with SARS-COV2 pneumonia at the time of their admission to a national referral hospital.

Methods: A cross-sectional, retrospective study was carried out in Peruvians diagnosed with SARS-COV2 pneumonia admitted to the "Dos de Mayo" National Hospital, between June and November 2020. Pneumonia due to other agents were excluded.

SARS-COV2 pneumonia was defined as: pulmonary TEM infiltrate + positive RT-PCR or positive rapid test (IgG and IgM). Alterations in the coagulation profile were determined by: prolonged prothrombin time ( $\mathrm{PT}>14 \mathrm{~s}$ ) and / or prolonged activated thromboplastin time (APTT > $35 \mathrm{~s}$ ) and / or fibrinogen $<200 \mathrm{mg} / \mathrm{dL}$. Additional variables such as age and sex were recorded.

The sample was selected consecutively. The data were collected and entered into a database and sent for analysis after quality control.

Frequencies and percentages were presented for the qualitative variables and summary measures for the quantitative variables. The data were processed RStudio version 1.3.1093.

Results: A total of 100 patients were included. Male sex was the most frequent (63\%) and the mean age was $54.74 \pm 16$ years. Seventy-one percent had PT prolongation and $36 \%$ had APTT prolongation. Only $5 \%$ had fibrinogen $<200 \mathrm{mg} / \mathrm{dL}$.

Conclusions: There is a high prevalence of alterations in the coagulation profile, predominantly prolonged PT. It is relevant to evaluate in prospective studies whether these changes can predict future bleeding or thrombotic events.

P0131 | SARS-CoV 2 and Venous Thromboembolism: A Risk beyond Thromboprophylaxis

C. Gumpel; L. Cinara; M.C. De Paoli; S. Ciarlo; A. Rocaspana;

J. Gnass; M. Bellantig; L. Welker; J.L. Fedele

Rosario Private Hospital, Rosario, Argentina

Background: It is known that SARS CoV-2 is associated with an increase in thrombotic events, specially venous thromboembolism (VTE). As a consequence there are many different thromboprophylaxis recommendations.

Aims: To evaluate the thrombotic complications in hospitalized patients with SARS CoV-2 and its relationship with thromboprophylaxis. Methods: Retrospective analysis of adults with SARS CoV-2 who needed to be hospitalized at Rosario Private Hospital between 0108-2020 to 31-10-2020. Results were analized with the R statistical software.

Results: 160 patients. 69 (43.13\%) women and 91 (56.87\%) men. Median age 63 (20 - 95) years old. 37 (23.12\%) were admitted to ICU. 20 (12.5\%) patients had a venous thromboembolic event; 9 (5.6\%) pulmonary embolism (PE), 9 (5.6\%) deep vein thrombosis (DVT) and 2 (1.25\%) were PE+DVT. All the patients were on heparin 
treatment; $75 \%$ on prophylactic dose and $25 \%$ on therapeutic dose. In a multivariate analysis the VTE risk wasn't associated with sex, age, renal insufficiency, previous VTE, history of anticoagulant treatment, tromboprophylaxis indication, and convalescent plasma treatment. The risk of VTE was significant associated with ICU admission. Mean D-Dimer (DD) level at the moment of hospitalization in patients without VTE was 0.82 ( $\mathrm{Cl} 0.57-1.08), 1.06(\mathrm{Cl} 0.52-2.6)$ in patients with DVT, $2.82(\mathrm{Cl} 0.23-5.4)$ in patients with $\mathrm{PE}$ and 3.82 $(\mathrm{Cl} 2.5-5.1)$ in patients with DVT+'PE.

VTE RISK IN SARS-CoV- 2 patients

\section{TABLE 1}

\section{ICU ADMISSION}

DD 2x normal range

DD $4 x$ normal range

DD $6 \times$ normal range*
OR $3.28(\mathrm{Cl} 1.2-8.7)$

OR $1.89(0.6-5 ., 6)$

OR 3.89 (1.2-13.3)

OR $5.44(1.3-22.6)$
*DD normal level: $\leq 0.50 \mathrm{mcg} / \mathrm{mL}$ fibrinogen equivalent units (FEU)

Conclusions: An increase in DD level at the moment of hospitalization was associated with an increase risk in VTE specially PE. In critical patients (ICU admission) the risk is higher. VTE is a worrying SARS-CoV 2 complication because of its occurence in spite of thromboprophylaxis.

P0132 | Mechanical Prosthetic Valve Thrombosis in COVID-19 Infection: Case Report

N. Diaconu; D. Lupu; T. Cuzor; A. Grosu

Institut of Cardiology, Chisinau, Moldova, Republic of

Background: Coronavirus disease 2019 (COVID-19) is associated with a coagulopathy favouring thrombosis over bleeding that imparts a poor prognosis. Mechanical prosthetic valve thrombosis is a life-threatening complication necessitating immediate intervention. The presenting signs and symptoms of this illness are somewhat variable, and often complicated to recognize, especially in patients with COVID-19.

Aims: We present a case of a 63-year-old woman with a clinical presentation of heart failure symptoms due to mechanical mitral valve thrombosis overlapping with mild COVID-19 pneumonia.

Methods: Clinical case presentation.

Results: The patient presented to the emergency unit with marked dyspnea, asthenia, oliguria, hypotension, oxygen desaturation (83\%), and right ventricular dysfunction. The primary diagnosis was made based on chest X-ray - bilateral pulmonary infiltration and tests suggestive of SARS-COV2 viral infection, including increased values of d-dimer- 5.43. After receiving the negative result of the PCR test at COVID-19, the patient was transferred to the cardiology department with suspicion of PE. EchoCG examinations establishedmitral mechanical valve dysfunction with a gradient of $56 \mathrm{~mm} \mathrm{Hg}$. Given the patient's critical condition we used alteplase and UFH, obtaining symptomatic relief and hemodynamic stability. We identified an extremely elevated D-dimer ( $>10 \mathrm{mcg} / \mathrm{mL}$ ), and computed tomography pulmonary angiography (CTPA) revealed an unexpected low thrombus burden. Research into the cause of thrombosis found low INR values (1.37) despite regular use of unchanged dose of warfarin, and immunological tests found anti-SARS-VOC 2 antibodies.

Conclusions: This case raises awareness to the catastrophic thrombosis of mechanical valve, presenting in an late phase of COVID-19 infection without the typical hyper-inflammation and severe lung injury associated with development of COVID-related coagulopathy. It also serves to inform on the critical role echocardiography has in the comprehensive evaluation and re-evaluation of hospitalized patients with COVID-19, and the importance of a multidisciplinary organized approach in clinical decision-making for this complex and poorly understood disease and its sequelae.

P0133 | IMPROVE Score and Coagulopathy Screening in Coronavirus Disease 2019 Patients

C. Syukur; H. Haroen; L.W.A. Rotty; P.F.C. Lasut; C. Hendrata; C.T.H. Kawengian

Sam Ratulangi University, Manado, Indonesia

Background: Coronavirus disease 2019 (COVID-19) has the possibility of activating systemic coagulation, leads to hypercoagulability condition and caused venous thromboembolism (VTE). Risk stratification measures hypercoagulability in COVID-19 to treat patients. Common coagulopathy laboratory findings in COVID-19 were changes in D-Dimer, prothrombin time (PT), activated partial thromboplastin time (aPTT), thrombocyte, and fibrinogen level.

Aims: This research wants to see the difference in the value of the coagulopathy screening examination between medium-high and low-risk VTE groups in COVID-19 patients.

Methods: Cross-sectional analytical observation done on confirmed COVID-19 patients. The risk assessment of VTE was assessed with IMPROVE score. Coagulopathy screening tests performed were DDimer, PT, aPTT, International Normalized Ratio (INR), thrombocyte, and fibrinogen. Statistical analysis was performed using the MannWhitney test.

Results: From the 42 confirmed COVID-19 patients, 12 patients (29\%) had medium-high VTE risk and 30 patients (71\%) had low. From the Mann-Whitney test, there were statistically significant differences in the D-Dimer, PT, INR, and thrombocyte value between groups of patients with medium-high and low VTE risk in patients [5.26 (17.21) vs $1.54(2.61) \mu \mathrm{g} / \mathrm{mL}, P=0.004 ; 15.85$ (2.03) vs 13.40 (1.40) seconds, $P=0.0001 ; 1.18(0.30)$ vs $1.02(0.26), P=0.004 ; 160$ (91.50) vs $\left.252.50(202.75) 10^{3} / \mu \mathrm{L}, P=0.003\right]$

Conclusions: There were statistically differences in the values of $D$ Dimer, PT, INR, and thrombocyte between groups of patients with medium-high and low VTE risk in patients with COVID-19. The patients with medium-high risk of VTE had higher values on D-Dimer, PT, and INR tests, while lower value was found on thrombocyte. 
P0134 | Reinforcement of Platelets Activity in Patients with Acute Myeloid Leukemia (AML) and Concomitant COVID-19 Infection

Z. Stupakova ${ }^{1}$; I. Dyagil ${ }^{1}$; O. Karnabeda ${ }^{2}$; H. Menzarar ${ }^{3}$

${ }^{1}$ State Institution "National Research Center for Radiation Medicine of NAMS of Ukraine", Kyiv, Ukraine; ${ }^{2}$ Bohomolets National Medical University, Kyiv, Ukraine; ${ }^{3}$ Cancer Center INNOVACIA, Lyutizh, Kiev Region, Ukraine

Background: The overall incidence of venous thromboembolism (VTE) in AML patients is high despite the thrombocytopenia and prophylaxis. Concomitant COVID-19 infection could may well make it worse.

Aims: The primary endpoint was an evaluation of functional activity of thrombocytes under the COVID-19 influence by measuring platelet count, platelets aggregation in the presence of ristocetin, collagen, adenosine diphosphate (ADP), von Willebrand factor: ristocetin cofactor activity [VWF:RCo] as a link of vascular haemostasis; routine coagulation tests - thrombin time, prothrombin time, APTT, Fibrinogen, Antithrombin III as a link of coagulation haemostasis.

Methods: We include 3 patients (2 males and 1 female) with newly diagnosed $A M L$ during induction treatment with concomitant COVID-19 infection and 5 patients with newly diagnosed AML during induction treatment, COVID-19 negative. The emergence of VTE events was assessed by a computed tomography.

Results: Three patients in the study group were experienced fatal VTE - two of them had pulmonary embolism (PE) and one mesenteric venous thrombosis (MVT). There were no VTE events in the control group.

In the comprehensive analysis we observed combined increasing in the rate of platelets aggregation in the presence of ristocetin, collagen and ADP higher than normal rates in three patients in the study group which was associated with VTE of different localisations. In the control group such elevation had not been observed.

We have monitored extremely increased levels of vWF:RCo $(217 \%$, $190 \%, 160 \%)$ in three patients in the study group, that soon was associated with fatal PE and MVT.

Conclusions: Based on these preliminary results we wanted to emphasize the pathologic changes in primary hemostasis as a result of COVID-19 infection in patients with AML. COVID-19 triggered activation of the haemostasis system might lead to increased consumption of clotting inhibitors as well as associated with extreme enhanced activation of platelets and need to be looked at in more depth.

\section{P0135 On the Issue of Thrombinemia in Moderate COVID-19}

N. Vorobyeva $^{1,2}$; A. Barteneva ${ }^{2}$; I. Lovkova ${ }^{2}$

1"National Medical Research Center for Hematology" (Northern

Branch) Ministry of Health of Russia, Arckhangelsk, Russian Federation;

${ }^{2}$ Northern State Medical University Ministry of Health of Russia,

Arckhangelsk, Russian Federation

Background: COVID-19 in hospital in Arkhangelsk.

Aims: The goal is to assess clinical and laboratory signs in moderately severe patients with COVID-19.

Methods: The study included patients hospitalized for COVID-19 -109 patients. On admission to the hospital, some clinical symptoms were assessed; CRP, LDH, ferritin, APTT, D-dimer, fibrinogen indicators and complete blood count (leukocyte, lymphocyte, platelet count.

Results: The average age of patients was Me [Q1-Q3] 62 [34-71] years. Upon admission to the hospital, a positive smear by the polymerase chain reaction method for the presence of coronavirus infection was determined in 60\% ( $n=66)$ of patients. Fever was reported in $60 \%(n=65)$, in $15 \%$ of cases, patients described chest pain $(n=$ $16), 44 \%$ of patients complained of shortness of breath $(n=49), 71 \%$ ( $n=77$ ) of patients complained of cough, sore throat and lack of sense of smell were observed in $4.6 \%(n=5)$. In the coagulogram, all patients showed an increased level of D-dimer - 4 [0.5-8.0] $\mathrm{mg} / \mathrm{L}$, the level of fibrinogen - 4 [2.6-7.5] g/L, APTT 41, 6 [24-54] s, There was a normal level of prothrombin time - 12 [10-17] s. The average level of leukocytes was 8.2 [3.8-10.5] * 109 / I, lymphocytes - 30 [2657] \%, platelets - 223 [170-339] * 109 / I, ESR - 30.9 [ 10-47.5] mm / h. The values of biochemical parameters - markers of inflammation were also above the reference range: CRP - 50.4 [0.4-237] $\mathrm{mg} / \mathrm{L}$, ferritin - $563 \mu \mathrm{g} / \mathrm{L}$, LDH - 638 [100-760] U / L.

Conclusions: In the initial period of the development of COVID-19 there was an increased level of thrombinemia, which indicated the presence of a risk of thromboembolic complications, but there was a tendency towards an increase in APTT.

\section{P0136 | Management of a Patient with Hemorragic and Thrombotic Risk Factors, a Brazilian Case Report}

N.d.F. Centuriao; A.A.R. Villarinho; V.F. Aranda; D.H.C. Campelo;

R.M.C. Penteado; J.C.d.C. Guerra

Hospital Israelita Albert Einstein, Sao Paulo, Brazil

Background: Snakebites comprise an important neglected public health problem that affects people worldwide. Hematologic abnormalities are the most common effects of snake envenoming specially the venom-induced consumption coagulopathy.

The novel coronavirus disease of 2019 (COVID-19) is caused by the severe acute respiratory syndrome coronavirus 2 (SARSCoV2). Venous thromboembolism (VTE) has emerged as an 
important complication in the management of hospitalized patients with COVID. Manage both situations might be challenging.

Aims: Describe the successful treatment of our group in conduct a patient with both hemorragic (Snake venom) and thrombotic risk factors ( COVID19).

Methods: Case report of a patient with both hemorragic and thrombotic risk factors.

Results: Female patient, 33 years old, had a snake envenomation in a waterfall in Brazil. She received, in the hospital close to the accident site, specific anti-venon sérum, Where the diagnosis of SARS-CoV-2 infection was made even though the patient was asymptomatic.At entrance, the patient underwent fasciotomy due to compartment syndrome in the left upper limb. However, it evolves with hemorrhagic shock in the postoperative period. Based on complementary exams, that revealed disseminated intravascular coagulation with hyperfibrinolyses (Figure 1).

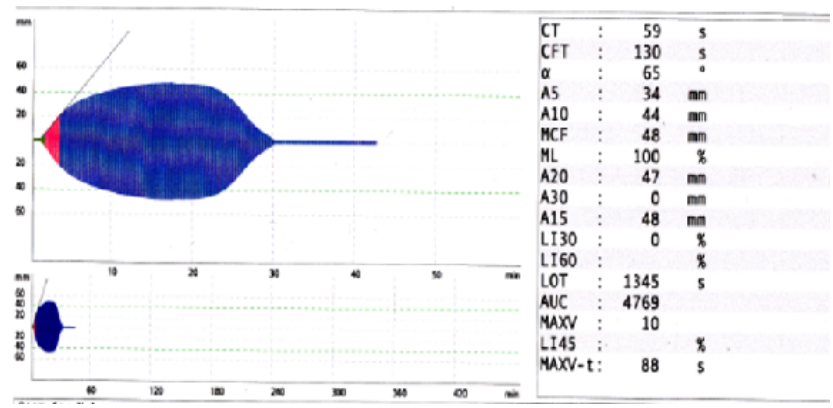

FIGURE 1 Patient's thromboelastography at entrance

She was treated with tranexamic acid, prothrombin complex, recombinant fibrinogen, vitamin $\mathrm{K}$, parenteral iron, and prescribed compressive curative, red blood cell and platelet transfusion.After reversed the coagulopathy, $48 \mathrm{~h}$ after initial measurements (Table 1), it was evidenced an increase in D-dimer (above $7650 \mathrm{ng} / \mathrm{ml}$ ) and fibrin degradation products (above 120). As patient had many risk factors for venous thromboembolism (immobilization, recent surgery and SARS-CoV-2) and following institutional protocols, we started prophylactic anticoagulation. Patient was discharged with progressive improvement after one month of hospitalization with instruction to maintain oral anticoagulation in prophylactic dose for more 30 days.

TABLE 1 Evolution of exams during treatment

\begin{tabular}{|c|c|c|c|c|c|c|c|c|}
\hline & $\mathrm{Hb}$ & $\mathrm{HCT}$ & WSC & Neut & Plat & RNI & aPTT & Fib \\
\hline $09 / 04$ & 8.8 & 25.8 & 8350 & 6939 & 86 & 1.63 & 1.67 & $<35$ \\
\hline 09/05 & 7.1 & 19.9 & 8080 & 5494 & 102 & 1.33 & 1.07 & 94 \\
\hline 09/06 & 8.2 & 22.8 & 6690 & 4683 & 109 & 1.14 & 0.88 & 128 \\
\hline $09 / 07$ & 8.3 & 24 & 7060 & 4660 & 143 & 1.06 & 0.85 & 181 \\
\hline
\end{tabular}

P0137 | Sepsis-induced Disseminated Intravascular Coagulation Secondary to COVID-19 and Baricitinib Treatment

U.R. Madera Sanchez; K. Cruz Lopez; P.J. Colon Ortiz;

S.A. Gonzalez Goyri

Hospital Universitario Ramon Ruiz Arnau, Bayamon, Puerto Rico

Background: COVID-19 possesses a high risk for coagulopathy, being Disseminated intravascular coagulation (DIC) described in few cases. Baricitinib is a selective inhibitor of Janus kinase 1 and 2 that is used in COVID-19 with reports of increased thrombotic events.

Aims: This case will serve as an awareness to screen for DIC in severe COVID-19 infection treated with baricitinib to reduce complications, mortality and improve patient outcomes.

Methods: A 68-year-old female with a known medical history of ischemic stroke, arterial hypertension, and diabetes mellitus type 2 admitted with pneumonia, COVID-19 infection, hypoxic respiratory failure on mechanical ventilation, acute kidney injury, and distributive shock. The patient was started on IV antibiotics, remdesivir, baricitinib, vasopressors, and thrombosis prophylaxis. Sputum culture, urine culture, and blood culture from admission were reported negative. WBC and c-reactive protein trending down. On day six patient was extubated, and vasopressors discontinued. On day eight patient became hypoxic and hemodynamically unstable, needing reintubation and vasopressors. The patient was pan-cultivated. On day 10 , sputum, blood, and urine culture reported E. coli ESBL positive. On day 14, the patient started oozing from the venipuncture site. Anticoagulation was discontinued. The International Society on Thrombosis and Haemostasis score for DIC was 5.

Results: The patient had two units of fresh frozen plasma transfused. After the transfusions, the coagulation panel showed an increase in PT, PTT, and D-dimer along with a decrease of fibrinogen level. Another unit of fresh frozen plasma was administered, but despite this treatment, the fibrinogen level kept in decreasing trend, and the patient started bleeding from the mouth and nose. Unfortunately, the patient passed away on day 15.

Conclusions: The use of institutional protocols and appropriate coagulation tests can make the difference in patient manejament with hemorragic and/or thrombotic deseases. 
Conclusions: We hypothesize that the baricitinib treatment in our patient predisposed to DIC by two mechanisms: by its inherent thrombotic effect and, superinfection leading to E. coli bacteremia, and multiorgan failure leading to DIC.

\section{P0138 | COVID-19 and Coagulation Abnormalities Diagnostic} Dilemma

N. Odeleye; N. Kennedy; P. Shinde

Homerton University Hospital, London, United Kingdom

Background: Low platelets and fibrinogen levels at term are associated with pre-ecplampsia and abruption of placenta whilst congenital hypofibrogenemia in pregnancy is extremely rare, all have potential significant haemorrhagic consequences. We present a case of antepartum haemorrhage (APH), hypofibrinogenemia and thrombocytopenia on a background of a COVID-19 diagnosis.

Aims: A 26 y, G3 P0 +2 miscarriages presented at 36 weeks with APH of $500 \mathrm{ml}$ and positive PCR for Covid-19. She had an epidural sited for labour analgesia, her subsequent blood tests revealed thrombocytopenia (87) and a hypofibrogenemia of $0.5 \mathrm{~g} / \mathrm{dl}$ despite normal platelets 12 weeks prior. She had an uneventful emergency caesarean necessitated by foetal compromise in labour (working diagnosis of placental abruption), 2 units of cryoprecipitate was transfused, blood loss was $600 \mathrm{ml}$ and no abruption found. We monitored her for neurological recovery from the epidural block and delayed removal of epidural catheter until her post-partum fibrinogen was $1.5 \mathrm{~g} / \mathrm{dl}$ and platelets 112 after discussion with haematology. She and her baby were discharged uneventfully on day two.

Methods: The cause for this patient's severe hypofibrogenemia and associated thrombocytopenia was unclear at the time.

Results: A normal platelet count earlier in her pregnancy suggests an acute or pregnancy related process. Pre-eclampsia and placental abruption were ruled out at c-section.

Conclusions: Coagulopathy associated with Coivd-19 remains a real possibility as our knowledge continues to evolve and expand, this has large implications for obstetric anaesthetic practice given that the status quo is to default to neuroaxial blockade to avoid intubation, the presenting APH is likely secondary to the COVID driven coagulopathy despite mild symptoms. In addition to measurement of platelets, routine measurement of PT, APTTR and fibrinogen levels should be routinely considered in all pregnant women with Covid-19 per the ISTH guidance to help in differential diagnosis, guide admission to hospital/intensive care and need for obstetric intervention.

\section{DIAGNOSTICS AND OMICS}

\section{Biomarkers of Thrombosis and Hemostasis}

LPB0012 | Effects of Liraglutide vs. Lifestyle Changes on sST2 and Galectin-3 in Obese Subjects with Prediabetes or Type 2 Diabetes after Comparable Weight Loss

R. Tripaldi ${ }^{1}$; P. Simeone ${ }^{1}$; R. Liani ${ }^{1}$; S. Ciotti ${ }^{1}$; A.E. Michelsen ${ }^{2}$; B. Halvorsen ${ }^{2}$; T. Ueland ${ }^{2}$; P. Aukrust ${ }^{2}$; A. Consoli ${ }^{1}$; F. Santilli ${ }^{1}$ ${ }^{1}$ University G. d'Annunzio of Chieti, Center for Advanced Studies and Technology (CAST), Chieti, Italy; ${ }^{2}$ Research Institute for Internal Medicine, Oslo University Hospital, Oslo, Norway

Background: Soluble suppression of tumorigenesis-2 (sST2) and Galectin-3 (Gal-3) have been associated with the pathogenesis of atherosclerosis and thrombosis. Their increased levels have a predictive role for the risk of cardiovascular death. Treatment with liraglutide, a glucagon-like peptide 1 analog, is associated with weight loss, improved glycemic control, and reduced cardiovascular risk.

Aims: The aim of this work is to assess whether liraglutide treatment may modulate SST2 and Gal-3 levels in patients with prediabetes or early T2DM independently of weight loss.

Methods: Forty metformin-treated obese subjects (BMI $\geq 30$ ) with prediabetes [impaired fasting glucose (IFG) or impaired glucose tolerance (IGT) or both $(n=24)$ ] or newly diagnosed T2DM ( $n=16$ ), were randomized to liraglutide or lifestyle counseling until achieving a comparable weight loss ( $7 \%$ of initial body weight). Sixteen subjects were used as controls. sST2 and Gal-3 were analyzed by ELISA. Thromboxane (TX)-dependent platelet activation as reflected in vivo by the urinary excretion of 11-dehydro- $\mathrm{TXB}_{2}$ was measured by radioimmunoassay method.

Results: SST2 levels were comparable between controls and patients with prediabetes $(P=0.988)$ or T2DM $(P=0.179)$ (Fig. 1A). Gal-3 levels were significantly higher in patients with IGT/IFG and T2DM as compared to controls ( $P<0.001$ for both) (Fig. 1B). Baseline sST2 plasma levels correlated directly with fasting insulin (Rho $=0.391, P=0.014$ ), total cholesterol (Rho $=0.372$, $P=0.020)$, VAT (Rho $=0.376, P=0.018)$ and HOMA-B (Rho $=0.435$ $P=0.006)$. Baseline Gal-3 plasma levels correlated inversely with waist to hip ratio $(\mathrm{WHR})(\mathrm{Rho}=-0.455, P=0.004)$ and haemoglobin (Rho $=-0.328, P=0.041$ ) and directly with IL-6 (Rho $=0.402$, $P=0.023)$. After weight loss, changes in SST2 correlated directly with changes in 11-dehydro- $\mathrm{TXB}_{2}(\mathrm{Rho}=0.346, P=0.033)$. $\mathrm{SST} 2$ levels were significantly reduced only in the liraglutide arm $(P=0.040)$ (Fig. 2) while Gal-3 levels were not affected by weight loss in either arm. 
Figure 1
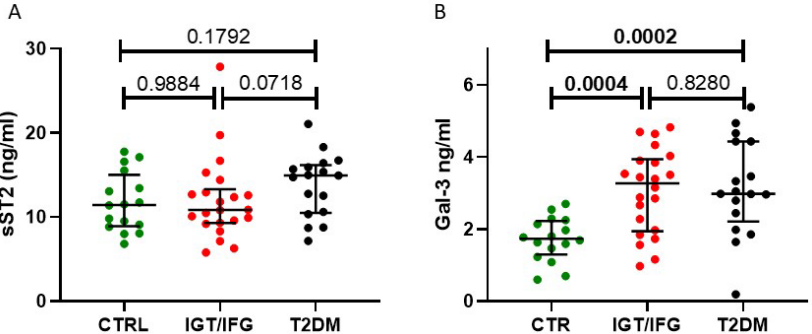

FIGURE 1 basal levels of sST2 (A) and Gal-3 (B) in controls, patients with IGT/IFG and T2DM

\section{Figure 2}

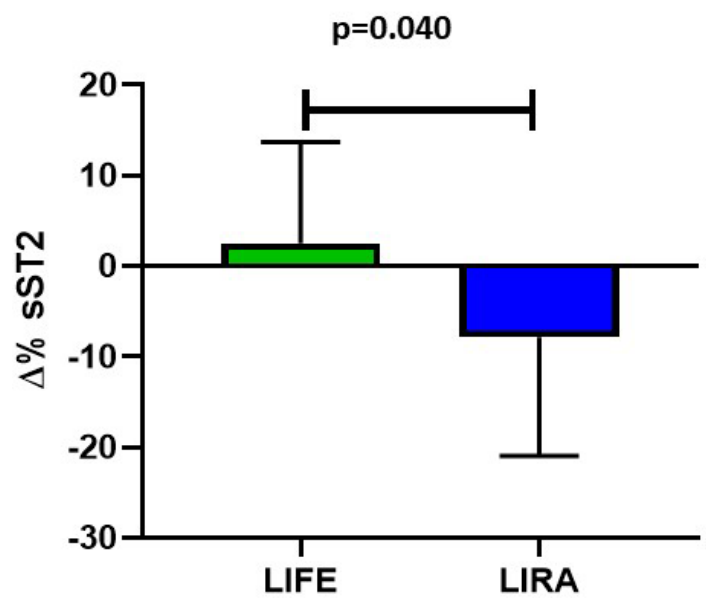

FIGURE 2 Changes in SST2 levels after liraglutide- or lifestyleinduced weight loss, in obese subjects with prediabetes and early type 2 diabetes. $P$ values (lifestyle vs. liraglutide) are for comparison of changes between groups.

Conclusions: Liraglutide-induced SST2 reduction suggests that in obese patients with prediabetes or early T2DM this drug may have a positive effect on atherosclerosis and thrombosis.

TABLE 1 OHP results of various sub-populations of VTE patients

\begin{tabular}{|c|c|c|c|c|c|c|c|c|c|}
\hline & $\begin{array}{l}\text { Normal } \\
\text { control }\end{array}$ & $\begin{array}{l}\text { All } \\
\text { subjects }\end{array}$ & $P$ & Malignancy & No malignancy & $P$ & $\begin{array}{l}\text { PE/DVT } \\
\text { with no } \\
\text { residual } \\
\text { PE }\end{array}$ & $\begin{array}{l}\text { PE/DVT } \\
\text { with } \\
\text { residual } \\
\text { PE }\end{array}$ & $P$ \\
\hline $\begin{array}{l}\text { No. } \\
\text { results }\end{array}$ & 143 & 208 & & 17 & 189 & & 74 & 13 & \\
\hline OFP\% & 80.1 & 73.62 & $<0.0001$ & 68.53 & 74.19 & 0.01 & 74.32 & 71.48 & 0.28 \\
\hline
\end{tabular}

PB0308 | Overall Haemostatic Potential Assay Can Detect Hypercoagulability and Hypofibrinolysis in Anticoagulated Patients Following Venous Thromboembolism

J. Wang ${ }^{1,2} ; \mathrm{P} \mathrm{Ho}^{1,2,3}$

${ }^{1}$ Northern Health, Epping, Australia; ${ }^{2}$ University of Melbourne, Parkville, Australia; ${ }^{3}$ Australian Centre for Blood Diseases, Melbourne, Australia

Background: The Overall Haemostatic Potential assay (OHP) is a global coagulation assay that measures fibrin generation and fibrinolysis potential. The OHP has been poorly studied among patients with VTE.

Aims: To characterise fibrin generation and fibrinolysis in anticoagulated VTE patients using the OHP assay.

Methods: Adult patients were recruited following a confirmed diagnosis of DVT and/or PE, and blood sampled whilst receiving therapeutic anticoagulation. Platelet poor plasma (PPP) was analysed by the OHP assay in which fibrin generation and fibrinolysis is measured spectrophotometrically at $405 \mathrm{~nm}$. The overall coagulation potential (OCP) is the area under the time-curve of PPP activated by thrombin. The OHP is determined by the addition of tPA (350 ng/ $\mathrm{mL}$ ) and thrombin to a parallel plasma sample. The overall fibrinolysis potential (OFP) is calculated by (OCP-OHP)/OCP $100 \%$.

Results: From Jan 2018 to Jan 2021, 208 patients were recruited. Compared with normal control patients (previous data), study subjects displayed significantly higher OCP, OHP, and reduced OFP (Table 1). There were no significant differences between provoked/ unprovoked or major/minor VTE. Patients with active malignancy, or subsequently diagnosed with malignancy showed higher OHP and reduced OFP compared to those without malignancy (Table 1). Patients with residual PEs had significantly higher OCP and OHP compared with those with none. No differences were found according to the presence of residual thrombus in those with DVT only. Patients receiving warfarin (Table 2) displayed significantly higher OCP and OHP compared to those treated with DOACs, which may be due to patient differences as displayed in Table 2 . 
TABLE 2 OHP results and patient characteristics of patients treated with warfarin vs DOACs

\begin{tabular}{llll} 
& Warfarin & DOACs & \\
& $N=37$ & $N=155$ & $P$-value \\
OCP, mean & 48.09 & 39.84 & $<0.001$ \\
OHP, mean & 13.08 & 10.49 & 0.006 \\
OFP, mean & 73.87 & 74.20 & 0.82 \\
Age & 55.90 & 56.00 & 0.96 \\
Weight & 113.27 & 89.18 & $<0.001$ \\
D-dimer & 0.44 & 0.56 & 0.68 \\
F8 & 196.06 & 146.97 & $<0.001$ \\
vWF Ag & 199.74 & 145.09 & $<0.001$ \\
Fibrinogen & 4.40 & 3.60 & $<0.001$ \\
\hline
\end{tabular}

Conclusions: Hypercoagulability and hypofibrinolysis were detected by the OHP assay in patients receiving anticoagulation after acute VTE. Individuals with malignancy, with residual PE and anticoagulated with warfarin had significantly hypercoagulable and/or hypofibrinolytic results. Further study is required to confirm the effect of anticoagulation on OHP, and the utility of using OHP to prognosticate for VTE recurrence.

PB0310 | Use of MRX PT DOAC in a Routine Laboratory Setting for Detection of Interfering DOACs in Thrombophilia Investigation

$\underline{\text { T. Lindahl }}^{1}$; M. Lund ${ }^{1} ;$ K. Arbring ${ }^{2}$; L. Onelö $v^{3}$

${ }^{1}$ Linköping University/ Dept of Biomedical and Clinical Sciences, Linköping, Sweden; ${ }^{2}$ Linköping University/ Dept of Heatlh and Medicine, Linköping, Sweden; ${ }^{3}$ Nordic Biomarker, Linköping, Sweden

Background: Direct oral anticoagulants (DOACs) may interfere with lupus anticoagulant assays (LAC) and cause falsely positive results. We have previously shown that the MRX PT DOAC test can be used to detect interfering DOACs in thrombophilia investigations (PB0598 ISTH 2020). For this extended and ongoing study, both laboratory data and clinical records are available. Thus anticoagulant treatment can be checked.

Aims: To evaluate if MRX PT DOAC can detect DOACs at concentrations interfering with coagulation analysis in thrombophilia investigations.

Methods:

1. MRX PT DOAC was analyzed at the time of thrombophilia investigation. Positive LA-dRVVT samples were reanalyzed after addition of DOAC-STOP. Clinical records were reviewed for ongoing anticoagulant treatment.

2. The literature was reviewed for concentrations at which DOACs interfere with LAC tests with focus on LA-dRVVT.
3. Data was collected from patient samples where both DOAC concentration and MRX PT DOAC were available to make sure that MRX PT DOAC detects DOACs at concentrations known to interfere.

4. The study was approved by the ethical committee, approval No 2020-04525.

Results: At present, 232 patients are evaluated, 18 of these were treated with DOACs (ten with apixaban, eight with rivaroxaban). All apixaban patients had a negative LA-dRVVT test. In the rivaroxaban patients the LA-dRVVT test was falsely positive in two patients due to rivaroxaban interference, in another four patients the LA-dRVVT test was truly positive while LA-dRVVT was negative in the final two. MRX PT DOAC detected $100 \%$ of falsely positive LA-dRVVT samples.

Hillarp et al 2020, reported interference of LAC test by rivaroxaban and apixaban at concentrations over $50 \mu \mathrm{g} / \mathrm{L}$ and $350 \mu \mathrm{g} / \mathrm{L}$, respectively.

Results confirmed that MRX PT DOAC detects DOACs at concentrations where interference with $L A C$ test could be an issue.

Conclusions: Our results indicate that the screening method MRX PT DOAC can detect the presence of interfering DOACs in patient samples during thrombophilia investigation.

\section{PB0311 | Circulating Biologic Markers of Endothelial} Dysfunction in Chronic Myeloproliferative Neoplasms

O. Smirnova; N. Silina; N. Korsakova; O. Golovina; E. Efremova; M. Fominykh; S. Voloshin; L. Papayan

Russian Research Institute of Hematology and Transfusiology, Saint Petersburg, Russian Federation

Background: Chronic myeloproliferative neoplasms (MPN) are stem cell disorders characterized by myeloid proliferation and increased blood cell counts. Large-vessel thrombosis and microvascular disturbances remains the main complication of MPN patients. The mechanisms leading to it are complex and include endothelial dysfunction. Aims: The aim of our study was to assess potential endothelial impairment in patients with MPN by measuring such biomarkers as ristocetin-cofactor von Willebrand's factor activity (vWF:RCo), antigen (vWF:Ag), and homocysteine (HCY).

Methods: We studied vWF:RCo activity (\%), vWF:Ag (\%) and HCY levels $(\mu \mathrm{mol} / \mathrm{l})$ in $173 \mathrm{MPN}$ patients and 68 healthy volunteers. Statistical analysis was performed by non-parametric methods (median (Me), 95\% confidence interval (95\% Cl), Mann-Whitney $U$ test and Spearman's rank correlation coefficient (Rs), Statistica 12.0), $P<0.05$ was considered statistically significant.

Results: The vWF:RCo activity and vWF:Ag were significantly higher in patients compared with controls, and no difference in the $\mathrm{HCY}$ levels was observed (data shown in the table). 
Table 1

\begin{tabular}{lll}
$\begin{array}{l}\text { Parameter (Me, } \\
\text { 95\% Cl) }\end{array}$ & Patients with MPN & Controls \\
vWF:RCo, \% & $105.0^{*}(50.0-270.3)$ & $N=68$ \\
vWF: Ag, \% & $130.0^{*}(71.1-381.7)$ & $107.0(63.0-144.3)$ \\
HCY, $\mu \mathrm{mol} / \mathrm{I}$ & $9.2(5.6-23.7)$ & $10.1(5.1-14.8)$ \\
\hline
\end{tabular}

${ }^{*} \mathrm{P}=0.001$ with controls.

Also, there was no relationship between these parameters and previous thrombotic complications history. In the same time, we found weak but significant correlation between $\mathrm{HCY}$ levels and both vWF:RCo (Rs = 0.21) and vWF:Ag (Rs=0.25), $P=0.03$.

Conclusions: The increase of vWF:RCo activity and vWF:Ag may reflect a chronic endothelial impairment and predispose to thromboembolic complications, although much molecular details still need to be clarified.

PB0313 | Profiling of Inflammatory Biomarkers and Coagulation Factors in End-stage Renal Disease

E. Bontekoe; A. Grewal; V. Bansal; M. Ben Hadj Tahar;

D. Hoppensteadt; J. Fareed

Loyola University Medical Center, Maywood, United States

Background: Early detection of chronic kidney disease (CKD) is warranted in preventing complications that develop into kidney failure and vascular complications. Currently, the detection of early stages of CKD is lacking predictive values, delaying the application of specific diagnostics and therapeutic interventions. End-stage renal disease (ESRD) patients are at risk for cardiovascular complications and vascular thrombosis, which are associated with numerous coagulation and fibrinolytic abnormalities.

Aims: Profiling inflammatory biomarkers and coagulation factors in ESRD patients may allow early onset detection and severity of kidney damage, furthering the understanding of the pathogenesis of this disease.

Methods: Citrated blood samples were collected from 95 confirmed ESRD patients undergoing maintenance hemodialysis under an IRB approved protocol. Biomarkers of inflammation and coagulation, including PAI-1, TAFI, CRP, MMP-9, MP, D-Dimer, Factor VII, IX, X, and XIII were measured using commercially available ELISA methods. Normal controls were comprised of commercially available male and female citrated plasma (George King Biomedical). All results were compiled as mean $\pm \mathrm{SEM}$, and percent change in comparison to normal human plasma.

Results: Biomarkers including CRP $(7 \mu \mathrm{g} / \mathrm{ml} \pm$ 0.6, 183\%), MMP-9 (88 ng/ml $\pm 8,58 \%)$ D-Dimer (1059 ng/ml \pm 138$)$, Factor IX (124\% \pm 3 , $23 \%)$, and Factor XIII (104\% $\pm 1,27 \%)$ showed significant increase when compared to normal plasma. Elevated CRP indicates severe inflammatory response and elevated D-Dimer indicates endogenous fibrin formation. MP and PAI-1 suggest endothelial dysfunction.
Conclusions: These results suggest the upregulation of inflammatory responses and the multifactorial pathophysiology of ESRD in the dysregulation of hemostasis. Profiling of inflammatory biomarkers and coagulation parameters may be helpful in the risk stratification management of ESRD patients.

\section{PB0314 | Clinical Relevance of JAK2V617F on Patients with} Philadelphia-negative Myeloproliferative Neoplasms

R. Frikha; H. Kamoun

University Hospital of Sfax, Sfax, Tunisia

Background: Thrombosis is a leading cause of morbidity and mortality in patients with Philadelphia chromosome-negative myeloproliferative neoplasms (MPNs), particularly polycythemia vera (PV) and essential thrombocythemia (ET). The acquired point mutation in the pseudokinase domain of JAK2 (JAK2V617F) in these disorders is variably associated with thrombosis

Aims: This study was aimed to investigate the incidence of JAK2V617F mutation in BCR-ABL negative patients with MPN and its relation with thrombosis.

Methods: The peripheral blood of patients was collected and the genomic DNA was exacted. Allele-specific polymerase chain reaction (AS-PCR) was used to amplify the exon 12 of JAK2 gene which harbours V617F mutation.

Results: The JAK2V617F mutation was detected in 29 patients out of 37 patients (78.4\%) among them 19 (79.2\%) with PV and 10 (76.9\%) ET patients, respectively.

Thrombocytosis was found both in patients with ET and PV $(58.3 \%$ and $23.8 \%$, respectively; $P=0.04$ ) mainly those who harbors JAK2V617F (26.9\%; $P=0.03)$.

Conclusions: Emphasis should be placed on the detection of JAK2 mutation in MPN, since JAK2V617F appears to be a risk-modifying mutation and increase the likelihood of thrombotic events in Tunisian patients mainly ET and PV.

\section{PB0315 | Proteome Analysis of Extracellular Vesicles from Patients with Myocardial Infarction and Relation to Heart Function: An Observational Study}

A. Khandagale ${ }^{1}$; S. Bergström Lind ${ }^{2}$; G. Shevchenko ${ }^{2}$; J. Westerbergh ${ }^{3}$; B. Lindahl ${ }^{1,3}$; A. Siegbahn ${ }^{4}$; C. Christersson ${ }^{1}$ ${ }^{1}$ Department of Medical Sciences, Cardiology, Uppsala University, Uppsala, Sweden; ${ }^{2}$ Department of Chemistry - BMC, Analytical Chemistry, Uppsala University, Uppsala, Sweden; ${ }^{3}$ UCR-Uppsala Clinical Research Center, Uppsala University, Uppsala, Sweden; ${ }^{4}$ Department of Medical Sciences, Clinical Chemistry and Science for Life Laboratory, Uppsala University, Uppsala, Sweden

Background: Acute myocardial infarction (MI) with plaque ruptureinduced thrombosis, coronary occlusion and subsequent ischemic 
injury, results in heart failure and is associated with poor prognosis. The emerging role of extracellular vesicles (EVs) in intercellular communication has stimulated renewed interest in exploring the potential application of EVs as tools for prognosis, and therapy in MI.

Aims: To analyze endogenous EVs proteome during $\mathrm{MI}$ and explore differences in EVs proteome from $\mathrm{MI}$ patients in relation to myocardial damage defined as left ventricular ejection fraction (LVEF).

Methods: 40 patients with MI, ST-elevation (STEMI) and non-STelevation (NSTEMI), were enrolled 2 days (mean) after the acute event. The EVs from plasma of patients and healthy controls (HC) were purified and characterized by Mass spectrometry based proteomic analysis. Proteins were identified and quantified using the database UniProt and software MaxQuant 1.5.1.2. The association between protein expression and continuous variables were analyzed using Spearman correlation test. For categorical variables, Wilcoxon rank sum test was used. The statistical analysis were made using $\mathrm{R}$ version 3.6.1. A $P$-value of $<0.05$ was considered statistically significant.

Results: 50 differentially expressed proteins from endogenous EVs were identified among MI patients compared to $\mathrm{HC}$ using MS analysis, of which 32 were up-regulated and 19 were down-regulated in MI patients as against HC. Statistical analysis comparing STEMI and NSTEMI patients revealed 4 differently expressed proteins. 22 proteins were found associated with LVEF function, when analyzed as a continuous variable, whereas 14 proteins were found to be differently expressed when LVEF was dichotomized in $\leq$ vs $>0.40$ (Figure \& Table).

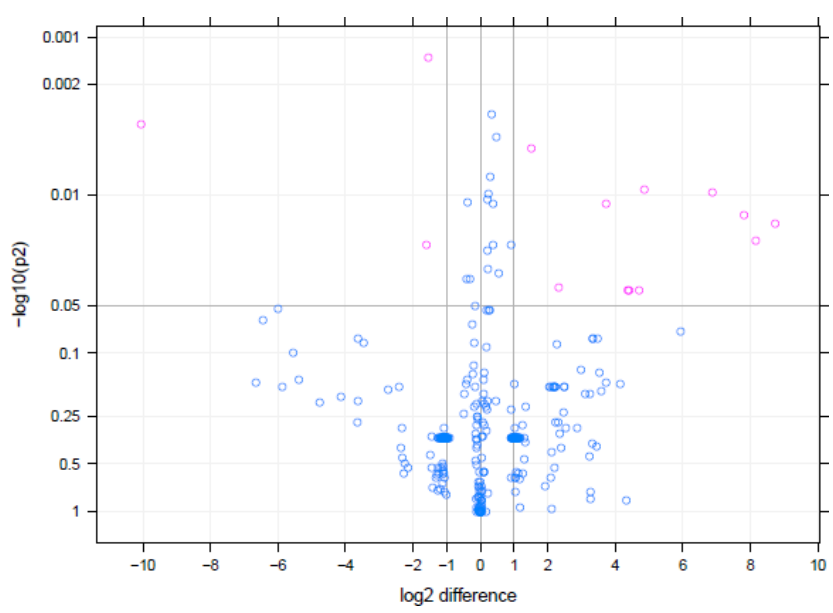

FIGURE 1 Volcano plot for categorical variable of LVEF; Mean log2 difference vs individual $P$-values from Wilcoxon rank sum test, LVEF $\leq$ vs $>0.40$

TABLE 1 Differently expressed EV proteins as defined in relation to LVEF

\begin{tabular}{|c|c|c|c|c|c|c|c|c|}
\hline $\begin{array}{l}\text { Proteins within } \\
\text { EVs }\end{array}$ & $\begin{array}{l}\text { LVEF } \\
\text { continuous } \\
\text { variable }\end{array}$ & $\begin{array}{l}\text { LVEF } \\
\text { dichotomized } \\
\text { in } \leq \text { vs }>0.40\end{array}$ & Proteins within EVs & $\begin{array}{l}\text { LVEF } \\
\text { continuous } \\
\text { variable }\end{array}$ & $\begin{array}{l}\text { LVEF } \\
\text { dichotomized } \\
\text { in } \leq \text { vs }>0.40\end{array}$ & Proteins within EVs & $\begin{array}{l}\text { LVEF } \\
\text { continuous } \\
\text { variable }\end{array}$ & $\begin{array}{l}\text { LVEF } \\
\text { dichotomized } \\
\text { in } \leq \text { vs }>0.40\end{array}$ \\
\hline Haptoglobin & $x$ & & $\begin{array}{l}\text { Fibrinogen gamma } \\
\text { chain }\end{array}$ & $x$ & & $\begin{array}{l}\text { Alpha-a- } \\
\text { antichymotrypsin }\end{array}$ & $x$ & \\
\hline $\begin{array}{l}\text { C-reactive } \\
\text { protein }\end{array}$ & $x$ & $x$ & Apolipoprotein M & $x$ & & $\begin{array}{l}\text { Inter-alpha-trypsin } \\
\text { inhibitor heavy } \\
\text { chain H3 }\end{array}$ & $x$ & $x$ \\
\hline C8A protein & $x$ & & $\begin{array}{l}\text { Apolipoprotein C-III } \\
\text { variant } 1\end{array}$ & $x$ & & $\begin{array}{l}\text { Alpha-1-acid } \\
\text { glycoprotein } 1\end{array}$ & $x$ & \\
\hline $\begin{array}{l}\text { Serum amyloid } \mathrm{A} \\
\text { protein }\end{array}$ & $x$ & $x$ & Ceruloplasmin & $x$ & & $\begin{array}{l}\text { Epididymis secretory } \\
\text { sperm binding } \\
\text { protein }\end{array}$ & $x$ & \\
\hline $\begin{array}{l}\text { Serum amyloid } \\
\text { A2 protein }\end{array}$ & $x$ & & Serotransferrin & $x$ & & GCT-A9 heavy chain & & $x$ \\
\hline Clusterin & $x$ & & APOL1 protein & $x$ & $x$ & $\begin{array}{l}\text { GCT-A4 light chain } \\
\text { variable region }\end{array}$ & & $x$ \\
\hline Fibulin-1 & $x$ & $x$ & Transthyretin & $x$ & & $\begin{array}{c}\text { Pigment epithelium- } \\
\text { derived factor }\end{array}$ & & $x$ \\
\hline 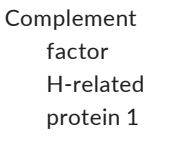 & $x$ & $x$ & $\begin{array}{l}\text { Von Willebrand } \\
\text { factor }\end{array}$ & $x$ & $x$ & $\begin{array}{l}\text { Vinculin isoform } \\
\text { CRA_c }\end{array}$ & & $x$ \\
\hline $\begin{array}{l}\text { Complement } \\
\text { component } \\
\text { C9 }\end{array}$ & $x$ & & $\begin{array}{l}\text { Lipopolysaccharide- } \\
\text { binding protein }\end{array}$ & $x$ & $x$ & $\begin{array}{l}\text { Filamin-A } \\
\text { IgG H Chain }\end{array}$ & & $\begin{array}{l}x \\
x\end{array}$ \\
\hline
\end{tabular}

Conclusions: Endogenous EVs from patients with recent MI with reduced LVEF harbor proteins known associated with heart failure as well as several other proteins that demand further investigation to define whether they actively modulate myocardial function or present as a result of the myocardial damage upon ischemia. 
PB0316 | Anti-annexinv Serum Levels and Haematological Indices in Sickle Cell Anaemia Patients in Vasooclusive Crisis and Steady States Seen at Lagos University Teaching Hospital Lagos Nigeria

D. Edewor-Okiyo ${ }^{1}$; A. Adediran²; A. Ogbenna ${ }^{1} ;$ O. Olowoselü ${ }^{1}$; A. Omolade ${ }^{3}$; S. Idogun ${ }^{3}$

${ }^{1}$ Lagos University Teaching Hospital, Lagos, Nigeria; ${ }^{2}$ Ben Carson School Of Medicine Babcock University, Lagos, Nigeria; ${ }^{3}$ University of Benin Teaching Hospital, Benin, Nigeria

Background: Annexin $\mathrm{V}$ is a glycoprotein with anticoagulant activity IgG and IgM Anti-AnnexinV antibodies have the ability to displace annexin $V$ from phospholipid surfaces and this has been linked with enhanced prothrombotic conditions such as vasoocclusive crisis, the hallmark of Sickle Cell Anaemia (SCA).

Aims: To determine the serum level of IgM and IgG antibodies to AnnexinV in SCA patients in crisis and steady states in order to assess the contribution of the procoagulant effect of Anti-AnnexinV to vasoocclusive crisis and its severity.

Methods: The study comprises of 90 SCA subjects; 35 subjects in vasoocclusive crisis (VOC), 35 subjects in steady states and 20 normal Haemoglobin (HbAA) control subjects. Anti-annexin $\mathrm{V}$ antibodies activity was determined with enzyme-linked immunosorbent assay (ELISA) KIT. Haematological parameters were analyzed using Haematology autoanalyser (SYSMEX).

Data analysis was done with the statistical package for social sciences version 21.

Results: The results showed that the prevalence of anti-annexinV IgG seropositivity was found to be $22.9 \%$ in crisis, $20.0 \%$ in steady state and $0.0 \%$ in controls. The seropositivity of IgM anti- annexin $\checkmark$ was significantly higher in steady state than in control $(P=0.015)$, but in VOC the seropositivity of anti annexin $\mathrm{V}$ IgM though higher than in controls was not statistically significant $(P=0.071)$.The mean platelet count in the study subjects were $287.8 \pm 125.1 \times 10^{9}$ cells $/ \mathrm{L}$, $288.4 \pm 132.6 \times 10^{9} \mathrm{cells} / \mathrm{L}$ and $179.5 \pm 56.4 \times 10^{9} \mathrm{cells} / \mathrm{L}$, the difference in mean platelet count was significantly lower in the control subjects than in the SCA groups $(P=0.002)$.

Conclusions: Serum levels of anti-annexin V IgM and IgG do not significantly differ between steady state and VOC. There is positive and significant correlation between the platelet count and IgM antiannexin $\mathrm{V}$ in patients with SCA in steady state.

TABLE 1 BMI; BLOOD COUNTOF STUDY SUBJECTS SCA in Steady State $n=35$ SCD in VOC $n=35$ Control $n=20 P$ value Mean \pm SD Mean \pm SD Mean \pm SD Age (years) $26.8 \pm 6.625 .8 \pm 5.226 .8 \pm 8.2$ 0.781 Height $(m) 1.68 \pm 0.091 .67 \pm 0.071 .69 \pm 0.090 .694$ Weight (kg) $56.5 \pm 11.254 .7 \pm 10.264 .8 \pm 6.50 .001 \mathrm{BMI}(\mathrm{kg} / \mathrm{m} 2) 20.1 \pm 3.8$ $19.6 \pm 3.122 .8 \pm 2.40 .002$ Stable PCV (\%) $23.1 \pm 3.722 .9 \pm 2.8 \mathrm{NA}$ $0.001 \mathrm{Hb}(\mathrm{g} / \mathrm{dl}) 8.7 \pm 1.97 .8 \pm 1.312 .5 \pm 1.40 .001 \mathrm{PCV}(\%) 24.2 \pm$ $3.621 .9 \pm 3.938 .0 \pm 4.60 .001 \mathrm{WBC}(\times 109 \mathrm{cell} / \mathrm{L}) 11.2 \pm 3.615 .8 \pm$ $6.85 .2 \pm 1.80 .001$ Platelet count (x109 cell/L) $287.8 \pm 125.1288 .4 \pm$ $132.6179 .5 \pm 56.40 .002$ Reference ranges: Male: $\mathrm{Hb}$ (13.5 to 18.0),
Female: $\mathrm{Hb}$ (11.5 to 16.4), male: PCV (40 to 54\%), Female: PCV (37 to $45 \%)(W B C(2,800$ to 8,500$)$, Platelet count $(150,000$ to 400,000$)$. TABLE 2 ANTI-ANNEXIN V, IgG AND IgM IN STEADY STATES, VOC AND CONTROL SUBJECTS SCA in Steady State $n=35$ SCA in VOC $n=35$ Control $n=20 P$ value Anti-Annexin V IgG: Mean \pm SD 23.3 $\pm 10.724 .4 \pm 16.519 .3 \pm 7.70 .390$ n (\%) n (\%) n (\%) Positive(>8 U/ $\mathrm{ml}$ ) Borderline $(5-8 \mathrm{U} / \mathrm{ml})$ Negative $(8 \mathrm{U} / \mathrm{ml}) 8$ (22.9) 7 (20.0) 0 (0.0) Borderline (5-8 U/ml) 1 (2.9) 3 (8.6) 3 (15.0) 0.128 Negative (8 U/ml). Anti-AnnexinVlgM levels (>8 U/ml) was detected in $8(22.9 \%)$ of the steady state subjects, 7 (20.0\%) of VOC subjects and none 0 (0.0\%) of Control group. The differences in prevalence of Anti-AnnexinV IgM was not statistically significant $(P=0.128)$ between the steady state and the control.

\section{PB0317 | Evaluation of Oxidative Stress, Thrombosis, and Inflammation in Patients with End-stage Osteoarthritis}

V. Jain; E. Bontekoe; F. Siddiqui; J. Liles; J. Fareed; W. Hopkinson Loyola University Medical Center, Maywood, United States

Background: Osteoarthritis (OA) is widely prevalent, as 14 million people suffer from knee osteoarthritis in the United States alone. Though attention has been paid to inflammation, processes that mediate thrombosis and oxidative stress have become increasingly important pathogenic factors. Integrated data on these 2 processes is not available in patients with osteoarthiritis.

Aims: Therefore, we determined circulating levels of biomarkers of oxidative stress and thromboinflammation in patients undergoing joint arthroplasty compared with normal population.

Methods: Deidentified venous blood was collected at 3 different draw dates (day 0, day 1 post-operatively, and day 5-7 postoperatively) from patients undergoing TJA due to $O A(n=73)$. Venous blood from 5 healthy volunteers were drawn. The samples were collected in vacutainer tubes containing EDTA, plasma was separated from blood via centrifugation, and then were frozen and thawed in batches for analysis. The samples were analyzed using ELISA kits for NLRP-3, Nitrotyrosine, MDA, ADMA; Chlorination assay was used for MPO. D-dimer, VWF, MPTF, PAI-1, NLP 9, fibronectin and CRP were also measured by using ELISA methods.

Data was analyzed using GraphPad Prism, and Microsoft Excel. Each variable was correlated using 2-tailed Spearman's rho correlation. Percent and fold change relative to EDTA control samples were calculated.

Results: Among the oxidative stress biomarkers, nitrotyrosine showed significantly higher levels and other biomarkers showed modest elevation. The thromboinflammatory biomarkers showed markedly higher levels in D-Dimer, CRP, MPTF, PAI-1 and variable modest increase in other biomarkers (Table 1). In addition, only 3 correlations showed significance (Nitrotyrosine with $\mathrm{VWF} P=0.042$, MDA with PAI-1 $P=0.011$, and MPO with NLRP-3 $P=0.045$ - data not shown), however they were very weakly correlated. 
TABLE 1 Tabulated Data

\begin{tabular}{|c|c|c|c|c|}
\hline \multirow[b]{2}{*}{ Onidosint Sirem Marken } & \multicolumn{2}{|c|}{ Osteoarteribs Paties Samples } & \multirow{2}{*}{$\frac{\text { ETIA Coetrol }}{\text { Averaps }}$} & \multirow{2}{*}{ Feld lecrease } \\
\hline & Averafe \& SEM & Median & & \\
\hline ADMA (ng/mi) & $7600+862$ & 50.04 & 5800 & 1.31 \\
\hline MDA (posolynt) & 42636.4932 & 30208 & 41848 & 1.02 \\
\hline MPO (aUn'lal) & $33.54 \div 0.64$ & 32.36 & 31.80 & 1.05 \\
\hline Nincononsine faM) & $498.13+122.44$ & 246.73 & 303.40 & 1.64 \\
\hline $\mathrm{NLRP3}(\mathrm{ng} / \mathrm{ml})$ & $42=0.13$ & 4.06 & 406 & 1.04 \\
\hline \multicolumn{5}{|l|}{ Inflammatory Elenarkers } \\
\hline DDect $(f y / m)$ & $4,962.81296 \times 5$ & $4,572.61$ & 115,05 & $\$ 2.14$ \\
\hline WWE (S) & $125.9 \div 3.36$ & $12 \times 21$ & 104.10 & 121 \\
\hline MP.TF (peinl) & $227+151$ & 2002 & 300 & 7.52 \\
\hline TAP (N) & $90.9 \pm 1.74$ & 100.13 & $1020 s$ & agy \\
\hline PALI ( $(\mathrm{ng}$ ml) & $49 x+43$ & 41.24 & 16.86 & 295 \\
\hline MMP.9 (neint) & $1149: 1223$ & 96.14 & $\pi 91$ & 1.47 \\
\hline Färocestin (ng)inl) & $270.7 m \cdot 4 t=11352 \cdot 14$ & 274,14330 & 25852960 & 1.05 \\
\hline CQP (ug mb) & $45.4 \div 7,13$ & 2059 & 319 & 15,17 \\
\hline
\end{tabular}

Conclusions: The substantial elevation in D-dimer and CRP with the role of thrombosis and inflammation in the pathogenesis of OA. In terms of correlating biomarkers, our study suggests three weak yet significant correlations.

PB0318 | Features of the Coagulation Potential of Blood in Patients with Endometriosis

I. Vasilenko ${ }^{1,2}$; R. Lifenko ${ }^{3}$; S. Gasparyan ${ }^{4}$; Z. Kardashova ${ }^{1}$;

E. Rusanova ${ }^{1}$

${ }^{1}$ M.F. Vladimirsky Moscow Regional Clinical and Research Institute (MONIKI), Moscow, Russian Federation; ${ }^{2}$ A.N. Kosygin Russian State University, Moscow, Russian Federation; ${ }^{3}$ City Clinical Hospital of the city of Pyatigorsk, Pyatigorsk, Russian Federation; ${ }^{4}$ Stavropol State Medical University, Stavropol, Russian Federation

Background: The survival and growth of endometrioid implants are associated with a number of pathogenetic factors: inflammatory process, dysregulation of immunity, apoptosis inhibition, activation of angiogenesis processes, and local hormonal impairments. Recently, the changes of indicators of coagulation and fibrinolysis were revealed in patients with endometriosis. However, the presence of hemostasis violations and their severity in female patients with endometriosis remain controversial.

Aims: We were made the effort to assess coagulation potential using test of Thrombodynamics.

Methods: Hemostasis indicators in peripheral blood of 29 patients with endometriomas (mean age $26.9 \pm 3.5$ years) were studied. Investigations were carried out using laboratory diagnostic system "Thrombodynamic register T-2". The process of spatio-temporal formation of fibrin clot was registered: in a series of pictures received (Pic.1), the dependence of the size and area of spontaneous clots on time was evaluated and the numerical parameters of coagulation activity.

Results: It was determined that in female patients with endometriomas, the shift to hypercoagulation was found as compared to norm. The trend to expressed growth of a series of indicators was revealed: the speed of clot growth of $35.1 \pm 9.1 \mathrm{mkm} / \mathrm{min}$ (normal growth $20-30 \mathrm{mkm} / \mathrm{min})$; initial speed of clot growth of $59.3 \pm 8.6$ $\mathrm{mkm} / \mathrm{min}$ (norm of $38-56 \mathrm{mkm} / \mathrm{min}$ ), stationary speed of clot growth of $33.2 \pm 7.9 \mathrm{mkm} / \mathrm{min}$ (normal $20-29 \mathrm{mkm} / \mathrm{min}$ ), and clot size of $1243 \pm 255 \mathrm{mkm}$ (normal $800-1200 \mathrm{mkm}$ ). In 7 female patients (24.1\%), the spontaneous clots were registered. The data obtained indicate a state of hypercoagulation in women with endometriosis and reflect a close relationship between coagulation and inflammation.

Conclusions: The data demonstrate alterations in hemostasis system of patients with endometriosis in direction of hypercoagulation connected with development of systemic inflammation. Thrombodynamic indices may be assessed not only as potential diagnostic markers but also as important characteristic for decoding of some pathogenetic aspects of endometriosis.

\section{BLOOD COMPONENTS AND MANAGEMENT}

\section{LPB0060 | The Incidence of Severe Infusion Reactions Associated with Common Iron Repletion Strategies}

A. Arastu $^{1}$; J. Cohen ${ }^{2}$; M. Oakes ${ }^{1}$; S. Olson ${ }^{3}$; B. Elstrott ${ }^{1}$; J. Aslan ${ }^{4}$; T. DeLoughery ${ }^{3} ;$ J. Shatzel ${ }^{3}$

${ }^{1}$ Oregon Health and Science University/Department of Medicine, Portland, United States; ${ }^{2}$ Oregon Health and Science University/ Department of Pharmacy, Portland, United States; ${ }^{3}$ Oregon Health and Science University/Department of Hematology-Oncology, Portland, United States; ${ }^{4}$ Oregon Health and Science University/Department of Biomedical Engineering, Portland, United States

Background: While generally very safe, little is known regarding the comparative rates of infusion reactions associated with various iron repletion strategies to aid the clinician in selecting the safest therapy.

Aims: To define the rate of infusion reactions necessitating the use of epinephrine for four frequently used intravenous (IV) iron repletion strategies (iron sucrose, iron dextran, ferumoxytol, and ferric carboxymaltose).

Methods: A multicenter retrospective analysis of IV iron administered between January 1, 2015 and December 31, 2019 at 6 ambulatory centers in Portland, Oregon. Each order was categorized as a unique infusion event independent of whether or not a patient received more than one infusion on the same date. A total of 33,005 iron infusions were administered of which 19,832 were iron sucrose (for 5,215 individual patients), 9,288 iron dextran (4,587 patients), 2,896 ferumoxytol (1,389 patients), and 989 ferric carboxymaltose (419 patients). Concomitant administration of infusion reaction medications (diphenhydramine, epinephrine, famotidine, or hydrocortisone) on the day of the IV iron infusion was recorded. A severe reaction was defined as requiring epinephrine.

Results: Among the 33,005 individual infusions, 3,639 (11.0\%) were associated with administration of at least one infusion reaction medication, 2,150 (10.8\%) in the iron sucrose group, 1,285 (13.8\%) in the iron dextran group, 160 (5.5\%) in the ferumoxytol group, and 
$44(4.5 \%)$ in the ferric carboxymaltose group. A subgroup analysis of per-patient epinephrine use, which is reserved for significant reactions, suggests that severe infusion reactions were uncommon:
Iron sucrose (1.55\% of patients received epinephrine), iron dextran (1.04\%) ferumoxytol (0.14\%) and ferric carboxymaltose (0\%).

TABLE 1 Infusion Events and Associated Reaction Rate

\begin{tabular}{|c|c|c|c|c|}
\hline & Infusion Events & Unique Patients & $\begin{array}{l}\text { Peri-Infusion Medication Use } \\
\text { (\% of Infusions) }\end{array}$ & $\begin{array}{l}\text { Severe Reactions } \\
\text { (\% of Patients) }\end{array}$ \\
\hline Iron Dextran & 9,288 & 4,587 & $1,285(13.8 \%)$ & 48 (1.04\%) \\
\hline Ferumoxytol & 2,896 & 1,389 & $160(5.5 \%)$ & $2(0.14 \%)$ \\
\hline
\end{tabular}

Conclusions: In this large multicenter analysis, severe infusion reactions were rare. Iron sucrose had the highest risk of infusion reaction requiring epinephrine, while iron dextran, ferumoxytol and ferric carboxymaltose had decreasingly lower risk, respectively.

PB0319 | Platelets Collected from Blood Diversion Pouches Are an Appropriate Alternative for Functional and Bioenergetic Analyses

O. Esparza ${ }^{1}$; G. Hernandez ${ }^{1}$; M. Kelher ${ }^{1}$; K. Kelly ${ }^{2}$; C. Silliman ${ }^{1}$;

L. Dumont ${ }^{2}$; P. Davizon-Castillo ${ }^{1}$

${ }^{1}$ University of Colorado Anschutz Medical Campus, Aurora, United

States; ${ }^{2}$ Vitalant Research Institute, Denver, United States

Background: The diversion of the first flow of blood into a pouch has been widely adopted in blood donation systems as a means to reduce whole-blood unit contamination from skin bacteria. Analyses of platelet biology require careful control of variables, including collection methods. The diversion pouch may serve as an alternative blood collection method to standard venipuncture, allowing a greater number of healthy blood bank donors to serve as research subjects.

Aims: To determine if the functional and bioenergetic profiles of platelets isolated from the diversion pouch are representative of platelets isolated from venipuncture.

Methods: Whole blood from the diversion pouch was collected into citrated tubes within two minutes of collection. Platelets were isolated and washed following standard protocols. Platelet activated fibrinogen binding site was determined at baseline and following activation (thrombin [0.075 IU/ml] and ADP [1.25 $\mu \mathrm{M}]$ ) with PAC-1 antibodies. The bioenergetic profile (aerobic and anaerobic) of washed platelets was determined by the 24-well format Seahorse extracellular flux analyzer.

Results: Platelets isolated from venipuncture and diversion pouch exhibit similar PAC-1 expression at baseline and with activation by thrombin and ADP (Figure 1). No differences in the bioenergetic profile were noted between both groups (Figure 2).
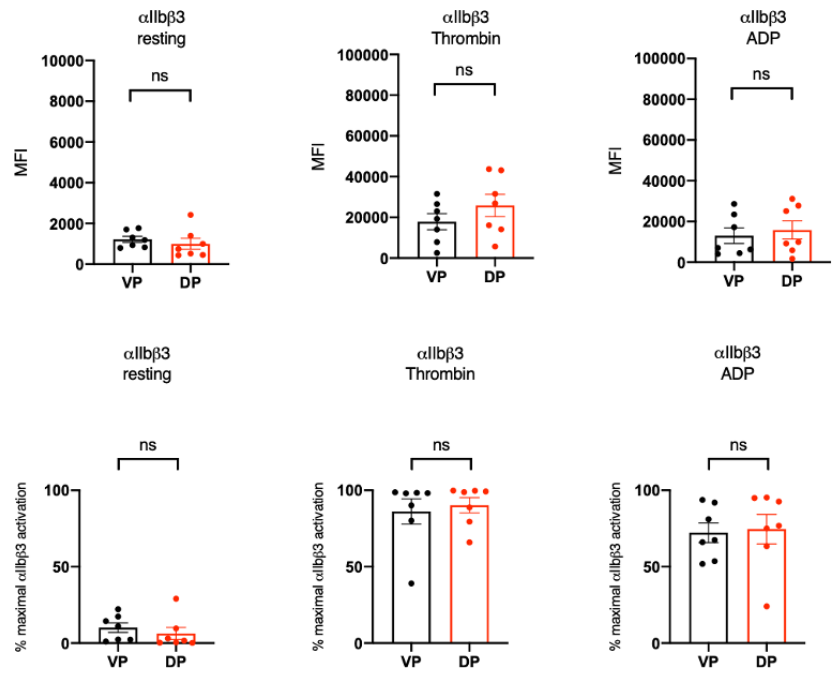

FIGURE 1 Platelet functional profile of whole blood samples obtained by venipuncture and diversion pouch are similar
A)

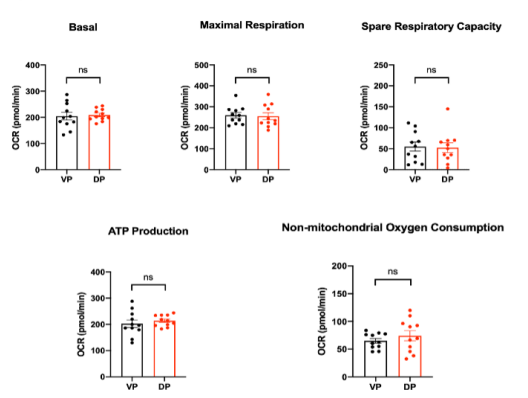

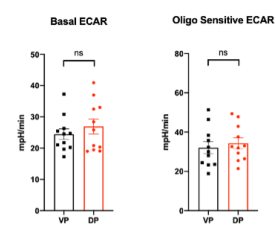

FIGURE 2 Platelet bioenergetic profile of whole blood samples obtained by venipuncture and diversion pouch are similar

Conclusions: Platelets isolated from the diversion pouch exhibit similar functional and bioenergetic profiles when compared to the standard venipuncture method. These findings suggest that 
platelets isolated from the diversion pouch are a suitable option for functional and bioenergetic testing analyses.

PB0320 | Calculation of Fresh Frozen Plasma Amount to Correct Prolonged International Normalized Ratio

L. Pinitsubsin ${ }^{1}$; Y. Chinthammitr ${ }^{2}$

${ }^{1}$ Department of Medicine, Faculty of Medicine Siriraj Hospital, Mahidol University, Bangkok, Thailand; ${ }^{2}$ Division of Hematology, Department of Medicine, Faculty of Medicine Siriraj Hospital, Mahidol University, Bangkok, Thailand

Background: Fresh frozen plasma (FFP) is the most widely-used blood component to correct prolonged International Normalized Ratio (INR). However, There is little knowledge about factors related to normalized INR and about the appropriate amount of FFP to correct prolonged INR.

Aims: To develop a formula to calculate the appropriate amount of FFP for correction of prolonged INR and analyze factors related to normalized INR.

Methods: Retrospective chart-review study includes 144 patients, aged $>18$ years, who had prolonged INR $(>1.5)$ and were transfused with FFP with INR value after transfusion between September 5 , 2019 and September 18, 2020. Ninety-six and 48 patients were in warfarin and non-warfarin groups, respectively. The following parameters were collected and analyzed: principal diagnosis, comorbidities, pre-transfusion INR (PreINR), post-transfusion INR (PostINR), $\triangle \mathrm{INR}$ (PreINR-PostINR), vitamin K given, amount of FFP, and date and time when blood samples were collected.

TABLE 1 Formulas of $\triangle I N R$ classified by subgroup

Subgroup
FFP 2 units
Warfarin user
Warfarin user and FFP 2 units
Non-warfarin user
Non-warfarin user and FFP 2 units
Warfarin user + FFP 2 units + Vit.K IV $>=5 \mathrm{mg}$
Warfarin user + FFP 2 units + Vit.K IV $<5 \mathrm{mg}$ or oral Vit.K or without
$\quad$ Vit.K
Non-warfarin user + FFP 2 units + Vit.K IV > = 5 mg
Non-warfarin user + FFP 2 units + Vit.K IV $<5$ mg or oral Vit.K or
$\quad$ without Vit.K

N

126

96

84

48

42

48

36

23

19
Results: The median (range) PreINR was 2.67 (1.50 - 14.64) and median (range) $\triangle I N R$ was 0.87 (-0.09 - 12.81). The mean (SD) amount of FFP was $2.2+0.6$ units. The median (range) time from FFP administration to PostINR testing were $4 \mathrm{~h}$ (0-19). The median (range) time between PrelNR and PostINR testings were $10 \mathrm{~h}$ (3-33). Vitamin $\mathrm{K}$ was given in 88 patients (oral 5 , intravenous 83 ). The formula $\triangle I N R$ after FFP 2 units $=\left(0.91 \times\right.$ PreINR) $-1.38\left(R^{2}=0.964\right.$ between predicted INR and actual improvement INR; $P<0.001)$. The factors correlated with normalized PostINR (INR $<1.5)$ were lower PreINR level (adjusted odds ratio $[\mathrm{aOR}]=0.64, P=0.017$ ), intravenous vitamin $\mathrm{K}$ ( $\mathrm{aOR}=1.74, P=0.025)$, and longer duration between PreINR and PostINR testings $(\mathrm{aOR}=1.30, P=0.001)$.

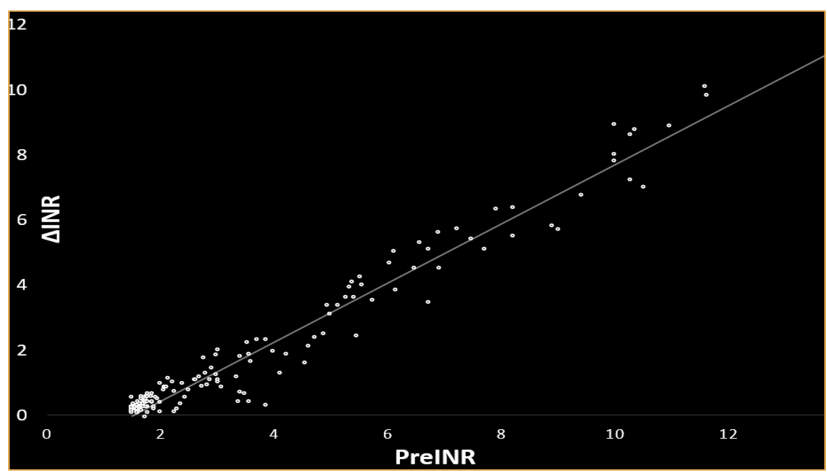

FIGURE 1 The relationship between PreINR and $\triangle I N R$ for patients who received 2 units of FFP $(N=126)$. Formula: $\Delta I N R=(0.91 \times$ PreINR) - $1.38\left(R^{2}=0.962\right)$

Conclusions: This formula may be used to predict INR after 2-unit FFP transfusion. Factors correlated with normalized INR after transfusion were lower pre-transfusion INR, intravenous vitamin $\mathrm{K}$, and longer duration between pre- and post-transfusion INR measurement. 
PB0321 | A Service Evaluation of Prothrombin Complex

Concentrate Use and Safe Administration Procedures in a Tertiary Hospital and Trauma Centre

M. Robinson; C. Hickman

Cambridge University Hospitals NHS Foundation Trust, Cambridge, United Kingdom

Background: Prothrombin complex concentrate (PCC) is a commonly used blood product to correct bleeding related to warfarin, as well as Direct Oral AntiCoagulants (DOACS). Additionally there is a suggestion that PCC can be used to correct coagulopathy related to acquired disease (e.g. hepatic disease). These products contain four clotting factors to replace those inhibited or absent. To date, there has been no published standard for the optimum time to administration.

Aims: For anticoagulant related haemorrhage, our centre has adopted a one hour "decision-to -needle" based on the short half-life of factor VII in PCC. We also permit use of PPC to correct coagulopathy in selected patients with acquired factor deficiencies. We reviewed our clinical practice of the prescribing of PCC for these two indications.

Methods: We reviewed the clinical records of every patient who administered PCC in our centre. The indication was reviewed. For patients with anticoagulant-induced major bleeding, time from decision to administration was recorded. For every case that missed the one hour target, a reason for variance was sought. For all patients, complication rates and survival data at 30 days was collected.

Results: Over one year PCC was administered in 99 occasions for anticoagulant-related bleeding Successful administration within an hour was only recorded in $14 \%$ of cases. The median time to administration was $111 \mathrm{~min}$. Reasons for delay are recorded in Table 1. PCC was administered on 69 occasions to correct acquired coagulopathy. There was no increased incidence of thrombosis or mortality related to PCC at 30 days.

TABLE 1 Reasons for delay in administration of PCC in haemorrhage

\begin{tabular}{|c|c|c|}
\hline Reason for delay & Occurrence & $\begin{array}{l}\% \text { of total } \\
\text { delays }\end{array}$ \\
\hline $\begin{array}{l}\text { Required confirmation with } \\
\text { haematology registrar }\end{array}$ & 7 & 11.1 \\
\hline Slow processing by laboratory* & 15 & 23.8 \\
\hline $\begin{array}{l}\text { Issues with no porters to collect the } \\
\text { ordered beriplex }\end{array}$ & 7 & 11.1 \\
\hline Incorrect dose ordered initially & 10 & 15.9 \\
\hline Miscommunication $^{* * *}$ & 3 & 4.76 \\
\hline $\begin{array}{l}\text { Clinical workload and unstable } \\
\text { patient }\end{array}$ & 17 & 27.0 \\
\hline Weight of patient & 1 & 1.59 \\
\hline Octaplex ordered instead of beriplex & 2 & 3.17 \\
\hline Nursing staff not trained to give ${ }^{* * * *}$ & 1 & 1.59 \\
\hline
\end{tabular}

Conclusions: Our data suggests that there is a failure to give PCC in a timely manner. We have devised a set of measures to improve administration. Our data also provides additional evidence that PCC is safe for use in acquired coagulopathies with no increase in morbidity or mortality.

PB0322 | Use of Blood Products and Hemostatic Agents during Massive Transfusions: Review of our Practice

G.S. Ene ${ }^{1}$; L. Edo Caballero²; N. Tamayo Ubeda ${ }^{2}$; A. Carpi Medina ${ }^{2}$; N. Palo Mauriz²; A. Villauvi Serra²; A. Bolivar Lopez ${ }^{1}$; P. Sanchez Coronado ${ }^{1}$; I. Sanchez Ron²; C. Raya Hinojosa²; J.C. Alvarez Garcia ${ }^{2}$

${ }^{1}$ Banc de Sang i Teixits, Barcelona, Spain; ${ }^{2}$ Hospital del Mar, Barcelona, Spain

Background: Massive bleeding(MB) is associated with coagulopathy and high mortality. Currently, there are diverse approaches to the management of MB. The main difference is the number of blood products in the transfusion packages, which include red blood cells(RBC), fresh frozen plasma(FFP) and platelet concentrates(PC). The use of hemostatic agents like fibrinogen concentrate(FC), tranexamic acid and prothrombin complex concentrate (PCC) has been shown to reduce mortality.Our MB protocol utilizes a ratio of $4 \mathrm{RBC}: 4 \mathrm{FFP}: 1 \mathrm{PC}$ in the initial transfusion package. Our hospital administrates approx. 12000 blood products/year. The anesthetics team is experienced with the use of Rotational thrombelastometry (ROTEM) in the management of MB.

Aims: This study aimed to determine the use of blood components and hemostatic agents during MB in our hospital.

Methods: We performed a retrospective review of $\mathrm{MB}$ cases from January 2018 to December 2020. Patient demographics, indications and laboratory records were analyzed. ROTEM guided transfusion was performed by the team of anesthesiologists managing the patient.

Results: During this period, a total of $108 \mathrm{MB}$ were detected, with an average of $36 /$ year. The medium age for these events was 57.18 y (range of 18-88 y) and included 49 females and 59 males. MB were associated with surgery(57), cirrhotic complications(27), trauma(20), obstetric bleeding(4).The blood products used were 850 RBC(average 7.87/patient) and 169 PC(average 1.56/patient). Of the 510 FFP units(average 4.72/patient) that were thawed, 411(average 3.84/patient) were used. The remaining units were discarded. Tranexamic acid was used in 70 patients and CCP in 13.There was a favorable outcome in 83 cases and 25 patients died from the major bleeding event.A decrease in MB events was recorded during the COVID pandemic. 
TABLE 1 Use of blood products and hemostatic agents

\begin{tabular}{lll} 
Blood products & Used & Average \\
RBC & 850 & $7.87 /$ patient \\
PC & 169 & $1.56 /$ patient \\
FFP & 411 & $3.84 /$ patient \\
Haemostatic agents & & \\
$\quad$ Fibrinogen concentrates & $227 \mathrm{gr}$ & $2.76 \mathrm{gr} /$ patient \\
$\quad$ Tranexamic acid & $89 \mathrm{gr}$ & $1.25 \mathrm{gr} /$ patient \\
\hline
\end{tabular}

Conclusions: The optimal way to resuscitate patients with MB remains unclear, and clinical trials are difficult to perform in this setting. We believe that uniform approach for the management of $M B$ is deleterious to the best use of blood products.

\section{CELLULAR THERAPIES}

LPB0106 | Enhancing Transfusable Platelets Using mRNA Therapy to Produce Exogenous Proteins

J. Leung ${ }^{1,2,3}$; C. Strong ${ }^{1,2,3} ;$ A. Hagner ${ }^{4} ;$ K. Badior $^{1,2,3}$;

D. Witzigmann ${ }^{1}$; L.J. Juang ${ }^{1,2,3} ;$ M. Robertson ${ }^{1,2,3}$; E. Jan ${ }^{1}$;

P. Zandstra ${ }^{2,4}$; P. Cullis ${ }^{1}$; C. Kastrup ${ }^{1,2,3}$

${ }^{1}$ University of British Columbia, Biochemistry and Molecular Biology,

Vancouver, Canada; ${ }^{2}$ University of British Columbia, Michael Smith

Laboratories, Vancouver, Canada; ${ }^{3}$ University of British Columbia,

Centre for Blood Research, Vancouver, Canada; ${ }^{4}$ University of British

Columbia, School of Biomedical Engineering, Vancouver, Canada

Background: Platelets are transfused therapeutically for hemostasis, and are an integral part of hemorrhage management. However, transfusions can be ineffective in the most severe cases of hemorrhage. In addition to hemostasis, platelets are a potential cell therapy in other applications, but development has been hindered by inadequate methods to control which proteins are expressed by platelets. For example, there are no methods to express exogenous proteins in transfusable platelets, which would expand their use to help treat the diseases they modulate. A method is therefore needed to modify transfusable platelets, and thus enhance their protein composition for specific applications.

Aims: To produce engineered, transfusable platelets to enhance their natural coagulability and functional repertoire by directly transfecting donor-derived platelets and megakaryocytes with mRNA via lipid nanoparticle (LNP)-mediated delivery. LNPs have already demonstrated clinical safety and efficacy for gene therapy, and cultured megakaryocytes provide an alternative source of cells that can be engineered to produce modified platelets.

Methods: As a proof-of-concept, megakaryocytes cultured from cord-blood hematopoietic stem cells were treated with GFPencoding mRNA-LNPs and induced to produce platelet-like particles, with nonGFP mRNA-LNPs as a control. Donor-derived platelets were also incubated with GFP or luciferase-encoding mRNA-LNPs, or LNPs incorporating the lipophilic tracer Dil. GFP expression and
LNP uptake were assessed following $24 \mathrm{~h}$ via flow cytometry and confocal microscopy; luciferase expression was assessed by measuring luminescence intensity normalized to total protein content.

Results: Megakaryocytes demonstrate successful transfection and produce GFP-positive plalelet-like particles. Nearly $100 \%$ of donorderived platelets appear to take up the LNPs, and demonstrate expression of both GFP and luciferase.
A)

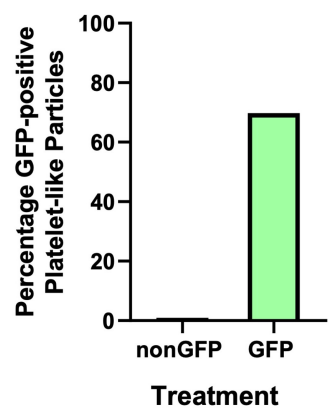

B)

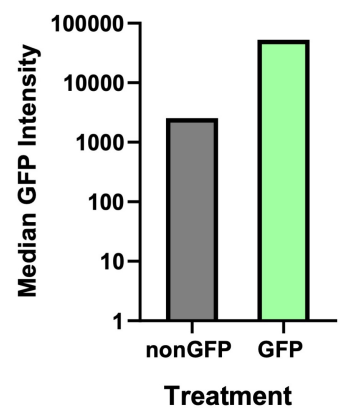

FIGURE 1 (A) Percentage-positive platelet-like particles expressing GFP and (B) corresponding change in median fluorescence intensity
A)

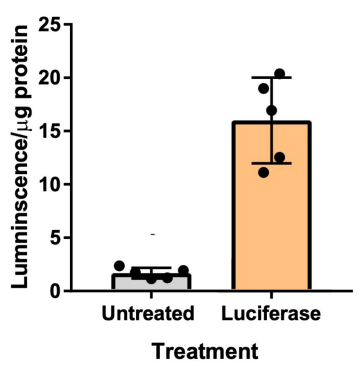

B)

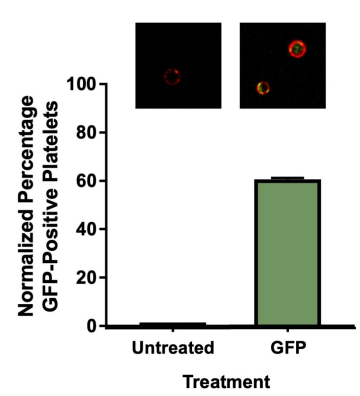

FIGURE 2 Donor-derived platelet mRNA-LNP expression of (A) luciferase and (B) GFP after $24 \mathrm{~h}$. Error bars represent SEM

Conclusions: Megakaryocytes and platelets can readily be transfected with LNPs to produce exogenous protein. Further optimization can eventually lead to the creation of a platform technology that in the long-term will allow platelets to deliver therapeutic proteins and yield more effective platelet products.

PB0325 | Hemostatic and Thrombogenic Properties of Lyophilized Human Platelets

H. Zhou ${ }^{1}$; A. Lee ${ }^{2}$; K. Moskowitz ${ }^{2}$;. Diacovo ${ }^{1}$

${ }^{1}$ University of Pittsburgh, Pittsburgh, United States; ${ }^{2}$ Cellphire Inc, Rockville, United States

Background: Thrombosomes ${ }^{\circledR}$ are lyophilized human platelet derivatives (LHP) currently under Phase 2 clinical trial for use in noncompressible hemorrhage, a condition with high mortality. The mechanism(s) of action in vivo have yet to be elucidated. Using humanized VWF HA1 transgenic mice, which have been previously 
reported to support human but not mouse platelet mediated hemostasis and thrombosis, we characterized the ability of LHP to support these critical biological processes.

Aims: To determine whether LHP (i) require VWF to support interactions at sites of vascular injury, (ii) can mediate platelet accrual in vivo, and (iii) can mitigate bleeding in response to trauma-induced injury.

Methods: An intravital model of laser-induced arteriole injury was employed to assess the behavior of fluorescently labeled LHP and/ or human and mouse platelets within the microcirculation of the cremaster muscle of VWF HA1 mutant and VWF KO animals. A standard tail bleeding assay was used to determine their hemostatic properties compared to freshly isolated human platelets.

Results: Intravital studies revealed that human platelets were able to form large thrombi in laser-injured arterioles of VWF HA1 mutant but not VWF KO mice (max thrombus size of 6,985 $\pm 697 \mu \mathrm{m}^{2}$ vs $185 \pm 110 \mu \mathrm{m}^{2}$, respectively; mean $\left.+\mathrm{SD} ; n=10\right)$. In contrast, LHP accumulated to a similar extent in damaged arterioles in either animal without forming occlusive thrombi (max thrombus size of $6,436 \pm 1,753 \mu \mathrm{m}^{2}$ vs 5,902 $\pm 1,694 \mu \mathrm{m}^{2}$, respectively; mean + SD; $n=10 ; P=0.22$ ). Of note, only surface adherent LHP in VWF HA1 mice could (i) recruit a significant number human platelets into the growing thrombus, and (ii) restore hemostasis in response to amputation of distal tail tip (bleeding times of $257 \pm 135 \mathrm{~s}$ vs $>600 \mathrm{~s}$ for PBS treated).

Conclusions: LHP can accumulate at sites of vascular injury independent of VWF and circulating platelets but require VWF to support effective hemostasis.

\section{EPIGENETICS, OMICS AND BIOINFORMATICS}

\section{PB0326 | In silico Methods of Structural Biology for Studying} (Not Only) Coagulation Proteins

\section{Z. Sovova; J. Suttnar; J.E. Dyr}

Institute of Hematology and Blood Transfusion, Praha 2, Czech

Republic

Background: Behavior of biomacromolecules is governed, inter alia, by their structure. Apart from widely used experimental methods for their characterization (X-ray diffraction, NMR, etc.), in silico methods become widely used in description of macromolecular since they are generally cheaper and can reveal features beyond the scope of experiment. Molecular dynamics (MD), Monte Carlo and numerous quantum calculations as well as molecular modelling and docking and various bioinformatics analyses are among the standard theoretical methods used in the field of life sciences to study molecules on atomistic scale.

Aims: The aim of this work is to propose techniques of molecular dynamics simulation and sequence analysis for coagulation studies and to demonstrate usage of these methods in characterization the effects of post-translational modifications (PTMs) on fibrinogen structure.

Methods: Molecular dynamics simulations of fibrinogen were performed in Gromacs software with Gromos 54a7 force field. PTMs were introduced into the truncated crystal structure $3 \mathrm{GHG}$ by Vienna-PTM 2.0 server. Sequence analysis was performed on sequences downloaded from NIH database.

Results: Molecular dynamics simulations revealed that PTMs can, but do not have to, influence secondary structure of fibrinogen and they can alter internal dynamic of the protein. On the example of MD simulation of pristine fibrinogen, we show that certain variations in secondary structure, those are not captured in the crystal structure, are native for the protein. We further revealed that various PTMs at the same position have different effect on fibrinogen behavior. Sequence analysis highlights the importance of proline in unfolded region of the $\gamma$ chain ( $\gamma$ Y68 - $\gamma$ M78) within the coiled-coil domain of fibrinogen.

Conclusions: In silico methods captures characteristics and behavior of biomacromolecules that is unreachable by experiments. These methods can be used for understanding and interpretation of experimental results in the field of structural biology. (Funded by MH CR, grant number 00023736.)

\section{PB0327 | The Prevalence of Gene Polymorphism of the Cytochrome P-450 and VKORC1 System among the Indigenous Population of the Arctic

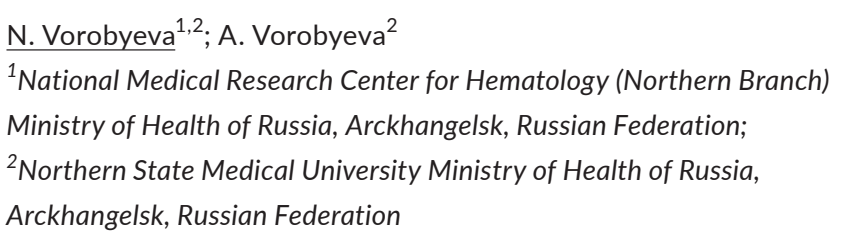

Background: The stability of indigenous ethnic groups to adverse conditions largely depends on the structure of their gene pool, the ability to metabolize foreign environmental substances. In this regard, the personification of pharmacotherapy taking into account the genetic characteristics of the system of biotransformation of genes of the cytochrome P-450 and VKORC1 family taking into account ethnic characteristics is a promising direction.

Aims: The purpose of the study was to analyze the prevalence of genotypes and allelic variant in the genes CYP2C9, CYP2C19, VKORC1 in the population of the indigenous ethnic group of the Arctic under conditions of permanent island living.

Methods: A transverse population study of an indigenous ethnic group living in the Arctic zone of Russia (Vaygach Island $-70^{\circ} 01^{\prime} \mathrm{N} 59^{\circ} 33$ ' E) was carried out during a scientific expedition (grant RFBR KOMFI (18-00-00478). Research - Nenets, research subject - genomic DNA obtained from peripheral venous blood leukocytes by the express method. The reported study was funded by RFBR according to the research project No18-00-00814-KOMFI (18-00-00478).

Results: According to the results of CYP2C9*2 (rs1799853) gene detection, it was revealed that the SS genotype was the most common variant (90.63\%). CYP2C9*3 (rs1057910) gene analysis identified 
only one AA genotype. According to the results of the VKORC1 G3730A (rs7294) gene study, two genotypes were identified - GG (56.25 (18) and GA(43.74 (14), while there was no homozygous allelic polymorphism for the pathological allele in the studied sample. Conclusions: The study showed the variability of the frequency distribution of genotypes and alleles of polymorphisms of the CYP2C9*2 (rs1799853), CYP2C9*3 (rs1057910), CYP2C19*2 (rs4244285), VKORC1G3730A (rs7294), VKORC1C1173T (rs9934438) genes in the population of the Arctic ethnic group, the wild-type allele with the highest homozygous CY9 genotype * 3 was noted in $100 \%$ of cases, which is a feature of the genotype of the Nenets of Vaigach island.

\section{LABORATORY DIAGNOSTICS}

\section{LPB0013 | Evaluation of Heparin-induced Thrombocytopenia Before and during the COVID-19 Pandemic at a US National Reference Laboratory}

E. Wong ${ }^{1}$; L. Worfolk ${ }^{1}$; L. Noh ${ }^{1}$; W. Wu ${ }^{1}$; M. Sahud ${ }^{2}$; F. Racke ${ }^{2}$; J. Dlott ${ }^{1}$

${ }^{1}$ Quest Diagnostics Nichols Institute, Chantilly, United States; ${ }^{2}$ Quest Diagnostics Nichols Institute, San Juan Capistrano, United States

Background: Heparin-induced thrombocytopenia (HIT) is a clinicopathologic diagnosis that can result in thrombotic complications in patients receiving heparin thromboprophylaxis. COVID-19-associated coagulopathy was recognized internationally in February 2020 and is now considered a prothrombotic state; multiple scientific societies recommend thromboprophylaxis in hospitalized COVID-19 patients. The major thromboprophylaxis agents are low molecularweight heparin (LMWH) and unfractionated heparin (UFH). Given this increased exposure to heparins and the thrombo-inflammatory nature of COVID-19, we investigated whether there was a change in positive rates of HIT antibody tests.

Aims: To determine if functional and antigenic HIT antibody positivity was affected by the COVID-19 pandemic

Methods: We conducted a retrospective review of HIT IgG enzymelinked immunosorbent assay (ELISA) and serotonin release assay (SRA) results from February to December of 2019 and the corresponding months in 2020. Positivity for HIT IgG ELISA was defined as an optical density $>0.300$ per manufacturer instructions. SRA positivity was defined as $\geq 20 \%$ release at low heparin concentration that coincided with $>50 \%$ reduction of serotonin release at high heparin concentration. Median monthly positivity rates of 2019 and 2020 were compared for HIT IgG ELISA, and for LMWH and UFH SRA. Nonparametric testing for statistical significance was performed by Mann-Whitney $U$ testing (two-tailed).

Results: From 2019 to 2020, testing increased for LMWH SRA (16.7\%), UFH SRA (11.2\%), and HIT IgG ELISA (56.1\%), indicating either possible increased exposure to heparin or suspicion for HIT. The median monthly percent positivity did not significantly differ between 2019 and 2020 for either SRA. However, the median monthly percent positivity was significantly lower in 2020 than in 2019 for IgG ELISA (See Table).

TABLE 1 Median (5th and 95th Percentile) Monthly Positivity Rates for HIT IgG ELISA and UFH and LMWH SRA in 2019 and 2020

\begin{tabular}{llll} 
HIT Assay & 2019 & 2020 & $P$ value \\
LMWH SRA & $12.9 \%(7.3-17.9 \%)$ & $13.5(7.9-18.8 \%)$ & 0.1499 \\
UFH SRA & $8.1 \%(6.4-11.0 \%)$ & $8.8 \%(6.8-10.7 \%)$ & 0.8415 \\
HIT IgG ELISA & $17.6 \%(14.6-21.1 \%)$ & $14.9 \%(10.4-17.7 \%)$ & 0.0048 \\
\hline
\end{tabular}

Conclusions: Exposure to either LMWH or UFH during the COVID-19 pandemic in 2020 was not associated with increased SRA positivity, and HIT IgG ELISA positivity decreased, despite increased clinical suspicion for HIT during 2020.
LPB0014 | Global Thrombosis Test: Occlusion by Coagulation or SIPA?

V. Clavería; P. Yang; M. Griffin; D. Ku

Georgia Institute of Technology, Atlanta, United States

Background: The Global Thrombosis Test (GTT) has been proposed as a point of care device to quantify thrombotic and thrombolytic status. Is GTT thrombosis is dominated by coagulation or high shearinduced platelet aggregation (SIPA)?

Aims: Demonstrate that GTT occludes predominantly by coagulation induced fibrin, blocked by heparin, and lysed by tPA.

Create alternative conditions for faster occlusion by SIPA, resistant to tPA.

Methods: Thrombus formed in the GTT was run with fresh human blood according to the Instructions and the Occlusion Time (OT) recorded without and with heparin or IPA. Occlusive thrombi were stained with 
Carstairs to detect fibrin or platelets. The assay was then modified to eliminate the low shear zone and coat with fibrillar collagen.

Results: The GTT formed thrombi in the gaps between the beads that were fibrin-rich. The OT was long at $526 \pm 188 \mathrm{~s}$, and was strongly lengthened by heparin ( $847 \pm 106 \mathrm{~s}, P<0.0001, n=12)$. tPA caused lysis (865s) in half the time of control (1660s, $P<0.005)$. In contrast, the modified assay created thrombi that were rich in platelets. Occlusion time was shortened to $217 \pm 71 \mathrm{~s}(P=0.0005)$ while heparin caused a slight increase to $244 \mathrm{~s}$. Both the control and tPA lysis times were prolonged beyond the control lysis time for the original GTT. The rapid attachment of platelets to fibrillar collagen and relative resistance to heparin and tPA created a SIPA-like thrombosis. Unfortunately, both the original GTT and modified assay exhibited large variability.

FIGURE 1 (a) GTT and modified GTT OT are shown for fresh and

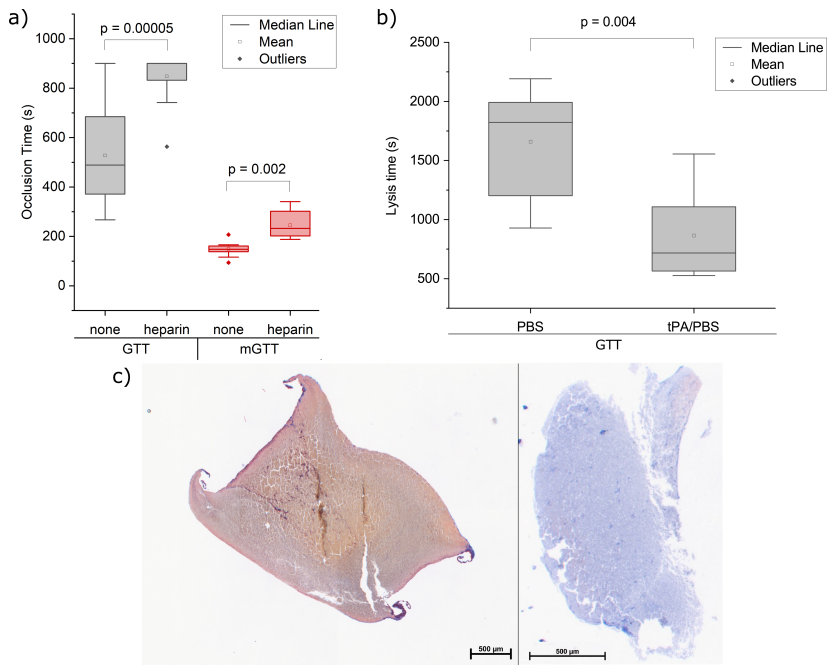

heparinized blood, (b) GTT lysis time with and without tPA is presented and (c) representative histology of clot harvested from the GTT (left) and modified GTT (right) devices using Carstairs' stain. Red indicates fibrin and blue platelets.

Conclusions: GTT occludes primarily from a clot that is rich in fibrin in the low-shear zone, in a longer time-scale of the coagulation cascade, and is affected strongly by heparin; despite the existence of a high shear gap in the system. The tubular test section could be modified to induce faster occlusion with a SIPA platelet-rich thrombus, that was relatively resistant to IPA.

\section{LPB0015 | Comparison of D-dimer values at peak and trough concentrations of direct oral anticoagulants: result of D-dimer testing is not affected by plasma drug concentration}

S. Margetić $^{1}$; I. Ćelap ${ }^{1} ;$ M. Razum ${ }^{1}$; R. Mihić ${ }^{1}$; S. Šupraha-Goreta ${ }^{2}$

${ }^{1}$ University Hospital Center Sestre Milosrdnice, Clinical Institute of Chemistry, Zagreb, Croatia; ${ }^{2}$ Faculty of Pharmacy and Biochemistry, University of Zagreb, Department of Biochemistry and Molecular Biology, Zagreb, Croatia
Background: Knowledge of the Effect of Direct Oral Anticoagulants (DOACs) Concentration in Plasma on D-dimer Values Has so far Been Insufficiently Examined

Aims: To investigate whether plasma concentration (peak vs. trough) of DOACs (dabigatran, rivaroxaban and apixaban) affects D-dimer values. Methods: The study included a total of 218 plasma samples including peak $(N=109)$ and trough $(N=109)$ DOACs levels in outpatients treated with standard drug doses (dabigatran $N=35,2 \times 150 \mathrm{mg}$ / day, rivaroxaban $N=37,1 \times 20 \mathrm{mg} /$ day and apixaban $N=37,2 \times 5$ $\mathrm{mg} /$ day) in steady-state and obtained during their regular clinical examination. Blood samples were taken on the same day to obtain both trough (immediately prior the next drug dose) and peak (two hours after drug administration) DOACs concentrations. Rivaroxaban and apixaban concentrations were determined using chromogenic anti-FXa assay (Innovance heparin, Siemens Healthineers, Germany) with drug specific calibrators (Hyphen Biomed, France). Dabigatran was measured using Innovance DTI assay (Siemens Healthineers, Germany). D-dimer concentration was determined by quantitative particle-enhanced immunoturbidimetric assay using monoclonal antibody (Innovance D-dimer, Siemens Healthineers, Germany). All measurements were performed on BCSXP analyzer (Siemens Healthineers, Germany). Statistical analysis was done using Wilcoxon test by MedCalc Statistical Software version 11.5.1. The study was funded by the Croatian Science Foundation as part of the research project IP-2016-06-8208.

Results: Concentrations of DOACs have shown significant differences between peak and trough levels $(P<0.001)$ for all three DOACs (Figure 1). On the contrary, concentrations of D-dimer did not show significant differences in samples with peak and trough concentrations of all three DOACs drugs $(P=0.852, P=0.274$ and $P=0.833$ ), as shown in Figures 1 and 2 .

\begin{tabular}{|c|c|c|c|c|}
\hline DOAC drug & $\begin{array}{c}\text { Peak conc. } \\
\text { ng/mL } \\
\text { Median (95\%Cl) } \\
\text { IQR }\end{array}$ & $\begin{array}{l}\text { Trough conc. } \\
\text { ng/mL } \\
\text { Median(95\% Cl) } \\
\text { IQR }\end{array}$ & $\begin{array}{c}\text { D-dimer at } \\
\text { peak conc. } \\
\text { (mg/L FEU) } \\
\text { Median }(95 \% \mathrm{Cl}) \\
\text { IQR }\end{array}$ & $\begin{array}{c}\text { D-dimer at } \\
\text { trough conc. } \\
\text { (mg/L FEU) } \\
\text { Median ( } 95 \% \mathrm{Cl} \text { ) } \\
\text { IQR }\end{array}$ \\
\hline $\begin{array}{c}\text { Dabigatran } \\
N=35\end{array}$ & $\begin{array}{c}154(112-186) \\
97-248\end{array}$ & $\begin{array}{c}70(59-92) \\
50-119\end{array}$ & $\begin{array}{c}0.35(0.28-0.62) \\
0.27-0.77\end{array}$ & $\begin{array}{c}0.37(0.26-0.62) \\
0.25-0.73\end{array}$ \\
\hline $\mathbf{P}$ & \multicolumn{2}{|c|}{$<0.001$} & \multicolumn{2}{|c|}{0.852} \\
\hline $\begin{array}{c}\begin{array}{c}\text { Rivaroxaban } \\
\mathrm{N}=37\end{array} \\
\end{array}$ & $\begin{array}{c}205(175-235) \\
133-243\end{array}$ & $\begin{array}{c}22(11-51) \\
9-81\end{array}$ & $\begin{array}{c}0.53(0.40-0.67) \\
0.34-0.89\end{array}$ & $\begin{array}{c}0.47(0.40-0.62) \\
0.37-0.81\end{array}$ \\
\hline $\mathbf{P}$ & \multicolumn{2}{|c|}{$<0.001$} & \multicolumn{2}{|c|}{0.274} \\
\hline $\begin{array}{c}\text { Apixaban } \\
\mathrm{N}=37\end{array}$ & $\begin{array}{c}184(154-211) \\
142-234\end{array}$ & $\begin{array}{c}109(84-129) \\
71-139\end{array}$ & $\begin{array}{c}0.53(0.46-0.72) \\
0.37-0.76\end{array}$ & $\begin{array}{c}0.55(0.42-0.74) \\
0.37-0.81\end{array}$ \\
\hline $\mathbf{P}$ & \multicolumn{2}{|c|}{$<0.001$} & \multicolumn{2}{|c|}{0.833} \\
\hline
\end{tabular}

FIGURE 1 Results of D-dimer testing at peak and trough concentrations of DOACs 

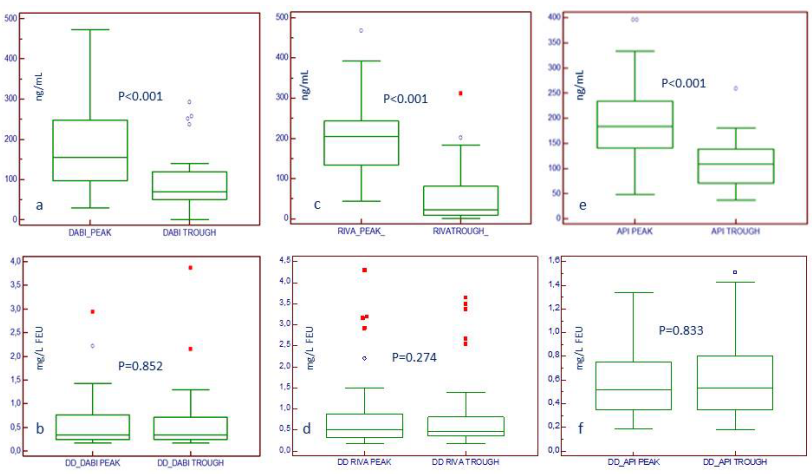

FIGURE 2 Graphic presentation of the differences between peak and trough concentrations of dabigatran (a), rivaroxaban (c) and apixaban (e) and D-dimer values at peak and trough plasma concentrations of dabigatran (b), rivaroxaban (d) and apixaban ( $f$ )

Conclusions: Concentration of DOACs in plasma does not affect Ddimer values. Our results clearly indicate that D-dimer testing can be performed at any time point in patients treated with all three DOACs without effect of drug concentration in plasma on the D-dimer test result.

LPB0061 | Impact of Characteristic Inhibition Kinetics of Different FVIII Inhibitors on the Inhibitor Titer Quantification in the Modified Nijmegen-Bethesda Assay

$\underline{\text { C. Ketteler }}^{1}$; I. Hoffmann ${ }^{1}$; S. Davidson ${ }^{2}$; A. Tiede ${ }^{3}$; N. Richter ${ }^{1}$

${ }^{1}$ Roche Diagnostics GmbH, Penzberg, Germany; ${ }^{2}$ Roche Diagnostics International Ltd, Rotkreuz, Switzerland; ${ }^{3}$ Department of Hematology, Hemostasis, Oncology and Stem Cell Transplantation, Hannover Medical School, Hannover, Germany

Background: The development of inhibitors to infused factor VIII (FVIII) in congenital hemophilia A (HA) patients display a serious complication. Moreover, FVIII inhibitors can occur due to an autoimmune disorder (acquired HA). The inhibitors are classified as type I and II according to their different kinetics. Routinely the inhibitor titer is quantified using the modified Nijmegen-Bethesda assay
(MNBA). The resulting titers are often difficult to compare due to the different criteria used for calculation.

Aims: To analyze the impact of different FVIII inhibitor kinetics on the titer calculation in the MNBA applying different criteria.

Methods: Two type I FVIII antibodies [4A4 (Green Mountain Antibodies), BO2C11 (Creative Biolabs)] and two type II FVIII antibodies [2-54 (Green Mountain Antibodies), ESH-8 (ImmBioMed)] were analyzed at different concentrations $(0.25-4 \mu \mathrm{g} / \mathrm{mL})$ with the MNBA using the cryocheck ${ }^{\mathrm{TM}}$ Factor VIII Inhibitor Kit (Precision BioLogic). FVIII residual activities (RAs) were measured in duplicates with a FVIII One-Stage Clotting Assay using a cobas t 511/711 analyzer in combination with the FVIII assay kit (Roche Diagnostics). The RAs were plotted against inhibitor dilution in a semi-log(x)-plot.

Results: The kinetics of four different FVIII antibodies were compared to the theoretical kinetic model (black lines), where every RA would lead to the same calculated titer result (Figure 1). As a result, only the inhibition kinetic of one analyzed antibody $4 \mathrm{~A} 4$ (C) was perfectly represented by the theoretical kinetic while the other kinetics showed significant deviations from the used model. These deviating inhibition kinetics (A/B and D) can have a significant impact on titer calculation when using different criteria (Table 1).
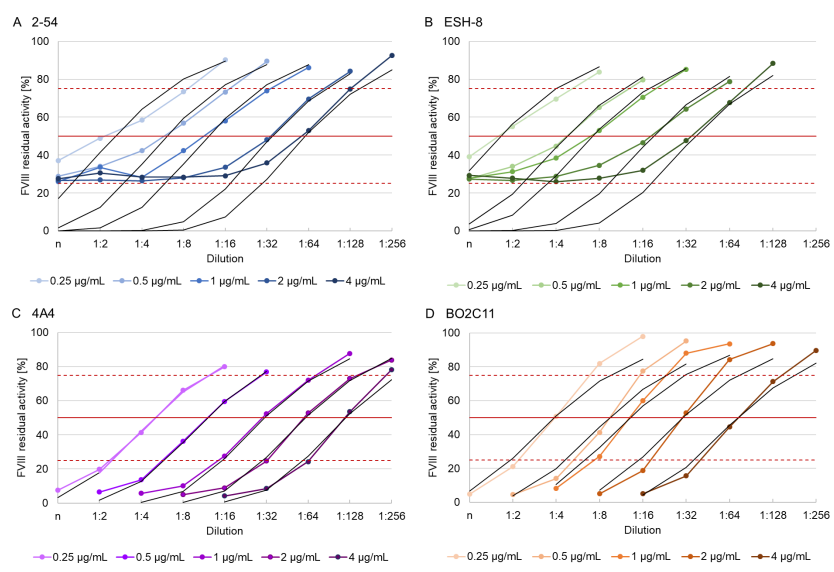

FIGURE 1 Inhibition kinetics of different FVIII inhibitors in relation to the theoretical kinetic.

TABLE 1 Overview and limitations of different criteria for titer quantification.

\section{Criteria*}

Number Reference

$$
1
$$

2

S. Kitchen et al., WFH 34 (2010) 132 (2019)
Inhibitor titer calculation

first dilution with RA > $25 \%$

Medicine and Hemostasis

3 mean value of all titers with RAs between 25 and $75 \%$

titer of the dilution closest to $50 \%$ RA
Limitations for inhibitors with deviating kinetics $^{* *}$

A/B: long plateau phase (> $25 \%$ RA) $\downarrow$ selected dilution with RA close to $25 \% \downarrow$ D: selected dilution with RA close to 25 $\% \uparrow$

A/B: long plateau phase (> $25 \%) \downarrow$ uneven distribution of values $\downarrow \uparrow$ D: uneven distribution of values $\downarrow \uparrow$ A/B, D: RAs far from $50 \% \downarrow \uparrow$ 


\section{Criteria $^{*}$}

\begin{tabular}{ll}
\hline Number & Reference \\
4 & B. Verbruggen \\
& et al., Thrombosis and \\
& haemostasis 74 (1995)
\end{tabular}

5
Inhibitor titer calculation

RAs (25-75 \%) were plotted versus dilution in a semi-log(y)-plot titer calculation with the concentration that gives $50 \%$ RA by extrapolation

sigmoidal regression of all RAs in a semi-log(x)-plot RA [\%] = bottom + (100 - bottom $) /(1+$ $10^{\wedge}(($ LogEC50 $-x) \cdot$ HillSlope $\left.)\right)$

titer calculation with the concentration that gives

$50 \%$ RA
Limitations for inhibitors with deviating kinetics $^{* *}$

D: no calculation possible when only one value is between $25-75 \%$

"Criteria 1-3 describe different strategies to select dilutions with RAs which can be used for a reliable inhibitor titer calculation. Criteria $4+5$ use a recalculation to determine the concentration that gives $50 \%$ RA in different ways.

${ }^{* *} A-D$ corresponding to the generated kinetics as presented in figure 1. Showing either a kinetic profile with a lower slope (e.g. A +B) or a higher slope (e.g. D) at $50 \%$ RA. $\downarrow \uparrow$ indicate a resulting over- or underestimation of the calculated titer depending on the criteria in combination with an outlined deviation of the kinetic profile.

Conclusions: The theoretical kinetic model of the MNBA does not perfectly reflect the inhibition profile of various different FVIII inhibitors. This consequently leads to differences in the calculated titer especially depending on the criteria used for quantification. COBAS and COBAS T are registered trademarks of Roche.

\section{LPB0062 | Smartphone-based Detection of Platelet Activation in Microfluidic Strips}

\section{Hodge; G. Little; A. Edwards; C. Jones}

University of Reading, Reading, United Kingdom

Background: To enable platelet function analysis in a wider range of settings, we used microfluidic technology coupled with standard smart-phone imaging, to develop a 'dip-stick' platelet function analyser capable of assessing platelet response to a range of agonists and anti-platelet drugs.

Aims: We aimed to demonstrate the device's capability of detecting platelet activation via ADP and thrombin stimulation in a reproducible manner.

Methods: 10-capillary Micro-Capillary Film (MCF) was coated internally with a hydrophilic polymer (PVOH) then loaded with ADP or thrombin. These capillaries were dipped into whole citrated blood for $30 \mathrm{~s}$. Formation of platelet aggregates impeded capillary rise, which was imaged with a smart-phone camera. Height of capillary rise was then analysed in ImageJ.

Results: Capillaries coated with thrombin or ADP showed a concentration dependant reduction in capillary rise. Optimisation of the coating and loading procedures has allowed a highly reproducible test, with an average intra-strip CV value below $8 \%$, and an average inter-strip CV value below $3 \%$, with no significant variance across strip batches, indicating a robust manufacturing procedure. In strips loaded with ADP, mean inhibition of capillary rise was $16.1 \%$, upon treatment of blood with cangrelor this decreased to $5.1 \%(P<0.05)$, indicating a detectable reduction in platelet aggregation.

Conclusions: We have developed a proof-of-principle 'dip-stick' platelet function analyser with reproducible capillary rise, demonstrable dose-dependent reduction in capillary rise in the presence of platelet agonists which can be reversed by pathway specific inhibition of platelet function. This demonstrates the potential utility of this device as a viable platelet assay.

\section{LPB0107 | Preoperative Measurement of Platelet Contribution to Clot Stiffness (PCS) with the Quantra ${ }^{\circledR}$ QPlus ${ }^{\circledR}$ System and Bleeding after Cardiac Surgery}

T.B Washburn Jr ${ }^{1}$; W. Houdijk ${ }^{2}$; R. Sudhagoni ${ }^{2}$

${ }^{1}$ Huntsville Hospital, Huntsville, United States; ${ }^{2}$ HemoSonics LLC, Charlottesville, United States

Background: PCS estimated by the Quantra QPlus System is associated with platelet count and ADP-dependent platelet function (1). It has been shown that preoperative ADP-dependent platelet function could predict post-operative bleeding in cardiac surgery and guides waiting time in clopidogrel-treated patients $(2,3)$. The association of preoperative PCS with postoperative blood loss is unknown.

1. Baryshnikova E, etal. J Cardiothorac Vasc Anesth. 2019;33:1590-8.

2. Ranucci M, et al. Br J Anaesth. 2014;113:970-6.

3. Mahla E, et al. Circ Cardiovasc Interv. 2012;5:261-9. 
Aims: Assessment of the correlation of preoperative PCS with platelet count and ADP-dependent platelet function and its association with postoperative blood loss in adult patients undergoing cardiac surgery.

Methods: Prospective observational study in 40 patients (34 male; mean age 64 years) on platelet $\mathrm{P}_{2} \mathrm{Y}_{12}$-inhibitors undergoing cardiac surgery (38 CABG, 2 other). Preoperative whole blood samples were tested for platelet count, PCS $(\mathrm{hPa})$ and ADP-dependent platelet function (TEG Platelet Mapping (TEG-PM): MA-ADP(mm) and \% ADP-inhibition; Verify Now PRU Test). Blood loss was measured by drain output with severe blood loss defined as $>1000 \mathrm{~mL}$ at $24 \mathrm{~h}$. Correlation between parameters was assessed by the Pearson correlation coefficient. Association with severe blood loss was assessed by receiver operating curve $(\mathrm{ROC})$ analysis.

Results: Mean (SD) 24-hour blood loss was 969 (406) mL, with 18 patients (45\%) showing severe blood loss. PCS demonstrated a weak correlation with platelet count $(r=0.42)$ and MA-ADP $(r=0.46)$ and a negligible correlation with \% ADP-inhibition ( $r=-0.21)$ and Verify Now PRU ( $r=0.29)$. Pre-surgical PCS and TEG-PM (MA-ADP) were significantly associated with severe blood loss at $1000 \mathrm{~mL}$ (Table 1).

TABLE 1 ROC analysis: preoperative platelet function parameters and severe blood loss (>1000 $\mathrm{mL}$ at $24 \mathrm{~h}$ ).

\begin{tabular}{lll} 
Parameter & AUC $(95 \% \mathrm{Cl})$ & $P$-value \\
PCS $(\mathrm{hPa})$ & $0.78(0.62-0.89)$ & 0.0002 \\
TEG-PM; MA-ADP $(\mathrm{mm})$ & $0.72(0.55-0.85)$ & 0.0117 \\
TEG-PM; \%ADP-Inhibition & $0.66(0.49-0.80)$ & 0.0729 \\
VerifNow PRU & $0.58(0.41-0.73)$ & 0.4166 \\
\hline
\end{tabular}

Conclusions: Pre-surgical PCS, rapidly assessed by the whole blood Quantra QPlus System, can predict severe post-operative blood loss. Larger studies are needed to validate whether pre-surgical PCS can guide the waiting time for surgery for patients on $\mathrm{P}_{2} \mathrm{Y}_{12}$-inhibitors.

LPB0108 | Usefulness of an Academic High-throughput Sequencing Panel to Detect Copy Number Variations in Patients with Syndromic Thrombocytopenia

S. Santos-Mínguez ${ }^{1}$; C. Miguel-García ${ }^{1}$; V. Ramírez-Maldonado ${ }^{1}$; I. Serramito-Gómez ${ }^{1}$; A. Marín-Quilez ${ }^{1}$; J. Matías-Martín ${ }^{1}$;

J.R. González-Porras ${ }^{1,2}$; H. González-García ${ }^{3}$; J. Huerta-Aragonés ${ }^{4}$; C. Blázquez-Goñi ${ }^{5}$; A. Rodríguez-Alén ${ }^{6}$; C. García-Diaz ;

E. Urbaneja-Rodríguez ${ }^{3}$; A. Hortal Benito-Sendín ${ }^{8}$; N. Revilla9; V. Palma-Barqueros ${ }^{9}$; J. Rivera ${ }^{9}$; J.M. Hernández-Rivas ${ }^{1,2}$; J.M. Bastida ${ }^{1,2}$; R. Benito ${ }^{1}$

${ }^{1}$ IBSAL, IBMCC, Universidad de Salamanca, CSIC, Centro de Investigación del Cáncer (CIC), Salamanca, Spain; ${ }^{2}$ Departamento de Hematología - Complejo Asistencial Universitario de Salamanca (CAUSA), Instituto de Investigación Biomédica de Salamanca (IBSAL),
Universidad de Salamanca (USAL), Salamanca, Spain; ${ }^{3}$ Servicio de Pediatría - Hospital Clínico Universitario de Valladolid, Valladolid, Spain; ${ }^{4}$ Servicio de Oncología y Hematología Pediátricas - Hospital Materno-Infantil Gregorio Marañón, Madrid, Spain; ${ }^{5}$ Servicio de Hematología - Hospital de Jerez, Cádiz, Spain; ${ }^{6}$ Servicio de Hematología y Hemoterapia - Hospital Virgen de la Salud, Toledo, Spain; ${ }^{7}$ Servicio de Hematología - Complejo Asistencial de Burgos, Burgos, Spain; ${ }^{8}$ Servicio de Pediatría - Complejo Asistencial de Zamora, Zamora, Spain; ${ }^{9}$ Servicio de Hematología y Oncología Médica - Hospital Universitario Morales Meseguer, Centro Regional de Hemodonación, Universidad de Murcia, IMIB-Arrixaca, CB15/00055-CIBERER, Murcia, Spain

Background: High-throughput sequencing (HTS) has allowed to increase the molecular diagnosis in patients with inherited thrombocytopenia (IT), mainly caused by single-nucleotide variants (SNVs). Syndromic IT could be caused by structural variants like Copy Number Variations (CNV), which are not usually detected by gene panels.

Aims: To design and apply an academic HTS panel for identifying CNVs in patients with syndromic IT.

Methods: A custom HTS panel was designed using the SureDesign Studio (Agilent) including 85 genes with relevance in ITs. Libraries were prepared following the SureSelectQXT-Target-Enrichment protocol (Agilent), and finally sequenced on an Illumina NextSeq platform. Data were processed using an in-house pipeline. CNV analysis was made using "Depth-Of-Coverage" method, assuming a linear correlation between the coverage depth and the number of copies. Lower reading depth than expected represented losses or deletions, while higher depth represented gains or amplifications. Molecular findings were validated with aCGH or MLPA.

Results: Clinical and biological characteristics are showed in Table 1. Patient 1 and 2 (P1, P2) showed a specific syndromic phenotype, while clinical suspicion of P3 and P4 was not clarified. In $\mathrm{P} 1$, thrombocytopenia-absent radius syndrome was previously diagnosed and HTS allowed us to identify a low frequency SNP which affects the 5'UTR region of RBM8A (c.-21 G>A; rs139428292) and a deletion on chromosome 1q. In P2, Di George syndrome (DGS) was clinically diagnosed and deletion of GP1BB and TBX1 confirmed a del22q. In P3, a syndromic thrombocytopenia was suspected and a deletion of GP1BB and TBX1 was detected, which led to confirm the diagnosis of incomplete DGS. Finally, In P4 with syndromic thrombocytopenia, a deletion on chromosome 21q which involved RUNX1 was identified (Figure 2). 
TABLE 1 Clinical and biological phenotype of patients with syndromic IT in whom CNVs have been analyzed

\begin{tabular}{|c|c|c|c|c|c|}
\hline Case & Age, gender/BS & Clinical symptoms & $\begin{array}{l}\text { Laboratory } \\
\text { phenotype }\end{array}$ & Suspected/final IT & CNVs \\
\hline 1 & $\begin{array}{l}45 \text { y, female/2: } \\
\text { heavy } \\
\text { menstrual } \\
\text { bleeding }\end{array}$ & $\begin{array}{l}\text { Bicuspid aortic valve and cow's } \\
\text { milk intolerance, bilateral } \\
\text { radioulnar agenesis, hip } \\
\text { dysplasia, other skeletal and } \\
\text { facial malformations }\end{array}$ & $\begin{array}{l}\mathrm{P}=84 \times 103 / \mathrm{mm} 3 \\
\mathrm{MPV}=13.8 \mathrm{fL} \\
\text { BF: large platelets }\end{array}$ & $\begin{array}{l}\text { Thrombocytopenia } \\
\text { with absent radius } \\
\text { syndrome }\end{array}$ & $\begin{array}{l}\text { RBM8A deletion, } \\
\text { del1q, and SNP } \\
\text { which affects } 5 \text { 'UTR } \\
\text { region }\end{array}$ \\
\hline 2 & $13 \mathrm{y}, \mathrm{male} / 0$ & $\begin{array}{l}\text { Hypertelorism, prognathism, } \\
\text { dental mal-occlusion, short } \\
\text { stature, psychomotor } \\
\text { retardation, bicuspid aortic } \\
\text { valve, umbilical hernia }\end{array}$ & $\begin{array}{l}\mathrm{P}=135 \times 103 / \mathrm{mm} 3 \\
\text { VPM }=13.7 \mathrm{fL} \\
\text { BF: large platelets }\end{array}$ & DiGeorge syndrome & $\begin{array}{l}\text { GP1BB and TBX1 } \\
\text { deletion, del22q }\end{array}$ \\
\hline 3 & $14 \mathrm{y}$, male/0 & $\begin{array}{l}\text { Hypertelorism, short stature, } \\
\text { learning difficulties, hearing } \\
\text { loss, infections, situs solitus }\end{array}$ & $\begin{array}{l}\mathrm{P}=79 \times 103 / \mathrm{mm} 3 \\
\mathrm{MPV}=12.8 \mathrm{fL} \\
\text { BF: large platelets }\end{array}$ & $\begin{array}{l}\text { Syndromic } \\
\text { thrombocytopenia/ } \\
\text { Incomplete DGS }\end{array}$ & $\begin{array}{l}\text { GP1BB and TBX1 } \\
\text { deletion, del22q }\end{array}$ \\
\hline 4 & $\begin{array}{l}1 \text { y, male/2: } \\
\text { epistaxis and } \\
\text { ecchymosis }\end{array}$ & $\begin{array}{l}\text { Bilateral sensorineural } \\
\text { deafness, corpus callosum } \\
\text { hypoplasia, cardiac and facial } \\
\text { malformations }\end{array}$ & $\begin{array}{l}\mathrm{P}=35 \times 103 / \mathrm{mm} 3 \\
\mathrm{MPV}=8 \mathrm{fL} \\
\text { BF: dysmorphic cells }\end{array}$ & $\begin{array}{l}\text { Syndromic } \\
\text { thrombocytopenia }\end{array}$ & $\begin{array}{l}\text { RUNX1 deletion } \\
\text { del21q }\end{array}$ \\
\hline
\end{tabular}

Abbreviators: P: platelet counts; MPV: mean platelet volume; BF: blood film; DGS: DiGeorge syndrome; BS: bleeding score according to ISTH-BAT.

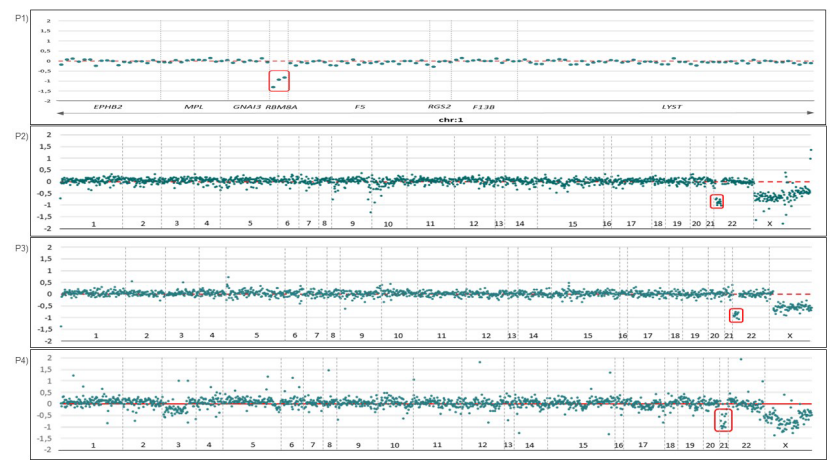

FIGURE 1 Representation of CNVs identified in 4 patients with syndromic ITs.

Conclusions: This academic HTS panel has allowed us to detect SNVs and CNVs. CNV analysis is particularly useful in genetic diagnosis of syndromic IT in which no conventional variants have been detected.

\section{LPB0109 | Full Automation of the Bethesda Assay}

T. Siegemund; A. Siegemund; H. Bönigk

MVZ Limbach Magdeburg, Magdeburg, Germany

Background: In 1974, a conference was held in Bethesda with the purpose to standardize inhibitor testing. Since then, researches have revealed the nature of clotting factor VIII, von Willebrand factor and how antibodies act on their targets. We have achieved automation of about every possible analyte in our labs. The Bethesda inhibitor assay remained unfazed. Therapeutic regimens stand or fall depending on the result of an assay that still shows unsatisfyingly high variability.
Aims: Full automation of the Bethesda assay, as presented here, is a prerequisite for standardization of an assay.

Methods: The Bethesda and the Nijmegen-Bethesda assay were adapted to the Atellica COAG360 analyzer (Siemens) for coagulation factors V, VII, VIII and IX. The determination of an inhibitor titer lasts between 15 and $130 \mathrm{~min}$. 38 samples obtained from patients with hemophilia were investigated in addition to commercial plasma samples. Results were compared to the manual method.

Results: Inhibitor potencies obtained with the fully automated method showed similar results compared to the manual method. Lowest interday variability was found for a FV inhibitor at $4 \%$ coefficient of variation, followed by a FVII inhibitor at 11\%. 10\% CV was found for a FIX inhibitor of $5 \mathrm{BU} / \mathrm{mL}$, while $24 \% \mathrm{CV}$ was observed at $1 \mathrm{BU} / \mathrm{mL}$. Eleven positive samples were investigated for FVIII yielding in CVs between 11 and 17\%. All negative samples were found negative, with one exception (likely a technical error). We successfully used the method for FIX inhibitors in external quality assessments for two years.

Conclusions: Automation of the Bethesda assay is possible and reliable. The presented approach significantly reduces time and effort in inhibitor testing. Automation contributes to standardization of inhibitor testing and may lead to lower inter-laboratory variability. An adaption to other diagnostic systems is possible for all but, unfortunately, FVIII inhibitors. 
PB0329 | Newly Developed Dilute Russell's Viper Venom Reagents for Lupus Anticoagulant Detection

$\underline{\text { L. Talon }^{1}}$; V. Fourneyron ${ }^{1}$; M. Tardieu ${ }^{1} ;$ A. Trapani ${ }^{1} ;$ T. Sinegre ${ }^{1}$;

A. Lebreton ${ }^{1,2}$

${ }^{1}$ Hématologie Biologique, CHU, Clermont-Ferrand, France; ${ }^{2}$ Centre de Ressources et Compétences des Maladies Hémorragiques Constitutionnelles, CHU, Clermont-Ferrand, France

Background: Dilute Russell's viper venom time (dRVVT) is one of the recommended assays for lupus anticoagulant (LA) detection. However, reagents variability affects diagnostic efficacy in LA testing and it is not clear whether all dRVVT reagents may be considered equivalent.

Aims: The objective of this study was to evaluate the analytical performances of a new formulation DVVtest/DVVconfirm (Horiba Medical) performed with the Yumizen G800 analyzer and to compare it with a currently available dRVVT assay performed on a STAR Max analyzer.

Methods: Within-run and between-run imprecision of DVVtest and DVVconfirm were evaluated using the low- and high-quality control plasmas (Latrol Normal and Abnormal, Horiba Medical). DVVtest/ DVVconfirm were evaluated for 62 healthy controls, 20 patients who met clinical criteria of antiphospholipid syndrome (APS) and were LA-positive when tested with Stago dRVV Screen/Confirm, and 39 patients with an autoimmune disease but without clinical criteria for APS and LA-negative with Stago dRVV Screen/Confirm. LA was considered positive if the screen and LA ratio were positive (cut-off provided by the manufacturers).

Results: Within- and between-run coefficients of variation were consistent with manufacturer's data (Table 1). Two controls, who were LA-negative with Stago dRVV reagents, had a positive LA testing with DVVtest/DVVconfirm. Two patients with clinical criteria for APS and LA-positive with Stago dRVV Screen/Confirm had a negative LA testing performed with DVVtest/DVVconfirm. For DVVtest/DVVconfirm assay performed on the Yumizen G800 analyzer, sensitivity, specificity and negative predictive value were of $90 \%, 97 \%$ and $97.8 \%$, respectively. Among the 39 patients who were LA-negative with Stago dRVV reagents, 6 were found positive with DVVtest/DVVconfirm including 2 patients with systemic lupus erythematosus and 2 patients with positive anticardiolipin and anti-beta 2 glycoprotein I antibodies, raising the question of the sensitivity/specificity of these assays.
Conclusions: The analytical performances of DVVtest and DVVconfirm reagents make it suitable for LA detection in clinical laboratories.

\section{PB0330 | Risk of Misdiagnosis for Patients Presenting the Closed Variants E1359K or V1360A in Exon 28 of VWF due to a Major Discordance on VWF Activity Results between the VWF:GplbM and VWF:GplbR Assays}

F. Lassalle $^{1}$; E. Jeanpierre ${ }^{1}$; C. Zawadzki ${ }^{1}$; C. Pouymayou ${ }^{2}$;

S. Castet ${ }^{3}$; Y. Huguenin ${ }^{3}$; R. Marlu ${ }^{4}$; A. Voyer ${ }^{5}$; P. Chamouni ${ }^{6}$;

J. Goudemand ${ }^{1} ;$ S. Susen ${ }^{1}$

${ }^{1}$ Institut d'Hématologie-Transfusion - CHU de Lille, Lille, France;

${ }^{2}$ Service d'Hématologie Biologique - Hôpital La Timone, Marseille, France; ${ }^{3} \mathrm{CHU}$ Bordeaux Pellegrin, Bordeaux, France; ${ }^{4}$ Laboratoire d'Hémostase - CHU de Grenoble, Grenoble, France; ${ }^{5}$ Laboratoire d'Hémostase - CHU Amiens, Amiens, France; ${ }^{6}$ Laboratoire d'Hématologie - CHU de Rouen, Rouen, France

Background: The diagnosis of von Willebrand disease (VWD) relies on measurements of von Willebrand factor antigen (VWF:Ag) and functional activity (VWF:Act). Measuring VWF:Act is based on the quantification of VWF binding to platelets through Gplba, with or without using ristocetin. Some polymorphisms are known to cause discordances between the different techniques, as the variant $\mathrm{D} 1472 \mathrm{H}$ that falsely decreases the ristocetin cofactor activity (VWF:Rco) by interacting with ristocetin.

Aims: To identify if other variants located in the A1 domain of VWF (containing the binding sites for Gplba) can interfere with the different techniques for measuring VWF:Act.

Methods: After being notified that 2 VWD type $2 \mathrm{M}$ patients (one carrying E1359K and the other one V1360A) completely normalized their ratios measured with the Acustar ${ }^{\circledR}$ VWF:GplbR assay (using ristocetin) comparing to the VWF:Rco agregometry technique, we decided to retest all the patients from the database of the French VWD Reference Center presenting the same variants. We tested in total 15 patients with the Acustar ${ }^{\circledR}$ assay and the Innovance ${ }^{\circledR}$ VWF:GplbM assay (no ristocetin).

TABLE 1 Imprecision of the DVVtest and DVVconfirm assays. Abn: abnormal; N: normal

\begin{tabular}{|c|c|c|c|c|c|c|c|}
\hline & & & $n$ & $\begin{array}{l}\text { Mean value } \\
\text { (seconds) }\end{array}$ & SD (seconds) & CV (\%) & $\begin{array}{l}\mathrm{CV}(\%) \\
\text { manufacturer }\end{array}$ \\
\hline \multirow[t]{3}{*}{ DVVtest } & Within-run & LAtrol Abn & 13 & 107.9 & 1.6 & 1.5 & 3.3 \\
\hline & \multirow[t]{2}{*}{ Between-run } & LAtrol Abn & 25 & 97.8 & 3.9 & 4.0 & 5.6 \\
\hline & & LAtrol N & 25 & 40.7 & 1.5 & 3.7 & 6.8 \\
\hline \multirow{3}{*}{ DVVconfirm } & Within-run & LAtrol N & 13 & 39.9 & 0.8 & 1.9 & 1.8 \\
\hline & \multirow[t]{2}{*}{ Between-run } & LAtrol Abn & 25 & 48.8 & 2.2 & 4.5 & 4.7 \\
\hline & & LAtrol N & 25 & 37.4 & 0.4 & 1.0 & 2.3 \\
\hline
\end{tabular}


Results: All the 15 patients have a low ratio with the Innovance ${ }^{\circledR}$ assay (mean $=0.20$, interval range $=<0.1-0.37$ ). Among them, 11 have a completely normal ratio with the Acustar ${ }^{\circledR}$ assay (mean $=0.86$ interval range $=0.70-1.21)$. Two patients have a very low ratio $(<0.1)$ with the 2 techniques including one who also has a VWD type 3 variant. The variants E1359K and V1360A, localized in the A1 domain, might interfere with the Acustar ${ }^{\circledR}$ assay, either with the ristocetin or with the chemiluminescence detection, rendering it insensitive to the binding defect.

Conclusions: This study is another proof about the complexity to measure VWF:Act and to choose the right technique. Due to their VWF genetics, the diagnosis of VWD for these patients could have been missed if only the Acustar ${ }^{\circledR}$ assay has been performed.

PB0331 | Evaluation of Activated Clotting Time (ACT) Results on a New POC Coagulation Analyzer

A. Leung; W. Lu; M. Martin; C. Smith; J. Diggs; S. Chen; P. Yu;

R. Bottenus

Instrumentation Laboratory, San Diego, United States

Background: The GEM Hemochron 100 is a point of care (POC) coagulation analyzer that performs individual ACT tests on fresh whole blood samples. It is the next generation Hemochron device following the on-market Hemochron Signature Elite. There are two ACT cartridges available for the system, ACT+ and ACT-LR, which demonstrate linear correlation to the anticoagulation effects of heparin from 1.0 up to $6.0 \mathrm{U} / \mathrm{mL}$ and 0 up to $2.5 \mathrm{U} / \mathrm{mL}$, respectively.

Aims: To demonstrate that ACT results reported by the GEM Hemochron 100, over the ACT+ and ACT-LR reportable ranges, are equivalent to the results given by the Hemochron Signature Elite. To evaluate the precision of the system for each assay.

Methods: Method comparison testing was performed to evaluate the GEM Hemochron 100 system, in a multi-site clinical study utilizing the Hemochron Signature Elite as the predicate device. Patient blood samples were tested in duplicate on paired instruments. Data analysis included Passing-Bablok regression, calculation of the Pearson product-moment correlation coefficient ( $r$ ), and determination of the percentage bias at medical decision levels. A liquid quality control (LQC) precision study was also performed, and fully nested variance components models were employed for analysis.

Results: Table 1 summarizes the method comparison study results and Table 2 summarizes the LQC precision study results.
TABLE 1 Clinical Method Comparison Summary, GEM Hemochron 100 vs. Hemochron Signature Elite

\begin{tabular}{|c|c|c|c|}
\hline Assay Type & $N$ & Metric & Result (95\% Cl) \\
\hline \multirow{2}{*}{$\begin{array}{l}\text { ACT + (Pooled, } \\
\quad \text { First } \\
\text { Replicate) }\end{array}$} & \multirow[t]{2}{*}{1279} & $\begin{array}{l}\text { Passing-Bablok } \\
\text { Slope }\end{array}$ & $1.04(1.03-1.06)$ \\
\hline & & $\begin{array}{l}\text { Correlation } \\
\quad \text { Coefficient, } r\end{array}$ & 0.95 \\
\hline \multirow{2}{*}{$\begin{array}{l}\text { ACT-LR (Pooled, } \\
\text { First } \\
\text { Replicate) }\end{array}$} & \multirow[t]{2}{*}{463} & $\begin{array}{l}\text { Passing-Bablok } \\
\text { Slope }\end{array}$ & $0.96(0.93-1.00)$ \\
\hline & & $\begin{array}{l}\text { Correlation } \\
\quad \text { Coefficient, } r\end{array}$ & 0.95 \\
\hline
\end{tabular}

TABLE 2 Liquid Quality Control (LQC) Precision Summary from 20 Day Study

\begin{tabular}{|c|c|c|c|c|}
\hline \multirow[b]{2}{*}{ LQC Level } & \multirow[b]{2}{*}{$N$} & \multirow[b]{2}{*}{ Mean } & \multicolumn{2}{|c|}{$\begin{array}{l}\text { Within Run } \\
\text { (Repeatability) }\end{array}$} \\
\hline & & & SD & $\% \mathrm{CV}$ \\
\hline $\begin{array}{l}\text { ACT-LR Level } \\
1\end{array}$ & 640 & 176.3 & 14.2 & $8.1 \%$ \\
\hline $\begin{array}{l}\text { ACT-LR Level } \\
\quad 2\end{array}$ & 640 & 294.5 & 16.8 & $5.7 \%$ \\
\hline ACT+Level 1 & 640 & 158.9 & 12.5 & $7.9 \%$ \\
\hline ACT+Level 2 & 640 & 447.7 & 10.4 & $2.3 \%$ \\
\hline
\end{tabular}

Conclusions: The GEM Hemochron $100 \mathrm{ACT}+$ and ACT-LR results are equivalent to the results given by the Hemochron Signature Elite. The assay systems yielded precision estimates in \%CV below $10 \%$ and can be used to assess response to unfractionated heparin in many clinical applications.

The clinical study did not require written Informed Consent in alignment with FDA guidance provided in 71 FR 23924. It was performed under the oversight of, and after approval by, the appropriate Institutional Review Boards.

\section{PB0332 | Endothelial Dysfunction in Patients with Ph-negative Myeloproliferative Neoplasms}

N. Korsakova; O. Golovina; N. Silina; E. Efremova; L. Papayan Russian Research Institute of Hematology and Transfusiology, SaintPetersburg, Russian Federation

Background: Thrombotic complications represent a leading cause of morbidity and mortality in patients with Ph-negative myeloproliferative neoplasms (MPN): polycythemia vera (PV), essential thrombocythemia (ET), and primary myelofibrosis (PMF). Endothelial dysfunction (ED) is supposed to play significant role in pathogenetic mechanisms of thrombosis in MPN. Considering the variety of ED forms and methods of its evaluation it is of great importance to reveal most informative ones for early procoagulant changes detection and prevention.

Aims: To evaluate ED in patients with MPN by different methods. 
Methods: The study included 49 patients with MPN (22 patients with ET, 17 with PV and 10 with PMF).Endothelial activation and damage plasma soluble marker von Willebrand factor (VWF) activity and level (VWF:Ag) were measured by standard techniques. The control group consisted of 68 healthy persons. Endothelial function estimation was also performed with EndoPAT 2000 apparatus by non-invasive peripheral arterial tonometry (PAT) method based on endothelial-dependent vasodilatation registration by digital pletysmography probes. In this setting endothelial functional activity is characterized by reactive hyperemia index (RHI) with ED supposed when $\mathrm{RHI} \leq 1.67,1.67<\mathrm{RHI} \leq 2.0$ lying in risk zone and $\mathrm{RHI}>2.0$ associated with good endothelial functioning. STATISTICA 6.0 was used, median (Me) and 95\% confidence interval (Cl) were calculated. Mann-Whitney test was applied, differences between groups considered statistically significant with $P<0.05$.

Results: The results of endothelial function evaluation are shown in Tables 1 and 2 . they interfere with many coagulation assays, depending on the type of DOAC and the assay principle. DOAC Stop ${ }^{\mathrm{TM}}$ is an activated charcoal mini-tablet that in vitro absorbs DOACs from plasma. Antithrombin (AT) assays are performed to detect those with antithrombin deficiency.

Aims: To investigate the effectiveness of DOAC Stop ${ }^{\mathrm{TM}}$ in removing DOACs from plasma and to confirm that DOAC Stop ${ }^{\mathrm{TM}}$ does not alter AT activity assessment.

Methods: The Hyphen AT assay principle is based on factor Xa inhibition by AT in the presence of heparin. This assay was used to assess AT activity before and after using DOAC Stop ${ }^{\mathrm{TM}}$ in plasma samples from patients anticoagulated with rivaroxaban $(n=27)$, apixaban ( $n=15)$, edoxaban $(n=2)$ and 47 individuals not receiving DOACs. DOAC anti-Xa levels were also determined before and after DOAC Stop ${ }^{\mathrm{TM}}$. Berichrom thrombin-based assay was used as an alternative method to compare AT activities of DOACs samples.

TABLE 1 VWF activity and level in patients with MPN and controls, $\mathrm{Me}(95 \% \mathrm{Cl})$

\begin{tabular}{llllll} 
& Controls $(n=68)$ & MPN $(n=49)$ & ET $(n=22)$ & PV $(n=17)$ & PMF $(n=10)$ \\
VWF activity, \% & $97.0(63.0-144.3)$ & $105.0(58.0-206.0)$ & $107.5(64.3-189.0)$ & $88.0(53.2-222.0)$ & $96.5(70.7-177.6)$ \\
VWF:Ag, \% & $107.0(74.6-161.7)$ & $125.4^{*}(67.4-239.7)$ & $124.8^{*}(84.8-234.9)$ & $118.7(61.1-203.1)$ & $126.8(88.9-257.4)$ \\
\hline
\end{tabular}

TABLE 2 Endothelial function evaluation using PAT method in patients with MPN

\begin{tabular}{lllll} 
& MPN $(n=49)$ & ET $(n=22)$ & PV $(n=17)$ & PMF $(n=10)$ \\
$\mathrm{RHI} \leq 1.67, n(\%)$ & $18(36.7 \%)$ & $7(31.8 \%)$ & $8(47.1 \%)$ & $3(30 \%)$ \\
$1.67<\mathrm{RHI} \leq 2.0, n(\%)$ & $13(26.5 \%)$ & $7(31.8 \%)$ & $1(5.9 \%)$ & $5(50 \%)$ \\
$\mathrm{RHI}>2.0, n(\%)$ & $18(36.7 \%)$ & $8(36.4 \%)$ & $8(47.1 \%)$ & $2(20 \%)$ \\
\hline
\end{tabular}

Conclusions: VWF activity and level in all groups of MPN patients exceeded controls reaching statistical significance for VWF:Ag in whole MPN group and in ET patients indicating high extent of endothelial activation and damage and coagulation activation in these patients. Nevertheless PAT method showed vasomotor ED approximately in one third of MPN patients, having higher incidence in patients with PV. These results suggest different methods of endothelial function evaluation are valuable to fully characterize endothelial abnormalities in MPN.

PB0333 | The Effect of DOAC Stop ${ }^{\mathrm{TM}}$ on Measuring

\section{Antithrombin Activity}

K. Mayger $^{1,2,3}$; N. De Jager ${ }^{1}$; E. Foxton ${ }^{1}$; A. Doyle ${ }^{2}$; B.J. Hunt ${ }^{2}$

${ }^{1}$ Viapath, London, United Kingdom; ${ }^{2}$ Guy's and St Thomas' NHS

Foundation Trust, London, United Kingdom; ${ }^{3}$ University of Portsmouth, Portsmouth, United Kingdom

Background: Direct Oral Anticoagulants (DOACs) are used widely for the treatment and prophylaxis of thromboembolism. However,
Statistical analysis was performed using the Wilcoxon signed rank test using SPSS version 26 . The statistical significance was considered when $P<0.05$.

Results: Table 1 shows DOACs concentrations post DOAC Stop ${ }^{\mathrm{TM}}$ were below the limit of detection $(<20 \mathrm{ng} / \mathrm{mL})$ for all except one rivaroxaban sample. AT activity levels differed significantly between patients on DOACs before and after DOAC Stop ${ }^{\mathrm{TM}}$ treatment $(P$ $<0.05$ ). Hyphen post DOAC Stop ${ }^{\mathrm{TM}}$ AT activity correlated positively with Berichrom $(r=0.98)$. Levels of AT activity were not statistically different in those not on DOACs pre and post DOAC Stop ${ }^{\mathrm{TM}}$ (Figure 1). 
TABLE 1 Effect of DOAC StopTM on Hyphen AT activity and anti-Xa assays in plasma samples from patients on direct Xa inhibitors and not anticoagulated with DOACs controls

\begin{tabular}{|c|c|c|c|c|c|}
\hline \multirow[b]{2}{*}{ Assay } & \multirow[b]{2}{*}{ Anticoagulant } & \multirow[b]{2}{*}{$n$} & \multirow{2}{*}{$\frac{\text { Pre DOAC Stop }}{\text { Median (range) }}$} & \multirow{2}{*}{$\frac{\text { Post DOAC Stop }}{\text { Median (range) }}$} & \multirow[b]{2}{*}{$P$ value } \\
\hline & & & & & \\
\hline Anti $X a$ activity [ng/mL] & DOACs & 44 & $148(0.9-518)$ & $<20\left(0-22^{*}\right)$ & N/A \\
\hline \multirow{4}{*}{$\begin{array}{l}\text { AT activity (Hyphen) Xa- } \\
\text { based [IU/dL] }\end{array}$} & DOACs & 44 & $116(54-151)$ & $100(48-119)$ & $<0.05$ \\
\hline & None & 34 & $99(44-138)$ & $100(42-141)$ & 0.980 \\
\hline & Other** & 13 & $90(52-120)$ & $90(51-121)$ & 0.753 \\
\hline & All non-DOACs & 47 & $98(44-138)$ & $98(42-141)$ & 0.966 \\
\hline
\end{tabular}

"One rivaroxaban sample gave value above the limit of detection: $20 \mathrm{ng} / \mathrm{mL}$ ** $\operatorname{warfarin} n=1$; UFH $n=5 ;$ LMWH $n=7$

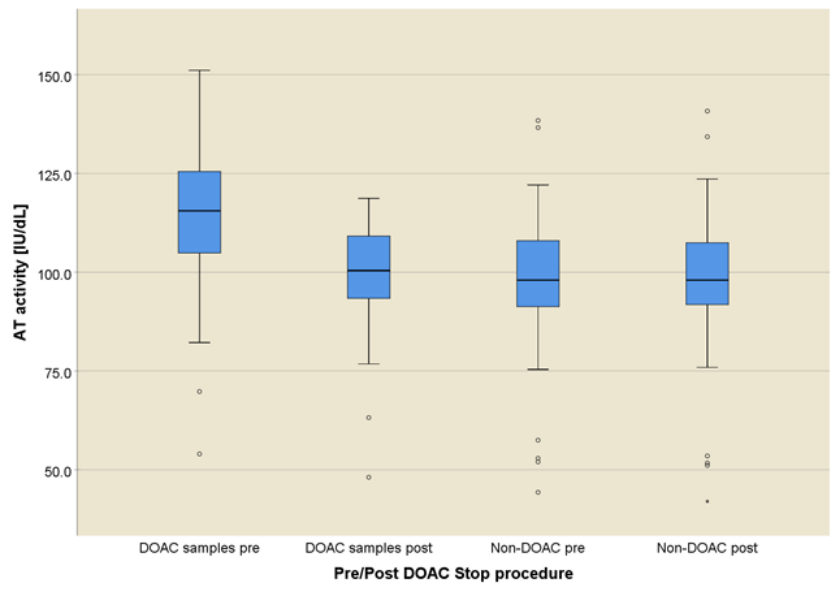

FIGURE 1 Evaluation of DOAC Stop efficacy. The box plots represent the medians, 25-75 percentile and minimum/maximum values for Hyphen factor Xa-based AT activity assay

Conclusions: DOAC Stop ${ }^{\mathrm{TM}}$ effectively remove DOACs from most but not all samples, therefore results should be interpreted with caution. DOAC Stop ${ }^{\mathrm{TM}}$ does not appear to affect AT activity measurement in plasma.

PB0334 | Use of Rotational Thromboelastometry (ROTEM) to Monitor Patients with Severe Haemophilia A on Emicizumab

A. Riddell; P. Lane; J. Kemp; T. Gandhi; P. Chowdary

Royal Free Hospital, London, United Kingdom

Background: Emicizumab (Hemlibra ${ }^{\circledR}$ ) prophylaxis does not usually require laboratory monitoring as an overall coagulation response cannot easily be shown. On occasion, it may be clinically useful to distinguish the effect of therapeutic emicizumab levels on global coagulation.

Aims: To evaluate use of ROTEM in patients during switch from factor VIII prophylaxis to emicizumab.

Methods: Fifteen patients (16-62 years-old) with severe HA were evaluated. Citrated whole blood (CWB) samples \pm corn trypsin inhibitor (CTI) were taken (day 0, week(s), 2, 4-6 and 12-24). Citrated platelet poor plasma was evaluated for APTT (s), and emicizumab levels $(\mu \mathrm{g} / \mathrm{mL})$ by modified one stage FVIII assay. ROTEM parameters CT, CFT and alpha angle (AA) were evaluated in CWB activated by calcium chloride (NATEM) and CWB+CTI activated with $0.12 \mathrm{pm}$ tissue factor (TF) (CWB-TF).

Results: Baseline FVIII levels ranged $<1 \mathrm{IU} / \mathrm{dL}$ to $54 \mathrm{IU} / \mathrm{dL}$ (trough levels), APTTs 33-159s, NATEM parameters CT (reference range(RR)371-797s), CFT (RR:147-443s) and $A A(R R: 37-69)$, results ranged from 647-5001s, 0-936s, and 0-58 respectively and CWB-TF test CT, CFT and AA results ranged from 517-1400s, 275-1041s and 31-47 respectively. Week2, 4/15 patients had trough FVIII levels present and by weeks4-6 one patient had a trough FVIII present, APTT results ranged from 23.7-30.9s, weeks4-6 results were 20.1-25.8s, and weeks12-24 - 19.7-25.6s. Week2 emicizumab levels ranged from $11-36 \mu \mathrm{g} / \mathrm{mL}$ rising to $48-96 \mu \mathrm{g} / \mathrm{mL}$ at weeks $4-6$, with no further increase at week12-24 sampling. Week 2:NATEM CT (range 532-1227s) and CFT (range 154-374s) decreased and angle increased (38-69) and by weeks4-6, CT and CFT decreased (range 441-951 and 146-327 respectively) and AA increased (range 42-65), whilst CTI CWB-TF ROTEM parameters CT, CFT and angle results for week2, 4-6 and 12-26 sampling were variable and overlapping with no overall reduction in CT and CFT or increase in AA parameters.

Conclusions: ROTEM NATEM assay showed normal global coagulation responses for CFT and AA parameters after weeks4-6 of emicizumab prophylaxis.

PB0335 | Ring Trial for Quality Assurance of the Manual Tilt Tube Technique for Prothrombin Time and INR Determinations in WHO Calibration Laboratories

A. van den Besselaar; C. van Rijn; C. Cobbaert

Leiden University Medical Center, Coagulation Reference Laboratory,

Department of Clinical Chemistry and Laboratory Medicine, Leiden,

Netherlands

Background: Many patients are still treated with vitamin $\mathrm{K}$ antagonists (VKA). Laboratory monitoring of VKA therapy depends on determination of the Prothrombin Time (PT) and calculation of the International Normalized Ratio (INR). A Reference Measurement System (RMS) is required to achieve traceability and longitudinal 
accuracy of PT/INR results. The RMS depends on the establishment of a Reference Measurement Procedure (RMP). A harmonized WHO Manual Tilt Tube Technique (MTT) has been proposed as RMP for the PT/INR. The harmonized MTT was developed in two workshops in which operators of three WHO calibration laboratories participated. Aims: Setting up Ring Trials to assess the performance of the harmonized MTT by laboratories involved in calibration of PT/INR systems. Methods: Two lyophilized control plasma samples (level 1 and level 2) were sent together with the WHO International Standards for thromboplastin (RBT/16 and rTF/16) to three calibration laboratories. The laboratories were instructed to analyze the control plasmas by 2 operators on 5 days in duplicate. Analysis of variance (ANOVA) was performed on the PT results.

Results: Mean PT results and within-operator coefficients of variation (CV) are shown in Table 1. The differences between the operators were statistically significant $(P<0.001)$. The PT results obtained by operators in laboratory \#3 were longer than those in the other laboratories, suggesting a systematic error. Transformation of PT results to INR is shown in Table 2. The INR results of laboratory \#3 were lower than those in the other laboratories.
PB0336 | Laboratory Assessment of DOAC Assays for FXa Inhibitors in the Presence of Reversal Agent Andexanet Alfa on the ACL TOP/TOP 50 Coagulation Analyzers

A. Khan ${ }^{1}$; K. Shishko ${ }^{1}$; G. Lü ${ }^{2}$; V. Nguyen ${ }^{1}$; A. Winkler ${ }^{1}$

${ }^{1}$ Instrumentation Laboratory, Bedford, United States; ${ }^{2}$ Portola, a subsidiary of Alexion, South San Francisco, United States

Background: Commercial DOAC assays for measuring FXa inhibitors (FXal) apixaban and rivaroxaban on the ACL TOP/TOP50 analyzers are available in many countries. These assays utilize the anti-FXa reagent with drug specific calibrators and controls to determine the drug concentration in patient plasma. In the presence of andexanet alfa (AnXa), a specific reversal agent for FXal, these assays show erroneous elevation of the measured anti-FXa activity. This is attributed to the large sample dilution in the assay setup (1:27), which causes dissociation of the FXal from AnXa due to a reversible binding equilibrium.

Aims: The aim of this study was to verify the analytical performance of a modified user-defined setting for the apixaban and rivaroxaban

TABLE 1 PT results obtained in Ring Trial

\begin{tabular}{|c|c|c|c|c|c|c|c|c|c|}
\hline & & $\begin{array}{l}\text { RBT/16, } \\
\text { level } 1\end{array}$ & $\begin{array}{l}\text { RBT/16, } \\
\text { level } 1\end{array}$ & $\begin{array}{l}\mathrm{RBT} / 16, \\
\text { level } 2\end{array}$ & $\begin{array}{l}\text { RBT/16, } \\
\text { level } 2\end{array}$ & rTF/16, level 1 & $\begin{array}{l}\text { rTF/16, } \\
\text { level } 1\end{array}$ & rTF/16, level 2 & $\begin{array}{l}\text { rTF/16, } \\
\text { level } 2\end{array}$ \\
\hline number & number & Mean PT (s) & CV (\%) & Mean PT (s) & CV (\%) & Mean PT (s) & CV (\%) & Mean PT (s) & CV (\%) \\
\hline 1 & 1 & 20.45 & 0.8 & 44.73 & 0.8 & 13.42 & 1.8 & 34.45 & 0.7 \\
\hline 2 & 1 & 20.64 & 1.4 & 45.71 & 1.0 & 13.53 & 2.0 & 34.24 & 3.0 \\
\hline 3 & 2 & 20.28 & 3.4 & 45.34 & 1.7 & 14.03 & 3.5 & 34.33 & 2.5 \\
\hline 4 & 2 & 20.46 & 2.4 & 45.31 & 2.5 & 14.06 & 1.1 & 33.84 & 2.6 \\
\hline 5 & 3 & 23.16 & 4.1 & 48.89 & 1.9 & 16.00 & 2.7 & 36.71 & 2.0 \\
\hline 6 & 3 & 24.15 & 4.2 & 48.76 & 2.2 & 16.82 & 3.5 & 39.06 & 3.8 \\
\hline
\end{tabular}

TABLE 2 Mean INR and between-operator coefficient of variation obtained in Ring Trial

\begin{tabular}{lllllllll} 
Control & & Mean INR, & Mean INR, & Mean INR & Mean INR, & $\begin{array}{l}\text { Mean INR, } \\
\text { Operator \#5 }\end{array}$ & $\begin{array}{l}\text { Mean INR, } \\
\text { Operator \#6 }\end{array}$ & $\begin{array}{l}\text { Between- } \\
\text { operator CV } \\
\text { (\%) }\end{array}$ \\
sample & Reagent & Operator \#1 & Operator \#2 & Operator \#3 & Operator \#4 & Oprat & 4.6 \\
Level 2 & RBT/16 & 2.57 & 2.62 & 2.64 & 2.61 & 2.47 & 2.34 & 2.55 \\
\hline
\end{tabular}

Conclusions: Despite harmonization of the MTT procedure, there were important differences in PT and INR results between calibration laboratories. Further efforts should be made to reduce these differences, e.g. by training of the operators in communal workshops. Regular Ring Trials should be continued for calibration laboratories using the harmonized MTT. tests on the ACL TOP/TOP50 analyzers with HemosIL Anti-Xa reagent and HemosIL Apixaban and Rivaroxaban calibrators.

Methods: The modified assay setup and calibration were optimized to minimize the sample dilution to 1:2.6 suitable for analyzing FXal in AnXa-containing samples. Ten plasma samples containing different concentrations of apixaban $(230,460,920 \mathrm{ng} / \mathrm{mL}, 0.5-2.0 \mu \mathrm{M})$ or rivaroxaban $(218,436,872 \mathrm{ng} / \mathrm{mL}, 0.5-2.0 \mu \mathrm{M})$ in the presence of AnXa at different molar ratios were tested (Table 1). The optimized calibration curves reported in the $10-100 \mathrm{ng} / \mathrm{mL}$ linear range. 
TABLE 1 Representative apixaban or rivaroxaban samples spiked with andexanet alfa

\begin{tabular}{|c|c|c|c|c|c|}
\hline \multirow[b]{2}{*}{ Sample ID } & \multirow[b]{2}{*}{ Total Apixaban (ng/mL) } & \multirow{2}{*}{$\begin{array}{l}\text { Total Rivaroxaban (ng/ } \\
\mathrm{mL} \text { ) }\end{array}$} & \multicolumn{3}{|c|}{ Molar Concentration } \\
\hline & & & FXal $(\mu \mathrm{M})$ & AnXa $(\mu \mathrm{M})$ & Molar Ratio \\
\hline 1 & 230 & 218 & 0.5 & 0 & 0 \\
\hline 2 & & & 0.5 & 0.25 & $1: 2$ \\
\hline 3 & 230 & 218 & 0.5 & 0.5 & $1: 1$ \\
\hline
\end{tabular}

Data was obtained from ten samples each in the apixaban and rivaroxaban panel with different concentrations of $\mathrm{FXal}$ at $0.5,1.0,2.0 \mu \mathrm{M}$ and andexanet (AnXa at 0- $4.0 \mu \mathrm{M}$ ). For clarity, only concentrations for Samples 1-4 are shown. Rivaroxaban MW = 435.9 Da; Apixaban MW = 459.5 Da; AnXa MW = 41 kDa; FXal- FXa inhibitor.

Results: The data generated with minimized sample dilution on the ACL TOP/TOP50 is comparable to the 1:2 dilution in the modified 96-well plate assay previously used in AnXa clinical studies. Representative data shown in Figure 2 demonstrates a dosedependent reversal of anti-FXa activity with increasing AnXa:FXal molar ratio. Similar results were observed in samples with proportionally higher FXal and AnXa concentrations (not shown).

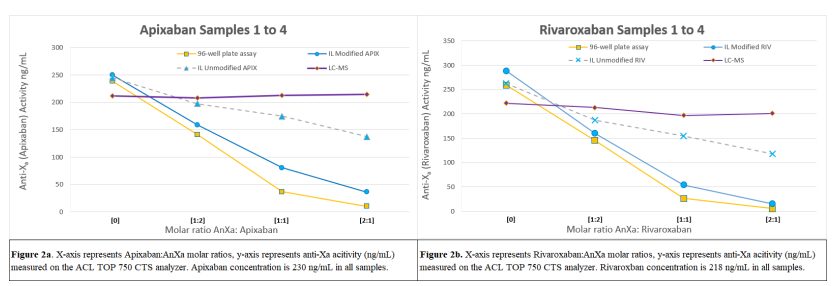

FIGURE 1 Representative data for the effect of andexanet on apixaban and rivaroxaban anti-FXa activity as measured by the modified user-defined setup on the ACL TOP/TOP50 analyzers

Conclusions: The modified tests for apixaban and rivaroxaban on the ACL TOP/TOP50 analyzers demonstrate dose-dependent percent reversal comparable to the 96 -well plate format. The modified user-defined setting could be used for measuring FXal in AnXacontaining samples.
PB0337 | Diagnosis of Disseminated Intravascular Coagulation in the Context of Human Immunodeficiency Viral (HIV) Infections in a South African Tertiary Care Setting

\section{E. Mayne; A. Mayne; S. Louw}

Faculty of Health Sciences, University of the Witwatersrand and

National Health Laboratory Service, Johannesburg, South Africa

Background: HIV infection is associated with an increased risk of abnormal thrombosis including microangiopathic thrombosis. Previously, HIV was not considered a direct cause of DIC but we have described it as a significant predisposing risk factor. A number of DIC scoring systems exist including the ISTH system which assigns points for prolongation of the prothrombin time (PT), presence of fibrin degradation products (D-dimers), reduced fibrinogen levels and thrombocytopaenia. Coagulation profiles may be deranged in HIV-infected patients in the absence of overt DIC. It becomes important, therefore, to describe the laboratory presentation in confirmed DIC in HIV-infected patients.

Aims: To evaluate the laboratory presentation of DIC in patients with and without HIV infection.

Methods: This study was approved by the human research ethics committee of the University of the Witwatersrand (M160389). ISTH DIC scores for all DIC screens between 2015 and 2016 were computed and patients with an ISTH score above 5 were included in the analysis. For the paired HIV-infected and HIV-uninfected patients, tests were recorded for the 4 parameters of the DIC score - PT, fibrinogen, D-dimers and platelets as well age. Since paired differences were normally distributed, a paired $t$-test for equality of means was performed with Stata ${ }^{\circledR}$-14.2. A P-value $<0.05$ was considered significant to reject equality of means.

Results: HIV-infected patients presented with DIC at a significantly younger age, significantly higher D-dimer levels, significantly prolonged PT values, lower fibrinogen levels, higher D-dimer levels and lower platelet counts than uninfected patients(Table 1). Clinically, the ISTH score was correspondingly higher in HIV-infected patients suggesting a more severe coagulopathy. 
TABLE 1 Comparison of the patient age and parameters of the ISTH DIC score between HIV infected and uninfected patients $(n=112)$.

\begin{tabular}{|c|c|c|c|c|c|c|c|c|c|c|}
\hline \multirow{2}{*}{$\begin{array}{l}\text { Parameter } \\
\text { (units) } \\
\text { HIV status }\end{array}$} & \multicolumn{2}{|c|}{ Age (years) } & \multicolumn{2}{|c|}{ Prothrombin Time (PT) } & \multicolumn{2}{|c|}{ Fibrinogen (g/l) } & \multicolumn{2}{|c|}{ D-dimer (mg/l) } & \multicolumn{2}{|c|}{ Platelet (×10\%/I) } \\
\hline & infected & uninfected & infected & uninfected & infected & uninfected & infected & uninfected & infected & uninfected \\
\hline Mean & 35.82 & 42.38 & 22.22 & 21.37 & 3.73 & 4.38 & 3.66 & 3.41 & 40.95 & 55.54 \\
\hline Variance & 114.56 & 410.68 & 66.84 & 75.33 & 2.67 & 5.87 & 7.95 & 9.76 & 1136.16 & 3167.85 \\
\hline$P$-value & $P<0.01$ & & $<0.01$ & & $<0.01$ & & $<0.01$ & & $<0.01$ & \\
\hline
\end{tabular}

Conclusions: HIV-infected patients show activation of the coagulation system even in the absence of overt DIC and this derangement becomes pronounced when DIC develops. This should be considered when applying DIC scoring systems in the context of HIV infection.

\section{PB0338 | The Red Blood Cell Paradox in Viscoelastic} Coagulation Tests - Hemoglobin Concentration as a Confounder for Test Reliability?

I. Pekrul $^{1,2}$; S. Adamowicz ${ }^{1}$; A. Giebl ${ }^{3} ;$ E. Kilger $^{1} ;$ M. Spannagl ${ }^{2}$; P. Möhnle 2,1 $^{2}$

${ }^{1}$ Ludwig-Maximilian University /Department of Anaesthesiology, Munich, Germany; ${ }^{2}$ Ludwig-Maximilian-University/Department of Transfusion Medicine, Cell Therapeutics and Haemostaseology, Munich, Germany; ${ }^{3}$ Augsburg University Clinic / Institute for Transfusion Medicine and Hemostaseology, Augsburg, Germany

Background: Viscoelastic testing (VT) is recommended for guidance of hemostatic treatment (HT) in acute bleeding. Whole-blood pointof-care methods are however limited by inter- and intra-observer variability and by the lack of the possibility for subsequent measurements for quality control.

Aims: Our aim was to develop a prediction model for bedside pointof-care VT fibrinogen clot firmness (MCF) from corresponding measurements in stored plasma samples.

Methods: We assessed 100 samples ( $n=77$ patients, $n=23$ healthy volunteers) with the ROTEM ${ }^{\mathrm{TM}}$ delta (Instrumentation Laboratory) and the ClotPro ${ }^{\mathrm{TM}}$ (Haemonetics) device. MCF was determined in whole blood and thawed plasma with the FIBTEM and the Fib-test assay. Clauss fibrinogen concentrations (analyzers/reagent: $\mathrm{ACL}$ TOP 500/Werfen/Fibrinogen-C/HemosIL and BCS XP/Siemens/ Multifibren U, Siemens) as well as a blood count (KX-21/Sysmex) were additionally measured. Correlations were analysed and a prediction model was developed using step-wise multiple regression.

Results: The fibrinogen content of the samples covered a broad range of the Clauss fibrinogen concentration measurement (median 250, IQR 127, $\mathrm{min} / \mathrm{max}$ 140-632). Strong correlations of whole blood with plasma in FIBTEM/Fibtest MCF $(r=0.795, P<0.001 / r=0.795$, $P<0.001)$ were observed. Multivariate analysis showed FIBTEM values in whole blood may be predicted from FIBTEM values in corresponding measurements in plasma samples solely $\left(r^{2}=0.69\right.$, n.s. while Fib-test MCF values in whole blood may be predicted taking into account plasma Fib-test MCF values and the hemoglobin value of the original sample as an independent variable, inversely related to $\operatorname{MCF}\left(r^{2}=0.906, P<0.001\right)$.

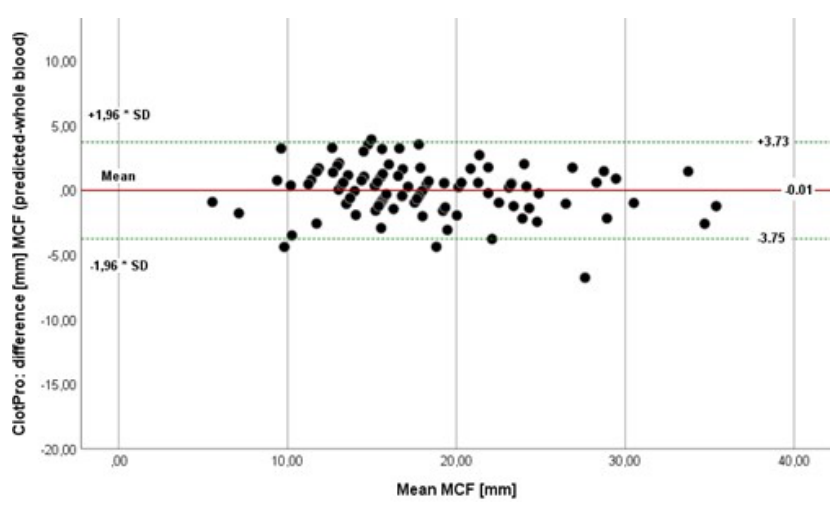

FIGURE 1 Bland-Altman plot showing the difference versus the average of MCF [mm] of the predicted MCF results and the MCF results of whole blood samples measured by the ClotPro ${ }^{\circledR}$ analyzer. Bias, upper and lower limits of agreement $( \pm 1.96$ SD (=1.91), green dotted lines) are represented. The red line indicates equality (difference $=0$ ). Multiple linear regression analysis was used to develop a model for predicting MCF [mm] in whole blood from MCF [mm] in thawed plasma, hemoglobin concentration, red blood cell count and Clauss fibrinogen concentration $(\mathrm{g} / \mathrm{L})$. The analysis identified the MCF $[\mathrm{mm}]$ in thawed plasma and the hemoglobin concentration as significant predictor variables $(p)$

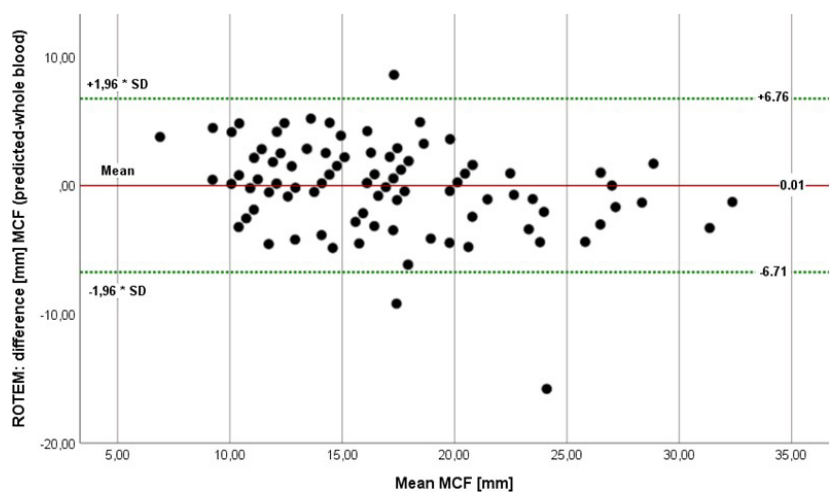

FIGURE 2 Bland-Altman plot showing the difference versus the average of MCF [mm] of the predicted MCF and the MCF results of 
whole blood samples measured by the ROTEM delta ${ }^{\circledR}$ analyzer. Bias, upper and lower limits of agreement $( \pm 1.96 \mathrm{SD}(=3.44)$, green dotted lines) are represented. The red line indicates equality (difference $=$ $0)$. Multiple linear regression analysis was used to develop a model for predicting MCF $[\mathrm{mm}]$ in whole blood from MCF $[\mathrm{mm}]$ in thawed plasma, haemoglobin concentration, red blood cell count and Clauss fibrinogen concentration ( $\mathrm{g} / \mathrm{L}$ ). The analysis identified the MCF [mm] in thawed plasma as the only predictor variable; the other variables were not significant in multiple regression analysis. The predicted FIBTEM-MCF was calculated with the following mathematical formula: FIBTEM pred. $=0.675 \times \mathrm{MCF}[\mathrm{mm}]+0.68$.

Conclusions: We developed a model to reliably predict MCF from stored plasma samples, taking into account plasma MCF and the hemoglobin concentration of the original sample. Our findings first serve to contribute to quality control for VT measurement in clinical practice and research and second point to the importance of hemoglobin as a significant factor of influence in VT measurements, potentially indicating the need for defining hemoglobin-dependent treatment cut-off values for fibrinogen replacement.

PB0339 | Quantra: The Influence of Hematocrit, Platelet Count, Ringer Lactate, and Acetylsalicylic Acid on Viscoelastic Properties of Clot Formation

A.-M. Hulshof ${ }^{1}$; R. van Oerle ${ }^{2}$; Y. Henskens ${ }^{1}$

${ }^{1}$ Maastricht University Medical Center+, Maastricht, Netherlands;

${ }^{2}$ Maastricht University, Maastricht, Netherlands

Background: Quantra is a novel point-of-care (POC) whole blood viscoelastic assay that applies Sonic Estimation of Elasticity via Resonance (SEER). In short, every few seconds ultrasound waves are used to initiate erythrocyte movement, monitor the consequent deviation of these erythrocytes, and determine the shear modulus. The shear modulus surges during clot formation as elasticity (illustrated by erythrocyte deviation) diminishes. The Qplus cartridge reports the parameters (abbreviation; reagents/formula) clot time (CT; kaolin), clot time heparinase (CTH; kaolin, heparinase), clot stiffness (CS tissue factor), fibrinogen contribution to clot stiffness (FCS; tissue factor, abciximab), clot time ratio (CTR; CT/CTH), and platelet contribution to clot stiffness (PCS; CS-FCS).

Aims: The applicability of a POC assay depends upon its susceptibility to differences in blood composition, including (but not limited to) hematocrit, platelet count, ringer lactate, and acetylsalicylic acid (ASA). We aimed to evaluate the influence of the aforementioned variables on Quantra parameters.

Methods: All experiments were performed in vitro with blood from healthy volunteers. Hematocrit $(n=1)$ and platelet count $(n=2)$ were modified within a range of, respectively, 0.2-0.7 L/L and 50$450 \times 10^{9} / \mathrm{L}$. Cell counts were not substantially altered (max deviation 12\%) after adjustment of hematocrit and platelet count. Whole blood volume was replaced with ringer lactate from 0 up to $60 \%$
( $n=1$ ) to mimic dilutional coagulopathy and healthy volunteers $(n=3)$ were spiked with $100 \mu$ M ASA

Results: Clot strength parameters were dependent upon hematocrit (negative association), platelet count (positive association), and dilution by ringer lactate (negative association). CT decreased when dilution with ringer lactate exceeded 50\%. Quantra parameters were unaffected by ASA-induced platelet inhibition.
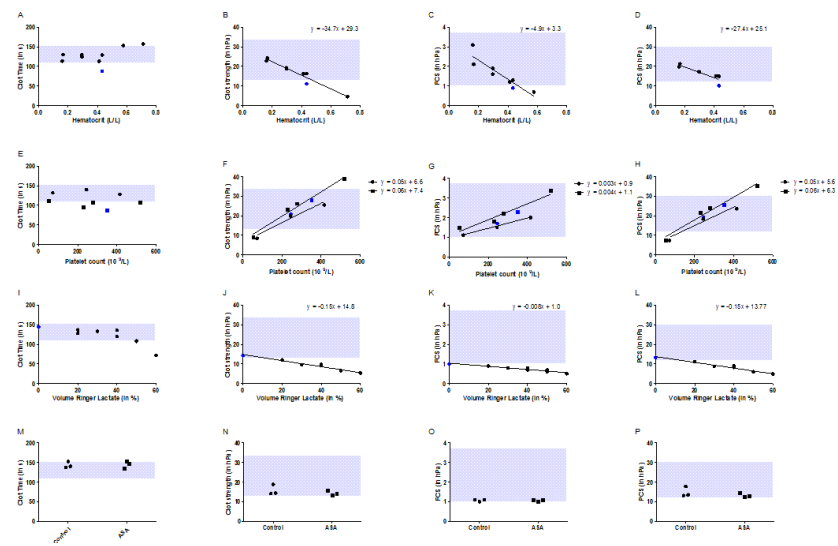

FIGURE 1 The influence of hematocrit (A-D) platelet count $(E-H)$, dilution with ringer lactate (I-L), and acetylsalicylic acid (ASA) (M-P) on Quantra Clot Time (A/E/I/M), Clot Strength (B/F/J/N), Fibrinogen Contribution to Clot Strength (FCS; C/G/K/O), Platelet Contribution to Clot Strength (PCS; D/H/L/P). Blue dots illustrate the unaltered (normal) healthy volunteer samples and reference ranges are presented as grey areas. In case of significant linear regression (p)

Conclusions: Viscoelastic assessment by Quantra is dependent upon hematocrit, platelet count, and dilution by ringer lactate. These variables should be taken into account when interpreting Quantra results in patients. Additionally, the results suggest Quantra is insensitive for ASA-induced platelet inhibition.

\section{PB0340 | The Impact of Inactive FVIII Antigen in Factor VIII Deficient Plasma on the Measurement of FVIII Inhibitors}

A. Wood ${ }^{1}$; N. Kesavan ${ }^{1}$; A. Sadeghi-Khomami; ${ }^{1} ;$ K. Black $^{1}$; M. Boylan ${ }^{1}$; R. Ni²; J. Della Maestra ${ }^{2}$; P. Erb²; D. Foulon ${ }^{2}$;

H. Hoogendoorn ${ }^{2}$

${ }^{1}$ Precision BioLogic Inc, Dartmouth, Canada; ${ }^{2}$ Affinity Biologicals Inc, Hamilton, Canada

Background: Congenital Factor VIII Deficient plasma (F8DP) is considered ideal for use in quantifying FVIII or FVIII inhibitors in hemophilia A patients as it provides a substrate that matches the intended patient sample matrix. Access to congenital F8DP is limited and has high donor variability. Manufactured F8DP from chemically or immuno-depleted normal plasma varies in comparison as some lack 
von Willebrand Factor (VWF) or contain significant inactive FVIII antigen.

Aims: To compare the performance of congenital F8DP (George King Bio-Medical Inc.), to manufactured F8DP (Instrumentation Laboratory), and to a newly developed manufactured F8DP with VWF (Precision BioLogic Inc.) in their application as a sample diluent in the Nijmegen Bethesda Assay (NBA).

Methods: Various commercial F8DP with VWF (including congenital F8DP) were compared by evaluating the VWF and FVIII content of each. Based on this comparison, representative F8DPs (with varying FVIII antigen content) were used as a sample diluent in the NBA to quantify FVIII inhibitor in low ( 1.5 BU) and mid ( 5 BU) FVIII inhibitor samples.

Results: Evaluated F8DPs contained normal levels of VWF (activity and antigen) and were deficient in FVIII activity $(<1 \%)$, but the FVIII antigen content varied. Precision BioLogic's F8DP was the only one that contained similar FVIII antigen to congenital F8DP (<1\%). Other suppliers contained higher FVIII antigen up to $>100 \%$.

FIGURE 1 displays a summary of the results $(n=20)$ when three F8DP's were used as a sample diluent to quantify FVIII inhibitors in two samples.

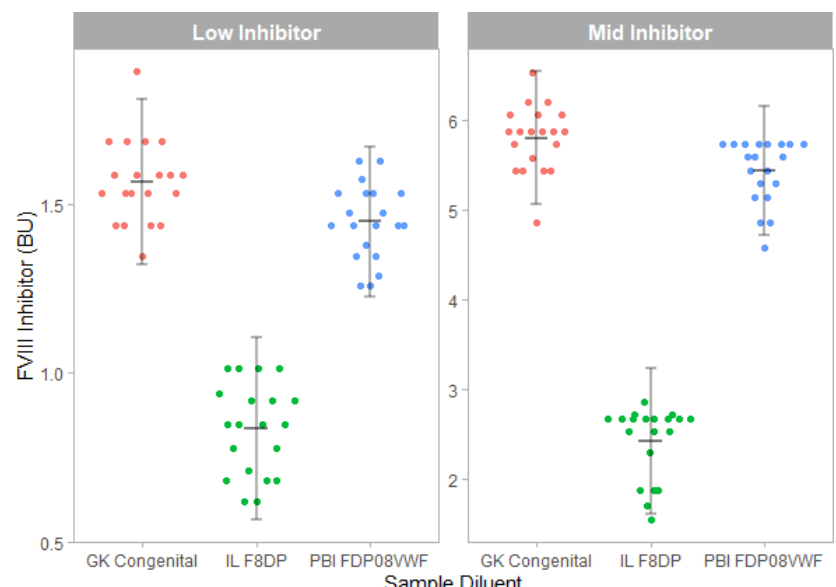

FIGURE 1 Commercial Factor VIII Deficient (with VWF) performance comparison when used as a sample diluent in NBA to quantify two FVIII inhibitor samples $(n=20)$.

Conclusions: Precision BioLogic's F8DP with VWF most resembles congenital F8DP, as it contains similar VWF and FVIII content. This makes it an ideal deficient plasma as a sample diluent in the NBA to quantify FVIII inhibitors. Other F8DPs could result in an underestimation of FVIII inhibitor due to significant inactive FVIII antigen presence.
PB0341 | Cost-effectiveness of a Stepwise versus an All-in-One Approach to Diagnose Mild Bleeding Disorders

F. Heubel-Moenen ${ }^{1}$; L. Ansems ${ }^{1}$; P. Verhezen ${ }^{2}$; R. Wetzels ${ }^{2}$; R. Straat ${ }^{2}$; Y. Henskens ${ }^{2}$; E. Beckers ${ }^{3} ;$ M. Joore ${ }^{4}$

${ }^{1}$ Department of Hematology, Internal Medicine, Maastricht University Medical Centre+, Maastricht University Medical Centre+, Maastricht, Netherlands; ${ }^{2}$ Central Diagnostic Laboratory, Unit for Hemostasis and Transfusion, Maastricht University Medical Centre+, Maastricht, Netherlands; ${ }^{3}$ Department of Hematology, Internal Medicine, Maastricht University Medical Centre+, Maastricht, Netherlands; ${ }^{4}$ Department of Clinical Epidemiology and Medical Technology Assessment (KEMTA), Maastricht University Medical Centre+, Maastricht, Netherlands

Background: The diagnostic work-up of patients referred for bleeding evaluation is based on a stepwise approach, in which bleeding history and results of limited screening laboratory tests guide further testing. This stepwise approach can be time consuming and burdensome for patients, leading to numerous hospital visits, venipunctures and travel costs. Furthermore, in half of the patients, no diagnosis is found. To improve this diagnostic strategy, the initial laboratory investigation can be extended.

Aims: To compare the costs, effectiveness, and patient burden of the standard stepwise diagnostic approach with an all-in-one diagnostic approach for patients referred for bleeding evaluation.

Methods: Adult patients referred to a hematologist for bleeding evaluation, were diagnosed according to the standard stepwise approach, and an all-in-one approach, in which platelet function tests, coagulation factors, and fibrinolysis tests were included in the initial laboratory investigation. For both approaches data was collected for: final diagnosis, number of doctor visits, number of hospital visits, number of venipunctures, number of multidisciplinary team (MDT) meetings, time to diagnosis, patients travel expenses and costs of all laboratory tests performed.

Results: 150 patients ( $80 \%$ female, mean age 46 years) were included. Results of both diagnostic approached are shown in Table 1. Compared to the stepwise approach, in the all-in-one approach 22 more bleeding disorders were diagnosed: 4 factor deficiencies, 10 platelet function disorders, and 8 cases of hyperfibrinolysis. Also, the number of doctor visits, venipunctures, MDT meetings and time to diagnosis, were significantly lower and shorter in the all-in-one approach. However, due to higher laboratory costs, the mean total costs per patient in the all-in-one approach were higher (difference $€ 397.06)$. 
TABLE 1 Effectiveness, costs and patient/healthcare burden of different approaches for bleeding evaluation

\begin{tabular}{|c|c|c|c|c|c|c|}
\hline Diagnostic algorithm & $\begin{array}{l}\text { Stepwise } \\
\text { approach }\end{array}$ & $\begin{array}{l}\text { All-in-one } \\
\text { approach }\end{array}$ & Diagnostic Algorithm & Stepwise approach & $\begin{array}{l}\text { All-in-one } \\
\text { approach }\end{array}$ & $P$-value \\
\hline \multicolumn{3}{|l|}{ Diagnosis, $n$ (\%) } & \multicolumn{4}{|c|}{ Total costs $(€)$, mean $(95 \% \mathrm{BCl})$} \\
\hline $\begin{array}{l}\text { Bleeding of unknown } \\
\text { cause }\end{array}$ & $47(31)$ & $47(31)$ & Total costs per patient & $\begin{array}{c}1.713 .34(1.513 .80- \\
1.901 .57)\end{array}$ & $\begin{array}{c}2.110 .40(1.194 .70- \\
226.38)\end{array}$ & 0.000 \\
\hline $\begin{array}{l}\text { Platelet function } \\
\text { disorder }\end{array}$ & $21(14)$ & $28(19)$ & \multicolumn{4}{|c|}{ Patient/healtcare burden, mean $(95 \% \mathrm{BCl})$} \\
\hline Factor deficiency & $9(6)$ & $9(6)$ & Number of doctor visits & $4(4-4)$ & $3(3-3)$ & 0.000 \\
\hline Von Willebrand disease & $7(5)$ & $6(4)$ & $\begin{array}{l}\text { Number of hospital } \\
\text { visits }\end{array}$ & $5(4-5)$ & $4(4-5)$ & 0.215 \\
\hline Hyperfibrinolysis & $9(6)$ & $11(7)$ & $\begin{array}{l}\text { Number of } \\
\text { venipunctures }\end{array}$ & $3(3-3)$ & $2(2-2)$ & 0.000 \\
\hline
\end{tabular}

Conclusions: For bleeding evaluation, an all-in one diagnostic approach provides health benefits (more diagnosis, reduction of patient burden and healthcare workload) at higher cost. These findings open the discussion to what the health benefit in bleeding evaluation is worth.

\section{PB0342 | A Novel Paper-based Test for Diagnostic of Fibrinogen} Levels on Whole Blood

\section{A.T. Sachetto $^{1}$; N.T. Picon ${ }^{2}$; C.B. Morgado ${ }^{2}$; M. L. Santoro ${ }^{1}$ \\ ${ }^{1}$ Instituto Butantan/Faculdade de Medicina da Universidade de São Paulo, São Paulo, Brazil; ${ }^{2}$ Instituto Butantan, São Paulo, Brazil}

Background: Fibrinogen is the most abundant coagulation factor on plasma, a key component of hemostasis and inflammation, and an acute-phase protein. In acquired conditions leading to hypofibrinogenemia - e.g., snakebite envenomation, trauma-induced coagulopathy, disseminated intravascular coagulation, sepsis and liver dysfunction -, the rapid determination of fibrinogen levels is essential to provide correct diagnosis and treatment to patients. Current assays for fibrinogen measurement have limitations, such as expensive costs, time-consuming manipulations and the requirement of centrifugation steps to obtain plasma samples. Therefore, to develop and validate new point-of-care diagnostic tests for fibrinogen assay is essential.

Aims: To develop a novel and rapid paper-based test to assay fibrinogen levels in whole blood, taking into consideration that during coagulation activation fibrinogen is clotted into a fibrin network that is semi-permeable to dye solutions.

Methods: Procedures involving animals were approved by IACUC. The flow assay cassette was constructed using a 3D printer, lateral flow plasma separation pads were used to separate red blood cells from plasma, and filter paper strips were the support for observing the migration distance of a methyl green dye solution. Mice were injected with saline (controls) or snake venom to evaluate hypofibrinogenemia. After $3 \mathrm{~h}$, whole blood $(30 \mu \mathrm{L})$ was collected from the lateral saphenous vein, and added to the diagnostic device. After $3 \mathrm{~min}$, the dye solution was added, and the travel distance was then recorded at 2, 5 and $10 \mathrm{~min}$.

Results: Envenomed mice had hypofibrinogenemia compared to control mice, and fibrinogen levels were inversely proportional to the travel distance of the dye $(r=-0.9, P<0.050)$.

Conclusions: The test was effective in detecting differences in fibrinogen levels in whole blood samples. We are currently evaluating the performance of this diagnostic test with other samples from F9, F8 and Vwf knockout mice, as well as in human blood samples. Funding: FAPESP, CNPq, CAPES, Fundação Butantan.

\section{PB0343 | Heparin Interference of the Thrombin Generation} Assay Can Be Eliminated by Heparinase Treatment

A. Weber ${ }^{1}$; M. LLusa ${ }^{2}$; H. Gritsch'; N.B Binder ${ }^{2}$

${ }^{1}$ Baxalta Innovations $\mathrm{GmbH}$, a Takeda Company, Wien, Austria;

${ }^{2}$ Technoclone, Wien, Austria

Background: Reversal of anticoagulation by vitamin $\mathrm{K}$ antagonists (VKAs) is obtained by administration of four-factor prothrombin complex concentrates (4F-PCCs). Thrombin generation assay (TGA) has been shown to be suitable for the in vivo evaluation of the hemostatic potency of 4F-PCCs. Commercially available 4F-PCCs contain heparin at different levels. Consequently, TGA results obtained for in vitro samples will be biased and may not fully reflect the clinical efficacy of 4F-PCCs observed. Removal of heparin by anion exchange adsorption has been described but this procedure could potentially alter the complex composition of 4F-PCCs. Heparin neutralization with protamine sulphate requires exact titration in order not to interfere with the determination of the hemostatic potency.

Aims: Description of a robust method to remove the inhibitory heparin effect on the TGA. 
Methods: A heparin-containing 4F-PCC sample was mixed with Heparinase and incubated for $5 \mathrm{~min}$ at room temperature. Then, the Technothrombin TGA (Technoclone) was done using the fully automated coagulation analyzer Ceveron ${ }^{\circledR}$ (Technoclone). Normal and VKA anticoagulated plasma was used at a final heparinase concentration of $1.25 \mathrm{U} / \mathrm{mL}$ at two trigger levels. The adequate heparinase concentration was determined by investigating a concentration series of heparinase, ranging from 0.25 to $2.5 \mathrm{U} / \mathrm{mL}$.

Results: Heparinase at a final concentration of $1.25 \mathrm{U} / \mathrm{mL}$ completely removed the heparin interference. At this heparinase level, there was only moderate influence on the characteristic TGA readouts lag time, peak thrombin, endogenous thrombin potential and velocity index for all conditions tested. After heparinase treatment, the heparincontaining 4F-PCC concentrate demonstrated dose-dependent TGA response, which was not the case when this treatment was omitted.

Conclusions: Enzymatic breakdown of heparin by heparinase efficiently removed the heparin interference observed during the in vitro TGA testing of 4F-PCCs. Heparinase treatment can easily introduced in existing test procedures and will increase the significance of data generated with TGA.

\section{PB0344 | Comparison of Peak Plasma Concentrations of Apixaban in Adolescent and Adult Patients}

I. Celap ${ }^{1}$; S. Margetic ${ }^{1}$; J. Obuljen ${ }^{2}$; J. Lenicek Krleza ${ }^{2}$; I. Linarić2 M. Razum ${ }^{1}$; R. Mihic ${ }^{1}$

${ }^{1}$ Department of Clinical Chemistry, Sestre Milosrdnice University Hospital Center, Zagreb, Croatia; ${ }^{2}$ Department of Laboratory Diagnostics, Children's Hospital Zagreb, Zagreb, Croatia

Background: Knowledge related to expected peak concentrations of apixaban in children and adolescent populations is very scarce, since it has only recently been approved for treatment in pediatric population. Aims: To compare peak plasma concentrations of apixaban in adolescents and adults.

Methods: Innovance heparin (Siemens Healthineers, Germany) calibrated with apixaban calibrators (Hyphen BioMed, France) was used for quantitative determination of apixaban concentration. The study included plasma samples from adult patients ( $N=80 ; 47-89 \mathrm{yrs}$ ) treated with standard dose $(2 \times 5 \mathrm{mg} /$ day $)$ and from adolescents $(N=59 ; 13-22$ yrs) treated with two dosing regimens: $2 \times 2.5 \mathrm{mg} /$ day $(N=38)$ and $2 \times 5$ $\mathrm{mg} /$ day $(\mathrm{N}=21)$. Blood samples were taken two to four hours after drug administration in order to obtain peak apixaban concentrations. The study was funded by the Croatian Science Foundation as part of the research project IP-2016-06-8208. Statistical analysis was done using Wilcoxon test by MedCalc Statistical Software version 11.5.1.

Results: Concentrations of apixaban showed significantly lower peak values in adolescents treated with $2 \times 2.5 \mathrm{mg} /$ day compared with those treated with $2 \times 5 \mathrm{mg} /$ day $(P=0.003)$, as well as compared with adults treated $2 \times 5 \mathrm{mg} /$ day $(P<0.001)$. On the contrary, peak concentrations between adolescents and adults treated with the same standard dose of $2 \times 5 \mathrm{mg} /$ day did not differ $(P=0.937)$ (Figures 1 and 2). In both populations, adolescents and adults, treated with the same standard dose of $2 \times 5 \mathrm{mg} /$ day, peak concentrations showed very good agreement with the published expected values in adult patients.

\begin{tabular}{|c|c|c|c|}
\hline & Adolescents & Adolescents & Adults \\
\hline Apixaban dose (mg/day) & $2 \times 2.5$ & $2 \times 5$ & $2 \times 5$ \\
\hline $\mathrm{N}$ & 38 & 21 & 80 \\
\hline $\begin{array}{c}\text { Peak concentration, } \mathrm{ng} / \mathrm{mL} \\
\text { Median }(95 \% \mathrm{Cl}) \\
\text { IQR }\end{array}$ & $\begin{array}{c}101(73-110) \\
69-124\end{array}$ & $\begin{array}{c}189(137-229) \\
120-232\end{array}$ & $\begin{array}{c}179(159-195) \\
141-224\end{array}$ \\
\hline \multirow{3}{*}{$P$} & \multicolumn{2}{|c|}{0.003} & \\
\hline & & \multicolumn{2}{|c|}{0.937} \\
\hline & \multicolumn{3}{|c|}{$<0.001^{*}$} \\
\hline $\begin{array}{c}\text { Expected published values } \\
(\mathrm{ng} / \mathrm{mL}) \text { in adults }\end{array}$ & \multicolumn{3}{|c|}{$\begin{array}{c}\text { Non valvular atrial fibrillation: } 171 ; 91-321 \\
\text { Treatment of PE/VTE: } 132 ; 59-302\end{array}$} \\
\hline
\end{tabular}

FIGURE 1 Results of apixaban concentrations in adolescents and adults
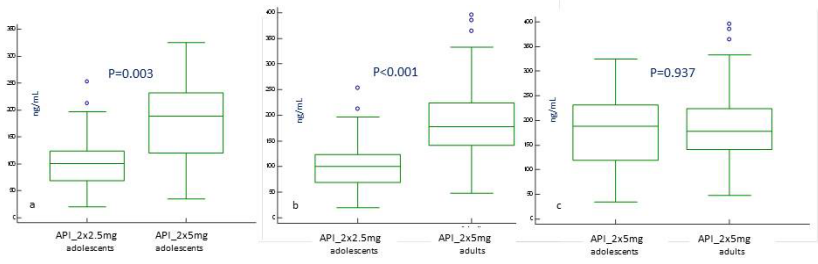

FIGURE 2 Differences between apixaban concentrations in adolescents and adults depending on dose regimens: adolescents $2 \times 2.5$ and $2 \times 5 \mathrm{mg} /$ day (a), adolescents $2 \times 2.5$ and adults $2 \times 5 \mathrm{mg} / \mathrm{day}$ (b) and adolescents $2 \times 5$ and adults $2 \times 5 \mathrm{mg} /$ day (c)

Conclusions: Peak plasma concentrations of apixaban in adolescents depend on the dose regimen ( $2 \times 2.5$ vs. $2 \times 5 \mathrm{mg} /$ day $)$ showing roughly a twice lower values in plasma of adolescents treated with a twice lower doses. Further, peak concentrations in adolescents treated with standard dose regimen of $2 \times 5 \mathrm{mg}$ /day are completely comparable with values in adult patients with the same dosing regimen as well as with the expected values published for adult patients.

\section{PB0345 | Development of a New ELISA Method for Anti-FVIIa} Antibody Measurement

C. Valsecchi ${ }^{1}$; L. Schiavone ${ }^{1}$; H. Behrouz ${ }^{2}$; Z. Ahsani ${ }^{2}$;

A. Saadatirad ${ }^{2}$; F. Peyvandi ${ }^{3}$

${ }^{1}$ Fondazione IRCCS Ca' Granda Ospedale Maggiore Policlinico, Angelo Bianchi Bonomi Hemophilia and Thrombosis Center and Fondazione Luigi Villa, Milan, Italy; ${ }^{2}$ Biopharmaceutical Research Center, AryoGen Pharmed Inc., Alborz University of Medical Sciences, Karaj, Iran, Islamic Republic of; ${ }^{3}$ Fondazione IRCCS Ca' Granda Ospedale Maggiore Policlinico, Angelo Bianchi Bonomi Hemophilia and Thrombosis Center and Fondazione Luigi Villa, and the Department of Pathophysiology and Transplantation, Università degli Studi di Milano, Milan, Italy

Background: Recombinant FVIla has been shown to be efficacious for treatment of bleeding in congenital and acquired hemophilia $A$ with inhibitor and in congenital FVII deficiency as well as in Glanzmann's 
thrombasthenia. As for other recombinant therapeutic proteins, administration of rFVIla may induce the development of anti-FVIla antibody that could be associated with a decrease of the treatment efficacy. Very limited data are available on development of anti-FVIla antibody. Surveillance of rFVIla immunogenicity is then required.

Aims: To develop a method for measuring the anti-FVIla antibody in patients treated with rFVIla.

Methods: An ELISA bridging method has been developed by using rFVIla (NovoSeven, Novo Nordisk) for both coating and detection. The best experimental conditions were a high-density of immobilized antigen, acid dissociation and long incubation time with plasma samples. Commercial (Affinity Biological) FVII deficient plasma and FVII Inhibitor plasma (4.5 and 67.1 BU) have been used as negative and positive controls with sheep anti-human FVII IgG used as a reference standard.

Results: Several experiments have been performed and a significant improvement in IgG recovery has been achieved with FVII depleted plasma containing $1 \mathrm{ug} / \mathrm{mL}$ rFVIla and 1.25 or $0.6 \mathrm{ug} / \mathrm{mL}$ of anti-human FVII IgG, by the acid dissociation and the long incubation time (Figure 1).
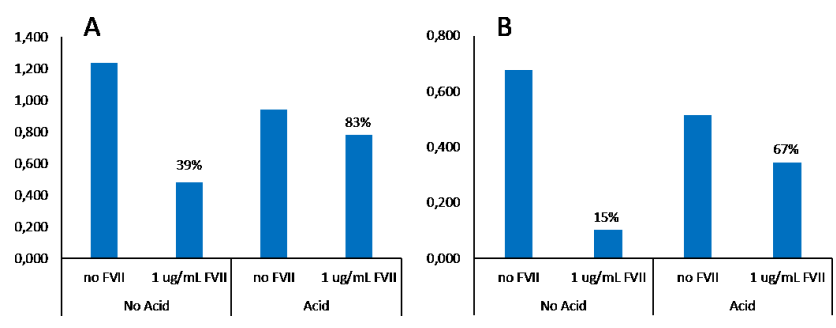

FIGURE 1 Improvement of IgG recovery obtained by acid dissociation with FVII depleted plasma containing $1 \mathrm{ug} / \mathrm{mL}$ of $\mathrm{rFVIla}$ and 1.25 (A) or $0.6 \mathrm{ug} / \mathrm{mL}$ (B) of anti-human FVII IgG

By increasing the concentration of immobilized antigen from 1 to to $5 \mu \mathrm{g} / \mathrm{mL}$ further improvement has been achieved in the IgG recovery (Figure 2).
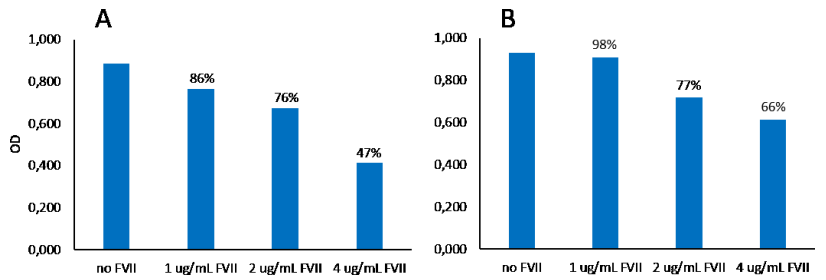

FIGURE 2 Higher IgG recovery was obtained by using NovoSeven at $5 \mathrm{ug} / \mathrm{mL}(\mathrm{B})$ instead of $1 \mathrm{ug} / \mathrm{mL}(\mathrm{A})$ in the coating step. Mixtures of FVII depleted plasma containing $1.25 \mathrm{ug} / \mathrm{mL}$ of anti-human FVII IgG and increasing amount of rFVIla were tested

Conclusions: Monitoring the patient immune-response to a therapeutic protein is important to assess the drug efficacy and its immunogenicity. However, the antibody detection could be complicated by the presence of the antigen in the plasma sample. Here we describe a novel method unaffected by the presence of rFVIla that could detect the incidence of the anti-FVIla antibodies during the treatment with recombinant FVIla.

PB0346 | Frequency and Clinical Significance of Different Types of Calreticulin (CALR) Mutations in Myeloproliferative Neoplasms (MPNs): A Cohort of Patients from Pakistan

R. Ahmed ${ }^{1}$; M. Rashid ${ }^{2}$; U. Zaidi ${ }^{2}$; T. Shmasi ${ }^{2}$

${ }^{1}$ University of Karachi, Karachi, Pakistan; ${ }^{2}$ National Institute of Blood

Diseases \& Bone Marrow Transplantation (NIBD \& BMT), Karachi,

Pakistan

Background: Most of the defined mutations in exon 9 of CALR gene are indels, with the exception of a few non-recurrent point mutations. More than 50 different CALR indels have been described including 52-bp deletion (p.L367fs*46) as type 1 and 5-bp TTGTC insertion (p.K385fs*47) as type 2 which are more frequent mutations. These different types of mutations in CALR exon 9 are thought to present different phenotypes. Aims: To investigate the frequency of different types of calreticulin mutations in classical MPNs and to study statistical difference in their presentation.

Methods: Bi-directional Sanger sequencing detected CALR exon 9 mutations in total 28 MPNs patients (ET = 19, PMF = 9).

Results: Among various CALR mutation types, type 1 was observed more common $(53.5 \% ; 15 / 28)$ in ET and PMF collectively compared to type 2 (35.7\%; 10/28). In ET type-1 CALR mutation was found in $47.3 \%$ (9/19) of patients and two of them had two novel scattered point mutations (c.1081C>G p.Gln361Glu and c.1086C>G p.Asp362Glu) at upstream and type-2 was in $42.1 \%$ (8/19) of patients. Mutations in ET other than type 1 and 2 were 10.5\% (2/19) comprising a novel double deletion of 10bp (c.1130_1139del p.Lys377ArgfsTer50) and 28bp (1195_1222del p.Glu399MetfsTer2) in a patient and one known CALR 12bp deletion (c.1214_1225del; p.Glu405_Asp408del) in another patient. In PMF type-1 CALR mutation was found in $66.6 \%$ (6/9) patients and type-2 in 22.2\% (2/9) of patients whereas one patient harbored novel homozygous CALR mutation (c.1139delA p.Glu380lyfsTer50).

There was no significant difference observed in terms of gender, mean age and haematological parameters of CALR positive type 1 and type 2 in ET and PMF.

\section{CALR Mutation Types}

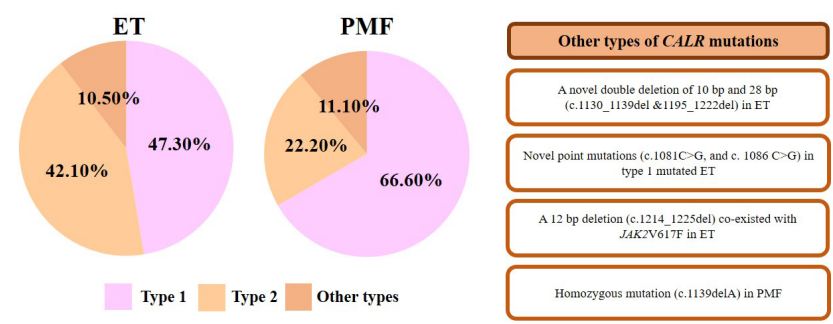

FIGURE 1 
TABLE 1

ET

$\begin{array}{ll} & \text { Type 1 } \\ \text { CALR +ve } & (N=9) \\ (N=19)(A) & (B)\end{array}$

Gender, No. (\%)

Male

Female

Age(in years)

Mean ( \pm SD)

Haemoglobin (g/

14 (73.7)

5 (26.3)

$41.8( \pm 12.9)$

7 (77.8)

$6(75)$

2 (22.2)

2 (25)

$40.4( \pm 14.7)$

$39.2( \pm 9.5)$

$11.7( \pm 2.4)$

$11.5( \pm 2.6)$

$12.3( \pm 2.0)$

$11.0(1.3)$

8 (88.9)

1 (11.1)

$51.8( \pm 14.1)$

$$
5 \text { (83.3) }
$$

1 (16.6)

$55.3( \pm 15)$

$11.2( \pm 1.5)$

$11.0( \pm 1.4)$

2 (100)

0.893

$0(0)$

$38.5( \pm 2.1)$

0.184

0.074

dl)

Mean ( \pm SD)

$$
9.9( \pm 4.2)
$$$$
9.98( \pm 5.57) \quad 9.4( \pm 3.10)
$$

$11.6( \pm 7.09) \quad 14.0( \pm 7.7)$

$7.07( \pm 2.0)$

0.805

0.280

0.433

Leukocyte count

$\left(\times 10^{9} / \mathrm{L}\right)$

Mean ( \pm SD)

Platelet count

1254

(×109/L)

Mean ( \pm SD)
$( \pm 602)$
1317

$( \pm 562)$
$472( \pm 432)$

$502( \pm 501)$
$535( \pm 370)$

0.490

0.936

0.002
Conclusions: This study reported a complex mutation, a novel double deletion of CALR and at upstream of type 1 CALR two novel single nucleotide variants.

PB0347 | Plasmatic Rivaroxaban Concentration Estimation through the Measurement of Anti Xa Activity Calibrated with Heparin Calibrators Using 3 Reactive/Instrument Systems: Multicenter Study

M. Martinuzzo $^{1,2}$; C. Duboscq ${ }^{3}$; M. Arias ${ }^{4}$; M.S. López $z^{1,2}$; E.S Viñuales ${ }^{5,2}$; D. Mezzarobba ${ }^{5}$; V. Privitera ${ }^{5}$; D. Penchasky ${ }^{5}$; F. Chuliber ${ }^{5}$; J. Ceresetto ${ }^{3}$; M. Gurfinkiel ${ }^{4}$; L. Barrera ${ }^{1,2}$ ${ }^{1}$ Hospital Italiano de Buenos Aires, Laboratorio Central, CABA, Argentina; ${ }^{2}$ Instituto Universitario del Hospital Italiano de Buenos Aires, CABA, Argentina; ${ }^{3}$ Hospital Británico de Buenos Aires, Hematología, CABA, Argentina; ${ }^{4}$ Hospital Dr. César Milstein, Laboratorio de Hematología, CABA, Argentina; ${ }^{5}$ Hospital Italiano de Buenos Aires, Hematología, CABA, Argentina

Background: Plasma rivaroxaban (RIVA) concentration cannot be evaluated through classic coagulation tests. AntiXa activity tests with specific calibrators, not widely available, are needed.

Aims: To correlate heparin calibrated (HC) antiXa activity (hybrid curve) obtained in 3 reactive/instrument systems with RIVA concentrations measured through antiXa test calibrated with specific calibrators. To compare antiXa-HC values calculated in the 3 systems for clinically relevant RIVA levels.
Methods: Multicenter study. Samples from center 1 were distributed in 2 others to measure antiXa-HC activity. Samples from 19 consecutive patients receiving RIVA at peak or through (8 -321 ng/ $\mathrm{mL}$ ), and 20 receiving enoxaparin (0.01-1.76 U/mL).

RIVA measurement: HemosIL Liquid antiXa(HEM) in ACLTOP, HemosIL Rivaroxaban Calibrators and Controls (IL, Instrumentation Laboratory).

AntiXa-HC Activity (hybrid curves)

(1) HEM, HemosIL Heparin Calbrator in ACLTOP;

(2) Innovance Heparin(INN), universal calibrators in Sysmex CS2500 (Siemens Health Care);

(3) STA-Liquid AntiXa(STA), HNF/HBPM calibrators in STACOMPACT MAX (Diagnostica STAGO).

Statistics: SPSS 23 software.

Results: Statistically significant correlation between AntiXa-HC and RIVA concentrations were found, Table 1. 
TABLE 1 Correlation and regression lines between anti Xa-HC assays and RIVA levels, and Anti Xa-HC in LMWH samples

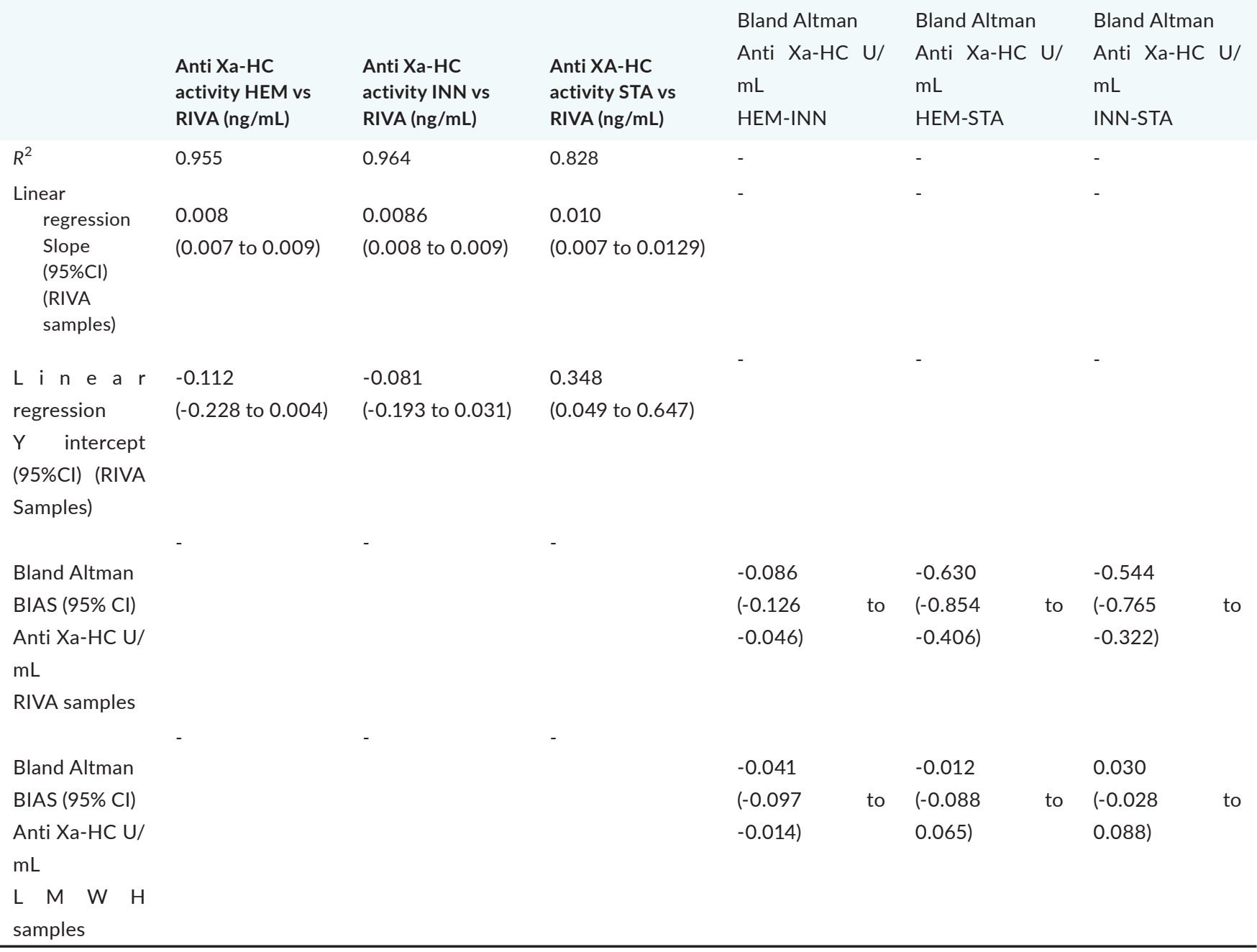

TABLE 2 Anti Xa-HC activity expected by linear regression equation at three different clinically relevant RIVA concentrations

\begin{tabular}{llll}
$\begin{array}{l}\text { RIVA concentration } \\
(\mathrm{ng} / \mathrm{mL})\end{array}$ & $\begin{array}{l}\text { Anti Xa-HC } \mathrm{HEM}(\mathrm{U} / \mathrm{mL}) \\
\text { value }(95 \% \mathrm{Cl})\end{array}$ & $\begin{array}{l}\text { Anti Xa-HC INN }(\mathrm{U} / \mathrm{mL}) \\
\text { value }(95 \% \mathrm{Cl})\end{array}$ & $\begin{array}{l}\text { Anti XA-HC STA }(\mathrm{U} / \mathrm{mL}) \\
\text { value }(95 \% \mathrm{Cl})\end{array}$ \\
30 & $0.13(0.01-0.24)$ & $0.18(0.06-0.26)$ & $0.64(0.35-0.94)$ \\
50 & $0.29(0.17-0.40)$ & $0.35(0.24-0.46)$ & $0.84(0.55-1.15)$ \\
200 & $1.49(1.37-1.60)$ & $1.64(1.52-1.75)$ & $2.35(1.05-2.65)$ \\
\hline
\end{tabular}

Linear regression equations AntiXa-HC to RIVA were similar for HEM and INN, but different for STA (Higher Y intercept). In RIVA samples, AntiXa-HC comparison between systems by Bland Altman curves yielded significant and important BIAS (Table 1) for STA-HEM and STA-INN. AntiXA-HC estimated by regression equations at three RIVA clincal relevant concentrations showed that values from STA are higher than HEM and INN(Table 2).

In LMWH samples, Anti Xa-HC correlation $R^{2}$ were $0.967,0.927$ and 0.959, $P<0.001$ for HEM-INN, HEM-STA and INN-STA, respectively. Paired analysis did not demonstrate significant BIAS(Table 1). Conclusions: LMWH concentration measured by each reactive/ instrument system are comparable using hybrid curves. However, in RIVA samples, each antiXa-HC system exert different results, close values with HEM and INN, but higher with STA. Knowledge of
antiXa-HC values for RIVA levels with common reagent/instrument systems could be clinically usefull in laboratories that do not have specific calibrators available. 
PB0348 | Comparison of Platelet Counts Using Impedance, Optical and CD61-immunoplatelet Methods in Thrombocytopenic Patients

T. Nguyen ${ }^{1}$; P.-L. Tran ${ }^{2} ;$ T. Tran ${ }^{1} ;$ T. Nguyen ${ }^{1} ;$ T. Nguyen ${ }^{1}$

${ }^{1}$ Cho Ray Hospital, Ho Chi Minh City, Vietnam; ${ }^{2}$ District 2 Hospital, Ho Chi Minh Clty, Vietnam

Background: In thrombocytopenic patients, platelet transfusion is a necessary treatment modality. However, it can potentially leads to transfusion-related complications. Consequently, appropriate evaluation of platelet counts is of utmost importance. There are multiple automated platelet analyzers commercially available, which usually employ one of the following counting methods: optical, impedance or immunoplatelet. However, there were several reports on the discrepancies between results obtained from these methods.

Aims: To assess the correlation between platelet counts obtained from analyzers using different counting methods (optical, impedance and immunoplatelet) in normal people and thrombocytopenic patients.

Methods: Blood samples were collected from normal people (platelet count at least $150 \times 10^{9} / \mathrm{L}$ ) and thrombocytopenic patients (platelet count less than $50 \times 10^{9} / \mathrm{L}$.) Blood smears were checked in thrombocytopenic samples for possible interferences (microcytosis, cell fragments, giant platelets.) The first counts were obtained using Advia 2120i optical analyzer (Siemens Healthcare $\mathrm{GmbH}$, Erlangen, Germany.) The samples were then reanalyzed using LH780 impedance analyzers (Beckmann Coulter, California, USA) and CELL-DYN Sapphire CD61 immunoplatelet analyzer (Abbott Laboratories, Illinois, USA.) The results obtained from different methods would then be compared.

Results: 20 normal and 154 thrombocytopenic samples were collected. 26 thrombocytopenic samples contained possible interferences. In normal people, the correlation coefficients $(R)$ between optical-impedance (R1), optical-immunoplatelet (R2) and impedanceimmunoplatelet (R3) methods were $0.96,0.98$ and 0.97 , respectively. In thrombocytopenic patients, R1, R2 and R3 were 0.94, 0.88 and 0.88 , respectively. In samples with interferences, R1, R2 and R3 were $0.93,0.66$ and 0.77 , respectively. Using Bland-Altman plots, it could be observed that both the optical and impedance methods usually overestimated platelet counts compared to the immunoplatelet method.

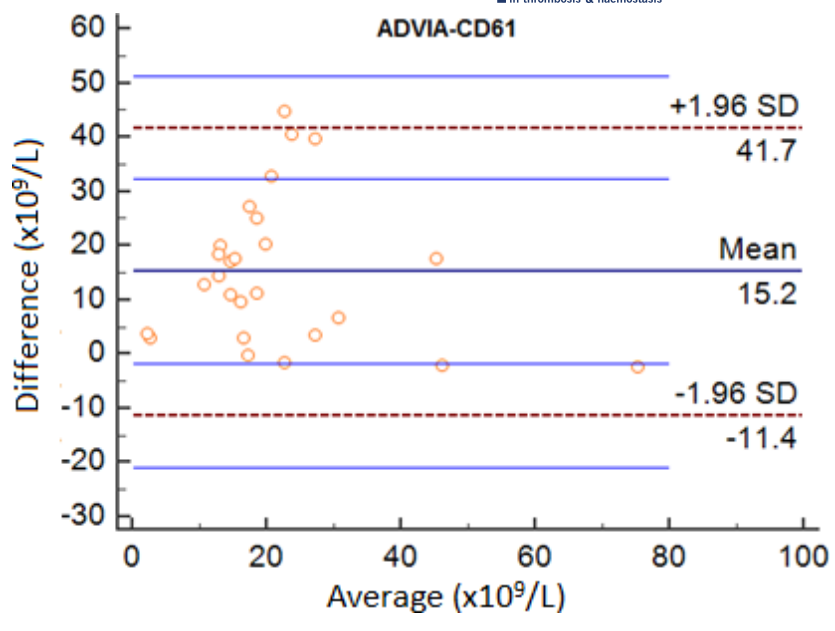

FIGURE 1 Bland-Altman plot describing the agreement between optical (Advia) and immunoplatelet (CD61) methods

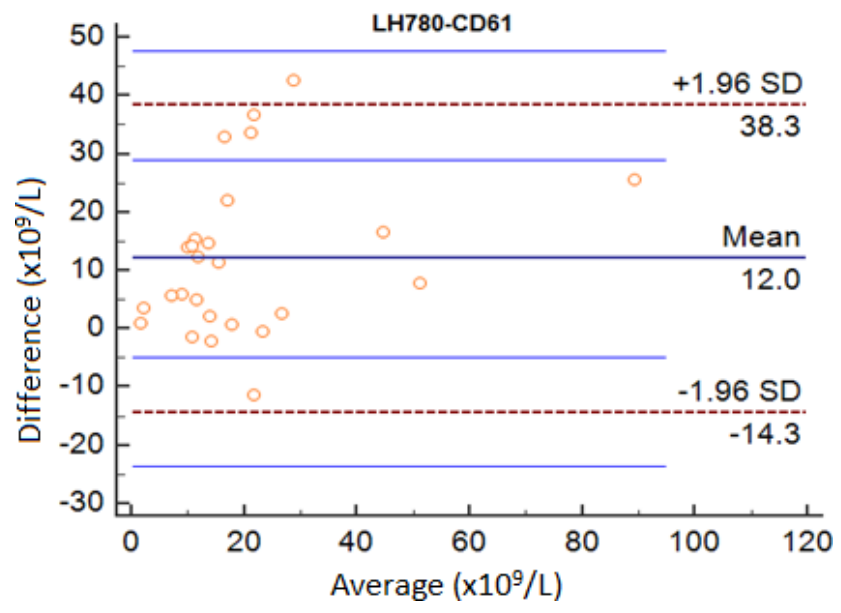

FIGURE 2 Bland-Altman plot describing the agreement between impedance (LH780) and immunoplatelet (CD61) methods

Conclusions: There were discrepancies between platelet counts obtained using optical, impedance and CD61-immunoplatelet methods in thrombocytopenic samples, especially those with interferences. These differences may be of clinical significance, since platelet counts are used as indicators for transfusions.

PB0349 | Assessment of Sample Stability after Freezing on ST Genesia System Using the STG-BleedScreen Application

J. Mullins ${ }^{1}$; D. White ${ }^{1}$ A. Carlo ${ }^{2}$; S. Cox-Morton ${ }^{1}$; S. Mac Donald ${ }^{1}$

${ }^{1}$ Cambridge University Hospital NHS Foundation Trust, Cambridge, United Kingdom; ${ }^{2}$ Diagnostica Stago, Asnières-sur-Seine, France

Background: Despite its entrance in the clinical laboratory thanks to automation, Thrombin generation (TG) assay remains mainly done in batch of frozen samples. Knowing the impact of freezing and the 
sample stability after freezing is critical to ensure the validity of results.

Aims: The study aims to assess the impact of freezing on TG measured on Haemophilia $A(H A)$, haemophilia $B(H B)$ and healthy individuals plasma samples, using STG-BleedScreen on ST Genesia, along with the sample stability for this assay after freezing at $-70^{\circ} \mathrm{C}$ or lower.

Methods: 8 severe HA, 2 severe HB and 10 healthy individuals were enrolled for the study after informed consent.

Plasma aliquots were prepared from their blood samples. One aliquot was tested fresh with STG-BLS immediately, while others were frozen at $-70^{\circ} \mathrm{C}$. Samples were tested 1 day (D1), 1 month, 2 months, 3 months, 6 months and 7 months after freezing.

Impact of freezing was assessed through paired-comparison tests on fresh and D1 samples, on Lag Time, Peak Height and Endogenous Thrombin Potential (ETP) parameters.

Evaluation of sample stability after freezing was assessed by analyzing the change over time of normalized results as described before (Douxfils J et al, JTH 2019).

Results: Impact of freezing is significant on ETP as shown on the $P$-values from Table 1.

TABLE 1 Impact of freezing is significant on ETP

$\begin{array}{llll}\text { P-values } & \begin{array}{l}\text { Lag time } \\ \text { absolute }\end{array} & \begin{array}{l}\text { Lag time } \\ \text { normalised }\end{array} & \begin{array}{l}\text { Peak Height } \\ \text { absolute }\end{array} \\ \text { Healthy } & 0.2633 & 0.8698 & 0.056 \\ \text { Haemophilia } & 0.6422 & 0.4706 & 0.0571\end{array}$

Graphical analysis of change over time on frozen samples show no statistical impact over the 7 months measured with a regression line never crossing the acceptance limits and showing a slope not statistically different from 0 .

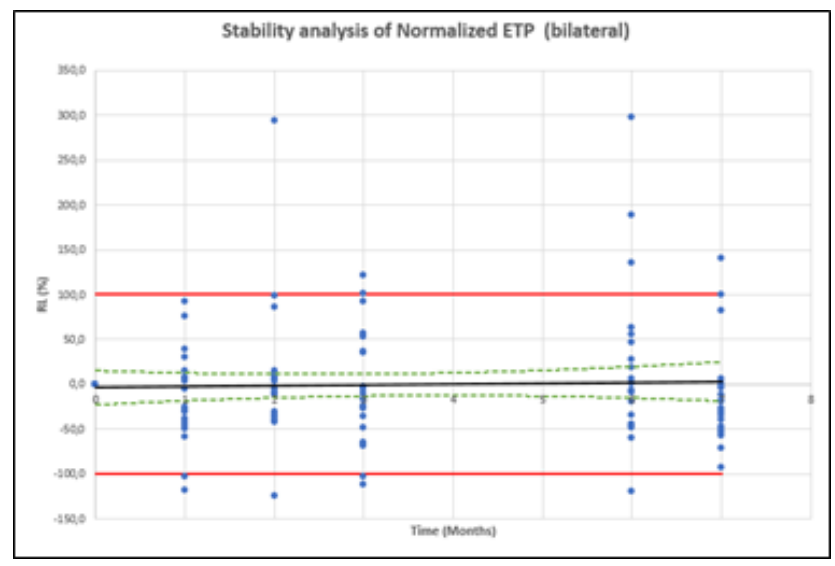

FIGURE 1 Stability analysis of Normalized ETP
Conclusions: Freezing plasma samples affects their TG when measured with STG-BleedScreen on ST Genesia. However, once frozen at $-70^{\circ} \mathrm{C}$ or lower, samples remain then stable over 6 months. Altogether this suggests the necessity to measure TG on plasma samples after a first freezing step at $-70^{\circ} \mathrm{C}$ to ensure consistency of results and analyses.

\section{PB0350 | Hemolysis Interference in the Anti-Xa Heparin Activity Assay}

K. Ruby ${ }^{1}$; L. Salvatore ${ }^{1}$; R. Lomachinsky ${ }^{1}$; D. Ornstein ${ }^{1,2}$; J. Khan ${ }^{1}$

${ }^{1}$ Department of Pathology and Laboratory Medicine, DartmouthHitchcock Medical Center, Lebanon, United States; ${ }^{2}$ Department of Medicine; Dartmouth-Hitchcock Medical Center, Lebanon, United States

Background: Catheter-based ventricular assist devices are increasingly used to support patients with end stage heart failure.
Anticoagulation to prevent device thrombosis often involves heparin infusions monitored by chromogenic anti-Xa assays. These devices produce hemolysis due to mechanical shearing of red blood cells, and hemolyzed specimens submitted for anti-Xa assays may be rejected due to anticipated test interference by free hemoglobin. In fact, the presence of free hemoglobin was shown to underestimate anti-Xa activity using the Stago liquid anti-Xa assay on Stago Compact analyzers with a concentration-dependent effect observed even in the absence of added heparin, suggesting that the mechanism of interference may be inherent in the assay. The effect of free hemoglobin on other chromogenic anti-Xa assay systems has not been reported. Aims: The purpose of this study was to investigate the effect of free hemoglobin on anti-Xa activity measurements using the HemosIL Liquid anti-Xa assay system.

Methods: Free hemoglobin was prepared by osmotic lysis of whole blood followed by centrifugation and dilution with OK buffer. Buffered hemoglobin was suspended in pooled normal plasma containing $0.0,0.3$ or $0.6 \mathrm{U} / \mathrm{mL}$ unfractionated heparin to final hemoglobin concentrations of $0-400 \mathrm{mg} / \mathrm{dL}$. The absorbance change ( $\mathrm{mAbs} / \mathrm{min}$ ) and residual factor Xa activity were determined in duplicate using the HemosIL Liquid anti-Xa assay on the ACL TOP 550 CTS Analyzer.

Results: Increasing concentrations of plasma hemoglobin produced no significant increase in the absorbance change slope (Figure 1) or 
decrease in anti-Xa activity (Figure 2 ) at heparin concentrations of $0.0,0.3$ and $0.6 \mathrm{U} / \mathrm{mL}$.

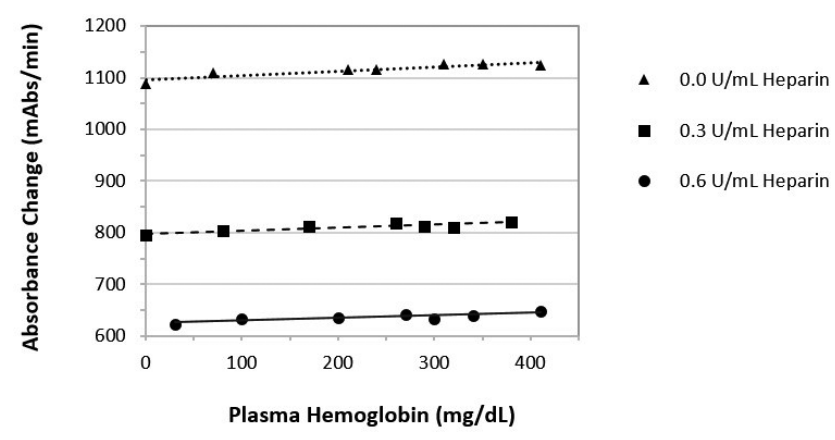

FIGURE 1 Effect of Plasma Hemoglobin on Average Absorbance Change

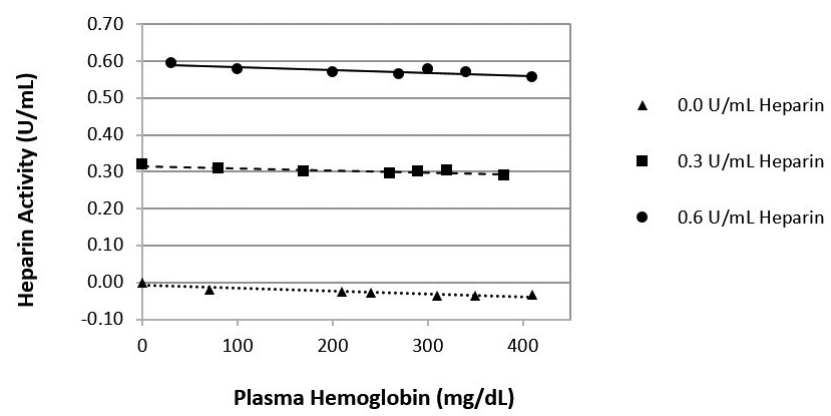

FIGURE 2 Effect of Plasma Hemoglobin on Anti-Xa Heparin Activity

Conclusions: In contrast to the Stago liquid anti-Xa assay, the presence of free hemoglobin did not result in a clinically significant underestimation of unfractionated heparin activity in the HemosIL Liquid anti-Xa assay. Evaluation of other marketed chromogenic anti-Xa assays is important, as interference from hemolysis may vary across different assay platforms.

\section{PB0351 | Measurement of Factor VIII in the Presence of} Emicizumab Is dependent on the chromogenic assay selected for use

M. Byrne; M. Doyle; K. Ryan; M. Lavin; J.S O'Donnell;

N.M O'Connell

National Coagulation Centre, Dublin, Ireland

Background: Prophylaxis of persons with Haemophilia A (PwHA) includes the nonfactor therapy, emicizumab; a humanised bispecific antibody with two antigen binding domains (Factor IX/IXa and Factor $\mathrm{X} / \mathrm{Xa}$ ), resulting in FIXa mediated activation of FX, eliciting the haemostatic response. Therapeutic Factor VIII (FVIII) concentrate must be measured by chromogenic substrate assay (CSA) with bovine components, avoiding interference by emicizumab. CSA may comprise human, partial bovine or fully bovine components.

Aims: To demonstrate that measurements of post-infusion FVIII concentrate in PwHA are influenced by the chromogenic assay used. Methods: In an in-vitro spiking study both congenital Factor VIII deficient plasma and a sample pool from PwHA treated with emicizumab (PwHA/emi) were spiked with Elocta to give a range of FVIII concentrations from 0.0-2.0 IU/ml. Factor VIII levels were measured by one stage clotting assay (OSCA) and/or by two CSA methods (Technoclone Technochrom ${ }^{\circledR}$ FVIII:C, partial bovine and HemosIL ${ }^{\circledR}$ Electrachrome ${ }^{\mathrm{TM}} \mathrm{FVIII}$, fully bovine) on the ACL Top 750. In addition, post-infusion FVIII levels were measured in samples from $\mathrm{PwHA}$ / emi $(n=38)$ treated with Elocta (PwHA/emi/Elocta) using both CSA. Results: FVIII levels in a range of Elocta spiked plasmas were higher by both CSA compared with the OSCA; (median difference Technochrom $^{\circledR}: 48 \%$, Electrachrome $\left.{ }^{\mathrm{TM}}: 17 \%\right)$.

FVIII levels in PwHA/emi spiked with a range of Elocta concentrations were higher with Technochrom ${ }^{\circledR}$ CSA compared with Electrachrome ${ }^{\mathrm{TM}}$ CSA (median difference $=29 \%$ ).

Post infusion samples $(n=38)$ from PwHA/emi/Elocta were assayed using both CSA. FVIII levels when measured by the Technochrom ${ }^{\circledR}$ assay were significantly higher than the Electrachrome ${ }^{\mathrm{TM}}$ assay (median difference 43\%, $P<0.05$ ).

Conclusions: Measured FVIII levels with the Technochrom ${ }^{\circledR}$ assay are higher when compared with the Electrachrome ${ }^{\mathrm{TM}}$ assay; this difference is magnified for PwHA/emi/Elocta patient samples. Measurement of FVIII in PwHA treated with Elocta in addition to emicizumab requires careful consideration when selecting the appropriate CSA to monitor this treatment.

\section{PB0352 | Normal Values in Thrombin Generation Parameters in} Hemorraghic Diseases

I. Tirado ${ }^{1}$; N. Vilalta ${ }^{1}$; L. Romero ${ }^{1}$; C. Vallvé ${ }^{1}$ I. Gich ${ }^{2}$; J.C. Souto ${ }^{1}$; J. Mateo ${ }^{1}$

${ }^{1}$ Hospital de la Santa Creu i Sant Pau. Servei d'Hematologia. Unitat d'Hemostàsia i Trombosi, Barcelona, Spain; ${ }^{2}$ Hospital de la Santa Creu i Sant Pau. Servei d'Epidemiologia, Barcelona, Spain

Background: Thrombin Generation (TG) assay is a comprehensive test that assesses thrombin formation during the coagulation process. ST-Genesia ${ }^{\circledR}$ is an automated system (STG, Diagnostica Stago) which provides a normalization of TG parameters against reference plasma in order to reduce technical variability.

Aims: To determine normal values for bleeding conditions with STGBleedScreen ${ }^{\circledR}$ (STG-BLS) test in a healthy population.

Methods: We tested: 44 healthy individuals: 19 women and 25 men aged between 22 and 85 years. STG-BLS test was performed with ST-Genesia ${ }^{\circledR}$ system. Parameters: Lag time (ratio), peak high (\%), time to peak (ratio), endogenous thrombin potential (ETP, \%), velocity index (Vel \%) and start tail (ratio) were analysed in absolute and normalized units. Normal reference intervals for STG-BLS 
parameters were calculated for all individuals and by sex and age ( $<50$ and $\geq 50$ years).

Results: Table 1 shows all normalized parameters. Although the variables do not show a normal distribution, mean values were similar to median values. Normalized variables showed lower variability than absolute values. In women aged $<50$, higher values were observed for peak height and ETP (Figure 1).

TABLE 1 Normalized Bleedscreen parameters

\begin{tabular}{|c|c|c|c|c|c|c|}
\hline $\begin{array}{l}\text { Normalized data } \\
(n=44)\end{array}$ & \begin{tabular}{|l} 
Lag time \\
(ratio)
\end{tabular} & Peak Height (\%) & \begin{tabular}{|l|} 
Time to \\
peak \\
(ratio)
\end{tabular} & ETP (\%) & Vel index (\%) & \begin{tabular}{|l} 
Start Tail \\
(ratio)
\end{tabular} \\
\hline $\operatorname{Men}(n=25)$ & $1,4 \pm 0,3$ & $\begin{array}{c}88,4 \pm 31,1 \\
83,1(66-107)^{*}\end{array}$ & $1,4 \pm 0,2$ & $\begin{array}{c}97,2 \pm 22,9 \\
97(83-116)^{*}\end{array}$ & $69,7 \pm 32,7$ & $1,15 \pm 0,1$ \\
\hline $\operatorname{Men}<50(\mathrm{n}=7)$ & $1,2 \pm 0.2$ & $\begin{array}{c}113,2 \pm 39,2 \\
100,4(76-147)^{*}\end{array}$ & $1,2 \pm 0,1$ & $\begin{array}{c}115,4 \pm 22,4 \\
118(89-133)^{*}\end{array}$ & $91,7 \pm 44,9$ & $1,1 \pm 0.1$ \\
\hline Men $250(n=18)$ & $1,5 \pm 0,3$ & $\begin{array}{r}78,7 \pm 21,8 \\
79,1(57-92)^{*}\end{array}$ & $1,4 \pm 0,2$ & $\begin{array}{c}90,1 \pm 19,4 \\
90,5(72-100)^{*}\end{array}$ & $61,1+22,8$ & $1,2 \pm .1$ \\
\hline Women $(n=19)$ & $1,3 \pm 0,3$ & $\begin{array}{c}122 \pm 46,4 \\
116,6(82-164)^{*}\end{array}$ & $1,2 \pm 0,2$ & $\begin{array}{c}120,7 \pm 31,7 \\
113,2(94-138)^{*}\end{array}$ & $116,7 \pm 68,1$ & $1,1 \pm 0,1$ \\
\hline Women <50 $(n=8)$ & $1,01 \pm 0,1$ & $\begin{array}{c}147,7 \pm 54,2 \\
143,3(103-204)^{*}\end{array}$ & $1,02 \pm 0,1$ & $\begin{array}{c}140,5 \pm 37,8 \\
141,9(108-179)^{*}\end{array}$ & $154,3 \pm 79,6$ & $1,01 \pm 0,1$ \\
\hline Women $\geq 50$ ( $n=11)$ & $1,4 \pm 0,3$ & $\begin{array}{c}103,4 \pm 30,3 \\
94,24(82-123)^{*}\end{array}$ & $1,33 \pm 0,2$ & $\begin{array}{c}106,2 \pm 16,3 \\
103,5(89-115)^{*}\end{array}$ & $89,4 \pm 44,2$ & $1,1 \pm 0,1$ \\
\hline
\end{tabular}

Mean values \pm SD. ${ }^{*}$ Median and interquartile range (25th and 75 th percentiles)

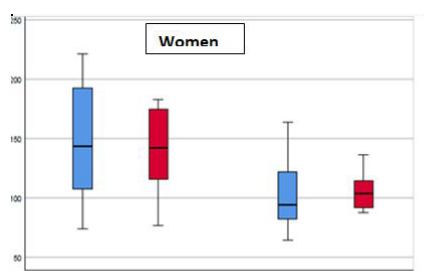

$<50$

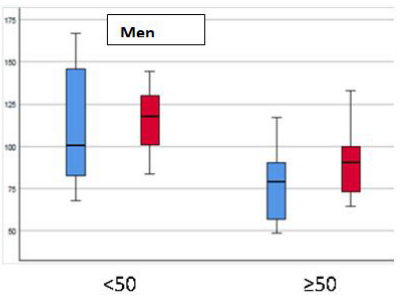

FIGURE 1 Peak height (blue) and ETP (red) by sex and age group

Conclusions: We found differences in Bleedscreen parameters according age and sex, for this reason we consider to establish normal values, subgroups of age and sex have to be taken into account.

PB0353 | Evaluation of a New Fully Automated ADAMTS13 Activity Assay

S. Geiter ${ }^{1}$; L. Wagner ${ }^{1}$; N. Binder ${ }^{1}$; U. Budde ${ }^{2}$

${ }^{1}$ Technoclone GmbH, Vienna, Austria; ${ }^{2}$ MEDILYS Laborgesellschaft $\mathrm{mbH}$, Hamburg, Germany

Background: ADAMTS13 activity is a unique marker for thrombotic thrombocytopenic purpura (TTP) which helps differentiate TTP patients from those suffering other more common thrombotic microangiopathies (TMA) and also less frequent conditions such as hemolytic uremic syndrome. In routine, a fully automated assay, delivering ADAMTS13 activity results within less than 30 min and without need to batch samples, spares the cost of unnecessary treatments and improves the overall outcome of the patient care.
Aims: The aim of this study was to evaluate the performance of the new fully automated ADAMTS13 activity assay in routine use by correlating the reults with those of the ADAMTS13 activity ELISA.

Methods: The Technofluor ADAMTS13 activity assay is based on a modified FRETS-VWF73-Fluorescence-Quenching-Substrate and can be performed fully automated on the coagulation analyzer Ceveron s100. A calibration curve is performed once per substrate lot. A method comparison study with 202 routine TMA patient samples was performed, comparing this new assay with ADAMTS13 activity ELISA. Both assays are calibrated against the WHO 1 st International Standard ADAMTS13 Plasma.

Results: Technofluor ADAMTS13 activity assay showed good correlation with ELISA ( $r$ 0.9246) assay, with the Passing-Bablok equation - Ceveron $(\mathrm{IU} / \mathrm{mL})=0.00575+1.113 \mathrm{ELISA}(\mathrm{IU} / \mathrm{mL})$. The study revealed high agreement with ELISA (Kappa 0.924) in classifying TTP patients with a deficiency of ADAMTS13 activity $<0.01 \mathrm{IU} / \mathrm{mL}$.

Conclusions: The fully automated ADAMTS13 activity assay showed a good correlation with the ELISA and excellent clinical performance for the diagnosis of severe ADAMTS13 deficiency. This fully automated ADAMTS13 activity assay is the optimal option for rapid evaluation of ADAMTS13 activity to confirm the diagnosis of acute clinical TTP in emergency settings.

PB0354 | Correlation between the Rosner Index with the Etiological Diagnosis of the Prolongation of the Activated Partial Thromboplastin Time (aPTT) in Asymptomatic Presurgical Patients

B.L. Diaz Jordan; V.B. Yepez Espinales; G. Lopez de la Osa Garcia Valdepenas General Hospital, Valdepenas, Spain

Background: aPTT prolongation in asymptomatic pre-operative patients (pts) is a common reason for haematology consultation. Rosner index (RI) is a guiding parameter for the aetiologic study of these pts.

Aims: The objective of this study is to assess the correlation between the percentage obtained in the calculation of the Rosner Index with the etiological study (determination of the deficit of coagulation factors and determination of nonspecific inhibitors) in asymptomatic patients with prolonged aPTT pending intervention.

Methods: A single-centre, retrospective analytical study that analysed (between 02/2017 and 02/2020) 74 pts with prolonged aPTT. The mixing test was performed and subsequent application of RI for group stratification ( $<12 \%$ suggesting factor deficiency, $12-15 \%$ undetermined, and $>15 \%$ suggesting inhibitor). In addition, FVIII, FIX, FXI, FXII, vWF and unspecific inhibitors diagnostic of antiphospholipid syndrome were measured bycoagulation technique and the results were correlated using the chi-square test and logistic regression.

Results: The median age was 60 years, $52.7 \%$ females. $16.2 \%$ did not show disorders in the measurement of factors and determination of inhibitors. $25.7 \%$ were diagnosed with intrinsic pathway coagulation factor deficiency, being factor XII deficit the most frequent (52.6\%). 
There was no statistically significant relationship between any of the factors and the Rosner index groups $(P=0.171)$. The presence of a nonspecific inhibitor was diagnosed in $58.1 \%$ of the participants. The lupus anticoagulant (present in $44.2 \%$ of the sample), demonstrated a relationship between the Rosner index and the presence of IgM anticardiolipin antibodies $(P=0.022)$ and $\lg G(P=0.007)$. When performing the logistic regression models, a relationship between a Rosner index $>15 \%$ (indicative of the presence of an inhibitor) and the simultaneous presence of lupus anticoagulant and IgM-lgG anticardiolipin antibodies was established $(P=0.003, \mathrm{OR}=4.33,95 \%$ Cl:1.43-13.08)

Conclusions: RI is auseful, easy-to-apply guiding parameter for the study of prolonged aPTT, with a statistically significant correlation in pts with unspecific inhibitors.

\section{PB0355 | Utility of the STG-Bleedscreen Assay in Clinical Routine}

T. Siegemund; A. Siegemund; H. Bönigk

MVZ Limbach Magdeburg, Magdeburg, Germany

Background: A bleeding diathesis can be caused by a variety of defects. A single factor level is not sufficient to predict the bleeding tendency in affected patients. Global assays, like the fully automated STG-Bleedscreen thrombin generation assay, may be better predictors of bleeding events and may help tailor treatment.

Aims: We aim to test the utility of the STG-Bleedscreen assay in diagnosis and monitoring of patients with bleeding disorders.

Methods: Patients with suspected or confirmed bleeding diathesis in our outpatient clinic were include in this study. Over 750 measurements were performed using STG-Bleedscreen on the ST Genesia analyzer (Stago). This includes about 100 samples from patients with hemophilia. Results are expressed as thrombin peak in nanomolar concentration, and normalized with a standard plasma (in \%).

Results: In average, patients with suspected bleeding diathesis (no hemophilia) showed a normal thrombin generation of $153 \pm 69 \mathrm{nM}$ (normalized $104 \pm 47 \%$ ). Nevertheless, patients with blood type $\mathrm{O}$ show significantly reduced thrombin generation accompanied with lower levels of FVIII and VWF.

Patients with haemophilia showed comparable reduction of thrombin generation: $53 \pm 31 \mathrm{nM}$ or $37 \pm 22 \%$ for haemophilia $\mathrm{A}$ and 48 $\pm 15 \mathrm{nM}$ or $32 \pm 10 \%$ for hemophilia B. A good correlation between factor VIII level and thrombin peak was observed. In patients receiving enhanced half-life products, thrombin generation clearly shows the prolonged protective effect.

Conclusions: The STG-Bleedscreen assay is useful in monitoring haemophilia and treatment. Especially in the monitoring of extended half-live products, the assay reflectss the prolonged protection. In patients with suspected bleeding diathesis, the assay might be able to separate patients with a defect in the plasmatic coagulation system. The STG-Bleedscreen assay is a fully automated assay. It can be easily implemented in daily routine, as it does not require high technical effort.

PB0356 | Activated charcoal can be used as an effective in vitro removal agent of direct oral anticoagulants dabigatran, rivaroxaban and apixaban in plasma of patients with protein $\mathrm{S}$ activity testing

S. Margetić ${ }^{1}$; I. Ćelap ${ }^{1}$; J. Buben ${ }^{2}$; M. Razum ${ }^{1}$; S. Šupraha-Goreta ${ }^{3}$

${ }^{1}$ University Hospital Center Sestre milosrdnice, Clinical Institute of Chemistry, Zagreb, Croatia; ${ }^{2}$ Faculty of Pharmacy and Biochemistry, University of Zagreb, Zagreb, Croatia; ${ }^{3}$ Faculty of Pharmacy and Biochemistry, Department of Molecular Biology and Biochemistry, University of Zagreb, Zagreb, Croatia

Background: As Other Coagulation Thrombophilia Assays, Testing of Protein S (PS) Activity Can also Be Influenced and Misinterpreted in Patients on Direct Oral Anticoagulants (DOACs)

Aims: To investigate the effect of DOACs on PS activity results and to evaluate the use of our own optimized method with activated charcoal $(A C)$ in removing potential interference.

Methods: The study included 45 plasma samples from patients treated with three DOACs (Table 1). DOACs concentrations were determined in native plasma. Then, using our method earlier optimized for other thrombophilia assays, $100 \mathrm{mg}$ of medical AC powder (Jadran Galenski laboratorij, Rijeka, Croatia) was added to $500 \mu \mathrm{L}$ plasma allowing DOACs adsorption for $10 \mathrm{~min}$. Treated samples were centrifuged $20 \mathrm{~min}$ at $1800 \times \mathrm{g}$ and DOACs concentrations and PS activity were measured in supernatant. Rivaroxaban and apixaban were determined using commercial anti-FXa assay (Siemens Healthineers, Germany) with drug specific calibrators (Hyphen Biomed, France). Dabigatran was measured using Innovance DTI assay (Siemens Healthineers, Germany). Protein S activity was determined using coagulometric method (Protein S Ac, Siemens Healthineers, Germany). All measurements were performed on BCSXP analyzer (Siemens Healthineers, Germany). Statistical analysis was done using Wilcoxon test by MedCalc Statistical Software version 11.5.1 (MedCalc Software, Belgium). The study was funded as part of Croatian Science Foundation research project IP-2016-06-8208.

Results: All three DOACs have shown significant interfering effect on PS activity results in terms of false increased values. In 37/45 of native plasma samples, results of PS activity were above upper limit of measuring range $(>130 \%)$ with significantly higher values in native than in $A C$ treated samples $(P=0.0001)$ (Figures 1 and 2). 


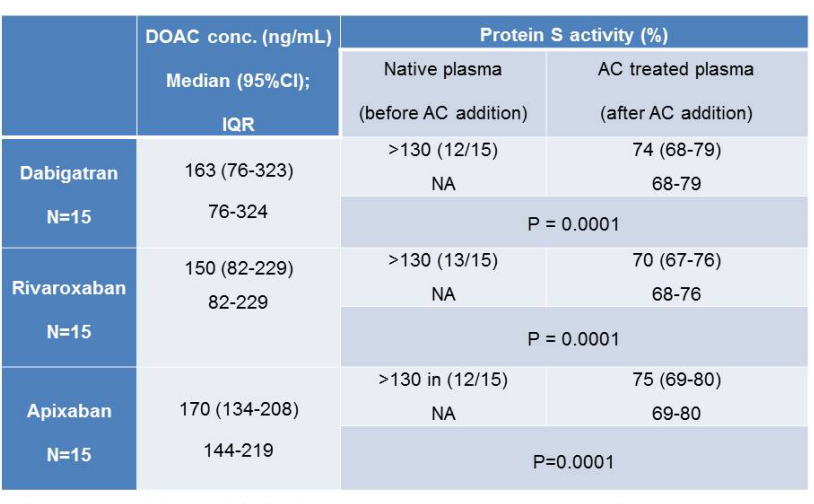

IQR = interquartle range; $A C=$ activated charcoal; $P<0.05$ was considered statistically significant

FIGURE 1 Results of protein S (PS) activity testing before and after addition of medical activated charcoal (AC) in patients treated with DOACs
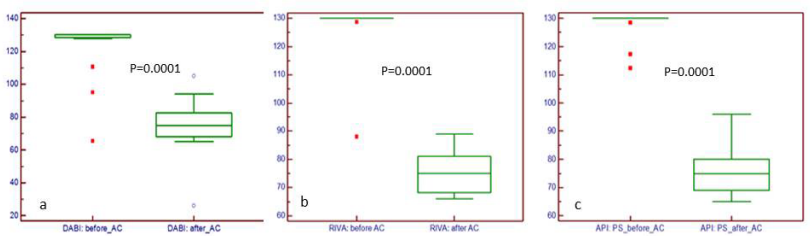

FIGURE 2 Differences between medians of protein S (PS) activities (\%) in dabigatran (a), rivaroxaban (b) and apixaban (c) native and activated charcoal $(\mathrm{AC})$ treated samples

Conclusions: All DOACs have strong interfering effect on protein $S$ activity in terms of possible false negative result. Our optimized method using medical AC has been found effective in overcoming interference of DOACs on PS activity testing.
PB0357 | Evaluation of Intra-site and Inter-site Precision of PT Determination with STA-NeoPTimal on Start Max Semi-automated Instrument

G. Le Goff ${ }^{1}$; M. Bouchain ${ }^{1}$; L. Chaillet ${ }^{2}$; J. Gautier ${ }^{3}$; V. Genty ${ }^{2}$;

S. Langlet ${ }^{1}$; A. Carlo ${ }^{4}$; J. Beltran ${ }^{1}$

${ }^{1}$ Diagnostica Stago, Gennevilliers, France; ${ }^{2}$ Amarok Biotechnologies, Saint-Malo, France; ${ }^{3}$ Cerba Xpert, Cergy, France; ${ }^{4}$ Diagnostica Stago,

Asnières-sur-Seine, France

Background: Prothrombin time (PT) is the first essential routine hemostasis parameter monitored in every laboratory. Consequently, ensuring its proper precision whatever the laboratory configuration is of utmost importance.

Aims: We evaluated the intra-site and inter-site precision of PT when determined with STA-NeoPTimal reagent used on the semiautomated instrument STart Max, following recommendations of EP05-A3 CLSI guidelines.

Methods: STA-NeoPTimal precision was evaluated on 6 unitary samples covering the whole measuring range: 2 normal, 3 from patients under vitamin $\mathrm{K}$ antagonist (VKA) and 1 deficient factor $\mathrm{V}$, using 3 batches of STA-NeoPTimal.

For single-site precision, a total of 40 runs with each batch of reagent were measured in duplicates on STart Max with 2 independent runs per day and 2 operators.

Multisite precision was determined on 3 sites, with 2 runs per day on 5 non-consecutive days, and 3 replicates per run.

All reagents and instruments were from Stago, Asnières-sur-Seine, France. 1 site was internal and 2 external.

For each STA-NeoPTimal batch used, the ISI used for INR determination was the one provided by the manufacturer. The mean normal prothrombin time used was the same for all sites, after local determination on the same normal samples.

Results: Results were calculated utilizing ANOVA tests.

TABLE 1 Intra-site precision - all lots combined

\begin{tabular}{|c|c|c|c|c|c|}
\hline Sample & $N$ & Mean (s) & within-lab precision CV(\%) & Mean (INR) & within-lab precision $\mathrm{CV}(\%)$ \\
\hline Normal 1 & 240 & 13.0 & 4.3 & / & / \\
\hline Normal 2 & 240 & 15.3 & 4.0 & / & / \\
\hline VKA 1 & 240 & 31.5 & 4.8 & 2.20 & 3.7 \\
\hline Deficient V & 240 & 49.0 & 10.0 & / & / \\
\hline VKA 2 & 240 & 61.0 & 6.1 & 4.17 & 5.2 \\
\hline VKA 3 & 240 & 89.6 & 7.3 & 6.04 & 6.7 \\
\hline
\end{tabular}

TABLE 2 Inter-site precision - all lots and sites combined

\begin{tabular}{|c|c|c|c|c|c|}
\hline Sample & $N$ & Mean (s) & Total precision CV(\%) & Mean (INR) & Total precision CV(\%) \\
\hline Normal 2 & 270 & 15.8 & 4.9 & / & / \\
\hline Deficient $\mathrm{V}$ & 270 & 49.4 & 9.7 & / & / \\
\hline VKA 2 & 270 & 62.5 & 7.0 & 4.27 & 6.0 \\
\hline
\end{tabular}


Conclusions: PT and INR determination with STA-NeoPTimal, on STart Max is very precise and reproducible at single site and multisite extent, over the whole measuring range. Besides, the intra-site precision confirmed the excellent between-operators precision. These results allow the safe implementation of the assay on this semi-automated technique.

\section{PB0358 | Analytical Performances of a New Immunoturbidimetric D-Dimer Assay}

$\underline{\text { L. Talon }}^{1}$; V. Fourneyron ${ }^{1}$; A. Trapani ${ }^{1}$; T. Sinegre ${ }^{1}$; A. Lebreton ${ }^{1,2}$

${ }^{1}$ Hématologie Biologique, CHU Clermont-Ferrand, Clermont-

Ferrand, France; ${ }^{2}$ Centre de Ressources et Compétences Maladies Hémorragiques Constitutionnelles, CHU Clermont-Ferrand, ClermontFerrand, France

Background: Widespread daily use of D-Dimer assay for exclusion of venous thromboembolism led to development of many assays. However, their usefulness depends on their reliability and performance. Aims: To evaluate the analytical performances of the Yumizen G DDi 2 assay (Horiba Medical) performed with the Yumizen G800 analyzer and to compare it with other available D-Dimer assays: Vidas D-Dimer Exclusion II on Vidas 3 (BioMérieux), Liatest D-Di Plus on STA-R Max (Diagnostica Stago), Innovance D-dimer on CS-2100i (Siemens Healthineers) and HemoSil D-Dimer HS500 on ACLTOP700 (Werfen). Methods: Within-run and between-run imprecision were evaluated using the low- and high-quality control plasmas. Limit of detection (LoD), limit of quantification (LoQ) and linearity were determined according to the Clinical and Laboratory Standards Institute. Interferences due to hemolysis, icterus or lipemia were evaluated by spiking plasmas with hemolysate, bilirubin or intralipids. Comparison with other available assays was evaluated with the Bland-Altman plot analysis.

Results: Within- and between-run coefficients of variations were comprised between $1.7 \%$ and $5.8 \%$ and $2.8 \%$ and $5.5 \%$ respectively (Table 1). The linearity of the assay was excellent up to $32700 \mathrm{ng} / \mathrm{mL}$ FEU. No impact of hemolysis and icterus was detected until a hemoglobin concentration of $10 \mathrm{~g} / \mathrm{L}$ and bilirubin concentration of $300 \mathrm{mg} / \mathrm{L}$. Lipemia interference seemed to underestimate $\mathrm{D}$-dimer level above an intralipid concentration of $5 \mathrm{~g} / \mathrm{L}$. Comparison study of Yumizen $\mathrm{G}$ DDi2 with other assays, performed with 66 consecutive fresh routine patients' plasmas demonstrated Spearman's rank correlation coefficients $>0.93$. Bland-Altman analysis revealed highly agreement between Yumizen G DDi 2 assay and other assays, especially with Vidas D-Dimer Exclusion II with a mean bias of $81.4 \mathrm{ng} / \mathrm{mL}$ FEU (Figure 1).
$1 \mathrm{~A}$

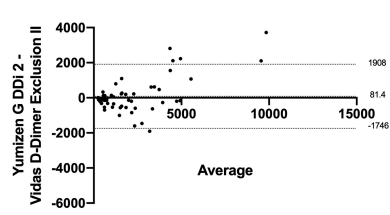

$1 \mathrm{C}$

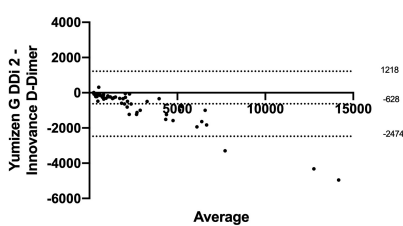

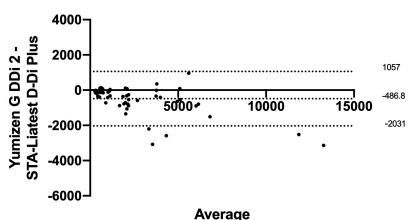

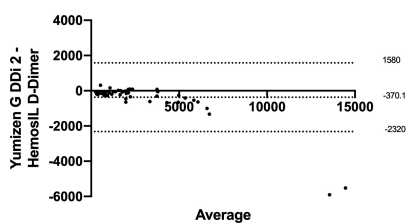

FIGURE 1 Bland Altman plots of D-Dimer values obtained with the Yumizen G DDi 2 assay with the Yumizen G800 analyzer and other available assays. The $x$ axis represents the mean of the measurements (ng/mL FEU) and the $Y$ axis represents the difference between measurements ( $\mathrm{ng} / \mathrm{mL}$ FEU). Continuous and dotted lines represent the bias and the lower and upper limits of agreement with a $95 \%$ confidence interval, respectively.

Conclusions: The analytical performances and the main technical features of the new Yumizen G DDi 2 assay make it a suitable method for the rapid quantification of D-dimer in clinical laboratories.

PB0359 | Next Generation Sequencing in Patients with Bleeding, Thrombotic and Platelet Disorders: A Single Centre Experience

N. Vilalta; I. Tirado; L. Romero; J.A. Millón; M. Carrasco;

M. Corrochano; J.C. Souto; J. Mateo

Hospital de la Santa Creu i Sant Pau, Servei d'Hematologia, Unitat d'Hemostàsia i Trombosi, Barcelona, Spain

Background: Inherited bleeding and thrombotic disorders are a heterogeneous group caused by DNA variants in a large number of loci. Their actual incidence is unknown because most of them are infra or misdiagnosed. Next generation sequencing (NGS) has provided information about diagnosis that impact on clinical management.

Aims: We are using NGS since 2019 and we show our findings below.

TABLE 1 Imprecision of the Yumizen G DDi 2 assay

\begin{tabular}{|c|c|c|c|c|c|c|}
\hline & & \multicolumn{4}{|c|}{ Mean value (ng/mL } & \multirow{2}{*}{$\begin{array}{l}\mathrm{CV}(\%) \\
\text { manufacturer }\end{array}$} \\
\hline & & $n$ & FEU) & $\mathrm{SD}$ (ng/mL FEU) & CV (\%) & \\
\hline \multirow[t]{2}{*}{ Within-run } & CTRL DDi I & 30 & 330 & 19 & 5.8 & 6.4 \\
\hline & CTRL DDi II & 30 & 1044 & 18 & 1.7 & 4.2 \\
\hline \multirow[t]{2}{*}{ Between-run } & CTRL DDi I & 32 & 500 & 27 & 5.5 & 3.5 \\
\hline & CTRL DDi II & 32 & 2015 & 55 & 2.8 & 4.0 \\
\hline
\end{tabular}


Methods: Our custom-gene panel (Nonacus) included 83 genes: 32 associated to bleeding disorders, 20 to thrombosis and 31 to platelet disorders (Table 1). Genes were selected according to previous evidence supporting their potential role in these pathologies. The sequencing results were processed by using a bioinformatic approach (Data genomics, Imegen) and assessed following the ACMG Guidelines.

TABLE 1 Genes included in our custom panel

\begin{tabular}{|l|l|l|l|l|}
\hline \multicolumn{5}{|l|}{ Bleeding associated genes } \\
\hline F12 & MCFD2 & FGG & SELP & CST3 \\
\hline F11 & F13A1 & KLKB1 & ORM1 & ABCC6 \\
\hline F10 & F13B & GGCX & COL3A1 & JAM3 \\
\hline F7 & FGA & VKORC1 & COL4A1 & ENG \\
\hline F5 & FGB & SERPINE1 & APP & ACVRL1 \\
\hline PLG & KNG1 & F2 & PLOD1 & SERPINF2 \\
\hline HPS3 & HPS5 & HPS6 & HPS7/DTNBP1 & AP3B1 \\
\hline HPS8/BLOC1S3 & ITGA2B & ITGB3 & HOXA11 & RBM8A \\
\hline CYCS & ANKRD26 & NBEA & FLI1/EWSR2 & PLAU \\
\hline TBXAS1 & RASGRP2 & TUBB1 & DIAPH1 & KDSR \\
\hline STXBP5 & \multicolumn{5}{l}{} \\
\hline Thrombosis associated genes & SLC44A2 & HRG & APOA1 \\
\hline PLAT & THPO & HPCA1 & PTPRD & C4BPA \\
\hline SERPIND1 & PROC & ABCA13- \\
\hline MPL & SERPINC1 & C4BPB & ZNF366 & ADAMTS13- \\
\hline & & & & ADAM \\
\hline THBD & STX2 & TSPAN15 & JAK-2 & \\
\hline Platelet disorders associated genes & HPS4 \\
\hline GP1BA & MYH9 & GFI1B & P2RY12 & PLA2G4A \\
\hline GP1BB & HPS1 & GP5 & ANO6 & \\
\hline GP9 & GP6 & NBEAL2 & & \\
\hline
\end{tabular}

Results: Ninety-seven patients were studied. We identified 50 mutations in 50 patients in 30 different genes: 13 (26\%) were pathogenic, $11(22 \%)$ probably pathogenic, $1(2 \%)$ probably benign, 14 (28\%) benign and 11 (22\%) mutations with unknown significance (Table 2). Considering pathogenic and probably pathogenic mutations, molecular diagnosis was established in 24 (48\%) patients.

In 20 out of 30 cases there was a prior clinical suspicion due to phenotypic conventional findings. But mutations in the HPS1, NBEAL, TUBB1, and SERPINE1 genes detected by NGS were very helpful in diagnosis.

TABLE 2 Genes and mutations identified

\begin{tabular}{|c|c|c|c|c|c|}
\hline Gene & Mutation & $\begin{array}{c}\text { Clinical } \\
\text { interpretation }\end{array}$ & Gene & Mutation & $\begin{array}{c}\text { Clinical } \\
\text { interpretation }\end{array}$ \\
\hline ACVRL & $c .2313 G \times A$ & Benign & GP9 & $C .182 A>B$ & Probabby pathogenic \\
\hline \multirow[t]{2}{*}{ ANKRD26 } & \multirow{2}{*}{ 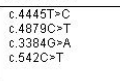 } & $\begin{array}{l}\text { Benigan } \\
\text { Benigan } \\
\text { Benign }\end{array}$ & ITGA2B & $\begin{array}{l}.1316>T \\
c .1424-1427 \mathrm{dup} \\
c .4390>0\end{array}$ & $\begin{array}{l}\text { Pathogenic } \\
\text { Pathogenic }\end{array}$ \\
\hline & & Denign & ITGB3 & 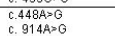 & $\begin{array}{l}\text { Unlnown significance } \\
\text { Probably pathogenic }\end{array}$ \\
\hline CYCS & $c .2951 A>B$ & Benign & MYH9 & $\begin{array}{l}0.3493 C \times T \\
.70<6\end{array}$ & $\begin{array}{l}\text { Pathouenic } \\
\text { Prophopic }\end{array}$ \\
\hline F2 & $c .1084 G \times A$ & Probabls pathogenic. & & & Probably pathogenic \\
\hline F5 & $c .3481 C>T$ & Pathogenic & NBEA & $c .7501 A>G$ & Benign \\
\hline F7 & $\begin{array}{l}c .805+33805+6 \mathrm{del} \\
c .9346=\mathrm{A}\end{array}$ & $\begin{array}{l}\text { Probably pathogenic } \\
\text { Probably pathogenic }\end{array}$ & NBEAL2 & $\begin{array}{l}\text { c.6318C>T } \\
c .1504 \_1707 \mathrm{del}\end{array}$ & $\begin{array}{l}\text { Benign } \\
\text { Pathogenic }\end{array}$ \\
\hline F11 & $\frac{1.9540, A}{c .1288 \theta \times T}$ & Probable pathogenic & SERPINE1 & 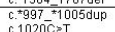 & Pathogenic \\
\hline FGA & $\begin{array}{l}C .532 C>C=C \\
\text { c. } 112 A>G\end{array}$ & $\begin{array}{l}\text { Pathogenic } \\
\text { Unknownth significance }\end{array}$ & SERPINC1 & $\begin{array}{l}\text { c.1109T>C } \\
\text { c.1218+1G } \Rightarrow A\end{array}$ & $\begin{array}{l}\text { Probably pathogenic } \\
\text { Pathogenic }\end{array}$ \\
\hline FGB & $\frac{C .1018 \geqslant T}{c .298 C \gg T}$ & Unknownow significance & PLG & $c .112 A>B$ & Pathogenic \\
\hline FGG & $\begin{array}{l}\text { C. } 1201 \mathrm{C}>\mathrm{T} \\
\text { c. } 1036 \mathrm{G} \times \mathrm{C}\end{array}$ & $\begin{array}{l}\text { Pathogenic } \\
\text { Probably pathogenic }\end{array}$ & PROC & 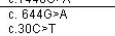 & $\begin{array}{l}\text { Probabbl pathogenic } \\
\text { Probably pathogenic }\end{array}$ \\
\hline & & Probable pathouenic & PLAU & $\frac{1.50 \times A}{c .172 G \approx A}$ & Denign \\
\hline GPI1B & $0.521 \mathrm{C} \gg \mathrm{A}$ & Unknown significance & THPO & $\begin{array}{l}0.994 C \times T \\
\end{array}$ & Unknown significance \\
\hline GP1BA & $0.129 \mathrm{G} \Rightarrow \mathrm{C}$ & Unknown significance & TBAXAS1 & $c .1352 \mathrm{C} \gg \mathrm{A}$ & Benign \\
\hline & & & TBXA2R & & Unknown significance. \\
\hline HOXA11 & $c . * 910 \mathrm{C} \gg \mathrm{T}$ & Benign & & $\begin{array}{l}c .1000>C \\
c .737 A>G\end{array}$ & $\begin{array}{l}\text { Benign } \\
\text { Unknown significance }\end{array}$ \\
\hline HPS1 & $c .972 \mathrm{del}$ & Pathogenic & TUBB1 & C.1267C>T & Pathogenic \\
\hline
\end{tabular}

Conclusions: A good phenotypic characterization is important in order to offer appropriate molecular diagnosis with custom-genepanels. In some cases, NGS is the only diagnosis tool.

By using NGS we found a high proportion (22\%) of mutations with unknown significance. To characterize their role in diseases, it would be necessary to perform functional studies.

A conclusive molecular diagnosis may impact clinical management.

PB0360 | The Effect of Trough Dabigatran Plasma Concentration on Rotational Thromboelastometry Parameters

M. Milić; J. Štanfel; K. Paradinović; V. Šerić

Clinical Hospital Centre Osijek, Osijek, Croatia

Background: A rapid assessment of the dabigatran anticoagulant effect is desirable for both dabigatran treated patients before emergent surgical procedures and for patients with hemorrhage or thrombosis. Specific assays for measuring dabigatran plasma concentration are not widely available. Rotational thromboelastometry (ROTEM) is a point-of-care testing device used to monitor hemostasis. Therefore, it would be useful to determine relative sensitivity of a ROTEM parameters to dabigatran.

Aims: To assess the effect of trough dabigatran plasma concentration on ROTEM parameters.

Methods: Blood was collected into 3.2\% Na-citrate tubes (BD Vacutainer) from 12 patients taking $150 \mathrm{mg}$ bd dabigatran for thromboprophylaxis. Viscoelastic analysis of whole blood was performed on ROTEM using EXTEM and INTEM tests (Werfen). Dabigatran levels in plasma were measured by ecarin chromogenic assay using STA-ECA II assay on the STA-R Evolution analyser (Stago).

Results: Mean dabigatran trough concentrations were $65.3 \mathrm{ng} /$ $\mathrm{ml} \pm 37.9$ SD. Spearman's coefficient of rank correlation (rho) was statistically significant between dabigatran concentration and $\mathrm{CT}$ EXTEM (rho $=0.832 ; \mathrm{Cl} 0.494$ to $0.952, P=0.005$ ) and CT INTEM (rho $=0.839 ; \mathrm{Cl} 0.512$ to $0.954, P=0.005$ ). There was no statistically significant correlation between dabigatran concentration and other ROTEM parameters: CFT, MCF and alpha angle. CT EXTEM was prolonged ( $>79 \mathrm{~s}$ ) in 9 out of 12 samples. CT INTEM was prolonged (>240 s) in 8 out of 12 samples. Both CT EXTEM and CT INTEM were normal in 2 out of 12 samples were dabigatran concentration were $<56 \mathrm{ng} / \mathrm{ml}$.

Conclusions: We demonstrated that trough dabigatran concentrations corelate well with CT EXTEM and CT INTEM. Normal levels for both CT EXTEM and CT INTEM suggest drug concentration levels below $56 \mathrm{ng} / \mathrm{ml}$. According to Eikelboom et al. most surgical procedures can safely be performed with dabigatran concentrations $<50$ $\mathrm{ng} / \mathrm{ml}$. Therefore, ROTEM tests EXTEM and INTEM can be useful for exclusion of higher levels of dabigatran. 
PB0361 | Effect and Stability of Dabigatran on Thrombin Generation over a Year in Elderly Patients with Atrial Fibrillation

T.R. Rocha ${ }^{1}$; A. Pastana ${ }^{2}$; P. Villaça $a^{1}$; E. D Amico ${ }^{1}$; P. Yu ${ }^{2}$;

I. Magalhães ${ }^{2}$; F.A. Cardozo ${ }^{2}$; G. Lustosa ${ }^{3}$; L. Franco ${ }^{3}$; B. Caramelli ${ }^{2}$; D. Calderaro $^{2}$

${ }^{1}$ University of São Paulo Medical School - Coagulation Laboratory - Hospital das Clínicas, Sao Paulo, Brazil; ${ }^{2}$ University of São Paulo Medical School - Interdisciplinary Medicine in Cardiology - Heart Institute (InCor), Sao Paulo, Brazil; ${ }^{3}$ Stago, Sao Paulo, Brazil

Background: Direct oral anticoagulants are first options for thromboembolism prevention in non-valvular atrial fibrillation. Little is known about their effect on thrombin generation (TG) parameters and there is no information about the stability of anticoagulation effect during treatment.

Aims: The first aim of this study is to analyze the effect of dabigatran on TG parameters and then to assess the stability of the identified parameters over time.

Methods: Our local ethics committee approved the protocol. We included 25 patients with atrial fibrillation and age above or equal to 70 yrs. scheduled to start dabigatran treatment. Blood samples for TG assay in automated system (ST GENESIA) were collected before (time A), 3 months (time B) and 12 months(time C) after dabigatran start. Comparisons between TG parameters in time $A$ and $C$ showed the effect of dabigatran, and comparisons between times $B$ and $C$ showed stability or the effect.

Results: Dabigatran significantly prolonged the Lag Time: $3.30 \pm$ 1.44 vs $1.67 \pm 0.49, P<0.001$, and the Time to Peak: $4.41 \pm 1.18$ vs $3.02 \pm 0.75, P<0.001$. There was no significant effect on Thrombin Peak ( $259.79 \pm 98.86$ vs $297.82 \pm 69.05, P=0.06$ ) or on Endogenous Thrombin Potential (950.68 \pm 365.28 vs $286.82, P=0.012$ ). The comparisons of LagTime ( $3.36 \pm 1.22$ vs $3.30 \pm 1.14, P=0.98)$ and Time to Peak ( $4.48 \pm 1.29$ vs $4.41 \pm 1.18$ and $P=0.99)$ between 3 and 12 months showed impressive stability (Figure1).
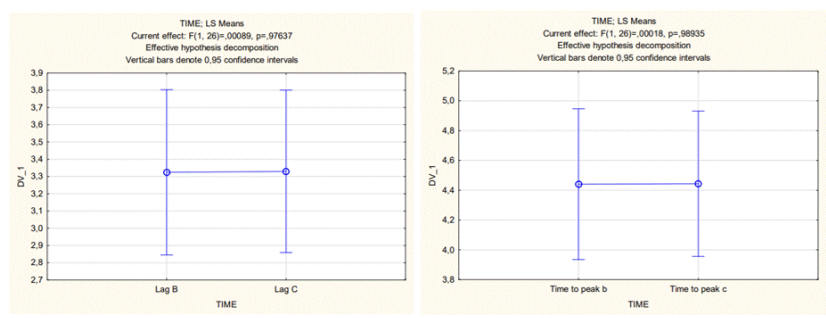

FIGURE 1 Comparison between LagTime and Time to Thrombin Peak in 3 and 12 months of dabigatran therapy

Conclusions: Dabigatran increased the Lag-Time and Time to Thrombin Peak in the TG ST-GENESIA assay and these effects remained impressively stable over the time.
PB0362 | In vitro Neutralization of Unfractionated Heparin for the Measurement of Thrombin Generation with the STGdrugScreen Reagent on ST Genesia

S. Fontaine ${ }^{1}$; M. Bourdin ${ }^{1}$; M.-A. Bildan ${ }^{1}$; O. Kerleau ${ }^{1}$; M. Lambert ${ }^{1}$; F. Depasse ${ }^{2}$; O. Mathieu ${ }^{1}$

${ }^{1}$ Diagnostica Stago, Recherche Prospective R\&D, Gennevilliers, France; ${ }^{2}$ Diagnostica Stago, Développement Clinique, Asnières-sur-Seine,

France

Background: The ST Genesia (STG) is a fully automated thrombin generation test (TGT) platform more standardized than the original Calibrated Automated Thrombogram (CAT) and designed to support clinical applications in the lab.

In some clinical indications such as prevention of thrombosis or ExtraCorporeal Membrane Oxygenation, patients may receive unfractionated heparin (UFH). TGT is adversely affected by UFH, thereby it cannot reflect basic haemostatic state (without the influence of heparin) in these patients, requiring UFH neutralization. Polybrene $(\mathrm{Pb})$ at $25 \mu \mathrm{g} / \mathrm{mL}$ has been shown to be effective for UFH neutralization in the original CAT system ${ }^{(1)}$.

Aims: Conceive an automated TGT test using the STG-DrugScreen reagent neutralizing $\mathrm{UFH}$ and allowing to restore basic TGT parameters.

Select between $\mathrm{Pb}$ and Heparinase I (Hep) the best neutralizing agent based on test performances: dose-response effect, neutralization performances on UFH-spiked samples and on board stability. Methods: Neutralizing agent ( $\mathrm{Pb}$ or Hep) was added at different concentrations to the reconstituted STG-DrugScreen reagent.

Normal Pooled Plasma (NPP) and twelve donors' leftover plasma samples were spiked with UFH. NPP was spiked with five different levels of sodium unfractionated heparin $(\mathrm{HNa})$ and two levels of calcium heparin ( $\mathrm{HCa}$ ) to cover the STA-Liquid anti-Xa measuring range of $0-1.08 \mathrm{IU} / \mathrm{mL}$ (Table 1 ). 
TABLE 1 Evaluation of neutralizing agents in STG-DrugScreen reagent with citrated plasma samples

\begin{tabular}{|c|c|c|c|c|}
\hline Performances & Sample & $\begin{array}{l}\text { UFH Anti-Xa } \\
\text { concentrations }\end{array}$ & Neutralizing agent & Analytical result \\
\hline Dose-response & NPP & $\leq 0.10 \mathrm{IU} / \mathrm{ml}$ & $\begin{array}{l}\mathrm{Pb}: 0-210 \mu \mathrm{g} / \mathrm{mL} ; \\
6 \text { levels } \\
\text { Hep: } 0-1.6 \mathrm{IU} / \mathrm{mL} ; \\
7 \text { levels }\end{array}$ & $\begin{array}{l}\text { Relative deviation to } \\
\text { STG-DrugScreen TGT } \\
\text { baseline }\end{array}$ \\
\hline $\begin{array}{l}\text { Neutralization } \\
\text { performance on } \\
\text { spiked NPP }\end{array}$ & Heparin-spiked plasmas & $\begin{array}{l}\text { HNa: } 0-1.08 \mathrm{IU} / \mathrm{mL} ; \\
6 \text { levels } \\
\text { HCa: } 0-1.07 \mathrm{IU} / \mathrm{mL} ; \\
3 \text { levels }\end{array}$ & $\begin{array}{l}\text { Pb: } 0-210 \mu \mathrm{g} / \mathrm{mL} \text {; } \\
6 \text { levels } \\
\text { Hep: } 0-1.6 \mathrm{IU} / \mathrm{mL} \text {; } \\
7 \text { levels }\end{array}$ & $\begin{array}{l}\text { Relative deviation to } \\
\text { STG-DrugScreen TGT } \\
\text { baseline }\end{array}$ \\
\hline $\begin{array}{l}\text { On-board stability } \\
\text { of modified } \\
\text { STG-DrugScreen }\end{array}$ & Heparin-spiked plasmas & $\begin{array}{l}\text { HNa: } 0-0.8 \mathrm{IU} / \mathrm{mL} ; \\
3 \text { levels }\end{array}$ & $\begin{array}{l}\mathrm{Pb}: 25 \mu \mathrm{g} / \mathrm{mL} \\
\text { Hep: } 1.2 \mathrm{IU} / \mathrm{mL}\end{array}$ & $\begin{array}{l}\text { Relative deviation of } \\
\text { STG-DrugScreen } \\
\text { TGT parameters at T4 } \\
\text { hours versus T0 }\end{array}$ \\
\hline $\begin{array}{l}\text { Neutralization } \\
\text { performance on } \\
\text { donor samples }\end{array}$ & $\begin{array}{l}\text { Donors' leftover plasma } \\
\text { samples spiked with Hna }\end{array}$ & $\begin{array}{l}\mathrm{HNa}: 0-0.8 \mathrm{IU} / \mathrm{mL} ; \\
12 \text { samples }\end{array}$ & $\mathrm{Pb}: 25 \mu \mathrm{g} / \mathrm{mL}$ & $\begin{array}{l}\text { Relative deviation to } \\
\text { STG-DrugScreen TGT } \\
\text { parameters before } \\
\text { versus after heparin } \\
\text { neutralization }\end{array}$ \\
\hline
\end{tabular}

Results: Table 2 provides summary of assay performances.

TABLE 2 Summary of performance evaluation

Performances
Dose-response
Neutralization performance on heparin-spiked
NPP
On board stability of modified STG-DrugScreen

Neutralization performance on donor samples

\section{Results}

$\mathrm{Pb}$ had no significant impact for $\mathrm{Pb}$ at concentrations $<150 \mu \mathrm{g} / \mathrm{mL}(<10 \%)$.

Hep had minimal impact (< $0 \%$ ) on TGT of NPP regardless to the tested concentrations.

$25 \mu \mathrm{g} / \mathrm{mL}$ of $\mathrm{Pb}$ or $1.2 \mathrm{IU} / \mathrm{mL}$ of Hep completely neutralized the effect of both $\mathrm{HNa}$ and HCa UFH on TGT.

With $25 \mu \mathrm{g} / \mathrm{mL}$ of $\mathrm{Pb}$ or $1.2 \mathrm{IU} / \mathrm{mL}$ of Hep, on board stability relative deviation to reference time was less than $8 \%$ for all parameters except $11 \%$ for NPP Lag Time with Hep. On board stability with $25 \mu \mathrm{g} / \mathrm{mL}$ of $\mathrm{Pb}$ was up to $4 \mathrm{~h}$.

Relative deviation to STG-DrugScreen was less than $8 \%$ with $25 \mu \mathrm{g} / \mathrm{mL}$ pf Pb, except for one donor sample which shifted to a more rapid TGT up to 14\% Lag Time reduction.
Conclusions: Our results show that $25 \mu \mathrm{g} / \mathrm{mL}$ of $\mathrm{Pb}$ or $1.2 \mathrm{IU} / \mathrm{mL}$ of Hep can completely neutralize the anticoagulant effect of UFH up to $1.08 \mathrm{IU} / \mathrm{mL}$. At these concentrations, TGT parameters measured in NPP were not impacted. Pb showed slightly better on-board stability than Hep.

These in vitro results indicate that $\mathrm{Pb}$ or Hep can be used added to STG-DrugScreen reagent to automatically neutralize UFH for TGT measurement on STG with a four-hour reagent on-board stability. This allows to abolish heparin interference and assess the basic TGT profile of a patient.

(1) Carlo A et al. ISTH 2009 PP-WE-164
PB0363 | Evaluation of Effect of Extracorporeal Near-infrared Laser-mediated Photobiomodulation Therapy on Endothelial Dysfunction Improvement Using a New B-mode Ultrasound Image Processing Software-based Automatic Method

$\underline{\text { H. Mehrad }}^{1,2}$; M. Farhoudi ${ }^{3}$

${ }^{1}$ Islamic Azad University, Basic Sciences Faculty, Department of Physics, Tabriz Branch, Tabriz, Iran, Islamic Republic of; ${ }^{2}$ Mehrad Research Lab, Division of Translational Development of Noninvasive Treatments, Tabriz, Iran, Islamic Republic of; ${ }^{3}$ Tabriz University of Medical Sciences, Neurosciences Research Center (NSRC), Tabriz, Iran, Islamic Republic of

Background: Endothelial cells form the barrier between vessels and tissue and control the flow of substances and fluid into and out of 
a tissue. Endothelial dysfunction is a type of non-obstructive artery disease in which the blood vessels constrict instead of dilating.

Aims: In this study, we developed an experimental near- infrared laser- mediated photobiomodulation therapy system, and investigated its effectiveness on inflammation reduction and improvement of endothelial dysfunction in the hamster common carotid artery model of early stage atherosclerosis, using a new computerized ultrasound image processing software.

Methods: Briefly, golden Syrian hamsters' right common carotid arteries in the treatment group, treated using extracorporeal near- infrared laser- mediated photobiomodulation therapy system $(\lambda=850$ $\mathrm{nm}, P=150 \mathrm{~mW}$ ). In order to evaluate endothelial-dependent relaxation, acetylcholine-mediated dilation (AMD) was measured during the infusion of acetylcholine at a rate of $0.5 \mu \mathrm{g} / \mathrm{kg} / \mathrm{min}$ and endothelial independent relaxation was evaluated by measuring nitroglycerin mediated dilation (NMD) during the infusion of nitroglycerin at a rate of $5 \mu \mathrm{g} / \mathrm{kg} / \mathrm{min}$. For off-line analysis, a computerized analysis method for evaluating instantaneous changes in the wall of the hamster carotid artery was used. For automatic measurement of the mean wall thickness and the percentage of luminal cross-sectional area of stenosis from longitudinal B-mode ultrasound images, two algorithms, i.e., maximum gradient and dynamic programming were composed and implemented. Reference points and cost function were based on dynamic programming and maximum gradient, respectively.

Results: Results from computerized B- mode ultrasound image processing software data showed significant differences in AMD between the treated and the non-treated hamsters $(P<0.05)$, whereas there were no significant differences in NMD between the treatment and normal groups $(P>0.05)$.

Conclusions: It is concluded that the new automatic method enables accurate and repeated evaluation of improvement of endothelial function and regression of early stage atherosclerosis using nearinfrared laser- mediated photobiomodulation therapy in this animal model.

PB0364 | A Comparison of Functional Methods with Absolute Quantification of Heparin Levels in Clinical Samples by Heparin Red Assay

E. Bontekoe $^{1}$; F. Siddiqui ${ }^{1}$; D. Hoppensteadt ${ }^{1}$; A. Kouta ${ }^{1}$; J. Fareed ${ }^{1}$; R. Kraemer ${ }^{2}$

${ }^{1}$ Loyola University Medical Center, Maywood, United States;

${ }^{2}$ Heidelberg University, Heidelberg, Germany

Background: Activated partial thromboplastin time (aPTT) method is widely used as a routine blood test and is commonly used for the monitoring of heparin levels in clinical patient samples. Heparin Red assay utilizes fluorescence for the direct and sensitive detection of the absolute level of heparin in plasma.

Aims: The purpose of this study is to compare functional activities (anticoagulant and anti-Xa levels) in clinical patient samples to the absolute levels of heparin to determine endogenous activity upon the therapeutic administration of this agent.

Methods: Plasma samples from patients treated with therapeutic dosage of heparin $(n=100)$ were collected from Loyola University Medical Center. Citrated blood samples were analyzed using aPTT clotting method, anti-Xa chromogenic assay and Heparin Red (Redprobes UG, Deutschland) assay relative to a commercially used heparin (Medefil) calibration curves. Normal controls were comprised of commercially available 25 male and 25 female citrated plasma samples (George King Biomedical, Overland Park, Kansas City).

Results: Marked increases were noted in aPTT (81.11 \pm 7.37 , normal $30.16 \pm 0.80 \mathrm{~s} ; P<0.001)$, anti-Xa (43.90 \pm 2.83 , normal $0 \pm 0$ $\%$ Inhibition; $P<0.001$ ), and Heparin Red recovered concentration $(2.90 \pm 0.16$, normal $0.07 \pm 0.03 \mathrm{ug} / \mathrm{ml} ; P<0.001)$ in clinical samples compared to normal controls. Significant correlations were observed between Heparin Red and other functional parameters studied.

Conclusions: These studies demonstrate that Heparin Red method is a reliable assay for the absolute quantification of circulating heparin level in plasma. Unlike the functional methods, which are also influenced by many endogenous factors, such as AT levels and variations in the clotting proteins, Heparin Red detects absolute amounts of circulating heparin in plasma which are not influenced by endogenous factors and other anticoagulant drugs.

PB0365 | Presentation of the Patient with Low Peak Dabigatran Levels in Plasma Suggests the Importance of Quantitative Measurement of DOAC Drugs in Clinical Decision Making and Treatment

S. Margetić ${ }^{1}$; I. Ćelap ${ }^{1}$; A. Lovrenčić-Huzjan²; M. Bosnar Puretić M. Roje Bedeković ${ }^{2}$; M. Razum ${ }^{1}$; R. Mihić ${ }^{1}$; S. Šupraha Goreta ${ }^{3}$

${ }^{1}$ Sestre Milosrdnice University Hospital Center/Department of Clinical Chemistry, Zagreb, Croatia; ${ }^{2}$ Sestre Milosrdnice University Hospital Center/Department of Neurology, Zagreb, Croatia; ${ }^{3}$ Faculty of Pharmacy and Biochemistry, University of Zagreb/Department of Biochemistry and Molecular Biology, Zagreb, Croatia

Background: In special clinical conditions quantitative measurement of direct oral anticoagulants (DOACs) in plasma, should be performed to help clinical decision making and treatment.

Aims: To present a case report of a patient treated with dabigatran in whom low peak drug concentrations in plasma suggested inadequate anticoagulation.

Methods: A 88 years old male was hospitalized due to recurrent symptoms of cerebral ischemia for last few days and brain ischemia was confirmed on CT scan. Before hospitalization, the patient was taking dabigatran (110 mg twice daily) due to persistent atrial fibrillation. Dabigatran was measured by Innovance DTI assay and rivaroxaban was measured by anti-FXa assay on BCSXP analyzer (Siemens Healthineers, Germany). The study was funded 
by the Croatian Science Foundation as a part of the research project IP-2016-06-8208.

Results: Peak (two hours after the last dose) concentrations of dabigatran measured at three consecutive days were 35, 16 and 19 $\mathrm{ng} / \mathrm{mL}$, whereas trough (before the next dose) concentrations were and $34,<5$ and $14 \mathrm{ng} / \mathrm{mL}$, respectively. Low peak dabigatran concentrations obtained in three consecutive measurements suggested anticoagulation undertreatment that might result with embolic complications. This observation led to the decision to replace dabigatran therapy with rivaroxaban $(1 \times 20 \mathrm{mg} /$ day $)$. Measurement of rivaroxaban plasma levels after the second day of administration has shown both peak and trough rivaroxaban concentrations within expected therapeutic values i.e., 215 and $30 \mathrm{ng} / \mathrm{mL}$, respectively. No new ischemic symptoms occurred and the patient was discharged home with rivaroxaban therapy. Results of laboratory testing are presented in Figure 1.

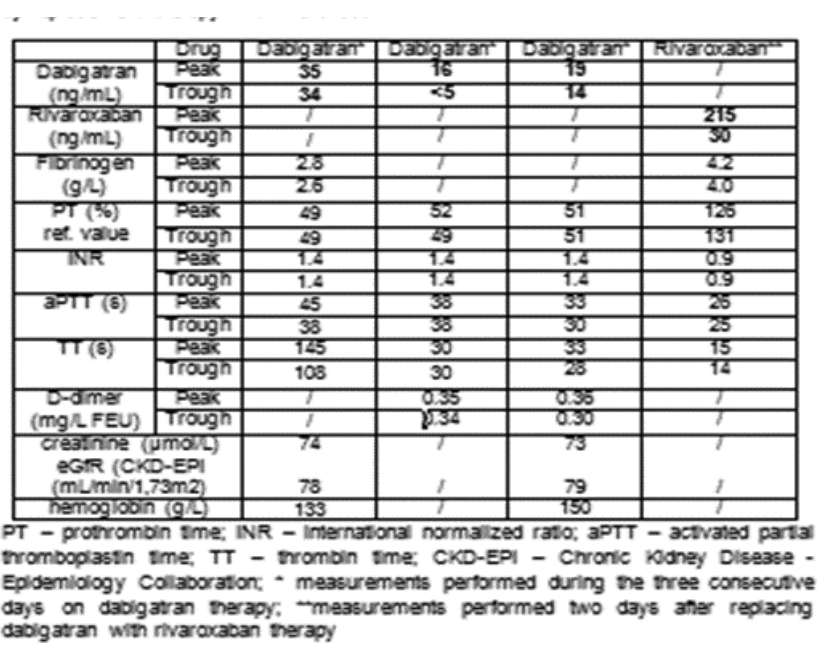

FIGURE 1 The results of laboratory testing in a patient treated with dabigatran followed by replacement therapy with rivaroxaban

Conclusions: Persistently low peak dabigatran concentrations could contribute to inadequate anticoagulation and consequent embolic complications in our patient. This case report strongly suggests the importance of quantitative measurement of DOACs levels in plasma in selected clinical conditions confirming as an effective approach in clinical decision making and treatment.
PB0366 | Multicenter Performance Evaluation and Reference Ranges for the Quantitative Determination of Factor XII Activity by One-stage Factor Assay on the Cobas t 711 Analyzer

M. Büchsel ${ }^{1}$; J. Creaghan²; U. Geisen ${ }^{1}$; P. Jilma ${ }^{3}$; R. Jones ${ }^{2}$;

S. Kitchen ${ }^{2}$; G. Rozsnyai ${ }^{4}$; P. Quehenberger ${ }^{3}$

${ }^{1}$ Institute for Clinical Chemistry and Laboratory Medicine, University Medical Center Freiburg, Freiburg, Germany; ${ }^{2}$ Sheffield Haemostasis and Thrombosis Centre, Sheffield, United Kingdom; ${ }^{3}$ Department of Laboratory Medicine, Medical University of Vienna, Vienna, Austria; ${ }^{4}$ Roche Diagnostics International Ltd, Rotkreuz, Switzerland

Background: Factor XII (FXII) initiates the intrinsic coagulation pathway. FXII deficiency is mostly asymptomatic; thus, discovery is usually by detection of a prolonged activated partial thromboplastin time (aPTT). We performed a multicenter evaluation of a one-stage clotting assay in combination with aPTT for the quantitative determination of FXII activity.

Aims: Evaluate the analytical performance of the FXII assay on the high throughput cobas t 711 analyzer (Roche Diagnostics International Ltd, Rotkreuz, Switzerland).

Methods: Performance evaluation at sites in Freiburg, Sheffield, and Vienna (September 2020-February 2021) used 3.2\% citrated, anonymized, ethically approved, residual routine or commercially purchased plasma samples. Five human plasma pools and two controls were used for determination of assay repeatability (one run/ site; $n=21$ replicates/sample), intermediate precision, and total reproducibility (five aliquots/sample/day for five days) and coefficients of variation (CVs) were calculated. Lot-to-lot variability (three reagent lots) and method comparison (versus Siemens Coagulation FXII Deficient Plasma [with Dade Actin FS] on Sysmex CS-5100 analyzer), using three sets of $\geq 120$ samples covering the assay measuring range, were evaluated by Passing-Bablok and Deming regression, respectively, and Pearson's $r$ calculated. The reference range was determined with three different reagent lots (Freiburg only) using fresh plasma samples from 199 healthy individuals aged $\geq 18$ years, not receiving anticoagulants.

Results: Repeatability for the FXII assay across all sites was excellent (CV, 0.8-3.2\%). High intermediate precision (CV, 1.5-5.1\%) and good total reproducibility (CV, 3.5-4.3\%) were demonstrated. The FXII assay showed excellent lot-to-lot variability (Pearson's $r$, 0.997-0.998;

Table 1) and good agreement with the comparator assay (Pearson's $r$, 0.967-0.989; Table 2). Among healthy individuals, $2.5^{\text {th }}$ and $97.5^{\text {th }}$ percentiles for FXII activity were: $52.7 \mathrm{IU} / \mathrm{dL}$ and $169.0 \mathrm{IU} / \mathrm{dL}$.

TABLE 1 Lot-to-lot comparisons for the Roche FXII assay on the cobas t 711 analyzer

$\begin{array}{llll}\text { Assay } & \text { FXII } & & \\ \text { Site } & \text { Freiburg } & \text { Sheffield } & \text { Vienna } \\ \text { Lot } & \text { A vs B } & \text { C vs A } & \text { B vs C } \\ \text { Sample, } n & 130 & 123 & 123\end{array}$




\begin{tabular}{llll} 
ABSTRACT & & & \\
\hline & FXII & & \\
Assay & 0.991 & 1.012 & 1.021 \\
Slope (Passing-Bablok) & 0.10 & 0.06 & 0.66 \\
$\begin{array}{l}\text { Intercept (Passing-Bablok) } \\
\text { Pearson's } r\end{array}$ & 0.998 & 0.997 & 0.997 \\
$\begin{array}{l}\text { Relative mean bias at 20 IU/ } \\
\quad \text { dL, \% }\end{array}$ & -0.4 & 1.5 & 5.4 \\
$\begin{array}{l}\text { Relative mean bias at 70 IU/ } \\
\text { dL, \% }\end{array}$ & -0.7 & 1.3 & 3.4 \\
\hline
\end{tabular}

TABLE 2 Method comparison between the Roche and Siemens FXII assays

\begin{tabular}{|c|c|c|c|}
\hline Assay & FXII & & \\
\hline Site & Freiburg & Sheffield & Vienna \\
\hline Lot & $A$ & C & B \\
\hline Sample, $n$ & 122 & 123 & 122 \\
\hline Slope (Deming) & 1.093 & 0.963 & 1.021 \\
\hline Intercept (Deming) & 1.14 & 1.52 & 0.58 \\
\hline Pearson's $r$ & 0.989 & 0.967 & 0.982 \\
\hline $\begin{array}{l}\text { Relative mean bias at } 20 \text { IU/ } \\
\text { dL, \% }\end{array}$ & 15.0 & 3.9 & 5.0 \\
\hline $\begin{array}{l}\text { Relative mean bias at } 70 \text { IU/ } \\
\quad d L, \%\end{array}$ & 11.5 & -0.6 & 3.3 \\
\hline
\end{tabular}

Conclusions: The robust analytical performance of the FXII assay on the cobas $\mathrm{t} 711$ analyzer supports its use in the routine clinical laboratory setting.

PB0367 | A Systematic Review and Meta-analysis of Performances of Prototype and Commercial DOAC Dipstick Tests for Detecting Direct Oral Anticoagulants in Patient Urine Samples - Increasing Confidence for Testing in Emergency Care

A. Martini ${ }^{1}$; R. Bauersachs ${ }^{2}$; J. Beyer Westendorf ${ }^{3}$; M. Crowther ${ }^{4}$; J. Douxfils ${ }^{5,6}$; I. Elalamy ${ }^{7}$ J. Harenberg ${ }^{8,9}$; S. Hetjens ${ }^{1}$; C. Weiss ${ }^{1}$

${ }^{1}$ Ruprecht-Karls-University Heidelberg, Department of Medical Statistics and Biomathematics, Medical Faculty Mannheim, Mannheim, Germany; ${ }^{2}$ Klinikum Darmstadt GmbH, Department of Vascular Medicine, Darmstadt, Germany; ${ }^{3}$ University Hospital Dresden, Department of Medicine I, Dresden, Germany; ${ }^{4}$ McMaster University, Department of Medicine, Hamilton, Canada; ${ }^{5}$ University of Namur, Department of Pharmacy, Namur, Belgium; ${ }^{6}$ Qualiblood S.A., Namur, Belgium; ${ }^{7}$, Hôpitaux Universitaires Est Parisien, Assistance Publique Hôpitaux de Paris, Paris, France; ${ }^{8}$ Heidelberg University, Heidelberg, Germany; ${ }^{9}$ DOASENSE GmbH, Heidelberg, Germany

Background: The DOAC Dipstick ${ }^{\mathrm{TM}}$ accurately determines the presence or absence of oral direct factor Xa (DXI) or oral direct thrombin inhibitors (DTI) in urine samples from patients treated with these classes of direct oral anticoagulants (DOACs). Several studies have investigated the performance of prototype and commercial dipsticks.
Aims: We aim to compare the performance of prototype and commercial dipsticks in a systematic review and meta-analysis to analyze the robustness and consistency of the data collected in these different studies.

Methods: We systematically searched PubMed (MEDLINE) and the Cochrane Library for relevant articles. Heterogeneity between studies was calculated using the chi-squared heterogeneity test and $\mathrm{I}^{2}$ index. A random effects model was used to compare data for prototype and commercial dipsticks.

Results: Four of 1081 publications were considered eligible for inclusion; three reported results on prototype dipsticks (DXI $n=658$, DTI $n=586$ ) and one on commercial dipsticks (DXI $n=451$, DTI $n=429$ ). There were no significant differences in sensitivity and specificity of DXI and DTI detection between prototype and commercial dipsticks. The pooled sensitivity and specificity was 0.968 (95\% Cl 0.956-0.978, $\left.P=0.1290, \mathrm{I}^{2} 47.1 \%\right)$ and 0.979 (95\% Cl 0.968$\left.0.992, P=0.1965, I^{2} 35.9 \%\right)$ respectively for detecting DXIs and $0.993\left(95 \% \mathrm{Cl} 0.986-0.997, P=0.1870, I^{2} 37.5 \%\right)$ and $0.993(95 \% \mathrm{Cl}$ $\left.0.986-0.997, P=0.7380, \mathrm{I}^{2} 0 \%\right)$ respectively for detecting DTIs. Simulation down to $1 \%$ prevalence showed a very high negative predictive value of 0.999 , indicating that the dipstick test may remain valid for detecting DOACs in patients' urine with acute medical conditions, and a still high positive predictive value between 0.238 and 0.590 for type of DOAC probably depending upon population and renal function.

Conclusions: Detection of DXI and DTI in patient urine samples was not significantly different between prototype and commercial DOAC dipsticks. This strengthens the reported results and should increase confidence to use of the DOAC Dipstick in emergency patient care and other medical indications.

PB0368 | Comparison of PT/INR Assessment with STANeoPTimal on an Automated and Semi-automated Test Platform

S. Savigny ${ }^{1}$; . Depasse $^{2} ;$ A. Veyradier ${ }^{3}$

${ }^{1}$ Diagnostica Stago, Gennevilliers, France; ${ }^{2}$ Diagnostica Stago, Asnièressur-Seine, France; ${ }^{3}$ APHP-Hôpital Lariboisière, Paris, France

Background: Prothrombin time (PT) is essential in the routine hemostasis panel. To ensure proper patient medical care continuity, but also to allow the test to be available whatever the laboratory structure and organization, demonstration of consistency of results between semi-automated and automated test application is compulsory.

Aims: The objective of this study was to show the correlation and agreement of PT/INR results with STA-NeoPTimal when determined on STA-R Max analyzer or with the newly developed application on STart-Max semi-automated system.

Methods: A total of 144 samples was tested in parallel with one batch of STA-NeoPTimal on STA-R Max and STart Max (all Stago, Asnières-sur-Seine, France). The sample set covered the measuring range from 10 to $90 \mathrm{~s}$ in PT $\mathrm{s}$ and from 1 to 6 in INR. It consisted in 
57 normal individual samples 64 samples from patients under VKA, 5 samples from patients with extrinsic factor pathway deficiency,

7 patients with multiple acquired deficiency and 11 samples from patients under heparin treatment.

The ISI used for INR determination were the ones provided by the while the mean normal prothrombin times (MNPT) were the one determined locally on 20 normal samples.

Results: The MNPT was of 13.6 s on STA-R Max and 14.8 s on STart Max.

No outlier was detected during the analysis of the comparison data. Passing-Bablok regression was performed to evaluate correlations. Slope, intercept and Pearson correlation coefficients of those regressions are displayed in Table 1.

TABLE 1 PT and INR determinations with STA-NeoPTimal: correlation between STart Max and STA-R Max

\begin{tabular}{|c|c|c|c|}
\hline \multirow{2}{*}{$\begin{array}{l}\text { STart Max } \\
\text { vs STA-R } \\
\text { Max }\end{array}$} & \multicolumn{2}{|c|}{$\begin{array}{l}\text { Passing-Bablok regression } \\
\text { equation } \\
y=a x+b\end{array}$} & \multirow{2}{*}{$\begin{array}{l}\text { Pearson correlation } \\
\text { coefficient }(r)\end{array}$} \\
\hline & Slope (a) & Intercept (b) & \\
\hline PT (s) & 1.10 & -0.40 & 0.998 \\
\hline INR & 0.92 & 0.08 & 0.998 \\
\hline
\end{tabular}

Conclusions: The correlation along the measuring range was excellent. Even if PT clotting times were slightly prolonged and INR values slightly lower on STart Max vs STA-R Max results, consistency of results was proven. PT/INR determination with STA-NeoPTimal can be safely measured either using its semi-automated or automated methodologies These results open the door for an easier usage of STA-NeoPTimal in any type of laboratory.

PB0369 | Effect of NETS from Different Models on Fibrin Polymerization Profile and Coagulation-fibrinolysis Balance

N.M.-b. Biatougou ${ }^{1}$; F. Mas ${ }^{2}$; B. Montpellier ${ }^{2}$; S. Mirshahi ${ }^{1}$; J. Soria ${ }^{3}$; G. Contant ${ }^{1}$

${ }^{1}$ Prospective Research, Diagnostica Stago, Gennevilliers, France;

${ }^{2}$ Biocytex, Hematology Dept, Research and Technology, Marseille, France; ${ }^{3}$ CAP-Paris Tech., INSERM U 1275, Hôpital Lariboisière, Paris,

France

Background: The Fibrin Polymerization (FP) and Coag-Lysis (CL) assays are fully automated for the turbidimetric measurement of the Fibrin Polymerization (1) and Coagulation/Fibrinolysis (2) profiles. Neutrophil Extracellular Traps (NETs) are known to modify the Fibrin structure and render it resistant to destruction (3).

Aims: To analyse the in vitro effect of NETs in FP and CL assays using spiking, inhibition and cells activation models.

Methods: DNA or Histone $3(\mathrm{H} 3)(70 \mu \mathrm{g} / \mathrm{mL} ; 50 \mu \mathrm{g} / \mathrm{mL})$ and their inhibition with DNAse or $\mathrm{H} 3$ inhibitor $(140 \mathrm{U} ; 50 \mu \mathrm{g} / \mathrm{mL})$ were respectively tested in normal pool plasma on FP and CL using PPP Reagent Low ${ }^{\circledR}$ with (CL) or without (FP) tissue Plasminogen Activator. Whole blood and isolated neutrophils from healthy donors were activated with LPS or PMA and Flow Cytometry analysis of leukocytes and FP/CL determinations were conducted. The presence of DNA from NETs was evidenced by confocal microscopy using Dapi and Picogreen.

Results: DNA had pro-coagulant and anti-fibrinolytic effects in FP and $\mathrm{CL}$, as opposed to $\mathrm{H} 3$ which had anti-coagulant, clot stabilisation and pro-fibrinolytic effects.

The effect of DNA was partially reversed by DNase, while Fibrin/ Lysis parameters were completely restored with the $\mathrm{H} 3$ inhibitor. In activation models, DNA was released and DNA-like effect was obtained in FP and CL.

A decreased size and DNA related fluorescence of neutrophils and an increased granularity, DNA fluorescence and TF/HLA-DR expression of monocytes, testified to their activation.

Conclusions: DNA released from cells activation modified FP and CL profiles, down regulating clot lysis. $\mathrm{H} 3$ effect was observed in spiking model only, as opposed to DNA's effect on FP and CL profiles due to its positive charges as shown in inhibition model. Therapeutic strategies could be optimized on these bases to enhance fibrinolysis in clots containing DNA and histones.

(1) Contant G. RPTH 2017.

(2) Pieters M. JTH 2018.

(3) Longstaff C. J. Biol. Chem. 2013.

PB0370 | Anticoagulated Patients Exhibit Intact Endogenous Thrombin Potential Using ST Genesia Unlike the Calibrated Automated Thrombogram

T. Helin ${ }^{1}$; M. Lemponen ${ }^{1}$; R. Lassila ${ }^{2}$; L. Joutsi-Korhonen ${ }^{1}$

${ }^{1}$ Coagulation Disorders Unit, HUS Diagnostics Center, HUSLAB, Helsinki University Hospital and University of Helsinki, Helsinki, Finland; ${ }^{2}$ Coagulation Disorders Unit, Helsinki University Hospital, Research Program Unit in Systems Oncology, University of Helsinki, Helsinki, Finland

Background: The thrombin generation (TG) assay is a feasible but labor-intensive method for detecting global coagulation. It enables comprehensive assessment of anticoagulation, while drug-specific assays only assess exposure. Traditionally, the Calibrated Automated Thrombogram $\left(\mathrm{CAT}^{\circledR}\right)$ has been used, however the ST Genesia ${ }^{\circledR}$ (Diagnostica Stago) allows automated evaluation.

Aims: We aimed to observe coagulation using the ST Genesia ${ }^{\circledR}$ and compare the data with those of $\mathrm{CAT}^{\circledR}$ in anticoagulated patients.

Methods: Altogether, In total, 43 frozen-thawed samples were studied using DrugScreen ${ }^{\circledR}$ to assess direct oral anticoagulants (DOACs), warfarin, and low molecular-weight heparin. Twenty samples (nine rivaroxaban, five apixaban, three warfarin, and three heparin) were also compared using $\mathrm{CAT}^{\circledR}$ (5 $\mathrm{pM}$ tissue factor). 
Results: TG reduction in DrugScreen ${ }^{\circledR}$ depended on the specific drug and modestly correlated with DOAC levels (lag time $R^{2}=0.36$; peak $R^{2}=0.50$, Figure). The best correlation was observed with Peak thrombin and rivaroxaban-specified anti-Xa activity $\left(R^{2}=0.60\right)$. When comparing ST Genesia ${ }^{\circledR}$ with $C A T^{\circledR}$, only the results for apixaban concorded $\left(R^{2}=0.97\right)$. Unlike CAT ${ }^{\circledR}$, ST Genesia ${ }^{\circledR}$ yielded a normal endogenous thrombin potential (ETP) in 77\% (24/31) FXainhibitor cases, and it failed to give readouts at INR $\geq 4.5$ and at anti-Xa $\geq 1.0 \mathrm{IU} / \mathrm{mL}$.

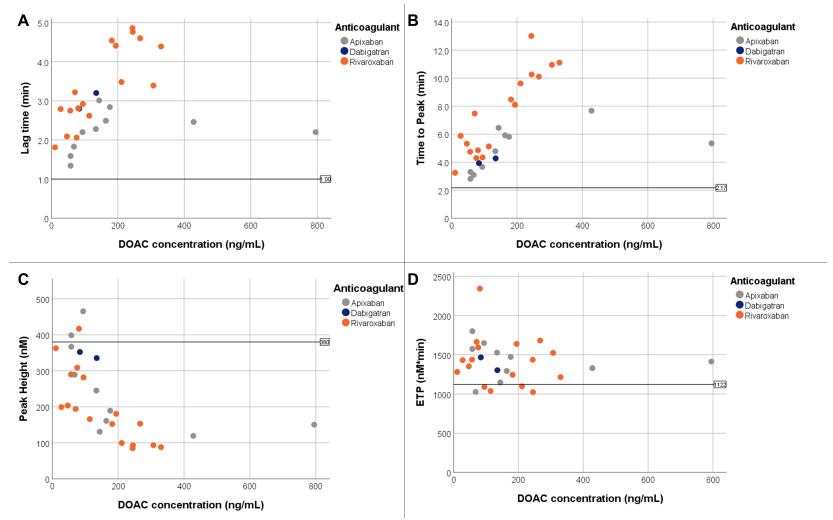

FIGURE 1 DOAC response in 31 samples as assessed using ST Genesia ${ }^{\circledR}$ DrugScreen ${ }^{\circledR}$. Increasing DOAC concentrations were correlated with $(\mathrm{A})$ prolonged Lag time $\left(R^{2}=0.33\right)$ and $(\mathrm{B})$ greater Time to peak $\left(R^{2}=0.53\right)$, and $(C)$ lower Peak $\left(R^{2}=0.45\right)$. (D) ETP responses varied widely $\left(R^{2}=0.08\right)$. The line shows the reference values reported in the literature.

Conclusions: The ST Genesia ${ }^{\circledR}$ data did not correlate with CAT $^{\circledR}$, but it was independently associated with INR, anti-Xa, and DOAC concentrations. The Lag time and Peak responses were similar; the major differences were that ST Genesia ${ }^{\circledR}$ showed no ETP effect of DOACs, and failed to give readout at high INR or anti-Xa activity.

These data have been accepted for publication in Research and Practice in Thrombosis and Haemostasis 23.1.2021.
PB0371 | Standardization and Automation of Lupus Anticoagulant Diagnosis Using a Complex Automated Algorithm

A. Sáez de Cámara Álvarez; M.A. Chang Azancot; C. Ponga Palacio; G. Mugertza Berastegi; U. Canibe Galindez; I. Ajuria Morentin Hospital Galdakao-Usansolo, Galdakao, Spain

Background: Lupus anticoagulants (LA) diagnosis consists in a complex multistep procedure difficult to standardize in laboratories.

Aims: To automate, standardize and find the most cost-effective solution to provide the best diagnosis for LA through a noninterventional observational study.

Methods: Forty samples with LA request were analyzed through three different protocols with Diagnostic Stago reagents and analyzer. Protocol (P) no algorithm (PO): phase 1 every day (DRVVscreen/confirm + APTTscreen then freezing positive samples for APTTscreen) and phase 2 one day/week (APTTconfirm). P1 Stago's automated algorithm ${ }^{1}$ in two days: phase 1 every day (DRVV Screen-mix-confirm + APTT screen-mix/freezing samples found positive mixing) and phase 2 one day/week (APTTconfirm). P2 algorithm by batch: complete algorithm one day/week freezing samples each day.

Accuracy in diagnosis along with, quantification of the number of steps, manual actions and processing time were collected for each step on every protocol. Reagent usage was also registered.

1: Florin L., et al. Int J Lab Hematol. 2019;1-6.

Results: 37 samples were found with the same diagnosis. Three samples were found positive for DRVV pathway only in PO but negative for P1/P2 (to be confirmed 12 weeks apart as per diagnostic guidelines). The percentage of human time saving per week vs PO was $1.2 \%$ for $\mathrm{P} 1$ and $47.9 \%$ for $\mathrm{P} 2$. The percentage of total processing time saved for the diagnosis of LA per week vs P0 was $2,5 \%$ for P1 and $33,4 \%$ for $\mathrm{P} 2$ (Table 1). The percentage of reagents vials saved with P2 compared to P0/P1 was 62,07\% (Table 2). Only P2 guaranteed double centrifugation of the samples. The algorithm ensured diagnostic standardization due to automatic test addition with associated comments.

TABLE 1 Workflow analysis for three different protocols (P0, P1 and P2). P0 No algorithm: Phase 1 every day (DRVV screen and confirm + APTT screen) and Phase 2 one day/week (APTT confirm). P1 Algorithm: Phase 1 every day (DRVV Screen-mix-confirm + APTT screen-mix) and Phase 2 one day/week (APTT confirm). P2 complete algorithm: one day a week freezing samples each day

\begin{tabular}{|c|c|c|c|c|c|c|c|}
\hline Table 1 & & $N^{\circ}$ Steps & $\begin{array}{l}\text { Total number of } \\
\text { manual actions }\end{array}$ & $\begin{array}{l}\text { Total sum of the } \\
\text { average sample } \\
\text { processing time } \\
\text { for each step } \\
\text { (min) }\end{array}$ & $\begin{array}{l}\text { Total sum of the } \\
\text { average human } \\
\text { processing time } \\
\text { for each step } \\
\text { (min) }\end{array}$ & $\begin{array}{l}\text { Total } \\
\text { processing } \\
\text { time per week } \\
(\mathrm{min})\end{array}$ & $\begin{array}{l}\text { Total human } \\
\text { processing time } \\
\text { per week (min) }\end{array}$ \\
\hline \multirow[t]{2}{*}{ PO } & Phase 1 & 5 & 7 & 72 & 3 & 1027.0 & 42 \\
\hline & $\begin{array}{l}\text { Phase } 1+\text { Phase } \\
\quad 2\end{array}$ & 12 & 21 & 199 & 7.3 & & \\
\hline \multirow[t]{2}{*}{ P1 } & Phase 1 & 5 & 5 & 70 & 3 & 1001.5 & 41.5 \\
\hline & $\begin{array}{l}\text { Phase } 1+\text { Phase } \\
\quad 2\end{array}$ & 12 & 18 & 196.5 & 7 & & \\
\hline
\end{tabular}




\begin{tabular}{|c|c|c|c|c|c|c|c|}
\hline Table 1 & & $\mathrm{~N}^{\circ}$ Steps & $\begin{array}{l}\text { Total number of } \\
\text { manual actions }\end{array}$ & $\begin{array}{l}\text { Total sum of the } \\
\text { average sample } \\
\text { processing time } \\
\text { for each step } \\
\text { (min) }\end{array}$ & $\begin{array}{l}\text { Total sum of the } \\
\text { average human } \\
\text { processing time } \\
\text { for each step } \\
\text { (min) }\end{array}$ & $\begin{array}{l}\text { Total } \\
\text { processing } \\
\text { time per week } \\
\text { (min) }\end{array}$ & $\begin{array}{l}\text { Total human } \\
\text { processing time } \\
\text { per week (min) }\end{array}$ \\
\hline P2 & $\begin{array}{l}\text { Complete } \\
\text { Algorithm }\end{array}$ & 9 & 13 & 114 & 3.5 & 684 & 21.9 \\
\hline
\end{tabular}

TABLE 2 Number of reagent vials used for each protocol

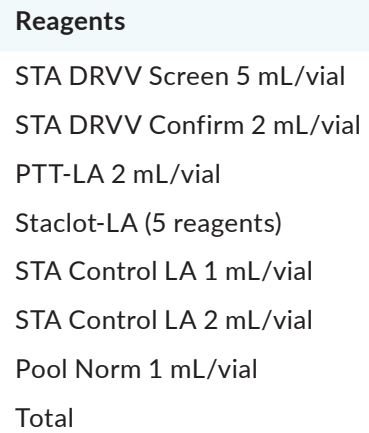

Conclusions: Working on batches of frozen samples with the Stago's automated algorithm demonstrated the most accurate, cost-efficient and standardized way of working for the diagnosis of LA.

\section{PB0372 | Evaluation of Silica Clotting Time and Hexagonal (II) Phase Phospholipid Neutralization for Lupus Anticoagulant} Detection

X.T. Gao ${ }^{1} ;$ R. Sumugod ${ }^{2} ;$ K. Carmichael ${ }^{2}$ P. Lindholm ${ }^{2}$; L. Gillard ${ }^{1}$; N. Lerret $^{1}$

${ }^{1}$ Rush University, Chicago, United States; ${ }^{2}$ Northwestern University Department of Pathology, Chicago, United States

Background: Lupus anticoagulant (LA) testing is essential for diagnosing antiphospholipid syndrome (APS) for which lifelong anticoagulant therapy is often indicated. The test is commonly ordered for patients with a history of thrombosis, recurrent spontaneous fetal loss and pre-operative patients with prolonged PTT. The current LA testing algorithm at our institution includes PTTLA; manual hexagonal (II) phase phospholipid neutralization (HPPN) assay and dilute Russell Viper Venom time (dRVVT) in addition to screening PT, aPTT and TT. The manual HPPN assay had high CV and low reproducibility in our laboratory. The silica clotting time (SCT) can be an automated alternative to PTTLA-HPPN assay.

Aims: To evaluate the concordance and reproducibility of automated SCT and PTTLA-HPPN assay.

Methods: Consecutive patient specimens were tested over a 2month period following current LA algorithm plus SCT. Automated coagulation tests were performed on ACL TOP 700 analyzer. The HPPN assay was performed on manual hemostasis analyzer. The cutoffs for positives were obtained from 20 normal donor specimens. Reproducibility for SCT and HPPN were assessed from 8 reactive samples.

Vials for P2/week
1
1
1
5
1
1
1
11

Results: 263 specimens were analyzed for comparison between PTTLA-HPPN and SCT. The SCT normalized ratio, PTTLA and HPPN assay positive test cut-off values were $1.21,43.0 \mathrm{~s}$ and $8.0 \mathrm{~s}$, respectively. Both SCT and PTTLA-HPPN assay were negative in 249 specimens, and both were positive in 3 specimens. In total, 252 results were concordant, and the overall percent agreement was $95.8 \%$. Positive percent agreement and negative percent agreement were $37.5 \%$ and $97.6 \%$, respectively. CV for HPPN assay ranged from $14.1 \%$ to $86.2 \%$ (Median: $41.3 \%$ ); CV for SCT ranged from $1.1 \%$ to 25.7\% (Median: 5.3\%).

Conclusions: LA negative results from SCT showed good agreement with PTTLA-HPPN, while discrepancies were found with some positive LA results. The SCT reproducibility was high with $5.3 \%$ median CV.

PB0375 | In vitro Neutralization of Low Molecular Weight Heparins for the Measurement of Fibrin Polymerization Profile and Coagulation-fibrinolysis Balance

M.-A. Bildan; M. Lambert; S. Fontaine; O. Mathieu; G. Contant Diagnostica Stago, Recherche Prospective R\&D, Gennevilliers, France

Background: The Fibrin Polymerization (FP) and the Coag-Lysis (CL) assays are fully automated, for the turbidimetric measurement of the Fibrin Polymerization profile and of the Coagulation-Fibrinolysis balance, on STA ${ }^{\circledR}$ - $R$ prototype. The FP is used to improve venous thromboembolism (VTE) diagnosis ${ }^{(1)}$ and the CL assay could help discriminate patients on their clot properties, in bleeding/thrombotic tendency ${ }^{(2)}$.

In some clinical indications such as prevention of thrombosis or VTE treatment, patients could receive low-molecular-weight heparin (LMWH). FP and CL are adversely affected by LMWH. Polybrene 
(Pb) and Heparinase I (Hep) have been shown to be effective for heparin neutralization ${ }^{(3)}$.

Aims: To design automated FP and CL assays, with reagent neutralizing $\mathrm{LMWH}$, allowing to restore Fibrin and/or Lysis parameters in samples from patients treated with LMWH. Select the best neutralizing agent based on tests performances.
Methods: FP and $C L$ were assessed on STA ${ }^{\circledR}$-R prototype using the PPP Reagent Low ${ }^{\circledR}$ with (CL) or without (FP) tissue Plasminogen Activator. The reagents were reconstituted with different concentrations of $\mathrm{Pb}$ or Hep (Table 1).

Normal Pooled Plasma (NPP) were spiked with different levels of tinzaparin, enoxaparin or dalteparin (Table 1) to cover an anti-FXa activity range up to $1.85 \mathrm{IU} / \mathrm{mL}$.

TABLE 1 Evaluation of neutralizing agents in FP and CL reagent with citrated plasma samples.

\begin{tabular}{|c|c|c|c|c|}
\hline Performances & Sample & LMWH Anti-Xa concentrations & Neutralizing agent & Analytical result \\
\hline Dose-response & NPP & $\leq 0.10 \mathrm{IU} / \mathrm{mL}$ & $\begin{array}{l}\text { CL:Pb: }[0-30] \mu \mathrm{g} / \mathrm{mL} ; 9 \text { levels } \\
\text { Hep: [0.07-0.27] IU/mL; } 4 \text { levels } \\
\text { FP:Pb: [0-85] } \mu \mathrm{g} / \mathrm{mL} ; 11 \text { levels } \\
\text { Hep: [0.13-0.27] IU/mL; } 3 \text { levels }\end{array}$ & $\begin{array}{l}\text { Relative deviation to } \mathrm{CL} \\
\text { and FP }\end{array}$ \\
\hline $\begin{array}{l}\text { Neutralization } \\
\text { performance on } \\
\text { spiked NPP }\end{array}$ & $\begin{array}{l}\text { Spiked } \\
\text { plasmas }\end{array}$ & $\begin{array}{l}\text { Tinzaparin: [0-0.89] IU/mL; } 5 \\
\quad \text { levels } \\
\text { Tinzaparin: [0-1.64] IU/mL; } 6 \\
\quad \text { levels } \\
\text { Dalteparin: [0-0.94] IU/mL; } 3 \\
\text { levels } \\
\text { Enoxaparin: }[0-0.94] \mathrm{IU} / \mathrm{mL} ; 3 \\
\text { levels }\end{array}$ & $\begin{array}{l}\text { CL: Hep: [0.07-0.27] IU/mL; } 4 \text { levels } \\
\text { FP:Hep: [0.13-0.27] IU/mL; } 3 \text { levels } \\
\text { CL:Pb: [0-10] } \mu \mathrm{g} / \mathrm{mL} ; 4 \text { levels } \\
\text { FP:Pb: [0-20] } \mu \mathrm{g} / \mathrm{mL} ; 6 \text { levels } \\
\text { CL and FP:Hep: } 0.13 \mathrm{IU} / \mathrm{mL}\end{array}$ & $\begin{array}{l}\text { Relative deviation to } \mathrm{CL} \\
\text { and FP }\end{array}$ \\
\hline $\begin{array}{l}\text { On board stability of } \\
\text { reagent }\end{array}$ & $\begin{array}{l}\text { Spiked } \\
\text { plasmas }\end{array}$ & $\begin{array}{l}\text { Tinzaparin: [0-0.63] IU/mL; } 2 \\
\text { levels }\end{array}$ & $\mathrm{CL}$ and FP:Hep: $0.13 \mathrm{IU} / \mathrm{mL}$ & $\begin{array}{l}\text { Relative deviation of T4 } \\
\text { hours to T0 }\end{array}$ \\
\hline
\end{tabular}

Results: Table 2 provides summary of assay performances.

TABLE 2 Summary of performance evaluation.

Performances
Dose-response
$\begin{gathered}\text { Neutralization performance on } \\ \text { spiked NPP }\end{gathered}$
intermediate reagent

\section{Results}

$\mathrm{Pb}$ had no impact on CL and FP assays of NPP at concentrations $\leq 10 \mu \mathrm{g} / \mathrm{mL}$ and $\leq 30 \mu \mathrm{g} / \mathrm{mL}$ respectively. Hep had no impact $(<10 \%)$ on CL and FP assays of NPP regardless to the tested concentrations.

$20 \mu \mathrm{g} / \mathrm{mL}$ of $\mathrm{Pb}$ neutralized the effect of tinzaparin up to $0.78 \mathrm{IU} / \mathrm{mL}$ on FP assays but modified lysis parameters of $\mathrm{CL}$ above $8.5 \mu \mathrm{g} / \mathrm{mL}$.

$0.13 \mathrm{IU} / \mathrm{mL}$ of Hep completely neutralized the effect of tinzaparin at $0.89 \mathrm{IU} / \mathrm{mL}$ but only up to $0.43 \mathrm{IU} /$ $\mathrm{mL}$ of dalteparin and enoxaparin on $\mathrm{CL}$ and FP assays.

On board stability with $0.13 \mathrm{IU} / \mathrm{mL}$ of Hep was up to $2 \mathrm{~h}$ on $\mathrm{CL}$ and FP assay with and without tinzaparin.
Conclusions: Our results show that $0.13 \mathrm{IU} / \mathrm{mL}$ of Hep can neutralize the anticoagulant effect up to $0.89 \mathrm{IU} / \mathrm{mL}$ for tinzaparin and up to $0.43 \mathrm{lU} / \mathrm{mL}$ for dalteparin and enoxaparin. At these concentrations, neutralization allowed to restore FP and CL parameters in plasma samples. Performance is reduced with $\mathrm{Pb}$.

These in vitro results indicate that Hep is the neutralizing agent of choice to abolish LMWH interference and assess FP or CL profile of patients under LMWH treatment.

(1) Contant G, et al. Res Pract Thromb Haemost 2017.

\footnotetext{
(2) Pieters M, et al. J Thromb Haemost. 2018.

(3) Hutt ED, et al. J Lab Clin Med 1972.
} 
PB0376 | Correlation between Plasma Levels, Anti-FXa Activity and Coagulation Parameters in Real-world Patients on Treatment with Edoxaban

M. Corrochano; R. Acosta-Isaac; S. Mojal; N. Grabalosa; M. Plaza;

B. Jiménez; J.C. Souto; MACACOD Proyect

Thrombosis and Haemostasis Unit, Hospital de la Santa Creu i Sant

Pau, Barcelona, Spain

Background: Previous studies have correlated an increased antifactor Xa (FXa) activity with increased levels of edoxaban plasma concentration; however, real-world data is scarce.

Aims: To analyze the relationship between edoxaban plasma concentrations, anti-FXa activity and basic coagulation tests (NCT04042155) and the effect of other relevant covariates (age, body mass index (BMI) and the glomerular filtration rate (GFR)).

Methods: We analyzed anti-FXa activity (HemosIL ${ }^{\circledR}$ Liquid Anti-Xa, Werfen) and edoxaban plasma concentration (HemoslL ${ }^{\circledR}$ Liquid Anti-Xa in conjunction with BIOPHEN ${ }^{\top M}$ edoxaban Calibrator) at the trough (pre-dose) and the peak ( $2 \mathrm{~h}$ after intake) of 250 patients with atrial fibrillation initiated with this treatment $(30 \mathrm{mg}$ or $60 \mathrm{mg}$ daily according to the product data sheet) between October 2019 and October 2020 in our center. Spearman's rho coefficient was used to assess correlations.

Results: The mean (SD) age of the patients analyzed was of 79.1 (7.1) years and $51 \%$ of them were women. Anti-FXa activity ranged widely (Trough: $0-0.71 \mathrm{IU} / \mathrm{ml}$; peak: $0.25-2.69 \mathrm{lU} / \mathrm{ml}$ ) as well as plasma concentrations (Trough: 3.50 - $138.60 \mathrm{ng} / \mathrm{ml}$; peak: 61.0 $464.90 \mathrm{ng} / \mathrm{ml}$ ). Anti-FXa activity showed a strong positive correlation with plasma concentrations at trough, peak (Figure 1) and also in the difference between this to values ( $r=0.863,0.970$ and 0.972 , respectively). From the trough to the peak PT was prolonged by a mean (SD) of 0.44 (0.19) seconds. This change was positively correlated with an increase of anti-FXa activity and edoxaban plasma concentrations ( $r=0.844$ and 0.853 respectively). We found no correlation with GFR, age or BMI.

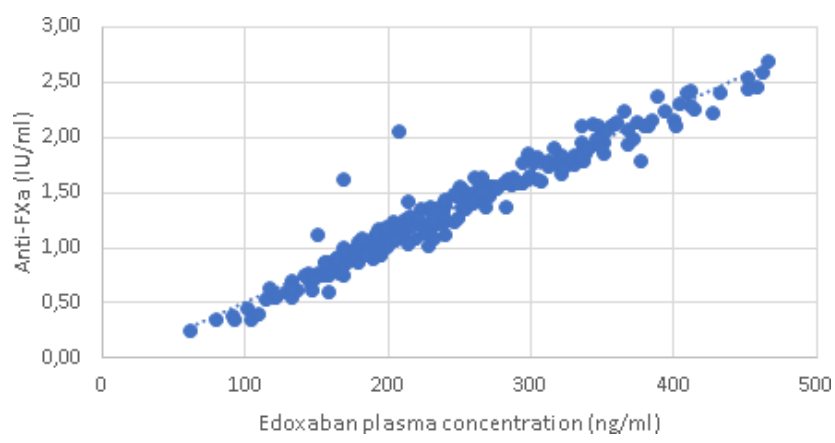

FIGURE 1 Correlation between Anti-FXa activity and edoxaban plasma concentration $2 \mathrm{~h}$ after intake (peak)

Conclusions: In real-world patients, plasma concentrations of edoxaban are highly correlated with the anti-factor Xa activity and
PT seems to have a modest positive correlation. This could be useful in day-to-day practice especially in urgent situations in which clinicians may want to be informed of DOAC drug concentrations.

\section{PB0377 | Immune Mediators in Pediatric Patients with Chronic} Kidney Disease

A. Silva $^{1}$; L. Perucci ${ }^{2}$; R. Silva ${ }^{1}$; L. Sousa ${ }^{1}$; A. Talvani ${ }^{2}$; A.P. Mota ${ }^{1}$;

L. Dusse ${ }^{1}$; P. Alpoim ${ }^{1}$

${ }^{1}$ Universidade Federal de Minas Gerais, Belo Horizonte, Brazil;

${ }^{2}$ Universidade Federal de Ouro Preto, Ouro Preto, Brazil

Background: Pediatric chronic kidney diseases (CDK) are mainly associated with kidneys congenital anomalies or urinary and glomerular tract diseases. IL-33 (or IL-1F11) is a cytokine with pro-inflammatory or regulatory roles, depending on the pathological context. CXCL-16 (CXC motif ligand 16) may function as chemoattractant of activated T cells, as an adhesion molecule, or as a scavenger receptor. Both, IL-33, CXCL-16 may have a dual role in the inflammatory response. IL-17 is a pro-inflammatory cytokine. Understanding the effects of cytokines on the onset and progression of pediatric CDK is crucial as new prognostic markers and maybe as alternative therapeutic targets.

Aims: Evaluating IL-33, CXCL16 and IL-17 plasma levels in CKD pediatric patients and healthy volunteers (control group).

Methods: This study was previously approved by the local Ethical Committee of The Federal University of Minas Gerais (\# 07513513.9.0000.5149) and consent was obtained in all cases. It included 38 CKD pediatric patients (6-18 years old) and 31 healthy volunteers matching age and sex. IL-33, CXCL16 and IL-17 plasma levels were determined by immunoassays.

Results: Median and interquartile range for IL-33 were $205.8 \mathrm{pg} / \mathrm{mL}$ (225.1) in CDK group and $334.42 \mathrm{pg} / \mathrm{mL}$ (261.53) in controls $(P=0.027)$, for CXCL-16, $168.44 \mathrm{pg} / \mathrm{mL}$ (112.3) in CKD group and $260.68 \mathrm{pg} / \mathrm{mL}$ (213.56) in controls $(P \leq 0.001)$ and for IL-17, $14.91 \mathrm{pg} / \mathrm{mL}(16.34)$ in CKD group and $55.04 \mathrm{pg} / \mathrm{mL}$ (45.87) in controls $(P \leq 0.001)$.

Table 1 show the statistical results of the cytokines.

TABLE 1 Statistical results of the cytokines.

\begin{tabular}{|c|c|c|c|}
\hline Parameters & CKD group & Control group & $P$ value \\
\hline IL-33 pg/mL & $\begin{array}{l}205.8 \\
\quad(225.1)\end{array}$ & $334.42(261.53)$ & 0.027 \\
\hline $\begin{array}{c}\text { CXCL-16 pg/ } \\
\mathrm{mL}\end{array}$ & $\begin{array}{l}168.44 \\
\quad(112.3)\end{array}$ & $260.68(213.56)$ & 0.001 \\
\hline IL-17 pg/mL & $\begin{array}{l}14.91 \\
\quad(16.34)\end{array}$ & $55.04(45.87)$ & 0.001 \\
\hline
\end{tabular}

Conclusions: Kidney diseases are complex and heterogeneous. Our findings suggest a compromised regulation of inflammatory process in CKD pediatric patients. However other studies are need to clarify how the citokynes are involved in the disease pathogenesis and mainly the CKD progress. 
PB0379 | Comparability of PT Determination with STANeoPTimal on STart and STart Max Semi-automated Instruments

G. Le Goff ${ }^{1}$; M. Bouchain ${ }^{1} ;$ A. Carlo²; J. Beltran ${ }^{1}$

${ }^{1}$ Diagnostica Stago, Gennevilliers, France; ${ }^{2}$ Diagnostica Stago, Asnièressur-Seine, France

Background: Prothrombin time (PT) is a cornerstone of the routine hemostasis panel. This means the test has to be available any time, in any laboratory, whatever the test platform. Quality management plans within clinical laboratories verify this consistency, but to ease this work, manufacturers can show the agreement by design.

Aims: The objective of this study was to show the correlation and agreement of PT/INR determination with STA-NeoPTimal when assessed on STart and STart Max semi-automated analyzers.

Methods: 86 individual samples, spreading the PT measuring range, were tested with one batch of STA-NeoPTimal on STart and STart Max analyzer in parallel (reagents and analyzers from Stago, Asnières-sur-Seine, France). This samples set included samples from patients under vitamin $\mathrm{K}$ antagonists or patients having any exogenous factor deficiency.

The ISI used for INR determination was the one provided by the manufacturer while the mean normal prothrombin time (MNPT) was the one determined locally.

Results: Linear regression was performed to evaluate correlations. Slope, intercept and Pearson correlation coefficients of those regressions are displayed in Table 1.
PB0380 | FVIII Stability, the Limiting Factor in Chromogenic Fix Kits?

A. Evenepoel; C. Gavard; S. Desmet; V. Deneys; M.-A. van Dievoet Cliniques Universitaires Saint-Luc, Department of Laboratory Haematology, Brussels, Belgium

Background: For the determination of nonacog-beta-pegol (N9-GP) Ezban et al recommend the use of chromogenic assays and certain chronometric tests, like SynthAfax ${ }^{\circledR}[1]$. N9-GP is a new recombinant factor IX (FIX) with an extended half-life. Chronometric FIX tests are based on the activated partial thromboplastin time also called onestage aPTT assays. Chromogenic tests will measure the amount of chromogenic substrate cleavage by FXa after addition of factor X, factor VIII (FVIII) and other activators. However, FVIII is known to be unstable and can lead to kit instability during usage.

Aims: This study evaluated precision claims for Biophen ${ }^{\circledR}$ FIX (chromogenic assay, Hyphen, France) stored at $-30^{\circ} \mathrm{C}$ and $-80^{\circ} \mathrm{C}$, and the SynthAfax ${ }^{\circledR}$ kit (chronometric assay, Werfen, Spain), stored at $2-8^{\circ} \mathrm{C}$. Additionally, we assessed their applicability in the laboratory.

Methods: CV\% was determined following CLSI protocol EP15-A2 using Werfen (normal - abnormal) and Hyphen controls (normal - diluted normal and abnormal). We used the minimal CV\% of $6.9 \%$ and desirable $C V \%$ of $4.6 \%$ as acceptance criteria and tested significance using the $\mathrm{Chi}^{2}$ test [2].

Results: Table 1 displays the results of the imprecision experiment. The obtained CV\% for SynthafAx and Biophen $\left(-80^{\circ} \mathrm{C}\right)$ did not differ

\section{TABLE 1}

\begin{tabular}{|c|c|c|c|c|}
\hline \multirow[b]{2}{*}{ STart vs STart Max } & \multicolumn{2}{|c|}{$\begin{array}{l}\text { Linear regression equation } \\
y=a x+b\end{array}$} & \multirow{2}{*}{$\begin{array}{l}\text { Pearson correlation coefficient } \\
\text { (r) }\end{array}$} & \multirow[b]{2}{*}{ Average bias } \\
\hline & Slope (a) & Intercept (b) & & \\
\hline PT (s) & 1.01 & -0.58 & 0.995 & $1 \%$ \\
\hline INR & 1.01 & -0.04 & 0.996 & $\begin{array}{l}-0.89 \% \text { to } 01 \% \text { depending on } \\
\text { INR levels }\end{array}$ \\
\hline
\end{tabular}

Conclusions: Results confirm a very high correlation and agreement of PT/INR determination with STA-NeoPTimal in its semi-automated methodology on both STart and STart Max instruments. These good results preclude easy method validation process and assay flexibility, for a possible instrument switch over time. significantly from the minimal CV\%, yet the desirable CV\% (4.6\%) is not achieved in most cases $(P<0.01)$. Total $C V \%$ of the Biophen kit stored at $-30^{\circ} \mathrm{C}$ differed significantly from the minimal CV\%, making these storage conditions unfit for routine use.

TABLE 1 Overview of the analytical variance (CV\%) - SynthAfax, chronometric assay; Biophen, chromogenic assay; CVwithin run, reproducibility; CVtotal, total AC, acceptance criterion.

\begin{tabular}{|c|c|c|c|c|c|c|c|c|c|c|c|c|}
\hline & \multicolumn{4}{|c|}{ SynthAfax: stored at $2-8^{\circ} \mathrm{C}$} & \multicolumn{4}{|c|}{ Biophen: stored at $-80^{\circ} \mathrm{C}$} & \multicolumn{4}{|c|}{ Biophen: stored at $-30^{\circ} \mathrm{C}$} \\
\hline & Level & $\begin{array}{l}\text { Result } \\
\text { (\%) }\end{array}$ & $A C$ & $P$-value & Level & $\begin{array}{l}\text { Result } \\
\text { (\%) }\end{array}$ & $\mathrm{AC}$ & $P$-value & Level & $\begin{array}{l}\text { Result } \\
\text { (\%) }\end{array}$ & $\mathrm{AC}$ & $P$-value \\
\hline \multirow[t]{3}{*}{$\mathrm{CV}_{\text {within run }}$} & Low & 3.8 & $4.6 \%$ & 0.73 & Low & 6.1 & 4.6 & 0.08 & Low & $8.0 \%$ & $6.9 \%$ & 0.20 \\
\hline & & & & & Intermediate & 4.9 & 4.6 & 0.34 & & & & \\
\hline & High & 2.2 & $4.6 \%$ & 0.99 & High & 5.5 & 4.6 & 0.17 & High & $3.1 \%$ & $4.6 \%$ & 0.91 \\
\hline
\end{tabular}


SynthAfax: stored at $2-8^{\circ} \mathrm{C}$

\begin{tabular}{lllll} 
& Level & $\begin{array}{l}\text { Result } \\
\text { (\%) }\end{array}$ & AC & P-value \\
\cline { 3 - 5 } CV $_{\text {total }}$ & Low & 8.1 & $6.9 \%$ & 0.23
\end{tabular}

Biophen: stored at $-80^{\circ} \mathrm{C}$

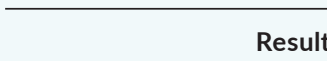

Level

Low

Intermediate

High

\section{Result}

(\%)

6.7
8.6

5.5
Biophen: stored at $-30^{\circ} \mathrm{C}$

$\begin{array}{llll}\text { High } \quad 7.4 \quad 6.9 \% & 0.33\end{array}$

AC

AC

P-value

6.9

0.49

6.9

0.15

\begin{tabular}{llll}
\hline Level & $\begin{array}{l}\text { Result } \\
(\%)\end{array}$ & AC & $P$-value \\
Low & $16.7 \%$ & $6.9 \%$ & $<0.01$
\end{tabular}

Conclusions: We obtained acceptable results with the Biophen chromogenic FIX assay when conserving reagents at $-80^{\circ} \mathrm{C}$. In comparison, better reproducibility was seen with the Synthafax chronometric kit. We hypothesize that a possible instability of FVIII in the chromogenic assay plays a role. This could compromise usability and cost-efficiency of chromogenic assays in clinical practice. Nevertheless, each laboratory should define kit precision and determine acceptability criteria for routine measurements.

\section{PB0381 | Newly Developed Ellagic Acid Based APTT Reagent} Showed High Lupus Anticoagulant Sensitivity

O. Kumano $^{1}$; D. Shimomura²; K. Kitano ${ }^{1}$; N. Arai ${ }^{1} ;$ T. Matsumoto ${ }^{3}$. ${ }^{1}$ Sysmex Corporation, Kobe, Japan; ${ }^{2}$ Tenri Hospital, Tenri, Japan; ${ }^{3}$ Tenri Health Care University, Tenri, Japan

Background: Activated partial thromboplastin time (APTT) is widely used as a screening test for that characterizes coagulation of the blood. Recently, new ISTH lupus anticoagulant (LA) detection guidance described that ellagic acid activator reagent might show acceptable sensitivity in some reagents.

Aims: The purpose of this study was to perform the basic evaluation of newly developed APTT reagent (New-APTT), which uses ellagic acid and synthetic phospholipids, and investigate the LA sensitivity. Methods: Four kinds of APTT reagents including New-APTT, current reagent with ellagic acid and two reagents prepared from silica activator were used. The within-run precision and the between-day precision were determined from 10 times measurements and 15 times measurements, five times a day over three days, in normal and abnormal controls, respectively. The comparative study against current ellagic acid reagent was performed using total 100 clinical samples including 37 LA-positive. LA sensitivity was evaluated among four APTT reagents in nine kinds of LA-positive controls by index of circulating anticoagulant (ICA) values calculated from the mixing tests. Results: The coefficient of variation \% (CV\%) in within-run and between-day precision were within $0.77 \%$ and $1.59 \%$ in both normal and abnormal controls, respectively. The correlation coefficient and the regression equation were 0.899 and $y=1.42 x-14.26$, respectively. The clotting times of New-APTT was more prolonged than those of current reagent in LA-positive and LA-positive with anticoagulant drug samples significantly. In LA sensitivity, the means of ICA values in New-APTT, current reagent and two silica reagents were 26.2, 26.7, 21.6 and 25.1, respectively. The significant difference was not observed among the reagents.
Conclusions: New-APTT showed an excellent analytical performance and had a high LA sensitivity comparable to silica reagents in LA-positive controls and clinical samples.

\section{PB0382 | Validation of Mixing Test Formula for the} Discrimination between Lupus Anticoagulant and Acquired Hemophilia A in New APTT Reagent

O. Kumano ${ }^{1}$; D. Shimomura ${ }^{2}$; K. Kitano ${ }^{1}$; N. Arai ${ }^{1}$; T. Matsumoto ${ }^{3}$. ${ }^{1}$ Sysmex Corporation, Kobe, Japan; ${ }^{2}$ Tenri Hospital, Tenri, Japan; ${ }^{3}$ Tenri Health Care University, Tenri, Japan

Background: The mixing test is considered useful for lupus anticoagulant (LA) diagnosis and is also recommended to differentiate between acquired hemophilia $\mathrm{A}(\mathrm{AHA})$ and factor deficiency. In our previous study, we proposed the mixing test formula using index of circulating anticoagulant (ICA) calculated from the clotting times of without incubation and $2 \mathrm{~h}$ incubation.

Aims: Recently, we have developed new APTT reagent prepared from ellagic acid as the activator and synthetic phospholipids. The purpose of this study was to validate the mixing test formula proposed in our previous study in that reagent.

Methods: We examined total 100 clinical samples including 37 LApositive, 4 factor deficiency, 51 anticoagulant drugs and 9 FVIII inhibitor. ICA values, calculated from the clotting times without incubation and after $2 \mathrm{~h}$ incubation, were defined as ICA immediate (ICAi) and ICA delated (ICAd), respectively. ICAd/ICAi and ICAd - ICAi were calculated as the mixing test formula. $132 \%$ and 6.4 established previously were used as the cut-off values in new APTT reagent for the validation, respectively.

Results: The ICAi values of LA-positive samples were significantly higher than those in another samples, and FVIII inhibitors samples as well as LA-positive showed the elevated ICAd values than those of factor deficiency and anticoagulant drugs samples. In ICAd/ICAi and ICAd - ICAi formulas, only FVIII inhibitor samples were elevated, and the sensitivity and specificity for FVIII inhibitor were $100 \%$ and $79.3 \%$ for ICA/ICAi and $100 \%$ and $94.6 \%$ for ICAd - ICAi, respectively.

Conclusions: ICAd/ICAi and ICAd - ICAi showed significantly higher values in FVIII inhibitor and acceptable sensitivity and specificity. It was confirmed that these formulas were worked well in new APTT reagent. These formulas were useful for the discrimination between LA and FVIII inhibitor and can contribute to the rapid diagnosis and treatment. 
PB0383 | Comparison of Visual Interpretation of the DOAC Dipstick Test with a Reader System for Rapid and Accurate Detection of Direct Oral Anticoagulants in Neurology and Cardiology - Interim Data from a Prospective Consecutive Comparative Study

S. Margetić ${ }^{1}$; I. Ćelap ${ }^{1}$; D. Delić-Brkljačić ${ }^{2}$ J. Harenberg ${ }^{3,4}$; A. Lovrenčić-Huzjan ${ }^{5}$; P. Mioč ${ }^{6}$ S. Šupraha Goreta ${ }^{1}$ Sestre milosrdnice University Hospital Center, Department of Clinical Chemistry, Zagreb, Croatia; ${ }^{2}$ Sestre Milosrdnice University Hospital Center, Department of Cardiovascular Diseases,, Zagreb, Croatia; ${ }^{3}$ Heidelberg University, Heidelberg, Germany; ${ }^{4}$ DOASENSE GmbH, Heidelberg, Germany; ${ }^{5}$ Sestre Milosrdnice University Hospital Center, Department of Neurology, Zagreb, Croatia; ${ }^{6}$ Sestre Milosrdnice University Hospital Center, Department of Cardiovascular Diseases, Zagreb, Croatia; ${ }^{7}$ Sestre Milosrdnice University Hospital Center, Faculty of Pharmacy and Biochemistry, Zagreb, Croatia

Background: Direct oral anticoagulants (DOACs) are detected in patient urine samples by DOAC Dipstick ${ }^{\mathrm{TM}}$ test based on color changes in pads that contain specific chemical components. However, identification of these color changes may vary between observers and the test instructions may be difficult to follow in emergency situations.

Aims: The objective of this ongoing study is to examine whether DOAC Dipstick results can be used to exclude the presence of clinically relevant concentrations of dabigatran, rivaroxaban and apixaban in patients admitted to a neurology and cardiology department for elective or emergency interventions.

Methods: In this interim analysis, we compared the inter-observer variability of DOAC Dipstick results obtained by medical personnel with those obtained by a semi-automatic reader. This single-center study will include between 120 and 180 patients with neurologic and cardiovascular diseases treated with rivaroxaban, apixaban and dabigatran who are admitted to Sestre Milosrdnice University Hospital Center in Zagreb. Factor Xa and thrombin inhibitor pads of the DOAC Dipstick were incubated in patient urine samples and evaluated visually by two independent observers and by a DOASENSE Reader. Here, we report interim descriptive results on variability of two observer and as compared to DOASENSE Reader results. We will compare plasma DOAC concentrations with DOAC Dipstick results after termination of the study.

Results: So far, 45 patients have been included (rivaroxaban $n=20$, apixaban $n=9$, dabigatran $n=10$, and switching from rivaroxaban to dabigatran $=6$ ). No differences in color interpretation for presence or absence of direct oral factor Xa or thrombin inhibitors have been detected between visual inspection of pads of the test strips between the two observers and the results obtained DOASENSE Reader.

Conclusions: These interim data suggest low inter-observer variability of visual evaluation of DOAC Dipstick colors for presence or absence of DOACs and indicate a very high agreement with results of DOASENSE Reader
PB0384 | Assessment of Low Response Rates to P2Y12 Inhibition in Cardiology Patients: Comparison of the VerifyNow P2Y12 and Anysis-P2Y12 Assay

S. Shin ${ }^{1}$; J. Piao ${ }^{1}$; Y.-W. Whang ${ }^{2}$; C.U. Choi ${ }^{2}$.

${ }^{1}$ Korea University, Seoul, Korea, Republic of; ${ }^{2}$ Korea University Guro

Hospital, Seoul, Korea, Republic of

Background: Analysis of responsiveness to P2Y12 therapy is vital to preventing thrombotic complications as well as hemorrhagic complications in the management of patients with cardiovascular diseases. Aims: This study aimed to evaluate a new Anysis-P2Y12 assay system comparing with VerifyNow-P2Y12 in cardiology patients and to analyze P2Y12 low-response rates between two devices with various cutoff values.

Methods: Overall, 125 citrated blood samples were collected from cardiology patients referred for antiplatelet response test. In Anysis assay, a test result was a blood migration distance (MD) until clogging of flow passage, which is comparable to both P2Y12 reaction units (PRU) and percent inhibition (\%INH) obtained using VerifyNow. The two devices were simultaneously used and compared.

Results: The MDs for without and with taking P2Y12 were $182 \pm 30$ and $264 \pm 12 \mathrm{~mm}$, respectively $(P<0.0001)$. Compared with VerifyNow (reference), the sensitivity and specificity of Anysis-200 was $96.8 \%$ and $88.7 \%$, respectively. The Cohen's kappa coefficient between the two devices was 0.761 , indicating a relatively high agreement. However, there was an apparent difference in the rates of low-response rates to P2Y12, which were 36.5\% for VerifyNow and $5.9 \%$ for Anysis, respectively. The different results might be caused by the difference in concentrations of ADP ( 20 uM vs. 2 uM) as an agonist used in their assays.

Conclusions: The present study confirmed that the microfluidic platelet function assay, Anysis-P2Y12 has accuracy and precision equivalent to that of, and significant agreement with, VerifyNow. The low-response rate of P2Y12 should be re-evaluated with assaying devices which adopt relatively low concentration of ADP as an agonist.

\section{PB0385 | Analytical Performance of the Novel Haema T4 Thromboelastograph}

M. Schoorl' ${ }^{1}$; T. Jansen ${ }^{1} ;$ J. van Pelt ${ }^{2}$

${ }^{1}$ Northwest Clinics, Alkmaar, Netherlands; ${ }^{2}$ U-Diagnostics B.V., Baarn, Netherlands

Background: The novel Haema T4 Thromboelastograph (MedCaptain Medical Technology Co., Shenzhen, Guangdong, China) is an analyzer for monitoring, measuring, and analyzing the coagulation process of a blood sample. With the qualitative and semi-quantitative test results an automatically generated prediction of the patient's blood coagulation condition is made.

Aims: In this study, the intra-assay, inter-assay, day-to-day precision, sample- and reagent stability, inter-lotnumber variation and reference 
values were established for the Kaolin Activation Test (Kaolin), Rapid Kaolin Test (R-Kaolin), Heparin assay (HEP), functional Fibrinogen assay (FIB) and platelet aggregation assay (AA+ADP) (MedCaptain).

Methods: Whole blood samples (Vacutainer ${ }^{\circledR} 0.109 \mathrm{M}$ Citrate) were used to perform all tests. A reference group of 25 blood donors (Sanquin, Amsterdam, The Netherlands) was used for establishing normal reference ranges.Thromboelastography controls I and II were used to establish day-to-day precision (MedCaptain).

Results: The biochemical reactions of the coagulation cascade and clot formation process are characterized by measurement of the first blood clot reaction time $(\mathrm{R})$, clot formation rate ( $\mathrm{K}$ and angle), maximum clot strength (maximum amplitude, MA) and heparin monitoring index (ACT) (Figure 1).
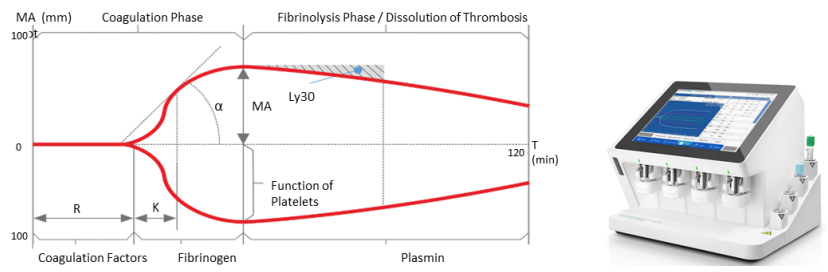

FIGURE 1 The biochemical reactions of the coagulation cascade and clot formation process are characterized by measurement of the first blood clot reaction time ( $R$ ), clot formation rate ( $K$ and angle), maximum clot strength (maximum amplitude, MA) and heparin monitoring index (ACT). In addition to the five main parameters the coagulation index $(\mathrm{Cl})$ may also contribute to the determination of the kinetics, hardness and stability of the blood clot. Using the fibrinogen assay, the Flev value represents the content of the functional fibrinogen which can be cross-linked into fibrin.

Intra-assay $(n=8)$, inter-assay $(n=8)$ and day-to-day precision ( $n=20$ ) of all five tests and parameters yielded appropriate results for $\mathrm{K}(\mathrm{CV}<15 \%)$, R, angle, MA and ACT (CV < 10\%). Blood samples were stable for maximum $2 \mathrm{~h}$ after collection at $20-25^{\circ} \mathrm{C}$. Reference ranges are demonstrated in Table 1.
Conclusions: In a laboratory setting, the novel Haema T4 Thromboelastograph yield good analytical performance for the Kaolin, R-Kaolin, HEP, FIB and AA\&ADP tests.

TABLE 1 Reference ranges for the kaolin, R-kaolin, Fibrinogen and AA\&ADP tests, established with a reference group of 25 blood donors (Sanquin, The Netherlands)

\begin{tabular}{|c|c|c|c|c|c|c|c|}
\hline & $\mathrm{ACT}(\mathrm{s})$ & $R(\min )$ & $\mathrm{K}(\mathrm{min})$ & Angle (deg) & $\mathrm{MA}(\mathrm{mm})$ & $\mathrm{Cl}$ & Flev (mg/dL) \\
\hline Kaolin & & $3.3-6.0$ & $0.8-2.0$ & $65-78$ & $55-72$ & $-0.1-3.8$ & \\
\hline R-Kaolin & 69-103 & $0-0.9$ & $0.5-1.5$ & $74-85$ & $44-82$ & $3.6-7.4$ & \\
\hline AA\&ADP & & $0.3-2.0$ & $1.1-2.2$ & $19-75$ & $5-15$ & $622-628$ & \\
\hline$-F$ & & $0.7-1.8$ & $1.5-2.9$ & $66-79$ & $55-69$ & $1.9-5.8$ & \\
\hline$-F+A D P$ & & $5.8-9.0$ & & $64-73$ & $60-68$ & $-1.9-1.5$ & \\
\hline - kaolin & & & & & & & \\
\hline
\end{tabular}

Test results did not change during the shelf life of the reagents (9 months).

When using three different reagent batch numbers, the mutual difference for all tests ranged for $\mathrm{K} 10-15 \%$, R 9-15\%, angle 2-5\%, MA 3-5\% and ACT 5-7\%. 
PB0386 | Effect of Warfarin on Thrombin Generation Parameters in Elderly Patients with Atrial Fibrillation

T.R. Rocha ${ }^{1}$; A. Pastana ${ }^{2}$; E. D`Amico ${ }^{1}$; P. Villaça ${ }^{1}$; P. Yu ${ }^{2}$;

I. Magalhães'; F.A. Cardozo ${ }^{2}$; L. Franco ${ }^{3}$; G. Lustosa ${ }^{3}$; B. Caramelli'; D. Calderaro ${ }^{2}$

${ }^{1}$ University of São Paulo Medical School - Coagulation Laboratory - Hospital das Clínicas, Sao Paulo, Brazil; ' University of São Paulo Medical School - Interdisciplinary Medicine in Cardiology - Heart Institute (InCor), Sao Paulo, Brazil; ${ }^{3}$ Stago, Sao Paulo, Brazil

Background: Despite the availability of direct oral anticoagulants, warfarin is still widely used and is the only option for patients with mechanical heart valves or end-stage renal disease.

Measurement of international normalized ratio (INR) for prothrombin time is the standard for warfarin-dosage adjustment and little is known about the value of thrombin generation (TG) test in this context.

Aims: We conducted this study to analyze the effect of warfarin on TG and the correlation between its parameters and INR.

Methods: Our local ethics committee approved the protocol. We included 25 patients on warfarin therapy for atrial fibrillation (AF), with age above or equal to 70 years, and 25 controls, with age above or equal to 70 years and $\mathrm{AF}$ without anticoagulant.

We measured thrombin generation using an automated system (ST Genesia) and its results were compared between groups. For warfarin-treated patients, we also measured INR and analyzed its relation to TG parameters: lag time, time to peak, peak, and endogenous thrombin potential (ETP).

Results: Warfarin significantly increased lag time: 2.83 (IQR: 2.574.48) vs 1.53 (IQR: 1.38-1.74), $P$ < 0.001 and time to peak: 4.29 (IQR: 3.96-6.14) vs 2.84 (IQR: $2.54-3.36$ ), $P<0.001$, and significantly decreased thrombin peak 108.8 (IQR: 83.67 - 139.25) vs 304.5 (IQR 245.85 - 329.10), $P<0.001$, and endogenous thrombin potential (ETP): 417.7 (IQR: 323.4 - 507.45) vs 1086 (IQR 870 - 1255), $P<0.001$. We observed a significant linear correlation between INR and ETP [ $\rho$ Spearman - $0.627(-0.823$ a -0.297) $P<0.001]$, INR and Peak [R Pearson -0.642 (-0.823 a -0.319) $P=0.001$, and no significant correlation between INR and Lagtime [ $\rho$ Spearman +0.297 $(-0.124$ a 0.627$) P=0.150$ ] or INR and time to Peak [ $\rho$ Spearman $+0.228(-0.196 a+0.580) P=0.273]$.

Conclusions: Although warfarin alters each parameter of the TG test, only ETP and thrombin peak significantly correlate with INR.
PB0387 | Prevalence of Hemolysis, Icterus and Lipemia in the Coagulation Laboratory: Database Analyses

F. Mil ${ }^{1}$; B. Girard ${ }^{1}$; A. Carlo ${ }^{2}$; D. Guimbard ${ }^{1}$

${ }^{1}$ Diagnostica Stago, Gennevilliers, France; ${ }^{2}$ Diagnostica Stago, Asnièressur-Seine, France

Background: The pre-analytical phase is the leading source of errors in laboratory diagnostics [Plebani M, Clin Biochem Rev 2012]. Presence of hemolysis $(\mathrm{H})$, icterus $(\mathrm{I})$ or lipemia $(\mathrm{L})$ is reported as one of the major causes of pre-analytical errors [Salvagno GL et al, JECP 2008]. Thanks to automation, detection of these interferences has improved and automate database analyses can allow assessment of prevalence of $\mathrm{H}, \mathrm{I}$ and $\mathrm{L}$ on samples actually tested.

Aims: Evaluate the frequency of the different indices of $\mathrm{H}, \mathrm{I}, \mathrm{L}$ perturbations on mechanical clot detection systems in coagulation laboratories.

Methods: The database was consolidated from anonymized raw test results obtained on Stago instruments equipped with Expert Preanalytical Check (EPC) module and transferred to Stago for further investigation.

From this dataset, we have quantified the proportion of samples with the different indices of $\mathrm{H}, \mathrm{I}, \mathrm{L}$ as calibrated in the EPC module. The $1^{\text {st }}$ index is not equivalent to absence of $\mathrm{H}, \mathrm{I}, \mathrm{L}$ but determines the lowest levels $(\mathrm{H} 1=\mathrm{Hb}<0.3 \mathrm{~g} / \mathrm{L}$; $\mathrm{I} 1=$ bilirubin $<1.5 \mathrm{mg} / \mathrm{dL}$; $\mathrm{L}=$ equivalent Intralipid ${ }^{\circledR}<160 \mathrm{mg} / \mathrm{dL}$ ).

Results: Our database contains a total of 8875084 HIL indices determinations.

75\% of the dataset comes from 191 instruments placed in France. Proportion of $\mathrm{H}, \mathrm{I}, \mathrm{L}$ indices in France (Fig 1 ) and in the rest of the world (Fig. 2) indicate that frequency of $L$ levels above 1 is very low $(<0.2 \%$ total), while $\mathrm{I}$ is more frequent in 8 to $9 \%$ of the samples and $\mathrm{H}$ in 7 to $8 \%$ of the samples.

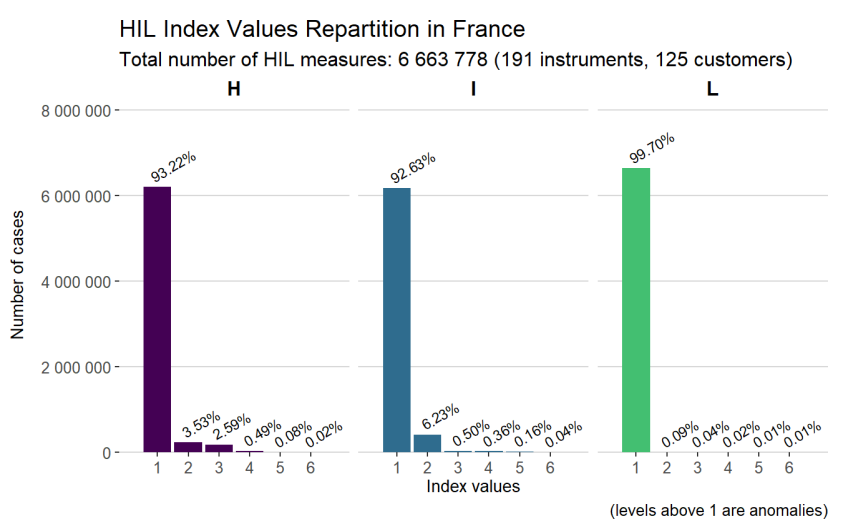

FIGURE 1 repartition of H, I, L indices in the 6663778 results coming from France. 
HIL Index Values Repartition in out of France Total number of HIL measures: 2211306 (106 instruments, 64 customers)

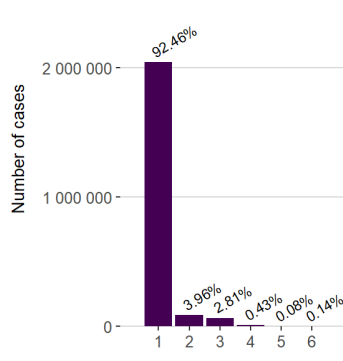

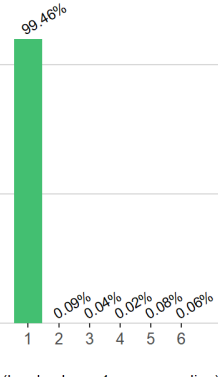

(levels above 1 are anomalies)
FIGURE 2 repartition of $\mathrm{H}, \mathrm{I}, \mathrm{L}$ indices in the 22211306 results coming from out of France.

Conclusions: Among the plasma samples effectively measured in the coagulation laboratory equipped with mechanical clot detection systems, icterus tends to be the most frequent interference. For these samples, optical clot detection between 400 and $540 \mathrm{~nm}$ should be avoided.

PB0388 | Main Blood Serum Metabolites Responsible for the Discrimination of Hospitalized Patients with Moderate Symptoms with or without COVID-19

L. Martins ${ }^{1}$; S. Martins' ${ }^{2}$ E. Braga ${ }^{1}$; D. Stanisic ${ }^{1}$; S. Montalvão ${ }^{2}$;

L. Da Silva'² S. Huber²; C. Bacchin²; N. Santos'²; T. Diaz ${ }^{3}$;

C. Wroclawski ${ }^{4}$; C. Filho ${ }^{4}$; L. Tasic ${ }^{2} ;$ J. Annichino-Bizzacchi ${ }^{2}$

${ }^{1}$ State University of Campinas - UNICAMP / Institute of Chemistry, Campinas, Brazil; ${ }^{2}$ State University of Campinas - UNICAMP, Campinas, Brazil; ${ }^{3}$ Anhembi Field Municipal Hospital, São Paulo, Brazil; ${ }^{4}$ Sirio Libanes Hospital, São Paulo, Brazil

Background: The variation in human blood serum metabolites resulting from an infection can improve diagnosis.

Aims: To map serum signatures of hospitalized symptomatic patients, positive or negative to SARS-CoV-2.

Methods: Patients $(n=64)$ admitted to Anhembi Field Municipal Hospital, a hospital set up for initial care to patients with moderate symptoms, were analyzed being discriminated in positive $(n=32)$ or negative. Age and gender were matched to ensure homogeneity in the basal metabolic rates. Three Nuclear Magnetic Resonance (NMR) data set were recorded on Bruker AVANCE III spectrometer for serum samples analyzed in MetaboAnalyst 5.0 software platform.

Results: The results for positive and negative patients were: mean age $54.92 \pm 12.41$ and $54.30 \pm 12.15$, and $50 \%$ female in each group. The ethnicity was $56.2 \%$ vs. $46.8 \%$ caucasian, $34.3 \%$ mixed race in both groups, and $9.3 \%$ vs. $12.5 \%$ black in positive and negative groups, respectively. BMI was $24 \pm 6.93$ vs. $33.5 \pm 7.85$ in comparison to positive and negative patients, respectively. In both groups $50 \%$ of patients presented alveolar infiltrate. Although the groups were not paired by comorbidities, they were homogeneous ensuring that the metabolic variation is due to COVID-19. Clinical symptoms were also remarkably similar between the groups (Table 1). The Partial Least Squares - Discriminant Analysis (PLS-DA) performed onto noesy1d data discriminated positively from negative patients (Figure 1.A). Also, it covered lower variance (Figure 1.B). Combining NMR techniques, it was possible to depict the main metabolites that distinguished the COVID-19 signatures. Alanine, glucose, cholesterol, and glutamine were increased, and lactate decreased (Figure 1.C.) in COVID-19.

TABLE 1 Patients description at the enrollment

$\begin{array}{ll}\text { Positive } & \text { Negative } \\ \text { SARS-CoV-2 } & \text { SARS-CoV-2 }\end{array}$

\section{Comorbidities}

$\begin{array}{lll}\text { Arterial Hypertension } & 17(53.1 \%) & 19(59.3 \%) \\ \text { Diabetes } & 9(28.1 \%) & 9(28.1 \%) \\ \text { Dyslipidemia } & 1(3.1 \%) & -\end{array}$

Dyslipidemia

$1(3.1 \%)$

Clinical Symptoms

\begin{tabular}{lll} 
Fever & $15(46.8 \%)$ & $14(43.7 \%)$ \\
Dry cough & $10(31.2 \%)$ & $10(31.2 \%)$ \\
Dyspnea & $10(31.2 \%)$ & $12(37.5 \%)$ \\
Myalgia & $6(18.7 \%)$ & $4(12.5 \%)$ \\
\hline
\end{tabular}
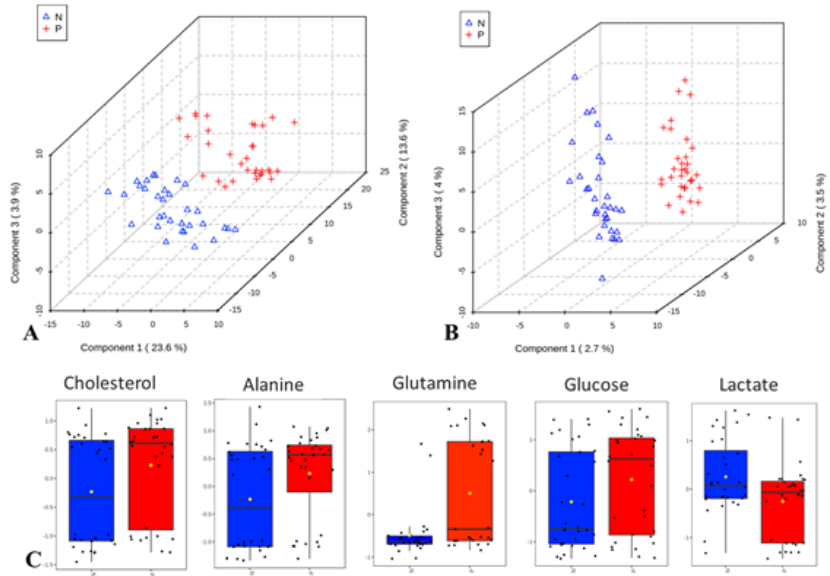

FIGURE 1 COVID-19 signatures observed trough $1 \mathrm{H}-\mathrm{NMR}$ of serum. A. PLS-DA scores in PC 1 vs. PC 2 vs. PC 3 of noesy1d spectra, with explained variances shown in brackets. B. Scores in PC 1 vs. PC 2 vs. PC 3 from CPMG data. SARS-CoV-2 qRT-PCR positive (P) group is indicated in red and negative $(\mathrm{N})$ group is indicated in blue. $\mathrm{C}$. Some of the main metabolites identified as responsible for distinguishing SARS-CoV-2 positive ( $P$, red) from negative ( $N$, blue) individuals, while levels of cholesterol, glucose, alanine, and glutamine were increased, lactate was decreased in $\mathrm{P}$ group.

Conclusions: These results suggest NMR as an excellent tool to differentiate hospitalized patients with moderate symptoms as COVID-19 positive or negative. 
Supported by FAPESP - 2018/06510-4 and 2016/14172-6, FAEPEX 2283/20 519.292, CNPq grant 401256/2020-0.

Approved by Ethics Research Committee of Unicamp.

PB0389 | Utility of Platelet Parameters in Pediatric Outpatient Setting: Experience from a Single Institution

R. Natesirinilkul; S. Manowong; P. Saihikam

Faculty of Medicine, Chiang Mai University, Chiangmai, Thailand

Background: Thrombocytopenia and thrombocytosis are two common abnormalities found in results of complete blood count (CBC). Unlike red blood cell (RBC) indices and reticulocyte count $(R C)$, the interpretation of platelet indices and immature platelet fraction (IPF) is less understandable in various number of platelet count (PLT) and the benefit of using those parameters in clinical setting particularly in pediatric population is inconclusive.

Aims: To find the way to interpret and the utility of platelet parameters in pediatric outpatient setting

Methods: A retrospective study was conducted to review the results of $\mathrm{CBC}$ and platelet indices including platelet distribution width (PDW), mean platelet volume (MPV) and immature platelet fraction (IPF) in Pediatric Clinic, Chiang Mai University Hospital between July and December 2020. The CBC, platelet indices and IPF were performed by Sysmex XN-350 ${ }^{\text {TM }}$.

Results: Among 237 CBC tests, there were 163 results with normal PLT, 45 with thrombocytosis (PLT > 450,000/ $\mathrm{mm}^{3}$ ) and 29 with thrombocytopenia (PLT < 150,000/ $\mathrm{mm}^{3}$ ). The PDW and MPV were shown only in 162 results (68\%). The median (min-max) PDW and MPV in reports of normal PLT [10.1 (7.3-25.6)\% and 9.9 (7.6-13.6) $\mathrm{fL}$, respectively] and thrombocytosis [10.3 (8.1-16.6)\% and 9.8 (8.112.9) $\mathrm{fL}$, respectively] were lower than in those of thrombocytopenia [13.6 (8.8-22.1)\% and 12 (8.5-13.8) fL, respectively], $(P=0.001$ and $<0.001$, respectively). Moreover, the median (min-max) IPF in reports of normal PLT [2 (0-9.6)\%] and thrombocytosis [1.8 (0.28.1)] was lower than one of thrombocytopenia [7.4 (1.3-39.6)\%], $(P<0.001)$. In thrombocytopenia group, the median PDW and IPF were higher in ones with increased consumption (19.1 and $12.8 \%$, respectively) rather than ones with underproduction (11.3 and $2.6 \%$, respectively) ( $P=0.018$ and 0.009 , respectively).

Conclusions: Though increased platelet indices and IPF are the findings in pediatric patients who have thrombocytopenia especially in ones who have thrombocytopenia due to increased consumption, the platelet indices are shown only two-third of $\mathrm{CBC}$ reports.
PB0390 | Comparison between Coagulation Factor Deficiencies versus Pharmacological Inhibition of Activated Coagulation Factors Using Clot Waveform Analysis

Y. Fujimori ${ }^{1}$; M. Wakui ${ }^{2}$; H. Katagiri ${ }^{1}$; Y. Ozaki ${ }^{1}$; Y. Kondo ${ }^{1}$; S. Oka ${ }^{1}$; T. Nakagawa ${ }^{1}$; S. Nakamura ${ }^{1}$; M. Murata ${ }^{2}$

${ }^{1}$ Keio University Hospital, Tokyo, Japan; ${ }^{2}$ Keio University, Tokyo, Japan

Background: Clot waveform analysis (CWA) has been reported to extend the interpretation of clotting time measurement. To date, effect of coagulation factor deficiencies and that of pharmacological anticoagulation have been quantitatively assessed by decrease in peak values in successive derivatives of the clotting reaction curves. In addition, abnormal waveforms have been reported to appear in patients with coagulation disorders or on warfarin therapy.

Aims: To make comparison between deficiency of coagulation factors and pharmacological inhibition of activated coagulation factors using CWA.

Methods: The samples of coagulation factor deficiency were prepared by adding normal pooled plasma to each factor deficient plasma (i.e., factor II, V, VIII, IX, X, XI and XII). The samples of pharmacological inhibition were prepared by spiking normal plasma with various anticoagulants. APTT measurement and APTT-CWA were performed using various APTT reagents and automated blood coagulation analyzers, CS5100 and CN6000 (Sysmex).

Results: Abnormal waveforms in first derivative curves were observed more clearly with decreasing activity of each coagulation factor except for factor II. The abnormality exhibited two patterns, i.e., the steepness of the first derivative curve changed either before or after reaching the peak. APTT reagents seemingly affected such appearances. The abnormal waveforms were not observed in plasma spiked with any anticoagulants. The parameters of CWA, i.e., maximum velocity, maximum acceleration, and maximum deceleration, appeared to make a hyperbolic curve with upward convex to drop to zero, dependently on decreasing coagulation factors. This feature is distinct from features of plasma spiked with anticoagulants.

Conclusions: The abnormal waveforms were induced by impaired usage of substrates (i.e., coagulation factors) but not by impaired usage of enzymes (i.e., activated coagulation factors). The appearance of abnormal waveforms potentially distinguishes coagulation abnormalities. Thus, findings obtained through CWA would provide insights into coagulopathy and anticoagulation.

PB0391 | Natural Fluorescence of Thrombin Substrate ZGly-Gly-Arg 7-amino-4-methylcoumarin: Considerations for Wavelength Choice

J. Jackson; L. Parunov; D. Monteil; M. Ovanesov

US Food and Drug Administration, Silver Spring, United States

Background: The improvement of the thrombin generation (TG) assay, since its development in the 1950s, have highlighted its 
potential to assess the hemostatic capacity in a variety of plasma samples. Indeed, the use of current fluorogenic thrombin substrates, such as Z-Gly-Gly-Arg 7-amino-4-methylcoumarin (ZGGR-AMC), have alleviated the turbidity issues encountered by its chromogenic predecessor, allowing for larger sample variety, such as platelet rich plasma, for testing. The commercial system, Calibrated Automated Thrombinography (CAT; Stago Diagnostics) performs measurements at an emission of $390 \mathrm{~nm}$ with an excitation of $460 \mathrm{~nm}$. Several reports have demonstrated the measurement of TG curves using additional substrates and emission/excitation spectra.

Aims: We sought to investigate the optimal spectra parameters for TG measurement, and the natural fluorescence properties of the thrombin substrate, ZGGR-AMC.

Methods: ZGGR-AMC and free AMC fluorescence was measured in a range of excitations and emission of $450 \mathrm{~nm}$.

Results: The AMC fluorophore has the highest fluorescence at an excitation of $340 \mathrm{~nm}$ and emission of $\sim 450 \mathrm{~nm}$. The thrombin substrate, ZGGR-AMC, possesses fluorescence properties, albeit shifted to the left of the excitation and emission spectra of free AMC molecule. The fluorescence of ZGGR-AMC interferes with the maximal AMC fluorescence when excited at $340 \mathrm{~nm}$ and emitted at 450 $\mathrm{nm}$, which is completely abrogated if the excitation is set at $380 \mathrm{~nm}$ and emission at $\sim 450 \mathrm{~nm}$.

Conclusions: These data provide evidence and corroborates the measurements of TG at an excitation $~ 380 \mathrm{~nm}$, but not lower than $360 \mathrm{~nm}$, providing the maximum AMC fluorescence for the assay readout while effectively denying the contamination of ZGGR-AMC substrate fluorescence. Measurements for TG performed at the optimal wavelength for AMC fluorescence (i.e., $340 \mathrm{~nm}$ ) runs the risk of detecting an overlapping signal from the thrombin substrate, potentially providing inaccurate results.

\section{PB0392 | Effect of Edoxaban on Laboratory Methods}

$\underline{\text { H. von Horn }}^{1,2}$; L. Bui ${ }^{3}$; A. Rasmuson ${ }^{1,2}$; J. Douxfils ${ }^{4}$;

J. Harenberg ${ }^{5,6}$; J. Antovic ${ }^{1,2} ;$ K. Christensen ${ }^{3}$

${ }^{1}$ Division of Clinical Chemistry, Karolinska University Hospital, Stockholm, Sweden; ${ }^{2}$ Department of Molecular Medicine and Surgery, Karolinska Institutet, Stockholm, Sweden; ${ }^{3}$ Department of Clinical Chemistry Central Hospital Karlstad, Karlstad, Sweden; ${ }^{4}$ University of Namur, Department of Pharmacy, Namur, Belgium; ${ }^{5}$ Heidelberg University, Heidelberg, Germany; ${ }^{6}$ DOASENSE GmbH, Heidelberg, Germany

Background: Direct acting oral anticoagulants (DOACs) are increasingly prescribed. Routine determination of DOACs is not considered necessary in general but may be required in the acute medical setting to rule out significant drug concentration. The prescription of edoxaban is expected to increase in patients with active cancer treated with anticoagulants due to atrial fibrillation.
Aims: We aim to investigate the effect of edoxaban in a real life setting on laboratory assays to rule out significant drug concentration with short turn-around time, including point of care tests.

Methods: A prospective consecutive study was performed in 50 patients treated with $30 \mathrm{mg}$ or $60 \mathrm{mg}$ od edoxaban at the anticoagulation clinic at Karlstad Central Hospital, Sweden, and blood and urine samples were taken at different times of the day at trough or peak levels of edoxaban. Plasma and urine samples were sent to the Karolinska University Laboratory for analyses of edoxaban concentration by chromogenic anti-factor Xa method and to the University of Namur to measure edoxaban concentration by liquid chromatography tandem mass spectrometry (LC-MS/MS). The POCT DOAC Dipstick (DOASENSE GmbH) was used to rule out drug concentration in patients' urine samples by visual and DOASENSE Reader analysis. Plasma concentration were compared to urine concentrations and quantitative results were dichotomized to compare with results from qualitative methods. Turnaround times for laboratory analysis of DOACs methods were compared. The study was approved by the local ethics committee and adheres to the declaration of Helsinki.

Results: To date, the first 10 patients have been included. The complete inclusion (or study) will be done by the end of June 2021.

Conclusions: The result of this study will generate reliable information on the metabolism of edoxaban and on rapid and accurate exclusion of significant amount of active edoxaban before acute interventions to avoid bleeding or to accelerate medical decisionmaking in major bleed.

PB0393 | Establishing Reference Values in Burkina Faso: A Study of Haemostasis Global Assays and Coagulation Factors Eight and Nine, Based on Blood Donors

K. Nebie ${ }^{1,2} ;$ S. Sawadogo ${ }^{1,2} ;$ j. Koulidiati ${ }^{3,4}$; W. Ouedraogo ${ }^{1}$;

D. Kima ${ }^{5}$; M. Nikiema ${ }^{4}$; S. Sawadogo ${ }^{6,7}$; D. Lasne ${ }^{8}$; A. Toure ${ }^{9}$;

E. Kafando ${ }^{1,5}$; GIH Study Group

${ }^{1}$ University Joseph KI-ZERBO, UFR SDS, Fundamental Sciences Department, Laboratory of Haematology, Ouagadougou, Burkina Faso; ${ }^{2}$ National Blood Centre, Ouagadougou, Burkina Faso; ${ }^{3}$ University Joseph KI-ZERBO, Ouagadougou, Burkina Faso; ${ }^{4}$ Yalgado Ouedraogo Teaching Hospital, Ouagadougou, Burkina Faso; ${ }^{5}$ Paediatric Teaching Hospital Charles de Gaulle, Ouagadougou, Burkina Faso; ${ }^{6}$ University Nazi Boni, National Institute for Medical Sciences, Bobo-Dioulasso, Burkina Faso; ${ }^{7}$ Souro Sanou Teaching Hospital, Bobo-Dioulasso, Burkina Faso; ${ }^{8}$ Necker Enfant Malade Teaching Hospital, Laboratory of General Hematology, Paris, France; ${ }^{9}$ University Cheikh Anta Diop, Dakar, Senegal

Background: The need to explore haemostasis is increasing in Burkina Faso with the changing health profile in the country, where, cardiovascular and metabolic diseases are emerging. However, interpretation of the results faces the absence of duly established reference values adapted to local populations. 
Aims: Our study aims to establish reference values for haemostasis global Assays (PT, aPPT, Fibrinogen) and FVIII and FIX levels.

Methods: We carried out a cross-sectional study from August 31 to October 222020 in the regional blood transfusion centre of Ouagadougou. Healthier Voluntary blood donors were included following CLSI guidelines. The sample size was 280 (140 males and 140 females). Inclusion criteria were, no current disease, no medical history or laboratory finding that can influence coagulation (smoking, inflammatory diseases, extreme obesity). A $5 \mathrm{ml}$ blood sample was drawn out in a citrated tube, centrifuged and the plasma been stored within $4 \mathrm{~h}$ at minus $80^{\circ} \mathrm{C}$. Later, we performed assays comprising PT, aPPT fibrinogen, FIX for all 280 subjects, and FVIII for only 140. The Sysmex ${ }^{\mathrm{TM}}$ CA660 coagulometer was used with Siemens ${ }^{\mathrm{TM}}$ reagents. Reference values were calculated using "Mean $\pm 2 X$ SD" or the "Central 95 percentile" when appropriate.

Results: Reference values were found to be [1.533-3.573] for fibrinogen, [67.72 - 116.57] for PT, [19.86 s - 28.74 s] for aPTT, [45.09\% - 149.4\%] for FIX, and [46.0\% - 174.6\%] for FVIII. FVIII was significatively lower in blood group $\mathrm{O}$ subjects compared to non-O blood groups (74.8\% to $93.5 \% ; P=0.005)$. Fibrinogen was higher in female than in male $(2.81 \mathrm{~g} / \mathrm{L}$ versus $2.30 \mathrm{~g} / \mathrm{L}, P<0.01)$.

Conclusions: The study demonstrates it is necessary to establish Reference values that fit local populations as lower limits of FVIII and FIX reference intervals appears to be low than expected and closer to minor haemophilia state.
PB0394 | Laboratory Testing in Patients Treated with Direct Oral Anticoagulants: Our Experience

G. Garcia-Donas; M. García; M. Bonete; A. Rodríguez

Hospital Virgen Macarena, Sevilla, Spain

Background: Direct oral anticoagulants (DOAC) do not require routine laboratory monitoring but it is useful to know the impact on coagulation assays in order to interpret the results specially in emergency situations as bleeding or urgent perioperative management.

Aims: The objective of our study is to determnine de DOAC concentration with specific test in control patients under different DOAC treatment and compare the results with routine coagulation assays in different moments, plasma concentration at peak $(3 \mathrm{~h}$ after drug intake) and through (just before intake).

Methods: Samples from volunteers patients with non-valvular atrial fibrillation under DOAC treatmente were collected at peak and through plasma concentrations. For all samples prothrombine time (PT) with Tromborel and activated partial thromboplastin time (APTT) with Actin-FS were measured. For anti-Xa DOAC anti-Xa activity (heparin calibrated) with Innovance Heparine and plasma concentration with STALiquid anti-Xa (Stago) was quantified, for Dabigatran thrombin time (TT) with Thromboclotin and the specific test STA-ECAll (Stago) were used. Results: 30 patients were included (59\% males, median age 71 years. The results for different test are seen in Table 1. Under Dabigatran TT was uncoagulable for all samples, and all PT and APTT were pathological. The most statistical correlation test with ditrect $\mathrm{FXa}$ inhibitor was anti-Xa (heparin calibrated) specially at throug (Fig. 1).S

TABLE 1 Results of coagulation test at through and peak moments under DOAC treatment

\begin{tabular}{|c|c|c|c|c|c|c|}
\hline & \multirow{2}{*}{\multicolumn{2}{|c|}{$\begin{array}{l}\text { APIXABAN n:15 } \\
\text { Median } \\
\text { (25th-75th percentile) }\end{array}$}} & \multirow{2}{*}{\multicolumn{2}{|c|}{$\begin{array}{l}\text { RIVAROXABAN n:10 } \\
\text { Median } \\
\text { (25th-75th percentile) }\end{array}$}} & \multirow{2}{*}{\multicolumn{2}{|c|}{$\begin{array}{l}\text { DABIGATRAN n:5 } \\
\text { Median } \\
\text { (25th-75th percentile) }\end{array}$}} \\
\hline & & & & & & \\
\hline $\begin{array}{l}\text { PT s } \\
\text { (normal range 10-14) }\end{array}$ & $\begin{array}{l}13.1 \\
(12.5-13.5)\end{array}$ & $\begin{array}{l}14.3 \\
(13.5-14.8)\end{array}$ & $\begin{array}{l}12.8 \\
(11.7-16.5)\end{array}$ & $\begin{array}{l}16.1 \\
(15.5-24.2)\end{array}$ & $\begin{array}{l}15.6 \\
(15.1-15.7)\end{array}$ & $\begin{array}{l}16.6 \\
(16.3-17.8)\end{array}$ \\
\hline $\begin{array}{l}\text { APTT ratio } \\
\text { (normal range } 0.8-1.2 \text { ) }\end{array}$ & $\begin{array}{l}1.15 \\
(1.1-1 . s 3)\end{array}$ & $\begin{array}{l}1.2 \\
(1.1-1.2)\end{array}$ & $\begin{array}{l}1.1 \\
(1.0-1.4)\end{array}$ & $\begin{array}{l}1.4 \\
(1.3-1.7)\end{array}$ & $\begin{array}{l}1.7 \\
(1.6-1.9)\end{array}$ & $\begin{array}{l}2.1 \\
(1.8-2.2)\end{array}$ \\
\hline $\begin{array}{l}\text { Anti-Xa IU/mL } \\
\text { (heparin calibrated) }\end{array}$ & $\begin{array}{l}0.56 \\
(0.36-0.78)\end{array}$ & $\begin{array}{l}1.79 \\
(1.25-2.01)\end{array}$ & $\begin{array}{l}0.25 \\
(0.05-0.73)\end{array}$ & $\begin{array}{l}1.70 \\
(1.63-1.75)\end{array}$ & & \\
\hline
\end{tabular}




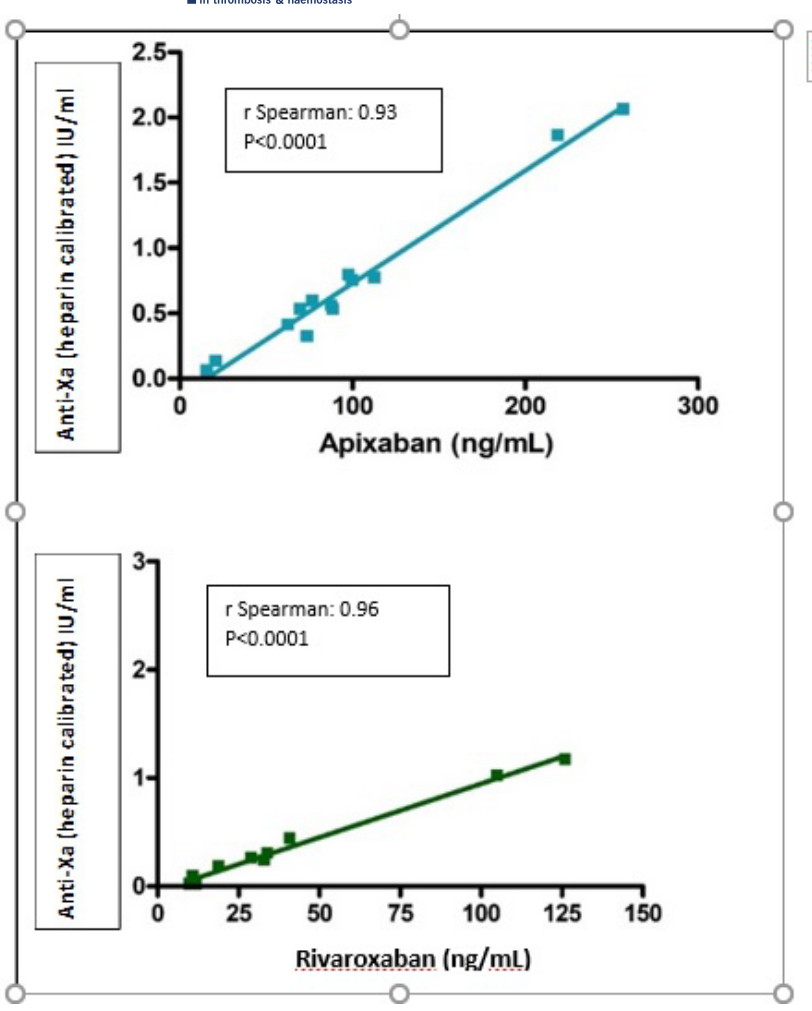

FIGURE 1 Correlation between anti-Xa (heparin calibrated) and DOAC levels at through moments

Conclusions: The results of DOAC concentration are similar to literature except for Dabigatran which are higher maybe because few samples.

We confirm Dabigatran is the DOAC that most modifies coagulation assays specially APTT and Apixaban nearly no modifies them.

The quatification of DOAC levels in plasma is more informative and useful than other coagulation parameter. In case to measure low plasma concentrations specific test calibrated are required but if these are not available for direct FXa inhibitors a heparin-calibrated chromogenic assay can be indicative of clinically relevant levels of a ditrect FXa inhibitor.

\section{NANOTECHNOLOGY AND NOVEL BIOMOLECULES}

\section{PB0395 | Protein S Gla Domain as Theranostic for Early Vascular Calcification}

A. Gentier; S. Agten; L. Schurgers; T. Hackeng

Department of Biochemistry, Cardiovascular Research Institute Maastricht (CARIM), University Maastricht, Maastricht, Netherlands

Background: Vascular calcification is implicated in severe pathologies such as atherosclerosis and chronic kidney disease. Early-stage calcification (microcalcification) is considered to be life-threatening both by increasing risk of plaque rupture and evading detection by conventional imaging techniques. In addition, no therapy exists to intervene with ectopic calcification. Calcium binding properties of coagulation factors are governed by post-translationally modified $\gamma$-carboxy glutamic acid (Gla) residues, hence the Gla-domain. Previous research has shown that some of these Gla-domains are able to interact with deleterious calcium-crystal formation. The Gladomain of protein $\mathrm{S}$ harbours the most Gla-residues of all vitamin K-dependent proteins.

Aims: To investigate if protein S Gla-domain can act as both tracer and therapy for microcalcification.

Methods: Protein S Gla domain was chemically synthesized and subsequently used for detection and inhibition of calcification in in vitro calcification assays. For this purpose, human vascular smooth muscle cells (hVSMCs) cultured under calcifying conditions were exposed to protein S Gla domain or a negative control protein S Glu domain. The ability to detect and interfere with calcification was determined using a combination of fluorescence microscopy and quantitative calcium phosphate crystal measurements.

Results: Protein S Gla domain selectively bound to microcalcifications in an in vitro setting allowing specific detection. Importantly, protein S Gla-domain interfered with initiation as well as progression of calcification of hVSMCs. Finally, at low concentrations, protein S Gla domain was used to follow progression of calcification in a time-dependent manner without interfering with the calcification process.

Conclusions: Protein S Gla-domain is a promising tool for detection and follow-up of early vascular calcification and has potential as a calcification inhibitor, due to its ability to interfere with calcification nucleation sites as well as progression of calcium crystals.

PB0396 | Single-cell Level Quantification of Surface Proteins in Platelets and Platelet-derived EVs Using SC-ICP-MS

E. Choi ${ }^{1}$; J. Shin ${ }^{1}$; S. Park ${ }^{1}$; J. Song ${ }^{2}$; T.H. Yoon ${ }^{1,3,4}$

${ }^{1}$ Hanyang University, Seoul, Korea, Republic of; ${ }^{2}$ Yonsei University, Seoul, Korea, Republic of; ${ }^{3}$ Center for Next Generation Cytometry, Seoul, Korea, Republic of; ${ }^{4}$ Institute of Next Generation Material Design, Seoul, Korea, Republic of

Background: Platelets play an important role in our blood system, such as hemostasis. However, their behaviors, such as mechanisms of their division process, are not well understood yet, due to the challenging nature of quantifying platelets and platelet-derived extracellular vesicles (EVs). Recently, mass cytometry, combining single cell inductively coupled plasma mass spectrometry (SC-ICP-MS) with immunostaining using antibodies tagged with a rare earth metal ions, is gaining more attention as a next-generation flow cytometry technique.

Aims: In this study, we have investigated the division process of platelet by quantifying their surface proteins using SC-ICP-MS 
technique and cross-checked with conventional flow cytometry with CFSE staining.

Methods: Platelets isolated from platelet-rich-plasma were stained with CFSE and incubated for 0,6 and $20 \mathrm{~h}$. Then, they were stained with CD41a and CD61, measured by SC-ICP-MS \& flow cytometry and compared.

Results: As the incubation time increase from 0 to 6 and $20 \mathrm{~h}$, enhancements in the number of platelets or platelet-derived EVs were observed, while the numbers of surface proteins per platelets or platelet-derived EVs were reduced. A single peak was observed at the incubation time of $\mathrm{O} \mathrm{h}$, but second peak with lower contents of surface proteins was found after $6 \mathrm{~h}$ of incubation and the number of cells with low protein contents increased significantly after $20 \mathrm{~h}$ of incubation. This temporal evolution of secondary peak with lower contents of surface proteins confirms that we are able to monitor the platelet division process in a single cell level via SC-ICP-MS technique.

Conclusions: Throughout this study, we have shown that the mass cytometry, or SC-ICP-MS, technique is able to quantitatively monitor division processes of platelet and platelet-derived EVs in a single cell mode and can be further used to investigate various properties of platelet and platelet-derived EVs.
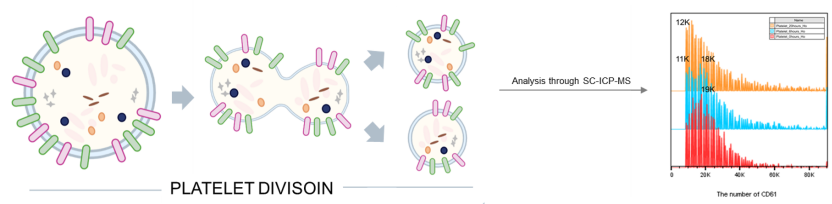

FIGURE 1 Quantification of surface protein in platelet using SC-ICP-MS

PB0397 | Development of an Anti-thrombotic and Antiinflammatory Coating for Medical Devices

K. Gonzalez ${ }^{1,2}$; K. Yu ${ }^{1,2}$; V. Lei ${ }^{1}$; E.M. Conway ${ }^{1,2}$;

J.N. Kizhakkedathu ${ }^{1,2,3}$

${ }^{1}$ Centre for Blood Research, University of British Columbia, Vancouver, Canada; ${ }^{2}$ Department of Pathology and Laboratory Medicine, University of British Columbia, Vancouver, Canada; ${ }^{3}$ School of Biomedical

Engineering, University of British Columbia, Vancouver, Canada

Background: There are several blood contacting medical devices that are widely used in medicine to enhance the quality of care. These include, for example, coronary stents, vascular grafts, and mechanical heart valves. A major problem that arises from use of such devices is a lack of biocompatibility that results in activation of enzyme cascades in the blood, leading to excess clotting and inflammation. This may lead to restrictions in blood flow through the device, device failure, embolism, and an increase in the risk of local and widespread infection. One strategy that has been employed to prevent clotting and inflammation on medical devices is the development of specialized surface coatings, inspired by natural anticoagulant systems. Our lab has recently developed an antifouling surface coating made from polydopamine and poly( $\mathrm{N}, \mathrm{N}$-dimethylacrylamide) (PDA/PDMA), to which modified proteins/molecules, such as heparin, may be covalently bound in predetermined orientations for optimal function. We and others have also shown that the lectin-like domain of thrombomodulin (TM-LLD) has profound anti-inflammatory properties.

Aims: This project aims to utilize the PDA/PDMA surface coating to conjugate heparin and TM-LLD, thereby developing a new and more effective anti-thrombotic and anti-inflammatory coating that may be used for a wide range of medical devices.

Methods: We have modified unfractionated heparin and TM-LLD at their terminal end, allowing them to be conjugated to the PDA/ PDMA coating. Prior to conjugation, activity of modified heparin in solution was evaluated by aPTT and activity of modified TM-LLD was evaluated by leukocyte adhesion and complement ELISA.

Results: We have confirmed that PDA/PDMA-conjugated modified heparin binds to antithrombin and thus likely retains its anticoagulant properties. Modified TM-LLD in solution dampens leukocyte adhesion and complement activation and is similarly being prepared for conjugation to the PDA/PDMA.

Conclusions: These constructs will be evaluated individually and in combination for their capacity to reduce thrombo-inflammation on the biomaterial surface.

\section{FIBRINOGEN, FIBRINOLYSIS AND PROTEOLYSIS}

\section{FIBRINOGEN AND FACTOR XIII}

LPB0016 | The Fibrin(ogen) $\gamma$-chain Integrin allb $\beta 3$ Binding Motif is Dispensable for Platelet Accumulation but Modifies Cross-linking in the Injured Liver

L.G. Poole ${ }^{1}$; D.J. Groeneveld ${ }^{1}$; H.M. Cline-Fedewa ${ }^{1}$; M.J. Flick ${ }^{2}$; J.P. Luyendyk ${ }^{1}$

${ }^{1}$ Michigan State University, East Lansing, MI, United States; ${ }^{2}$ University of North Carolina at Chapel Hill, Chapel Hill, United States

Background: Hepatic fibrin(ogen) deposition and platelet accumulation are hallmarks of liver injury driven by acetaminophen (APAP) overdose. Prior studies indicate that platelets exacerbate early necrosis and inhibit repair of the injured liver. However, the mechanisms mediating initial platelet accumulation in the injured liver are not understood.

Aims: We tested the hypothesis that hepatic platelet accumulation in the APAP-injured liver is mediated by fibrin(ogen) engagement of the platelet integrin $a_{11 b} \beta_{3}$. 
Methods: Male wild-type mice and mice expressing normal levels of a mutant fibrinogen incapable of engaging integrin $\alpha_{l \mid l} \beta_{3}$ through the c-terminal domain of the fibrinogen $\gamma$ chain ( $F i b \gamma^{\Delta 5}$ mice) were challenged with APAP (300 mg/kg, i.p.). Hepatic platelet accumulation, liver necrosis, and hepatic fibrin(ogen) deposition were assessed 6 and $24 \mathrm{~h}$ after APAP challenge.

Results: Hepatocellular necrosis, fibrin(ogen) deposition and platelet accumulation were evident in APAP-challenged wild-type mice at both time points. In contrast to our hypothesis, hepatic platelet accumulation was modestly increased in Fiby ${ }^{\Delta 5}$ mice compared to wild-type mice at both time points. Hepatocellular injury was initially similar in wild-type and Fiby ${ }^{\Delta 5}$ mice after APAP challenge but increased significantly in APAP-challenged Fiby ${ }^{\Delta 5}$ mice at $24 \mathrm{~h}$, as indicated by increased plasma alanine aminotransferase activity. Surprisingly, immunolabeling revealed a unique pattern of fibrin(ogen) deposits in livers of APAP-challenged Fiby ${ }^{\Delta 5}$ mice compared to wild-type mice. Hepatic fibrin(ogen) deposits generally filled necrotic lesions in APAP-challenged wild-type mice, but appeared in patches throughout the lesion in Fiby ${ }^{\Delta 5}$ mice. Moreover, fibrinogen $\gamma$-chain cross-linking was unique in livers of APAP-challenged Fiby ${ }^{\Delta 5}$ mice, favoring accumulation of high molecular weight cross-linked $\gamma$-chain complexes ( 220 and $350 \mathrm{kDa})$.

Conclusions: The results indicate that hepatic platelet accumulation in the APAP-injured liver is not driven by the fibrin(ogen) $\gamma$ chain-integrin $a_{11 b} \beta_{3}$ motif. Rather, the results suggest that the fibrin(ogen) $\gamma$-chain integrin $\alpha_{11 \mathrm{~b}} \beta_{3}$ binding motif modifies intrahepatic fibrin(ogen) deposition and cross-linking during acute hepatocellular necrosis.

LPB0017 | Activation Mechanism Dependent Surface Exposure of Cellular FXIII on Platelets and Platelet Microparticles: Immunofluorescence and Immune Electron Microscopic Study

L. Somodi ${ }^{1}$; H. Bárdos ${ }^{2}$; I. Beke Debreceni ${ }^{3}$; G. Kis ${ }^{4}$;

J. Kappelmayer ${ }^{3}$; M. Antal ${ }^{4}$; L. Muszbek ${ }^{1}$

${ }^{1}$ University of Debrecen, Faculty of Medicine/Department of Laboratory Medicine, Division of Clinical Laboratory Science, Debrecen, Hungary; ${ }^{2}$ University of Debrecen, Faculty of Medicine, Department of Public Health and Epidemiology, Debrecen, Hungary; ${ }^{3}$ University of Debrecen, Faculty of Medicine/Department of Laboratory Medicine, Debrecen, Hungary; ${ }^{4}$ University of Debrecen, Faculty of Medicine/ Department of Anatomy, Histology and Embryology, Debrecen, Hungary

Background: The cellular form of coagulation factor XIII (cFXIII) is a dimer of two potentially active A subunits (FXIII-A2). cFXIII is of cytoplasmic localization and amounts to $3 \%$ of the total platelet protein. Coinciding activation of platelet collagen and the PAR1 receptor by convulxin (CVX) and thrombin (THR) transpose cFXIII (Mitchell et al. Blood 2014;124:3982-90) and phosphatidylserine (PS) to the surface of activated platelets.
Aims: To compare the surface exposure of cFXIII and PS on platelets undergoing receptor-mediated (CVX+THR) and non-receptormediated (Ca2+-ionophore) activation and on platelet microparticles shed from activated cells.

Methods: Gel-filtered platelets were stimulated by CVX+THR or $\mathrm{Ca} 2+-$ ionophore (A23187). Platelets and platelet derived MPs were identified by anti-CD41a antibodies. In immunfluorescent studies FXIII-A was labeled by rabbit anti-human FXIII-A and DyLight 488-labeled horse anti-rabbit IgG, annexin $\mathrm{V}$ was conjugated to Alexa Fluor 568. For double immunogold labeling rabbit anti-FXIII-A and mouse anti-CD41a were followed by goat-anti-rabbit IgG conjugated to $15 \mathrm{~nm}$ gold particles and goat-anti-mouse lgG conjugated to $10 \mathrm{~nm}$ gold particles.

Results: Following activation by CVX+THR in over half of platelets and platelet MPs PS and CFXIII became transposed to the outer membrane surface. The majority of PS-positive MPs also showed FXIII-A positivity. Most of the surface exposed cFXIII accumulated in a cap-like structure. Larger microparticle with intact CD41a positive membrane showed weak FXIII positivity while smaller particles were CD41a negative and strongly labeled for cFXIII. Non-receptor mediated activation triggered by $\mathrm{Ca} 2+-$ ionophore resulted PS positive platelets and MPs, however neither the cells nor the formed MPs expressed FXIII-A on their surface.

Conclusions: Surface exposure of PS and cFXIII on both platelets and microparticles was induced by receptor-mediated activation. Transposition of PS and cFXIII to the membrane surface requires different mechanisms. Elevation of intracellular $\mathrm{Ca} 2+$ concentration is sufficient for PS transposition but insufficient to expose cFXIII.

\section{LPB0063 | Epigenome-wide Association Study of DNA Methylation in Blood and Circulating Fibrinogen Levels}

J. Hahn ${ }^{1}$; J. Bressler ${ }^{1}$; M.R. Brown ${ }^{1} ;$ M. Fornage ${ }^{1,2} ;$ J. Bell ${ }^{3}$; D.I. Boomsma ${ }^{4}$; A. Dehghan ${ }^{5}$; A. Domingo-Relloso ${ }^{6,7}$; X. Guo ${ }^{8}$; A.D. Johnson ${ }^{9,10}$; M. Kleber ${ }^{11}$; D.L. McCartney ${ }^{12}$; P.-E. Morange ${ }^{13}$; N.L. Smith ${ }^{14,15,16}$; A. Teumer ${ }^{17}$; W. Zhao ${ }^{18}$; A.C. Morrison ${ }^{1}$; P.S. de Vries $^{1}$; CHARGE Epigenetics and Hemostasis Working Groups ${ }^{1}$ Human Genetics Center, Department of Epidemiology, Human Genetics, and Environmental Sciences, School of Public Health, The University of Texas Health Science Center at Houston, Houston, United States; ${ }^{2}$ Institute of Molecular Medicine, McGovern Medical School, The University of Texas Health Science Center at Houston, Houston, United States; ${ }^{3}$ Department of Twin Research and Genetic Epidemiology, King's College London, London, United Kingdom; ${ }^{4}$ Department of Biological Psychology, Neuroscience Campus Amsterdam, VU University, Amsterdam, Netherlands; ${ }^{5}$ Department of Epidemiology and Biostatistics, Imperial College London, London, United Kingdom; ${ }^{6}$ Department of Chronic Diseases Epidemiology, National Center for Epidemiology, Carlos III Health Institute, Madrid, Spain; ${ }^{7}$ Department of Environmental Health Sciences, Columbia University Mailman School of Public Health, New York, United States; ${ }^{8}$ Statistical Genetics, Institute for Genomics and Population Sciences, 
The Lundquist Institute and Department of Pediatrics at HarborUCLA Medical Center, Torrance, United States; ${ }^{9}$ Division of Intramural Research, Population Sciences Branch, National Heart, Lung and Blood Institute, Bethesda, United States; ${ }^{10}$ The Framingham Heart Study, Framingham, United States; ${ }^{11}$ Vth Department of Medicine, Medical Faculty Mannheim, Heidelberg University, Mannheim, Germany; ${ }^{12}$ Centre for Genomic and Experimental Medicine, University of Edinburgh, Western General Hospital, Edinburgh, United Kingdom; ${ }^{13}$ Aix-Marseille University, INSERM, INRAE, C2VN, Marseille, France; ${ }^{14}$ Department of Epidemiology, University of Washington, Seattle, United States; ${ }^{15}$ Kaiser Permanente Washington Health Research Institute, Seattle, United States; ${ }^{16}$ Seattle Epidemiologic Research and Information Center, Department of Veterans Affairs Office of Research and Development, Seattle, United States; ${ }^{17}$ Institute for Community Medicine, University Medicine Greifswald, Greifswald, Germany; ${ }^{18}$ Department of Epidemiology, University of Michigan, Ann Arbor, United States

Background: DNA methylation of cytosine-phosphate-guanine $(\mathrm{CpG})$ sites across the genome is an epigenetic mechanism that regulates levels of gene expression. Fibrinogen plays an essential role in blood coagulation and inflammatory pathways. Inter-individual differences in DNA methylation may be associated with varying levels of fibrinogen. No epigenome-wide association study (EWAS) of circulating fibrinogen levels has been performed to date.

Aims: To perform an EWAS to examine an association between blood DNA methylation levels and circulating fibrinogen levels to better understand its biological and pathophysiological actions.

Methods: We performed an EWAS of circulating fibrinogen levels in 18,037 participants representing 13 studies from the CHARGE consortium. DNA methylation in blood of 12,904 participants was measured using the Illumina $450 \mathrm{~K}$ array, and of 5,133 using the $850 \mathrm{~K}$ array. This sample included individuals of European $(n=11,357)$ and African ( $n=4,007)$ ancestries, American Indians $(n=2,293)$ and Hispanics $(n=380)$. Significant associations were identified using Bonferroni corrected P-values, and they were cross-replicated. A sensitivity analysis was performed within the ARIC study comparing models with and without C-reactive protein (CRP) adjustment to examine a potential confounding effect of inflammation.

Results: We identified 208 and 87 significant CpG sites associated with fibrinogen from the $450 \mathrm{~K}(P<1.03 \mathrm{E}-7)$ and $850 \mathrm{~K}$ arrays $(P<5.78 \mathrm{E}-8)$, respectively. There were 78 associations from the $450 \mathrm{~K}$ analysis that replicated in the $850 \mathrm{~K}$ analysis, and 26 vice versa. After accounting for the overlapping sites, there were 83 replicated $\mathrm{CpG}$ sites located in 61 independent loci. Examples of genes located near these CpG sites were SOCS3, AIM2, and SBNO2, which are involved in inflammatory pathways. The sensitivity analysis showed the associations for all 83 replicated CpG sites were attenuated after CRP adjustment, although many remained significant.

Conclusions: We identified $83 \mathrm{CpG}$ sites associated with fibrinogen levels. These associations are partially driven by the inflammatory pathway shared by both fibrinogen and CRP.
LPB0110 | FXIIla-mediated Fibrin $\gamma$-chain Crosslinks Are Critical for Fibrin Fiber Stiffness and Resistance to Rupture

$\underline{\text { T. Feller }}^{1}$; C. Duval ${ }^{1}$; S.D.A. Connell ${ }^{2}$; R.A.S. Ariëns ${ }^{1}$

${ }^{1}$ University of Leeds, Leeds Institute of Cardiovascular and Metabolic

Medicine, Leeds, United Kingdom; ${ }^{2}$ University of Leeds, School of

Physics, Leeds, United Kingdom

Background: The fibrin network, which provides the scaffold for the blood clot, is a biopolymer with multiscale structural hierarchy leading to complex mechanical behaviour. As the last step of clot formation, activated coagulation factor XIII (FXIIla) crosslinks the $a-$ and $\gamma$-chains of fibrin, thus increasing clot stiffness.

Aims: To investigate how the presence/absence of $\gamma$-crosslinks alters fibrin mechanics in a novel murine model (FGG3X), we compared the stress-strain behaviour of wild type (WT) and $\gamma Q 398 N / Q 399 N /$ K406R fibrinogen (FGG3X), unable to undergo $\gamma-\gamma$ crosslinking.

Methods: Clots of plasma-purified FGG3X or WT murine fibrinogen were made on a striated surface $(0.5 \mathrm{mg} / \mathrm{ml}$ fibrinogen, 0 or $10 \mu \mathrm{g} / \mathrm{ml}$ rhFXIII-A $A_{2}, 0.5 \mathrm{U} / \mathrm{ml}$ thrombin, $5 \mathrm{mM} \mathrm{CaCl}{ }_{2}$ ). Individual fibrin fibers crossing between ledges were stretched with atomic force microscopy (AFM). Stress vs. strain curves were used for further analysis, where stress and strain were calculated from the lateral deflection and y-position of the cantilever, respectively.

Results: Compared with crosslinked FGG3X fibers, crosslinked WT fibers ruptured at significantly higher stress (2.2-fold, $P<0.001)$, had 1.6-fold higher initial stiffness at low $(<150 \%)$ strains $(P<0.05)$, stiffened more (1.3-fold, $P<0.01)$, reached a higher stiffness before rupture (2.8-fold, $P<0.001)$ and had higher toughness (2.1-fold, $P<0.01$ ). Both uncrosslinked WT and FGG3X fibers showed similar parameters as the crosslinked FGG3X fibers.

Conclusions: In the presence of $\gamma-\gamma$ crosslinks, individual fibrin fibers were less prone to rupture, as seen from the increased toughness, rupture stress and rupture stiffness of crosslinked WT fibers compared with crosslinked FGG3X fibers. Thus, when $\gamma$-chain crosslinks are present, fibers are much stronger and less prone to rupture. These findings may have important implications for clot fragmentation and thus thromboembolic diseases such as pulmonary embolism or cardioembolic stroke.

\section{LPB0111 | Molecular Interactions of Coagulation Factor XIII B Subunit}

$\underline{\text { K. Pénzes }}^{1}$; B. Bécsi ${ }^{2}$; F. Erdődi²; L. Muszbek ${ }^{1}$

${ }^{1}$ University of Debrecen, Faculty of Medicine, Department of Laboratory Medicine, Division of Clinical Laboratory Science, Debrecen, Hungary; ${ }^{2}$ University of Debrecen, Faculty of Medicine, Department of Medical Chemistry, Debrecen, Hungary

Background: Coagulation factor XIII (FXIII) consists of two potentially active $A$ subunits and two inhibitory/protective $B$ subunits (FXIII$A_{2} B_{2}$ ). FXIII-B is in excess, half of it circulates in non-complexed form. It contains $8.5 \%$ carbohydrate; the glycan part has a role in keeping 
FXIII- $A_{2} B_{2}$ in the circulation. A major polymorphism of FXIII-B is due to a novel splice acceptor site in intron $\mathrm{K}(\mathrm{IK})$ and the replacement of $10 \mathrm{C}$-terminal amino acids by 25 novel amino acids. FXIII-B is associated to fibrinogen through fibrinogen $\gamma$-chain. Peak 1 fibrinogen (P1F; $85 \%$ of plasma fibrinogen) contains two $\gamma \mathrm{A}$-chains, peak 2 fibrinogen (P2F) also posseses alternatively spliced $\gamma$ '-chain.

Aims: To evaluate whether the glycan residue and FXIII-B IK polymorphism influence the binding of FXIII-B to FXIII-A and how FXIII-B and its polymorphic variant binds to $\mathrm{P} 1 \mathrm{~F}$ and $\mathrm{P} 2 \mathrm{~F}$.

Methods: Binding studies between proteins and large peptides were carried out by surface plasmon resonance technique. Association rate constant (ka), dissociation rate constants (kd), and equilibrium dissociation constants $(\mathrm{Kd})$ were calculated. Association of a short $\gamma \mathrm{A}$-chain peptide to FXIII-B was investigated by isothermal titration calorimetry (ITC).

Results: Deglycosylated FXIII-B (DG-FXIII-B) showed decreased affinity to FXIII-A. The Kd for the binding of native and IK FXIII-B variant to FXIII-A were comparable. Interestingly, FXIII-B as analyte also bound to the ligand FXIII-B ( $\left.\mathrm{Kd}=2.75 \times 10^{-8}\right)$. FXIII-B bound to P2F with somewhat higher affinity than to $\mathrm{P} 1 \mathrm{~F}\left(\mathrm{Kd}=2.89 \times 10^{-8}\right.$ versus $\left.\mathrm{Kd}=5.03 \times 10^{-7}\right)$. Using ITC, we confirmed the results of Byrnes et al. (Blood 2016;128:1969-78) demonstrating that the C-terminal peptide of $\gamma \mathrm{A}$-chain, which is also present in the $\gamma^{\prime}$-chain binds to FXIII-B $\left(\mathrm{Kd}=2.40 \times 10^{-8}\right)$. However, the sulfated peptide part of the $\gamma^{\prime}$-chain also showed relatively high affinity toward FXIII-B $\left(\mathrm{Kd}=7.42 \times 10^{-8}\right)$.
LPB0112 | Monocytes Expose Factor XIII-A and Stabilize Thrombi against Fibrinolytic Degradation

F. Alshehri $^{1,2}$

${ }^{1}$ University of Aberdeen, Union street Aberdeen, United Kingdom;

${ }^{2}$ Institiute of Medical Science, Aberdeen, United Kingdom

Background: Factor XIII (FXIII) is a transglutaminase (TG) that promotes thrombus stability by cross-linking fibrin. FXIII exists as a cellular form, a homodimer of the A subunits (FXIII-A) and it is found in abundance within circulating platelets and monocytes.

Aims: To investigate externalization of FXIII-A on monocytes and its role in extracellular crosslinking reactions.

Methods: Isolated human monocytes or THP-1 cells $\left(6 \times 10^{5}\right.$ cells/ $\mathrm{ml}$ ) were treated $\pm 20 \mathrm{ng} / \mathrm{ml}$ interleukin-4 (IL-4); $20 \mathrm{ng} / \mathrm{ml}$ interleukin-10 (IL-10); or $100 \mathrm{ng} / \mathrm{ml}$ lipopolysaccharide (LPS) for $24 \mathrm{~h}$ FXIII-A exposure and activity were detected using a FITC-labelled anti-FXIII-A antibody $(40 \mu \mathrm{g} / \mathrm{ml})$ or the fluorescent amine donor substrate, TAMRA ( $40 \mu \mathrm{g} / \mathrm{ml}$ ) by flow cytometer and imaged by confocal microscopy. Pooled normal plasma or factor XIII deficient plasma + FITC-labelled fibrinogen $(45 \mu \mathrm{g} / \mathrm{ml})$ was re-calcified with $\mathrm{CaCl}_{2}(10.9$ $\mathrm{mM}) \pm$ stimulated monocytes or THP-1 cells $\left(3 \times 10^{5}\right.$ cells $\left./ \mathrm{ml}\right) \pm 1 \mathrm{mM}$

TABLE 1 Surface plasmon resonance results

\begin{tabular}{lllll} 
Ligand & Analite & ka (1/Ms) & kd (1/s) & Kd (M) \\
FXIII-B & rFXIII-A & $1.17 \times 10^{5} \pm 2.36 \times 10^{4}$ & $7.64 \times 10^{-4} \pm 9.31 \times 10^{-5}$ & $6.80 \times 10^{-9} \pm 1.86 \times 10^{-9}$ \\
IK-FXIII-B & rFXIII-A & $1.03 \times 10^{5} \pm 3.16 \times 10^{4}$ & $7.81 \times 10^{-4} \pm 7.21 \times 10^{-5}$ & $8.43 \times 10^{-9} \pm 3.62 \times 10^{-9}$ \\
DG-FXIII-B & rFXIII-A & $7.32 \times 10^{4} \pm 3.24 \times 10^{4}$ & $2.11 \times 10^{-3} \pm 8.76 \times 10^{-4}$ & $3.06 \times 10^{-8} \pm 8.63 \times 10^{-9}$ \\
FXIII-B & FXIII-B & $2.28 \times 10^{4} \pm 1.37 \times 10^{4}$ & $5.29 \times 10^{-4} \pm 9.14 \times 10^{-5}$ & $2.75 \times 10^{-8} \pm 9.89 \times 10^{-9}$ \\
Peak1 fibrinogen & FXIII-B & $1.47 \times 10^{4} \pm 9.32 \times 10^{3}$ & $2.79 \times 10^{-3} \pm 2.79 \times 10^{-3}$ & $5.03 \times 10^{-7} \pm 7.68 \times 10^{-7}$ \\
Peak2 fibrinogen & FXIII-B & $1.11 \times 10^{5} \pm 1.29 \times 10^{5}$ & $1.89 \times 10^{-3} \pm 2.06 \times 10^{-4}$ & $2.89 \times 10^{-8} \pm 1.12 \times 10^{-7}$ \\
IK-FXIII-B & Peak1 fibrinogen & $3.76 \times 10^{3} \pm 8.32 \times 10^{2}$ & $1.22 \times 10^{-2} \pm 1.36 \times 10^{-3}$ & $3.36 \times 10^{-6} \pm 6.29 \times 10^{-7}$ \\
IK-FXIII-B & Peak2 fibrinogen & $4.45 \times 10^{4} \pm 8.92 \times 10^{4}$ & $1.31 \times 10^{-3} \pm 2.33 \times 10^{-3}$ & $8.17 \times 10^{-7} \pm 1.97 \times 10^{-6}$ \\
$\gamma^{\prime}$ sulfated peptide & FXIII-B & $1.75 \times 10^{4} \pm 6.70 \times 10^{3}$ & $1.17 \times 10^{-3} \pm 3.80 \times 10^{-5}$ & $7.42 \times 10^{-8} \pm 2.48 \times 10^{-8}$ \\
$\gamma^{\prime}$ non-sulfated peptide & FXIII-B & $1.95 \times 10^{4} \pm 2.25 \times 10^{4}$ & $1.27 \times 10^{-3} \pm 2.29 \times 10^{-4}$ & $1.68 \times 10^{-7} \pm 1.78 \times 10^{-7}$ \\
\hline
\end{tabular}

Conclusions: The results suggest that the glycan structure of FXIII-B contributes to its binding to FXIII-A and FXIII-B associates to both types of fibrinogen chains. 
a TG inhibitor. Thrombi were bathed in tissue plasminogen activator $(1 \mu \mathrm{g} / \mathrm{ml}$ ) and sampled every $30 \mathrm{~min}$ for $4 \mathrm{~h}$ and fluorescence read (Ex 485 nm Em 528 nm).

Results: IL-10 stimulation significantly increased the number of FXIII-A positive monocytes compared to unstimulated cells (21.2 \pm $3.4 \%$ vs $7.9 \pm 2.1 \% P<0.05$ ). FXIII-A activity was augmented 2.5 -fold with IL-4 and IL-10 stimulation compared to resting cells. Basal levels of FXIII-A antigen expression were higher in THP-1 cells with a negligible impact of stimulation. However, FXIII-A activity on the surface of THP-1 cells was significantly increased with all stimuli compared to resting cells. FXIII-A antigen and activity were expressed on the external membrane of stimulated monocytes and THP-1 cells. However, the distribution with LPS stimulation produced a distinct punctate pattern around the cell periphery. IL-4 or IL-10 activated monocytes and THP-1 cells stabilized FXIII-depleted.

Conclusions: Activated monocytes and THP-1 cells externalize and retain active FXIII-A on their membrane thereby stabilizing thrombi against fibrinolytic degradation.

PB0399 | Biomechanics, Thermodynamics and Mechanisms of Rupture of Fibrin Clots

V. Barsegov ${ }^{1}$; V. Tutwiler ${ }^{2}$; F. Maksudov ${ }^{1}$; R. Litvinov ${ }^{2}$; J. Weisel ${ }^{2}$

${ }^{1}$ Department of Chemistry, University of Massachusetts, Lowell, United States; ${ }^{2}$ Department of Cell and Developmental Biology, University of Pennsylvania Perelman School of Medicine, Philadelphia, United States

Background: Fibrin is a unique biomaterial and a major component and mechanical determinant of hemostatic blood clots and obstructive thrombi.

Aims: Here, we explored the rupture of blood clots, emulating thrombus breakage by stretching fibrin gels with single-edge cracks. Methods: We employed tensile testing to collect the forcedisplacement response of fibrin gels with controlled defects, and scanning electron microscopy and transmission electron microscopy to visualize fibers rupture

Results: The stress-strain profiles display the weakly non-linear regime I of the gel due to alignment of fibrin fibers; linear elastic regime II owing to reversible stretching of fibers; and the rupture regime III for large deformations, during which irreversible breakage of fibers occurs. These dynamic mechanical regimes correlate with structural changes in the fibrin network. To model the stress-strain curves, we developed the Fluctuating Spring model, which maps the fibrin alignment, elastic network stretching, and cooperative rupture of coupled fibrin fibers into a theoretical framework to calculate stress as a function of strain.

Conclusions: Cracks render network rupture stochastic. The free energy change for fiber deformation and rupture decreases with the crack size, thereby making the network rupture more spontaneously, but mechanical cooperativity due to the inter-fiber coupling strengthens the fibrin network. These results provide a basis for understanding of blood clot breakage that underlies thrombotic embolization. The Fluctuating Spring model can be used to characterize the dynamics of mechanical deformation of other protein networks.
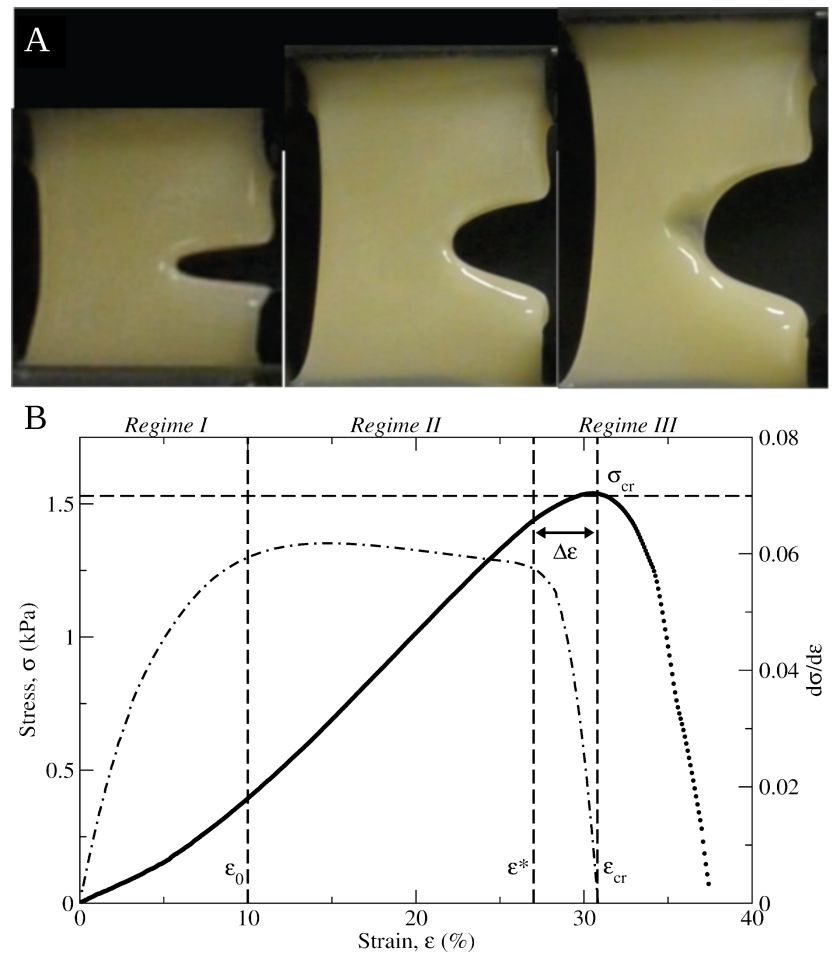

FIGURE 1 Panel A: Snapshots of fibrin gel sample $(30 \mathrm{~mm} \times 30 \mathrm{~mm}$ $\times 6.5 \mathrm{~mm}$ ) with a 12-mm single-edge crack at the times that correspond to the dynamic mechanical regimes I-III. Panel B: Stress-strain and slope-strain profiles showing non-linear regime I of deformation due to fibrin fibers alignment at the characteristic strain, linear regime II of elastic deformation due to fibers stretching, and rupture regime III due to fibers rupture at the strain threshold. The critical stress of $1.53 \mathrm{kPa}$ and critical strain of $31 \%$ are attained when all fibers have ruptured.

PB0400 | Fibrinogen B Arg448Lys and Factor XIII Val34Leu Polymorphisms Are Associated with Prothrombotic Fibrin Clot Properties in Patients with Acute Pulmonary Embolism

A. Klajmon $^{1}$; J. Chmiel ${ }^{2}$; M. Ząbczyk ${ }^{2,1}$; J. Natorska ${ }^{2,1}$; A. Undas ${ }^{2,1}$

${ }^{1}$ John Paul II Hospital, Cracow, Poland; ${ }^{2}$ Institute of Cardiology, Jagiellonian University Medical College, Cracow, Poland

Background: Pulmonary embolism (PE) remains a major cause of mortality. Prothrombotic fibrin clot properties in acute PE are associated with eightfold increased risk of death. To our knowledge, no reports assessed the impact of fibrinogen and factor XIII (FXIII) polymorphisms on plasma fibrin clot features in acute PE. 
Aims: We investigated whether a fibrinogen alpha chain (FGA) Thr312Ala, fibrinogen beta chain (FGB) Arg448Lys and FXIII Val34Leu polymorphisms are associated with fibrin clot properties assessed on admission in acute PE patients.

Methods: In 126 normotensive acute PE patients (aged $58 \pm 14$ years) we assessed 3 genetic polymorphisms FGA rs6050 C_-_2892877_10, FGB rs1800790 C__7429790_20 and factor XIII rs5958 C___1639938_20 in relation to fibrin clot permeability $\left(K_{s}\right)$, clot lysis time (CLT), and endogenous thrombin potential (ETP).

Results: Patients with the SNP Arg448Lys in the FGB $(n=40,31.7 \%$, 4 homozygous) compared to non-carriers had $15.7 \%$ reduced $\mathrm{K}_{\mathrm{s}}$ ( $P=0.0003), 11.1 \%$ prolonged CLT $(P=0.019)$, and $10.9 \%$ higher ETP ( $P=0.011$ ). The FXIII 34Leu allele ( $n=49,38.9 \%, 9$ homozygous) was associated with $18.3 \%$ reduced $\mathrm{K}_{\mathrm{s}}(P<0.0001)$, $9.8 \%$ prolonged $\operatorname{CLT}(P=0.004)$, and $9.2 \%$ higher ETP $(P=0.018)$ compared to noncarriers, also after adjustment for fibrinogen (all $P<0.01$ ). There was no impact of the FGA Thr312Ala polymorphism on fibrin clot properties or ETP (all $P>0.05$ ). The Arg448Lys polymorphism explained $13 \%$ of the variance in $\mathrm{K}_{\mathrm{s}}$ and17\% in CLT, while FXIII 34Leu allele explained $25 \%$ of the variance in $\mathrm{K}_{\mathrm{s}}$ and $23 \%$ in CLT.

Conclusions: Fibrinogen B Arg448Lys and FXIII Val34Leu polymorphisms are associated with formation of more compact fibrin networks in acute PE patients, suggesting that despite heightened thrombin generation and inflammation typical of acute thromboembolism the 2 common generic variants have detectable effect on clot properties, similar to those reported in subjects at risk of venous thrombosis.

This work was supported by the Polish National Science Centre (UMO-2015/B/NZ5/02215).

\section{PB0401 | Decreased Hepatic Fibrin(ogen) Deposition in a} Mouse Model of Failed Liver Regeneration Is Not Associated with Systemic Coagulation Changes

D. Groeneveld; L.G. Poole; H. Cline-Fedewa; K.S. Baker;

J.P. Luyendyk

Michigan State University, East Lansing, United States

Background: Partial hepatectomy $(\mathrm{PHx})$ is a common surgical procedure to remove liver tumors and is possible because of the liver's unique regenerative capacity. Failure of the liver to regenerate leads to serious complications including post-hepatectomy liver failure (PHLF). Platelets rapidly accumulate in the liver remnant following $\mathrm{PHx}$ in rodents and humans and are a critical driver of liver regeneration. We have recently shown that rapid hepatic fibrin(ogen) deposition following standard (i.e., $2 / 3^{\text {rd }}$ ) $\mathrm{PHx}$ in mice drives platelet accumulation and liver regeneration. Importantly, patients who ultimately developed PHLF did not show an increase in hepatic fibrin(ogen) deposition after resection. The mechanisms for this failure are poorly understood, in part, because standard PHx in mice is not associated with failed liver regeneration.
Aims: We tested the hypothesis that early coagulation activation and hepatic fibrin(ogen) deposition is reduced in a mouse model of failed liver regeneration.

Methods: Sham surgery, $2 / 3^{\text {rd }} \mathrm{PHx}$ and $90 \% \mathrm{PHx}$ were performed in wild-type mice and samples were collected 30 min after.

Results: $\mathrm{PHx}$-induced hepatic fibrin(ogen) deposition was reduced in mice undergoing $90 \% \mathrm{PHx}$ compared to $2 / 3^{\text {rd }} \mathrm{PHx}$. This reduction was accompanied by a reduction in hepatic platelet accumulation. Interestingly, in proof-of-concept studies, administration of purified human fibrinogen increased hepatic fibrin(ogen) deposition and hepatic platelet accumulation after $90 \%$ PHx. Surprisingly, reduced hepatic fibrin(ogen) deposition in $90 \% \mathrm{PHx}$ was not associated with systemic changes in coagulation. Plasma fibrinogen and thrombinantithrombin (TAT) levels, a marker of coagulation activation, were not different between both $\mathrm{PHx}$ groups. Plasma D-dimer levels, suggestive of fibrin degradation, were also not different.

Conclusions: The results suggest that local regulation of hepatic fibrin(ogen) deposition fails in mice after $90 \% \mathrm{PHx}$ which may ultimately lead to failed regeneration. Moreover, our results suggest that fibrinogen supplementation may have pro-regenerative potential by rescuing defective hepatic fibrin(ogen) deposition in a model of failed liver regeneration.

PB0402 | A Patient with Congenital Hypofibrinogenemia who Showed Embryo Implantation, Continuation of Pregnancy, and Delivery Following Continuous Transfusion of Dry Human Fibrinogen Preparations

Y. Seki $^{1}$; T. Ushiki ${ }^{2}$; M. Masuko ${ }^{3}$; J. Takizawa ${ }^{3}$; H. Sone ${ }^{3}$; N. Okumura ${ }^{4}$

${ }^{1}$ Niigata University/Department of Hematology, Uonuma Institute of Community Medicine, Minami-Uonuma, Japan; ${ }^{2}$ Niigata University/ Blood transfusion, Niigata, Japan; ${ }^{3}$ Niigata University/Department of Hematology, Niigata, Japan; ${ }^{4}$ Shinsyu University/Department of Health Science, Matsumoto, Japan

Background: We report a patient with congenital hypofibrinogenemia showing embryo implantation, continuation of pregnancy, and delivery following the continuous transfusion of dry human fibrinogen preparations.

Aims: [Case report] A 38-year old woman underwent embryo transfer (pre-pregnancy fibrinogen [Fbg] activity and antigen levels: 33 and $91 \mathrm{mg} / \mathrm{dL}$, respectively). During the $2^{\text {nd }}$ embryo transfer (failure), the Fbg level just after Fbg supplementation was $145 \mathrm{mg} / \mathrm{dL}$ and 66 $\mathrm{mg} / \mathrm{dL}$ on day 5 . During the third attempt (successful), we supplemented $4 \mathrm{~g}$ of Fbg 2 days before the embryo transfer (day 0 ) and at days 2,7 , and 12 for stabilizing the endometrium. The Fbg levels at implantation were maintained at $150 \mathrm{mg} / \mathrm{dL}$. In the early phase of pregnancy, we supplemented $4 \mathrm{~g}$ of $\mathrm{Fbg}$ (thrice/2 weeks) regularly; Fbg levels were maintained at $>150 \mathrm{mg} / \mathrm{dL}$. From the $32^{\text {nd }}$ week of pregnancy, the baby gained weight and the Fbg levels decreased. Then, we supplemented $3 \mathrm{~g}$ of Fbg (thrice/week); Fbg levels were 
maintained at $130-150 \mathrm{mg} / \mathrm{dL}$. Because the Fbg levels decreased from the $34^{\text {th }}$ week, we supplemented $13 \mathrm{~g}$ of Fbg/week. A child was born in the $35^{\text {th }}$ week via cesarean operation.

Methods: [Gene analysis] Genetic anlysis of the DNA base sequences of the PCR products of the $A a, B \beta$, and $\gamma$ chains confirmed heterozygosity, with the nucleotides TGT (normal, wild-type) and CGT (morph), which encoded the $326^{\text {th }}$ amino acid in the $\gamma$ chain of exon 8. Further, cysteine (normal, wild-type) was replaced with arginine (morph); thus far, no studies have reported this finding.

Results: [Discussion] Although Fbg is not reported to be necessary during implantation in natural pregnancies, herein, implantation was successful in the presence of Fbg levels $>150 \mathrm{mg} / \mathrm{dL}$ during embryo transfer.

Conclusions: Fbg levels > $150 \mathrm{mg} / \mathrm{dL}$ may be necessary for implantation during embryo transfer in patients with congenital hypofibrinogenemia.

PB0403 | Thermal Shift Assay to Probe Melting of Thrombin, Fibrinogen, Fibrin Monomer and Fibrin: Gly-Pro-Arg-Pro Induces a Fibrin Monomer-like State in Fibrinogen

\section{J. Crossen; S. Diamond}

University of Pennsylvania, Philadelphia, United States

Background: Thrombin activates fibrinogen and binds the fibrin $\mathrm{E}$ domain $\left(K_{d} \sim 2.8 \mathrm{mM}\right)$ and the splice variant $\gamma^{\prime}$-domain $\left(\mathrm{K}_{\mathrm{d}} \sim 0.1 \mathrm{mM}\right)$. Previous studies have shown that thrombin becomes "trapped" within fibrin fibers and will remain in the clot for extended periods of time after buffer wash.

Aims: We investigated if the loading of D-Phe-Pro-Argchloromethylketone inhibited thrombin (PPACK-thrombin) onto fibrin could enhance fibrin stability. We also confirmed previous DCS analysis of fibrin(ogen) with thermal shift assay (TSA) and looked at thermal stability of fibrin monomers in comparison to GPRP-bound fibrinogen

Methods: A 384-well plate TSA with SYPRO-orange provided melting temperatures $\left(T_{m}\right)$ of thrombin, PPACK-thrombin, fibrinogen, fibrin monomer, and fibrin.

Results: As indicated by large increases in $\mathrm{T}_{\mathrm{m}}$, calcium led to protein stabilization ( 0 vs. $2 \mathrm{mM} \mathrm{Ca}^{2+}$ ) for fibrinogen $\left(54.0\right.$ vs. $62.3^{\circ} \mathrm{C}$ ) and fibrin (62.3 vs. $72.2^{\circ} \mathrm{C}$ ). As expected, fibrin was more stable than fibrinogen $\left( \pm \mathrm{Ca}^{2+}\right)$. Active site inhibition with PPACK dramatically increased the $T_{m}$ of thrombin (58.3 vs. $78.3^{\circ} \mathrm{C}$ ). Treatment of fibrinogen with fibrin polymerization inhibitor GPRP increased fibrinogen stability by $\Delta \mathrm{T}_{\mathrm{m}}=9.3^{\circ} \mathrm{C}$, similar to the $\Delta \mathrm{T}_{\mathrm{m}}$ when fibrinogen was converted to fibrin monomer $\left(\Delta \mathrm{T}_{\mathrm{m}}=8.8^{\circ} \mathrm{C}\right)$ or to fibrin $\left(\Delta \mathrm{T}_{\mathrm{m}}=10.4\right.$ ${ }^{\circ} \mathrm{C}$ ). Addition of PPACK-thrombin at high 5:1 molar ratio to fibrin (or fibrinogen) had little effect on fibrinogen or fibrin $T_{m}$ values, indicating that thrombin binding does not detectably stabilize fibrin via a putative bivalent $\mathrm{E}$-domain to $\gamma^{\prime}$-domain interaction.

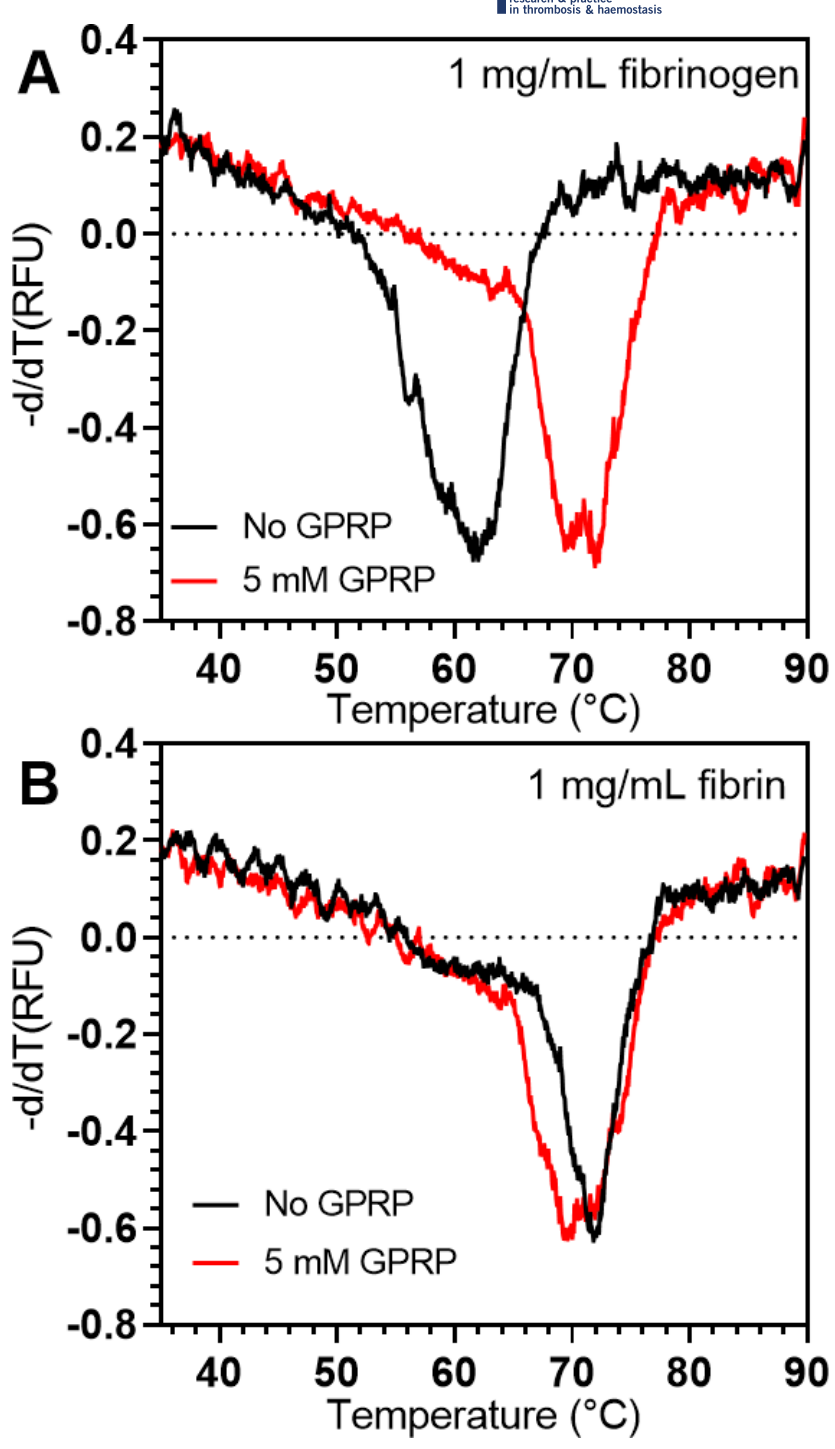

FIGURE 1 Representative fibrinogen and fibrin melt curve derivative with and without GPRP. $1 \mathrm{mg} / \mathrm{mL}$ fibrinogen, 5x SYPRO, $2 \mathrm{mM} \mathrm{Ca2+}$ in each well. (A) Fibrinogen comparison. No GPRP (black) and $5 \mathrm{mM}$ GPRP (red). (B) Fibrin (50 nM thrombin). No GPRP (black) and 5 mM GPRP (red).

TABLE 1 Summary of shift in Tm measurements from reference fibrinogen on each well plate. $N=3$ plates, $n=9$ wells

$\begin{array}{ll}\text { Sample } & \text { Average } \Delta \\ \text { Fibrinogen } & 0.0 \pm 0.4 \\ \text { Fibrinogen; } 5 \text { mM GPRP } & 9.3 \pm 0.4 \\ \text { Fibrin; } 5 \text { mM GPRP } & 8.8 \pm 0.4 \\ \text { Fibrin } & 10.4 \pm 0.4 \\ \text { Fibrinogen; 300 nM PPACK-thrombin } & -0.3 \pm 0.4 \\ \text { Fibrin; 300 nM PPACK-thrombin } & 9.7 \pm 0.2\end{array}$

Conclusions: TSA was a sensitive assay of protein stability and detected:

(1) the effects of calcium-stabilization,

(2) thrombin active site labeling,

(3) fibrinogen conversion to fibrin, and 
(4) GPRP induced changes in fibrinogen stability being essentially equivalent to that of fibrin monomer or polymerized fibrin. The assay can be used in future experiments to determine fibrinogen interactions in which a small volume is required due to rarity of reagents.

PB0404 | Strength and Deformability of Fibrin Clots: Biomechanics, Thermodynamics, and Mechanisms of Rupture

V. Tutwiler ${ }^{1,2}$; F. Maksudov ${ }^{3}$; R.I. Litvinov ${ }^{1,4}$; J.W. Weisel ${ }^{1}$;

V. Barsegov ${ }^{3}$

${ }^{1}$ University of Pennsylvania, Philadelphia, United States; ${ }^{2}$ Rutgers University, Piscataway, United States; ${ }^{3}$ University of Massachusetts -Lowell, Lowell, United States; ${ }^{4}$ Kazan Federal Research University, Kazan, Russian Federation

Background: Fibrin is the major mechanical component of blood clots and plays a critical role in 1) the formation of a strong, hemostatic seal to prevent bleeding and 2) the propensity for thrombotic embolization. While the viscoelastic mechanical properties of fibrin have been well studied, these properties are distinct from the ability of a material to resist rupture. Aims: To determine the structure-function relationship of fibrin rupture. Methods: To assess the dynamics of mechanical deformations/resistance to rupture, we performed tensile testing experiments on plasma blood clots with a single edge crack (Figure 1) and correlated with ultrastructural studies (Figure 2). We developed a Fluctuating Spring model, which maps the changes in fiber alignment, stretching of the elastic network, and sequential rupture of coupled fibrin fibers with cooperativity on the strain-scale into a theoretical framework.
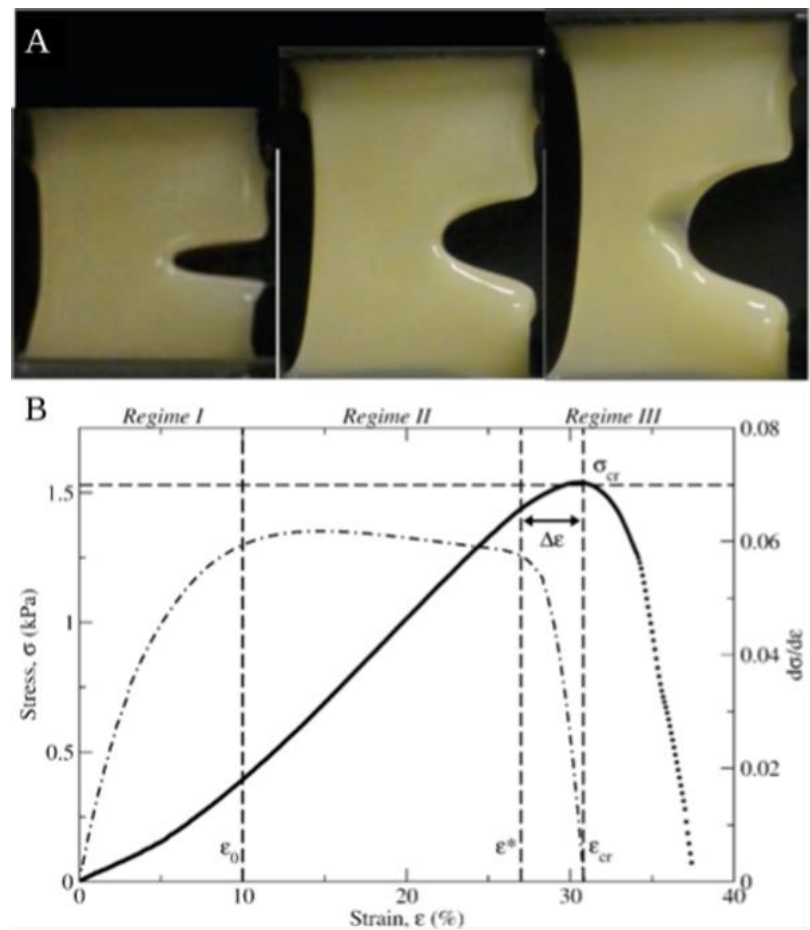

FIGURE 1 Experimental stress-strain analysis of the dynamics of deformation and rupture of a plasma clot with single-edge crack.
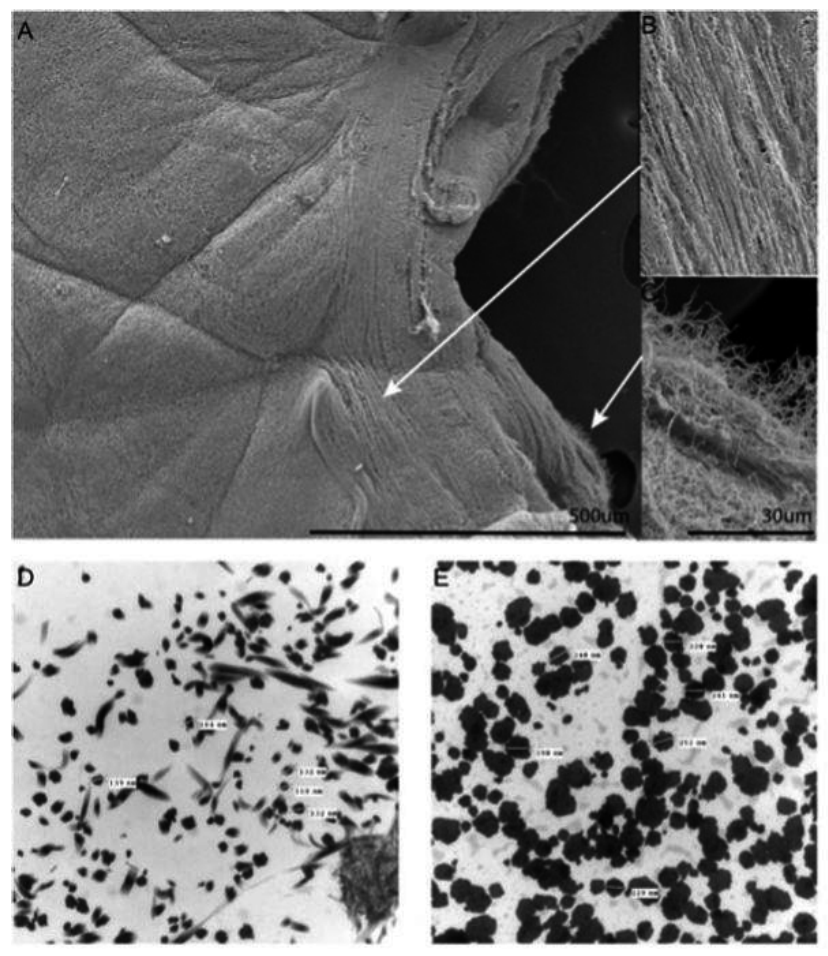

FIGURE 2 Scanning and transmission electron microscopy of plasma clots

Results: Through examination of experimental stress-strain curves and the fibrin ultrastructure, we determined that fibrin undergoes three distinct deformation regimes during the rupture process (Figure 1). We show that for fibrin gels formed under these conditions, the critical strain is $34-45 \%$ and the critical stress is $1.5-22$ $\mathrm{kPa}$. Our theoretical framework reveals that the presence of a crack or defect in the fibrin network renders them more stochastic. We show that the free energy associated with the fiber deformation and rupture is inversely related to the crack length; therefore, increasing crack length makes network rupture more spontaneous. By contrast, inter-fiber connectivity reinforces the fiber network, making rupture less likely to occur. Microscopy and theoretical results reveal that the fibrin network forms multifiber threads during the extension process; when strain exceeds the limits of the thread extensibility, the fibrin begins to rupture cooperatively.

Conclusions: The results obtained provide a fundamental understanding of blood clot breakage that underlies thrombotic embolization. 
PB0406 | Application of the ISTH-BAT In Congenital Fibrinogen Disorders

$\underline{\text { S. Mohsenian }^{1} \text {; O. Seidizadeh }}{ }^{2}$; A. Azarkeivan ${ }^{1}$; M. Jazebi ${ }^{3}$;

S. Moazezi ${ }^{3}$.

${ }^{1}$ Blood Transfusion Research Center, High Institute for Research and Education in Transfusion Medicine, Tehran, Iran, Islamic Republic of;

${ }^{2}$ Fondazione IRCCS Ca' Granda Ospedale Maggiore Policlinico, Angelo Bianchi Bonomi Hemophilia and Thrombosis Center and Fondazione Luigi Villa, Milan, Italy; ${ }^{3}$ Iranian Comprehensive Hemophilia Care Center, Tehran, Iran, Islamic Republic of

Background: The ISTH bleeding assessment tool (ISTH-BAT) was developed to aid the evaluation of suspected patients for bleeding disorders.

Aims: To assess the utility of ISTH-BAT in diagnosis, determining the bleeding severity in congenital fibrinogen disorders (CFDs) and diagnosing them according to the new ISTH classification.

Methods: A total of 70 (40 female, 30 male) Iranian patients with CFDs and 31 adult healthy controls (15 female, 16 male) were selected. Routine coagulation laboratory tests such as prothrombin time (PT), activated partial thromboplastin time (APTT), and thrombin time (TT) were performed. The fibrinogen antigen level (Fg:Ag) and its functional activity were measured using an immunoturbidimetric assay and the Clauss method (Fg:C), respectively. The ratio of Fg:C/Fg:Ag was calculated and ISTH-BAT was used to assess the bleeding score (BS) of patients and healthy controls.

Results: Laboratory and clinical parameters are summarized in Table 1. Patients were identified according to the new classification: 1A (afibrinogenemia, $n=37$ ), 2B (moderate hypofibrinogenemia, $n=6$ ), 2C (mild hypofibrinogenemia, $n=2$ ), 3A (dysfibrinogenemia, $n=19$ ), $4 \mathrm{~B}$ (moderate hypodysfibrinogenemia, $n=2$ ), 4C (mild hypodysfibrinogenemia, $n=4)$. Sixty-seven (95.7\%) patients experienced bleeding events and only 3 (4.3\%) patients with dysfibrinogenemia were asymptomatic. Each group of patients showed a statistically higher median BS than healthy controls (Fig. 1). Overall median BS of all patients was 4 (range 0-9) and was statistically significantly higher than healthy controls (median 1 , range $0-6, P<0.002$ ).

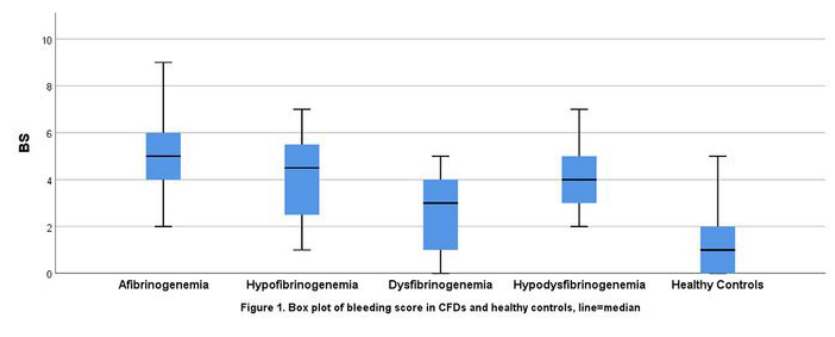

FIGURE 1 Comparison of bleeding scores (BS) in patients and healthy controls

The most common clinical symptoms in patients with CFDs were cutaneous bleeding (56\%), menorrhagia (39\%), and umbilical cord bleeding (29\%). Umbilical cord bleeding was observed only in afibrinogenemia and one afibrinogenemia patient experienced eye bleeding. Miscarriage was reported in three patients (two dysfibrinogenemia, one hypodysfibrinogenemia), and two women (afibrinogenemia) had an ovarian cyst rupture.

Conclusions: This study showed that ISTH-BAT is a useful tool for the diagnosis of CFDs and identifies those patients who need further investigations.

PB0407 | Effect of Flow and Shear Rate on Structural Properties and Fibrinolysis of the Fibrin Network

J.J. de Vries; I. El-Idrioui; D.C. Rijken; M.P. de Maat

Erasmus Medical Center, Rotterdam, Netherlands

Background: Flow and shear rate highly influence structural characteristics of the fibrin network. This will affect thrombus stability, and thus disease burden and mortality in cardiovascular disease. In addition, the structure of the fibrin matrix affects susceptibility to fibrinolysis and therefore may influence the efficiency of fibrinolytic therapy.

Aims: To study the effect of flow and shear rate on structural properties and fibrinolysis rate of the fibrin network.

TABLE 1 Laboratory and clinical characteristics of patients (median range)

\begin{tabular}{|c|c|c|c|c|c|c|c|c|c|}
\hline $\begin{array}{l}\text { Classification of } \\
\text { disorders }\end{array}$ & $N(\%)$ & Age & $\begin{array}{l}\text { Sex } \\
(M / F)\end{array}$ & $\mathrm{Fg}: \mathrm{C}(\mathrm{mg} / \mathrm{dl})$ & $\mathrm{Fg}: \mathrm{Ag}(\mathrm{mg} / \mathrm{dl})$ & $\mathrm{PT}(\mathrm{s})$ & PTT (s) & $\mathrm{TT}(\mathrm{s})$ & BS \\
\hline Afibrinogenemia & 37 (53\%) & $13(1-39)$ & $20 / 17$ & Undetectable & Undetectable & $>60$ & $>120$ & $>60$ & $5(2-9)$ \\
\hline Hypofibrinogenemia & $8(11 \%)$ & $23(2-34)$ & $3 / 5$ & $89(75-117)$ & $110(73-134)$ & $17(11-19)$ & $38(31-47)$ & $23(23-29)$ & $4.5(1-6)$ \\
\hline Dysfibrinogenemia & $19(27 \%)$ & $34(4-63)$ & $5 / 14$ & $50(25-78)$ & $312(228-370)$ & $15(12-20)$ & $36(32-47)$ & $35(19-46)$ & $3(0-5)$ \\
\hline Hypodysfibrinogenemia & $6(9 \%)$ & $27(8-61)$ & $2 / 4$ & $43(40-90)$ & $125(76-156)$ & $14(11-20)$ & $36(30-42)$ & $25(17-39)$ & $4(2-7)$ \\
\hline Normal range & - & - & - & $154-475$ & $184-334$ & $10-13$ & $18-28$ & $14-19$ & $\begin{array}{l}\text { male } \leq 3 \\
\text { female } \leq 5 \\
\text { children } \leq \\
2\end{array}$ \\
\hline
\end{tabular}


Methods: We used a flow model in which plasma clots were formed under flow conditions with venous shear rates (from 50/s to $250 / \mathrm{s}$ ) or under static conditions. Plasma was supplemented with fluorescently labelled fibrinogen and mixed with thrombin (final concentration $1 \mathrm{U} / \mathrm{ml}$ ) and calcium (17 $\mathrm{mM}$ ) just before entering the flow chamber. Fibrin clots were formed in the flow chamber and Z-stacks were recorded using a Leica SP5 microscope. The diameter and orientation of fibrin fibers formed at different shear rates were measured using custom-written and ImageJ software. After clot formation, $500 \mathrm{ng} / \mathrm{ml}$ tPA in plasma was perfused over the clots and the amount of degraded fibrin, measured as fluorescence in aliquots from the outlet tube, was measured to determine time to $50 \%$ lysis. Results: Clots formed under flow had significantly thicker fibers and increased longitudinal orientation compared to clots formed under static conditions (Figure 1). Increasing shear rate further increased fiber thickness, while this did not significantly affect fiber orientation. No differences between clots formed at different shear rates were found in the time to $50 \%$ fibrinolysis.
A

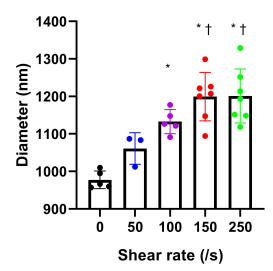

B

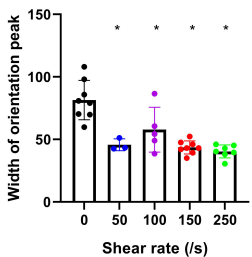

C

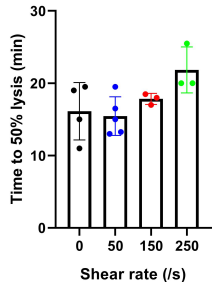

FIGURE 1 Effect of flow and shear rate on fiber diameter (A), orientation (B) and fibrinolysis (C). Width of peak is inversely related to the degree of orientation. Differences were tested using one-way ANOVA with multiple comparisons. ${ }^{*}$ means significant difference with $0 / \mathrm{s} ;{ }^{\dagger}$ means significant difference with $50 /$ s. Every dot represents one clot.

Conclusions: Flow and variations in venous shear rate affect structural properties of the fibrin network, while there was no significant effect of shear rate on fibrinolysis. These results further elucidate the mechanism through which flow affects properties of the fibrin network.

PB0408 | A Case of Congenital Afibrinogenemia with Two Successful Spontaneous Delivery after Recurrent Pregnancy Losses

M. Mastanzade; M.M. Özbalak; S. Kalayoğlu Beşışık

Istanbul University, Istanbul Medical Faculty, Department of Internal Medicine, Division of Hematology, Istanbul, Turkey

Background: Patients with congenital hypofibrinogenemia/afibrinogenemia $(\mathrm{CH} / \mathrm{CA})$ maybe with various bleeding symptoms proportional to the low values of functional and antigenic fibrinogen (FIB) or may have thrombotic complications. FIB is important in maintaining placenta integrity by supporting cytotrophoblast.

Aims: We present a case of congenital afibrinogenemia with two favored pregnancy outcomes.

Methods: .

Results: Case: A 31-year-old woman with excessive uterine bleeding and recurrent vulvar hematomas had prolonged aPTT/PT (APTT:>160 s, PT:108 s) and low FIB (<25 mg/dl) with normal d-dimer level. CA was thought of as a diagnosis and cryoprecipitate were given as an emergent replacement. Further evaluation could not be made: The patient was lost of follow-up. She admitted 6 years later as being in the 5 th gestational week. She described 5 early pregnancy losses and had a highly consanguineous family(Fig. 1). One of her 6 siblings had also CA. Regular replacement therapy to keep the FIB level $>75 \mathrm{mg} / \mathrm{dL}$ with FIB concentrates or cryoprecipitate infusions throughout pregnancy was started. On the 36th week, with a FIB level of $150 \mathrm{mg} / \mathrm{dl}$, a spontaneous vaginal delivery successfully occurred. The infant appeared well, had normal vital signs, and good perfusion. Bodyweight was $2950 \mathrm{~g}$ with an Apgar score of 9/10. Fibrinogen replacement was discontinued. Two years later, she was pregnant again on the 5 th week. She commenced regular replacement therapy for increasing FIB. The second pregnancy was also ended favorably by spontaneous vaginal delivery. The infant's body weight was $3210 \mathrm{~g}$. No postpartum complications were experienced. The patient continued to breastfeed for one year period after her first delivery. She continues breastfeeding after her 2nd delivery without any replacements.

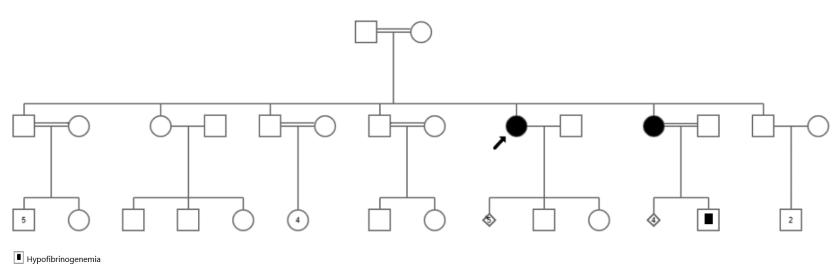

FIGURE 1 Pedigree of the patient

Conclusions: $\mathrm{CH} / \mathrm{CA}$ or dysfibrinogenemia may result in obstetric complications such as first-trimester pregnancy loss, hemorrhage, placental abruption, and thrombosis. Our main strategy was FIB replacement from the beginning of the pregnancy until delivery with a target FIB level of $60-100 \mathrm{mg} / \mathrm{dL}$ to manage $\mathrm{CH} / \mathrm{CA}$ patients. 
PB0409 | Clinical and Molecular Characterization of Iranian Patients with Congenital Qualitative Fibrinogen Disorders

$\underline{\text { S. Mohsenian }}{ }^{1}$; A. Azarkeivan ${ }^{1}$; M. Mirakhorli ${ }^{2}$; M. Jazebi ${ }^{2}$;

O. Seidizadeh ${ }^{3}$.

${ }^{1}$ Blood Transfusion Research Center, High Institute for Research and Education in Transfusion Medicine, Tehran, Iran, Islamic Republic of;

${ }^{2}$ Iranian Comprehensive Hemophilia Care Center, Tehran, Iran, Islamic Republic of; ${ }^{3}$ Fondazione IRCCS Ca' Granda Ospedale Maggiore

Policlinico, Angelo Bianchi Bonomi Hemophilia and Thrombosis Center and Fondazione Luigi Villa, Milan, Italy

Background: Congenital fibrinogen disorders (CFDs) are caused by mutations in FGA, FGB, FGG genes and are classified as quantitative (afibrinogenemia, hypofibrinogenemia) and qualitative (dysfibrinogenemia, hypodysfibrinogenemia) fibrinogen defects.

Aims: To identify the mutations that cause CFDs and to examine the genotype effect on clinical phenotype in patients with CFDs.

Methods: Thirteen Iranian patients with CFDs (Dysfibrinogenemia, $n=10$; hypodysfibrinogenemia, $n=2$ and Afibrinogenemia, $n=1$ ) within 9 unrelated families were included. Gene sequencing was performed following a polymerase chain reaction (PCR) amplification of FGA, FGB, FGG genes. Routine and specific laboratory tests such as fibrinogen antigen ( $\mathrm{Fib}: \mathrm{Ag}$ ) and Clauss fibrinogen assay (Fib:C) were performed and ISTH Bleeding Assessment Tool (ISTHBAT) was also evaluated.

Results: Patients' characteristics were summarized in Table 1. We identified 6 different but previously reported mutations located on FGA exon 2 (61\%), exon 4 (8\%), exon 5 (8\%), and FGG exon 8 (23\%). Variants are including p.Arg38Thr, p.Arg35His/Cys in FGA exon 2, p.Val145Asp in FGA exon 4, p.Gly316GlufsX105 in FGA exon 5, and p.Arg301Cys in FGG exon 8. In patient 5 we also identified two polymorphisms in FGA: c.510+37C>T (intron 4) and p.Thr312Ala (exon 5). Functional consequences of the former variant are unclear, while the last one is reported to be associated with the risk of venous thrombosis, but our patient has not experienced thrombosis. In patients with dysfibrinogenemia, two hotspot mutations (Arg35 and Arg301) were identified in 60\%: p.Arg35His (20\%), p.Arg35cys (10\%), and p.Arg301Cys (30\%), and the remaining patients (40\%) had p.Arg38Thr mutation. The p.Arg301Cys was observed in both dysfibrinogenemia and hypodysfibrinogenemia in the same affected family members. The overall median bleeding score (BS) was 4 (range $0-5$ ) and it was 3.5 (range 0-5) in dysfibrinogenemia. Cutaneous bleeding (64\%) and menorrhagia (43\%) were the common bleeding manifestations.

TABLE 1 Characteristics of Patients

\begin{tabular}{|c|c|c|c|c|c|c|c|c|}
\hline Family & Patient & $\begin{array}{l}\text { Age at } \\
\text { diagnosis/ } \\
\text { sex }\end{array}$ & $\begin{array}{l}\text { Fib:C /Fib:Ag } \\
\text { (mg/dl) }\end{array}$ & Phenotype & Gene/Exon & Protein & $\begin{array}{l}\text { Genotype } \\
\text { status }\end{array}$ & BS \\
\hline \multirow{2}{*}{ I } & 1 & $63 \mathrm{y} / \mathrm{F}$ & $46 / 331$ & & & & & 0 \\
\hline & 2 & $19 \mathrm{y} / \mathrm{F}$ & $55 / 311$ & & & & & 5 \\
\hline IV & 5 & $25 \mathrm{y} / \mathrm{F}$ & $60 / 261$ & Dysfibrinogenemia & $\mathrm{FGG} / 2$ & p.Arg35His & Hetero & 1 \\
\hline V & 6 & $50 \mathrm{y} / \mathrm{F}$ & $27 / 370$ & Dysfibrinogenemia & $\mathrm{FGA} / 2$ & p.Arg35cys & Hetero & 4 \\
\hline \multirow[t]{3}{*}{ VI } & & & & Dysfibrinogenemia & $\mathrm{FGA} / 2$ & p.Arg38Thr & Hetero & \\
\hline & 7 & $8 \mathrm{y} / \mathrm{M}$ & $35 / 289$ & & & & & 0 \\
\hline & 8 & $40 \mathrm{y} / \mathrm{M}$ & $41 / 317$ & & & & & 1 \\
\hline VII & 11 & $18 \mathrm{y} / \mathrm{F}$ & $<20 / 139$ & Hypodysfibrinogenemia & & & & 3 \\
\hline VIII & 12 & $3 y / F$ & $65 / 156$ & Mild hypodysfibrinogenemia & $\mathrm{FGA} / 4$ & p.Val145Asp & Hetero & 4 \\
\hline IX & 13 & $6 \mathrm{~d} / \mathrm{F}$ & undetectable & Afibrinogenemia & $\mathrm{FGA} / 5$ & p.Gly316GlufsX105 & Homo & 5 \\
\hline
\end{tabular}

Conclusions: We found several mutations with variable BS indicating that there was a weak genotype-phenotype correlation in CFDs.
FIBRINOLYTIC FACTORS AND INHIBITORS

LPB0018 | Role of the "Mobile Loop" (Residues 142-150) in the Intrinsic Instability of TAFla 
Background: Thrombin-activatable fibrinolysis inhibitor (TAFI) is a plasma zymogen implicated in regulating the balance between coagulation and fibrinolysis and is a molecular link between coagulation and inflammation. TAFla, formed by cleavage by thrombinthrombomodulin, has the unusual property of being intrinsically unstable, with a half-life at body temperature of 8-15 min; spontaneous TAFla inactivation is a key mechanism regulating TAFla activity. While TAFla inactivation involves a conformational change, the nature of this change is unknown. Two regions of the enzyme have been implicated in the intrinsic instability: (1) the "dynamic flap" (residues 297-350) whose mobility is restricted by the activation peptide or by competitive inhibitors of TAFla; and (2) the "mobile loop" (residues 142-150) whose mobility (in the bovine enzyme) is reduced by binding of tick carboxypeptidase inhibitor.

Aims: Whether the mobile loop impacts TAFI instability is unknown. We therefore constructed recombinant variants of human TAFI in which the mobile loop residues were mutated individually to their cognate residues in the stable pancreatic carboxypeptidase $B$ (five variants in total); additionally, we deleted the extra residue in TAFI $(\Delta 142)$ or replaced the entire mobile loop with the corresponding region from the pancreatic enzyme $(\triangle \mathrm{ML})$.

Methods: Recombinant TAFI variants were expressed in baby hamster kidney cells and purified by immunoaffinity chromatography. TAFla was formed by incubation with thrombin-thrombomodulin, and then incubated at $37^{\circ} \mathrm{C}$; timed aliquots were removed and residual TAFla activity was measured using the substrate $\mathrm{N}$-(4methoxyphenylaxoformyl)-Arg (AAFR).

Results: All TAFI variants were activated at similar rates by thrombinthrombomodulin and cleaved AAFR at similar rates, although the $\triangle 142$ and $\triangle M L$ variants showed approximately $75 \%$ and $50 \%$ activity, respectively. No differences in the half-lives of the variant enzymes at $37^{\circ} \mathrm{C}$ were observed compared to wild-type TAFla.

Conclusions: The mobile loop (residues 142-150) in human TAFla does not impact the intrinsic instability of the enzyme.

LPB0064 | The Role of Activated Thrombin Activatable Fibrinolysis Inhibitor on Thrombus Stability in Venous Thromboembolism

J.Chessum ${ }^{1,2}$; S. Shaya ${ }^{1,2}$; S. Cerroni ${ }^{2,3} ;$ D. Rajab ${ }^{1} ;$ J. Zhou ${ }^{2} ;$
J.C.M. Meijers ${ }^{4,5}$; J. Weitz ${ }^{1,2,3,6}$; P. Gross ${ }^{1,2,3} ;$ P. Kim ${ }^{1,2,3}$
${ }^{1}$ McMaster University, Hamilton, Canada; ${ }^{2}$ Thrombosis and
Atherosclerosis Research Institute, Hamilton, Canada; ${ }^{3}$ Department
of Medicine, McMaster University, Hamilton, Canada; ${ }^{4}$ Department
of Experimental Vascular Medicine, Amsterdam UMC, University of
Amsterdam, Amsterdam, Netherlands; ${ }^{5}$ Department of Molecular
Hematology, Sanquin Research, Amsterdam, Netherlands; ${ }^{6}$ Department
of Biochemistry and Biomedical Sciences, McMaster University,
Hamilton, Canada

Background: Thrombin-activatable fibrinolysis inhibitor (TAFI) levels have been correlated with the risk of thrombosis. However, the role of TAFI in venous thromboembolism (VTE) remains uncertain.
Aims: To determine the effect of TAFI on venous thrombus composition and stability in a murine model.

Methods: Male and female wild-type mice (WT) or TAFI-deficient mice $\left(\mathrm{Cpb2}^{-/-}\right)$were used. Thrombosis in the isolated femoral vein was induced with $\mathrm{FeCl}_{3}$. The thrombus, labeled with an antibody to platelets, was imaged using fluorescent intravital videomicroscopy at intervals to quantify embolization. Between intervals, thrombus montage images were used to quantify thrombus size. After 2-hours, organs were harvested and examined by histology. Composition of venous thrombi and pulmonary emboli were quantified using fluorescent antibodies against platelet GPIb and fibrin. Pulmonary embolism (PE) burden was assessed by counting the emboli in the lungs. Large emboli were defined as emboli having sizes greater than 4 standard deviations of average emboli size.

Results: Venous thrombus size increased over time only in male $\mathrm{Cpb2}^{-/-}$and female WT mice. Male $\mathrm{Cpb2}^{-/-}$mice had 3.7-fold and 5.8-fold more total and large embolic events compared with male WT mice, respectively. Compared with male WT mice, female WT and $\mathrm{Cpb2}^{-/-}$mice had 10.4-fold and 9.4-fold more total embolic events, respectively, and 8.8-fold and 13.5-fold more large embolic events, respectively. Male $\mathrm{Cpb2}^{-/-}$mice had 1.5 -fold greater PE burden compared with male WT mice, while female $\mathrm{Cpb2^{-/- }}$ had a 2.1fold greater PE burden than female WT mice. The compositions of clots also differed. $\mathrm{Cpb}^{-/-}$mice had more fibrin-rich emboli compared with WT. In venous thrombi, $\mathrm{Cpb2^{-/- }}$ mice exhibited greater platelet presence compared with WT.

Conclusions: Loss of TAFI contributes to thrombus instability, which translates to increased PE burden. Female mice appear to be at greater risk of $\mathrm{PE}$ than male mice.

PB0410 | Tranexamic acid for Hyperacute Primary Intracerebral Haemorrhage ( $\mathrm{TICH}-3$ ): Protocol for a Multinational Phase 3 Placebo-controlled Randomised Trial

M. Desborough ${ }^{1,2} ;$ R. Al-Shahi Salman ${ }^{3} ;$ T. Coats $^{4} ;$ R. Dineen ${ }^{5}$; T. England ${ }^{6}$; D. Havard ${ }^{5}$; T. Hepburn 7 S. Hodgson ${ }^{5}$; M. James ${ }^{6}$; A. Montgomery ${ }^{6}$; C. Rick ${ }^{6}$; I. Roberts ${ }^{8}$; T. Robinson ${ }^{4}$; C. Roffe ${ }^{9}$; D. Werring ${ }^{10}$; P. Bath ${ }^{5}$; N. Sprigg ${ }^{5}$ ${ }^{1}$ Oxford University Hospitals NHS Foundation Trust, Oxford, United Kingdom; ${ }^{2}$ University of Oxford, Oxford, United Kingdom; ${ }^{3}$ Centre for Clinical Brain Sciences, University of Edinburgh, Edinburgh, United Kingdom; ${ }^{4}$ University of Leicester, Leicester, United Kingdom; ${ }^{5}$ Stroke, Division of Clinical Neuroscience, University of Nottingham, Nottingham, United Kingdom; ${ }^{6}$ University of Nottingham, Nottingham, United Kingdom; ${ }^{7}$ Clinical Trials Unit, University of Nottingham, Nottingham, United Kingdom; ${ }^{8}$ London School of Hygiene and Tropical Medicine, London, United Kingdom; ${ }^{9}$ Keele University, Keele, United Kingdom; ${ }^{10}$ National Hospital for Neurology and Neurosurgery, London, United Kingdom

Background: Intracerebral haemorrhage $(\mathrm{ICH})$ can be devastating with high early mortality and disability in survivors, with outcome related to volume of haematoma and haematoma growth. 
Tranexamic acid (TXA) reduces mortality in other bleeding conditions when given early and reduced haematoma growth in previous study, $\mathrm{TICH}-2$.

Aims: To assess the clinical effectiveness of TXA after ICH and determine whether TXA should be used in clinical practice.

Methods: Pragmatic phase III prospective blinded randomised placebo-controlled trial.

Setting: Emergency departments, acute stroke units across the UK and worldwide. Estimated 100 UK sites, 65 non-UK sites.

Participants: Adult patients with $\mathrm{ICH}$ confirmed on brain imaging within $4.5 \mathrm{~h}$ of symptom onset. Exclusion: Contraindication for TXA, patient known to be taking anti-coagulation, Glasgow coma scale $(G C S)<5$, estimated haematoma volume $(\mathrm{HV})>60 \mathrm{mls}$, palliative care.

Intervention: intravenous TXA $2 \mathrm{~g}$ given as $1 \mathrm{~g}$ bolus in $100 \mathrm{~m}$ normal saline $0.9 \%$ infusion over $10 \mathrm{~min}$ and $1 \mathrm{~g}$ infusion in $250 \mathrm{ml}$ normal saline $0.9 \%$ over $8 \mathrm{~h}$ or placebo (normal saline $0.9 \%$ ) administered by an identical regimen. Randomisation will be to TXA vs. placebo in a 1:1 ratio.

Primary outcome: mortality at 7 days,

Secondary outcome: modified Rankin Scale at (mRS) 180 days.

Timelines for delivery: 7.25 years project; 3 month develop protocol ready for submissions, 9 month set up to obtain regulatory approvals, team recruitment and produce the trial treatment, 5.25 years participant recruitment in the UK, 4.75 years in International sites, 6 months final follow-up, 6 months data clean, analysis and dissemination.

Results:

FIGURE 1 Study flow chart. \$ standard imaging on arrival to hospi-

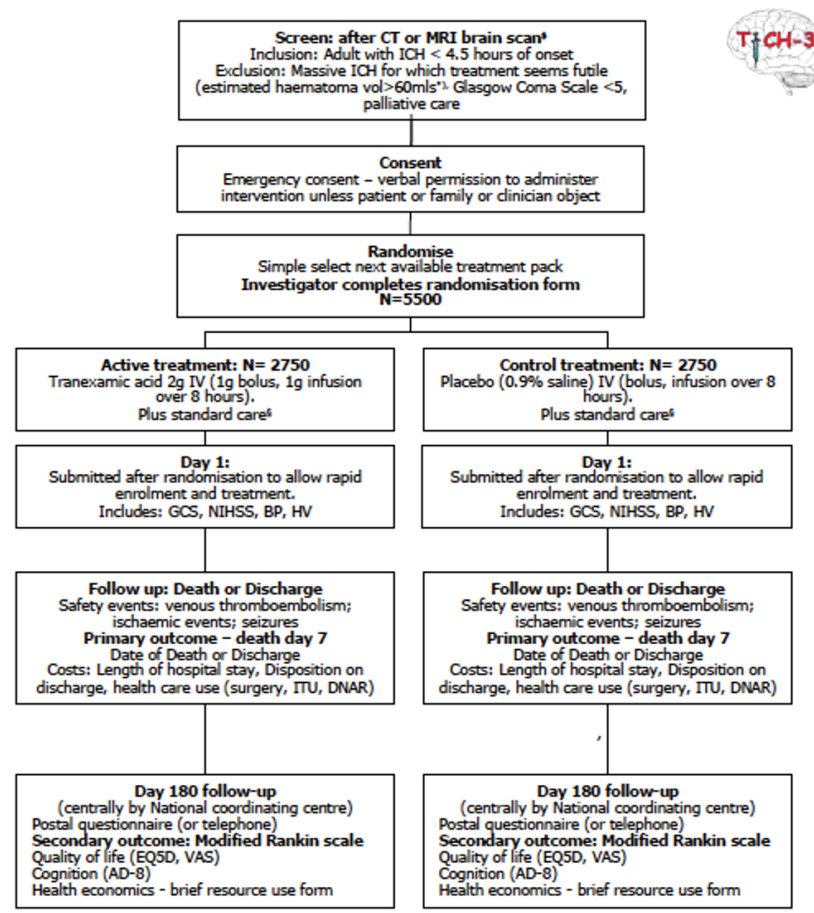

tal for all suspected acute stroke patients; *estimated haematoma volume $\S$ standard care for ICH including blood pressure lowering and admission to stroke unit as indicated National coordinating centre is Nottingham in the UK International follow-up will be coordinated and delivered by the countries National Coordinator Abbreviations: $\mathrm{ICH}$ Intracerebral haemorrhage, IV intravenous, NIHSS National Institute Health Stroke Scale, HV haematoma volume, DNAR do not attempt resuscitation; GCS Glasgow coma scale, Blood Pressure, ITU intensive care unit, EQ5D EuroQuol 5 Domain, VAS visual analogue scale, AD-8 The Washington University Dementia Screening Test

Conclusions: TXA is affordable, widely available, already utilised in other fields (trauma, gynaecology, cardiology) worldwide. If the trial confirms that TXA is effective, TXA could be rapidly implemented into the clinical pathway for $\mathrm{ICH}$ and has the potential for global impact reducing mortality.

\section{PB0411 | Utility of Lysis Time (Global Fibrinolytic Capacity)} Assay for Detection of Hyperfibrinolysis in Trauma

A. Rossetto $^{1,2}$; T. Torres ${ }^{2}$; S. Platton ${ }^{2}$; P. Vulliamy ${ }^{1,2}$; R. Davenport ${ }^{1,2}$ ${ }^{1}$ Centre for Trauma Sciences, Blizard Institute, Queen Mary University of London, London, United Kingdom; ${ }^{2}$ Barts Health NHS Trust, London, United Kingdom

Background: Rotational thromboelastometry (ROTEM), a viscoelastic assay used for rapid detection of hyperfibrinolysis, has limited sensitivity to identify moderate or low levels of fibrinolytic activation. Injured patients with low/normal levels of EXTEM maximum lysis (ML) have been shown to have biomarker evidence of hyperfibrinolysis with associated poor clinical outcomes.

Aims: To compare the diagnostic performance of global fibrinolytic capacity/lysis time assay (LT) and ROTEM EXTEM ML in detecting fibrinolytic activity, the effect of tranexamic acid (TXA), and whether LT can predict early mortality.

Methods: We performed an exploratory analysis of a prospective cohort of adult trauma patients and healthy donors at a single UK major trauma centre. LT was measured in plasma according to manufacturer's protocol. Hyperfibrinolysis was defined as $M L>15 \%$ or $\mathrm{LT}<=30 \mathrm{~min}$ and hypofibrinolysis as $\mathrm{ML}<5 \%$ or $\mathrm{LT}>60 \mathrm{~min}$.

Results: Compared to healthy donors ( $n=19$ ), non-TXA-treated trauma patients ( $n=60$ ) had evidence of shortened LT indicative of hyperfibrinolysis (median LT: 30 (IQR 26-36) vs 43 (IQR 40-47), $P<0.001)$. All patients who received TXA $(n=19)$ had prolonged LT with complete abolition of fibrinolysis. Only 2 patients had hyperfibrinolysis on ROTEM, both of whom displayed markedly shortened LT. However, 45\% (26/58) of the non-TXA-treated patients without overt ROTEM-hyperfibrinolysis had short LT (hyperfibrinolysis), with all observed mortality and massive transfusions occurring in the group with rapid LT but ML low/normal. Only one patient with low ML had prolonged LT (hypofibrinolysis). LT showed increased accuracy compared to $\mathrm{ML}$ in predicting 24-hour mortality (AUROC $0.98,95 \% \mathrm{Cl} 0.96-1.00$, vs $0.61,95 \% \mathrm{Cl} 0.40-0.81, P=0.001$ ). 
Conclusions: This analysis found LT to be superior to ROTEM in identifying hyperfibrinolysis and predicting outcome. Trauma patients were characterised by a shift towards a hyperfibrinolytic state on admission, with hypofibrinolysis an extremely rare occurrence. Future studies to compare LT performance with gold standard biomarkers of fibrinolysis are required.

\section{PB0412 | Tranexamic Acid to Reduce Bleeding in Patients} Treated with Non-Vitamin K Oral Anticoagulants Undergoing Dental Extraction: The EXTRACT-NOAC Randomized Clinical Trial

A. Ockerman ${ }^{1}$; I. Miclotte ${ }^{1}$; M. Vanhaverbeke ${ }^{2} ;$ T. Vanassche ${ }^{2}$;

A. Belmans ${ }^{1} ;$ J. Vanhove ${ }^{3}$;. Meyns ${ }^{4} ; \mathrm{N}_{\text {. Nadjmi }}{ }^{5,6}$;

G. Van Hemelen ${ }^{6}$; P. Winderickx ${ }^{6}$; R. Jacobs ${ }^{1,7}$; C. Politis ${ }^{2}$;

P. Verhamme ${ }^{2}$.

${ }^{1}$ University of Leuven, Leuven, Belgium; ${ }^{2}$ University Hospitals Leuven, Leuven, Belgium; ${ }^{3}$ Regional Hospital Heilig Hart Leuven, Leuven, Belgium; ${ }^{4}$ General Hospital St-Jan Genk, Genk, Belgium; ${ }^{5}$ University of Antwerp, Antwerp, Belgium; ' ${ }^{6}$ MACK Association, AZ Monica Antwerp, Antwerp, Belgium; ${ }^{7}$ Karolinska Institutet, Stockholm, Sweden

Background: Oral bleeding after dental extraction in patients on non-vitamin $\mathrm{K}$ oral anticoagulants (NOACs) is a frequent problem.

Aims: We investigated whether $10 \%$ tranexamic acid (TXA) mouthwash decreases post-extraction bleeding in patients treated with non-vitamin $\mathrm{K}$ oral anticoagulants.

Methods: The EXTRACT-NOAC study is a randomized, double blind, placebo-controlled, multi-center, clinical trial, approved by the Medical Ethics Committee of University Hospitals Leuven (ClinicalTrials.gov NCT03413891). Patients gave informed consent and were randomly assigned to $10 \%$ TXA or placebo mouthwash, and were instructed to use the mouthwash prior to dental extraction, and three times a day for three days thereafter. The primary outcome was the number of patients with any post-extraction oral bleeding up to day 7. Secondary outcomes include the number and type of bleeds. The primary outcome was analyzed using a chisquare test. The effect of treatment was estimated by the risk ratio. Results: 218 patients were included in the full analysis set of which 106 patients were assigned to TXA (74.8 ( \pm 8.8$)$ years; 81 men) and 112 to placebo $(72.7$ ( \pm 10.7 ) years; 64 men). Post-extraction bleeding occurred in 28 (26.4\%) patients in the TXA group and in 32 (28.6\%) patients in the placebo group (relative risk, 0.92; 95\% confidence interval $[\mathrm{Cl}], 0.60$ to $1.42 ; P=.72$ ). There were 46 bleeds in the TXA group and 85 bleeds in the placebo group (rate ratio, $0.57 ; 95 \% \mathrm{Cl}$, 0.31 to $1.05 ; P=.07$ ), Figure 1. Extraction-related bleeds (rate ratio $0.56 ; 95 \% \mathrm{Cl}, 0.32$ to 0.96 ) and delayed bleeds (rate ratio, 0.32; $95 \%$ $\mathrm{CI}, 0.12$ to 0.89 ) were lower in the TXA group.

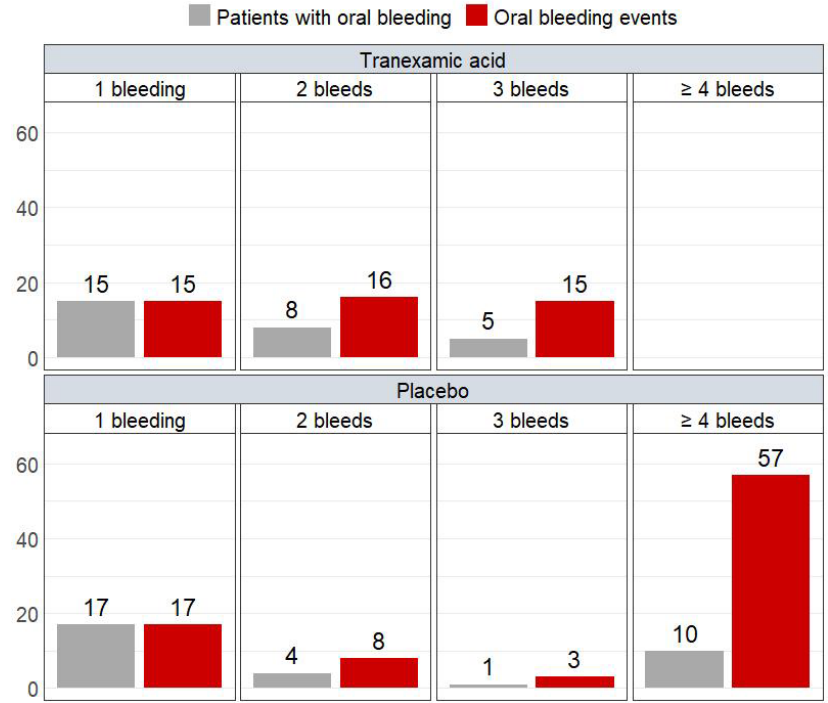

FIGURE 1 Post-extraction oral bleeds in patients who suffered from one, two, three or $\geq$ four bleeds.

Conclusions: In patients on NOACs undergoing dental extracting, TXA mouthwash did not reduce the number of patients with postextraction oral bleeding. TXA may reduce the number of extractionrelated and delayed bleeds.

PB0413 | The Effect of Lower-leg Trauma and Knee Arthroscopy on the Natural Anticoagulant and Fibrinolytic Systems

C. Touw ${ }^{1}$; B. Nemeth ${ }^{1}$; R. van Adrichem ${ }^{1}$; W. Lijfering ${ }^{1}$; A. van Hylckama Vlieg ${ }^{1}$; R. Nelissen ${ }^{1}$; T. Lisman²; S. Cannegieter ${ }^{1}$ ${ }^{1}$ Leiden University Medical Center, Leiden, Netherlands; ${ }^{2}$ University Medical Center Groningen, Groningen, Netherlands

Background: Despite thromboprophylaxis, patients with lower-leg cast immobilization and patients undergoing knee arthroscopy are at high risk of venous thromboembolism (VTE). Hence, exploration of new preventive strategies is necessary. For this, it is of interest to what extent the natural anticoagulant and fibrinolytic systems contribute to VTE.

Aims: To measure the effect of (1) lower-leg trauma and (2) knee arthroscopy on plasma levels of natural anticoagulant and fibrinolytic factors.

Methods: Plasma samples were provided by participants of the POTCAST (Prevention Of Thrombosis after Lower Leg Plaster Cast) and POT-KAST (Prevention Of Thrombosis after Knee Arthroscopy) trials. Samples were collected upon lower-leg trauma and before/after arthroscopy (<4 h), respectively. Clot lysis time (CLT) was measured in in a selection of patients who did not develop VTE (lower-leg trauma $N=289$, arthroscopy $N=293$ ) by monitoring plasma clot formation and subsequent lysis in the presence of exogenous tPA. Plasma levels of antithrombin, free protein S, protein C, plasminalpha-2-antiplasmin complexes, plasminogen activator inhibitor-1, t-PA and D-dimer were quantified in subsets of these patients. For aim 1, measurements in lower-leg injury patients were compared with preoperative measurements of arthroscopy patients (controls), expressed as mean differences (or mean ratios if In-retransformed) 
and adjusted for age, sex, BMI and comorbidities. For aim 2, pre- and postoperative measurements of arthroscopy patients were compared, i.e., paired mean changes.

Results: Ex vivo fibrinolytic potential was elevated in lower-leg injured and knee arthroscopy patients, however, in vivo activation of fibrinolysis was not affected. Plasma levels of antithrombin increased moderately after lower-leg trauma, while anticoagulant factors decreased following knee arthroscopy.

TABLE 1 Aim 1: Natural anticoagulant factor levels, clot lysis time and fibrinolytic factor levels in patients with lower-leg injury compared with subjects without lower-leg injury

\begin{tabular}{|c|c|c|c|c|c|c|}
\hline & $\begin{array}{l}\text { Lower-leg } \\
\text { injury, number } \\
\text { of patients }\end{array}$ & $\begin{array}{l}\text { Lower-leg injury, mean } \\
\text { (SD) }\end{array}$ & $\begin{array}{l}\text { No lower-leg } \\
\text { injury, number } \\
\text { of patients }\end{array}$ & $\begin{array}{l}\text { No lower-leg injury, } \\
\text { mean (SD) }\end{array}$ & $\begin{array}{l}\text { Mean difference/ } \\
\text { ratio }(95 \% \mathrm{Cl})^{\dagger}\end{array}$ & $\begin{array}{l}\text { Adj. mean } \\
\text { difference/ratio } \\
(95 \% \mathrm{Cl})^{\dagger^{*}}\end{array}$ \\
\hline Antithrombin (\%) & 89 & $113.6(13.1)$ & 88 & $109.6(10.6)$ & $4.0(0.5$ to 7.6$)$ & $4.8(1.2$ to 8.4$)$ \\
\hline Free protein S (\%) & 89 & $102.6(20.5)$ & 88 & $103.6(17.9)$ & $-0.9(-6.7$ to 4.8$)$ & $-1.6(-7.1$ to 3.9$)$ \\
\hline Protein C (\%) & 89 & $123.7(24.1)$ & 88 & $125.6(21.4)$ & $-1.9(-8.6$ to 4.9$)$ & $-0.8(-7.3$ to 5.7$)$ \\
\hline Clot lysis time (min) & 289 & $70.0(15.7)$ & 293 & $77.8(23.7)$ & $-7.7(-11.0$ to -4.5$)$ & $-5.1(-8.2$ to -2.0$)$ \\
\hline $\begin{array}{l}\text { PAP complexes (ng/ } \\
\mathrm{mll}^{\ddagger}\end{array}$ & 67 & 296.1 (269.5 to 325.4 ) & 28 & 254.0 (203.0 to 317.9$)$ & $1.2(0.9 \text { to } 1.4)^{\dagger}$ & $1.1(0.9 \text { to } 1.4)^{\dagger}$ \\
\hline tPA (ng/ml) & 67 & $6.2(2.9)$ & 28 & $7.3(5.4)$ & $-1.1(-2.8$ to 0.6$)$ & $-1.4(-3.1$ to 0.3$)$ \\
\hline PAl-1 $(\mathrm{ng} / \mathrm{ml})^{\ddagger}$ & 67 & 1.1 (0.9 to 1.3 ) & 28 & 1.1 (0.8 to 1.6$)$ & $-0.4(-1.0 \text { to } 0.1)^{\dagger}$ & $-0.6(-1.1 \text { to }-0.1)^{\dagger}$ \\
\hline
\end{tabular}

PAP: plasmin-alpha-2-antiplasmin; tPA: tissue plasminogen activator; PAI-1: plasminogen activator inhibitor-1.

Adjusted for age, sex, body mass index and comorbidities.

${ }^{\dagger}$ Mean ratios are denoted in the table with this symbol and in italics.

${ }^{\ddagger} \mathrm{D}$-dimer, PAP and PAI-1 were not normally distributed and therefore In-retransformed, resulting in geometric means with $95 \% \mathrm{Cls}$ and mean ratios.

TABLE 2 Aim 2: Pre- and postoperative natural anticoagulant factor levels, clot lysis time and fibrinolytic factor levels in patients who underwent knee arthroscopy

\begin{tabular}{lllll} 
& Number of patients & & Postoperative, mean & $\begin{array}{l}\text { Paired mean change } \\
\text { (95\%Cl) }\end{array}$ \\
Antithrombin (\%) & 88 & $109.6(10.6)$ & $101.7(10.9)$ & $-7.8(-9.5$ to -6.0$)$ \\
Free protein S (\%) & 88 & $103.6(17.9)$ & $99.4(17.8)$ & $-4.3(-5.8$ to -2.7$)$ \\
Protein C (\%) & 88 & $125.6(21.4)$ & $117.3(20.3)$ & $-7.9(-9.4$ to -6.4$)$ \\
Clot lysis time (min) & 277 & $77.8(23.7)$ & $65.1(14.1)$ & $-13.3(-15.4$ to -11.2$)$ \\
D-dimer ${ }^{\dagger}$ & 277 & $231.8(209.7$ to 256.1$)$ & $212.0(193.5$ to 232.3$)$ & $-56.1(-101.3$ to -11.0$)$ \\
PAP complexes $(\mathrm{ng} / \mathrm{ml})^{\dagger \S}$ & 28 & $254.0(203.0$ to 317.9$)$ & $247.9(201.4$ to 305.3$)$ & $-32.3(-276.6$ to 211.9$)$ \\
tPA $(\mathrm{ng} / \mathrm{ml})$ & 28 & $7.3(5.4)$ & $6.8(5.6)$ & $-0.4(-3.3$ to 2.4$)$ \\
PAI-1 $(\mathrm{ng} / \mathrm{ml})^{\dagger}$ & 28 & $1.1(0.8$ to 1.6$)$ & $0.8(0.5$ to 1.1$)$ & $-0.7(-1.0$ to -0.3$)$ \\
\hline
\end{tabular}

PAP: plasmin-alpha-2-antiplasmin; tPA: tissue plasminogen activator; PAI-1: plasminogen activator inhibitor-1.

*Due to lack of plasma or inadequate sample quality, anticoagulant factors were not measured in postoperative samples of three patients and clot lysis time was not measured in postoperative samples of 16 patients.

${ }^{\dagger} \mathrm{D}$-dimer, PAP and PAI-1 were not normally distributed and therefore In-retransformed, resulting in geometric means with $95 \% \mathrm{Cls}$.

Conclusions: Ex vivo fibrinolytic potential increases after lower-leg trauma and knee arthroscopy, which however does not lead to elevated in vivo fibrinolysis. Anticoagulant factor levels decrease after knee arthroscopy, while they are not clearly affected by lower-leg trauma.

\section{PB0414 I EACA Loaded Platelets Sustain Clots More Efficiently then Free EACA}

S. Xu; D. Sheik; K. Moskowitz

Cellphire, Inc., Rockville, United States

Background: During bleeding treatment, epsilon aminocaproic acid (EACA) is often given to prevent clot breakdown. Platelets have recently been investigated as drug delivery vehicles that naturally target and aggregate at injury sites. Platelets can be stabilized by cryopreservation or lyophilization. Cryopreserved and lyo-preserved platelets/platelet derived hemostatic agents are currently entering phase 2 clinical trials. Herein we generate a stabilized platelet formulation wherein platelets loaded with EACA effectively preserve clots during maturation as a proof of principle for such formulations to be applied as a novel treatment option for bleeding episodes.

Aims: Assess the in vitro efficacy of using EACA loaded platelets to prevent clot breakdown.

Methods: Pooled apheresis platelets were incubated in the presence or absence of $100 \mathrm{mM}$ EACA in for $3 \mathrm{~h}$ at $37^{\circ} \mathrm{C}$ to create loaded and unloaded platelets. Platelets were cryopreserved using DMSO. Unloaded platelets with free EACA were used as control samples. Thromboelastography was used to measure the clot strength. 
Samples were mixed with $500 \mathrm{U} / \mathrm{mL}$ tissue plasminogen activator (tPA). Clot formation was initiated by $0.01 \mathrm{M} \mathrm{CaCl}_{2}$. Duplicate samples were run in all groups. LY30 is the percentage decrease in clot strength $30 \mathrm{~min}$ after maximum amplitude.

Results: $140 \mu \mathrm{g} / \mathrm{mL}$ Free EACA, a therapeutically relevant concentration, showed an LY30 in the presence of tPA of 3.5\%. Platelet loaded EACA required the equivalent of $5 \mu \mathrm{g} / \mathrm{mL}$ to reverse $\mathrm{tPA}$ effect to the same degree as free EACA, reaching 2.7\% LY30. Similarly, $5 \mu \mathrm{g} / \mathrm{mL}$ of cryopreserved platelet loaded EACA reached $2.1 \% \mathrm{LY} 30$.

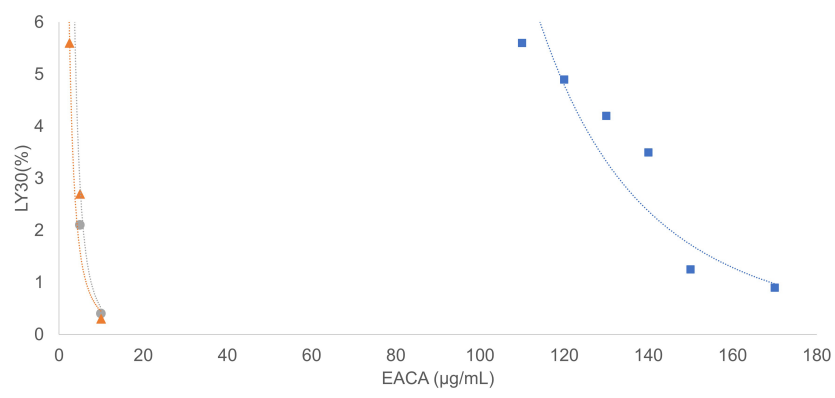

FIGURE 1 Concentration-effect curves for epsilon aminocaproic acid (EACA) in the form of free drug solution (blue squares), platelet loaded (orange triangles), and cryopreserved platelet loaded (grey circles). Each data point represents the mean of 2 duplicate assays. LY30 is the percentage decrease in clot strength 30 min after Thromboelastography maximum amplitude, with $100 \%$ corresponding to complete lysis and $0 \%$ corresponding to absence of lysis.

Conclusions: EACA Loaded into platelets can achieve correction of tPA-induced coagulopathy at approximately 30X lower concentrations than free EACA. Cryopreserved EACA loaded platelets have the same effect. These data represent a proof of principle of using platelets as carriers to increase the efficiency of antifibrinolytic drug in bleeding treatment.

PB0416 | Fibrinolytic Disorders as Cause of Bleeding in Patients with an Increased Bleeding Tendency

L. Valke $^{1,2}$; D. Meijer ${ }^{3}$; L. Nieuwenhuizen ${ }^{2,4}$; B. Laros-van Gorkom $^{1,2}$; N. Blijlevens ${ }^{1}$; W. Van Heerde ${ }^{2,5}$; S. Schols ${ }^{1,2}$

${ }^{1}$ Department of Hematology, Radboud University Medical Center, Nijmegen, Netherlands; ${ }^{2}$ Hemophilia Treatment Center, Nijmegen-Eindhoven-

Maastricht, Netherlands; ${ }^{3}$ Department of Laboratory Medicine, Laboratory of Hematology, Radboud University Medical Center, Nijmegen, Netherlands; ${ }^{4}$ Department of Hematology, Internal Medicine, Maxima Medical Center, Veldhoven, Netherlands; ${ }^{5}$ Enzyre BV, Nijmegen, Netherlands

Background: Analysis of fibrinolytic disorders (FD) is challenging in patients with an increased bleeding tendency, resulting in an expected underdiagnosis of these patients.

Aims: Identifying a FD diagnosis in patients with an increased bleeding tendency.
Methods: Retrospective study of patients with a BAT-score $\geq 10$ in whom fibrinolytic parameters were measured between January 2016-February 2020 in our Hemophilia Treatment Center. Laboratory phenotypic characterization consists of measuring plasminogen activator inhibitor type-1 (PAI-1) antigen and activity level, a2-antiplasmin level, tissue plasminogen activator (tPA), euglobulin clot lysis time (ECLT) before and after venous occlusion. Clinical characterization was performed by analyzing BAT-score, clinical phenotype, and treatment plans from electronic patient files of patients who agreed of data-usage for research purposes. Therefore, no institutional approval was necessary. Descriptive statistics were used, numbers are shown as median (interquartile range).

Results: In total 160 patients were included; 98 (61\%) without, 62 (39\%) with FD, see table. Demographics were equal between groups, median age 49 (17-76) versus 38 (15-78) and most patients were female (91\% and $87 \%$ ). Mean total BAT-scores did not differ (9.3 versus 9.7, Figure 1a), nor did BAT-score subcategories (Figure 1b). FD were subdivided in 33 patients with increased ECLT ratio, 25 patients with low PAI-1 antigen and activity level, and 4 patients with both abnormalities, none had decreased a2-antiplasmin level (table). tPA was higher in patients with FD. The decision to establish a general treatment plan (GTP) was individualized by the physician. The GTP of the majority (56\%) of FD patients consisted of tranexamic acid monotherapy, while the GTP of most patients without FD (74\%) contained DDAVP and/or thrombocyte transfusion.
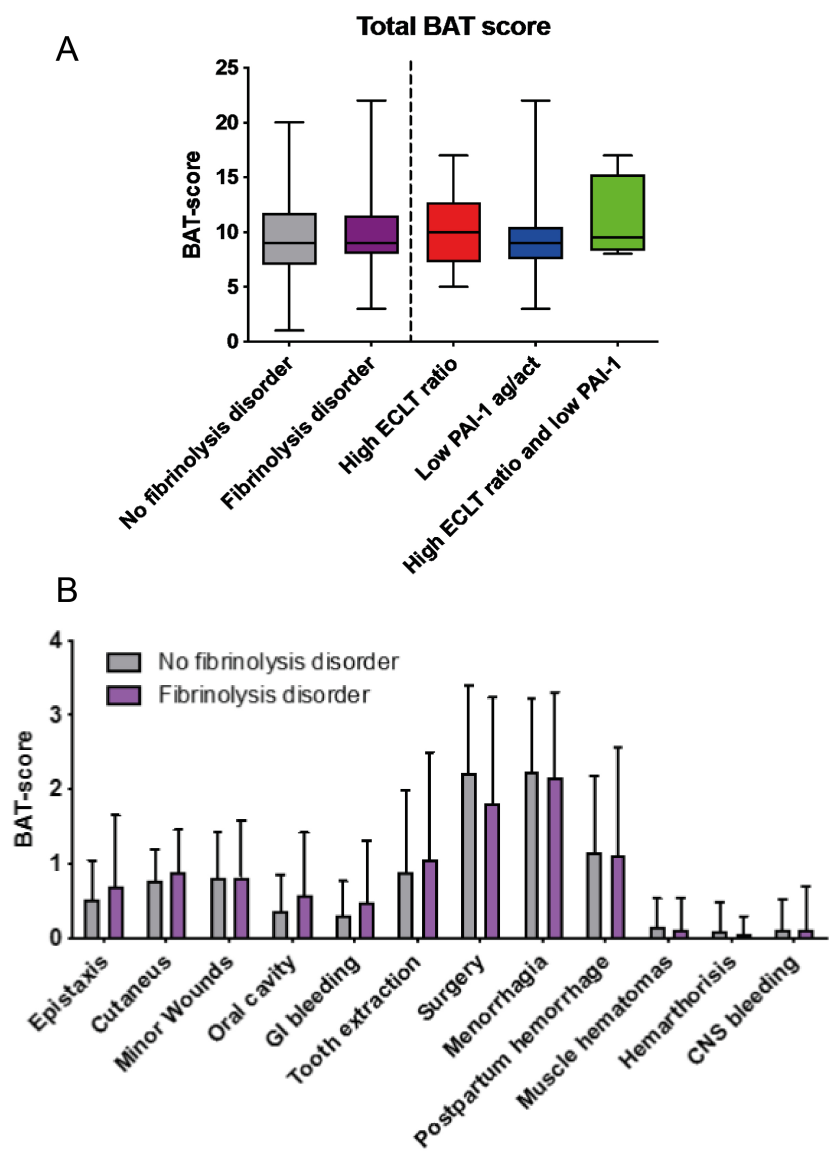

FIGURE 1 (A) Total BAT-score and (B) BAT score according to subcategorie in patients with and without fibrinolytic disorder. Box represents interquartile range and whiskers range 
TABLE 1 Laboratory results of fibrinolytic parameters of patients included in the study.

\begin{tabular}{|c|c|c|c|c|c|c|}
\hline Parameter\# & $\begin{array}{l}\text { All patients } \\
(n=160)\end{array}$ & $\begin{array}{l}\text { No fibrinolytic } \\
\text { disorder }(n=98)\end{array}$ & $\begin{array}{l}\text { Fibrinolytic } \\
\text { disorder } \\
(n=62)^{\$}\end{array}$ & $\begin{array}{l}\text { Increased ECLT } \\
\text { ratio }(n=33)^{\dagger}\end{array}$ & $\begin{array}{l}\text { Low PAI-1 ag and } \\
\text { act }(n=25)^{\dagger}\end{array}$ & $\begin{array}{l}\text { Increased ECLT } \\
\text { ratio and low } \\
\text { PAI-1 ag and act } \\
(n=4)^{\dagger}\end{array}$ \\
\hline $\begin{array}{l}\text { ECLT before in } \\
\text { minutes }\end{array}$ & $270(170->300)$ & $>300(250->300)$ & $175(145-230)$ & $220(155-285)$ & $165(130-180)$ & $155(140-170)$ \\
\hline $\begin{array}{c}\text { ECLT after in } \\
\text { minutes }\end{array}$ & $78(33-150)$ & $125(80-260)$ & $30(22-50)$ & $23(20-30)$ & $55(40-80)$ & $20(18-25)$ \\
\hline ECLT ratio & $2.9(1.8-5.5)$ & $2.2(1.2-3.3)$ & $6.2(3.0-8.9)$ & $8.6(6.3-11.4)$ & $2.9(2.2-3.7)$ & $7.5(6.1-8.3)$ \\
\hline $\mathrm{tPA}$ in IU/ml & $2.0(1.2-3.1)$ & $1.5(1.1-2.2)$ & $3.1(2.0-6.1)$ & $5.9(3.1-9.5)$ & $2.2(1.4-2.5)$ & $5.8(5.5-5.8)$ \\
\hline $\begin{array}{l}\text { PAI-1 antigen in } \\
\mathrm{ng} / \mathrm{ml}\end{array}$ & $7.9(4.4-14.2)$ & $11.7(7.3-18.3)$ & $3.7(<2.5-7.4)$ & $6.8(5.0-12.2)$ & $<2.5(<2.5-2.7)$ & $<2.5(<2.5-3.0)$ \\
\hline $\begin{array}{l}\text { PAI-1 activity in } \\
\mathrm{ng} / \mathrm{ml}\end{array}$ & $1.1(<1.0-2.6)$ & $1.9(1.0-4.4)$ & $<1.0(<1.0-1.0)$ & $<1.0(<1.0-1.7)$ & $<1.0(<1.0-<1.0)$ & $<1.0(<1.0-<1.0)$ \\
\hline
\end{tabular}

\#All numbers are given as median (interquartile range).

\$These include patients with an increased ECLT ratio and low PAI-1 antigen and activity level, causing the range to include normal values.

${ }^{\dagger}$ ECLT ratio increased if $\geq 5.7$, PAl-1 deficiency if PAl-1 antigen $\leq 3.4 \mathrm{ng} / \mathrm{ml}$ and PAl-1 activity $\leq 1.0 \mathrm{ng} / \mathrm{ml}$, alpha- 2 antiplasmin decreased if activity level $<80 \%$.

ECLT: euglobulin clot lysis time; tPA: tissue-plasminogen activator; PAI-1: plasminogen activator inhibitor-1.

Conclusions: Analysis of FD in patients with a BAT-score $\geq 10$ has a high diagnostic yield. Treatment of FD can be targeted, leading to less consumption of unnecessary coagulation products, with lower treatment costs, and a smaller chance of adverse events.

PB0417 | Assessment of Cardiometabolic Biomarkers in Young Adults with Normal Body Weight and Obesity

V. Chulkov; E. Gavrilova; V. Chulkov; N. Vereina; S. Sinitsin;

S. Martynov; P. Tkachenko; E. Lenets

South Ural State Medical University, Chelyabinsk, Russian Federation

Background: Plasminogen activator inhibitor-1 (PAI-1) as the main regulator of fibrinolysis, has been shown to be elevated in metabolic syndrome and generally is correlated with the number of components of metabolic syndrome. However, it is important to investigate its relationship to cardiovascular biomarkers in various metabolic phenotypes.

Aims: To evaluate the association of adipokines and fibrinolytic markers in young adults with normal body weight and obesity.

Methods: A cross-sectional study included 251 patients: group 1 healthy metabolic profile with normal body weight $(n=62)$; group 2 - unhealthy metabolic profile with normal body weight $(n=57)$; group 3 - healthy metabolic profile and obesity $(n=16)$; group 4 - unhealthy metabolic profile and obesity $(n=116)$. Statistical significance was defined as $P<0.05$.

Results: The concentration of serum leptin $(\mathrm{ng} / \mathrm{ml})$ was higher in group 4 (37.9 [13.0-63.4]) vs group 1 (10.0 [5.1-15.5]); group 2 (9.5 [5.5-21.5]); group 3 (25.7 [4.7-37.2]), $\mathrm{p}_{4-1.2 .3}<0.05$. The lowest concentrations of adiponectin $(\mu \mathrm{g} / \mathrm{ml})$ were found in patients with obesity - group 3 (7.9 [4.9-10.2]) and group 4 (7.9 [5.2-12.4]) compare to group 1 (9.1 [7.5-12.2] and group 28.7 [7.2-10.5], $\left.\mathrm{p}_{3.4-1.2}<0.05\right)$. Individuals in group 4 had higher concentrations of PAI-1 $(\mathrm{ng} / \mathrm{ml})$ : 464.5 [285.1-628.5] vs 308.2 [211.7-415.7] - group 1; 342.3 [222.2490.0] - group 2; 371.0 [273.1-621.0] - group 3, $\mathrm{p}_{4-1}<0.001$. Overall, there was a significant relationship between the concentration of PAI-1 and levels of systolic (Spearman $r=0.337, P=0.04$ ) and diastolic blood pressure $(r=0.314, P=0.022)$ in group 1 . The most significant linear correlations among metabolically unhealthy obese individuals were obtained between diastolic blood pressure and fibrinogen levels $(r=0.346, P<0.001)$, as well as body mass index and serum leptin ( $r=0.521, P<0.001)$.

Conclusions: In the present study, we found that young individuals with unhealthy metabolic profile and obesity had significantly higher levels of leptin, fibrinogen, PAI-1 and lower levels of adiponectin than their counterparts with normal body weight. 
PB0418 | Prolonged Tourniquet Application in the Hind Limb of Rats Is Associated with Fibrinolysis

J. Chung ${ }^{1,2}$; O. Mian ${ }^{3,2}$; A.K. Chan ${ }^{4,2}$; D. Matino ${ }^{4,2}$

${ }^{1}$ University of Toronto, Toronto, Canada; ${ }^{2}$ Thrombosis \& Atherosclerosis Research Institute, Hamilton, Canada; ${ }^{3}$ University of Ottawa, Ottawa, Canada; ${ }^{4}$ McMaster University, Hamilton, Canada

Background: Despite the pervasive use of tourniquets in major orthopedic surgeries, its effectiveness and associated complications remains a controversial topic. Our previous study has shown prolonged tourniquet application in the hind limb of rats to be associated with elevated D-Dimer (DD) levels. Tourniquets are effective in controlling intraoperative blood loss. However, complications such as thromboembolic events, with a symptomatic incidence of $10 \%$, are a major postoperative complication for patients associated with increased morbidity and mortality.

Aims: To evaluate the effects of prolonged tourniquet application on hematologic parameters such as Von Willebrand factor (VWF), tissue plasminogen activator (TPA) and DD, in Sprague Dawley Rats (SD).

Methods: Male SD rats weighing $\sim 325 \mathrm{~g}$ were anesthetized under isoflurane. Experimental animals $(n=4)$ received a tourniquet standardized to 38 newtons for 3-hours around the hind-leg, at the proximal femur. Control animals $(n=4)$ did not receive tourniquet application. After $3 \mathrm{~h}$, the tourniquet was removed on experimental animals and blood was collected from the tail vein of all subjects. All parameters were measured using respective Asserachrom antigenic kits (Diagnostic Stago), an enzyme immunoassay by ELISA method.

Results: The experimental animals demonstrated elevated levels of DD, VWF and TPA. The mean DD value for experimental and control animals was $289.3 \mathrm{ng} / \mathrm{mL}$ and $67.37 \mathrm{ng} / \mathrm{mL}$, respectively $(P=0.0035)$. The mean TPA value for experimental and control animals was $11.4 \mathrm{ng} / \mathrm{mL}$ and $1.0 \mathrm{ng} / \mathrm{mL}$, respectively $(P=0.0005)$. The mean VWF value for experimental and control animals was $3.4 \%$ and $2.9 \%$, respectively $(P=0.19)$.

\section{TPA and DD Concentrations}

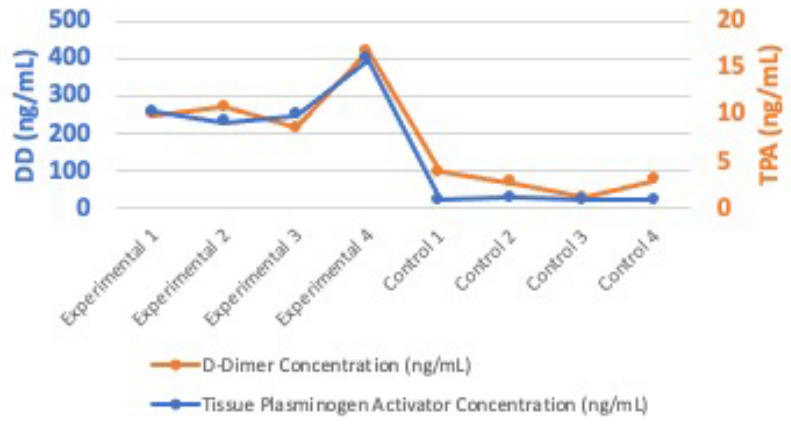

Von Willebrand Factor: AG Level (\%)

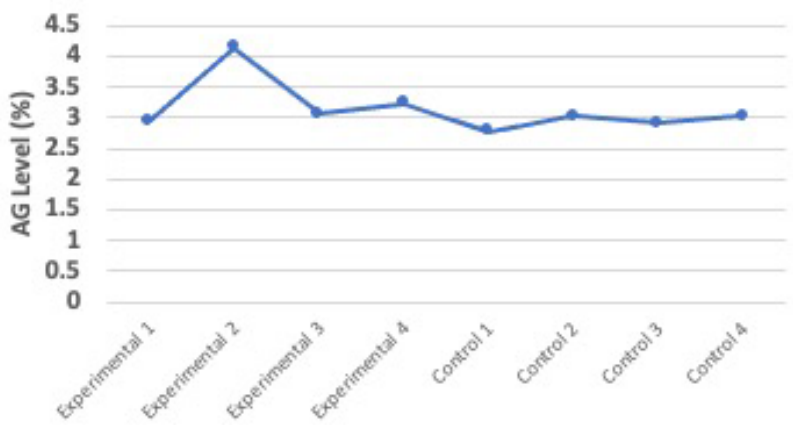

FIGURE 1 D-Dimer (DD), Tissue Plasminogen Activator (TPA) and Von Willebrand Factors (VWF) levels in animals with prolonged tourniquet application (experimental) and control animals without tourniquet application. Mean differences in DD and TPA are statistically significant.

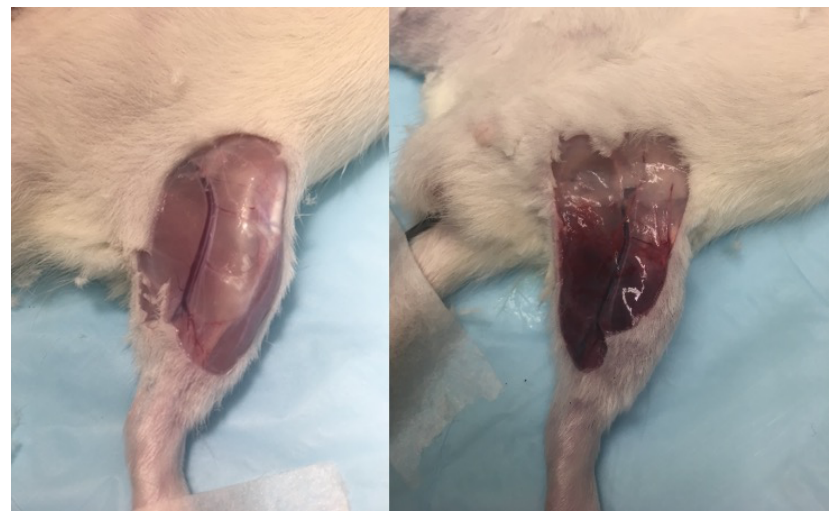

FIGURE 2 Right: Hind limb of experimental animal after 3 anaesthetized hours of prolonged tourniquet application. Vascular and muscular tissue damage visible. Left: Hind limb of control animal after 3 anaesthetized hours of no tourniquet application.

Conclusions: The significant elevations in TPA and DD suggest the activation of the fibrinolytic system in animals receiving prolonged tourniquet application. This animal model aligns with our previous work to suggest the direct effects of tourniquet application on activation of the coagulation cascade. Further research is required to study the pathophysiology and development of venous 
thromboembolic events, using diagnostic imaging methods, following prolonged tourniquet application.

PB0419 | Thromboelastography for the Evaluation and Management of Patients with Traumatic Brain Injury

J. Dias ${ }^{1}$; J. Hartmann ${ }^{1}$; J. Cannon ${ }^{2}$

${ }^{1}$ Haemonetics Corporation, Braintree, United States; ${ }^{2}$ Perelman School of Medicine at the University of Pennsylvania, Philidelphia, United

States

Background: Traumatic brain injury (TBI) is frequently associated with coagulopathy and increased mortality risk. Thromboelastography can provide rapid coagulation assessment and may be particularly useful in distinguishing coagulapathic TBI (CTBI) profiles and severity, aiding goal-directed treatment of CTBI patients.

Aims: To conduct a systematic review and outcomes analysis to assess the utility of thromboelastography for evaluation and management of CTBI patients.

Methods: A systematic literature search was performed on PubMed and EMBASE to identify clinical studies of adult patients with TBI assessed using thromboelastography. Articles were screened using predefined inclusion and exclusion criteria. Demographic, diagnostic, laboratory, treatment, and outcome data were extracted for comparison and an aggregate analysis performed to compare the effect of CTBI management with thromboelastography versus conventional coagulation assays (CCA) on 28-day mortality.

Results: Searches identified 303 non-duplicate publications with seven additional manuscripts identified by the authors. After screening and review, 29 publications were identified with relevant data on the use of thromboelastography in CTBI patients. Results showed that thromboelastography identified hypercoagulable patterns in TBI patients. Notably, the K-value, $a$ angle and MA values consistently differed from established reference ranges. Furthermore, thromboelastography parameters differed between TBI subtypes; penetrating TBI was associated with increased likelihood of coagulopathy, evidenced by significant differences in all TEG parameters (except R time), compared with blunt TBI. Thromboelastography assays were also able to predict a range of clinical outcomes including mortality, neurosurgical intervention, and bleeding complications, with evidence for improved outcomes following thromboelastography guided blood transfusion in TBI patients. Two studies were identified for an aggregate analysis, and demonstrated a significant mortality benefit of using viscoelastic testing to guide resuscitation in TBI patients compared to CCA (Figure 1).

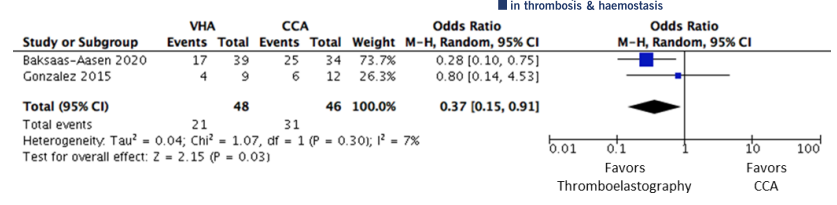

FIGURE 1 Effect of thromboelastography guided treatment on mortality in TBI patients

Conclusions: Thromboelastography may have an important role in both the diagnosis and management of patients with TBI. Further studies are needed to define optimal use.

\section{PB0420 | Effect of Alpha2-plasmin Inhibitor C-terminal Forms on in vitro Clot Lysis}

B. Baráth; R. Orbán-Kálmándi; Z. Bagoly; É. Katona

Division of Clinical Laboratory Science, Faculty of Medicine, University of Debrecen, Debrecen, Hungary

Background: Alpha2-plasmin inhibitor (A2PI) is the main inhibitor of plasmin. The secreted C-terminally intact form of A2PI (PB-A2PI) is proteolytically cleaved in the circulation. The C-terminally cleaved form lacks the plasminogen-binding site (NPB-A2PI), it remains an active plasmin inhibitor, but reacts more slowly with plasmin. It has not been studied in detail whether the truncated form is incorporated into the clot and affects clot lysis.

Aims: To investigate the effect of the incorporation of PB-A2PI and NPB-A2PI into fibrin clots on the lysis of the clot.

Methods: Citrated plasma samples from 86 healthy individuals were clotted by thrombin and calcium. Total-A2PI and PB-A2PI levels were measured by sandwich ELISAs from the original plasma and the extruded serum samples, and the concentration of NPB-A2PI was calculated from these values. An in vitro clot lysis assay was performed using platelet poor plasma samples. Clotting and subsequent lysis were induced by adding human tissue factor, rt-PA and $\mathrm{CaCl}_{2}$. Optical density was measured at $340 \mathrm{~nm} 37^{\circ} \mathrm{C}$ for $300 \mathrm{~min}$. Curves were analyzed using the Shiny app software.

Results: Total-A2PI, PB-A2PI and NPB-A2PI levels (mean \pm SD) in the plasma samples were $64.3 \pm 7.5 \mathrm{mg} / \mathrm{L}, 43.5 \pm 4.7 \mathrm{mg} / \mathrm{L}$ and $20.8 \pm 5.5 \mathrm{mg} / \mathrm{L}(32.0 \pm 6.1 \%)$, respectively. $43.6 \pm 5.9 \%$ of the plasma PB-A2PI and $62.2 \pm 12.4 \%$ of the plasma NPB-A2PI was found to be incorporated into the clot resulting in an elevated ratio of PB-A2PI to NPB-A2PI in the serum as compared to the ratio in the plasma. Plasma total-A2PI, NPB-A2-PI levels and the amount of incorporated NPB-A2PI showed significant correlation with $50 \%$ clot lysis time (CLT50) $(r=0.313, P=0.003 ; r=0.387, P<0.001$ and $r=0.256, P=0.017$, respectively), while PB-A2PI levels did not correlate $(r=0.053, P=0.629)$ with lysis parameters.

Conclusions: Our results suggest that non-covalent incorporation of the C-terminally truncated NPB-A2PI may have an effect on clot lysis and it should be further investigated. 
PB0421 | Assessment of DOACs Effect on Clot Formation and Fibrinolysis Using the FibWave

J. Evrard $^{1}$; V. Maloteau ${ }^{1}$; J.M. Dogné ${ }^{1}$; J. Douxfils ${ }^{2}$

${ }^{1}$ University of Namur, Namur, Belgium; ${ }^{2}$ Qualiblood, Namur, Belgium

Background: Direct oral anticoagulants (DOACs) are widely used for the prevention and treatment of thromboembolic events and are known to impact coagulation assays and enhance FX-dependent fibrinolysis and plasmin generation.

Simultaneous assessment of the coagulation and fibrinolysis processes could facilitate the global understanding of the pharmacodynamics of DOACs. We have previously reported the performance of the FibWave to assess the impact of DOACs on the clot formation process. In this study the FibWave has been adapted in order to measure both the clot formation and fibrinolysis processes simultaneously.

Aims: To evaluate the suitability of the FibWave for assessing the impact of DOACs on coagulation and fibrinolysis simultaneously.

Methods: DOACs were spiked at final concentrations from 0 to 250 $\mathrm{ng} / \mathrm{mL}$ in normal pooled plasma. The fibrin clot formation and the fibrinolysis were measured on the FibWave using a mixture of tissue factor, phospholipids and tissue plasminogen activator (tPA).

Results: In absence of tPA, the most influenced parameters in clot formation were the FW-Max ${ }_{2}$ FW-Min ${ }_{2}$ and FW-Ttpeak for factor $\mathrm{Xa}$ (FXa) inhibitors and FW-Ttpeak for dabigatran.

The presence of tPA did not impact significantly the parameters of the clot formation. FXa inhibitors showed a reduction of maximum fibrinolysis velocity $\left(\left|\mathrm{FW}-\mathrm{Min}_{1}\right|\right)$ while dabigatran showed a prolongation of fibrinolysis time (FW-TFib). The time length between fibrinolysis and coagulation velocities (FW-TFib - Ttpeak) was reduced for all anticoagulants (Figure 1).
A

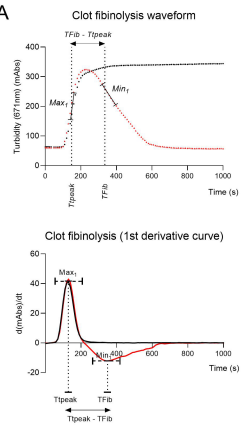

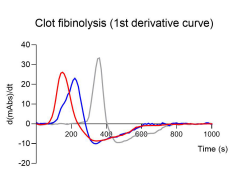

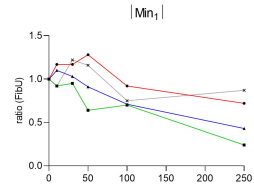

- NPP with PPA

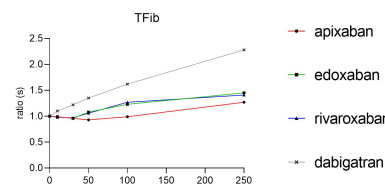

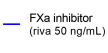

- $\underset{\substack{\text { dabigatran } \\(50 \mathrm{ng} / \mathrm{mL})}}{ }$
TFib- Theak

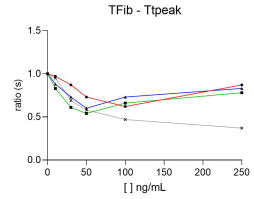

FIGURE 1 Schematic curve of initial and first derivative of FibWave (A) and impact of DOACs on fibrinolytic parameters with tPA (B). (A) Curves show the clot formation and fibrinolysis in absence (black curves) and in presence (red curves) of tPA on normal pool plasma
(NPP). The fibrinolytic parameters are presented on initial and 1st derivative curves. The last figure shows the clot fibrinolysis waveform in presence of FXa inhibitor (rivaroxaban, $50 \mathrm{ng} / \mathrm{mL}$ ) and dabigatran $(50 \mathrm{ng} / \mathrm{mL}$ ). (B) The figure represents the impact of DOACs on the maximum fibrinolysis velocity (|FW-Min1|), the fibrinolysis time (FW-TFib) and the time length between fibrinolysis and coagulation velocities (FW-TFib - Ttpeak). The FW-fibrinolysis time (FW-TFib) (in seconds) represents the time when the maximal fibrinolysis velocity is reached.

Conclusions: Thanks to its capacity to assess coagulation and fibrinolysis, the FibWave parameters could be useful for understanding global haemostatic potential and evaluating the bleeding risk from the perspective of fibrinolysis.

PB0422 | Thrombolysis and Anti-cancer Activity of Koji-Natto (Kojikinase)

H. Sumi $^{1}$; T. Mitsuo ${ }^{2}$; C. Yatagai ${ }^{1}$

${ }^{1}$ Kurashiki University of Science and the Arts, Kurashiki, Japan; ${ }^{2}$ Mitsuo Clinic, Tokyo, Japan

Background: This research started when I learned that shochu lees (jiuqu) is optimal for enhancing the production effect of nattokinase, which is a thrombolytic enzyme.

Aims: The properties of this excellent novel thrombolytic enzyme will be described below. Koji-Natto (Kojikinase) showed not only a strong thrombolysis, vitamin $\mathrm{K}_{2}$ and polyamine contents, but also strong anti-cancer activity in HeLa cell culture system.

Methods: Thrombolytic activity, hydrolyzing for amide substrate, analysis of vitamin $\mathrm{K}_{2}$ and polyamine by HPLC were performed as described previously.

The anti-cancer activity was measured by culturing human HeLa cells and the necrosis efficiency of the cells. The number of cells was measured at an absorbance of $660 \mathrm{~nm}$.

Results: The fermented product was yellow to white, had little stickiness and odor, and was dried at $50^{\circ} \mathrm{C}$ to obtain a clean solid product.

It was found that the amidase activity is stronger against synthetic substrate I than synthetic substrate II (Sub I/Sub II = 2.04), and that nattokinase also reacts with anti-nattokinase serum.

Kojikinase had an activity of $110 \mathrm{IU} / 100 \mathrm{~g}$ dry weight. It was also found that a considerable amount of vitamin $\mathrm{K}_{2}(800 \mu \mathrm{g} / 100 \mathrm{~g})$ and polyamine $(4,300 \mu \mathrm{g} / 100 \mathrm{~g})$ were accumulated in the fermented product.

It was confirmed that when Kojikinase was added to the HeLa cell culture system, it strongly suppressed thrombolytic activity and completely necrotized HeLa cells at about $5 \mathrm{mg} / \mathrm{ml}$.

Conclusions: This method is characterized by not using soybeans at all. Therefore, according to the definition of natto, Koji-Natto is not natto. Jiuqu has been a technique for saccharification that can be dusty in Japan since ancient times, and has been widely used for making sake and miso. This method is a method of producing an 
extremely rare physiologically active substance using it. In particular, the strong inhibitory effect of Kojikinase on HeLa cells will be an important issue in the future.

PB0423 | Evaluation of the Lysis Timer to Investigate 'Global Fibrinolysis Capacity' in Plasma

M. Bareille ${ }^{1}$; M. Hardy ${ }^{2}$; B. Chatelain ${ }^{1}$; T. Lecompte ${ }^{3}$; F. Mullier ${ }^{1}$

${ }^{1}$ Université Catholique de Louvain, Hematology laboratory, Namur Thrombosis and Hemostasis Center (NTHC), CHU UCL Namur, Yvoir, Belgium; ${ }^{2}$ Université Catholique de Louvain, Anesthesiology Department, Namur Thrombosis and Hemostasis Center (NTHC), CHU UCL Namur, Yvoir, Belgium; ${ }^{3}$ Departments of Medicine, Division of Angiology and Haemostasis, Geneva University Hospitals, and Geneva Platelet Group (GpG), Faculty of Medicine, University of Geneva, Geneva, Switzerland

Background: There is currently no universal and standardized test available for plasma fibrinolytic activity. The 'global fibrinolysis capacity' (GFC) assay performed with the Lysis Timer instrument (SD Innovation; reagents and internal quality controls from Hyphen Biomed) does not have the euglobulin clot lysis time (ECLT) related preanalytical constraints.

Aims: Our primary aims were i) to investigate the influence of the temperature used to keep the samples after sampling $\left(4^{\circ} \mathrm{C}\right.$ versus ambient temperature); ii) and that of a freezing-thawing cycle; and iii) to establish a preliminary reference range for GFC. Our secondary aim was to compare results of GFC with ECLT.

Methods: Influence of sample storage temperature and a freezingthawing cycle were studied with fresh plasma samples from 30 healthy adult volunteers. Reference range was established on fresh plasma samples from 21 healthy adult volunteers. Method comparison was performed with 77 samples (healthy volunteers $n=34$, patients with personal history of bleedings $n=43$ ).

Results: There was no difference between GFC assessed in plasma samples processed on melting ice or at room temperature $(P=0.17)$, whereas GFC assessed in frozen-thawed plasma samples was prolonged when compared to fresh samples $(P=0.002)$ (Figure 1).
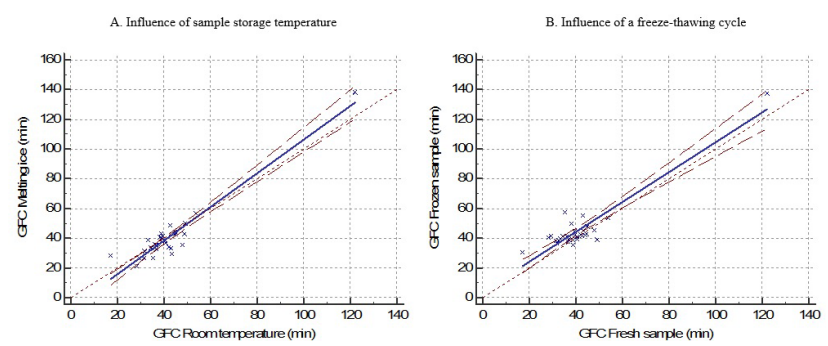

FIGURE 1 Influence of preanalytical factors (A. Sample storage temperature; B. Freeze-thawing cycle)
Preliminary reference interval ranged from $29(90 \% \mathrm{Cl}=[27-32])$ to $50(90 \% \mathrm{Cl}=[46-53])$ minutes.GFC and ECLT were weakly correlated $(\rho=0.229, P=0.04)$, with a poor agreement $(\mathrm{K}=0.03,95 \% \mathrm{Cl}$ $=[-0.154-0.221])($ Table 1$)$.

TABLE 1 Agreement table between euglobulin clot lysis time (ECLT) and global fibrinolysis capacity (GFC)

\begin{tabular}{|c|c|c|c|}
\hline & GFC & & \\
\hline ECLT & $\begin{array}{l}\text { hyperfibrinolysis } \\
\text { (n) }\end{array}$ & $\begin{array}{l}\text { within } \\
\text { reference } \\
\text { range }(n)\end{array}$ & $\begin{array}{l}\text { hypofibrinolysis } \\
\text { (n) }\end{array}$ \\
\hline hyperfibrinolysis (n) & 0 & 9 & 0 \\
\hline $\begin{array}{c}\text { within reference } \\
\text { range }(n)\end{array}$ & 0 & 52 & 11 \\
\hline hypofibrinolysis (n) & 0 & 3 & 2 \\
\hline
\end{tabular}

Conclusions: Sample storage temperature had no influence on the result. Reference ranges need to be established for fresh and frozen samples. Shorter than 1-hour reference interval enables the use of GFC in emergency cases. Finally, GFC and ECLT showed a weak correlation and a poor agreement, likely explained by plasma absence of fibrinolysis inhibitors such as PAI-1 in the euglobulin fraction.

\section{PB0424 | Thrombolytic Activity of Pearl-like Tempeh}

H. Sumi $^{1}$; Y. Yanagisawa ${ }^{2}$; S. Naito ${ }^{1}$; C. Yatagai ${ }^{1}$

${ }^{1}$ Kurashiki University of Science and the Arts, Kurashiki, Japan; ${ }^{2}$ Chiba Institute of Science, Choshi, Japan

Background: Tempeh is a fermented soybean food produced by Rhizopus oligosporus, but it is known to have weaker thrombolytic activity than natto produced by Bacillus subtilis natto. However, tempeh is easier to eat because it has no odor or stickiness compared to natto, which is the same fermented food.

Aims: In this study, we report that we were able to obtain pearl-like tempeh with high thrombolytic activity by shaking culture of tempeh with a high-concentration substrate.

Methods: Rhizopus oligosporus purchased from Akita Konno Co., Ltd. was used, and standing culture was carried out as usual. In addition, some attempted shaking culture of the substrate at a high concentration. That is, $200 \mathrm{ml}$ of polypeptone $\mathrm{S}$ was used as a substrate in a $500 \mathrm{ml}$ Erlenmeyer flask in a shaking incubator (Yamato IM41), and the cells were cultured at $37^{\circ} \mathrm{C}$ for 10 days at $100 \mathrm{rpm}$.

Results: As a result of shaking culture, the color of the medium changed from yellow to black at a substrate concentration of 2.5 to $60 \%$, and in each case, a clean yellow pearl-like tempeh with a diameter of 0.2 to $1.0 \mathrm{~cm}$ was obtained. The substance was durable and stable even when washed with water, and a clean dried product could be obtained by freeze-drying. Thrombolytic activity peaked at $2.5 \%$ and $30-40 \%$ substrate concentrations. However, it was found that $70 \%$ or more of the thrombolytic activity was inactivated when this product was heat-dried at $50^{\circ} \mathrm{C}$ or higher. 
Conclusions: Pearl-like tempeh could be obtained. The thrombolytic system of tempeh is fairly complex and may develop over time under more anaerobic constant conditions. This is because we have confirmed that tempeh bacteria significantly increase tyramine and cadaverine under more anaerobic conditions in petri dishes. New functional foods, especially the anti-cancer activity of pearl-like tempeh, are promising in the future.

\section{PLASMINOGEN ACTIVATION IN THE CNS AND IMMUNITY}

LPB0065 | Mutation of Sortase A Cleavage Site and Potential Impact on Human Plasminogen Binding by PAM

B. Readnour; Y. Ayinuola; V. Ploplis; F. Castellino

University of Notre Dame, South Bend, United States

Background: Plasminogen-binding group A streptococcal M-like protein (PAM) is the primary protein responsible for binding human plasminogen $(\mathrm{hPg})$ in several Group A streptococcus (GAS) strains. PAM is bound to the cell wall through sortase $A(S r t A)$ interaction with the LPXTG motif. The inactivation of SrtA has shown a reduction in the binding activity of PAM to $\mathrm{hPg}$. Mutating the amino acid in the fourth position of the LPXTG motif has shown reduced affinity of SrtA to cleave the peptides in vitro.

Aims: Investigating the impact of inhibiting SrtA cleavage of PAM through mutation of the LPXTG motif in GAS.

Methods: LPSXG peptides were tested using FRET pairs incubated with SrtA protein and LPSYG was shown to have the lowest activity. AP53 GAS strain containing a mutated PAM protein was generated. Cell wall fractions were separated, and relative abundance was determined by western blot. Binding of hPg by PAM was determined by whole cell ELISA. Cell membrane fractions were collected and tested with fluorescent peptides to determine cleavage rates.

Results: The LPSYG peptide showed reduced activity with SrtA protein in vitro. When tested in GAS cells, the PAM mutants were attached to the cell wall and bound to hPg at the same level as wild type (Figure 1).

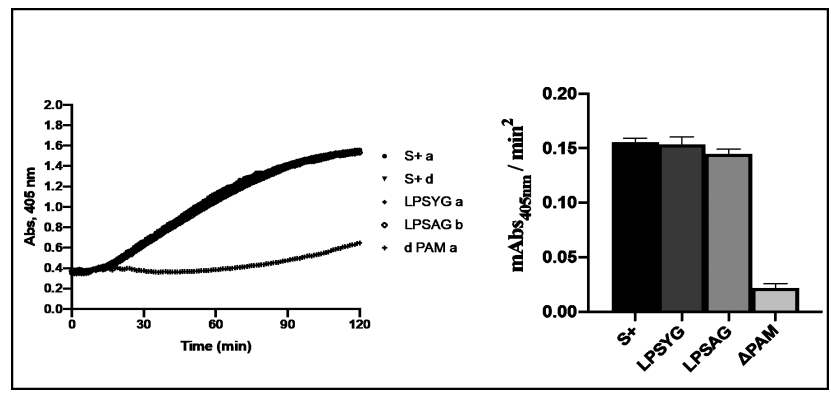

FIGURE 1 SK2b-mediated activation of hPg-stimulation by whole cell AP53(S+), LPSYG, LPSAG, and delta PAM.

Cell membrane fractions were tested with fluorescent peptides and the LPSYG peptide was cleaved at significantly higher rates than LPSTG (Figure 2).

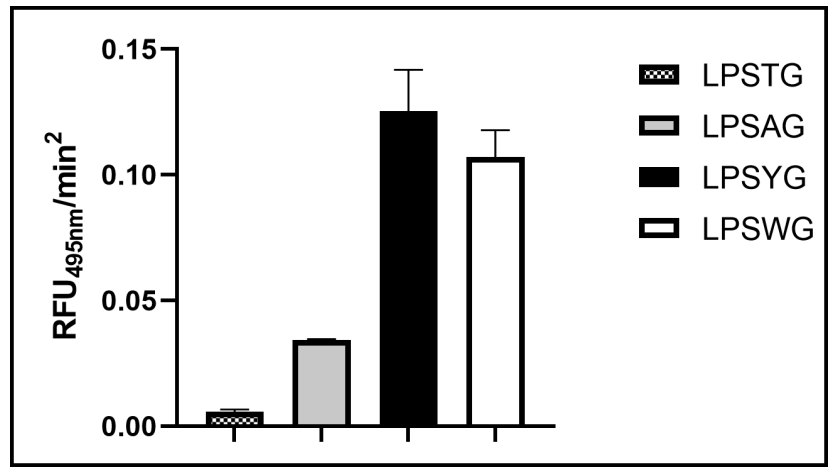

FIGURE 2 Cleavage of Dabcyl-LPSXG-EDANS fluorescent peptides by AP53 cell membrane fractions.

Conclusions: In vitro SrtA demonstrated no interaction with the LPSYG peptide. When mutated into GAS, PAM showed similar binding affinity to hPg. Cell membrane fractions were added to the peptides and LPSYG was cleaved at higher rates than LPSTG. This result suggests another protein can recognize the LPSYG motif and cleave in place of SrtA.

\section{LPB0113 | Streptococcal Surface Enolase (SEN) Is} Overshadowed by Plasminogen-binding Group A Streptococcal Mprotein (PAM) for Human Plasminogen Acquisition and Activation

S. Tjia-Fleck; Y. Ayinuola; Z. Liang; V. Ploplis; F. Castellino University of Notre Dame, Notre Dame, United States

Background: Human plasmin (hPm) proteolytic activity is utilized by various pathogenic bacteria, such as Group A Streptococcus (GAS), to increase their dissemination and pathogenicity. GAS is a humanselective Gram-positive coccus bacterium that utilizes the function of $\mathrm{hPm}$ through the expression of surface human plasminogen $(\mathrm{hPg})$ receptors. Once hPg is recruited to the cell surface, it is converted into active hPm. There are at least three $\mathrm{hPg}$ receptors on the GAS cell surface with different binding strengths to hPg. Plasminogenbinding Group A Streptococcal M-protein (PAM) is typically found on skin-tropic Pattern D GAS strains, such as AP53. PAM binds hPg with a very high affinity ( $\mathrm{Kd} \sim 1 \mathrm{nM})$ and is able to stimulate $\mathrm{hPg}$ activation in the presence of GAS-secreted streptokinase (SK) and hPg activators such as urokinase (uPA). Unlike PAM, another hPg receptor, streptococcal surface enolase (SEN), is found on the surface of most GAS strains and its importance to $\mathrm{hPg}$ acquisition in skin-tropic GAS strain is not known, especially when PAM is also present. 
Aims: This study presents the results of an investigation on the contribution of SEN in $\mathrm{hPg}$ binding and activation.

Methods: Analytical ultracentrifugation, isothermal titration calorimetry, and flow cytometry were used in this study.

Results: The data generated showed that the $45 \mathrm{kDa}$ monomer unit of recombinant SEN oligomerizes to form a highly stable octamer in solution. The octameric SEN forms a complex with $\mathrm{hPg}$, characterized by a Kd of $\sim 100 \mathrm{nM}$. Although SEN tightly binds hPg, it does not stimulate $\mathrm{hPg}$ activation by SK or UPA. Whole GAS cell studies showed that the surface expression of SEN is diminished upon targeted deletion of the PAM gene. hPg binding to AP53 cells emphasizes a lower affinity of SEN for hPg compared to PAM.

hPg Activator Mediated Activation
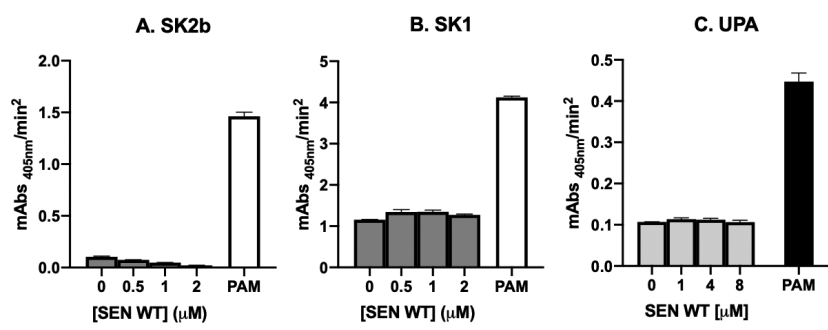

FIGURE $1 \mathrm{hPg}$ activator mediated plasminogen activation in the presence of plasminogen receptors PAM and SEN.

\section{Flow Cytometry - Anti SEN and hPg Binding}

\section{A. Cell Surface SEN}

\section{B. Whole Cell Binding to $\mathrm{hPg}$}
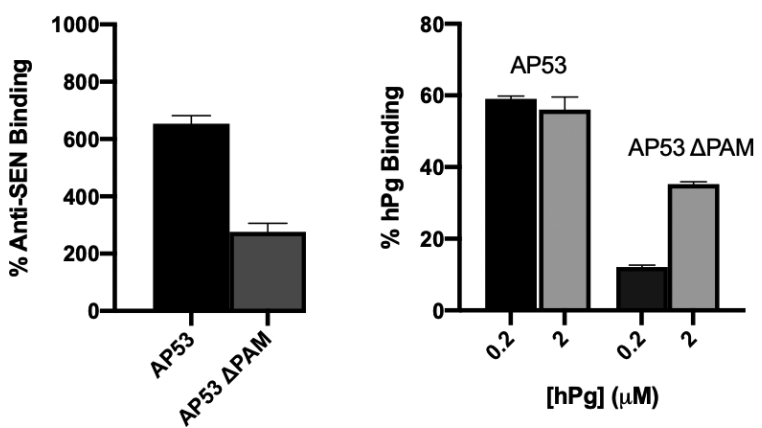

FIGURE 2 Flow cytometry for SEN level in the cell surface and the $\mathrm{hPg}$ binding using anti SEN.

Conclusions: This study strongly suggests that SEN plays a secondary role as $\mathrm{hPg}$ receptor in skin-tropic GAS strains.
PB0425 | Changes in Plasminogen Binding and Cell Surface Properties in Streptococcus pyogenes in the Absence of Sortase A Are Due to Disruptions in Localization of M-protein

\section{B. Russo $^{1,2}$; V. Ploplis ${ }^{1,2} ;$ F. Castellino ${ }^{1,2}$}

${ }^{1}$ W.M. Keck Center for Transgene Research, University of Notre Dame, Notre Dame, United States; ${ }^{2}$ Department of Chemistry and Biochemistry, University of Notre Dame, Notre Dame, United States

Background: Group A Streptococcus (GAS) is a human specific pathogen that conscripts the fibrinolytic system to increase its survival and dissemination in the body. In skin-trophic strain AP53 this disruption requires cell wall-anchored plasminogen $(\mathrm{hPg})$-binding GAS $M$-protein (PAM) that is anchored via the transpeptidase, sortase $A$ (srtA). Deletion of srtA results in localization of PAM to the cell membrane and the supernatant, a reduction of whole cell $\mathrm{hPg}$ binding and activation, and disruption of surface properties. The degree to which membrane-bound PAM in the absence of srtA in GAS strain AP53 is able to bind hPg for activation, and whether changes to other cell surface properties are due to protein localization or transcription, have not been established.

Aims: To further understand the role of srtA in determining cell surface properties and ability to bind hPg for activation in GAS strain AP53.

Methods: An AP53/ $\Delta$ srtA mutant was generated by routine techniques. An isogenic complemented strain and a $\Delta p a m$ strain were also generated for comparison. Digestion with PlyC and ultracentrifugation were used to isolate cell fractions. Spectrophotometry was used to quantify bacterial capsule and hydrophobicity. hPg activation assays were performed using a colorimetric reporter. Quantitative RT-PCR was performed to compare transcriptional changes.

Results: Membrane fractions from AP53/ $\Delta$ stA, but not the wild type or complement, activated hPg. In AP53/AsrtA, transcription of PAM was unchanged. AP53/ $\Delta$ srtA had lower hydrophobicity than wild type but no change in the amount of capsule. 


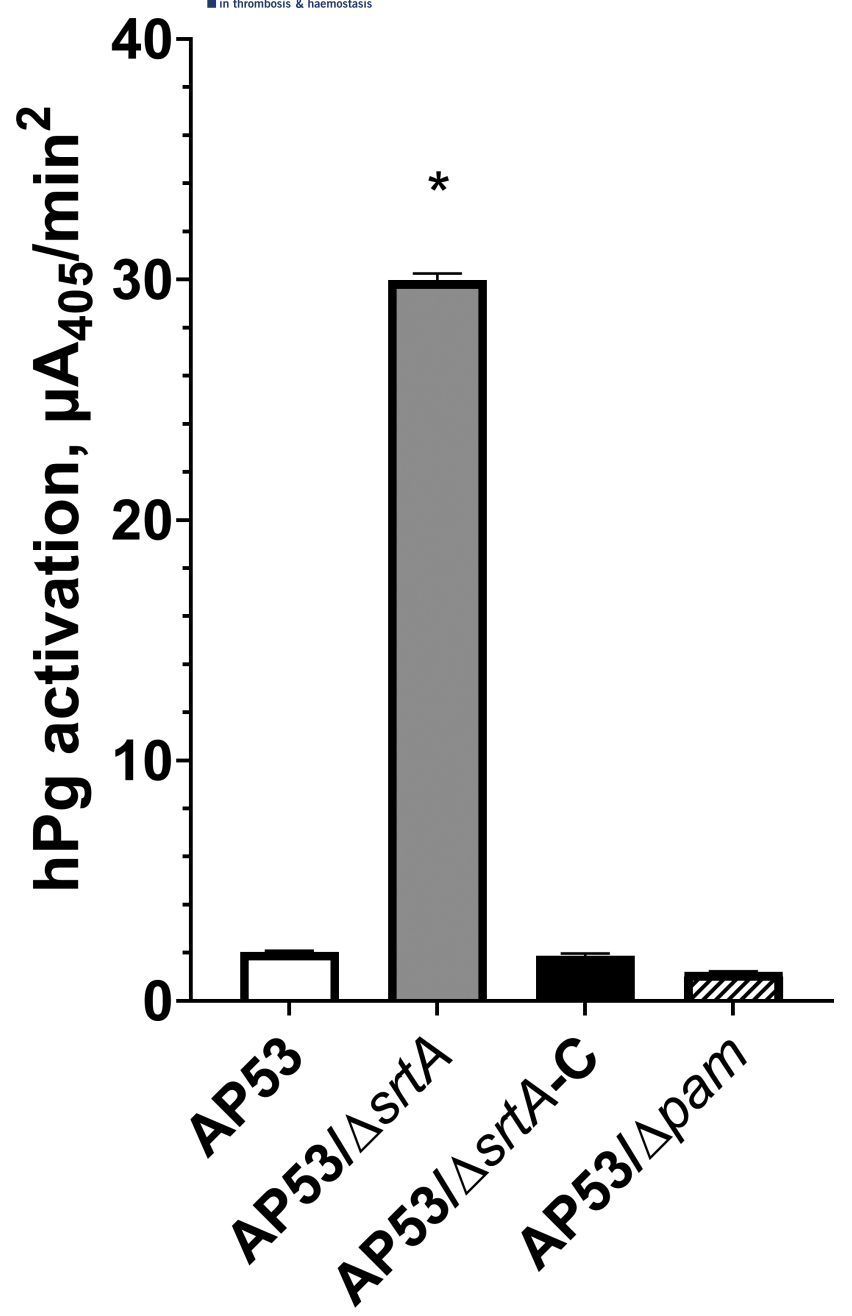

changes in the amount of capsule and in transcription suggest that observed changes in hydrophobicity and $\mathrm{hPg}$ binding and activation when srtA is deleted are due to disruption of PAM surface anchoring rather than changes in transcription.

\section{THROMBOLYTIC THERAPY}

LPB0066 | Decreased Clot Burden is Associated with Factor XIII Val34Leu Polymorphism and Better Functional Outcomes in Acute Ischemic Stroke Patients Treated with Intravenous Thrombolysis

I. Szegedi ${ }^{1}$; R. Orbán-Kálmándi ${ }^{2} ;$ A. Nagy ${ }^{3}$; F. Sarkady ${ }^{2}$; N. Vasas ${ }^{4}$; M. Sik ${ }^{4}$; L. Lánczi ${ }^{4}$; E. Berényi ${ }^{4}$; L. Oláh ${ }^{1}$; L. Csiba ${ }^{5}$ Z. Bagoly ${ }^{6}$

${ }^{1}$ University of Debrecen, Faculty of Medicine, Department of Neurology, Debrecen, Hungary; ${ }^{2}$ University of Debrecen, Faculty of Medicine, Department of Laboratory Medicine, Division of Clinical Laboratory Sciences, Debrecen, Hungary; ${ }^{3}$ University of Debrecen, Faculty of Public Health, Department of Preventive Medicine, Debrecen, Hungary; ${ }^{4}$ University of Debrecen, Faculty of Medicine, Department of Radiology, Debrecen, Hungary; ${ }^{5}$ University of Debrecen, Faculty of Medicine, Department of Neurology, MTA-DE Cerebrovascular and Neurodegenerative Research Group, Debrecen, Hungary; ${ }^{6}$ University of Debrecen, Faculty of Medicine, Department of Laboratory Medicine, , Division of Clinical Laboratory Sciences, MTADE Cerebrovascular and Neurodegenerative Research Group, Debrecen, Hungary

FIGURE $1 \mathrm{hPg}$ Activation by Cell Membrane

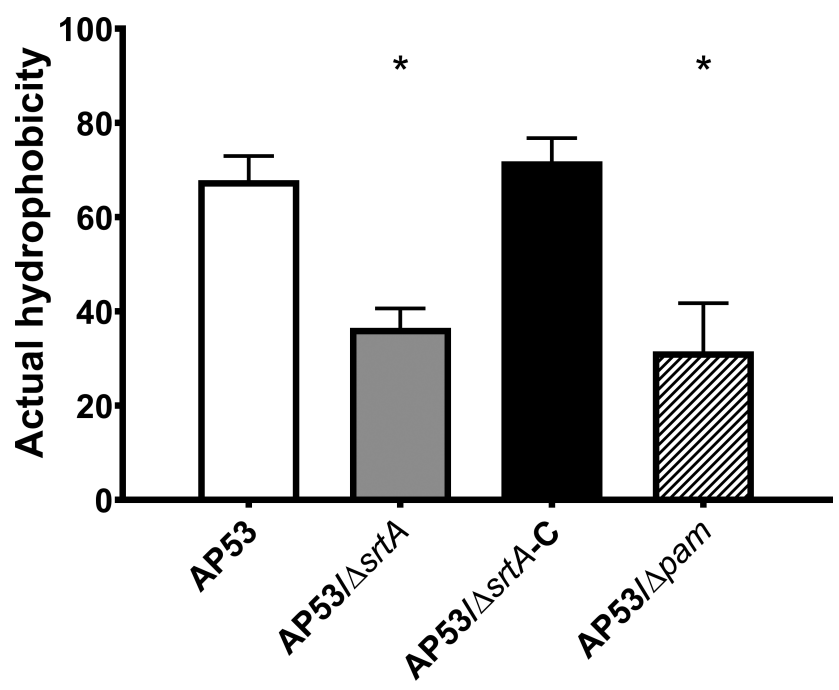

FIGURE 2 Hydrophobicity at Mid-Logarithmic Phase

Conclusions: Our data support the hypothesis that PAM retained in the cell membrane retains its ability to bind $\mathrm{hPg}$ and is responsible for whole cell hPg binding and activation. Additionally, lack of

Background: Intravenous thrombolysis using recombinant tissue plasminogen activator remains the mainstay treatment of acute ischemic stroke (AIS), although endovascular treatment is becoming standard of care in case of large vessel occlusions (LVO).

Aims: Here we aimed to evaluate how thrombus burden affects the outcome of the intravenous thrombolysis and studied the association between a semiquantitative CT angiography (CTA) grading system, the clot burden score (CBS), and various fibrinolysis parameters. Methods: In this single-centered, prospective observational casecontrol study, 200 anterior circulation AIS patients receiving intravenous thrombolysis without thrombectomy were enrolled: 100 AIS patients with LVO (CBS 0-9) were matched with 100 age- and sexmatched AIS patients without LVO (CBS 10). Fibrinogen, a2-plasmin inhibitor, plasminogen, factor XIII activity, and D-dimer were assessed from blood samples taken on admission and $24 \mathrm{~h}$ after thrombolysis, and FXIII-A Val34Leu was genotyped. CBS was calculated using admission CTA imaging. Short-term outcomes were defined based on the change in NIHSS by day 7, long-term outcomes were assessed according to the modified Rankin scale at 3 months post-event.

Results: Poor outcomes were significantly more frequent in the CBS 0-9 group. Plasminogen activity on admission was significantly higher in the CBS 0-9 group. In a univariate analysis, a significant protective effect of the Leu34 allele against developing larger clots (CBS 0-9) 
could be demonstrated (OR:0.519; 95\%Cl:0.298-0.922, $P=0.023$ ). Multivariate regression analysis revealed that larger thrombus burden (CBS 0-9) is an independent predictor of short- and longterm functional outcomes (mRS 3-6: OR: 2.5; 95\%Cl:1.179-5.306, $P=0.017$ ) while such effect of the studied fibrinolysis parameters could not be demonstrated.

Conclusions: CBS was found to be a significant independent predictor of thrombolysis outcomes. FXIII-A Leu34 carrier status was associated with smaller thrombus burden, which is consistent with the in vitro described whole blood clot mass reducing effects of the allele, but the polymorphism had no effect on thrombolysis outcomes.

PB0426 | Co-delivery of Tissue Plasminogen Activator and Plasminogen Using Magnetically Powered Microwheels

D. Disharoon ${ }^{1}$; D. Marr ${ }^{1}$; K. Neeves ${ }^{2}$

${ }^{1}$ Colorado School of Mines, Golden, United States; ${ }^{2}$ University of Colorado Denver, Aurora, United States

Background: We reported that tissue plasminogen activator (tPA) immobilized on magnetically powered microwheels can increase the rate of fibrinolysis by five-fold compared to therapeutic concentrations of tPA (Tasci et al., Small, 2017). This rate increase is due to the accumulation and penetration of microwheels into a thrombus. However, at these high tPA concentrations, the availability of plasminogen becomes the rate-limiting step in fibrinolysis.

Aims: To co-deliver tPA and plasminogen on microwheels to overcome plasminogen depletion and hasten fibrinolysis.

Methods: Mesoporous silica nanoparticles (MSN) and tPA were coupled to $1 \mu \mathrm{m}$ superparamagnetic beads (Dynabeads ${ }^{\mathrm{TM}}$ ) via biotinstreptavidin binding (Fig. 1). The MSN were loaded with plasminogen and their release kinetics were measured by spectroscopy. The MSN decorated beads were subject to a rotating magnetic field (8 $\mathrm{mT}, 10 \mathrm{~Hz}$ ) to induce the self-assembly of wheel-like disks that rolling. Fibrinolysis of a plasma clot $(4.5 \mathrm{nM}$ thrombin, $20 \mathrm{mM} \mathrm{CaCl}$ ) was measured by optical microscopy.

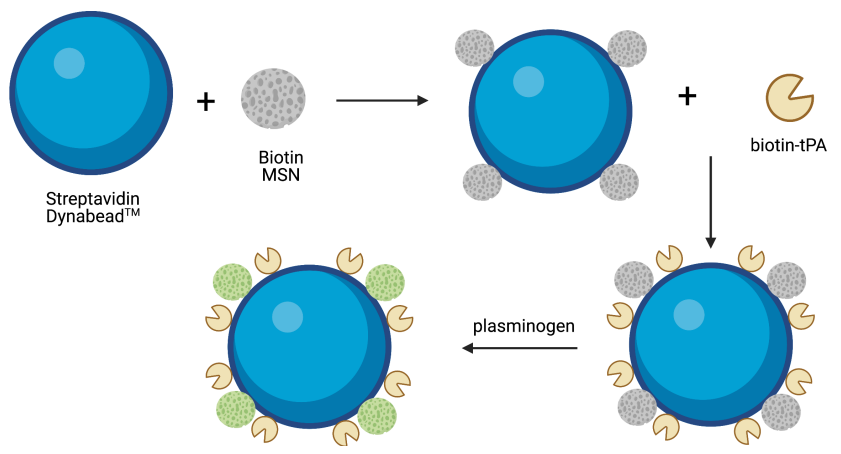

FIGURE 1 Synthesis of plasminogen-tPA-beads

Results: Plasminogen-tPA-beads accumulated the clot and were estimated to achieve a local tPA and plasminogen concentrations of
$40 \mathrm{nM}$ and $2.5 \mu \mathrm{M}$, respectively. Pgn-tPA-microwheels driven in a direct line motion into the plasma clot, lyse fibrin at a rate of $13.6 \pm$ $1.8 \mu \mathrm{m} / \mathrm{min}$ compared to $2.5 \pm 0.7 \mu \mathrm{m} / \mathrm{min}$ for tPA-microwheels, and $0.4 \pm 0.1 \mu \mathrm{m} / \mathrm{min}$ for $40 \mathrm{nM}$ tPA (Fig. 2). Pgn-tPA-microwheels driven in corkscrew motion penetrate into clot, further accelerating lysis to $21.0 \pm 2.9 \mu \mathrm{m} / \mathrm{min}$ (Fig. 2).

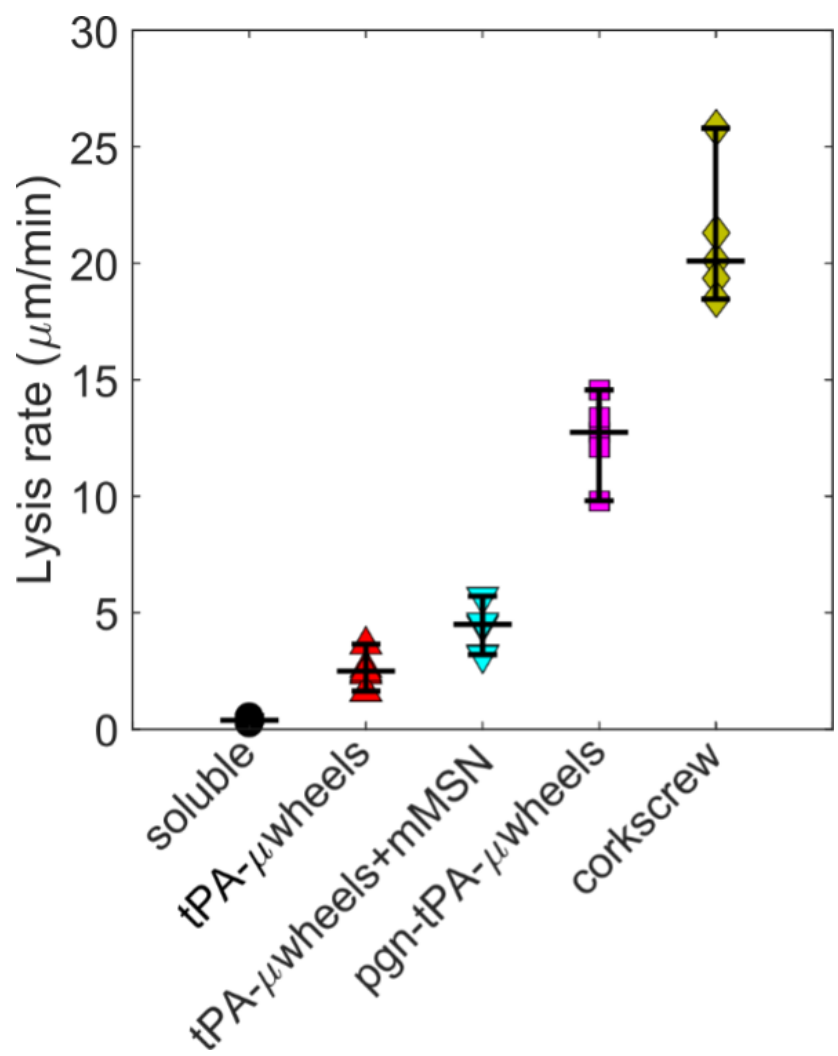

FIGURE 2 Fibrinolysis rates for tPA- and plasminogen-tPAmicrowheels for direct and corkscrew motion.

FIGURE 2 Lysis rates for different microwheel configurations

Conclusions: The co-delivery of plasminogen and tPA on microwheels overcomes substrate limited fibrinolysis, resulting in over a five-fold increase in lysis rate compared to tPA alone. When combined with a corkscrew motion, wherein microwheels can bore their way into a clot, the lysis rate is further enhanced. These data establish the utility of combining enzyme and substrate delivery as a thrombolysis strategy. 
PB0427 | P2Y12 Inhibitors Administration in Intracranial Stent Procedure, the Usefulness of Monitoring

O. Duranteau; M. Drir; L. Abdenour; V. Degos

La Pitié Salpétrière, Paris, France

Background: The implantation of stents in cerebral arteries must be preceded by the administration of antiplatelets agents based on a dual therapy with Aspirin (Kardegic ${ }^{\circledR}$ ) and a P2Y12 inhibitor Devices can monitor the action of the P2Y12 inhibitor, such as the Multiplate ${ }^{\circledR}$ which can predict the phenomenon called "high ontreatment platelet reactivity ».

Aims: This study focuses on the use of platelet function monitoring in the context of a prescription of clopidogrel and its possible replacement by ticagrelor.

Methods: We performed an observational retrospective cohort monocentric study from January 2017 to June 2020.

Patients received a prescription for 5 days of clopidogrel $(75 \mathrm{mg}$ per day) before the procedure. The day before the procedure, if adenosine diphosphate (ADP) test result was above 300 area under the curve on multiplate, patients were considered as resistant to clopidogrel and Ticagrelor was introduced (dosage $=180 \mathrm{mg}$ ).

We collected ADP test results from Multiplate ${ }^{\circledR}$ at the anaesthesiologist consultation and the day before the surgery

The primary endpoint was the occurrence of thromboembolic or haemorrhagic events for one month postoperatively.

Results: 104 patients were included, and all benefitted from the treatment of an intracerebral aneurysm. 76 patients were classified as respondent to clopidogrel regarding local protocol and received clopidogrel. 28 had to be switched from clopidogrel to ticagrelor according to local protocol. Eight patients under clopidogrel (10.5\%) had an ischemic event and 3 under ticagrelor (11\%) during the 30 days post-operative period. No patient had a haemorrhagic event under clopidogrel and 2 under ticagrelor (7.5\%) during the same period. The difference was not statistically significant regarding ischemic or haemorrhagic event and treatment $(P$-value $=0.055)$.

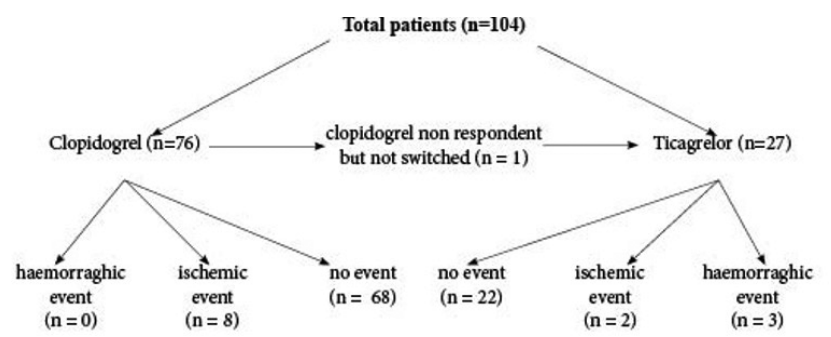

FIGURE 1 Flow Chart
ADP Test evolution regarding drug administration and events

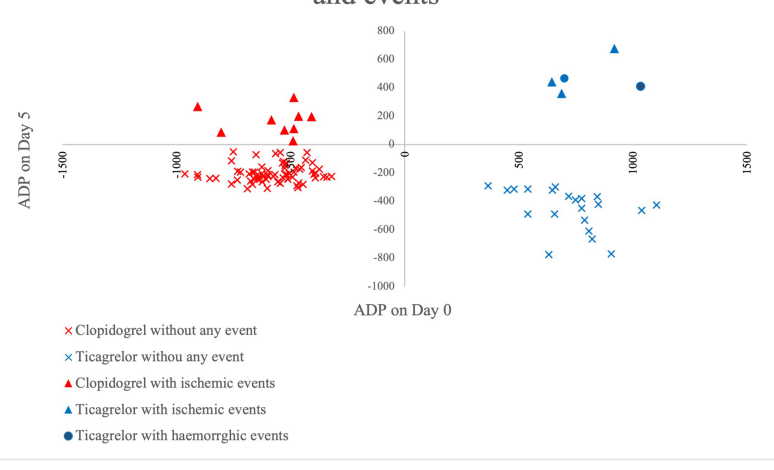

FIGURE 2 ADP test evolution (on Day 0 and on Day 5) regarding drug administration and events

Conclusions: The use of a platelet function monitoring device helps reduce the risk of treatment complications (ischemic or haemorrhagic). The definition of a threshold below 300 AUC could likely result in improved morbidity at 30 days.

\section{PB0428 | Producing if Tenecteplase with High Activity}

D. Chashchinova ${ }^{1}$; V. Leonov ${ }^{2} ;$ X. Smolova ${ }^{2}$; D. Kudlay ${ }^{1}$

${ }^{1}$ JSC GENERIUM, Volginsky Settlement, Russian Federation; ${ }^{2}$ LLC IBC GENERIUM, Volginsky Settlement, Russian Federation

Background: Tenecteplase is widely used thrombolytic drug, approved for the treatment of acute myocardial infarction. Biological activity of tenecteplase is related to glycoforms of a biomolecule, such as content of I/II glycosylation types and sialic acids content. Wide variety of CQA results in difficulties in developing biosimilar to tenecteplase.

Aims: Developing of a method for preparation of the tenecteplase drug, taking into account the content of isoforms.

Methods: Production of Tenecteplase performed in CHO-M cells in fed-batch mode. Cells of producer clones were placed in Erlenmeyer flasks with BalanCD medium. From the third to the eighth day of cultivation, feed supplements were added to the medium to $4 \%$ of the medium volume. Glucose level in the medium was maintained at $3 \mathrm{~g} / \mathrm{l}$. Content of sialic acids was determined spectrophotometrically by the resorcinol method. Biological activity of tenecteplase was determined in two ways: in samples of the culture fluid - using the Human tPA Chromogenic AssaySense Activity Assay Kit (AssayPro); in samples of purified protein - by fibrin clot lysis assay.

Results: During the initial clone selection process reduced fibrinolytic activity and hypersialyation of the tenecteplase produced by leader clones was found. Linear regression analysis showed that tenecteplase with more than 5 sialic acid molecules in $\mathrm{N}$-linked oligosaccharides had activity below the target range. Decrease in the viability of cell culture reduce the content of sialic acids in oligosaccharides of tenecteplase by $13 \%$ and increase activity by $14-15 \%$. Addition of ammonium chloride up to $20 \mathrm{mM}$ in stationary phase 
of cultivation decreases sialic acid content by $29-34 \%$ and increases activity by $17 \%$.

Conclusions: Biological activity screening and glycosylation pattern evaluation in clone selection process allow selecting clone that produces high-active form of tenecteplase. Attention to the content of glycosylation variants on early stage of process development allows to get a product with high biosimilarity to the original drug.

PB0429 | Cangrelor Long Term Prescription, Several Scenarios: A Case Report Series

O. Duranteau; L. Abdennour; M. Drir; F. Clarencon; V. Degos La Pitié Salpétrière, Paris, France

Background: Cangrelor, a P2HY12 inhibitor, with a half-life is about 3 to $5 \mathrm{~min}$, a clearance of $50 \mathrm{~L} / \mathrm{h}$ and an off set about $1 \mathrm{~h}$. Dose infusion protocol is the same, ie: $30 \mu \mathrm{g} / \mathrm{kg}$ bolus, followed by $4 \mu \mathrm{g} /$ $\mathrm{kg} / \mathrm{min}$ during 2 to $4 \mathrm{~h}$ maximum with a bridge with another P2Y12.

Aims: However, long-term prescription of Cangrelor is not describe in the literature. Four experiences situations with cangrelor longterm prescription following flow-diverter or stent-assisted coiling procedure in neurosurgical environment are described.

Methods: As the cangrelor use is off label in intracranial stenting, all the indications were discussed collegially through a multidisciplinary discussion (neurosurgeon, neuro-intensivist, neuro-radiologist).

Results: Case 1: Cangrelor-ticagrelor switch was organised with Ticagrelor administered to the patient $30 \mathrm{~min}$ before the stop of Cangrelor. Six hours after a cangrelor-ticagrelor switch, the arteriography made for a brutally coma showed an extension of the thrombosis of the branches covered by the flow diverter with vasospasm of the left M1 treated by a chemical dilatation.

Case 2: Multiples therapeutic windows for external ventricular drain changeset and removal with non-complications.

Case 3: No therapeutic windows, but a shock colic was developed, with vasospasm of the splanchnic arterial network at day 22.

Case 4: Multiples therapeutic windows, cangrelor IV line was unplugged during $3 \mathrm{~h}$ with no clinical consequences for the patient Conclusions: Cangrelor long term administration is part of the solution of stent or coil implantation in neurosurgical patients. Its use needs an ICU environment with frequent clinical exam. The infusion process has to be done by trained paramedical staff. The therapeutics windows or Ticagrelor-bridge periods have to be under high surveillance. With all these restrictions, cangrelor could be part of future therapeutic arsenal with complicated cases mixing neurosurgery and interventional neuroradiology.
PB0430 | Subcasular Hepatic Hematoma as Thrombolysis Complication. Case Report

D.A. Madrigal-Campos ${ }^{1}$; B.d.C. Madrigal-Sanchez ${ }^{2}$

${ }^{1}$ ISSSTE, Chihuahua, Mexico; ${ }^{2}$ Baptist Medical Center, San Antonio, United States

Background: Even though primary angioplasty is the suggested treatment for early revascularization, thrombolysis is still used as well. Even though thrombolysis has a lower risk of complications, they may still occur.

Aims: Thrombolysis complication.

Methods: This was the case for a male 68-year-old patient with smoking as the only cardiac risk factor. He was diagnosed by clinical, electrocardiogram and cardiac enzymes with inferior acute myocardial infarction. Due to primary angioplasty being inaccessible at that moment, it was decided to thrombolysis with streptokinase. Treatment with antiplatelet agents, anticoagulants and anti-ischemic drugs was provided as recommended. One day later, the patient presented epigastric and mesogastric pain, abdominal rigidity and hypoactive bowel sounds. Low blood pressure, tachycardia and oliguria were also present. A sonogram was performed in which free intraperitoneal fluid and hepatic subcapsular hematoma were detected. The hepatic subcapsular hematoma was confirmed in the abdominal CT scan[Ed1] . After a surgery consultation, it was decided to keep the patient under observation. The use of anticoagulants and antiplatelet agents was suspended, and the patient underwent NPO[Ed2] and hydroelectrolytic therapy. The patient showed improvement within two days and was discharged a week later.

Results: Case report.

Conclusions: Acute coronary syndromes have several complications, such as those related to fibrinolysis. Hepatic hematoma is considered to be a major complication of fibrinolysis. In the literature, we only found five cases of hepatic hematoma with hepatic rupture secondary to thrombolysis and CPR. Our patient did not present CPR or hematologic alterations. 


\section{HEMOPHILIA AND RARE BLEEDING DISORDERS}

\section{ACQUIRED HEMORRHAGIC COAGULATION DISORDERS}

\section{PB0431 | The Use of Emicizumab for Acquired Hemophilia A at US Hemophilia Treatment Centers}

J. Poston ${ }^{1}$; R. Kruse-Jarres ${ }^{2}$

${ }^{1}$ University of Vermont, Burlington, United States; ${ }^{2}$ University of Washington, Seattle, United States

Background: Acquired hemophilia $\mathrm{A}(\mathrm{AHA})$ is a severe bleeding disorder from an autoantibody against factor VIII (FVIII) with high morbidity and mortality from bleeding and complications from immunosuppression. Outcomes could be improved with adequate hemostatic prophylaxis tailored to the outpatient setting that limits bleeds and allows reduced intensity immunosuppression. Emicizumab, a bispecific antibody that mimics FVIII, has revolutionized prophylaxis for congenital hemophilia A with inhibitors, but the role in AHA is unknown.

Aims: We sought to understand how emicizumab is used for AHA at US hemophilia treatment centers (HTCs).

Methods: One adult hematologist per HTC was surveyed on their center's use of emicizumab for AHA. Pediatric hematologists were excluded given the negligible incidence of pediatric AHA.

Results: Adult hematologists at 87 US HTCs were queried. All 32 respondents reported experience treating AHA; combined, 358 patients with AHA were treated at the 32 HTCs within the last 5 years. 10 respondents (31\%) have given emicizumab to a total of 40 patients with AHA. The majority (90\%) used emicizumab as second line therapy Emicizumab was started for: convenience compared to other hemostatic agents, ongoing bleeding despite other agents, desire to minimize immunosuppression, and failed response to immunosuppression. Most (70\%) were able to delay/decrease immunosuppression after starting emicizumab. Breakthrough bleeding on emicizumab occurred in 2/40 patients. No HTCs discontinued emicizumab for adverse events. HTCs without experience with emicizumab treated fewer patients with AHA in the last 5 years (average 8 vs 17 patients). The majority (86\%) would consider emicizumab if safety data in AHA was available.

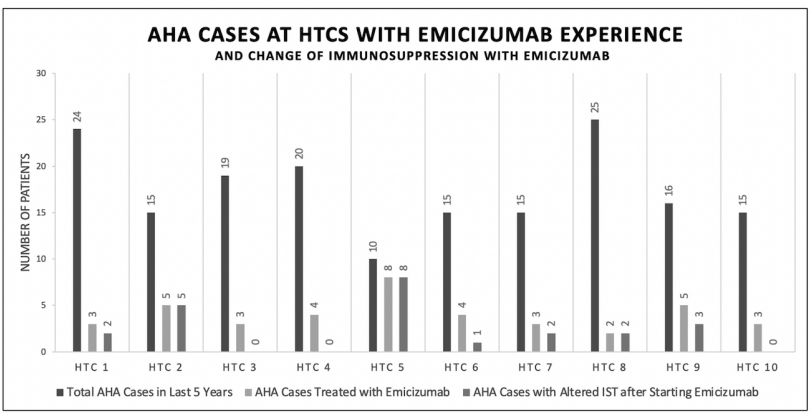

FIGURE 1 AHA Cases at HTCs with emicizumab experience

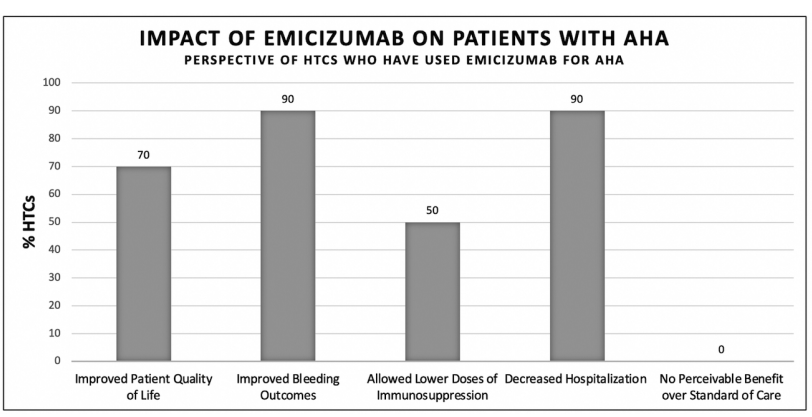

FIGURE 2 Impact of emicizumab on patients with AHA

Conclusions: Emicizumab could radically improve AHA outcomes by providing outpatient hemostatic prophylaxis that reduces bleeding, allows lower intensity immunosuppression, and may reduce costs. A minority of US HTCs are already using emicizumab for AHA. Additional safety and dosing data are needed to clarify the role of emicizumab.

\section{PB0432 | Development of a Computable Phenotype for} Hospital-associated Bleeding: The Medical Inpatients Thrombosis and Hemostasis (MITH) Study

M. Gergi ${ }^{1}$; K. Wilkinson ${ }^{1}$; I. Koh ${ }^{1}$; J. Munger ${ }^{1}$; H. Al-Samkari ${ }^{2}$; N. Smith ${ }^{3}$; N.S. Roetker ${ }^{4}$; M. Cushman ${ }^{1}$; C. Holmes ${ }^{1}$; N. Zakai ${ }^{1}$ ${ }^{1}$ Larner College of Medicine, University of Vermont, Burlington, United States; ${ }^{2}$ Massachusetts General Hospital, Harvard Medical School, Boston, United States; ${ }^{3}$ University of Washington, Seattle, United States; ${ }^{4}$ Hennepin Healthcare Research Institute, Minneapolis, United States

Background: Bleeding events are a critical outcome that must be accurately identified in observational and studies of hospitalized patients. Given major limitations in the use of diagnostic billing codes alone, manual chart review is often required for accurate identification of bleeding events. Validated algorithms are needed for accurate, non-manual identification of bleeding events in electronic health record (EHR) systems. Aims: We developed and preliminary validated an algorithm to detect bleeding in hospitalized patients, ie, a "computable phenotype". Methods: We developed a "computable phenotype", or specialized algorithm, to screen EHR medical records for bleeding events. We included all admissions to the University of Vermont (UVM) Medical Center between 2010-19. Components of the phenotype included international classification of disease (ICD)-9 and ICD-10 discharge diagnoses that were present on admission, laboratory values, current procedure terminology codes, and use of transfusion support. Clinically relevant non-major bleeding (CRNMB) and major bleeding (MB) were defined according to ISTH definitions. To improve accuracy, separate algorithms were developed by bleeding site (Table). Results: 


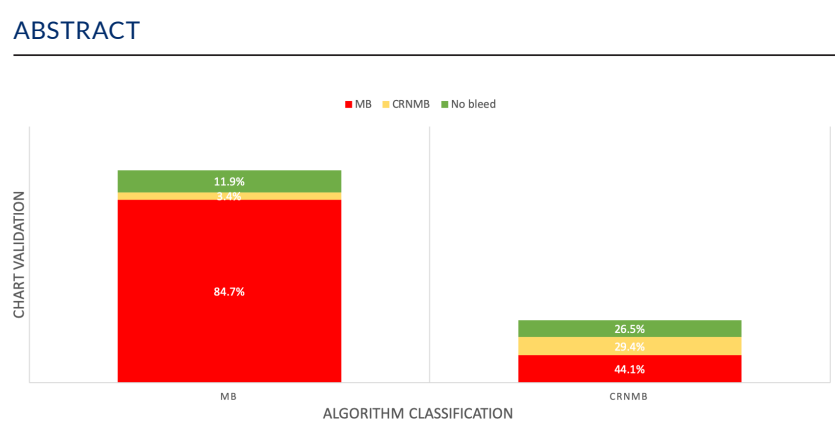

FIGURE 1118 charts identified by the algorithm as MB were reviewed: 100 were confirmed as MB, 4 had CRNMB and in 12 hospitalizations a bleed could not be identified. 34 charts identified by the algorithm as CRNMB were reviewed: 15 of them were labeled as MB, 10 confirmed as CRNMB and in 9 hospitalizations no bleed was identified

TABLE 1 Specific sites of bleeding as detected by Electronic Health Record compared to chart validation

\begin{tabular}{llllllllll} 
& GI & GU & GYN & ICH & Pericardial & Retroperitoneal & Intra-ocular & Nasal & Miscellaneous \\
Total validated & 29 & 14 & 9 & 10 & 7 & 13 & 7 & 10 & 37 \\
Accurate validation & $22(75.9 \%)$ & $6(42.8 \%)$ & $7(77.8 \%)$ & $9(90 \%)$ & $7(100 \%)$ & $9(69.2 \%)$ & $3(42.8 \%)$ & $3(30 \%)$ & $23(62.1 \%)$ \\
$\begin{array}{c}\text { No bleed } \\
\quad \text { identified/ }\end{array}$ & 7 & 4 & 2 & 1 & 0 & 1 & 3 & 5 & 10 \\
$\quad \begin{array}{l}\text { Unclear } \\
\begin{array}{c}\text { Bleeding } \\
\text { associated to a } \\
\text { different site }\end{array}\end{array}$ & 1 & 4 & 0 & 0 & 0 & 3 & 1 & 2 & 4 \\
\hline
\end{tabular}

Among 62,468 admissions, our algorithm identified 10,202 inhospital bleeding events; 4,650 were CRNMB and 5,552 were MB. On manual validation of 150 charts; the algorithm positive predictive validity (PPV) was $86 \%(100 / 116)$ for MB and $29 \%$ (10/34) for CRNMB, with an overall PPV of 84\% (126/150) for any bleeding event. The algorithm performed well for all bleeding sites (Table). Manual review determined that $41 \%(15 / 34)$ CRNMB events were MB events. (Figure).

Conclusions: We developed a computable phenotype for bleeding which can be applied to EHR systems. The computable phenotype was $86 \%$ specific for major bleeding and able to identify the bleeding site. This computable phenotype however, requires further validation and will serve as basis in future studies.

\section{PB0433 | Clinical Characteristics of Acquired Haemophilia A Patients in 5 European Countries}

$\underline{\text { I. Palau }}^{1}$; S. Brown ${ }^{1} ;$ A. Rosli ${ }^{2} ;$ E. Seth ${ }^{2}$

${ }^{1}$ Ipsos, London, United Kingdom; ${ }^{2}$ Ipsos, Kuala Lumpur, Malaysia

Background: Acquired haemophilia $\mathrm{A}(\mathrm{AHA})$ is a rare bleeding phenotype, usually associated with autoimmune disease, where patients experience bleeding with unknown family history of bleeding disorder.

Aims: This study aims to provide an overview of clinical characteristics observed in patients with acquired haemophilia in France, Germany, Italy, Spain and UK.
Methods: Ipsos' Haemophilia Therapy Monitor is a multi-country, multi-centre online medical chart review study of patients with haemophilia $A(H A)$ and haemophilia $B(H B)$ conducted from November 2020 to January 2021. De-identified data on patients in France, Germany, Italy, Spain and UK were collected from treating physicians recruited from an access panel; physicians were screened for duration of practice in their specialty and minimum caseload managed ( 5 $\mathrm{HA}$ and $1 \mathrm{HB}$ moderate-severe patients in the last six months). 183 sampled physicians provided data on 1212 HA patients, of which 97 had AHA.

Results: Across the 97 AHA patients, the main comorbidities were metabolic disorder (46\%), lung and heart disease (19\%) and musculoskeletal conditions (14\%).

$35 \%(n=34)$ of the AHA patients experienced target joints prior to their most recent visit to the haematologist, and reported the most prevalent locations as being knees $(62 \%, n=21)$, elbows $(29 \%$, $n=10)$ and wrists $(21 \%, n=7)$.

$68 \%(n=66)$ of patients experienced bleeds in the last 12 months; spontaneous bleed $(42 \%, n=28)$ and trauma $(38 \%, n=25)$ were the most frequent cause of bleeding reported.

All AHA patients had inhibitors currently detected. Increased dose of factor VIII (48\% of AHA pts) and by-passing agents (46\%) were the main treatment approach in these patients.

Conclusions: Results from this present study highlight the presence of other autoimmune comorbidities, along with bleeds and target joints, experienced by AHA patients. Additional research would yield further understanding of other clinical aspects of this rare bleeding disorder. 
PB0434 | Efficacy of Rituximab for the Treatment of Acquired Hemophilia A: A Systematic Review

S. Moonla ${ }^{1}$; A. Suriyasathaporn ${ }^{1}$; K. Kiatsoonthon ${ }^{1}$;

C. Chai-Adisaksopha ${ }^{1,2}$

${ }^{1}$ Faculty of Medicine, Chiang Mai University, Chiang Mai, Thailand;

${ }^{2}$ Division of Hematology, Department of Internal Medicine, Faculty of

Medicine, Chiang Mai University, Chiang Mai, Thailand

Background: Acquired hemophilia $\mathrm{A}(\mathrm{AHA})$ is a disease characterized by the presence of autoantibody against the factor VIII. Bleeding control and inhibitor eradication are major goals for the treatment of acquired hemophilia A. There is limited data on the rituximab used as an inhibitor eradication.

Aims: To determine the efficiency and safety of rituximab for treating acquired hemophilia $A$.

Methods: We conducted a systematic review and individual patientdata analysis by searching PubMed, EMBASE, and Scopus databases from Inception to February 2021. Studies were included if they were case reports, case series, cross-sectional studies, case-control studies, or cohort studies concerning the utilization of rituximab on the treatment of acquired hemophilia A. We excluded studies that lacked individual patient data. Primary outcomes were a response rate and all-cause mortality.

Results: A total of 105 studies involving 186 patients with acquired hemophilia A were included in the analysis. Males were $56.6 \%$. The median age was 71 years (interquartile range; IQR, 56-78). Median factor VIII clotting activity (FVIII: C) was $0.02 \%$ (IQR 0-2), and the median FVIII inhibitor was 31 Bethesda unit/mL (IQR 10-128). Rituximab was used as a single agent in 23 patients (12.4\%). The rest of the patients received rituximab in combination with either corticosteroid cytotoxic agents, mycophenolate mofetil, or others. Overall response rate was $85.0 \%$ (complete remission $72.6 \%$, partial remission $12.4 \%$ ). Rituximab treated as first-line therapy was associated with a higher rate of complete remission as comparing to second-line therapy, $82.1 \%$ and $64.7 \%$, respectively (Table 1 ). The median time to respond was 76 days (IQR 30-149). Twenty-seven patients (14.4\%) died. Of these, 10 patients died from infectious complications.

TABLE 1 Response comparing line of rituximab therapy

\begin{tabular}{lll} 
& \multicolumn{2}{l}{ Response } \\
\cline { 2 - 3 } therapy & Complete remission & Partial remission \\
First-line therapy & $69(82.14 \%)$ & $5(5.95 \%)$ \\
$\begin{array}{l}\text { Second-line } \\
\text { therapy }\end{array}$ & $66(64.71 \%)$ & $18(17.65 \%)$ \\
\hline
\end{tabular}

Conclusions: This study showed that rituximab monotherapy or in combinations were effective for the inhibitor eradication therapy in patients with acquired hemophilia A. Further prospective studies investigating the efficacy of rituximab in acquired hemophilia are urgently needed.
PB0435 | Hemostatic Evaluation by Global Coagulation Assays in Patients with Acquired Hemophilia A: Analysis of Multi Centers in Japan

M. Takeyama ${ }^{1}$; S. Furukawa ${ }^{1,2}$; K. Yada ${ }^{1,3} ;$ K. Ogiwara $^{1}$; T. Onishi ${ }^{1}$; M. Shima ${ }^{1}$; K. Nogami ${ }^{1}$

${ }^{1}$ Department of Pediatrics, Nara Medical University, Kashihara, Japan;

${ }^{2}$ The Course of Thrombosis and Hemostasis Molecular Pathology, Nara Medical University, Kashihara, Japan; ${ }^{3}$ The Course of Hemophilia Education, Nara Medical University, Kashihara, Japan

Background: The coagulation function and clinical phenotype in acquired hemophilia $\mathrm{A}(\mathrm{AHA})$ of ten do not correlate with the levels of factor VIII activity (FVIII:C) and FVIII inhibitor.

Aims: To evaluate the coagulation potential in AHA by clot waveform analysis (CWA) and thrombin and plasmin generation assays (T/P-GA). Methods: We analyzed the plasma samples from 59 AHA patients by CWA and T/P-GA.

Results: The medians (range) of FVIII:C and FVIII inhibitor titer at the admission were $3.3(<1.0-12.5) \mathrm{IU} / \mathrm{dL}$ and $63.0(2.4-3,872) \mathrm{BU} / \mathrm{mL}$, respectively. The medians clot time and maximum coagulation velocity (|min1|) values calculated by CWA on the day of admission were 110 sec and 1.0, respectively, which were markedly less than those in the normal plasmas (30.2 sec, and 3.6, respectively). The peak thrombin and peak plasmin values calculated by T/P-GA on the day of admission were $24.7 \mathrm{nM}$ and $9.5 \mathrm{nM}$, respectively, which were also markedly less than those in the normal plasmas ( $242 \mathrm{nM}$, and $15.3 \mathrm{nM}$, respectively). Furthermore, we evaluated the coagulation potentials during the clinical course in 39 cases (on the days $0,14,28,56$, and 93). The parameters of CWA were improving according to the elevation of FVIII:C and the decreasing of FVIII inhibitor. The peak thrombin was slowly improving on day 56 but still low comparing to the that of normal plasma. Peak plasmin was recovered to the same value as the congenital severe HA patients $(12.7 \mathrm{nM})$ on day 14 and the values remained the same low level. Peak plasmin was finally recovered to normal level on day 93.

Conclusions: The low values of peak thrombin and peak plasmin at the onset might show the severe bleeding symptom in AHA, and the quick improvement of peak plasmin would suggest the recovery symptom of coagulation potential.

PB0436 | NSAIDs prescription use and subsequent risk of upper gastrointestinal bleeding: a time-trend analysis

A. Bedene; E.L. van Dorp; F.R. Rosendaal; A. Dahan; W.M. Lijfering Leiden University Medical Center, Leiden, Netherlands

Background: The prevalence of nonsteroidal anti-inflammatory drugs (NSAIDs) prescriptions has decreased since 2013 in the Netherlands following the advice of postoperative pain prescription guidelines.

Aims: We aimed to investigate whether the incidence of upper gastrointestinal bleeding-one of the adverse events of NSAIDs 
use - has decreased between 2013-2018 in a nation-wide population study.

Methods: We investigated individual patient data of 17 million residents of the Netherlands, from national statistics on medication prescription, hospital admission and mortality between 2013 and 2018. The cumulative incidence of severe upper gastrointestinal bleeding (either fatal or requiring hospital admission) over two calendar years' time was calculated and the relative risk of severe upper gastrointestinal bleeding was compared between 2018-2017, 2016-2015 and 2014-2013 (reference cohort). Demographic differences were adjusted with logistic regression.
Results: The cumulative incidence of severe upper gastrointestinal bleeding did not decrease throughout the study period (Table 1). A similar result was shown when the analysis was restricted to those who did not receive NSAIDs or antithrombotic agents prescription (age- and sex-adjusted odds ratio, 1.29 [95\% Cl, 1.26-1.32], comparing 2017-2018 with 2013-2014) (Table 1). In a post hoc analysis we observed that the risk of severe upper gastrointestinal bleeding was higher in men than in women in all age groups, except in the young, where women had a 1.5-1.9 fold higher risk than men (Figure 1). The latter suggests an over-the-counter of NSAIDs use, since their use is far more prevalent in young women than men due to alleviate menstrual pain.

TABLE 1 Risk of incident upper gastrointestinal bleeding, the Netherlands, cohorts 2013-2014, 2015-2016, and 2017-2018

\begin{tabular}{|c|c|c|c|c|c|c|}
\hline & Year & No. & Total No. & $\begin{array}{l}\text { Cumulative } \\
\text { incidence, per } \\
100,000 \text { inhabitants } \\
(95 \% \mathrm{Cl})\end{array}$ & $\begin{array}{l}\text { Odds ratio }(95 \% \\
\text { Cl) }\end{array}$ & $\begin{array}{l}\text { Odds ratio }(95 \% \\
\text { Cl)* }\end{array}$ \\
\hline \multirow[t]{3}{*}{$\begin{array}{l}\text { Incident upper gastrointestinal } \\
\text { bleeding }\end{array}$} & 2013-2014 & 34071 & 17112982 & $\begin{array}{l}199.09 \\
\quad(196.99-201.22)\end{array}$ & 1.00 (reference) & 1.00 (reference) \\
\hline & 2015-2016 & 42732 & 17269164 & $\begin{array}{l}247.45 \\
\quad(245.11-249.80)\end{array}$ & $1.24(1.23-1.26)$ & $1.21(1.20-1.23)$ \\
\hline & 2017-2018 & 45516 & 17473459 & $\begin{array}{l}260.49 \\
\quad(258.11-262.89)\end{array}$ & $1.31(1.29-1.33)$ & $1.25(1.24-1.27)$ \\
\hline \multirow{3}{*}{$\begin{array}{l}\text { Incident upper gastrointestinal } \\
\text { bleeding in those without } \\
\text { prescription for NSAIDs }\end{array}$} & 2013-2014 & 23029 & 13018126 & $\begin{array}{l}176.90 \\
\quad(174.63-179.20)\end{array}$ & 1.00 (reference) & 1.00 (reference) \\
\hline & $2015-2016$ & 29562 & 13362796 & $\begin{array}{l}221.23 \\
\quad(218.72-223.76)\end{array}$ & $1.25(1.23-1.27)$ & $1.21(1.19-1.23)$ \\
\hline & 2017-2018 & 32503 & 13737729 & $\begin{array}{l}236.60 \\
\quad(234.04-239.18)\end{array}$ & $1.34(1.32-1.36)$ & $1.26(1.24-1.28)$ \\
\hline \multirow{3}{*}{$\begin{array}{l}\text { Incident upper gastrointestinal } \\
\text { bleeding in those without } \\
\text { either prescription for } \\
\text { NSAIDs nor antithrombotic } \\
\text { agents }\end{array}$} & 2013-2014 & 10837 & 11678227 & $92.80(91.07-94.56)$ & 1.00 (reference) & 1.00 (reference) \\
\hline & $2015-2016$ & 14062 & 11935659 & $\begin{array}{l}117.82 \\
\quad(115.88-119.78)\end{array}$ & $1.27(1.24-1.30)$ & $1.24(1.21-1.27)$ \\
\hline & 2017-2018 & 15338 & 12229473 & $\begin{array}{l}125.42 \\
\quad(123.45-127.42)\end{array}$ & $1.35(1.32-1.39)$ & $1.29(1.26-1.32)$ \\
\hline
\end{tabular}

Abbreviations: $\mathrm{Cl}$, confidence interval; NSAIDs, nonsteroidal anti-inflammatory drugs. All medications were identified through prescription reimbursement data, based on their ATC codes. Upper gastrointestinal bleeding cases were identified based on ICD-10CM codes in the hospital admission and death registry per two calendar years. *adjusted for: age (categorized), and sex. 
A

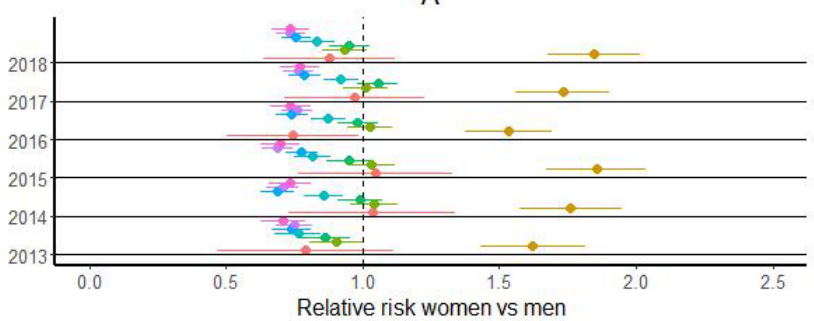

B

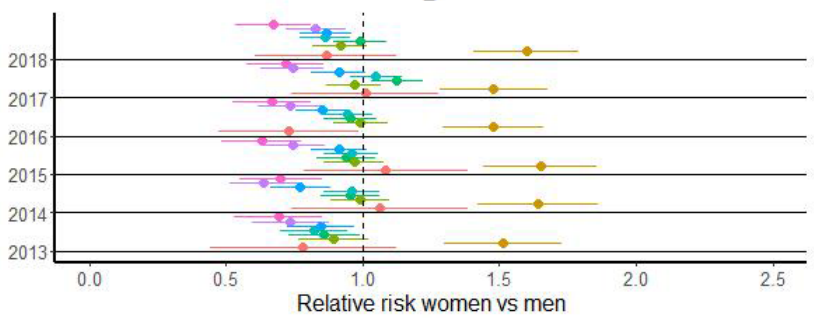

C

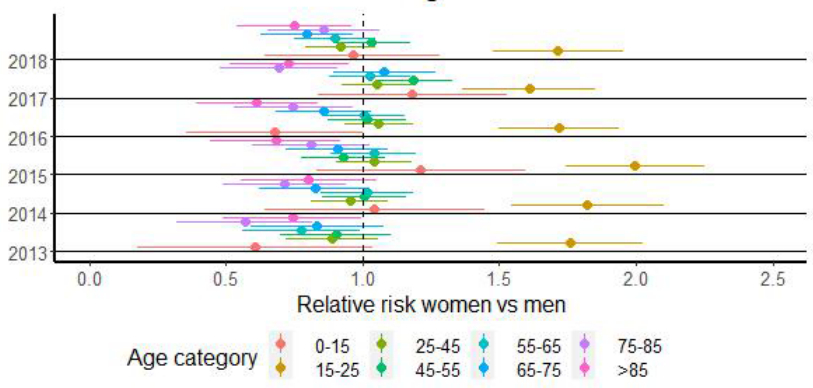

FIGURE 1 An annual time-trend analysis of incident upper gastrointestinal bleeding in three different clinical scenarios stratified by age and sex, the Netherlands, from 2013 to 2018 Figure shows crude relative risk of women vs men of upper gastrointestinal bleeding per calendar year in four different scenarios: A) total population, B) individuals who did not receive a prescription for NSAIDs nor antithrombotic agents, C) individuals without competing risks, which was defined as having received a prescription for NSAIDs (ATC code: M01A), antithrombotic agents (B01A), anticancer medication (L01, L02), systemic corticosteroids (H02A, H02B), drugs for stomach-acid related disorders (A02A, $\mathrm{A} 02 \mathrm{~B}, \mathrm{A02X}$ ), antidepressants (N06A), antihypertensives (C02, $\mathrm{C03}, \mathrm{C07}, \mathrm{C08}, \mathrm{C09}$ ), antidiabetic medication (A10).defined as having received a prescription for NSAIDs (ATC code: M01A), antithrombotic agents (B01A), anticancer medication (L01, L02), systemic corticosteroids ( $\mathrm{HO2} \mathrm{A}, \mathrm{HO2B})$, drugs for stomach-acid related disorders (A02A, A02B, A02X), antidepressants (N06A), antihypertensives (C02, C03, C07, C08, C09), antidiabetic medication (A10)

Conclusions: The decrease in the prevalence of NSAIDs prescriptions has not led to a decrease in the incidence of severe upper gastrointestinal bleeding.

\section{PB0437 | Why Measure von Willebrand Factor Levels for} Biological Follow-up of Acquired Haemophilia A Patients?

M. Coulongeat ${ }^{1}$; T. Baglo ${ }^{2}$; L. Faucheux ${ }^{2}$; C. Vayne ${ }^{2,3}$; F. Maillot ${ }^{1}$; N. Ferreira-Maldent ${ }^{1}$; C. Pouplard ${ }^{2,3}$

${ }^{1}$ Department of Internal Medicine, University Hospital of Tours, Tours, France; ${ }^{2}$ Department of Haemostasis, University Hospital of Tours, Tours, France; ${ }^{3}$ EA 7501, University of Tours, Tours, France

Background: FVIII and von Willebrand factor antigen (vWFAg) levels are similar in healthy subjects and the FVIII/VWF ratio is therefore > 0.7. In acquired haemophilia $A(A H A)$, this ratio decreases during the acute phase and its normalization could predict a favorable outcome. Aims: The objective of our study was to analyses how evolved the FVIII/vWF ratio during the follow-up of AHA patients according to their clinical evolution.

Methods: We retrospectively included 45 AHA patients. Complete remission (CR) was defined in a patient on corticosteroid therapy (CST) receiving $\leq 15 \mathrm{mg} /$ day prednisone without further immunosuppressive therapy (IST) with anti-FVIII $<0.6 \mathrm{BU} / \mathrm{mL}$ and FVIII $>70 \%$. Partial remission (PR) was defined in patients with anti-FVIII $<0.6 \mathrm{BU} / \mathrm{mL}$ and FVIII $>70 \%$, but always on IST. Relapse was defined when anti-FVIII titre became $>0.6 \mathrm{BU} / \mathrm{mL}$ after previous remission. Eight patients were excluded from the final analysis due to insufficient biological data.

Results: Of the 37 patients, 24 showed remission, including 16 with CR and 8 who relapsed after a median of 103 days. Thirteen others did not exhibit remission after a median follow-up of 97 days. Among the 13 patients without initial remission, FVIII levels remained below $15 \%$, while vWFAg levels were above $150 \%$, leading to a dramatic decrease in the FVIII/vWF ratio.

Conversely, in the 16 patients with CR, APTT and FVIII levels normalized over time, as did vWFAg levels (between 150 and 300\% in the acute phase) and the FVIII/vWF ratio.

For the 8 patients with remission followed by relapse, the FVIII/ vWF ratio remained lower during the remission (ratio $=0.556$ [0.248-0.985]), and then decreased significantly during the relapse (ratio $=0.023$ [0.002-0.17]; $P=0.029$ ).

Conclusions: Our study shows that the evolution of the FVIII/vWF ratio during $\mathrm{AHA}$ is highly predictive of the clinical course. A decrease of the ratio could be a sensitive marker of relapse in patients with an apparent remission of AHA. 
PB0438 | Incidence Comparison and The Antiplatelet Effect on Bleeding Risk Between Chronic Kidney Disease Undergoing Haemodialysis and Peritoneal Dialysis: A Meta-analysis of Cohort Studies

\section{B.P. Putra ${ }^{1}$; F.N. Putra ${ }^{2}$}

${ }^{1}$ Medical Doctor, Blitar, Indonesia; ${ }^{2}$ Faculty of Medicine Universitas Airlangga, Surabaya, Indonesia

Background: Chronic kidney disease (CKD) patients undergoing haemodialysis (HD) and peritoneal dialysis (PD) have increased bleeding risk based on the previous limited studies. The HD patients tend to have higher bleeding risk compared with PD patients, although the results were still inconclusive. Almost CKD patients receive antiplatelet therapy that affect the bleeding risk, yet the result is inconsistent.

Aims: This study aims to measure the incidence comparison and the platelet effect on bleeding risk between HD and PD patients.

Methods: We did both hand-searching and comprehensive literature searching in online databases of Pubmed, ScienceDirect, and The Cochrane Library, to include all relevant studies from 2000 until 2021. This study follows the PRISMA guideline. We included all cohort studies that compare and access the bleeding risk between HD and PD patients. Bias risk were accessed by using The NewcastleOttawa Scale for cohort study. We performed analysis to provide pooled risk ratio (RR) with $95 \%$ confidence interval ( $\mathrm{Cl}$ ) using fixedeffect heterogeneity test.

Results: We included 5 cohort studies that met our inclusion criteria. Patients undergoing HD have significant incremental upper gastrointestinal bleeding (GIB) risk compared with the PD patients (pooled RR $=1.24,95 \% \mathrm{Cl} 1.12-1.37, P<0.0001, \mathrm{I}^{2}=0 \%$ ). The overall bleeding risk also showed that HD patients have significantly in creased bleeding risk when combined with other bleeding events, such as lower GIB and non-traumatic subdural haemorrhage (pooled $\mathrm{RR}=1.27,95 \% \mathrm{Cl} 1.16-1.38, P<0.00001, \mathrm{I}^{2}=0 \%$ ). The antiplatelets administration slightly increase the bleeding risk among CKD patients although the result is no statistically significant (pooled $\mathrm{RR}=1.05,95 \% \mathrm{Cl} 0.87-1.26, P=0.60, \mathrm{I}^{2}=0 \%$ ).

Conclusions: The HD patients have increased bleeding risk compared with PD patients and the antiplatelet use does not increase the bleeding risk among CKD patients. However, further studies are needed to establish the causalities.
PB0439 | Rutin Succinate Acts as an Anticoagulant and Inhibits Snake Venom Metalloproteinases in an Acute Thrombohemorrhagic Disorder Model

A.T.A. Sachetto; M.L. Santoro

Instituto Butantan/Faculdade de Medicina da Universidade de São

Paulo, São Paulo, Brazil

Background: Rutin is an anti-inflammatory and anticoagulant flavonoid, which inhibits protein disulfide isomerase (PDI). The venom of the Brazilian pit viper (Bothrops jararaca) is a complex mixture of components, in which snake venom metalloproteinases (SVMP) distinguishes themselves in abundance and high toxicity, and are involved hemostatic disturbances evoked by snakebites. SVMP present procoagulant, proinflammatory, hemorrhagic and lethal activities, and therefore molecules that inhibit these activities are important to improve the treatment of thrombo-hemorrhagic diseases, as snakebite envenomation.

Aims: To investigate the effects of rutin succinate, a water-soluble form of rutin, on coagulation parameters and venom activities, in vitro and in vivo.

Methods: Procedures involving animals were approved by IACUC. The activity of rutin succinate was evaluated on coagulation tests using murine plasma or bovine fibrinogen, on the reducing activity of PDI, and also on proteolytic and coagulant activities of the venom in vitro. In vivo, venom was injected i.p. in C57BL/6 mice alone or pre-incubated with rutin succinate or o-phe (1,10-phenantroline, an SVMP inhibitor) to address the potential of rutin succinate to inhibit the toxic and lethal activities of the venom in vivo.

Results: Rutin succinate prolonged prothrombin time, activated partial thromboplastin time and thrombin time and interacted with PDI and fibrinogen, altering their structures and activities. Rutin succinate was more effective than o-phe to inhibit SVMP, decreasing venom procoagulant activity on plasma or fibrinogen, and venom proteolytic activity on gelatin, casein and fibrinogen. SVMP inhibition by rutin succinate or o-phe completely prevented venominduced lethality and hemorrhage. Nevertheless, rutin succinate showed a much broader effect, and also improved venom-induced thrombocytopenia and hypofibrinogenemia in mice.

Conclusions: Rutin succinate is a water-soluble compound from rutin, showing anticoagulant and snake venom metalloproteinasesinhibiting properties. It has a great potential as a treatment for snake envenomation, and possibly other thrombo-hemorrhagic diseases. Funding: FAPESP, CNPq, CAPES, Fundação Butantan. 
PB0440 | Life-threatening Bleeding in Acquired Factor V Inhibitor Can Be Controlled Combining Platelet Transfusion and Recombinant Activated Factor VII Administration

V. Robles; C.A. Dávalos; L.M. Serrano; J. Torres; R. Fernández;

P. Chamorro; M.P. Chávez; C. Fernández

Hematology Department. Hospital Universitario de Cabueñes, Gijón, Spain

Background: Acquired factor (F) $\vee$ deficiency (AFVD) is a rare and potentially fatal coagulopathy with limited reported cases. Treatment aims to control bleeding and autoantibody eradication. Control of bleeding is challenging as commonly used hemostatic agents are not useful due to the low FV content. Herein we present a case of AFVD which combined use of recombinant activated factor VII (rFVIla, Novoseven ${ }^{\circledR}$; off-label use) and platelet transfusion (PTT) were effective to control a life-threatening hemorrhage.

Aims: Description of the use of combined therapy with PTT (as FVsource) and rFVIla to support hemostatic effect to control bleeding in a severe case of AFVD.

Methods: Data were obtained prospectively after diagnosis and consent of the patient.

Results: A 77-year-old male with cirrhosis and several comorbidities presented severe hematuria, hemoglobin $(\mathrm{Hb})=10.9 \mathrm{~g} / \mathrm{dL}$, significant prothrombin time and activated partial thromboplastin time (aPTT) prolongation, normal fibrinogen plasma levels and negative lupus anticoagulant (Table 1). FV levels in the mixed test was $0 \%$ with a $2.4 \mathrm{BU} / \mathrm{mL}$ inhibitor titer. rFVIla $70 \mu \mathrm{g} / \mathrm{kg} / 3 \mathrm{~h}$ and methylprednisolone at a dose of $1.5 \mathrm{mg} / \mathrm{kg} /$ day were started. After 6 days of treatment and persistence of bleeding, PTT ( 1 pool/12 h) was administrated (5 pools in total). Hematuria disappeared after $48 \mathrm{~h}$ of PTT, stabilizing $\mathrm{Hb}$ thus PTT was suspended and gradual decrease of rFVIla was also initiated until suspension 2 days later. At discharge, INR remained increased, with normal aPTT ratio and fibrinogen but $0.87 \mathrm{BU} / \mathrm{mL}$ inhibitor titer. After 18 days of corticosteroid therapy, inhibitor disappeared, and tapering was initiated. Patients is currently asymptomatic on prednisone tapering and oral vitamin $\mathrm{K} / 24 \mathrm{~h}$. INR (1.44) remains prolonged and $\mathrm{FV}=53 \%$
Conclusions: PTT combined with rFVIla hemostatic support were effective to control severe bleeding in a case of AFVD. Early use of PPT as FV source might improve outcome in this rare acquired condition.

PB0441 | To Treat or Not? Remission Induction of Acquired von Willebrand Syndrome Secondary to Chronic Lymphocytic Leukemia: A Case Report

R. Khalife ${ }^{1}$; A. Aw ${ }^{1}$; L. Duffett ${ }^{1}$; O. Radhwi ${ }^{1}$; L. Lacasse ${ }^{1}$;

L. Huebsch ${ }^{1}$; M. Bowman ${ }^{2}$; P. James ${ }^{2}$; A. Tinmouth ${ }^{1}$

${ }^{1}$ Department of Medicine (Hematology) and The Ottawa Hospital, University of Ottawa, Ottawa, Canada; ${ }^{2}$ Department of Medicine, Queen's University, Kingston, Canada

Background: Acquired von Willebrand syndrome (AVWS) is an underdiagnosed bleeding disorder that can be associated with hematological malignancies, such as chronic lymphocytic leukemia (CLL). The management of AVWS remains empirical and relies heavily on dissemination of clinical experiences.

Aims: We present an elderly male with asymptomatic CLL who developed AVWS. Our objective is to highlight the importance of treating the underlying cause despite the lack of other guideline-driven treatment indications.

Methods: Case report.

Results: A 92-year-old male presented with recurrent epistaxis requiring hospitalization. In the preceding 18-month period, he had 16 visits to hospital with mucocutaneous bleeding, primarily epistaxis, without prior personal or family history of bleeding. Two years prior to this presentation, he was diagnosed with asymptomatic, Rai stage 0, CLL. Laboratory testing showed a low VWF antigen (VWF:Ag; $0.06 \mathrm{U} / \mathrm{mL} ; \mathrm{N}: 0.40-1.75)$, undetectable VWF activity $(<0.1 \mathrm{U} / \mathrm{mL}$, $\mathrm{N}: 0.45-1.80$ ), low factor VIII level (FVIII; $0.13 \mathrm{U} / \mathrm{mL}, \mathrm{N}: 0.50-1.49$ ), a significantly elevated VWF propeptide to antigen ratio (VWFpp/ VWF:Ag) of 17.0 (N:0.9-1.45), and weakly positive anti-VWF total IgG antibodies. Daily infusions of plasma-derived VWF concentrate

TABLE 1 Laboratory parameters; PT: prothrombin time, aPTT: activated partial thromboplastin time, FV: factor V, UNK: unknown value

\begin{tabular}{|c|c|c|c|c|c|c|c|c|}
\hline Parameter & At admission & Day +1 & Day +2 & Day +4 & Day +9 & Day +12 & Day +18 & Day +25 \\
\hline $\mathrm{Hb}(\mathrm{g} / \mathrm{dL})$ & 16.1 & 13.4 & 11.5 & 10.9 & 10.8 & 11.6 & 13.8 & 14.8 \\
\hline $\begin{array}{l}\text { Platelets }\left(\times 10^{3} /\right. \\
\qquad \mu \mathrm{L})\end{array}$ & 168 & 154 & 159 & 206 & 178 & 111 & 82 & 81 \\
\hline aPTT (s) & 214 & 195 & 111 & 86 & 49 & 42 & 31 & 27 \\
\hline PT (s) & 68 & 58 & 39 & 38 & 25 & 27 & 21 & 18 \\
\hline $\begin{array}{l}\text { Fibrinogen (mg/ } \\
\quad \mathrm{dL} \text { ) }\end{array}$ & 432 & 459 & 401 & 312 & 405 & 443 & 297 & 208 \\
\hline FV (\%) & UNK & UNK & 2 & 3 & 10 & 11 & 24 & 53 \\
\hline $\begin{array}{l}\text { Inhibitor titer } \\
\qquad(\mathrm{BU} / \mathrm{mL})\end{array}$ & UNK & UNK & 2.4 & 3.6 & 0.5 & 0.9 & 0.0 & UNK \\
\hline
\end{tabular}


helped control his bleeding temporarily. The patient failed a trial of corticosteroids and IVIG. Therefore, the clinical decision was to treat his CLL with Obinutuzumab and Chlorambucil for 6 cycles, which resolved his bleeding symptoms and lymphocytosis. Upon treatment completion, testing showed normalization in VWF:Ag (1.14 U/mL), VWF activity (1.33 U/mL), FVIII (1.50 U/mL), and VWFpp/VWF:Ag ratio (0.9). Twenty-two months after therapy, he remains in remission without evidence of AVWS or lymphocytosis.

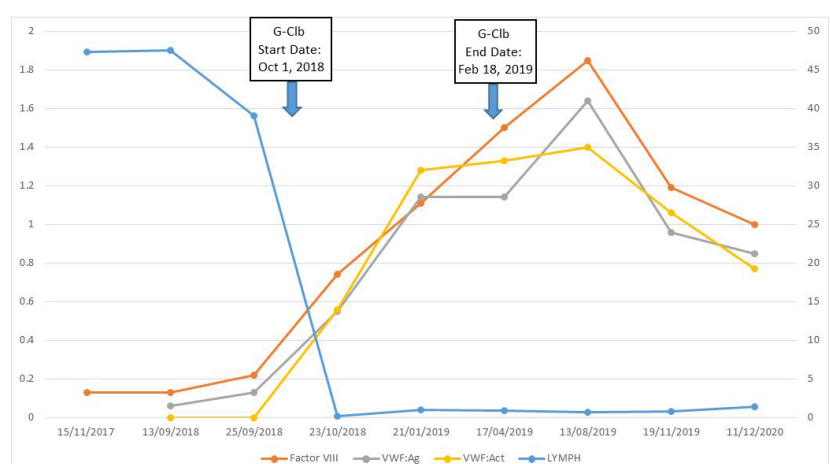

FIGURE 1 Graphic representation of FVIII (IU/mL), von Willebrand factor antigen (VWF:Ag; IU/mL), and von Willebrand factor activity (VWF:Act; IU $/ \mathrm{mL}$ ) in association with the absolute lymphocyte count (LYMPH; x 109/L) and Obinutuzumab and Chlorambucil (G$\mathrm{Clb})$ chemoimmunotherapy treatment

Conclusions: This is the first report demonstrating remission induction in both AVWS and CLL following therapy with Obinutuzumab and Chlorambucil. We suspect Obinutuzumab played a particular role in inducing remission compared to corticosteroids and IVIG. In the setting of asymptomatic hematologic malignancies, the benefit of treating AVWS with chemoimmunotherapy may outweigh the risks as it can reduce significant bleeding-related morbidity.

PB0442 | Acquired Haemophilia A - Data from a Regional Blood Center in Rio de Janeiro, Brazil

$\underline{\text { P.H.J. Santos }}^{1}$; M.H. Cerqueira ${ }^{1}$; M.M. Loureiro ${ }^{2}$

${ }^{1}$ HEMORIO, Rio de Janeiro, Brazil; ${ }^{2}$ Federal University of Rio de Janeiro, Rio de Janeiro, Brazil

Background: Acquired haemophilia $\mathrm{A}(\mathrm{AHA})$ is a rare severe condition that occurs in people with variable age. Neutralizing autoantibodies are formed against factor VIII (FVIII), causing spontaneous or trauma-induced bleeding. Immunosuppressive therapy (IST) results in remission in most patients despite high morbidity and mortality.

Aims: Long-term follow-up data from the haemophilia centre of Rio de Janeiro (HEMORIO) were analysed to assess baseline characteristics and prognostic factors in patients with AHA.

Methods: This is a retrospective cohort study in which all patients with laboratory confirmed AHA admitted to HEMORIO, the regional public blood center located in Rio de Janeiro, Brazil, were included.
AHA was defined as FVIII inhibitor titres (IT) $\geq 0.6$ Bethesda Units (BU) and levels of FVIII $<50 \mathrm{UI} / \mathrm{dL}$. Complete remission (CR) was defined as FVIII inhibitor titres $<0.6 \mathrm{BU}$. Major bleeding was defined according to the ISTH criteria.

Results: Between 1990 and 2020, 35 patients met the inclusion criteria. Mean age was 58 years, and $51 \%$ was female. No underline cause was found in $82 \%$ of them. Major bleeding occurred in $31 \%$, and haemostatic treatment was given to $82 \%$ patients. All subjects underwent IST, and monotherapy with oral steroids was the treatment of choice (71\%). In $75 \%$ of the patients, pre-treatment IT was $>20 \mathrm{BU}$, and $57 \%$ had FVIII levels $<1 \%$. Seventeen per cent did not achieve CR and, among those, $83 \%$ had initial IT > 20 BU and $66 \%$ had initial FVIII levels $<1 \%$. No one died during observation time.

Conclusions: AHA is a rare disease. In the present study, most of patients who did not achieve CR had high IT and very low FVIII levels, which suggests the prognostic role of these tests.

PB0443 | Acquired Hemophilia A Presenting as Spontaneous Intramuscular Hematomas

S. Ahmed; E. Joseph; K. Loughry

Advocate Christ Medical Center, Oak Lawn, United States

Background: Acquired Hemophilia $\mathrm{A}(\mathrm{AHA})$ is a rare autoimmune bleeding disorder where autoantibodies are directed against clotting factors, most commonly factor VIII. The etiology is poorly understood but it is most commonly associated with pregnancy/postpartum status, malignancy and other autoimmune disorders.

Aims: Case Presentation.

Methods: Our patient is an 84-year-old female with a history of hypertension (HTN), hyperlipidemia (HLD) and left total hip arthroplasty (THA), who presented with progressively worsening pain and bruising to her left posterolateral thigh of 5 weeks duration. Her laboratory studies were significant for Partial Thromboplastin Time (PTT) of 80, hemoglobin of 5.7, platelet count of 650,000 and C reactive protein of 6.3. Computed Tomography of the Pelvis and lower extremity revealed multiple low-attenuation, large hematomas (range: 7-8 cm), which were evidently expanding muscle groups of the posterior thigh, triggering concerns for compartment syndrome. Results: The workup for the elevated PTT resulted in a positive mixing study, a low factor VIII assay, and an elevated factor VIII inhibitor level using the Bethesda assay. A clinical diagnosis of Acquired Autoimmune Hemophilia A was established. Treatment was then initiated with low-dose corticosteroids and Cyclophosphamide. Factor VIII inhibitor levels peaked at 124 Bethesda Units (BU). Subsequently, Rituximab was added due to first-line treatment refractoriness. Factor VIII inhibitor levels then decreased to $24 \mathrm{BU}$ and continued to downtrend. 


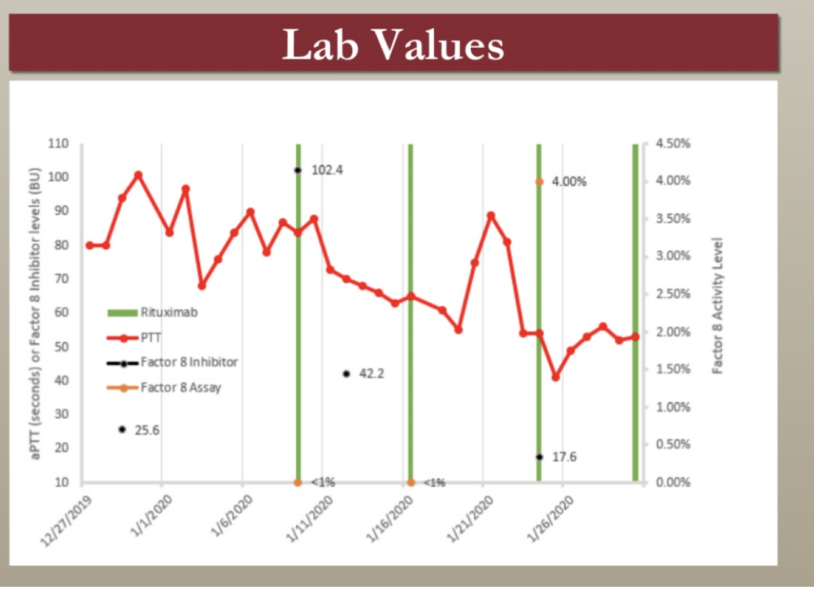

FIGURE 1 Factor 8 Activity vs. Inhibitor Levels Throughout Admission

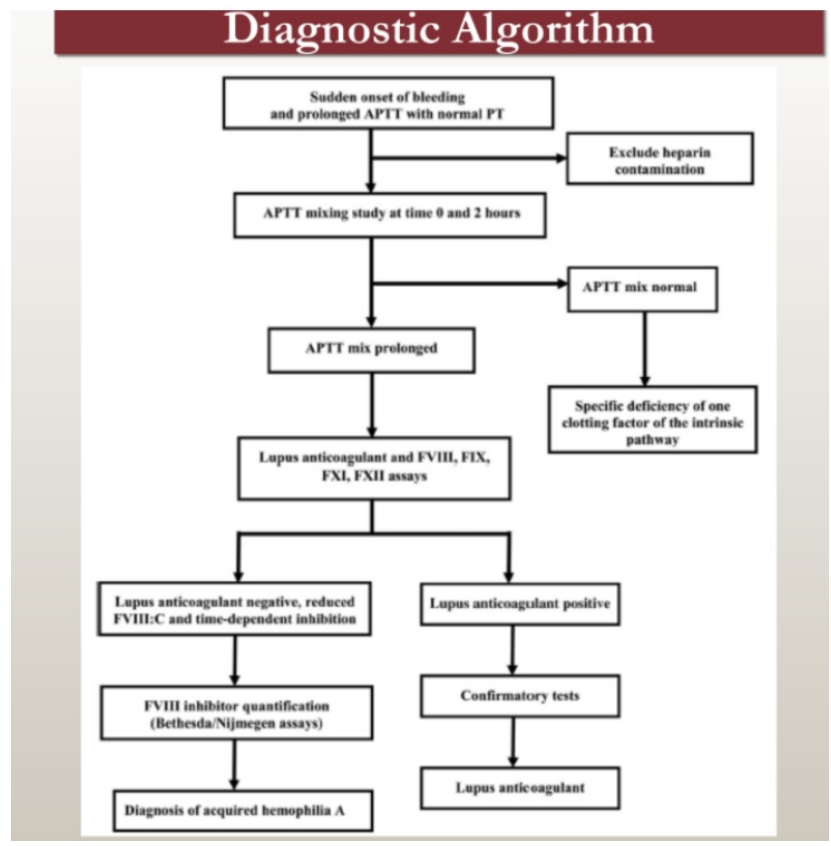

FIGURE 2 Diagnostic Algorithm of Acquired Hemophilia A

Conclusions: Acquired hemophilia $\mathrm{A}$ is a rare autoimmune disorder with uncertain etiology. One of the major complications resulting from factor VIII assay activity less than $1 \%$ is spontaneous hematoma formation. Improvements in Factor VIII assay typically will not be seen until inhibitor levels are below $10 \mathrm{BU}$. Interestingly, the observed $80 \%$ decline in the inhibitor level occurred after only one dose of Rituximab, suggesting a possible synergistic effect between Cyclophosphamide and corticosteroid. There are currently no definitive guidelines governing treatment of $\mathrm{AHA}$.
PB0444 | Refractory Acquired Hemophilia A (Even More) Complicated with SARS-CoV-2 Infection

C. Gomez del Castillo Solano; M. Fernández Docampo;

T. Lado Cives; M.T. Fernández Fernández; D. Martínez Señarís;

A. Hevilla Carmona; M.F. López Fernández

Complexo Hospitalario Universitario A Coruña, A Coruña, Spain

Background: Acquired hemophilia A (AHA) is caused by autoantibodies against coagulation factor VIII (FVIII). Treatment focuses on both eradicating the inhibitor and treating and preventing bleeding episodes. SARS-CoV-2 infection causes a high mortality hipercoagulable state.

Aims: Review AHA case with high risk of bleeding and thrombotic complications.

Methods: We present a patient with refractory AHA treated with immunosupression, SARS-CoV-2 infection and iliopsoas hematoma treated with recombinant porcine FVIII (rpFVIII).

Results: 73 year old male was admitted because of ecchymosis, hematuria and anemia ( $\mathrm{Hb} 8.5 \mathrm{~g} / \mathrm{dL}$ ). Coagulation tests showed APTT ratio 2.12, FVIII:C $1.8 \mathrm{UI} / \mathrm{dL}$ and anti-FVIII $156 \mathrm{BU}$. He was diagnosed with AHA and put on rFVIla, prednisone and rituximab. Three weeks later he developed severe respiratory insuffiency, bilateral pneumonia and severe acute immflamatory response with a positive PCR test for SARS-CoV-2. Last dose of rituximab was postponed and treatment with metilprednisolone, tocilizumab, lopinavir/ritonavir, hidroxicloroquine and prophylactic heparin (LMWH) was started. He was enrolled on a clinical trial with remdesivir. 4 days later he related acute pain on the right hip with inability to walk. CT scan showed iliopsoas hematoma. Due to high thrombotic risk of using bypass agents, a request for rpFVIII (susoctocog alfa - Obizur ${ }^{\circledR}$ ) was made. After SARS-CoV2 infection was resolved, treatment with mofetil mycophenolate was started (no response to previous treatment). Inhibitor title is $<0.4 \mathrm{~B}$. FVIII levels remain above $50 \mathrm{UI} / \mathrm{dL}$

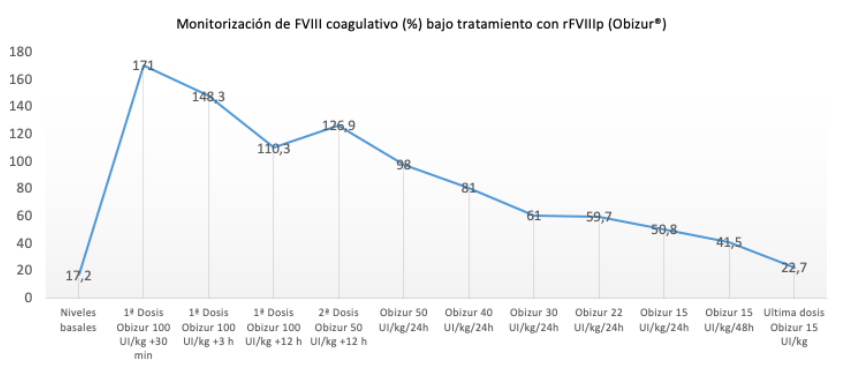

FIGURE 1 FVIII levels while treatment with rpFVIII

Conclusions: Patients with AHA and SARS-CoV-2 infection are at high risk of bleeding and thrombotic events, complicating treatment with bypass agents.

RpFVIII, allows to extend time between infusions, to monitor levels of FVIII. It is a good option to treat bleeding episodes without adding thrombotic risk.

After tocilizumab administration a reduction on the inhibitor title was seen. It may be have a roll in refractory AHA. 
The use of LMWH is not well stablished for patients with Covid19 and $\mathrm{AHA}$.

TABLE 1 Clinical manifestations and laboratory diagnosis

\begin{tabular}{|c|c|c|}
\hline & Patient $\mathrm{N}^{\circ} 1$ & Patient $\mathrm{N}^{\circ} 2$ \\
\hline Age (years) /sex & $42 \mathrm{~F}$ & $27 \mathrm{~F}$ \\
\hline $\begin{array}{l}\text { Evolution } \\
\text { (months) }\end{array}$ & 2 & 4 \\
\hline History & $\begin{array}{l}\text { Bronchial } \\
\text { asthma } \\
\text { G3P3 }\end{array}$ & $\begin{array}{l}\text { No history } \\
\text { G1P1 }\end{array}$ \\
\hline $\begin{array}{l}\text { Bleeding } \\
\text { symptoms }\end{array}$ & $\begin{array}{l}\text { Extensive } \\
\text { ecchymosis }\end{array}$ & $\begin{array}{l}\text { Extensive ecchymosis } \\
\text { Gingivorrhagia } \\
\text { Right Knee hemarthrosis }\end{array}$ \\
\hline $\begin{array}{l}\text { APTT }(\mathrm{T}) \mathrm{sec} / \\
\text { rosner index }\end{array}$ & $59(31) / 15$ & $62(29) / 16$ \\
\hline FVIII or IX & VIII: $0.5 \%$ & VIII<1\% \\
\hline $\begin{array}{l}\text { Titer of inhibiteur } \\
\text { UB }\end{array}$ & 19.2 & 83 \\
\hline
\end{tabular}

\section{Patient $\mathrm{N}^{\circ} 3$}

$36 \mathrm{~F}$

immediate post-partum

No history

G1P1

Extensive ecchymosis
Hematoma
Hemoperitunium
81.5(30)
VIII:1\%

Extensive ecchymosis

32

\section{Patient $\mathrm{N}^{\circ} 4$}

$18 \mathrm{~F}$

8

Aopecia

PRP injection

\section{G5P3}

2 abortion

Extensive ecchymosis

Muscle Hematoma

Hemoperitineum

$73(31) / 27.5$

$69(31) / 27.4$

VIII:2\%

IX: $23 \%$

VIII: $234 \%$

PRP: platelet-rich plasma, F: Female.

TABLE 2 Etiology and managment of acquired hemophilia

\begin{tabular}{|c|c|c|c|c|c|}
\hline & Patient $\mathrm{N}^{\circ} 1$ & Patient $\mathrm{N}^{\circ} 2$ & Patient $\mathrm{N}^{\circ} 3$ & Patient $\mathrm{N}^{\circ} 4$ & Patient $\mathrm{N}^{\circ} 5$ \\
\hline Substantive treatment & Corticoisteroid & Corticoisteroid & $\begin{array}{c}\text { Corticoisteroid / } \\
\text { Anti-CD20 }\end{array}$ & Corticoisteroid & Corticoisteroid \\
\hline Evolution & $\begin{array}{l}\text { No bleeding } \\
\text { NormaL APTT } \\
\text { ACC(-) }\end{array}$ & $\begin{array}{l}\text { No bleeding } \\
\text { NormaL APTT } \\
\text { ACC(-) }\end{array}$ & $\begin{array}{l}\text { No bleeding } \\
\text { NormaL APTT } \\
\text { ACC(-) }\end{array}$ & $\begin{array}{l}\text { No bleeding } \\
\text { NormaL APTT } \\
\text { ACC(-) }\end{array}$ & $\begin{array}{l}\text { No bleeding } \\
\text { Prolonged APTT (8 } \\
\text { months) }\end{array}$ \\
\hline
\end{tabular}

Conclusions: Acquired postpartum hemophilia A represents the most frequent etiology in our series; the time to onset is very variable, ranging from the immediate postpartum up to 4 months.
Despite its rarity, we diagnosed acquired hemophilia B from the postpartum period, the aetiological context found several immune disorders (autoimmune hemolytic anemia, sarcoidosis).the value of 
a multidisciplinary collaboration in its management and an adequate etiological treatment

PB0446 | Acquired Hemophilia after Chemotherapy: Oxaliplatin and Capecitabine, a Case Report

M. Mijares; K. Pena; L. Carmona; V. Vega; L. Puente; S. Aranda

Hospital San Juan de Dios, Santiago, Chile

Background: Acquired hemophilia $\mathrm{A}(\mathrm{AHA})$ has been described in elderly patients, pregnancy, puerperium, autoimmune disorders and associated to cancer. Some reviews report $\mathrm{AH}$ after drugs. We present a case of AHA secondary to chemotherapy, oxaliplatin and capecitabine, a 62-year-old female, who in May 2010 was admitted with septic shock due to perforated sigmoid colon tumor, multiorgan failure and activated partial thromboplastin test (APTT) 43.9 (normal 23.6-35.0 seconds). She received fresh frozen plasma and cryoprecipitate with improvement. Laparotomy with colostomy was made and biopsy reported: moderately differentiated T3 sigmoid adenocarcinoma, N1a, which invaded to pericolic adipose and lymphovascular tissue, surgical margins free of neoplasm, positive lymph nodes: 1 of 13. Computed tomography (CT) without signs of secondary spread. The oncology committee decides to administer chemotherapy, XELOX, oxaliplatin and capecitabine, she received 3 cycles. Three weeks after the last cycle, she presented spontaneous bruising and ecchymoses in the extremities, doppler eco: myofascial tear between soleus and gastrocnemius with hematic collection in lower right limb. APTT: 57.4 seconds without correction, Factor VIII 3\% (normal 50-150\%) and FVIII inhibitor titer 8 BU. She was treated with prednisone $1 \mathrm{mg} / \mathrm{kg} / \mathrm{day}$ and cyclophosphamide $1.5 \mathrm{mg} / \mathrm{kg} /$ day, for 4 weeks, together with activated recombinant Factor VII $90 \mu \mathrm{g} / \mathrm{kg}$ every 6 hours/ 1 day, then due to low availability of agents, activated prothrombin complex concentrate was indicated, doses: $62 \mathrm{U} / \mathrm{kg} /$ day, for 4 days. She had good clinical and laboratory evolution. A new CT scan and bone scintigraphy were performed without evidence of neoplasia; because of this we think that she had an AHA secondary to chemotherapy, oxaliplatin and capecitabine and that situation has not been reported.

Aims: Present a acquired hemophilia case report.

Methods: Review of clinical and laboratory date.

Results: We are presenting an acquired hemophilia induced by chemotherapy.

Conclusions: We have an hemophilia acquired secondary to chemotherapy, oxaliplatin and capacitabine, no reported so far.
PB0447 | Acquired Hemophilia A - Misdiagnosis and Possible Hazards: A Case Report

D. Carneiro-Leão ${ }^{1}$; C. Santos ${ }^{1}$; J. Sousa Silva ${ }^{2}$; F. Correia ${ }^{1}$;

S. Nobre Fernandes ${ }^{1}$; M. Carvalho ${ }^{1}$; M. Lopes ${ }^{1}$; C. Koch ${ }^{1}$

${ }^{1}$ Congenital Coagulopathies Reference Centre, Centro Hospitalar

Universitário de São João, E.P.E., Porto, Portugal; ${ }^{2}$ Department of Immunohemotherapy, Hospital Central do Funchal, Funchal, Portugal

Background: Acquired hemophilia $\mathrm{A}(\mathrm{AHA})$ is a rare bleeding disorder due to development of inhibitors against factor VIII which leads to bleeding. AHA tends to occur in elderly patients with comorbidities and polypharmacy, and it is underdiagnosed/misdiagnosed in real-world clinical practice.

Aims: A case-report of AHA initially misdiagnosed.

Methods: A 74-years-old man came to the emergency department on December 2020, with a $48 \mathrm{~h}$ history of right thigh pain, local tenderness, swelling, redness and induration. He had history of trauma of right knee 5 days before, without visible wound. He also reported asthenia, dizziness and hematuria episodes. A diagnosis of deep venous thromboembolism (DVT) was assumed and treatment with anticoagulation proposed, but not started, as hemoglobin $(\mathrm{Hb})$ was $5.3 \mathrm{~g} / \mathrm{dL}$ and patient received 2 red blood cell (RBC) units. The ultrasound of thigh pointed towards a cellulitis, and ceftriaxone has been started. Due to the uncertainty of the diagnosis, a venous ecodoppler was performed and revealed absence of DVT. In parallel, urology discarded that mild hematuria was the cause of this severe anemia.

The patient was admitted and on day 4 , he received 2 more RBC units due to $\mathrm{Hb}: 6.3 \mathrm{~g} / \mathrm{dL}$. On same day, a routine coagulation profile showed an isolated prolonged aPTT: 95.7", which did not correct in mixing tests. Further study revealed FVIII level $<1 \%$ and 5 BU of FVIII inhibitor. A subsequent careful physical exam exposed several hematomas (torso, face, arms and right thigh).

Results: Patient was treated with prednisolone $(1 \mathrm{mg} / \mathrm{Kg} /$ day) and bypassing agent (aPCC $50 \mathrm{U} / \mathrm{Kg}$ tid, and then bid until $\mathrm{Hb}$ stabilization) and was discharged on day 14 (FVIII: $23 \%$ and $\mathrm{Hb}: 9.2 \mathrm{~g} / \mathrm{dL}$ ).

Conclusions: Although AHA is rare, medical teams should consider this diagnosis, facing exacerbated symptoms due to mild trauma. As this patient was admitted in hard pandemic times, maybe overload of work was an issue to delay diagnosis. 


\section{PB0448 | Acquired Hemophilia A: Pediatric Case Report}

L. Velazquez Marmolejo; A.M. Moreno Gonzalez; L.M. Munoz Juarez Diaz; M.J. Garcia Tevera; B.L. Ventura Lopez; García Tevera María José, Moreno González Aida Mashenka, Muñoz Juárez Díaz Lucia Mariana, Velázquez Marmolejo Lizette, Ventura López Brenda Lissette.

Hospital Infantil de Mexico Federico Gomez, Ciudad de México, Mexico

Background: Acquired hemophilia $\mathrm{A}$ is a disorder of rare entity, resulting in spontaneous bleeding in individuals with no history of bleeding disorders. Symptoms in general are related to spontaneous bleeding in the skin, muscles, soft tissues and mucous membranes. The most common type is secondary to coagulation factor VIII in hibitors. It is a disease with a high mortality rate without adequate treatment. The basis of the diagnosis is the detection of low concentrations of FVIII and the presence in plasma of an inhibitor whose action depends on the time. Acquired hemophilia typically presents in middle age and beyond. It rarely arises in childhood we report the case of a 16-year-old female patient in hospital Infantil de México Federico Gomez.

Aims: Present a rare clinic case in pediatrics.

Methods: Description of clinical case and review of a clinical record. Results: The patient had an abnormal uterine bleeding associated with aregenerative hypochromic microcytic anemia with prolonged aPTT evaluated by endocrinology for probable polycystic ovary syndrome, bleeding episodes persisted, and factor VIII was dosed on two occasions, reporting less than $40 \%$, not have an associated family history or history of bleeding, a factor VIII inhibitor is indicated which reports $9.54 \mathrm{UB}$, so immunosuppressive treatment is started with prednisone at $1 \mathrm{mg} / \mathrm{kg} /$ day, 3 weeks after steroids new levels of factor VIII are taken that report $40.7 \%$ and factor VIII inhibitor that report $0 \cup B$.

Conclusions: Acquired hemophilia is significantly rarer than the inherited form. It rarely arises in childhood, where the presence of an inhibitor on laboratory screening of a previously healthy child is much more likely caused by autoimmune disease.

TABLE 1 Case description

$\begin{array}{ll}\text { Gender } & \mathrm{F} \\ \text { Age [years] } & 71 \\ \text { Myeloma status } & \text { De novo } \\ \text { M protein type } & \text { IgG lambda } \\ \text { Symptoms } & \text { Muscle hematomas, } \\ & \text { subcutaneous hematomas } \\ \text { On AHA diagnosis } & \text { hemoglobin [g/dl] } \\ & \text { serum M protein } \\ & \text { FVIII [\%] } \\ \text { Inhibitor titer }[\mathrm{BU} / \mathrm{ml}]\end{array}$

PB0449 | Acquired Hemophilia A as a Complication of Multiple Myeloma

A. Hoppe; J. Rupa-Matysek; L. Gil

Department of Hematology and Bone Marrow Transplantation, Poznan University of Medical Sciences, Poznan, Poland

Background: Acquired hemophilia $\mathrm{A}(\mathrm{AHA})$ is a rare bleeding disorder. Multiple myeloma (MM) is a rare cause of AHA. Management of bleeding control and inhibitor eradication remains controversial.

Aims: To report the challenge of bleeding treatment and inhibitor eradication management in MM patients with AHA.

Methods: We report two case studies of AHA in MM patients.

Results: Case 1: A female (71years) with unremarkable medical history was admitted to hospital due to soft tissue hematomas. Laboratory tests revealed normocytic anemia, prolonged APTT failed to correct in mixing study. AHA diagnosis was made with FVIII $=9 \%$, inhibitor titer $=7.8 \mathrm{BU} / \mathrm{ml}$. Patient received immunosuppressive treatment (IST) and aPCC for bleeding control. After 3 weeks we observed partial resolution of hematomas. During diagnostic workup serum electrophoresis revealed IgG monoclonal protein- $41.5 \mathrm{~g} / \mathrm{l}$, trephine biopsy showed $70 \%$ monoclonal plasmocytes, also multiple bone fractures were identified by computed tomography. We diagnosed MM R-ISS2 and administered VCD regimen as a combination of IST and anti-myeloma treatment. After 1 cycle, patient achieved very good partial response of MM and complete remission (CR) of AHA-Table1.

Case 2: A female (70years) was admitted to hospital due to large subcutaneous hematomas. She has a history of light kappa chain MM, diagnosed -2016, treated with autologous stem cell transplantation -2017. In 10/2019 she developed progression treated with lenalidomide and dexamethasone. After 3 cycles she achieved partial remission (PR), then therapy was withdrawn due to ocular surgery. On admission: anemia and persistent APTT prolongation on mixing tests, $\mathrm{FVIII}<1 \%$, inhibitor titer $=108 \mathrm{BU} / \mathrm{ml}$. MM relapse with serum kappa light chain $290 \mathrm{mg} / \mathrm{I}$ was confirmed. Patient received lenalidomide in combination with IST and bleeding control with aPCC. Two months later she achieved CR of AHA with PR of MM-Table 1.

\section{Case 1}

$\mathrm{F}$

70

Relapse

Kappa light chain

Subcutaneous hematomas

6.7 IgG lambda M protein $41.5 \mathrm{~g} / \mathrm{I}$ 9 7.8
Case 2

6.2

kappa free light chain $290 \mathrm{mg} / \mathrm{ml}$ $<1$ 108 


\section{research \& prectice
in thrombosis \& haemostasis}

Treatment

Inhibitor eradication treatment

After treatment

Time to AHA response [days]

\section{Case 1}

aPCC $100 \mathrm{IU} / \mathrm{kg}$ bw in 2 separated doses

Prednisone $1 \mathrm{mg} / \mathrm{kg}$ bw, VCD (bortezomib $1.3 \mathrm{mg} / \mathrm{m}^{2}$ $1,4,8,11 d$, cyclophosphamide $500 \mathrm{mg} / \mathrm{w}$, dexamethasone 40mg 1,4,8,11d; cycle 21d). FVIII [\%] Inhibitor titer [BU/ml]

AHA response

$M$ protein

$\mathrm{MM}$ response
21

78

$<0.5 \mathrm{BU} / \mathrm{ml}$

CR

$3.9 \mathrm{~g} / \mathrm{I}$

VGPR
Case 2

aPCC $2001 \mathrm{l} / \mathrm{kg}$ bw in 3 separated doses

Prednisone $1 \mathrm{mg} / \mathrm{kg}$ bw, Cyclophosphamide $1.5 \mathrm{mg} /$ $\mathrm{kg} / \mathrm{d}$, Lenalidomide $25 \mathrm{mg} / \mathrm{d}$

60

62

$<0.5 \mathrm{BU} / \mathrm{ml}$

CR

$58.2 \mathrm{mg} / \mathrm{ml}$

$\mathrm{PR}$

Abbreviations: F - female, M protein - monoclonal protein, AHA - acquired hemophilia A, MM - multiple myeloma, aPCC - activated Prothrombin Complex Concentrate, bw - body weight, CR - complete response, VGPR - very good partial response, PR - partial response.

Conclusions: Acquired hemophilia A can occur both in the onset and relapse of multiple myeloma. Combination of IST and anti-myeloma therapy is efficient in inhibitor eradication.

\section{P0142 | The Case of Acquired Haemophilia A}

O. Stasyshyn; V. Krasivska

SI IPKTM UNAMS, Lviv, Ukraine

Background: Acquired haemophilia is an rare hemorrhagic disorders Aims: We are representing the case of acquired haemophilia $A$ and analyze the timeliness and adequacy of treatment.

Methods: The subject of the study was acquired haemophilia A

Results: Female, 33 years old, pregnancy II, urgent delivery. There was excessive bleeding in the early postpartum period, hemostatic therapy was provided. She hospitalized three times with recurrences of uterine bleeding. Vacuum aspiration of the walls of the uterine cavity was performed twice. After the third recurrence, laparotomy and extirpation of the uterus was performed. Despite intensive care, the bleeding continued. Three relaparotomies were performed, the source of bleeding was not found, there was excessive bleeding from the operating field. The total blood loss was $24447 \mathrm{ml}$. Intensive infusion-transfusion therapy was continued, which gave unstable hemostasis. The patient received $12060 \mathrm{ml}$ of fresh-frozen plasma, $15130 \mathrm{ml}$ of erythrocytes, $600 \mathrm{ml}$ of albumin $10 \%, 20$ doses of cryoprecipitate, $16000 \mathrm{U}$ of APCC, $8 \mathrm{mg}$ of rVIla, $8000 \mathrm{U}$ of PCC. 16.1 $\mathrm{BU} / \mathrm{ml}$ of inhibitor FVIII, FVIII <1.0\%, APTT - $146.1 \mathrm{~s}$ was detected on day 37. Within 30 days, blood loss was $10608 \mathrm{ml}$; patient received $23420 \mathrm{ml}$ of fresh-frozen plasma, $2080 \mathrm{ml}$ of erythrocytes, $17 \mathrm{mg}$ of rVIla, $19000 \mathrm{U}$ of APCC, $6000 \mathrm{U}$ of PCC, 61 dose of cryoprecipitate, $50000 \mathrm{IU}$ of FVIII. Immunosuppressive therapy (prednisolone $1-1.5 \mathrm{mg} / \mathrm{kg} /$ day) was started on day 67 and lasted for 12 months. Bleeding stopped completely 10 months after delivery, and after 14 months the inhibitor was not detected, factor levels and APTT returned to normal.

Conclusions: Recurrent postpartum hemorrhage requires early diagnosis of coagulation factors inhibitors, which will ensure specific transfusion and immunosuppressive therapy, avoidance of serious consequences, including extirpation of the uterus and reduce the economic costs of treatment.

P0145 | When Therapy with Anticoagulant and Antiplatelet Agents Hides an Acquired Coagulopathy - A Case Report

E. Parcelas ${ }^{1}$; F. Francisco ${ }^{1}$; M.C. Lobo ${ }^{2} ;$ J. Esteves $^{2}$

${ }^{1}$ Centro Hospitalar Lisboa Ocidental, Hospital de São Francisco Xavier, Lisbon, Portugal; ${ }^{2}$ Centro Hospital de Lisboa Ocidental, Hospital de Egas Moniz, Lisbon, Portugal

Background: Acquired Hemophilia $\mathrm{A}(\mathrm{AHA})$ is a rare hemorrhagic autoimmune disease caused by the presence of autoantibodies against factor VIII (FVIII). In most cases, bleeding episodes at presentation are spontaneous and severe, with no previous family or personal history associated. However, in some cases, there is mild bleeding. More than $50 \%$ of the cases are idiopathic, but there are several conditions associated, namely: autoimmune diseases, solid tumors, lymphoproliferative diseases and pregnancy. AHA is more common in the elderly with comorbidities and it has a high mortality, associated to the underlying disease, to bleeding or to treatment complications. There is controversy regarding the ideal treatment, but it should include: replacement therapy, bypassing agents or other hemostatic approaches; and inhibitors eradication.

Aims: .

Methods: .

Results: Case Description: A 82 year-old Caucasian patient with multiple comorbidities and medicated with two antiplatelet drugs and rivaroxaban was admitted in the emergency room (ER), twice in a few days, due to gingival hemorrhage. A week later, the family practitioner sent him back with anemia and raised aPTT. He also developed macroscopic hematuria, which was assumed in the context of a cystitis. With a functional FVIII of $0.1 \%$, patient was hospitalized and started on prednisolone. The diagnosis of AHA was confirmed by the presence of anti-FVIII inhibitors and the treatment was continued, with good response. No etiology was attributed until now. 
TABLE 1 Chronological events and blood tests - ER

\begin{tabular}{|c|c|c|c|c|c|c|c|}
\hline $\begin{array}{l}\text { Chronological } \\
\text { events and blood } \\
\text { analysis results } \\
\text { (part 1) }\end{array}$ & May 11 (ER) & May 13 (ER) & May 18 & $\begin{array}{l}\text { Family } \\
\text { Practitioner } \\
\text { (May 22) }\end{array}$ & May 23 (ER) & $\begin{array}{l}\text { May 27-28 } \\
\text { (ER) }\end{array}$ & June 1 (ER) \\
\hline Events & $\begin{array}{l}\text { Admitted in } \\
\text { the ER due } \\
\text { to gingival } \\
\text { bleeding. } \\
\text { Managed } \\
\text { with local } \\
\text { noninvasive } \\
\text { treatment } \\
\text { by plastic } \\
\text { surgery. }\end{array}$ & $\begin{array}{l}\text { Admitted in } \\
\text { the ER due } \\
\text { to gingival } \\
\text { bleeding. } \\
\text { Managed } \\
\text { with local } \\
\text { noninvasive } \\
\text { treatment } \\
\text { by plastic } \\
\text { surgery. }\end{array}$ & $\begin{array}{l}\text { Seen by the } \\
\text { Dentist } \\
\text { - local } \\
\text { treatment. } \\
\text { Before } \\
\text { the small } \\
\text { surgery, it } \\
\text { was stopped } \\
\text { one of the } \\
\text { antiplatelet } \\
\text { agents and } \\
\text { rivaroxaban. }\end{array}$ & $\begin{array}{l}\text { Persistant mild } \\
\text { gingival } \\
\text { bleeding in } \\
\text { the same } \\
\text { spot. Not } \\
\text { taking } \\
\text { one of the } \\
\text { antiplatelet } \\
\text { agents and } \\
\text { rivaroxaban. }\end{array}$ & $\begin{array}{l}\text { No more } \\
\text { bleeding. } \\
\text { Decided to } \\
\text { discharge } \\
\text { and keep } \\
\text { those two } \\
\text { medications } \\
\text { suspended } \\
\text { until new } \\
\text { evaluation. }\end{array}$ & $\begin{array}{l}\text { Macroscopic } \\
\text { hematuria } \\
\text { and } \\
\text { dizziness. } \\
\text { Assumed } \\
\text { cystitis } \\
\text { and sent } \\
\text { home with } \\
\text { antibiotics. }\end{array}$ & $\begin{array}{l}\text { The patient was } \\
\text { started on } \\
\text { Prednisolone } \\
40 \mathrm{mg} / \text { day } \\
(\sim 0.5 \mathrm{mg} / \mathrm{kg} / \\
\text { day). Waiting } \\
\text { to be } \\
\text { admitted to } \\
\text { the medical } \\
\text { yard. }\end{array}$ \\
\hline Hemoglobin & & & & $9.3 \mathrm{~g} / \mathrm{dl}$ & $10.5 \mathrm{~g} / \mathrm{dl}$ & $9.3 \mathrm{~g} / \mathrm{dl}$ & $9.9 \mathrm{~g} / \mathrm{dl}$ \\
\hline $\begin{array}{l}\text { Activated partial } \\
\text { thromboplastin } \\
\text { time (aPTT) }\end{array}$ & & & & 71.5 seconds $(\mathrm{s})$ & $86.8 \mathrm{~s}$ & $108.6 \mathrm{~s}$ & $105.6 \mathrm{~s}$ \\
\hline Thrombin time (PT) & & & & & $\begin{array}{r}11.4 \text { seconds } \\
\text { (INR 1.0) }\end{array}$ & & \\
\hline aPTT FS & & & & & & $60.9 \mathrm{~s}$ & \\
\hline Functional FVIII & & & & & & $\begin{array}{l}0.1 \% \text { (only } \\
\text { available a } \\
\text { few days } \\
\text { later) }\end{array}$ & $0.8 \%$ \\
\hline $\begin{array}{l}\text { Rivaroxaban blood } \\
\quad \text { value }\end{array}$ & & & & & & $<10 \mathrm{ng} / \mathrm{ml}$ & \\
\hline $\begin{array}{l}\text { anti-FVIII inhibitor } \\
\text { title }\end{array}$ & & & & & & & 65 UB \\
\hline
\end{tabular}

TABLE 2 Chronological events and blood tests - Medical Yard and Consultations

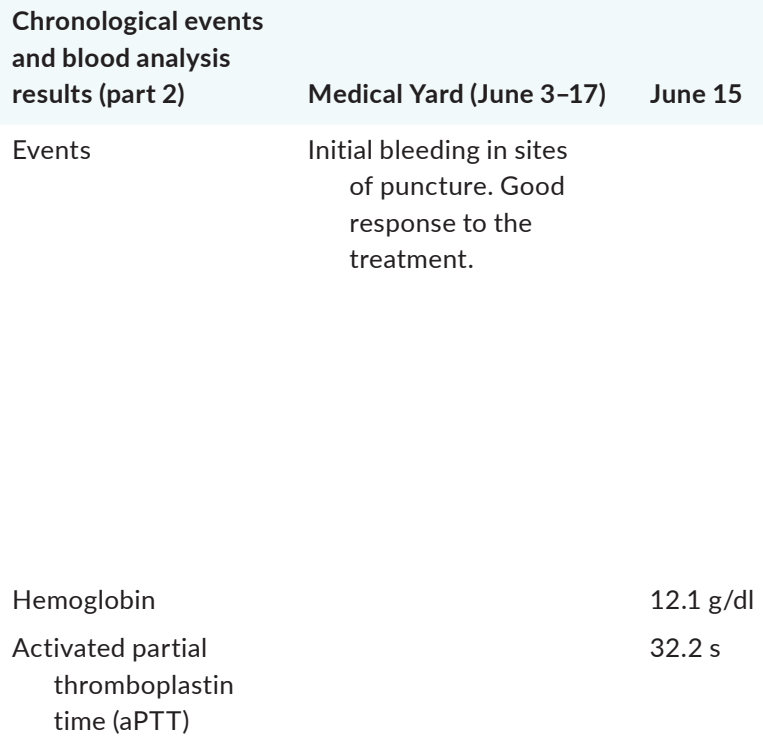

Conclusions: This patient had an uncommun mild bleeding presentation of AHA. Even on antiplatelet and/or anticoagulation therapy, in the presence of persistent bleeding, it is crucial to procede to coagulation blood tests and a complete blood count. The functional FVIII result is also important and should be more time efficient in al laboratories. It is essential to be alert and train ER doctors in the management of coagulopathies (specially high mortality diseases), as the presentation can be highly variable and subtle. 


\section{DISSEMINATED INTRAVASCULAR COAGULATION}

PB0450 | A Prospective Comparison Study of the Diagnosis of Disseminated Intravascular Coagulation (DIC) Comparing Between ISTH DIC Scoring system and General Practice in Prediction of 28-day Mortality

T. Thamgrang; C. Sirisuksakun; K. Prayongratana

Department of Medicine, Phramongkutklao Hospital and College of Medicine, Bangkok, Thailand

Background: DIC is one of the most common complicated conditions in medical practice. The International Society of Thrombosis and Haemostasis (ISTH) DIC scoring system was developed to guide in diagnosis of DIC. However, there was no study in Thailand about the prognostic values of this scoring system when comparing to general practice in prediction of mortality.

Aims: To compare the diagnosis of DIC by using ISTH DIC scoring system and general practice to predict 28-day mortality in terms of sensitivity, specificity and accuracy.

Methods: The prospective cohort study was conducted. 150 in patients complicated with clinical suspicious of DIC were enrolled. The diagnosis of DIC by general practice was performed by hematologists in Phramongkutklao Hospital. DIC score was calculated for each patient by using ISTH scoring system. The sensitivity, specificity, accuracy and survival analysis of the patients were evaluated.

Results: In prediction of 28-day mortality, ISTH DIC scoring system had a better specificity and accuracy when comparing to general practice; $68.7 \%$ and $61.3 \%(P$-value $=0.183) ; 72.0 \%$ and $52.0 \%$ $(P$-value $=0.004)$, respectively. However, the sensitivity of general practice was better; $80.0 \%$ and $62.0 \%$ ( $P$-value $=0.047)$. Survival analyses by using DIC scoring system showed that patients with overt-DIC group had a statistically significant lower rate of survival at 28 days when comparing to non-overt DIC group; $55.88 \%$ and $73.53 \%$ ( $P$-value $=0.002$. . However, by using general practice method, the rate of survival at 28 days in patients with DIC group was not significantly different from non-DIC group; $63.97 \%$ and 66.91\% $(P$-value $=0.064)$. (Figure 1$)$.

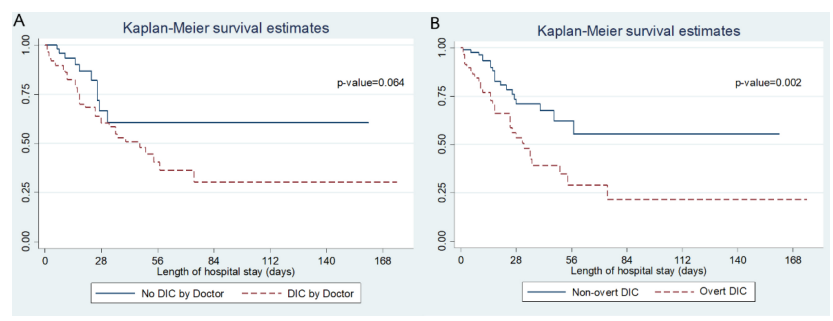

FIGURE 1 Kaplan-Meier analysis of survival in patients with DIC and non-DIC. Figure 1A demonstrated general practice method; Figure 1B demonstrated ISTH DIC scoring system method
Conclusions: The diagnosis of DIC by ISTH DIC scoring system had better accuracy and specificity in prediction of 28-day mortality, however, it had less sensitivity when comparing to general practice. In addition, ISTH DIC score could have a better power of discrimination between survivors and non-survivors in patients with DIC.

\section{HEMOPHILIA - BASIC}

PB0451 | Hemostatic Effect of Tranexamic Acid Combined with Emicizumab in Prophylactic Setting in Severe Hemophilia A: A Preclinical Study

Y. Dargaud $^{1}$; N. Enjolras ${ }^{2}$; M. Janbain ${ }^{3}$; R. Bolbos ${ }^{4}$; M. Brevet ${ }^{5}$; J.-C. Bordet ${ }^{2}$

${ }^{1}$ University of Lyon - Lyon Hemophilia Treatment Center, Lyon, France; ${ }^{2}$ UR4609-University of Lyon, Lyon, France; ${ }^{3}$ Tulane School of Medicine, Hematology, New Orleans, United States; ${ }^{4}$ CERMEP, Centre d'Etude et de Recherche Multimodal et Pluridisciplinaire, Lyon, France; ${ }^{5} \mathrm{Cy} P a t h$, Villeurbanne, France

Background: Hemophilia is characterized by a compromised hemostatic response with delayed development of a clot and the formation of clots that are vulnerable to fibrinolysis. Emicizumab is a bispecific antibody that mimics activity of activated FVIII and increases haemostatic capacity to the level of moderate-to-mild haemophilia A. It is indicated for prophylaxis in hemophilia A patients with and without inhibitors. Despite its impressive clinical performance, breakthrough-bleeding events may still occur and management of these bleeds is challenging especially in patients with inhibitors due to increased thrombotic risk when adding bypassing therapy.

Aims: We proposed to study, in factor VIII knockout mice(FVIII-KO), whether hemostasis is improved with the addition of tranexamic acid (TxAc) to emicizumab.

Methods: FVIII-KO mice received prophylaxis with emicizumab or emicizumab+TxAc before trauma. E Emicizumab $1.5 \mathrm{mg} / \mathrm{kg}$ and TxAc $0.25 \mathrm{mg} / 25 \mathrm{~g}$ was given via IV injection. A second retro-orbital IV injection containing human FIX and FX (both $100 \mathrm{U} / \mathrm{kg}$ ) was given 24 hours later and 5 minutes before the tail amputation or knee trauma. After knee trauma, joint bleeding was assessed using magnetic resonance imaging, histological analysis of the knee to compare the hemostastic efficacy of the two prophylactic strategies. Thrombin generation(TG) was measured and clots obtained with TG experiment were analyzed by scanning electron microscopy (SEM).

Results: In FVIII-KO mice, blood loss after tail clip was lower after prophylaxis with emicizumab+TxAc compared to emicizumab, with no statistical significance $(P>0.05)$. However, MRI results and histological analysis of knee joints showed that the addition of TXA significantly decreased joint bleeding ( 3 mice had joint bleed and one mice died in the group treated with emicizumab $(n=6)$, while 1 mice only had bleeding in the group receiving emicizumab+TxAc). SEM analysis to explore the effect of TxAc on fibrin clot quality is currently ongoing. 
Conclusions: Our results suggest a potential benefit of TxAc when used in combination with emicizumab in prophylactic settings.

PB0452 | Real-world Clinical and Patient-centric Outcomes in People with Haemophilia A in France: Findings from the CHESS II Study

A. Shaikh ${ }^{1}$; T. Burke ${ }^{1,2}$; C. Hawes ${ }^{3}$; G. Duport ${ }^{4}$; J. O'Hara ${ }^{1,2}$;

C. Camp ${ }^{3}$

${ }^{1}$ HCD Economics, Daresbury, United Kingdom; ${ }^{2}$ Faculty of Health and Social Care, University of Chester, Chester, United Kingdom; ${ }^{3}$ BioMarin Europe, London, United Kingdom, ${ }^{4}{ }^{\prime}$ 'Association Française des Hémophiles (AFH), Paris, France

Background: Haemophilia A (HA; factor VIII [FVIII] deficiency), characterised by prolonged trauma-related and/or spontaneous intra-articular bleeding events, is associated with adverse impacts on physical functioning and quality of life (QoL). The breadth of clinical and patient-relevant outcomes/experiences of people with HA (PWHA) in France - and variation between those with mild, moderate and severe $\mathrm{HA}$ - is not well understood.
Aims: To examine demographic, clinical and patient-centric outcomes across condition severity among adult PWHA in France, as captured in the 'Cost of Haemophilia in Europe: a Socioeconomic Survey - II' (CHESS II) study.

Methods: Data for non-inhibitor PWHA living in France were extracted from CHESS II and stratified based on clinician-reported baseline endogenous FVIII level. Participant demographics, clinical outcomes (annualized bleeding rate (ABR), incidence of target joints (ISTH definition) and joints exhibiting symptoms of chronic damage ('problem' joints)), treatment regimen and QoL (EQ-5D-5L index score) were assessed. Outcomes by HA severity were compared using descriptive statistics. Study methodology and interpretation of results were informed by a representative from I'Association Française des Hémophiles (AFH) patients' organisation.

Results: Sixty PWHA were included in the analysis (mild $n=10$, moderate $n=19$, severe $n=31$ ) with a mean age of 26 years. Forty-five percent of participants were full-time students; for the remainder of the cohort, full-time employment decreased with increasing HA severity (Table 1). Prophylaxis regimens were most common for participants with severe HA. Low incidence was observed for all clinical outcomes, and only target joint incidence increased with increasing severity of HA. EQ-5D-5L index scores were similar across subgroups (Table 2).

TABLE 1 Participant demographics by HA severity

\begin{tabular}{|c|c|c|c|c|}
\hline Severity of HA & Mild $(n=10)$ & Moderate $(n=19)$ & Severe $(n=31)$ & TOTAL $(n=60)$ \\
\hline Age, mean $\pm S D$ & $25.10 \pm 4.43$ & $23.68 \pm 4.89$ & $28.48 \pm 12.99$ & $26.40 \pm 10.05$ \\
\hline $\begin{array}{l}\text { Body Mass Index (BMI), } \\
\text { mean } \pm \text { SD }\end{array}$ & $23.35 \pm 2.65$ & $25.03 \pm 1.96$ & $23.83 \pm 2.18$ & $24.13 \pm 2.25$ \\
\hline \multicolumn{5}{|l|}{ Employment status, $n$ (\%) } \\
\hline Employed full time & $6(60 \%)$ & $6(32 \%)$ & $6(19 \%)$ & $18(30 \%)$ \\
\hline Employment part-time & $0(0 \%)$ & $1(5 \%)$ & $2(6 \%)$ & $3(5 \%)$ \\
\hline Self-employed & $0(0 \%)$ & $0(0 \%)$ & $2(6 \%)$ & $2(3 \%)$ \\
\hline Unemployed & $1(10 \%)$ & $0(0 \%)$ & $1(3 \%)$ & $2(3 \%)$ \\
\hline Student & $3(30 \%)$ & $8(42 \%)$ & $16(52 \%)$ & 27 (45\%) \\
\hline Other & $0(0 \%)$ & $4(21 \%)$ & $4(13 \%)$ & $8(14 \%)$ \\
\hline
\end{tabular}

TABLE 2 Treatment strategy, clinical and patient-centric outcomes by HA severity

\begin{tabular}{|c|c|c|c|c|}
\hline Severity of HA & Mild $(n=10)$ & Moderate $(n=19)$ & Severe $(n=31)$ & TOTAL $(n=60)$ \\
\hline $\begin{array}{l}\text { Receiving factor replacement } \\
\text { therapy, } n(\%)\end{array}$ & $3(30 \%)$ & $5(26 \%)$ & 31 (100\%) & $39(65 \%)$ \\
\hline Primary on-demand & $3(100 \%)$ & $3(60 \%)$ & $8(26 \%)$ & $14(36 \%)$ \\
\hline Primary prophylaxis & $0(0 \%)$ & $0(0 \%)$ & $2(6 \%)$ & $2(5 \%)$ \\
\hline Secondary on-demand & $0(0 \%)$ & $2(20 \%)$ & $7(23 \%)$ & $9(23 \%)$ \\
\hline Secondary prophylaxis & $0(0 \%)$ & $0(0 \%)$ & $14(45 \%)$ & $14(36 \%)$ \\
\hline Annualised bleed rate, mean \pm SD & $1.40 \pm 0.97$ & $1.06 \pm 0.94$ & $2.94 \pm 2.35$ & $2.10 \pm 2.01$ \\
\hline Target joints, mean \pm SD & $0.10 \pm 0.32$ & $0.11 \pm 0.46$ & $0.68 \pm 1.05$ & $0.40 \pm 0.85$ \\
\hline Problem joints, mean \pm SD & $0.10 \pm 0.32$ & $0.00 \pm 0.00$ & $0.19 \pm 0.48$ & $0.12 \pm 0.37$ \\
\hline EQ-5D-5L, $n$; mean \pm SD & $3 ; 0.98 \pm 0.02$ & $8 ; 0.96 \pm 0.04$ & $22 ; 0.96 \pm 0.06$ & $33 ; 0.96 \pm 0.05$ \\
\hline
\end{tabular}


Conclusions: Real-world data from CHESS II provides preliminary insights into the clinical outcomes and QoL of PWHA in France. Participants enrolled comprised a young cohort of PWHA with low reported incidence of clinical outcomes and high QoL. Further data is needed to contextualize the burdens experienced by PWHA in France.

\section{PB0453 | Mitigation of Injection Site Reactions after} Subcutaneous Administration of Dalcinonacog Alfa (DalcA) in Hemophilia B Using Preclinical Models

N. Le Moan ${ }^{1}$; L. Kelly ${ }^{1}$; E. Merle²; P. Descargues²; N. Gaudenzio²; H. Gagnon ${ }^{3}$; A. Chatterji ${ }^{1}$; G.E. Blouse ${ }^{1}$

${ }^{1}$ Catalyst Biosciences, South San Francisco, United States; ${ }^{2}$ Genoskin, Inc, Salem, United States; ${ }^{3}$ Phenoswitch Biosciences, Sherbrooke,

Canada

Background: Prophylactic subcutaneous (SQ) administration of dalcinonacog alfa (DalcA), an engineered recombinant factor IX (FIX) variant with 22-fold enhanced potency compared with BeneFIX, provides stable and protective therapeutic FIX levels in individuals with Hemophilia B. Phase 1 and 2b studies demonstrated DalcA was well tolerated and efficacious, showing sustained FIX levels and no bleeding events from therapy start through washout. However, some subjects reported mild-to-moderate injection site reactions (ISR) consisting of pain and/or redness following initial SQ injections.

Aims: We investigated the underlying ISR mechanism after SQ DalcA administration.

Methods: We examined cutaneous microanatomic, cellular, and proteomic changes in the HypoSkin ${ }^{\circledR}$ injectable human skin biopsy platform (Genoskin, Salem, MA, USA) and Göttingen minipigs.

Results: Daily DalcA SQ injections for 14 days induced erythema and redness in minipigs comparable with observed human ISR. Ex vivo studies in HypoSkin showed that DalcA stimulated mast cell degranulation within 4 hours. In vitro studies further demonstrated that DalcA activates the Mas-related G protein-coupled receptor X2 (MRGPRX2) expressed by mast cells and associated with dermal ISR to a similar level as wild-type recombinant FIX. Since cationic drugs can stimulate MRGPRX2, we examined DalcA effect on MRGPRX2 activation when presented in alternative formulation buffers. Formulation buffers with higher $\mathrm{pH}$ and/or tween content reduced in vitro MRGPRX2-mediated cell activation, suggesting that modifying the DalcA formulation buffer may reduce in-vivo mast cell degranulation and downstream signaling of pain and dermal inflammation. A biomarker signature predictive of minipig ISR was generated from the cross comparison of cutaneous proteomes of minipig and ex vivo human skin models.

Conclusions: This approach of integrating ex vivo and in vitro studies with a defined biomarker signature coupled with a drug product stability profile facilitated identification of an improved formulation buffer expected to lower the risk of clinical ISR with SQ DalcA injection without impacting DalcA product quality.

PB0454 | Impact of Adherence to Prophylactic Treatment on Real-world Outcomes in Adults with Severe Haemophilia: A CHESS II Analysis in Europe

T. Burke ${ }^{1,2}$; S. Asghar ${ }^{1}$; J. O'Hara ${ }^{1,2}$; D. Yee ${ }^{3}$;. Kar $^{3}$

${ }^{1}$ HCD Economics, Daresbury, United Kingdom; ${ }^{2}$ University of Chester, Chester, United Kingdom; ${ }^{3}$ Freeline Therapeutics, Stevenage, United Kingdom

Background: Haemophilia is a genetic bleeding disorder characterised by prolonged trauma-related or spontaneous bleeding events and associated chronic complications such as synovitis, pain, and, eventually, destruction of the joint. Life-long adherence to prophylactic treatment with factor replacement is required for protection from bleeds and improved haemophilia-related clinical outcomes.

Aims: To investigate the relationship between real world adherence to prophylaxis and long-term clinical outcomes and hospitalisations in adults with severe haemophilia across Europe.

Methods: Physician reported data were extracted from the CHESS II dataset, a prevalence-based cross-sectional, burden-of-illness study in adults with mild, moderate, and severe haemophilia A and B throughout Europe ( $n=1337)$. The cohort analysed consisted of people with severe haemophilia receiving prophylaxis, without a current inhibitor diagnosis ( $n=467)$. 'Full adherence' (FA) was defined as missing fewer than $15 \%$ of annual factor infusions $(n=385)$ and 'moderate or low adherence' (MLA) was defined as missing $\geq 15 \%$ of annual factor infusions $(n=82$ ). Clinical outcomes evaluated were level of chronic pain (none, low, moderate, or high), joint health (a composite measure of no joint issues: absence of both problem joints [PJ] and target joints [TJ]) and annual hospitalisations related to haemophilia.

Results: Full adherence to a prescribed prophylactic regimen was associated with superior clinical outcomes in adults with haemophilia. Those with FA had lower levels of chronic pain, better joint health (higher proportion with $0 \mathrm{TJ} / \mathrm{PJ}$ ) and fewer annual hospitalisations related to haemophilia, compared to those with MLA (Table 1). 
TABLE 1 Adherence to prophylactic treatment and real-world outcomes in haemophilia

$\begin{array}{ll}\text { Full adherence } & \text { Moderate/Low } \\ (n=385) & \text { adherence }(n=82)\end{array}$

Joint health, $n$ (\%)

$0 \mathrm{TJ} / \mathrm{PJ}$

Zero haemophilia-related 272 (70.6) 32 (39.0) hospitalisations, $n$ (\%)

Haemophilia-related hospitalisations, mean (SD, median)

Chronic pain, $n$ (\%)

No pain

89 (23.1)

10 (12.2)

Mild pain

$183(47.5)$

Moderate pain

$100(26.0)$

26 (31.7)

Severe pain

$13(3.4)$

Conclusions: This study suggests that adherence to current haemophilia prophylactic regimens remains a challenge and this contributes to significant morbidity as evidenced by real-world data. Gene therapy approaches, which eliminate the need for adherence to factor supplementation regimens, may have the potential to provide more consistent levels of factor and therefore improved patient outcomes.

PB0455 | Immune Complex Formation of Factor VIII (FVIII) with Inhibitors is Modulated by von Willebrand Factor (vWF)

\section{O. Oleshko ${ }^{1}$; U. Curth ${ }^{2}$; A. Tiede ${ }^{1}$; S. Werwitzke ${ }^{1}$}

${ }^{1}$ Department of Haematology, Haemostaseology, Oncology and Stem Cell Transplantation, Hannover Medical School, Hannover, Germany;

${ }^{2}$ Institute for Biophysical Chemistry, Hannover Medical School,

Hannover, Germany

Background: Formation of neutralizing anti-FVIII antibodies (inhibitors) due to FVIII replacement in hemophilia A patients leads to therapy failure. Moreover, immune reaction could be potentially augmented by FVIII-containing immune complexes (FVIII-IC) binding to low affinity Fc gamma receptor IIB (CD32) and/or C1q initiating thereby FVIII internalization by antigen-presenting cells. VWF is considered a possible immuno-protective factor in inhibitors formation, but there is little information on the vWF influence on potentially harmful FVIII-IC characteristics.

Aims: The aim of this study is to analyze the effect of VWF on FVIII-IC characteristics concerning their size and CD32 and C1q binding.

Methods: For FVIII-IC generation, two types of inhibitors were used: anti-FVIII murine sera and a mix of 7 purchasable monoclonal anti-FVIII IgG antibodies. Plasma-derived vWF was incubated with recombinant human FVIII followed by inhibitors addition and examination by in-house ELISA and analytical ultracentrifugation with fluorescence detection. Data analysis was performed using SEDFIT (a diffusion-deconvoluted differential sedimentation coefficient distribution model, c(s)) and GraphPadPrism software.

Results: FVIII-IC but not monomeric IgG were bound by CD32 and C1q. As demonstrated in both assays, vWF suppressed binding in a dose-related manner independently on used inhibitors type. Under conditions of 60 -fold FVIII excess, vWF reduced binding by $70 \%$, while application of VWF at physiologically relevant ratios almost fully prevented FVIII-IC CD32 and C1q binding. In the presence of VWF, we observed incorporation of fewer inhibitors into FVIII-IC, as indicated by increased amounts of free lgG, but also increased size of FVIII-IC as indicated by faster sedimenting complexes.

Conclusions: VWF impedes FVIII-IC binding to effector molecules as Fc gamma receptor and $\mathrm{C} 1 \mathrm{q}$ possibly via concealment of the IgG Fcfragments. The ability of VWF to alter the FVIII-IC characteristics is indicative of its potential role for the regulation of anti-FVIII immune response and for achieving immune tolerance.

PB0456 | Real-world Clinical and Patient-centric Outcomes in People with Haemophilia A in Spain: Findings from the CHESS II Study

A. Shaikh ${ }^{1}$; T. Burke ${ }^{1,2}$; C. Hawes ${ }^{3}$; J. O'Hara ${ }^{1,2}$; C. Camp ${ }^{3}$

${ }^{1}$ HCD Economics, Daresbury, United Kingdom; ${ }^{2}$ Faculty of Health and Social Care, University of Chester, Chester, United Kingdom; ${ }^{3}$ BioMarin Europe, London, United Kingdom

Background: Haemophilia A (HA; factor VIII [FVIII] deficiency), characterised by prolonged trauma-related and/or spontaneous intra-articular bleeding events, is associated with adverse impacts on physical functioning and quality of life (QoL). The breadth of clinical and patient relevant outcomes/experiences of people with HA (PWHA) in Spain - and variation between those with mild, moderate and severe $\mathrm{HA}$ - is not well understood.

Aims: To examine demographic, clinical and patient-centric outcomes across condition severity among adult PWHA in Spain, as captured in the 'Cost of Haemophilia in Europe: a Socioeconomic Survey - II' (CHESS II) study.

Methods: Data for non-inhibitor PWHA living in Spain were extracted from CHESS II and stratified based on clinician-reported baseline endogenous FVIII level. Participant demographics, annualized bleeding rate (ABR), incidence of target joints (ISTH definition) and joints exhibiting symptoms of chronic damage ('problem' joints), treatment regimen and QoL (EQ-5D-5L index score) were assessed. Outcomes by HA severity were compared using descriptive statistics.

Results: One hundred and eighty-seven PWHA were included in the analysis (mild $n=33$, moderate $n=66$, severe $n=88$ ). The proportion of participants in full-time employment declined with increasing $\mathrm{HA}$ severity; reporting of part-time employment increased with increasing severity (Table 1). Participants with mild/moderate HA received on-demand FVIII; prophylaxis regimens were most common in participants with severe HA. Mean ABR, incidence of target joints and EQ-5D-5L index score increased with severity. Incidence of problem joints was highest in participants with moderate HA (Table 2). 
TABLE1 Participant demographics by HA severity

Severity of HA
Age, mean \pm SD
Body Mass Index (BMI), mean \pm SD
Employment status, $n$ (\%)
Employed full time
Employment part-time
Self-employed
Unemployed
Student
Other

$\begin{array}{llll}\text { Mild }(n=33) & \text { Moderate }(n=66) & \text { Severe }(n=88) & \text { TOTAL }(n=187) \\ 43.58 \pm 17.03 & 40.77 \pm 16.08 & 39.44 \pm 14.28 & 40.64 \pm 15.42 \\ 25.08 \pm 2.45 & 24.63 \pm 2.65 & 24.76 \pm 2.75 & 24.77 \pm 2.65 \\ 20(61 \%) & 25(38 \%) & 31(35 \%) & 76(41 \%) \\ 2(6 \%) & 8(12 \%) & 22(25 \%) & 32(17 \%) \\ 3(9 \%) & 1(2 \%) & 4(5 \%) & 8(4 \%) \\ 1(3 \%) & 4(6 \%) & 3(3 \%) & 8(4 \%) \\ 4(12 \%) & 7(11 \%) & 9(10 \%) & 20(11 \%) \\ 3(9 \%) & 21(32 \%) & 19(22 \%) & 43(23 \%)\end{array}$

TABLE 2 Treatment strategy, clinical and patient-centric outcomes by HA severity

\begin{tabular}{lllll} 
Severity of HA & Mild $(n=33)$ & Moderate $(\boldsymbol{n}=66)$ & Severe $(\boldsymbol{n}=88)$ & TOTAL $(\boldsymbol{n}=187)$ \\
Receiving factor replacement therapy, $n(\%)$ & $8(24 \%)$ & $25(38 \%)$ & $88(100 \%)$ & $121(65 \%)$ \\
Primary on-demand & $8(100 \%)$ & $20(80 \%)$ & $37(42 \%)$ & $65(54 \%)$ \\
Primary prophylaxis & $0(0 \%)$ & $0(0 \%)$ & $10(11 \%)$ & $10(8 \%)$ \\
Secondary on-demand & $0(0 \%)$ & $5(20 \%)$ & $6(7 \%)$ & $11(9 \%)$ \\
Secondary prophylaxis & $0(0 \%)$ & $0(0 \%)$ & $35(40 \%)$ & $35(29 \%)$ \\
Annualised bleed rate, mean \pm SD & $1.09 \pm 0.96$ & $2.77 \pm 3.57$ & $4.58 \pm 10.65$ & $3.34 \pm 7.73$ \\
Target joints, mean \pm SD & $0.06 \pm 0.24$ & $0.53 \pm 0.83$ & $1.00 \pm 1.30$ & $0.67 \pm 1.08$ \\
Problem joints, mean \pm SD & $0.33 \pm 0.48$ & $0.86 \pm 1.18$ & $0.78 \pm 1.12$ & $0.73 \pm 1.07$ \\
EQ-5D-5L, $n$; mean \pm SD & $15 ; 0.87 \pm 0.19$ & $28 ; 0.81 \pm 0.15$ & $55 ; 0.77 \pm 0.19$ & $98 ; 0.80 \pm 0.18$ \\
\hline
\end{tabular}

Conclusions: The CHESS II study enrolled a large cohort of PWHA in Spain and provides an important resource for exploring differences in clinical outcomes and QoL across the spectrum of HA severity. This analysis observed increasing clinical burden and lower QoL associated with greater condition severity. Collection of further country-specific data will help to validate these findings and provide further insight into the differences observed in the present study.

PB0457 | Frequency of Dosing and Presence of Endogenous VWF Influences FVIII Inhibitor Development

M. Cormier; K. Nesbitt; P. Batty; C. Hough; D. Lillicrap

Queen's University, Kingston, Canada

Background: Patients with hemophilia A (HA) are deficient in Factor VIII (FVIII) and are treated with FVIII replacement therapy. The frequency of dosing in pediatric patients can vary, and it is unknown whether this influences inhibitor development. Furthermore, the role of von Willebrand factor (VWF) in modifying FVIII immunogenicity is controversial.

Aims: To determine if the frequency of dosing and/or the presence of endogenous murine VWF influences the anti-FVIII immune response in HA mice.

Methods: Mice were treated with four retro-orbital injections of recombinant human FVIII (total dose: $0.2 \mu \mathrm{g}$ ) in parallel to saline treated controls on one of four dosing schedules - weekly, biweekly or once daily (with or without lipopolysaccharide [LPS] on Day 0). Littermate control mice (age and sex) were used (cohorts, $n=2-$ 4). Experiments were performed in two C57Bl6 severe HA mouse models: FVIII knockout $\left(\mathrm{FVIII}^{-/}\right)$and a FVIII-VWF double knockout $\left(\mathrm{FVIII}^{-/} \mathrm{VWF}^{-/}\right)$. Blood sampling was performed on days 7,17 and 24 after the first infusion with anti-FVIII immunoglobulin (IgG) measured by enzyme linked immunosorbent assay (Figure 1). \#

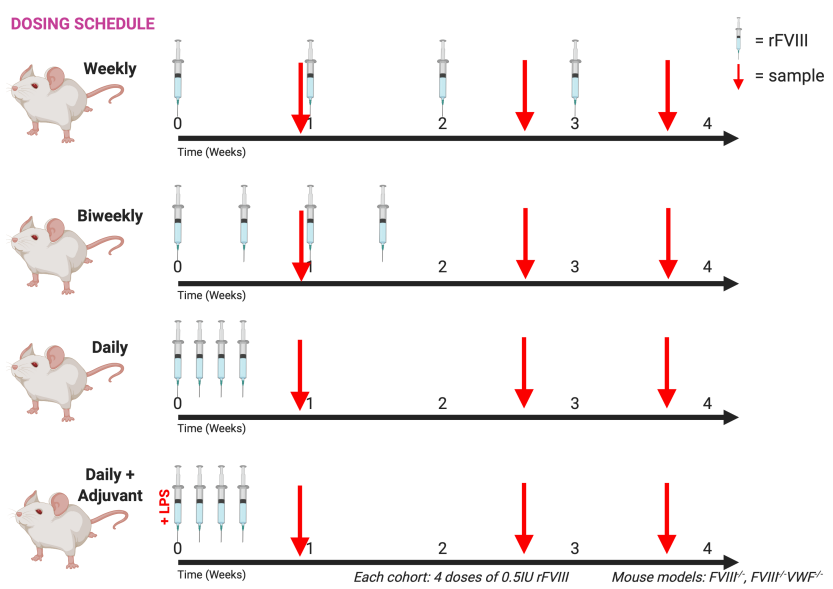

FIGURE 1 Factor VIII Dosing Schedules 
Results: Anti-FVIII IgG was first detected at day 17 or day 7-17 for FVIII-/- mice and FVIII-/-VWF-/- mice, respectively. Peak antibody titers were measured at day 24. FVIII-/- mice treated weekly generated the highest antibody titers (median titer $=5120$ ), with all other more frequent dosing schedules resulting in lower titers (median titers $=40-320$ ). For FVIII-/-VWF-/- mice, anti-FVIII IgG appeared the earliest (day 7) and with the greatest magnitude (median titer $=1600$ ) in mice administered FVIII daily with one LPS injection in comparison to the three other schedules (median titer $=0-160$ ). Except when an adjuvant was present, the immune response in FVIII-/-VWF-/- mice was less than or comparable to FVIII-/- mice.

Conclusions: The frequency at which FVIII is delivered as well as the presence of VWF and/or an adjuvant influence FVIII immunogenicity.

PB0458 | Dose Selection of Marzeptacog Alfa (Activated) in Children with Hemophilia: A Population Pharmacokinetic Exposure Matching Strategy

A. Faraj ${ }^{1}$; R. van Wijk ${ }^{1}$; L. Neuman ${ }^{2}$; S. Desai ${ }^{2}$; G.E Blouse ${ }^{2}$;

T. Knudsen ${ }^{2}$; U.S. Simonsson ${ }^{1}$

${ }^{1}$ Uppsala Universitet, Uppsala, Sweden; ${ }^{2}$ Catalyst Biosciences, South

San Francisco, United States

Background: Marzeptacog alfa (activated) (MarzAA), a novel human variant recombinant Factor VIla is in clinical development for subcutaneous (SQ) administration for on-demand or prophylactic use in hemophilia $A(H A)$ or $B(H B)$ with inhibitors and other inherited bleeding disorders.

Aims: To support the pediatric trial dose selection for SQ MarzAA in children with $\mathrm{HA} / \mathrm{HB}$ with inhibitors through simulations.

Methods: A previously developed population pharmacokinetic (popPK) model in adults including allometric scaling was used to inform the dose for the pediatric Phase 3 trial design. The relationship between clearance (CL) and bodyweight (BW) was studied. Concentration-time profiles were simulated for 1000 virtual pediatric subjects in several age categories assessing different doses. Median and $95 \%$ prediction intervals (PI) of the area-under-the-curve over 24 hours (AUCO-24) and maximum concentrations (Cmax) were derived to identify the dosing regimen that would best match the adult exposure of $60 \mu \mathrm{g} / \mathrm{kg}$ given on-demand at 3-hourly intervals.

Results: The relationship between MarzAA CL and BW was found to be non-linear with higher $\mathrm{CL} / \mathrm{kg}$ at lower BW (Figure 1), with most of the non-linear trend below $14 \mathrm{~kg}$, corresponding to the median BW in children of 2 years of age as described in The National Health and Nutrition Examination Survey (NHANES) database.

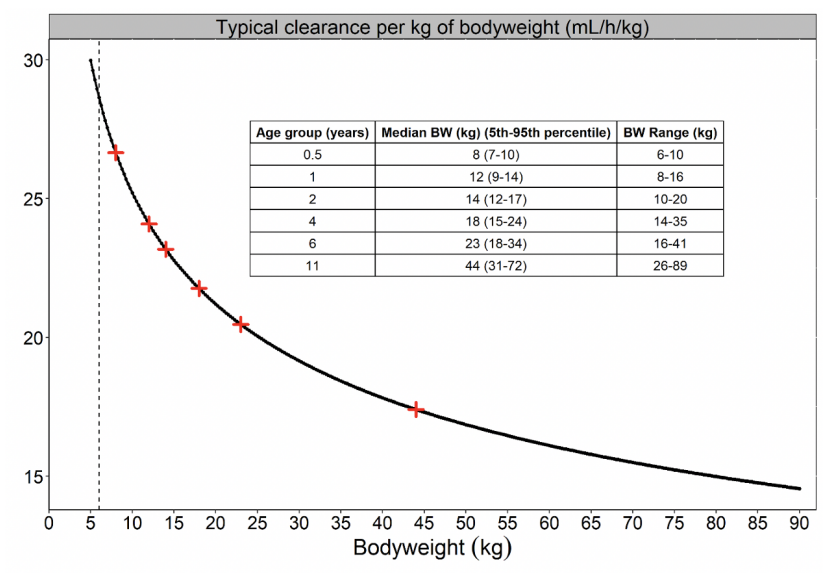

Figure 1. Predicted bodyweight-adjusted clearance versus a range of bodyweights $(5-75 \mathrm{~kg})$ based on the adult population pharmacokinetic model with allometric scaling. The dashed line is the median bodyweight in children of 0.5 years of age from the NHANES database. The table shows the median and spread statistics of bodyweight for each age group. The red crosses illustrate the median bodyweight in each age group from the NHANES database.

\section{FIGURE 1}

Across all age groups using a single $60 \mu \mathrm{g} / \mathrm{kg}$ dose, the lower bound of the $95 \% \mathrm{PI}$ of AUC0-24h in pediatrics was only slightly lower compared to that of adults, and a similar pattern was observed for Cmax (Figure 2), indicating comparable exposure across pediatric age groups compared to adults.
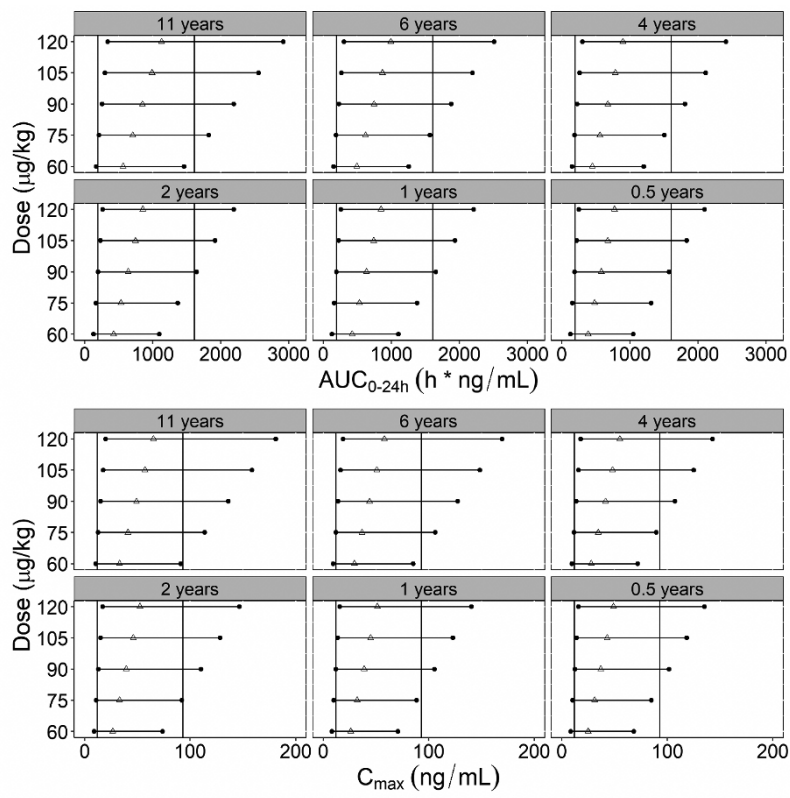

Figure 2. Predictions of area-under-the-curve over 24 hours (AUC $C_{0.2 a h}$ ) and maximum concentrations $\left(\mathrm{C}_{\mathrm{max}}\right)$ in the pediatric population for different age groups and dose levels given as a single dose. The triangles and horizontal lines are the media administered as a single SQ injection. All predictions were based on simulation of 1000 children per are group per dose level

\section{FIGURE 2}

Conclusions: The simulations support selecting a SQ dose of $60 \mu \mathrm{g} /$ $\mathrm{kg}$ MarzAA in a clinical trial with pediatric subjects with $\mathrm{HA} / \mathrm{HB}$ with inhibitors. 
PB0459 | Optimization of Thrombin Generation for Hemophilia A

T. van de Berg ${ }^{1} ;$ E. Beckers ${ }^{2} ;$ T. Hackeng ${ }^{1}$

${ }^{1}$ Cardiovascular Research Institute Maastricht, Department of Biochemistry, Maastricht, Netherlands; ${ }^{2}$ Department of Hematology, MUMC+, Maastricht, Netherlands

Background: Hemophilia A (HA) is a bleeding disorder characterized by decreased or absent FVIII. Clinical analysis of coagulation potential in this patient population is classically based on APTT based FVIII assays. Although both the one-stage FVIII assay and the chromogenic FVIII assay can measure FVIII concentrations reliably these types of assays only give insight on the initiation of coagulation. Global coagulation assays, like thrombin generation (TG), can be used to measure the full coagulation spectrum of initiation, amplification and propagation. However the frequently used, commercially available, TG kits lack sensitivity for measurements of hemophilia plasma within the lower FVIII ranges (below 20\%).

Aims: We aim to optimize the sensitivity of the TG-assay for measurements in hemophilia A patients, especially in the lower FVIII ranges.

Methods: In order to minimize patient specific sensitivity a hemophilia A pool plasma was created. Analysis of the influence of preanalytical variables, like contact activation inhibitors, on the assay was performed. Initiation of coagulation by different reagents was compared for sensitivity towards factor FVIII titrations in patient plasma. Other assay variables like phospholipids and temperature were adjusted to increase sensitivity even further.

Results: Commonly used tissue factor (TF) initiated TG alone at varying concentrations was unable to significantly differentiate in FVIII levels below $20 \%$. In contrast, TG activation with low concentrations of TF in presence of FXla appeared to be highly sensitive for FVIII changes both in high and low ranges. Additionally, a representative baseline TG-curve in severe HA plasma could only be produced using this dual TF/FXla-activation.

Conclusions: TF/FXla dual activation thrombin generation increased assay sensitivity in severe hemophilia plasma, allows for dosedependent measurements in all FVIII ranges and provides a solid baseline curve that can be used for further clinical evaluation of coagulation potential and possibly therapeutic monitoring in hemophilia A.

PB0460 | Influence of Activated Factor XI on Simultaneous Thrombin Generation and Fibrin Clot Lysis in Factor Deficiencies

I. Tarandovskiy; W.C. Chang; M. Ovanesov

US Food and Drug Administration, Silver Spring, United States

Background: Activation of coagulation cascade involves activation of factor (F) XI and activated FXI (FXla) was found in plasma of patients with thrombotic conditions and other conditions. Global hemostasis parameters are usually investigated in response to tissue factor (TF) trigger and there is limited-investigation of additional effect that can be caused by presence of FXla.

Aims: To evaluate the influence of FXla on fluorogenic thrombin generation (TG), clot formation and lysis under conditions of reduced FV, FVII, FVIII or FIX.

Methods: A dual channel fluorescence and turbidity microplate reader was used to simultaneously observe TG and clot formation and lysis, respectively. FV-, FVII-, FVIII-, and FIX-deficient plasmas were supplemented with their respective factors in the range of concentrations from 0.01 to $1 \mathrm{IU} / \mathrm{ml}$. Coagulation was triggered with TF with or without FXla in the presence of tissue plasminogen activator. Thrombin peak height (TPH) and the times to clot formation (ClotT), thrombin peak (PeakT) and clot lysis (LysisT) were obtained.

Results: In FVII- and FIX-deficient plasmas, FXla addition increased the responses of TPH to FVIII and FIX in the concentrations of respective factors higher than $0.01 \mathrm{lU} / \mathrm{ml}$. In FV- and FVII-deficient plasmas, FXla changed TG, clot formation and lysis parameters at the concentrations of FV and FVII below $0.01 \mathrm{IU} / \mathrm{ml}$. In FVII-deficient plasma, FXla blocked the responses of ClotT, PeakT and LysisT to FVII and the correlation between ClotT and LysisT. The correlations between TPH and LysisT in FVIII- and FIX-deficient plasmas were weakened by FXla. In FV-deficient plasma, there was no correlation between TG, clot formation and lysis parameters with or without FXla.

Conclusions: Addition of FXla to TF trigger reveals new relationships between TG and lysis parameters, offering improved differentiation of coagulopathies associated with coagulation factor deficiencies.

\section{PB0461 | Monocytes Express Different Cytokines upon Stimulations with a Variety of FVIII but Not FIX Concentrates}

S. Scatigna; A. Orlowski; E. Salzmann-Manrique; C. Königs;

A. Schmidt

University Hospital Frankfurt, Frankfurt am Main, Germany

Background: Patients with haemophilia A or B are treated by factor replacement. Inhibitors arise in a substantial number of patients. Risk factors for inhibitor development are being discussed including a different risk profile for different types of concentrates.

Aims: To analyze the cytokine production in immune cell subsets from buffy coats of 9 healthy individuals upon stimulation with different FVIII and FIX products.

Methods: Each $1 \times 10^{6}$ PBMCs have been stimulated with FVIII and FIX concentrates in the presence of anti-CD28 antibody. Myelinoligodendrocyte glycoprotein (MOG) was used as autoantigen control. Concentrations within product groups were adjusted by international units, the MOG control was used in a comparable molar concentration. Following incubation for $16 \mathrm{~h}$, cells were classified into CD8+, CD4+, CD19+, CD14+ cells by flow cytometry. Friedman test or Wilcoxon signed-rank test were used to assess differences between the distributions of the paired groups as appropriate. 
Results: Among analysed cells, major differences have been observed for CD14+ monocytes: FIX concentrates were mostly comparable to the MOG control, except for a significantly lower IL-10 production in response to pFIX ( $P=0.008)$. Comparison of FVIII concentrates showed moderate to high differences for GM-CSF, IL-10, and IL-6 production (see figure). Lowest IL-6-, IL-10-, and GM-CSF-producing monocytes were found for pFVIII and differed significantly $(P<0.001)$ from the MOG control, but percentages were also significantly lower for rFVIII-2nd and rFVIII3rd. Interestingly, cytokines after stimulation with rFVIIIFc were comparable to the MOG control.
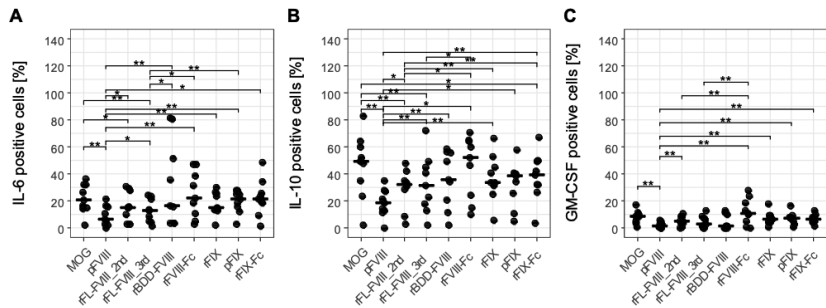

FIGURE 1 Percentage of cytokine-positive monocytes following stimulation with different FVIII concentrates and MOG

Conclusions: The immune reaction of PBMCs from healthy individuals towards FIX concentrates is very similar to the MOG control, whilst the response towards different FVIII concentrates generally differs from the response to MOG, with rFVIII-Fc being an exception, inducing comparable amounts of cytokine-producing monocytes. As regulatory as well as pro-inflammatory cytokines have been analysed, the role and clinical relevance needs to be addressed in the future.

PB0462 | Monocytes Show Differences in Cytokine Expression after Stimulation with FVIII Concentrates Compared to FIX or MOG

S. Scatigna; A. Orlowski; E. Salzmann-Manrique; C. Königs; A. Schmidt

University Hospital Frankfurt, Frankfurt am Main, Germany

Background: Acquired haemophilia $\mathrm{A}$ is a rare but severe autoimmune disorder with factor VIII as autoantigen. Hardly any cases of acquired haemophilia $B$ with an immune response against factor IX are reported. Also, in congenital haemophilia A and B, different rates of anti-drug antibodies are known acknowledging different genetic and environmental risk factors, which are not fully understood.

Aims: To analyze the production of cytokines in PBMCs of healthy individuals upon stimulation with FVIII or FIX compared to an autoantigen control.

Methods: Each $1 \times 10^{6}$ PBMCs from buffy coats of 9 healthy individuals were stimulated with concentrates of FVIII and FIX in the presence of costimulating anti-CD28 antibody. Myelin-oligodendrocyte glycoprotein (MOG) was used as autoantigen control. Concentrations within product groups were adjusted by international units, the MOG control was used in a comparable molar concentration. Following incubation, cytokine-positive cells were measured by flow cytometry. Friedman test or Wilcoxon signed-rank test was used to assess differences between the distributions of the paired groups as appropriate. Results: Stimulation with different FVIII and FIX concentrates resulted in highly significant differences in cytokine production of monocytes for IL-6, IL-10, and GM-CSF and was lowest for pFVIII pFVIII (medians $7 \%, 19 \%$, and $1 \%$ respectively). Here, values differed significantly from MOG, pFIX, rFIX, and rFIX-Fc. Additionally, rFL-FVIII-2nd resulted in lower amounts of IL-10 positive monocytes as rFIX and rFIX-Fc.
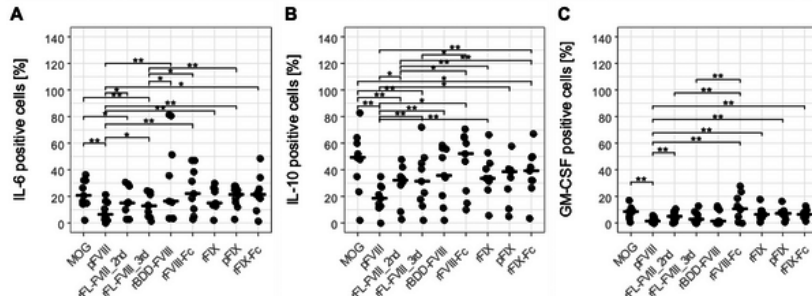

FIGURE 1 Percentages of cytokine-positive monocytes following stimulations with FVIII and FIX concentrates

Conclusions: The immune system of healthy individuals reacts differently towards FVIII and FIX, whereas this difference is most prominent for pFVIII. The reason for this is unknown. pFVIII contains vWF but also traces of immunomodulatory molecules which potentially are present in pFIX as well. As pFVIII-stimulation results in a reduction of regulatory as well as pro-inflammatory cytokine production, the impact on an autoimmune response has to be further elucidated.

PB0463 | Real-world Outcomes in People with Severe Hemophilia B Receiving FIX Prophylaxis across Europe: A CHESS II Analysis

T. Burke ${ }^{1,2} ;$ S. Asghar ${ }^{1}$; J. O'Hara ${ }^{1,2} ;$ M. Chuang ${ }^{3} ;$ E. Sawyer $^{3} ; \mathrm{N}^{2} \mathrm{Li}^{3}$ ${ }^{1}$ HCD Economics, Daresbury, United Kingdom; ${ }^{2}$ University of Chester, Chester, United Kingdom, ${ }^{3}$ uniQure Inc, Lexington, United States

Background: People with severe hemophilia B (HB) continue to experience bleeds and joint morbidity, despite therapeutic advancement and proliferation of prophylactic factor IX (FIX) therapy across Europe. Aims: The objective of this research is to examine real-world outcomes of severe hemophilia B across Europe, among people receiving FIX prophylaxis.

Methods: We report 12 months' outcomes using CHESS II ('Cost of Hemophilia in Europe: a Socioeconomic Survey II'), a retrospective burden-of-illness study conducted in 2019-2020. People with nonsevere HB, FIX inhibitors, or treated on-demand were excluded from the analysis. Results are presented as mean (standard deviation) or $\mathrm{N}$ (\%). 
Results: Of 132 people with severe HB in CHESS II, 75 subjects met the eligibility criteria and were included in the analysis. The mean age (years) and weight (kg) were 36.17 (15.97) and 77.27 (11.07). Respectively, 42 and 33 subjects received standard and extended half-life (SHL and EHL) FIX. Many experienced joint health issues, while the mean annualized bleeding rate was 2.40 (2.14).

The mean annual total medical cost per patient was $€ 235,723$ (€154,953), of which FIX therapy accounted for $98 \%$ of total cost. Mean annual FIX consumption was 245,175 IU $(144,903)$ and the overall cost of FIX was $€ 232,328$ (€153,979). Average SHL FIX consumption and cost were 286,969 IU $(153,211)$ at $€ 186,528(€ 116,403)$, while average EHL FIX consumption and cost were 191,680 IU $(115,180)$ and €290,620 (€176,665), respectively. Mean EQ-5D-5L score was 0.67 (0.21), annual non-medical costs were $€ 1,997$, and indirect costs were $€ 8,973$ (among a subset of subjects who completed the patient-reported outcome survey: $N=40$ ).

Conclusions: Despite recent treatment advancements, this analysis of CHESS II shows that burden and unmet needs remain among people with severe HB treated with FIX prophylaxis in Europe.

PB0464 | Real-world Clinical and Patient-centric Outcomes in People with Haemophilia A in Italy: Findings from the CHESS II Study

A. Shaikh ${ }^{1}$; T. Burke ${ }^{1,2}$; C. Hawes ${ }^{3}$; A. Lupi ${ }^{4}$; J. O'Hara ${ }^{1,2}$; C. Camp ${ }^{1}$ HCD Economics, Daresbury, United Kingdom; ${ }^{2}$ Faculty of Health and Social Care, University of Chester, Chester, United Kingdom; ${ }^{3}$ BioMarin Europe, London, United Kingdom; ${ }^{4}$ FedEmo (Federazione delle Associazioni Emofilici), Milan, Italy

Background: Haemophilia A (HA; factor VIII [FVIII] deficiency), characterised by prolonged trauma-related and/or spontaneous intra-articular bleeding events, is associated with adverse impacts on physical functioning and quality of life (QoL). The breadth of clinical and patient-relevant outcomes/experiences of people with HA (PWHA) in Italy - and variation between those with mild, moderate and severe $\mathrm{HA}$ - is not well understood.

Aims: To examine demographic, clinical and patient-centric outcomes across condition severity among adult PWHA in Italy, as captured in the 'Cost of Haemophilia in Europe: a Socioeconomic Survey - II' (CHESS II) study.

Methods: Data for non-inhibitor PWHA living in Italy were extracted from CHESS II and stratified based on clinician-reported baseline endogenous FVIII level. Participant demographics, annualized bleeding rate (ABR), incidence of target joints (ISTH definition) and joints exhibiting symptoms of chronic damage ('problem' joints), treatment regimen and QoL (EQ-5D-5L index score) were assessed. Outcomes by HA severity were compared using descriptive statistics. Study methodology and interpretation of results were informed by a representative from the Italian Federation of Haemophilia Associations (FedEmo) patients' organisation.

Results: Two hundred and thirty-two PWHA were included in the analysis (mild $n=34$, moderate $n=76$, severe $n=122$ ). The proportion of participants in full-time employment was lowest in severe HA (26\%; Table 1). Replacement FVIII was received by less than one third of participants with mild and moderate HA; prophylaxis regimens were most commonly used by participants with severe $H A$. Mean $A B R$, incidence of target joints and problem joints and EQ-5D-5L index score (0.94-0.78) all increased with severity of HA (Table 2).

TABLE 1 Participant demographics by HA severity

\begin{tabular}{|c|c|c|c|c|}
\hline Severity of HA & Mild $(n=34)$ & Moderate $(n=76)$ & Severe $(n=122)$ & TOTAL $(n=232)$ \\
\hline Age, mean $\pm S D$ & $36.59 \pm 12.03$ & $41.41 \pm 13.96$ & $38.54 \pm 13.53$ & $39.19 \pm 13.51$ \\
\hline $\begin{array}{l}\text { Body Mass Index (BMI), } \\
\quad \text { mean } \pm \mathrm{SD}\end{array}$ & $24.65 \pm 2.42$ & $24.48 \pm 2.82$ & $24.27 \pm 2.45$ & $24.39 \pm 2.57$ \\
\hline \multicolumn{5}{|l|}{ Employment status, $n$ (\%) } \\
\hline Employed full time & $16(47 \%)$ & 40 (53\%) & $32(26 \%)$ & 88 (16\%) \\
\hline Employment part-time & $2(6 \%)$ & $9(12 \%)$ & $19(16 \%)$ & $30(22 \%)$ \\
\hline Self-employed & $9(26 \%)$ & $8(11 \%)$ & $26(21 \%)$ & $43(30 \%)$ \\
\hline Unemployed & $1(3 \%)$ & $4(5 \%)$ & $11(9 \%)$ & $16(4 \%)$ \\
\hline Student & $4(12 \%)$ & $10(13 \%)$ & $21(17 \%)$ & 35 (10\%) \\
\hline Other & $2(6 \%)$ & $5(7 \%)$ & 13 (11\%) & $20(9 \%)$ \\
\hline
\end{tabular}


TABLE 2 Treatment strategy, clinical and patient-centric outcomes by HA severity

\begin{tabular}{|c|c|c|c|c|}
\hline Severity of HA & Mild $(n=34)$ & Moderate $(n=76)$ & Severe $(n=122)$ & TOTAL $(n=232)$ \\
\hline $\begin{array}{l}\text { Receiving factor replacement } \\
\text { therapy, } n(\%)\end{array}$ & $10(29 \%)$ & $21(28 \%)$ & $122(100 \%)$ & $153(66 \%)$ \\
\hline Primary on-demand & 9 (90\%) & $16(76 \%)$ & $47(39 \%)$ & 72 (47\%) \\
\hline Primary prophylaxis & $0(0 \%)$ & $0(0 \%)$ & $22(18 \%)$ & $22(14 \%)$ \\
\hline Secondary on-demand & 1 (10\%) & $5(24 \%)$ & $5(4 \%)$ & $11(7 \%)$ \\
\hline Secondary prophylaxis & $0(0 \%)$ & $0(0 \%)$ & 48 (39\%) & 48 (31\%) \\
\hline Annualised bleed rate, mean \pm SD & $1.06 \pm 0.89$ & $2.91 \pm 2.80$ & $3.94 \pm 3.17$ & $3.18 \pm 2.99$ \\
\hline Target joints, mean \pm SD & $0.12 \pm 0.33$ & $0.30 \pm 0.65$ & $0.75 \pm 0.85$ & $0.51 \pm 0.77$ \\
\hline Problem joints, mean \pm SD & $0.15 \pm 0.44$ & $0.70 \pm 0.82$ & $1.02 \pm 1.04$ & $0.79 \pm 0.95$ \\
\hline EQ-5D- $5 \mathrm{~L}, n$; mean \pm SD & $19 ; 0.94 \pm 0.07$ & $24 ; 0.81 \pm 0.12$ & $63 ; 0.78 \pm 0.20$ & $106 ; 0.82 \pm 0.17$ \\
\hline
\end{tabular}

Conclusions: The CHESS II study enrolled a large cohort of PWHA in Italy and provides an important resource for exploring differences in clinical outcomes and QoL across the spectrum of HA severity. This analysis observed greater clinical impairment and lower QoL with declining baseline FVIII levels.

PB0465 | Thrombin Generation by Genesia in Patients with Haemophilia A and Inhibitors during Immune Tolerance Induction

T. Szanto $^{1,2}$; E. Lehtinen ${ }^{1}$; M. Lemponen ${ }^{2}$; R. Lassila ${ }^{1,2}$

${ }^{1}$ Unit of Coagulation Disorders, Department of Hematology, Helsinki University Hospital Comprehensive Cancer Center, University of Helsinki, Helsinki, Finland; ${ }^{2}$ Research Program in Systems Oncology in Faculty of Medicine, University of Helsinki, Helsinki, Finland

Background: Inhibitor formation complicates hemophilia treatment and requires immune tolerance induction (ITI) to get rid of the hightiter inhibitors (> $5 \mathrm{BU} / \mathrm{ml}$ ).

Aims: To assess the hemostatic response to FVIII replacement during ITI by studying thrombin generation (TG) related to traditional FVIII and inhibitor levels.

Methods: Seven hemophilia A patients undergoing ITI were studied. FVIII product was plasma-derived in one and recombinant in 6 patients with the dosing $100 \mathrm{lU} / \mathrm{kg}$ od. Patients underwent a laboratory assessment after 72-h wash-out period followed by a prospective observation of 12 months and a maximum of 36 months. TG by Genesia/STG-BleedScreen and inhibitor titers (BU/ml) by Nijmegen Bethesda assay were measured. FVIII and TG before and after FVIII infusions were periodically repeated during follow-up (at week 3 , and at 3, 6 and 12 months). ABR (annual bleeding rate) and QoL (quality of life) were assessed.

Results: During the ITI, half of the patients achieved complete response within 6 months, one within 36 months, while two other patients achieved partial response. The peak inhibitor titer during ITI varied between 0.6 to $717 \mathrm{BU} / \mathrm{mL}$ (median 34). Genesia detected the hemostatic response to FVIII and ITI outcome. Post-infusion FVIII levels (30 min) correlated positively with ETP at week 3 (FVIII:C: $r=0.9$ and $P=0.02$; FVIII chromogenic: $r=0.9$ and $P=0.04$ ), and both with peak thrombin (FVIII:C: $r=0.8$ and $P=0.04$; FVIII chromogenic: $r=0.9$ and $P=0.02$ ) and ETP (FVIII:C: $r=0.9$ and $P=0.03$; FVIII chromogenic: $r=0.8$ and $P=0.04$ ) at 6 months. In contrast, inhibitor titers correlated negatively with post-infusion peak height $(r=-0.9$ and $P=0.02)$ and ETP $(r=-0.9$ and $P=0.003)$ at 3 months, and lag time at 6 and 12 months $(r=-0.9, P=0.03$ and $r=-0.9, P=0.01)$. ABR and QoL improved.

Conclusions: TG by Genesia distinguished different responses with a high sensitivity in inhibitor patients undergoing FVIII replacement.

PB0466 | GEPHARD - The PUP Cohort of the 'Standing Commission Paediatrics of the Society for Thrombosis and Haemostasis Research' - A Progress Report

C. Bildingmaier ${ }^{1}$; M. Olivieri ${ }^{1}$; M. Türknetz ${ }^{2}$; S. Schultze-Strasser ${ }^{2}$; C. Escuriola Ettingshausen ${ }^{3}$; K. Kentouche ${ }^{4}$; B. Zieger ${ }^{5}$; K. Kurnik ${ }^{1}$; W. Eberl ${ }^{6}$; C. Königs ${ }^{2}$; GEPHARD study group

${ }^{1}$ LMU Munich, Munich, Germany; ${ }^{2}$ University Hospital Frankfurt,

Frankfurt, Germany; ${ }^{3}$ HZRM Haemophilia Center Rhine Main,

Mörfelden-Walldorf, Germany; ${ }^{4}$ University Hospital Jena, Jena, Germany; ${ }^{5}$ University Hospital Freiburg, Freiburg, Germany; ${ }^{6}$ Klinikum Braunschweig, Braunschweig, Germany

Background: Approximately 40-60 newborns are newly diagnosed with haemophilia A or B every year in Germany. For prevention of bleeding and sequelae including haemophilic arthropathy, the standard treatment of PUPs is prophylaxis with clotting factor concentrates, although non-factor therapies are used increasingly. The optimal time or regimen for starting prophylactic therapy to avoid the development of joint disease or neutralising antibodies to clotting factors is under debate.

Aims: In Germany, data on incidence, treatment, clinical course and outcome of newly diagnosed haemophilia patients, have not been documented yet.

Methods: The German Paediatric Haemophilia Research Database (GEPHARD) includes all children and adolescents ( $<18$ years) that have been diagnosed with haemophilia A or B (FVIII or FIX levels $<25 \%)$ after January $1^{\text {st }}, 2017$. This prospective registry is open to all 
centres in Germany and collects variables related to diagnosis and therapy concentrating on outcome in haemophilia, including inhibitor development, offers quality assurance and serves as a base for future studies.

Results: The database has been established on the same platform as PedNet to allow international collaborations and data analyses. Since January $1^{\text {st }} 2017,252$ children have been enrolled from 37 participating centres. Out of these, 203 children were diagnosed with haemophilia $A$. The median age of diagnosis of children with severe phenotype $(n=127)$ is 0.38 years, with moderate $(n=16) 0.04$ years and 1.5 years with mild phenotype $(n=59)$. Thirty-seven children were diagnosed with haemophilia B. The median age of diagnosis of children with severe phenotype $(n=25)$ is 0.17 years, with moderate $(n=7) 2.75$ years and 3.21 years with mild phenotype $(n=6)$. By establishing new ways of data collection, such as remote documentation longitudinal documentation to collect and analyse clinical parameters can continue despite the pandemic.

Conclusions: GEPHARD has been established successfully and has adopted to the pandemic. Further results on novel treatment strategies are expected in 2021.

\section{PB0467 | Effects of von Willebrand Factor on Plasma}

\section{Haemostasis}

\section{Kluft; K. Van Leuven; C. Krijnen; P. De Kruijf}

Good Biomarker Sciences, Sassenheim, Netherlands

Background: Mechanisms of VWF in vessel wall and platelet interactions are well documented. In addition, VWF also modulates plasma coagulation and fibrinolysis.

Aims: We evaluated the effects of VWF in plasma ROTEM, in standard one-stage clotting assay and in plasma clot growth with the Thrombodynamics (Hemacore).

Methods: APTT was with silica reagent. Rotem analysis after recalcification recorded maximal clot firmness; with thrombodynamics both growth of fibrin and formation of thrombin was followed. Deficient plasma's for VWF and Factor VIII, and normal plasma were obtained from commercial sources. Added VWF was Willefact.

Results: With normal physiological concentrations, factor VIII is approximately for 50\% reversibly bound to VWF. In the complex, Factor VIII clotting activity is inhibited. Assay with APTT shows about 2-fold higher Factor VIII when VWF is absent. This was used for a modification of factor analysis with lower detection limit valuable in severe haemophilia patients.

In the thrombodynamic system, no effect was observed on thrombin generation at the tissue factor surface and on the rate of clot growth from $0-100 \%$ VWF. However, a clear increase in thrombin generated further away from the surface (intrinsic thrombin generation dependent upon factor XI, IX and VIII) was seen with an increase in thrombin amplitude of $250 \%$ for 100 - 0\% VWF. This thrombin formation is considered to produce increased thrombomodulin
(TM)-dependent TAFI activation and concerns a potential of clot protection against lysis in TM-rich area's in VWF deficiency.

ROTEM recalcifation analysis of VWF deficient plasma shows a low rigidity, which increases dose-dependently after addition of VWF.

Conclusions: Taken these effects together it suggests that plasma coagulation in VWF deficiency results in relatively more rapid coagulation due to larger effects of factor VIII, more clot stabilization in TM-rich environment, and a lower rigidity. These opposite effects may modulate the bleeding profile.

PB0468 | Examination and Validation of a Patient-centric Joint Metric: "PROBLEM JOINT"; Empirical Evidence from the CHESS Paediatrics Dataset

T. Burke ${ }^{1}$;. Rodriguez Santana ${ }^{1}$; P. Chowdary ${ }^{2}$; R. Curtis ${ }^{3}$; K. Khair ${ }^{4}$; M. Laffan ${ }^{5}$; P. McLaughlin²; D. Noone ${ }^{1}$; B. O'Mahony ${ }^{6}$; J. Pasi ${ }^{7}$; M.W Skinner ${ }^{8} ;$ J. O'Hara ${ }^{1}$

${ }^{1}$ HCD Economics, Daresbury, Warrington, United Kingdom; ${ }^{2}$ Katherine Dormandy Haemophilia Centre and Thrombosis Unit, Royal Free London NHS Foundation Turst, London, United Kingdom; ${ }^{3}$ Hematology Utilization Group Study (HUGS), Walnut Creek, United States;

${ }^{4}$ Haemnet, London, United Kingdom; ${ }^{5}$ Centre for Haematology, Imperial College London, London, United Kingdom; ' Irish Haemophilia Society, Dublin, Ireland; ${ }^{7}$ Royal London Haemophilia Centre, Barts and The London School of Medicine and Dentistry, London, United Kingdom; ${ }^{8}$ Institute for Policy Advancement Ltd, Washington, United States

Background: Haemophilia is characterized by spontaneous hemarthrosis that often develop into chronic synovitis, pain, and eventually destruction of the joint.

Clinical metrics of joint morbidity, e.g., 'Target joint', are prevalent, accepted and relevant, though measures focused on bleeding activity are arguably becoming less sensitive to patient burden as current treatment strategies look to eradicate hemarthrosis.

'Problem Joint' (PJ) is a joint morbidity metric defined by an Expert Reference Group as having chronic joint pain and/or limited range of movement due to chronic synovitis and/or haemophilic arthropathy, with or without persistent bleeding.

Aims: Test the sensitivity of PJs as a patient relevant metric in children.

Methods: Data on PJs, demographics, clinical and socio-economic variables were captured within the 'Cost of Haemophilia in Men: Socioeconomic Survey' a family of datasets containing over 4,000 people with haemophilia. The paediatric European cohort (CHESS Paeds) comprised the focus of this piece. Statistical analysis explored the association of PJ count and location with respect to quality of life (EQ-5D-Y), and parent caregiving duties. Individuals with inhibitors were excluded.

Results: This cohort $(N=198)$ recorded a mean age of 11.2. Around $19 \%$ had one or more PJs. Weight bearing PJs were more prevalent; $12 \%$ had $1+$ weight bearing PJs vs. $8.5 \%$ non-weight bearing. EQ-5D and PJ count showed a negative trend (see Figure 1). Mean EQ-5D 
score was 0.71 for those with 0 PJs ( $N=160), 0.60$ for 1 PJ $(N=30)$ and 0.47 for $2+$ PJs $(N=8)$. Similarly, an increase in PJs lead to a greater caregiver burden: an average of 19.2 hours/week for those with 0 PJ $(N=124)$ vs. 30.8 for $1+P J s(N=29)$.

Figure 1: Mean EQ-5D by number of Problem Joints (PJ) $(\mathrm{N}=198)$

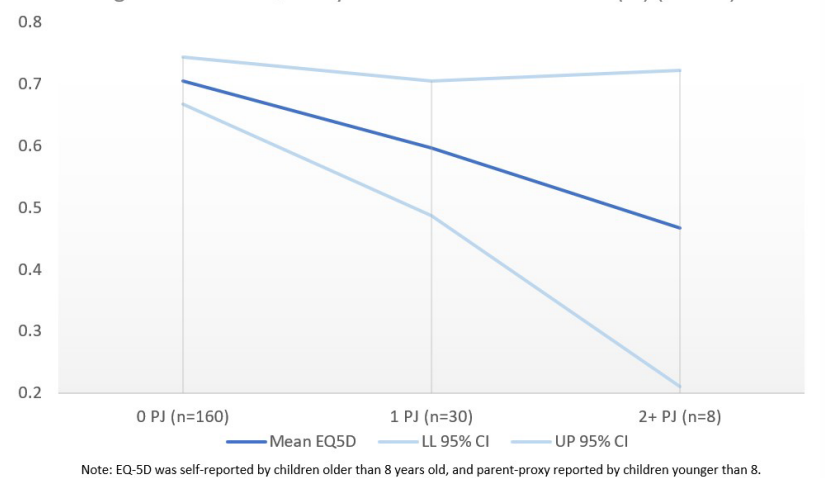

FIGURE 1 Mean EQ-5D by number of Problem Joints (PJ) - N = 198

Conclusions: An increase in the number of PJs was associated with increasing humanistic burden in children. The PJ definition assesses from a patient relevant perspective. Further work will evaluate the appropriateness of the measure in a wider cohort.

\section{PB0469 | Dose Selection for Subcutaneous Marzeptacog Alfa} (Activated) in Subjects with Factor VII Deficiency Using Population Pharmacometric Clinical Trial Simulations

A. Faraj ${ }^{1}$; S. Desai ${ }^{2}$; L. Neuman ${ }^{2}$; G.E Blouse ${ }^{2}$; T. Knudsen ${ }^{2}$;

U.S. Simonsson ${ }^{1}$

${ }^{1}$ Uppsala Universitet, Uppsala, Sweden; ${ }^{2}$ Catalyst Biosciences, South

San Francisco, United States

Background: Marzeptacog alfa (activated) (MarzAA) is a novel increased potency rFVIla being developed for subcutaneous (SQ) treatment of bleeding in FVII deficiency (FVIID) and other bleeding disorders.

Aims: To conduct clinical trial simulations to support dose selection for SQ MarzAA in a Phase 1/2 trial in FVIID.

Methods: A population pharmacokinetic (popPK) model based on clinical data from subjects with hemophilia was adapted for dose selection in FVIID. Clinical trial simulations following different doses were conducted in 1000 virtual FVIID subjects by baseline adjusting FVIla levels to 0 and assuming lower FVIla recovery (specifically $43.5 \%$ of that observed in Hemophilia A). Assuming that MarzAA is at least 5-fold more potent than wild type FVIla, secondary PK parameters and the expected time-to-target of $2 \mathrm{ng} / \mathrm{mL}$ and percentage of the population above target at different time-points were derived.
Results: A single dose of 20, 30 or $60 \mu \mathrm{g} / \mathrm{kg}$ resulted in median Cmax (and $80 \%$ prediction intervals (PI)) of 4.8 (2.4-9.7), 7.3 (3.7-14.5) or 14.5 (7.3-29.0) ng/mL, respectively. The median and $80 \% \mathrm{PI}$ of timeto-target following a single dose of 20,30 or $60 \mu \mathrm{g} / \mathrm{kg}$ were $1.5(0.8-$ 3.2), 1.2 (0.6-2.3) and $0.8(0.4-1.5)$ hours, respectively (Table 1).

TABLE 1

Table 1. Time to reach target levels in ( $\geq 2 \mathrm{ng} / \mathrm{mL}$ ) in individuals with FVIID.

\begin{tabular}{|c|c|}
\hline $\begin{array}{c}\text { Regimen } \\
\text { (Dosing time point, hours) }\end{array}$ & $\begin{array}{c}\text { Time in hours } \\
\text { Median (80\% PI) }\end{array}$ \\
\hline $20 \mu \mathrm{g} / \mathrm{kg}(\mathrm{t}=0)$ & $1.5(0.8-3.2)$ \\
\hline $30 \mu \mathrm{g} / \mathrm{kg}(\mathrm{t}=0)$ & $1.2(0.6-2.3)$ \\
\hline $60 \mu \mathrm{gg} / \mathrm{kg}(\mathrm{t}=0)$ & $0.8(0.4-1.5)$ \\
\hline
\end{tabular}

A single dose of $20 \mu \mathrm{g} / \mathrm{kg}$ resulted in $80 \%, 90 \%$ and $68 \%$ of the population being above target at 3, 6, and 24 hours post dosing, respectively. Doses of 30 or $60 \mu \mathrm{g} / \mathrm{kg}$ expanded the proportion of population above target to $89 \%$ and $98 \%$ at 24 hours post dosing, respectively (Table 2 ).

\section{TABLE 2}

Table 2. Percentage of the simulated FVIID population above target $(2 \mathrm{ng} / \mathrm{mL})$ after varying SQ marzeptacog alfa (activated) doses.

\begin{tabular}{|c|c|c|c|}
\hline & \multicolumn{3}{|c|}{$\begin{array}{r}\text { Percentage of population above target for three different doses } \\
\text { given as a single SQ injection }\end{array}$} \\
\hline Time after dose (hours) & $20 \mu \mathrm{g} / \mathrm{kg}$ & $\mathbf{3 0 ~ \mu g / k g}$ & $60 \mu \mathrm{g} / \mathrm{kg}$ \\
\hline 1 & $20 \%$ & $35 \%$ & $68 \%$ \\
\hline 3 & $80 \%$ & $92 \%$ & $99 \%$ \\
\hline 6 & $90 \%$ & $97 \%$ & $100 \%$ \\
\hline 9 & $92 \%$ & $98 \%$ & $100 \%$ \\
\hline 12 & $92 \%$ & $98 \%$ & $100 \%$ \\
\hline 24 & $68 \%$ & $89 \%$ & $98 \%$ \\
\hline 48 & $29 \%$ & $53 \%$ & $87 \%$ \\
\hline & $6.1 \%$ & $18 \%$ & $56 \%$ \\
\hline
\end{tabular}

Conclusions: The clinical trial simulations indicated that hemostasis may be achieved with the proposed doses and that good exposure levels may be sustained for 24 hours even in the $20 \mathrm{ug} / \mathrm{kg}$ dose group. The Phase $1 / 2$ trial (NCT04548791) is currently enrolling participants. 
PB0470 | Predicting the Duration of Recombinant Factor VIla Effect on Tail Bleeding by the Thrombin Generation Assay in Hemophilia A Mice

\section{S. Surov $^{1,2} ;$ M. Ovanesov ${ }^{1}$}

${ }^{1}$ Center for Biologics Evaluation and Research, U.S. Food and Drug

Administration, Silver Spring, United States; ${ }^{2}$ Center for Theoretical

Problems of Physicochemical Pharmacology, Moscow, Russian

Federation

Background: Recombinant factor VIla (rFVIla) is used for treatment of hemophilia patients with inhibitors and other indications. The mechanism of rFVIla action is mediated by the platelet phospholipids (PL) and tissue factor (TF). Traditional laboratory assays have not been useful in guiding rFVIla therapy, and the PL- and/or TF-triggered thrombin generation (TG) assay was proposed for this purpose.

Aims: Study the predictive value of plasma levels of FVIla activity and TG assays for the effect of rFVIla treatment on tail bleeding in FVIII knockout mice.

Methods: Mice were treated with $5 \mathrm{mg} / \mathrm{kg}$ of $\mathrm{rFVIla}$ and blood was collected 5, 30, 60 minutes and 2 hours (2h), 4h, 8h, 16h and 24h after drug administration. TG was triggered with combinations of $\mathrm{PL}$, TF and human or mouse soluble TF (sTF). FVIla activity was measured with an sTF-based clotting StaClot assay. In a parallel tail clip study, bleeding was evaluated for 30 minutes after injury.

Results: Tail bleeding studies demonstrated gradual weakening of rFVIla effect between 5 minute and $1 \mathrm{~h}$ points, with no hemostatic effect by $2 \mathrm{~h}$ after administration. All TG assays showed difference in thrombin peak height readouts between the $1 \mathrm{~h}$ and $2 \mathrm{~h}$ time points. The peak height responses of sTF+PL and TF+PL triggered TG assays continued to decline until $16 \mathrm{~h}$ after administration, and for the remaining TG assays - until $4 \mathrm{~h}$ to $8 \mathrm{~h}$. The greatest gradient in thrombin peak responses at $1 \mathrm{~h}$ time point was provided by the $\mathrm{PL}$ trigger.

Conclusions: TG assay may be suitable for evaluation of the duration of hemostatic action of rFVIla. PL-triggered TG assay offers better correlation with the loss of hemostatic effect which may be explained by the primarily PL-dependent activity of human rFVIla in mice.

Disclosure: This paper is an informal communication and represents the authors' best judgment. These comments do not bind or obligate FDA.
PB0471 | Comparative Recovery of FVIII Antigen with Various FVIII Therapeutic Concentrates Using an Improved $2^{\text {nd }}$ Generation ELISA Kit

R. Ni ${ }^{1}$; P. Erb ${ }^{1}$; J. Della Maestra ${ }^{1} ;$ H. Atkinson ${ }^{2} ;$ D. Foulon ${ }^{1}$; K.M Black ${ }^{3}$; H. Hoogendoorn ${ }^{1}$; D. Matino ${ }^{2,4}$

${ }^{1}$ Affinity Biologicals, Hamilton, Canada; ${ }^{2}$ Thrombosis and Atherosclerosis Research Institute, Hamilton, Canada; ${ }^{3}$ Precision BioLogic, Dartmouth, Canada; ${ }^{4}$ Department of Medicine, McMaster University, Hamilton, Canada

Background: In recent years, the treatment of hemophilia A has trended toward the use of truncated forms of FVIII where most or all of the B-domain has been deleted (BDD-FVIII), while FVIII coagulant activity is retained. The use of BDD-FVIII constructs present challenges for existing commercial FVIII antigen kits, where their recognition can be reduced. A new $2^{\text {nd }}$ generation ELISA kit (F8PLUS-AG) was developed with the intent to provide a more uniform recognition of native, full-length, and BDD-FVIII preparations, while using a WHO-traceable plasma-based calibrator. The method and assay performance of the $1^{\text {st }}$ (FVIII-AG) and $2^{\text {nd }}$ generation (F8PLUS-AG) kits are similar with regard to sensitivity $(0.008 \mathrm{IU} / \mathrm{mL})$ and noninterference by von Willebrand factor. The distinguishing feature of the new kit is the improved recognition of BDD-FVIII.

Aims: To assess the $2^{\text {nd }}$ generation antigen kit (F8PLUS-AG) in the quantification of various therapeutic FVIII products currently available.

Methods: In this study, plasma-derived (pdFVIII), recombinant fulllength (rfIFVIII) and BDD-FVIII replacement products were reconstituted and spiked into congenital FVIII-deficient plasma to achieve activity levels of $1.0,0.3$, and $0.05 \mathrm{IU} / \mathrm{mL}$ based on the product assigned activity potency. FVIII antigen was measured in both $1^{\text {st }}$ and $2^{\text {nd }}$ generation kits and expressed as a ratio of antigen to the expected activity.

Results: Both antigen kits gave similar recoveries for pdFVIII and rfIFVIII, which were consistent with the expected activity. The exception was porcine rFVIII, which was not recognized with either of these kits (Table 1). 
TABLE 1 Ratios (FVIII Antigen : Expected FVIII Activity) of pdFVIII, rfIFVIII, PEGylated FVIII, and porcine rFVIII as determined using 1st generation (FVIII-AG) and 2nd generation (F8PLUS-AG) antigen kits.

\begin{tabular}{|c|c|c|c|c|c|c|c|}
\hline FVIII Sample & Description & \multicolumn{3}{|c|}{ FVIII Ag:Ac Ratio by $1^{\text {st }}$ Gen (FVIII-AG) Kit } & \multicolumn{3}{|c|}{ FVIII Ag:Ac Ratio by New $2^{\text {nd }}$ Gen (F8PLUS-AG) Kit } \\
\hline NPP & $\begin{array}{l}\text { Normal Pooled } \\
\text { Plasma }\end{array}$ & 1.50 & & & 1.33 & & \\
\hline pdFVIII-2 & $\begin{array}{c}\text { Plasma-Derived } \\
\text { FVIII/VWF } \\
\text { Complex }\end{array}$ & 0.72 & 0.63 & 0.62 & 1.04 & 0.83 & 0.84 \\
\hline rfIFVIII-1 & $\begin{array}{l}\text { Recombinant } \\
\text { Full-Length } \\
\text { FVIII }\end{array}$ & 1.43 & 1.40 & 1.34 & 1.16 & 1.00 & 1.02 \\
\hline rfIFVIII-PEG & $\begin{array}{l}\text { PEGylated } \\
\text { Recombinant } \\
\text { Full-Length } \\
\text { FVIII }\end{array}$ & 1.37 & 1.26 & 1.06 & 1.22 & 0.97 & 0.90 \\
\hline $\begin{array}{l}\text { rFVIII- } \\
\text { Porcine }\end{array}$ & $\begin{array}{l}\text { Recombinant } \\
\text { Porcine FVIII } \\
\text { Sequence }\end{array}$ & not detectable & not detectable & not detectable & not detectable & not detectable & not detectable \\
\hline
\end{tabular}

The BDD-FVIII products tested in the $1^{\text {st }}$ generation kit were significantly under-recovered, confirming earlier observations. In contrast, these samples tested in the F8PLUS-AG kit demonstrated considerably higher recoveries, with antigen values more in line with the expected activity values (Table 2).

TABLE 2 Ratios (FVIII Antigen : Expected FVIII Activity) of five different BDD-FVIII products determined using 1st generation (FVIII-AG) and 2nd generation (F8PLUS-AG) antigen kits.

\begin{tabular}{|c|c|c|c|c|c|c|c|}
\hline \multirow{2}{*}{$\begin{array}{l}\text { FVIII Sample } \\
\text { NPP }\end{array}$} & \multirow{2}{*}{$\begin{array}{l}\text { Description } \\
\text { Normal Pooled Plasma }\end{array}$} & \multicolumn{3}{|c|}{ FVIII Ag:Ac Ratio by $1^{\text {st }}$ Gen (FVIII-AG) Kit } & \multicolumn{3}{|c|}{$\begin{array}{l}\text { FVIII Ag:Ac Ratio by New } 2^{\text {nd }} \text { Gen } \\
\text { (F8PLUS-AG) Kit }\end{array}$} \\
\hline & & 1.50 & & & 1.33 & & \\
\hline \multicolumn{2}{|c|}{ Expected FVIII Activity Values } & $1.00 \mathrm{IU} / \mathrm{mL}$ & $0.30 \mathrm{IU} / \mathrm{mL}$ & $0.05 \mathrm{IU} / \mathrm{mL}$ & $1.00 \mathrm{IU} / \mathrm{mL}$ & $0.30 \mathrm{IU} / \mathrm{mL}$ & $0.05 \mathrm{IU} / \mathrm{mL}$ \\
\hline BDDrFVIII-1 & $\begin{array}{l}\text { Recombinant B-Domain } \\
\text { Deleted FVIII }\end{array}$ & 0.30 & 0.28 & 0.26 & 0.79 & 0.70 & 0.74 \\
\hline BDDrFVIII-2 & $\begin{array}{l}\text { Recombinant B-Domain } \\
\text { Deleted FVIII }\end{array}$ & 0.26 & 0.21 & 0.20 & 0.81 & 0.67 & 0.66 \\
\hline BDDrFVIII-3 & $\begin{array}{l}\text { Recombinant B-Domain } \\
\text { Deleted FVIII }\end{array}$ & 0.26 & 0.20 & 0.20 & 0.78 & 0.60 & 0.52 \\
\hline BDDrFVIII-4 & $\begin{array}{l}\text { Recombinant B-Domain } \\
\text { Deleted FVIII }\end{array}$ & 0.32 & 0.28 & 0.24 & 0.87 & 0.70 & 0.66 \\
\hline BDDrFVIII-5 & $\begin{array}{l}\text { Recombinant B-Domain } \\
\text { Deleted FVIII }\end{array}$ & 0.27 & 0.22 & 0.20 & 0.71 & 0.57 & 0.56 \\
\hline
\end{tabular}

Conclusions: We verified significantly improved recognition of various BDD-FVIII constructs using the $2^{\text {nd }}$ generation antigen kit, demonstrating its versatility in modern hemophilia research. 
PB0472 | Results of Thrombin Generation Assay in Patients Treated with Emicizumab

B. Baran $^{1}$; E. Stefanska-Windyga ${ }^{1}$; A. Buczma ${ }^{1}$; E. Odnoczko ${ }^{1}$; J. Windyga ${ }^{2}$

${ }^{1}$ Institute of Haematology and Transfusion Medicine, Warszawa, Poland; ${ }^{2}$ Institute of Haeamatology and Transfusion Medicine, Warszawa, Poland

Background: Emicizumab (EMI) is a recombinant, humanized bispecific antibody that mimicking the cofactor function of FVIII. Although the use of EMI does not require routine laboratory monitoring, there are clinical situations (e.g. critical bleeding) when evaluation of the patient's hemostatic status may prove useful. EMI therapy interferes with standard coagulation tests and makes laboratory monitoring challenging.

Aims: To investigate the role of thrombin generation assay (TGA) in monitoring the hemostatic potential of emicizumab in hemophilia $A$ patients.

Methods: We analyzed 50 plasma samples from 13 hemophilia A (including 8 with FVIII inhibitor) patients on emicizumab. All patients samples were collected in steady state, just before the next dosage. Laboratory work-up included plasma emicizumab concentration (using a modified one-stage Factor VIII assay on the BCS XP analyzer [Siemens ${ }^{\circledR}$ ] with an emicizumab-specific calibrator and controls [ ${ }^{2}$ Diagnostics]) and TGA determined in platelet-poor plasma on Ceveron ${ }^{\circledR}$ alpha with RC low reagent (Technoclone $\left.{ }^{\circledR}\right)$. The patients' TGA results were compared with those in two control groups [43 patients (aged 18-79) with confirmed mild hemophilia A (MHA) and 21 healthy male (aged 21-63)].

Results: Individual patient EMI concentration were within the range $26.6 \pm 2.41$ to $67.1 \pm 6.16 \mu \mathrm{g} / \mathrm{mL}$. Mean TGA peak thrombin (TGA-pt) was lower in EMI patients than in healthy controls $(42.70 \pm 1.70$ vs $92.20 \pm 11.74$, respectively) while it was comparable to the TGA-pt results of the MHA patient group $(42.70 \pm 1.70$ vs $43 \pm 11.74 \mathrm{nM}$, respectively). In the sample with the lowest concentration of EMI $(24.1 \mu \mathrm{g} / \mathrm{mL})$ TGA-pt was $31.6 \mathrm{nM}$ and in the sample with the highest concentration $(73.5 \mu \mathrm{g} / \mathrm{mL})-46.00 \mathrm{nM}$. No breakthrough bleeds was reported in patients during our study.

Conclusions: TGA seems to be a useful laboratory tool in monitoring of EMI therapy. Peak thrombin parameter is particularly useful in evaluating hemostasis in patients on EMI prophylaxis. There was positive correlation between EMI concentration and TGA-pt.
PB0473 | Clinical Characteristics and Incidence of Inhibitors in Previously Untreated Children with Haemophilia: An Update of the Hemfil Cohort Study

L.L. Jardim ${ }^{1,2}$; M. Antônio Portugal Santana3 ${ }^{3}$; D. Gonçalves Chaves $^{4}$; L. Werneck Zucheratto ${ }^{1}$; M. Hermida Cerqueira ${ }^{5}$; C. Santos Lorenzato ${ }^{6}$; V. Franco $;$; J. van der Bom²; S. Meireles Rezende $^{1}$

${ }^{1}$ Faculty of Medicine, Universidade Federal de Minas Gerais, Belo Horizonte, Brazil; ${ }^{2}$ Department of Clinical Epidemiology, Leiden University Medical Centre, Leiden, Netherlands; ${ }^{3}$ Fundação HEMOMINAS, Belo Horizonte, Brazil; ${ }^{4}$ Fundação HEMOMINAS, Belo Horizonte, Brazil; ${ }^{5}$ Hemorio, Rio de Janeiro, Brazil; ${ }^{6}$ HEMEPAR, Curitiba, Brazil; ${ }^{7}$ HEMOSC, Florianopolis, Brazil

Background: Inhibitors are the most serious complication in patients with haemophilia $\mathrm{A}(\mathrm{HA})$ and $\mathrm{B}(\mathrm{HB})$. A complete understanding of inhibitor development is still lacking.

Aims: To update the clinical characteristics and the cumulative incidence of inhibitor development in the HEMFIL cohort study.

Methods: The HEMFIL is a Brazilian prospective cohort study on risk factors for inhibitor development. We include children with HA and $\mathrm{HB}$ before the first infusion of any factor concentrate and follow up until 75 exposure days (ED) or inhibitor development. Time to inhibitor development was calculated by Kaplan-Meier cumulative incidence with cumulative ED as the time variable.

Results: Previously, the Hemfil study reported a cumulative incidence of $36 \%$ [95\% Cl,26\%-49\%] for all inhibitors, $27 \%$ (95\%Cl,18\%-40\%) for high-titer and $13 \%(95 \% \mathrm{Cl}, 6-24 \%)$ for low-titre inhibitors in a population of 70 included children under exclusive use of a thirdgeneration recombinant FVIII [Advate ${ }^{\circledR}($ Shire, USA)] (Jardim, et al 2018). Currently, 120 children were included, of whom 104 with $\mathrm{HA}$ and 16 with $\mathrm{HB}$. A total of 10 patients with $\mathrm{HB}$ have completed 75ED and no patient developed inhibitor. Of all patients with HA, $89 \%$ are severe/moderately severe (FVIII:C $<2 \%$ ). Inhibitor was detected in 33/85 (38\%) patients of which $22(69 \%)$ were high-titre and 53/85 (62\%) patients reached 75ED without inhibitors. Currently, the cumulative incidence of inhibitors in HA patients at 75ED is 35\% (95\% Cl,26\%-46\%) for all inhibitors, 25\% (95\%Cl,17\%-36\%) for hightiter and $13 \%(95 \% \mathrm{Cl}, 8 \%-23)$ for low-titer inhibitors. The median time for inhibitor development remains 14ED (IQR,7-21) with a median age of 13 months (IQR,10-17). The median weight at inclusion is $9 \mathrm{~kg}(I Q R, 8-12)$. 
TABLE 1 Baseline characteristics of children with HA

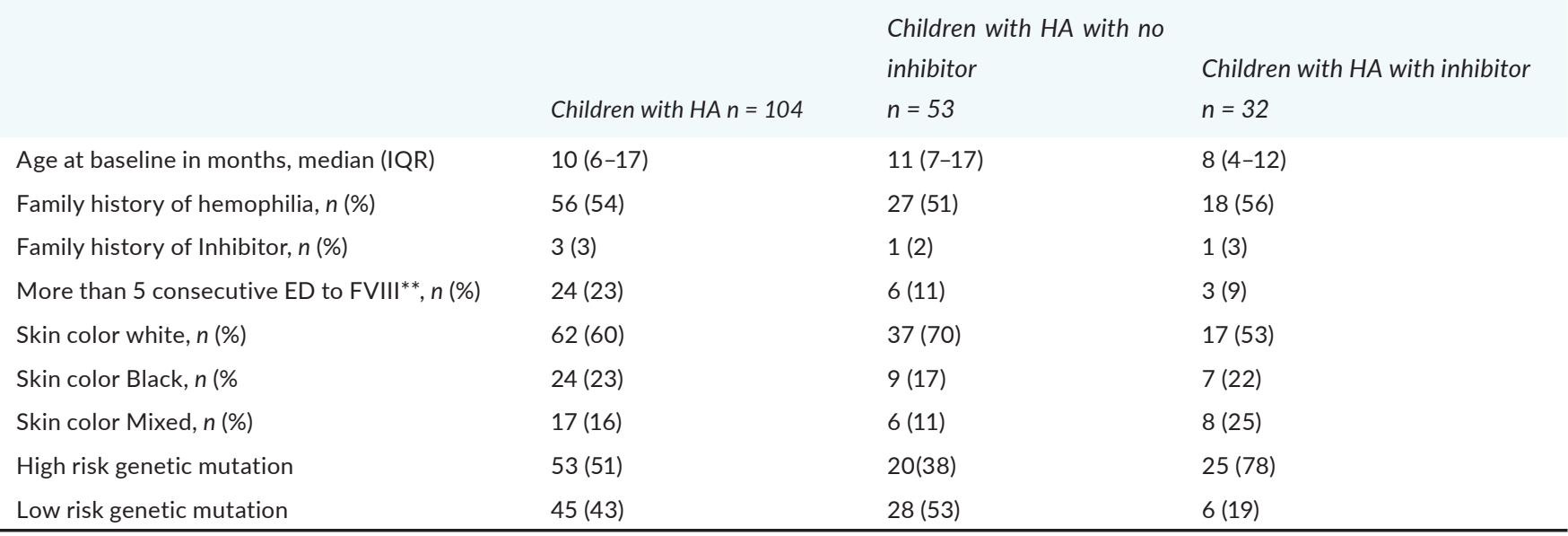

Conclusions: The cumulative incidence of inhibitor development under the exclusive use of a third-generation recombinant FVIII concentrate remains constant 3 years after the first report, inclusion of additional patients and a longer follow-up. This reinforces the robustness of these data.

PB0474 | Is Standardisation the Key? FVIII Inhibitor Results: A UK National External Quality Assessment Scheme for Blood Coagulation (UK NEQAS BC) Exercise

A. Lowe; S. Kitchen; I. Jennings; I. Walker

UK NEQAS BC, Sheffield, United Kingdom

Background: FVIII inhibitors are immunoglobulins that bind to FVIII molecules reducing the function of the FVIII protein to perform its job, leaving the patient with bleeding episodes. Clinical identification of the presence of FVIII inhibitors has to be confirmed by laboratory testing.

Aims: To assess the large range of results from centres of a UK NEQAS BC FVIII inhibitor exercise by investigating different aspects of the FVIII inhibitor methodology.

Methods: Two lyophilised plasma samples from a donor with haemophilia A, identified as having an inhibitor to FVIII were distributed with different code numbers (S20:04 \& S20:05) so that it was not apparent the two samples were identical. Participants were invited to perform a FVIII inhibitor assay using test methods in routine use in their laboratory. Methodology details were requested from centres including;

1. Assay used for measurement

2. Plasma sample dilutions

3. Diluent medium

4. FVIII inhibitor methodology

Results: 84 centres returned results of the FVIII inhibitor methodologies used

Bethesda method $(n=25)$ FVIII inhibitor range $=0.0--7.6 \mathrm{Bu} / \mathrm{ml}$

Nijmegen-Bethesda method $(n=24)$ FVIII inhibitor range $=0.44--$ $4.8 \mathrm{Bu} / \mathrm{ml}$

Non-classified method $(n=35)$ FVIII inhibitor range $=0.00--4.8 \mathrm{Bu}$ / $\mathrm{ml}$

26 different types of dilution combination were used, ranging from single dilution (1in1) to greater than 9 (1in2, 1in 4, 1in8, 1in16, 1in32, 1in64, 1 in128 up to 1 in2048) FVIII inhibitor results from 82 participants according to what diluent was used

\section{TABLE 1}

\begin{tabular}{lllll} 
Type of diluting medium & N & Median FVIII inhibitor (BU/ml) & FVIII inhibitor results (BU/ml) & CV (\%) \\
FVIII deficient plasma & 24 & 2.3 & $0.00-4.80$ & 200.9 \\
Buffer & 53 & 2.9 & $0.00-7.60$ & 195.4 \\
Not stated & 5 & 3.3 & $1.97-4.00$ & 196.7 \\
\hline
\end{tabular}


There was highly significant correlation $\left(R^{2}=0.72, P<1.00\right)$ between results obtained on the two samples indicating intra-lab precision and some consistency in how methods were applied locally

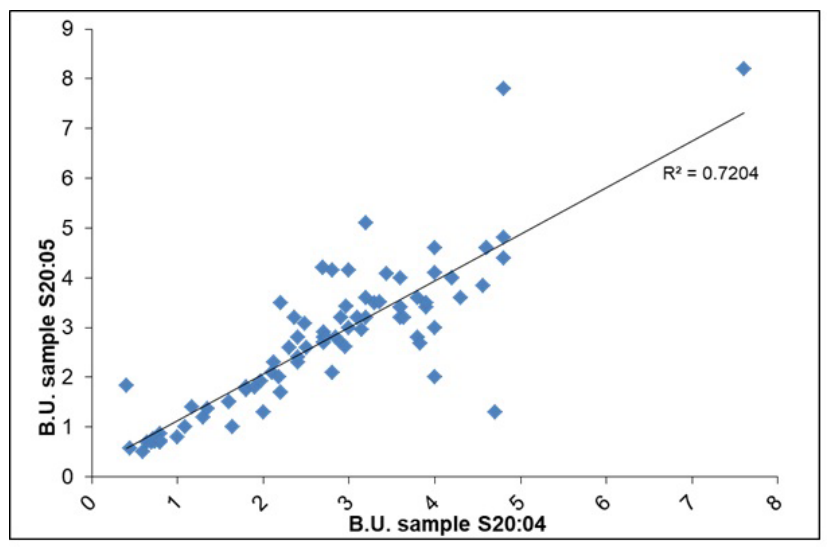

\section{FIGURE 1}

Conclusions: There was considerable variation throughout the FVIII inhibitor assay methods used by participants, with no clear pattern or trend to help explain the wide range of results. The data demonstrate that there is a requirement for appropriate standardisation of the FVIII inhibitor assay to reduce misdiagnosis and potential inappropriate clinical management.

\section{PB0475 | Spectrum of F8/F9 Gene Mutations in the PedNet Cohort}

V. Labarque ${ }^{1}$; M.-E. Mancuso ${ }^{2}$; M. Kartal-Kaess ${ }^{3}$; R. Ljung ${ }^{4}$; T.S. Mikkelsen ${ }^{5}$; N. Gretenkort Andersson ${ }^{6}$; PedNet Study Group ${ }^{1}$ University Hospitals Leuven, Leuven, Belgium; ${ }^{2}$ IRCCS Humanitas Research Hospital, Milan, Italy; ${ }^{3}$ Inselspital, University Hospital, University of Bern, Bern, Switzerland; ${ }^{4}$ Lund University, Lund, Sweden; ${ }^{5}$ Aarhus University Hospital, Aarhus, Denmark; ${ }^{6}$ Skåne University Hospital, Lund, Sweden

Background: Hemophilia $A(H A)$ and $B(H B)$ are caused by mutations in the F8 or F9 gene. Online databases (e.g. CHAMP/CHBMP or EAHAD) list over 4,200 unique pathogenic variants. However, selective reporting may influence the distribution of mutations reported in these databases. Aims: We aimed to determine the spectrum of F8 and F9 gene mutations in an international prospective cohort of unselected patients (PedNet Registry).

Methods: The PedNet Registry collects clinical, genetic, and phenotypic data prospectively in children with hemophilia. A total of 2,304 patients registered by January 2020 were included. Data on type and severity of hemophilia and on molecular genetic analysis of F8 and F9 gene variants (HGVS nomenclature) were analyzed.

Results: Genetic information was available on 1,548 out of 1,926 HA patients (80\%) and 279 out of $378 \mathrm{HB}$ patients (74\%). No abnormalities were found in the respective genes in $32 \mathrm{HA}$ and $5 \mathrm{HB}$ patients
( $2 \%$ each). The distribution of mutations in severe patients is shown in figure 1.

In severe HA ( $n=1,089$ ), we identified 586 intron 22 inversions (54\%), 178 frameshift (16\%; mostly exon 14), 125 missense (11\%; mostly exons 23, 26), 98 nonsense ( $9 \%$; mostly exon 14), and 37 splice site mutations (3\%), 28 large structural changes (2.5\%), 17 intron 1 inversions (2\%) and 3 small structural changes $(0.3 \%$; in exons $15,22,24)$.

In severe HB ( $n=140)$, we found mostly missense $(n=65 ; 46 \%)$ and nonsense mutations ( $n=28 ; 20 \%$ ), but also large structural changes ( $n=17 ; 12 \%)$, frameshift ( $n=13 ; 9 \%$ ), promoter and splice site mutations ( $n=6 ; 4 \%$ each), and small structural changes $(n=3 ; 2 \%)$. In HB,

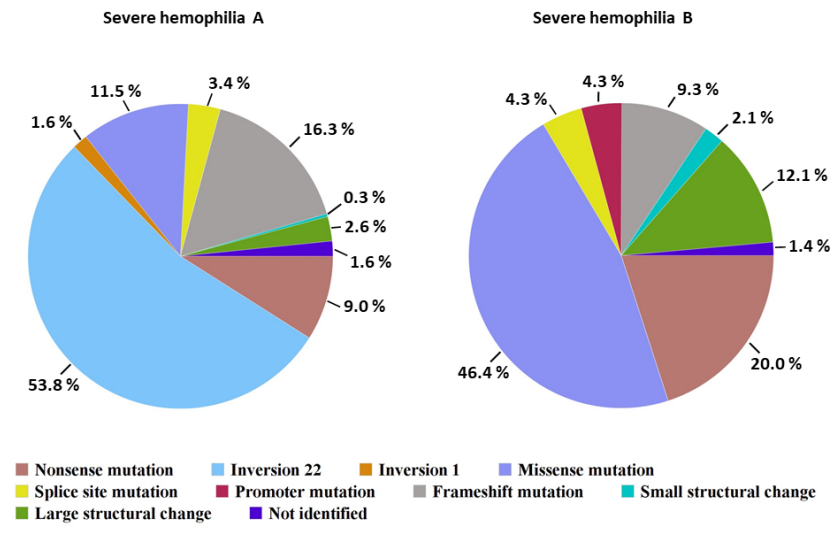

FIGURE 1 Distribution of mutations in severe hemophilia patients

the genetic variants were spread throughout the F9 gene.

Conclusions: In conclusion, we report a wide spectrum of F8 and F9 mutations in a multicenter large cohort of unselected patients, providing a population based reference which could be useful in future studies.

PB0476 | Association between Factor VIII Deficiency and Low Bone Mineral Density in Children with Hemophilia

O.U. Galindo-Aguilar ${ }^{1}$ P.A. García-Hernández²; J.Á. Garza ${ }^{3}$; D. Garay-Mendoza ${ }^{4}$; M. Ortiz-Castillo ${ }^{5}$; A. González-Martínez A.J. Bautista-Gómez ${ }^{5}$; G. González-Martínez ${ }^{5}$; J.F. Molina-de la Garza $^{5}$; L. Villarreal Martínez ${ }^{5}$

${ }^{1}$ Pediatric Department, Hospital Universitario "Dr. José Eleuterio González", Monterrey, Mexico; ${ }^{2}$ Endocrinology Service, Hospital Universitario "Dr. José Eleuterio González", Monterrey, Mexico; ${ }^{3}$ Sports Medicine and Rehabilitation, Hospital Universitario "Dr. José Eleuterio González", Monterrey, Mexico; ${ }^{4}$ Department of Traumatology and Orthopedics, Hospital Universitario "Dr. José Eleuterio González", Monterrey, Mexico; ${ }^{5}$ Hematology Service, Hospital Universitario "Dr. José Eleuterio González", Monterrey, Mexico

Background: It has been observed that patients with hemophilia have a tendency to have low bone mineral density (BMD). It is 
generally accepted that the etiology is the low physical activity due to the hemophilic arthropathy that they suffer. Several studies have recently proposed that the deficiency of factor VIII is independently correlated with the reduction of the BMD, even in the absence of hemorrhage and hemophilic arthropathy.

Aims: To assess the BMD in children regarding the treatment of the patients, and to determine the difference of BMD between patients with moderate and severe hemophilia.

Methods: This is an observational, descriptive, cross-sectional pilot study in which we included a total of 22 patients between ages 3 to 15. Patients were divided into 2 groups: severe and moderate hemophilia. Each patient was submitted to a bone densitometry and they were compared to 68 controls from a historic database.

Results: Seventeen patients had severe hemophilia and five were moderate. The spine Z-Score in the moderate group was -1.8800 and in the severe group was -0.4471 with a $P$ value of 0.008 . Four patients receiving on demand therapy were compared with the control group, with a mean hip Z-Score of -2.10 and -0.77 respectively, with a $P$ value of 0.032 .

Conclusions: We found that the spine Z-Score of patients with severe hemophilia was significantly higher than in the moderate group $(P=0.008$ ) (Table 1). We also observed that patients that were on demand therapy had significantly lower hip Z-Score than healthy children (Table 2). No correlation could be demonstrated between the amount of factor VIII and low bone mineral density in children with hemophilia. These results should be further complemented with a larger sample size. This suggests the possibility that the lack of FVIII alone may not be responsible for BMD reduction.

TABLE 1 Comparison of densitometry testing between moderate and severe hemophilia

\begin{tabular}{llll} 
& $\begin{array}{l}\text { Moderate } n=5 \\
\text { Mean (SD) }\end{array}$ & $\begin{array}{l}\text { Severe } n=17 \\
\text { Mean (SD) }\end{array}$ & P value \\
Hip Z-Score & $-1.84(1.193)$ & $-1.15(1.214)$ & 0.324 \\
Spine Z-Score & $-1.8800(0.8318)$ & $-0.4471(0.8063)$ & $0.008^{*}$ \\
\hline
\end{tabular}

TABLE 2 Comparison of densitometry testing between patients with on demand therapy and controls

\begin{tabular}{llll} 
& $\begin{array}{l}\text { Demand } n=4 \\
\text { Mean (SD) }\end{array}$ & $\begin{array}{l}\text { Control } n=68 \\
\text { Mean (SD) }\end{array}$ & P value \\
Hip Z Score & $-2.10(1.445)$ & $-0.77(1.157)$ & 0.032 \\
Spine Z Score & $-0.6000(1.4159)$ & $-0.5662(1.0676)$ & 0.947 \\
\hline
\end{tabular}

PB0477 | Differences in Thrombin Generating Capacity in Severe Haemophilia A

C. Hamid $^{1,2}$; J. Dunster ${ }^{3}$; E. Aradom ${ }^{1}$; J. McVey ${ }^{4}$; P. Chowdary ${ }^{1,2}$

${ }^{1}$ Katharine Dormandy Haemophilia and Thrombosis Centre, Royal Free Hospital/ NHS Foundation Trust, London, United Kingdom; ${ }^{2}$ University College London, London, United Kingdom; ${ }^{3}$ University of Reading, Reading, United Kingdom; ${ }^{4}$ University of Surrey, Guildford, United Kingdom

Background: It is surmised that differences in coagulation phenotype contribute to the variable bleeding phenotype in severe haemophilia A (SHA)

Aims: We measured thrombin generation (TGA) in a cohort of SHA in the absence of FVIII:C utilizing three concentrations of tissue factor (TF).

Methods: Venous blood $(20 \mathrm{~mL})$ was collected from 25 SHA patients and 6 normal donors (NDs) $3.2 \%$ citrated Sarstedt Monovette ${ }^{\mathrm{TM}}$ tube Numbrecht, CTI $18 \mu \mathrm{g} / \mathrm{mL}$, Cambridge Biosciences) and triple spun platelet poor plasma (PPP) prepared. Addition of $20 \mathrm{BU} / \mathrm{mL}$ FVIII:C antibody (Cambridge Biosciences) reduced FVIII:C activity to $<1 \mathrm{IU} / \mathrm{dL}$. TGA was triggered with $0.5,1$ and 2 pM TF (Innovin ${ }^{\circledR}$ Siemens UK) and 4uM phospholipid (Rossix, Quadratech Diagnostics Ltd). $20 \mu \mathrm{L}$ of trigger and $80 \mu \mathrm{L}$ of PPP were pre-incubated for 5 min at $37^{\circ} \mathrm{C}$ and $20 \mu \mathrm{L}$ FluCa $\left(0.1 \mathrm{M} \mathrm{CaCl}_{2}\right.$ /Fluorogenic substrate, Thrombinoscope BV) initiated TGA (Hemker method with calibrator). (Ethical approval, Katharine Dormandy coagulation research plasma bank, REC No:14/YH/1272).

Results: Patients demonstrated decreased TF concentration dependent thrombin generating capacity compared to NDs. 0.5, 1 and 2pM TF, SHA and NDs, Median (5-95\% quantiles) respectively. Lagtime (mins) 9.5(5.2-23.1), 5.7(3.7-11.3), 3.3(2.7-7.1); 6.1(4.4-9.2), 3.7(3.4-4.8), 3.3(2.5-3.6). ttPeak (mins) 50.2(19.6-62.7), 28.5(18.353.2), 15.8(8.4-28.8); 43.6(26.2-55.0), 17.7(11.0-24.7), 12.1(8.416.9). Peak (nM Flla) 6.9(1.6-15.2), 12.0(4.6-22.5), 27.7(10.2-53.0); 9.1(6.2-13.0), 20.7(10.3-30.0), 66.6(55.1-97.1). SHA results showed a highly significant correlation between Lagtime and ttPeak, $R=0.83$ (0.5TF) $P=0.01,0.85$ (1TF) $P=0,0.61$ (2TF) $P=0$. Peak and ETP (endogenous thrombin potential) showed weak correlation with these parameters (data not shown).

Conclusions: TG in SHA demonstrates less TF dependent thrombin generating capacity than normal donors in the absence of FVIII:C activity with greater variation in response. Lack of correlation between ETP and Peak with Lagtime and ttPeak suggest that the latter are independent variables that may contribute to phenotypic differences. 
PB0478 | Laboratory Verification of a Chromogenic Factor IX Assay Kit

J. Tange; M. Sridharan; N. Heikal; D. Chen; R. Pruthi

Mayo Clinic, Rochester, United States

Background: One-stage clotting FIX assays (OSA) are used for diagnosis of hemophilia $B$ and monitoring clotting factor concentrate (CFC). Selected extended half-life CFC require chromogenic assay (CSA), which are not US FDA approved.

Aims: We present our in-house validation of a CSA.

Methods: The ROX FIX, (Rossix AB, Sweden) was optimized on the IL-ACL TOP 700. Following CLSI guidelines (CLSI EP28, EP05, EP15, EP09C, EP06). Assays were performed on CFC (ALPROLIX ${ }^{\circledR}$ and $\left.I_{\text {IDELVION }}{ }^{\circledR}\right)$ spiked in FIX deficient plasma $(n=32)$, proficiency testing samples $(n=3)$ and waste de-identified patient plasma samples $(n=33)$. Linearity was performed on one commercial normal pool sample and 3 de-identified individual patient samples with elevated OSA (in triplicate at a minimum of 10 different dilutions with diluent buffer). Reference range was established following CLSI guidelines ( $n=120$ ). Analytical sensitivity was carried out on diluent buffer, FIX deficient plasma, and control material diluted to approximately $1 \%$; each material had multiple replicates tested on multiple lots of reagent.

Results: Reference range was established at 65-140\% and analytical sensitivity was achievable down to $1 \%$ (CV:4.9\%). Reportable range was established at 1-200\%. Intra/inter CV's $\leq 6 \%$ were obtained for results ranging 9.8 - 107.2\% (Table 1). Accuracy results compared with the FIX OSA (Figure 1): All Data $\left(r^{2}=0.874\right.$, slope $=0.984$, avg $\%$ diff $=3.5 \%$ ); Known Assigned and Patient Samples $\left(r^{2}=0.958\right.$, slope $=0.893$, avg\% diff $=-8.1 \%) ;$ ALPROLIX ${ }^{\circledR}\left(r^{2}=0.990\right.$, slope $=1.205$, avg $\%$ diff $\left.=-6.6 \%\right) ;$ IDELVION $^{\circledR}\left(r^{2}=0.995\right.$, slope $=2.035$, avg\% diff $\left.=46.3 \%\right)$. Linearity average $r^{2}=0.998$ and average slope $=1.02$ for all samples with results ranging from $0.7-174.8 \%$.

Conclusions: Our CSA kit validation efforts met acceptance criteria for non-spiked patient samples. Data demonstrates the CSA assay can be optimized to provide reliable FIX activity estimates for baseline and ALPROLIX ${ }^{\circledR}$ with an acceptable CV and lower limit of detection of $1 \%$ activity. Additional studies are needed to further understand differences between the OSA and CSA when IDELVION ${ }^{\circledR}$ is present.

TABLE 1 Intra and Inter Precision

\begin{tabular}{lcc}
\hline & Mean & \%CV \\
\hline \multirow{3}{*}{ Intra } & $105.4 \pm 3.50$ & $3.3 \%$ \\
& $37.5 \pm 1.05$ & $2.8 \%$ \\
& $10.9 \pm 0.35$ & $3.2 \%$ \\
\hline \multirow{3}{*}{ Inter } & $107.2 \pm 4.19$ & $3.9 \%$ \\
& $37.6 \pm 1.76$ & $4.7 \%$ \\
& $9.8 \pm 0.59$ & $6.0 \%$ \\
\hline
\end{tabular}

Table 1. Intra and Inter Precision

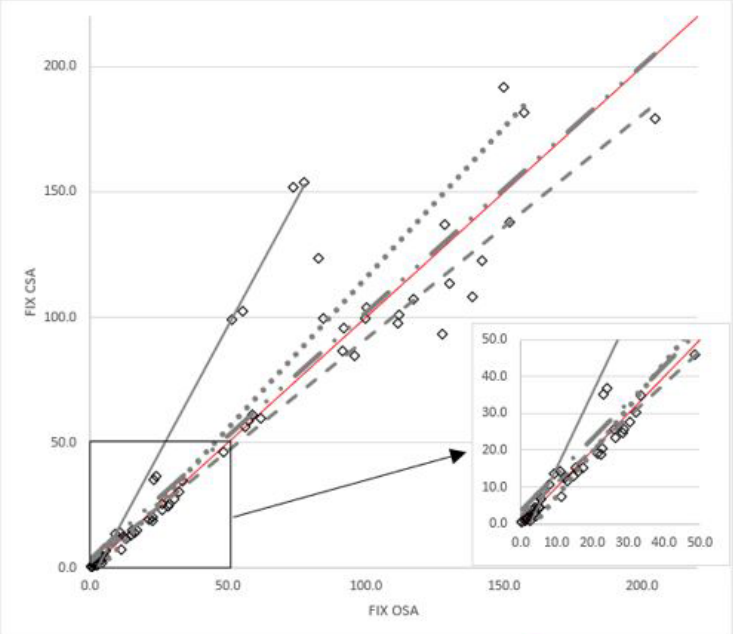

Figure 1. Regression analysis of accuracy studies: $(-)$ Line of Reference; () All Data Points; $(-\cdots)$ All Data Regression Line $\left(r^{2}=0.874\right.$, slope $=0.984$, avg \% diff $=3.5 \%$ ); (- - Known Assigned and Patient Samples Regression Line $\left(r^{2}=0.958\right.$, slope $=0.893$, avg $\%$ diff $\left.=-8.1 \%\right) ;(\cdots)$ Spiked ALPROLIX ${ }^{\odot}$ Samples Regression Line $\left(r^{2}=0.990\right.$, slope $=1.205$, avg \% diff $\left.=-6.6 \%\right) ;(-)$ Spiked IDELVION ${ }^{\circledR}$ Samples Regression Line $\left(r^{2}=0.995\right.$, slope $=2.035$, avg $\%$ diff $\left.=46.3 \%\right)$

FIGURE 1 Regression Analysis of Accuracy Studies

PB0479 | Real-world Clinical and Patient-centric Outcomes in People with Haemophilia A in the United Kingdom: Findings from the CHESS II Study

A. Shaikh ${ }^{1}$; T. Burke ${ }^{1,2}$; C. Hawes ${ }^{3}$; W. McKeown ${ }^{4}$; D. Morgan ${ }^{4}$; J. O'Hara ${ }^{1,2}$; C. Camp ${ }^{3}$

${ }^{1}$ HCD Economics, Daresbury, United Kingdom; ${ }^{2}$ Faculty of Health and Social Care, University of Chester, Chester, United Kingdom; ${ }^{3}$ BioMarin Europe, London, United Kingdom; ${ }^{4}$ The UK Haemophilia Society, London, United Kingdom

Background: Haemophilia A (HA; factor VIII [FVIII] deficiency), characterised by prolonged trauma-related and/or spontaneous intra-articular bleeding events, is associated with adverse impacts on physical functioning and quality of life (QoL). The breadth of clinical and patient-relevant outcomes/experiences of people with HA (PWHA) in the United Kingdom (UK) - and variation between those with mild, moderate and severe HA - is not well understood.

Aims: To examine demographic, clinical and patient-centric outcomes across condition severity among adult PWHA in the UK, as captured in the 'Cost of Haemophilia in Europe: a Socioeconomic Survey - II' (CHESS II) study.

Methods: Data for non-inhibitor PWHA living in the UK were extracted from CHESS II and stratified based on clinician-reported baseline endogenous FVIII level. Participant demographics, annualized bleeding rate (ABR), incidence of target joints (ISTH definition) and joints exhibiting symptoms of chronic damage ('problem' joints), treatment regimen and QoL (EQ-5D-5L index score) were assessed. Outcomes by HA severity were compared using descriptive statistics. Study methodology and interpretation of results were informed by representatives from the UK Haemophilia Society. 
Results: Sixty-nine PWHA were included in the analysis (mild $n=11$, moderate $n=22$, severe $n=36$ ). The proportion of participants in full-time employment decreased with increasing HA severity (Table 1 ). The majority of participants received on-demand treatment regimens. Mean ABR, incidence of problem joints and $E Q$ 5D-5L index score were all suggestive of greater impairment with increasing HA severity. No similar pattern was observed in target joint outcomes (Table 2).

TABLE 1 Participant demographics by HA severity

\begin{tabular}{|c|c|c|c|c|}
\hline Severity of HA & Mild $(n=11)$ & Moderate $(n=22)$ & Severe $(n=36)$ & TOTAL $(n=69)$ \\
\hline Age, mean \pm SD & $43.82 \pm 18.31$ & $29.68 \pm 10.41$ & $27.78 \pm 8.38$ & $30.94 \pm 12.31$ \\
\hline $\begin{array}{l}\text { Body Mass Index (BMI), } \\
\quad \text { mean } \pm \mathrm{SD}\end{array}$ & $24.50 \pm 3.36$ & $23.93 \pm 5.38$ & $24.54 \pm 4.42$ & $24.34 \pm 4.55$ \\
\hline \multicolumn{5}{|l|}{ Employment status, $n(\%)$} \\
\hline Employed full time & $4(36 \%)$ & 4 (18\%) & $3(8 \%)$ & $11(16 \%)$ \\
\hline Employment part-time & $1(9 \%)$ & $5(23 \%)$ & $9(25 \%)$ & $15(22 \%)$ \\
\hline Self-employed & $2(18 \%)$ & $5(23 \%)$ & $14(39 \%)$ & $21(30 \%)$ \\
\hline Unemployed & $0(0 \%)$ & $2(9 \%)$ & $1(3 \%)$ & $3(4 \%)$ \\
\hline Student & $1(9 \%)$ & 2 (9\%) & 4 (11\%) & $7(10 \%)$ \\
\hline Other & $3(27 \%)$ & $4(18 \%)$ & $5(14 \%)$ & $12(17 \%)$ \\
\hline
\end{tabular}

TABLE 2 Treatment strategy, clinical and patient-centric outcomes by HA severity

\begin{tabular}{lllll} 
Severity of HA & Mild $(n=11)$ & Moderate $(n=22)$ & Severe $(n=36)$ & TOTAL $(n=69)$ \\
$\begin{array}{l}\text { Receiving factor replacement } \\
\quad \text { therapy, } n(\%)\end{array}$ & $4(36 \%)$ & $14(64 \%)$ & $36(100 \%)$ & $54(78 \%)$ \\
Primary on-demand & $4(100 \%)$ & $8(57 \%)$ & $6(17 \%)$ & $18(33 \%)$ \\
Primary prophylaxis & $0(0 \%)$ & $0(0 \%)$ & $8(22 \%)$ & $8(15 \%)$ \\
Secondary on-demand & $0(0 \%)$ & $6(43 \%)$ & $13(36 \%)$ & $19(35 \%)$ \\
Secondary prophylaxis & $0(0 \%)$ & $0(0 \%)$ & $9(25 \%)$ & $9(17 \%)$ \\
Annualised bleed rate, mean \pm SD & $1.18 \pm 0.87$ & $3.00 \pm 1.54$ & $4.28 \pm 1.77$ & $3.38 \pm 1.93$ \\
Target joints, mean \pm SD & $0.00 \pm 0.00$ & $0.18 \pm 0.50$ & $0.11 \pm 0.40$ & $0.12 \pm 0.40$ \\
Problem joints, mean \pm SD & $0.00 \pm 0.00$ & $0.18 \pm 0.39$ & $0.36 \pm 0.49$ & $0.25 \pm 0.43$ \\
EQ-5D-5L, $n$; mean \pm SD & $3 ; 0.88 \pm 0.10$ & $8 ; 0.81 \pm 0.18$ & $4 ; 0.61 \pm 0.06$ & $15 ; 0.77 \pm 0.17$ \\
\hline
\end{tabular}

Conclusions: Data from the CHESS II study provides important insights into the clinical experience and QoL of people with mild, moderate and severe HA in the UK. A pattern of increased burden in clinical outcomes and QoL was observed with declining baseline FVIII levels. This study highlights the importance of real-world data in understanding the burden across the spectrum of HA in the UK setting.

PB0480 | Usefulness of Global Coagulation Assays to Monitor Emicizumab Therapy

D. Bertaggia Calderara; M.G Zermatten; A. Aliotta; A.P Batista Mesquita Sauvage; L. Alberio

Division of Hematology and Central Hematology Laboratory, Lausanne University Hospital (CHUV) and University of Lausanne (UNIL),

Lausanne, Switzerland

Background: Emicizumab is a bispecific antibody mimicking the action of coagulation factor VIII, approved for treatment of patients affected by Hemophilia A, without or with inhibitors. Very low concentrations of emicizumab are sufficient to normalize the activated partial thromboplastin time (APTT) in treated patients, thus conventional APTT-based assays are not useful to monitor emicizumab therapy. Here we studied thrombin generation (TG) and fibrin clot formation (FCF) profiles in two adult patients without inhibitors starting emicizumab treatment.

Aims: Our aim was to investigate whether global-coagulation-assays (GCA) could be used to monitor non-factor replacement therapy with emicizumab.

Methods: TG was measured with Calibrated-AutomatedThrombogram and with the automated ST Genesia assays (Stago, Asnières-sur-Seine, France). FCF was measured with the innovative Thrombodynamics Analyzer (LLC Hemacore, Moscow, Russia), which monitors the spatio-temporal (tissue factor-dependent and -independent) dynamics of coagulation. The patients received a weekly dose of Hemlibra ${ }^{\circledR}$ (Hoffmann-La Roche, Switzerland) (3 $\mathrm{mg} / \mathrm{kg}$ per body weight from week (W) 1 to $\mathrm{W} 4$, and $1.5 \mathrm{mg} / \mathrm{kg}$ from W5 onwards). Response to treatment was monitored weekly for two 
months. Analyses of TG and FCF were performed in platelet poor plasma in presence of TF and phospholipids.

Results: In both patients we observed that: i) emicizumab improved TG and FCF compared to baseline; ii) TG normalized after two weeks of treatment; FCF normalized already after one week; iii) both TG and FCF reached a plateau (starting at W4) that lasted until the end of the monitoring. Of note, we observed during emicizumab therapy specific individual coagulation profiles, which correlated with the clinical response.

Conclusions: According to this limited experience, emicizumab seems to improve the hemostatic potential in an individual-specific and "all-or-nothing" manner. TG/FCF assays seem to be promising tools to evaluate the degree of correction exerted by emicizumab on hemostasis, thus possibly personalizing patient treatment. Further investigations are needed to confirm this observation.

PB0481 | Analysis of the Percentage of Persons with Severe Hemophilia A as an Indicator of Care

E. Tootoonchian ${ }^{1}$; J. Stonebraker ${ }^{2}$; M. El Ekiaby ${ }^{3}$; E. Gouider ${ }^{4}$; A. Iorio ${ }^{5}$; M. Makris ${ }^{6}$; J. O'Hara ${ }^{7}$; M. Recht ${ }^{8}$; G. Pierce ${ }^{1}$; D. Coffin ${ }^{1}$ ${ }^{1}$ World Federation of Hemophilia, Montreal, Canada; ${ }^{2}$ North Carolina State University, Raleigh, United States; ${ }^{3}$ Shabrawichi Hospital, Cairo, Egypt; ${ }^{4}$ Université Tunis El Manar, Tunis, Tunisia; ${ }^{5}$ McMaster University, Hamilton, Canada; ${ }^{6}$ University of Sheffield, Sheffield, United Kingdom; ${ }^{7} \mathrm{HCD}$ Economics, London, United Kingdom; ${ }^{8}$ ATHN, Rochester, United States

Background: Hemophilia diagnosis and care varies globally and is dependent on healthcare infrastructure, availability of resources and economic levels.

Aims: To assess diagnosis and care capabilities we analyzed the percentage of severe vs. mild and moderate hemophilia in persons with hemophilia A (PWHA), as reported to the World Federation of Hemophilia (WFH) Annual Global Survey (AGS).

Methods: The AGS is an annual cross-sectional survey. Each year the WFH National Member Organizations (NMOs) are asked to fill out the survey including questions on identified patients and level of hemophilia care. We calculated the percentage of male PWHA with severe disease and explored the change over time and in various economical settings using the gross national income.

Results: In 2010, the percentage of severe PWHA was higher in high income countries (48\%) than those in low-income ones (25\%) and the percentage of those with unknown severity was higher in lowincome countries (Figure 1). Latest survey results in 2019 showed that the proportion of PWHA with severe hemophilia in upper middle- and lower-income countries is similar to those in high income countries. However, the proportion of mild and moderate patients identified remains lower in low-income countries (Figure 2).
Figure 1. Severity of hemophilia A in males by GNI - 2010

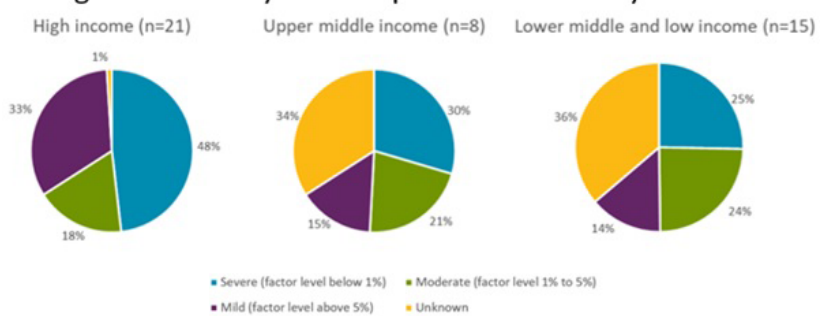

FIGURE 1 Severity of hemophilia A in males by GNI - 2010

Figure 2. Severity of hemophilia A in males by GNI - 2019

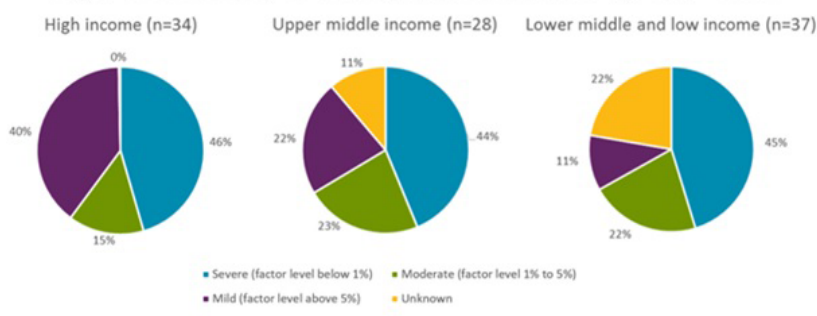

FIGURE 2 Severity of hemophilia A in males by GNI - 2019

Conclusions: The analysis of this indicator shows the change in diagnostic capabilities over time and by economic levels. In both years, as economic status increased, accurate diagnosis improved. Over time, the data shows that diagnosis and reporting in lower income countries have become more accurate, as the percentage of severe disease increased, and percentage of unknown severity reduced. Furthermore, there has been improvement in diagnosis of mild patients in upper middle countries, but more progress needs to be made in lower middle- and low-income countries. In conjunction with other indicators, the proportion of PWHA with severe disease can inform health care development and support planning and decision-making.

PB0482 | Evaluation of Primary Haemostasis in Children and Young Adults with Haemophilia

M. Kürschner ${ }^{1}$; J. Lohse ${ }^{1}$; F. Boiti ${ }^{1}$; O. Tiebel ${ }^{2}$; R. Knöfler ${ }^{1}$

${ }^{1}$ Department of Pediatric Haemostaseology, Faculty of Medicine, Technical University Dresden, Dresden, Germany; ${ }^{2}$ Department of Clinical Chemistry and Laboratory Medicine, Faculty of Medicine, Technical University Dresden, Dresden, Germany

Background: Patients with haemophilia present individual differences in bleeding tendency despite similar levels of the clotting factors VIII (FVIII:C) and IX (FIX:C). In this context the additional presence of hereditary coagulopathies such as thrombophilia, deficiencies of other coagulation factors and impairment of primary haemostasis is discussed in the literature. 
Aims: Analyzing primary haemostasis parameters mainly in pediatric patients with haemophilia $A$ and $B$ in comparison to a pediatric control group.

Methods: Specimens from 38 haemophilia patients (33 with haemophilia A: severe $n=24$, moderate $n=1$, mild $n=8$ and 5 with haemophilia B: severe $n=3$, moderate $n=1$, mild $n=1$ ) with a median age of 11 years (range: 1 to 32 years) and 30 healthy controls (median 5.5 years ranging from 1 to 19 years) were evaluated. Platelet function was investigated by impedance aggregometry and luminometry in whole blood, by light transmission aggregometry and flow cytometry in platelet rich plasma (PRP). Furthermore, the PFA $100{ }^{\circledR}$ closure times, factor VIII- and IX-activities, von Willebrand factor parameters, hemogram, and blood group were determined. Informed consent was obtained.

Results: Patients showed a significant elevated maximal aggregation induced by arachidonic acid (final concentration: $0.5 \mathrm{mM}$ ) and collagen $(1 \mu \mathrm{g} / \mathrm{ml})$ in whole blood. In PRP a significantly reduced ADP-induced $(5 \mu \mathrm{M})$ maximal aggregation was detected in the haemophilia group. A reduced CD63 expression induced by ADP was significantly more often seen in the patient's group (50\%) compared to controls (22\%). CD63 expression by TRAP and mepacrine release showed no differences between the two groups. Patients had significantly lower platelet counts (median: $266.500 / \mu \mathrm{l}$ ) compared to controls $(312.000 / \mu \mathrm{l}$.) No significant differences between the groups were detected for all other parameters.

Conclusions: Our data demonstrate only minor and most likely clinically non-relevant differences in primary haemostasis between patients with haemophilia and healthy controls.

The study was supported by an unrestricted grant from Intersero ${ }^{\circledR}$.
PB0483 | New Likely Pathogenic Variants in Three Spanish Patients with Mild Hemophilia A and B: Importance of Genetic Counseling

M. Civeira $^{1}$; R. Lahoz-Alonso ${ }^{2}$; R. González-Tarancón ${ }^{2}$; N. Fernández-Mosteirín ${ }^{1}$; J.M. Calvo-Villas ${ }^{1}$; S. Izquierdo-Alvarez ${ }^{2}$ ${ }^{1}$ Hospital Universitario Miguel Servet, Hematology and Hemotherapy Department, Zaragoza, Spain; ${ }^{2}$ Hospital Universitario Miguel Servet, Genetics Department, Zaragoza, Spain

Background: Mild hemophilia A ( $\mathrm{HA})$ and $B(\mathrm{HB})$ are $\mathrm{X}$-linked recessive hereditary diseases, characterized by factor VIII and IX deficiency, respectively. Patients with mild or moderate hemophilia mostly present specific mutations in F8 gene (Xq28) or F9 (Xq22), detectable by direct sequencing. More than 4000 mutations have been detected so far and genotype analysis should be offered in selected patients. Therefore, genetic assessment is important to provide reproductive options.

Aims: We report three new likely pathogenic variants in three male Spanish patients. Case 1 presented asymptomatic prolonged preoperatory aPTT and the others were studied due to hemorrhagic complications of surgery. All presented isolated reduced factor VIII or IX activity levels.

Methods: Molecular genetic studies: DNA was extracted from peripheral blood. Coding exons and adjacent intronic regions of F8 and F9 gene were amplified by polymerase chain reaction (PCR) and sequenced on Applied Biosystems ${ }^{\circledR}$ 3500DX Genetic Analyzer. The results were checked in HGMD, ClinVar, dbSNP, gnomAD, and 1000G international databases and different in silico prediction algorithms (PolyPhen-2; SIFT; Mutation Taster; Align GVGD, REVEL...) were consulted.

Results: Three new missense variants were found (table 1). These variants have not been previously reported in the literature or in any of the databases consulted. They do affect the physical-chemical characteristics of the protein (in silico prediction). Therefore, they are classified as likely pathogenic. Because of this, we extended the study to first-degree relatives; finding carriers that were not diagnose at that time (Figure 2). A 20-year-old daughter (case 1) and a sister (case 2 ) received genetic counseling. 
TABLE 1 Genotypic results of the three patients. In silico prediction algorithms used in the three cases: PolyPhen-2, SIFT, Mutation Taster and Align GVGD. *In patient 3 the following algorithms were also utilized: FATHMM, FATHMM-MKL, DEOGEN2, MVP, MetaSVM, MetaIR, DANN, Mut. Assessor, SIFT, SIFT4G, Provean, REVEL and GERP.

\begin{tabular}{|c|c|c|c|c|c|c|c|c|}
\hline \multirow[b]{2}{*}{ Patients } & \multirow[b]{2}{*}{$\begin{array}{l}\text { Hemophilia } \\
\text { type }\end{array}$} & \multirow{2}{*}{$\begin{array}{l}\text { Age at } \\
\text { diagnose } \\
\text { (years) }\end{array}$} & \multirow[b]{2}{*}{$\begin{array}{l}\text { Factor activity } \\
\text { (\%) }\end{array}$} & \multicolumn{3}{|c|}{ DNA variations } & \multirow[b]{2}{*}{ Genotype } & \multirow[b]{2}{*}{$\begin{array}{l}\text { In silico } \\
\text { prediction }\end{array}$} \\
\hline & & & & Gene & Exon & $\begin{array}{l}\text { Amino acid } \\
\text { (nucleotide) change }\end{array}$ & & \\
\hline P1 & A & 42 & 13.3 & F8 & 4 & $\begin{array}{l}\text { p.Val181Leu } \\
\quad \text { (c.541G>T) }\end{array}$ & hemizygous & $4 / 4$ \\
\hline P2 & A & 55 & 10.5 & F8 & 3 & $\begin{array}{l}\text { p.Asp101Glu } \\
\quad \text { (c.303T>A) }\end{array}$ & hemizygous & $4 / 4$ \\
\hline P3 & B & 30 & 23.2 & F9 & 7 & $\begin{array}{l}\text { p.Glu259Gly } \\
\text { (c.776A>G) }\end{array}$ & hemizygous & $12 / 16^{*}$ \\
\hline
\end{tabular}
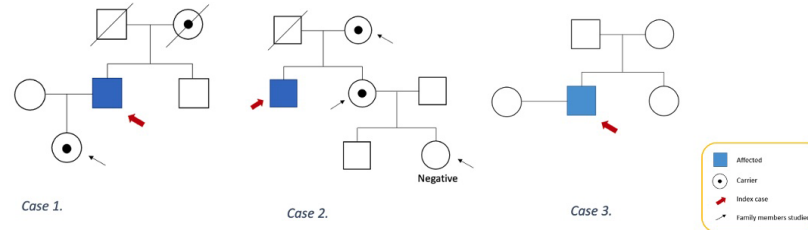

FIGURE 1 Pedigree of three index cases and family members studied with mild hemophilia. ${ }^{*}$ Case 3 . Segregation analyses on the parents could not be performed.

Conclusions: These new genetic variants confirm the clinical suspicion of mild hemophilia. The molecular genetic analysis in the family members helps finding asymptomatic carriers so we can provide them genetic counseling. Future reproductive options will be considered in the youngest patient and any family members if needed.

PB0484 | Is Zero Bleeds a Realistic Goal for Hemophilia Patients in the Real World Setting?

A. Adramerina; A. Teli; S. Symeonidis; A. Nianiu; M. Economou 1st Pediatric Department, School of Medicine, Faculty of Health Sciences, Aristotle University of Thessaloniki, Thessaloniki, Greece

Background: Recent additions to the hemophilia prophylaxis success story are extended half-life products (EHLs) and non-factor replacement therapies. As a consequence, prophylaxis now ambitiously aims at achieving zero bleeds in patients, instead of simply converting their phenotype from severe to moderate.

Aims: To evaluate progress made in achieving treatment goals over a 3 year period in the real world setting of a pediatric hemophilia center.

Methods: Records of severe hemophilia patients on prophylaxis were retrospectively studied with regards to age, product type, annualized bleeding rate (ABR) and joint ABR for years 2018, 2019 and 2020.
Results: In 2018, 21 patients with a mean age of 12.4 (5-18) years were evaluated, out of which 16 (76.2\%) were on standard half-life products (SHLs), 2 (9.5\%) on EHLs, whereas 3 (14.3\%) switched from SHL to EHL during the study year. Mean ABR was $4.5(0-13)$ and mean AjBR 3.2 (0-11). In 2 (9.5\%) patients zero bleeds were reported. In 2019, 22 patients with a mean age of 11.9 (3-18) years were evaluated, out of which 13 (59\%) were on SHLs, 5 (22.7\%) on EHLs, whereas 2 (9\%) switched during study year. Emicizumab was administered to 2 (9\%) patients. Mean ABR was $4(0-10)$ and mean AjBR 3 (0-9). Zero bleeds were reported in 4 patients (18.3\%).

In 2020, 26 patients with a mean age of 10.5 (1-18) years were evaluated. In total, 14(53.8\%) were on SHLs, 8 (30.7\%) on EHLs, 1 (3.84\%) switched from SHL to EHL and 3 received emicizumab (11.5\%). Mean ABR was $1.8(0-8)$ and mean AjBR $1.1(0-8)$. Zero bleeds were reported in 6 patients (23\%).

Conclusions: Improvement in offered prophylaxis is attainable with the use of new therapies. However, the ultimate goal of zero bleeds is still difficult to achieve in the real world setting.

\section{PB0485 | Laboratory Monitoring of Emicizumab in Treated}

\section{Patients - How Does APTT Compare?}

\section{A. McCormick}

Viapath Analytics, London, United Kingdom

Background: Emicizumab comprises a bispecific monoclonal antibody mimicking the function of FVIIla by binding FIXa and FX leading to formation of FXa and has the advantage of a half-life of approx. 30 days versus $12 \mathrm{hr}$ for FVIII. The differing mode of action of emicizumab results in overestimation of its activity using existing one stage FVIII assay (OSA) and chromogenic FVIII assay (CSA). The use of CSA with bovine reagents is unaffected by emicizumab presence and is useful for estimation of baseline FVIII. A diluted onestage assay employing emicizumab calibrator $\left(r^{2}\right.$ diagnostics $)$ has been validated for use in our laboratory to provide a more accurate estimation of circulating emicizumab levels in treated patients. 
Aims: To assess data obtained from patients undergoing emicizumab treatment using a modified one-stage assay employing commercial emicizumab calibrator with APTT results.

Methods: The one-stage emicizumab assay, bovine chromogenic FVIII assay and APTT (ratio and seconds) were measured. The APTT results were compared with emicizumab levels in patients where baseline FVIII were found to be $<1 \mathrm{IU} / \mathrm{dL}$

Results: The APTT ratio was found to have been reduced below 1 in all patients treated with measurable emicizumab. A broad correlation was observed between emicizumab levels and APTT.

Conclusions: The most efficient method of monitoring emicizumab therapy combines the use of bovine based CSA to measure endogenous FVIII and a modified dilute OSA calibrated using commercial emicizumab reagent. However this combination of assays may not be available to all laboratories and many may rely on the APTT as a general monitor of therapy. Whilst not ideal, an APTT ratio $<1$ may give a broad indication of the presence of therapeutic levels of emicizumab.

PB0486 | Characteristics of Anti-drug Antibody against Emicizumab Analyzed Using Hemophilia A Patient-derived Plasma Samples

R. Kawasaki ${ }^{1}$; M. Kaneda ${ }^{2}$; M. Noguchi ${ }^{1}$; N. Matsumoto ${ }^{1}$; H. Abe ${ }^{1}$; Y. Tashiro ${ }^{1}$; Y. Inokuchi ${ }^{1}$; H. Yasuno ${ }^{1}$; T. Soeda ${ }^{1}$; Y. Yoshimura ${ }^{1}$; T. Oka ${ }^{2}$

${ }^{1}$ Chugai Pharmaceutical Co., Ltd., Kamakura, Japan; ${ }^{2}$ Sapporo Tokushukai Hospital, Sapporo, Japan

Background: Emicizumab is a humanized bispecific monoclonal antibody that bridges activated factor IX and factor $X$ to mimic the function of factor VIII (FVIII). It suppresses the bleeding tendency in hemophilia A patients with or without FVIII inhibitors. At Sapporo Tokushukai Hospital, we experienced a case of an adult FVIII inhibitor-positive hemophilia A patient in whom treatment with emicizumab was discontinued owing to increased bleeding frequency and prolonged activated partial thromboplastin time. Because of these signs, we suspected decrease in the efficacy of emicizumab.

Aims: To analyze the mechanisms underlying the decrease in the efficacy of emicizumab, we analyzed the factors affecting the pharmacological effect of emicizumab in the plasma of the patient.

Methods: Residual plasma from blood samples that had been collected from the patient for clinical testing were used to measure the following items: concentration of emicizumab in the plasma, determined by enzyme-linked immunosorbent assay; anti-drug antibody (ADA) against emicizumab, detected by electrochemiluminescence (ECL); and neutralizing activity against emicizumab, measured by a modified Bethesda method using emicizumab-spiked FVIII-deficient plasma.

Results: Before emicizumab treatment was discontinued, emicizumab concentration in the plasma was $1.75 \mu \mathrm{g} / \mathrm{mL}$ after the first bleeding was observed, and ADA was detected. After discontinuation of emicizumab treatment, no emicizumab was detected in the plasma. The ECL signal derived from ADA increased transiently following discontinuation of emicizumab and then attenuated. Neutralizing activity against emicizumab was observed in the plasma after the discontinuation of emicizumab treatment.

Conclusions: The appearance of ADA decreased the concentration of emicizumab in the plasma, leading to a reduction in its efficacy. This indicates that the ADA had neutralizing activity against emicizumab.

PB0487 | Real-world Clinical and Patient-centric Outcomes in People with Haemophilia A in Germany: Findings from the CHESS II Study

A. Shaikh ${ }^{1}$; T. Burke ${ }^{1,2}$; C. Hawes ${ }^{3}$; T. Becker ${ }^{4}$; S. Brandt ${ }^{4}$; J. O'Hara ${ }^{1,2}$; C. Camp ${ }^{3}$

${ }^{1}$ HCD Economics, Daresbury, United Kingdom; ${ }^{2}$ Faculty of Health and Social Care, University of Chester, Chester, United Kingdom; ${ }^{3}$ BioMarin Europe, London, United Kingdom; ${ }^{4}$ Interessengemeinschaft Hämophiler e.V., Trier, Germany

Background: Haemophilia A (HA; factor VIII [FVIII] deficiency), characterised by prolonged trauma-related and/or spontaneous intra-articular bleeding events, is associated with adverse impacts on physical functioning and quality of life (QoL). The breadth of clinical and patient-relevant outcomes/experiences of people with HA (PWHA) in Germany - and variation between those with mild, moderate and severe $\mathrm{HA}$ - is not well understood.

Aims: To examine demographic, clinical and patient-centric outcomes across condition severity among adult PWHA in Germany, as captured in the 'Cost of Haemophilia in Europe: a Socioeconomic Survey - II' (CHESS II) study.

Methods: Data for non-inhibitor PWHA living in Germany were extracted from CHESS II and stratified based on clinician-reported baseline endogenous FVIII level. Participant demographics, annualized bleeding rate (ABR), incidence of target joints (ISTH definition) and joints exhibiting symptoms of chronic damage ('problem' joints), treatment regimen and QoL (EQ-5D-5L index score) were assessed. Outcomes by HA severity were compared using descriptive statistics. Study methodology and interpretation of results were informed by representatives from the German patients' organisation IGH.

Results: Forty-seven PWHA were included in the analysis (mild $n=10$, moderate $n=16$, severe $n=21$ ). The proportion of participants in full-time employment decreased with increasing HA severity (Table 1). Two-thirds of participants with severe HA received prophylaxis FVIII regimens. Mean ABR and incidence of target joints and problem joints were suggestive of greater impairment with increasing severity of HA. EQ-5D-5L index scores showed a potential downward trend (Table 2). 
TABLE 1 Cohort demographics by HA severity

\begin{tabular}{|c|c|c|c|c|}
\hline Severity of HA & Mild $(n=10)$ & Moderate $(n=16)$ & Severe $(n=21)$ & TOTAL $(n=47)$ \\
\hline Age, mean \pm SD & $40.70 \pm 11.14$ & $44.75 \pm 15.72$ & $39.95 \pm 11.07$ & $41.74 \pm 12.76$ \\
\hline $\begin{array}{l}\text { Body Mass Index (BMI), } \\
\quad \text { mean } \pm \text { SD }\end{array}$ & $24.69 \pm 1.84$ & $24.73 \pm 2.62$ & $25.45 \pm 3.13$ & $25.04 \pm 2.70$ \\
\hline \multicolumn{5}{|l|}{ Employment status, $n$ (\%) } \\
\hline Employed full time & $8(80 \%)$ & $12(75 \%)$ & $14(67 \%)$ & $34(72 \%)$ \\
\hline Employment part-time & $0(0 \%)$ & $1(6 \%)$ & $2(10 \%)$ & $3(6 \%)$ \\
\hline Self-employed & $1(10 \%)$ & $1(6 \%)$ & $4(19 \%)$ & $6(13 \%)$ \\
\hline Unemployed & $0(0 \%)$ & $0(0 \%)$ & $0(0 \%)$ & $0(0 \%)$ \\
\hline Student & $1(10 \%)$ & $1(6 \%)$ & $0(0 \%)$ & $2(4 \%)$ \\
\hline Other & $0(0 \%)$ & $1(6 \%)$ & $1(5 \%)$ & $2(4 \%)$ \\
\hline
\end{tabular}

TABLE 2 Treatment strategy, clinical and patient-centric outcomes by HA severity

$\begin{array}{lllll}\begin{array}{l}\text { Severity of HA } \\ \text { Receiving factor replacement } \\ \quad \text { therapy, } n(\%)\end{array} & \text { Mild }(n=10) & \text { Moderate }(n=16) & \text { Severe }(n=21) & \text { TOTAL }(n=47) \\ \text { Primary on-demand } & 3(30 \%) & 4(25 \%) & 21(100 \%) & 28(78 \%) \\ \text { Primary prophylaxis } & 3(100 \%) & 4(100 \%) & 7(33 \%) & 14(50 \%) \\ \text { Secondary on-demand } & 0(0 \%) & 0(0 \%) & 8(38 \%) & 8(29 \%) \\ \text { Secondary prophylaxis } & 0(0 \%) & 0(0 \%) & 0(0 \%) & 0(0 \%) \\ \text { Annualised bleed rate, mean } \pm \text { SD } & 0(0 \%) & 0(0 \%) & 6(29 \%) & 6(21 \%) \\ \text { Target joints, mean } \pm \text { SD } & 0.70 \pm 0.48 & 1.25 \pm 1.57 & 1.86 \pm 1.53 & 1.40 \pm 1.44 \\ \text { Problem joints, mean } \pm \text { SD } & 0.10 \pm 0.32 & 0.19 \pm 0.40 & 0.43 \pm 0.81 & 0.26 \pm 0.61 \\ \text { EQ-5D-5L, } n \text {; mean } \pm \text { SD } & 2 ; 0.97 \pm 0.04 & 4 ; 0.90 \pm 0.08 & 0.57 \pm 1.25 & 0.34 \pm 0.89 \\ \end{array}$

Conclusions: Real-world data from CHESS II provides preliminary insights into the clinical outcomes and QoL of people with mild, moderate and severe HA in Germany. A low incidence of clinical outcomes was reported, though a pattern of increased clinical burden with declining baseline FVIII levels was observed. Additional QoL data could provide insight into how clinical outcomes impact upon daily functioning and lived experiences of PWHA in Germany.

PB0488 | Exploring the Level of Congruence between Patientand Haematologist-reporting of Anxiety and Depression in People with Haemophilia A

T. Burke ${ }^{1,2}$; A. Shaikh ${ }^{1}$; G. Pedra ${ }^{1}$; C. $\underline{\text { Hawes }}^{3}$; C. Camp ${ }^{3}$; J. O'Hara ${ }^{1}$ ${ }^{1} \mathrm{HCD}$ Economics, Daresbury, United Kingdom; ${ }^{2}$ Faculty of Health \& Social Care, University of Chester, Chester, United Kingdom; ${ }^{3}$ BioMarin Europe, London, United Kingdom

Background: Hemophilia A (HA; factor VIII deficiency) is a chronic bleeding disorder characterized by prolonged trauma-related or spontaneous bleeding events into joint spaces and muscle tissue. While psychosocial challenges are a recognized issue faced by people with hemophilia A (PWHA), there is limited research on the reporting of such challenges in real-world data.
Aims: To explore the level of congruence between patient and hematologist reporting of anxiety and depression in PWHA using data from the 'Cost of Hemophilia in Europe: a Socioeconomic Survey - II' (CHESS II) study.

Methods: Data for PWHA without active inhibitors was extracted from CHESS II, a retrospective burden-of-illness study in 787 adult males with hemophilia. Diagnoses of anxiety or depression, as reported by the respondent hematologist, were grouped into a single "anxiety/depression" indicator. Patient-reported anxiety/depression was determined via the respective dimension of the EQ-5D-5L using two approaches: any level of impairment greater than "none" $(\geq 2)$; and any level greater than "slight problems" $(\geq 3)$. For both approaches, congruence between patient and hematologist reporting was assessed.

Results: Two hundred fifty-eight PWHA completed the EQ-5D-5L and were included in this analysis. Patient-reported anxiety/depression was observed in $n=132$ (51.2\%) and $n=34$ (13.2\%) subjects for the two anxiety/depression dimension levels $\geq 2$ and $\geq 3$, respectively, compared to $n=45$ (17.4\%) reported by respondent hematologists. Congruence in patient and hematologist reporting occurred on $n=139(53.9 \%, \geq 2)$ and $n=199(77.1 \%, \geq 3)$ occasions, respectively. Conclusions: Congruence between patient and hematologist reporting of anxiety/depression is greater when the level of patientreported impairment is more severe; however, some PWHA experience impairment related to anxiety/depression that is not 
reported by their hematologist. PWHA and health care professionals should be encouraged to openly discuss any mental health-related challenges as part of a multidisciplinary approach to managing HA Future research would benefit from utilizing tools specifically designed to assess mental health.

PB0490 | Properties of FVIII Concentrate Were Obtained by the Method of Dye-ligand Affinity Chromatography

N. Shurko; T. Danysh; V. Novak

State Institution "Institute of Blood Pathology and Transfusion

Medicine NAMS of Ukraine", Lviv, Ukraine

Background: The factor VIII (FVIII) concentrate should have to high specific activity, viral security, stability and don't cause the appearance of anti-FVIII antibodies. It's known that the use of FVIII concentrate with a high content of factor von Willebrande (vWF) reduces the risk of development of anti-FVIII antibodies.

Aims: to investigate and describe the main properties of the FVIII concentrate.

Methods: The Fresh Frozen Plasma was used as initial material. The activity of FVIII was determined using a coagulation test that measures the partial thromboplastin time; vWF - the cofactor activity of rystomycin; the total concentration of proteins - the Bradford method; the albumine - with bromcresol green. The ion-exchange and dye-ligand affinity chromatography have been performed.

Results: We have been developed a method of purification of FVIII by the methods ion-exchange and dye-ligand affinity chromatography. The obtained concentrate of FVIII contain VWF $(3.07 \pm 0.18 \mathrm{IU} / \mathrm{ml})$ and albumine $(0.67 \pm 0.02 \times 10-1 \mathrm{mg} / \mathrm{ml})$. The both of these proteins are used as stabilizers of its activity. It's known that some researchers suggest using of vWF as a natural stabilizer of FVIII activity and doesn't require the addition of stabilizers. In our case, the resulting drug additionally contains these two proteins. The researches of the stability of FVIII concentrate have been demonstrated the following: the activity of FVIII in solution is reduced by $20 \%$ after $6-8$ hours, by $40 \%$ - after 24 hours and by $90 \%$ after 48 hours at room temperature. The activity of the freezing factor is fully preserved during the month, after three months of storage it decreases by $10 \%$, after a year of storage - by $20-25 \%$. Lyophilization of the concentrate ensures the preservation of its activity for two years at $+(4 \div 8)^{\circ} \mathrm{C}$.

Conclusions: We have been developed a new method purification of FVIII which doesn't require the additional proteins as stabilizers.
PB0491 | A Review Following Implemented Training for Health Care Practitioners in Primary Care Settings of Immunisation Practice in Children with Bleeding Disorders

M. Eales ${ }^{1}$; A. Wilkinson ${ }^{1}$; S. Pool ${ }^{2}$; G.W Hall ${ }^{1}$; N. Bhatnagar ${ }^{1}$

${ }^{1}$ Oxford Comprehensive Care Centre Oxford University Hospital, Oxford, United Kingdom; ${ }^{2}$ Oxford University Hospital, Oxford, United Kingdom

Background: In 2019, the Paediatric Haemophilia Team at Oxford Haemophilia Comprehensive Care Centre completed an audit to examine patients/parents experience of immunisation practice within primary care $(P C)$ settings. It was highlighted that further training for health care practitioners (HCP) would be beneficial to improve patient/parent experience when immunisations were due. The Paediatric Haemophilia Team conducted bespoke training for different teams of HCP's on 2 occasions and also created an immunisation patient information leaflet.

Aims: The purpose of this research is to confirm if patient/parent experience has improved over the last 2 years and if training HCP's on immunisation practice is helpful and indicated as important practice for Haemophilia centre's in the UK.

Methods: Two different questionnaires were produced of 10 questions each. The first questionnaire was sent to parents and patients. The second was sent to HCP's who received immunisation training. Results: Preliminary data shows that this questionnaire received a response of $26 \%$. These initial responses have shown that current immunisation experience has improved for our patient group. Parents have revealed that anxiety around planning for their child's immunisation is common. Parents also felt that further training was still required for GP's and practice nurses in PC settings.

The second survey has an initial response of $25 \%$. The results indicated that training indeed was helpful and that further education on paediatric patients with bleeding disorders was beneficial. The questionnaire also highlighted the need for HCPs to have further training on subcutaneous injections.

Conclusions: This review of patient/parent experience and also experience of HCP's emphasised that there remains considerable variability in the delivery of immunisations to children with bleeding disorders in primary care. Areas of good practice were identified and training will continue to be offered to other health professionals within the bleeding disorder community. 
PB0492 | Clinical Profile and Bleeding Outcome of Pediatric Hemophilia A: The Royal Hospital's Experience, Oman

A. Al Rawahi ${ }^{1}$; F. Al Riyami ${ }^{2}$; I. Al Ghaithi ${ }^{3}$; H. Al Shukaili ${ }^{4}$; M. Al Abri ${ }^{4}$; A. Al Rawahi ${ }^{5}$

${ }^{1}$ Oman Medical Speciality Board, Pediatric Program, Muscat, Oman;

${ }^{2}$ Oman Medical Speciality Board, Muscat, Oman; ${ }^{3}$ Consultant, Department of Pediatric Hematology/Oncology, Royal Hospital, Ministry of Health, Muscat, Oman; ${ }^{4}$ Medical Student, Sultan Qaboos University, Muscat, Oman; ${ }^{5} \mathrm{Head}$ of Research and Studies, Oman Medical Speciality Board, Muscat, Oman

Background: Haemophilia A is an X-linked inherited bleeding disorder caused by deficiency of coagulation factor VIII. Affected patients are predisposed to variety of bleeding events. There are limited number of publications regarding clinical characteristics and bleeding outcome of pediatric Hemophilia A in Oman.

Aims: This study aims to describe the clinical profile and outcome of Omani children with haemophilia A at the Royal Hospital, the largest referral centre in Oman, from 2006 to 2019.

Methods: This is a retrospective descriptive cohort study that includes all Omani children, younger than 13 years old, diagnosed with Hemophilia A and followed up at the Royal Hospital. Patients data was retrieved from their electronic chart system (AIShifa). Collected data includes age at presentation, factor VIII level, treatment, complications and bleeding episodes. Statistical analysis was performed using SPSS 24.0 (SPSS, Inc, Chicago, Illinois).

Results: Forty-four male patients were included with a mean age of 1 year (range 1 day - 9 years) at presentation. The mean period of follow up was 7.9 (SD 3.6) years. Twenty fours patients (55\%) were diagnosed during screening test performed in response to a positive family history. The two most common complaints at presentation were muscle bleeding 14 (30\%) and post circumcision-bleeding 5 (11\%). According to factor's level at diagnosis, 2 (5\%) patients had mild disease, 29 (66\%) patients had moderate disease, and 13 (30\%) patients had severe disease. Twenty-eight patients (64\%) were on regular prophylaxis. Ten (23\%) patients developed target joint and six (14\%) developed factor VIII inhibitors. The mean ABR outcome was 1.8, 4.6, and 4.6 for the mild, moderate, and severe patients respectively (Table 1 ).

TABLE 1 Summary of clinical phenotype and outcome for the cohort

\begin{tabular}{llll} 
& Mild & Moderate & Severe \\
No. (\%) & $2(5 \%)$ & $29(66 \%)$ & $13(30 \%)$ \\
Mean duration of follow up & 8.0 years & 8.5 years & 6.7 years \\
Prophylaxis treatment No. (\%) & $1(50 \%)$ & $19(66 \%)$ & $8(62 \%)$ \\
Inhibitor development & $0(0 \%)$ & $3(10 \%)$ & $3(23 \%)$ \\
Mean ABR & 1.8 & 4.6 & 4.6 \\
Patients with at least one target joint (\%) & $0(0 \%)$ & $7(23 \%)$ & $3(20 \%)$ \\
\hline
\end{tabular}

PB0493 | Acute Myelogenous Leukemia in a Patient with Mild Haemophilia A. A Case Report at Hospital Infantil de México Federico Gómez

L.M. Muñoz Juárez Díaz; A.M. Moreno Gonzalez; L. Velázquez Marmolejo Hospital Infantil de México Federico Gómez, Mexico, Mexico

Background: Haemophilia A is an X-linked bleeding disorder in which the deficiency of coagulation factor VIII (FVIII) results in spontaneous bleeding. Haemophilia A (HA) represents $80 \%$ of total patients and it affects 1 in 5,000 male births worldwide. The association with other blood disorders is rare.

An infrequent finding in this type of patients is the development of acute myelogenous leukemia (AML) that is worth reporting, although cases of haemophilia and other malignancies have been reported in HIV-positive subjects, it represents a challenge.

Aims: Report the case of a Mexican patient with mild haemophilia A and acute myeloid leukemia (M2) at the time of presentation.

Methods: A 16-year-old male with known mild haemophilia A with diagnosis at 11 months old with episodic treatment, he received FVIII concentrates twice. He presented with headache, disseminated purpuric lesions, pallor, asthenia and swelling of left temporomandibular joint.

Laboratories revealed haemoglobin $8.9 \mathrm{~g} / \mathrm{dL}$, total leucocyte count $350,100 / \mathrm{mm}^{3}$, differential count polymorph $0 \%$, lymphocyte $2 \%$, blasts $98 \%$, platelet count 35,000 .

Bone marrow smear with $98 \%$ myeloid blasts. Immunophenotype: myeloperoxidase 100\%, CD13 80.2\%, CD33 85.5\%, CD117 99\%, CD71 90\%, HLA/DR 96.2\%, CD7 74.7\% with diagnosis of acute myeloid leukemia M2.

The patient is receiving chemotherapy according to NOPHO-AML or bone marrow aspiration) the patient received recombinant FVIII concentrate $40 \mathrm{UI} / \mathrm{Kg}$ without any bleedings.

Results: None

Conclusions: Haemophilia is a rare disease and associated with AML represents a challenge. Literature has described cases of adult patients 93 protocol. Previous to any procedure (intrathecal chemotherapy

Conclusions: The clinical phenotype of Omani children is apparently milder than what is published in the literature as there is higher percentage of moderate Hemophilia A but with a similar clinical outcome. 
with haemophilia who developed HIV-associated malignancies or patients with leukemia developing an acquired hemophilia due to an inhibitor, however, the incidence of leukemia in patients with previous diagnosis of hemophilia and its treatment are important to report. The challenge of treatment because of the risk of bleeding and its complications is important because there is no consensus in the literature.

\section{HEMOPHILIA - CLINICAL}

\section{LPB0067 | Drastic Decrease in FVIII Response after Repeated}

Daily Desmopressin Administrations in Peri-operative Non-severe Hemophilia A Patients: Final Results of Tachyphylaxis in the DAVID and Little DAVID Study

L.G.R. Romano ${ }^{1}$; L.M. Schütte ${ }^{1}$; R.M. van Hest ${ }^{2}$; K. Meijer ${ }^{3}$; B.A.P. Laros - van Gorkom ${ }^{4}$; L. Nieuwenhuizen ${ }^{5}$; J. Eikenboom ${ }^{6}$; F.C.J.I. Heubel-Moenen ${ }^{7}$; N. Uitslager ${ }^{8}$; M. Coppens ${ }^{9}$;

K. Fijnvandraat ${ }^{10}$; M.H.E. Driessens ${ }^{11}$; S. Polinder ${ }^{12}$;

M.H. Cnossen ${ }^{13}$; F.W.G. Leebeek ${ }^{1}$; R.A.A. Mahtôt ${ }^{2}$; M.J.H.A. Kruip ${ }^{1}$, On behalf of the SYMPHONY Consortium

${ }^{1}$ Department of Hematology, Erasmus MC, Erasmus University Medical Center, Rotterdam, Netherlands; ${ }^{2}$ Department of Hospital Pharmacy and Clinical Pharmacology, Amsterdam University Medical Centers - University of Amsterdam, Amsterdam, Netherlands; ${ }^{3}$ Department of Hematology, University Medical Center Groningen, Groningen, Netherlands; ${ }^{4}$ Department of Hematology, Radboud University Medical Center, Nijmegen, Netherlands; ${ }^{5}$ Department of Hematology, Máxima Medical Center, Veldhoven, Netherlands; ${ }^{6}$ Department of Internal Medicine, Section of Thrombosis and Hemostasis, Leiden University Medical Center, Leiden, Netherlands; ${ }^{7}$ Department of Hematology, Maastricht University Medical Center+, Maastricht, Netherlands; ${ }^{8}$ Van Creveldkliniek, University Medical Center Utrecht, Utrecht, Netherlands; ${ }^{9}$ Department of Hematology, Amsterdam University Medical Centers - University of Amsterdam, Amsterdam, Netherlands, ${ }^{10}$ Department of Pediatric Hematology, Amsterdam University Medical Centers - University of Amsterdam, Emma Children's Hospital, Amsterdam, Netherlands, ${ }^{11}$ Netherlands Hemophilia Patient Society (NVHP), Nijkerk, Netherlands, ${ }^{12}$ Department of Public Health, Erasmus MC, Erasmus University Medical Center, Rotterdam, Netherlands,

${ }^{13}$ Department of Pediatric Hematology, Erasmus MC - Sophia Children's Hospital, Rotterdam, Netherlands

Background: Non-severe hemophilia A patients can peri-operatively be treated with desmopressin or factor VIII (FVIII) concentrate. Desmopressin increases FVIII levels which if sufficient can be used for (minor) procedures. Data is limited on tachyphylaxis, a decreased response after repeated desmopressin administration.

Aims: To study desmopressin response in hemophilia A patients after repeated peri-operative daily doses of desmopressin and to investigate extent of tachyphylaxis.
Methods: Hemophilia A patients responsive to desmopressin undergoing a (minor) procedure were included. Historical results from a desmopressin test administration were collected from patients' medical files. Desmopressin was administered once-a-day intravenously $(0.3 \mu \mathrm{g} / \mathrm{kg})$ in 30 minutes on day of surgery (D0) and maximally two days onwards (D1 and D2). Blood samples were collected before start and \pm ca. 20-30 minutes after end of infusion. The FVIII increase was calculated as the difference between the FVIII level before and after desmopressin on the respective day. Response to repeated desmopressin administration (tachyphylaxis) between consecutive days and comparison between DO and desmopressin test was calculated as a percentage, dividing latter and earlier mentioned FVIII increases with each other. Local ethics committee approval was obtained and patients provided written informed consent.

Results: Patient characteristics and treatment days of 21 procedures are described in Table 1. Figure 2 shows the increase of FVIII after desmopressin test and treatment. Median percentage of tachyphylaxis was: D1/D0 42.9\% ( $n=19$; IQR 31.1\% - 51.9\%), D2/D0 33.3\% ( $n=12$; IQR 23.2\% - 46.3\%) and 88.5\% ( $n=12$; IQR 66.4\% - 109.1\%) D2/D1. Median percentage of desmopressin test/D0 was $95 \%$ ( $n=21$; IQR $81.8 \%-135.2 \%$ ).

TABLE 1 Patient characteristics

\begin{tabular}{ll}
$\begin{array}{l}\text { Characteristic } \\
\text { Hemophilia severity }\end{array}$ & $\begin{array}{l}\text { Number (\%) / median } \\
\text { [interquartile range] }\end{array}$ \\
Moderate & $1(4.8)$ \\
Mild & $20(95.2)$ \\
Lowest FVIII level (IU/ml) & $0.15[0.08-0.19]$ \\
Consecutive days desmopressin & \\
$<2$ days & $2(9.1)$ \\
2 days & $7(31.8)$ \\
3 days & $13(59.1)$ \\
\hline
\end{tabular}

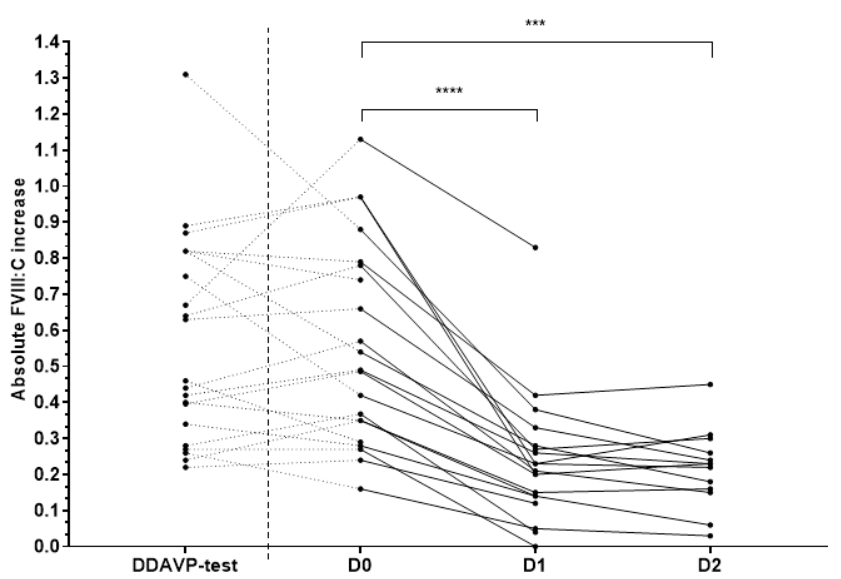

FIGURE 1 Absolute FVIII:C increase (IU/ml) in prior DDAVP test and response after first (D0) and repeated doses (D1, D2). $P<$ $0.001=^{* * *}$ and $P<0.0001={ }^{* * * *}$ 
Conclusions: Repeated daily desmopressin administration in the perioperative setting results in a drastic decrease of more than $50 \%$ in FVIII response, especially when comparing first with second desmopressin administration. Desmopressin test response predicted the response to the first pre-operative desmopressin administration accurately.

LPB0114 | Safety of FEIBA and Emicizumab (SAFE): Dose Escalation Study Evaluating the Safety of in vivo Administration of Activated Prothrombin Complex Concentrate in Hemophilia A Patients with Inhibitors on Emicizumab

H. Kizilocak ${ }^{1}$; E. Marquez-Casas ${ }^{1}$; J. Malvar ${ }^{1}$; G. Young ${ }^{2}$

${ }^{1}$ Children's Hospital Los Angeles, Los Angeles, United States; ${ }^{2}$ Children's Hospital Los Angeles, Keck School of Medicine, University of Southern California, Los Angeles, United States

Background: Hemophilia A (HA) patients on emicizumab still experience breakthrough bleeding and may need treatment with activated prothrombin complex concentrate (aPCC). A concomitant drug reaction between emicizumab and aPCC resulting in thrombotic events was noted in the HAVEN 1 study which led to a reduction in the use of aPCC. Previous in vitro studies demonstrated excess thrombin generation (TG) when aPCC was spiked into simulated hemophilia inhibitor plasma with emicizumab.

Aims: To determine TG of in vitro spiking and in vivo administration of aPCC at escalating concentrations/doses in patients on emicizumab.

Methods: Patients with severe HA with inhibitors who are currently on emicizumab had TG performed at baseline and after spiking clinically relevant concentrations of aPCC in vitro into blood samples. Then, the same patients had TG performed at baseline and following an infusion of escalating doses of aPCC $(5-75 \mathrm{U} / \mathrm{kg})$ in the in vivo portion of the study. Once a patient's endogenous thrombin potential (ETP) was $\geq 90 \%$ of the pooled normal plasma's ETP, no further escalation was done.

Results: Nine patients with severe HA and inhibitors currently on emicizumab were enrolled in the study. The summary statistics are provided in Table 1.

TABLE 1 The summary statistics

\begin{tabular}{llll} 
& $\begin{array}{l}\text { Dose of aPCC at } \\
\text { final visit }\end{array}$ & Lag time & ETP \\
Patient number & $50 \mathrm{U} / \mathrm{kg}$ & 4.17 & 724.62 \\
SAFE-01 & $50 \mathrm{U} / \mathrm{kg}$ & 4.1 & 986.16 \\
SAFE-02 & $75 \mathrm{U} / \mathrm{kg}$ & 4 & 1415.16 \\
SAFE-03 & $50 \mathrm{U} / \mathrm{kg}$ & 4.94 & 1113.72 \\
SAFE-04 & $25 \mathrm{U} / \mathrm{kg}$ & 3.64 & 1274.32 \\
SAFE-05 & $10 \mathrm{U} / \mathrm{kg}$ & 5.00 & 1221.72 \\
SAFE-06 & $75 \mathrm{U} / \mathrm{kg}$ & 4.1 & 897.43 \\
SAFE-07 & $75 \mathrm{U} / \mathrm{kg}$ & 3.1 & 741.61 \\
SAFE-08 & $75 \mathrm{U} / \mathrm{kg}$ & 3.1 & 623.95 \\
SAFE-09 & & & \\
\hline
\end{tabular}

* Patient moved out of state, was eligible for the last visit but could not be enrolled.†Patient reached maximum dose of $75 \mathrm{IU} / \mathrm{kg}$ of aPCC.
Conclusions: This study demonstrates that spiking experiments of aPCC and emicizumab may be misleading. The in vitro portion of the study demonstrated that clinically relevant concentrations of aPCC resulted in excessive TG, however in vivo administration of aPCC to the same patients demonstrated significantly different results, with most of the patients (66\%) having normal (not excessive) TG at the approved doses of aPCC (Figure 1). In conclusion, this data suggests that a single licensed dose of aPCC is safe for most patients on emicizumab and importantly calls into question the validity of in vitro spiking studies using TG in this setting.

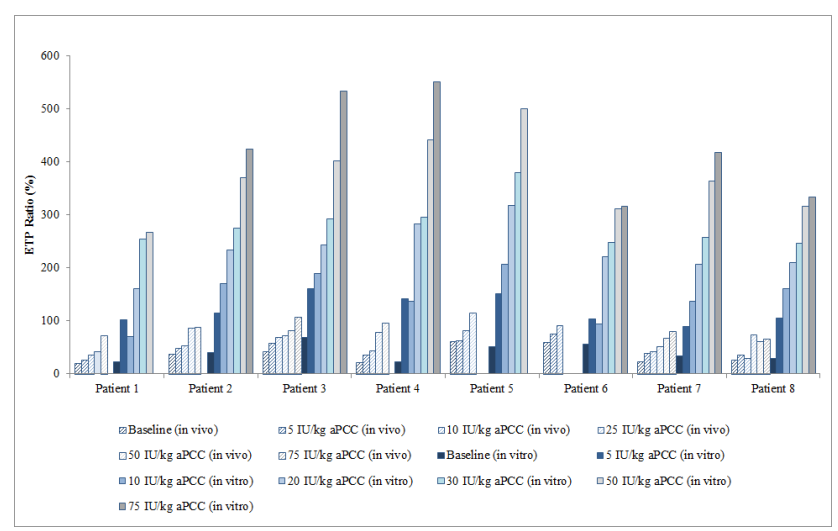

FIGURE 1 Comparison of in vitro and in vivo administration of aPCC

$\begin{array}{lll} & \begin{array}{l}\text { Ratio of Patient's } \\ \text { ETP to Pooled } \\ \text { Normal Plasma's ETP }\end{array} & \text { Adverse events } \\ \text { Peak thrombin } & * 71.85 \% & \text { No } \\ 47.7 & * 88.45 \% & \text { No } \\ 62.73 & 106.74 \% & \text { No } \\ 90.95 & 96.13 \% & \text { No } \\ 70.75 & 115.79 \% & \text { No } \\ 82.53 & 92.15 \% & \text { No } \\ 66.66 & \dagger 80.49 \% & \text { No } \\ 60.28 & \dagger 66.52 \% & \text { No } \\ 49.4 & \dagger 55.96 \% & \text { No } \\ 47.89 & & \end{array}$


LPB0115 | High Impact and Severe Outcomes of Intracranial Hemorrhage $(\mathrm{ICH})$ in an Italian Population of Adult Mild Hemophiliac Patients: Data from the EMO.REC Registry

E. Zanon ${ }^{1}$; S. Pasca ${ }^{1}$; F. Demartis ${ }^{2}$; A. Tagliaferri ${ }^{3}$; C. Santoro ${ }^{4}$; I. Cantori ${ }^{5}$; A.C. Molinari ${ }^{6}$; C. Biasioli ${ }^{7}$; A. Coppola ${ }^{3}$; G. Castaman ${ }^{2}$; S. Linari' ${ }^{2}$; M. Luciani ${ }^{8}$; G. Sottilotta ${ }^{9}$ I. Ricca ${ }^{10}$; B. Pollio ${ }^{10}$; A. Borchiellini ${ }^{11}$; A. Tosetto ${ }^{12}$; P. Simioni ${ }^{1}$; F. Peyvandi ${ }^{13}$ ${ }^{1}$ Padua University Hospital, Padua, Italy; ${ }^{2}$ Careggi University Hospital, Florence, Italy; ${ }^{3}$ Parma University Hospital, Parma, Italy; ${ }^{4}$ Umberto I University Hospital, Rome, Italy; ${ }^{5}$ Hospital of Macerata, Macerata, Italy; ${ }^{6}$ Gaslini Children Hospital, Genoa, Italy; ${ }^{7}$ Cesena Hospital, Cesena, Italy; ${ }^{8}$ Bambin Gesù Children Hospital, Rome, Italy; ${ }^{9}$ Hospital of Reggio Calabria, Reggio Calabria, Italy, ${ }^{10}$ Regina Margherita Children Hospital, Turin, Italy, ${ }^{11}$ Le Molinette Hospital, Turin, Italy, ${ }^{12}$ S. Bortolo Hospital, Vicenza, Italy, ${ }^{13}$ Milan University Hospital, Milan, Italy

Background: Patients with mild hemophilia are usually considered to be at low-risk of bleeding, but the increase in mean age, associated with some concomitant diseases such as hypertension, and the absence of prophylactic treatment puts them at risk of severe or even fatal bleeding. To date, data in this regard are still scarce.

Aims: To assess the impact of $\mathrm{ICH}$ in an Italian population of adult mild patients with hemophilia $(\mathrm{PWH})$ in terms of risk factors, incidence, treatments and outcomes.

Methods: All data of mild PWH with ICH were retrospectively/prospectively collected at 13 Italian Hemophilia Centers, and subsequently analyzed and compared with the moderate/severe subjects also present in our EMO.REC Registry.

Results: 31 adult patients with ICH were recorded, of whom $45.2 \%$ had mild hemophilia. The incidence/year $\times 1000$ mild hemophilicacs was 0.24 , IC 95\% (0.14-0.40). Mean age at diagnosis was 62.5years, higher than non-mild patients (45.0years). In $92.9 \%$ of cases, ICHs were spontaneous. Hypertension was reported in $85.7 \%$ of mild patients, while only in $23.5 \%$ of non-mild $(P<0.05)$. At the time of $\mathrm{ICH}$ only $7.1 \%$ of mild subjects were on prophylaxis versus $29.4 \%$ of the moderate/severe. A treatment with factor concentrates was administered for at least three weeks to $78.5 \%$ of mild subjects. $50 \%$ of mild PWH underwent neurosurgery. Mortality rate in moderatesevere was almost twice as high as in mild subjects ( $52.9 \%$ vs $28.5 \%$ ). Prophylaxis was maintained life-long only in $20 \%$ of mild survivors, $45.5 \%$ of mild survivors had a permanent disability. One on-demand patient had an $\mathrm{ICH}$ recurrence.

Conclusions: The impact of $\mathrm{ICH}$ in adult mild $\mathrm{PWH}$ is high, with severe outcomes. The correction of risk factors such as hypertension, is not enough to prevent an onset. The prophylaxis is not usual in mild hemophiliacs, innovative strategic therapies could be necessary to reduce the hemorrhagic incidence in this group of patients.
PB0494 | Evolution of Emicizumab Plasma Concentrations and aPTT rratio in 183 Treated Patients with Severe Haemophilia A. A Real Life and Multi-centre Analysis

C. Pouplard $^{1,2}$; C. Nougier ${ }^{3}$; C. Flaujac ${ }^{4}$; C. Ternisien ${ }^{5}$; C. Auditeau ${ }^{4}$; M. Donnard ${ }^{6}$; F. Grand ${ }^{7}$; E. Jeanpierre ${ }^{8}$; V. Proulle ${ }^{9}$; S. Timsit ${ }^{10}$; L. Sattler ${ }^{11}$; Y. Repesse ${ }^{12}$; A. Ryman ${ }^{13}$; N. Hezard ${ }^{14}$; D. Lasne ${ }^{10}$; BIMHO Study Group on behlaf of GFHT

${ }^{1}$ University Hospital of Tours, Tours, France; ${ }^{2}$ University of Tours, EA 7501, Tours, France; ${ }^{3}$ University Hospital of Lyon, Lyon, France; ${ }^{4}$ Hospital of Versailles, Versailles, France; ${ }^{5}$ University Hospital of Nantes, Nantes, France; ' University Hospital of Limoges, Limoges, France; ${ }^{7}$ University Hospital of Poitiers, Poitiers, France; ${ }^{8}$ University Hospital of Lille, Lille, France; ${ }^{9}$ APHP, Hopital Cochin, Paris, France, ${ }^{10}$ APHP, Hopital Necker, Paris, France, ${ }^{11}$ University Hospital of Strasbourg, Strasbourg, France, ${ }^{12}$ University Hospital of Caen, Caen, France, ${ }^{13}$ University Hospital of Bordeaux, Bordeaux, France,

${ }^{14}$ University Hospital of Marseille, Marseille, France

Background: Measurement of emicizumab plasma concentration is available in laboratories but usual values are not well defined as well as variations in aPTT ratio.

Aims: To evaluate emicuzumab concentrations $\left(\mathrm{EmC}^{\circ}\right)$ and aPTT variation in a large cohort of severe hemophilia $A(H A)$ patients.

Methods: 183 severe HA patients (whose $69<12$ years) with $(n=57)$ or without $(n=126)$ inhibitors were included in 13 centres. EmC ${ }^{\circ}$ were assessed during ( $n=67$ ) or after the loading phase (LP) with different regimen: $1.5 \mathrm{mg} / \mathrm{kg}$ weekly $(n=92), 3.0 \mathrm{mg} / \mathrm{kg}$ biweekly $(n=21)$ or $6 \mathrm{mg} / \mathrm{kg}$ monthly $(n=3)$.

Results: Median $\mathrm{EmC}^{\circ}$ increased from $17.7 \mu \mathrm{g} / \mathrm{ml}$ in the first week to $50.4 \mu \mathrm{g} / \mathrm{ml}$ at the end of the LP (Figure). During the maintenance phase (MP), the median concentration remained stable around 50.9 $\mu \mathrm{g} / \mathrm{mL}$ (10th-90th percentiles: 31.1-77.2 $\mu \mathrm{g} / \mathrm{ml}, n=143$ ). EmC ${ }^{\circ}$ did not vary according to the regimen $(50.9 \mu \mathrm{g} / \mathrm{mL}(\mathrm{IQR} 27.0 \mu \mathrm{g} / \mathrm{mL})$ and $53.8 \mu \mathrm{g} / \mathrm{mL}$ (IQR $27.8 \mu \mathrm{g} / \mathrm{mL}$ ) with one injection weekly ( $n=115$ ) or every two weeks $(n=26)$ respectively). The aPTT ratio shortened during the $\operatorname{LP}(0.86)$ and then during the MP $(0.79, P<0.0001)$. Interestingly, $\mathrm{EmC}^{\circ}$ were significantly lower during MP in children than in adults/adolescents ( 47.4 vs $56.1 \mu \mathrm{g} / \mathrm{ml}, P=0.036$ ). 22 patients had bleeding events during the $\operatorname{LP}(n=6)$ or the MP $(n=16)$, but $\mathrm{EmC}^{\circ}$ were not significantly different than in non-bleeders $(50.7$ vs $49 \mu \mathrm{g} / \mathrm{mL}$ ). Moreover, $\mathrm{EmC}^{\circ}$ were lower than the $10^{\text {th }}$ percentile in 2 of 16 bleeders during MP compared to 10/86 in those without bleeding (NS). 


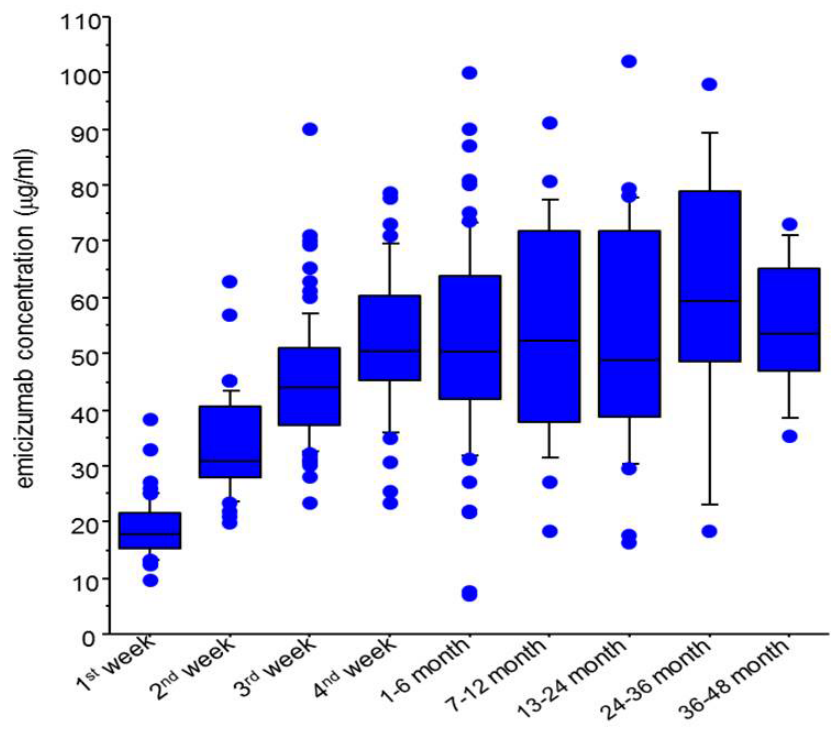

FIGURE 1 Evolution of emicizumab concentration during treatment

Conclusions: Our results confirm that $\mathrm{EmC}^{\circ}$ are quite variable in treated HA patients. This variability must be taken into account for interpretating $\mathrm{EmC}^{\circ}$ in case of suspicion of anti-drug antibody or lack of compliance.

PB0495 | Emicizumab in Obese Adults with Hemophilia A Pooled Data from Three Phase III Studies (HAVEN 1, 3 and 4)

M. Recht ${ }^{1,2} ;$ J. Mahlangu ${ }^{3} ;$ M. Minhas ${ }^{4}$; B. Trzaskoma ${ }^{4}$;

M. Shah ${ }^{4} ;$ G. Young ${ }^{5}$

${ }^{1}$ American Thrombosis and Hemostasis Network, Rochester, United States; ${ }^{2}$ Oregon Health \& Science University, Portland, United States; ${ }^{3}$ University of the Witwatersrand and National Health Laboratory Service, Johannesburg, South Africa; ${ }^{4}$ Genentech, Inc., South San Francisco, United States; ${ }^{5}$ Children's Hospital Los Angeles, Keck School of Medicine, University of Southern California, Los Angeles, United States

Background: Modelling indicates that body weight is an important predictor of emicizumab pharmacokinetics. In the HAVEN studies, actual weight-based dosing provided efficacious bleed prevention, but the impact of obesity (body mass index $[\mathrm{BMI}] \geq 30 \mathrm{~kg} / \mathrm{m}^{2}$ ) was not assessed.

Aims: To compare annualized bleed rate ( $A B R$ ) and emicizumab trough concentrations among obese and non-obese adult persons with hemophilia $\mathrm{A}(\mathrm{PwHA})$ in HAVEN 1, 3, and 4 in a post-hoc analysis.

Methods: Informed consent and ethics committee approval were obtained before the HAVEN studies. Inclusion criteria for this analysis were: $\geq 18$ years of age; with or without FVIII inhibitors; $\geq 24$ weeks on emicizumab; baseline BMI available. Treated ABRs were analyzed across the study period (using negative binomial regression) and in 24-week intervals (unadjusted) until up-titration for those who were up-titrated. Mean emicizumab trough plasma concentrations, measured at scheduled time points during each study, were analyzed.

Results: At database cut-off (15 May 2020), 44 (17\%) and 216 (83\%) adult $\mathrm{PwHA}$ with $\mathrm{BMI} \geq 30 \mathrm{~kg} / \mathrm{m}^{2}$ and $\mathrm{BMI}<30 \mathrm{~kg} / \mathrm{m}^{2}$ were included and had 94.29 and 522.97 patient-years of emicizumab exposure, respectively. Across the study period, adjusted ABRs were 1.48 (95\% confidence interval [Cl]: 0.82-2.68) and 1.40 (95\% Cl: 1.06-1.85) for adults with $\mathrm{BMI} \geq 30 \mathrm{~kg} / \mathrm{m}^{2}$ and $<30 \mathrm{~kg} / \mathrm{m}^{2}$, respectively. ABRs were consistent across the two groups over time (Table 1). Efficacious trough concentrations were sustained in both groups regardless of dosing regimen, with concentrations $>30 \mu \mathrm{g} / \mathrm{mL}$. In the once weekly dosing cohort, mean (standard error) emicizumab trough concentration was 51.47 (3.04) $\mu \mathrm{g} / \mathrm{mL}$ and 53.21 (1.18) $\mu \mathrm{g} / \mathrm{mL}$ at Week 5 following loading dosing, for adults with $\mathrm{BMI} \geq 30 \mathrm{~kg} / \mathrm{m}^{2}(n=27)$ and $<30 \mathrm{~kg} / \mathrm{m}^{2}$ ( $\left.n=137\right)$, respectively (Figure 1$)$.

Conclusions: Efficacious emicizumab trough concentrations were maintained in obese and non-obese PwHA in HAVEN 1, 3, and 4, and treated ABRs were similar in both groups.

TABLE 1 Unadjusted annualized bleed rates for treated bleeds among adult PwHA with and without FVIII inhibitors with measured baseline BMI and $\geq 24$ weeks on emicizumab in HAVEN 1, HAVEN 3 , or HAVEN 4 , grouped by baseline BMI $\left(\geq 30 \mathrm{~kg} / \mathrm{m}^{2}\right.$ or $\left.<30 \mathrm{~kg} / \mathrm{m}^{2}\right)$ over time $(N=260)$
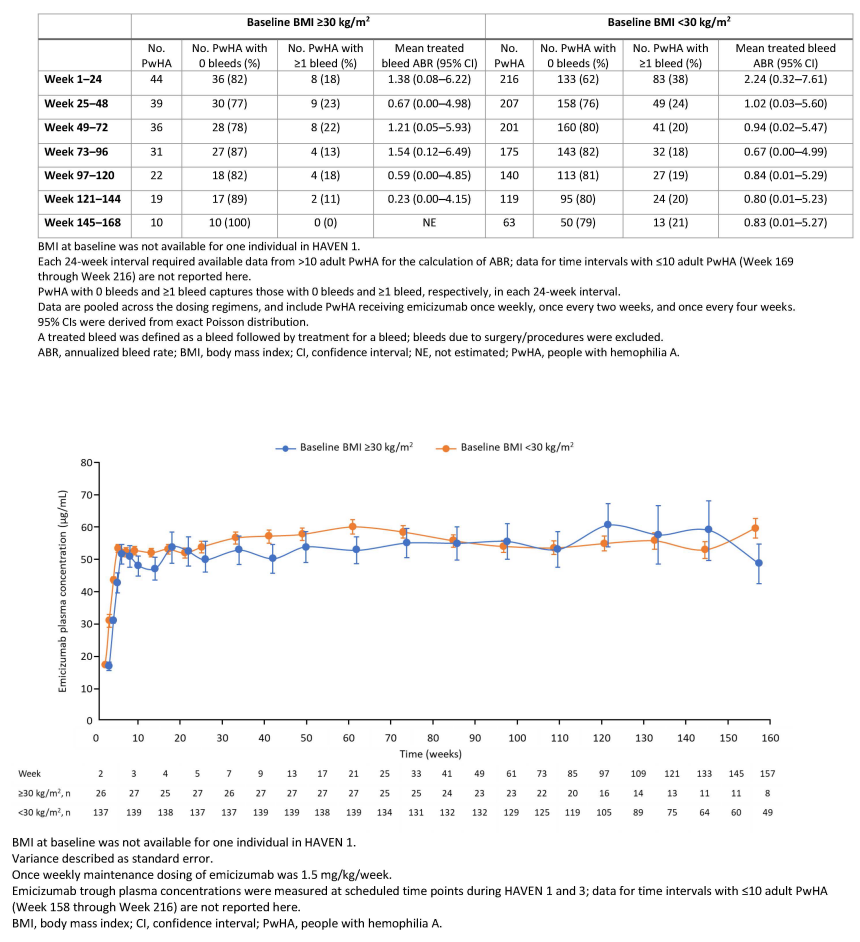

FIGURE 1 Mean (standard error) emicizumab trough concentrations of PwHA with and without FVIII inhibitors with measured baseline weight and $\geq 24$ weeks receiving emicizumab once weekly in HAVEN 1 or HAVEN 3 , grouped by baseline BMI $\left(\geq 30 \mathrm{~kg} / \mathrm{m}^{2}\right.$ or $<30$ $\left.\mathrm{kg} / \mathrm{m}^{2}\right)$ over time $(\mathrm{N}=166)$ 
PB0496 | Increased Prevalence of Age-related Comorbidities and Health Service Utilization among Adult Men with Hemophilia in Alberta, Canada

A.U. Alam; C. Wu; H. Sun

University of Alberta, Edmonton, Canada

Background: Improvements in treatment strategies over time have led to increased life expectancy of patients with hemophilia (PWH). Consequently, age-related comorbidities become increasingly relevant and pose challenges in management.

Aims: We aimed to evaluate the prevalence of age-related comorbidities and health service utilization among adult men with hemophilia compared to the general population.

Methods: We conducted a retrospective cohort study using linked administrative data. Cases of hemophilia were identified in Alberta, Canada from 2012 to 2019 with a validated case definition and were age and sex-matched (1:3) with population controls. Comorbidities were identified by presence of at least 2 international classification of diseases (ICD) codes. Research ethics approval was obtained.

Results: We identified 206 and 36 male adult patients with hemophilia A (HA) and hemophilia B (HB), respectively. Thirty (12.6\%) were over age 65 . There was a significantly higher prevalence of hypertension (HA: $14.1 \%$ vs $6.6 \%$ ), hyperlipidemia (HA: $2.4 . \%$ vs $0.3 \%$ ), liver diseases (HA: $13.1 \%$ vs $0.8 \%$, HB: $22.2 \%$ vs $0.0 \%$ ) and malignancy (HA: $5.8 \%$ vs $2.3 \%$, HB: $16.7 \%$ vs $2.8 \%$ ) in hemophilia cases than controls (Table 1). However, prevalence of cardiovascular and other major organ diseases was not significantly different from the general population for both types of hemophilia. Hemophilia was associated with significantly higher rates of hospitalization (HA: $53.4 \%$ vs $18.8 \%$, HB: $50.0 \%$ vs $17.6 \%$ ), intensive care unit admission (HA: $5.8 \%$ vs $1.1 \%$ ), emergency department visit (HA: $83.5 \%$ vs $68.4 \%$, HB: $83.3 \%$ vs $63.3 \%$ ), and longer surgical admission length of stay (HA: 15.7 vs 7.4 days) (Table 2).

TABLE 1 Prevalence of comorbidities among adult men with hemophilia compared with age- and sex-matched control population

\begin{tabular}{|c|c|c|c|c|c|c|}
\hline & \multicolumn{3}{|c|}{ Hemophilia A } & \multicolumn{3}{|c|}{ Hemophilia B } \\
\hline Hypertension & $29(14.1)$ & $41(6.6)$ & 0.001 & $2(5.6)$ & $6(5.6)$ & 0.99 \\
\hline Hyperlipidemia & $5(2.4)$ & $2(0.3)$ & 0.01 & $0(0.0)$ & $1(0.9)$ & 0.99 \\
\hline Obesity & $4(1.9)$ & $3(0.5)$ & 0.07 & $0(0.0)$ & $0(0.0)$ & $\mathrm{N} / \mathrm{A}$ \\
\hline Renal disease & $3(1.5)$ & $6(1.0)$ & 0.69 & $0(0.0)$ & $1(0.9)$ & 0.99 \\
\hline \multicolumn{7}{|c|}{ Cardiovascular or coronary artery diseases } \\
\hline Coronary artery diseases & $9(4.4)$ & $18(2.9)$ & 0.21 & $1(2.8)$ & $3(2.8)$ & 0.99 \\
\hline Cerebrovascular diseases & $8(3.9)$ & $12(1.9)$ & 0.12 & $1(2.8)$ & $3(2.8)$ & 9.99 \\
\hline Venous thromboembolism & $0(0.0)$ & $10(1.6)$ & 0.07 & $0(0.0)$ & $2(1.9)$ & 0.99 \\
\hline Malignancy & $12(5.8)$ & $14(2.3)$ & 0.02 & $6(16.7)$ & $3(2.8)$ & 0.008 \\
\hline Chronic pulmonary disease & $9(4.4)$ & $13(2.1)$ & 0.08 & $0(0.0)$ & $2(1.9)$ & 0.99 \\
\hline Liver disease & $27(13.1)$ & $5(0.8)$ & $<0.001$ & $8(22.2)$ & $0(0.0)$ & $<0.001$ \\
\hline Neurological disease & $3(1.5)$ & $12(1.9)$ & 0.77 & $1(2.8)$ & $3(2.8)$ & 0.99 \\
\hline
\end{tabular}

${ }^{a}$ Based on chi square or fisher's exact test (as appropriate).

TABLE 2 Comparison of health service utilization of adult men with hemophilia and age and sex-matched control population

\begin{tabular}{|c|c|c|c|c|c|c|}
\hline & \multicolumn{3}{|l|}{ Hemophilia A } & \multicolumn{3}{|l|}{ Hemophilia B } \\
\hline Intensive care admission & $12(5.8)$ & $11(1.8)$ & 0.002 & $1(2.8)$ & $3(2.8)$ & 0.99 \\
\hline $\begin{array}{l}\text { Emergency department } \\
\text { visit }\end{array}$ & $172(83.5)$ & $423(68.4)$ & $<0.001$ & 30 (83.3) & $68(63.0)$ & 0.02 \\
\hline Ambulatory orthopedic & $56(27.2)$ & $62(10.0)$ & $<0.001$ & $7(19.4)$ & $9(8.3)$ & 0.12 \\
\hline
\end{tabular}
surgery visits 


\begin{tabular}{|c|c|c|c|c|c|c|}
\hline & \multicolumn{3}{|l|}{ Hemophilia A } & \multicolumn{3}{|l|}{ Hemophilia B } \\
\hline & $\begin{array}{l}\text { Case } N=206 \\
(\%)\end{array}$ & Control N = $618(\%)$ & $P$ value $^{a}$ & Case $N=36(\%)$ & Control $N=108(\%)$ & $P$ value $^{a}$ \\
\hline $\begin{array}{l}\text { Orthopedic surgery } \\
\text { inpatient admission }\end{array}$ & $22(10.7)$ & $10(1.6)$ & $<0.001$ & $1(2.8)$ & $2(1.9)$ & 0.99 \\
\hline $\begin{array}{l}\text { Hematology inpatient } \\
\text { admissions }\end{array}$ & $11(5.3)$ & $0(0.0)$ & $<0.001$ & $1(2.8)$ & $0(0.0)$ & 0.25 \\
\hline
\end{tabular}

${ }^{a}$ Based on chi square or fisher's exact test (as appropriate) for categorical variable and t-test for continuous variable;

${ }^{\mathrm{b}}$ Length of stay.

${ }^{\mathrm{c}}$ Standard deviation.

Conclusions: Despite advanced care, hemophilia is associated with higher acute care utilization than the general population. PWH have higher prevalence of malignancies and cardiovascular risk factors without increased risk of vascular diseases. Future studies are needed to examine the reasons for increased health service utilization.

PB0498 | Prevalence of Hypertension in Hemophilia Patients: A Systematic Review

A. Ter-Zakarian ${ }^{1}$; K. Al Banaa ${ }^{1}$; R. Barnes ${ }^{1}$; A. von Drygalski ${ }^{1,2}$

${ }^{1}$ University of California San Diego, San Diego, United States; ${ }^{2}$ The

Scripps Research Institute, La Jolla, United States

Background: The prevalence of hypertension in persons with hemophilia (PWH) is reported to be higher compared to the general population in many, but not all, studies examining cardiovascular risk factors in PWH. As life expectancy of PWH increases, an improved understanding of hypertension in $\mathrm{PWH}$ is critical.

Aims: To evaluate the role of HTN and associated risk factors in PWH by performing a systematic review of published literature.

Methods: We searched five medical electronic databases for articles on hypertension in hemophilia: PubMed, Web of Science, Embase, Trip and MedNar, screening articles published up to July 30, 2020. ATZ and KAB screened study titles and abstracts, following "Meta-analysis of Observational Studies in Epidemiology" guidelines. We extracted data on hypertension prevalence in both PWH and non-hemophilia comparator populations, hemophilia type and severity, Hepatitis C (HCV) and/or Human Immunodeficiency Virus infection, obesity, diabetes and smoking. Estimates of prevalence and odds ratio (OR) were combined using random-effects models. Heterogeneity in prevalence and OR estimates was examined by fitting meta-regression models.

Results: From 5167 articles, 32 met eligibility criteria; we considered only independent studies, excluding those without numerical data.
Hypertension prevalence estimates varied from $5 \%$ to $76 \%$; overall prevalence was $39 \%$ (95\% Cl: 31,47$)$. Large studies ( $\geq 100$ subjects) showed lower prevalence estimates $(P=0.025)$. Age $(P=0.102$, Figure 1$)$, obesity $(P=0.013)$ and smoking $(P=0.097)$ in univariate models also explained some variation in prevalence. Meta-regression yielded an overall OR of 1.45 (95\% Cl: 1.12, 1.87) for hypertension in PWH ( $n=16$ studies, Figure 2). OR estimates varied from 0.32 to 3.22 , but didn't vary with sample size or risk factors except HCV $(P=0.003)$.

Figure 1.

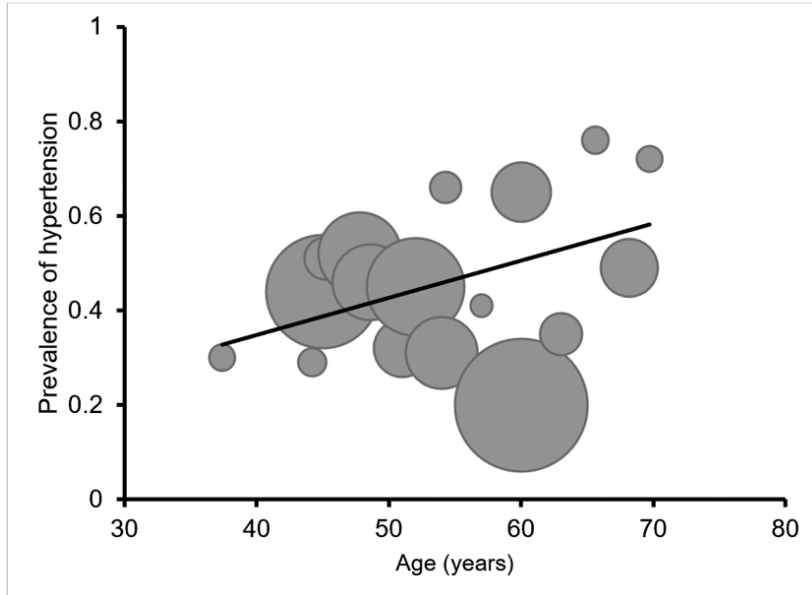

FIGURE 1 Meta-regression model illustrating the association between the prevalence of hypertension and median age in each study. The size of the bubbles is proportional to the precision of each study. The slope of the regression line, $b=0.007(95 \% \mathrm{Cl}$ : $-0.002,0.016, P=0.102$ ) 
Figure 2.

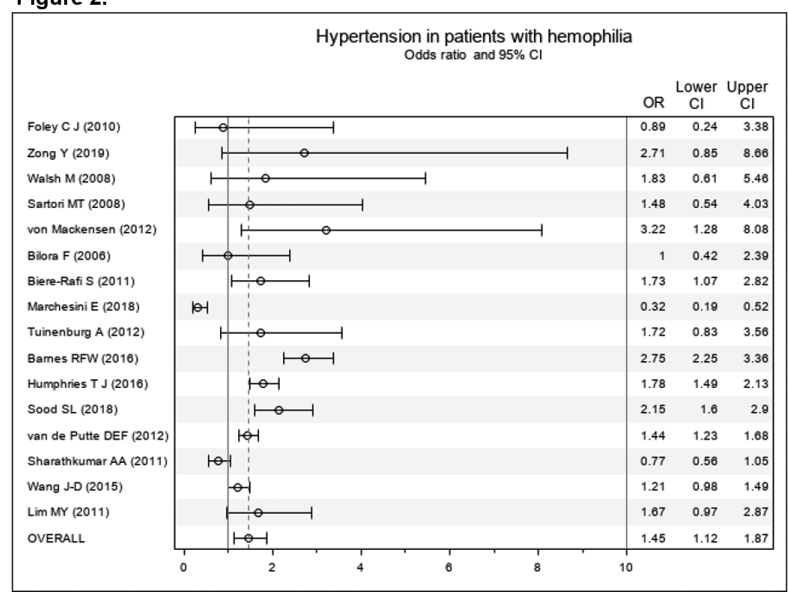

FIGURE 2 Forest plot showing odds ratios for 16 studies. The overall OR was 1.45 (95\% Cl: 1.12, 1.87)

Conclusions: PWH have a higher prevalence of hypertension compared to the general population, not explained by the usual cardiovascular risk factors.

PB0499 | Understanding the burden of Care for Haemophilia in the UK: Direct Medical Costs and Associated Predictors from the CHESS I and II Studies

I. Woollacott ${ }^{1} ;$ A. Chhabra ${ }^{1} ;$ J. Brownrigg ${ }^{1} ;$ T. Burke $^{2}$;

A. Shaikh ${ }^{2}$; J. O'Hara²; J. Godfrey ${ }^{3}$; M. Laffan ${ }^{4}$

${ }^{1}$ Pfizer, Surrey, United Kingdom; ${ }^{2}$ HCD Economics, Daresbury, United

Kingdom; ${ }^{3}$ JG Zebra Consulting Ltd, London, United Kingdom; ${ }^{4}$ Centre

for Haematology, Imperial College London, London, United Kingdom
Background: Although haemophilia A (HA) and haemophilia B (HB) pose a significant economic burden on healthcare systems, little is known about the direct medical costs (DMC), and associated predictors, in people with haemophilia (PWH) in the United Kingdom (UK). Aims: To analyse the DMC and determine the associated predictors in PWH in the UK, using data from the 'Cost of Haemophilia in Europe: a Socioeconomic Survey' (CHESS) I and II retrospective burden-of-illness studies.

Methods: Data for PWH in the UK were extracted from CHESS I (adult males with severe haemophilia across the UK, France, Germany, Italy, and Spain); and CHESS II (adult males with all severities from 8 European countries including the UK). Twelve-month DMC (excluding factor replacement therapy) were calculated using publicly available unit cost data for the UK. A generalised linear model was used to model DMC, including patient demographic and clinical predictors.

Results: Four hundred PWH were included (HA: 316 (79\%), mean annual per patient $\mathrm{DMC}=£ 3,992$; HB: 84 (21\%), mean annual per patient $\mathrm{DMC}=£ 5,075$ ); mean age of 35.12 (standard deviation (SD), 15.38) (Table 1). The mean difference in DMC between HA and HB patients was $£ 1,083(P=0.83$ ). In the regression, haemophilia subtype was not significantly associated with DMC (average marginal effect at the mean: $£ 58, P=0.92$ ). Both 'problem' joints (PJs: chronically damaged joints) and annual bleed rate (ABR) were significantly associated with DMC in haemophilia overall. DMC increased by $£ 1,976$ per increase in PJ count. Compared with those with an $A B R$ of ' 0 ', additional DMC in ABR subgroups of '1-5', '6-10', '11+' were $£ 2,053, £ 3,028$, and $£ 6,347$, respectively (Table 2 ).

TABLE 1 Descriptive statistics: cohort characteristics

\begin{tabular}{|c|c|c|c|c|c|c|c|}
\hline Variable & Total & HA & HB & Variable (cont.) & Total & HA & HB \\
\hline Age (mean $\pm S D)$ & $35.12 \pm 15.38$ & $34.50 \pm 15.20$ & $37.44 \pm 15.88$ & $\begin{array}{c}\text { 'Problem' joints, } \\
\text { n/total (\%) }\end{array}$ & & & \\
\hline Yes & $16 / 400(4 \%)$ & $14 / 316(4 \%)$ & $2 / 84(2 \%)$ & 1 & $\begin{array}{l}99 / 400 \\
(25 \%)\end{array}$ & $\begin{array}{r}75 / 316 \\
(24 \%)\end{array}$ & $24 / 84$ (29\%) \\
\hline Yes & $37 / 400(9 \%)$ & $30 / 316(9 \%)$ & $7 / 84(8 \%)$ & $\begin{array}{l}\text { Annual bleed } \\
\text { rate, } A B R \\
\text { (mean } \pm S D \text { ) }\end{array}$ & $3.84 \pm 4.83$ & $3.50 \pm 4.19$ & $5.12 \pm 6.60$ \\
\hline $\begin{array}{l}\text { Other comorbidities, } \\
\text { n/total (\%) }\end{array}$ & & & & $\begin{array}{l}\text { Therapy } \\
\text { regimen, } n / \\
\text { total (\%) }\end{array}$ & & & \\
\hline & & & & Prophylaxis & $\begin{array}{r}195 / 400 \\
(49 \%)\end{array}$ & $\begin{array}{r}154 / 316 \\
(49 \%)\end{array}$ & 41/84 (49\%) \\
\hline
\end{tabular}


TABLE 2 Regression: average marginal effects of direct medical costs (DMC)

\begin{tabular}{|c|c|c|c|}
\hline Covariate & Coefficient, $P$ value & Covariate (cont.) & Coefficient, $P$ value (cont.) \\
\hline Age & $10.73, P=0.51$ & $\begin{array}{l}\text { Annual bleed rate, } A B R \text { ('O ABR' } \\
\text { reference) }\end{array}$ & \\
\hline Haemophilia subtype (subtype B vs. A) & $58.39, P=0.92$ & $1-5$ & $2,053.04, P<0.01$ \\
\hline Inhibitor (current inhibitor present) & $4,040.80, P=0.07$ & $6-10$ & $3,028.24, P<0.01$ \\
\hline Blood-borne viruses (present) & $-823.70, P=0.29$ & $11+$ & $6,346.84, P<0.01$ \\
\hline Other comorbidities (present) & $728.96, P=0.17$ & $\begin{array}{l}\text { Therapy regimen ('No treatment' } \\
\text { reference) }\end{array}$ & \\
\hline \multirow[t]{2}{*}{ 'Problem' joints (number of joints: $0-14$ ) } & $1,976.33, P<0.01$ & On-demand & $2,115.57, P<0.01$ \\
\hline & & Prophylaxis & $2,768.66, P<0.01$ \\
\hline
\end{tabular}

Conclusions: Chronic joint damage and frequency of bleeding events are significantly associated with DMC in PWH in the UK. This highlights the economic importance of optimising joint health and minimising bleeding events in managing $\mathrm{PWH}$.

PB0500 | Inhibitor Development upon Switch from Plasmaderived to Recombinant FVIII in PUPs with Severe Haemophilia A: Preliminary Analysis from the PUP-SWITCH Study

S. Miri ${ }^{1}$; R.K. Kavakli ${ }^{2}$; S. Halimeh ${ }^{3}$; G. Nicolò ${ }^{4}$; D. Gürlek Gökçebay $^{5}$; N.Y. Özbek ${ }^{5}$; T. Celkan ${ }^{6}$; M. Karimi ${ }^{7}$; A. Shahsavani ${ }^{7}$; B.T. Gunes ${ }^{8}$; B. Atabay ${ }^{8}$; Z. Kaya ${ }^{9}$; Y. Ay ${ }^{10}$; Y. Oymak ${ }^{11}$; S. Akbayram ${ }^{12}$; B. Yılmaz ${ }^{13}$; E. Kazanci ${ }^{14}$; P.M. Mannucci ${ }^{4}$; F.R. Rosendaal ${ }^{15}$; F. Peyvandi ${ }^{16}$; PUP-SWITCH Study Group ${ }^{1}$ Università degli Studi di Milano, Department of Biomedical Sciences for Health, Milan, Italy; ${ }^{2}$ Ege Hemophilia Center, Izmir, Turkey; ${ }^{3}$ Coagulation and Thrombosis Centre, Duisburg, Germany; ${ }^{4}$ Fondazione IRCCS Ca' Granda Ospedale Maggiore Policlinico, Angelo Bianchi Bonomi Hemophilia and Thrombosis Center, Milan, Italy; ${ }^{5}$ Pediatric Hematology and Oncology, Ministry of Health Ankara City Hospital, Ankara, Turkey; ${ }^{6}$ Department of Pediatric Hematology and Oncology, Cerrahpaşa Faculty of Medicine, İstanbul University, Istanbul, Turkey; ${ }^{7}$ Hematology Research Center, Shiraz University of Medical Sciences, Shiraz, Iran, Islamic Republic of; ${ }^{8}$ Tepecik Education and Research Hospital, Izmir, Turkey; ${ }^{9}$ Department of Pediatrics, Medical School Of Gazi University, Ankara, Turkey, ${ }^{10}$ Department of Pediatric Hematology, Pamukkale University Faculty of Medicine, Denizli, Turkey, ${ }^{11}$ Department of Pediatric Hematology, Dr Behcet Uz Children's Hospital, Izmir, Turkey, ${ }^{12}$ Department of Pediatric Hematology-Oncology, Gaziantep University Faculty of Medicine, Gaziantep, Turkey, ${ }^{13}$ Division of Pediatric Hematology-Oncology, Marmara University, Istanbul, Turkey, ${ }^{14}$ University of Health Sciences Turkey, Pediatric Hematology and Oncology, Bursa, Turkey, ${ }^{15}$ Department of Clinical Epidemiology, Leiden University Medical Center, Leiden, Netherlands, ${ }^{16}$ Università degli Studi di Milano, Department of Pathophysiology and Transplantation, Milan, Italy

Background: In 2016, the SIPPET study reported that in previously untreated patients (PUPs) with severe haemophilia $A(H A)$ the choice of plasma-derived FVIII (pdFVIII) products in the first 50 exposure days (EDs) is associated with a significantly lower incidence of inhibitors compared to recombinant FVIII (rFVIII) products. ${ }^{1}$ However, the choice of FVIII product class after this high-risk period has been subject of debate. It has been established that switching products in multi-transfused patients (> $150 \mathrm{EDs}$ ) does not affect the risk of inhibitor development which becomes approximately 100 times lower in these previously treated patients (PTPs).

Aims: To investigate whether a switch from pdFVIII to rFVIII in PUPs after the early high-risk period would follow the expected low-risk pattern of inhibitor incidence as previously reported in PTPs, or a novel peak of inhibitors would appear.

Methods: We designed a survey on the ISTH REDCap platform ${ }^{2}$ to investigate the rate of novel inhibitors after the switching of PUPs from pdFVIII to rFVIII between 50 and 150 EDs (Figure 1.A). Centres who did the switch after the SIPPET publication were identified and invited to participate in this observational cohort study on PUPs under 6 years old with severe HA, who have been switched according to this strategy.

Figure 1. A. Study design

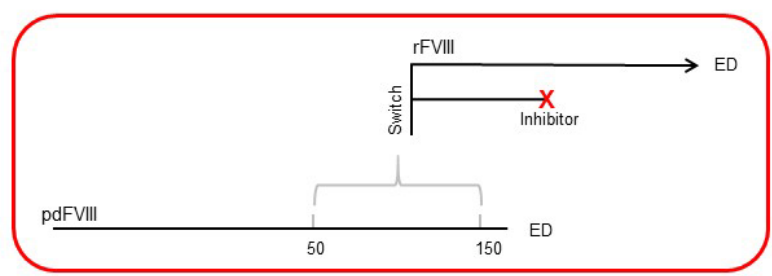

Figure 1. B. Flowchart

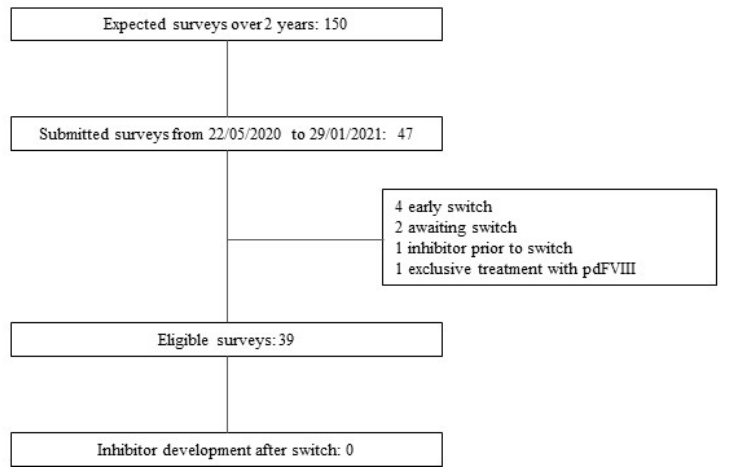

FIGURE 1 Study design and flowchart 
Results: To date, 11 Turkish centres, 1 Iranian centre, and 1 German centre have participated in this study. Fourty-seven surveys were analysed, among which 39 patients satisfied all eligibility criteria (Figure 1.B). Patients were switched at a median of 60 (54 - 71) EDs and received a median of 111 (84 - 222) EDs with rFVIII after the switch. None of these 39 patients developed an inhibitor after the switch (Table 1).

TABLE 1 Switch-related data in the 39 eligible patients

\begin{tabular}{|c|c|c|c|c|c|c|c|}
\hline \multirow{2}{*}{$\begin{array}{l}\text { Eligible } \\
\text { patients } \\
n\end{array}$} & \multicolumn{2}{|c|}{ ED of switch } & \multicolumn{2}{|c|}{ EDs with rFVIII after switch } & \multicolumn{2}{|c|}{$\begin{array}{l}\text { Total EDs with rFVIII at survey } \\
\text { retrieval }\end{array}$} & \multirow{2}{*}{$\begin{array}{l}\text { Inhibitor after } \\
\text { switch } \\
\text { n }\end{array}$} \\
\hline & Median & IQR & Median & IQR & Median & IQR & \\
\hline 39 & 60 & $54-71$ & 111 & $84-222$ & 178 & $144-279$ & 0 \\
\hline
\end{tabular}

Conclusions: Preliminary results from the first 39 patients enrolled in the PUP-SWITCH study did not show an additional risk of inhibitor incidence upon switch from pdFVIII to rFVIII.

PB0501 | Real-world Use of FVIII in People with Severe Hemophilia A without Inhibitor on Prophylaxis, Comparing Switched to rFVIII-Fc versus Non-switched Patients: A Multicentre, Retrospective, Non Interventional, before-after Study with the French Nationwide Claims Database (MOTHIF-II)

V. Horvais ${ }^{1}$; N. Drillaud ${ }^{1}$; M. Sigaud ${ }^{1}$; M. Fouassier ${ }^{1}$;

C. Ternisien ${ }^{1}$; Y. Repesse ${ }^{2}$; B. Guillet ${ }^{3}$; S. Bayart ${ }^{3}$; P. Beurrier ${ }^{4}$;

L. Ardillon ${ }^{5}$; Y. Gruel'; J.-B. Valentin ${ }^{5}$; S. Bauer ${ }^{6}$; B. Pan-Petesch;

V. Cussac ${ }^{8}$; J. Rose ${ }^{8}$; M. Pennetier ${ }^{1}$; M. Trossaërt ${ }^{1}$, BERHLINGO Group

${ }^{1}$ Nantes University Hospital, Nantes, France; ${ }^{2}$ Caen University Hospital, Caen, France; ${ }^{3}$ Rennes University Hospital, Rennes, France; ${ }^{4}$ Angers University Hospital, Angers, France; ${ }^{5}$ Tours University Hospital, Chambray-les-Tours, France; ${ }^{6}$ Tours University Hospital, Chambraylès-Tours, France; ${ }^{7}$ Brest University Hospital, Brest, France; ${ }^{8}$ Le Mans Hospital, Le Mans, France

Background: Based on the results of clinical studies, factor VIII (FVIII) with extended half-life (EHL) is supposed to have an impact on the consumption of FVIII and the management of prophylaxis, without reducing clinical efficacy. What about real-life?

Aims: The MOTHIF-II study explored FVIII prophylaxis in severe patients with Haemophilia A without inhibitor (pwSHA) in Western France, further to the avaibility of the efmoroctocog alfa (rFVIII-Fc). Methods: We investigated pwSHA followed in 7 centres of the BERHLINGO Network, before and after the rFVIII-Fc supply $(2 \times 12$ months-study, T1 07/2015-06/2016 versus T2 07/2017-06/2018). Data were extracted from both the BERHLINGO and the French nationwide claims databases (SNDS). We compared the prescriptions and real consumptions of standard FVIII versus rFVIII-Fc for matched patients.

Results: The prescriptions of 156 patients treated on prophylaxis on both periods were analyzed, whom 54\% have switched to rFVIII-Fc $(n=85)$. Patients treated three times a week saw their injection frequency decrease significantly after the switch, to a frequency of two injections per week (Fischer's exact test, $a=5 \%, P=0.0001$ ). As seen in Table 1, for 129 patients with weight information, the prescribed quantities of FVIII did not vary from period to period, regardless of FVIII (220,965 vs 218,602 IU/patient/year, Wilcoxon signed-rank test, $\mathrm{a}=5 \%, P=0.459)$. Over T2, the weekly dose of FVIII/kg prescribed decreased significantly when considering all patients $(-5.5 \%$ between $\mathrm{T} 1$ and T2), with the overall decrease being primarily due to patients treated with $\mathrm{rFVIII-Fc}$ alone $(83.1 \mathrm{IU} / \mathrm{kg} / \mathrm{w}$ versus $75.2 \mathrm{IU} / \mathrm{kg} / \mathrm{w}$, $P=0.048)$. FVIII delivery data from SNDS is currently being analyzed.

TABLE 1 Description of prescribed FVIII before and after switch to rFVIII-Fc (matched patients, according to the type of FVIII prescribed on T2)

Table 1: matched patients, on prophylaxis over T1 \& T2, weight information noted $(n=129 / 156)$

T1

July 2015 - June 2016

\begin{tabular}{|c|c|}
\hline $\begin{array}{l}\text { Weight in kgs } \\
\quad(\text { mean } \pm \mathrm{sd})\end{array}$ & $51.4 \pm 24.0$ \\
\hline $\begin{array}{l}\text { Prescribed FVIII } \\
\text { (IU/patient/ } \\
\text { year) } \\
\text { (mean } \pm \mathrm{sd})\end{array}$ & $207,529 \pm 120,685$ \\
\hline $\begin{array}{l}\text { Prescribed } \\
\text { FVIII (IU/ } \\
\text { kg/week/ } \\
\text { patient) }\end{array}$ & $81.0 \pm 30.5$ \\
\hline
\end{tabular}

Standard FVIII, then EHL $\mathrm{N}=29$ patients

$237,328 \pm 127,163$ FVIII over T2

$53.6 \pm 26.8$

$88.2 \pm 25.9$

Standard FVIII only over T2 $\mathrm{N}=62$ patients

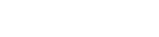

rFVIII-Fc only over
T2
$\mathrm{N}=38$ patients

$57.1 \pm 25.5$

$230,400 \pm 113,055$

$220,965 \pm 119,781$
Total

$\mathrm{N}=129$ patients

$53.5 \pm 25.0$ ) 
Table 1: matched patients, on prophylaxis over T1 \& T2, weight information noted $(n=129 / 156)$

$\mathrm{T} 2$

July 2017-June 2018

\begin{tabular}{|c|c|}
\hline $\begin{array}{l}\text { Weight in kgs } \\
\qquad(\text { mean } \pm \mathrm{sd})\end{array}$ & $55.1 \pm 21.3$ \\
\hline $\begin{array}{l}\text { Prescribed FVIII } \\
\text { (IU/patient/ } \\
\text { year) }\end{array}$ & $211,135 \pm 106,154$ \\
\hline
\end{tabular}

$76.9 \pm 28.3$

Prescribed
Standard FVIII only over T2 $\mathrm{N}=62$ patients FVIII (IU/ $\mathrm{kg} /$ week/ patient)

(mean \pm SD)
Standard FVIII, then EHL FVIII over T2

$\mathrm{N}=29$ patients

$56.3 \pm 25.4$

TOTAL: $232,208 \pm 98,809$

(52 w)

=STD: $137,267 \pm 81,772$

(30 w)

+EHL: $94,941 \pm 61,534$

(22 w)

TOTAL: $86.7 \pm 29.3(52 \mathrm{w})$

$=$ STD: $86.4 \pm 31.1(30 \mathrm{w})$

and EHL: $86.6 \pm 29.8(22 \mathrm{w})$
rFVIII-Fc only over $\mathrm{T} 2$

$\mathrm{N}=38$ patients

$61.5 \pm 23.5$

$220,402 \pm 91,138$

$218,602 \pm 99,884$

Total

$\mathrm{N}=129$ patients

$57.3 \pm 22.9$

$75.2 \pm 38.0^{\mathrm{a}}$

$78.6 \pm 31.7^{b}$

${ }^{a}$ Wilcoxon signed-rank test, significative, $a=5 \%(P=0.048)$.

${ }^{b}$ Wilcoxon signed-rank test, significative, $a=5 \%(P=0.006)$.

Conclusions: MOTHIF-II shows a reduction in the dosing frequency, but a slight decrease in the amount of FVIII prescribed. More data are needed to provide a real in-depth analyse, especially with a comparative with real FVIII dispensations.

PB0502 | Prospective, Open-label, Multicentre Phase II Study (PeKaFIX) to Evaluate the Pharmacokinetic Parameters of a Plasma Derived Factor IX Concentrate and Build a Pharmacokinetic Bayesian Model

\section{Negrier; on behalf of the PekaFIX study Group}

Haematology Department, Louis Pradel University Hospital, University Claude Bernard, Lyon, France

Background: Pharmacokinetic-guided personalisation of prophylactic regimens has the potential to optimise factor IX (FIX) therapy in patients with haemophilia $\mathrm{B}$ by enabling the regimen to be adapted to the needs of the individual patient.

Aims: The primary objective was to describe the pharmacokinetic parameters of a FIX concentrate $\left(\right.$ OCTANINE ${ }^{\circledR} \mathrm{F}$, OCTAFIX ${ }^{\circledR}$; Octapharma). Secondary objectives included the development of a Bayesian population pharmacokinetic model.
Methods: The PeKaFIX study was a prospective, open-label, multicentre phase II study in previously treated patients with severe haemophilia B. A dose of $75 \pm 5 \mathrm{IU} / \mathrm{kg}$ was administered after a washout period of $\geq 96$ hours. FIX levels were measured at 13 timepoints (pre-dose and 0.167, 0.5, 1, 3, 6, 9, 12, 24, 32, 48, 72 and 96 hours post-dose). Pharmacokinetic data were used to build a Bayesian population pharmacokinetic model to predict FIX pharmacokinetics based on sparse samples. A full pharmacokinetic sampling design (13 samples) was used as reference and compared with 9 sparse sampling designs ( 3 or 4 samples).

Results: The study enrolled 15 patients (10 adults and 5 children) across 8 centres. FIX profiles were adequately described by a 2-compartment model, with first-order distribution and elimination, and a zero-order production. The mean $( \pm \mathrm{SD})$ terminal half-life of OCTANINE ${ }^{\circledR}$ was 35 hours $( \pm 3.6)$ and mean $( \pm$ SD) time to $5 \%$ FIX was 93.8 hours ( \pm 19.8 ). The Bayesian model showed a good agreement between the observed and predicted FIX levels at 48, 72, 96 , 120 hours and the predicted time-to-reach FIX levels of 5, 4 and 3\% based on sparse sampling data.

Conclusions: OCTANINE ${ }^{\circledR}$ has a terminal half-life of 35 hours after a single administration. The FIX model enables sparse sampling data to be used to individualise OCTANINE ${ }^{\circledR}$ prophylaxis and suggests that some patients could be treated with less frequent injections to maintain a trough level above $1 \%$.

\section{TABLE 1 PeKaFIX Study Group}

Investigator

Claire Berger

Stéphane Vanderbecken

Cécile Stoven

Fabienne Genre-Volot

Philippe Nguyen

\section{Affiliation}

Pediatric Hematology Division, University Hospital, St-Etienne, France

Hematology Division, Felix Guyon Hospital, St-Denis, La Réunion, France

Pediatric Division, South Hospital, St-Pierre, La Réunion, France

Hematology Division, Bocage University Hospital, Dijon, France

Hematology Division, Robert Debré University Hospital, Reims, France 
Investigator

Philippe Beurrier

Brigitte Tardy

Claude Negrier

Emilie Henin

\section{Affiliation}

Hematology Division, University Hospital, Angers, France

Hematology Division, University Hospital, St-Etienne, France

Haematology Department, Louis Pradel University Hospital, University Claude Bernard, Lyon, France

Calvagone, Liergues, France
PB0503 | Safety and Efficacy of Concizumab Prophylaxis Following a Switch from rFVIIa on-demand Treatment: Subanalysis Results from the Phase 2 Explorer4 Trial in Patients with Hemophilia A or B with Inhibitors

A. Shapiro ${ }^{1}$; K. Cepo ${ }^{2}$; S.M. Tønder ${ }^{3}$; G. Young ${ }^{4}$; V. Jiménez-Yuste ${ }^{5}$ ${ }^{1}$ Indiana Hemophilia \& Thrombosis Center, Indianapolis, United States; ${ }^{2}$ Biopharm Medical \& Science, Novo Nordisk A/S, Søborg, Denmark; ${ }^{3}$ Biostatistics Biopharm, Novo Nordisk A/S, Søborg, Denmark; ${ }^{4}$ Keck School of Medicine, University of Southern California, Los Angeles, United States; ${ }^{5}$ Haematology Department, La Paz University Hospital, Madrid, Spain

Background: Explorer4 (NCT03196284) was a phase 2 trial to establish clinical proof-of-concept for subcutaneous, once-daily concizumab (anti-tissue factor pathway inhibitor [TFPI] monoclonal antibody) in bleed prevention in hemophilia A/B with inhibitors (HAwl/HBwl).

Aims: Assess safety and efficacy in patients who switched from recombinant activated FVII (rFVIIa) on-demand treatment in the explorer4 main part to daily concizumab prophylaxis in the extension.

Methods: Patients, randomized 2:1, received daily concizumab prophylaxis/rFVIla on-demand during the explorer4 main part ( $\geq 24$ weeks). During the extension (56-94 weeks), on-demand patients switched to $0.15 \mathrm{mg} / \mathrm{kg}$ concizumab prophylaxis (following a loading dose of 0.5 $\mathrm{mg} / \mathrm{kg}$ ), with the possibility to escalate to 0.20 and $0.25 \mathrm{mg} / \mathrm{kg}$ (if $\geq 3$ spontaneous treated bleeds occurred within 12 weeks). Annualized bleeding rate (ABR) was estimated based on a negative binomial regression with log-exposure time as offset and treatment arm as a factor. Results are presented at the last concizumab dose level.

Results: Eight patients (6 HAwl; $2 \mathrm{HBwl}$ ) were included in this subanalysis. Two patients remained on $0.15 \mathrm{mg} / \mathrm{kg}$ concizumab; 3 escalated to $0.20 \mathrm{mg} / \mathrm{kg}$ and 3 to $0.25 \mathrm{mg} / \mathrm{kg}$. One patient fulfilled protocol-defined lack of efficacy criteria on $0.25 \mathrm{mg} / \mathrm{kg}$ and was withdrawn. Estimated mean ABR (95\% confidence interval [CI]) was $19.2(10.7 ; 34.2)$ vs $4.9(2.4 ; 10.0)$ for on-demand treatment and concizumab prophylaxis, respectively. Estimated mean spontaneous and joint bleed ABR $(95 \% \mathrm{Cl})$ decreased from $17.0(9.7 ; 29.8)$ to 2.5 (1.1; 5.7), and from $14.8(8.1 ; 26.9)$ to $3.0(1.3 ; 6.9)$, respectively. Three patients experienced zero bleeds on their last concizumab dose level (exposure time: 212, 115, 303 days). There were no safety signals following concizumab switching.

Conclusions: Safety and efficacy for explorer4 patients who switched from rFVIla on-demand to concizumab prophylaxis were consistent with main/extension part trial results, with longer-term efficacy maintained and a favorable safety profile while on concizumab.

PB0504 | Emicizumab Treatment in Pediatric Haemophilia A Patients: >1 Year Safety Based on Real-world Data from the PedNet Cohorts

G. Kenet ${ }^{1}$; B. Nolan² ; J. Oldenburg ${ }^{3}$; J. Motwani ${ }^{4}$; R. D' Oiron ${ }^{5}$; C. Male ${ }^{6}$; H. Chambost ${ }^{7}$; K. Fischer ${ }^{8}$; C. Van Geet ${ }^{9}$; H.M. van den Berg $^{10}$, PedNet Study Group

${ }^{1}$ National Hemophilia Center Sheba Medical center, Tel Hashomer \& Amalia Biron Research Institute of Thrombosis \& Hemostasis, Tel Aviv, Israel; ${ }^{2}$ Department of Paediatric Haematology, Our Lady's Children's Hospital for Sick Children Crumlin, Dublin, Ireland; ${ }^{3}$ Institut für Experimentelle Hämatologie und Transfusionsmedizin, Universitätsklinikum, Bonn, Germany; ${ }^{4}$ Department of Haematology, The Children's Hospital, Birmingham, United Kingdom; ${ }^{5}$ Centre de Référence pour le Traitement des Maladies Hémorragiques (CRTH), Hôpital Bicêtre, Paris, France; ${ }^{6}$ Department of Paediatrics, Medical University Hospital of Vienna, Vienna, Austria; ${ }^{7}$ La Timone Children's Hospital, Center for Bleeding Disorders \& Aix Marseille Univ, INSERM, INRA, C2VN, Marseille, France; ${ }^{8}$ Van Creveld Kliniek, University Medical Center Utrecht, Utrecht, Netherlands; ${ }^{9}$ Catholic University of Leuven, Campus Gasthuisberg, Service of Pediatric Haematology, Leuven, Belgium, ${ }^{10}$ PedNet Haemophilia Research Foundation, Baarn, Netherlands

Background: Emicizumab is a bispecific humanised antibody, approved for treatment of patients with haemophilia A (HA). Data from paediatric patients were limited at the time of marketing authorization. Moreover, a different coagulation system in very young infants may contribute to unexpected side effects.

Aims: To evaluate safety and efficacy of Emicizumab prophylaxis in children.

Methods: The PedNet Registry includes all children with haemophilia born since January 1st 2000 (NCT02979119), diagnosed and treated in one of the 33 participating centres in Europe, Canada and Israel. For this analysis, data were collected from the moment children switched to Emicizumab until 1 January 2021, on total number of bleeds, additional treatment for bleeds, trauma and surgery as well as adverse events (AE)

Results: A total of 141 patients with HA (134 severe, 6 moderate and 1 mild) were included, of whom 79 (56\%) had inhibitors. Twentyeight children were below 2 years of age. The median treatment 
period in months (IQR) was 17.9 (7.9-28.1) for children with inhibitors and 3.8 (2.3-9.0) months for those without. A total of 82 patients (58\%) reported zero bleeds. Details on bleeds are described in the table and figure. Major bleeds were more frequent in inhibitor patients; this might be due to longer follow-up for inhibitor patients. Three adverse events were reported: one patient had a local skin reaction, one patient developed antibodies against Emicizumab, and one child died from unrelated problems.

TABLE 1 Inhibitor and non-inhibitor patients from start emicizumab to January 2021

$\begin{array}{llll} & \text { Inhibitor } & \text { Non-inhibitor } & \text { All patients } \\ \text { Number (N) of patients } & 79 & 61 & 141^{\wedge} \\ \text { Median age at start emicizumab, months (IQR) } & 80.5(26.1-138.0) & 71.5(33.9-128.2) & 75.5(27.6-135.5) \\ \text { N start emicizumab before 2 years of age } & 17 & 11 & 28 \\ \text { median treatment duration, months (IQR) } & 17.9(7.9-28.1) & 3.8(2.3-9.0) & 9.8(3.6-19.8) \\ \text { N patients with bleeds } & 42 & 16 & 58 \\ \text { N reported major* joint \& soft tissue bleeds } & 28 & 11 & 39 \\ \text { N reported major \& minor joint bleeds } & 44 & 5 & 15 \\ \text { N reported minor** soft tissue bleeds } & 134 & 49\end{array}$

"Major bleed: pain, swelling, limitation of motion and failure to respond within 24 hours of treatment

** Minor bleed: mild pain, minimal swelling, minimal restriction of motion, resolving within 24 hours

^ 1 patient with unknown inhibitor status.

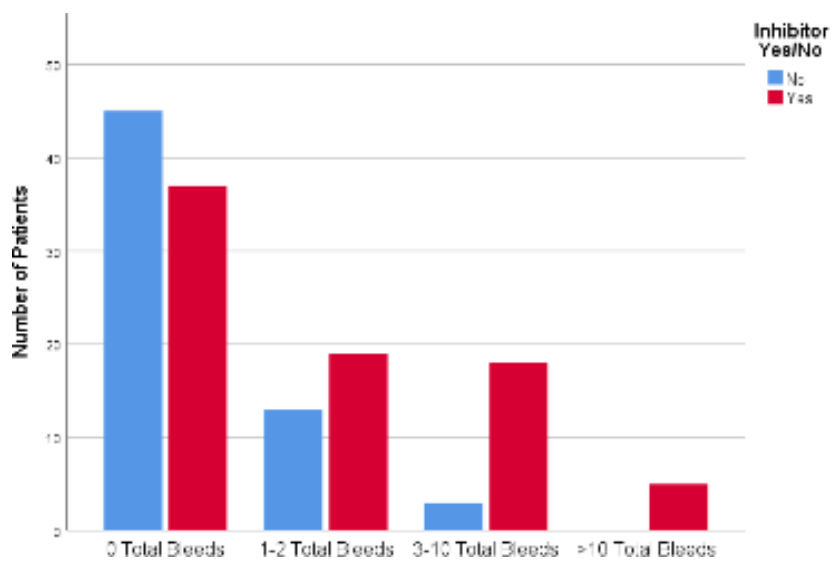

FIGURE 1 Total bleeds per category and inhibitor status

Conclusions: Emicizumab prophylaxis provided excellent bleed control. Follow up for non-inhibitor patients is still short. One patient developed anti-drug antibodies.
PB0505 | Incorporating Patient Reported Outcomes into Clinical Practice: A 360-degree Clinical Evaluation for Better Care and Treatment of People with Hemophilia through myWBDR and myPROBE

T. Youttananukorn ${ }^{1}$; D. Coffin ${ }^{1}$; S. Diop ${ }^{2}$; C. Hermans ${ }^{3}$; B. Konkle ${ }^{4}$; C. Lambert ${ }^{5}$; D. Noone ${ }^{6}$; J. O'Hara ${ }^{7}$; G. Pierce ${ }^{1}$; M.W Skinner ${ }^{8,9}$; A. Iorio ${ }^{10}$ ${ }^{1}$ World Federation of Hemophilia, Montreal, Canada; ${ }^{2}$ Cheikh Anta Diop University and Centre National de Transfusion Sanguine, Dakar, Senegal; ${ }^{3}$ Division of Haematology, Cliniques universitaires Saint-Luc, Université catholique de Louvain (UCLouvain), Leuven, Belgium; ${ }^{4}$ Bloodworks Northwest, Seattle, United States; ${ }^{5}$ Division of Haematology, Cliniques universitaires Saint-Luc, Université catholique de Louvain (UCLouvain), Brussels, Belgium; ${ }^{6}$ European Hemophilia Consortium, Brussels, Belgium; ${ }^{7}$ HCD Economics, London, United Kingdom; ${ }^{8}$ Institute for Policy Advancement Ltd, Washington DC, United States; ${ }^{9}$ Department of Health Research Methods, Evidence, and Impact, McMaster University, Ontario, Canada, ${ }^{10}$ McMaster University, Hamilton, Canada

Background: The World Federation of Hemophilia (WFH) World Bleeding Disorders Registry (WBDR) is designed to fill gaps in hemophilia care and knowledge that exist globally. The WBDR is a tool for clinicians to collect real world data on patient clinical care and quality of life, and a tool to empower people with hemophilia (PWH) to manage their own care and treatment. The WFH will be introducing myWBDR - a mobile application for PWH participating in the WBDR through the participating hemophilia treatment centers.

Aims: To collect bleed and other patient-reported outcome (PRO) data in the WBDR. 
Methods: myWBDR is designed to track bleeds, associated pain, treatments, and health status using EQ-5D-5L and the Patient Reported Outcomes, Burdens, and Experiences (PROBE) questionnaires (Table). Initially, myWBDR will be available in English, French, Hindi, Spanish, and Vietnamese.

TABLE 1 myWBDR and Patient-Reported Outcome Data

\section{Hemophilia Treatment}

myWBDR

Bleed history: Location of bleed, frequency, access to treatment at time of bleed.

Pain level: Pain associated with a bleed event.

Treatment record: infusion log, dose, frequency, reason for treatment, location of treatment received.

\begin{tabular}{|c|c|}
\hline Hemophilia Health-related Quality of Life & General Health Status \\
\hline myPROBE & EQ-5D-5L \\
\hline Pain: chronic/acute, interference, occurrence & Mobility \\
\hline $\begin{array}{l}\text { Independence: limitations, impact on activities of } \\
\text { daily living }\end{array}$ & Self-care \\
\hline Education: attainment, attendance & Usual Activities \\
\hline $\begin{array}{l}\text { Employment: duration, underemployment, } \\
\text { attendance }\end{array}$ & Pain / Discomfort \\
\hline Family Life: marriage, children & Anxiety / Depression \\
\hline Mobility: assistance required, impairment & VAS (visual analog scale) \\
\hline
\end{tabular}

Results: myWBDR is undergoing field testing in non-WBDR PWH and will follow with a testing cohort of PWH within the WBDR. Implementation will be regional, starting with 5 countries (2 regions) in Q2 2021. myWBDR is a simple tool, allowing users to record accurate bleeds and treatment in under 1 minute. The inclusion of the EQ-5D-5L and PROBE questionnaires, both available in a large number of languages, will allow users to track changes in their health status over time. Both myWBDR and myPROBE include an off-line feature so data can be entered at anytime and offer simple data visualization on bleeds (number, location), level of pain and health-status. Conclusions: Integration of PRO via the myWBDR will allow PWH to play an active role in their care and treatment. With the 360-degree data, researchers can better understand hemophilia and work to improve quality of care and treatment for $\mathrm{PWH}$ around the world. A data dashboard for $\mathrm{PWH}$ to visualize and compare their personal data is in development.

PB0506 | Interim Analysis of the PREVENT Study: Real World Prospective Data from Children and Adolescents with Haemophilia A or B Treated with Recombinant Factor VIII Fc (rFVIIIFc) or Recombinant Factor IX Fc (rFIXFc)

C. Heller $^{1}$; C. Bidlingmaier ${ }^{2}$; C. Escuriola ${ }^{3}$; N. Hagedorn ${ }^{4}$;

J. Oldenburg ${ }^{5}$; J. van den Boom ${ }^{6}$; H. Malmström ${ }^{7}$ E. Santagostino ${ }^{8}$;

A. Tiede ${ }^{9}$

${ }^{1}$ Department of Pediatric Hematology/Oncology/Haemostaseology,

University Hospital of Frankfurt, Frankfurt, Germany; ${ }^{2}$ Dr. von Hauner's Children's Hospital, Paediatric Haemophilia Centre, Munich, Germany; ${ }^{3}$ Haemophilia Centre Rhein Main (HZRM), MörfeldenWalldorf, Germany; ${ }^{4}$ Charité - Universitätsmedizin Berlin, Department of Pediatrics, Division of Oncology and Hematology, Berlin, Germany; ${ }^{5}$ Institute of Experimental Haematology and Transfusion Medicine, University Clinic Bonn AöR, Bonn, Germany; ${ }^{6}$ Swedish Orphan Biovitrum $\mathrm{GmbH}$, Martinsried, Germany; ${ }^{7}$ Swedish Orphan Biovitrum AB,
Stockholm, Sweden; ${ }^{8}$ Swedish Orphan Biovitrum AG, Basel, Switzerland; ${ }^{9}$ Department of Hematology, Haemostaseology, Oncology and Stem Cell Transplantation, Hannover Medical School, Hannover, Germany

Background: Safety and efficacy of rFVIIIFc and rFIXFc were established in phase 3 trials. The PREVENT study prospectively evaluates real-world usage and effectiveness of rFVIIIFc and rFIXFc in Germany over 24-months in patients with haemophilia $\mathrm{A}(\mathrm{PwHA})$ or $\mathrm{B}(\mathrm{PwHB})$. Aims: Providing first interim analysis of the paediatric population ( $<18$ years) with at least 9 months of prospective observation.

Methods: PREVENT (NCT03055611) was approved by medical ethics committees. Informed consent was obtained from all patients/ caregivers. Primary endpoints are annualised bleeding rate (ABR), injection frequency and factor consumption. Secondary endpoints include reason for initiating rFVIIIFc or rFIXFc treatment. The interim analysis (data cut September 24, 2020) used descriptive statistics.

Results: The analysis included 55 PwHA and $10 \mathrm{PwHB}$, with median (range) age of 8.0 (0-17) and 11.5 (3-15) years, respectively. Forty-nine (89\%) PwHA and 6 (60\%) PwHB were severe. Inhibitor history prior to rFVIIIFc/rFIXFc initiation was reported in 12 (21.8\%) severe PwHA and none among PwHB. No inhibitors had been detected at time of data cut. Median ABR and joint ABR were low for PwHA and PwHB (table 1), during a median (range) follow-up of 18.9 (9.3-23.3) and 20.1 (10.523.7) months, respectively. For this follow-up period, the proportion of zero joint bleeds were $63.6 \%$ in $\mathrm{PwHA}$ and $40.0 \%$ in $\mathrm{PwHB}$. Median injection frequency and factor consumption are shown in table 1.

Most patients and physicians were satisfied or highly satisfied with the treatment (table 1).

Main reason for initiating rFVIIIFc or rFIXFc treatment was to reduce injection frequency while maintaining protection from bleeds (table 2). 
TABLE 1 Clinical outcomes and treatment of paediatric patients on prophylaxis with rFVIIIFc or rFIXFc

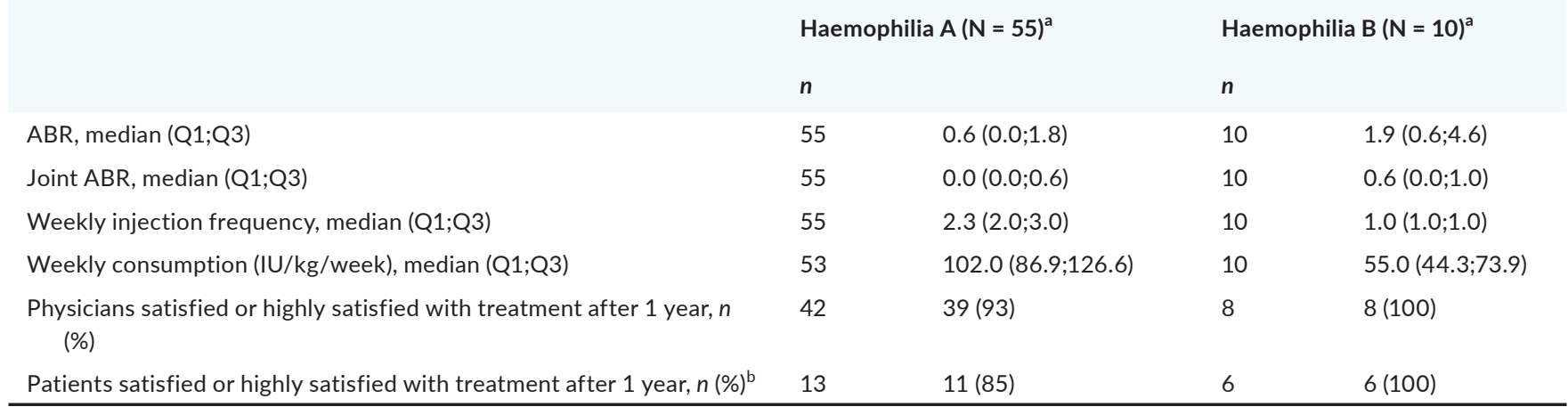

${ }^{a}$ For some of the outcomes data were not available for all patients at the interim data cut.

${ }^{b}$ Completion of all patient-reported outcomes was optional.

TABLE 2 Reason for treatment initiation with rFVIIIFc or rFIXFc

\begin{tabular}{cll} 
& $\begin{array}{l}\text { Haemophilia A } \\
(\mathrm{N}=55)\end{array}$ & $\begin{array}{l}\text { Haemophilia B } \\
(\mathrm{N}=10)\end{array}$ \\
$\begin{array}{c}\text { Improve protection } \\
\text { from bleeds }\end{array}$ & $12(21.8 \%)$ & $1(10.0 \%)$ \\
$\begin{array}{c}\text { Improve protection } \\
\text { to increase } \\
\text { physical activity } \\
\text { level }\end{array}$ & $1(1.8 \%)$ & $0(0.0 \%)$ \\
$\begin{array}{c}\text { Reduce injection } \\
\text { frequency while } \\
\text { maintaining } \\
\text { protection from } \\
\text { bleeds }\end{array}$ & $42(76.4 \%)$ & \\
\hline
\end{tabular}

Conclusions: Treatment with rFVIIIFc or rFIXFc in children and adolescents provided a high level of protection from bleeds with low injection frequency. This interim analysis of the real-world use of rFVIIIFC and rFIXFc for up to 2 years reinforces the results of the pivotal trials.

PB0507 | Hemophilia A Patients with Factor VIII Inhibitor Feature Reduced Levels of CD4+CD25+CD127- Treg Cells and Plasma IL-35

Z.Y. Yan; Z. Yan; H. He; Y. Xie; L. Wang; W. Wu; S. Chen; Y. Yan;

J. Yang

North China University of Science and Technology Affiliated Hospital, Tangshan, China

Background: Regulatory T cells (Tregs) play a crucial role in the tolerance of FVIII. CD4 ${ }^{+}$CD $25^{+}$CD127Treg cells, IL-35, IL-10 and TGF- $\beta$ may be involved in the formation of inhibitors in patients with hemophilia A.

Aims: To determine the expression of Tregs, IL-35, IL-10 and TGF- $\beta$ in patients with hemophilia $A$ and to explore its possible mechanism in the formation of inhibitors.

Methods: This study included 43 patients with hemophilia A(6 with factor VIII inhibitors and 37 without inhibition of factor VIII) and 20 healthy controls. Among the 43 patients in the hemophilia A group,13 were diagnosed with mild or moderate hemophilia $A$,whereas 30 were severe.
The expression rate of $\mathrm{CD} 4^{+} \mathrm{CD} 25^{+} \mathrm{CD} 127$ Tregs in peripheral blood was detected by flow cytometry, while the levels of IL-35, IL-10 and TGF- $\beta$ in plasma were detected by enzyme-linked immunosorbent assay (ELISA).

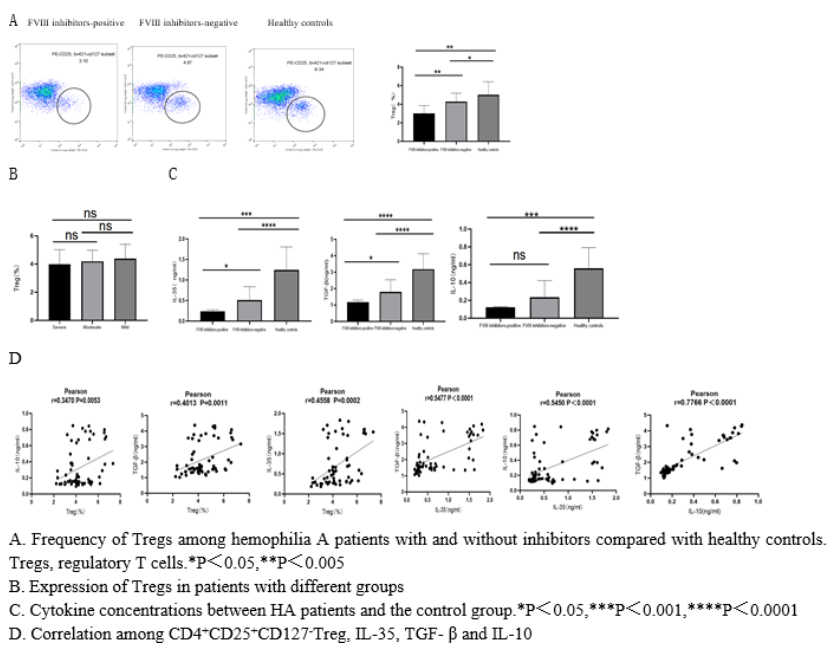

FIGURE 1 Hemophilia A patients with factor VIII inhibitor feature reduced levels of CD4+CD25+CD127- Treg cells and plasma IL-35

Results: In comparison of healthy controls, hemophilia A patients featured decreased levels of Tregs, for both FVIII inhibitor-positive and -negative group, where the inhibitor-positive group showed the lowest level of Tregs. However, there were no significant differences in the number of Tregs between different disease severity(Fig1 B).Plasma IL-35 and TGF- $\beta$ concentrations were lower in the inhibitorpositive patients compared to inhibitor-negative patients. The level of plasma IL-10 in patients with hemophilia A was significantly lower than that in healthy controls, but there was no significant difference between inhibitor-positive group and inhibitor-negative group. The level of $\mathrm{CD} 4^{+} \mathrm{CD} 25^{+} \mathrm{CD} 127$ Treg cells in peripheral blood was positively correlated with the levels of IL-35, TGF- $\beta$ and IL-10.Serum IL-35 level was positively correlated with serum IL-10 and TGF- $\beta$ (Fig).

Conclusions: The formation of inhibitors in patients with hemophilia A may be related to the abnormality of $\mathrm{CD} 4^{+} \mathrm{CD} 25^{+} \mathrm{CD} 127$ Treg cells, and cytokines IL-35 and TGF- $\beta$ may also be involved. 
PB0508 | Clinical Outcomes after Joint Surgery in Patients on Turoctocog Alfa Pegol Prophylaxis

A. Tiede $^{1}$; S. Shivamurthy ${ }^{2}$; P. Chowdary ${ }^{3}$

${ }^{1}$ Hannover Medical School, Hanover, Germany; ${ }^{2}$ Novo Nordisk, Bengaluru, India; ${ }^{3}$ Royal Free Hospital, London, United Kingdom

Background: Joint damage in patients with hemophilia (PWH) may require surgical intervention. However, surgery effects on bleeding rates are unclear.

Aims: To investigate the effect of joint surgery on clinical outcomes (joint bleeding rates and patient-reported outcomes [PROs]), in PWH A on prophylaxis with turoctocog alfa pegol (N8-GP).

Methods: Patients in the Pathfinder 2 clinical study received N8-GP prophylaxis and enrolled in the Pathfinder 3 study for their surgeries. Patients returned to Pathfinder 2 post-surgery and continued prophylaxis until end-of-study. Pre-surgery (immediately before surgery) and post-surgery (at Pathfinder 2 end-of-study) joint annualized bleeding rates (jABRs) were calculated collectively across all reported joint locations. Joint-health-related PROs were derived from patient records. Results: Data from 41 joint surgeries in 30 patients were analyzed using a datamining approach and descriptive analysis. Pre-surgery mean jABR was similar in the surgery patients vs 146 non-surgery patients. In surgery patients, mean jABR decreased pre-surgery to post-surgery (1.33 to $0.37, P=0.03$ ). In all but three patients, jABR improved or remained the same post-surgery (Table 1 ).
Joint-health-related PROs were based on responses to the HaemA-QoL AU1.0 questionnaire before and after surgery. The questionnaire reported whether patients had experienced the outcomes in the preceding 4 weeks.

Haem-A-QoL, Haemophilia Quality of Life Questionnaire for Adults; PRO, patient-reported outcome.

Conclusions: Combined analysis of Pathfinder 2 and 3 permitted assessment of joint bleeding and PROs after joint surgery. Mean jABR significantly declined post-surgery and mobility parameters improved in patients whose pre- and post-surgery jABR was 0 . However, these findings should be interpreted with caution. A general decline in jABR was observed in the pathfinder 2 cohort, whose patients were on N8-GP prophylaxis; effects in patients after surgery cannot be attributable solely to surgery.

TABLE 1 Numbers of patients and surgeries in each jABR outcome group

\begin{tabular}{|c|c|c|c|c|c|}
\hline & \multicolumn{5}{|c|}{ Post-surgery vs pre-surgery jABR } \\
\hline Number of patients ${ }^{\dagger}$ & 13 & 13 & 3 & 3 & 30 \\
\hline Number of surgeries & 17 & 18 & 3 & 3 & 41 \\
\hline
\end{tabular}

" Of the three patients whose jABR worsened, all had longstanding arthropathy, and two underwent surgery for implant pain and bilateral trigger thumbs, respectively. ${ }^{\dagger}$ Two patients were counted twice because of multiple surgeries with different effects on jABR. jABR, joint annualized bleed rate.

In the three patients whose jABR was stable at 1 (all with multiple joint arthropathy), post-surgery bleeds were mostly at non-operated sites. Two of the three patients whose jABR increased underwent surgery for prosthetic pain and bilateral trigger thumbs, resolution of which may not ameliorate overall longstanding arthropathy. Mobility parameters often improved in patients whose jABR was 0 pre-surgery and post-surgery (Table 2 ).

TABLE 2 Effect of surgery on PRO scores in patients whose jABR was 0 before and after surgery (13 patients; 18 surgeries)

\begin{tabular}{|c|c|c|c|c|c|}
\hline PRO parameter & \multicolumn{3}{|c|}{ Count of surgeries } & Not collected & Tota \\
\hline Painful to move & 13 & 1 & 4 & - & 18 \\
\hline Difficulty to walk as far as wanted & 10 & 4 & 4 & - & 18 \\
\hline
\end{tabular}


PB0509 | Does Pharmacokinetic-guided Prophylaxis with Antihemophilic Factor (Recombinant) Improve Bleeding Rates over Standard Prophylaxis? Real-world Data from the AHEAD German Study

R. Klamroth ${ }^{1}$; A. Huth-Kühne ${ }^{2}$; K. Kurnik ${ }^{3}$; C. Escuriola-

Ettingshausen ${ }^{4}$; S. Regensburger ${ }^{5}$; L. Tang ${ }^{6}$; J. Norton7;

A. Fernandez ${ }^{6}$; J. Oldenburg ${ }^{8}$

${ }^{1}$ Department for Internal Medicine, Vascular Medicine and

Haemostaseology, Vivantes Klinikum im Friedrichshain, Berlin,

Germany; ${ }^{2}$ SRH Kurpfalzkrankenhaus and Haemophilia Centre

Heidelberg $\mathrm{GmbH}$, Heidelberg, Germany; ${ }^{3}$ Dr. von Haunersches

Kinderspital Klinikum der Universität München, Munich, Germany;

${ }^{4}$ Hämophilie-Zentrum Rhein Main GmbH, Mörfelden-Walldorf,

Germany; ${ }^{5}$ Takeda Pharma Vertrieb GmbH \& Co. KG, Berlin, Germany;

${ }^{6}$ Takeda Pharmaceuticals International AG, Zürich, Switzerland;

${ }^{7}$ Takeda Development Center Americas, Inc., Lexington, United States;

${ }^{8}$ Institute for Experimental Hematology and Transfusion Medicine,

Bonn University Clinic, Bonn, Germany

Background: The German Antihemophilic factor (recombinant) (rAHF) Hemophilia $\underline{A}$ (HA) outcome Database (AHEAD) study (DRKS00000556) is a non-interventional prospective multicenter real-world study that assesses long-term effectiveness and safety of rAHF in patients with HA.
Aims: To describe hemostatic effectiveness of rAHF administered as standard prophylaxis (SP) and individualized pharmacokineticguided prophylaxis (PKP) in moderate or severe HA.

Methods: The 7th interim analysis describes results from the safety analysis set (SAS) (data cutoff: Jun 30, 2020). Patients were followed over 7 years; 4 years of follow-up are available for SP versus PKP, because day 1 was reset after patients adopted PKP. Key outcomes included annualized bleeding rates (ABRs [all bleeds]), annualized joint bleeding rates (AJBRs), rAHF consumption, factor VIII (FVIII) trough levels, and adverse events (AEs). Ethics committee approval and patients' informed consent were obtained.

Results: Of 322 patients in SAS (severe HA, $n=258$; moderate HA, $n=64$ ), 250 received prophylaxis (SP and PKP), 67 on-demand treatment, and 5 immune tolerance induction. Median ABRs were lower in patients receiving PKP versus SP over years 1-3. Similar median AJBRs were observed between groups, although the range was wider in patients receiving SP. A higher proportion of patients receiving PKP versus SP had an ABR or AJBR of zero over years 1-3 (Table 1). Mean annualized total dose over years 1-4 was $5260-5437 \mathrm{IU} / \mathrm{kg}$ for SP and 4545-5769 IU/kg for PKP. Mean (median [Q1-Q3]) target FVIII troughs for patients with moderate $\mathrm{HA}$ at years $1(n=2)$ and $2(n=1)$ were 2.2 (2.2[1.0-3.4]) and 1.0 (1.0[NA]). For severe HA, mean (median [Q1-Q3]) FVIII troughs for years $1(n=14)$ and $2(n=7)$ were 4.4 (3.5[2-5]) and 3.4 (3.0[1.5-5.5]). Treatment-related AEs occurred in $31 / 322$ (9.6\%) patients; serious treatment-related AEs, 23/322 (7.1\%).

TABLE 1 Bleeding Rates and Consumption of rAHF in Patients Receiving SP Versus PKP*

\begin{tabular}{|c|c|c|c|c|c|c|c|c|}
\hline & Year 1 & & Year 2 & & Year 3 & & Year 4 & \\
\hline Bleeding rates & $\begin{array}{l}\text { SP } \\
n=246\end{array}$ & $\begin{array}{l}\text { PKP† } \\
n=24\end{array}$ & $\begin{array}{l}\text { SP } \\
n=229\end{array}$ & $\begin{array}{l}\text { PKP† } \\
n=12\end{array}$ & $\begin{array}{l}\text { SP } \\
n=211\end{array}$ & $\begin{array}{l}\text { PKP† } \\
n=3\end{array}$ & $\begin{array}{l}\text { SP } \\
n=190\end{array}$ & $\begin{array}{l}\text { PKP† } \\
n=1\end{array}$ \\
\hline Median ABR (Q1-Q3) & $2(0-6)$ & $0(0-2)$ & $2(0-4)$ & $0(0-0.5)$ & $1(0-5)$ & $0(0-1)$ & $1(0-4)$ & $3(3-3)$ \\
\hline $\begin{array}{l}\text { Median AJBR } \\
\text { (Q1-Q3) }\end{array}$ & $0(0-2)$ & $0(0-0.5)$ & $0(0-2)$ & $0(0-0)$ & $0(0-2)$ & $0(0-1)$ & $0(0-2)$ & $1(1-1)$ \\
\hline $\begin{array}{c}\% \text { patients with } \\
A B R=0\end{array}$ & 36 & 63 & 36 & 75 & 37 & 67 & 48 & 0 \\
\hline $\begin{array}{c}\% \text { patients with } \\
\text { AJBR }=0\end{array}$ & 58 & 75 & 59 & 83 & 56 & 67 & 64 & 0 \\
\hline Consumption & $\begin{array}{l}\text { SP } \\
n=246\end{array}$ & $\begin{array}{l}\text { PKP* } \\
n=24\end{array}$ & $\begin{array}{l}\text { SP } \\
n=232\end{array}$ & $\begin{array}{l}\text { PKP* } \\
n=14\end{array}$ & $\begin{array}{l}S P \\
n=215\end{array}$ & $\begin{array}{l}\text { PKP* } \\
n=3\end{array}$ & $\begin{array}{l}\text { SP } \\
n=195\end{array}$ & $\begin{array}{l}\text { PKP* } \\
n=1\end{array}$ \\
\hline $\begin{array}{l}\text { Median (Q1-Q3) } \\
\text { annualized total } \\
\text { dose, IU/kg }\end{array}$ & $\begin{array}{l}4737 \\
\left(\begin{array}{l}3143- \\
6789)\end{array}\right.\end{array}$ & $\begin{array}{l}4986 \\
\left(\begin{array}{l}3601- \\
7125)\end{array}\right.\end{array}$ & $\begin{array}{l}4805 \\
\left(\begin{array}{l}3283- \\
6592)\end{array}\right.\end{array}$ & $\begin{array}{l}4827 \\
\left(\begin{array}{l}3900- \\
5778)\end{array}\right.\end{array}$ & $\begin{array}{l}4727 \\
\left(\begin{array}{l}3033- \\
6556)\end{array}\right.\end{array}$ & 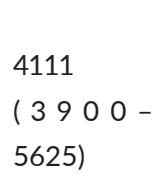 & 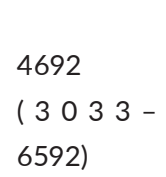 & $\begin{array}{l}5769 \\
\text { (NA) }\end{array}$ \\
\hline
\end{tabular}

NA, not available; Q, quartile.

*All patients receiving prophylaxis started on SP but could be switched to PKP.

${ }^{\dagger}$ Patients with HA using the FDA-approved mobile app that allows patients to track treatment with rAHF, view estimated factor VIII levels, and share data with healthcare provider. 
Conclusions: These real-world data show improved bleeding rates with PKP versus SP with similar rAHF consumption.

PB0510 | Updates on the Atlanta Protocol Cohort: Two-year Institutional Outcomes on Immune Tolerance Induction in Children with Hemophilia A and Inhibitors on Emicizumab

\section{G. Batsuli ${ }^{1}$; K. Zimowski ${ }^{1}$; K. Tickle ${ }^{2}$; S.L. Meeks ${ }^{1}$; R.F. Sidonio ${ }^{1}$}

${ }^{1}$ Emory University and Children's Healthcare of Atlanta, Atlanta, United States; ${ }^{2}$ Children's Healthcare of Atlanta, Atlanta, United States

Background: Remarkable reductions in bleeding events among individuals with hemophilia $A$ and inhibitors receiving the bispecific antibody emicizumab have generated debates on the utility of immune tolerance induction (ITI). Yet, ITI remains the only method shown to eradicate inhibitors. In an effort to combine the bleeding protection of emicizumab with inhibitor eradication, we previously reported inhibitor outcomes at $<1$ year of 7 pediatric patients with hemophilia A and inhibitors who underwent concomitant ITI with factor VIII (FVIII) 50-100 IU/kg/dose 2-3 times weekly on emicizumab prophylaxis, termed the Atlanta Protocol. In this report, we provide 2-year follow-up data on outcomes.

Aims: To describe inhibitor status and FVIII pharmacokinetics in a cohort of pediatric patients after 2 years on the Atlanta Protocol.

Methods: Chart review of electronic medical records was performed under IRB approval.

Results:

TABLE 1 Two year follow-up of the original cohort on the Atlanta protocol

\begin{tabular}{|c|c|c|c|c|c|c|c|c|c|}
\hline Age & $\begin{array}{l}\text { Baseline } \\
\text { FVIII } \\
\text { Activity } \\
(\%)\end{array}$ & $\begin{array}{l}\text { Historical } \\
\text { Peak } \\
\text { Inhibitor } \\
\text { Titer (BU) }\end{array}$ & $\begin{array}{l}\text { Initial FVIII ITI } \\
\text { Regimen on } \\
\text { Emicizumab }\end{array}$ & $\begin{array}{l}\text { Last } \\
\text { Inhibitor } \\
\text { Titer } \\
\text { (CBU) }\end{array}$ & $\begin{array}{l}\text { FVIII } \\
\text { Recovery of } \\
\text { Expected } \\
(\%)\end{array}$ & $\begin{array}{l}\text { Estimated } \\
\text { Half-Life } \\
\text { (hr) }\end{array}$ & Tolerance Status & $\begin{array}{l}\text { Time from } \\
\text { FVIII ITI Start } \\
\text { to Tolerance } \\
\text { Status* (mo) }\end{array}$ & $\begin{array}{l}\text { Current FVIII } \\
\text { Regimen on } \\
\text { Emicizumab }\end{array}$ \\
\hline $4 y$ & $<1$ & 5.5 & $\begin{array}{l}\mathrm{rFVIII} 100 \mathrm{IU} / \mathrm{kg} \\
3 x / \mathrm{wk}\end{array}$ & 1.4 & $<66$ & $<6$ & Failed & 26 & $\begin{array}{l}\text { N/A - ITI } \\
\text { discontinued }\end{array}$ \\
\hline $4 y$ & $<1$ & 30.7 & $\begin{array}{l}\mathrm{rFVIII} 100 \mathrm{IU} / \mathrm{kg} \\
3 x / \mathrm{wk}\end{array}$ & $<0.6$ & 100 & $12-13$ & Success & 28 & $\begin{array}{l}\text { rFVIII } 50 \text { IU/kg 2x/ } \\
\text { week }\end{array}$ \\
\hline $4 y$ & $<1$ & 2.0 & $\begin{array}{l}\mathrm{rFVIII} 100 \mathrm{IU} / \mathrm{kg} \\
3 x / \mathrm{wk}\end{array}$ & $\begin{array}{l}<0.6 \\
7.1\end{array}$ & $\begin{array}{l}75 \\
<66\end{array}$ & $\begin{array}{l}10 \\
<6\end{array}$ & $\begin{array}{l}\text { Successful } \\
\text { (Inhibitor } \\
\text { Relapse) }\end{array}$ & $\begin{array}{l}20 \\
(28)\end{array}$ & $\begin{array}{c}\text { rFVIII-Fc } 100 \mathrm{IU} / \mathrm{kg} \\
\text { 3x/week }\end{array}$ \\
\hline 11 y & 3 & 4.1 & $\begin{array}{l}\text { BDD rFVIII } 100 \text { IU/ } \\
\text { kg 3x/wk }\end{array}$ & $N / A^{\#}$ & $\mathrm{~N} / \mathrm{A}^{\#}$ & $\mathrm{~N} / \mathrm{A}^{\#}$ & $\begin{array}{l}\text { N/A }{ }^{\#} \text { (Lost to } \\
\text { follow-up) }\end{array}$ & $\mathrm{N} / \mathrm{A}^{\#}$ & $\begin{array}{l}\text { N/A - ITI } \\
\quad \text { discontinued }{ }^{\#}\end{array}$ \\
\hline $14 y$ & $<1$ & 198 & $\begin{array}{c}\mathrm{rFVIII-Fc} 100 \mathrm{IU} / \mathrm{kg} \\
3 x / \text { wk }\end{array}$ & $<0.6$ & 100 & 9 & Successful & 14 & $\begin{array}{c}\text { rFVIII-Fc } 50 \mathrm{IU} / \mathrm{kg} \\
1 \mathrm{x} / \text { week }\end{array}$ \\
\hline $4 y$ & $<1$ & 9.1 & $\begin{array}{l}\mathrm{pdFVIII} 50 \mathrm{IU} / \mathrm{kg} \\
3 x / \mathrm{wk}\end{array}$ & 9.6 & $<66$ & $<6$ & Failed & 24 & $\begin{array}{l}\text { N/A - ITI } \\
\text { discontinued }\end{array}$ \\
\hline $6 y$ & $<1$ & 7.4 & $\begin{array}{l}\mathrm{rFVIII} 100 \mathrm{IU} / \mathrm{kg} \\
3 x / \mathrm{wk}\end{array}$ & $<0.6$ & 100 & 6 & Partial & 25 & $\begin{array}{c}\text { rFVIII } 100 \mathrm{IU} / \mathrm{kg} \\
1 \mathrm{x} / \text { week }\end{array}$ \\
\hline
\end{tabular}

BDD, B domain deleted; BU, Bethesda unit; CBU, chromogenic Bethesda unit; hr, hour; FVIII, factor VIII; ITI, immune tolerance induction; ml, millileters; mo, months; N/A, not applicable; pdFVIII, plasma-derived FVIII; rFVIII, recombinant FVIII; wk, week.

${ }^{*}$ Time between initiation of Atlanta Protocol to determination of tolerance status impacted by delays in inhibitor testing and restricted in-person clinical visit as a result of the COVID-19 pandemic.

\#Limited data available due to patient being lost to follow-up after a move out of state. Current FVIII regimen reflects status on last communication.
The 7 patients in the original cohort now have a median age of 4 years (range 4-14 years), previously 2 years (range 1.8-12 years). There is limited data on 1 patient lost to follow-up (moved away). Of the 6 remaining patients, 3 (50\%), 1 (17\%), and 2 (33\%) achieved successful, partial, and failed tolerance, respectively, determined at a mean of $22.8 \pm 5.1$ months from initiation of the Atlanta Protocol (Table 1). One successfully tolerized patient had an inhibitor relapse with a high titer inhibitor 8 months after successful tolerance and following a decrease in FVIII infusions of $50 \mathrm{lU} / \mathrm{kg}$ from thrice to twice weekly. This individual remains on ITI after switching FVIII concentrates. The patient with partial tolerance was classified as such due to a shortened half-life. All 7 patients remain on emicizumab prophylaxis, but 3 patients discontinued concurrent FVIII ITI. There were no thrombotic events.

Conclusions: Similar to traditional ITI, inhibitor relapse and failed tolerance are potential outcomes of ITI per the Atlanta Protocol that are difficult to predict. 
PB0511 | Efficacy and Safety of Emicizumab Prophylaxis in Severe Haemophilia A without Inhibitors: A Report from the UK Haemophilia Centre Doctors' Organisation (UKHCDO)

C. Wall ${ }^{1}$; H. Xiang ${ }^{2}$; B.P Palmer ${ }^{2}$; E. Chalmers ${ }^{3}$; P. Chowdary ${ }^{4}$; P.W Collins ${ }^{5}$; S. Fletcher ${ }^{6}$; D.P Hart'; G.W Hall ${ }^{8}$; R. Liesner ${ }^{9}$; S. Shapiro ${ }^{6}$; D. Stevensen ${ }^{10}$; K. Talks ${ }^{11}$; C.RM Hay ${ }^{1}$, United Kingdom Haemophilia Centre Doctors' Organisation

${ }^{1}$ Manchester Royal Infirmary, Manchester, United Kingdom; ${ }^{2}$ UK National Haemophilia Database, Manchester, United Kingdom; ${ }^{3}$ Royal Hospital for Sick Children, Glasgow, United Kingdom; ${ }^{4}$ Royal Free Hospital, London, United Kingdom; ${ }^{5}$ University Hospital of Wales, Cardiff, United Kingdom; ${ }^{6}$ Churchill Hospital, Oxford, United Kingdom; ${ }^{7}$ Royal London Hospital, London, United Kingdom; ${ }^{8}$ Oxford Children's Hospital, Oxford, United Kingdom; ${ }^{9}$ Great Ormond Street Hospital, London, United Kingdom, ${ }^{10}$ Kent and Canterbury Hospital, Canterbury, United Kingdom, ${ }^{11}$ Royal Victoria Infirmary, Newcastle upon Tyne, United Kingdom

Background: Real-world reports of outcomes of emicizumab prophylaxis in severe haemophilia A (SHA) without inhibitors are limited.

Aims: To evaluate the efficacy and safety of emicizumab across the UK Methods: This prospective, observational study used registry and Haemtrack (HT) patient reported outcome data from patients prescribed emicizumab between 01/08/2019-30/09/2020. Short-term efficacy (ABR, AJBR and bleed-free proportion) were determined and compared with prior FVIII prophylaxis (within-person analysis) in patients with $\geq 6$ months HT data before and after switching. Adverse events (AE) were adjudicated by the Co-Morbidities Working Party.

Results: Emicizumab was prescribed to 378 subjects, median (IQR) age 36.0 (31.0; 45.5), including $83<18$ years -15 were previously untreated and 18 minimally treated patients. Within-person comparison in 179 with $\geq 6$ months pre/post switching HT data (including 40 using rFVIIIFc prophylaxis), demonstrated significant reduction in bleeding rates with emicizumab (table). The overall bleed-free proportion increased from $37 \%$ to $75 \%$ in the 36 weeks before and after switching $(P<0.001)$. A sub-analysis of the $44 / 179$ who reported bleeding after switching showed a higher median (IQR) preswitch $A B R$ of $5.21(2.20 ; 16.0)$ reducing to $1.90(1.52 ; 3.67)$ post; a change of $-3.22(-10.0 ;-0.36),(P<0.001)$. AEs, reported in 9/378 (2.4\%), occurred predominantly during loading and included: grade 1-2 cutaneous reactions in three, severe headaches in three (resulting in drug cessation in one) and poor efficacy, unrelated to antidrug antibodies, in one person who reverted to rFVIIIFc prophylaxis. Recurrence of a FVIII inhibitor (Bethesda negative for $\sim 4$ years) was reported after 6 months emicizumab therapy without factor VIII exposure. One death, unrelated to emicizumab, was reported. No thrombotic or microangiopathic events occurred.

TABLE 1 Within-person analysis of emicizumab with prior prophylaxis

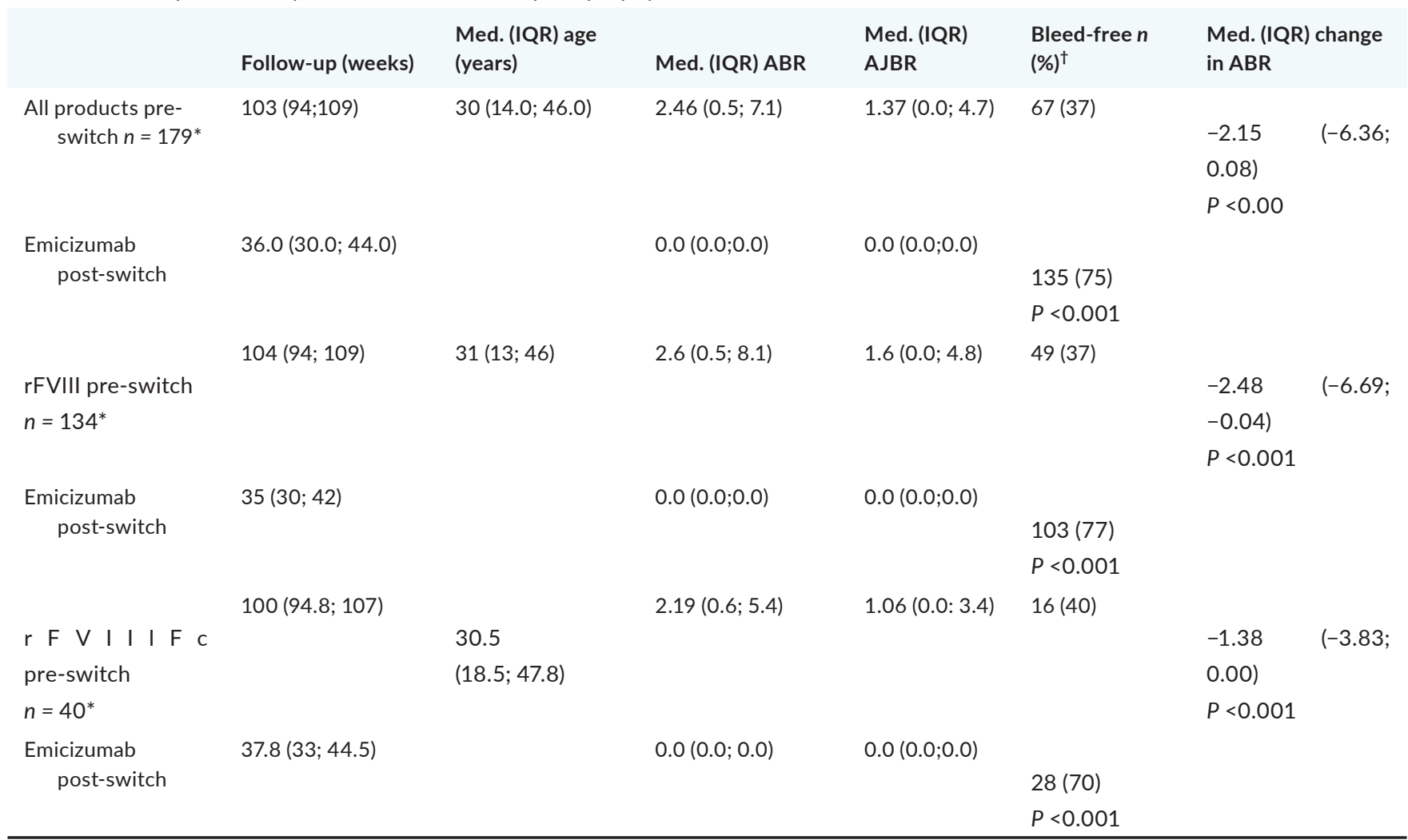

${ }^{*} n=5$ with mixed $r F V I I I$ and $r F V I I I F c$ during the study period were not included in the sub-analysis by factor replacement type

${ }^{\dagger}$ follow-up periods pre and post matched to median 36 weeks for comparison of bleed-free rates 


\section{Conclusions:}

Significant reductions in Haemtrack reported bleeding events were observed after changing to emicizumab prophylaxis in UK clinical practice. The safety profile appears acceptable but ongoing pharmacovigilance is required to determine long-term outcomes.

PB0512 | Association of Physical Activity with Bleeding Frequency in Children with Hemophilia A: A CHESS PAEDs Study Analysis

R. Ofori-Asenso $^{1}$; F. Nissen ${ }^{2}$; M. Silkey ${ }^{2}$; T. Xü ${ }^{2}$ H. YamaguchiSuita $^{3}$; D. Nosaka ${ }^{3}$; M. Aizenas ${ }^{2}$; O. Meier ${ }^{2}$; T. Burke ${ }^{4,5}$; J. O'Hara ${ }^{4,5}$

${ }^{1}$ Roche Products Ltd, Welwyn Garden City, United Kingdom; ${ }^{2} \mathrm{~F}$. Hoffmann-La Roche Ltd, Basel, Switzerland; ${ }^{3}$ Chugai Pharmaceutical Co. Ltd, Tokyo, Japan; ${ }^{4}$ HCD Economics, Warrington, United Kingdom; ${ }^{5}$ University of Chester, Chester, United Kingdom

Background: Limited published data characterizing the relationship between participation in physical activity (PA) and frequency of bleeding in children with hemophilia $\mathrm{A}(\mathrm{CwHA})$ exist.

Aims: To examine the association between participation in PA and annualized bleed rate ( $A B R$ ) among $\mathrm{CwHA}$ in a real-world setting.

Methods: Data from the Cost of Haemophilia across EuropeSocioeconomic Survey in Paediatric Population (CHESS PAEDs) study, conducted in France, Germany, Italy, Spain, and the United Kingdom during 2017, were utilized. Following ethics approval, hemophilia specialists provided information on CwHA they treated, including demographics, treatment type, and clinical outcomes. Patients completed quality-of-life-related questionnaires and reported activity levels (directly or via caregivers).

Results: Overall, $138 \mathrm{CwHA}$ who reported participation in PA were included. The mean \pm standard deviation hours spent per week on PA were $23.8 \pm 24.8$ and $10.8 \pm 11.3$ for moderate and severe patients, respectively (Table 1). Mean ABRs were $4.3 \pm 7.9$ and $6.9 \pm 18.7$ for moderate and severe patients, respectively, and $9.19 \pm 27.2$ and $5.75 \pm 13.7$ for on-demand and prophylaxis patients, respectively. CwHA who engaged in vigorous PA were similar in age and BMI to those who did not, but had a higher proportion of on-demand users. The mean ABR was higher in CwHA who engaged in vigorous PA $(8.0 \pm 21.0)$ compared to those who participated in only light and/or moderate

TABLE 1 Characteristics of patients who participated in physical activities

Table 1. Characteristics of patients who participated in physical activities

\begin{tabular}{|c|c|c|c|c|c|}
\hline & \multicolumn{2}{|c|}{ Severity } & \multicolumn{2}{|c|}{ Treatment approach } & \multirow[b]{2}{*}{$\begin{array}{c}\text { Overall } \\
\text { (N=138) }\end{array}$} \\
\hline & $\begin{array}{c}\text { Moderate } \\
(\geq 1 \text { and } \leq 5 \text { (10/dL } \\
(\mathrm{N}=32)\end{array}$ & $\begin{array}{c}\text { Severe } \\
(\leqslant 1 U / d L) \\
(N=106)\end{array}$ & $\begin{array}{l}\text { On-demand }{ }^{*} \\
(\mathrm{~N}=26)\end{array}$ & 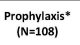 & \\
\hline Mean age, years (SD) & $10.6(4.1)$ & $10.5(4.0)$ & $10.2(3.7)$ & $10.7(4.1)$ & $10.5(4.0)$ \\
\hline Mean BMI, kg/ $/ \mathrm{m}^{2}(S D)$ & $19.7(5.3)$ & $22.8(23.5)$ & $28.7(43.2)$ & $20.8(10.0)$ & $22.0(20.8)$ \\
\hline Proportion on prophylaxis", n (\%) & $23(71.9)$ & $85(80.2)$ & $0(0.0)$ & $108(100.0)$ & $108(78.3)$ \\
\hline Proportion using on-demand*, $n$ (\%) & $6(18.8)$ & $20(18.9)$ & $26(100.0)$ & $0(0.0)$ & $26(18.8)$ \\
\hline \multicolumn{6}{|l|}{$\overline{\text { ABR [bleeds/year] }}$} \\
\hline Mean (SD) & $4.3(7.9)$ & $6.9(18.7)$ & $9.2(27.2)$ & $5.8(13.7)$ & $6.3(16.9)$ \\
\hline Median (IQR) & $2.0(1.0-3.0)$ & $3.0(2.0-4.0)$ & $2.0(2.0-3.8)$ & $3.0(2.0-4.0)$ & $3.0(2.0-4.0)$ \\
\hline Mean hours of physical activity per week (SD) & $23.8(24.8)$ & $10.8(11.3)$ & $13.5(10.5)$ & $13.5(17.7)$ & $13.8(16.3)$ \\
\hline $\begin{array}{l}\text { Particicates in only light/moderate (no } \\
\text { vigiorous) } \mathrm{PA}^{\mathrm{A}} \text {. n (\%) }\end{array}$ & $10(31.3)$ & $42(39.6)$ & $5(19.2)$ & $47(43.5)$ & $52(37.7)$ \\
\hline
\end{tabular}

TABLE 2 Frequency of bleed according to therapy adaption prior to participation in physical activities*

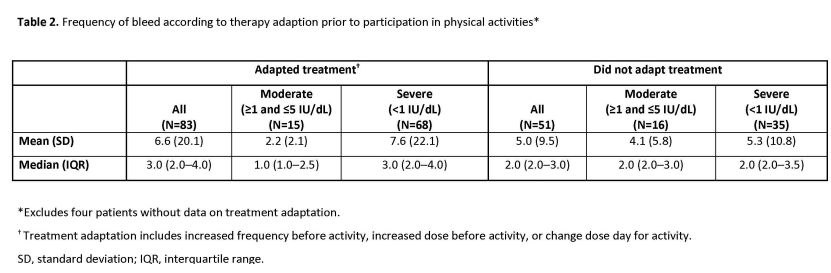

PA (3.5 \pm 3.7$)$; however, the median $A B R$ and proportion with zero bleeds were similar. There was no clear trend in ABR between $\mathrm{CwHA}$ who adapted their treatment prior to engaging in PA compared to those who did not (Table 2).

Conclusions: This 2017 study suggests a slightly higher bleed frequency in CwHA with higher participation in PA, which could indicate that, although $78 \%$ of all $\mathrm{CwHA}$ were receiving prophylaxis, the treatment was suboptimal in real-world settings. However, these findings should be interpreted with caution considering the small sample and potential confounders.

PB0513 | Developing Predictive Models of rFVIII-Fc Half Life for Personalized Prophylaxis in Patients with Hemophilia A

C.-Y. Chang ${ }^{1,2}$; S.-W. Lai ${ }^{3}$; Y.-L. Liu ${ }^{1,2}$; J.-R. Tsai ${ }^{4}$; C.-H. Tsai ${ }^{5}$; C.-N. Cheng ${ }^{6}$; S.-H. Hu' ; J.-T. Ku ${ }^{8}$; Y.-C. Chen ${ }^{9,7}$

${ }^{1}$ Department of Pediatrics, College of Medicine, School of Medicine, Taipei Medical University, Taipei, Taiwan, Province of China; ${ }^{2}$ Hemophilia Center, Division of Pediatric Hematology/Oncology, Taipei Medical University Hospital, Taipei, Taiwan, Province of China; ${ }^{3}$ Hemophilia Care and Research Center, Division of Hematology/ Oncology, Tri-ServiceGeneral Hospital, National Defense Medical Center, Taipei, Taiwan, Province of China; ${ }^{4}$ Division of Hematology/ Oncology, Taipei Medical University Hospital, Taipei, Taiwan, Province of China; ${ }^{5}$ Division of Hematology/Oncology, Cheng Hsin General Hospital, Taipei, Taiwan, Province of China; ${ }^{6}$ Department of Pediatrics, National Cheng Kung University Hospital, Taipei City, Taiwan, Province of China; ${ }^{7}$ Hemophilia Care and Research Center, Tri-Service General Hospital, Taipei, Taiwan, Province of China; ${ }^{8}$ Division of Pediatric Hematology/Oncology, Taipei Medical University Hospital, Taipei, Taiwan, Province of China; ${ }^{9}$ Division of Hematology/Oncology, TriServiceGeneral Hospital, National Defense Medical Center, Taipei, Taiwan, Province of China

Background: Pharmacokinetics (PK) of extended half-life products is important for individualized prophylaxis of patients with hemophilia A (PwHA). Great variation in real world of rFVIII-Fc half life have been reported in some developed countries.

Aims: We aimed to develop a predictive model of $r F V I I I-F c$ half life in PwHA.

Methods: Totally 50 PwHA on rFVIII-Fc replacement therapy were enrolled from two hemophilia centers. Clinical data, including age, BW, BMI, Hct, ABO blood group, von Willebrand factor (VWF) levels, inhibitor history, HCV status, and individual PKs calculated by 
WAPPS-hemo were collected from charts reviewed retrospectively. Linear regression by SAS software was used for searching out predictors of rFVIII-Fc half life from all the covariates.

Results: The mean age was $34.6 \pm 15.9$ y/o (8-64). The mean Hct was 43.8\% (29.9-52.6\%). Twenty-one patients were blood-group $\mathrm{O}$, and 29 were non-O. Mean VWF:Ag was 112.5 $\pm 54.1 \%$ (50-294.7\%). Mean ratio of VWF:activity or Rco over VWF:Ag (defined as VWF:Quality) was 0.93 (0.67-1.21). For PwHA of non-O group and $\mathrm{O}$ group, rFVIII-Fc half lives were $22.83 \pm 6.46 \mathrm{~h}$ and $16.26 \pm 4.61 \mathrm{~h}$, respectively $\left(P<0.001^{* * *}\right)$ and VWF:Ag levels were $132.10 \pm 63.72 \%$ and $86.93 \pm 19.30 \%$, respectively $\left(P<0.01^{* *}\right)$. After multivariate linear regression analysis, for all blood groups, VWF:Ag, HCV infection, and Hct were identified as positive predictors and $\mathrm{O}$ blood group and inhibitor history as negative predictors of $\mathrm{rFVIII-Fc}$ half life. For non-O group, BMI was an extra positive predictor. For $\mathrm{O}$ group, BW was an extra positive predictor and VWF:Quality was an extra negative predictor. Three predictive equations were developed (Table 1), explaining $51.97 \%, 75.17 \%$, and $66.38 \%$ of all variability in rFVIII-Fc half-life for $\mathrm{PwHA}$ of non-O group, $\mathrm{O}$ group, and all blood groups, respectively. Conclusions:

Several predictors of rFVIII-Fc half life were searched out and prediction models were well developed. We believe they will be useful for physician to individualize prophylaxis of $\mathrm{PwHA}$ on $\mathrm{rFVIII-Fc.}$
Dormandy Haemophilia Centre and Thrombosis Unit, Royal Free London NHS Foundation Trust, London, United Kingdom; ' Institute of Clinical Hemostaseology and Transfusion Medicine, Saarland University and University Hospital, Homburg, Saar, Germany; ${ }^{7}$ Haematology Department, La Paz University Hospital, Madrid, Spain; ${ }^{8}$ Department of Hematology, Ege University Children's Hospital, Izmir, Turkey; ${ }^{9}$ Department of Transfusion Medicine, Nagoya University Hospital, Nagoya, Japan, ${ }^{10}$ The Hemophilia Centre, Department of Haematology, Aarhus University, Aarhus, Denmark, ${ }^{11}$ Department of Immunohematology and Department of Molecular Hemostasis, Institute of Experimental Hematology and Transfusion Medicine, University Clinic, Bonn, Germany, ${ }^{12}$ Department of Haematology, Haemophilia and Thrombosis Unit, University Hospital Centre Zagreb, Medical School of Osijek and Medical School of Zagreb, Zagreb, Croatia,

${ }^{13}$ Indiana Hemophilia \& Thrombosis Center, Indianapolis, United States,

${ }^{14}$ Department of Pathology, Microbiology and Immunology, Vanderbilt University Medical Center, Nashville, United States, ${ }^{15}$ Keck School of Medicine, University of Southern California, Los Angeles, United States

Background: The anti-tissue factor pathway inhibitor (TFPI) monoclonal antibody concizumab is in phase 3 clinical development as a once-daily, subcutaneous prophylaxis for hemophilia A/B (HA/HB)

TABLE 1 Predictive equations of $\mathrm{rFVIII-Fc} \mathrm{half} \mathrm{life} \mathrm{by} \mathrm{multivariate} \mathrm{linear} \mathrm{regression} \mathrm{analysis}$

Blood groups

1) For hemophilia A patients with non-O blood group

Adjusted-R2

2) For hemophilia A patients $O$ blood group

Adjusted-R2

3) For hemophilia A patients all blood groups
Predictive equation of $\mathrm{rFVIII-Fc}$ half life

$-0.81245+0.62874 *(\mathrm{BMI})+0.06065 *(\mathrm{VWF}: \mathrm{Ag} \%)$

0.5197

$-0.68276+0.13301 *(\mathrm{VWF}: \mathrm{Ag} \%)+0.26831 *(\mathrm{BW})-1.16586 *(\mathrm{BMI})$ +16.02271 * (VWF:Quality ${ }^{\dagger}$ )

0.7517

$-1.75958+0.07243 *(\mathrm{VWF}: \mathrm{Ag} \%)-3.84423 *$ (Inhibitor history $\left.{ }^{\ddagger}\right)+2.98525 *$ $\left(\mathrm{HCV}_{\text {infection }}^{\ddagger}\right)-2.82626 *\left(\mathrm{O}\right.$ blood group $\left.{ }^{\#}\right)+0.30393 *($ Hct\%)

0.6538

Adjusted-R2

${ }^{\dagger}, \mathrm{VWF}$ :Quality is defined as ratio of VWF:activity or Rco over VWF:Ag; ${ }^{\ddagger}$, positive is 1 and negative $=0$; ${ }^{\#}, \mathrm{O}$ group is 1 and non-O group is 0 .

PB0514 | Longer-term Efficacy and Safety of Concizumab Prophylaxis in Hemophilia A and Hemophilia A/B with Inhibitors: Results from the Main and Extension Parts of Concizumab Phase 2 Trials

J. Astermark $^{1}$; P. Angchaisuksiri²; G. Benson ${ }^{3}$; G. Castaman ${ }^{4}$; P. Chowdary ${ }^{5}$; H. Eichler ${ }^{6}$; V. Jiménez-Yuste ${ }^{7}$; K. Kavakli ${ }^{8}$; T. Matsushita ${ }^{9}$; L. Hvitfeldt Poulsen ${ }^{10}$; J. Oldenburg ${ }^{11}$; S. Zupancic Salek $^{12}$; A. Shapiro ${ }^{13}$; A.P. Wheeler ${ }^{14}$; G. Young ${ }^{15}$

${ }^{1}$ Center for Thrombosis and Haemostasis, Lund University, Skåne University Hospital, Malmö, Sweden; ${ }^{2}$ Division of Hematology, Department of Medicine, Ramathibodi Hospital, Mahidol University, Bangkok, Thailand; ${ }^{3}$ Department of Haematology, Belfast Health and Social Care Trust, Belfast, Northern Ireland, United Kingdom; ${ }^{4}$ Center for Bleeding Disorders and Coagulation, Department of Oncology, Careggi University Hospital, Florence, Italy; ${ }^{5}$ Katharine with/without inhibitors. We present results from the combined main and extension parts of the concizumab explorer4 (NCT03196284) and explorer5 (NCT03196297) phase 2 trials.

Aims: The objective of the extension part of the trials was to assess long-term efficacy and safety of concizumab in $\mathrm{HA} / \mathrm{HB}$ with inhibitors (HAwl/HBwl) (explorer4) and in severe HA (explorer5).

Methods: Both trials comprised a main ( 224 weeks) and an extension part (up to 102 weeks in total). Patients were treated with $0.15 \mathrm{mg} / \mathrm{kg}$ concizumab with potential dose escalation to 0.20 and $0.25 \mathrm{mg} / \mathrm{kg}$ if they experienced $\geq 3$ treated spontaneous bleeds within 12 weeks. Endpoints included annualized bleeding rate (ABR), change in coagulation-related parameters, number of adverse events (AEs) and anti-drug antibody (ADA) occurrence during the trial. ABR was estimated based on a negative binomial regression with log of exposure time to concizumab in main and extension part as offset and haemophilia type as a factor (explorer4). Results are presented at the last concizumab dose level. 
Results: During the trials, $36 \mathrm{HA}, 15 \mathrm{HAwl}$ and $10 \mathrm{HBwl}$ patients were exposed to concizumab. Concizumab efficacy (Fig.1) was maintained in $\mathrm{HA}, \mathrm{HAwl}$ and $\mathrm{HBwl}$ patients in the trial extension parts. Ddimer and prothrombin fragment 1+2 increases were observed with increasing concizumab concentrations in some patients, reflecting concizumab's hemostatic effect. The majority of ADAs observed were low-titer and transient with no observed clinical effect. There were no AEs leading to withdrawal, no thromboembolic events, and no deaths during the main and extension parts of either trial.

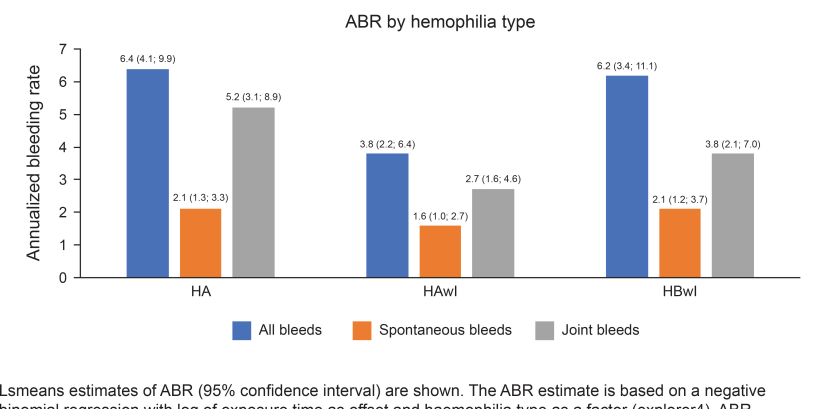
binomial regression with log of exposure time as offset and haemophilia type as a factor (explorer4). ABR,
annualised bleeding rate; $H A$, hemophilia A; HAwl, hemophilia A with inhibitors; $H B w l$, hemophilia B with inhibitors

FIGURE 1 ABRs (treated bleeding episodes on last dose level) by hemophilia type during the main and extension parts of the concizumab explorer4 (HAwl, HBwl) and explorer5 (HA) trials

Conclusions: Results from the extension parts of concizumab phase 2 trials were consistent with main-part results, supporting its development as a once-daily, subcutaneous treatment across all hemophilia subtypes.

PB0515 | Reviewing HJHS as a Tool to Assess Outcome Measures in Patients with Haemophilia A in Australia

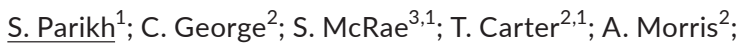

C. Cramey ${ }^{4}$; K. McElroy ${ }^{5}$; H. $\operatorname{Tran}^{6,1}$

${ }^{1}$ Australian Haemophilia Centre Directors' Organisation, Melbourne, Australia; ${ }^{2}$ Perth Children's Hospital, Perth, Australia; ${ }^{3}$ Launceston General Hospital, Tasmania, Australia; ${ }^{4}$ Royal Adelaide Hospital, Adelaide, Australia; ${ }^{5}$ Queensland Children's Hospital, South Brisbane, Australia; ${ }^{6}$ The Alfred Hospital, Melbourne, Australia

Background: It is widely recommended that management of patients with haemophilia include the use of standardised joint assessment tools. The tool incorporated into the Australian Bleeding Disorders Registry (ABDR) is the UK MOD-HJHS, a validated but time-intensive tool of high sensitivity.

Aims: To characterise clinician practices regarding the use of HJHS in routine assessment of patients with Haemophilia $A(H A)$ in Australia; to identify potential barriers to HJHS tool usage via ABDR; and to examine relationships between HJHS score in individual patients and other treatment and outcome measures.

Methods: Demographic data was derived from the ABDR for HA patients from whom consent had been obtained. Data was obtained on severity, treatment regimen, product usage, inhibitor status and age for patients with at least one HJHS recorded.
Results: There were 547 HA patients (368 severe) with 1169 HJHS in the ABDR. 53.5\% (368/687) of severe HA patients in Australia had at least one HJHS. 36.7\% (429/1169) of HJHS assessments were performed in a paediatric setting, median baseline age 11years (8-14). Median baseline HJHS of severe HA patients on prophylaxis for at least 6months was $1(0-3)$ for paediatric and $11(3-28)$ for adult patients. $293 \mathrm{HA}$ patients prescribed prophylaxis had more than one HJHS with median observation time of 14 months (11-25) and median delta HJHS $0(-2,2)$. Results demonstrated that high titre inhibitor, non-adherence and joint bleeds/surgery had significant impact on longitudinal HJHS. All HJHS were performed by physiotherapists (around 55\% in clinics, $45 \%$ in outpatient/outreach settings). HJHS was usually recorded on paper at assessment and then later transcribed into ABDR.

Conclusions: This study provided the first baseline assessment of joint health data for HA patients in Australia, and an initial understanding of how individual and treatment factors may influence long-term joint outcomes. It also provided insights into practical limitations of the HJHS tool.

PB0516 | Sports Injuries in Dutch People with Hemophilia: Results of a 12 Months' Prospective Study

O. Versloot ${ }^{1}$; J. Blokzijl ${ }^{1}$; M. Schuuring ${ }^{2}$; M. Timmer ${ }^{1}$; M. Bartels ${ }^{1,2}$; W. van Beers ${ }^{1}$; K. van Galen ${ }^{1}$; I. Kremer Hovinga ${ }^{1}$; R. Schutgens ${ }^{1}$; M. Suijker ${ }^{1}$; P. van der Valk ${ }^{1}$; L. van Vulpen ${ }^{1}$; J. van der net ${ }^{2}$; K. Fischer ${ }^{1}$ ${ }^{1}$ van Creveldkliniek, UMC Utrecht, Utrecht, Netherlands; ${ }^{2}$ Wilhelmina Childrens' Hospital, UMC Utrecht, Utrecht, Netherlands

Background: During the last decades, sports participation has become a prominent aspect of life in people with hemophilia (PWH). However, data on the risk of sports injuries are lacking.

Aims: To assess sports injuries according to severity in Dutch PWH and compare the results to the general population (GP).

Methods: For 12 months, sports injury data and bleeds following sports injury were prospectively collected in PWH aged 6-50 without inhibitors who played sports at least once weekly. A sports injury was defined as an injury sustained during sports participation, limiting future sports participation. Injuries were compared according severity and to general population data. High-risk sports were defined as the highest two categories ("moderate to high risk" and "high risk") of the National Hemophilia Foundation classification. Groups were compared using Chi square.

Results: Data were collected on 125 participants. Mean age was $25.3 \pm 12.0$, $90 \%$ had hemophilia A, including $48 \%$ severe (all on prophylaxis) and $52 \%$ non-severe (5\% on prophylaxis, mean factor levels $15 \pm 8 \mathrm{lU} / \mathrm{dl}$ ). A total of 87 sports injuries were reported by 51 (41\%) participants, including 31 (31/60: 52\%) with severe and 20 (20/65: $31 \%)$ with non-severe hemophilia $(P<0.01)$. The majority of injuries (59\%) occurred during high-risk sports. Participants with severe hemophilia reported higher injury rates at 6.3 (95\% Cl: 4.9-8.1) inj/1000 hrs exposure, compared to those with non-severe hemophilia (1.9 (1.3-2.7) inj/1000 hrs exposure; $P=0.02$ ). Participants administered extra coagulation after 59 (68\%) injuries. Overall, 26/87(30\%) [FK(1] 
[OV2] of injuries in 20/125 (16\%) participants resulted in a bleed, independent of severity (severe: $30 \%$, non-severe: $30 \%$ ).

Conclusions: During follow-up, $41 \%$ of PWH had a sports injury, and $16 \%$ suffered an injury induced bleed. Sports injury rates were increased in severe hemophilia patients, but the proportion of injuries resulting in bleeds was not.

PB0517 | Soccer Is Popular and Relatively Safe for Children and Young Adults with Hemophilia: Experiences from the Netherlands

O. Versloot $^{1}$; J. Blokzijli ${ }^{1}$; M. Schuuring ${ }^{2} ;$ M. Timmer ${ }^{1}$; M. Bartels ${ }^{1,2}$; W. van Beers ${ }^{1}$; K. van Galen ${ }^{1}$; I. Kremer Hovinga ${ }^{1}$; M. Suijker ${ }^{1}$;

R. Schutgens ${ }^{1}$; P. van der Valk ${ }^{1}$; L. van Vulpen ${ }^{1}$; J. van der $\mathrm{Net}^{2}$; K. Fischer ${ }^{1}$

${ }^{1}$ van Creveldkliniek, UMC Utrecht, Utrecht, Netherlands; ${ }^{2}$ Wilhelmina Childrens' Hospital, UMC Utrecht, Utrecht, Netherlands

Background: Despite ongoing controversy about injuries, soccer is the most popular sport in young Dutch persons with hemophilia (PWH). Especially data regarding injury-risk are lacking.

Aims: To assess injuries in Dutch PWH playing soccer according to age and severity, compared to the general population (GP).

Methods: The data were part of a prospective study assessing sports participation and injuries in PWH aged 6-50 without inhibitors. Soccer participation was assessed at baseline using the Modifiable Activities Questionnaire. Injuries were recorded during a 12 months' follow-up. A sports injury was defined as an injury sustained during sports participation, limiting future sports participation. Participants indicated whether an injury resulted in a bleed. Participation and injuries were assessed according to age and severity and compared to GP data.

Results: Out of 125 participants (48\% severe, prophylaxis: 100\%; non-severe: $5 \%$ on prophylaxis with mean factor levels $15 \pm 8 \mathrm{IU} / \mathrm{dl}$ ), 39 played soccer (31\%). More children than adults played soccer (66 vs. $22 \%$; $P<0.01$ ), nobody beyond the age of 33 played soccer (figure 1). Soccer participation ( $27 \%$ vs. $35 \%$ ) and exposure (3.3 vs. 3.5 $\mathrm{hrs} / \mathrm{wk}$ ) were similar between severe and non-severe hemophilia. A total of 26 soccer injuries were reported by 18 participants (overal $46 \%$, severe hemophilia: 19/26 (73\%)), of which 4 resulted in bleeds (3 joint, 1 muscle) that needed FVIII treatment (overall 10\%, all severe). Table 1 shows a lower injury rate than the GP. People with severe hemophilia had a higher injury rate than those with non-severe hemophilia.

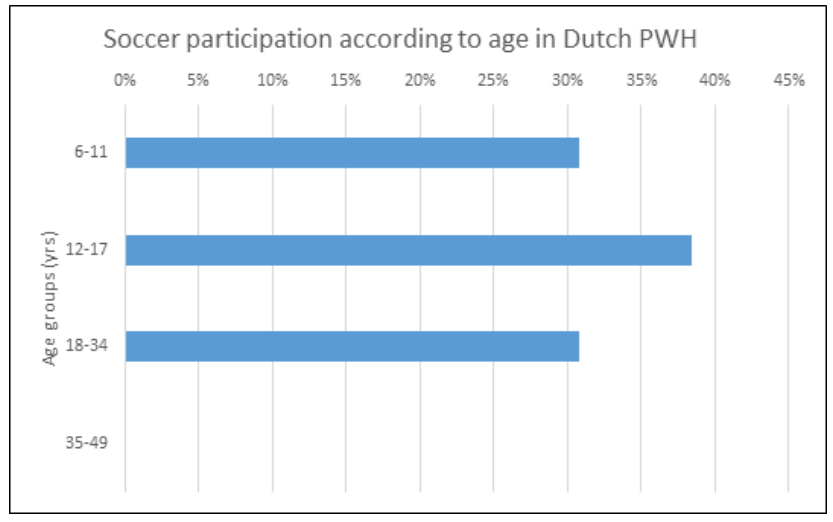

FIGURE 1: Sports participation according to age in Dutch people with hemophilia

TABLE 1 Soccer participation and injury characteristics ( $n(\%))$; median (IQR))

\begin{tabular}{llll} 
& Overall & Severe & Non-severe \\
Participants $(n)$ & 124 & $59(48 \%)$ & $65(52 \%)$ \\
Patients playing soccer $(n(\%))$ & $39 / 124(31 \%)$ & $16 / 59(27 \%)$ & $23 / 65(35 \%)$ \\
Weekly & $3.5(2.8-4.2)$ & $3.3(2.0-4.7)$ & $3.5(2.8-4.2)$ \\
Exposure (hrs/wk) & & & - \\
Patients with any soccer injury & $18 / 39(46 \%)$ & $14 / 18(78 \%)$ & $5 / 18(22 \%)$ \\
Number of soccer injuries observed & 26 & $19 / 26(73 \%)$ & $7 / 26(27 \%)$ \\
Soccer injury rate (inj./1000 hrs) & $4.3(2.9-6.3)$ & $7.7(4.9-12.1)$ & $2.0(0.8-4.2)$ \\
soccer injury resulting in bleed & $4 / 26$ & $4 / 19$ & $0 / 7$ \\
Patients with a bleed following soccer injury & $4 / 39(10 \%)$ & $4 / 16$ & $0 / 23$ \\
\hline
\end{tabular}

Conclusions: Soccer is the most popular sport among Dutch PWH up to 33 years of age. Overall, $46 \%$ suffered an injury during followup, resulting in a bleed in $10 \%$. Despite prophylaxis, people with severe hemophilia had a higher injury rate $(7.7 \mathrm{inj} / 1000 \mathrm{hrs})$ than those with non-severe hemophilia (2.0 inj/1000 hrs). Overall, soccer was relatively safe in Dutch $\mathrm{PWH}$. 
PB0518 | Treatment Patterns and Clinical Outcomes among Patients with Hemophilia A Treated with Factor VIII Replacement Therapies and Nonfactor Therapy: An Assessment of US Realworld Data

A. Adeyemi ${ }^{1}$; A. Cockerham ${ }^{1}$; R. Preblick ${ }^{1}$; A. Swenson ${ }^{2}$; J. Dumont ${ }^{1}$

${ }^{1}$ Sanofi, Waltham, MA, United States; ${ }^{2} \mathrm{OM} 1$, Inc., Boston, MA, United

States

Background: Recombinant factor VIII Fc fusion protein (rFVIIIFc) was the first extended half-life (EHL) factor approved for adults and children with hemophilia A (HA). Despite availability of rFVIIIFc, other EHL factors, and non-factor therapy, many patients remain on standard half-life (SHL) factor VIII (FVIII).

Aims: Describe characteristics and clinical outcomes of patients with HA treated with EHL or SHL factors, or emicizumab.

Methods: This was a longitudinal, retrospective, observational study of males with HA treated with SHL, EHL, both SHL and EHL factor, and/or emicizumab (January 2013-May 2020). Data originated from the US-based OM $1^{\circledR}$ Real-World Data Cloud (OM1, Inc., Boston, MA, USA), derived from patient-level healthcare claims and electronic medical records. Eligibility criteria included $\geq 1$ diagnosis code for HA and $\geq 1$ prescription/administration of an SHL, EHL, or emicizumab in the study period, with no evidence of inhibitors during follow-up. Index date was date of product (SHL, EHL, or emicizumab) initiation within the study period. Continuous medical activity $\geq 12$ months pre-index date was required. Variables included patient demographics, hemophilia-related treatment patterns, and clinical outcomes.

Results: Most patients with HA on SHL (74\% of 457), EHL (rFVIIIFc and other EHLs; $70 \%$ of 321), rFVIIIFc (EHL subgroup; 65\% of 205), and emicizumab ( $63 \%$ of 118 ) were aged $\geq 12$ years. In patients receiving rFVIIIFc or emicizumab, mean monthly all-factor consumption post-index date was $55 \%$ and $65 \%$ less, respectively, compared with pre-index consumption. For EHLs overall, mean all-factor consumption was unchanged (Table 1). Limited pre-index data presented a challenge comparing factor consumption in the SHL group. FVIII consumption was reported in $47 \%(n=56)$ of emicizumab-treated patients. Use of rFVIIIFc, EHLs, or emicizumab was associated with decreased annualized bleed rate (ABR; 34\%, 35\%, 26\%). ABR did not improve with SHL (Table 2).

Conclusions: rFVIIIFc, EHL overall, and emicizumab were associated with lower ABR during follow-up, and rFVIIIFc with lower consumption.

TABLE 1 FVIII consumption in the pre- and post-index periods among male patients with hemophilia A with available consumption data*,†,‡,§

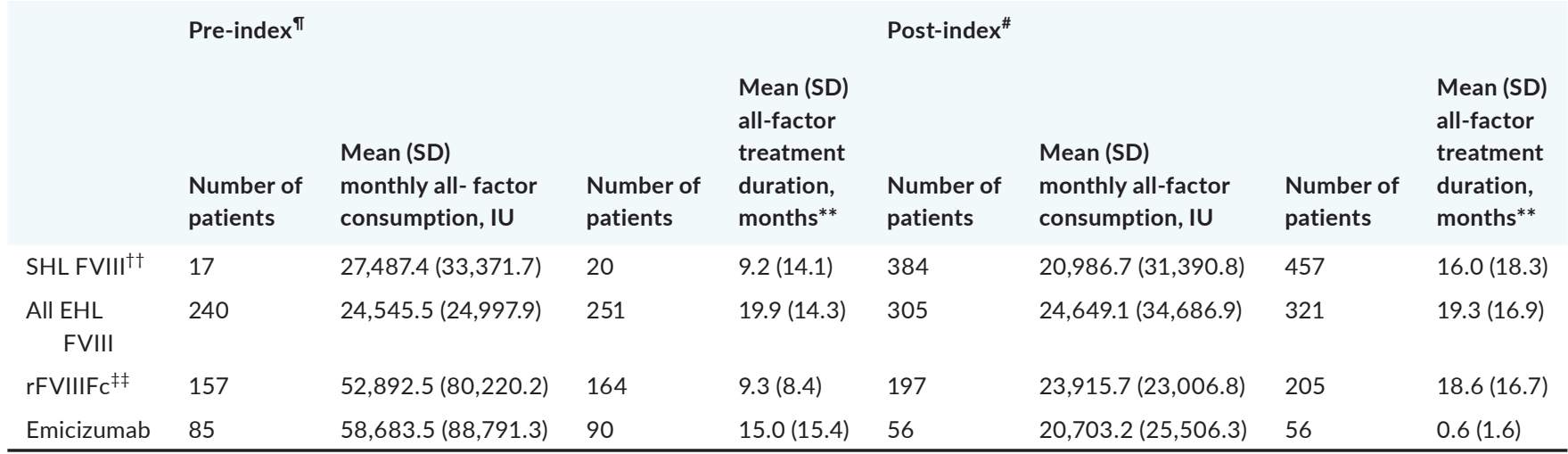

EHL, extended half-life; FVIII, factor VIII; rFVIIIFc, recombinant factor VIII Fc fusion protein; SD, standard deviation; SHL, standard half-life.

*Pre-index corresponds to the period $\geq 12$ months before the index date.

${ }^{\dagger}$ Patients included had pharmacy claims in the pre- and post-index periods and lacked evidence of inhibitors (based on receipt of bypassing agents or evidence of immune tolerance induction, identified as patients receiving 3 times the median factor consumption for their age group for 6 consecutive months [allowing for a 1-month grace period] during follow-up).

${ }^{\ddagger}$ Factor consumption data were not available for all patients; therefore, patient numbers differ between pre- and post-index, as well as between those with reported factor consumption and those with reported treatment duration.

${ }^{\S}$ Factor consumption normalized to patient weight is not included, as limited available data were considered unlikely to produce valid estimates.

"Pre-index corresponds to the interval from first observed encounter to initiation of the specified product, excluding date of initiation of the specified product.

\#Post-index corresponds to the interval from initiation of the specified product to the study end date or the patient's last observed encounter, whichever is earlier.

**Treatment duration based on patients with pharmacy claims.

${ }^{+\dagger}$ No data on factor consumption were available in the pre-index period for most patients in the SHL group. The remainder were receiving EHL FVIII in the pre-index period.

${ }^{\ddagger \ddagger} \mathrm{rFVIIIFC}$ is a subgroup of the EHL FVIII group. 
TABLE 2 ABR pre- and post-index periods among male patients with hemophilia A having $\geq 6$ months of exposed follow-up documented in medical records*,†,‡,§

\begin{tabular}{llll} 
& & Pre-index ${ }^{\pi}$ & Post-index $^{\#}$ \\
& Number of patients & Mean (SD) ABR & Mean (SD) ABR \\
SHL FVIII & 251 & $0.4(1.7)$ & $0.41(1.90)$ \\
All EHL FVIII & 215 & $0.4(2.4)$ & $0.26(1.90)$ \\
rFVIIIFc* & 144 & $0.5(2.9)$ & $0.33(2.27)$ \\
Emicizumab & 68 & $0.5(3.5)$ & $0.37(2.08)$ \\
\hline
\end{tabular}

ABR, annualized bleed rate; EHL, extended half-life; EMR, electronic medical record; FVIII, factor VIII; rFVIIIFc, recombinant factor VIII Fc fusion protein; SD, standard deviation; SHL, standard half-life.

*Pre-index corresponds to the period $\geq 12$ months prior to the index date.

${ }^{\dagger}$ Patients included had pharmacy claims in the pre- and post-index periods and lacked evidence of inhibitors (based on receipt of bypassing agents or evidence of immune tolerance induction, identified as patients receiving 3 times the median factor consumption for their age group for 6 consecutive months [allowing for a 1-month grace period] during follow-up).

${ }^{\ddagger} A B R$ was estimated based on the number of unique bleeding events per patient per year during exposed person-time requiring treatment in a healthcare setting. Unique bleeding events were identified using a previously reported approach (Shrestha A, et al. Haemophilia. 2017;23(4):e267-e275) with claims for bleeding events for the same body part that occur within 7 days collapsed into one bleeding event. Bleeding diagnosis codes for different body parts with different start dates were considered separate events.

${ }^{\S}$ Measures are based on real-world data and not all bleeding events will have been captured by the OM1 data source if they were not submitted as a claim or documented in the EMR (eg, bleeds treated at home); thus, ABR will be underestimated.

"Pre-index corresponds to the interval from first observed encounter to initiation of the specified product, excluding date of initiation of the specified product.

"Post-index corresponds to the interval from initiation of the specified product to the study end date or the patient's last observed encounter, whichever is earlier.

** rFVIIIFC is a subgroup of the EHL FVIII group.

PB0520 | The Efficacy and Prognosticator for Inhibitor Eradication in Hemophilia A Boys with High-titer Inhibitors Using a Low-dose Immune Tolerance Induction Strategy (with or without Immunosuppressants): Data from the National Center for Children's Health of China

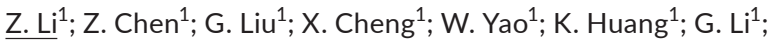
Y. Zhen ${ }^{1}$; X. Wu ${ }^{1}$; S. Cai ${ }^{1}$; M.-C. Poon ${ }^{2}$; R. Wu ${ }^{1}$

${ }^{1}$ Beijing Children's Hospital affiliated to Capital Medical University, Beijing, China; ${ }^{2}$ University of Calgary Cumming School of Medicine, Calgary, Canada

Background: The low-dose immune tolerance induction (ITI) \pm immunosuppressants (IS) as a cost-saving strategy to eradicate hemophilia A inhibitors has been used in recent years in China. However, the prognostic/predictive factors for success are unclear.
Aims: To investigate the efficacy and prognostic factors of this ITI strategy.

Methods: This is a single-center prospective observational cohort study with patients enrolled from September 2016 to September 2019. Nighty-six boys with FVIII level $<0.02 \mathrm{IU} / \mathrm{ml}$ and high-titer inhibitors were treated with low-dose ITI \pm IS according to inhibitor titer and ITI response. We defined "Success" as undetectable inhibitor after 2 consecutive measurements plus normal FVIII recovery. Results: Forty-five patients used ITI-alone and 51 patients used ITI-IS. For the whole cohort, success was achieved in $71.9 \%(69 / 96)$ in a median 9.9 (IQR 6.5-16.8) months. The Highest Peak Titer (HPT), in the combined historical and ITI periods was the most powerful predictor of ITI-success. The cut-off levels were 64BU [odds ratio (OR) 40.0] for the whole cohort, and 141.3BU (OR 54.7) for the ITI-IS group. Good predictors include also the monthly bleeding rate $<0.46$ (OR 8.3) during ITI for the whole cohort, and age $<4.8$ years (OR 5.0) at ITI initiation for the ITI-IS patients.

TABLE 1 The coefficient estimates of the prediction model and ORs of different factors included in the model of all patients

\begin{tabular}{llllll} 
& Coeffective & & & & \\
Factor & Estimate & SE & K-value & P-value & OR (95\% Cl) \\
Highest peak titer $\geq 64.0 \mathrm{BU}$ & 3.6883 & 0.7754 & 22.6247 & $<0.0001$ & $39.976(8.745,182.740)$ \\
Monthly bleeding rate $\geq 0.46$ & 2.1196 & 0.7130 & 8.8378 & 0.0030 & $8.328(2.059,33.687)$ \\
\hline
\end{tabular}

TABLE 2 The coefficient estimates of the prediction model and ORs of different factors included in the model of patients on ITI-IS

\begin{tabular}{llllll} 
Factor & Coeffective Estimate & SE & K-value & P-value & OR (95\% Cl) \\
Highest peak titer $\geq 141.3 \mathrm{BU}$ & 4.0019 & 1.1508 & 12.0940 & 0.0005 & $54.702(5.734-521.819)$ \\
Age at ITI initiation $\geq 4.8$ year-age & 1.6028 & 0.8165 & 3.8536 & 0.0496 & $4.967(1.003-24.605)$ \\
\hline
\end{tabular}

Abbreviations: OR, odds ratio; SE, standard error; ITI-IS, immune tolerance induction (ITI) therapy using FVIII together with immunosuppressive agents (IS). 
Conclusions: The $71.9 \%$ overall Success rate using this low-dose ITI strategy is comparable to those of other ITI protocols. An HPT $<64 \mathrm{BU}$ predicted Success, and $>141 \mathrm{BU}$ predicted failure even with additional IS treatment. Bleeding control during ITI and initiating ITI at a young age were also important factors for Success.

PB0521 | Final Analysis of the STASEY Trial: A Single-arm, Multicenter, Open-label, Phase III Clinical Trial Evaluating the Safety and Tolerability of Emicizumab Prophylaxis in Persons with Hemophilia A (PwHA) with Factor (F)VIII Inhibitors

V. Jiménez-Yuste ${ }^{1}$; F. Peyvandi ${ }^{2}$; R. Klamroth ${ }^{3}$; G. Castaman ${ }^{4}$; C. Shanmukhaiah ${ }^{5}$; S. Rangarajan ${ }^{6}$; J. García Chavez ${ }^{7}$; R. Martinez ${ }^{8}$; G. Kenet ${ }^{9}$; H. Alzahrani ${ }^{10}$; S. Robson ${ }^{11}$; C. Schmitt ${ }^{11}$; A. Kiialainen ${ }^{11}$; O. Meier ${ }^{11}$; M. Ozelo ${ }^{12}$

${ }^{1}$ Hospital Universitario La Paz, Autónoma University, Madrid, Spain;

${ }^{2}$ IRCCS Fondazione Ca' Granda Ospedale Maggiore Policlinico, Angelo Bianchi Bonomi Hemophilia and Thrombosis Center and Department of Pathophysiology and Transplantation, University of Milan, Milan, Italy; ${ }^{3}$ Comprehensive Care Haemophilia Treatment Centre, Vivantes Klinikum, Berlin, Germany; ${ }^{4}$ Careggi University Hospital, Florence, Italy; ${ }^{5}$ Department of Haematology, Seth GS Medical College and King Edward Memorial Hospital, Mumbai, India; ${ }^{6}$ Fortis Hospitals Ltd, Mumbai, India; ${ }^{7}$ Unidad de Investigación en Enfermedades Hematologicas, Hospital de Especialidades CMN La Raza, IMSS, Mexico City, Mexico; ${ }^{8}$ UMAE Hospital De Especialidades CMNSXXI, Mexico City, Mexico; ${ }^{9}$ Sheba Medical Center, Tel Hashomer, Israel, ${ }^{10}$ King Faisal Specialist Hospital and Research Centre, Riyadh, Saudi Arabia, ${ }^{11} \mathrm{~F}$. Hoffmann-La Roche Ltd, Basel, Switzerland, ${ }^{12}$ Hemocentro UNICAMP, University of Campinas, Campinas, Brazil

Background: Emicizumab replaces the function of missing activated FVIII in PwHA, thereby restoring hemostasis.

Aims: Report the final analysis from STASEY (NCT03191799), a phase III trial assessing the safety of emicizumab prophylaxis in PwHA with FVIII inhibitors.

Methods: Informed consent and ethics committee approval were acquired. PwHA age $\geq 12$ years with FVIII inhibitors received subcutaneous emicizumab $3 \mathrm{mg} / \mathrm{kg}$ once weekly (QW) for 4 weeks, followed by $1.5 \mathrm{mg} / \mathrm{kg}$ QW for up to 2 years. The primary objective was to evaluate the safety of emicizumab (adverse events [AEs]; including thromboembolic events [TEs], thrombotic microangiopathies [TMAs], and hypersensitivity reactions). Secondary objectives included assessing the efficacy of emicizumab (annualized bleed rates [ABRs]), and anti-drug antibody (ADA) development.

Results: At date of last participant's last visit (19-November-2020), 193 PwHA (median age [range]: 28.0 [12-80] years) had received $\geq 1$ dose of emicizumab, thus forming the safety-evaluable population. Median (range) treatment duration was 103.1 (1.1-108.3) weeks. Emicizumab was well-tolerated (Table 1). The most common AEs were arthralgia ( $n=33,17.1 \%)$, nasopharyngitis $(n=30,15.5 \%)$, and headache ( $n=29,15.0 \%$ ). No new TEs were reported since the two in the interim analyses (myocardial infarction; hypertrophic clot). Emicizumab-related AEs were reported in 35 (18.1\%) participants; most frequently, injection-site reactions ( $n=19,9.8 \%$ ). Further to the fatality reported at the first interim analysis, one death was reported (abdominal compartment syndrome; deemed unrelated to emicizumab). Five PwHA received activated prothrombin complex concentrate, with no associated TMAs or TEs. Ten (5.2\%) participants developed ADAs, five (2.6\%) were neutralizing in vitro. Mean ABR for treated bleeds was 0.5 , with $82.6 \%$ of participants having zero treated bleeds (Table 2).

Conclusions: The safety profile of emicizumab demonstrated in the HAVEN clinical trials was confirmed in a large, diverse population of PwHA with FVIII inhibitors, with no new safety signals and the majority of PwHA having zero treated bleeds.

TABLE 1 Safety summary (safety-evaluable population) of the STASEY study final analysis

Table 1: Safety summary (safety-evaluable population) of the STASEY study final analysis

\begin{tabular}{|c|c|}
\hline Adverse event (AE) & $\begin{array}{c}\text { Emicizumab } \\
1.5 \mathrm{mg} / \mathrm{kg} / \mathrm{week} \\
\mathrm{N}=193\end{array}$ \\
\hline Total number of $\mathrm{AE}$ & 800 \\
\hline Number of people with hemophilia $A$ (PwHA) with $\geq 1$ event, $n$ (\%) & $163(84.5)$ \\
\hline Fatal $\mathrm{AE}^{*}$ & $2(1.0)$ \\
\hline Serious AE & $31(16.1)$ \\
\hline $\mathrm{AE}$ leading to treatment withdrawal ${ }^{\dagger}$ & $1(0.5)$ \\
\hline AE leading to dose modification or interruption ${ }^{\ddagger}$ & $4(2.1)$ \\
\hline AE leading to study discontinuation $\$$ & \\
\hline Grade $\geq 3 \mathrm{AE}$ & $39(20.2)$ \\
\hline Study treatment-related $A E^{\pi}$ & $35(18.1)$ \\
\hline Injection-site reaction (ISR) ${ }^{\star \star}$ & \\
\hline \multicolumn{2}{|l|}{ Number of PwHA with AEs of interest, $n(\%)$} \\
\hline Systemic hypersensitivity/anaphylactic/anaphylactoid reaction & \\
\hline Thromboembolic event (TE) ${ }^{+t}$ & $2(1.0)$ \\
\hline TE associated with activated prothrombin complex concentrate (aPCC) & \\
\hline & \\
\hline Thrombotic microangiopathy (TMA) & \\
\hline TMA associated with aPCC and emicizumab\# & \\
\hline \multicolumn{2}{|l|}{ Most common AEs ( $\geq 10 \%$ of PwHA), $n(\%)$} \\
\hline Arthralgia & $33(17.1)$ \\
\hline Nasopharyngitis & 30 (15.5) \\
\hline Headache & $29(15.0)$ \\
\hline ISR $\$ \$$ & \\
\hline Pyrexia & $21(10.9)$ \\
\hline \multicolumn{2}{|l|}{ Number of PwHA with anti-drug antibodies (ADAs), $n$} \\
\hline ADAs & $10(5.2)$ \\
\hline Neutralizing ADAs in vitro ${ }^{* *}$ & \\
\hline \multicolumn{2}{|c|}{ 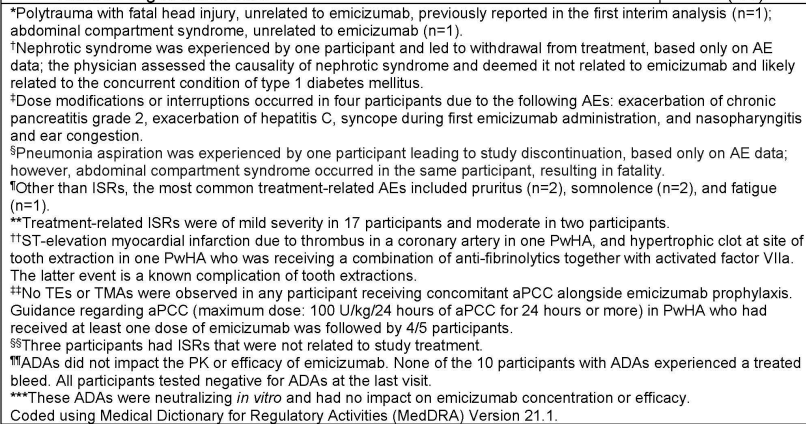 } \\
\hline
\end{tabular}


TABLE 2 Efficacy summary (intent-to-treat population) of the STASEY study final analysis

Table 2: Efficacy summary (intent-to-treat population) of the STASEY study final analysis

\begin{tabular}{|c|c|}
\hline Annualized bleed rate $(A B R)^{*}$ & $\begin{array}{c}\text { Emicizumab } \\
1.5 \mathrm{mg} / \mathrm{kg} / \mathrm{week} \\
\mathrm{N}=195\end{array}$ \\
\hline \multicolumn{2}{|l|}{ Treated bleeds $^{\dagger}$} \\
\hline Mean ABR, model based ${ }^{\ddagger}$ (95\% confidence interval [CI]) & $0.5(0.27-0.89)$ \\
\hline Median ABR, calculated (interquartile range [IQR]) & $0.0(0.00-0.00)$ \\
\hline People with HA (PwHA) with zero bleeds, $n(\%)$ & $161(82.6)$ \\
\hline \multicolumn{2}{|l|}{ All bleeds } \\
\hline Mean ABR, model based ${ }^{\ddagger}(95 \% \mathrm{Cl})$ & $1.1(0.80-1.47)$ \\
\hline Median ABR, calculated (IQR) & $0.0(0.00-1.01)$ \\
\hline PwHA with zero bleeds, $\mathrm{n}(\%)$ & $107(54.9)$ \\
\hline \multicolumn{2}{|l|}{ Treated spontaneous bleeds ${ }^{\dagger}$} \\
\hline Mean ABR, model based ${ }^{\ddagger}(95 \% \mathrm{Cl})$ & $0.3(0.15-0.73)$ \\
\hline Median ABR, calculated (IQR) & $0.0(0.00-0.00)$ \\
\hline PwHA with zero bleeds, $n(\%)$ & $174(89.2)$ \\
\hline \multicolumn{2}{|l|}{ Treated joint bleeds $^{\dagger \S}$} \\
\hline Mean ABR, model based ${ }^{\ddagger}(95 \% \mathrm{Cl})$ & $0.4(0.15-0.86)$ \\
\hline Median ABR, calculated (IQR) & $0.0(0.00-0.00)$ \\
\hline PwHA with zero bleeds, $\mathrm{n}(\%)$ & $176(90.3)$ \\
\hline \multicolumn{2}{|l|}{ Treated target joint bleeds ${ }^{\dagger \S \|}$} \\
\hline Mean ABR, model based ${ }^{\ddagger}(95 \% \mathrm{Cl})$ & $0.2(0.07-0.68)$ \\
\hline Median ABR, calculated (IQR) & $0.0(0.00-0.00)$ \\
\hline PwHA with zero bleeds, $\mathrm{n}(\%)$ & $183(93.8)$ \\
\hline \multicolumn{2}{|c|}{$\begin{array}{l}\text { "The Bleed and Medication Questionnaire was completed by participants/caregivers via an electronic } \\
\text { handheld device. Bleed definitions were based on International Society on Thrombosis and Haemostasis } \\
\text { criteria (Blanchette VS, et al., J Jhromb Haemost 2014;12:1935-9). } \\
\text { tTreated bleeds were defined as a bleed directly followed by a hemophilia medication reported as a } \\
\text { treatment for bleed, without an intervening bleed and irrespective of the time between the treatment and the } \\
\text { preceding bleed. If multiple bleeds occurred on the same calendar day, the subsequent treatment was } \\
\text { considered to apply to each of these multiple bleeds. Bleeds due to surgery/procedure were excluded. } \\
\text { tCalculated using the negative binomial regression method. } \\
\text { 5Joint bleeds are defined as bleeds with type reported at joint' in combination with } \geq 1 \text { of the following } \\
\text { symptoms: increased swelling or warmth of the skin over the joint; increasing pain; decreased range of } \\
\text { motion or difficulty in using the joint compared with baseline. } \\
\text { "Target joints were defined as major joints (e.g. hip, elbow, wrist, shoulder, knee, and ankle) in which } \geq 3 \\
\text { bleeding events occurred over a 24-week period. }\end{array}$} \\
\hline
\end{tabular}

PB0522 | Final Results of RelTIrate - A Prospective Study of Rescue Immune Tolerance Induction (ITI) with Recombinant Factor VIII Fc (rFVIIIFc) in Patients who Have Failed Previous ITI Attempts

C. Königs ${ }^{1}$; S. Meeks ${ }^{2}$; M. Löfqvist ${ }^{3} ;$ J. Dumont $^{4}$; L. Leickt ${ }^{3}$;

S. Nayak ${ }^{3}$; S. Lethagen ${ }^{3}$

${ }^{1}$ Department of Pediatrics and Adolescent Medicine, Frankfurt University Hospital, Frankfurt am Main, Germany; ${ }^{2}$ Aflac Cancer and Blood Disorders Center, Department of Pediatrics, Emory University School of Medicine and Children's Healthcare of Atlanta, Atlanta, United States; ${ }^{3}$ Swedish Orphan Biovitrum AB, Stockholm, Sweden; ${ }^{4}$ Sanofi, Cambridge, United States

Background: Immune tolerance induction (ITI) is the gold standard for inhibitor eradication in haemophilia A (HA). Non-clinical and clinical data indicate potential benefits of rFVIIIFC in ITI.

Aims: RelTIrate (NCT03103542) was designed to prospectively evaluate rescue ITI with rFVIIIFc in previous ITI failures.

Methods: RelTIrate, a prospective, interventional, multicentre, open-label study, enrolled severe HA inhibitor patients, who failed previous ITI attempts. Primary objective: ITI outcome with rFVIIIFc (200 IU/kg/day) within a 60-week time limit. Stringent criteria defined previous failures and ITI success. Anti-drug antibody (ADA) characteristics and immune cell responses were evaluated.

Results: The 16 enrolled patients had multiple risk factors for poor ITI outcome and long duration of previous ITI. Seven patients withdrew prematurely (due to adverse events [2], physician/patient decision [3], other [2]). Of 9 patients completing the ITI period, 4 reached confirmed negative inhibitor titre $(<0.6 \mathrm{BU} / \mathrm{mL})$ (figure 1) within 19 (11-60) weeks (median [range]). Three of these also reached an incremental recovery $>66 \%$ of expected, 2 of which reached a $t^{1} / 2>7$ hours ( 1 did not have all criteria simultaneously). One patient maintained all three success criteria and experienced no relapse. No unanticipated safety concerns were identified.

Flow cytometry immunophenotyping indicated an increase in regulatory T cells (CD4 ${ }^{+} \mathrm{CD} 25^{+} \mathrm{CD} 127^{\text {low) }}$ (figure 2 ). ADA titre decreased in 11 patients. IgG subclasses and epitope targeting were also characterised.
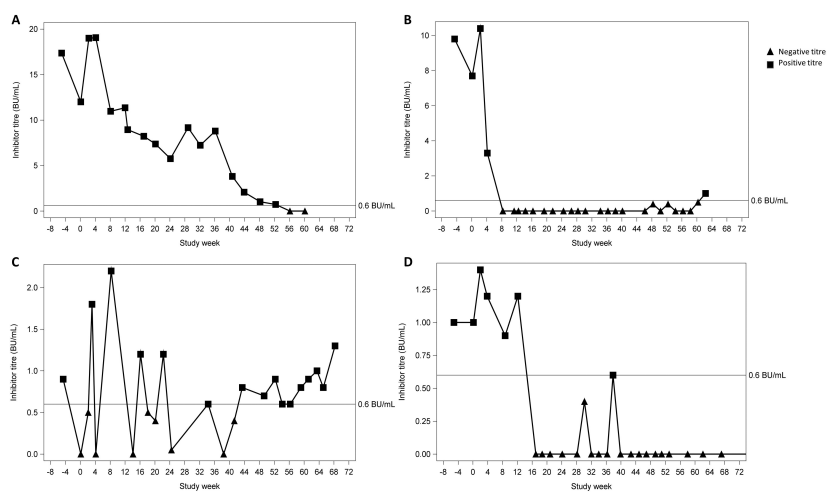

FIGURE 1 Inhibitor titres (local lab) in four patients achieving negative inhibitor titres (66\%; C: Negative inhibitor titre + IR $>66 \%$ $+t \frac{1}{2}>7 \mathrm{~h}$, but not simultaneously; $\mathrm{D}$ : Negative inhibitor titre + IR $>66 \%+t^{1} / 2>7 h=$ Complete ITI success. NB: the BU scale on the $\mathrm{Y}$-axis differs between subjects. BU, Bethesda units; ITI, Immune tolerance induction; IR, Incremental recovery

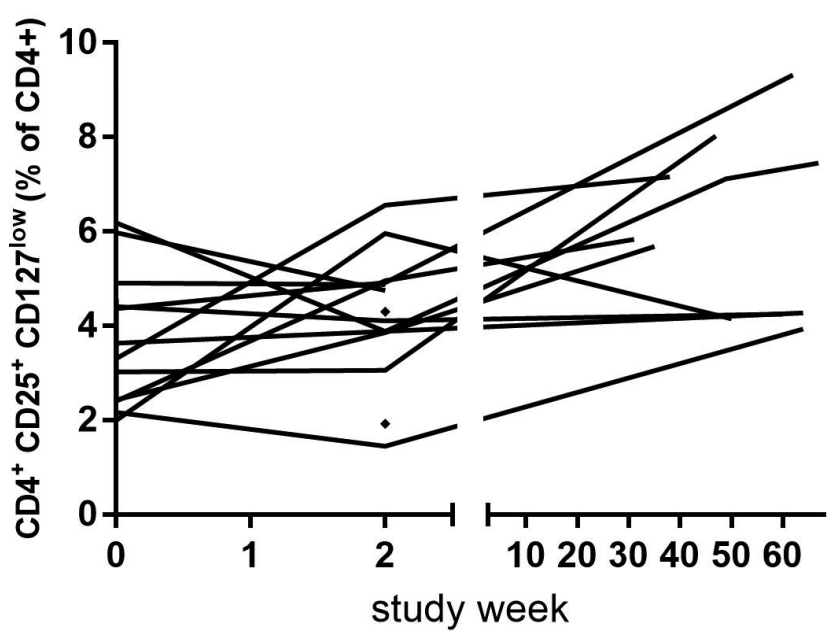

FIGURE 2 Regulatory T cells (\% CD4+CD25+CD127low of CD4+ T cells) in patients with available cell analysis data $(n=15)$.

Conclusions: RelTIrate is the first prospective study of ITI with an extended half-life FVIII product in previous ITI failures. ReITIrate evaluated rFVIIIFC ITI with an ambitious study design, within a limited time-period. Benchmarking with other studies is difficult, due to small study populations, differences in risk profiles, ITI intensity, duration and definition of previous failure and success criteria. Immunophenotype analysis supports the potential development of immune tolerance markers in some patients. The outcomes indicate a potential clinical benefit of rFVIIIFc for ITI in this difficult-to-treat population. 
PB0523 | Hemophilia Treatment in 2021: Choosing the 'Optimal' Treatment Using an Integrative, Patient-orientated Approach to Shared Decision-making between Patients and Clinicians

C. Hermans ${ }^{1}$; G. Benson ${ }^{2}$; G. Dolan ${ }^{3}$; H. Eichler ${ }^{4}$; V. JiménezYuste $^{5}$; C. Königs ${ }^{6}$; S. Lobet ${ }^{1}$; M.E. Mancuso ${ }^{7}$; D. Noone ${ }^{8}$; D. Pollard ${ }^{9}$; S. Zupančić Šalek ${ }^{10}$

${ }^{1}$ Haemostasis and Thrombosis Unit, Division of Haematology, Cliniques Universitaires Saint-Luc, Brussels, Belgium; ${ }^{2}$ Haemophilia and Thrombosis Centre, Belfast City Hospital, Belfast, Ireland; ${ }^{3}$ Centre for Haemostasis and Thrombosis, St Thomas' Hospital, London, United Kingdom; ${ }^{4}$ University and University Hospital of Saarland, Institute of Clinical Haemostaseology and Transfusion Medicine, Homburg (Saar), Germany; ${ }^{5}$ Hospital Universitario La Paz, Unidad de Coagulopatías, Servicio de Hematología, Autonoma University, Madrid, Spain; ${ }^{6}$ Klinische und Molekulare Hämostaseologie, Klinik für Kinder- und Jugendmedizin, Universitätsklinikum, Frankfurt, Germany; ${ }^{7}$ Center for Thrombosis and Hemorrhagic Diseases, Humanitas Clinical and Research Center - IRCCS, Rozzano, Milan, Italy; ${ }^{8}$ Irish Haemophilia Society, Dublin, Ireland; ${ }^{9}$ Katharine Dormandy Haemophilia \& Thrombosis Centre, Royal Free London NHS Foundation, London, United Kingdom, ${ }^{10}$ University Hospital Centre Zagreb, School of Medicine, University of Osijek and Medical School University of Zagreb, Zagreb, Croatia

Background: The mainstay of hemophilia treatment is to enhance hemostasis sufficiently to prevent bleeds typically through prophylaxis, the standard of care. Personalized treatment rather than "one-size-fits-all" protocols can result in better outcomes. With the current range of replacement and non-replacement treatment options, treatment decision-making is now a multifaceted process of identifying the most appropriate approach for each patient.

Aims: To develop practical algorithms for facilitating treatment decisions between clinicians and patients in a way that allows selection of the optimal approach to prevent bleeds.

Methods: A multidisciplinary expert panel (11 healthcare professionals from seven European countries) convened across three virtual meetings (September-October 2020) to discuss the practicalities of developing treatment algorithms for different patient groups. Developing the algorithm involved: agreement upon general principles to guide algorithm development; identify, refine and summarize variables that influence treatment choices in hemophilia; utility of an algorithm and patient tool to facilitate and guide treatment option choice that meet each patient's expectations, aspirations and priorities.

Results: The experts identified a list of key patient variables considered applicable to children, young adult, adult and elderly patients. An algorithm was developed using five identified objective variables: bleeding phenotype, musculoskeletal status, treatment adherence, venous access and lifestyle (Figure 1) aiming at improving patient care. A complementary patient-focused tool was also hypothesized, to help explore patients' expectations regarding their treatment. With this tool, the patient and clinician would independently rank each variable according to importance so that treatments would be 'weighted' against each variable, facilitating a shared decisionmaking process.

Conclusions: This algorithm and patient treatment tool should help select optimal treatment options. The chosen option should be as efficient as possible for bleeding prevention and take into account patients' clinical needs and expectations explored in a shared decision-making process for modern hemophilia management.

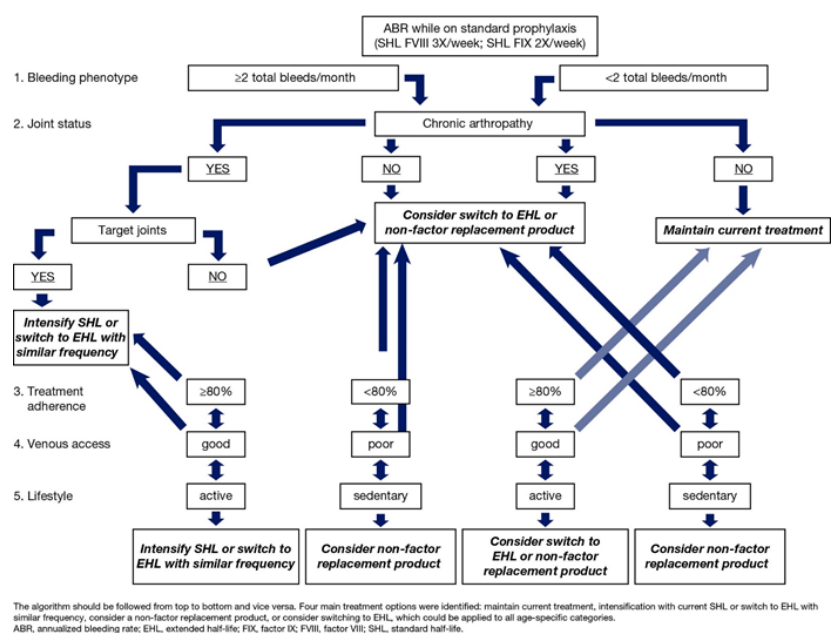

FIGURE 1 Algorithm based on objective clinical variables for guiding treatment decisions in hemophilia

PB0524 | Efficacy and Safety of rIX-FP: A Longitudinal Analysis of Patients Treated across the PROLONG-9FP Clinical Trials Program

I. Pabinger ${ }^{1}$; T. Lissitchkov ${ }^{2}$; A. Nagao ${ }^{3}$; L.M. Lepatan ${ }^{4}$;

Y. Li ${ }^{5}$; W. Seifert ${ }^{6}$; M.E. Mancuso 7,8

${ }^{1}$ Clinical Division of Haematology and Haemostaseology, Medical Clinic I, Medical University Vienna, Vienna, Austria; ${ }^{2}$ Department of Coagulation Disorders and Anemia, Specialized Hospital for Active Treatment Joan Pavel, Sofia, Bulgaria; ${ }^{3}$ Department of Hematology, Ogikubo Hospital, Tokyo, Japan; ${ }^{4}$ Perpetual Succour Hospital, Cebu, Philippines; ${ }^{5}$ CSL Behring, King of Prussia, United States; ${ }^{6}$ CSL Behring, Marburg, Germany; ${ }^{7}$ Fondazione IRCCS Ca' Granda, Ospedale Maggiore Policlinico, Milan, Italy; ${ }^{8}$ Center for Thrombosis and Hemorrhagic Diseases, Humanitas Clinical and Research Center - IRCCS, Milan, Italy

Background: Regular prophylactic replacement with factor IX (FIX) is the recommended treatment for patients with severe hemophilia B. rIX-FP, a fusion protein linking recombinant FIX with albumin, has been shown to be efficacious and well tolerated with prolonged dosing intervals of up to 21 days.

Aims: Assess the long-term clinical outcomes in previously-treated patients (PTPs) treated with rIX-FP. 
Methods: Data from PTPs participating across four studies in the PROLONG-9FP clinical program were included in an integrated analysis to evaluate the long-term efficacy and safety of rIX-FP. Dosing intervals were up to 14 days $(50-75 \mathrm{lU} / \mathrm{kg}$ ) if well-controlled, with no spontaneous bleeding in the previous 4 weeks, on the 7-day regimen (25-50 IU/kg); patients $\geq 18$ years could switch to a 21 -day regimen $(100 \mathrm{lU} / \mathrm{kg})$ if well-controlled on a 14-day regimen. Patients were included in analyses across multiple dose cohorts if they received $\geq 12$ weeks treatment.

Results: Ninety-nine PTPs participated across four PROLONG-9FP clinical studies. Patients receiving a 7- $(n=92), 10-(n=24), 14-$ $(n=51)$, or 21-day $(n=11)$ dosing regimen received treatment for a mean (SD) of 726.0 (543.4), 765.5 (516.9), 821.5 (529.8) and 654.5 (333.78) days, respectively; bleeding rates are shown in Table 1. Median (Q1, Q3) annualized bleeding rates were 1.57 (0.00, 4.46), $1.37(0.40,4.97), 1.61(0.30,4.24)$ and $0.32(0.00,2.48)$ for patients treated with a 7-, 10-, 14-, or 21-day regimen, respectively. Mean (SD) age was 25.6 (17.11); $27.3 \%$ were pediatric patients $(0-<12$ years), $8.1 \%$ were adolescents ( $\geq 12-<18$ years), and $64.6 \%$ were adults ( $\geq 18-65$ years). No safety concerns were observed. dosed according to label, clinicians are obligated to discard or overdose medication due to discrepancies between calculated dose and vial content

Aims: The aim of this study was to compose a cost-efficient emicizumab maintenance dosing regimen using Monte Carlo simulation based on vial size, patient friendly intervals and patient characteristics such as bodyweight, while striving for similar plasma concentrations as observed in clinical trials.

Methods: Monte Carlo simulations were used to investigate alternative dosing regimens in patients weighing 3 to $150 \mathrm{~kg}$. Tested dosing regimens were based on bodyweight, vial content, injection volume, and dosing interval. Simulated regimens were targeted to achieve median emicizumab plasma concentrations at steady state $\left(\mathrm{C}_{\mathrm{av}, \mathrm{ss}}\right)$ of 40-60 (90\% range: $25-95) \mu \mathrm{g} / \mathrm{ml}$. The efficiency of the alternative dosing regimens was calculated in mg emicizumab saved and costs saved per patient per year.

Results: In Table 1, a summary of the soon to be published full dosing scheme for children and adults is depicted. This proposed alternative dosing regimen achieved similar target emicizumab $\mathrm{C}_{\mathrm{av}, \mathrm{ss}}$ levels compared to the registered weekly maintenance dosing of 1.5

TABLE 1 Bleeding rates across the PROLONG 9-FP clinical program

\begin{tabular}{lllll} 
Regimen & 7 day & 10 day & 14 day & 21 day \\
N & 92 & 24 & 51 & 11 \\
AsBR, median (Q1, Q3) & $0.00(0.00,1.36)$ & $0.13(0.00,1.46)$ & $0.29(0.00,1.88)$ & $0.00(0.00,0.45)$ \\
ABR, median (Q1, Q3) & $1.57(0.00,4.46)$ & $1.37(0.40,4.97)$ & $1.61(0.30,4.24)$ & $0.32(0.00,2.48)$ \\
Monthly infusions, mean (SD) & $4.3(0.2)$ & $3.0(0.2)$ & $2.2(0.1)$ & $1.5(0.0)$ \\
Dose per infusion, mean (SD) & $48.8(11.5)$ & $70.8(8.3)$ & $73.1(7.5)$ & $100.6(3.6)$ \\
\hline
\end{tabular}

$A B R$, annualized bleeding rate; AsBR, annualized spontaneous bleeding rate.

Conclusions: Treatment with rIX-FP was associated with low bleeding rates; no safety concerns were identified. Extended dosing regimens with rIX-FP may be suitable for selected patients provided they are well-controlled on a shorter rIX-FP dosing interval.

PB0525 | Emicizumab Dosing in Children and Adults with Hemophilia A: Simulating a User-friendly and Cost-efficient Regimen

L.H. Bukkems ${ }^{1}$; K. Fischer ${ }^{2}$; I. Kremer-Hovinga ${ }^{2}$; A.A. Donners ${ }^{3}$; K. Fijnvandraat ${ }^{4}$; R.E. Schutgens ${ }^{2}$; M.H. Cnossen ${ }^{5}$; R.A. Mathôt ${ }^{1}$

${ }^{1}$ Amsterdam University Medical Centers, Amsterdam, Netherlands;

${ }^{2}$ Van Creveldkliniek, University Medical Center Utrecht, University Utrecht, Utrecht, Netherlands; ${ }^{3}$ University Medical Center Utrecht, Utrecht, Netherlands; ${ }^{4}$ Amsterdam UMC, University of Amsterdam, Emma Children's Hospital, Amsterdam, Netherlands; ${ }^{5}$ Erasmus University Medical Center - Sophia Children's Hospital Rotterdam, Rotterdam, Netherlands

Background: Emicizumab is a humanized bispecific monoclonal antibody used for the treatment of hemophilia A. When emicizumab is $\mathrm{mg} / \mathrm{kg}$ with a median deviation of less than $2 \mu \mathrm{g} / \mathrm{mL}$ in $78 \%$ of the bodyweight categories. Compared to dosing $1.5 \mathrm{mg} / \mathrm{kg}$ every week, alternative dosing saved $€ 59,058$ (range: $€ 0$ - €118,114) per year in children weighing between 12 and $55 \mathrm{~kg}$. For adults weighing 56-95 kg median cost savings were $€ 59,058$ (range: $€ 0-€ 88,586$ ). When the alternative dosing regimen is applied, costs are saved in 53 $60 \%$ of the patients (Figure 1 ). 
TABLE 1 Summary of alternative emicizumab maintenance dosing regimen and resulting average steady state emicizumab levels per bodyweight category based on Monte Carlo simulations while striving for plasma concentrations as observed in HAVEN trials. The simulated results are compared to the registered maintenance dosing scheme of $1.5 \mathrm{mg} / \mathrm{kg}$ per week in steady state.

\begin{tabular}{lllll}
$\begin{array}{l}\text { Bodyweight } \\
(\mathrm{kg})\end{array}$ & Alternative maintenance dose & $\begin{array}{l}\text { Injection } \\
\text { volume }(\mathrm{ml})\end{array}$ & $\begin{array}{l}\text { Median average emicizumab level } \\
- \text { alternative dose }(\mu \mathrm{g} / \mathrm{mL})(90 \% \\
\text { range })\end{array}$ & $\begin{array}{l}\text { Median average emicizumab } \\
\text { level - } 1.5 \mathrm{mg} / \mathrm{kg} \text { every week } \\
(\mu \mathrm{g} / \mathrm{mL})(90 \% \mathrm{range})\end{array}$ \\
$12-16$ & $60 \mathrm{mg}$ every 3 weeks & 0.4 & $44.2(26.8-72.8)$ & $46.9(29.0-75.9)$ \\
$17-25$ & $60 \mathrm{mg}$ every 2 weeks & 0.4 & $45.9(27.6-76.5)$ & $49.0(30.4-78.9)$ \\
$26-35$ & $90 \mathrm{mg}$ every 2 weeks & 1.4 & $48.1(28.7-79.7)$ & $50.0(30.6-81.0)$ \\
$36-45$ & $120 \mathrm{mg}$ every 2 weeks & 0.8 & $49.5(29.5-81.6)$ & $50.3(30.3-82.1)$ \\
$46-55$ & $150 \mathrm{mg}$ every 2 weeks & 1.0 & $50.0(29.8-82.6)$ & $50.6(30.4-83.1)$ \\
$56-65$ & $90 \mathrm{mg}$ every week & 1.4 & $50.5(30.0-83.5)$ & $51.0(30.5-83.8)$ \\
$66-75$ & $105 \mathrm{mg}$ every week & 0.7 & $50.9(30.3-84.3)$ & $51.3(30.6-84.5)$ \\
$76-85$ & $120 \mathrm{mg}$ every week & 0.8 & $51.4(30.6-85.1)$ & $51.6(30.8-85.2)$ \\
$86-95$ & $135 \mathrm{mg}$ every week & 1.7 & $51.8(30.8-85.7)$ & $51.9(30.9-85.7)$ \\
\hline
\end{tabular}

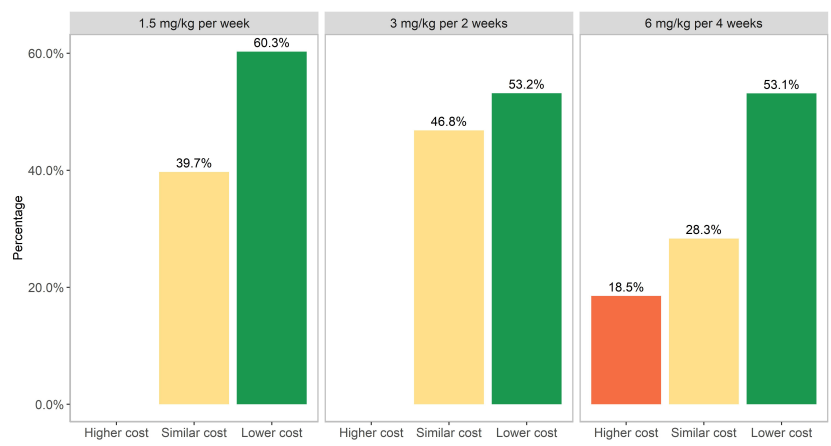

FIGURE 1 Percentage of patients in the virtual population (3-150 $\mathrm{kg}$ ) in which the alternative dose dosing scheme will result in lower, similar expense or higher costs compared to the registered dosing schemes $(1.5 \mathrm{mg} / \mathrm{kg}$ week, $3 \mathrm{mg} / \mathrm{kg}$ per week or $6 \mathrm{mg} / \mathrm{kg}$ per week). The cost savings depend on the bodyweight of the patient and registered dosing scheme to which the alternative dosing scheme is compared.

Conclusions: This alternative maintenance dosing scheme - applicable in hemophilia A patients on emicizumab prophylaxis - reduces financial costs, avoids medication spillage and is patient friendly without loss of efficacy.

PB0526 | Fitusiran Population Pharmacokinetic and Pharmacodynamic (PopPK/PD) Modeling to Support Revised Dose, Dosing Regimens \& Dose Mitigation Scheme

P. Bhagunde ${ }^{1}$; S. Ge ${ }^{1}$; S. Iqbal' ${ }^{2}$; B. Mei ${ }^{2}$; S. Andersson ${ }^{2}$; V. Kanamaluru' ${ }^{1}$; Q. Lu ${ }^{1}$

${ }^{1}$ Sanofi, Bridgewater, United States; ${ }^{2}$ Sanofi, Cambridge, United States

Background: Fitusiran is an investigational, subcutaneously administered, small interference RNA therapeutic that targets antithrombin
(AT) and restores thrombin generation sufficient to rebalance hemostasis in people with hemophilia A/B, with/without inhibitors.

Aims: This analysis aimed to characterize the AT dynamics by PopPK/PD model and predict dosing regimens to mitigate the risk of vascular thrombotic events.

Methods: AT activity data from phase 1 (NCT02035605) and phase 2 (NCT02554773) studies in healthy subjects and people with hemophilia A or B, with or without inhibitors were used to develop the PopPK/PD model. The model was externally validated using AT activity data from phase 3 studies (NCT0341710, NCT03417245 and NCT03549871). The PopPK/PD model was further used to simulate AT activity at various dosing regimens in 1000 virtual patients.

Results: The PopPK/PD dataset included 1752 AT activity observations from 45 subjects. A modified indirect response (Figure 1) PopPK/PD model described the dynamics and variability in AT activity reasonably well. Simulations showed that with starting dose of 50 mg Q2M, >90\% patients ( $10^{\text {th }}$ percentile trough-AT activity $=15.3 \%$ ) would achieve AT activity $>15 \%$; the median peak AT activity is projected to be $32.4 \%$, indicating that $~ 50 \%$ patients may have AT activity $>35 \%$. For these patients, a dose escalation to $50 \mathrm{mg}$ QM would be required to achieve AT activity $<35 \%$. Amongst patients switching to $50 \mathrm{mg}$ QM regimen, if any patients still have peak AT activity $>35 \%$, another escalation to $80 \mathrm{mg}$ QM is recommended.

Conclusions: The AT activity profile and inter-patient variability were well characterized by PopPK/PD model. Simulations using this model supported the thrombosis risk mitigation scheme to start with a subcutaneous dose of fitusiran at $50 \mathrm{mg}$ Q2M, if needed escalate to $50 \mathrm{mg}$ QM, and in limited cases, to further escalate to $80 \mathrm{mg}$ QM, based on AT levels. 


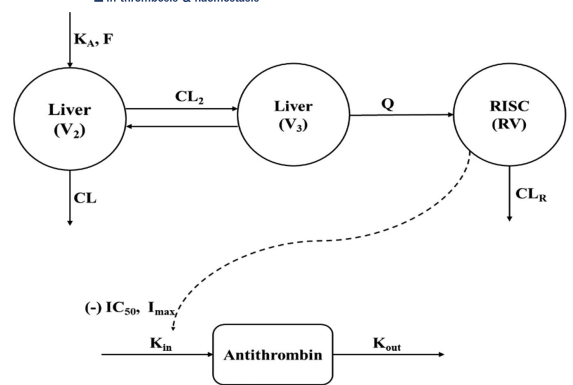

Parameters: $\mathrm{K}_{\mathrm{A}}=$ absorption rate; $\mathrm{F}=$ bioavailability; $\mathrm{V}_{2}=$ volume of liver central compartment; $\mathrm{V}_{3}=$ volume of liver peripheral compartment; $\mathrm{CL}=$ clearance from liver central compartment; $\mathrm{CL}_{2}=$ intercompartmental clearance; $\mathrm{Q}=$ clearance into RISC compartment; $\mathrm{RV}=\mathrm{RISC}$ volume; $\mathrm{CL}_{\mathrm{R}}=$ clearance out of RISC compartment; $K_{\text {in }}=$ AT production rate; $K_{\text {out }}=$ AT elimination rate; $I_{\max }=$ maximum inhibition of AT; IC $5_{00}=$ RISC concentration for $50 \%$ of maximum effect

FIGURE 1 Schematic of the PopPK/PD model

PB0527 | Postoperative Bleeding Complications in Patients with Hemophilia Undergoing Major Orthopedic Surgery: A Prospective, Multicenter Observational Study

T.W. Buckner $^{1}$; B. Kleiboer ${ }^{2}$; M. Layer ${ }^{2}$; L. Cafuir ${ }^{3}$; A. Cuker ${ }^{4}$; M. Escobar ${ }^{5}$; M.E. Eyster ${ }^{6}$; E. Kraut ${ }^{7}$; A.D. Leavitt ${ }^{8}$; S.R. Lentz ${ }^{9}$; D. Quon ${ }^{10}$; M. Ragni ${ }^{11}$; D. Thornhill ${ }^{1}$; M. Wang ${ }^{1}$; N.S. Key ${ }^{2}$

${ }^{1}$ University of Colorado School of Medicine, Aurora, United States;

${ }^{2}$ University of North Carolina School of Medicine, Chapel Hill, United States; ${ }^{3}$ Emory University School of Medicine, Atlanta, United States; ${ }^{4}$ Perelman School of Medicine, University of Pennsylvania, Philadelphia, United States; ${ }^{5}$ McGovern Medical School, University of Texas Health Science Center at Houston, Houston, United States; ${ }^{6}$ Penn State Hershey Medical Center, Hershey, United States; ${ }^{7}$ The Ohio State University Wexner Medical Center, Columbus, United States; ${ }^{8}$ University of California, San Francisco School of Medicine, San Francisco, United States; ${ }^{9}$ The University of lowa Carver College of Medicine, lowa City, United States, ${ }^{10}$ Orthopaedic Hemophilia Treatment Center, Orthopaedic Institute for Children, Los Angeles, United States, ${ }^{11}$ University of Pittsburgh Medical Center, Pittsburgh, United States

Background: Chronic hemophilic arthropathy develops in persons with hemophilia (PWH) after recurrent intra-articular bleeding. Joint replacement surgery may provide relief from intractable pain and improve quality of life in PWH who have end-stage arthropathy.

Aims: To determine the risk of bleeding during the immediate postoperative hospitalization period following total hip (THA) and total knee arthroplasty (TKA).

Methods: We conducted a multicenter, prospective, observational cohort study at 14 Hemophilia Treatment Centers (HTCs) in the United States. We included subjects with hemophilia A or B of all severities undergoing THA or TKA who were at least 18 years old. All clinical treatment decisions were made per local standards of care. All subjects provided informed consent. Clinical information describing treatments, evaluations, procedures, and outcomes were abstracted from medical records in real time. We defined major bleeding as that which occurred in a critical site, resulted in a decrease in hemoglobin of $\geq 2 \mathrm{~g} / \mathrm{dL}$ over any 24-hour period, resulted in transfusion of $\geq 2$ units of packed red blood cells ( $p$ RBC) during hospitalization, or led to death. The impact of clinical factors on the likelihood of bleeding was examined using univariate logistic regression analyses.

Results:

Figure 1: Major bleeding events ${ }^{*}$ during hospitalization following 131 total hip and knee arthroplasty procedures in men with hemophilia $A$ or $B$.

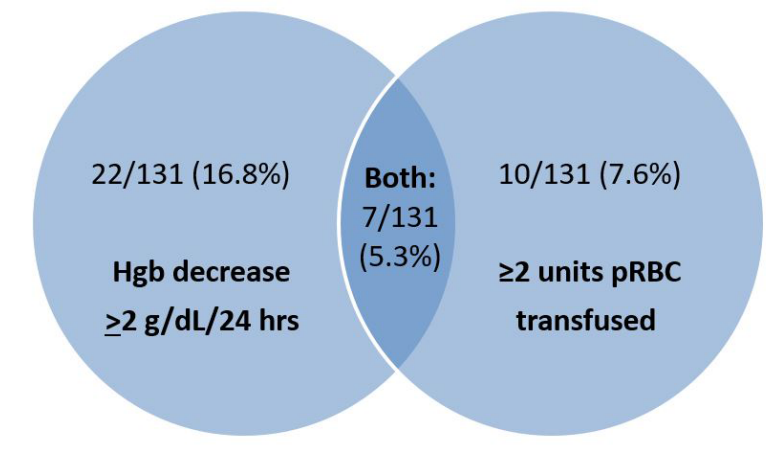

*There were zero bleeds into critical sites and no fatal bleeding in the study.

FIGURE 1 Major bleeding events during hospitalization following 131 total hip and knee arthroplasty procedures in men with hemophilia A or B.

TABLE 1 Relationship of clinical factors potentially related to bleeding risk in men with hemophilia A or B during hospitalization following THA or TKA: univariate logistic regression.

\section{Variable}

Procedure Type

Active Inhibitor*

Clotting Factor Administration Type
Number with Major Bleeding, $n /$ total (\%)

$\begin{array}{ll}\text { THA: 15/33 (45.5) } & \text { TKA: 24/98 (24.5) } \\ \text { Present: 6/12 (50) } & \text { Not Present: 33/119 (27.7) } \\ \text { Continuous Infusion: } & \text { Bolus Only: 24/75 (32) }\end{array}$
$13 / 46$ (28.3)
Odds Ratio (variable in second column is referent value)

95\% Confidence Interval $P$ vlaue

$1.10-5.85$ 0.03

2.44

$0.73-8.13$ 0.15 1.20

$0.53-2.67$ 0.67 


\begin{tabular}{|c|c|c|c|c|c|}
\hline Variable & \multicolumn{2}{|c|}{ Number with Major Bleeding, $n /$ total (\%) } & \multirow{2}{*}{$\begin{array}{l}\text { Odds Ratio (variable } \\
\text { in second column is } \\
\text { referent value) } \\
0.48\end{array}$} & \multirow{2}{*}{$\begin{array}{l}95 \% \text { Confidence } \\
\text { Interval } \\
0.20-1.18\end{array}$} & \multirow{2}{*}{$\begin{array}{l}P \text { vlaue } \\
0.11\end{array}$} \\
\hline $\begin{array}{l}\text { Use of Antifibrinolytic } \\
\text { Medication }\end{array}$ & AF used: 8/38 (21.1) & AF not used: $31 / 93$ (33.3) & & & \\
\hline $\begin{array}{l}\text { Use of Pharmacologic } \\
\text { Thromboprophylaxis }\end{array}$ & $\begin{array}{l}\text { Yes (LMWH): 2/7 } \\
(28.6)\end{array}$ & No: $37 / 124$ (29.8) & 0.88 & $0.16-4.72$ & 0.88 \\
\hline
\end{tabular}

* Active inhibitor defined as use of bypass agent as primary hemostatic medication during / after procedure.

THA=total hip arthroplasty; TKA=total knee arthroplasty; $\mathrm{AF}=$ antifibrinolytic; $\mathrm{LMWH=low}$ molecular weight heparin

131 procedures (98 TKA and 33 THA) were performed. Of these, 39 (29.8\%) were associated with major bleeding, including $46 \%$ of those undergoing THA and 25\% TKA. Half of those with active inhibitors (6 of 12) experienced a major bleed. Neither use of continuous clotting factor infusion nor pharmacologic thromboprophylaxis was associated with differences in bleeding risk. Use of concomitant antifibrinolytic medication was associated with a non-statistically significant reduction in bleeding rate from $33 \%$ to $21 \%$ of procedures.

Conclusions: The risk of bleeding during the immediate postoperative period after THA and TKA is significant, despite factor replacement. These data suggest the need for standard guidelines for postoperative hemostasis in $\mathrm{PWH}$ undergoing these procedures.

PB0528 | Assessment of the Bleeding Phenotype in Patients with Non-severe Hemophilia According to the ISTH Bleeding Assessment Tool (BAT)

F. Kloosterman ${ }^{1}$; A. Zwagemaker ${ }^{1}$; S.C. Gouw ${ }^{1,2}$; C.N. Bagot ${ }^{3}$; P. Brons ${ }^{4}$; M. Cnossen ${ }^{5}$; P. Collins ${ }^{6}$; S. Jackson ${ }^{7}$; K. Meijer ${ }^{8}$; M.J.H.A. Kruip ${ }^{9}$; F.W.G. Leebeek ${ }^{9}$; B. Laros-van Gorkom ${ }^{10}$; C. Male ${ }^{11}$; I. Pabinger ${ }^{12}$; S. Shapiro ${ }^{13}$; M. Coppens ${ }^{14}$; J.G. van der Bom $^{15} ;$ K. Fijnvandraat ${ }^{1,16}$, DYNAMO Study Group

${ }^{1}$ Amsterdam UMC, University of Amsterdam, Emma Children's Hospital, Pediatric Hematology, Amsterdam, Netherlands; ${ }^{2}$ Department of Clinical Epidemiology, Leiden University Medical Center, Leiden, Netherlands; ${ }^{3}$ Department of Haematology, Glasgow Royal Infirmary, Glasgow, United Kingdom; ${ }^{4}$ Department of Pediatric Hemato-Oncology, Radboud University Medical Center, Nijmegen, Netherlands; ${ }^{5}$ Department of Pediatric Hematology, Erasmus University Medical Center - Sophia Children's Hospital, Rotterdam, Netherlands; ${ }^{6}$ Arthur Bloom Haemophilia Centre, University Hospital of Wales School of Medicine, Cardiff, United Kingdom; ${ }^{7}$ Inherited Bleeding Disorders Program of BC - Adult Division St. Paul's Hospital, Vancouver, Canada; ${ }^{8}$ Department of Hematology, University Medical Center Groningen, Groningen, Netherlands; ${ }^{9}$ Department of Hematology, Erasmus MC, Erasmus University Medical Center, Rotterdam, Netherlands, ${ }^{10}$ Department of Hematology, Radboud University Medical Center, Nijmegen, Netherlands, ${ }^{11}$ Department of Pediatrics, Medical University of Vienna, Vienna, Austria, ${ }^{12}$ Clinical Division of Hematology and Hemostaseology, Medical University of Vienna, Vienna, Austria, ${ }^{13}$ Department of Haematology, Churchill Hospital, Oxford University Hospitals NHS Foundation, Oxford, United Kingdom, ${ }^{14}$ Department of Vascular Medicine, Amsterdam University Medical Centres, Amsterdam, Netherlands, ${ }^{15}$ Center for Clinical Transfusion Research, Sanquin-Leiden University Medical Center, Leiden, Netherlands, ${ }^{16}$ Department of Molecular Cellular Hemostasis, Sanquin Research and Landsteiner Laboratory, Amsterdam, Netherlands
Background: The International Society of Thrombosis and Haemostasis Bleeding Assessment Tool (ISTH-BAT) was developed to objectively quantify bleeding symptoms in patients with a bleeding disorder. Quantitative data on of the bleeding phenotype in patients with non-severe hemophilia is currently lacking. The use of the ISTH-BAT may provide insight into the extent of reported bleeding in this population.

Aims: To describe the bleeding phenotype in patients with nonsevere hemophilia according to the ISTH-BAT.

Methods: The DYNAMO study is a multicenter international cohort study that includes patients with non-severe hemophilia $A$ and $B$ (baseline FVIII/FIX 2-35 IU/dL) aged 12-55 years. Data were collected from medical files and participants completed questionnaires including the ISTH-BAT. ISTH-BAT scores were compared between factor levels, severities and hemophilia type. Abnormal scores were defined as $\geq 4$. Medical ethical approval and written consent was obtained (ClinicalTrials.gov: NCT03623295).

Results: We report preliminary data on 106 patients (89 HA, $17 \mathrm{HB}$ ) who completed the questionnaire (response rate $60 \%$ ). The median age was 38 years (IQR 25-50) and the median factor level was $11 \mathrm{IU} /$ $\mathrm{dL}$ (IQR 5-16). The median ISTH-BAT score in our population was 9 (IQR 4-15), with $82 \%$ of our population reporting an abnormal bleeding score. Patients with moderate and mild hemophilia had median ISTH-BAT scores of 13 (IQR 9-16) and 7 (IQR 4-13) respectively. An inverse association was observed between the ISTH-BAT scores and the baseline factor level (Figure 1). 


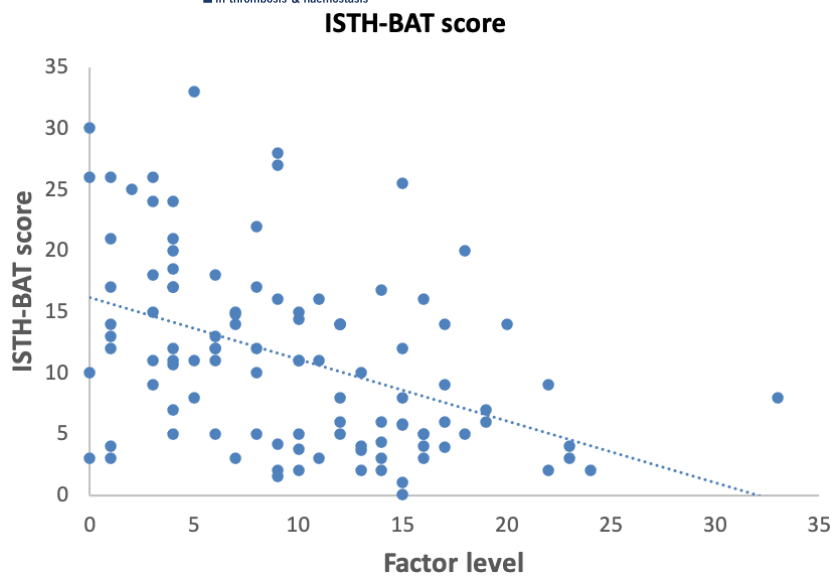

FIGURE 1 Scatterplot of the ISTH-BAT score against the factor level with a line of best fit shown. Spearman's coefficient and $P$ values are presented

ISTH-BAT scores were similar between hemophilia A and B. Joint bleeds, muscle bleeds and bruises were scored most frequently: $59 \%$, $63 \%$ and $60 \%$ respectively (Figure 2), with $54 \%$ and $49 \%$ of patients reporting sub-scores $\geq 3$ for joint and muscle bleeds respectively.

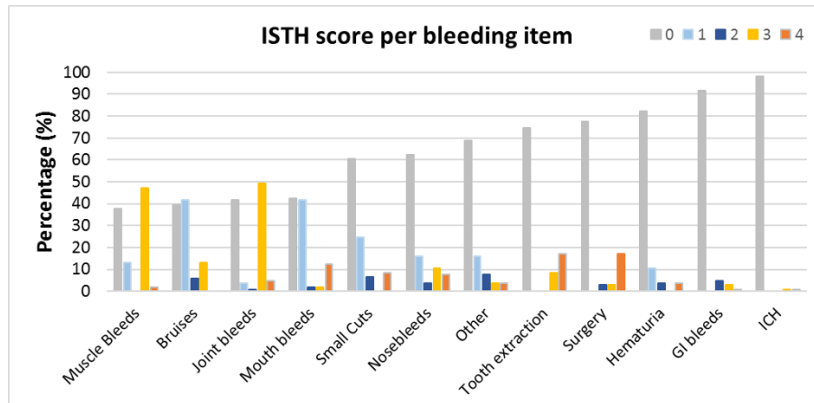

FIGURE 2 ISTH sub-scores per bleeding item, ranging from 0-4 ( 0 = no bleeding 4 = bleeding requiring intensive treatment)

Conclusions: Abnormal bleeding scores were seen in $82 \%$ of the patients, and the median ISTH-BAT score of 9, demonstrating a considerable burden of disease in patients with non-severe hemophilia.

PB0529 | Changes in Treatment Patterns in Patients with Severe Haemophilia - a Longitudinal Analysis of the Austrian Haemophilia Registry

D. Kraemmer ${ }^{1}$; V. Ritschl ${ }^{2}$; J. Rejtö후 C. Feistritzer ${ }^{3}$; S. Gallistl ${ }^{4}$; S. Jäger ${ }^{5}$; N. Jones ${ }^{6}$; A. Kurringer ${ }^{7}$; P. Neumeister ${ }^{8} ;$ J. Rettl'; G. Schuster ${ }^{10} ;$ R. Schwarz ${ }^{11}$; M. Sohm ${ }^{12}$; W. Streif ${ }^{13} ;$ T. Schindl ${ }^{14}$; T. Stamm ${ }^{2}$; K. Thom ${ }^{15}$; C. Male ${ }^{15}$; I. Pabinger ${ }^{1}$; C. Ay ${ }^{1}$

${ }^{1}$ Clinical Division of Haematology and Haemostaseology, Department of Medicine I, Medical University of Vienna, Vienna, Austria; ${ }^{2}$ Section for Outcomes Research, Center for Medical Statistics, Informatics, and Intelligent Systems, Medical University of Vienna, Vienna, Austria;
${ }^{3}$ Department of Internal Medicine - Haematology and Oncology, Medical University of Innsbruck, Innsbruck, Austria; ${ }^{4}$ Department of General Paediatrics and Adolescent Medicine, Medical University of Graz, Graz, Austria; ${ }^{5}$ Third Medical Department, Paracelsus Medical University, Salzburg, Austria; ' ${ }^{6}$ Department of Paediatrics, Paracelsus Medical University, Salzburg, Austria; ${ }^{7}$ Department of Paediatrics, LKH Bregenz, Bregenz, Austria; ${ }^{8}$ Division of Haematology, Department of Internal Medicine, Graz, Austria; ${ }^{9}$ Klinikum Klagenfurt am Wörthersee, Klagenfurt, Austria, ${ }^{10}$ Austrian Red Cross, Blood Transfusion Service for Upper Austria, Linz, Austria, ${ }^{11}$ Department of Paediatrics and Adolescent Medicine, Kepler University Hospital, Sankt Pölten, Austria, ${ }^{12}$ Department of Paediatrics, Universitätsklinikum Sankt Pölten, Sankt Pölten, Austria, ${ }^{13}$ Department of Paediatrics, Medical University of Innsbruck, Innsbruck, Austria, ${ }^{14}$ Austrian Haemophilia Society, Vienna, Austria, ${ }^{15}$ Department of Paediatrics, Medical University of Vienna, Vienna, Austria

Background: The last decade has seen significant advances in treatment options of haemophilia.

Aims: The Austrian Haemophilia Registry is a nationwide registry collecting data on all patients with haemophilia cared for in its participating centres. Here, we investigated how treatment of severe haemophilia has changed over the last decade in Austria.

Methods: We included all patients with severe haemophilia recorded between 2009 and 2019 (338). We excluded those with positive inhibitors $(50,14.8 \%)$, immune tolerance induction therapy (34, $10.1 \%)$, or receiving inhibitor bypassing agents $(21,6.2 \%)$. The final study cohort consisted of 278 patients (82.2\%). Loss to follow-up was defined as no visit in the last 5 years.

Results: Patients with severe haemophilia (mean age, 24.3 years [ \pm 16.8 years]; $92.1 \%$ haemophilia $A ; 7.9 \%$ haemophilia $B$ ) were followed for a median observation period of 9.2 years $\left(25^{\text {th }}-75^{\text {th }}\right.$ percentile, 5.0-10.5 years; total observation time, 2,514.9 years) during which 37 (13.3\%) patients died or were lost to follow-up. The proportion of patients on prophylaxis increased from initially $72.1 \%$ to $85.0 \%$ (Figure 1A). Following marketing authorisation, the end of the decade saw a sharp increase in treatment with extended half-life products (35.7\%), whereas the use of plasma-derived (pd) and recombinant standard half-life products declined to their lowest levels in 2019 (15.4\% and 45.1\%, respectively) (Figure 1B). In patients with haemophilia A aged $<18$ years, however, an initially decreasing usage of pd products reversed again after 2015. In fact, in this age group, we recorded the highest rate of treatment with pd products in the two very last years (24.0\% and $20.5 \%$, respectively) (Figure 2 ), possibly reflecting the impact of the SIPPET study on the initial treatment strategy for children with haemophilia. 


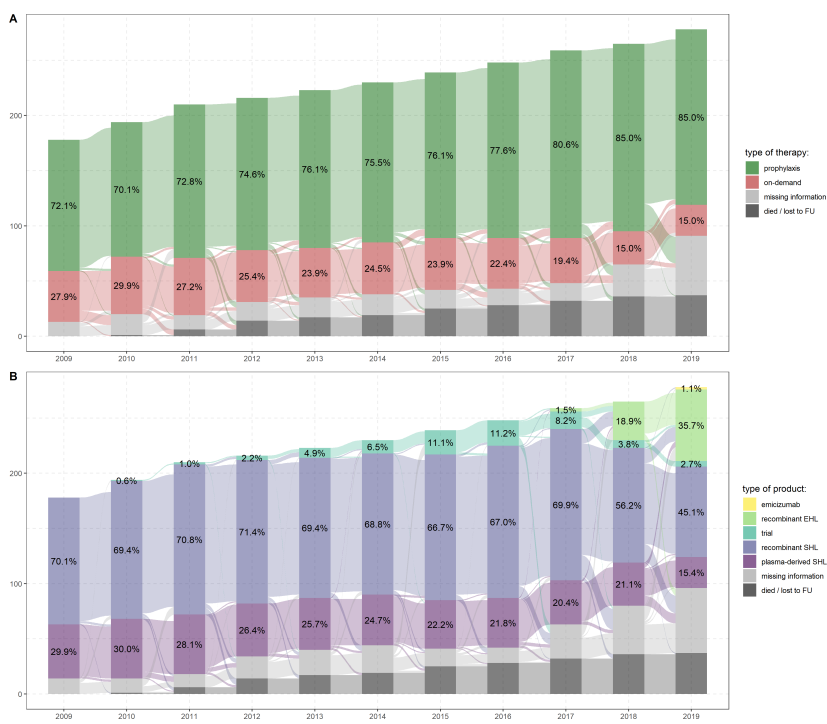

FIGURE 1 Changes in treatment regimens (A) and types of products used (B) in patients with severe haemophilia. Depicted percentages restricted to patients for whom information on therapy was available in the respective year. Missing information denotes missing registry visits where information on therapy regimen or type of product used could not be derived from a later visit. A majority of patients participating in clinical trials, denoted as type of product "trial", received EHL products (28, 87.5\%). EHL: extended half-life; FU: follow-up; SHL: standard half-life

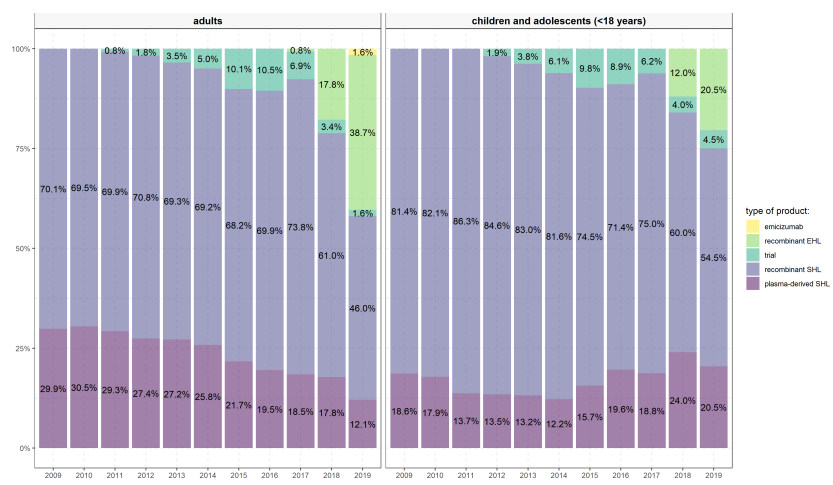

FIGURE 2 Percentages of types of products used per year in patients with severe haemophilia A above and below 18 years. Depicted percentages refer to patients for whom information on therapy was available in respective year. A majority of patients participating in clinical trials, denoted as type of product "trial", received EHL products (28, $87.5 \%)$. EHL: extended half-life; SHL: standard half-life

Conclusions: Our study provides an overview of changes in haemophilia therapy in Austria over the recent decade, reflecting significant advances in therapeutic options.
PB0530 | Adoption of Emicizumab for Hemophilia A in Europe: Data from the 2020 European Association for Haemophilia and Allied Disorders Survey

E. Krumb $^{1}$; K. Fijnvandraat ${ }^{2}$; M. Makris ${ }^{3}$; F. Peyvandi ${ }^{4,5}$; A. Ryan ${ }^{6}$; A. Athanasopoulos ${ }^{6}$; C. Hermans ${ }^{1}$

${ }^{1}$ Hemostasis and Thrombosis Unit, Division of Hematology, Cliniques Universitaires Saint-Luc, Université Catholique de Louvain, Brussels, Belgium; ${ }^{2}$ Amsterdam UMC, Emma Children's Hospital, University of Amsterdam, Amsterdam, Netherlands; ${ }^{3}$ Sheffield Haemophilia and Thrombosis Centre, University of Sheffield, Sheffield, United Kingdom; ${ }^{4}$ Angelo Bianchi Bonomi Hemophilia and Thrombosis Center, IRCCS Fondazione $\mathrm{Ca}$, Granda Ospedale Maggiore Policlinico, Milan, Italy; ${ }^{5}$ Department of Pathophysiology and Transplantation, University of Milan, Milan, Italy; ${ }^{6}$ European Association for Haemophilia and Allied Disorders (EAHAD), Brussels, Belgium

Background: Emicizumab is a bispecific monoclonal antibody, mimicking coagulation factor (F) VIII by linking factor IXa and X and thereby restoring steady-state hemostasis in patients with hemophilia A. It is administered subcutaneously and has been approved for use in hemophilia A patients with and without inhibitors.

Aims: To assess availability, acceptance and safety of emicizumab for hemophilia A in Europe.

Methods: An online questionnaire was sent to 144 European Haemophilia Comprehensive Care Centers (EHCCC) and European Haemophilia Treatment Centers (EHTC). The survey was open from November 17, 2020 to January 31, 2021. Descriptive statistics were performed using SPSS version 27.

Results: Forty-six physicians from 21 countries responded. Emicizumab is reimbursed for all patients with inhibitors and the majority of patients without inhibitors. Reducing treatment burden was the main reason to switch both inhibitor and non-inhibitor patients to emicizumab. An annualized bleeding rate (ABR) of zero could be achieved in most patients with inhibitors on emicizumab $(72.9 \%)$. Inhibitor titers were regularly monitored in $78.4 \%$ of inhibitor patients on emicizumab and chromogenic FVIII assay was not available in $27.0 \%$ of centers. Hemostasis was satisfactory in the majority of minor $(93.7 \%)$ and major $(90.7 \%)$ surgical procedures performed while on emicizumab. In the 35 centers that responded to the question, a total of four patients on emicizumab have died to date, although none of these deaths were directly linked to emicizumab. The Covid-19 pandemic did not have a considerable impact on the adoption of emicizumab in most centers (64.9\%). 


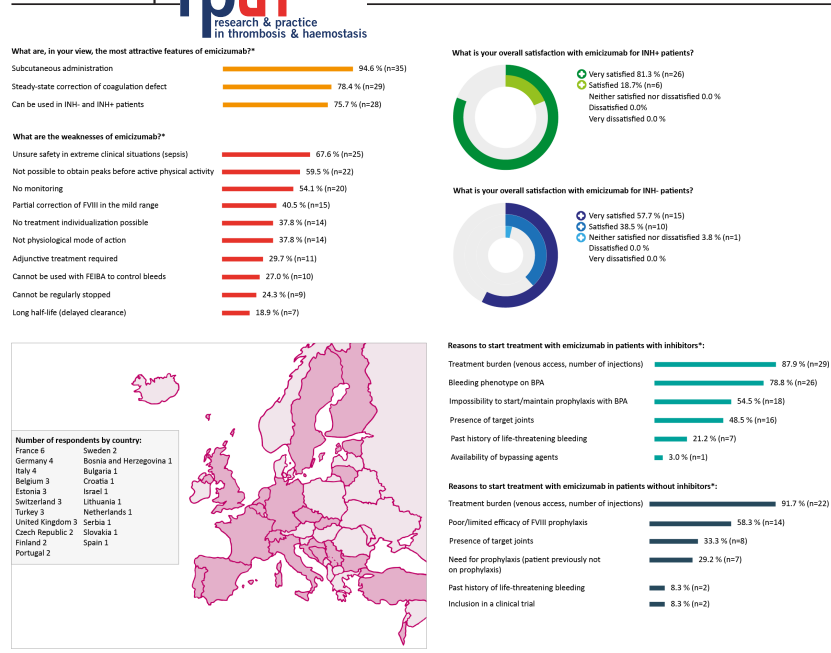

FIGURE 1 From top left to bottom right: benefits and shortcomings of emicizumab, physicians' satisfaction with emicizumab, number of respondents by country and reasons for switching patients to emicizumab; * multiple selections were allowed; BPA = bypassing agents, FVIII = coagulation factor VIII, INH = inhibitors

Conclusions: Three years after its rollout in Europe, emicizumab is widely available for both inhibitor and non-inhibitor patients. Satisfaction of physicians with emicizumab is very good. Unmet needs include availability of laboratory assays and guidelines for cotreatment during surgery and in case of breakthrough bleeding.

\section{PB0531 | Use of Desmopressin in the Treatment of Bleeding in} Non-severe Hemophilia A

A. Zwagemaker $^{1}$; F.R. Kloosterman ${ }^{1}$; S.C. Gouw ${ }^{1,2}$; E.A.M. Beckers ${ }^{3}$; S. Boyce ${ }^{4}$; P. Collins ${ }^{5}$; J. Eikenboom ${ }^{6}$; S. Shapiro ${ }^{7}$; S. Jackson ${ }^{8}$; M.J.H.A. Kruip ${ }^{9}$; B. Laros-Van Gorkom ${ }^{10}$; L. Nieuwenhuizen ${ }^{11}$; I. Pabinger ${ }^{12}$; M. Coppens ${ }^{13}$; J.G. Van der Bom ${ }^{2,14}$;

G. Castaman ${ }^{15}$; K. Fijnvandraat ${ }^{1,16}$, DYNAMO Study Group

${ }^{1}$ Amsterdam UMC, University of Amsterdam, Emma Children's

Hospital, Pediatric Hematology, Amsterdam, Netherlands;

${ }^{2}$ Department of Clinical Epidemiology, Leiden University Medical Center, Leiden, Netherlands; ${ }^{3}$ Department of Hematology, Maastricht University Medical Centre, Maastricht, Netherlands; ${ }^{4}$ Department of Haematology, University Hospital Southampton, Southampton, United Kingdom; ${ }^{5}$ Arthur Bloom Haemophilia Centre, University Hospital of Wales School of Medicine, Cardiff University, Cardiff, United Kingdom; ${ }^{6}$ Department of Internal Medicine, Division of Thrombosis and Hemostasis, Leiden University Medical Center, Leiden, Netherlands; ${ }^{7}$ Department of Haematology, Churchill Hospital, Oxford University Hospitals NHS Foundation, Oxford, United Kingdom; ${ }^{8}$ Inherited Bleeding Disorders Program of BC - Adult Division St. Paul's Hospital Vancouver, British Columbia, Vancouver, Canada; ${ }^{9}$ Department of Hematology, Erasmus MC, Erasmus University Medical Center, Rotterdam, Netherlands, ${ }^{10}$ Department of Hematology, Radboud University Medical Center, Nijmegen, Netherlands, ${ }^{11}$ Department of Hematology, Maxima Medical Center Eindhoven, Eindhoven, Netherlands, ${ }^{12}$ Clinical Division of Hematology and Hemostaseology,
Medical University of Vienna, Vienna, Austria, ${ }^{13}$ Department of Vascular Medicine, Amsterdam University Medical Centres, Amsterdam, Netherlands, ${ }^{14}$ Center for Clinical Transfusion Research, Sanquin-Leiden University Medical Center, Leiden, Netherlands, ${ }^{15}$ Department of Oncology, Center for Bleeding Disorders, Careggi University Hospital, Florence, Italy, ${ }^{16}$ Department of Molecular Cellular Hemostasis, Sanquin Research and Landsteiner Laboratory, Amsterdam, Netherlands

Background: Desmopressin is used in non-severe hemophilia A to increase endogenous factor VIII (FVIII). Large inter-individual variation in desmopressin response has been recognized and therefore it may not always be effective. Due to its benefits in comparison to FVIII concentrates, including lower costs and lack of inhibitor risk, it is an important alternative treatment modality. Little is known on real world clinical use of desmopressin compared to FVIII concentrates in the treatment of bleeds.

Aims: To describe the clinical use of desmopressin and FVIII concentrate in non-severe hemophilia A.

Methods: The DYNAMO study is an international, multicentre cohort study that includes patients with non-severe hemophilia $A$ (FVIII 2-35 IU/dL) aged 12-55 years. Data were collected on desmopressin test response and treatment of bleeding episodes over the past 10 years. Treatment product was analysed according to desmopressin response and FVIII level. Medical ethics approval and written consent was obtained (ClinicalTrials.gov:NCT03623295).

Results: We included 146 patients with non-severe hemophilia $A$ in this preliminary analysis. The median age was 36 years (IQR 24-49) and the median FVIII level was 11 IU/dL (IQR 5-17). The desmopressin test peak response was $<30 \mathrm{IU} / \mathrm{dL}$ (inadequate) in $9(6 \%)$ patients, between $30-50 \mathrm{IU} / \mathrm{dL}$ (adequate) in 37 (25\%) patients and $>50 \mathrm{IU} / \mathrm{dL}$ (excellent) in 74 (51\%) patients. In 26 (18\%) patients the desmopressin response was unknown/not tested. Of all bleeds, 594 (74\%) were treated with FVIII concentrates, 94 (12\%) with desmopressin and 75 (9\%) with a combination. Figure 1 and table 1 present treated bleeds per FVIII level and desmopressin response. The majority of bleeds were joint and muscle bleeds (57\%) and these were mainly treated with FVIII concentrates (81\%) or a combination of FVIII concentrates and desmopressin (12\%).

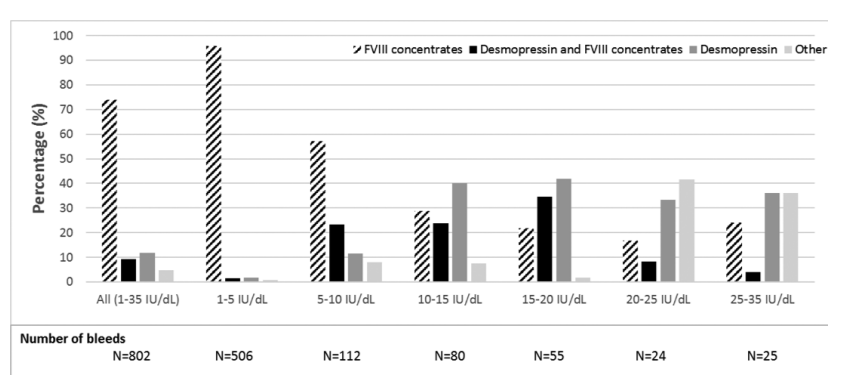

FIGURE 1: Percentage of bleeds treated with FVIII concentrate, desmopressin, a combination of both and other treatment modalities (i.e. tranexamic acid) according to baseline FVIII activity. 
TABLE 1 Bleeds treated with desmopressin, FVIII or a combination of both per response category.

\begin{tabular}{|c|c|c|c|c|c|}
\hline $\begin{array}{l}\text { Desmopressin response } \\
\text { class (n patients) }\end{array}$ & Inadequate $(n=9)$ & Adequate $(n=37)$ & Excellent $(n=74)$ & $\begin{array}{l}\text { Unknown/not tested } \\
(n=26)\end{array}$ & Total $(n=146)$ \\
\hline $\begin{array}{l}\text { Moderate patients, } n \text { (\% } \\
\text { of response class) }\end{array}$ & $7(78)$ & $13(35)$ & $6(8)$ & $18(69)$ & $44(30)$ \\
\hline Total number of bleeds & 72 & 162 & 187 & 381 & 802 \\
\hline $\begin{array}{l}\text { Bleeds per patient, } \\
\text { median (IQR) }\end{array}$ & $3(2-13)$ & $3(1-5)$ & $2(0-3)$ & $6(2-18)$ & $2(1-5)$ \\
\hline \multicolumn{6}{|c|}{ Treatment of bleeds (\% of total bleeds) } \\
\hline FVIII & $71(99)$ & $85(52)$ & $73(39)$ & $365(96)$ & $594(74)$ \\
\hline FVIII and desmopressin & - & $33(20)$ & $41(22)$ & $1(0)$ & $75(9)$ \\
\hline Desmopressin & - & $37(23)$ & $54(29)$ & $3(1)$ & $94(12)$ \\
\hline $\begin{array}{l}\text { Other (i.e. tranexamic } \\
\text { acid) }\end{array}$ & $1(1)$ & $7(4)$ & $19(10)$ & $12(3)$ & $39(5)$ \\
\hline
\end{tabular}

Conclusions: Desmopressin response was adequate or excellent in $76 \%$ of patients and was used in $21 \%$ of the bleeding episodes in the total population.

PB0532 | Evaluation of Liver Health after Fidanacogene Elaparvovec Gene Therapy: Data from Study Participants with up to 5 Years of Follow-up

L.A. George ${ }^{1,2}$; S.K. Sullivan ${ }^{3}$; J.E. Rasko ${ }^{4}$; A. Giermasz ${ }^{5}$; B.J. Samelson-Jones ${ }^{1}$; J.M. Ducore ${ }^{5}$; J.M. Teitel ${ }^{6}$; C. E. McGuinn ${ }^{7}$; D. Rybin $^{8}$; J.E. Murphy ${ }^{9}$; I. Winburn ${ }^{10}$; A. Chhabra ${ }^{9}$; J. Rupon ${ }^{11}$

${ }^{1}$ The Children's Hospital of Philadelphia, Philadelphia, United States;

${ }^{2}$ Perelman School of Medicine at the University of Pennsylvania, Philadelphia, United States; ${ }^{3}$ Mississippi Center for Advanced Medicine, Madison, United States; ${ }^{4}$ Royal Prince Alfred Hospital, SLHD, Sydney, Australia; ${ }^{5}$ University of California Davis, Sacramento, United States; ${ }^{6}$ St. Michael's Hospital, University of Toronto, Toronto, Canada;

${ }^{7}$ Weill Cornell Medical College, New York, United States; ${ }^{8}$ Pfizer Inc., Cambridge, United States; ${ }^{9}$ Pfizer Inc., New York, United States, ${ }^{10}$ Pfizer Ltd, Surrey, United Kingdom, ${ }^{11}$ Pfizer Inc., Collegeville, United States

Background: Adeno-associated virus (AAV)-based gene therapy for the treatment of hemophilia has generally targeted hepatocytes. As such, liver health is an important aspect of screening assessments for these studies. The hemophilia population has a high percentage of patients with prior hepatitis C (HCV) and/or B (HBV) virus infections, which places additional importance on better understanding the role of AAV gene therapy on long-term liver health.

Aims: Evaluate liver health following fidanacogene elaparvovec, a hemophilia B gene therapy that utilizes a hepatotropic AAV vector.

Methods: Fidanacogene elaparvovec was administered to 15 adult males with moderately severe/severe hemophilia B as part of a phase 1/2a study and a subsequent long-term follow-up study (LTFU). Informed consent was obtained from all participants and both studies were reviewed and approved by an ethics committee. Patients with a history of HCV and/or HBV were eligible for study entry if liver health parameters were met. After completing the phase $1 / 2 a$ study, 14/15 patients were enrolled in the LTFU for an additional follow-up of 5 years. One participant withdrew from LTFU study 3 years post- infusion.

Results: Ten of 14 participants who continued into the LTFU study had a history of resolved HCV, and seven participants also had prior HBV. With follow up ranging from 32 to 60 months, abnormalities on annual liver ultrasound included fatty liver in one participant. There were no significant elevations in plasma alfa-fetoprotein. Most common findings have been mild sustained elevations of alanine aminotransferase of uncertain etiology. FIX activity levels have remained relatively stable in these patients.

Conclusions: With up to 5 years of follow up, fidanacogene elaparvovec continues to be generally well tolerated. Long-term impacts to overall liver health currently appear to be mild in patients with moderately severe/severe hemophilia B. Longer term monitoring is ongoing to consolidate this initial result.

PB0533 | A single center study of bone mineral density in adult patients with severe Haemophilia A and correlation with markers of bone metabolism

H. Ivanova ${ }^{1}$; Z. Grudeva-Popova ${ }^{1}$; T. Deneva ${ }^{1} ;$ N. Mateva $^{2}$

${ }^{1}$ UMHAT "St George ", Plovdiv, Bulgaria; ${ }^{2}$ Medical University Plovdiv,

Plovdiv, Bulgaria

Background: Osteopenia and osteoporosis leading to increase rate of fractures are now well recognised comorbidities in haemophilia patients. The pathogenesis is multifactorial and includes decreased thrombin generation as a factor of impaired osteoblastic differentiation as well as increased osteoclastic activity.

Aims: To evaluate BMD of severe haemophilia A adult patients and to assess possible correlation between serum levels of circulating remodeling biomarkers -OPG, RANKL, CTX, osteocalcin, sclerostin and VitD level. Methods: 26 male subjects (median age $42.88 \pm 11.73$ with $\mathrm{BMI}$ $24.86 \pm 3.59)$ affected with severe haemophilia A were enrolled 
Patients with chronic liver disease and HCV were excluded. In addition 33 age -matched controls(median age $38.69 \pm 12.69$ with BMI $22.61 \pm 2.84$ ) were recruited. All subjects provided written informed consent and all procedures were approved by local review board. Biomarkers of bone metabolism were tested with ELISA assay and BMD was investigated with DEXA of two zones -lumbar spine and total hip. Data was analysed statistically using SPSS package.

Results: Patients with haemophilia had lower LS-BMD - $0.955 \pm 0.145$ vs $1.118 \pm 0.079, P=0.05$ and TH-BMD $-0.840 \pm 0.147$ vs $0.951 \pm$ $0.075, P=0.05$ comparing with controls, respectively $\mathrm{TH}$ T-score of $-1.41 \pm 0.91$ vs $0.4 \pm 0.49, P=0.05$ and LS-T-score of $-1.16 \pm 1046$ vs $.14 \pm 0.72, P=0.05 .66 .6 \%$ of patients $<50$ years had osteopenia and $8.3 \%$-osteoporosis. Among those $>50$ years $50 \%$ experience osteopenia and $20 \%$ osteoporosis. They had significantly higher OPG (123.69 \pm 107.05 vs $41.98 \pm 18.95, P=0.05)$ and lower $\operatorname{SRANKL}(23.49 \pm 29.39$ vs $131.32 \pm 201.27, P=0.05$ ) levels, sRANKL/OPG ratio $0.27 \pm 0.35$ vs $5.28 \pm 10.01, P=0.05$ with positive correlation between $S R A N K L$ and BMD and T score of lumbar spine, $P=0.001$.

Conclusions: Higher circulating osteoprotegerin levels in hemophilia patients with osteopenia/osteoporosis are reflection of increased bone turnover and a compensatory response to excessive osteoclast activity. Our results suggest that SRANKL level and ratio can be used as a predictor of BMD but further investigation will be necessary.

PB0534 | Dutch Overweight People with Hemophilia Report More Limitations in Activities, but No Association with Sports

O. Versloot ${ }^{1}$; E. van Balen ${ }^{2}$; S. Hassan²; S. Schols ${ }^{3}$; F. Leebeek ${ }^{4}$; M. Coppens ${ }^{5}$; J. Eikenboom ${ }^{2}$; L. Hooimeijer ${ }^{6}$; L. van Vulpen ${ }^{1}$; C. Smit ${ }^{2}$; M. Driessens ${ }^{7}$; J. van der Net $^{8}$; S. Gouw ${ }^{5,2}$; K. Fischer ${ }^{1}$, HiN6 steering group

${ }^{1}$ van Creveldkliniek, UMC Utrecht, Utrecht, Netherlands; ${ }^{2}$ Leiden University Medical Center, Leiden, Netherlands; ${ }^{3}$ Radboud UMC, Nijmegen, Netherlands; ${ }^{4}$ Erasmus MC, Rotterdam, Netherlands;
${ }^{5}$ Amsterdam UMC, Amsterdam, Netherlands; ${ }^{6}$ UMC Groningen, Groningen, Netherlands; ${ }^{7}$ Netherlands Haemophilia Patient Society, Nijkerk, Netherlands; ${ }^{8}$ Wilhelmina Childrens' Hospital, UMC Utrecht, Utrecht, Netherlands

Background: Overweight ( $\mathrm{BMl}>25)$ is a serious issue in European countries. Physical activity is advised to control body weight and promote healthy lifestyle. People with hemophilia ( $\mathrm{PWH}$ ) active in sports are expected to report less overweight and obesity.

Aims: To assess the association between BMI, limitations in activities and sports participation in $\mathrm{PWH}$.

Methods: Data was collected during the cross-sectional nationwide study 'Haemophilia in the Netherlands' (HiN6). HiN6 included questionnaires concerning body composition (height, weight) and sports participation (self-reported limitations ((Pediatric) Hemophilia Activities List (Ped)HAL)) and Modifiable Activities Questionnaire (MAQ)). Data were presented as median (interquartile range). Body weight was grouped as 'normal weight' or 'overweight' (adults: BMI>25, children: BMI over age specific limit). Groups were compared using Chi-square testing.

Results: BMI was available for 664 adults (median age: 51 (35-62)) and 130 children (age: 9 (5-13)). Most participants had hemophilia A (87\%; 37\% severe, $86 \%$ prophylaxis; non-severe: $4.8 \%$ prophylaxis). Median BMI in adults was 25.3 (23.1-27.9) and 16.7 (15.018.7 ) in children, indicating $52 \%$ of adults and $13 \%$ of children were overweight. Table 1 shows weight distribution in PWH and the GP. Patients with severe hemophilia (39\%) reported less overweight than those with non-severe hemophilia (50\%). Fewer PWH reported severe obesity ( $\mathrm{BMI}>30$ ) than in the GP in both adults ( $0.8 \%$ vs. $13.5 \%)$ and children ( $0 \%$ vs $2 \%$ ). BMI was not associated with sports participation in adults (rho: -0.08 ) and children (rho: 0.09) or weekly sports exposure $(r h o=-0.02)$. Figure 1 shows the association between BMI and self-reported limitations in activities: BMI was associated with self-reported limitations in activities $(P<0.01$; see figure 1$)$.

TABLE 1 Distribution of weight and overweight in Dutch persons with hemophilia and the general population

\begin{tabular}{|c|c|c|c|c|c|}
\hline & \multirow[b]{2}{*}{ Overall } & \multicolumn{2}{|c|}{ People with Hemophilia } & \multicolumn{2}{|l|}{ General Population } \\
\hline & & Normal overweight & Overweight* & Normal overweight & Overweight* \\
\hline Adults & 664 & $47.7 \%$ & $52.3 \%$ & $42.7 \%$ & $56.30 \%$ \\
\hline Children & 130 & $86.9 \%$ & $13.0 \%$ & $87.0 \%$ & $13.00 \%$ \\
\hline Severe & 294 & $60.5 \%$ & $39.4 \%$ & & \\
\hline Non-severe & 495 & $50.1 \%$ & $49.9 \%$ & & \\
\hline \multicolumn{6}{|c|}{ Sports characteristics } \\
\hline Sports & 638 & $55.9 \%$ & $43.2 \%$ & & \\
\hline Adults & 664 & $50.8 \%$ & $40.1 \%$ & & \\
\hline Children & 130 & $86.6 \%$ & $13.3 \%$ & & \\
\hline
\end{tabular}




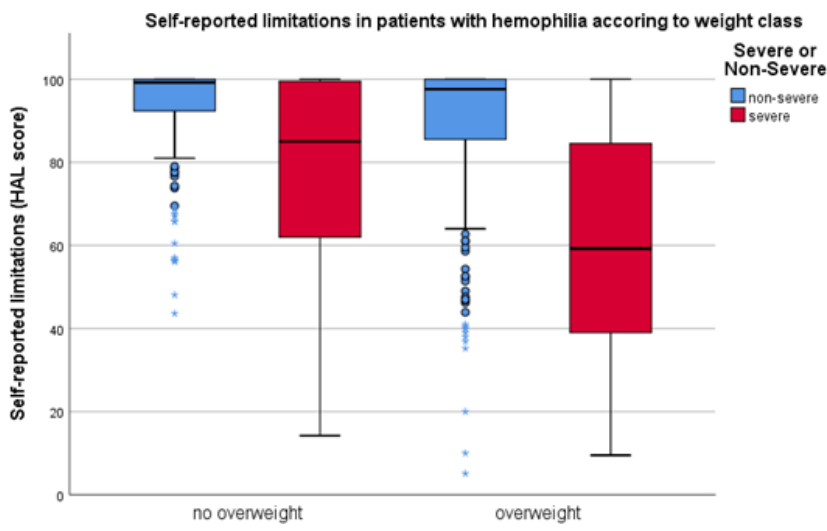

FIGURE 1 Self-reported limitations in activities increase according to weight categories in Dutch people with hemophila

Conclusions: Distribution of BMI between PWH and GP was comparable. However, BMI was not associated with sports participation in Dutch PWH. Overweight PWH reported more limitations than those with healthy weight. This could be cause or consequence.

\section{PB0535 | Long-term Prophylaxis with Simoctocog Alfa in} Children

A. Klukowska ${ }^{1}$; M. Jansen ${ }^{2}$; S. Knaub ${ }^{3}$; R.J. Liesner ${ }^{4}$

${ }^{1}$ Department of Pediatrics, Hematology and Oncology, Warsaw Medical University, Warsaw, Poland; ${ }^{2}$ Octapharma Pharmazeutika Produktionsgesellschaft m.b.H., Vienna, Austria; ${ }^{3}$ Octapharma AG, Lachen, Switzerland; ${ }^{4}$ Haemophilia Comprehensive Care Centre, Great Ormond Street Hospital for Children, NHS Foundation Trust, NIHR GOSH BRC, London, United Kingdom

Background: In children with severe haemophilia A, FVIII prophylaxis prevents life-threatening bleeds, protects from joint damage and enables daily activities. Simoctocog alfa $\left(\right.$ Nuwiq $\left.^{\circledR}\right)$ has proven efficacy and safety in the prevention and treatment of bleeds in children and adults, and is associated with a low rate of inhibitor development in previously untreated patients.

Aims: To assess the efficacy and safety of long-term prophylaxis with simoctocog alfa in children.

Methods: This is a pooled analysis of children with severe haemophilia A enrolled in the prospective GENA-15 and GENA-13 studies. GENA-15 and GENA-13 were long-term extension studies of the GENA-05 (NuProtect) and GENA-03 studies in previously untreated and previously treated children, respectively. Prophylaxis duration was defined as the time from first until last prophylactic dose.

Results: 97 children were enrolled in the extension studies and 96 were included in these analyses. One child who had undergone immune tolerance induction was excluded. The median (IQR) age at prophylaxis start was $3(1-6)$ years, and the median (IQR) duration of prophylaxis was 359 (300-509) exposure days over 36.4 (31.640.9) months. The median (IQR) annualised bleeding rate (ABR) was $0.31(0-1.08)$ for spontaneous bleeds and $1.81(0.58-3.94)$ for all bleeds; $44.8 \%$ of patients experienced no spontaneous bleeds over a median of 3 years. A total of 763 bleeds were treated with simoctocog alfa. Most (86.5\%) were managed with 1 or 2 infusions, and $85.7 \%(649 / 757)$ of rated bleeds were treated successfully (excellent or good). None of the 96 patients on long-term prophylaxis withdrew from the studies due to an adverse event and there were no thrombotic events.

Conclusions: Simoctocog alfa is efficacious and well-tolerated for long-term prophylaxis in children with severe haemophilia $A$ and is an attractive long-term therapeutic option for children.

PB0536 | Results from the Eptacog Beta in the Treatment of Bleeding Episodes of all Severities in Pediatric Hemophilia A or B Patients with Inhibitors

G. Castaman $^{1}$; S.W. Pipe ${ }^{2}$; M. Callaghan ${ }^{3}$; J. Ducore ${ }^{4}$; M. Escobar ${ }^{5}$; C. Hermans ${ }^{6}$; J. Mahlangu ${ }^{7}$; U. Nowak-Göttl ${ }^{8}$; J. Journeycake ${ }^{9}$; S. Meeks ${ }^{10}$; M. Recht ${ }^{11}$; M. Wang ${ }^{12}$; G. Young ${ }^{13}$; N. Chambron ${ }^{14}$; A. AL-Sabbagh ${ }^{15}$; D. Bonzo ${ }^{15}$; C. Macie ${ }^{16}$; I.S. Mitchell ${ }^{17}$; A. Shapiro ${ }^{18}$ ${ }^{1}$ Careggi University Hospital, Florence, Italy; ${ }^{2}$ University of Michigan, Ann Arbor, MI, United States; ${ }^{3}$ Central Michigan University, Detroit, MI, United States; ${ }^{4}$ University of California Davis, Sacramento, CA, United States; ${ }^{5}$ University of Texas Health Science Center, Houston, TX, United States; ${ }^{6}$ Saint-Luc University Hospital, Brussels, Belgium; ${ }^{7}$ University of the Witwatersrand, Johannesburg, South Africa; ${ }^{8}$ University Medical Center Schleswig-Holstein, Kiel, Germany; ${ }^{9}$ University of Oklahoma Health Sciences Center, Oklahoma City, OK, United States, ${ }^{10}$ Aflac Cancer and Blood Disorders and Emory University, Atlanta, GA, United States, ${ }^{11}$ American Thrombosis \& Hemostasis Network and Oregon Health \& Science University, Portland, OR, United States, ${ }^{12}$ University of Colorado Denver, Aurora, CO, United States, ${ }^{13}$ Children's Hospital Los Angeles, Los Angeles, CA, United States, ${ }^{14}$ LFB, Les Ulis, France, ${ }^{15}$ LFB-USA, Framingham, MA, United States, ${ }^{16}$ HEMA Biologics, Louisville, KY, United States, ${ }^{17}$ Gloval, Broomfield, CO, United States, ${ }^{18}$ Indiana Hemophilia \& Thrombosis Center, Indianapolis, IN, United States

Background: Management options for bleeding episodes (BEs) in pediatric patients with hemophilia A or B with inhibitors (PwHABI) are limited. Suboptimal treatment has negative musculoskeletal consequences.

Aims: We assessed the efficacy and safety of eptacog beta (EB; LFB and HEMA Biologics), a new bypassing agent (recombinant FVIla) in treating $\mathrm{BEs}$ in pediatric $\mathrm{PwHABI}$.

Methods: PERSEPT 2 was a randomized, crossover, international, phase 3 study of EB in pediatric PwHABI. Patients received either an initial dose (ID) of 75 or $225 \mu \mathrm{g} / \mathrm{kg}$ EB followed by per protocol dosing of $75 \mu \mathrm{g} / \mathrm{kg}$ at prespecified intervals (determined by clinical response). The primary efficacy endpoint using the EMA definition was the proportion of BEs of all severity (mild, moderate and severe) with 'good' or 'excellent' response at $12 \mathrm{~h}$ after the initial dose. Secondary/tertiary efficacy endpoints included time to response, 
number of administrations, pain relief at $12 \mathrm{~h}$, bleeding recurrence within $24 \mathrm{~h}$, and responses at $24 \mathrm{~h}$.

Results: Twenty-five patients (range 1-11 years; median 5.0 years) received EB treatment for 549 BEs (546 mild/moderate, 3 severe). Of these, $239 \mathrm{BEs}(67 \%)$ treated with $75 \mu \mathrm{g} / \mathrm{kg}$ ID regimen (IDR) and $310 \mathrm{BEs}(63 \%)$ treated with $225 \mu \mathrm{g} / \mathrm{kg}$ IDR achieved hemostatic response within $12 \mathrm{~h}$; median time to response was 9 and $12 \mathrm{~h}$, respectively. Response was achieved with a median of 3 doses with $75 \mu \mathrm{g} /$ $\mathrm{kg}$ IDR and 2 doses with the $225 \mu \mathrm{g} / \mathrm{kg}$ IDRs. Pain was relieved within 12 hours in the vast majority of cases (>90\%). Most BEs (>98\%) had no recurrence, and the success proportion at $24 \mathrm{~h}$ was $>97 \%$ for both IDRs (Table 1). EB was well tolerated. No thromboembolic, allergic, or treatment-related adverse events were reported. No neutralizing antibodies to EB were observed.

TABLE 1 Primary, Secondary and Tertiary Efficacy Endpoints ( $N=549$ BEs, Regardless of Severity*)

\begin{tabular}{|c|c|c|}
\hline & \multicolumn{2}{|c|}{ Initial Dose Regimen at the time of $\mathrm{BE}$} \\
\hline & $\begin{array}{l}75 \mu \mathrm{g} / \mathrm{kg} \\
(\mathrm{N}=23)\end{array}$ & $\begin{array}{c}225 \mu \mathrm{g} / \mathrm{kg} \\
(\mathrm{N}=24)\end{array}$ \\
\hline \multirow[t]{2}{*}{ Number of BEs } & 239 & 310 \\
\hline & \multicolumn{2}{|c|}{ Success proportion $[95 \% \mathrm{Cl}]$} \\
\hline Primary endpoint & & \\
\hline BEs with 'good' or 'excellent' response at $12 \mathrm{~h}$ & $66.7 \%[53.3,80.0]$ & $62.5 \%[50.0,75.0]$ \\
\hline \multicolumn{3}{|l|}{ Secondary/tertiary endpoints } \\
\hline BEs with successful pain relief at $12 \mathrm{~h}$ & $92.8 \%[85.6,100.0]$ & $90.2 \%[82.7,97.7]$ \\
\hline BEs without recurrence of bleeding within $24 \mathrm{~h}$ & $99.2 \%[97.6,100.0]$ & $98.1 \%[96.1,100.0]$ \\
\hline BEs not requiring alternative treatment & $97.5 \%[91.5,100.0]$ & $97.7 \%[93.8,100.0]$ \\
\hline BEs with 'good' or 'excellent' response at $24 \mathrm{~h}$ & $97.4 \%[91.3,100.0]$ & $97.7 \%[94.1,100.0]$ \\
\hline
\end{tabular}

Conclusions: Results from PERSEPT 2 provide clinically meaningful evidence that EB is an effective and safe therapeutic option for the treatment of BEs in pediatric $\mathrm{PwHABI}$.

PB0537 | Real-world Experience in Introducing Emicizumab Prophylaxis for Adults with Haemophilia A without Inhibitors

N. Larkin; E. Singleton; J. Benson; N. Boland; J. Cleary; S. Brady; D. Gorman; N. Flynn; F. Ryan; M. Mc Gowen; S. Roche; C. Bergin; B. Mary; K. Ryan; M. Lavin; J.S O Donnell; N. O Connell

St James Hospital, Dublin 8, Ireland

Background: Prophylaxis to prevent spontaneous bleeding and joint disease is the standard of care for people with severe Haemophilia A (PWSHA) in Ireland. In October 2019, Emicizumab became available as an option in Ireland for prophylaxis for PWSHA without inhibitors. Aims: To offer Emicizumab for prophylaxis in PWSHA without in hibitors, having regard for clinical considerations, safety, personal choice and treatment outcomes.

Methods: PWSHA ( $n=91$ ) were informed by letter of the availability of Emicizimab as a prophylaxis option. Dedicated clinics were set up to enable adequate time for in-depth clinical review and collaborative discussion about the potential benefits or risks in switching. A standardised process was developed to support patients and the multidisciplinary team and to ensure a seamless and safe transition to Emicizumab for those who chose to change prophylaxis regimen.

Results: To date, 35 patients have been reviewed at the dedicated clinic and 25 have switched to Emicizumab prophylaxis. Of the remaining 10 patients, seven chose not to switch following consultation, two were non-compliant with recording of home treatment (an exclusion for switching) and one is due to start Emicizumab shortly. Of the patients who switched to Emicizumab, 24/25 have severe Haemophilia A, one patient has moderate Haemophilia A with a severe bleeding phenotype. Fortnightly maintenance doses are the most common treatment frequency (21/25 patients) with the remaining four patients on weekly maintenance doses.

Since switchover, seven breakthrough bleeds were reported, with five requiring factor replacement (three trauma and two spontaneous bleeds).

Conclusions: A standardised clinical review and consultation process enabled shared decision making with PWSHA regarding their choice of prophylaxis, in line with personal health care goals. $20 \%$ of those reviewed chose not to switch to Emicizumab, for clinical reasons or personal choice. Patients receiving Emicizumab prophylaxis demonstrate low bleed rates and improved treatment frequency.

PB0538 | Efficacy and Safety Analysis of the Use of rIX-FP in Patients with Hemophilia B: A Prospective, Non-interventional, Surveillance Study from Germany

J. Oldenburg'; ;. Holzhauer²; S. Wenning ${ }^{3}$; M. Olivieri' ${ }^{4}$;

C. Pfrepper ${ }^{5}$

${ }^{1}$ University Clinic Bonn, Institute of Experimental Haematology and Transfusion Medicine, Bonn, Germany; ${ }^{2}$ Charité University Medicine, Department of Pediatric Hematology and Oncology, Berlin, Germany; ${ }^{3}$ SRH Kurpfalzkrankenhaus, Heidelberg, Germany; ${ }^{4}$ Paediatric Thrombosis and Haemostasis Unit, Paediatric Haemophilia Center, LMU, Munich, Germany; ${ }^{5}$ Division of Hemostaseology, University Hospital Leipzig, Leipzig, Germany

Background: rIX-FP, a long-acting fusion protein, previously demonstrated safety and efficacy in patients with hemophilia B in clinical trials. Data from routine clinical practice on the effectiveness and safety of rIX-FP are required.

Aims: To obtain data on clinical outcomes in patients treated with rIX-FP during routine clinical practice in Germany.

Methods: A non-interventional study was initiated in Germany in March 2018. All patients with hemophilia B are eligible for enrolment. Patients are followed for 2-3 years or until 100 exposure days and are treated with rIX-FP up to every 14 days and routinely monitored every 3-12 months.

Results: At the interim data cut-off, 26 October 2020, 63 patients including one female were enrolled across 22 sites. One patient has since withdrawn due to lack of treatment satisfaction and another 
requested to withdraw but has remained on rIX-FP prophylaxis. Enrolled patients range in age from 1-80 years and have been observed for mean (SD) 7.4 (6.82) months. Mean rIX-FP consumption is lower or within the EU recommended initial dosing range for rIXFP (35-50 IU/kg/week) for all age groups (adults, $32.3 \mathrm{IU} / \mathrm{kg} /$ week; adolescents, $38 \mathrm{IU} / \mathrm{kg} /$ week; pediatrics, $43 \mathrm{IU} / \mathrm{kg} /$ week). The effectiveness of treatment was rated good or excellent in $87.3 \%$ of cases. Mean annualized bleeding rates (ABR) were $1.1(n=18), 1.1(n=2)$, and $2.4(n=10)$ for adult, adolescent, and pediatric patients, respectively. Median spontaneous ABR was 0.0 in adult and pediatric patients. Thirty-seven adverse events (AEs) have been reported, none of which were related to rIX-FP. Eight previously untreated patients have been treated; no inhibitors or drug-related AEs have been reported. To date, no patients have developed inhibitors.

Conclusions: Data from this study indicate that rIX-FP prophylaxis is well tolerated in routine clinical practice in Germany. Further studies analyzing patients treated with rIX-FP in other countries are ongoing.

PB0539 | Is Zero Bleeds in PWH an Achievable Goal? 3 Years of Emicizumab in Persons with Hemophilia A and Inhibitors in a Portuguese Hemophilia Treatment Centers

S. Campaniçơ ${ }^{1}$; F. Rodrigues ${ }^{1}$; A. Pereira ${ }^{1}$; E. Rocha ${ }^{2}$; G. Cevadinha ${ }^{2}$; C. Catarino ${ }^{1}$

${ }^{1}$ Centro Hospitalar Universitário Lisboa Norte, Lisbon, Portugal;

${ }^{2}$ Centro Hospitalar Universitário do Algarve, Faro, Portugal

Background: In Portugal, emicizumab, although only approved for persons with hemophilia $A$ with inhibitors ( $\mathrm{PwHAi}$ ), changed the mindset of prophylaxis in haemophilia.

Aims: To evaluate the clinical efficacy of emicizumab prophylaxis in PwHAi followed at our center.

Methods: Data on PwHAi on prophylaxis with emicizumab for the last 3 years, were retrospectively retrieved from the medical records. Other than annual bleeding rates (BR), an evaluation of episodes responsible for visits to the emergency room (ER) and for hospitalization was also done.

Results: Since January 2018, emicizumab was started in 9 patients (8 children, 1 adult) and the median duration of therapy was 29 months (18-38). Age at initiation of emicizumab ranged from 4 months to 27 years, median 6.5years. 3 patients had received immune tolerance induction (ITI) without success and were on prophylaxis with bypassing agents (BPA). On emicizumab, no patient experienced spontaneous bleeding and 7 patients had no treated bleeding events. The ABR with emicizumab was $0.3(0-2)$ vs 10.4 (1-24) with prior BPA, representing a $97 \%$ reduced risk of bleeding. 2 patients were occasionally treated with rFVIla for trauma (hemarthrosis; epistaxis; traumatic lip bleeding; 2 muscle hematomas and head trauma), and 1 and for prevention of bleeding before 1 minor procedure. In the period between 2016 and 2017, 3/5 of our PWHi had at least one hospitalization and were responsible for $22 \%$ of all hospital admissions in PWSevereH. However, since the beginning of emicizumab, there were no hospitalizations.
Regarding visits to the ER, before 2018 PWHi accounted for about $10 \%$ of these visits, but this also changed after prophylaxis with emicizumab, with only 6 visits in this 3-year period.

Conclusions: In our study, we find that the efficacy of emicizumab is well demonstrated by the low BR, as well as by the almost inexistent visits to the ER and by the lack of hospitalizations.

\section{PB0540 | Feasibility of Measuring through PROMIS Computer Adaptive Tests (CATs) and Short Forms (SFs) in Haemophilia - Preliminary Data}

I.A. Kuijlaars $^{1}$; L. Teela ${ }^{2}$; J.J. Muis ${ }^{2,3}$; E.S. van Hoorn ${ }^{4}$; L. van Vulpen $^{5}$; M. Timmer ${ }^{5}$; M. Coppens ${ }^{6}$; S.C. Gouw ${ }^{3,7}$; M. Peters ${ }^{3}$; M.J. Kruip ${ }^{8}$; M.H. Cnossen ${ }^{9}$; L. Haverman ${ }^{2}$; K. Fischer ${ }^{5}$

${ }^{1}$ University Medical Center Utrecht, Utrecht, Netherlands; ${ }^{2}$ Amsterdam University Medical Centers, University of Amsterdam, Emma Children's Hospital, Child and Adolescent Psychiatry \& Psychosocial Care, Amsterdam Reproduction and Development, Amsterdam Public Health, Amsterdam, Netherlands; ${ }^{3}$ Emma Children's Hospital, Amsterdam University Medical Centers, University of Amsterdam, Pediatric Hematology, Amsterdam, Netherlands; ${ }^{4}$ Erasmus MC, University Medical Center Rotterdam, Department of Public Health, Rotterdam, Netherlands; ${ }^{5}$ Van Creveldkliniek, University Medical Center Utrecht, Utrecht University, Utrecht, Netherlands; ${ }^{6}$ Department of Vascular Medicine, Amsterdam Cardiovascular Sciences, Amsterdam University Medical Centers, University of Amsterdam, Amsterdam, Netherlands; ${ }^{7}$ Department of Clinical Epidemiology, Leiden University Medical Center, Leiden, Netherlands; ${ }^{8}$ Erasmus MC, University Medical Center Rotterdam, Department of Hematology, Rotterdam, Netherlands; ${ }^{9}$ Erasmus MC, University Medical Center Rotterdam, Department of Pediatric Oncology and Hematology, Rotterdam, Netherlands

Background: Patient Reported Outcomes Measurement Information System (PROMIS $\left.{ }^{\circledR}\right)$ is a set of universal, person-centered item banks to evaluate physical, mental and social health. PROMIS Computer Adaptive Tests (CATs) have shown better reliability and more efficient outcome assessment than standard questionnaires (legacy instruments). Aims: To evaluate the feasibility of PROMIS CATs and short forms (SFs) in adult persons with haemophilia (PWH).

Methods: Adult PWH who participated in the Haemophilia in the Netherlands-6 (HiN-6) study were invited by email. Nine PROMIS item banks were completed electronically: 'pain interference', 'physical function', 'depression', 'anxiety', 'ability to participate in social roles and activities', 'fatigue' and 'satisfaction with social roles and activities' as CATs, and 'self-efficacy for managing medications and treatment' and 'self-efficacy for managing symptoms' as SFs. All item banks score 0-100, with a mean around 50 and thresholds for abnormal/limited scores vary from 0.5SD-1SD according to guidelines. The number of questions answered and floor and ceiling effects were determined to evaluate the feasibility.

Results: Overall, 142/373 of invited PWH (mean age 47y [range 1879], $49 \%$ severe haemophilia, $46 \%$ received prophylaxis) responded. 
'Pain interference' (33\%) and 'physical function' (38\%) were most frequently scored as abnormal/limited. 'Satisfaction with social roles and activities' (11\%) and 'self-efficacy for managing symptoms' (9\%) were least frequently scored as abnormal/limited. For the CATs mean number of questions answered per item bank varied from 5 (range 3-12) for 'satisfaction with social roles and activities' to 9 (range 5-12) for 'anxiety', without floor and ceiling effects. For the self-efficacy SFs, lowest scores were reported in 1-2\% and highest scores in $22-28 \%$.

Conclusions: PROMIS CATs are feasible and could lower burden of outcome assessment by reducing the number of questions needed to assess various aspects of health compared to legacy instruments. The next step is to determine construct validity of PROMIS questionnaires through comparison with $\mathrm{HiN}-6$ legacy instruments.

PB0541 | Warfarin Resistance Is Rare but Has Heterogeneous Origin in an Anticoagulation Clinic Population

$\underline{\text { R. Peck }}{ }^{1}$; K. Burley ${ }^{1}$; L. FitzGibbon ${ }^{1}$; C. Doherty ${ }^{2}$; S. Bacon ${ }^{3}$;

R. Thorne ${ }^{2}$; A. Mumford ${ }^{1}$

${ }^{1}$ University of Bristol, Bristol, United Kingdom; ${ }^{2}$ University Hospital NHS Foundation Trust, Bristol, United Kingdom; ${ }^{3}$ North Bristol NHS Trust, Bristol, United Kingdom

Background: Warfarin remains an essential anticoagulant for some patients with high thrombotic risk and requires meticulous dose monitoring.

Aims: We performed a snapshot survey of 2,093 patients in the warfarin clinics at two UK hospitals in January 2021 to investigate the prevalence and aetiology of high warfarin dose requirement.

Methods: In both clinics, venous blood INRs were determined using Innovin thromboplastin and Sysmex CA1500 coagulometers. Warfarin dosing was based on RAID or DAWN algorithms.

Results: The median daily warfarin dose was $4.9 \mathrm{mg}$ (range $0.64-100 \mathrm{mg}$ ). Five (0.24\%) patients had warfarin resistance (WR; warfarin dose $>25$ $\mathrm{mg} /$ day). Four of these cases (recurrent thrombosis $n=3$; mechanical heart valve $=1$ ) initially achieved stable therapeutic anticoagulation with warfarin $<10 \mathrm{mg} /$ day but then required escalating doses to achieve target INR. Plasma warfarin levels were all within therapeutic range of 0.7-2.3 mg/l indicating acquired pharmacokinetic WR. In two cases, this could be linked to established interacting co-medications.

The remaining case was a 43-year-old male who required anticoagulation after mitral valve repair. INR-guided warfarin dosing rapidly escalated to $35 \mathrm{mg} /$ day at which point the INR was 1.3. Despite this sub-therapeutic INR, the plasma warfarin level was $3.03 \mathrm{mg} / \mathrm{I}$ indicating pharmacodynamic WR. Analysis of VKORC1 that encodes the warfarin target Vitamin $\mathrm{K}$ epoxide reductase subunit 1 (VKORC1) revealed the heterozygous missense c.85G>T variant predictive of a p.Val29Leu substitution. This likely disrupts VKORC1 warfarin binding interface I (Leu22-Val29) and was shown by others in a cell line model to increase the warfarin $I C_{50}$ by more than five-fold. c.85G $>T$ is one of 26 missense variants in VKORC1 previously associated with
WR in humans, but which do not diminish VKORC1 function in the Vitamin K pathway.

Conclusions: Our findings indicate that WR remains a rare phenotype but has heterogeneous origin. We highlight the utility of measuring plasma warfarin level and VKORC1 genetic testing to assist diagnosis.

PB0542 | Looking Beyond Fixed-Dose Rurioctocog Alfa Pegol Prophylaxis: Post Hoc Analysis of PK-guided Regimens from the PROPEL Phase 3 Study

C. Escuriola-Ettingshausen ${ }^{1}$; M. Escobar ${ }^{2}$; J. Windyga ${ }^{3}$; B. Zulfikar ${ }^{4}$; S.D. Tangada ${ }^{5}$; W. Engl ${ }^{6}$; I. Honauer ${ }^{7}$; H.-Y. Lee ${ }^{7}$; R. Klamroth ${ }^{8}$ ${ }^{1}$ Hämophilie-Zentrum Rhein Main, Mörfelden-Walldorf, Germany; ${ }^{2}$ University of Texas Health Science Center at Houston, Houston, United States; ${ }^{3}$ Department of Hemostasis Disorders and Internal Medicine, Institute of Hematology and Transfusion Medicine, Warsaw, Poland; ${ }^{4}$ Department of Pediatric Hematology, Istanbul University Oncology Institute, Istanbul, Turkey; ${ }^{5}$ Baxalta US Inc., a Takeda Company, Cambridge, United States; ${ }^{6}$ Baxalta Innovations $\mathrm{GmbH}, a$ Takeda Company, Vienna, Austria; ${ }^{7}$ Baxalta GmbH, a Takeda Company, Zürich, Switzerland; ${ }^{8}$ Department for Internal Medicine, Vascular Medicine and Haemostaseology, Vivantes Klinikum im Friedrichshain, Berlin, Germany

Background: PROPEL primary data showed that targeting $8-12 \%$ factor VIII (FVIII) troughs results in fewer bleeds vs 1-3\% troughs, which suggests personalized dosing can help control/prevent bleeding in patients without compromising the efficacy/safety of rurioctocog alfa pegol (TAK-660, BAX 855).

Aims: To explore the impact of pre-study treatment regimen (ondemand FVIII [OD] or prophylactic FVIII [PPx]) and annualized bleeding rate (ABR) on the efficacy of TAK-660 prophylaxis targeting $1-3 \%$ or $8-12 \%$ FVIII troughs.

Methods: Design, primary outcomes, and overall safety of PROPEL (NCT0285960) were previously reported; ethics committee approvals and patient informed consent were obtained. Patients aged 12-65 years with $A B R \geq 2$ were randomized to 12 months' pharmacokinetic (PK)-guided prophylaxis targeting $1-3 \%$ or $8-12 \%$ FVIII troughs (first 6 months: treatment adjustment). Subgroups analyzed: patients on previous OD, PPx with average $A B R<5$ before study start, and PPx with $A B R \geq 5$ before study start. Outcomes in second 6 months: point estimates $(95 \% \mathrm{Cl})$ of mean for $\mathrm{ABR}$ and proportion of patients with zero ABR (total [all bleeds], spontaneous, spontaneous joint, injury related).

Results: In the $8-12 \%$ arm, total, spontaneous, and spontaneous joint ABRs were lower in all subgroups versus the 1-3\% arm (Table 1). Injury-related ABR was lower in the $8-12 \%$ arm among previous OD patients and patients on previous $\mathrm{PPx}$ with $\mathrm{ABR}<5$, but similar between arms in patients on previous PPx with $A B R \geq 5$. In the $8-12 \%$ arm, the proportion of patients with zero total $A B R$ was higher versus the $1-3 \%$ arm, regardless of pre-study regimen or ABR (Table 2). 
TABLE 1 Point estimate $(95 \% \mathrm{Cl})^{*}$ of mean for $\mathrm{ABR}$ in second 6 months stratified by pre-enrollment regimen and $\left.\mathrm{ABR}(\mathrm{PPAS})^{\dagger}\right)$.

\begin{tabular}{|c|c|c|c|c|c|c|}
\hline \multirow[b]{3}{*}{ Parameter } & \multicolumn{2}{|c|}{$\begin{array}{l}\text { On-demand FVIII treatment before } \\
\text { study (OD) }\end{array}$} & \multicolumn{4}{|c|}{ Prophylactic FVIII treatment before study (PPx) } \\
\hline & \multirow[b]{2}{*}{$\begin{array}{l}\text { FVIII trough } \\
\text { level } 1-3 \%\end{array}$} & \multirow[b]{2}{*}{$\begin{array}{l}\text { FVIII trough } \\
\text { level } 8-12 \%\end{array}$} & \multicolumn{2}{|c|}{$\begin{array}{l}\text { Average } A B R<5 \text { in } 12 \text { months } \\
\text { before enrollment }\end{array}$} & \multicolumn{2}{|c|}{$\begin{array}{l}\text { Average } A B R \geq 5 \text { in } 12 \text { months } \\
\text { before enrollment }\end{array}$} \\
\hline & & & $\begin{array}{l}\text { FVIII trough } \\
\text { level } 1-3 \%\end{array}$ & $\begin{array}{l}\text { FVIII trough } \\
\text { level } 8-12 \%\end{array}$ & $\begin{array}{l}\text { FVIII trough } \\
\text { level } 1-3 \%\end{array}$ & $\begin{array}{l}\text { FVIII trough level } \\
8-12 \%\end{array}$ \\
\hline Patients, $n$ & 13 & 13 & 19 & 14 & 20 & 16 \\
\hline Total ABR & $1.8(1.0-3.3)$ & $0.3(0.1-1.2)$ & $2.3(1.4-3.8)$ & $0.5(0.2-1.5)$ & $3.5(2.2-5.5)$ & $2.3(1.3-4.2)$ \\
\hline Spontaneous ABR & $0.8(0.3-1.9)$ & $0.1(0.0-1.0)$ & $1.7(0.8-3.3)$ & $0.3(0.1-1.2)$ & $2.1(1.1-4.2)$ & $1.1(0.4-2.6)$ \\
\hline Spontaneous joint ABR & $0.8(0.3-2.0)$ & 0.0 & $1.1(0.5-2.2)$ & $0.1(0.0-1.0)$ & $1.4(0.6-3.4)$ & $0.9(0.3-2.6)$ \\
\hline Injury-related ABR & $0.9(0.3-2.4)$ & $0.2(0.0-1.2)$ & $0.5(0.2-1.2)$ & $0.2(0.0-0.9)$ & $1.3(0.6-2.6)$ & $1.2(0.5-2.8)^{\ddagger}$ \\
\hline
\end{tabular}

$\mathrm{ABR}$, annualized bleeding rate; $\mathrm{Cl}$, confidence interval; $\mathrm{FVIII}$, factor VIII; PPAS, per-protocol analysis set.

${ }^{*}$ Point estimates and $95 \% \mathrm{Cls}$ were obtained from a generalized linear model fitting a negative binomial distribution.

${ }^{\dagger}$ Patients in the PPAS completed the second 6 months of prophylaxis with no significant protocol deviations.

${ }^{\ddagger}$ One patient experienced 5 knee-related injuries in 6 months that contributed to the injury-related ABR in this subgroup.

TABLE 2 Point estimate $(95 \% \mathrm{Cl})$ of proportion of patients with zero bleeds in second 6 months stratified by pre-enrollment regimen and $A B R$ (PPAS*).

\begin{tabular}{|c|c|c|c|c|c|c|}
\hline \multirow[b]{3}{*}{ Parameter } & \multicolumn{2}{|c|}{$\begin{array}{l}\text { On-demand FVIII treatment before } \\
\text { study (OD) }\end{array}$} & \multicolumn{4}{|c|}{ Prophylactic FVIII treatment before study (PPx) } \\
\hline & \multirow[b]{2}{*}{$\begin{array}{l}\text { FVIII trough } \\
\text { level } 1-3 \%\end{array}$} & \multirow[b]{2}{*}{$\begin{array}{l}\text { FVIII trough } \\
\text { level } 8-12 \%\end{array}$} & \multicolumn{2}{|c|}{$\begin{array}{l}\text { Average } A B R<5 \text { in } 12 \text { months } \\
\text { before enrollment }\end{array}$} & \multicolumn{2}{|c|}{$\begin{array}{l}\text { Average } A B R \geq 5 \text { in } 12 \text { months } \\
\text { before enrollment }\end{array}$} \\
\hline & & & $\begin{array}{l}\text { FVIII trough } \\
\text { level } 1-3 \%\end{array}$ & $\begin{array}{l}\text { FVIII trough } \\
\text { level } 8-12 \%\end{array}$ & $\begin{array}{l}\text { FVIII trough } \\
\text { level } 1-3 \%\end{array}$ & $\begin{array}{l}\text { FVIII trough } \\
\text { level } 8-12 \%\end{array}$ \\
\hline Patients, $n$ & 13 & 13 & 19 & 14 & 20 & 16 \\
\hline $\begin{array}{l}\text { Patients with total } \\
\qquad A B R=0, \%\end{array}$ & $46.2(19.2-74.9)$ & $84.6(54.6-98.1)$ & $42.1(20.3-66.5)$ & $71.4(41.9-91.6)$ & $35.0(15.4-59.2)$ & $50.0(24.7-75.3)$ \\
\hline $\begin{array}{l}\text { Patients with } \\
\text { spontaneous } \\
\text { ABR }=0, \%\end{array}$ & $69.2(38.6-90.9)$ & $92.3(64.0-99.8)$ & $57.9(33.5-79.7)$ & $85.7(57.2-98.2)$ & $55.0(31.5-76.9)$ & $68.8(41.3-89.0)$ \\
\hline $\begin{array}{l}\text { Patients with } \\
\text { spontaneous joint } \\
\qquad A B R=0, \%\end{array}$ & $69.2(38.6-90.9)$ & 100.0 (NA) & $63.2(38.4-83.7)$ & $92.9(66.1-99.8)$ & $65.0(40.8-84.6)$ & $81.3(54.4-96.0)$ \\
\hline $\begin{array}{l}\text { Patients with injury- } \\
\quad \text { related } A B R=0, \%\end{array}$ & $69.2(38.6-90.9)$ & $92.3(64.0-99.8)$ & $73.7(48.8-90.9)$ & $85.7(57.2-98.2)$ & $65.0(40.8-84.6)$ & $56.3(29.9-80.2)$ \\
\hline
\end{tabular}

$\mathrm{ABR}$, annualized bleeding rate; $\mathrm{Cl}$, confidence interval; FVIII, factor VIII; NA, not applicable; PPAS, per-protocol analysis set.

*Patients in the PPAS completed the second 6 months of prophylaxis with no significant protocol deviations.

Conclusions: Irrespective of pre-study regimen/ABR, targeting 8$12 \%$ FVIII troughs lowered bleeding rates. In patients on pre-study PPx with $A B R \geq 5,50 \%$ and $35 \%$ in the $8-12 \%$ and $1-3 \%$ arms, respectively, achieved zero total bleeds. Across all pre-study PPx patients, $81-93 \%$ and $63-65 \%$ in the $8-12 \%$ and $1-3 \%$ arms, respectively, achieved zero spontaneous joint bleeds. 
PB0543 | Thrombin Generation Capacity of Patients with Severe Haemophilia Receiving Prophylaxis (HOPE Study)

S. Meunier ${ }^{1}$; A. Lienhart ${ }^{1}$; M. Trossaert ${ }^{2}$; V. Gay ${ }^{3}$; C. Berger ${ }^{4}$;

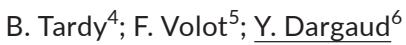

${ }^{1}$ Lyon Haemophilia Treatment Center, Lyon, France; ${ }^{2}$ Nantes

Haemophilia Treatment Center, Nantes, France; ${ }^{3}$ Chambery

Haemophila Treatment Center, Chambery, France; ${ }^{4}$ Saint Etienne

Haemophilia Treatment Center, Saint Etienne, France; ${ }^{5}$ Dijon

Haemophilia Treatment Center, Dijon, France; ${ }^{6}$ University of Lyon -

Lyon Hemophilia Treatment Center, Lyon, France

Background: Prophylaxis is the gold standard for the management of patients with severe haemophilia (PWSH) to avoid joint bleeds and other serious bleeding events. However, there is no international recommendation on optimal prophylactic regimen and dosing is still a crucial issue. We have previously reported that thrombin generation assay (TGA) better reflects the clinical bleeding tendency of PWSH and the test may be a better indicator of the efficacy of prophylaxis compared to FVIII:C.

Aims: The main objective was to compare trough TG capacity and FVIII levels in PWSH A and compare these two laboratory results with the number of spontaneous joint bleeds occurred during the last 6 months of prophylaxis.

Methods: This is an open label, multicentre, prospective cohort study including 34 PWSH A on prophylaxis with standard half-life FVIII concentrates. Trough TG and FVIII:C were measured and clinical data were collected.

Results: During the study period, nine patients (40\% with hemophilic arthropathy) had spontaneous bleeds whilst on prophylaxis and 25 patients had no bleed. The mean trough FVIII:C of all patients was $2.18 \pm 2.9 \mathrm{IU} / \mathrm{dL}$. Trough FVIII:C levels were not significantly different in the group who did not experienced spontaneous bleed (2.6 $\pm 3.3 \mathrm{IU} / \mathrm{dL}$ ) compared to patients who presented spontaneous bleeds $(1 \pm 1.4 \mathrm{IU} / \mathrm{dL} ; P>0.05)$. We observed a tendency for lower ETP levels in the small group of 9 patients who had spontaneous bleeds while they were on prophylaxis (316 $\pm 100 \mathrm{nM}$.min) compared to those who had no bleeding $(500 \pm 379$ nM.min). Fisher exact test showed that trough ETP level>516 nM.min had excellent negative predictive value for spontaneous bleeding risk (NPV $=100 \%$ $(95 \% \mathrm{Cl}=71.5-100) ; P=0.01)$ with a sensitivity $100 \%(95 \% \mathrm{Cl}=66-$ $100)$ and specificity $44 \%(95 \% \mathrm{Cl}=24.4-65)$.

Conclusions: TGA may be valuable additional laboratory tool to FVIII:C, for the monitoring of prophylaxis in PWSH A and to personalize their treatment regimens.
PB0544 | Eptacog Beta (Factor VIla, Recombinant) Provides Rapid Bleed Resolution and Pain Relief in Adult and Adolescent Patients with Hemophilia A or B with Inhibitors

W. Miesbach ${ }^{1}$; C. Hermans ${ }^{2}$; S.W. Pipe ${ }^{3}$; J. Journeycake ${ }^{4}$; J. Ducore'; M. Escobar ${ }^{6}$; M. Wang7; D. Quon ${ }^{8}$; L. Boggio9; N. Chambron ${ }^{10}$; A. AL-Sabbagh ${ }^{11}$; D. Bonzo ${ }^{11}$; C. Macie ${ }^{12}$; I.S. Mitchell ${ }^{13} ;$ J. Mahlangu ${ }^{14}$

${ }^{1}$ Goethe University Hospital, Frankfurt, Germany; ${ }^{2}$ Saint-Luc University Hospital, Brussels, Belgium; ${ }^{3}$ University of Michigan, Ann Arbor, MI, United States; ${ }^{4}$ University of Oklahoma Health Sciences Center, Oklahoma City, OK, United States; ${ }^{5}$ University of California Davis, Sacramento, CA, United States; ${ }^{6}$ University of Texas Health Science Center, Houston, TX, United States; ${ }^{7}$ University of Colorado Denver, Aurora, CO, United States; ${ }^{8}$ Orthopaedic Hemophilia Treatment Center, Los Angeles, CA, United States; ${ }^{9}$ Rush University Medical Center, Chicago, IL, United States, ${ }^{10}$ LFB, Les Ulis, France, ${ }^{11}$ LFB-USA, Framingham, MA, United States, ${ }^{12} \mathrm{HEMA}$ Biologics, Louisville, $\mathrm{KY}$, United States, ${ }^{13}$ Gloval, Broomfield, CO, United States, ${ }^{14}$ University of the Witwatersrand, Johannesburg, South Africa

Background: Rapid bleed control in patients with hemophilia $A / B$ with inhibitors (PwHABI) is critical to maintain normal musculoskeletal structure and function.

Aims: We evaluated the efficacy and safety of eptacog beta (EB; LFB/HEMA Biologics) for the treatment of bleeding episodes (BEs) in adult/adolescent ( $\geq 12$ years of age) $\mathrm{PwHABI}$.

Methods: PERSEPT 1 was a randomized, crossover, phase 3 study. Patients received either an initial dose (ID) of 75 or $225 \mu \mathrm{g} / \mathrm{kg}$ EB followed by per protocol dosing of $75 \mu \mathrm{g} / \mathrm{kg}$ at prespecified intervals (determined by clinical response). The primary efficacy endpoint (PEE) using the EMA definition was the proportion of BEs of all severity (mild, moderate and severe) with 'good' or 'excellent' responses at $12 \mathrm{~h}$. Other efficacy endpoints were time to response, number of administrations, bleeding recurrence, responses at $24 \mathrm{~h}$ and pain assessments at $12 \mathrm{~h}$ (Visual Analogue Scale).

Results: $465 \mathrm{mild} /$ moderate and 3 severe BEs were treated in 27 PwHABI. Hemostatic response (PEE) was achieved in $94 \%$ of BEs in the $225 \mu \mathrm{g} / \mathrm{kg}$ ID regimen (IDR; $n=216$ ) and $86 \%$ in the $75 \mu \mathrm{g} / \mathrm{kg}$ IDR ( $n=252$ ) within $12 \mathrm{~h}$. The median time to response was $3 \mathrm{~h}$ and $6 \mathrm{~h}$ respectively in mild/moderate BEs. Hemostasis was achieved at $12 \mathrm{~h}$ for the 3 severe BEs. A single dose of $225 \mu \mathrm{g} / \mathrm{kg}$ achieved response in $81.3 \%$ of BEs compared to $29 \%$ with the $75 \mu \mathrm{g} / \mathrm{kg}$ dose regardless of bleed severity (Figure 1). 
Pain was relieved in $\geq 89 \%$ of cases by $12 \mathrm{~h}$ for both IDRs. The success proportion at $24 \mathrm{~h}$ was $>98 \%$; most BEs had no recurrence or need for alternative treatment. Other efficacy results are detailed in Table 1. No thromboembolic, allergic, or anti-EB antibodies were observed.

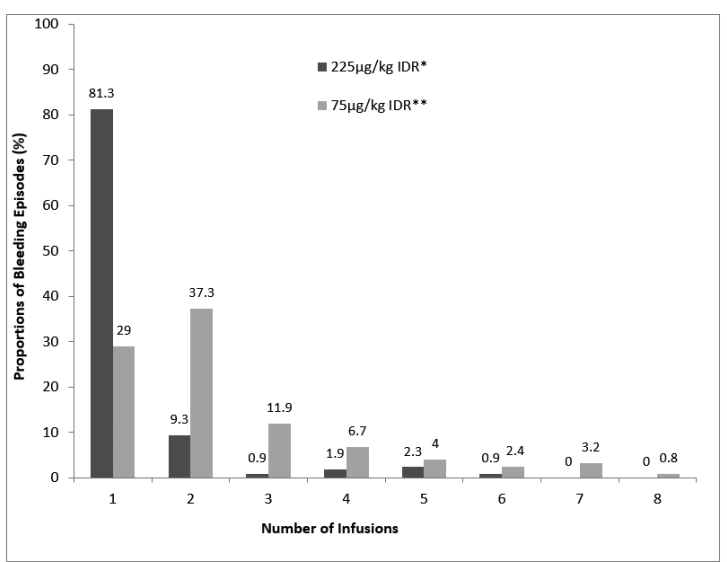

$B E$, bleeding episode; IDR, initial dose regimen; $92 h$, every 2 hours; $q 3 h$, every 3 hours.

*225 $\mu \mathrm{g} / \mathrm{kg}$ IDR: $225 \mu \mathrm{g} / \mathrm{kg}$ followed if necessary, after gh by $75 \mu \mathrm{g} / \mathrm{kg} q 3 \mathrm{~h}$, for mild or moderate BEs and by $75 \mu \mathrm{g} / \mathrm{kg}$ q2 $\mathrm{h}$ after 6h for severe BEs.

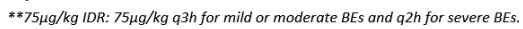

FIGURE 1 Successfully Treated Bleeding Episodes (Includes Mild/Moderate and Severe Bleeds) by Number of Infusions and Treatment Regimen

TABLE 1 Primary, Secondary and Tertiary Efficacy Endpoints ( $N=468$ BEs, Regardless of Severity*)

\begin{tabular}{|c|c|c|}
\hline & \multicolumn{2}{|c|}{ Initial Dose Regimen at the time of BE } \\
\hline & $\begin{array}{l}75 \mu g / k g \\
(N=25)\end{array}$ & $\begin{array}{c}225 \mu \mathrm{g} / \mathrm{kg} \\
(\mathrm{N}=25)\end{array}$ \\
\hline \multirow[t]{2}{*}{ Number of BEs } & 252 & 216 \\
\hline & \multicolumn{2}{|c|}{ Success proportion $[95 \% \mathrm{CI}]$} \\
\hline $\begin{array}{l}\text { Primary endpoint } \\
\text { BEs with good or excellent response at } 12 \mathrm{~h}\end{array}$ & $85.7 \%[75.0,96.4]$ & $93.8 \%[88.9,98.6]$ \\
\hline Secondary/Tertiary endpoints & & \\
\hline BEs with successful pain relief at $12 \mathrm{~h} * *$ & $92.8 \%[84.8,98.4]$ & $89.9 \%[82.2,97.6]$ \\
\hline BEs without recurrence of bleeding within $24 \mathrm{~h}$ & $100.0 \%[100.0,100.0]$ & $99.5 \%[98.6,100.0]$ \\
\hline BEs not requiring alternative treatment & $98.4 \%[95.9,100.0]$ & $99.5 \%[98.6,100.0]$ \\
\hline BEs with good or excellent response at $24 \mathrm{~h}$ & $96.7 \%[93.3 \%, 100.0]$ & $98.6 \%[98.6,100.0]$ \\
\hline
\end{tabular}

Conclusions: EB is an effective and safe bypassing agent that provides a new treatment option for BEs in adult/adolescent PwHABI. A single administration of $225 \mu \mathrm{g} / \mathrm{kg}$ EB offers rapid $B E$ resolution in the vast majority of patients.
PB0546 | Ultrasound Assessment of the Joint Status in Hemophilia Patients under Prophylaxis Using the Hemophilia Early Arthropathy Detection with Ultrasound (HEAD-US) Score

I. Ricca ${ }^{1}$; B. Pollio ${ }^{1}$; R. Albiani ${ }^{1}$; C. Martinoli ${ }^{2}$

${ }^{1}$ Centro di Riferimento Regionale per le Malattie Emorragiche e

Trombotiche Ereditarie in Età Pediatrica - Città della Salute e della

Scienza di Torino, Turin, Italy; ${ }^{2}$ DISSAL University of Genova - UO

Radiologia, IRCCS Policlinico San Martino, Genoa, Italy

Background: Progressive arthropathy is the main cause of morbidity in hemophilia. Prophylaxis significantly reduces acute bleeds but subclinical disease may still occur. The ultrasound detection of early joint damage may contribute to optimize prophylaxis.

Aims: To assess the joint status in a series of hemophilia patients under prophylactic treatment by means of the Hemophilia Early Arthropathy Detection with Ultrasound (HEAD-US) system.

Methods:

Starting from January 2016, we prospectively performed the ultrasound examination of the elbows, the knees and the ankles in a series of 39 consecutive hemophilia patients under prophylactic treatment (Table). Ultrasound assessments were performed during the annual follow-up visit. When synovial hypertrophy was detected, an additional ultrasound study was planned 6 months later than the baseline examination. 
TABLE 1 Patients' characteristics.

\begin{tabular}{|c|c|c|c|c|}
\hline Diagnosis ( $n^{\circ}$ of patients) & severe HA (27) & moderate HA (4) & severe HB (8) & Total (39) \\
\hline median age (range) & 14 yrs (3-37) & 9 yrs (6-13) & 12 yrs (4-33) & 14 yrs (3-37) \\
\hline prophylaxis with SHL products & 8 & 3 & 1 & 12 \\
\hline prophylaxis with EHL products & 13 & 1 & 7 & 21 \\
\hline $\begin{array}{l}\text { prophylaxis with Emicizumab ( } n^{\circ} \text { of patients } \\
\text { with/without inhibitors) }\end{array}$ & $6(2 / 4)$ & n.a. & n.a. & 6 \\
\hline
\end{tabular}
with/without inhibitors)

$\mathrm{HA}=$ hemophilia $\mathrm{A} ; \mathrm{HB}$ = hemophilia $\mathrm{B} ; \mathrm{SHL}$ = standard half-life; $\mathrm{EHL}$ = extended half-life; .a.= not applicable.

Results: A total of 105 ultrasound examinations (630 joints) using the HEAD-US protocol were performed. At the first examination, 11 patients under primary prophylaxis (5 severe HA; 2 moderate HA, $4 \mathrm{HB}$ ) had HEAD-US score $=0$, whereas 5 patients with severe arthropathy (4 HA; $1 \mathrm{HB}$ ) presented worse HEAD-US scores related to osteochondral damage. The median HEAD-US score at baseline was 3. A second follow-up examination was performed in 35 patients (29 HA, 6 HB) with a median follow-up of 28 (range 6-60) months. Out of the 7 patients with HEAD-US score $=0$ evaluable for followup, 5 (2 severe HA, 1 moderate HA and 2 HB treated with rVIIIFc, moroctocog alfa and rIX-FP respectively) didn't change joint status and HEAD-US score during follow-up (median 44 months, range 17$60)$, whereas 2 patients with severe HA treated with SHL products worsen their synovial HEAD-US subscore. The follow-up results of patients with HEAD-US>0 is illustrated in the.

Figure. Three-years follow-up of patients with arthropathy: total HEAD-US score (A) and synovial HEAD-US subscore (B)
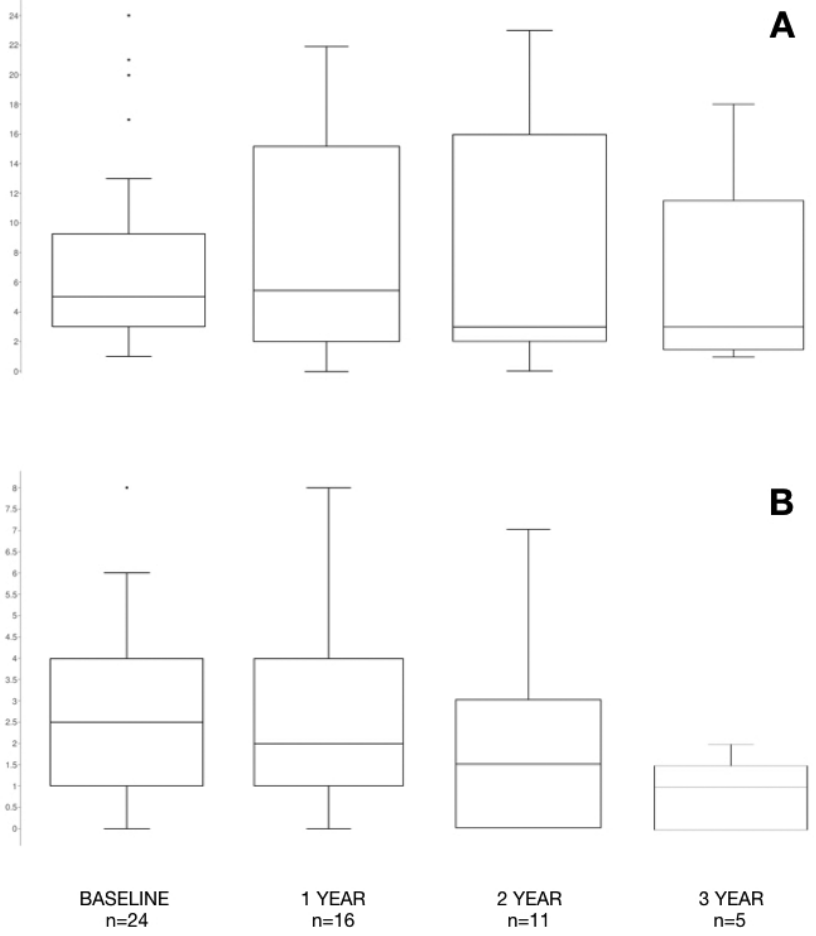

B

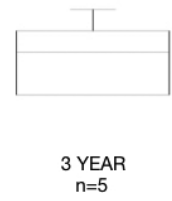

FIGURE 1 Three-years follow-up of patients with arthropathy
Conclusions: Ultrasound performed with the HEAD-US protocol may help clinicians to monitor synovial hypertrophy and osteochondral damage during prophylaxis. Although preliminary, our data suggest progressive reduction of active joint disease during optimized prophylaxis.

PB0547 | The Safety of Eptacog Beta in Hemophilia Patients with Inhibitors in Adult, Pediatric, and Perisurgical Settings

J. Mahlangu ${ }^{1} ;$ M. Escobar ${ }^{2} ;$ M. Callaghan ${ }^{3} ;$ J. Ducore $^{4}$;

P. De Moerloose ${ }^{5}$; C. Hermans ${ }^{6}$; G. Castaman ${ }^{7}$; J.-F. Schved ${ }^{8}$;

J. Journeycake ; C. Leissinger ${ }^{10}$; J. Luck ${ }^{11}$; D. Quon ${ }^{11}$; M. Recht ${ }^{12}$; R. Sidonio $\mathrm{Jr}^{13}$; A. Srivastava ${ }^{14}$; M. Wang ${ }^{15}$; G. Young ${ }^{16}$; D. Bonzo ${ }^{17}$; N. Chambron ${ }^{18}$; C. Kessler ${ }^{19}$

${ }^{1}$ University of the Witwatersrand, Johannesburg, South Africa;

${ }^{2}$ University of Texas Health Science Center, Houston, United States;

${ }^{3}$ Central Michigan University, Detroit, United States; ${ }^{4}$ University of California Davis, Sacramento, United States; ${ }^{5}$ University hospitals of Geneva HUG, Geneva, Switzerland; 'Saint-Luc University Hospital, Brussels, Belgium; ${ }^{7}$ Careggi University Hospital, Florence, Italy; ${ }^{8} \mathrm{CHRU}$ Montpellier, Montpellier, France; ${ }^{9}$ University of Oklahoma Health Sciences Center, Oklahoma City, United States, ${ }^{10}$ Tulane University, New Orleans, United States, ${ }^{11}$ Orthopaedic Hemophilia Treatment Center, Los Angeles, United States, ${ }^{12}$ American Thrombosis $\&$ Hemostasis Network and Oregon Health \& Science University, Portland, United States, ${ }^{13}$ Aflac Cancer and Blood Disorders and Emory University, Atlanta, United States, ${ }^{14}$ Christian Medical College, Vellore, India, ${ }^{15}$ University of Colorado Denver, Aurora, United States, ${ }^{16}$ Children's Hospital Los Angeles, Los Angeles, United States, ${ }^{17}$ LFBUSA, Framingham, United States, ${ }^{18}$ LFB, Les Ulis, France, ${ }^{19}$ Georgetown University, Washington D.C., United States

Background: Eptacog beta (EB; LFB, HEMA Biologics), is a recombinant FVIla shown to be safe and effective in the treatment of bleeding episodes (BEs) in patients with hemophilia A/B with inhibitors (PwHABI).

Aims: We report on the overall pooled safety data from three pivotal prospective Phase 3 studies (PERSEPT 1, 2, and 3) using EB in adult, pediatric, and perisurgical settings in PWHABI.

Methods: Twenty-seven subjects in PERSEPT 1 (ages 12-54) and 25 in PERSEPT 2 (ages 1-11) treated BEs with an initial dose of 75 or $225 \mu \mathrm{g} / \mathrm{kg}$ EB followed by per protocol dosing of $75 \mu \mathrm{g} / \mathrm{kg}$ at 
prespecified intervals (determined by clinical response). Twelve PERSEPT 3 subjects (ages 2-56) received an initial perioperative dosing of $75 \mu \mathrm{g} / \mathrm{kg}$ (for minor procedures) or $200 \mu \mathrm{g} / \mathrm{kg}$ (for major surgeries) with subsequent $75 \mu \mathrm{g} / \mathrm{kg}$ doses given intraoperatively and post-operatively as per protocol. Descriptive statistics were used for data analyses.

Results: Sixty PwHABI received 3,388 doses of EB during 1,087 exposure episodes (associated with BEs, invasive procedures, postoperative treatments, or pharmacokinetic assessments). Of $133 \mathrm{ad}$ verse events (AEs), 10 were treatment-related and 7 were serious $A E s$ (SAEs). None of those 7 SAEs were considered treatment-related: acute tonsillitis, subarachnoid hemorrhage, intracranial bleed, paresis, bloody stool/dysentery, blood loss anemia, and gastrointestinal hemorrhage. One death occurred due to blood loss anemia that was deemed unlikely related to EB treatment by the independent PERSEPT 3 Data Monitoring Committee. AE distribution by type and by study is shown in Table 1. Overall, EB was well-tolerated; no allergic, hypersensitivity, anaphylactic, or thrombotic events occurred. No neutralizing anti-EB antibodies were detected (Table 1).

TABLE 1 Safety and Immunogenicity Data by Study

\begin{tabular}{|c|c|c|c|c|c|}
\hline & \\
\hline & & $\begin{array}{l}\text { PERSEPT } 1 \\
\text { (27 patients) }\end{array}$ & $\begin{array}{c}\text { PERSEPT } 2 \\
\text { (25 patients) }\end{array}$ & $\begin{array}{c}\text { PERSEPT } 3 \\
\text { (12 patients) }\end{array}$ & $\begin{array}{c}\text { All studies } \\
\text { (60 patients) })^{*}\end{array}$ \\
\hline TEAEs & $\begin{array}{l}\text { Number of AEs; } n \\
\text { Number of subjects with AEs; } \mathrm{N}(\%){ }^{* * *} \\
\text { Number of SAEs; } \mathrm{n} \\
\text { Number of subjects with SAEs; } \mathrm{N}(\%) * *\end{array}$ & $\begin{array}{c}27 \\
12(44.4) \\
2 \\
1(3.7) \\
\end{array}$ & $\begin{array}{c}70 \\
17(68.0) \\
3 \\
2(8.0) \\
\end{array}$ & $\begin{array}{c}36 \\
10(83.3) \\
2 \\
1(8.3) \\
\end{array}$ & $\begin{array}{c}133 \\
39(65) \\
7 \\
4(6.7) \\
\end{array}$ \\
\hline \begin{tabular}{|l|l|} 
Treatment- \\
Related AEs
\end{tabular} & $\begin{array}{l}\text { Number of AEs; } \mathrm{n}^{* * *} \\
\text { Number of subjects with AEs; } \mathrm{N}(\%)^{* * *}\end{array}$ & $\begin{array}{c}7 \\
2(7.4)\end{array}$ & $\begin{array}{c}0 \\
0(0)\end{array}$ & $\begin{array}{c}3^{6} \\
1(8.3)\end{array}$ & $\begin{array}{c}10 \\
3(5.0)\end{array}$ \\
\hline \multicolumn{2}{|c|}{ Immunogenicity; NFF } & 0 & 0 & 0 & 0 \\
\hline \multicolumn{6}{|c|}{$\begin{array}{l}A E \text {, adverse event; } E B \text {, eptacogo beta; } S A E \text {, serious adverse event; } T E A E \text {, treatment-emergent adverse event, defined os any } A E \text { that occurred following } \\
\text { initial } E B \text { administration regardless of whether the } A E \text { is drug relatede. }\end{array}$} \\
\hline \multicolumn{6}{|c|}{$\begin{array}{l}\text { *PERSEPT 1, PERSEPT } 2 \text {, and P PESEEPT } 3 \text { collectively errolled } 64 \text { subjects; however, as two subjects from PERSEPT } 1 \text { and two subjects from PERSEPT } 2 \text { also } \\
\text { participated in in PERSET } 3 \text {, in total } 60 \text { individuals participated in the three trials. }\end{array}$} \\
\hline \multicolumn{6}{|c|}{${ }^{* *}$ The percentage of subjects experiencing AEs, relative to the number of subjects in each trial (or trial subgroup), is shown in parentheses. } \\
\hline \multicolumn{6}{|c|}{ 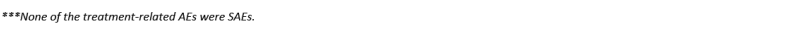 } \\
\hline \multicolumn{6}{|c|}{ 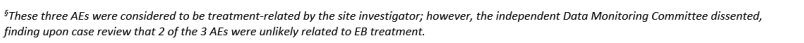 } \\
\hline
\end{tabular}

Conclusions: The pooled safety analysis of the PERSEPT studies established a favorable EB safety profile for the treatment of BEs and perioperative management in $\mathrm{PwHABI}$. No thrombotic events were observed, and no neutralizing anti-EB antibodies were found.

PB0548 | The Clinical Bleeding Phenotype of Patients with Nonsevere Hemophilia A and B

A. Zwagemaker ${ }^{1}$; F. Kloosterman ${ }^{1}$; M. Coppens ${ }^{2}$; C.N. Bagot ${ }^{3}$; E.A.M. Beckers ${ }^{4}$; S. Boyce ${ }^{5}$; P. Brons ${ }^{6}$; M.H. Cnossen ${ }^{7}$;

J. Eikenboom ${ }^{8}$; F.W.G. Leebeek ${ }^{9}$; C. Male ${ }^{10}$; K. Meijer ${ }^{11}$; L. Nieuwenhuizen ${ }^{12}$; S.C. Gouw ${ }^{1,13}$; J.G. van der Bom ${ }^{14}$; G. Castaman ${ }^{15}$; K. Fijnvandraat ${ }^{1,16}$; DYNAMO Study Group ${ }^{1}$ Amsterdam UMC, University of Amsterdam, Emma Children's Hospital, Pediatric Hematology, Amsterdam, Netherlands; ${ }^{2}$ Department of Vascular Medicine, Amsterdam University Medical Centres, Amsterdam, Netherlands; ${ }^{3}$ Department of Haematology, Glasgow Royal Infirmary, Glasgow, United Kingdom; ${ }^{4}$ Department of Hematology, Maastricht University Medical Centre, Maastricht, Netherlands; ${ }^{5}$ Department of Haematology, University Hospital Southampton,
Southampton, United Kingdom; ${ }^{6}$ Department of Pediatric HematoOncology, Radboud University Medical Center, Nijmegen, Netherlands; ${ }^{7}$ Department of Pediatric Hematology, Erasmus University Medical Center - Sophia Children's Hospital, Rotterdam, Netherlands;

${ }^{8}$ Department of Internal Medicine, Division of Thrombosis and Hemostasis, Leiden University Medical Center, Leiden, Netherlands; ${ }^{9}$ Department of Hematology, Erasmus MC, Erasmus University Medical Center, Rotterdam, Netherlands, ${ }^{10}$ Department of Pediatrics, Medical University of Vienna, Vienna, Austria, ${ }^{11}$ Department of Hematology, University Medical Center Groningen, Groningen, Netherlands,

${ }^{12}$ Department of Hematology, Maxima Medical Center Eindhoven, Eindhoven, Netherlands, ${ }^{13}$ Department of Clinical Epidemiology, Leiden University Medical Center, Leiden, Netherlands, ${ }^{14}$ Center for Clinical Transfusion Research, Sanquin-Leiden University Medical Center, Leiden, Netherlands, ${ }^{15}$ Department of Oncology, Center for Bleeding Disorders, Careggi University Hospital, Florence, Italy, ${ }^{16}$ Department of Molecular Cellular Hemostasis, Sanquin Research and Landsteiner Laboratory, Amsterdam, Netherlands

Background: Hemophilia is an inherited hemorrhagic disorder, characterized by bleeding episodes, mainly in joints and muscles. Although around half of all patients have non-severe hemophilia, most research has focused on severe patients. Detailed information on the clinical phenotype in non-severe hemophilia is largely lacking. This information is urgently required as presently novel treatment modalities change the phenotype of severe hemophilia into nonsevere hemophilia.

Aims: To gain insight in the clinical bleeding phenotype of patients with non-severe hemophilia A and B.

Methods: The DYNAMO study is an observational multicenter cohort study including patients with non-severe hemophilia $A$ and $B$ (FVIII/FIX 2-35 IU/dL) aged 12-55 years. Data were collected from medical files on lifetime and past 10-year treated bleeding history. We calculated annualized bleeding rates (ABRs) and annualized joint bleeding rates (AJBRs). Results were compared between mild and moderate hemophilia using a Mann-Whitney $U$ test. The study was approved by medical ethics committees and written consent was obtained (ClinicalTrials.gov: NCT03623295).

Results: Currently, we have included 242 patients in the DYNAMO study. We report preliminary data on the first 186 patients. The database will be closed in May 2021 and final results will be presented at the congress. Patients had a median age of 35 years (IQR 24-48). The median factor level was $10 \mathrm{IU} / \mathrm{dL}$ (IQR 4-16). In our population, $81 \%$ had a positive lifetime history for any bleeding episode treated with factor concentrate and $50 \%$ for joint bleeding (Table 1 ). 
TABLE 1 Bleeding history, cumulative bleeds and ABR's for the total population and sub-categorized per mild and moderate hemophilia.
Total population
Mild hemophilia
$(\mathrm{N}=186)$
Moderate hemophilia $(N=60)$
$(\mathrm{N}=126)$
Moderate vs. mild
(P-value)

Lifetime bleeding history, $n(\%)$

\begin{tabular}{|c|c|c|c|c|}
\hline $\begin{array}{l}\geq 1 \text { bleed treated with } \\
\text { factor concentrate }\end{array}$ & $150(81 \%)$ & $55(92 \%)$ & $95(75 \%)$ & 0.008 \\
\hline $\begin{array}{l}\geq 1 \text { joint bleed treated } \\
\text { with factor } \\
\text { concentrate }\end{array}$ & $92(50 \%)$ & $42(72 \%)$ & $50(40 \%)$ & $<0.001$ \\
\hline \multicolumn{5}{|c|}{ Cumulative treated bleeds over the last 10 years, median (IQR) } \\
\hline Treated bleeds & $2(1-5)$ & $4(2-14)$ & $2(0-4)$ & $<0.001$ \\
\hline Treated joint bleeds & $0(0-2)$ & $1(0-4)$ & $0(0-1)$ & $<0.001$ \\
\hline \multicolumn{5}{|c|}{ Annualized treated bleeding rates, median (IQR) } \\
\hline Treated ABR & $0.2(0.1-0.5)$ & $0.6(0.2-1.4)$ & $0.2(0.0-0.3)$ & $<0.001$ \\
\hline Treated AJBR & $0.0(0.0-0.2)$ & $0.1(0.0-0.4)$ & $0.1(0.0-0.1)$ & $<0.001$ \\
\hline
\end{tabular}

The median ABR was 0.2 (IQR 0.1-0.5) for the total population and was inversely associated with baseline factor level (Figure 1).

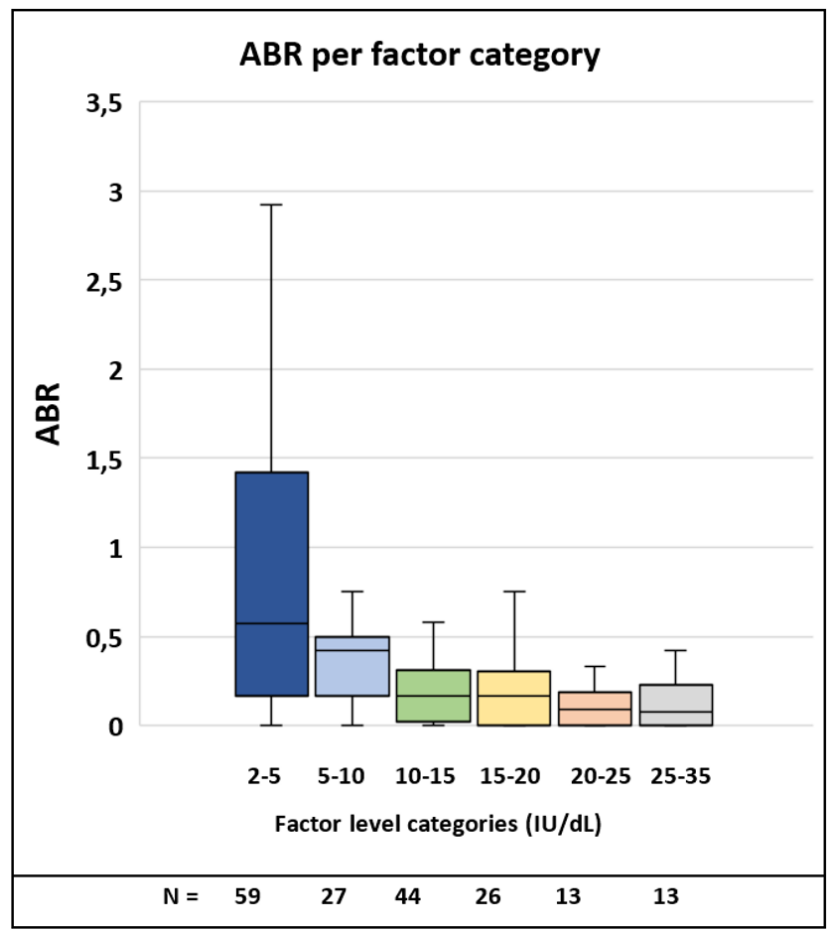

FIGURE 1 Treated ABR according to baseline factor levels categories presented in boxplots (Box representing median and IQR)

Despite lower ABRs for mild patients, this group still experienced a median of 0.2 treated bleeding events per year compared to a median of 0.6 for moderate patients.

Conclusions: Despite low annualized bleeding rates, half of this nonsevere hemophilia population has suffered a treated hemarthrosis.
PB0549 | Immunogenicity and Safety of Simoctocog Alfa in Previously Treated Patients Switching to Simoctocog Alfa in the GENA Clinical Trial Programme

M.J. Manco-Johnson ${ }^{1}$; R.J. Liesner ${ }^{2}$; A. Tiede ${ }^{3}$

${ }^{1}$ University of Colorado Anschutz Medical Campus, Aurora, United States; ${ }^{2}$ Great Ormond Street Hospital for Children NHS Trust Haemophilia Centre, NIHR GOSH BRC, London, United Kingdom; ${ }^{3}$ Hannover Medical School, Hannover, Germany

Background: Simoctocog alfa (Nuwiq ${ }^{\circledR}$ ) is a 4th generation recombinant FVIII (rFVIII) produced in a human cell line. Simoctocog alfa has been evaluated in nine completed prospective clinical studies in adult and paediatric previously treated patients (PTPs) with severe haemophilia A.

Aims: To assess immunogenicity and safety of simoctocog alfa in PTPs enrolled in the GENA clinical trial programme.

Methods: All participants had previously received FVIII for at least 50 exposure days. Pooled immunogenicity and safety data were analysed. In all studies, inhibitory antibodies were measured by the Bethesda assay, with an inhibitor titre $\geq 0.6$ Bethesda units $(\mathrm{BU}) / \mathrm{mL}$ classified as a positive inhibitor test. All treatment-related adverse events recorded across all studies were identified.

Results: A total of 310 paediatric or adult PTPs were switched to simoctocog alfa and received treatment. Of the 310 individuals, 119 (38.4\%) switched from plasma-derived FVIII (pdFVIII) products, 151 (48.7\%) from rFVIII, 36 (11.6\%) had received both pdFVIII and rFVIII, and $4(1.3 \%)$ switched from an unknown FVIII. Patients received a total of 54,112 infusions during the studies. FVIII inhibitors did not develop in any patient after switching to simoctocog alfa. A total of 15 treatment-related adverse events were recorded in 9 (2.9\%) patients. Only one (0.3\%) patient had an AE (mild pyrexia that resolved) that was classified as serious because the patient was hospitalised. 
Conclusions: None of the 310 PTPs who switched to simoctocog alfa developed an inhibitor and very few treatment-related adverse occurred during extensive exposure.

PB0550 | Real-world Joint Bleeding Rates in Patients with Congenital Hemophilia and Inhibitors: Final Data from the FEIBA Global Outcome (FEIBA GO) Study

J. Windyga ${ }^{1}$; C. Hermans ${ }^{2}$; P.A. Holme ${ }^{3}$; C. Escuriola-

Ettingshausen ${ }^{4}$; A.R. Cid ${ }^{5}$; K. Khair ${ }^{6}$; J. Oldenburg ${ }^{7}$; J. Botha ${ }^{8}$; N. Kemenyash ${ }^{8}$; M. Kokot-Kierepa ${ }^{8}$; C. Négrier ${ }^{9}$

${ }^{1}$ Department of Hemostasis Disorders and Internal Medicine, Institute of Hematology and Transfusion Medicine, Warsaw, Poland; ${ }^{2}$ Division of Haematology, Cliniques universitaires Saint-Luc, UCLouvain, Belgium; ${ }^{3}$ Department of Haematology, Oslo University Hospital and Institute for Clinical Medicine, University of Oslo, Oslo, Norway; ${ }^{4}$ HämophilieZentrum Rhein Main GmbH, Mörfelden-Walldorf, Germany; ${ }^{5}$ Unidad de Hemostasia y Trombosis, Hospital Universitario y Politécnico La

Fe, València, Spain; ${ }^{6}$ Centre for Outcomes and Experience Research in Children's Health, Illness and Disability (ORCHID), Great Ormond Street Hospital for Children, London, United Kingdom; ${ }^{7}$ Institute of Experimental Hematology and Transfusion Medicine, Bonn University Clinic, Bonn, Germany; ${ }^{8}$ Takeda Pharmaceuticals International AG, Zürich, Switzerland; ${ }^{9}$ Louis Pradel Cardiology Hospital, University Claude Bernard, Lyon, France

Background: The development of inhibitory antibodies is a major complication of factor replacement therapy in patients with congenital hemophilia, with the risk of joint arthropathy in patients with recurrent joint bleeding. The long-term real-world effectiveness and safety of activated prothrombin complex concentrate (aPCC; Baxalta US Inc, a Takeda company, Lexington, MA, USA) have been assessed in patients with congenital hemophilia $A$ or $B$ with high-responding inhibitors ( $\mathrm{PwHI}$ ) in the FEIBA Global Outcome study (FEIBA GO).

Aims: To assess the effectiveness of prophylactic and on-demand aPCC therapy on joint health in $\mathrm{PwHI}$ and its safety.

Methods: FEIBA GO (EUPAS6691) was a post-authorization, prospective, observational, non-interventional, multicenter cohort study. Male PwHI diagnosed and prescribed treatment with aPCC (physician's discretion) before study entry were eligible; planned observational period was 4 years. Ethics committee approval and patient consent were obtained. Data are from the safety analysis set.

Results: Fifty PwHI who were enrolled between September 3, 2014 and December 19, 2017 received aPCC prophylaxis $(n=37)$ or ondemand treatment $(n=13)$ at 25 sites in 11 countries (hemophilia A: $n=49$; hemophilia B: $n=1$ ). Overall annualized joint bleeding rate (AJBR) was lower in patients receiving prophylaxis than in those receiving on-demand therapy (Table 1). Overall, 11 (29.7\%) and 4 (33.3\%) PwHIs receiving prophylaxis or on-demand therapy had zero joint bleeds, respectively. Among PwHIs receiving prophylaxis or ondemand therapy, zero joint bleeds occurred in 6/18 PwHIs with 2-4 years' follow-up and in $2 / 7$ PwHIs with $>4$ years' follow-up. A total of 177 and 31 adverse events were reported in $70.0 \%(28 / 40)$ and $76.9 \%$ (10/13) of $\mathrm{PwHI}$ receiving prophylaxis or on-demand therapy, respectively. Most frequently reported events were infections and musculoskeletal disorders (Table 2). No thrombotic microangiopathy was reported.

TABLE 1 AJBR by treatment group and by follow-up period*

\begin{tabular}{|c|c|c|c|c|c|c|}
\hline & \multicolumn{2}{|c|}{ By treatment regimen* } & \multicolumn{2}{|c|}{ Patients completing study*†‡ } & \multicolumn{2}{|c|}{ By follow-up period ${ }^{\S}$} \\
\hline & $\begin{array}{l}\text { Prophylaxis } \\
(n=37)\end{array}$ & $\begin{array}{l}\text { On-demand } \\
(n=13)\end{array}$ & $\begin{array}{l}\text { Prophylaxis } \\
(n=6)\end{array}$ & $\begin{array}{l}\text { On-demand } \\
(n=5)\end{array}$ & $2-4$ years & $>4$ years \\
\hline $\begin{array}{l}\text { Median (range) age } \\
\text { at baseline, years }\end{array}$ & $15.0(2-71)$ & $36.0(5-65)$ & $17.5(3-71)$ & $40.0(33-65)$ & - & - \\
\hline AJBR & $n=37$ & $n=12$ & $n=8$ & $n=4$ & $n=18$ & $n=7$ \\
\hline Median (range) & $2.3(0-28.4)$ & $6.3(0-20.9)$ & $0.5(0-19.9)$ & $2.7(0-16.3)$ & $3.0(0-8.6)$ & $4.8(0-19.9)$ \\
\hline
\end{tabular}

‡Overall follow-up for 11 patients completing study: 44.4 patient-years.

* Patients could appear in $>1$ regimen group (prophylaxis or on-demand).

${ }^{\dagger}$ Includes 4 patients (prophylaxis, $n=2$; on-demand, $n=2$ ) who had a completion visit as they had $>47$ months of follow-up but did not reach

48-months' follow-up.

$\S$ For all treatment regimens. 
TABLE 2 Most frequently reported adverse events*

\begin{tabular}{lll} 
& $\begin{array}{l}\text { Treatment regimen at time of } \\
\text { event }\end{array}$ \\
$\begin{array}{ll}\text { Prophylaxis } \\
(\%) \text { of patients, } \boldsymbol{n} \text { of events }\end{array}$ & $\begin{array}{l}\text { On-demand } \\
(\boldsymbol{n}=\mathbf{n}=13)\end{array}$ \\
$\begin{array}{l}\text { Nasopharyngitis } \\
\text { Infections (not specified) }\end{array}$ & $6(15.0) 8$ & $3(23.1) 7$ \\
$\begin{array}{l}\text { Venous device-related } \\
\text { infection }{ }^{\dagger}\end{array}$ & $5(12.5) 9$ & 0 \\
Hemarthrosis & $4(10.0) 6$ & $1(7.7) 1$ \\
Pain in extremity & $2(5.0) 2$ & $2(15.4) 2$ \\
Synovitis & $4(10.0) 5$ & 0 \\
\hline
\end{tabular}

* In $\geq 10 \%$ of patients receiving either regimen; patients can appear in $>1$ regimen group (prophylaxis or on-demand).

${ }^{\dagger}$ Central venous catherization reported as pre-existing condition in 1 patient in the prophylaxis group ( 3 events) and as an adverse event in 2 patients in the prophylaxis group ( 2 events).

Conclusions: These real-world data further support the use of ondemand and prophylactic aPCC therapy to maintain low levels of recurrent joint bleeding in $\mathrm{PwHI}$.

\section{PB0551 | Real-world Usage of rFIXFc in Sweden: A Report from} the Swedish National Registry for Bleedings Disorders

A. Olsson ${ }^{1}$; L. Myrin-Westesson ${ }^{1}$; F. Baghaei ${ }^{1}$; M. Holmström ${ }^{2,3}$;

E. Olsson ${ }^{2}$; M. Magnusson ${ }^{2,4,5}$; S. Ranta ${ }^{4}$; J. Astermark ${ }^{6}$;

N. Gretenkort Andersson ${ }^{7} ;$ J. Thanner ${ }^{8} ;$ J. Szamosi ${ }^{8} ;$ K. Sennfält ${ }^{8}$

${ }^{1}$ Region Västra Götaland, Sahlgrenska University Hospital, Department of Medicine, Gothenburg, Sweden; ${ }^{2}$ Coagulation Unit, Department of Hematology, Karolinska University Hospital, Stockholm, Sweden; ${ }^{3}$ Dept of Health, Medicine and Caring Sciences, Linköping University, Linköping, Sweden; ${ }^{4}$ Pediatric Coagulation Unit, Astrid Lindgren Children's hospital, Karolinska University hospital, Stockholm, Sweden; ${ }^{5}$ MMK and CLINTEC, Karolinska Institutet, Stockholm, Sweden; ${ }^{6}$ Department of Translational Medicine and Department of Hematology, Oncology and Radiation Physics, Lund University, Skåne University Hospital, Lund, Sweden; ${ }^{7}$ Department for Paediatric Haematology and Oncology, Children's Hospital, University Hospital Skåne and Department of Clinical Sciences, Paediatrics, Lund University, Lund, Sweden; ${ }^{8}$ Swedish Orphan Biovitrum AB, Stockholm, Sweden

Background: The current standard of care for patients with moderate and severe haemophilia $B(\mathrm{HB})$ is prophylactic treatment with factor IX (FIX). In Sweden, patients with haemophilia are provided centralized care at one of the three centers in Gothenburg, Malmö or Stockholm. All centers are using a web-based national quality registry for bleeding disorders.

Aims: To use real-world data to describe HB patients being on prophylaxis and to compare treatment and outcomes in patients before and after switching from a standard half-life (SHL) FIX product to extended half-life recombinant FIX Fc fusion protein (rFIXFc). Methods: Patient characteristics and treatment data from the Swedish haemophilia registry were collected and assessed for all $\mathrm{HB}$ patients aged $\geq 13$ years being treated prophylactically for $\geq 6$ months at the cut-off date (November 18, 2020). A retrospective within-patient comparison of prescribed dose, patient-reported factor consumption, number of injections, and annualised bleeding rate (ABR) of patients who switched from SHL FIX to rFIXFc, with $\geq 6$ months documented prophylactic treatment pre- and post-switch was performed.

Results: Out of $38 \mathrm{HB}$ patients receiving rFIXFc treatment the majority (35 patients; 92\%) were on prophylactic treatment (Figure 1). In within-patient analyses before and after switching to rFIXFc median (IQR) follow-up time was 609.0 (426.5-657.5) days ( $n=19$ ); median (IQR) prescribed dose decreased by $42 \%$ from 77.4 (42.5-82.0) to 45.0 (37.0-63.4) IU/kg/week ( $n=14$ ); median (IQR) patient-reported factor consumption decreased by $37 \%$ from 68.1 (45.9-91.2) to 42.8 (37.8-50.7) IU/kg/week ( $n=16$ ); median (IQR) number of injections per week decreased from $2.0(2.0-3.2)$ to 1.0 (1.0-1.0) ( $n=17)$; median (IQR) ABR was 0.0 (0.0-1.0) on SHL FIX and 0.0 (0.0-0.0) on $\operatorname{rFIXFc}(n=18)$ (Table 1$)$

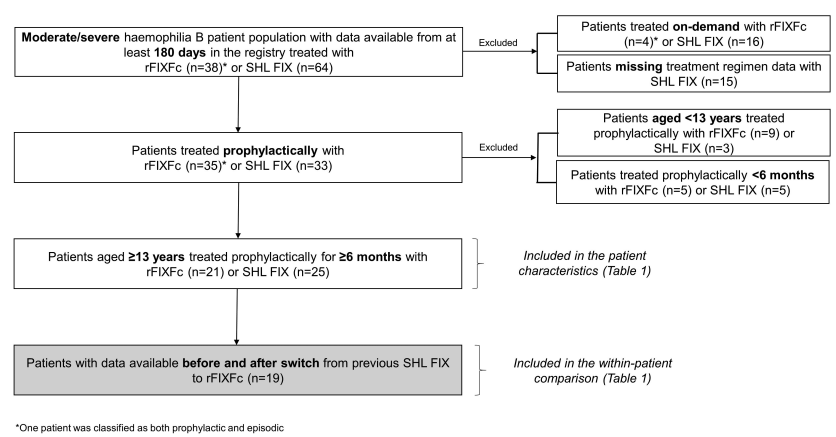

FIGURE 1 Study population

TABLE 1 Analyses of the haemophilia B population aged $\geq 13$ years treated prophylactically for $\geq 6$ months

\begin{tabular}{|c|c|c|}
\hline $\begin{array}{l}\text { Patient } \\
\text { characteristics }\end{array}$ & SHL FIX $(n=25)$ & $\operatorname{rFIXFc}(n=21)$ \\
\hline \multicolumn{3}{|c|}{ Severity of haemophilia; $n(\%)$} \\
\hline Severe & $22(88.0)$ & $13(61.9)$ \\
\hline Moderate & $3(12.0)$ & $8(38.1)$ \\
\hline \multicolumn{3}{|l|}{ Age (years) } \\
\hline Mean (SD) & $46.3(22.7)$ & $39.5(18.9)$ \\
\hline Median (IQR) & $48.0(24.0-65.0)$ & $34.0(26.0-53.7)$ \\
\hline \multicolumn{3}{|c|}{ Age distribution (years); $n$ (\%) } \\
\hline $13-17$ years & $0(0.0)$ & $2(9.5)$ \\
\hline $18-49$ years & $13(52.0)$ & $13(61.9)$ \\
\hline$\geq 50$ years & $12(48.0)$ & $6(28.6)$ \\
\hline
\end{tabular}




\begin{tabular}{|c|c|c|}
\hline $\begin{array}{l}\text { Within-patient } \\
\text { comparison }\end{array}$ & SHL FIX $(n=19)$ & $\mathrm{rFIXFc}(n=19)$ \\
\hline \multicolumn{3}{|c|}{ Prescribed prophylactic dose; IU/kg/week } \\
\hline Mean (SD) & $69.8(27.5)$ & $51.8(20.1)$ \\
\hline Median (IQR) & $77.4(42.5-82.0)$ & $45.0(37.0-63.4)$ \\
\hline Missing; $n$ & 5 & 5 \\
\hline \multicolumn{3}{|c|}{ Patient-reported consumption; IU/kg/week } \\
\hline Mean (SD) & $67.5(34.3)$ & $46.9(15.2)$ \\
\hline Median (IQR) & $68.1(45.9-91.2)$ & $42.8(37.8-50.7)$ \\
\hline Missing; $n$ & 3 & 3 \\
\hline \multicolumn{3}{|c|}{ Number of injections per week; $n$} \\
\hline Mean (SD) & $2.3(0.8)$ & $1.1(0.3)$ \\
\hline Median (IQR) & $2.0(2.0-3.2)$ & $1.0(1.0-1.0)$ \\
\hline Missing; $n$ & 2 & 2 \\
\hline \multicolumn{3}{|c|}{ Annualized bleeding rates (ABRs) } \\
\hline Mean (SD) & $1.1(2.1)$ & $0.2(0.7)$ \\
\hline Median (IQR) & $0.0(0.0-1.0)$ & $0.0(0.0-0.0)$ \\
\hline Missing; $n$ & 1 & 1 \\
\hline
\end{tabular}

Conclusions: Swedish real-world data suggest that patients on prophylaxis switching from a SHL FIX product to rFIXFc reduced factor consumption and injection frequency with an at least maintained level of bleed protection.

PB0552 | B-MORE, Baseline Analysis from a 24-month Prospective, Non-interventional, Multicentre Study on Real-world Effectiveness and Usage of Recombinant Factor IX Fc (rFIXFc) in Haemophilia B

G. Castaman ${ }^{1}$; S. Ranta ${ }^{2}$; D. Allsup ${ }^{3}$; H. Glosli ${ }^{4}$; M. Saleh ${ }^{5}$;

Å. Carlsheimer ${ }^{6}$; A. Francke ${ }^{6}$; E. Santagostino ${ }^{7}$

${ }^{1}$ Center for Bleeding Disorders and Coagulation, Careggi University Hospital, Florence, Italy; ${ }^{2}$ Pediatric Coagulation Center, Astrid Lindgren Children's Hospital, Karolinska University Hospital, Stockholm, Sweden; ${ }^{3}$ Haematology, Hull University Teaching Hospital Trust And Centre for Atherothrombosis and Metabolic Disease, Hull York Medical School, Hull, United Kingdom; ${ }^{4}$ Division of Pediatric and Adolescent Medicine, Oslo University Hospital, Oslo, Norway; ${ }^{5}$ King Faisal Specialist Hospital and Research Centre, Riyadh, Saudi Arabia; ${ }^{6}$ Swedish Orphan Biovitrum AB, Stockholm, Sweden; ${ }^{7}$ Swedish Orphan Biovitrum AG, Basel,

Switzerland

Background: Safety and efficacy of rFIXFc, an extended half-life FIX, were established in phase 3 trials in patients with haemophilia $\mathrm{B}(\mathrm{HB} ; \leq 2 \mathrm{IU} / \mathrm{dL}$ FIX) across age-groups. These trials showed low annualised bleeding rates (ABR) with long-term prophylaxis, and wellestablished efficacy in treatment of bleeds and during surgery. The B-MORE study will evaluate real-world effectiveness and usage of rFIXFc in $\mathrm{HB}$ patients in Europe and Middle East (ME).

Aims: To describe interim baseline data for patients treated with rFIXFc enrolled into the ongoing B-MORE (NCT03901755) study.
Methods: B-MORE is a 24-month prospective, non-interventional, multicentre study approved by relevant ethics committees. This first interim analysis was planned at inclusion of at least 50 patients. Eligible patients were prescribed on-demand or prophylactic rFIXFc treatment prior to or at enrolment and provided informed consent. At enrolment, baseline characteristics, initial rFIXFc prescription data and 12-month retrospective data prior to rFIXFc switch were collected, including $A B R$ and joint $A B R$.

Results: At data cut-off (4th December 2020), 62 patients were enrolled ( 1 female) at 13 centres (Europe and ME). Baseline patient characteristics are shown in Table 1 . Thirty-five patients were aged $<18$ years (median 8 , range 1-13).

Prior to rFIXFc initiation, median (IQR) ABR and joint ABR were 1.0 (0.0-1.9) and $0.0(0.0-1.0)$, respectively, in patients previously on prophylaxis $\geq 6$ months $(n=36$ ) with standard half-life FIX administered a median of two injections/week.

At rFIXFc initiation, the majority of patients $(56 / 62,90.3 \%)$ received rFIXFc prophylactically; most commonly weekly (71.4\%) or every 14 days (12.5\%), see Table 2.

TABLE 1 Baseline demographic data and characteristics

\begin{tabular}{|c|c|}
\hline At enrolment & $N=62$ \\
\hline Age [years], median (range) & $11.7(1-81)$ \\
\hline$<18$ years, $n(\%)$ & $35(56.5)$ \\
\hline$\geq 18$ years, $n(\%)$ & $27(43.5)$ \\
\hline \multicolumn{2}{|l|}{ Severity of haemophilia, $n$ (\%) } \\
\hline Severe & $47(75.8)$ \\
\hline Moderate & $12(19.4)$ \\
\hline Mild & $2(3.2)$ \\
\hline Missing & $1(1.6)$ \\
\hline \multicolumn{2}{|l|}{$\begin{array}{l}\text { At least one target joint at enrolment, } \\
\qquad n(\%)\end{array}$} \\
\hline Yes & $2(3.2)$ \\
\hline No & $58(93.6)$ \\
\hline Missing & $2(3.2)$ \\
\hline \multicolumn{2}{|l|}{ Prior to rFIXFc initiation, $n(\%)$} \\
\hline \multicolumn{2}{|l|}{ History of inhibitors } \\
\hline Yes $^{1}$ & $1(1.6)$ \\
\hline No & $60(96.8)$ \\
\hline Missing & $1(1.6)$ \\
\hline \multicolumn{2}{|l|}{ Treatment regimen } \\
\hline Prophylaxis regimen ${ }^{2}$ & $42(67.7)$ \\
\hline On-demand regimen & $9(14.5)$ \\
\hline Previously untreated patients (PUPs) ${ }^{3}$ & $7(11.3)$ \\
\hline Missing & $4(6.5)$ \\
\hline \multicolumn{2}{|c|}{$\begin{array}{l}{ }^{2} \text { ABR and injection frequency prior to } \mathrm{rFIXFc} \text { initiation were only } \\
\text { analysed for patients with available data of } \geq 6 \text { months on previous FIX } \\
\text { prophylaxis ( } n=36 \text { ) } \\
{ }^{3} \text { All with severe haemophilia }\end{array}$} \\
\hline
\end{tabular}


TABLE 2 rFIXFc treatment regimen and prophylactic dosing interval at initiation

$\begin{array}{ll}\begin{array}{l}\text { rFIXFc treatment regimen at initiation, } \\ n(\%)\end{array} & \mathrm{N}=62 \\ \text { Prophylaxis } & 56(90.3) \\ \text { On-demand } & 6(9.7) \\ \text { Prescribed prophylactic dosing } & \mathrm{N}=56 \\ \quad \text { frequency, } \boldsymbol{n}(\%) & \\ \text { Every } 5 \text { days } & 1(1.8) \\ \text { Every } 7 \text { days } & 40(71.4) \\ \text { Every } 8 \text { days } & 2(3.6) \\ \text { Every } 10 \text { days } & 5(8.9) \\ \text { Every } 14 \text { days } & 7(12.5) \\ \text { Missing } & 1(1.8)\end{array}$

Conclusions: Interim baseline data from the ongoing B-MORE study provides insights into demographics and clinical characteristics of HB patients treated with rFIXFc. There was a wide age range, with a large proportion of paediatric patients (7 PUPs). The vast majority of patients initiated rFIXFc on a prophylactic regimen with once weekly or longer dosing intervals.

\section{PB0553 | Tissue Factor Pathway Inhibitor (TFPI) Levels and} Bleeding Risk in Patients with Severe Hemophilia A or B

B. Tardy-Poncet ${ }^{1,2,3} ;$ A. Montmartin ${ }^{3} ;$ F. Genre-Volot ${ }^{4}$;

H. Chambost ${ }^{5}$; E. De Maistre ${ }^{4}$; P. Morange ${ }^{5}$; L. Ardillon ${ }^{6}$; Y. Dargaud ${ }^{7}$; A. Lienhart ${ }^{7}$; P. Nguyen ${ }^{8}$; B. Frotscher ${ }^{9}$; B. Wibaut ${ }^{10}$; C. Derory ${ }^{2}$; M. Delolme ${ }^{1}$; B. Tardy ${ }^{2}$

${ }^{1}$ Haemophilia Treatment Center, Saint-Etienne, France; ${ }^{2}$ INSERM CIC 1408, FCRIN-INNOVTE, Saint-Etienne, France; ${ }^{3}$ INSERM U 1059 SAINBIOSE, Saint-Etienne, France; ${ }^{4}$ Haemophilia Treatment Center, Dijon, France; ${ }^{5}$ Haemophilia Treatment Center, Marseille, France; ${ }^{6}$ Haemophilia Treatment Center, Tours, France; ${ }^{7}$ Haemophilia Treatment Center, Lyon, France; ${ }^{8}$ Haemophilia Treatment Center, Reims, France; ${ }^{9}$ Haemophilia Treatment Center, Vandoeuvre-lès-Nancy, France, ${ }^{10}$ Haemophilia Treatment Center, Lille, France

Background: Bleeding severity in hemophilic patients, reflecting low levels of thrombin generation (TG), can vary widely between patients with the same coagulation factor deficiency level, possibly reflecting differences in tissue factor pathway inhibitor (TFPI).

Aims: To compare TFPI levels in patients with severe hemophilia A (HA) and severe hemophilia $B(H B)$ and to investigate the relationships between TG potential and bleeding, between TG potential and TFPI level and between TFPI level and bleeding.

Methods: Data on bleeding episodes recorded during follow-up visits over 5-10 years were collected and used to calculate annualized hemarthrosis rate (AHR) and annualized bleeding rate (ABR). Free TFPI levels (Asserachrom Free TFPI, Stago) and basal TG parameters were determined in platelet-poor plasma (PPP) and platelet-rich plasma (PRP) using the CAT system.
Results: Mean TFPI levels did not differ significantly between HA $(n=34)$ and HB $(n=19)$. Mean values in PPP of endogenous thrombin potential (ETP) and thrombin peak (Peak) were higher at TFPI levels $<9.4 \mathrm{ng} / \mathrm{mL}$ than at TFPI levels $>14.3 \mathrm{ng} / \mathrm{mL}(E T P=287.4 \pm$ 11.1 vs $142.6 \pm 69.1 \mathrm{nmol} ; P=0.002)$ (Peak $=12.4 \pm 5.6$ vs $5.1 \pm 2.6$ nmol; $P=0.001$ ) respectively. In patients on demand treatment, a statistically significant inverse relationship between ETP in PRP was only seen with AHR. In patients on prophylaxis no association was found between basal TG parameters neither with ABR nor AHR. An association was observed between AHR or ABR and TFPI levels, only in patients on prophylaxis.

Conclusions: Bleeding tendency depends on the basal TG only in patients without prophylactic treatment.

Moreover, TFPI levels influences the bleeding tendency for patients receiving a prophylactic treatment only. In other words, the difference of bleeding tendency in severe hemophilia patients on prophylaxis can be explain by different TFPI levels.

\section{PB0554 | The Impact of PK-guided Prophylaxis on Clinical} Outcomes and Resource Utilization in Hemophilia A Patients: Real-world Evidence From the CHESS II Study

E. Ferri Grazzi ${ }^{1}$; T. Burke ${ }^{1,2}$; J. O'Hara' ${ }^{1,2}$ S.X Sun ${ }^{3}$

${ }^{1}$ HCD Economics Ltd, Daresbury, United Kingdom; ${ }^{2}$ University of Chester, Chester, United Kingdom; ${ }^{3}$ Shire Human Genetic Therapies, Inc., a Takeda Company, Cambridge, United States

Background: Individualizing treatment dose and frequency using pharmacokinetic (PK)-guided prophylaxis can help achieve and maintain targeted factor VIII (FVIII) trough levels in patients with hemophilia A.

Aims: To describe and compare clinical and resource use outcomes between patients with hemophilia A treated with or without PKguided dosing using data from the Cost of Haemophilia in Europe: $A$ Socioeconomic Survey (CHESS) II database.

Methods: CHESS II was a cross-sectional, retrospective, prevalencebased, burden-of-illness study using data collected across 8 European countries from November 2018 to July 2019. Patients eligible for this analysis were male, $\geq 18$ years of age, and diagnosed with congenital hemophilia A of any severity. The clinical endpoints analyzed were annualized bleeding rate (ABR), joint deterioration (presence and number of problem/target joints), and occurrence of joint surgeries. Resource utilization endpoints included the number of hematologist consultations and bleed-related hospitalizations or emergency department admissions. Data were stratified according to the use of PK-guided dosing.

Results: A total of 155 patients on prophylactic treatment had available FVIII trough level data. Mean (SD) age was 38.6 (14.4) years. Of these, a specific FVIII trough level was targeted in 72 (46.5\%) patients and 16 (22.2\%) received PK-guided dosing. Patients receiving a PK-guided dose had a mean (SD) ABR of 1.6 (1.1) and a mean (SD) number of target joints of 0.6 (0.8) compared with 4.4 (2.8) and 
1.2 (1.5), respectively, for patients whose dose was not PK-guided

(Table 1). The mean (SD) number of hematologist consultations was 6.2 (2.9) for patients receiving PK-guided dosing versus 11.8 (5.7) for those who were not. A higher proportion of patients required hospitalization during their lifetime if they did not receive PK-guided dosing compared with those who did (Table 2).

TABLE 1 ABR and joint health outcomes in patients with available PK information $(n=72)$

\begin{tabular}{lll} 
& PK-guided dosing Yes $(\boldsymbol{n}=16)$ & PK-guided dosing No $(\boldsymbol{n}=\mathbf{5 6})$ \\
ABR, mean (SD) & $1.6(1.1)$ & $4.4(2.8)$ \\
Presence of target joints, $\boldsymbol{n}$ (\%) & $7(43.8)$ & $30(53.6)$ \\
Number of target joints, mean (SD) & $0.6(0.8)$ & $1.2(1.5)$ \\
Presence of problem joints, $\boldsymbol{n}$ (\%) & $11(68.8)$ & $29(51.8)$ \\
Number of problem joints mean (SD) & $1.1(0.9)$ & $0.9(1.2)$ \\
\hline
\end{tabular}

$\mathrm{ABR}$, annualized bleeding rate; NS, not significant; $\mathrm{PK}$, pharmacokinetic.

TABLE 2 Bleed-related hospitalizations in patients with available PK information ( $n=72$ )

$\begin{array}{lll} & \text { PK-guided dosing Yes }(n=16) & \text { PK-guided dosing No }(n=56) \\ \begin{array}{l}\text { Proportion of patients needing hospitalization during their } \\ \quad \text { lifetime, } n(\%)\end{array} & 10(62.5) & 49(87.5) \\ \begin{array}{l}\text { Proportion of patients needing hospitalization in the } 12 \text { months } \\ \quad \text { prior to data collection, } n \text { (\%) }\end{array} & 7(43.8) & 27(48.2) \\ \begin{array}{l}\text { Details for hospitalizations occurring in the } 12 \text { months prior to } \\ \quad \text { data collection, mean (SD) }\end{array} & & 0.0(0.2) \\ \text { Number of day cases }(n=72) & 0.2(0.5) & 1.0(0.0) \\ \text { Number of days }(n=4) & 1.5(0.7) & 0.8(1.1) \\ \text { Number of ward hospitalizations }(n=72) & 0.3(0.4) & 5.8(4.1) \\ \text { Number of days on ward }(n=31) & 5.2(4.0) & 0.1(0.3) \\ \text { Number of ICU hospitalizations }(n=72) & 0.1(0.3) & 1.5(0.6) \\ \text { Number of days in ICU }(n=5) & 1.0(-) & \end{array}$

ICU, intensive care unit; NS, not significant; PK, pharmacokinetic.

Conclusions: This analysis provides real-world evidence suggesting a beneficial impact of PK-guided dosing for prophylaxis on clinical and health resource utilization outcomes.

PB0555 | Identify Clinical and Laboratory Phenotypes of People with Hemophilia in Southwest Nigeria

T. Adeyemo $^{1}$; A. Bolarinwa ${ }^{1}$; L. Adiat ${ }^{1}$; A. Olatinwo ${ }^{1}$; O. Opadeyi ${ }^{1}$; O. Otokiti ${ }^{1}$; T. Youttananukorn ${ }^{2}$; D. Coffin ${ }^{2}$

${ }^{1}$ Lagos University Teaching Hospital, Lagos, Nigeria; ${ }^{2}$ World Federation of Hemophilia, Montreal, Canada

Background: Previously Lagos University Teaching Hospital Haemophilia Treatment Center (HTC) has over 60 people with hemophilia (PWH) but severity level and inhibitor status were mostly unknown. Majority of PWH cannot afford cost of test. Clinical management were based on clinical history and trial with factor VIII and IX concentrate. We applied for funding from Research Support Program of World Federation of Hemophilia World Bleeding Disorders Registry (WBDR), which enabled us to identify severity and inhibitor status.
Aims: To offer diagnostic and inhibitor testing to $\mathrm{PWH}$ and to complete clinical data.

Methods: Our research was cross-sectional study. In Q4 2018, we had $12 \mathrm{PWH}$ registered in the WBDR. We conducted educational sessions and outreach to encourage all PWH to attend our HTC for accurate diagnosis of their hemophilia type, severity and inhibitor status. We used the fund to pay for storage of testing materials, diagnostic kits and laboratory charges.

Results: We were able to accurately determine hemophilia type, severity level, and inhibitor status for 60 additional PWH. In Q1 2021, we have clinical data of 79 male $\mathrm{PWH}$; $67 \mathrm{PWH}$ were diagnosed with Hem A, 9 with Hem B and 3 are awaiting results. For Hem A, 43, 14 and 2 were diagnosed as severe, moderate and mild, and 8 are waiting to run the severity test. For Hem B, 4 and 1 were identified as severe and moderate, and 4 are waiting to test severity. Eight of 77 (10\%) (7 Hem A, $1 \mathrm{Hem} \mathrm{B)} \mathrm{PWH} \mathrm{were} \mathrm{diagnosed} \mathrm{with} \mathrm{an} \mathrm{inhibitor}$ (mixing study).

Conclusions: The fund allowed us to identify, diagnose and provide clinical data of PWH in Southwest Nigeria. We use the WBDR to store data, which is useful when the National Registry does not exist. With complete and easy to access data set through the WBDR, we have better interaction with the $\mathrm{PWH}$. 
PB0556 | Randomized Clinical Trial of PK-guided Treatment of Desmopressin Combined with FVIII Concentrate versus Only FVIII Concentrate in Peri-operative Hemophilia A Patients: Final Results of the Little DAVID Study

L.G.R. Romano ${ }^{1}$; L.M. Schütte ${ }^{1}$; R.M. van Hest ${ }^{2}$; K. Meijer ${ }^{3}$;

B.A.P. Laros - van Gorkom ${ }^{4}$; F.C.J.I. Heubel-Moenen ${ }^{5}$; M.H.E. Driessens ${ }^{6}$; S. Polinder'; M.H. Cnossen ${ }^{8}$; F.W.G. Leebeek ${ }^{1}$; R.A.A. Mathôt ${ }^{2}$; M.J.H.A. Kruip ${ }^{1}$; On behalf of the SYMPHONY Consortium

${ }^{1}$ Department of Hematology, Erasmus MC, Erasmus University Medical Center, Rotterdam, Netherlands; ${ }^{2}$ Department of Hospital Pharmacy and Clinical Pharmacology, Amsterdam University Medical Centers - University of Amsterdam, Amsterdam, Netherlands; ${ }^{3}$ Department of Hematology, University Medical Center Groningen, Groningen, Netherlands; ${ }^{4}$ Department of Hematology, Radboud University Medical Center, Nijmegen, Netherlands; ${ }^{5}$ Department of Hematology, Maastricht University Medical Center+, Maastricht, Netherlands; ${ }^{6}$ Netherlands Hemophilia Patient Society (NVHP), Nijkerk, Netherlands; ${ }^{7}$ Department of Public Health, Erasmus MC, Erasmus University Medical Center, Rotterdam, Netherlands; ${ }^{8}$ Department of Pediatric Hematology, Erasmus MC - Sophia Children's Hospital, Rotterdam, Netherlands

Background: Patients with non-severe hemophilia A can be treated peri-operatively with desmopressin or factor VIII (FVIII) concentrate. Desmopressin leads to increase of FVIII levels, however, in terpatient variability is high. If insufficient, FVIII concentrate is used.
Desmopressin combined with FVIII concentrate is not common practice, but can overcome downsides of monotherapy (2020 WFH guideline). Furthermore, desmopressin is less expensive than concentrate and combined treatment could save costs.

Aims: To compare efficacy and FVIII consumption of peri-operative pharmacokinetic (PK)-guided combination treatment (desmopressin and FVIII concentrate) versus PK-guided FVIII concentrate monotherapy in non-severe hemophilia A patients undergoing small procedures.

Methods: Non-severe hemophilia A patients responsive to desmopressin undergoing a minor intervention were included in this twoarmed randomized clinical trial. In the standard treatment (control) arm only a PK-guided dose of FVIII concentrate was administered. In the intervention arm desmopressin was administered intravenously $(0.3 \mu \mathrm{g} / \mathrm{kg})$ with additionally, based on earlier reported desmopressin response, a PK-guided FVIII concentrate dose. FVIII trough and peak levels were determined in both arms. A predicted FVIII level was considered accurate if difference between measured and predicted FVIII level was $\leq 0.2 \mathrm{IU} / \mathrm{ml}$. Local ethics committee approval was obtained and patients provided written informed consent.

Results: Patient and procedure characteristics are described in Table 1. All FVIII peak responses after desmopressin (peak desmopressin response) were predicted accurately. For peak of FVIII concentrate (intervention) 5/6 (83.3\%) and peak of FVIII concentrate (control) 5/6 (83.3\%) measurements were also accurate (Figure 2). Combination treatment resulted in $\sim 47 \%$ lower FVIII concentrate consumption at loading dose $(P=0.009)$.

TABLE 1 Patient and procedure characteristics

Number (\%) / median [interquartile range]

Characteristic

Control (FVIII) $(n=6)$

Intervention (combination) $(n=6)$

Hemophilia severity

Moderate

1 (16.7)

Mild

5 (83.3)

Age (years)

Lowest measured FVIII (IU/ml)

32 [26.3-49]

$0.12[0.10-0.13]$

Study loading dose FVIII (IU)

3750 [3375-4000]

Performed procedure

Oral and maxillofacial

$5(83.3)$

2 (33.3)

Gastro-intestinal

Lumbar puncture

Biopsy/excision

Bleeding complications
1 (16.7)

1 (16.7)

1 (16.7)

2 (33.3)

$1(16.7)$ 


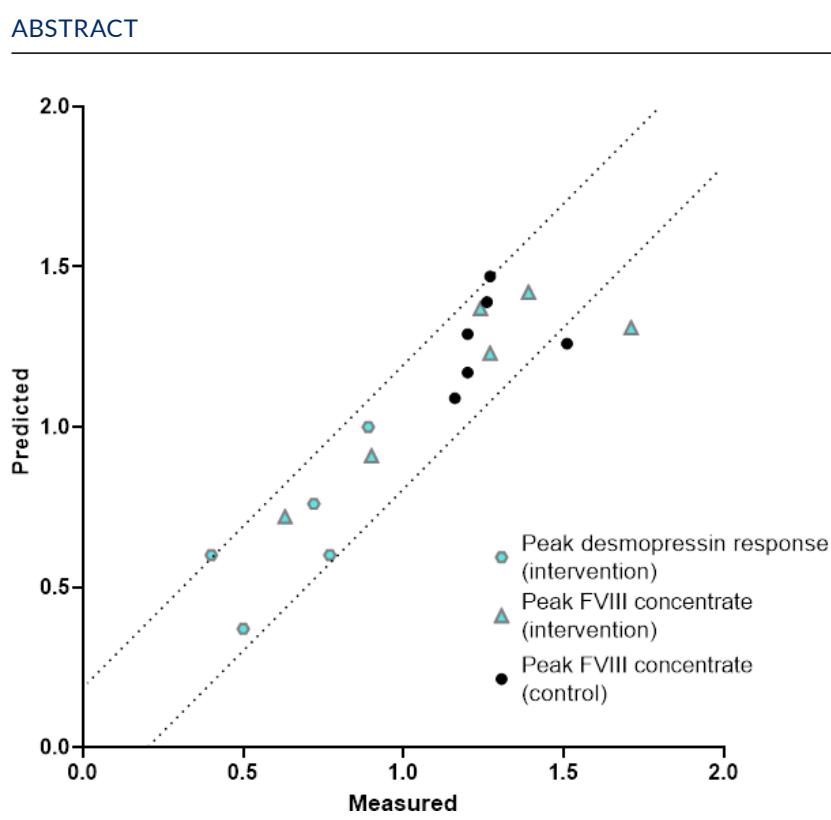

FIGURE 1 Comparison of measured versus predicted FVIII:C levels (IU/ml) in control and intervention arm. Dotted lines signify $\pm 0.2 \mathrm{IU} / \mathrm{ml}$. For one patient in the intervention arm, peak of desmopressin response was unknown

Conclusions: Efficacy of peri-operative combination treatment of desmopressin and FVIII concentrate under PK-guidance was similar to PK-guided FVIII concentrate monotherapy in non-severe hemophilia A patients. Furthermore, significantly less FVIII concentrate was used with combination therapy. Therefore, combination therapy should be considered as treatment of choice for small procedures in non-severe hemophilia A.

PB0558 | The Devil is in the Detail: An Irish Approach to Switching to Emicizumab Prophylaxis in an Adult Comprehensive Care Centre (CCC)

E. Singleton; N. Larkin; M. Mc Gowan; S. Roche; C. Bergin; M. Byrne; K. Ryan; M. Lavin; J. O Donnell; N. O Connell National Coagulation Centre, St James's Hospital, Dublin, Ireland

Background: Product switches can be extremely challenging for Haemophilia care providers, requiring intensive planning and organisation between multiple stakeholders. In October 2019, Emicizumab became available for prophylaxis in People with Severe Haemophilia A (PWSHA) without inhibitors in Ireland.

Aims: To develop a pathway to identify and assess patients who are eligible to consider a switch to Emicizumab and to ensure that switching is undertaken in an efficient, safe and cost effective manner.

Methods: A management pathway (table 1) was developed for each stage of the evaluation and switching process.
TABLE 1 Pathway to Switch to Emicizumab
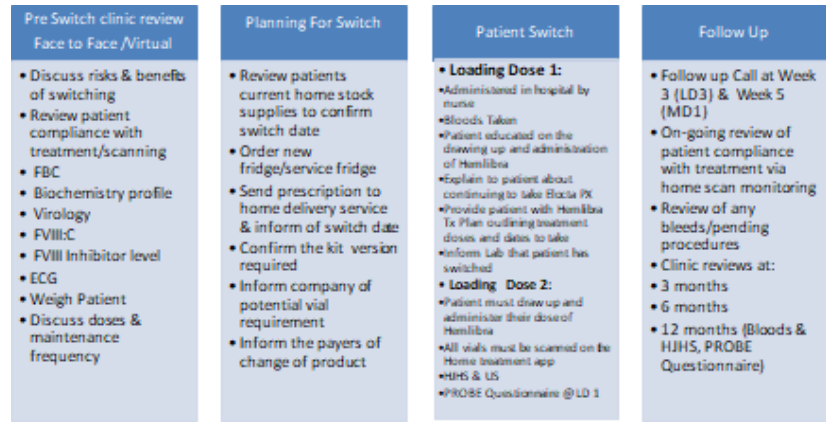

Results: To date, 35 PWSHA have been reviewed at clinic, of whom 25 have switched to Emicizumab prophylaxis. Missed, incorrect or delayed doses occurred on eight occasions and were more common in people using a fortnightly maintenance dosing schedule and in the first six weeks of switching. An additional follow up phone call with the Clinical Nurse Specialists at week three was implemented when the time of transition to maintenance dosing was identified as a risk period for incorrect dosing.

One episode of product wastage occurred due to incorrect storage of Emicizumab post switch. There was no wastage of rFVIII concentrate associated with the switchover process.

Conclusions: Successful switching of patients to Emicizumab, with low incidence of incorrect dosing or product wastage, is achieved with a well-coordinated and collaborative multidisciplinary approach.

\section{PB0559 | Predicted FVIII Consumption of BAY 94-9027} Compared with Standard- and Extended-half-Life FVIII Products in Patients with Severe Hemophilia A

A. Solms ${ }^{1}$; A. Shah ${ }^{2}$; E. Berntorp ${ }^{3} ;$ A. Tiede ${ }^{4} ;$ A. Iorio $^{5}$; A. Bröker ${ }^{6}$; M.E. Mancuso ${ }^{7}$

${ }^{1}$ Bayer, Berlin, Germany; ${ }^{2}$ Bayer Healthcare, New Jersey, United States; ${ }^{3}$ Lund University, Lund, Sweden; ${ }^{4}$ Hannover Medical School, Hannover Germany; ${ }^{5}$ McMaster University, Hamilton, Canada; ${ }^{6}$ Bayer, Wuppertal, Germany; ${ }^{7}$ Humanitas Research Hospital, Milan, Italy

Background: Previously conducted head-to head $(\mathrm{H} 2 \mathrm{H})$ studies based on an intra-individual cross-over design in patients with severe hemophilia $A$, compared the pharmacokinetic (PK) parameters between BAY 94-9027 and three other recombinant factor VIII (rFVIII) products. In all studies, BAY 94-9027 showed improved PK versus the comparator.

Aims: To use population PK (popPK) modeling of available $\mathrm{H} 2 \mathrm{H}$ PK data to predict weekly FVIII consumption of different regimens of BAY 94-9027 (Jivi), compared with antihemophilic factor (recombinant) formulated with sucrose (Kogenate-FS), rFVIIIFc (Elocta) and BAX 855 (Adynovi). 
Methods: Three H2H studies compared PK of BAY 94-9027 with antihemophilic factor (recombinant) FS, rFVIIIFc or BAX 855. For each study, the respective individual PK parameters from popPK modeling were used to predict steady-state FVIII trough (pre-dose) levels for each product for different prophylaxis regimens. Using these predicted trough levels, the individual dose required to maintain the patients consistently above threshold level of clinical interest, such as 3 IU/dL, was determined. The respective predicted weekly FVIII (IU/kg) consumption was calculated depending on the regimen, and the ratio of the predicted weekly dose for BAY 94-9027 versus the comparator was estimated.

Results: Overall, 49 patients were included in this analysis $(n=14$, antihemophilic factor [recombinant] FS; $n=17$, rFVIIIFc; $n=18$, BAX 855). Targeting a threshold of $3 \mathrm{IU} / \mathrm{dL}$ in patients on a prophylaxis regimen of twice a week required lower median doses by approximately 58\%, 33\% and 35\% for treatment with BAY 94-9027 compared to treatment with antihemophilic factor (recombinant) FS, rFVIIIFC and BAX 855 respectively (Table 1). Other target thresholds and regimens will be presented.

TABLE 1 Predicted FVIII consumption of PK tailored treatment with BAY 94-9027 compared with standard- and extended-half-life rFVIII products targeting a FVIII trough level of $3 \mathrm{IU} / \mathrm{dL}$ on a prophylaxis regimen of twice a week

\begin{tabular}{lllll} 
Product & $\mathrm{n}$ & $\begin{array}{l}\text { Weekly consumption, IU/kg, } \\
\text { median }\end{array}$ & Ratio, median & Reduction with Jivi, \% \\
$\begin{array}{l}\text { BAY 94-9027 } \\
\begin{array}{l}\text { Antihemophilic factor } \\
\text { (recombinant) FS }\end{array}\end{array}$ & 14 & 64.6 & 0.416 & 58 \\
BAY 94-9027 & 14 & 175 & & 0.666 \\
rFVIIIFc & 17 & 96.6 & & 33 \\
BAY 94-9027 & 17 & 166 & 0.647 & 35 \\
BAX 855 & 18 & 79.2 & & \\
\hline
\end{tabular}

Conclusions: These dose predictions reveal the potential of BAY 94-9027 in reducing FVIII consumption compared with other rFVIII products, while maintaining the same FVIII trough level, and thereby similarly efficient protection from bleeds.

PB0560 | Time from the Inhibitor Detection to the Start of Immune Tolerance Induction and the Outcome of Immune Tolerance Induction: Results from the Brazilian Immune Tolerance (BrazIT) Study

R.M. Camelo $^{1,2}$; M.M. Dias ${ }^{1}$; C. Caram-Deelder ${ }^{2,3}$; S. Gouw ${ }^{4}$; L.P. de Magalhaes ${ }^{1}$; L.W. Zuccherato ${ }^{1}$; L.L. Jardim ${ }^{1,2}$; J. van der Bom $^{2,3}$; S.M. Rezende ${ }^{1}$

${ }^{1}$ Faculty of Medicine of the Universidade Federal de Minas Gerais, Belo Horizonte, Brazil; ${ }^{2}$ Department of Clinical Epidemiology, Leiden University Medical Centre, Leiden, Netherlands; ${ }^{3}$ Jon J van Rood Centre for Clinical Transfusion Research, Sanquin/LUMC, Leiden, Netherlands; ${ }^{4}$ Department of Paediatric Haematology, Emma Children's Hospital, Amsterdam UMC, University of Amsterdam, Amsterdam, Netherlands

Background: Immune tolerance induction (ITI) is the treatment of choice for eradication of anti-factor VIII (FVIII) antibodies (inhibitors) in people with inherited haemophilia $\mathrm{A}$ and inhibitors ( $\mathrm{PwHAi}$ ). Previous studies (most deriving from registries) have shown that a longer time elapsed between inhibitor development and the start of ITI $\left(\Delta \mathrm{t}_{\text {inhi-ITI }}\right)$ associates with a lower ITI success rate among PwHAi.

Aims: We aimed to evaluate this association among PwHAi included in the BrazlT Study.
Methods: PwHAi ( $n=133$ ) were enrolled from 15 haemophilia treatment centres in Brazil. PwHAi were treated according to the Brazilian ITI Protocol, using a low-dose regimen (50 IU/kg three times weekly). Upon absence of a decline in inhibitor titre, a highdose regimen (100 IU/kg daily) was indicated. Total success was defined as a negative inhibitor titre $(<0.6 \mathrm{BU} / \mathrm{mL})$ and a normal FVIII pharmacokinetics (recovery $66 \%$ or higher of expected values, and half-life of $6 \mathrm{~h}$ or higher). Partial success was defined as inhibitor titre between 0.6 and $2 \mathrm{BU} / \mathrm{mL}$; and/or abnormal FVIII pharmacokinetics (recovery below $66 \%$ of expected values; and/or half-life less than 6 h). Patients who did not achieve success parameters after 33 months were considered as failure.

Results: The median ages at inhibitor diagnosis and at ITI start were 3.6 years [IQR,1.6-7.2] and 7.0 years [IQR,2.6-19.2], respectively. PwHAi were stratified among four $\Delta \mathrm{t}_{\text {inhi-ITI }}$ quartiles: first $(0.00$ to 0.63 year), second (0.64 to 1.75 years), third (1.76 to 9.48 years), and fourth quartile ( 9.49 to 24.52 years). The overall success rate was $67.7 \%(90 / 133)$ of which first, second, third and fourth were $69.7 \%$ (23/33 patients), 69.7\% (23/33 patients), 64.7\% (22/34 patients), and fourth $66.7 \%$ (22/33 patients), respectively $(P=0.965)$.

Conclusions: In this study, about $70 \%$ of PwHAi responded to the Brazilian ITI Protocol. A longer $\Delta \mathrm{t}_{\text {inhi-ITI }}$ was not associated with a lower success rate of ITI in PwHAi. 
PB0561 | Impact of Prophylactic Treatment on Employment and Disability in Persons with Hemophilia

${ }_{\text {P.A. Holme }}^{1}$; C. Qvigstad ${ }^{1}$; R.C. Tait ${ }^{2}$

${ }^{1}$ Department of Haematology, Oslo University Hospital, Institute of Clinical Medicine, University of Oslo, Oslo, Norway; ${ }^{2}$ Royal Infirmary, Glasgow, United Kingdom

Background: Hemophilic arthropathy and other hemophiliaassociated co-morbidities may increase the likelihood of unemployment due to disability in people with hemophilia (PWH).

Aims: To assess the association between employment status and hemophilia treatment after adjusting for clinically relevant variables, potentially affecting employment status in $\mathrm{PWH}$ using baseline data from the ADVANCE study which aimed to assess the incidence of cardiovascular disease in older $\mathrm{PWH}$.

Methods: Twenty hemophilia centers from 15 European countries recruited 791 participants aged 40 years and over with hemophilia A or B by August 2018. Analyses included descriptive statistics and logistic regression models.

Results: Employment status was available for 756 of 791 participants (85\% hemophilia A) aged 40-88 years (mean 54 years). Employment status was self-assigned by each participant into one of six categories ( $n, \%$, mean age): full-time work (386, 51\%, 50y), part-time work (71, 9\%, $54 y)$, early retirement $(46,6 \%, 56 y)$, retired (150, 20\%, 67y), unemployed disabled (64, 8\%, 52y), and unemployed not disabled (39, 5\%, 51y). In the regression analysis, we compared PWH in full-time employment with those unemployed due to disability, yielding a sample of 407 persons. In multivariable logistic regressions, age $(O R=1.1, P<0.01)$, severe hemophilia (OR = 11.7, $\mathrm{P}<0.001)$, current smoker (OR = 2.4, P 0.01), and chronic liver disease $(O R=1.9, P=0.05)$ were associated with higher odds of being unemployed due to disability, while prophylactic treatment $(O R=0.4, P=0.01)$ was negatively associated with unemployment due to disability. There was no association with type of hemophilia, BMI, HIV+, or inhibitor (previous or current). The presence of a target joint was associated with unemployment due to disability (OR 2.2, $P=0.01)$ in univariate analysis, but not in multivariable logistic regressions.

Conclusions: Adjusting for the severity of hemophilia, PWH on prophylactic treatment are less likely to be unemployed due to disability than those with on demand treatment.

TABLE 1 Demographics

\begin{tabular}{|c|c|c|c|c|c|}
\hline & Severe FVIII deficiency & $\begin{array}{l}\text { Severe FIX } \\
\text { deficiency }\end{array}$ & $\begin{array}{l}\text { Moderate FVIII } \\
\text { deficiency }\end{array}$ & $\begin{array}{l}\text { Moderate FIX } \\
\text { deficiency }\end{array}$ & Total \\
\hline$n=$ & 28 & 9 & 5 & 1 & 43 \\
\hline range & $18-72$ & $20-73$ & $35-60$ & - & $18-73$ \\
\hline \multicolumn{6}{|l|}{ Treatment Regimens } \\
\hline \multirow[t]{2}{*}{ Treatment type } & rFVIIIFc $n=13$ & rFIXFc $n=9$ & rFVIIIFc $n=4$ & rFIXFc $n=1$ & \\
\hline & emicizumab $n=15$ & & no data $n=1$ & & \\
\hline
\end{tabular}

PB0562 | User Evaluation of a Home-treatment Recording Smartphone App

M. O'Donovan ${ }^{1,2}$; N. Larkin ${ }^{1}$; E. Singleton ${ }^{1}$; J. Benson ${ }^{1}$;

F. McGroarty ${ }^{1}$; J. Farrelly ${ }^{3}$; C. Bergin ${ }^{1}$; R. Bird ${ }^{1}$; C. Buckley ${ }^{1}$; J.S. O'Donnell' ${ }^{1}$; K. Ryan ${ }^{1}$; N.M. O'Connell ${ }^{1,2}$

${ }^{1}$ National Coagulation Centre, St James's Hospital, Dublin, Ireland;

${ }^{2}$ School of Medicine, Trinity College Dublin, Dublin, Ireland; ${ }^{3}$ rrish Haemophilia Society, Dublin, Ireland

Background: Prophylaxis is the standard of care for many people with severe bleeding disorders (PWSBD) and is based on home self-administration of replacement therapy. Accurate home record keeping is needed to optimise clinical care provided by the coagulation team. In Ireland, PWSBD are encouraged to use a specifically designed smartphone app (MPro HX, Crimson Tide). This app facilitates real-time medication barcode scanning at home to perform safety checks, using the GS1 datamatrix barcode as required by the Falsified Medicines Directive.

Aims: To evaluate patient recording of home-treatments, and perceived advantages and disadvantages of the smartphone app.

Methods: PWSBD (> 18 years) on home treatment, excluding those on clinical trials, were identified from the National Haemophilia Electronic Health Record (EHR). A questionnaire to explore key areas and themes was developed by the clinical team in collaboration with patient representatives. This anonymised patient survey was posted to PWSBD on home treatment and responses analysed. Results: Surveys were sent to 138 PWSBD, with response rate of $31 \%$ (Table 1). $88 \%$ use the app for recording home-treatments. Most were familiar with benefits of scanning treatment pre-administration, including alerts for expired (87\%), incorrect (62\%) or recalled (57\%) treatments, but only $50 \%$ scan treatment pre-administration. Users were aware that information recorded in the app is transferred to their EHR (93\%) and permits review of home-treatments in clinic (88\%). Almost all respondents (92\%) felt this improves management of their bleeding disorder and makes them more involved in treatment decisions (83\%). Challenges in using the app included difficulty scanning barcodes (57\%) and internet connectivity (30\%) but overall, $87 \%$ found the app was straightforward to use. 
Conclusions: This novel home-treatment smartphone app contains important safety features, including real-time scanning of hometreatments which can identify recalled or incorrect treatments. PWSBD are positive and well-educated about the benefits of recording treatments using this app.

PB0563 | Genetic and Clinical Determinants for the Outcome of Immune Tolerance Induction in Severe Hemophilia A - Preliminary Results

A. Abdi ${ }^{1}$; I. Oomen ${ }^{1,2}$; S.Q. Nagelkerke ${ }^{3,4}$; K. Fischer ${ }^{5}$; M. Carcao ${ }^{6}$; G.E. Rivard7; N. Rydz ; J. Eikenboom $;$; A.B. Mohseny ${ }^{9}$; S. Schols ${ }^{10}$; F.W.G. Leebeek ${ }^{11}$; J. Geissler ${ }^{3}$; M.W. Tanck ${ }^{12}$; D. Lillicrap ${ }^{13}$; J. Voorberg ${ }^{2}$; T.W. Kuijpers ${ }^{3,4}$; K. Fijnvandraat ${ }^{1,2}$; S.C. Gouw ${ }^{1,14}$; International GO-ITI Study Group

${ }^{1}$ Amsterdam UMC, University of Amsterdam, Emma Children's Hospital, Pediatric Hematology, Amsterdam, Netherlands; ${ }^{2}$ Department of Molecular and Cellular Hemostasis, Sanquin Research and Landsteiner Laboratory, Amsterdam, Netherlands; ${ }^{3}$ Department of Blood Cell Research, Sanquin Research and Landsteiner Laboratory, Amsterdam, Netherlands; ${ }^{4}$ Department of Immunology and Infectious Diseases, Emma Children's hospital, Amsterdam University Medical Center, Amsterdam, Netherlands; ${ }^{5}$ Department of Hematology, Van Creveldkliniek, University Medical Center Utrecht, Utrecht, Netherlands; ${ }^{6}$ Division of Hematology/Oncology, Department of Pediatrics, Hospital for Sick Children, University of Toronto, Toronto, Canada; ${ }^{7}$ Division of Hematology/Oncology, Department of Pediatrics, Centre Hospitalier Universitaire Sainte-Justine, Université de Montréal, Montréal, Canada; ${ }^{8}$ Department of Hematology and Hematologic Malignancies, Foothills Medical Center, Calgary, Canada; ${ }^{9}$ Department of Internal Medicine, division of Thrombosis and Hemostasis, Leiden University Medical Center, Leiden, Netherlands, ${ }^{10}$ Department of Hematology, Radboud University Medical Center, Hemophilia Treatment Center Nijmegen-Eindhoven-Maastricht, Nijmegen, Netherlands, ${ }^{11}$ Department of Hematology, Erasmus University Medical
Center, Rotterdam, Netherlands, ${ }^{12}$ Department of Epidemiology and Data Science, Amsterdam University Medical Center, Amsterdam, Netherlands, ${ }^{13}$ Department of Pathology and Molecular Medicine, Queen's University, Kingston, Canada, ${ }^{14}$ Department of Clinical Epidemiology, Leiden University Medical Center, Leiden, Netherlands

Background: Despite the prophylactic efficacy of emicizumab, eradicating inhibitors to restore the efficacy of factor VIII (FVIII) is a desirable treatment goal for patients with severe hemophilia A (SHA) and inhibitors, as this enables treatment of bleeding episodes with FVIII concentrates. However, since immune tolerance induction (ITI) is a burdensome and costly treatment, it is important to identify determinants for ITI success to individualize ITI treatment. Perhaps this has never been so crucial as now as many patients/clinicians may feel that it may not be worth trying ITI if emicizumab is available.

Aims: The aim of this study is to identify genetic/clinical determinants for ITI outcome in patients with SHA.

Methods: Dutch and Canadian patients with SHA (FVIII: $P<0.01 \mathrm{IU} /$ $\mathrm{mL}$ ) who underwent ITI were included. The primary outcome was successful ITI, defined by a negative inhibitor titer and an adequate clinical response to standard doses of FVIII. We analyzed both clinical and genetic determinants (FCGR2A (p.His166Arg, p.GIn62Trp, c.777+1G>A), FCGR2B (p.lle232Thr, promotor haplotypes 2B.1/2B.4), FCGR2C (p.GIn57Ter, promotor haplotypes 2B.1/2B.2), FCGR3A (p.Val176Phe), FCGR3B (haplotypes NA1/NA2), CTLA-4 (rs5742909-318C/T) and TNFa (rs1800629 -308G/A)). Proportions of determinants were compared between patients with ITI success and failure in univariate analysis.

Results: 76 patients were included. Baseline characteristics are in Table 1. Most patients were Caucasian and 46/76 had the intron22 inversion mutation. 62 (82\%) patients achieved ITI success. Risk factors for ITI failure were $>3$ months between inhibitor detection and ITI start, pre-ITI inhibitor titer $>5 \mathrm{BU} / \mathrm{ml}$ and peak inhibitor titer $>200 \mathrm{BU} / \mathrm{ml}$ (Table 2). We did not find an association between ethnicity, F8 genotype and the investigated genetic variations with ITI outcome.

TABLE 1 Baseline characteristics

Characteristics

F8 genotype, $n$ (\%)

Ethnicity, $n$ (\%)

FVIII product at start ITI, $n$ (\%)FVIII product at start ITI, $n(\%)$

Interval inhibitor detection - ITI start, weeks (IQR)

Age at start ITI, years (IQR)

Inhibitor titer, BU/mI (IQR)
Intron-22 inversion

Caucasian

Recombinant

Plasma-derived

At first detection

Pre-ITI

Peak
Total cohort $(n=76)$

46 (61)

52 (68)

41 (54)

32 (31)

$8.1(1.0-28.5)$

2 (1-4)

4.7 (1.3-14.9)

$5.8(2.6-20.0)$

25.3 (4.7-194.3) 
TABLE 2 Clinical determinants for ITI outcome

\begin{tabular}{|c|c|c|c|c|}
\hline Determinants & & ITI success $(n=62)$ & ITI failure $(n=14)$ & RRR failure [Cl] \\
\hline \multirow{2}{*}{$\begin{array}{l}\text { Inhibitor titer at first } \\
\text { detection }\end{array}$} & $<5 \mathrm{BU} / \mathrm{ml}$ & 33 & 6 & $*$ \\
\hline & $>5$ & 23 & 6 & 1.35 [0.48-3.75] \\
\hline \multirow{2}{*}{$\begin{array}{r}\text { Interval between inhibitor } \\
\text { detection and ITI start }\end{array}$} & $<3$ months & 36 & 3 & * \\
\hline & $>3$ & 25 & 8 & 3.15 [0.91-10.93] \\
\hline \multirow[t]{2}{*}{ Pre-ITI inhibitor titer } & $<5 \mathrm{BU} / \mathrm{ml}$ & 28 & 2 & * \\
\hline & $>5$ & 30 & 10 & 3.80 [0.89-15.86] \\
\hline \multirow[t]{2}{*}{ Peak inhibitor titer } & $<200 \mathrm{BU} / \mathrm{ml}$ & 52 & 5 & $*$ \\
\hline & $>200$ & 10 & 7 & 4.69 [1.71-12.91] \\
\hline
\end{tabular}

${ }^{*}$ Reference group

Conclusions: Our results confirm that clinical determinants are associated with ITI outcome but did not show a role of genetic variation in FCGR, CTLA-4 and TNFa genes on ITI outcome. The cohort will be expanded to $200-250$ patients.

PB0564 | Real World Data on Haemophilia A Patients Switching to rFVIIIFc Treatment in Greece. Interim Results from the Multicenter Observational Study TOOL

O. Katsarou ${ }^{1}$; A. Kouramba ${ }^{1}$; E. Nomikou ${ }^{2}$;

G. Kampelos ${ }^{2}$; S. Vakalopoulou ${ }^{3}$; H. Moka ${ }^{3}$; M. Economou ${ }^{4}$;

V. Gourtsa ${ }^{4}$; E. Michalopoulou ${ }^{5}$; H. Pergantou ${ }^{5}$

${ }^{1}$ Blood Tranfsusion Centre and National Reference centre for Congenital Bleeding Disorders Laiko General Hospital, Athens, Greece;

${ }^{2}$ Blood Bank and Hemophilia Unit, Hippokration General Hospital of Athens, Athens, Greece; ${ }^{3}$ Haematology Unit - Haemophilia Centre of Northern Greece, 2nd Propedeutic Department of Internal Medicine, Hippokration General Hospital of Thessaloniki, Aristotle University of Thessaloniki, Thessaloniki, Greece, ${ }^{4} 1$ st Pediatric Department, Hippokration General Hospital, Aristotle University of Thessaloniki, Thessaloniki, Greece; ${ }^{5}$ Haemostasis Unit, Haemophilia Centre, 'Aghia Sophia' Children's Hospital, Athens, Greece

Background: Phase III pivotal studies have proven the safety and efficacy of extended half-life agents. However, studies under everyday clinical practice conditions in national health care environments are still limited, although they can offer valuable information.

Aims: Aim of this study is to collect data on outcomes of severe and moderate haemophilia A patients, who switched from prophylactic or on demand treatment with standard half-life factor VIII to rFVIIIFc.

Methods: This is a multicenter observational study, in all 5 haemophilia centres in Greece. Until interim data lock point, 59 patients were enrolled and included in the current analysis. 45 patients had already completed the 12 months of follow-up after switch.

Results: Mean(range) age of patients was 31(6-56) years and $50(84.7 \%)$ had severe haemophilia A. For patients on prophylaxis before and after the switch ( $n=42)$, there was statistically significant decrease in meanABR from 3.1 to $1.5(P=0.002)$, while the percentage of patients with zero bleeds increased from $35.7 \%$ to $62.2 \%$., Of total bleeds in patients started rFVIIIFc, 56.8\% were spontaneous vs $76.6 \%$ before switch. Decrease in ABR was more profound in patients $(n=8)$ transferred from on demand to prophylaxis (mean8.0 before switch to 0.7 after, $P=0.047$ ). Significant decrease in ABR was also noted in the subgroup of patients $(n=21)$ on secondary prophylaxis (4.4 before switch to 2.0 after, $P=0.008$ ), compared to the subgroup ( $n=20$ ) on primary prophylaxis 2.2 before to 0.9 after, $P=0.153$ ). Joint health improved after switch (mean HJHS score: 14.9 before to 11.7 after, $P=0.008$ ), as was thelevel of pain experienced by the patients (mean VAS score: 4.9 before to 3.5 after, $P<0.001$ ). Nine adverse events, none of them related to rFVIIIFc and no inhibitors were observed.

Conclusions: These interim data from the first multicenter study concerning people with haemophilia A in Greece, are encouraging for the safety and effectiveness of newer drugs with improved pharmacokinetics.

PB0565 | Emicizumab in Pediatric Hemophilia: A Single-center Retrospective Study

C. Cohen; R. Diaz

Baylor College of Medicine/Texas Children's Hospital, Houston, United States

Background: Emicizumab use as prophylaxis in pediatric hemophilia A (HA) is increasing due to improved ease of administration and less frequent dosing schedule compared to factor VIII or bypassing agent infusions. Data on the pediatric experience with emicizumab outside of clinical trials, however, is limited.

Aims: To determine the safety and efficacy of emicizumab prophylaxis in pediatric HA within our institution.

Methods: Retrospective review on patients under 21 years of age with HA who have been treated with emicizumab as prophylaxis at Texas Children's Hospital.

Results: A total of 28 patients received emicizumab, 96.4\% $(n=27)$ of which had severe HA. Emicizumab initiation occurred at a median age of 6.7 years (range $0.2-20.4$ ) with all receiving an every-otherweek maintenance dosing schedule. The median length of time 
patients were on emicizumab at the time of data collection was 18.5 months (range 2-26). A history of inhibitors was present in $46.4 \%$ ( $n=13), 38.5 \%(n=5)$ of which had undergone immune tolerance prior to initiating emicizumab. The mean annualized bleeding rate (ABR) declined from 3.61 (range $0-15$ ) over the 12-24 months prior to initiating emicizumab to 0.44 (range $0-4)$ after $(P=0.0002$; paired $\mathrm{t}$-test). Seven patients underwent a surgical procedure (5 port removals, one lower extremity orthopedic procedure, and one arteriovenous fistula takedown). All were treated with continuation of emicizumab and peri-operative factor replacement with one minor post-operative bleeding event (hematoma at the site of a port removal). $14.3 \%(n=4)$ patients experienced mild injection site side effects. One patient with a history of multiple deep venous thromboses experienced a symptomatic cephalic vein thrombosis following arteriovenous fistula takedown, there were no other thrombotic complications. All patients but one opted to remain on emicizumab. Conclusions: The experience at our center demonstrates that emicizumab is a safe and effective prophylactic option for patients with $\mathrm{HA}$ both with and without inhibitors.

PB0566 | In vitro Analysis of Coagulation Function in Emicizumab-treated Hemophilia A Patients with Inhibitors under Immune Tolerance Induction Treatment

Y. Nakajima ${ }^{1}$; H. Tonegawa ${ }^{1}$; M. Noguchi-Sasaki ${ }^{2}$; K. Nogami ${ }^{1}$

${ }^{1}$ Nara Medical University/Department of Pediatrics, Kashihara, Japan;

${ }^{2}$ Chugai Pharmaceutical Co., Ltd., Kamakura, Japan

Background: Emicizumab prophylaxis substantially reduces bleeding episodes in patients with hemophilia A and factor (F)VIII inhibitor (PwHA-inh). The main therapeutic approach to eradicate FVIII inhibitor in PwHA is immune tolerance induction treatment (ITI). Concomitant therapy with ITI and emicizumab prophylaxis could provide additional benefits but remains to be investigated.

Aims: We have assessed coagulation potential in an in vitro simulated model of ITI for PwHA-inh and emicizumab.

Methods: FVIII-deficient plasma was incubated with anti-A2 and anti-C2 monoclonal antibodies (0,1, and $10 \mathrm{BU} / \mathrm{mL}$ each). Emicizumab ( $50 \mu \mathrm{g} / \mathrm{mL}$; therapeutic dose) was spiked in together with bypassing agents (BPAs): activated prothrombin complex concentrates (1.3 U/ $\mathrm{mL}$; corresponded to $50 \mathrm{IU} / \mathrm{kg})$, recombinant FVIla $(2.2 \mu \mathrm{g} / \mathrm{mL} ; 90$ $\mu \mathrm{g} / \mathrm{kg})$, or plasma-derived FVIIa/FX $(1.5 \mu \mathrm{g} / \mathrm{mL} ; 60 \mu \mathrm{g} / \mathrm{kg})$, and FVIII (100 and $200 \mathrm{IU} / \mathrm{dL} ; 50$ and $100 \mathrm{lU} / \mathrm{kg}$, respectively). These samples were analyzed by mixed ellagic acid and tissue factor-triggered clot waveform analysis, and adjusted-|min1| (Ad|min1|) was calculated.

Results: Ad|min1| levels in FVIII-deficient high titre inhibitor plasmas $(10 \mathrm{BU} / \mathrm{mL})$ were increased in the presence of emicizumab with various concentrations of FVIII concomitant with each BPA, but remained within the normal range even under conditions of maximal coagulation potentials. Those in FVIII-deficient low titre inhibitor plasmas $(1 \mathrm{BU} / \mathrm{mL})$ together with BPA and FVIII (200 IU/dL) showed values near or beyond the normal range, but a half concentration of rFVIla based on the half-life in blood showed within the normal range. In contrast, Ad|min1| values in non-inhibitor samples incorporating emicizumab, FVIII (100 and $200 \mathrm{IU} / \mathrm{dL}$ ) and each BPA were far beyond the normal range, but the coagulation potential in copresence of FVIII (100 IU/dl) and a half concentration of rFVIla remained within the normal range.

Conclusions: The results may support the feasibility of concurrent ITI in emicizumab-treated PwHA-inh.

PB0567 | Efficacy of Reduced-dose Emicizumab in Haemophilia A with Inhibitors: Real World Experience in East Malaysia

A.S.O. Tang; T.S. Leong; C.T. Ko; L.P. Chew

Sarawak General Hospital, Kuching, Malaysia

Background: Sarawak, the largest state in Malaysia, reported prevalence (per 100,000 males) of 2.46 cases for severe haemophilia A (HA). The development of inhibitory alloantibodies results in impairment of quality of life (QoL). The advent of emicizumab offers the potential for a paradigm shift in haemophilia treatment.

Aims: We aimed to study the clinical outcome in our patients who received emicizumab at a reduced dose due to the paucity of data.

Methods: This was a case series of three severe HA patients with inhibitors treated with emicizumab in East Malaysia between June 2018 and January 2021. The primary endpoint was a reduction of bleeding events. The secondary endpoints included: (a) number of breakthrough bleeds with emicizumab at a reduced dose; (b) QoL assessed using Haemo-QoL-A questionnaire; and (c) adverse events. Results: The median duration of treatment was 117 weeks, ranging from 90 to 132 weeks. Two had achieved zero bleeding events after receiving emicizumab. One had an episode of haemarthrosis which fully resolved after emicizumab administration without requiring any bypassing agents. Two patients, who required walking aids prior to emicizumab, were able to ambulate independently after emicizumab commencement. In resource-restricted setting (after the end of compassionate programme), emicizumab dose was tapered down (Figure 1). Following dose reduction, one had an episode of haematuria, whereas no bleeding event was observed in two others. QoL assessment showed no statistical difference after dose reduction (Figure 2; $P$-value 0.877; paired sample t-test). None had any adverse events. No thromboembolic events were reported. 


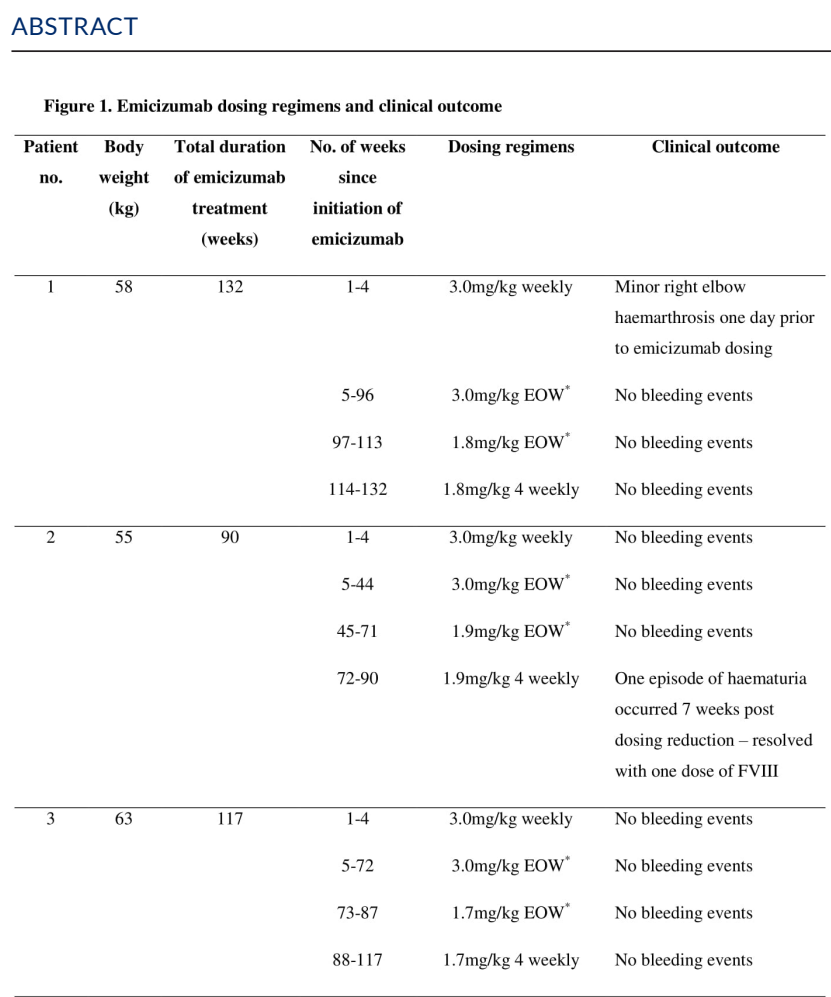

FIGURE 1 Emicizumab dosing regimens and clinical outcome

\begin{tabular}{|c|c|c|c|c|c|c|c|}
\hline & $\begin{array}{l}\text { Before } \\
\text { emicizumab } \\
\text { (A)* }\end{array}$ & $\begin{array}{l}\text { After } 24 \\
\text { weeks of } \\
\text { emicizumab } \\
\text { (B)* }\end{array}$ & $\begin{array}{l}\text { Difference } \\
(B-A)^{*}\end{array}$ & $\begin{array}{l}p^{-} \\
\text {value }^{+}\end{array}$ & $\begin{array}{l}24 \text { weeks } \\
\text { after } \\
\text { emicizumab } \\
\text { dose } \\
\text { reduction } \\
(\text { C)* }\end{array}$ & $\begin{array}{l}\text { Difference } \\
(\mathrm{C}-\mathrm{B})^{*}\end{array}$ & $p$-value ${ }^{+}$ \\
\hline Physical & 11.87 & 77.77 & 65.90 & 0.010 & 79.48 & 1.71 & 0.569 \\
\hline functioning & $( \pm 8.44)$ & $( \pm 3.87)$ & $( \pm 11.23)$ & & $( \pm 7.46)$ & $( \pm 4.37)$ & \\
\hline Role functioning & $\begin{array}{l}29.03 \\
( \pm 13.74)\end{array}$ & $\begin{array}{l}81.73 \\
( \pm 11.06)\end{array}$ & $\begin{array}{l}52.70 \\
( \pm 15.86)\end{array}$ & 0.029 & $\begin{array}{l}80.61 \\
( \pm 8.59)\end{array}$ & $\begin{array}{l}-1.21 \\
( \pm 2.78)\end{array}$ & 0.529 \\
\hline Worry & $\begin{array}{l}46.67 \\
( \pm 39.46)\end{array}$ & $\begin{array}{l}84.00 \\
( \pm 10.58)\end{array}$ & $\begin{array}{l}37.33 \\
( \pm 34.49)\end{array}$ & 0.202 & $\begin{array}{l}80.00 \\
( \pm 8.00)\end{array}$ & $\begin{array}{l}-4.00 \\
( \pm 4.00)\end{array}$ & 0.225 \\
\hline $\begin{array}{l}\text { Consequences of } \\
\text { bleeding }\end{array}$ & $\begin{array}{l}17.13 \\
( \pm 15.04)\end{array}$ & $\begin{array}{l}75.20 \\
( \pm 14.67)\end{array}$ & $\begin{array}{l}58.07 \\
( \pm 26.52)\end{array}$ & 0.063 & $\begin{array}{l}73.33 \\
( \pm 12.88)\end{array}$ & $\begin{array}{l}-1.87 \\
( \pm 4.37)\end{array}$ & 0.529 \\
\hline Emotional impact & $\begin{array}{l}47.80 \\
( \pm 23.38)\end{array}$ & $\begin{array}{l}73.30 \\
( \pm 5.77)\end{array}$ & $\begin{array}{l}25.50 \\
( \pm 18.92)\end{array}$ & 0.144 & $\begin{array}{l}71.11 \\
( \pm 5.09)\end{array}$ & $\begin{array}{l}-2.19 \\
( \pm 1.92)\end{array}$ & 0.184 \\
\hline $\begin{array}{l}\text { Treatment } \\
\text { concern }\end{array}$ & $\begin{array}{l}37.80 \\
( \pm 42.26)\end{array}$ & $\begin{array}{l}64.40 \\
( \pm 36.75)\end{array}$ & $\begin{array}{l}26.60 \\
( \pm 23.04)\end{array}$ & 0.184 & $\begin{array}{l}75.56 \\
( \pm 13.88)\end{array}$ & $\begin{array}{l}11.16 \\
( \pm 36.71)\end{array}$ & 0.652 \\
\hline $\begin{array}{l}\text { Overall } \\
\text { transformed score }\end{array}$ & $\begin{array}{l}31.72 \\
( \pm 12.95)\end{array}$ & $\begin{array}{l}76.10 \\
( \pm 2.27)\end{array}$ & $\begin{array}{l}44.38 \\
( \pm 10.68)\end{array}$ & 0.019 & $\begin{array}{l}76.68 \\
( \pm 3.83)\end{array}$ & $\begin{array}{l}0.58 \\
( \pm 5.67)\end{array}$ & 0.877 \\
\hline
\end{tabular}

FIGURE 2 Health-related quality of life scores by 6 domains (Haemo-QoL-A)

Conclusions: The results demonstrated that emicizumab has a favourable safety profile with encouraging efficacy in HA patients with inhibitors, even at a reduced dose. This is not standard of care, but due to cost constraints in developing countries, it is worth conducting future studies for validation of this modified regime. rothicher

PB0568 | Pharmacokinetic-guided Prophylaxis Improved Clinical Outcomes in 46 Pediatric Patients with Severe Hemophilia A

H. Kun; L. Gang; Z. Yingzi; W. Xinyi; C. Zhenping; W. Runhui Beijing Children's Hospital, Capital Medical University, Beijing, China

Background: Due to the inter-individual variability of PK parameters, the traditional weight-based dosing regimen could lead to under/overdosage. PK-guided prophylaxis could be an optimized therapy choice.

Aims: To investigate the clinical outcomes of PK-guided prophylaxis in 46 hemophiliac boys

Methods: Forty-six boys with severe hemophilia A were enrolled. After a single-dose infusion(50IU/kg) of Kovaltry, the PK tests were performed using a five-point assay, and PK parameters were calculated by WinNonlin software. The dosing regimen and bleeding rates(ABR, annual bleeding rate; AJBR, annual joint bleeding rate; ASBR, annual spontaneous bleeding rate) were collected by their daily record during the observation period( 6 months before and after PK test or dosing regimen adjustment respectively). The adjustment was based on PK evaluation, bleeding details, doctor's advice, and patients' choice.

Results: The age distribution of our population was $7.41 \pm 3.30$ years old. In our patients, 23 patients had their trough level escalated and 5 patients had it reduced, while the other 18 patients kept previous dosing regimen. In the patients without change in dosing regimen, the trough level was $4.0 \pm 2.41 \mathrm{IU} / \mathrm{dL}$ and the bleeding rates were similar after PK tests. For patients with higher trough level after adjustment, higher dose[29.41(23.81, 35.71) vs. 20(16.67,27.78), $P<0.01]$ and frequency[3.0(3.0,3.5) vs. 3.0(2.0, 3.0), $P<0.05]$ were observed as well as higher trough level[2.1(1.0,3.4) vs. 1.0(0.5,2.0), $P<0.001]$. Reduced ABR[2(2.6) vs. 8(4,10), $P<0.001]$, AJBR[2(0,4) vs. $4(0,8)$, $P<0.01]$ and $\operatorname{ASBR}[0(0,2)$ vs. $4(0,4), P<0.01]$ were showed. In the other 5 patients with trough level reduced, lower infusion frequency[2.33(3.0,3.5) vs. 3.0(2.17, 2.67), $P<0.05]$ and weekly FVIII consumption[58.25 vs. $75 \mathrm{IU} / \mathrm{kg} /$ week, $P<0.05$ ] were observed. In these patients, no statistical difference was found in $\operatorname{ABR}[0(0,2)$ vs. $1(0,2), P=0.62]$, AJBR $[0(0,3)$ vs. $0(0,4), P=0.63]$ and $\operatorname{ASBR}[0(0,2)$ vs. $0(0,1), P=0.39]$. 
TABLE 1 Dosing regimens and trough FVIII level during the adjustment

\begin{tabular}{|c|c|c|c|c|}
\hline & & $\begin{array}{l}\text { Trough FVIII level escalated } \\
(\mathrm{N}=23)\end{array}$ & $\begin{array}{l}\text { Trough FVIII level } \\
\text { reduced }(\mathrm{N}=5)\end{array}$ & $\begin{array}{l}\text { Dosing regimen } \\
\text { unchanged }(\mathrm{N}=18)\end{array}$ \\
\hline \multirow[t]{2}{*}{ Dose(IU/kg) } & Pre-adjustment & $20.0(16.67,27.78)$ & 25.0(13.31,29.17) & \multirow[t]{2}{*}{$24.63(18.36,32.53)$} \\
\hline & $P$ value & $<0.01$ & 0.26 & \\
\hline \multirow{2}{*}{$\begin{array}{l}\text { Frequency(infusions per } \\
\quad \text { week) }\end{array}$} & Pre-adjustment & $3.0(2.0,3.0)$ & $3.0(3.0,3.5)$ & \multirow[t]{2}{*}{$3.5(3.0,3.5)$} \\
\hline & $P$ value & $<0.05$ & $<0.01$ & \\
\hline \multirow[t]{3}{*}{ Trough FVIII levels (IU/dL) } & Pre-adjustment & $1.0(0.5,2.0)$ & $3.1(1.55,5.2)$ & \multirow[t]{3}{*}{$4.0 \pm 2.41$} \\
\hline & Post-adjusted & $2.1(1.0,3.4)$ & $1.9(0.95,2.7)$ & \\
\hline & $P$ value & $<0.001$ & 0.07 & \\
\hline
\end{tabular}

TABLE 2 Bleeding rates before and after adjustment

\begin{tabular}{|c|c|c|c|c|}
\hline bleeding rates & & $\begin{array}{l}\text { Trough FVIII level } \\
\text { escalated( } N=23)\end{array}$ & $\begin{array}{l}\text { Trough } \mathrm{FVIII} \mathrm{level} \\
\text { reduced }(\mathrm{N}=5)\end{array}$ & $\begin{array}{l}\text { Dosing regimen unchanged } \\
(N=18)\end{array}$ \\
\hline \multirow[t]{2}{*}{ annual bleeding rate } & Pre-adjustment & $8(4,10)$ & $1(0,2)$ & $0(0,0)$ \\
\hline & Pvalue & $<0.001$ & 0.62 & 0.57 \\
\hline \multirow[t]{2}{*}{ annual joint bleeding rate } & Pre-adjustment & $4(0,8)$ & $0(0,4)$ & $0(0,0)$ \\
\hline & Pvalue & $<0.01$ & 0.63 & $>0.99$ \\
\hline \multirow{3}{*}{$\begin{array}{l}\text { annual spontaneous bleeding } \\
\text { rate }\end{array}$} & Pre-adjustment & $4(0,4)$ & $0(0,1)$ & $0(0,0)$ \\
\hline & Post-adjustment & $0(0,2)$ & $0(0,2)$ & $0(0,0)$ \\
\hline & Pvalue & $<0.01$ & 0.39 & 0.75 \\
\hline
\end{tabular}

Conclusions: PK-guided prophylaxis could help hemophiliac patients improve quality of life by reducing bleeds with affordable FVIII concentrate consumption as well as decreasing infusion frequency with similar bleeds.

\section{PB0569 | The Role of Familial Mediterranean Fever in} Hemophilia: Should We Tested?

S. Kirkiz; Z. Kaya; S. Gönen; Ü. Koçak

Gazi University School of Medicine, Ankara, Turkey

Background: Familial Mediterranean fever (FMF) is a heterogenous autoinflammatory genetic disorder characterized by periodic fever, peritonitis, and synovitis, which can be associated with several disorders including autoimmune or systemic vasculitis. However, FMF in patients with hemophilia has not been reported previously. Both disorders result in arthritis.

Aims: We aim to investigate the frequency and clinical characteristics of FMF in patients with severe hemophilia.

Methods: This cross-sectional study included 28 patients with severe hemophilia (24 hemophilia A and 4 hemophilia B). Clinical and laboratory data were recorded in patients with hemophilia. All cohorts were genetically tested for FMF by Pyrosequencing ${ }^{\mathrm{TM}}$. The diagnosis of FMF is based on Tel-Hashomer clinical criteria and/ or genetic results. This study was approved by the Institutional Review Board.

Results: Of the 28 individuals, seven (25\%) were diagnosed with genetically confirmed FMF. Five different heterozygous mutations including M694V ( $n=2), \mathrm{E} 148 \mathrm{Q}(n=2), \operatorname{V726\mathrm {A}}(n=1), \mathrm{P} 369 \mathrm{~S}(n=1)$, $\mathrm{E} 148 \mathrm{Q} / \mathrm{K} 695 \mathrm{R}(n=1)$ were identified in these patients. The median ages of them were 18 years (2-42 years). Seven patients with severe hemophilia were receiving prophylaxis. Among them, four were symptomatic for FMF cases. The first case was a 10-year-old boy with hemophilia B with recurrent fever, abdominal pain, and arthritis who was diagnosed with FMF according to the Tel-Hashomer criteria and positive M694V heterozygous mutation and started colchicine. His symptoms recovered. The remaining three older cases with hemophilia A were positive family history for FMF. They had recurrent arthritis without fever. No bleeding was detected in their joint ultrasound during several attacks. The number of joint bleedings in mutation-positive patients with FMF was significantly lower than in mutation-negative patients with FMF $(P<0.05)$. 
Conclusions: Our data indicate that screening for FMF may be useful in patients with severe hemophilia who develop arthritis without prominent bleeding and have a positive family history in many Mediterranean countries, including Turkey.

PB0570 | First Interim Analysis from the Observational HEMPOWR Study Evaluating Effectiveness and Safety of Real-world Treatment with Damoctocog Alfa Pegol in Previously Treated Patients with Hemophilia A

M. Reding ${ }^{1}$; M. Teresa Alvarez Román ${ }^{2} ;$ M. Sanabria ${ }^{3} ;$ G. Castaman $^{4}$; M. Janbain ${ }^{5}$; T. Matsushita ${ }^{6}$; K. Meijer ${ }^{7}$; K. Schmidt ${ }^{8}$; J. Oldenburg 9

${ }^{1}$ University of Minnesota Medical Center, Minneapolis, Minnesota, United States; ${ }^{2}$ Hospital Universitario La Paz, Madrid, Spain; ${ }^{3}$ Bayer, Basel, Switzerland; ${ }^{4}$ Careggi University Hospital, Florence, United Kingdom; ${ }^{5}$ Tulane School of Medicine, New Orleans, Louisiana, United States; ${ }^{6}$ Nagoya University Hospital, Nagoya, Japan; ${ }^{7}$ University Medical Center Groningen, Groningen, Netherlands; ${ }^{8}$ Bayer, Berlin, Germany; ${ }^{9}$ University Clinic Bonn, Bonn, Germany

Background: Damoctocog alfa pegol (BAY 94-9027) is an extendedhalf-life PEGylated recombinant factor VIII product, approved for use in previously-treated patients (PTPs) aged $\geq 12$ years with hemophilia $A$. Aims: HEM-POWR (NCT03932201) is a multicenter, noninterventional, open-label, prospective phase IV trial investigating routine clinical use of damoctocog alfa pegol. Planned enrollment is $\geq 200$ PTPs with mild, moderate, or severe hemophilia A. The 6month interim safety analysis is presented.

Methods: Eligible patients include all PTPs receiving damoctocog alfa pegol on-demand or as prophylaxis, according to local label who provided informed consent. Primary outcomes include total bleeding events and annualized bleeding rate. Secondary outcomes include long-term safety, joint health, and patient-reported outcomes. Analyses will be exploratory, stratified by prophylaxis regimen and hemophilia severity. Ethical approval was obtained for all sites.

Results: At data cut-off (21 October), 39 PTPs were enrolled; 38 patients comprised the safety analysis set (one patient withdrew consent), including $8 \mathrm{mild} /$ moderate patients (Table 1). Most patients ( $n=32$ ) had received damoctocog alfa pegol within 12 months of baseline for variable time periods (mean [SD]=354.6 [614.02] days). Median (Q1;Q3) dose was 3000 (2000;3650) IU/infusion; the most common dosing regimen being twice-weekly prophylaxis (44\%). 26/32 patients provided information about Factor VIII treatment within 12 months prior to initiation of damoctocog alfa pegol. In this group, the most common dosing frequency was three times weekly (data available for 24 patients). A group of six damoctocog alfa pegol-naïve patients had a comparatively worse bleeding history (Table 2 ). There were no study-drug-related treatment-emergent adverse events, nor inhibitor development. Two patients discontinued. Remote follow-up visits were made due to Covid-19; initial visits may now be conducted remotely.

TABLE 1 Demographics, Baseline Characteristics, and Exposure

Patients in SAF ( $n)$

Region /country of recruitment ( $n$, \%)

Europe / Japan / United States of

America

Age at signing informed consent (median; Q1, Q3, y)

History of inhibitors $(n, \%)$

Severe / moderate / mild hemophilia at diagnosis $(n, \%)$

Patients with at least one concomitant disease ( $n, \%)$

Hemarthrosis / pain / psychiatric disorders

Patients with at least one concomitant medication $(n, \%)$

Observation days (mean, SD, range)

SAF, safety analysis set; SD, standard deviation; 1 patient excluded (missed dose).

TABLE 2 Bleeding History 12 months prior to enrollment

$\begin{array}{llll}\begin{array}{l}\text { Number of reported bleeds within } 12 \\ \text { months prior to enrollment (median, } \\ \text { Q1, Q3) }\end{array} & \begin{array}{l}\text { Naïve to damoctocog alfa pegol } \\ (\mathrm{N}=6)\end{array} & \begin{array}{l}\text { Pre-treated with damoctocog alfa } \\ \text { pegol }(\mathrm{N}=32)\end{array} & \begin{array}{l}\text { Total (N = 38) } \\ \text { Total bleeds }\end{array} \\ \begin{array}{l}\text { Spontaneous } \\ \text { Srauma }\end{array} & 3.5(1.0,10.0) & 0.0(0.0,1.0) & 0.0(0.0,4.0) \\ \text { Joint } & 0.0(0.0,1.0) & 0.0(0.0,0.0) & 0.0(0.0,1.0) \\ \text { Spontaneous joint } & 3.5(1.0,10.0) & 0.0(0.0,0.0) & 0.0(0.0,1.0) \\ \text { Trauma joint } & 3.0(1.0,10.0) & 0.0(0.0,1.0) & 0.0(0.0,2.0) \\ & 0.0(0.0,0.0) & 0.0(0.0,1.0) & 0.0(0.0,1.0)\end{array}$

Note: Patients were enrolled at their initial visit, at which baseline data was recorded. 
Conclusions: This first interim analysis of the HEM-POWR study provides real-world evidence that damoctocog alfa pegol is welltolerated in routine clinical use in patients with mild, moderate, or severe disease.

PB0571 | Health Care Resource Use (HRU) among Adult Persons with Hemophilia B (PwHB) and Concurrent Joint Disease (JD) in the United States

L.E. Markson ${ }^{1}$; L. Young ${ }^{2}$; L. Ban ${ }^{3}$; Y. Chen ${ }^{1}$; R. Zaha ${ }^{4}$; P.F. Fogarty ${ }^{1}$

${ }^{1}$ Pfizer Inc., Collegeville, United States; ${ }^{2}$ Pfizer Ltd, Walton Oaks, United Kingdom; ${ }^{3}$ PPD Ltd, Beijing, China; ${ }^{4}$ Evidera, Bethesda, United States

Background: Frequency of HRU can be an indicator of disease burden.

Aims: Describe HRU in a cohort of adult PwHB with administrative claims for JD, regardless of cause.

Methods: PharMetrics Plus data from January 2010-April 2020 were used to study PwHB with no evidence of an inhibitor or nonmild liver disease. The cohort included males aged 19-64 years prescribed factor IX replacement during the study period and no evidence of bypassing agent use. JD was defined by relevant diagnosis/ surgery codes in $\geq 1$ inpatient claim, or in $\geq 2$ outpatient claims $\geq 30$ days apart. Severity of hemophilia was not available. HRU statistics account for differential follow-up.

Results: In total, 237 PwHB had HRU for JD. Mean age was 39.1 years (SD, 13.7). Of these, 167 (70.5\%) had no coded diagnoses from 20 prespecified conditions of interest. Mild chronic liver disease, depression, and anxiety were reported in $8.4 \%, 7.6 \%$, and $6.8 \%$, respectively. The median follow-up from first JD diagnosis was 26.4 months (interquartile range, 13.1-48.7). Among the $237 \mathrm{PwHB}$ $10.7 \%$ had $\geq 1$ joint surgery in the first 12 months following a JD diagnosis, with the highest joint surgery rate of $19.1 \%$ in those aged 31-44 years compared with $4.8 \%$ in those aged $19-30$ years and $10.1 \%$ in those aged $45-64$ years. For arthropathy-related inpatient admissions, the cumulative incidence risk at 12 months of follow-up was $8.0 \%$. Mean length of stay was 4.6 days (SD, 4.1 ). The incidence rate of arthropathy-related emergency department visits was 24.3 per 100 person-years (95\% Cl, 20.9-28.3); of physical therapy visits was 59.4 per 100 person-years ( $95 \% \mathrm{Cl}, 53.8-65.4$ ); and of pharmacy claims for pain relief medications was 133.4 per 100 person-years (95\% Cl, 125.0-142.3).

Conclusions: The observed rates of HRU in this cohort of PwHB and concurrent JD provides insight into the disease burden encountered for this population.
PB0572 | Health Care Resource Use (HRU) among Adult Persons with Hemophilia A (PwHA) and Concurrent Joint Disease (JD) in the United States

L.E. Markson ${ }^{1}$; P.F. Fogarty ${ }^{1}$; L. Ban ${ }^{2}$; Y. Chen ${ }^{1}$; R. Zaha ${ }^{3}$; L. Young ${ }^{4}$

${ }^{1}$ Pfizer Inc., Collegeville, United States; ${ }^{2}$ PPD Ltd, Beijing, China;

${ }^{3}$ Evidera, Bethesda, United States; ${ }^{4}$ Pfizer Ltd, Walton Oaks, United Kingdom

Background: Frequency of HRU can indicate disease burden.

Aims: Describe HRU in a cohort of adult PwHA with administrative claims for JD, regardless of cause.

Methods: PharMetrics Plus claims data from Jan 2010-April 2020 were used to study PwHA with no evidence of an inhibitor or nonmild liver disease. The cohort included males aged 19-64 years prescribed factor VIII replacement during the study period and no evidence of bypassing agent use. JD was defined by relevant diagnosis/surgery codes in $\geq 1$ inpatient claim, or in $\geq 2$ outpatient claims $\geq 30$ days apart. Severity of hemophilia was not available. HRU statistics account for differential follow-up.

Results: In total, 738 PwHA had HRU for JD. Mean age was 35.8 yrs (SD, 12.9 years). Of these, 68\% $(n=502)$ had no coded diagnoses from a list of 20 prespecified conditions of interest. Mild chronic liver disease, HIV/AIDS, depression, and anxiety were reported in $10.7 \%, 7.9 \%, 7.3 \%$, and $6.0 \%$, respectively. The median follow-up from first JD diagnosis was 24.3 months (interquartile range, 12.245.2). Cumulative incidence of joint surgery in the first 12 months of follow-up was highest, at $19.2 \%$ in those aged $45-64$ years compared with $12.0 \%$ in those aged $31-44$ years and $13.9 \%$ in those aged 19-30 years .For arthropathy-related inpatient admissions, the cumulative incidence risk at 12 months of follow-up was $10.3 \%$. Mean length of stay was 4.4 days (SD, 4.5). The incidence rate of arthropathy-related emergency department visits was 14.2 per 100 person-years (95\% Cl, 12.7-16.0); of physical therapy visits was 51.6 per 100 person-years $(95 \% \mathrm{Cl}, 48.5-54.9)$, and of pharmacy claims for pain relief medications was 161.2 per 100 person-years $(95 \% \mathrm{Cl}$, 155.7-167.0).

Conclusions: The observed rates of HRU in this cohort of PwHA and comorbid JD provides insight into the disease burden encountered for this population. 
PB0573 | Patients with Severe Hemophilia A under Middledosed Prophylaxis Benefit from Multi-disciplinary Team Management, an Experience

Z. Shiqiu ${ }^{1}$; C. Rong ${ }^{1}$; G. Hua ${ }^{1}$; L. Jia ${ }^{1}$; G. Jing ${ }^{1}$; Y. Ming ${ }^{2}$; Z. Hong ${ }^{2}$; Y. $\operatorname{Lin}^{2} ;$ Z. Xin ${ }^{3}$

${ }^{1}$ Department of Hematology, the Third People's Hospital of Chengdu, Chengdu, China; ${ }^{2}$ Department of Ultrasonography, the Third People's Hospital of Chengdu, Chengdu, China; ${ }^{3}$ Department of Physical Medicine and Rehabilitation, the Third People's Hospital of Chengdu, Chengdu, China

Background: For hemophilia patients, comprehensive care promotes both physical and psychological health and quality of life while decreasing morbidity and mortality. MDT management plays a key role in comprehensive care.

Aims: Explore the benefit of MDT model in improving the treatment effect of severe hemophilia A patients under middle-dosed prophylaxis.

Methods: 24 severe hemophilia patients under middle-dosed prophylaxis were included in the observational before-after self-control study. We defined the data collected from November 2016 to June 2017 which did not implement MDT management as control group and that from July 2017 to December 2020 as the experimental group. The nurse collected the bleeding rates. The musculoskeletal sonologists evaluated joints based on HEAD-US-C scanning protocol every 3 month and physiotherapist completed HJHS questionnaire half a year. We performed pair Wilcoxon test with SPSS 22.0 on the before-after groups to compare the $A B R, A J B R$, ultrasound score, HJHS score and FVIII consumption.

Results: After MDT management, the ABR decreased from 18.9 to $8.9(P<0.000)$, AJBR dropped from 13.5 to $6.9(P=0.003)$. (figure 1$)$

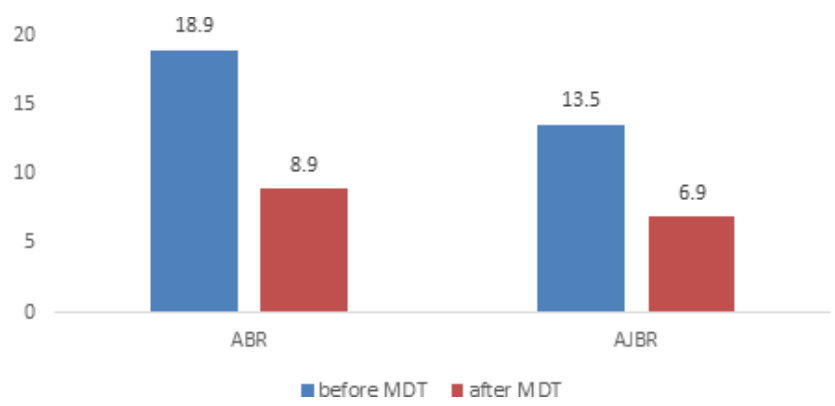

FIGURE 1 The ABR and AJBR score before and after MDT

Along with the decrease of bleeding rates, the HJHS score improved in 22 (92.0\%) patients and the average HJHS score decreased $51.6 \%$ which demonstrated better joint function. However, the ultrasonic score showed an deteriorating trend, all the US score evidently increased. (table 2)

From the perspective of FVIII consumption, the weekly dosage lifted from $33.6 \mathrm{IU} / \mathrm{kg} /$ week to $39.8 \mathrm{IU} / \mathrm{kg} /$ week $(P=0.0067)$.
TABLE 1 HEAD-US-C score before and after MDT HEAD-US-C score

\begin{tabular}{llll} 
& Before MDT & After MDT & P value \\
Knee & 6.86 & 10.17 & 0.001 \\
Ankle & 6.17 & 10.54 & 0.003 \\
Elbow & 7.79 & 10.20 & 0.004 \\
\hline
\end{tabular}

Conclusions: The 3-year period MDT experience prompt severe hemophilia A patients benefit from comprehensive care as bleeding rates decreased and joint function improved. With multidisciplinary management, the FVIII consumption showed upward trend statistically, but still within the range of middle-dosed prophylaxis. However, the deterioration in ultrasonic score demonstrate middle-dosed prophylaxis was still not enough to prevent progress of synovitis or arthropathy. Standard prophylaxis or individualized prophylaxis based on pharmacokinetics parameters could further benefit hemophilia A patients.

PB0574 | Adherence to Prophylaxis and Recording of Hometreatment Following a Switch to Extended Half-life Clotting Factor Concentrate

M. O'Donovan $^{1,2}$; C. Bergine ${ }^{1}$; N. Larkin ${ }^{1}$; E. Singleton ${ }^{1}$; J. Benson ${ }^{1}$; F. McGroarty ${ }^{1}$; L. Jeffrey ${ }^{3}$; R. Bird ${ }^{1}$; C. Buckley ${ }^{1}$; K. Ryan ${ }^{1}$; J.S. O'Donnell $^{1}$; N.M. O'Connell ${ }^{1,2}$

${ }^{1}$ National Coagulation Centre, St James's Hospital, Dublin, Ireland; ${ }^{2}$ School of Medicine, Trinity College Dublin, Dublin, Ireland; ${ }^{3}$ Crimson Tide, Kent, United Kingdom

Background: Prophylaxis and bleeding episodes are typically selfmanaged by people with haemophilia (PWH). Prophylaxis adherence and accurate recording of clotting factor concentrate (CFC) home-treatments is vital to optimise outcomes and clinical review by the haemophilia team. In Ireland, PWH are encouraged to use a haemophilia-specific app (MProHX, Crimson Tide) to record home-treatments.

Aims: To evaluate adherence to prophylaxis and adherence to recording home-treatments after switching to extended half-life (EHL) CFC.

Methods: PWH who switched to EHL-CFC were identified from the National Haemophilia Electronic Health Record. Data on CFC dispensed, prophylaxis prescriptions and smartphone treatment records were collected for one-year post switchover. Adherence to prophylaxis was estimated as percentage of prescribed prophylaxis dispensed. Patient adherence to recording all home-treatments (episodic and prophylaxis) was estimated as percentage of CFC dispensed which was recorded. Patient adherence to recording only prophylaxis was estimated as percentage of prescribed prophylaxis which was recorded.

Results: 102 PWH, median age 36 years were included (Table 1). 
High rates of adherence to prophylaxis were demonstrated. Median prescribed prophylaxis dispensed was 101\% with EHL-FVIII and $112 \%$ with EHL-FIX.

Lower rates of adherence to recording home-treatments was demonstrated. Median CFC dispensed which was recorded (surrogate marker of adherence to recording all home-treatments) was $81 \%$ for EHL-FIX but only 52\% for EHL-FVIII. Median prescribed prophylaxis recorded (surrogate marker for adherence to recording prophylaxis) was $84 \%$ for EHL-FIX and $47 \%$ for EHL-FVIII.

TABLE 1 Demographics and treatment regimens

\begin{tabular}{|c|c|c|c|c|}
\hline & & Age, median (years) & & \\
\hline & $N=$ & range & EHL-CFC prophylaxis & EHL-CFC on demand \\
\hline Complete cohort & 102 & $\begin{array}{l}36 \\
17-75\end{array}$ & 98 (96\%) & $4(4 \%)$ \\
\hline \multicolumn{5}{|l|}{ FIX deficiency } \\
\hline Severe FIX deficiency & 24 & $\begin{array}{l}42 \\
17-63\end{array}$ & 24 (100\%) & $0(0 \%)$ \\
\hline \multicolumn{5}{|l|}{ FVIII deficiency } \\
\hline Severe FVIII deficiency & 71 & $\begin{array}{l}33 \\
18-75\end{array}$ & 70 (99\%) & $1(1 \%)$ \\
\hline Moderate FVIII deficiency & 6 & $\begin{array}{l}48 \\
33-59\end{array}$ & $3(50 \%)$ & $3(50 \%)$ \\
\hline Mild FVIII deficiency & 1 & 38 & $1(100 \%)$ & $0(0 \%)$ \\
\hline
\end{tabular}

Conclusions: Estimated adherence to prophylaxis with EHL-CFC is excellent among people with haemophilia $\mathrm{A}$ and $\mathrm{B}$ in this cohort. However, adherence to recording home-treatments demonstrates lower rates, particularly among people with haemophilia A. This may suggest a need for greater patient education regarding the benefits of recording prophylaxis and episodic treatment of bleeding events. Adherence is challenging to quantify even with sophisticated CFC track and trace systems, and haemophilia-specific apps.

PB0575 | The Potential Benefits of Physical Exercises for Reducing Pain in Adult Haemophilic Arthropathy Patients: A Meta-analysis of Randomized Controlled Trials

B.P. Putra ${ }^{1}$; F.N. Putra ${ }^{2}$

${ }^{1}$ Medical Doctor, Blitar, Indonesia; ${ }^{2}$ Faculty of Medicine Universitas

Airlangga, Surabaya, Indonesia

Background: Haemophilia patients tend to develop kinesiophobia to avoid movement pain that may lead to physical inactivity, decreased quality of life, and poor mental health. Recent studies suggested that physical exercise can give pain improvement in adult haemophilic arthropathy patients although the results were still inconsistent.

Aims: This study aims to measure the efficacies of physical exercise for reducing pain in adult haemophilic arthropathy patients.
Methods: We did comprehensive online searching databases of Pubmed, ScienceDirect, and The Cochrane Library, to include all relevant studies from January 2000 until January 2021. This study was conducted according to the PRISMA guideline. We included all randomized controlled trials (RCTs) that compared the efficacies between physical exercise and control groups then accessed the changes of pain scale in adult haemophilic arthropathy patients. Physical exercises included in this study are programmed sport therapy (PST), mechanical therapy (MT), and educational therapy (ET). We used the Cochrane Risk-of-bias (RoB 2) tool for accessing bias risks. We conducted analysis to provide standard mean difference (SMD) with 95\% confidence interval $(\mathrm{Cl})$ using fixed-effect heterogeneity test.

Results: We included 6 RCTs met our eligibility criteria. The MT group shows significant pain reduction compared with the control group $\left(\mathrm{SMD}=1.22,95 \% \mathrm{Cl} 0.67\right.$ to $\left.1.76, P<0.0001, \mathrm{I}^{2}=0 \%\right)$. Both PST (SMD $=0.35,95 \% \mathrm{Cl}-0.10$ to $0.79, P=0.13, \mathrm{I}^{2}=0 \%$ ) and $\mathrm{ET}$ groups (SMD $=0.22,95 \% \mathrm{Cl}-0.24$ to $0.68, P=0.34, I^{2}=0 \%$ ) also give pain improvement although not statistically significant. Thus, the overall analysis suggests that physical exercises provide significant pain reduction in adult haemophilic arthropathy patients compared with the control group (SMD $=0.53,95 \% \mathrm{Cl} 0.25$ to $0.80, P=0.0002$, $\mathrm{I}^{2}=28 \%$ ).

Conclusions: Physical exercises have potential benefits for reducing pain in adult haemophilic arthropathy patients. However, further studies are needed to establish the efficacy. 
PB0576 | Impact of COVID-19 Pandemic on the Joint Bleeding Pattern and Prophylactic Management of Hemophilia Patients: A Single Centre Study in a Tertiary Care Centre of Eastern India

A. Chatterjee; A.K. De; M. Bera; S. Roy; A. Chowdhury

Midnapore Medical College and Hospital, Midnapore, India

Background: Patients with hemophilia if not treated adequately can lead to permanent joint disease. The global pandemic SARS COVID 19 had many consequences in the management of hemophilia patients which are of great concern, especially in developing countries like India.

Aims: This study aims to assess the impact of COVID 19 pandemic on the joint bleeding pattern and prophylactic management of hemophilia patients.

Methods: A longitudinal study was done on 32 boys of age ranging from 4 to 18 years attending the Hemophilia day care centre of Pediatrics department of Midnapore Medical College and Hospital from March 2020 to October 2020.

Results: Knee joint was predominantly affected by hemarthrosis followed by elbow joint, ankle joint and shoulder joint. Out of total, 90.62\% patients had developed target joints. Knee joint was the predominant target joint in $59.37 \%$ cases. No significant change in the joint bleeding pattern was observed before and after COVID 19 Annualised bleeding rate was found to be worsened during the pandemic which was statistically significant ( $P$ value $<0.0001$ ). Seventy eight percent patients had more than 4 weeks of absenteeism from taking prophylactic factor therapy during the pandemic. The reason for being absent was due to non-availability of transport in $60 \%$ cases, non-availability of factor in $24 \%$ cases and financial reasons in $12 \%$ cases.

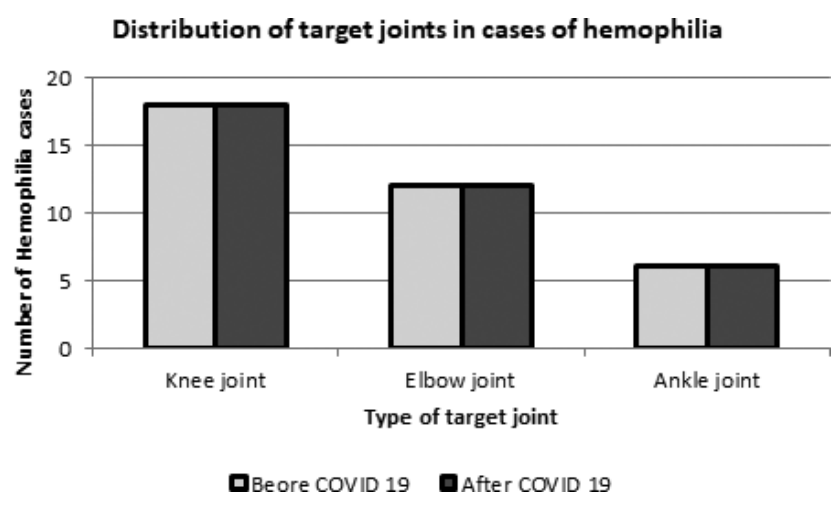

FIGURE 1 Distribution of target joints in cases of hemophilia
Annualised bleeding rate before and after covid 19

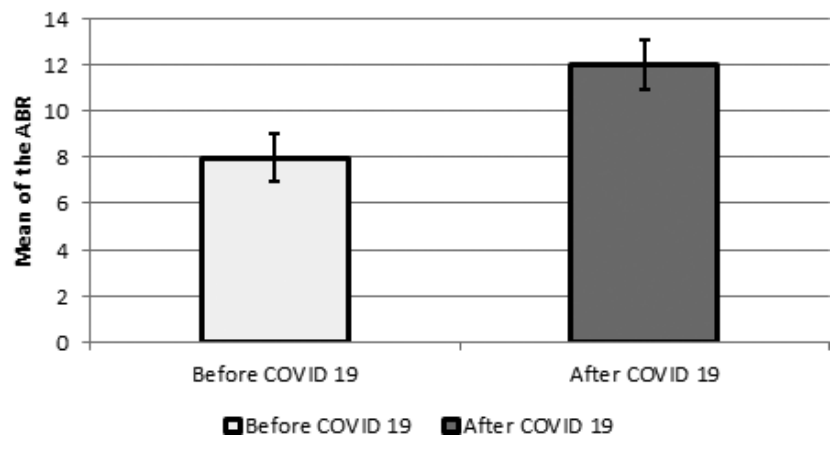

FIGURE 2 Annualised bleeding rate before and after COVID 19

Conclusions: Joint bleeding episodes worsened during the COVID 19 pandemic. Hemophilic patients and their families faced a lot of challenges during this period in getting the prophylactic factor therapy mainly due to non-availability of transport and also due to nonavailability of factor and financial reasons in few cases.

PB0577 | Real-world Annual Bleeding Rate in People with Severe Hemophilia A without Inhibitor on Prophylaxis, Comparing Switched to rFVIII-Fc versus Non-switched Patients: A Multicentre, Retrospective, Non interventional, before-after Study with the French BERHLINGO Database (MOTHIF-II)

$\underline{\text { V. Horvais }}^{1}$; N. Drillaud ${ }^{1}$; M. Sigaud ${ }^{1}$; M. Fouassier ${ }^{1}$; C. Ternisien ${ }^{1} ;$ Y. Repesse $^{2}$; B. Guillet ${ }^{3}$; S. Bayart ${ }^{3}$; P. Beurrier ${ }^{4}$; L. Ardillon ${ }^{5}$; Y. Gruel ${ }^{5}$; J.-B. Valentin ${ }^{5}$; S. Bauer ${ }^{6}$; B. Pan-Petesch7; V. Cussac ${ }^{8}$; J. Rose ${ }^{8}$; M. Pennetier ${ }^{1}$; M. Trossaërt ${ }^{1}$, BERHLINGO Group

${ }^{1}$ Nantes University Hospital, Nantes, France; ${ }^{2}$ Caen University Hospital, Caen, France; ${ }^{3}$ Rennes University Hospital, Rennes, France; ${ }^{4}$ Angers University Hospital, Angers, France; ${ }^{5}$ Tours University Hospital, Chambray-les-Tours, France; ${ }^{6}$ Tours University Hospital, Chambraylès-Tours, France; ${ }^{7}$ Brest University Hospital, Brest, France; ${ }^{8}$ Le Mans Hospital, Le Mans, France

Background: According to the pivotal studies, factor VIII (FVIII) with extended half-life (EHL) efmoroctocog alfa rFVIII-Fc could influence the management of prophylaxis, while maintaining good clinical efficacy on the occurrence of hemorrhagic events. What about in reality?

Aims: The MOTHIF-II study explored FVIII prophylaxis and the impact on the annual bleeding rate (ABR) in severe patients with Haemophilia A without inhibitor (pwSHA) in Western France, following the availibility of rFVIII-Fc.

Methods: We investigated pwSHA followed in 7 French centres via the BERHLINGO research database, before and after the rFVIII-Fc supply ( 2 × 12 months, T1 07/2015-06/2016 versus T2 07/2017$06 / 2018$ ). We compared the ABR and the amounts of FVIII needed to treat hemorrhagic events for matched patients.

Results: A total of 88 patients had a complete traceability logbook for both study periods. As described in Table 1, no significative 
difference was globally observed in the ABR. Nevertheless, for switched patients (rFVIII-Fc only on T2), a significant decrease of the ABR and of the amount of FVIII injected to treat bleeding events was noted (ABR from 6.6 to 4.0 and $40 \%$ drop in FVIII required <> Wilcoxon signed-rank test, $a=5 \%, P=0.003$ for $A B R \& P=0.028$ for FVIII). Over T2, the ABR of switched patients became similar to that of patients on standard FVIII (4.0 versus 3.3). For switched patients, the hemorrhagic profile was also different, with a higher $A B R$ at baseline and a significantly bigger proportion of hemarthroses $(48 \%$ of total treated events versus $29 \%$ for non-switched people). Their annual bleeding joint rate (ABJR) was three times higher at the outset, the switch to rFVIII-Fc leading to its significant decrease from 3.2 to 1.9 (See Table 2 - Wilcoxon signed-rank test, $a=5 \%, P=0.033$ for ABJRs).

TABLE 1 Description of ABR and FVIII administered for bleeding events in matched patients, before and after switch to rFVIII-Fc (according to the type of FVIII prescribed over T2)

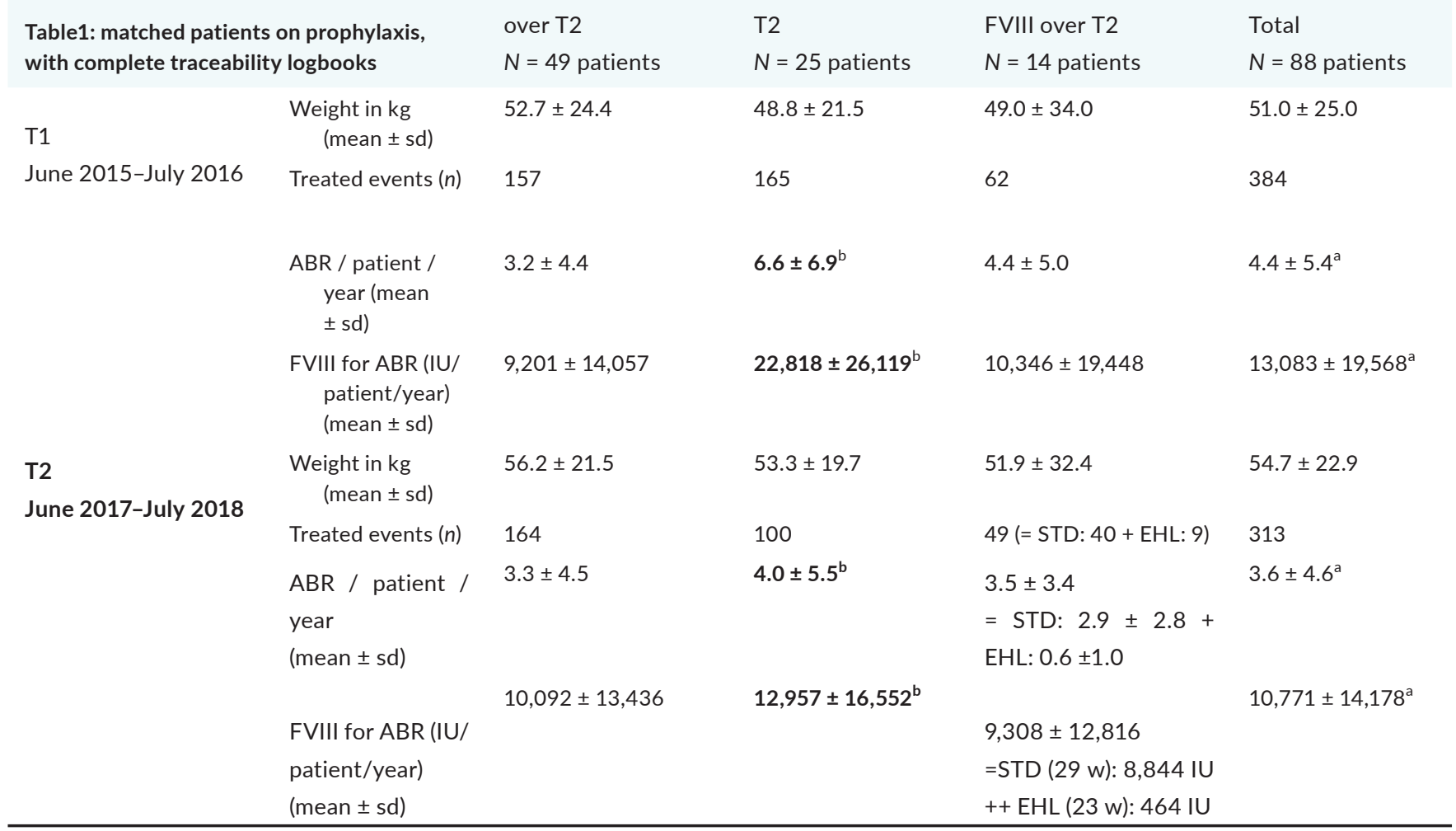

${ }^{a}$ Wilcoxon signed-rank test, non significative $a=5 \%$.

${ }^{b}$ Wilcoxon signed-rank test, significative $a=5 \%(P=0.003$ for $A B R \& P=0.028$ for administered FVIII). 
TABLE 2 ABR and ABJR in matched patients (switched to rFVIII-Fc versus non-switched), according to the type of FVIII prescribed over T2

Table 2: ABR and ABJR in matched patients (switched to rFVIII-Fc versus non-switched), according to the type of FVIII prescribed over T2

Patients with $\mathrm{ABR}=0(n)$

Patients with $\mathrm{ABR} \neq 0$ (n) / Total of treated bleeding events $(n)$

$\Rightarrow A B R$

- Whom patients treated for hemarthrosis / total of treated hemarthrosis $\Rightarrow A B J R$
Standard FVIII only over T2

$\mathrm{N}=49$ patients

$\mathrm{T} 1=07 / 2015$

$06 / 2016$

14

35 / 157

$\mathrm{ABR}=3.2^{\mathrm{a}}$

$17 / 46$

ABJR $=0.9^{a}$
T2 = 07/2017-06/2018

15

34 / 164

$A B R=3.3^{a}$
rFVIII-Fc only over T2

$N=25$ patients

$\mathrm{T} 1=07 / 2015-06 / 2016$

3

22 / 165

$\mathrm{ABR}=6.6^{\mathrm{b}}$
$T 2=07 / 2017-06 / 2018$

7

18 / 100

$\mathrm{ABR}=4.0^{\mathrm{b}}$

${ }^{a}$ Wilcoxon signed-rank test, non significative $a=5 \%$ (ABR vs ABR \& ABJR vs ABJR)

${ }^{b}$ Wilcoxon signed-rank test, significative $a=5 \%(P=0.003$ for $A B R s$ and $P=0.033$ for $A B J R s)$

Conclusions: Our study showed a significant improvement in the hemorrhagic phenotype in patients treated with rFVIII-Fc. Only reallife studies are likely to show such results.

PB0578 | Evaluating the Effectiveness of Recombinant Factor VIII Fc Fusion Protein (rFVIIIFc) in Adolescents and Children with Hemophilia A in the Real World in Japan: Interim Analysis of the Multicenter, Observational Fc Adolescent and Children Treatment Study (FACTs)

M. Kobayashi ${ }^{1}$; K. Nogami ${ }^{2}$; Y.-D. Park ${ }^{3}$; M. Shiraishi ${ }^{4}$; M. Takatoku ${ }^{4}$; T. Matsushita ${ }^{5}$

${ }^{1}$ Hiroshima University, Hiroshima, Japan; ${ }^{2}$ Nara Medical University, Nara, Japan; ${ }^{3}$ BOKU Children's Clinic, Osaka, Japan; ${ }^{4}$ Sanofi, Tokyo, Japan; ${ }^{5}$ Nagoya University, Aichi, Japan

Background: The Phase 3 A-LONG/Kids A-LONG and ASPIRE extension studies evaluated efficacy and safety of extended half-life recombinant factor VIII Fc fusion protein (rFVIIIFc). No patients in Japan were enrolled in Kids A-LONG or previously untreated patients (PUPs) A-LONG studies. The prospective, multicenter, observational FACTs study is designed to evaluate effectiveness of rFVIIIFc in adolescents and children with hemophilia A (Part 1) and summarize the experience of immune tolerance induction (ITI; Part 2) in Japan.

Aims: Present results from the second interim analysis of FACTs Part 1. Methods: Data from patients $<18$ years of age with hemophilia A treated with $\mathrm{rFVIIIFc}$ were collected at baseline (including retrospective prestudy data) and every 6 months for up to 2 years. The regimen was at the investigator's discretion. Study participants provided written informed consent and local research ethics committees approved the study.

Results: As of July 4, 2020, 91/100 enrolled patients (from 62 centers) were included in this interim analysis; 16 (18\%) were PUPs, 81 had >6 months' follow-up, and 35 completed 2 years' follow-up (Table 1). Prior to the study, 5/10 patients with a history of inhibitors underwent ITI with factor VIII (FVIII). Sixty-five (71\%) patients had received prophylactic treatment with FVIII; of those, 29 (45\%) received FVIII treatment at least 3 times/week. During the study, 49 patients on prophylaxis received rFVIIIFc 2 times/week. Overall, injection frequency was maintained or lower on study with rFVIIIFc compared with pre-study (Table 2). Median spontaneous annualized bleed rate (ABR) on study was 0 (interquartile range: $0-0$ ). Three patients developed inhibitors (PUPs, $n=2$; minimally treated patient, $n=1$ ) with titers of 3.0, 0.8, and $5.2 \mathrm{BU} / \mathrm{mL}$, respectively, and started ITI with rFVIIIFc.

Conclusions: In FACTs Part 1, rFVIIIFc prophylaxis was associated with reduced infusion frequency and low $A B R$ in young Japanese patients, consistent with findings with rFVIIIFc in previous global studies.

TABLE 1 Patient characteristics

$\begin{array}{ll} & \text { All patients }(\mathrm{N}=91) \\ \begin{array}{c}\text { Median (range) age at rFVIIIFc start, } \\ \text { years }\end{array} & 6.7(0-17.2) \\ \text { tSevere (<1 IU/dLt FVIII) } & \\ \text { Moderate (1-5 IU/dL FVIII) } & 61(67.0) \\ \text { Mild (5-40 IU/dL FVIII) } & 22(24.2) \\ \text { Unknown } & 6(6.6) \\ & 2(2.2)\end{array}$

FVIII exposure prior to rFVIIIFc treat- $16(17.6)$

ment (ED), $n$ (\%) $6(6.6)$

0

$6(6.6)$

1-3

$7(7.7)$

4-20

$8(8.8)$

21-100

$47(51.6)$

$101-150$

$1(1.1)$

$\geq 151$

Not available

Family history of inhibitor, $\mathbf{n}(\%)$

8 (8.8)

ED, exposure day; FVIII, factor VIII; rFVIIIFc, recombinant factor VIII Fc fusion protein. 
TABLE 2 Previously treated patients' prophylactic regimens in the pre-study and on study periods*

\begin{tabular}{|c|c|c|c|c|c|c|c|c|c|}
\hline & & On stu & VIIIFc & & & & & & \\
\hline & & $\begin{array}{l}\text { Every } \\
\text { other } \\
\text { day } \\
(n=2)\end{array}$ & $\begin{array}{l}3 \text { times } \\
\text { weekly } \\
(n=10)\end{array}$ & $\begin{array}{l}2 \text { times } \\
\text { weekly } \\
(n=42)\end{array}$ & $\begin{array}{l}\text { Every } \\
3 \text { days } \\
(n=2)\end{array}$ & $\begin{array}{l}\text { Every } \\
4 \text { days } \\
(n=0)\end{array}$ & $\begin{array}{l}\text { Every } \\
5 \text { days } \\
(n=0)\end{array}$ & $\begin{array}{l}\text { Weekly } \\
(n=1)\end{array}$ & $\begin{array}{l}\text { Not } \\
\text { available } \\
(n=1)\end{array}$ \\
\hline Pre-study & $\begin{array}{l}\text { Every other day } \\
\qquad(n=5)\end{array}$ & 1 & 0 & 4 & 0 & 0 & 0 & 0 & 0 \\
\hline FVIII & $\begin{array}{l}3 \text { times weekly } \\
\quad(n=24)\end{array}$ & 0 & 8 & 15 & 1 & 0 & 0 & 0 & 0 \\
\hline & $\begin{array}{l}2 \text { times weekly } \\
\quad(n=19)\end{array}$ & 0 & 1 & 17 & 1 & 0 & 0 & 0 & 0 \\
\hline & $\begin{array}{c}\text { Every } 3 \text { days } \\
\quad(n=2)\end{array}$ & 0 & 0 & 1 & 0 & 0 & 0 & 0 & 1 \\
\hline & $\begin{array}{c}\text { Every } 4 \text { days } \\
\quad(n=0)\end{array}$ & 0 & 0 & 0 & 0 & 0 & 0 & 0 & 0 \\
\hline & $\begin{array}{l}\text { Every } 5 \text { days } \\
\quad(n=0)\end{array}$ & 0 & 0 & 0 & 0 & 0 & 0 & 0 & 0 \\
\hline & $\begin{array}{l}\text { Weekly } \\
(n=7)\end{array}$ & 1 & 0 & 5 & 0 & 0 & 0 & 1 & 0 \\
\hline & $\begin{array}{c}\text { Not available } \\
\quad(n=1)\end{array}$ & 0 & 1 & 0 & 0 & 0 & 0 & 0 & 0 \\
\hline
\end{tabular}

FVIII, factor VIII; rFVIIIFc, recombinant factor VIII Fc fusion protein.

*Includes patients with data available pre- and post-baseline $(n=58)$.

PB0579 | Evaluation of Real-world Effectiveness of BAY 818973 to Treat Hemophilia A: An Analysis within the ATHNdataset

C. $\mathrm{He}^{1}$; M. Chandler ${ }^{1}$; M. Recht ${ }^{1}$; J. Charlet ${ }^{2}$; T. Moulton ${ }^{2}$; P. Vashi ${ }^{2}$

${ }^{1}$ American Thrombosis \& Hemostasis Network, Rochester, United

States; ${ }^{2}$ Bayer US LLC, Whippany, United States

Background: The ATHNdataset is a HIPAA-compliant, de-identified database sponsored by The American Thrombosis and Hemostasis Network. Over 40,000 people with a bleeding or clotting disorder, followed at U.S. hemophilia treatment centers, had their health information submitted. This nationally representative sample includes 15,747 hemophilia A patients. BAY $81-8973$ (Kovaltry ${ }^{\circledR}$ ) is a recombinant, full length FVIII product indicated for prophylactic and on demand treatment of bleeding events associated with hemophilia A. Aims: Evaluate the effectiveness of BAY 81-8973 to treat hemophilia $A$ in a real-world setting.

Methods: The ATHNdataset was queried for patients being treated with BAY 81-8973 either prophylactically or on demand. Collected data included baseline demographics, treatment history, comorbidities, and bleed rates. The data were captured via patient charts between January 1, 2010 and October 31, 2020.

Results: At data cutoff, 295 patients were treated with BAY 81-8973, of which 198 (67\%) had severe hemophilia A, 95 (32\%) had mild/ moderate disease (2 had unknown severity), and 288 (98\%) were males. In this population 27 (9\%) patients had a documented history of inhibitors. The mean age of patients was 30 years (median $=27.98$ ) with 37 patients (12.5\%) under the age of 12. Patients had an average of 2.4 years of therapy with BAY $81-8973$, and most of them (224/295, 76\%) had $>12$ months of experience.

270 (91.5\%) patients had documented bleed rates, including patients treated by prophylaxis or on-demand regimens, with 224 (75.9\%) on treatment for $>12$ months. Table 1 captures the overall annualized bleed rates ( $A B R s$ ), spontaneous $A B R$, and the proportion of patients with zero bleeds during the observation period. Of this group, $84.3 \%$ of patients were on prophylaxis for $>12$ months. 
TABLE 1 Evaluation of bleeding rates between all patients who were treated with BAY 81-8973 (stratified based on duration of therapy and prophylactic treatment regimens

\section{All Patients}

Full Analysis Group ( $n=270$ )

\section{Overall ABR}

Mean \pm SD

Median (Q1 - Q3)

$0.59 \pm 2.22$

$0(0-0.33)$

Spontaneous ABR

Mean \pm SD

$0.28 \pm 1.44$

Median (Q1 - Q3)

$0(0-0)$

Proportion of patients with 0 bleeds

\begin{tabular}{lll}
$\mathrm{N}$ & 201 & 114 \\
$\%$ & $74.4 \%$ & $74.5 \%$ \\
\hline
\end{tabular}

$>12$ months treatment

\section{Prophylactic Patients} ( $n=153$ )

Full Analysis Group

( $n=224$ )

Prophylactic Patients ( $n=129$ )

$0.62 \pm 2.55$

$0(0-0.22)$

$0.31 \pm 1.72$

$0(0-0)$

$0.20 \pm 0.78$

$0(0-0)$
$0(0-0)$

$0.39 \pm 1.22$

$0(0-0.23)$

$0.15 \pm 0.48$

93

$72.1 \%$

Conclusions: The data show that BAY 81-8973 effectively prevents bleeding events in the real-world. These data should be interpreted with caution owing to limitations of real-world studies.

PB0580 | Population Pharmacokinetic Modeling of Factor Concentrates in Hemophilia: A Detailed, Clinical Overview and Evaluation of Best Practice

M.H. Goedhart ${ }^{1}$; L.H. Bukkems ${ }^{2}$; C.M. Zwaan ${ }^{1}$; R.A. Mathot ${ }^{2}$; M.H. Cnossen ${ }^{1}$; for the OPTI-CLOT study group and SYMPHONY consortium.

${ }^{1}$ Erasmus MC Sophia Children's Hospital, Rotterdam, Netherlands;

${ }^{2}$ Amsterdam University Medical Centers, Amsterdam, Netherlands

Background: The accuracy of pharmacokinetic (PK)-guided dosing of factor FVIII (FVIII) and factor FIX (FIX) in hemophilia A and B patients depends on the clinical and laboratory data used to construct a real life population PK model, besides the individual PK profile of the patient.

Aims: This review aims to provide a detailed overview of the data used to construct population PK models for FVIII and FIX concentrates as reported in literature. Also, we do suggestions for best practice, in order to support physicians in their choices which model bests suit the individual patient.

Methods: An literature search was performed; publications describing prophylactic population PK models for FVIII and FIX based on original patient data and constructed using non-linear mixed-effect modeling (NLMEM) were included. The following data was collected: detailed demographics of study populations, hemophilia severity, type of product, assessed and included covariates, laboratory specifications and validation of models. Included models were scored according to our personal recommendations of best practice, specifically scoring the quality of data documentation.

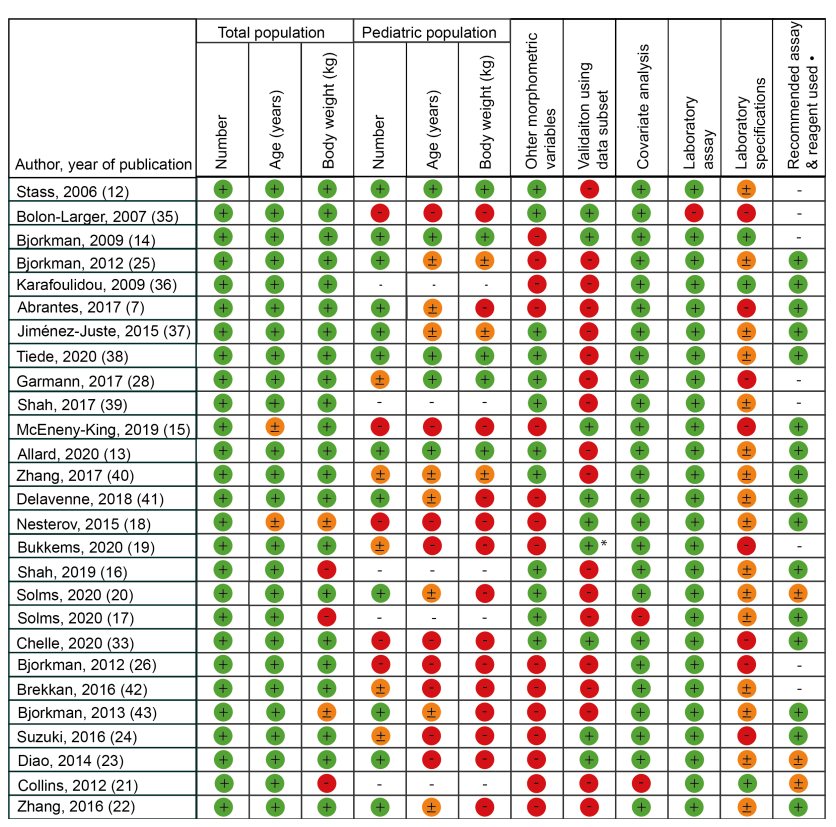

FIGURE 1 Evaluation of included models according to our recommendations of best practice, specifically on the quality of data documentation. Results presented as complete (green + ), incomplete (orange \pm ), absent (red -) or not applicable ( -). - According to UK guidelines Grey et al. Haemophilia 2020. * Bukkems et al. have externally validated the model of Nesterov et al.

Results: Respectively, twenty population PK models for FVIII and seven for FIX concentrates were retrieved. Although most models $(22 / 27)$ included pediatric patients, only four reported detailed demographics. The wide range of body weights suggested that overweight and obese adults were indeed represented. However, other morphometric variables (mostly BMI) were reported in only 12/27 publications. Twenty-six models reported the assay applied to measure factor levels, whereas only 16 models named reagents used. 
Eight models were internally validated using a data subset. Figure 1 depicts evaluations of best practice in our opinion.

Conclusions: This overview presents a wide variation in detail of included clinical and laboratory data used to construct currently available population PK models. We give recommendations to support best practice to increase the reliability of PK-guided prophylactic dosing of factor concentrates in hemophilia A and B.

PB0581 | Intracranial Haemorrhage (ICH) in Egyptian Children with Haemophilia A and B: A 15 Year Retrospective Single Center Study

\section{Abdelwahab}

Cairo University, Cairo, Egypt

Background: Intracranial haemorrhage $(\mathrm{ICH})$ is one of the potential life threatening complications in person with haemophilia (PAH) that can result in long-term morbidity. There are very few reports in Africa and Egypt especially in children.

Aims: Our study will assess the prevalence, risk factors and outcome of $\mathrm{ICH}$ in $\mathrm{PAH}$.

Methods: This is a retrospective study on all PAH from 2006 to 2021 We collected data on all children who had presented with $\mathrm{ICH}$ to Cairo University Paediatric Hospital. ICH was confirmed by clinical and computed tomographic imaging data. Clinical data were extracted at baseline and during the follow-up.

Results: 18/ 250 (6.8\%) PWH (15 HA, 12 severe whether by factor activity and/or phenotypically, 1 moderate, 2 mild; 3HB, 1 mild, 2 severe) with a mean age of 9.4 years who developed cerebral bleeds at a mean age of 3 years were enrolled. $58.8 \%$ were a product of consanguineous marriage. $\mathrm{ICH}$ was the initial presenting symptom in $33.3 \%$. 6 children had inhibitors ( 3 high titre) that nearly all developed after the onset of the cerebral bleed. Symptoms of ICH included pallor in $44.4 \%$, vomiting in $55.6 \%$, seizures and disturbed consciousness level in $77.8 \%$ and squin $t$ in $11 \%$. Our studied cohort developed 23 episodes of cerebral bleeds mainly post-traumatic (70\%), including 15 subdural, 7 intracerebral/intraventricular, and 1 brainstem. Mortality and morbidity is usually associated with late presentation to hospital $82 \%$ were managed conservatively with replacement therapy for 1 to 3 weeks. 22.2\% died of severe brain insult, $15.4 \%$ developed a cognitive impairment with hydrocephalic changes and visual impairment in one, 1 squint. Eight children are currently on biweekly secondary prophylaxis.

Conclusions: $\mathrm{ICH}$ is not a rare complication in our paediatric Egyptian cohort with an unfavourable outcome. Young age, lack of primary prophylaxis and trauma are the risk factors in our cohort.

\section{PB0582 | Reducing the Time of HAL and pedHAL Assessment}

I.A. Kuijlaars ${ }^{1}$; J. van der Net $^{2}$; V. Bouskill ${ }^{3}$; P. Hilliard ${ }^{4}$; A. Juodyte ${ }^{5}$; K. Khair ${ }^{6}$; R.E. Schutgens ${ }^{1}$; S.S. Trakymiene ${ }^{5}$; K. Fischer ${ }^{1}$

${ }^{1}$ Van Creveldkliniek, University Medical Center Utrecht, Utrecht University, Utrecht, Netherlands; ${ }^{2}$ Center for Child Development, Exercise and Physical Literacy, University Medical Center Utrecht, Utrecht University, University Children's Hospital, Utrecht, Netherlands; ${ }^{3}$ Department of Nursing, Division of Hematology/Oncology, The Hospital for Sick Children, Toronto, Canada; ${ }^{4}$ Child Health Evaluative Sciences, The Hospital for Sick Children, Toronto, Canada; ${ }^{5}$ Clinic of Children's Diseases, Faculty of Medicine, Vilnius University, Vilnius, Lithuania; ${ }^{6}$ Centre for Outcomes and Experience Research in Children's Health, Illness and Disability (ORCHID), Great Ormond Street Hospital for Children NHS Foundation Trust, London, United Kingdom

Background: The Haemophilia Activities List (HAL/pedHAL) assesses self-reported limitations in activities and participation in patients with haemophilia. Clinicians and researchers reported some items to be non-informative.

Aims: To determine which items of the (ped)HAL are redundant allowing for modifications and shorter versions of the questionnaires. Methods: For the pedHAL (7 domains/53 items/optimum score: $100)$, a secondary analysis was performed on pooled data of six published studies in children. Redundant items were identified in seven steps: floor and ceiling effects, proportions of missing and 'notapplicable' responses, inter-item correlations, component loadings in factor analysis, internal consistency and item-total correlations. After removing non-informative items, a pedHAL ${ }_{\text {short }}$ was created and pedHAL and pedHAL $\mathrm{L}_{\text {short }}$ sum scores were compared. For the HAL (7 domains/42 items/optimum score: 100), a similar procedure was followed with pooled data of two published studies in adults.

Results: For the pedHAL, data on 315 children (median age 12 [range 4-18], $87 \%$ severe haemophilia, $80 \%$ on prophylaxis) were evaluated. Median (IQR) pedHAL sum score was 97 (88-100). The stepwise procedure resulted in a ped $\mathrm{HAL}_{\text {short }}$ of 22 items with a median sum score of 97 (87-100). For the HAL, data on 420 adults with haemophilia (median age 30 [range 18-80], 88\% male, 43\% severe haemophilia, $61 \%$ on prophylaxis) were evaluated. Median (IQR) HAL sum score was 65 (56-89). The stepwise procedure resulted in a $\mathrm{HAL}_{\text {short }}$ of 18 items with a median sum score of 63 (54-87).

Conclusions: This clinimetric study resulted in $>50 \%$ reduction of the length of the pedHAL and HAL. The 22-item pedHAL $L_{\text {short }}$ and 18item $\mathrm{HAL}_{\text {short }}$ reduce patient burden and are expected to capture accurate information on activities and participation. The next step will be to validate both pedHAL $\mathrm{H}_{\text {short }}$ and $\mathrm{HAL}_{\text {short }}$ in other populations. 
PB0583 | Left Atrial Appendage Occlusion for the Management of Atrial Fibrillation in Persons with Hemophilia: A Case Report and Systematic Review of the Literature

M.Y. Lim; M.Y. Abou-Ismail

University of Utah, Salt Lake City, United States

Background: The management of atrial fibrillation (AF) in persons with hemophilia is a challenge as antithrombotic therapy, the standard of care, is usually contraindicated. Left atrial appendage occlusion (LAAO) is an alternative option for patients with a contraindication to antithrombotic therapy.

Aims: We present the case of a person with mild hemophilia A (HA) and $A F$ who underwent successful LAAO with short-term warfarin therapy, and a systematic review of existing cases in the literature.

Methods: Case report and a systematic search in Embase, Scopus and PubMed for existing cases in the literature.
Results: A 79-year-old man with mild HA (baseline factor VIII (FVIII) levels $9 \%$ ) presented with a transient ischemic attack (TIA) secondary to rate-controlled AF. He was previously on low-dose aspirin with no bleeding complications. He did not require treatment for his TIA as his neurologic symptoms resolved within 24 hours. His CHADS2-VASc risk score was 7 and HAS-BLED score was 5. LAAO was recommended along with short-term warfarin therapy for 6 weeks post-procedure. He received FVIII replacement therapy with a target of $100 \%$ pre-procedure. Post-procedure, warfarin (goal INR 2-3) was initiated without a heparin bridge. Aspirin was discontinued. Post-procedure, he received bolus FVIII infusions to maintain trough FVIII levels of $30 \%$ while on warfarin therapy. At 6 weeks, warfarin and factor replacement therapy were discontinued, and low-dose aspirin was resumed. He remains well at 12-months follow-up with no bleeding or thrombotic complications.

A systematic review of the literature identified an additional 8 cases of persons with hemophilia who underwent LAAO. The clinical characteristics and outcomes of these cases are shown in Table 1.

TABLE 1 Clinical characteristics and outcome of persons with hemophilia undergoing left atrial appendage occlusion

\begin{tabular}{|c|c|c|c|c|c|c|c|c|c|}
\hline $\begin{array}{l}\text { Author } \\
\text { (year) }\end{array}$ & $\begin{array}{l}\mathrm{CHA}_{2} \mathrm{DS}_{2-}^{-} \\
\text {VASc }\end{array}$ & $\begin{array}{l}\text { Age/ } \\
\text { Gender }\end{array}$ & $\begin{array}{l}\text { Prophylaxis } \\
\text { therapy }\end{array}$ & $\begin{array}{l}\text { Factor } \\
\text { deficiency }\end{array}$ & Diagnosis & $\begin{array}{l}\text { Replacement } \\
\text { therapy during and } \\
\text { post-implantation }\end{array}$ & $\begin{array}{l}\text { Anti- } \\
\text { thrombotic } \\
\text { therapy post- } \\
\text { intervention }\end{array}$ & $\begin{array}{l}\text { Short-term } \\
\text { outcome }\end{array}$ & $\begin{array}{l}\text { Duration of } \\
\text { follow-up }\end{array}$ \\
\hline $\begin{array}{l}\text { Cheung } \\
\qquad(2013)\end{array}$ & 6 & $73 \mathrm{M}$ & None & Factor VIII (8\%) & $\begin{array}{l}\text { CABG, stroke, } \\
\text { carotid } \\
\text { endarterectomy, } \\
\text { AF. Had } \\
\text { previously } \\
\text { tolerated SAPT } \\
\text { (Aspirin or } \\
\text { Clopidogrel) } \\
\text { without any } \\
\text { bleeding } \\
\text { complications }\end{array}$ & $\begin{array}{l}\geq 100 \% \text { pre- } \\
\text { procedure, } \\
\text { then } \geq 80 \% \\
\text { trough for } 3 \\
\text { days, then } \\
>30 \% \text { while on } \\
\text { DAPT }\end{array}$ & $\begin{array}{l}\text { Aspirin } 75 \\
\text { mg and } \\
\text { clopidogrel } \\
75 \text { mg for } 6 \\
\text { weeks }\end{array}$ & $\begin{array}{l}\text { TOE at } 6 \text { weeks } \\
\text { showed well- } \\
\text { sealed device. } \\
\text { Aspirin and } \\
\text { factor infusion } \\
\text { discontinued, } \\
\text { continued } \\
\text { clopidogrel } \\
\text { life-long }\end{array}$ & 9 months \\
\hline $\begin{array}{l}\text { Bhatti } \\
\qquad(2019)\end{array}$ & 3 & $60 F$ & None & Factor IX (15\%) & $\begin{array}{l}\text { Paroxysmal AF, Sick } \\
\text { sinus syndrome, } \\
\text { s/p pacemaker } \\
\text { implantation, } \\
\text { TIA }\end{array}$ & $\begin{array}{l}82 \mathrm{U} / \mathrm{kg} \text { of factor } \\
\text { pre-procedure, } \\
\text { then } 67 \mathrm{U} / \\
\mathrm{kg} \text { on POD } \\
\# 1 \text {, then } 20 \\
\mathrm{U} / \mathrm{kg} \text { every } \\
\text { other day to } \\
\text { maintain levels } \\
>30 \%\end{array}$ & $\begin{array}{l}\text { Warfarin } \\
\text { (INR 2-3) }\end{array}$ & $\begin{array}{l}\text { TOE at } 30 \text { days } \\
\text { showed well- } \\
\text { sealed device } \\
\text { with no leak. } \\
\text { Warfarin and } \\
\text { factor infusion } \\
\text { discontinued }\end{array}$ & 6 months \\
\hline $\begin{array}{l}\text { Guray } \\
\qquad(2019)\end{array}$ & 3 & $67 \mathrm{M}$ & $\begin{array}{l}\text { On prophylaxis } \\
\text { to keep } \\
\text { FVIII at } 10 \%\end{array}$ & Factor VIII (NR) & $\mathrm{AF}$ & $\begin{array}{l}\text { Factor pre- } \\
\text { procedure and } \\
\text { post-procedure }\end{array}$ & $\begin{array}{l}\text { Aspirin } 81 \\
\text { mg and } \\
\text { clopidogrel } \\
75 \mathrm{mg} \text { for } \\
1 \text { month } \\
\text { with factor } \\
\text { infusion, } \\
\text { then only } \\
\text { aspirin } \\
81 \text { mg for } \\
2 \text { more } \\
\text { months }\end{array}$ & $\begin{array}{l}\text { TOE at } 6 \text { weeks } \\
\text { showed } \\
\text { well-sealed } \\
\text { device. All } \\
\text { antithrombotic } \\
\text { therapy } \\
\text { discontinued at } \\
3 \text { months }\end{array}$ & 1 year \\
\hline
\end{tabular}




\begin{tabular}{|c|c|c|c|c|c|c|c|c|c|}
\hline $\begin{array}{l}\text { Author } \\
\text { (year) }\end{array}$ & $\begin{array}{l}\mathrm{CHA}_{2} \mathrm{DS}_{2-} \\
\text { VASc }\end{array}$ & $\begin{array}{l}\text { Age/ } \\
\text { Gender }\end{array}$ & $\begin{array}{l}\text { Prophylaxis } \\
\text { therapy }\end{array}$ & $\begin{array}{l}\text { Factor } \\
\text { deficiency }\end{array}$ & Diagnosis & $\begin{array}{l}\text { Replacement } \\
\text { therapy during and } \\
\text { post-implantation }\end{array}$ & $\begin{array}{l}\text { Anti- } \\
\text { thrombotic } \\
\text { therapy post- } \\
\text { intervention }\end{array}$ & $\begin{array}{l}\text { Short-term } \\
\text { outcome }\end{array}$ & $\begin{array}{l}\text { Duration of } \\
\text { follow-up }\end{array}$ \\
\hline \multirow[t]{2}{*}{$\begin{array}{l}\text { Coppola } \\
\qquad(2020)\end{array}$} & 3 & $M$ & $\begin{array}{l}\text { Tertiary } \\
\text { prophylaxis }\end{array}$ & $\begin{array}{l}\text { Factor VIII } \\
\qquad(<1 \%)\end{array}$ & $\mathrm{AF}$ & $\begin{array}{l}\geq 80 \% \text { pre- } \\
\text { procedure } \\
\text { and for } 12 \\
\text { hours post- } \\
\text { procedure, } \\
\text { then continued } \\
\text { factor infusion } \\
\text { to maintain } \\
\text { trough }>5 \%\end{array}$ & Clopidogrel & $\begin{array}{l}\text { Withdrew } \\
\text { clopidogrel } \\
\text { after } 2 \text { months } \\
\text { due to severe } \\
\text { epistaxis and } \\
\text { joint bleeds }\end{array}$ & NR \\
\hline & 3 & $M$ & $\begin{array}{l}\text { Tertiary } \\
\text { prophylaxis }\end{array}$ & $\begin{array}{l}\text { Factor VIII } \\
\qquad(<1 \%)\end{array}$ & $\mathrm{AF}$ & $\begin{array}{l}\geq 80 \% \text { pre- } \\
\text { procedure } \\
\text { and for } 12 \\
\text { hours post- } \\
\text { procedure, } \\
\text { then continued } \\
\text { factor infusion } \\
\text { to maintain } \\
\text { trough }>5 \%\end{array}$ & Clopidogrel & NR & NR \\
\hline \multirow[t]{3}{*}{$\begin{array}{l}\text { Toselli } \\
\qquad(2020)\end{array}$} & 3 & $76 \mathrm{M}$ & $\begin{array}{l}\text { Tertiary } \\
\text { prophylaxis }\end{array}$ & $\begin{array}{l}\text { Factor VIII } \\
\qquad(<1 \%)\end{array}$ & Asymptomatic AF & $\begin{array}{l}>60 \% \text { pre- } \\
\text { procedure, } \\
\text { then resumed } \\
\text { usual } \\
\text { prophylaxis }\end{array}$ & $\begin{array}{l}300 \mathrm{mg} \\
\text { clopidogrel } \\
\text { loading } \\
\text { dose, then } \\
\text { clopidogrel } \\
75 \mathrm{mg}\end{array}$ & $\begin{array}{l}\text { Within the first } \\
\text { few weeks, } \\
\text { FVIII dosing } \\
\text { was increased } \\
\text { due to minor } \\
\text { hemarthrosis } \\
\text { and epistaxis. } \\
\text { TOE at } 3 \\
\text { months showed } \\
\text { well-sealed } \\
\text { device. } \\
\text { Clopidogrel } \\
\text { discontinued. } \\
\text { Usual factor } \\
\text { prophylaxis } \\
\text { dosing resumed }\end{array}$ & 20 months \\
\hline & 4 & $73 M$ & None & $\begin{array}{l}\text { Factor IX } \\
\qquad \text { (Moderate) }\end{array}$ & $\begin{array}{l}\text { CABG, ischemic } \\
\text { cardiomyopathy, } \\
\text { residual heart } \\
\text { failure with } \\
\text { LVEF } 40 \%, \\
\text { paroxysmal AF }\end{array}$ & $\begin{array}{l}40 \mathrm{U} / \mathrm{kg} \text { of factor } \\
\text { infused } \\
\text { pre-procedure }\end{array}$ & $\begin{array}{l}\text { DAPT for } 3 \\
\text { months, } \\
\text { then SAPT } \\
\text { life-long }\end{array}$ & $\begin{array}{r}\text { TOE at } 6 \text { months } \\
\text { showed well- } \\
\text { sealed device }\end{array}$ & 12 months \\
\hline & 5 & $79 M$ & $\begin{array}{l}\text { Tertiary } \\
\text { prophylaxis }\end{array}$ & $\begin{array}{l}\text { Factor VIII } \\
\qquad(<1 \%)\end{array}$ & TIA, recurrent AF & $\begin{array}{l}>65 \% \text { pre- } \\
\text { procedure, } \\
\text { then resumed } \\
\text { usual } \\
\text { prophylaxis }\end{array}$ & $\begin{array}{l}\text { Clopidogrel } 75 \\
\text { mg }\end{array}$ & $\begin{array}{l}\text { Cardiac CT at } 3 \\
\text { months showed } \\
\text { optimal device } \\
\text { position, } \\
\text { clopidogrel } \\
\text { discontinued }\end{array}$ & NR \\
\hline $\begin{array}{l}\text { This case } \\
\text { (2021) }\end{array}$ & 7 & $79 M$ & None & Factor VIII (9\%) & $\begin{array}{l}\mathrm{CAD}, \mathrm{s} / \mathrm{p} \mathrm{PCl}, \mathrm{AF} \mathrm{s} / \mathrm{p} \\
\text { AVN ablation } \\
\text { and CRT-P } \\
\text { placement, } \\
\text { stroke (on } \\
\text { aspirin } 81 \mathrm{mg} \text { ) }\end{array}$ & $\begin{array}{l}>100 \% \text { pre- } \\
\text { procedure, } \\
\text { then continued } \\
\text { factor infusion } \\
\text { to maintain } \\
\text { trough }>30 \%\end{array}$ & $\begin{array}{l}\text { Warfarin } \\
\text { (INR 2-3) }\end{array}$ & $\begin{array}{l}\text { TOE at } 6 \text { weeks } \\
\text { showed well- } \\
\text { sealed device. } \\
\text { Warfarin } \\
\text { discontinued, } \\
\text { remained on } \\
\text { aspirin } 81 \mathrm{mg}\end{array}$ & 12 months \\
\hline
\end{tabular}

Conclusions: Our case report and systematic review demonstrate that with appropriate hemostatic management, LAAO can be a safe and successful strategy for reducing the long-term thromboembolic risk of persons with hemophilia and AF. 


\section{PB0584 | Patients with Hemophilia B with Inhibitors in China}

X. Dou; X. Wang; R. Yang

State Key Laboratory of Experimental Hematology, National Clinical

Research Center for Blood Diseases, Institute of Hematology \& Blood

Diseases Hospital, Chinese Academy of Medical Sciences \& Peking

Union Medical College, Tianjin, China

Background: The development of inhibitors is a serious complication of coagulation factor IX (FIX) replacement therapy in patients with hemophilia $B(\mathrm{HB})$, occurring in approximately $1.5-3 \%$ in $\mathrm{HB}$. Currently, only less than 200 HB patients with inhibitors were reported all over world.

Aims: This study aimed to describe clinical characteristics of $\mathrm{HB}$ patients with inhibitors registered all over China.

Methods: Patient information was collected from the National Hemophilia Registration System in China from the year 2011 to 2021. Detailed data were also acquired by telephone follow-up on each patient.

Results: A total of 19 HB patients(16 had severe and 3 had moderate $\mathrm{HB}$ ) with inhibitors were included in this study. The median age at diagnosis of FIX inhibitors was 10 (range, 2-43) years, and four patients had a family history of HB. Seventeen patients developed high-titer inhibitor, and the other two had low-titer inhibitor. Eleven (57.9\%) patients used to be allergic to FIX products, while most (10 patients) had mild allergic reactions, such as itching and urticaria. Only one patient had dyspnea and chill. After the diagnosis of inhibitors, seven of the 19 patients underwent immune tolerance induction (ITI). The median age at the initiation of ITI was 7 (range, 2-14) years. Three (42.9\%) patients achieved a continuous negative titer successfully, and another two (28.6\%) patients developed nephrotic syndrome during ITI. In those 16 patients who didn't do or had a failure of ITI, seven patients switched to rFVIla for bypass treatment, six patients combined PCC and rFVIla, and three patients still used PCC for bleeding control.

Conclusions: We described the clinical features of HB patients with inhibitors in China retrospectively. The development of inhibitors in $\mathrm{HB}$ is related to allergic reactions/anaphylaxis, and ITI is more difficult in HB.

\section{PB0585 | Mental Health Status of Hemophilia Carriers in China}

D. Cui; Q. Hu

Tongji Hospital, Tongji Medical College, Huazhong University of Science and Technology, Wuhan, China

Background: Hemophilia carriers are prone to suffer from mental health problems, but there are still very limited data of mental health status of hemophilia carriers in China.

Aims: This study was to investigate mental health status of hemophilia carriers in China.
Methods: Hemophilia carriers were asked to complete the Symptom Check-list 90 (SCL-90, China/Mandarin version) from April 2018 to April 2020. Each item is rated on a five-point scale, with 1-5 representing the severity of the symptoms from "none" to "severe". When the score of an item is equal or greater than 2, this item is considered a positive item. If the total score exceeds 160 , number of positive items exceeds 43 , or any factor score exceeds 2 , it is considered the participants have psychological symptoms. The measurement data were expressed as mean ( \pm standard deviation) and the $t$ test was performed for comparison between groups which indicated hemophilia carriers and Chinese population norms.

Results: A total of 71 valid questionnaires were collected. The total mean score, the global severity index and the number of positive items of SCL-90 for surveyed hemophilia carriers were 173.03 ( \pm $60.80), 1.92$ ( \pm 0.68 ), and 46.04 ( \pm 22.55 ), respectively, all of which were markedly higher than those in the Chinese population norms ( $P<0.001) .73 .2 \%$ of the respondents $(n=52)$ were identified with psychological symptoms. The pooled estimates of all the nine factors, including somatization, obsession-compulsion, interpersonalsensitivity, depression, anxiety, hostility, phobic anxiety, and paranoid ideation among hemophilia carriers, were significantly higher than those in the general population $(P<0.001)$. Obsessivecompulsion and depression were most common and severe factors, and their rates were $63.4 \%$ and $54.9 \%$, respectively.

Conclusions: The situation of psychological health of hemophilia carriers in China is worrying, and it is urgent to take some effective measures to improve their mental health.

\section{PB0586 | A Qualitative Study on the Experiences of Hemophilia} Carriers before, during and after Pregnancy

M.C. Punt ${ }^{1}$; L. Teela ${ }^{2}$; K. Fischer ${ }^{1}$; K.W.M. Bloemenkamp ${ }^{3}$; A.T. Lely $^{3}$; M.H.E. Driessens ${ }^{4}$; L. Pekel ${ }^{4}$; L. Haverman ${ }^{2}$; K.P.M. van Galen ${ }^{1}$.

${ }^{1}$ Van Creveldkliniek, University Medical Center Utrecht, Utrecht University, Utrecht, Netherlands; ${ }^{2}$ Emma Children's Hospital Amsterdam UMC, University of Amsterdam, Psychosocial Department, Amsterdam, Netherlands; ${ }^{3}$ Department of Obstetrics, Birth Centre Wilhelmina's Children Hospital, Division Woman and Baby, Utrecht University, Utrecht, Netherlands; ${ }^{4}$ Dutch Society of Hemophilia Patients (NVHP), Nijkerk, Netherlands

Background: Hemophilia carriers (HCs) face considerable hemostatic and psychological challenges during reproduction, though no recent qualitative studies have been published. Up-to-date knowledge on reproductive phases in the current era of hemophilia treatment and assessment of the psychosocial impact can assist healthcare professionals during conception and childbirth.

Aims: To assess the HCs' perspective on healthcare, as well as the psychological impact in the current era of hemophilia treatment during all reproductive phases: preconception, pregnancy, childbirth and the postpartum period. 
Methods: The study was approved by the medical ethics committee and informed consent was obtained. Focus group discussions (FGDs) and semi-structured interviews were conducted among HCs in January/February 2020 until data saturation was reached. All sessions were recorded, transcribed verbatim and analyzed by two independent researchers through thematic content analysis using MAXQDA ${ }^{\circledR}$ software. The results were then discussed within the research team until consensus was reached. The emerged themes were shared with and reviewed by the HCs and members of the Dutch hemophilia patient organization.
Results: Fifteen HCs were included during 3 FGDs and 4 interviews. Five central themes emerged: 1) communication by healthcare professionals, 2) lack of knowledge, 3) feeling insecure, 4) autonomy and 5) family experiences with hemophilia (Table 1). Desired improvements in care mainly concern counselling during preconception and pregnancy. This includes timely access to comprehensive information during each consecutive phase, healthcare professionals accepting HCs' choices and healthcare provision tailored to the HC's family experience with hemophilia.

TABLE 1 Overview of main results

\begin{tabular}{|c|c|c|c|c|c|}
\hline & & \multicolumn{4}{|l|}{ Phase } \\
\hline & & Preconception & Pregnancy & Childbirth & Postpartum \\
\hline \multirow{2}{*}{ Themes } & Feeling insecure & $\begin{array}{l}\text { Upcoming choices } \\
\text { during consecutive } \\
\text { phases }\end{array}$ & $\begin{array}{l}\text { - Potentially affected } \\
\text { child } \\
\text { - Safer in CCC } \\
\text { - Deciding on PND }\end{array}$ & $\begin{array}{l}\text { - Distance to CCC } \\
\text { - Lack of HCP experi- } \\
\text { ence with HCs } \\
\text { - Mode of delivery } \\
\text { - Maternal and neo- } \\
\text { natal (m/f) bleeding } \\
\text { risk }\end{array}$ & $\begin{array}{l}\text { - Testing baby }(\mathrm{m} / \mathrm{f}) \\
\text { - Maternal and neo- } \\
\text { natal }(\mathrm{m} / \mathrm{f}) \text { bleeding } \\
\text { risk }\end{array}$ \\
\hline & Family experiences & $\begin{array}{l}\text { - Severity of } \\
\text { hemophilia and } \\
\text { coping of family } \\
\text { determines the } \\
\text { attitude towards } \\
\text { pregnancy }\end{array}$ & $\begin{array}{l}\text { - Severity of } \\
\text { hemophilia and } \\
\text { family attitudes } \\
\text { influence PND and } \\
\text { decisions regarding } \\
\text { TOP }\end{array}$ & $\begin{array}{l}\text { - Home delivery } \\
\text { possible }\end{array}$ & $\bullet-$ \\
\hline
\end{tabular}

Legend: $\mathrm{HCPs}=$ Healthcare professionals; $\mathrm{HCs}$, hemophilia carriers; $\mathrm{CCCs}=$ Comprehensive Care Centers; $\mathrm{PND}=$ prenatal diagnosis; $\mathrm{m}=$ male;

$f=$ female; TOP $=$ Termination of pregnancy. 
Conclusions: In recent years, hemophilia treatment has seen major advances, which could impact general and reproductive care for $\mathrm{HCs}$. HCs indicate that reproductive care would benefit from a more personal and informative approach. Healthcare professionals could use these insights to adapt their consultations to meet the needs of these women when (preparing for) having children.

PB0587 | Posttraumatic Stress Disorder and Posttraumatic Stress Symptoms Among Adults with Hemophilia A and B

$\underline{\text { A. Stahl }}{ }^{1}$; K. Barnett ${ }^{2} ;$ A. Wilson ${ }^{3}$; H.S. Park ${ }^{1} ;$ M. Geary ${ }^{1}$; S. Ren ${ }^{4}$; D. Neuberg $^{4}$; A. Parnes ${ }^{1}$

${ }^{1}$ Brigham and Women's Hospital, Boston, United States; ${ }^{2}$ Mount Sinai Hospital, New York City, United States; ${ }^{3} \mathrm{M}$ Health Fairview, Minneapolis, United States; ${ }^{4}$ Dana-Farber Cancer Institute, Boston, United States

Background: Studies show that the prevalence of broad illnessinduced (cancer, cardiovascular disease, stroke) post-traumatic stress disorder (PTSD) ranges from 12-15\%, but little is known about the prevalence of PTSD or post-traumatic stress symptoms (PTSS) among hemophilia patients. We hypothesized that hemophilia patients are particularly at risk for PTSD and PTSS given their bleeding diathesis, factor treatment and prophylaxis requirements, and history of treatment viral contamination.

Aims: 1. Determine the prevalence of PTSD and PTSS in hemophilia $A$ and $B$ patients.

2. Characterize the traumatic experiences of hemophilia patients.

Methods: Using REDCap, a validated self-administered questionnaire containing the PTSD checklist for DSM-5 (PCL-5 survey) was offered to all adult hemophilia patients age $20+$ at the time of their annual comprehensive visits to their Hemophilia Treatment Centers (HTC). Three large academic HTCs (Boston Hemophilia Center, MA; Mount Sinai Hemophilia Center, NY; M Health Fairview Center for Bleeding and Clotting Disorders, MN) participated in the study. Descriptive statistics were used to analyze the data. This study was approved by the Institutional Review Boards.

Results:

TABLE 1 Patient Gender, Age, and Education by Post-Traumatic Stress in Hemophilia

\begin{tabular}{lllll} 
& & & \multicolumn{2}{l}{ Neither PTSD or } \\
Mild hemophilia & PTSD & PTSS Excluding PTSD & PTSS & $19(26 \%)$ \\
Moderate hemophilia & & $6(21 \%)$ & $13(37 \%)$ & $11(15 \%)$ \\
Severe hemophilia & $1(13 \%)$ & $5(17 \%)$ & $5(14 \%)$ & $42(58 \%)$ \\
Full-time work & $7(88 \%)$ & $18(62 \%)$ & $17(49 \%)$ & $47(65 \%)$ \\
Part-time work & $4(50 \%)$ & $17(59 \%)$ & $26(74 \%)$ & $4(6 \%)$ \\
Unemployed due to hemophilia & $1(13 \%)$ & $1(3 \%)$ & $2(6 \%)$ & $5(7 \%)$ \\
Unemployed not due to hemophilia & $2(25 \%)$ & $4(14 \%)$ & $5(14 \%)$ & $14(19 \%)$ \\
Student & & $7(24 \%)$ & $1(3 \%)$ & $1(3 \%)$ \\
Retired & & & & $1(1 \%)$ \\
\hline
\end{tabular}

TABLE 2 Hemophilia Severity and Patient Employment by Post-Traumatic Stress in Hemophilia

\begin{tabular}{lllll} 
& PTSD & PTSS Excluding PTSD & Neither PTSD or PTSS & Total \\
$n$ & 8 & 29 & 35 & 72 \\
Male & $8(100 \%)$ & $27(93 \%)$ & $31(87 \%)$ & $66(92 \%)$ \\
Female & 0 & $2(7 \%)$ & $4(13 \%)$ & $6(8 \%)$ \\
Age (Median, Range) & $38(22-59)$ & $40(24-70)$ & $33(22-76)$ & $36.5(22-76)$ \\
Education & & & & $1(1 \%)$ \\
Did not complete high school & & $1(3 \%)$ & $9(26 \%)$ & $23(32 \%)$ \\
Completed high school & $5(63 \%)$ & $9(31 \%)$ & $17(49 \%)$ & $32(44 \%)$ \\
Completed college & $2(25 \%)$ & $13(45 \%)$ & $9(26 \%)$ & $16(22 \%)$ \\
Master's or higher & $1(13 \%)$ & $6(21 \%)$ & & \\
\hline
\end{tabular}

So far, a total of 72 patients responded to the survey including 42 severe hemophilia patients, 11 moderate, and 19 mild (see Tables 1 and 2). 8 (11\%) patients had a positive screen for PTSD. 7 out of 42 (17\%) severe hemophilia patients had a positive screen for PTSD. 37 out of 72 (51\%) patients surveyed could identify a traumatic incident related to their hemophilia and are experiencing PTSS or PTSD. 25 out of 42 (60\%) severe hemophilia patients could identify a traumatic incident related to their hemophilia and are experiencing PTSS or PTSD. 
Conclusions: These preliminary results demonstrate a high prevalence of PTSD and PTSS in hemophilia patients. Prevalence appears to increase with disease severity. This data verifies the demand for psychosocial services in the bleeding disorders community and improvements in hemostatic therapies.

\section{PB0588 | The FIX Gene Mutation Analysis of Hemophilia B in China Central and Western Regions}

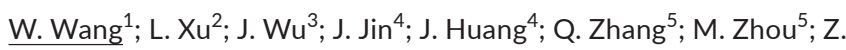
$\mathrm{Xu}^{6} ; \mathrm{Y}$. Huang ${ }^{6}$; J. Peng ${ }^{7}$; Q. $\mathrm{Hu}^{1}$

${ }^{1}$ Tongji Hospital, Tongji Medical College of Huazhong University of Science and Technology, Wuhan, China; ${ }^{2}$ Anhui No. 2 Province People's Hospital, Hefei, China; ${ }^{3}$ First Affiliated Hospital, University of Science and Technology of China, Hefei, China; ${ }^{4}$ The Affiliated Hospital of Guizhou Medical University, Guiyang, China; ${ }^{5}$ Chengdu Women's and Children's Central Hospital, Chengdu, China; ${ }^{6}$ Jiangxi Provincial Children's Hospital, Nanchang, China; ${ }^{7}$ Xiangya Hospital, Central South University, Changsha, China

Background: Hemophilia $B(\mathrm{HB})$ is a rare disorder that is complex to diagnose and manage. It's an X-linked congenital bleeding disorder caused by FIX gene mutations.

Aims: 1. To understand the genotype and molecular pathogenesis of HB patients in China central and western regions;

2. To analyze the relationship between gene mutation and bleeding phenotype and inhibitor formation.

Methods: From March 2019 to January 2021, 123 patients with HB (mild 11, moderate 57, severe 55) were enrolled in six hemophilia centers. We analyzed FIX gene by Sanger sequencing and multiplex ligation-dependent probe amplification (MLPA). SPSS 22.0 was used to analyze the data.

Results: 1. A total of 92 FIX gene mutations were detected in 123 patients with $\mathrm{HB}$, with the detection rate of $99.2 \%(122 / 123)$;

2 . The most common type of FIX gene mutation was point mutation (84.6\%), among which missense mutation was the dominant type (65.4\%). Serine protease region was the mutation hot spot (53.2\%);

3. This study found 13 novel mutations and enriched the mutation database;

4. The mutation types of patients with mild to moderate HB were mainly missense mutation, accounting for $81.8 \%$ and $68.4 \%$, respectively; In severe HB, missense mutation (36.4\%) and nonsense mutation (31.0\%) were the main mutation types, and indel, duplication, insertion mutation were only found in severe HB patients;

5.9 out of 110 (8.2\%) HB patients were inhibitor positive, with genotype including 1 large deletion, 6 missense mutations, 1 small deletion and 1 nonsense mutations.

Conclusions: 1 . The detection rate of FIX gene mutation in this study was $99.2 \%$, and point mutation was the most common mutation type; Serine protease region was the hot spot of mutations;

2. HB patients showed significant genotype-phenotype heterogeneity;
3. The prevalence of inhibitors was $8.2 \%$.

correspondence: Qun Hu

This work was funded by Pfizer.

PB0589 | Impact of Switching to Recombinant Factor IX Fc Fusion Protein Prophylaxis: A Qualitative Study

M. O'Donovan ${ }^{1,2}$; P. Byrne ${ }^{1}$; B. O'Mahony ${ }^{3,4}$; K. Ryan ${ }^{1}$; N.M. $\mathrm{O}^{\prime}$ Connell ${ }^{1,2}$; D. Hevey ${ }^{5}$

${ }^{1}$ National Coagulation Centre, St James's Hospital, Dublin, Ireland;

${ }^{2}$ School of Medicine, Trinity College Dublin, Dublin, Ireland; ${ }^{3}$ Irish Haemophilia Society, Dublin, Ireland; ${ }^{4}$ Trinity College Dublin, Dublin, Ireland; ${ }^{5}$ School of Psychology, Trinity College Dublin, Dublin, Ireland

Background: In Ireland, in 2017 all people with severe haemophilia B (PWSHB) switched from standard half-life (SHL) factor IX (FIX) to prophylaxis with recombinant factor IX Fc fusion protein ( $\mathrm{FIXFc).}$

Aims: To gain insight into the lived experience of PWSHB following three years of rFIXFc prophylaxis.

Methods: Key areas and themes for evaluation were identified by a focus group. A pilot study to investigate feasibility and applicability of questions was performed. PWSHB ( $\geq 18$ years) who switched to rFIXFc prophylaxis were then invited to participate in a qualitative study. Online semi-structured interviews were carried out by two psychologists, audio recorded, transcribed and subsequently analysed for common themes.

Results: Areas identified from the focus group for exploration included symptom change, impact on life, experience of treatment change, agency and sense of self. Fourteen PWSHB, median age 52 years (range 20-73 years), who switched from episodic treatment $(n=3)$ or prophylaxis $(n=11)$ with SHL-FIX to rFIXFc prophylaxis participated.

Preliminary analysis revealed a number of key themes characterising participants' experience of the treatment change. A more "Secure Self" emerged with rFIXFc prophylaxis regimen: participants reported less worry about bleeds, which produced a greater sense of safety, security and control over daily living. This sense of security underpinned the development of the "Confident self": patients were not only more confident in managing their haemophilia and bleeds, but more confident in being active and engaged. The change was experienced as a shift in focus as participants now experienced "Illness in background, life in foreground": worries about bleeds are still present but they are more in the back of the mind, and the patients' focus is now on pursuing valued activities.

Conclusions: Preliminary analysis suggests that switching to rFIXFc prophylaxis has resulted in a positive patient experience. Additional analysis will provide further insight into the transition. 
PB0590 | Efficacy of Simoctocog Alfa in Previously Untreated Patients with Severe Haemophilia A: Final Results from the NuProtect Study

\section{E.J. Neufeld ${ }^{1}$; R.J. Liesner ${ }^{2}$}

${ }^{1}$ St. Jude Children's Research Hospital, Memphis, United States;

${ }^{2}$ Haemophilia Comprehensive Care Centre, Great Ormond Street Hospital for Children, NHS Foundation Trust, NIHR GOSH BRC, London, United Kingdom

Background: Inhibitors are the most serious treatment-related complication of haemophilia A and develop in up to $40 \%$ of previously untreated patients (PUPs). The NuProtect study assessed the immunogenicity, efficacy and safety of simoctocog alfa, a 4th generation recombinant FVIII produced in a human cell line, in 108 PUPs with haemophilia A. High-titre inhibitors developed in $16 \%$ of patients. NuProtect is the largest prospective study of a single FVIII product in true PUPs to date.

Aims: To assess the efficacy of simoctocog alfa for the prevention and treatment of bleeding in PUPs.

Methods: NuProtect was a prospective, multinational, open-label, non-controlled phase III study of 108 PUPs of any age and ethnicity with severe haemophilia A. Patients received simoctocog alfa for 100 exposure days or up to 5 years for prophylaxis, surgical prophylaxis or on-demand treatment. Informed consent was obtained from patients/caregivers and the study was approved by all relevant independent ethics committees and institutional review boards.

Results: In PUPs who received continuous prophylaxis for $\geq 24$ weeks ( $n=50$ ), the mean (SD) annualised bleeding rate (ABR) was 0.54 (1.07) for spontaneous bleeding episodes (BEs) and 3.61 (3.82) for all BEs. The efficacy of continuous prophylaxis was rated as excellent or good in $100 \%$ of patients for spontaneous BEs and $96 \%$ for all BEs. A total of 808 BEs in 85 patients were treated during inhibitorfree periods, of which 502 (62.1\%) occurred in 51 patients during ondemand treatment. Efficacy in treating BEs was rated as excellent or good in $92.9 \%$ of 804 rated BEs. The majority of BEs (92.3\%) were controlled with 1 or 2 infusions. Efficacy in surgical prophylaxis was rated as excellent or good in $94.7 \%$ (18/19) of procedures.

Conclusions: Simoctocog alfa was efficacious for the prevention and treatment of BEs in PUPs with haemophilia A, including during surgery.
PB0591 | Development of a Scale in the Diagnosis and Monitoring of Arthropathy in Patients with Hemophilia Using Ultrasound (US) and MRI: Initial Report

M. Walentowska-Janowicz ${ }^{1}$; J.B. Ćwikła ${ }^{2,3}$; M. Brzewski ${ }^{1}$; M. Matysiak $^{4}$; P. Łaguna ${ }^{4}$

${ }^{1}$ Departament of Pediatric Radiology, Medical University of Warsaw, Warsaw, Poland; ${ }^{2}$ Diagnostic and Therapeutic Center - Gammed, Warsaw, Poland; ${ }^{3}$ Department of Cardiology and Internal Medicine, School of Medicine University of Warmia and Mazury, Olsztyn, Poland; ${ }^{4}$ Department of Oncology, Hematology and Bone Marrow Transplantation and Pediatrics Medical University of Warsaw, Warsaw, Poland

Background: Hemophilic arthropathy is a fundamental clinical problem in patients with hemophilia.

Aims: The purpose of this study was to develop a scale for the severity of hemophilic arthropathy based on MRI and US to evaluate the degree of joint involvement, as well as to determine the persistent active synovitis in patients after previous radiosynovectomy to select patients to further therapy approach.

Methods: The study group consists of 9 patients with examinations of 15 joints. All affected joints were evaluated in MRI using 3T system (Siemens; G) with dedicated protocol. Additional each joint was examined using US to assess synovial overgrowth and increase vascularization with utilization of $\mathrm{mSMI}{ }^{\circledR}$ software (Cannon; J), which was used to improve the detection of low flow rate in case of synovitis without use of contrast medium. All patients had at least single session of RS, overall 32 RS were performed. Both techniques were used to objective evaluation of the potential regression after previous RS and as imaging follow-up..

Results: MRI indicated advanced hemophilic arthropathy with features of cartilage destruction in 10 joints, clinically all subjects with MRI positive scans had joint stiffness, additional in 3 cases bone marrow edema were observed without accompanying advanced arthropathy.

Thickened and overgrowth of synovium were detected in 8 cases of MRI. In US respectively all cases with synovial overgrowth were seen in the same localization as in MRI and all of them showed features of increased vascularization in mSMI, indicated active persistent synovitis. In 4 cases the vascularization and very high flow was seen in $\mathrm{mSMI}$, rest of them had moderate vascular flow.

Conclusions: MRI With Very High Contrast Resolution Of Images Is An Excellent Method To Detect Structural Lesions In Affected Joint In Patients With Hemophilic Arthropathy After RS.

The Study Was Carried Out Thanks To A Grant From The SOBI Company. 
PB0592 | Assessment of Thrombin Generation on Severe Haemophilia A Patients in the Clinical Laboratory

J. Mullins ${ }^{1}$; D. White ${ }^{1}$; S. Cox-Morton ${ }^{1}$; A. Marin ${ }^{2}$; M. Gironella ${ }^{2}$; F. Bosch $^{2}$; S. Franco ${ }^{2}$; A. Tosetto ${ }^{3}$ S. Mac Donald ${ }^{1}$

${ }^{1}$ Cambridge University Hospital NHS Foundation Trust, Cambridge, United Kingdom; ${ }^{2}$ Hematology Department of Vall d'Hebrón University Hospital, Barcelona, Spain; ${ }^{3}$ Hemophilia and Thrombosis Center,

Hematology Department, San Bortolo Hospital, Vicenza, Italy

Background: While Thrombin Generation (TG) measurement was extensively studied by the international scientific community, and especially in haemophilia, results are so far inconsistent due to poor standardization of TG assays.

The ST Genesia is a fully automated analyzer which provides standardized quantitative measurement of TG in plasma. In association with this instrument, the STG-BleedScreen reagent is a trigger containing low concentration of tissue factor and targets patients with bleeding risk, such as haemophiliacs.

Aims: The study aims at evaluating the ability of TG measured with STG-BleedScreen to discriminate severe Haemophilia A (HA) patients from healthy individuals in a multicentric study.

Methods: A total of 63 severe HA patients and 86 healthy individuals were enrolled on three sites: Cambridge, Vicenza and Barcelona. The study was approved by the local Ethics Committee and all the subjects gave their informed consent prior to enrolment.

Platelet Free Plasma aliquots were prepared from their blood samples and stored for a maximum of 6 months at $-70^{\circ} \mathrm{C}$ prior to analysis on ST Genesia with the STG-BleedScreen. The TG results were compared between HA and healthy groups for absolute and normalized Lag Time (LT), Peak Height (PH) and Endogenous Thrombin Potential (ETP) by using a null hypothesis significance test.

Results: The statistical analysis was performed site by site and by pooling all centers. Table 1 summarizes the mean of each parameter per group.

TABLE 1 Mean LT, PH and ETP parameters (absolute and normalized) for each group and each study site

\begin{tabular}{|c|c|c|c|c|c|c|c|c|c|c|}
\hline \multirow[b]{2}{*}{ Center } & & \multicolumn{2}{|l|}{ LT } & \multicolumn{3}{|l|}{$\mathrm{PH}$} & \multicolumn{4}{|l|}{ ETP } \\
\hline & & Healthy & Severe HA & \multicolumn{2}{|c|}{ Healthy } & Severe HA & \multicolumn{2}{|c|}{ Healthy } & \multicolumn{2}{|c|}{ Severe HA } \\
\hline Cambridge & & $\begin{array}{l}\text { Abs: } 2.72 \mathrm{~min} \\
\text { Norm: } 1.18\end{array}$ & $\begin{array}{l}\text { Abs: } 2.66 \mathrm{~min} \\
\text { Norm: } 1.11\end{array}$ & $\begin{array}{l}\text { Abs: } \\
\text { nM } \\
\text { Norn }\end{array}$ & $\begin{array}{l}103.60 \\
66.42 \%\end{array}$ & $\begin{array}{l}\text { Abs: } 27.31 \mathrm{nM} \\
\text { Norm: } 18.19 \%\end{array}$ & $\begin{array}{l}\text { Abs: } \\
\text { nM.n } \\
\text { Norm }\end{array}$ & $\begin{array}{r}741.0 \\
5.81 \%\end{array}$ & $\begin{array}{l}\text { Abs: } \\
\text { nM.m } \\
\text { Norm }\end{array}$ & $\begin{array}{r}331.9 \\
0.14 \%\end{array}$ \\
\hline \multirow[t]{2}{*}{ Vicenza } & $\begin{array}{l}n \\
\text { mean }\end{array}$ & 30 & 20 & 30 & & 20 & 30 & & 20 & \\
\hline & & $\begin{array}{l}\text { Abs: } 2.57 \mathrm{~min} \\
\text { Norm: } 1.17\end{array}$ & $\begin{array}{l}\text { Abs: } 2.18 \mathrm{~min} \\
\text { Norm: } 0.96\end{array}$ & $\begin{array}{l}\text { Abs: } \\
\text { Norn }\end{array}$ & $\begin{array}{l}\text { L.15nM } \\
110.15 \%\end{array}$ & $\begin{array}{l}\text { Abs: } 31.31 \mathrm{nM} \\
\text { Norm: } 22.78 \%\end{array}$ & $\begin{array}{l}\text { Abs: } \\
\text { nM.n } \\
\text { Norm }\end{array}$ & $\begin{array}{l}828.6 \\
0.49 \%\end{array}$ & $\begin{array}{l}\text { Abs: } \\
\text { nM.m } \\
\text { Norm }\end{array}$ & $\begin{array}{r}377.2 \\
7.63 \%\end{array}$ \\
\hline \multirow[t]{2}{*}{ All sites } & $\begin{array}{l}n \\
\text { mean }\end{array}$ & 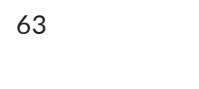 & 86 & 63 & & 86 & 63 & & 86 & \\
\hline & & $\begin{array}{l}\text { Abs: } 2.76 \text { min } \\
\text { Norm: } 1.17\end{array}$ & $\begin{array}{l}\text { Abs: } 2.55 \mathrm{~min} \\
\text { Norm: } 0.98\end{array}$ & $\begin{array}{l}\text { Abs: } \\
\text { nM } \\
\text { Norm }\end{array}$ & $\begin{array}{r}110.30 \\
72.31 \%\end{array}$ & $\begin{array}{l}\text { Abs: } 33.96 \mathrm{nM} \\
\text { Norm: } 23.83 \%\end{array}$ & $\begin{array}{l}\text { Abs: } \\
\text { nM.n } \\
\text { Norm }\end{array}$ & $\begin{array}{r}801.1 \\
4.14 \%\end{array}$ & $\begin{array}{l}\text { Abs: } \\
\text { nM.m } \\
\text { Norm }\end{array}$ & $\begin{array}{r}412.3 \\
8.66 \%\end{array}$ \\
\hline
\end{tabular}

LT, PH and ETP were significantly decreased in the haemophiliac population in comparison with the healthy individuals for both absolute and normalized results $(P<0.05)$. These significant differences were observed on all sites as well as in the multicentric analysis.

Conclusions: The STG-BleedScreen used on the ST Genesia is able to discriminate Severe HA patients from Healthy individuals consistently across clinical laboratories. This opens the door for the system to be used in severe HA patients management. 


\section{PB0593 | Promising Prospective Data on the Effect of Ankle} Joint Distraction in Hemophilic Arthropathy: Three Year Follow-up

E. van Bergen; L. van Vulpen; H. Vogely; P. de Kleijn; W. Foppen; P. van Roermund; R. Schutgens; S. Mastbergen; F. Lafeber UMC Utrecht, Utrecht, Netherlands

Background: Hemophilic ankle arthropathy (HAA) causes morbidity in (young) hemophilia patients. If conservative treatment fails, major surgical interventions like arthrodesis are indicated. An alternative treatment to postpone these interventions and maintain joint mobility is desired. In osteoarthritis, joint distraction was a good jointpreserving alternative. Retrospective evaluation of three patients with HAA treated with ankle joint distraction (AJD) showed evident clinical and radiological improvements.

Aims: To gather prospective data on clinical efficacy and tissue structure changes of AJD in HAA.

Methods: This ongoing first prospective study included patients $\geq 18$ and $\leq 55$ years with severe complaints of HAA, insufficiently responding to conservative treatment. AJD was performed over ten weeks using an external frame, applying $5 \mathrm{~mm}$ distraction. Data on clinical efficacy (questionnaires and performance tests) and tissue structure changes (X-ray and MRI) were evaluated.

Results:
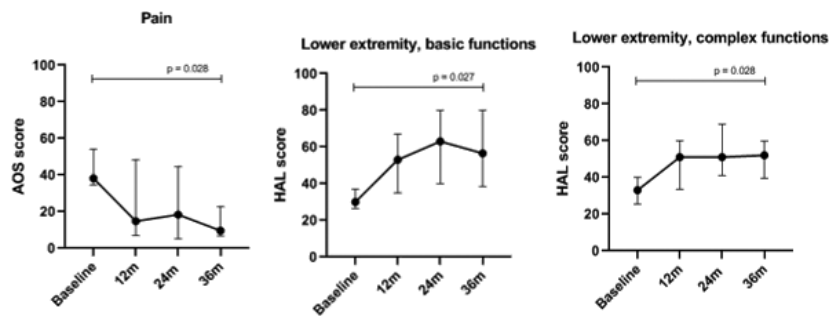

FIGURE 1 The AOS score is a valid instrument to measure pain related to ankle arthritis. Zero means 'no pain', hundred means 'worst pain imaginable'. The HAL score is a valid instrument to measure the impact of hemophilia on self-perceived functional abilities. Zero represents 'poor functional status' and hundred represents 'the best functional status'. Data over the course of three years is represented here as median scores. $m=$ months after distraction

Three-year follow-up data is available for eight patients. Over three years, ankle pain, as evaluated by the Ankle Osteoarthritis Scale (AOS) reduced significantly and a similar downward trend was seen in pain as evaluated by the Visual Analogue Scale (VAS). Functional status, assessed by the Hemophilia Activities List (HAL) and performance tests, significantly improved over time (figure 1). There were no significant changes in range of motion (ROM), indicating that joint mobility was retained among patients. MRI showed structural improvements in the ankle joints of these patients (figure 2). The main complication during treatment was pin tract infection $(n=6)$, commonly seen with external frames and effectively treated with a short course of antibiotics. There were no adverse bleeding events. Conclusions: This explorative study shows that AJD in HAA results in decreased pain, improved function and tissue repair for a prolonged time. This joint-preserving treatment may have a great impact, especially in the relatively young hemophilia population. Prolonged follow-up is needed to determine the duration of efficacy in HAA.

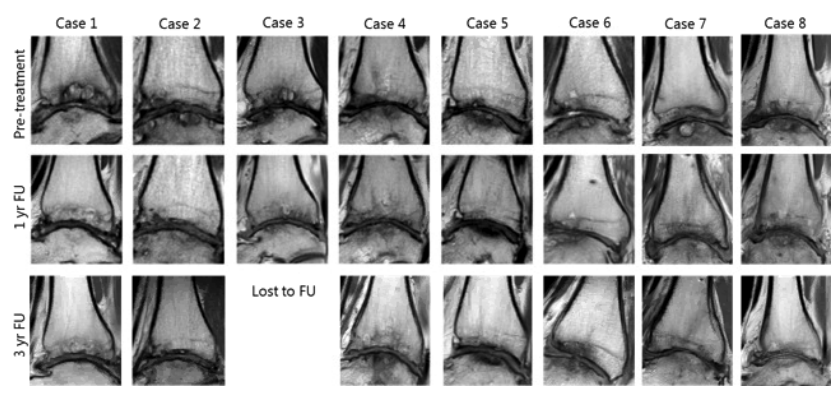

FIGURE 2 Structural changes (decrease in subchondral cysts and edema) on MRI. Sagittal images at baseline and one and three year after AJD

PB0594 | Years ofPprophylaxis with rFVIII-Fc in Haemophilia in a Portuguese Haemophilia Center: Did Doctors and Patients See a Change?

S. Campaniço; F. Rodrigues; A. Pereira; J. Amorim; C. Correia; L. Parusnikova; C. Catarino Centro Hospitalar Universitário Lisboa Norte, Lisbon, Portugal

Background: In recent years, several studies suggested that extended half-life FVIII products allowed a reduced infusion frequency and a lower clotting factor consumption (CFC), while improving compliance, outcomes and patient's quality of life (QoL).

Aims: The objective of this study was to evaluate the impact, in prophylaxis optimization and consumption, of switching our patients from standard half-life (SHL) products to rFVIII-Fc.

Methods: We retrospectively evaluated, for 12 months before and after the switching, data on prophylaxis regimen, focusing on prophylactic infusion frequency, all and joint bleeding ( $A B R$ a jABR) and CFC. Reasons for switching were also analyzed.

Results: Since october 2018, 25 severe and 1 moderate hemophilia patients, mean age 25 years (range: $5-68$ ), were switched to rFVIIIFc. Reducing the burden of IV infusion and improving protection and adherence were considered the main reasons for switching. Two patients had an history of inhibitors, and 1 was submitted to a successful immune tolerance induction with rFVIII-Fc. Patients started prophylaxis with the previous dose and prophylaxis regimen was then adapted to individual pharmacokinetic (PK) profiles and clinical needs.

For adults and adolescents, the dosing interval lengthened for $87.5 \%$ of subjects. $7 / 26$ (46\%) patients were dosed every 3 days; $7 / 26$ (27\%) 
twice a week and 5/26 (19\%) every 4 days. In 4 children < 12 years prophylaxis was possible ever 3 days or twice a week. Mean ABR and mean jABR were lower while on prophylaxis with rFVIII-Fc (3.54 vs 0.96 and 2.31 vs 0.58 , respectively). Number of patients with zero bleeds was higher with rFVIII-Fc than with SHL (4/26 vs $16 / 26)$. After switching to rFVIII-Fc concentrates, the mean annual CFC dropped $25 \%$.

Conclusions: We observed a clear reduction in weekly infusion frequency; a lower annual CFC but more important prophylaxis was efficacious across all ages and was associated with lower ABRs.

\section{PB0595 | Emicizumab in Acquired Hemophilia A: A Scoping}

\section{Review}

W. Shi; M. Crowther; K. Kim

McMaster University, Department of Medicine, Hamilton, Canada

Background: Emicizumab is a newly FDA approved antibody for bleeding prophylaxis in congenital hemophilia $\mathrm{A}(\mathrm{CHA})$ with or without inhibitors. Acquired hemophilia $A(A H A)$ is a rare form of hemophilia A where antibodies against endogenous factor VIII develops, resulting in a phenotype similar to $\mathrm{CHA}$ with inhibitors. Current guidelines on hemostatic agents in AHA recommends use of recombinant factor VIIa and replacement factor VIII. Given the shared pathophysiology between $\mathrm{CHA}$ with inhibitors and AHA, we hypothesize that there may be clinical evidence supporting the use of emicizumab in AHA.

Aims: perform a systematic literature search of all available evidence on the use of emicizumab in AHA in a clinical setting and assess its efficacy as a prophylactic and hemostatic agent.

Methods:

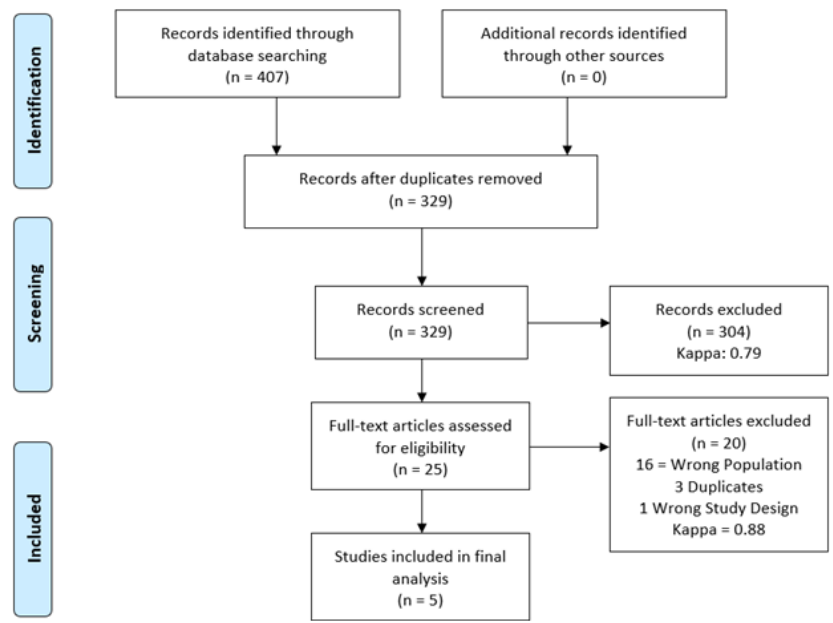

FIGURE 1 Flow diagram of the literature search of the literature

The scoping review was conducted following the PRISMA-P protocol. The search strategy was designed in consultation with a medical librarian to capture the widest scope of available evidence in Ovid MEDLINE, Embase and Web of Science, excluding only non-clinical study types. Title and abstract screening were performed by two independent reviewers followed by data extraction with a predetermined extraction form.

Results:

\begin{tabular}{|c|c|c|c|c|c|c|}
\hline \multirow[t]{2}{*}{ Study } & \multicolumn{6}{|c|}{ Population } \\
\hline & Age & Sex & $\begin{array}{l}\text { Inhibitor } \\
\text { Levels } \\
\text { (BU) }\end{array}$ & $\begin{array}{l}\text { FVIII activity } \\
(\%)\end{array}$ & Comorbidities & $\begin{array}{l}\text { Indication/Site of } \\
\text { Bleeding }\end{array}$ \\
\hline Al-Banaa 2019 & 87 & $\mathrm{~F}$ & $>100$ & $<1 \%$ & $\begin{array}{l}\text { Atrial fibrillation } \\
\text { Gl bleeding } \\
\text { Anemia }\end{array}$ & $\begin{array}{l}\text { Muscle(chest wall and } \\
\text { pelvic hematoma) }\end{array}$ \\
\hline Dane 2019 & 72 & M & 409 & Undetectable & $\begin{array}{l}\text { NSTEMI } \\
\text { Bullous pemphigoid }\end{array}$ & $\begin{array}{l}\text { Peri-procedural } \\
\text { hemostasis (PCl) }\end{array}$ \\
\hline $\begin{array}{l}\text { Elommersfield } \\
2019\end{array}$ & 21 & $\mathrm{~F}$ & 40 & $<0.25 \%$ & $\begin{array}{l}\text { Autoimmune hemolytic } \\
\text { anemia } \\
\text { Immune } \\
\text { thrombocytopenia } \\
\text { Epidermolysis bullosa } \\
\text { Acquisita verrucae }\end{array}$ & $\begin{array}{l}\text { Musculoskeletal } \\
\text { (Upper and lower limb) }\end{array}$ \\
\hline Knoebl 2019* & $\begin{array}{l}62.3 \\
(50- \\
78)\end{array}$ & $\begin{array}{l}2 \mathrm{M} \\
2 \mathrm{~F}\end{array}$ & $\begin{array}{l}54.3 \\
(3.1-84)\end{array}$ & $<1 \%$ & Not reported & $\begin{array}{l}\text { Patients (1-2): post- } \\
\text { op bleeding } \\
\text { Patient 3: major } \\
\text { bleeding } \\
\text { Patient 4: skin } \\
\text { (hematoma) }\end{array}$ \\
\hline Mohnle 2019 & 83 & M & 36 & $<1 \%$ & $\begin{array}{l}\text { CHF NYHA III } \\
\text { Atrial fibrillation } \\
\text { CKD Stage III } \\
\text { Previous DVT and PE }\end{array}$ & Diffuse hematomas \\
\hline
\end{tabular}

FIGURE 2 Summary of study details and population characteristics. *This was the case series and only the mean and range of the patient data was reported. $\mathrm{BU}=$ Bethesda units

407 journal articles were identified in the initial search. After screening, four case reports and one case series totaling five studies were included in data extraction. Four males and four females totaling eight patients with a mean age of 64 were included in this review. All patients had severe AHA with high titer FVIII inhibitors and FVIII activity below $1 \%$. All patients received emicizumab after achieving initial hemostasis with replacement or bypass therapy. Additional data on outcomes will be presented at the conference.

Conclusions: Our findings suggest there is limited clinical evidence of emicizumab prophylaxis in AHA based on a few case reports, demonstrating an important knowledge gap for this area. Additional research and guidelines are needed to guide clinical care. 
PB0596 | Usefulness of Clot Waveform Analysis as a Perioperative Monitoring in Arthroscopic Synovectomy to a Pediatric Patient with Hemophilia A and Inhibitor Receiving Emicizumab

K. Mizumachi $^{1}$; Y. Tsumura ${ }^{2}$; Y. Nakajima ${ }^{1}$; K. Ko ${ }^{2}$; K. Nogami ${ }^{1}$

${ }^{1}$ Department of Pediatrics, Nara Medical University, Kashihara, Japan;

${ }^{2}$ Department of Hematology/Oncology, Saitama Children's Medical

Center, Saitama, Japan

Background: Emicizumab prophylaxis reduces bleeding in patients with hemophilia $\mathrm{A}$ and inhibitors (PwHA-I). A small number of cases have been reported describing global coagulation assays for monitoring hemostasis during concomitant treatment with bypassing agents in emicizumab-treated $\mathrm{PwHA}-\mathrm{I}$ undergoing surgery. However, coagulation potential during the perioperative period in these circumstances remains to be assessed.

Aims: To assess global coagulation potential for the perioperative hemostatic management of emicizumab-treated PwHA-I.

Methods: Arthroscopic synovectomy in one pediatric PwHA-I receiving emicizumab prophylaxis was performed during concomitant treatment with recombinant factor VIla ( $\mathrm{FFVIIa).} \mathrm{Coagulation} \mathrm{potential} \mathrm{was} \mathrm{assessed}$ using modified-clot waveform analysis (CWA) triggered with a reagentmixture containing tissue factor and ellagic acid, and thrombin generation assays (TGA) triggered by tissue factor were utilized. Maximum velocity (Ad|min1|) and clot time in CWA, and peak thrombin, endogenous thrombin potential (ETP) and time-to-peak in TGA were calculated.

Results: A bolus infusion of $\mathrm{rFVIla}(80 \mu \mathrm{g} / \mathrm{kg})$ was administered immediately before surgery, and continued at the same dose every 3 hours on day 1 , every 4 hours on day 2 , and 6 hours day 3 , respectively. Treatment with rFVIla was discontinued on day 4 . No perioperative bleeding or thrombotic events were observed. Clot time in CWA and time-to-peak in TGA were shortened than the normal upper limits and ETP in TGA barely reached the lower normal limits, indicating that these parameters appeared unlikely to reflect coagulation potential in the patient. In contrast, measurements of Ad|min1| (5.26 at baseline level) were increased to 6.55-7.55, and remained within or near to the normal range (mean $\pm \mathrm{SD} ; 6.7 \pm 0.6$ ) during concomitant therapy with rFVIla, suggesting good hemostatic management. Changes in peak thrombin in TGA paralleled the Ad|min1| results.

Conclusions: Ad|min1| in modified-CWA and peak thrombin in TGA provide useful data for assessing coagulation potential for the perioperative hemostatic management of emicizumab-treated PwHA-I.

\section{PB0597 | Telemedicine in Hemophilia: A Comprehensive Review}

C.L Li ${ }^{1} ;$ K. Strike ${ }^{2,3}$; D. Matino ${ }^{2,4}$; M. Bhatt ${ }^{1,2} ;$ K. Decker ${ }^{2}$; A.K. Chan ${ }^{1,2}$ ${ }^{1}$ Department of Pediatrics, McMaster University, Hamilton, Canada;

${ }^{2}$ Hamilton Niagara Regional Hemophilia Program, Hamilton Health Sciences, Hamilton, Canada; ${ }^{3}$ School of Rehabilitation Science,

Faculty of Health Sciences, McMaster University, Hamilton, Canada; ${ }^{4}$ Department of Medicine, Division of Hematology \& Thromboembolism, McMaster Children's Hospital, Hamilton, Canada
Background: Patients with hemophilia require regular assessments and physical examinations. The COVID-19 pandemic has resulted in the rapid adoption of telemedicine to enable virtual consultations and reduce hospital visits. However, the process of virtual consultations is new to many hemophilia clinics. A better understanding of best practices in telemedicine is important to ensure optimal quality of care for patients with hemophilia.

Aims: To summarize the current literature on the use of direct-toconsumer telemedicine for patients with hemophilia and to describe the effectiveness and potential limitations of the technology and methods used.

Methods: A comprehensive search was conducted in MEDLINE and EMBASE databases using the terms "hemophilia" AND "telemedicine" and their concept synonyms. There were no time or language restrictions. Title, abstracts, and full texts were screened. Included articles involved telemedicine interventions to facilitate clinical services directly between patients and providers without the use of third-party personnel. The primary outcome was the satisfaction of providers and patients. Secondary outcomes included economic considerations and clinical outcomes. Data were extracted based on study-specific, patient-specific, intervention-specific, and outcome-specific data.

Results: Of the 925 articles screened, 6 articles were identified and summarized (Figure 1).

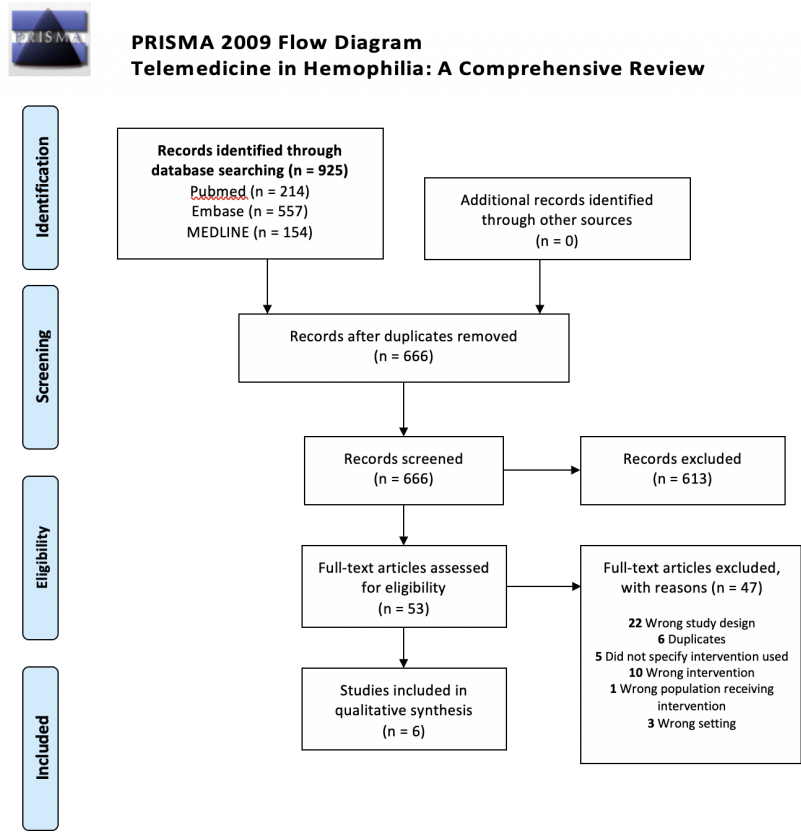

FIGURE 1 PRISMA Flow Diagram of Study Selection

Three articles described telemedicine within the context of COVID-19. Technologies used included telephone calls, videoconferencing, text messaging, and email. All studies involved a multidisciplinary team. Telemedicine in hemophilia care was found to positively impact the patient experience. Providers were satisfied with telemedicine. Telemedicine was economically beneficial and positively impacted patient outcomes. However, none of the articles 
reported on how telemedicine was specifically used to perform assessments during the virtual consultation process.

Conclusions: The positive reception of telemedicine by patients and providers combined with the limited information available on methods of virtual assessments necessitates the development of a standard telemedicine guideline that can help providers learn how to best incorporate telemedicine to improve quality of care.

PB0598 | Demographic and Clinical Characteristics of Patients with Hemophilia A in Brazil: Real-world Data from the 6th Interim Analysis of the AHEAD International Study

M.H. Cerqueira ${ }^{1}$; C. Ferreira ${ }^{2}$; C. Lorenzato ${ }^{3}$; L.E.M. Carvalho ${ }^{4}$; L.C Oliveira de Oliveira ${ }^{5}$; I. Pinto ${ }^{6}$; A. Prezotti ${ }^{7}$ J. Norton ${ }^{8}$; L. Tang9; M.C Ozelo ${ }^{10}$

${ }^{1}$ Instituto Estadual de Hematologia Arthur de Siqueira Cavalcanti (HEMORIO), Rio de Janeiro, Brazil; ${ }^{2}$ Hemocentro de Porto Alegre (HEMORGS), Porto Alegre, Rio Grande do Sul, Brazil; ${ }^{3}$ HEMEPAR, Curitiba, Paraná, Brazil; ${ }^{4}$ Centro de Hematologia e Hemoterapia do Ceará (HEMOCE), Fortaleza, Ceará, Brazil; ${ }^{5}$ Hemocentro Ribeirão Preto, Universidade de São Paulo, Ribeirão Preto, São Paulo, Brazil; ${ }^{6}$ Fundação HEMOPA, Belém, Pará, Brazil; ${ }^{7}$ Centro de Hemoterapia e Hematologia do Espírito Santo (HEMOES), Vitória, Espírito Santo, Brazil; ${ }^{8}$ Takeda Development Center Americas, Inc., Lexington, MA, United States;

${ }^{9}$ Takeda Pharmaceuticals International AG, Zürich, Switzerland,

${ }^{10}$ Hemocentro UNICAMP, Department of Internal Medicine, School of Medical Sciences, University of Campinas, Campinas, São Paulo, Brazil
Background: The international Antihemophilic factor (recombinant) (rAHF) Hemophilia A (HA) outcome Database (AHEAD) study is assessing the long-term effectiveness and safety of rAHF in patients with $\mathrm{HA}$ in real-world clinical practice.

Aims: To report demographic and clinical characteristics at screening of the AHEAD Brazil patient subset.

Methods: AHEAD is a prospective, non-interventional, multicenter study (NCT02078427) that includes patients of any age with HA (factor VIII $\leq 5 \%$ ). The study was approved in Brazil on July 14, 2015. Ethics committee approval and patients' informed consent were obtained. The primary endpoint is joint health outcomes evaluated by Gilbert score (pain, 0-3; bleeding, 0-3; physical exam, 0-12). Investigators can choose Hemophilia Joint Health Score (HJHS) as an alternative to Gilbert Score. Secondary endpoints include annualized bleeding rates (ABRs; all bleeds), annualized joint bleeding rates (AJBRs), and safety endpoints. Here, we report demographic and clinical characteristics at screening from the safety analysis set (SAS) for patients in the AHEAD Brazil subset. These data were extracted from the 6th interim analysis of the AHEAD International study (data cutoff: July 15, 2019).

Results: The AHEAD Brazil SAS included 203 patients (median [range] age, 13.0 [0-43] years): 190 received prophylaxis (median [range] age, 14.0 [0-43] years), 2 received on-demand treatment (median [range] age, 12.0 [0-24] years), and 11 patients with inhibitors received immune tolerance induction (ITI; median [range] age, 12.0 [3-34] years). All patients were male (Table 1). At screening, bleeding events had occurred in 130 (68.4\%) prophylaxis patients, 1 (50.0\%) on-demand patient, and 4 (36.4\%) patients receiving ITI (Table 2). Bleeding events recorded at screening had occurred up to 12 months prior to screening.

TABLE 1 Patient demographics at screening

Prophylaxis $(n=190)$

On-demand $(n=2)$

$1(50.0)$

$0(0.0)$

Asian

$98(51.6)$

$3(1.6)$

$0(0.0)$

Black or African American

$27(14.2)$

$62(32.6)$

$1(50.0)$

Hemophilia A severity, $n$ (\%)

Factor VIII 1-5\%

Factor VIII $<1 \%$

\section{5 (18.4)}

155 (81.6)
ITI $(n=11)$

$6(54.5)$

$0(0.0)$

$3(27.3)$

2 (18.2)

2 (18.2)

9 (81.8)
Total $(N=203)$

$105(51.7)$

3 (1.5)

$30(14.8)$

$65(32.0)$

37 (18.2)

$166(81.8)$

TABLE 2 Gilbert score, HJHS, and bleeding rates at screening*

$\begin{array}{llll} & \text { Prophylaxis } & \text { On-demand } & \text { ITI } \\ \text { Average Gilbert score (all joints), } n & 35 & - & 1 \\ \text { Mean } \pm \text { SD } & 1.7 \pm 1.6 & & 1.0 \\ \text { Median (range) } & 1.0(0.0-5.0) & 0 & 1.0(1.0-1.0) \\ \text { HJHS: Global Gait Score, } n & 86 & - & 8 \\ \text { Mean } \pm \text { SD } & 1.6 \pm 1.6 & & 1.8 \pm 1.5 \\ \text { Median (range) } & 1.0(0.0-4.0) & 2 & 1.0(0.0-4.0) \\ \text { ABR, } n & 190 & & 11\end{array}$


Prophylaxis

$3.3 \pm 4.8$

Mean \pm SD

Median (range)

AJBR, $† n$

Mean \pm SD

Median (range)
On-demand

$5.0 \pm 7.1$

$5.0(0.0-10.0)$

2

$4.5 \pm 6.4$

4.5 (0.0-9.0)
ITI

$4.0 \pm 7.9$

$0.0(0.0-26.0)$

11

$2.6 \pm 5.6$

$0.0(0.0-19.0)$

*Bleedings occurring during regimens of $<90$ days were not used for this analysis. Bleedings recorded at screening had occurred up to 12 months prior to screening. Patients with $>1$ regimen during a study year (switch patients) were counted for each regimen; bleeding events were only counted for the regimen during which they occurred.

${ }^{\dagger}$ Owing to missing information for joint/non-joint classification, AJBRs may be underestimated.

Conclusions: The AHEAD Brazil subset, which is part of the larger AHEAD International study, included mostly patients receiving prophylaxis. Future analyses will investigate the clinical outcomes of patients in the AHEAD Brazil subset.

PB0599 | Prospective, Non-interventional Study with a Cohort to Assess Real-world Effectiveness (RWE) and Safety of Emicizumab in Persons with Hemophilia A (PwHA) without Inhibitors in Spain, Hemolution Cohort

M.T. Álvarez Román ${ }^{1}$; I. Soto ${ }^{2}$ H. de la Corte ${ }^{1}$; P. Bosh ${ }^{3} ;$ Á. Lavandeira $^{4}$; C. Alvarez ${ }^{5}$; I. Guerra ${ }^{5}$

${ }^{1}$ Service of Hematology, Hospital Universitario La Paz, Madrid, Spain, Madrid, Spain; ${ }^{2}$ Service of Hematology, Hospital Universitario Central de Asturias, Oviedo, Spain; ${ }^{3}$ Service of Hematology, Hospital Universitario La Fe, Valencia, Spain, Valencia, Spain; ${ }^{4}$ Person with Hemophilia A, Madrid, Spain, Madrid, Spain; ${ }^{5}$ Medical Department, Roche Farma, Madrid, Spain, Madrid, Spain

Background: Haemophilia A (HA) is a disorder characterized by deficiency in clotting FVIII that affects daily lives of patients and caregivers. Emicizumab is a humanized, bispecific, monoclonal antibody, which substitutes missing activated FVIII.

Aims: Effectiveness and safety on emicizumab in clinical practice. Methods: Hemolife study initiated in March 2020, describes the socio-occupational impact of HA without inhibitors in patients and caregivers, in terms of quality of life, activity, and productivity/absenteeism, among others. At that time, both COVID-19 pandemic and emicizumab approval for PwHA without inhibitors happened.

A multidisciplinary Scientific Committee of experts in HA, rethought Hemolife study to include a cohort of patients treated with emicizumab (Hemolution), resulting in a more complete and stronger
RWE study, and avoiding logistical duplications that a separate study would mean to centers and patients.

2020 has been a year for adaptation, efficiency and re-design; also, in RWE context.

100 PwHA will be followed for 12 months within Hemolife. At any time, a patient prescribed with emicizumab may be enrolled in Hemolution cohort, followed for at least 8 months after initiation of emicizumab to collect efficiency and safety data (Figure 1).

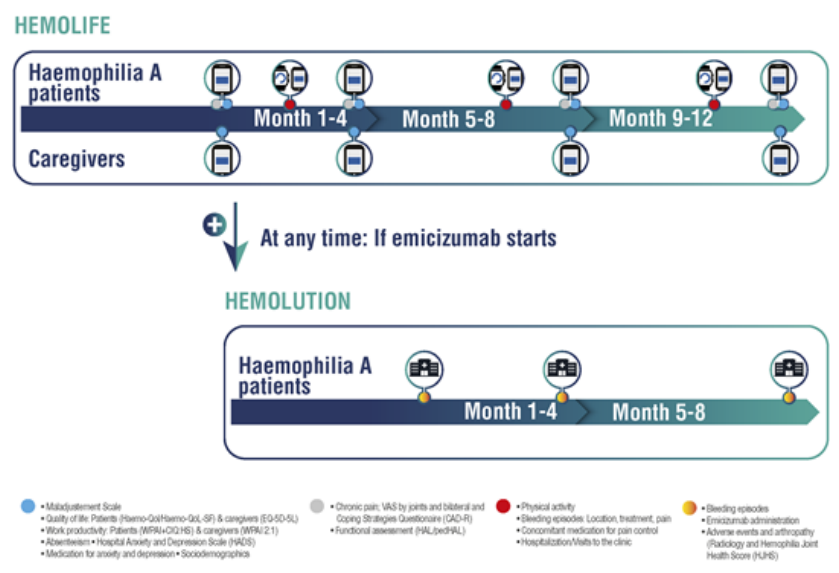

\section{FIGURE 1}

Finally, Hemolife involves telemedicine; in a pandemic setting, Hemolution could also benefit from this live data collection and may shape a new and more dynamic form of care in HA that avoids being in the hospital.

Results: Ongoing study. Protocol was submitted and approved by authorities/ethics committee's (Table 1). 


\section{TABLE 1}

\begin{abstract}
Region
Andalucía

Aragón

Asturias

Castilla La Mancha \& Castilla León
\end{abstract}

Galicia

Islas Baleares \& Islas Canarias

Madrid

Navarra

\section{Hospital}

Hospital Universitario Virgen del Rocío

Hospital Regional Universitario de Málaga

Hospital Puerta del Mar de Cádiz

Hospital Universitario Reina Sofía

Hospital del SAS Jerez de la Frontera

Hospital Virgen de las Nieves

Hospital Universitario Miguel Servet

Hospital Universitario Central de Asturias

Hospital Universitario General de Toledo

Hospital Universitario de Guadalajara

Hospital del Río Hortega

Hospital Universitario de Salamanca

Hospital de la Santa Creu i Sant Pau

Hospital Sant Joan de Déu Barcelona

Hospital Universitario Vall d'Hebron

CHUAC - Complexo Hospitalario Universitario A Coruña

Complejo Hospitalario Universitario de Vigo

Hospital Universitari Son Espases

Hospital Universitario de Gran Canaria Doctor Negrín

Hospital Universitario La Paz

Complejo Hospitalario de Navarra
Conclusions: By this novel approach we will gather information on emicizumab in clinical practice and provide relevant information to the Haemophilia community, contributing to improve patient care in HA.

\section{PB0600 | Cost-effectiveness of rFVIII Single-chain in an Italian} Population of Hemophilia A Patients: The Veneto Experience

E. Zanon ${ }^{1}$; S. Pasca ${ }^{1}$; P. Radossi ${ }^{2}$; E. Bonetti ${ }^{3}$; A.C. Giuffrida ${ }^{3}$; A. Tosetto $^{4}$

${ }^{1}$ Padua University Hospital, Padua, Italy; ${ }^{2}$ Istituto Oncologico Veneto (IOV), Castelfranco Veneto, Italy; ${ }^{3}$ Verona University Hospital, Verona, Italy; ${ }^{4}$ S. Bortolo Hospital, Vicenza, Italy

Background: Primary prophylaxis started early at the dosage of 25$40 \mathrm{IU} / \mathrm{kg}$ three times/week is considered the gold standard for the treatment of patients with severe hemophilia, improving their quality of life (QoL) and reducing bleeds. In the last years different new concentrates have been marketed, allowing a reduction of annual bleeding rate (ABR) with concomitant improvement of compliance to treatment.

Aims: The aim of this study was to compare the differences between the treatment with the single chain rFVIII, lonoctocog-alpha, and octocog-alpha in terms of total, joint and spontaneous $A B R$, number of infusions and dosage in a group of patients with hemophilia A (HA).

Methods: All patients with HA, referred to four Hemophilia Centers (Padova, Vicenza, Castelfranco Veneto, Verona) belonging to the same Italian region (Veneto), previously receiving octocog-alpha $\left(2^{\text {nd }}\right.$ generation), and subsequently switched to lonoctocog-alpha were considered in our study. Statistical analysis were performed considering one year of treatment for any type of treatment.

Results: Twenty-one previously treated patients (PTPs), 10-59 years-old were switched to lonoctocog-alpha, 13/21 of them were previously on prophylaxis with octocog-alpha. $81 \%$ had severe hemophilia, $5 \%$ moderate, while three (13\%) were mild young subjects in prophylactic treatment as practice sport activity. $72.6 \%$ were adults ( $\geq 14$ years). Five PTPs on demand were put on prophylaxis with $\mathrm{rFVIII}$ single-chain. Mean ABR were decreased after switch, while the weekly median number of infusion remained unchanged, 2 (range 1-3). A comparison head to head between the 13 patients on prophylaxis with the two different $\mathrm{rFVIII}$ concentrates are reported in Figure 1. 


\begin{tabular}{|l|c|c|c|c|c|c|}
\hline & \multicolumn{2}{|c|}{$\begin{array}{c}\text { Prophylaxis (N=13) } \\
\text { octocog-alpha }\end{array}$} & \multicolumn{3}{c|}{$\begin{array}{c}\text { Prophylaxis (N=13) } \\
\text { rFVIII Single-Chain }\end{array}$} \\
\hline & $\begin{array}{c}\text { Eose single } \\
\text { infusion (IU/kg) }\end{array}$ & $\begin{array}{c}\text { Estimated annual } \\
\text { consumption (IU/kg) }\end{array}$ & $\begin{array}{c}\text { Dose single } \\
\text { infusion (IU/kg) }\end{array}$ & $\begin{array}{c}\text { Estimated annual } \\
\text { consumption (IU/kg) }\end{array}$ \\
\hline Min & 15,6 & & 812,5 & 15,6 & \multicolumn{2}{|c|}{$1.425,8$} \\
\hline Max & 38,5 & & $5.032,3$ & 44,4 & \multicolumn{2}{|c|}{$5.032,3$} \\
\hline Mean & $\mathbf{2 7 , 5}$ & & $\mathbf{3 . 2 4 9 , 2}$ & $\mathbf{3 1 , 6}$ & & $\mathbf{3 . 6 3 1 , 6}$ \\
\hline & $\mathbf{A B R}$ & $\mathbf{A j B R}$ & $\mathbf{A s B R}$ & $\mathbf{A B R}$ & $\mathbf{A j B R}$ & AsBR \\
\hline Min & 0 & 0 & 0 & 0 & 0 & $\mathbf{0}$ \\
\hline Max & $\mathbf{5}$ & 3 & 3 & 2 & 2 & $\mathbf{1}$ \\
\hline Mean & $\mathbf{1 , 2 3}$ & $\mathbf{0 , 8 5}$ & $\mathbf{0 , 5 4}$ & $\mathbf{0 , 5 4}$ & $\mathbf{0 , 3 1}$ & $\mathbf{0 , 2 3}$ \\
\hline
\end{tabular}

FIGURE 1 Comparison head to head between 13 prophylaxis with octocog-alpha and lonoctocg-alpha. ABR: annual bleeding rate; $A j B R$ : annual joint bleeding rate; $A s B R$ : annual spontaneous bleeding rate

Conclusions: In our study the switch to rFVIII single-chain was proved to be more effective compared to prophylaxis with octocogalpha in all patients. The 13 patients already on prophylaxis with octocog-alpha reduced their ABR by $50 \%$ after the switch, while the annual consumption of the product remained similar as was the cost.

PB0601 | Estimation of Secondary Prophylaxis Effectiveness in Patients with Severe Form of Hemophilia A

$\underline{\text { T. Talako }}^{1}$; H. Kuzmich ${ }^{2}$; V. Zmachinsky ${ }^{1}$; A. Uss ${ }^{2}$

${ }^{1}$ Belorusian State Academy of Postgraduate Education, Minsk, Belarus;

${ }^{2}$ Minsk Scientific and Practical Center for Surgery, Transplantology and

Hematology, Minsk, Belarus

Background: Prophylactic administration of antihemophilic drugs is preferable compared to the treatment "on demand" among pediatric patients with hemophilia. The effectiveness of prophylactic treatment in adult patients requires clarification.

Aims: To evaluate the effectiveness of secondary prophylaxis with coagulation factor (F) VIII in adult patients with severe hemophilia A. Methods: The study included 12 patients with severe hemophilia A. Prophylaxis included the administration of FVIII at a dose of 30-40 IU / kg body weight 3 times a week for 6 months. Prior to and during the 6 months of the study, monthly, all patients underwent an assessment of coagulogram, level of FVIII and the presence of an inhibitor to it, the severity of pain (VAS), joint's status (Gilbert's score and Hemophilic Joint Health Score 2.1 (HJHS Total score)), bleeding episodes per month and the need for additional administration of the FVIII.

Results: A statistically significant decrease of VAS index and Gilbert's score was detected 1 month after the start of prophylaxis and after 2, 3, 4, 5, and 6 months they were significantly less than the initial values. The highest decrease of VAS index was revealed after 6 months of prophylaxis: $27.5(17.5-37.5) \mathrm{mm}$, the largest decrease of Gilbert's score - after 4 months: 3.0 (2.0-4.5) and it remained the same after 5 and 6 months of prevention. After 3 months of prophylaxis a significant decrease of HJHS Total score was established, which continued monthly until the end of prevention. The greatest decrease in HJHS Total score was observed after 5 months of prevention - $3.5(2.0-5.0)$ and it remained the same after 6 months of prevention.

Conclusions: Secondary prohpylaxis with affects both the pain syndrome and the functional state of the joints, which indicates not only the possibility, but also the validity of its implementation in adult patients with severe hemophilia A.

PB0602 | Emicizumab: What Can Patients Expect Regarding Hemostasis, Bleeding and Sports Capacity?

B. Boulden Warren; D. Thornhill; L. Jacobson; C. Baird; D. Hill; C. Ng; T. Buckner; M. Wang; M. Manco-Johnson

University of Colorado, Aurora, United States

Background: Emicizumab is a bispecific monoclonal antibody that activates factor $X$ in the absence of factor VIII (FVIII). Since its approval (FDA 2017; EMA 2019), there has been rapid adoption of emicizumab by persons with hemophilia $\mathrm{A}(\mathrm{PwHA})$ of all severities, with and without inhibitors, owing to convenient subcutaneous administration, less frequent dosing and reduced bleeding.

Aims: To determine real-world data on hemostatic, physical activity participation and bleeding outcomes of emicizumab in PwHA.

Methods: This was a retrospective analysis of clinical and laboratory data from an approved consented cohort study of hemophilia. All PwHA prescribed emicizumab for 6 months or more on whom a research blood sample was available were included in the analysis. Clinical data including FVIII baseline, inhibitor status, time on emicizumab, regular activities rated using the US National Hemophilia Foundation Sports Risk rating scale, bleeding, and treatment history were obtained by chart review. Chromogenic FVIII (FVIII:Chr) activities were assayed using human (Hyphen BioMed) and bovine (Chromogenix, to exclude infused FVIII) substrates. Thrombin generation endogenous thrombin potential (ETP) was measured using the calibrated automated thrombogram (CAT, Diagnostica Stago).

Results: 46 PwHA used emicizumab for a mean of 9.3 (standard deviation 7) months. Table 1 displays coagulation results. PwHA severe had a greater absolute increase in FVIII:Chr compared with mild $(P=0.006)$, but both achieved similar levels. The absolute change in thrombin generation was greater for severe $(P<0.001)$ than moderate $(P=0.01)$ or mild $(P=.36)$ but all achieved similar results. Table 2 displays activities performed and bleeding events on emicizumab. PwHA severe engaged actively but experienced a lower bleeding rate compared with mild or moderate. 
TABLE 1 Laboratory Assessment of Hemostatic Improvement with Emicizumab

\begin{tabular}{|c|c|c|c|c|}
\hline Participants & All $(n=46)$ & Mild $(n=5)$ & Moderate $(n=10)$ & Severe $(n=31)$ \\
\hline Inhibitors & 14 & 1 & 0 & 13 \\
\hline Activities: Level $1 \& 1.5$ & 42 & 4 & 10 & 28 \\
\hline Activities Level $2 \& 2.5$ & 31 & 7 & 5 & 19 \\
\hline Activities Level 3 & 4 & 0 & 2 & 2 \\
\hline Bleeds: Major/Fracture/Head & 18 & 1 & 6 & 11 \\
\hline Joint & 52 & 6 & 11 & 33 \\
\hline Muscle & 11 & 1 & 6 & 4 \\
\hline Soft Tissue & 15 & 2 & 1 & 12 \\
\hline Bleeds per Month & 0.19 & 0.30 & 0.33 & 0.14 \\
\hline
\end{tabular}

TABLE 2 Activities and Bleeds in PwHA on Emicizumab

\begin{tabular}{|c|c|c|c|}
\hline Hemophilia Severity & Mild $(n=5)$ & Moderate $(n=10)$ & Severe $(n=28)$ \\
\hline \multicolumn{4}{|c|}{ Chromogenic FVIII with Human Reagents (\% of normal, mean (SD)) } \\
\hline Baseline & $10.2(3.9)$ & $3.6(2.1)$ & $0.4(0.3)$ \\
\hline Change & $17.0(8.3)$ & $13.8(7.4)$ & $24.1(9.1)$ \\
\hline Level Achieved & $27.3(9.9)$ & $17.4(7)$ & $24.5(9.1)$ \\
\hline \multicolumn{4}{|c|}{ Thrombin Generation (CAT ETP, ETP) (\% of normal, mean (SD)) } \\
\hline Baseline & $44.6(23)$ & $32.1(13.7)$ & $26.2(18.2)$ \\
\hline Change & $11.4(24.6)$ & $23.7(18.1)$ & $34.0(24.7)$ \\
\hline Level Achieved & $56.0(9.1)$ & $55.2(12.2)$ & $56.4(20.4)$ \\
\hline
\end{tabular}

Conclusions: PwHA on emicizumab demonstrated improved Chr:FVIII and CAT:ETP, and had active life-styles with low bleeding rates. PwHA mild had smaller improvement with equivalent hemostatic achievement but higher bleeding rates. Emicizumab efficacy in prevention of activity-related bleeding requires further study.

\section{PB0603 | Update on Real Life Use of Emicizumsb in Pediatric} Patients without Inhibitors

\section{S. Heine; N. Graf}

Universitätskinderklinik, Homburg, Germany

Background: Emizicumab has been licensed in Germany for non inhibitor patients for two years now. We have treated the first PUP in Germany, he has had no treated bleed now for two years, we report on further 11 patients with two more PUPs (age range 4 months to $15 \mathrm{yrs}$ ). Aims: We describe the successful use in a broad range of pediatric patients with severe hemophilia A including 3 PUPs and with varying challenges. We want to point out real life concerns in the treatment regarding compliance, difficulties in dosing due to the limited vial size and in the necessity of additional factor dosing in case of non severe bleeding.

Methods: We describe 12 pediatric patients with the dosing schemes, extended dosing intervals due to vial size, the subsequent Emicizumab levels, the bleeding events and when we chose to add factor, and the overall satisfaction of the patients and their families. Results: We report the successful outcomes in the treatment with Emicizumab. We report 2 port removals with additional factor, one dose given in case of head trauma and one traumatic muscle bleed needing additional factor.

The levels taken after adjusted dosing (every 3 weeks, every ten days e.g.) according to vial size were in the desired range.

Conclusions: We advocate the use of Emicizumab especially in PUPs. Our oldest PUP has been treated now for 2 years thus saving himself 312 iv injections, the the other two PUPs would have needed ports. We have hardly seen bleeds needing factor, we advocate a personalized dosing scheme due to vial size. Questions remain regarding when to treat a bleed and when to wait, whether to try to tolerize a PUP. A very important issue is the complete lack of educational material for families as all the books available do not include Emizicumab.

PB0604 | Can Plasminogen Activator Inhibitor 1 and Thrombin Activitable Fibrinolysis Inhibitor Explain Bleeding Phenotype in Hemophilia A?

$\underline{\text { S. Elhefnawy }}^{1}$; M. Abd Elrehim ${ }^{2} ;$ M. Elhawy ${ }^{2}$.

${ }^{1}$ Shebin Elkom Educational Hospital, Shebin Elkom, Egypt; ${ }^{2}$ Menoufia, Shebin Elkom, Egypt

Background: Patients with hemophilia A display varied bleeding phenotypes not correlated with degree of deficiency of factor VIII level. previous studies also releaved that premature clot lysis occur in plasma deficient in components of intrinsic pathway.So, bleeding in Hemophilia may be due to enhanced fibrinolysis $n$ addition to factor VIII deficiency. Aims: We aimed to find correlation between bleeding variations in hemophilic patients and their level of Plasminogen Activatable 
Fibrinolysis Inhibitor 1(PAI-1) and Thrombin Activatable Fibrinolysis Inhibitor (TAFI) levels as antifibrinolytic factors.

Methods: Case control study was conducted on 26 malechildren patients diagnosed with Hemophilia A and 14 apparently healthy male children subjects with matched age as a control group.

The International Society of Thrombosis Bleeding Assessment Tool (ISTH/BAT) was used to assess bleeding score in patients. Patients are divided according to their bleeding score into intense and non-intense haemorrhagic groups. Plasminogen Activator Fibrinolysis Inhibitor (PAI1) and Thrombin Activatable Fibrinolysis Inhibitor (TAFI) levels were measured by enzyme-linked immunosorbent assay (ELIZA).

Results: As compared to controls, hemophilic patients had significantly high bleeding score, low PAI 1 level and high TAFI level. There was no significant correlation between bleeding score by ISTH/BAT and patient severity. PAI 1 and TAFI level have no significant correlation with patient severity. We found significant correlation between PAI-1 level and bleeding intensity, however we couldn't able to detect such correlation between TAFI level and bleeding intensity.

Conclusions: We concluded that there are significant correlation between patients and control as regard PAI-1 and TAFI levels. That means presence of enhanced fibrinolysis in hemophilic.PAI-1 significantly correlated with bleeding intensety, while TAFI didn't correlate with it.

PB0605 | Recommendations for Intramuscular COVID-19 Vaccination in Patients with Hemophilia

C. Pfrepper ${ }^{1}$; K. Holstein ${ }^{2}$; C. Koenigs ${ }^{3}$; C. Heller ${ }^{3}$; M. Krause ${ }^{4}$; M. Olivieri' ; C. Bidlingmeier ${ }^{5}$; M. Sigl-Kraetzig ${ }^{6}$; J. Wendisch ${ }^{7}$; R. Klamroth $^{8}$; S. Halimeh ; J. Oldenburg ${ }^{10}$; S. Horneff ${ }^{10}$; H. Richter ${ }^{11}$; I. Wieland $^{12} ;$ A. Tiede ${ }^{12}$

${ }^{1}$ University Hospital Leipzig, Leipzig, Germany; ${ }^{2}$ University Medical Center Hamburg-Eppendorf, Hamburg, Germany; ${ }^{3}$ Frankfurt University Hospital, Pediatric Hemostaseology, Frankfurt, Germany; ${ }^{4}$ Deutsche Klinik für Diagnostik, Wiesbaden, Germany; ${ }^{5}$ Pediatric Thrombosis and Hemostasis Unit, Pediatric Hemophilia Centre, Dr. von Hauner Children's Hospital, LMU Munich, Munich, Germany; ${ }^{6}$ Hemostasis Center, Blaubeuren, Germany; ${ }^{7}$ Health Department of the City of Dresden, Vaccination Centre, Dresden, Germany; ${ }^{8}$ Vascular Medicine and Haemostaseology, Vivantes Klinikum im Friedrichshain, Berlin, Germany; ${ }^{9}$ Gerinnungszentrum Rhein Ruhr, Duisburg, Germany, ${ }^{10}$ Bonn University Hospital, Bonn, Germany, ${ }^{11}$ Muenster Hemostasis Center, Muenster, Germany, ${ }^{12}$ Hannover Medical School, Hannover, Germany

Background: Currently available COVID-19 vaccines were developed for intramuscular injection and efficacy may not be ensured when given subcutaneously. In Germany most traditional vaccines were recommended to inject subcutaneously in patients with hemophilia to avoid intramuscular bleeds.

Aims: To develop practical guidance for COVID-19 vaccination in people with hemophilia.
Methods: The Delphi methodology was used to develop consensus recommendations. An initial, structured list of recommendations was prepared by a steering committee and evaluated by 34 hemophilia experts. Consensus was defined as $\geq 75 \%$ agreement (score 7 or higher on a scale of 1 to 9); strong consensus was defined $\geq 95 \%$ agreement. After 3 rounds of refinement, a final list of statements was compiled.

Results: Consensus was achieved that COVID-19 vaccines licensed only for intramuscular injection should also be administered through this route in people with hemophilia. Prophylactic factor replacement should be given before vaccination in patients with moderate or severe hemophilia, preferentially given on the day of vaccination, and with a maximum interval between prophylaxis and vaccination of $24 \mathrm{~h}$ (factor VIII and conventional factor IX concentrates) or $48 \mathrm{~h}$ (half-life extended factor IX). Strong consensus was also achieved that patients with mild hemophilia and residual activity $>10 \%$ and patients on prophylaxis with emicizumab do not need factor replacement before vaccination. Swelling, erythema and hyperthermia after vaccination are not always signs of bleeding but should prompt consultation of the hemophilia care center. In case of injection site hematoma, patients should receive replacement therapy until symptoms disappear. Adjunctive therapies of injection site hematoma include rest, cooling and antiphlogistic/analgetic therapy as needed.

Conclusions: Using the Delphi methodology, strong consensus was achieved on recommendations for intramuscular COVID-19 vaccination after replacement therapy for people with hemophilia depending on disease severity.

PB0606 | Emicizumab in the Treatment of Adult Haemophilia Patients Real-world Data

J. Windyga ${ }^{1}$; J. Zdziarska ${ }^{2}$; E. Stefanska-Windyga ${ }^{3} ;$ E. Chmielewska $^{4}$; K. Chojnowski ${ }^{5}$; M. Jamrozik ${ }^{6}$; J. Kozinska ${ }^{7}$ J. RupaMatysek $^{8}$; G. Semenczuk ${ }^{9}$; E. Zarzycka ${ }^{10}$; A. Buczma ${ }^{1}$ ${ }^{1}$ Department of Hemostasis Disorders and Internal Medicine, Institute of Hematology and Transfusion Medicine, Warsaw, Poland; ${ }^{2}$ Department of Hematology, Jagiellonian University Medical College, Kraków, Poland; ${ }^{3}$ Outpatient Clinic for Persons with Disorders of Hemostasis, Institute of Hematology and Transfusion Medicine, Warsaw, Poland; ${ }^{4}$ Department of Hematology, Pomeranian Medical University, Szczecin, Poland; ${ }^{5}$ Institute of Hemostasis, Department of Hematology, Medical University of Lodz, Łódź, Poland, Lodz, Poland; ${ }^{6}$ Department of Hematology and Bone Marrow Transplantation, Medical University of Silesia, Katowice, Poland; ${ }^{7}$ Department and Clinic of Hematolooncology and Bone Marrow Transplantation, Medical University of Lublin, Lublin, Poland; ${ }^{8}$ Department of Hematology and Bone Marrow Transplantation, Poznan University of Medical Sciences, Poznan, Poland; ' ${ }^{9}$ Department of Hematology, Nicolaus Copernicus Hospital, Torun, Poland, ${ }^{10}$ Department of Hematology and Transplantology, Medical University of Gdańsk, Gdansk, Poland

Background: Emicizumab has been used in the management of Polish patients with haemophilia A complicated by inhibitors (PWHI) 
since 2020 (not including clinical trials). So far a total of 42 pediatric and adult PWHI have been put on emicizumab prophylaxis.

Aims: The aim was to present the interim analysis of the efficacy and safety of emicizumab in 20 adult PWHI.

Methods: Every patient starting emicizumab prophylaxis had to report in real-time the data on emicizumab injections and breakthrough bleeding episodes via dedicated app (Emibaza $\left.{ }^{\circledR}\right)$. In case of trauma, bleeding episodes requiring additional hemostatic therapy or other adverse events patients were instructed to consult the local Haemophilia Treatment Centre (HTC).

Results: The interim analysis comprised 20 PWHI aged 23-70 (median 39) years. All were high responders with maximum historical inhibitor titer towards factor (F) VIII ranging from 7 to 7,000 BU/ ml. 19/20 (95\%) patients reported severe arthropathy. Annualized bleeding rates requiring haemostatic therapy (ABR-HT) in the year preceding emicizumab prophylaxis ranged from 6 to 80 (median 19). At the time of analysis the median duration of emicizumab prophylaxis in the group was 10 months (5-11). 10/20 (50\%) patients received emicizumab once- a week and 10/20 (50\%) every two weeks. During emicizumab administration 2/20 (10\%) patients experienced a total of 3 bleeds ( 2 bleeds were treated BPA and one required no haemostatic therapy). $3 / 20$ (15\%) patients on emicizumab required minor surgical interventions (dental extractions); one patient received 4 doses of recombinant activated factor VII (rFVIIa), another - one dose of rFVIla, and the third required no additional haemostatic therapy. No deaths, thromboembolic or thrombotic microangiopathy episodes were observed.

Conclusions: Switching from by-passing agents to emicizumab significantly reduced $A B R-H T$ in adult persons with haemophilia $A$ complicated by FVIII inhibitor with no safety concerns.

PB0607 | Chinese Real-world Experience of Product Switching in Severe Hemophilia Patients: from rFVIII-FS(Kogenate $\left.{ }^{\circledR}\right)$ to BAY 81-8973 (Kovaltry®)

C. Rong ${ }^{1}$; Z. Shiqiu ${ }^{1}$; G. Hua ${ }^{1}$; L. Jia ${ }^{1}$; G. Jing ${ }^{1}$; Y. Ming ${ }^{2}$; Z. Hong ${ }^{2}$; Y. $\operatorname{Lin}^{2} ;$ Z. $\mathrm{Xin}^{3}$

${ }^{1}$ Department of Hematology, the Third People's Hospital of Chengdu, Chengdu, China; ${ }^{2}$ Department of Ultrasonography, the Third People's Hospital of Chengdu, Chengdu, China; ${ }^{3}$ Department of Physical Medicine and Rehabilitation, the Third People's Hospital of Chengdu, Chengdu, China

Background: Both rFVIII-FS(Kogenate $\left.{ }^{\circledR}\right)$ and BAY 81-8973 (Kovaltry ${ }^{\circledR}$ ) are unmodified, full-length recombinant factor VIII (FVIII) indicated for prophylaxis and treatment of bleeds in patients with haemophilia A (HA). Both rFVIII's safety and efficacy were established in clinical studies, however the real-world data of product switch in China is scarce.

Aims: Explore the efficacy and safety after product switching from rFVIII-FS(Kogenate ${ }^{\circledR}$ ) to BAY 81-8973 (Kovaltry ${ }^{\circledR}$ ) in Chinese realworld clinical setting.
Methods: 24 patients under regularly follow-up with middledosed prophylaxis regimen were included. Data include annual bleeding rate(ABR), annual joint bleeding rate(AJBR), ultrasound score(HEAD-US-C) and HJHS for six index joints(bilateral elbow, knee and ankle) was collected. All patients switch their product from rFVIII-FS(Kogenate ${ }^{\circledR}$ ) to BAY 81-8973 (Kovaltry ${ }^{\circledR}$ ) since 2020 May. The data from 2019 January to 2020 December was used to conduct paired Wilcoxon test with SPSS 22.0.

Results: After product switching, the ABR significantly decreased from 8.9 to $5.9(P=0.003)$ and AJBR significantly declined from 6.9 to $4.3(P=0.012)$. (figure 1$)$.

While the mean score of HJHS decreased 51.5\% (from 25.7 to 16.9, $P=0.006$ ), the US score slightly lifted $10.3 \%$ after the switching. All patients are willing to accept the switch. No difference of the FVIII consumption was found. No inhibitor formation during the switch.

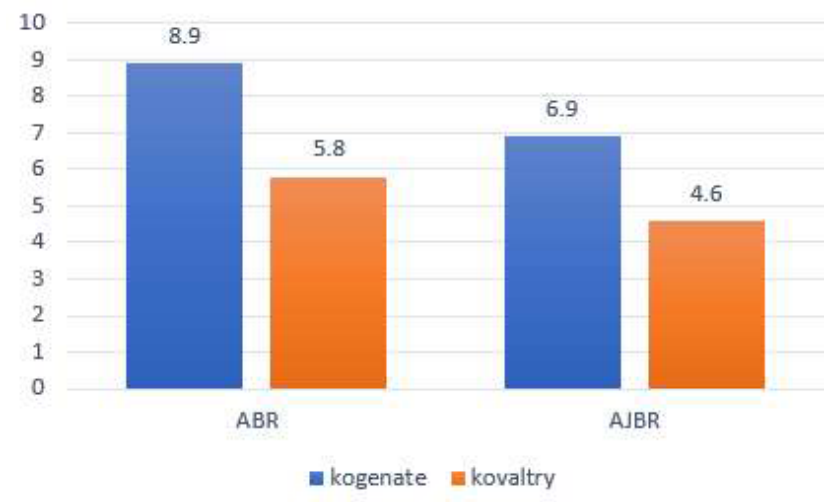

FIGURE 1 The ABR and AJBR score before and after product switching

Conclusions: All these 24 patients benefit from the product switching (from rFVIII-FS(Kogenate $\left.{ }^{\circledR}\right)$ to BAY 81-8973 (Kovaltry $\left.{ }^{\circledR}\right)$ ) with less bleeds and better joint function while the joint structure is not reversable under intermediate-dose prophylaxis. With no extra FVIII consumption needed, BAY 81-8973(Kovaltry ${ }^{\circledR}$ ) showed optimal efficiency which may contributed to its superior pharmacokinetic profiles. Further studies focus on PK comparison of these two products in Chinese real-world setting are needed.

PB0608 | MOdern Treatment of Inhibitor-PositiVe PATiEnts with Haemophilia A - MOTIVATE (www.motivate-study.com)

C. Escuriola Ettingshausen ${ }^{1}$; R.F. Sidonio, Jr. ${ }^{2}$

${ }^{1}$ HZRM Hämophilie Zentrum Rhein Main, Mörfelden-Walldorf, Germany; ${ }^{2}$ Aflac Cancer and Blood Disorders Center, Emory University, Atlanta, United States

Background: Immune tolerance induction (ITI) is the only proven method to eradicate inhibitors against FVIII in haemophilia A patients. While emicizumab and bypassing agents may be used to 
control bleeding in patients with inhibitors, they do not eradicate the inhibitor.

Aims: To capture different approaches to the management of patients with haemophilia A and inhibitors, document current ITI approaches from real-world clinical experience, and evaluate the efficacy and safety of ITI, emicizumab prophylaxis, and ITI with emicizumab prophylaxis.

Methods: The investigator-initiated, international MOTIVATE study (NCT04023019; EudraCT 2019-003427-38) is investigating in real-life clinical practice the management of patients with haemophilia A and inhibitors. Participants of any age with haemophilia A of any severity and inhibitors will be observed for up to five years. The treatment approaches evaluated (Figure 1) are standard ITI with FVIII (Group 1), FVIII ITI with emicizumab prophylaxis (Group 2) and prophylaxis with emicizumab or bypassing agents (Group 3). Participants are not randomised and all treatments are at the discretion of the physician. The primary endpoints are success of ITI and annual bleeding rates. Optional sub-studies will explore factors that may influence ITI outcome and the impact of different treatment approaches on aspects of patient health, including joint and bone health and the risk of thrombotic events.

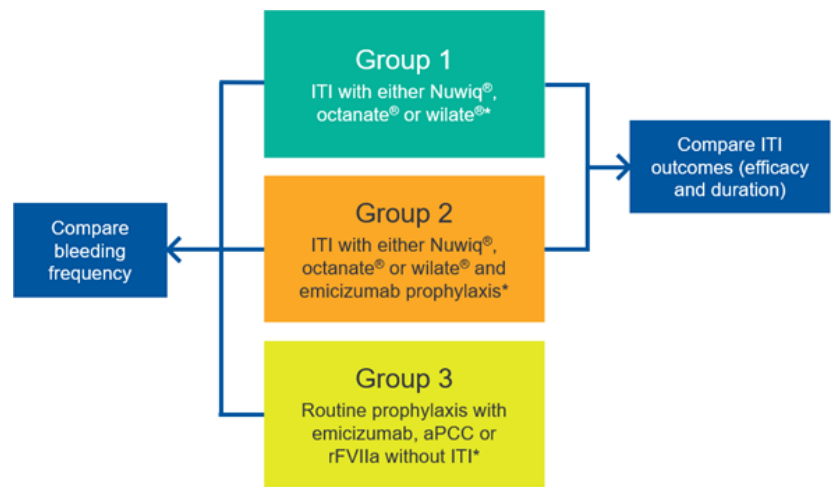

FIGURE 1 MOTIVATE study design

Results: Target enrolment is 120 patients. As of 1 March 2021, 15 patients have been enrolled at seven study sites in Croatia, France, Germany and Switzerland. A total of 35 sites have been selected so far in 13 counties, namely the US, Canada, UK, Finland, France, Germany, Switzerland, Croatia, Norway, Spain, Sweden, Colombia and Taiwan.

Conclusions: The MOTIVATE study is collecting real-world data on current approaches for management of patients with inhibitors. These data will provide guidance to healthcare professionals for the optimal management of patients with haemophilia A and inhibitors.
PB0609 | Rationale and Design of the "International Registry on the Symptomatic Hemophilia A/B Carriers"

S. Pasca $^{1}$; R. Sidonio Jr. ${ }^{2}$; E. Grandone ${ }^{3}$; E. Zanon ${ }^{1} ;$ M. Othman ${ }^{4}$

${ }^{1}$ Padua University Hospital, Padua, Italy; ${ }^{2}$ Emory University, Atlanta, United States; ${ }^{3}$ IRCSS Casa Sollievo della Sofferenza, San Giovanni Rotondo (FG), Italy; ${ }^{4}$ Queen's University Kingston, Ontario, Canada

Background: Although the first reports concerning women with hemophilia date back to the 1950s, not much is known about the real worldwide burden of this hemorrhagic disease in females, the incidence of symptomatic women, their treatment and their quality of life.

Aims: The first aim of our Registry is to establish how many women with hemophilia A and B are actually followed in the different Hemophilia Centers around the world and how many of these are symptomatic. Our secondary aim is to characterize the bleeding to better understand how hemophiliac pathology occurs in the female sex and what are the clinical and psychological outcomes.

Methods: All hemophilia A and B carriers of any age (obligate or geneticall confirmed) can be included in this Registry through the e-CRF reported in the specific RedCap platform. All Hemophilia Centers who treat or have treated women and girls with hemophilia (with and without FVIII/FIX deficiency) are encouraged to participate at our Registry. The e-CRF will be divided into different sections: 1 . Demographic; 2. Laboratory; 3. Genetics; 4. Medical history (not related to hemophilia); 5. Clinical history (related to hemophilia; ISTH BAT); 6. Pharmacologic history (related to hemophilia); 7. Quality of life (EQ-5D-5L). Each of the sections will also be divided into several sub-sections. All data will be collected both in retrospective terms (without time limitation) and in prospective terms (from the study start date to the closing date of the data collection).

Results: The Registry has just been activated (February 2021), therefore no data is available yet.

Conclusions: From our registry we expect to answer the difficult questions regarding the complex impact of hemophilia on women, but which can lead to physical and mental disabilities, with a consequent increase in costs for the entire society.

PB0610 | Intracranial Hemorrhage in Hemophiliac Patients at Chiang Mai University Hospital: A 10-year Retrospective Study

J. Khamyuang; P. Charoenkwan; L. Satitsamitpong; S.

Sittipreechacharn; C. Chai-Adisaksopha; R. Natesirinilkul

Faculty of Medicine, Chiang Mai University, Chiangmai, Thailand

Background: Intracranial hemorrhage $(\mathrm{ICH})$ is one of the lifethreatening bleeding events in hemophiliac patients resulting in high mortality rate and neurological sequelae. Although the guideline of treatment of hemophiliac patients with $\mathrm{ICH}$ is established, the management of hemophiliac patients with $\mathrm{ICH}$ in the developing countries is challenging. 
Aims: To determine prevalence and outcome following treatment of $\mathrm{ICH}$ in hemophilia patients at Chiang Mai University (CMU) Hospital.

Methods: This study retrospectively reviewed ICH among hemophilic patients at CMU Hospital over a period of 10 years (2009-2019).

Results: Nine patients (six with hemophilia A and three with hemophilia B) with 14 events of ICH from a total of 105 hemophiliac patients were enrolled. The prevalence of nine percent of hemophiliac patients. Eight of 14 events were reportedly traumatic. Five ICH events in three patients [36 and $33 \%$ of events and patients, respectively] were took care under Department of Pediatrics. One patient had significant inhibitor prior to the ICH event while one patient was treated with secondary low-dose prophylaxis. Two events of $\mathrm{ICH}$ were treated with factor concentrates alone, while other 12 events were treated with blood components, including cryoprecipitate or fresh frozen plasma or combined with factor concentrates including recombinant activated factor VII in the patient with inhibitor. Only one adult patient with $\mathrm{ICH}$ passed away despite receiving replacement therapy and surgical intervention resulting in the mortality rate at $11 \%$ in this cohort. Median total factor replacement (combination of various products) in hemophilia A and hemophilia B were 721 and 945 unit/kg/event, respectively. Only two pediatric patients had been treated with secondary prophylaxis treatment after acute events.

Conclusions: Despite proper replacement therapy with the limited resources and surgical intervention, $\mathrm{ICH}$ was still the emergency bleeding events with high mortality rate among hemophiliac patients in this cohort especially in those who had treated with on demand treatment.

PB0611 | Clinicopathological Parameters and Influence of Blood Group and von Willebrand Factor (vWF) Levels on Residual Factor VIII (FVIII) Levels in Haemophilia A Patients

D. Ray; N. Kumar; J. Ahluwalia; R. Das; C. Hans; A. Jain; P. Malhotra;

D. Bansal

Postgraduate Institute of Medical education and Research, Chandigarh, India

Background: The clinical phenotype of Haemophilia A (HA) does not always correlate with the severity of HA. Similarly, the presence of inhibitors does not necessarily portend a higher bleeding-risk. This paradox between clinical and laboratory findings may be partially attributed to nongenetic modifiers like blood-group and vWF which are known to influence FVIII levels in healthy individuals.

Aims: To assess the bleeding-risk in HA patients with and without inhibitors; and the effect of blood-group and vWF on residual FVIII levels across the severity spectrum of HA.

Methods: Records of 600 consecutive HA patients attending a north-Indian tertiary centre from 2010-2020 were retrospectively reviewed. Quantitative data was compared using t-test. Categorical data was compared using chi-square test or Cochran-Armitage test for trend (as applicable). Correlations was assessed using Spearman's correlation.
Results: Mild, moderate, and severe HA were present in 96 (16\%), $246(41 \%)$ and 258 (43\%) patients, respectively. Overall, the commonest site of bleeding was intra-articular (89\%). No difference in the site of bleed was noted between the three severity subgroups. Risk of spontaneous bleeding was significantly lower in mild HA (33.3\%) compared to moderate (45.12\%) and severe HA (47.6\%, $P=0.03)$.

Inhibitors were present in 35 (5.8\%) patients. Blood-group A patients had higher odds of developing inhibitors [OR 3.02, 95\% Cl 1.1-8.8, $P=0.04]$. Patients with inhibitors had a significantly higher risk of intracranial haemorrhage compared to those without inhibitors $(20 \%$ vs $4.1 \%, P=0.03$ ).

No correlation was observed between vWF and residual FVIII level across the severity spectrum of HA. In mild HA, a statistically nonsignificant trend to higher residual levels of FVIII were seen in bloodgroups $\mathrm{A}$ and $\mathrm{B}$. No such correlation was observed in moderate or severe HA.

Conclusions: Unlike healthy individuals, vWF and blood-group does not influence residual FVIII levels across the severity spectrum of HA.

PB0612 | Prevalence of Hematuria in Patients with Hemophilia $A$ and $B$ and its Association with Comorbidities: Experience from a Brazilian Center

D. Bittencourt Siqueira; M. D'Auria Jacomassi; L. Bassolli;

C. Rothschild; E. Okazaki; V. Rocha; P. Villaca

Service of Haematology, Transfusion and Cell Therapy. Hospital das

Clínicas, Faculty of Medicine, Sao Paulo University (FM-USP), São

Paulo, Brazil

Background: Hemophilia A and B are X-linked inherited bleeding disorders. Hematuria is a common manifestation among patients, resulting in a worsening of quality of life. Results of previous studies regarding the association between hematuria and hemophilia have been inconsistent, leading to the need for further analysis.

Aims: Determine the prevalence of macroscopic or microscopic hematuria in patients with hemophilia, as well as its association with comorbidities.

Methods: Transversal unicenter study with adult patients ( $\geq 18$ years old) bearing hemophilia A or B under active outpatient clinic in a reference center over the year of 2019. Hematuria was defined as any macroscopic episode or $\geq 3$ red blood cells per high power field in urine analysis. Using simple and multivariable logistic analysis, age, diagnosis type, disease severity, comorbidities and creatinine clearance have been studied as potential characteristics associated with the prevalence of hematuria.

Results: In total, 179 male patients were included. Mean age was 26 years ( $s d=15$ ), the majority of them were diagnosed with hemophilia $A(76 \%)$ and $66 \%$ presented a severe disease. Despite our young population, the prevalence of comorbidities was high, mainly hypertension (10\%), dyslipidemia (10\%), diabetes (5\%) and obesity (10\%). The prevalence of hematuria was $39 \%(95 \% \mathrm{Cl} 32 \%-47 \%)$, of these a half presented at least one macroscopic episode. Age (OR = 1.03[1.01-1.05]), 
hemophilia $B(O R=0.47[0.21-0.99])$, hypertension $(O R=3.88[1.45$ 11.55]), obesity (OR $=2.92[1.11-8.24])$ and creatinine clearance $(\mathrm{OR}=0.99[0.98-1.00])$ were associated with hematuria in univariable analysis. No independent factors were found in multivariable analysis. Conclusions:

Hematuria is common among patients with hemophilia and further studies are needed to elucidate the relationship between hematuria and increased morbidity.

PB0613 | Thrombin Generation in Hemophilia A Patients after Infusion of Recombinant and PlasmadDerived Factor VIII Concentrates

O. Smirnova; O. Matvienko; V. Burakov; V. Soldatenkov; K.

Komissarov; L. Papayan

Russian Research Institute of Hematology and Transfusiology, Saint

Petersburg, Russian Federation

Background: The increasing number of factor VIII (FVIII) products allows patients and healthcare professionals to adjust treatment to individual needs. The major advantage of recombinant FVIII (rVIII) products compared with plasma-derived FVIII (pdFVIII) products is related to product safety, and potentially they have better hemostatic effectiveness.

Aims: The aim of our study was to compare the FVIII activity and thrombin generation (TG) parameters in patients with hemophilia $A$ after rVIII and pdFVIII administration as well as with normal range.

Methods: 38 patients with hemophilia A had the minor surgeries under two FVIII products - human, plasma-derived cogulation FVIII concentrate $(n=28)$ and human cell line recombinant FVIII $(n=10)$. The laboratory support included the monitoring of FVIII activity (FVIII, $\%$ ) and TG test before and in an hour after FVIII infusion. The patients had not received any treatment for 72 hours prior to the study. TG was measured in platelet poor plasma at Fluoroscan Ascent fluorimeter using TF 1pM. The following parameters were analyzed: endogenous thrombin potential (ETP, $\mathrm{nM}^{*} \mathrm{~min}$ ) and the maximum concentration of thrombin (Peak, nM). The control group comprised 16 healthy volunteers. Statistical analysis was performed by non-parametric methods (median (Me), 95\% confidence interval $(95 \% \mathrm{Cl}$ ) and Mann-Whitney $\mathrm{U}$ test, Statistica 12.0). $P<0.05$ was considered statistically significant. Results: The results are summarized in the table.

\section{TABLE 1}

\begin{tabular}{lllll} 
& & & & \\
Parameter (Me, 95\% CI) & Patients after pdFVIII & Patients after rFVIII & Controls \\
FVIII activity, \% & Basal & $\mathrm{N}=28$ & $\mathrm{~N}=10$ & $\mathrm{~N}=16$ \\
& In an hour & $1,8(0,1-36,2)$ & $2,0(0,4-37,2)$ & $100,5(66,-182,8)$ \\
ETP, nM*min & Basal & $79,5(25-117,0)$ & $128,5^{*}(79,3-176,0)$ & $1021,3(704,9-1400,9)$ \\
Peak, nM & In an hour & $350,5(49,4-1185,0)$ & $234,9(81,1-640,0)$ & $994,5(364,1-1111,0)$ \\
& Basal & $908,7(504,6-1736,9)$ & $10,8(3,4-43,6)$ & $69,4(47,2-143,9)$ \\
\hline
\end{tabular}

* $-P=0,0001$ with pdFVIII.

Despite FVIII activity increased significantly in patients after rFVIII compare to pdFVIII administration, the TG parameters were the same in both groups. Also, we found that thrombin generaton in patients after each FVIII product restored to normal level.

Conclusions: rVIII products can sighnificantly increase FVIII levels when infused at the same dosing schedule as pdFVIII products, but TG assay after $\mathrm{rVIII}$ and pdFVIII infusion showed comparable results. So rVIII and pdFVIII products equally can be effectively used to prevent and treat bleeding episodes in hemophilia patients.
PB0614 | Pharmacokinetics-adjusted Dosing of Rurioctocog Alfa Pegol (Adynovi) in Adult Patients with Haemophilia A

J. Treliński ${ }^{1}$; K. Chojnowski ${ }^{1}$; M. Robak ${ }^{1}$; A. Mital ${ }^{2}$; E. Zarzycka ${ }^{2}$; M. Bober $^{3}$; J. Dziamska ${ }^{3}$; T. Woźny ${ }^{3}$; D. Urbaniak-Kujda ${ }^{4}$; I. Prajs ${ }^{4}$; M. Podolak-Dawidziak ${ }^{4}$

${ }^{1}$ Department of Haemostasis and Haemostatic Disorders, Medical University of Łódź, Łódź, Poland; ${ }^{2}$ Department of Haematology and Transplantology, Medical University of Gdańsk, Gdański, Poland; ${ }^{3}$ Department of Haematology and Internal Medicine MSWA Hospital of Poznań, Poznań, Poland; ${ }^{4}$ Department of Haematology, Blood Neoplasms and Bone Marrow Transplantation, Medical University of Wroctaw, Wroctaw, Poland

Background: Rurioctocog alfa pegol (Adynovi) is a recombinant factor VIII (FVIII) concentrate conjugated to polyethylene glycol to 
reduce clearance and extend time in circulation. Due to large interindividual variability in pharmacokinetics (PK) of FVIII products treatment optimization may require $\mathrm{PK}$-adjusted dose and schedule personalization. myPKFiT app was designed to allow PK evaluation in hemophilia $A(H A)$ patients treated with Adynovi.

Aims: Evaluation of PK-adjusted dosing of Adynovi in adult HA patients.

Methods: Ten adult HA patients (median age 38 years) from four centers in Poland who participated in BAX855 trial and were treated with Adynovi as a part of Named Patient Access were included. All participants received prophylaxis with Adynovi in PK-adjusted doses and were followed up for 30 months. In each patient, PK parameters (T1/2, time to $1 \%$, through level) were established using myPKFiT software. All bleeding episodes during the follow-up period were recorded. Informed consent has been obtained from all participants. Results: PK-adjusted dosing allowed to decrease Adynovi dose in al patients (mean $4870 \mathrm{IU}$ vs $3386 \mathrm{IU}$ ). In 6 out of 10 patients, injection frequency was significantly lowered, and the time between injections was extended from 48 hrs to 72 hrs $(n=3), 84$ hrs $(n=2)$ or 96 hrs $(n=1)$. During 30 months of follow-up, median annual bleeding rate dropped from 30 (on demand) to 0 (prophylaxis), and 6 out of 10 patients did not experience any bleeds during the study period. The median through level of factor VIII was $2.5 \%$ (range $2.0 \%$ to $6.5 \%$ ).

Conclusions: Our results confirmed the efficacy of prophylactic treatment with Adynovi in heavily burdened adult HA population. The PK-adjusted dosing allows injection frequency reduction without compromising treatment efficacy and myPKFiT app is an accurately and conveniently designed tool allowing dosage personalization in patients treated with Adynovi. Low bleeding rate resulted from increased through level of factor VIII.

PB0615 | Prostate Surgery in Patients with Hemophilia: A Monocentric Real-life Experience

$\underline{\text { N. Drillaud }}^{1}$; B. Mesnard ${ }^{2}$; M. Sigaud ${ }^{1}$; M. Fouassier ${ }^{1}$; C. Ternisien ${ }^{1}$; M.-C. Bene ${ }^{3}$; J. Branchereau ${ }^{2}$; M. Trossaërt ${ }^{1}$

${ }^{1}$ Department of Haemostasis, Nantes University Hospital, Nantes, France; ${ }^{2}$ Department of Urology, Nantes University Hospital, Nantes, France; ${ }^{3}$ Department of Haematology, Nantes University Hospital, Nantes, France

Background: Prostate surgery is a common procedure with a high risk of bleeding, especially in patients with hemophilia $(\mathrm{PwH})$. It requires upstream preparation and collaboration between the surgeon, anesthetist and hematologist to prevent excessive bleeding. Despite this, hemorrhagic complications are common and sometimes difficult to manage.

Aims: To describe the real-life experience of a French center and evaluate bleeding complications of prostate surgery in $\mathrm{PwH}$.

Methods: Data collected between 1997 and 2020 were extracted from an institutional care database (NHEMO/BERHLINGO). All PwH requiring prostate biopsies, radical prostatectomy, adenomectomy,
TransUrethral Resection of the Prostate (TURP) or laser-vaporization were included.

Patients were treated with either desmopressin or recombinant factor VIII or IX depending on the type of haemophilia and response to desmopressin.

Results: Fifty-two procedures were performed on 32 patients. Twenty-eight patients had mild, 1 moderate and 1 severe haemophilia A and 2 had mild haemophilia B. No patients had inhibitor.

Prostate biopsies: only $1 / 26$ patient needed a blood transfusion for rectorrhagia one day after the procedure while residual factor VIII (FVIII:C) was normal (83 IU/dL).

Prostatectomy: 2/11 patients required re-hospitalisation and additional FVIII infusion due to abnormal haematuria at days 11 and 15. TURP: 2/5 patients required additional FVIII infusion due to bleeding when the scabs fell off at days 11 and 12 and one required clot removal one day after surgery.

Laser-vaporization: 3/7 patients required additional FVIII infusion due to bleeding when the scabs fell off from day 9 to 13 , among whom 2 required surgical revision.

Finally, no bleeding complication was observed in the 3 cases of adenomectomy. FVIII:C values are detailed in the figure.
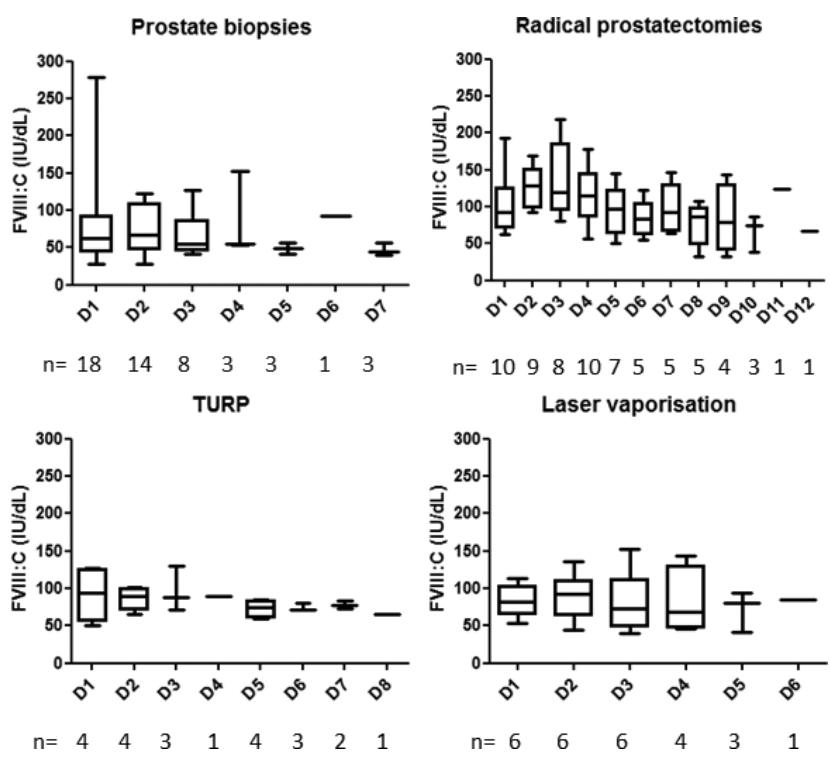

FIGURE 1 FVIII:C levels over time in different types of prostate surgery. $\mathrm{D}=$ day after the first FVIII infusion. Each boxplot represents the interquartile range with median value (horizontal line); vertical bars indicate the minimum and maximum levels.

Conclusions: One night hospitalisation followed by treatment at home seems reasonable for prostate biopsies. TURP and laservaporization are at higher risk of bleeding and require normal haemostasis for 15 days. Alternatives such as radio-embolization look promising. 
PB0616 | Association of Individual Pharmacokinetics-guided Regimen in Hemophilia A Patients and FVIII Consumption: A Single-center Study

Q. Hong ${ }^{1}$; C. Shu Qin ${ }^{1}$; Z. Wen Jing ${ }^{2}$; H. Xiao Hua ${ }^{3}$; C. Yong Fang ${ }^{1}$; Y. Yu Hua ${ }^{1} ; \mathrm{X}$. Xing $\mathrm{Yi}^{4}$

${ }^{1}$ Department of Hematology, Panyu Central Hospital, Guangzhou, China; ${ }^{2}$ Department of Ultrasound, Panyu Central Hospital, Guangzhou, China; ${ }^{3}$ Department of Rehabilitation, Panyu Central Hospital, Guangzhou, China; ${ }^{4}$ Department of Clinical Laboratory, Panyu Central Hospital, Guangzhou, China

Background: Individualized treatment can be tailored by requirement of hemophilia patients. Pharmacokinetics (PK)-guided individualized treatment is important to avoid disability .

Aims: The aim of this study was to analyze whether PK-guided individual regimen impacts the cost of treatment.

Methods: 17 patients from our center's registry were included in this study, which divided into individualized group $(n=8)$ and nonindividualized group $(n=9)$ according to different treatment regimens after PK analysis. All patients received a single dose infusion of FVIII after a 72-hour washout period, post-infusion blood sample were collected at $9 \mathrm{~h}$ and $15 \mathrm{~h}$, and the concentration of FVIII was detected. WAPPS system was used to calculate individual pharmacokinetic parameters. Amount of recombinant factor VIII usage, health-related quality of life (HRQoL) scores, number of joint bleeds, HJHS scores of joint rehabilitation and 6 major joint ultrasound scale scores (0-14 points) were collected in both groups for 6 months before and after PK test. SPSS 20.0 statistical software was used to analyze the differences in efficacy between and within groups using 2 Independent Samples Tests, and a significant difference was expressed at $P<0.05$.

Results: 17 patients had a median age of $21(8-57)$ years and a median baseline FVIII:C activity of $0.6(0.1-1.1) \%$. The number of bleeding $(P=0.020)$ and joint ultrasound scores $(P=0.014)$ were significantly different in two groups, especially in the right elbow $(P=0.006)$. Although non-significantly difference was shown in HRQOL scores, the individualized group was significantly improved in social functioning $(P=0.031)$. Also there was no significant increase in FVIII consumption before and after individualized treatment.

Conclusions: Individualized treatment is expected to improve the quality of life by significantly reducing joint bleeds and improving social skills without significantly increasing the cost of additional FVIII consumption.
PB0617 | Comprehensive Genotype Characterization in Nonsevere Hemophilia A. Mutational Profile and Assessment of the Bleeding Phenotype in Argentine Patients

L. Rossetti ${ }^{1}$; A.I. Woods ${ }^{2}$; A. Blanco ${ }^{3}$; J. Paiva ${ }^{3}$; V. Marchione ${ }^{1}$; C.P. Radic $^{1}$; M.M. Abelleyro ${ }^{1}$; B. Ziegler ${ }^{1}$; K. Waisman ${ }^{1}$; M.L. Romero ${ }^{3}$; M.M. Casinelli ${ }^{3}$; A. Sánchez-Luceros ${ }^{2,3} ;$ C. De Brasi ${ }^{1,4}$ ${ }^{1}$ Instituto de Medicina Experimental (IMEX), CONICET-Academia Nacional de Medicina (ANM), Ciudad Autónoma de Buenos Aires, Argentina; ${ }^{2}$ IMEX, CONICET-Academia Nacional de Medicina, Laboratorio de Hemostasia y Trombosis, Ciudad Autónoma de Buenos Aires, Argentina; ${ }^{3}$ Instituto de Investigaciones Hematológicas, Academia Nacional de Medicina, Hemostasia y Trombosis, Ciudad Autónoma de Buenos Aires, Argentina; ${ }^{4}$ Instituto de Investigaciones Hematológicas Mariano R Castex (IIHEMA), ANM, Ciudad Autónoma de Buenos Aires, Argentina

Background: Mild-moderate (non-severe, NS) Hemophilia A (HA) associates with minor reduction in factor VIII (FVIII:C) (1-40IU/dL) and involve about $60 \%$ of HA cases worldwide. Despite the importance of NS-HA, patients are rarely genotyped, particularly in developing countries. Clinical manifestations and laboratory phenotype are frequently mistaken with VWD2N.

Aims: Characterize the F8-genotype and phenotype, exclude VWD2N, in a series of Argentine patients with FVIII:C 1-40IU/dL.

Methods: Thirty-eight unrelated-families including 50 individuals (probands and relatives) were studied. The bleeding phenotype was obtained from clinical records.

Laboratory: FVIII:C (one-stage method), VWF:Ag (ELISA), VWF:RCo (aggregometry). Mixing studies of the patient/control plasmas to evaluate the presence of inhibitors were undertaken by APTT and VWF:RCo.

Genotyping: peripheral blood leukocyte-extracted genomic-DNA was mutational screened by PCR-amplification of all coding and regulatory regions of the F8 followed by conformation sensitive gel electrophoresis (CSGE) and selected-amplimers were characterized by Sanger sequencing. Exons 17-27 of the VWF were PCR-amplified and directly Sanger-sequenced.

Results: 
TABLE 1 F8-genotype and the associated phenotype in the Argentinian population with NS-HA

\begin{tabular}{|c|c|c|c|c|c|c|}
\hline $\begin{array}{l}\text { Patients } \\
\mathrm{N}+(\%)\end{array}$ & F8 Domain & $\begin{array}{l}\text { F8 } \\
\text { Disease-causing variants } \dagger \dagger\end{array}$ & $\begin{array}{l}\text { FVIII:C } \\
\mathrm{X} \pm \text { SD }\end{array}$ & VWF:Ag & VWF:RCo & $\begin{array}{l}\text { Major bleeding (\% of } \\
\text { patients) }\end{array}$ \\
\hline $3(8)$ & A1 & $\begin{array}{l}\text { c. } 601+5 \mathrm{G}>\mathrm{A} \\
\text { c. } 602 \mathrm{G}>\mathrm{A} P .(\mathrm{Gly} 201 \mathrm{Glu}) \\
\text { c.671-28_671-17del }\end{array}$ & $16 \pm 10.8$ & $85 \pm 10.8$ & $77 \pm 10.8$ & 66.6 \\
\hline $17(45)$ & A2 & 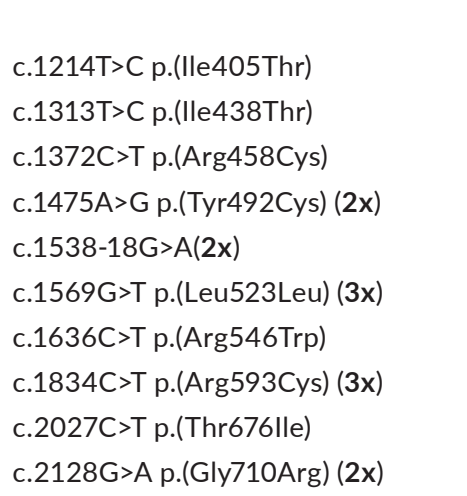 & $15.4 \pm 8.9$ & $77 \pm 13.5$ & $75 \pm 16$ & 40 \\
\hline $1(2.5)$ & a3 & c.5096A>T; p.(Tyr1699Phe) & 20 & 76 & 90 & 0 \\
\hline & B & c.3637delA p.(Ile1213Phefs*5) (A run) & 3 & 70 & 65 & 100 \\
\hline $7(18)$ & A3 & $\begin{array}{l}\text { c.5428T>C p.(Ser1810Pro) }(2 x) \\
\text { c.5879G }>\text { T p.(Arg1960Leu) } \\
\text { c.5912A > p p.(Asn1971Ser) } \\
\text { c.5954G >A p.(Arg1985GIn) (2x) } \\
\text { c.5981T>C p.(Leu1994Pro) }\end{array}$ & $22.6 \pm 9.7$ & $108 \pm 22$ & $90 \pm 10.9$ & 42.8 \\
\hline $3(8)$ & $\mathrm{C} 1$ & $\begin{array}{l}\text { c. } 6301 C>\text { G p.(His2101Asp) } \\
\text { c. } 6505 C>\text { T p.(Arg2169Cys) } \\
\text { c. } 6443 \text { A > G p.(Asn2148Ser) }\end{array}$ & $30 \pm 17.6$ & $103 \pm 23.4$ & $78 \pm 5.5$ & 100 \\
\hline $6(16)$ & $\mathrm{C} 2$ & 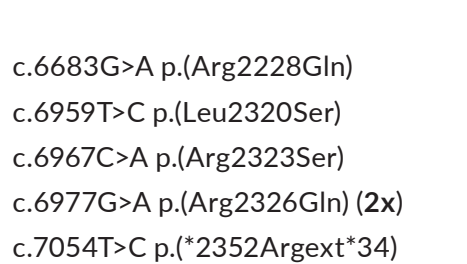 & $9.3 \pm 5.2$ & $75 \pm 19.6$ & $76 \pm 25.8$ & 40 \\
\hline
\end{tabular}

†N: number of families per specific F8 genotype; ††Human Genome Variant Society (HGSV) recommended nomenclature; RefSeq F8: NG_011403.1; F8 mRNA: NM_000132.3, FVIII Protein: NP_000123.1.

A HA-causative F8-genotype was identified in all 38 families including 28 pathogenic variants (PV) (Table 1). Eighteen (47\%) of them showed 8 recurrent PV (range 2-3 families/PV), whereas the remnant 20 (53\%) showed non-recurrent (private) F8-PV (Figure 1). No variants were found in VWF. Patients with major hemorrhages: 48.6\%. Laboratory phenotype is shown in Table 1. 


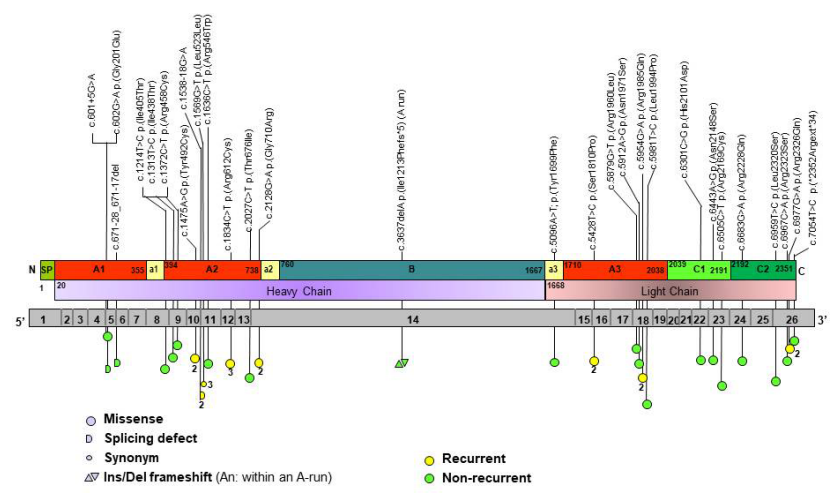

FIGURE 1 Molecular distribution of F8 variants characterized in our series of NS-HA patients.

Conclusions: Our practical approach is adequate to characterize the PV in NS-HA patients and relatives. The confirmed diagnosis of NS-HA allows an appropriate treatment and genetic counseling for $X$-linked inheritance highlighting the role of genetic testing and phenotypic assays. Combined HA+VWD2N was excluded in all patients. The predominance of missense PV (30/38 cases, 79\%) mostly targeting the FVIII-A2-domain and the high frequency of recurrent PVs found in NS-HA may reflect a reduced mutational loss due to the relatively conserved reproductive fitness of probands hence preserving the gene pool in the population.

PB0618 | Genetic and Non-genetic Determinants for the Outcome of Immune Tolerance Induction in Patients with Hemophilia A and Inhibitors - A Systematic Review

I. Oomen ${ }^{1,2}$; R. Mesquita Camelo ${ }^{3}$; S. Meireles Rezende ${ }^{3}$; J. Voorberg $^{2}$; M.E. Mancuso ${ }^{4}$; J. Oldenburg; ${ }^{5}$ M. Carcao ${ }^{6}$; D. Matino $^{7}$; D. Lillicrap ${ }^{8}$; K. Fischer ${ }^{9}$; K. Fijnvandraat ${ }^{1,2}$; S.C. Gouw ${ }^{1,10}$, International GO-ITI study group

${ }^{1}$ Amsterdam UMC, University of Amsterdam, Emma Children's Hospital, Pediatric Hematology, Amsterdam, Netherlands; ${ }^{2}$ Department of Molecular and Cellular Hemostasis, Sanquin Research and Landsteiner Laboratory, Amsterdam, Netherlands; ${ }^{3}$ Department of Internal Medicine, Faculty of Medicine, Federal University of Minas Gerais, Belo Horizonte, Belo Horizonte, Brazil; ${ }^{4}$ Center for Thrombosis and Hemorrhagic Diseases, IRCCS Humanitas Research Hospital, Rozzano, Milan, Italy; ${ }^{5}$ Department of Internal Medicine, University Clinic Bonn, University of Bonn, Bonn, Germany; ${ }^{6}$ Division of Hematology/Oncology, Department of Pediatrics, Hospital for Sick Children, University of Toronto, Toronto, Canada; ${ }^{7}$ Department of Medicine, McMaster University, University of Hamilton, Hamilton, Canada; ${ }^{8}$ Department of Pathology and Molecular Medicine, Queen's University, Kingston, Canada; ${ }^{9}$ Department of Hematology, Van Creveldkliniek, University Medical Center Utrecht, Utrecht, Netherlands, ${ }^{10}$ Department of Clinical Epidemiology, Leiden University Medical Center, Leiden, Netherlands

Background: Immune tolerance induction (ITI) is the only therapy to eradicate anti-factor VIII antibodies (inhibitors) in patients with hemophilia A. However, this burdensome and costly treatment fails in $10-40 \%$. In order to determine whether to attempt ITI, it is important to identify determinants for ITI success.

Aims: This systematic review aims to assess:

(i) the genetic determinants for ITI success and

(ii) the non-genetic determinants for ITI success in patients with hemophilia A.

Methods: A comprehensive literature search was conducted on February 17 2021, in the MEDLINE and Embase databases using the following search terms; hemophilia and immune tolerance. Cross-sectional, longitudinal and cohort studies and registries reporting on determinants for ITI outcome in patients with congenital hemophilia A who underwent ITI therapy were eligible for inclusion. Data were extracted on study, patient and treatment characteristics, ITI outcome definitions and genetic and non-genetic determinants associated with ITI success or failure. Two independent reviewers performed the study selection, data extraction and methodological quality assignment using an adapted checklist of the Joanna Briggs Institute for cross-sectional and cohort studies. Studies were classified as high-quality when $\geq 5 / 9$ criteria were met. Incidence of ITI success were reported for each study.

In case of limited heterogeneity, pooled effect estimates of individual determinants will be assessed.

Results: The literature search yielded 1021 unique papers. After title and abstract screening, 54 papers were selected. Full-text screening is currently ongoing. Final results of this systematic review are expected in July.

Conclusions: This systematic review will summarize the evidence on genetic and non-genetic determinants for ITI outcome in patients with congenital hemophilia A. We expect that these results will provide more insight into factors influencing the outcome of ITI.

PB0619 | Patient and Healthcare Professional Perspectives on the Use of a Personal Health Record in Hemophilia Care: A Qualitative Study

$\underline{\text { M.R Brands }}^{1}$; J.J Muis ${ }^{2}$; M. Driessens ${ }^{3} ;$ F.JM van der Meer ${ }^{4}$; S. Meijer $^{3}$; M. de Jong ${ }^{3}$; F. Bellinck ${ }^{4}$; G. Goedhart ${ }^{4,5}$; J.G van der Bom ${ }^{6}$; M.H Cnossen ${ }^{7}$; L. Haverman ${ }^{2}$; K. Fijnvandraat ${ }^{1,8}$; S.C Gouw ${ }^{1,6}$

${ }^{1}$ Amsterdam UMC, University of Amsterdam, Emma Children's Hospital, Pediatric Hematology, Amsterdam, Netherlands; ${ }^{2}$ Amsterdam UMC, University of Amsterdam, Emma Children's Hospital, Psychosocial Department, Amsterdam, Netherlands; ${ }^{3}$ Dutch Association of Hemophilia-Patients (NVHP), Nijkerk, Netherlands; ${ }^{4}$ HemoNED Foundation, Leiden, Netherlands; ${ }^{5}$ Department of Thrombosis and Hemostasis, Leiden University Medical Center, Leiden, Netherlands; ${ }^{6}$ Department of Clinical Epidemiology, Leiden University Medical Center, Leiden, Netherlands; ${ }^{7}$ Erasmus University Medical Center, Erasmus University Rotterdam, Sophia Children's Hospital, Pediatric Hematology, Rotterdam, Netherlands; ${ }^{8}$ Department of Molecular Cellular Hemostasis, Sanquin Research and Landsteiner Laboratory, Amsterdam, Netherlands

Background: Currently, data on patient-relevant health outcomes is collected to enable personalized treatment and shared decision-making for people with hemophilia. However, information is fragmented across 
hospital information systems, digital treatment diaries and questionnaire portals, hampering integrated care. We intend to develop a nationwide digital personal health record for patients to manage and share relevant medical information, for which perspectives of people with hemophilia and healthcare professionals are crucial.

Aims: To assess attitudes towards, and expectations, requirements and concerns of a personal health record for hemophilia care in the Netherlands, by people with hemophilia, their caretakers and healthcare professionals.

Methods: Nineteen adult and pediatric patients with hemophilia $A$ or $\mathrm{B}$ and their caretakers, as well as 18 multidisciplinary healthcare professionals (e.g. (pediatric) hematologists, nurses, pharmacists, psychologists and physiotherapists) participated in this qualitative, semi-structured interview study. Perspectives of patients and healthcare professionals were compared.

Results: Most patients using prophylaxis or frequent on-demand therapy and most healthcare professionals have a positive attitude regarding a personal health record. Patients not using prophylaxis consider it less beneficial for personal use.

All participants do advocate for its development. They expect it would enhance patient safety, by facilitating the digital exchange of health data and enabling proxy access. Patients especially anticipate its positive effects on patient empowerment, by managing health data and integrated access to digital apps. Healthcare professionals underline the importance of an up-to-date medication overview and unified display of patient-relevant health outcomes.

Participants expressed their concerns about privacy, interoperability, responsibility, ownership of data and its suitability for patients with few (digital) self-management skills.

Conclusions: People with hemophilia using prophylaxis or frequent on-demand therapy, their caretakers and healthcare professionals have a positive attitude towards a personal health record facilitating integrated hemophilia care and patient's self-management. They raised important concerns that will be addressed in the tool we are currently developing.
PB0620 | Haemophilia A Genotyping in Patients with

Discrepancy in FVIII Activity Levels Measured with One-stage and Chromogenic Assays

L. Rossetti ${ }^{1}$; V. Marchione ${ }^{1}$; M. Arias ${ }^{2}$; E. Sueldo ${ }^{2}$; C.P. Radic ${ }^{1}$; B. Ziegler $^{1}$; M.M. Abelleyro ${ }^{1}$; K. Waisman ${ }^{1}$; A. Baqués ${ }^{3}$; C. De Brasi ${ }^{1,4}$

${ }^{1}$ Instituto de Medicina Experimental (IMEX), CONICET-Academia Nacional de Medicina (ANM), Ciudad Autónoma de Buenos Aires, Argentina; ${ }^{2}$ Laboratorio de Hematología. Hospital Dr. César Milstein, Ciudad Autónoma de Buenos Aires, Argentina; ${ }^{3}$ Servicio de Hemofilia. Hospital Dr.César Milstein, Ciudad Autónoma de Buenos Aires, Argentina; ${ }^{4}$ Instituto de Investigaciones Hematológicas Mariano $R$ Castex (IIHEMA), ANM, Ciudad Autónoma de Buenos Aires, Argentina

Background: In approximately one-third of patients with mildmoderate haemophilia $A(H A)$, there is a discrepancy between the results of FVIII activity (FVIII:C) measured by one-stage-assay (OSA) and chromogenic-substrate-assays (CSA), phenomenon which has been associated with particular missense variants.

Aims: Characterize the F8-genotype in persons with HA (PwHA) from Argentina showing discrepancy between FVIII:C assays and compare them with those reported in the International F8-variant database EAHAD (European Association for Haemophilia and Allied Disorders, https://f8-db.eahad.org/).

Methods: PwHA samples ( $\mathrm{N}=5$ with OSA ranging mild-moderate phenotypes, from 4 families) that showed discrepancy in the measurement of FVIII:C were included. OSA and CSA FVIII:C was determined using two reagent/coagulometer systems, IL/ACL Top 300 and Siemens/Sysmex CS-2500.

Genotyping: peripheral blood leukocyte-extracted genomic-DNA was mutational screened by PCR-amplification of all coding and regulatory regions of $F 8$ followed by conformation sensitive gel electrophoresis (CSGE) and selected-amplimers were characterized by Sanger sequencing. Pathogenicity of F8-variants was classified according the ACMG criteria.

Results:

TABLE 1 Biochemical \& Molecular features of an Argentinian series of patients with HA showing discrepancy in FVIII:C assays vs EAHAD

\begin{tabular}{|c|c|c|c|c|c|}
\hline $\begin{array}{l}\text { Patient\# } \\
\text { Age [years] }\end{array}$ & $\begin{array}{l}\text { F8 region / } \\
\text { FVIII-Domain }\end{array}$ & F8-variants ${ }^{\dagger}$ & $\begin{array}{l}\text { Variant type / } \\
\text { ACMG-class }^{\dagger \dagger}\end{array}$ & $\begin{array}{l}\text { FVIII:C } \\
\text { [\%] OSA }\end{array}$ & $\begin{array}{l}\text { FVIII:C } \\
\text { [\%] CSA }\end{array}$ \\
\hline $\begin{array}{l}\# 1^{\dagger \dagger \dagger} \\
48\end{array}$ & exon 21 / C1 & $\begin{array}{l}\text { c. } 6246 C>A \\
\text { p. }(\text { Ser2082Arg) }\end{array}$ & $\begin{array}{l}\text { Missense / } \\
\text { pathogenic }\end{array}$ & $2-5$ & $1-2.5$ \\
\hline
\end{tabular}

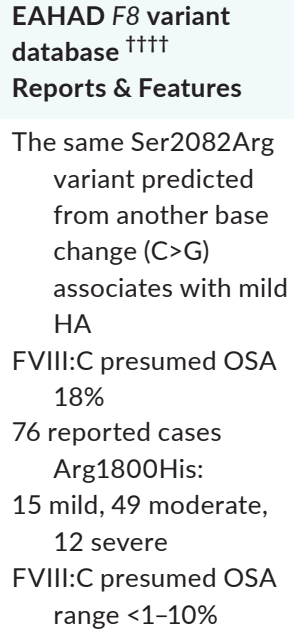




\begin{tabular}{|c|c|c|c|c|c|}
\hline $\begin{array}{l}\text { Patient\# } \\
\text { Age [years] }\end{array}$ & $\begin{array}{l}\text { F8 region / } \\
\text { FVIII-Domain }\end{array}$ & F8-variants ${ }^{\dagger}$ & $\begin{array}{l}\text { Variant type / } \\
\text { ACMG-class }^{\dagger \dagger}\end{array}$ & $\begin{array}{l}\text { FVIII:C } \\
\text { [\%] OSA }\end{array}$ & $\begin{array}{l}\text { FVIII:C } \\
\text { [\%] CSA }\end{array}$ \\
\hline $\begin{array}{l}\# 3 \\
50\end{array}$ & $\begin{array}{l}\text { exon } 16 \text { / A3 } \\
\text { exon } 14 \text { / B }\end{array}$ & $\begin{array}{l}\text { c. } 5508 \mathrm{G}>\mathrm{T} \\
\text { p. }(\text { Trp1836Cys) } \\
\text { c. } 3780 \mathrm{C}>\mathrm{G} \\
\text { (rs1800292) } \\
\text { p.(Ser1288=) }\end{array}$ & $\begin{array}{l}\text { Missense / } \\
\text { likely_ } \\
\text { pathogenic } \\
\text { Synonymous / } \\
\text { benign }\end{array}$ & $8-10$ & $2-4$ \\
\hline $\begin{array}{l}\# 4^{\dagger \dagger \dagger \dagger} \\
51\end{array}$ & $\begin{array}{l}\text { exon } 18 \text { / A3 } \\
\text { intron } 18 \text { / A3 } \\
\text { exon 14/ B }\end{array}$ & $\begin{array}{c}\text { c. } 5981 \mathrm{~T}>\mathrm{C} \\
\text { p. }(\text { Leu1994Pro) } \\
\text { c. } 5998+91 \mathrm{~T}>\mathrm{A} \\
\text { rs4898352 } \\
\text { c. } 3864 \mathrm{~A}>\mathrm{C} \text { rs1800292 } \\
\text { p. }(\text { Ser1288=) }\end{array}$ & $\begin{array}{l}\text { Missense / } \\
\text { pathogenic } \\
\text { Intronic/ benign } \\
\text { Synonymous/ } \\
\text { benign }\end{array}$ & $2-5$ & $0.9-1$ \\
\hline $\begin{array}{l}\# 5^{\dagger+\dagger \dagger} \\
47\end{array}$ & $\begin{array}{l}\text { exon } 18 \text { / A3 } \\
\text { intron } 18 \text { / A3 } \\
\text { exon } 14 \text { / B }\end{array}$ & $\begin{array}{l}\text { c. } 5981 \mathrm{~T}>\mathrm{C} \\
\text { p. }(\text { Leu1994Pro }) \\
\text { c. } 5998+91 \mathrm{~T}>\mathrm{A} \\
\mathrm{rs} 4898352 \\
\text { c. } 3864 \mathrm{~A}>\mathrm{C} \text { rs } 1800292 \\
\text { p. }(\text { Ser1288 }=)\end{array}$ & $\begin{array}{l}\text { Missense / } \\
\text { pathogenic } \\
\text { Intronic / benign } \\
\text { Synonymous / } \\
\text { benign }\end{array}$ & $2.2-5$ & $0-1$ \\
\hline
\end{tabular}
EAHAD F8 variant database ${ }^{\text {t†† }}$ Reports \& Features
Trp1836Cys was not reported.

${ }^{\dagger}$ Human Genome Variant Society (HGSV) recommended nomenclature; RefSeq F8: NG_011403.1; F8 mRNA: NM_000132.3, FVIII Protein: NP_000123.1.

${ }^{+\dagger}$ Variant pathogenicity classification recommended by the American College of Medical Genetics and Genomics (ACMG) (Richards et al, Genet Med 2015, 17: 405-24): (i) pathogenic, (ii) likely_pathogenic, (iii) uncertain_significance, (iv) likely_benign, (v) benign. The HA causative variant is indicated in boldface.

${ }^{+1+}$ Showed a FVIII inhibitor of high response (9.7 BU/dL).

${ }^{+1+\dagger}$ Patients \#4 and \#5 are full brothers (equal parents).

${ }^{+\dagger+\dagger}$ F8-variant database EAHAD (European Association for Haemophilia and Allied Disorders, http://f8-db.eahad.org/), accessed online 08/03/2021.

The HA-causative F8-genotype was identified in all 5 PwHA (Table 1) and classified as missense-type: one located in FVIII-C1-Domain and 4 in FVIII-A3-Domain. None of them was previously associated with assay discrepancy in EAHAD. Two of them were not reported (c.6246C>A/p.(Ser2082Arg); c.5508G>T/p.(Trp1836Cys)), whereas the reported ones were associated with different (mild-moderatesevere) phenotypes but only based on presumed OSA (Table 1).

Conclusions: Thus far, in EAHAD database, only 28 missense HAcausative variants were associated with FVIII:C assay discrepancy, and none of them matched with our 5 cases from Argentina, which showed different phenotype severities in EAHAD when were reported. Our data may reveal some degree of misclassification of phenotype severity in $\mathrm{HA}$, which may be associated with the rare application of CSA to measure FVIII:C complementing OSA levels. Consequently, our findings reinforce the convenience to base the initial diagnosis of non-severe-HA phenotype on the results of both, one-stage and chromogenic assays.

PB0621 | Influence of Pregnancy on the Pharmacokinetic Profile of a Severe Hemophilia A Female

L. Costa; A. Ferreira; M. Coutinho; M. Pereira; E. Cruz; S. Morais Centro de Coagulopatias Congénitas, Centro Hospitalar Universitário do Porto (CHUP), Porto, Portugal

Background: Since the availability of Bayesian population-based pharmacokinetic (PK) platforms, estimation of individual PK became widespread. Individual PK assessment considers patient variables (e.g. age, weight, blood group, von Willebrand factor [vWF]), but the gender influence, at the best of our knowledge, is unknown. PK information on women is still missing, especially in pregnant women with hemophilia.

Aims: To describe the PK profile of a female with severe HA during pregnancy.

Methods: We performed PK assessments outside and during the pregnancy, using both myPKFiT (Takeda Pharmaceuticals, Japan) and WAPPS-Hemo (McMaster University, Ontario, Canada) platforms. We also reviewed the clinical data present in patient's electronic records. Whenever possible, FVIII levels were measured by one stage assays (OSA) [Siemens Healthineers, Marburg, Germany] and chromogenic assays (CSA) [HemosIL Electrachrome, Werfen, USA]. Balanced estimates of terminal half-lives ( $t 1 / 2)$ were compared among those two assays. Analysis was performed using tStudent test.

Results: The patient was on prophylaxis with $\mathrm{Advate}{ }^{\circledR}$, twice weekly previously $(20 \mathrm{lU} / \mathrm{kg})$ and during pregnancy $(25 \mathrm{lU} / \mathrm{kg})$ until gestational age of 31 weeks, when she intensified prophylaxis to 3 times/ week $(21.5 \mathrm{IU} / \mathrm{kg})$.

The $t^{1 / 2}$ were $17 \mathrm{~h}$ and $10.5 \mathrm{~h}$ before pregnancy, and $24.3 \mathrm{~h}$ and $18.3 \mathrm{~h}$ at 31 weeks of gestation, using OSA and CSA, respectively. We observed an increase in FVIII levels and mainly a marked vWF rise during pregnancy (1.80 to $3.27 \mathrm{IU} / \mathrm{mL})$. Patient reached half-lives $\left(\mathrm{t}^{1 / 2}\right)$ at 36 weeks of gestation of 20h (WAPPS-Hemo) and 17h (myPKFiT) by OSA. 
Conclusions: Outside pregnancy, the higher $t \frac{1}{2}$ in the patient compared to the reference mean $\mathrm{t} 1 / 2$ of Advate $\AA(12.4 \pm 2.0 \mathrm{~h}$, Malhangu, 2014), raises the question of whether it is due to individual PK differences or it is dependent of the female gender. The increased $t \frac{1}{2}$ during pregnancy confirms what was expected and accompanies the rising of vWF during pregnancy.

PB0622 | AOZORA Study: Long-Term Safety and Joint Health in Pediatric Patients with Hemophilia A without FVIII Inhibitors Receiving Emicizumab - Protocol for a Multicenter, Open-label, Phase IV Clinical Study

M. Shima ${ }^{1}$; H. Takedani ${ }^{2}$; K. Kitsukawa ${ }^{3}$; M. Taki ${ }^{4}$; A. Ishiguro ${ }^{5}$; A. Nagao $^{6}$; H. Yamaguchi-Suita ${ }^{7}$ Y. Kyogoku'; S. Yoshida ${ }^{7}$; K. Nogami ${ }^{1}$. ${ }^{1}$ Department of Pediatrics, Nara Medical University, Kashihara, Japan; ${ }^{2}$ Department of Joint Surgery, IMSUT Hospital, The University of Tokyo, Tokyo, Japan; ${ }^{3}$ Department of Radiology, St. Marianna University School of Medicine, Kawasaki, Japan; ${ }^{4}$ Department of Pediatrics, St Marianna University School of Medicine, Kawasaki, Japan; ${ }^{5}$ Division of Hematology, National Center for Child Health and Development, Tokyo, Japan; ${ }^{6}$ Department of Blood Coagulation, Ogikubo Hospital, Tokyo, Japan; ${ }^{7}$ Chugai Pharmaceutical Co., Ltd, Tokyo, Japan

Background: Hemophilic arthropathy is a major complication of hemophilia A (HA). Factor (F)VIII prophylaxis has limitations with regard to maintaining long-term joint health. An alternative treatment, emicizumab, is a recombinant monoclonal antibody that bridges activated FIX and FX, replacing the function of FVIII in persons with hemophilia A (PwHA). Emicizumab has demonstrated efficacy, along with good tolerability and safety, in the treatment of HA throughout phase III clinical trials; however, data on long-term joint health are lacking.

Aims: AOZORA is a multicenter, open-label, phase IV clinical study designed to evaluate the long-term safety and joint health effects of emicizumab in pediatric PwHA without FVIII inhibitors.

Methods: Thirty participants $<12$ years old, weighing $>3 \mathrm{~kg}$, with a diagnosis of severe congenital HA, without FVIII inhibitors, were to be enrolled. Key inclusion and exclusion criteria are shown in Table 1. Participants could enter AOZORA as either emicizumabnaïve or following on from the HOHOEMI phase III study (Shima et al. Haemophilia 2019) (Figure 1); participants will receive emicizumab for 313 weeks. Magnetic resonance imaging (MRI) will be performed every 3 years to evaluate early joint changes and the progression of hemophilic arthropathy by International Prophylaxis Study Group (IPSG) score. The Hemophilia Joint Health Score will be used to evaluate joint function. The study is registered at www.clini caltrials.jp (JapicCTI-194701).

Results: Patient characteristics for 30 participants, 20 newly enrolled from AOZORA and 10 transferred from $\mathrm{HOHOEMI}$, and baseline MRI IPSG scores for 29 participants will be presented. Soft tissue pathological changes, such as synovial hypertrophy, were observed in 13/29 cases; osteochondral changes were observed in one case.
Conclusions: The findings from this study will add to the safety and efficacy data accumulated from previous clinical trials, providing a more complete long-term profile, beyond bleeding rates, for emicizumab.

TABLE 1 AOZORA key inclusion and exclusion criteria

\begin{tabular}{|c|c|c|}
\hline Inclusion & & Exclusion \\
\hline - & $\begin{array}{l}\text { Written informed consent for study } \\
\text { participation from the PwHA's legally } \\
\text { acceptable representative; where } \\
\text { possible, written informed consent from } \\
\text { the participant }\end{array}$ & $\begin{array}{l}\text { - Inherited or acquired bleeding disorder } \\
\text { other than HA }\end{array}$ \\
\hline - & $\begin{array}{l}\text { Age }<12 \text { years at the time of informed } \\
\text { consent }\end{array}$ & - Current receipt of ITI therapy \\
\hline - & Body weight of $>3 \mathrm{~kg}$ & $\begin{array}{l}\text { - Previous (within the past } 12 \text { months) or } \\
\text { current treatment for or signs of TE } \\
\text { disease }\end{array}$ \\
\hline - & $\begin{array}{l}\text { Ability to comply with scheduled study } \\
\text { visits, treatment plans, laboratory tests, } \\
\text { and other procedures }\end{array}$ & $\begin{array}{l}\text { - History of clinically significant } \\
\text { hypersensitivity associated with } \\
\text { monoclonal antibody therapies }\end{array}$ \\
\hline - & $\begin{array}{l}\text { Diagnosis of severe (endogenous FVIII } \\
\text { level }<1 \% \text { ) congenital } \mathrm{HA}\end{array}$ & - Prior receipt of emicizumab* \\
\hline - & $\begin{array}{l}\text { Negative results for FVIII inhibitors (<0.6 } \\
\text { BU/mL) in the most recent assay within } 8 \\
\text { weeks prior to enrollment }\end{array}$ & $\begin{array}{l}\text { Currently receiving an investigational } \\
\text { drug; receipt of an investigational drug to } \\
\text { treat or reduce the risk of hemophilic } \\
\text { bleeds within } 5 \text { half-lives of last drug } \\
\text { administration; or receipt of a non-HA- } \\
\text { related investigational drug within the last } \\
30 \text { days or } 5 \text { half-lives }\end{array}$ \\
\hline - & $\begin{array}{l}\text { Adequate hematologic function at the time } \\
\text { of screening (WBC count } \geq 100 \times 10^{9} \\
\text { cells/L, hemoglobin } \geq 8 \mathrm{~g} / \mathrm{dL}[4.97 \\
\text { mmol/L]) }\end{array}$ & $\begin{array}{l}\text { - Any other reason that, in the judgment of } \\
\text { the investigator, would render the } \\
\text { participant unsuitable for inclusion }\end{array}$ \\
\hline - $\begin{array}{c}A \\
s \\
s \\
s\end{array}$ & $\begin{array}{l}\text { Adequate hepatic function at the time of } \\
\text { screening (total bilirubin } \leq 1.5 \times \text { age- } \\
\text { specific ULN, AST and ALT } \leq 3 \times \text { age- } \\
\text { specific ULN) }\end{array}$ & \\
\hline $\begin{aligned} &- A \\
& 5 \\
& s \\
& r \\
& \vdots\end{aligned}$ & $\begin{array}{l}\text { Adequate renal function at the time of } \\
\text { screening (serum creatinine } \leq 1.5 \times \text { age- } \\
\text { specific ULN). Creatinine clearance }>70 \\
\mathrm{~mL} / \mathrm{min} / 1.73 \mathrm{~m}^{2} \text { if the serum creatinine is } \\
\geq 1.5 \times \cup L \mathrm{~N}\end{array}$ & \\
\hline
\end{tabular}

Figure 1. Summary of the AOZORA study design

A

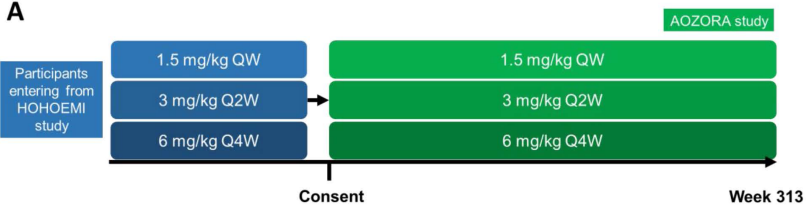

B

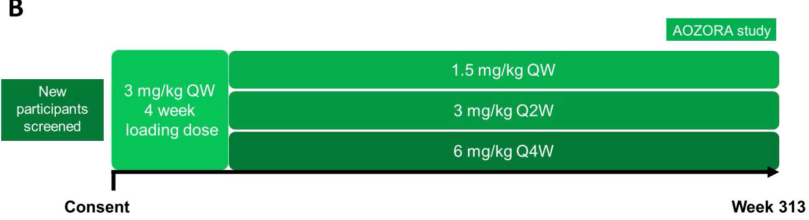

A) Participants entering AOZORA from the HOHOEMI study will receive one of the three permitted doses for a total of 313 weeks from the date of first emicizumab administration; this treatment period is inclusive of the emicizumab administered within HOHOEMI. B) The treatment period for is inclusive of the emicizumab administered within HOHOEMI. B) The treatment period for followed by either of the three dose regimens until Week 313 . The emicizumab treatment regimen may be modified to a different approved dosing regimen.

QW, weekly; Q2W, every 2 weeks; Q4W, every 4 weeks.

FIGURE 1 Summary of the AOZORA study design 
PB0623 | AKATSUKI Study: A Prospective, Multicenter, Phase IV Study to Evaluate the Safety of Emicizumab Under and Immediately after Immune Tolerance Induction (ITI) Therapy in Persons with Congenital Hemophilia A (PwHA) with Factor (F) VIII Inhibitors

T. Matsushita ${ }^{1}$; N. Suzuki ${ }^{1}$; A. Nagao ${ }^{2}$; C. Nagae ${ }^{3}$; H. YamaguchiSuita ${ }^{4}$; Y. Kyogoku ${ }^{4}$; A. loka ${ }^{4}$; K. Nogami ${ }^{5}$

${ }^{1}$ Nagoya University Hospital, Nagoya, Japan; ${ }^{2}$ Ogikubo Hospital, Tokyo, Japan; ${ }^{3}$ St Marianna University School of Medicine, Kawasaki, Japan; ${ }^{4}$ Chugai Pharmaceutical Co., Ltd, Tokyo, Japan; ${ }^{5}$ Nara Medical University, Kashihara, Japan

Background: Emicizumab is a recombinant monoclonal antibody that bridges FIXa and FX, replacing the function of FVIIla. Emicizumab has demonstrated efficacy and a favorable safety profile in PwHA with/without FVIII inhibitors. For all PwHA developing persistent FVIII inhibitors (PwHAwI), ITI therapy for inhibitor eradication is recommended. However, since PwHAwl on ITI therapy were excluded from the emicizumab clinical development program, there are currently limited safety data for emicizumab treatment under/immediately after ITI therapy in PwHAwl. Accordingly, there is a need to collect safety/efficacy data on this concomitant treatment strategy in a clinical setting. The present study was planned as no such study has been conducted in Japan.

Aims: To evaluate the safety of emicizumab under/immediately after ITI therapy in PwHAwI. Here we report details of the study protocol. Methods: This open-label, non-randomized, interventional, multicenter study in Japan (jRCTS041200037) of emicizumab in PwHAwl receiving ITI therapy (Figure) aims to enroll 20 participants; informed consent and approvals will be obtained prior to study start. The primary endpoint is to comprehensively evaluate safety (adverse events [mainly thromboembolic events] and abnormal laboratory values) over time. Secondary endpoints are the number of bleeds requiring coagulation factor treatment, the number of participants achieving a partially successful ITI therapy response, FVIII inhibitor titers under/immediately after ITI therapy, quality of life, and time to achieve negative FVIII inhibitor titers and partial success in PwHAwl starting ITI therapy after study enrollment. Partial success is defined as a negative FVIII inhibitor titer plus normal FVIII recovery; for success, a normal FVIII half-life is required, but due to the participants' burden, half-life assessment based on full pharmacokinetic analysis is not mandatory (Table).

Results: This registered study is ongoing.

Conclusions: This study will evaluate the safety of emicizumab prophylaxis administered under/immediately after ITI therapy in PwHAwl, providing reference data to inform treatment strategies in this study population.
Figure. AKATSUKI study design

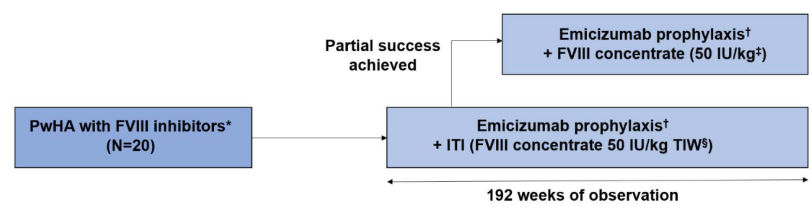

*PwHA receiving ITI therapy and/or emicizumab prophylaxis at study enrolment are eligible for participation.

'Emicizumab prophylaxis will be administered according to the dosing regimen approved in Japan.

FThe dosing regimen is as follows: $50 \mathrm{IU} / \mathrm{kg} \mathrm{QW}$ for the first 24 weeks and then $50 \mathrm{IU} / \mathrm{kg}$ Q2W for the next 24 weeks, after which the FVIII concentrate may be discontinued at the investigator's discretion. The dose of FVIII concentrate may be adjusted by $\pm 20 \%$.

§ A dosing frequency of twice per week is permitted when using extended half-life FVIII concentrates. The dose of FVIII concentrate may be adjusted by $\pm 20 \%$.

FVIII, factor VIII; ITI, immune tolerance induction; PwHA, persons with hemophilia A; QW once per week; Q2W, once every two weeks; Q4W, once every four weeks; TIW, three times per week.

FIGURE 1 AKATSUKI study design

TABLE 1 Assessment criteria for partial and complete success

Table. Assessment criteria for partial and complete success

\begin{tabular}{|l|r|}
\hline & Assessment criteria \\
\hline Negative FVIII inhibitor titer ${ }^{\star \dagger}$ & $\begin{array}{r}\text { FVIII inhibitor titer }<0.6 \text { BU on two consecutive occasions } \\
\text { at least 2 weeks apart }\end{array}$ \\
\hline $\begin{array}{l}\text { Normal FVIII recovery }{ }^{* \dagger} \\
\text { Normal FVIII half-life }{ }^{\dagger}\end{array}$ & $\begin{array}{r}\text { FVIII recovery } \geq 66 \% \text { of predicted value on two consecutive } \\
\text { occasions at least } 2 \text { weeks apart } \\
\text { concentrates or } \geq 7 \text { hours for extended half-life FVIII } \\
\text { concentrates }\end{array}$ \\
\hline${ }^{*}$ Criteria for partial success. & \\
\hline
\end{tabular}

'Criteria for complete success; however, due to the participants' burden, FVIII half-life assessment based on full pharmacokinetic analysis is not mandatory.

BU, Bethesda Unit; FVIII, factor VIII.

PB0624 | A Bleed Suppression Efficacy of Emicizumab in Patients with Hemophilia A whose Therapies Switched from Conventional Prophylaxis

T. Fujii; N. Yamasaki; T. Inoue; T. Fujii

Division of Transfusion Medicine, Hiroshima University Hospital, Hiroshima, Japan

Background: Emicizumab is an asymmetrical bispecific antibody that mimics the cofactor function of activated factor VIII (FVIIla). In 2019, clinical usage of emicizamab was approved for hemophilia A regardless with or without inhibitor in Japan. Because most adult and adolescent patients have already received prophylactic treatment by factor concentrate, their bleeding episodes are suppressed enough. However, in case the switch from the conventional therapy 
to emicizumab administration in such patients, the bleed suppression efficacy has not been well-known.

Aims: Our objective is to clarify an efficacy of emicizumab in patients with hemophilia A(PWHAs) without inhibitor whose therapies switched from conventional prophylaxis.

Methods: We calculated respective annualized bleeding rates (ABR) and annualized joint bleeding rates (AJBR) by patient diaries of 11 PWHAs without inhibitor whose therapies switched from conventional prophylaxis to emicizumab administration. Then, the ABRs and the AJBRs were compared before and after switching to emicizumab. On the other hand, trough levels of factor VIII and factor VIII equivalent activities of patients' plasma were respectively measured by chromogenic assay (CGA).

Results: Nine of 11 had severe hemophilia and the others had moderate. The observation periods of conventional prophylaxis and emicizumab administrations were 4.00 years and 1.08 years(median). The median ABR and AJBR during prophylaxis was 3.33 and 1.06 . The median values of $A B R$ and AJBR after switching emicizumab was 2.01 and 1.00. Most trough levels of Factor VIII activity at conventional prophylaxis were higher than respective baseline (median $3 \mathrm{IU} / \mathrm{dL}$ ). However, their FVIII equivalent values at steady state by regulary emicizumab administrations were $15.7 \mathrm{IU} / \mathrm{dL}$ (median, minmax; 13.8-19.7).

Conclusions: Even when switching from conventional prophylaxis to emicizumab administration, their bleedings were still suppressed. Besides, their FVIII equivalent values seemed to be higher than their trough levels of FVIII activity at conventional prophylaxis.

PB0625 | Primary Prophiliaxis was Associated with a Lower Risk of Joint Damage in Colombian Men Patients with Hemophilia A

G.A. Diaz Mosquera ${ }^{1}$; S. Sarmiento Doncel ${ }^{2}$; J.M. Cortes ${ }^{1}$; F.J. Meza $^{1}$; J.E. Peña ${ }^{1}$; C. Agudelo Rico ${ }^{3}$; N. Ramirez Plazas ${ }^{1}$; C.A. Montaño Mejia ${ }^{1}$; I.A. Perdomo Amar²; A. Maza Villadiego ${ }^{1}$; A. Parrado Rey ${ }^{1}$

${ }^{1}$ Integral Solutions SD, Bogota, Colombia; ${ }^{2}$ Integral Solutions, Bogota, Colombia; ${ }^{3}$ Integral Solutions Research, Bogota, Colombia

Background: Scientific evidence describes that primary prophylaxis can reduce the incidence of hemophilic arthropathy and its impact on quality of life. In Colombia, starting with Law 100 of 1993, a comprehensive social security regime was implemented; in the case of hemophilia, coverage went from 15\% in 1993 to $98 \%$ in 2012. In 2003, prophylaxis was generalized, achieving that in 2015 more than $90 \%$ of patients had treatment regardless of their age.

Aims: The objective was to evaluate the association between primary prophylaxis and hemophilic arthropathy in Colombian men with hemophilia A (HA).

Methods: Retrospective descriptive study with a total of 90 patients with severe / moderate HA without inhibitors who were distributed into two groups: primary and secondary / tertiary prophylaxis, in a health service provider institution (Integral Solutions SD).
Results: The median age was 20.5 years, the median at diagnosis 1 year, the dose of FVIII used was 27.8 (IU / Kg / dose) with a significant difference between primary (25.0) and secondary prophylaxis / tertiary (28.6). There were no differences in the frequency of prophylaxis application. The ABR of the cohort was 0.81 without significant differences between the 2 groups. The prevalence of hemophilic arthropathy in the study cohort was $42.2 \%(n=38)$ with a significant difference ( $P$ value 0.00 ) between primary $(0.0 \%)$ versus secondary / tertiary prophylaxis (55.1\%). A greater tendency to have arthropathy was observed in patients diagnosed before 2004, the date on which prophylaxis was widely implemented in Colombia. Conclusions: Giving patients early prophylaxis with factor VIII concentrates can prevent not only bleeding, but also joint damage, in turn generating a better quality of life. This decrease in joint damage could be evidenced after prophylaxis with coverage and efficacy was implemented in Colombian patients starting in 2004.

PB0626 | Feasibility of Integrating PROBE (Patient Reported Outcomes, Burdens and Experiences) into a Clinical Care Pathway to Facilitate Transformation to a Value-based Health Care Model in Hemophilia

S. Jackson ${ }^{1,2}$; B. Sheridan ${ }^{2}$; D. Gue $;$ S. Lyons ${ }^{3} ;$ R. Waines ${ }^{3} ;$ T. Ireland $^{3}$; D. Pete ${ }^{4}$; A. Iorio ${ }^{5,4}$; L. Walsh ${ }^{4}$; M. Skinner ${ }^{6}$; F. Germini ${ }^{7,8}$ ${ }^{1}$ University of British Columbia / St. Paul's Hospital, Vancouver, Canada; ${ }^{2}$ British Columbia Provincial Bleeding Disorders Program - Adult Division, St. Paul's Hospital, Vancouver, Canada, ${ }^{3}$ Vancouver, Canada; ${ }^{4}$ Health Information Research Unit, Department of Health Research Methods, Evidence and Impact (HEI), McMaster University, Hamilton, Canada; ${ }^{5}$ Department of Health Research Methods, Evidence and Impact, Department of Medicine, McMaster University, Hamilton, Canada; ${ }^{6}$ Institute for Policy Advancement Ltd, Washington, United States; ${ }^{7}$ Department of Medicine, McMaster University, Hamilton, Canada; ${ }^{8}$ Health Information Research Unit, Department of Health Research Methods, Evidence and Impact (HEI), Hamilton, Canada

Background: The PROBE survey was developed by patients, hemophilia caregivers and methodological experts as a stand-alone crosssectional instrument with multistep validation and control group data. While not developed for use in clinics, it measures important value-based health outcomes including health status, mobility, independence, acute/chronic pain and overall quality of life.

Aims: We report initial results from a feasibility project using PROBE during routine care to enable patients to provide outcome measures to clinical teams and improve quality, value and experience of hemophilia care.

Methods: Three patients from the British Columbia Adult Bleeding Disorders Provincial Program were identified to guide the BC steering group and the McMaster PROBE database teams. The steering group endorsed including 6 additional vocational survey items to the 30 question PROBE instrument. An anonymized link was sent 1-2 weeks pre-appointment and upon completion, patients were given 
the option to share survey results with the clinical team. All patients were sent an evaluation form post-visit.

Results: 25 survey links were sent and 17 patients completed in full. 4 had technical issues sharing the survey and 13 independently completed and shared the survey. 7 patients completed a 10 question evaluation post-survey with $86 \%$ indicating PROBE covered all areas that they felt were important to address, $100 \%$ were willing to take it again, and $86 \%$ felt the PROBE responses had a very beneficial or beneficial impact on the team members ability to have a meaningful discussion during the visit.

Conclusions: It is feasible to embed PROBE into routine care from a patient and clinician perspective for longitudinal single patient or population-based outcome tracking. Next steps include enhancing the interface for clinician viewing, allowing patients to trend their results over time and allowing the option for patients to compare their results to selected peer groups within the National/Global PROBE system.

PB0627 | PRactical Utilisation of Octapharma FVIII Concentrates in Previously Untreated and Minimally Treated Haemophilia A Patients Entering Routine Clinical Treatment with Nuwiq $^{\circledR}$, octanate ${ }^{\circledR}$ or wilate ${ }^{\circledR}$ - The Protect-NOW Study

J. Oldenburg ${ }^{1}$; P.M. Vera ${ }^{2}$; S. Halimeh ${ }^{3}$; R. Klamroth ${ }^{4}$; S. Lowndes ${ }^{5}$; M. Jansen ${ }^{6}$

${ }^{1}$ University Clinic Bonn, Institute of Experimental Haematology and Transfusion Medicine, Bonn, Germany; ${ }^{2}$ Hospital General Universitario de Alicante, Alicante, Spain; ${ }^{3}$ Gerinnungszentrum Rhein-Ruhr, Duisburg, Germany; ${ }^{4}$ Vivantes Klinikum im Friedrichshain, Berlin, Germany; ${ }^{5}$ Octapharma AG, Lachen, Switzerland; ${ }^{6}$ Octapharma Pharmazeutika Produktionsgesellschaft m.b.H., Vienna, Austria

Background: Optimal management of previously untreated patients (PUPs) and minimally treated patients (MTPs) with severe haemophilia A (FVIII:C <1\%) must address numerous concerns to ensure good long-term outcomes. The efficacy, safety and immunogenicity of Nuwiq ${ }^{\circledR}$, octanate ${ }^{\circledR}$ and wilate ${ }^{\circledR}$ in PUPs have been investigated in clinical trials; the incidence of high-titre inhibitors was $16 \%, 8 \%$ and $11 \%$ with Nuwiq ${ }^{\circledR}$, octanate ${ }^{\circledR}$ and wilate ${ }^{\circledR}$, respectively.

Aims: Protect-NOW is an ongoing, non-interventional, prospective and retrospective study. Protect-NOW aims to investigate the effectiveness and overall safety, including immunogenicity, of Nuwiq ${ }^{\circledR}$, octanate ${ }^{\circledR}$ and wilate ${ }^{\circledR}$ in routine clinical practice for the prevention and treatment of bleeding events in PUPs and MTPs with severe haemophilia A. Optional sub-studies will assess risk factors associated with inhibitor development.

Methods: It is planned to enrol 140 PUPs and MTPs ( $<5$ previous exposure days [EDs] with other FVIII products) of any age and ethnicity for 100 EDs or up to 3 years after ED1. Treatment is at the discretion of the investigator. The primary objectives are effectiveness in the prevention and treatment of bleeding events, as well as overall safety, including inhibitor development. Measurement of inhibitor activity is recommended every 3-4 EDs until ED20 and every 10-12 EDs thereafter. Secondary objectives are to assess treatment utilisation patterns (including dosage and frequency of administration) and effectiveness in surgical prophylaxis.

Results: As of March 2021, 37 patients have been enrolled in the study from 13 sites in Belarus, Canada, France, Germany, Italy, Lithuania, Spain and the United Kingdom, with additional sites to be initiated.

Conclusions: Protect-NOW is collecting clinically relevant information on the outcomes of PUPs and MTPs treated with Octapharma's FVIII products in a real-world setting.

\section{PB0628 | A Study of Psychosocial Issues for Mothers of Children} with Hemophilia

A. Nakao; J. Yamanouchi

Ehime University, Toon, Japan

Background: An innovative therapeutic management of hemophilia has been implemented at many hospitals in Japan. However, psychosocial support for hemophilia patients and their families is limited because there are few hemophilia treatment centers which can provide multidisciplinary care and support in Japan. Our hospital is a non-hemophilia treatment center and only medical doctors have seen patients with hemophilia. In 2016, we formed a hemophilia care team. The team contributed to hemophilia health care, and resolved diverse medical and psychosocial issues.

Aims: This study reports on the assessment of psychosocial issues in mothers of children with hemophilia.

Methods: We evaluated 25 mothers (median age of 41 (range: 27-55)) of children with hemophilia. A clinical psychologist had interviews with patients and/or families independently of doctor's medical examination, and measured mood and emotion state by POMS2 (Profile of Mood States Second Edition). The results of the POMS2 were compared with those of 24 mothers (median age of 42 (range: 35-61 years)) who had children without chronic diseases. Results: The main issues were remorse for their children with hemophilia and their husband's family, anxiety about patient's bleeding and genetic problems of offspring. Reflecting these results, the mothers of children with hemophilia had higher levels of confusion and depression than the control group. In addition, we found that there was unhealthy mood in the mothers who have patients with serious problems. They need a psychosocial care. One year later, Total Mood Disturbance is getting better.

Conclusions: By supports of hemophilia care team, the healthrelated quality of life in hemophilia patients and families was greatly improved. We would like to continue to provide supports for the psychosocial issues of mothers of children with hemophilia. 
PB0629 | Clinical Assessment with the Evaluation of Joint Disease and Haemostatic Treatment in Patients with Severe Haemophilia B

A. Buczma ${ }^{1}$; E. Odnoczko²; B. Baran ${ }^{2}$; A. Gwozdowska ${ }^{1}$; J. Zawilski ${ }^{3}$; J. Windyga ${ }^{1}$

${ }^{1}$ Department of Hemostasis Disorders and Internal Medicine in Institute of Hematology and Transfusion Medicine, Warsaw, Poland; ${ }^{2}$ Department of Hemostasis and Metabolic Disorders in Institute of Hematology and Transfusion Medicine, Warsaw, Poland; ${ }^{3}$ Interlab-

Fizjoterapia, Poznań, Poland

Background: Severe type of haemophilia B (HB) is defined by undetectable FIX plasma level activity $(<1 \mathrm{IU} / \mathrm{ml}$ ) and characterized by spontaneous bleeds into joints and muscles. Without proper treatment, recurrent intraarticular bleeds inevitably lead to joint damage, resulting in function impairment and major disability.

Aims: The study goal was to perform a clinical analysis of severe HB patients including disease complications and treatment regimens, evaluation of haemophilic arthropathy and degree of of joints' function impairment.

Methods: Study group consisted of 53 severe HB patients aged 12 74 (median 36) years. Retrospective and prospective clinical data were collected using dedicated questionaries. The Hemophilia Joint Health Score (HJHS) v.2 scale was used to evaluate the degree of joint disease. The examination included function measurement of ankles, knees and elbows, together with gait assessment.

Results: In the study group 22/53(41,5\%) had life-threatening bleeding episodes including 9 (17\%) intracranial haemorrhages. Spontaneous intraarticular bleeds were reported by $50 / 53(94,3 \%)$ and intramuscular bleeds by $40 / 53(75 \%)$ patients. The mean HJHS score (joints) was $25.57 \pm 20.47$ (median 23) and gait impairment was 2 points. Due to advanced haemophilic arthropathy, 22/53(42\%) patients underwent orthopaedic surgery, $11(21 \%)$ had at least one alloplasty. Prospectively collected data revealed that $37 / 52(71,2 \%)$ patients were prophylactic infusers, 10(19,2\%) were treated "on demand" and 5(9,62\%) on demand with occasional prophylactic FIX infusions. The mean annualized bleeding rate was $2.53 \pm 6.54$ for prophylactic users, and $15.4 \pm 13.00$ for "on demand" group.

Conclusions: Our study confirmed that severe haemophilia B patients are at high risk of bleeding, including life-threatening haemorrhages unless prophylactic therapy is applied. Study analysis indicated connection between the lack of optimal treatment and advanced joint desease and demonstrated that switching from "on demand" to prophylaxis was beneficial even in adult patients with preexisting severe arthropathy.
PB0630 | Benefits of Prophylactic Emicizumab in Enhancing Immune Tolerance Induction in a Hemophiliac Boy with Very High Inhibitor Titer

N. Sirachainan ${ }^{1}$; A. Chuansumrit ${ }^{1}$; S. Parapakpenjune ${ }^{2}$; P. Wongwerawattanakoon ${ }^{3}$; S. Lertthammakiat ${ }^{4}$; P. Kadegasem ${ }^{1}$; W. Sasanakul ${ }^{1}$

${ }^{1}$ Department of Pediatrics, Faculty of Medicine Ramathibodi Hospital, Mahidol University, Bangkok, Thailand; ${ }^{2}$ Department of Pediatrics, Maharat Nakhon Ratchasima Hospital, Nakhon Ratchasima, Thailand; ${ }^{3}$ Department of Nursing, Faculty of Medicine Ramathibodi Hospital, Mahidol University, Bangkok, Thailand; ${ }^{4}$ Department of Pediatrics, Chakri Naruebodindra Medical Institute, Samut Prakan, Thailand

Background: Approximately $20-30 \%$ of severe hemophilia A patients develop inhibitor, causing more frequent bleeding episodes. Immune tolerance induction (ITI) therapy is a mandatory treatment option. During ITI, patients have bleeding risk, as a result, prophylaxis with bypassing agents has been reported. Emicizumab, a bispecific antibody to factor IXa and factor X, has been used as prophylactic treatment among hemophilia A patient with inhibitor.

Aims: To report the benefit of prophylactic emicizumab in enhancing ITI in a boy with severe hemophilia A and high inhibitor titer.

Methods: A descriptive report.

Results: Case report: A 2-year-old boy with severe hemophilia A developed inhibitor of 16.9 Bethesda units $(B U)$ after receiving factor VIII concentrates to treat 2 subdural hemorrhages. At 10 years of age, he exhibited extensive intracerebral bleeding inducing obstructive hydrocephalus requiring bilateral ventriculostomy. He required aPCC 82,500 units, rFVIla $140 \mathrm{mg}$, and plasma exchange with high dose factor VIII concentrates (54,530 units) to control bleeding resulting in being bedridden. The ITI was initiated at the inhibitor of $140 \mathrm{BU}$ and historic peak inhibitor of 3,360 BU with EHL factor VIII 100 units / kg 3 times weekly. The peak inhibitor during ITI was 380 $\mathrm{BU}$ with one of each episode of intramuscular and joint bleeding. The inhibitor titer was $17.2 \mathrm{BU}$ at the end of the first year of ITI. Then, $3 \mathrm{mg} / \mathrm{kg}$ weekly for 4 doses and $6 \mathrm{mg} / \mathrm{kg}$ every 4 weeks of emicizumab prophylaxis was added in the second year of ITI. The inhibitor further declined to $0.6 \mathrm{BU}$ at the end of third year of ITI. He finally achieved a partially successful ITI at the end of 3.5 years of ITI. His factor VIII:C recovery was $60 \%$ and half-life at 5.5 hours. Conclusions: Additional emicizumab resulting in zero bleeding was helpful in enhancing ITI in a hemophilia boy with very high inhibitor titer. 
PB0631 | Standard Prophylactic Regimen Is Three Times and Twice a Week in Hemophilia A and B, Respectively. The Low Dose Tailoring Method of Prophylaxis Has Been Introduced for Patients with Hemophilia

M. Karimi ${ }^{1}$; A. Parsa ${ }^{1}$; S. Haghpanah ${ }^{1}$; F. Sadat Rajaie Ramsheh ${ }^{1} ;$ M. Bordbar $^{1}$; N. Bolandparvaz ${ }^{2}$; A. Bazrafshan ${ }^{1}$; H. Tavoosi ${ }^{2}$ ${ }^{1}$ Hematology Research Center, Shiraz University of Medical Sciences, Shiraz, Islamic Republic of Iran; ${ }^{2}$ Shiraz Hemophilia Comprehensive Center, Dastgheib Hospital, Shiraz University of Medical Sciences,

Shiraz, Islamic Republic of Iran

Background: Standard prophylactic regimen is three times and twice a week in hemophilia A and B, respectively. The low dose tailoring method of prophylaxis has been introduced for patients with hemophilia.

Aims: To evaluate the efficacy and safety of low dose modified prophylaxis, once per week in patients with severe hemophilia.

Methods: In this retrospective study, 53 patients less than 15 years with severe hemophilia (45 hemophilia A and 8 hemophilia B) who were involved in standard prophylactic regimen during 2015-2018 were investigated. All patients underwent prophylaxis with dose of $25 \mathrm{lU} / \mathrm{kg}$ once per week and increased to twice or 3 times a week if any of these conditions occurred:

1. Three episodes of hemarthrosis in past three months

2. Four episodes of important soft tissue bleeding in past three months

3. Any episode of a life-threatening and major bleeding without history of trauma

Results: All studied patients were negative for factor inhibitor. Mean age of diagnosis and mean age of starting infusion were $1.60 \pm 1.41$ (range: $1-7$ ) and $2.71 \pm 2.21$ (range: $1-7$ ) respectively. Mean age of starting prophylaxis was $6.15 \pm 3.9$ (range: $1-14$ ) years. The duration of prophylaxis was $47.09 \pm 11.12$ months. Nine patients discontinued prophylaxis and were excluded. Mean annual bleeding rate in the remained patients $(n=44)$ significantly decreased from $18.5 \pm$ 10.3 episode before prophylaxis to $5.7 \pm 3.7$ episode after prophylaxis $(P<0.001)$.

Number of patients received primary, secondary, and tertiary prophylaxis were 17 (32.1\%), 33 (62.3\%), and 3 (5.7\%) respectively

Conclusions: Prophylactic treatment in patients with severe hemophilia is shown to significantly reduce the numbers of bleeding episodes, arthropathy and costs of hospitalization. Our results showed modified prophylaxis treatment as a first choice in patients with severe hemophilia, especially in developing countries facing with limitation of financial and medical resources would be an ideal optional treatment.
PB0632 | Emicizumab Therapy for Neonatal Intracranial Hemorrhage

A. Dunn ${ }^{1,2}$

${ }^{1}$ Nationwide Children's Hospital, Columbus, United States; ${ }^{2}$ The Ohio State University College of Medicine, Columbus, United States

Background: Data on emicizumab-kxwh use for neonates with hemophilia and birth related intracranial hemorrhage is lacking.

Aims: To present the use of emicizumab for care of a newborn with severe hemophilia $\mathrm{A}(\mathrm{HA})$ and birth related intracranial hemorrhage. Methods: Chart review was performed for clinical, demographic, laboratory and radiologic details.

Results:

A Black male infant weighing 3420 grams was born to a 19 year old G1P1 mother whose pregnancy was complicated by late prenatal care and a family history of HA. She had two brothers with severe HA due to Intron 22 inversion, both with history of high-titer inhibitors. One achieved tolerance with a plasma derived, von Willebrand containing FVIII concentrate, the other failed multiple attempts at tolerance. Delivery was complicated by pre-eclampsia, chorioamnionitis, shoulder dystocia, and meconium stained fluids leading to a C-section. A head ultrasound prompted by downward gaze was concerning for bleeding. After loading with plasma-derived FVIII, an MRI demonstrated right temporoparietal subgaleal hematoma and bilateral subdural hemorrhages. He was continued on concentrate twice daily with goal trough of $80 \%$. Inhibitor testing at DOL 7 was negative and he began loading with Emicizumab at $3 \mathrm{mg} / \mathrm{kg}$ weekly. Daily bovine chromogenic FVIII levels ranged from 55-114\%. On DOL 11 an MRI showed reduction in size of all hemorrhages. He did well clinically and the downward gaze improved. On DOL 19 he had his third loading dose of Emicizumab. The FVIII was discontinued and he was discharged. He returned to clinic one week later for his 4th loading dose. He continued out-patient maintenance dosing of $1.5 \mathrm{mg} / \mathrm{kg} /$ week. On DOL 54, MRI showed complete resolution of the hemorrhages. He experienced no injection site reactions and no bleeding since discharge.

Conclusions: Emicizumab was used safely and successfully to treat intracranial hemorrhage in an infant with severe hemophilia A who was at increased risk of inhibitor development.

PB0633 | Compare the Efficacy and Safety of Low-dose and Intermediate-dose Prophylactic Therapy in Adult Patients with Severe Hemophilia A, a Self-control Study

h. 高华; R. Chen; S. Zhou

Department of Hematology, The Third People's Hospital of Chengdu, Chengdu, China

Background: WFH guideline recommend any type of prophylaxis regimen- standard, intermediate-dose or low-dose prophylaxis- is 
better than on-demand treatment for hemophilia patients. The latter two regimens are generally applied in adult patients in China.

Aims: To compare the efficacy and safety of low-dose and intermediate-dose prophylaxis in adult patients with severe hemophilia A.

Methods: Sixteen adult patients with severe hemophilia A who received low-dose prophylaxis treatment then transited to intermediate-dose prophylaxis under regular follow-up in hemophilia clinic of Chengdu third people's Hospital were included. The data of annual bleeding rate(ABR), the annual joint bleeding rate(AJBR), the total ultrasonic score (HEAD-US-C) and HJHS score of bilateral elbow joint, knee joint and ankle joint were collected retrospectively. We conduct paired Wilcoxon test with these data by SPSS 22.0.

Results: Sixteen adult patients with severe hemophilia A had a median age of 30 years (20-37 years). The average FVIII consumption per year is $1182 \mathrm{IU} / \mathrm{kg}$ during low-dose prophylaxis period and 1767 $\mathrm{IU} / \mathrm{kg}$ during intermediate-dose prophylaxis period respectively. The average $A B R$ reduced from 21 to $12(P=0.001)$ and the average AJBR declined from 18 to $10(P=0.002)$ after regimen adjustment. The average total ultrasound score showed no significantly difference between low-dose(26.9) and intermediate-dose (31.81) prophylaxis. The average HJHS score demonstrate a significant reduction from 43. 69 points during the low-dose prophylactic treatment period to 37. 31points during the intermediate-dose prophylactic treatment $(P=0.001)$. No FVIII inhibitors occurred after 1 year of intermediate-dose prophylaxis.

Conclusions: Intermediate-dose prophylaxis can significantly reduce the $A B R$ and AJBR in adult patients with severe hemophilia $A$. Although the joint structure damage is not reversable, the joint function can be improved. Medium dose prophylaxis does not increase the risk of FVIII inhibitors in patients with severe hemophilia A.
PB0634 | Effectiveness, Safety and Cost Assessment of Emicizumab in Clinical Practice

A. Riera; A. Plaza; N. Pagès; M. Masip; P. Riera; M. Carrasco; J. Mateo; N. Vilalta

Hospital de la Santa Creu i Sant Pau, Barcelona, Spain

Background: Emicizumab is a humanized bispecific monoclonal antibody that bridges activated factors IX and $X$ to restore the function of factor VIII. It is indicated for prophylaxis of bleeding episodes in patients with haemophilia $A$.

Aims: To describe the effectiveness and safety profile of emicizumab in patients with haemophilia A in clinical practice. We also evaluated the economic impact of emicizumab in comparison with previous treatments.

Methods: A retrospective and descriptive study was conducted involving patients treated with emicizumab between July 2020 and February 2021 in a tertiary hospital. Data were obtained from medical and pharmacy claim records. We collected demographic and clinical variables (sex, age, presence of FVIII inhibitor and haemophilic arthropathy) and related to previous and current therapy (dosification, number of bleeding events and adverse effects). We considered the wholesale acquisition cost of pharmacologic treatments within the Spanish public healthcare system.

Results: Five patients were included. All of them were men with a mean age of $32(20-48)$ and haemophilic arthropathy. Only one patient (No.3) presented FVIII inhibitors at baseline. Mean number of days under emicizumab treatment was 125 (56-224). Main outcomes are depicted at Table 1.

\section{TABLE 1}

\begin{tabular}{|c|c|c|c|c|c|c|}
\hline Patient & \multicolumn{3}{|l|}{ Previous treatment } & \multicolumn{3}{|c|}{ Emicizumab treatment } \\
\hline 2 & Efmoroctocog & 3000 UI/48h & 1 & $300 \mathrm{mg} / 2 \mathrm{w}$ & 161 & 0 \\
\hline 3 & $\begin{array}{r}\text { von Willebrand } \\
\text { factor/FVIII }\end{array}$ & 3000 UI/48h & 0 & $195 \mathrm{mg} / 2 \mathrm{w}$ & 126 & 0 \\
\hline 4 & $\begin{array}{l}\text { Efmoroctocog } \\
\text { Moroctocog }\end{array}$ & $\begin{array}{l}3000 \mathrm{Ul} / 48-72 \mathrm{~h} \\
3000 \mathrm{UI} / 48 \mathrm{~h}\end{array}$ & 1 & $270 \mathrm{mg} / 2 \mathrm{w}$ & 56 & 0 \\
\hline 5 & $\begin{array}{l}\text { PEGylated- } \\
\text { Damoctocog }\end{array}$ & $3000 \mathrm{UI} / 72 \mathrm{~h}$ & 0 & $127 \mathrm{mg} / \mathrm{w}$ & 58 & 1 \\
\hline
\end{tabular}

Concerning effectiveness, four patients achieved clinical response. However, in one patient (No.5) emicizumab treatment was stopped due to lack of effectiveness (haematuria) and a severe injection site reaction. The other four patients did not experience any remarkable side effects.
As for costs, emicizumab treatment was more cost-effective in comparison to previous treatments. Due to confidentiality reasons, we are not allowed to disclose the exact acquisition costs.

Conclusions: Our results suggest that emicizumab is a cost-effective treatment for haemophilia A. Despite the favourable safety profile observed in clinical trials, our results show that severe adverse 
effects can appear and contribute to treatment discontinuation. Further studies are warranted to establish the effectiveness and safety in clinical practice.

PB0635 | Hemophilia and Concussion Outcome Study (HeCos): Neurological Status of Children with Hemophilia - Prevalence and Relevance of Mild Traumatic Brain Injury

A. Berghuber ${ }^{1}$; C. Bidlingmaier ${ }^{1}$; M. Marx ${ }^{1} ;$ S. Lardschneider ${ }^{1} ;$ L. Wiederer $^{1}$; M. Olivieri ${ }^{1}$; S. Juranek ${ }^{1}$; S. von Mackensen ${ }^{2}$; K. Kurnik ${ }^{1}$; M. Bonfert ${ }^{1}$

${ }^{1}$ Dr. von Haunersches Children Hospital, Ludwig-Maximilians-

Universität, Munich, Germany; ${ }^{2}$ University Medical Centre HamburgEppendorf Department of Medical Psychology, Hamburg, Germany

Background: Mild traumatic brain injury (mTBI) is a common pediatric incident. Despite this, only sparse data exist on the prevalence and consequences of mTBI in patients with hemophilia (PWH). mTBI itself may impact a child's development, as has been reported for children without hemophilia. Since traumatic injuries, including mTBI, are often well documented for patients with hemophilia as incidents leading to factor substitution are generally documented in the patients substitution diaries, PWH could form an interesting and well documented patient group to study $\mathrm{mTBI}$ and its possible consequences.

Aims: This study aims at documenting the prevalence of $\mathrm{mTBI}$ in $\mathrm{PWH}$ and at reporting on the neurological status of $\mathrm{PWH}$.

Methods: Cross-sectional study, including 50 PWH aged 4-17 years. Investigation of motor profiency (Hemophilia Joint Health Score, BOT -2 Bruininks-Oseretzky Test, HEP-Test-Q), neurological status (mild neurological dysfunction, posturography, gait analysis dual task gait paradigma) and psychological aspects (Haemo-QoL Questionnaire, Child Behaviour Checklist). Patient records and substitution diaries are reviewed to document the following: head injury and its consequences (i.e. factor substitution, medical presentation, inpatient monitoring, imaging), documented further bleeds, including joint bleeds, medical history including treatment and complications. The study was approved by the local IRB and informed consent was obtained from all participants and their caregivers.

Results: The study is still ongoing. Until abstract submission retrospective chart analysis was performed for all eligible patients and $n=20$ patients have been clinically evaluated. Until presentation at the congress $n=40$ participants are anticipated to be included. Their data will be presented.

Conclusions: Based on the findings the significance of regular neuropediatric/developmental status assessments within care programs for patients suffering hemophilia may be discussed: the clinical outcome after head injury in PWH may be closely followed to provide rehabilitation or specialized therapy (i.e. occupational therapy) on an early stage.

PB0636 | Discrepancies in Non-severe Hemophilia A. Experience in a Hemophilia Center in Argentina

E. Sueldo ${ }^{1}$; R. Porsella ${ }^{1}$; L.C.R. Rossetti ${ }^{2}$; V. Marchione ${ }^{2}$; P. Radic ${ }^{2}$; C. De Brasi ${ }^{2}$; A. Baques ${ }^{1}$; M. Arias ${ }^{1}$

${ }^{1}$ Hospital Dr. Cesar Milstein, Caba, Argentina; ${ }^{2}$ Instituto de Medicina Experimental (IMEX), CONICET-Academia Nacional de Medicina (ANM), Caba, Argentina

Background: Diagnosis of hemophilia $A$ is generally based on the measurement of plasma factor VIII activity using the one-stage assay (FVIII:OSA) or the two-stage chromogenic substrate assay (FVIII:CSA). The results of these methods show considerable discrepancy in about one-third of non-severe hemophilia A patients. This can lead to failure in diagnosing HA or incorrectly classifying the severity of the disease. Aims: To evaluate the frequency of discrepancies between FVIII:OSA and FVIII:CSA, in the diagnostic classification of Patients with Hemophilia.

Methods: From 2012 to 2020, 107 patients with bleeding disorders were diagnosed as Hemophilia A (HA). Hemostasis laboratory studies were performed (Prothrombin time (PT), Activated Partial thromboplastin time (aPTT), Thrombin Time (TT), aPTT 1:1 mix, FVIII:OSA, FVIII:CSA, $v W: A g, v W: C o R)$. Patients were initially categorized into the different clinical categories by FVIII:OSA (Severe Hemophilia A: $\mathrm{SHA}<0.01 \mathrm{IU} / \mathrm{mL}$, Moderate Hemophilia A: mHA 0.01-0.05 IU/mL, Mild Hemophilia A: $\mathrm{miHA} 0.05-0.40 \mathrm{IU} / \mathrm{mL}$ ). FVIII Assay discrepancy was defined as a twofold or greater difference between the results of two assays. (FVIII:OSA/ FVIII:CSA $\leq 0.5$; FVIII:CSA/FVIII:OSA $\geq 2.0$ ), correlating it with the clinical phenotype. Genotyping: PCR-amplification followed by conformation sensitive gel electrophoresis (CSGE) and Sanger sequencing.

Results: 78 Patients were diagnosed as SHA, 29 Patients as nonsevere HA. 5 Patients with non-severe HA showed disagreement between the methods (Table 1 ).

TABLE 1 Patients with discrepancies

\begin{tabular}{|c|c|}
\hline Patients & F8-genotype variants \\
\hline 1 & c.6246C>A p.(Ser2082Arg) \\
\hline 2 & $\begin{array}{l}\text { c. } 5399 \mathrm{G}>\mathrm{A} \text { p. }(\text { Arg1800His) c.3780C }>\mathrm{G}(\mathrm{rs} 1800291) \\
\text { p.(Asp1260Glu) }\end{array}$ \\
\hline \multirow[t]{2}{*}{3} & c.5508G>T p.(Trp1836Cys) \\
\hline & c. $3780 \mathrm{C}>\mathrm{G}(\mathrm{rs} 1800292)$ p.(Ser1288=) \\
\hline
\end{tabular}

\section{FVIII:OSA}

2-5

$2-2.9$

$8-10$
FVIII:CSA

$1-2.5$

$0.8-1$

$2-4$
OSA/CSA

2.0

$2.5-2.9$

c.3780C > G (rs1800292) p.(Ser1288=) 
Patients F8-genotype variants

c.5981T>C p.(Leu1994Pro)

c. $5998+91 T>A$ rs4898352

c.3864A >C rs1800292 p.(Ser1288=)

5

c.5981T>C p.(Leu1994Pro)

FVIII:OSA

2-5

2.2-5
FVIII:CSA

0.9-1

$0.8-1$
OSA/CSA

2.2-5.0

c. $5998+91 \mathrm{~T}>\mathrm{A}$ rs 4898352

c. $3864 A>C$ rs1800292 p.(Ser1288=)

Conclusions: $6 \%$ of patients with $\mathrm{AH}$ showed discrepancies, being $17 \%$ of patients with non-severe Hemophilia A. A lower frequency was found with respect to the bibliography. We conclude that nonsevere haemophilia patients could benefit if chromogenic assay is in cluded in diagnosing and classification on severity haemophilia, and it also could help to individualize group of patients that could benefit from early replacement treatment.

PB0637 | Real World Experience with Emicizumab with Hemophilia A Treated in Private Practice in Mexico

M.T. Pompa Garza ${ }^{1}$; A. Arias Aranda ${ }^{2}$; R. Miranda Madrazo ${ }^{3}$; N. Padilla Duron ${ }^{4}$; C.S. Gómez González ${ }^{5}$

${ }^{1}$ Universidad Autonoma de Nuevo Leon, Monterrey, Mexico;

${ }^{2}$ Universidad La Salle, Mexico City, Mexico; ${ }^{3}$ Hospital Español, Mexico City, Mexico; ${ }^{4}$ Hospital General de Occidente, Guadalajara, Mexico;

${ }^{5}$ Hospital del Niño y la Mujer, Queretaro, Mexico

Background: Emicizumab is a biespecific, humanized, monoclonal antibody that restores the function of missing FVIIla in patientes with hemophilia A (PwHA) .In Mexico, Emicizumab is approved for $\mathrm{PwHA}$ with and without inhibitors, but the experience in $\mathrm{PwHA}$ with special characteristics is limited.

Aims: The objective of this retrospective study was to describe our real-world experience in prescribing Emicizumab prophylaxis in 10 PwHA with special characteristics treated in private practice in Mexico.

Methods: Eligible patients for our retrospective analysis included patients with severe hemophilia A treated with Emicizumab in ptivate practice in Mexico between September 2019 to December 2020. Data collected included: age, bleeding, and treatment history before and after initiation on Emicizumab, the reason for switching, comorbidities, and adverse events.

Results: We presented the data of ten patients, median age 8 years, all males, who attended our clinics. All of them received Emicizumab for more than 4 months. Nine switched from FVIII to Emicizumab and 8, 9 were on prophylaxis or ITI with either rFVIII or pdFVIII. Patient 10 was a PUP and received FVIII secondary to bleeding and he started on Emicizumab afterward. Patient 1, received ITI for a year without success, he is currently on Emicizumab + ITI and he reported 0 bleeds since then. Patient 4 has obesity and autism and patients 6-8 are siblings, all of their caregivers reported improved adherence with the SQ therapy started. Patient 8 has been treated with cryoprecipitates and he has HIV, HCV, and chronic arthropathy. All patients improve their bleeding rate, $80 \%$ have 0 bleeds. No significant adverse events were observed.

Conclusions:

TABLE 1 Demographics and Results

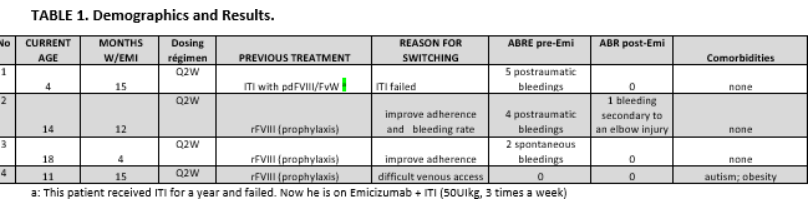

TABLE 2 Demographics and Results Cont.

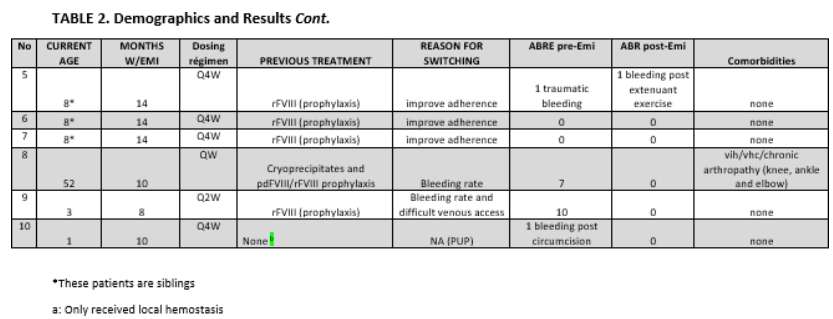

Prophylaxis with Emicizumab was efficacious and well-tolerated in our PwHA with special characteristics. All of them improve their bleeding rate and adherence. More data are needed on the use of Emicizumab, particularly in PUPs and patients with comorbidities concerning safety and efficacy.

PB0638 | Low-dose Prophylaxis in Severe Hemophilia A: A Single-center Study

M. Borhany ${ }^{1}$; M. Abid ${ }^{1}$; R. Ahmed ${ }^{2}$; A. Jamal ${ }^{2}$; S. Zafar ${ }^{1}$; R. Nadeem ${ }^{2}$ ${ }^{1}$ National Institute of Blood Disease, Karachi, Pakistan; ${ }^{2}$ Hemophilia Welfare Society Karachi, Karachi, Pakistan

Background: Prophylaxis is not practiced in Pakistan primarily due to non-affordability of clotting factor concentrates, which is why the majority of these patients are treated by on demand administration of factor VIII concentrates to stop bleeding. 
Aims: To access low dose prophylaxis (LDP) for the management of severe Hemophilia A (HA) patients.

Methods: A prospective observational study was conducted from 2018 to 2020. Sixteen children with severe HA (ages 1-10) were enrolled for prophylaxis ( 250 units or $20 \mathrm{lU} / \mathrm{kg}$ twice weekly) and observed. APTTbased inhibitor screening was done at baseline and intervals as needed. The two scales used for the evaluation of joint assessment were; the Functional Independence Score (FISH) and the Hemophilia Joint Health Score (HJHS). Annual bleeding rate was also documented.

Results: A total of 16 patients were recruited in the LDP study with a median age of $5.5(\mathrm{IQR}=5.5)$ years. Joint assessment scoring of hemophilia patients is mentioned in Table 1. Annual bleeding rate before prophylaxis was observed as minor bleeding in 9 (56.2\%) patients and major bleeding in 7 (43.7\%). After prophylaxis, 9 (56.2) patients had no bleeding while $6(37.5 \%)$ reported minor bleeding. One (6.25\%) patient, however, witnessed major bleeding. The annual joint bleeding was improved after two years of prophylaxis as illustrated in Figure 1. Overall, 16 patients received $1005750 \mathrm{IU} / \mathrm{kg} /$ year of prophylaxis while 10 (62.5) received $44000 \mathrm{IU} / \mathrm{kg} /$ year as on-demand treatment. Absenteeism from school was reduced from 6 (37.5\%) to 1 (6.25\%) patient after prophylaxis. No history of hospitalization and inhibitor development was observed. Furthermore, ultrasound imaging showed no degeneration in joints prior to or after receiving prophylaxis.

TABLE 1 Joint assessment scoring on 2 different scales in a hemophilia patients

\begin{tabular}{llll} 
& Before & After & P-value \\
FISH & 28 & 32 & 0.002 \\
$\begin{array}{l}\text { HJHS + Joint specific score+ } \\
\text { GGS }\end{array}$ & 3.13 & 1.60 & 0.077 \\
\hline
\end{tabular}

Note Global gait score (GGS), Functional Independence Score (FISH), Hemophilia Joint Health Score (HJHS).

Figure 1: Annual joint bleed rate (AJBR) in Hemophilia A patients

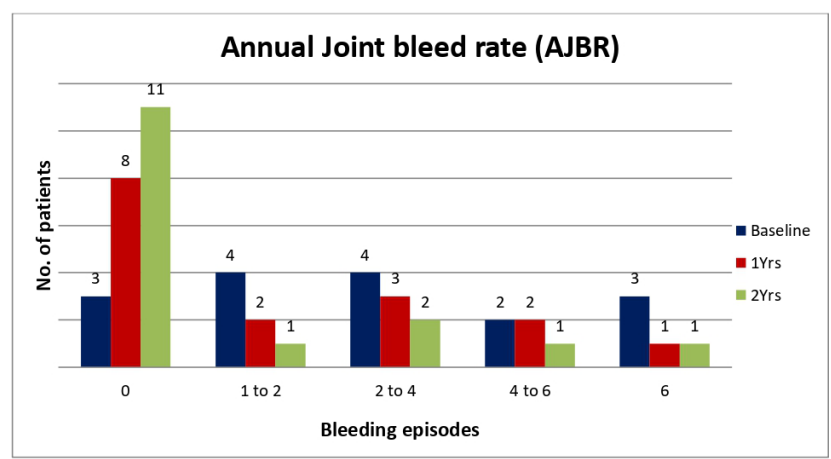

FIGURE 1 Annual joint bleed rate (AJBR) in Hemophilia A patients

Conclusions: Functional Independence Score in hemophilia improved after treatment as annual bleeding rate reduced. This in turn improved the quality of life of severe HA patients on LDP.
PB0639 | Off-pump Coronary Artery Bypass Graft Management in a Severe Hemophilia B Patient

G. Barillari; A. Poz; A. Bertone; M.E. Mosanghini

Azienda Sanitaria Universitaria Friuli Centrale, Udine, Italy

Background: Hemophilia B (HB) is a rare X-linked congenital bleeding disorder characterized by a deficiency of coagulation factor IX (FIX). There is evidence that people with hemophilia develop atherosclerosis at similar rates to those in the general population; moreover, they also may develop ischemic heart disease.

Aims: The aim of this clinical case is to highlight that a good management of $\mathrm{HB}$ patients undergoing coronary artery bypass graft (CABG) is essential for successful intervention.

Methods: A 58-year-old man suffering from severe $\mathrm{HB}(\mathrm{FIX}<1 \%)$ reported symptomatic retrosternal burning when walking in cold weather. He presented many comorbidities, as arterial hypertension, type 2 diabetes mellitus, hyperlipidemia, overweight. Coronary angiography highlighted obstructive coronary artery disease with two-vessel critical stenosis. In consideration of the patient's ischemic and hemorrhagic risk, CABG without extracorporeal circulation (off-pump, OP) was performed, through skeletonized left internal mammary artery on anterior interventricular artery. $6000 \mathrm{UI}$ of albutrepenonacog, a recombinant FIX fused with albumin, were administered intravenously one hour before the procedure, together with tranexamic acid at $500 \mathrm{mg} / \mathrm{hour}$ as a continuous infusion. After OP-CABG, tranexamic acid was administered 1 gram every 8 hours, for seven days. Albutrepenonacog 4000 IU was infused in the third post-surgery day, and, afterwards, once a week to maintain FIX levels above $50 \%$. One unit of concentrated red blood cells was transfused. No hemorrhagic or thrombotic events were documented. Acetylsalicylic acid $75 \mathrm{mg}$ /day was prescribed.

Results: Four months of cardiological rehabilitation were carried out, with progressive subjective improvement and tolerance to effort. After a 6-month follow-up, ischemic heart disease was clinically stable.

Conclusions: This report shows that a good management of coronary artery disease in HB patients is possible. It requires close cooperation between hemophilia and cardiology teams, and should be individualized in terms of hemophilia therapy and choice of the best treatment for the disease.

PB0640 | A Prospective Cohort Study on Disabilities in Patients with Haemophilia Using Orthopaedic Scores

M. Bajracharya; B.S. Poudyal; S. Marahatta; S. Rayamajhi; V.

Prajapati; C.D.K. Mahato

Civil Service Hospital, Kathmandu, Nepal

Background: Hemophilia is an X-linked recessive disorder which may be inherited or arise from spontaneous mutation. Nepal is a developing country, patients with hemophilia $(\mathrm{PWH})$ are unable to purchase clotting factors. Patients are unable to receive prophylaxis and treatment. Disability becomes an inevitable consequence. 
Aims: Our study aims to highlight the need to provide coagulation factor concentrates (CFC) in sufficient amounts to prevent disability. Methods: Total number of PWH enroll in the World Bleeding Disorder Registry is 405.50 of them were enrolled in our study. Patients with Hemophilia A and B, age ranging from $10-55$ years with mild, moderate and severe hemophilia were selected by simple random sampling. Joint assessment was done using HJHS, FISH and WOMAC score. Radiological assessment of joint damage was done by Pettersson score. Results: Most of PWH enrolled in our study were unable to perform their daily activities (walking on flat surface, climbing up the stairs) like healthy individuals. Six out of 50 PWH (12\%) enrolled in this study needed crutches to walk, $24 \%(n=12)$ had difficulty in performing self-care activities like eating, bathing, dressing, 34\% ( $n=17$ ) of PWH experience daily pain. Proportion of PWH who experienced bleeding episodes one or more times per month was $80 \%(n=45)$ while those who were able to receive factor for their bleeds was $50 \%$ ( $n=25)$, which can be related to the lack of funding for CFC. Average family income annually (approximately 1500USD) shows that it is almost impossible to purchase clotting factors. HJHS, which was designed for children and adolescent, has been found to be significantly applicable in adult PWH. WOMAC score, which was used for osteoarthritis, has been used in Hemophilia and has positive correlation with FISH, HJHS and Pettersson.

Conclusions: Due to lack of CFCs, PWH in Nepal have frequent bleeds, which has led to a high prevalence of disability.

PB0641 | Patient-centered Digital Health Interventions and their Effects on Health Outcomes: A Systematic Review

M.R Brands ${ }^{1}$; S.C Gouw ${ }^{1,2}$; M. Beestrum ${ }^{3}$; M. Driessens ${ }^{4}$; R.M. Cronin $^{5}$; K. Fijnvandraat ${ }^{1,6}$; S.M Badawy ${ }^{3,7}$

${ }^{1}$ Amsterdam UMC, University of Amsterdam, Emma Children's Hospital, Pediatric Hematology, Amsterdam, Netherlands; ${ }^{2}$ Department of Clinical Epidemiology, Leiden University Medical Center, Leiden, Netherlands; ${ }^{3}$ Feinberg School of Medicine at Northwestern University, Chicago, United States; ${ }^{4}$ Dutch Association of Hemophilia-Patients (NVHP), Nijkerk, Netherlands; ${ }^{5}$ The Ohio State University, Columbus, United States; ${ }^{6}$ Department of Molecular Cellular Hemostasis, Sanquin Research and Landsteiner Laboratory, Amsterdam, Netherlands; ${ }^{7}$ Ann and Robert H. Lurie Children's Hospital of Chicago, Chicago, United States

Background: Congenital bleeding disorders have a large impact on patient's lives due to the high disease burden. Patient-centered digital health interventions, such as patient portals and personal health records, can significantly help engage and empower patients with chronic health conditions, including congenital bleeding disorders. Highly engaged patients have higher knowledge of their disease, more self-efficacy, better self-management, and improved clinical outcomes. However, this relationship is not well-studied and has not yet been investigated thoroughly in congenital bleeding disorders.
Aims: Our aim is to evaluate the most recent evidence for the effects of patient-centered digital health interventions on health outcomes and patient satisfaction, specifically for non-hospitalized children and adults with chronic health conditions, including congenital bleeding disorders.

Methods: We searched MEDLINE, Cochrane Library, CINAHL, EMBASE and PsycINFO databases between 2000 and November 2020. PRISMA guidelines were followed. Two independent reviewers screened titles, abstracts and full-text papers. Eligible studies were original full-text research papers that evaluated an intervention with a digital portal function, intended for non-hospitalized patients with a chronic health condition. Patients with a high disease burden are a subgroup of additional interest. Primary outcomes included clinical and self-reported outcomes. Secondary outcomes included healthcare utilization, treatment adherence, patient satisfaction, feasibility and acceptability.

Results: Our search yielded 7242 unique records. After title and abstract screening, 298 records remained for full-text screening. These are currently being analyzed. Final data will be presented at the conference.

Conclusions: We expect that these results will provide more insight in how patient-centered digital health interventions are utilized and leveraged to improve relevant health outcomes among patients with congenital bleeding disorders.

PB0642 | Joint Health Improvement Following a Switch to Prophylaxis with rVIII-SingleChain: A Patient Case

O. Benítez Hidalgo ${ }^{1}$; M.F. Martinez Garciaㄹ; N. Miranda Pinzon²; E. Ibañez Gomez ${ }^{1}$; N. Blazquez Gomez ${ }^{1}$; M. Suito Alcantara ${ }^{3}$ ${ }^{1}$ Hemophilia Unit, Hematology Department, Vall d'Hebron Hospital, Barcelona, Spain; ${ }^{2}$ Hematology Department, Vall d'Hebron Hospital, Barcelona, Spain; ${ }^{3}$ Hemostasis Unit, Hematology Department, Vall d'Hebron Hospital, Barcelona, Spain

Background: Joint arthropathy can significantly impact the quality of life of patients with hemophilia, and often leads to the need for joint replacement. Early use of prophylactic factor replacement can reduce or prevent arthropathy; however, some joint bleeds may still occur. rVIII-SingleChain is a long-acting novel recombinant FVIII (rFVIII) with improved pharmacokinetic and pharmacodynamic properties compared with standard-acting rFVIII.

Aims: To describe the case of a 49-year-old male with severe hemophilia A, hemophilic arthropathy, and target joints who switched from prophylaxis with plasma-derived von Willebrand factor (VWF)/ FVIII (pdVWF/FVIII) to rVIII-SingleChain.

Methods: A patient treated prophylactically with pdVWF/FVIII twice-weekly since March 2018 was referred for orthopedic consultation and assessed using HEAD-US. He presented with a high annualized bleeding rate (ABR) and annualized joint bleeding rate (AJBR) despite high treatment adherence. He was deemed a good candidate for switching to rVIII-SingleChain to reduce his bleeding frequency. 
Results: In February 2018, assessment using HEAD-US showed damage to the articular surfaces in the cartilage in both ankles and both elbows and in the bone in both ankles and the left elbow; synovitis was observed in both ankles. In July 2019, the patient switched to rVIIISingleChain prophylaxis every four days. Compared with baseline, after 1 year of treatment, adherence to the regimen was improved (92.9\% vs. 100\%) and both ABR and AJBR significantly improved ( 4.61 vs. 0 and 4.61 vs. 0 , respectively). A further assessment using HEAD-US in November 2020 showed that synovitis was no longer present in the right ankle. All target joints were considered resolved following the switch. The patient is satisfied with his treatment and has not required any extra infusions despite an active lifestyle.

Conclusions: This case report demonstrates an improvement in joint health following the switch to rVIII-SingleChain in a patient with severe hemophilia $\mathrm{A}$ and significant joint damage.

PB0643 | Urosurgical Management in Mild Hemophilia with Inhibitor: A Case Report

L. Phillips ${ }^{1}$; M. Movassaghi ${ }^{2}$; C.P. Carpenter ${ }^{2}$; D. Kaur ${ }^{1}$

${ }^{1}$ Department of Pediatric Hematology, Oncology \& SCT, Morgan Stanley Childrens Hospital, Columbia University Irving Medical Center, New York, United States; ${ }^{2}$ Department of Urology, Morgan Stanley Childrens Hospital, Columbia University Irving Medical Center, New York, United States

Background: Optimal urosurgical perioperative hemostasis management in patients with hemophilia $A$ and inhibitors on emicizumab is not well established.
Aims: Discuss peri-operative hemostatic management in a hemophilia patient with inhibitor on emicizumab undergoing major surgery.

Methods: We present a 6-year-old boy with a factor VIII level of $\sim 35 \%$ by one-stage assay, identified after developing bilateral scrotal hematomas following orchidopexy surgery. Genetic testing confirmed diagnosis of hemophilia A with a novel single point missense mutation c.1649G >A, Arg531His. He re-presented at 8 years old with extensive perineal hematoma and was treated with daily recombinant factor eight ( $r F V I I I)$ replacement. However hematuria persisted and he was found to have a urethral fistula communicating with the hematoma. Treatment with rFVIII was extended while awaiting surgical fistula repair, during which time he developed a high-titer inhibitor (11.2 BU). Therapy was switched to emicizumab 4 weekly loading doses, followed by maintenance monthly dosing. The patient underwent urethroplasty, cystoscopy and open cystotomy with suprapubic tube placement, with emicizumab dose 2.5 weeks prior to surgery. Inhibitor level at time of surgery was $10 \mathrm{BU}$. Post-operative hematuria with passage of clots was not controlled despite high frequency dosing of rFVIla, and ultimately required limited FEIBA doses for bleeding cessation and systemic antifibrinolytic therapy accompanied by continuous bladder irrigation (Table 1). Emicizumab was switched to weekly maintenance 10 days post-operatively. Until suprapubic catheter was removed, patient continued on daily rFVIla and aminocaproic acid. There were no thrombotic events.

TABLE 1 Perioperative hemostatic agents in case patient's urologic surgery

\begin{tabular}{|c|c|c|c|c|}
\hline Time & Single dose & Q3H & Q4H & Q6H \\
\hline Preoperative & $\begin{array}{l}\text { rFVIla } 100 \text { mcg/kg } \\
\text { rFVIla } 50 \text { mcg/kg }\end{array}$ & & & \\
\hline Intraoperative & $\mathrm{rFVIla} 50 \mathrm{mcg} / \mathrm{kg} \times 2$ & & & \\
\hline POD 0 & & & & rFVIIa 15 mcg/kg \\
\hline POD 1 & & & & rFVIla 50 mcg/kg \\
\hline POD 2 & & & rFVIIa 75-100 mcg/kg & \\
\hline POD 3 & & & rFVIla 100 mcg/kg & \\
\hline POD 4 & & rFVIla 100 mcg/kg & & \\
\hline POD 5 & FEIBA $25 \mathrm{IU} / \mathrm{kg} \times 2$ & & & \\
\hline POD 6 & FEIBA $25 \mathrm{IU} / \mathrm{kg} \times 2$ & & & \\
\hline POD 7-9 & & & rFVIIla $100 \mathrm{mcg} / \mathrm{kg}$ & Aminocaproic acid 50 mg/kg \\
\hline POD 10 & Emicizumab 1.5 mc/kg & & rFVIIla $100 \mathrm{mcg} / \mathrm{kg}$ & Aminocaproic acid $50 \mathrm{mg} / \mathrm{kg}$ \\
\hline POD 11 & FEIBA $25 \mathrm{IU} / \mathrm{kg} \times 2$ & & & Aminocaproic acid $50 \mathrm{mg} / \mathrm{kg}$ \\
\hline POD 12 & & & rFVIIla $100 \mathrm{mcg} / \mathrm{kg}$ & Aminocaproic acid $50 \mathrm{mg} / \mathrm{kg}$ \\
\hline \multicolumn{5}{|l|}{ POD 13-16 } \\
\hline & & & & rFVIla 100 mcg/kg \\
\hline & & & & Aminocaproic acid $50 \mathrm{mg} / \mathrm{kg}$ \\
\hline
\end{tabular}




\section{Time}

POD 17

Single dose

Emicizumab $1.5 \mathrm{mc} / \mathrm{kg}$

POD 18-35*

POD 36-38^

rFVIla $100 \mathrm{mcg} / \mathrm{kg}$
Q3H

Q4H
Q6H

rFVIla $100 \mathrm{mcg} / \mathrm{kg}$

Aminocaproic acid $50 \mathrm{mg} / \mathrm{kg}$

rFVIla $100 \mathrm{mcg} / \mathrm{kg}$

Aminocaproic acid $50 \mathrm{mg} / \mathrm{kg}$

once daily

POD = post-operative day

*Suprapubic catheter removed POD 35.

${ }^{\wedge} \mathrm{PICC}$ line removed POD 38.

Results: In major urologic surgery, rFVIla and emicizumab proved insufficient for hemostasis. Our patient required additional bypassing agent FEIBA and systemic antifibrinolytic therapy for adequate hemostasis.

Systemic antifibrinolytic therapy can be safely used if concurrent urologic support for bladder irrigation and manual clot removal is available.

Conclusions: Hemostasis following major urologic surgery in a hemophilia A patient with inhibitor on emicizumab required additional agents aside from rFVIIa.

PB0644 | Consistency Analysis and Data Validation Regarding Hospitalization among Hemophilic Patients from the Hemophilia Center of the Hematology Service of HC FMUSP - (2008-2018)

A.C. Guersoni $^{1}$; P. Villaça ${ }^{2}$; V. Rocha ${ }^{2}$; E. Trindade ${ }^{3}$; O.D. Luiz ${ }^{4}$

${ }^{1}$ University of São Paulo, Collective Health, São Paulo, Brazil;

${ }^{2}$ University of São Paulo, Hematology, São Paulo, Brazil; ${ }^{3}$ University of São Paulo Medical School, Health Technology Assessment, São Paulo, Brazil; ${ }^{4}$ University of São Paulo, Collective Health,, São Paulo, Brazil

Background: Prophylaxis treatment for Severe Hemophilic patients has been implemented in Brazil since 2011. Its implementation logistics used the system organized by the Ministry of Health $(\mathrm{MoH})$ two years before: - the national registry of patients with inherited coagulopathies and other bleeding disorders, Hemovida Web Coagulopatias* (HWC). The HCW registry compiles profile of patients, diagnosis, treatments and complications (http://coagulopat iasweb. datasus.gov.br).

Aims: The consistency analysis for validation of the data regarding hospitalizations among hemophilic patients from the HC FMUSP. They were hospitalized in the in Public Hospitals from the Health Secretary of the São Paulo State (SES-SP).

Methods: Amid patients with Hemophilia, registered at HWC as under treatment at FMUSP ( $n=267)$, 92\% $(n=246)$ were followed from 2008 to 2018, and 30\% were hospitalized ( $n=73$ ) according to the SES-SP registries. The consistency analysis applied data validation through current HC FMUSP medical records review regarding the hospitalization diagnosis, length of stay and procedures realized. We estimated HCW performance comparing the periods before and after the implementation of the Brazilian primary prophylaxis treatment for severe patients.

Results: From 246 followed at HC FMUSP (2008-2018), 73 patients were hospitalized in this period. They were diagnosed at the mean age of 5,7 years (range: $0-46,8$ years).

TABLE 1 Number of patients hospitalized in 2008 and 2018, and the ages.

\begin{tabular}{lll} 
& 2008 & 2018 \\
$\begin{array}{l}\text { Patients Hospitalized } \\
\text { (number/year) }\end{array}$ & 13 & 6 \\
Age (years in $\quad \begin{array}{l}15 \text { year } \\
\text { (range: 0,8-52,9) }\end{array}$ & $\begin{array}{l}\text { (range: 2,4-62,9) } \\
\text { avarage) }\end{array}$ & \\
min - max & & \\
\hline
\end{tabular}

TABLE 2 Hospitalization/year, age and causes of the Hemophilic inpatients from Hemophilia Center of the Hematology Service of HC FMUSP, in period $\mathrm{A}(2008-2011)$ and $\mathrm{B}$ (2012-2018)

2008-2011 (A)

Hospitalizations/year (min-max)

Age
$6,7(3,8-9,5)$

15 year (average) (range: 0,8-52,9)
2012-2018 (B)

$3,2(1-6,2)$

22 year (average) (range: 2,4-62,9) 
2008-2011 (A)

Main causes of Hospitalizations
1- Factor infusion

2- Bruise or hemorrhage

3- Trauma or Traumatic brain injury

4- Upper digestive bleeding,

5- Hematuria
2012-2018 (B)

1- Factor infusion (in pediatric patients)

2- Mild traumatic brain injury

3- Fractures and arthroplasty.
Conclusions: The registered dates of hospital admission at SUS varied: some patients went to other hospitals some were registered by the municipal or state managers.

Although some discrepancies there were 52\% decrease in hospitalization, probably due to the implementation of the primary prophylaxis program in Hemophilia, since 2011.

The hospitalizations causes were similar to literature In period B most hospitalizations for factor administration ( $<5$ years), while parents were trained for home infusion. Brain injuries $(<8 y)$ arthroplasties in older.

PB0645 | Real-world Experience of rIX-FP Prophylaxis at Dosing Intervals of up to 14 Days in a Pediatric Patient with Hemophilia B during the COVID-19 Pandemic

\section{B.L. Díaz Jordan ${ }^{1}$; T. Cebanu²; M.T. Álvarez Román ${ }^{2}$}

${ }^{1}$ Servicio de Hematología, Hospital General de Valdepeñas, Ciudad

Real, Spain; ${ }^{2}$ Unidad de Hemostasia, Servicio de Hematología, Hospital

Universitario La Paz, Madrid, Spain

Background: rIX-FP, a recombinant factor IX (rFIX) linked with recombinant human albumin, has demonstrated efficacy in prophylactic regimens up to every 21 days in patients with hemophilia $B$.

Aims: To describe real world experience for a rural pediatric patient during the COVID-19 pandemic.

Methods: Demographic, clinical (HEAD-US and Hemophilia Joint Health Score) and pharmacokinetic (PK) data were collected for up to one year following a switch from rFIX to rIX-FP.

Results: In May 2018, a 3-year-old patient (moderate hemophilia B, FIX: $2.7 \mathrm{IU} / \mathrm{dL}$ ) experienced hemarthrosis in the left knee and initiated prophylaxis with rFIX (40 IU/kg 2×/week). In March 2020, the patient switched to weekly rIX-FP prophylaxis (40 IU/ $\mathrm{kg}$ ), due to difficulties with venous access and accessing the clinic during the pandemic. Trough levels of $7.7 \%$ two weeks post-infusion and $5.1 \%$ 20 days post-infusion were maintained. Functional and joint assessment on day 90 of rIX-FP treatment showed no change, and no FIX inhibitors developed. On day 95 of treatment, a post-traumatic otorrhagia subsided after a single $40 \mathrm{IU} / \mathrm{kg}$ dose of rIX-FP. On day 120 , the treatment interval was extended to 10 days without complications. After 160 days, the patient was diagnosed with an asymptomatic SARS-CoV-2 infection and self-isolated at home for 14 days with no changes to prophylactic treatment. On day 200, after a new PK study (trough levels of $6.9 \%$ at 14 days post-infusion), the prophylaxis interval was prolonged to $70 \mathrm{IU} / \mathrm{kg}$ every 14 days without incident. With 14-day dose intervals, monthly consumption reduced by
$56.2 \%$ and the number of infusions by $75 \%$ compared with the initial rFIX treatment, without affecting efficacy or adherence.

Conclusions: Switching to rIX-FP in this pediatric patient reduced the number of administrations and clinic visits without affecting efficacy or adherence, a fact that is relevant in the current context of the pandemic.

PB0646 | Clinical and Pharmacokinetic Assessment after Switching to an Extended Half-life Product for Prophylaxis in Haemophilia B

K.I. Álvarez Tosco; M.Á. Ocaña Gómez; A.M. Ríos de Paz; M.D. De Dios García; M.C. Mesa Lorenzo; J. Merino Alonso Hospital Universitario Nuestra Señora de Candelaria, Santa Cruz de Tenerife, Spain

Background: Hemophilia concentrates' pharmacokinetics (PKs) have important variability between patients, for this reason standard doses often result far from goal plasmatic concentrations. Prophylactic treatment efficacy, which has become the gold-standard in patients with moderate or severe hemophilia, is based on plasmatic concentrations which depends on several variables: genotype, fenotype, bleeding events (intensity, location, frequency), lifestyle, age, weight and physical activity, among others. PK-guided approach is necessary in these patients to personalize each treatment.

Aims: Clinical and pharmacokinetic assessment of hemophilia B patients with prophylactic treatment, who have been switched from standard recombinant products (SHL) to extend half-life recombinant factors (EHL).

Methods: PKs were assessed in four hemophilia B patients with prophylactic treatment based on SHL who were switched in December 2020 to EHL factors. Data was collected from medical records and PK software, results were compared to prior prophylaxis regimen.

Results:

The patients, all males, three diagnosed with severe and one with moderate hemophilia $\mathrm{B}$ had been receiving infusions of SHL productos, mean doses of $40 \mathrm{UI} / \mathrm{kg}$ twice a week, and with trough levels from 2 to $5 \%$. After switching to EHL factors through levels achieved were from 5 to $12 \%$ with doses of 35-50 UI/kg each week or each two weeks. However, all patients had joint bleeding and ache after switching. 
Patient 1

a) $\mathrm{SHI}$

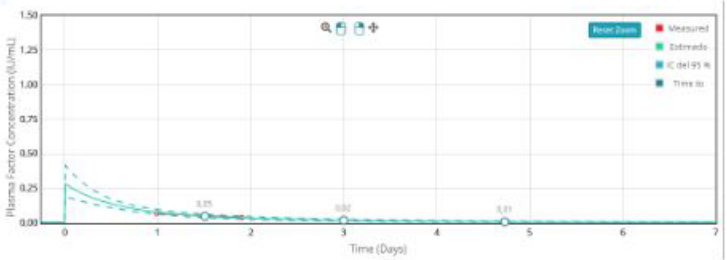

b) $\mathrm{EHL}$

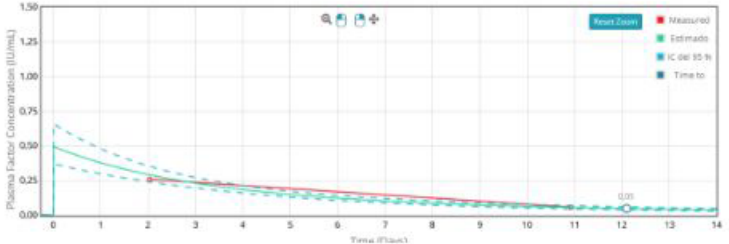

FIGURE 1 Patient 1

Patient 2

a) $\mathrm{SHL}$

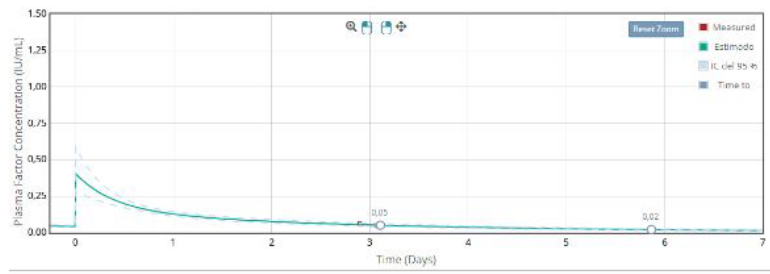

b) EHL

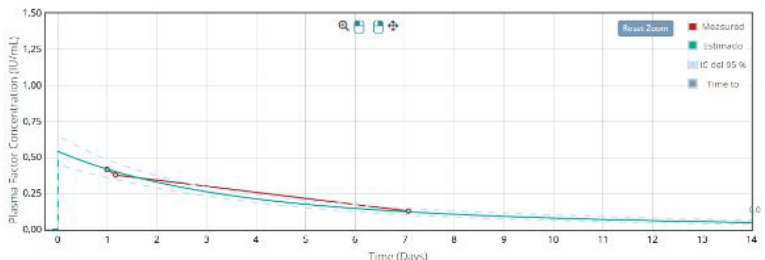

FIGURE 2 Patient 2

Conclusions: Switch to EHL was associated with an improvement in PK profile and dosification without improving clinical outcome. These results raised questions about the convenience of PK-guided approaches in these treatments.

\section{PB0647 | Inhibitors in Non Severe Haemophilia A (NS-HA): A}

\section{Case Report}

M. R. Lopez; E. Lopez Ansoar; O. Dominguez Muñiz; A. Nieto

Vazquez; C. Albo Lopez

Hospital Alvaro Cunqueiro, Vigo, Spain

Background: The incidence of inhibitors in NS-HA was estimated between $3-13 \%$ but certain F8 genotypes have been identified as associates with higher inhibition.There are limited data about optimal therapeutic approach to eradicate inhibitors in NS-HA patients.

Aims: Explain our strategy for suppression of inhibitor antif8 in mild HA patient who developed an inhibitor 2 months after treatment of spontaneous epidural spinal hematoma that required intensive replacement with F8 Concentrate (CF8) (ReFacto $\left.{ }^{\circledR}\right)$ and Desmopressin, and decompressive laminectomy

Methods: Patient, seventeen, Afro-Caucasian, mild HA (F8: C 15\%). The mutation is c.6301 C> G (exon 22) that involves change from aa to p. His2101Asp (fC1 domain). Respondent to Desmopressin. Two months after hematoma and having carried out negative inhibitor controls (2), he went to Urgencies for pain in right iliac fossa of 4 days; Abdominal echography showed retroperitoneal hematoma; Analytically, aPTT 2.13 (0.7-1.3) was observed. For a longer aPTT than usual we repeated coagulation (Table 1 ) and showed HIGH-titer inhibitor antiF8 (12,8 Bethesda Units, uBT).

\section{TABLE 1}

\begin{tabular}{|l|l|l|}
\hline & Results & Normal \\
\hline APTT ratio & 2.27 & $0.7-1.3$ \\
\hline $\begin{array}{l}\text { APTT mix 1:1 Normal } \\
\text { Plasma }\end{array}$ & 36.3 seconds (Control 30) & \\
\hline PT ratio & 1.2 & $0.8-1.2$ \\
\hline Fibringen Clauss & $318 \mathrm{mg} / \mathrm{dl}$ & $150-400$ \\
\hline FVIII:C & $3,6 \mathrm{IU} / \mathrm{dl}$ & $50-200$ \\
\hline Inhibitor AntiFVIII & $12,80 \mathrm{uBT}(1: 32)$ & $<0.6$ \\
\hline FVIII chromogenic & $3,2 \mathrm{IU} / \mathrm{dl}$ & \\
\hline
\end{tabular}

Results: Hematoma was treated with Novoseven ${ }^{\circledR}, 7$ days. We began ITI (Induction to Inmuno-Tolerance) at the same time with Immunosuppression (IS) (Prednisone $1 \mathrm{mg} / \mathrm{Kg} /$ day, 5 weeks and AntiCD20 Antibody 375 mg / m2 / weekly, 4 weeks). 1 month after AntiCD20, a negative inhibitor titer ( $<0.6 \mathrm{uBT})$ was observed. Continuous progressive decrease of Prednisone (10 mg by week) until suspension (2 months). He recovered the response to Desmopressin (Table 1). We made pharmacokinetic study with 30 IU/Kg CF8 (Fandhi $\left.{ }^{\circledR}\right)\left(\right.$ WAPPS Hemo $\left.{ }^{\circledR}\right)$, to certify the success: recovery $2.25 / /$ half life 8,25 hours.

\section{TABLE 2}

\begin{tabular}{|c|c|c|c|c|c|}
\hline & ât rate & $\begin{array}{l}\text { Aptrt mix } \\
\text { fcontrol } \\
\text { (ueconds) }\end{array}$ & Ot ratio & Fince $[\mathbf{S}]$ & $\begin{array}{c}\text { Antionil } \\
\text { [जaT) }\end{array}$ \\
\hline one & 1.45 & $31 / 28$ & 1.19 & 16.5 & $<0.40$ \\
\hline A Hove 00 & 2.11 & & 1.15 & 88.2 & \\
\hline $2 \mathrm{H}$ & 1.06 & & 1.10 & 110 & \\
\hline $4 H$ & 0.97 & & 1.14 & 1343 & \\
\hline
\end{tabular}

Conclusions: This is succesfully ITI in NS-HA with IS. Inhibitors in NS-HA are a relevant clinical problem being imperative to learn more about treatment regimens aimed at eradicating it. IS seems a valid option, as reported by Kempton in a cohort of 32 patients. 


\section{PB0648 | Infectious Complications in Hemophilia Patients}

$\underline{\text { H. Khachatryan }}^{1,2,3}$; G. Tamamyan ${ }^{1,2}$; I. Melnichenko ${ }^{1,2}$; L. Sahakyan $^{1}$; L. Petrosyan ${ }^{1}$; S. Danelyan ${ }^{1}$; S. Hovhannisyan ${ }^{1,2}$; A. Movsisyan $^{1,2}$; L. Hambardzumyan ${ }^{1,2}$; N. Sargsyan ${ }^{4,2,3}$

${ }^{1}$ Hematology Center after Prof. R.H. Yeolyan, Yerevan, Armenia;

${ }^{2}$ Yerevan State Medical University, Yerevan 0025, Armenia;

${ }^{3}$ Hemophilia Treatment Center, Yerevan, Armenia; ${ }^{4}$ Hematology Center named Prof. R.H. Yeolyan, Yerevan, Armenia

Background: Replacement therapy increases factor VIII plasma level, which temporarily corrects its deficiency in the blood plasma and prevents bleeding. Replacement therapy has, in the past and now, influenced the treatment of patients. Before the use of modern factors, treatment for patients was traditionally with fresh frozen plasma, which has never been virally inactivated. Most people with hemophilia who treated with fresh frozen plasma were infected with the HIV virus, Hepatitis C, Hepatitis B and other blood-borne viruses.

Aims: The aim of the study is to analyze the incidence of infectious complications depending on the treatment and the severity of the disease. Important to investigate the prevalence of hepatitis among patients treated with fresh frozen plasma and VIII, IX concentrates, their distribution by age groups, by immunogenic complications.

Methods: The study was based on the registration data of the Armenian Hemophilia Center. Conducted a retrospective analysis of 271 patients (4.85\% younger than 18 years of age) indicated the outpatient cards with HCV infection, HbSAg- in, HBcore-on in education. The data obtained were processed by statistical methods SPSS, x2 and Student.

Results: According to the data obtained, infectious complications were registered only in patients of the I group. Infectious complications were found in 71 (33\%) of 212 examined adult patients. Hepatitis C prevailed - 62 cases (87\%) among the observed infectious complications; hepatitis B was detected in 8 patients (11\%); hepatitis $B$ and $C$ were combined in 1 case (2\%).

Conclusions: The use of factors in all types of severity excludes infection complications. The immunologic complications, the development of inhibitors, joint complications, in mild forms of diseases include a low percentage. Experience has shown that mild to moderate diseases of the disease go unnoticed. They have more frequent infectious complications, joint diseases, and therefore more frequent bleeding. Such a group goes unnoticed.
PB0649 | From Stroke Treatment, through a Tongue Bite, until Intensive Care Unit: The Challenge of Antithrombotic Treatment in People with Haemophilia. A Case Report

S. Teixeira' ${ }^{1}$ I. Machado ${ }^{1}$; D. Gonçalves ${ }^{1}$; J.P. Silva ${ }^{2}$; S. Fernandes ${ }^{1}$; M. Lopes ${ }^{1}$; M. Carvalho ${ }^{1}$; D. Aranda ${ }^{1}$; C. Koch ${ }^{1}$

${ }^{1}$ Congenital Coagulopathies Reference Centre, Centro Hospitalar

e Universitário de São João, Porto, Portugal; ' 2 Department of Imunnohemotherapy, Hospital Central do Funchal, Funchal, Portugal

Background: The life expectancy of people with hemophilia $(\mathrm{PWH})$ has been increasing. Age-related conditions became a challenge, including the management of thrombotic diseases, to minimize the bleeding risk and ensure sufficient anticoagulation and antiaggregation.

Aims: Present a case report to highlight the issues associated with treatment of PWH with thrombotic events, complicated by hemorrhage.

Methods: A 76-year-old male with moderate hemophilia A (FVIII-4\%) with history of dyslipidemia, acute myeloid leukemia and amputation of inferior lower limb due to pseudo-tumor, came into the emergency department (ED) on January 2021, due to left hemiparesis and dysarthria. He underwent endovascular revascularization under factor replacement therapy. After discharge, double antiaggregation (acetylsalicylic acid and ticagrelor) was decided as he had a new stenosis of cerebral vessels. Three weeks later, he went into the ED of local hospital, due to a tongue bite with a small hematoma and swelling on site and was discharged with indication to apply ice and cold diet. As the edema and hematoma increased, he returned to that hospital. In this moment, reference center was contacted and patient was transferred.

Results: On admission into the ED, he presented edema of tongue with active bleeding and inframandibular/upper neck hematoma. He initiated FVIII concentrate and after evaluation by otorhinolaryngology, an oozing bleeding was observed and nasotracheal intubation was performed to protect airway. In intensive care unit, the patient needed packed red blood cells transfusion and FVIII replacement. FVIII levels, hemorrhagic manifestations and thrombotic risk were evaluated in order to guide further treatment. He was discharged with only aspirin and secundary prophylactic treatment.

Conclusions: The optimal treatment to manage PWH needing antithrombotic therapy remains a challenge. It is important to be aware of the bleeding phenotype of the patient, factor level and thrombotic risk, debating all these factors in a multidisciplinary team in order to delineate the best strategy. 
P0140 | Analysis of Hospitalization Factors of Hemophilia: 12 Years of Experience in a Single Center

C. Qu; F. Xue; W. Liu; L. Chen; L. Zhang; R. Yang

State Key Laboratory of Experimental Hematology, National Clinical Research Center for Blood Diseases, Institute of Hematology \& Blood Diseases Hospital, Chinese Academy of Medical Sciences \& Peking Union Medical College, Tianjin, China

Background: The hospital utilization and medical resource usage of hemophilia patients is closely related to the burden on China's healthcare system.

Aims: This study was based on a single center in China and was conducted to comprehensively assess the hospitalization factors of hemophilia A or B patients.

Methods: We retrospectively analyzed clinical characteristics, diagnosis, inhibitor status, causes and duration of hospitalizations and medical costs of 323 hospitalizations in which hemophilia must be considered as the main cost factor during hospitalization from January 2009 to December 2020 from the Institute of Hematology and Blood Diseases Hospital in Tianjin, China. Non-parametric statistics were used and $P<0.05$ was considered statistically significant. Results:

TABLE 1 Causes of hospitalizations

\begin{tabular}{lllll} 
& $\begin{array}{l}\text { Severe hemophilia without } \\
\text { inhibitor }\end{array}$ & $\begin{array}{l}\text { Moderate or mild hemophilia } \\
\text { without inhibitor }\end{array}$ & Inhibitor patients & 298 \\
Minor bleed & 62 & 164 & 72 & 53 \\
Major bleed & 10 & 28 & 15 & 14 \\
Pseudotumor & 2 & 9 & 3 & 1 \\
Surgery & 0 & 9 & 6 & 24 \\
Other & 9 & 9 & 97 & 399 \\
Total & 83 & 219 & & 10 \\
\hline
\end{tabular}

There were 265 hospitalizations with hemophilia A and 58 hospitalizations with hemophilia B. Seventy-eight hospitalizations were positive for inhibitor. Cause of most hospitalizations was minor bleeding (Table 1). The median length of hospitalization time was 5 (range 1 745) days. The cost of clotting factor concentrates is a major burden in the economic management of in-patients with hemophilia . Total cost in a single hospitalization of hemophilia $A$ is about twice as much as hemophilia B. Expenditure of drugs in hemophilia $A$ is two to three times more than that of hemophilia B. Total cost and drug cost in hospitalizations of patients with inhibitors were about two times more than these without. When normalized by weight, total cost of non-adults was significantly higher than adults $(P=0.002)$. The positive rates were $1.8 \%$ for hepatitis $B$ virus and $12.7 \%$ for hepatitis $C$ virus in 283 hospitalizations screened for blood-borne infections.

Conclusions: For hemophilia, the main factor of cost during hospitalization is clotting factor concentrates. Diagnosed with hemophilia $A$ and inhibitor positive increase the global cost.
P0141 | Successful Immune Tolerance Induction with High Dose Simoctocog Alfa in Hemophilia A Adult Patient with High Responder Inhibitor: A Case Report

S.-N. Cheng ${ }^{1}$; L.H.-L. Yu²; A.J.-H. Lin ${ }^{2}$; L.T. Wang ${ }^{1}$

${ }^{1}$ Hemophilia and Rare Disease Treatment Center, Tungs' Taichung MetroHarbor Hospital, Taichung, Taiwan, Province of China; ${ }^{2}$ Medical Affairs Department, Panco Healthcare Co., Ltd., a PharmaEssentia Company, Taipei, Taiwan, Province of China

Background: Immune tolerance induction (ITI) is the only method to eradicate inhibitors. A number of host- and treatment-related variables were identified as predictors of ITI success. Simoctocog alfa is a recombinant coagulation factor VIII (rFVIII) product, which harbors a fully sulphated Tyr1680. This contributes to a higher von Willebrand factor binding affinity than other rFVIII products. Simoctocog alfa is produced in a human cell line without chemical modification or protein fusion, with the goal of reducing immunogenicity. These characteristics may be beneficial on ITI outcome.

Aims: We report a case of Taiwanese hemophilia $A(H A)$ adult with high responder inhibitors and other unfavourable prognostic factors who underwent high-dose Simoctocog alfa ITI and 18-month follow-up.
Methods: The 48-year-old severe HA patient, who developed high-titre inhibitors to another recombinant FVIII concentrate, was treated with high dose (200IU/kg/day) simoctocog alfa. He was diagnosed at age 18 due to repeated joint bleeds since infancy. $\mathrm{He}$ has been treated with bypassing agents on-demand. He has nonsense mutation (Location: A5536 ->T; Lys-> Termination on domain A3, exon 16) and several other unfavourable prognostic factors: age, historical peak $>300 \mathrm{BU}$ and inhibitor history $>25$ years.

Results: As figure 1 shows, patient's inhibitor titre was 4.3BU at ITI initiation and peaked at $167 \mathrm{BU}$ at week 2. It showed a progressive decline to $23.4 \mathrm{BU}$ at week 4 , and became undetectable at week 14. In consideration of adequate response, we reduced the dose to $100 \mathrm{IU} / \mathrm{kg} /$ day at week 14 . At decision time point $9^{\text {th }}$ month, suggested by the FIT group, the inhibitor titer still remained undetectable. At week $54^{\text {th }}$, we changed the dose to $50 \mathrm{IU} / \mathrm{kg} / \mathrm{day}$. At 18 months, a 72-h washout pharmacokinetic evaluation was conducted, showing $64 \%$ and $19.6 \% \mathrm{FVIII}$ at 0.5 and $7 \mathrm{hrs}$, respectively. 


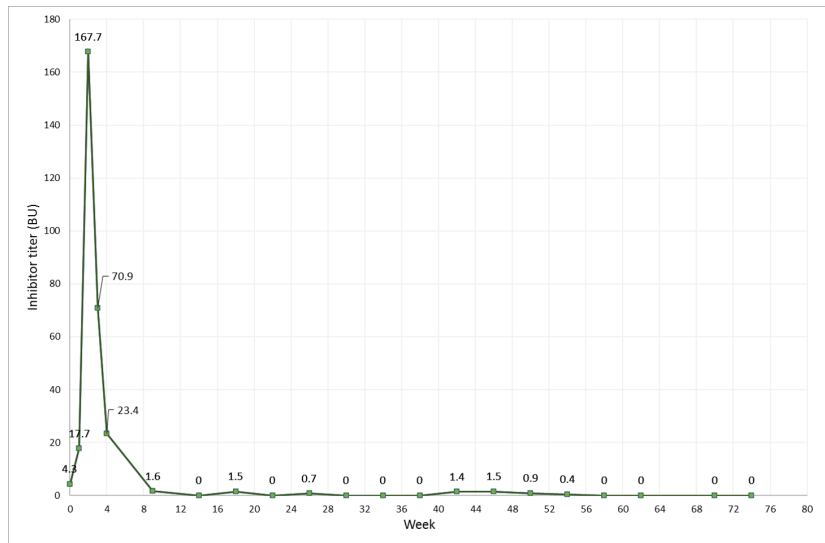

FIGURE 1 Inhibitor titer during 18-month ITI regimen.

Conclusions: Simoctocog alfa may be an effective option for hemophilia A adult patient with high-responder inhibitor and other unfavourable prognostic factors.

P0146 | TSUBASA Study: An Observational Study of Emicizumab to Evaluate Physical Activity, Bleeding Events, Quality of Daily Life, and Safety in People with Hemophilia A without FVIII Inhibitors

K. Nogami ${ }^{1}$; T. Fujii ${ }^{2}$; A. Sawada ${ }^{3} ;$ A. Nagao ${ }^{4}$; C. Nagae ${ }^{5}$; N. Suzuki ${ }^{6}$; M. Nojima ; D. Nosaka ${ }^{8}$; T. Nakamura ${ }^{8}$; S. Yoshida ${ }^{8}$; K. Amano ${ }^{9}$ ${ }^{1}$ Nara Medical University, Department of Pediatrics, Nara, Japan; ${ }^{2}$ Hiroshima University Hospital, Division of Transfusion Medicine/ Hemophilia Treatment Center, Hiroshima, Japan; ${ }^{3}$ Hyogo College of Medicine, Division of Hematology, Department of Respiratory Medicine and Internal Medicine, Hyogo, Japan; ${ }^{4}$ Ogikubo Hospital, Department of Blood Coagulation, Tokyo, Japan; ${ }^{5}$ St. Marianna University School of Medicine, Department of Pediatrics, Kanagawa, Japan; ${ }^{6}$ Nagoya University Hospital, Department of Transfusion Medicine, Aichi, Japan; ${ }^{7}$ Institute of Medical Science, University of Tokyo, Center for Translational Research/Division of Advanced Medicine Promotion, Tokyo, Japan; ${ }^{8}$ Chugai Pharmaceutical Co., Ltd, Tokyo, Japan; ${ }^{9}$ Tokyo Medical University, Department of Laboratory Medicine, Tokyo, Japan

Background: Several clinical studies conducted worldwide have shown that Emicizumab is effective and tolerable in people with hemophilia A ( $\mathrm{PwHA}$ ) regardless of its use in $\mathrm{PwHA}$ with/without factor VIII (FVIII) inhibitors. Although physical activity is recommended for PwHA, little information is available on the relationship of bleeding to physical activity in PwHA on Emicizumab. Additionally, the experience with administering Emicizumab to infant patients is limited; thus, more safety data should be accumulated in the real-world setting.

Aims: To evaluate the relationship between exercise status and bleeding, the quality and content of daily life, and the safety of Emicizumab in PwHA without FVIII inhibitors.
Methods: We were enrolling PwHA without FVIII inhibitors scheduled to begin prophylactic treatment with Emicizumab. During 97 weeks after the enrollment, the status of bleeding in patients treated with Emicizumab that are attributable to exercise, the quality and content of daily life and physical activity data extracted from electronic patient-reported outcomes systems and wearable activity tracker will be evaluated by acquiring patient-reported exercise status.

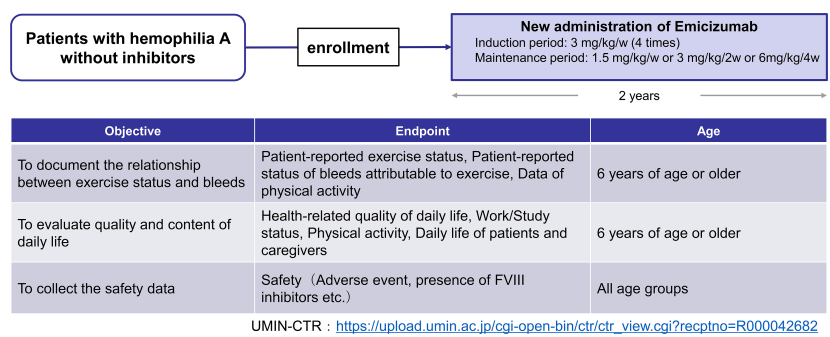

FIGURE 1 Study design

Results: Until October 2020, 65 patients (including <2 years old: 8 , 2-5 years old: 4, 6-17 years old: $10, \geq 18$ years old: 43 ) were enrolled. Regarding the severity, 56 patients were severe and 8 patients were moderate, 1 patient were unknown. And 30 patients, 168 exercise events were reported from $\geq 6$ years old patients evaluated for the exercise status. At this interim analysis, patient characteristics, status of patient exercise and bleeding, and safety information are reported.

Conclusions: This study will clarify the effects of Emicizumab on the lives of hemophilia patients and provide important information for selecting treatments for PwHA without FVIII inhibitors. Acquiring information on demographically diverse patients (especially infant patients) in clinical settings provide particularly valuable data for starting or continuing Emicizumab treatment.

P0149 | The Detection of Hemophilia C in a 66-year-Old Patient with Postoperative Urinary Bleeding

I. Melnichenko ${ }^{1,2} ;$ T. Arakelyan ${ }^{1} ;$ N. Sargsyan ${ }^{2,3}$; L. Sargsyan ${ }^{1,2} ;$ S. Danielyan $^{4}$; G. Tamamyan ${ }^{1,2}$; H. Khachatryan ${ }^{2,3}$

${ }^{1}$ Pediatric Cancer and Blood Disorders Center of Armenia, Hematology Center after Prof. R.H. Yeolyan, Yerevan, Armenia; ${ }^{2}$ Department of Pediatric Oncology and Hematology, Yerevan State Medical University, Yerevan, Armenia; ${ }^{3}$ Armenian Hemostasis and Thrombosis Center, Hematology Center after Prof. R.H. Yeolyan, Yerevan, Armenia; ${ }^{4}$ Hematology Center after Prof. R.H. Yeolyan, Yerevan, Armenia

Background: Hemophilia C or Rosenthal syndrome caused by the factor of XI (FXI) deficiency and known as plasma thromboplastin antecedent deficiency belongs to a mild form of hemophilia. Hemophilia $\mathrm{C}$ is considered a rare disease requiring further investigations. 
Aims: To describe a clinical case of acquired hemophilia $\mathrm{C}$ in a patient in Armenia who previously underwent two surgeries without bleeding complications and coagulopathy.

Methods: A retrospective review of the patient's medical history was performed.

Results: A 66-year-old male presented with frequent urination complaints with decreased urine output, dysuria, and sometimes a feeling of incomplete bladder emptying. The prostate incision for sclerosis, TUR biopsy of the prostate and bladder were performed. Bleeding from the urethra observable on the first day after surgery was stopped with conservative measures. On the sixth day after the surgery, the patient had more severe and intense bleeding from the urethra. The problem was solved by suprapubic cystostomy. The blood tests showed high-normal activated plasma thromboplastin time-88.9s (normal range: $26-40 \mathrm{~s}$ ), prothrombin time-13.5s (normal range: 11.9 - 15.4s), and internationalized normal ratio-1.2. Factors VIII, IX, and XIII levels were within the normal range-98\%, 102\%, and $82 \%$, respectively. Whereas the FXI level reduced to $3.9 \%$ (normal range: $60-150 \%$ ), thereby helping to diagnose intermediate $\mathrm{FXI}$ deficiency. Transitory antibodies against FXI were developed. Though the patient didn't receive anti-hemophilia therapy and had FXI deficiency, he had no bleeding disorders during last five years.

Conclusions: The deficiency of FXI can develop at any age, and the cause of it can only be revealed on the basis of reliable data on the detection of genetic mutations. The temporary appearance of antibodies and their current absence did not allow confirming the immune nature of hemophilia.

\section{HEMOPHILIA GENE THERAPY}

\section{LPB0019 | Expression of Adenovirus-mediated Human} Coagulation Factor IX Gene in SD Rat Adipose Mesenchymal Stem cells

Z. Yan; Q. Sun; Y. Xie; L. Wang; S. Chen; W. Wu; Y. Yan; J. Yang North China University of Science and Technology Affiliated Hospital, Tangshan, China

Background: The high accessibility and low immunogenicity of adipose-derived stem cells (ADSCs) make them a promising cell model to study hemophilia B gene therapy.

Aims: To explore the possibility of transfecting ADSCs with recombinant adenoviral vector containing human coagulation factor IX gene (FIX), and using them as target cells for hemophilia $B(H B)$ gene therapy.

Methods: ADSCs were isolated (from inguinal fat tissues of SD rats) and cultured (using collagenase I digestion method). A recombinant adenovirus vector carrying FIX was transfected into ADSCs: F9 group: (Ad-hFIX was used to transfect ADSCs), F9, group: (one generation after passage of Ad-hFIX was used to transfect ADSCs),

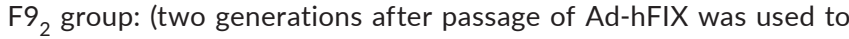
transfect $\mathrm{ADSCs}$ ), $\mathrm{F9}_{3}$ group: (three generations after passage of
Ad-hFIX was used to transfect ADSCs), Empty group: (Ad-GFP was used to transfect ADSCs), Blank group: (DMEM was used instead of adenovirus to transfect ADSCs). The morphology and transfection efficiency of ADSCs after transfection were observed by inverted fluorescence microscope, while the expression of hFIX antigen, gene and protein was detected by ELISA, RT-PCR and western blot. Results:
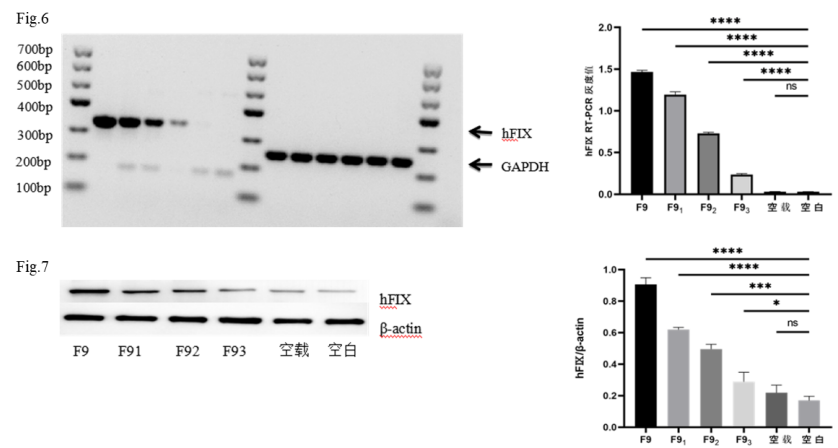

Fig6: The expression of hFIX gene was detected by RT-PCR

Fig7: The expression of hFIX protein in ADSCs detected by Western blot

FIGURE 1 Expression of adenovirus-mediated Human Coagulation Factor IX Gene in SD Rat Adipose Mesenchymal Stem cells
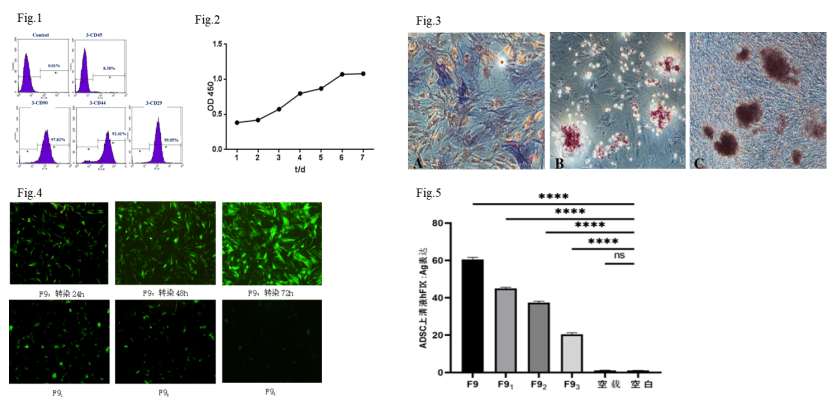

Fig.1 Cultivated to the thrid genetation of ADSCs in FCM instrument testing surface antigen. Fig. 2 Growth curve of third generation ADSC.

Fig. 3 Induction and identification of $\mathrm{ADSCS}$ (Inverted microscope, $\times 100$ ). A: ALP staining after osteogenic induction for 7 days; $\mathrm{B}$ : Oil Red $\mathrm{O}$ staining after adipogenic induction for 14 days; $\mathrm{C}$ : Alizarin red staining after osteogenic induction for 30

作 Fig 5 The expression of cell supernatant FIX:Ag by ELISA.

FIGURE 2 Expression of adenovirus-mediated Human Coagulation Factor IX Gene in SD Rat Adipose Mesenchymal Stem cells

ADSCs were extracted successfully. Inverted fluorescence microscope well observed the morphology and transfection efficiency of ADSCs cells after transfection. The expressions of hFIX antigen, gene and protein were detected after the transfection of recombinant adenovirus vector into SD rat ADSCs, as illustrated by the significant differences in comparison with those from the blank control group $(\mathrm{P}<0.05)$.

Conclusions: 1. Adenovirus carrying hFIX gene can effectively transfect ADSCs of SD rats. 2. ADSCs transfected with recombinant adenovirus can still express hFIX after subculture. ADSCs may serve as vector cells for hemophilia B gene therapy. 
LPB0020 | Etranacogene Dezaparvovec (AAV5-Padua hFIX Variant, AMT-061), an Enhanced Vector for Gene Transfer in Adults with Severe or Moderate-severe Hemophilia B: 2.5 Year Data from a Phase 2b Trial

E. Gomez ${ }^{1}$; A. Giermasz ${ }^{2}$; G. Castaman ${ }^{3}$; N.S Key ${ }^{4}$; S.U Lattimore ${ }^{5}$; F.W. Leebeek ${ }^{6}$; W. Miesbach ${ }^{7} ;$ M. Recht ${ }^{5}$; A. von Drygalski ${ }^{8}$;

E.K Sawyer ${ }^{9}$; S.W. Pipe ${ }^{10}$

${ }^{1}$ Phoenix Children's Hospital, Phoenix, United States; ${ }^{2}$ University of California Davis, Sacramento, United States; ${ }^{3}$ Azienda Ospedaliera Universitaria Careggi, Florence, Italy; ${ }^{4}$ University of North Carolina, Chapel Hill, United States; ${ }^{5}$ Oregon Health \& Science University, Portland, United States; ${ }^{6}$ Erasmus University Medical Center, Rotterdam, Netherlands; ${ }^{7}$ University Hospital Frankfurt, Frankfurt, Germany; ${ }^{8}$ University of California San Diego, La Jolla, United States, ${ }^{9}$ uniQure Inc, Lexington, United States, ${ }^{10}$ University of Michigan, Ann Arbor, United States

Background: Etranacogene dezaparvovec, an investigational gene therapy for hemophilia $B(\mathrm{HB})$, comprises an adeno associated virus serotype 5 (AAV5) vector containing a codon-optimized Padua variant human factor IX (FIX) transgene with liver-specific promoter.

Aims: We previously demonstrated that a single dose of etranacogene dezaparvovec provides sustained FIX activity in the mildto-normal range for up to two years. Updated results through 30 months of follow-up will be presented.

Methods: A Phase $2 b$, open-label, single-dose, single-arm, multicenter trial in adult HB participants $(n=3)$. All participants received a single intravenous dose of etranacogene dezaparvovec $\left(2 \times 10^{13}\right.$ $\mathrm{gc} / \mathrm{kg}$ ) and are being followed for 5 -years. The primary endpoint was FIX activity at Week 6. Secondary endpoints include bleeds, use of FIX replacement, laboratory parameters, joint health, and adverse events (AEs) (NCT03489291).

Results: Participants had FIX $\leq 1 \%$, required routine FIX prophylaxis, and had neutralizing antibodies to AAV5 at baseline. Following treatment with etranacogene dezaparvovec, FIX activity rapidly increased to a mean of $31 \%$ at Week 6 . By 2 years, mean FIX activity further increased to $44.2 \%$, with FIX activity levels of $45 \%, 36 \%$ and $52 \%$ in participants 1-3 respectively. No relationship between treatment response and presence of anti-AAV5 NAbs at BL was observed. One participant experienced a spontaneous mild bleed and self-administered a single infusion of FIX replacement in year 2. No clinically significant elevations in liver enzymes occurred and no participant required steroids related to treatment. As reported previously, one participant experienced 2 mild AEs possibly-related to treatment shortly after dosing and one participant underwent hip surgery. No participant developed inhibitors to FIX. Data up to 2.5 years will be presented.

Conclusions: Patients in this Phase $2 \mathrm{~b}$ etranacogene dezaparvovec trial have shown sustained FIX activity into the mild-to normal range over 2 years, enabling discontinuation of routine prophylaxis, irrespective of neutralizing antibodies to AAV5 at baseline.
LPB0021 | Practical Application of FIX-Padua Field Study Results Enables a Comparison of FIX:C Results Across AAV Gene Therapy Trials Independent of FIX:C Assay Reported

E. Shehu ${ }^{1}$; J.H. Foley ${ }^{1}$; E. Gray ${ }^{2}$; A. Riddell ${ }^{3,4}$; A. Goodale ${ }^{1}$, I-M. Yu ${ }^{1}$; J. Little ${ }^{1}$; D. Shattock ${ }^{1}$; S. Kitchen ${ }^{5}$; P. Chowdary ${ }^{3,4}$; A. Nathwani ${ }^{3,4}$; R. Corbau ${ }^{1}$

${ }^{1}$ Freeline, Stevenage, United Kingdom; ${ }^{2}$ National Institute for Biological Standards and Control, Potters Bar, United Kingdom; ${ }^{3}$ Katharine Dormandy Haemophilia and Thrombosis Centre, Royal Free Hospital, London, United Kingdom; ${ }^{4}$ University College London, London, United Kingdom; ${ }^{5}$ Department of Coagulation, Royal Hallamshire Hospital, Sheffield, United Kingdom

Background: Factor IX-Padua (FIX-R338L) is a FIX variant with an approximate 8-fold higher specific activity than wild-type FIX. Previous studies have shown discrepancies in results between different FIX assay methodologies when measuring FIX-Padua transgene product. To support the development of verbrinacogene setparvovec (FLT180a), an investigational AAV gene therapy for the treatment of haemophilia $B(\mathrm{HB})$ that expresses the gain-of-function FIX-Padua variant, an international multicentre field study was conducted to characterise FIX-R338L activity across 15 commonly used FIX activity (FIX:C) assays. Results showed greater than 3-fold variation in FIX-R338L activity depending on the assay used.

Aims: To demonstrate the applicability of a FIX-Padua activity "Calculator," which enables meaningful comparison of FIX:C results from various $\mathrm{HB}$ gene therapy trials across the commonly used assays.

Methods: FIX:C assay results from the FIX-R338L field study were indexed to SynthASil ${ }^{\mathrm{TM}} \mathrm{FIX}: \mathrm{C}$ results to generate FIX:C ratios. These ratios (the Calculator) were used to predict FIX:C results across tested assays using clinical samples containing the FIX-R338L variant. Predicted results were compared to actual assay results.

Results: Comparison of FLT180a ROX Factor IX ${ }^{\mathrm{TM}}$ chromogenic assay data with those predicted by our Calculator using input SynthASil values revealed a strong positive correlation $(r=0.94, P<0.0001)$. Previously published SynthASil FIX:C results of FIX-Padua transgene product (Robinson et al 2018) were input into our Calculator to predict results for ACTIN-FSL ${ }^{\mathrm{TM}}$, CK Prest $^{\mathrm{TM}}$, PTT Automate ${ }^{\mathrm{TM}}$ and ROX FIX:C assays (Table). There was a strong positive correlation between all published clinical assay results $(r>0.85, P<0.01)$ and calculated results with average coefficient of variation ranging from $3.3 \%$ (ROX Factor IX) to $11 \%$ for CK Prest. 
TABLE 1 Predicted FIX-R338L activity relative to SynthASil across common one-stage and chromogenic FIX activity assays. The 3-fold range in FIX activity for FIX-R338L is exemplified by TriniCLOT aPTT HS vs. Biophen FIX

\begin{tabular}{llllll} 
Assay & \multicolumn{5}{c}{ nominal \% FIX:C, relative to SynthASil } \\
TriniCLOT aPTT HS & 6 & 25 & 62 & 125 & 187 \\
Cephascreen & 6 & 25 & 61 & 123 & 184 \\
CK Prest & 5 & 21 & 54 & 107 & 161 \\
SynthASil & 5 & 20 & 50 & 100 & 150 \\
ACTIN-FS & 5 & 20 & 50 & 100 & 150 \\
Pathromtin & 4 & 16 & 40 & 80 & 119 \\
ACTIN-FSL & 4 & 15 & 37 & 73 & 110 \\
ROX FIX & 2 & 9 & 22 & 43 & 65 \\
Biophen FIX & 2 & 8 & 21 & 42 & 63 \\
\hline
\end{tabular}

Conclusions: FIX:C ratios derived from our FIX-R338L field study permit direct, reliable comparison of FIX:C assay results. Our Calculator enables estimation of activity results, which facilitates a meaningful comparison of different investigational FIX-R338Lbased HB gene therapies.

\section{LPB0022 | Global Seroprevalence of Pre-existing Immunity} against AAV Serotypes in People with Hemophilia A

R. Klamroth ${ }^{1}$; G. Hayes ${ }^{2}$; T. Andreeva ${ }^{3}$; T. Suzuki ${ }^{4}$; B. Hardesty ${ }^{5}$; M. Shima ${ }^{6}$; T. Pollock ${ }^{7}$; P. Slev; J. Oldenburg ${ }^{8}$; M.C Ozelo ; S.-M. Castet $^{10}$; J. Mahlangu ${ }^{11}$; F. Peyvandi ${ }^{12}$; R. Kazmi ${ }^{13}$; A.D Leavitt ${ }^{14}$; M. Callaghan $^{15}$; B. Pan-Petesch ${ }^{16}$; D. Quon ${ }^{17}$; M. Li ${ }^{2}$; W.Y. Wong ${ }^{2}$ ${ }^{1}$ Comprehensive Care Haemophilia Treatment Center, Vivantes Klinikum im Friedrichshain, Berlin, Germany; ${ }^{2}$ BioMarin Pharmaceutical Inc., Novato, United States; ${ }^{3}$ Municipal Center of Hemophilia Therapy, St. Petersburg, Russian Federation; ${ }^{4}$ Ogikubo Hospital, Tokyo, Japan; ${ }^{5}$ Indiana Hemophilia and Thrombosis Center, Indianapolis, United States; ${ }^{6}$ Nara Medical University, Kashihara, Japan; ${ }^{7}$ ARUP Laboratories, Salt Lake City, United States; ${ }^{8}$ Universitätsklinikum
Bonn, Bonn, Germany; ${ }^{9}$ Hemocentro UNICAMP, Department of Internal Medicine, School of Medical Sciences, University of Campinas, Campinas, Brazil, ${ }^{10}$ Centre de Ressources et de Compétence des Maladies Hémorragiques Constitutionnelles, CHU de Bordeaux, Bordeaux, France, ${ }^{11}$ Haemophilia Comprehensive Care Centre, University of the Witwatersrand and National Health Laboratory Service, Johannesburg, South Africa, ${ }^{12}$ Angelo Bianchi Bonomi Hemophilia and Thrombosis Center, Fondazione IRCCS Ca' Granda Ospedale Maggiore Policlinico, Milan, Italy, ${ }^{13}$ Department of Haematology, Southampton University Hospital, Southampton, United Kingdom, ${ }^{14}$ Departments of Medicine and Laboratory Medicine, University of California San Francisco, San Francisco, United States, ${ }^{15}$ Division of Pediatric Hematology/Oncology, Central Michigan University, Detroit, United States, ${ }^{16}$ Centre Hospitalier Régional Universitaire de Brest, Hôpital A. Morvan, Brest, France, ${ }^{17}$ The Orthopedic Hemophilia Treatment Center, Los Angeles, United States

Background: Adeno-associated virus (AAV)-mediated gene therapy may provide durable protection from bleeding events and reduced treatment burden to people with hemophilia A (PwHA). However, pre-existing immunity against AAV may limit treatment success. Global data on the prevalence of AAV serotypes are limited.

Aims: We determined the prevalence of pre-existing immunity against AAV2, AAV5, AAV6, AAV8, and rh10 capsids among PwHA from 9 countries.

Methods: BMN 270-901 was a prospective study conducted in PwHA in Brazil, France, Germany, Italy, Japan, Russia, South Africa, the UK, and the USA. Plasma samples were collected from participants who provided informed consent. Antibodies against each serotype were detected using validated, electrochemiluminescentbased enzyme-linked immunosorbent assays. To evaluate changes in antibody titers over time, $20 \%$ of participants were retested at 3 and 6 months.

Results: The study enrolled 546 participants: 478 adults (aged $\geq 18$ years) and 68 adolescents ( $<18$ years). Global seroprevalence of antibodies on day 1 are displayed in Table 1.

TABLE 1 Global seroprevalence of antibodies on day 1

\begin{tabular}{|c|c|c|c|c|c|c|c|c|c|c|}
\hline AAV Serotype & Global & Brazil & France & Germany & Italy & Japan & Russia & $\begin{array}{l}\text { South } \\
\text { Africa }\end{array}$ & UK & USA \\
\hline AAV2 +/total (\%) & $\begin{array}{l}300 / 513 \\
(58.5 \%)\end{array}$ & $\mathrm{N} / \mathrm{A}$ & $\begin{array}{l}52 / 86 \\
\quad(60.5 \%)\end{array}$ & $\begin{array}{l}43 / 89 \\
\quad(48.3 \%)\end{array}$ & $\begin{array}{l}9 / 20 \\
\quad(45.0 \%)\end{array}$ & $\begin{array}{l}36 / 83 \\
\quad(43.4 \%)\end{array}$ & $\begin{array}{l}58 / 91 \\
\quad(63.7 \%)\end{array}$ & $\begin{array}{l}53 / 56 \\
\quad(94.6 \%)\end{array}$ & $\begin{array}{l}11 / 17 \\
\quad(64.7 \%)\end{array}$ & $\begin{array}{r}38 / 71 \\
(53.5 \%)\end{array}$ \\
\hline AAV5 +/total (\%) & $\begin{array}{l}188 / 540 \\
(34.8 \%)\end{array}$ & $\begin{array}{l}7 / 26 \\
\quad(26.9 \%)\end{array}$ & $\begin{array}{l}32 / 86 \\
\quad(37.2 \%)\end{array}$ & $\begin{array}{l}25 / 89 \\
\quad(28.1 \%)\end{array}$ & $\begin{array}{l}8 / 20 \\
\quad(40.0 \%)\end{array}$ & $\begin{array}{l}25 / 84 \\
\quad(29.8 \%)\end{array}$ & $\begin{array}{l}42 / 91 \\
\quad(46.2 \%)\end{array}$ & $\begin{array}{l}29 / 56 \\
\quad(51.8 \%)\end{array}$ & $1 / 17(5.9 \%)$ & $\begin{array}{l}19 / 71 \\
\quad(26.8 \%)\end{array}$ \\
\hline AAV6 +/total (\%) & $\begin{array}{l}250 / 513 \\
(48.7 \%)\end{array}$ & $\mathrm{N} / \mathrm{A}$ & $\begin{array}{l}47 / 86 \\
\quad(54.7 \%)\end{array}$ & $\begin{array}{l}39 / 89 \\
\quad(43.8 \%)\end{array}$ & $\begin{array}{l}8 / 20 \\
\quad(40.0 \%)\end{array}$ & $\begin{array}{l}26 / 83 \\
(31.3 \%)\end{array}$ & $\begin{array}{l}51 / 91 \\
\quad(56.0 \%)\end{array}$ & $\begin{array}{l}45 / 56 \\
(80.4 \%)\end{array}$ & $7 / 17(41.2 \%)$ & $\begin{array}{l}27 / 71 \\
\quad(38.0 \%)\end{array}$ \\
\hline AAV8 +/total (\%) & $\begin{array}{l}234 / 513 \\
(45.6 \%)\end{array}$ & $\mathrm{N} / \mathrm{A}$ & $\begin{array}{l}39 / 86 \\
\quad(45.3 \%)\end{array}$ & $\begin{array}{l}38 / 89 \\
\quad(42.7 \%)\end{array}$ & $\begin{array}{l}8 / 20 \\
\quad(40.0 \%)\end{array}$ & $\begin{array}{l}32 / 83 \\
\quad(38.6 \%)\end{array}$ & $\begin{array}{l}49 / 91 \\
\quad(53.8 \%)\end{array}$ & $\begin{array}{l}39 / 56 \\
\quad(69.6 \%)\end{array}$ & $7 / 17(41.2 \%)$ & $\begin{array}{l}22 / 71 \\
\quad(31.0 \%)\end{array}$ \\
\hline rh10 +/total (\%) & $\begin{array}{l}236 / 513 \\
(46.0 \%)\end{array}$ & $N / A$ & $\begin{array}{l}45 / 86 \\
(52.3 \%)\end{array}$ & $\begin{array}{l}33 / 89 \\
(37.1 \%)\end{array}$ & $\begin{array}{l}10 / 20 \\
(50.0 \%)\end{array}$ & $\begin{array}{l}25 / 83 \\
(30.1 \%)\end{array}$ & $\begin{array}{l}\text { 48/91 } \\
\quad(52.7 \%)\end{array}$ & $\begin{array}{l}41 / 56 \\
(73.2 \%)\end{array}$ & $9 / 17(52.9 \%)$ & $\begin{array}{l}25 / 71 \\
\quad(35.2 \%)\end{array}$ \\
\hline
\end{tabular}

Data are shown for participants with non-missing assessments.

+, positive; AAV, adeno-associated virus; N/A, not available. 
Considerable geographic variability was observed in the prevalence of pre-existing antibodies against each serotype, but the percentage of participants positive for AAV5 was consistently the lowest among serotypes and across the countries studied. A greater percentage of adult participants were positive for AAV5 antibodies (36\%) when compared with adolescents (29\%). Comparative analyses of AAV serostatus in non-hemophilic individuals in select countries showed similar rates of seropositivity, as would be expected for viruses en demic to the human population. Serostatus and antibody titer were generally stable over the 6-month sampling period.

Conclusions: Among PwHA, pre-existing immunity against AAV serotypes varied across serotypes and regions, but global seropositivity was lowest for AAV5 and highest for AAV2. As clinical trials of AAV-mediated gene therapies progress, the presence of antibodies against the various AAV serotypes may become an increasingly important eligibility consideration.

PB0650 I A Novel Strategy for Platelet-specific Gene Therapy for the Treatment of Hemophilia A via Intranasal Delivery Lentiviral Vectors Containing Factor VIII

C.W. Rementer; C. Li; C.-Y. Chen; C.H. Miao

Seattle Children's Research Institute, Seattle, United States

Background: Hemophilia A (HemA) is a disease caused by absent or deficient FVIII protein. Gene therapy targeting hematopoietic stem cells is an attractive approach to treating this disease. Emerging evidence points to the lung being a reservoir for hematopoietic progenitors/stem cells (HSCs), in addition to bone marrow. Our previous data demonstrated that specific promoter GP1ba (G) can drive external factor VIII (FVIII) gene and achieve long-term expression of FVIII protein in megakaryocytes by transducing HSCs in mice.

Aims: Deliver a lentiviral vector (LV) intranasally into HemA mice to correct FVIII deficiency and improve coagulation.

Methods: HemA mice were conditioned with $\mathrm{N}$-acetylcysteine and dexamethasone to reduce lung mucus and the immune response, respectively. They were then administered a LV with a titer of $1.1 \times 10^{9}$ ifu/mL intranasally. The LV contained a megakaryocyte-specific Gp1ba-promoter driven FVIII gene to target expression to platelets. Results: Using flow cytometry, we found that IN delivery of GFP containing LVs $\left(2.5 \times 10^{9} \mathrm{ifu} / \mathrm{mL}\right)$ produced up to $0.075 \%$ positive GFP expressing-platelets after 4 days. When a vector containing the FVIII gene (G-F8-LV) was delivered intranasally $\left(2.5 \times 10^{9} \mathrm{ifu} / \mathrm{mL}\right)$, FVIII expression in platelets was detectable by ELISA at a level of $0.4 \mathrm{mU} / 10^{8}$ platelets. Additionally, rotational thromboelastometry (ROTEM) assays using whole blood from the gene therapy group showed that the mice had functional improvements at days 7 and 30 post-treatment.

Conclusions: These data demonstrate that we have succeeded in producing FVIII expressing platelets and partially corrected HemA phenotype in mice via intranasal delivery of G-FVIII-LVs in the lung. The benefit of FVIII expression and storage in platelet a-granules is to protect FVIII from naturalizing antibodies and to decrease the possibility of inducing inhibitory antibodies. The IN delivery of LVs encoding FVIII gene targeting lung would be a promising option for therapeutic treatment of hemophilia patients.

\section{PB0651 | The Impact of Platelet allb33 on the Hemostatic Efficacy of Platelet-derived FVIII Gene Therapy}

$\underline{\text { H. Yu }}^{1,2}$; J.A. Schroeder ${ }^{1,2}$; J.G. Mattson ${ }^{2} ;$ Q. Shi ${ }^{1,2,3,4}$

${ }^{1}$ Department of Pediatrics, Medical College of Wisconsin, Milwaukee, United States; ${ }^{2}$ Blood Research Institute, Versiti, Milwaukee, United States; ${ }^{3}$ Children's Research Institute, Milwaukee, United States; ${ }^{4}$ MACC Fund Research Center, Milwaukee, United States

Background: Our previous studies have demonstrated that platelettargeted FVIII (2bF8) expression is effective in rescuing the bleeding phenotype of $\mathrm{FVIII}^{-/-}$mice even in the presence of anti-FVIII inhibitors. We showed that VWF is essential for the clinical efficacy of platelet gene therapy in the inhibitor model, but GPIb is not.

Aims: To investigate how platelet aggregation impacts the clinical efficacy of platelet-FVIII in $\mathrm{FVIII}^{-/-}$mice.

Methods: We used anti-mouse integrin allb $\beta 3$ antibody (Leo.H4) to inhibit platelet aggregation. Hemostatic properties of platelet-FVIII were assessed by ROTEM and native whole blood TGA (nWB-TGA) analysis of whole blood and $\mathrm{FeCl}_{3}$-induced carotid artery injury model.

Results: In 2bF8/FVIII ${ }^{-/-}$mice, platelet-FVIII levels were $6.89 \pm 0.65$ $\mathrm{mU} / 10^{8}$ platelets, with all parameters from ROTEM analysis comparable to those in WT control mice. However, all parameters including clotting time, clot formation time, maximal clot formation, $a$-angle, and $\mathrm{A} 10$ were significantly impaired in the presence of Leo. H4 compared to native samples from $2 \mathrm{bF} 8 / \mathrm{FVIII}^{-/-}$mice $(8.5 \pm 1.96 \mathrm{~min}$, $3.1 \pm 0.41 \mathrm{~min}, 67.25 \pm 2.63 \mathrm{~mm}, 55.75 \pm 3.59^{\circ}, 44.25 \pm 4.03 \mathrm{~mm}$ in native samples versus $10.68 \pm 1.77 \mathrm{~min}, 13.5 \pm 3.11 \mathrm{~min}, 28.5 \pm 0.58 \mathrm{~mm}$, $33 \pm 2.83^{\circ}, 18.25 \pm 1.26 \mathrm{~mm}$ in those with Leo.H4, respectively). In contrast, in WT mice, only a-angle and A10 were significantly tempered in the presence of Leo.H4. Interestingly, in $2 \mathrm{bF} 8 / \mathrm{FVIII}^{-/-}$mice, peak thrombin and endogenous thrombin potential determined by TGA were significantly enhanced in the presence of Leo.H4 compared to native samples $(58.4 \pm 23.72 \mathrm{nM}$ and $600.8 \pm 185.83 \mathrm{nM}$ vs. $97.93 \pm 13.89 \mathrm{nM}$ and $921.03 \pm 72.95 \mathrm{nM}$, respectively), but there were no significant differences in other parameters. Leo.H4 did not impact the hemostatic parameters determined by nWB-TGA in WT mice. Complete occlusion occurred in the $\mathrm{FeCl}_{3}$-induced carotid injury in all 2bF8 and WT mice, but none occluded when Leo.H4 was infused.

Conclusions: allb $\beta 3$-mediated platelet aggregation is critical in maintaining the hemostatic functions and clinical efficacy of plateletderived FVIII in hemophilia A. 
PB0652 | Evolution of AAV Vector Gene Therapy is Ongoing In Hemophilia. Will the Unique Features of BAY 2599023 Address the Outstanding Needs?

S.W. Pipe ${ }^{1}$; C. Hay ${ }^{2}$; J. Sheehan ${ }^{3}$; T. Lissitchkov ${ }^{4}$; M. Coppens ${ }^{5}$; H. Eichler ${ }^{6}$; S. Weigmann ${ }^{7}$; F. Ferrante ${ }^{8}$

${ }^{1}$ University of Michigan, Ann Arbor, United States; ${ }^{2}$ Manchester University Department of Haematology, Manchester, United Kingdom; ${ }^{3}$ University of Wisconsin-Madison, Madison, United States; ${ }^{4}$ National Specialized Hospital for Active Treatment of Haematologic Diseases, Sofia, Bulgaria; ${ }^{5}$ Amsterdam University Medical Centers, University of Amsterdam, Amsterdam, Netherlands; ${ }^{6}$ Institute of Clinical Haemostaseology and Transfusion Medicine, Saarland University, Homburg, Germany; ${ }^{7}$ Bayer, Wuppertal, Germany; ${ }^{8}$ Bayer, Basel, Switzerland

Background: Ongoing phase 3 gene therapy trials for hemophilia A show promise but unpredictable factor (F)VIII expression of uncertain durability. Gene therapy must evolve to meet patient expectations of a durable, efficacious and safe treatment. Thus, BAY 2599023 (AAVhu37.hFVIIIco) comprises an adeno-associated virus vector with capsid serotype hu37 (AAVhu37) and codon-optimized B-domain-deleted human FVIII (hFVIIIco), with packaging and expression driven by a transthyretin promoter/enhancer combination. Aims: Report safety and FVIII activity achieved to date in this firstin-human, dose-finding study of BAY 2599023.

Methods: This phase 1/2, open-label study (NCT03588299) includes males aged $\geq 18$ years with severe hemophilia $A,>150$ exposure days to FVIII products, no history of FVIII inhibitors, and no detectable pre-existing neutralizing antibodies to AAVhu37. Patients received a single intravenous infusion of BAY 2599023. Primary endpoints were adverse events (AEs), serious AEs (SAEs) and AEs/SAEs of special interest (S/AESIs). Secondary endpoint was FVIII activity over time. Informed patient consent and ethics committee approval were obtained.

Results: Three cohorts of $\geq 2$ patients each $(N=8)$ were enrolled sequentially (Figure 1).

Figure 1. BAY 2599023 dose escalation

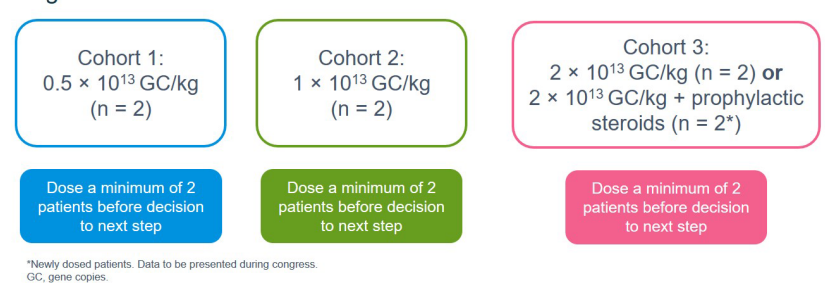

FIGURE 1 BAY 2599023 dose escalation

At data cutoff (January 2021), FVIII activity data were available for the first 6 patients (Figure 2). BAY 2599023 delivered sustained FVIII expression levels for up to 21 months, with evidence of bleed protection. Of these 6 patients, 3 (Cohorts $2[n=1]$ and $3[n=2]$ ) developed AESIs: asymptomatic elevations in alanine aminotransferase, managed reactively with corticosteroids. No SAEs have been reported. Two additional patients recently enrolled in Cohort 3 are being treated prophylactically with corticosteroids. Data from all 8 patients, up to 27 months, will be presented.

Figure 2. FVIII levels (chromogenic ${ }^{\star}$ [BDD plasma]) by patient over time ${ }^{\dagger}$ in Cohorts 1,2 and $3\left(n=6^{\ddagger}\right)$

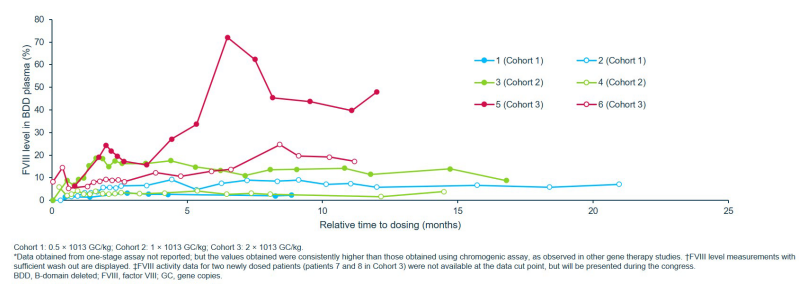

FIGURE 2 FVIII levels (chromogenic* [BDD plasma]) by patient over time† in Cohorts 1, 2 and 3 ( $n=6 \ddagger)$

Conclusions: BAY 2599023 was designed to enhance efficacy and durability of FVIII expression with a favorable safety profile. All patients with evaluable data have shown effective, sustained FVIII levels, with no SAEs, making BAY 2599023 a key candidate in the evolution of gene therapy in hemophilia $A$.

\section{PB0653 | 52 Week Efficacy and Safety of Etranacogene} Dezaparvovec in Adults with Severe or Moderate-severe Hemophilia B: Data from the Phase 3 HOPE-B Gene Therapy Trial

S.W Pipe ${ }^{1}$; F.W. Leebeek ${ }^{2}$; M. Recht ${ }^{3}$; N.S Key ${ }^{4}$; S. Lattimore ${ }^{3}$; G. Castaman ${ }^{5}$; E.K Sawyer ${ }^{6,7}$; S. Verweij ${ }^{6,7}$; V. Colletta ${ }^{6,7}$; D. Cooper ${ }^{6,7}$; R. Dolmetsch ${ }^{6,7}$; W. Miesbach ${ }^{8}$, HOPE-B Investigators ${ }^{1}$ University of Michigan, Ann Arbor, United States; ${ }^{2}$ Erasmus University Medical Center, Rotterdam, Netherlands; ${ }^{3}$ Oregon Health \& Science University, Portland, United States; ${ }^{4}$ University of North Carolina, Chapel Hill, United States; ${ }^{5}$ Center for Bleeding Disorders and Coagulation, Careggi University Hospital, Florence, Italy, ${ }^{6}$ uniQure Inc, Lexington, United States, ' uniQure BV, Amsterdam, Netherlands; ${ }^{8}$ University Hospital Frankfurt, Frankfurt, Germany

Background: The investigational gene therapy for hemophilia B (HB) etranacogene dezaparvovec comprises an adeno-associated virus serotype 5 (AAV5) vector containing a codon-optimized Padua variant human factor IX (FIX) transgene with a liver specific promoter. Aims: A Phase 3 trial (HOPE-B; NCT03569891) is underway to further assess efficacy and safety of etranacogene dezaparvovec in adults with $\mathrm{HB}$.

Methods: An open-label, single-dose, single-arm, international trial in adult males with severe or moderate-severe $\mathrm{HB}(\mathrm{FIX} \leq 2 \%)$ on routine FIX prophylaxis and with/without pre-existing neutralizing antibodies (NAbs) to AAV5. A $\geq 6$ month lead-in period preceded a single dose of etranacogene dezaparvovec $\left(2 \times 10^{13} \mathrm{gc} / \mathrm{kg}\right)$. The co-primary 
endpoints were FIX activity at 26 and 52wks and 52wk annualized bleeding rate (ABR). Secondary endpoints included adverse events (AEs), and reactive use of steroids.

Results: 54pts were dosed and completed 26wks of follow-up. During lead-in, 38pts (70\%) had 123 bleeds (42 spontaneous; 66 traumatic; 15 other), despite prophylaxis. Post treatment with etranacogene dezparvovec, at 26wks, mean (SD; min, max) change in FIX activity from $B L$ was $+36.0 \%(19.7 ; 0,96.1, P<0.0001)$ and $52 / 54$ participants had successfully discontinued routine prophylaxis. 23/54 pts had pre-existing AAV5 NAbs; no correlation with FIX activity was seen up to a titer of $678.2 ; n=52, R^{2}=0.078$; one participant with a NAb titer of 3212.3 did not respond and an additional participant received a partial dose and remained on prophylaxis. Relative to lead-in, total bleeds reduced by $83 \%$ and treated bleeds reduced by $91 \%$ at 26 wks. Treatment-related AEs occurred in 37 participants, most of which (81.5\%) were mild. One year data on FIX activity, ABR, use of FIX replacement and safety will be presented. Conclusions: Following a single dose of etranacogene dezaparvovec, FIX activity increased, without the need for prophylactic immunosuppression, into the mild-to-normal range at $26 \mathrm{wks}$ in pts with severe/moderately severe $\mathrm{HB}$.

PB0654 | Clinical Translatable Preconditioning for Platelet Gene Therapy in Murine Hemophilia A with Inhibitors

Y. Chen ${ }^{1,2}$; J. Schroeder ${ }^{1}$; J. Li ${ }^{1,2} ;$ W. Jing ${ }^{1} ;$ J. Hu ${ }^{2}$; Q. Shi ${ }^{1}$

${ }^{1}$ Medical College of Wisconsin, Versiti Blood Research Institute, Children's Research Institute, Milwaukee, United States; ${ }^{2}$ Department of Hematology, Fujian Institute of Hematology, Fujian Provincial Key Laboratory of Hematology, Fujian Medical University Union Hospital, Fuzhou, China

Background: We have demonstrated that platelet-FVIII gene therapy restores hemostasis in Hemophilia A (HA) mice with antiFVIII inhibitors under total body irradiation (TBI) preconditioning. Currently, Fludarabine along with busulfan (FB) as preconditioning has been shown to be highly effective for hematopoietic stem cell transplantation (HSCT) in the clinic.

Aims: To evaluate if FB works as a preconditioning approach for platelet gene therapy of HA with inhibitors.

Methods: Fludarabine was administered on days -7 through -4 , and busulfan was given on days -2 and -1 into rhF8-primed HA mice. A TBI regimen was used as a parallel control. Platelet FVIII expression was introduced by transplantation of $2 \mathrm{bF} 8$ lentivirus (2bF8LV)transduced HSCs. Animals were analyzed by FACS, quantitative PCR, FVIII assays, and tail bleeding test.

Results: All recipients achieved more than 55\% donor-derived leukocytes at 20 weeks after HSCT with not significantly difference between the two groups. The copy number of the 2bF8 cassette and the platelet-FVIII in the FB group were not significantly different compared to the TBI group $(0.93 \pm 0.17$ vs $0.79 \pm 0.11$ copy/cell and $4.44 \pm 1.14$ and $5.16 \pm 3.19 \mathrm{mU} / 10^{8}$ platelets, respectively). The bleeding phenotype was rescued in 2bF8LV-transduced recipients. Notably, anti-FVIII antibody titers declined with time under both preconditioning. The half-life of inhibitor was no significant difference between the two groups. When the titer dropped to undetectable, recipients were challenged with rhF8. There were no detectable anti-FVIII inhibitors in the transduced recipients from either the FB or TBI group. In contrast, all the untransduced control mice produced inhibitors. These data demonstrated that immune tolerance was established in 2bF8LV-transduced primed-HA mice.

Conclusions: FB preconditioning successfully introduced therapeutic levels of platelet FVIII expression, leading to phenotypic correction and immune tolerance induction in rhF8-primed HA mice. Our data suggest that this approach may be a promising clinically translatable strategy for gene therapy of hemophilia A with inhibitors.

PB0655 | Development of Ordinary Differential Equation-based aPTT and Thrombin Generation Models for Characterizing FIXPadua Gain of Function

T. Orfeo $^{1}$; M. Gissel ${ }^{1}$; S.J. Everse ${ }^{1}$; E. Shehu²; R. Corbau ${ }^{2}$; J.H. Foley ${ }^{2}$

${ }^{1}$ Department of Biochemistry, University of Vermont, Colchester, United States; ${ }^{2}$ Freeline, Stevenage, United Kingdom

Background: Adeno-associated virus (AAV) gene therapies for haemophilia $B$ are being developed to produce sustained increases in factor IX (FIX) serum levels in treated patients. A hyperfunctional FIX variant (FIXPadua, FIX-R338L) is being utilized across gene therapy programmes to achieve FIX activity levels within the normal range (50\%-150\%). Despite the broad usage of FIX-R338L in AAV gene therapies, the mechanisms underlying the FIX-R338L gain of function are not yet fully understood. Aims: To report the development of computational models for characterizing differences in activity between FIX-R338L and FIX-WT.

Methods: Published data on the kinetic constants and respective thrombin generation of FIX-R338L (Samelson-Jones et al 2019) and FIX-R338A (a related variant; Chang et al 1998) and aPTT data were used in concert with published ordinary differential equation-based models of aPTT and thrombin generation to guide the development of accurate FIX-R338L computational models.

Results: Computational modelling showed that $\mathrm{K}_{\mathrm{M}}$ and $\mathrm{k}_{\text {cat }}$ differences between the FIX-R338L and FIX-WT derived intrinsic tenases are sufficient to account for observed functional differences between the two variants. To achieve good correspondence with both aPTT and thrombin generation data, the model suggests that catalytic efficiency $\left(\mathrm{k}_{\mathrm{cat}} / \mathrm{K}_{\mathrm{M}}\right)$ of FIX-R338L tenase must be $\sim$-fold greater than that of FIX-WT tenase. The modelling suggests that both a lower $\mathrm{K}_{\mathrm{M}}$ and a greater $\mathrm{k}_{\text {cat }}$ are required to capture the FIXR338L gain of function observed in empirical aPTT and thrombin generation data. Computational model outputs are consistent with our empirical findings in clinically relevant FIX assays showing that factor X, but not factor VIII, impacts FIX-R338L activity estimates. Conclusions: Results to date suggest that factor $X$ levels affect FIXR338L activity as measured by clinically relevant FIX assays. Iterative 
evaluation of $\mathrm{K}_{\mathrm{M}}$ and $\mathrm{k}_{\mathrm{cat}}$ for FIX-R338L led to refinement of computational models that will serve as powerful tools for understanding the mechanisms that contribute to FIX-R338L gain of function.

PB0656 | Targeted CRISPR/Cas9-mediated Gene Addition to a Safe Harbor in Placental Cells for the Treatment of Hemophilia A

R.M. Ramamurthy ${ }^{1}$; M. Rodriguez ${ }^{1}$; D. Meares ${ }^{2}$; A. Farland ${ }^{2}$; A. Atala ${ }^{1}$; C.B. Doering ${ }^{3}$; H.T. Spencer ${ }^{3}$; C.D. Porada ${ }^{1}$;

G. Almeida-Porada ${ }^{1}$

${ }^{1}$ Fetal Research and Therapy Program, Wake Forest Institute for Regenerative Medicine, Winston-Salem, United States; ${ }^{2}$ Wake Forest School of Medicine, Winston-Salem, United States; ${ }^{3}$ Aflac Cancer and Blood Disorders Center, Children's Healthcare of Atlanta and Department of Pediatrics, Emory University, Atlanta, United States

Background: Gene therapy is a promising approach for treating monogenic diseases such as hemophilia $\mathrm{A}(\mathrm{HA})$. Considering the heterogeneity of FVIII mutations, a "gene addition" approach to knock-in a functional fVIII transgene would be more efficient compared to gene editing, and CRISPR/Cas9 can be used to achieve location-specific insertion of a fVIII transgene into a genomic safe harbor with less off-target effects in comparison to lentiviral transduction, which can cause insertional mutagenesis.

Aims: To insert the bioengineered fVIII transgene "IcoET3" into the AAVS1 site in human placental stem cells (PLCs) and human liverderived endothelial cells (HLECs) and assess and compare their subsequent levels of FVIII production and to confirm integration at the AAVS1 site and check for off-target effects.

Methods: CRISPR/Cas9-mediated gene addition was achieved with co-transfection of the plasmids pCas-Guide-AAVS1 and pAAVS1-puroEF1a-IcoET3. Genomic insertion, mRNA expression, and clotting activity was assessed using PCR, RT-qPCR and aPTT assay, respectively.

Results:

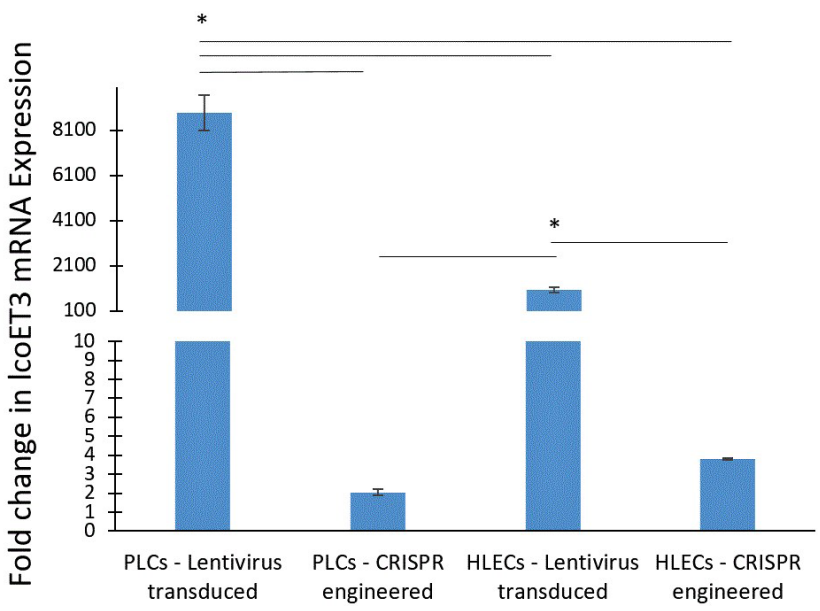

FIGURE 1 RT-qPCR results show fold change in expression of IcoET3. Delivery of IcoET3 through a lentiviral vector resulted in robust expression

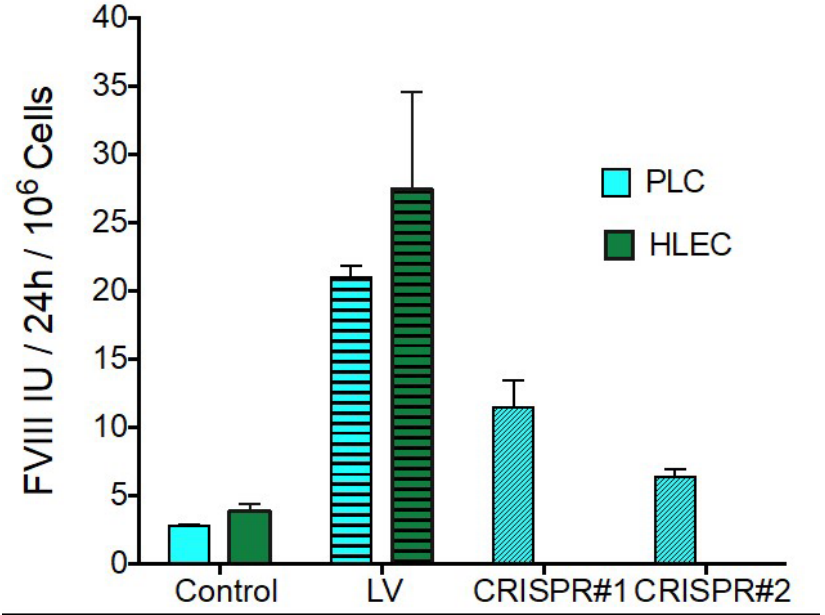

FIGURE 2 aPTT assay shows PLCs transduced with lentivirus (LV) encoding IcoET3 secreted significantly more FVIII than CRISPR/ Cas9-engineered PLCs and control PLCs

RT-qPCR with IcoET3-specific primers confirmed expression of the bioengineered fVIII transgene in both HLECs and PLCs (Figure 1). aPTT assay demonstrated that the CRISPR/Cas9-engineered PLCs secreted significantly more FVIII:C than engineered HLECs and control PLCs (Figure 2). However, lentivirus transduced cells produced the highest levels of expression and protein. NGS to confirm successful insertion at the AAVS1 site and off-target effect analysis is currently underway.

Conclusions: The levels of FVIII:C produced by PLCs following CRISPR/Cas9-mediated knock-in of the bioengineered IcoET3 fVIII transgene were sufficient to be in the therapeutic range, showing its potential as a cell-gene delivery platform to treat HA. The ability to successfully edit HLECs in vitro represents an important first step to establishing the feasibility of using CRISPR/Cas9 to mediate geneediting in HLECs in vivo, achieving FVIII expression within the cells that serve as the natural site of synthesis of this protein and thereby correcting HA.

PB0657 | Novel Hyperactive FIX Variants and their Potential for Hemophilia B Therapy

A.-K. Urbanowitz ${ }^{1}$; P. Milanov ${ }^{1}$; P. Quade-Lyssy ${ }^{1}$; D. Abriss ${ }^{1}$;

E. Seifried ${ }^{1} ;$ J. Schüttrumpf ${ }^{2}$; J. Schwäble ${ }^{1}$

${ }^{1}$ Goethe University Hospital Medical School/Institute for Transfusion Medicine and Immunohematology, Frankfurt, Germany; ${ }^{2}$ Biotest AG, Dreieich, Germany

Background: Adeno-associated-virus (AAV) based gene transfer has shown potential to provide sustained Factor IX (FIX) activity at low levels in severe hemophilia B, preventing spontaneous bleeding and reducing FIX consumption. However, further enhancement was hampered by dose-dependent liver toxicity, most likely due to cytotoxic T-cells directed against transduced hepatocytes or cellular stress by 
high protein expression. The hyperactive FIX Padua variant enhanced efficacy but the issue of dose-limiting liver toxicity remained.

Aims: Two FIX variants, based on FIX-Padua, with further enhanced intrinsic activity and one of them with decreased collagen IV binding were evaluated for their potential to enhance efficacy after AAVbased gene transfer and to allow novel strategies of recombinant FIX substitution in hemophilia B.

Methods: Recombinant FIX variants were tested in-vivo for pharmacokinetic and pharmacodynamic parameters and their immunogenic potential. The FIX variants were further tested in AAV-based gene transfer for efficacy and safety, particularly with regard to thrombotic events.

Results: Both novel FIX variants showed 1.5-fold increased activity compared to FIX Padua. Collagen type IV binding had no impact on enzymatic or functional activity of the protein. Instead, reduction of collagen IV binding dramatically increased the bioavailability in hemophilia $B$ mice after intravenous or subcutaneous injection but also enhanced the elimination of the protein. In AAV gene therapy, the reduced collagen type IV binding variant has no additional effect. Risk assessments of the new hyperactive variants in hemophilia B mice showed neither increased immunogenicity due to the introduced mutations, nor increased propensity to thrombosis due to further increased intrinsic activity.

Conclusions: Our new hyperactive FIX variants harbor increased activity compared to FIX-Padua, promising enhanced efficiency in AAV-based gene therapy for hemophilia B without increasing therapy-related toxicity. In addition, decreased collagen type IV binding improves bioavailability and bears potential for alternative substitution strategies, such as subcutaneous application.

PB0658 | Preferences of People with Hemophilia A and B for Treatments Including Gene Therapies in the US: A Discrete Choice Experiment

M. Witkop ${ }^{1}$; G. Morgan ${ }^{2}$; J. O'Hara ${ }^{2,3}$; M. Recht ${ }^{4,5}$; T.W. Buckner ${ }^{6}$; D. Nugent ${ }^{7}$; R. Curtis ${ }^{8}$; B. O'Mahony ${ }^{9,10}$; M.W. Skinner ${ }^{11,12}$; B. Mulhern ${ }^{13} ;$ M. Cawson ${ }^{2} ;$ T. Ali ${ }^{14} ;$ E. Sawyer ${ }^{14} ; \mathrm{N}^{2} \mathrm{Li}^{14}$

${ }^{1}$ National Hemophilia Foundation, New York, United States; ${ }^{2} \mathrm{HCD}$

Economics, Daresbury, United Kingdom; ${ }^{3}$ Faculty of Health and Social Care, University of Chester, Chester, United Kingdom; ${ }^{4}$ Oregon Health \& Science University, Portland, United States; ${ }^{5}$ American Thrombosis \& Hemostasis Network, Rochester, United States; ${ }^{6}$ Hemophilia and Thrombosis Center, University of Colorado School of Medicine, Aurora, United States; ${ }^{7}$ Department of Pediatrics and Division of Hematology at CHOC Children's Hospital, Center for Inherited Blood Disorders, Orange, United States; ${ }^{8}$ Hematology Utilization Group Studies, Los Angeles, United States; ${ }^{9}$ Irish Haemophilia Society, Dubline, United Kingdom, ${ }^{10}$ Trinity College, Dublin, United Kingdom, ${ }^{11}$ Institute for Policy Advancement, Ltd, Washington, United States, ${ }^{12}$ McMaster University, Hamilton, Canada, ${ }^{13}$ Centre for Health Economics Research and Evaluation, University of Technology Sydney, Sydney, Australia, ${ }^{14}$ uniQure Inc, Lexington, United States

Background: Evidence of patient preferences for novel hemophilia treatment such as gene therapies is limited. Understanding which treatment attributes are important for people with hemophilia $A$ (PWHA) and $\mathrm{B}$ (PWHB) will help to inform the value of gene therapy. Aims: To examine treatment preferences, including gene therapies, of PWH using a discrete choice experiment (DCE).

Methods: An online survey was conducted among US male adults with severe or moderate hemophilia, recruited from patient panels including the National Hemophilia Foundation Community Voices in Research platform. In the DCE, each participant completed 14 choice tasks selecting their preferred option from hypothetical pairs of treatment scenarios with varying attribute levels. A total of six attributes were developed based on a systematic literature review and semi-structured interviews with patient advocates and clinical experts. Responses were excluded for inconsistencies using a dominated choice task and completion time. Random parameters logit models (which assumes preference heterogeneity) were used to estimate the preference weights and the relative attribute importance (RAI) in PWHA and PWHB.

Results: The DCE was completed by $183 \mathrm{PWH}$ with a mean (SD) age of 39.3 (13.8) years, with 120 PWHA and 63 PWHB. The two most important attributes were 'Dose frequency and durability' (RAI: PWHA 23\%; PWHB 35\%) and 'Effect on overall annual bleeding rate (ABR)' (RAI: PWHA 32\%; PWHB 29\%), the former being more important for PWHB. They were followed by 'Uncertainty regarding short-term or long-term significant safety issues' (RAI: PWHA and PWHB both $17 \%$; all three attributes contributing to $70-80 \%$ of RAI). The attributes 'Impact on activity of daily life/physical activity', 'Transformative/mental health impact' and 'Post treatment, possibility to undergo minor surgery without need for factor therapy' contributed to the remaining $20-30 \%$ of RAl.

Conclusions: The results from this novel application of DCE informs the value that patients place on different aspects of gene therapies.

\section{PB0659 | Management of Infusion Reactions: Lessons from the Phase 3 HOPE-B Gene Therapy Trial of Etranacogene Dezaparvovec in Adults with Hemophilia B}

M. Recht ${ }^{1}$; F.W. Leebeek ${ }^{2}$; W. Miesbach ${ }^{3}$; N.S Key ${ }^{4}$; S. Lattimore ${ }^{1}$; G. Castaman ${ }^{5}$; E.K. Sawyer'; D. Cooper ${ }^{6}$; V. Colletta ${ }^{6}$; S.W. Pipe, HOPE-B Investigators

${ }^{1}$ Oregon Health \& Science University, Portland, United States; ${ }^{2}$ Erasmus University Medical Center, Rotterdam, Netherlands; ${ }^{3}$ University Hospital Frankfurt, Frankfurt, Germany; ${ }^{4}$ University of North Carolina, Chapel Hill, United States; ${ }^{5}$ Center for Bleeding Disorders and Coagulation, Careggi University Hospital, Florence, Italy, ' uniQure Inc, Lexington, United States; ${ }^{7}$ University of Michigan, Ann Arbor, United States

Background: The Phase 3 HOPE-B trial (NCT03569891) utilized etranacogene dezaparvovec, an investigational gene therapy for hemophilia $B(\mathrm{HB})$ comprising an adeno-associated virus serotype 5 (AAV5) vector containing a codon-optimized Padua variant human factor IX (FIX) transgene with a liver specific promoter. 
Aims: Here, adverse events of special interest reported as related to the investigational drug occurring on day of dosing are described. Methods: Following a lead-in period of $\geq 6$ months, 54 adult males with $\mathrm{HB}(\mathrm{FIX} \leq 2 \%)$ with or without neutralizing antibodies (NAbs) to AAV5 received a single dose of etranacogene dezaparvovec $\left(2 \times 10^{13} \mathrm{gc} / \mathrm{kg}\right)$ and completed 26 weeks of follow up.

Results: Seven of 54 (13\%) participants experienced a total of 9 infusion-related reactions. Of the 9 reactions, 5 were mild and 4 were moderate, and they occurred in participants with AAV5 NAbs at baseline (5/7; titer range 23-3212) and in those without (2/7). All events were reported on the day of infusion and most (8/9) resolved on the same day. The first occurrence (a moderate suspected hypersensitivity reaction) occurred after approximately $10 \%$ of the dose of etranacogene dezaparvovec was administered; the drug was withdrawn and the participant received intravenous corticosteroids and antihistamines. Subsequent occurrences were managed through a combination of temporarily interrupting or slowing the etranacogene dezaparvovec infusion and/or supportive treatment with steroids/antihistamines. Three mild reactions (e.g. hives, itchiness, headache and dizziness) in 3 participants required no supportive treatment; this included 1 participant with a baseline AAV5 NAb titer of 3212.

Conclusions: Infusion-related reactions occurred in 7/54 participants (13\%), regardless of the presence or absence of AAV5 NAbs, the majority of which were mild and resolved on the same day. Mild reactions were successfully managed by decreasing the infusion rate. Temporary infusion interruption, supportive treatment with antihistamines and steroids, and a subsequent slower infusion rate are recommended for moderate reactions.

PB0660 | Exigency: The Real-world Impact of Gene Therapy on the Lives of People with Haemophilia and their Families. An Interim Report

$\underline{\text { S. Fletcher }}^{1}$; S. Chaplin²; M. Holland ${ }^{2}$; K. Jenner ${ }^{2}$; K. Khair ${ }^{2}$

${ }^{1}$ Oxford Haemophilia and Thrombosis Centre, Oxford, United Kingdom;

${ }^{2}$ Haemnet, London, United Kingdom

Background: Gene therapy for haemophilia has the potential to change the life experience of individuals and their families. Few studies have sought to explore the impact of gene therapy on individuals and their families.

Aims: The aim of the Exigency study is to capture the real-life experience of gene therapy in people with haemophilia and their families. Methods: Participants who had undergone gene therapy were recruited by specialist centres, social media and word of mouth referral. Each participant and a family member when available took part in a semi-structured qualitative interview which was recorded, transcribed verbatim and analysed thematically.

All elements of the study were reviewed by local statutory authorities and informed consent was sought in all cases.
Results: To date, nine participants (eight with haemophilia $A$ and one with haemophilia B), mean age 43.1 years (range 23-68 years), along with a family member, took part in a single qualitative interview online $(n=8)$, or in person $(n=1)$. Mean time since transfection was 1.89 years (range $1-5$ years).

Five major themes have emerged: freedom; altruism; side effects of immunosuppression; loss of factor expression; and loss of control.

Conclusions: Most of those interviewed saw participation in gene therapy as a positive experience: it had freed them from the personal burden of haemophilia and furthered treatment options for the wider haemophilia community. Participants however reported being unprepared for the side effects of immunosuppression. Some also reported feeling unsupported and as having little control over what was happening as their factor levels became the focus of the process.

The results so far suggest that psychosocial support needs to be integrated into gene therapy programmes to help participants and families process their experiences both positive and negative. Interviews are ongoing to confirm these early data and to identify further themes.

\section{PB0661 | The World Federation of Hemophilia Gene Therapy Registry}

B.A. Konkle ${ }^{1,2}$; D. Coffin ${ }^{3}$; M. Naccache ${ }^{2}$; C. Clark ${ }^{4}$; L.A. George A. Iorio ${ }^{6}$; W. Miesbach ${ }^{7}$; D. Noone ${ }^{8}$; F. Peyvandi ${ }^{9,10}$; S.W. Pipe ${ }^{11}$; M. Recht ${ }^{12}$; M. Skinner ${ }^{13}$; L.A. Valentino ${ }^{14}$; J.N. Mahlangu ${ }^{15,16}$; G.F. Pierce ${ }^{2}$

${ }^{1}$ Bloodworks Northwest, Seattle, United States; ${ }^{2}$ World Federation of Hemophilia, Montreal, Canada; ${ }^{3}$ World Federation Hemophilia, Montreal, Canada, ${ }^{4}$ IInternational Society on Thrombosis and Haemostasis, Carrboro, United States; ${ }^{5}$ The Children's Hospital of Philadelphia, Philadelphia, United States; ${ }^{6}$ McMaster University, Hamilton, Canada; ${ }^{7}$ University Hospital Frankfurt, Frankfurt, Germany; ${ }^{8}$ European Haemophilia Consortium, Brussels, Belgium; ${ }^{9}$ Department of Pathophysiology and Transplantation, Università degli Studi di Milano, Milan, Italy, ${ }^{10}$ Angelo Bianchi Bonomi Hemophilia and Thrombosis Center, Fondazione IRCCS Ca' Granda Ospedale Maggiore Policlinico, Milan, Italy, ${ }^{11}$ C. S. Mott Children's Hospital, Ann Arbor, United States, ${ }^{12}$ American Thrombosis and Hemostasis Network, Rochester, United States, ${ }^{13}$ Institute for Policy Advancement Ltd, Washington, United States, ${ }^{14}$ National Hemophilia Foundation, New York, United States, ${ }^{15}$ International Society on Thrombosis and Haemostasis, Scientific and Standardization Committee (SSC), Carrboro, United States, ${ }^{16}$ University of the Witwatersrand, Johannesburg, South Africa

Background: Gene therapy for hemophilia is an evolving therapeutic modality, with many challenges and unresolved questions that will not be fully answered at the completion of current ongoing clinical trial programs.

Aims: The World Federation of Hemophilia (WFH) Gene Therapy Registry (GTR) is being developed to collect long-term data on 
safety, and variability and durability of efficacy on all people with hemophilia (PWH) who receive gene therapy.

Methods: A Steering Committee (SC) composed of health care professionals, patient advocates, industry representatives, and regulatory agency liaisons oversees the project. The WFH GTR is a prospective, observational, and longitudinal registry. All PWH who receive gene therapy, via clinical trial or post-approval, will be invited to participate. The WFH GTR's goal is to enroll $100 \%$ of eligible PWH globally.

Results: Through an iterative process and following guidance of the European Medicines Agency and the US Food and Drug Administration, the WFH GTR SC has developed a core data set (Konkle et. al 2020). Data will be collected at 3, 6, 9, 12, 18, and 24 months post-gene therapy infusion and annually thereafter. Data will be captured directly via participating hemophilia treatment centres ( $\mathrm{HTC}$ ) or through data linkage with existing hemophilia registries who meet outlined criteria. Accordingly, data from US patients will be collected via the ATHN and transferred directly into the GTR. Outreach to European HTCs began in the Fall 2020. Policies governing data usage are being developed by a scientific advisory board comprised of relevant patient and physician advocates.

Conclusions: The WFH GTR will ensure that rare adverse events, in a small patient population over a large geographical area, will be detected. The many unknowns of gene therapy for hemophilia make it critical that the bleeding disorders community ensures all patients are followed over their lifetimes.

PB0662 | Responses to Anxiety and Depression Items in People with Hemophilia who Tested a Patient Reported Outcome Measure: The coreHEM MH Study

\section{E. Clearfield ${ }^{1}$; H.-Y. Chang ${ }^{1}$; T. Majid ${ }^{2}$; M.W. Skinner ${ }^{3}$}

${ }^{1}$ Center for Medical Technology Policy, Baltimore, United States;

${ }^{2}$ Erickson School of Aging Studies, University of Maryland, Baltimore County, Baltimore, United States; ${ }^{3}$ Institute for Policy Advancement Ltd, Washington, DC, United States

Background: coreHEM Mental Health (coreHEM MH) builds on the coreHEM core outcome set for hemophilia gene therapy (Iorio Haemophilia 2018). coreHEM MH seeks to develop a contentvalidated, patient-reported outcome measure (PROM) to measure mental health $(\mathrm{MH})$ outlook in patients who receive gene therapy, a coreHEM core outcome. Once fully developed, the coreHEM MH PROM can be used to measure changes in $\mathrm{MH}$ outlook in hemophilia gene therapy trials.

Aims: To compare the anxiety/depression domain of the EQ-5D-3L and the draft anxiety and depression items of the coreHEM $\mathrm{MH}$ PROM.

Methods: A conceptual framework and draft PROM were developed following a background literature review. The framework included living with/treating hemophilia and aspects of $\mathrm{MH}$ outlook that may change after gene therapy. Concepts were categorized into five domains: stigma, anxiety, depression, life interference, and identity. Thirty participants with severe or moderate hemophilia A or B participated in concept elicitation interviews and completed the PROM, a demographic survey, and the EQ-5D-3L.

Results: For EQ-5D-3L, 43\% reported they were not anxious or depressed. 56\% reported being moderately or extremely anxious or depressed (Table). Mean EQ-5D VAS score indicating participant "health today" was 68.5 (range 20-99). For the coreHEM MH PROM, those who reported they were not anxious or depressed on the EQ$5 \mathrm{D}-3 \mathrm{~L}$ had higher mean scores in both the PROM anxiety (7 items) and depression domains (10 items) compared to those who reported they were "moderately" or "extremely" anxious or depressed in the EQ-5D-3L (Figure). For the coreHEM MH PROM a higher percent indicates a better $\mathrm{MH}$ outlook.

TABLE 1 EQ-5D-3L Responses: Anxiety/Depression Domain

\begin{tabular}{ll} 
Anxiety/Depression "Today" & Number (Percent) \\
I am not anxious or depressed & $13(43 \%)$ \\
I am moderately anxious or depressed & $16(53 \%)$ \\
I am extremely anxious or depressed & $1(3 \%)$ \\
\hline
\end{tabular}

Mean Scores for coreHEM MH Anxiety and Depression Items by EQ-5D-3L Responses

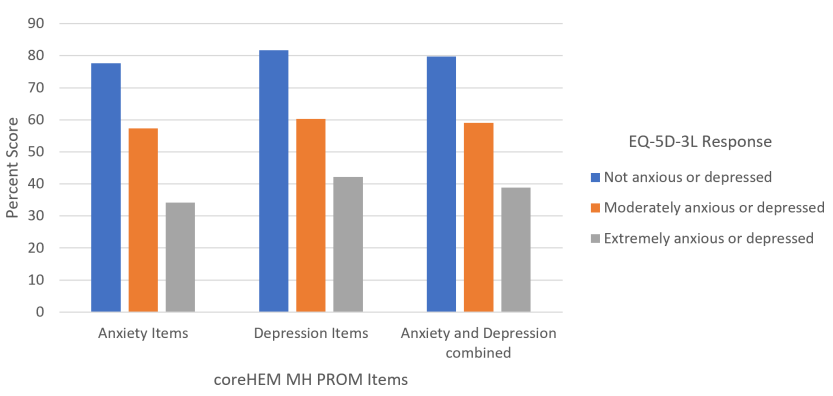

FIGURE 1 Mean Scores for coreHEM MH Anxiety and Depression Items by EQ-5D-3L Response

Conclusions: Domain scores for anxiety and depression on the coreHEM MH PROM follow the expected pattern aligning with the EQ$5 \mathrm{D}-3 \mathrm{~L}$ levels. Thematic analysis will be performed on the qualitative interview data to refine the conceptual framework and PROM. This work is funded by Bayer, BioMarin, Pfizer, Sanofi, Spark, Takeda, and uniQure. 
PB0663 | A Savvy Approach to Clinical Trial Recruitment for the SAAVY (Seroprevalence of AAV AntibodY) Study in the Era of COVID-19: Designing for a Prospective, Observational Study in the United States during a Global Pandemic

L.A. Valentino' ${ }^{1}$ M. Vaghela ${ }^{2}$; M. Lauw ${ }^{2}$; G. Dela Cerda²; M. Jones²; D. Hinds ${ }^{2}$; V. Newman ${ }^{2}$; F. Leal-Pardinas ${ }^{2}$; D. Rotellini ${ }^{1}$; K. Schafer ${ }^{3}$; G. Pierce ${ }^{4}$; S. Pipe ${ }^{5,6}$

${ }^{1}$ National Hemophilia Foundation, New York, United States; ${ }^{2}$ BioMarin Pharmaceutical Inc., Novato, United States; ${ }^{3}$ University of California Hemostasis \& Thrombosis Center, Sacramento, United States; ${ }^{4}$ World Federation of Hemophilia, Montreal, Canada; ${ }^{5}$ Medical and Scientific Advisory Council to the National Hemophilia Foundation, New York, United States; ${ }^{6}$ Departments of Pediatrics and Pathology, University of Michigan, Ann Arbor, United States

Background: Adeno-associated virus (AAV)-mediated gene therapy is being investigated as a treatment for people with hemophilia $A$ (PwHA). Commonly studied AAV serotypes include AAV5, AAV6, and AAV8. Pre-existing immunity against these $A A V s$ restricts patient eligibility yet, published data on AAV seroprevalence and seroconversion rate in $\mathrm{PwHA}$ are limited.

Aims: To describe design and recruitment methods used for the SAVVY study. This study is designed to characterize AAV antibody prevalence and titers, evaluate changes in antibody titer over time, and examine factors that may influence antibody positivity, titer, and seroconversion. Study design and recruitment methods seek to minimize the need for in-person study interactions during the COVID-19 pandemic.

Methods: SAVVY is a patient-centered, decentralized, prospective, observational study involving blood draws at 2 time points. The target sample size of 1,000 PwHA represents approximately $5 \%$ of PwHA in the US. SAVVY employs a user-friendly mobile application, provides remote recruitment through e-consent, and uses a network of $1,800+$ laboratories throughout the US for biospecimen sample collection. This approach minimizes patient travel, reduces potential exposure to COVID-19, and removes burden from HTC staff to perform study assessments. Recruitment has leveraged various forms of virtual outreach to physicians, HTC staff, and patient advocacy groups; direct-to-patients are being used to create awareness of the study and its scientific aims. See Table 1.

TABLE 1 Recruitment Outreach

\begin{tabular}{ll} 
Recruitment Efforts & $\begin{array}{l}\text { Community Awareness } \\
\text { Campaigns }\end{array}$ \\
SAAVY website & HCP Educational Webinars \\
SAAVY mobile application & SAAVY Advisor Videos \\
Partner email outreach & Infographic Brochures \\
Online recruitment [advertising] & $\begin{array}{c}\text { Patient Advocacy group } \\
\text { initiatives }\end{array}$ \\
\hline
\end{tabular}

Results: First patient enrollment occurred within four months of final protocol; 41 patients were enrolled within 2 weeks of activation.
At the time of abstract submission, 105 patients had consented to participate.

Conclusions: The COVID-19 pandemic disrupted the progress of clinical studies necessary for the advancement of treatments. Adaptability to existing conditions is critical. Careful study design and multi-modal engagement with the hemophilia community can facilitate the conduct of studies, minimize risks associated with COVID-19, and may enhance patient experience and clinical trial recruitment.

PB0664 | The Importance of Patient Preferences and Other Patient-Centric Metrics in Developing and Evaluating New Haemophilia Treatments

T. Burke ${ }^{1,2}$; S. Asghar ${ }^{1}$; J. O'Hara ${ }^{1,2}$; D. Yee ${ }^{3}$ S. $\mathrm{Kar}^{3}$

${ }^{1}$ HCD Economics, Daresbury, United Kingdom; ${ }^{2}$ University of Chester, Chester, United Kingdom; ${ }^{3}$ Freeline Therapeutics, Stevenage, United Kingdom

Background: Life-long prophylactic treatment with factor replacement therapy is standard of care for patients with severe or moderately severe haemophilia to prevent bleeding episodes, especially haemarthroses. Gene therapy is an investigational treatment that has the potential to result in sustained endogenous levels of clotting factor within the normal range, thus offering potentially transformative benefits including a functional cure for haemophilia.

While conventional clinical metrics offer some insight into the benefits of gene therapy for patients, they can fail to account fully for more holistic aspects of health or evaluate what is most important to patients. Regulators and pharmaceutical appraisal bodies have thus issued guidance to incorporate patient preference data into development programs, which could better characterise what a 'functional cure' could mean to patients.

Aims: To summarise current research into haemophilia patient preferences related to emerging pharmaceutical modalities such as gene therapy.

Methods: Desktop research was undertaken to identify current literature on patient preferences in haemophilia, with particular attention to gene therapy. The focus of this search was patient preference studies such as discrete choice experiments (DCEs) and qualitative analyses to assess costs, benefits, and trade-offs associated with new therapies.

Results: There exist few published studies that explore haemophilia patient preferences. Previous studies in haemophilia using the DCE framework have reported that patients had a strong preference for infrequent dosing, non-invasive route of administration, and protection against bleeding. Only one publication cited aspects of gene therapy in the aims and attributes of the analysis.

Conclusions: An analysis of the literature confirms a need to conduct further research to identify outcomes that are most important to haemophilia patients and their preferences regarding gene therapy. Transformative innovations such as gene therapy may offer 
expansive benefits beyond the reach of traditional clinical outcome measures, and further research to understand how gene therapy may address patients' needs is warranted.

\section{MANAGEMENT OF BLEEDING AND TRAUMA}

\section{LPB0068 | Efficacy of Parenteral Formulations of Desmopressin in the Treatment of Bleeding Disorders: A Systematic Review}

S. McKinlay ${ }^{1}$; S. Sreeraman ${ }^{1}$; O. Motalo ${ }^{1} ;$ A. Li ${ }^{2} ;$ M. Crowther ${ }^{1}$

${ }^{1}$ McMaster University, Hamilton, Canada; ${ }^{2}$ University of Ottawa, Ottawa, Canada

Background: Desmopressin (DDAVP) is a proven therapy for bleeding disorders, however, the therapeutic efficacy of different formulations has yet to be systematically analyzed.

Aims: This study investigates whether subcutaneous (SC) DDAVP provides equivalent hemostatic efficacy to intravenous (IV) desmopressin, particularly in patients with mild to moderate bleeding tendencies from hemophilia A or von Willebrand disease (vWd).

TABLE 1 Studies investigating hemostatic efficacy in healthy patients

$\begin{array}{lll}\text { Study } & \begin{array}{l}\text { Number of } \\ \text { Participants }(n)\end{array} & \text { Patient Population } \\ \text { Kohler, } 1986 & 10 & \text { Healthy patients }\end{array}$

Kohler, 1988

10

Healthy patients

MacGregor, 1988

6

Healthy patients

t-PA, t-PA:Ag, FVIII, FVIII:Ag, vWF:Ag, PAI

\section{Main Outcomes of Interest}

FVIII:C, FXII, HMW-kininogen levels, ristocetin cofactor (vWF:Ag); also measured half-life, FVIII:Ag

FVIII:Ag, t-PA, leukocyte count, bioavailability
Törnebohm, 1992
Methods: We searched PubMed, EMBASE, MEDLINE, Cochrane, and CINAHL databases for observational studies and randomized controlled trials, which compared the hemostatic efficacy of parenteral formulations of desmopressin in healthy patients, or those livfortial inclusion in our study. To systematically vWF activity.

Results: The search strategy yielded a total of 5519 studies. Twelve studies met the inclusion criteria and were included in the review. All (Table 1) and seven out of ure hemostatic efficacy.

The average number of participants per study was 39 . 
TABLE 2 Studies investigating hemostatic efficacy in patients with hemophilia A or vWd

\begin{tabular}{|c|c|c|c|c|}
\hline Study & $\begin{array}{l}\text { Number of } \\
\text { Participants (n) }\end{array}$ & Patient Population & $\begin{array}{l}\text { Main Outcomes of } \\
\text { Interest }\end{array}$ & Main Conclusions \\
\hline Kohler, 1984 & 53 & $\begin{array}{l}\text { Mild to moderate } \\
\text { hemophilia A and mild } \\
\text { vWd }\end{array}$ & $\begin{array}{l}\text { FVIII:C, FVIIIR:Ag, } \\
\text { FVIII:Rcof }\end{array}$ & $\begin{array}{l}\text { SC administration offered a consistent and } \\
\text { reliable hemostatic response in place of IV } \\
\text { administration. }\end{array}$ \\
\hline De Sio, 1985 & 26 & $\begin{array}{c}\text { Mild to moderate } \\
\text { hemophilia A }\end{array}$ & FVIII:C & $\begin{array}{l}\text { The response to DDAVP after } 60 \text { minutes } \\
\text { did not statistically differ between } \\
\text { formulations. }\end{array}$ \\
\hline Ghirardini, 1987 & 59 & $\begin{array}{l}\text { Mild hemophilia and Type } \\
1 \mathrm{vWd}\end{array}$ & FVIII:C & $\begin{array}{l}\text { There were no statistically significant } \\
\text { differences between IV or either SC } \\
\text { formulations (concentrated vs. diluted). }\end{array}$ \\
\hline Mannucci, 1987 & 14 & $\begin{array}{c}\text { Mild to moderate } \\
\text { hemophilia A }\end{array}$ & $\begin{array}{l}\text { FVIII:C; measured peak } \\
\text { DDAVP, time to peak } \\
\text { levels, time curve, } \\
\text { and half-life }\end{array}$ & $\begin{array}{l}\text { No significant difference in mean FVIII:C levels } \\
\text { between IV and SC. No differences in } \\
\text { half-life or AUC values between IV and SC } \\
\text { formulations. }\end{array}$ \\
\hline Grana, 1990 & 11 & $\begin{array}{l}\text { Hemophilia } \mathrm{A}, \mathrm{vWd} \text {, or } \\
\text { a primary platelet } \\
\text { aggregation disorder }\end{array}$ & FVIII:Ag, FVIII:C & $\begin{array}{l}\text { No significant difference in the mean increase } \\
\text { in FVIII:Ag between IV and SC formulations. } \\
\text { Hemostatic response and correction of } \\
\text { bleeding time was comparable between IV } \\
\text { and SC forms. }\end{array}$ \\
\hline Jensen, 1997 & 22 & Mild hemophilia A & FVIII:C, vWF:Ag, APTT & $\begin{array}{l}\text { No significant difference in response of FVIII:C } \\
\text { between routes of administration. }\end{array}$ \\
\hline Knofler, 2012 & 80 & Hemophilia A or carriers & FVIII:C & $\begin{array}{l}\text { The magnitude of FVIII:C response between IV } \\
\text { and SC forms was relatively equal ( } P \text {-value } \\
\text { not reported). }\end{array}$ \\
\hline Loomans, 2018 & 169 & Moderate hemophilia A & FVIII:C & $\begin{array}{l}\text { There was no significant difference between } \\
\text { formulations in vWF:Ag levels, IV had a } \\
\text { significantly higher peak FVIII:C }(P<0.001) \text {. }\end{array}$ \\
\hline
\end{tabular}

APTT = activated partial thromboplastin time; FVIII:Ag = factor VIII antigen; FVIII:C = factor VIII activity; FVIII:Rcof = factor VIII ristocetin cofactor; FVIIIR:Ag = factor VIII related antigen; $\mathrm{VWF}: \mathrm{Ag}=$ von Willebrand factor antigen.

Conclusions: This is the first systematic review to date to evaluate the hemostatic efficacy of parenteral DDAVP formulations. Our study shows that IV and SC administration of DDAVP appears to result in a near equivalent rise of laboratory hemostatic markers; however, these findings are limited by the overall under-sampling of primary studies, necessitating larger and more recent randomized controlled trials to provide further clarification.

LPB0069 | Self-Propelled Hemostatic Powder Safely Achieves and Maintains Hemostasis in a Live Porcine Model of Upper Gastrointestinal Bleeding

$\underline{\text { N. Ali-Mohamad }}^{1,2} ;$ M. Cau ${ }^{1,2,3} ;$ J. Baylis ${ }^{1,2,3}$;

F. Donnellan ${ }^{4,5}$; C. Kastrup ${ }^{1,2,6}$

${ }^{1}$ Michael Smith Laboratories, The University of British Columbia,

Vancouver, Canada; ${ }^{2}$ Centre for Blood Research, The University

of British Columbia, Vancouver, Canada; ${ }^{3}$ School of Biomedical

Engineering, The University of British Columbia, Vancouver, Canada;

${ }^{4}$ Division of Gastroenterology, The University of British Columbia,

Vancouver, Canada; ${ }^{5}$ Vancouver General Hospital, Vancouver, Canada;

${ }^{6}$ Department of Biochemistry and Molecular Biology, The University of British Columbia, Vancouver, Canada
Background: Upper gastrointestinal bleeding (UGIB) is a serious complication of bleeding disorders. Currently, clip placement and BICAP cautery are used to manage these bleeds but are technically challenging procedures to perform during active bleeding. New endoscopic hemostatic sprays that coat the bleed site and act as mechanical bandages have rebleeding rates up to $30 \%$ and display poor efficacy against severe cases of UGIB. A novel hemostatic powder has been developed that effectively propels thrombin ("propelled thrombin powder") against blood flow and increases its delivery into sites of injury.

Aims: To test the efficacy and safety of propelled thrombin hemostatic powder for achieving lasting hemostasis in heparinized porcine models.

Methods: Five anesthetized and heparinized pigs underwent an endoscopic mucosal snare resection of the stomach wall to trigger an ulcer bleed. Propelled thrombin powder was sprayed using compressed carbon dioxide through a 7 FR catheter, inserted through the instrument channel of the gastroscope. The time to initial hemostasis from the initial application of propelled thrombin powder and the amount of powder delivered were measured. Pigs underwent a rescope 72 hours post-procedure and gross necropsy and histopathology were performed.

Results: All pigs achieved initial hemostasis in $5.4 \pm 1.2 \min (n=5)$ with less than $5 \mathrm{~g}$ of powder applied. At 72 hours post-procedure, 
rescope showed no rebleeding in any animals. There were no signs of foreign bodies or thromboembolism during an extensive gross necropsy and histopathology of all organs examined. There was no tissue damage due to propelled thrombin powder identified around the ulcer site and coagulation parameters were comparable to baseline. Conclusions: Propelled thrombin powder successfully stopped bleeding and prevented rebleeding in five ulcer bleeds. Propelled thrombin may be a valuable tool for rapidly halting UGIB in patients with bleeding disorders in the future. Further studies comparing efficacy to other modalities are required.

PB0665 | Off-label Use of Emicizumab in Persons with Acquired Hemophilia A and von Willebrand Disease: A Scoping Review of the Literature

V. Mathew Thomas ${ }^{1}$; M.Y. Lim²

${ }^{1}$ University of Connecticut, Farmington, United States; ${ }^{2}$ University of Utah Health, Salt Lake City, United States

Background: Since the approval of emicizumab, a bispecific, FVIIImimetic antibody, for use in persons with congenital hemophilia $A$ in 2018, there have been increasing case reports of off-label use of emicizumab in other bleeding disorders, including acquired hemophilia A (AHA) and von Willebrand disease (VWD).

Aims: To describe the clinical presentation and outcomes of off-label emicizumab use in AHA and VWD.

Methods: We conducted a comprehensive search in Pubmed, EMBASE, and Scopus up to January 17, 2021. The following criteria were applied to the studies identified in the initial search: Patients had a diagnosis of AHA or VWD; and the study reported on the outcome of emicizumab use.

Results: The flowchart for the studies included in the scoping review and the primary reasons for study exclusion are described in Figure 1. Twelve studies ( 9 full text, 3 conference abstracts) were included in the final review, for a total of 24 patients (20 AHA, 4 VWD). Of the $20 \mathrm{AHA}$ patients, 17 received emicizumab for active bleeds and 3 for surgical prophylaxis. The median duration of emicizumab use was 60 days (range: $12-180$ days). There were no adverse events reported related to emicizumab use in AHA patients. All 4 VWD patients had type 3 VWD and received emicizumab for active bleeds. 3 of the 4 patients continue to be on emicizumab indefinitely, and the fourth for a total duration of 12 months. There were no adverse events reported related to emicizumab use in VWD patients. Hemostasis was achieved in all patients (AHA and VWD) in whom emicizumab was used.
66 citations identified through

PubMed, EMBASE and Scopus

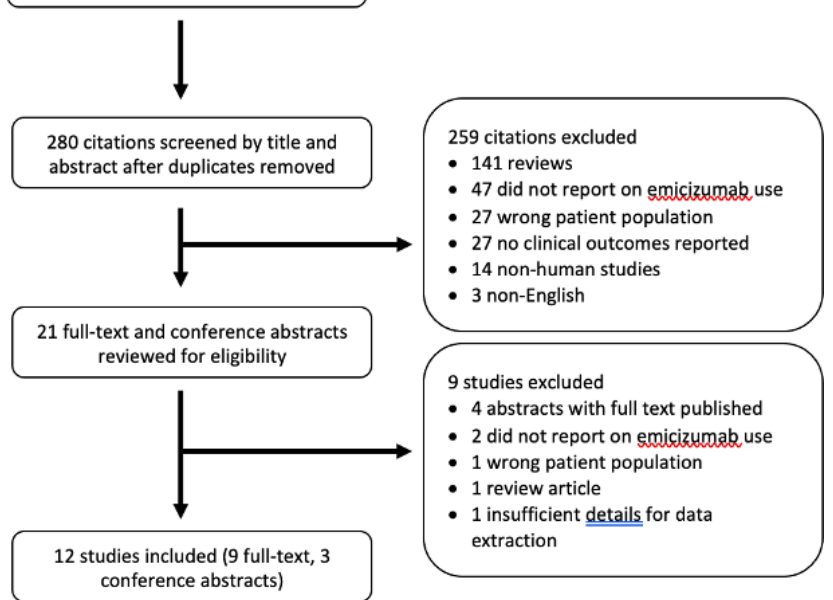

FIGURE 1 Flowchart for the studies included in the scoping review

Conclusions: Based on published case reports and case series, emicizumab appears to be an effective hemostatic therapy for AHA and VWD. Larger confirmatory randomized controlled trials are needed to confirm these findings.

\section{PB0666 | Functional Transitory Anti-emicizumab Antibody Creating Bleeding Episodes}

F. Peyvandi ${ }^{1}$; S. Braham ${ }^{2}$; R. Gualtierotti ${ }^{1}$; S. Arcudi ${ }^{3}$; L. Schiavone ${ }^{2}$; C. Novembrino ${ }^{2}$; C. Valsecchi ${ }^{2}$

${ }^{1}$ Fondazione IRCCS Ca' Granda Ospedale Maggiore Policlinico, Angelo Bianchi Bonomi Hemophilia and Thrombosis Center and Fondazione Luigi Villa, and the Department of Pathophysiology and Transplantation, Università degli Studi di Milano, Milan, Italy; ${ }^{2}$ Fondazione IRCCS Ca' Granda Ospedale Maggiore Policlinico, Angelo Bianchi Bonomi Hemophilia and Thrombosis Center and Fondazione Luigi Villa, Milan, Italy; ${ }^{3}$ Department of Pathophysiology and Transplantation Università degli Studi di Milano, Milan, Italy

Background: The development of anti-drug antibody in patients with Hemophilia A treated with emicizumab is a rare event. Nevertheless, as already described, patients may develop neutralizing anti-emicizumab antibody which could be associated with unexpected bleeding.

Aims: To describe a new case of partially neutralizing antiemicizumab antibody, developed in a Hemophilia A patient with no FVIII inhibitor treated with emicizumab which disappeared with continuous use of drug.

Methods: Case: The patient is a 33-years-old male with severe HA without FVIII inhibitor, previously in prophylaxis with $2000 \mathrm{U}$ of recombinant FVIII every other day. His replacement therapy was switched to emicizumab receiving the initial four standard loading doses with $3 \mathrm{mg} / \mathrm{kg} /$ week followed by the $1.5 \mathrm{mg} / \mathrm{kg} /$ week regimen. After receiving the $5^{\text {th }}$ dose, the patient developed an acute bleeding episode at his right elbow confirmed with ultrasound. This acute 
bleeding required additional treatment of FVIII. Emicizumab plasma level and development of anti-drug antibody has been controlled.

Results: The bleeding episode in our patient at $5^{\text {th }}$ dose of emicizumab was associated with a modest reduction of emicizumab concentration from 49 to $31.7 \mathrm{ug} / \mathrm{mL}$ and presence of anti-emicizumab antibody tested with western blot analysis on total IgG fraction. Since the neutralization was not complete the patient has continued his regular administration using emicizumab and a reduced dose of FVIII (4 total infusions) with a close clinical (ultrasound) and laboratory (emicizumab level and anti-emicizumab antibody) monitoring. The intensity of anti-drug antibody was reduced at $7^{\text {th }}$ dose and completely disappeared at $8^{\text {th }}$ dose with a gradual recovery of emicizumab concentration up to $41 \mathrm{ug} / \mathrm{mL}$ in patient's plasma.

Conclusions: During the initial phase of emicizumab treatment $(<20$ exposure days) patients need particular attention with an accurate clinical and laboratory monitoring. Some transitory anti-drug antibody could have a functional effect creating bleeding episodes, and this need to be properly treated.

PB0667 | Differential Neutralization of Apixaban, Betrixaban, Edoxaban, and Rivaroxaban by Andexanet Alfa as measured by Whole Blood Thromboelastographic Analysis

S. Mehrotra $^{1}$; D. Hoppensteadt ${ }^{1}$; W. Jeske ${ }^{1}$; O. Iqbal ${ }^{1}$; A. Tafur ${ }^{2}$; B. Lewis ${ }^{1} ;$ J. Fareed ${ }^{1}$

${ }^{1}$ Loyola University Medical Center, Maywood, United States; ${ }^{2}$ North Shore University Health Systems, Evanston, United States

Background: The purpose of this study was to investigate the anticoagulant effects of currently available oral Anti-Xa agents such as Apixaban, Betrixaban, Edoxaban, and Rivaroxaban and their relative neutralization by AA in terms of such thromboelastographic parameters as $\mathrm{R}, \mathrm{K}$, Angle and $\mathrm{MA}$.

Aims: Analysis was carried out in whole blood using thromboelastography (TEG) using the TEG 5000 Hemostasis System (Haemonetics Corp, Massachusetts).

Methods: Blood was drawn from healthy donors in individual groups ( $n=5-10$ ) into $3.2 \%$ citrated tubes. In the TEG cup for testing, 0.2 $\mathrm{M} \mathrm{CaCl}_{2}$, saline (with a filler and control), each of the individual $\mathrm{FXa}$ Inhibitors at a final concentration (FC) of $1 \mathrm{ug} / \mathrm{mL}, \mathrm{AA}$ at $\mathrm{FC}$ of both $100 \mathrm{ug} / \mathrm{mL}$ and $50 \mathrm{ug} / \mathrm{mL}$ were tested. TEG parameters such as Rtime, K-time, angle and MA were measured.

Results: Edoxaban showed the strongest anticoagulant effects in both $\mathrm{R}$ and $\mathrm{K}$ time followed by Betrixaban, then Rivaroxaban which were very similar in their anticoagulant effects, with Apixaban showing the weakest anticoagulant effect. Andexanet alpha produced varying levels of neutralization of the anti-Xa agents which are tabulated on Table 1.

\section{TABLE 1}

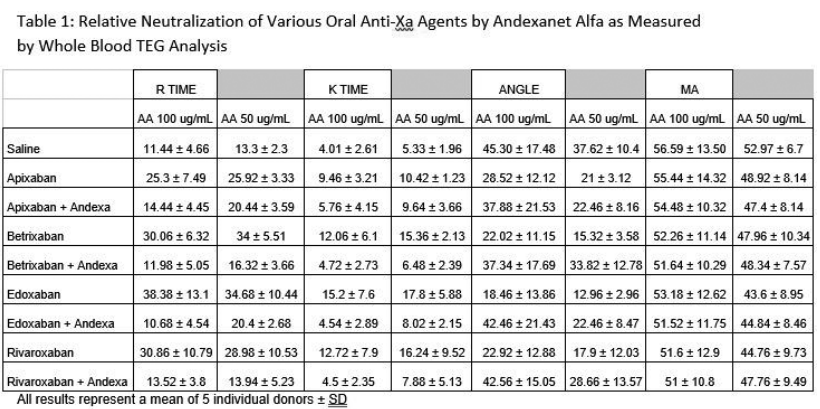

Conclusions: All of the four anti-Xa agents produced measurable anticoagulant activities at $1 \mathrm{ug} / \mathrm{mL}$ as measured by the TEG parameters. Edoxaban exhibited the strongest anticoagulant effect followed by Betrixaban and Rivaroxaban whereas Apixaban showed much weaker anticoagulant effects. AA FC $=100 \mathrm{ug} / \mathrm{mL}$ showed much stronger, consistent, and complete neutralization effects of all of the 4 FXa Inhibitors when compared to $A A$ at $F C=50 \mathrm{ug} / \mathrm{mL}$. These results strongly suggest that regardless of the variable anticoagulant effect exhibited by the FXa Inhibitors, AA at FC $=100 \mathrm{ug} / \mathrm{mL}$ fully neutralized the anticoagulant effects of this agent as measured by the TEG parameters. AA is shown to be the most effective in neutralizing Betrixaban in R-Time and K-Time at both concentrations. $A A$ was seen to neutralize Apixaban the least.

\section{PB0668 | Global Octim ${ }^{\circledR}$ Shortage: Experience in Strasbourg}

J. Wimmer ${ }^{1}$; A.-C. Gerout ${ }^{2}$; L. Sattler ${ }^{1}$; L. Grunebaum ${ }^{1}$; O. Feugeas ${ }^{3}$; D. Desprez ${ }^{3}$

${ }^{1}$ Hematology Laboratory, University Hospital, Strasbourg, France;

${ }^{2}$ Pharmacy Department, University Hospital, Strasbourg, France;

${ }^{3}$ Regional Hemophilia Treatment Center, University Hospital, Strasbourg, France

Background: Desmopressin is administered nasally $\left(\operatorname{Octim}^{\circledR}\right)$, intravenously (Minirin ${ }^{\circledR}$ ) or subcutaneously (Octostim ${ }^{\circledR}$ ). On July 2020, the French National Agency for Medicines and Health Products Safety published a withdrawal for all batches of Octim ${ }^{\circledR}$ currently distributed.

Aims: This situation prompted us to prescribe Octostim ${ }^{\circledR}$ to patients who usually use $\operatorname{Octim}^{\circledR}$ to treat their bleeds at least once a month or to newly diagnosed patients.

Methods: The treatment regimen is as follows: injection of $15 \mu \mathrm{g} /$ $\mathrm{mL}$ for patients weighing less than $75 \mathrm{~kg}$ and $30 \mu \mathrm{g} / \mathrm{mL}$ for those weighing 75 to $100 \mathrm{~kg}$. At first, 15 patients underwent a therapeutic trial with Octostim ${ }^{\circledR}$ then they were taught how to administer the subcutaneous injection properly. The cohort was composed of 4 patients with minor haemophilia A, 3 haemophiliacs with inhibitor, 1 carrier of haemophilia, and 7 patients with Von Willebrand disease (VWD) including 4 patients who never used desmopressin because 
their diagnosis was established in August 2020. The average (minmax) age was 32 years (17-75) with a sex-ratio (M/F) of 0.9.

Results: All patients responded favourably to Octostim ${ }^{\circledR}$ with an average increase in factor VIII activity (FVIII) of $249 \%$ and Von Willebrand factor activity (VWF:Rco) of $216 \%$ for patients with VWD. Comparatively, after an Octim ${ }^{\circledR}$ administration to the same patients, the average increase in FVIII and VWF:Rco were respectively $234 \%$ and $211 \%$. These results suggest a bioequivalence between the 2 galenic forms. During the trial, 2 patients reported pain with a burning sensation at the injection site. After 3 months, patients were contacted for follow-up and only 3 of them have actually used Octostim ${ }^{\circledR}$ as self-treatment, respectively for a menometrorrhagia, a tooth extraction and a lipectomy.

Conclusions: With a storage temperature of $+4^{\circ} \mathrm{C}$, the use of Octostim ${ }^{\circledR}$ is much more difficult and inappropriate for an emergency but constitute a good alternative for Octim ${ }^{\circledR}$ responsive patients.

PB0669 | Thromboelastography to Monitor Periprocedural Hemostasis in Advanced Liver Disease: A Systematic Review and Meta-analysis

J. Hartmann ${ }^{1}$; J. Dias ${ }^{1}$; E. Pivalizza²; G. Garcia-Tsao ${ }^{3}$

${ }^{1}$ Haemonetics Corporation, Braintree, United States; ${ }^{2}$ UT Health

McGovern Medical School, Houston, United States; ${ }^{3}$ Yale School of

Medicine, New Haven, United States

Background: Patients with cirrhosis often have abnormal hemostatic profiles, with increased risk of hemorrhage. Thromboelastography can be used to give a rapid assessment of the coagulation status and may be beneficial for patients with liver disease experiencing bleeding or undergoing invasive procedures or surgery.

Aims: To determine if the use of thromboelastography to monitor hemostasis, compared with standard of care, reduces blood product use or leads to changes in adverse events or mortality, in adult patients with liver disease.

Methods: Literature searches were conducted in the MEDLINE (Pubmed), Cochrane and EMBASE databases, for randomized control trials (RCTs) comparing patient management with thromboelastography (intervention) versus standard of care (control) in adult patients with liver disease, using pre-defined search terms and inclusion/exclusion criteria. Primary outcome was transfusion of a) fresh frozen plasma (FFP) and b) platelet units. Additional outcomes included blood product utilization, adverse events and mortality. Meta-analysis methods were used to combine study results, where possible. The study was registered in the PROSPERO database (CRD42020192458).

Results: The database search returned 191 non-duplicate results, with 29 manuscripts given a full text screen, and 5 RCTs included in the meta-analysis. Significantly lower use of platelets (primary outcome; $P=0.04)$, blood products $(P<0.001)$, FFP + platelets $(P<0.001)$ and cryoprecipitate $(P<0.001)$ were seen in the intervention group (Figure 1). Mortality was not statistically different between groups and was reported at different timepoints in each study, making data consolidation difficult (Table 1). Two studies reported adverse events ( $n=1$ in the control group for both). One study reported serious transfusion-related reactions, experienced by fewer patients in the intervention group $(30.6 \%$ vs $74.5 \%$; $P<0.001)$.

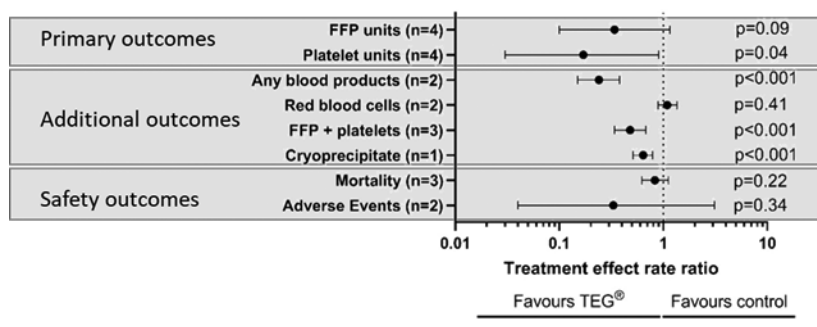

' $n$ ' refers to the number of studies with data available for selected outcome measure; Mortality defined as mortality within the period $40-90$ days only

FIGURE 1 Treatment effect for primary and secondary outcomes

TABLE 1 Individual reported mortality rates and timepoints across all five studies

\begin{tabular}{|c|c|c|c|}
\hline Study & $\begin{array}{l}\text { Timepoint (following surgery/ } \\
\text { intervention) }\end{array}$ & $\begin{array}{l}\text { Mortality in } \\
\text { intervention group, } n(\%)\end{array}$ & Mortality in control group, $n(\%)$ \\
\hline De Pietri et al. Hepatology 2016 & 90 days & $8(26.6)$ & $7(23.3)$ \\
\hline Kumar et al. Hepatology 2020 & 42 days & $27(55.1)$ & $31(66.0)$ \\
\hline $\begin{array}{l}\text { Rout et al. Journal of clinical gastroenterology } \\
\qquad 2020\end{array}$ & 6 weeks & $4(13.3)$ & $8(26.7)$ \\
\hline $\begin{array}{l}\text { Wang et al. Transplantation proceedings } \\
\quad 2010\end{array}$ & 3 years & $12(85.8)$ & $11(78.6)$ \\
\hline
\end{tabular}

Conclusions: Thromboelastography-guided management of patients with liver disease who are bleeding or undergoing invasive procedures or surgery can lead to reduced use of certain blood products, and may also result in reduction of serious transfusionrelated reactions. 
PB0670 | Success of Online CME at Improving Knowledge, Competence, and Confidence around Guideline-directed Management of Oral Anticoagulant-related Urgent Bleeding

M. Harris; J. Spyropoulos; A. Carothers; C. Padbury

Medscape Education, New York, United States

Background: Patients who experience oral anticoagulant (OAC)related major bleeding are not always appropriately managed, resulting in poor outcomes. Clinicians need an update on the most recent reversal recommendations and strategies to appropriately manage OAC-related urgent bleeding.

Aims: We sought to determine if online continuing medical education (CME) could improve the knowledge/competence and confidence of emergency medicine physicians and cardiologists regarding guideline-directed management of OAC-related urgent bleeding.

Methods: This CME intervention comprised of a 30-minute online video-based roundtable discussion among experts in the management of OAC-related major bleeding. Responses to 3 multiplechoice, knowledge/competence questions and 1 self-efficacy, 5 -point Likert scale confidence question were analyzed using a repeated pairs pre-/post-assessment study design. A Chi-square test ( $P<.05$ is considered significant) assessed pre- to post-activity change. Confidence improvement was measured using the percentage of physicians with measurable increases in confidence. Data were collected from September through October 2019.

Results: In total, 369 emergency medicine physicians and 521 cardiologists were included in this study. Overall, there were significant knowledge/competence improvements among participants from pre- to post-assessment.
Conclusions: This study demonstrates the success of online, CMEaccredited, video-based roundtable discussion on significantly improving knowledge/competence and increasing confidence of emergency medicine physicians and cardiologists related to the guideline-directed management of urgent OAC-related bleeding. Continued educational gaps were identified for future educational targets.

\section{PB0671 | Extended Half-life Products for Perioperative Hemostasis in Major Orthopedic Surgery: Real-world Data from the LASH Study}

L.P. Solimeno ${ }^{1}$; R. Gualtierotti ${ }^{2}$; J. Acquati Lozej $^{1} ;$ S. Arcudi ${ }^{2}$; E. Biguzzi ${ }^{3}$; S.M. Siboni ${ }^{3}$; C. Carulli ${ }^{4}$; F. Peyvandi ${ }^{3}$

${ }^{1}$ Ortho-Trauma Department and Angelo Bianchi Bonomi Hemophilia and Thrombosis Center, Fondazione IRCCS Ca' Granda, Ospedale Maggiore Policlinico, Milan, Italy; ${ }^{2}$ Angelo Bianchi Bonomi Hemophilia and Thrombosis Center, Fondazione IRCCS Ca' Granda, Ospedale Maggiore Policlinico, Department of Pathophysiology and Transplantation, Università degli Studi di Milano, Milan, Italy; ${ }^{3}$ Angelo Bianchi Bonomi Hemophilia and Thrombosis Center, Fondazione IRCCS Ca' Granda, Ospedale Maggiore Policlinico, Milan, Italy; ${ }^{4}$ Department of Health Sciences, Orthopaedic Unit, University of Florence, Florence, Italy

Background: Hemophilia A and B lead to disability and early need of prosthetic surgery. Extended half-life product (EHL) recently introduced allow to reach higher trough levels with a reduced number of infusions and may allow cost saving in the course of invasive procedures. EHL have demonstrated to be effective and safe for the

\section{TABLE 1}

\begin{tabular}{|c|c|c|}
\hline Topic & $\begin{array}{l}\text { Emergency Medicine Physicians ( } \mathrm{N}=369 \text { ) } \\
\text { Absolute \% improvement (pre-assessment vs } \\
\text { post-assessment; } P \text {-value) }\end{array}$ & $\begin{array}{l}\text { Cardiologists }(\mathrm{N}=521) \\
\text { Absolute \% improvement (pre-assessment vs } \\
\text { post-assessment; } P \text {-value) }\end{array}$ \\
\hline $\begin{array}{l}\text { Knowledge regarding the burden of OAC- } \\
\text { related bleeding in the emergency } \\
\text { department setting }\end{array}$ & $45 \%$ (19\% pre vs 63\% post; $P<0.001)$ & $45 \%$ (23\% pre vs $68 \%$ post; $P<0.001)$ \\
\hline $\begin{array}{l}\text { Competence related to selecting the most } \\
\text { appropriate guideline-recommended } \\
\text { approach to manage patients with OAC- } \\
\text { related urgent bleeding }\end{array}$ & $16 \%$ (57\% pre vs $67 \%$ post; $P<0.05)$ & $23 \%$ (51\% pre vs $67 \%$ post; $P<0.0001)$ \\
\hline
\end{tabular}

Additionally, $46 \%$ of emergency medicine physicians and $48 \%$ of cardiologists had a measurable increase in confidence in their ability to manage OAC-related urgent bleeding.

This study also uncovered significant knowledge/competence gaps among physicians, as $33 \%$ of emergency medicine physicians and $33 \%$ of cardiologists failed to select the appropriate guideline-based approach to manage OAC-related major bleeding and $28 \%$ of emergency medicine physicians and $24 \%$ of cardiologists still remain at only a rating of 1 to 2 on a scale of 1 to 5 in their confidence managing OAC-related urgent bleeding. prevention and treatment of bleeds during major and minor surgery in clinical trials. However, real-world data are still scarce.

Aims: Our aim was to describe our experience with patients with hemophilia $A$ and $B$ treated with extended half-life products for perioperative hemostasis in major orthopedic surgery (LASH study). Methods: We recruited 10 patients ( 8 adults, 2 children) affected by hemophilia A ( $n=7 ; 6$ severe, 1 moderate) and by hemophilia B ( $n=3 ; 2$ severe, 1 moderate), referring to the Orthopedic Surgery Unit and the Angelo Bianchi Bonomi Hemophilia and Thrombosis Center of Milan to undergo major orthopedic surgery. Informed 
consent was obtained by participants or their parents and the LASH study was approved by the local ethics committee. Data on perioperatory rFVIII-Fc or rFIX-Fc consumption and pain and satisfaction were collected over a mean follow-up of 11.4 months.

Results: Demographic and clinical characteristics of the patients and details and outcomes of the surgical procedures are reported in table 1. Pain and satisfaction during follow-up are reported in figure $1 \mathrm{~A}$ and $\mathrm{B}$. No thrombotic events were reported and none of the patients experienced inhibitor occurrence during the follow-up.

TABLE 1 Demographic and clinical characteristics of the patients included in the study $(n=10)$ and details and outcomes of the 10 major orthopedic surgical procedures

Median (Interquartile range)

Age

Type of surgery

Hemophilia A

preoperative FVIII plasma levels

preoperative rFVIII-Fc UI

post-infusion FVIII plasma levels

post-surgical FVIII plasma levels

total perioperative $\mathrm{UI}$

consumption

total costs of hemostatic treat-

ment (n.UI* 0.62€)

\section{Hemophilia B}

$8 \%(6 \%-33 \%)$

preoperative FIX plasma levels

preoperative rFIX-Fc UI

$53(46-76) \mathrm{UI} / \mathrm{kg}$

$82 \%(61 \%-94 \%)$

$50 \%(50 \%-57 \%)$

post-infusion FIX plasma levels

post-surgical FVIII plasma levels

$34,000(18,000-49,000)$ UI

total perioperative UI $34,340(18,180-49,490) €$

consumption

total costs of hemostatic treat-

ment (n.UI*1.01€)

Length of hospitalisation
Median (Interquartile range)

Other perioperative treatments 7 patients

postoperative tranexamic acid 1 patients

thromboprophylaxis

Days of postoperative treatment $\quad 5(2.5-8)$
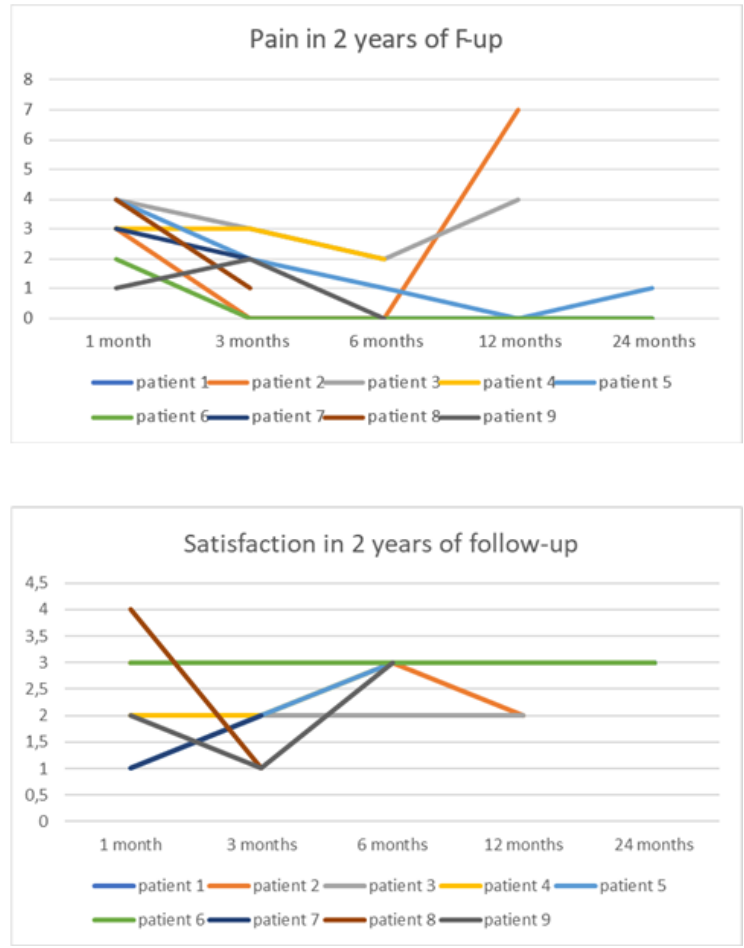

FIGURE 1 Pain (A) and satisfaction (B) reported by patients over a 2-year follow-up

Conclusions: In summary, in 10 major orthopedic surgery, rFVIIIFc and rFIXFc demonstrated efficacious hemostasis and a good safety profile, with no inhibitor occurrence and no thrombotic events reported. rFVIIIFc and rFIXFc consumption during major orthopedic surgeries was similar or reduced compared to SHL products. Perioperative pain and satisfaction were acceptable.

Further studies on larger samples and head-to-head studies are needed to confirm our findings.
6 (3.25-8.00) days

Min-max 1-40 days 
PB0672 | Late Prophylaxis in Severe Hemophilia A: Effect on Functional Abilities

M. Sousa-Pimenta ${ }^{1,2}$; L. Soares Miranda $a^{1}$ J. Ferreira ${ }^{1}$; A. Barros ${ }^{1} ;$ M. Coutinho $^{1}$; S. Morais ${ }^{1}$; E. Cruz ${ }^{1}$

${ }^{1}$ Centro de Coagulopatias Congénitas, Centro Hospitalar Universitário do Porto (CHUP), Porto, Portugal; ${ }^{2}$ Serviço de Hemato-Oncologia do Instituto Português de Oncologia do Porto, Porto, Portugal

Background: Primary prophylaxis in severe Hemophilia A (HA) has established benefits in preventing arthropathy. If started later in life, when arthropathy is already present, prophylaxis may have an impact in decreasing bleedings and subsequently improving functional activities. Aims: Study the impact of tertiary prophylaxis on adults with severe $\mathrm{HA}$ and pre-existing joint disease in bleeding rates and in autoperception of functional activities.

Methods: Ten adults with severe HA (median age 49; 26-59) changed from on demand to prophylaxis therapy. Two of them have less than 35 years-old and 7 had previous prosthetic replacement of joints. Annualized bleeding rate (ABR), annualized joint bleeding rate (AJBR) and functional activities self-assessed (HAL-Hemophilia Activities List questionnaire) were evaluated during on demand therapy (in 2016) and thereafter on prophylaxis (in 2020). Results of HAL for each patient were normalized for a maximum score of 100 corresponding to the best function.

Results: Patients in prophylactic therapy exhibited a significant decrease in ABR (mean rate of 33 [6;85] before prophylaxis and 6 [0;14] with prophylaxis) and AJBR (mean rate of 29 [6;81] before prophylaxis and 5.2 [0;13] afterwards).

Overall, median results of HAL questionnaires before and after the institution of prophylaxis, although not statistically significant, showed a tendency for improvement (57 to 62 overall score, respectively). Higher scores were obtained for the 2 younger patients analyzed (72 before prophylaxis and 74 after prophylaxis).

Conclusions: Prophylaxis versus on demand in adults with HA leads to a clear bleeding reduction. These may be reflected in the residual increase in functional ability auto-perception evaluated by the HAL questionnaire. Our results although subtle, probably due to a smal number of patients and a short period of evaluation, corroborate the fact that prophylaxis started later in life, although reducing dramatically the bleeding rates, may not improve established joint disease but may ameliorate general functional activities.

PB0673 | Decreased Annual Bleeding Rate in Paediatric Patients with Hemophilia a and Inhibitors Treated with Emicizumab vs Bypassing Agents Experience at the National Institute of Pediatrics, Mexico City, Mexico

M. Nunez Toscano; N. Lopez-Santiago; M.d.L. Gonzalez Pedroza;

R. Paredes Aguilera; G. Tavera Rodriguez; A. Monsivais Orozco; K. Maldonado Silva; A. Bravo Lindoro

Instituto Nacional de Pediatria, Mexico City, Mexico
Background: According to WFH, patients with Hemophilia A should receive factor VIII prophylaxis, as we know that patients with severe disease their unreplacement bleeding rate leads to early joint damage. The development of inhibitors in patients with haemophilia A occurs around $30 \%$ within the first 50 exposures to FVIII concentrate with fatal consequences, there were few therapeutic options for patients with hemophilia and inhibitors who did not undergo ITI; the advent of bypassing agents such as FVIIa and CCPa, on-demand management, improved the bleeding rate.

Emicizumab, a monoclonal antibody that mimics the function of factor VIII has been one of the real and most effective alternatives of recent years in the management of patients with Hemophilia A and inhibitors. In 2017 Oldenburg et al. reported their results with the use of emicizumab with a $79 \%$ decrease in annual bleeding rate (ABR) vs patients using the bypassing agent.

Aims: Described the ABR reduce with emicizumab

Methods: Review ABR pre/post emicizumab

Results: Seven patients with severe hemophilia $A$ and high response titer inhibitors were treated with emicizumab, impregnation dose for 4 weeks and maintenance every 2 weeks, 71\% presented lifethreatening bleeding before emicizumab. After the monoclonal, $93 \%$ decrease joint $A B R$ was observed. During the impregnation period, $28.6 \%(2 / 7)$ presented joint hemorrhage that resolved with 1 dose of FVIla. All patients have continued with successful rehabilitation. A patient presented a CNS hemorrhage in the last impregnation week, which required VIla for 10 days discharged without complications.

\begin{tabular}{|c|c|c|c|c|c|c|c|c|c|c|}
\hline ID & $\begin{array}{c}\text { UB } \\
\text { MAX } \\
\text { TITER }\end{array}$ & $\begin{array}{l}\text { BLEEDING/ } \\
\text { YEAR }\end{array}$ & $\begin{array}{l}\text { ABR } \\
\text { PQE } \\
\text { EMAI }\end{array}$ & $\begin{array}{c}\text { JOIN } \\
\text { DAMAGE }\end{array}$ & $\begin{array}{c}\text { MAJOR } \\
\text { CNS } \\
\text { BLEED }\end{array}$ & $\begin{array}{c}\text { VII } \\
\text { DOSE } \\
\text { PRE }\end{array}$ & $\begin{array}{c}\text { DOSE } \\
\text { APPC } \\
\text { PRE }\end{array}$ & $\begin{array}{c}\text { POST } \\
\text { EMI } \\
\text { BLEEDS }\end{array}$ & $\begin{array}{c}\text { VII } \\
\text { DOSE } \\
\text { POST } \\
\text { EMI }\end{array}$ & $\begin{array}{l}\text { ABR } \\
\text { POST } \\
\text { EMI }\end{array}$ \\
\hline 1 & 84.5 & 15 & 15 & $Y$ & 1 & 164 & 0 & 1 & 1 & 1 \\
\hline 2 & 2600 & 0 & 0 & $\mathrm{Y}$ & 0 & 109 & 0 & 0 & 2 & 0 \\
\hline 3 & 37 & 15 & 15 & $Y$ & 1 & 4 & 184 & 0 & 0 & 0 \\
\hline 4 & 109 & 1 & 1 & $\mathrm{Y}$ & 0 & 50 & 9 & 0 & 0 & 0 \\
\hline 5 & 60 & 2 & 2 & $Y$ & 1 & 0 & 23 & 1 & 7 & 1 \\
\hline 6 & 8.5 & 3 & 3 & $Y$ & 2 & 72 & 28 & 1 & 40 & 1 \\
\hline 7 & 75 & 4 & 4 & $Y$ & 1 & 2 & 55 & 0 & 3 & 0 \\
\hline
\end{tabular}

FIGURE 1 Annual bleeding rate

Conclusions: Emicizumab significantly reduced the joint annual rate bleeding.Currently assume, bleeding events occurred in the early stages of emicizumab treatment.The use of emicizumab is effective in the hemophilia A with inhibitors management of joint bleeds and has allowing an effective rehabilitation.

Full protection for major bleeding such as CNS is not complete clear, and more studies and patients are required. 


\section{NOVEL BIOTHERAPEUTICS IN HEMOPHILIA}

LPB0116 | An Accurate and Simple Liquid Chromatography Tandem Mass-spectrometric Method for the Quantification of Emicizumab in People with Hemophilia A

A. Donners; L. Gerencsér; K. van der Elst; K. Fischer; R. Urbanus; M. El Amrani

University Medical Center Utrecht, Utrecht, Netherlands

Background: Emicizumab is approved for people with hemophilia A ( $\mathrm{PwHA}$ ) with a body-weight-based dose regimen independent of laboratory monitoring. Guidelines, however, recommend measuring the emicizumab plasma concentration when suspecting neutralizing anti-drug antibodies. In addition, monitoring can be useful in shared decision-making, adherence checks, and for research purposes.

Aims: The objective was to validate a liquid chromatography tandem mass-spectrometry (LC-MS/MS) method for the quantification of emicizumab in plasma of PwHA.

Methods: Sample preparation for LC-MS/MS analysis was performed by protein precipitation with ammonium sulphate and by trypsin digestion. A signature peptide of emicizumab was used for quantification. A stable isotopically labeled internal standard with an amino acid-sequence matching the chosen signature peptide, was added to correct for loss during sample preparation and eliminate matrix effect during analysis. The signature peptide of emicizumab was separated chromatographically from other tryptic peptides and measured with tandem mass-spectrometry. Cross validation with 48 remnant samples from PwHA was performed with a modified and calibrated ( $r^{2}$ Diagnostics) one stage-clotting assay (OSA). The LC-MS/MS method was validated in accordance with the European Medicines Agency (EMA) guideline on bioanalytical method validation.

Results: The calibration curve of emicizumab was linear over a concentration range from $4-512 \mu \mathrm{g} / \mathrm{mL}$ with an $\mathrm{R}^{2}$ of 0.999 . Within-run and between-run precision and accuracy were well within acceptance criteria (Table 1). Samples were stable for three freeze and thaw cycles. Lower limit of quantification had a signal-to-noise ratio of 88 (criterion $>5$ ). The numerical values for all other parameters were well within acceptance criteria as well. Cross validation demonstrated a strong correlation between the two methods, despite their fundamental differences (Figure 1).
Figure 1.

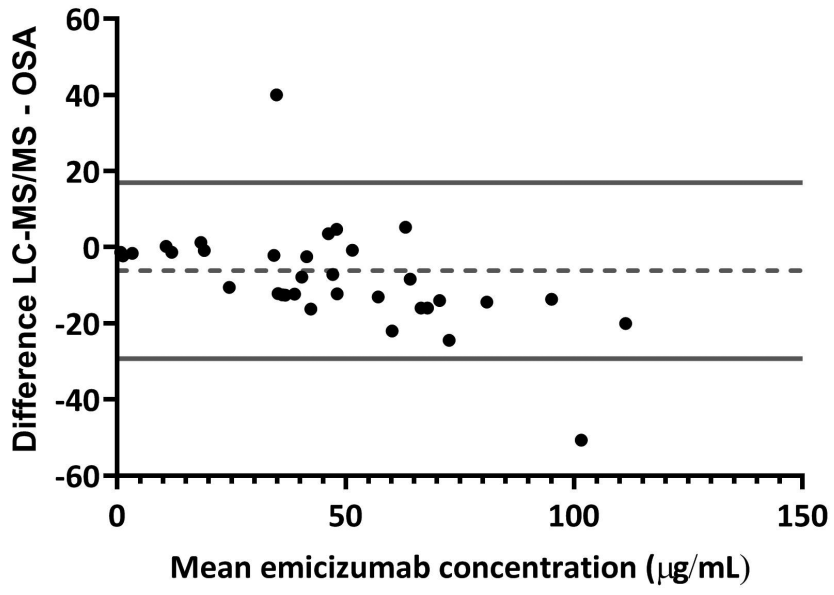

FIGURE 1 Bland-Altman plot

Conclusions: We present the first, rapid, and robust LC-MS/MSmethod for quantification of emicizumab in plasma of PwHA. A full validation of the analytical method, with cross validation, was performed successfully in accordance with the EMA guideline.

\section{LPB0117 | The Association of Emicizumab and rFVIla for Major Orthopaedic Surgery}

C. Carulli ${ }^{1}$; S. Linari ${ }^{2}$; L. Pieri ${ }^{2}$; G. Castaman ${ }^{2}$

${ }^{1}$ Orthopaedic Clinic, University of Florence, Careggi University Hospital, Florence, Italy; ${ }^{2}$ Center for Bleeding Disorders and Coagulation, Careggi University Hospital, Florence, Italy

Background: The association of emicizumab and recombinant activated FVII ( $r F V I l a)$ has been recently proposed for surgical procedures in persons with Haemophilia A (PWHA) and inhibitors. However, only isolated experiences of major orthopaedic surgery have been to date reported.

TABLE 1 Summary of precision and accuracy validation parameter results

\begin{tabular}{llllll} 
Quality Control $(\mathrm{QC})$ samples & Concentration $(\mu \mathrm{g} / \mathrm{mL})$ & Precision $(\% \mathrm{CV})$ & \multicolumn{2}{l}{ Accuracy (bias \%) } & Acceptance criteria \\
& & Within-run & Between-run & Overall \\
Lower limit of quantification & 4 & 4.9 & 7.4 & 6.1 & $<20$ \\
Low & 10 & 4.2 & 4.5 & -4.1 & $<15$ \\
Medium & 200 & 2.4 & 2.8 & -3.8 & $<15$ \\
High & 400 & 2.1 & 3.4 & 1 & $<15$ \\
\hline
\end{tabular}


Aims: This is the report about the first experience on major orthopaedic surgery with several procedures at a single Haemophilia centre in PWHA and inhibitors.

Methods: Between 2018 and 2020, three PWHA and high titer inhibitors underwent 5 major orthopaedic surgeries: one above-theknee amputation and a Total Knee Arthroplasty (TKA) in a 56-years old subject; a Total Hip Arthroplasty (THA) in a 59-years old patient; a partial revision knee arthroplasty (rTKA) and an acetabular revision on a failed THA in a 49-years old subject. Visual Analogic Scale (VAS), Haemophilic Joint Health Score (HJHS), and a postoperative radiologic study were used for patients' evaluation. The prophylaxis was performed by a regimen of weekly emicizumab and bolus infusions of $\mathrm{rFVIla}[\mathrm{c} 1$ ] (preoperative bolus of $90 \mu \mathrm{g} / \mathrm{kg}$ before anaesthesia, followed by infusions for 2 weeks after surgery).

[c1]Qui andrebbe inserito lo schema come già suggerito in altro abstract - WFH?).

Results: All patients were successfully treated by a single surgeon, without any intra- or post-operative complications and with an effective bleeding control. No signs of hypercoagulabity or thrombotic microangiopathy were observed, clinically and by using specific laboratory markers (schistocytes, platelet count, LDH, and D-dimer). All patients were regularly rehabilitated at the same hospital. The mean follow-up is 15.7 months (range: 5-24). No adverse event was recorded at the latest evaluation. All patients reported satisfaction, pain reduction, and improved VAS and HJHS scores.

Conclusions: Major orthopaedic surgery conducted by emicizumab and rFVIla in PWHA and inhibitors showed excellent clinical outcomes and an effective bleeding control. A larger number of procedures and longer follow-up periods are required to validate such protocol.

\section{PB0674 | The Effect of rFVIla and Mim8 Combination in Hemophilia A-like Blood}

M. Ezban; K. Jensen; J. Lund

Novo Nordisk A/S, Måløv, Denmark

Background: Mim8 - a novel, human bispecific antibody that mimics factor (F)VIIla - was designed for subcutaneous prophylactic treatment of patients with hemophilia $\mathrm{A}(\mathrm{HA})$ with and without inhibitors. In both, in vitro and HA mouse models, Mim8 has demonstrated superior hemostatic properties to a sequence identical analogue (SIA) of emicizumab. Combination of bispecific antibodies and activated prothrombin complex concentrates (aPCC) can cause synergistic effects, increasing the risk of thrombotic events. No increased safety risk has been shown for the combination of biAbs and recombinant activated FVII (rFVIIa).

Aims: Here, we measure the effects of Mim8+rFVIla or aPCC in HAlike blood using different global assay conditions.

Methods: Blood from healthy donors $(\mathrm{N}=5)$ was made HA-like through addition of FVIII-neutralizing antibody. Mim8 (0-190 nM plasma concentrations) was added to the blood/plasma combined with $\mathrm{rFVIIa}(0-270 \mu \mathrm{g} / \mathrm{kg}$ dose equivalent) or $1 \mathrm{IU}$ aPCC. EmicizumabSIA+aPCC was used as a comparator. Clot formation was measured by thromboelastography (TEG) after recalcification with $0.011 \mathrm{M}$ $\mathrm{Ca}^{2+}$. Thrombin generation assay (TGA) was initiated by $0.008 \mathrm{U} / \mathrm{mL}$ FXla or $1 \mathrm{pM}$ tissue factor (TF).

Results: The effects of Mim8+rFVIla on TEG parameters were additive and lower in magnitude than the effects observed with Mim8+aPCC or emicizumab-SIA+aPCC, which had synergistic effects. (Figure A). In FXla-triggered TGA, Mim8 increased thrombin generation in a concentration-dependent manner, a response unaffected by rFVIla addition. Supplementing either Mim8 or emicizumab-SIA with aPCC significantly increased thrombin generation (Figure B). In TF-triggered TGA, Mim8+rFVIla combination appeared to have effects slightly greater than the sum of their individual contributions (possibly caused by TF-rFVIla interaction). However, thrombin peak was significantly lower than observed for emicizumab-SIA+aPCC (Figure C).

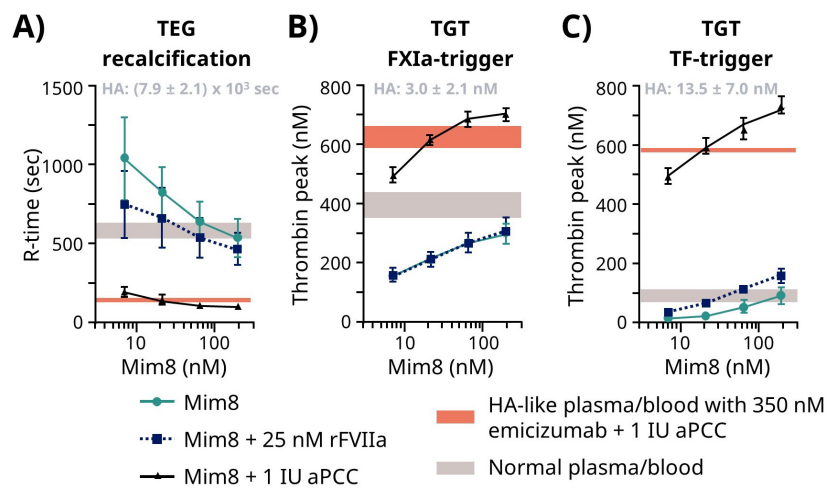

FIGURE 1 Clot formation and thrombin generation in HA-like blood supplemented with Mim8+25 nM rFVIla, Mim8+1 IU aPCC or 350 nM emicizumab-SIA+1 IU aPCC. A) TEG R-time in HA-like blood supplemented with Mim8+25 nM rFVIla, Mim8+1 IU aPCC or 350 nM emicizumab-SIA+1 IU aPCC. Thrombin peak of TGA triggered using B) FXla or C) TF in HA-like blood spiked with Mim8+25 nM rFVIIa, Mim8+1 IU aPCC or 350 nM emicizumab-SIA+1 IU aPCC. Error bars represent standard deviation. HA, hemophilia A; rFVIla, recombinant activated factor VII; FXIa, activated factor XI; SIA, sequence identical analogue; TEG, thromboelastography; TGA, thrombin generation assay; TF, tissue factor.

Conclusions: Mim8+25 nM rFVIla combination resulted in nearnormal thrombin generation and clot formation at most Mim8 concentrations tested, effects far lower in magnitude than those observed for Mim8+aPCC or emicizumab-SIA+aPCC combination. 
PB0675 | Administration of fVIII Protein through Human Placental Cells Reduces the Risk of an Antibody Response in Juvenile Sheep

B. Trevisan ${ }^{1}$; M. Rodriguez ${ }^{1}$; S. George ${ }^{1}$; J. Shields ${ }^{2}$; S. Lankford ${ }^{1}$; R. Combs ${ }^{3}$; M. Gautreaux ${ }^{4}$; J. Owen ${ }^{3}$; A. Atala ${ }^{1}$; C.B. Doering ${ }^{2}$; H.T. Spencer ${ }^{2}$; C.D. Porada ${ }^{1}$, MG. Almeida-Porada ${ }^{1}$ ${ }^{1}$ Fetal Research and Therapy Program, Wake Forest Institute for Regenerative Medicine, Winston Salem, United States; ${ }^{2}$ Aflac Cancer and Blood Disorders Center, Children's Healthcare of Atlanta and Dpt. of Pediatrics, Emory University, Atlanta, United States; ${ }^{3}$ Special Hematology Laboratory, Wake Forest School of Medicine, Winston Salem, United States; ${ }^{4} \mathrm{HLA} /$ Immunogenetics and Immunodiagnostics Laboratories, Wake Forest School of Medicine, Winston Salem, United States

Background: Current hemophilia A treatments consist of fVIII protein infusions, but up to $30 \%$ of patients develop inhibitors to $\mathrm{fVIII}$, rendering subsequent treatments ineffective, and placing the patient at risk of life-threatening bleeding events.

Aims: To develop a cell/gene-therapy product using mesenchymal placental cells (PLC) to enable the delivery of a bioengineered fVIII protein (mcoET3) without eliciting antibody formation.

Methods: PLC were transduced with a lentiviral vector (PLC-mcoET3) to produce high levels of fVIII/mcoET3 $\left(4.9-6 \mathrm{IU} / 10^{6} \mathrm{cells} / 24 \mathrm{hr}\right.$; VCN: 0.5). PLC-mcoET3 were administered to juvenile sheep: 1) intravenously (IV), weekly, for three weeks at a dose to provide 20IU/ kg ET3 each $24 \mathrm{hr}(n=3)$; or 2) a single intraperitoneal (IP) infusion of PLC-mcoET3/kg to provide 60IU/kg ET3 each $24 \mathrm{hr}$. As a control/ reference group, we administered 5 weekly IV injections of $20 \mathrm{IU} / \mathrm{kg}$ recombinant ET3 $(n=3)$ protein or hfVIII protein $(n=3)$. FVIII activity was evaluated by aPTT throughout 15 weeks. Formation of ET3/ fVIII specific IgG and IgM antibodies was evaluated by ELISA, HLA antibodies assessed with a Luminex assay, and $\mathrm{Th}_{1}$ response with ELISpot assays.

Results: All sheep receiving hfVIII and ET3 protein developed fVIII/ ET3-specific IgG antibodies, with titers of 1:20-1:245 that persisted up to 15 weeks. Sheep receiving PLC-mcoET3 IV and IP had $34 \%$ and $31 \%$ increases in FVIII activity from baseline, respectively, at week 15, demonstrating that PLC-mcoET3 persisted in treated animals. Recipients receiving IV cells did not develop ET3-specific IgM or IgG antibodies. ET3-specific IgG antibodies (titers of 1:20-1:245) were detected in $66 \%$ of IP recipients. However, in contrast to those that received proteins, the antibodies declined/disappeared by week 5. ET3-reactive $T h_{1}$ cells were not present in any of the treated animals. In addition, none of the animals developed anti-HLA antibodies to PLC-mcoET3.

Conclusions: These results show that PLC-mcoET3 can provide an increase in FVIII levels while minimizing immunogenicity.
PB0676 | Tailoring the Effect of Antithrombin-targeting Therapy in Hemophilia A Using in Silico Thrombin Generation

$\underline{\text { R. de Laat-Kremers }}{ }^{1}$; M. Ninivaggi ${ }^{1}$; I. van Moort ${ }^{2}$; M. de Maat ${ }^{2}$; B. de Laat ${ }^{1}$

${ }^{1}$ Synapse Research Institute, Maastricht, Netherlands; ${ }^{2}$ Erasmus University Medical Center, Rotterdam, Netherlands

Background: New treatment strategies for hemophilia A are emerging. A novel treatment approach is the restoration of the balance between pro- and anticoagulants, by inhibiting natural anticoagulants such as antithrombin (AT) or protein C.

Aims: We used in silico modelling to investigate the effect of AT reduction on thrombin generation (TG) and the hemostatic balance in hemophilia A patients.

Methods: 26 hemophilia A patients and 27 age- and sex-matched control subjects were enrolled in this study. TG was measured with the CAT method using PPP reagent low. Thrombin dynamics analysis was used to extract prothrombin conversion curves from the TG data and to perform in silico experiments to study the effect of AT reduction on TG in hemophilia patients. Each individual prothrombin conversion curve was used to simulate a series of 14 TG curves, by varying the AT level from $100 \%$ to $2.5 \%$. TG parameters peak height, ETP and velocity index were quantified.

Results: AT reduction partially normalized TG dose-dependently in all patients. Peak height, ETP and velocity index increased dosedependently. The average thrombin peak height was $41 n M \pm 24 n M$ in hemophilia patients at $100 \%$ AT. An AT reduction of $95 \%$ could restore TG in hemophilia patients up to an average peak height of $192 \pm 94 \mathrm{nM}$, although the variation between patients was high. At $100 \%$ AT, ETP was $570 \mathrm{nM} \cdot \mathrm{min} \pm 281 \mathrm{nM} \cdot \mathrm{min}$ on average in hemophilia patients, and a $95 \%$ AT reduction increased the ETP to 6100 $\pm 3212 \mathrm{nM} \cdot \mathrm{min}$. The velocity index of $4.6 \mathrm{nM} / \mathrm{min} \pm 3.3 \mathrm{nM} / \mathrm{min}$ at $100 \%$ AT in hemophilia patients was increased to $21.7 \pm 13.1 \mathrm{nM} /$ min after $95 \%$ reduction of AT. 
A
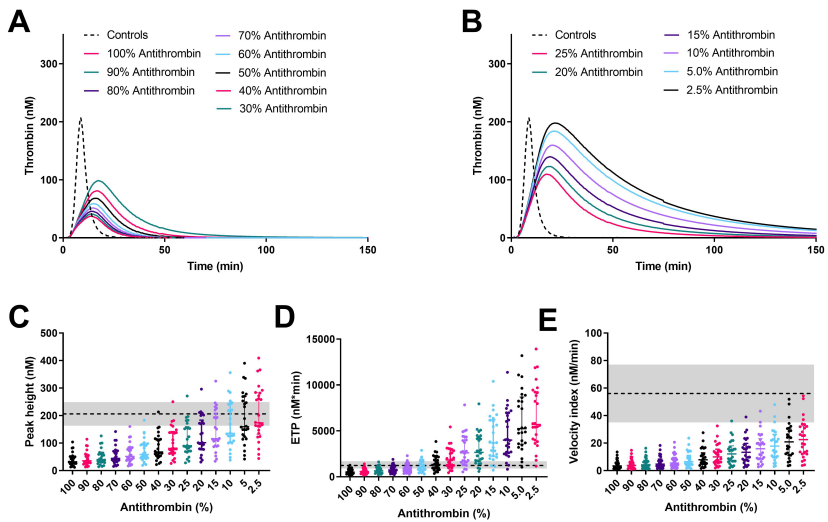

E

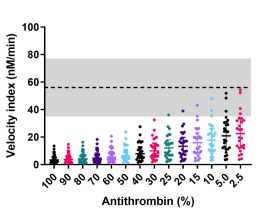

FIGURE 1 The in silico effect of antithrombin targeting in hemophilia A on thrombin generation. (A) The average TG curve of the hemophilia A patients at the original plasma AT level (100\%) in silico generated average thrombin generation curves of the same patients at $90,80,70,60,50,40$, and $30 \%$ AT. (B) The average in silico generated average thrombin generation curves of the hemophilia A patients at 25, 20, 15, 10, 5, and 2.5\% AT. The generated TG curves were quantified by their peak height (C), ETP (D) and velocity index (E). References ranges in the healthy subject group are plotted as grey box with the mean value in healthy subjects indicated as a dashed line

Conclusions: An AT reduction of 95\% led to almost normal TG peak height in most patients. The large variation in response to AT reduction indicates that tailoring AT targeting therapeutics could be beneficial.

PB0677 | Real-world Experience of Emicizumab Treatment Using Entire Vials Only

K. Fischer; A.A. Donners; R.T Urbanus; I.C. Kremer Hovinga;

L.F. van Vulpen; P.R van der Valk; K.P. van Galen; N. Uitslager; R.E. Schutgens University Medical Center Utrecht, Utrecht, Netherlands

Background: Emicizumab, a subcutaneous factor VIII mimetic, was recently approved for severe hemophilia A patients. According to label, maintenance dosing is $6 \mathrm{mg} / \mathrm{kg} / 4$ weeks, administered every 1, 2 or 4 weeks. Based on the reports of linear pharmacokinetics, a maintenance dose of $6 \mathrm{mg} / \mathrm{kg} / 4$ weeks using entire vials only while varying the dosing intervals, was adopted at the Van Creveldkliniek in the Netherlands.

Aims: To evaluate emicizumab plasma concentrations and bleeding control on a maintenance dosing strategy using entire vials at 7 to 28-days' intervals.

Methods: In a single-center study, all patients receiving emicizumab maintenance treatment for $>2$ months with emicizumab through concentrations available were eligible. Loading doses were exactly dosed according to the label. Maintenance treatment was $6 \mathrm{mg} /$ $\mathrm{kg} / 4$ weeks using entire vials at 7-28 days' intervals. According to local protocol, emicizumab concentrations were measured after $2 / 3$ and 12 months of maintenance treatment using a validated mass spectrometry-method. Bleeding data were extracted from patient files. Patients were instructed to contact the center in case of (suspected) bleeding.

Results: During the study period 33 patients had received a median of 157 days (P25-P75 (IQR) 112-391) of emicizumab maintenance treatment. Median age at treatment start was 17 years (IQR 6-51), median weight $66 \mathrm{~kg}$ (IQR 22-83), 30\% had inhibitors and $88 \%$ severe haemophilia A. Median emicizumab dose was $5.9 \mathrm{mg} / \mathrm{kg} / 4$ weeks (IQR 5.6-6.2), administered in intervals of 7-28 days, with $42 \%$ using other intervals than 1-2x/week (Table 1). Median emicizumab concentrations were $73 \mu \mathrm{g} / \mathrm{ml}$ (IQR 47-82). Only 5/33 patients (15\%) had concentrations $<35 \mu \mathrm{g} / \mathrm{ml}$, including 3 confirmed non-adherent patients. Bleeding control was excellent: $79 \%$ had zero bleeds during follow-up, independent of emicizumab concentrations.

TABLE 1 Doses and intervals used during emicizumab maintenance dosing

\begin{tabular}{|c|c|c|c|c|c|c|c|c|c|}
\hline & 7 days & 8 days & 9 days & 12 days & 14 days & 18 days & 20 days & 21 days & 28 days \\
\hline $30 \mathrm{mg}$ & 1 & & & & & & & & \\
\hline $60 \mathrm{mg}$ & 2 & & & 1 & 1 & 2 & 1 & 2 & \\
\hline $120 \mathrm{mg}$ & 3 & & & & & & & & \\
\hline $150 \mathrm{mg}$ & 2 & 4 & 1 & & 1 & & & & \\
\hline $210 \mathrm{mg}$ & & & & & 1 & & & & \\
\hline $255 \mathrm{mg}$ & & & & & 1 & & & & \\
\hline Total & $14(42 \%)$ & 5 (15\%) & $1(3 \%)$ & $1(3 \%)$ & $5(15 \%)$ & $2(6 \%)$ & $1(3 \%)$ & $3(9 \%)$ & $1(3 \%)$ \\
\hline
\end{tabular}


Conclusions: Dosing emicizumab using entire vials with varying intervals to achieve $6 \mathrm{mg} / \mathrm{kg} / 4$ weeks resulted in efficacious concentrations of emicizumab and excellent bleeding control. This dosing strategy can be applied to avoid spillage.

PB0678 | Cost-effectiveness Analysis of the Brazilian Immune Tolerance Induction Protocol with Recombinant Factor VIII and Prophylaxis with Bypassing Agents or Emicizumab

R.M. Camelo ${ }^{1}$; S.M. Rezende ${ }^{1}$; J. Álvares-Teodoro ${ }^{2}$

${ }^{1}$ Faculty of Medicine, Universidade Federal de Minas Gerais, Belo Horizonte, Brazil; ${ }^{2}$ Faculty of Pharmacy, Universidade Federal de Minas Gerais, Belo Horizonte, Brazil

Background: Treatment of people with haemophilia A and inhibitors (PwHAi) has improved considerably in Brazil since the introduction of immune tolerance induction (ITI) in 2012. Recently, emicizumab (EMI) was incorporated by the Brazilian Public Healthcare System (SUS) for prophylaxis of PwHAi who failed ITI.

Aims: We aimed to compare the costs and the outcomes of the Brazilian ITI Protocol (BIP) with recombinant factor VIII concentrate (FVIII; a-octocog) and prophylaxis with bypassing agents (BPA; ITI+BPA) or EMI (ITI+EMI), using a decision-three model, from the SUS (payer) perspective.

Methods: The BIP recommends starting ITI at low-dose rFVIII regimen (50IU/kg 3x/week) for all PwHAi and, upon poor response, increasing $\mathrm{rFVIII}$ dose to $100 \mathrm{IU} / \mathrm{kg} /$ day. BPA can be prescribed to treat or prevent bleeding during ITI. Outcomes were success (inhibitor titre $<2 \mathrm{BU} / \mathrm{mL}$ and FVIII responsiveness) and failure. The success rate of the BIP was $71 \%$. In this analysis, the price of EMI was considered as the amount paid by the Ministry of Health in the last purchase, which is almost twice as high as the price proposed by the industry for incorporation.

TABLE 1 Model assumptions. rFVIla, activated recombinant factor VII; EMI, emicizumab; ITI, immune tolerance induction; aPCC, partially activated prothrombine complex concentrate; rFVIII, recombinant factor VIII, a-octocog.

\begin{tabular}{|c|c|c|}
\hline Parameter & ITI+EMI & ITI+BPA \\
\hline Age and mean body weight at ITI start & 2 years $/ 15 \mathrm{~kg}$ & 2 years $/ 15 \mathrm{~kg}$ \\
\hline Prophylaxis regimen & $\begin{array}{l}\mathrm{EMI} \text { attack } 3.0 \mathrm{mg} / \mathrm{kg} / \mathrm{wk} \text { for } 4 \text { weeks and } \\
\text { maintenance } 1.5 \mathrm{mg} / \mathrm{kg} / \mathrm{wk}\end{array}$ & $\begin{array}{l}\text { aPCC (50\%) } 75 \text { U/kg q48h or rFVIla (50\%) } 90 \\
\mu \mathrm{g} / \mathrm{kg} \mathrm{q} 24 \mathrm{~h}\end{array}$ \\
\hline Mean total treated bleedings (success/failure) & $0.54 / 0.93$ & $6 / 16$ \\
\hline Episodic regimen & rFVIla $180 \mu \mathrm{g} / \mathrm{kg} /$ bleeding & $\begin{array}{l}\text { aPCC (for those on aPCC prophylaxis) } 262.5 \mathrm{U} / \\
\mathrm{kg} / \text { bleeding or } \mathrm{rFVIla} \text { (for those on rFVIla } \\
\text { prophylaxis) } 180 \mathrm{\mu g} / \mathrm{kg} / \text { bleeding }\end{array}$ \\
\hline $\begin{array}{l}\text { Need of increase of rFVIII regimen (success/ } \\
\text { failure) }\end{array}$ & $14 \% / 74 \%$ & $14 \% / 74 \%$ \\
\hline $\begin{array}{l}\text { Proportion of the duration of each rFVIII } \\
\text { regimen, when increase is needed } \\
\text { (low-dose:high-dose) }\end{array}$ & $50 \%: 50 \%$ & $50 \%: 50 \%$ \\
\hline Mean ITI duration (success/failure) & 1.8 year/3.1 years & 1.8 year/3.1 years \\
\hline Success rate & $71 \%$ & $71 \%$ \\
\hline Products costs (US\$/unit)rFVIII cost & 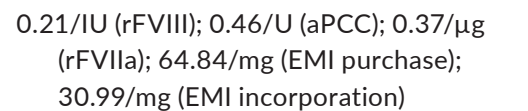 & $\begin{array}{l}0.21 / \mathrm{IU} \text { (rFVIII); 0.46/U (aPCC); 0.37/Mg (rFVIIa); } \\
64.84 / \mathrm{mg} \text { (EMI purchase); 30.99/mg (EMI } \\
\text { incorporation) }\end{array}$ \\
\hline
\end{tabular}

Results: In the cost-effectiveness analysis, the costs were US $\$ 414,773.84$ (ITI+BPA) and US\$243,244.25 (ITI+EMI), generating an additional cost of US\$171,529.59 for PwHAi receiving ITI. ITI+BPA resulted in 8.25 bleeds over ITI+EMI, and each additional bleed cost, US $\$ 20,799.28$. By deterministic sensitivity analysis, the most impacting variable to the incremental cost was the EMI price: if the incorporation proposed price was used, the savings generated using EMI could reach US\$254,319.20 for each PwHAi on ITI.

TABLE 2 Cost-effectiveness analysis of the Brazilian Immune Tolerance Induction Protocol using a-octocog and prophylaxis with bypassing agents or emicizumab. BPA, bypassing agent; EMI, emicizumab; ITI, immune tolerance induction.

\begin{tabular}{|c|c|c|c|c|c|}
\hline Treatment & Cost (US\$) & $\begin{array}{l}\text { Incremental cost } \\
\text { (US\$) }\end{array}$ & $\begin{array}{l}\text { Total treated } \\
\text { bleedings }\end{array}$ & $\begin{array}{l}\text { Incremental treated } \\
\text { bleedings }\end{array}$ & $\begin{array}{l}\text { Incremental cost } \\
\text { per treated bleeding } \\
\text { (US\$) }\end{array}$ \\
\hline \multicolumn{6}{|c|}{ Current EMI price (US\$ 64.84/mg) } \\
\hline ITI+EMI & $243,244.25$ & & 0.65 & & \\
\hline ITI+BPA & $414,773.84$ & $171,529.59$ & 8.9 & 8.25 & $20,799.28$ \\
\hline
\end{tabular}




\begin{tabular}{|c|c|c|c|c|c|}
\hline \multicolumn{6}{|c|}{1 in thrombosis \& haemostasis } \\
\hline Treatment & Cost (US\$) & $\begin{array}{l}\text { Incremental cost } \\
\text { (US\$) }\end{array}$ & $\begin{array}{l}\text { Total treated } \\
\text { bleedings }\end{array}$ & $\begin{array}{l}\text { Incremental treated } \\
\text { bleedings }\end{array}$ & $\begin{array}{l}\text { Incremental cost } \\
\text { per treated bleeding } \\
\text { (US\$) }\end{array}$ \\
\hline \multicolumn{6}{|c|}{ Previously proposed EMI price (US\$ 30.99/mg) } \\
\hline ITI+EMI & $160,454.64$ & & 0.65 & & \\
\hline ITI+BPA & $414,773.84$ & $254,319.20$ & 8.9 & 8.25 & $30,838.16$ \\
\hline
\end{tabular}

Conclusions: In conclusion, our model showed ITI+BPA is more expensive and people in this arm are more likely to experience bleeding than ITI+EMI. The inclusion of EMI in the BIP can reduce costs for the SUS and bleedings among PwHAi.

PB0679 | Measuring the Chromogenic FVIII Mimetic Activity of the New Bispecific Antibody, Mim8, in Artificially Spiked Severe Haemophilia A Plasma

A. Bowyer ${ }^{1}$; M. Ezban ${ }^{2}$; S. Kitchen ${ }^{1}$

${ }^{1}$ Sheffield Haemophilia and Thrombosis Centre, Sheffield, United

Kingdom; ${ }^{2}$ Novo Nordisk A/S, Måløv, Denmark

Background: Mim8, a new bispecific antibody, facilitates activation of factor (F)X by binding FIXa and FX. This FVIII mimetic was designed for the subcutaneous prophylactic treatment of patients with haemophilia A (HA) with or without inhibitors. Chromogenic substrate assays (CSAs) are used to monitor the FVIII activity (FVIII:C) of patients with $\mathrm{HA}$.
Aims: We evaluated the reliability of Mim8 measurements made using plasma-calibrated FVIII CSA and the necessity of productspecific calibrator for accuracy.

Methods: Mim8 was spiked into severe HA plasma at $0-20 \mu \mathrm{g} / \mathrm{mL}$. Mim8 mimetic FVIII activity (FVIII:C) was measured on two days by 5 CSA kits (Table 1) using either a Sysmex CS-5100 or an ACL TOP 700. Calibration curves were constructed using human plasma standards. A modified Hyphen CSA was evaluated on three days using a Mim8 reference product $(10.26 \mu \mathrm{g} / \mathrm{mL})$ as calibrator and $1 / 80$ plasma dilution

(Table 2)

Results: Mimetic FVIII:C of $33.2 \mathrm{IU} / \mathrm{dL}$ was observed at the lowest Mim8 concentration $(1 \mu \mathrm{g} / \mathrm{mL})$ and increased concurrently with Mim8 concentration, with the Hyphen CSA calibrated using human plasma. Siemens CSA was insensitive to even $20 \mu \mathrm{g} / \mathrm{mL}$. Some mimetic FVIII:C was observed above $5 \mu \mathrm{g} / \mathrm{mL}$ with the Coamatic CSA, whilst some mimetic FVIII:C was measured at $1-2 \mu \mathrm{g} / \mathrm{mL}$ Mim8 using Precision Biologic and Rossix CSA (Table 1).

The between-day coefficient of variance of a modified Hyphen CSA calibrated with Mim8 was $<3 \%$ for each Mim8 concentration. Recovery of $20 \%$ or less from the target Mim8 concentration was observed for all concentrations evaluated (Table 2).

TABLE 1 Mim8 mimetic FVIII activity measured using chromogenic substrate assays and standard human plasma calibrator; *lower dilution required; FVIII:C, factor VIII activity

Hyphen

FVIII:C

(IU/dL)

Source of protein in kit; analyser; dilution

$\begin{array}{cl}\text { dilution } & \text { Human } \\ & \text { CS510 } \\ \text { Mim8 } 1 \mu \mathrm{g} / \mathrm{mL} & 33.2 \\ \text { Mim8 } 2 \mu \mathrm{g} / \mathrm{mL} & 70.0 \\ \text { Mim8 } 5 \mu \mathrm{g} / \mathrm{mL} & 148.3 \\ \text { Mim8 } 8 \mu \mathrm{g} / \mathrm{mL} & 212.4 \\ \text { Mim8 } 10 \mu \mathrm{g} / \mathrm{mL} & 247.0 \\ \text { Mim8 } 15 \mu \mathrm{g} / \mathrm{mL} & 332.7 \\ \text { Mim8 } 20 \mu \mathrm{g} / \mathrm{mL} & 389.1 \\ \text { Mim8 reference } & 242.9 \\ 10.26 \mu \mathrm{g} / \mathrm{mL} & \end{array}$

Siemens FVIII:C

(IU/dL)

Bovine FX

FIXa; Bovine

CS5100; $1 / 30$

$<3$

$<3$

$<3$

$<3$

$<3$

$<3$

$<3$

$<3$
Coamatic ${ }^{\circledR}$ FVIII:C

(IU/dL)

Bovine FX

FIXa;

Bovine FIXa; $A C L$ TOP $700 ; 1 / 15$

$<1$

$<1$

$<1$

1.3

2.0

2.5

5.2

3.3
Precision Biologic

Rossix FVIII:C

(IU/dL)

FVIII:C

(IU/dL)

Bovine FX

Bovine FX

Human FIXa; ACL TOP 700; 1/60* TOP 700; 1/30*

$1.7 *$

$2.5^{*}$

5.0

7.5

9.2

17.7

19.7

10.4 
TABLE 2 Mim8 concentration measured using modified Hyphen chromogenic substrate assay and Mim8 product-specific calibrator

\begin{tabular}{|c|c|c|c|c|}
\hline & $\begin{array}{l}\text { Mean Mim8 concentration ( } \mu \mathrm{g} / \\
\mathrm{mL} \text { ) }\end{array}$ & Standard deviation & $\begin{array}{l}\text { Coefficient of variance } \\
\text { (\%) }\end{array}$ & $\begin{array}{l}\text { Difference from expected } \\
\text { target (\%) }\end{array}$ \\
\hline Mim8 $0 \mu \mathrm{g} / \mathrm{mL}$ & $<0.6$ & 0.01 & 43.3 & not calculated \\
\hline $\operatorname{Mim} 82 \mu \mathrm{g} / \mathrm{mL}$ & 1.9 & 0.04 & 2.2 & -5.2 \\
\hline Mim8 $5 \mu \mathrm{g} / \mathrm{mL}$ & 5.2 & 0.06 & 1.1 & 2.9 \\
\hline 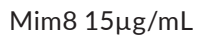 & 16.6 & 0.02 & 0.1 & 10.6 \\
\hline Mim8 20 $\mu \mathrm{g} / \mathrm{mL}$ & 21.1 & 0.17 & 0.8 & 5.7 \\
\hline $\begin{array}{r}\text { Mim8 reference } \\
10.26 \mu \mathrm{g} / \mathrm{mL}\end{array}$ & 11.2 & 0.10 & 0.8 & 8.7 \\
\hline
\end{tabular}

Conclusions: Human IXa/X and hybrid bovine X/human IXa CSAs (Table 1) demonstrated sensitivity to the presence of Mim8. Bovine $\mathrm{IXa} / \mathrm{X}$ CSA were largely insensitive to Mim8 and can likely be used to accurately measure additional recombinant FVIII therapy or FVIII inhibitors in patients treated with Mim8. Mim8 concentration can be accurately determined in a modified Hyphen CSA with Mim8 calibrator.

PB0680 | Measuring the FVIII Mimetic Activity of the New Bispecific Antibody, Mim8, in Severe Haemophilia A Plasma Using APTT and One-stage FVIII Assays

A. Bowyer ${ }^{1}$; M. Ezban ${ }^{2}$; S. Kitchen ${ }^{1}$

${ }^{1}$ Sheffield Haemophilia and Thrombosis Centre, Sheffield, United Kingdom; ${ }^{2}$ Novo Nordisk A/S, Måløv, Denmark

Background: Mim8 is a new human bispecific antibody designed for the subcutaneous prophylactic treatment of patients with haemophilia A (HA) with or without inhibitors. Mim8 mimics the function of activated factor (F)VIII by binding FIXa and FX, to facilitate activation of FX. Mim8 can influence the results of the one-stage activated partial thromboplastin time (APTT)-based FVIII clotting assays (OSA). Therefore, modification of OSA is needed to assess Mim8 drug concentration levels.

Aims: We investigated APTT and OSA using 7 reagents in severe HA (SHA) plasma spiked with Mim8. OSA were calibrated using both human plasma and product-specific calibrators.

Methods: Mim8 was spiked into SHA plasma at $0-20 \mu \mathrm{g} / \mathrm{mL}$. APTT and human plasma calibrated (1/10 dilution) OSA were evaluated on either a Sysmex CS5100 or ACL TOP 700 analyser. Modified OSA were evaluated on three days with each APTT reagent using a Mim8 reference product (at $10.26 \mu \mathrm{g} / \mathrm{mL}$ ) as calibrator and $1 / 80$ plasma dilution. Results: The APTT with all reagents was shortened to within normal limits at $1 \mu \mathrm{g} / \mathrm{mL}$ Mim8 (Table 1). Standard mimetic FVIII activity OSA were $>250 \mathrm{IU} / \mathrm{dL}$ at Mim8 concentrations above $1 \mu \mathrm{g} / \mathrm{mL}$ (data not shown).

Mim8 reference product calibrated modified OSA demonstrated some reagent variation (Table 2). However, the between-day coefficient of variance was $<20 \%$ for all reagents and Mim 8 concentrations. The recovery of Mim8 compared to expected target concentration was within 22\% for all reagents and Mim8 concentrations.

TABLE 1 APTT of severe haemophilia A plasma spiked with Mim8

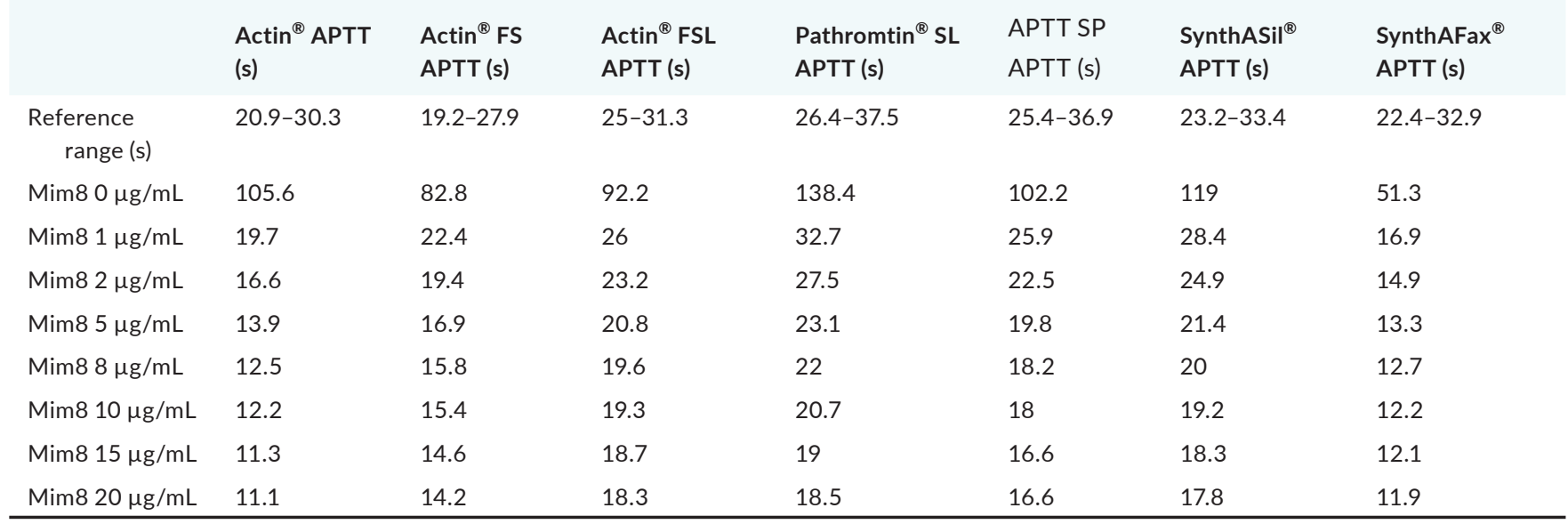


TABLE 2 Mim8 concentration in spiked severe haemophilia A plasma measured using modified one-stage assays and product-specific standard

\begin{tabular}{|c|c|c|c|c|c|c|c|}
\hline & $\begin{array}{l}\text { Actin }^{\circledR} \\
(\operatorname{Mim} 8 \mu \mathrm{g} / \\
\mathrm{mL})\end{array}$ & $\begin{array}{l}\text { Actin }{ }^{\circledR} \text { FS } \\
(\mathrm{Mim} 8 \mu \mathrm{g} / \\
\mathrm{mL})\end{array}$ & $\begin{array}{l}\text { Actin }{ }^{\circledR} \text { FSL } \\
\text { (Mim8 } \mu \mathrm{g} / \\
\mathrm{mL})\end{array}$ & $\begin{array}{l}\text { Pathromtin }{ }^{\circledR} \\
\text { SL } \\
\text { (Mim8 } \mu \mathrm{g} / \mathrm{mL} \text { ) }\end{array}$ & $\begin{array}{l}\text { APTT SP } \\
\text { (Mim8 } \mu \mathrm{g} / \\
\mathrm{mL})\end{array}$ & $\begin{array}{l}\text { SynthASil }^{\circledR} \\
\text { (Mim8 } \mu \mathrm{g} / \\
\mathrm{mL})\end{array}$ & $\begin{array}{l}\text { SynthAFax }{ }^{\circledR} \\
\text { (Mim8 } \mu \mathrm{g} / \\
\mathrm{mL} \text { ) }\end{array}$ \\
\hline $\operatorname{Mim} 80 \mu \mathrm{g} / \mathrm{mL}$ & $<0.6$ & $<0.6$ & $<0.6$ & $<0.6$ & $<1$ & $<1$ & $<1$ \\
\hline Mim8 $1 \mu \mathrm{g} / \mathrm{mL}$ & 0.97 & 1.11 & 0.90 & 0.93 & 0.98 & 1.22 & 1.08 \\
\hline Mim8 $2 \mu \mathrm{g} / \mathrm{mL}$ & 2.00 & 2.19 & 1.97 & 2.20 & 2.16 & 2.24 & 2.28 \\
\hline Mim8 $5 \mu \mathrm{g} / \mathrm{mL}$ & 4.80 & 4.97 & 4.70 & 4.87 & 5.14 & 4.97 & 5.37 \\
\hline $\operatorname{Mim} 88 \mu \mathrm{g} / \mathrm{mL}$ & 8.10 & 8.16 & 8.23 & 8.03 & 8.56 & 8.09 & 8.47 \\
\hline Mim8 $10 \mu \mathrm{g} / \mathrm{mL}$ & 9.93 & 9.95 & 9.87 & 9.80 & 9.89 & 10.00 & 10.12 \\
\hline Mim8 $15 \mu \mathrm{g} / \mathrm{mL}$ & 15.77 & 16.24 & 16.10 & 15.73 & 14.93 & 15.47 & 15.38 \\
\hline Mim8 $20 \mu \mathrm{g} / \mathrm{mL}$ & 20.73 & 22.38 & 22.97 & 20.73 & 20.25 & 19.61 & 21.38 \\
\hline $\begin{array}{l}\text { Mim8 reference product } \\
10.26 \mu \mathrm{g} / \mathrm{mL}\end{array}$ & 10.40 & 10.55 & 10.23 & 10.40 & 10.07 & 10.23 & 11.14 \\
\hline
\end{tabular}

Conclusions: The APTT with all reagents was shortened to within normal limits at the lowest Mim8 concentration studied; this corresponded to falsely elevated FVIII:C measurements using standard OSA. Standard OSA should not be performed in patients treated with Mim8. A modified OSA calibrated using a Mim8 calibrator enabled accurate measurement of Mim8 drug concentration and was reproducible with all APTT reagents studied.

PB0681 | Emicizumab Assays: One Stage vs. Chromogenic Stage Assay

M. Stuart; D. Chen; R. Pruthi

Mayo Clinic, Rochester, United States

Background: Emicizumab is administered at a fixed dose for prophlaxis of hemorrhage in hemophilia A, monitoring drug levels is not indicated. Measurement of drug levels may be useful in patients experiencing breakthrough bleeding episodes for assessment of compliance or development of an anti-drug antibody. On therapy trough levels have been reported to range from 35 to 55 micrograms/mL (Pipe et al, Lancet Haematol 2019;6: e295-305).

Aims: Compare analytical performance characteristics of one stage (OSA) and chromogenic (CSA) Emicizumab assays.

Methods: A modified FVIII:C OSA using HemosIL ${ }^{\circledR}$ SynthASil Reagent (Instrumentation Laboratory [IL], Bedford, MA) and CRYOcheck ${ }^{\text {TM }}$ Factor VIII Deficient Plasma (F8DP) (Precision BioLogic, Dartmouth, NS, Canada) and human reagent based FVIII:C CSA assay (Hyphen Biomed, Neuville-sur-Oise, France) and a drug specific calibrator $\left(r^{2}\right.$ Diagnostics, South Bend, IN, USA) were developed on the ACL TOP 700 (IL).

Results: Precision of OSA and CSA was $<10 \% \mathrm{CV}$ for two levels of control [repeatability $(n=9)$ and within laboratory assessments $(n=20)$ ]. Accuracy compared to known (emicizumab spiked) targets $(n=9)$ : OSA yielded: $m=1.05, b=-0.021, R^{2}=0.99 . C S A$ yielded: $m=0.81$, $b=-2.78, R^{2}=0.97$. Overall deviation from assigned value: OSA $=2 \%$ and CSA $=-31 \%$. Assays performed on patient samples demonstrated a similar negative bias. LoB, mean of F8DP ( $n=5$, target $<1.0 \mathrm{mcg} / \mathrm{mL}$ ) for OSA was $0.18 \mathrm{mcg} / \mathrm{mL}$, CSA was $1.16 \mathrm{mcg} / \mathrm{mL}$. LoQ mean $/ \% C V$ of dilute control ( $n=5$, target $4.88 \mathrm{mcg} / \mathrm{mL}$ ) for OSA was $4.86 \mathrm{mcg} / \mathrm{mL} / 1.1 \%$ and CSA $2.62 \mathrm{mcg} / \mathrm{mL} / 3.2 \%$. Residual FVIII (>10\%) artifactually raises emicizumab level in CSA more than the OSA. Preanalytic heat inactivation was examined; emicizumab recovery was not consistently maintained.

TABLE 1 Precision

\begin{tabular}{r|cccc}
$\begin{array}{c}\text { Precision } \\
\text { Emicizumab (mcg/mL) }\end{array}$ & \multicolumn{2}{|c}{ OSA } & \multicolumn{2}{c}{ CSA } \\
Control 1 & Control 2 & Control 1 & Control 2 \\
\hline Repeatability Mean & 24.3 & 74.6 & 17.4 & 58.1 \\
\hdashline Within Laboratory Mean & 25.9 & 75.9 & 17.5 & 56.2 \\
\hline Repeatability SD & 1.6 & 2.6 & 0.9 & 2.5 \\
\hdashline Within Laboratory SD & 1.1 & 3.9 & 1.3 & 5.8 \\
\hline Repeatability \%CV & $6.70 \%$ & $3.50 \%$ & $5.20 \%$ & $4.30 \%$ \\
\hdashline Within Laboratory \%CV & $4.20 \%$ & $5.20 \%$ & $7.30 \%$ & $10.40 \%$
\end{tabular}

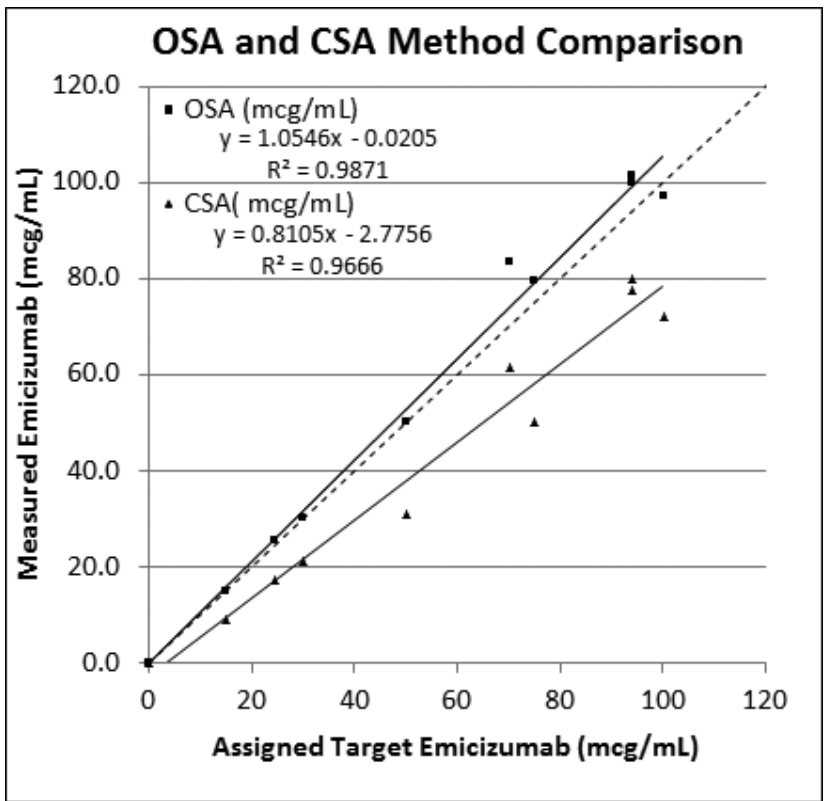

FIGURE 1 Accuracy 
Conclusions: Although both assays have acceptable precision, the OSA has superior performance characteristics. Heat inactivation of sample is not reliable solution for accurate measurement of emicizuamb in the presence of FVIII for either method.

PB0682 | A Systematic Review on the Pharmacokinetics and Associated Pharmacodynamics of Emicizumab in Humans

A. Donners ${ }^{1}$; C. Rademaker ${ }^{1}$; L. Bevers ${ }^{1}$; A. Huitema ${ }^{1,2,3}$;

R. Schutgens ${ }^{1} ;$ A. Egberts ${ }^{1,4} ;$ K. Fischer ${ }^{1}$

${ }^{1}$ University Medical Center Utrecht, Utrecht, Netherlands; ${ }^{2}$ Netherlands Cancer Institute, Amsterdam, Netherlands; ${ }^{3}$ Princess Máxima Center for Pediatric Oncology, Utrecht, Netherlands; ${ }^{4}$ Utrecht Institute for Pharmaceutical Sciences, Faculty of Science, Utrecht University, Utrecht, Netherlands

Background: Emicizumab is an effective, new treatment for people with hemophilia $\mathrm{A}$ ( $\mathrm{PwHA}$ ) and, comparable to other therapeutic monoclonal antibodies, its financial costs are substantial. The approved dosing regimens are body weight-based without the necessity of dose adjustments based on laboratory monitoring. This assumes a clear dose-concentration-response relationship with low variability due to other factors than body weight.

Aims: To investigate this assumption, a systematic review on the pharmacokinetics (PK) and associated pharmacodynamics (PD) of emicizumab in humans was conducted.

Methods: The database Embase, Pubmed and CENTRAL were searched up to November $15^{\text {th }} 2020$ to identify studies on PK data of emicizumab in humans. Study, subject, PK and PD outcome data were synthesized. Exposure-effect modelling was performed using non-linear least squares regression in an Emax model with trough plasma concentrations and annualized bleed rate (ABR) of treated bleeds, all model-based by means of binomial regression.

Results: The 15 included studies on PK of emicizumab in humans reported data on 140 volunteers and $467 \mathrm{PwHA}$ including children (aged 0 to $<12$ years), adolescents/adults ( $>12$ years), both with and without FVIII inhibitors. Emicizumab demonstrated a clear linear dose-concentration profile already at low doses of $0.3 \mathrm{mg} / \mathrm{kg} /$ week (Figure 1). The inter-individual variability of trough plasma concentrations was moderate (32\%) and similar across various subgroups, such as FVIII inhibitor status, age, dose interval. The EC $_{50}$ was estimated at $1.47 \mu \mathrm{g} / \mathrm{ml}$ in the Emax model. The effectiveness plateau of the concentration-response relationship was clearly established, and all ABR observations resided herein with mean trough concentrations of $\sim 50 \mu \mathrm{g} / \mathrm{ml}$. The control of treated bleeds did not further improve above emicizumab trough plasma concentrations of $\sim 30$ $\mu \mathrm{g} / \mathrm{ml}$, potentially enabling lower dosing in a considerable proportion of PwHA (Figure 2).
Figure 1.

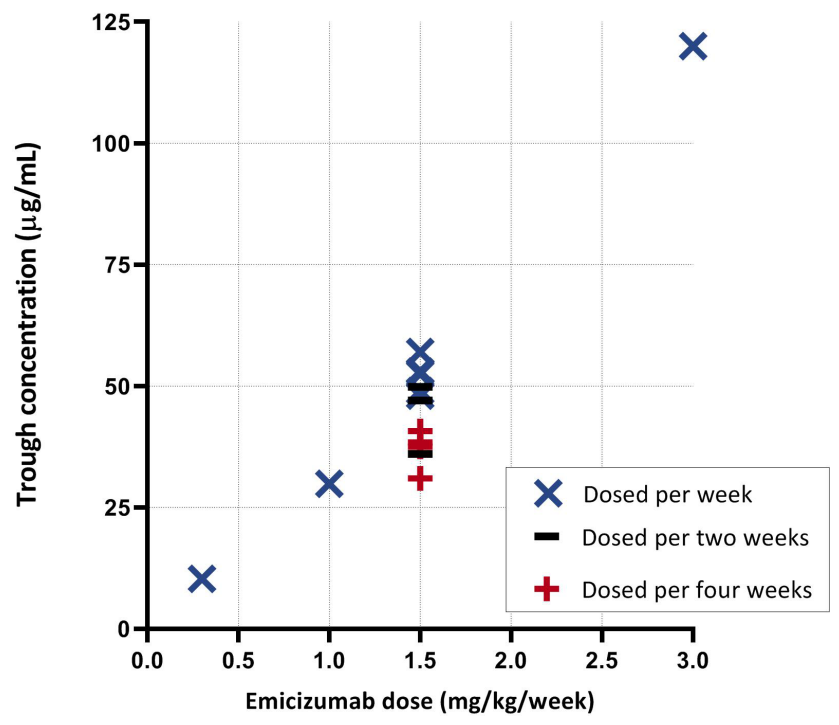

FIGURE 1 Linear dose-concentration relationship of emicizumab in people with hemophilia $A$

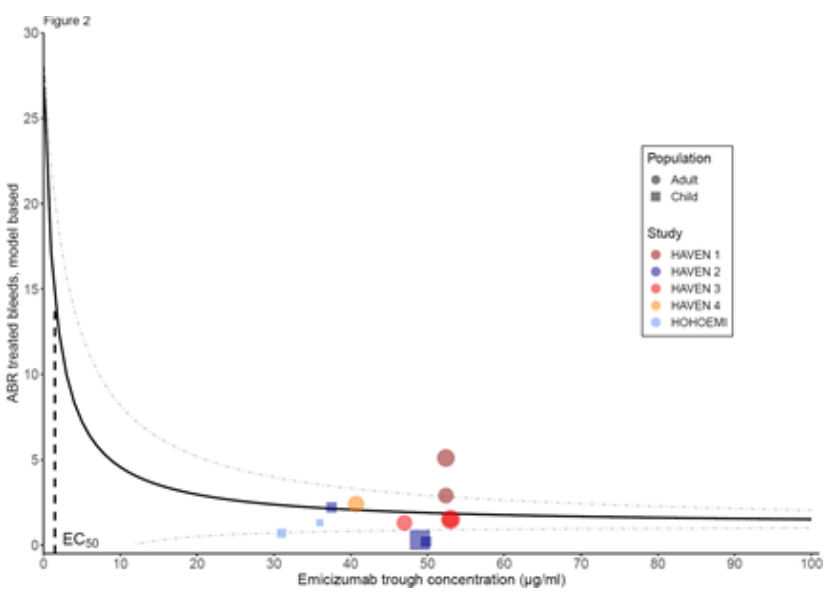

FIGURE 2 Emax model with concentration-response relationship of emicizumab in people with hemophilia A

Conclusions: This systematic review supports body-weight-based dosing of emicizumab in PwHA. Individualized dosing by monitoring the concentration may be more cost-effective.

PB0683 | Twelve Months of Emicizumab Prophylaxis in a Woman with Mild Hemophilia A and Inhibitor: Effectiveness, Safety and Economic Outcome

R.M. Camelo ${ }^{1}$; E.S. Casaretto ${ }^{2}$; M.D. Castro Tenório ${ }^{2}$; N. Dantas$\mathrm{Silva}^{3}$; S.S. de Figueiredo ${ }^{2}$; J. Álvares-Teodoro ${ }^{4}$

${ }^{1}$ Faculty of Medicine, Universidade Federal de Minas Gerais, Belo Horizonte, Brazil; ${ }^{2}$ Hemocentro da Paraíba (HEMOÍBA), João Pessoa, Brazil; ${ }^{3}$ HEMOMINAS Foundation, Belo Horizonte, Brazil; ${ }^{4}$ Faculty of Pharmacy, Universidade Federal de Minas Gerais, Belo Horizonte, Brazil

Background: The development of inhibitor in people with HA $(\mathrm{PwHA})$ can increase the annual bleeding rate (ABR), and hemostasis 
with bypassing agents is not as predictable as with factor VIII (FVIII). Emicizumab is an alternative for such cases.

Aims: We report the first Brazilian woman on emicizumab treatment.

Methods: Medical files were reviewed.

Results: Due to an increase in the partial thromboplastin time at 9 years, she was referred from another center, where she had been treated for Melnick-Needles syndrome. Her FVIII activity was 10.0\%. On-demand intravenous FVIII was started (peripheral venous access was difficult to provide due to her body structure). She developed a high-response inhibitor two years later, but immune tolerance induction was not tried because central venous access could not be performed. She was kept on self-infused intravenous activated recombinant factor VII ( $r F$ VIla) episodic treatment.

Between Jan/28/2019 and Jan/28/2020, her ABR was 11.0. Her Functional Independence Score in Hemophilia (FISH) was 21. Total rFVIla consumption was $375 \mathrm{mg}$. In Jan/28/2020, at 28 years, she received subcutaneous emicizumab attack of $3.0 \mathrm{mg} / \mathrm{kg}$ once weekly for 4 weeks, and $1.5 \mathrm{mg} / \mathrm{kg}$ weekly as maintenance. She self-infused emicizumab at home. From Jan/28/2020 to Jan/28/2021, ABR was 0.0 and her FISH increased to 30 , although she did not receive emicizumab during 5 consecutive weeks on May-Jun/2020 and 2 consecutive weeks on Jul/2020. Puncture site or thrombotic events were not reported. Her estimated annual consumption of emicizumab was $2,520 \mathrm{mg}$ (attack+maintenance during the first year), or 2,340mg, for each additional year without attack doses. The estimated annual cost of treatment increased from US $\$ 180,903.22$ (US\$482.41/ mg rFVIla, purchased by the Brazilian Ministry of Health in 2018) to US $\$ 206,551.80-222,440.40$ (US $\$ 88.27 / \mathrm{mg}$ emicizumab, weighted mean of the first 6 purchases from the state Secretary of Health).

Conclusions: Prophylaxis with emicizumab was effective and safe, although treatment costs increased by $14 \%-23 \%$.

PB0684 | Emi and Me: The Real-world Impact of Emicizumab on the Lives of People with Haemophilia A with Inhibitors and their Families

S. Fletcher ${ }^{1}$; M. Holland ${ }^{2}$; K. Jenner ${ }^{2}$; K. Khair ${ }^{2}$

${ }^{1}$ Oxford Haemophilia and Thrombosis Centre, Oxford, United Kingdom;

${ }^{2}$ Haemnet, London, United Kingdom

Background: Compared with people with Haemophilia A, people with haemophilia $\mathrm{A}$ and inhibitors $(\mathrm{PWHi})$ have been disproportionately affected by their condition, suffering more orthopaedic complications, bleeding and pain. The advent of emicizumab as a prophylactic treatment has led to a reduction in bleed frequency and a significant improvement in overall quality of life. No research at this time has examined the nature of this improvement.

Aims: The Emi and Me study aims to capture the real-life experience of using emicizumab for PWHi and their families.

Methods: Participants were recruited through treatment centres, social media and word of mouth. Each participant and a family member, if available, took part in a semi-structured qualitative interview.
All interviews were recorded, transcribed verbatim and analysed thematically.

All elements of the study were reviewed by local statutory authorities and informed consent was sought from all participants.

Results: 15 participants, mean age 27.2 years (range 8-63 years), along with a family member, participated in a single qualitative interview online $(n=13)$, by telephone $(n=1)$ or in person $(n=1)$. Mean time on emicizumab was 2.22 years (range $1-5$ years).

Six themes emerged: a reduction in bleed frequency; a reduction in treatment burden; an increased sense of freedom (for both $\mathrm{PWHi}$ and family members); decreased pain; enhanced wellbeing; and unachieved potential.

Emicizumab prophylaxis has delivered significant improvements in the lives of the participants. Despite this, some participants felt that pre-existing physical disabilities, as well as a lack of physiotherapy support has prevented them achieving the levels of functional ability they expected these improvements to facilitate.

Conclusions: This study suggests a more detailed understanding of the impact of treatments on the lives of PWHi and their families is required. Such an understanding is needed to determine patients' true needs and may also help to identify more appropriate treatment outcomes to guide medical management.

PB0685 | Twelve Months of Emicizumab Prophylaxis in a Severe Hemophilia A Man with Inhibitor who Failed Immune Tolerance Induction: Effectiveness, Economic, and Safety Outcomes

$\underline{\text { R.M. Camelo }}^{1}$; T.C. de Medeiros ${ }^{2}$; D.G.B. de Albuquerque ${ }^{2}$; N. Dantas-Silva ${ }^{3} ;$ J. Álvares-Teodoro ${ }^{4}$

${ }^{1}$ Faculty of Medicine, Universidade Federal de Minas Gerais, Belo Horizonte, Brazil; ${ }^{2}$ Hemocentro do Rio Grande do Norte (HEMONORTE), Natal, Brazil; ${ }^{3}$ HEMOMINAS Foundation, Belo Horizonte, Brazil; ${ }^{4}$ Faculty of Pharmacy, Universidade Federal de Minas Gerais, Belo Horizonte, Brazil

Background: Emicizumab is an effective non-replacement prophylactic drug for people with $\mathrm{HA}$ and inhibitor (PwHAi), although its safety and treatment costs have not been clarified yet. In 2019, the Brazilian Ministry of Health started the process to incorporate it in the national protocol of PwHAi who failed immune tolerance induction (ITI).

Aims: We aimed to describe the first Brazilian PwHAi who was treated with emicizumab.

Methods: Medical files were reviewed. The Brazilian registry of persons with hemophilia A receiving emicizumab (Emicizumab CasesEMCase Study) was approved centrally by the Committee on Ethics in Research of the Universidade Federal de Minas Gerais (CAAE 10664919.6.0000.5149) and registered in the Brazilian Registry of Clinical Trials (RBR-57rnpz).

Results: We report a 28-year-old man who received emicizumab for one year. He was diagnosed as severe HA (factor VIII activity $0.9 \%$ ) when he was born. He had been treated exclusively on demand, even 
after developing a high-response inhibitor at the age of 20 years. Three years later, ITI was prescribed for 32 months with plasmaderived FVIII and prophylaxis with bypassing agent. He failed ITI, and prophylaxis with recombinant activated factor VII (rFVIla) 250 kIU 3x/week was maintained.

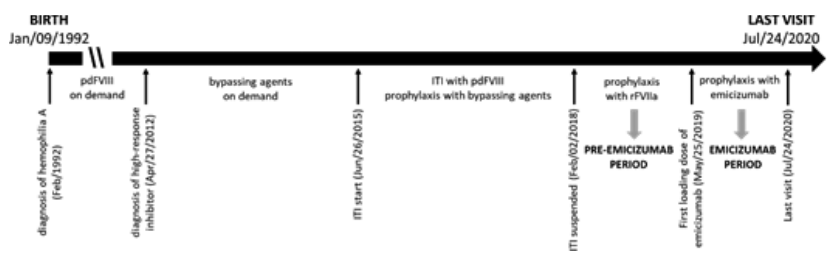

FIGURE 1 Patient history and the periods which were evaluated in this case report. pdFVIII, plasma-derived factor VIII; ITI, immune tolerance induction; rFVIIa, recombinant activated factor VII

Between Feb/27/2018 and Jul/24/2019, the annualized bleeding rate was 2.1 episodes/y. Mean consumption of rFVIla was 2,771 \pm $772 \mathrm{kIU} / \mathrm{month}$. On Jul/25/2020, emicizumab was loaded with 3.0 $\mathrm{mg} / \mathrm{kg}$ once weekly for 4 weeks, maintaining $1.5 \mathrm{mg} / \mathrm{kg}$ weekly thereafter. Mean consumption of emicizumab from Jul/25/2019 to $J u l / 24 / 2020$ was $366.92 \pm 93.40 \mathrm{mg} / \mathrm{month}$, with no bleeding episode requiring factor replacement. The mean monthly treatment cost reduced from US\$34,590.00 $\pm 8,903.00 /$ month, in the preemicizumab period (Feb/27/2018 to Jul/24/2019), to US\$25,736.25 $\pm 6,677.04$ /month, during the emicizumab period (Jul/25/2019 to Jul/24/2020). No thrombotic event was suspected/reported.

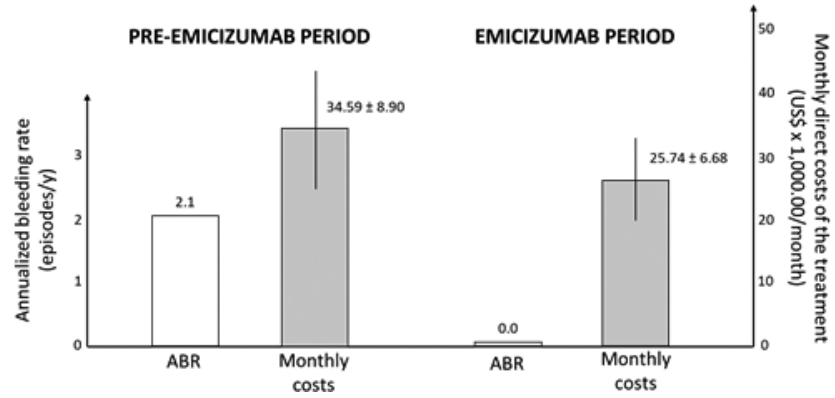

FIGURE 2 Annualized treated-bleeding rates (ABR) and monthly direct treatment costs before (pre-emicizumab period, from Feb/27/2018 to Jul/24/2019) and during prophylaxis with emicizumab (emicizumabe period, from Jul/25/2019 to Jul/24/2020). Annualized treated-bleeding rates (bars in white; leftsided $y$-axis) were calculated as a proxy annual number of bleeding episodes requiring replacement treatment for hemostasis during the evaluated periods. The monthly direct treatment costs (bars in grey; right-sided $y$-axis) were calculated according to the prices of the respective products in United States dollar currency of the day of purchase. They were expressed by mean \pm standard deviations.

Conclusions: In this case, prophylaxis with emicizumab was effective and safe. The annualized direct costs reduced about $25 \%$ after switching from prophylaxis with rFVIla to emicizumab.
PB0686 | Do New Therapies alter the Treatment of Hemophilia Patients?

H. Duda; B. Haschberger; J. Hesse; H. Windecker; A. Hilger;

C. Keipert

Paul-Ehrlich-Institut, Langen, Germany

Background: Hemophilia is a rare bleeding disorder that requires treatment for life. While therapeutic options were limited in the past, multiple new hemophilia therapies have reached marketing authorization in recent years.

Aims: The aim of this evaluation is to analyze specific aspects of data collected in the German Hemophilia Registry (Deutsches Hämophilieregister, dhr) for the impact of these new therapies on the treatment of German hemophilia patients.

Methods: To identify changes in total factor consumption or shifts in product class preferences, the per-capita factor consumption of relevant patient groups was calculated and correlated with the market access of novel products for the past decade in Germany.

Results: Preliminary results suggest an interesting shift in selected aspects of hemophilia treatment in Germany due to the availability of new therapeutic options.

Conclusions: Data collected in the German hemophilia registry support the contemporary investigation of real time data to elaborate on patient-orientated supply.

PB0687 | Successful Experience of Emicizumab Prophylaxis in a Child with Severe Hemophilia A and Inhibitor without Previous Immune Tolerance Induction

R.M. Camelo ${ }^{1}$; M. Palis ${ }^{2}$; M.A.F. de Cerqueira ${ }^{2}$; N. Dantas-Silva ${ }^{3}$; J. Álvares-Teodoro ${ }^{4}$

${ }^{1}$ Faculty of Medicine, Universidade Federal de Minas Gerais, Belo Horizonte, Brazil; ${ }^{2}$ Hemocentro do Piauí (HEMOPI), Teresina, Brazil; ${ }^{3}$ HEMOMINAS Foundation, Belo Horizonte, Brazil; ${ }^{4}$ Faculty of Pharmacy, Universidade Federal de Minas Gerais, Belo Horizonte, Brazil

Background: Emicizumab has been approved recently in Brazil for prophylaxis of children with hemophilia A and inhibitors. However, before the incorporation, patients accessed the medicine by judicialization.

Aims: We aimed to describe the 8-month experience of emicizumab prophylaxis in a boy with severe hemophilia A and inhibitor.

Methods: Patient medical files were reviewed.

Results: He was diagnosed at 7 months after a large subcutaneous hematoma developed during venous puncture. Prophylaxis was not started due to poor venous access and frequent subcutaneous/muscular hematomas when venous punctures were performed. Central venous device was not implanted, because the patient did not have prompt access to a Vascular Surgeon. High-response inhibitor (17.5BU/ $\mathrm{mL}$ ) was diagnosed at 1.5 year, after 20 exposure days. Immune tolerance induction or bypassing agent prophylaxis were not indicated due 
to the difficulty of his venous route. In the following 9 months, his annualized bleeding rate was 17.3 , including subcutaneous/muscular hematomas after venous punctures. He has been hospitalized many times to treat muscular bleedings (gluteus, thighs, and calves). After judicialization, emicizumab was initiated by four attacking doses $(3.0 \mathrm{mg}$ / $\mathrm{kg} / \mathrm{wk}$ ) and maintenance thereafter ( $1.5 \mathrm{mg} / \mathrm{kg} / \mathrm{wk})$. Emicizumab was stopped for 8 weeks, because the Health Secretary did not purchase the product. The patient had hemarthrosis in the right knee, which was treated with recombinant activated factor VII. Emicizumab was resumed as the maintenance regimen. No treated bleeding, puncture site event, nor thrombosis have been reported during emicizumab treatment. He did not receive clotting factors to treat many bruises, one jugal mucosa hematoma after biting, and one subgaleal hematoma after falling, which solved spontaneously.

Conclusions: We conclude that emicizumab was safe and effective as prophylaxis, and the boy has been benefitting from maintaining its use. We expect to start immune tolerance induction as soon as he has better vein access.

\section{RARE BLEEDING DISORDERS}

\section{LPB0070 | Efficacy and Safety of Human Fibrinogen} Concentrate for Treatment of on-demand Bleeding in Adolescent and Pediatric Patients with Congenital Fibrinogen Deficiency: Results from the FORMA-02 and FORMA-04 Clinical Trials

C. Djambas-Khayat ${ }^{1,2} ;$ S. Lohade ${ }^{3} ;$ F. D'Souza ${ }^{4}$; L. Shamanur ${ }^{5}$; O. Zekavat'; I. Kruzhkova7; B. Schwartz ${ }^{8}$; C. Solomon; F. Peyvandi ${ }^{9,1}$

${ }^{1}$ Department of Pathophysiology and Transplantation, Università degli Studi di Milano, Milan, Italy; ${ }^{2}$ Hotel Dieu de France Hospital, Beirut, Lebanon; ${ }^{3}$ Sahyadri Specialty Hospital, Pune, India; ${ }^{4}$ St. John's Medical College Hospital, Bangalore, India; ${ }^{5}$ S.S Institute of Medical Science and Research Center, Davangere, India; ${ }^{6}$ Hematology Research Center, Nemazee Hospital, Shiraz University of Medical Sciences, Shiraz, Iran, Islamic Republic of; ${ }^{7}$ Octapharma AG, Lachen, Switzerland; ${ }^{8}$ Octapharma, Hoboken, United States; ${ }^{9}$ Fondazione IRCCS Ca' Granda Ospedale Maggiore Policlinico, Angelo Bianchi Bonomi Hemophilia and Thrombosis Center and Fondazione Luigi Villa, Milan, Italy

Background: Human fibrinogen concentrate (HFC) is administered for on-demand bleeding episode (BE) treatment in patients with congenital fibrinogen deficiency (CFD): a rare disorder characterized by a lack of/low levels of functional fibrinogen.

Aims: Data is reported on the efficacy and safety of HFC for ondemand BE treatment from two Phase 3 studies in adolescent and pediatric patients ( $<18$ years) with CFD.

Methods: FORMA-02 and FORMA-04 were international, multicenter, prospective, open-label, uncontrolled Phase 3 studies of HFC (Fibryga ${ }^{\circledR}$, Octapharma) efficacy and safety in adult/adolescent and pediatric patients with CFD. Hemostatic efficacy was assessed by the investigators and adjudicated by an Independent Data
Monitoring and Endpoint Adjudication Committee (IDMEAC) using an objective 4-point scale (excellent; good; moderate; none). All adverse events (AEs) were recorded.

Results: Six adolescent (12-17 years) and 8 pediatric (0-11 years) patients received HFC for on-demand BE treatment. HFC was administered for 11 adolescent BEs (all minor) and for 10 pediatric $B E s$; 8 of which were minor and 2 were major BEs (intraperitoneal bleed from spleen and knee/thigh bleed). The median (range) total dose per BE was $78.57 \mathrm{mg} / \mathrm{kg}$ (37.78-91.30) for adolescents and 73.91 $\mathrm{mg} / \mathrm{kg}$ (47.45-262.50) for pediatric patients. Overall hemostatic efficacy was rated successful (excellent/good rating) for $100 \%$ of BEs by the IDMEAC.

Thirteen AEs occurred in 7 (50\%) of the 14 patients receiving HFC for BE treatment. Ten AEs were mild, 2 were moderate, and 1 was severe (psoas hematoma), but was not related to treatment. No allergic/hypersensitivity reactions or deaths were observed.

Conclusions: Across two Phase 3 clinical trials, HFC was efficacious for on-demand BE treatment in adolescent and pediatric patients with CFD, which is comparable to adult patients with CFD. Hemostatic efficacy of HFC was comparable for adolescent and pediatric patients, with a favorable safety profile.

\section{PB0688 | High-throughput Screening for Pharmacological Enhancers of Mutated Factor VII Activity}

E. Andersen ${ }^{1,2}$; M.C. Mowinckel ${ }^{1,2} ;$ B. Stavik ${ }^{1,2}$; P.M. Sandset ${ }^{1,2,3}$; M.E. Chollet ${ }^{1,2}$

${ }^{1}$ Oslo University Hospital, Department of Haematology, Oslo, Norway; ${ }^{2}$ Oslo University Hospital, Research Institute of Internal Medicine, Oslo, Norway; ${ }^{3}$ University of Oslo, Institute of Clinical Medicine, Oslo, Norway

Background: Replacement therapy requiring intravenous injection is the mainstay of treatment for congenital factor (F) VII deficiency. As such, there is an unmet need for new therapeutic strategies using other delivery mechanisms. Drug repurposing allows for efficient discovery and implementation of new treatments, and identification of pharmacological enhancers of FVII variant activity would be of clinical importance.

Aims: To identify clinically approved drugs that enhance the activity of the FVII variant p.Q160R.

Methods: High-throughput screening was conducted using a library of $>1500$ FDA-approved drugs. The primary screen was performed in conditioned medium from $\mathrm{CHO}-\mathrm{K} 1$ cells transiently expressing wild-type (wt) or variant (p.Q160R) FVII. Equal amounts of FVII antigen from conditioned medium were loaded onto the assay plates. Samples were incubated with library drugs for 1.5 hours (h) at $37^{\circ} \mathrm{C}$. FVII activity was analyzed using Biophen FVII assay and absorbance measured at $405 \mathrm{~nm}$ for $2 \mathrm{~h}, 37^{\circ} \mathrm{C}$. Data were analyzed using KNIME software. Positive hits were verified in patient plasma. 
Results: The initial velocity $\left(\mathrm{V}_{0}\right)$ and absorbance $\left(\mathrm{A}_{405}\right)$ at $2 \mathrm{~h}$ at various compound concentrations were calculated. The primary screen of conditioned media identified the orally available histone deacetylase inhibitor Abexinostat and the inhaled surfactant Tyloxapol as enhancers of the FVII variant with an $\mathrm{EC}_{50}$ of $\sim 1$ or $\sim 3 \mu \mathrm{M}$, respectively. The 2 hits were verified in plasma from 7 p.Q160R variant patients. Tyloxapol showed a dose-response effect in plasma from all patients with an $\mathrm{EC}_{50}$ of $\sim 1.5-2 \mu \mathrm{M}$. Abexinostat demonstrated a dose-response effect in 6 of 7 patients with an $\mathrm{EC}_{50}$ of $\sim 2 \mu \mathrm{M}$.

Conclusions: This proof-of-concept study demonstrates that drug repurposing may be feasible for novel treatment of FVII deficiency. Clinically approved drugs can be quickly channeled for use in clinical trials or serve as templates for discovery of novel drugs for this disease.

PB0689 | GGCX Mutations Lead to Different $\gamma$-carboxylation Status of Vitamin K Dependent Coagulation Proteins

S. Ghosh $^{1}$; J. Mueller ${ }^{1}$; A. Biswas ${ }^{1}$; K. Höning ${ }^{2}$; V. Hornung ${ }^{3}$;

F. Forin ${ }^{1} ;$ M. Watzka ${ }^{1}$, k. J. Czogalla ${ }^{1}$; J. Oldenburg ${ }^{1}$

${ }^{1}$ Institute of Experimental Haematology and Transfusion Medicine, Bonn, Germany; ${ }^{2}$ Institute of Molecular Medicine, Bonn, Germany;

${ }^{3}$ Gene Center and Department of Biochemistry, Ludwig-Maximilians-

Universität, Munich, Germany

Background: Vitamin K Dependent Coagulation Factor Deficiency type 1 (VKCFD1) is a rare bleeding disorder caused by mutations in $\gamma$-glutamyl carboxylase (GGCX) gene, which is characterized by spontaneous bleeding due to under $\gamma$-carboxylated vitamin $\mathrm{K}$ dependent (VKD) clotting factors.

Aims: The aim of this study is to categorize 22 reported GGCX mutations into responders and non-responders to vitamin $\mathrm{K}\left(\mathrm{K}_{1}\right)$ treatment with respect to VKD clotting factors.

Methods: The cDNAs of GGCX together with F2, F7, F9, F10 or Protein $C(P C)$ were cloned into a bicistronic vector, which was transfected into $G_{G C X}{ }^{-/-}$cells, and treated with different $K_{1}$ concentrations to determine $\gamma$-carboxylation by ELISA. Moreover, an in silico GGCX model was generated to visualize the localization of heterogeneous mutations.

Results: Elevated $K_{1}$ concentrations increase $\gamma$-carboxylation of VKD clotting factors for most mutations. GGCX:p.(M174R) and GGCX:p.(F299S) show loss-of-function in $\gamma$-carboxylation for all clotting factors at any $\mathrm{K}_{1}$ concentration. Mutations GGCX:p.(R83P), GGCX:p.(R83W), GGCX:p.(D153G), GGCX:p.(L394R), GGCX:p. (H404P) and GGCX:p.(S300F) are low responding mutations, which showed reduced levels of $\gamma$-carboxylation compared to wild-type at high $\mathrm{K}_{1}$ concentration. Certain mutants show a differential effect on $\gamma$-carboxylation. GGCX:p.(R485P) $\gamma$-carboxylates FX, FVII and FIX as wild-type, whereas FII is $\gamma$-carboxylated to a lower level. Mutation GGCX:p.(G558R) and GGCX:p.(T591K) cannot $\gamma$-carboxylate FX to any level, whereas it increases slightly for FII. These differential mutations are located close to the propeptide binding site in our GGCX in silico model.

Conclusions: Therapy with $\mathrm{K}_{1}$ will lead to normal coagulation for most mutations. Low responding mutations are difficult to treat with $\mathrm{K}_{1}$. Therefore, patients with low responding mutation in homozygous form or in combination with a loss-of-function mutation should be treated with Prothrombin Complex Concentrate. The close location of GGCX:p.(R485P), GGCX:p.(G558R), and GGCX:p.(T591K) to the propeptide binding site in our GGCX in silico model might be responsible for the differential responses to the clotting factors.

\section{GGCX mutations}

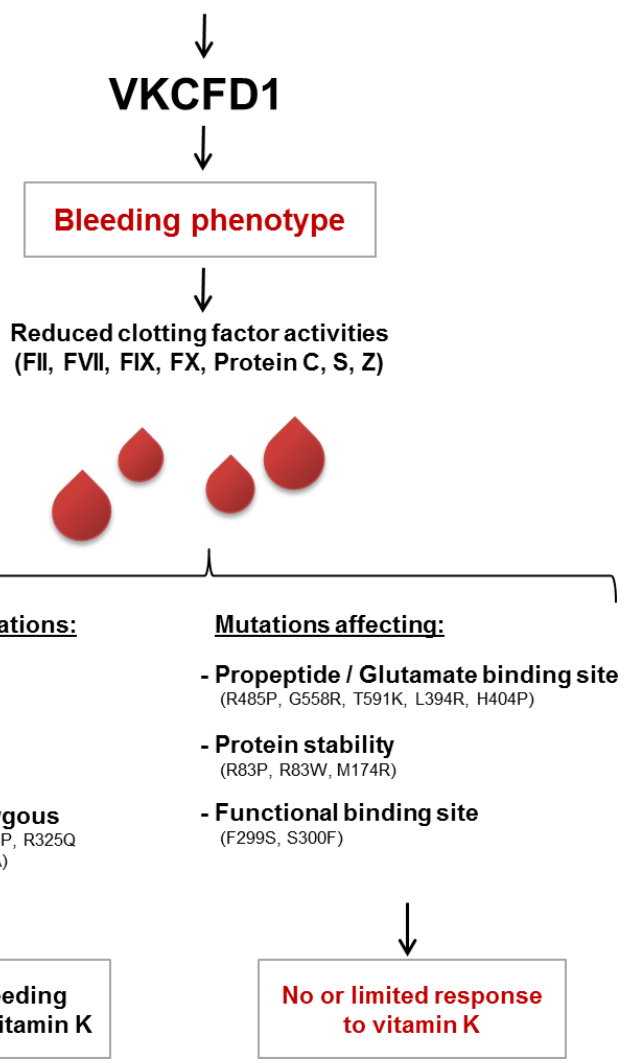

FIGURE 1

PB0690 | Concurrent Use of Tranexamic Acid and Hormonal Therapy: Management of Heavy Menstrual Bleeding in Women with Inherited Bleeding Disorders

A. Epstein; O. Turan; R. Abdul-Kadir

Royal Free Hospital, London, United Kingdom

Background: Hormonal therapy and Tranexamic acid (TXA) are often used as a first line therapy for the management of heavy menstrual bleeding (HMB).

Aims: The aim of this study is to review effectiveness and safety of their concurrent use in women with bleeding disorders. 
Methods: Retrospective data collected from electronic patient records of 87 women managed with TXA and hormonal therapy for HMB. Data collected included demographics, indication, dosage, duration of use of TXA and hormonal therapy, side effects and response to treatment.

Results: The median age was 20-29 (range 10-54) years, 23(26\%) were overweight or obese. None had a personal or family history of VTE. Von Willebrand disease was the commonest bleeding disorders affecting 31 (36\%) women. The bleeding disorder was classified a severe, moderate and mild in 24(35\%), 13(22\%) and 26(43\%). Median pictorial blood assessment chart score was 137. 19(22\%) had iron deficiency anaemia and $7(8 \%)$ had uterine fibroids.

The median duration for the combination therapy was 4 years with $34(39 \%)$ of women using the treatment for more than 5 years. Hormonal therapies used were combined oral contraceptives in 42 (48\%) women, LNG-IUS in 14 (16\%), cyclical progesterone in $16(18 \%)$, desogestrel $75 \mathrm{mcg}$ in 9 (10\%).81 (93\%) of women used TXA only during the period; 41(47\%) required TXA use for more than 7 days a month.

Median PBAC score at 6 months of treatment was 71 and $77(89 \%)$ women rated the combined therapy as effective. Surgical intervention reported in 8 (9\%) women. Treatment discontinuation of the combined therapy was reported in $4(5 \%)$ due to side effects; but 10 (11\%) women discontinued only hormonal therapy use due to irregular spotting or hormonal side-effects. No VTE reported in any women.

Conclusions: Concurrent use of TXA and hormonal therapy is safe, effective and well tolerated in women with bleeding disorders with $\mathrm{HMB}$.

PB0691 | Unclassified Bleeding Disorders and Inherited Platelet Function Disorders: Response to Desmopressin Challenge

M. Desborough ${ }^{1}$; J. Alamelu² $;$ S. Austin ${ }^{1}$; G. Ling ${ }^{1}$; E. Foxton ${ }^{3}$;

A. McCormick ${ }^{3}$; G. Dolan ${ }^{1}$; B. Madan ${ }^{1}$

${ }^{1}$ Centre for Haemostasis and Thrombosis, Guy's and St Thomas' NHS

Foundation Trust, London, United Kingdom; ${ }^{2}$ Everlina Children's

Hospital, London, United Kingdom; ${ }^{3}$ Diagnostic Haemostasis

Laboratory, Viapath, London, United Kingdom

Background: People with unclassified bleeding disorders and mild platelet function disorders are at risk of bleeding when undergoing surgery or invasive dental work. The optimal treatment has not been established.
Aims: The aim of this study was to assess changes in platelet function, Von Willebrand Factor (VWF) and Factor VIII for patients with platelet function disorders and unclassified bleeding disorders following a desmopressin challenge and to assess the incidence of bleeding when they underwent invasive procedures and were treated pre-operatively with desmopressin.

Methods: Participants were included if they were registered at the Centre for Haemostasis and Thrombosis, St Thomas' Hospital, London, UK, with a diagnosis of unclassified bleeding disorder or platelet function disorder, and had a desmopressin challenge between May 2009 and May 2020.

For desmopressin trials, blood samples were taken at baseline prior to desmopressin $0.3 \mathrm{mcg} / \mathrm{kg}$ infusion and 30-60 minutes after the infusion. For surgery, desmopressin $0.3 \mathrm{mcg} / \mathrm{kg}$ subcutaneously 60-90 minutes pre-operatively was given in combination with tranexamic acid 1 gram three times a day. Data were compared using Wilcoxon sign rank test. A p-value $<0.05$ was considered statistically significant.

Results:
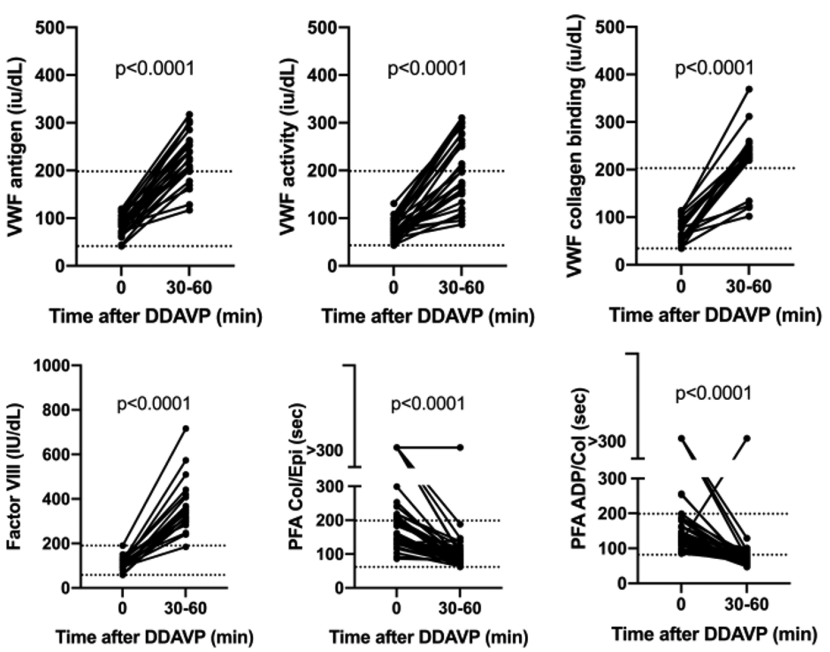

FIGURE 1 Changes in VWF antigen ( $n=26)$, VWF activity $(n=27)$, VWF collagen binding $(n=18)$, Factor VIII chromogenic $(n=21)$, PFA100 collagen/ADP $(n=41)$ and PFA100 collagen/epinephrine ( $n=41$ ) before and 30-60 minutes after administration of subcutaneous desmopressin $0.3 \mathrm{mcg} / \mathrm{kg}$. Dotted lines represent normal range. Comparisons with Wilcoxon sign rank with $P$ 
TABLE 1 Baseline characteristics. ISTH BAT - International Society on Thrombosis and Haemostasis Bleeding Assessment Tool, PPH - postpartum haemorrhage, VWF - Von Willebrand Factor.

$\begin{array}{lll}\text { Characteristic } & & \text { Median (IQR) or } n \text { (\%) } \\ \text { Age (years) } & & 40 ; 28 \text { to } 52 \\ \text { Gender } & \text { Male } & 2(5 \%) \\ & \text { Female } & 40(95 \%) \\ \text { Family history of bleeding disorder } & \text { First degree relative } & 24(57 \%) \\ & \text { Second degree relative } & 2(5 \%) \\ & \text { No family history } & 16(38 \%) \\ \text { ISTH BAT score } & & 9.5(6 \text { to } 12) \\ \text { Previous bleeding symptoms } & \text { Bleeding after dental extraction } & 13(31 \%) \\ & \text { Surgical bleeding } & 20(48 \%) \\ & \text { Menorrhagia } & 39(98 \%), n=40 \\ & \text { Post-partum haemorrhage } & 12(30 \%), n=40 \\ \text { Diagnosis } & \text { Unclassified bleeding disorder } & 20(48 \%) \\ \text { Baseline blood tests } & \text { Platelet function disorder } & 22(52 \%)\end{array}$

Forty-two patients were included (Table 1). Responses to desmopressin are outlined in Figure 1. Overall VWF antigen increased by 50 to $380 \%$ compared to baseline (Figure 1).

23/42 (55\%) patients who had a desmopressin challenge, went onto to have surgical procedures and were treated with desmopressin before surgery. The 23 patients underwent 48 invasive procedures: eight tooth extractions, 23 minor surgeries and 17 major surgeries. There were no bleeding complications and no instances of arterial thrombosis, venous thrombosis or hyponatraemic seizures.

Conclusions: Patients with unclassified bleeding disorders and platelet function disorders both had a consistent improvement in VWF levels and reduction in PFA100 closure time with desmopressin challenge. We found no instances of significant bleeding when desmopressin was given pre-operatively.
PB0692 | Population Parmacokinetics of rFXIII (Catridecacog) and Clinical Outcomes of Prophylaxis in an Italian Population of Patients with Factor XIII Deficiency

E. Zanon ${ }^{1}$; S. Pasca ${ }^{1}$; G. Sottilotta ${ }^{2}$; A.C. Molinari ${ }^{3}$; L. Banov ${ }^{3}$; A. Ferretti ${ }^{4}$; P. Di Gregorio ${ }^{5}$; B. Pollio ${ }^{6}$; S.M. Siboni ${ }^{7}$; R. Palla ${ }^{7}$; F. Peyvandi ${ }^{7}$; M. Pizzuti ${ }^{8}$; L.D. Notarangelo ${ }^{9}$; P. Cojutti ${ }^{10}$; C. Biasoli ${ }^{11}$; P. Simioni ${ }^{1}$; F. Pea ${ }^{10}$

${ }^{1}$ Padua University Hospital, Padua, Italy; ${ }^{2}$ Hospital of Reggio Calabria, Reggio Calabria, Italy; ${ }^{3}$ Gaslini Children Hospital, Genoa, Italy;

${ }^{4}$ Umberto I University Hospital, Rome, Italy; ${ }^{5}$ SS. Annunziata Hospital, Chieti, Italy; ${ }^{6}$ Regina Margherita Children Hospital, Turin, Italy; ${ }^{7}$ Milan University Hospital, Milan, Italy; ${ }^{8}$ S. Carlo Hospital, Potenza, Italy; ${ }^{9}$ ASST Spedali Civili of Brescia, Brescia, Italy, ${ }^{10}$ Udine University Hospital, Udine, Italy, ${ }^{11}$ Cesena Hospital, Cesena, Italy

Background: FXIII deficiency is a very rare coagulation disorder. Bleeds are usually mucocutaneous, but life-threatening intracranial hemorrhages (ICHs) can occur in cases of severe disease. Currently the best treatment consists in the use of FXIII replacement concentrates in prophylaxis since birth. Data concerning the use of catridecacog in the real-life is scarce.

Aims: The primary end-point is to compare the different PK-profiles of $r F X I I I$ using a population statistics model, secondary end-point is to evaluate the clinical outcomes of prophylaxis with rFXIIII.

Methods: This study enrolled all patients presenting FXIII deficiency treated with catridecacog at ten Italian Hemophilia Centers. 
PK-profiles were evaluated at the Pharmacology Department of Udine University Hospital. All clinical data and outcomes were collected and analyzed.

Results: Overall 20 patients with FXIII deficiency were collected, $75 \%$ presenting severe disorder. 11/20 were females. Mean age at diagnosis was 15 years (range birth-74 years). 60\% had a known family disorder. Pharmacokinetics was assessed in 16/20 of cases before starting prophylaxis. Mean age at PK-assessment was 36 years (6-74 years), mean dose of rFXIII infused for PK evaluation was $31.5 \mathrm{IU} / \mathrm{kg}$ (25-50 IU/kg), Figure 1.

\begin{tabular}{|c|c|c|}
\hline Parameter & Value (RSE\%) & Median $(95 \% \mathrm{CI})$ of Bootstrap \\
\hline \multicolumn{3}{|l|}{ Fixed-effect } \\
\hline $\mathrm{CL}(\mathrm{mL} / \mathrm{h} / \mathrm{kg})$ & $0.163(7.08)$ & $0.133(0.122-0.158)$ \\
\hline $\mathrm{V}_{1}(\mathrm{~mL} / \mathrm{kg})$ & $40.5(6.41)$ & $39.97(37.7-41.1)$ \\
\hline $\mathrm{Q}(\mathrm{mL} / \mathrm{h} / \mathrm{kg})$ & $0.124(12.3)$ & $0.124(0.107-0.134)$ \\
\hline $\mathrm{V}_{2}(\mathrm{~mL} / \mathrm{kg})$ & $14.7(38.3)$ & $32.41(13.53-51.44)$ \\
\hline$t_{1 / 2}$ (days) & $11.95^{\mathrm{a}}$ & - \\
\hline $\mathrm{V}_{\mathrm{ss}}(\mathrm{mL} / \mathrm{kg})$ & $56.45^{\mathrm{a}}$ & - \\
\hline \multicolumn{3}{|c|}{ Between-Subject Variability } \\
\hline$\omega \mathrm{CL}(\%)$ & $21.8(29.4)$ & $19.5(14.8-22.7)$ \\
\hline$\omega V_{1}(\%)$ & $21.6(26.7)$ & $15.2(12.1-20.2)$ \\
\hline$\omega \mathrm{V}_{2}(\%)$ & $59.0(45.6)$ & $36.43(30.9-77.2)$ \\
\hline \multicolumn{3}{|l|}{ Residual Variability } \\
\hline a (constant, IU/mL) & $0.0577(28.9)$ & $0.0007(0.0004-0.039)$ \\
\hline b (proportional, \%) & $0.123(14.8)$ & $0.365(0.212-0.408)$ \\
\hline \multicolumn{3}{|l|}{ Correlation } \\
\hline $\operatorname{Corr}\left(V_{1}, C L\right)$ & $-0.908(23.4)$ & $-0.930(-0.989--0.900)$ \\
\hline \multicolumn{3}{|c|}{$\begin{array}{l}\mathrm{CI} \text {, confidence interval; CL, total body clearance; } \mathrm{V}_{1} \text {, central volume of distribution; } \mathrm{Q} \text {, inter } \\
\text { compartmental clearance; } \mathrm{RSE} \text {, relative standard error; } \mathrm{V}_{2} \text {, peripheral volume of distribution; } \mathrm{V}_{\mathrm{ss}} \text {, volume } \\
\text { of distribution at steady-state; } \mathrm{t}_{1 / 2} \text {, terminal half-life }\end{array}$} \\
\hline
\end{tabular}

FIGURE 1 Summary of population pharmacokinetic parameters

Individual steady-state rFXIII concentration-time profiles of 16 evaluated patients is represented in Figure 2. Prophylaxis was subsequently started on $65 \%$ of patients at a mean dosage of $33.1 \mathrm{IU} / \mathrm{kg}$ (range 25.0-80.0 IU/kg), on average every 4.0 weeks (range 3.0-8.0 weeks). During a mean follow-up of 43 months, one ileo-psoas hematoma which quickly resolved, one muscular hematoma, and two minor surgeries were reported. One severe patient who remained on demand treatment experienced a severe $\mathrm{ICH}$.

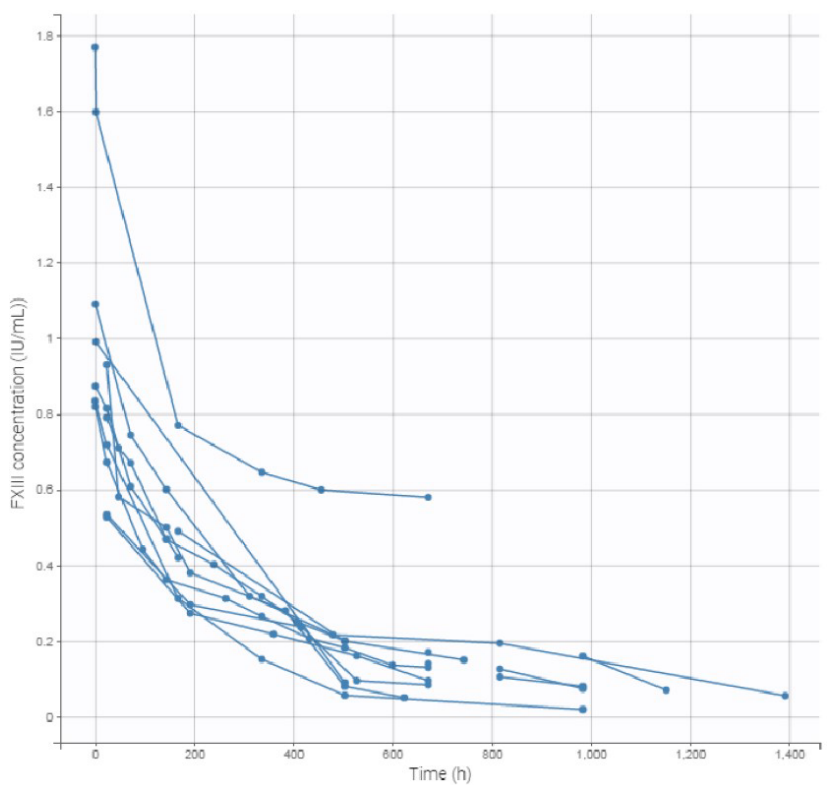

FIGURE 2 Individual steady-state rFXIII concentration-time profiles of 16 evaluated patients

Conclusions: The pharmacokinetics population obtained was similar to that reported in the MENTOR studies but dose used and infusion timing are in some cases different. Efficacy and safety of rFXIII was proven in all patients. The role of prophylaxis to prevent severe hemorrhagic events was also highlighted.

\section{PB0693 | Clinical and Genetic Analysis of Nine Cases of Hereditary Factor V Deficiency}

D. Zhang ${ }^{1}$; X. Zhang ${ }^{2}$; R. Yang ${ }^{1}$

${ }^{1}$ State Key Laboratory of Experimental Hematology, National Clinical Research Center for Blood Diseases, Institute of Hematology \& Blood Diseases Hospital, Chinese Academy of Medical Sciences \& Peking Union Medical College, Tianjin, China; ${ }^{2}$ The Hematology Department of Zhongnan Hospital of Wuhan University, Wuhan, China

Background: Factor $\mathrm{V}$ congenital deficiency is a rare hereditary disease, it exposes patients to hemorrhagic risk, with high morbi-mortality.

Aims: To analyze the clinical phenotype and molecular pathogenesis of 9 patients with hereditary FV deficiency.

Methods: Nine patients with hereditary FV deficiency who were admitted to the Institute of Hematology \& Blood Diseases Hospital from April 1999 to September 2019 were analyzed: activated partial thromboplastin time (APTT), prothrombin time (PT) and FV procoagulant activity $(\mathrm{FV}: \mathrm{C})$ were measured for phenotypic diagnosis; highthroughput sequencing was used for F5 gene mutations screening; Sanger sequencing was used to confirm candidate variants and parental carrying status; Swiss-model was used for three-dimensional structure analysis; ClustalX-2.1 software was used for homologous analysis. 
Results: The FV:C of 9 patients ranged from 0.1 to 10.6. Among them, 8 patients had a history of hemorrhage, with kin/mucosal bleeding was the most common symptom ( 3 cases, $37.5 \%$ ), while 1 case had no bleeding symptom. There were 5 homozygotes and 4 compound heterozygotes. A total of 12 pathogenic or likely pathogenic mutations were detected, of which c.6100C>A/p.Pro2034Thr, c.6575T>C/p.Phe2192Ser, c.1600_1601delinsTG/p. Gln534*, c. $4713 \mathrm{C}>\mathrm{A} / \mathrm{p}$. Tyr1571* and c.952+5G $>\mathrm{C}$ were reported for the first time.

Conclusions: The newly discovered gene mutations enriched the F5 gene mutation spectrum which associated with hereditary FV deficiency. High-throughput sequencing could be an effectively method for the detection of F5 gene mutations.

PB0694 | Monitoring Rare Bleeding Disorders and their Response to Therapeutic Treatments with a Microchip Flowchamber Assay

P. Acuña; E. G. Arias-Salgado; E. Monzón Manzano; M. Martín Salces; M.T. Álvarez Román; M.I. Rivas Pollmar; S. García Barcenilla; T. Cebanu; E. Gonzalez Zorrilla; N. Butta; V. Jiménez Yuste Service of Hematology, Hospital Universitario La Paz, Madrid, Spain, Madrid, Spain

Background: The management of congenital rare bleeding disorders (RBDs) and of von Willebrand disease (VWD) is difficult due to the wide spectrum of clinical phenotypes and to several procoagulant responses to different treatments.

Aims: We aimed to test the usefulness of a microchip flow-chamber system (T-TAS $\left.{ }^{\circledR}\right)$ for analyzing hemostatic and coagulation status in RBDs and type-3 VWD, and the response to treatments with replacement factor and with a non-factor therapy, an anti-TFPI neutralizing antibody (clone-2021).

Methods: We recruited one patient with FVII deficiency (5\% FVII), one with FXI deficiency (<15\% FXI), and one with FX deficiency ( $5 \%$ $\mathrm{FX}$ ), representative, respectively, of anomalous extrinsic, intrinsic and common coagulation pathways. One patient with deficit of FXIII (5\% FXIII), other with type-3 VWD (0\% VWF / 0.4\% FVIII) and five healthy controls were also included.

Microchips coated with either collagen/thromboplastin [atherome (AR)-chip] or collagen [platelet (PL)-chip] were used to evaluate fibrin-rich platelet thrombus formation or platelet thrombus formation.

Results: Using AR-chips, deficiencies of FVII (Fig.1A) and FXI (Fig.1B) caused an anomalous clot formation. In vitro treatment of samples with the corresponding replacement factor restored coagulation profile in FVII and FXI deficiencies. Anti-TFPI corrected the deficiencies of these factors although less effectively. FX deficiency practically prevented clot development and treatment with PCC or anti-TFPI scarcely improved it (Fig.1C). Lack of FXIII did not affect clot formation (Fig. 1D).
The impairment in coagulation (Fig.2A) and primary haemostasis (Fig.2B) observed in type-3 VWD was partially corrected by pdFVIII/VWF, whereas anti-TFPI was almost ineffective.

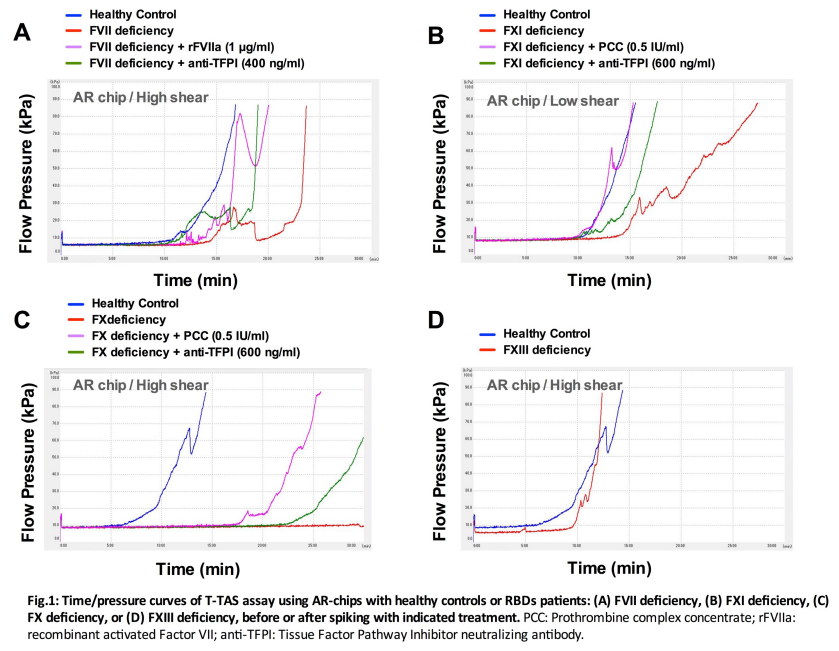

FIGURE 1 Time/pressure curves of T-TAS assay using AR-chips with healthy controls or RBDs patients: (A) FVII deficiency, (B) FXI deficiency, (C) FX deficiency, or (D) FXIII deficiency, before or after spiking with indicated treatment
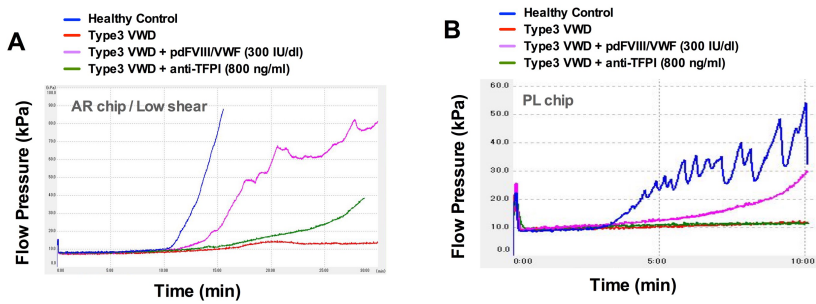

Fig.2: Time/pressure curves of T-TAS assay using AR-chip (A) or PL-chip (B) with healthy controls or type-3 VWD patient, before or after spiking with indicated treatment. pdFVIII/VWF: plasma-derived Factor VIII - Von Willebrand Factor; anti-TFPl: Tissue Facto

FIGURE 2 Time/pressure curves of T-TAS assay using AR-chip (A) or PL-chip (B) with healthy controls or type-3 VWD patient, before or after spiking with indicated treatment

Conclusions: Analysis with a flow chamber-based assay to measure thrombus formation in vitro may be useful for the evaluation of the coagulation profile in RBDs and VWD and for monitoring effects of therapeutic treatments. Anti-TFPI was effective for correcting FVII and FXI deficiencies but have poor effects for amending FX deficiency and VWD. 
PB0695 | Outcomes of a Bleeding Disorders Diagnostic Multidisciplinary Team Meeting for Diagnosing Unclassified Bleeding Disorder / Bleeding of Unknown Cause

W. Thomas ${ }^{1}$; M. Ali ${ }^{1}$; A. Arora ${ }^{2}$; J. Prabhakaran ${ }^{2}$; K. Abu-Zeinah ${ }^{2}$; D. White ${ }^{1}$; S. MacDonald ${ }^{1}$; E. Symington ${ }^{1}$.

${ }^{1}$ Cambridge University Hospitals NHS Foundation Trust, Cambridge, United Kingdom; ${ }^{2}$ University of Cambridge School of Clinical Medicine, Cambridge, United Kingdom

Background: Unclassified bleeding disorder (UBD) is 'a clear bleeding tendency but normal haemostatic investigations'. There is growing recognition of UBD; $2.6 \%$ of patients registered with the UK Haemophilia Center Doctors Organisation have UBD. We register such patients with our center so we can give haemostatic advice for menorrhagia, childbirth and invasive procedures. There are no diagnostic guidelines for UBD so in 2019 we setup a monthly multidisciplinary team (MDT) meeting, attended by doctors/nurses/clinical scientists, to standardise patient registration with UBD/bleeding disorders.

Aims: Review of the bleeding disorders MDT outcomes at our center from May 2019-November 2020.

Methods: A database was held of the patient details, condensed MCMDM-1 VWD bleeding score (BS) and the outcome of the MDT discussion (e.g. register or discharge) and notes reviewed for further information. The project had approval as a service evaluation.

Results: 116 patients were discussed; 35 registered as UBD, 36 with a conventional haemostatic disorder (mild coagulation factor deficiencies/VWD/platelet function disorders) and 45 discharged with re-assurance that they did not have a significant bleeding tendency and results are summarised in table 1 . The BS of the patients was significantly different across groups $(P<0.01)$ and for individual comparisons between groups the difference was not significant between patients discharged without a diagnosis of a bleeding disorder and those with a conventional haemostatic defect found (mean bleeding score $4.3 \vee 5.8$ respectively), however between those 2 groups and the UBD group the BS was significantly different $(P<0.001)$; the mean BS was 11.1 in UBD patients.

TABLE 1 Characteristics of patients that have been discussed at the MDT

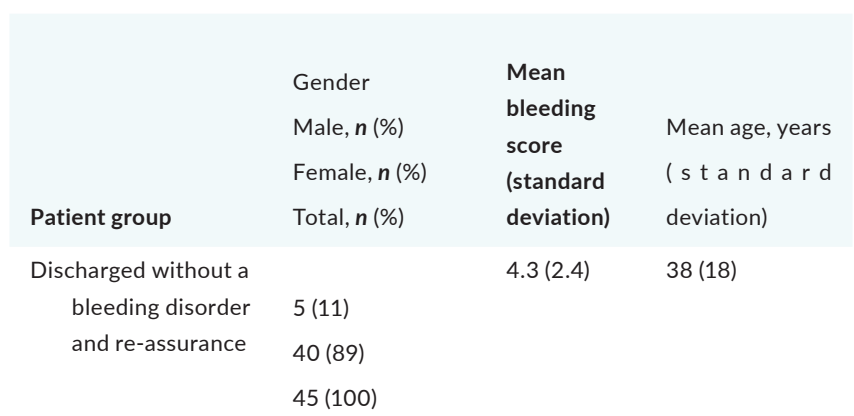

\begin{tabular}{|c|c|c|c|}
\hline Patient group & $\begin{array}{l}\text { Gender } \\
\text { Male, } \boldsymbol{n}(\%) \\
\text { Female, } \boldsymbol{n}(\%) \\
\text { Total, } \boldsymbol{n}(\%)\end{array}$ & $\begin{array}{l}\text { Mean } \\
\text { bleeding } \\
\text { score } \\
\text { (standard } \\
\text { deviation) }\end{array}$ & $\begin{array}{l}\text { Mean age, years } \\
\text { ( } \mathrm{s} \mathrm{t} \text { a } \mathrm{n} \mathrm{d} \text { a r d } \\
\text { deviation) }\end{array}$ \\
\hline $\begin{array}{l}\text { Regsitered with a } \\
\text { conventional } \\
\text { haemostatic } \\
\text { disorder }\end{array}$ & $\begin{array}{l}7(19) \\
29(81) \\
36(100)\end{array}$ & $5.8(3.4)$ & 37 (18) \\
\hline $\begin{array}{l}\text { Registered as } \\
\text { unclassified } \\
\text { bleeding disorder }\end{array}$ & $\begin{array}{l}1(3) \\
34(97) \\
35(100)\end{array}$ & $11.1(4.4)$ & 45 (13) \\
\hline
\end{tabular}

Conclusions: UBD patients have higher BS than patients with conventional haemostatic defects probably due to bias in registration patterns (i.e. the bar for registration is 'higher'). A BS of 4 may be considered significant however this was the mean BS of patients discharged after investigation with re-assurance highlighting the limitations diagnostically of the BS in UBD.

PB0696 | Implementation of a National Reference Service for Activity Testing, Typing, and Genotyping of Hereditary Factor XIII Deficiency (Project BR-13)

$\underline{\text { R. Camelo }}^{1}$; J. Moreira ${ }^{2}$; L.I. Pires Filho ${ }^{3}$; V. Franco ${ }^{4}$; C.B. Ferreira ${ }^{5}$; L.C.C.M. de Souza ${ }^{6}$; M.D. Tenório; S. Figueiredo ${ }^{7}$; S. Lopes ${ }^{8}$

${ }^{1}$ Faculty of Medicine of the Universidade Federal de Minas Gerais, Belo Horizonte, Brazil; ${ }^{2}$ HEMOMINAS Foundation, Belo Horizonte, Brazil; ${ }^{3}$ Hemocentro do Piauí (HEMOPI), Teresina, Brazil; ${ }^{4}$ Hemocentro de Santa Catarina (HEMOSC), Florianópolis, Brazil; ${ }^{5}$ Hemocentro do Rio Grande do Sul (HEMORGS), Porto Alegre, Brazil; ${ }^{6}$ Hemocentro do Amapá (HEMOAP), Macapá, Brazil; ${ }^{7}$ Hemocentro da Paraíba (HEMOÍBA), João Pessoa, Brazil; ${ }^{8}$ Laboratory of Genetics and Molecular Biology of the Universidade Estadual da Paraíba (UEPB), Campina Grande, Brazil

Background: Hereditary factor XIII deficiency (FXIIID) is a rare autosomal recessive disease, characterized by late bleeding, including intracranial hemorrhage. Accurate diagnosis and early institution of prophylaxis with FXIII concentrate are the key to a normal survival of the person with FXIIID. However, the diagnosis of FXIIID is challenging since conventional coagulation tests are normal. Clot solubility tests are not standardized and have low sensitivity, and are not recommended as routine screening, although they are the first test - and perhaps the only test - in several hemostasis laboratories. Quantitative FXIII activity tests are recommended, but many laboratories are not properly equipped. Mutation research complements the diagnosis. For these reasons, it is recommended to carry out the diagnosis in reference laboratories.

Aims: The Project BR-13 aims at implementing a center specialized in the diagnosis of FXIIID at the Hemocentro da Paraíba (Blood Center 
of Paraíba; HEMOÍBA, João Pessoa/Brazil) and at the University of the State of Paraíba (UEPB, Campina Grande/Brazil).

Methods: All index cases previously diagnosed by urea clotting assays will be tested for FXIII activity and typing of deficiency A or B (HEMOÍBA), as well as analyzing the disease-related mutation (UEPB). Clinical data will be reviewed from the medical records using a validated form. Based on the heredogram of index cases, firstdegree relatives will be investigated in the same way.

Results: In 2015, there were 64 people with FXIIID registered by the Brazilian Ministry of Health (prevalence of 1:3 million inhabitants). We expect to include 32 index cases initially, and then 64 firstdegree relatives. The centers involved so far have 37 people with FXIIID, from four of the five regions in Brazil.

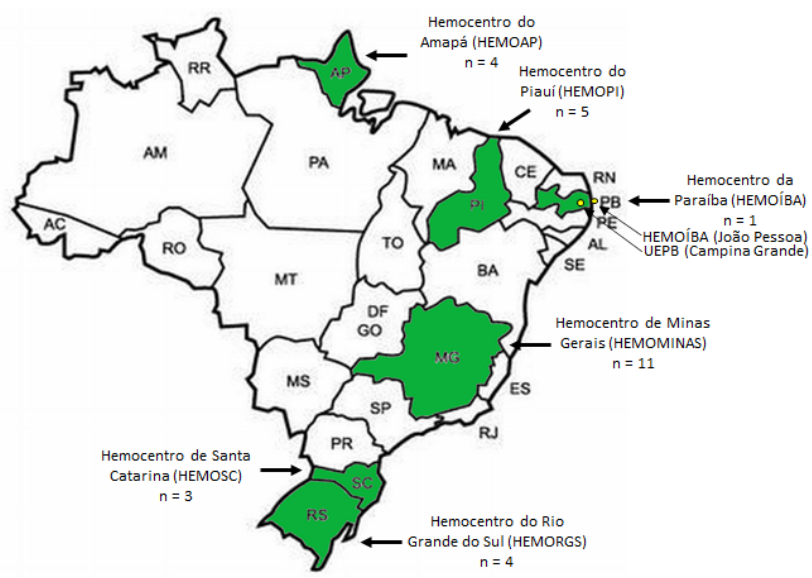

FIGURE 1 Map of Brazilian states highlighting the treatment centers included in the Project BR-13. Included treatment centers were depicted in green. Each center is responsible for the treatment of the patients who live in the respective state. The number ( $n$ ) of people with FXIII deficiency registered at each center was shown. The centers responsible for the project tests were depicted in yellow circles. [UEPB, Universidade Estadual da Paraíba]

Conclusions: We intend to create a national reference diagnostic center, to accurately investigate new cases and to guarantee an adequate treatment follow-up.

PB0697 | Factor XI Deficiency in Pregnant Women: A Genotype to Phenotype Correlation and Analysis

G. Sacchi de Camargo Correia ${ }^{1}$; S. Rajeeve ${ }^{2}$; L. Cytryn ${ }^{1}$

${ }^{1}$ Icahn School of Medicine at Mount Sinai West and Morningside, New York, United States; ${ }^{2}$ Icahn School of Medicine at Mount Sinai, New York, United States

Background: Factor XI deficiency (FXIDef) is an otherwise rare bleeding disorder that commonly affects Ashkenazi and Iraqi Jews; homozygous prevalence is 1:450, and heterozygosity prevalence is 8\%. Pregnancy in FXIDef may pose a potential increased bleeding risk during delivery.

Aims: In our Hematology Consultation Service, we reviewed and analyzed genotypes and phenotypes of pregnant women with FXIDef. Methods: We identified 39 patients with FXIDef evaluated between October 2016 and February 2020. Diagnosis was established on routine genetic screening during pregnancy. Comparisons between FXI Activity levels (FXIAct) at first visit and near term, and between genotypes, were made with two-sample T-test. Anesthetic modalities and routes of delivery were extracted from the record.

Results: In the sample, 61.54\% presented mutation c.901T>C, p.F301L (c.901); 28.20\% had mutation c.403G>T, p.E135X (c.403); and $10.26 \%$ had 4 different mutations. In the c.901 group, $87.5 \%$ showed Jewish ancestry, while $72.73 \%$ in the c.403 group presented this ancestry. No statistically significant differences in FXIAct at first visit $(P=0.43)$, or at near term $(P=0.45)$, were seen between the commonest mutations. Comparing FXIAct within the same genotype between the first appointment and near term again showed no significant difference. One patient (c.901) received prophylactic intervention before delivery (tranexamic acid). No other patients received hemostatic interventions. Route of delivery did not differ between genotypes. See Table-1 and Figure-2 for further data.

TABLE 1 Epidemiological, Hematologic and Delivery Data

\begin{tabular}{|c|c|c|}
\hline & $\begin{array}{l}\text { c.901T>C, } \\
\text { p.F301L }(n=24)\end{array}$ & $\begin{array}{l}\text { c403G>T, } \\
\text { p.E135X }(n=11)\end{array}$ \\
\hline Mean Age (years) & $33.12 \pm 3.94$ & $33.91 \pm 3.83$ \\
\hline $\begin{array}{l}\text { Number of homozygous } \\
\text { patients }(n ; \%)\end{array}$ & $1 ; 4.2 \%$ & $0 ; 0 \%$ \\
\hline $\begin{array}{l}\text { Mean FXI activity on } \\
1^{\text {st }} \text { appointment } \\
\text { (\%) (normal range: } \\
50-150 \%)\end{array}$ & $54.92 \pm 18.68$ & $68.00 \pm 51.69$ \\
\hline $\begin{array}{l}\text { Mean aPTT on 1st } \\
\text { appointment (s) } \\
\text { (normal range: } \\
24.6-34.7 \mathrm{~s} \text { ) }\end{array}$ & $30.90 \pm 3.19$ & $31.85 \pm 2.51$ \\
\hline $\begin{array}{l}\text { Mean INR on 1st } \\
\text { appointment (normal } \\
\text { range: } 0.9-1.1 \text { ) }\end{array}$ & $0.97 \pm 0.07$ & $1 \pm 0.04$ \\
\hline $\begin{array}{l}\text { Mean FXI activity near } \\
\text { term (\%) (normal } \\
\text { range: } 50-150 \%)\end{array}$ & $55.26 \pm 23.13$ & $50.11 \pm 11.13$ \\
\hline $\begin{array}{l}\text { Mean aPTT at birth } \\
\text { (s) (normal range: } \\
24.6-34.7 \mathrm{~s} \text { ) }\end{array}$ & $30.04 \pm 2.16$ & $30.48 \pm 2.54$ \\
\hline $\begin{array}{l}\text { Neuraxial Anesthesia } \\
\qquad(n ; \%)\end{array}$ & $15 ; 62.5 \%$ & $5 ; 45.45 \%$ \\
\hline $\begin{array}{l}P \text {-Value comparing FXIA } \\
\text { on } 1^{\text {st }} \text { appointment } \\
\text { and near term }\end{array}$ & 0.96 & 0.29 \\
\hline
\end{tabular}

Conclusions: No significant differences in FXIAct or clinical presentation were noted between genotypes in our series, in which 
Factor XI Mutations - Percentages

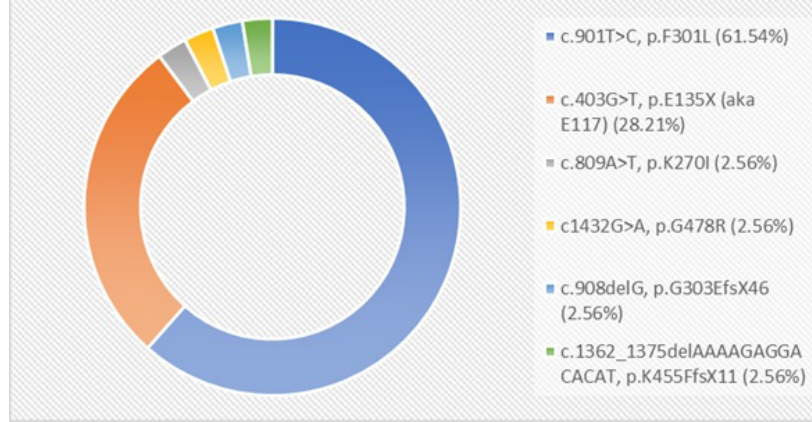

FIGURE 1 Factor XI Mutations Profile

all patients but one were heterozygous. Genotypes correlate with ethnicity, with mutations c.901 and c.403 associated with Jewish ancestry. Comparing our data with other cohorts may allow for broadening of the finding that different genotypes do not affect presentation during pregnancy. Nonetheless, monitoring for FXIDef allows for prophylactic treatment in cases of increased bleeding risk. Therefore, genetic tests are valuable for counseling and diagnostic purposes.

PB0698 | Human Fibrinogen Concentrate for Bleeding Prophylaxis During Surgery in Adult, Adolescent and Pediatric Patients with Congenital Fibrinogen Deficiency: Results from the FORMA-02 and FORMA-04 Clinical Trials

C. Djambas-Khayat ${ }^{1}$; S. Lohade ${ }^{2}$; F. D'Souza ${ }^{3}$; L. Shamanur ${ }^{4}$; O. Zekavat ${ }^{5}$; I. Kruzhkova ${ }^{6}$; B. Schwartz'; C. Solomon ${ }^{6}$ ${ }^{1}$ Hotel Dieu de France Hospital, Beirut, Lebanon; ${ }^{2}$ Sahyadri Specialty Hospital, Pune, India; ${ }^{3}$ St. John's Medical College Hospital, Bangalore, India; ${ }^{4}$ S.S Institute of Medical Science and Research Center, Davangere, India; ${ }^{5}$ Hematology Research Center, Nemazee Hospital, Shiraz University of Medical Sciences, Shiraz, Iran, Islamic Republic of; ${ }^{6}$ Octapharma AG, Lachen, Switzerland; ${ }^{7}$ Octapharma, Hoboken, United States

Background: In patients with rare congenital fibrinogen deficiency (CFD), human fibrinogen concentrate (HFC) can be administered as prophylaxis prior to and during surgery to prevent excessive blood loss.

Aims: Data is reported on patients from two Phase 3 clinical studies using HFC to prevent surgical bleeding.

Methods: FORMA-02/FORMA-04 were international, multicenter prospective, open-label Phase 3 studies on the efficacy and safety of HFC (Fibryga ${ }^{\circledR}$, Octapharma) for surgical bleeding prophylaxis in adult/adolescent and pediatric CFD patients. Hemostatic efficacy assessments were adjudicated using objective criteria by an Independent Data Monitoring Committee (IDMEAC), and overall hemostatic efficacy was assessed as success/failure for each surgery.
Results: Eleven patients ( 3 pediatric: $<12$ years; 1 adolescent: 12 years; 7 adults: $\geq 18$ years) received HFC in 14 surgeries. Two surgeries were classified as major: 1 adult (right eye enucleation with socket reconstruction) and 1 pediatric (splenectomy). All remaining surgeries were minor. A lower mean \pm SD HFC dose was administered in adults prior to surgery compared to younger patients (pediatrics: $78.53 \pm 27.96 \mathrm{mg} / \mathrm{kg}$; adolescent: $127.91 \mathrm{mg} / \mathrm{kg}$; adults: $68.98 \pm 5.74$ $\mathrm{mg} / \mathrm{kg}$ ). Figure 1 and Figure 2 show HFC dosing and fibrinogen activity for minor surgeries requiring single-dose treatment (target fibrinogen level $=100 \mathrm{mg} / \mathrm{dL}$ ) in an adult and pediatric patient, respectively. Four minor adult surgeries required $>1$ HFC dose (2-5 total doses; mean \pm SD total dose: $132.87 \pm 29.06 \mathrm{mg} / \mathrm{kg}$ ). The adult/ pediatric major surgeries required 8/6 HFC infusions, respectively (loading doses: $102.56 \mathrm{mg} / \mathrm{kg}$ and $53.00 \mathrm{mg} / \mathrm{kg}$; total doses: 225.33 $\mathrm{mg} / \mathrm{kg}$ and $450.00 \mathrm{mg} / \mathrm{kg}$ ). All surgeries (100\%) were rated successful by the IDMEAC. One serious adverse event was considered possibly related to treatment; portal vein thrombosis in the pediatric patient undergoing splenectomy. No allergic/hypersensitivity reactions or deaths were observed.

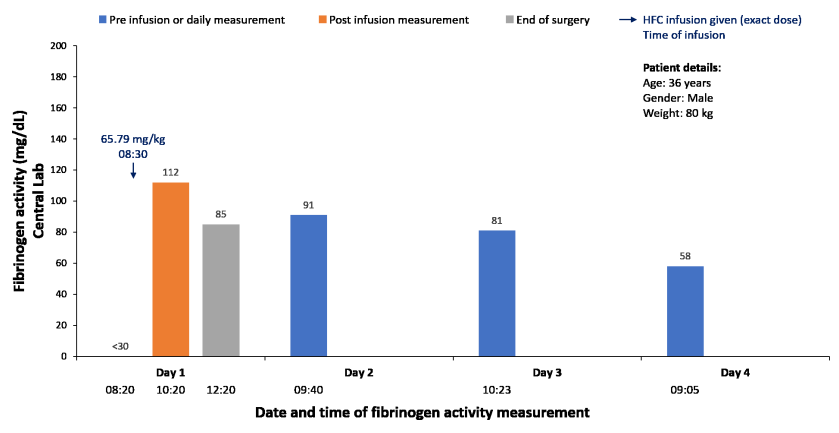

FIGURE 1 Fibrinogen activity (central lab measurements) and single HFC dose administered in a successfully treated adult patient undergoing minor surgery

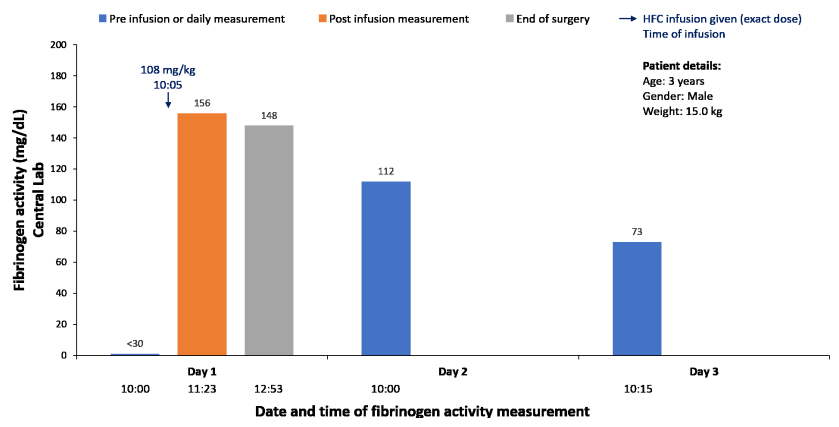

FIGURE 2 Fibrinogen activity (central lab measurements) and single HFC dose administered in a successfully treated pediatric patient undergoing minor surgery 
Conclusions: In conclusion, HFC administration for bleeding prophylaxis during minor/major surgery was efficacious for the ultra-rare CFD in adult, adolescent and pediatric patients.

PB0699 | Subcutaneous Marzeptacog Alfa (Activated) is Effective Treatment of Bleeding in FVII Deficient Rats

T. Knudsen ${ }^{1}$; P.B Johansen ${ }^{1}$; L. Neuman ${ }^{1}$; H. Levy ${ }^{1}$; J. Reckless²; G.E Blouse ${ }^{1}$

${ }^{1}$ Catalyst Biosciences, South San Francisco, United States; ${ }^{2}$ RxCelerate Limited, Cambridgeshire, United Kingdom

Background: Marzeptacog Alfa (Activated) (MarzAA), a novel high potency rFVIla, is being developed for subcutaneous (SQ) treatment of bleeding in Factor VII deficiency (FVIID) and other rare bleeding disorders. Patients with a severe phenotype can have life threatening bleeding and reduced quality of life.

Aims: To investigate the effect of SQ MarzAA on bleeding after tail vein transection (TVT) in a rat model of FVIID.

Methods: Sprague Dawley rats were treated with intravenous (IV) vehicle or warfarin $0.3 \mathrm{mg} / \mathrm{kg}$ to induce FVIID. Blood samples were obtained 1 to 4 hours after dosing to assess prothrombin time (PT) and activated partial thromboplastin time (aPTT) and 3 hours after dosing was chosen to investigate SQ MarzAA treatment (Table 1). Rats were placed under isoflurane anesthesia for the TVT procedure. Rats were treated with increasing doses of SQ MarzAA at either two hours before or one minute after TVT. The tail was briefly immersed in saline and then a lateral vein transected at $5 \mathrm{~mm}$ in diameter. Subsequent bleeding was monitored for 60 minutes and total blood loss quantified by measurement of hemoglobin in the saline.

Results: Dosing with warfarin induced a window when the PT was significantly prolonged while the aPTT was minimally affected (Table 1); this period represents a functional FVIID without overtly inducing concurrent defects of other factors traditionally affected by warfarin, including prothrombin, Factors IX and X.

\section{TABLE 1}

Table 1.PT and aPTT change after IV treatment with vehicle or $0.3 \mathrm{mg} / \mathrm{kg}$ warfarin. Means and $95 \%$ C.I., $N=3$.

\begin{tabular}{|l|c|c|c|c|c|}
\hline Treatment & Time $(\mathrm{h})$ & PT $(\mathrm{sec})$ & P vs. vehicle & aPTT $(\mathrm{sec})$ & P vs. vehicle \\
\hline Vehicle & - & $26.4(24.0-28.8)$ & - & $15.9(15.4-16.4)$ & - \\
\hline & 1 & $29.4(27.7-31.1)$ & ns & $16.1(15.4-16.9)$ & ns \\
0 & 2 & $41.8(38.2-45.5)$ & $<0.0001$ & $17.1(14.8-19.3)$ & ns \\
& 3 & $71.2(61.7-80.7)$ & $<0.0001$ & $19.7(18.0-21.4)$ & $<0.001$ \\
& 4 & $126.0(121.6-130.5)$ & $<0.0001$ & $28.7(25.7-31.7)$ & $<0.0001$ \\
\hline
\end{tabular}

SQ MarzAA administered prior to TVT reduced blood loss in a dose dependent manner (Figure 1) and was completely normalized at the highest dose. Notably, SQ MarzAA also decreased blood loss when used on-demand 1 minute after injury.

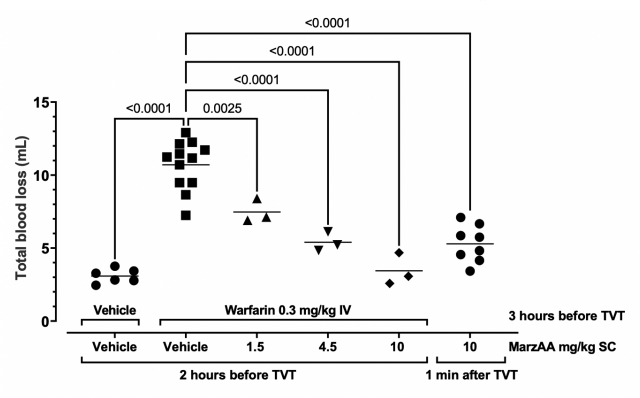

Figure 1. Effect of SQ MarzAA on total blood loss in rats treated with intravenous vehicle or warfarin $0.3 \mathrm{mg} / \mathrm{kg}$ three hours before tail vein transection. One-way ANOVA with adjustments of the $P$ values according to Dunnet. Means with individual values.

\section{FIGURE 1}

Conclusions: SQ MarzAA successfully treated induced bleeding in FVIID rats. These results provide robust nonclinical evidence that SQ MarzAA has the potential for prophylaxis or on-demand treatment of bleeding in patients with FVIID.

\section{PB0700 | Phenotype-Genotype Relationship in Patients with Hereditary FXI Deficiency}

\section{Albayrak ${ }^{1}$; D. Albayrak ${ }^{2}$; H. Gürkan ${ }^{3}$; S. Demir ${ }^{3}$}

${ }^{1}$ Ondokuz Mayıs University Medical Faculty Pediatric Hematology Department, Samsun, Turkey; ${ }^{2}$ Samsun Medicalpark Hospital, Samsun, Turkey; ${ }^{3}$ Trakya University Medical Faculty, Edirne, Turkey

Background: Hereditary factor XI (FXI) deficiency is a rare bleeding disorder caused by mutations in the F11 gene, also called hemophilia C, and is autosomal recessively inherited. To date, more than 220 mutations in the F11 gene have been identified in patients with FXI deficiency.

Aims: The aim of this study is to determine the phenotype-genotype relationship of our patients diagnosed with hereditary Factor XI deficiency in our clinic.

Methods: Patients with factor XI activity below $20 \%$ were accepted as severe factor XI deficiency. Those with $20-70 \%$ factor XI activity were considered as mild factor XI deficiency. Families were informed about genetic transmission and family screening was performed. Approval was obtained from the ethics committee of our hospital for this study. Factor F11 gene mutation was studied in 14 families. Results: In our clinic, hereditary factor XI deficiency was detected in 255 patients. Eighty-five of our patients were diagnosed with severe factor XI deficiency. Ten patients were evaluated as homozygous and four patients were evaluated as compound heterozygous. 30 of the family members were heterozygous. 58 mutant alleles were detected in 44 patients in total. 23 of the patients were male and 21 were female. The most common mutation was $P$. Ala109Thr and was found in $71 \%(41 / 58)$ of the mutated alleles. The second most common mutation, p. Ala43Thr, was found in 9\% (5/58) of the mutated alleles. The third most common mutations were with p. Glu135Ter, c.325 + 1G>A, p. Thr150Met, p. Phe61Cys, p. Thr208Lys and p. 
Thr60, each of which was found in 3.3\% (2/58) of the mutated alleles. Cleft palate and cleft lip were detected in one patient.

Conclusions: Eight different mutations were identified in 14 families with severe inherited FXI deficiency. In this study, p.Ala109Thr frequency of mutations in $71 \%$, has been found at high frequency in the northern region of Turkey.

\section{PB0701 | Evaluation of Coagulation Profile in FVII Deficiency with Inhibitor Using Global Coagulation Tests: A Case Report}

M.I. Rivas Pollmar; E. Garcia Arias-Salgado; M.T. Alvarez Roman; E. Monzon Manzano; P. Acuña Butta; M. Martin Salces; S. Garcia Barcenilla; T. Cebanu; E. Gonzalez Zorrilla; N. Butta Coll; V. Jimenez Yuste

Hospital Universitario La Paz. Idi-Paz, Madrid, Spain

Background: FVII deficiency is considered a rare bleeding disorder. Development of FVII inhibitors is very uncommon and represents a serious complication. Management of bleeding episodes in patients with inhibitors is a challenge in the treatment of this disorder.

Aims: Evaluation with global assays of the hemostatic state of a patient with FVII deficiency and inhibitor with hemorrhagic and thrombotic complications.

Methods: Blood samples were collected with CTI and were assessed by thromboelastometry (ROTEM) and thrombin generation test (TGT). ROTEM was performed with low concentration of tissue factor (EXTEM diluted_1:50000). TGT was triggered with PPP-Low. Functional FVII and inhibitor levels were also evaluated.

Results: Two year-old girl diagnosed with severe FVII deficiency (FVII 7\%) on prophylaxis with rFVIla since first month of life due to an intracranial haemorrhage. At 6 months she developed inhibitor against FVII (9.8 BU). As shown in Fig.1, ROTEM and TGT showed that FVII deficiency with inhibitor caused a delayed clotting time (CT) and thrombin generation Lag-time (LT). At the age of 23 months she was admitted at hospital because of anemization and Port-A-Cath infection. Prophylaxis treatment was suspended due to thrombosis of peripheral venous access, but global tests continued showing an hypocoagulable state with delayed CT and LT. After 7 days without prophylaxis and high inhibitor titer (59 BU), she developed a new intracranial haemorrhage, needing treatment with continuous rFVIla infusion, immunoglobulines and rituximab to erradicate the inhibitor. Hemostatic parameters improved during the administration of these drugs and inhibitor levels decreased. After restarting prophylaxis due to resolution of bleeding episodes, inhibitor and hypocoagulable state were observed again.

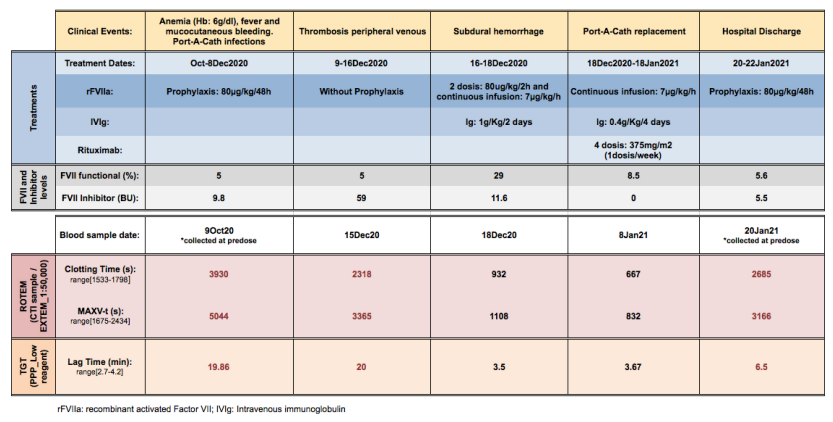

FIGURE 1 Follow up of laboratory parameters

Conclusions: This case shows the relevance of monitoring hemostasis in FVII deficiency patients with global coagulation assays especially in presence of bleeding and thrombotic events.

PB0702 | Hereditary Haemorrhagic Telangiectasia (HHT) or Rendu-Osler Weber Disease as an Important Rare Haemorrhagic Disorder in Costa Rica: A Report of Costa Rica

L. Salazar-Sanchez ${ }^{1}$; K. Leiva-Gabriel ${ }^{1}$; B.I. Hernandez-Salazar ${ }^{1}$; C. Salazar-Chacon ${ }^{2}$

${ }^{1}$ University of Costa Rica/Medicine School, San José, Costa Rica;

${ }^{2}$ College of Physicians and Surgeons, San José, Costa Rica

Background: Hereditary hemorrhagic telangiectasia (HHT) or Rendu-Osler-Weber disease is an autosomal dominant disease, penetrance related with age and with an important variability in the manifestations clinics among affected individuals, including between patients of the same family. The vascular manifestations are the presence in skin and mucous membranes telangiectasias, recurrent and spontaneous epistaxis and arteriovenous malformations on different organs.

Aims: The main objective of this investigation is to obtain epidemiological information and clinical data of patients with HHT and determine its prevalence in Costa Rica.

Methods: Data was obtained from the referral main hospitals in San Jose, Costa Rica. It was analyzed from October 1989 until 2017. Statistical support was provided by the Medical School of the University of Costa Rica utilizing the Epi.Info. 7.1 (CDC, Atlanta USA) package. The basic data were collected in a questionnaire, following the criteria of Curaçao.

Results: Approximate frequency of 2.51 cases per year was obtained, where age average of the patients was 61years, with a diagnosis of over 40 years. Geographically, $83.3 \%$ of the cases were reported in the province of San José, $8.3 \%$ in Puntarenas and $4.16 \%$ in two areas (Limón and Alajuela), Figure 1.The main reason for consultation was a history of heavy bleeding, and chronic persistent epistaxis in most patients, or upper gastro-intestinal bleeding. All cases were treated with iron replacement and/or blood products transfusion, and some with antifibrinolytic therapy or anticoagulation. 


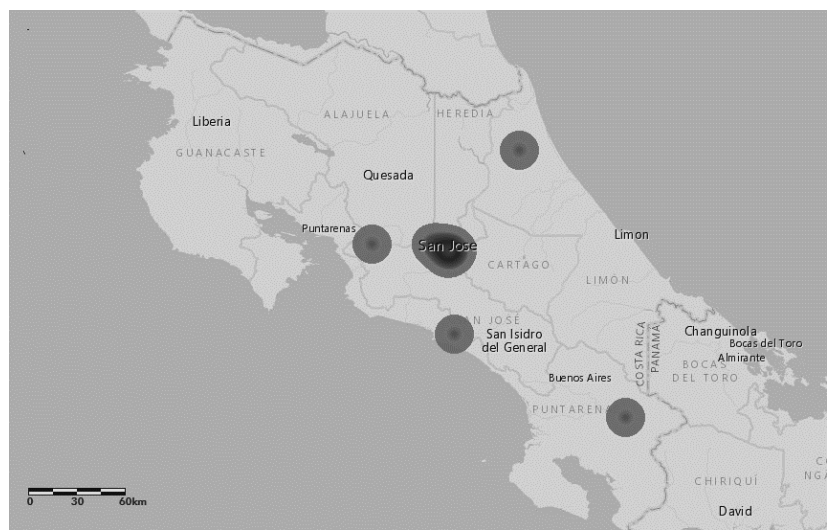

FIGURE 1 Geographic distribution of reported cases of Hereditary Hemorrhagic Telangiectasia, according to percentage distribution in provinces of Costa Rica

Conclusions: This is the first study of HHT in Costa Rica, there is a significant underreporting of the disease. There is no record of the care received by HHT patients at the first and second levels of care in the rest of the country. It is important to spread knowledge and educate health personnel about this condition, to try to reduce their underreporting and improve the quality of life of patients.

PB0703 | A Review of Beighton Scores Recorded for All Patients with a Bleeding Tendency Registered at One Haemophilia Centre without Another Formal Bleeding Disorder Diagnosis

J. Clark; D. Stephensen; C. Dodd; K. Elliott

Kent Haemophilia Centre, Canterbury, United Kingdom

Background: Disorders of collagen are known to be associated with a mild bleeding tendency, and generalised joint hypermobility is common in collagen disorders and can be objectively assessed with measures such as the Beighton score, with a score of 5 or more being positive for hypermobility. Previous studies have shown that the prevalence of hypermobility in a bleeding disorder clinic is approximately $24 \%$, compared to control population prevalence of $2 \%$. Aims: Review proportion of patients with unexplained bleeding tendency or platelet defect who have been assessed for hypermobility with a Beighton score.

Methods: Review of Haemophilia Centre registry for all patients registered between 2015 and 2020 with a diagnosis of either a platelet defect or unexplained bleeding tendency, and comparison with records of Beighton score assessment.

Results: 146patients were registered to our haemophilia centre with a diagnosis of either platelet defect or unexplained bleeding tendency between 2015 and 2020. 26 (17.8\%) of this total had been assessed with a Beighton score, and a total of 19 (13.0\%) were found to have a current or historical Beighton score $>/=5$. 77 patients were registered with a diagnosis of unexplained bleeding tendency, of which 19 (24.7\%) were assessed with a Beighton score, and a total of $13(16.9 \%)$ of the total were found to have a Beighton score $>/=5$
Conclusions: Our data shows that there is an increased proportion of patients with a bleeding tendency with hypermobility compared to that expected in the normal population, roughly in line with other bleeding clinic prevalence, suggesting that an underlying collage disorder is common and may not be recognised as a contributor to a bleeding tendency, opening up potential for more routine assessment in this context.

PB0704 | Congenital Factor XI Deficiency: A Single-center Retrospective Analysis of 80 Cases

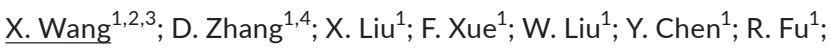
L. Zhang ${ }^{1,2,3}$; R. Yang ${ }^{1,2,3}$

${ }^{1}$ State Key Laboratory of Experimental Hematology, National Clinical Research Center for Hematological Disorders, Institute of Hematology and Blood Diseases Hospital, Chinese Academy of Medical Sciences and Peking Union Medical College, Tianjin, China; ${ }^{2}$ CAMS Key Laboratory of Gene Therapy for Blood Diseases, Tianjin, China; ${ }^{3}$ Tianjin Laboratory of Blood Disease Gene Therapy, Tianjin, China; ${ }^{4}$ Tianjin Sino-US Diagnostics Co., Tianjin, China

Background: Congenital factor XI (FXI) deficiency is an autosomal incomplete recessive hereditary hemorrhagic disease caused by $\mathrm{FXI}$ gene(F11) mutation. The global incidence rate is about $1 / 1$ million, with the highest incidence among Jews. Most of the patients have no symptoms or mild bleeding symptoms. The treatment included antifibrinolytic drugs, fresh frozen plasma, FXI concentrate and recombinant FVIla. Aims: To analyze the clinical manifestation, laboratory examination, treatment and prognosis of patients with congenital FXI deficiency. Methods: The clinical data of 80 patients with congenital FXI deficiency in Institute of Hematology \& Blood Diseases Hospital, Chinese Academy of Medical Sciences from September 2006 to October 2020 were analyzed retrospectively.

Results: Among the 80 patients, there were 33 males (41.3\%) and 47 females (58.8\%), with a median age of 32 (2-66) years. Twenty-eight cases (35.0\%) had bleeding events, including 11 cases of spontaneous bleeding (13.8\%), 9 cases of ecchymosis or bleeding after skin trauma (11.3\%), 9 cases of postoperative bleeding (11.3\%). Among the female patients, there were 11 cases of menorrhagia (23.4\%) and 1 case of bleeding after vaginal delivery (2.1\%). Laboratory examination were characterized by prolonged activated partial thromboplastin time (APTT), normal prothrombin time (PT), and decreased FXI activity (FXI:C). Nine patients (11.3\%) were tested for FXI gene (F11) with 11 mutations. Twenty-seven patients (33.8\%) received fresh frozen plasma(FFP)treatment, 15 patients (18.8\%) were received for prophylaxis with no bleeding occurred during and after operation.

Conclusions: Most patients with congenital FXI deficiency have no or mild bleeding symptoms. There was no significant correlation between FXI:C and the severity of bleeding symptoms, and there was a well consistency between FXI:C and F11 homozygous or heterozygous mutation type. Prophylactic infusion of FFP can effectively reduce the risk of operative bleeding. 
PB0705 | Increased Risk for Re-bleeding after Hemostatic Challenges in Patients with a Mild to Moderate Bleeding Tendency

D. Mehic ${ }^{1}$; G. Neubauer ${ }^{1}$; A. Kaider ${ }^{2}$; C. Ay ${ }^{1}$; I. Pabinger ${ }^{1}$;

J. Gebhart ${ }^{1}$

${ }^{1}$ Clinical Division of Haematology and Haemostaseology, Department of Medicine I, Medical University of Vienna, Vienna, Austria; ${ }^{2}$ Center for Medical Statistics, Informatics and Intelligent Systems, Vienna, Austria

Background: There is a lack of data on the occurrence of re-bleeding after hemostatic challenges in patients with mild bleeding disorders (MBDs) or bleeding of unknown cause (BUC).

Aims: To investigate re-bleeding rates and risk factors for re-bleeding in patients with MBD or BUC, who underwent surgery, tooth extraction or childbirth.
Methods: 253 of 639 patients with a mild bleeding tendency (39.6\%), who were included in the Vienna bleeding biobank (VIBB, EC: 603/2009, Gebhart et al. JTH. 2018) were available for a followup to record re-bleeding rates after hemostatic challenges using a standardized questionnaire.

Results: Of the 253 patients, 95 (37.5\%) had a MBD and 158 (62.5\%) were BUC patients. The mean (standard deviation, SD) age, bleeding score (BS) at study inclusion, and time to follow-up of the patients were 44.7 (15.9) years, 6.0 (3.2), and 36.0 (19.2) months.

At follow-up, 23/74 (31.1\%) patients had a re-bleeding after surgery, $13 / 28(46.4 \%)$ after tooth extraction, and 1/3 (33.3\%) woman had a re-bleeding after childbirth (Table 1). Patients with bleeding after surgery and tooth extraction at study inclusion had a significant increased risk for re-bleeding of the same manifestation (Table 1). There was no difference between patients with MBD or BUC (data not shown).

TABLE 1 Association of re-bleeding rates with bleeding symptoms at inclusion in patients with invasive procedures

\begin{tabular}{|c|c|c|c|c|c|c|c|}
\hline Symptom & Total & $\begin{array}{l}\text { Bleeding at } \\
\text { inclusion }\end{array}$ & $\begin{array}{l}\text { Re-Bleeding } \\
\text { at FU }\end{array}$ & $\begin{array}{l}\text { No bleeding at } \\
\text { inclusion }\end{array}$ & $\begin{array}{l}\text { Bleeding } \\
\text { at FU }\end{array}$ & $P^{*}$ & $\mathrm{BHC}$ \\
\hline & $\mathrm{N}$ & $\mathrm{N}$ & $n(\%)$ & $\mathrm{N}$ & $n(\%)$ & & \\
\hline $\begin{array}{l}\text { Bleeding after tooth } \\
\text { extraction } \dagger\end{array}$ & 55 & 28 & $13(46.4)$ & 27 & $3(11.1)$ & 0.004 & $<.05$ \\
\hline
\end{tabular}

FU, follow-up; BHC, Bonferroni-Holm-correction; ${ }^{*} \chi 2$ test or Fisher's exact.

$\dagger$ including patients, who had surgery or tooth extraction in the follow-up period, respectively

$\S$ including all women who gave birth in the follow-up period

In multivariable analysis, a higher BS at baseline and blood group $\mathrm{O}$ were independent risk factors for re-bleeding in patients with hemostatic challenges (Table 2).

TABLE 2 Univariate and multivariable logistic regression of risk factors for re-bleeding in patients with invasive procedure (surgery, tooth extraction and child birth)

\begin{tabular}{|c|c|c|c|c|c|c|c|}
\hline & \multirow[b]{2}{*}{ Unit } & \multicolumn{3}{|c|}{ Univariate } & \multicolumn{3}{|c|}{ Multivariable } \\
\hline & & OR & $95 \% \mathrm{Cl}$ & $P$ & OR & $95 \% \mathrm{Cl}$ & $P$ \\
\hline Sex & Female vs. male & 3.18 & $0.90-11.21$ & 0.072 & 3.33 & $0.89-12.43$ & 0.074 \\
\hline $\begin{array}{c}\text { Vicenza BS at } \\
\text { baseline }\end{array}$ & 1.0 & 1.18 & $1.06-1.31$ & 0.003 & 1.16 & $1.03-1.31$ & 0.015 \\
\hline Diagnosis & non-BUC vs. BUC & 1.34 & $0.65-2.75$ & 0.426 & & & \\
\hline $\begin{array}{l}\text { Positive family } \\
\text { history }\end{array}$ & No vs. yes & 0.57 & $0.27-1.19$ & 0.131 & & & \\
\hline Follow up time & 1.0 year & 0.88 & $0.70-1.11$ & 0.278 & & & \\
\hline
\end{tabular}


Conclusions: Patients with a mild to moderate bleeding tendency with previous bleeding after invasive procedures have a significant risk for re-bleeding, independent of a diagnosis of a bleeding disorder, especially in patients with blood group $\mathrm{O}$ and a high bleeding score at inclusion. These findings encourage prophylactic treatment when patients face hemostatic challenges such as surgery or tooth extractions.

PB0706 | ROTEM in Combination with Bleeding History - Can it Serve as a Screening Test for Factor XIII Deficiency in Patients with Suspected Bleeding Disorder?

T. Geevar; R.G. Dave; T. David V; G. K C; R. Vijayan; A. Samuel;

S. Singh; J. John Mammen; S. Chandran Nair

Christian Medical College, Vellore, India

Background: Routine coagulation screening tests are not useful in the diagnosis of Factor XIII (FXIII) deficiency. Though, clot solubility test can be used as a screening test, the test is time consuming and lacks sensitivity. Global viscoelastic test like Rotational thromboelastometry (ROTEM) is the only rapid point of care test to assess hyperfibrinolysis.

Aims: To determine the diagnostic performance of bleeding symptoms, ISTH bleeding assessment tool (BAT) and ROTEM parameters in the diagnosis of FXIII deficiency.

Methods: This was a retrospective study wherein patients with FXIII deficiency among patients referred with a suspected bleeding disorder from October 2016 - October 2020 were included. Eighty consecutive patients with no intrinsic abnormality were recruited as controls. Diagnosis was confirmed after a detailed coagulation workup.

Results: 34 patients with FXIII deficiency were identified during the study period. The median (IQR) of FXIII activity and antigen levels of patients were 5.9 (4.3-8) and 0 (0-2.5) respectively. All patients had significant BAT score with a median (IQR) of 8 (5-11). The mean maximum clot firmness (MCF) mm was significantly lower [48.5 \pm 10.1 vs 63.2 \pm 4.8; $P=<0.0001]$, and Maximum Lysis (ML)\% was significantly higher [20.7 \pm 9.1 vs $8.3 \pm 3.9 ; P=<0.0001]$ in patients than controls. FXIII deficiency patients with $\mathrm{ML}<15 \%$ had significantly higher Fibrinogen levels (mg/dL) compared to those with ML $\geq 15 \%$ [Mean: $325 \pm 83$ vs $250.5 \pm$ 71; $P=0.024]$ which could have contributed to the lower $M L \%$. The sensitivity and specificity of different variables are shown in Figure. Presence of either increased lysis in ROTEM or umbilical stump bleeding had a sensitivity of $97.1 \%$. When both umbilical stump bleeding and increased lysis was present, the specificity was $100 \%$.

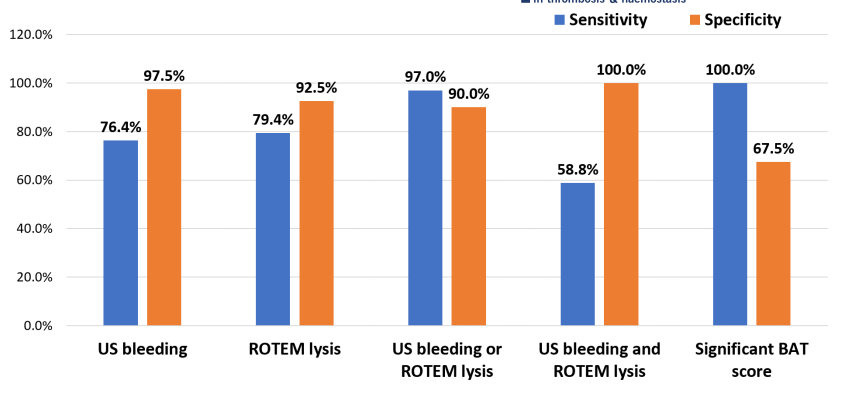

FIGURE 1 Sensitivity and specificity of umbilical stump (US) bleeding, increased lysis ( $M L>15 \%$ ) in ROTEM and BAT score in the diagnosis of Factor XIII deficiency

Conclusions: A combination of bleeding history, specifically umbilical stump bleeding and increased lysis in ROTEM can serve as a useful screening test for FXIII deficiency.

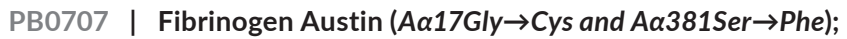
Identification of a Novel Fibrinogen Mutation Causing Dysfibrinogenaemia

\section{J. Rigano}

Alfred Health, Melbourne, Australia

Background: Fibrinogen is encoded by the FGA, FGB and FGG genes on chromosome $4 \mathrm{q}$. The $340 \mathrm{kDa}$ glycoprotein is organised as two identical heterotrimers consisting of $A \alpha, B \beta$ and $\gamma$ chains. Fibrinogen abnormalities can be acquired or congenital disorders. Mutations causing hypofibrinogenaemia and/or dysfibrinogenaemia phenotypically give rise to haemorrhage and/or thrombosis.

Aims: This report describes two novel fibrinogen mutations in a woman causing dysfibrinogenaemia with a bleeding phenotype.

Methods: A 51-year-old female presented for investigation of menorrhagia with a history of mild bleeding. Ten other family members were also investigated. Coagulation assays were performed using HemosIL ${ }^{\circledR}$ reagents on the ACL TOP CTS 500 analyser (Instrumentation Laboratory; Werfen). Fibrinopeptide release assays, HPLC fibrinogen, SDS-PAGE, whole protein time-of-flight mass spectrometry (TOF MS) and DNA sequencing were performed to assess the physical, functional and molecular properties of fibrinogen. Results: Patient showed a low Fibrinogen-Clauss with prolonged Thrombin and Reptilase Time. Six out of ten family members demonstrated similar results. Fibrinopeptide release assays showed delayed polymer formation and decreased release of fibrinopeptide A. Released peptides were quantitated by HPLC resulting in a reduced $A: B$ ratio. SDS-PAGE, Whole protein TOF MS revealed abnormal masses of $\mathrm{Aa}$ chains. DNA sequencing analysis showed patient was heterozygous for three sequence changes in Aa chain; Aa312Thr $\rightarrow$ Ala polymorphism and two novel mutations Aa17Gly $\rightarrow$ Cys and Aa381Ser $\rightarrow$ Phe. Aa17Gly $\rightarrow$ Cys was the cause of decreased fibrinopeptide $A$ release and Aa381Ser $\rightarrow$ Phe was the cause delayed polymer formation both contributing to dysfibrinogenaemia. Aa312Thr $\rightarrow$ Ala is a known benign polymorphism. DNA analysis of ten family members confirmed 
the two mutations were co-inherited on the same FGA allele. This novel double mutation variant was named fibrinogen Austin.

Conclusions: Here reported was the identification of two novel fibrinogen mutations in a woman causing dysfibrinogenaemia. Investigation of extended family members revealed the mutations were co-inherited on the same FGA allele.

\section{PB0708 | Rare Bleeding Disorders in Children. Towards} Establishing a Local Registry

D. Florinskiy; D. Fedorova; A. Poletaev; E. Seregina; A. Pshonkin;

\section{P. Zharkov}

Dmitry Rogachev National Research Center of Pediatric Hematology, Oncology and Immunology, Moscow, Russian Federation

Background: Rare bleeding disorders are a heterogeneous group of hereditary diseases characterized by a quantitative or qualitative deficiency of blood coagulation factors[1-2]; Prevalence in the general population varying between 1 in 500000 for FVII deficiency and 1 in 2 million for FXIII, FII deficiencies[3-4]; In comparison with haemophilia epistaxis, umbilical cord bleeding occur more frequently, while hemarthrosis, muscle bleeding occur more rarely [1].

Aims: Analysis of clinical manifestations in children with various rare bleeding disorders observed in our centers in outpatient consultative unit and short-term Hospital Department in 2017 - 2019.

Methods: Retrospective analysis of medical records; 47 patients (018 y.o.) with rare bleeding disorders; Bleeding history was analyzed with Pediatric Bleeding Questionnaire;

Results: Analysis of bleedings: $40 \%$ of patients appeared asymptomatic; Most common bleedings: skin bleeding, epistaxis, tooth bleeding; High percent of joint bleeding - 7 patients; Most severe bleedings were seen at VKCFD, Afibrinogenemia and Factor VII deficiency.

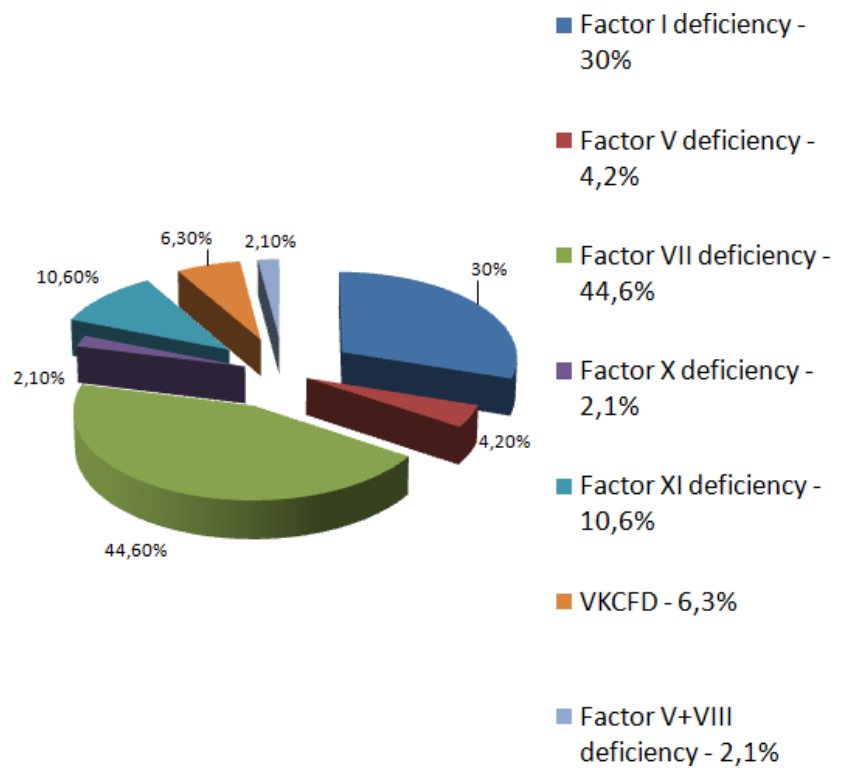

FIGURE 1 Distribution of rare bleeding disorders

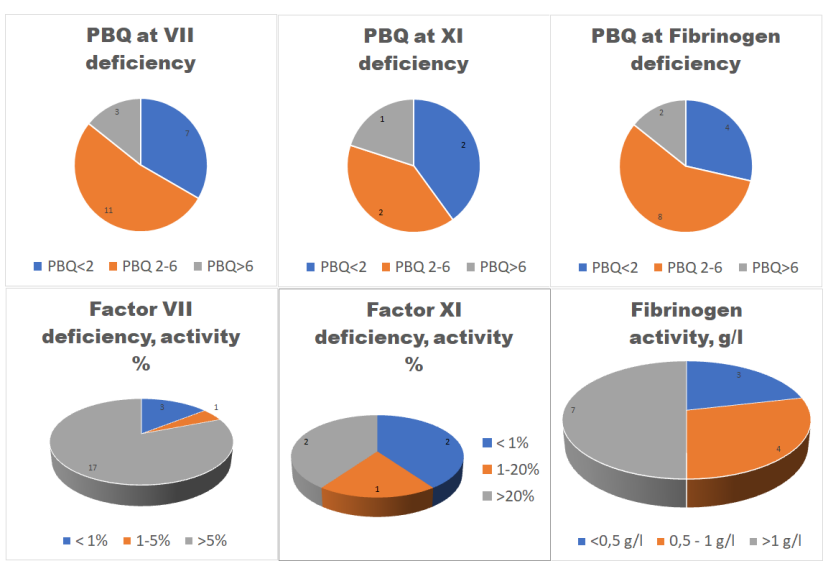

FIGURE 2 PBQ and activity by fibrinogen, Factor VII, Factor XI deficiencies

Conclusions: The prevalence of one or another rare bleeding disorder is connected to ethnic characteristics of the population and is not always proportional to the expected frequency; More data is required to establish the local characteristics of the spread of rare bleeding disorders and to develop adequate diagnostic criteria; References:

1. Mannucci PM, et al. Blood 2004;104:1243-1252.

2. Peyvandi F, et al. Blood Transfus. 2008;6(Suppl. 2):s45-s48.

3. Shapiro AD, et al. Am J Prev Med. 2011;41(6 Suppl. 4):S324-331. Kearon C, et al. Chest. 2012 ;141(2 Suppl):e419S-96S.

4. Peyvandi F, et al. J Thromb Haemost. 2012;10(4):615-621.

PB0710 | Hemorrhagic Adventitious Bursitis of the Foot in a Patient with Congenital Type 3 von Willebrand Disease

R. Gualtierotti ${ }^{1}$; S.M. Stella ${ }^{2}$; E. Biguzzi ${ }^{3}$; S.M. Siboni ${ }^{3}$; S. Arcudi ${ }^{1}$; L.P. Solimeno ${ }^{3}$; F. Payvandi ${ }^{1}$

${ }^{1}$ Angelo Bianchi Bonomi Hemophilia and Thrombosis Center, Fondazione IRCCS Ca' Granda, Ospedale Maggiore Policlinico, Department of Pathophysiology and Transplantation, Università degli Studi di Milano, Milan, Italy; ${ }^{2}$ Advanced Musculoskeletal Ultrasound SIUMB School of Pisa, Department of Advanced Biomedical Sciences, School of Specialization in Sports Medicine, University of Pisa, Santa Chiara University Hospital, Pisa, Italy; ${ }^{3}$ Angelo Bianchi Bonomi Hemophilia and Thrombosis Center, Fondazione IRCCS Ca' Granda, Ospedale Maggiore Policlinico, Milan, Italy

Background: Musculoskeletal ultrasound (MSK-US) in patients with congenital bleeding disorders is a valid tool for the differential diagnosis of acute joint pain. MSK-US can also help identifying less frequent periarticular complications of bleeding disorders.

Aims: Hemorrhagic adventitious bursitis of a subcutaneous bursa over the II-III metatarsal heads was found in a 57-year-old patient with type 3 Von Willebrand disease (VWD) -basal FVIII plasma 1\%, VWF:Ag $<3 \%$ and VWF:RCo $<6 \%$ - and arthropathy of the ankles and left elbow, requiring prophylaxis with FVIII/VWF (Haemate-P) 
2000 UI twice weekly. Furthermore, she is affected by systemic lupus erythematosus for which she is treated chronically with a low daily dose of prednisone. She has normal BMI (19.5).

Methods: The patient referred to the Angelo Bianchi Bonomi Hemophilia and Thrombosis Center for pain in the forefoot after walking and jogging in the mountains. The MSK-US examination was performed with a Philips Affinity 50 ultrasound machine with a 5-12 MHz linear probe.

Results: The physical examination showed swelling and pain of the forefoot compatible with collapse of the distal transverse foot arch (DTFA) (Figure 1) confirmed with MSK-US examination of the dorsal and plantar forefoot. Figure 2 shows a longitudinal scan (A) and a transverse scan (B) of a subcutaneous hemorrhagic bursitis over the II and III metatarsophalangeal joints of the left foot. The patient was treated with Haemate-P 2000 UI every other day for a week with benefit.

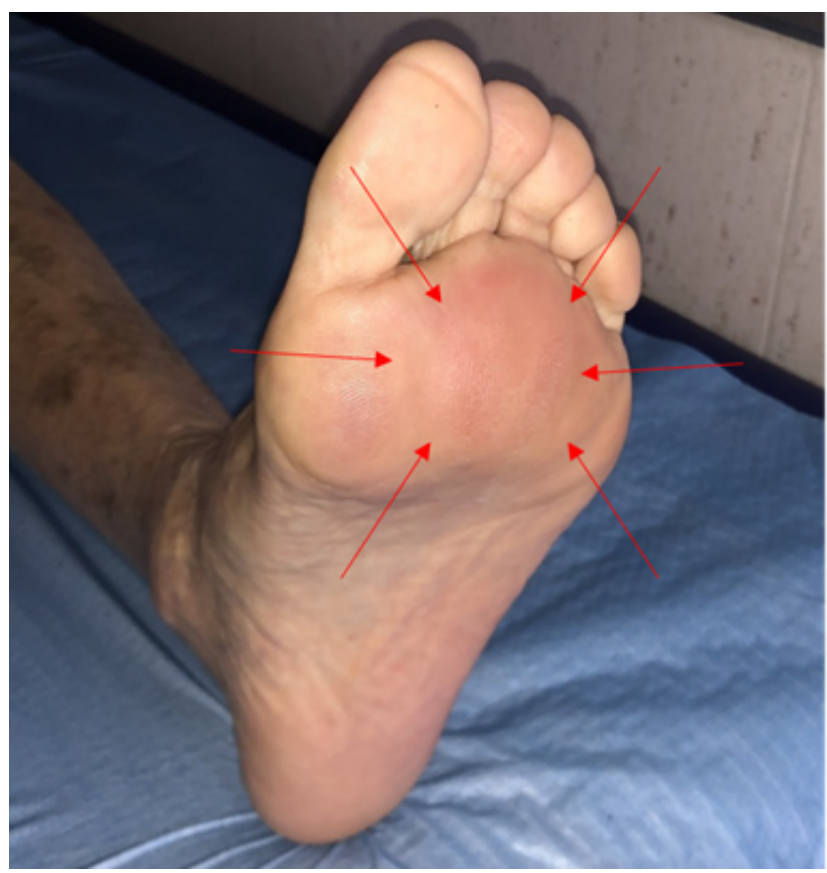

FIGURE 1 Plantar view of the left foot of the patient showing the swelling of the distal portion of the forefoot caused by collapse of the distal transverse foot arch (red arrows)

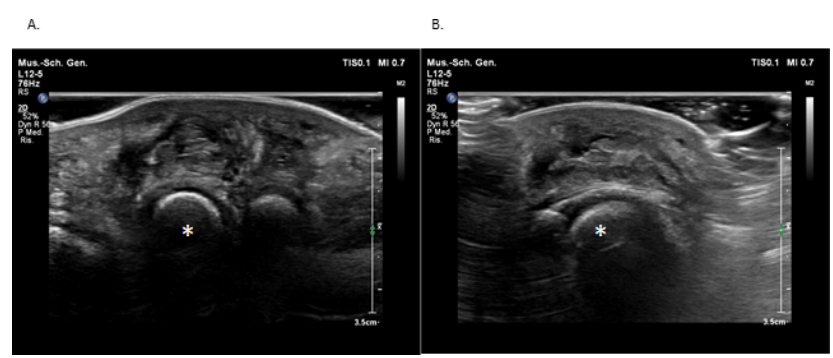

FIGURE 2 Longitudinal scan (A) and a transversal scan (B) of the subcutaneous bursa with dishomogeneous content compatible with hemorrhagic effusion, fibrin clots and synovial hyperplasia over the II metatarsophalangeal joint of the left foot. The II metatarsal head (asterisk) is visible
Conclusions: Serous bursae are normally present at the plantar side of the I and $V$ metatarsal bones (also known as Lenoir's bursae) in subjects with a normal DTFA. The subcutaneous bursa we described can be considered as the equivalent of these bursae formed as a consequence of collapse of the DTFA for repeated trauma eventually leading to bleeding and inflammation (hemorrhagic bursitis).

\section{PB0711 | Registry of Patients with Congenital Bleeding}

\section{Disorders from a Single Centre: Clinical and Analytical}

\section{Characteristics}

A. Lorenzo Jambrina; A. Torres Tienza; M. Mosquera Tapia; S. Marcellini Antonio; A. García Mateo; J.A. Queizán Hernández Hospital General de Segovia, Segovia, Spain

Background: Congenital congenital bleeding disorders (CBD) comprise a heterogeneous group of diseases that affect hemostasis, in patients with a family history of bleeding.

Aims: Describe clinical and analytical characteristics of the patients in our center with CBD.

Methods: We retrospectively reviewed all factor assays (II, V, VII, VIII, IX, X and XI) performed in our center from 02/09/2009 to 08/31/2020. 3911 assays performed, in 594 cases level was below the reference range. Subsequently, we review medical histories of each patient and hematology paper medical records.

Results:

TABLE 1 Clinical characteristics of the patients in our center with CBD

\begin{tabular}{|c|c|c|c|c|c|c|}
\hline Clinical variables & FV & FVII & FVIII & FIX & FX & FXI \\
\hline Patients & 15 & 51 & 12 & 18 & 15 & 59 \\
\hline Families & 2 & 4 & 2 & 4 & 3 & 8 \\
\hline $\begin{array}{l}\text { Age at diagnosis } \\
\text { (median) }\end{array}$ & 14 & 37 & 0 & 14 & 35 & 38 \\
\hline Cause of study & 1 & 0 & 0 & 3 & 0 & 1 \\
\hline - Bleeding history & 4 & 7 & 7 & 10 & 6 & 10 \\
\hline - Family study & 9 & 44 & 3 & 3 & 9 & 48 \\
\hline - Prolonged aTTP/PT & 1 & 0 & 2 & 2 & 0 & 0 \\
\hline \multicolumn{7}{|l|}{ - Unknown } \\
\hline ISTH BAT score $\geq 1$ & 4 & 6 & 7 & 5 & 1 & 3 \\
\hline Spontaneous bleeding & 2 & 6 & 7 & 5 & 1 & 3 \\
\hline Surgeries & 10 & 42 & 3 & 9 & 6 & 46 \\
\hline $\begin{array}{r}\text { Perioperative } \\
\text { treatment }\end{array}$ & 4 & 15 & 3 & 4 & 2 & 16 \\
\hline $\begin{array}{c}\text { Postoperative } \\
\text { bleeding }\end{array}$ & 2 & 0 & 0 & 0 & 0 & 2 \\
\hline
\end{tabular}


TABLE 2 Analytical variables of the patients in our center with CBD

\begin{tabular}{|c|c|c|c|c|c|c|}
\hline Analytical variables & FV & FVII & FVIII & FIX & FX & FXI \\
\hline Patients & 15 & 51 & 12 & 18 & 15 & 59 \\
\hline Median factor level (\%) & 49 & 41 & - & - & 48 & 43 \\
\hline Factor range (\%) & $28-59$ & $13-57$ & $0-43$ & $0-55$ & $36-57$ & $1-58$ \\
\hline Confirmation deficiency & 8 & 32 & 10 & 13 & 4 & 23 \\
\hline Thrombopenia $(<100.000 / \mathrm{mm} 3)$ & 1 & 2 & 0 & 0 & 0 & 0 \\
\hline Thrombocytosis (> 450.000/mm3) & 0 & 0 & 0 & 0 & 0 & 0 \\
\hline Prothrombin activity (<70\%) & 10 & 33 & 0 & 0 & 8 & 1 \\
\hline PT (>15 s) & 13 & 47 & 0 & 0 & 9 & 2 \\
\hline aPTT (> $36 \mathrm{~s})$ & 13 & 1 & 11 & 18 & 4 & 54 \\
\hline
\end{tabular}

We found 168 patients with factor deficiencies. Patients with von Willebrand Disease and thrombopathies were excluded. Tables 1 and 2 shows the clinical and analytical characteristics of our patients. 7 patients had combined factor deficiencies. Most frequent disorder was $\mathrm{FXI}(35 \%), \mathrm{FV}(55 \%)$ and $\mathrm{FX}(47 \%)$ deficiencies. Only one patient was diagnosed with dysprothrombinemia, no patient factor FXIII deficiency. Median factor levels (excluding hemophiliac patients) was $45 \%$, the range of factor levels was highly variable. 3 patients presented thrombopenia. Factor deficiency was confirmed in 90 patients with a second assay test. 23 families were identified. Median age range at diagnosis was O(hemophilia A)-38(FXI), $55 \%$ were male and $45 \%$ female. Most frequent cause of study was alterations in coagulation tests. 28 patients had ISTH BAT $\geq 15$ patients had severe bleeding. 9 patients are receiving prophylaxis. 118 surgeries were performed, in 44 cases patients received treatment (factor, FFP, desmopressin) and there was no bleeding. In the other group 3 patients had severe bleeding, but not fatal.

Conclusions: Our data reproduce clinical and analytical characteristics of the registries of patients with CBD previously reported.

In our series there is probably a familial underdiagnosis. Family history is essential and greater awareness is necessary.

Analysis of registry of patients with CBD from each center is essential to improve the quality of care for our patients.
PB0712 | Quality of Life of People with Hereditary Factor XIII Deficiency Treated at a Reference Centre

A. Drumond ${ }^{1} ;$ R. Camelo ${ }^{2}$

${ }^{1}$ HEMOMINAS Foundation, Belo Horizonte, Brazil; ${ }^{2}$ Faculty of

Medicine, Universidade Federal de Minas Gerais, Belo Horizonte, Brazil

Background: Hereditary factor XIII deficiency (FXIIID) is a rare recessive autosomal disease. Accurate diagnosis and early start of FXIII prophylaxis are key to prevent abnormal bleedings.

Aims: To describe the effectiveness and the safety of FXIII prophylaxis, and the health-related quality of life (EuroQol-5 Dimension or EQ-5D) of people with FXIIID (PwFXIIID) on prophylaxis treated at HEMOMINAS, Belo Horizonte/Brazil.

Methods: PwFXIIID on FXIII prophylaxis were invited. Clinical data were reviewed using a standardized file. Subjects self-administered the EQ-5D, at study entry.

Results: Of the 11 PwFXIIID registered, 9 (82\%) signed the Consent Form (Table 1). Prophylactic regimens ranged from 9.1 to $18.8 \mathrm{IU} /$ kg monthly. Since the start of prophylaxis, 5/9 and 3/9 patients reported spontaneous or post-traumatic bleeding which required FXIII infusion, respectively. No arterial/venous thrombotic events were reported during the prophylaxis period.

TABLE 1 Characteristics of people with hereditary factor XIII deficiency. (PDM, pregnancies, deliveries, and miscarriages ---, missing; N/A, not applied)

\begin{tabular}{|c|c|c|c|c|c|c|c|c|c|}
\hline Variable\Patient\# & \#1 & \#2 & \#3 & $\# 4$ & \#5 & \#6 & \#7 & \#8 & \#9 \\
\hline $\begin{array}{l}\text { Age at study entry } \\
\quad \text { (year) }\end{array}$ & 43 & 23 & 23 & 19 & 50 & 14 & 28 & 27 & 30 \\
\hline $\begin{array}{l}\text { Parents } \\
\text { consanguinity }\end{array}$ & no & no & yes & --- & no & yes & yes & yes & yes \\
\hline Sex & female & male & female & female & male & female & female & male & male \\
\hline $\mathrm{PD}$ (mode) $\mathrm{M}$ & $\begin{array}{l}\text { P2D2(caesarean) } \\
\text { M0 }\end{array}$ & N/A & PODOMO & PODOMO & N/A & PODOMO & $\begin{array}{l}\text { P1D1(caesarean) } \\
\text { M0 }\end{array}$ & N/A & $\mathrm{N} / \mathrm{A}$ \\
\hline $\begin{array}{l}\text { Age at diagnosis } \\
\quad \text { (year) }\end{array}$ & 32 & 4 & 2 & 7 & 48 & 13 & 6 & 1 & 2 \\
\hline $\begin{array}{l}\text { Age at start of } \\
\text { prophylaxis } \\
\text { (year) }\end{array}$ & 33 & 20 & 2 & 16 & 48 & 13 & 23 & 3 & 15 \\
\hline
\end{tabular}




\begin{tabular}{|c|c|c|c|c|c|c|c|c|c|}
\hline \multicolumn{9}{|l|}{ ABSTRACT } & 533 of 972 \\
\hline Variable\Patient\# & $\# 1$ & \#2 & $\# 3$ & $\# 4$ & $\# 5$ & \#6 & $\# 7$ & $\# 8$ & $\# 9$ \\
\hline $\begin{array}{l}\text { Treated } \\
\text { spontaneous } \\
\text { bleedings } \\
\text { during } \\
\text { prophyaxis }\end{array}$ & 3 & 1 & 4 & 0 & 4 & 0 & 2 & 0 & 0 \\
\hline $\begin{array}{l}\text { Treated post- } \\
\text { traumatic } \\
\text { bleedings } \\
\text { during } \\
\text { prophylaxis }\end{array}$ & 1 & 0 & 0 & 0 & 0 & 2 & 1 & 0 & 0 \\
\hline
\end{tabular}

Only 3/9 patients reported both "no problems" in the 5 dimensions of EQ-5D and high levels of QoL in the EQ-VAS (Table 2). One patient reported "some problem" or "extreme problem" in all EQ-5D dimensions, and a low level of EQ-VAS. While "self-care" was usually considered as "no problem" by most patients (8/9), "pain/discomfort" and "anxiety/depression" were reported as problematic by almost half of them.

TABLE 2 EQ-5D of people with hereditary factor XIII deficiency. (EQ-5DC), EuroQoL quality of life questionnaire with 5 dimensions; VAS, visual analogue scale)

\begin{tabular}{|c|c|c|c|c|c|c|c|c|c|}
\hline \multicolumn{10}{|l|}{ Quality of life \ } \\
\hline Patient\# & \#1 & \#2 & \#3 & \#4 & \#5 & \#6 & \#7 & \#8 & \#9 \\
\hline Mobility & $\begin{array}{l}\text { some } \\
\text { problem }\end{array}$ & no problem & no problem & no problem & no problem & no problem & no problem & no problem & $\begin{array}{l}\text { some } \\
\text { problem }\end{array}$ \\
\hline Self-care & $\begin{array}{l}\text { some } \\
\text { problem }\end{array}$ & no problem & no problem & no problem & no problem & no problem & no problem & no problem & no problem \\
\hline Usual activities & $\begin{array}{l}\text { some } \\
\text { problem }\end{array}$ & no problem & $\begin{array}{l}\text { some } \\
\text { problem }\end{array}$ & no problem & no problem & no problem & no problem & no problem & $\begin{array}{l}\text { some } \\
\text { problem }\end{array}$ \\
\hline Pain/disconfort & $\begin{array}{l}\text { extreme } \\
\text { problem }\end{array}$ & no problem & $\begin{array}{l}\text { some } \\
\text { problem }\end{array}$ & $\begin{array}{l}\text { some } \\
\text { problem }\end{array}$ & no problem & no problem & no problem & no problem & $\begin{array}{l}\text { some } \\
\text { problem }\end{array}$ \\
\hline $\begin{array}{l}\text { Anxiety/ } \\
\text { depression }\end{array}$ & $\begin{array}{l}\text { extreme } \\
\text { problem }\end{array}$ & no problem & $\begin{array}{l}\text { some } \\
\text { problem }\end{array}$ & no problem & $\begin{array}{l}\text { some } \\
\text { problem }\end{array}$ & $\begin{array}{l}\text { extreme } \\
\text { problem }\end{array}$ & no problem & no problem & no problem \\
\hline VAS & 10 & 80 & 90 & 90 & 80 & 90 & 97 & 90 & 80 \\
\hline
\end{tabular}

Conclusions: To our knowledge, this is the first report about EQ-5D evaluation among PwFXIIID on prophylaxis, which is the standard treatment with a good safety profile. In general, QoL was well perceived, although some patients reported severe symptoms in few or all domains. Unfortunately, we could not find a cause of this profile, since clinical-outcome characteristics were too heterogenous. We are elaborating future registries involving patients from other Brazilian states, to better clarify both treatment characteristics and QoL of PwFXIIID. We believe QoL should be formally evaluated in PwFXIIID, and the multiprofessional team should try to help him/her to overcome the reported troubles.
PB0713 | Impact of Inherited Bleeding Disorders on Pregnancy and Postpartum Hemorrhage

\section{N. Fernández Mosteirín; J.M. Calvo Villas}

Haematology and Haemotherapy Department. Universitary Hospital Miguel Servet, Zaragoza, Spain

Background: Women with inherited bleeding disorders (IBD) are at risk of bleeding complications associated with pregnancy.

Aims: To evaluate the incidence of bleeding complications and obstetric outcomes in women with IBD.

Methods: Retrospective medical records review of the pregnant women with IBD from 2005-2020 in our hospital. Demographic, clinical and therapeutic features were registered.

Results: 39 pregnancies among 25 patients were included. Table 1 shows factor deficiency type and number of pregnancies. Median age at pregnancy: 38 years (range: $33-47$ ), median age at diagnosis: 30 years (range: $2-41$ ). Diagnosis of IBD during or after pregnancy in 3 patients (12\%). Six patients (24\%) reported history of bleeding (all showed heavy menstrual bleeding), only one patient (2A VWD) showed a positive ISTH-BAT score. 9 (23.1\%) previous miscarriages 
were observed in 7 patients. Delivery plan was available in 38 pregnancies. Regarding delivery: 31 vaginal ( 2 instrumented), 7 cesarean section and 1 therapeutic abortion (fetal malformation). Regional block was performed in 31 pregnancies without reported complications. Regarding factor level 9 patients reached normal levels in 12 pregnancies. Hemostatic treatment was administered before delivery and/or regional block in 12 pregnancies (Table 2). Postpartum antifibrinolytic treatment lasts 3-5 days in 6 patients and 4 weeks in the GT patient. No thromboembolic complications were observed. Three patients (7.7\%) developed PPH: 2 primary (FVII deficiency and SHB carrier) and 1 secondary (2A VWD). No significantly differences between age and risk of PPH were observed and TXA didn't significantly reduce the risk of secondary PPH $(P=0.303)$. No bleeding complications were observed among newborns.

\section{TABLE 1}

Table 1.

\begin{tabular}{|lcccc|}
\hline IBD type & Ne patients (8) & Ne Pregnancies (8) \\
\hline SHA carrier & 3 & $12 \%$ & 5 & $12.8 \%$ \\
\hline MHA carrier & 4 & $16 \%$ & 7 & $18 \%$ \\
\hline SHB carrier & 4 & $16 \%$ & 5 & $12.8 \%$ \\
\hline WWD & 3 & $12 \%$ & 5 & $12.8 \%$ \\
\hline FV deficiency & 1 & $4 \%$ & 2 & $5.1 \%$ \\
\hline FVII deficiency & 5 & $20 \%$ & 8 & $20.5 \%$ \\
\hline FX deficiency & 1 & $4 \%$ & 1 & $2.6 \%$ \\
\hline FXI deficiency & 3 & $12 \%$ & 5 & $12.8 \%$ \\
\hline GT & 1 & $4 \%$ & 1 & $2.6 \%$ \\
\hline \multicolumn{1}{c}{ Total } & & 25 & \multicolumn{3}{c}{39} \\
\hline
\end{tabular}

SHA: Severe Haemophilia A, MHA: Mild Haemophilia A, SHB: Severe Haemophilia B, GT: Glanzmann Thrombastenia

TABLE 2 Hemostatic treatment

Table 2. Hemostatic treatment

\begin{tabular}{|c|c|c|c|}
\hline Type of IBD & Bleeding history & Factor level (\%) & Treatment \\
\hline SHB carrier & No & FIX $39.6 \%$ & Recombinant FIX \\
\hline Type 2A VWD & $\begin{array}{c}\text { Yes } \\
\text { (HMB, epistaxis and upper } \\
\text { gastrointestinal bleeding) }\end{array}$ & $\begin{array}{c}\text { VWF:Ag 138\% } \\
\text { VWF:RCo 17.6\% } \\
\text { FVIII } 99.2 \%\end{array}$ & pdFVIII/VWF + TXA \\
\hline Type 2A VWD & $\begin{array}{c}\text { Yes } \\
\text { (HMB, epistaxis and upper } \\
\text { gastrointestinal bleeding) }\end{array}$ & $\begin{array}{c}\text { VWF:Ag } 94.6 \% \\
\text { VWF:RCo } 34.3 \% \\
\text { FVIII } 88.6 \%\end{array}$ & pdFVIII/VWF + TXA \\
\hline Type 1 VWD & No & $\begin{array}{c}\text { VWF:Ag } 60.4 \% \\
\text { VWF:RCo } 36.9 \% \\
\text { FVIIII } 104.8 \%\end{array}$ & pdFVIII/VWF + TXA \\
\hline GT & $\begin{array}{c}\text { Yes } \\
\text { (HMB and upper } \\
\text { gastrointestinal bleeding) }\end{array}$ & - & rFVlla + TXA \\
\hline Mild FV deficency & No & $36.6 \%$ & FFP \\
\hline Mild FV deficency & No & $45.5 \%$ & FFP \\
\hline FXI deficiency & No & $2 \%$ & $F F P+T X A$ \\
\hline FXI deficiency & No & $2 \%$ & $F F P+T X A$ \\
\hline FXI deficiency & No & $3 \%$ & $F F P+T X A$ \\
\hline FXI deficiency & No & $36.4 \%$ & TXA \\
\hline FXI deficiency & No & $46.4 \%$ & TXA \\
\hline
\end{tabular}

FFP: Fresh frozen plasma, HMB: heavy menstrual bleeding, pdFVIII/VWF: plasma derived FVII/VWF, rFVIII: activated recombinant FVII, TXA: tranexamic acid
Conclusions: Risk of PPH is increased in women with IBD. A multidisciplinary team approach with individualized delivery plan including recommended mode of delivery, methods of pain relief, prophylactic hemostatic treatment when it is required and need of antifibrinolytic therapy to prevent maternal and newborn bleeding complications, can minimize these risks.

PB0714 | Successful Prophylactic Use of Coagulation Factor X in an Infant Patient in the Home Environment

C. Miller; Y. Lantz; B. Prosser; T. Walton

Soleo Health, Frisco, United States

Background: Factor $\mathrm{X}$ deficiency is a rare bleeding disorder that requires long-term management. The female infant discussed within this case study, Patient $A$, was diagnosed with severe factor $X$ deficiency two days after birth. Therapy with prothrombin complex concentrate (PCC) was initiated until factor $X$ concentrate was approved by the insurance provider. After initially receiving twice weekly factor $X$ concentrate infusions at her local pediatric Hemophilia Treatment Center (HTC) for prophylaxis of bleeding episodes, infusion therapy was transitioned to the home environment at one-month old.

Aims: The purpose of this case study is to highlight the successful long-term, effective use of factor $X$ concentrate in a female infant patient in the home environment.

Methods: A retrospective review of medical records was performed by a multidisciplinary team. The review included customized clinical assessments specific to bleeding disorders that were performed by the specialty infusion pharmacy, as well as progress notes, nursing visit assessments, and hospital and physician office visit notes from June 9, 2017 through January 23, 2020.

Results: During the 30 months that Patient $A$ received factor $X$ concentrate infusions in the home environment, she experienced zero bleeding episodes and zero adverse reactions. The family became independent with twice weekly infusions within two months of initiating services through the specialty infusion pharmacy. Several catheter events, such as occlusions and breakages, did occur; however, no events were related to catheter infections. Patient A's underlying factor $X$ deficiency did cause her to suffer a brain injury either during or before birth, but physical limitations were resolved with physical and occupational therapy.

Conclusions: Factor $\mathrm{X}$ concentrate is an effective targeted therapy for factor $X$ deficiency that is well-tolerated. With proper use and frequent monitoring through customized clinical assessments, factor $X$ deficiency can be successfully managed in the home environment at any age. 
PB0715 | Safe and Effective Prophylaxis with Plasma-derived Factor X Concentrate in an Elderly Patient with Hereditary Factor $X$ Deficiency

M. Reding ${ }^{1}$; S. Peltier ${ }^{1} ;$ K. Clark ${ }^{2}$

${ }^{1}$ University of Minnesota Medical Center / Center for Bleeding and Clotting Disorders, Minneapolis, United States; ${ }^{2}$ Bio Products Laboratory, Durham, United States

Background: Coagulation factor $X$, human is a plasma-derived factor $X$ concentrate indicated to treat and prevent bleeding in individuals with hereditary factor $X$ deficiency (HFXD). The ages of patients in whom data was collected in the initial registration trials and postmarketing studies ranged from 0-58 years. To date, there have been no reports on treatment of elderly ( $>65$ years) patients with plasmaderived factor $\mathrm{X}(\mathrm{pdFX})$ concentrate.

Aims: To report the experience with pdFX concentrate in the prophylactic treatment of HFXD in a patient $>65$ years.

Methods: Data was collected on a 71-year-old female with HFXD receiving prophylactic treatment with pdFX.

Results: Although suspected to have some type of bleeding disorder since age 2 , this individual was not diagnosed with HFXD until age 20. Initial treatment included fresh frozen plasma on-demand for acute bleeding episodes. Subsequent treatment included threefactor prothrombin complex concentrate (PCC), starting in the 1990s. Prophylactic treatment was started at age 57 after she developed a spontaneous subdural hematoma. She was transitioned from PCC to pdFX prophylaxis in November 2019 to more directly address her factor $X$ deficiency and to avoid increasing other clotting factor levels above the normal range. She is currently receiving pdFX 21 units/kg weekly, which has been well tolerated and she has experienced no bleeding issues since starting this therapy.

Conclusions: This is the first reported experience of prophylactic treatment of an elderly patient with pdFX. Additional details of this case including laboratory data will be presented. The data supports the successful prophylactic treatment of elderly patients and the need to continue to collect data in this population of patients with this rare bleeding disorder.

PB0716 | Congenital Factor Vii Deficiency and Vacterl Association: An Unusual Combination

A. Ramirez; C. Lince-Rivera; L. Florez; A. Infante; Y. Vargas

Pontifical Xavierian University, Bogotá, Colombia

Background: Congenital factor VII deficiency is an autosomal recessive rare bleeding disorder, affecting 1 in every 500,000 people. It has never been reported to be combined with VACTERL association. Aims: Case report of a newborn with congenital factor VII deficiency and congenital malformations consistent with VACTERL association. Methods: The authors present a case report and a literature review of the topic.
Results: A preterm male neonate is delivered at 35 weeks of gestation, non-consanguineous parents, an antenatal diagnosis of severe polyhydramnios and duodenal atresia. At birth, with low weight, anterior anus and hypospadias. He was scheduled for surgical correction for duodenal atresia and anorectal malformation, coagulation tests revealed prolonged prothrombin time, he received two doses of vitamin $\mathrm{K}$ with persistence of prolongation. Studies confirmed moderate factor VII deficiency. He received activated recombinant factor VII ( $\mathrm{rFVIla}$ ) (30 mcg/kg) from the preoperative period, continuing every 4 hours for 48 hours. There were no bleeding complications in the postoperative care or during follow-up care. In genetics consult evaluation, VACTERL association was confirmed.

Conclusions: Factor VII deficiency is a rare bleeding disorder, with clinical heterogeneity and different degrees of severity, requiring high clinical suspicion as well as adequate interpretation of coagulation tests. The evidence for rFVIla in newborns with congenital FVII deficiency is very limited, therefore, the most important predictive tool is family and personal history of bleeding. Prophylaxis with recombinant factor VII is not commonly used and it should only be considered in patients at high risk of bleeding or severe bleeding. No other case reports or associations were found between clotting factor deficiencies, such as factor VII deficiency and congenital malformations like VACTERL association.

PB0717 | Clinical Profile and Bleeding Outcome of Pediatric Rare Bleeding Disorders: The Royal Hospital's Experience, Oman

M. AL Saadi ${ }^{1}$; H. AL Hasani ${ }^{1}$; A. Al Roshdi ${ }^{1}$; A. Al Amri ${ }^{1}$;

I. Al Ghaithi ${ }^{2}$

${ }^{1}$ Royal Hospital, Muscat, Oman; ${ }^{2}$ Royal Hospital, Consultant, Pediatric Hematology/Oncology, Muscat, Oman

Background: Rare bleeding disorders are group of inherited deficiencies of coagulation factors including, fibrinogen, factor II, FV, FVII, FX, FXI, and FXIII deficiency. They are largely inherited by autosomal recessive genetics and more common in areas with a high rate of consanguineous marriages.

Aims: To study the bleeding profile and outcome for Omani pediatric patients with Rare Bleeding Disorders in the period between 2010-2020.

Methods: This is a retrospective descriptive cohort study that includes all Omani children, less than 13 years of

age, who are diagnosed with rare bleeding disorders at Royal Hospital.

Patient's data was retrieved from their electronic chart system (AIShifa). Statistical analysis was performed using SPSS 24.0

Results: Forty one patients were included, with a mean age of 4 years (range between 1 day to 12 years) at presentation. There were 24 (58.5\%) males. The most common presenting complaint was mucocutaneous bleeding (31.7\%), however, 22 (53.7\%) patients were diagnosed pre- operation. Based on factor level at diagnosis, 34 (83\%) patients have factor VII deficiency, 4 (9.7\%) patients have 
factor XIII deficiency, 1 (2.4\%) patient with fibrinogen deficiency, and 1 patient (2.4\%) has factor $X$ deficiency. In term of treatment, only 4 (9.4\%) patients required blood transfusion for active bleeding, 15 (36.6\%) patients were treated on demand with tranexamic acid, and $3(7.3 \%)$ patients were on regular prophylaxis with tranexamic acid. The mean period of follow up was 3.15 years. The majority (87.8\%) of patients had no active bleeding, and only 2 (4.9\%) patients developed severe bleeding. The mean ABR outcome was 0.2, 0.5 and 7 for patients with FVII, FXIII and FX respectively.

Conclusions: Omani children with rare bleeding disorders are commonly diagnosed incidentally. They are usually do not require active treatment because their bleeding outcome is apparently favourable.

PB0718 | Familial Multiple Coagulation Factor Deficiencies (FMCFDs); A Rare Case of Combined Deficiency of Factor V and Factor VIII (F5F8D)

\section{J. Rigano}

Alfred Health, Melbourne, Australia

Background: FMCFDs are characterised by the presence of more than one coagulation factor deficiency arising from a genetic defect or defects and transmissible as a familial trait. The three subgroups of disorders include FMCFDs arising from: (i) co-inherited single coagulation factor deficiencies (ii) a single genetic defect (iii) cytogenetic abnormalities. F5F8D is one of the most common FMCFDs caused by mutations in either the LMAN1 or MCFD2 genes responsible for the transportation of FV and FVIII from the ER to the Golgi for post-translational modification and secretion into the circulation. Aims: To describe a case of F5F8D in a child that was not initially diagnosed at presentation of bleeding.

Methods: A 3-year-old girl requiring treatment for post-operative infection and debridement presented with intermittent bleeding. She had recently required sutures from a lower lip bleed caused by a fall and previously had an upper lip injury which bled for three days. The initial PT and APTT were markedly prolonged. IV vitamin $\mathrm{K}$ was administered with slight improvement in APTT only She received Prothrombinex ${ }^{\circledR}$ with slight improvement in PT and normalised APTT. NovoSeven ${ }^{\circledR}$ was given to cease bleeding prior to discharge. Two weeks later the PT and APTT were consistently markedly prolonged.

Results: Initial PT and APTT suggested common pathway factor deficiency hence vitamin $\mathrm{K}$ administration. PT and APTT mixing studies corrected immediately and after 2 hours incubation at $37^{\circ} \mathrm{C}$ excluding an inhibitor. Factor assays revealed deficient levels of FV and FVIII explaining the ineffective response of vitamin $\mathrm{K}$ and Prothrombinex ${ }^{\circledR}$. Repeat analysis confirmed FV and FVIII deficiencies. Patients present with prolonged bleeding following trauma or surgery. Bleeding episodes are treated on demand with DDAVP, FVIII concentrates and FFP.
Conclusions: Here reported was the detection of F5F8D in a child who presented with bleeding following trauma and prolonged PT and APTT.

\section{PB0719 | Inherited Factor XIII Deficiency: A Mild and a Severe} Case Reports

A.C. Gusmão ${ }^{1}$; N.N.S. Magalhães ${ }^{1}$; R.D.M. Almeida ${ }^{1}$; A.C.A. Santos $^{2}$; T.S. Espósito ${ }^{2}$; L.O.W. Rodrigues ${ }^{3}$; O.F. Santos ${ }^{4}$; J.A.S. Lopes $^{4}$; D.O.W. Rodrigues ${ }^{5,2}$

${ }^{1}$ Faculdade de Ciências Médicas e da Saúde de Juiz de Fora, Juiz de Fora, Brazil; ${ }^{2}$ Centro Universitário Presidente Antônio Carlos - FAME, Juiz de Fora, Brazil; ${ }^{3}$ Carleton University, Ottawa, Canada; ${ }^{4}$ Universidade Federal de Juiz de Fora, Juiz de Fora, Brazil; ${ }^{5}$ Fundação Hemominas, Juiz de Fora, Brazil

Background: Inherited Factor XIII (FXIII) Deficiency is a rare autosomal recessive disorder of hemostasis, with estimated incidence of one per two million people and higher prevalence in consanguineous marriage. Possible clinical manifestations include: intracranial hemorrhage, umbilical cord bleeding at birth, hematoma, miscarriages and menorrhagia.

Aims: To present two different clinical cases with FXIII Deficiency. Methods: Case 1: A 32-year-old man was referred to a hematology service with the main complaint of coagulation disturb. The patient reported splenectomy post trauma and denied major bleeding after tooth extraction. In September 2020, the patient had extensive spontaneous hematoma on the right thigh when he received a transfusion of cryoprecipitate, despite normal coagulation tests. He has family's history for bleeding disorder.

Case 2: A 35 year-old female was referred to hematological investigation due to a bleeding disorder. The patient reported increased umbilical cord bleeding at birth, which required blood transfusion. After a traumatic brain injury in a mild car accident in 2012, the patient evolved with intracranial bleeding, with platelet unities transfusion. Nevertheless, the laboratory screening tests, showed normal results for coagulation disorders. Family history was negative for coagulation disorder

Results: Both patients were O positive blood type. The diagnostic laboratory tests are described in Table 1. It was still performed complete factor dosages (Factors X, XI, XII), research on inhibitors, whole blood count and lupus anticoagulant, with all the results within the normal range. In Case 2, the radiographic image is illustrated in Figure 1. The Case 1 patient is under control and has regular follow ups, while the Case 2 patient receives monthly prophylactic treatment with FXIII. 
TABLE 1 Laboratory Tests Results - Case 1 and Case 2. Source: The authors

\begin{tabular}{|c|c|c|c|}
\hline Laboratory Tests & Case 1 & Case 2 & $\begin{array}{l}\text { Reference } \\
\text { Values }\end{array}$ \\
\hline $\begin{array}{l}\text { Activated Partial } \\
\text { Thromboplastin Time } \\
\text { (aPTT) }\end{array}$ & $28 "$ & $34 "$ & $28 "-38 "$ \\
\hline Prothrombin Activity (PA) & $96 \%$ & $100 \%$ & $100 \%$ \\
\hline $\begin{array}{l}\text { Platelets Count, Platelet } \\
\text { Aggregation Curve (ADP, } \\
\text { Ristocetin, ADR, collagen) }\end{array}$ & Normal & Normal & Normal \\
\hline Fibrinogen & $\begin{array}{c}450 \mathrm{mg} / \\
\mathrm{dL}\end{array}$ & $280 \mathrm{mg} / \mathrm{dL}$ & $\begin{array}{r}175-450 \\
\mathrm{mg} / \mathrm{dL}\end{array}$ \\
\hline FVIII coagulation assay & $128.80 \%$ & $100 \%$ & $50-150 \%$ \\
\hline FIX coagulation assay & $115.80 \%$ & $120 \%$ & $50-130 \%$ \\
\hline FXIII coagulation assay & $12.70 \%$ & $<1 \%$ & $70-140 \%$ \\
\hline $\begin{array}{l}\text { Von Willebrand Factor and } \\
\text { Ristocetin Cofactor }\end{array}$ & $\begin{array}{r}183 \% / \\
98 \%\end{array}$ & $63 \% / 112 \%$ & $60-240 \%$ \\
\hline
\end{tabular}

Subtitle: ADP: adenosine diphosphate, ADR: adrenalin

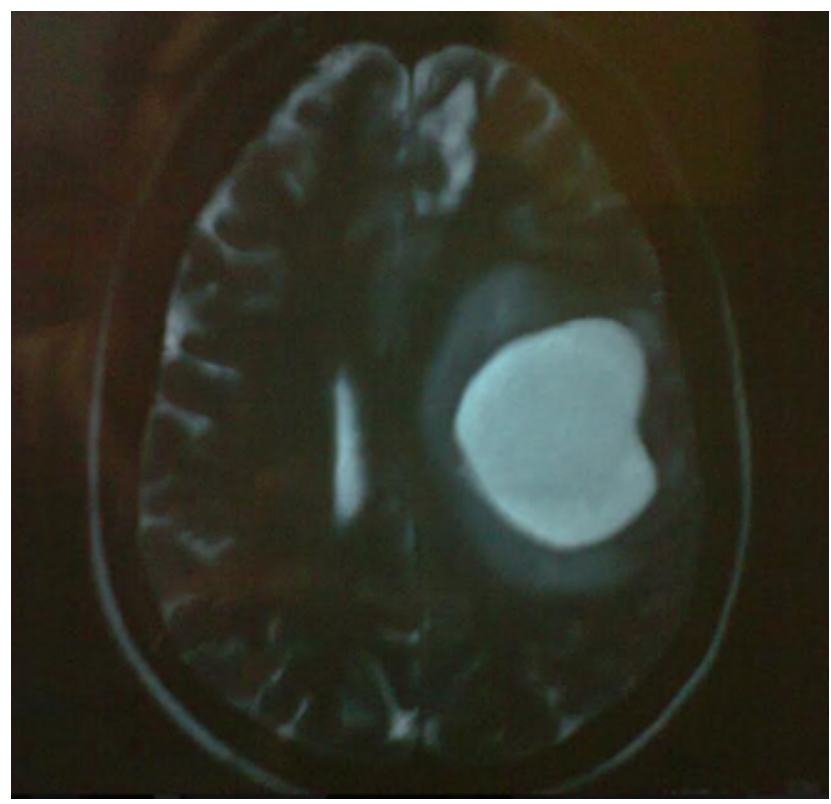

FIGURE 1 Brain Nuclear Magnetic Resonance. Subtitle: Subacute intraparenchymal hematoma measuring $5.5 \times 4.5 \mathrm{~cm}$ located in the subcortical white substance, promoting mass effect with erasure of the cortical grooves, partial collapse of the left lateral ventricle and deviation of the midline structures to right by $0.6 \mathrm{~cm}$

Conclusions: The diagnosis of FXIII Deficiency in patients with major bleeding should be considered if the coagulation tests are normal. Quantitative determination of FXIII activity, antigenic assays and molecular studies is required.
PB0720 | Epidemiology of Severe Rare Congenital Bleeding Disorders at a Bleeding Disorders Reference Center in Spain

M.F. Martinez Garcia ${ }^{1}$; O. Benitez Hidalgo ${ }^{1}$; I. Corrales Insa ${ }^{2}$;

S. Franco Palacios ${ }^{3}$; M. Fernandez Caballero ${ }^{4}$; M. Suito Alcantara ${ }^{5}$; V. Cortina Giner ${ }^{6}$; M. Gironella Mesa ${ }^{7}$; F. Bosch Albareda ${ }^{8}$

${ }^{1}$ Hemophilia Unit, Vall d'Hebron University Hospital, Barcelona, Spain; ${ }^{2}$ Banc de Sang i Teixits, Barcelona, Spain; ${ }^{3}$ Experimental Hematology, Vall d'Hebron Institute of Oncology (VHIO), Barcelona, Spain;

${ }^{4}$ Hematology Laboratory, ICO-Badalona, Hospital Germans Trias i Pujol, Josep Carreras Leukaemia Research Institute, Autonomous University of Barcelona, Badalona, Spain; ${ }^{5}$ Hemostasis Unit, Vall d'Hebron University Hospital, Barcelona, Spain; ${ }^{6}$ Hemostasis Laboratory, Hematology Department, Vall d'Hebron Hospital, Barcelona, Spain; ${ }^{7}$ Hematology Department, Vall d'Hebron Hospital, Barcelona, Spain; ${ }^{8} \mathrm{Head}$ of Hematology Department, Vall d'Hebron Hospital, Barcelona, Spain

Background: Rare bleeding disorders (RBD) constitute $5 \%$ of the total congenital bleeding disorders, although the number could be higher, due to the presence of undiagnosed asymptomatic patients.

Aims: The objective of this study was to analyze the prevalence and characteristics of patients with severe RBD, followed in our center. Methods: We collected data of the patients with RBD with factor levels $<20 \%$ or fibrinogen $<1 \mathrm{~g} / \mathrm{dL}$ visited in our center between January 2014 and December 2019.

Results: A total of 92 patients were analyzed. The median age was 24.4 years (range $0-89$ ), and $54 \%$ were male patients. The most frequent RBD in our population was the FVII deficiency (38\%), followed by the FXI deficiency and fibrinogen deficit with $25 \%$ and $15 \%$ respectively. Regarding the diagnostic reason, the most frequent cause was a preoperative analysis (46\%) and only $19.5 \%$ reported bleeding symptoms at the diagnosis and $25 \%$ presented at least one episode of spontaneous bleeding during the follow-up. The $63 \%$ had received replacement treatment mostly as prophylaxis prior to invasive procedures. Twenty-two percent of our population had a pathological ISTH-BAT score, and of these, $70 \%$ were female patients. A genetic study was carried out in $66 \%$ of patients finding a Missense mutation as the most frequent mutation in our series.

Conclusions: The distribution of RBD in our center is similar than reported in the literature. The majority of RBD have been diagnosed from a preoperative analysis and this has allowed a preparation prior to surgical interventions thus avoiding bleeding complications. Despite having levels classified as severe, most patients (83\%), do not have a pathological bleeding phenotype according to the ISTHBAT scale, and it is striking that most of the patients who have a pathological score are women (70\%). 
PB0721 | Thromboembolic Disease in Severe Factor V (FV) Deficiency: A Case Report: "Anticoagulating the Anticoagulable"

M. R. Lopez; E. Lopez Ansoar; A. Nieto Vazquez; C. Albo Lopez Hospital Alvaro Cunqueiro, Vigo, Spain

Background: Congenital FV deficiency is a bleeding disorder, with mild to severe hemorrhagic symptoms and low prevalence in population. Patients with significant symptoms show very low or unmeasurable plasma FV levels and are usually homozygous or compound heterozygous for mutations in FV gene. The only therapy is freshfrozen plasma (FFP). Thromboembolic events have not been described -to our knowledge- in these patients and no guidelines are published to guide the treatment, if it were possible

Aims: Report an unusual case of severe FV Deficiency with jugular vein thrombosis after port-a-Cath insertion and our management.

Methods: Female, 78 years, severe FV deficiency (FV:C 1-2\%): Multiple admissions for bleedings of variable severity. She was admitted with hematoma in left biceps femoris, starting FFP (10 ml / $\mathrm{Kg} / \mathrm{12}$; target trough level: 15-20\%); Given the poor venous accesses, on 4th day, we requested placement of central venous catheter (Port-a-Cath). 72 hours after, she presented swelling on right neck, erythema and pain. On suspicion of thrombosis, ECODoppler was performed and showed thrombosis of right internal jugular vein without involvement of catheter.

Results: We maintain FFP (trough level FV 15-20\%) and started anticoagulation: Enoxaparin ${ }^{\circledR}$, first dose $1 \mathrm{mg} / \mathrm{Kg}$ weight followed by $0.5 \mathrm{mg} / \mathrm{Kg} / 12$ hours (AntiXa level close to 0.5 units / ml, between therapeutic and prophylactic range and simultaneous empirical antibiotic therapy, 10 days, (not identifying germ in cultures). The treatment was carried out for 10 days, well tolerance, without adverse events. 3 months after discharge, ECOdoppler showed complete resolution of thrombus.

Conclusions: It's unusual venous thrombosis in severe FV deficiency and hemorrhagic phenotype, probably influenced by placement of central catheter, without being able to rule out associated infection, managed with minimal anticoagulation. Given the rarity, it's interesting to report it since it is difficult to generate any scientific evidence to establish management recommendations.
PB0722 | Safety and Efficacy of Fibrinogen Concentrate in Congenital Fibrinogen Deficiency: A Post-marketing Observational Study

B. Schwartz ${ }^{1}$; C. Solomon ${ }^{2}$; S. Knaub ${ }^{2}$; F. Peyvandi ${ }^{3,4}$

${ }^{1}$ Octapharma, Hoboken, United States; ${ }^{2}$ Octapharma AG, Lachen, Switzerland; ${ }^{3}$ Fondazione IRCCS Ca' Granda Ospedale Maggiore Policlinico, Angelo Bianchi Bonomi Hemophilia and Thrombosis Center and Fondazione Luigi Villa, Milan, Italy; ${ }^{4}$ Department of Pathophysiology and Transplantation, Università degli Studi di Milano, Milan, Italy

Background: Congenital fibrinogen deficiency (CFD) is a rare disease associated with severe and/or frequent bleeding episodes (BEs). Human fibrinogen concentrate (HFC) has been demonstrated to restore hemostasis in the event of such bleeding.

Aims: To collect real-world evidence from clinical practice concerning the safety and efficacy of HFC for treatment of acute BEs in CFD. Methods: FORMA-07 is a prospective, non-interventional, multicenter observational study in patients of any age with a documented diagnosis of congenital afibrinogenemia or hypofibrinogenemia expected to require on-demand BE treatment with HFC. The study will examine this purified, plasma-derived, double virus inactivated/eliminated, lyophilized HFC (Fibryga ${ }^{\circledR}$, Octapharma). This Octapharma-sponsored study will seek approval from independent ethics committees prior to study start. Freely given, written informed consent will be obtained from all patients at screening. Patients with other bleeding disorders, including acquired fibrinogen deficiency and dysfibrinogenemia; history of anti-fibrinogen inhibitors; or participating in concurrent clinical studies will be excluded. Study outline is shown in Figure 1.

FORMA-07 will investigate the use of fibrinogen concentrate in treatment of CFD, focusing on the safety profile, including serious adverse drug reactions (ADRs), and ADRs of special interest (i.e., incidence of thromboembolic ADRs and anaphylaxis). Hemostatic efficacy, assessed by the investigator using a 4-point objective scale within the first 2-24 hours following treatment, will also be assessed. The study aims to enroll a minimum of 25 patients treated with HFC over 7 years to describe 105 BEs (at least 10 major BEs in 10 patients). 


\section{Patients:}

Patients of any age expected to require treatment with a commercial HFC for a BE*

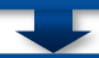

Treatment:

Patients treated according to current routine clinical practice

HFC individually dosed as recommended by locally

approved package insert to achieve recommended target

plasma fibrinogen level dependent on the severity of

bleeding (minor or major)

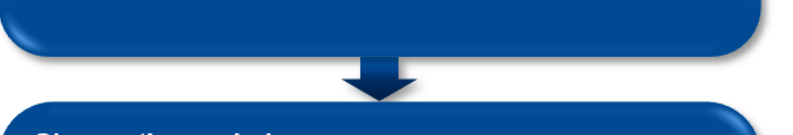

Observation period:

From the first dose of HFC administered, for up to 28 days after treatment

- Efficacy of HFC for achieving hemostasis assessed by the investigator within 2 to 24 hours following HFC administration

- Patients will be contacted 7 and 28 days after treatment in a telephone interview

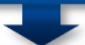

\section{Study completion:}

Study may be considered completed at the end of the observational period or when a minimum of 10 patients have at least one documented major BE

*Screening can be completed on the same day as treatment $\mathrm{BE}$, bleeding episode; HFC, human fibrinogen concentrate

\section{FIGURE 1 FORMA-07 Study outline}

Results: FORMA-07 has commenced and is currently enrolling. Completion is expected by Q4 2027. Results will be monitored on an ongoing basis.

Conclusions: Real-world evidence from routine clinical use will further assess the safety profile of HFC, supporting clinical study data on the use of this HFC for bleeding treatment in patients with congenital fibrinogen deficiency.

\section{PB0723 | Evaluation of FXI-deficiency Treatment in Pediatric} Patients: A Case-series

D. Muzafar Gani ${ }^{1,2} ;$ K. Strike ${ }^{3,4} ;$ K. Decker ${ }^{4}$; T. Almonte ${ }^{4}$; M.D Bhatt $^{4,5}$; D. Matino ${ }^{4,6}$; A.K. Chan ${ }^{4,5}$

${ }^{1}$ Royal College of Surgeons in Ireland - MUB, Bahrain, Bahrain; ${ }^{2}$ McMaster University, Hamilton, Canada; ${ }^{3}$ School of Rehabilitation Science, Faculty of Health Sciences, McMaster University, Hamilton, Canada; ${ }^{4}$ Hamilton Niagara Regional Hemophilia Program, Hamilton Health Sciences, Hamilton, Canada; ${ }^{5}$ Department of Pediatrics, McMaster University, Hamilton, Canada; ${ }^{6}$ Department of Medicine, Faculty of Health Sciences, McMaster University, Hamilton, Canada

Background: FXI-deficiency is a rare inherited bleeding disorder caused by mutation(s) in Factor 11 gene. Affected individuals may be asymptomatic or have prolonged bleeding following trauma or surgery. The available treatments include plasma, FXI concentrate (FXIc), and tranexamic acid (TXA). Currently, FXIc is not approved in Canada but can be accessed via the Health Canada Special Access Program.

Aims: To evaluate the procedural policies and clinical practices surrounding the treatment of FXI-deficiency at the Hamilton-Niagara Hemophilia Treatment Centre.

Methods: A retrospective chart review was performed on pediatric patients diagnosed with FXI-deficiency between 2000-2020. Data on patient demographics, family/bleeding history, presenting complaint, FXI-levels, and treatment recommendations were obtained. One investigator extracted all available data. Any missing data were collected by two other investigators. Accuracy of the data set was verified by the Principal Investigator.

Results: Five FXI-deficient patients were identified, 3 females and 2 males, between the ages of 5 and 16 . Most patients did not require hemostatic intervention and were originally referred to the clinic due to a positive family history of FXI-deficiency. All patients were recommended plasma for the treatment of acute bleeding/before surgical procedures, with some dosage variability. Four patients were recommended TXA for dental procedures/epistaxis. None of the patients were treated with FXIc. During the search process, it was noted that the Hamilton Health Science policy for FXIc use has not been updated since March of 2014, and the current recommended guidelines $(15-50 \mathrm{lU} / \mathrm{Kg}$ ) differ from that of Canadian Blood Services and may pose thrombotic risk.

Conclusions: Management of FXI-deficiency was fairly consistent and appropriate, given the rarity of the condition. Policy pertaining to FXIc dosage must be updated immediately to not exceed dosing in commonly accepted guidelines and revised annually. Clinical audits and periodic review of policy library may help improve healthcare practices surrounding FXI-deficiency.

\section{PB0724 | Severe Congenital Factor XIII-A Deficiency: A Clinical} Case

I. Delgado; A. Tugues; C. Marzo; E. Vicente; A. Garcia; A. Ferrero; M.E. Rivero; A. Luaña; C.E. Chavez; M. Teixido; A. Ruiz; R. Santoja; T. Garcia

Department of Hematology, Hospital Universitario Arnau de Vilanova, Lleida, Spain

Background: Congenital FXIII-A deficiency is a rare bleeding disorder that affects one individual in 1-2 million. It is inherited in an autosomal recessive pattern and it can occur due to mutation in either FXIII-A (type 2) or FXIII-B (type 1) genes. FXIII-A (type 2) defect accounts for $95 \%$ of all FXIII bleeding disorders.

Severe congenital FXIII deficiency causes severe bleeding events, with delayed type umbilical cord bleeding the first classic clinical sign of disease and the intracranial haemorrhage being the major 
cause of death. Therefore, early diagnosis and prophylaxis to prevent this life-threatening bleed is the key.

Aims: We present a case of a 4-year-old boy with severe congenital FXIII deficiency.

Methods: The diagnosis of Factor XIII deficiency had based upon identification of characteristic symptoms, a detailed patient and family history, a thorough clinical evaluation, and a variety of specialized laboratory tests (FXIII activity assay, inhibitor assay, and molecular diagnosis).

Results: Our patient, a 4-year-old boy with history of umbilical bleeding in the neonatal period and prolonged bleeding after trauma, showed a frequent easy bruising. The basic haemostatic study was normal. Nevertheless, laboratory examination revealed that Factor XIII activity was only $1 \%$ and ruled out the presence of anti-FXIII antibodies. In addition, molecular diagnosis revealed two heterozygous mutations of FXIII-A gene. Accordingly, the patient started on regularly plasma-derived FXIII concentrate application every three weeks. The objective is to maintain levels of Factor XIII $>5 \%$. No bleeding has occurred in the three months since then.

Conclusions: Awareness of clinical symptoms indicative of this disorder is paramount to ensure early diagnosis. The majority of patients with FXIII levels $<1 \%$, are a great risk of severe spontaneous bleeding. Early diagnosis and prophylaxis to prevent life-threatening bleeding is the key, as well as to individualize prophylaxis in patients with levels $>1 \%$.

PB0725 | Successful Pregnancy in a Patient with Factor XIII Deficiency and Recurrent Miscarriages

T. Surapaneni ${ }^{1}$; H. Vadlamani ${ }^{1} ;$ M. Sukayogula ${ }^{1}$; C. Ranjith ${ }^{2}$; A. Aribandi ${ }^{2}$

${ }^{1}$ Fernandez Foundation, Hyderabad, India; ${ }^{2}$ American Oncology Institute, Hyderabad, India

Background: Factor XIII deficiency can present with severe bleeding, intracranial haemorrhages (30\%), poor wound healing and spontaneous abortions.

Aims: To present management strategy of a patient with Factor XIII deficiency with Recurrent Miscarriages.

Methods: Hematological, biochemical and imaging studies were done routinely. APLA screen, Coagulation workup like PT, APTT, factor VIII, VWFAg, urea clot lysis assay and factor XIII levels were evaluated.

Results: A 27years old woman, had 3rd degree consanguineous marriage had history of 3 consecutive abortions all less than 10 weeks of gestation, came to our institute after 4th spontaneous conception, registered at 7th week of gestation. Clinical Exome sequencing by next generation sequencing, found pathogenic variant of Exon 2 mutation at F13A1 transcript level, homozygous in nature and consistent with Factor XIIIA deficiency.

At 7th week of gestation she gave history of spotting, managed conservatively. Her Factor XIII Ag level was $1.9 \%$ and she received 4units of Fresh frozen plasma(FFP). At 14weeks of gestation she was admitted for threatened miscarriage, ultrasound abdomen showed live fetus managed conservatively and discharged. In view of risk of bleeding she was planned for prophylactic FFP transfusion every 2 weeks. As she had allergic reactions to FFP, she was switched to cryoprecipitate -5 units every 15 days. she had regular antenatal followup visits with the obstetrician.

At $36+5$ weeks of gestation she delivered a $3.1 \mathrm{~kg}$ weight female baby by LSCS. During post-partum period, she developed atonic PPH which was managed conservatively. She received 2 days of 5units of cryoprecipitate each day and was discharged on 5th postpartum day in a stable condition.

Conclusions: We successfully used prophylactic FFP \& cryoprecipitate in a pregnant patient with factor XIII deficiency and recurrent miscarriages, who had a successful pregnancy outcome with out any complications.

PB0726 | Bleeding and Thrombosis in One Patient Management of Challenging Disorders of Hemostasis

L. Stanciakova ${ }^{1}$; M. Dobrotova ${ }^{1}$; P. Holly ${ }^{1}$; M. Brunclikova ${ }^{1}$; T. Bolek ${ }^{2}$; M. Samos ${ }^{2}$; P. Kubisz ${ }^{1}$; J. Stasko ${ }^{1}$

${ }^{1}$ National Center of Hemostasis and Thrombosis, Department of Hematology and Transfusion Medicine, Comenius University in Bratislava, Jessenius Faculty of Medicine in Martin, Martin University Hospital, Martin, Slovak Republic, Martin, Slovakia; ${ }^{2}$ Department of the Internal Medicine I, Comenius University in Bratislava, Jessenius Faculty of Medicine in Martin, Martin University Hospital, Martin, Slovak Republic, Martin, Slovakia

Background: Despite the typical phenotype of the bleeding coagulopathies, these disorders do not prevent the affected patients from the development of the thromboembolism. The major contributing factors for thrombosis in these cases are the traditional risk factors, such as advanced age, arterial hypertension, hyperlipidemia, smoking, immobilization, surgery, pregnancy, and also the coexistence of the thrombophilic state, substitution treatment and the use of bypassing agents.

Aims: To discuss the management of the coincidence of bleeding and thrombosis in several patients.

Methods: The authors present two case reports of the patients with bleeding coagulopathy who developed thromboembolic complications and report on the successful management of their challenging clinical states.

Results: The antithrombotic treatment was administered without any complications. The patient with coagulation factor VII deficiency and iliofemoral deep vein thrombosis plus subsequent combined central retinal arterial and venous occlusion is presented. Moreover, the authors describe the management of the patient with hemophilia $A$ and venous thrombosis, who subsequently developed arterial thrombosis. 
Conclusions: Older patients with bleeding disorder of hemostasis have prothrombotic risk factors comparable with that of general population. In the concomitant existence of several prothrombotic risk factors, the risk of the development of thrombosis is increased. Generally, there is a lack of large studies supporting the universal guidelines for such situations. Therefore, the combination of the substitution and antithrombotic treatment should be individualized. The project complies with the Declaration of Helsinki and the informed consent in each patient is obtained.

Acknowledgements: Authors thank the support of the projects of the Scientific Grant Agency (Vega)1/0549/19 and Agency for the Support of Research and Development APVV-16-0020.

\section{PB0727 | The Paradoxal Association beetween Congenital FVI} Deficiency and Thromboembolism

I. krichen ${ }^{1}$; N. Moussa ${ }^{2}$; Y. Elloumi ${ }^{1}$; F. Megdich ${ }^{1}$; W. Fekih ${ }^{2}$;

Y. Kammoun ${ }^{1}$; S. Kammoun ${ }^{2}$; C. Kallel ${ }^{1}$

${ }^{1}$ Laboratory of Hematology, Hospital University Habib Bourguiba, Sfax, Tunisia; ${ }^{2}$ Department of Pneumology, Hospital University Hedi Chaker, Sfax, Tunisia

Background: Congenital factor FVII deficiency is the most wideprad of the rare bleeding disorders . Clinical features are extremely variable ranging from mild cutaneo-mucosal bleeding to lethal cerebral haemorrhges and are often not correleted with FVII coagulant activity (FVII:C). Conversely, some patients can develop arterial or venous thrombosis.

Aims: The purpose of this study is to report thromboembolism in a FVII deficieny's patient.

Methods: case report description.

Results: A 23 year-old woman was admitted to the department of pneumology due to respiratory distress and tachycardia. Her medical history included diabetes diagnosed since 6 months ago. The patient reported an exertional dyspnea that had progressed for months. A CT angiography of the chest showed a pulmonary hypertension and absence of pulmonary embolism. The pulmonary hypertension was confirmed by transthoracic echocardiogram (PAPS $=55 \mathrm{mmHg}$ ). An etiological investigation turned out to be negative. In fact, familial pulmonary hypertension history, congenital heart disease, connectivity and portal hypertension were discarded.

The haemostasis tests revealed an isoleted prolonged prothrombin time of 24,3 seconds. Level of FVII activity assayed by a rabbit brain reagent was $4 \%$. Neverless, she had no history of significant bleeding history.

Notably, the patient had not a deficiency of one of the natural inhibitors of coagulation (PS $=70 \%, P C=84 \%, A T=100 \%$ ). No resistance to activated protein $\mathrm{C}$ ( $\mathrm{rAPC}$ ) was found .Screening for antiphospholipid antibodies was negative.

Thus, arterial pulmonary hypertension was potentially secondary to chronic thromboembolism. In this regard, about $3 \%$ of patients with FVII deficiency can develop chronic thromboembolism.
The patient was discharged with diuretic and long-term oxygen. Conclusions: Thrombotic events are uncommon in congenital bleeding disorders. Among the rare coagulation disorders, FVII deficiency has been reported to be more frequently associated with thrombosis. The clinician must be aware that thrombosis can occur in FVII deficiency under certain provoked circumstances and severe deficiency does not protect against thrombosis.

\section{P0147 | Management of Acquired Hemophilia A: A Case-report}

J. Cabral $^{1}$; M. Afonso ${ }^{1}$; L. Costa ${ }^{2}$; B. Malheiro ${ }^{3} ;$ M. Calheiros ${ }^{1}$; A.P. Barbosa ${ }^{1}$; C. Calaza ${ }^{1}$; M. Majar ${ }^{3}$; G. Carvalho ${ }^{3}$; A. Marques ${ }^{1}$ ${ }^{1}$ Immunohemotherapy Service, Hospital de Braga, Braga, Portugal; ${ }^{2}$ Intensive Care Medicine, Hospital de Braga, Braga, Portugal; ${ }^{3}$ Clinical Pathology Service, Hospital de Braga, Braga, Portugal

Background: Acquired hemophilia $\mathrm{A}(\mathrm{AHA})$ is a rare bleeding disorder caused by autoantibodies against clotting factor VIII (FVIII). Identified on patients with no previous personal or family history of bleeding and an isolated prolonged activated partial thromboplastin time (aPTT).

Prompt diagnosis of this disorder is essential to control hemorrhage and suppress the inhibitor appropriately.

Aims: Describing bleeding control in a patient with AHA.

Methods: Review a clinical case.

Results: A 66-year-old man, previously healthy and no history of bleeding, was submitted to a laparoscopic cholecystectomy, complicated by peritonitis after iatrogenic small bowel perforation.

Six months later, he had an abdominal wall abscess with enterocutaneous fistula. Abscess drainage and later bowel transit reconstruction were performed.

Ten days postoperatively, he presented anastomosis dehiscence and intra-abdominal hemorrhage, with necessity of hematoma drainage and abdominal packing for uncontrollable bleeding.

Screening coagulation tests showed a prolonged aPTT (67.9"), a normal prothrombin time and fibrinogen.

A mixing study resulted in failure of aPTT correction (Rosner index: $18 \%)$, suggesting the presence of a factor inhibitor. Factors levels: FVIII 17\%, FIX 139.5\%, FXI 62.9\%.

AHA etiological investigation started.

The patient was initially treated with plasma-derived coagulation FVIII concentrate due to unavailability of alternative therapy and initiated corticosteroid therapy. In D2, the packing was removed and 4 draining tubes were inserted under recombinant activated factor VII $(90 \mu \mathrm{g} / \mathrm{kg}$ every 3 hours) and transfusion support, with significant improvement of the hemorrhagic episode. In D12, he switched to anti-inhibitor coagulant complex (FEIBA).

Although the hemorrhagic episodes were successfully controlled, and the inhibitor title lowered, the patient had gradual health status deterioration by the infectious condition dying 19 days after the diagnosis of AHA. 
Conclusions: Identifying the etiology of $\mathrm{AHA}$ and treating underlying co-morbidities, as well as controlling bleeding and infections associated to immunosuppression, are key to reduce morbidity and mortality in AHA.

P0148 | Cocktail of Inherited Hemostatic and Red Cell Disorders in Unrelated Families of Interior Sindh

A. Naz ${ }^{1} ;$ M. Khanzada ${ }^{2}$

${ }^{1}$ Liaquat University of Medical and Health Sciences, Diagnostic and Research Lab, Jamshoro, Pakistan; ${ }^{2}$ Liaquat University of Medical and Health Sciences, Diagnostic and Research Lab, Hyderabad, Pakistan

Background: Non-availability of medical and diagnostic facilities of hematological disorders in rural areas of interior Sindh even in urban except Karachi multiple patients still unexplored their diagnosis. Around 10 hematologists are designated in the 33 million population of interior Sindh. Even these peoples did not actually know that they are suffering from some disorder and they marring with each other cousin marriage is very common in their families and in childhood age they had a high occurrence of inherited hematological disorders. Aims: This pilot project with them to reduce disease occurrence in the new generation and also reduce the incidence of inherited blood disorders.

Methods: Total of 18 families (104 members) was screened and identified in the Diagnostic and Research lab, Hyderabad through structured questionnaires during visits of patients for the hematological opinion of index patient from Civil hospital and interior Sindh. Most of the families were unrelated and belong to different casts of Sindhi, Pushto, and Balochi. First-line and second-line hemostatic, hematological, and biochemical investigations were done. Whole Exome sequencing was done in some patients. Simple descriptive was applied for the analysis of data.

Results: Only 3 families were identified as defects in the VWF gene and Fibrinogen both in the same member. Few members are affected. Rest of the 5 families identified as defected F.XIII, red cell disorders like folate deficiency and iron deficiency like beta-thalassemia minor and major in the same member. The median age group was 10 years old. The majority of daughters were born with only head, neck, and limb and died within 2 years of after birth.

Conclusions: Many novel findings identified in these families but the management of these patients quite miserable. As per published reports, a cocktail of these types of multiple hematological disorders and phenotypic expressions not documented.
P0150 | Severe Hemophilia C in a 70-year-old Woman: A Clinical Case

J.A.S. Lopes ${ }^{1}$; R.D.M. Almeida ${ }^{2}$; A.C. Gusmão²; N.N.S. Magalhães ${ }^{2}$; O.F. Santos ${ }^{1}$; A.C.A. Santos ${ }^{3}$; T.S. Espósito ${ }^{3}$; L.O.W. Rodrigues ${ }^{4}$; D.O.W. Rodrigues ${ }^{5,3}$

${ }^{1}$ Universidade Federal de Juiz de Fora, Juiz de Fora, Brazil; ${ }^{2}$ Faculdade de Ciências Médicas e da Saúde de Juiz de Fora, Juiz de Fora, Brazil; ${ }^{3}$ Centro Universitário Presidente Antônio Carlos - FAME, Juiz de Fora, Brazil; ${ }^{4}$ Carleton University, Ottawa, Canada; ${ }^{5}$ Fundação Hemominas, Juiz de Fora, Brazil

Background: Factor XI deficiency (Hemophilia C) is a rare bleeding disorder that was first described in 1953 by Rosenthal el at. in patients who experienced severe bleeding after dental extractions. The estimated prevalence is about 1 in 1 million with increased prevalence among Ashkenazi Jews (8-9\%). Patients are generally classified into three categories based on the factor XI levels: severe $(<15-20 \%$ of normal), intermediate (20-40\%) and mild (>40\%). Distinct from hemophilia A and B, FXI deficiency usually presents as post-traumatic bleeding and rarely manifests as spontaneous bleeding.

Aims: To report a case of severe Hemophilia $C$ in a 70 -year-old woman.

Methods: A 70-year-old woman was referred to Fundação Hemominas Juiz de Fora, Brazil due to gingivorrhagia and recurrent epistaxis. She reports having already received blood transfusions due to massive bleeding after surgeries, including cholecystectomy, cesarean section and hysterectomy. The patient is the sixth daughter of a seven children family. Her mother, three siblings and one nephew also have coagulation disorders, unknown Jewish descendancy.

Results: The laboratory study showed normal whole blood count, screening tests for coagulation disorders showed normal platelet count and an increase of activated partial thromboplastin time (aTTP). A plasma dosage of coagulation factors was performed, with results shown in Table 1 . With the reduction of factor $\mathrm{XI}$ activity levels (3.59\%), the increase of aTTP and the normality of other tests, the diagnosis of Hemophilia $\mathrm{C}$ was established. A family study was performed with heredogram, shown in Figure 1.

TABLE 1 Tests for investigation and diagnosis.

$\begin{array}{lll}\begin{array}{l}\text { Laboratory Tests } \\ \begin{array}{l}\text { Activated Partial } \\ \text { Thromboplastin } \\ \text { Time }\end{array}\end{array} & \text { Patient's Results } & \begin{array}{l}\text { Reference } \\ \text { Ranges }\end{array} \\ \begin{array}{l}\text { Platelet Agregation } \\ \text { Curve with ADP, }\end{array} & 25-35^{\prime \prime} \\ \begin{array}{l}\text { Collagen and } \\ \text { Ristocetin }\end{array} & \text { Normal } & \text { Normal } \\ \begin{array}{l}\text { Fibrinogen } \\ \text { Prothrombin Activity }\end{array} & 100 \% & \\ \text { FVII Coagulation Assay } & 80 \% & 60-130 \%\end{array}$




\begin{tabular}{lll} 
ABSTRACT & \\
\hline Laboratory Tests & Patient's Results & $\begin{array}{l}\text { Reference } \\
\text { Ranges }\end{array}$ \\
FVIII Coagulation Assay & $70 \%$ & $50-150 \%$ \\
FIX Coagulation Assay & $80 \%$ & $60-130 \%$ \\
FXI Coagulation Assay & $3.59 \%$ & $60-130 \%$ \\
Von Willebrand Factor & $88 \% / 80 \%$ & $50-150 \% /$ \\
$\quad \begin{array}{l}\text { and Ristocetin } \\
\text { Cofactor }\end{array}$ & $50-150 \%$ \\
\hline
\end{tabular}

Source:The Authors

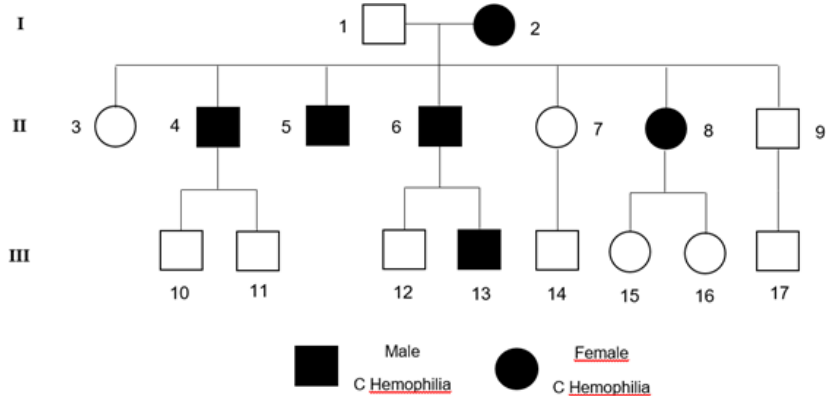

FIGURE 1 Heredogram of the patient's family.

Conclusions: Hemophilia $\mathrm{C}$ is a rare bleeding disorder with a wide variability in clinical presentation. Adequate diagnosis is essential to minimize severe hemorrhagic conditions, specially in surgical procedures. Family screening is recommended if a member has FXI deficiency and prevalence may vary in Brazil due to the wide miscegenation.

\section{HEMOSTATIC SYSTEM IN CANCER, INFLAMMATION AND IMMUNITY}

\section{COAGULATION PROTEINS BEYOND HEMOSTASIS}

LPB0023 | Role of Tissue Factor in Delayed Bone Repair Induced by Diabetic State in Mice

$\underline{\text { K. Tatsumi }}{ }^{1,2}$; H. Ehara ${ }^{2}$;. Takafuji ${ }^{2}$; N. Kawao ${ }^{2}$; M. Ishida ${ }^{2}$;

K. Okada ${ }^{2}$; N. Mackman ${ }^{3}$; M. Shima'; H. Kaji ${ }^{2}$

${ }^{1}$ Nara Medical University, Kashihara, Japan; ${ }^{2}$ Kindai University Faculty of Medicine, Osaka-Sayama, Japan; ${ }^{3}$ University of North Carolina at Chapel Hill, Chapel Hill, United States

Background: Tissue factor (TF) is the primary activators of the extrinsic coagulation protease cascade. Although TF is related to various pathological states, the roles of TF in bone metabolism are unknown. Aims: To examine the roles of TF in delayed bone repair induced by diabetic state in mice using wild-type (WT) and low TF-expressing (LTF) mice.

Methods: Diabetes was induced by daily intraperitoneal injections of streptozotocin at a dose of $50 \mathrm{mg} / \mathrm{kg}$ BW for 4 days. Mice with blood glucose levels greater than $300 \mathrm{mg} / \mathrm{dL}$ were considered diabetic. At 2 weeks after confirming the induction of diabetes, a bone

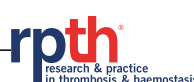

defect surgery was performed in the right femur of the mice. The repair of the defect was serially monitored with quantitative computed tomography (qCT).

Results: A prolonged diabetic state significantly reduced total and trabecular bone mineral density (BMD) as well as cortical bone thickness both in WT and LTF mice; these BMD parameters were similar between WT and LTF mice with or without STZ treatment. A diabetic state delayed bone repair following bone injury on the femoral bone in WT mice. Interestingly, a LTF state was associated with further delay in bone repair in diabetes. In in vitro experiments, TF significantly decreased receptor activator of nuclear factor-kB ligand (RANKL)-induced osteoclast formation and osteoclastogenic gene expression in RAW264.7 cells. On the other hand, TF did not affect the gene expression levels of runt-related transcription factor 2 (Runx2) and Osterix as well as alkaline phosphatase activity in mouse primary osteoblasts.

Conclusions: LTF state is associated with enhanced bone repair delay induced by diabetic state in mice. Moreover, TF suppressed osteoclast formation in vitro. TF-induced suppression of bone remodeling might be involved in the protective effects of TF on delayed bone repair induced by a diabetic state.

LPB0024 | Coagulation-cancer Crosstalk; Von Willebrand Factor Contributes to Pro-invasive and Pro-angiogenic Properties in Breast Cancer Cells

S. Patmore ${ }^{1}$; S. Pal Singh Dhami ${ }^{1}$; J. Castle ${ }^{2}$; C. C. Kirwan ${ }^{2,3}$; J. S. O'Donnell ${ }^{1}$; J. M. O'Sullivan ${ }^{1}$

${ }^{1}$ Irish Centre for Vascular Biology, School of Pharmacy and Biomolecular Science, Royal College of Surgeons in Ireland, Dublin, Ireland; ${ }^{2}$ Manchester Cancer Research Centre, The University of Manchester, Manchester, United Kingdom; ${ }^{3}$ The Nightingale Centre, Manchester University Foundation Trust, Southmoor Road, Wythenshawe, Manchester, United Kingdom

Background: Von Willebrand Factor (VWF) is a glycoprotein critical for normal haemostasis. Recently, novel roles for VWF have been identified in cancer progression including angiogenesis and metastasis. Elevated plasma VWF levels have been reported in several cancer cohorts. These levels correlate not only with risk of venous thromboembolism but presence of metastasis.

Aims: Consequently, we aim to elucidate the potential role of VWF in promoting breast cancer metastasis.

Methods: Human breast cells used in this study included, MCF-7, MDA-MB-231 and MCF-10A. Interaction of recombinant VWF with breast cancer cells (BCCs) was assessed under static and shear conditions. BCC adhesion to human endothelial cells was quantified by flow cytometry. Live cell imaging was used to assess BCC invasion and angiogenesis.

Results: Increased plasma VWF levels were observed in patients with metastatic breast cancer compared to healthy controls (264.7IU/ 
dL vs 101.4IU/dL). Human VWF bound in a dose-dependent manner to MDA-MB-231 and MCF-7 BCCs. VWF-BCC adhesion was enhanced by ristocetin suggesting that VWF-A1 domain conformational unfolding may enhance BCC adhesion. In support of this, BCCs displayed significant binding to VWF under conditions of shear stress. VWF adhesion to BCCs enhanced tumour cells binding to the endothelium 2-fold compared to untreated cells, suggesting that VWF may facilitate initial steps in transendothelial migration. Interestingly, presence of VWF promoted the invasion of MDAMB-231 cells across an extracellular matrix barrier by $79.6 \%$ versus control cells. Furthermore, binding of VWF to MDA-MB-231 cells induced upregulation of Epidermal Growth Factor receptor and endothelial trans-differentiation thus forming vascular-like networks within BCCs. Importantly, this vasculogenic mimicry of BCCs is associated with tumour angiogenesis and progression in patients.

Conclusions: Collectively, these findings provide insights into nove biological roles for VWF in tumour progression, particularly in promoting pro-invasive and pro-angiogenic effects in BCCs and may help identify novel anti-metastatic therapeutic targets in the crosstalk between coagulation and cancer.

\section{LPB0071 | Coagulation Factor 5 (F5) Is an Estrogen-responsive Gene in Breast Cancer Cells}

M. Seierstad Andresen ${ }^{1} ;$ M. Sletten ${ }^{1}$; P.M. Sandset ${ }^{2,1}$; N. Iversen ${ }^{1}$; B. Stavik ${ }^{* 1} ;$ M. Tinholt*1

${ }^{1}$ Oslo University Hospital, Oslo, Norway; ${ }^{2}$ University of Oslo, Oslo, Norway

Background: Coagulation factor $(\mathrm{F}) \mathrm{V}$ is an influential regulator of blood coagulation and has been shown to be increased in breast tumors. Cancer contributes to activation of the coagulation system leading to an imbalance in the hemostatic system. Most breast tumors express estrogen receptor (ER) where estrogen signaling plays an important role.

Aims: To explore the molecular association between estrogens and FV in ERa positive breast cancer cells.

Methods: MCF-7 cells were treated with $17-\beta$-estradiol (E2) or $17-\beta$-ethinylestradiol (EE2) alone or in combination with the ER antagonist fulvestrant. ERa was also transiently knocked down prior to stimulation with estrogen. F5 mRNA and FV protein levels were measured using qRT-PCR and Western blotting. Potential estrogen responsive elements (ERE) within the $F 5$ promoter were identified by bioinformatic analysis. The F5 5'-flanking region containing wild type or mutated EREs were cloned into a luciferase reporter gene vector and transfected into MCF-7 cells prior to estrogen stimulation. Kaplan-Meyer survival analysis of F5 mRNA in ER positive tumors from breast cancer patients in the Kaplan-Meier plotter database were performed.

Results: The expression of F5 mRNA and FV protein levels were significantly increased in cells after stimulation with the natural E2 or synthetic EE2, in a dose- and time-dependent manner. Treatment with fulvestrant or knockdown of ERa counteracted this upregulation. Significantly increased luciferase activity was observed in E2 treated cells transfected with the F5 wild type promoter construct containing the potential ERE binding sites. This effect was abolished when all three ERE sites were destroyed. High F5 mRNA levels in ER positive tumors were associated with increased relapse-free survival in a clinical breast cancer material.

Conclusions: We demonstrate that F5 is an estrogen responsive gene in breast cancer cells expressing ERa and that high F5 levels in ER positive breast cancer was associated with relapse-free survival.

\section{PB0728 | Antigenic Levels of von Willebrand Factor Predict Complications and Mortality in Patients with Liver Cirrhosis}

M. El Horri ${ }^{1}$; I. Khachaa ${ }^{1}$; A. Cheikh Khelifa ${ }^{1}$; A. Berrah ${ }^{1}$; M. Baghdadi ${ }^{1}$; A. Moueden ${ }^{2}$; D. Benlaldj ${ }^{2}$; F. Seghier ${ }^{2}$

${ }^{1}$ Military University Hospital of Oran, Oran, Algeria; ${ }^{2}$ Hospital University Center of Oran, Oran, Algeria

Background: Recently von willebrand factor has been identified as a marker of portal hypertension. The elevation of antigenic levels of this marker in liver cirrhosis, have led scientists to suggest that von willebrand factor could be a good prognostic marker, and thus bring improvements to the current prognostic scores which suffer from limitations since they do not take into account portal hypertension.

Aims: We aimed to assess the prognostic value of von-Willebrand factor, in the prediction of complications and mortality in patients with liver cirrhosis.

Methods: Patients with different stages of liver cirrhosis were recruited. vWF-Ag levels were measured by immuno-turbidimetric assay. Clinical and biological data were determined at study entry. During follow-up, complications of liver cirrhosis, death or transplantation were recorded.

Results: One hundred and fourty-five patients with liver cirrhosis (CPS-A 39\%, B 42\%; C 19\%; 51\% male; mean age 58 years; $64.8 \%$ with decompensated liver cirrhosis) were included.

During a median follow-up of 14 months ( $\mathrm{Cl}$ : 12-18), recurrence of decompensation was observed in in 65 (44.8\%) patients and 35 (24.1\%) patients deceased.

Raised levels of vWF-Ag were associated with recurrence of decompensation $(P=0.0001)$ and death $(P=0.001)$.

The cut-off value of $\geq 253 \%$ for vWF-Ag, predicts relapse, with Sensitivity of $82.3 \%$ and specificity of $68 \%$. While the cut-off value of $\geq 324 \%$ for vWF-Ag, predicts mortality, with Sensitivity of $91.4 \%$ and specificity of $75.2 \%$.

This cut-off value defined two different populations of patients with a highly different survival probability: 38\% after 12 months if VWF-Ag was $\geq 324 \%$, compared to $97 \%$ for those who had a VWF-Ag < 324\%, $(P=0.0001)$, with a HR = 20.8 [CI 95\%: $6.3-68.1]$. 
TABLE 1 Prognostic value of VWF-Ag in the prediction of decompensation recurrence and mortality in patients with liver cirrhosis

\begin{tabular}{|c|c|c|c|c|}
\hline & \multicolumn{2}{|c|}{ Recurrence of decompensation } & \multicolumn{2}{|l|}{ Death } \\
\hline & No recurrence & Recurrence & Survivors & Dead \\
\hline $\mathrm{N}$ & $80(55.2 \%)$ & $65(44.8 \%)$ & $110(75.9 \%)$ & 35 (24.1\%) \\
\hline vWF-Ag mean (\%) & 243 & 405 & 273 & 455 \\
\hline vWF-Ag IQR (\%) & {$[173-300]$} & {$[308-462]$} & [190 - 325] & {$[361-516]$} \\
\hline$P$ & 0,0001 & & 0,001 & \\
\hline \multicolumn{5}{|l|}{ Cut-off values } \\
\hline & \multicolumn{2}{|c|}{ Cut-off value : $253 \%$ AUC $=0.855$} & \multicolumn{2}{|c|}{ Cut-off value : $324 \%$ AUC $=0.873$} \\
\hline & \multicolumn{2}{|c|}{ Se: $92.4 \%$ / Sp 68.9\% } & \multicolumn{2}{|c|}{ Se $91.4 \%$ / Sp $75.2 \%$} \\
\hline & \multicolumn{2}{|c|}{ PPV 70.7\% / NPV 91.8\% } & \multicolumn{2}{|c|}{ PPV 54\% / NPV 96.5\% } \\
\hline
\end{tabular}

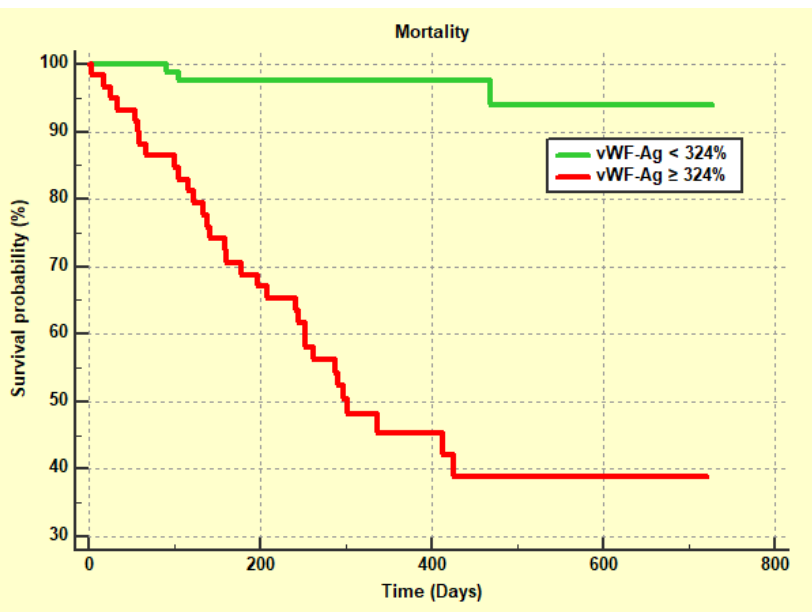

FIGURE 1 Kaplan Meier curve showing survival probability in patients with liver cirrhosis according to VWF-Ag cut-off value of $324 \%$

Conclusions: vWF-Ag may become a valuable marker for the prediction of mortality in patients with liver cirrhosis.

PB0729 | Dual Effect of Hepsin Expression on Tumor Phenotype and Prothrombotic Potential of Colorectal Cancer Cells and Inhibition by Venetoclax

M.C. Rodenas-Bleda ${ }^{1}$; J. Peñas-Martínez ${ }^{2,1}$; D. Zaragoza-Huesca ${ }^{2,1}$;

C. Ortega-Sabater ${ }^{2,1}$; J. Peña-García ${ }^{3}$; S. Espín ${ }^{1}$; G. Ricote ${ }^{1}$; F. Ayala-de la Peña ${ }^{1,2}$; G. Luengo-Gil ${ }^{1,2}$; A. Nieto ${ }^{1}$; F. García-Molina ${ }^{2}$; V. Vicente ${ }^{1,2}$; F. Bernardi ${ }^{4}$; H. Pérez-Sánchez ${ }^{3}$; A. Carmona-

Bayonas $^{1,2}$; I. Martínez-Martínez ${ }^{1}$

${ }^{1}$ IMIB, Murcia, Spain; ${ }^{2}$ University of Murcia, Murcia, Spain; ${ }^{3}$ UCAM Catholic University of Murcia, Murcia, Spain; ${ }^{4}$ University of Ferrara, Ferrara, Italy

Background: The effect of hepsin levels on colorectal cancer has not been explored, despite of being associated with a worse prognosis in other tumors.
Aims: Here, for first time, we aimed to demonstrate the contribution of hepsin level to a worse prognosis in colorectal cancer and the prediction by virtual screening and identification of FDA-approved drugs as potential hepsin blockers.

Methods: In silico screening of 2037 compounds approved by the FDA was performed, and selected compounds were analyzed for their hepsin inhibition. The hepsin level effect was investigated on cell migration, invasion, proliferation and key cancer signaling pathways activation. The hepsin procoagulant function was determined by the thrombin generation assay. Hepsin levels were analyzed in plasma of resected and metastatic patients and mutant KRAS association was evaluated.

Results: Our results demonstrate that hepsin overexpression caused increased migration and invasion of Caco- 2 cells. This overexpression resulted in higher phosphorylation of Erk1/2 and STAT3, associated with worse prognosis. In fact, our study showed a higher hepsin expression in metastatic patients, which was associated to mutant KRAS. The higher hepsin expression in Caco-2 cells also leaded to a higher thrombin generation in plasma, underlying a potential hypercoagulant state. Among the screened FDA-approved drugs, venetoclax showed the most potent effect as hepsin inhibitor and reduced the metastatic and prothrombotic phenotype of colorectal cancer cells.

Conclusions: Collectively, our results demonstrate that elevated hepsin levels may be indicative of a more aggressive and prothrombotic tumor phenotype and demonstrates an antitumor role of venetoclax as a hepsin inhibitor. This constitutes a foundation for molecular targeted therapy for colorectal cancer and other hepsinoverexpressing tumors.

PB0730 | Comparison of the Relative PAR1, PAR2 and PAR3 Cleavage Profiles by Thrombin, APC, Factor Xa and Factor VIla

A. Yamashita ${ }^{1,2}$; M. Taki ${ }^{2}$ J.H. Griffin ${ }^{1}$; L.O. Mosnier ${ }^{1}$

${ }^{1}$ Department of Molecular Medicine, The Scripps Research Institute, La Jolla, United States; ${ }^{2}$ Department of Pediatrics, St. Marianna University School of Medicine, Kawasaki, Japan

Background: Thrombin, factor VIla (FVIla), FXa, and activated protein $C$ (APC) affect multiple cellular functions via protease activated receptors (PAR). 
Aims: To help understand differences between these proteases, their relative potencies for cleaving PAR1, PAR2, and PAR3 were determined.

Methods: PAR sensitivities to cleavage were determined using HEK293 cells expressing EPCR and secreted embryonic alkaline phosphatase (SEAP)-PAR1, SEAP-PAR2, and SEAP-PAR3.

Results: In the presence of EPCR, dose-response curves for APC and FXa cleavage of PAR1 and PAR3 were similar. Each required a 100 -fold higher concentration for $50 \%$ cleavage $\left(E_{50}\right)$ compared to thrombin for PAR1 cleavage $\left(\mathrm{EC}_{50}=36.0,49.8\right.$ and $0.3 \mathrm{nmol} / \mathrm{L}$ for APC, FXa and thrombin, respectively) and a 16-fold higher concentration for PAR3 cleavage $\left(E_{50}=20.1,19.2\right.$ and $1.22 \mathrm{nM}$ for APC, $\mathrm{FXa}$ and thrombin, respectively). Notably, thrombin and FXa cleave PAR1 at Arg41 whereas APC cleaves at Arg46; APC and FXa cleave PAR3 at Arg41 whereas thrombin cleaves at K38. FVIla showed minor cleavage of PAR1 at $>100 \mathrm{nM}$ and no cleavage of PAR3. Doseresponse curves for APC and FVIla PAR2 cleavage at Arg36 in the presence of EPCR were similar but they required a 20 -fold higher concentration compared to $\mathrm{FXa}\left(\mathrm{EC}_{50}=15.5,349\right.$ and $369 \mathrm{nM}$ for FXa, APC and FVIla, respectively). Mutation of positively charged residues in the PAR2 N-terminus (R31Q, K34Q, K41Q and K51Q) revealed strikingly different effects for FXa, APC and FVIla. Notably $\mathrm{K} 34 \mathrm{Q}$ improved PAR2 cleavage by $\mathrm{FXa} 4$-fold $\left(\mathrm{EC}_{50}=3.7 \mathrm{nM}\right)$ but reduced cleavage by APC or FVIla 2 to 4 -fold $\left(E_{50}=1431\right.$ and $779 \mathrm{nM}$ for APC and FVIla, respectively).

Conclusions: Cleavages of PAR1, PAR2 and PAR3 by thrombin, APC, $\mathrm{FXa}$ and FVIla vary greatly with respect to relative sensitivities based on dose-responses, EPCR-dependency, and cleavage sites. These remarkable variabilities help explain how these proteases use the same receptor systems to modulate diverse cellular functions.

PB0731 | Activated Protein C Impacts Survival and Activity of Mast Cells and Neurons via the Modulation of Nuclear Factor-кB Activation

\section{Gorbacheva $^{1,2} ;$ A. Ivanova ${ }^{1}$}

${ }^{1}$ Pirogov Russian National Research Medical University, Moscow, Russian Federation; ${ }^{2}$ Lomonosov Moscow State University, Moscow, Russian Federation

Background: Brain injury is associated with neuroinflammation, neurodegeneration, and also blood coagulation with thrombin formation and generation of activated protein C (APC).

Aims: In the present study we investigated the ability of APC to modulate the production of IL-1b and IL- 6 by mast cells (MC), MC survival and NF-KBp65 translocation into MC nucleus in acute inflammation in rats and at glutamate(Glu)- induced toxicity in cultura neurons.

Methods: MC were isolated from the peritoneal cavity of thioglycolate broth-induced acute inflammation rats by Ficoll density gradient. IL-1b, 6 were analyzed using ELISA Kit. The translocation of
NF-kBp65 to nucleus was analyzed by antiNF-кBp65 antibodies. MTT-test was used to evaluate cell viability.

Results: Thioglycolate injection induced inflammatory response with the rise of IL-1 $\beta$ and IL-6. MC IL-6 production was 1.3, 1.8 and 1.9-fold decreased by single intraperitoneal injection of $5 \mathrm{nM} \mathrm{APC}$ at $0.5,1.5$ and $4 \mathrm{~h}$ of inflammation, respectively. The enhanced production of IL-1 $\beta$ in MC under action of APC was also decreased. APC protected $\mathrm{MC}$ from death at $30 \mathrm{~min}$ of acute inflammation. The development of inflammation in rats led to activation of NF-KBp65. APC blocks the translocation of NF-kBp65 into the nucleus of MC. The maximal effect of APC was revealed in $1.5 \mathrm{~h}$ after induction of inflammation. APC at concentrations as low as 1-2 nM inhibits the translocation of NF-KBp65 into the nucleus of cultured rat hippocampal neurons, induced by $100 \mathrm{M}$ Glu or $50 \mathrm{nM}$ thrombin. The blocking effect of APC on NF-kBp65 translocation was observed at 1 and $4 \mathrm{~h}$ after cell treatment with Glu. The binding of APC to EPCR/ PAR-1 is required to control NF-KB activation at Glu-toxicity and acute inflammation in rats.

Conclusions: Our data indicate that APC provides not only antiapoptotic protection (in case of Glu-toxicity), but also antineuroinflammatory activity via decrease the nuclear level of NF-kBp65 in cells

PB0732 | Acute Promyelocytic Leukaemia with Anti Phospholipid Antibody Syndrome - Double Trouble

S.L. Koulmane Laxminarayana; S. Belurkar Kasturba Medical College, Manipal, Manipal Academy of Higher Education, Udupi, India

Background: Antiphospholipid syndrome (APS) is an acquired systemic autoimmune disorder characterized by venous, arterial and microvascular thrombosis and adverse pregnancy outcomes and has persistent autoantibodies against phospholipids and protiens. Acute Promyelocytic Leukaemia (APL) affected patients may present with features of disseminated intravascular coagulation due to the presence of procoagulant factors secreted by the abnormal promyelocytes. It is associated with thrombocytopenia, hypofibrinogenemia, prolonged prothrombin time and partial thromboplastin time. These factors increase the risk of bleeding which could be fatal. Coexistence of both these diseases is difficult to diagnose and treat.

Aims: To describe a rare co-occurrence of APL in a patient with APS. Methods: A 28 year old male presented with bleeding per rectum. He was a known case of APS diagnosed 2 years back. He had repeated episodes of deep vein thrombosis of leg with positive lupus anticoagulant on two different occasions 12 weeks apart. He was treated with low molecular weight heparin and followed by warfarin. He was stable until the present visit. There was no hepatosplenomegaly or lymphadenopathy or icterus.

Results: 


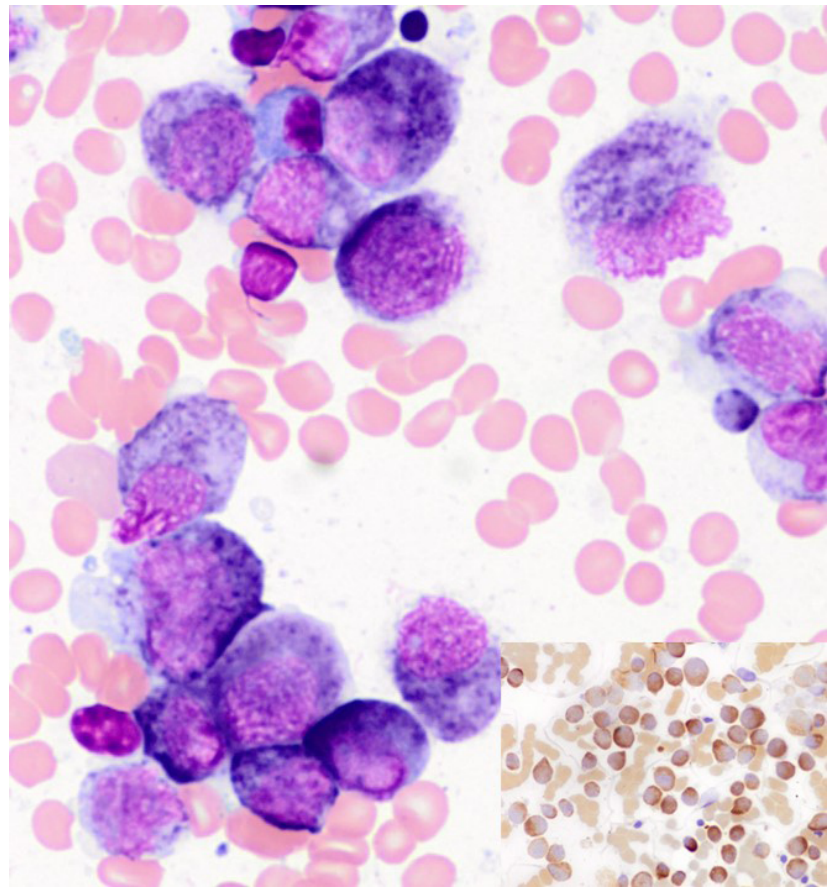

FIGURE 1 Bone marrow aspirate, Leishman stain. 40x. Abnormal promyelocytes with azurophilic granules; inset shows strongly positive MPO in abnormal cells

TABLE 1 Summary of lab investigations

\begin{tabular}{lll} 
& $\begin{array}{l}\text { Observed } \\
\text { value }\end{array}$ & $\begin{array}{l}\text { Reference } \\
\text { range }\end{array}$ \\
Haemoglobin $\mathrm{g} / \mathrm{dL}$ & 12.3 & $13.5-16.5$ \\
Total Leukocyte Count X $10^{3} / \mu \mathrm{L}$ & 1.6 & $4.5-10.5$ \\
Platelet count X $10^{3} / \mu \mathrm{L}$ & 22 & $150-450$ \\
Prothrombin time (PT) in seconds & 52.4 & 10.9 \\
INR & 2.2 & - \\
PT Test+control in seconds & 12.4 & - \\
PT Test+ aged serum in seconds & 14.4 & - \\
PT test + adsorbed plasma in & 48.7 & - \\
$\quad$ seconds & & \\
Activated partial thromboplastin & 43.7 & 25.2 \\
time (APTT) in seconds & & - \\
APTT Test+ control in seconds & 27.7 & - \\
APTT Test + aged serum in & 24.7 & - \\
$\quad$ seconds & & \\
APTT Test + adsorbed plasma in & 45 & \\
seconds & & \\
Dilute Russel's viper Venom test/ & 1.8 & \\
control ratio & & \\
\hline
\end{tabular}

Peripheral smear did not show any abnormal cells. Bone marrow examination showed abnormal promyelocytes with large orange red granules in the cytoplasm. Myeloperoxidase was strongly positive on cytochemistry (Figure 1). Investigations are summarized in table 1. Cytogenetic and molecular testing confirmed $t(15 ; 17)$ and $P M L-$ RARA. Hence, the patient was diagnosed to have concurrent APL in a case of APS and was treated with arsenic trioxide. Postinduction bone marrow showed remission with features of erythroid regeneration. All the coagulation parameters improved and patient is currently on warfarin.

Conclusions: Coexistence of a thrombotic disorder like APS with APL may render the management difficult as these patients are at high risk of disseminated intravascular coagulation and bleeding diathesis. Treatment of APL with arsenic trioxide may normalize coagulation parameters.

PB0733 | Plasma D-dimer and Fibrinogen Levels Correlates with Tumour Size and Disease Progression in Nigerian Breast Cancer Patients

O. Izuegbuna $^{1}$; O. Agodirin ${ }^{2}$; H. Olawumi²; S. Olatoke ${ }^{2}$

${ }^{1}$ LAUTECH Teaching Hospital, Ogbomoso, Nigeria; ${ }^{2}$ University of Ilorin, Ilorin, Nigeria

Background: Breast cancer is a major cause of mortality in women. D- dimer and fibrinogen have been reported to be elevated in cancer patients, and both have predictive value in cancer management. This study aimed to evaluate the role of D-dimer, fibrinogen as well as Ddimer/ fibrinogen ratio (DFR) in predicting lymph node metastasis, and their correlation with some clinicopathologic parameters.

Aims: The aim of this study was to evaluate the role of D-dimer, fibrinogen as well as D-dimer/ fibrinogen ratio (DFR) in predicting lymph node metastasis, and their correlation with some clinicopathologic parameters.

Methods: 45 breast cancer patients and 50 apparently healthy sexmatched controls from the University of Ilorin Teaching Hospital were enrolled in this study. D-dimer and fibrinogen were assayed using an immunoturbidimetric latex assay and Fibrinogen determination kit- Clauss method respectively. Prothrombin time (PT) and Activated partial thromboplastin time (APTT) were determined using commercially prepared reagents according to the manufacturer's instructions. Various clinical and histopathologic parameters were also determined.

Results: Plasma D-dimer and fibrinogen were found to be significantly higher than controls $(P<0.001)$. APTT was significantly shorter than the controls $(P=0.001)$. $D$-dimer and fibrinogen were also significantly positively correlated with ECOG, disease stage, lymph node involvement and tumour size. The cut-off value of the $D$ - dimer, fibrinogen and DFR in the breast cancer patients were determined to be $1.18 \mathrm{mg} / \mathrm{ml}, 4.47 \mathrm{~g} / \mathrm{l}$, and 0.262 based on the ROC curve, and the sensitivity and specificity for lymph node involvement were $85.7 \%$ and $40 \%, 82.9 \%$ and $50 \%, 74.3 \%$ and $40 \%$ respectively. On multivariate analysis, D-dimer and fibrinogen were found to be independently related to lymph node involvement $(P=0.002$; $P=0.042$ ).

Conclusions: This study shows that plasma D- dimer and fibrinogen levels are elevated in breast cancer patients, and both are markers of disease progression especially lymph node involvement. 
TABLE 1 Variables $\chi^{2} \rho$ Study (\%) Control (\%) Age groups (Years) $4.7750 .189<354$ (8.9) 13 (26.0) 35 - 5026 (57.8) 23 (46.0) 51 - 65 8 (17.8) 8 (16.0) $\geq 667$ (15.6) 6 (12.0) Level of education 5.1480 .161 None 10 (22.2) 4 (8.0) Primary 6 (13.3) 4 (8.0) Secondary 11 (24.4) 17 (34.0) Tertiary 18 (40.0) 25 (50.0) Occupation 2.0140 .116 White collar 16 (35.6) 25 (50.0) Others 29 (64.4) 25 (50.0) BMI 8.845 0.031 Underweight 1 (2.2) 0 (0.0) Normal 17 (37.8) 31(62.0) Overweight 15 (33.3) 15 (30.0) Obese 12 (26.7) 4 (8.0) SBP 8.231 0.016 Normal 18 (40.0) 33 (66.0) Pre HTN 17 (37.8) 14 (28.0) Hypertensive 10 (22.2) 3 (6.0) DBP 4.329 0.115 Normal 40 (88.90 45 (90.0) Pre HTN 0 (0.0) 3 (6.0) Hypertensive 5 (11.1) 2 (4.0)

TABLE 2 Variable Study, $n=45$ Control, $n=50$ P-value D-Dimer Mean \pm SD Median IR $2520.47 \pm 1784.22196 .02018 .0370 .08 \pm$ $182.9350318 .5<0.001$ Fibrinogen Mean \pm SD Median IR $6.27 \pm$ $2.16 .513 .252 .02 \pm 0.81 .781 .24<0.001$ PT Mean \pm SD Median IR $12.89 \pm 1.513213 .12 \pm 1.31320 .534$ APTT Mean \pm SD Median IR $34.31 \pm 4.736438 .08 \pm 4.13870 .001$

\section{PB0734 | Malignant Clonal Cell Proliferation in Multiple} Myeloma and the Hypercoagulable State

T. Indran ${ }^{1}$; G. Gerotziafas ${ }^{2}$; J. Fareed ${ }^{3} ;$ A. Spencer ${ }^{1}$

${ }^{1}$ The Alfred Hospital, Melbourne, Australia; ${ }^{2}$ Sorbonne Universite, Paris, France; ${ }^{3}$ Loyola University Medical Centre, Maywood, United States

Background: The mechanism for hypercoagulability in Multiple Myeloma is unclear and confounded by the use of immunomodulatory drugs (IMiDs) such as Lenalidomide, which contribute to the prothrombotic effect despite thromboprophylaxis.

Aims: We aim to demonstrate the changes in coagulation profile with Daratumumab, Lenalidomide and Dexamethasone (DRd) and its correlation with plasma cell disease burden in MM using serum paraprotein (SPEP) and the serum free light chain assay (SFLC).

Methods: Data was obtained from $16 \mathrm{MM}$ patients who were Velcade refractory and received treatment with DRd at The Alfred Hospital, Melbourne from April 2019 to August 2020. Statistical analysis was performed at two time points, i) post 1-2 cycles of DRd and ii) post 3-4 cycles of DRd using descriptive statistics and the Wilcoxon Sign Rank Test with $P$ values of $<0.05$ indicating statistical significance.

Results:

7 patients had complete coagulation profiles at both time points and 9 patients with coagulation profiles after 3-4 cycles DRd. The Wilcoxon Sign Rank with imputed median differences allowed the inclusion of the 9 patients, increasing the sample size to a total of 16 (Table 1).

The reduction in the median fibrinogen level from $5.4 \mathrm{~g} / \mathrm{L}(2.8-8)$ to $3.98 \mathrm{~g} / \mathrm{L}(2.3-5.1)(P=0.001)$ was statistically significant with an increase in Thrombin Clotting Time $(P=0.005)$. This correlated with SPEP reduction from $10.8 \mathrm{~g} / \mathrm{L}(6-13)$ to $7.6 \mathrm{~g} / \mathrm{L}(2-13)(P=0.007)$. SFLC assay values reduced from median values of $82.8 \mathrm{mg} / \mathrm{L}(8.1-$ $415.7)$ to $54.6 \mathrm{mg} / \mathrm{L}(0.8-338.6)$ but was not statistically significant $(P=0.084)$
TABLE 1 Wilcoxon Sign Rank Test with median imputed difference comparing coagulation profiles, serum paraprotein and serum free light chains for 7 patients post 1-2 cycles Drd and 16 patients post 3-4 cycles Drd

\begin{tabular}{|c|c|c|c|c|}
\hline Test & $\begin{array}{l}\text { Normal } \\
\text { range }\end{array}$ & $\begin{array}{l}\text { Post } 1-2 \text { cycles } \\
\text { median (min-max), } \\
n=7 \text { patients }\end{array}$ & $\begin{array}{l}\text { Post } 3-4 \text { cycles } \\
\text { median }(\text { min-max }), n= \\
16 \text { patients }\end{array}$ & $\begin{array}{l}{ }^{*} \mathrm{p} \text { value } \\
<0.05\end{array}$ \\
\hline PT & $11-16 s$ & $13.6(11.9-16.6)$ & $12.7(11.7-14.1)$ & 0.006 * \\
\hline PTT & $28.6-38 \mathrm{~s}$ & $30.2(25.1-37.4)$ & $29.7(25.4-39.1)$ & 0.587 \\
\hline Fibrinogen & $2.0-4.0 \mathrm{~g} / \mathrm{L}$ & $5.4(2.8-8)$ & $3.9(2.3-5.1)$ & $0.001^{\star}$ \\
\hline TCT & $14-23 s$ & $15.7(14.4-18.4)$ & $16.1(14.6-18.8)$ & $0.005^{*}$ \\
\hline SPEP & $\mathrm{g} / \mathrm{L}$ & $10.8(6-13)$ & $7.6(2-14)$ & $0.007^{\star}$ \\
\hline SLFC & $\mathrm{mg} / \mathrm{L}$ & $82.8(8.1-415.7)$ & $54.6(0.8-338.6)$ & 0.084 \\
\hline
\end{tabular}

Conclusions: This analysis shows a coagulation response to treatment correlating with a decline in SPEP after 3-4 cycles of DRd. A larger study is required to confirm these findings and better understand the role of clonal plasma cells and hypercoagulability.

\section{PB0735 | Fibrinogen Binding to Macrophages Attenuates} Antiviral Responses to dsRNA

D. Martinez; M.J. Flick; S. Antoniak

UNC Blood Research Center, Chapel Hill, United States

Background: Fibrinogen is an acute-phase protein in response to viral infections. In addition, viral replication leads to dsRNA generation. CD11b/CD18 (Mac-1), a receptor for fibrinogen, has been identified as a surface receptor for extracellular dsRNA. The binding of dsRNA to macrophage Mac-1 resulted in increased cytokine expression in vitro and in vivo.

Aims: We investigate if fibrinogen acts as an immunosuppressant by reducing the proinflammatory response of macrophages to the dsRNA mimetic poly I:C.

Methods: To analyze the effect of fibrinogen binding to macrophages, murine macrophagic cells (RAW264.7) were preincubated with or without fibrinogen $(50 \mu \mathrm{g} / \mathrm{mL})$ for $1 \mathrm{hr}$ prior to poly I:C $(5 \mu \mathrm{g} /$ $\mathrm{mL}$ ) stimulation under serum-free conditions. The release of TNFa and IL- 6 into the media was analyzed by ELISA 24 hrs after stimulation. Activation of the p38 MAPK pathway over $4 \mathrm{hrs}$ in response to poly I:C stimulation with or without fibrinogen preincubation was analyzed by Western blot.

Results: Fibrinogen preincubation of RAW264.7 macrophages reduced TNFa and IL- 6 release in the media to $52 \%$ and $56 \%$, respectively, of the poly I:C stimulation alone $(P<0.05)$. Importantly, fibrinogen binding to macrophages alone did not induce any cytokine release. The reduction in poly I:C-mediated cytokine release from cells preincubated with fibrinogen was associated with reduced activation of the $\mathrm{p} 38 \mathrm{MAPK}$ pathway. 
Conclusions: Our results indicate that fibrinogen binding to macrophages reduced dsRNA-induced responses in macrophages. This suggests that during viral infections fibrinogen might act as an immunosuppressant to dampen proinflammatory responses to dsRNA.

PB0737 | An Assessment of Some Inflammatory and Haemostatic Proteins as Biomarkers for Vaso-occlusive Crises in Sickle Cell Anaemia Patients

O. Izuegbuna $^{1}$; I. Durotoye ${ }^{2}$; H. Olawumi ${ }^{2}$; I. Okpala ${ }^{3}$; K. Ernest ${ }^{2}$

${ }^{1}$ LAUTECH Teaching Hospital, Ogbomoso, Nigeria; ${ }^{2}$ University of Ilorin, Ilorin, Nigeria; ${ }^{3}$ University of Nigeria, Nsukka, Nigeria

Background: Sickle cell anaemia is associated with inflammatory and haemostatic abnormalities. C- reactive protein (CRP), absolute neutrophil count (ANC) as well as Factor VIII and von Willebrand factor (VWF) are known drivers of inflammation and haemostasis. The aim of this study, therefore, was to assess the role of VWF, Factor VIII, ANC and CRP as markers of vaso-occlusive crisis (VOC) in sickle cell anaemia patients.

Aims: The aim of this study, therefore, was to assess the role of VWF, Factor VIII, ANC and CRP as markers of vaso-occlusive crisis (VOC) in sickle cell anaemia patients.

Methods: A total of $97 \mathrm{HbSS}$ patients, 50 in steady-state and 47 in crises were studied at the University of Ilorin Teaching Hospital, while $50 \mathrm{HbAA}$ apparently well, age-matched persons served as controls. The mean CRP, ANC, VWF and Factor VIII in VOC were determined and compared to steady-state patients using the student $\mathrm{t}$-test. Association between the biomarkers were determined using the Spearman correlation test. A one way ANOVA was done to determine the mean difference across the pain scale of the different biomarkers.

Results: In a total of 97 patients evaluated, the mean values of CRP, ANC, VWF and Factor VIII were significantly higher in VOC than in steady-state $(P<0.001)$. ANC, VWF and Factor VIII were positively correlated with CRP. CRP level was significant in the ANOVA test. In the multivariate analysis, ANC was the best predictor of VOC.

Conclusions: These data highlight the clinical relevance of inflammation and haemostasis in VOC. Increased ANC and CRP could be early predictors of VOC and thus have potential use as biomarkers of early VOC.

TABLE 1 Parameters SCA (steady state) SCA (VOC) Controls Age Hct WBC Platelets ANC ALC NLR PLR PT APTT VWF Factor VIII HsCRP $19.10 \pm 11.5723 .12 \pm 3.6310 .81 \pm 2.50^{* *} 395.86 \pm 106.74^{* *}$ $5.18 \pm 1.29^{* *} 4.47 \pm 1.69^{* *} 1.28 \pm 0.45^{* *} 100.66 \pm 46.20^{* *} 11.68 \pm$ $1.6724 .53 \pm 4.76^{* *} 156.38 \pm 38.66^{* *} 162.64 \pm 34.32^{* *} 7.12 \pm 2.63^{* *}$ $17.74 \pm 7.3921 .73 \pm 4.1518 .05 \pm 3.25275 .45 \pm 84.8211 .02 \pm 2.82$ $5.29 \pm 1.092 .16 \pm 0.7554 .08 \pm 18.6511 .77 \pm 0.8221 .49 \pm 2.28$ $216.55 \pm 34.05205 .85 \pm 30.3316 .12 \pm 3.0618 .52 \pm 8.2639 .77 \pm$ $3.024 .69 \pm 1.18226 .62 \pm 40.121 .99 \pm 0.512 .21 \pm 0.610 .93 \pm 0.21$ $109.98 \pm 36.9311 .24 \pm 0.8729 .98 \pm 2.4873 .86 \pm 29.2272 .31 \pm 8.19$ $3.88 \pm 1.18$
TABLE 2 Biomarker B SE Wald P value OR (95\% CI) WBC Platelets ANC ALC NLR PLR APTT VWF Factor VIII HsCRP 1.192 -0.020 4.432 $0.4084 .419-0.055-0.2430 .0430 .0430 .8540 .2810 .0051 .749$ 0.1540 .9630 .0120 .0720 .0080 .0090 .18018 .03818 .5106 .424 $6.96521 .05720 .69311 .49926 .21821 .26522 .601<0.001<0.001$ $0.0110 .008<0.001<0.001<0.001<0.0013 .295(1.900-5.712)$ 0.980 (0.971-0.989) $84.123(2.732-2590.576) 1.503(1.111-2.034)$ 82.979 (12.570-547.757) 0.946 (0.924-0.969) 0.784 (0.681-0.903) 1.044 (1.027-1.062) 1.044 (1.025-1.064) 2.348 (1.651-3.338)

\section{PB0738 | Biomarkers of Inflammation and Thrombosis in} Hepatocellular Carcinoma

A. Farooqui; A. Kulkarni; D. Hoppensteadt; D. Van Thiel; F. Siddiqui; J. Fareed

Loyola University Medical Center, Maywood, United States

Background: Hepatocellular carcinoma (HCC) is the sixth most common cancer worldwide. The most common causes of HCC are hepatitis B or C virus infections, alcoholic liver disease, nonalcoholic fatty liver disease, biliary diseases, metabolic disorders, drugs, toxins, and genetic conditions. Chronic inflammation and consequent regeneration enhance mutagenesis in hepatocytes. The inflammation of hepatic cells results in the secretion of cytokines and chemokines as well as lysis of infected cells, which causes much of the liver injury. Interleukins (IL) are a group of cytokines that were first seen to be expressed by white blood cells and stimulate inflammation; this stimulation of the immune response by interleukins can lead to thrombosis.

Aims: The purpose of this study is to analyze different Interleukins, their concentration, and role in the development of thrombosis in HCC.

Methods: Citrated blood samples from 36 patients with confirmed diagnosis of HCC were collected from the hospital and frozen at $-80 \mathrm{C}$. Controlled plasma samples were comprised of 49 samples collected from normal healthy individuals. Biomarkers of inflammation including IL-1b, IL-2, IL-4, IL-6 and IL-10 were measured using commercially available kits (R\&D laboratories, Minneapolis, MN). All results were compiled as mean \pm SEM.

Results: In comparison to normal subjects, patients with HCC showed variable increases in the parameters study. IL6 and 8 showed the most pronounced increase in comparison to other interleukins. There was no correlation between IL-6 and IL-8 levels.

Conclusions: These results demonstrate that interleukin levels are elevated in patients with ACC in comparison to normal samples IL-6 and IL-8 showed the most pronounced increase. This elevation of interleukins potentially contributes to the pathogenesis of HCC. Thus, interleukins provide a prognostic biomarker panel for HCC. 


\section{COMPLEMENT AND HEMOSTATIC SYSTEM}

LPB0118 | Clinically Meaningful and Long-term Improvements in Fatigue with the C3 Inhibitor Pegcetacoplan In Paroxysmal Nocturnal Hemoglobinuria: Post-hoc Analyses from the PEGASUS Study Week 48

D. Cella ${ }^{1}$; S.P. Sarda ${ }^{2}$; R. Hsieh ${ }^{3}$; J. Fishman²; Z. Hakimi ${ }^{4}$; K. Cutts ${ }^{3}$; W.R. Lenderking ${ }^{5}$

${ }^{1}$ Northwestern University, Chicago, United States; ${ }^{2}$ Apellis

Pharmaceuticals, Inc, Waltham, United States; ${ }^{3}$ Evidera, Bethesda, United States; ${ }^{4}$ Sobi, Stockholm, Sweden; ${ }^{5}$ Evidera, Waltham, United States

Background: PEGASUS was a phase 3, randomized, open-label, active-comparator-controlled study in 80 adults with paroxysmal nocturnal hemoglobinuria (PNH) with $\mathrm{Hb}<10.5 \mathrm{~g} / \mathrm{dl}$ while on eculizumab (ECU) therapy. After 16 weeks, pegcetacoplan (PEG) was superior to ECU in improving $\mathrm{Hb}$ levels and fatigue. ECU was switched to PEG after randomized controlled period (Figure 1).

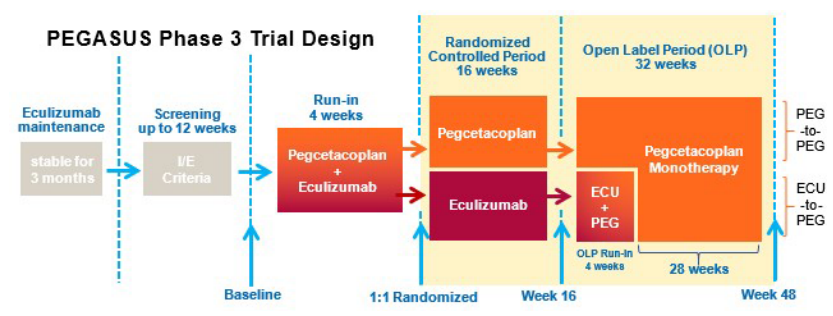

Aims: This analysis evaluated changes in fatigue through week 48. Methods: Fatigue was assessed with the Functional Assessment of Chronic Illness Therapy-Fatigue (FACIT-F) scale. Mean changes by treatment, including patients with intercurrent events (ICE), from baseline to week 16, and week 16 to week 48 are described. Spearman correlations assessed validity. Responder analysis used thresholds of clinically important differences of 3-, 4-, or 5-point responders. Item-level analysis was conducted to assess consistency and persistence of effect.

Results: FACIT-F total score was significantly correlated with hemoglobin ( $r=0.47, P<0.0001)$ at week 16 . At week 48, both PEG-toPEG and ECU-to-PEG groups had near equal FACIT-F total scores (PEG-to-PEG: 40.6 and ECU-to-PEG: 42.5), maintaining the statistically significant correlation $(r=0.32, P<0.05)$ indicating strong treatment effect with PEG. PEG-to-PEG group showed a high percentage of responders early in treatment, by week 16 , and remained high (less fatigue) from week 16 to week 48. In contrast, ECU-toPEG group showed a worsening of fatigue between baseline and Week 16, which then improved to the level of the PEG group after they were switched to PEG (Table 1). Both the experience (5-item) and impact (8-item) subsets of the FACIT-F reflected this pattern.

FIGURE 1 PEGASUS Phase 3 Trial Design

TABLE 1 Mean Changes and Percent Responders by Treatment Group for FACIT-F Score

\begin{tabular}{|c|c|c|c|c|}
\hline \multirow{2}{*}{ Treatment Group } & \multicolumn{4}{|c|}{ Responder Threshold* } \\
\hline & $\triangle$ Score & $\geq 3 \mathrm{MCID}$ & $\geq 4 \mathrm{MCID}$ & $\geq 5 \mathrm{MCID}$ \\
\hline \multicolumn{5}{|c|}{ Baseline to Week 16 (including RIP) ${ }^{\dagger}$} \\
\hline PEG $(n=36)$ & $10.3(11.03)$ & $30(83.3 \%)$ & $29(80.6 \%)$ & $26(72.2 \%)$ \\
\hline \multicolumn{5}{|l|}{ Week 16 to Week 48} \\
\hline PEG-to-PEG $(n=28)$ & $-0.5(7.38)$ & $8(28.6 \%)$ & $6(21.4 \%)$ & $4(14.3 \%)$ \\
\hline ECU-to-PEG $(n=28)$ & $10.9(10.86)$ & 23 (82.1\%) & 22 (78.6\%) & 21 (75.0\%) \\
\hline
\end{tabular}

* increase of 3 points on the FACIT-F is considered clinically meaningful.

${ }^{\dagger}$ analysis performed on all available data regardless of intercurrent events.

PEG: pegcetacoplan, ECU: eculizumab. MCID: Minimal Clinically Important Difference. SD: standard deviation. RIP: Run in period, where patients received both eculizumab and pegcetacoplan.

Conclusions: Findings showed clinically meaningful improvements in Fatigue among patients taking PEG, continuously through Week 48 in PEGASUS, and in those who switched from ECU. The effect was consistent across all the items of FACIT-F demonstrating that PEG meaningfully reduces fatigue levels and maintains this effect over time by increasing $\mathrm{Hb}$ levels. 


\section{PB0739 | Acquired Isolated FVII Deficiency in Oncological}

\section{Patients}

N. García-León; M.T. Calderón-López; S. Gómez-Arevalillo Hidalgo;

P. Martín-Serrano Martín; A. Matilla-García

Hospital Central de la Defensa, Gómez Ulla, Madrid, Spain

Background: Acquired isolated factor VII (FVII) deficiency is a rare bleeding disorder and it can be associated with an oncological disease. Aims: To investigate the acquired isolated FVII deficiency associated with malignancy.

Methods: We analyzed FVII deficiency found in the tests performed in our hospital from 2018 to 2020. Inclusion criteria were isolated FVII defect of less than $55 \%$ of normal, normal level of other vitamin $\mathrm{K}$-dependent clotting factors, no history of liver disease and negative coagulation pattern in the family. All these patients had at least 2 samples with a prolonged PT and a normal PT mixing study.

Results: 43 patients had an isolated FVII deficiency and met the inclusion criteria. 6 had an infection, 21 patients had a recent history of malignancy and 16 had no relation with any disease.

The 21 oncological patients had a normal prothrombin time (PT) before the diagnosis of the malignant disease and none of them had bleeding history. 12 of these patients died during the first year after the diagnosis of the FVII deficiency.

Median age was 65 (range 16-91), 16 were men and 5 women. The mean level of FVII was $42 \%$ (range $21,4-51 \%$ )

The most frequent tumor involved was genitourinary and the histological type, adenocarcinoma.

Only 2 out of 21 patients had bleeding symptoms and in both cases was a moderate hematuria associated with bladder tumor.

TABLE 1 Tumors involved

\begin{tabular}{ll}
\hline DIGESTIVE & 5 \\
GENITOURINARY & 7 \\
THYROID & 1 \\
PULMONARY & 4 \\
HEMATOLOGICAL & 2 \\
BONE & 1 \\
SOFT TISSUE & 1 \\
\hline
\end{tabular}

TABLE 2 Histological types of tumors

\begin{tabular}{ll}
\hline ADENOCARCINOMA & 9 \\
SARCOMA & 3 \\
CLEAR CELL CARCINOMA & 1 \\
PAPILAR CARCINOMA & 1 \\
FIBROUS HISTIOCYTOMA & 1 \\
MESENQUIMAL & 1 \\
WALDESTROM MACROGLOBULINEMIA & 1 \\
HODGKIN LYMPHOMA & 1 \\
NOT HISTOLOGICAL RESULT & 3 \\
\hline
\end{tabular}

Conclusions: Acquired isolated FVII deficiency is a rare defect, which appears to be associated with several conditions, especially sepsis and tumors. This indicates the need for a careful investigation of even a mild prolongation of prothrombin time, especially in oncological patients.

\section{PB0740 | Coagulopathy in Multiple Myeloma}

K. Chasakova ${ }^{1}$; L. Slavik ${ }^{2}$; D. Starostka ${ }^{1}$; J. Úlehlová ${ }^{2}$;

T. Papajik ${ }^{2}$; J. Minařík ${ }^{2}$

${ }^{1}$ Department of Clinical Hematology, Hospital in Havirov, Havirov, Czech Republic; ${ }^{2}$ Department of Hemato-oncology, University Hospital and Faculty of Medicine and Dentistry, Palacký University Olomouc, Olomouc, Czech Republic

Background: Multiple myeloma (MM) is a lymphoproliferative disease characterized by clonal proliferation and accumulation of neoplastic plasma cells, the presence of monoclonal immunoglobulin and osteolytic skeletal involvement.

Some of hemostasis disorders are attributed to M-lg interactions with blood clotting factors (acquired von Willebrand's disease, acquired hemophilia $A$ or deficits of other coagulation factors, circulating anticoagulant, hyperviscosity, amyloidosis and lupus anticoagulant) or with platelets (acquired thrombocytopathy), MIg-independent effects (thrombocytopenia, other thrombocytopathies, DIC, immobility and hypercalcaemia).

Aims: The aim of our work is to detect abnormalities of coagulation in patients with newly diagnosed MM suitable for intensive chemotherapy depending on the activity of the disease, which predispose patients to thrombotic resp. bleeding complication in MM.

Methods: We included 113 patients with newly diagnosed multiple myeloma in this study. Patients with MM were examined by coagulation tests detecting both bleeding and thrombotic tendency: PT, APTT, TT, fibrinogen, antithrombin, D-dimers (D-Dim), levels of coagulation factors (II, V, VII, X, VIII, IX, XI and XII), vWF, lupus anticoagulant, protein $C$, protein $S$, resistance to activated protein $C$. We also monitored plasma cell counts and serum paraprotein levels in these patients.

Results: All markers were evaluated (average value, standard deviation) to the disease activity defined by the paraprotein level resp. number of plasma cells. A significant correlation was found between D-Dim and paraprotein $P=0.0031$ resp. plasma cells $P=0.0006$ and between vWF vs. paraprotein $P=0.0053$ not between plasma cells $P=0.42$, which is interesting.

Conclusions: In newly diagnosed patients with $\mathrm{MM}$, we recommend increased attention to the level of D-Dim and vWF, especially in patients with higher disease activity in order to estimate possible bleeding or thrombotic complications, for which long term observation is needed.

Supported by grant LF-2021-001 and MH CZ - DRO (FNOI, 00098892). 
PB0741 | Elucidating the Relationship between Thromboinflammatory Biomarkers and Blood Cellular Indices in Lymphoma Patients

M. Jaradeh ${ }^{1}$; N. Baig ${ }^{1}$; E. Bontekoe ${ }^{1}$; D. Antic ${ }^{2}$; J. Fareed ${ }^{1}$

${ }^{1}$ Loyola University Medical Center, Maywood, United States;

${ }^{2}$ University of Belgrade, Belgrade, Serbia

Background: Although thrombo-inflammatory biomarkers and blood cellular indices have been reported in the pathogenesis and tumorigenesis in lymphoma patients, their interrelationship has not been fully elucidated.

Aims: In this study, we aimed to determine the relationship of thrombo-inflammatory biomarkers and blood cellular indices in lymphoma patients compared to normal controls.

Methods: Ninety-nine citrated blood samples were collected from histologically-confirmed lymphoma patients at the Hematology Clinic, University of Belgrade, Serbia. Normal controls were comprised of plasma obtained from 50 healthy volunteers. These samples were analyzed for the circulating levels of plasminogen activator inhibitor (PAI-1), D-Dimer, factor XIII, C-reactive protein (CRP), microparticles (Mp), Von Willebrand factor (vWF), total protein $S$, urokinase-type plasminogen activator (UPA), tumor necrosis factor (TNF), beta2-glycoprotein I (B2GPI), and fibronectin utilizing commercially available ELISA methods. Thrombin generation profile (TGA) was measured using a fluorometric kinetic assay. Platelets, leukocytes, lymphocytes, and neutrophils were measured in conjunction with the complete blood profile. Such cellular indices as neutrophil/lymphocyte, platelet/neutrophil, and platelet/lymphocytes were compiled.

Results: We found a statistically significant difference in lymphoma growth compared to control in levels of PAI-1, DDimer, factor XIII, CRP, microparticles, vWF, UPA, TNF, B2GPI, fibronectin, and peak thrombin when compared to normals (all $P<0.001$ ). In relating thrombo-inflammatory biomarkers to cellular indices, we found the platelet to leukocyte ratio correlated to TNF and fibronectin ( $R=-0.31$ and -0.53 , respectively) and the platelet to neutrophil ratio correlated to factor XIII and B2GPI (R values $=0.40$ and 0.40 , respectively). Wide variations were noted in the neutrophil/lym phocyte ratio with marginal correlations with some of the thromboinflammatory biomarkers.

Conclusions: When compared to normals, lymphoma patients appear to have a significantly altered thrombo-inflammatory biomarker profile that has notable correlations to blood cellular indices. These findings warrant clinical outcome analysis and the relevance of the thrombo-inflammatory biomarkers and cellular indices in a larger cohort in subsets of lymphoma populations.

\section{INFECTION AND HEMOSTATIC FACTORS}

LPB0119 | Impact of Vancomycin-induced Shift of the Gut Microbiome in Gram Negative Direction on Plasma Factor VIII:C Levels: Results from a Randomized, Controlled Trial

G. Grimnes ${ }^{1,2} ; \underline{\text { S. Bhoelan }}{ }^{1,3} ;$ K. Hindberg ${ }^{1} ;$ M. Davids ${ }^{4}$; M. Nieuwdorp ${ }^{4,5,6}$; T.E. Mollnes ${ }^{1,7,8}$; A. Michelsen ${ }^{9,10}$; T. Ueland ${ }^{1,9,10}$; S. Braekkan ${ }^{1,2}$; J.-B. Hansen ${ }^{1,2}$; V. Tichelaar ${ }^{1,3,11}$

${ }^{1}$ Thrombosis Research Center (TREC), Department of Clinical Medicine, UiT - The Arctic University of Norway, Tromsø, Norway; ${ }^{2}$ Division of Internal Medicine, University Hospital of North Norway, Tromsø, Norway; ${ }^{3}$ Department of Haematology, University Medical Centre Groningen and University of Groningen, Groningen, Netherlands; ${ }^{4}$ Department of Vascular Medicine, Amsterdam UMC location AMC, University of Amsterdam, Amsterdam, Netherlands; ${ }^{5}$ Diabetes Center, Department of Internal Medicine, Amsterdam UMC location VUmc, VU Amsterdam, Amsterdam, Netherlands; ' Wallenberg Laboratory, University of Gothenberg, Gothenberg, Sweden; ${ }^{7}$ Research Laboratory, Nordland Hospital, Bodø, Norway; ${ }^{8}$ Department of Immunology, Oslo University Hospital and K.G. Jebsen IRC, University of Oslo, Oslo, Norway; ${ }^{9}$ Research Institute of Internal Medicine, Oslo University Hospital, Rikshospitalet, Oslo, Norway, ${ }^{10}$ Faculty of Medicine, University of Oslo, Oslo, Norway, ${ }^{11}$ Certe Thrombosis Service, Groningen, Netherlands

Background: Dysbiosis might be a source of systemic inflammation and subsequent increased coagulation activity by translocation of lipopolysaccharides from gram-negative bacteria to the systemic circulation.

Aims: To investigate whether a vancomycin induced gram-negative shift of the microbiome influences systemic inflammation and plasma factor (F)VIII activity.

Methods: We performed a randomized controlled trial including healthy adult volunteers. Participants were randomized to seven days of oral vancomycin treatment or to no intervention. Feces and blood were sampled at baseline (TO) and the day after end of intervention (T1). The gut microbiome composition was assessed before and after intervention. Plasma FVIII activity (FVIII:C) and high-sensitivity (hs)CRP, cytokines, complement activation and zonulin as a marker for intestinal permeability were measured. The differences between T1 and T0 were analysed. All subjects provided informed consent. The study was approved by the Regional Committee for Medical and Health Research Ethics and the Norwegian Data Inspectorate.

Results: Fourty-three participants were included, 21 in the intervention group and 22 in the control group (mean age 26.7 vs. 25.0 years and 14 vs. 15 females respectively). Vancomycin intake significantly reduced gut microbiome diversity and increased the abundance of gram-negative bacteria. Change in FVIII:C in the intervention group was $+4 \mathrm{lU} / \mathrm{dL}$ vs. $-6 \mathrm{IU} / \mathrm{dL},(P=0.01)$ in the control group. A similar change was observed for log-transformed hs-CRP (+0.21 vs. -0.25 , 
$P=0.04)$ The cytokines and complement activation markers remained similar in the two groups. No differences in zonulin levels were found.

Conclusions: Although vancomycin intake did not affect intestinal permeability in this healthy population, our findings support the hypothesis that a vancomycin-induced gram-negative shift in the gut microbiome could lead to an increase in plasma FVIII:C and CRP levels.

PB0743 | Von Willebrand Factor Propeptide to Antigen Ratio: Evidence of Endothelial Activation in HIV Infected Pregnancy

E. Schapkaitz ${ }^{1}$; E. Libhaber ${ }^{1}$; B. Jacobson ${ }^{1}$; M. Meiring ${ }^{2}$; H. Büller ${ }^{3}$ ${ }^{1}$ University of Witwatersrand, Johannesburg, South Africa; ${ }^{2}$ University of Free State and National Health Laboratory Service, Bloemfontein, South Africa; ${ }^{3}$ University of Amsterdam, Amsterdam, Netherlands

Background: An increased incidence of venous thrombo-embolism (VTE) has been observed in human immunodeficiency virus (HIV) infected pregnant women in the era of antiretroviral therapy (ART). The extent to which endothelial activation contributes among virologically- suppressed HIV-infected pregnant women has not been studied. The plasma ratio of von Willebrand factor (VWF) propeptide to VWF antigen, which declines in normal pregnancy, has been identified as a useful marker of endothelial activation.

Aims: To assess the VWF propeptide to antigen ratio in all three trimesters of pregnancy and its relationship with HIV viral load (VL) and ADAMTS13

Methods: A cross-sectional analysis was performed in 89 HIVinfected virologically-suppressed patients on ART for 5 [7] years, 63 HIV-infected patients with VL $>50$ copies/ml and 85 matched HIVuninfected women. Testing was measured in the first (6-12 weeks), second (13-26 weeks) and third trimesters (27-39 weeks). The study protocol was approved by the ethics committee of the University of the Witwatersrand (M-181018).
Results:

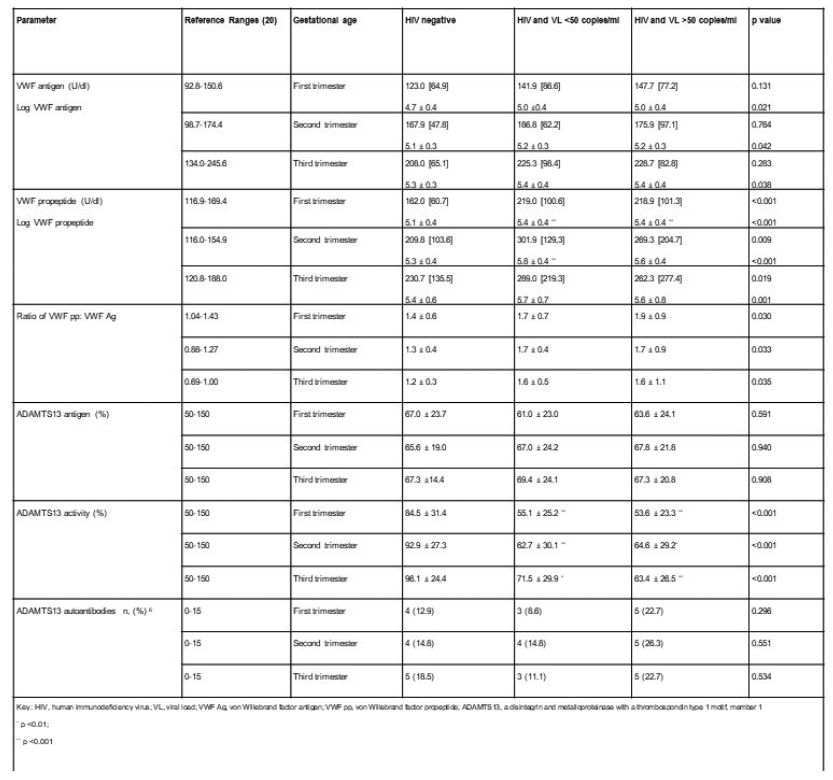

FIGURE 1 Comparison of von Willebrand Factor associated parameters between HIV-uninfected, HIV-infected with virologicalsuppression and HIV-infected with VL $>50$ copies/ml study groups

Elevated measurements of VWF propeptide to antigen ratio were observed, in the first, second and third trimesters, in the HIV-infected group with $\mathrm{VL}>50$ copies $/ \mathrm{ml}(1.9 \pm 0.9,1.7 \pm 0.9,1.6 \pm 1.1)$ and the HIV-infected virologically-suppressed group $(1.7 \pm 0.7,1.7 \pm 0.4,1.6$ \pm 0.5 ) as compared to the HIV-uninfected group (1.4 $\pm 0.6,1.3 \pm 0.4$, $1.2 \pm 0.3, P<0.05)$. This was associated with a modest reduction in ADAMTS13 activity per trimester in the HIV-infected group with VL $>50$ copies/ml (53.6 $\pm 23.3 ; 64.6 \pm 29.2 ; 63.4 \pm 26.5)$ and the HIVinfected virologically-suppressed group $(55.1 \pm 25.2 ; 62.7 \pm 30.1 ; 71.5$ $\pm 29.9 \%)$ as compared to the HIV-uninfected group (84.5 \pm 31.4 ; $92.9 \pm$ 27.3; $96.1 \pm 24.4, P<0.001)$. Antibodies directed against ADAMTS13 were not significantly increased in HIV infected pregnancies $(P<0.05)$. There were no VTE cases in pregnancy or up to six-weeks postpartum. Conclusions: HIV-infected virologically suppressed pregnant patients show persistent endothelial activation.

PB0744 | Long Term Trends of Coagulation Parameters in People Living with HIV-1 Treated with Combined Antiretroviral Therapy

$\underline{\text { S. Bhoelan }^{1}}$; J. Borjas Howard ${ }^{1}$; V. Tichelaar ${ }^{1,2}$; W. Bierman ${ }^{3}$; K. Meijer ${ }^{1}$

${ }^{1}$ Department of Haematology, University Medical Centre Groningen and University of Groningen, Groningen, Netherlands; ${ }^{2}$ Certe Thrombosis Service, Groningen, Netherlands; ${ }^{3}$ Department of Internal Medicine, Division of Infectious Disease, University Medical Centre Groningen and University of Groningen, Groningen, Netherlands

Background: Human immunodeficiency (HIV) infection causes a procoagulant state. However, haemostatic trends after long term treatment with combination antiretroviral therapy (CART) are unclear. 
Aims: To longitudinally describe long term coagulation parameters in people living with HIV on CART.

Methods: We followed forty male HIV-1 infected subjects starting cART. Plasma levels of procoagulant parameters, factor VIII (FVIII), von Willebrand factor (vWF) and D-dimer, and anticoagulant parameter Protein S (PS), were measured before start and three months, one and nine years later. We adjusted for cardiovascular risk factors (age, smoking and hypertension) at baseline as confounding explanatory variables. All subjects provided informed consent. The study was approved by the medical ethics committee of University Medical Centre Groningen.

Results: At baseline, mean age was $44 \pm 12$ years and median CD4/ CD8-ratio 0.24 (IQR 0.17-0.36). Procoagulant parameters were markedly elevated and PS was in the lower range of normal. At nine years CD4/CD8-ratio improved to median 0.87 (IQR 0.74-1.31).

At three months, the procoagulant parameters were decreasing (FVIII -37.5\%[95\% Cl -53.9, -21.2], vWF -52.7\%[-77.9, -27.6], Ddimer $-239.5 \mu \mathrm{g} / \mathrm{L}[-376.9,-102.2])$. This trend continued moderately until one year. Conversely, from one to nine years an increase was observed (FVIII 15.6\%[4.5, 26.7], vWF 1.2\%[-13.3, 15.7], Ddimer $102.4 \mu \mathrm{g} / \mathrm{L}[-33.3,238.1])$. After correction for age, smoking and hypertension, this increase was reversed (FVIII -6.5\%[-23.5, 10.5], vWF -10.4\%[-33.6, 12.7], D-dimer -9.1 $\mu \mathrm{g} / \mathrm{L}[-254.3,236.2])$. PS remained stable during the first year and slightly increased from one to nine years (total 12.2\%[4.2, 20.2], free 13.2\%[6.2, 20.2]).

In the first year, changes in mainly the procoagulant parameters were correlated to CD4/CD8 ratio improvement. After this period, this correlation was no longer present.
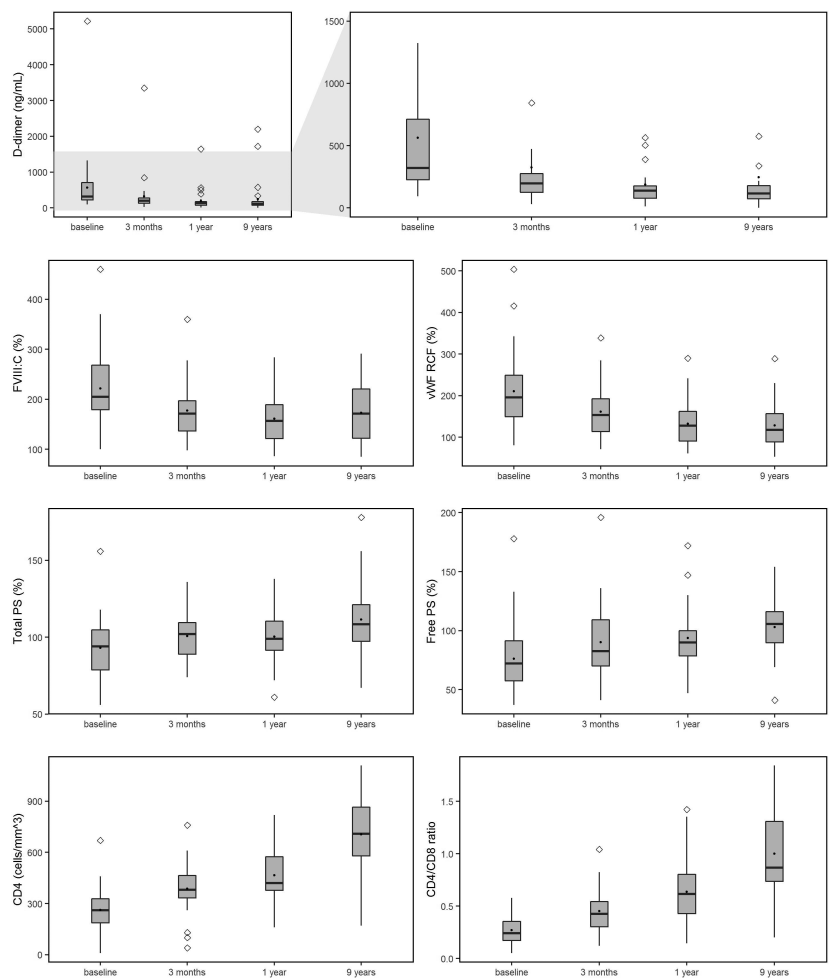

FIGURE 1 Trends of coagulation and immune activation parameters over time

TABLE 1 Crude (Model 1 ) and multivariable (Model 2) analysis of changes $(\Delta)$ in activity of procoagulant parameters 1 year vs. 9 years (t) with a $95 \%$ confidence interval

$\begin{array}{lll}\text { Model } & & \Delta \mathrm{FVIII} \\ 1 & \mathrm{t} & 15.6(4.5,26.7) \\ 2 & \mathrm{t} & -6.5(-23.5,10.5) \\ & \text { Age } & -0.3(-1.2,0.6) \\ & \text { Smoking } & 18.4(-1.6,38.5) \\ & \text { Hypertension } & 44.0(15.4,72.6)\end{array}$

$\Delta \mathrm{vWF}$

$1.2(13.3,15.7)$

$-10.4(-33.6,12.7)$

$0.1(-1.2,1.3)$

$-0.6(-27.9,26.7)$

$52.9(14.0,91.9)$
$\Delta$ D-dimer

$102.4(-33.3,238.1)$

$-9.1(-254.3,236.2)$

$8.3(-5.2,21.8)$

$134.4(-154.1,422.9)$

$49.6(-362.2,461.5)$
Conclusions: Immune reconstitution by CART restores the procoagulant state in HIV during the first year. On long term this restoration is reversed despite on-going immune reconstitution. This seems to be related to cardiovascular risk factors.
PB0745 | Endothelial Protein C Receptor (EPCR)-dependent Sequestration of $P$. falciparum Erythrocyte Membrane Protein 1 (PfEMP1)-derived Fluorescent Microspheres in the Brain Vasculature of a Humanized Mouse

R. Aiolfi' ${ }^{1}$; P.J. Cowan²; H. Gock ${ }^{2}$; H. Weiler ${ }^{3}$; L. Turner ${ }^{4}$;

T. Lavstsen ${ }^{4}$; L.O. Mosnier ${ }^{1}$

${ }^{1}$ The Scripps Research Institute, La Jolla, United States; ${ }^{2}$ The University of Melbourne, Melbourne, Australia; ${ }^{3}$ Blood Research Institute, Milwaukee, United States; ${ }^{4}$ Centre for Medical Parasitology, University of Copenhagen, Copenhagen, Denmark

Background: PfEMP1 variants containing EPCR binding CIDRa1 domains are associated with severe malaria complications. The 
inhibition of activated protein C (APC) binding to EPCR by CIDRa1 domains and thereby APC's cytoprotective activities is considered to be a major contributing factor for the development of cerebral malaria (CM). However, purified CIDRa1 domains administered to a humanized EPCR (hEPCR) mouse accumulated predominantly in the lung with only minor accumulation in the brain, raising new questions how infected erythrocytes sequester in the brain vasculature. Aims: To characterize the specific EPCR-dependent sequestration of CIDRa1.1-derivatized $2 \mu \mathrm{m}$ fluorescent microspheres, mimicking infected erythrocytes, in the brain vasculature in a hEPCR mouse model. Methods: Streptavidin coated fluorescent microspheres were labeled with biotinylated anti-V5 antibody and V5-tagged CIDRa1 domains and administered in the carotid artery of wild-type and hEPCR mice.

Results: In vivo, injection of CIDRa1.1+ microspheres in the ascendant carotid artery resulted in significant accumulation of beads into the brain microvasculature of hEPCR transgenic mice compared to WT. The carotid artery inoculation resulted in a dose and time dependent sequestration of CIDRa1.1+ microspheres peaking at 1 hour post injection. Accumulation was predominant in the brain hemisphere fed by the injected carotid artery even though microsphere distribution was also observed in the contralateral one especially in hEPCR transgenic mice.

Conclusions: In vivo, accumulation of CIDRa1.1+ microspheres in the brain of mice expressing hEPCR was much more efficient than that of purified CIDRa1.1 domains. This model provides an effective method for the analysis of EPCR-targeting treatments during severe malaria and to determine the effect of brain edema, a hall mark of cerebral malaria, on the EPCR-dependent sequestration of infected erythrocytes in the brain vasculature.

\section{PLATELETS AND CANCER}

LPB0072 | Aspirin vs P2RY12-Inhibitors to Reduce Cancer Associated Thrombosis and Tumor Growth in Digestive Cancers

A.L. Palacios Acedo ${ }^{1}$; S. Mezouar ${ }^{1}$; D. Mege ${ }^{1,2}$; L. Crescence ${ }^{1}$; C. Dubois ${ }^{1}$; L. Panicot-Dubois ${ }^{1}$

${ }^{1}$ C2VN INSERM 1263, Marseille, France; ${ }^{2}$ Department of Digestive Surgery, Timone University Hospital, Marseille, France

Background: Platelet function can be "kidnaped" by cancer cells to support tumor growth; causing alterations in the delicate hemostatic equilibrium. This leads to a four-fold risk of thrombotic events in cancer patients, which in turn, portend poor prognosis. We previously demonstrated that anti-P2RY12 drugs inhibit thrombosis associated with cancer and formation of metastasis in pancreatic cancer models.

Aims: (1) To determine the expression of P2RY12 across different digestive cancers (colorectal and pancreatic) and demonstrate its effect in vitro.
(2) To characterize the effects of known antiplatelet drug clopidogrel (platelet P2RY12 inhibitor) on cancer associated thrombosis and cancer growth in vivo.

(3) To compare the effects of clopidogrel and aspirin in pancreatic cancer progression.

Methods: We used flow cytometry and immunohistochemistry; along with molecular biology techniques like RT-qPCR, and cDNA sequencing techniques to determine P2YR12 presence in primary tissue and cell lines. The effect of P2RY12 on cancer cell lines was determined using wound healing, proliferation tests and transwell culture. Syngeneic ectopic and orthotopic mouse cancer models using pancreatic cancer cell line PANC02, coupled with intravital microscopy. Results: Various cancer cell lines express functional P2RY12, which when activated significantly increases cell migration and proliferation. Differential tumor P2RY12 expression could explain different procoagulation status of patients. Clopidogrel treatment resulted in better survival rates with smaller primary tumors and less metastasis than aspirin treatment. Clopidogrel was more effective than aspirin at dissolving spontaneous endogenous thrombi in our orthotopic cancer mouse model. Conclusions: Pancreatic adenocarcinomas express P2RY12, which gives the tumor proliferative advantages. The power to slow cancer spread of P2RY12 inhibitors warrants further studies to include it in current pancreatic cancer treatment panels. Clopidogrel can be used to target and prevent Trousseau's syndrome with fewer side effects than aspirin.

\section{LPB0120 | Correlation between Procoagulant Platelets and Hypercoagulability in Lung Cancer Patients}

M. Schreuder $^{1,2}$; E.E. Ramsay ${ }^{1,2}$; H. Campbell ${ }^{3}$; J. Simes ${ }^{1}$; M. Boyer ${ }^{4}$; A. Joshua ${ }^{5,6,7}$; V. Chin ${ }^{5,6,7}$; J. Young ${ }^{8,9,10}$; B. Brown ${ }^{2}$; V.M. Chen ${ }^{3,11}$; P.J. Hogg 2,12

${ }^{1}$ The University of Sydney, NHMRC Clinical Trials Centre, Sydney, Australia; ${ }^{2}$ Sydney Catalyst, The University of Sydney, Sydney, Australia; ${ }^{3}$ ANZAC Research Institute, The University of Sydney, Sydney, Australia; ${ }^{4}$ Chris O'Brien Lifehouse, Sydney, Australia; ${ }^{5} \mathrm{St}$ Vincent's Hospital, Sydney, Australia; ${ }^{6}$ The Kinghorn Cancer Centre, Sydney, Australia; ${ }^{7}$ Garvan Institute of Medical Research, Sydney, Australia; ${ }^{8}$ The University of Sydney, School of Public Health, Sydney, Australia; ${ }^{9}$ Sydney Local Health District, RPA Institute of Academic Surgery, Sydney, Australia, ${ }^{10}$ Sydney Local Health District, Surgical Outcomes Research Centre (SOuRCe), Sydney, Australia, ${ }^{11}$ Concord Repatriation General Hospital, Department of Haematology, Sydney, Australia, ${ }^{12}$ Centenary Institute, The University of Sydney, Sydney, Australia

Background: Thrombotic events are a major cause of morbidity and mortality in cancer. The procoagulant subset of platelets have an essential role in triggering coagulation and thrombosis by facilitating surface-dependent reactions. Here, we hypothesize that procoagulant platelet levels could have prognostic significance in cancer, guiding clinical decisions and minimising cancer-associated thrombotic events. 
Aims: Investigate the role of procoagulant platelets in lung cancer and evaluate their prognostic value to identify lung cancer patients that are at increased risk for thrombosis.

Methods: Serial, citrated blood samples were collected from a prospective cohort of patients with lung cancer enrolled in the EnRICH (Embedding Research (and Evidence) In Cancer Healthcare) Program, a flagship program of Sydney Catalyst and The University of Sydney. Whole blood was stimulated with platelet agonists (thrombin and collagen) to reveal procoagulant platelets. Samples were labelled and analysed by flow cytometry.

Results: Interim analyses revealed that lung cancer patients ( $n=117$ ) have significantly higher levels of procoagulant platelets at time of cancer diagnosis relative to healthy volunteers $(n=55)$ and reached peak levels at 6 months after diagnosis. Moreover, lung cancer patients had elevated D-dimer levels at diagnosis compared to healthy controls. Significant associations were found between the level of procoagulant platelets and the Khorana score and thrombotic events during follow-up and trends were observed correlating the formation of procoagulant platelets with cancer progression and overall survival. Conversely, procoagulant platelet levels were comparable in patients with or without a history of thrombosis. Interestingly, procoagulant platelet formation correlated with neutrophil and monocyte count, but not with lymphocyte count.

Conclusions: Collectively, our data suggest that procoagulant platelets correlate with cancer-associated thrombosis and poor prognosis. The increased procoagulant platelet capacity of lung cancer patients may provide novel insights into the risk of thrombotic events in cancer patients. Further collection of data will help to characterise the pathophysiology of thrombosis in cancer.

\section{LPB0121 | Quantitative and Qualitative Changes in Platelet} Traits of Sunitinib-treated Patients with Renal Cell Carcinoma in Relation to Circulating Sunitinib Levels: A Proof-of-Concept Study

B.M. Tullemans ${ }^{1}$; S.L. Brouns ${ }^{1}$; F. Swieringa ${ }^{1}$; S. Sabrkhany ${ }^{1}$; F.W. van den Berkmortel ${ }^{2}$; N.A. Peters ${ }^{3}$; P. de Bruijn ${ }^{4}$; S.L. Koolen ${ }^{4,5}$; J.W. Heemskerk ${ }^{1}$; M.J. Aarts ${ }^{6}$; M.J. Kuijpers ${ }^{1}$ ${ }^{1}$ Cardiovascular Research Institute Maastricht, Department of Biochemistry, Maastricht University, The Netherlands, Maastricht, Netherlands; ${ }^{2}$ Department of Medical Oncology, Zuyderland Medical Centre, Sittard-Geleen, Netherlands; ${ }^{3}$ Department of Internal Medicine, Sint Jans Gasthuis, Weert, Netherlands; ${ }^{4}$ Department of Medical Oncology, Erasmus MC Cancer Institute, Erasmus University Medical Centre, Rotterdam, Netherlands; ${ }^{5}$ Department of Pharmacy, Erasmus University Medical Centre, Rotterdam, Netherlands; ${ }^{6}$ Department of Medical Oncology, Maastricht University Medical Centre+, Maastricht, Netherlands

Background: Tyrosine kinase inhibitors (TKIs), such as sunitinib, are used for cancer treatment, but may also affect platelet count and function with possible hemostatic consequences.
Aims: We investigated how patient treatment with the TKI sunitinib affected quantitative and qualitative platelet traits as a function of the sunitinib level and the occurrence of bleeding.

Methods: The study was approved by the medical ethical committee of Maastricht University Medical Center+ $\left(\mathrm{MUMC}^{+}\right)$and full informed consent was obtained from all participants. Blood was collected from 20 metastatic renal cell carcinoma (mRCC) patients before treatment, and at 2 weeks, 4 weeks and 3 months after sunitinib administration. We measured blood cell counts, platelet aggregation, and the concentrations of sunitinib, as well as its $\mathrm{N}$-desethyl metabolite, in plasma, serum and isolated platelets. Progression of disease (PD) and bleeding were monitored after 3 months.

Results: In sunitinib-treated $\mathrm{mRCC}$ patients, concentrations of $(\mathrm{N}$ desethyl)sunitinib in plasma and serum were highly correlated. In the patients' platelets the active metabolite levels was relatively increased as compared to sunitinib. On average, a continued reduction in platelet count was observed on-treatment, which was significantly related to the inhibitor levels in plasma/serum. Principal component and correlational analysis showed that the ( $\mathrm{N}$-desethyl) sunitinib levels in plasma/serum were linked to a reduction in both platelet count and collagen-induced platelet aggregation. The reduced aggregation was in part associated with reported bleeding, but did not correlate to PD.

Conclusions: The sunitinib-induced reduction in quantitative and qualitative platelet traits may reflect the effective sunitinib levels in the patient. These results may serve as a proof-of-principle for other TKI-related drugs, where both platelet count and functions are affected, which could be used for dose adjustments.

This work was funded by Pfizer as an Investigator-Initiated Research grant to M.J.E.K. (Tracking Number WI209458).

PB0746 | Tumor Cell-induced Platelet Aggregation is Sustained by Platelet Entrapment into the Fibrin Network Formed by Thrombin

I. Tesakov ${ }^{1}$; A. Filkova ${ }^{2,3,4}$; A. Deputatova ${ }^{4}$; A. Korneichuk ${ }^{4}$; S. Obydennyi ${ }^{2,3}$; I. Kireev ${ }^{5}$; M. Panteleev ${ }^{2,3,4}$; A. Sveshnikova ${ }^{2,3,4}$

${ }^{1}$ Lomonosov Moscow State University, Faculty of Medicine, Moscow, Russian Federation; ${ }^{2}$ Center for Theoretical Problems of Physicochemical Pharmacology RAS, Moscow, Russian Federation; ${ }^{3}$ Dmitry Rogachev National Medical Research Center of Pediatric Hematology, Oncology and Immunology, Moscow, Russian Federation; ${ }^{4}$ Lomonosov Moscow State University, Faculty of Physics, Moscow, Russian Federation; ${ }^{5}$ Belozersky Institute of Physico-Chemical Biology, Lomonosov Moscow State University, Moscow, Russian Federation

Background: Cancer cells are known to induce platelet aggregation in vitro in the presence of small amounts of plasma. Thrombin is considered to be the key platelet activator in this process. In several studies, it was shown that tumor-cell induced platelet aggregation (TCIPA) is not completely suppressed by integrin $\alpha_{11 \mathrm{~b}} \beta_{3}$ antagonists. 
Thus, the molecular mechanisms that underlie aggregate formation remain unclear and have been the focus of recent research.

Aims: The aim of this study is to investigate the mechanisms of platelet aggregation induced by cancer cells in vitro.

Methods: Human breast cancer cell line MCF-7 was maintained in DMEM. Washed human platelets (WP) were prepared by centrifugation from human citrated blood. Platelet aggregation was performed in a light transmission aggregometer Biola LA-220. Flow cytometry, electron and confocal fluorescence microscopy were utilized to characterize the aggregating suspension.

Results: Tumor cells induced washed platelet aggregation (TCIPA) in concentration of at least 104 cells $/ \mathrm{ml}$ in presence of platelet-free plasma (PFP, 1\%). Apyrase, monafram and aspirin had no effect on TCIPA, while hirudin prevented it completely. Prostaglandin E1 reduced maximum aggregation in a dose-dependent manner, but did not suppress it completely. No aggregation of PFA-fixed platelets was observed, while a $100 \%$ aggregation was observed for PFAfixed platelets with fibrinogen at their surface. Flow cytometry, fluorescent and electron microscopy revealed normal platelet activation responses to happen during TCIPA. However, flow cytometry and confocal microscopy analyses did not reveal formation of heteroaggregates and direct cancer cell-platelet interaction.

Conclusions: We suggest that TCIPA in the presence of PFP starts with thrombin generation triggered by cancer cells. Although thrombin can induce platelet activation, this process is not necessary for the observed platelet aggregation.

The study was supported by Russian Science Foundation (grant 20-45-01014).

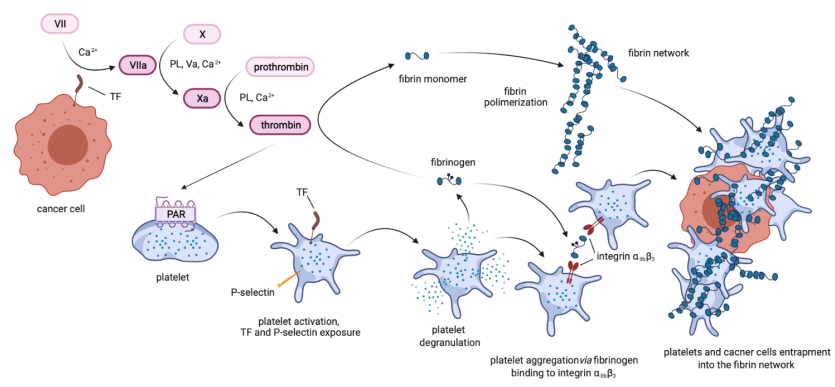

FIGURE 1 Molecular mechanisms of TCIPA. TCIPA is mediated by both fibrinogen binding to integrin allb $\beta 3$ and platelets and cancer cells entrapment into the fibrin network. TF - tissue factor, PAR protease-activated receptors, PL - phospholipids
PB0747 | Platelets Promoted Malignancy Involving Exosomal HMGB1

J.-D. Wang ${ }^{1}$; C.-J. Chen ${ }^{1}$; S.-L. Liao ${ }^{1}$; S.-W. Huang ${ }^{2}$

${ }^{1}$ Taichung Veterans General Hospital, Taichung, Taiwan, Province of China; ${ }^{2}$ China Medical University Hospital, Taichung, Taiwan, Province of China

Background: Reciprocal crosstalk between platelets and malignancies underscores the clinical importance of anti-platelet therapy in cancer patient treatment. Clinical and experimental studies demonstrate that anti-platelet therapy reduces incidence of malignancies and malignant progression by targeting platelet receptors, interfering with platelet granule release, or inhibiting platelet-specific enzymes.

Aims: Investigate the reciprocal crosstalk between platelets and cancer cells as well as the potential involvement of HMGB1 using anti-platelet therapy.

Methods: Human chronic myeloid leukemia K562 cell-differentiated megakaryocytes, human bladder cancer T24 cells, murine platelets, murine Lewis Lung Carcinoma (LLC) cells, and C57BL/6-LLC tumorbearing mice models were established and subjected to anti-platelet drug treatment. The reciprocal crosstalk between platelets and cancer cells as well as the potential involvement of HMGB1 were further investigated using dipyridamole.

Results: In this study, we found that human chronic myeloid leukemia K562 cell-differentiated megakaryocytes and murine platelets produced bioactive substances and these are released into the extracellular space, partly in their exosomal form. High-Mobility Group Box-1 (HMGB1) is a type of exosomal cargo and anti-platelet drug aspirin and dipyridamole interfered with its incorporation into the exosomes. Those released substances and exosomes, along with exogenous HMGB1, promoted cancer cell survival and protected cells from doxorubicin cytotoxicity. In tumor-bearing model established using murine Lewis Lung Carcinoma (LLC) cells and C57BL/6 mice, the tumor suppressive effect of dipyridamole correlated well with decreased circulating White Blood Cells, soluble P-Selectin, TGF-b1, exosomes, and exosomal HMGB1, as well as tumor platelet infiltration. Exosome release inhibitor GW4869 exhibited suppressive effects, as well. The suppressive effect of dipyridamole on cancer cell survival was paralleled by a reduction of HMGB1/Receptor for Advanced Glycation End-Products axis, and proliferation- and migration-related b-catenin, Yes-Associated Protein-1, Runt-Related Transcription Factor 2, and TGFb1/Smad signals.

Conclusions: Exosomes and exosomal HMGB1 appear to have roles in platelet-driven cancer malignancy, and represent targets of antiplatelet drugs in anti-cancer treatment. 
PB0748 | Anti-platelet Drugs and Risk of Intracranial Hemorrhage in Patients with Metastatic Brain Tumors

E. Miller $^{1}$; R. Patell ${ }^{2}$; E. Uhlmann ${ }^{3}$; S. Ren ${ }^{4}$; H. Southard ${ }^{2}$; P. Elavalakanar ${ }^{2}$; G. Weber ${ }^{5}$; D. Neuberg ${ }^{4}$; J. Zwicker ${ }^{2}$ ${ }^{1}$ Beth Israel Deaconess Medical Center, Department of Internal Medicine, Boston, United States; ${ }^{2}$ Beth Israel Deaconess Medical Center, Department of Hematology and Hematologic Malignancies, Boston, United States; ${ }^{3}$ Beth Israel Deaconess Medical Center, Department of Neurology, Boston, United States; ${ }^{4}$ Dana Farber Cancer Institute, Department of Data Science, Boston, United States; ${ }^{5}$ Harvard Medical School, Department of Bioinformatics, Boston, United States

Background: Intracranial hemorrhage (ICH) is frequent in patients with intracranial malignancy but data are lacking on the risk of $\mathrm{ICH}$ with antiplatelet agents in patients with brain metastases.

Aims: We investigated whether patients with metastatic brain tumors receiving antiplatelet agents had an increased risk of ICH compared to a matched cohort.

Methods: We performed an Institutional Review Board approved retrospective matched cohort study of patients with brain metastases at primary tumor diagnosis treated at our center between 2010-2019. Patients with brain metastases treated with antiplatelet agents (aspirin and P2Y12 inhibitors) were matched with patients not receiving antiplatelet agents. The primary endpoint was cumulative incidence of $\mathrm{ICH}$ at one year after primary tumor diagnosis accounting for death as a competing risk. Intracranial hemorrhage was classified as trace $\left(<1 \mathrm{~cm}^{3}\right)$, measurable $\left(1-10 \mathrm{~cm}^{3}\right)$, or major (symptomatic or $>10 \mathrm{~cm}^{3}$ ) by a neuro-oncologist blinded to cohort allocation.

Results: Study population included 392 patients with brain metastases (134 patients exposed to antiplatelet agents). Most common malignancies were non-small cell lung cancer (74.0\%), small cell lung cancer (9.9\%), melanoma (4.6\%), and renal cell carcinoma (4.3\%). Cumulative incidence of any ICH at 1 year was $19.3 \%(95 \% \mathrm{Cl}, 14.1-$ 24.4\%) in patients not receiving anti-platelet agents compared with $22.5 \%(95 \% \mathrm{Cl}, 15.2-29.8 \%$, Gray test $\mathrm{P}=0.22)$ in those receiving antiplatelet agents. Cumulative incidence of major $\mathrm{ICH}$ was $5.4 \%$ (95\% Cl, 2.6-8.3\%) among controls compared with $5.5 \%(95 \% \mathrm{Cl}$ 1.5-9.5\%, Gray test $P=0.8$ ) in those receiving antiplatelet agents. Antiplatelet agent use did not significantly increase the risk of all $\mathrm{ICH}$ (HR 1.4, 95\% Cl 0.9 - 2.1) or major ICH (HR 1.1, 95\% Cl 0.5 - 2.6); inclusion of anticoagulation in the model did not alter the risk.

Conclusions: In patients with brain metastases, administration of antiplatelet agents was not associated with an increased incidence of $\mathrm{ICH}$.
PB0749 | Native Rotational Thromboelastometry in Chemotherapy Induced Thrombocytopenia

A.-M. Hulshof $^{1}$; F. Heubel-Moenen ${ }^{1}$; F. Tjahjadi ${ }^{2}$; C. Saelmans ${ }^{2}$; T. van de Berg ${ }^{2}$; E. Beckers ${ }^{1}$; T. Hackeng ${ }^{2}$; Y. Henskens ${ }^{1}$

${ }^{1}$ Maastricht University Medical Center+, Maastricht, Netherlands;

${ }^{2}$ Maastricht University, Maastricht, Netherlands

Background: Native rotational thromboelastometry (NATEM) is a viscoelastic assay in which the patient sample is solely re-calcified in absence of an activator of hemostasis. NATEM reflects the endogenous hemostatic potential, which might show subtle interindividual variations. Patients with severe chemotherapy induced thrombocytopenia (CIT) present with lower levels of red blood cells, platelets and leukocytes. Prophylactic platelet transfusions are indicated to prevent bleeding in patients with platelet count below $10 \times 10^{9} / \mathrm{L}$. However, not all patients bleed despite low platelet count and unnecessary platelet transfusions are unwarranted. NATEM might aid in identifying CIT patients at risk for bleeding.

Aims: We aimed to evaluate differences between CIT patients and healthy volunteers in whole blood (WB) and platelet poor plasma (PPP) NATEM assessment.

Methods: CIT patients with a platelet count of $\leq 50 \times 10^{9} / \mathrm{L}$ were included prior to platelet transfusion. Thermostable inhibitor of contact activation (TICA) was added to the blood withdrawal tube to prevent contact activation during sampling for NATEM assessment. NATEM was performed in WB and PPP of 17 and 4 CIT patients. In subsequent experiments, platelets were depleted from healthy volunteers $(n=22)$ in vitro to mimic CIT.

Results: Routine assays such as PT, aPTT and EXTEM clotting time (CT) were within reference range. CIT patients had significantly prolonged NATEM CT (WB:2826[2345-3508] and PPP:7193[6406-7194]) compared to healthy volunteers (WB:1414[1311-1758] and PPP:2869 [2358-3636]; $P<0.001$ and $P=0.005)$. Artificial platelet depletion in healthy volunteers did not replicate the NATEM CT prolongation observed in CIT patients. 
A

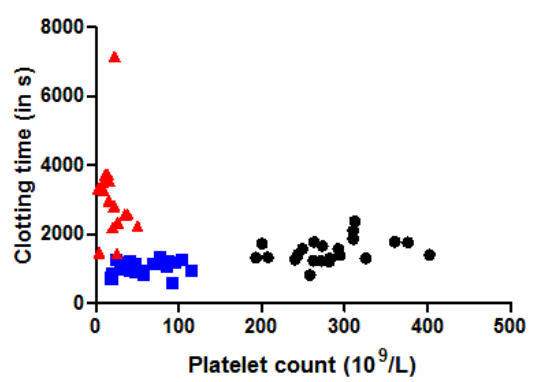

- HV

- Thrombocytopenic HV

- CIT patient
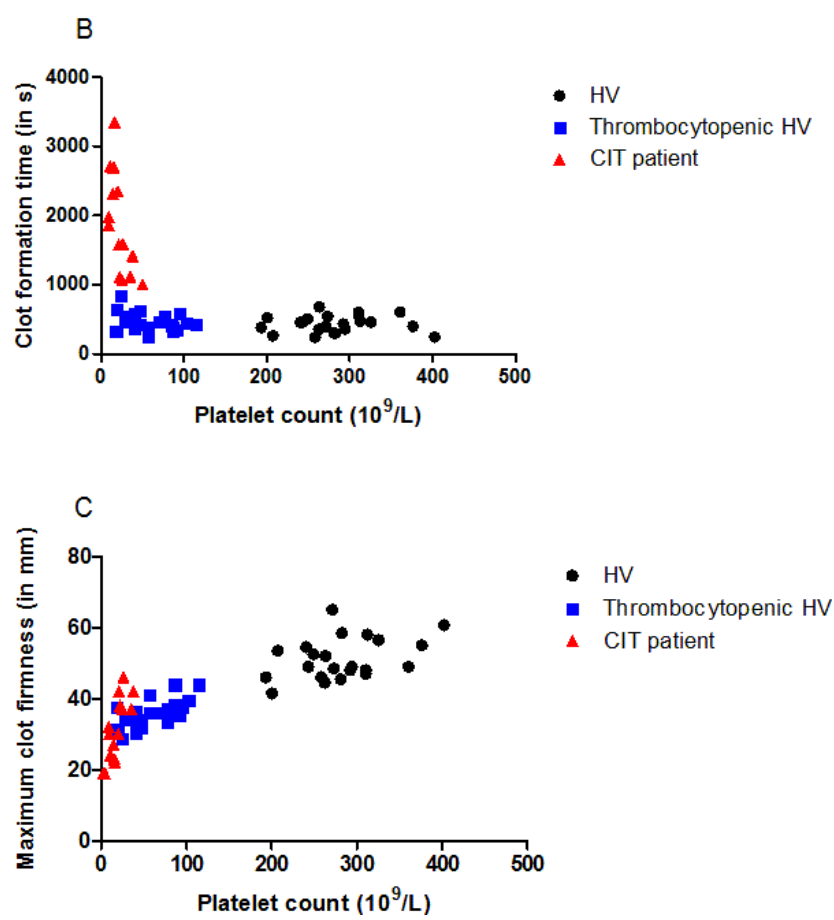

FIGURE 1 NATEM clotting time (A), clot formation time (B) and maximum clot firmness $(C)$ assessment in healthy volunteers $(\mathrm{HV})$, healthy volunteers after manual platelet depletion (thrombocytopenic HV) and patients with chemotherapy induced thrombocytopenia (CIT patient)

Conclusions: CT according to NATEM is prolonged in WB of CIT patients, consistent with a bleeding phenotype. The similar effect in PPP and lack of effect in thrombocytopenic healthy volunteers suggests that markers other than low platelet counts contribute to bleeding risks in CIT patients. Future research should focus on the applicability of NATEM in the identification of alternative risk factors and its use to predict bleeding.

PB0750 | Correlation between Serum PDGF-CC Levels with Clinical Stage of Colorectal Cancer

E. Sundarita; M. Syahrir; N. Djamaludin; Y.D. Andayani; Z. Zulaikha; E. Syahriana

Universitas Sriwijaya, Palembang, Indonesia

Background: Platelets are the blood component that is most responsible for the hemostasis and coagulation processes, but now it is proven that platelets also play a role in cancer progression, one of which is colorectal cancer. An important component released by platelets when activated by cancer cells is platelet-derived-growth-factor, which is responsible for angiogenesis, so it is thought to be an important biomarker in estimating the clinical stage of colorectal cancer.

Aims: To analyze the correlation between serum PDGF-CC levels and the clinical stage of colorectal cancer.

Methods: This study was an observational correlation analysis study with a cross-sectional design.

Results: From the 70 study subjects involved, 40 subjects were stage I-III (nonmetastatic) and 30 subjects were stage IV (metastatic). From the various platelet indices analyzed in this study, statistical significance was obtained between serum platelets, NLR, CEA, and PDGF-CC with clinical stage of colorectal cancer, with a $P$-value of 0.02; 0.00; 0.04; and 0.02. Correlation analysis test with Pearson test on PDGF-CC and clinical stage of colorectal cancer showed a strong positive correlation, with $P$-values of 0.03 and $r 0.79$.

Conclusions: The results of this study indicate that serum PDGF-CC can serve as a promising biomarker for the diagnosis of colorectal cancer.

PB0751 | A Role of Possible Synergistic Effect of P Selectin Glycoprotein Ligand 1 Polymorphism and Antiphosholipid Antibodies on Thrombosis in Myeloproliferative Neoplasia Patients

R. Dambrauskiene $\dot{e}^{1} ;$ R. Gerbutavičius $^{1}$;

R. Vadeikiené ${ }^{1}$; R. Gerbutavičius ${ }^{2}$; R. Ugenskiené $\dot{1}^{1}$;

M. Rudžianskiené $\dot{1}^{1}$; E. Juozaityte $\dot{e}^{1}$; D. Remeikiene $\dot{e}^{1}$;

R. Gerbutavičiené ${ }^{3}$; R. Paukštaitien $\dot{e}^{4}$

${ }^{1}$ Institute of Oncology, Lithuanian University of Health

Sciences, Oncology and Haematology Clinic, Kaunas, Lithuania;

${ }^{2}$ Augenarztpraxis Dres. Kortüm, Ludwigsburg, Germany; ${ }^{3}$ Department of Drug Technology and Social Pharmacy, Lithuanian University of Health Sciences, Kaunas, Lithuania; ${ }^{4}$ Lithuanian University of Health Sciences, Department of Physics, Mathematics and Biophysics, Kaunas, Lithuania

Background: The morbidity and mortality of BCR-ABL negative myeloproliferative neoplasms (polycythaemia vera, essential thrombocyhaemia and primary myelofibrosis) is highly dependent on thrombotic complications. In myeloproliferative neoplasia patients 
circulating platelets are already activated and they secrets higher levels of surface Pselectin and tissue factor. Anti- $\beta 2$ glycoprotein 1 antibodies ( $32 \mathrm{GP} 1$ ) bind to the $\beta 2 \mathrm{GP} 1$ receptors on the platelet surface, activating them that is important for thrombus formation. Antiphospholipid antibodies activates endothelial cells, whose dysfunction also plays an important role in the pathogenesis of thrombosis in BCR-ABL negative myeloproliferative neoplasia.

Aims: To evalute the antiphospholipid antibodies in relationship $\mathrm{P}$ Selectin Glycoprotein Ligand 1 (PSGL1) polymorphism on the development of thrombosis with other risk factors in patients with MPN

Methods: A total of 105 MPN patients were included in this retrospective study. 58 of the MPN patients were diagnosed with essential thrombocythemia (ET), 41 with polycythemia vera (PV), 6 with myelofibrosis (MF). Clinical data about the

development of thrombosis was collected. PSGL1 polymorphisms was determined from peripheral blood samples using PCR method. In electrophoresis the size of A allele was 558 bp, B allele - 528 bp. Data analysis was performed using

"IBM SPSS Statistics 23". There were two different selective and one confirmatory test for the LA determination. The detection of antibodies against $\beta 2$-glycoprotein-1 ( $\beta 2 \mathrm{GP} 1$ ) was performed using a chemiluminescent method. The detection of antibodies against cardiolipin ( $\mathrm{aCL}$ ) was performed ELISA using commercial Aeskulisa Cardiolipin-GM (AESKU.DIAGNOSTICS, Germany).

Results: Arterial thrombotic complications were present in 38 (42.2\%) of those with MPN. These patients were diagnosedwith transient ischaemic attack (TIA), ischaemic stroke or myocardial infarction. aPL together with PSGL1 polymorphism were significant more frequently found in arterial thrombosis MPN patients compared with MPN patients who didn'texperienced thrombosis $(21.1 \%$ and $5.8 \% ; P<0.029$ ).

Conclusions: Myeloproliferative neoplasm's patients with antiphospholipid antibodies and PSGL1 polymorphism may have the increased risk of thrombosis development.

PB0752 | Chemotherapy Induced Thrombocytopenia: Is it Inducing Additional Social, Economic and Clinical Burden?

N. Anwar; N. Fatima; H.-u.- Mujtaba; T. Shamsi

National Institute of Blood Diseases and Bone Marrow Transplantation, Karachi, Pakistan

Background: Chemotherapy induced thrombocytopenia (CIT) is perilous and may complicate the chemotherapeutic treatments resulting in extended hospitalization, bleeding, transfusion and the economic burden. Typically patients with hematological malignancies (HM) are at high risk of bleeding as well as thrombosis which may lead to poor overall survival and disease outcome. Our center is a referral institute for blood disorders where a significant number of patients undergo chemotherapeutic treatment having frequently encountered malignancies as leukemia, lymphoma and myeloma.
Aims: This study was designed to observe the incidence of CIT and its clinical, social and economical consequences.

Methods: This was a retrospective cohort study conducted at NIBD PECHS campus from January-December 2019. Study population comprised of patients with HM receiving chemotherapy. The incidence of CIT and its resultant clinical, social and economic consequences were evaluated.

Results: A total of 63 chemotherapeutic cycles for HM were analyzed. Median and range of age of patients was 42(9-75years) with predominance of males $47(75 \%)$. The overall incidence of CIT was $18 \%$. The odds ratio for leukemia protocol regimen observing CIT was higher 2.557 (95\% Cl: 1.565-4.177) than non leukemic regimen $0.267(95 \% \mathrm{Cl}: 0.075-0.950)$. The median length of hospital stay was $4(1-14)$ days, bleeding was present in 6(16\%) patients and median of 3.5 units of platelet were transfused. Voluntary donations were only 5(3\%). The approx cost of CIT related admissions was 30,365PKR (US\$196) per patient.

Conclusions: In conclusion, CIT may lead to additional clinical, social and economic burden on patients. In a developing country where voluntary donations are scarce and people are vulnerable to get treatment of primary disease, these social affairs are also an additive burden. Additional measures like education for voluntary donations, blood camps for patients residing in remote areas and prophylactic tranexamic acid are needed to be formerly addressed especially in leukemia patients to minimize the consequences of CIT.

PB0753 | Association of Platelet Count and Coagulopathy before, during and Post Chemotherapy: Is it Worth Doing?

N. Anwar; N. Fatima; H.-u. Mujtaba; T. Shamsi

National Institute of Blood Diseases and Bone Marrow Transplantation, Karachi, Pakistan

Background: Patients with hematological malignancies are at high risk of venous thromboembolism and bleeding that may have profound impact on treatment outcome and survival. As coagulopathy in hematological malignancies is a predictor of thrombotic and bleeding complications, by adding the intellectual observation of platelet count, we may segregate patients and identify the susceptibility to either thrombosis or bleeding apparently.

Aims: The study was designed to evaluate the significance of association of platelet count with prothrombin time (PT) and activated partial thromboplastin time (APTT) pre, during and post chemotherapy. Methods: A retrospective cohort was studied at NIBD PECHS campus during 06 months period. Study population comprised of hematological malignant patients. PT, APTT and platelet count, pre, pro and post chemotherapy was assessed. Mann-Whitney $U$ test and spearman correlation was applied to observe the association.

Results: A total of 52 cases were recruited, out of which 34 cases were analyzed based on inclusion criteria. Median age and range of patients was 30(13-68 years) with male predominance. Out of 34 cases, $14(41.2 \%)$ were on active chemotherapy, 11(32.4\%) were post 
chemotherapy and in 9(26.5\%) cases chemotherapy was not started. Overall PT and APTT was abnormal in 21(61.8\%) and 24(70.6\%) cases respectively. The association between platelet and count and PT and APTT is shown in table : $1 \& 2$. Spearman correlation revealed moderate negative correlation of platelet and APTT during chemotherapy and moderate negative correlation between platelet and PT before and post chemotherapy.

Conclusions: We concluded from this study that prolonged PT was associated with low platelet count and may be beneficial in taking diligent clinical decisions before starting chemotherapy. Additionally, risk of bleeding and thrombosis may be ruled out by comparing the platelet count with coagulopathy in patients undergo chemotherapy. This will not only improve treatment outcome but also lessen the unnecessary financial burden on patients.

\section{PB0754 | Antiplatelet and Cytostatic Properties of Nilotinib} Synthetic Analogues

L.M. Pechlivani ${ }^{1}$; N. Ntemou ${ }^{2}$; P. Voulgari ${ }^{2}$; D. Pantazi ${ }^{1}$;

K. Skobridis ${ }^{2}$; A.D. Tselepis ${ }^{1}$

${ }^{1}$ Department of Chemistry, Atherothrombosis Research Centre, Laboratory of Biochemistry, University of loannina, loannina, Greece;

${ }^{2}$ Department of Chemistry, Section of Organic Chemistry and

Biochemistry, University of loannina, loannina, Greece

Background: Nilotinib is a Bcr-Abl tyrosine kinase inhibitor used to treat various malignancies. We had previously demonstrated that Nilotinib exhibits an antiplatelet activity (Pantazi D, et al. DDDT. 2019;13:4225-38).

Aims: We synthesized new Nilotinib analogues in an effort to improve the drug antiplatelet potency, while maintaining or enhancing its cytostatic properties.
Methods: Three novel Nilotinib analogues, containing the following modifications at the final phenyl ring, were designed, synthesized and fully characterized:

1. In all analogues, the $\mathrm{CF}_{3}$ group at the 5-position was removed, whereas the 4-methylimidazolyl ring at the 3-position was replaced by a $\mathrm{NO}_{2}$ group.

2. A halogen atom, $\mathrm{F}$ or a $\mathrm{Cl}$, was incorporated at the 4-position (analogue-2 and analogue-3, respectively). Light Transmittance Aggregometry (LTA) in the presence of Nilotinib or its analogues was performed in platelet-rich plasma (PRP) activated with Arachidonic acid (AA), ADP or TRAP-6. Confluent hepatoma HepG2 cells in culture were incubated for $48 \mathrm{~h}$ with Nilotinib or its analogues. The cell cycle was studied by Flow Cytometry, after cell staining with propidium iodide. Data are expressed as means \pm SD and statistical analysis was performed by Two-way ANOVA.

Results: Nilotinib and its analogues, significantly inhibited platelet aggregation induced by $\mathrm{AA}$, the analogues 1 and 2 being more potent than Nilotinib. The $\mathrm{IC}_{50}$ values and the threshold concentrations are shown in Table 1. A slight non-significant inhibition by all compounds was observed when ADP or TRAP- 6 were used as agonists. In HepG2 cells, Nilotinib significantly increased the \%gated cells in M1 phase, whereas all analogues reduced the \%gated cells in M1 phase and increased the \%gated cells in M3 phase (Table 2).

TABLE 1 The inhibitory effect of Nilotinib and its analogues on AAinduced platelet aggregation in PRP

\begin{tabular}{lll} 
Compounds & $\mathrm{IC}_{50}$ values $(\mu \mathrm{M})$ & $\begin{array}{l}\text { Threshold } \\
\text { concentration }(\mu \mathrm{M})\end{array}$ \\
Nilotinib & $3.94 \pm 2.09$ & $10.00 \pm 2.83$ \\
Analogue-1 & $1.50 \pm 1.08$ & $2.87 \pm 1.31$ \\
Analogue-2 & $0.57 \pm 0.21$ & $3.00 \pm 1.41$ \\
Analogue-3 & $5.45 \pm 6.11$ & $8.33 \pm 10.12$ \\
\hline
\end{tabular}

TABLE 2 The effects of Nilotinib and its analogues on the cell cycle phases of confluent hepatoma HepG2 cells $\%$ Gated cells

\begin{tabular}{llllll} 
Cell cycle phases & Control & Nilotinib $(10 \mu \mathrm{M})$ & Analogue-1 $(10 \mu \mathrm{M})$ & Analogue-2(10 $\mu \mathrm{M})$ & Analogue-3 $(10 \mu \mathrm{M})$ \\
$\mathrm{M} 1(\mathrm{G} / \mathrm{G} 1)$ & $85.14 \pm 2.84$ & $87.36 \pm 2.66^{*}$ & $79.96 \pm 4.53^{*, * *}$ & $81.59 \pm 1.19^{*, * *}$ & $78.35 \pm 3.51^{*, * *}$ \\
M2(S) & $4.62 \pm 1.01$ & $3.93 \pm 1.05$ & $5.38 \pm 1.19$ & $5.62 \pm 0.27$ & $6.39 \pm 1.03^{* *}$ \\
M3(G2/M) & $9.84 \pm 1.92$ & $8.18 \pm 1.85$ & $13.84 \pm 3.03^{*, * *}$ & $12.25 \pm 0.85^{*, * *}$ & $13.26 \pm 2.20^{*, * *}$ \\
\hline
\end{tabular}

${ }^{*} P<0.05$ and ${ }^{* *} P<0.05$ compared with control and Nilotinib, respectively.

Conclusions: There is considerable potential to develop synthetic Nilotinib analogues with potent antiplatelet activity and improved cytostatic properties to treat cancer and to prevent cancerassociated thrombosis.

PB0755 | Characteristics and Management of a Series of TAFRO Syndrome

$\underline{\mathrm{X} . \mathrm{Ye}}$

Second Affiliated Hospital of Guangzhou Medical University, Guangzhou, China

Background: TAFRO syndrome as a rare subtype of multi-center Castleman disease is characterized by Thrombocytopenia, Anasarca, myelofibrosis, Renal dysfunction, Organomegaly, lymphadenopathy 
and a histopathological pattern of atypical Castleman's disease. It is always fatal if not managed appropriately.

Aims: To present 6 cases of TAFRO syndrome and describe their clinical characteristics and our management experience for the disease. Methods: 6 patients were admitted during June, 2016 and July, 2020. 3 were male and 3 were female. The age ranged from 42 to 65 years old. All present with high fever unresponsive to antibiotics therapy, lymphadenopathy and splenomegaly. 2 cases had a history of multi-center Castleman disease respectively 2 to 3 years before and were admitted due to recurrence. The other 4 cases received lymph node biopsy and were pathologically diagnosed Castleman disease after admission. All had progressive thrombocytopenia, renal and liver insufficiency, pleural effusion, ascites or pericardial effusion. Bone marrow cellularity were hyperproliferative.

Results: One patient relapsed after $\mathrm{CHOP}$ regimen chemotherapy 3 years before received cyclosporin $\mathrm{A}$ and tocilizumab treatment. Another one patient only received COP regimen chemotherapy. Four patients received COP regimen chemotherapy due to poor constitutional condition at presentation and then $\mathrm{RCHOP}$ regimen after improved condition. 3 patients received cyclosporin A maintenance therapy after chemotherapy. All were responsive to the treatment. Five patients had sustained response and in complete remission. One lost follow-up after one course of therapy.

Conclusions: TAFRO syndrome is always misdiagnosed and may be the result of delayed treatment for multi-center Castleman disease. Careful physical examination to look for lymphadenopathy and lymph node biopsy pathologic analysis is important for timely diagnosis and management. Most of the cases are responsive to rituximab based combined chemotherapy. Cyclosporin A and tocilizumab may be treatment of choice for some patients.

PB0756 | Chronic Myeloid Leukemia with Extreme Thrombocytosis as Unusual Initial Presentation

\section{N. Petkova}

Military Medical Academy, Hematology Clinic, Sofia, Bulgaria

Background: Chronic myeloid leukemia (CML) is a myeloproliferative neoplasm (MPN) with abnormal fusion gene, characterized with an usually distinct initial presentation of neutrophilic leukocytosis and splenomegaly.

Aims: Case report of a CML patient with initial extreme thrombocytosis and treatment approach.

Methods: A 74-year-old man was admitted in the clinic for diagnostic work-up and therapy because of high platelet count. He was asymptomatic, thrombocytosis was incidentally detected.

Results: OBlood count results were: Hb-93g/l, WBC-17 × 109/I, Plt-2467 × 109/I; blood smear displayed Sg-76\%, Ly-17\%, Mo-4\%, Eo-1\%, Ba-2\%, marked increase in platelets with giant and hypogranulated forms. He had mild hepatomegaly, but no splenomegaly. Bone marrow was hypercellular with myeloid hyperplasia, $2 \%$ myeloblasts and increased number of megakaryocytes with clustering and monolobated forms, indicative of MPN/CML. Features were suggestive of essential thrombocytosis, but molecular analysis was negative for typical mutations. Philadelphia chromosome $t(9 ; 22)$ (q34;q11) was detected in all bone-marrow metaphases analyzed, proved with positive BCR/ABL1 FISH analysis result. Subsequently RT-PCR analysis for BCR/ABL1 rearrangement showed the presence of typical b3a2 fusion gene. The patient was diagnosed with $\mathrm{CML}$, chronic phase, associated with extreme thrombocytosis. Considering the high platelets count that is usually associated with high thrombotic risk or bleeding potential due to platelet dysfunction in MPN, complete tests of hemostasis were performed. They showed normal platelet function, $100 \%$ aggregation and $0 \%$ inhibition with ADP and arachidonic acid, coagulation tests were within reference ranges. Target therapy with nilotinib $2 \times 300 \mathrm{mg}$ was started and clopidogel $75 \mathrm{mg}$ was added. After one course of nilotinib treatment, the patient's platelets were reduced to normal levels-358 $\times 109 / \mathrm{l}$. He achieved deep molecular response (DMR) at 3 months nilotinib therapy. The patient even experienced mild COVID-19 infection without complications. His DMR remains stable.

Conclusions: Both morphologic and cytogenetic combined with molecular analysis are required for correct MPN-type diagnosis, which is important, as the disease outcome is based on proper initial therapy.

PB0757 | Chemotherapy-induced Thrombocytopenia and Platelet Transfusions in Hematology/Oncology Practice

$\underline{\text { L. Hambardzumyan }}^{1,2}$; A. Movsisyan ${ }^{1,2} ;$ M. Badikyan ${ }^{2,3}$; N. Martirosyan ${ }^{2,3}$; H. Khachatryan ${ }^{1,2}$; A. Sevoyan²; H. Grigoryan ${ }^{1,1,2}$; N. Martirosyan ${ }^{2,4}$; G. Tamamyan ${ }^{1,2,4}$; S. Danielyan ${ }^{2}$ ${ }^{1}$ Yerevan State Medical University, Department of Pediatric Oncology and Hematology, Yerevan, Armenia; ${ }^{2}$ Hematology Center after Prof. R.H. Yeolyan, Yerevan, Armenia; ${ }^{3}$ Yerevan State Medical University, Department of Hematology, Yerevan, Armenia; ${ }^{4}$ Pediatric Cancer and Blood Disorders Center of Armenia, Hematology Center after Prof. R.H. Yeolyan, Yerevan, Armenia

Background: Chemotherapy-induced thrombocytopenia (CIT) is a common complication of cancer therapy and platelet transfusions are the most frequently used treatment option, however, the management strategies of CIT among different specialists vary significantly.

Aims: The aim of our study was to evaluate the management practices of CIT with platelet transfusions by hematologists and oncologists in Armenia.

Methods: A survey was conducted among the physicians of the Hematology Center after Prof. R.H. Yeolyan

Results: From 38 respondents 15 were adults' hematologists, 7 oncologists, 16 pediatric hematologists/oncologists. 66\% (25) of them had $\leqslant 6$ years of professional experience, $16 \%(6) \geqslant 10$ years, and $18 \%(7) \geqslant 20$ years. 
$13.2 \%(5)$ of physicians considered thrombocytopenia when platelet count falls below $180 \times 10^{9} / \mathrm{L}, 42.1 \%(16)<150 \times 10^{9} / \mathrm{L}, 15.8 \%(6)$ $<140 \times 10^{9} /$ L, 21.1\% (8) $<100 \times 10^{9} /$ L. All 38 physicians were favoring prophylactic transfusions over on-demand transfusions. For $73.3 \%$ (11) of adults' hematologists, $57.1 \%$ (4) of adults' oncologists, and $50 \%$ (8) of pediatricians $10 \times 10^{9} / \mathrm{L} \mathrm{PLT}$ count was threshold for transfusing afebrile (non-APL) patients. $81.3 \%$ (13) of adults' hematologists, $57.1 \%$ (4) of adults' oncologists and $73.3 \%$ (11) of pediatricians would transfuse febrile (non-APL) patients when PLT $<20 \times 10^{9} / \mathrm{L}$. Afebrile and febrile adults with APL would get transfusions by $75 \%$ (12) and $73.3 \%$ (11) of hematologists when PLT $<20 \times 10^{9} / \mathrm{L}$, respectively. 56.3\% (9) of pediatricians would transfuse both afebrile and febrile patients with APL when PLT $<50 \times 10^{9} /$ L. 53\% (20) mentioned $24 / 7$ access to platelet products.

$67 \%$ of adults' hematologists and oncologists mentioned that the cost of platelet products affects their decision on making prophylactic transfusion because patients have to pay out-of-pocket.

Conclusions: Our cohort physicians prefer PLT transfusions for prophylaxis not only on-demand. When it comes to febrile (non-APL) patients the majority of them prioritize $20 \times 10^{9} / \mathrm{L}$ over $10 \times 10^{9} / \mathrm{L}$ as threshold. Their decision on making a transfusion is mainly affected by financial factors.

TABLE 1 Frequency according to age, sex and type of neoplasia

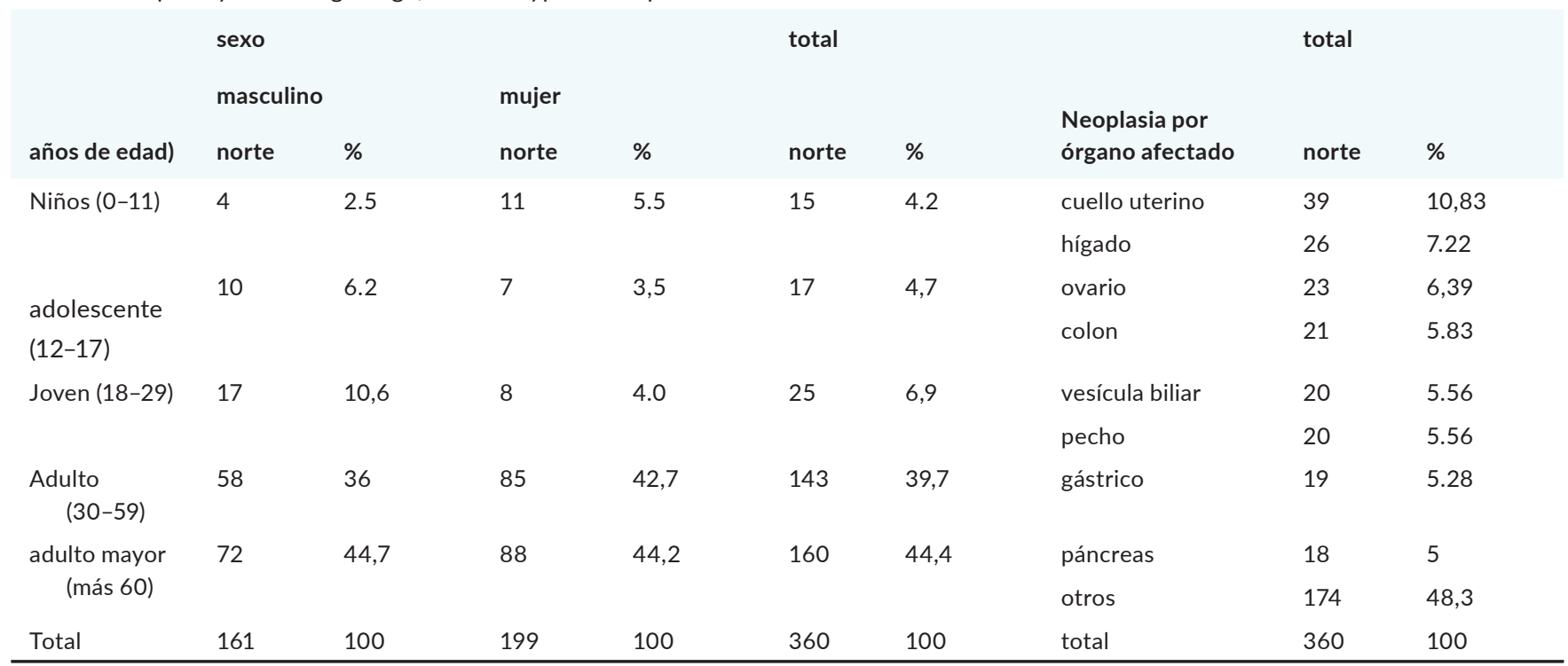

PB0758 | Hemostatic Evaluation by Thromboelastography in Patients from a Peruvian Oncologic Center

L.F. Motta Rosada ${ }^{1}$; R.M. Rodriguez Torres ${ }^{1}$; N.V. Herrera Valverde ${ }^{2}$

${ }^{1}$ Universidad Nacional Mayor de San Marcos, Lima, Peru; ${ }^{2}$ Instituto Nacional de Enfermedades Neoplásicas, Surquillo, Peru

Background: A prothrombotic state in oncologic patients represent a high risk problem, so evaluation of hemostasis is relevant for their monitoring. Thromboelastography (TEG) evaluates coagulation and fibrinolysis functionality and identifies altered phase that contributes to treatment decision.

Aims: The study aim is to evaluate hemostasis by TEG in patients with a diagnosis of neoplasia in a Peruvian oncology center.

Methods: A descriptive cross-sectional study was performed, with a review of two years (2017-2018) reports. TEG parameters were categorized into decreased, normal and increased according reference intervals. The software used was STATA.

Results: 360 reports were included, patients with antithrombotic therapy and double reports were excluded. Female cases reached $55 \%$, being cérvix cancer the most frequent diagnosis with $11 \%$ (see Table 1). For TEG parameters, $\mathrm{R}(4-8$ seconds) and $\mathrm{K}(1-4$ minutes) were categorized normal above $70 \%$ of cases, while alpha angle $\left(47^{\circ}\right.$ $\left.74^{\circ}\right)$, MA (55-73 mm) and $\mathrm{Cl}(-3$ to +3$)$ showed mostly frequencias between normal and increased categories (see graphic 1). 
Graphic 1. Frecuency distribution of TEG parameters acording to categories

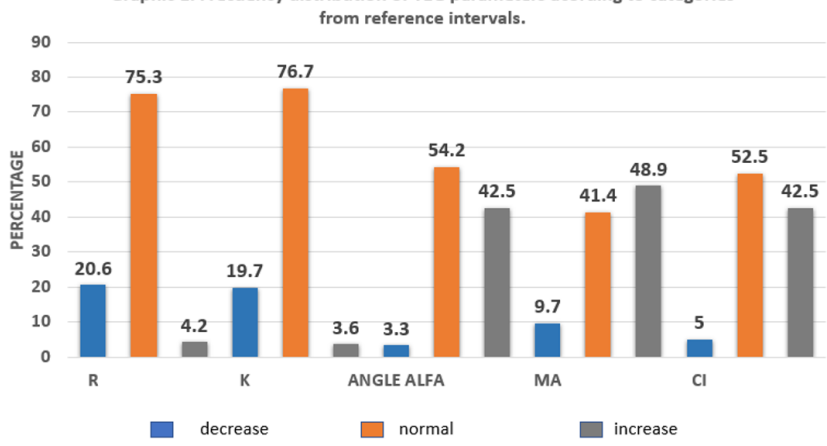

FIGURE 1 Frequency distribution of TEG parameters according to categories from reference intervals

Conclusions: Hemostatic evaluation by TEG in oncologic patients demostrated a hypercoagulable state due increased MA (related to platelets) in $48,9 \%$ of cases and alpha angle (clot formation velocity) in $42,5 \%$.

P0151 | Essential Thrombocythemia with Deep Vein Thrombosis and Recurrent Gangrene Pedis

\section{H. Gustian}

Andalas University, Padang, Indonesia

Background: Essential thrombocythemia (ET) is a myeloproliferative neoplasm with rare incidence, a prevalence of 30/100.000 population. the disorder is characterized by increase of platelet with tendency for thrombosis (micro vascular and macro vascular) and bleeding. the median age at diagnosis is 65 to 70 , but can occur at any age. the ratio of male to female is approximately $2: 1$. Thrombocytosis can occur because of other diseases (reactive thrombocytosis) or without any other causes (primary thrombocytosis / clonal), which requires additional tests to know the cause of thrombocytosis.

Aims: Case report essential thrombocythemia with deep vein thrombosis and recurrent gangrene pedis.

Methods: Case repot.

Results: A 54-year-old man with blackened of both feet since 4 months ago. The patient complained of stabbing pain than happens intermittently. Furthermore, there was swelling and pain on the right feet since one month ago. This patient had a history of left toe amputation twice due to gangrene; the fifth finger on the left feet two years ago and the first finger on the left feet a year ago. Physical examination found splenomegaly. The laboratory tests found hemoglobin $10 \mathrm{gr} / \mathrm{dl}$, leukocytes $34.080 / \mathrm{mm}^{3}$, thrombocytes $3.032 .000 /$ $\mathrm{mm}^{3}$ and giant platelets, multinucleated, and grape-like shapes was found at the peripheral blood smear. Doppler ultrasound found partial DVT on the right leg. Bone marrow examination results: multinucleated megakaryocytes, grouped platelets and cytoplasm area. Test result of JAK2V617F is positive. Final diagnosis: essential thrombocythemia with DVT and recurrent gangrene pedis. Treatment of this patient is anti coagulants (heparin followed by warfarin), anti aggregation (aspilet $80 \mathrm{mg}$ daily) and hydroxyurea $500 \mathrm{mg}$ twice per day howed clinical and laboratory improvement.

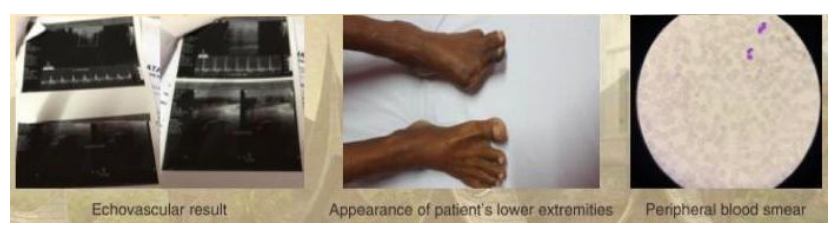

\section{FIGURE 1}

Conclusions: We reported a 54 years old male with diagnosis essential thrombocythemia with deep vein thrombosis and recurrent gangrene digiti pedis. Patient was medicated with anti coagulant, anti aggregation, and hydroxyurea which gives good outcome.

\section{PLATELETS AND INFECTION}

\section{LPB0073 | Platelet Function Is Inhibited by Dengue Virus}

MA. Mosso-Pani ${ }^{1}$; M.I. Salazar ${ }^{2}$; N. Corona-de-la-Peña ${ }^{3}$

${ }^{1}$ Oakland University, Rochester, United States; ${ }^{2}$ Instituto Politécnico Nacional, Ciudad de México, Mexico; ${ }^{3}$ IMSS Hospital General Regional 1 Dr. Carlos Mac Gregor Sánchez Navarro, Ciudad de México, Mexico

Background: There are many very important events that condition the progression to severe dengue, one of them is thrombocytopenia. Increasing evidence supports the fact that platelet dysfunction, in patients with dengue, could be due to direct interaction between platelets and dengue virus. We hypothesize that direct interaction induces changes in platelet function and contributes to pathogenesis. Aims: To evaluate DENV2 effects on platelet function.

Methods: Blood samples from healthy volunteers were collected and the platelet fraction was obtained. Purified DENV2 particles were incubated with platelets for $2 \mathrm{~h}$ at $37^{\circ} \mathrm{C}$. Morphological changes, activation, aggregation, mitochondrial function, and surface markers expression were tested in platelets.

Results: We found that DENV2 induces conspicuous morphological changes on platelets not induced by other arboviruses. We found an increase in CD62P (8979 \pm 1189 vs $1723 \pm 160 \mathrm{MFI} P<0.05)$ and a reduction in CD42b (4131 \pm 936 vs. $39428 \pm 5685$; $P<0.05)$ expression on the platelet surface; reduction in agonist-induced platelet aggregation ( $30 \pm 10$ vs $71 \pm 9 \% ; P<0.05$ ) with assiociated increases intracellular NO production ( $0.4 \pm 0.2$ vs $0.07 \pm 0.01 ; P<0.05)$; reduced thrombin-induced oxidative phosphorylation $(45 \pm 6.69$ vs $348 \pm 26.9$ ngatomO $/ \mathrm{min} / 10^{9}$ platelets; $\left.P<0.0001\right)$ and $\mathrm{PI} 3 \mathrm{~K} /$ Akt signaling $(P<0.001)$. Surprisingly, rDC-SIGN treatment reverts DENV2 effects on platelet activation $(9693 \pm 1033$ vs $2379 \pm 390$ $\mathrm{MFI} ; P<0.01$ ). 
Conclusions: Our data suggest a model where DENV2 inhibits thrombin-induced oxidative phosphorylation through PI3K/Akt signaling pathway inhibition. These changes induce, by short-term interaction of platelets with DENV2, a direct effect over platelet function and may be correlated with the clinical manifestations observed during severe dengue complications, such as thrombocytopenia and hemorrhages. The impact of these changes in signaling and immune response remains to be determined to propose specific therapeutic strategies.

LPB0074 | Platelet Transfusion Does Not Prevent Experimental Polymicrobial-induced Septic Shock in Mice

Y. Rabouel $^{1}$; S. Magnenat ${ }^{1}$; F. Lefebvre ${ }^{1}$; X. Delabranche ${ }^{2}$;

C. Gachet ${ }^{1}$; B. Hechler ${ }^{1}$

${ }^{1}$ Etablissement Français du Sang, INSERM UMR-S1255, Strasbourg,

France; ${ }^{2}$ Hôpitaux Universitaires de Strasbourg, Anesthésie,

Réanimation et Médecine Périopératoire, Strasbourg, France

Background: The specific role of platelets in the course of sepsis is not yet fully understood, probably related to the paradox of platelets being potentially beneficial but also deleterious via their thrombotic functions.

Aims: To evaluate the impact of experimental thrombocytopenia on the pathogenesis of polymicrobial-induced septic shock in mice and to investigate whether transfusion of platelets, either fully functional or with impaired hemostatic properties, might have beneficial effects.

Methods: Septic shock was induced by cecal ligation and puncture (CLP); sham-operated animals were used as control. Experimental depletion of circulating platelets was induced by intravenous injection of a rat anti-mouse GPIba monoclonal antibody. Transfusion of either native platelets, platelets treated with the antiplatelet drugs acetylsalicylic acid (ASA) and clopidogrel, or GPIlbllla-deficient platelets treated with clopidogrel and ASA was performed $4 \mathrm{~h}$ after CLP surgery.

Results: Experimental depletion of circulating platelets negatively affected the pathogenesis of septic shock in mice, worsening systemic inflammation, coagulopathy, organ damage and mortality, raising the question of whether a higher platelet count could protect against septic shock. Transfusion of either fully functional platelets or platelets with combined treatment with ASA and clopidogrel, with or without additional GPIIbIlla deficiency, $4 \mathrm{~h}$ after CLP surgery, afforded an immediate return of circulating platelet counts to their initial values before surgery. However, transfusion of each of the three types of platelets did not prevent arterial hypotension, inflammatory response, coagulopathy and organ damage during septic shock.

Conclusions: Experimental depletion of circulating platelets negatively affects polymicrobial septic shock, while platelet transfusion has no significant beneficial effect. Transfusion of platelets may nevertheless be safely used to treat profound thrombocytopenia associated with bleeding during sepsis, without having any deleterious impact by fueling sepsis.

\section{PB0759 | Platelets Show a Distinct Response in a Controlled Human Malaria Infection (CHMI) Model}

A. Singh ${ }^{1}$; A. Witzemann ${ }^{1}$; L. Pelzl ${ }^{1}$; I. Marini ${ }^{1}$; F. Rigoni ${ }^{1}$; J. Zlamal ${ }^{1}$; A. Kreidenweiss ${ }^{2}$; B. Mordmüller ${ }^{2}$; P. Kremsner ${ }^{2}$; T. Bakchoul ${ }^{1,3}$

${ }^{1}$ Institute for Clinical and Experimental Transfusion Medicine (IKET), University Hospital of Tuebingen, Tuebingen, Germany; ${ }^{2}$ Institute for Tropical Medicine, University Hospital of Tuebingen, Tuebingen, Germany; ${ }^{3}$ Centre for Clinical Transfusion Medicine, University Hospital of Tuebingen, Tuebingen, Germany

Background: Low platelet count is a common clinical finding during early stages of malaria infection in humans. Despite growing evidence of platelets playing a crucial role in malaria pathophysiology, mechanisms leading to the decreased platelet numbers in malaria infections are still poorly understood.

Aims: In this study, we utilized a controlled human malaria infection (CHMI) model established for vaccine clinical trial to investigate several platelet parameters during early stages of Plasmodium falciparum (Pf) infection.

Methods: We used flow cytometry to analyze circulating platelets in healthy, malaria-naïve, adult volunteers undergoing $\mathrm{CHMI}$ using infectious Pf sporozoites. Volunteers were followed daily until parasites were detected in the circulation by RT-qPCR. The following parameter were analyzed: Platelet count, mean platelet volume (MPV), platelet desialylation, the expression of CD62P as a-granule and CD63 as a $\delta$-granule marker, respectively. The platelet responsiveness was investigated by determining the platelet activation markers after incubation with the agonist adenosine diphosphate (ADP), or thrombin receptor-activating peptide (TRAP).

Results: We found a significant drop in platelet count of all malaria positive participants 10 days after Pf-inoculation. Interestingly, MPV was found to be lower in malaria positive subjects and relatively decreased from day 8 onwards. On the other hand, increased platelet desialylation was observed in malaria positive subjects. Flow cytometry analysis showed that platelets of a subgroup of participants were pre-activated as indicated by the higher CD62P expression compared to control blood donors. No significance differences were observed in platelet markers CD42a, CD63 as well as in cell viability and apoptosis.

Conclusions: In conclusion, our study shows that early malaria infection leads to a significant thrombocytopenia in infected patients during $\mathrm{CHMI}$. The higher incidence of desialylated platelets and decreased MPV among infected participants indicate that the cleavage of sialic acid on platelets, and probably megakaryocytes, might be involved in the pathophysiology of malaria-induced thrombocytopenia. 
PB0760 | PSGL-1 is Important for Platelet-monocyte Aggregate Formation and Monocyte Maturation in Lentiviral Infection

C. Lyons $^{1,2}$; E. Shirk ${ }^{1}$; S. Vijay ${ }^{1} ;$ A. Feng ${ }^{1}$; M.R. Richardson ${ }^{1}$; Z. Gholizadeh ${ }^{1}$; B. Carlson ${ }^{1}$; S. Guerrero-Martin ${ }^{1,2}$; K. Metcalf Pate ${ }^{1,2}$

${ }^{1}$ Johns Hopkins University School of Medicine, Baltimore, United States; ${ }^{2}$ Massachusetts Institute of Technology, Cambridge, United States

Background: Platelet-monocyte aggregate (PMA) formation contributes to thrombocytopenia in early HIV infection. PMAs mature into macrophages, and infected macrophages are an important barrier to cure. Canonically, monocyte P-selectin glycoprotein ligand 1 (PSGL-1) binds platelet P-selectin to form PMAs. In a non-infectious disease context, platelet binding to monocytes has further been shown to drive maturation of classical monocytes (CD14+ CD16-) into intermediate (CD14+ CD16+) and non-classical (CD14- CD16+) monocytes. Aims: We utilized the EcoHIV mouse model of HIV to interrogate the importance of PSGL-1 in PMA formation as well as changes in surface marker expression of monocytes during infection.

Methods: Age-matched wild-type C57BI/6J mice and PSGL-1 knockout mice were infected with EcoHIV or mock-infected with saline and euthanized at four endpoints (days 3, 9, 11, and 15 post-infection). Blood was collected via cardiac venipuncture and flow cytometry performed to interrogate PMA formation and monocyte maturation. Results: In comparison to wild-type mice, PSGL-1 knockout mice formed significantly fewer PMAs (classical $P<0.0001$, intermediate $P<0.0001$, and non-classical monocytes $P=0.007$ ) throughout acute infection. Wild-type mice exhibited an increase in classical monocytes (Ly6C+CD43-) with corresponding decrease in intermediate (Ly6C+CD43+) and non-classical (Ly6C-CD43+) monocytes during acute infection, while PSGL-1 knockout mice instead demonstrated a large increase in intermediate monocytes $(P<0.0001)$ with little to no change in classical $(P<0.0001)$ and non-classical monocytes $(P<0.0001)$.

Conclusions: We conclude that PSGL-1 is important for the formation of PMAs and the maturation of monocytes in the context of EcoHIV infection in mice, and that modulation of these interactions are an intriguing target for control of monocyte maturation in HIV.

PB0761 | Phenotypic Changes in Platelets during Measles Virus Infection of Rhesus Macaques (Macaca Mulatta)

S. Guerrero-Martin ${ }^{1,2} ;$ E. Shirk $^{1}$; J. Brockhurst ${ }^{1,3} ;$ N. Peart

Akindele ${ }^{1,3}$; J. Villano ${ }^{1}$; D. Griffin ${ }^{1,3}$; K. Metcalf Pate P.1 $^{2,1}$

${ }^{1}$ Johns Hopkins University School of Medicine, Baltimore, United States; ${ }^{2}$ Massachusetts Institute of Technology (MIT), Cambridge, United States; ${ }^{3}$ Johns Hopkins Bloomberg School of Public Health, Baltimore, United States

Background: Vaccination efforts have reduced the number of global measles virus (MeV)-related deaths 73\% from 2000-2018; however, there were still over 140,000 people who died as the result of measles in 2018. Although rare, the measles, mumps, and rubella (MMR) vaccine is a recognized cause of immune thrombocytopenia (ITP), with an incidence of about 2.5 cases per 100,000 vaccinations, and has been an observed complication of wildtype $\mathrm{MeV}$ infection, as well. Rhesus macaques (Macaca Mulatta) develop disease similar to humans and provide an excellent model for investigating the pathogenesis of $\mathrm{MeV}$ infection and have been utilized to study changes in platelets following other types of infection.

Aims: There are few studies of platelet function during $\mathrm{MeV}$ infection and, to the authors' knowledge, no studies of changes in platelet surface marker expression or on platelet-leukocyte aggregate formation following wildtype $\mathrm{MeV}$ infection have been conducted.

Methods: To investigate changes in platelets during wildtype $\mathrm{MeV}$ pathogenesis, we obtained whole blood samples from MeV-infected rhesus macaques ( $N=5$ ) prior to and following wildtype $\mathrm{MeV}$ inoculation, and assessed platelet numbers and surface markers through hematology and flow cytometry.

Results: All monkeys showed an increase in circulating platelet numbers following $\mathrm{MeV}$ infection, accompanied by an increase in mean platelet volume. Further, we noted an increase in the active form of gpllb/IIla (PAC-1) and MHC class II (HLA-DR) expression on the surface of platelets and an elevation in platelet aggregation with nonclassical monocytes (CD14low/-, CD16+).

Conclusions: These data show that there are changes in platelet production and in platelet activation levels during wildtype $\mathrm{MeV}$ infection. Therefore, MeV-infected rhesus macaques may provide a model for obtaining a more robust understanding of the etiology and mechanisms underlying changes in platelet number and function during wildtype $\mathrm{MeV}$ infection and during the response to the liveattenuated virus present in the MMR vaccine.

PB0762 | Effect of Social Stress on Innate Immune Activation in an SIV-infected Pigtail Macaque (Macaca nemestrina) Model of Acute HIV Infection

N. Castell ${ }^{1}$; S. Guerrero-Martin ${ }^{1,2}$; E.N. Shirk ${ }^{1}$; L.H. Ruben ${ }^{1}$; S.E. Queen ${ }^{1}$; M. Li' ; B. Bullock ${ }^{1}$; D. Graham ${ }^{1}$; M.C. Zink ${ }^{1}$; L. Gama ${ }^{1,3}$; R.J. Adams ${ }^{1}$; J.E. Clements ${ }^{1}$; J.L. Mankowski ${ }^{1}$; K.A. Metcalf Pate ${ }^{1,2}$ ${ }^{1}$ Johns Hopkins University, Baltimore, United States; ${ }^{2}$ Massachusetts Institute of Technology, Cambridge, United States; ${ }^{3}$ National Institutes of Health, Bethesda, United States

Background: Simian immunodeficiency virus (SIV) infected pigtail macaques (Macaca nemestrina) are a common model for AIDS pathogenesis. Similar to HIV, disease progression is affected by both internal (host and pathogen) and external factors.

Aims: Because the deleterious impact of chronic stress on the immune response is well established, we hypothesized that singly housed macaques would have a less robust innate immune response post-inoculation than those housed in stable conspecific pairs. Two 
cell populations critical to innate immune function, platelets and monocytes, were assessed.

Methods: A retrospective analysis of acute infection data from juvenile male pigtailed macaques $(n=76)$ was performed. Complete blood count and staining with P-selectin, CD40L, HLA-ABC, CD14, and CD16 fluorochrome antibodies was performed on whole blood. Flow cytometry was analyzed by a single blinded researcher. Mixed effects regression models assessed the influence of housing status from baseline up to 14 days post-inoculation.

Results: Consistent with previous findings, SIV infection induces a transient thrombocytopenia which recovers by day 14 postinoculation. By day 10, platelet activation was increased in socially housed macaques as evidenced by increased P-selectin and MHC-I expression, without an increase in CD40L. No significant changes were present in singly housed animals at day 10 , although P-selectin increased by day 14 . Though all groups had an elevation in monocyte count following infection, only singly housed animals demonstrated a transient decrease on day 7. Animals that were socially housed showed a significantly greater rate of classical monocyte proliferation at all post-infection time points.

Conclusions: The immunomodulatory effects of chronic social stress significantly alter the innate immune response to acute retroviral in fection, with singly housed animals less able to respond appropriately to viral challenge. This variable should be considered in study design both to reduce inter-animal variability and for its translational significance for people with HIV.

PB0763 | Incidence and Correlation of Thrombocytopenia to Blood Stream Infections; Does this Correlation Really Matter?

N. Anwar; N. Fatima; H.-u.- Mujtaba; T. Shamsi

National Institute of Blood Diseases and Bone Marrow Transplantation, Karachi, Pakistan

Background: Thrombocytopenia is a serious clinical consequence of certain blood and immunological disorders, tumors, drugs, and infections. Enormous literature is available addressing the incidence of thrombocytopenia following sepsis and viral infections. However there is scant data revealing the association of blood stream infections with thrombocytopenia.

Aims: This study was designed to observe the impact of blood cultures on platelet count and also to find out the association between type of microbial organism and severity of thrombocytopenia.

Methods: An observational study was conducted at NIBD PECHS campus from July to December 2019 after institutional review board approval. The incidence of platelet count $\left(\times 10^{9} / \mathrm{L}\right)$ of $<10,<50$ and $<100$ were observed in patients having positive blood cultures. Chi square or Fisher's exact test was used to observe the association and $P$ value $<0.05$ was considered significant.

Results: A total of 94 blood cultures were analyzed and correlated with the presence of thrombocytopenia. Forty four (47\%) cultures were positive. The frequently encountered gram negative organism was Pseudomonas aeruginosa 16(43\%) and gram positive was Staphylococcus aureus 3(43\%). The incidence of thrombocytopenia in patients with positive and negative blood cultures were $39 \%$ and $20 \%$ respectively at platelet count of $<10(P=0.046)$. For platelet count $\left(\times 10^{9} / \mathrm{L}\right)<50$ and $<100$, it was not found significant. However, The incidence of thrombocytopenia with respect to specific gram negative or positive organism was not found significant.

Conclusions: In our study, positive blood cultures were more inclined to have platelet count of $<10 \times 10^{9} / \mathrm{L}$ but there was no significant impact of type of organism on platelet count. Thrombocytopenia is one of the grave and inevitable consequence of infections which may lead to mortality and morbidity. Future prospective studies are needed to fill the void in this aspect.

\section{PB0764 | Platelet Involvement and its Association with Mortality in COVID-19 Patients at a Safety-net Hospital}

L.F. Gonzalez-Mosquera ${ }^{1}$; S. Gomez-Paz ${ }^{1}$; E. Lam ${ }^{1}$; D. CardenasMaldonado $^{1}$; J. Fogel ${ }^{2}$; V. Adi ${ }^{1}$; S. Rubinstein ${ }^{1}$

${ }^{1}$ Nassau University Medical Center, East Meadow, United States;

${ }^{2}$ Brooklyn College, Brooklyn, United States

Background: COVID-19 has shown to affect the hematologic system especially platelets. Some studies showed its association with increased mortality. However, there are not enough studies done with a predominantly minority population.

Aims: Evaluate the impact of the involvement of platelets, white blood cells including absolute neutrophil count (ANC), and hemoglobin in COVID-19 patients and their association with mortality and length of stay (LOS).

Methods: This is a retrospective study of 475 patients with confirmed positive COVID-19 infection and hematologic laboratory derangements, including platelet count abnormalities, at a safety-net hospital in the metropolitan New York City area. We defined platelet peak as defined the highest platelet count level during admission and platelet nadir as the lowest platelet count level during admission. We also analyzed their effect on mortality and length of stay (LOS) using multivariate logistic regression and linear regression respectively. IBM SPSS Statistics version 26 was used for the analyses.

Results: Of a total of 475 patients, 363 had platelet count involvement (thrombocytopenia: $206 \mathrm{k} / \mathrm{mm}^{3}$, thrombocytosis: $157 \mathrm{k} / \mathrm{mm}^{3}$ ). The mean platelets peak was $350.7 \mathrm{k} / \mathrm{mm}^{3}$ and the mean platelets nadir was $191.6 \mathrm{k} / \mathrm{mm}^{3}$. Higher platelet peak (OR:0.995, 95\% $\mathrm{Cl}$ 0.992-0.997, $P<0.001)$ and higher hemoglobin on admission were associated with decreased mortality. We did not find an association between platelets nadir and mortality. Higher platelet peak had increased $\operatorname{LOS}(B=0.001, \mathrm{SE}=<0.001, P<0.001)$, and those with higher platelet nadir had decreased LOS $(B=-0.001, S E=<0.001$, $P<0.001)$. Increased (ANC) (OR:1.20; 95\% Cl:1.02-1.42, $P<0.05$ ), and days to hematologic involvement was associated with higher mortality. 
TABLE 1 Multivariate analysis of the effect of hematologic involvement on mortality

\begin{tabular}{lll}
$\begin{array}{l}\text { Hematologic } \\
\text { parameters }\end{array}$ & Odds ratio $(95 \% \mathrm{Cl})$ & $P$-value \\
Platelets peak & $0.995(0.992,0.997)$ & $P<0.001$ \\
Platelets nadir & $1.00(0.999,1.01)$ & $P>0.05$ \\
White blood cell peak & $0.70(0.08,6.30)$ & $P>0.05$ \\
White blood cell nadir & $0.93(0.06,15.59)$ & $P>0.05$ \\
$\begin{array}{l}\text { Absolute neutrophil } \\
\quad \text { count nadir }\end{array}$ & $1.20(1.02,1.42)$ & $P<0.05$ \\
$\begin{array}{l}\text { Hemoglobin admission } \\
\text { Hemoglobin nadir }\end{array}$ & $0.77(0.60,0.98)$ & $P<0.05$ \\
$\begin{array}{l}\text { Days to hematology } \\
\text { involvement }\end{array}$ & $1.25(0.94(1.42,13.90)$ & $P>0.05$ \\
\hline
\end{tabular}

Conclusions: Increased platelet peak and increased hemoglobin on admission were associated with decreased mortality, and higher platelet peak had increased LOS. High ANC and increased days to any hematologic involvement were associated with increased mortality. Hematologic parameters, including platelet count, can be used by clinicians to better risk stratify COVID-19 patients with hematologic involvement and tailor therapies to potentially improve patient outcomes.

\section{PLATELETS AND INFLAMMATION}

PB0765 | Human Myeloperoxidase Enhances Thrombin Induced Phosphatidylserine Exposure in Human Platelets through Tyrosine Kinase/Calcium/Potassium Channels-mediated Pathway

A Kokhan ${ }^{1}$, F Balabin $^{2,3}$, I Gorudko ${ }^{1}$, A Sokolov ${ }^{4,5}$,

A Sveshnikova ${ }^{2,3,6}$ E Shamova $^{1}$

${ }^{1}$ Belarusian State University, Minsk, Belarus; ${ }^{2}$ Center for Theoretical

Problems of Physicochemical Pharmacology, Russian Academy of

Sciences, Moscow, Russian Federation; ${ }^{3}$ National Medical Research

Centre of Pediatric Hematology, Oncology and Immunology named after Dmitry Rogachev, Moscow, Russian Federation; ${ }^{4}$ Institute of Experimental Medicine, St. Petersburg, Russian Federation; ${ }^{5}$ Federal Research and Clinical Center of Physical-Chemical Medicine of Federal Medical Biological Agency, Moscow, Russian Federation; ${ }^{6}$ Lomonosov Moscow State University, Moscow, Russian Federation

Background: There is a close relationship between inflammation and coagulation in multiple diseases. Revealing the mechanisms of molecular and cellular connections between these systems is necessary for the development of new therapeutic strategies. One of the local mediators of inflammation is myeloperoxidase (MPO), hemeperoxidase contained mainly in neutrophils. MPO generates cytotoxic oxidants and modulates cellular signal cascades by binding to cellular surfaces. MPO was shown to potentiate platelet aggregation and $\mathrm{Ca}^{2+}$-response, so it may contribute to pathological thrombosis.
Aims: This study was designed to investigate MPO effect on platelet procoagulant activity and to disclose new mechanisms of MPO action.

Methods: Platelets were isolated from citrated blood from healthy volunteers. Single channel ionic currents were registered using patch-clamp technique in cell-attach configuration. Voltage-gated potassium channels were inactivated by patch hyperpolarization. Platelet calcium responses were measured using TIRF microscope by observing CalBryte 590-AM fluorescence. Phosphatidylserine (PS) exposure was measured by flow cytometry using annexin $\mathrm{V}$-Alexa Fluor 647.

Results: MPO (100 nM-400 nM), incubated with platelets for 15 min, enhanced thrombin-induced PS exposure on platelet surface in a dose-dependent manner without any influence on the number of PS-positive platelets induced by ADP or collagen-related peptide. This effect of MPO was associated with increased frequency (2fold) and duration of calcium oscillations and with opening of potassium channels ( 20 pS). Charybdotoxin and genistein canceled the observed effects, revealing the role of tyrosine kinases and $\mathrm{Ca}^{2+}$-induced potassium channels. Although the receptor for MPO on platelet surface is unknown, obtained results indicate that MPO is involved in $\mathrm{Ca}^{2+}$-signaling through activation of tyrosine kinases, probably through ITAMs.

Conclusions: This study reveals new mechanisms of MPO modulating effect on platelet function at sites of inflammation, leading to procoagulant platelet formation. Obtained results might be of potential pharmacological interest in inflammatory and thrombotic disorders.

Work was partly supported by grant B20R-215.

\section{PB0767 | Selected Platelet Parameters in Patients with Rheumatoid Arthritis at Total Hip Arthroplasty}

A.S. Ershov ${ }^{1,2}$;.P. Antropova ${ }^{1,3}$; E.A. Volokitina ${ }^{1}$; L.P. Evstigneeva ${ }^{1,2}$ ${ }^{1}$ Ural State Medical University, the Ministry of Health of the Russian Federation, Ekaterinburg, Russian Federation; ${ }^{2}$ Sverdlovsk Regional Clinical Hospital, Ekaterinburg, Russian Federation; ${ }^{3}$ Institute of High-Temperature Electrochemistry of the Ural Branch of the Russian Academy of Science, Ekaterinburg, Russian Federation

Background: Platelets link with inflammation. However, data describing the effect of rheumatoid arthritis (RA) on platelet morphofunctional parameters are contradictory.

Aims: The aim of the study was to determine the effect of rheumatoid arthritis on platelet parameters at primary total hip arthroplasty (THA).

Methods: The prospective study included 23 patients with RA and 21 patients with osteoarthritis (OA) underwent total hip arthroplasty. The groups had no significantly differ in gender, age, stage of the disease, concomitant pathology, type of surgical intervention, prosthesis fixation method, implantation system type. Crystalloids were carried out intraoperatively in all patients according standard 
scheme. There were no blood transfusions. Blood indicators were studied using the Sysmex XT-4000i automatic hematology analyzer. Samples were analyzed within 1 hour after venipuncture to exclude platelet swelling. "Statistica" data analysis package was used for statistical processing.

Results: According Table 1, before THA red blood cell count and the level of hemoglobin were lower in the RA group against OA group; platelet count was higher in the RA group in comparison to OA group, but MPV in RA group was lower than in OA group. After surgery, there were no differences in red blood cells and hemoglobin levels in groups. The decreased level of red blood cells was significantly less in RA group compared with OA group; the decrease in hemoglobin level was also less in the RA group (Figure).

In patients with RA, there is a positive correlation between leukocyte count and platecrit (Spearman's $\rho=0.48 ; P<0.05$ ) and negative correlation between white blood cell count and MPV $(\rho=-0.43$; $P<0.05)$

\section{PROTEASES AND CANCER}

PB0768 | The Intact Heavy Chain of Plasminogen Supresses Angiogenesis More Strongly than the Known Angiostatin

K. Gershkovich $^{1}$; L. Mukhametova ${ }^{2}$; R. Aisina ${ }^{2}$

${ }^{1}$ Emanuel Institute of Biochemical Physics of RAS, Moscow, Russian Federation; ${ }^{2}$ Lomonosov Moscow State University, Moscow, Russian Federation

Background: It is known that plasminogen heavy chain degradation products containing K1-3, K1-4 and K1-4.5 kringle domains, called angiostatins, inhibit angiogenesis and tumor growth. Angiostatin K1-4.5 is the most effective inhibitor of endothelial cell proliferation, neovascularization and tumor growth.

TABLE 1 Hematological parameters in patients with RA and OA before and 1 day after total hip arthroplasty. Results are presented as median [interquartile range]

\begin{tabular}{|c|c|c|c|c|c|c|}
\hline \multirow[b]{2}{*}{ Parameter } & \multirow{2}{*}{$\begin{array}{l}\text { Before surgery } \\
\text { RA }\end{array}$} & \multicolumn{5}{|c|}{1 Day after surgery } \\
\hline & & OA & & $P$ & RA & $P$ \\
\hline $\begin{array}{l}\text { Red blood cells }\left(\times 10^{6} /\right. \\
\quad \mu \mathrm{L})\end{array}$ & $4.37[4.10 ; 4.68]$ & $4.75[4.30 ; 4.98]$ & 0.086 & $3.65[3.23 ; 4.01]$ & $3.71[3.27 ; 4.03]$ & 0.839 \\
\hline Hemoglobin (g/L) & $133.0[119.0 ; 136.0]$ & $140.0[124.0 ; 146.0]$ & 0.031 & 107.0 [103.0; 113.0] & $107.0[102.0 ; 116.0]$ & 0.228 \\
\hline Platelets $\left(\times 10^{3} / \mu \mathrm{L}\right)$ & $257.0[233.0 ; 306.0]$ & 219.0 [178.0; 262.0] & 0.026 & 213.0 [174.0; 267.0] & $170.0[146.0 ; 225.0]$ & 0.025 \\
\hline
\end{tabular}

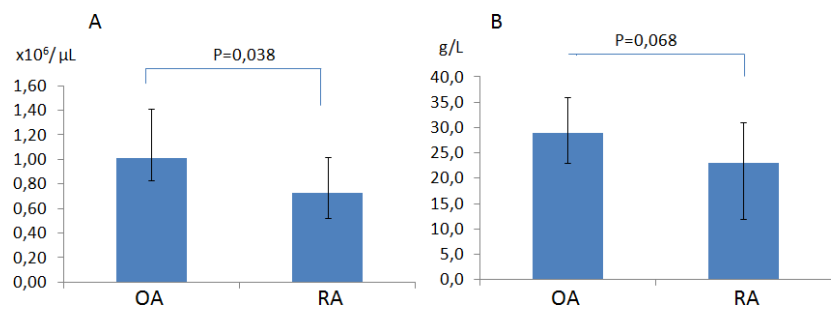

FIGURE 1 Loss of red blood cells (A) and hemoglobin (B) at total hip arthroplasty in patients with osteoarthritis (OA) and rheumatoid arthritis (RA). Results are presented as median [interquartile range]

Conclusions: In RA patients requiring THA, platelet parameters are associated with leukocyte count and possibly inflammation. Platelets morpho-functional features in RA may contribute to a lower loss of red blood compared to patients with OA at surgery.
Aims: Comparison of inhibitory effect of K1-4.5 and a new K1-5 fragment containing intact kringle 5 on the Glu-plasminogen $(\mathrm{Pg}) \mathrm{ac}-$ tivation by its activators and on corneal neovascularization.

Methods: Intact K1-5 and K1-4.5 were prepared from human plasminogen. Their influence on Pg activation kinetics by tPA (in presence of soluble fibrin) and uPA was measured by conjugated method. Results: Fragments K1-4.5 and K1-5 did not affect the amidolytic activity of plasmin, tPA and uPA, but inhibited the Pg activation by uPA and tPA in dose-dependent manner. The $\mathrm{k}_{\mathrm{Pg}}$ and $\mathrm{K}_{\mathrm{Pg}}$ values of $\mathrm{Pg}$ activation in absence of the fragments were $0.02 \mathrm{~s}^{-1}$ and 0.52 $\mu \mathrm{M}$ for $\mathrm{UPA}$ and $0.086 \mathrm{~s}^{-1}$ and $0.023 \mu \mathrm{M}$ for tPA, respectively. Both fragments had no effect on $\mathrm{K}_{\mathrm{Pg}}$ and increased $\mathrm{K}_{\mathrm{Pg}}$ of $\mathrm{Pg}$ activation by uPA, whereas they reduced $\mathrm{k}_{\mathrm{Pg}}$ and increased $\mathrm{K}_{\mathrm{Pg}}$ of Pg activation by tPA. Inhibition constants by K1-4.5 and K1-5 were 0.59 and 0.12 $\mu \mathrm{M}$ for $\mathrm{UPA}$ and 0.4 and $0.05 \mu \mathrm{M}$ for tPA, respectively. Experiments on the use of these fragments in the treatment of rabbit eyes burn have shown that K1-5 suppresses corneal neovascularization more strongly than K1-4.5.

Conclusions: The K1-4.5 and K1-5 displace Pg from uPA-Pg and tPA-Pg complexes and from fibrin surface in case of tPA. Absence of 
protease domain in these fragments leads to decreasing the concentrations of uPA-Pg and tPA-Pg-fibrin activator complexes and consequently reducing the plasmin generation rate. Owing the kringle 5 integrity, K1-5 stronger inhibits $\mathrm{Pg}$ activation and angiogenesis, than K1-4.5. Intact K1-5 is promising for suppressing endothelial cells proliferation and neovascularization.

PB0769 | Anti-cancer Activity of Tempeh - Spermine Can Induce the Necrosis of HeLa Cells

H. Sumi ${ }^{1}$; T. Mitsuo ${ }^{2}$; C. Yatagai ${ }^{1}$

${ }^{1}$ Kurashiki University of Science and the Arts, Kurashiki, Japan; ${ }^{2}$ Mitsuo Clinic, Tokyo, Japan

Background: Tempeh is an Indonesian fermented soybean food with a history of more than 500 years, but its strong antioxidant or antibacterial substances are attracting attention.

Aims: We report that spermine, one of the polyamines contained in tempeh, has a fairly strong anti-cancer activity.

Methods: Rhizopus oligosporus from Akita Konno Co. Ltd., and soymilk powder from Imuraya Foods Co. Ltd.. Thrombolytic activity, vitamin $\mathrm{K}_{2}$ and polyamine were determined as described previously. For HeLa cell culture, E-MEM medium containing 10\% fetal bovine serum (FBS) was autoclaved at $121^{\circ} \mathrm{C}$ for $15 \mathrm{~min}$ and then sterilized by filtration. The cells were seeded on a 96-well plate at $3 \times 10^{4}$ cells $/ \mathrm{cm}^{2}$ and cultured at $37^{\circ} \mathrm{C}$ under $5 \% \mathrm{CO}_{2}$ conditions until they became confluent. $50 \mu \mathrm{l}$ of the diluted solution of each sample was added to HeLa cells for $24 \mathrm{hr}$ (1st) and $48 \mathrm{hr}$ (2nd). The number of cells was measured at an absorbance of 600nm (Yatagai et al., Pathophysiol. Haemost. Thromb., 36, 227, 2009).

Results: When the thrombolytic activity of the HeLa cell culture medium was determined, t-PA release was 17.7 times more active in natto than in control. That is, it was found that natto causes strong t-PA release from HeLa, whereas tempeh strongly suppresses t-PA release. Next, when the effect of adding spermine on t-PA expression in HeLa cells was investigated, it was found that thrombolytic activity was clearly suppressed in both 1 st medium and 2 nd medium. It was found that the suppression rate of spermine was remarkably high at the concentration of $1 \mu \mathrm{mol} / \mathrm{ml}$ and the cancer cells had been completely necrotic. On the other hand, in contrast, the effect was negative in the addition experiment of spermidine or putrescine.

Conclusions: HeLa cells are known as t-PA producing cells, but spermine strongly suppressed them, and complete necrosis of cancer cells was confirmed.

\section{NURSES AND ALLIED HEALTH}

PB0770 | Effect of Aroma Hand Massage on the Plasma Concentration of Plasminogen Activator Inhibitor-1 Produced by Endothelial Cells in Healthy Subjects

T. Hayashi $^{1}$; N. Akita ${ }^{2}$; A. Tamada ${ }^{1}$

${ }^{1}$ Mie Prefectural Collage of Nursing, Tsu city, Japan; ${ }^{2}$ Suzuka

University of Medical Science, Suzuka-city, Japan

Background: Endothelial cells express various fibrinolytic factors such as tissue plasminogen activator ( $t-P A)$ and plasminogen activator inhibitor (PAI-1), which play an important role in adequate blood clot formation. Hand massage is a common nursing technique, and previously it has been reported that aroma hand massage has various effects, such as a sedative effect, on the autonomic nervous system. However, a preventive effect of aroma hand massage against thrombosis has not been reported.

Aims: In the present study, to evaluate the effect of aroma hand massage on prevention of thrombosis, changes in plasma concentrations of PAI-1, which is produced by endothelial cells and functions as a physiological inhibitor to t-PA, were measured before and after aroma hand massage for different lengths of time.

Methods: In these experiments two percent of rosemary $(400 \mu \mathrm{l})$ was used for each hand massage. We divided healthy volunteers into $5 \mathrm{~min}, 10 \mathrm{~min}$ and $20 \mathrm{~min}$ aroma hand massage groups, and obtained plasma samples from the subjects of each group before, immediately after, $10 \mathrm{~min}$ after and $20 \mathrm{~min}$ after each aroma hand massage. Plasma PAI-1 concentration was measured using a commercially available ELISA kit.

Results: For 5 min and 10 min aroma hand massage, plasma PAI-1 concentration was not significantly changed at any time point as compared with before aroma hand massage. However, in the case of $10 \mathrm{~min}$ after $20 \mathrm{~min}$ aroma hand massage, plasma PAI-1 concentration was significantly decreased as compared with before aroma hand massage.

Conclusions: These results suggest that $20 \mathrm{~min}$ aroma hand massage reduced PAI-1 expression from endothelial cells and/or secretion of PAI-1 from endothelial cells, and aroma hand massage has a preventive effect against thrombosis.

\section{PB0772 | The Importance of Follow up Post PE}

\section{Roberts}

University of Glamorgan, Pontyprydd, United Kingdom

Background: Pulmonary embolism (PE) is an acute and potentially fatal condition. The incidence of PE increases with age. Mortality rates can be as high as $25 \%$ if untreated however, with anticoagulant therapy, this rate decreases to about $2-8 \%$ in the 3 months following 
diagnosis. The benefit of early anticoagulation to improve mortality and decrease recurrent thromboembolism is well proven. A study by Konstantinides, et al (2014) found that patterns of patient follow up post PE differ greatly and firm evidence to provide guidance is lacking.

Aims: NICE Guidelines state that patients should be discharged with adequate monitoring of anticoagulant treatment and that the patient is provided with sufficient information and education. The purpose of this work was to determine if patients are provided with this in formation upon discharge and to determine how this service can be improved.

Methods: The British Thoracic Society Guidelines (2018) state that patients should have a formal review during the first week after discharge to ensure therapeutic compliance along with the absence of complications. Discussion was held between Haematology Consultant and Anticoagulation Staff to improve the quality of service provided to patients discharged on anticoagulation following a PE. It was decided that Anticoagulation Nurse Specialists would contact patients, within a week of discharge, to determine their understanding of their medication and offer support if needed. A protocol was created outlining important aspects of care. Patients would be contacted and questions were asked regarding their medication, discharge and information received.

Results: Results showed patients were often not provided with key information. $28 \%$ stated no advice was received and $47 \%$ received no written information on discharge.

Conclusions: Patients greatly appreciated the call and many further issues were noted such as missing or incorrect information regarding dosing and education. This service will be continued to improve patient safety.

\section{PB0773 | Depression and Anxiety Screening in a Pediatric Coagulation Disorder Program: A Brief Description of a 7-week Pilot}

\section{Braunreiter; E. Marietta; S. Pentoney; L. Vanderwal;}

B. Kurt; J. Bowden

Helen DeVos Children's Hospital at Spectrum Health, Grand Rapids, United States

Background: Children with chronic health conditions have higher rates of mental illness compared to peers without health conditions. Ghanizadeh et al. reported a $6 \%$ rate of major depressive disorder in children diagnosed with hemophilia. Adults who experienced a venous thromboembolism report significant stress and anxiety.

The U.S. Preventive Services Task Force recommends screening for major depressive disorder in patients 12 to 18 years of age in the primary care setting. Our Pediatric Coagulation Disorder Program piloted a depression and anxiety screening protocol for 7 weeks. We surveyed providers to assess the screening process.
Aims: To identify gaps of the mental health screening process for continued process improvement.

Methods: The 4-item Patient Health Questionnaire for anxiety and depression (PHQ-4), PHQ 9-item depression module, Generalized Anxiety Disorder 7-item scale (GAD-7), and Columbia-Suicide Severity Rating Scale (C-SSRS) were utilized. (Figure 1) Electronic medical records of patients $\geq 12$ years were reviewed to determine if a screening was performed. An anonymous electronic survey was emailed to providers inquiring about the screening process.

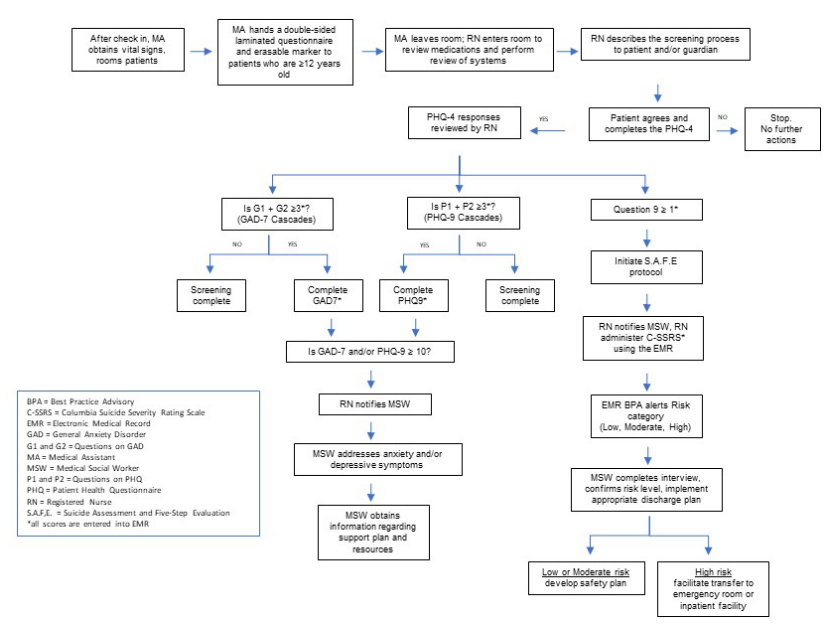

FIGURE 1 Pediatric Coagulation Disorder Program Mental Health Screening Process

Results: 70 patients were seen during the 7 -week pilot. 38 were > 12 years of age. 33 (87\%) were screened, 2 did not require a screen (screened within the last two weeks, seeing psychologist on the same visit), 2 refused, and 1 was missed. Two patients scored $\geq 1$ on question 9 of PHQ- 4 which is the suicide indicator. The S.A.F.E protocol was initiated on one of these occasions. Urgent referrals were not required during this 7 -week pilot. 11 providers were emailed the survey. $82 \%$ of providers completed the survey. (Figure 2)

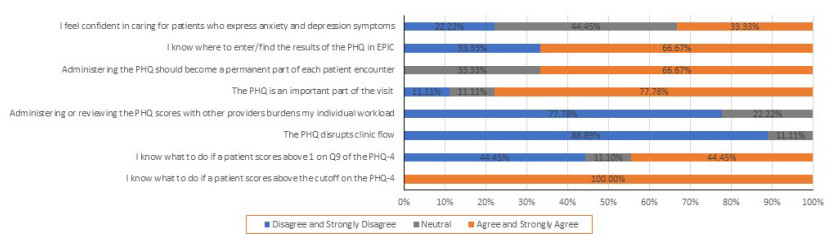

FIGURE 2 Provider Survey Results

Conclusions: Majority of providers responded that the mental health screening was important, not disruptive nor a burden. Future initiatives will aim to impart knowledge and skills to providers in order for them to be confident in caring for patients who express anxiety or depressive symptoms. 
PB0774 | Development of Decision-making Process to Support Equitable Participant Selection in Pediatric Hemophilia Trials

K. Hamilton; K. Strike; K. Decker; R. Goldsmith; T. Almonte; W. Xie; M.D Bhatt; A.K. Chan

McMaster Children's Hospital, Hamilton, Canada

Background: As clinical trials have limited enrollment, it is vital to establish objective methods for subject selection to address medical needs and to ensure equitable access to potentially life-altering therapeutic options.

Aims: To describe our preliminary steps in the development of an objective clinical decision-making process for the selection of pediatric patients with hemophilia for participation in therapeutic trials. Methods: This clinical decision-making process was developed using an iterative design with our inter-disciplinary team.

Results: The team identified need to structure the process with a numerical matrix in an attempt to minimize subjectivity. Random selection was also discussed. The first iteration identified areas of unmet clinical needs including venous access, bleeding, joint health. The second iteration included the addition of social and treatment support needs including home infusion and transportation. Following review, the team recognized the need to include patient safety measures such as adherence to treatment recommendations and attendance. The final step addressed barriers to participation. These barriers were identified in consultation with team members and Equity/Diversity Specialists for equitable inclusion of participants from marginalized or racialized communities, those who may experience reduced family medical literacy, interest in, access to or enrollment on previous clinical trials. Throughout this process the team recognized the importance of inter-disciplinary participation and the need for a defined structure to generate discussion.

Conclusions: This was our first attempt at developing a decision support process which included a numerical matrix followed by structured team discussion, to address equity for participation in pediatric clinical trials. Next steps will involve further consultation with Equity-Diversity Specialists and other stakeholders. This approach is an ongoing iterative process and may be applicable to clinical trials in rare chronic diseases; other therapeutic areas may find random selection sufficient to mitigate selection bias and equity of opportunity.

PB0775 | Clinical Factors Predicting Decrease in Hemoglobin Levels during Preoperative Autologous Blood Donation by Patients Undergoing Major Orthopedic Surgery

S. Fujii; Y. Watanabe; A. Ito

Asahikawa Medical University Hospital, Asahikawa, Japan

Background: To identify patients whose hemoglobin $(\mathrm{Hb})$ levels can decrease after autologous blood donation may contribute to effective and safe autologous blood transfusion.
Aims: In this study we aimed to determine the correlations of baseline laboratory markers before autologous blood donation with decrease of $\mathrm{Hb}$ levels after first blood drawing.

Methods: Patients undergoing major orthopedic surgery consisting of 35 males and 111 females were enrolled. $\mathrm{Hb}$ change was defined as the decrease or differences of $\mathrm{Hb}$ from first $400 \mathrm{ml}$ blood drawing. Laboratory data were obtained in the hospital laboratory. Data were analyzed using Steel-Dwass multiple tests and Wilcoxon rank sum test (Mann-Whitney $U$ test).

Results: Body height, body weight and body mass index (BMI) had no correlations with $\mathrm{Hb}$ change. Gender showed no significant correlation to change of $\mathrm{Hb}$. Higher levels of baseline red blood cells (RBC), $\mathrm{Hb}$,hematocrit $(\mathrm{Ht})$ correlated with lower $\mathrm{Hb}$ levels after donation. Higher levels of RBC and $\mathrm{Ht}$ exhibited lower $\mathrm{Hb}$ recovery rate. Reticulocyte (Ret) levels before blood donatiuon negatively correlated with $\mathrm{Hb}$ change. Ret levels before blood donation positively correlated with $\mathrm{Hb}$ recovery amount and $\mathrm{Hb}$ recovery rate. In contrast, serum iron,unsaturated iron binding capacity,total iron binding capacity,ferritin,estimated glomerular filtration rate, and non-transferrin binding iron levels had no correlations with $\mathrm{Hb}$ change, $\mathrm{Hb}$ recovery amount and $\mathrm{Hb}$ recovery rate.

Conclusions: The results suggested that Ret can be used as a predictor for estimating $\mathrm{Hb}$ levels of donors after blood donation. Donors with higher $\mathrm{Hb}$ and $\mathrm{Ht}$ would have greater decrease of $\mathrm{Hb}$ than donors with lower $\mathrm{Hb}$ and $\mathrm{Ht}$.

\section{PEDIATRICS}

\section{BLEEDING IN NEONATES AND CHILDREN}

\section{LPB0122 | Severe FXI Deficiency a Pediatric Cohort}

A.A. Barg; S. Levy; E. Avishai; A. Dvir; N. Kornbrot; R. Dardik;

G. Kenet

Sheba Medical Center and Tel Aviv University, Tel Aviv, Israel

Background: Factor XI (FXI) deficiency is a rare bleeding disorder in which bleeding usually occurs only following surgery or trauma. To date no studies examining the presentation and complications of this disorder in the pediatric population have been conducted.

Aims: To evaluate the presentation, hemostatic phenotype and complications in a cohort of children aged 0-18 years diagnosed with severe FXI deficiency

Methods: After IRB approval, electronic medical records were screened to identify children with severe FXI deficiency (defined as a FXI level $=15 \%$, compatible with homozygous or compound heterozygous mutations), followed at our tertiary center. Data regarding reasons for referral, bleeding symptoms, surgeries outcome and complications were evaluated. 
Results: Twenty-two children whose median age at diagnosis was 7 years (range: 1 day- 16 years) comprised the study cohort. In $68 \%$ evaluation was performed either due to family history or preoperatively.

Seven patients presented with bleeding symptoms: triggered intracranial hemorrhage $(\mathrm{ICH} ; 2 / 7)$, excessive bleeding following trauma or post-surgical procedures and gastrointestinal bleeding. Another $\mathrm{ICH}$ was encountered during follow up. Only one child presented with major bleeding following teeth extraction.

Four Major surgeries were safely performed among children in our cohort assisted by plasma transfusions and tranexamic acid. Sixteen male patients underwent circumcision as neonates. Excessive bleeding post circumcision was noted in two patients, one bled despite the administration of prophylactic tranexamic acid.

Interestingly, 2 children sustained triggered thrombotic complications, both resolved without anticoagulant therapy.

Conclusions: This study describes the bleeding phenotype associated with severe FXI deficiency in a pediatric cohort. The significant incidence of $\mathrm{ICH}$ as well as the occurrence of thrombosis in children with severe FXI deficiency warrants attention and should be further evaluated in prospective larger cohorts.

PB0776 | A Retrospective Review of Diagnosis and Management of Heavy Menstrual Bleeding and Co-morbidities in Patients Seen in Young Women's Blood Clinic

D. Desai; M. Joshi; R. Upadhyay; D. Costescu; M. Bhatt

McMaster University, Hamilton, Canada

Background: Menorrhagia is prevalent in $37 \%$ of adolescents and associated with co-morbidities like bleeding disorders (BD), iron deficiency anemia (IDA), and mood disorders. $\mathrm{MCH}$ is the first tertiary care centre in Ontario to start a multidisciplinary Young Women's Blood Clinic (YWBC) staffed by a gynaecologist, hematologist, and nurse to provide diagnosis and management of menorrhagia.

Aims: The purpose of this study is to evaluate the prevalence of $B D$ and IDA in adolescents (<18 years) with menorrhagia seen in YWBC. Additionally, management and outcomes will be reported.

Methods: This is a retrospective cohort study between July 2017 to June 2020. Patient records were reviewed for demographics, laboratory parameters, management plans, and outcomes.

Results: 80 new patients (mean age: 14.7 years) with menorrhagia were seen in the monthly YWBC during the study period. 4 patients were referred with pre-existing BDs (2 vWD, 1 factor XI deficiency, 1 factor VII deficiency), while 3/76 (4\%) were newly diagnosed (3 vWD). On presentation, 25 (31\%) had IDA, while 40 patients (51\%) had iron deficiency without anemia. 54 patients were treated with oral iron alone, while 11 required IV iron and 10 required blood transfusion. Combined oral contraceptive pills were the most common management option, followed by tranexamic acid and IUD. After a median follow-up of $\mathbf{2 0 . 5}$ months, the majority of patients showed an improvement in menorrhagia.
Conclusions: Menorrhagia can be debilitating and requires coordinated multidisciplinary care to diagnose and manage adequately.

PB0777 | Blood Transfusion is Associated with Mortality among Neonates with Congenital Diaphragmatic Hernia on ECMO Support

Y. Yang ${ }^{1}$; S.H. Gowda ${ }^{1,2}$; J.L. Hagan ${ }^{2}$; L. Hensch ${ }^{1,2}$; J. Teruya ${ }^{1,2}$; C.J. Fernandes ${ }^{1,2}$; S.-K.R. Hui ${ }^{1,2}$

${ }^{1}$ Baylor College of Medicine, Houston, United States; ${ }^{2}$ Texas Children's Hospital, Houston, United States

Background: Transfusion support is frequently needed to maintain adequate hemostasis on ECMO, especially in neonates with immature coagulation system and surgical intervention. However, there is also evidence suggesting transfusion in early life is associated with decreased survival.

Aims: To evaluate transfusions among other risk factors during the peri-operative period of congenital diaphragmatic hernia $(\mathrm{CDH})$ repair and their associations with mortality.

Methods: Retrospective chart review of all neonates with $\mathrm{CDH}$ who underwent surgical repair while on ECMO. We reviewed medical records over a period of 10 years from $1 / 2010$ to 12/2020. Transfusion records include red cells, plasma, platelets and cryoprecipitate. Definitions of each study period are shown in Figure 1. Logistic regression and the Wilcoxon rank sum test were used to investigate associations with survival status.

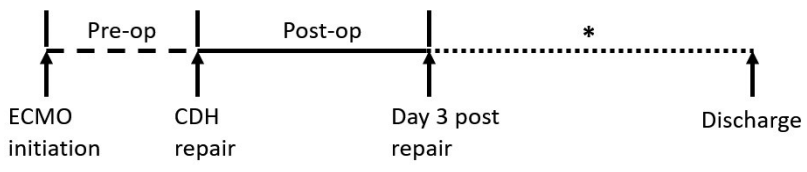

Pre-op period: Defined as from the day of ECMO initiation to the day before $\mathrm{CDH}$ repair surgery. Post-op period: Defined as the day of $\mathrm{CDH}$ repair and 3 days post repair.

*: Mortality and hemorrhagic complication were collected in the period from the day of ECMO initiation to discharge.

FIGURE 1 Definition of the study periods

Results: Sixty-two patients met the inclusion criteria. Only cryoprecipitate transfusion in the pre-op period is associated with increased mortality [OR $2.15,95 \% \mathrm{Cl}: 1.05,4.38]$. In the post-op period, increased total number of blood products [OR 1.08, 95\% Cl: 1.00 , 1.17] and platelet transfusions [OR $1.42,95 \% \mathrm{Cl}: 1.06,1.90]$ are associated with increased mortality while other blood components are not. ECMO duration [OR 1.17, $95 \% \mathrm{Cl}$ : 1.05, 1.30] but not circuit change is associated with increased mortality. Major hemorrhagic complications had the strongest association with mortality [OR 10.98, 95\% Cl: 3.27, 36.91]. Gestational age, birth weight, 1 and 5 minutes Apgar score, sex, blood type, right vs left $\mathrm{CDH}$, veno-venous vs veno-arterial ECMO, duration of ECMO before $\mathrm{CDH}$ repair, surgical bleeding during $\mathrm{CDH}$ repair were not significantly associated with survival. 
Conclusions: Transfusion of cryoprecipitate and platelets are associated with increased mortality for our cohort of patients. Major bleeding has the strongest association with mortality. Our data sug gests that maintaining a robust hemostatic state while limiting unnecessary transfusion should be the cornerstone of $\mathrm{CDH}$-related ECMO management.

\section{PB0778 | Emergency Department Care in Pediatric and} Adolescent Patients Presenting with Abnormal Uterine Bleeding

N. Kendel $^{1}$; J. Stanek ${ }^{1}$; F. Haamid ${ }^{1}$; J. Powers ${ }^{2}$; S. O'Brien ${ }^{1}$

${ }^{1}$ Nationwide Children's Hospital, Columbus, United States; ${ }^{2}$ Texas Children's Hospital, Houston, United States

Background: Twenty percent of females presenting with abnormal uterine bleeding (AUB) may have an underlying bleeding disorder. For many, AUB is their initial presenting symptom. Prior work evaluating the inpatient management of adolescents admitted for AUB found that Black and Hispanic girls were overrepresented and that hemostatic evaluations were inconsistent. Patterns of emergency care in these patients have not been well studied.

Aims: 1. Assess patterns in hematologic evaluation of girls presenting to the emergency department (ED) with AUB.

2. Determine if girls from racial minority groups are disproportionately over-represented within this population.

Methods: This was a retrospective study utilizing the Pediatric Health Information System (PHIS) database, which contains clinical and resource utilization data for 51 children's hospitals in the United
States. ED encounters from October 2015-September 2020 for females 8-21 years with a diagnosis code of AUB were evaluated. Demographic information, additional diagnostic codes, and laboratory evaluations were collected. Data were descriptively summarized, and comparisons made using nonparametric methods.

Results: During the study period, 24,091 unique patients with AUB were seen in the ED; $24.8 \%$ were admitted and excluded from further analysis. Median age was 16.3 years (IQR 14.1-17.8 years). Despite comprising only $25 \%$ of all ED encounters in PHIS during the study period, Black girls accounted for $34.4 \%$ of AUB encounters (Table 1). Return ED visits were common (17.1\% returning within 30 days; $28.0 \%$ returning within 90 days). Most encounters (65.1\%) included evaluation for anemia versus only $6.9 \%$ for iron deficiency and $26.6 \%$ for hemostatic disorders. Laboratory evaluations differed in White versus Black females (Table 2).

TABLE 1 Patient demographics

$\begin{array}{ll}\text { Characteristic } & N(\%) \\ \text { Unique Patients } & 18,160 \\ \text { Race } & \\ \text { White } & 7,160(39.4) \\ \text { Black } & 6,238(34.4) \\ \text { Other/Unknown } & 4,762(26.2) \\ \text { Presenting Symptoms } & \\ \text { Abdominal pain } & 3,755(20.7) \\ \text { Headache } & 924(5.1) \\ \text { Dizziness } & 877(4.8)\end{array}$

TABLE 2 Racial disparities in frequency of laboratory evaluations

\begin{tabular}{|c|c|c|c|c|}
\hline Lab Evaluation & $\begin{array}{l}\text { All Patients } \\
n=18,160\end{array}$ & $\begin{array}{l}\text { White Patients } \\
n=7,160\end{array}$ & $\begin{array}{l}\text { Black Patients } \\
N=6.238\end{array}$ & $P$-value \\
\hline $\mathrm{CBC}$ or Hemoglobin & $11,902(65.5)$ & $4,995(69.8)$ & $3,669(58.8)$ & $<.001$ \\
\hline $\begin{array}{l}\text { Prothrombin Time/Partial } \\
\text { Thromboplastin Time }\end{array}$ & $4,597(25.3)$ & $2,061(28.8)$ & $1,238(19.8)$ & $<.001$ \\
\hline Von Willebrand Antigen & $1,584(8.7)$ & $765(10.7)$ & $404(6.5)$ & $<.001$ \\
\hline $\begin{array}{l}\text { Von Willebrand Ristocetin Cofactor } \\
\text { Activity }\end{array}$ & $1,553(8.6)$ & $692(9.7)$ & $429(6.9)$ & $<.001$ \\
\hline Factor VIII (Assay or Antigen) & $1,700(9.4)$ & $821(11.5)$ & $438(7.0)$ & $<.001$ \\
\hline Platelet Aggregation & $240(1.3)$ & $110(1.5)$ & $63(1.0)$ & 0.007 \\
\hline
\end{tabular}

Conclusions: In a national cohort of pediatric patients presenting with AUB to the ED, most were evaluated for anemia and one-fourth for an underlying hemostatic disorder. Black girls were overrepresented in this population and less likely to have hematologic evaluations performed. 
PB0779 | Specificity and Sensitivity of Pediatric Bleeding Questionnaire (PBQ) in Identifying Patients Requiring further Laboratory Evaluation

T. Yafoshkina; D. Florinskiy; K. Voronin; D. Polokhov; A. Poletaev;

E. Seregina; D. Fedorova; P. Zharkov

Dmitry Rogachev National Research Center of Pediatric Hematology,

Oncology and Immunology, Ministry of Health of the Russian

Federation, Moscow, Russian Federation

Background: To standartize the collection and quantify bleeding symptoms, in 2009 Bowman and colleagues developed PBQ, but its role in identifying patients reauiring futher laboratory evaluation is poorly understood.

Aims: To access specificity and sensitivity of PBQ in identifying the laboratory cause of bleeding in children.

Methods: We have performed a retrospective analysis of medical records of 346 patients (0-17 years), who attended Outpatient Consultative Clinic of our tertiary care Centre because of excessive bleeding symptoms excepting thrombocytopenia from 2017 to 2019. Translated PBQ forms were filled with a doctor, and then all the patients had a laboratory evaluation to verify a bleeding disorder (CBC, PT, APPT, TT, Fibrinogen, vWF:RCo, LTA with ristocetin, collagen, adrenalin, ADP, platelet cytofluorometry). Specificity and sensitivity, of $\mathrm{PBQ}$ points in evaluation of any test alteration were calculated using ROC-analysis (XLSTAT, Addinsoft).

Results:

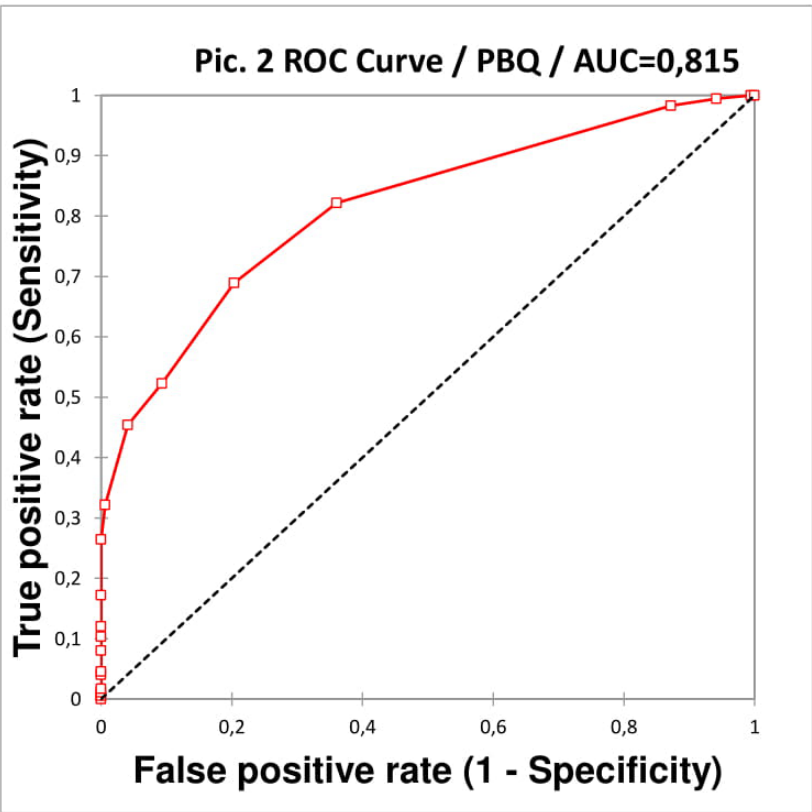

FIGURE 1 The PBQ points in groups of children with various bleeding disorders

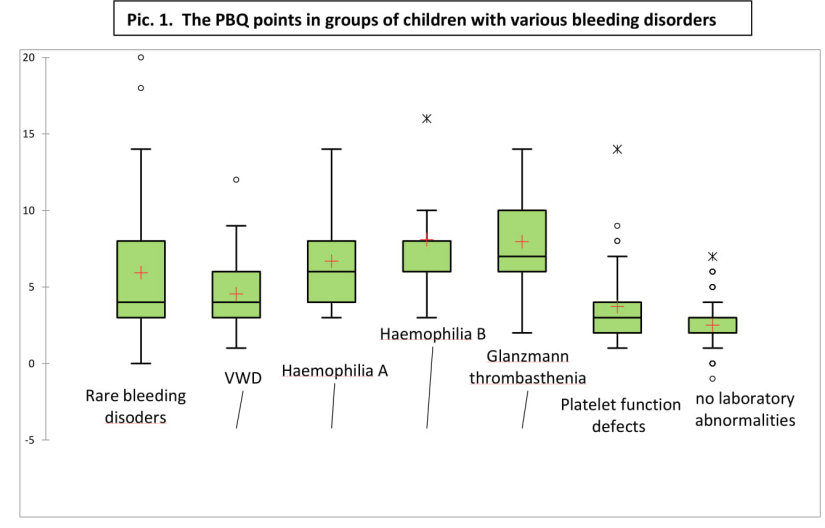

FIGURE 2 ROC Curve / PBQ / AUC = 0,815

There was a variability in the scores obtained between children in groups; it was also noted for different children with the same diagnosis (pic.1). If a positive bleeding score was defined as $\geq 2$; the sensitivity of the method was $98 \%$, the specificity was $12 \%$. The best ratio of sensitivity and specificity (69\% and $79 \%$, respectively) was achieved when the positive bleeding score was $\geq 4$ points (pic 2 ).

Conclusions: In our cohort, using local screening test battery, the majority of patients with $\mathrm{PBQ}=2$ had no laboratory confirmation, but if $P B Q \geq 4$ was used as a cutoff, a substantial part of mild bleeding disorders could be potentially misdiagnosed. We found PBQ was handy and useful to collect and standardize bleeding symptoms, but can't be used as a tool, which could alter our decision to test or not to test.

Reference: Bowman M., et al. JTH;2009;7(8):1418-1421.

PB0780 | The Distribution of Anti-platelet Membrane Glycoprotein Antibodies Subtypes in Children with Primary Immune Thrombocytopenia and its Relationship with Therapeutic Effect

R. Li; R. Yang

Chinese Academy of Medical Sciences \& Peking Union Medical College, Tianjin, China

Background: Anti-platelet membrane glycoprotein antibody is the main cause of platelet destruction, and IVIG combined with glucocorticoids is the standard regimen.

Aims: To study the distribution of anti-GP subtypes in children with primary immune thrombocytopenia, and to evaluate the the relationship between anti-GP subtypes and the therapeutic effect.

Methods: A retrospective analysis of 320 children with ITP from September 2013 to April 2019 was determined. They were divided into newly diagnosed ITP group (127 cases), persistent ITP group (101 cases) and chronic ITP group (92 cases). Monoclonal antibodyspecific platelet antigen fixation (MAIPA) was used to detect antiGP IIb/IIla and anti-GP la/Ila in each group, and the relationship between the distribution of anti-GP subtypes and the therapeutic effect was analyzed. 
Results: The positive rates of platelet membrane glycoprotein antibody in the newly diagnosed ITP group, persistent ITP group, and chronic ITP group were $40.9 \%, 22.8 \%$, and $26.1 \%$, respectively, the difference was statistically significant $(P=0.006)$. The positive rate of anti-GP IIb/IIla, and anti both GP IIb/IIla and GP la/ IIla were $19.7 \%$ and $10.2 \%$ in the newly diagnosed ITP group, which showed no statistical significance compared with the persistent group (14.9\%, 3\%) and chronic group (10.9\%, 5.4\%) ( $\mathrm{P}$ values were 0.201 and 0.078 , respectively).The effective rate of IVIG combined with glucocorticoids in anti-GP IIb/IIla positive children (94.6\%) and antibody negative patients $(96.3 \%)$ showed no statistically significant difference $(P=0.458)$. The effective rate of IVIG combined with glucocorticoids in anti-GP Ilb/IIla and GP la/ IIla both positive patients (78.6\%) was lower than negative patients (97.3\%), and the difference was statistically significant $(P=0.003)$.

Conclusions: The positive rate of anti-GP Ilb/IIla, and anti-GP Ilb/ IIla and GP la/ IIla were higher in newly diagnosed pediatric ITP, but wit no statistical significance. Patients with anti-GP IIb/IIla and GP la/Ila both negative responded well to the standard regimen of IVIG combined with glucocorticoids.

\section{PB0781 | A Conservative Therapy Spontaneous Intracranial} Hemorrhage in a Pediatric Patient with Severe Acquired Aplastic Anemia

M.-M. Deveza ${ }^{1}$; A. Rodrigues ${ }^{1} ;$ T. Quaresma $^{1}$; C. Peixoto ${ }^{1}$;

A. Ferrão ${ }^{2}$

${ }^{1}$ Centro Hospitalar Universitário Lisboa Norte, EPE -

Immunohematherapy Department, Lisboa, Portugal; ${ }^{2}$ Centro Hospitalar Universitário Lisboa Norte, EPE -Pediatric Department, Lisboa, Portugal

Background: Aplastic anaemia (AA) is a quantitative deficiency of haematopoiesis responsible of pancytopenia with poor bone marrow. AA is often acquired, infectious or iatrogenic. It can be also constitutional and sometimes idiopathic.

The bleeding is the firth cause of death. Intracranial haemorrhage $(\mathrm{ICH})$ is an almost always fatal complication associated with AA.

$\mathrm{ICH}$ in children is a rare but often disabling disease, leading to high rates of morbidity and mortality in this population.

Aims: To treat conservatively a spontaneous $\mathrm{ICH}$ in a paediatric patient with severe acquired $A A$.

Methods: A 12-year-old boy, from Angola, without relevant background and a diagnosis of a very severe acquired AA (confirmed by bone marrow examination) was admitted to our hospital. After multiple bleeding episodes and, febrile neutropenia without isolated agent, he suffered a spontaneous subdural haematoma, with no indication for emergent surgical drainage. We decide to start an optimization of the medical therapeutics: eltrombopag during 68 days, Immunoglobulin $1 \mathrm{~g} / \mathrm{kg} /$ day plus methylprednisolone (1mg/ $\mathrm{kg}$ /day), as well as coagulation management with platelets, FVII, FXIII and fibrinogen concentrates expecting to maintain the following targets: platelets count $\geq 50000 / \mu \mathrm{L}, \mathrm{FVII}>50 \%, \mathrm{FXII} \geq 60 \%$, and fibrinogen $\geq 200 \mathrm{mg} / \mathrm{dl}$ levels, until CT image resolution. After clinical and CT image stabilization the platelet target changed to $\geq 20.000$ / $\mu \mathrm{L}$.

Results: After 3 months, the haematoma was completely reabsorbed and the patient referred for allogeneic transplant with hematopoietic progenitors cells.

Conclusions: Thrombocytopenia, regardless of cause, is a risk factor for the development of spontaneous subdural haematoma. Detailed history and physical examination as well as monitoring of clotting factors (Fibrinogen, FVII and FXIII) is critical to the management of subdural haematoma in the context of AA.

A conservative treatment of an $\mathrm{ICH}$ in a children with $\mathrm{AA}$ was achieved by the correction of the thrombocytopenia and the coagulation factors levels, accompanied by essential general measures and symptomatic treatment.

PB0782 | Children with Bleeding and Clotting Disorders: Reviewing Quality of Life Tools

S. Jones ${ }^{1,2}$; J. Bushe ${ }^{1}$; H. David ${ }^{1}$; C. Dinh ${ }^{1}$; K. Kolosidhi ${ }^{1}$; D. Rai ${ }^{1}$; F. Newall ${ }^{2,1}$

${ }^{1}$ University of Melbourne, Department of Nursing, Calton, Australia;

${ }^{2}$ The Royal Children's Hospital, Parkville, Australia

Background: Haemophilia and anticoagulation therapy have a significant effect on children's quality of life (QoL) due to an increased risk of bleeding, repeated painful procedures and multiple hospital visits. Hence, the tools used to assess QoL in these children must accurately represent their lived experiences.

Aims: This scoping review aimed to identify the existing QoL tools for children with haemophilia or receiving anticoagulation therapy and evaluate the validity, reliability and responsiveness of each tool. Methods: A comprehensive search was undertaken using MEDLINE, CINAHL and Embase. Studies reporting the development or validation of QoL tools in children (aged 0-18) with haemophilia or receiving anticoagulation were included. The search was limited to articles published in English within the last 20 years. The Joanna Briggs Institute Methodology for Scoping Reviews was followed for methodological quality and data extraction. The COnsensusbased Standards for the selection of health status Measurement Instruments (COSMIN) checklist was used for evaluating the validity, reliability and responsiveness of the QoL tools.

Results: Initial searches identified 423 studies, but 16 studies were included for evaluation. Five QoL tools were identified, consisting of four haemophilia specific and one anticoagulant specific tool. Each tool demonstrated validity and reliability to an extent but were limited by poor methodological quality. The Haemo-QoL and KIDCLOT PAC QL( tools demonstrated the best methodological quality.

Conclusions: Currently available QoL tools for children with haemophilia or receiving anticoagulation are appropriate, yet all have discrete methodological limitations. In an era where clinical outcomes of treatment are recognized to provide only a partial measure of the 
success of therapy, the limitations of these tools need to be acknowledged and efforts to optimize the accurate measurement of QoL implemented. Further research is required to report on measurement error and construct validity of paediatric QoL for both disorders.

PB0783 | Use of Single Photon Emission Computed Tomography and Arthrocentesis in the Management of Hip Hemarthrosis in Hemophilia A

J. Mooney; P. Moorehead; H. Martin; A. Pickles

Memorial University, St. John's, Canada

Background: Hip hemarthrosis in patients with hemophilia are rare. Due to hip anatomy, the associated increased intracapsular pressure can compromise femoral head perfusion, resulting in avascular necrosis (AVN). Unfortunately, typical imaging may miss early hypoperfusion. Conventional treatment includes intravenous factor infusion, bed rest, and pain control. Arthrocentesis is controversial.

Aims: We present a case of hip hemarthrosis in a patient with hemophilia $A$ that highlights the novel use of bone scan with single photon emission computed tomography (SPECT) for early detection of femora head ischemia, and the possible role of arthrocentesis in management. Methods: .

Results: Case: Thirteen-year-old male with hemophilia A presented with hip pain. US detected a hip hemarthrosis. He remained unresponsive to typical treatment. Bone scan with SPECT revealed femoral head hypoperfusion. The patient underwent arthrocentesis and experienced immediate symptom improvement. Repeat bone scan with SPECT demonstrated increased blood flow to the affected femoral head.

Discussion: Nuclear medicine imaging may help detect early ischemic changes in hip hemarthrosis. Bone scan with SPECT in our patient allowed for early identification of hypoperfusion and procedural intervention, which normalized femoral head perfusion. It is possible that decreased perfusion can be identified early with the use of this imaging, creating the opportunity to intervene and prevent adverse outcomes. Our case also demonstrates the utility of arthrocentesis for hip hemarthrosis that are unresponsive to conventional treatment. This can provide immediate symptom relief, improved profusion, and an expedited recovery. There have been no documented adverse outcomes with this procedure.

Conclusions: Management of hip hemarthrosis in patients with hemophilia should be expedited to prevent morbidity. If there is no clinical improvement after $24-48$ hours of treatment, arthrocentesis should be considered, with appropriate factor replacement therapy. We also encourage the consideration of medical imaging to assess femoral head perfusion, particularly in cases with severe or unresponsive symptoms, or large joint effusions.

\section{THROMBOSIS IN NEONATES AND CHILDREN}

\section{LPB0026 | How to Use Thrombolytic Agents in the Treatment of Neonatal Thrombosis: A Systematic Review}

R. Leong ${ }^{1}$; J. Patel ${ }^{2}$; N. Samji ${ }^{3}$; B. Paes ${ }^{3,4}$; A.KC Chan ${ }^{4}$;

J.-A. Petropoulos ${ }^{5}$; M.D Bhatt ${ }^{4}$

${ }^{1}$ Contributed equally as co-first authors, McMaster University, Faculty of Health Sciences, Hamilton, Canada; ${ }^{2}$ Contributed equally as co-first authors, McMaster University, Faculty of Science, Hamilton, Canada; ${ }^{3}$ McMaster University, Department of Medicine, Hamilton, Canada; ${ }^{4}$ McMaster University, Department of Pediatrics, Hamilton, Canada;

${ }^{5}$ McMaster University, Faculty of Health Sciences, Hamilton, Canada

Background: Among children, neonates have the highest incidence of thrombosis with recent studies reporting up to 15 per 1000 neonatal intensive care unit (NICU) admissions. Thrombolytic agents are used for management of life and/or organ threatening thrombosis in neonates. There is limited literature evaluating dosing, monitoring, efficacy and safety of thrombolytic use in the neonatal population.

Aims: To cumulatively summarize published literature on dosing, administration, monitoring, and duration recommendations proposed by primary studies on tissue plasminogen activator (tPA), streptokinase and urokinase treatment in neonates ( $\leq 28$ days).

Methods: A systematic literature search was conducted using OVID Medline, Embase, Web of Science, CINAHL, and EBM databases from database inception until June 20, 2020. The initial search yielded 6,411 articles, of which 23 were included for review.

Results: Out of 23 included studies, 15, 4, and 1 studies exclusively reported on the use of tPA, streptokinase, and urokinase, respectively. In total tPA use was reported in 195 patients, streptokinase in 58 patients and urokinase in 14 patients. The reported dosing range for tPA was 0.01-0.6 mg/kg/h, streptokinase was $50-2000 \mathrm{U} / \mathrm{kg} / \mathrm{h}$, and urokinase was $1000-20,000 \mathrm{U} / \mathrm{kg} / \mathrm{h}$ with duration ranging from 30 minutes up to 30 days. Complete and partial resolution rates are reported to be $66-71 \%$ and $20-22 \%$, respectively, with recurrence risk in $0-11 \%$. The bleeding risk is significant at $18-44 \%$ and mortality reported in $11-15 \%$ of neonates. The dosing, efficacy and safety are summarized in Table 1.

TABLE 1 Summary of dosing, efficacy and safety from 23 included studies. Each variable is reported from only the data that was available in the studies

$\begin{array}{lllll} & \text { Parameter } & \text { tPA } & \text { Streptokinase } & \text { Urokinase } \\ \text { Numbers } & \text { Studies } & 16 & 7 & 3 \\ & \text { Patients } & 195 & 58 & 14 \\ \text { Dosing \& Duration } & \text { Dosing } & 0.01-0.6 \mathrm{mg} / \mathrm{kg} / \mathrm{h} & 50-2000 \mathrm{U} / \mathrm{kg} / \mathrm{h} & 1000-20,000 \mathrm{U} / \mathrm{kg} / \mathrm{h} \\ & \text { Duration } & 30 \text { minutes }-30 \text { days } & 1-11 \text { days } & 6 \text { hours }-9 \text { days }\end{array}$




\begin{tabular}{|c|c|c|c|c|}
\hline & Parameter & tPA & Streptokinase & Urokinase \\
\hline \multirow[t]{2}{*}{ Efficacy } & Recurrence risk & $7.4 \%(2 / 27)$ & $0 \%(0 / 10)$ & $11.1 \%(1 / 9)$ \\
\hline & Partial resolution & $20.0 \%(19 / 95)$ & $22.4 \%(11 / 49)$ & $21.4 \%(3 / 14)$ \\
\hline Safety & Bleeding risk & $18.2 \%(27 / 148)$ & $20.7 \%(12 / 58)$ & $44.4 \%(4 / 9)$ \\
\hline
\end{tabular}

Conclusions: This is the first study to objectively summarize the efficacy and safety of thrombolytic agents in neonates. Overall, thrombolytic use is associated with up to $69 \%$ complete resolution of thrombosis and $11 \%$ recurrence risk. The reported bleeding risk is about $28 \%$, which is much higher compared to other anticoagulants. Larger prospective studies are required to elucidate effective dosing regimens and further clarify efficacy and safety of thrombolytic agents.

LPB0027 | Systematic Review on Neonatal Portal Vein Thrombosis

$\underline{\text { M. Alhaddad }}{ }^{1}$; N. Radadia ${ }^{1}$; A.K. Chan ${ }^{1}$; B. Paes ${ }^{1}$; S. Williams ${ }^{2}$; M.D Bhatt ${ }^{1}$

${ }^{1}$ McMaster University, Hamilton, Canada; ${ }^{2}$ The Hospital for Sick Children, Toronto, Canada

Background: Neonatal portal vein thrombosis (PVT) is increasingly recognized with medical advances in neonatal care. The goal of treatment is avoiding long-term complications such as portal hypertension (PHN). However, the exact incidence and utility of anticoagulation remains unknown.

Aims: To perform a systematic literature review on neonatal PVT to identify the incidence, risk factors, management, and outcomes.

Methods: A search of Pubmed, EMBASE, CINAHL, and Web of science databases was conducted for publications from 1946-2019 and downloaded into Covidence ${ }^{\odot}$. Studies with $\geq 5$ neonates $(\leq 28$ days of age) were included.

Results: 2956 articles were retrieved; 51 were included in the final analysis after abstract and full-text screening by 2 authors. Pooled PVT incidence was 1.1/100,000 live births (1 study), 16.6/1000 neonatal intensive care unit admissions (2 studies), and 10.6/100 umbilical venous catheter (UVC) insertions (12 studies). In studies with available information, sepsis and thrombophilia occurred in $33.2 \%$ and $14.7 \%$, respectively. Other risk factors for PVT included prematurity, congenital anomalies, and blood transfusion through the UVC. Ten studies reported management and outcome for 561 subjects. Anticoagulation therapy was used in $36.9 \%$. Complete or partial resolution was observed in $78.3 \%$, PVT was stable in $20.6 \%$, and thrombus progression occurred in $1.2 \%$ after a median followup of 6 weeks to 16.6 months. In 2 studies examining the utility of anticoagulation, no improvement in partial or complete thrombus resolution was described (OR $0.77,95 \% \mathrm{Cl} 0.45-1.31$ ). Two studies reported PHN in $0.45 \%$ and $3 \%$ after follow-up of 5 and 37.2 months, 4 studies reported no PHN after a follow-up of 1.5-16.6 months.

Conclusions: There is a need for larger, well-conducted, multicenter prospective studies in neonates with PVT, to elucidate the risk factors for PHN after long-term follow-up, preferably >5years. Once known, this may improve both management and surveillance strategies for those at risk.

LPB0028 | Difference is Risk Factors and Clinical Outcomes in Pediatric Patients with Deep Venous Thrombosis as Compared to Pulmonary Embolism

S. Suryaprakash ${ }^{1}$; C. Sabo ${ }^{2}$; A. Farooqi ${ }^{3}$; M. Rajpurkar ${ }^{2,3,4}$

${ }^{1}$ Department of Pediatrics, Childrens hospital of Michigan, Detroit, United States; ${ }^{2}$ Department of Hematology, Childrens hospital of Michigan, Detroit, United States; ${ }^{3}$ Central Michigan University College of Medicine, Mt Pleasant, United States; ${ }^{4}$ Wayne State University,

Detroit, United States

Background: Pulmonary embolism (PE) and deep venous thrombosis (DVT) are considered to be in the same spectrum of venous thromboembolism (VTE). Risk factors for the development and outcomes after each may differ.

Aims: To evaluate the clinical characteristics, clinical and laboratory risk factors, and outcomes of pediatric patients with isolated DVT as compared to PE (PE with or without DVT).

Methods: In this retrospective single-center cohort study, we included consecutive patients $<21$ years with radiologically confirmed PE or DVT between Jan 1, 2001, and Nov 4, 2020. Clinical and laboratory risk factors, treatment modalities, and outcomes were compared between the two. Statistical analysis was carried out using SPSS version 26

Results: Of the 827 patients, 729 presented with DVT and 98 with $P E$. There was a bimodal age distribution in both groups. Univariate analysis showed that PE patients were more likely to be female, obese, have a history of oral contraceptives use, surgery, immobilization, and systemic lupus erythematosus whereas DVT patients were more likely to have a history of catheterization or dehydration $(P<0.05)$. PE patients were more likely to have a high $D$-dimer, factor VIII, fibrinogen, Von Willebrand factor, positive anticardiolipin antibodies, lupus anticoagulant, anti-nuclear antibody (ANA), at presentation and on follow up $(P<0.05)$. On preliminary logistic regression, patients with PE had a high risk for recurrent VTE (OR 
2.592, 95\% confidence interval [Cl] 1.249-5.380). Other risk factors associated with recurrence were catheterization (OR $2.987,95 \% \mathrm{Cl}$ 1.668-5.348), high factor VIII (OR 3.466, 95\% CI 1.063-11.304), and positive ANA (OR 5.926, (5\% Cl 1.083-34.423) at follow up.

Conclusions: Pediatric PE patients have unique risk factors and outcomes as compared to those with DVT. This finding needs to be confirmed in larger studies.

LPB0075 | Treatment of Catheter-related Arterial Thrombosis in Children: A 15-year Single Center Experience

O. Crameri ${ }^{1} ;$ M. Rizzi ${ }^{2}$; M. Albisetti ${ }^{1}$

${ }^{1}$ University Children's Hospital, Zürich, Switzerland; ${ }^{2}$ University Hospital CHUV, Lausanne, Switzerland

Background: Catheter-related arterial thrombosis (CAT) is a recognized complication in children. However, very little information is available on the optimal treatment of CAT.

Aims: To report a single-center experience with the treatment of pediatric CAT.

Methods: Children diagnosed with extremity indwelling catheter (EIC)- or CC-related CAT between 2002 and 2017 were included in this study. Collected data included age, underlying disorders, location of CAT, radiologic results, and treatment modalities.

Results: A total of 242 CAT developed in 224 children. Of these, 125 (52\%) were EIC-related and 117 (48\%) were CC-related CAT. Children with EIC-related CAT were significantly younger that children with CC-related CAT (median age, 0.66 vs 1.58 months, $P=0.02$ ). Treatment modalities included heparin (UFH and/or LMWH) alone in 60 (25\%) of the 242 CAT, ASS alone in $6(2 \%)$, heparin followed by ASS in 171 (71\%), heparin followed by vitamin $\mathrm{K}$ antagonist (VKA) in $4(1.5 \%)$, and VKA alone in a single case (0.5\%). In 24 (40\%) of 60 cases, heparin alone was administered for $\leq 4$ weeks and in 62 (36\%) of 171 cases, heparin followed by ASS was administered for $\leq$ 3 months. Overall, complete resolution of thrombosis was observed in $173(71.5 \%)$, partial resolution in $13(5.4 \%)$, and no resolution in 56 (23.1\%) of the 242 CAT. No statistical significance in the resolution rate was observed between treatment groups $(P=0.23)$. Of note, in $66 \%$ of cases, complete resolution occurred at a median of 18 days (range 4-44) with heparin alone. A switch from heparin to ASS in children with partial or no resolution of CAT did not increase the resolution rate at follow-up. No thrombus progression/recurrence were observed.

Conclusions: Heparin is an efficient treatment modality for pediatric CAT. Long-term, subsequent treatment with ASS does not increase the resolution rate.
LPB0076 | Cerebral Sinovenous Thrombosis and Asparaginase Re-exposure in Patients Aged 1 to 45 Years with Acute Lymphoblastic Leukemia: A NOPHO ALL2008 Study

M.T. Skipper ${ }^{1}$; C.U. Rank ${ }^{2,3}$; L. Andrés-Jensen ${ }^{4}$; K. Schmiegelow ${ }^{4}$; B.K. Albertsen ${ }^{1,5}$; R. Tuckuviene ${ }^{4,6}$; NOPHO Thrombosis and Haemostasis Working group

${ }^{1}$ Pediatrics and Adolescent Medicine, Aarhus University Hospital, Aarhus, Denmark; ${ }^{2}$ Pediatric Oncology Research Laboratory, Rigshospitalet, University of Copenhagen, Copenhagen, Denmark; ${ }^{3}$ Department of Hematology, Rigshospitalet, University of Copenhagen, Copenhagen, Denmark; ${ }^{4}$ Department of Paediatrics and Adolescent Medicine, Rigshospitalet, Copenhagen, Denmark; ${ }^{5}$ Department of Clinical Medicine, Aarhus University, Aarhus, Denmark; ${ }^{6}$ Section of Paediatric Haematology \& Oncology, Rigshospitalet, Copenhagen, Denmark

Background: Cerebral sinovenous thrombosis (CSVT) is a serious complication of acute lymphoblastic leukemia (ALL) therapy. Asparaginase (ASP), a cornerstone in ALL treatment, is associated with increased risk of CSVT. CSVT-related premature truncation of ASP may impact survival rates.

Aims: To explore

- incidence of ASP truncation after CSVT in ALL patients

- potential clinical and imaging factors predictive of ASP truncation

- CSVT sequelae

Methods: We included patients $(1-45$ years, $n=2400)$ treated according to the NOPHO ALL2008 protocol from 2008/7-2018/10. Data were retrospectively collected from medical charts. Informed consent was obtained and the study was approved by the local ethics committees.

Results: We identified 45 patients with CSVT, corresponding to a prevalence of $1.9 \%$ (95\%-Cl:1.4-2.5\%). Patients with CSVT were older (33\% adolescents/10-17.9 years; $22 \%$ adults/ $\geq 18$ years), and $22 \%$ had CNS leukemia at ALL diagnosis, compared with patients without thromboembolism (16\%, $14 \%$ and $12 \%$, respectively) $(P<0.01)$. Three patients (7\%) died due to CSVT.

Twenty-nine patients (66\%) were re-exposed to ASP a median of 33 days after CSVT, all under anticoagulation cover. Thirty-four (83\%) had $\geq 1$ ASP dose omitted. Patients re-exposed and non-re-exposed had a median of 2 and 6 doses omitted, respectively. Two patients (4\%) experienced re-thrombosis after ASP re-exposure. No rethrombosis among non-re-exposed patients. Re-exposed patients performed better on the Modified Rankin Scale (mRS) compared to non-re-exposed (2.0 versus 2.7, $P<0.05$ ) at CSVT diagnosis. No neurological findings predicted re-exposure. CSVT-score by Zubkov AY et al. quantifying CSVT extension showed no difference among reexposed and non-re-exposed patients at CSVT diagnosis (2.3 versus $2.9, P=0.16)$ nor at last follow-up $(0.6$ versus $0.8, P=0.21)$. No difference was detected applying $\mathrm{mRS}$ among re- and non-re-exposed patients ( 0.38 and $0.45, P=0.08$ ) at last follow-up (mean 5.8 years). 
Conclusions: Although $66 \%$ of ALL patients diagnosed with CSVT were re-exposed to ASP, a low recurrence of thrombosis (4\%) was detected.

LPB0077 | Management of Localized Intravascular Coagulopathy with Rivaroxaban in Children with Venous Malformations: Report of 6 Cases

F.B. Belen Apak ${ }^{1}$; F. Nabili ${ }^{2}$; F. Sarialioglu ${ }^{1}$

${ }^{1}$ Baskent University Medical Faculty Department of Pediatric Hematology and Oncology, Ankara, Turkey; ${ }^{2}$ Baskent University Medical Faculty Department of Pediatrics, Ankara, Turkey

Background: Venous malformations (VMs) contain dilated venous vessels and are complicated with the activation of coagulation. Localized intravascular coagulation (LIC) may cause microthrombi in the lesion, therefore causing an increase in the dimension of the lesion and causing pain. Moreover, the localized activation of coagulation can lead to a consumptive coagulopathy characterized by increased $\mathrm{D}$-dimers and decreased fibrinogen and platelets leading to bleeding tendency. Low molecular weight heparin (LMWH) therapy is an effective therapy in restoring normal coagulation in VM patients, however, the lifelong requirement of subcutaneous use is not easy in small children. Here we report, the first use of rivaroxaban, a direct oral anticoagulant drug in 6 pediatric patients with coagulopathy of venous malformations.

Aims: The aim of the study was to evaluate the clinical responses and coagulation laboratory tests of the patients under rivaroxaban therapy.

Methods: Between March 2020-December 2020, 6 pediatric patients aged between 2-12 years with VM and LIC have been transited to rivaroxaban therapy as they had been using LMWH for 3-6 months. Approval for off-label use of rivaroxaban from the Ministry of Health and informed consent was obtained for each patient. Platelet counts, fibrinogen and D-dimer levels, and clinical findings of the patients were retrospectively analyzed before and after rivaroxaban therapy. Clinical response was defined as a decrease in pain and strain over the VM.
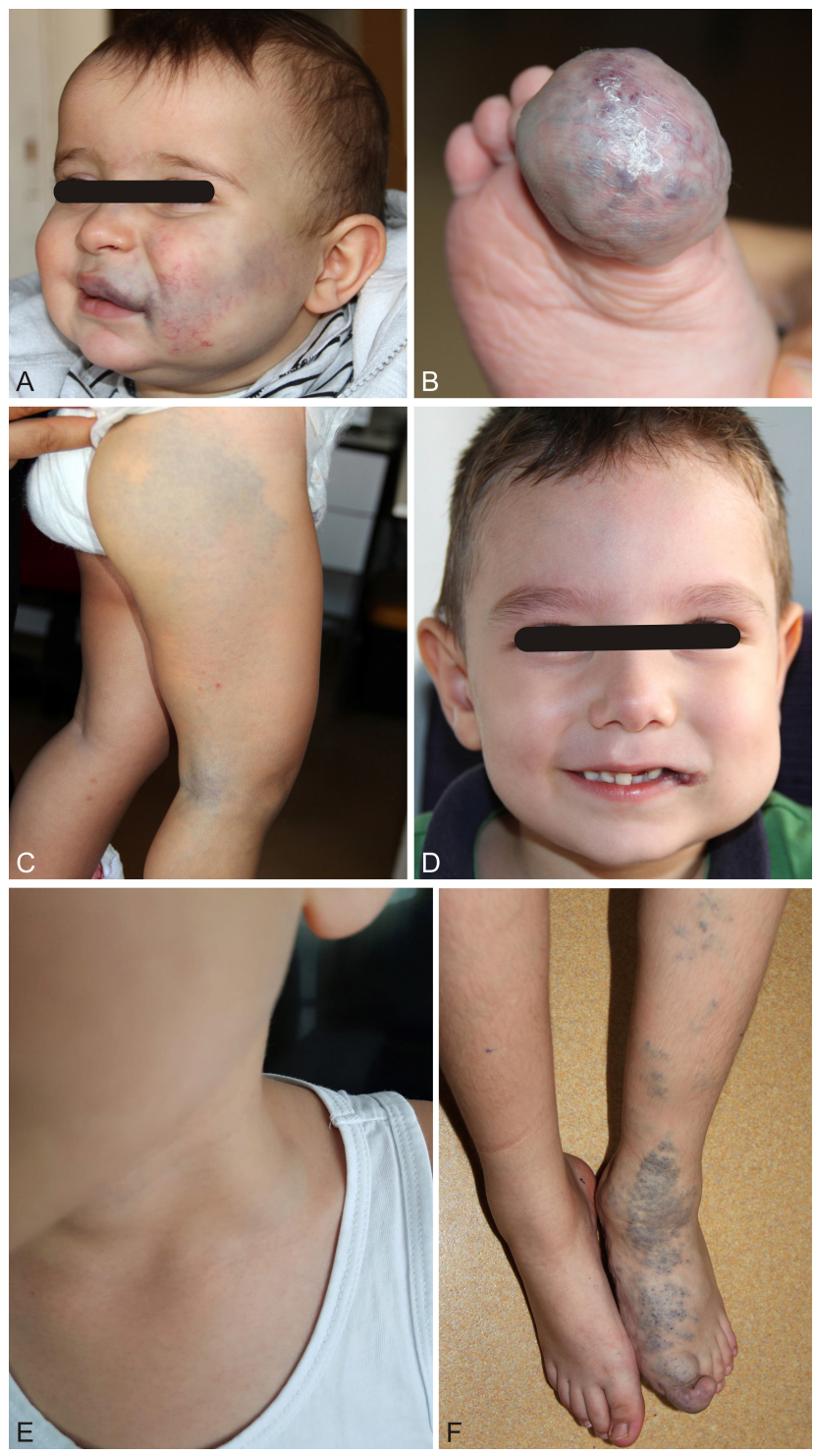

FIGURE 1 Lesions of patients with vascular malformations at the beginning of rivaroxaban therapy

\section{Results:}

The mean age of the patients was 6.33 (2-11.5 ages). The mean ddimer levels significantly decreased after a median time of 3 months of (2-8 months) rivaroxaban therapy. The clinical, laboratory findings of the patients and responses to therapy are given in Table 1. The lesions before therapy were given in Figure 1. All of them showed clinical responses and no bleeding regarding VM.

TABLE 1

\begin{tabular}{|c|c|c|c|c|c|c|c|c|c|}
\hline Patients & Age & Sex & $\begin{array}{l}\text { Region of venous } \\
\text { malformation }\end{array}$ & Previous medications & $\begin{array}{l}\text { Rivaroxaban } \\
\text { dose }\end{array}$ & $\begin{array}{l}\text { D-dimer- } \\
\text { before } \\
\text { therapy }\end{array}$ & $\begin{array}{l}\text { D-dimer- } \\
\text { after therapy }\end{array}$ & $\begin{array}{l}\text { Clinical } \\
\text { response }\end{array}$ & $\begin{array}{l}\text { Side } \\
\text { effects }\end{array}$ \\
\hline 1 & 2 & M & Left cheek & Sirolimus, LMWH & $2 \times 5 \mathrm{mg}$ & 1.14 & 0.24 & + & None \\
\hline 2 & 4 & $\mathrm{~F}$ & $\begin{array}{l}\text { Right toe, left lower } \\
\quad \text { leg }\end{array}$ & $\begin{array}{l}\text { Sirolimus, } \\
\text { propranolol, } \\
\text { LMWH }\end{array}$ & $2 \times 5 \mathrm{mg}$ & 2.08 & 0.22 & + & None \\
\hline 3 & 5 & M & $\begin{array}{l}\text { Left lower and higher } \\
\text { leg, left thigh }\end{array}$ & Propranolol, LMWH & $2 \times 5 \mathrm{mg}$ & 0.96 & 0.21 & + & None \\
\hline
\end{tabular}




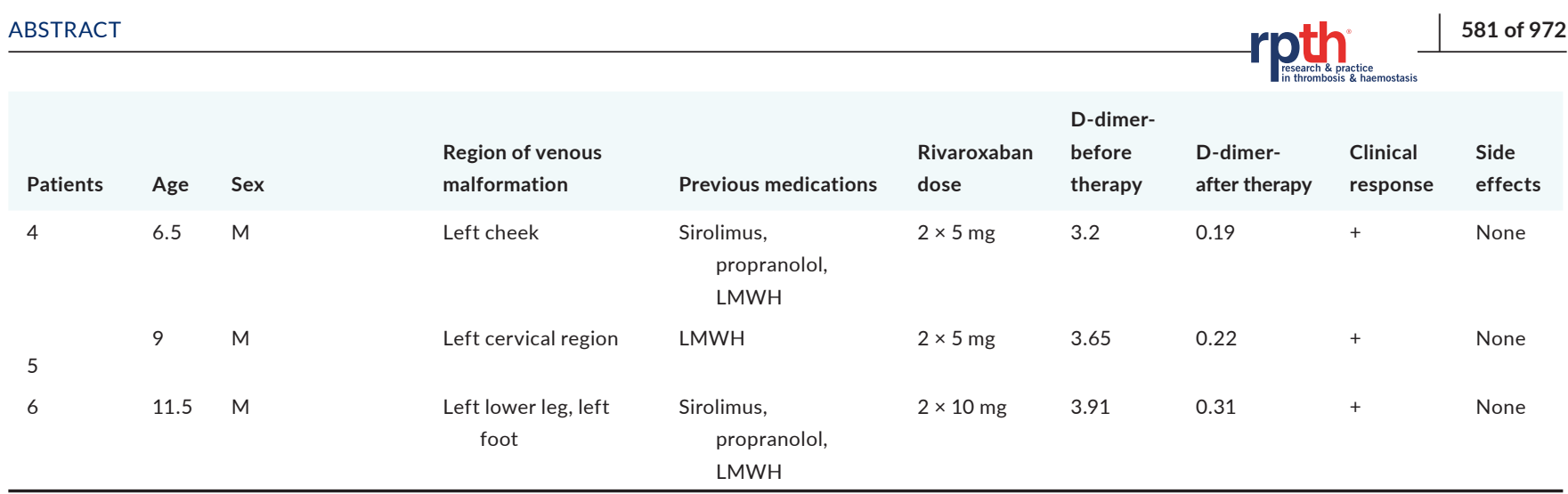

Conclusions: Rivaroxaban therapy can be safely used in LIC of venous malformations in children.

\section{LPB0078 | Intracranial Involvement and Neurologic} Manifestations in Lemierre Syndrome: Analysis of 712 Patients

L. Valerio $^{1}$; T. Nicoletti ${ }^{2}$; G. Corsi ${ }^{3}$; S. Granziera ${ }^{4}$; M. Jankowski ${ }^{1}$; A. Pecci ${ }^{5}$; C. Sacco ${ }^{6}$; F. Zane ${ }^{7}$; S.V. Konstantinides ${ }^{1}$; S. Barco ${ }^{8}$ ${ }^{1}$ Center for Thrombosis and Hemostasis, University Medical Center of the Johannes-Gutenberg University, Mainz, Germany; ${ }^{2}$ Institute of Neurology, Catholic University of the Sacred Heart, Rome, Italy; ${ }^{3}$ Department of Clinical, Integrated and Experimental Medicine (DIMES), University of Bologna, Bologna, Italy; ${ }^{4}$ Department of Geriatrics, San Giovanni e Paolo Hospital, Venice, Italy; ${ }^{5}$ Department of Internal Medicine, IRCCS Policlinico San Matteo Foundation and University of Pavia, Pavia, Italy; ${ }^{6}$ Center for Thrombosis and Hemorrhagic Diseases, Humanitas Clinical and Research CenterIRCCS, Rozzano (Milan), Italy; ${ }^{7}$ Department of General Medicine, Hospital of Sondrio, Sondrio, Italy; ${ }^{8}$ Angiology Clinic, University Hospital Zurich, Zurich, Switzerland

Background: Lemierre syndrome is a rare septic thrombophlebitis following a head-neck infection in children, adolescents, and young adults. Intracranial involvement in these patients is frequent, a major prognostic factor for early complications when observed at presentation, and among the main causes of death and long-term sequelae. However, with available information limited to case reports and small series, intracranial involvement in this condition has never been explored systematically.

Aims: To systematically describe the patterns of intracranial involvement and neurological symptoms in Lemierre syndrome.

Methods: We analyzed 712 patients described between 2000 and 2017 and identified through systematic search of grey and published literature. We distinguished three types of intracranial involvement: cerebral venous sinus thrombosis, intracranial suppuration, and arterial stroke or carotid damage. In these patients, we characterized neurological manifestations, clinical and demographic characteristics, and rate of death or clinical sequelae.

Results: Among 712 patients, 187 (26.3\%) had intracranial involvement, including 146 (78.1\%) with cerebral vein thrombosis, most often in the lateral sinus ( $N=93$ ), jugular bulb $(N=76)$ or cavernous sinus ( $N=51$; Figure 1$)$; 81 (43.3\%) with intracranial suppurations; and 22 (11.8\%) with arterial involvement. At least two forms of involvement were found in 51 patients (27\%; Figure 2). Neurological symptoms were most commonly focal neurologic signs (57/187, $30 \%)$ followed by headache $(47 / 187,25 \%)$ and decreased consciousness (37/187, 19\%). Compared with patients without intracranial involvement, these patients had similar sex distribution, but were slightly younger, more likely to have otomastoiditis as primary infection, and less likely to have previously consulted a general practitioner. Patients with intracranial involvement were more likely to die ( $8.0 \%$ vs. $1.5 \%)$ and to have sequelae at discharge ( $8.6 \%$ vs. $1.7 \%$ ).

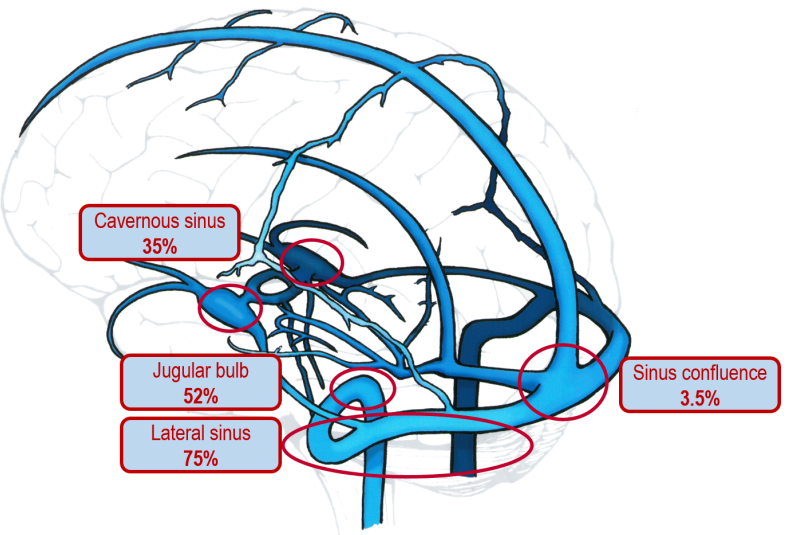

FIGURE 1 Thrombosis location in 146 patients with Lemierre syndrome and cerebral venous sinus thrombosis. 


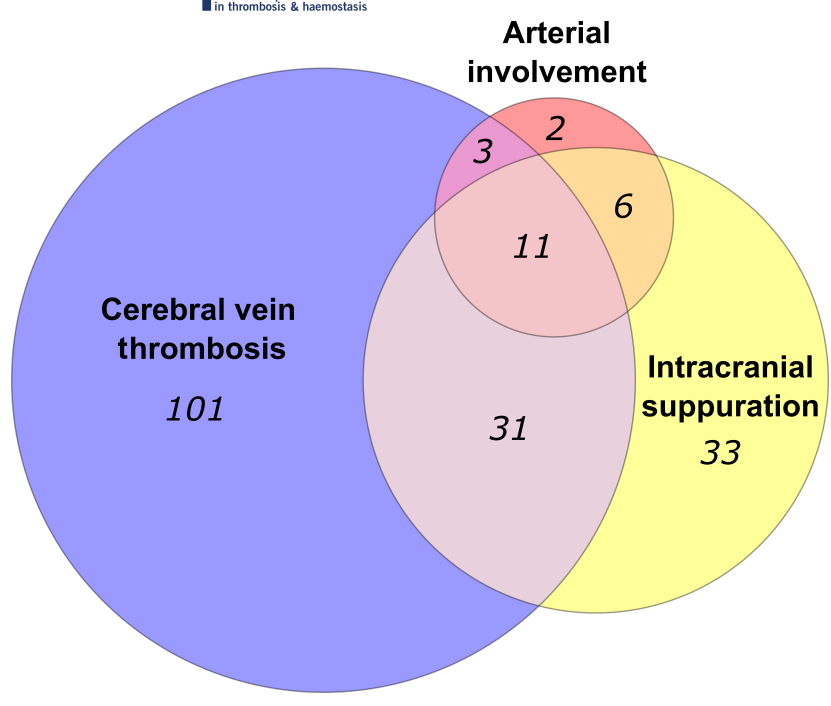

FIGURE 2 Proportional area plot of the patterns of intracranial involvement in 187 of 712 patients with Lemierre syndrome

Conclusions: Systematic neurologic assessment at presentation and in-hospital neurologic monitoring should be considered in Lemierre syndrome. Pending confirmation from ongoing research, any pattern of intracranial involvement should prompt the search of others.

LPB0123 | Thrombotic Complications in a Cohort of Children with Myeloproliferative Neoplasms

N. Kucine $^{1} ;$ V. Vanderpoel ${ }^{2} ;$ K. Shimano ${ }^{2}$

${ }^{1}$ Weill Cornell Medicine, New York, United States; ${ }^{2}$ UCSF Benioff Children's Hospital, San Francisco, United States

Background: Thrombotic events are well-known complications of the BCR-ABL-negative, classical myeloproliferative neoplasms (MPNs). Both arterial and venous thromboses can occur, especially splanchnic vein thromboses (including Budd-Chiari syndrome). There are various potential risk factors, including JAK2V617F, the most common MPN driver mutation, yet height of platelet count has been shown to not correlate with thrombosis risk. While older age is an established risk factor, thrombotic events have been reported in pediatric MPN patients, but the available literature is limited.

Aims: To describe the thrombotic events in a pediatric MPN cohort. Methods: We reviewed charts of our pediatric MPN cohort for data around thrombotic events, which included demographic information, type of thrombotic event, blood counts at time of event, and mutational status. All subjects are enrolled on an IRB-approved observational study where consent/assent was obtained.

Results: In a cohort of 42 children with MPNs, 6 (14.3\%) had a thrombotic event. Five subjects were female, and five were adolescents when thrombosis developed. Five subjects had a JAK2V617F mutation, while one was negative for any known mutation. One subject had a stroke, one had a cerebral venous sinus thrombosis and pulmonary embolism, and four had Budd-Chiari syndrome. Two subjects developed their thrombotic events after diagnosis of their
MPN, while four subjects' thrombotic events occurred at MPN presentation. Five subjects had thrombocytosis (but none had extreme thrombocytosis - platelets $>1000 \times 10^{9} / \mathrm{L}$ ), four had polycythemia, and four had leukocytosis at the time of their thrombotic events.

Conclusions: Our data confirms the limited available literature showing that children with MPNs can develop thrombotic complications. In our cohort, children who developed thromboses were predominantly female and JAK2V617F-mutation-positive. Budd-Chiari syndrome was the most common form of thrombosis. Further study is needed to better understand the pathophysiology and natural history of thromboses in our young patients with MPNs.

LPB0124 | Low Hemoglobin as a Biomarker for Paediatric Sinus Thrombosis

B. Moiz; S. Altaf; R. Devi Ukrani; A. Arif; I. Akbar; M. Wahhaab Sadiq

Aga Khan University, Karachi, Pakistan

Background: Cerebral venous sinus thrombosis (CVST) is rare in children. Its risk factors and outcome are largely unknown in low-middle income countries. Some case reports have shown presence of anemia in pediatric CVST as a probable risk factor.

Aims: This study was done to determine the frequency of anemia in patients having pediatric CVST and to observe its association with the severity of disease.

Methods: Patients between age 0 to 18 years of both genders diagnosed with sinus thrombosis during 2011 to 2020 were identified through international classification of diseases (ICD 9). Data was collected through in-house computerized system and SPSS version 19 was used for analysis.

Results: Of 143492 patients who were admitted, 32 (21 males and 11 females) with a median (IQR) age of 4.5 years (0-16) had CVST. This is equivalent to 2.2 CVST events $/ 10,000$ pediatric admissions. Overall mortality was $3.4 \%$. Primary underlying disorders were infections (59\%), hematological neoplasms (12.5\%), thrombotic thrombocytopenic purpura (3\%) and antiphospholipid syndrome (3\%). Significant laboratory findings were anemia (65\%), low protein S (19\%), low protein C (19\%), low antithrombin III (6\%) and activated protein $C$ resistance (25\%). Mean $( \pm S D)$ hemoglobin in anemic patients was $9.0 \mathrm{~g} / \mathrm{dL}( \pm 2.3)$. SARS-COV2 was negative in patients tested in 2020. Regression analysis showed a positive association of anemia with multiple sinus involvement $(P 0.009)$ but anemia had no association with the duration of symptoms ( $P$ 0.344), hospital stay $(P$ $0.466)$, age ( $P$ 0.863) or gender ( $P$ 0.542) of the patients.

Conclusions: Low hemoglobin was observed in a significant proportion of patients with pediatric CVST. Anemia may be an important biomarker along with other clinical variables in the risk assessment of sinus thrombosis in children. Future studies may unveil the role of anemia in coagulopathy and more specifically in pediatric CVST. 


\section{LPB0125 | Characteristics of Pediatric Oncology Patients with} Tumour Thrombus

N. Samji; A.F. Fajardo; A.K. Chan; M.D Bhatt

McMaster University, Hamilton, Canada

Background: Intravascular tumour extension is a rare but important complication of pediatric solid tumours. There is limited information regarding characteristics and outcomes of pediatric patients with tumour thrombus.

Aims: To look at incidence, risk factors, management, and outcomes of pediatric oncology patients with tumour thrombus.

Methods: A retrospective analysis was conducted on patients aged $<18$ years diagnosed with solid tumours from $2000-2020$ at McMaster Children's Hospital. Data collection included: demographics (age, gender, cancer details, staging, pathology report, treatment, outcomes), and tumour thrombus details (imaging, anticoagulation use, resolution, recurrence, complications of anticoagulation, thromboembolic phenomena).
Results: Of 347 patients identified with solid tumours, 53 were excluded due to lack of data. 294 were included: Neuroblastoma (67), Wilms tumour (WT) (40), renal cell carcinoma (2), Hepatoblastoma (15), germ cell tumour (GCT) (24), Ewing sarcoma (26), osteosarcoma (32), rhabdomyosarcoma (32), rhabdoid tumour (4), desmoplastic small round cell tumour (3), and other (49). Median age was 5.68 years. Tumour thrombus was identified in 10 patients (10/294; $3.4 \%)$, with WT having the highest incidence (12.5\%). Vascular involvement included inferior vena cava (IVC) $(n=8)$, pulmonary vein branches $(n=1)$, sigmoid sinus $(n=1)$, isolated renal vein $(n=1)$, combined renal vein and IVC $(n=4)$. One patient had $>1$ site involved. All patients with tumour thrombus were high risk or had metastatic disease except in WT (Table 1). Anticoagulation was initiated prior to surgical resection in four cases (40\%) and post-operatively in three cases (30\%). Progression of thrombus was identified in one patient. IVC thrombus recurrence was noted post-operatively in 3 patients with WT.

TABLE 1 Characteristics of patients with and without solid tumour thrombus

\begin{tabular}{|c|c|c|c|}
\hline Disease & Risk Factors & $\begin{array}{l}\text { Patients with Tumour } \\
\text { Thrombus }\end{array}$ & $\begin{array}{l}\text { Patients without Tumour } \\
\text { thrombus }\end{array}$ \\
\hline \multirow[t]{8}{*}{ Neuroblastoma } & Age (median in years) & 2.47 & 2.07 \\
\hline & Male gender & $0 / 2(0 \%)$ & $36 / 65$ (55.4\%) \\
\hline & High Risk Group & $2 / 2(100 \%)$ & $30 / 65(46.2 \%)$ \\
\hline & Outcome & $1 / 2(50 \%)$ & $14 / 65(21.5 \%)$ \\
\hline & Deceased & $1 / 2(50 \%)$ & $5 / 65(7.7 \%)$ \\
\hline & On-therapy & $0 / 2(0 \%)$ & $46 / 65(70.1 \%)$ \\
\hline & Remission & $1 / 2(50 \%)$ & $11 / 65$ (16.9\%) \\
\hline & Relapsed/Progression & & \\
\hline \multirow[t]{7}{*}{ Wilms } & Age (median) & 4.13 & 3.09 \\
\hline & Male Gender & $3 / 5(60 \%)$ & $23 / 35(65.7 \%)$ \\
\hline & Metastatic disease & $1 / 5(20 \%)$ & 5/35 (14.3\%) \\
\hline & Outcome & $0 / 5(0 \%)$ & $3 / 35(8.6 \%)$ \\
\hline & Deceased & 5/5 (100\%) & $32 / 35(91.4 \%)$ \\
\hline & Remission & $1 / 5(20 \%)$ & $5 / 35$ (14.3\%) \\
\hline & Relapsed/Progression & & \\
\hline \multirow[t]{7}{*}{ Hepatoblastoma } & Age (median) & 1.05 & 2.78 \\
\hline & Male gender & $1 / 1(100 \%)$ & $10 / 14(71.4 \%)$ \\
\hline & High Risk Group & $1 / 1(100 \%)$ & $10 / 14(71.4 \%$ \\
\hline & Outcome & $1 / 1(100 \%)$ & $4 / 14$ (28.6\%) \\
\hline & Deceased & $0 / 1(0 \%)$ & $9 / 14(64.2 \%)$ \\
\hline & Remission & $1 / 1(100 \%)$ & $3 / 14$ (21.4\%) \\
\hline & Relapsed/Progression & & \\
\hline
\end{tabular}




\begin{tabular}{|c|c|c|c|}
\hline Disease & Risk Factors & $\begin{array}{l}\text { Patients with Tumour } \\
\text { Thrombus }\end{array}$ & $\begin{array}{l}\text { Patients without Tumour } \\
\text { thrombus }\end{array}$ \\
\hline \multirow[t]{7}{*}{ GCT } & Age (median) & 9.2 & 13.4 \\
\hline & Male Gender & $1 / 2(50 \%)$ & $10 / 22(45.4 \%)$ \\
\hline & High Risk Group & $2 / 2(100 \%)$ & $5 / 22(22.7 \%)$ \\
\hline & Outcome & $0 / 2(0 \%)$ & $0 / 22(0 \%)$ \\
\hline & Deceased & $2 / 2(100 \%)$ & $22 / 22(100 \%)$ \\
\hline & Remission & $0 / 2(0 \%)$ & $0 / 22(0 \%)$ \\
\hline & Relapsed/Progression & & \\
\hline
\end{tabular}

Conclusions: This is the first study to document incidence of tumour thrombus in a large group of pediatric solid tumour patients. In our cohort, tumour thrombus was identified in neuroblastoma, WT, hepatoblastoma, and GTC patients. Risk factors, optimal management, and effect on overall patient outcome require further clarification.

PB0784 | A 5-Year Study of Arterial and Venous Thromboses in Greek Infants, Children and Adolescents. Comparison of Data Before and during the Pandemic Period

L. Ioannidou; A. Dettoraki; A. Michalopoulou; Z. Kapsimali; S. Saslis; M. Mazarakis; H. Pergantou

Haemophilia Centre, Haemostasis and Thrombosis Unit, Aghia Sophia Children's Hospital, Athens, Greece

Background: Arterial and venous thromboses are rare in childhood but have rising incidence due to longer survival of chronic patients, catheters' use and increased sensitivity of diagnostic tools. Coronovirus pandemic could induce further increase considering the thrombotic complications of the virus, decreased mobility or restricted access to health care.

Aims: The aims of this study were to provide data regarding thromboses in childhood within a 5 -year period and identify possible distinct characteristics of cases diagnosed within the first year of the pandemic.

Methods: Data were retrospectively collected from January 2016 to December 2020, for children aged 28 days-18 years old, with both venous and arterial thromboses, in a reference Thrombosis Centre in Greece. Patients with cancer-associated thrombosis or perinatal ischemia were excluded.

Results: Overall, 214 children were reported with thromboses [mean age: 6.1 \pm 5.1 years, $133(62.1 \%)$ males], with rising incidence per year. The most statistically significant ( $P$ : 0.024$)$ increase, by $51 \%$, was in 2020 in comparison to 2019 (74 compared to 49 new cases respectively). Most thromboses diagnosed in 2020 (55.1\%) manifested in the central nervous system, with equal presence of arterial ischemic strokes and cerebral sinus venous thromboses (CSVT), followed by deep vein thromboses (DVT) of extremities (33.3\%), visceral venous thromboses (7.2\%) and pulmonary embolism (2.9\%), similar to previous years. Only two patients were found positive for coronavirus.
Infection was the commonest underlying condition in all years (23.7\% of total), whereas $16.1 \%$ of total cases were central venous line-related DVT and $14.7 \%$ were CSVT secondary to otitis media or acute mastoiditis. Frequencies of thrombophilia mutations concerning homozygosity for MTHFR and heterozygosity for FV Leiden and FII20210A were 7\%, 5.6\% and 3.3\% respectively.

Conclusions: Incidence of thromboses was increased by more than $50 \%$ in 2020 but further research is needed to elucidate whether this increase is directly or indirectly related to the pandemic.

\section{PB0785 | Utility of Antithrombotic Therapy in Pediatric Portal Vein Thrombosis after Endovascular Procedure}

K. Na; R. Diaz; J. Hernandez; H. Justino; C. Cohen; S. Sartain;

Y. Lee-Kim

Baylor College of Medicine, Houston, United States

Background: Native portal vein thrombosis (PVT) in children, that is not associated with liver transplantation, is often chronic and associated with bleeding risks due to the development of portal hypertension and esophageal varices, making anticoagulation challenging. While there are case series describing surgical interventions, standard practice for and outcomes after antithrombotic therapies have not been established.

Aims: We report 20 cases of pediatric patients with native PVT, not associated with liver transplant, to describe the outcomes after antithrombotic therapy.

Methods: A retrospective chart review was conducted of 20 pediatric PVT patients referred to interventional radiology who underwent at least one attempt at percutaneous recanalization at Texas Children's Hospital from 2014 to 2019.

Results: There were 8 cases of initially unsuccessful recanalization; $4(50 \%)$ of these received antithrombotics. Of the 12 who had successful recanalization, 11 (92\%) received antithrombotics. Of the 15 on antithrombotic therapy after recanalization attempt, 5 (30\%) received antiplatelet agents alone, $1(1 \%)$ received anticoagulation alone, $3(20 \%)$ received anticoagulants transitioned to antiplatelet therapy, and $6(40 \%)$ received a combination of antiplatelet and anticoagulant therapies. Of these 15 patients, 11 (73\%) patients had recurrence/progression of PVT and 5 (33\%) reported bleeding ( 2 with gastrointestinal (GI) bleeding, 2 with bruising, 1 with both 
GI bleeding and bruising). Of the 5 not receiving antithrombotics, 2 (40\%) patients had bleeding prior to procedure, precluding antithrombotic therapy, but the other 3 (60\%) had no bleeding.

Conclusions: In our case series of native PVT, there was no significant association of antithrombotic therapy preventing recurrence or progression of PVT after angioplasty/recanalization. GI bleeding and bruising occurred in one-third of patients while on antithrombotic therapy.

PB0786 | Analysis of Novel Risk Factors for Arterial or Venous Thrombosis in Neonates

K. Bhatia ${ }^{1}$; S. Solanki ${ }^{2}$; A.KC Chan ${ }^{3}$; B. Paes ${ }^{3}$; M.D Bhatt ${ }^{3}$

${ }^{1}$ SUNY Downstate Health Sciences University, Brooklyn, United States;

${ }^{2}$ McMaster University, Hamilton, Canada; ${ }^{3}$ McMaster Children's

Hospital, McMaster University, Hamilton, Canada

Background: Among children, neonates have the highest incidence of thrombosis. Multiple risk factors for thrombosis have been reported including central vascular catheters (CVCs), sepsis, and prematurity. Non-O blood group is associated with increased risk of thrombosis in adults and pediatric leukemia patients. However, it has not been studied as a potential risk factor in neonates.
Aims: To evaluate blood group as a risk factor for neonatal thrombosis.

Methods: Retrospective chart review of neonatal thrombosis diagnosed in a single neonatal intensive care unit (NICU) between January-2014 and September-2018, using the Canadian Neonatal Network database. Data on demographics, thrombosis details, blood group and hematocrit were extracted. A multiple imputation model was used to input missing data. The association between risk factors and thrombosis is reported as odds ratio (OR) and compared using Fisher's exact test. Risk factors with a $P$-value $<0.1$ were included in a multiple logistic regression model; $P<0.05$ was considered statistically significant.

Results: Of 4860 NICU admissions, 186 were associated with thrombosis (38/1000) involving 195 sites (Portal vein, 117; Inferior vena cava, 24; Deep venous except IVC 18; Cardiac, 12; Cerebral sinus venous, 8; Renal vein, 1; and Arterial, 15). On univariate analysis, birth weight $<2500$ grams, prematurity (GA <37 weeks), CVCs, culture positive sepsis, respiratory distress syndrome (RDS), hematocrit and $\mathrm{O}$ blood group were statistically significant risk factors for thrombosis (Table 1). On multivariate analysis, CVCs, culture positive sepsis and RDS remained significant with adjusted ORs of 6.38, 5.16, and 2.41 , respectively.

TABLE 1 Univariate and multivariate analysis results (Significant $P$ values are bolded)

\begin{tabular}{|c|c|c|c|c|}
\hline & Univariate analysis & & Multivariate analysis & \\
\hline Variable & Odds Ratios (95\% Cl) & Pvalue & $\begin{array}{l}\text { Adjusted Odds Ratios (95\% } \\
\text { Cl) }\end{array}$ & Pvalue \\
\hline Maternal Chorioamnionitis & $1.25(0.76-2.08)$ & 0.374 & - & - \\
\hline Maternal gestational diabetes & $1.12(0.75-1.66)$ & 0.581 & - & - \\
\hline Maternal hypertension & $1.29(0.87-1.92)$ & 0.200 & - & - \\
\hline Birth Weight $<2500 \mathrm{~g}$ & $1.63(1.21-2.19)$ & 0.001 & $0.82(0.50-1.37)$ & 0.458 \\
\hline Prematurity & $1.57(1.15-2.14)$ & 0.004 & $0.83(0.48-1.40)$ & 0.478 \\
\hline Central catheters & 7.16 (4.93-10.39) & $<0.001$ & $6.38(4.38-9.31)$ & $<0.001$ \\
\hline Surgery & $1.05(0.62-1.77)$ & 0.851 & - & - \\
\hline Non-O blood group & $0.74(0.55-1.00)$ & 0.050 & $0.75(0.55-1.02)$ & 0.067 \\
\hline
\end{tabular}

Conclusions: Unlike adult and pediatric studies, O blood group approached statistical significance as a risk factor for neonatal thrombosis on univariate analysis. In multivariate analysis, however, previously known risk factors of CVCs, sepsis and RDS remained significant. Further study is required to elucidate the role of blood group as a potential risk factor for neonatal thrombosis. 
PB0787 | Efficacy and Safety of Dabigatran in the Treatment and Secondary Prevention of Venous Thromboembolism in Children with Cerebral Venous and Sinus Thrombosis

L. Brandão ${ }^{1,2}$; I. Tartakovsky ${ }^{3}$; J. Halton ${ }^{4}$; L. Bomgaars ${ }^{5}$;

E. Chalmers ${ }^{6}$; L. Mitchell ; M. Luciani ${ }^{8}$; I. Gergei ${ }^{3,9}$; E. Kleine ${ }^{10}$; M. Brueckmann ${ }^{3,9} ;$ M. Albisetti ${ }^{11}$

${ }^{1}$ The Hospital for Sick Children, University of Toronto, Toronto, Canada; ${ }^{2}$ Dalla Lana School of Public Health, University of Toronto, Toronto, United States; ${ }^{3}$ Boehringer Ingelheim International $\mathrm{GmbH}$, Ingelheim, Germany; ${ }^{4}$ University of Ottawa, Ottawa, Canada; ${ }^{5}$ Department of Pediatrics, Texas Children's Cancer Center, Baylor College of Medicine, Houston, United States; ${ }^{6}$ Royal Hospital for Children, Glasgow, United Kingdom; ${ }^{7}$ University of Alberta, Edmonton, Canada; ${ }^{8}$ Pediatric Hematology/Oncology Department, Pediatric Hospital Bambino Gesù, Rome, Italy; ${ }^{9}$ Faculty of Medicine Mannheim of the University of Heidelberg, Mannheim, Germany, ${ }^{10}$ Biostatistics and Data Sciences, Boehringer Ingelheim Pharma GmbH \& Co. K.G., Ingelheim, Germany,

${ }^{11}$ Hematology Department, University Children's Hospital, Zürich, Switzerland

Background: Dabigatran etexilate (DE) has shown noninferiority versus standard of care (SOC) for treatment of acute venous thromboembolism (VTE), and a favorable safety profile in secondary VTE prevention in children. Efficacy and safety of DE in children with cerebral venous and sinus thrombosis (CVST) has not yet been evaluated.

Aims: Subgroup analyses evaluating DE for treatment and secondary prophylaxis of VTE in children with CVST, from two pediatric trials. Methods: In the DIVERSITY trial, children with objectively confirmed VTE initially treated with heparin were randomized (2:1) to receive up to 3 months of DE or SOC. Primary composite efficacy endpoint: complete thrombus resolution and freedom from VTE recurrence and VTE-related death. A secondary VTE prevention trial treated children with DE for up to 12 months. Eligible children had VTE treated with SOC for $\geq 3$ months or had completed either treatment in DIVERSITY. Primary endpoints included VTE recurrence and bleeding events (BEs).

Results: In DIVERSITY, 26 children (9.7\%) had CVST. Within this subset, $45.0 \%$ of children treated with DE versus $16.7 \%$ treated with SOC achieved the primary endpoint, whereas $45.9 \%$ versus $44.0 \%$, respectively, met the primary endpoint in the non-CVST subgroup (Table 1). Parameters of thrombus resolution and VTE recurrence (reflecting those of the primary endpoint) and BEs are shown in Table 1. Treatment by CVST interaction was not tested statistically due to the limited sample size. In the secondary prevention trial (24 patients with CVST), there was no recurrent VTE in patients with CVST and overall, few major/clinically relevant nonmajor BEs (Table 2).

Conclusions: These subgroup analyses, in a small cohort of children with CVST, do not appear to contradict the efficacy and safety profile of DE versus SOC for treatment of acute VTE demonstrated in the overall trial, and show a favorable safety profile of DE for secondary VTE prevention.
TABLE 1 Acute VTE treatment trial: outcomes for CVST subgroup analysis

\begin{tabular}{|c|c|c|c|c|}
\hline \multirow{2}{*}{$\begin{array}{l}\text { DIVERSITY trial } \\
\text { CVST subgroup analysis }\end{array}$} & \multicolumn{2}{|c|}{ CVST yes, $\mathrm{N}=26$} & \multicolumn{2}{|c|}{ CVST no, $\mathrm{N}=241$} \\
\hline & $\mathrm{DE}$ & soc & DE & soc \\
\hline Efficacy endpoints (randomized set), N & 20 & 6 & 157 & 84 \\
\hline $\begin{array}{l}\text { Primary endpoint: Complete thrombus } \\
\text { resolution, freedom from recurrent VTE, } \\
\text { freedom from VTE-related death, } \mathrm{n}(\%)\end{array}$ & $9(45.0)$ & $1(16.7)$ & $72(45.9)$ & $37(44.0)$ \\
\hline \multicolumn{5}{|l|}{$\begin{array}{l}\text { Residual thrombotic burden at day } 84 \text { or end } \\
\text { of therapy }\end{array}$} \\
\hline Thrombus progression, ${ }^{*} \mathrm{n}(\%)$ & 0 & $1(16.7)$ & $5(3.2)$ & $3(3.6)$ \\
\hline Stabilization, n (\%) & $2(10.0)$ & $2(33.3)$ & $9(5.7)$ & $8(9.5)$ \\
\hline Partial resolution, $\mathrm{n}(\%)$ & $8(40.0)$ & $2(33.3)$ & $49(31.2)$ & $23(27.4)$ \\
\hline Complete resolution, $n(\%)$ & $9(45.0)$ & $1(16.7)$ & $72(45.9)$ & $37(44.0)$ \\
\hline Missing & $1(5.0)$ & 0 & $22(14.0)$ & $13(15.5)$ \\
\hline $\begin{array}{l}\begin{array}{l}\text { Free from recurrent VTE or mortality related } \\
\text { to VTE, } \mathrm{n}(\%)\end{array} \\
\end{array}$ & $20(100.0)$ & $5(83.3)$ & $150(95.5)$ & $78(92.9)$ \\
\hline \begin{tabular}{|l|} 
Partial or complete resolution and free from \\
recurrent VTE or mortality related to VTE, $n(\%)$ \\
\end{tabular} & $17(85.0)$ & $3(50.0)$ & $120(76.4)$ & $59(70.2)$ \\
\hline On-treatment any bleeding (treated set), $\mathrm{N}$ & 20 & 6 & 156 & 84 \\
\hline Any bleeding, $\mathrm{n}(\%)$ & $5(25.0)$ & $4(66.7)$ & $33(21.2)$ & $18(21.4)$ \\
\hline Major bleeding, ${ }_{1} \mathrm{n}(\%)$ & $1(5.0)$ & $1(16.7)$ & $3(1.9)$ & $1(1.2)$ \\
\hline CRNM bleeding, $\mathrm{n}(\%)$ & 0 & 0 & $2(1.3)$ & $1(1.2)$ \\
\hline
\end{tabular}

TABLE 2 Secondary VTE prevention trial: outcomes for CVST subgroup analysis

\begin{tabular}{|l|c|c|c|c|c|c|c|c|}
\hline $\begin{array}{l}\text { Secondary prevention trial } \\
\text { CvST subgroup analysis }\end{array}$ & \multicolumn{9}{|c|}{ CVST yes } & \multicolumn{5}{c|}{ CVST no } \\
\hline & Total & $\begin{array}{c}\text { Birth to } \\
<2 \text { yrs }\end{array}$ & $\begin{array}{c}2 \text { to } \\
<12 \text { yrs }\end{array}$ & $\begin{array}{c}12 \text { to } \\
<18 \text { yrs }\end{array}$ & Total & $\begin{array}{c}\text { Birth to } \\
<2 \text { yrs }\end{array}$ & $\begin{array}{c}2 \text { to } \\
<12 \text { yrs }\end{array}$ & $\begin{array}{c}12 \text { to } \\
<18 \text { rs }\end{array}$ \\
\hline $\begin{array}{l}\text { Number of children (treated } \\
\text { set) }\end{array}$ & 24 & 1 & 14 & 9 & 189 & 8 & 29 & 152 \\
\hline $\begin{array}{l}\text { Recurrent VTE at } 12 \text { months, } \\
n(\%)\end{array}$ & 0 & 0 & 0 & 0 & $3(1.6)$ & 0 & 0 & $3(2.0)$ \\
\hline $\begin{array}{l}\text { Any bleeding events at } 12 \\
\text { months; } n(\%)\end{array}$ & $4(16.7)$ & 0 & $2(14.3)$ & $2(22.2)$ & $44(23.3)$ & $1(12.5)$ & $2(6.9)$ & $41(27.0)$ \\
\hline$\quad$ Major bleeding, $n(\%)$ & 0 & 0 & 0 & 0 & $3(1.6)$ & 0 & 0 & $3(2.0)$ \\
\hline$\quad$ CRNM bleeding, $n(\%)$ & $1(4.2)$ & 0 & $1(7.1)$ & 0 & $2(1.1)$ & 0 & $1(3.4)$ & $1(0.7)$ \\
\hline $\begin{array}{l}\text { *No patients had intracranial bleeding. } \\
\text { CRNM, clinically relevant nonmajor; CVST, cerebral venous and sinus thrombosis; VTE, venous thromboembolism. }\end{array}$ \\
\hline
\end{tabular}

PB0788 | Outcomes of Prophylactic Enoxaparin against Venous Thromboembolism in Hospitalized Children

E. Bennett ${ }^{1}$; C. Delgado Corcoran ${ }^{2}$; C. Heyrend ${ }^{2}$; R. Wilcox ${ }^{2}$; C. Pannucci ${ }^{3}$; V. Faustino ${ }^{4}$

${ }^{1}$ University of Arkansas for Medical Sciences/Arkansas Children's Hospital, Little Rock, United States; ${ }^{2}$ University of Utah/Primary Children's Hospital, Salt Lake City, United States; ${ }^{3}$ Plastic Surgery Northwest, Spokane, United States; ${ }^{4}$ Yale School of Medicine/Yale New Haven Children's Hospital, New Haven, United States

Background: Hospitalized children are at increased risk of healthcare acquired venous thromboembolism (HA-VTE). Studies have shown that prophylactic enoxaparin may prevent HA-VTE in hospitalized older children, but not in hospitalized infants $<1$ year old. Other factors may be associated with HA-VTE despite prophylactic enoxaparin.

Aims: To characterize the biochemical and clinical outcomes of hospitalized children who received prophylactic enoxaparin.

Methods: We conducted a single-center, retrospective study of hospitalized children $<18$ years old who received prophylactic enoxaparin against HA-VTE. Children received age-based enoxaparin dose, which was adjusted to achieve anti-Xa level of $0.2-0.5 \mathrm{IU} / \mathrm{mL}$. Primary biochemical and clinical outcomes were achievement of goal 
anti-Xa range and development of HA-VTE, respectively. Secondary clinical outcome was development of clinically relevant bleed.

Results: We analyzed 194 children composed of $13(7 \%)$ infants and 181 (93\%) older children. Anti-Xa levels were drawn in 9 (69.2\%) infants and 107 (59.1\%) older children ( $P=0.47)$. Goal anti-Xa range was achieved in $6(66.7 \%)$ infants and $88(82.2 \%)$ older children $(P=0.37)$. Median number of anti-Xa levels prior to goal was 2 (interquartile range, IQR: 2,3$)$ in infants and 1 (IQR: 1,2$)$ in older children $(P=0.01)$. HA-VTE developed in $2(15.4 \%)$ infants and 9 (5.0\%) older children $(P=0.16)$. Personal history of VTE was associated with HA-VTE (odds ratio, OR: 11.98; 95\% confidence interval, $\mathrm{Cl}: 2.30$, 62.32). Infant status (OR: $6.25 ; 95 \% \mathrm{Cl}: 0.99,39.53$ ) and prophylactic enoxaparin started within 3 days after hospital admission (OR: 0.25; $95 \% \mathrm{Cl}: 0.06,1.07)$ were marginally associated with HA-VTE. Bleeds developed in none $(0 \%)$ of the infants and in $4(2.2 \%)$ older children.
Figure 1. Distribution of anti-Xa levels after the initial enoxaparin dose ( $A$ ) and the number of measurements prior to achievement of goal range (B) among hospitalized children who received prophylactic enoxaparin

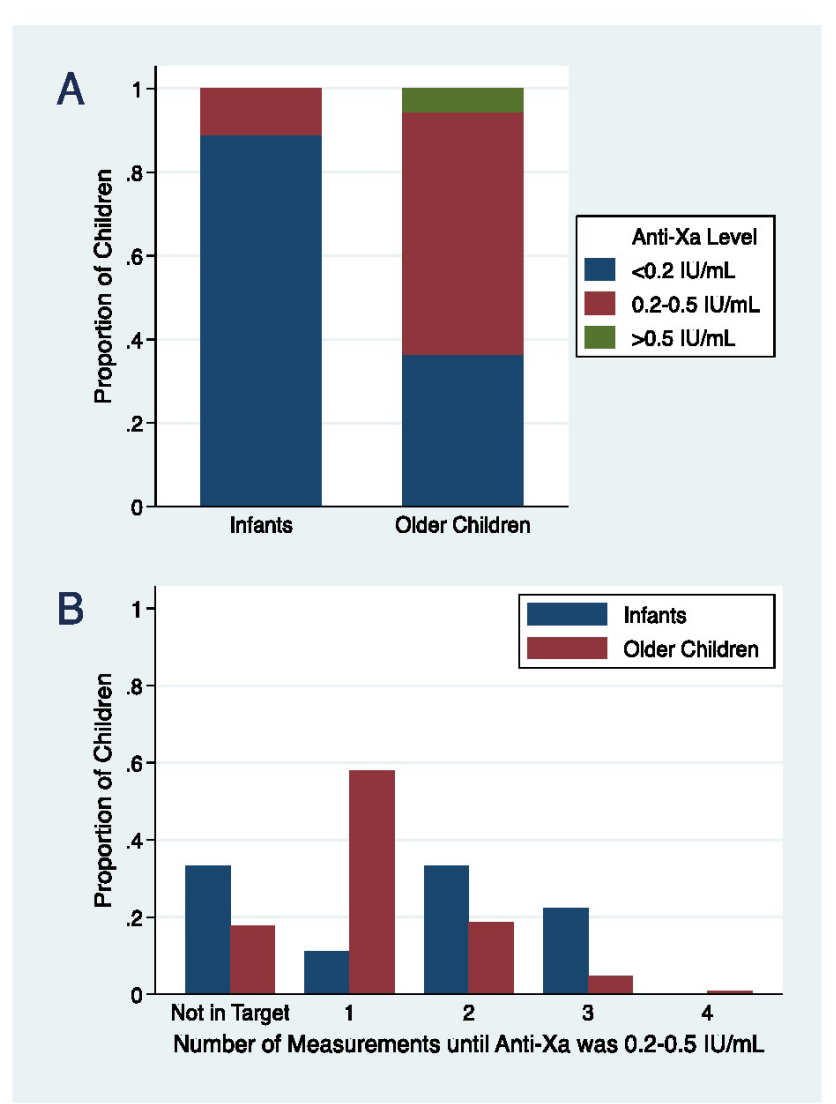

FIGURE 1 Distribution of Anti-Xa Levels after the Initial Enoxaparin Dose (A) and the Number of Measurements Prior to Achievement of Goal Range (B) among Hospitalized Children who received Prophylactic Enoxaparin

TABLE 2 Factors Associated with Healthcare-Acquired Venous Thromboembolism (VTE) among Hospitalized Children who received Prophylactic Enoxaparin

\begin{tabular}{|c|c|c|c|c|}
\hline Variable & Unadjusted Odds Ratio & $\begin{array}{l}95 \% \text { Confidence } \\
\text { Interval }\end{array}$ & Adjusted Odds Ratio & $\begin{array}{l}95 \% \text { Confidence } \\
\text { Interval }\end{array}$ \\
\hline Infant & 3.47 & $0.67,18.07$ & 6.25 & $0.99,39.53$ \\
\hline Obesity & 0.91 & $0.19,4.38$ & & \\
\hline Personal history of VTE & 7.25 & $1.64,32.06$ & 11.98 & $2.30,62.32$ \\
\hline Congenital Heart Disease & 2.49 & $0.49,12.58$ & & \\
\hline Cancer & 3.06 & $0.75,12.47$ & & \\
\hline Surgery & 1.3 & $0.38,4.4$ & & \\
\hline Trauma & 0.4 & $0.08,1.92$ & & \\
\hline $\begin{array}{l}\text { Admission to the Intensive Care } \\
\text { Unit }\end{array}$ & 3.5 & $0.73,16.63$ & & \\
\hline Sequential compression device & 1.26 & $0.37,4.27$ & & \\
\hline Central venous catheter & 5.92 & $1.25,28.19$ & & \\
\hline Patient on other anticoagulant & 1.47 & $0.30,7.23$ & & \\
\hline Early prophylaxis & 0.27 & $0.07,1.04$ & 0.25 & $0.06,1.07$ \\
\hline Starting dose & 9.37 & $0.92,95.27$ & & \\
\hline Hemoglobin & 1.13 & $0.89,1.44$ & & \\
\hline
\end{tabular}




\begin{tabular}{|c|c|c|c|c|}
\hline Variable & Unadjusted Odds Ratio & $\begin{array}{l}\text { 95\% Confidence } \\
\text { Interval }\end{array}$ & Adjusted Odds Ratio & $\begin{array}{l}95 \% \text { Confidence } \\
\text { Interval }\end{array}$ \\
\hline Anti-Xa Measured & 0.54 & $0.16,1.84$ & & \\
\hline
\end{tabular}

Conclusions: HA-VTE developed in hospitalized children despite prophylactic enoxaparin. HA-VTE among infants seemed related to delay in achieving goal anti-Xa range. Early administration of therapeutic enoxaparin may prevent HA-VTE in infants and in those with personal history of VTE.

PB0789 | Management of Iliofemoral Deep Vein Thrombosis (DVT) in Pediatric Patients in the Context of May-Thurner Syndrome (MTS)

$\underline{\text { V. Patel }}^{1}$; N. Stein ${ }^{2}$; F. Elias ${ }^{3}$; A.K. Chan ${ }^{4}$; M. Bhatt ${ }^{4}$

${ }^{1}$ McMaster University, Hamilton, Canada; ${ }^{2}$ McMaster University

Department of Radiology, Hamilton, Canada; ${ }^{3}$ McMaster University

Department of Vascular Surgery, Hamilton, Canada; ${ }^{4}$ McMaster

University Department of Pediatrics, Hamilton, Canada

Background: Children with May-Thurner Syndrome (MTS), classified as compression of iliac vein by iliac artery, have higher likelihood of experiencing lower extremity deep vein thrombosis (LEDVT). There is limited pediatric literature describing MTS associated LEDVT.

Aims: To describe presentation, diagnosis, management and outcomes of MTS associated LEDVT in the pediatric population.

Methods: Retrospective chart review of patients 18 years of age with MTS associated LEDVT seen at McMaster Children's Hospital between 2008-2020.

Results: During the study period, there were 16 patients ( $M: 5$, F: 11 , median age: 15.5 years, range: $13-18$ years) with MTS and LEDVT. Common presenting symptoms were pain, swelling, and skin changes -observed in 15(94\%), 12(75\%), and 6(37.5\%), patients respectively. There were $2(12.5 \%)$ patients who presented with pulmonary embolism confirmed on imaging. Common risk factors were thrombophilia (68.8\%), OCP (56.25\%), surgery (12.5\%), obesity (6.25\%), and pro-longed non-ambulatory period (6.25\%). Among the 11 patients with thrombophilia, most common conditions were factor $\mathrm{V}$ Leiden mutation (27.3\%), prothrombin gene mutation (18.2\%), protein C deficiency (9.1\%), and protein S deficiency (9.09\%). All patients were initially managed with anticoagulation alone, with low molecular weight heparin (81\%) being most common agent. One patient was treated with catheter directed thrombolysis on recurrence. One patient (6.3\%) had clot recurrence. Out of remaining 15 patients,14 had available follow-up imaging. Complete resolution, partial resolution, and stable thrombosis was observed in $3(21.4 \%)$ 6(42.9\%), and 5(35.7\%), respectively. The median treatment length was 7.4 months, while 6 patients (37.5\%) were on indefinite longterm anticoagulation for preventing recurrence.

Conclusions: MTS associated LEDVT commonly presents in adolescent age group and is commonly associated with thrombophilia. Like adults, the risk of recurrence was low in our small cohort of pediatric patients with anticoagulation therapy for MTS associated LEDVT.

PB0790 | Management of Antithrombotic Treatment and Thrombohaemorragic Events in Children under Berlin Heart Excor Devices

M. Arguello-Tomas ${ }^{1}$; R. Gomez-Antonio ${ }^{1}$; M.J Santiago ${ }^{1}$; G. Pérez Rus ${ }^{1,2}$; J.L. Diez-Martin ${ }^{2,3,1}$; C. Pascual Izquierdo ${ }^{1,3}$ ${ }^{1}$ Gregorio Marañón Hospital, Madrid, Spain; ${ }^{2}$ Complutense University of Madrid, Madrid, Spain; ${ }^{3}$ Instituto de Investigación de Salud Gregorio Marañon, Madrid, Spain

Background: Berlin-Heart EXCOR (BHE) is a ventricular assist device (VAD) used as bridge to cardiac transplantation. Thrombohaemorragic events (THE) are the most life-threatening complications, so close monitorization is needed.

Aims: Our goal was describing THE and antithrombotic monitoring results in our experience.

Methods: Eighteen paediatric patients undergoing BHE between 2009-2020 were identified. One patient receiving cardiac transplant the same day that BHE was implanted was excluded of statistical analysis. Median age was 15.9 months (4.6-43.6) and $66.7 \%$ were male. Three-hundred and ninety-seven haemostatic controls (blood count, basic and special coagulation tests) based in our protocol (Figure 1) were included: every 24 hours first week, every 3-7 days from then and at the time and in previous 48 hours before any THE.

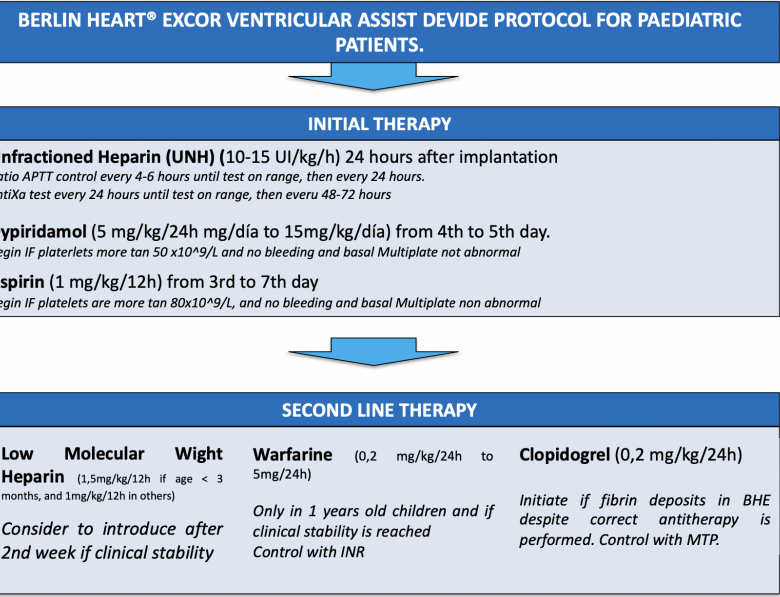

FIGURE 1 Berlin Heart EXCOR protocol for paediatric patients from our centre 
Results: Haemostasia tests controls are resumed in Table 1 Anticoagulation based on low molecular weight heparin (LMWH) was the longest used anticoagulation therapy $(P=0.002)$, and a lower rate of thrombocytopenia $(P=.0019)$ and higher rate $(P=0.011)$ and days $(P=0.002)$ under therapeutical range was observed. Only $38.3 \%$ of Multiplate ${ }^{\circledR}$ tests results were in correct range, both similar in ADP and ASPI tests.

Forty THE occurred with a median of 2THE (1-3) per patient. Bleeding was the most frequent (52.5\%), followed by BHE thrombosis (30\%). Clinical thrombosis was the less frequent (17.5\%); all of them were ischemic stroke. Bleeding was the earliest complication complication ( $P=0.0023$ ). Previous to each THE, just in $42.9 \%$ there was a haemostatic state in blood controls that explained the event, so other factors not related to haemostasis must be considered.

The $66.7 \%$ of patients were successfully bridged to cardiac transplantation. Five patients (27.8\%) died while on a device, but only one because a THE (bleeding).
Results: The JAK2 V617F mutation was positive in 16 patients (18.6\%). The CALR mutations were found in 3 of 62 patients (4.8\%). None of them was MPL-mutated. Surprisingly, although bone marrow histology was consistent with a diagnosis of ET in all the 67 cases without driver mutations, a spontaneous normalization of the platelet count occurred in 7 cases (10.4\%). We found that several factors might be 'true ET'-specific, including palpable splenomegaly, presence of immunophenotypic abnormality (mainly showing reduced CD38 expression on myeloid blast cells and reduced CD36 expression on nucleated red blood cells), positive endogenous erythroid colonies, subnormal erythropoietin, and presence of molecular markers other than driver mutations. Severe splenomegaly was a risk factor of myelofibrosis transformation in children. The Efficacy and safety between pegylated interferon and regular interferon were compared for the first time in childhood patients. Better tolerance was seen in children using pegylated interferon. Furthermore,

TABLE 1 Anticoagulant and haemostasis tests controls in our patients. Results are medians, interquartile range and percentages

\begin{tabular}{llll} 
Anticoagulant & Unfractioned Heparin & Low-Molecular Weight Heparin & Warfarin \\
Days from BHE implantation & $1(1-1,5)$ & $9(7.5-31.5)$ & $8(6-15)$ \\
Duration (days) & $13(6.5-28)$ & $34(2-94)$ & $25(1-32)$ \\
Days to first test on range & $3(3-4.5)$ & $1(1-8)$ & $2(1-6)$ \\
Normal range values & RAPTT 2-2.5 & Axa 0.6-1 & INR 2-3 \\
Tests on range & $48.1 \%$ & $67.2 \%$ & $53.5 \%$ \\
\hline
\end{tabular}

Conclusions: Protocolized anticoagulation and antiplatelets monitoring allows focused management of these patients and could have diminished life-threating THE. LWMH could offer better therapeutical stability, but not less THE.

PB0791 | Essential Thrombocythemia in Children: A Retrospective, Real-world Study Conducted in China

R. Fu; D. Zhang; H. Li; F. Xue; X. Liu; Y. Chen; W. Liu; R. Yang; L. Zhang

State Key Laboratory of Experimental Hematology, National Clinical Research Center for Blood Diseases, Institute of Hematology \& Blood Diseases Hospital, Chinese Academy of Medical Sciences \& Peking Union Medical College, Tianjin, China

Background: Essential thrombocythemia (ET) is rare in pediatric patients, with uncertain criteria for diagnosis, prognosis and treatment strategies in real-world clinical practice.

Aims: The study aimed at trying to optimize the diagnostic, prognostic and therapeutic regimens based on existing evidence in pediatric ET.

Methods: A total of 86 pediatric patients (age $\leq 16$ years) with an initial diagnosis of ET according to the 2016 WHO diagnostic criterion were enrolled. The immunophenotype of bone marrow mononuclear cells and mutations in 137 genes associated with myeloid malignancies were analyzed in 55 children. pegylated interferon was still effective and tolerant in 8 children that were resistant or intolerant for regular interferon.

Conclusions: Based on the 2016 WHO diagnostic criterion for ET, several 'true ET'-specific features might be considered for the diagnostic screening of childhood ET. Prognostic factors for children are different from that for adult patients. Pegylated interferon has an extensive prosperity in childhood ET.

PB0792 | VTE Incidence in Children with Different Blood Disorders: Data from a Large Monocenter Retrospective Cohort Study

P.A Zharkov; D.A Evstratov; K.A Voronin; D.V Fedorova; A.V Pshonkin

Dmitry Rogachev National Medical Research Center of Pediatric Hematology, Oncology and Immunology, Moscow, Russian Federation

Background: We have previously reported (Fedorova DV et al., ISTH2019 PB 0852; PB 0853; Morozova DS et al., ISTH2019, PB 0856) frequency analysis of VTE incidence in hospitalized children with different blood disorders, which has several limitations. To improve the quality of research, here we report cumulative incidence rates (CIR) of VTE, asymptomatic or incidental (aVTE) and symptomatic (sVTE) in this cohort of patients.

Aims: to analyze incidence of VTE in hospitalized children with different blood disorers. 
Methods: Data of 533 VTE cases in 1962 hospitalized children aged 0-17 years with objectively confirmed diagnosis of ALL, AML, lymphoma, histiocytosis (Hc) and aplastic anemia (AA) was retrospectively analyzed. All objectively confirmed VTE episodes were collected and presented as CIR that was divided to total (VTE), aVTE and sVTE. Statistical analysis was performed using R, The R Project 4.0.3 Software.

Results:

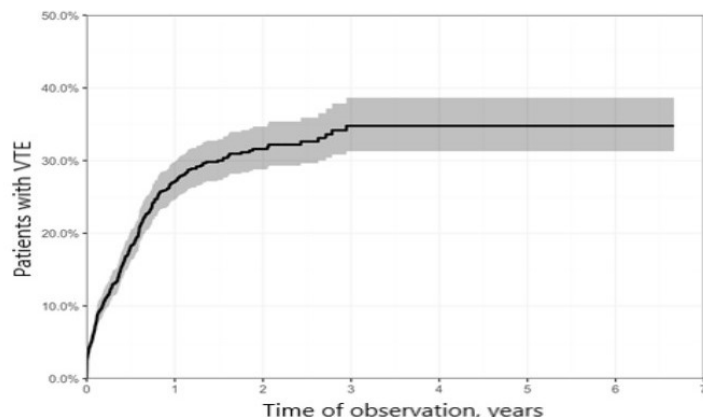

Pic. 1A. CIR of all VTE episodes

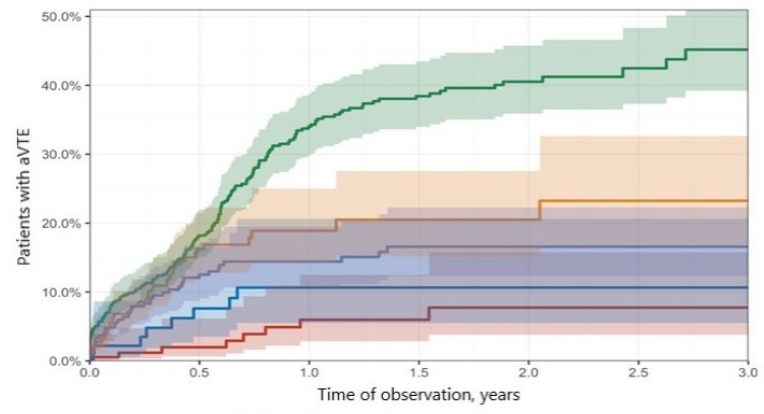

Pic. 1B. CIR of aVTE episodes

- AA - HC - Lymphoma- ALL - AML

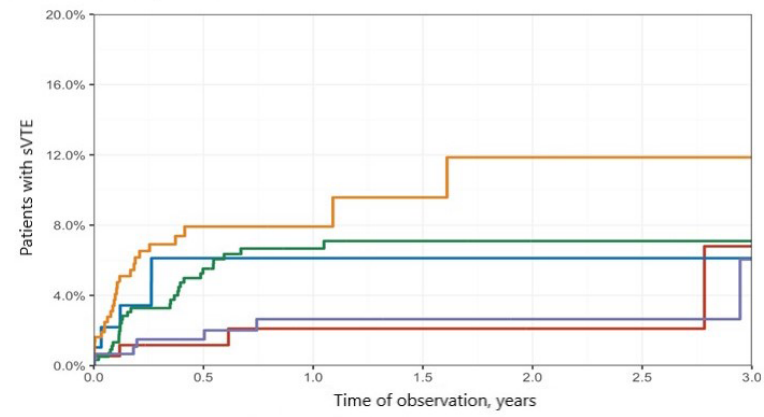

Pic. 1C. CIR of sVTE episodes

$={ }^{\mathrm{AA}}=\mathrm{Hc}=\mathrm{Lymphoma}=\mathrm{ALL}=\mathrm{AML}$

FIGURE 1 CIR of VTE in Children with Different Blood Disorders

3-year CIR of VTE is found to be $34,8 \%(95 \% \mathrm{Cl} 31,3-38,7 \%$, Pic. 1A.). Most of the VTE episodes were presented as aVTE (3-year $\mathrm{CIR}=28,9 \%$; $95 \% \mathrm{CI} 25,7-32,5 \%)$ and 3-year CIR of sVTE was more than 3 times less (3-year $\mathrm{CIR}=8,2 \%$; 95\% CI 5,8-11,5\%). While highest aVTE rates were found in children with ALL and lymphomas, followed by AML, Hc and AA (Pic. 1B.), the majority of sVTE episodes were diagnosed in lymphoma, followed by $A L L, H c, A M L$ and AA. More than a half of sVTE episodes were diagnosed in the first
6 months of treatment, with majority of episodes in children with lymphoma, $\mathrm{Hc}$ and $\mathrm{AML}$ diagnosed in first 3 months (Pic. 1C.).

Conclusions: 3-year CIR of VTE in hospitalized children with different blood diseases is as high as $34,8 \%(95 \% \mathrm{Cl} 31,3-38,7 \%$, Pic 1A.) and is presented mainly with aVTE. The highest CIR of sVTE episodes were found in patients with lymphomas with majority of episodes diagnosed in first 3 months of treatment.

PB0793 | In Children Receiving Long-term Anticoagulation Decreased Bone Mineral Density is Associated with Pubertal Development

K. Thom; C. Male; F. Haufler; A. Raimann

Medical University Vienna, Childrens Hospital, Vienna, Austria

Background: Children with chronic diseases are at increased risk of secondary osteoporosis. In adults, long-term anticoagulation (LTA) including Vitamin $\mathrm{K}$ antagonist (VKA) treatment has been associated with an increased risk of osteoporosis and hip fractures. Risk factors of impaired skeletal health and the role of LTA on bone metabolism during the vulnerable phase of linear bone growth in children remain poorly understood.

Aims: To assess bone mineral density (BMD) and to identify risk factors for decreased BMD in children with chronic diseases receiving LTA.

Methods: Children under LTA were included in a cross-sectional approach to assess age, height and sex-adjusted BMD on a Hologic QDR 4500. Further investigations included anthropometric and biochemical markers for skeletal metabolism, and carboxylationspecific osteocalcin as marker for Vitamin $\mathrm{K}$ status.

Results: 39 children (age 4-18 years) were included, 31 (79\%) on VKA and 8 (21\%) on direct oral anticoagulants. Mean BMD was decreased for both lumbar spine (-0.7SDS) and total body less head (TBLH; -1.32SDS). Earlier pubertal stages were strongly associated with decreased BMD-TBLH (-1.9SDS; $P=0.01$ ). Additionally, low body mass index (BMI) correlated significantly with reduced BMD $\left(R^{2}\right.$ 0.24; $\left.P=0.003\right)$. Neither LTA type, intensity nor treatment duration were associated with $\mathrm{BMD}$ alterations. Interestingly, the undercarboxylated osteocalcin fraction was strongly correlated with patients' age $\left(R^{2} 0.28 ; P<0.001\right)$ but not with BMD. Vitamin $D$ deficiency was detected in $26 \%$ of patients with significantly lower values after onset of puberty $(-34.5 \% ; P=0.03)$.

Conclusions: In children with chronic diseases under LTA we found substantially reduced BMD. While type of anticoagulation was not associated with BMD, low BMI and delayed progression of puberty represent important risk factors for decreased BMD in children. Awareness of potential modifiers of BMD and high rates of Vitamin $D$ deficiency in pubertal patients could contribute to improve bone health in this vulnerable patient group. 


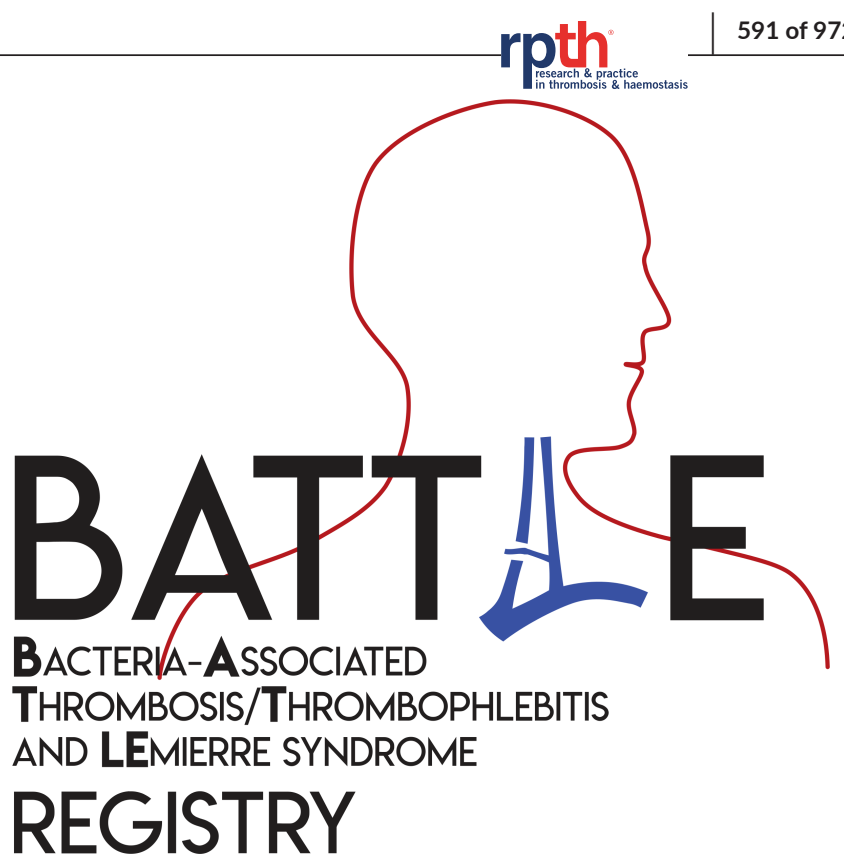

PB0794 | The Bacteria-associated Thrombosis,

Thrombophlebitis and LEmierre Syndrome (BATTLE) Registry:

Background and Rationale

L. Valerio ${ }^{1}$; G. Corsi ${ }^{2}$; T. Sebastian ${ }^{3}$; S. Barco ${ }^{3}$

${ }^{1}$ Center for Thrombosis and Hemostasis, University Medical Center of the Johannes-Gutenberg University, Mainz, Germany; ${ }^{2}$ Department of Clinical, Integrated and Experimental Medicine (DIMES), University of Bologna, Bologna, Italy; ${ }^{3}$ Angiology Clinic, University Hospital Zurich, Zurich, Switzerland

Background: Bacteria-associated (septic) thrombophlebitis and especially its head/neck form, Lemierre syndrome, which affects children, adolescents and young adults typically causing jugular vein thrombosis, are still associated with considerable fatality and long-term sequelae. Yet, available evidence is limited to case reports or series and is therefore fragmented, susceptible to bias, and inadequate to guide clinical management. Aims: To increase the level of evidence on Lemierre syndrome and septic thrombophlebitis, support the development of guidelines and consensus statements, and ultimately improve care for these patients.

Methods: The Lemierre Study Group designed the Bacteria-Associated Thrombosis, Thrombophlebitis and LEmierre syndrome (BATTLE) registry specifically to (i) fill the gaps in amount and quality of evidence on the continuum of Lemierre syndrome and bacterial thrombophlebitis by addressing reporting, detection and case-mix bias, (ii) obtain information useful to guide management, and (iii) comply with current international guidelines on quality, standardization and security of patient registries, including the FAIR framework and the European Commission Joint Research Centre's recommendations.

Results: The BATTLE registry is an independent, investigator-initiated and academically sponsored, noninterventionist, multicenter, international, ambispective study. Inclusion criteria are (1) invasive bacterial infection, (2) primary infection in the head/neck (Lemierre syndrome subgroup) or abdominopelvic district, and (3) thrombosis or embolism in location anatomically consistent with the primary infection focus (Table). Data entry is electronic only through the website www.battl e-registry.com (Figure). The electronic case report form is designed by and for physicians, includes the European Commission's set of common data elements for Rare Disease Registration with no patient identifiers to ensure compliance with local ethical requirements, and covers patient history, clinical course until death or discharge, and long-term follow-up including assessment of functional status and quality of life.

FIGURE 1 Logo of the BATTLE registry

Conclusions: The innovative features of the BATTLE registry may contribute to supporting clinical management for patients with a group of rare but severe acute thromboembolic disorders.

PB0796 | Results of a Multinational Survey of Diagnosis, Practices and Expertise in the Management of Venous Thromboembolism (VTE) in Pediatric Patients with Sickle Cell Disease (SCD)

\section{Betensky ${ }^{1}$; R. Kumar ${ }^{2}$; N.A. Goldenberg ${ }^{3}$}

${ }^{1}$ Johns Hopkins School of Medicine and Johns Hopkins All Children's Hospital, St. Petersburg, United States; ${ }^{2}$ Boston Children's Hospital, Boston, United States; ${ }^{3}$ Johns Hopkins School of Medicine and Johns Hopkins All Children's Hospital, St. Petersburg, United States

Background: Pediatric-specific data are lacking to guide recommendations for prevention and management of VTE in pediatric SCD. To date, experience and expert opinion in this area has not been reported.

TABLE 1 Inclusion criteria of the BATTLE registry

Bacteria-associated thrombophlebitis

Lemierre syndrome subgroup
1. Invasive bacterial infection

clinically or microbiologically diagnosed

2. Primary non-vascular infection not in head/ neck district

anatomically consistent with venous

thromboembolism

based on history or objective diagnosis

3. Thrombosis and/or septic embolism

in vein anatomically consistent with location

of primary non-vascular infection

objectively diagnosed
2. Primary non-vascular infection in head/neck district based on history or objective diagnosis

3. Thrombosis and/or septic embolism in head/neck vein objectively diagnosed 
Aims: We sought to characterize the experience and management practices of VTE in pediatric SCD via a multinational online survey of pediatric hematologists. We hypothesized there is substantial variability in preferences on the type and duration of anticoagulant therapy and thromboprophylaxis.

Methods: A Qualtrics ${ }^{\mathrm{TM}}$ survey was emailed to pediatric hematologists members of the International Society on Thrombosis and Haemostasis, and the Hemostasis and Thrombosis Research Society (January-February 2021). Descriptive statistics were used to summarize results.

Results: The response rate was $42 \%$ (141 surveys emailed, 58 total responses, 52 complete responses analyzed). Table 1 shows the responders characteristics. Two-thirds (68\%) of physicians treated at least one patient with SCD-associated VTE during the preceding 12 months. Ninety-eight percent reported to "always" use anticoagulation for symptomatic VTE, 78\% for asymptomatic pulmonary embolism (PE) and 56\% for asymptomatic deep venous thrombosis (DVT). Table 2 shows preferred agents for VTE treatment. Lowmolecular-weight heparin was the preferred agent used for prevention of hospital-acquired VTE. Duration of therapy varied by VTE type, $95 \%$ of physicians prescribed 6 weeks-3 months for provoked DVT and $67 \%$ for provoked PE with the remaining $1 / 3$ treating for 6-12 months. For unprovoked VTE, 62\% treated for 6-12 months, while $25 \%$ prescribed a shorter 6 weeks- 3 months course. The most challenging issue identified was determining the optimal duration and intensity of anticoagulation for secondary prophylaxis.

TABLE 1 Responders characteristics

\begin{tabular}{l|c|}
\hline Table 1. Characteristics & Total $\mathbf{n = 5 2 ( 1 0 0 \% )}$ \\
\hline Years in practice & \\
$<5$ years & $14(27)$ \\
5 - 10 years & $12(23)$ \\
$>10$ years & $26(50)$ \\
\hline Primary Practice Setting & \\
Academic Adult and Pediatric & $12(23)$ \\
Academic Pediatric only & $37(72)$ \\
Community Adult and Pediatric & $2(4)$ \\
Community Pediatric only & $1(2)$ \\
\hline Country & \\
USA & $47(91)$ \\
Other & $5(10)$ \\
\hline Number of patients with SCD followed at & \\
primary institution & \\
$<100$ & $9(18)$ \\
100 - 499 & $18(35)$ \\
$>500$ & $25(48)$ \\
\hline Number of patients with SCD and VTE treated & \\
during the preceding 12 months & \\
None & \\
1 - 2 & $17(33)$ \\
3 - 5 & $24(47)$ \\
$>6$ & $10(20)$ \\
n= number; SCD= sickle cell disease; VTE= venous \\
thromboembolism Other= Canada, Netherlands \\
\hline
\end{tabular}

TABLE 2 Physicians anticoagulant agent of preference for SCDassociated VTE by Age group and clinical setting

$\begin{array}{lll}\begin{array}{ll}\text { Agent and Setting } \\ \text { Inpatient setting }\end{array} & \begin{array}{l}\text { Age Group 0-<18 } \\ \text { years 18 - 21 years }\end{array} \\ \text { Unfractionated Heparin no. (\%) } & 6(12) & 6(12) \\ \text { Low Molecular Weight Heparin no. (\%) } & 50(97) & 33(64) \\ \text { Warfarin no. (\%) } & 1(2) & 1(2) \\ \text { Direct oral anticoagulant no. (\%) } & 2(4) & 18(35) \\ \text { Outpatient setting } & & \\ \text { Low molecular weight heparin no. (\%) } & 48(93) & 10(20) \\ \text { Warfarin no. (\%) } & 3(6) & 1(2) \\ \text { Direct oral anticoagulant no. (\%) } & 9(18) & 40(77)\end{array}$

Conclusions: This survey demonstrates variability in practice patterns in the management of SCD-related VTE, and identifies that optimal duration and intensity of secondary thromboprophylaxis is unclear. These findings highlight the need for cooperative multicenter studies to identify VTE prognostic factors and outcomes of pediatric SCD-related VTE, to inform future interventional studies.

\section{PB0797 | Once a Day Compared to Twice a Day Enoxaparin} in Children with Venous Thromboembolism: A Single Center Experience

N. Montanez ${ }^{1}$; A. Gibson ${ }^{2}$; K. Addy ${ }^{1}$; S.S. Hashmi ${ }^{3}$; D. Brown ${ }^{1}$; N. Rodriguez ${ }^{1}$; N. Menon ${ }^{1}$; L. Srivaths ${ }^{1}$

${ }^{1}$ University of Texas Health and Science Center of Houston, McGovern Medical School, Gulf States Hemophilia and Thrombophilia Center, Houston, United States; ${ }^{2}$ University of Texas MD Anderson Cancer Center, Pediatric Hematology Oncology Fellow, Houston, United States; ${ }^{3}$ University of Texas Health and Science Center of Houston, McGovern Medical School, Houston, United States

Background: Once a day enoxaparin (ODE) is considered standard of care (SOC) for venous thromboembolism (VTE) in adults, with few pediatric studies showing variable clinical results. We sought to review our center's experience with ODE in treating pediatric VTE and to compare its efficacy with twice a day enoxaparin (TDE), considered SOC. Aims: To compare efficacy of ODE to TDE in pediatric VTE. Methods: A retrospective chart review of children with VTE treated with ODE or TDE from 2016-2021 was undertaken, with IRB approval and waiver of consent. Data about patient demographics, VTE, treatment and complications were collected.

Results: Seventy-one children with VTE met the eligibility criteria; 39 (55\%; median age $0.99 \mathrm{yrs}$; range $0-17$; 44\% F) were treated with ODE (median dose $1.5 \mathrm{mg} / \mathrm{kg}$; IQR 1.2-2.0) and 32 (44\%; median age $0.46 \mathrm{yrs}$; range $0-17 ; 38 \% \mathrm{~F}$ ) were treated with TDE (median dose 1.1 $\mathrm{mg} / \mathrm{kg}$; IQR 1.0-1.4). ODE patients were initially treated with TDE (median 14 d; IQR 10-14), before switching to ODE. Most common site of VTE was extremity, more common in ODE than TDE (ODE $N=21,54 \%$ vs. TDE $N=16,23 \%, P=0.033$ ) and most common 
risk factor was central line (ODE $N=29,74 \%$ vs. TDE $N=19,60 \%$ ) for both cohorts. There was no statistically significant difference in the total duration of therapy ( $\geq 3 \mathrm{mo}$; ODE $95 \%$, TDE $91 \% ; P=0.276$ ). Bleeding complications were rare (ODE: $N=2,5 \%$; TDE N = 5, 15.6\%; $P=0.231)$. ODE and TDE had no difference in outcome $(P=1.000)$, with all patients having resolved, improved, or stable VTE.

Conclusions: ODE for pediatric VTE therapy appears to be equally efficacious as TDE, with $100 \%$ achieving favorable outcome in both cohorts. Less frequent injections with ODE may also potentially improve patient adherence. Larger randomized trials between ODE and TDE will help validate our center's observation.

\section{PB0798 | Validation of a de novo Paediatric Warfarin Nomogram}

S. Jones ${ }^{1,2,3}$; P. Monagle ${ }^{1,4,3} ;$ A. Allan ${ }^{2} ;$ S. Chen ${ }^{2} ;$ K. Dawson ${ }^{2}$; A. Kuzmitsky ${ }^{2}$; M. Pham-Crepps ${ }^{2}$; E. Renner-Hahn²; A. Greenway ${ }^{1}$; F. Newall ${ }^{1,2,3}$

${ }^{1}$ The Royal Children's Hospital, Department of Clinical Haematology, Parkville, Australia; ${ }^{2}$ University of Melbourne, Department of Nursing, Calton, Australia; ${ }^{3}$ Murdoch Children's Research Institute, Parkville, Australia; ${ }^{4}$ University of Melbourne, Calton, Australia

Background: Warfarin therapy in children is impacted by many variables, including diet and concurrent illness. To support the implementation of a self-management program within a paediatric anticoagulation service, a paediatric-specific warfarin nomogram was needed. A literature review revealed no published paediatric nomograms therefore a de novo nomogram was developed drawing upon the hospital's warfarin management evidence-based guideline.

Aims: This study aimed to validate a paediatric warfarin nomogram. Methods: A retrospective audit of electronic medical records compared the dosing and international normalised ratio (INR) retest decisions made by haematology clinicians to the dosing and retesting recommended by a de novo warfarin nomogram at a tertiary pediatric hospital. Children (aged six months-18 years) on warfarin therapy for longer than six months were included. Data was collected between September 2019 and February 2020. Descriptive data analysis was performed and the study was approved by the hospital human research ethics committee. Results: Warfarin dosing and INR retest decisions made by haematology clinicians for 25 children were included, equating to 396 INR tests. The nomogram matched $79.3 \%$ of clinicians dosing decisions and $23.5 \%$ of INR retest decisions. $53 \%$ of the recommended retest dates were earlier than the nomogram's recommendation. In the INR 2.0-3.0 group, $62.5 \%$ of INR results were within the TTR and $83.0 \%$ of dosing decisions matched the nomogram. In the INR 3.04.0 group, only $43.5 \%$ of INR results were within the target range and the nomogram matched $65.9 \%$ of dosing decisions.

Conclusions: These preliminary results suggest this de novo paediatric nomogram is reliable and valid tool for warfarin dosing, as recommended warfarin doses matched the majority of clinicians' dosing decisions. Dosing decisions and INR retesting recommendations from the nomogram most accurately matched haematology team decisions when the INR was in range. Further analysis is needed confirm the validity of the nomogram in children with unstable INRs.

PB0799 | Pediatric Thromboembolism in Chiang Mai University Hospital: A 10-year Review

P. Rahoorak; A. Jaengkrajang; K. Mankan; P. Wongwai;

P. Pisanuwong; K. Fanhchaksai; R. Natesirinilkul

Faculty of Medicine, Chiang Mai University, Chiangmai, Thailand

Background: Thromboembolism (TE) is less prevalent in pediatric population than adult population. However, the incidence of pediatric TE has been increasing over the last decades. Although the risk factors of pediatric TE were published numerously, most reports were studied in Western countries which the settings were quite different from Thai population.

Aims: To find the current incidence of TE and to determine the risk factors of TE in Thai pediatric population.

Methods: A retrospective case-control study was performed in Faculty of Medicine, Chiang Mai University between 1 January 2008 and 31 December 2018. All patients with TE were diagnosed by imaging studies and categorized as the case group while the age- and gender-matched pediatric inpatients without TE were categorized as the control group. The demographic data and the risk factors of TE were collected.

Results: We enrolled 129 cases and 300 controls. Fifty-six per cent were male and the median age was 1.8 years (min-max, 0-19.5) but both parameters did not differ between both groups. The incidence of TE was 3: 1,000 pediatric admission including 74 with venous TE, 54 with arterial TE and one with combined venous and arterial TE. The incidence of TE was highest in patients aged between 1 month and 1 year. Surgery, heart disease, trauma and center venous catheter insertion were the risk factors of TE in this study $(P=0.006,0.021$, $0.011,<0.001$, respectively). Moreover, patients who had shock, requiring inotropic drugs and receiving steroids also had higher risks of TE development $(P=<0.001,<0.001$ and 0.009 , respectively) Conclusions: The incidence of TE in inpatient pediatric population is prevalent in this study. Several risk factors associate with TE in pediatric patients. Therefore, TE risk-stratified protocol and TE prophylaxis should be further investigated to prevent inpatient pediatric population who are at risk of TE development.

\section{PB0800 | Factors, Determining Thrombus Recanalization in Children with Different Blood Disorders}

P.A Zharkov; D.A Evstratov; K.A Voronin; D.V Fedorova;

A.V Pshonkin

Dmitry Rogachev National Medical Research Center of Pediatric Hematology, Oncology and Immunology, Moscow, Russian Federation

Background: VTE is a frequent complication of treatment in hospitalized children, but factors, potentially influencing thrombus recanalization rates are still unknown. 
Aims: To assess the factors that determine recanalization in children with symptomatic(sVTE) and asymptomatic VTE(aVTE).

Methods: Data of 533 VTE in 1962 hospitalized children aged 0-17 years with ALL, AML, lymphoma, histiocytosis(Hc) and aplastic anemia(AA) was retrospectively analyzed. Information regarding clinical symptoms, thrombus size, type, intensity and time of antithrombotic treatment(ATT) and thrombus recanalization (full/partial) were collected. VTE episodes were divided in SVTE and aVTE. ATT(performed/not performed), therapy quality(correct/incorrect), therapy time(1 month incremental) and diagnosis were referred to ALL cases and added to multivariant logistic regression model( $R$, the $R$ Project, 4.0.3). ATT was considered as correct in cases when: It was started in 3 days after VTE evaluation; Thrombolytic therapy or surgical thrombectomy performed in cases of life/organ-threating VTE; UFH or LMWH was prescribed in non- life/organ-threating VTE; Close radiographic monitoring with risk reevaluation was performed in cases when there were absolute contraindications for ATT.

Results: In our mixed subset of patients such parameters as therapy, its duration or quality did not show any statistically significant impact on the rate of recanalization in patients with aVTE. In this group, when referred to ALL, OR of recanalization depended only on diagnosis and was highest in patients with lymphoma(7,63; 95\% Cl 3,09-23,13, $P<0.001)$, followed by $\mathrm{AML}(3,15 ; 95 \% \mathrm{Cl}$ 1,39-7,69, $\mathrm{P}=0,008)$. As for $\mathrm{VVTE}$ cases, diagnosis of lymphoma and the fact of correct therapy increased the chance of thrombus recanalization in $4,41(95 \% \mathrm{Cl} 1,31-18,62, \mathrm{P}=0,025)$ and 4,35 times $(95 \% \mathrm{Cl} 1,44-13,83, P=0,01)$, respectively.

Conclusions: Patients with lymphoma have a better prognosis for aVTE and sVTE recanalization, while correct ATT increases the chance of favorable outcome only in the sVTE cases. Summarizing our previously discovered data, it seems that cases of aVTE could potentially have another nature and does not respond to ATT.

PB0801 | A Rare Case of Thrombotic Storm in HenochSchonlein Purpura

A.K. Baylon; H.P. Tan; M.L. Naranjo; C. Fernandez; R. Anastacio; C.R. Castro

Hematology Center, National Children's Hospital, Quezon City, Philippines

Background: Henoch Schonlein Purpura (HSP) is a common vasculitis in children. Thrombotic events rarely occur. In the extreme end of the spectrum of thrombosis, a condition called thrombotic storm was seen to occur in a patient with HSP.

Aims: This case report aims to discuss the clinical course and management of thrombotic storm as a complication of Henoch-Schonlein Purpura in a female adolescent patient.

Methods: This a case report of a female adolescent patient presenting with thrombotic storm as a complication of Henoch-Schonlein Purpura.
Results:
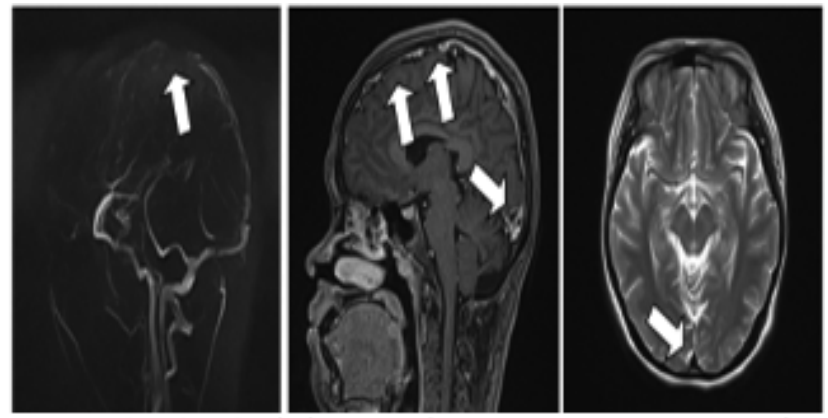

Figures 1a, 1b, 1c (left to right): MRA showing paucity of contrast flow in the superior sagittal sinus (1a); T1 MPRAGE showing multiple filling defects within the anterior and posterior portions of the superior sagittal sinus (1b); T2 axial showing an "empty delta sign" signifying a hypointense thrombus (1c)

FIGURE 1 (a-c) MRAs

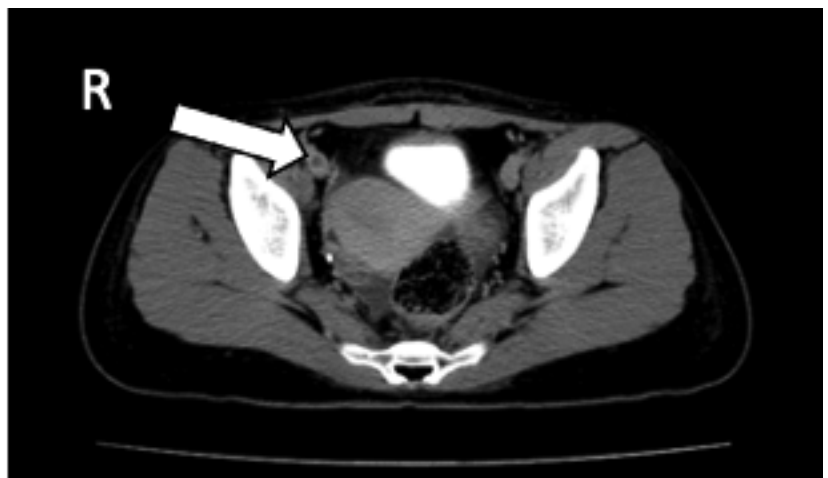

Figure 3. Contrast abdominal CT scan showing a partial luminal filling defect on the right common iliac vein, reflective of a thrombus formation

FIGURE 2 Contract abdominal CT scan

A previously healthy 14 year old female was diagnosed with HSP and unexpectedly developed multiple sites of thrombosis (deep venous thrombosis, superior sagittal sinus thrombosis and transverse sinus thrombosis). This led to the suspicion of a rare, accelerated thrombotic event known as thrombotic storm. Despite being on enoxaparin, she went on to develop common iliac vein thrombosis, which strengthened the diagnosis of thrombotic storm. Anticoagulation was continued, after which, there was marked clinical improvement. Anticoagulation was continued after discharge, with noted resolution of DVT on the second month of treatment. Further improvement was seen with resolution of common iliac vein and superior sagittal sinus thrombosis on the seventh month of anticoagulation and resolution of transverse sinus thrombosis on the $16^{\text {th }}$ month of anticoagulation. The patient remains to be asymptomatic.

Conclusions: Thrombotic storm occurring as a complication of a vasculitic disease strengthens the link between inflammation and thrombosis. Since there is no specific test to diagnose thrombotic 
storm, the clinician's awareness of this syndrome is key, so that treatment is started immediately upon recognition of the symptoms. Once the thrombotic cascade resolves, prognosis is excellent, however lifelong anti-coagulation is recommended to prevent recurrent thrombosis.

PB0802 | Inherited Antithrombin Deficiency in Childhood: A Multicenter National Study

A. Unuvar ${ }^{1}$; N. Sarper ${ }^{2}$; U. Demirsoy ${ }^{2}$; I. Eker ${ }^{3} ;$ N. Eroglu ${ }^{3} ;$ Z. Kaya ${ }^{4}$; D. Aslan ${ }^{4}$; N.O. Karadas ${ }^{5}$; K. Kavakli ; N. Ozbek ${ }^{6}$; N. Yarali ${ }^{6}$; A. Canbolat'; T. Celkan ${ }^{8}$; M. Evim ; S. Karaman ${ }^{1}$; H. Oren ${ }^{10}$

${ }^{1}$ Istanbul University, School of Medicine, Division of Pediatric Hematology \& Oncology, Istanbul, Turkey; ${ }^{2}$ Kocaeli University School of Medicine, Kocaeli, Turkey; ${ }^{3}$ Afyonkarahisar University School of Medicine, Afyonkarahisar, Turkey; ${ }^{4}$ Gazi University School of Medicine, Ankara, Turkey; ${ }^{5}$ Ege University School of Medicine, Izmir, Turkey; ${ }^{6}$ Ankara City Hospital, Ankara, Turkey; ${ }^{7}$ Medeniyet University School of Medicine, Istanbul, Turkey; ${ }^{8}$ Istanbul University-Cerrahpasa School of Medicine, Istanbul, Turkey; ${ }^{2}$ Uludag University School of Medicine, Bursa, Turkey, ${ }^{10}$ Dokuz Eylul University School of Medicine, Izmir, Turkey

Background: Inherited antithrombin (AT) deficiency (IATD) is a rare but strong risk factor for development of serious thrombosis. It is a challenging condition due to very limited data in childhood.

Aims: The aim of this study was to evaluate the clinical characteristics and outcome in children with IATD by a multicenter national study.

Methods: A questionnaire form was sent to collect the children with IATD (AT activity levels< $70 \%$ ) to all Pediatric Hematology\&Oncology centers on behalf of the Hemostasis, Thrombosis and Hemophilia Subcommittee of Turkish Society of Pediatric Hematology.

Results: Nine centers reported the detailed data of 19 children (12girls, 7boys) with IATD (2 patients were sibling). Sixteen patients were admitted with acute thrombosis and 3 asymptomatic children $(10,17,19$ years) for follow-up due to the family history.The youngest age for the first thrombosis was 8 days, and the oldest was 17.5 years. The age distribution had two peaks: Newborns/infants and adolescents. The lowest AT level was $<5 \%$, and the highest was $52 \%$. The family history was positive in 11 of 19 children, and the parents with consanguineous marriage was present in 5.Fourteen patients had DVT, and two patients had arterial thrombosis. The DVT sites of 14 patients were as follows:lower extremity DVT ( $n: 5)$, CSVT ( $n$ : $4)$, renal vein $(n: 3)$, rom left hepatic veins to vena cava inferior ( $n: 1)$, upper extremity DVT $(n: 1)$. Nine patients had acquired risk factors for thrombosis such as infections, catheters, trauma, andone patient was at the 10th day of post-partum period. All patients are alive, 2 new patients are on the treatment of acute thrombosis. During the follow-up period, thrombosis complications developed as follows: PTS (n:4), extremity amputation, unilateral nephrectomy and bowel resection, renal atrophy, mild psychomotor retardation, hemiparesis, osteonecrosis in each patient.In addition, the recurrence with pulmonary embolism developed in 3 patients. Mutation analyses could be done for 7 children.Ten of 14 patients are on thrombosis prophylaxis with different agents.

Conclusions: In conclusion, acute and long-term management of IATD are very important. Patients and families should be informed of the additional risk factors which further increase the recurrence of thrombosis.

\section{PB0803 | Resolution Rates of Provoked Deep Vein Thrombosis and Pulmonary Embolism in Pediatric Patients Managed at a Tertiary Care Center}

M. Dhaliwal; A. Chan; M. Bhatt

McMaster University, Hamilton, Canada

Background: The incidence of deep vein thrombosis (DVT) and pulmonary embolism (PE) is increasing in children, provoked by multiple risk factors such as central vascular catheters (CVCs), cancer, infection, and estrogen therapy. Like adults, pediatric patients with provoked DVT/PE are treated with anticoagulation for 3 months as long as risk factors are resolved. There is limited evidence regarding resolution rates in pediatric patients at the end of 3-month treatment.

Aims: To determine rates of complete resolution (CR), partial resolution (PR), and stable thrombosis (ST) in pediatric patients with provoked DVT/PE.

Methods: Retrospective chart review of pediatric patients with DVT/PE diagnosed in or admitted to McMaster Children's Hospital between 2008 and 2020. Data extraction included demographics, thrombosis diagnosis, management, and follow-up imaging.

Results: 150 patients were diagnosed with 171 clots (Upper extremity DVT: 78, lower extremity DVT: 56, PE: 37). Median age was 13 years and male to female ratio was 0.83:1. Of the DVT patients, 85 had occlusive DVT and 49 had non-occlusive DVT. Common provoking factors were CVCs (55.3\%), infection (29.3\%), surgery (27.3\%), and oral contraceptives (16\%). The most frequently used anticoagulants were low-molecular-weight heparin (90\%), direct oral anticoagulants (5.3\%), and warfarin (3.3\%). Two patients had surgical thrombectomy. Out of 110 patients that had a 3-month follow up, the rates of CR, PR, and ST were $56.4 \%, 30.9 \%$, and $12.7 \%$, respectively. Three patients (2\%) had recurrent thrombosis.

Conclusions: The rate of $\mathrm{CR}$ in pediatric patients with provoked DVT/PE is comparable to adults. Larger prospective studies are required to validate findings. 
PB0804 | Acquired von Willebrand Disease in a Child as Leading Symptom of Systemic Lupus Erythematosus

F. Achini-Gutzwiller ${ }^{1}$; K. Ruchti ${ }^{1}$; G. Spartà ${ }^{1}$; K. Ruoss ${ }^{1}$; S. Prader ${ }^{1}$; J. Pachlopnik Schmid ${ }^{1}$; J.A. Kremer ${ }^{2}$; S. Palmer Sarott ${ }^{1}$; M. Albisetti ${ }^{1}$

${ }^{1}$ University Children's Hospital, Zürich, Switzerland; ${ }^{2}$ Bern University Hospital, University of Bern, Bern, Switzerland

Background: In children, acquired von Willebrand disease (AvWD) is an extremely rare bleeding disorder mainly related to critical clinical conditions. Congenital heart diseases associated with increased shear stress and myeloproliferative neoplasia with thrombocytosis are the most prevalent causes of AvWD in children. Single cases of paediatric AvWD associated with rare autoimmune disorders including systemic lupus erythematosus (SLE) and Hashimoto's thyroiditis have been reported. However, pathophysiological mechanisms potentially linking the development of AvWD to acquired immune dysregulation as well as clinical implications in children remain incompletely defined.

Aims: We report clinical and laboratory findings of a child with treatment-refractory $V W D$ type 3-like AvWD as early manifestation of SLE and explore immunological mechanisms underlying this particular condition.

Methods: Case report.

Results: A 12-year-old female patient with no history of bleeding disorders presented with moderate thrombocytopenia and hemodynamic relevant anemia (hemoglobin $48 \mathrm{~g} / \mathrm{l}$ ) due to hypermenorrhea. Further laboratory investigations revealed markedly reduced FVIII $(0.08 \mathrm{U} / \mathrm{ml})$, von Willebrand Factor (vWF) ristocetin cofactor (0.07 U/ml), vWF activity (0.08 U/ml) and vWF antigen (0.03 U/ml). Non-neutralizing anti-vWF IgG were found while screening for further autoimmunity showed an enhanced antinuclear antibody (ANA) titer (1:640). Malignancy was excluded. Extended immunological analyses ruled out primary and acquired immunodeficiency. Initial response to infusion of plasma-derived vWF and immunoglobulins was poor. Menorrhagia was controlled with combined hormonal contraceptive pill and tranexamic acid. Six weeks later, the ANA titer increased and the patient developed proteinuria in nephrotic range while maintaining good renal function. Renal full-house SLE nephropathy was histologically confirmed. Following first-line treatment with intravenous steroid pulse and oral maintenance therapy with mycophenolate mofetil and steroids, vWF levels increased significantly.

Conclusions: Severe AvWD is a rare manifestation of SLE which frequently presents with serious features in children. Early recognition of this singular hemostatic phenomenon may significantly impact treatment and prognosis of the underlying autoimmune disorder.
PB0805 | Preliminary Results of the Adventh-pediatric Registry in a Southeastern Brazilian City

T. Mello ${ }^{1}$; S. Rigatto ${ }^{1} ;$ R. Vilela ${ }^{1} ;$ M. Veríssimo ${ }^{2}$; T. Rodrigues ${ }^{1}$;

G. Nunes ${ }^{1}$; S. Huber ${ }^{1}$; V. Pinheiro ${ }^{1}$; J. Pires ${ }^{2}$; S. Montalvão;

J. Annichino-Bizzacchi ${ }^{1}$

${ }^{1}$ State University of Campinas - UNICAMP, Campinas, Brazil; ${ }^{2}$ Boldrini Hospital, Campinas, Brazil

Background: There is a lack of epidemiological data regarding pediatric venous thromboembolism (VTE) in Brazil.

Aims: A registry to evaluate the incidence of VTE in hospitalized children (0 to 16 years) in a southeastern Brazilian city.

Methods: A prospective multicenter study initially involving two tertiary hospitals, a pediatric cancer (Boldrini) and a nursery with ICU (Clinical Hospital, UNICAMP), in Campinas, SP-Brazil. All hospitalized patients were evaluated daily, through access to each patient's electronic medical record and those with a diagnosis of VTE by an objective method were included. All patients with a central venous catheter (CVC) were carefully observed and should undergo doppler ultrasound (US) with any sign of VTE. There was no search for asymptomatic VTE. Results: During Sept2018 to Feb2021, 971 children were hospitalized and 21 cases of VTE were diagnosed, an incidence of 2.1/100 hospital admissions. Clinical parameters were described in table 1. VTE was located in the upper ( $n=8 / 38.1 \%$ ) or lower limbs ( $n=10 / 47.6 \%)$, portal vein ( $n=1 / 4.8 \%$ ), and pulmonary embolism ( $n=1 / 4,8 \%$ ). Regarding presentation, 6(28.6\%) were incidental, 2(9.5\%) malfunctioning CVC and 13(61.9\%) with common signs of VTE. VTE was associated with CVC in 10 (47.6\%) cases. The mean time between CVC insertion and VTE diagnosis was 22,1 days ( \pm 43.2). The mean time between symptoms/diagnosis was $2,5( \pm 2.5)$ days. Risk factors for VTE were found in $95 \%$ of the children (table 2). Corticosteroids and asparaginase were the medications more commonly used in VTE patients $(33.3 \%$ and $4.8 \%$ vs. $22.1 \%$ and $0.1 \%$, respectively). Therapeutic anticoagulation was used only in 13 $(61.9 \%)$ patients, because of thrombocytopenia in those with cancer.

TABLE 1 Demographic and risk factors associated with VTE in children with and without VTE

\begin{tabular}{lll} 
Patients & Non VTE (N = 950) & VTE (N = 21) \\
Mean Age \pm SD & $6.6 \pm 5.3$ & $6.4 \pm 5.3$ \\
Sex (F/M) & $399 / 551$ & $7 / 14$ \\
Hospital & Boldrini 138 & Boldrini 9 \\
& HC nursery 603 & HC nursery 6 \\
& HC ICU 209 & HC ICU 6 \\
Bed Immobilization & $612(64.4 \%)$ & $12(57.1 \%)$ \\
Surgery during & $351(36.9 \%)$ & $4(19.0 \%)$ \\
$\quad$ Hospitalization & 32 & 0 \\
$\begin{array}{l}\text { Trauma } \\
\text { CVC }\end{array}$ & $262(27.6 \%)$ & $8(38.1 \%)$ \\
Hospitalization Time & $33.3 \pm 704.4$ & $11.8 \pm 33.3$ \\
(days) & & $14(66.7 \%)$ \\
ICU & $307(32.3 \%)$ & \\
\hline
\end{tabular}


TABLE 2 Risk factors associated with VTE

$\begin{array}{ll}\text { Patient's Risk Factors } & \\ \text { Cancer } & 4(19.0 \%) \\ \text { Gastrointestinal } & 2(9.5 \%) \\ \text { Congenital Heart Disease } & 2(9.5 \%) \\ \text { Kidney Disease } & 2(9.5 \%) \\ \text { Neurological Disease } & 1(4.8 \%) \\ \text { Others } & 3(14.3 \%)\end{array}$

Thrombosis Risk Factor

Immobilization

$9(42.8 \%)$

Infection

$14(66.7 \%)$

Surgery or Intravascular procedure

ICU

$8(38.1 \%)$

CVC

$10(47.6 \%)$
Conclusions: Preliminary data from the first Brazilian VTE pediatric registry indicate it is prevalent among hospitalized children, and largely associated to CVC.

Supported by a grant of FAPESP, number 2016/14172-6.

Approved by Ethics Research Committee of Unicamp.

PB0806 | A Prospective Data Collection Utilizing the International Pediatric Thrombosis Network (IPTN) to Understand the Epidemiology for the Development of Neonatal Renal Vein Thrombosis

G. Woods ${ }^{1}$; J. Motwani ${ }^{2}$; N. Sirachainan ${ }^{3} ;$ R. Berrueco ${ }^{4}$; C.H. van Ommen ${ }^{5}$; A.KC Chan ${ }^{6}$

${ }^{1}$ Aflac Cancer and Blood Disorders Center at Children's Healthcare of Atlanta and Emory University School of Medicine, Atlanta, United States; ${ }^{2}$ Birmingham Children's Hospital, Birmingham, United Kingdom; ${ }^{3}$ Ramathibodi Hospital and Mahidol University, Bangkok, Thailand; ${ }^{4}$ Hospital Sant Joan de Déu, Barcelona, Spain; ${ }^{5}$ Erasmus MC Sophia, Rotterdam, Netherlands; ${ }^{6}$ McMaster University, Hamilton, Canada

Background: Renal vein thrombosis is the most prevalent noncatheter associated neonatal thrombosis. Macroscopic hematuria, palpable abdominal mass, and thrombocytopenia are cardinal symptoms with prematurity, perinatal asphyxia, and inherited thrombophilia identified as common risk factors. Despite this knowledge, the epidemiology of neonatal renal vein thrombosis (nRVT) is still not completely understood and optimal management strategies are unclear.

Aims: We aimed to collect prospective international data to clarify the epidemiology and risk factors associated with nRVT and to describe appropriate management strategies, understand long term renal sequelae and identify risk factors for recurrent nRVT.

Methods: The International Pediatric Thrombosis Network (IPTN) registry was used to collect prospective data on $\mathrm{nRVT}$ including thrombosis characteristics, presenting symptoms, risk factors, and management.

Results: Three patients (two females) have been described in the registry. All were born prematurely, with gestational ages of 27, 33, and 35 weeks, had macroscopic hematuria as their only symptom, and were diagnosed by doppler ultrasound. Two patients had right nRVT with one having inferior vena cava extension, and one had a left nRVT. Two were treated with therapeutic anticoagulation for at least 3 months. The right nRVT without IVC extension had a history of a previous venous thromboembolism, recurrence in the contralateral renal vein and was treated with therapeutic anticoagulation for 8 months. Thrombophilia testing for this patient was unremarkable. The two right nRVT had renal atrophy at one year follow up.

Conclusions: Neonatal RVT are rare events with variable clinical characteristics and management strategies. An international registry to collect data could optimize the ability to understand nRVT risk factors and appropriate management approaches. Encouraging the international pediatric thrombosis community to utilize the IPTN (https://redcap.isth.org/surveys/?s=FPEDLXEF9A) to collect patient data would aid in the development of much needed international guidelines for nRVT management.

PB0807 | A Case of a Pediatric Patient with Protein S Heerlen Polymorphism and Deep Venous Thrombosis

M. Kacar; M. Bhatt

McMaster University, Hamilton, Canada

Background: Hereditary Protein S (PS) deficiency is an autosomal dominant disorder with increased risk of venous thromboembolism (VTE), prevalent in $0.5 \%$ of the general population. The PS Heerlen polymorphism is a rare mutation at codon 501 of the PS gene that was initially considered a variant of uncertain significance but has since been shown to have a reduced levels of free PS.

Aims: Description of PS Heerlen polymorphism in an adolescent with deep venous thrombosis.

Methods: Collection of clinical data from Hamilton Health Sciences Medical record.

Results: A female patient, 14-year-old, presented with history of left lower leg fullness was diagnosed with deep vein thrombosis. The patient recently had prolonged airline travel and was using combined oral contraceptives. Anticoagulation with low molecular weight heparin was initiated. MRI venogram showed a narrowed left common iliac vein with mild compression of the proximal left common iliac vein by right common iliac artery, consistent with May Thurner syndrome. After 9 months of anticoagulation, a pro-thrombotic work up was initiated, including antithrombin III, protein C, Factor V Leiden, anticardiolipin antibodies, prothrombin gene mutation, which were normal. Free PS was decreased, $0.60 \mathrm{U} / \mathrm{mL}$ (some pediatric ranges reported, but not established). Peripheral blood was sent for DNA 
sequencing of Protein S, positive for heterozygous missense mutation variant, c.1501T>C, p.(Ser501Pro), (Heerlen polymorphism) in exon 13. The patient has continued on prolonged anticoagulation.

Conclusions: The association between PS Heerlen and VTE is controversial. In the pediatric population, PS deficiency alone generally increases risk of VTE 3x. This patient case indicates that Heerlen polymorphism is a potential contributing risk factor for VTE, in combination with other prothrombotic states, such as oral contraceptives and anatomical variants (May Thurner). Pediatric patients with this polymorphism should be considered to have a genetic basis of PS deficiency and managed accordingly, including genetic counseling and consideration of secondary thromboprophylaxis.
PB0808 | Catastrophic Thrombotic Syndrome in 8 Year Old Boy:

\section{Clinical Case Report}

Y. Kashpurenko; K. Vilchevska; I. Bakhchyvandzhy

National Specialized Children'S Hospital 'Ohmatdyt', Center of

Hemostasis Pathology, Kiev, Ukraine

Background: Catastrophic thrombotic syndromes are characterized by rapid onset of multiple thromboembolic occlusions affecting diverse vascular beds. Patients with hypercoagulability may present with a single thrombosis and subsequently develop progressive thromboses at other sites and develop them rapidly over days to weeks.

Aims: To present the case of fatal thrombotic syndrome in a young boy.

Methods:

TABLE 1 Clinical presentation and treatment

\begin{tabular}{|c|c|c|c|c|c|c|c|}
\hline $\mathrm{Hb}$ & $99 \mathrm{~g} / \mathrm{L}$ & $\begin{array}{l}\text { D-dimer }(\mathrm{N}:<0.5 \mathrm{mcg} / \\
\mathrm{mL})\end{array}$ & 19,7 & $\begin{array}{l}\text { Ferritin (N: 7-140 } \\
\mathrm{mcg} / \mathrm{L} \text { ) }\end{array}$ & 382,5 & $\begin{array}{l}\text { Circulating immune } \\
\text { complexes (N:50- } \\
90 \mathrm{IU})\end{array}$ & 76,8 \\
\hline RBC & $4,56 \times 10^{12} / \mathrm{L}$ & $\begin{array}{l}\text { Anthi thrombin III } \\
\text { (N: 80-120\%) }\end{array}$ & $104 \%$ & $\begin{array}{l}\text { Folate } \\
(\mathrm{N}: 2,3-12 \mathrm{ng} / \mathrm{ml})\end{array}$ & 9,06 & $\mathrm{RF}$ & negative \\
\hline WBC & $9,2 \times 10^{9} / \mathrm{L}$ & Protein C (N:70-140\%) & $92 \%$ & $\begin{array}{l}\text { Homocysteine } \\
\text { (N:5-15 } \mu \mathrm{mol} / \mathrm{L})\end{array}$ & 15 & $\begin{array}{l}\text { Factor II mutation } \\
\quad(20210 \mathrm{G}>\mathrm{A})\end{array}$ & negative \\
\hline CRP & $64,6 \mathrm{mg} / \mathrm{L}$ & $\begin{array}{l}\text { Plasminogen } \\
(\mathrm{N}: 72-126 \%)\end{array}$ & $92 \%$ & $\begin{array}{l}\text { beta2-Glykoprotein } \\
\text { Ig G }\end{array}$ & negative & $\begin{array}{c}\text { MTHFR (C677T) } \\
\text { mutation }\end{array}$ & negative \\
\hline $\begin{array}{l}\text { aPTT } \\
\text { (N:26-36 sec) }\end{array}$ & 33,4 & $\begin{array}{l}\text { Inhibitor plasminogen } \\
\text { (N:89-112\%) }\end{array}$ & $91 \%$ & Lupus anticoagulant & negative & t-PA mutation & negative \\
\hline INR & 1,28 & $\begin{array}{l}\text { von Willebrand factor } \\
\qquad(\mathrm{Ag})(\mathrm{N}: 61-157 \%)\end{array}$ & $220,8 \%$ & ANA-Screen & negative & $\begin{array}{l}\text { PAI-1 mutation } \\
\text { ( } 6755 \mathrm{G}>4 \mathrm{G} \text { ) }\end{array}$ & heterozygous \\
\hline Fbg & $2,54 \mathrm{~g} / \mathrm{L}$ & $\begin{array}{l}\text { Factor XII } \\
\text { (N:50-150\%) }\end{array}$ & $112 \%$ & c-ANCA & negative & $\begin{array}{l}\text { TNFa } \\
\text { (G308A) }\end{array}$ & heterozygous \\
\hline
\end{tabular}

374,1

TABLE 2 Laboratory investigation

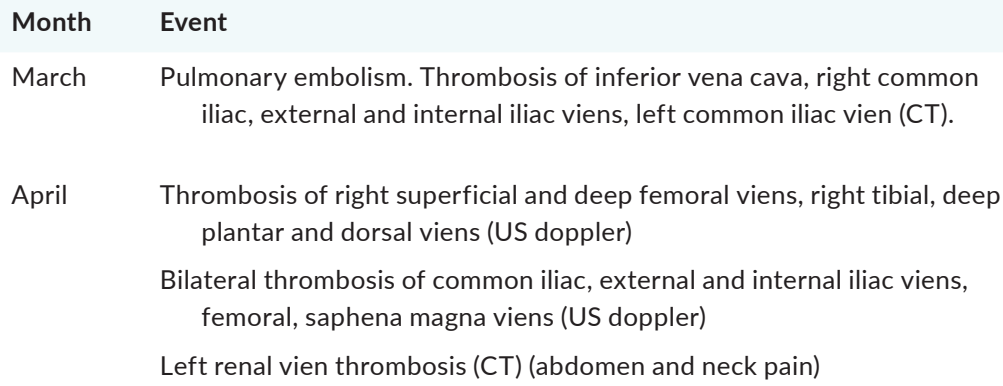

March Pulmonary embolism. Thrombosis of inferior vena cava, right common iliac, external and internal iliac viens, left common iliac vien (CT).

April Thrombosis of right superficial and deep femoral viens, right tibial, deep plantar and dorsal viens (US doppler)

Bilateral thrombosis of common iliac, external and internal iliac viens, femoral, saphena magna viens (US doppler)

Left renal vien thrombosis (CT) (abdomen and neck pain)

Treatment

tPA

IV unfractioned heparin infusion (UFH)

Continued on UFH

Continued on UFH

Continued on UFH 
Month Event

May

Thrombosis of left v.cephalica, v.axilaris, v.subclavia (US doppler)

Thrombosis of small vessels of the sigmoid colon with ischemia of the rectum (CT)

June

Extensive pulmonary embolism (CT)
Treatment

Warfarin per/os

Subcutaneous low molecular weight heparin (LMWH)

Subcutaneous low molecular weight heparin (LMWH)

$\mathrm{tPA}+\mathrm{UFH}$
In a previously healthy 8-year-old boy had been developed pain in the left side, legs, breathlessness, increased body temperature to 38,5C. Few days before trauma - falling from a tree. He had no hypercoaugulable disorders and other illnesses, family history was negative too. On examination reduced air entry was identified on the back lower lobes of the lung. His chest $x$-ray showed bilateral chest infiltrates. CT scan of the chest, abdomen and pelvis showed extensive bilateral pulmonary embolism with bilateral polysegmental lower lobe pneumonia, bilateral hemothorax, areas of thrombosis including inferior vena cava, right common iliac, external and internal iliac viens, left common iliac vien. Clinical presentation and treatment during staying at hospital - Table 1. Regular laboratory testing didn't show any significant abnormalities - Table 2.

Results: While receiving anticoagulation therapy, there was positive dynamic with $50 \%$ of recanalization previous located thromboses (by US doppler) and improving general well-being. But thrombotic syndrome continued to progress with complication by extensive pulmonary emboli. Despite systemic tPA and anticoagulation therapy the patient died.

Conclusions: We have described a fatal case of a young boy who developed multiple acute arterial and venous thrombotic events within 3 month. Catastrophic thrombotic syndrome is an infrequent but potentially fatal presentation of VTE in children. Generally recommended that patients with catastrophic macrovascular thrombotic events must be treated with a long-term antithrombotic therapy.

PB0809 | Severe Thrombosis Progression Despite Anticoagulation - Case Report of Antithrombin Deficiency

T. Quaresma ${ }^{1}$; A. Parente Freixo ${ }^{1}$; M.M. Deveza ${ }^{1}$; C. Peixoto ${ }^{1}$; J. Amorim ${ }^{1}$; A.J Fernandes ${ }^{2}$; M.J. Pereira ${ }^{2}$; Í.R. Oliveira ${ }^{2}$; M.J. Palare ${ }^{3}$; M. Galvão ${ }^{1} ;$ Á. Beleza ${ }^{1}$

${ }^{1}$ Centro Hospitalar Universitário Lisboa Norte - Imunohemoterapia, Lisbon, Portugal; ${ }^{2}$ Centro Hospitalar Universitário do Algarve -

Pediatria, Faro, Portugal; ${ }^{3}$ Centro Hospitalar Universitário Lisboa Norte - Pediatria, Lisbon, Portugal

Background: We report a case of a 16-year-old female suffering from unilateral left leg swelling and pain, with positive Homan's sign. She had started oral contraceptive one month before and had no family history of venous thromboembolic events(VTE). Ultrasonography with Doppler(UD) showed large thrombosis on left common iliac vein. Anticoagulation with enoxaparin $(1 \mathrm{mg} / \mathrm{kg} / \mathrm{q} 12 \mathrm{~h})$ was started.
On the following day the edema and pain got worse and a new UD and computed tomography venography showed thrombosis progression to the inferior vena cava reaching iliac-femoral-popliteal veins.

Aims: To evaluate and manage severe and refractory deep vein thrombosis(DVT).

Methods: We collected blood at expected peak level of enoxaparin (4 hours after administration) that showed anti-factor Xa plasma level of $0,01 \mathrm{U} / \mathrm{mL}$, on chromogenic assay, and a functional assay for plasma antithrombin activity (AT-heparin cofactor assay) of $38 \%$. There were no other thrombophilic defects except for an heterozigoty for Factor $\mathrm{V}$ Leiden (FVL) variant.

Results: In this context of thrombotic event at young age and decreased level of AT, the Antithrombin deficiency diagnosis was established. Treatment with antithrombin concentrate was started, targeting to a range between $80-120 \%$ of AT activity(Fig.1) while keeping the patient on enoxaparin, which lead to a significant clinical improvement. AT function and anti-Factor Xa were monitored(Fig.2). The patient was discharged with rivaroxaban $20 \mathrm{mg} /$ day.

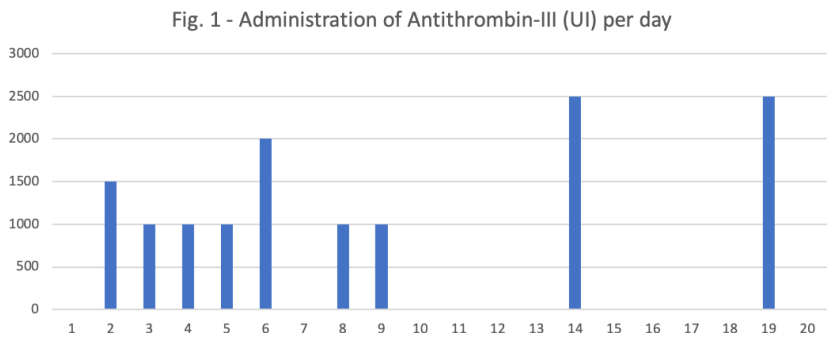

FIGURE 1 Administration of Antithrombin-III (UI) per day

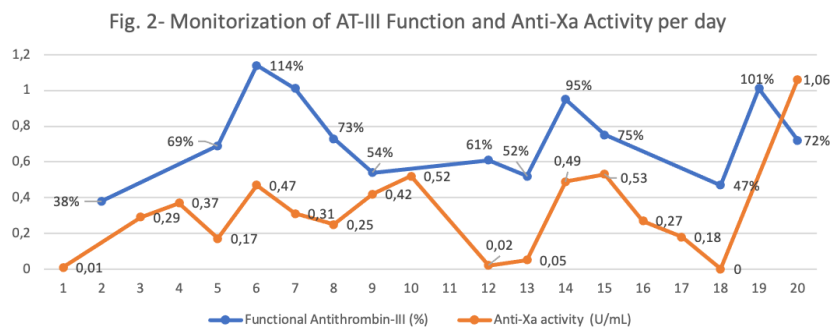

FIGURE 2 Monitorization of AT-III Function and Anti-Xa Activity per day 
Conclusions: Inherited Antithrombin deficiency is a rare condition, representing a risk factor for VTE. Two other conditions (estrogenic treatment and heterozigoty for FVL) are contributing factor for VTE. Although thrombophilia screening is not usually recommended during the acute phase of VTE clinicians must stay aware of AT deficiency while ruling out other causes for children/adolescent DVT, especially when anticoagulation with heparins fails.

Rivaroxaban was preferred for better patient adherence, supported by recent data on its efficacy and safety in pediatric venous thromboembolism.

PB0810 | A Comprehensive Review of Tumour Thrombus in Pediatric Wilms Tumour Patients

K. Kwok; A.K. Chan; M.D Bhatt; N. Samji

McMaster University, Hamilton, Canada

Background: Intravascular extension to the inferior vena cava and right atrium is a well-recognized complication of Wilms tumour (WT), with incidence of $4-10 \%$ and $0.7-1 \%$, respectively. Neoadjuvant chemotherapy is commonly used to decrease the size of the tumour thrombus prior to resection in cases of extensive vascular extension. Complications of intravascular tumour thrombus include pulmonary embolism, disease progression despite chemotherapy, and death.

Aims: Explore the utility, efficacy, and safety of anticoagulation in patients with intravascular extension of WT.

Methods: Searches conducted in PubMed used the following terms: Wilms*, anticoagula*, tumor thrombus, intracaval, and intra-atrial. Studies were included if patients had WT with intravascular extension. Data was retrieved from 8 case reports, 2 case series, and 14 retrospective analyses, one of which was abstract-only.

Results: Intravascular extension of WT was reported in 482 cases (6.9\%). Pulmonary embolism (PE) was described in four patients (retrohepatic[1], intracaval[1], and intra-atrial[2]) across three studies. Disease progression was reported in 13 patients in four studies and death was reported in 30 cases across all studies. Of 24 retrieved articles, two reported anticoagulant use adjunctively with chemotherapy: a retrospective analysis in 9/29 patients and one case report. The only documented complication was minor bleeding in one patient (10\%). Majority of cases did not report concurrent use of anticoagulation, apart from instances when patients underwent cardiopulmonary bypass or postoperatively after inferior vena cava manipulation.

Conclusions: Tumor thrombosis is an important complication in pediatric WT patients, however minimal literature exists addressing optimal management strategies including the utility of anticoagulation therapy. Literature suggests that renal vein thrombosis can lead to kidney damage. Use of anticoagulation may be considered to prevent propagation of the clot to the contralateral kidney. Risk of bleeding and of acquired von Willebrand Disease must also be considered prior to anticoagulation therapy. As limited literature is available, further study is needed.
PB0811 | Prevalence of Venous Thrombembolic Events in the Czech Pediatric Population - Epidemiological Study

T. Kuhn $^{1,2}$

${ }^{1}$ University Hospital Ostrava, Ostrava-Poruba, Czech Republic;

${ }^{2}$ University of Ostrava, Faculty of Medicine, Ostrava, Czech Republic

Background: Venous thrombolism (VTE) is uncommon in pediatric age. Published data confirm increasing evidence of rising incidence of pediatric VTE. There is a worldwide effort to establish the national and international databases and registries of of pediatric VTEs.

Aims: Based on our own clinical observations to describe the incidence of severe venous thrombosis in a representative sample of the Czech pediatric population.

Methods: Single study center survey in the university hospital settings which serves as a regional pediatric hematology centre for the population of more than 300 thousands of inhabitants with population of 62 thousands children. The task was to identify during 17 years (2004-2020) collection period patients aged 0-18 years with diagnosis pulmonary embolism (PE) (ICD 10 code - I 26.0), cerebral sinovenous thrombosis (CSVT) (167) and thrombotic events of the extremities (I 80, I82, 187). The survey was performed from the hospital registry.

Results: 109 patients with age span from 3 days till 17,99 years were detected having at least one episode of VTE. 16 (15\%) patients with PE (M:F ratio 0,33:1), median age 16,7 yrs

22 (20\%) patients with CSVT (M:F ratio 1,44:1), median age 6,8 yrs, and $71(65 \%)$ patients with thromosis in lower extremities (M:F ratio 0,92:1), median age 15,2 yrs

The annual incidence of pediatric VTE in our survey was 6,4 cases per year which accounts for $0,01 \%$ of the pediatric population.

Conclusions: This work is the first analysis of the VTE incidence in the Northern Moravia region. Together with similar studies from other cooperating Czech pediatric hematology centers it has a potential to establish the Czech national database of pediatric venous thrombembolic events.

PB0812 | A Case Report and Comprehensive Review of Acquired Purpura Fulminans in Children

S. Zarb $^{1}$; A.K. Chan ${ }^{2}$; V. Price ${ }^{3}$; M.D Bhatt ${ }^{2}$

${ }^{1}$ McMaster University, Hamilton, Canada; ${ }^{2}$ Division of Pediatric Hematology/Oncology, Department of Pediatrics, McMaster Children's Hospital, McMaster University, Hamilton, Canada; ${ }^{3}$ Division of Pediatric Hematology/Oncology, Department of Pediatrics, IWK Health Center, Dalhousie University, Halifax, Canada

Background: Acquired purpura fulminans (APF) is a rare limb and life-threatening thrombotic disorder characterized by purpuric skin lesions and associated with deficiency of anticoagulant proteins $\mathrm{C}$ and/or S. 
Aims: To describe the successful treatment of APF with protein $C$ concentrate in a child and discuss incidence, risk factors, management, and outcomes of APF in children based on a comprehensive literature review.

Methods: Case details were obtained from patient records after ethics approval. For literature review, PubMed database was searched for articles from inception to 2020 using terms "acquired purpura fulminans" AND "children".

Results: A 3-year-old previously healthy male was admitted to pediatric intensive care unit with septic shock and purpura on multiple digits. Protein $C$ level of $0.16 \mathrm{U} / \mathrm{mL}$ (normal $>0.70 \mathrm{U} / \mathrm{mL}$ ) revealed the diagnosis of APF in the context of respiratory syncytial virus and S. aureus pneumonia. APF was initially managed with fresh frozen plasma (FFP), followed by protein $C$ concentrate (Ceprotin) once available, to keep protein $C$ level trough $>0.50 \mathrm{U} / \mathrm{mL}$, for 13 days. Patient was treated with concurrent enoxaparin for 3 months. The purpuric lesions started to improve on day $3-4$, with complete resolution by 1 month. Patient recovered fully with no complications. The incidence of APF is 1 in 20,000 children. Risk factors include viral, bacterial (N. meningitidis being most common), or fungal infections as well as liver dysfunction, congenital heart disease, galactosemia, and antiphospholipid antibodies. Protein $\mathrm{C}$ replacement therapy, if started in a timely fashion ( $<24$ hours from diagnosis), has been shown to reduce amputation rates and mortality in children with APF in retrospective studies. There are no prospective cohort or randomized controlled trials to date.

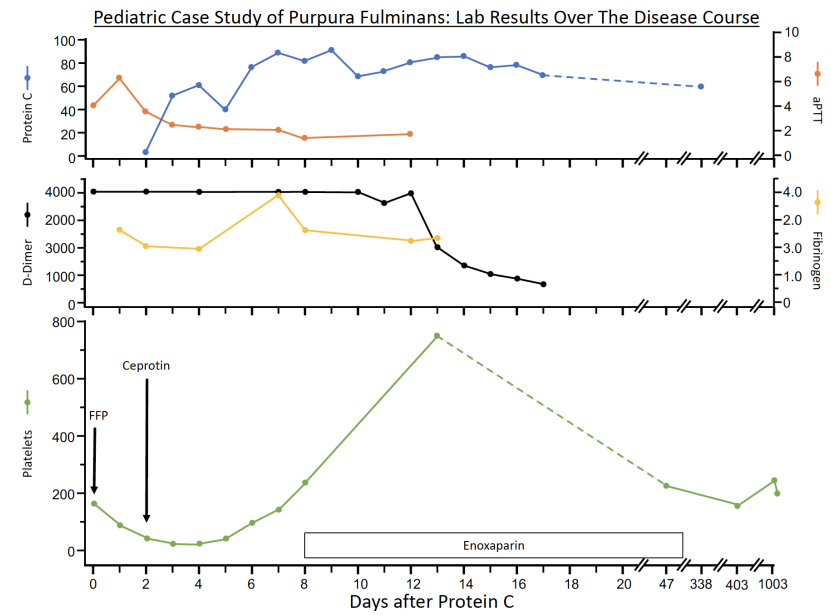

FIGURE 1 Pediatric Case Study of Purpura Fulminans: Lab Results Over The Disease Course

Conclusions: APF is important to recognize and manage promptly to prevent amputation and reduce mortality risk. Special access authorization required for protein $\mathrm{C}$ concentrate can delay initiation of treatment.
PB0813 | Identification of a Common PROC Gene Mutation in Children with Moyamoya Disease Using Whole Exome Sequencing

N. Sirachainan ${ }^{1}$; P. Pongphitcha ${ }^{1}$; Y. Okuno ${ }^{2}$; L. Thampratankul ${ }^{1}$;

C. Khongkhatithum ${ }^{1}$; P. Komvilaisak ${ }^{3}$; T. Tim-Aroon ${ }^{1}$; D. Songdej ${ }^{1}$; D. Wattanasirichaigoon ${ }^{1}$

${ }^{1}$ Faculty of Medicine Ramathibodi Hospital, Mahidol University, Bangkok, Thailand; ${ }^{2}$ Medical Genomics Center, Nagoya University Hospital, Nagoya, Japan; ${ }^{3}$ Faculty of Medicine Khon Kaen University, Khon Kaen, Thailand

Background: Moyamoya is characterized by stenosis of internal carotid artery and compensatory developing collateral vessels. It is more common in Asian countries compared to the Western countries. The incidence is around 2-3:100,000 population per year. There are bimodal distributions: age $<10$ years and 45-49 years. Moyamoya is classified into moyamoya syndrome and disease. Moyamoya syndrome associates with underlying conditions, such as sickle cell disease, neurofibromatosis type 1 , and post-radiation, while moyamoya disease does not. The etiologies causing abnormal vascular formation are unknown; therefore, this study is to report the results of whole exome sequencing (WES) in pediatric patients diagnosed with moyamoya.

Aims: To identify genetic abnormality in children with moyamoya Methods: Children aged <18 years, diagnosed with moyamoya by MRI/MRA study, were studied. After informed consent, WES was performed by using the SOPHiA DDM ${ }^{\circledR}$ platform (Sophia Genetics, SA). The demographic data, such as age, gender, treatment, and outcome were recorded.

Results: A total of 11 patients, female: male 1.8:1, median (range) age at diagnosis 8.2 (1.7-12.6) years, were enrolled. Moyamoya disease was diagnosed in seven patients. Moyamoya syndrome consisted of two a-thalassemia, one of each with Williams syndrome and craniopharyngioma post-radiation. The results of WES identified three out of seven patients (42.8\%) with moyamoya disease having pathogenic variants as follow; two patients (28.6\%) with heterozygous PROC gene mutation (c.565G>T, p.R189W) and one patient (14.3\%) with heterozygous RNF213 mutation (c.14429G>A, R4810K). One of the two with heterozygous p.R189W mutation also had homozygous HBB c.79G>A, p.E27K, causing mild anemia. There was no pathogenic variant in patients with moyamoya syndrome.

Conclusions: PROC gene mutation, causing low level of protein C, had shown to be a common pathogenic variant of moyamoya disease in Thai children. The allele frequency of c.565G $>$ T was 0.14 , while the reported allele frequency in Thai population was 0.03 . 
PB0814 | The Correlation of Vascular Platelet Hemostasis

Disorders and Typical Autoantibodies in Hepatic Cirrhosis at the Outcome of Autoimmune Hepatitis in Children

\section{E. Konovalova; A. Lavrova; M. Presniakova}

Federal State Budgetary Educational Institution of Higher Education Research Medical University of Volga Region of the Russian Ministry of Public Health, Nizhny Novgorod, Russian Federation

Background: According to current literature data endothelial dysfunction is one of pathogenesis factors in systemic autoimmune diseases. However, the role of autoantibodies in the development thereof in children with autoimmune hepatitis (AIH) has scarcely been studied.

Aims: To assess vascular platelet hemostasis disorders and their correlation with typical autoantibodies in hepatic cirrhosis at the $\mathrm{AlH}$ outcome in children.

Methods: 31 children with hepatic cirrhosis at the $\mathrm{AlH}$ outcome were studied. To assess haemostatic disorders, there were determined the platelet count, the maximal platelet aggregation rate and degree (adenosine phosphate inductor (ATP) - $1.25 \mathrm{mkg} / \mathrm{ml}$, collagen - $2.0 \mathrm{mg} / \mathrm{ml}$ ), von Willebrand factor activity (VWF), endothelin-1 concentration. There was determined the concentration of autoantibodies. The control group $(n=15)$ consisted of children included in health groups I or II. Mann-Whitney U-test, Spearman's rank correlation coefficient. Quantitative data are herein presented as Me (Q1;Q3). To determine the capability of laboratory parameters for predicting an increase in autoantibodies, the ROC curve was constructed with identifying AUC, the identification of diagnostic sensitivity (DS), specificity (DSp). The study was approved by the Local Ethics Committee (Dec.5,2017). All the study participants signed their informed consent. Funding: the research was completed under the Governmental Assignment for 2017-2020.

Results: Changes in the hemostatic system were characterized in all the patients by proneness to thrombocytopenia, higher platelet aggregation activity and endothelial thrombogenicity (Tab.1). High concentrations of autoantibodies responsible for autoimmune inflammation in the liver were associated with vascular platelet hemostasis disorders (Tab.2). High antinuclear antibodies concentrations in the blood were associated with activity of VWF $\geq 200.0 \%$ a (AUC:0.75; DS-83\%, DSp-69\%).

Conclusions: The VWF activity can be used to assess the autoimmune status in hepatic cirrhosis at the AlH outcome in children. The data obtained prove the relationship of autoantibodies with the development of endothelial dysfunction and thrombocytopenia in hepatic cirrhosis at the AlH outcome in children.

TABLE 1 Vascular platelet hemostasis changes (Me (Q1;Q3)) (Note: $n$-number of observations, $p$ - significance level)

\begin{tabular}{|c|c|c|c|}
\hline Indices & $\begin{array}{l}\text { Study group } \\
n=31\end{array}$ & $\begin{array}{l}\text { Control group } \\
n=15\end{array}$ & $P$ \\
\hline Platelets, $\left(10^{9} / \mathrm{L}\right)$ & $150(117 ; 212)$ & $311.0(266.0 ; 349.0)$ & 0.000 \\
\hline Platelet aggregation degree (ATF inductor), (\%) & $54.5(30.2 ; 67.8)$ & $22.3(14.8 ; 24.2)$ & 0.001 \\
\hline Platelet aggregation rate (ATF inductor), (\%/min) & $43.9(31.6 ; 55.4)$ & $29.9(17.8 ; 36.5)$ & 0.003 \\
\hline Platelet aggregation degree (collagen inductor), (\%) & $73.1(66.2 ; 84.7)$ & $61.8(55.4 ; 70.3)$ & 0.023 \\
\hline Platelet aggregation rate (collagen inductor), (\%/min) & $59.1(40.7 ; 73.7)$ & 33. $(18.5 ; 58.4)$ & 0.032 \\
\hline Endothelin-1, (fmol/ml) & $1.8(0.8 ; 5.1)$ & $0,6(0.2 ; 0.6)$ & 0000 \\
\hline VWF, (\%) & $167.5(149.0 ; 267.0)$ & $76.6(58.9 ; 105.4)$ & 0.000 \\
\hline
\end{tabular}

TABLE 2 Correlation relationship between hemostasis system indices and the concentration of typical autoantibodies (Spearman's method) (Note: $r$ - Spearman's rank correlation coefficient, $p$ - correlation relationship reliability)

$\begin{array}{llll}\text { Autoantibodies } & \text { Hemostasis system indices } & \text { Spearman's rank correlation coefficient }(r) & P \\ \text { Anti-liver kidney microsomal antibodies (type 1) } & \text { Platelets } & -0.448 & 0.013 \\ \text { Antibodies to soluble liver antigen } & \text { VWF } & 0.514 & 0.024 \\ & \text { Endothelin - 1 } & 0.458 & 0.037 \\ \text { Antinuclear antibodies } & \text { VWF } & 0.619 & 0.004\end{array}$


P0154 | Heterozygous FV Leiden Mutation Associated with Multiple Cerebral Venous Thrombosis in Childhood. A Case Report

Y. Larfi; N. Ait Hamouda; M. Agha; D. Dahlouk

Central Hospital of Army of Algeria, Algiers, Algeria

Background: The usually asymptomatic heterozygous FV Leiden mutaion is often diagnosed in adulthood where it is responsible of thromboembolic disease, especially when aassociated whith other contributing risk factors. We propose to report the case of a 22 month-old carrying this mutation who presented spontaneous multiple cerebral venous thrombosis.

Aims: Highlight the very heterozygous clinical expression of FVL which depends on many known or unknown risck factors.

Methods: - Medical imaging by cerebral magnetic reasoning imaging - Phenotypic and genotypic screening for thrombophilia.

- Family survey

Results: We report a clinical case of a 22-month-old girl referred to the laboratory for thrombophilia screening. In personal history, the patient was born premature at 33 weeks of amenorrhea, the onset of the disease was marked by an infectious syndrom whith diarrhea complicated by dehydration followed by neurological symptoms (drowsiness and walking disorders). Brain MRI showed massive venous thrombosis of the cortical veins, the sagittal sinus and the trocular of the right sinus. In family history, there aren't any venous or arterial thromboembolic disease, however, the mother presented 8 early abortions. screening for thrombophilia showed on the one hand, normal levels of plasma inhibitors, AT, PC, PS. The screening of antiphospholipid antibodies and lupus anticoagulants was negative. on the other hand, the sreening of the APCR was positif by phenotypic test confirmed by SSP-PCR (heterozygous FVL).the screening of the G20210A mutation of FII was negative.

Family investigation found the same mutation in her mother, the father and the two brothers were normal.

Conclusions: This is an unusual observation, the patient was treated by oral anticoagulant VKA.The follow up during 4 years was favorable without any recurrence.

There is an interest in making wider screening of thrombophilia to detect possible associations.

\section{PLATELET DISORDERS, VON WILLEBRAND DISEASE AND THROMBOTIC MICROANGIOPATHIES}

\section{ACQUIRED THROMBOCYTOPENIAS}

PB0815 | Time Course of Response to Fostamatinib, an Oral Spleen Tyrosine Kinase (SYK) Inhibitor for the Treatment of Immune Thrombocytopenia (ITP)

M. Sholzberg ${ }^{1}$; D. Arnold ${ }^{2}$; D. Liles ${ }^{3}$; R. Numerof ${ }^{4}$; M. Boxer ${ }^{5}$

${ }^{1}$ Department of Hematology and Oncology, St Michael's Hospital, University of Toronto, Toronto, Canada; ${ }^{2}$ Michael G. DeGroote School of Medicine, McMaster University, Hamilton, Canada; ${ }^{3}$ Divison of Hematology-Oncology, East Carolina University, Greenville, United States; ${ }^{4}$ Department of Medical Affairs, Rigel Pharmaceuticals, Inc, South San Francisco, United States; ${ }^{5}$ Arizona Oncology Associates, PC, Tucson, United States

Background: Immune thrombocytopenia (ITP) is a heterogeneous disease characterized by low platelet counts and increased bleeding risk. Platelet loss is mediated by a spleen tyrosine kinase (SYK) dependent pathway in macrophages. Fostamatinib is an oral SYK inhibitor approved for the treatment of ITP. In phase 3 clinical studies, fostamatinib was shown to both decrease bleeding risk and improve platelet counts, with $54 \%$ of patients achieving platelet counts $\geq 50,000 / \mu \mathrm{L}$.

Aims: To evaluate the time course of response and adverse effects associated with fostamatinib over one year.

Methods: In a post-hoc analysis, clinical endpoints were assessed in 3-month increments over the first year of the phase 3 studies. Patients with data for the full year were included. Patients received fostamatinib at 100-150 mg BID. 
Results:

TABLE 1 Characteristics of Patients who Received Fostamatinib Treatment for $>1$ Year and

\begin{tabular}{|c|c|c|}
\hline & $\begin{array}{c}\text { Patients on } \\
\text { fostamatinib } \geq 1 \\
\text { year } \\
(n=58)\end{array}$ & $\begin{array}{c}\text { Patients on } \\
\text { fostamatinib }<1 \\
\text { year } \\
(n=88)\end{array}$ \\
\hline $\begin{array}{l}\text { Duration of fostamatinib treatment, } \\
\text { months } \\
\qquad \text { Median (range) }\end{array}$ & $44.7(12.3-61.7)$ & $4.9(<1-10)$ \\
\hline $\begin{array}{l}\text { Age, years } \\
\text { Median (range) }\end{array}$ & $49(23-88)$ & $57(20-85)$ \\
\hline $\begin{array}{l}\text { Sex } \\
\quad \text { Female, } \mathbf{n}(\%)\end{array}$ & $38(66)$ & $49(56)$ \\
\hline $\begin{array}{c}\text { Time since ITP diagnosis, years } \\
\text { Median (range) }\end{array}$ & $8.2(<1-43.7)$ & $8.5(<1-53)$ \\
\hline Persistent ITP, n (\%) & $4(6.9)$ & $6(6.8)$ \\
\hline Chronic ITP, n (\%) & $54(93.1)$ & $82(93.2)$ \\
\hline $\begin{array}{c}\text { Number of prior ITP meds } \\
\text { Median (range) }\end{array}$ & $4(1-42)$ & $6(1-32)$ \\
\hline $\begin{array}{l}\text { Prior ITP Management, } \mathrm{n}(\%) \\
\text { Corticosteroids } \\
\text { Thrombopoietin-receptor agonists } \\
\text { Rituximab } \\
\text { Splenectomy }\end{array}$ & $\begin{array}{l}57(98.3) \\
25(43.1) \\
11(19) \\
17(29.3)\end{array}$ & $\begin{array}{c}80(90.9) \\
43(49.4) \\
36(40.9) \\
34(39)\end{array}$ \\
\hline $\begin{array}{l}\text { Baseline platelet count }(/ \mu \mathrm{L}) \\
\text { Median }\end{array}$ & 20,000 & 14,000 \\
\hline
\end{tabular}

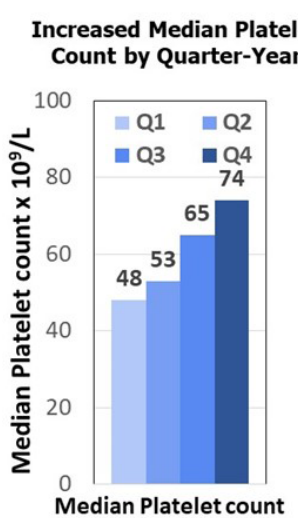

Decreased Incidence of Bleeding Events and Rescue Therapy by Quarter-Year

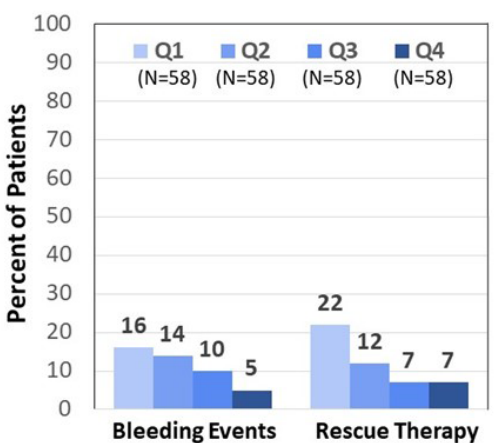

FIGURE 1 Clinical endpoints assessed in 3-month increments in the 58 patients with data for all 4 quarters of the first year of treatment

Characteristics of the 58 patients who continued treatment for $\geq 1$ year differed from those with $<1$ year of treatment with regard to the number of prior treatments and baseline platelet count (see table). The patients treated for $\geq 1$ year had a response rate of $91 \%$ $(53 / 58)$ (achieved a platelet count $\geq 50,000 / \mu \mathrm{L}), 71 \%$ (41/58) within 12 weeks.

Among those treated for $\geq 1$ year $(n=58)$, over the first year of treatment, median platelet counts continued to increase per each 3month period (see figure), and the incidence of bleeding events and use of rescue therapy decreased with continued treatment (see figure). Also, among these patients, the incidence of common adverse events (diarrhea [38\%] and hypertension [28\%]) decreased over each 3-month period of treatment.

Conclusions: In these post-hoc analyses of ITP patients with $\geq 1$ year of treatment, fostamatinib provided durable and progressive clinical benefit with diminishing adverse events over time.

PB0816 | Exploring the Immune-related Biomarkers for Predicting the Efficacy of Rituximab Treatment in Patients with Chronic Immune Thrombocytopenia

X. Wang ${ }^{1,2,3} ;$ H. Li $i^{1,2,3} ;$ X. Liu ${ }^{1} ;$ F. Xue ${ }^{1} ;$ W. Liu ${ }^{1} ;$ Y. Chen ${ }^{1} ;$ R. Fu ${ }^{1}$; L. Zhang ${ }^{1,2,3} ;$ R. Yang ${ }^{1,2,3}$

${ }^{1}$ State Key Laboratory of Experimental Hematology, National Clinical Research Center for Hematological Disorders, Institute of Hematology and Blood Diseases Hospital, Chinese Academy of Medical Sciences and Peking Union Medical College, Tianjin, China; ${ }^{2}$ CAMS Key Laboratory of Gene Therapy for Blood Diseases, Tianjin, China; ${ }^{3}$ Tianjin Laboratory of Blood Disease Gene Therapy, Tianjin, China

Background: Immune thrombocytopenia (ITP) is an autoimmune disease caused by immune intolerance. Rituximab is a monoclonal antibody for therapeutic B-cell depletion, which has been used as second-line therapy in chronic ITP with up to $60 \%$ response rates. However, considering its high cost and complications, it is necessary to find biomarkers to predict clinical efficacy to help clinicians make better choices.

Aims: To explore the immune-related biomarkers for predicting response to rituximab in patients with chronic ITP.

Methods: We prospectively included 30 patients with chronic ITP in our center from May 2019 to Nov 2020. We examined the phenotypes of peripheral blood lymphocytes, monocytes, and T helper (Th) cells in 4 hours cultured peripheral blood mononuclear cells in vitro stimulated by PMA/ ionomycin. Efficacy was evaluated 3 months after rituximab treatment. Optimal cutoff values were established using ROC analysis.

Results: Among 30 patients, there were 9 males and 21 females, with a median age of 30 (11-57) years. Eighteen patients showed response ( $\mathrm{PLT} \geq 30 \times 10^{9} / \mathrm{L}$ after treatment). Comparing immune status before rituximab therapy, naïve B cells /CD19+B lymphocyte(Bn/B) in the effective group was significantly higher than that in the ineffective group [(68.9 \pm 9.8$) \%$ vs $(57.6 \pm 11.6) \%, P=0.024]$. Memory B cells/ $\mathrm{CD} 19+\mathrm{B}$ lymphocyte $(\mathrm{Bm} / \mathrm{B})$ had a lower level in the effective group compared with the ineffective group [(29.5 \pm 9.9$) \%$ vs $(41.7 \pm 11.5) \%$, $\mathrm{P}=0.014]$. Significantly difference was observed in the ratio of Th1/ Th2 between the effective and the ineffective group [(8.0 \pm 4.1$) \%$ vs $(13.3 \pm 5.2) \%, P=0.012]$. Patients were divided further using an optimal cut-off $\mathrm{Bn} / \mathrm{B}$ of $62.7 \%, \mathrm{Bm} / \mathrm{B}$ of $37.0 \%$ and Th1/Th2 of 9.8 . The model had an AUC of 0.910 (95\% Cl 0.778-1.000). 


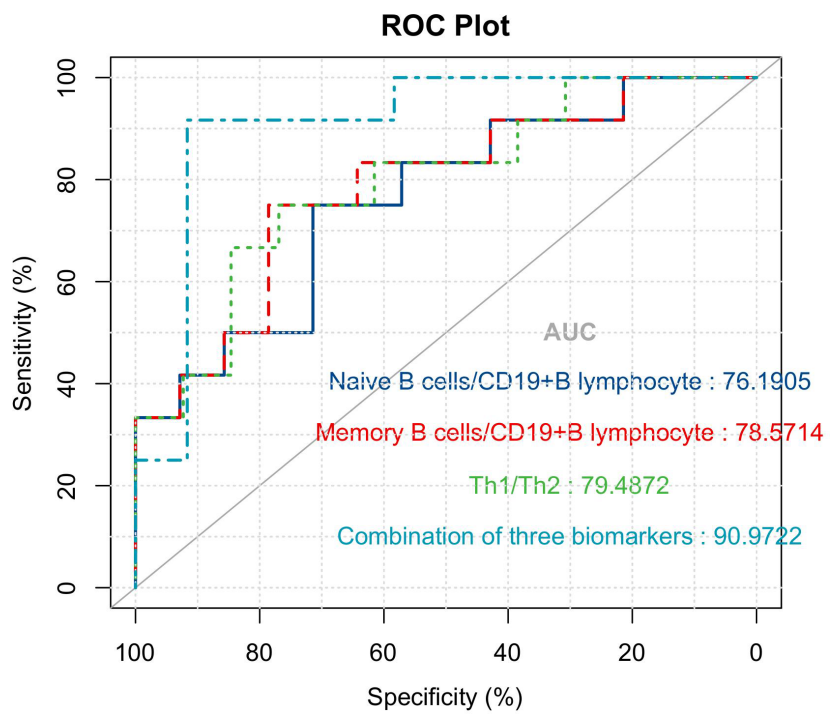

FIGURE 1 ROC curve for predicting the efficacy of rituximab

Conclusions: ITP patients with $\mathrm{Bn} / \mathrm{B}>62.7 \%, \mathrm{Bm} / \mathrm{B}<37.0 \%$ and Th1/ Th2 $<9.8$ tend to achieve response by rituximab treatment. Our study proposed a model for predicting the efficacy of rituximab, which is helpful to assist clinicians in making treatment decisions.

PB0817 | Platelet Apoptosis and Autophagy in Pediatric Immune Thrombocytopenia: New Mechanistic Insights

T. Stein; R. Hinselmann; C. Gowin; D. Greber; S. Shllaku; N. Gölz;

M. Schmugge; F.D. Franzoso

University Childrens' Hospital Zurich, Division of Hematology,

University of Zürich, Zürich, Switzerland

Background: Immune thrombocytopenia (ITP) is an autoimmune disease that can cause severe bleeding symptoms and it can occur in both pediatric and adult patients. Antiplatelets antibodies, T-cell mediated destruction of platelets and impaired megakaryopoiesis leading to a reduced platelet formation by loss of autophagy have been described as possible etiologies of the low platelet counts that hallmark this disorder. We have previously reported an increased phosphatidylserine exposure and caspase- 3 in platelets from acute ITP patients, suggesting that platelet apoptosis is involved into the ITP pathogenesis.

Aims: In our present study, we aimed to identify ITP platelets' clearance mechanisms that control the interplay between the apoptotic and autophagic machinery.

Methods: We performed an apoptosis proteomic profiling of 35 genes (R\&D Systems) and we validated our results by RT-qPCR and immunoblotting.

Results: We could demonstrate by apoptosis proteomic profiling significantly increased expression levels of some apoptotic genes such as clusterin (CLU), pro-caspase 3, catalase, TRAILR1/DR4, Bax, $\mathrm{Bad}$ and $\mathrm{Bcl}-2$ compared to healthy controls in platelet-rich plasma (PRP) from 10 ITP patients. We could validate by both RT-qPCR and immunoblotting that CLU, a stress-activated chaperone, is significantly increased in both acute and chronic ITP. Further, we investigated specific autophagy and ER-autophagy markers by Western blot and RT-qPCR. We could observe that the mRNA expression of autophagy-initiation (Beclin-1), -autophagosome (p62) and -lysosome (LAMP1) markers was up to 3-fold significantly increased in acute and chronic ITP patients. mRNA levels of ER-autophagy genes CLIMP63 and FAM134b were significantly increased whereas ATL3 and REEP4 showed significantly decreased levels.

Conclusions: Our results indicate an impairment in the modulation of apoptosis and autophagy pathways in ITP platelets. We assume that targeting apoptosis and/or autophagy pathway genes could lead to potential identification of new biomarkers and therapies against this autoimmune disorder, and thereby, could it provide an augmented quality of life of ITP patients.

PB0818 | Therapeutic Landscape in Primary Immune Thrombocytopenia (ITP) over the Last Three Decades in the United Kingdom (UK Adult Primary ITP Registry Project)

$\underline{\text { P. Raheja }}{ }^{1}$; H. Miah ${ }^{1} ;$ A. Miah ${ }^{1} ;$ N. Cooper ${ }^{2} ;$ M. Scully ${ }^{3} ;$ T. Biss ${ }^{4}$; S. Chattree ; D. Allsup ${ }^{6}$; G. Evans ${ }^{7}$; S. Pavord ${ }^{8}$; G. Matthias ${ }^{9}$; C. Bagot ${ }^{10}$; Q. Hill ${ }^{11}$; C. Bradbury ${ }^{12}$; V. Martlew ${ }^{13}$; S. Johns ${ }^{14}$; L. Taylor ${ }^{1}$; A. Newland ${ }^{1}$; D. Provan ${ }^{1}$; V. McDonald ${ }^{1}$; On behalf of the United Kingdom adult primary ITP registry

${ }^{1}$ Barts Health NHS trust, London, United Kingdom; ${ }^{2}$ Hammersmith Hospital, London, United Kingdom; ${ }^{3}$ University College Hospital, London, United Kingdom; ${ }^{4}$ Royal Victoria Infirmary, Newcastle, United Kingdom; ${ }^{5}$ Sunderland Royal Hospital, Sunderland, United Kingdom; ${ }^{6}$ Castle Hill Hospital, Cottingham, United Kingdom; ${ }^{7}$ Kent and Canterbury Hospital, Canterbury, United Kingdom; ${ }^{8}$ Churchill Hospital, Oxford, United Kingdom; ${ }^{9}$ Queen Alexandra Hospital, Harlow, United Kingdom, ${ }^{10}$ Glasgow Royal Infirmary, Glasgow, United Kingdom, ${ }^{11}$ Leeds Teaching Hospital, Leeds, United Kingdom, ${ }^{12}$ University Hospitals Bristol, Bristol, United Kingdom, ${ }^{13}$ Royal Liverpool and Broadgreen University Hospital Trust, Liverpool, United Kingdom,

${ }^{14}$ Royal Cornwall Hospital, Cornwall, United Kingdom

Background: Due to its low incidence and heterogeneous nature, treatment practices in ITP vary considerably between centres nationally and internationally.

Aims: The UK adult ITP registry is a large multicentre registry, whose aim is to use real world data to describe diagnostic workup, clinical features, understand the natural history of disease and evaluate current UK practice.

Methods: Adults (>18 years old) diagnosed with primary ITP were included. Data was collected retrospectively from 2007 to July 2020. A total of 4116 patients were recruited across 73 sites in the UK, of which we have complete data on 3261 . The patients were grouped into thee time brackets for analysis according to their date of ITP diagnosis: 1989-1998; 1999-2008; 2009-2020. 
Results: The median age at diagnosis was 52 and $56 \%$ were female. The majority of the patients were diagnosed from 2009-2020 (77\%). Of all the patients, $29 \%$ did not receive treatment. Of those treated, corticosteroids were the most commonly used agent and one third only received steroids as their treatment. Most of these were initially treated with prednisolone (87\%), $12.9 \%$ with dexamethasone and $4.8 \%$ with methylprednisolone. Intravenous immunoglobulin was required in around $39 \%$ of patients.

In the last decade, the discovery of Rituximab has prompted its increased use as a steroid-sparing agent (23.5\% of patients from 2009 to 2020). Thrombopoyetin receptor analogues (TPO-RA) have been introduced recently their uptake has increased in the last decade. Mycophenolate mofetil (MMF) has also gained popularity from $0.5 \%$ to $19.5 \%$. Leading to a decreased use of other immunosuppressants with more undesirable effects (Table 1). Splenectomy fell from $65.4 \%$ in the first bracket to $4.3 \%$ from $2009-2020$.

Conclusions: This registry helps portray treatment trends in ITP. Overall, steroids have been used consistently as first line of treatment. While, the use of rituximab, TPO-RAs and MMF have increased in the last decade.

PB0819 | Length of Thrombopoietin Receptor Agonist (TPO-RA) Treatment and Persistence in Immune Thrombocytopenia (ITP): Real World United States Claims Analyses

M. Vredenburg; S. Kolodny; M. Wojdyla; H. Boyer; T. Darden Dova Pharmaceuticals, Durham, United States

Background: The recent ASH Guidelines and International Consensus Report for the management of ITP suggest consideration of TPO-RAs as subsequent therapy following an insufficient response to first-line treatments, especially if seeking a durable platelet response.

Aims: To understand length of therapy (LOT) and persistence (PER) of treatment in adult ITP patients in US, treated with an FDAapproved TPO-RA (avatrombopag (AVA), eltrombopag (ELT) or romiplostim (ROMI).

Methods: Inclusion in the analyses required a D69.3 or D69.49 ICD10 diagnostic code for ITP and $\geq 1$ first-initiated claim for ELT or ROMI during the 2019 calendar year from the Symphony Health Claims Database and from a closed system of specialty pharmacies (CVS, Accredo, Biologics, Kroger, PharmaCord, Panther) from August 2019 through December 2019 for AVA.

LOT for each TPO-RA was determined by calculating the time between the first claim/shipment date and the last claim/shipment date while adding on the number of days of supply provided in the last claim/shipment.

PER for each TPO-RA was determined by claims occurring in specific months. Unlike LOT, PER did not add the number of days of supply to the last evaluable claim/shipment.
Results: LOT and PER for each TPO-RA are shown below (Table 1). LOT was highest in AVA patients, regardless of whether a conservative single claim/shipment or $\geq 2$ claims/shipments were used. PER for each individual month reached was highest for AVA, with the median AVA patient reaching 10 months of treatment.

TABLE 1 Length of Therapy (LOT) and Persistence of Treatment (PER) for TPO-RAs in Adult ITP

\begin{tabular}{|c|c|c|c|c|c|c|c|c|c|c|c|c|}
\hline \multicolumn{13}{|c|}{ Length of Therapy (LOT) } \\
\hline TPO-RA & \multicolumn{6}{|c|}{$\begin{array}{l}\text { Months of Therapy } \\
\text { (1 claim/shipment) }\end{array}$} & \multicolumn{6}{|c|}{$\begin{array}{l}\text { Months of Therapy } \\
\text { (2+ claims/shipments) }\end{array}$} \\
\hline AVA $(n=171)$ & \multicolumn{6}{|c|}{7.7} & \multicolumn{6}{|c|}{9.3} \\
\hline ELT $(n=1,400)$ & \multicolumn{6}{|c|}{6.5} & \multicolumn{6}{|c|}{8.2} \\
\hline ROMI (n=1,283) & \multicolumn{6}{|c|}{5.5} & \multicolumn{6}{|c|}{6.4} \\
\hline \multicolumn{13}{|c|}{ Persistence of Treatment (PER) } \\
\hline & \multicolumn{12}{|c|}{ Proportion (\%) of Patients by Month Reached } \\
\hline TPO-RA & 1 & 2 & 3 & 4 & 5 & 6 & 7 & 8 & 9 & 10 & 11 & 12 \\
\hline AVA $(n=171)$ & 100 & 80 & 71 & 66 & 63 & 60 & 57 & 56 & 52 & 51 & 47 & 43 \\
\hline ELT $(n=1,400)$ & 100 & 75 & 66 & 60 & 55 & 50 & 47 & 43 & 40 & 37 & 34 & 29 \\
\hline ROMI $(n=1,283)$ & 100 & 78 & 63 & 55 & 51 & 46 & 42 & 38 & 35 & 32 & 30 & 28 \\
\hline
\end{tabular}

Conclusions: AVA demonstrated a greater length of treatment and higher rates of persistence than other TPO-RAs, possibly due to a variety of variables: route of administration, convenience, patient preference, efficacy, side effects, insurance coverage. These results should be interpreted with caution. Claims data using matched methodologies were utilized; however, due to AVA's commercial availability occurring in July 2019, slightly different time frames were evaluated.

PB0820 | Altered Hemostatic Potential in Adults with Primary ITP

T. Schramm ${ }^{1}$; J. Machacek ${ }^{1}$; M. Fillitz ${ }^{2}$; B. Dixer ${ }^{2}$; D. Mehic ${ }^{1}$; S. Koder ${ }^{1}$; C. Ay ${ }^{1}$; I. Pabinger ${ }^{1}$; J. Gebhart ${ }^{1}$

${ }^{1}$ Department of Medicine I, Clinical Division of Haemotology and Haemostaseology, Medical University of Vienna, Vienna, Austria; ${ }^{2}$ Department of Internal Medicine, Hanusch Krankenhaus, Vienna, Austria

Background: Primary immune thrombocytopenia (ITP) patients clinically show heterogeneous bleeding phenotypes and partially increased thromboembolic risk, which are not necessarily associated to platelet counts.

Aims: To investigate the global hemostatic potential in adult ITP patients in comparison to thrombocytopenic- and healthy controls and its relation to the clinical phenotype.

Methods: A thrombin generation assay (TGA) and a plasma clot formation and lysis assay (CLA) were performed in duplicates from citrated platelet poor plasma of ITP patients, non-immunological thrombocytopenic controls after chemotherapy (TC) and age- and 
sex-matched healthy controls (HC). Written consent was obtained (EC 1843/2016).

Results: 88 ITP patients (71\% chronic, 11\% persistent, 17\% acute, median bleeding score 1 (IQR 0-3)), 19 TC and 159 HC were investigated (Table 1). In TGA, after adjustment for sex, age, BMI and fibrinogen, ITP patients had a significantly longer lag time and TTP in comparison to TC and lower peak thrombin compared to HC (Table 2). In CLA, ITP patients had a lower clot formation rate as well as lower clot density than TC and longer lag time, lower clot formation rate and longer time to peak than $\mathrm{HC}$. Clot lysis was most affected in ITP patients and much slower than in TC and HC (Table 2).
The bleeding score correlated weakly with time to peak in TGA only (Spearman rho 0.220, $P=0.041$ ). In the four patients with history of thrombosis the pattern of TGA and CLA was not different compared to ITP without thrombotic events.

Conclusions: Thrombin generation and clot formation was mildly impaired and clots were lysed slower in ITP patients in comparison to $\mathrm{HC}$ and TC, without an association with bleeding severity or history of thrombosis. Whether the slow rate of lysis in ITP patients could indicate counter-regulatory mechanisms in ITP patients remains to be further investigated.

TABLE 1 Demographic and laboratory data of patients with primary ITP ( $n=88)$ compared to thrombocytopenic controls $($ TC) $(n=19)$ and healthy controls $(\mathrm{HC})(n=159)$

\begin{tabular}{|c|c|c|c|c|c|c|c|c|}
\hline & $n$ & Primary ITP & $n$ & TC & $n$ & $\mathrm{HC}$ & $\begin{array}{l}\text { Primary ITP } \\
\text { vs TC, } P\end{array}$ & $\begin{array}{l}\text { Primary ITP } \\
\text { vs HC, } P\end{array}$ \\
\hline Female, $n(\%)$ & 88 & $57(64.8)$ & 19 & $10(52.6)$ & 159 & $107(67.3)$ & 0.321 & 0.688 \\
\hline Age, years, mean \pm SD & 88 & $43.9 \pm 17.0$ & 19 & $51.7 \pm 14.7$ & 159 & $44.4 \pm 13.8$ & 0.042 & 0.497 \\
\hline $\begin{array}{l}\text { BMI, median (25th-75th } \\
\text { percentile) }\end{array}$ & 84 & $\begin{array}{l}25.4 \\
\quad(22.9-29.7)\end{array}$ & 18 & $\begin{array}{c}27.3(22.8- \\
29.9)\end{array}$ & 159 & $23.4(21.1-25.7)$ & 0.997 & $<0.001$ \\
\hline $\begin{array}{l}\text { Platelet counts, } \times 10^{\wedge} 9 / \mathrm{L} \\
\text { (25th-75th percentile) }\end{array}$ & 86 & $58(30-119)$ & 19 & $59(18-71)$ & 159 & $252(217-293)$ & 0.322 & $<0.001$ \\
\hline $\begin{array}{l}\text { Fibrinogen, } \mathrm{mg} / \mathrm{dl}(25 \mathrm{th}- \\
\text { 75th percentile) }\end{array}$ & 85 & $\begin{array}{l}306 \\
\quad(272.5-359)\end{array}$ & 19 & $\begin{array}{l}427 \\
\quad(315-488)\end{array}$ & 159 & $297(258-331)$ & $<0.001$ & 0.086 \\
\hline Previous thrombosis, $n$ (\%) & 74 & $4(5.4)$ & 18 & $3(16.7)$ & 159 & $0(0)$ & 0.106 & 0.108 \\
\hline $\begin{array}{l}\text { Bleeding score (25th-75th } \\
\text { percentile) }\end{array}$ & 88 & $1(0-3)$ & 18 & $2(1-4)$ & 105 & $0(0-0)$ & 0.198 & $<0.001$ \\
\hline $\begin{array}{l}\text { Disease duration, months } \\
\text { (25th-75th percentile) }\end{array}$ & 88 & $61(9-130)$ & na & - & na & - & - & - \\
\hline
\end{tabular}

Abbreviations: BMI, Body mass index; Bleeding Score was assed according to the ISTH ITP SMOG Index, n, number of patients of whom data are available; na, not applicable

Acute ITP, 0-3 months; persistent ITP, 3-12 months; chronic ITP, >12 months.

TABLE 2 Thrombin generation parameters and Clot formation and lysis parameters in patients with primary ITP ( $n=88$ ) compared to thrombocytopenic controls $(n=19)$ and healthy controls $(n=159)$. Data are shown in median and interquartile range (25th-75th percentile); CLA: 157 healthy controls.

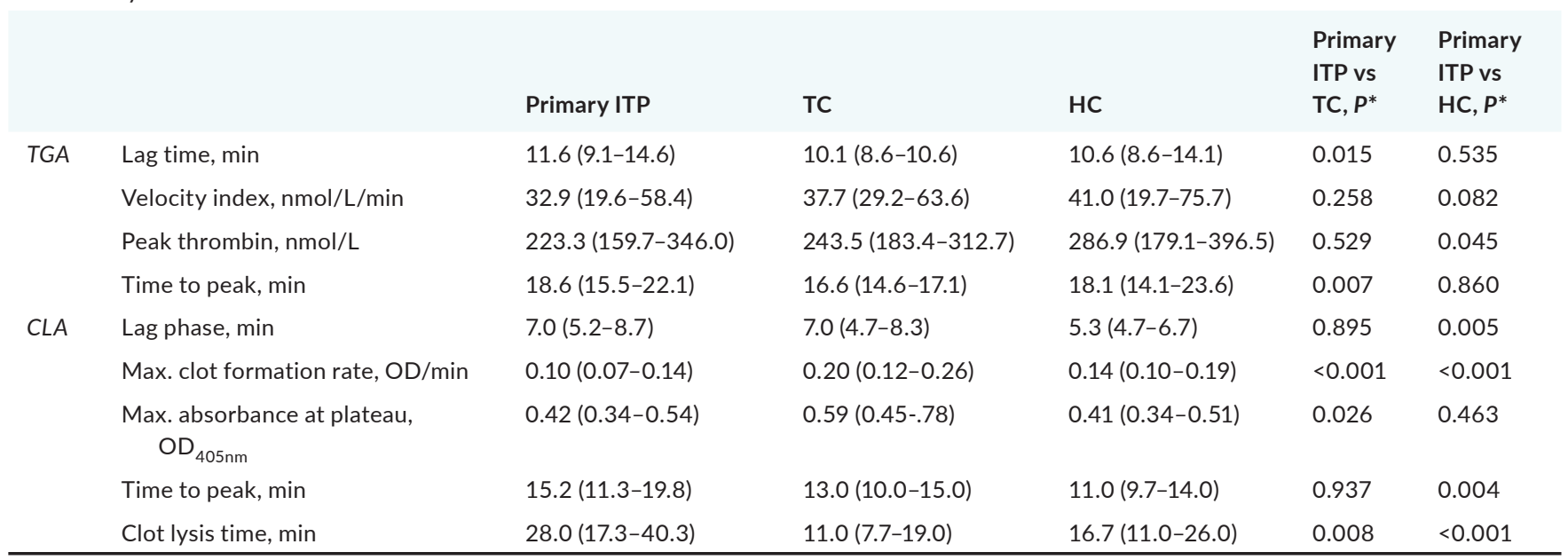


PB0821 | A Multicenter Double-blind, Double-dummy, Randomized Study of rhTPO vs Eltrombopag in the Treatment of Chinese Immune Thrombocytopenia

H. Mei ${ }^{1}$ M. Xu ${ }^{1}$; G. Yuan ${ }^{2}$; F. Zhu ${ }^{3}$; J. Guo ${ }^{4}$; R. Huang ${ }^{5}$; J. Qin ${ }^{6}$; T. Lv²; F. Qin ${ }^{3}$; H. Cai ${ }^{4}$; P. Yin ${ }^{7}$; T. Qin $;$ Y. Hu ${ }^{1}$

${ }^{1}$ Institute of Haematology, Union Hospital, Tongji Medical College, Huazhong University of Science and Technology, Wuhan, China; ${ }^{2}$ Department of Hematology, Xiangyang Central Hospital, Affiliated Hospital of Hubei University of Arts and Science, Xiangyang, China; ${ }^{3}$ Institute of Haematology, Loudi Central Hospital, Loudi, China;

${ }^{4}$ Institute of Haematology, Yichang Central People's Hospital, Yichang, China; Institute of Haematology, the First Affiliated Hospital of Nanchang University, Nanchang, China; ${ }^{6}$ Institute of Haematology, People's Hospital, Shiyan, China; ${ }^{7}$ Department of Epidemiology \& Statistics, School of Public Health, Tongji Medical College, Huazhong University of Science \& Technology, Wuhan, China

Background: Both recombinant human thrombopoietin (rhTPO) and eltrombopag are recommended second-line drugs for the treatment of immune thrombocytopenia (ITP).

Aims: This double-blind, double-dummy controlled study aims to explore which drugs are more suitable for the rapid increase of platelets in Chinese ITP patients.

Methods: 96 patients were 1:1 randomly assigned to receive eltrombopag $25 \mathrm{mg} /$ day or rhTPO $300 \mathrm{U} / \mathrm{kg}$ for 2 weeks.

Results:
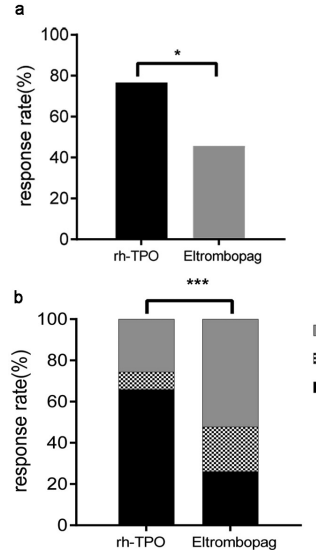
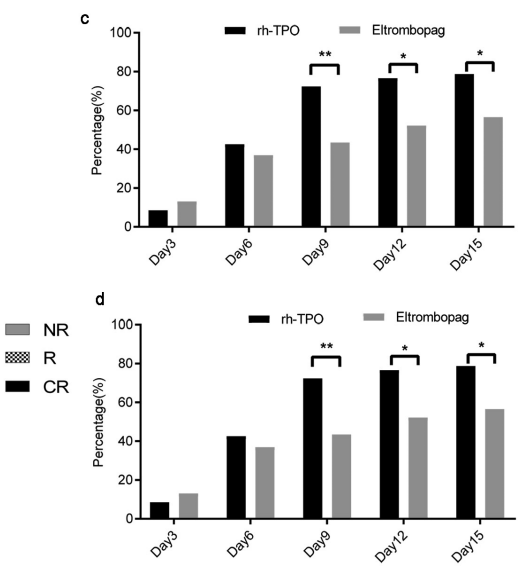

FIGURE 1 Response rate. The proportion of patients achieving platelet counts $50 \times 109 / \mathrm{L}$ or more at day 15 (a); the proportion of patients achieving CR, R or NR at day 15 (b); CR: platelet count $\geq 100 \times 109 / \mathrm{L}$ and absence of bleeding; R: platelet count $\geq 30 \times 109 / \mathrm{L}$ and at least 2 -fold increase of the baseline platelet count and absence of bleeding; NR: platelet count not achieved $50 \times 109 / \mathrm{L}$ or 2 -fold increase of the baseline platelet count or with bleeding. The proportion of patients whose platelets reached $\geq 50 \times 109 / \mathrm{L}$ at least once during the treatment (c); the proportion of patients whose platelets increased twice more than baseline at least once during the treatment $(\mathrm{d}) .{ }^{*} P<0.05 ;{ }^{* *} P<0.005 ;{ }^{* * *} P<0.001$.
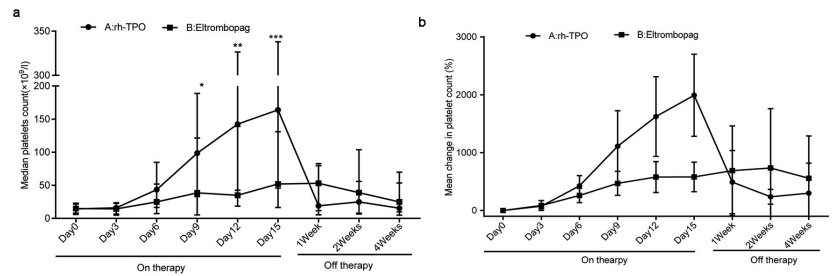

FIGURE 2 Median platelet counts (a) and mean changes in platelet counts (b) at every visit. Median platelet counts at every visit are shown with IQR, and mean changes in platelet counts from baseline at every visit are shown with 95\% Cls; ${ }^{*} \mathrm{P}<0.05$; $^{* *} \mathrm{P}<0.005$; $^{* *} \mathrm{P}<0.001$.

The primary endpoint was the proportion of patients achieving platelet counts $\geq 50 \times 10^{9} / \mathrm{L}$ at day 15 . Secondary endpoints included the platelet response, time to response, and adverse events during treatment. The primary endpoint was achieved in $75 \%(36 / 48)$ of patients in the rhTPO group and $43.75 \%(21 / 48)$ in the eltrombopag group, $P=0.003$. Complete response was achieved in $64.58 \%$ of patients in the rhTPO group vs $25.00 \%$ of patients in the eltrombopag group. The proportion of patients whose platelets increased twice more than baseline or reached $\geq 50 \times 10^{9} / \mathrm{L}$ at least once was higher in the rhTPO group on days 9,12 , and 15 . The time to increase the platelets twice more than the baseline $(P=0.048)$ or achieve the platelets $\geq 50 \times 10^{9} / L(P=0.048)$ was shorter in the rhTPO group. However, after treatment, the platelets dropped to the baseline within 1 week in the rhTPO group, while platelets dropped slowly in the eltrombopag group. Adverse events have no significant difference between the two groups.

Conclusions: Compared to the rhTPO, the dose of $25 \mathrm{mg}$ eltrombopag may be insufficient to rapidly increase platelets to a safe level in Chinese ITP patients. This study is registered with ClinicalTrials. gov, number NCT03771378.

PB0822 | Platelet Activation and Function in Adult Patients with Primary Immune Thrombocytopenia

J. Machacek ${ }^{1}$; L. Buresch ${ }^{1}$; D. Mehic ${ }^{1}$; T. Schramm ${ }^{1}$; M. Fillitz ${ }^{2}$;

B. Dixer ${ }^{2}$; T. Flasch ${ }^{1}$; T. Anderle ${ }^{1}$; A. Rath ${ }^{1}$; C. Ay ${ }^{1}$; I. Pabinger ${ }^{1}$;

J. Gebhart ${ }^{1}$

${ }^{1}$ Medical University of Vienna, Vienna, Austria; ${ }^{2}$ Hanusch Krankenhaus, Vienna, Austria

Background: Immune thrombocytopenia (ITP) is associated with a heterogeneous clinical presentation, which cannot be predicted by the platelet count. Platelet activation might influence the clinical presentation of ITP. Aims: To investigate platelet activation in a cohort of 51 adult patients with primary ITP and 18 age- and sex-matched non-immunological thrombocytopenic controls (TPC).

Methods: Patients were included in two haematological centers after written informed consent (EC1843/2016). Soluble P-selectin (sPsel) levels and platelet function by flow cytometry, uninitiated and after addition of agonists were assessed. 
Results: Patient characteristics are shown in Table 1. All analyses were adjusted for platelet counts. Median sPsel levels [25-75 percentile] and non-activated CD62P were increased in ITP compared to TPC (sPsel 31.7 [23.3-52.2] and 14.5 [5.1-23.9], $P=0.002$; CD62P 0.0 [0.0-9.5] and 0.0 [0.0-0.0], $P=0.044$; Figure 1). ITP patients under treatment had higher sPsel-levels than untreated patients (44.3 [30.7-61.2] and 28.2 [19.6-47.5], $P=0.013$ ), whereas there was no difference in other parameters of platelet function. TPORA-treated patients had higher median sPsel and lower PAC-1 ADP than untreated patients (sPsel 60.5 [33.8-70.0] and 27.3 [17.347.2], $P=0.019$; PAC-1 ADP 40.8 [18.1-75.7] and 157.6 [63.9267.5], $P=0.017$ ), while there was no difference in comparison to corticosteroid-treated patients (sPsel 38.6 [24.8- 60.9], $P=0.181$ and PAC1-ADP 90.7 [23.1-366.1], $P=0.784$ ). Splenectomized patients tended towards higher sPsel-levels compared to nonsplenectomized (46.4 [30.4-70.6] and 30.6 [17.6- 50.4], $P=0.056$ ). Previous thrombosis had no impact on sPsel-levels or platelet activation markers. Bleeding severity, assessed by the ISTH-ITP-BAT showed a weak correlation with unactivated $C D 62 P(B=0.34$, $P=0.016$ ), whereas there was no correlation with sPsel or other parameters of platelet activity.

TABLE 1 Patients' clinical and laboratory characteristics

\begin{tabular}{lll} 
& ITP & \\
& $n$ & $n 1$ \\
Gender, female & 51 & 35 \\
Current ITP treatment & 51 & \\
none & & 33 \\
Corticosteroids & & 6 \\
TPO-RA & 50 & 9 \\
others & 50 & 3 \\
Previous thrombosis & $n$ & 4 \\
Splenectomy & 51 & 12 \\
& 51 & median \\
Age, years & 51 & 77 \\
Platelet count, x10 $\%$ L & 51 & 113 \\
Duration of disease, months & & 2 \\
BS SMOG-Index, total & 50 \\
\hline
\end{tabular}
68.6

64.7

11.8

17.7

5.9

8

24

25-75 percentile

$32-55$

41-139

40-162

$0-6$
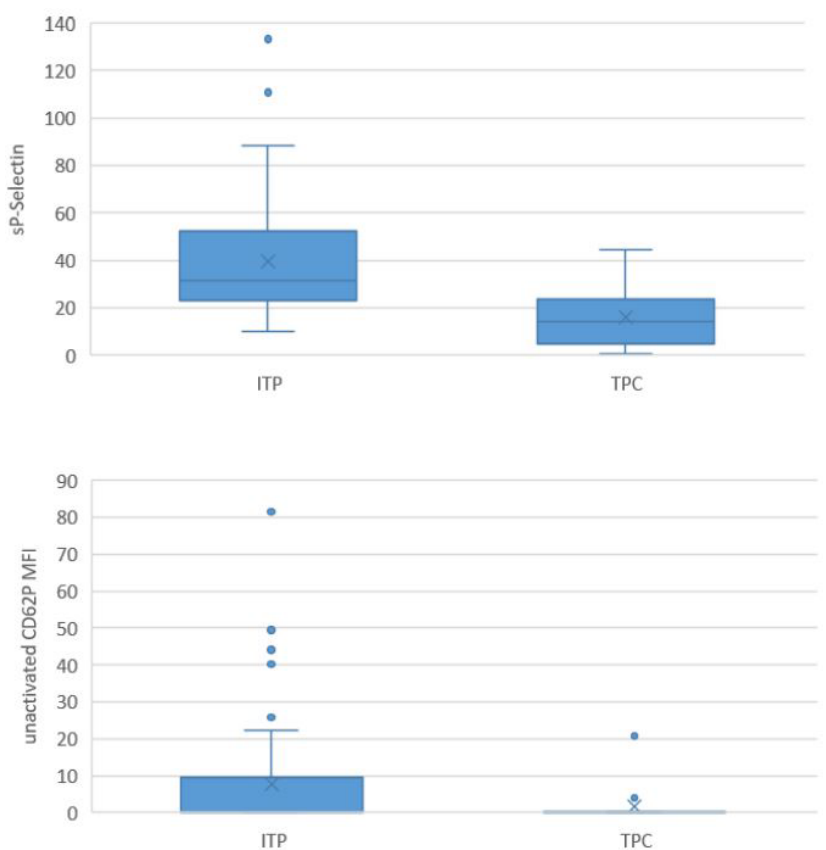

FIGURE 1 Comparison of sP-selectin $(\mathrm{ng} / \mathrm{ml})$ and unactivated CD62P MFI (median fluorescence intensity) between ITP patients and non-immunological thrombocytopenic controls (TPC)

TPC

$\begin{array}{lll}n & \mathrm{n} 1 & \text { \% } \\ 18 & 9 & 50 \\ 18 & \mathrm{Na} & \text { na }\end{array}$

Conclusions: ITP-patients have increased sPsel, especially under TPO-RA-treatment, whereas there was no clear pattern of platelet hyperreactivity. There was no association or correlation of platelet activation markers with previous thrombosis or bleeding severity. 
PB0823 | Switching from Eltrombopag or Romiplostim to Avatrombopag in Immune Thrombocytopenia: A Multicenter Study of U.S. ITP Referral Centers

H. Al-Samkari ${ }^{1,2}$; D. Jiang ${ }^{3,4} ;$ T. Gernsheimer ${ }^{3,4} ;$ H. Liebman ${ }^{5}$; S. Lee ${ }^{5}$; M. Wojdyla ${ }^{6}$; M. Vredenburg ${ }^{6}$; A. Cuker ${ }^{7}$

${ }^{1}$ Massachusetts General Hospital, Boston, United States; ${ }^{2}$ Harvard Medical School, Boston, United States; ${ }^{3}$ University of Washington, Seattle, United States; ${ }^{4}$ Seattle Cancer Care Alliance, Seattle, United States; ${ }^{5}$ University of Southern California, Los Angeles, United States; ${ }^{6}$ Dova Pharmaceuticals, a wholly owned subsidiary of Swedish Orphan Biovitrum, Durham, United States; ${ }^{7}$ University of Pennsylvania, Philadelphia, United States

Background: Avatrombopag is an oral thrombopoietin receptor agonist (TPO-RA) approved for treatment of immune thrombocytopenia (ITP). Data describing effectiveness of avatrombopag following treatment with other TPO-RAs is limited.

Aims: Evaluate ITP treatment outcomes in patients switching from eltrombopag/romiplostim to avatrombopag.

Methods: We retrospectively evaluated all adults with ITP switched from eltrombopag or romiplostim to avatrombopag at four U.S. tertiary ITP referral centers from July 2019 through December 2020 who were treated with avatrombopag for $\geq 2$ months.

Results: 45 patients were included, with a median (range) age of 60 (21-87) years; $53 \%$ were female. Mean avatrombopag treatment duration was 9 months. At avatrombopag initiation, patients had ITP for a mean of 8.3 years with a mean (range) of 4.8 (2-10) prior ITP treatments. The reason for switching to avatrombopag was convenience in $51 \%$, ineffectiveness of romiplostim/eltrombopag in $31 \%$, and adverse event on romiplostim/eltrombopag in $18 \%$.

Platelet Outcomes: In all patients, the median platelet count (PIt) on eltrombopag or romiplostim was $45 \times 10^{9} / \mathrm{L}$ vs. $114 \times 10^{9} / \mathrm{L}$ on avatrombopag $(P<0.0001)$; in patients switched for ineffectiveness of romiplostim/eltrombopag, it was $28 \times 10^{9} / \mathrm{L}$ on romiplostim/ eltrombopag vs. $88 \times 10^{9} / \mathrm{L}$ on avatrombopag $(P=0.025)$, FIGURE. Regardless of the indication for switching, most patients achieved a complete response (PIt $\geq 100 \times 109 / L$ ) on avatrombopag (TABLE).

Concomitant Medications/Rescue Therapy: Of the 19 patients who required concomitant corticosteroids while on romiplostim/eltrombopag, 12 (63\%) discontinued steroids, 6 (32\%) reduced steroid dose, and none increased steroid dose after switching to avatrombopag. 15 patients (33\%) required rescue in the year prior to switching versus 9 (20\%) following the switch.

Avatrombopag Discontinuation: 2 patients (4\%) discontinued for adverse events (headache, portal vein thrombosis) and 1 (2\%) discontinued for lack of response.
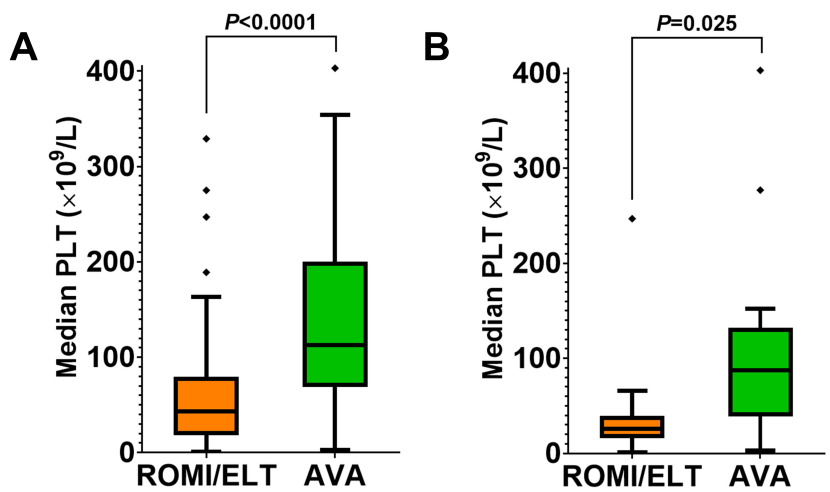

FIGURE 1 Median platelet counts for each patient prior to switch (during treatment with romiplostim or eltrombopag) vs. following the switch to avatrombopag. For each patient, the median platelet count is the median of the most recent 3 platelet counts measured while receiving that agent. (A) All patients $(N=45)$. (B) Patients switched due to ineffectiveness of romiplostim or eltrombopag ( $N=14)$. One patient with median PIt $585 \times 109 /$ L on avatrombopag omitted from both graphs to preserve graph resolution

Conclusions: In a heavily-pretreated chronic ITP population, avatrombopag was effective following therapy with romiplostim or eltrombopag, with high response rates even in patients with inadequate response to a prior TPO-RA.

\section{PB0824 | Refractory Immune Thrombocytopenia}

(ITP): The Combination of Thrombopoietin Analogs and Immunosuppressants, Experience in a Single Spanish Center

I. Sánchez Bazán; S. Martín Téllez; F.J. López Jaime; M.I. Muñoz Hospital Regional de Málaga, Málaga, Spain

Background: A small proportion of patients with immune thrombocytopenia (ITP) do not respond to conventional treatments, they may benefit from combined therapy with thrombopoietin analogs and immunosuppressants.

Aims: To describe our experience in the treatment of refractory ITP with combination of TPO-RA and immunosuppressants, with focus on response and safety.

Methods: We study adults with refractory ITP considering refractoriness not reaching platelets higher than $30 \times 10^{\wedge} 9 / \mathrm{L}$ or corticosteroid-dependence. All patients were diagnosed and treated in the same center with combined treatment after failure

TABLE 1 Rates of platelet response following switch to avatrombopag in the absence of rescue therapy (counts were disqualified if

\begin{tabular}{lllll} 
& \multicolumn{2}{l}{ Reason for Switch from Eltrombopag or Romiplostim to Avatrombopag } \\
Platelet Count Threshold & All Patients $(\mathbf{N}=45)$ & Ineffectiveness ( $\mathbf{N}=14)$ & Convenience $(\mathbf{N}=23)$ & Adverse Event $(\mathbf{N}=\mathbf{8})$ \\
$\geq 30 \times 10^{9} / \mathrm{L}$ & $42 / 45(93 \%)$ & $12 / 14(86 \%)$ & $23 / 23(100 \%)$ & $7 / 8(88 \%)$ \\
$\geq 50 \times 10^{9} / \mathrm{L}$ & $42 / 45(93 \%)$ & $12 / 14(86 \%)$ & $23 / 23(100 \%)$ & $7 / 8(88 \%)$ \\
$\geq 100 \times 10^{9} / \mathrm{L}$ & $39 / 45(87 \%)$ & $10 / 14(71 \%)$ & $22 / 23(96 \%)$ & $7 / 8(88 \%)$ \\
\hline
\end{tabular}


of monotherapy. We consider complete response a platelet count higher than $100 \times 10^{\wedge} 9 / \mathrm{L}$ and response by a platelet count $30-100$ $\times 10^{\wedge} 9 / \mathrm{L}$, according to the international recommendations. We report adverse events of 6 weeks after the combination (infections requiring hospital admission and thromboembolic events as deep vein thrombosis and pulmonary embolism).

Results: We analyzed 13 adult patients, 73\% female, median age 52 (range 18-85). Two had secondary ITP, eleven had primary ITP. Four had previous Splenectomy, not performed in 9 because of contraindication or patient refusal. At the time of combination, median platelet count was $18 \times 10^{\wedge} 9 / \mathrm{L}(4-33)$, median duration of ITP was 28 months (2-216). The combination therapy includes a thrombopoetin analog (romiplostim or eltrombopag) with an immunosuppressant, azathioprine in $70 \%$ of them and corticosteroids in $30 \%$. Nine patients $(69 \%)$ respond to the combination. Seven reached complete response, durable after a median follow up of 23 months. In fact, two cases maintain response after discontinuation. Just one did not respond and three remain cortico-dependent. The median time response was 14 days (7-20). Two patients experienced a thromboembolic event and just one had an infection. Combination was well tolerated in all of them but one.

Conclusions: Patients with refractory ITP, may benefit from treatment combinations with an acceptable safety profile. More studies are needed to support this conclusions.

PB0825 | Immature Platelet Fraction in Systemic Lupus Erythematosus-Related Thrombocytopenia: Potential Predictor of Platelet Response to Treatment?

T. Nguyen; D. Truong-Pham; T. Huynh; T. Nguyen; T. Tran; L. PhanNguyen; G. Nguyen-Tran; T. Tran

Cho Ray Hospital, Ho Chi Minh City, Vietnam

Background: Immature platelet fraction (IPF) has been shown to be a useful marker in thrombocytopenia cause identification. Moreover, there are reports showing that IPF can predict platelet recovery and response to treatment in various conditions, including immune thrombocytopenia (ITP.) However, in patients with thrombocytopenia related to systemic lupus erythematosus (SLE), IPF range and usefulness in response prediction is not well-studied.

Aims: Identify the range of IPF in SLE-related thrombocytopenia patients and its value as a predictor for early platelet response to treatment.

Methods: Newly diagnosed SLE patients with platelet counts $<50 \times 10^{9} / \mathrm{L}$ were recruited to our study. All patients received methylprednisolone, hydroxychloroquine and an immunomodulating agent. Blood samples were collected on the first day of treatment and 48 hours later for complete blood count and IPF testing. Patients who received platelet transfusion during the 48-hour follow-up were excluded. Also, SLE Disease Activity Index (SLEDAI) was evaluated in each patient at the start of treatment.

Results: 16 patients were recruited in this preliminary study. Mean baseline platelet count and IPF values were $32.63 \pm 22.97 \times 10^{9} / \mathrm{L}$ and $19.16 \pm 17.96 \%$, respectively. The $95 \%$ confidence interval $(95 \%$ $\mathrm{CI}$ ) of IPF was $9.59-28.73 \%$. Baseline IPF did not significantly correlate with baseline platelet count and SLEDAI $(P>0.05$.) However, baseline IPF had a significant correlation with platelet count change after 48 hours $(P<0.01$, correlation coefficient 0.68$)$ (Figure 1$)$

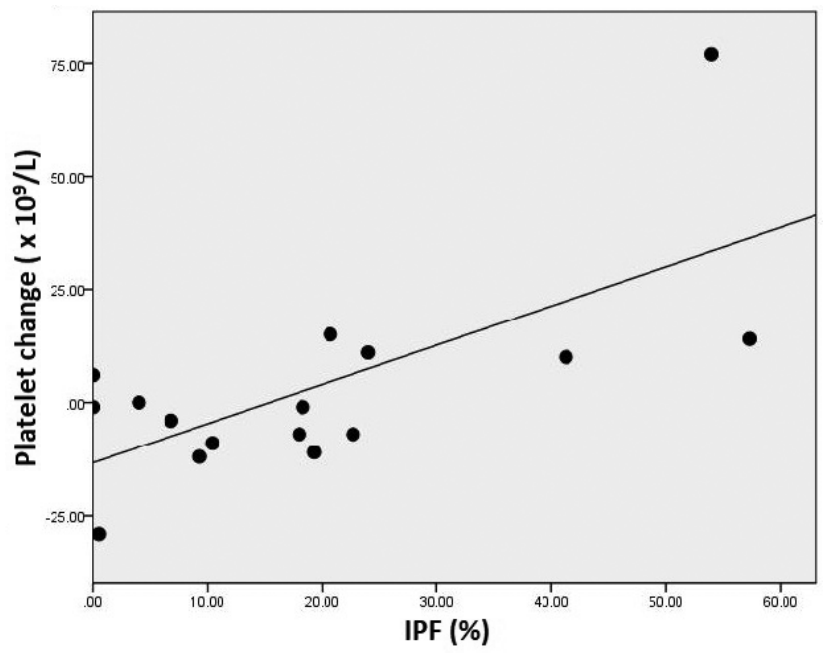

FIGURE 1 Correlation between baseline IPF and platelet change after 48 hours

Conclusions: Our early data shows that unlike ITP, in which hyperdestruction the main etiology of platelet consumption, SLE-related thrombocytopenia may involve multiple mechanisms, as the $95 \%$ $\mathrm{Cl}$ of IPF was wide and IPF did not correlate with baseline platelet count and SLEDAI. Finally, IPF seems to be a promising predictor for early platelet response for treatment in this patient population. More data is required to confirm these early findings. 
PB0826 | Off-label Use of Thrombopoietin Receptor Agonists: Case Series and Review of the Literature

M. Capecchi ${ }^{1}$; F. Serpenti ${ }^{2}$; J. Giannotta ${ }^{3}$; L. Pettine ${ }^{4}$; G. Reda ${ }^{4}$; W. Barcellini ${ }^{4}$; B. Fattizzo ${ }^{5}$;. Martinelli ${ }^{6}$; A. Artoni ${ }^{6}$; F. Peyvandi ${ }^{7}$ ${ }^{1}$ Fondazione IRCCS Ca' Granda Ospedale Maggiore Policlinico, Angelo Bianchi Bonomi Hemophilia and Thrombosis Center, Università degli Studi di Milano, Department of Biomedical Sciences for Health, Milan, Italy; ${ }^{2}$ Fondazione IRCCS Ca' Granda Ospedale Maggiore Policlinico, Angelo Bianchi Bonomi Hemophilia and Thrombosis Center, Università degli Studi di Milano, Department of Oncology and Onco-Hematology, Milan, Italy; ${ }^{3}$ Fondazione IRCCS Ca' Granda Ospedale Maggiore Policlinico, Hematology Unit, Università degli Studi di Milano, Department of Oncology and Onco-Hematology, Milan, Italy; ${ }^{4}$ Fondazione IRCCS Ca' Granda Ospedale Maggiore Policlinico, Hematology Unit, Milan, Italy; ${ }^{5}$ Università degli Studi di Milano, Department of Oncology and Onco-Hematology, Fondazione IRCCS Ca' Granda Ospedale Maggiore Policlinico, Hematology Unit, Milan, Italy; ${ }^{6}$ Fondazione IRCCS Ca' Granda Ospedale Maggiore Policlinico, Angelo Bianchi Bonomi Hemophilia and Thrombosis Center, Milan, Italy; ${ }^{7}$ Fondazione IRCCS Ca' Granda Ospedale Maggiore Policlinico, Angelo Bianchi Bonomi Hemophilia and Thrombosis Center, Università degli Studi di Milano, Department of Pathophysiology and Transplantation, Milan, Italy

Background: Since their license in 2008 for use in immune thrombocytopenic purpura, thrombopoietin receptor agonists (TPO-RA), due to their favorable efficacy and safety profile, are becoming candidates for the management of thrombocytopenia in different settings.

Aims: The study aimed to report the response rates and safety profile of the TPO-RA used off-label.

Methods: Observational retrospective study including consecutive patients aged $>18$ years and receiving off-label TPO-RA at our hospital between January 2010 and June 2020.

Results: A total of 81 patients have been treated with a TPO-RA (eltrombopag or romiplostim) at our center. Sixty-seven patients received the TPO-RA for in-label conditions - ITP (54 patients) or aplastic anemia (13 patients) - while 14 patients received a TPO-RA for unlabeled conditions

(Figure 1). Among the latter group, 4 patients had a transplantassociated thrombocytopenia, 8 suffered from myelodysplastic syndrome and 2 had thrombocytopenia associated to a lymphoproliferative neoplasm. All patients were treated with eltrombopag, except for one who received romiplostim. Figure 2 shows median platelet trends over time among the different groups. Overall, TPO-RA proved effective in all these conditions achieving responses also in heavily pretreated patients. Overall response rate (ORR) was $100 \%$ in patients with thrombocytopenia after transplantation and in those with lymphoproliferative diseases and $75 \%$ in patients with myelodysplastic syndromes. Median duration of therapy was 285 days (range 93-1513 days). Four patients (29\%) discontinued treatment, because of lack of response $(n=2)$ or a sustained response $(n=2)$. No grade 3-4 adverse events occurred, particularly no thrombosis.

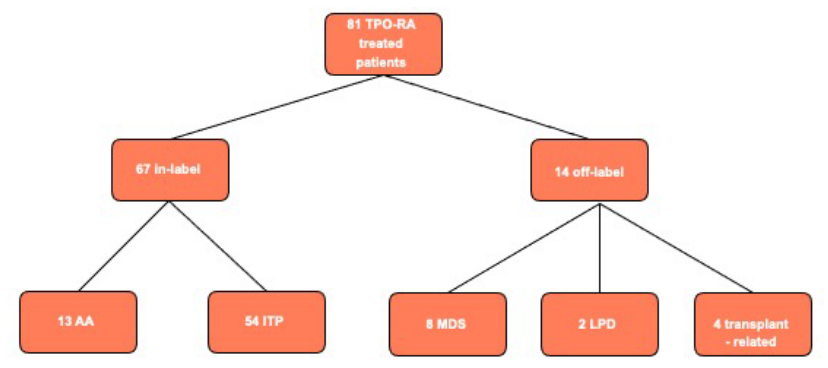

FIGURE 1 Flowchart of thrombopoietin receptor agonist (TPORA) treated patients at our Center in the last 10 years. Aplastic anemia (AA) refers to aplastic anemia patients treated in second line after failure of immunosuppressive therapy as for current drug indications; we had no off-label AA patient treated in first line. MDS, myelodysplastic syndrome; LPD, lymphoproliferative disease; ITP, autoimmune thrombocytopenia.

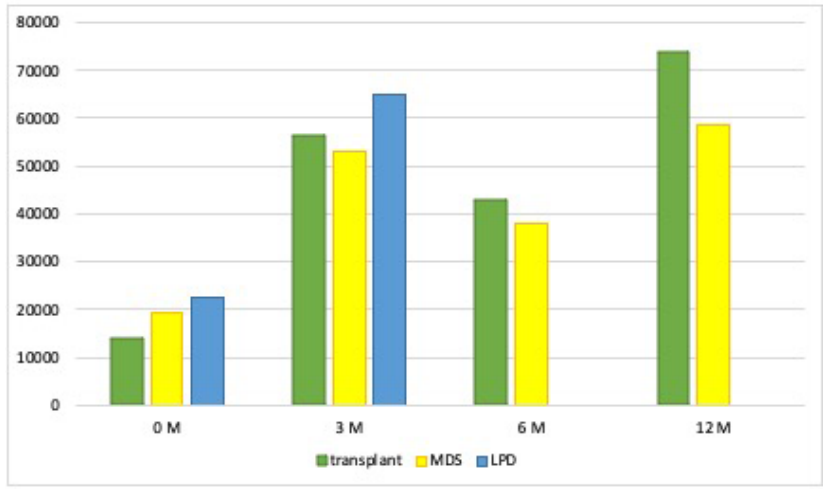

FIGURE 2 Median platelet count at baseline, 3, 6 and 12 months per group of patients. At 6 and 12 months no data are reported for the lymphoproliferative patients because the median follow-up was inferior to 6 months in this group. LPD, lymphoproliferative disease; MDS, myelodysplastic syndrome; $M$, months.

Conclusions: In our real-life experience, TPO-RA were effective and safe and proved of value in the challenging management of patients with refractory thrombocytopenia associated with different conditions. 
PB0827 | Fatigue in Chronic Immune Thrombocytopenia

Patients: Validation of the Checklist Individual Strength and Comparison to Other Chronically III Populations

W.E.M. van Dijk ${ }^{1}$; I.A.R. Kuijlaars ${ }^{1}$; M.H. Suijker ${ }^{2}$; K.P.M. van Galen ${ }^{1}$; R.E.G. Schutgens ${ }^{1}$

${ }^{1}$ University Medical Center Utrecht, Utrecht, Netherlands; ${ }^{2}$ Wilhelmina KinderZiekenhuis, Utrecht, Netherlands

Background: Patients with immune thrombocytopenia (ITP), a condition characterized by thrombocytopenia due to immune-mediated destruction of platelets, frequently report concomitant fatigue.

Aims: (1) To validate a well-known fatigue-specific questionnaire, the Checklist Individual Strength (CIS), in ITP patients.

(2) To assess the presence of fatigue and compare this with other chronically-ill populations.

Methods: In a single-center cross-sectional study, patients with chronic ITP patients aged $\geq 16$ years, who were currently treated or had a platelet count $<100 * 10 \% / \mathrm{L}$ were included. Patients completed the entire CIS20 (subscales: fatigue (CIS8), concentration, motivation and activity), and the FACIT-F, a fatigue-specific questionnaire validated in ITP. Internal consistency was assessed by Cronbach's a ( $a$; $\geq$ 0.8). Construct validity was assessed by a Spearman's correlation $(r)$ with FACIT-F. We considered $r \leq-0.7$ a strong correlation (negative due to reverse scoring in the two questionnaires). The cut-off for severe fatigue is a score of $\geq 35$ on the CIS8. Descriptive statistics included percentages or medians (interquartile range). Comparative data from other chronically ill populations were extracted from the literature.

Results: We assessed 55 ITP patients (62\% female, age 44 years (29-58)), of whom $23 \%$ received treatment at time of assessment. Median platelet count was $63^{*} 10^{9} / \mathrm{L}\left(46-92^{*} 10^{9} / \mathrm{L}\right)$. The internal consistency was good for both the CIS8 and the CIS20 ( $a$ for both: 0.96 (95\%-CI 0.94-0.97)). The correlation with the FACIT-F was also strong for both ( $r-0.86$, and -0.85$)$. 32 ITP patients (58\%) were severely fatigued. This proportion is higher than in neuralgic amyotrophy, renal transplantation, rheumatoid arthritis, and type 1 diabetes (39-44\%), but lower than in Ehlers-Danlos syndrome, chronic pancreatitis, primary Sjögren, multiple sclerosis (68-77\%).

Conclusions: Both the CIS20 and the CIS 8 are valid in ITP. Severe fatigue in ITP patients is common, comparable with other chronically ill populations.
PB0828 | Autoimmune Platelet Function Disorders: Systematic Review of Acquired Glanzmann Thrombasthenia and Acquired Delta Storage Pool Disease Cases

A. Ferretti ${ }^{1} ;$ M. Bacci ${ }^{2} ;$ M. Marchetti ${ }^{3} ;$ M.A. Alberelli ${ }^{1}$; A. Falanga ${ }^{4}$; C. Lodigiani ${ }^{5}$; E. De Candia ${ }^{6}$

${ }^{1}$ IRCCS Policlinico Universitario A. Gemelli Foundation, Rome, Italy; ${ }^{2}$ Humanitas Clinical and Research Center-IRCCS, Milan, Italy; ${ }^{3}$ Department of Immunohematology and Transfusion Medicine, Hospital Papa Giovanni XXIII, Bergamo, Italy; ${ }^{4}$ Università di Milano Bicocca, School of Medicine and Surgery, Monza, Italy; ${ }^{5}$ Humanitas University Department of Biomedical Sciences, Milan, Italy; ${ }^{6}$ Department of Translational Medicine and Surgery, Catholic University of Rome, Rome, Italy

Background: Acquired platelet function disorders (PFD) are rare bleeding diseases that should be suspected in all patients (pts) with unexplained recent mucocutaneous bleeding onset, no previous history of bleedings, normal coagulation test and platelet count.

Aims: We revised the literature of the acquired PFD caused by autoimmune mechanisms (aPFDs).

Methods: We queried the electronic databases PubMed, Embase, Scopus and Google Scholar for "acquired Glanzmann Thromboasthenia" (aGT), "acquired delta storage pool disease" (adSPD) retrieving all articles reporting aPFDs sustained by autoimmune mechanism until December 2020.

Results: We found 44 cases of aGT, 12 cases of adSPD and 47 cases of autoimmune PFDs of uncertain/mixed diagnosis. APFDs can be primary or secondary to other diseases. Among aGT, 10 cases were primary, 17 were associated to lymphoproliferative diseases $(5 \mathrm{HL}, 7$ NHL, 1 ALL, 1 HCL, 1 MM, 4 MGUS/paraprotein), 4 were described in renal and heart transplant recipients receiving immunosuppressive therapy, 13 cases had autoimmune disorders, including ITP. Among adSPD, 7 cases were associated with autoimmune/connective tissue disorders (2 SLE, 1 RA, 2 unspecified connective tissue diseases), 4 cases were associated with lymphoproliferative disorders (1 HCL, 2 CLL, 1 Waldenstrom disease), only one case was a primary adSPD. Autoimmune PFDs may develop in patients splenectomized for ITP (13 cases). Fourteen out of 44 aGT cases and 3 out of 11 adSPD cases had ITP before or after aPFD. Treatments included hemostatic therapies for the control of bleedings, treatment of primary disease, if present, and immunosuppressive treatment for the eradication of antibodies. Response to treatments was variable and unpredictable. Conclusions: Autoimmune PFDs are rare syndromes whose diagnosis is challenging and might be underestimated. A rapid diagnosis and appropriate therapy are crucial to stop bleedings. So far, therapies have been personalized based on clinical phenotype (major/minor bleedings), clinical needs (surgery, anemia) and underlying disease

TABLE 1 Legend

$\begin{array}{ll}\mathrm{HL} & \text { Hodgkin's lymphoma } \\ \mathrm{NHL} & \text { non Hodgkin's lymphoma } \\ \text { ALL } & \text { acute lymphoblastic leukemia }\end{array}$




\begin{tabular}{ll} 
HCL & hairy cell leukemia \\
MM & multiple myeloma \\
SLE & systemic lupus erythematosus \\
RA & rheumatoid arthirits \\
CLL & chronic lymphocytic leukemia \\
\hline
\end{tabular}

PB0829 | The Spectrum of Immune Thrombocytopenia with COVID-19 Infection

\section{Muhsen; J. Petkova; L. Rice}

Dept of Medicine, Division of Hematology, Houston Methodist Hospital, Weill Cornell Medical College, Houston, United States

Background: Thrombocytopenia has substantial prognostic impact with COVID infection, and there are numerous potential causes. A September 2020 review uncovered 45 reported cases of ITP (immune thrombocytopenia). We have seen several such patients.

Aims: To provide insight into features of COVID-associated ITP from three of our patients, highlighting differences from current literature. Methods: This is a case series of three patients with variable clinical presentations of COVID-associated ITP.

Results: (1) A 58 year old man underwent cardiac and later autologous stem cell transplant for AL amyloidosis beginning 2013. He developed COVID pneumonia November 2020, hospitalized two days. Severe thrombocytopenia emerged one month later (platelets $1,000 /$ ul). Responses have been poor to high dose corticosteroids, repeated IVIG, and thrombopoietin agonists (TPOs), platelets still $4,000 /$ ul after two months. (2) A 44 year old woman was treated for ITP in 2002 and had multiple subsequent relapses. For two years on fostamatanib, she had stable partial remission (platelets 50,000/ul) when she experienced one day of fever, cough, and tested positive for COVID. She required hospitalization five days later for petechiae and oral hemorrhagic bullae. Platelets remained 1,000/ul for three weeks despite steroids, IVIG, androgens, TPOs, plasma exchanges, platelet transfusions, azathioprine, vincristine and zanubrutinib. Platelets recovered after six weeks. (3) An asymptomatic 78 year old woman with chronic diabetic nephropathy had incidentally found platelets $8,000 / u l$. Single digit platelets persisted despite aggressive therapy. After two weeks, she developed tremors, unsteady gait, and positive COVID tests. Thrombocytopenia (and neurologic problems) resolved after one month. All patients had blood smears and bone marrows typical for ITP and lacked indications of alternative diagnoses (high d-dimers, suspicious medications).

Conclusions: ITP is common with COVID. It can complicate mild disease or be its first manifestation, and can exacerbate pre-existing ITP. It can be highly refractory to therapy, but is usually transient.
PB0830 | Efficacy and Safety of Efgartigimod PH20

Subcutaneous in Adult Patients with Primary Immune Thrombocytopenia: ADVANCE SC, a Global Phase 3 Clinical Trial in Progress

C. Broome ${ }^{1}$; V. McDonald ${ }^{2}$; S. Jain ${ }^{3}$; S. Babu ${ }^{4} ;$ E. Oliva ${ }^{5}$; W. Parys ${ }^{6}$; A. Hultberg ${ }^{6}$; K. De Beuf ${ }^{6}$; D. Gandini' ${ }^{6}$ Y. Miyakawa ${ }^{7}$; W. Ghanima ${ }^{8}$ ${ }^{1}$ Georgetown University, Washington, United States; ${ }^{2}$ Barts Health NHS Trust, London, United Kingdom; ${ }^{3}$ RUSH University Medical Center, Chicago, United States; ${ }^{4}$ Fort Wayne Medical Oncology and Hematology, Inc, Fort Wayne, United States; ${ }^{5}$ Haematology Unit, Grande Ospedale Metropolitano, Reggio Calabria, Italy, ${ }^{6}$ argenx, Ghent, Belgium; ' Saitama Medical University Hospital, Saitama, Japan; ${ }^{8}$ Departments of Medicine, Hematology-Oncology, and Research, Østfold Hospital Trust, Kalnes, and the Department of Hematology, Oslo University Hospital and Institute of Clinical Medicine, University of Oslo, Oslo, Norway

Background: Efgartigimod, an FcRn antagonist, was well tolerated compared to placebo and induced a rapid reduction of total IgG levels, which was associated with clinically relevant increases in platelet counts, and a reduced proportion of patients with bleeding in the Phase 2 trial in patients with primary ITP (Newland AC. Am J Hematol. 2020;95:178-187. NCT03102593), warranting further evaluation in Phase 3 clinical trials. A subcutaneous (SC) formulation has been developed to offer additional flexibility and convenience for patients.

Aims: ADVANCE SC, a Phase 3, multicenter, randomized, doubleblinded, placebo-controlled trial (NCT04687072), will evaluate the efficacy and safety of efgartigimod $\mathrm{PH} 20$ administered $\mathrm{SC}$ in adults with persistent or chronic ITP.

Methods: Eligible patients must have a mean platelet count $<30 \times 10^{9} /$ L over at least 3 qualifying evaluations and have received at least 2 prior ITP treatments or 1 prior and 1 concurrent treatment, with response to at least one. Patients will enter a 24-week treatment period and receive either efgartigimod (1,000 mg) coformulated with $\mathrm{PH} 20$ or matching placebo (randomization 2:1), administered weekly from visits 1 to 4 and then either weekly or every other week from visits 5 to 16 , as determined by platelet counts. Dosing schedule will be fixed from visits 17 to 24 . Permitted concurrent ITP treatments include corticosteroids, oral immunosuppressants, dapsone/danazol, fostamatinib and/or oral TPO-RAs.

Results: The primary endpoint is the proportion of patients with a sustained platelet count response $\left(\geq 50 \times 10^{9} / \mathrm{L}\right.$ for at least 4 of the 6 visits between study weeks 19 and 24). Secondary endpoints include safety and tolerability, bleeding severity, quality of life and patient-reported outcome measures, and the immunogenicity and pharmacokinetic/pharmacodynamic effects of efgartigimod.

Conclusions: Recruitment is ongoing in Asia-Pacific, Europe, Japan, Latin America, the Middle East, Africa and USA. Trial participants will be eligible for continuation into ADVANCE $\mathrm{SC}^{+}$, a long-term open-label extension trial. 
PB0831 | Prevalence of Thrombotic Events and Risk Factors in Patients with Primary Immune Thrombocytopenia

A. Doblas-Marquez; F.-J. Lopez-Jaime; S. Martin-Tellez; I. SanchezBazan; M.-I. Muñoz-Perez

Hospital Universitario Regional de Malaga, Malaga, Spain

Background: Primary immune thrombocytopenia (ITP) is an inherited autoimmune disorder characterised by peripheral platelet destruction and abnormally low platelet production.

Although the typical symptoms of this disease are bleeding events, a thrombophilic disorder has been described, with a higher risk of thrombosis than in the general population. This higher incidence has been associated with cardiovascular risk factors, such as arterial hypertension ( $\mathrm{HT}$ ), diabetes mellitus (DM), dyslipidemia (DL), smoking, advanced age and a previous history of thrombosis.

Aims: To analyze and evaluate the thrombotic events in patients with ITP in our center.
Methods: Observational, retrospective, single-center study in adult patients older than 18 years diagnosed with ITP.

Arterial thrombotic events were defined as stroke and myocardial infarction (MI).

Venous thrombotic events were considered pulmonary embolism (PE) and deep vein thrombosis (DVT).

Results: A total of 75 patients have been recruited and 11 thrombotic events were described highlighting that 5 thrombotic events occurred with a platelet count lower than $50 \times 10^{9} / \mathrm{L}$. Most arterial thrombotic events were in therapeutic abstinence (80\%), however all patients with venous thrombosis events were on TPO analogs treatment (table 1).

In our series, the most important risk factor for presenting a thromboembolic event was the previous history of thrombosis, achieving statistical significance both the previous history of arterial thrombosis $(P=0.006)$ and venous $(P=0.007)$.

However, we did not find significant differences in other cardiovascular risk factors, possibly due to the limited sample size.

TABLE 1 Resume of the thrombotic events. Art: Arterial thrombosis; Elt: Eltrombopag; Ev1: First thrombosis; Ev2: Second thrombosis; Rom: Romiplostim; Ven: Venous thrombosis

\begin{tabular}{|c|c|c|c|c|c|c|c|c|c|}
\hline \multirow{2}{*}{$\begin{array}{l}\text { Patients with } \\
\text { thrombotic events } \\
n=8 / 75 \text { (10.7\%) }\end{array}$} & \multicolumn{2}{|c|}{ Thrombotic events $n=11$} & \multicolumn{2}{|c|}{$\begin{array}{l}\text { Platelets counts } \\
\times 10^{9} / \mathrm{L}\end{array}$} & \multicolumn{2}{|c|}{ Treatment } & \multirow[b]{2}{*}{ Esplenectomy } & \multicolumn{2}{|c|}{$\begin{array}{l}\text { Previous } \\
\text { thrombosis }\end{array}$} \\
\hline & Art & Ven & Ev 1 & Ev 2 & Ev 1 & Ev 2 & & Art & Ven \\
\hline Patient 2 & Yes & & 33 & & Rom & & No & Yes & Yes \\
\hline Patient 3 & Yes & & 45 & & No & & No & No & No \\
\hline Patient 5 & & Yes/Yes & 365 & 365 & Rom & Rom & No & Yes & Yes \\
\hline Patient 6 & Yes & & 40 & & No & & Yes & Yes & No \\
\hline Patient 7 & & Yes/Yes & 49 & 53 & Elt & Elt & No & Yes & Yes \\
\hline Patient 8 & Yes & & 429 & & No & & Yes & No & No \\
\hline
\end{tabular}

Conclusions: - Our results show a high prevalence of thrombosis in ITP patients, even despite presenting low platelet counts.

- The ratio of arterial and venous thrombosis was the same, however the use of TPO analogues was more associated with venous than arterial thrombosis.

- In our series, the greatest risk factor for suffering a thromboembolic event were having a previous history of thrombosis, despite being on antiplatelet or anticoagulant treatment.

\section{PB0832 | Drug Induced Purpura in Autologous Hematopoietic} Stem Cell Transplantation - A Case Report

T. Quaresma ${ }^{1}$; A. Rodrigues ${ }^{1}$; A. Garçãoํㅜㄹ C. Malcata ${ }^{2}$; A. Silva Martins ${ }^{3}$; A. Cristina Alho ${ }^{3}$; M. Galvão ${ }^{1}$

${ }^{1}$ Centro Hospitalar Universitário Lisboa Norte - Imunohemoterapia, Lisbon, Portugal; ${ }^{2}$ Instituto Português de Sangue e Transplantação - CST Lisbon, Lisbon, Portugal; ${ }^{3}$ Centro Hospitalar Universitário Lisboa Norte - Hematologia, Lisbon, Portugal

Background: Autologous Hematopoietic stem cell transplantation (AHSCT) is a standard of care in fit multiple myeloma (MM) patients aged $<70$ years. After AHSCT the pre-engraftment period may last 10-12 days and is characterized by severe pancytopenia. Platelets count may decline as low as $5-10 \times 10^{9} / \mathrm{L}$, translating into mucosal hemorrhage and petechiae. However, thrombocytopenic purpura is not a common presentation. 
Aims: To manage, diagnose and treat purpura during the preengraftment period of AHSCT.

Methods: We report the case of a 68-year-old woman diagnosed with MM IgG Kappa. She was treated with 6 cycles of lenalidomide, bortezomib and dexamethasone (VRD). Peripheral Blood Stem Cells were collected by leukapheresis after cycle three. Five months later she was admitted to AHSCT and started conditioning with melphalan $200 \mathrm{mg} / \mathrm{m} 2$ followed by infusion of $3.36 \times 10^{6} / \mathrm{kg}$ CD34+ cells on day 0 (D0). On D11 post-infusion she presented fever, dyspnea and hypoxemia. The blood count showed hemoglobin of $11.9 \mathrm{~g} / \mathrm{dL}$, leukocyte count of $0.1 \times 10^{9} / \mathrm{L}$ and platelet count of $11 \times 10^{9} / \mathrm{L}$. She was transfused with platelet concentrated pool and empirical antibiotic treatment with amikacin and piperacillin-tazobactam was started. On D12 she presented with acute generalized purpuric lesions.

Results: On Laboratory testing, using solid phase tecnhique, antibodies binding to platelets were positive, as well as in the presence of piperacilin-tazobactam. The tests in the presence of the remaining drugs (amikacin, aciclovir and fluconazol) were negative. ELISA test was negative for auto and alloantibodies. Purpuric lesions disappeared after piperacilin-tazobactan discontinuation and antibiotic replacement. Other causes of thrombocytopenia were excluded.

Conclusions: We present a case of acute onset of generalized purpura in the pre-engraftment period post-AHSCT. The presence of drug-dependent platelet antibodies has clarified the diagnosis, with clinical improvement after antibiotic replacement. When purpura occurs in patients treated with AHSCT, apart from testing for drug induced reaction, immunization against platelet's antigens must always be excluded.

PB0833 | Treatment of Immune Thrombocytopenia (ITP) in the COVID-19 Era: Fostamatinib, an Oral Spleen Tyrosine Kinase (SYK) Inhibitor

N. Cooper ${ }^{1}$; A. Charania ${ }^{2}$; A. Hart ${ }^{3}$; C. Ademokun ${ }^{3}$; R. Numerof ${ }^{4}$ ${ }^{1}$ Imperial College Healthcare NHS Trust, Hammersmith Hospital, London, United Kingdom; ${ }^{2}$ Epsom and St Helier University Hospitals NHS Trust, Epsom, United Kingdom; ${ }^{3}$ Imperial College Healthcare NHS Trust, London, United Kingdom; ${ }^{4}$ Department of Medical Affairs, Rigel Pharmaceuticals, Inc, South San Francisco, United States

Background: Management of ITP became increasingly challenging during the COVID-19 pandemic. Immunosuppressive treatments increase susceptibility of patients to COVID-19. Therapies increasing the thrombotic risk are suboptimal due to coagulopathy observed with COVID-19. The need to minimize office visits to limit potentia viral exposure renders intravenous administration or injections less suitable. Consequently, ITP management requires careful patientcentric consideration during the pandemic.

Fostamatinib is a potent oral SYK inhibitor that abrogates SYKmediated destruction of platelets and may abrogate SYK-mediated thromboinflammation.
Aims: To evaluate fostamatinib as an ITP treatment during the COVID-19 era.

Methods: Review of safety, immunotoxicology, and mechanism of action and administration for fostamatinib.

Results: Preclinical studies demonstrated intact innate and humoral responses to immune challenges in fostamatinib (R406) treated rodents. ${ }^{1}$ In ITP clinical trials, the incidence of adverse events (including infections) was somewhat higher with fostamatinib vs placebo ( $83 \%$ vs $75 \%$ ), which is consistent with the 2.4 -fold increase in exposure to fostamatinib vs. placebo ( 29 vs. 12 patient-exposure years, respectively). No patients had opportunistic infections. The rate of thromboembolic events with fostamatinib (0.7\% over 5 years) was low compared with similar studies with other ITP treatments (2.6$8.9 \%$ over $2-8$ years). Office visits can be minimized due to oral administration of fostamatinib and simplified titration: fostamatinib is initiated at $100 \mathrm{mg}$ BID and increased to $150 \mathrm{mg}$ BID after 4 weeks if needed. Thrombocytosis was uncommon (1.4\% over 5 years).

Conclusions: Fostamatinib is an immunomodulatory treatment for ITP that may lower the risk of thrombosis. The need for office visits may be reduced due to oral administration, simplified titration, and infrequency of thrombocytosis. Fostamatinib is an appropriate option for the treatment of ITP in the COVID-19 era.

Zhu Y, et al. Toxicol Appl Pharmacol. 2007;221(3):268-77

PB0834 | Idiopathic Thrombocytopenic Purpura: Conventional Prednisolone vs High-dose Dexamethasone

C. Lewis $^{1,2} ;$ Z. Ng ${ }^{1,3}$; C. Grove ${ }^{1,2,3}$

${ }^{1}$ Department of Haematology, Sir Charles Gardiner Hospital, Nedlands, Australia; ${ }^{2}$ University of Western Australia, Faculty of Medicine and Pharmacology, Nedlands, Australia; ${ }^{3}$ PathWest Laboratory Medicine, Nedlands, Australia

Background: Idiopathic thrombocytopenic purpura (ITP) is an autoimmune condition treated with corticosteroids. Traditionally long-term prednisolone (PDN) has been used. Recently high-dose dexamethasone (HD-DXM), 40mg for four days, reported higher rates of complete response (CR) and similar sustained remission (SR). HD-DXM therefore became our first line treatment for acute ITP in 2016.

Aims: To compare outcomes, including response, retreatment, use of additional therapies and bleeding, for treatment naïve ITP patients managed with conventional PDN versus short course HD-DXM at our institution.

Methods: Retrospective review of all patients with treatment naïve ITP at Sir Charles Gairdner Hospital from January 1st 2014 to December 31st 2018. Partial response (PR) and CR were defined as rise in platelet count $>30 \times 10^{9} / \mathrm{L}$ and $>100 \times 10^{9} / \mathrm{L}$ respectively, with at least a two-fold increase from baseline. SR required maintenance for six months. HD-DXM cycles were 10 days, PDN cycles 28 days long. 
Results: 44 patients were identified. Initial treatment was PDN in 21, HD-DXM in 19 and four received other therapies. Response to cycle 1 (C1) treatment was $90 \%$ in the PDN ( $81 \%$ CR, 43\% SR) and $88 \%$ in the HD-DXM cohort ( $59 \%$ CR, 35\% SR). In C1, 16 patients received intravenous immunoglobulin (IVIG). Eight patients were retreated with HD-DXM: two achieved response, five switched to PDN/alternate therapies and one never responded. Overall, 66\% achieved SR with additional therapy required (41\%). $20.5 \%$ were lost to follow-up or died. Bleeding rate was similar between PDN and HD-DXM cohorts (11 actionable bleeds each). Two HD-DXM patients had major gastrointestinal haemorrhage, resulting in one death.

TABLE 1 First cycle data
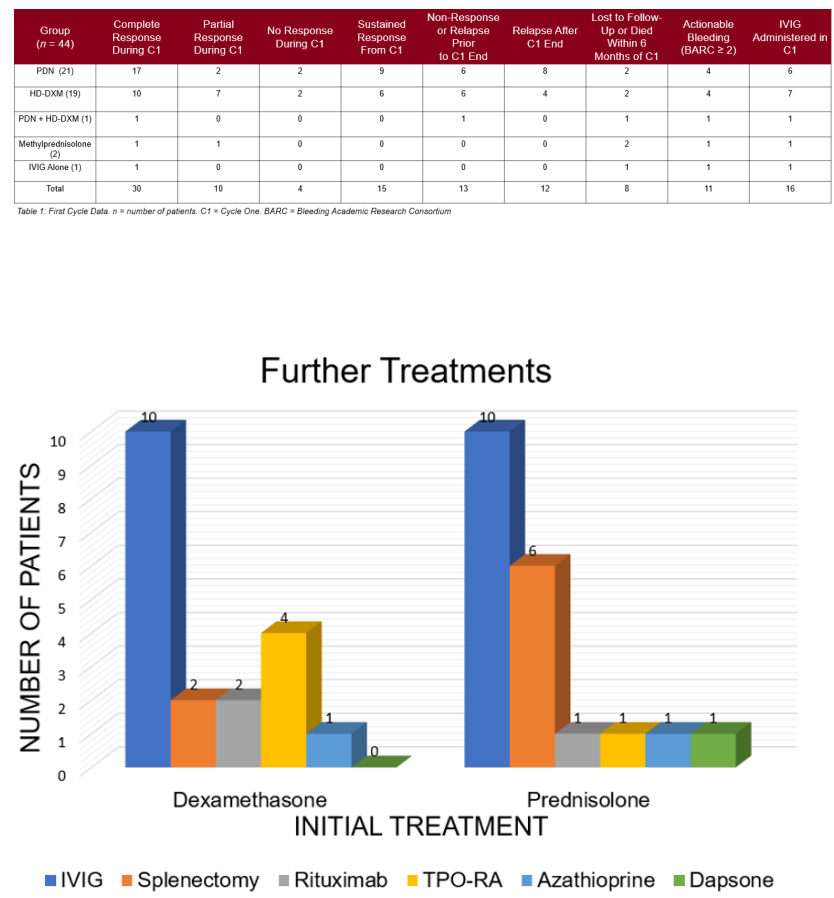

Figure 1: Subsequent alternative treatments, at any time, HD-DXM and PDN cohorts. $I V I G=$ intravenous immunoglobulin, TPO-RA = thrombopoietin receptor agonist

FIGURE 1 Subsequent alternative treatments in HD-DXM and PDN cohorts

Conclusions: Similar rates of response were seen between cohorts. Most patients who were retreated with HD-DXM did not respond to HD-DXM alone. Many patients received early IVIG without waiting for steroid response and yet there was still a high rate of clinically actionable bleeding.
PB0835 | Serum Complement Levels in Immune

Thrombocytopenia: Relation with Clinical Presentation and Treatment Response

S. Pérez -León; B. Galmés; G. Puguet; A. Gutiérrez; J.M. SánchezRaga; M. Canaro-Hyrnik Son Espases University Hospital, Palma de Mallorca, Spain

Background: The complement system activation is one of the mechanisms behind platelet destruction in immune thrombocytopenia, but its role in the clinical setting is still limited.

Aims: Characterized $\mathrm{C} 3, \mathrm{C} 4$, and $\mathrm{CH} 50$ levels from in ITP patients and its relation with the clinical presentation and treatment response.

Methods: A retrospective observational study in a teaching hospital with specific ITP outpatient consults. Complement levels were measured in any moment of the disease course through turbidimetric immunoassays. The relations between complements levels and characteristics of the disease and the response to treatments were evaluated with regression analysis.

Results:

\section{FIGURE 1}

Complememt levels and response to first line treatment

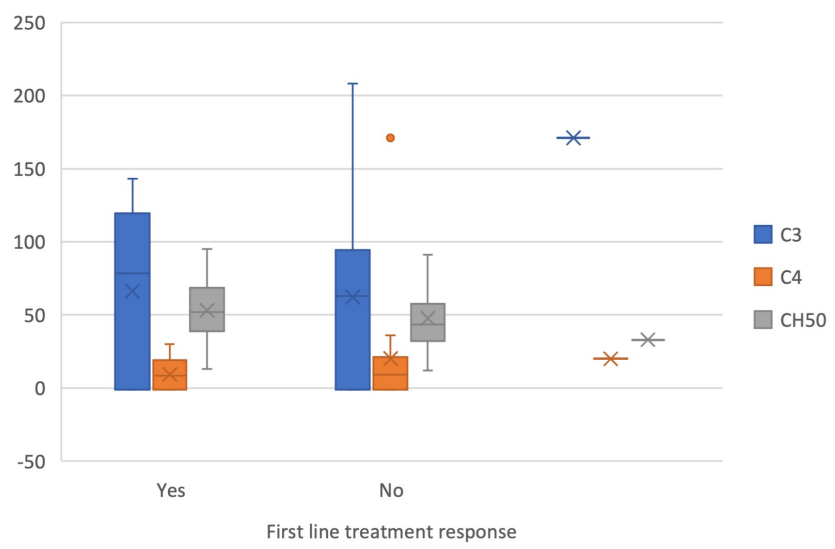

FIGURE 2

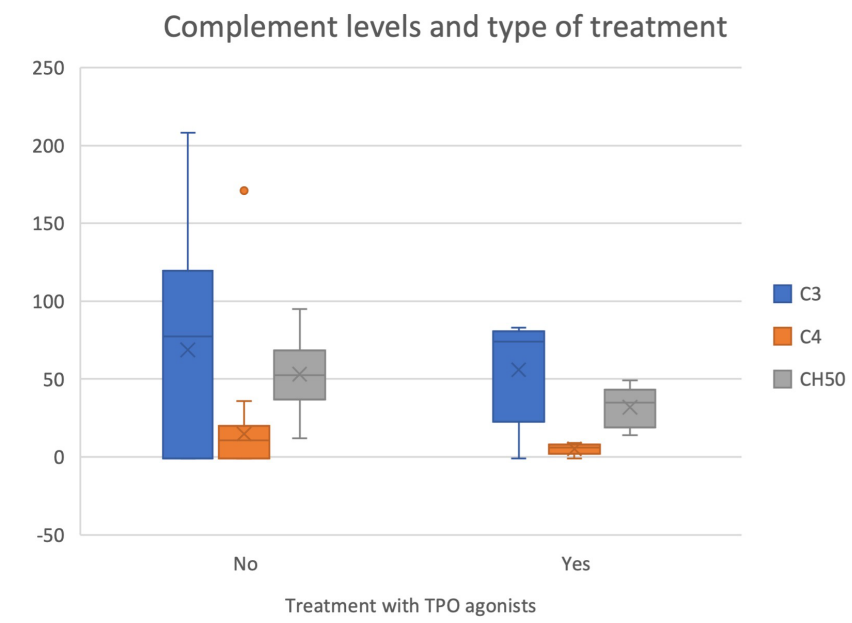


Forty-three ITP patients with complement C3, C4, CH50 were included. Medium age was 44 years (16.99). In the moment when complement levels were measured $70 \%$ of patients were not receiving any treatment. There were $16 \%$ of previously splenectomized patients. Medium C3: 103,5 (34-28), medium C4: 145 (4-171), with $75 \%$ of the patients with levels of $\mathrm{C} 4$ below the normal range. Medium CH50 49 (12-95). Not responders to first line treatment had lower $C 3$ levels ( $P$ : 0.018). Patients receiving treatment had lower mean C4 (P: 0.039) and lower C3 (P: 0.065). In multivariable analyses patients receiving TPO agonist had lower levels of $\mathrm{C} 3, \mathrm{C} 4$, and $\mathrm{CH} 50$ ( $P$ : 0.024; 0.019 y 0.054). There were no relation between complement level and severity of bleeding, refractory disease or splenectomy status.

Conclusions: ITP patients had lower levels of C4, especially those on active treatment. Patients not responders to first line treatments had lower levels of C3. Those on treatment with thrombopoietin receptor agonists had lower levels of $\mathrm{C} 3, \mathrm{C} 4$, y CH50. Further prospective studies are needed to evaluate the role of complement in the current clinical practice and with the emergent complement- directed therapies.

\section{P0155 | Dentoalveolar Procedures in Immune} Thrombocytopenia; Systematic Review and Practical Recommendations

W.E.M. van Dijk ${ }^{1}$; R.J.J. van Es ${ }^{1}$; M.E.P. Correa ${ }^{2}$; R.E.G. Schutgens ${ }^{1}$; K.P.M. van Galen ${ }^{1}$

${ }^{1}$ University Medical Center Utrecht, Utrecht, Netherlands; ${ }^{2}$ University of Campinas, Campinas, Brazil

Background: Dentoalveolar procedures in immune thrombocytopenia (ITP) pose a risk of bleeding, due to thrombocytopenia, and infection, due to immunosuppressive treatments.

Aims: To systematically review the safety and management of dentoalveolar procedures in ITP patients in order to create practical recommendations.

Methods: Pubmed, Embase, Cochrane and Cinahl were searched. All original studies with surgical and non-surgical dentoalveolar procedures (including tooth extractions, scaling, and periodontal and endodontic surgery) in adult and pediatric patients with known primary ITP at the time of procedure were included. Details on bleeding- and infection-related outcomes were recorded, including the therapeutic strategies. Clinically relevant bleeding was defined as needing treatment or medical attention.

Results: Eighteen articles were included, of which twelve case reports/series. Overall, the quality of the available evidence was poor. Outcomes and administered therapies (including hemostatic therapies and prophylactic antibiotics) were not systematically reported. At least 118 dentoalveolar procedures in 94 ITP patients were described. The range of preoperative platelet count was $2-412 * 10^{9} / \mathrm{L}$. Two clinically relevant bleedings were reported in the same patient, of which one life-threatening. Strategies used to minimalize the risk of bleeding were heterogeneous and included therapies to increase platelet count, antifibrinolytics, local measures and minimal invasive techniques. Reports on the occurrence of bleedings due to anesthetics or infection were lacking.

Conclusions: It appears that clinically relevant bleeding complications after dentoalveolar procedures in ITP patients occur rarely, based on low quality data. Reported therapeutic strategies to prevent bleedings are heterogeneous. Prospective and controlled studies are needed to evaluate safety of dentoalveolar procedures in patients with low platelet counts and to investigate the efficacy of therapeutic interventions. We propose practical recommendations to manage dentoalveolar procedures in ITP patients based on the best available evidence and our single-center clinical experience.

\section{P0157 | Self-Administration of Romiplostim in Patients with Chronic Immune Thrombocytopenia Compared with Administration by a Healthcare Provider: A Single Center Experience}

F.J. López Jaime; A. Doblas Márquez; E. Calavia Aranda; I. Sánchez Bazán; A. Montaño Brioso Unidad de Hemostasia y Trombosis, Hospital Universitario Regional de Málaga, IBIMA, Málaga, Spain

Background: Primary immune thrombocytopenia (ITP) is a disorder characterized by low platelet counts $\left(<100 \times 10^{9} / \mathrm{L}\right)$, which has a significant negative impact on quality of life, work productivity and emotional well-being of patients. Romiplostim is a thrombopoietin receptor agonist used for the treatment of adults with refractory ITP that is administered weekly subcutaneously to increase platelet production. In the EU, the self-administration (SA) of romiplostim by patients and caregivers was approved, demonstrating comparable levels of efficacy and safety to when administered by healthcare professionals (HCP).

Aims: To examine and compare the efficacy and safety of romiplostim SA group with HCP group.

Methods: Retrospective, observational and single-center study of adult patients with ITP treated with romiplostim. The efficacy endpoints included percentage of patients and weeks with platelets within the target range of $50-200 \times 10^{9} / \mathrm{L}$ without the use of any rescue medication and percentage of patients with platelet counts $<20 \times 10^{9} / \mathrm{L}$ or $>400 \times 10^{9} / \mathrm{L}$. Safety was assessed by the incidence of treatment-related adverse events.

Results: A total of 33 patients (64.5\% women) were treated with romiplostim. The median age was 63 and 54.4 years in the SA $(n=21)$ and HCP $(n=10)$ groups, respectively. In both groups, more than $90 \%$ of patients achieved $\geq 1$ platelet response. The median of treatment duration was 106 vs 46 weeks with a similar proportion of weeks with a platelet response (49.0\% vs $47.15 \%)$. Rescue medication was used in $57.9 \%$ and $37.5 \%$ of patients. Treatment discontinuation was successful in $71.4 \%$ and $75 \%$. Two patients in the SA 
group had deep vein thrombosis (DVT), although both had a normal platelet count at the time of thrombosis.

TABLE 1 Clinical Parameters of patients with primary immune thrombocytopenia treated with romiplostim

\begin{tabular}{|c|c|c|c|c|c|}
\hline \multirow[b]{2}{*}{ Parameter/Group: } & \multicolumn{2}{|c|}{$\begin{array}{l}\text { HEALTHCARE } \\
\text { PROFESSIONAL } \\
(n=10)\end{array}$} & \multicolumn{2}{|c|}{$\begin{array}{l}\text { AMBULATORY } \\
\text { TREATMENT } \\
(n=21)\end{array}$} & \multirow[b]{2}{*}{$P$} \\
\hline & $\mathrm{N}$ & Freq (\%) & $N$ & Freq (\%) & \\
\hline Prior splenectomy & 4 & 40 & 4 & 19 & 0.652 \\
\hline $\begin{array}{l}\text { Response (at least } \\
\text { once) }\end{array}$ & 9 & 90 & 21 & 100 & 0.323 \\
\hline $\begin{array}{l}<20,000 \text { platelets (at } \\
\text { least once) }\end{array}$ & 6 & 66.7 & 16 & 76.2 & 0.666 \\
\hline $\begin{array}{c}>400,000 \text { platelets } \\
\text { (at least once) }\end{array}$ & 4 & 44.4 & 13 & 61.9 & 0.443 \\
\hline Rescue treatment & 3 & 37.5 & 11 & 57.9 & 0.420 \\
\hline $\begin{array}{c}\text { Concurrent ITP } \\
\text { therapy }\end{array}$ & 1 & 12,5 & 10 & 50 & 0.099 \\
\hline $\begin{array}{l}\text { Treatment } \\
\text { discontinuation }\end{array}$ & 6 & 75 & 15 & 71.4 & 1.000 \\
\hline $\begin{array}{l}\text { Adverse events } \\
\text { (DVT) }\end{array}$ & 0 & 0 & 2 & 9.5 & 1.000 \\
\hline
\end{tabular}

Conclusions: In adults with ITP who received romiplostim, SA was comparable to HCP administration in terms of efficacy and safety profiles, suggesting that SA may reduce patients' need for clinic visits and improve their quality of life.

\section{ADAMTS13 and TTP}

LPB0079 | Evaluation of the Different Platelet-dependent von Willebrand Factor Activity Assays Capacity to Assess the in vivo Inhibitory Effect of Caplacizumab on the VWF-platelet Interaction

$\underline{\text { P. Colpani }}^{1}$; L. Baronciani ${ }^{1}$; C. Novembrino ${ }^{1}$; I. Mancini ${ }^{2}$; G. Cozzi ${ }^{1}$; P. De Leo ${ }^{1}$; E. Galbiati ${ }^{1}$; M. Boscarino ${ }^{2}$; A. Artoni ${ }^{1}$; F. Peyvandi ${ }^{1,2}$

${ }^{1}$ Fondazione IRCCS Ca' Granda Ospedale Maggiore Policlinico, A. Bianchi Bonomi Hemophilia and Thrombosis Center and Fondazione Luigi Villa, Milan, Italy; ${ }^{2}$ Department of Pathophysiology and Transplantation, Università degli Studi di Milano, Milan, Italy

Background: Caplacizumab is an anti-von Willebrand Factor (VWF) humanized Nanobody ${ }^{\circledR}$ for the treatment of acquired Thrombotic Thrombocytopenic Purpura (aTTP). This nanobody binds to the VWF A1-domain and sterically prevents its interaction with the platelet glycoprotein Iba (GPIba). The measurement of the plateletdependent VWF activity may be used for therapeutic drug monitoring of caplacizumab. This activity, usually measured with the VWF ristocetin cofactor assay, nowadays can be assessed with alternative methods. However, it's unclear whether there are differences in the evaluation of the inhibitory effect of caplacizumab using these assays.
Aims: To evaluate if the most common commercially available platelet-dependent VWF activity assays were equally capable to assess the in vivo inhibitory effect of caplacizumab on the VWF-GPIba interaction.

Methods: We identified 14 patients with an acute episode of aTTP and a positive clinical response to caplacizumab. Patients were evaluated, along with 14 normal controls matched for sex/age, for the VWF antigen (VWF:Ag) and platelet-dependent VWF activity. We measured VWF:RCo (ristocetin and platelets), VWF:GPIbR (ristocetin and recombinant [r]GPIba) using a turbidimetric and a chemiluminescent assays, VWF:GPIbM (gain-of-function rGPIba without ristocetin) and VWF:Ab (monoclonal antibody directed against the GPIba binding epitope of VWF).

Results: The assays behaved differently in these patients, while consistent results were obtained in controls. Patients VWF activity was undetectable using VWF:RCo and VWF:GPIbM assays (the lowest activity/VWF:Ag ratios), whereas the VWF:Ab assay gave the highest activity/VWF:Ag ratios. Both VWF:GPIbR assays showed reduced activity/VWF:Ag ratios, although not to the extent of the VWF:RCo and VWF:GPIbM (Table and Figure).

TABLE 1 VWF:GPIbR* immunoturbidimetric assay; VWF:GPIbR** chemiluminescent assay; + For these analyses, test results below the lower limits of quantification were set at half that value (i.e. 6 IU/dL for VWF:RCo, 3 IU/dL for the VWF:GPIbR*, $0.25 \mathrm{IU} / \mathrm{dL}$ for VWF:GPIbR** and 2 IU/dL for VWF:GPIbM)

$$
\begin{array}{ll}
\text { aTTP patients } & \text { Controls } \\
(\mathrm{N}=14) & (\mathrm{N}=14) \\
87[37,137] & 132[90,210]
\end{array}
$$

\section{VWF:Ag}

Median [Min, Max]

$6^{+}[6,6]$

$115[67,197]$

VWF:RCo

Median [Min, Max]

VWF:GPIbR*

$26^{+}[3,39]$

$102[68,172]$

Median [Min, Max]

$29^{+}[0.25,47]$

$99[70,152]$

VWF:GPIbR ${ }^{* *}$

Median [Min, Max]

$$
2^{+}[2,2]
$$

VWF:GPIbM

Median [Min, Max]

$$
63[17,90]
$$

VWF:Ab

Median [Min, Max] 


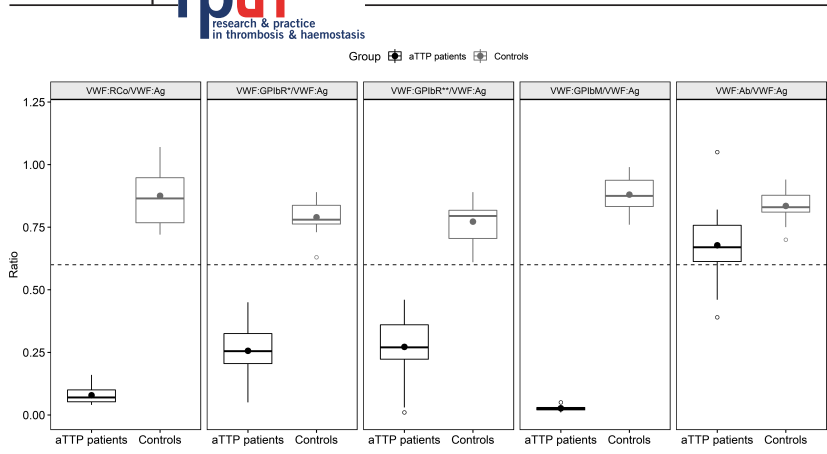

FIGURE 1 Distribution of activity/VWF:Ag ratios in aTTP patients and controls using 5 different platelet-dependent VWF activity assays. VWF:GPIbR* immunoturbidimetric assay; VWF:GPIbR** chemiluminescent assay. Each box-plot represents the interquartile range with median value (horizontal line). Close circles indicate the mean value, the open circles indicate the outliers. The broken line indicates the cut-off value of 0.6 . Study groups were compared using the non-parametric Mann-Whitney test: the median comparison between aTTP patients vs controls for every ratio is statistically significant $(P$

Conclusions: The VWF:RCo and VWF:GPIbM appear to be the most suitable assays for the therapeutic drug monitoring of caplacizumab in aTTP patients. Both VWF:GPIbR assays could be useful, but the physicians should be aware of their limited capacity to detect the inhibitory effect of caplacizumab. The VWF:Ab assay should not be used for this purpose.

PB0836 | Melanoma Triggers Severe Thrombotic

Thrombocytopenic Purpura and Results in a High Mortality Rate in adamt13/- Zebrafish

\section{Zheng ${ }^{1}$; L. Cao ${ }^{2}$; X.L. Zheng ${ }^{1}$}

${ }^{1}$ Department of Pathology and Laboratory Medicine, University of Kansas Medical Center, Kansas City, United States; ${ }^{2}$ Department of Pathology, The University of Alabama at Birmingham, Birmingham, United States

Background: Thrombotic thrombocytopenic purpura (TTP) is caused by deficiency of plasma ADAMTS13 activity. However, ADAMTS13 deficiency alone is often not sufficient to cause acute TTP; additional environmental factors may be necessary to trigger the disease.

Aims: Present study aims to determine if malignancy triggers TTP and whether malignancy-induced TTP depends on plasma ADAMTS13 and its substrate von Willebrand factor (VWF) in zebrafish models. Methods: Zebrafish melanoma (ZMEL) cells expressing mCherry were subcutaneously injected into transgenic zebrafish (cd41/GFP and gata1/dsRed) at age of 6-10 weeks (wt, $a 13^{-/}, v^{\prime} f^{/-}$, and $a 13^{-\%}$, $\left.v w f^{\prime-}\right)$, three days after total body gamma irradiation; tumor growth, blood cells count, and survival rate were determined. All mutant zebrafish were generated in our laboratory using CRISPR/Cas9 technique and bred for multiple generations before use.

Results: Our results demonstrated that $a 13^{-/-}$zebrafish developed a higher tumor burden, metastasized earlier and more wide-spread, developed more severe and persistent thrombocytopenia (Fig. 1A \& 1B), and exhibited a higher and earlier mortality rate (Fig. 2A) than the wt zebrafish after irradiation and inoculation of ZMEL cells. Surprisingly, the incidence and severity of thrombocytopenia (i.e. TTP) (Fig. 1C \& 1D) and the early mortality rate (Fig. 2B) in $a 13^{\%}$ $\mathrm{vwf}^{-/-}$zebrafish were dramatically reduced compared with those in $a 13^{\%}$ zebrafish after irradiation and inoculation of ZMEL cells.
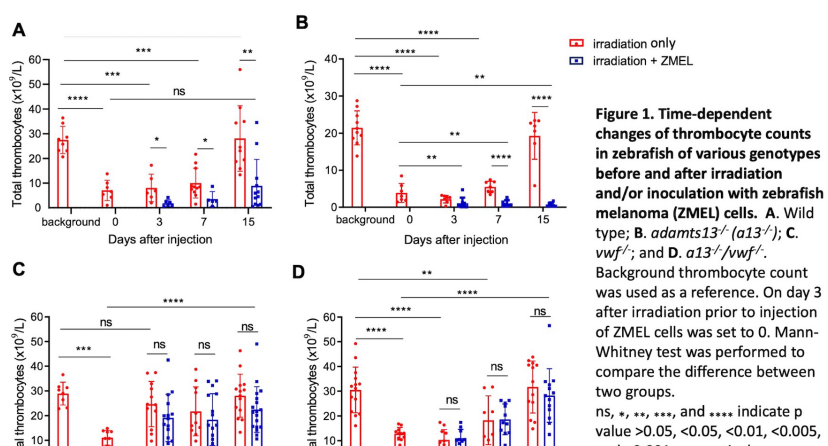

was used as a reference. On day 3 of ZMEL cells was set to 0 . MannWhitney test was performed to compare the difference between two groups.

$n s, *, \ldots, \ldots$, and $\ldots, \ldots$ indicate $p$ value $>0.05,<0.05,<0.01,<0.005$

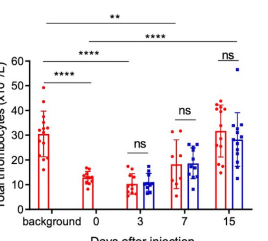
and $<0.001$, respectively.

FIGURE 1 Time-dependent changes of thrombocyte counts in zebrafish of various genotypes before and after irradiation and/or inoculation with zebrafish melanoma (ZMEL) cells.
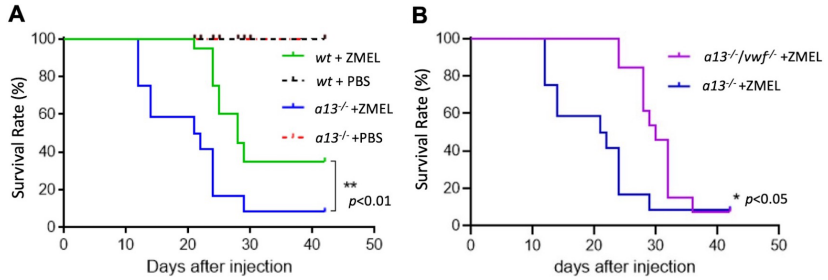

Figure 2. Kaplan-Meier survival analysis of zebrafish with various genotypes after irradiation and inoculation with zebrafish melanoma (ZMEL) cells. A. Comparison of the percentage of survival between wild type $(w t)$ and adamts $13 \%\left(a 13^{\%}\right)$ zebrafish after irradiation and inoculation of ZMEL cells or PBS. B. Comparison of the percentage of survival between vwf and $a 13 \% / v w f /$ zebrafish after irradiation and inoculation of ZMEL cells. Log-rank test was performed for statistical significance. Here, $*$ and $* *$ indicate $p<0.05$ and $<0.01$, respectively.

FIGURE 2 Kaplan-Meier survival analysis of zebrafish with various genotypes after irradiation and inoculation with zebrafish melanoma (ZMEL) cells.

Conclusions: Melanoma-induced thrombocytopenia (or TTP) and early death are highly dependent on both ADAMTS13 and VWF in zebrafish. Our findings provide scientific basis for targeting the ADAMTS13/VWF axis as a novel therapeutic strategy for malignancy-induced TTP. 
PB0837 | Design of a Phase 3, Randomized, Controlled Study of Prophylactic and On-demand Treatment with Recombinant ADAMTS13 for Patients with Severe Congenital Thrombotic Thrombocytopenic Purpura

N. Jain; C. Marquez; L. Martell

Baxalta US Inc., a Takeda Company, Cambridge, United States

Background: Congenital thrombotic thrombocytopenic purpura (cTTP) is a rare and life-threatening microvascular disease caused by ADAMTS13 deficiency. A recombinant ADAMTS13 (TAK-755 [BAX 930]; Baxalta US Inc., a Takeda company, Lexington, MA, USA) is being developed for use as on-demand and prophylactic ADAMTS13 replacement for patients with TTP.

Aims: We report the design (including recent updates) of a phase 3 , prospective, randomized, controlled, open-label, multicenter, crossover study to assess the safety and efficacy of TAK-755 for the prevention and treatment of acute episodes of TTP in patients with severe cTTP (NCT03393975).

Methods: This study will include $\sim 57$ patients (aged 0 to 70 years) with severe congenital ADAMTS13 deficiency (defined as plasma ADAMTS13 activity <10\%), randomized into 1 of 2 treatment sequences (TAK-755 then standard of care [SoC] or reverse) in the prophylaxis cohort. The prophylaxis treatment comprises 3 periods, 2 crossover pharmacokinetic (PK)/pharmacodynamic (PD) assessments (with a washout period of 14 [ \pm 2$]$ days), and 1 end-of-study PK assessment (Figure). The enrollment approach is consistent for all age groups. Patients will have the option to receive at-home TAK-755 infusions. Patients in the on-demand cohort will be randomized to receive treatment with SoC or TAK-755. The primary outcome is the incidence of acute TTP episodes among patients receiving either TAK-755 or SoC prophylactically. Secondary outcomes include the proportion of acute events responding to TAK-755 without requiring the use of another ADAMTS13-replacing agent, time to resolution of clinical symptomatology, incidence of adverse events, and the effect of immunogenicity on the PK/PD profile of ADAMTS13.

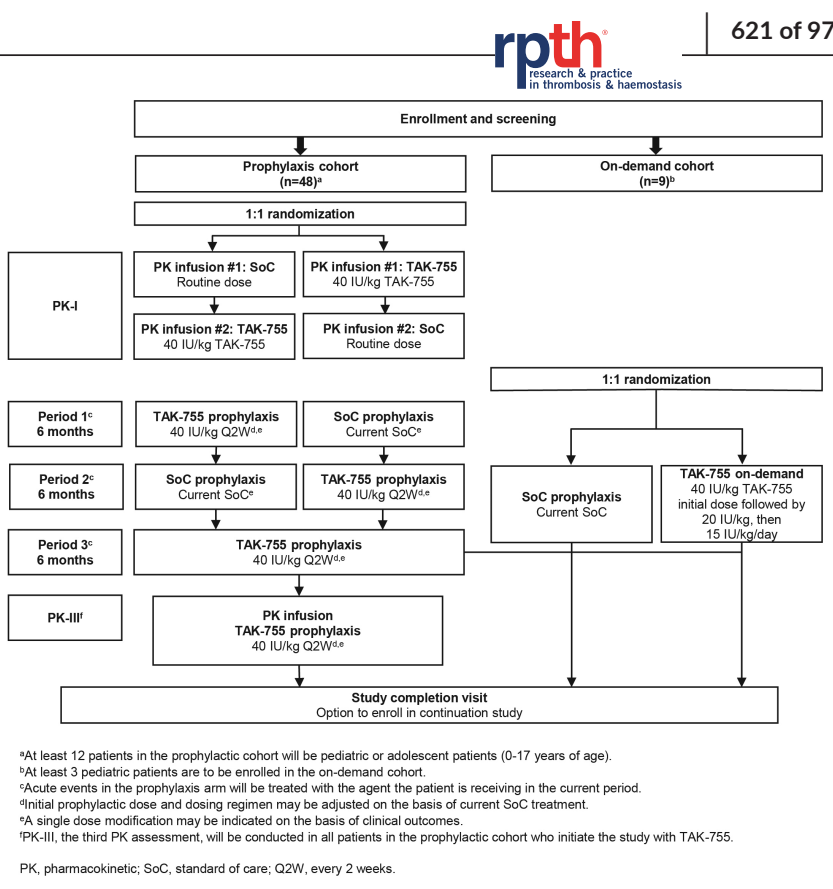

FIGURE 1 Study design

Results: The study initiated in October 2017; the estimated completion date is January 2024.

Conclusions: This study will provide data on the safety and efficacy of TAK-755 as ADAMTS13 replacement therapy in patients with cTTP.

\section{PB0838 | Caplacizumab Rapidly Inhibits VWF-platelet} Interaction: Pharmacodynamic Data from Healthy Volunteers and Patients with aTTP

F. Callewaert ${ }^{1}$; J. Minkue Mi Edou ${ }^{2}$; R. de Passos Sousa ${ }^{3}$

${ }^{1}$ Sanofi Genzyme, Diegem, Belgium; ${ }^{2}$ Sanofi Genzyme, Ghent, Belgium;

${ }^{3}$ Sanofi Genzyme, Lisbon, Portugal

Background: Caplacizumab targets the A1 domain of von Willebrand factor (VWF) and inhibits VWF-platelet interaction. In clinical trials in patients with aTTP, the $10 \mathrm{mg}$ dosing regimen of caplacizumab completely blocked VWF-mediated platelet adhesion within 24 hours.

Aims: To further characterize the speed of action of caplacizumab. Methods: VWF activity data (ristocetin cofactor [RICO] assay) from a Phase 1 study with caplacizumab in healthy White and Japanese volunteers (single intravenous [IV] or subcutaneous [SC] $10 \mathrm{mg}$ dose; $n=16$ per group), and from the Phase 2 TITAN study in a subset of patients ( $n=12$ ) with RICO sampling at 5-10 minutes, 3-6 hours, and 8-24 hours after the IV loading dose were included in this analysis. RICO inhibition to $<20 \%$ reflects full neutralization of VWF-platelet binding by caplacizumab. Informed consent was obtained from all study participants.

Results: Complete inhibition of RICO activity was achieved in 15/16 (94\%) healthy subjects at 1 hour after caplacizumab IV dosing, and in all participants at 3 hours after dosing (Table). With the $10 \mathrm{mg}$ 
SC dose, RICO activity $<20 \%$ was achieved in half of subjects (8/16) after 1 hour and in all subjects after 3 hours. RICO remained suppressed for 24 hours in 30/32 volunteers after a single IV or SC dose and started to recover thereafter.

In TITAN, Day 1 RICO activity values were available for 11/12 patients; 8/11 (72.7\%) achieved RICO <20\% within 5-10 minutes after the first IV loading dose, and the remaining 3 (27.3\%) patients after
3-6 hours. In 8/12 patients with available data, RICO remained $<20 \%$ at $8-24$ hours after the IV loading dose.

Conclusions: Caplacizumab, through its IV loading dose, induces rapid and sustained inhibition of VWF-platelet interaction, starting within minutes in most patients, which is essential in a lifethreatening disease like aTTP.

TABLE 1 Effect of caplacizumab on RICO activity in the Healthy Volunteer Study

\section{Caplacizumab dosing regimen}

$10 \mathrm{mg}$ IV

Analysis time point at Day $1{ }^{\text {a }}$

$n$ (\%)

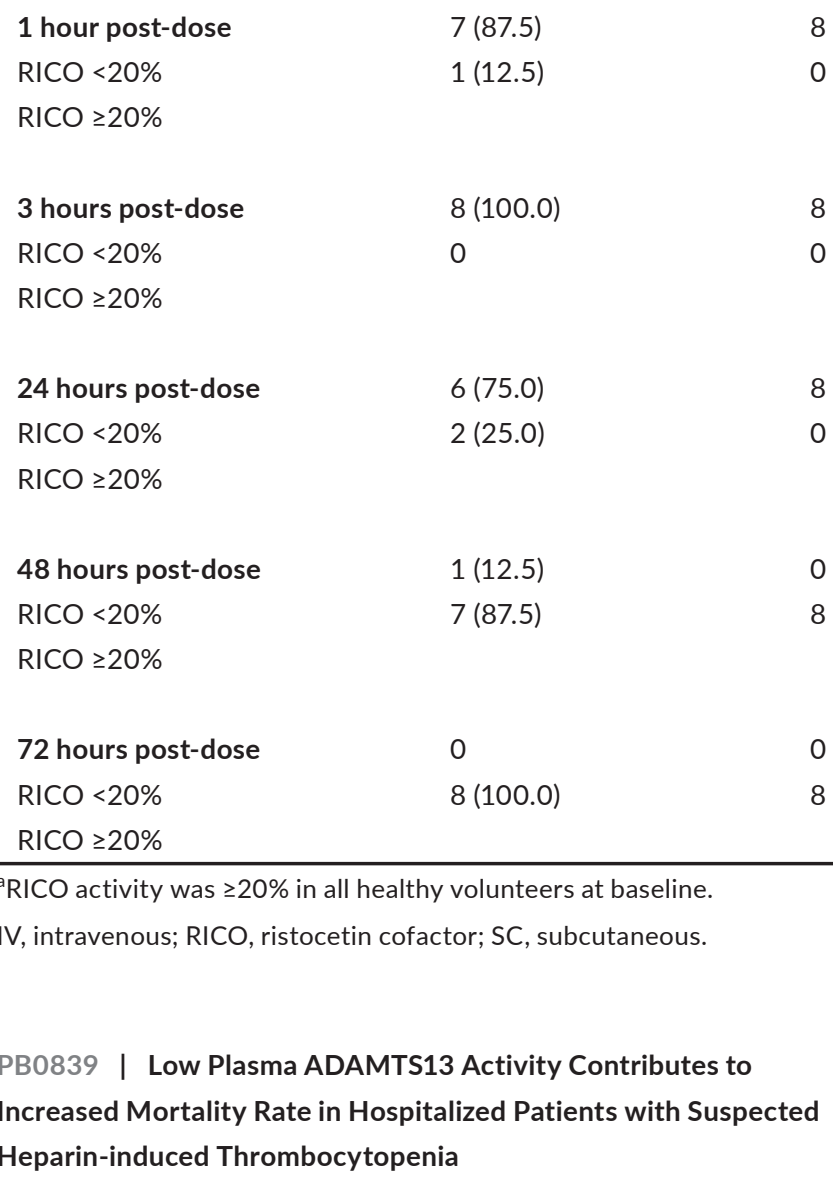

M. Chan ${ }^{1}$; X.L. Zheng ${ }^{2}$

${ }^{1}$ Department of Pathology, The University of Alabama at Birmingham, Birmingham, United States; ${ }^{2}$ Department of Pathology and Laboratory Medicine, University of Kansas Medical Center, Kansas City, United States

Background: Heparin-induced thrombocytopenia (HIT) is a lifethreatening thrombotic complication, resulting from platelet activation by antibodies against heparin/platelet factor- 4 complexes. However, the contribution of the ADAMTS13 and von Willebrand factor (VWF) to the disease outcomes in hospitalized patients with suspected HIT is not known.
$10 \mathrm{mg} \mathrm{SC}$

Japanese $(n=8)$

White $(n=8)$

Japanese $(n=8)$

$8(100.0)$

$4(50.0)$

$4(50.0)$

$4(50.0)$

$4(50.0)$

8 (100.0)

$8(100.0)$

0

$8(100.0)$

0

$8(100.0)$

8 (100.0)

8 (100.0)

$0 \quad 0$

0

$5(62.5)$

$6(75.0)$

8 (100.0)

$3(37.5)$

$2(25.0)$

0

0

8 (100.0)

$8(100.0)$
Aims: To determine plasma levels and clinical significance of ADAMTS13 activity, VWF antigen and activity in hospitalized patients with suspected HIT.

Methods: 261 hospitalized patients with suspected HIT at the University of Alabama at Birmingham between 2012 and 2019 were retrospectively enrolled for testing their plasma levels of ADAMTS13 activity, VWF antigen, and VWF activity.

Results: Of 261 cases, 87 (33.3\%) were positive for enzyme immunoassay (EIA+) and 174 (66.7\%) were EIA-. Of 87 EIA+ cases, 31 (35.6\%) were positive for serotonin-releasing assay (SRA+) but 56 (64.4\%) were SRA-. Comparing with those in healthy controls, plasma ADAMTS13 activity was significantly lower $(P<0.001)$ but plasma VWF antigen and activity $(P<0.0001)$ were significantly higher in all patients with suspected HIT. No statistically significant difference was detected among three groups in plasma ADAMTS13 activity, VWF 
antigen, and activity. Low plasma ADAMTS13 activity (<50th percentile) was highly predictive for a 90-day mortality rate $(P<0.0001)$. The factors associated with low ADAMTS13 activity were platelets count fall $>=50 \%$, pulmonary embolism, sepsis, and heart failure $(P<0.001)$. Among those with HIT, the percentage of thrombotic events and low ADAMTS13 activity was higher than that without HIT $(P=0.035)$ but such a difference was not detected in patients without HIT $(P=0.239)$. FIGURE 1 Plasma ADAMTS13 activity (A), VWF antigen(B), VWF collagen binding activity $(C)$, and the ratio of ADAMTS13 activity to VWF antigen (D), the ratio of ADAMTS13 activity to VWF activity $(E)$, and the ratio of VWF activity to VWF antigen $(F)$ in patients with EIA+/SRA+, EIA+/SRA-, EIA- and healthy controls (normal). The data was showed as the individual values (dots), the median, and the interquartile range. Here, ns, ${ }^{* * *}$, and ${ }^{* * * *}$ indicate $P$ values $\geq 0.05$

\section{A}

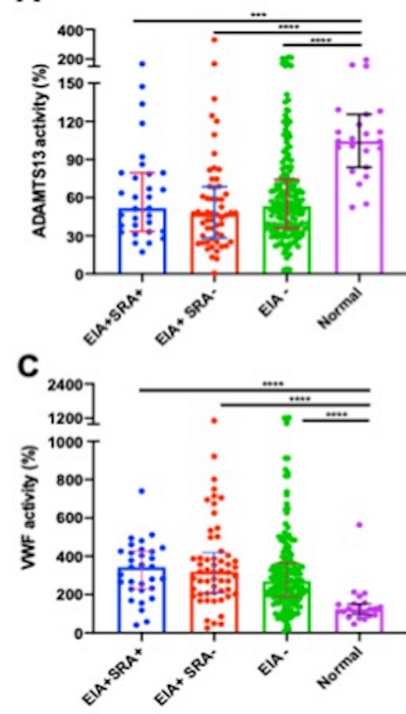

E
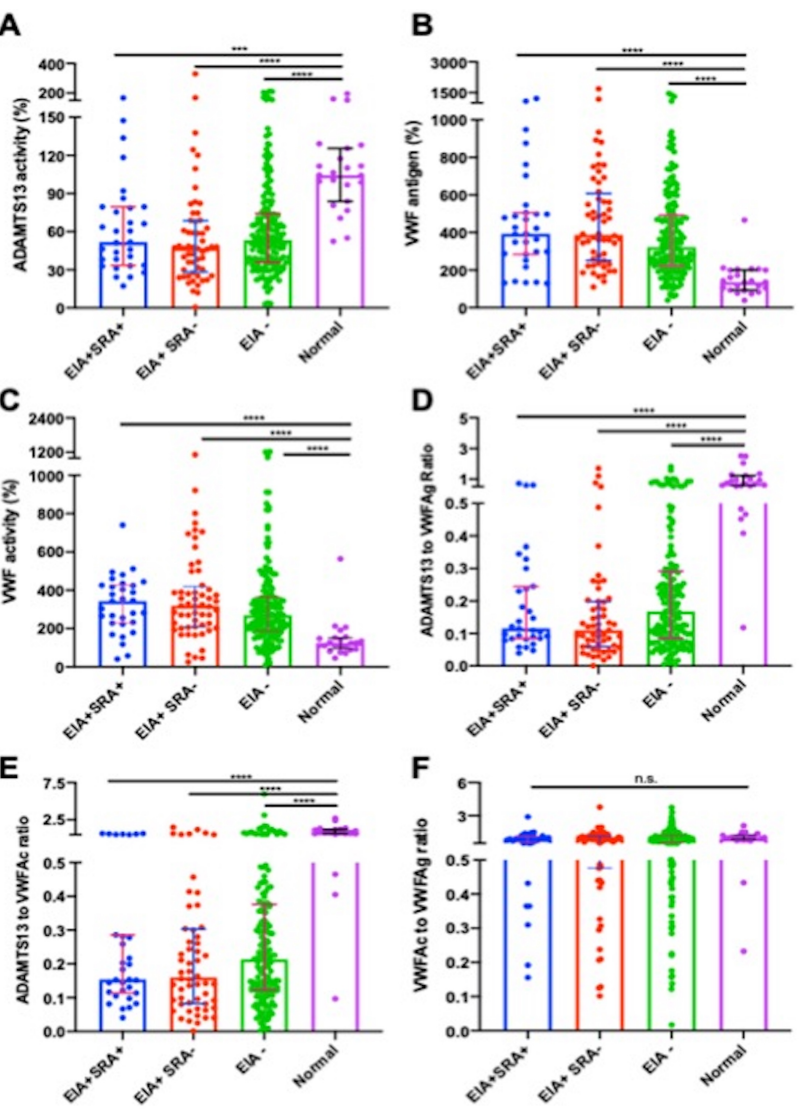

Fig. 1. Plasma ADAMTS13 activity (A), WWF antigen(B), VWF collagen binding activity (C), and the ratio of ADAMTS13 activity to VWF antigen (D), the ratio of ADAMTS13 activity to VWF activity(E), and the ratio of WWF activity to WWF antigen $(F)$ in patients with EIA+/SRA+, EIA+/SRA-, EIA- and healthy controls (normal). The data was showed as the individual values (dots), the median, and the interquartile range. Here, $\mathrm{ns}, \ldots$, and .... indicate $p$ values $\geq 0.05$, $<0.001$, and $<0.0001$, respectively.

FIGURE 1 Plasma ADAMTS13 activity (A), VWF antigen(B), VWF collagen binding activity $(C)$, and the ratio of ADAMTS13 activity to VWF antigen (D), the ratio of ADAMTS13 activity to VWF activity(E), and the ratio of VWF activity to VWF antigen ( $F)$ in patients with EIA+/SRA+, EIA+/SRA-, EIA- and healthy controls (normal). The data was showed as the individual values (dots), the median, and the interquartile range. Here, ns, ${ }^{* * *}$, and ${ }^{* * * *}$ indicate $P$ values $\geq 0.05$
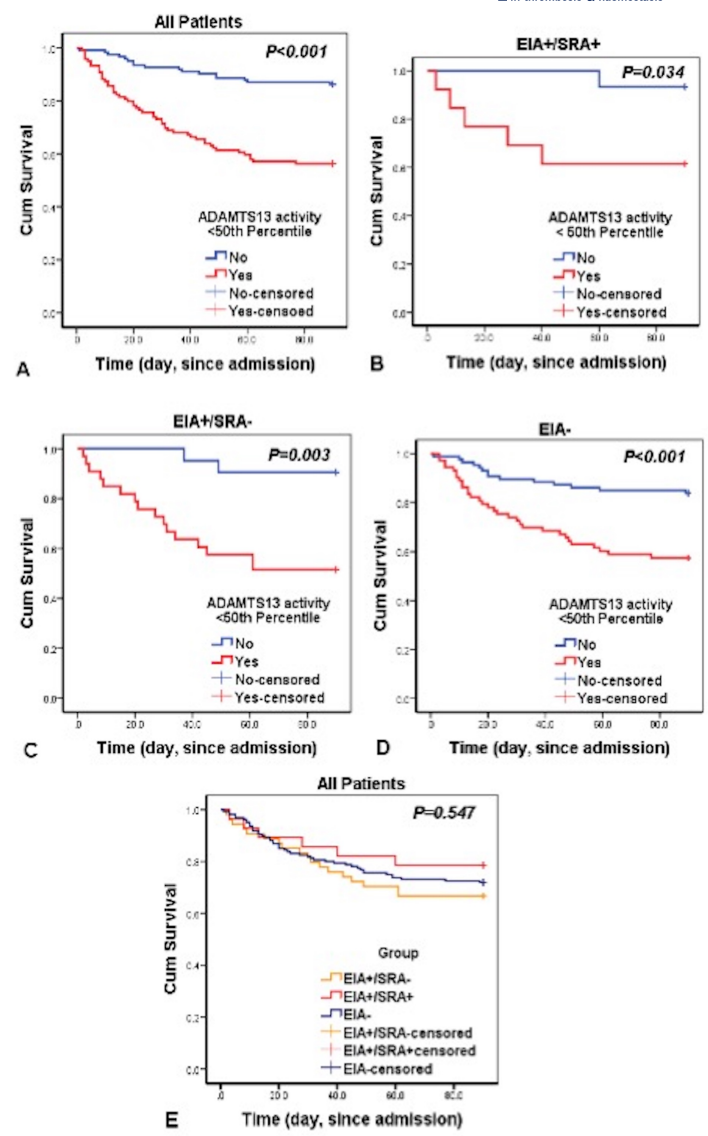

Fig. 2. Kaplan-Meier analysis. 90-day mortality rate in all patients with suspected HIT (A), EIA+/SRA+ (B), EIA+/SRA- (C), and EIA- (D) stratified based on plasma ADAMTS 13 activity $<50^{\text {th }}$ percentile vs. $\geq 50^{\text {th }}$ percentile. Also, 90 -day mortality in all patients with suspected HIT and stratified based on the HIT test results (E).

FIGURE 2 Kaplan-Meier analysis. 90-day mortality rate in all patients with suspected HIT (A), EIA+/SRA+ (B), EIA+/SRA- (C), and EIA- (D) stratified based on plasma ADAMTS13 activity

Conclusions: These results demonstrate that relative deficiency of plasma ADAMTS13 activity in hospitalized patients with suspected HIT is prevalent, and such relative deficiency of plasma ADAMTS13 may contribute to thrombotic complications and in-hospital mortality in these patients.

\section{PB0840 | Plasma BiolD of ADAMTS13}

H. Madarati; K. Singh; T. Sparring; C. Kretz

McMaster University, Hamilton, Canada

Background: Currently, the only known role of ADAMTS13 is in hemostasis, in its proteolytic activity to its only known substrate VWF. Having only one substrate, and one role, is unlikely as other ADAMTS proteases have multiple substrates and/or roles. Few studies tried to explore additional roles for ADAMTS13 outside of hemostasis and have eluted to that possibility. 
Aims: However, it is unclear whether these roles are directly dependent on the activity of ADAMTS13 or they are a result of the size of VWF multimers distribution.

Methods: Here, we have adapted the novel technique BiolD to be used in-vitro in plasma, to map the interactome of ADAMTS13.

Results: Our yield was an observation of 199 unique proteins, 108 of which were significant, and 6 of which are extracellular and of interest, including the auto-labelling of ADAMTS13, VWF and potential novel interactions with vitronectin or plasminogen. Validation studies using surface plasmon resonance (SPR) disregarded the interaction with vitronectin, and confirmed the interaction with plasminogen.

Conclusions: Further analysis using the SPR eluded to the possible interaction of ADAMTS13 to Kringle-domains of plasminogen, but not to proteins like prothrombin.

PB0841 | The Value of a Rapid, On-demand ADAMTS13 Activity Assay for the Diagnosis of Thrombotic Thrombocytopenic Purpura

A. White ${ }^{1}$; R. Martin ${ }^{1}$; K. Sew ${ }^{2}$; A. Stucke ${ }^{3}$ R. Cook $^{1}$

${ }^{1}$ Economist Intelligence Unit, London, United Kingdom; ${ }^{2}$ Economist Intelligence Unit, Singapore, Singapore; ${ }^{3}$ Economist Intelligence Unit, New York, United States

Background: Thrombotic thrombocytopenic purpura (TTP) is a rare thrombotic microangiopathy (TMA), characterized by ADAMTS13 activity $<10 \%$. ADAMTS13 activity assays are typically performed in specialized and reference laboratories with a turnaround time of several days. First-line treatment for TTP, therapeutic plasma exchange (TPE), is initiated while results are pending, as mortality is high if untreated. The HemosIL AcuStar ADAMTS13 Activity assay is automated and on-demand, with results available in under an hour This could reduce unnecessary TPE use in patients who do not have TTP, and therefore reduce hospital costs.

Aims: To estimate the hospital budget impact in the US, UK and France of using a rapid, on-demand ADAMTS13 activity assay.

Methods: We modelled a scenario where the rapid, on-demand assay is used in adults with TMA versus a scenario where it takes 3 days to receive results. The treatment pathways and model variables were based on published literature plus surveys and interviews with 5 clinicians from the 3 countries. The model includes patients with TTP, atypical hemolytic uremic syndrome (aHUS), Shiga toxinproducing E. coli-associated HUS (STEC-HUS), and other TMA diagnoses. The model includes costs for ADAMTS13 activity assays and TPE for 3 days.

Results: Model results suggest that if an on-demand, rapid ADAMTS13 activity assay is used, US, UK and French hospitals could save $\$ 18 \mathrm{~m}, £ 1.2 \mathrm{~m}$ and $€ 1.6 \mathrm{~m}$ annually, respectively. The saving equates to $\$ 10,788, £ 3,497$ and $€ 4,700$ per patient with TMA in the US, UK and France. The model is most sensitive to the proportion of TMA cases which are not TTP or HUS (61\% in the base case); if fewer patients have 'other' diagnoses, cost savings reduce.
Conclusions: In patients with TMA, use of a rapid, on-demand ADAMTS13 activity assay such as the HemosIL AcuStar ADAMTS13 Activity assay has the potential to be cost saving for hospitals.

\section{PB0842 | HIV is Not the Trigger for HIV-associated TTP}

M. Meiring ${ }^{1}$; M. Khemisi ${ }^{2}$.

${ }^{1}$ University of the Free State, Bloemfontein, South Africa; ${ }^{2}$ University of the WItwatersrand, Johannesburg, South Africa

Background: Thrombotic thrombocytopenic purpura (TTP) is a potentially fatal thrombotic microangiopathic disorder that might occur secondary to human immunodeficiency virus (HIV) infection. The pathogenesis involves a deficiency of the von Willebrand factor (VWF) cleaving protease ADAMTS13 and the presence of antiADAMTS13 autoantibodies.

Aims: Compare ADAMTS13 level and activity in HIV associated TTP patients to an HIV positive non-TTP control group.

Methods: Plasma from fifty-nine (59) patients diagnosed with HIVassociated TTP and hundred (100) HIV positive plasma samples from HIV infected patients without TTP were used as controls. ADAMTS13 levels and activities were performed (Technoclone kits). Results: The ADAMTS13 antigen levels of HIV-associated TTP patient plasma samples ranged from $0 \%-48 \%$, including 8 samples having undetectable ADAMTS13 antigen levels of $0 \%$. The ADAMTS13 activity levels were all under $10 \%$. Hundred (100) control plasma samples from HIV positive patients without TTP had ADAMTS13 antigen levels ranging from $36 \%-130 \%$ with $~ 85 \%$ in the normal range of $50 \%-160 \%$ and $15 \%$ with slightly low levels of $36 \%-50 \%$. The ADAMTS13 activity levels detected in this control group of patients with HIV infection but without TTP ranged from $25 \%-116 \%$, with $11 \%$ being below $50 \%$ (25-49\%), and $89 \%$ in the normal range (50-150\%). The ADAMTS13 antigen and ADAMTS13 activity levels of the HIV-associated TTP group and the HIV positive control group were compared and differs statistically significantly with a $P$-value of $<0.05$.

Conclusions: Fifteen, 15 (15\%) of the 100 HIV positive cohort plasma samples had slightly reduced ADAMTS13 levels ranging from 36 to $50 \%$ (normal range is from $50-150 \%$ ). It is suggested that the synthesis of metalloproteases, such as ADAMTS13 protein, might be decreased in HIV positive due to micronutrient deficiencies. However, HIV might not be the trigger to HIV-associated TTP. Reduced ADAMTS13 antigen and activity levels have only pathophysiological relevance in HIV-associated TTP. 
PB0843 | The Clinical Burden of Congenital and Immunemediated Thrombotic Thrombocytopenic Purpura: A

Retrospective Cohort Analysis

A. Satija ${ }^{1}$; S. Tzivelekis ${ }^{2}$; E. Swallow ${ }^{1}$; O. Patterson-Lomba ${ }^{1}$;

A. Briggs ${ }^{1}$; E. Yim ${ }^{1}$; B. Mellgard ${ }^{3}$

${ }^{1}$ Analysis Group, Inc., Boston, United States; ${ }^{2}$ Shire PIc, a Takeda

Company, Boston, United States; ${ }^{3}$ Baxalta US Inc., a Takeda Company,

Cambridge, United States

Background: Thrombotic thrombocytopenic purpura (TTP) is a rare blood disorder, classified as either congenital (cTTP) or immunemediated (iTTP). A significant proportion of patients with TTP suffer from comorbidities and impaired functioning.

Aims: To describe patient characteristics, comorbidities, and treatments associated with the management of TTP episodes.

Methods: A retrospective database analysis was conducted using insurance claims data collected between Q1 2009 and Q1 2019 from the IBM Watson Health MarketScan ${ }^{\circledR}$ Commercial Claims and Encounters database and the Medicare Supplemental and
Coordination of Benefits database. Patients had $\geq 2$ diagnosis claims for thrombotic microangiopathy (ICD-9=446.6; ICD-10=M31.1) and $\geq 1$ TTP-related visit at which treatment with either plasma exchange (PEX) or plasma infusion (PI) was given. Patients were stratified by TTP subtype on the basis of treatment received (iTTP cohort = only PEX; cTTP cohort = only PI; unclassified cohort = PEX and PI). Descriptive statistical analyses were conducted for patient-level demographics, clinical characteristics, and treatments.

Results: Data from 1174 TTP patients were analyzed. Mean (SD) age at baseline was $37.8(20.2)$ years for patients with cTTP and 49.1 (17.6) years for ITTP. $67.3 \%$ of patients with iTTP and $56.4 \%$ with cTTP were female. Mean (SD) baseline Charlson Comorbidity Index (CCI) scores by subtype were iTTP = 1.6 (2.3), cTTP $=0.9$ (1.3), and unclassified $=1.0$ (1.6). Renal disease, chronic pulmonary disease, and diabetes were the most common comorbidities contributing to baseline $\mathrm{CCl}$ score (Table 1). In the iTTP cohort, $88.4 \%$ of patients had $\geq 1$ TTP-related inpatient visit versus $28.2 \%$ for the cTTP cohort. Comorbid conditions present at TTP-related visits were more prevalent in the inpatient setting. Treatments used during TTP-related visits are shown in Table 2.

TABLE $1 \mathrm{CCl}$ component comorbid conditions occurring in $\geq 10 \%$ of patients in any cohort during the baseline period

$\begin{array}{llll}\text { Condition } & \operatorname{iTTP}(\boldsymbol{n}=805) & \mathrm{cTTP}(\boldsymbol{n}=39) & \text { Unclassified }(\boldsymbol{n}=330) \\ \begin{array}{l}\text { Any malignancy, including leukemia and } \\ \quad \text { lymphoma }\end{array} & 95(11.8) & 2(5.1) & 25(7.6) \\ \text { Cerebrovascular disease } & 71(8.8) & 7(17.9) & 63(19.1) \\ \text { Chronic pulmonary disease } & 105(13.0) & 3(7.7) & 30(9.1) \\ \text { Mild liver disease } & 74(9.2) & 4(10.3) & 28(8.5) \\ \text { Mild to moderate diabetes } & 101(12.5) & 2(5.1) & 43(13.0) \\ \text { Renal disease } & 145(18.0) & 10(25.6) & 63(19.1) \\ \text { Rheumatologic disease } & 85(10.6) & 2(5.1) & 26(7.9)\end{array}$

Values are number of patients (\%). Because a diagnosis code to distinguish TTP subtypes is not available, an algorithm was developed. The iTTP cohort included patients treated with PEX on the index date and not treated with PI at any point. The cTTP cohort included patients treated with PI on the index date and not treated with PEX at any point. The unclassified cohort included patients treated with both PEX and PI.

The index date was defined as the start date of the first TTP-related visit during the analysis period at which treatment with PEX or PI was given. The baseline period was defined as the 6 months prior to the index date.

Clinical conditions were defined according to ICD-9 and ICD-10 codes.

TABLE 2 Patient-level treatments received during TTP-related visits

\begin{tabular}{llll} 
Treatment & iTTP $(n=805)$ & cTTP $(n=39)$ & Unclassified $(n=330)$ \\
PEX + corticosteroids & $45(5.6)$ & $0(0.0)$ & $121(36.7)$ \\
PEX + rituximab & $19(2.4)$ & $0(0.0)$ & $80(24.2)$ \\
PEX + other or unclassified biologics & $0(0.0)$ & $0(0.0)$ & $1(0.3)$ \\
PI + rituximab & $0(0.0)$ & $2(5.1)$ & $76(23.0)$ \\
Cryoprecipitate-reduced PI & $0(0.0)$ & $1(2.6)$ & $62(18.8)$ \\
Frozen or fresh-frozen PI & $0(0.0)$ & $38(97.4)$ & $299(90.6)$ \\
Solvent/detergent-treated PI & $0(0.0)$ & $0(0.0)$ & $4(1.2)$ \\
PEX + PI at the same visit & $0(0.0)$ & $0(0.0)$ & $320(97.0)$ \\
\hline
\end{tabular}

Values are number of patients (\%). 
Conclusions: This retrospective database analysis is one of the first to classify patients into distinct iTTP and CTTP cohorts, and highlights the considerable clinical burden of disease and the long-term consequences for organ involvement.

PB0844 | Loss of Diagnostic Utility of D-dimers in Secondary Thrombotic Thrombocytopenic Purpura (TTP) in Patients with Human Immunodeficiency Virus (HIV) Infection

S. Louw; A. Mayne; E.S. Mayne

University of the Witwatersrand (WITS), National Health Laboratory Service (NHLS), Johannesburg, South Africa

Background: The two commonest microangiopathies in HIV infected patients in South Africa are acquired thrombotic thrombocytopenic purpura (TTP) and disseminated intravascular coagulation (DIC). The microthrombi in TTP are rich in von Willebrand factor (VWF) and platelets, with those in DIC consisting predominantly of fibrin. The treatment of these two conditions is different and accurate initial diagnosis is essential. Research suggest that $D$-dimes are not elevated in acquired TTP and together with preserved activated Partial Thermoplastic Time (aPTT) and antithrombin (AT) are useful in distinguishing acquired TTP and DIC in HIV-uninfected patients.

Aims: To determine the diagnostic utility of 3 routine parameters, aPTT, D-dimers and AT, in distinguishing between acquired TTP and DIC in HIV-infected patients.

Methods: This study approval human research ethics committee of the University of the Witwatersrand (M160134). aPTT, D-dimer and AT results of patients with HIV-associated TTP were compared

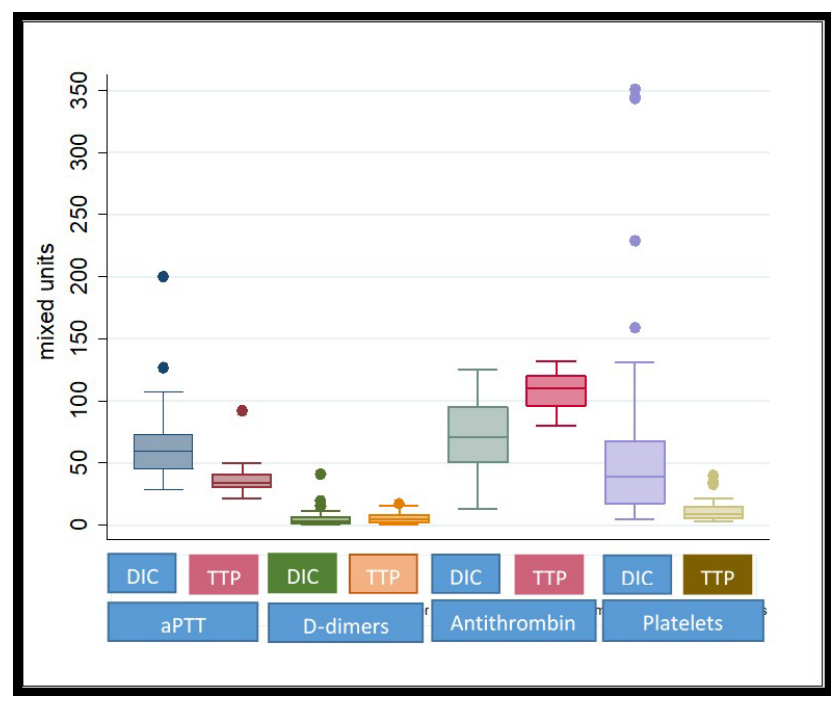

FIGURE 1 Boxplots - HIV-infected patients with DIC or acquired TTP : Paired tests for aPTT, D-dimers, antithrombin and platelet count $(n=53)$. DIC, disseminated intravascular coagulation; TTP, thrombotic thrombocytopenic purpura; aPTT, activated Partial Thromboplastin Time. (Dots above Boxplots represent outlier results).

with HIV infected patients with laboratory evidence of overt uncompensated DIC. Results were analysed using STATA and for nonparametric parameters, a Mann-Wilcoxon analysis was performed.

Results: The aPTT, AT levels and platelet count were significantly different between HIV-infected patients with DIC compared with HIV acquired TTP ( $P$-values $<0.0001)$. D-dimer levels in HIV-infected patients with TTP were, however, substantially elevated and were not statistically different from HIV infected patients with DIC.

TABLE 1 Two-sample Wilcoxon rank-sum (Mann-Whitney) test: DIC vs TTP

\begin{tabular}{|c|c|c|c|c|}
\hline $\begin{array}{l}\text { Parameter (normal } \\
\text { reference range) }\end{array}$ & aPTT (31-48 seconds) & D-dimer (0-0.25 mg/L) & $\begin{array}{l}\text { Antithrombin (80-120 } \\
\text { IU/dL) }\end{array}$ & Platelets $\left(186-454 \times 10^{9} / \mathrm{L}\right)$ \\
\hline z-score & 6.619 & -1.826 & -6.336 & 6.397 \\
\hline$P$-value & $<0.0001$ & 0.0678 & $<0.0001$ & $<0.0001$ \\
\hline Conclusion: & $\begin{array}{l}\text { Significantly prolonged } \\
\text { in DIC compared } \\
\text { to TTP }\end{array}$ & $\begin{array}{l}\text { No significant } \\
\text { difference between } \\
\text { DIC and TTP }\end{array}$ & $\begin{array}{l}\text { Significantly reduced in } \\
\text { DIC compared with } \\
\text { TTP }\end{array}$ & $\begin{array}{l}\text { Significantly reduced in TTP } \\
\text { compared with DIC }\end{array}$ \\
\hline
\end{tabular}

Conclusions: The elevated D-dimer levels in HIV infected patients with acquired-TTP probably reflects inflammation and local activation of the coagulation system related to endothelial damage. D-dimer levels are therefore not useful in distinguishing between acquired TTP and DIC in HIV-infected patients.
PB0845 | Evaluation of the Local Tolerability of Recombinant ADAMTS13 Following Subcutaneous Injection in Rabbits

J. Blank; J. McNulty; J. Nunes

Takeda Pharmaceuticals International Co., Cambridge, United States

Background: Thrombotic thrombocytopenic purpura (TTP) is a rare clotting disorder caused by deficiency in the von Willebrand factor (VWF) cleaving enzyme ADAMTS13 (a disintegrin and metalloproteinase with a thrombospondin type 1 motif, member 13). ADAMTS13 cleavage of VWF multimers decreases VWF-associated platelet aggregation activity. Recombinant (r)ADAMTS13 (TAK755) is currently under clinical investigation as an intravenous 
enzyme replacement therapy for patients with congenital (c)TTP and immune-mediated (i)TTP. Subcutaneous administration could provide a more convenient approach, potentially increasing treatment compliance, expanding self-administration, and improving patient quality of life.

Aims: To evaluate local subcutaneous tolerability of the current intravenous formulation of rADAMTS13 in rabbits and create an animal model to assess the potential risk of the subcutaneous administration route.

Methods: This study complied with all applicable sections of the Animal Welfare Act, and was approved by the facility's Institutional Animal Care and Use Committee. Eight New Zealand White rabbits were subcutaneously injected with $300 \mathrm{IU} / \mathrm{mL}$ of rADAMTS13 in a volume of $1 \mathrm{~mL}$ on the right dorsal side and with $0.9 \%$ sodium chloride (currently used as the vehicle for intravenous administration) on the left dorsal side as a control. Local tolerance was evaluated for up to 5 days following administration using the Draize dermal scoring system. Upon completion of the in-life observations (day 2 or 5), rabbits were euthanized and the injection sites were macroscopically evaluated at necropsy and prepared for microscopic evaluation by a veterinary pathologist.

Results: No abnormal behavioral changes were observed during the study, including at the time of injection. Purple discoloration and/ or edema were observed at both the treatment site $(n=2 / 8)$ and control site $(n=1 / 8)$, and were attributed to the injection procedure. No treatment-related histopathological alterations were observed.

Conclusions: A single subcutaneous injection of rADAMTS13 was not associated with treatment-related in-life findings, dermal observations, or histopathologic findings in rabbits.

PB0846 | Thirty-five Years of Follow up of a Patient with Congenital Thrombotic Thrombocytopenic Purpura

M. de Oliveira; C. Casais; C. Gonçalves; E. Cruz; M. Coutinho;

M. Pereira; J. Coutinho; S. Morais

Centro Hospitalar Unviersitário do Porto, Porto, Portugal

Background: Congenital thrombotic thrombocytopenic purpura (cTTP) is a rare, life-threatening disease caused by variants in the ADAMTS13 gene, encoding ADAMTS13, a metalloprotease involved in the cleavage of ultra-large von Willebrand factor multimers. Patients with this chronic relapsing disease may need long life prophylactic plasma therapy to maintain a minimum of ADAMTS13 activity level.

Aims: To describe thirty-five years of follow-up of clinical evolution and treatment of a woman with CTTP, from the diagnosis to the present day.

Methods: Retrospective analysis of clinical records.

Results: A 2-year-old girl, born of first-degree cousins, was referred to our hospital for hemolytic anemia and thrombocytopenia triggered by an infectious event. At the age of 3 , the girl was admitted with a similar process and the diagnosis of TTP was suspected, following impressive recover after transfusion with Fresh Frozen
Plasma (FFP). After subsequent relapses, a prophylactic regimen with regular infusions of FFP (10-15 $\mathrm{ml} / \mathrm{kg}$ every two to three weeks) was instituted. The diagnosis of CTTP was confirmed years later by documenting severe ADAMTS13 deficiency $(<1 \%)$ in the absence of anti-ADAMTS13 antibodies, and a homozygous variant (c.2074C>T) in the ADAMTS13 gene. At 20-years-old, she presented with a urinary infection, complicated with acute kidney failure requiring haemodialysis. During the age of 26 , she became pregnant, twice, but had miscarriages at 7 and 22 weeks of pregnancy. The kidney function deteriorated during her life, regardless of plasma prophylaxis and at 35-years-old the patient developed stage IV chronic kidney failure. Upon computed tomography brain scan following a transient ischaemic stroke, multiple past strokes, all of which were asymptomatic, were detected.

Conclusions: The incidence of cerebrovascular events is significantly lower in CTTP patients on regular prophylactic therapy. However, despite life-long prophylaxis, silent deterioration of the brain and kidney function occurred, highlighting the need for more effective forms of replenishing ADAMTS13 levels.

\section{PB0848 | Validating Lactate Dehydrogenase (LDH) as a Component of the PLASMIC Predictive Tool (PLASMIC-LDH)}

C.C.K. Liam ${ }^{1,2,3}$; J.Y.-H. Tiao ${ }^{1,2}$; Y.Y. Yap ${ }^{3}$; J. Sathar ${ }^{3}$; Y.L. Lee ${ }^{4}$; S. McRae ${ }^{5}$; A. Davis ${ }^{6}$; J. Curnow ${ }^{7} ;$ R. Bird ${ }^{8}$; P. Choi ${ }^{9}$; P. Angchaisuksiri ${ }^{10}$; S.L. Tien ${ }^{11}$; J.C.M. Lam ${ }^{12}$; D. Oh ${ }^{13}$; J.S. Kim ${ }^{14}$; S.-S. Yoon ${ }^{15}$; R. Wong ${ }^{16}$; S. Macpherson ${ }^{17,18}$; E. Merriman ${ }^{19,20}$; R.I. Baker ${ }^{1,2}$

${ }^{1}$ Perth Blood Institute, West Perth, Australia; ${ }^{2}$ Western Australian Centre for Thrombosis and Haemostasis (WACTH), Murdoch, Perth, Australia; ${ }^{3}$ Department of Haematology, Hospital Ampang, Ampang, Malaysia; ${ }^{4}$ Centre for Clinical Trials, Hospital Ampang, Ampang, Malaysia; ${ }^{5}$ Northern Cancer Service, Launceston, Australia; ${ }^{6}$ The Alfred Hospital, Melbourne, Australia; ${ }^{7}$ Westmead Hospital, Westmead, New South Wales, Australia, Westmead, Australia; ${ }^{8}$ Princess Alexandra Hospital, Woolloongabba, Australia; ${ }^{9}$ The Canberra Hospital, Canberra, Australia, ${ }^{10}$ Ramathibodi Hospital, Mahidol University, Bangkok, Thailand, ${ }^{11}$ Singapore General Hospital, Singapore City, Singapore, ${ }^{12} K K$ Women's and Children's Hospital, Singapore City, Singapore,

${ }^{13} \mathrm{CHA}$ Bundang Medical Center, CHA University, Seongnam, Korea, Republic of, ${ }^{14}$ Yonsei University College of Medicine, Seoul, Korea, Republic of, ${ }^{15}$ Seoul National University Hospital, Seoul, Korea, Republic of, ${ }^{16}$ The Chinese University of Hong Kong, Prince of Wales Hospital, Shatin, N.T, Hong Kong, SAR of China, ${ }^{17}$ Department of Haematology, Christchurch Hospital, Christchurch, New Zealand, ${ }^{18}$ Canterbury District Health Board, Christchurch, New Zealand,

${ }^{19}$ North Shore Hospital, Auckland, New Zealand, ${ }^{20}$ Waitemata District Health Board, Auckland, New Zealand

Background: The PLASMIC score is a convenient tool for predicting severe thrombotic thrombocytopenic purpura (TTP) with ADAMTS-13 activity of $<10 \%$. Lactate dehydrogenase (LDH) 
is widely used as a marker of haemolysis in TTP monitoring and could be a useful alternative for the lysis marker component of the PLASMIC score.

Aims: The purpose of this study is to validate the PLASMIC score in a multi-centre Asia Pacific region and examine $\mathrm{LDH}$ as a putative replacement lysis marker at presentation.

Methods: Records of patients with suspected thrombotic microangiopathy (TMA) in the Asia-Pacific Microangiopathy and Thrombosis (APMAT) Network biobank. The ADAMTS 13 activity with clinical and laboratory components of the PLASMIC score was obtained. Patients with incomplete results of ADAMTS-13 activity and/or PLASMIC scores were excluded. In all patients, the PLASMIC score and an alternative PLASMIC-LDH score, in which LDH replaced the traditional markers of lysis were calculated. We generated a receiver operator characteristics (ROC) curve and compared the predictive ability of each model.

Results: Of the 72 patients reviewed, 46 patients fulfilled the inclusion criteria of which 34 had ADAMTS 13 activity $<10 \%$. When dichotomized at intermediate-high risk (scores 5-7) and low risk (scores $0-4$ ), the PLASMIC score had a sensitivity of $97.1 \%$, a specificity of $58.3 \%$ with a positive predictive value (PPV) of $86.8 \%$, and negative predictive value (NPV) of $87.5 \%$. The PLASMIC-LDH had a sensitivity of $97.1 \%$, a specificity of $33.3 \%$ with a PPV of $80.5 \%$, and an NPV of $80.0 \%$.

Conclusions: Our study validated the original PLASMIC score as an excellent predictive tool for severe TTP in the APMAT cohort and may be used in patients presenting with TMA. Substituting LDH into the score (PLASMIC-LDH), did not significantly impair the predictive ability of the PLASMIC score, albeit with markedly reduced specificity. Further validation studies will enhance the modified predictive score.

PB0849 | TTP during Pregnancy: Acquired or Late-Onset Congenital? A Diagnostic Challenge

S. Demeester; N. De Beule; C. Orlando; A. De Becker; K. Jochmans Universitair Ziekenhuis Brussel, Brussels, Belgium

Background: Thrombotic thrombocytopenic purpura (TTP) is a rare thrombotic microangiopathy, characterised by thrombocytopenia, haemolytic anemia and possible organ damage. The disease is caused by a severely reduced activity of von Willebrand factorcleaving protease ADAMTS13. This is due to the presence of an inhibitory autoantibody in the acquired form or to mutations in the ADAMTS13 gene in the rare inherited form.

Aims: In July 2020, a 20 weeks pregnant 31-year-old woman presented with general discomfort.

Methods: Laboratory investigations were suggestive for TTP with severe thrombocytopenia and haemolytic anemia with presence of schistocytes.

Results: ADAMTS13 activity was $3 \%$, confirming TTP. Treatment with daily plasma exchange (PEX), caplacizumab and corticosteroids showed rapid normalization of laboratory and clinical parameters. PEX could be stopped after 5 days. From week 2, rituximab was administered once weekly for 4 weeks. Unfortunately, the baby deceased in utero at week 22 of gestation.

ADAMTS13 autoantibodies could not be identified, neither by Bethesda assay, nor by direct antibody ELISA. ADAMTS13 remained low during the next 2 months, therefore caplacizumab was continued. After 2 months, treatment was discontinued due to reimbursement modalities.

One week later, the patient relapsed clinically. Treatment with PEX, caplacizumab and corticosteroids was restarted. Shortly thereafter, results of the genetic analyses showed two mutations in the ADAMTS13 gene: c.3179C>T p.(Arg1060Trp), associated with late onset congenital TTP (cTTP), and c.84G>A p.(Trp28*), a possible novel mutation linked to cTTP. Genetic analysis in the parents confirmed our patient as compound heterozygous. Therapy was switched to 2-weekly fresh frozen plasma administration. Today, no relapse occurred and interval of infusion is extended to a 3-weekly regimen.

Conclusions: In conclusion, we suggest that pregnant women presenting with TTP should be screened for inherited TTP, even in the absence of a history of TTP-like symptoms. Our experience demonstrates that good clinical responses can be obtained with caplacizumab in cTTP.

PB0850 | Delayed Diagnosis of Congenital Thrombotic Thrombocytopenic Purpura Presenting as Recurrent Cryptogenic Strokes

M. Desancho; M. Beltrami Moreira

Weill Cornell Medicine/New York Presbyterian Hospital, New York, United States

Background: Congenital Thrombotic Trombocytopenic Purpura (cTTP) Is a Rare Disorder Caused by ADAMTS13 Deficiency and Constitutes a Rare Cause of Strokes

Aims: To recognize cTTP in the differential diagnosis of cryptogenic stroke. 
Methods:

TABLE 1 Hematologic and imaging results

\begin{tabular}{|c|c|c|c|c|c|c|}
\hline & 2001 & 2002 & 2006 & April 2020 & August 2020 & February 2021 \\
\hline $\begin{array}{l}\text { Hemoglobin } \\
\text { 11.7-16 mg/ } \\
\text { dL }\end{array}$ & & 12.5 & 12.8 & 12.8 & 11.5 & 12.7 \\
\hline $\begin{array}{l}\text { Platelet count } \\
\qquad \begin{array}{l}(150-450 \\
\times 10^{3} \text { cells/ } \\
\mu \mathrm{L})\end{array}\end{array}$ & & $\begin{array}{l}142 \\
199\end{array}$ & 244 & $\begin{array}{l}172 \\
149 \\
131\end{array}$ & $\begin{array}{l}38 \\
43 \\
55\end{array}$ & 174 \\
\hline $\begin{array}{l}\text { Protein C } \\
\text { activity } \\
\text { (70-130\%) }\end{array}$ & & $58 \%$ & $89 \%$ & & & \\
\hline $\begin{array}{l}\text { Protein S activity } \\
\quad(62-163 \%)\end{array}$ & & $60 \%$ & $55 \%$ & & & \\
\hline $\begin{array}{c}\text { Protein S Ag free } \\
\quad(70-160 \%)\end{array}$ & & & $60 \%$ & & & \\
\hline $\begin{array}{l}\text { ADAMTS13 } \\
\text { activity }\end{array}$ & & & & & $\begin{array}{c}<5 \% ; 9 \% \text { after } \\
4 \text { plasma } \\
\text { exchanges }\end{array}$ & 5 \\
\hline Imaging studies & $\begin{array}{l}\text { MRI: Subacute } \\
\text { left basal ganglia } \\
\text { infarct and acute } \\
\text { left parietal lobe } \\
\text { infarct } \\
\text { Normal trans- } \\
\text { e s o p h a g e a I } \\
\text { echocardiogram }\end{array}$ & $\begin{array}{l}\text { MRI: old left } \\
\text { parietal, left } \\
\text { caudate, and } \\
\text { right frontal } \\
\text { (subcortical/ } \\
\text { parasagittal) } \\
\text { infarcts }\end{array}$ & & $\begin{array}{l}\text { MRI: acute } \\
\text { infarction } \\
\text { in the right } \\
\text { superior } \\
\text { frontal gyrus } \\
\text { and subacute } \\
\text { infarct at } \\
\text { the right } \\
\text { inferior frontal } \\
\text { gyrus with } \\
\text { hemorrhagic } \\
\text { conversion }\end{array}$ & $\begin{array}{l}\text { СT: Acute right } \\
\text { middle } \\
\text { cerebral infarct }\end{array}$ & \\
\hline $\begin{array}{l}\text { Antithrombotic } \\
\text { agent }\end{array}$ & Aspirin low-dose & $\begin{array}{r}\text { Warfarin INR } \\
\text { target 2-3 }\end{array}$ & $\begin{array}{l}\text { Enoxaparin } \\
\text { to allow } \\
\text { for testing } \\
\text { protein } C \\
\text { and S }\end{array}$ & $\begin{array}{c}\text { Warfarin INR } 1.8 \\
\text { on admission }\end{array}$ & $\begin{array}{c}\text { Apixaban } 5 \mathrm{mg} \\
\text { twice daily }\end{array}$ & $\begin{array}{c}\text { Apixaban } 5 \mathrm{mg} \\
\text { twice daily }\end{array}$ \\
\hline Other & & & & & Plasma exchange & $\begin{array}{c}\text { Plasma infusions } \\
\text { bi-monthly }\end{array}$ \\
\hline
\end{tabular}

\section{Case report}

Results:

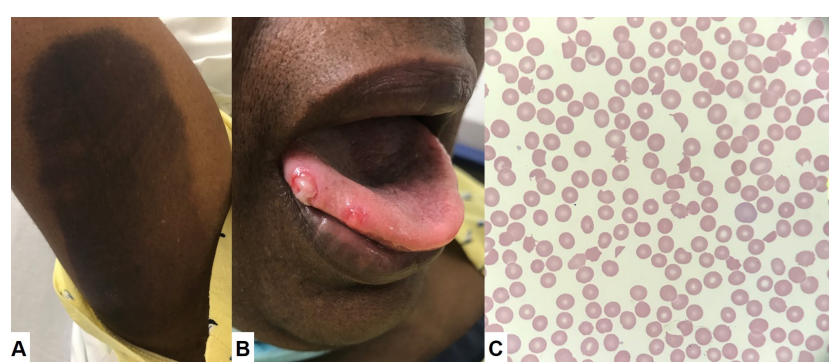

FIGURE 1 Physical Exam Findings and Peripheral Blood Smear: A) Spontaneous ecchymosis on medial/posterior aspect of the right upper arm. B) Tongue ulceration with fibrinous base, initially noted as tongue ecchymosis. C) Peripheral blood smear 100x magnification, demonstrating 4-5 schistocytes/HPF, polychromasia, and thrombocytopenia.
A 58-year-old woman was referred to Hematology in August 2020 after developing lingual bleeding and thrombocytopenia (38,000 cells $/ \mathrm{mm}^{3}$ ). She had suffered a stroke at age 39 , a transient ischemic attack at age 40 and another stroke at age 57. She was diagnosed with mild protein $\mathrm{S}$ deficiency and managed with warfarin. Her second stroke occurred after she had viral symptoms, but SARS-CoV-2 RT-PCR was negative. On admission her INR was subtherapeutic (1.8) and she was switched from warfarin to apixaban. Admission complete blood count (CBC) was normal. However, 4 days later, her platelet count was 131,000 cells $/ \mathrm{mm}^{3}$. (Table 1).

Physical exam revealed an ecchymosis on the right arm and a lingual lesion (Figure $1 \mathrm{~A}-\mathrm{B}$ ). Hemoglobin was $11.4 \mathrm{~g} / \mathrm{dl}$, platelets 55,000 cells $/ \mathrm{mm}^{3}$, reticulocyte $2.37 \%, \mathrm{LDH} 624 \mathrm{mg} / \mathrm{dl}$, and haptoglobin $92 \mathrm{mg} / \mathrm{dl}$. Five schistocytes per high-power field were evident on peripheral blood smear (Figure 1C). She was hospitalized with 
presumptive diagnosis of thrombotic thrombocytopenic purpura (TTP). Forty eight hours later, she developed new left sided weakness and dysarthria. Magnetic resonance imaging revealed a right medial cerebral artery ischemic stroke. ADAMTS13 was $<5 \%$ with negative inhibitor. Plasma exchange therapy was performed with normalization in platelet count. ADAMTS13 gene sequencing revealed homozygous mutation $3070 \mathrm{~T}>\mathrm{G}$ (exon 24). On follow-up, the patient has minimal neurological sequelae. $C B C$ remains normal, and she receives bimonthly plasma infusions as source of ADAMTS-13.

Conclusions: This patient's subtle hematologic findings underscore the high degree of suspicion required to recognize cTTP as a cause of cryptogenic strokes. ADAMTS13 replacement may prevent recurrences. Peripheral blood smear led to the accurate diagnosis of this unrecognized cause of cryptogenic strokes.

PB0851 | PK/PD Modeling and Simulations Highlight the Importance of the Intravenous Loading Dose and Daily Dosing Regimen with Caplacizumab for Patients with aTTP

T. Sou $^{1}$; F. Callewaert ${ }^{2}$; R. de Passos Sousa ${ }^{3}$; M.L. Sargentini-Maier ${ }^{1}$

${ }^{1}$ Sanofi Genzyme, Ghent, Belgium; ${ }^{2}$ Sanofi Genzyme, Diegem, Belgium;

${ }^{3}$ Sanofi Genzyme, Lisbon, Portugal

Background: For acquired thrombotic thrombocytopenic purpura (aTTP), the dosing recommendation of caplacizumab is a loading dose of $10 \mathrm{mg}$ intravenous (IV) bolus prior to therapeutic plasma exchange (TPE), followed by $10 \mathrm{mg}$ daily subcutaneous (SC) doses starting from the end of the first TPE and for $\geq 30$ days after the last daily TPE.

Aims: To use pharmacokinetic (PK) and pharmacodynamic (PD) simulations to evaluate the standard regimen of caplacizumab regarding the effect of the IV loading dose prior to TPE and the frequency of the maintenance $\mathrm{SC}$ dosing regimen after TPE.

Methods: An integrated PK/PD model was previously developed to describe the interaction between caplacizumab and von Willebrand factor (VWF) using nonlinear mixed effects modeling. The model was used to simulate PK and PD profiles for different scenarios (Table).

Results: The simulations suggest that the IV loading dose appears to be essential for rapid and sustained suppression of VWF levels during the initial hours of treatment. Omitting the IV loading dose, or administering a SC loading dose, leads to a delay in achieving optimal exposure required for the inhibition of VWF-platelet interactions.

In addition, the simulations confirm that VWF levels are sensitive to plasma caplacizumab concentrations. Non-daily dosing in the post-TPE period decreases drug exposure and leads to higher and more fluctuating VWF levels, which could expose patients with aTTP to the risk of suboptimal or incomplete inhibition of microthrombi formation. The effects are apparent with every second day dosing and become even more pronounced with every third day dosing.
Conclusions: The simulations confirm the importance of the IV loading dose of caplacizumab prior to TPE and the maintenance of a daily SC dosing regimen after TPE in ensuring that consistent and sufficient drug exposure and neutralization of VWF are achieved in patients with aTTP.

TABLE 1 Caplacizumab and TPE dosing scenarios

\begin{tabular}{lll} 
Scenario & Loading dose & After TPE dosing \\
1 & IV & Every day \\
2 & None & Every day \\
3 & SC & Every day \\
4 & IV & Every second day \\
5 & IV & Every third day \\
\hline
\end{tabular}

IV, intravenous; SC, subcutaneous; TPE, therapeutic plasma exchange.

P0156 | A Refractory Thrombotic Thrombocytopenic Purpura Case Complicated by Hepatic Sinusoidal Obstruction Syndrome and Successful Management with Defibrotide

S. Erdem ${ }^{1}$; M. Mastanzade ${ }^{1}$; S. Altay-Dadin²; M. Yanasik ${ }^{3}$;

S. Kalayoglu-Besisik ${ }^{1}$

${ }^{1}$ Istanbul University, Istanbul Medical Faculty, Department of Internal Medicine, Division of Hematology, Istanbul, Turkey; ${ }^{2}$ Istanbul University, Istanbul Medical Faculty, Department of Internal Medicine, Therapeutic Apheresis Unit, Istanbul, Turkey; ${ }^{3}$ Istanbul University, Istanbul Medical Faculty, Blood Transfusion Center, Istanbul, Turkey

Background: The clinical spectrum of immune-mediated thrombotic thrombocytopenic purpura (iTTP) is based on microvascular occlusion caused by platelet-rich thrombi.

Aims: We present a refractory iTTP case complicated by hepatic sinusoidal obstruction syndrome (SOS) and managed with defibrotide.

Methods: Case:

Results: A 36-year-old woman was admitted to the emergency department with weakness and bruises. She was pale, subicteric, and had ecchymoses. She had mild anemia (hemoglobin, $9.2 \times 10^{9} / \mathrm{L}$ ) and severe thrombocytopenia (platelet count, $7 \times 10^{9} /$ ) with normal hemorrhagic diathesis tests. With increased serum lactate dehydrogenase and indirect bilirubin level associated with decreased haptoglobin and red cell fragmentation on peripheral smear, iTTP was diagnosed. The direct antiglobulin test was negative. Daily therapeutic plasma exchange (TPE), and steroid (methylprednisolone: $1 \mathrm{~g} /$ day for 3 days and then $1 \mathrm{mg} / \mathrm{kg} /$ day) were started. On the second day during TPE, she developed tonic-clonic seizures and transferred to Intensive care unit as intubated. On the 5th day of TPE and steroid, serum LDH was persistently elevated with a mild increase in platelet count $\left(16 \times 10^{9} / \mathrm{L}\right)$. Rituximab was started $(375 \mathrm{mg} / \mathrm{m} 2 /$ week). Hepatomegaly and generalized edema as pleural effusion and ascites with an increase in serum direct bilirubin $(2.94 \mathrm{mg} / \mathrm{dL})$ added to the clinical picture. Hepatic SOS was suspected and defibrotide infusion $(25 \mathrm{mg} / \mathrm{kg} /$ day) was started. At that time the ADAMTS-13 
activity was achieved as being $0.001 \mathrm{U} / \mathrm{mL}$ with an inhibitör level of $>90 \mathrm{U} / \mathrm{mL}$. TPE was continued twice-daily with cryopoor plasma. In one-week, fluid retention gradually disappeared. On the 11th day, the platelet count reached the normal level. The patient could be extubated and transferred to the hematology ward. She is still confused and has disorientation and cooperation problems.

Conclusions: The pathogenesis of SOS is multifactorial but begins with the activation of sinusoidal endothelial cells. The disease can be mortal. In our case, it could be the consequence of iTTP or had an undetermined trigger. Defibrotide is given protective effects toward microvascular injury.

P0161 | Refractory Thrombotic Thrombocytopenic Purpura - A Case Report

A. Aribandi $^{1}$; C. Ranjith ${ }^{1}$; S. Sushma ${ }^{2}$; T. Surapaneni ${ }^{2}$

${ }^{1}$ American Oncology Institute, Hyderabad, India; ${ }^{2}$ Fernandez

Foundation, Hyderabad, India

Background: Thrombotic thrombocytopenic purpura (TTP) is a rare life-threatening thrombotic microangiopathy characterized by microangiopathic hemolytic anemia, thrombocytopenia, fever, Neurologic abnormalities, and acute renal insufficiency.

Aims: To present the diagnosis and management of a case of refractory thrombotic thrombocytopenic purpura.

Methods: Hematological and biochemical studies were done .ADAMS 13 activity \& antibodies were measured in our case to detect TTP.

Results: 27 year old lady, G3A2, at 21 weeks 6 days, was diagnosed to have Intrauterine fetal demise, so termination of pregnancy was done and she was advised LMWH for 6 weeks post-delivery. On PND 3 she presented with $\mathrm{C} / \mathrm{O}$ giddiness and burning micturition. On evaluation her CBC was $6 \mathrm{gm} / \mathrm{dl}$ and platelets were $20000 / \mathrm{mm}^{3}$. Peripheral smear showed schistocytes with thrombocytopenia. PT $\&$ APTT were normal. Empirical diagnosis of TTP was made and she was started on steroids and plasmapheresis. ADAMS 13 activity was $<3$ and ADAMTS inhibitor was $>32$. She received in total, 4 doses of Rituximab (weekly) \& 2 doses of weekly iv vincristine $(2 \mathrm{mg})$ along with 29 cycles of plasmapheresis and was discharged when her platelet count was $1,66,000 / \mathrm{mm} 3$. She was again readmitted after 10 days with platelet count $18,000 / \mathrm{mm} 3$. Rpt ADAMTS activity was $-<3$ (n-68-163), ADAMTS inhibitor was >1.5 $(n-<0.4)$. She received 5 cycles of plasmapheresis along with cyclosporine, which was added as a last resort. She was discharged on oral steroids and oral cyclosporin $100 \mathrm{mg}$ bd .Platelet count at the time of discharge was $2,05,000 / \mathrm{mm} 3$. She continues to be in remission post cyclosporine and is in follow up till now.

Conclusions: We present an unusual case of Thrombotic thrombocytopenic purpura, which was refactory to the usual treatments, i.e plasmapheresis, rituximab and vincristine. In our case cyclosporine was effective along with plasmapheresis, so cyclosporine can be used in case of refractory TTP for remission.

\section{ANTIPLATELET THERAPY}

PB0852 | Desmopressin for Reversal of Antiplatelet Drugs in Stroke due to Haemorrhage (DASH): Second Interim Report on Recruitment from a Phase II Double Blind Randomised Controlled Trial

M. Desborough ${ }^{1,2,3} ;$ R. Al-Shahi Salman ${ }^{4}$; S. Stanworth ${ }^{1,2,5}$; D. Havard ${ }^{6}$; P.M Brennan ${ }^{4}$; R. Dineen ${ }^{6}$; T. Coats $^{7}$; T. Hepburn ${ }^{8}$; L. Woodhouse ${ }^{6}$; P. Bath ${ }^{6}$; N. Sprigg ${ }^{6}$

${ }^{1}$ Department of Haematology, John Radcliffe Hospital, Oxford, United Kingdom; ${ }^{2}$ Oxford Clinical Research in Transfusion Medicine, University of Oxford, Oxford, United Kingdom; ${ }^{3}$ Centre for Haemostasis and Thrombosis, Guy's and St Thomas' NHS Foundation Trust, London, United Kingdom; ${ }^{4}$ Centre for Clinical Brain Sciences, University of Edinburgh, Edinburgh, United Kingdom; ${ }^{5} \mathrm{NHS}$ Blood and Transplant, Oxford, United Kingdom; ${ }^{6}$ Stroke, Division of Clinical Neuroscience, University of Nottingham, Nottingham, United Kingdom; ${ }^{7}$ Department of Cardiovascular Sciences, University of Leicester, Leicester, United Kingdom; ${ }^{8}$ Clinical Trials Unit, University of Nottingham, Nottingham, United Kingdom

Background: Intracerebral haemorrhage caused approximately 3 million deaths worldwide in 2015. Pre-stroke antiplatelet drug use is associated with a $27 \%$ relative increase in one-month case fatality compared to patients not using antithrombotic drugs.

Aims: We aim to assess the feasibility of administering desmopressin or placebo to patients with antiplatelet associated intracerebral haemorrhage to inform the design of a definitive trial.

Methods: In the ongoing DASH trial, we aim to include 50 patients within 24 hours of spontaneous intracerebral haemorrhage onset, associated with oral antiplatelet drug(s) use in the preceding seven days (figure 1). This time window was initially set at 12 hours but was increased on 01 December 2019 to maximise recruitment. Patients are randomised (1:1) to receive intravenous desmopressin 20 ug in $50 \mathrm{ml}$ sodium chloride $0.9 \%$ infused over 20 minutes or matching placebo. Feasibility outcomes include proportion of patients approached being randomised, number of patients receiving allocated treatment, rate of recruitment, and adherence to treatment and follow up. Secondary outcomes include change in intracerebral haemorrhage volume at 24 hours; early mortality < 28 days, death or dependency at day 90 , serious adverse events up to day 90 . 
Results:

TABLE 1 Characteristics of participants in the DASH trial.

\begin{tabular}{ll}
\hline $\begin{array}{l}\text { Number of patients randomised } \\
N\end{array}$ & 34 \\
\hline Age, years & $76.9(10.3)$ \\
Mean (standard deviation) & $20(59.0)$ \\
Sex & \\
Male (\%) & $9[4,17]$ \\
Time from symptom onset to randomisation & \\
$\quad$ (hours) & \\
Median [interquartile range] & $1[0,2]$ \\
Pre-stroke modified Rankin Scale & \\
Median [interquartile range] & $15[13,15]$ \\
Glasgow Coma Scale & \\
Median [interquartile range] & $156(22.9)$ \\
Systolic blood pressure (mmHg) & \\
Mean (standard deviation) & \\
Aspirin & $23(68)$ \\
$N$ (\%) & $14(41)$ \\
Clopidogrel & \\
$N$ (\%) & $1(3)$ \\
Dipyridamole & \\
$N$ (\%) &
\end{tabular}

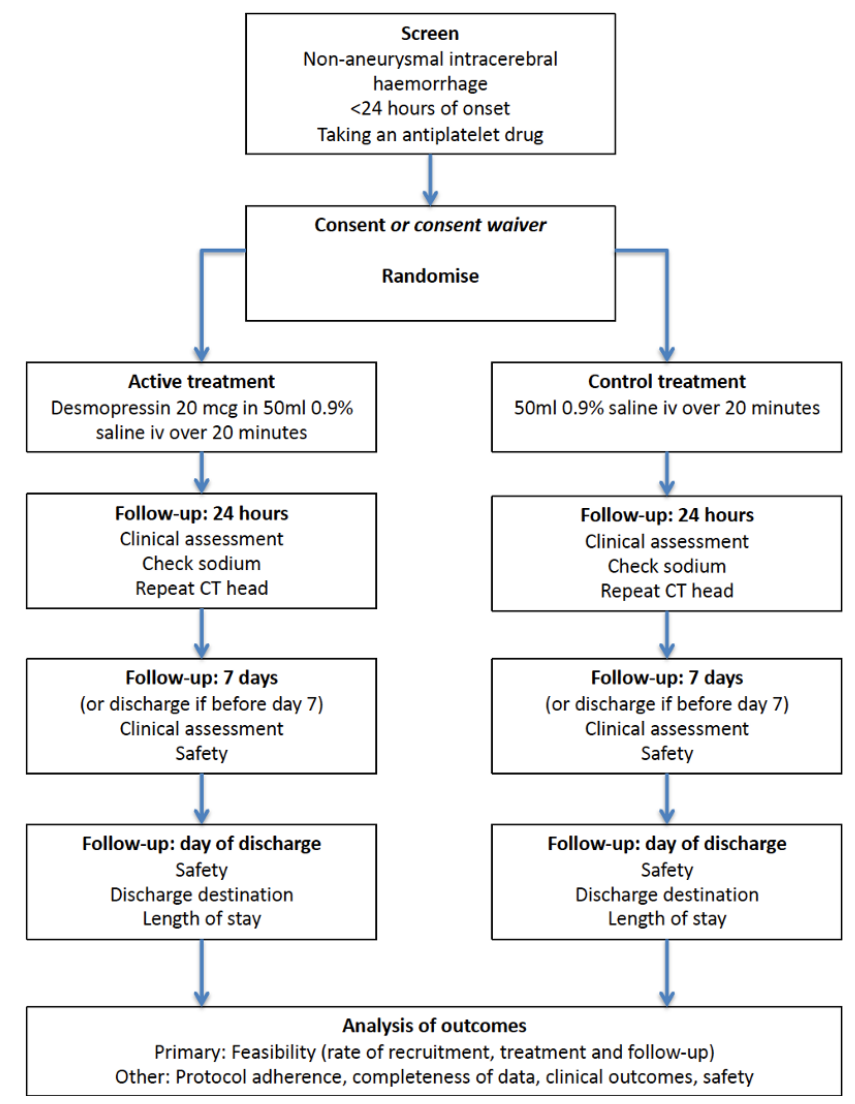

522 potential patients with intracerebral haemorrhage have been

FIGURE 1 Trial summary screened between 15 February 2019 and 15 January 2021 at 10 UK acute stroke centres. 34 patients (out of a planned 50 patients) have been recruited so far (table 1). Median time from symptom onset to administration of desmopressin or placebo was 9 hours (interquartile range 4 to 17 hours). The most common reasons patients were excluded were not being on an antiplatelet drug (268/522; $51 \%)$; presentation after the inclusion time window (62/522; 12\%) or presentation at times when research staff were not available (49/522; 9\%).

\begin{abstract}
Conclusions: This is an ongoing feasibility trial, which will inform the design of a definitive trial.
\end{abstract}

http://dash-1.ac.uk

PB0853 | Arginine-containing Peptides and Acetylsalicylic Acid: Evaluation of Antiplatelet Effects

M. Grigorjeva; T. Obergan; T. Shubina; L. Lyapina

Lomonosov Moskow State University, Moscow, Russian Federation

Background: Many pharmacological drugs of different structures (carbohydrate, peptide, protein) with antiplatelet action are known. Directed influence of these agents may to reduce pathologically increased platelet aggregation. Earlier it was shown that regulatory glyproline peptides exhibit anticoagulant and antiplatelet effects in the organism. Also, the amino acid arginine reduces the possibility of blood clots improving endothelial function and reducing platelet aggregation.

Aims: To study the antiplatelet effects of arginine-containing glyproline peptides and acetylsalicylic acid (aspirin) in a comparative aspect.

Methods: All experiments were conducted on male Wistar rats in accordance with the Helsinki Declaration of the world Medical Association on humane treatment of animals and the Geneva Convention «Internetional Guiding Principals for Biomedical 
Involving Animals». One of the peptides (100 mkg/kg) Lys-ArgArg-Lys-Pro-Gly-Pro (KRRKPGP), Lys-Lys-Arg-Arg-Pro-Gly-Pro (KKRRPGP), Arg-Lys-Lys-Arg-Pro-Gly-Pro (RKKRPGP), Arg-Pro-GlyPro (RPGP) and aspirin ( $1 \mathrm{mg} / \mathrm{kg}$ ) was administered to rats intragastric for 7 days. Blood for research were collected from the v.jugular $20 \mathrm{~h}$ after the last drugs administration. The measurement of platelet aggregation was started upon the addition of 10-6 M ADP in rich platelet blood plasma (Born method).

Results: It was found that $20 \mathrm{~h}$ after the last of administration of KRRKPGP, KKRRPGP, RKKRPGP and RPGP, platelet aggregation decreased in rats by $29 \%, 34 \%, 49 \%$ and $19 \%$, respectively, compared with saline control group (100\%). The injection of aspirin lead to platelet aggregation decrease by $24 \%$ vs. control. So, RKKRPGP had the most pronounced antiplatelet effects in rat organism.

Conclusions: Thus, the present research showed that the studied drugs have significant antiplatelet effect due to decrease activation of platelet haemostasis due to reduced platelet aggregation. Besides, the antiplatelet effects of the studied peptides are comparable to the action of the well-known agent aspirin. We assume that regulatory arginine-containing glyproline oligopeptides can be attributed to a perspective antiplatelet agents without side effects.

\section{HIT}

PB0854 | Variable Serotonin Release Assay Pattern and Characteristics of PF4-specific Antibodies in Heparin-Induced Thrombocytopenia, and Clinical Impact

N. Charuel ${ }^{1,2}$; J. Rollin ${ }^{1,3}$; Y. Gruel ${ }^{1,3}$; E.-A. Guéry ${ }^{3}$; M.-A. May ${ }^{4,5}$; C. Pouplard ${ }^{1,3} ;$ C. Vayne ${ }^{1,3}$

${ }^{1}$ EA 7501 GICC, University of Tours, Tours, France; ${ }^{2}$ Diagnostica Stago, Asnières-Sur-Seine, France; ${ }^{3}$ Department of Haemostasis, University Hospital of Tours, Tours, France; ${ }^{4}$ Department of Cardiovascular Surgery, University Hospital of Tours, Tours, France; ${ }^{5}$ Department of Anesthesiology, University Hospital of Tours, Tours, France

Background: The diagnosis of Heparin-induced thrombocytopenia (HIT) often necessitates functional assays to demonstrate in vitro that antibodies to platelet factor 4 (PF4) are activating platelets, typically only in the presence of therapeutic heparin $(\mathrm{H})$ concentrations ("classical" pattern). More rarely, HIT samples activate platelets even without heparin ("atypical" pattern). However, the clinical significance of such a profile is unclear.

Aims: We aimed to analyze the clinical and biological course of HIT patients according to their platelet activation pattern in serotonin release assay (SRA) and the main characteristics of PF4-specific antibodies.

Methods: We enrolled 74 patients with definite HIT under heparin treatment, and exhibiting in SRA either a "classical" ( $n=62)$, or "atypical" pattern $(n=12)$. Titers of IgG to PF4/H complexes and PF4 alone were measured by ELISA in 41 selected patients, and results were analyzed according to the SRA pattern, and bioclinical features.
Results: Higher anti-PF4/H IgG titers were measured in patients with an "atypical" SRA (median OD 2.52 vs. 1.94 in those with a "classical" pattern, $P<0.001)$. Patients of both groups had similar platelet count (PC) nadir and time to recovery, but those with an "atypical" SRA developed more thrombotic events (66.7 vs. 33.9\%, $P=0.05)$. Significant levels of anti-PF4 IgG (OD >0.4) were detected in both groups ( $38 \%$ and $58 \%$, respectively). But whatever the SRA pattern, a lower PC nadir (median: 30 vs. $54 \mathrm{G} / \mathrm{L}, P=0.007$ ) and a longer $P C$ recovery time (median: 6 vs. 3 days, $P=0.01$ ) were evidenced in patients with anti-PF4 antibodies, compared to those with anti-PF4/H IgG only.

Conclusions: An atypical SRA pattern with elevated anti-PF4/H IgG titers appears to be associated with an increased risk of thrombosis in HIT. IgG antibodies to PF4 alone may contribute to more severe and persistent thrombocytopenia, and their detection could be useful in clinical practice.

PB0855 | Diagnosing Heparin-induced Thrombocytopenia Using Machine Learning Algorithms: First Data of the TORADI-HIT Study

H. Nilius ${ }^{1}$; J.-D. Studt ${ }^{2}$; D.A. Tsakiris ${ }^{3}$; A. Greinacher ${ }^{4}$; A. Mendez ${ }^{5}$; A. Schmidt ${ }^{6}$; W.A. Wuillemin ${ }^{7}$; B. Gerber ${ }^{8}$; P. Vishnu ${ }^{9}$; L. Graf ${ }^{10}$; T. Bakchoul ${ }^{11}$; M. Nagler ${ }^{1}$

${ }^{1}$ University of Bern / University Institute of Clinical Chemistry, Bern, Switzerland; ${ }^{2}$ University and University Hospital Zurich / Division of Medical Oncology and Hematology, Zurich, Switzerland; ${ }^{3}$ Basel University Hospital / Diagnostic Haematology, Basel, Switzerland;

${ }^{4}$ Universitätsmedizin Greifswald / Institut für Immunologie und Transfusionsmedizin, Greifswald, Germany; ${ }^{5}$ Kantonsspital Aarau / Department of Laboratory Medicine, Aarau, Switzerland; ${ }^{6}$ City Hospital Waid and Triemli / Institute of Laboratory Medicine and Clinic of Medical Oncology and Hematology, Zurich, Switzerland; ${ }^{7}$ Cantonal Hospital of Lucerne and University of Bern / Division of Hematology and Central Hematology Laboratory, Lucerne, Switzerland; ${ }^{8}$ Oncology Institute of Southern Switzerland / Clinic of Hematology, Bellinzona, Switzerland; ${ }^{9} \mathrm{CHI}$ Franciscan Medical Group / Division of Hematology, Seattle, United States, ${ }^{10}$ Cantonal Hospital of St Gallen, St Gallen, Switzerland, ${ }^{11}$ University Hospital of Tübingen / Centre for Clinical Transfusion Medicine, Tübingen, Germany

Background: Diagnosing heparin-induced thrombocytopenia (HIT) at the bedside is challenging, and current diagnostic algorithms expose patients to a considerable risk of overtreatment and delayed diagnosis.

Aims: We conducted a prospective multicenter study detailedly acquiring clinical and laboratory variables to assess the diagnostic performance of these variables and to develop an easy-to-apply clinical prediction model. 
Methods: Consecutive patients with suspected HIT were included in 11 study centers and detailed clinical data were collected. Heparininduced platelet activation assay (HIPA; reference standard) and various immunoassays were conducted at the central laboratory. Variables with a $P$-value $<0.05$ for each level in a multivariable logistic regression were selected for the final model. Using $75 \%$ of the patients, logistic regression, penalized logistic regression, two random forest, and gradient boosting machine models were trained. The models were evaluated on the remaining 25\% (validation set). The performance of the model with the best c-statistic was then compared to the current clinical practice.

Results: To date, we enrolled 1'182 patients with suspected HIT; the prevalence of HIT was $9.3 \%$. Variables selected for the final model were: platelet nadir, use of unfractionated heparin, timing of thrombocytopenia, presence of other causes of thrombocytopenia, and immunoassay test result. Applied to the validation set and using an IgG-specific ELISA, the c-statistic of the random forest model was 98.8\% (95\% confidence interval [Cl]: 97.7, 99.9), the sensitivity was 96.0\% (95\% Cl: 79.6, 99.8) and the specificity 97.3\% (95\% Cl: 93.0 98.1). In contrast, the sensitivity of the currently recommended diagnostic algorithm was $80.0 \%(95 \% \mathrm{Cl}: 59.3,93.2)$, and the specificity $89.1 \%$ (95\% Cl: 84.6, 92.6).

Conclusions: Using detailed clinical and laboratory data and machine-learning algorithms, we developed and validated an accurate clinical prediction model for the diagnosis of HIT. This model has the potential to relevantly reduce overtreatment and delayed diagnosis in clinical practice.
Heparin-induced platelet activation assay (HIPA)

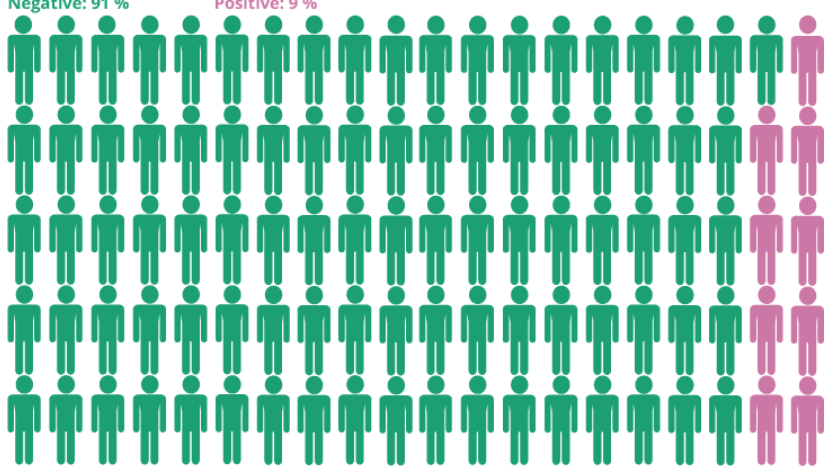
Current diagnostic algorithm (4T-Score/ELISA)

True negative: $81 \% \quad$ False positive: $10 \% \quad$ False negative: $2 \% \quad$ True positive: $7 \%$

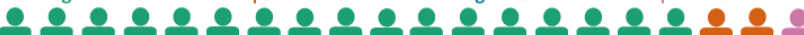

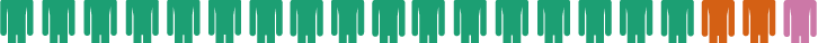

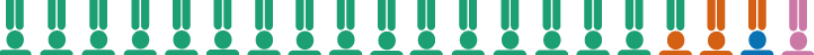

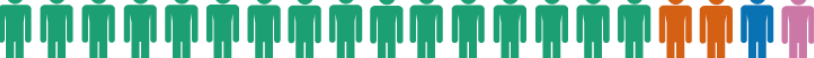

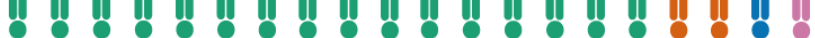

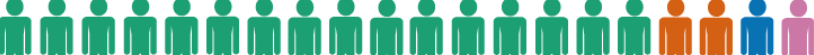

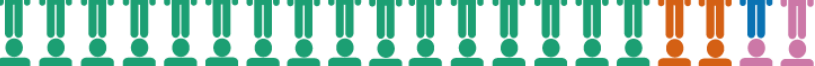

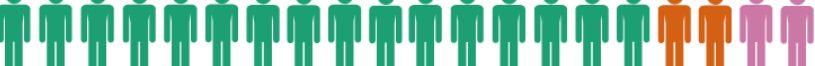

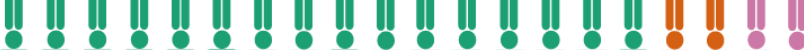

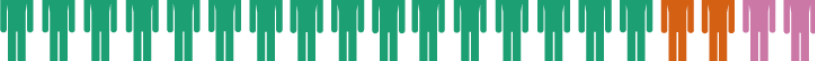
TORADI-HIT algorithm (random forest model)

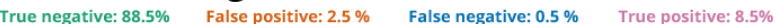
True negative: $88.5 \%$ False positive: $2.5 \%$ False negative: $0.5 \%$

FIGURE 1 Diagnostic accuracy of a random-forest-based clinical prediction model in contrast to the currently recommended diagnostic algorithm. Proportions of false negatives, false positives, true positives, and true negatives are shown

PB0856 | Minimal Role of the Alternative Pathway in Complement Aactivation by HIT Immune Complexes

$\underline{\text { A. Barnes }}^{1}$; S. Khandelwal ${ }^{1}$; S. Sartoretto ${ }^{1}$; S. Myoung ${ }^{1}$; S. Francis ${ }^{1}$; G. Lee ${ }^{1}$; L. Rauova²; D. Cines ${ }^{3}$; J. Skare ${ }^{4}$; B. Garcia ${ }^{5}$; G. Arepally ${ }^{1}$ ${ }^{1}$ Duke University Medical Center, Durham, United States; ${ }^{2}$ Children's Hospital of Philadelphia, Philadelphia, United States; ${ }^{3}$ Hospital of University of Pennsylvania, Philadelphia, United States; ${ }^{4}$ Texas A\&M University, Bryan, United States; ${ }^{5}$ East Carolina University, Greenville, United States

Background: Ultra-large immune complexes (ULICs) consisting of IgG, platelet factor 4 and heparin $(\mathrm{P}+\mathrm{H})$ initiate complement $\left(C^{\prime}\right)$ activation by the classical pathway $(\mathrm{CP})$ in heparin induced 
thrombocytopenia (HIT; PMID: 7412786). We recently demonstrated that inhibition of the CP markedly attenuates cellular activation by HIT ULICs independent of FcyRIIA.

Aims: Previous studies (PMID: 15544620) indicate that the alternative pathway (AP) amplifies C' activation by the CP by $>80 \%$, indicating a potentially important adjunctive form of intervention in HIT. Therefore, we compared inhibitors of AP and CP by HIT ULICs in whole blood (WB).

Methods: WB was preincubated with inhibitors of: a) the CP (BBK32, a borrelial protein inhibitor of $\mathrm{C} 1 \mathrm{r}$ ), b) AP (anti-factor B antibody; aFB $A b$, or Factor $D(f D)$ Alexion, Boston, MA) or $c$ ) combined AP/CP (C1esterase inhibitor, C1-INH, Berinert, CSL Behring; or soluble complement receptor 1, sCR1, Alexion) prior to adding $\mathrm{P}+\mathrm{H}$ and either a HIT-like monoclonal antibody, KKO, HIT IgG or isotype controls for 1 hour at $37^{\circ} \mathrm{C}$ followed by $10 \mathrm{mM}$ EDTA to quench further $\mathrm{C}^{\prime}$ activation. $C^{\prime}$ activation products (sC5b-9) and neutrophil degranulation (MMP9) were measured by immunoassay. AP inhibition was confirmed using a modified Wieslab assay (PMID 26579461).

Results:

Figure 1

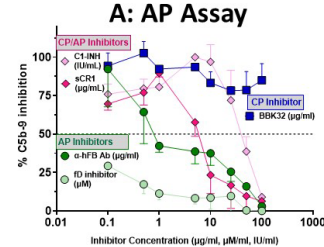

C: CP Assay (HIT IgG)

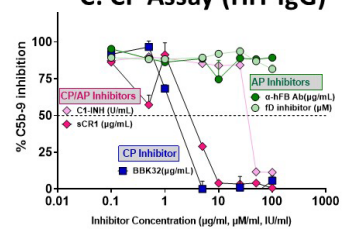

FIGURE 1 Effects of alternative and classical pathway inhibitors on complement activation by HIT antibodies

AP inhibitors, aFB Ab and fD inhibitor, potently inhibit AP (Figure 1A) activation in a modified Wieslab assay, whereas CP/AP inhibitors $C 1$ INH and sCR1 show modest inhibition and BBK32 had no effect. However, with KKO or HIT ULICs in WB, BBK32, C1-INH and SCR1potently blocked generation of sC5b-9 (Figure 1B/1C), whereas AP inhibitors had no effect. Similarly, AP inhibitors minimally inhibited MMP9 release compared to the CP inhibitor, BBK32 (Figure 1D).

Conclusions: Activation of the AP pathway provides little amplification of CP activity by HIT ULICs. Future studies of $C^{\prime}$ inhibition in HIT should focus on inhibition of the $\mathrm{CP}$ as potential adjunctive therapy for HIT.
PB0857 | A Multicenter Retrospective Evaluation of Direct Oral Anticoagulants for the Treatment of Heparin Induced Thrombocytopenia

K. Davis ${ }^{1}$; J. Sebaaly ${ }^{2}$; L. Wooten ${ }^{3}$; C. Khouli ${ }^{4}$; A. Mihm ${ }^{1}$; S. Nisly ${ }^{1,5}$ ${ }^{1}$ Wake Forest Baptist Health, Winston Salem, United States; ${ }^{2}$ ProCE, LLC, Charlotte, United States; ${ }^{3}$ Advent Health, Orlando, United States; ${ }^{4}$ Indiana University Health, Indianapolis, United States; ${ }^{5}$ Wingate University School of Pharmacy, Wingate, United States

Background: The direct oral anticoagulants (DOACs) represent an off-label, but potential alternative to traditional therapies for the treatment of heparin induced thrombocytopenia (HIT). Literature evaluating DOACs for HIT remains limited, with most studies being small retrospective cohorts and case series.

Aims: To evaluate the efficacy and safety of DOACs in patients with a diagnosis of laboratory confirmed HIT.

Methods: A multicenter retrospective cohort study of adult patients with a diagnosis of HIT treated with apixaban, rivaroxaban, or dabigatran between January 1, 2013 and January 1, 2020 was performed. Patients with an intermediate or high pre-test probability for HIT and a positive anti-platelet factor 4 /heparin complex assay, latex immunoturbidimetric assay (LIA), or serotonin release assay (SRA) were included for analysis. The primary outcome was the composite of newly diagnosed venous or arterial thromboembolism, gangrene, or severe limb ischemia requiring amputation at 3 months following DOAC initiation. Secondary outcomes included major bleeding as defined by the International Society on Thrombosis and Haemostasis. This study was approved by local institutional review boards with the requirement for informed consent waived.

Results: Seventy-seven patients from four healthcare systems were included. The median 4Ts score was 5 (interquartile range: 4.5-6) and $38(49.4 \%)$ patients had a diagnosis of HIT with thrombosis (Table 1). The most frequently used DOAC was apixaban $(n=51)$ followed by rivaroxaban $(n=24)$ and dabigatran $(n=2)$. Sixty three $(81.8 \%)$ patients received parenteral non-heparin anticoagulation prior to DOAC initiation. Median platelet count at the time of DOAC initiation was $126,000 /$ Liter. Nine (11.7\%) patients experienced the primary outcome of HIT related thrombotic events (Table 2). Of the 14 patients who exclusively received DOAC therapy, none experienced the primary outcome. Major bleeding occurred in 5 (6.5\%) patients.

TABLE 1 Baseline characteristics

\begin{tabular}{ll}
\hline${ }^{*}$ Age, years & $63(53-69.5)$ \\
Female, $n(\%)$ & $33(42.9)$ \\
Hospital location at time of diagnosis & \\
Medical floor, $n(\%)$ & $39(50.6)$ \\
Intensive care unit, $n(\%)$ & $38(49.4)$ \\
${ }^{*}$ Platelet nadir, $\times 10 \% / L$ & $54(35.5-72.5)$ \\
${ }^{*}$ Platelet count at DOAC initiation, $x 10^{9} / \mathrm{L}$ & $126(102-219)$ \\
${ }^{*} 4$ Ts score & $5(4.5-6)$ \\
HIT with thrombosis, $n(\%)$ & $38(49.4)$ \\
\hline
\end{tabular}

*Expressed as median (interquartile range) 
TABLE 2 Outcomes

\begin{tabular}{|c|c|c|c|}
\hline & $\begin{array}{l}\text { Total } \\
(n=77)\end{array}$ & $\begin{array}{l}\text { Received parenteral therapy } \\
\text { prior to DOAC initiation } \\
(n=63)\end{array}$ & $\begin{array}{l}\text { No parenteral } \\
\text { therapy prior to } \\
\text { DOAC initiation } \\
(n=14)\end{array}$ \\
\hline Platelet recovery, yes, $n$ (\%) & $61(79.2)$ & $52(82.5)$ & $9(64.3)$ \\
\hline${ }^{*}$ Time to platelet recovery, days & $5(3-8)$ & $5(3.3-8)$ & $4(2.5-9.5)$ \\
\hline *Length of Stay, days & $19(9.5-28)$ & $20(10-29)$ & $15.5(8-25.8)$ \\
\hline Major bleeding, $n(\%)$ & $5(6.5)$ & $4(6.3)$ & $1(7.1)$ \\
\hline Clinically relevant non-major bleeding, $n(\%)$ & $9(11.7)$ & $7(11.1)$ & $2(14.3)$ \\
\hline
\end{tabular}

*Expressed as median (interquartile range)

Conclusions: In this retrospective cohort study, DOACs were associated with low rates of thrombotic and hemorrhagic events for the treatment of HIT.

PB0859 | PF4/Polyanion ELISA-negative Antibodies to PF4 and Non-PF4 Targets Can Mediate Platelet Activation and Cause Heparin-induced Thrombocytopenia (HIT)

B. Singh ${ }^{1}$; C. Jones ${ }^{2}$; D.A. Garcia ${ }^{3}$; N. Leung ${ }^{1}$; R.S. Go ${ }^{1}$; R. Leger ${ }^{1}$; N. Heikal ${ }^{1}$; R. Pruthi ${ }^{1}$; D. Chen ${ }^{1}$; A. Padmanabhan ${ }^{1}$

${ }^{1}$ Mayo Clinic, Rochester, United States; ${ }^{2}$ Retham Technologies, Milwaukee, United States; ${ }^{3}$ University of Washington School of Medicine, Seattle, United States

Background: While antibodies to Platelet Factor 4 (PF4) are the hallmark of HIT, antibodies to other platelet granule proteins such as Interleukin-8 and Neutrophil-activating peptide-2 have been described.

Aims: An index PF4/Polyanion ELISA ("ELISA")-negative HIT patient and a cohort of additional patients suspected of HIT were evaluated for non-PF4 platelet-activating antibodies using a novel assay that assesses Platelet Releasate-dependent Platelet Activation ("RPA").

Methods: Donor platelets were activated with Thrombin receptoractivating peptide to produce platelet "releasate". Platelets were incubated with releasate and patient sera from 176 HIT-suspected patients, and $P$-selectin expression was measured. Samples with

TABLE 1 Summary of serological features of HIT cases

$\begin{array}{rlllr}\text { N } & \text { Patient } & \text { HIT ELISA } & \text { SRA } & \text { PEA } \\ 1 & \text { Index Case } & \text { Neg } & \text { Neg } & \text { Neg } \\ 2 & \text { HIT 1 } & \begin{array}{l}\text { Weak Pos } \\ (0.7 \text { OD) }\end{array} & \text { Pos } & \text { Neg } \\ & & & \end{array}$

Neg $\geq 50 \% \mathrm{p}$-selectin expression and/or those that were ELISA-positive were evaluated in the PF4-dependent P-selectin expression assay (PEA). Studies were approved by the institutional ethics committee. Results: The index HIT patient (4Ts score $=7$ ) was ELISA-/PEA-/ SRA- while the RPA was positive (97\%) and inhibited to $2 \%$ with high dose heparin (Table 1). In testing of 176 sera, one sample each was RPA+/PEA- (HIT 1, red circle, Fig 1A) and RPA-/PEA+ (green circle), and interestingly, two ELISA- patients were RPA+/PEA+ (HIT 2 \& 3 blue circles). RPA+/PEA- and RPA-/PEA+ samples were retested in the PEA and RPA against the same target platelets to exclude donor platelet variability as a cause for the initial results. HIT 1 produced reproducible results. This patient had delayed-onset HIT, was PEAand demonstrated IgG-mediated and heparin-inhibitable platelet activation (Fig 1B). Use of PF4-depleted releasate did not impact $p$ selectin expression. Both ELISA- cases had a clinical picture consistent with HIT and demonstrated PF4-dependent platelet activation in the PEA and inhibition with high-dose heparin (100U/mL; Table 1).

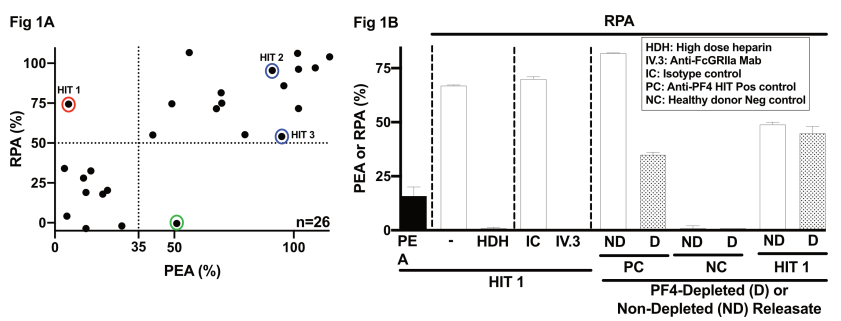

FIGURE 1 (A) RPA and PEA studies on HIT patient cohort; (B) RPA studies on patient HIT 1 


\begin{tabular}{|c|c|c|c|c|c|c|c|c|}
\hline \multicolumn{8}{|c|}{ ABSTRACT } & 637 of 972 \\
\hline$N$ & Patient & HIT ELISA & SRA & PEA & RPA & $\begin{array}{l}\text { HDH (100U/ } \\
\mathrm{mL})\end{array}$ & $\begin{array}{l}\text { RPA } \\
\text { (PF4-Depleted) }\end{array}$ & Conclusion \\
\hline 3 & HIT 2 & Neg & Neg & Pos (80\%) & Pos $(96 \%)$ & $\begin{array}{l}\text { Inhibited (PEA: } \\
1 \%)\end{array}$ & $\mathrm{N} / \mathrm{A}$ & ELISA/SRA-neg HIT \\
\hline
\end{tabular}

Conclusions: The RPA can facilitate detection of clinically-relevant PF4 and non-PF4 specific platelet-activating antibodies. The study also revealed that false-negative ELISA results may be obtained in some cases possibly due to non-exposure of physiologically-relevant PF4 neoepitopes in that assay.

PB0860 | Incidence of Heparin Induced Thrombocytopenia 2009-2017: Analysis of the National Inpatient Sample Database

Y. Hayashi-Tanner; M. Gaddam; S. Frankki; A. Borgert; L. Rosenstein Gundersen Health System, La Crosse, United States

Background: Heparin induced thrombocytopenia (HIT) prevalence ranges from $0.1-5.0 \%$. HIT is associated with increased healthcare cost, length of stay (LOS) and complications, including thrombosis and bleeding. The 4T Score, published in 2006, is a risk-stratifying tool that guides HIT testing and diagnosis. Use of direct oral anticoagulants (DOACs) has also become routine during this time. We seek to analyze HIT incidence trends in hospitalized patients during the DOAC and 4T era.

Aims: Assess HIT incidence in admissions reported in the National Inpatient Sample (NIS). Secondary aims include assessment of HIT associated complications, LOS, and total hospital charges amongst these groups.

Methods: A population-based assessment of the incidence of HIT and associated complications was performed for years 2009-2017 using the NIS. Poisson regression was used to examine associations between HIT incidence rates and relevant diagnosis categories for thrombotic complications.

Results:

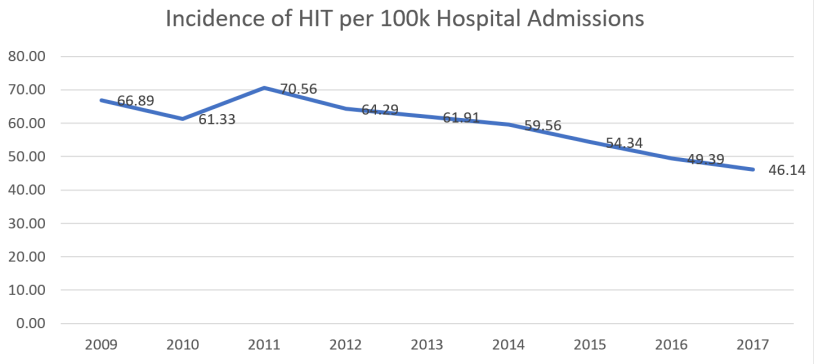

FIGURE 1 HIT Incidence 2009-2017

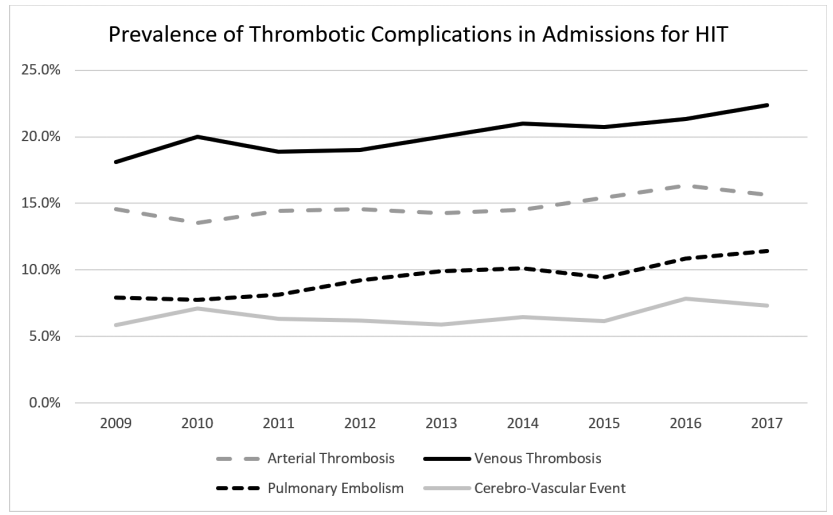

FIGURE 2 Thrombotic Complications in HIT

We identified 265,400,000 patients from 2009-2017, with 157,941 diagnosed with HIT. Median age was 67 years, LOS 10 days, and $\$ 92,234$ total hospital charge compared to 59 years, 3 days, and $\$ 24,104$ in non-HIT patients, respectively. No association with gender was found. Between 2009-2017, incidence of HIT significantly decreased (figure 1), a trend seen in all risk groups: surgical, trauma, and non-surgical. Prevalence of thrombotic complications have increased in HIT patients during the same time, while remaining stable in non-HIT patients (Figure 2).

Conclusions: Decreasing incidence of HIT over time and in all categories (including surgical) argues against DOACs causing the drop, as bypass patients still require exclusive use of heparin. One possible explanation is improved clinical identification of HIT following 4T Score implementation. This is further supported by the increased prevalence of thrombotic complication amongst HIT patients over time compared to stable findings in non-HIT patients. We may be more accurate in our diagnosis, which is important given the downstream costs and complications of HIT. 
PB0861 | Incidence of Heparin-induced Thrombocytopenia in Patients Treated with Mechanical Circulatory Support: Results from a Prospective Study

A. Gaupp ${ }^{1}$; O. Hidiatov ${ }^{1}$; S. Nowak-Harnau ${ }^{2}$; A. Straub $b^{3,4}$; H. Häberle ${ }^{3}$; P. Rosenberger ${ }^{3}$; T. Bakchoul ${ }^{1,2}$; K. Althaus ${ }^{1,2}$

${ }^{1}$ Transfusion Medicine, Medical Faculty of Tuebingen, University Hospital of Tuebingen, Tuebingen, Germany; ${ }^{2}$ Centre for Clinical Transfusion Medicine, University Hospital of Tuebingen, Tuebingen, Germany; ${ }^{3}$ Department of Anesthesiology and Intensive Care Medicine, University Hospital of Tuebingen, Tuebingen, Germany;

${ }^{4}$ Klinik für Anästhesie, Intensiv-, Notfall- und Schmerzmedizin Oberschwabenklinik, St. Elisabethen Klinikum, Ravensburg, Germany

Background: Extracorporeal circulations (ECC) are increasingly used in critically ill patients with respiratory or cardiac failure. Patients with extracorporeal membrane oxygenations (ECMO) receive continuous infusion of unfractionated heparin (UFH) for circuit patency and are at high risk for heparin induced thrombocytopenia (HIT). The diagnosis of HIT is, however, very challenging in critically ill patients undergoing ECC. In addition, switching anticoagulation to non-heparin agents in thrombocytopenic patients is associated with increased bleeding risk.

Aims: To assess the incidence and risk factors of HIT among patients under ECC.

Methods: Consecutive clinical and laboratory data of patients undergoing ECC were prospectively collected. Blood samples were taken at day 0, 1, 6 and 10 after ECC implementation. Patients with history of coagulation and/or platelet disorders were excluded. Diagnosis of HIT was made by using the 4Tscore, the Platelet factor 4 (PF4)/heparin IgG EIA and the functional assay (HIPA). HIT was defined as a positive EIA and HIPA.

Results: From 56 patients with ECC, 31 patients received venoarterial (va) ECMO, 14 patients veno-venous (vv) ECMO and 11 patients LVAD. All patients received UFH. In 61\% patients ECC could be explanted, $66 \%$ of the patients were discharged from hospital. Within 10 days $88 \%$ showed bleeding and $54 \%$ thrombotic events.

According to the $4 \mathrm{~T}-\mathrm{Score} 5 \%, 14 \%, 66 \%$, and $65 \%$ had clinically suspicion of HIT (score $>3$ ) at day $0,1,6$ and 10, respectively. Seroconversion (new PF4/heparin IgG-antibodies) was found in 23\% and $42 \%$ patients at day 6 and 10, respectively. The Frequency of HIT was estimated to be $3.57 \%$ and $4 \%$ at day 6 and 10 .

Conclusions: Incidence of clinically relevant HIT with ECC is low despite the high prevalence of thrombocytopenia (95\%) and IgG seroconversion (42\%). Diagnosis of HIT requires confirmation platelets activating antibodies in a functional assay to avoid overdiagnosis of HIT.
PB0862 | Urgent Cardiac Surgery in a Patient with Heparin Induced Thrombocytopenia

M. Romić ${ }^{1}$; D. Mišura ${ }^{1}$; G. Tomac $^{2}$; M. Čikeš ${ }^{1,3}$; H. Gašparović ${ }^{1,4}$; A. Boban ${ }^{1,5}$

${ }^{1}$ School of Medicine, University of Zagreb, Zagreb, Croatia;

${ }^{2}$ Department of Transfusion Medicine and Transplantation Biology, University Hospital Centre Zagreb, Zagreb, Croatia; ${ }^{3}$ Department of Cardiovascular Diseases, University Hospital Centre Zagreb, Zagreb, Croatia; ${ }^{4}$ Department of Cardiac Surgery, University Hospital Center Zagreb, Zagreb, Croatia; ${ }^{5}$ Division of Hematology, Department of Internal Medicine, University Hospital Centre Zagreb, Zagreb, Croatia

Background: Heparin induced thrombocytopenia (HIT) requires immediate replacement of heparin with non-heparin anticoagulants. Nevertheless, anticoagulation during cardiac surgery necessitates administration of unfractionated heparin, and the management of patients with positive HIT antibodies may be challenging if urgent surgery is needed.

Aims: We present a case of a 57-year-old male patient with heart failure treated with veno-arterial extracorporeal membrane oxygenation and the need for an urgent upgrade to a paracorporeal, surgically placed left ventricular assist device (LVAD) shortly after detection of high-titer HIT antibodies.

Methods: The patient had ischemic cardiomyopathy, arterial hypertension and diabetes. The acutization of heart failure was provoked by refractory ventricular arrhythmias following the amputation of the left toe due to gangrene. Following re-amputation of the left foot, thrombocytopenia was observed and HIT was verified by ELISA. Heparin was then replaced by fondaparinux, followed by the normalization of the platelet count.

The planned cardiac surgery included anticoagulation with unfractionated heparin. As preparation for the surgery, five procedures of plasma exchange were performed to remove HIT antibodies from the circulation. The surgery was done after two consecutive negative HIT antibodies tests, with additional infusion of intravenous gamma globulins (lvlg) given immediately before the procedure.

Results: The cardiac surgery procedure went uneventful regarding thrombotic events and hemostasis, while a right ventricular assist device was needed in addition to the planned LVAD. Postoperative anticoagulant treatment was continued with fondaparinux. No rise in HIT antibodies or platelet drop was described after the procedure, not later during follow up. Cardiac transplantation was done a month later with intraoperative administration of unfractionated heparin. No thrombocytopenia nor the anamnestic response of HIT was described after transplant, either.

Conclusions: The combination of plasma exchange and Ivlg resulted in negative HIT antibodies and enabled safe, life-saving cardiac surgery procedures with unfractionated heparin in a patient with a recent history of high-titer HIT. 
PB0863 | A Puzzling HIT History: HIT Associated with Fondaparinux or True Autoimmune HIT?

N. Sillamy ${ }^{1} ;$ C. Vayne ${ }^{1,2}$; J. Rollin ${ }^{1,2} ;$ M. Desailly ${ }^{1} ;$ M. Navarro ${ }^{3}$; J.-B. Valentin ${ }^{1}$; C. Pouplard ${ }^{1,2} ;$ Y. Gruel ${ }^{1,2}$

${ }^{1}$ Regional University Hospital Centre Tours, Department of Haemostasis, Tours, France; ${ }^{2}$ University of Tours, EA 7501 GICC, Tours, France; ${ }^{3}$ Regional University Hospital Centre Tours, Department of Dermatology, Tours, France

Background: Fondaparinux-associated HIT is a rare event, with PF4specific IgG antibodies detected in most affected patients, as in typical heparin-induced thrombocytopenia. However, this complication is also considered as an autoimmune HIT syndrome (aHIT), with antibodies bridging PF4 tetramers and inducing platelet activation, in the absence of heparin.

We recently managed a patient with fondaparinux-associated HIT, with features different from those associated with aHIT, suggesting an anti-PF4 immunization comparable to that involved in classical HIT.

Aims:

Methods:

Results: An 87-year-old woman developed recurrent superficial venous thrombosis, and fondaparinux was initiated $(7.5 \mathrm{mg} / \mathrm{d})$. Ten days later, she was hospitalized for fainting and extensive thigh hematoma. Fondaparinux was stopped because of active bleeding of the femoral artery, and reintroduced at lower dose $(2.5 \mathrm{mg} / \mathrm{d}) 3$ days after embolization. Thirteen days later, platelet count (PC) fell (decrease $>50 \%$ ), and although no thrombosis was observed, HIT was suspected 6 days later because no other cause of thrombocytopenia was present (4T's score: 5). Fondaparinux was replaced by rivaroxaban (10 mg/d). Anti-PF4/H IgG antibodies were detected (ELISA Immucor ${ }^{\circledR}$; OD: 1.6), and HIT was confirmed by serotonin release assay (SRA), which was strongly positive with UFH, but negative with fondaparinux.

Fondaparinux-associated HIT was then supported by PC recovery two days after fondaparinux withdrawal. However, as no platelet activation was demonstrated with fondaparinux in vitro, a spontaneous HIT syndrome was also evoked. But a role for atypical anti-PF4 antibodies able of promoting platelet activation without heparin was excluded, as such antibodies were not detected and no platelet activation was induced without heparin in SRA. Another pathogenic process could involve the release of heparin-like molecules from endothelial glycocalyx due to extensive vascular injury induced by the severe hematoma developed by the patient. This autoheparinization process could partly explain the hemorrhagic phenotype and the cross-reactivity observed with UFH in SRA.

Conclusions:
PB0864 | Successful Treatment with Edoxaban in Heparininduced Thrombocytopenia with Thrombosis: A Case Report

M. Porres-Aguilar ${ }^{1}$; F.A. Grimaldo-Gómez ${ }^{2}$; C. Jerjes-Sánchez ${ }^{3,4}$; G.A. Altamirano-Solorzano ${ }^{2}$; M.C. Guerrero de León ${ }^{5,6}$; R. IzaguirreÁvila²; D. Schuller'; R. Prieto ${ }^{8}$; D. Mukherjee ${ }^{9}$

${ }^{1}$ Department of Medicine, Division of Hospital Medicine; Texas Tech University Health Sciences Center, El Paso, United States; ${ }^{2}$ Department of Hematology, Instituto Nacional de Cardiología 'Ignacio Chávez', Mexico City, Mexico; ${ }^{3}$ Tecnológico de Monterrey, Escuela de Medicina y Ciencias de la Salud, Monterrey, Mexico; ${ }^{4}$ Instituto de Cardiología y Medicina Vascular, TecSalud, Monterrey, Mexico; ${ }^{5}$ Department of Internal Medicine and Critical Care, Hospital de Ginecología y Obstetricia 'Ignacio Morones Prieto', Monterrey, Mexico; ${ }^{6}$ UMAE 23, Instituto Mexicano del Seguro Social (IMSS), Monterrey, Mexico; ${ }^{7}$ Department of Medicine, Texas Tech University Health Sciences Center, El Paso, United States; ${ }^{8}$ Division of Hematology, Texas Tech University Health Sciences Center, El Paso, United States; ${ }^{9}$ Division of Cardiovascular Diseases; Texas Tech University Health Sciences Center, El Paso, United States

Background: Heparin-induced thrombocytopenia with thrombosis (HITT) represents a rare immunologic drug reaction, provoking a paradoxical hypercoagulable state, inducing life-threatening venous thromboembolism (VTE) and/or arterial thrombosis.

Aims: Herein, we describe a case of confirmed HITT successfully treated with edoxaban.

Methods: N/A

Results: A 56-year-old man underwent distal colorectal mass resection surgery; upon discharge he was sent home with subcutaneous dalteparin for extended thromboprophylaxis. On postoperative day 8 , patient returned to the hospital due to new-onset right sided pleuritic chest discomfort, associated with worsening dyspnea, and hypoxemia, and was subsequently diagnosed with acute right sided lobar pulmonary embolism. Initial platelet count was $245 \times 10^{3}$ / $\mu \mathrm{L}$. Within 24 hours of admission, platelet count dropped to 138 $\times 10^{3} / \mu \mathrm{L}$, and patient was switched to fondaparinux. His "4Ts" pretest scoring system was estimated at 6 points. His platelet factor- 4 (PF-4) dependent ELISA and serotonin-release assay were strongly positive, confirming the diagnosis of HITT. Patient had a remarkable clinical and laboratory improvement by hospital day 5 , with platelet count increased to $190 \times 10^{3} / \mu \mathrm{L}$; thus, it was decided to transition to edoxaban therapy at a dose of $60 \mathrm{mg}$ daily. During follow-up, platelet count completely recovered to $240 \times 10^{3} / \mu \mathrm{L}$ at 3 months, without any VTE recurrence nor significant bleeding events, despite colorectal adenocarcinoma diagnosis (see Figure). 


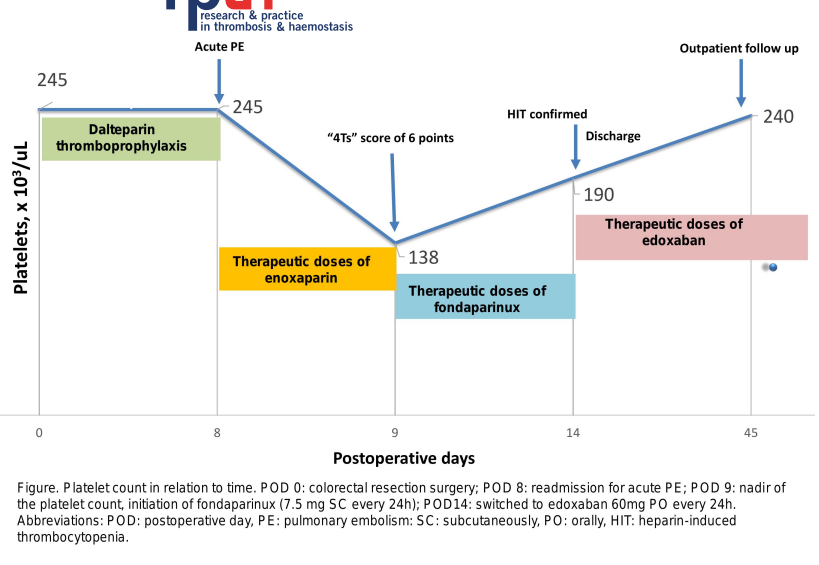

FIGURE 1 Platelet count in relation to time of clinical events

Conclusions: To our knowledge, this is only the second case describing successful therapeutic outcomes. Edoxaban has predictable pharmacokinetic and pharmacodynamic profiles and exhibit no interaction with PF-4, being an attractive therapeutic alternative for HITT. Further research with prospective randomized trials is required to evaluate its efficacy and safety in HITT patients.

HUS

PB0865 | Role of Soluble C5b-9 (sC5b-9) in Dosing Adjustments of Anti-complement Therapy during Pregnancy in a Patient with History of Atypical Hemolytic Uremic Syndrome (aHUS)

C. Dos Santos ${ }^{1}$; G. Greloni ${ }^{2}$; M.F. Alberto ${ }^{3}$; A. Sánchez-Luceros ${ }^{3}$ ${ }^{1}$ IMEX-CONICET-ANM, Buenos Aires, Argentina; ${ }^{2}$ Hospital Italiano de Buenos Aires, Buenos Aires, Argentina; ${ }^{3}$ IIHEMA-Academia Nacional de Medicina, Buenos Aires, Argentina

Background: Complications of pregnancy in women with aHUS include preeclampsia. This case study is a 35 -year-old carrier of genetic complement anomalies, with GMNC3 and aHUS diagnosis, currently treated with eculizumab (1200 mg/two weeks).

Aims: To monitor clinical and laboratory parameters of the patient during her second pregnancy.

Methods: Routine laboratory and complement testing (ELISA) were performed: (i) total complement activity (IMTEC, Human Diagnostics), (ii) plasmatic sC5b-9 (BD biosciences) and (iii) C5b-9 formation on endothelial cell line HMEC-1 in presence of human serum (home-made cell-based ELISA).

Results: At 24 weeks (w) of gestation, signs of hypertensive crisis included headaches and vomiting for which magnesium sulfate was administered. Between 36-37w, patient suffered nauseas, headaches and blurred vision, and delivered a healthy live birth $(2.630 \mathrm{~kg})$ following caesarean section. Eculizumab treatment was associated with low levels of total complement activation $(\leq 20 \mathrm{U} / \mathrm{mL})$ during pre- and post-natal period. Interestingly, plasmatic sC5b-9 increased significantly between $32 \mathrm{w}$ of gestation and first week puerperium (fig 1). However, eculizumab administration (900 mg) was given weekly during first month postpartum (fig 1).

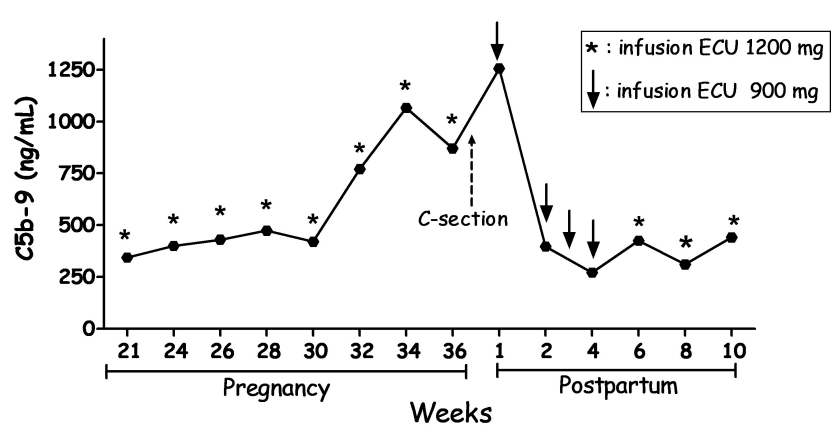

FIGURE 1 Timeline of soluble C5b-9 levels in patient plasma during her pregnancy and postpartum. Eculizumab (ECU) dosing regimen was indicated by asterisk $\left({ }^{*}\right)$ or black arrow.

Additionally, we quantified complement activation on HMEC-1 surface induced by cell incubation with patient serum. C5b-9 formation on HMEC-1 was significantly higher in presence of serum from pregnant patient compared with serum from patient postpartum (fig 2A). Complex formation of $\mathrm{C} 5 \mathrm{~b}-9$ during puerperium was similar to complex formed with a pooled human serum from 42 healthy donors (PS). Heat-inactivated ( $\mathrm{HI}$ ) serum at $56^{\circ} \mathrm{C}$ from $34 \mathrm{w}$ of gestation, corresponding to peak levels of plasmatic $\mathrm{s} C 5 \mathrm{~b}-9$, showed remnant complement activation on HMEC-1, whereas HI PS demonstrated complement inhibition (fig 2B).

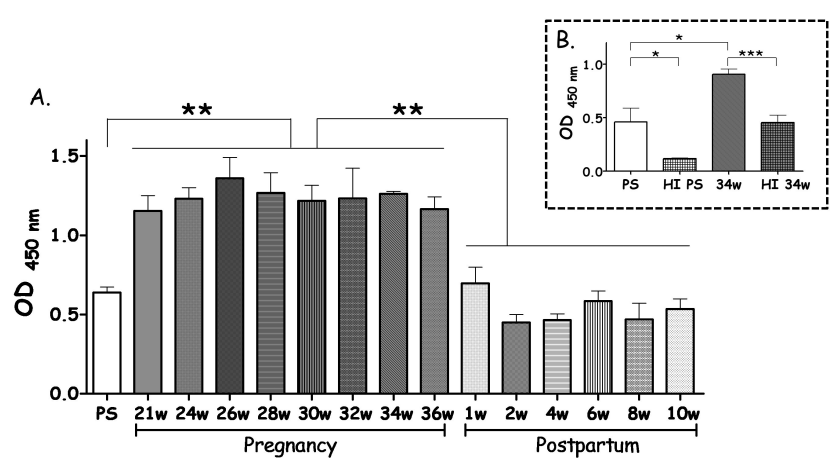

FIGURE 2 Detection of C5b-9 complex formation on endotelial cell line HMEC-1 using a cell-based ELISA assay. A. Serum from patient during her pregnancy and postpartum as well as from pooled human donors (PS) were incubated with HMEC-1 and complement membrane attack complex was detected using an anti-human $\mathrm{C} 5 \mathrm{~b}$ 9 antibody. ${ }^{* *} P$

Conclusions: Observations from this aHUS case study suggest that monitoring levels of sC5b-9 during pregnancy could offer insight in dose adjustment of anti-C5 therapy. Further studies are needed to explore other pathological pathway mechanisms involved in C5b-9 complex formation on cell surface during pregnancy. 


\section{INHERITED THROMBOCYTOPENIAS}

\section{LPB0080 | Clinical and Biological Assessment of the}

Second Pedigree Affected with X-linked GATA-1 Related

Thrombocytopenia and Blood Group Lutheran Null

A. Rodriguez-Alén ${ }^{1}$; V. Palma-Barqueros ${ }^{2}$; N. Rollón-Simón ${ }^{1}$; N. Revilla ${ }^{3}$; N. Bohdan² ${ }^{2}$. Padilla²; A. Zamora-Cánovas²; A. MarínQuílez $^{4}$; A. Sánchez-Fuentes²; J.R. González-Porras ${ }^{4}$; V. Vicente ${ }^{2}$; J. Cuesta ${ }^{1}$; M.L. Lozano ${ }^{2}$; J.M. Bastida ${ }^{4,5}$; R. Pozo ${ }^{2,5}$

${ }^{1}$ Hospital Virgen de la Salud, Complejo Hospitalario de Toledo, Toledo, Spain; ${ }^{2}$ Servicio de Hematología y Oncología Médica, Hospital Universitario Morales Meseguer, Centro Regional de Hemodonación, Universidad de Murcia, IMIB-Arrixaca, CIBERER-U765, Murcia, Spain; ${ }^{3}$ Hospital Universitario Ramón y Cajal, Madrid, Spain; ${ }^{4}$ Department of Hematology, Complejo Asistencial Universitario de Salamanca (CAUSA), Instituto de Investigación Biomédica de Salamanca (IBSAL), Universidad de Salamanca (USAL), Salamanca, Spain; ${ }^{5}$ Grupo Español de Alteraciones Plaquetarias Congénitas (GEAPC), Murcia, Spain

Background: Few known germline variants in the $\mathrm{X}$-linked transcription factor GATA-1 give rise to heterogeneous thrombocytopenia, dyserythropoietic anemia and platelet dysfunction. The c.1240T>C [p.X414R] variant resulting in 42 amino acid extralarge GATA-1, was found in a single family with mild macrothrombocytopenia and the rare X-linked blood group Lutheran (Lu) a- b- (Lu null) standing for lack of Lu antigen on erythrocytes.

Aims: To explore and confirm the association of GATA-1 p.X414R variant and the Lu null phenotype.

Methods: Two patients (Index case [IC] and son [S]) with lifelong macrothrombocytopenia and moderate bleeding enrolled the Spanish Project of Inherited Platelet Disorders. Clinical assessment, platelet phenotyping and DNA analysis were undertaken.

Results: The IC parents were first cousins. His mother, who died of sudden death from cardiomyopathy, and other maternal relatives had macrothrombocytopenia. The IC suffered from intraparenchymal hematoma following exertional syncope, required several surgery interventions due to neurological complications and platelet transfusions. A hypertrophic septal cardiomyopathy was discovered. His thrombocytopenic son has lifelong epilepsy and his untreated thrombocytopenia improved over the years. Complete blood cell analysis confirmed severe and moderate macrothrombocytopenia in IC and S ( 1 and $73 \times 10^{9} / \mathrm{l}$; non-recordable MPV). Large platelets were observed in stained blood smear. Flow cytometric studies showed large platelets and about 50\% increased platelet expression of major glycoproteins (Ib/IX, Ilb/IIla la and GPVI). Agonist-induced fibrinogen binding and $\alpha$ - and $\delta$-granule secretion were unaffected. High throughput sequencing gene panel and Sanger confirmation, identified IC and $\mathrm{S}$ as hemizygous carriers for the rare GATA- 1 variant p.X414R. Serological phenotyping of $S$ red blood cells corroborated the Lua-/Lub- phenotype. His non-thrombocytopenic mother, sister and brother, expressed Lua-/Lub+ on red blood cells. IC died before red cell analysis could be performed.
Conclusions: We report the second pedigree with thrombocytopenia associated to the GATA-1 p.X414R variant, consolidating the relationship of this rare variant with Lu null phenotype.

PB0866 | The Copenhagen Founder Variant GP1BA c.58T>G is Causal of Monoallelic Bernard-Soulier Syndrome

E. Leinøe $^{1}$; N. Broens ${ }^{1}$; A.O. Rasmussen ${ }^{2}$; M. Gabrielaite ${ }^{2}$; S. Rosthoej ${ }^{3}$; E. Zetterberg ${ }^{4}$; S.R. Ostrowski ${ }^{5}$; M. Rossing ${ }^{2}$

${ }^{1}$ Department of Haematology, Rigshospital University Hospital, Copenhagen, Denmark; ${ }^{2}$ Genomic Medicine, Rigshospitalet University Hospital, Copenhagen, Denmark; ${ }^{3}$ Department of Pediatrics, Aalborg University Hospital, Aalborg, Denmark; ${ }^{4}$ Clinical Coagulation Research Unit, Department of Translational Medicine, Lund University, Malmö, Sweden; ${ }^{5}$ Department of Clinical Immunology, Copenhagen, Denmark

Background: Heterozygous carriers of Bernard-Soulier syndrome (BSS) often have normal platelet counts. However, BSS can be inherited as an autosomal dominant trait associated with mild macrothrombocytopenia.

Aims: We present the results of genetic, phenotypic and cosegregation analyses of fourteen families with monoallelic BSS (mBSS).

Methods: DNA was analyzed by whole genome sequencing (WGS). A flow cytometric diagnosis of mBSS using Kaluza Flow Cytometry Software v. 2.1 (Beckman Coulter) was made by calculating the ratio between median expressions of CD42b and CD41a in percentages of healthy subjects. Identity by descent of the genomes was estimated with PLINK 1.9 after initial pruning of sites in linkage disequilibrium. Thrombopoietin (TPO) concentrations were analyzed by a commercially available solid phase sandwich ELISA test.

Results: We analyzed 106 patients referred for inherited thrombocytopenia (IT) by WGS and identified fourteen probands suspected of mBSS associated macrothrombocytopenia (13\%). Peripheral blood smears from the probands demonstrated macrothrombocytes and the relative ratio of CD42b/CD41a was $<1$ (median 0.52, range 0.40 - 0.67). Co-segregation analyses were performed among 46 family members whereof 31 patients carried a heterozygous variant associated with mBSS. Median platelet count was $91 \times 10^{9} / \mathrm{L}$ (range 44-131). We found significant ISTH BAT scores in two of the fourteen probands. Six probands carried the same heterozygous variant in GP1BA (c.58T>G, p.Cys20Gly). No cryptic relatedness was found across their genomes, and they shared a $2.0 \mathrm{Mb}$ region on chromosome 17 . The $c .58 \mathrm{~T}>\mathrm{G}$ variant likely prevents the formation of the disulfide bond between Cys20 and Cys33, thereby disrupting the local stability of the protein secondary structure. TPO levels in patients were normal (median $16.5 \mathrm{AU} / \mathrm{ml}$ ), not significantly different $(P>0.05)$ from family members with wild type (median $17 \mathrm{AU} / \mathrm{ml}$ ). 
Conclusions: Monoallelic BSS is the most frequent form of IT in the Copenhagen Region and this may be due to a founder variant in GP1BA (c.58T>G, p.Cys20Gly).

PB0867 | Investigations of the Impact of Sialidase-mediated Changes on Bleeding Using a Humanized in vivo Mouse Model

L. Pelzl ${ }^{1}$; A. Singh ${ }^{1}$; J. Zlamal ${ }^{1}$; I. Marini ${ }^{1} ;$ K. Althaus ${ }^{1,2} ;$ T. Bakchoul ${ }^{1,2}$

${ }^{1}$ Institute for Clinical and Experimental Transfusion Medicine, Medical

Faculty of Tuebingen, University Hospital of Tuebingen, Germany,

Tuebingen, Germany; ${ }^{2}$ Centre for Clinical Transfusion Medicine ZKT

$\mathrm{GmbH}$, Tuebingen, Germany

Background: Immune thrombocytopenia (ITP) is a bleeding disorder due to multiple alterations of the immune system leading to increased platelet (PLT) clearance and bleeding. Murine antibodies against GPIb have been shown to mediate PLT destruction in the liver.

Aims: To establish a humanized mouse model to study and investigate antibody -mediated destruction of human PLTs in vivo and to assess the impact of desialylation on bleeding.

Methods: Murine PLTs were depleted using a monoclonal antibody against CD42b in a NOD/SCID mouse model. After 24 hours, the $\%$ of circulating murine PLTs was determined by flow cytometry (FC). Human PLTs were injected in the tail vain with or without preincubation with a sialidase and their survival was determined after 1-5 hours by FC.

Results: A drastic reduction of the murine PLTs in the mouse circulation $(<5 \%)$ was observed after injection of CD42b antibody without affecting animal survival. Next, the human PLTs were injected in the mouse blood stream via tail vein and approximately $90 \%$ of circulating PLTs were from human origin. To investigate the impact of desialylation on PLT clearance and function, human PLTs were injected upon incubation with sialidase. Interestingly, a fast reduction of the PLT survival compared to control was observed (\% human PLT survival after 2 hours, mean \pm SEM: sialidase incubation vs. buffer: $28 \pm 5 \%$ vs. $45 \pm 3 \%, P=0.0191$ ). Higher number of human PLTs were detected after desialylation in the liver compared to the spleen. The bleeding time in the humanized mouse model was tested after murine PLTs depletion. In particular, a significant reduction of the bleeding time was observed after injection of human PLTs upon depletion. Conclusions: Our finding suggests the use of this mouse model as potential tool for different experimental approaches in order to investigate human platelet disorders.
PB0868 | Assigning Pathogenicity to a Novel de Novo ACTN1 Variant in a Child with Macrothrombocytopenia: A Case Description And Review of Previously Identified Variants

L. FitzGibbon ${ }^{1}$; V. Bouskill ${ }^{2}$; M. Carcao ${ }^{2}$; L. Li ${ }^{3}$; F.G Pluthero ${ }^{3}$; W.H. Kahr ${ }^{2,3,4}$; S.K Westbury ${ }^{1,3}$

${ }^{1}$ School of Cellular and Molecular Medicine, University of Bristol, Bristol, United Kingdom; ${ }^{2}$ Division of Haematology/Oncology, The Hospital for Sick Children, Toronto, Canada; ${ }^{3}$ Cell Biology Program, Research Institute, The Hospital for Sick Children, Toronto, Canada; ${ }^{4}$ Department of Biochemistry, University of Toronto, Toronto, Canada

Background: Rare, monoallelic missense variants in ACTN1 (encoding a-actinin-1, ACTN1) are associated with the non-syndromic disorder ACTN1-related thrombocytopenia (A-RT). Variants affecting the rod domain of ACTN1 may be associated with a milder platelet phenotype. ACTN1 is an ISTH Tier 1 thrombocytopenia gene included in diagnostic next generation sequencing (NGS) panels for thrombocytopenia. As a result, many variants of unknown significance (VUS) are identified that can be difficult to resolve.

Aims: 1 . To report a novel de novo ACTN1 rod domain variant in a child with isolated macrothrombocytopenia.

2. To use computational techniques to predict the pathogenicity of this novel variant and previously reported missense variants affecting the rod domain.

Methods: Extended evaluation of the pedigree was completed at the Hospital for Sick Children, Toronto with research ethics board approval (REB number 1000007948). A systematic search of the literature, HGMD Professional and the ClinVar database was performed to identify previous reports of rare missense variants in the rod domain of ACTN1 associated with thrombocytopenia. Structural modelling of variants was performed using Chimera.

Results: Clinical and laboratory results are provided in Figure 1. NGS of a 31-gene thrombocytopenia panel revealed a heterozygous ACTN1 variant (c.1861G>A, p.(Glu621Lys)) classified by the reporting laboratory as a VUS. No other credible variants were identified. Sanger sequencing of parental DNA was consistent with a de novo variant. Computational tools produced conflicting predictions of pathogenicity for this variant and several previously reported variants affecting the rod domain of ACTN1 (Figure 2). Despite additional investigations, the variant remains a VUS. 


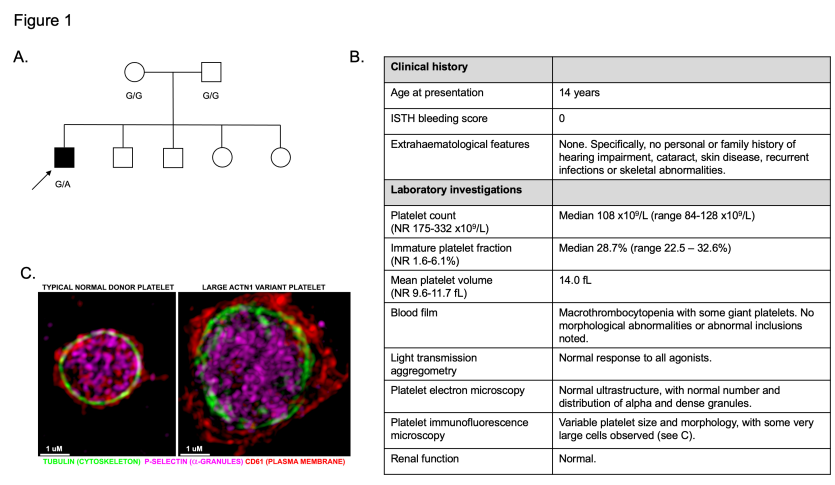

FIGURE 1 Clinical and laboratory features. A) Pedigree diagram. The proband is shown with an arrow. Black symbol indicates thrombocytopenia. White symbol indicates normal platelet count. $\mathrm{G}$, reference allele. A, variant allele. B) Key clinical and laboratory data. NR, local normal range. C) Immunofluorescence structured illumination microscopy images of a typical normal donor platelet (left) and a large patient platelet (right) with $>4 \mathrm{X}$ normal cell volume (maximum intensity renders; scale same for both images).

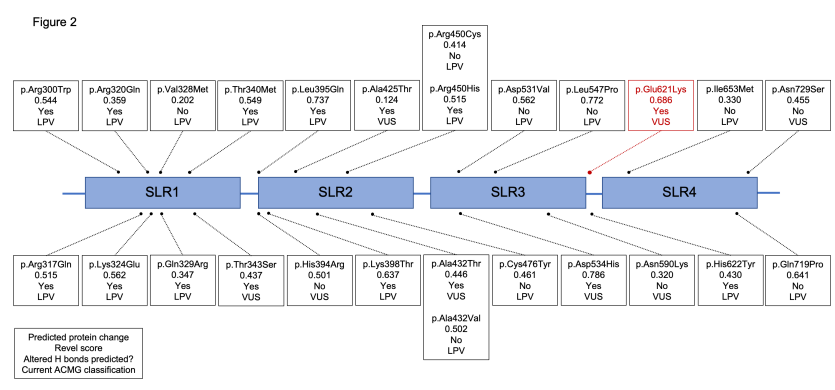

FIGURE 2 Variants affecting the rod domain of ACTN1 associated with thrombocytopenia. Diagrammatic representation of the rod domain of ACTN1 and position of the 26 missense variants associated with thrombocytopenia. The novel variant reported here is shown in red. The predicted amino acid substitution is given for each variant with reference to the canonical transcript ENST00000193403.10. The Revel score ranges from 0-1, with higher scores predicting more deleterious variants. The effect on hydrogen bond formation was predicted using Chimera. The current classification of the variant was taken from depositions in ClinVar as at 3rd March 2021. ACMG, American College of Medical Genetics and Genomics. LPV, likely pathogenic variant. SLR, spectrin-like repeat. VUS, variant of unknown significance.

Conclusions: Prediction of pathogenicity is particularly challenging where pedigrees are small or uninformative, where conditions lack pathognomonic features and where computational methods produce conflicting results. Development of experimental and computational tools alongside continued case reporting should be encouraged in order to improve the clinical utility of NGS.
PB0869 | Targeted Next Generation Sequencing for the Diagnosis of Patients with Inherited Thrombocytopenias

O. Gilad ${ }^{1}$; O. Steinberg - Shemer ${ }^{1}$; O. Dgany ${ }^{2}$; T. Krasnov ${ }^{2}$; S. Noy

- Lotan ${ }^{2}$; J. Yacobovich ${ }^{1}$; H. Tamary ${ }^{1,2}$

${ }^{1}$ Schneider Children's Medical Center of Israel, Petach Tikva, Israel;

${ }^{2}$ Pediatric Hematology Laboratory, Felsenstein Medical Research

Center, Petach Tikva, Israel

Background: Recent identification of the molecular basis of many forms of inherited thrombocytopenia (IT) suggests that this is a heterogeneous group of disorders affecting megakaryocytic differentiation and/or platelets production. Various forms of the disorder differ in bleeding tendency, non-hematological manifestations and the risk of malignant transformation. Next generation sequencing (NGS) techniques are offering accurate cost-effective molecular diagnosis of those disorders, which is essential for appropriate therapy and follow-up.

Aims: We used targeted NGS to diagnose IT in patients referred to us because of persistent or congenital thrombocytopenia with or without family history of thrombocytopenia and with no features of myelodysplastic syndrome.

Methods: Genetic diagnosis was performed by using a customized targeted NGS panel including of 45 genes known when mutated to cause IT.

Results: Genetic diagnosis was achieved in 18 out of 31 patients (58.1\%). Of those, six (33.3\%) patients were diagnosed with MYH9related disorder, two had mutations in ACTN1, one in NBEAL2, one in ITG3B, one in GALE and one in CYCS gene. Six patients were diagnosed as having inherited predisposition to MDS/AML (4 with ANKD26 mutations, one with ETV6 and one with RUNX1 mutation). Molecular diagnosis of the propositus led to a genetic diagnosis of IT in other family members. Interestingly, four patients out of 8 originally diagnosed as having chronic ITP, were found to have IT.

Conclusions: In our cohort, MYH9-related disorders were found in a third of the patients who were molecularly diagnosis as having IT. Those patients should be carefully monitored, as they are prone to develop kidney failure later in life. A third of the patients were found to have predisposition to MDS/AML. They should be followed carefully for signs of MDS and stem cell transplantation should be considered prior to development of AML. Further molecular analysis of patients with IT is indicated to optimize treatment and follow up. 
PB0870 | Disturbed Platelet Activation in Children with ANKRD26-associated Thrombocytopenia

D. Polokhov ${ }^{1}$; D. Fedorova ${ }^{1}$; A. Pshonkin ${ }^{1} ;$ A. Ignatova ${ }^{1}$; E. Ponomarenko'; M. Aleksenko ${ }^{1}$; I. Mersiyanova ${ }^{1}$; E. Seregina ${ }^{1}$; K. Voronin $^{1}$; A. Poletaev ${ }^{1}$; E. Raykina ${ }^{1}$; M. Panteleev ${ }^{1,2,3,4}$; P. Zharkov ${ }^{1}$. ${ }^{1}$ Federal Research and Clinical Centre of Pediatric Hematology, Oncology and Immunology, Moscow, Russian Federation; ${ }^{2}$ Center for Theoretical Problems of Physicochemical Pharmacology, Moscow, Russian Federation; ${ }^{3}$ Faculty of Physics, Moscow State University, Moscow, Russian Federation; ${ }^{4}$ Faculty of Biological and Medical Physics, Moscow Institute of Physics and Technology, Dolgoprudny, Russian Federation

Background: The mechanisms of platelet dysfunction in patients with ANKRD26 associated thrombocytopenia (ANKRD26-AT) are stil poorly understood.

Aims: The aim of this work is to detect possible morpho-functional disorders of platelets in children with ANKRD26-AT by flow cytometry with activation.

Methods: 8 children with median age of 6 years (range 1.5 to 15 years) with confirmed ANKRD26-AT by Next Generation Sequencing (NGS) were examined. The platelet count ranged from 29 to $172 \times 10^{9}$ / L, with a median of $60 \times 10^{9}$ / L. The severity of hemorrhagic manifestations was assessed on a standardized scale (Pediatric Bleeding Questionnaire, $\mathrm{PBQ}$ ) and it ranged from 0 to 5 score, with a median of 3.5 score. We investigated morphology of platelets by FSC and SSC, CD42b receptor and phosphatidylserine-positive platelets, at rest and after activation. Platelet activation was performed with a CRP + TRAP mixture. Comparison was carried out with the results of examination of 26 apparently healthy children (control group - CG) with median age of 7 years (range 2 to 15 years).

Results:
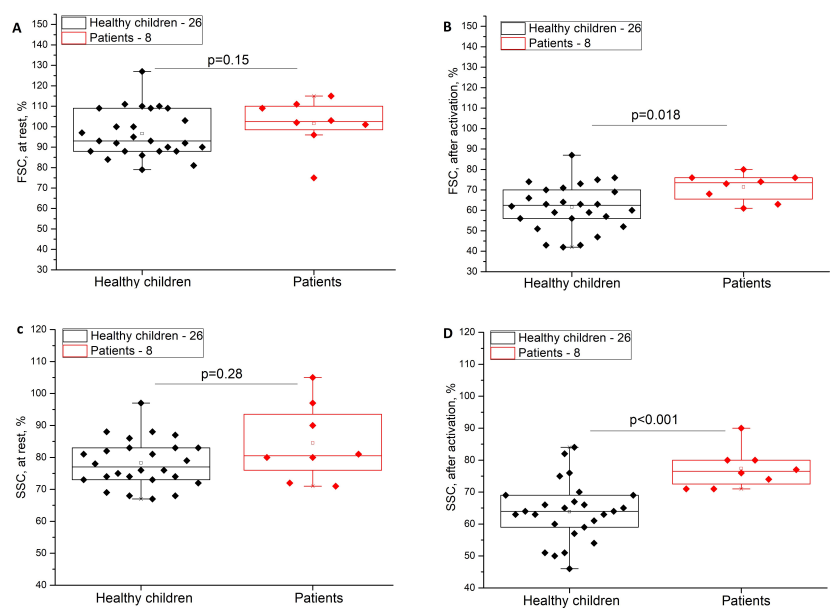

FIGURE 1 A-D. Integral assessment of morphological characteristics of platelet size and granularity at rest and after activation
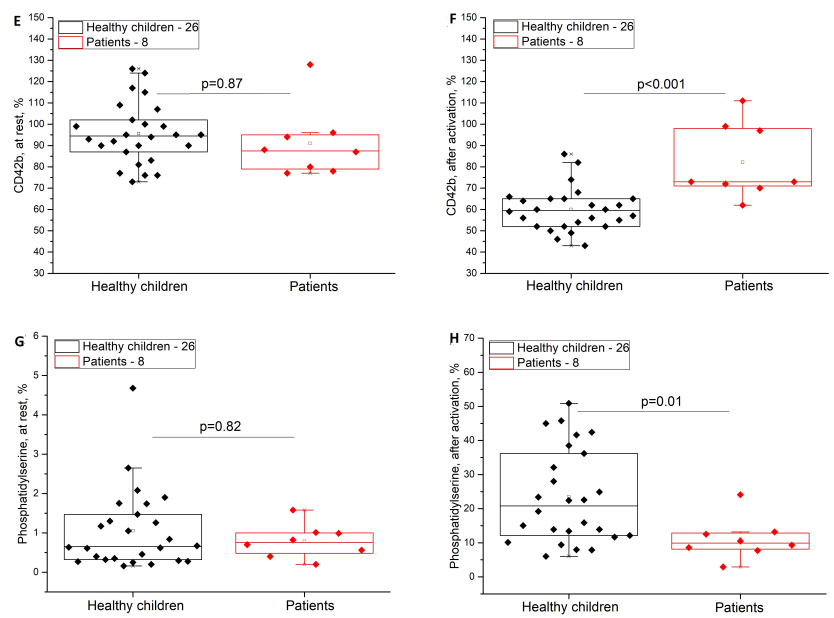

FIGURE $2 \mathrm{E}-\mathrm{H}$. Differences in CD42b receptor density and proportion of phosphatidylserine-positive platelets.

At rest, CG-platelets did not show significant differences by FSC, SSC, CD42b and procoagulant platelet count $(P>0.05)$ compared with patients (fig. A, C, E and G, respectively). After activation, ANKRD-26 platelets demonstrated an increased platelet size (FSC, $P=0.018$, fig. B) and granularity (SSC, $P<0.001$, fig. D), an increased density of $C D 42 b(P<0.001$, fig. $F)$ and a decrease in the proportion of procoagulant platelets $(P=0.01$, fig. $H)$.

Conclusions: These changes potentially indicate disturbed mechanisms of activation and shape changes of platelets in patients with ANKRD26-AT.

\section{PB0871 | Evaluation of the Sialidase Inhibitor Oseltamivir in GNE-associated Thrombocytopenia}

M. Fager Ferrari' ${ }^{1}$; K.I. Smolag ${ }^{2}$; E. Zetterberg ${ }^{1}$; E. Leinoe ${ }^{3}$;. T. Ek ${ }^{4}$; A.M. Blom ${ }^{2}$; M. Rossing ${ }^{5}$; M. Martin ${ }^{2}$

${ }^{1}$ Clinical Coagulation Research Unit, Department of Translational Medicine, Lund University, Malmö, Sweden; ${ }^{2}$ Section of Medical Protein Chemistry, Department of Translational Medicine, Lund University, Malmö, Sweden; ${ }^{3}$ Department of Hematology, Rigshospitalet National University Hospital, Copenhagen, Denmark; ${ }^{4}$ Children's Cancer Center, Queen Silvia Children's Hospital, Gothenburg, Sweden; ${ }^{5}$ Center for Genomic Medicine, Rigshospitalet National University Hospital, Copenhagen, Denmark

Background: GNE encodes UDP-N-acetyl-glucosamine-2epimerase/ $\mathrm{N}$-acetylmannosamine kinase, the rate limiting enzyme of sialic acid biosynthesis. Biallelic variants in GNE have recently been associated with severe isolated macrothrombocytopenia, attributed to an increased clearance of desialylated platelets. Interestingly, treatment with the sialidase inhibitor oseltamivir has been reported to increase platelet counts in conditions such as immune thrombocytopenia (ITP) and influenza. We present a case of a 17-year-old boy (the proband) with severe congenital macrothrombocytopenia (platelet counts $<10 \times 10^{9} / \mathrm{L}$ ). Whole genome sequencing revealed two previously undescribed compound heterozygous variants in 
GNE (c.416_426del, p.lle139Argfs*4 and c.1352G>A, p.Arg451GIn). The proband was otherwise healthy, with no signs of GNE myopathy. Aims: To investigate the consequences of the identified variants in GNE and evaluate the effect of oseltamivir in GNE-associated thrombocytopenia.

Methods: Sialylation of platelets, granulocytes, lymphocytes and monocytes was determined by flow cytometry in the proband and healthy controls ( $n=5)$, using Sambucus nigra lectin (SNA) and Maackia amurensis lectin II (MAL II). Platelet sialylation was reassessed in the proband following treatment with oseltamivir (75 mg twice daily, offlabel use). Informed consent was obtained from all participants. The study was approved by the regional ethical committee.

Results: Sialylation of platelets and leukocytes was markedly decreased in the proband compared with the healthy controls, consistent with a deleterious effect of the compound heterozygous variants in GNE (Figure 1). Platelet sialylation was persistently decreased after 18 days of treatment with oseltamivir, and no clinically significant elevation of the platelet counts could be observed (Figure 1, Figure 2).

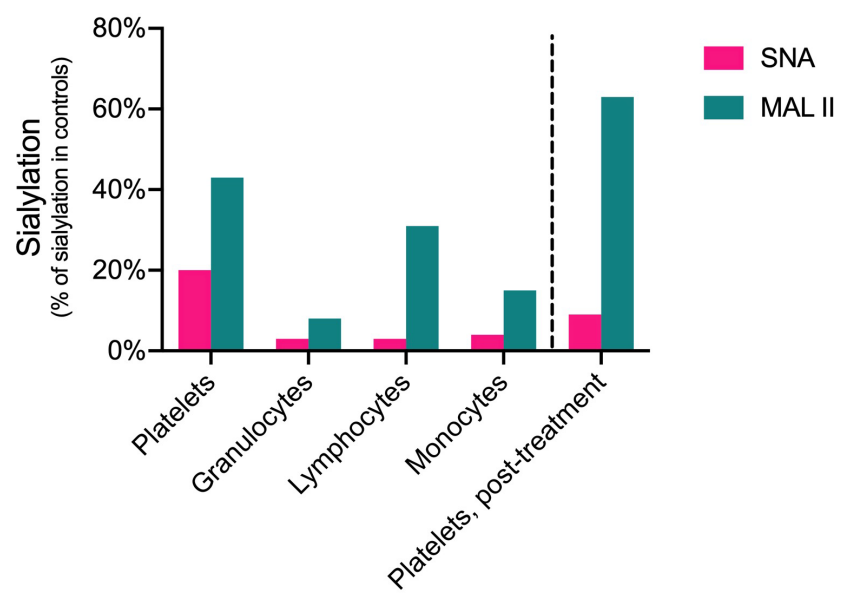

FIGURE 1 Sialylation of platelets and leukocytes in the proband. Results are presented as percentages of the median value in healthy controls $(n=5)$.

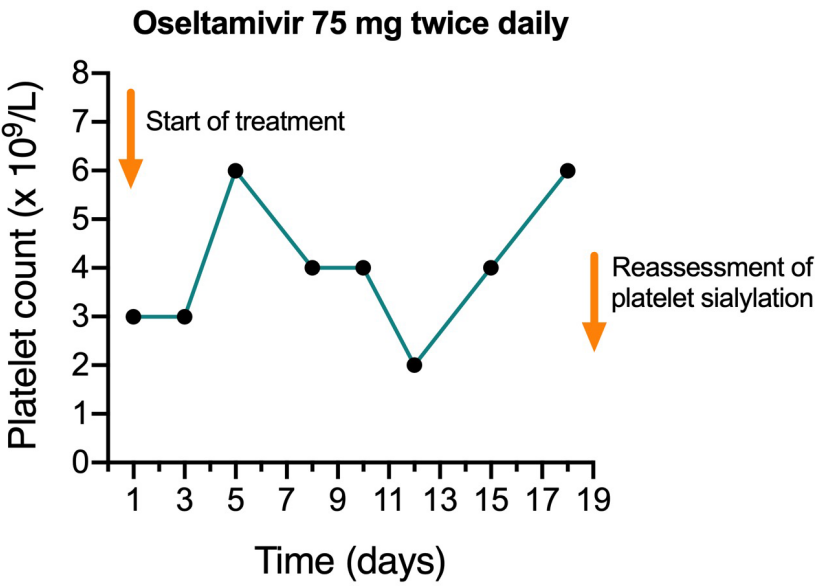

FIGURE 2 Platelet counts in the proband during treatment with oseltamivir. No clinically significant effect was observed after 17 days of treatment.
Conclusions: We report two compound heterozygous variants in GNE causing severe macrothrombocytopenia, as a result of decreased platelet sialylation. Treatment with oseltamivir did not prove to be effective for mitigating the GNE-associated thrombocytopenia identified in the patient.

PB0872 | Immunofluorescence Staining of Blood Smears for the Diagnostics of Platelet Disorders: A Single-center Experience in a Pediatric Hospital

N. Podoplelova $^{1,2}$; E. Popova ${ }^{3,1}$; P. Zharkov²; D. Fedorova ${ }^{2}$;

A. Greinacher ${ }^{4}$; M. Panteleev ${ }^{1,2,3}$

${ }^{1}$ Center for Theoretical Problems of Physicochemical Pharmacology, Moscow, Russian Federation; ${ }^{2}$ Dmitry Rogachev National Medical Research Center of Pediatric Hematology, Oncology and Immunology, Moscow, Russian Federation; ${ }^{3}$ Lomonosov Moscow State University, Moscow, Russian Federation; ${ }^{4}$ Institut für Immunologie und Transfusionsmedizin, Universitätsmedizin Greifswald, Greifswald, Germany

Background: The method of immunofluorescence staining of blood smears is a recently developed (Greinacher et al. J Thromb Haemost 2017; 15: 1511-1521) approach of remote diagnostics of various platelet pathologies including MYH9 disorders/MYH9-related disease, biallelic Bernard-Soulier syndrome, Glanzmann thrombastenia, and gray platelet syndrome, and others. We report here experience of introducing this method in the National Research Center of Pediatric Hematology, Oncology and Immunology named after Dmitry Rogachev (Moscow, Russia), which is the main national pediatric hematology hospital that provides diagnosis and therapy to children with blood disorders throughout the country.

Aims: Our study aimed to transfer this relatively labor-intensive and skill-sensitive methodology, to introduce in the routine laboratory practice, and to perform its validation.

Methods: Blood smears from healthy donors and patients were prepared using the standard method established worldwide, air-dried, and storage. Citrate anticoagulated blood was used for preparation. For immunofluorescence labeling was used primary antibodies (Myosin, LAMP 1 (H5G11), LAMP 2 (H4B4), VWF, P-Selectin (CD62P), CD63 (delta-granules), Ib/IX CD42a (FMC25), Ilb/IIla CD41 P2, $\beta 1$-Tubulin (2.1.), $\alpha$-Tubulin (RM113)) and fluorescence labeling secondary antibodies. Blood smears were assessed by immunofluorescence microscopy.

Results: Patients with different platelet disorders (9 patients with MYH9 disorder, 7 patients with Wiskott-Aldrich syndrome, 2 patients with Bernard-Soulier syndrome, 2 patients Hermansky-Pudlak syndrome,1 patient with gray platelets syndrome, 1 patient with Glanzmann thrombastenia) confirmed by the genetic analysis and clinical symptoms were assessed. In addition to the immunofluorescence assay, each patient was analyzed platelet function by flow cytometry and aggregation by standard methods. For all patients, 
we were able to identify the abnormalities by immunofluorescence associated with established diagnosis.

The study was supported by the grant of the President of the Russian Federation.

Conclusions: In this work it was determined that the immunofluorescence staining of blood smears enables us to receive reproducible results and implement the diagnostic of platelet disorders.

PB0873 | Clinical Application of Genetic Testing in Patients with Not Acquired Thrombocytopenia: One Center Experience

P. Silva de Tena ${ }^{1}$; M. Argüello-Tomás ${ }^{1}$; C. Pascual Izquierdo ${ }^{1,2,3}$; J.L. Díez-Martín ${ }^{1,2,3}$; C. Martinez Laperche ${ }^{1,2,3}$; G. Perez Rus ${ }^{1,2,3}$

${ }^{1}$ Hospital General Universitario Gregorio Marañón, Madrid, Spain;

${ }^{2}$ Gregorio Marañón Health Research Institute, Madrid, Spain;

${ }^{3}$ Universidad Complutense de Madrid, Madrid, Spain

Background: Knowledge about inherited thrombocytopenia has been expanded in recent years due to the introduction of new sequencing genetic analysis. There are patients with misdiagnosed thrombocytopenia that could benefit of these new techniques.

Aims: To evaluate the applicability of a next generation sequencing (NGS) based gene panel in the diagnosis of suspected inherited thrombocytopenia in clinical practice.

Methods: A prospective descriptive study was designed and 30 participants were included. Patients were previously diagnosed with not specified thrombocytopenia who fulfilled next inclusion criteria: not acquired thrombocytopenia with no previous normal range platelet count and without secondary causes of thrombocytopenia. Medical and family history, physical examination and blood test analysis including peripheral blood smear were recorded. Two hundred platelets were evaluated in every blood smear and platelet size, granulation and vacuolization were described The NGS gene panel was performed to all patients in peripheral blood. Tested genes are shown in Figure 1. Results are presented as medians, maximum, minimum and percentages. Informed consents were required for all patients.

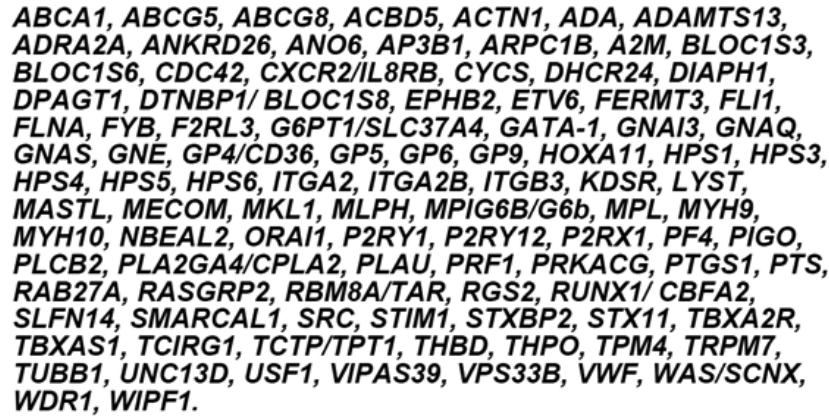

FIGURE 1 Included genes in next generation sequencing panel
Results: Main characteristics of selected patients are registered in Table 1. Fourteen subjects (47\%) had family history of thrombocytopenia and only 1 patient (3\%) had family history of hematologic malignancy. None of the patients presented syndromic symptoms. Former haemorrhagic complications were reported in 9 patients (30\%) and 10 (33\%) participants received previous treatment for immune thrombocytopenia without adequate response. Six (20\%) subjects presented large platelets in blood smear.

The NGS panel proved that 12 out of the 30 patients (40\%) had some genetic mutation related to inherited thrombocytopenia with a total of 15 gene alterations identified.

TABLE 1 Main characteristics of selected patients and test results

\begin{tabular}{|c|c|}
\hline Age, years & $51(22-86)$ \\
\hline \multicolumn{2}{|l|}{ Sex (number, \%) } \\
\hline Male & $13(43 \%)$ \\
\hline Female & $17(57 \%)$ \\
\hline Family background (number, \%) & $14(47 \%)$ \\
\hline Thrombocytopenia & $16(53 \%)$ \\
\hline Hematologic malignancy & $1(3 \%)$ \\
\hline Haemorrhagic symptoms (number, \%) & 9 (30\%) \\
\hline Platelet count (platelet/ $\mu \mathrm{L}$ ) & $76000(10000-126000)$ \\
\hline Platelet volume (fl) & $11,7(8,3-13,9)$ \\
\hline Previously treated patients (number, \%) & $10(33 \%)$ \\
\hline \multicolumn{2}{|l|}{ Type of treatment (number, \%) } \\
\hline Glucocorticoids & $10(33 \%)$ \\
\hline Intravenous Immunoglobulins & $5(17 \%)$ \\
\hline Splenectomy & $0(0 \%)$ \\
\hline Rituximab & $3(10 \%)$ \\
\hline Thrombopoietin analogs & $1(3 \%)$ \\
\hline Others & $3(10 \%)$ \\
\hline \multicolumn{2}{|l|}{ Peripheral blood smear (number, \%) } \\
\hline Large/giant platelets & $6(20 \%)$ \\
\hline Hypogranular platelets & $3(10 \%)$ \\
\hline Vacuolated platelets & $5(17 \%)$ \\
\hline $\begin{array}{l}\text { NGS gene mutation positive patients } \\
\text { (number, \%) }\end{array}$ & $12(40 \%)$ \\
\hline Gene alterations (number, \%) & $15(100 \%)$ \\
\hline ITGA2B/ITGB3 & $4(27 \%)$ \\
\hline RUNX1 & $2(13 \%)$ \\
\hline MYH9 & $2(13 \%)$ \\
\hline GFI1B & $2(13 \%)$ \\
\hline NBEAL2 & $2(13 \%)$ \\
\hline GP1BA & $1(7 \%)$ \\
\hline TUBB1 & $1(7 \%)$ \\
\hline ADAMST13 & $1(7 \%)$ \\
\hline
\end{tabular}

Conclusions: In our one-center experience, an adequate selection of patients allowed to diagnose an important group of subjects with inherited thrombocytopenia using a NGS based gene panel. In clinical 
practice, identifying these patients could avoid unnecessary immunosuppressive treatments and improve follow-up strategies.

\section{PB0874 | Evolution over 50 Years of a Patient with Undiagnosed} Gray Platelet Syndrome

A. Peleteiro Raíndo ${ }^{1}$; E. Mellid Fernández ${ }^{1}$; A. De Andrés y Jacob ${ }^{1}$; A. Abuin ${ }^{1}$; J.Á. Díaz Arias ${ }^{1}$; E. Fontanes Trabazo ${ }^{1}$; M.D. Vilariño López $^{1}$; A. Mosquera Orgueira ${ }^{1}$; N. Alonso Vence ${ }^{1}$; L. Bao Pérez ${ }^{1}$; P. Cadahía Fernández ${ }^{1}$; R. Ferreiro Ferro ${ }^{1}$; P. Melero

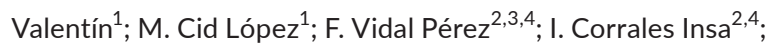
J.L. Bello López ${ }^{1}$

${ }^{1}$ University Hospital of Santiago de Compostela, Santiago de Compostela, Spain; ${ }^{2}$ Coagulopaties Congènites, Banc de Sang i Teixits (BST), Barcelona, Spain; ${ }^{3}$ Centro de Investigación Biomédica en Red de Enfermedades Cardiovasculares (CIBERCV), Instituto Carlos III (ISCIII), Madrid, Spain; ${ }^{4}$ Medicina Transfusional, Vall d'Hebron Institut de Recerca, Universitat Autònoma de Barcelona (VHIR-UAB), Barcelona, Spain

Background: Inherited platelet disorders result from functional abnormalities that cause failure of platelet adhesion, activation or aggregation. They are rare but clinically important because they are associated with hemorrhagic complications; furthermore their final diagnosis is often difficult to establish. Specifically, the Gray Platelet Syndrome (GPS) is characterized by defective production of alpha granules in platelets and it may be caused by different mutations in genes like NBEAL2 and, rarely, GFI1B. Nowadays, the existence of new molecular diagnostic techniques such as next generation sequencing (NGS) has allowed us to identify a new mutation in the GFI1B by whole exome sequencing (WES).

Aims: Our objective is to revise the diagnosis of a patient with longstanding constitutional thrombopenia who has been refractory to traditional treatments and was finally diagnosed with a GPS. We also revised his available family members in order to detect GPS features in them.

Methods: We revised patient's medical and family history (Figure 1), including initial diagnosis (morphological assessment, flow cytometry analysis, bleeding score), treatments and it was performed a WES by NGS with the finding of an autosomal dominant mutation in GFI1B (Heterozygous mutation in exon 6: c.737G>A, p.Arg246GIn).

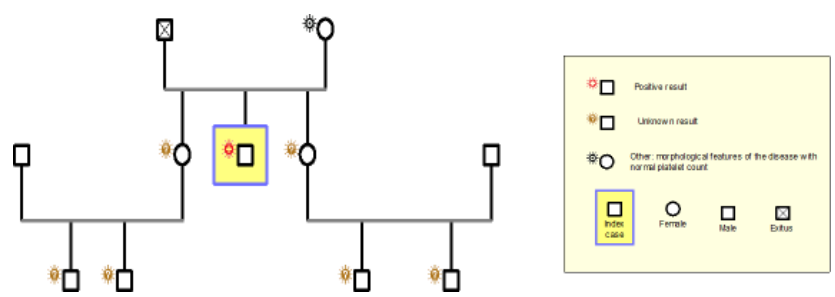

FIGURE 1 Family Tree
Results: Since the patient had been initially labelled as inmune thrombocytopenia, there was no response to inmunosupresive treatments (prednisone, cyclophosphamide, vincristine, immunoglobulins and splenectomy), which is concordant with the present diagnosis of GPS. There is also no progression to myelofibrosis or platelet sensitization after the transfusions received. We found that not all direct relatives had clinical involvement, but there were morphological features of the disease (Figure 2).

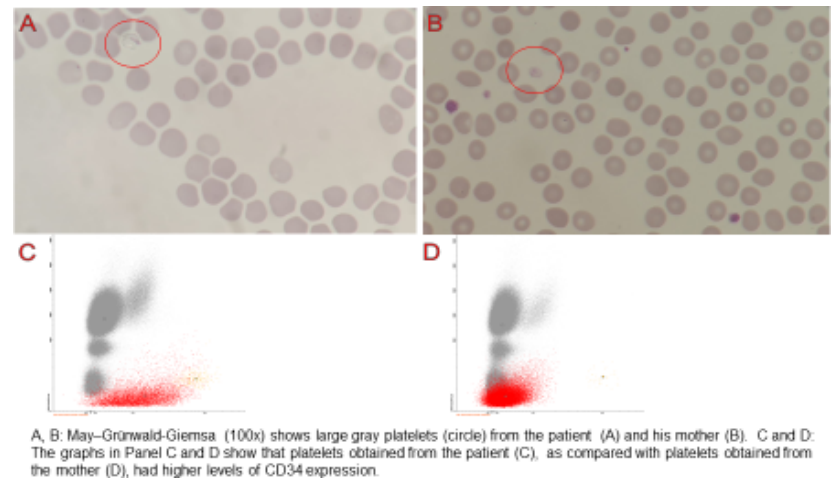

FIGURE 2 Characteristics of Autosomal Dominant Gray Platelet Syndrome

Conclusions: New molecular diagnostic techniques has allowed us to make a correct diagnosis. An early identification of the causing mutation could have avoided potentially damaging treatments as splenectomy.

Extension of the family study could shed light on the genotypephenotype correlation, and confirm a possible incomplete penetrance profile.

PB0875 | The Analysis of Platelet Functional Activity in Patients with Thrombocytopenia

M. Reshetova ${ }^{1}$; A. Poletaev ${ }^{1}$; E. Seregina ${ }^{1,2}$; D. Polokhov ${ }^{1}$

${ }^{1}$ Dmitry Rogachev National Medical Research Center of Pediatric Hematology, Oncology and Immunology, Moscow, Russian Federation; ${ }^{2}$ Center for Theoretical Problems of Physicochemical Pharmacology, Moscow, Russian Federation

Background: LTA, developed by Born, remains the "gold standard" diagnostic test for platelet function disorders, however, this method has limitations to application because it does not allow reliable assessment of patients with thrombocytopenia-the number of cells under study in a sample should be at least fifty thousand. Consequently, platelet functional activity needs to be assessed in patients with unspecified thrombocytopenia and hemorrhagic events. 
Aims: Studying the possibility of using the light transmission fluctuation method (LTF) to assess the platelet functional activity in patients with thrombocytopenia.

Methods: The study included 15 children with thrombocytopenia referred for bleeding assessments.In order to prepare samples of PRP plasma, the blood from patients was centrifuged at $1000 \mathrm{rpm}$ for 10 minutes, followed by measuring the number of platelets in the test sample. Plasma of 10 healthy volunteers was studied as a control group. The density of 20,000 platelets $/ \mu \mathrm{L}$ in the test samples was achieved by mixing PRP with PPP plasma which were centrifuged in three stages. LTA was studied using an ALAT-2 laser aggregation analyzer with 4 inducers:ADP, adrenaline, ristocytin, collagen. Results: Plasma samples from healthy volunteers showed little or no response according to the Born method. According to the light transmission fluctuation method(LTF), the formation of aggregates for ADP was from 1.1 to 4.3c.u.(average value 2.93c.u.), adrenalinefrom 1 to 6.2 c.u. (3.69c.u.), ristomycin-from 1.2 to 9.4c.u.(4.6c.u.), collagen-from 5.5 to 17.1 c.u. (10.5c.u.). The number of platelets in the studied samples varied from 0 to 50 cells/I (23.6cells/I). Patients showed a more pronounced response to inducers-13 of 15 patients had a response to ristocytin and collagen according to the Born method. Three patients had a response to ADP according to the Born method. There was no adrenaline based on the Born method. According to the LTF method, the response to the addition of the inducer was similar to normal.

Conclusions: Patients with thrombocytopenia have platelet reactivity compared to healthy volunteers. The LTF method makes it possible to assess the aggregation ability with a significant platelet decrease, however, additional tests are required for a more detailed study.

\section{NON HUS/TTP MICROANGIOPATHIES}

PB0876 | P2Y 12 Receptor Antagonists and Drug-induced Thrombotic Microangiopathies: A Systematic Review of the Primary Evidence

J.C Ho ${ }^{1}$; A. Eshaghpour ${ }^{1}$; S. Ge ${ }^{2}$; R. Foote ${ }^{1}$; M. Crowther ${ }^{3}$

${ }^{1}$ Michael G. DeGroote School of Medicine, McMaster University, Hamilton, Canada; ${ }^{2}$ Temerty School of Medicine, University of Toronto, Toronto, Canada; ${ }^{3}$ Department of Medicine, McMaster University, Hamilton, Canada

Background: Drug-induced thrombotic microangiopathies (DITMAs) have been associated with thienopyridine antiplatelets such as ticlopidine, discontinued in many markets for this reason, as well as clopidogrel. Clinical use of the thienopyridine prasugrel, and the non-thienopyridines ticagrelor and cangrelor, has increased and it is unclear whether they confer a risk for TMAs.
Aims: To establish if there is a relationship between contemporary $\mathrm{P}_{2} \mathrm{Y}_{12}$ receptor antagonists and TMAs.

Methods: We searched MEDLINE, Embase, and Scopus systematically from inception to November 30, 2020 for reports presenting patients receiving $\mathrm{P}_{2} \mathrm{Y}_{12}$ receptor antagonists for any indication who developed TMA after exposure. We included only Englishlanguage case reports and series, and other primary observational patient-level data. Two reviewers independently screened articles at title/abstract and full-text levels. The primary outcome was the causality likelihood judged by the World Health OrganizationUppsala Monitoring Centre (WHO-UMC) causality assessment scale. Secondary clinical outcomes such as mortality, use of plasma exchange, and medication restart were determined. Risk of bias was assessed using appropriate validated scales.

Results: A total of 45 unique cases of $\mathrm{P} 2 \mathrm{Y}_{12}$ receptor antagonistassociated TMAs were described in 33 reports between 20002020. The implicated agent was clopidogrel in 41 (91\%), ticagrelor in $2(4 \%)$, both clopidogrel and ticagrelor in $1(2 \%)$, and prasugrel in 1 (2\%). No cases involved cangrelor. WHO-UMC causality ratings were "Probable/likely" in 35 (78\%), "Possible" in 7 (16\%), "Unlikely" in $1(2 \%)$, and "Unassessable/Unclassifiable" in 2 (4\%). Plasma exchange was started in 40 (89\%) cases, 6 (13\%) died, and a P2Y 12 receptor inhibitor was restarted in 6 (13\%). Whether restart occurred was unspecified in 2 (4\%) cases.

Conclusions: Clopidogrel, ticagrelor, and prasugrel are all potential rare causes of TMA. Most cases responded to withdrawal of the agent and plasma exchange. More exploration of this issue, using population-based data, is indicated.

PB0877 | Vitamin B-12 Deficiency and Pseudo Thrombotic Microangiopathy: A Case Series

C. Jones ${ }^{1}$; R. Kumar ${ }^{2}$

${ }^{1}$ University of Louisville School of Medicine Internal Medicine Residency Program, Louisville, United States; ${ }^{2}$ University of Louisville Division of Medical Oncology and Hematology; Brown Cancer Center, Louisville, United States

Background: Vitamin B12 (cobalamin) deficiency, defined as a serum cobalamin level $<200 \mathrm{pg} / \mathrm{mL}$, is a common problem. Most clinicians are aware that cobalamin deficiency causes megaloblastic anemia, hypersegmented neutrophils, and subacute combined degeneration of the dorsal spinal column. However, many general clinicians are unaware that cobalamin deficiency can cause pseudo-thrombotic microangiopathy (TMA). Psuedo-TMA presents similarly to the lifethreatening condition thrombotic thrombocytopenic purpura (TTP), with laboratory evidence of hemolytic anemia. However, it is distinguished from TTP by very high levels of lactate dehydrogenase (LDH) and reticulocytopenia. Due to the extraordinarily high mortality rate of untreated TTP and its overlap in presentation with pseudo-TMA, pseudo-TMA is often treated with plasmapheresis, when simple vitamin replacement is what's needed. 
Aims: We present 3 cases of pseudo-TMA secondary to cobalamin deficiency. Our case series is important because although this pathology is known, it is not widely taught in basic medical education, leading to incorrect treatment with expensive and risky plasmapheresis.

Methods:

TABLE 1

\section{Case 1}

90 year old female with fatigue, weakness, anorexia, and lethargy. Found to have pernicious anemia with an intrinsic factor 100 times the upper limit of normal. Treated with 5 days of IM cyanocobalamin $1000 \mathrm{mcg}$, followed by daily sublingual tablets for life.

*** [It is important to note that ALL 3 cases had complete resolution of symptoms, with near normal labs on 3 month follow up after vitamin B12 replacement.]

\section{Case 2}

32 year old male with shortness of breath for two weeks. TTP incorrectly diagnosed, so he had urgent plasmapheresis. He developed hives from the procedure, and it was stopped. Found to have severe B12 deficiency, and received 4 days of IM cyanocobalamin $1000 \mathrm{mcg}$, followed by daily sublingual tablets for life.

\section{Case 3}

A 35 year old male with a history of alcohol abuse had fatigue, fevers, chills, and diarrhea. He had resolution of his GI symptoms, but persistent fatigue so he sought medical attention. Found to have severe macrocytic anemia and markedly elevated anti-parietal and anti-intrinsic antibodies. He received 5 days of IM cyanocobalamin $1000 \mathrm{mcg}$, followed by monthly injections.

Results:

TABLE 2

\begin{tabular}{|c|c|c|c|c|c|c|}
\hline PERTINENT LABS & Case 1 Presentation & $\begin{array}{l}\text { Case } 1 \\
\text { Discharge }\end{array}$ & $\begin{array}{l}\text { Case } 2 \\
\text { Presentation }\end{array}$ & Case 2 Discharge & Case 3 Presentation & Case 3 Discharge \\
\hline Hemglobin (g/dL) & 3.6 & 9.5 & 5.7 & 8.4 & 6.4 & 8.3 \\
\hline $\mathrm{MCV}(\mathrm{HI})$ & 104.9 & 91.9 & 108.8 & 100.2 & 125.7 & 108.6 \\
\hline $\begin{array}{l}\text { Reticulocyte Index } \\
\text { (RI) }\end{array}$ & 0.48 & 2.0 & 1.0 & 1.04 & 0.59 & 3.4 \\
\hline ADAMTS 13 & $>50 \%$ & N/A & $77.1 \%$ & N/A & $73 \%$ & N/A \\
\hline Cobalamin (pg/mL) & $<50$ & $>1,500$ & $<50$ & 2,079 & $<50$ & 1,918 \\
\hline
\end{tabular}

Conclusions: All three cases had the following similarities: schistocytes, undetectable haptoglobin, thrombocytopenia, normal renal function, significantly high levels of LDH (>2000 IU/L), and recitulocytopenia, with a low reticulocyte index $(\mathrm{RI}<2)$. These findings highlight how difficult it can be to distinguish TTP from psudeo-TMA, but also feature differences. The high LDH and low RI can be explained from a hypo-proliferative bone marrow response from insufficient cobalamin levels. None of the patients had a low ADAMTS13 level, nearly excluding TTP since ADAMTS13 activity less than $10 \%$ is a relatively specific finding in TTP. The risk of an adverse event in plasmapheresis is nearly $30 \%$, and the cost exceeds $\$ 100,000$ in the setting of TTP. This unnecessary risk and enormous financial burden can be eliminated if replacement of cobalamin is given in a timely manner. Our hope is that this case series prevents erroneous treatment in the future. 


\section{PLATELET ANTAGONISTS AND NOVEL THERAPEUTICS}

PB0878 | Development of a Novel "Arterial Thrombosis-on-achip" Microfluidic Device

$\underline{\text { J. Berry }}^{1}$; F.J. Peaudecerf ${ }^{2}$; N.A. Masters ${ }^{3}$; K.B. Neeves ${ }^{3}$;

R.E. Goldstein ${ }^{4}$; M.T. Harper ${ }^{1}$

${ }^{1}$ Department of Pharmacology, University of Cambridge, Cambridge,

United Kingdom; ${ }^{2}$ Department of Civil, Environmental, and Geomatic

Engineering, ETH Zürich, Zürich, Switzerland; ${ }^{3}$ Department of

Bioengineering, Department of Pediatrics, Section of Hematology,

Oncology, and Bone Marrow Transplant, Hemophilia and Thrombosis

Center, University of Colorado, Aurora, United States; ${ }^{4}$ Department of

Applied Mathematics and Theoretical Physics, University of Cambridge, Cambridge, United Kingdom

Background: Myocardial infarction is triggered by occlusion of coronary arteries by platelet-rich thrombi. Development of new antiplatelet drugs to prevent myocardial infarction depends on accurate models of thrombosis. In vivo animal models produce variable results and only have limited relevance to human disease. Few in vitro models using human blood generate occlusive thrombi; those that do generate occlusive thrombi do not allow quantitive assessment of antithrombotic compounds.

Aims: Development of a novel "arterial thrombosis-on-a-chip" microfluidic system that allows quantitative measurement of occlusion time.

Methods: A microfluidic chip was iteratively designed, and fabricated using soft lithography. Within the chip, a collagen and tissue factor patch triggers thrombosis in whole human blood flowed at arterial shear. Thrombus growth is monitored using confocal microscopy. Occlusion time is measured in a simple, robust way using a balance.

Results: Initial experiments confirmed that addition of a bifurcation into a microfludic chip allows occlusion to occur. However, further analysis highlighted that this occlusion can be caused by off-site coagulation, obscuring the effect of anti-platelet drugs. We therefore designed a microfluidic device that generates biologically relevant occlusive thrombi by quenching downstream coagulation. We validated our device by using the approved anti-platelet drug eptifibatide, demonstrating that our device can be used to monitor the effect of antithrombotic drugs on occlusion time in an unbiased manner.

Conclusions: We have developed a novel arterial thrombosis-ona-chip device that allows biologically relevant occlusive thrombi to form, and that can be used to assess the effect of anti-thrombotic compounds on occlusion time.
PB0879 | Structure-based Design of Cyclic Glycoprotein Iba-derived Peptides Affecting Platelet Interaction with von Willebrand Factor under Shear Conditions

J. Hrdinova ${ }^{1,2}$; D.I. Fernández ${ }^{1}$; B. Ercig ${ }^{1,2}$; B.M. Tullemans ${ }^{1}$; D.P. Suylen ${ }^{1}$; S.M. Agten ${ }^{1}$; K. Jurk ${ }^{3}$; T.M. Hackeng ${ }^{1}$; K. Vanhoorelbeke ${ }^{4}$; J. Voorberg ${ }^{2}$; C.P. Reutelingsperger ${ }^{1}$; K. Wichapong ${ }^{1}$; J.W.M. Heemskerk ${ }^{1}$; G.A.F. Nicolaes ${ }^{1}$ ${ }^{1}$ Department of Biochemistry, Cardiovascular Research Institute Maastricht, Maastricht University, Maastricht, Netherlands;

${ }^{2}$ Department of Molecular and Cellular Hemostasis, Sanquin-Academic Medical Center, Amsterdam, Netherlands; ${ }^{3}$ Center for Thrombosis and Hemostasis (CTH), University Medical Center of the Johannes Gutenberg University Mainz, Mainz, Germany; ${ }^{4}$ Laboratory for Thrombosis Research, Interdisciplinary Research Facility Life Sciences, Katholieke Universiteit Leuven Campus Kulak Kortrijk, Kortrijk, Belgium

Background: Plasma von Willebrand factor (VWF) circulates in a compact form unable to bind platelets. At high arterial shear stress or when immobilized to collagen, VWF undergoes a conformational change to expose the VWF A1 domain, which allows binding to platelet glycoprotein (GP)Iba present in the GPIb-V-IX complex resulting in shear-dependent thrombus formation. Interference with the VWF A1 domain-GPIba interaction is of therapeutic interest for many cardiovascular diseases, e.g. in immune thrombotic thrombocytopenia purpura (iTTP).

Aims: For this purpose, we aimed to design and test novel peptidebased drugs.

Methods: Cyclic peptides were designed in silico, mimicking the region in GPIba that binds to the exposed VWF A1 domain or to the A1 domain complexed with botrocetin. Peptides with lowest binding free energy (BFE) were chemically synthesized: a monocyclic monoORbIT peptide and a bicyclic bi-ORbIT peptide. Flow cytometry and citrated whole blood high shear stress microfluidic assays were used to assess the peptides inhibitory effect.

Results: Both peptides interfere with ristocetin- and botrocetininduced VWF binding to GPIb-V-IX as assessed by flow cytometry. Anti-VWF A1 domain antibody, CLB-RAg35, was used as a positive control. In whole-blood microfluidics assays at high shear stress, CLB-RAg35 suppressed stable platelet adhesion and markedly abrogated the formation of thrombi. Both peptides mimicked these phenotypic changes, albeit to a lesser extent than CLB-RAg35. Using mono-ORbIT as a template for an improved generation of peptides resulted in design and development of opt-mono-ORbIT, which showed higher inhibitory activity than the previous peptides.

Conclusions: Our data prove that a structure-based design of peptides can result in physiologically relevant peptide-based inhibitors, even for convoluted complexes such as GPIba-VWF A1. To counteract bleeding side effects observed in iTTP treatment with caplacizumab, controlled interference of VWF-GPIb complex formation may provide an alternative way of suppression of pathological thrombus formation at high shear stress. 
PB0880 | A Novel Dual AntiPlatelet and AntiCoagulant APAC: Interaction Between Platelet Factor 4 (PF4) and APAC Decreases Functional Activity

\section{Nevzorov ${ }^{1}$; A. Jouppila²; R. Lassila ${ }^{3,4}$}

${ }^{1}$ University of Helsinki, Helsinki, Finland; ${ }^{2}$ Clinical Research Institute $\mathrm{HUCH}$, Helsinki, Finland; ${ }^{3} \mathrm{Helsinki}$ University Hospital, Comprehensive Cancer Center, Department of Hematology, Coagulation Disorders Unit, and Research Program in Systems Oncology, Faculty of Medicine, Helsinki University, Helsinki, Finland; ${ }^{4}$ Aplagon Ltd., Helsinki, Finland

Background: APAC is a heparin proteoglycan mimetic with dual AntiPlatelet and AntiCoagulation activities. It inhibits collagen- and thrombin-induced platelet aggregation and prolongs coagulation times. APAC targets to damaged vascular sites co-localizing with collagen and von Willebrand factor (VWF) and diminishes arterial occlusion in several thrombosis models. APAC also VWF-dependently reduces platelet and fibrin deposition on collagen and tissue factor surfaces.

Upon activation, platelets release procoagulant factors including platelet factor 4 (PF4), which neutralizes negatively charged heparin-like glycosaminoglycans. PF4 as a chemokine has also well-established role in inflammation and heparin-induced thrombocytopenia.

Aims: We assessed the effect of PF4-APAC interaction by coagulation and platelet aggregation in vitro and the structure-function relationship of APAC after dissociation of the heparin-protein complex. Methods: APAC-spiked samples, \pm PF4, were studied in human citrated-plasma and platelet rich-plasma for APTT and TT, and collagen-induced $(0.5 \mu \mathrm{g} / \mathrm{mL})$ aggregation, respectively. In addition, APAC was reduced with dithiothreitol (DTT) to release the heparin and to assess subsequent activity after dissociation.

Results: APAC and unfractionated heparin (UFH, 0.5-1.5 $\mu \mathrm{g} / \mathrm{mL}$; $n=3-5$ ) prolonged the clotting times by $\geq 1$. 8 -fold and $\geq 1.2$-fold, respectively. APAC was at least 1.3 -fold (APTT) and 1.5-fold (TT) more potent anticoagulant than UFH. DTT-treatment decreased the anticoagulant potency of APAC to the level of UFH. PF4 (0.25-3.25 $\mu \mathrm{g} / \mathrm{mL}$ ) diminished the anticoagulant properties of both APAC and UFH. In collagen-induced platelet aggregation, APAC concentrationdependently $(0.5-10 \mu \mathrm{g} / \mathrm{mL} ; n=4)$ inhibited platelets unlike UFH. Again, PF4 (1.6-3.2 $\mathrm{\mu g} / \mathrm{mL})$ reduced anti-aggregatory effects of APAC.

Conclusions: We confirmed that APAC is more potent antiplatelet and anticoagulant agent than UFH in platelet aggregation and clotting time analysis. PF4 reversed APAC's activity, demonstrating its avid binding to heparin conjugate. Interestingly, after dissociating the heparin chains of APAC, the anticoagulant potency matched with UFH. Overall, the spatial organization of heparin chains supports both the anticoagulant and antiplatelet effects of APAC.
PB0881 | Thrombotic Microangiopathies (TMA): In vivo Evidence for Platelet Activation and Endothelial Cell Injury, and Potential for Therapeutic Intervention with Defibrotide

S. Elhadad ${ }^{1}$; S. Subrahmanian ${ }^{2}$; J. Ahamed ${ }^{2}$; J. Laurence ${ }^{1}$

${ }^{1}$ Weill Cornell Medicine, New York, United States; ${ }^{2}$ Oklahoma Medical Research Foundation, Oklahoma City, United States

Background: Endothelial cell (EC) activation and injury and platelet activation characterize thrombotic thrombocytopenic purpura (TTP) and atypical hemolytic uremic syndrome (aHUS). We found that $5 \mu \mathrm{g} / \mathrm{ml}$ defibrotide inhibits TMA plasma-mediated caspase 8 activation of EC, an initial step in apoptotic injury (ASH 2019, Abstract 3676), but defibrotide was reported to inhibit agonist-induced platelet activation only at clinically unachievable doses of $100-1000 \mu \mathrm{g} /$ ml (ASH 2019, Abstract 3614).

Aims: (1) Evaluate biomarkers of platelet activation and EC injury in TMA plasmas;

(2) determine whether clinically relevant defibrotide concentrations block agonist-mediated platelet activation.

Methods: (1) Biomarkers for platelet activation (platelet factor 4 (PF4), $\beta$-thromboglobulin ( $\beta$-TG)) and EC injury (von Willebrand factor (VWF) antigen) were measured in TMA patient plasmas ( 9 aHUS, 8 TTP) by ELISA.

(2) Washed human platelets were incubated with the PAR-1 agonist peptide RUJL or ADP $(2 \mu \mathrm{M})$, alone or with $5 \mu \mathrm{g} / \mathrm{ml}$ defibrotide. Platelet aggregation was quantified by light transmission aggregometry.

Results:

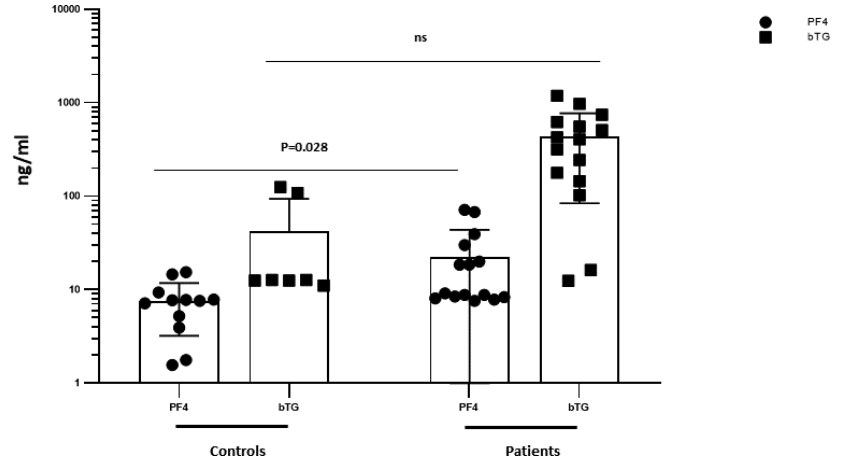

FIGURE 1 PF4 and B-thromboglobulin levels in plasmas of acute TMA patients vs. controls

(1) A significant increase in PF4 levels was seen in TMA patients $(n=15)$ vs. healthy controls ( $n=12)$ (Fig. 1). A significant difference in $\beta$-TG levels was not seen in TMA patients $(n=15)$ vs. controls ( $n=7$ ). The $\beta$-TG:PF4 ratio, a marker of in vivo platelet activation (Ann Rheum Dis 2005;64:484), was >2 in TMA and control plasmas, indicating some in vitro activation, but much more highly elevated 
in TMA (ratio $=19.4)$ vs. control plasmas (ratio $=5.6)(P=0.0058)$. vWF antigen levels were not significantly different in patients vs. controls.

(2) Defibrotide blocked platelet aggregation induced by both RUJL and $A D P$ at $5 \mu \mathrm{g} / \mathrm{ml}$ (Fig. 2).

Conclusions:
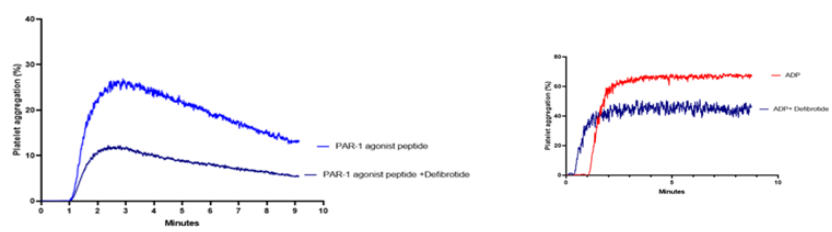

FIGURE 2: Effect of defibrotide on PAR-1 agonist and ADP induced platelet aggregation

The ability of defibrotide to block TMA plasma-mediated EC injury, shown previously, and now platelet activation has implications for TMA treatment as well as in progressive COVID-19, which presents features characteristic of TMAs and vaso-occlusive disease (J Thromb Haemost 2020:18:3106; Lancet Haematol 2020;7:e575).

PB0882 | Lyophilized Human Platelets Support Thrombosis Unlike Normal Platelets in the Presence of GPIIb/IIla Antagonists

M. Dickerson; K. Moskowitz

Cellphire Inc., Rockville, United States

Background: GPIIb/IIla inhibitors block platelet fibrinogen and vWF receptors thereby reducing clotting. In an emergency, these agents must be overcome to stop bleeding.

Aims: The aim of this study was to determine if Thrombosomes $₫$, a lyophilized human platelet (LHP) hemostatic agent under clinical development, were resistant to the effect of GPIIb/IIla antagonists and therefore restore hemostasis associated with GPIIb/IIla antagonist mediated bleeding.

Methods: GPIIb/IIla inhibitors representative of different classes of therapeutic molecules, an antibody (AP-2), a cyclic heptapeptide (eptifibatide) and a non-peptide small molecule (tirofiban) were tested for their ability to inhibit aggregation of donor and platelet rich plasma (PRP). Doses of each drug shown to inhibit aggregation of platelets were used to treat PRP and inhibit thrombus formation on the T-TAS ${ }^{\circ}$ flow system over thromboplastin-collagen coated microcapillaries. Single donor platelets (standard of care for drug reversal) and LHP were compared for their ability to recover loss of occlusion in the presence of each drug.

Results: Unlike fresh platelets, LHP were shown to be nonresponsive to ADP by aggregometry. At therapeutic doses, each drug was shown to inhibit ADP stimulated PRP aggregation. T-TAS ${ }^{\circledR}$ occlusion time of PRP was approximately 15 minutes and increased to 25-30 minutes with drug treatment. Conversely, each inhibitor had no effect on the occlusion time of LHP of 15 minutes. When PRP treated with each GPIIb/IIla inhibitors was dosed with LHP, occlusion times returned to normal (11-14 minutes). Dosing with equal number of single donor platelets did not recover normal occlusion. Conclusions: LHP, unlike normal platelets, were resistant to GPIIb/ IIla antagonists. In vitro studies reveal that LHP can be used to recover the anti-thrombotic effect of GPIIb/IIla inhibitors, potentially allowing continued drug compliance during procedures and as a possible treatment for bleeding while on GPIIb/IIla antagonist therapy.

\section{PLATELET FUNCTION DISORDERS, ACQUIRED}

\section{LPB0029 | Distinct Clonal Mutation Patterns between JAK2} V617F Positive and CALR-mutated Essential Thrombocythemia

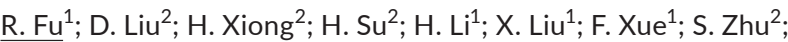
R. Yang ${ }^{1} ;$ T. Cheng ${ }^{1}$; L. Zhang ${ }^{1}$

${ }^{1}$ State Key Laboratory of Experimental Hematology, National Clinical Research Center for Blood Diseases, Institute of Hematology \& Blood Diseases Hospital, Chinese Academy of Medical Sciences \& Peking Union Medical College, Tianjin, China; ${ }^{2}$ BGI-Shenzhen, Shenzhen, China

Background: Single-cell sequencing of hematopoietic stem cells (HSCs) provides an unprecedented opportunity to directly reveal origins of driver mutations in essential thrombocythemia (ET)-a unique model for dissecting clonal architecture of tumors.

Aims: The study aimed at revealing the clonal mutation patterns of ET with different driver mutations.

Methods: Whole-exome sequencing of bulk bone marrow cells from $33 \mathrm{ET}$ patients were conducted, and targeted region sequencing of single HSCs from two representative patients was performed, one with JAK2 V617F and the other one with the CALR mutation.

Results: Seventeen patients (51.5\%) had the JAK2 V617F mutation, four (10.8\%) harbored the CALR mutations. More somatic non-silent mutations were observed in JAK2 V617F+ patients than in CALRmutated patients $(P=0.048)$. Mutations with known roles in myeloid neoplasms were more likely to co-occur with JAK2 V617F. Single-HSC sequencing revealed that not every possible key mutation identified in bulk cells was an 'essential mutation'. In the JAK2

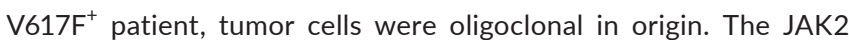
V617F mutation occurred after the LIN54, TET2, and DNMT3A mutations. These pre-JAK2 mutations randomly occurred and continually accumulated in almost all cells from the HSC pool. JAK2 V617F occurred in cells with at least one of the pre-JAK2 mutations. As a consequence, a cluster of cells with these four mutations in every cell were emerged and acquired a growth advantage. Unlike JAK2 V617F, the CALR mutation was an early event, and the clone containing a single CALR mutation acquire a survival advantage. JAK2 V617F+ cell amplification mainly occurred in the HSC compartment in ET30, but CALR-mutated cells were mainly amplified in HPCs in ET35. This observation helps explain why JAK2 V617F causes 
erythrocytosis, granulocytosis, and thrombocytosis, while the CALR mutations mainly cause thrombocytosis.

Conclusions: Single-HSC sequencing provides direct evidence for revealing subject-specific key mutations and clonal evolution patterns in patients with ET.

PB0883 | Importance of Platelet's Sialic Acid Residues on Haemostasis and Immune System of Patients with Immune Thrombocytopenia

N.V. Butta Coll ${ }^{1}$; M.T. Álvarez Román ${ }^{1}$; E. Monzón Manzano${ }^{1}$; M. Martín Salces ${ }^{1}$; M.I. Rivas Pollmar ${ }^{1}$; E. García Arias-Salgado ${ }^{1}$; P. Acuña ${ }^{1}$; S. García Barcenilla ${ }^{1}$; E. González Zorrilla; T. Cebanu; V. Jiménez Yuste Service of Hematology, Hospital Universitario La Paz, Madrid, Spain

Background: Immune thrombocytopenia (ITP) is an autoimmune disease characterised by a low platelet count with a deterioration of the regulatory compartment (regulatory $\mathrm{T}$ [Treg] and regulatory B [Breg] cells). Nevertheless the initial event leading to antiplatelet autoimmunity remains unclear.

Aims: The aim of our study was to analyse the role of sialic acid of platelet glycoproteins to better understand their role in platelet function and in the development of ITP.

Methods: This observational, prospective and transversal study included 82 patients with chronic primary ITP and 115 healthy controls. Our local Ethics Committee approved the protocol, and participants signed informed consent.

Platelet activation markers, loss of sialic acid (binding of Ricinus comunnis agglutinin, RCA), caspase activity; and detection of peripheral blood mononuclear cells subsets, were evaluated by flow cytometry.

Neuraminidase (NEU) activity in serum was determined with the substrate 20-(4-methylumbelliferyl)-a-D-N- (MUNANA).

Results: Loss of sialic acid from platelet surface was a key player for reducing platelet count (Figure 1A) and platelet's capacity of activation, as shown by the negative correlation between RCA binding and ability of fibrinogen receptor to be activated (PAC1 binding) and agonist-induced degranulation (P-selectin and CD63 exposure, Figure 1B) with TRAP. Loss of sialic acid was accompanied by increased platelet caspase activities(Figure 1C).

Loss of sialic acid from platelet surface was due to the enhancement of neuraminidase activity in patients with ITP (Figure 2A). ITP patients with the lowest exposure of sialic acid showed the most pronounced decrease in LTregand the higher plasmablasts count (Figure 2B).
A
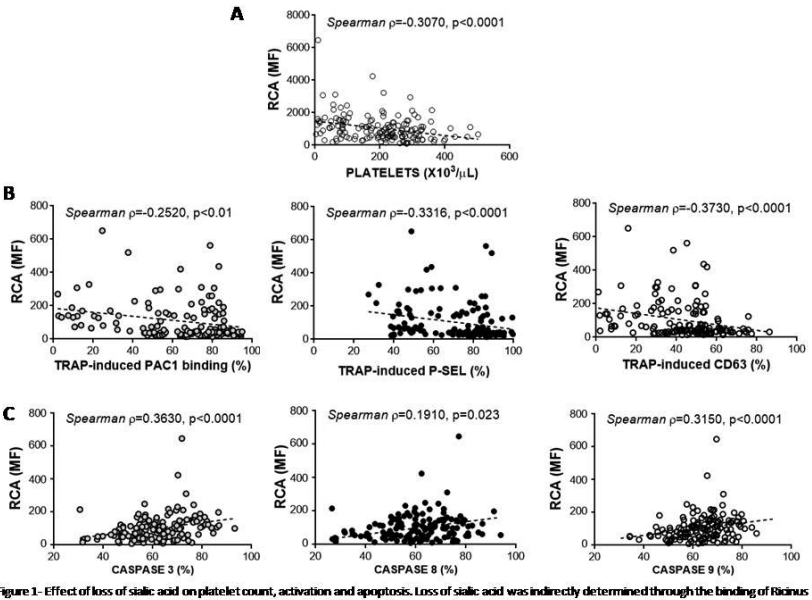

FIGURE 1: Effect of loss of sialic acid on platelet count, activation and apoptosis

$\mathbf{A}$
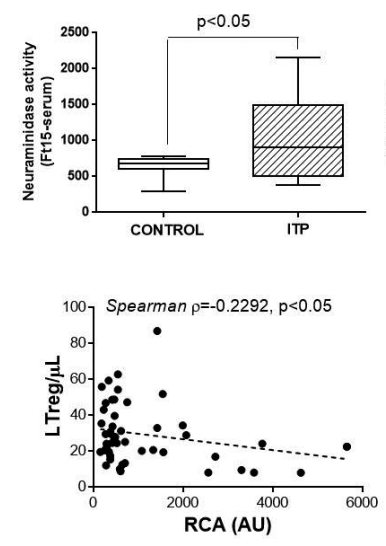

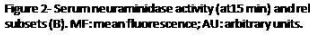
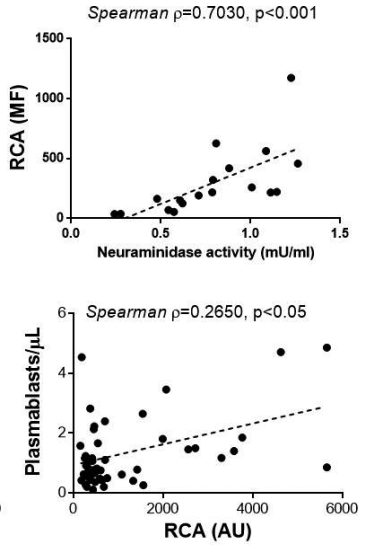

FIGURE 2: Serum neuraminidase activity (at15 $\mathrm{min}$ ) and relationship between loss of sialic acid and neuraminidase activity $(A)$ and LTreg and plasmablasts subsets (B)

Conclusions: Loss of sialic acid from platelets induced an impairment of their ability to be activated and an increased apoptosis, accompanied by a diminished LTreg population and a higher plasmablast subset. These observations posited loss of sialic from platelets as aetiopathogenic agents in ITP.

Research funded by FIS-Fondos FEDER PI19/00772 and Platelet Disorder Support Association 
PB0884 | Evaluation of an Automated Platelet Aggregation Method for the Detection of Patients with Congenital or Acquired Platelet Function Defects

A. Lecchi ${ }^{1}$; S. La Marca ${ }^{1}$; L. Padovan ${ }^{1}$; M. Boscarino ${ }^{2}$; A. Artoni ${ }^{1}$; N. Arai ${ }^{3}$; S. Shinohara ${ }^{3}$; M. Capecchi ${ }^{4}$; F. Peyvandi ${ }^{1,2}$

${ }^{1}$ Fondazione IRCCS Ca' Granda Ospedale Maggiore Policlinico, Angelo Bianchi Bonomi Hemophilia and Thrombosis Center, Milan, Italy, Milano, Italy; ${ }^{2}$ Università degli Studi di Milano, Department of Pathophysiology and Transplantation, Milan, Italy, Milano, Italy; ${ }^{3}$ Engineering 1, Sysmex Corporation, Kobe, Japan; ${ }^{4}$ Università degli Studi di Milano, Department of Biomedical Sciences for Health, Milano, Italy

Background: Abnormalities of platelet function are associated with an increased risk of bleeding. Light transmission aggregometry (LTA) is the gold standard and the most widely used laboratory method to screen patients with suspected abnormalities of primary hemostasis. LTA is a time-consuming and technically challenging method, so that an automated LTA-based method for use on Sysmex CS-2400, a high-performance automated coagulation analyzer, has been recently developed.

Aims: To evaluate the diagnostic capacity of CS-2400 with Revohem aggregation reagents panel for the diagnosis of platelet disorders and for monitoring the effect of anti-platelet therapy.

Methods: Platelet aggregation induced by adenosine diphosphate (ADP), arachidonic acid (AA), collagen and epinephrine was performed according to ISTH guidelines, on Sysmex CS-2400 analyzer and on lumi-light-transmission aggregometry (Chrono-log,Mascia Brunelli, IT).

Light transmission was recorded and aggregation response to all agonists was analysed and compared to controls.

To evaluate platelet response in patients on anti-platelet therapy, we used the "Platelet Aggregation Level (PAL)" on CS-2400, which decreases with platelet aggregation activity reduction. A-PAL and C-PAL were calculated from the wave obtained using two concentrations of ADP and collagen respectively.

Results: Ten patients with primary secretion defects (PSD), 11 patients on anti-platelet therapy and 10 healthy controls were enrolled in the study.Patients with PSD showed reduced median of \% lighttransmission compared to controls using each agonist. Data obtained with CS-2400 showed a low intra-group variability for each agonist except epinephrine (Figure 1).

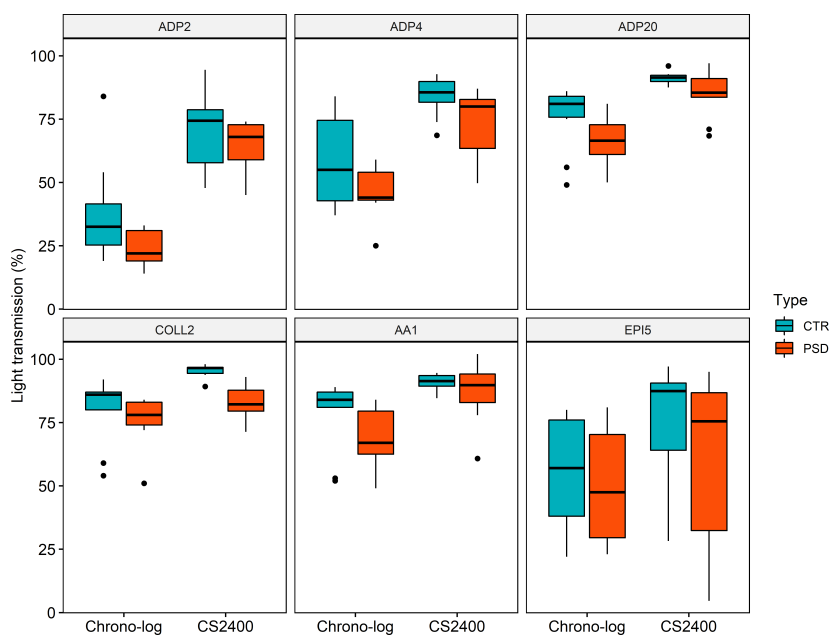

FIGURE $1 \%$ of light transmission in control group and PSD patients. Distribution of \% of light transmission according to different groups of patients. The box plots represent the interquartile ranges, the solid horizontal line inside each box plot is the median value and the vertical bars delimit the minimum and maximum values of the distribution. The black circles identify outliers.

Patients on anti-platelet therapy showed statistically significant reduced ( $P=0.001$ ) median of \% light-transmission compared to controls with ADP 4-20 $\mu \mathrm{M}$ and AA $1 \mathrm{mM}$. In these patients, A-PAL and C-PAL scores were lower than controls (Figure 2).

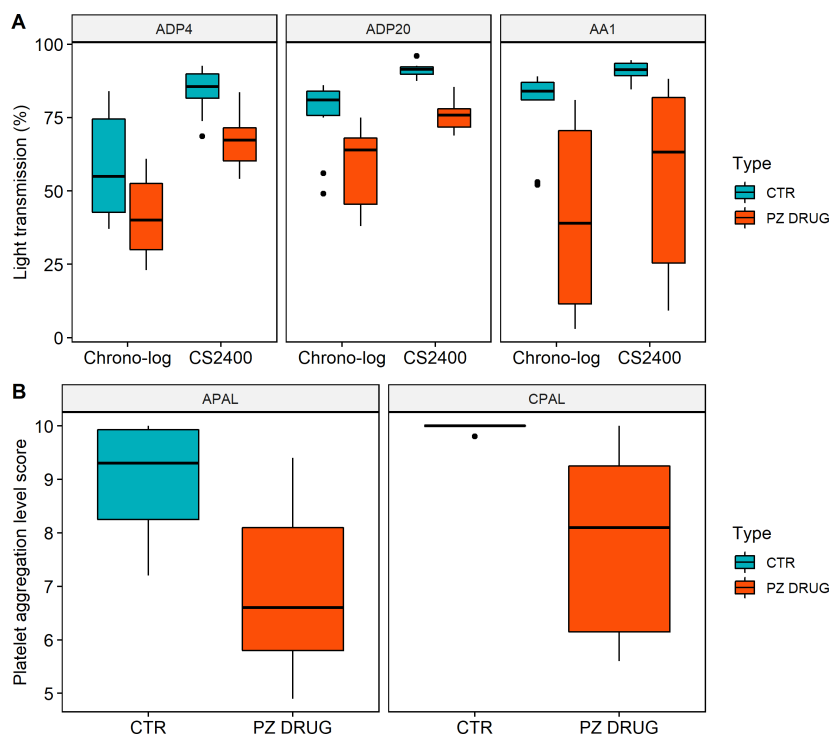

FIGURE $2 \%$ of light transmission in control group and patients on anti-platelet therapy (panel A). C-PAL and A-PAL scores in control group and patients on anti-platelet therapy (panel B). Distribution of $\%$ of light transmission (Panel A) and PAL score (panel B) according to different groups of patients. The box plots represent the interquartile ranges, the solid horizontal line inside each box plot is the median value and the vertical bars delimit the minimum and maximum values of the distribution. The black circles identify outliers 
Conclusions: Milan preliminary data using CS-2400 analyzer showed a good diagnostic capacity for PSD patients and a good performance in evaluating the aggregation response in patients on anti-platelet therapy.

PB0885 | Frequent Platelet Dysfunction and Fibrinolysis in Patients with Intracerebral Hemorrhage

$\underline{\text { P. Lindholm }}{ }^{1}$; H. Kwaan ${ }^{2}$; I. Weiss ${ }^{2}$; A. Naidech ${ }^{3}$

${ }^{1}$ Northwestern University Department of Pathology, Chicago, United

States; ${ }^{2}$ Northwestern University Department of Hematology, Chicago, United States; ${ }^{3}$ Northwestern University Department of Neurology, Chicago, United States

Background: Intracerebral hemorrhage (ICH) has significant morbidity and mortality. Improving patient outcomes with $\mathrm{ICH}$ depends on our ability to recognize hematoma expansion (HE). The altered hemostatic functions have not yet been clearly defined. We hypothesize that changes in coagulation parameters, platelet function and fibrinolytic system are present and can provide biomarkers for the progress of $\mathrm{HE}$, and furthermore may lead to targeted interventions to improve outcomes.

Aims: Determine hemostatic mechanisms occurring in $\mathrm{ICH}$ patients with $\mathrm{HE}$ using platelet function, coagulation activation and fibrinolysis assays.

Methods: Patients with acute ICH are studied after consent within the first 12 hours onset. Platelet function, coagulation factors and fibrinolysis activity are measured by thromboelastography (TEG) and ROTEM viscoelastic assays (VEA). Platelet function is also tested by PFA-100, and VerifyNow Aspirin and $\mathrm{P}_{2} \mathrm{Y}_{12}$ point-of-care (POC) assays. Coagulation activation is measured by Thrombin Generation Assay and Tissue Factor activity. Fibrinolysis is assayed by Plasmin/ antiplasmin (PAP) complex, Fibrinogen, D-Dimer and PAI-1 activity.

Results: Seventeen patients who presented with acute $\mathrm{ICH}$ and hypertension were consented. Eleven had basal ganglia or thalamus lesions; 4 cortical bleeding; 1 pontine and 1 cerebellar hemorrhage. Platelet dysfunction with inhibited ADP-induced aggregation was shown by TEG platelet mapping in 11/17 patients, with mean inhibition of $33.1 \%$ (range 6.8-96.5). Platelet dysfunction by POC assay was observed in 13 patients and was not accounted for by antiplatelet medication. EXTEM assay showed increased fibrinolysis in 6 cases. Five of 10 patients tested had elevated D-dimer, mean 1142 $\mathrm{ng} / \mathrm{mL} \mathrm{D}$-DU (range 443 to 3040). Fibrinogen levels were normal in 5 available cases.

Conclusions: This is the first evidence of platelet dysfunction and fibrinolysis in patients with acute ICH using VEA and POC testing. Platelet dysfunction, coagulation activation and fibrinolysis are in vestigated as potential biomarkers for $\mathrm{ICH}$ hematoma expansion.
PB0888 | Acquired 8-Storage Pool Disorder Co-existing with Acquired Factor V Deficiency in Myelodysplastic Syndrome / Myeloproliferative Neoplasm

R. Dave; J. Mammen; T. Geevar; J. Rasalam; R. Vijayan; A. Samuel; S. Singh; S. Nair; L. Mathew Christian Medical College and Hospital, Vellore, India

Background: Acquired $\delta$-Storage pool disorder(SPD) is frequently associated with myelodysplastic syndrome/myeloproliferative neoplasms(MDS/MPN) possibly resulting from chromosomal alterations in megakaryocyte lineage causing decreased dense granules production. Patients with MPN may also have acquired Factor $V$ deficiency either due to Factor $V$ adsorption on myeloid-megakaryocyte mass, hepatic synthetic dysfunction or inhibitors. Acquired SPD and factor deficiency may co-exist in patients with MDS/MPN, timely diagnosis of both being essential to offer appropriate therapeutic intervention at the time of bleeding.

Aims: To describe co-existence of acquired $\delta$-SPD and acquired factor $\mathrm{V}$ deficiency in a 14 years old child with MDS/MPN.

Methods: Informed consent was taken from parents. ISTHBleeding Assessment Tool(BAT) was used to objectively score the bleeding symptoms. Complete blood counts(CBC), Prothrombin Time(PT), Activated Partial thromboplastin time(APTT), mixing studies, Fibrinogen, Modified Ivy's bleeding time(BT), Closure time on Platelet function analyzer-200 (PFA-200), Ristocetin cofactor assay(vWF:RCo), light transmission aggregometry(LTA), lumiaggregometry, mepacrine uptake/release assay, CD63 expression after agonist stimulation by flow cytometry and one-stage clotbased factor assays were performed.

Results: Patient had elevated BAT score of 4 with recent onset epistaxis and ecchymosis. CBC revealed low hemoglobin $(6.9 \mathrm{gm} / \mathrm{dl})$, elevated WBC count $\left(76.4 \times 10^{9} / \mathrm{L}\right)$, mild thrombocytopenia $\left(83 \times 10^{9} / \mathrm{L}\right)$ with myeloid left shift, increased blasts(9\%), hypogranular myeloids and platelet anisocytosis(Figure1). Bone marrow examination was consistent with MDS/MPN with cytogenetics showing monosomy 7. PT and APTT were prolonged, correcting on mixing studies. Factor $V$ was mildly reduced(20\%) while other coagulation factors and vWF:RCo were normal. Although BT, PFA-200 closure time and LTA were normal, lumi-aggregometry showed absent ATP release. Markedly reduced Mepacrine uptake/release(Figure2) and markedly reduced CD63 expression upon convulxin stimulation confirmed the diagnosis of $\delta$-SPD. 


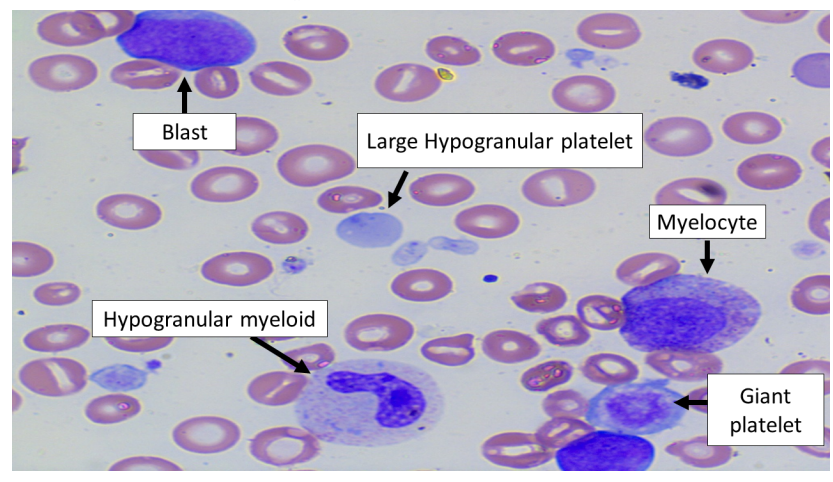

FIGURE 1 Peripheral smear showing blast, myelocyte, hypogranular myeloid, platelet anisocytosis and anisogranularity.
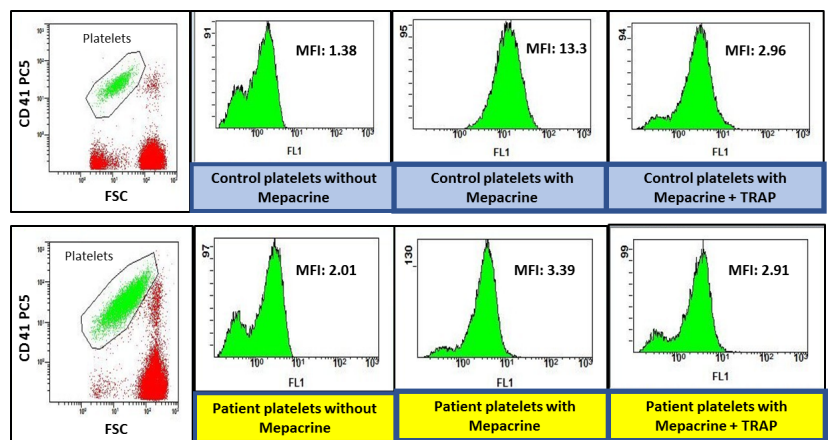

FIGURE 2 Mepacrine Uptake/Release Assay showing markedly reduced Mepacrine uptake/ release for the patient as compared to the control

Conclusions: Acquired $\delta$-SPD may co-exist with acquired factor $\mathrm{V}$ deficiency in patients with MDS/MPN. Hence, lumi-aggregometry and mepacrine uptake/release assay should be performed in patients with MDS/MPN having bleeding symptoms despite normal screening tests for primary hemostasis and normal LTA.

PB0889 | Emergency Splenectomy for Immune Thrombocytopenia (ITP) Patients with Life-threatening Bleeding: A Report of Three Cases and Review of the Literature

M. Yang ${ }^{1} ;$ Z. Zhou ${ }^{2}$

${ }^{1}$ The Second Clinical College, Kunming Medical University, Kunming, China; ${ }^{2}$ Department of Hematology, the Second Affiliated Hospital of Kunming Medical University, Kunming, China

Background: Immune thrombocytopenia (ITP) is a benign bleeding disease characterized by immune-mediated increased platelet destruction and/or thrombocytopenia. life-threatening bleeding (e.g., intracranial, gastrointestinal, and severe hemoptysis) can lead to serious complications and even death in ITP patients. Emergency Splenectomy treatment is available for these patients failure to respond to other medications.
Aims: To emphasize the importance of emergency splenectomy for immune thrombocytopenia patients with life-threatening bleeding.

Methods: We report the case details of three patients who had acute lethal hemorrhage of ITP:

case 1 (165/ male) severe hemoptysis;

case 2 (33/ female) and

case 3 (13/ female) intracranial hemorrhage.

The patient in Case 1 with chronic ITP improved hemoptysis after bronchial artery embolization and his platelet count recovered 2 days after splenectomy. But he went on to develop antiphospholipid syndrome at 5 months after operation.

Case 2 the patient underwent urgent emergency splenectomy due to left temporal lobe hemorrhage and recovered platelet count one day after surgery. But she was diagnosed secondary epilepsy after hemorrhage. One month after the operation, She returned to hospital for rituximab ( $375 \mathrm{mg} / \mathrm{m}^{2} \times 4$ weekly) treatment with thrombocytopenia and menorrhagia. She remained complete response (CR) during a two-year follow-up. Case 3 a girl underwent emergency splenectomy for intracerebral hemorrhage, and suffered a cardiopulmonary arrest shortly subsequently. Eventually, she died for increased intracranial hemorrhage.

Results: In our cases, except case 3, other patients successful performed emergency splenectomy and recovered the platelet count.

Conclusions: In literature, reports of ITP responding to emergency splenectomy in patients with life-threatening bleeding have been reported, but the peculiarity of our cases are that platelet count recovery has been obtained with emergency splenectomy in adult and the relapsed patient can benefit from rituximab after operation. However, the choice in children patient should be more cautious.

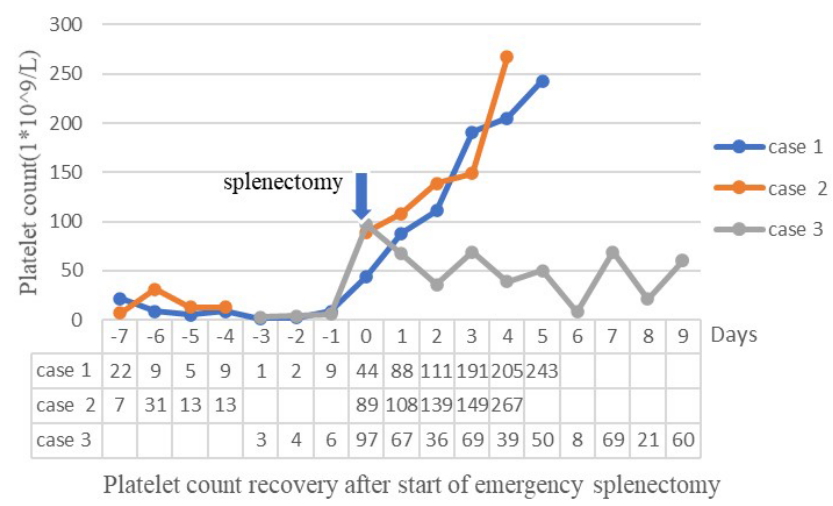

FIGURE 1 Platelet count recovery after start of emergency splenectomy 
PB0890 | Aspirin Resistance after Total Hip or Knee Arthroplasties

N. van Oosterom ${ }^{1,2} ;$ M. Barras ${ }^{1,2} ;$ N. Cottrell ${ }^{1} ;$ R. Bird ${ }^{2}$

${ }^{1}$ The University of Queensland, Brisbane, Australia; ${ }^{2}$ Princess Alexandra Hospital, Brisbane, Australia

Background: Aspirin is frequently used for venous thromboembolism (VTE) prophylaxis after total hip and knee arthroplasties (THA) TKA). However, the effectiveness and safety are unknown when used in patients with risk factors for aspirin resistance (AR) including diabetes, obesity, and elderly, which are also risk factors for THA/TKA and VTE. In cardiovascular disease, AR has been linked to poorer patient outcomes with a AR incidence rate of $28 \%$; but research is lacking post-THA/TKA.

Aims: To investigate the incidence of AR in patients post-THA/TKA who are diabetic, obese and/or elderly.
Methods: A prospective cohort study of obese (body mass index (BMI) $\left.{ }^{3} 30 \mathrm{~kg} / \mathrm{m}^{2}\right)$, diabetic and/or elderly ( ${ }^{3} 65$ years) patients prescribed aspirin for VTE prophylaxis post-THA/TKA in a major Australian tertiary hospital. Blood samples were collected after ${ }^{3} 7$ days of aspirin therapy to conduct a platelet function analyser (PFA-100) and full blood count. Adherence was confirmed verbally and through tablet count. Patients with thrombocytopenia, taking an anticoagulant, or non-adherence were excluded. Ethics approval was granted by Metro South HREC and patients provided informed consent. Funding was provided by a Metro South Research Support Scheme grant.

Results: Thirty-four patients were included, 19 (55.9\%) THA and 15 (44.1\%) TKA, with a mean $( \pm$ SD) age of $66.5( \pm 10.6)$ years, BMI of $31.6( \pm 5.8) \mathrm{kg} / \mathrm{m}^{2}$, and $20(58.8 \%)$ were male. Six-weeks of aspirin $150 \mathrm{mg}$ daily on discharge was the most used regimen ( $n=18,52.9 \%)$ with approximately 17 doses prior to analysis. The incidence of AR (Table 1 ) was $52.9 \%(n=18)$, of which, $4(22.2 \%)$ were diabetic, 11 (61.1\%) obese, and 11 (61.1\%) elderly participants.

TABLE 1 Summary of aspirin resistance risk factors and laboratory results

Total Population $(n=34,100 \%)$

$\mathrm{BMI} \geq 30\left(\mathrm{~kg} / \mathrm{m}^{2}\right)$

$23(100 \%)$

no. (\%)

Age $\geq 65$ (years)

no. (\%)

Diabetes

no. (\%)

$147.1( \pm 31.9)$

Population without aspirin resistance

$(n=16,47.1 \%)$

$12(\%)$

$10(\%)$

$21(100 \%)$

$8(100 \%)$

$4(50.0)$

Aspirin Dose (mg/day)

mean $( \pm S D)$

$16.0( \pm 7.5)$

Number of aspirin doses

taken

no. (\%)

Platelet count $\left(\times 10^{9} \mathrm{~L}\right)$

mean $( \pm S D)$

Haematocrit

mean $( \pm S D)$

PFA (s)

$169.1( \pm 60.5)$

(Collagen/Epinephrine)

mean $( \pm S D)$

PFA (s)

$92.7( \pm 31.1)$

$95.4( \pm 31.9)$

$71( \pm 7.0)$
$11(\%)$

$4(50.0 \%)$

Population with aspirin resistance $(n=18,52.9 \%)$

$11(\%)$

$144.4( \pm 15.7)$

$16.6( \pm 8.5)$

$424.3( \pm 102.5)$

$0.38( \pm 0.04)$

$132.8( \pm 26.3)$

(Collagen/Adenosine

diphosphate)

mean $( \pm S D)$ 
Conclusions: In this small sample, over half the patients had AR. Aspirin may not be an appropriate agent for VTE prophylaxis for patients who are obese, have diabetes or are elderly. Further research is required to confirm the clinical implications of $A R$ in this cohort.

\section{P0159 | Tetralogy of Fallot with Thrombolytic Ongoing Processes: A Case Report}

B. Badlou ${ }^{1} ;$ H. Najafpour ${ }^{2}$.

${ }^{1}$ BBAdvies and Research, Zeist, Netherlands; ${ }^{2}$ BiomedixBiotechkaniro Co., Tehran, Iran, Islamic Republic of

Background:Wereportararecase of unrepaired Tetralogy_Pantalogy of Fallot (TOF_POF) in a 20 years old girl, who presented with cyanotic finger tops appearance, ongoing thrombolytic processes and remarkable thrombocytopenia $(1,2)$, heart ventricular septal defect (VSD), and might atrial septal defect (ASD), anxiety, sleep disorders, nightmares, and limited social life. Thrombocytopenia in cyanotic heart disease patients, although well known, is not adequately understood. These kind of TOF/POF patients are suffering of cyanotic blue blood circulation with erythrocytosis.

Aims: The aim was to prevent chronic thrombocytopenia which might be result of chronic compensation process selected by brainheart axis to prevent premature thrombotic processes.

Methods: Owing to her growth retardation, unknown infections, poor cardiopulmonary status, the patient did not undergo surgery, and she only got medications and treatments with only symptomatic supportive therapy. We used novel developed technologies so called SONAPS to prevent further Erythrocytosis simultaneously inhibit Thrombocytopenia, without any platelet concentrates transfusion. Results: Using the combination of detection by the Novel developed inhouse technology so-called SONAPS, Hematologic - and auricular medicine approaches, we succeeded to affect her ongoing thrombolytic processes, erythrocytosis, decreased her heart arrhythmic aspects, increased oxygenation of whole body, while she claimed that somehow cyanotic aspects of her skin and fingers appearance were decreased, after 24 hours. All clinical results were checked up again with new clinical and basic research testing i.e. clinical feature of fatigue, tachycardia, and ECG testing. The exact mechanism of these phenomenal reconciliation need more investigation.

Conclusions: Our inhouse developed approaches saved life of a patient with cyanotic TOF POF disorders, who suffered from critical thrombocytopenia and erythrocytosis. Our approached novel system is working. We are looking for collaborations to carry out our model system, globally.
P0162 | Anti-lymphoma Therapy Improves Thrombocytopenia in a Young Patient with Hodgkin Lymphoma: A Case Report

M. Yang ${ }^{1}$; Z. Zhou ${ }^{2}$

${ }^{1}$ The Second Clinical College, Kunming Medical University, Kunming, China; ${ }^{2}$ Department of Hematology, The Second Affiliated Hospital of Kunming Medical University, Kunming, China

Background: Immune thrombocytopenia (ITP)can be observed in lymphoproliferative disorders, most commonly chronic lymphocytic leukemia (CLL), the minority of cases are reported in patients with Hodgkin lymphoma (HL). Some studies estimated that the prevalence of ITP with HD is $0.2 \%$ to $1 \%$. And the prognostic significance of its presence is unclear.

Aims: Our report broadens the knowledge about the clinical and therapeutic management of patients with Hodgkin lymphoma.

Methods: A 31-year-old female five years ago accepted cervical lymph node biopsy, and diagnosed with classical Hodgkin lymphoma with an interfollicular pattern. She just received 2 cycles of ABVD, and later refused regular treatment and reexamination. About 57 months after her initial treatment, she had multiple hospitalized for gum bleeding, petechiae and epistaxis with platelets of $5,000 / \mu \mathrm{L}$. A subsequent bone marrow aspiration showed increased immature megakaryocytes. After a week, she was referred to our hospital with complaint of epistaxis. CT showed extensive cervical lymphadenopathy. She was treated by oral prednisolone $45 \mathrm{mg} /$ day and platelet transfusion. A repeat cervical lymph node biopsy a week later was consistent with lymphocyte-rich classical Hodgkin lymphoma (LRCHL). She subsequently underwent chemotherapy in combination with 2 cycles of ABVD therapy. In January 2021, the patient achieved a significant increase in platelet count and decrease in lymph nodes' size. We will schedule a detailed follow-up, in order to monitor the immune dysregulation.

Results: This report documents a significant recurrence of lymphoma in the presence of ITP in HL patient. Anti-lymphoma therapy could achieve platelet count recovery.

Conclusions: In this case, the patient's ITP is regarded as paraneoplastic syndrome. Only proper combination chemotherapy treatment of the underlying lymphoma can successfully control the ITP, and curtail unnecessary transfusions to a patient.

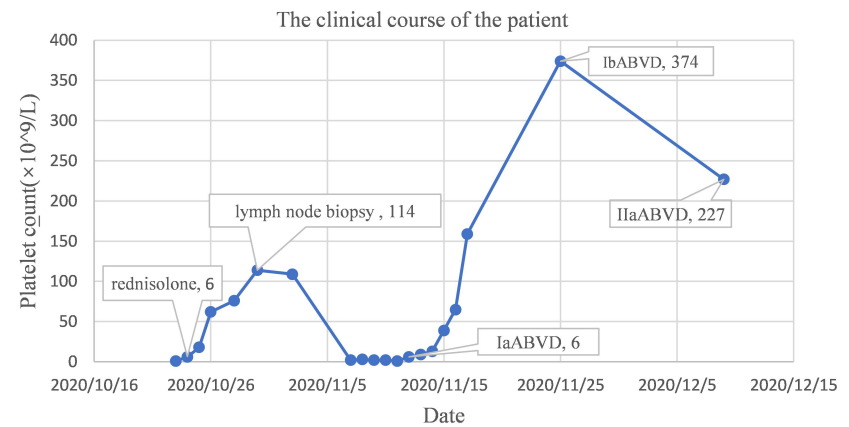

FIGURE 1 The clinical course of the patient 


\section{PLATELET FUNCTION DISORDERS, HEREDITARY}

LPB0081 | Low Adhesion and Interaction Forces of Myh9 Mutant Platelets Lead to Impaired Clot Retraction and Unstable Thrombus Formation

J. Baumann ${ }^{1}$; L. Sachs' ${ }^{2}$ Z Z. Nagy ${ }^{1}$; I. Schoen ${ }^{3}$; A. Greinacher ${ }^{2}$; O. Otto $^{4,5}$; R. Palankar ${ }^{2}$; M. Bender ${ }^{1}$.

${ }^{1}$ Institute of Experimental Biomedicine - Chair I, University Hospital and Rudolf Virchow Center, Würzburg, Germany; ${ }^{2}$ Institute for Immunology and Transfusion Medicine, University Medicine Greifswald, Greifswald, Germany; ${ }^{3}$ Irish Centre for Vascular Biology, Royal College of Surgeons in Ireland, Dublin, Ireland; ${ }^{4}$ Center for Innovation Competence, Humoral Immune Reactions in Cardiovascular Diseases, University of Greifswald, Greifswald, Germany; ${ }^{5}$ German Centre for Cardiovascular Research e.V., Greifswald site, University Medicine Greifswald, Greifswald, Germany

Background: The contractile protein non-muscle myosin heavy chain IIA, encoded by the MYH9 gene, binds to filamentous actin and generates biomechanical forces. Heterozygous defects in this gene lead to different autosomal dominant syndromes in humans, which are characterized among others by macrothrombocytopenia and a mild to moderate bleeding tendency.

Aims: We hypothesized that reduced platelet force generation is responsible for the increased bleeding risk in MYH9 patients.

Methods: We analyzed three mouse lines each with one point mutation in the Myh9 gene at the positions 702,1424 , or 1841 , which have been described to recapitulate defects found in patients. We characterized the basic platelet function and tested the biophysical properties of the mutant platelets with atomic force spectroscopy and micropost arrays.

Results: Myh9 mutant mice displayed a macrothrombocytopenia, but only slightly altered glycoprotein expression. allb $\beta 3$ integrin activation and P-Selectin surface exposure of mutant platelets was overall comparable to controls. The capacity to assemble actin after activation was partially reduced in Myh9 mutant platelets, although the Gto $\mathrm{F}$-actin ratio was unaltered in resting platelets. Phosphorylation of the myosin light chain after activation with thrombin was strongly reduced. In line with this, biophysical analysis revealed that Myh9 mutant platelets generate lower adhesion forces to collagen, lower interaction forces between platelets and reduced traction forces when spread on fibrinogen-coated micropost arrays. Clot retraction of mutant samples was delayed, further reflecting less force generation of Myh9 mutant platelets. Finally, we observed more unstable thrombi, when blood of Myh9 mutant mice was perfused ex vivo over collagen fibers.

Conclusions: We show that Myh9 mutant platelets generate lower forces. These data suggest that reduced platelet-substrate and platelet-platelet forces lead to the increased bleeding tendency found in MYH9 patients. We are currently testing platelets from humans with MYH9 mutations to test whether they show the same changes as mouse platelets.
LPB0082 | Enrichment of Variants in Platelet Genes in Patients with Bleeding of Unknown Cause

M. Bowman ${ }^{1}$; J. Grabell ${ }^{1}$; A.D. Paterson ${ }^{2}$; M.-C. Poon ${ }^{3}$; S. Jackson $^{4,5}$; D. Lillicrap ${ }^{6}$; G. Haller ${ }^{7}$; B. Sadler ${ }^{8}$; P. James ${ }^{1}$ ${ }^{1}$ Department of Medicine, Queen's University, Kingston, Canada; ${ }^{2}$ Program in Genetics \& Genome Biology, The Hospital for Sick Children, Toronto, Canada; ${ }^{3}$ University of Calgary Cumming School of Medicine, Southern Alberta Rare Blood and Bleeding Disorders Comprehensive Care Program, Calgary, Canada; ${ }^{4}$ Division of Hematology, St. Paul's Hospital, Vancouver, Canada; ${ }^{5}$ Department of Medicine, University of British Columbia, Vancouver, Canada; ${ }^{6}$ Department of Pathology, Queen's University, Kingston, Canada; ${ }^{7}$ Department of Neurosurgery, Washington University School of Medicine, St. Louis, United States; ${ }^{8}$ Department of Pediatrics, Washington University School of Medicine, St. Louis, United States

Background: Despite extensive laboratory investigations, 50\% of patients seen in hematology clinics with a significant bleeding history remain undiagnosed. These patients are referred to as bleeders of unknown cause (BUC). Understanding the underlying pathogenesis would inform management.

Aims: To use whole exome sequencing (WES) to identify pathogenic variants associated with a significant bleeding history in BUC.

Methods: Adults ( $\geq 18$ years) referred to tertiary care hematology clinics for query bleeding disorder were recruited. Research Ethics Board approval and informed consent from all participants was obtained. Detailed hemostatic investigations were carried out including CBC, aPTT, PT, VWF levels, coagulation factor levels, and platelet function testing. Bleeding scores (BS) were obtained using the Condensed MCMDM-1VWD bleeding questionnaire. To prioritize sequencing, patients with a positive BS ( $\geq 4)$, with a positive family history of bleeding and a non-diagnostic work-up were sent for WES. To enrich for potentially causal variants, we compared the frequency of rare ( $\leq 1 \%$ in gnomAD) variants with a ClinVar assignment of pathogenic or likely pathogenic, and rare stop-gain, frameshift or splice-site variants in platelet genes from the ThromboGenomics panel (version 3) in cases versus controls.

Results: 540 patients were recruited and 86 met the criteria to have WES performed ( 81 females, 5 males; median age $=42$ years; range $18-73$ years). In 37 of the 86 patients, 39 rare variants were identified in 19 platelet genes. 34 were rare missense variants and five were potentially causal. The potentially causal variants, BS and platelet investigations for the five patients are shown in Table 1. When compared to control exomes ( $n=22,344$ ) this difference was statistically significant (Fisher's exact $P=0.02$, Odds Ratio $=1.94$ ). 
TABLE 1 Variants in platelet genes classified as pathogenic identified in five BUC

\begin{tabular}{|c|c|c|c|c|c|c|c|c|c|}
\hline Patient ID & $\begin{array}{l}\text { Bleeding } \\
\text { score }\end{array}$ & $\begin{array}{l}\text { Platelet } \\
\text { count } \\
\left(\times 10^{\wedge} 9 / \mathrm{L}\right)\end{array}$ & $\begin{array}{l}\text { Platelet } \\
\text { aggregation } \\
\text { studies }\end{array}$ & $\begin{array}{l}\text { Gene } \\
\text { symbol }\end{array}$ & Gene name & Associated disease & Inheritance & $\begin{array}{l}\text { Amino Acid } \\
\text { Change }\end{array}$ & Genotype \\
\hline GWC-101 & 4 & 264 & normal & HPS1 & $\begin{array}{l}\text { HPS1, biogenesis } \\
\text { of lysosomal } \\
\text { organelles } \\
\text { complex } 3 \\
\text { subunit } 1\end{array}$ & $\begin{array}{l}\text { Hermansky-Pudlak } \\
\text { syndrome }\end{array}$ & $A R$ & p.Pro324fs & Heterozygous \\
\hline GWK-023 & 13 & 177 & normal & ITGA2B & $\begin{array}{l}\text { integrin subunit } \\
\text { alpha } 2 b\end{array}$ & $\begin{array}{l}\text { Platelet-type } \\
\text { bleeding } \\
\text { disorder 16, } \\
\text { Glanzmann } \\
\text { thrombasthenia }\end{array}$ & $\mathrm{AD} / \mathrm{AR}$ & p.GIn852fs & Heterozygous \\
\hline GWK-195 & 4 & 170 & normal & ITGA2B & $\begin{array}{l}\text { integrin subunit } \\
\text { alpha } 2 b\end{array}$ & $\begin{array}{l}\text { Platelet-type } \\
\text { bleeding } \\
\text { disorder } 16, \\
\text { Glanzmann } \\
\text { thrombasthenia }\end{array}$ & $\mathrm{AD} / \mathrm{AR}$ & p.Arg889fs & Heterozygous \\
\hline GWK-182 & 6 & 146 & normal & MYH9 & $\begin{array}{l}\text { myosin heavy } \\
\text { chain } 9\end{array}$ & May-Hegglin & $A D$ & p.Glu1350D & Heterozygous \\
\hline GWC-063 & 9 & 209 & normal & NBEAL2 & neurobeachin like 2 & $\begin{array}{l}\text { Gray platelet } \\
\text { syndrome }\end{array}$ & AR & p.Arg2187fs & Heterozygous \\
\hline
\end{tabular}

Conclusions: In BUC, rare Clinvar pathogenic variants and rare frameshift variants in genes related to platelet function were identified. The clinical significance of these variants requires further investigation.

LPB0126 | Whole Blood Impedance Aggregometry: A Usefool Tool but Still Not the Gold Standard

S. Ellouze $^{1}$; C. Lavenu-Bombled ${ }^{2}$; A. Perrier-Cornet ${ }^{2}$; S. Combe ${ }^{2}$; A. Blandinieres ${ }^{2}$; T. Lambert ${ }^{3}$; R. D'Oiron ${ }^{3}$; V. Proulle ${ }^{1}$

${ }^{1}$ Service Hématologie Biologique, Hôpital Cochin, APHP, Université de Paris, Paris, France; ${ }^{2}$ Service Hématologie Biologique, Hôpital Bicêtre, APHP, Université Paris Saclay, Le Kremlin Bicêtre, France; ${ }^{3}$ Centre de Référence pour le Traitement des Maladies Hémorragiques Hôpital Bicêtre, APHP, Le Kremlin Bicêtre, France
Background: Light transmission aggregometry (LTA) is the gold standard to diagnose inherited platelet function disorders (IPFD) and von Willebrand disease type 2B (VWD2B). LTA is a time-consuming method requiring large blood volumes. In contrast, whole blood impedance aggregometry (WBIA) is a quicker and simpler method requiring limited volume of blood.

Aims: We evaluate the ability of WBIA to identify already diagnosed IPFD and VWD2B. We also compare the results of both methods in patients referred for unexplained bleeding tendency.

Methods: One hundred sixty patients were studied (Table 1). LTA was performed on platelet-rich plasma according to international recommendations (SSC/ISTH). WBIA was performed on hirudinanticoagulated blood using the Multiplate ${ }^{\circledR}$ analyzer (Roche) according to manufacturer's instructions. Normal ranges were calculated in 30 healthy individuals.

TABLE 1 Characteristic of the 160 patients studied. VWD: von Willebrand disease

\begin{tabular}{lllll} 
& $N=$ & Gendre (F/M) & Age (years \pm SD) & Platelet Count $\left(\times 10^{9} \mathrm{~L}^{-1} \pm \mathrm{SD}\right)$ \\
Glanzmann Thrombasthenia & 18 & $10 / 18$ & $31 \pm 17$ & $146 \pm 83$ \\
Bernand-Soulier Syndrome & 3 & $2 / 1$ & $24 \pm 3$ & $27 \pm 6$ \\
MYH9-related disease & 7 & $1 / 6$ & $21 \pm 18$ & $61 \pm 24$ \\
VWD type 2B & 14 & $2 / 5$ & $36 \pm 21$ & $146 \pm 83$ \\
Platelet-type VWD & 3 & $22 / 7$ & $35 \pm 20$ & $190 \pm 25$ \\
VWD & 29 & $1 / 0$ & $28 \pm 16$ & $295 \pm 188$ \\
Gray Platelet Syndrome & 1 & $0 / 1$ & 37 & 41 \\
Scott Syndrome & 1 & $0 / 1$ & 28 & 208 \\
Wiscott-Aldrich Syndrome & 1 & $62 / 21$ & 46 & 57 \\
Unexplained bleeding tendency & 83 & $15 / 15$ & $29 \pm 18$ & $234 \pm 90$ \\
Healthy individuals & 30 & & $39 \pm 14$ & $245 \pm 80$ \\
\hline
\end{tabular}


Results: WBIA correctly diagnosed all patients with Glanzmann Thrombasthenia, Bernard-Soulier Syndrome, platelet-type VWD as well as $13 / 14$ patients with VWD2B, whatever the platelet count. Thrombocytopenic patients with MYH9-related disease had subnormal results with all agonists. However, VWD patients were poorly detected (15/29, 51.7\%). Among 83 patients referred for unexplained bleeding tendency, WBIA and LTA gave concordant results in 48/83 (57.8\%) patients: 29 had normal results and 19 had abnormal results with one or more agonists using both methods. Discrepant results were observed in $35 / 83(42.1 \%)$ patients (Table 2 ).

TABLE 2 Agreement between WBIA and LTA in patients referred for unexplained bleeding tendency $(n=83)$. PPV: positive predictive value. NPV: negative predictive value

\section{LTA}

\begin{tabular}{llll} 
WBIA & Abnormal & Normal & PP \\
Abnormal & 19 & 30 & 0.38 \\
Normal & 5 & 29 & \\
\hline
\end{tabular}

$\begin{array}{llll}\text { PPV } & \text { NPV } & \text { Sensitivity } & \text { Specificity } \\ 0.38 & 0.85 & 0.79 & 0.49\end{array}$

Conclusions: Our study shows that WBIA $1 /$ is able to properly characterize patients with severe IPFD and VWD2B, including those with thrombocytopenia 2 / is less sensitive/specific in detecting mild platelet abnormalities compared to LTA. The negative predictive value of 0.85 suggests that it might be a useful quick and easy-to use screening test while it cannot avoid further testing by LTA in patients with unexplained bleeding tendency.

LPB0127 | Defective Platelet Endocytosis of Albumin in RUNX1 Haplodeficiency Associated with Altered Caveolin-dependent Albumin Trafficking in Megakaryocytic Cells

F. Del Carpio-Cano ${ }^{1}$; G. Mao ${ }^{1}$; MA. Alam ${ }^{1}$; J. Wurtzel ${ }^{2}$;

L. Goldfinger ${ }^{2}$; A.K. Rao ${ }^{1}$

${ }^{1}$ Sol Sherry Thrombosis Research Center, Lewis Katz School of Medicine at Temple University, Philadelphia, United States; ${ }^{2}$ Cardeza Foundation for Hematologic Research, Thomas Jefferson University, Philadelphia, United States

Background: Heterozygous germline RUNX1 mutations lead to thrombocytopenia and impaired platelet function and granule contents. Our previous studies in a patient with RUNX1 mutation showed that platelet albumin was decreased (Sun et al, Blood 103: 948-54, 2004). Human platelet a-granules contain numerous proteins, some synthesized (PF4 and VWF) and others incorporated by endocytosis (fibrinogen, albumin and IgG) by megakaryocytes (MK). Aims: To understand the mechanisms leading to decreased platelet albumin and granule defects in RUNX1 haplodeficiency (RHD).

Methods: We studied endocytosis of fluorescent-labeled albumin, fibrinogen and IgG in platelet suspensions (0-90 min) and in PMAtreated megakaryocytic HEL cells (up to 24 hours) using flow cytometry and immunofluorescence microscopy. We studied alterations in caveolin-1.

Results: In platelets, protein uptake was time- and concentrationdependent. Uptake of albumin, fibrinogen and IgG was decreased in two patients (father and daughter) with RHD (c.352-1 G>T) (mean fluorescent intensity $<50 \%$ of normal). In HEL cells, uptake of albumin and fibrinogen was time- and concentration-dependent. On
siRNA RUNX1 knockdown, uptake/retention of albumin and fibrinogen over 24 hrs was higher than in control siRNA cells. Caveolin-1 and flotillin-1, two caveolae-associated membrane proteins linked to endocytosis, were increased on immunoblotting. Caveolin-1 downregulation (siRNA) in RUNX1-deficient HEL cells abrogated the increase in uptake of albumin, but not fibrinogen. On immunofluorescence microscopy staining of immobilized HEL cells albumin (but not fibrinogen) colocalized with CAV-1 in wild type cells at $30 \mathrm{~min}$; this colocalization was disrupted on RUNX1 knockdown.

Conclusions: Platelets with RUNX1 haplodeficiency have defective endocytosis of albumin, fibrinogen and IgG. With RUNX1 knockdown, in MK cells there is caveolin-1 upregulation, increased albumin uptake and defective caveolin-dependent trafficking of albumin. These studies provide insights into diverse mechanisms leading to a-granule abnormalities and handling of proteins by platelets and megakaryocytes in RHD. (Supported by Grants HL-109568, HL137376, HL137207).

PB0891 | Noonan Syndrome Bleeding Diathesis: Coagulation Factor Deficiencies and/or Platelet Function Disorders?

M. Daniel ${ }^{1}$; J.-C. Bordet ${ }^{1}$; S. Girard ${ }^{1}$; A. Putoux ${ }^{2}$; S. Le Quellec ${ }^{2}$

${ }^{1}$ Hospices Civils de Lyon, Lyon, France; ${ }^{2}$ University Claude Bernard Lyon I, Lyon, France

Background: Noonan Syndrome (NS) is a rare genetic disorder characterized by several morphological anomalies, and bleeding diathesis for which causes remain unclear.

Aims: We aimed to characterize the bleeding phenotype of patients with NS using coagulation and platelet functions assays.

Methods: In our center, 26 patients with NS, irrespective of their genotype, were screened for bleeding risk. Bleeding phenotype was scored using the ISTH Bleeding assessment tool. Prothrombin time, activated partial thromboplastin time, as well as coagulation factors including factor II, V, VII, X, VIII, IX, XI, and von Willebrand factor were measured. Platelet count, morphology, and function were extensively assessed. Light-transmission on blood smear, and transmission electron microscopy (TEM) on whole mount and ultrathin 
section of platelets, were performed. Platelet activation in response to various platelets agonists was studied using light-transmission aggregometry (LTA). In addition, platelet surface glycoprotein, CD62P, PAC1, and fibrinogen binding expressions were measured using flow cytometry analysis (FCM).

Results:
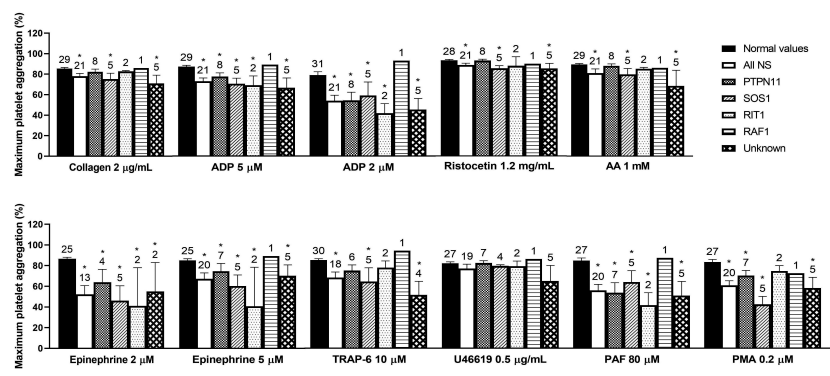

FIGURE 1 Maximum platelet aggregation after platelet activation by various agonists using LTA in patients with NS compared to normal values. ${ }^{*} \mathrm{p}$

8/26 (31\%) patients had significant hemorrhagic diathesis, with cutaneous and/or mucosal bleedings. 6/26 (23\%) patients had up to 3 factor levels below the normal laboratory values, but above the corresponding hemostatic threshold. Platelet count, blood smear, glycoprotein expression, and whole-mount TEM were normal for all patients. However, TEM studying ultrathin section of platelets showed elongated platelets with reduced alphatocrite in 11/26 (65\%) patients. All patients with NS presented a lower maximum aggregation intensity compared to normal values (Figure 1), and a lower CD62P, PAC1 and fibrinogen binding expressions, irrespective of the agonist used for platelet activation. The most frequent and the more pronounced defects were observed after TRAP, and ADP-induced platelet activation. Conclusions: Platelet function defects were the most frequent disorders observed in patients with NS. Therefore, we recommend platelet functions to be screened systematically in such patients to adapt the best therapeutic strategy for preventing bleeds during surgical procedures.

\section{PB0892 | Platelet Dysfunction in Noonan Syndrome}

S. Sorrentino $^{1}$; I. Lazzareschi ${ }^{2,3} ;$ M. Capurso ${ }^{2} ;$ R. Onesimo ${ }^{2} ;$ C. Leone $^{2}$; A. Romano ${ }^{2}$; M. Mele ${ }^{2}$; G. Zampino ${ }^{2,3}$; E. De Candia ${ }^{1}$

${ }^{1}$ Unità Malattie Emorragiche e Trombotiche, Fondazione Policlinico Universitario Agostino Gemelli IRCSS, Rome, Italy; ${ }^{2}$ UOC Pediatria, Fondazione Policlinico Universitario Agostino Gemelli IRCSS, Rome, Italy; ${ }^{3}$ Dipartimento di Scienze della Vita e della Sanità Pubblica, Università Cattolica del Sacro Cuore, Rome, Italy

Background: Noonan Syndrome (NS) is an autosomal dominant genetic disorder with multiple anomalies, including bleeding diathesis.
Although the bleeding phenotype is mild, surgical management is often required including procedures with high bleeding risk. CBC, $\mathrm{PT}$, aPTT and $\mathrm{F} \mathrm{XI}$ are recommended at diagnosis, however platelet function abnormalities were rarely reported in these patients.

Aims: To charachterize hemostatic and platelet function abnormalities in NS patients.

Methods: PT, aPTT were determined with IL Werfen automated coagulation analyzer. ISTH-BAT was administered to patients. Platelet function was investigated using light transmission aggregometry (LTA) and PRP stimulated by ADP, collagen, epinephrine, PAR1 activating peptide (AP). Maximal aggregation (MA, \%) for all agonist and lag phase (LP, seconds) for collagen were measured. Platelet secretion was investigated by flow cytometry using MoAbs directed against CD63 and CD62p on ADP and PAR1-AP stimulated platelets. Mean fluorescence intensity (MFI) was measured.

Results:
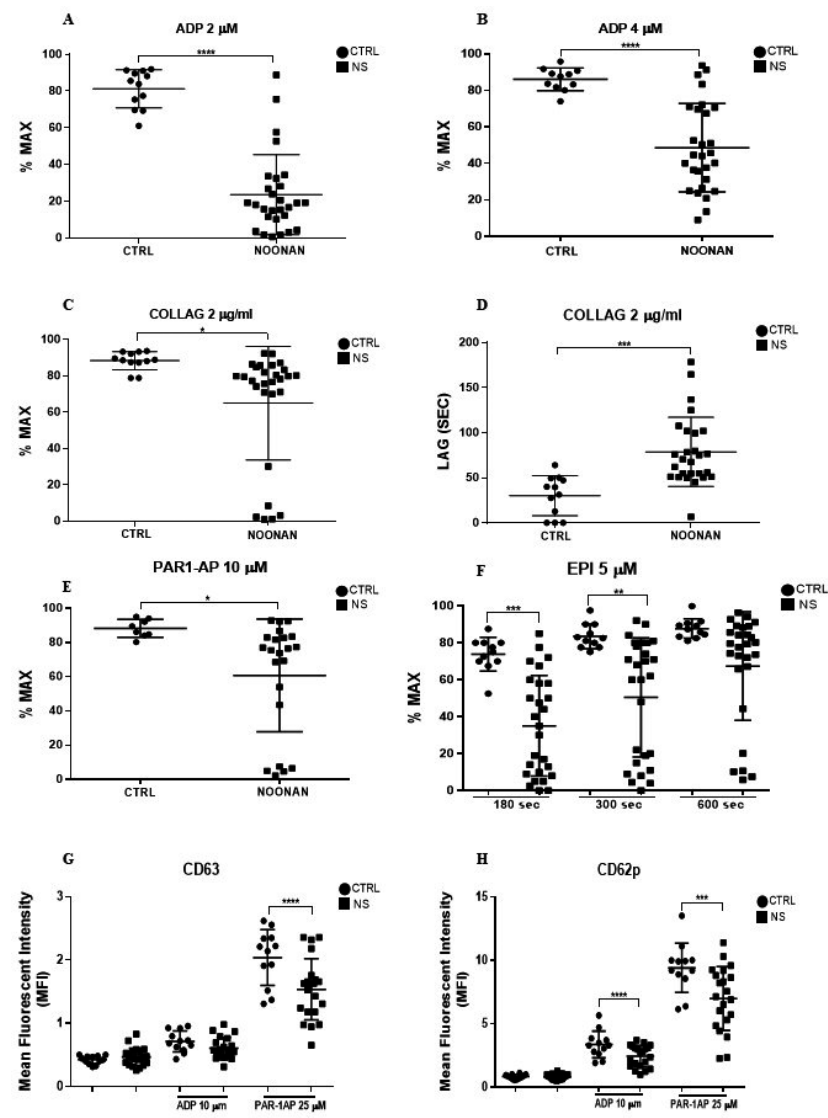

FIGURE 1 A-F: Maximal aggregation (MA \%) to ADP $2 \mu \mathrm{M}$, ADP 4 $\mu \mathrm{M}$, collagen $2 \mu \mathrm{g} / \mathrm{ml}$, epinephrine $5 \mu \mathrm{M}$, PAR1-AP $10 \mu \mathrm{M}$ in patients with Noonan syndrome and controls. G-H: Flow cytometry analysis for of platelet secretion markers (CD63 and CD62p) after stimulation with ADP $10 \mu \mathrm{M}$ and PAR1-AP $10 \mu \mathrm{M}$. A-E: One-Way ANOVA, followed by Bonferroni's test, was used to determinate significant differences. F-H: A two-tailed t-test was used to determinate significant differences. ${ }^{* * * *} P<0.0001 ;{ }^{* * *} P<0.001 ;{ }^{* *}$ $P<0.01$; $^{*} P<0.05$. 
TABLE 1 General characteristics of the study population, ISTH-BAT bleeding score, coagulation tests and genetic testing.

\begin{tabular}{|c|c|c|}
\hline & Patients with NS ( $n=24)$ & Controls $(n=14)$ \\
\hline Male/Female & $11 / 13$ & $1 / 11$ \\
\hline Age at bood sampling (median and range) & $13.9(0-34)$ & $1(0-11) ; 33(12-55)$ \\
\hline Bleeding Score (median and range) age $0-11$ yrs & $0.5(0-8)$ & NA \\
\hline Bleeding Score (median and range) age 12-34 yrs & $3(0-14)$ & NA \\
\hline Platelet count (mean and range ${ }^{*} 10^{\wedge} 3$ ) & $224(148-316)$ & $216(147-262)$ \\
\hline Abnormal PT, $\mathrm{n}(\%)$ & $1(4,16 \%)$ & 0 \\
\hline Prolonged aPT, $n(\%)$ & $7(29,16 \%)$ & 0 \\
\hline Abnormal fibrinogen levels, $n(\%)$ & $1(4,16 \%)$ & $1(7,14 \%)$ \\
\hline \multicolumn{3}{|c|}{ NS gene mutation, $n(\%)$} \\
\hline PTNPN11 & $18(75 \%)$ & 1 \\
\hline sos1 & $2(8,33 \%)$ & 1 \\
\hline KRAS & $1(4,16 \%)$ & 1 \\
\hline SHOC & $1(4,16 \%)$ & 1 \\
\hline Not found & $2(8,33 \%)$ & 1 \\
\hline
\end{tabular}

The study population included 24 patients and 14 controls. Platelet count, PT and fibriongen were not significantly different in the 2 groups, $30 \%$ of NS had aPTT prolongation (Table 1). Noonan patients had significant reduction of platelet aggregation with ADP $2 \mu \mathrm{M}$ (91.6\% with MA <60\%), ADP $4 \mu \mathrm{M}$ (79.1\% with MA<60\%), collagen 2 $\mu \mathrm{g} / \mathrm{ml}(41.6 \%$ with $\mathrm{MA}<75 \%, 87 \%$ with $\mathrm{LP}>50 \mathrm{sec})$, epinephrine $5 \mu \mathrm{M}$ (87\% with $\mathrm{MA}<60 \%$ at $180 \mathrm{sec}, 62.5 \%$ with MA $<70 \%$ at $300 \mathrm{sec}$ ), PAR1-AP (45.8\% with $M A<75 \%)$. A significant reduced expression of activation-dependent markers CD63 and CD62p was found after PAR1-AP stimulation, and of CD62p after ADP stimulation. (Fig.1).

Conclusions: In the present cohort, large majority (>90\%) of Noonan pts showed platelet dysfunction, whereas their platelet count was normal and only $30 \%$ of NS had aPTT prolongation. Platelet secretion defect is suggested by activation-dependent CD63 and CD62p marker exposure. Platelet dysfunction is the most frequent hemostasis abnormality in NS.

PB0893 | The Glanzmann's Thrombasthenia Registry (GTR): Safety of Platelet Therapy in Patients with Glanzmann's Thrombasthenia, with a Focus on Changes in alloimmunization Status

M.-C.Poon ${ }^{1,2}$; R. d'Oiron ${ }^{3}$; S. Baby ${ }^{4}$; R.B Zotz ${ }^{5,6}$; G. Di Minno ${ }^{1}$ Departments of Medicine, Pediatrics and Oncology, University of Calgary, Calgary, Alberta, Canada; ${ }^{2}$ Southern Alberta Rare Blood and Bleeding Disorders Comprehensive Care Program, Foothills Medical Centre, Calgary, Alberta, Canada; ${ }^{3}$ Centre de Référence de l'Hémophilie et des Maladies Hémoragiques Constitutionnelles, Hôpital Bicêtre, APHP, Université Paris Saclay, Le Kremlin-Bicêtre, France; ${ }^{4}$ Biostatistics \& Programming GD GBS, Novo Nordisk Service Centre India Private Ltd, Bangalore, India; ${ }^{5}$ Institute for Laboratory Medicine, Blood Coagulation and Transfusion Medicine (LBT), Düsseldorf, Germany; ${ }^{6}$ Department of Hemostasis, Hemotherapy and Transfusion Medicine, Heinrich Heine University Medical Centre, Düsseldorf, Germany; ${ }^{7}$ Department of Clinical Medicine and Surgery, Regional Reference Center for Coagulation Disorders, Federico II University, Naples, Italy

Background: Glanzmann's Thrombasthenia (GT) is a rare, inherited, autosomal-recessive platelet disorder resulting in defective glycoprotein (GP) Ilb-IIla. Treatment outcome and platelet transfusion safety data are limited due to the rarity of GT. The international, prospective, observational GT registry (GTR; NCT01476423), which assessed the effectiveness and safety of recombinant activated factor FVII ( $\mathrm{rFVIIa)} \mathrm{in} \mathrm{GT,} \mathrm{recorded} \mathrm{treatment} \mathrm{data} \mathrm{including} \mathrm{rFVIla} \mathrm{and}$ platelet transfusion from 218 patients (10 May 2007-16 December 2011). Data presented here do not affect the GTR primary analysis results.

Aims: Describe GTR sub-analysis results evaluating platelet-based therapy safety in GT patients.

Methods: This sub-analysis identified GTR patients with a recorded change in anti-platelet antibody and platelet refractoriness status (alloimmunization) following treatment including rFVIla, platelets and antifibrinolytics alone or in combination, and adverse events (AEs) following platelet-based therapies. No statistical comparisons were conducted.

Results: $34 / 218$ (16\%) patients had $\geq 1$ instance of platelet refractoriness. Platelet antibodies were reported in 65/218 (30\%) patients. Alloimmunization status change was recorded in $5 / 218$ patients (Table 1). One patient experienced an antibody status change. Four patients experienced a platelet-refractoriness status change: one in whom rFVIla-based treatments were recorded as only partially effective, and another with GPIIb-IIla antibodies. In the 5 patients, platelet-based regimens were used in the majority of admissions, regardless of the presence/absence of antibodies/refractoriness. The majority of admission records were incomplete (Table 1). In 581 admissions, there were $24 \mathrm{AEs}$ in 10 patients who received platelets alone, platelets and other hemostatics or other hemostatics alone; one AE was serious (generalized hives) (Table 2). 
TABLE 1 Demographics of GTR patients with a recorded change in anti-platelet antibody and platelet refractoriness status (alloimmunization) following treatment including rFVIla, platelets and antifibrinolytics alone or in combination

\begin{tabular}{|c|c|c|c|c|c|c|c|}
\hline Patient & Sex & Age at admission & $\begin{array}{l}\text { Previous } \\
\text { treatments }\end{array}$ & Antibody status & $\begin{array}{l}\text { Platelet refractory } \\
\text { status }\end{array}$ & $\begin{array}{l}\text { Treatment } \\
\text { administered }\end{array}$ & $\begin{array}{l}\text { Effectiveness to stop } \\
\text { bleeding }\end{array}$ \\
\hline \multirow[t]{3}{*}{1} & $M$ & 47 & Single & HLA - & NA & N7POH & Effective \\
\hline & & 56 & platelets, HLA & HLA + & NA & $\mathrm{P}$ & NA \\
\hline & & 57 & & HLA + & NA & $\mathrm{POH}$ & NA \\
\hline \multirow[t]{5}{*}{2} & M & 2 & Standard plate- & NA & Negative & $\mathrm{N7OH}$ & Effective \\
\hline & & 2 & lets, Single donor & NA & Negative & N7POH & Effective \\
\hline & & 2 & platelets & NA & Positive & $\mathrm{N7OH}$ & Effective \\
\hline & & 3 & & NA & Positive & $\mathrm{N7OH}$ & Effective \\
\hline & & 3 & & GPIIb/IIIa + & Positive & $\mathrm{N7OH}$ & Effective \\
\hline \multirow[t]{15}{*}{3} & $M$ & 3.3 & Standard plate- & NA & Negative & $\mathrm{POH}$ & NA \\
\hline & & 3.3 & RBCs, & NA & Negative & $\mathrm{P}$ & NA \\
\hline & & 3.8 & antifibrinolytic & NA & Negative & $\mathrm{OH}$ & NA \\
\hline & & 7.1 & & NA & Negative & $\mathrm{P}$ & NA \\
\hline & & 8.5 & & NA & Negative & $\mathrm{P}$ & NA \\
\hline & & 8.6 & & NA & Negative & N7 & Effective \\
\hline & & 9 & & NA & Positive & $\mathrm{N7OH}$ & NA \\
\hline & & 9 & & NA & Positive & N7POH & NA \\
\hline & & 10 & & NA & Positive & N7POH & Partially effective \\
\hline & & 10 & & NA & Positive & N7 & Partially effective \\
\hline & & 10 & & NA & Positive & $\mathrm{N7OH}$ & Partially effective \\
\hline & & 10 & & NA & Positive & N7 & Partially effective \\
\hline & & 10.3 & & NA & NA & NA & Partially effective \\
\hline & & 11 & & NA & NA & NA & Partially effective \\
\hline & & 11 & & NA & NA & NA & Effective \\
\hline \multirow[t]{3}{*}{4} & $M$ & 0.9 & Standard plate- & NA & Negative & $\mathrm{OH}$ & NA \\
\hline & & 1 & lets, RBCs & NA & Negative & $\mathrm{POH}$ & NA \\
\hline & & 1 & & NA & Positive & $\mathrm{POH}$ & NA \\
\hline \multirow[t]{21}{*}{5} & $\mathrm{~F}^{*}$ & 5 & Standard plate- & NA & Positive & $\mathrm{POH}$ & NA \\
\hline & & 5 & lets, $\quad$ RBCs, & NA & NA & NA & NA \\
\hline & & 5 & NovoSeven ${ }^{\circledR}$ & NA & NA & NA & NA \\
\hline & & 5 & & NA & NA & NA & NA \\
\hline & & 6 & & NA & NA & NA & NA \\
\hline & & 6 & & NA & Positive & $\mathrm{POH}$ & NA \\
\hline & & 6 & & NA & Positive & $\mathrm{POH}$ & NA \\
\hline & & 6 & & NA & Positive & $\mathrm{POH}$ & NA \\
\hline & & 6 & & NA & Positive & $\mathrm{P}$ & NA \\
\hline & & 6 & & NA & Positive & $\mathrm{POH}$ & NA \\
\hline & & 6 & & NA & Positive & $\mathrm{POH}$ & NA \\
\hline & & 6 & & NA & Positive & $\mathrm{POH}$ & NA \\
\hline & & 6 & & NA & Positive & N7POH & NA \\
\hline & & 6 & & NA & Positive & $\mathrm{POH}$ & NA \\
\hline & & 6 & & NA & Positive & $\mathrm{POH}$ & NA \\
\hline & & 6 & & NA & Positive & $\mathrm{POH}$ & NA \\
\hline & & 6 & & NA & Positive & $\mathrm{POH}$ & NA \\
\hline & & 6 & & NA & Positive & $\mathrm{POH}$ & NA \\
\hline & & 6 & & NA & Positive & $\mathrm{POH}$ & NA \\
\hline & & 6 & & NA & Positive & $\mathrm{POH}$ & NA \\
\hline & & 6 & & NA & Positive & $\mathrm{POH}$ & NA \\
\hline
\end{tabular}




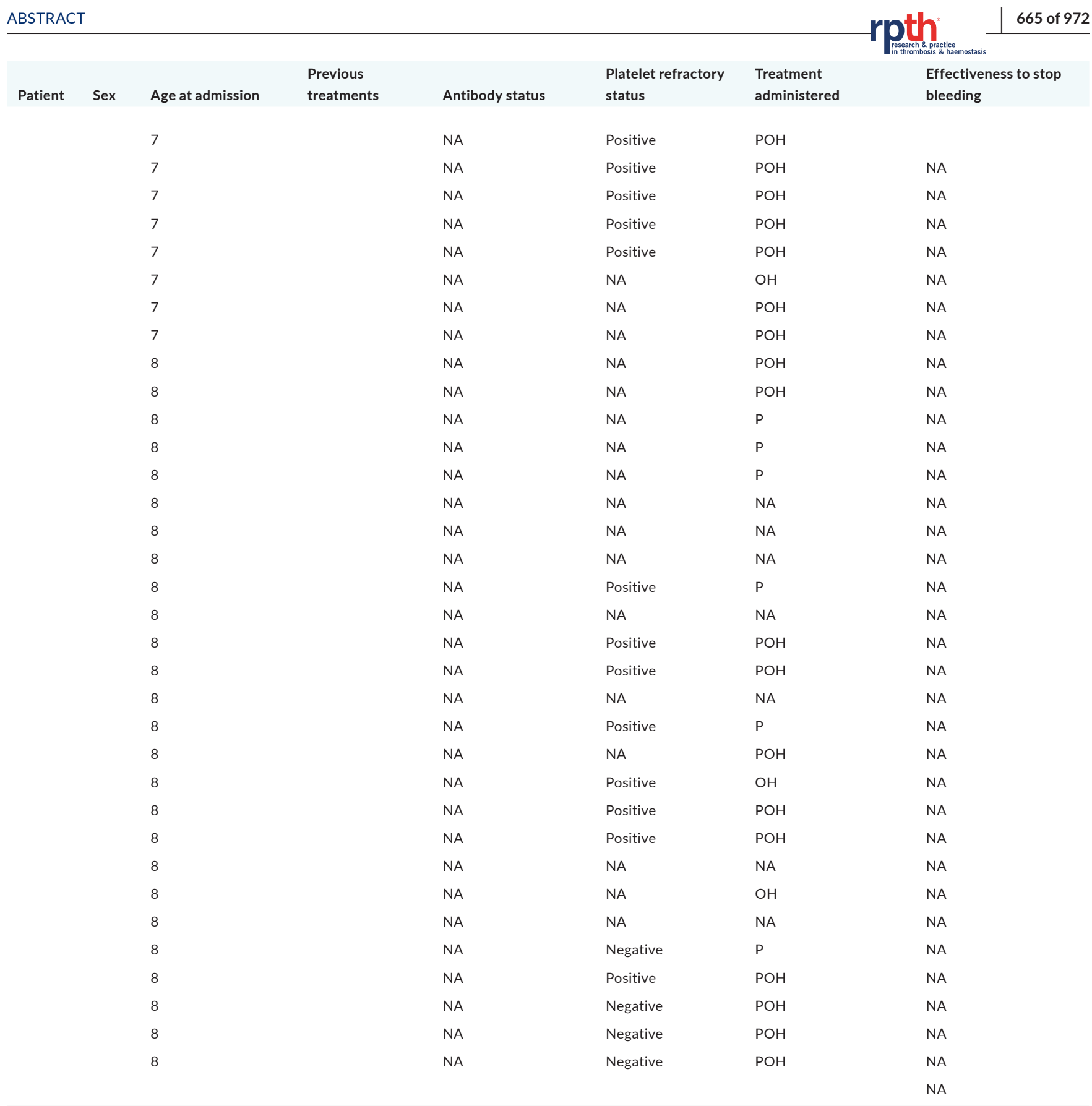

*Patient was not pregnant.

GPIIb/IIla, glycoprotein IIb/IIla; GPIIb/IIla +, GPIIb/IIla positive; GTR, Glanzmann's Thrombasthenia Registry; HLA, human leukocyte antigen; HLA -, HLA negative; HLA ,+ HLA positive; N7, NovoSeven ${ }^{\circledR}$; N7OH, NovoSeven ${ }^{\circledR}$ and other hemostatic treatment; N7POH, NovoSeven ${ }^{\circledR}$, platelets and other hemostatic treatment; NA, not available; $\mathrm{OH}$, other hemostatic treatment (mostly antifibrinolytics); P, platelets; POH; platelets and other hemostatic treatment; RBCs, red blood cells; rFVIla, recombinant activated factor VII (NovoSeven ${ }^{\circledR}$ ) 
TABLE 2 Adverse events occurring during admissions of patients in the GTR who were not treated with rFVIla either for a bleeding episode or for treatment related to surgery

\begin{tabular}{clll} 
& $\begin{array}{l}\text { Number of } \\
\text { patients }\end{array}$ & $\begin{array}{l}\text { Number of } \\
\text { admissions }\end{array}$ & $\begin{array}{l}\text { Number of } \\
\text { adverse events }\end{array}$ \\
152 & 581 & 24 \\
$\begin{array}{c}\text { Adverse } \\
\text { events, } \mathrm{n}\end{array}$ & $10^{*}$ & 16 & $1^{\dagger}$ \\
$\begin{array}{c}\text { Serious } \\
\text { adverse } \\
\text { events, } \mathrm{n}\end{array}$ & 1 & 1 & \\
$\begin{array}{c}\text { Non-serious } \\
\text { adverse } \\
\text { events, } \mathrm{n}\end{array}$ & 10 & 15 & 23 \\
\hline
\end{tabular}

*One patient experienced non-serious adverse events and a serious adverse event resulting in a total of 10 patients reporting adverse events.

${ }^{\dagger}$ Generalized hives; treatment related to a bleeding episode.

GTR, Glanzmann's Thrombasthenia Registry; rFVIla, recombinant activated factor VII (NovoSeven $\left.{ }^{\circledR}\right)$

Conclusions: Platelet transfusions may lead to AEs and treatment failure due to platelet refractoriness. However, in GT patients, routine platelet antibody testing is often not available due to the specialized nature of testing.

PB0894 | Modelling Studies to Characterize a Novel Diseasecausing Variant in the GP1BA Gene Related to Bernard Soulier Syndrome

D.M Primrose ${ }^{1}$; A.I. Woods ${ }^{2}$; M.F Alberto ${ }^{3}$; J. Paiva ${ }^{3}$; M. Asencio ${ }^{3}$; M.M Casinelli ${ }^{3}$; A.N Blanco ${ }^{3}$; A. Sánchez-Luceros ${ }^{3,2}$

${ }^{1}$ Higher School of Engineering, Informatics and Agri-food Sciences, University of Morón, Morón City, Argentina; ${ }^{2}$ Laboratory of Hemostasis and Thrombosis, IMEX-CONICET-National Academy of Medicine. Buenos Aires City, Argentina, Ciudad Autonoma de Buenos Aires, Argentina; ${ }^{3}$ Departament of Hemostasis and Thrombosis, Hematological Research Institute, National Academy of Medicine, Ciudad Autonoma de Buenos Aires, Argentina

Background: We described a novel disease-causing variant (DCV) p.Tyr231Cys in the GP1BA gene related to recessive BSS in a patient with no bleeding symptoms and 50\% CD42b-expression. Homology modelling was used to describe the effects of this DCV on proteinprotein interaction.

Aims: To study effect of the GPIba-p.Tyr231Cys on the interaction of GPIba with VWF-A1 domain by homology modelling.

Methods: The model GPIba-p.Tyr231Cys was made by replacing Tyr231 by Cys in wild-type-GPIba (Swiss-PDBviewer). Docking between VWF-A1 and GPIba-p.Tyr231Cys was obtained (PatchDock) and compared to WT-GPIba-VWF-A1. Hydrogen-bonds and rootmean-square deviation (RMSD) between $a$-carbon chains were obtained (UCSF Chimera).
Results: GPIba-Tyr231 is found in the a2-helix, nearby two disulphide-bonds: C225-C264; C227-C280. No new disulphidebonds are predicted in GPIba-p.Tyr231Cys. GPIba-Tyr231 forms intramolecular hydrogen-bonds with Phe208, Trp235 and Glu228.In p.Cys231Tyr, hydrogen-bond with Phe208 is lost and a new bond is formed with lle203. The bond with Trp235 is maintained and is still present with Glu228 but at a larger distance, consequently, weaker. In silico-model of wild-type-GPIba-VWF-A1 (Fig-1A) shows differences with GPIba-p.Tyr231Cys-VWF-A1(Fig-1B). Total RMSD between models is $38.05 \AA$ ( $0.65 \AA$ between GPIba portions; $15.07 \AA$ between VWF-A1 domains) (Fig-1C). The change Tyr231Cys causes a slight alteration in the conformation of GPIba ( $0.65 \AA$ ) respecting wild-type-GPIba. This change does not affect neighboring residues but alters the structure of GPIba at the site that acts as a hinge, increasing the distance of the b-switch region of GPIba (flexible loop at Val243-Ser257). This modifies the interaction with VWF-A1, changing hydrogen-bonds between these molecules (Table 1).

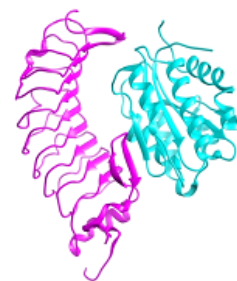

B

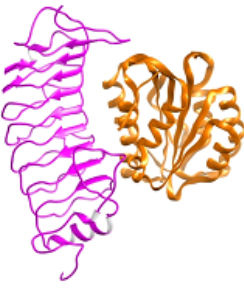

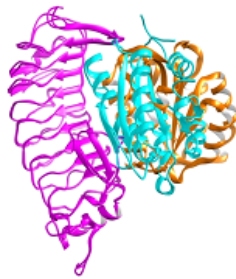

FIGURE 1 Hypothetical model of GPIba with the A1 domain of VWF. A) A1 (cian) with wild-type GPIba (magenta); B) A1 (orange) with mutated GPIba-p.Tyr231Cys; C) overlap of both models 
TABLE 1 Predicted hydrogen-bonds distance between wild-type-GPIba and the VWF-A1 domain and between GPIba-p.Tyr231Cys and the VWF-A1 domain

\begin{tabular}{|c|c|c|c|}
\hline Wild-type-GPIba & Residue on GPIba & Residue on VWF-A1 domain & H-bond Distance/Å \\
\hline \multirow[t]{9}{*}{ GPIba-Tyr231Cys } & Lys253 & Ala1327 & 3.0 \\
\hline & & & 3.2 \\
\hline & Met255 & Ser1325 & 3.0 \\
\hline & & & 2.9 \\
\hline & $\mathrm{G} \ln 143$ & Lys1371 & 1.7 \\
\hline & & Ile1368 & 3.6 \\
\hline & Glu241 & His1322 & 3.2 \\
\hline & Asp251 & Arg1336 & 3.4 \\
\hline & & Ser1338 & 3.2 \\
\hline
\end{tabular}

Conclusions: In-silico predictions show that GP1BA-p.Tyr231Cys modifies the tertiary structure of GPIba affecting the interaction with VWF-A1, explaining its negative effect that influences in both platelet count and size and in 50\% expression of GPIb as observed in our patient. The change Tyr231Cys could be preventing the conformational change of the $b$-switch from compact to extended thus altering the VWF-GPIba binding.

\section{PB0895 | Light Transmission Aggregometry (LTA) on TA-8V $\left(\right.$ Stago $\left.{ }^{\circledR}\right)$ : What about Normal Values?}

A. Dericquebourg ${ }^{1}$; M. Daniel ${ }^{2}$; J.-C. Bordet ${ }^{1}$; F. Sobas ${ }^{1}$; C.

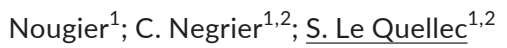

${ }^{1}$ Service d'Hématologie Biologique, Centre de Biologie et Pathologie Est, Hospices Civils de Lyon, Lyon, France, ${ }^{2}$ Unité d'Hémostase Clinique, Hôpital Cardiologique Louis Pradel, Hospices Civils de Lyon, Lyon,

France

Background: Diagnosis of platelet function disorders relies on lighttransmission aggregometry (LTA) using a panel of agonists. Hence, assessment of normal values and laboratory performances of the reagents used are critical for clinical practice.
Aims: The aim was to evaluate the performances of collagen, ADP, arachidonic acid, TRAP-6, and ristocetin reagents (Stago ${ }^{\circledR}$, Asnièressur-Seine, France) using LTA method in our laboratory.

Methods: LTA was performed on TA-8V aggregometer (SD-Medical, Stago ${ }^{\circledR}$ ) on citrated platelet-rich plasma (PRP) obtained from 30 consenting healthy volunteers (NCT04269603), using 5 different agonists at several final concentrations: $2 \mu \mathrm{g} / \mathrm{mL}$ collagen, 2 and $5 \mu \mathrm{M}$ ADP , $1 \mathrm{mM}$ arachidonic acid, 10 $\mu \mathrm{M}$ TRAP- 6 and $1.2 \mathrm{mg} / \mathrm{mL}$ ristocetin.

Normal values, intra-assay variability (CVintra, \%) and inter-operator variability for maximal aggregation intensity (Imax, \%) were measured after platelet activation by all agonists. Reagent stability was tested over a period of 2 months after reconstitution, sampling, and storage prior to use.

Results: Normal values for Imax ranged from $>30 \%$ for $2 \mu \mathrm{M}$ ADP- to $>80 \%$ for arachidonic acid- and ristocetin-induced platelet activation. Intra-assay variability was good, being below $8 \%$ in all conditions for platelet activation (range 2.7-7.8\%). These results were in line with manufacturer's normal values (when available; table1). Inter-operator variability showed an agreement of $>80 \%$ (range 84.6-100\%) for all agonists tested, with regards to Imax values, and reached $100 \%$ when aggregation curves were examined by a blinded expert biologist. All reagents were stable for 2 months.

TABLE 1 Maximum aggregation intensity (Imax) normal values and intra-assay variability (CV) of various agonist-induced platelet activation using TA-8V light-transmission aggregometer

\begin{tabular}{|c|c|c|c|c|c|c|}
\hline Agonist & \multicolumn{2}{|c|}{ Imax normal values, \% } & \multicolumn{2}{|l|}{ CVintra, \% } & \multicolumn{2}{|c|}{$\begin{array}{l}\text { Stability after reconstitution } \\
\text { and sampling, months (storage } \\
\text { temperature) }\end{array}$} \\
\hline Collagen $2 \mu \mathrm{g} / \mathrm{mL}$ & $>66$ & $>73$ & 5.8 & 2.7 & ND & $2\left(2-8^{\circ} \mathrm{C}\right)$ \\
\hline ADP $2 \mu \mathrm{M}$ & $>47$ & $>30$ & 6.4 & 7.8 & ND & $2\left(-20^{\circ} \mathrm{C}\right)$ \\
\hline Arachidonic acid $1 \mathrm{mM}$ & $>68$ & $>80$ & 3.8 & 4.2 & ND & $2\left(-20^{\circ} \mathrm{C}\right)$ \\
\hline TRAP-6 $10 \mu \mathrm{M}$ & $>69$ & $>66$ & 4.2 & 5.7 & ND & $2\left(-20^{\circ} \mathrm{C}\right)$ \\
\hline Ristocetin $1.2 \mathrm{mg} / \mathrm{ml}$ & ND & $>80$ & ND & 2.5 & ND & $2\left(-20^{\circ} \mathrm{C}\right)$ \\
\hline
\end{tabular}

ND: non determined. 
Conclusions: We provided normal range values and a study design for the assessment of laboratory performance of LTA method using collagen-, ADP-, arachidonic acid-, TRAP-6-, and ristocetin-agonists for platelet activation. The low CVintra, the good agreement between operators, and the 2-months period stability for all reagents tested, make them suitable for routine screening of platelet function disorders.

PB0896 | A Novel Hemizygous Variant in GATA1 Associated with Bleeding Diathesis and Platelet Dysfunction in Two Unrelated Patients

J.M. Bastida ${ }^{1,2,3,4}$; D. Boeckelmann ${ }^{5,6}$; V. Palma-Barqueros ${ }^{7,8}$; M. Wolter ${ }^{5,6}$; M.L Lozano ${ }^{7,8}$; H. Glonnegger ${ }^{5,6}$; R. Benito ${ }^{2,3}$; F.H Schilling $^{9}$; N. Morgan ${ }^{10}$; K. Freson ${ }^{11}$; J. Rivera ${ }^{7,8,4}$; B. Zieger ${ }^{5,6}$ ${ }^{1}$ Complejo Asistencial Universitario de Salamanca (CAUSA), Salamanca, Spain; ${ }^{2}$ Instituto de Investigación Biomédica de Salamanca (IBSAL), Salamanca, Spain; ${ }^{3}$ Universidad de Salamanca (USAL), Salamanca, Spain; ${ }^{4}$ Grupo Español de Alteraciones Plaquetarias Congénitas (GEAPC), SETH, Spain; ${ }^{5}$ Division of Pediatric Hematology and Oncology Medical Center, Freiburg, Germany; ' University of Freiburg, Faculty of Medicine, Freiburg, Germany; ${ }^{7}$ Hospital Universitario Morales Meseguer, Centro Regional de Hemodonación, Murcia,
Spain; ${ }^{8}$ Universidad de Murcia, IMIB-Arrixaca, CIBERER-U765, Murcia, Spain; ${ }^{9}$ Children's Hospital, Kantonsspital Luzern, Lucerne, Switzerland; ${ }^{10}$ Institute of Cardiovascular Sciences, College of Medical and Dental Sciences, University of Birmingham, Birmingham, United Kingdom; ${ }^{11}$ Center for Molecular and Vascular Biology, Department of Cardiovascular Sciences, University of Leuven, Leuven, Belgium

Background: The GATA1 transcription factor is essential for normal erythropoiesis and megakaryocytic development. Germline GATA1 pathogenic variants (PV) are typically associated with X-linked thrombocytopenia, dyserythropoietic anemia and/or platelet dysfunction. Phenotype/genotype correlation is important to better understand the diversity of rare GATA1 PV.

Aims: To characterize bleeding and platelet phenotype in two patients with a novel GATA1 variant identified by high throughput sequencing (HTS)

Methods: Two male index patients, (G1, 14 y; S2, 46 y), who presented with lifelong bleeding tendency have been investigated. Bleeding score (BS) was recorded with ISTH-BAT. Platelet phenotyping included full blood count (FBC), aggregometry (LTA), flow cytometry (FC), and electron microscopy (EM). Molecular analysis was performed by HTS panel. Results: Patient characteristics are shown in table 1.

\section{TABLE 1}

\section{Patient}

Bleeding symptoms

FBC:

$\operatorname{RBC}(\mathrm{T} / \mathrm{L})$

$\mathrm{Hb}(\mathrm{g} / \mathrm{dL})$

$\mathrm{MCV}(\mathrm{fL})$

Platelet (G/L)

MPV (fL)

LTA:

Collagen $(2.0 \mu \mathrm{g} / \mathrm{mL})$

Collagen $(10 \mu \mathrm{g} / \mathrm{mL})$

CRP $(5.0 \mu \mathrm{g} / \mathrm{mL})$

Ristocetin $(1.2 \mathrm{mg} / \mathrm{mL})$

$\operatorname{ADP}(4 \mu \mathrm{M}) /(5 \mu \mathrm{M})$

$\operatorname{ADP}(20 \mu \mathrm{M}) /(10 \mu \mathrm{M})$

Epinephrine $(8 \mu \mathrm{M}) /(5 \mu \mathrm{M})$

Epinephrine $(40 \mu \mathrm{mol} / \mathrm{L})$

Arachidonic $(0.3 \mathrm{mg} / \mathrm{mL})$

Arachidonic (1.5 mM)

TRAP (25 uM)

\section{German 1 (G1)}

Epistaxis, ecchymosis, prolonged bleeding after dental surgery, impaired wound healing

$3.73(\mathrm{~N}: 3.7-5.5)$

$11.5(\mathrm{~N}: 11-15)$

97 (N: 70-90)

256 (N: 100-436)

7.9 (N: 7-12)

$41(\mathrm{~N}>70)$

$61(\mathrm{~N}>85)$

$12(N>70)$

$37(\mathrm{~N}>70)$

$6(\mathrm{~N}>70)$

$8(\mathrm{~N}>70)$

$46(\mathrm{~N}>70)$
Spanish 2 (S2)

Epistaxis, ecchymosis, prolonged bleeding after dental surgery (required profylaxis with tranexamic acid and DDVAP) impaired wound healing

4.1 (N: 3.5-5.8)

12.8 ( N: 13-18)

$99.3(79-100)$

$123,215,134$ (N: 150-400)

10.9 (N: 6.8-11.8)

$2(\mathrm{~N}>85)$

$33(\mathrm{~N}>85)$

$15(n>85)$

$93(\mathrm{~N}>85)$

$54(\mathrm{~N}>85)$

$67(N>85)$

$35(\mathrm{~N}>85)$

$2(\mathrm{~N}>85)$

$73(\mathrm{~N}>85)$

$61(N>85)$ 
TABLE 2

Patient

FC

GP expression (GPIb/IX, GPIIb/

IIla and GPVI)

CD62

CD63

EM
German 1 (G1)

Normal

Severely decreased

Moderate decreased

Not conducted
Spanish 2 (S2)

Normal

Severely decreased

Moderate decreased
BS, for both patients, was 10. FBC showed mild anemia and anisocytosis and poikilocytosis in both patients, and normal platelet count in G1 but variable in S2 ranging from 123-215 G/L in multiple analysis. In both patients, LTA showed severe impaired response to Col, ADP, Epi, TRAP and CRP but normal expression of platelet glycoproteins. FC revealed severely decreased agonist-induced alpha and moderate dense granules secretion. EM (S2) also showed moderated alpha-granule defect. HTS panel identified a novel missense variant (c.865C $>$ T; p.His289Tyr) in GATA1 classified as a likely PV that is absent in gno$\mathrm{mAD}$ population database. This variant is located in the C-terminal $\mathrm{Zn}$ finger domain and disrupts a highly conserved amino acid residue. G1 mother's, a heterozygous carrier, presented with menorrhagia (BS = 2). Five females in the $\mathrm{S} 2$ pedigree were also heterozygous carriers of the variant but only two of these present with a bleeding diathesis.

Conclusions: The GATA-1 p. His289Tyr variant resulted in mild anemia, impaired platelet aggregation and secretion in hemizygous carriers. This is the first variant located in the GATA-1 C-terminal Zn-finger associated with platelet dysfunction and bleeding.

PB0897 | Utility of Modified Ivy's Bleeding Time and Closure Time on Platelet Function Analyzer-200 as a Screening Tool to Identify Platelet Function Disorders

R. Dave; T. Geevar; J. Mammen; G. Chellaiya; A. Samuel; R. Vijayan;

S. Singh; S. Nair

Christian Medical College and Hospital, Vellore, India

Background: Modified Ivy's Bleeding time(BT) is low cost but skillbased, invasive and operator-dependent screening test for platelet function defects(PFD). Platelet Function Analyzer-200 (PFA-200) is a pseudo-physiological system wherein citrated whole blood is drawn at high shear through a small aperture in membrane coated with collagen/epinephrine or collagen/ADP, causing platelet adhesion and aggregation occluding the aperture. Time from the start of the test until occlusion of the aperture is the Closure Time(CT). Prolonged CT indicates primary haemostatic defect.

Aims: To assess the performance of modified Ivy's BT and PFA-200 CT as screening tests for PFD.

Methods: Patients referred to our institution for bleeding workup from January 2016-January 2021 were included after informed consent. Detailed workup was done by complete blood count, BT, PFA-200 CT using Collagen/ADP and Collagen/Epinephrine cartridges, light transmission aggregometry, lumi-aggregometry, flow cytometry, mepacrine uptake/release assay, Prothrombin time, Activated partial thromboplastic time, Fibrinogen, Factor Assays and Ristocetin Cofactor assay. Patients with coagulation factor deficiency or Von Willebrand Disease were excluded. Patients with PFD were included, while patients with no haemostatic defect after detailed workup $(n=120)$ were taken as controls.

Results: Total of 498 patients were included out of which 67 had Bernard Soulier Syndrome(BSS), 208 had Glanzmann Thrombasthenia(GT),103 had mild PFD(storage pool defect / signal transduction defect / secretion defect) and 120 patients with no haemostatic defect were taken as controls. Overall, CT on PFA-200 Collagen/Epinephrine had highest sensitivity(98.6\%) and negative predictive value(NPV)(96\%) as screening tool for PFD. Sensitivity and NPV of BT, PFA-200 using Collagen/

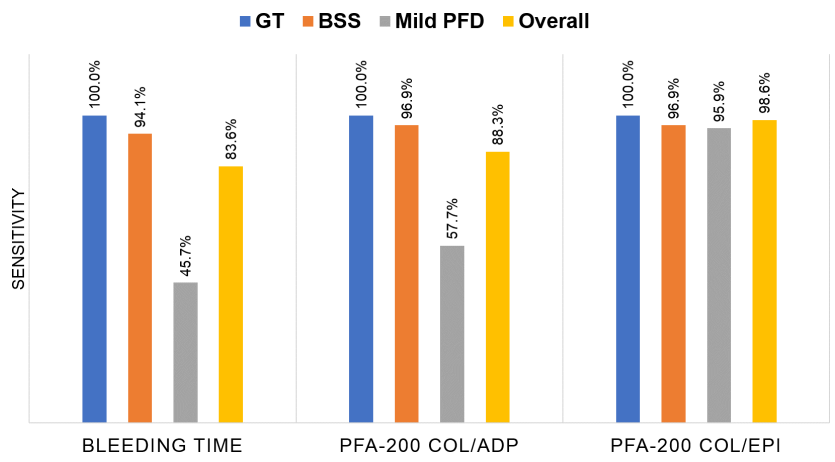

ADP and Collagen/Epinephrine was maximum(100\%) for identification of GT followed by BSS and least for mild PFDs.(Figure1,2)

FIGURE 1 Sensitivity of Modified Ivy's Bleeding Time, Closure Time on PFA-200 Collagen/ADP cartridge (COL/ADP) and Collagen/ Epinephrine cartridge (COL/EPI) for identification of Glanzmann Thrombasthenia (GT), Bernard Soulier Syndrome (BSS), Mild Platelet function defects (PFD) and Overall platelet function disorders 
$\square$ GT $=$ BSS Mild PFD Overall

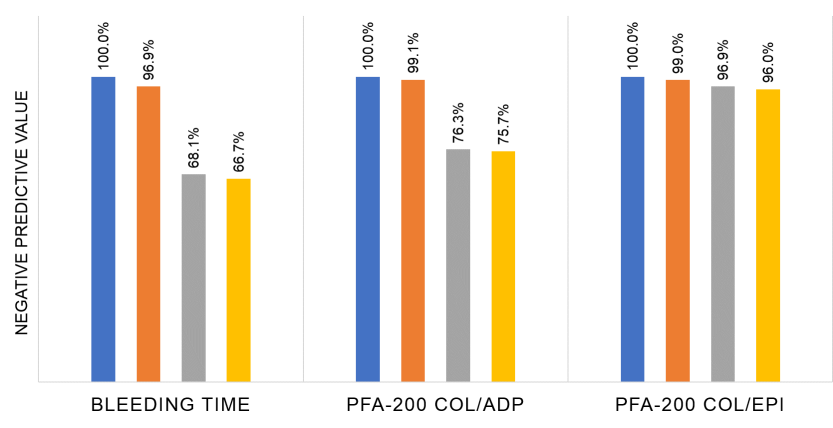

FIGURE 2 Negative Predictive Value of Modified Ivy's Bleeding Time, Closure Time on PFA-200 Collagen/ADP cartridge (COL/ADP) and Collagen/Epinephrine cartridge (COL/EPI) for identification of Glanzmann Thrombasthenia (GT), Bernard Soulier Syndrome (BSS), Mild Platelet function defects (PFD) and Overall platelet function disorders

Conclusions: Sensitivity and NPV for BT, PFA-200 CT for Collagen/ Epinephrine as well Collagen/ADP were comparable for identification of severe platelet function defects (GT,BSS), but CT on Collagen/ Epinephrine had maximum sensitivity and NPV as a screening tool for identification of mild PFD.

PB0899 | Investigation of the Epidemiology of Inherited Platelet Disorders in Patients Attending the Royal Hospital, Muscat, Oman

R. Al Ghaithi ${ }^{1}$; S. Al Hashami ${ }^{1}$; N. Al Amri ${ }^{1}$; S. Al Shiyadi ${ }^{1}$; R. Al Lawati $^{1}$; R. Al Busaidi ${ }^{1}$; H. Ambusaidi ${ }^{1}$; S. Al Lamki ${ }^{2}$; M. Al Yahyai ${ }^{1}$; M. Al Riyami ${ }^{1}$; M. Al Musalhi ${ }^{1}$; I. Al Salmi ${ }^{3}$

${ }^{1}$ Department of Haematology and Blood Transfusion, The Royal Hospital, Ministry of Health, Muscat, Oman; ${ }^{2}$ National Genetic Centre, The Royal Hospital, Ministry of Health, Muscat, Oman; ${ }^{3}$ Department of Neurology, The Royal Hospital, Ministry of Health, Muscat, Oman

Background: Inherited platelet disorders (IPDs) are a heterogeneous group of disorders associated with bleeding diatheses of varying severities. Light Transmission Aggregometry (LTA) is the most widely used method for the diagnosis of IPDs which monitor the responses of platelet to a panel of agonists. IPDs are considered to be rare with a frequency of 1: 10000 of healthy population. To date, very little is known about the prevalence of IPD within the Omani population. Aims: We therefore aimed to investigate the epidemiology of IPD

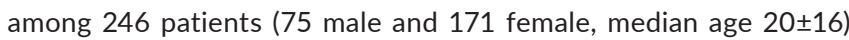
referred to the Royal Hospital between 2006 and 2020.

Methods: Data files of 246 patients were analyzed for the following parameters: LTA (ADP, Epinephrie, Arachidonic acid, Collagen and ristocetin) PFA-100 closure times (CT) and flow cytometry. Patients were categorized on the basis of the observed defects.

Results: Overall, IPD was diagnosed in 50(20.3\%) patients, in which, 15 patients were identified with secretion defects, 12 patients with Gi defects, 8 patients with Glanzmann's thrombasthenia (GT), 4 patients with cyclooxygenase (Cox) defects, 7 patients with multiple defects and 4 patients with other defects. Platelet count and Platelet Mean Volume (mean \pm SD) in patients' whole blood were $273 \pm 146$ $x 10^{3} / \mu \mathrm{L}$ and $8.7 \pm 2 \mathrm{fl}$, respectively. PFA-100 was tested in $36 / 50$ patients identified to have IPD of which $69 \%$ (25) gave abnormal CT. Flow cytometry results tested on patients with GT showed lack of expression of CD41 and CD61 on platelet surface.

Conclusions: Our current study revealed that secretion defect is the most common type of IPDs among Omani patients. The number of patients identified with GT in this study is in line with previously reported studies within the GCC countries.

\section{PB0900 | Hemorrhagic Phenotype in Pediatric Patients with} Glanzmann Thrombasthenia (GT)

D. Fedorova; E. Seregina; A. Poletaev; A. Pshonkin; P. Zharkov Dmitry Rogachev National Medical Research Center of Pediatric Hematology, Oncology and Immunology, Moscow, Russian Federation

Background: GT is one of the most severe platelet functional disorders. Platelet transfusion and rFVIla are available therapeutic options for severe bleeding. Nevertheless, there is no effective conventional prophylaxis of bleeding in GT patients nowadays.

Aims: To assess bleeding phenotype in pediatric GT patients and compare it with phenotype of other hereditary hemorrhagic disorders.

Methods: We used ISTH Bleeding Assessment Tool (ISTH BAT) and Pediatric Bleeding Questionnaire (PBQ) scales for assessment and documenting of hemorrhagic symptoms in 25 children ( 8 boys and 17 girls) with GT. We compared bleeding scores of GT patients with two other groups. The first group included children with von Willebrand disease (overall 35 patients, 19 - type 1, 13 - type 2, 3 - type 3), and the second group included 38 children with other platelet functional disorders.

Results: Median age at admission to our tertiary hospital was 5 years (IQR 1.5;7) in patients with GT. All patients had bleeding in more than one site.

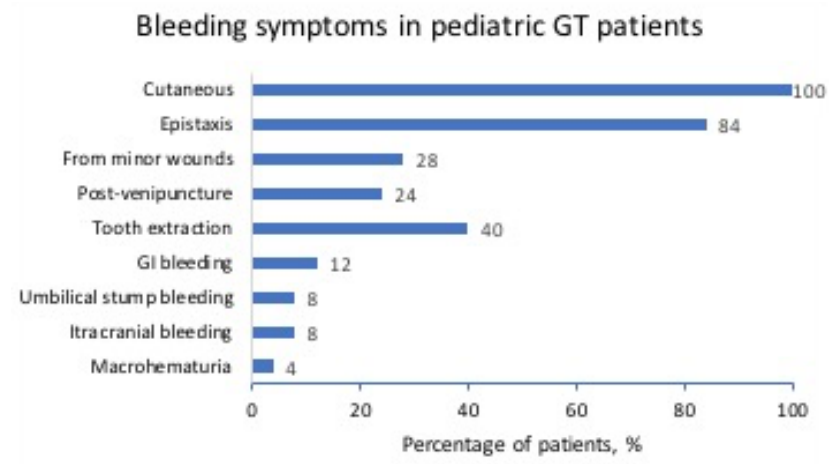

FIGURE 1 Bleeding symptoms in pediatric GT patients

All 8 school-age patients reported missing school because of bleeding episodes. Median age of onset of hemorrhagic symptoms was 1 month (IQR 0;6). Twenty-three (92\%) patients required emergency 
medical care for management of bleeding episodes. Twelve (48\%) patients received blood transfusion at least once. Median bleeding score was 8 (IQR 6.5;11.5) according to the ISTH BAT and 7 (IQR
5.5;10.5) according to the PBQ. Scores on both scales were significantly higher in GT patients compared to the other two groups $(P<0.05$ in pair-wise comparisons).

TABLE 1 Bleeding severity in patients with GT, other inherited functional platelet disorders (IFPD) and von Willebrand disease (vWD)

\begin{tabular}{|c|c|c|c|c|}
\hline Parameters & $\mathrm{GT}(n=25)$ & Other IPFD ( $n=38)$ & $\operatorname{vWD}(n=35)$ & $P$-value \\
\hline $\begin{array}{l}\text { Median age at admission (IQR), } \\
\text { months }\end{array}$ & $64(20 ; 88)$ & $77(39 ; 186,25)$ & $72(36 ; 120)$ & .13 \\
\hline Median PBQ score (IQR) & $7(5.5 ; 10.5)$ & $3(2 ; 5)$ & $4(2 ; 5.25)$ & $<.00001$ \\
\hline Min/max PBQ score & $2 / 14$ & $1 / 16$ & $1 / 12$ & - \\
\hline Median ISTH BAT score (IQR) & $8(6.5 ; 11.5)$ & $3(2 ; 5)$ & $4(2 ; 5.25)$ & $<.00001$ \\
\hline Min/max ISTH BAT score & $3 / 15$ & $1 / 17$ & $4(2 ; 5.25)$ & - \\
\hline$>1$ bleeding sites, $\mathrm{n}(\%)$ & $25(100)$ & $24(63)$ & $25(71)$ & .0007 \\
\hline Iron deficiency, $\mathrm{n}(\%)$ & $17(68)$ & $14(37)$ & $15(43)$ & .04 \\
\hline Emergency medical care, $\mathrm{n}(\%)$ & $23(92)$ & $17(45)$ & $14(40)$ & .00003 \\
\hline Blood transfusions or replacement & $13(52)$ & $8(21)$ & $12(34)$ & .03 \\
\hline
\end{tabular}

We could not demonstrate significant correlation between age and ISTH BAT and PBQ scores ( $P=0.58$ and 0.4 , accordingly) in GT patients. Probably, correlation was not significant because of absence of adolescent girls in our group.

Conclusions: Patients with GT demonstrate severe bleeding phenotype since first months of life. Majority of pediatric GT patients need emergency medical care for bleeding management.

PB0901 | Impact of Iron Deficiency Anemia on Bleeding Management in Pediatric Patients with Bernard-Soulier Syndrome and Glanzmann Thrombasthenia: A Single-institution Analysis

A. Lee $^{1} ;$ G. Batsuli ${ }^{1,2}$

${ }^{1}$ Emory University, Atlanta, United States; ${ }^{2}$ Children's Hospital of Atlanta, Atlanta, United States

Background: For pediatric patients with Bernard-Soulier Syndrome (BSS) and Glanzmann Thrombasthenia (GT), frequent and severe bleeding events (SBE) can lead to chronic blood loss and secondary iron deficiency anemia (IDA). SBE are primarily treated on-demand with platelet transfusions or recombinant activated factor VII (rFVIla) infusions. The impact of instituting prophylactic administration of platelets and/or rFVIla on bleeding and bleeding-associated complications is unclear.

Aims: To evaluate bleeding outcomes and management in pediatric patients with BSS or GT.

Methods: A retrospective chart-review of patients with BSS/GT followed at our pediatric hemophilia treatment center between $2007-$ 2019 was conducted.

Results: We identified 14 patients with a diagnosis of BSS $(n=2)$ or GT $(n=12)$. Annualized bleeding rates ranged from 0.1-18.4 events/ year, but $93 \%$ patients had at least one SBE. The most common bleeding symptoms were epistaxis and oral bleeding. Patients were treated with on-demand rFVIla infusions (7\%), platelets (7\%), or a combination of platelets and rFVIla (57\%) for bleeds. Approximately $86 \%$ of patients had IDA requiring iron replacement (11 oral and 3 intravenous) or packed red blood cell transfusions (pRBC, 7/14). Due to recurrent SBE resulting in refractory IDA, 3 patients (21\%) received rFVIla prophylaxis at 90 micrograms/kilogram 2-3 times/ week for 15-31 months. Patients started on rFVIla prophylaxis had a median hemoglobin of $9.77 \mathrm{~g} / \mathrm{dL}(8.0-10.7 \mathrm{~g} / \mathrm{dL}$ ) compared to $11.65 \mathrm{~g} / \mathrm{dL}(8.4-13.8 \mathrm{~g} / \mathrm{dL})$ for on-demand treated patients. In these 3 patients, median hemoglobin and ferritin increased by $1.28 \mathrm{~g} / \mathrm{dL}$ $(0.7-2.5 \mathrm{~g} / \mathrm{dL})$ and $14.63 \mathrm{mcg} / \mathrm{dL}(0.23-42.9 \mathrm{mcg} / \mathrm{dL})$, respectively. One patient on thrice-weekly rFVIla prophylaxis also required platelet transfusions every 2 weeks to further prevent epistaxis.

Conclusions: IDA is an important indicator of bleeding severity in pediatric patients with inherited bleeding disorders. Routine monitoring for IDA may help identify patients with BSS/GT that could benefit from prophylaxis regimens to reduce bleed burden.

PB0903 | Identification of ADP P2y12 Receptor Defect by Functional Assays Using Algorithmic Approach - A Case Series

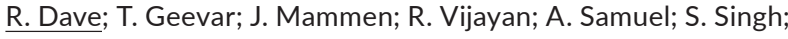
S. Nair

Christian Medical College and Hospital, Vellore, India

Background: Gi-coupled platelet P2Y12 receptor for ADP plays an important role in platelet function. Patients with inherited P2Y12 defect present with mild-moderate muco-cutaneous bleeding. Stepwise algorithmic approach using platelet function tests can help identify ADP P2Y12 receptor defect. 
Aims: To report findings in patients with ADP P2Y12 receptor $\operatorname{defect}(n=7)$ diagnosed using algorithmic approach.

Methods: Patients presenting with bleeding symptoms from May 2017 to January 2021 were evaluated after informed consent using stepwise algorithm (Figure1). Patients diagnosed with P2Y12 receptor defect were included. ISTH-Bleeding Assessment tool(BAT) was used to score bleeding symptoms. Screening tests for Primary hemostasis were Complete blood counts, modified Ivy's bleeding time and closure time(CT) on Platelet function analyzer-200 (PFA-200) using Collagen/ADP, Collagen/ Epinephrine and P2Y cartridges. Light Transmission Aggregometry(LTA) and lumi-aggregometry were performed for patients with abnormal screening tests. P2Y12 defect was suspected when ADP, even at high concentrations $(20 \mu \mathrm{M})$ was unable to induce full, irreversible platelet aggregation. P2Y12 defect was confirmed using vasodilator-stimulated phosphoprotein-phosphorylation(VASP-P) flow-cytometric assay and VerifyNow-P2Y12 assay(VN-P2Y12).

\begin{tabular}{|l|l|}
\hline $\begin{array}{l}\text { Patients presenting with muco-cutaneous } \\
\text { bleeding }\end{array}$ \\
Questionnaire based ISTH-BAT score calculation \\
$\qquad \begin{array}{l}\text { Tests to identify and exclude patients with } \\
\text { Coagulation factor deficiency or Von } \\
\text { Willebrand Disease : } \\
\text { Baseline investigations: } \\
\text { 1) Prothrombin Tine } \\
\text { 2) Activated Partial Thromboplastin Time } \\
\text { 3) Thrombin Time } \\
\text { 4) Von Clauss Fibrinogen } \\
\text { 5) Ristocetin Co-factor Assay } \\
\text { 6) Factor Assays }\end{array}$ \\
\hline
\end{tabular}

Screening tests for Primary Hemostasis:

1) Platelet Count and morphology

2) Bleeding Time (BT)

3) Closure time on PFA-200 (Collagen/ADP, Collagen/Epinephrine)
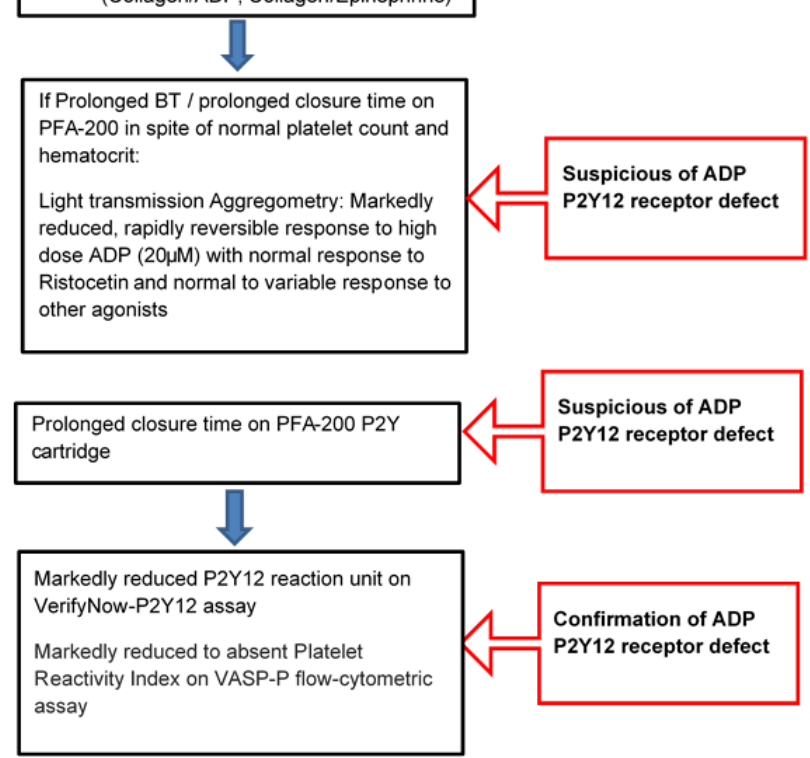

FIGURE 1 Algorithmic approach to diagnose ADP P2Y12 receptor defect.
Results: The median(IQR) age of patients was 11 years(4-38) with male:female ratio of 1:2.5. ISTH-BAT score ranged from 3-8(Median:6) with elevated ISTH-BAT score in $6 / 7$ patients. All cases had normal platelet count(IQR 254-290 × 10\%/L). Bleeding time was prolonged in 6/7 patients. PFA-200 CT for Collagen/ ADP and P2Y cartridges was prolonged in all cases, while Collagen/ Epinephrine CT was prolonged in 6 patients. LTA showed markedly reduced, rapidly reversible aggregation in response to high concentration $(20 \mu \mathrm{M})$ ADP (figure 2 ) with normal ATP release in all cases. $\mathrm{VN}-\mathrm{P} 2 \mathrm{Y} 12$ platelet reactivity test showed markedly reduced $\mathrm{P} 2 \mathrm{Y} 12$ Reaction Units. VASP-P flow-cytometric assay revealed 0\% platelet reactivity index in all cases, confirming the diagnosis of P2Y12 defect.

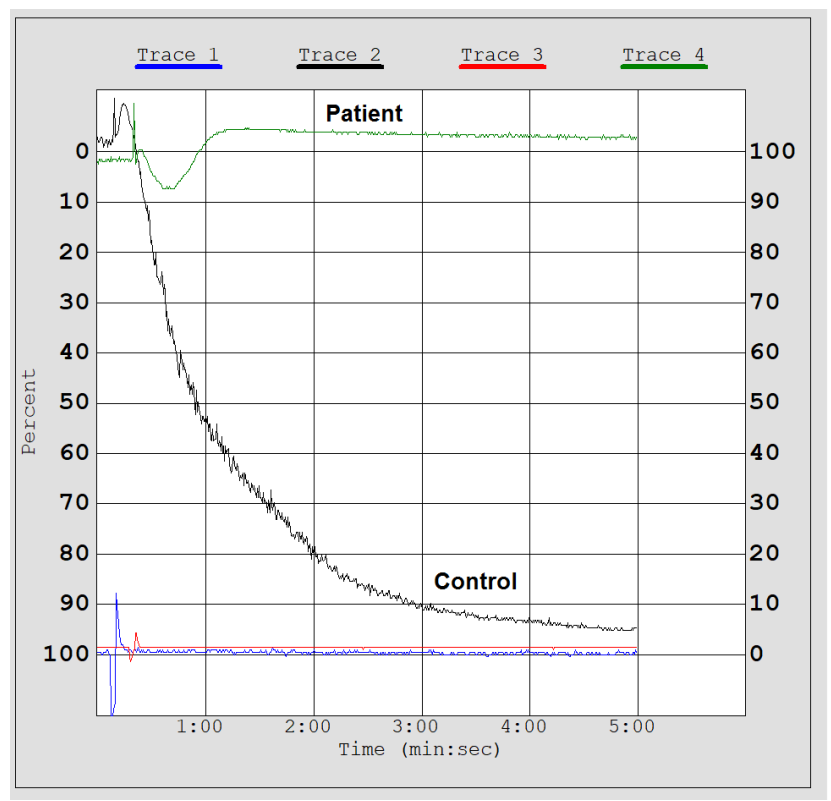

FIGURE 2 Light Transmission aggregometry showing markedly reduced, rapidly reversible aggregation in response to high concentration $(20 \mu \mathrm{M})$ ADP in a patient with ADP P212 receptor defect as compared to control

Conclusions: ADP P2Y12 receptor defect can be identified using functional assays and should be suspected in patients with mildmoderate muco-cutaneous bleeding and markedly reduced response to high dose $\operatorname{ADP}(20 \mu \mathrm{M})$. 
PB0904 | A Novel Disease-causing Variant in the GP1BA Gene Related to Bernard Soulier Syndrome

$\underline{\text { A.I. Woods }}{ }^{1}$; M.F. Alberto ${ }^{2}$; D.M. Primrose ${ }^{3}$; J. Paiva ${ }^{4}$; M. Asencio ${ }^{4}$; M.M. Casinelli ${ }^{4}$; A.N Blanco ${ }^{4}$; A. Sánchez-Luceros ${ }^{4,5}$

${ }^{1}$ Laboratory of Hemostasis and Thrombosis, IMEX-CONICET-National Academy of Medicine, Ciudad Autonoma de Buenos Aires, Argentina; ${ }^{2}$ Departament of Hemostasis and Thrombosis, Hematological Research Institute, National Academy of Medicine, Ciudad Autonoma de Buenos Aires, Argentina; ${ }^{3}$ Higher School of Engineering, Informatics and Agri-food Sciences, University of Morón, Morón City, Argentina; ${ }^{4}$ Department of Hemostasis and Thrombosis, Hematological Research Institute, National Academy of Medicine, Ciudad Autonoma de Buenos Aires, Argentina; ${ }^{5}$ Laboratory of Hemostasis and Thrombosis, IMEXCONICET-National Academy of Medicine. Buenos Aires City, Argentina, Ciudad Autonoma de Buenos Aires, Argentina

Background: Bernard Soulier syndrome (BSS) is a rare autosomal bleeding disorder caused by homozygous or compound heterozygous disease-causing variants (DCV) in any of the genes encoding for glycoprotein-Ib (GPIb) (GP1BA, GP1BB) and GPIX (GP9) of the platelet GPIb-IX-V-complex. The typical form is recessive (biallelic) with severe bleeding and moderate macrothrombocytopenia.

Heterozygous patients are usually asymptomatic with slight macrothrombocytopenia, reduced GPIb-IX-V expression, slightly reduced ristocetin-induced platelet aggregation (RIPA), and considered BSS carriers.

Aims: To describe a novel DCV responsible for recessive BSS.

Methods: Tests performed: platelet count; coagulation profile including factor FVIII:C; VWF:Ag; VWF:RCo; platelet aggregation $(2 \mu \mathrm{M}$-ADP, $1 \mu \mathrm{M}$-epinephrine, $1 \mu \mathrm{g} / \mathrm{mL}$-collagen and $1 \mathrm{mM}$ arachidonic acid).

GPIb-IX and GPIIb-IIla expression was analyzed by flow-cytometry using specific monoclonal antibodies (CD42b, CD41 and CD61).

Genomic DNA was extracted from peripheral blood. GP1BA was amplified by PCR and sequenced (Sanger methodology).

Genome Aggregation Database, Human Gene Mutation Database, Leiden Open Variation Database and Varsome were accessed to check the registry of variants.

Results: Male (56-yrs) (ISTH-SSC-BAT $=0$ ) was evaluated after obtaining written informed consent. He showed reduced platelets count $\left(121 \times 10^{9} / \mathrm{L}\right)$, macroplatelets (mean platelet volume $=10.9 \mathrm{fL}$; normal-range $=7-10.5 \mathrm{fL}$ ), $1.2 \mathrm{mg} / \mathrm{mL}-\mathrm{RIPA}=$ slightly normal with latency period, normal platelet aggregation, clotting and fibrinolytic systems.

Flow-cytometry: $50 \%$ of expression with CD42b; normal expression with both CD41 and CD61.

GP1BA sequence analysis: a novel missense substitution c.692A>G $\rightarrow$ p.Tyr231Cys in heterozygocity, predicted as damaging by PolyPhen-2, SIFT, Mutation-Taster, Provean and Varsome. I-Mutant predicted p.Tyr231Cys as large decrease of stability; p.Tyr231Cys was not found in 100 controls, without entries in the variant databases and was deposited in the LOVD at: http://www. lovd.nl/GP1BA.

Conclusions: We report a patient with no bleeding symptpms, mild macrothrombocytopenia, $50 \%$ of GPIb expression and heterozygous p.Tyr231Cys in the GP1BA, with negative effect influencing in both platelet count and size and in the expression of the GPIb. It appears to show recessive inheritance for BSS.

This patient was diagnosed as carrier of BSS.

P0160 | Platelet Hyperaggregability and Migraine Headache - A Single Centre Experience

M. Brunclikova ${ }^{1}$; L. Stanciakova ${ }^{1}$; J. Ivankova ${ }^{1}$; M. Skerenova ${ }^{2}$; T. Simurda ${ }^{1}$; M. Dobrotova ${ }^{1}$; P. Holly ${ }^{1}$; I. Skornova ${ }^{1}$; P. Kubisz ${ }^{1}$ J. Stasko ${ }^{1}$

${ }^{1}$ Comenius University in Bratislava, Jessenius Faculty of Medicine in Martin, Martin University Hospital, National Centre of Haemostasis and Thrombosis, Department of Haematology and Transfusiology, Martin, Slovakia; ${ }^{2}$ Comenius University in Bratislava, Jessenius Faculty of Medicine in Martin, Department of Clinical Biochemistry, Martin, Slovakia

Background: Platelet hyperaggregability triggered by low concentrations of platelet agonist adenosine diphosphate (ADP) or epinephrine (EPI) or both, named as sticky platelet syndrome (SPS) may be responsible for the development of wide spectrum of thrombotic events in various parts of the circulation. The most common presentation of arterial occlusions are considered the cerebral arteries with clinical manifestations including transitory ischemic attack (TIA) or ischemic stroke (IS). Apart from the stroke and TIA, the other clinical presentation of the syndrom due to the changes in cerebrovascular system is the migraine headache. It is necessary to take the SPS into account in differential diagnosis of the underlying cause of unexplained headache, especially in patients younger than 40 years with positive family history of migraine and thrombosis.

Aims: The aim of this study was to provide insight into the background of the events in cerebrovascular circulation caused by platelet hyperaggregability, especially with a focus on transient changes with manifestation of the migraine.

Methods: The authors analyzed the presence of platelet hyperaggregability in patients with a history of the migraine headache and described individual types of SPS and further characteristics in these patients.

Results: In this pilot study, the authors found the increased incidence of platelet hyperaggregability induced by ADP and/or EPI in the patients with migraine. In the selected groups of individuals, they also assessed the different subtypes of SPS.

Conclusions: Platelet hyperaggregability can be a serious risk factor for the development of thrombotic events and therefore we should take it into account not only in differential diagnosis of the arterial thrombosis but also unexplained headaches especially in younger patients. 


\section{VON WILLEBRAND FACTOR BIOLOGY}

LPB0030 | Investigating Impact of von Willebrand Factor Deficiencies on the Biogenesis of Weibel-Palade Bodies and Endothelial Cells Signaling Pathways

H. Yadegari $^{1,2}$; M.A. Jamil ${ }^{2}$; N. Marquardt ${ }^{2}$; O. El-Maarri ${ }^{2}$; J. Oldenburg ${ }^{2}$

${ }^{1}$ University Clinic Bonn, Bonn, Germany; ${ }^{2}$ University Clinic Bonn/ Institute of Experimental Hematology and Transfusion Medicine, Bonn, Germany

Background: This study included two type 3 von Willebrand disease (VWD) index patients (IPs), characterized by a virtual absence of plasma von Willebrand factor (VWF), but due to disparate molecular pathogenic mechanisms. In IP-I, almost no production of VWF mRNA was contributed to the disease pathogenesis, demonstrated by quantitative reverse transcription assay. Whereas, IP-II carried a heterozygous in-frame large deletion of exons 4-34 of the VWF gene, which had a dominant-negative impact on VWF biosynthesis by affecting multimer elongation, confirmed by multimer analysis.

Aims: This study aimed to elucidate the impact of these VWF deficiencies on Weibel-palade bodies (WPBs) formation, trafficking of the WPBs inflammatory factors, and cellular signaling pathways using patient-derived blood outgrowth endothelial cells (BOECs).

Methods: Immunostaining of BOECs and imaging of the cells using an Apotome.2 microscope was conducted to visualize VWF as well as WPBs inflammatory cargos angiopoietin-2 (Ang2) and P-selectin. Moreover, whole-transcriptome RNA-sequencing of the BOECs and Ingenuity Pathway Analysis was done.

Results: The IP-I BOECs were devoid of WPBs, with altered trafficking of the WPBs inflammatory proteins. In IP-II, WPBs were formed but abnormal. Ang2 was mainly accumulated in the nucleus of the IP-II BOECs, showing a complete lack of Ang2/VWF co-localization. The co-localization of VWF/P-selectin in IP-II BOECs was reduced but still detected. The RNA-sequencing of the IP-II BOECs showed an upregulation in genes of the WPBs proteins, e.g. P-selectin, IL-6, IL8, and CXCL1, as well as significant alterations of canonical pathways related to inflammatory responses, extracellular organization, and angiogenesis. While, in IP-I no deviations in the expression of WPBs inflammatory cargos were observed, and changes in cellular canonical pathways were less prominent compared with IP-II.

Conclusions: Different VWF deficiencies may affect WPBs biogenesis and trafficking of the WPBs inflammatory proteins in a different way, which in turn may influence cellular signaling pathways discretely.
PB0905 | Mutations at p.C1190 in VWF D3 Retain VWF in the ER in VWD 2A Patient-derived ECFCs

I. Van Moort ${ }^{1}$; P.E. Bürgisser ${ }^{1}$; M.W. Swinkels ${ }^{1}$; S. Yildiz ${ }^{1}$; F. Atiq ${ }^{1}$; F.W. Leebeek ${ }^{1}$; R. Bierings; for the SYMPHONY consortium Erasmus MC, University Medical Center Rotterdam, Rotterdam, Netherlands

Background: The bulk of von Willebrand factor (VWF) in plasma is synthesized in endothelial cells. During biosynthesis, VWF dimerizes in the ER and multimerizes in the Golgi via various intra- and interchain cysteines in the CK and D3 domain, respectively. Mutations in the D3 domain can lead to von Willebrand's disease (VWD) type $2 \mathrm{~A}$, which is characterized by a striking loss of high molecular weight (HMW) VWF multimers in plasma. Whether this arises due to reduced half-life in circulation or is caused by biosynthetic defects is incompletely understood.

Aims: To study VWF trafficking and secretion in endothelial colony forming cells (ECFCs) from VWD 2A patients with mutations in the D3 domain.

Methods: ECFCs were isolated from venous blood of healthy control donors and three patients from the Willebrand in the Netherlands (WiN) cohort with heterozygous c.3569 G>A (p.C1190Y) and c.3568 $\mathrm{T}>\mathrm{C}$ (p.C1190R) mutations in VWF. Secretion of VWF by ECFCs was determined by ELISA and multimer assay while morphology of intracellular compartments was assessed using confocal microscopy. The study was approved by the medical ethical committee of the Erasmus $\mathrm{MC}$ and all patients gave informed consent.

Results: Unstimulated (basal and constitutive) release of VWF was $\sim 50 \%$ lower in patient ECFCs compared to healthy ECFCs. In addition, patient ECFCs secreted less VWF after $\mathrm{Ca}^{2+}$ - and cAMP-dependent stimulation, although equal amounts of VWF were present intracellularly. A large pool of intracellular VWF colocalized with protein disulfide isomerase-positive spherical structures (Figure 1), suggestive of retention in the ER. In addition, patient ECFCs no longer secreted HMW VWF multimers, resembling the loss of HMW multimers in their plasma.
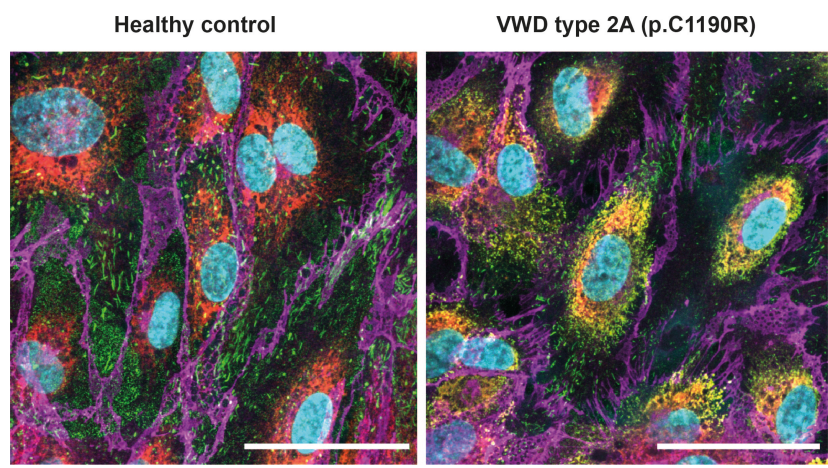

FIGURE 1 Mutations in the D3 domain of VWF cause VWF ER retention in patient-derived ECFCs. ECFCs stained for VWF (green), VE-Cadherin (magenta), protein disulfide isomerase (red) and DAPI (blue). 
Conclusions: These findings suggest that mutations in the D3 domain of VWF (p.C1190R and p.C1190Y) cause VWD as a result of VWF ER retention.

PB0906 | Evaluation of von Willebrand Factor (VWF) Replacement on in vivo Angiogenesis in VWF-deficient Mice

E. Ocran ${ }^{1}$; K. Nesbitt ${ }^{2}$; M. Hinds ${ }^{1}$; O. Rawley ${ }^{2}$; M. Bowman ${ }^{1}$;

D. Lillicrap ${ }^{2}$; P. James ${ }^{1}$

${ }^{1}$ Queen's University, Medicine, Kingston, Canada; ${ }^{2}$ Queen's University, Pathology and Molecular Medicine, Kingston, Canada

Background: Gastrointestinal (GI) bleeding from angiodysplasia is a common problem in patients with inherited and acquired abnormalities of von Willebrand Factor (VWF) and can be challenging to manage. Recent studies have demonstrated a negative regulatory role of VWF in angiogenesis.

Aims: To examine the effect of VWF replacement on in vivo angiogenesis in a mouse model of VWF-deficiency.

Methods: The Matrigel plug assay was performed in 14 to 16 -week old C57BI/6 VWF knockout (KO) mice of both genders $(N=9)$ that expressed VWF antigen (VWF:Ag) levels of $\geq 20 \mathrm{U} / \mathrm{ml}$ at 48 -hours following hydrodynamic injection with wild type (WT) murine VWF cDNA.

On day eight post-hydrodynamic injection, Matrigel mixed with fibroblast growth factor (FGF) and vascular endothelial growth factor (VEGF) was injected subcutaneously in the right back flank of each mouse. In the left back flank, an equal volume of Matrigel with phosphate buffered saline (PBS) was injected as a control. After 14 days, plugs were harvested and processed for hematoxylin and eosin (H\&E) and immunohistochemical staining (IHC). Retro-orbital (RO) sampling was performed at specific timepoints during the 14 day incubation period, to assess VWF:Ag and multimer structure (Figure 1A).

Results: VWF:Ag dropped to undetectable levels by day 12 (day 5 of Matrigel incubation) following hydrodynamic injections (Figure 1B). While VWF multimers were observed, high molecular weight multimers (HMWM) were absent and there was loss of intermediate MWM with time. (Figure 1 C\&D). Matrigel plugs supplemented with FGF and VEGF showed increased vascularization $\left(55 \pm 30\right.$ cells $\left./ \mathrm{mm}^{2}\right)$ compared to PBS controls ( $15 \pm 14$ cells $/ \mathrm{mm}^{2} ; P<0.01$; Figure $2 \mathrm{C}$ ).

Conclusions: Although hydrodynamic VWF replacement was successful but short-lived in VWF-deficient mice, liver expressed murine VWF was predominantly low MWM and did not prevent angiogenesis in the Matrigel plug assay. Further research is needed to evaluate the role of VWF in in-vivo angiogenesis.

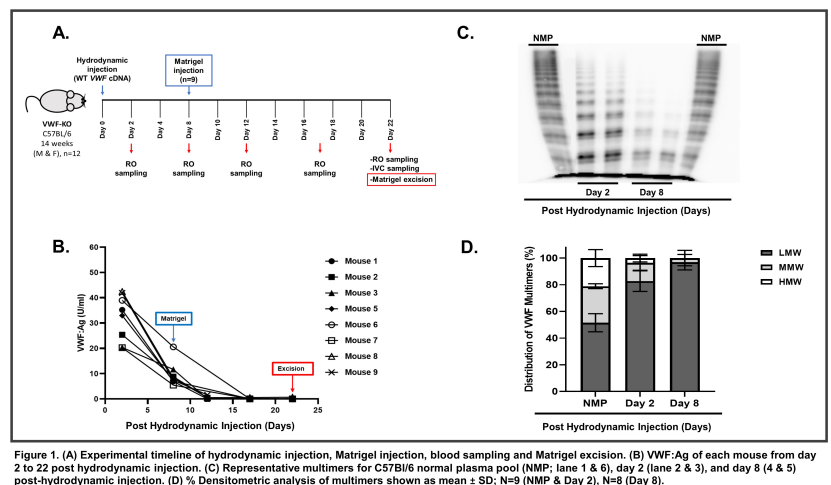

FIGURE 1 (A) Experimental timeline (B) VWF:Ag levels (C) VWF Multimers and (D) Densitometric analysis of multimers

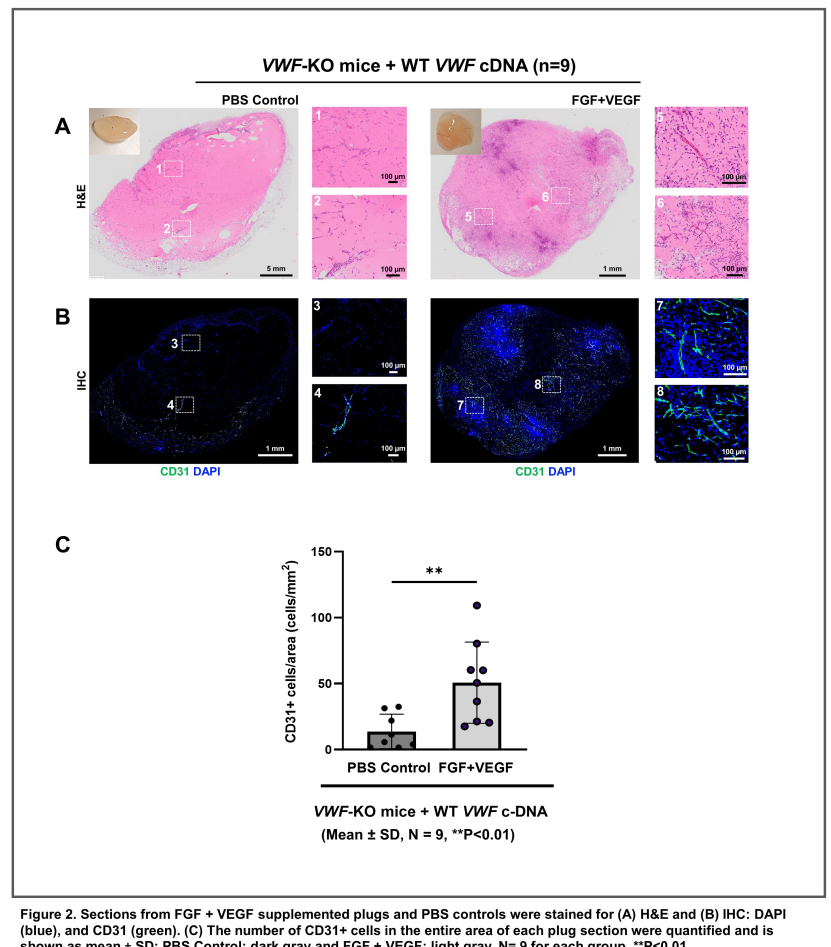

FIGURE 2 (A) H\&E (B) IHC images and (C) Endothelial cell (CD31+ staining) quantification of whole plugs from VWF-KO mice

PB0907 | Agglomeration and then Capture within 10 ms Creates Shear-induced Platelet Aggregation Controlled by von Willebrand Factor Concentration

Z. Liu; C. Bresette; C. Aidun; D. Ku

Georgia Institute of Technology, Atlanta, United States

Background: Shear-induced platelet aggregation (SIPA) under elevated shear rates $(\sim 10,0001 / \mathrm{s})$ is a major hallmark of occlusive arterial thrombosis. SIPA specific to elevated shear rates is independent of platelet activation while exclusively controlled by von Willebrand Factor (VWF). Current in vitro and in vivo assays lack resolution to 
capture the sub-cellular dynamics of SIPA within ultra-fast time scale of $10 \mathrm{~ms}$ and pathologically high shear rates. Prior in silico thrombosis models tend to ignore the presence of VWF.

Aims: Develop an in silico biophysical model to create SIPA, where the dynamics and interaction of individual VWF polymers and platelets are resolved directly.

Methods: The dynamics of platelet and VWF are computed through a multiscale blood flow solver. The GP1b-A1 binding kinetics are created from single-molecule measurements. The times to form a platelet agglomerate that is then captured at the wall are used as the end points for quantifying the rate of SIPA. A microfluidic thrombosison-a-chip platform is used to validate the in silico predictions.

Results: Results show that platelet agglomerates form in the flow prior to surface adherence (Figure A). Subsequent capture of the agglomerate creates a SIPA process within $\sim 10 \mathrm{~ms}$, equivalent to the transit time through an atherosclerotic stenosis. Increasing soluble VWF concentration by 20x in the model leads to 2 3x increase SIPA rates. This matches the increase in occlusion rates observed in vitro. (Figure B).
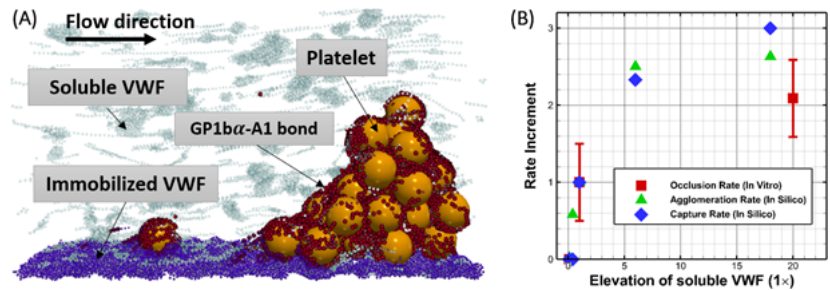

FIGURE 1 (A) In silico formation of a platelet aggregate entangled by VWF polymers through forming GP1ba-A1 bonds on a thrombogenic surface pre-coated with immobilized VWF. (B) Increment of SIPA rate (in terms of agglomeration rate and capture rate) or occlusion rate with respect with the elevation of soluble VWF concentration

Conclusions: A multiscale in silico model for high shear-induced platelet aggregation pertinent to arterial thrombosis is developed and validated against in vitro experiments of macroscopic occlusion rates. The in silico thrombotic model can be used to precisely design VWF-targeting drugs to control agglomeration or capture of platelets in arterial thrombosis.

PB0908 | Developing a Model for Studying von Willebrand Disease with hiPSC-derived Endothelial Cells

S. de Boer; R. Dirven; B. Laan; J. Eikenboom

Leiden University Medical Centre, Leiden, Netherlands

Background: Several recognized protocols exist to differentiate human induced pluripotent stem cells (hiPSCs) into endothelial cells (hiPSC-ECs). Even though these hiPSC-ECs mimic primary ECs very accurately, round Weibel-Palade bodies (WPBs) and low von Willebrand factor (VWF) production indicate an immature EC phenotype of the hiPSC-ECs. However, to be used as a proper model, these cells require a mature EC phenotype. Exposure to a histone deacetylase inhibitor (HDACi) during differentiation may cause remodeling of the gene expression profile and may improve in vitro maturation of hiPSC-ECs.

Aims: To improve the maturity of the EC phenotype of hiPSC-ECs by the addition of a HDACi during and after differentiation.

Methods: Peripheral blood mononuclear cells from three healthy donors were reprogrammed into hiPSCs and subsequently differentiated into ECs. At different timepoints, the HDACi sodium butyrate was added at concentrations ranging from $0.25-2.5 \mathrm{mM}$. VWF production and secretion of the hiPSC-ECs was measured, together with the expression of VWF related transcription factors.

Results: The addition of sodium butyrate did not have an effect on VWF secretion, neither at basal levels nor after histamine stimulation of the hiPSC-ECs. However, when measured in the cell lysates, a small increase in VWF production was seen, which was confirmed by confocal microscopy. Even though an increase in the number of WPBs was observed, these organelles still lacked the tubular shape and remained visible as round immature structures as shown previously in hiPSC-ECs. qPCR analysis did show an increase in the expression level of several VWF related transcription factors after the addition of sodium butyrate, leading to an increase in VWF expression and production.

Conclusions: Even though the addition of sodium butyrate increases the production of VWF in hiPSC-ECs, possibly through increased expression of transcription factors, the hiPSC-EC phenotype remains immature. However, the effects of hyperacetylation during EC differentiation and maturation needs to be investigated more.

PB0909 | Age-specific Changes in von Willebrand Factor Multimers in Healthy Children and Adults

N. Letunica ${ }^{1}$; S. Van Den Helm¹; R. Barton ${ }^{1,2,3}$; V. Karlaftis ${ }^{1,2}$; P. Monagle $\mathrm{e}^{1,2,3}$; V. Ignjatovic ${ }^{1,2}$

${ }^{1}$ Haematology Research Laboratory, Murdoch Children's Research Institute, Melbourne, Australia; ${ }^{2}$ Department of Paediatrics, The University of Melbourne, Melbourne, Australia; ${ }^{3}$ Department of Clinical Haematology, Royal Children's Hospital, Melbourne, Australia

Background: Age-specific differences in the concentration and function of several haemostatic proteins have been investigated previously and are encompassed under the concept of Developmental Haemostasis. However, few studies have investigated the effect of age and development on von Willebrand Factor (vWF) and its multimers. This is critical for accurate diagnosis and management of neonates and children with haematological complications, such as von Willebrand disorders.

Aims: To investigate age-specific changes in vWF and its multimers in a healthy population. 
Methods: Blood samples were obtained via clean venepuncture from 20 healthy neonates and 60 healthy children undergoing elective surgery (e.g. circumcision) and citrated plasma was stored for batch testing. vWF concentration and activity were measured using the STA R Max ${ }^{\circledR}$ analyser and Stago reagents, STA ${ }^{\circledR}$ vWF antigen (vWF:Ag) and STA ${ }^{\circ}$ Ristocetin cofactor activity (vWF:RCo) (Diagnostica Stago, France). vWF multimers were analysed using the Hydragel 11 vWF Multimer assay (Sebia, France). Results are expressed as mean with 95\% confidence intervals and were analysed using an one-way ANOVA followed by Dunnett's test to correct for multiple comparisons.

Results: Mean values and reference intervals according to age are presented in Table 1. The mean values for vWF low molecular weight multimer (Imwm) and vWF intermediate molecular weight multimer (imwm) differ significantly between neonates and adults.

TABLE 1 Patient demographic data and mean values with age-specific reference ranges for vWF ristocetin cofactor activity ( $V W F: R C o$ ), $v W F$ antigen (vWF:Ag), vWF low molecular weight multimer (Imwm), vWF intermediate molecular weight multimer (imwm) and vWF high molecular weight multimer (hmwm). Test results are expressed as percentage (\%)

\begin{tabular}{|c|c|c|c|c|c|c|}
\hline & Neonates (h) & $\leq 2$ years & $>2-5$ years & $6-10$ years & $11-17$ years & Adults \\
\hline subjects (n) & 20 & 22 & 11 & 8 & 19 & 20 \\
\hline age range & 24 to 96 & 0 to 1 & 2 to 4 & 9 to 10 & 11 to 17 & 20 to 54 \\
\hline sex & $10 \mathrm{M} / 10 \mathrm{~F}$ & $18 \mathrm{M} / 4 \mathrm{~F}$ & $5 \mathrm{M} / 6 \mathrm{~F}$ & $5 \mathrm{M} / 3 \mathrm{~F}$ & $10 \mathrm{M} / 9 \mathrm{~F}$ & $7 \mathrm{M} / 13 \mathrm{~F}$ \\
\hline mean & 83.1-121.0 & $60.7-87.2$ & $71.5-106.3$ & 68.3-103.9 & 81.2-113.2 & 78.1-107.3 \\
\hline \multicolumn{7}{|l|}{$95 \% \mathrm{Cl}$} \\
\hline vWF:Ag & 112.6 & 83.8 & 98.8 & 91.3 & 89.8 & 101.9 \\
\hline mean & $96.3-128.9$ & $71.0-96.5$ & $82.2-115.4$ & $70.2-112.3$ & 68.9-110.5 & $88.6-115.2$ \\
\hline mean & $17.1-22.7$ & $15.7-19.7$ & $14.5-22.1$ & 13.9-17.1 & $12.7-18.1$ & $14.0-17.0$ \\
\hline \multicolumn{7}{|l|}{$95 \% \mathrm{Cl}$} \\
\hline vWF imwm & $27.5^{*}$ & $27.7^{*}$ & $26.9^{*}$ & 30.5 & 28.4 & 30.5 \\
\hline mean & $25.5-29.6$ & $26.5-28.8$ & $24.7-29.1$ & $28.3-32.7$ & $26.9-29.8$ & $29.1-31.8$ \\
\hline \multicolumn{7}{|l|}{$95 \% \mathrm{Cl}$} \\
\hline vWF hmwm & 52.6 & 54.6 & 54.8 & 54.0 & 56.2 & 54.0 \\
\hline mean & $48.3-56.8$ & $52.6-56.7$ & $49.7-59.8$ & $51.4-56.6$ & $53.1-59.3$ & $51.8-56.3$ \\
\hline
\end{tabular}

${ }^{*} P$-value significantly different from adults $(P=<0.05) \cdot \mathrm{h}=$ hours

Conclusions: This study confirms that vWF multimers vary significantly with age, emphasising the importance of developing age-specific reference ranges, to correctly diagnose neonates and children with haematological complications. Our findings highlight that age-specific differences that exist physiologically are not detected using less sensitive measures that, in this case, do not account for the specific forms of the VWF multimers. Our findings are different to previously published work, potentially related to differences in neonatal subjects (gestation and health status) or methodological differences. Further studies are required to establish a gold standard for vWF multimer testing. 
PB0910 | Differential Release of VWF and VWF-propeptide from Platelet Alpha-granules

M. Swinkels ${ }^{1}$; J. Slotman ${ }^{2}$; A. Houtsmuller ${ }^{2}$; F. Leebeek ${ }^{1}$;

J. Voorberg ${ }^{3,4}$; G. Jansen ${ }^{1}$; R. Bierings ${ }^{1}$

${ }^{1}$ Department of Hematology, Erasmus MC, University Medical Center Rotterdam, Rotterdam, Netherlands; ${ }^{2}$ Optical Imaging Center, Department of Pathology, Erasmus MC, University Medical Center Rotterdam, Rotterdam, Netherlands; ${ }^{3}$ Molecular and Cellular Hemostasis, Sanquin Research and Landsteiner Laboratory, Amsterdam University Medical Center, University of Amsterdam, Amsterdam, Netherlands; ${ }^{4}$ Experimental Vascular Medicine, Amsterdam University Medical Center, University of Amsterdam, Amsterdam, Netherlands

Background: Platelets bud off from megakaryocytes into the circulation and contain different types of granules. Alpha-granules contain many hemostatic proteins, including Von Willebrand Factor (VWF) and a processed part of the protein, VWF-propeptide (VWFpp). While multimerization, storage and release of VWF has been extensively studied in endothelial cells, regulation in megakaryocytes and platelets is unclear. Studying these processes in platelets will help us better understand how this key hemostatic protein contributes to adequate hemostasis from different compartments.

Aims: To characterize the storage and release of VWF and VWFpp in platelet alpha-granules.

Methods: Healthy platelets were stimulated with PAR-1 activating peptide (PAR-1-ap). We employed super-resolution light microscopy and image analysis to generate quantitative imaging data. Slides were stained for alpha-tubulin, VWF and VWFpp, SPARC or fibrinogen. Data are normalized to resting platelets as percentage of granule numbers \pm SEM.

Results: We observed extensive, but not perfect ( 85-95\%) overlap in VWFpp+ and VWF+ granules in hundreds of resting platelets, implying that these proteins are stored in similar eccentric fashion in platelet alpha-granules (Figure 1).
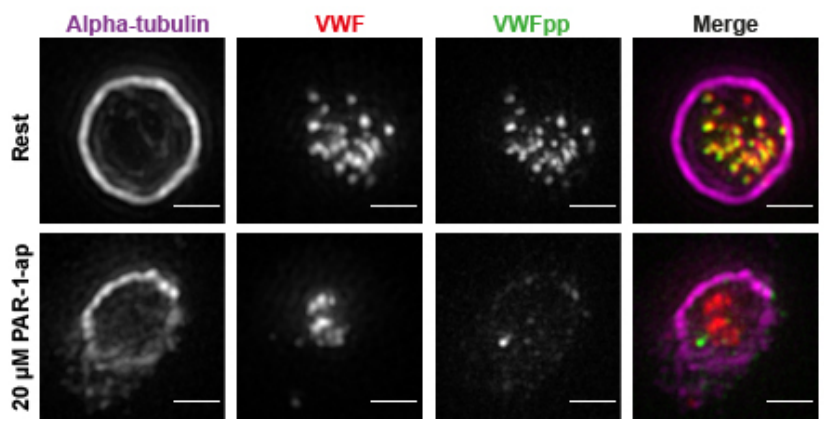

FIGURE 1 Distribution of VWF and VWFpp in resting and stimulated platelets

Representative images of immunostained platelets. Scale bar: $1 \mu \mathrm{m}$ In comparison to unstimulated platelets $(100 \pm 1.7 \%$ granules over 3 donors), we observed less VWFpp+ (64.3 $\pm 3.1 \%)$ and VWF+ granules $(75.3 \pm 1.4 \%)$ at $0.6 \mu \mathrm{M}$ of PAR-1-ap, suggesting rapid release of a subset of granules (Figure 2). Higher concentrations of PAR-1-ap triggered more pronounced differential release of VWFpp $(14.7 \pm 1.6 \%$ at $20 \mu \mathrm{M}, P<0.0001)$ compared to VWF $(62.4 \pm 1.4 \%$, $P=0.03)$. Release of other alpha-granule proteins was intermediate at $20 \mu$ M PAR-1-ap (SPARC: $37.8 \pm 1.4 \%$, fibrinogen $48.1 \pm 2.9 \%$; $P<0.001$ ), providing further evidence for differential exocytosis of alpha-granule cargo.
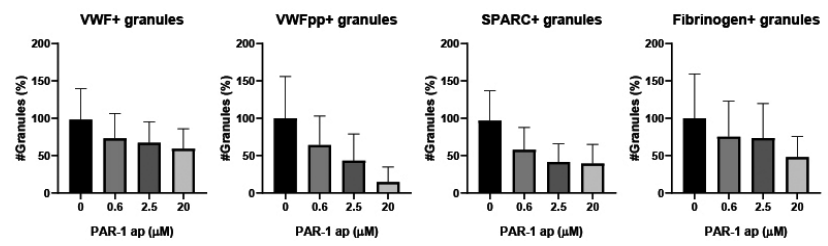

FIGURE 2 Quantification of differential alpha-granule cargo release Quantitative platelet granule numbers under PAR-1 stimulation

Conclusions: Our findings show that VWF and VWFpp, in contrast to endothelial cells, are differentially released from activated platelets. This may affect how platelet-derived VWF and VWFpp contribute to hemostatic clots.

PB0911 | Low Temperature Induces von-Willebrand Factor Expression via Increased Early Growth Response 1 Transcriptional Activity in Splenic Sinusoidal Endothelial Cells

K. Horioka ${ }^{1,2}$; H. Tanaka ${ }^{3} ;$ K. Okaba ${ }^{1} ;$ N. Ishii ${ }^{1} ;$ A. Motomura ${ }^{1}$; H. Inoue ${ }^{1}$; K. Alkass $^{2}$; H. Druid ${ }^{2}$; D. Yajima ${ }^{1}$

${ }^{1}$ Department of Legal Medicine, International University of Health and Welfare, Narita, Japan; ${ }^{2}$ Department of Oncology-Pathology, Karolinska Institutet, Stockholm, Sweden; ${ }^{3}$ Division of Tumor Pathology, Department of Pathology, Asahikawa Medical University, Asahikawa, Japan

Background: von Willebrand factor (VWF) is a large plasma glycoprotein that plays an important role in hemostasis by forming molecular bridges with platelets following vascular injury. Previously, we reported that hypothermia enhanced vWF production in the spleen, which resulted in the activation of the platelet pool in a hypothermiainduced murine model. However, the mechanisms that regulate vWF expression under hypothermic conditions remain unclear.

Aims: In this study, we determined gene expression changes induced by cold stimulation in human SSEC and aimed to identify the molecular basis of hypothermia-induced VWF expression.

Methods: Human splenic endothelial cells (HSEC) were incubated at $20^{\circ} \mathrm{C}$ for 1 hour. Total RNA was extracted from the cells, and cDNA microarray gene expression analysis was performed. Genes that may be associated with vWF expression in low-temperature culture conditions were then selected for further analysis. 
Results: Gene expression analysis showed that low-temperature conditions increased the expression of FOS and EGR1. We then hypothesized that these factors upregulate VWF mRNA expression in HSEC. The transcriptional inhibitors of EGR1 significantly inhibited vWF mRNA expression in HSEC cultured at a low temperature.

Conclusions: Our analysis revealed that low temperatures enhance the gene expression of EGR1, which transcriptionally increases vWF expression. This acute-phase reaction may play an important role in platelet activation in the spleen during hypothermia.

PB0912 | Palmitate Increases Gene Expression and Stimulated Secretion of von Willebrand Factor in Human Umbilical Vein Endothelial Cells

A.K. Seliga; K. Zabłocki; J. Bandorowicz-Pikuła

Nencki Institute of Experimental Biology, Polish Academy of Sciences, Warsaw, Poland

Background: Palmitic acid is one of the most abundant saturated fatty acid in the plasma of obese patients. Obesity which finally leads to the insulin resistance and type 2 diabetes results in a dysfunction of vascular endothelium and proinflammatory response. In human with type 2 diabetes or insulin resistance higher level of secreted von Willebrand Factor (vWF) is associated with an elevated risk of cardiovascular disease.

Aims: The aim of the study was to test whether in vitro palmitate treatment of Human Umbilical Vein Endothelial Cells (HUVEC) affects gene expression, secretion and protein level of VWF.

Methods: HUVECs were treated with palmitate complexed with BSA or BSA alone as a control. TaqMan RT-qPCR was performed to examine vWF, P-selectin, CD63 genes expression in the control and palmitatetreated cells. HUVECs also were treated with histamine and forskolin to compare using ELISA test a basal and stimulated secretion of vWF. Western Blot was employed to analyze vWF protein level in cell lysates. One-way ANOVA statistical analysis was performed.

Results: Incubation of HUVECs with palmitate $(100 \mu \mathrm{M})$ resulted in the increased vWF gene expression in comparison to BSA exposed controls after $24 \mathrm{~h}$ of treatment, while P-selectin and CD63 transcripts level remained unchanged. Moreover, $48 \mathrm{~h}$ treatment of the cells with palmitate increased histamine- and forskolin-stimulated secretion of VWF, without influencing the basal secretion.
Furthermore, the level of the mature protein form of vWF was elevated in the palmitate-treated cells.

Conclusions: In vitro incubation of HUVECs with palmitate increases vWF gene expression and protein vWF. Moreover it increases histamine- and forskolin stimulated secretion of vWF. These results suggest a potential link between obesity and dysfunction of vascular endothelium. This work was supported by a grant number 2016/23/B/NZ3/03116 from the National Science Center, Poland, and by statutory funds from the Nencki Institute of Experimental Biology.

PB0913 | Prevailing c.2435delC and Other VWF Gene Defects in Russian Patients with von Willebrand Disease Type 3. Four New Pathogenic Variants Found

D. Chernetskaya ${ }^{1}$; E. Likhacheva ${ }^{1}$; F. Perina ${ }^{2}$; O. Pshenichnikova ${ }^{1}$; V. Surin ${ }^{1}$; N. Zozulya ${ }^{1}$

${ }^{1}$ National Medical Research Center of Hematology Ministry of Health of Russia, Moscow, Russian Federation; ${ }^{2}$ Center of Children Oncology and Hematology, Sverdlovsk Region Clinical Hospital for Children No. 1, Ekaterinburg, Russian Federation

Background: Type 3 of von Willebrand disease (vWD) demands two pathogenic variants (compound or homozygous) in the vWF gene. This type is associated with the most severe disease symptoms and almost total lack of von Willebrand factor in the bloodstream and, as a consequence, low FVIII value too.

The vWF gene consists of 52 exons and lies in the 12th chromosome. Aims: We aimed to find pathogenic variants in the vWF gene, which could lead to observed symptoms.

Methods: We used the Sanger method to get sequences of vWF exons, using primers of our design for all the exons and exon-intron junctions, except for the first one. We chose 13 patients with VWF:RCo value $\leq 5 \%$ and FVIII:C $\leq 10 \%$, which describe vWD type 3 . Results: The deletion c.2435delC (Zhang, 1992) occurred in three patients in a homozygous state and in eight patients as a compound with another pathogenic variant (Table 1). In one case, no other disruptions were found, except for c.2435delC in a heterozygous state. The only patient without c. $2435 \mathrm{delC}$ had the following pathogenic variants: c.6970delG (new) in the 40th exon and c.2968-2 A>G (new) in intron before the 23rd exon (Table 1).

\section{Conclusions:}

TABLE 1 The pathogenic variants, were found in compound with c. $2435 \mathrm{delC}$

$\begin{array}{lllllll}\text { Patient No } & \begin{array}{l}\text { Pathogenic } \\ \text { variant (1) }\end{array} & \text { Exon number (1) } & \text { Reference (1) } & \begin{array}{l}\text { Pathogenic } \\ \text { variant (2) }\end{array} & \text { Exon number (2) } & \text { Reference (2) } \\ 1 & \text { c. } 2435 \mathrm{delC} & 18 & \text { Zhang, 1992 } & \text { p.Arg273Trp } & 7 & \text { Allen, 2000 } \\ 2 & \text { c. } 2435 \mathrm{delC} & 18 & \text { Zhang, 1992 } & \text { c.6029delC } & 35 & \text { New } \\ 3 & \text { c. } 2435 \mathrm{delC} & 18 & \text { Zhang, 1992 } & \text { p.Arg1659Stop } & 28 & \text { Zhang, } 1992 \\ 4 & \text { c. } 2435 \mathrm{delC} & 18 & \text { Zhang, 1992 } & \text { p.Ala2178Ser } & 37 & \text { Goodeve, } 2007 \\ 5 & \text { c. } 2435 \mathrm{delC} & 18 & \text { Zhang, 1992 } & \text { p.Arg373Stop } & 10 & \text { Baronciani, 2000 } \\ 6 & \text { c. } 2435 \mathrm{delC} & 18 & \text { Zhang, 1992 } & \text { p.Cys1101Arg } & 25 & \text { Gadisseur, 2009 }\end{array}$




\begin{tabular}{|c|c|c|c|c|c|c|}
\hline Patient № & $\begin{array}{l}\text { Pathogenic } \\
\text { variant (1) }\end{array}$ & Exon number (1) & Reference (1) & $\begin{array}{l}\text { Pathogenic } \\
\text { variant (2) }\end{array}$ & Exon number (2) & Reference (2) \\
\hline 7 & c. $2435 \mathrm{delC}$ & 18 & Zhang, 1992 & p.Thr791Met & 18 & Gaucher, 1991 \\
\hline 8 & c. $2435 \mathrm{delC}$ & 18 & Zhang, 1992 & c.6457insA & 37 & New \\
\hline 9 & c.6970delG & 40 & New & c. $2968-2 A>G$ & 23 & New \\
\hline
\end{tabular}

Deletion c.2435delC is prevailing for type 3 of vWD in the Russian population, it was found in 12 patients out of 13 . It is common in the world population, but we didn't expect it to be that prevailing.

The patient with only heterozygous c.2435delC and no other changes could have a large heterozygous deletion, which could not be found by Sanger sequencing.

In total, we found four different missense and two nonsense variants, one in the splicing area, three deletions, and one insertion. All pathogenic variants, except for c.2435delC, occurred only once. New (not mentioned in HGMD, EAHAD and NCBI) pathogenic variants were c.6457insA, c.6029delC, c.2968-2 A>G and c.6970delG.
P0158 | Von Willebrand Factor Multimer Distribution Analysis in a Group of Patients Diagnosed with von Willebrand Disease

E. Wojtasinska; O. Krupinska; M. Malachowska; A. Szczepaniak; J. Rupa-Matysek; L. Gil

Dept. of Haematology and Bone Marrow Transplantation Karol

Marcinkowski University of Medical Sciences, Poznan, Poland

Background: von Willebrand factor (vWF) multimer (MM) analyses are required for von Willebrand disease (vWD) classification and to distinguish among subtypes.

Aims: Was to analyse the vWF multimers distribution using the HYDRAGEL VW multimer assay (HS/11VWM, Sebia) in a group of 69 patients diagnosed with von Willebrand disease.

\section{Methods:}

TABLE 1

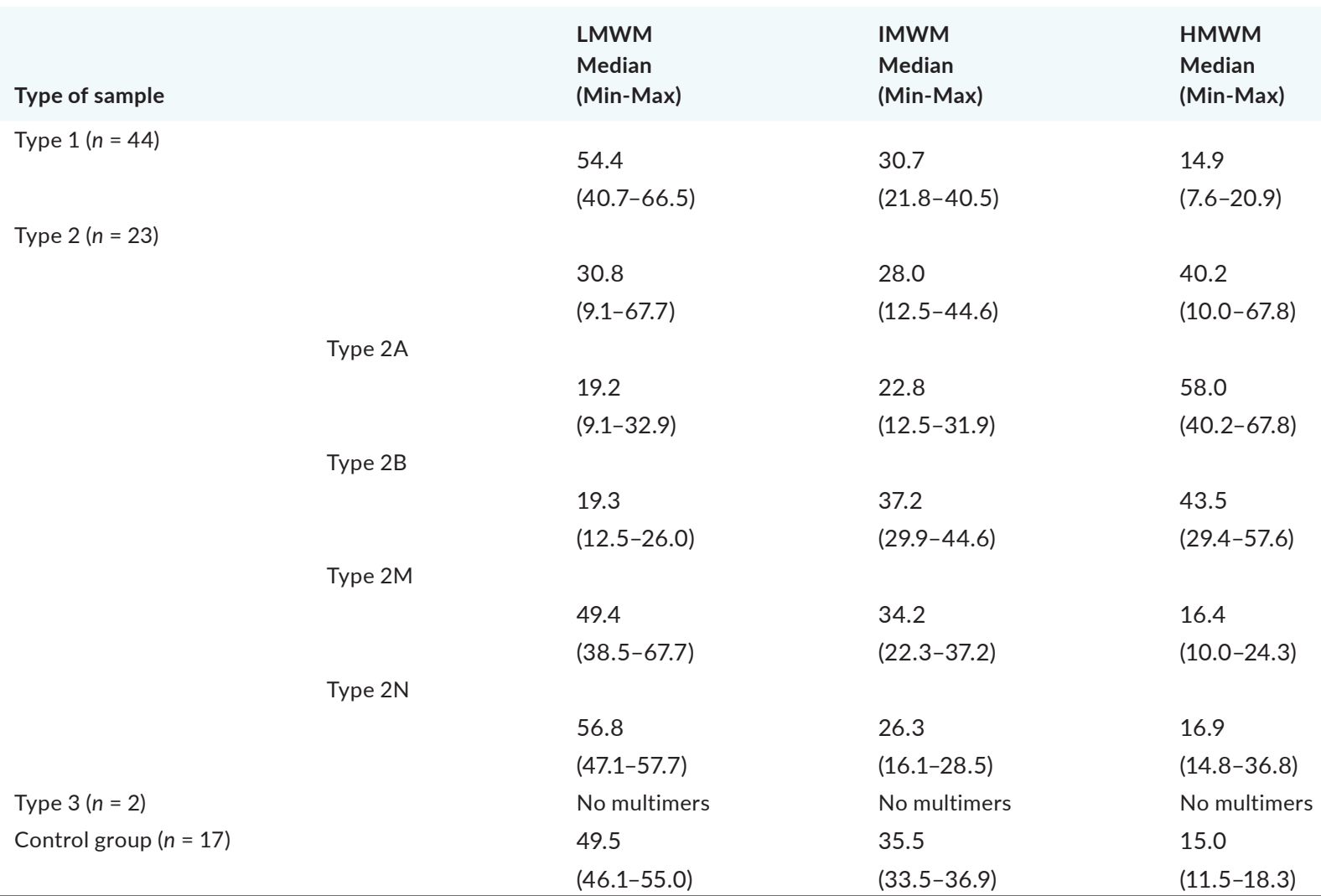

69 patients (52 female) with a median age of 42 (range 18-83) were classified into three main types and four subtypes of type 2 , according to the ISTH/SSC, using the following tests: vWF antigen (vWF:Ag), factor VIII clotting activity (FVIII:C), vWF ristocetin cofactor activity ( $v W F: R C o)$, ristocetin-induced platelet aggregation (RIPA), vWF collagen binding activity (vWF:CBA), ACLTop 300. Type 1: 44pts (63.8\%), type 2: 23 pts (33.3\%), type 2A: 11 pts (16\%), type $2 \mathrm{~B}: 2 \mathrm{pts}(2.9 \%)$, type $2 \mathrm{M}: 5 \mathrm{pts}(7.2 \%)$, type $2 \mathrm{~N}$ : $5 \mathrm{pts}(7.2 \%)$, type $3: 2 \mathrm{pts}(2.9 \%)$. The control group consisted of 17 normal healthy adults. Analysis of vWF multimers distribution was made using a HS/11VWM assay (Sebia). 
Results:

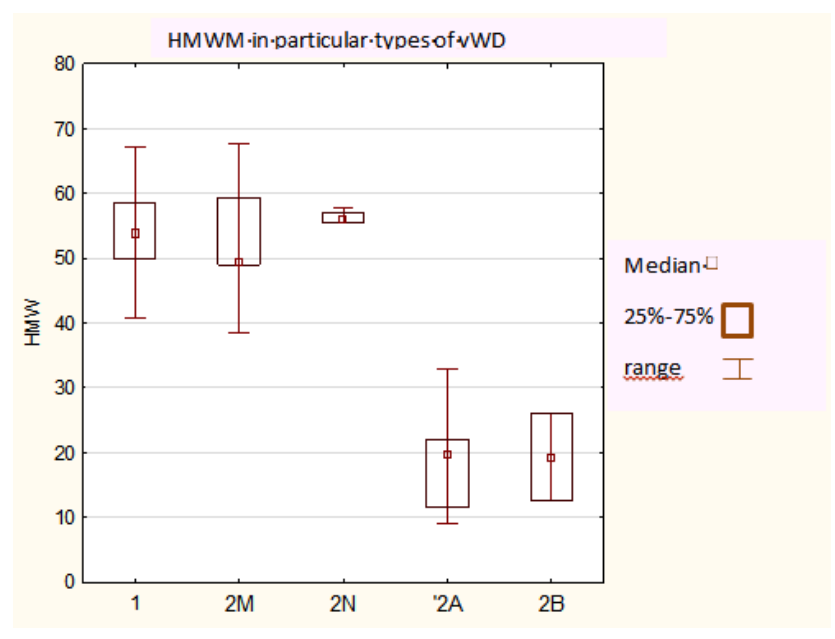

FIGURE 1

First we visually evaluated proteins separated into a gel into characteristic bands, then we defined the multimer fractions (LMWM low molecular weight multimers - peak 1-3; IMWM - intermediate molecular weight multimers - peak 4-7; HMWM >7) using Phoresis software. Densitometric quantification of the fractions were also conducted. A statistically significant difference was observed when comparing HMWM in the group of the patients with type 1 and type $2(P<0.0001)$. We also noticed significant differences in HMWM distribution when comparing patients with Type 1 and $2 \mathrm{~A}$ $(P<0.0001) ; 2 \mathrm{~A}$ and $2 \mathrm{M}(P=0.0351) ; 2 \mathrm{~A}$ and $2 \mathrm{~N}(P=0.0058)$. No difference was found in group of the patients classified as type 1 , type $2 \mathrm{M}$ and type $2 \mathrm{~N}(P=0.8569)$.

Conclusions: Multimer analysis using the HS/11VWM assay (Sebia) can be proposed as a screening test that helps to make an accurate distinction between normal multimer distribution (types $1,2 \mathrm{M}, 2 \mathrm{~N}$ ) and absence of multimers (type $2 \mathrm{~A}$ ).

\section{VWF AND VON WILLEBRAND FACTOR DISORDERS - CLINICAL CONDITIONS}

\section{LPB0031 | Activity and Cleavage of von Willebrand Disease Type 2B Variants}

$\underline{\text { M. Brehm }^{1}}$; Y. Yildiz ${ }^{2}$ T. Obser ${ }^{1}$; A. Mojzisch ${ }^{1} ;$ S. Peine ${ }^{3} ;$ S. Schneppenheim $^{4}$; U. Budde ${ }^{4}$; R. Schneppenheim ${ }^{5}$

${ }^{1}$ University Medical Center Hamburg-Eppendorf / Dermatology, Hamburg, Germany; ${ }^{2}$ Marienkrankenhaus, Hamburg, Germany;

${ }^{3}$ University Medical Center Hamburg-Eppendorf / Transfusion Medicine, Hamburg, Germany; ${ }^{4}$ MEDILYS Laborgesellschaft $\mathrm{mbH}$, Hamburg, Germany; ${ }^{5}$ University Medical Center Hamburg-Eppendorf / Pediatric Hematology and Oncology, Hamburg, Germany

Background: Von Willebrand disease (VWD) is the most common hereditary bleeding disorder. Subtype 2B (VWD2B) is caused by gain-of-function (GOF) mutations in the VWF-A1-domain inducing increased binding to platelet glycoprotein (GP)lba, inducing spontaneous platelet binding leading to thrombocytopenia. Further, variable reduction of von Willebrand factor (VWF) high molecular weight multimers (HMWM) and increased ADAMTS13 cleavage can occur. Aims: Aim of this study was the identification of underlying mutations in 113 patients with suspected VWD2B and functional characterization of the identified variants with respect to GPIba binding, multimer status and ADAMTS13 cleavage.

Methods: VWF exon 28 was sequenced in patient DNA samples for diagnostic purpose. VWF:GPIba binding was measured by an ELISA employing a recombinant GPIba peptide as capture component at multiple Ristocetin concentrations. Degradation of the variants by ADAMTS13 was measured employing a modified light transmission aggregometry (LTA) assay. Washed, recalcified platelets were mixed with the recombinant $2 \mathrm{~B}$ proteins. After complex formation, their degradation by ADAMTS13 was detected by increase in turbidty.

Results: Genetic analysis revealed 15 different mutations in our patient cohort, two of which were previously associated with VWD2M (p.Arg1315Cys, p.Val1279lle). Increased GPIba binding was confirmed for the remaining 13 variants. Degradation of 2BVWFplatelet-complexes by ADAMTS13, counterintuitively, revealed that some variants exhibit decreased sensitivity for proteolytic cleavage in simulated circulation.

Conclusions: Summarizing, we characterized VWD2B variants found in a cohort of 113 patients. The used ELISA proved to be applicable to differentiate $2 B$ variants from other types of VWD and the absence of patient platelets prevents false positive results due to platelet type-VWD. Additionally, our data indicate that increased proteolysis of some variants does not arise from enhanced degradation of circulating 2BVWF-platelet-complexes but more likely occurs at the surface of endothelial cells during secretion. Our data could increase understanding of VWD2B disease phenotypes.

LPB0032 | Genetic Characterization of von Willebrand Disease Type 2 in Milan Cohort Patients

O. Seidizadeh ${ }^{1}$; L. Baronciani ${ }^{1}$; M.T. Pagliari ${ }^{1}$; G. Cozzi ${ }^{1}$; P. Colpani ${ }^{1}$; S.M. Siboni ${ }^{1}$; E. Biguzzi ${ }^{1}$; F. Peyvandi ${ }^{1,2}$

${ }^{1}$ Angelo Bianchi Bonomi Hemophilia and Thrombosis Center, Fondazione IRCCS Ca' Granda Ospedale Maggiore Policlinico and Luigi Villa Foundation, Milan, Italy; ${ }^{2}$ Università degli Studi di Milano, Department of Pathophysiology and Transplantation, Milan, Italy

Background: Von Willebrand disease (VWD) type 2 is caused by qualitative defects of von Willebrand factor (VWF) for binding to glycoprotein Ib, collagen, or factor VIII.

Aims: Genetic characterization of a large VWD type 2 cohort in Milan.

Methods: We enrolled 311 patients (female/male $=173 / 138$ ) from 172 unrelated families with VWD type 2 diagnosis. Patients were characterized with full laboratory phenotype tests and their 
diagnosis was confirmed by target genetic analysis using Sanger sequencing following the ISTH guidelines.

Results: Patients were diagnosed with type $2 \mathrm{~A}(n=94), 2 \mathrm{~B}(n=84)$, $2 \mathrm{M}(n=105), 2 \mathrm{~N}(n=25)$ and 3 patients remain unclassified [Fig 1 ].

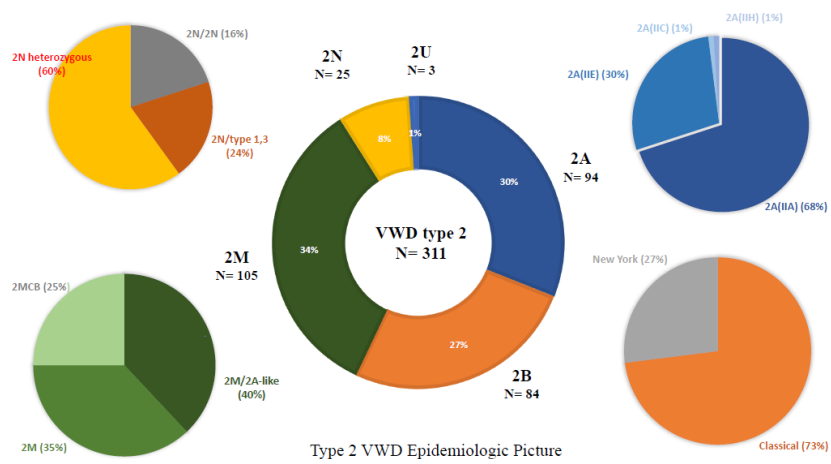

FIGURE 1 The epidemiologic picture and frequency of different VWD type 2

Eighty-three different VWF variants including 9 novels (p.L893R, p.C1126Y, p.C1142F, p.L1281R, p.R1379H, p.R1426P, p.L1657P, p.S1731L, p.C2557Y) were found. Most patients were heterozygous for a single variant $(n=249)$, whereas 35 cases had 2 mutations: 4 were homozygous, 16 compounds heterozygous (in trans), and 15 in cis position. Twenty-seven patients had $\geq 3$ variants, all due to gene conversion except one. Among the eighty-three distinct variants identified, five mutation types were observed: missense ( $n=65$, $78.3 \%)$, gene conversion ( $n=12,14.5 \%$ ), synonymous ( $n=1,1.2 \%$ ), deletion ( $n=4,4.8 \%$ ) and splice ( $n=1,1.2 \%$ ). In type $2 \mathrm{~A}, 59 \%$ of mutations were located in the A2 domain (IIA), $26 \%$ and $7.5 \%$ were respectively at the $\mathrm{D} 3$ and $\mathrm{A} 1$ domains (IIE). In type $2 \mathrm{~B}$, the variants were at $\mathrm{A} 1$ domain (85\%) and at the D3-A1 junction (15\%). In type $2 \mathrm{M}, 77 \%$ were located at the $\mathrm{A} 1$ domain, whereas $23 \%$ were at $A 3$ domain. In type $2 \mathrm{~N}$, all patients had p.R854Q ( $\mathrm{D}^{\prime}$ domain) in either homozygous, heterozygous (carrier), or compound heterozygous with VWF quantitative variants. The common mutations for each VWD type 2 are shown in red in Figure 2.

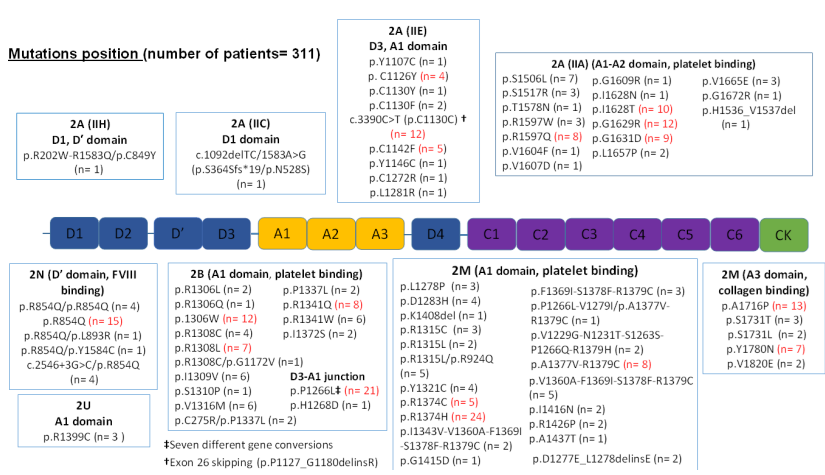

FIGURE 2 Location of type 2 VWD mutations on the pro-VWF coding region
Conclusions: > Genetic analysis of a large cohort of VWD type 2 in Milan showed that the vast majority of patients (88.4\%) had missense variants located in specific domains in each type.

LPB0128 | Phase 3 Trial Results: Prophylaxis with Recombinant von Willebrand Factor in Patients with Severe von Willebrand Disease

F.WG Leebeek ${ }^{1}$; F. Peyvandi ${ }^{2}$; M. Escobar ${ }^{3}$; A. Tiede ${ }^{4}$; G.

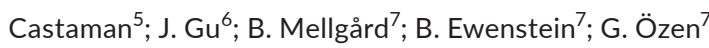

${ }^{1}$ Department of Hematology, Erasmus MC, University Medical Center, Rotterdam, Netherlands; ${ }^{2}$ Angelo Bianchi Bonomi Hemophilia and Thrombosis Center, Fondazione IRCCS Ca' Granda Ospedale Maggiore, Policlinico, University of Milan, Milan, Italy; ${ }^{3}$ University of Texas Health Science Center at Houston, Houston, United States; ${ }^{4}$ Hannover Medical School, Department of Hematology, Hemostasis, Oncology and Stem Cell Transplantation, Hannover, Germany; ${ }^{5}$ Center for Bleeding Disorders and Coagulation, Careggi University Hospital, Florence, Italy; ${ }^{6}$ Shire US Inc., a Takeda Company, Lexington, United States; ${ }^{7}$ Baxalta US Inc., a Takeda Company, Cambridge, United States

Background: Patients with severe von Willebrand disease (VWD) may benefit from prophylaxis with recombinant von Willebrand factor (rVWF, vonicog alfa; Baxalta US Inc., a Takeda company, Lexington, MA, USA) to reduce frequency of spontaneous bleeding events (BEs) requiring VWF treatment.

Aims: Investigate efficacy and safety of rVWF prophylaxis.

Methods: Prospective, open-label, non-randomized, multicenter, phase 3 study (NCT02973087, EudraCT 2016-001478-14). Eligible patients were aged $\geq 18$ years, had severe VWD (VWF ristocetin cofactor activity $<20 \mathrm{IU} / \mathrm{dL}$ ) requiring $\mathrm{VWF}$ therapy to manage BEs in past year (on-demand [prior OD arm] or plasma-derived VWF [pdVWF] prophylaxis [switch arm]), and no VWF or factor VIII inhibitors or history of thromboembolic events. Planned prophylactic rVWF treatment duration was $\geq 1$ year: prior OD patients started with $50 \pm 10 \mathrm{IU} / \mathrm{kg}$ twice weekly; starting dose for switch patients was based on prior pdVWF weekly VWF dose and dosing frequency (1-3 times weekly; maximum $80 \mathrm{lU} / \mathrm{kg} /$ infusion). Primary endpoint was annualized bleeding rates (ABRs) for treated, spontaneous BEs during rVWF prophylaxis. Informed consent and ethics approval were obtained.

Results: 23 enrolled patients received rVWF prophylaxis (prior OD arm: $n=13$; switch arm: $n=10) ; 18 / 23(78.3 \%)$ patients had type 3 VWD. Over the 12-month study period, 11/13 (84.6\%) prior OD patients and $7 / 10(70.0 \%)$ switch patients had a treated, spontaneous ABR (sABR) of zero, whereas, historically, 13/13 prior OD and 1/10 switch patients had an $S A B R>2$. The $S A B R$ was reduced by $91.5 \%$ on study compared with historical SABR in prior OD patients, and by $45.0 \%$ in switch patients (model-based analysis; Table 1). Benefit-risk profile was maintained, with no newly identified risks (Table 2). 
TABLE 1 Primary efficacy analysis: comparison of on-study SABR through month 12 vs historical SABR using a negative binomial model (full analysis set)

\begin{tabular}{|c|c|c|}
\hline Time period Statistic & $\begin{array}{l}\text { Prior OD arm* } \\
(n=13)\end{array}$ & $\begin{array}{l}\text { Switch } \operatorname{arm}^{\dagger} \\
(n=10)\end{array}$ \\
\hline $\begin{array}{l}\text { Historical } \\
\text { Number of treated } \\
\quad \text { spontaneous BEs } \\
\text { sABR }(95 \% \mathrm{Cl})\end{array}$ & $\begin{array}{l}201 \\
6.54(2.52,17.00)\end{array}$ & $\begin{array}{l}50 \\
0.51(0.04,6.31)\end{array}$ \\
\hline $\begin{array}{l}\text { On-study (while } \\
\text { receiving } \\
\text { prophylactic } \\
\text { rVWF) } \\
\text { Number of treated } \\
\text { spontaneous BEs } \\
\text { sABR }(95 \% \mathrm{Cl})\end{array}$ & $\begin{array}{l}9 \\
0.56(0.15,2.05)\end{array}$ & $\begin{array}{l}18 \\
0.28(0.02,3.85)\end{array}$ \\
\hline $\begin{array}{l}\text { Comparison (on- } \\
\text { study vs historical } \\
\text { sABR) } \\
\text { sABR on- } \\
\text { study:historical } \\
\text { ratio }(95 \% \mathrm{Cl}) \\
\text { sABR percentage } \\
\text { change from } \\
\text { historical }\end{array}$ & $\begin{array}{l}0.09(0.02,0.35) \\
91.5 \% \text { reduction }\end{array}$ & $\begin{array}{l}0.55(0.09,3.52) \\
45.0 \% \text { reduction }\end{array}$ \\
\hline
\end{tabular}

$\mathrm{BE}$, bleeding episode; OD, on-demand; rVWF, recombinant von Willebrand factor; SABR, spontaneous annualized bleeding rate (ABR for spontaneous bleeds).

sABR was the number of spontaneous bleeds divided by the observation period in years. Only BEs treated with VWF infusions are included. Six BEs of unknown cause (4 historical [all in prior OD group] and 2 on-study [switch group]) were counted as spontaneous bleeds for this analysis.

Percentage change in SABR was calculated directly from the SABR ratio (RR): $100 \times(R R-1)$.

*Patients who were treated on-demand with any VWF during the 12-month period prior to enrolling into this study to receive prophylaxis with rVWF.

tPatients who were treated prophylactically with a plasmaderived VWF during the 12-month period prior to enrolling into this study to receive prophylaxis with rVWF.
TABLE 2 Safety overview

\begin{tabular}{|c|c|c|}
\hline & $\begin{array}{l}\text { Prior OD arm } \\
(n=13)\end{array}$ & Switch arm $(n=10)$ \\
\hline All AEs & 10 (76.9) / 26 & $7(70.0) / 15$ \\
\hline Serious AEs & $1(7.7) / 1$ & $2(20.0) / 2$ \\
\hline AEs related to $\mathrm{rVWF}^{*}$ & $1(7.7) / 1$ & 0 \\
\hline $\begin{array}{l}\text { Serious AEs related } \\
\text { to } r V W F\end{array}$ & 0 & 0 \\
\hline $\begin{array}{l}\text { AEs leading to rVWF } \\
\text { discontinuation* }\end{array}$ & $1(7.7) / 1$ & 0 \\
\hline Severe AEs & $2(15.4) / 3$ & $1(10.0) / 1$ \\
\hline $\begin{array}{c}\text { AEs of special } \\
\text { interest }^{\dagger}\end{array}$ & $1(7.7) / 1$ & $1(10.0) / 1$ \\
\hline
\end{tabular}

$\mathrm{AE}$, adverse event; OD, on-demand.

Values are number (\%) of patients with events / number of events. ${ }^{*}$ Headache of moderate severity, considered possibly related to rVWF by investigator (the only AE considered related to study treatment), led to rVWF discontinuation and study withdrawal.

${ }^{\dagger} \mathrm{AE}$ of special interest (hypersensitivity reactions, thromboembolic events, development of inhibitors) were identified by broad standardized MedDRA queries (SMQ) search and immunogenicity laboratory results. 1 event of non-serious, non-severe purpura was attributed to trauma and considered unrelated to rVWF and 1 event of non-serious, non-severe rash pruritic was considered unrelated to rVWF; no inhibitors to VWF or FVIII were developed.

Overall number of exposure days (ED) for rVWF in Takedasponsored clinical trials increased from $\sim 450$ days in previously completed trials to $>2200$ days upon completion of this study.

Conclusions: These findings suggest that rVWF prophylaxis can effectively reduce SABR in patients previously treated OD with VWF products and maintains at least the same level of hemostatic control in patients who switch from prophylaxis with pdVWF to rVWF, with a favorable safety profile.

PB0915 | von Willebrand Factor (VWF) Multiplex Activity Assay Differentiation of Low VWF/Type 1 von Willebrand Disease (VWD) and Variant VWD: Analysis from the Comparative Effectiveness in the Diagnosis of VWD

J.C. Roberts ${ }^{1}$; P.A. Christopherson ${ }^{2}$; M.D. Tarantino ${ }^{1}$;

S.E. Gonzales ${ }^{1}$; P.A. Morateck ${ }^{2}$; C.L. Perry ${ }^{2}$; V.H. Flood ${ }^{2}$;

T.C. Abshire ${ }^{2}$; R.R. Montgomery ${ }^{2}$; Z.P. Investigators ${ }^{2}$

${ }^{1}$ Bleeding \& Clotting Disorders Institute, Peoria, United States; ${ }^{2}$ Versiti

Blood Research Institute, Milwaukee, United States

Background: VWD diagnosis is challenging. We developed an ELISA-based VWF Multiplex Activity Assay (VWF-MAA) (Roberts JC, et al. Blood 2016)* to address this concern; however, ability of 
the VWF-MAA to discriminate between low VWF/type 1 VWD, variant $\mathrm{VWD}$, and normal subjects is unknown.

Aims: To evaluate the VWF-MAA and its ability to differentiate between low VWF/type 1 VWD, variant VWD, and normal subjects in individuals undergoing an initial laboratory evaluation for bleeding. Methods: 177 plasma samples from the Zimmerman Program: Comparative Effectiveness in the Diagnosis of VWD were evaluated on the VWF-MAA at the Bleeding \& Clotting Disorders Institute (BCDI). Samples were obtained from 11 Centers across the US and Canada. VWF:Ag ratio of $<1.7$ was considered "low VWF/type 1 VWD" as this approximates $<51 \mathrm{U} / \mathrm{dL}$ when compared to $30 \%$ control plasma where control ratio is 1. Data were compared to the Versiti (VBRI) research laboratory and the local Center (LC) assigned diagnosis.

Results: The majority of patients evaluated were white, non-Hispanic, and female with mean and median age in adolescence. Table 1 and
Table 2 show data. Overall, 129/177 (72.9\%) were correctly assigned as normal (non-VWD), low VWF/type 1, or variant VWD compared to the VBRI assigned diagnosis. VWF-MAA assigned non-VWD accurately in 29/57 (50.9\%) samples, and low VWF/type 1 accurately in 93/110 (84.6\%) samples. Considering LC diagnosis where there was agreement with VWF-MAA and not VBRI diagnosis, low VWF/ type 1 was accurate in 105/110 (95.5\%) samples. For variant VWD, 4 type 2A VWD, 2 type 2B VWD, and 1 type $1 C$ VWD samples were accurately assigned.

Conclusions: We demonstrate the VWF-MAA has utility in differentiating low VWF/type 1 VWD, variant VWD, and normal subjects in individuals undergoing initial hemostatic testing. Comparing data from multiple hemostasis laboratories highlights challenges associated with current VWD diagnosis.

*Blood. 2016 May 19;127(20):2472-80.

TABLE 1 Versiti Blood Research Institute and Local Center Laboratory Studies

VBRI Laboratory Studies

\begin{tabular}{|c|c|c|c|c|c|c|}
\hline & $\begin{array}{l}\text { VWF:Ag } \\
\text { U/mL }\end{array}$ & $\begin{array}{l}\text { VWF:GPIbM } \\
\text { U/mL }\end{array}$ & $\begin{array}{l}\text { VWF:CB3 } \\
\mathrm{U} / \mathrm{mL}\end{array}$ & $\begin{array}{l}\text { VWF:Ag } \\
\text { U/mL }\end{array}$ & $\begin{array}{l}\text { VWF:RCo } \\
\text { U/mL }\end{array}$ & $\begin{array}{l}\text { Factor VIII activity } \\
\mathrm{U} / \mathrm{mL}\end{array}$ \\
\hline $\begin{array}{l}\text { Overall } \\
\text { Mean (St Dev) }\end{array}$ & $56.9(23.7)$ & 70.1 (39.1) & $71.5(32.2)$ & $55.8(23.3)$ & $48.2(22.8)$ & $80.1(26.6)$ \\
\hline $\begin{array}{l}\text { Overall } \\
\text { Median (Range) }\end{array}$ & $53(18-157)$ & $62(7-283)$ & $68.5(5-208)$ & $52(10-151)$ & $45(7-178)$ & $76(14-220)$ \\
\hline $\begin{array}{l}\text { Non-VWD } \\
\text { Mean (St Dev) }\end{array}$ & $90(28.6)$ & 94.5 (42.2) & $103.7(33.7)$ & $81.5(26.6)$ & $69.3(31.1)$ & 96.7 (31.3) \\
\hline $\begin{array}{l}\text { Non-VWD } \\
\text { Median (Range) }\end{array}$ & $71(50-157)$ & $80(50-257)$ & $92(61-208)$ & $72(51-151)$ & $55(23-178)$ & $90.4(52-220)$ \\
\hline $\begin{array}{l}\text { Low VWF } \\
\text { Mean (St Dev) }\end{array}$ & $52.7(11.5)$ & $65.5(21.4)$ & $68.5(16.8)$ & $51.2(10.2)$ & $45.5(9.7)$ & $79.4(20.0)$ \\
\hline $\begin{array}{l}\text { Low VWF } \\
\text { Median (Range) }\end{array}$ & $53(31-92)$ & $61(32-154)$ & $67.5(36-150)$ & $49.5(31-77)$ & 44 (29-98) & $77(40-166)$ \\
\hline $\begin{array}{l}\text { Type } 1 \text { VWD } \\
\text { Mean (St Dev) }\end{array}$ & $30.2(7.6)$ & $33.9(9.0)$ & $35.2(12.3)$ & $29.8(8.9)$ & $24.1(6.8)$ & 60.9 (19.3) \\
\hline $\begin{array}{l}\text { Type } 1 \text { VWD } \\
\text { Median (Range) }\end{array}$ & $30(18-48)$ & $33.5(17-51)$ & $36(13-58)$ & $29(10-48)$ & $25(13-39)$ & $61.5(14-101)$ \\
\hline $\begin{array}{l}\text { Variant VWD } \\
\text { Mean (St Dev) }\end{array}$ & $42.2(23.1)$ & $72.6(95.1)$ & $18.5(9.0)$ & $37.7(22.5)$ & $18.3(10.0)$ & $54.8(25.9)$ \\
\hline $\begin{array}{l}\text { Variant VWD } \\
\text { Median (Range) }\end{array}$ & $33(20-98)$ & $36(7-283)$ & $21.5(5-32)$ & $28(21-97)$ & $19.8(7-42)$ & $51(27-123)$ \\
\hline
\end{tabular}

Local Center Laboratory Studies 
TABLE 2 VWF-MAA Non-VWD vs Low VWF/Type 1 VWD

\begin{tabular}{llllll} 
Non-VWD & & \multicolumn{3}{l}{ Low VWF/Type 1 VWD } \\
VWF:Ag & Median (Range) & Mean (St Dev) & VWF:Ag & Median (Range) & Mean (St Dev) \\
OD Ratio & $2.22(1.73-3.97)$ & $2.33(0.46)$ & OD Ratio & $1.26(0.37-1.69)$ & $1.24(0.3)$ \\
\hline
\end{tabular}

PB0916 | Increased Cleavage of VWF by ADAMTS13 Might Reduce High-molecular-weight VWF Multimers, Leading to Acquired von Willebrand Syndrome in Patients with Essential Thrombocythemia

M. Kubo ${ }^{1,2}$; H. Kashiwagi ${ }^{3}$; H. Yagi ${ }^{4}$; Y. Seki ${ }^{5}$; A. Hasegawa ${ }^{2}$; H. Tanaka ${ }^{2}$; I. Amano ${ }^{2}$; Y. Tomiyama ${ }^{6}$; M. Matsumoto ${ }^{1}$ ${ }^{1}$ Department of Blood Transfusion Medicine, Nara Medical University, Kashihara, Japan; ${ }^{2}$ Department of Hematology, Nara Medical University, Kashihara, Japan; ${ }^{3}$ Department of Hematology and Oncology, Osaka University, Suita, Japan; ${ }^{4}$ Department of Hematology and Oncology, Nara Prefecture General Medical Center, Nara, Japan; ${ }^{5}$ Department of Hematology, Uonuma Institute of Community Medicine, Niigata University Medical and Dental Hospital, Minamiuonuma, Japan; ${ }^{6}$ Department of Blood Transfusion, Osaka University, Suita, Japan

Background: Essential thrombocythemia (ET) is a BCR/ABL1negative myeloproliferative neoplasm characterized by thrombocytosis and an elevated incidence of thrombosis. Paradoxically, when platelet count is markedly increased, bleeding is often observed. Extreme thrombocytosis is associated with reduced von Willebrand factor (VWF) large multimers. This condition is known as acquired von Willebrand syndrome.

Aims: We investigated whether VWF degradation by ADAMTS13 is enhanced in ET patients.

Methods: VWF antigen (Ag), VWF multimers, and ADAMTS13 activity were analyzed in $50 \mathrm{ET}$ patients before and after starting treatment. VWF multimers were divided into three classes (high-, medium-, and low-molecular weight multimers [HMW-, MMW-, and LMW-VWFMs]), and ratios of prevalence of each class in each patient to that in healthy subjects (multimer index) was calculated using densitometric analysis. VWF-degradation product (DP) was measured by ELISA, using a monoclonal antibody that specifically recognizes $\mathrm{Y} 1605$ at the C-terminal boundary of the VWF A2 domain (a determinant of cleavage by ADAMTS13).

Results: Fifty ET patients were divided into low platelet $\left(<750 \times 10^{3} /\right.$ $\mu \mathrm{l}, n=28$ ) and high platelet $\left(\geq 750 \times 10^{3} / \mu \mathrm{l}, n=22\right)$ cohorts. Compared to the low platelet group, the high platelet group showed a significant reduction in their HMW-VWFM index and an increase in their LMWVWFM index. The VWF-DP/Ag ratio was significantly higher in the high platelet group than in the low platelet group (Fig 1). Of the 50 patients, 25 received cytoreduction therapy (hydroxyurea, anagrelide, and busulfan). The group that received cytoreduction therapy had significantly lower platelet counts, a higher HMW-VWFM index, a lower LMW-VWFM index, and a lower VWF-DP/Ag ratio than the group that did not receive cytoreduction therapy (Table 1 ).
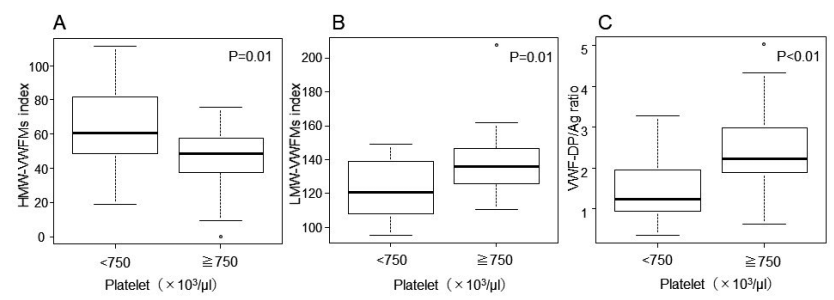

Fig1. HMW-VWFMs index, LMW-VWFMs index, or VWF-DP/Agratio in the low platelet group $\left(<750 \times 10^{3} / \mu 1, n=28\right)$ and the high platelet group $\left(\geq 750 \times 10^{3} / \mu 1, n=22\right)$. (A),(B) As compared to the low platelet group, the high platelet group showed a significant reduction in their HMW-VWFMs index ( $P=0.01)$ and an increase in their LMW-VWFMs index $(P=0.01)$. (C) The VWF-DPIAg ratio was significantly higher in the high platelet group than in the low platelet Con molecular-weight von Willebrand factor multimers: VWF-DP, von Willebrand factor-degradation product

FIGURE 1 HMW-VWFMs index, LMW-VWFMs index, or VWF-DP/ $\mathrm{Ag}$ ratio in the low platelet group and the high platelet group

TABLE 1 Comparison of 25 patients who received cytoreduction therapy with 25 patients who did not received cytoreduction therapy

\begin{tabular}{|c|c|c|c|}
\hline & $\begin{array}{l}\text { Cytoreduction therapy group } \\
(n=25)\end{array}$ & $\begin{array}{l}\text { No cytoreduction therapy } \\
\text { group }(n=25)\end{array}$ & $P$ value \\
\hline Age, years median (IQR) & $75(67-78)$ & $65(40-71)$ & $<0.01$ \\
\hline JAK2 V617F mutation, n(\%) & $11 / 22(50 \%)$ & $12 / 23(52 \%)$ & 1.0 \\
\hline WBCs, / $\mu \mathrm{L}$ median(IQR) & $6300(5400-7700)$ & $9000(7600-10400)$ & $<0.01$ \\
\hline VWF:Ag, \% median(IQR) & 117.7(104.1-149.0) & $94.0(53.5-111.3)$ & $<0.01$ \\
\hline HMW-VWFMs index, \% median(IQR) & $66.5(49.0-80.0)$ & $48.4(32.3-55.1)$ & $<0.01$ \\
\hline VWF-DP/Ag ratio, median(IQR) & $1.14(0.91-1.89)$ & $2.16(1.90-3.15)$ & $<0.01$ \\
\hline ADAMTS13 activity, $\%$ median(IQR) & $53.3(42.2-68.4)$ & $65.3(48.7-81.4)$ & 0.09 \\
\hline
\end{tabular}


Conclusions: In ET patients with pronounced thrombocytosis, increased cleavage of the Tyr1605-Met1606 bond in the VWF A2 domain lead to a reduction in HMW-VWFM. This condition can be ameliorated using cytoreduction therapy.

PB0917 | Pharmacokinetics/Pharmacodynamics (PK/PD) of Recombinant von Willebrand Factor (Vonicog Alfa) in Adult Patients with von Willebrand Disease (VWD) during Prophylactic Treatment

A. Iorio $^{1}$; F. Leebeek ${ }^{2}$; S. Susen ${ }^{3}$; A. Shapiro ${ }^{4}$; G. Özen ${ }^{5}$;

B. Mellgård ${ }^{5}$; Y. Wang ${ }^{6}$

${ }^{1}$ McMaster University, Hamilton, Canada; ${ }^{2}$ Erasmus University Medical Center, Rotterdam, Netherlands; ${ }^{3}$ Lille University Hospital, Lille, France; ${ }^{4}$ Indiana Hemophilia and Thrombosis Center, Indianapolis, United States; ${ }^{5}$ Baxalta US Inc., a Takeda Company, Cambridge, United States; ${ }^{6}$ Shire Human Genetic Therapies Inc., a Takeda Company, Cambridge,

United States

Background: Data on PK/PD of rVWF (vonicog alfa; Baxalta US Inc., a Takeda company, Lexington, MA, USA) following repeated dosing for prophylactic treatment of bleeding in VWD are limited.

Aims: To evaluate PK/PD parameters following 1 year of prophylaxis with rVWF.

Methods: PK/PD samples were collected from a phase 3, openlabel, international, multicenter study of rVWF prophylaxis in adult patients with severe VWD (NCT02973087). Patients transitioning from on-demand treatment with any VWF (Prior OD arm) or prophylaxis with plasma-derived VWF (Switch arm) received rVWF prophylaxis for $\geq 1$ year; most had type 3 VWD. Ethics committee approval and informed consent were obtained. PK/PD samples following single (at baseline in Prior OD patients) and multiple dosing were analyzed using noncompartmental methods for VWF:ristocetin cofactor (VWF:RCo) and factor VIII activity (FVIII:C) (Figure 1).

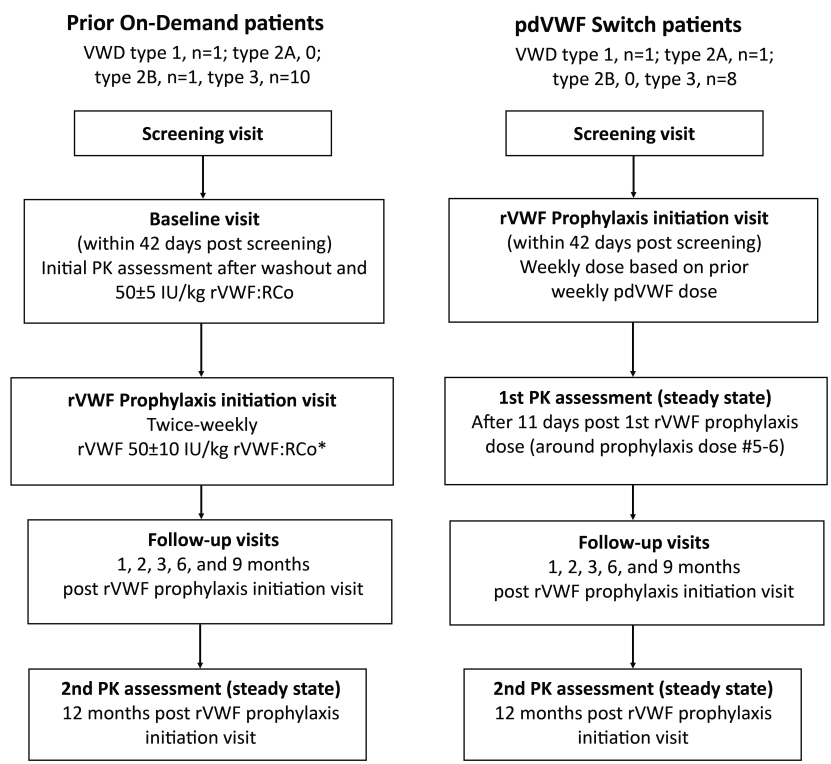

FIGURE 1 Schedule of PK assessments. *Prophylactic dose for Prior On-Demand patients could be increased up to $80 \mathrm{lU} / \mathrm{kg}$. Prior OnDemand patients: patients who were treated on demand with any VWF during the 12-month period prior to enrolling into this study to receive prophylaxis with rVWF. pdVWF Switch patients: patients who were treated prophylactically with a pd VWF during the 12month period prior to enrolling into this study received prophylaxis with rVWF. pd, plasma-derived von Willebrand factor, PK, pharmacokinetics; rVWF, recombinant von Willebrand factor; VWF:RCo, VWF:ristocetin cofactor.

Results: In Prior OD patients $(N=12)$, following a single intravenous dose after washout (50 $\pm 5 \mathrm{IU} / \mathrm{kg}$ VWF:RCo), geometric least squares mean (GeoLSMean) of VWF:RCo maximum plasma concentration $\left(C_{\max }\right)$ was $72.7 \mathrm{lU} / \mathrm{dL}$, and area under the curve zero to infinity $\left(A \cup C_{\text {inf }}\right.$ ) was $1113 \mathrm{IU}$ *h/dL. Following 1 year of twiceweekly prophylaxis $\left(50 \pm 10 \mathrm{IU} / \mathrm{kg}\right.$ VWF:RCo), $\mathrm{C}_{\max }$ and AUC over

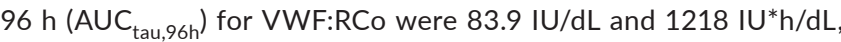
respectively. The corresponding FVIII:C GeoLSMeans were: $85.6 \mathrm{IU} /$ $\mathrm{dL}\left(\mathrm{C}_{\max }\right)$ and $4466 \mathrm{IU} \mathrm{H}^{*} \mathrm{dL}\left(\mathrm{AUC}_{0 \text {-tlast }}\right)$ initially, and $93.6 \mathrm{IU} / \mathrm{dL}\left(\mathrm{C}_{\max }\right)$ and $5453 \mathrm{IU} * \mathrm{~h} / \mathrm{dL}\left(\mathrm{AUC}_{\text {tau }, 96 \mathrm{~h}}\right.$ ) at study completion. Trough FVIII:C levels increased from $3.83 \mathrm{IU} / \mathrm{dL}$ (baseline) to $18.7 \mathrm{IU} / \mathrm{dL}$ after 1 year prophylaxis. In Switch patients ( $=10$ ), VWF:RCo and FVIII:C were generally comparable between initial steady state and following 1 year of rVWF prophylaxis.

Conclusions: PK for VWF:RCo were stable over 1 year of rVWF prophylaxis. In Prior OD patients, FVIII:C trough levels increased almost 5-fold from baseline to steady state. Following long-term prophylaxis, increases in FVIII:C trough levels were maintained for 1 year in rVWF-treated patients. 
PB0918 | Low VWF Level due to Heterozygous p.P1127S Mutation of VWF: Clinical Phenotype and Biochemical Effects

M. Tardugno ${ }^{1} ;$ M. Sacco ${ }^{2} ;$ S. Lancellotti ${ }^{2} ;$ F. Bernardi ${ }^{3} ;$ M. Pinotti ${ }^{3}$; A. Branchini ${ }^{4}$; E. De Candia ${ }^{5}$; L. Di Gennaro ${ }^{2}$; M. Basso ${ }^{2}$; B. Giusti ${ }^{6}$; G. Castaman 7 R. De Cristofaro ${ }^{1}$

${ }^{1}$ Università Cattolica S. Cuore - Facoltà di Medicina e Chirurgia 'A. Gemelli', Rome, Italy; ${ }^{2}$ Fondazione Policlinico Universitario 'A. Gemelli' IRCCS/Servizio Malattie Emorragiche e Trombotiche, Rome, Italy; ${ }^{3}$ Dipartimento di Scienze della Vita e Biotecnologie, Università di Ferrara, Ferrara, Italy; ${ }^{4}$ Dipartimento di Scienze della Vita e Biotecnologie, Universit 'a di Ferrara, Ferrara, Italy; ${ }^{5}$ Università Cattolica S. Cuore - Facoltà di Medicina e Chirurgia 'A. Gemelli', Rome, Italy; ${ }^{6}$ Dipartimento di Medicina Sperimentale e Clinica, Università di Firenze, Laboratorio Genetico Molecolare, Firenze, Italy; ${ }^{7}$ Centro Malattie Emorragiche e della Coagulazione, Dipartimento di Oncologia, Ospedale Universitario Careggi, Firenze, Italy

Background: A 21-year-old Italian woman (Blood Group A$\mathrm{Rh}+$ ) presented a thigh hematoma after minor trauma. She had VWF:Ag = $34.3 \mathrm{U} / \mathrm{dL}, \mathrm{VWF}: \mathrm{RCo}=32.8 \mathrm{U} / \mathrm{dL}$, and FVIII = $55.3 \mathrm{IU} /$ $\mathrm{dL}$. The patient was a carrier of the heterozygous missense mutation c.C3379>T (exon 25) of the VWF gene, never described before. This mutation, absent in her father, was found in her 54-year-old mother, who didn't present hemorrhagic disorders and VWF:Ag $=60 \mathrm{U} / \mathrm{dL}$. The mutation causes the p.P1127S substitution in the D3 domain of the mature VWF molecule.

Aims: To deeper examine the molecular pathogenesis of this mild form of type-1-like VWD, the aim of this study is to characterize this mutation phenotypically and functionally.

Methods: VWF:Ag and VWF:RCo were measured by chemiluminescence assays, while FVIII-Activity by chromogenic assay. FVIII binding (VWF:VIIIB) and pro-peptide levels (VWF:pp) were analyzed by ELISA assays; ADAMTS13-Activity by FRETS; VWF multimeric pattern by SDS-agarose-gel electrophoresis; ristocetin-induced plateletaggregation by the Born-assay; molecular modeling was performed using the I-TASSER threading modeling server. Recombinant expression of WT and p.P1127S mutant was performed by using HEK-293 cells.

Results: The heterozygous p.P1127S mutation was clinically associated with a similar decrease of both VWF:Ag and VWF:Act levels. The infusion of $0.3 \mu \mathrm{g} / \mathrm{Kg}$-BW desmopressin normalized the VWF levels, although the decrease of their value was faster $\left(t_{1 / 2}=7.6 \mathrm{~h}\right)$ than in isogroup normal subjects $\left(t_{1 / 2}=11.6 \mathrm{~h}\right)$. The basal VWF:pp/ VWF:Ag ratio was equal to 1.3. The VWF multimers, VWF-FVIIIbinding and ADAMTS-13 level were normal. Th p.P1127S mutant was expressed like the WT construct, both in the medium and HEK293 lysates. Ristocetin-induced-platelet-aggregation was normal. Molecular-modeling revealed a more open conformation in the mutant than in WT-form.

Conclusions: The p.P1127S mutation causes a conformational change that accelerates the clearance of VWF, but not its synthesis and secretion. To further investigate all mechanistic, structural, and functional features of this VWF mutant, biophysical and biochemical studies are ongoing in our laboratory.

PB0919 | Prophylactic Subcutaneous Emicizumab-kxwh in Adults and Children with Symptomatic Type 3 von Willebrand Disease

A. Pawar ${ }^{1}$; K. Braunstein ${ }^{2}$; J. Michals ${ }^{1}$; K. Vo ${ }^{1}$; K. Schafer ${ }^{1}$

${ }^{1}$ University of California Davis Health, Sacramento, United States;

${ }^{2}$ Emory Saint Joseph's Hospital, Atlanta, United States

Background: Type 3 von Willebrand disease (type 3 VWD) is the rarest and most severe form of $\mathrm{VWD}$, with almost total or near total lack of VWF. This also leads to a deficiency of factor VIII, which can no longer be protected by VWF. Current treatment for patients with type 3 VWD consists of on-demand infusions of plasma derived FVIII/VWF combinations or recombinant VWF factor. Prophylaxis is not standard of care. The FDA has approved emicizumab-kxwh, a subcutaneously administered, humanized, bispecific, monoclonal antibody to FIXa and FX that substitutes FVIIla function, for prophylaxis in patients with hemophilia A of all ages. Since type 3 VWD also has low FVIII, we report the successful, novel, prophylactic use of emicizumab-kxwh in four individuals with type 3 VWD including 2 children and 2 adults.

Aims: Reports of significant improvement in symptoms in patients with type 3 VWD after institution of emicizimab-kxwh prophylaxis. Methods: Case reports of two adult female patients with type 3 VWD who suffered from a lifetime of complications associated with severe hemorrhagic events requiring multiple hospitalizations, infusions of factor concentrate, and blood transfusions. Started prophylaxis with emicizumab-kxwh in the spring/summer of 2019.

Two pediatric patients aged 2 and 6 years, hospitalized multiple times for significant bleeding after minor childhood traumas. They had been treated with multiple doses of factor VIII/VWF concentrates and even recombinant FVIla. Initiated prophylaxis with emicizumab-kxwh.

Results: Significant improvement in the symptoms of all patients and the adults' perception of quality of life.

Conclusions: Subcutaneous emicizumab-kxwh prophylaxis in symptomatic patients with type 3 VWD was effective. As more substituting and rebalancing therapies in hemostasis become available, guidelines for prophylaxis in bleeding disorders like type 3 VWD will change. Multicenter trials about efficacy and safety as well as patient-reported outcomes (PRO) will greatly help in formulating the guidelines. 
PB0920 | Investigating Pathomolecular Mechanisms von Willebrand Disease Variants Located in a Domains of the von Willebrand Factor

H. Yadegari $^{1}$; A. Biswas ${ }^{1}$; S. Sadangi ${ }^{1}$; J. Oldenburg ${ }^{1}$

${ }^{1}$ University Clinic Bonn/Institute of Experimental Hematology and Transfusion Medicine, Bonn, Germany

Background: In our cohort of von Willebrand disease (VWD) patients, we found four missense substitutions located either in the A1 domain linker (p.Cys1227Arg), A1 region (p.Leu1288Arg and p.Leu1340Arg), or A2 domain (p.Val1524Gly).

Aims: This study aimed to characterize the impact of these missense variants on VWF conformations, biosynthesis, and functions.

Methods: The full-length wild-type (wt) or mutant VWF cDNA were expressed in HEK293T cells. Quantitative and qualitative assessments (GPIba binding and multimer analysis) of the VWF secreted into the medium were performed. The structural impact of the VWF variants was assessed by homology modeling.

Results: Homozygous expression of the p.Cys1227Arg and p.Val1288Arg demonstrated a substantial reduction in VWF secretion, $18.7 \%$ and $33.5 \%$ of $w t$, respectively, with loss of large multimers. The co-transfection of the p.Cys1227Arg/wt improved the expression but still showed reduced secretion and loss of large multimers. However co-transfection of the p.Leu1288Arg/wt corrected secretion and multimer profile but still showed diminished binding to GPIba. The homozygous and heterozygous expression of the variant p.Leu1340Arg showed only a slight reduction in VWF secretion, $77 \%$ and $81 \%$ of wt, respectively, but impaired binding to GPIba severely. Interestingly, the p.Val1524Gly did not affect VWF secretion in both single and coexpression studies but showed multimers with triplet structures (and loss of large multimers), which could be cleaved by endogenously produced ADAMTS13 in HEK293T. Homology modeling showed that p.Val1524G, result in easier access of the ADAMTS13 cleavage site by facilitating the unfolding of the $\mathrm{A} 2$ domain.

Conclusions: We demonstrated that variants p.Cys1227Arg and p.Val1288Arg affected the multimerization and secretion, besides interfering with platelet binding, whereas variant p.Leu1340Arg impaired the binding to GPIba, but did not affect the multimerization markedly. Furthermore, we showed the gain of function variant p.Val1524Gly in the A2 domain caused a structural alignment that leads to the accessibility of the ADAMTs13 cleavage site.
PB0921 | Sensitivity of ISTH Bleeding Assessment Tool, Bleeding Time and PFA-200 in the Diagnosis of von Willebrand Disease

T. Geevar ${ }^{1}$; R. Gautam Dave ${ }^{1}$; R. Vijayan ${ }^{1}$; A. Samuel ${ }^{1}$;

S. Singh ${ }^{1}$; J. John Mammen ${ }^{1}$; S. Chandran Nair ${ }^{1}$

${ }^{1}$ Christian Medical College, Vellore, Vellore, India

Background: Bleeding time (BT) and PFA (Platelet Function Analyser)-100/200 are screening tests for primary hemostatic disorders. The diagnosis of von Willebrand disease (VWD) in low- and medium-income countries (LMIC) is challenging due to cost and lack of laboratory infrastructure. Since PFA-100/200 is expensive, BT is the only screening test for VWD diagnosis in most laboratories in LMIC.

Aims: To determine the diagnostic performance of ISTH Bleeding assessment tool (BAT), BT and PFA-200 in the diagnosis of VWD in a tertiary centre in South India

Methods: This was a retrospective study of VWD patients who presented to a tertiary hospital in South India from January 2012 to March 2019. 188 consecutive patients with no intrinsic abnormality were included as controls. Final diagnosis was made after correlating with history and laboratory tests including BT, PFA-200 with Collagen/ADP (PFA-ADP) and Collagen/Epinephrine (PFA-EPI), Activated Partial Thromboplastin time, Factor VIII, Ristocetin cofactor assay (vWF:RCo), Von Willebrand antigen (VWF:Ag), Collagen binding assay (where applicable) and parental evaluation (where applicable) in both patients and controls. Bleeding time was performed only by trained and experienced technical staff.

Results: A total of 444 cases with VWF:Ag $<50 \mathrm{U} / \mathrm{dL}$ were included in the study. The distribution and results are summarised in Table. The proportion of severe (VWF:RCo <10\%), moderate (VWF:RCo 10-30\%) and mild (VWF:RCo >30\%) was 70\%, $19 \%$ and $11 \%$ respectively. The overall sensitivity/positive predictive value (PPV) of BT and PFA-200 (either COL/ADP or COL/EPI prolonged) was $72.3 \% / 99.6 \%$ and $91.8 \% / 96.1 \%$ respectively. The sensitivity of each subtype is shown in Figure. There was a good inverse correlation between BT vs VWF:RCo $(r=-0.66)$, PFA-ADP vs VWF:RCo $(r=-0.71)$ and PFA-EPI vs VWF:RCo $(r=-0.63)$. 
TABLE 1 Distribution of VWD and summary of laboratory characteristics and BAT score in different subtypes of VWD. *BT reference range (RR) : 2-6 minutes, upper limit (UL) 15 minutes; \# PFA-ADP RR: 68-142 secs, UL 300 secs; \$PFA-EPI RR: 89-167 secs, UL 300 secs

\begin{tabular}{|c|c|c|c|c|c|c|c|}
\hline & $\begin{array}{l}\text { Number of } \\
\text { cases (\%) } \\
N=444\end{array}$ & $\begin{array}{l}\text { vWF:RCo (\%) } \\
\text { Median } \\
\text { (IQR) } \\
N=444\end{array}$ & $\begin{array}{l}\text { vWF:Ag (U/ } \\
\text { dL) } \\
\text { Median (IQR) } \\
N=444\end{array}$ & $\begin{array}{l}\text { Median } \\
\text { BAT score } \\
\text { (IQR) } \\
N=395\end{array}$ & $\begin{array}{l}\text { BT (mins) } \\
\text { Median (IQR) } \\
\text { [\% with maximal } \\
\text { prolongation] } \\
N=351\end{array}$ & $\begin{array}{l}\text { PFA-ADP }^{\#}(\mathrm{~s}) \\
\text { Median (IQR) } \\
\text { [\% with maximal } \\
\text { prolongation] } \\
N=245\end{array}$ & $\begin{array}{l}\text { PFA-EPI CT }{ }^{\$}(\mathrm{~s}) \\
\text { Median (IQR) } \\
\text { [\% with maximal } \\
\text { prolongation] } \\
N=251\end{array}$ \\
\hline \multirow[t]{3}{*}{ Type 3 VWD } & 215 (48.4\%) & 0 & 0 & $7(4-10)$ & & & \\
\hline & & & & & $15(15-15)$ & $268.5(227-300)$ & $277(246-300)$ \\
\hline & & & & & [98\%] & [100\%] & [100\%] \\
\hline \multirow[t]{3}{*}{ Type 2 VWD } & $123(27.7 \%)$ & & & $4(2-5)$ & & & \\
\hline & & 10.4 & 33.8 & & $9(4.5-15)$ & $284(210-300)$ & $292(241-300)$ \\
\hline & & $(0-19)$ & $(23-47)$ & & {$[35 \%]$} & [90.5\%] & [92\%] \\
\hline \multirow[t]{4}{*}{ Type 1 VWD } & 69 (15.5\%) & & & $4(3-8)$ & & & \\
\hline & & 7 & 10.3 & & $8.5(3.25-15)$ & $293(258-300)$ & $299.5(262-300)$ \\
\hline & & $(0-14)$ & $(5-17)$ & & [48\%] & [87.5\%] & [90.5\%] \\
\hline & & & & $3(2-4)$ & & & \\
\hline Low VWF:Ag & 37 & 38.4 & 42.5 & & $3.25(2.5-4)$ & $113(98-139)$ & $154(142-200)$ \\
\hline$(30-50 \mathrm{u} / \mathrm{dL})$ & (8.3\%) & $(33-46)$ & $(36-46)$ & & [0\%] & [5\%] & [16\%] \\
\hline
\end{tabular}

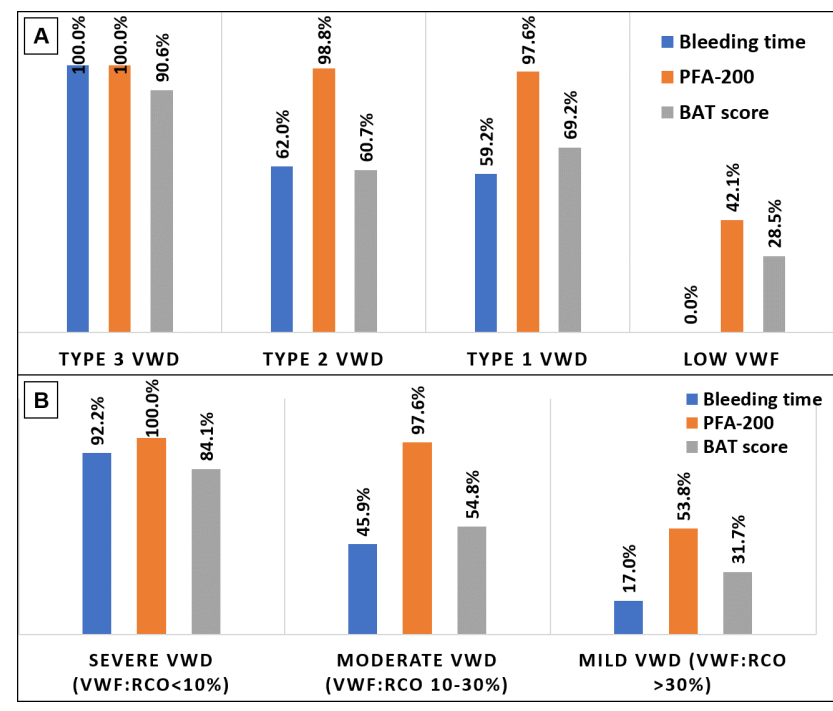

FIGURE 1 Sensitivity of Bleeding time, PFA-200 and BAT score in VWD classified based on A) subtypes of VWD, and B) severity of VWD

Conclusions: PFA-200 is very sensitive to VWD. BT has good sensitivity and was comparable to PFA-200 in severe VWD cases. When performed under standardised conditions, BT will help in the evaluation of VWD in LMIC.
PB0922 | Utility of a New, Rapid Automated von Willebrand Factor (VWF) Multimer Assay for the Diagnosis of von Willebrand Disease

J. Brodard ${ }^{1}$; R. Rubin ${ }^{2}$; D. Baumgarnter ${ }^{2}$; M. Reusser ${ }^{2}$; J.A Kremer; Hovinga $^{2}$

${ }^{1}$ Universtiy Clinic of Haematology Inselspital Bern, Bern, Switzerland;

${ }^{2}$ University Clinic of Haematology, Inselspital Bern, Bern, Switzerland

Background: The VWF multimer (VWF:MM) assay is an important tool while evaluating patients with suspected Von Willebrand Diseases (VWD), but results are rarely available in a timely manner. A rapid, automated standardized assay was developed to overcome this important limitation.

Aims: We evaluated the utility of this new method for the diagnosis of VWD in clinical practise.

Methods: Frozen plasma samples with known VWF:Activity and VWF:Ag levels of 88 consecutive patients having in-house VWF:MM as part of their evaluation for suspected VWD between 2017-2020 were available for study. The HYDRAGEL VWF:MM was performed using the 5- or 11 VWF multimer kits (Sebia, France). VWD diagnosis was based on the patient's bleeding score (ISTH-BAT), VWF:Activity, VWF:Ag, factor VIII:C, multimer distribution and densitometry, and compared to the historic in-house VWF:MM and VWD diagnosis.

Results: Overall agreement of VWD diagnosis between the in-house and the automated VWF:MM was 74\% (concordant in 65/88 patients, including 3 patients with undefined VWD), with VWD ruled out in 13 patients, VWD type 1 in 11 , type $2 A(I I A) / 2 B$ in 24 , type $2 \mathrm{~A}(\mathrm{IIE})$ in 29 , type $2 \mathrm{M}$ in 2 , type $2 \mathrm{~N}$ in 1 , and acquired Von Willebrand syndrome (AVWS) in 15 patients, respectively. Discrepant findings pertained primarily to VWD $2 \mathrm{M}$ and AVWS, which showed a large heterogeneity in the multimer distribution. Furthermore, three 
patients with defined VWD type 2A subtypes were not identified with HYDRAGEL VWF:MM, among them was a genetically confirmed VWD type 2A(IID). Results were available within one working day with the HYDRAGEL VWF:MM with implemented densitometry improved the interpretation and detection of slight structural variations that were not apparent by visual interpretation of the in-house VWF:MM.

Conclusions: HYDRAGEL VWF:MM analysis is a rapid, sensitive and standardized assay, which can be run as an initial VWF:MM screen when evaluating patients with a possible VWD.

PB0923 | Clinical, Economic, and Quality of Life Burden Associated with von Willebrand Disease (VWD) in Adults and Children: Systematic and Targeted Literature Reviews

G. Castaman ${ }^{1}$; N. Jansen ${ }^{2}$; S. Santos ${ }^{2}$; E. Berntorp ${ }^{3}$

${ }^{1}$ Center for Bleeding Disorders and Coagulation, Department of Oncology, Careggi University Hospital, Firenze, Italy; ${ }^{2} \mathrm{CSL}$ Behring, Hattersheim am Main, Germany; ${ }^{3}$ Clinical Coagulation Unit, Lund University, Malmö, Sweden

Background: Patients with VWD experience bleeds that can affect health-related quality of life (HRQoL) and may have long-term consequences. The magnitude of this burden on patients is not yet fully elucidated.

Aims: Estimate the prevalence and burden of clinical complications associated with VWD (types 1, 2, and 3), economic burden, and HRQoL of affected individuals.

Methods: Embase ${ }^{\circledR}$, MEDLINE $^{\circledR}$, and the Cochrane Library were searched (database inception-September 1, 2020) for studies on VWD, including conference abstracts (2017-2020). All study designs were considered. References were screened by 2-3 independent reviewers.

Results: Data are reported for patients with VWD from North American, European, and non-European countries. In 16 clinical burden studies (Figure 1) including 72,450 patients of all ages with VWD, the most reported bleeding symptoms were menorrhagia (2-95\%, mean [SD] 46.2\% [39.1]; $n=7$ studies), epistaxis (12-80\%, 45.0\% [33.5]; $n=5)$, and easy bruising (46-65\%, 55.5\%; $n=2)$. In 25 cost and resource use studies $(79,885$ patients of al ages with VWD), costs varied widely; higher costs for patients with VWD compared to patients with non-VWD disorders were reported. Patients with bleeding complications accrued higher costs regardless of VWD type. In two US studies, patients with VWD and severe complications (i.e. major bleeding) were more likely to have inpatient admission and emergency room visits. In 17 HRQoL studies (5,730 patients of all ages with VWD), QoL was assessed with the SF-36 ( $n=6)$, VWD-QoL $(n=1)$, and EuroQol-5 Dimension ( $n=2$ ) scales; all studies assessing treatment impact on VWD (except one) reported an improvement in single or multiple components of HRQoL scales.

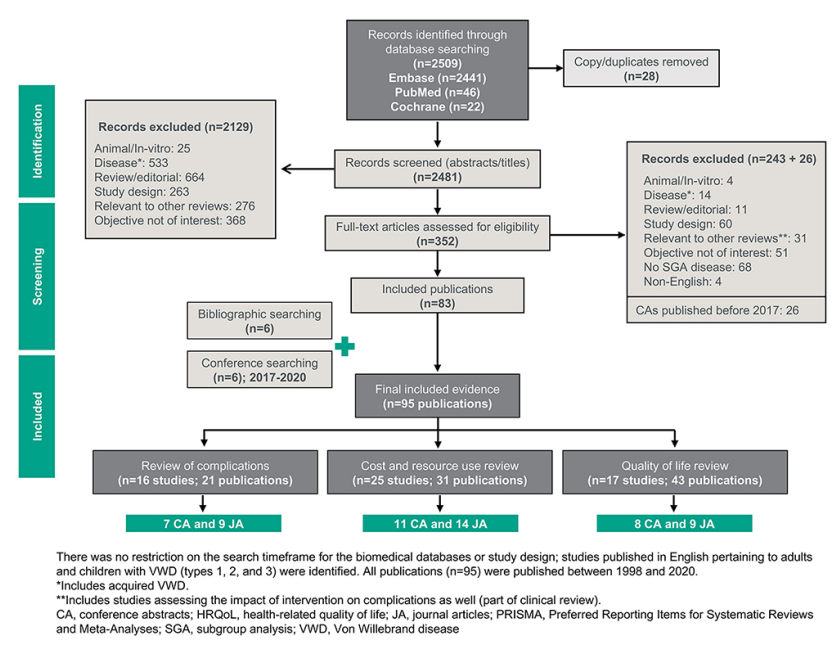

FIGURE 1 PRISMA diagram for the clinical complications associated with VWD, economic burden of disease, and HRQoL

Conclusions: Despite available data being highly variable and mostly published as conference abstracts, patients with VWD experience a high disease burden, which warrants further systematic investigation including cost consequence analysis, with the ultimate purpose of improving QoL.

\section{PB0924 | Burden of Illness in Patients with von Willebrand Disease: A Systematic Review}

P. Du ${ }^{1} ;$ A. Bergamasco²; T. Arredondo-Bisono²; Y. Moride ${ }^{2,3,4}$; F. Truong Berthoz ${ }^{5}$; G. Özen ${ }^{6}$; S. Tzivelekis ${ }^{7}$

${ }^{1}$ Millennium Pharmaceuticals, Inc., a Takeda Company, Cambridge, United States; ${ }^{2}$ YolaRX Consultants, Paris, France; ${ }^{3}$ Faculty of Pharmacy, Université de Montréal, Montréal, Canada; ${ }^{4}$ Rutgers, The State University of New Jersey, New Brunswick, United States; ${ }^{5}$ Baxalta $\mathrm{GmbH}$, a Takeda Company, Zürich, Switzerland; ${ }^{6}$ Baxalta US Inc., a Takeda Company, Cambridge, United States; ${ }^{7}$ Shire PIc, a Takeda Company, Boston, United States

Background: von Willebrand disease (VWD) is associated with multiple bleeding-related complications, which negatively impact quality of life (QoL) and result in high healthcare resource utilization (HCRU) in symptomatic patients. More research is required on VWD burden of illness to inform VWD care.

Aims: To describe bleeding-related complications, QoL, and HCRU in patients with VWD.

Methods: A systematic review (PROSPERO CRD42020197674) of observational human studies conducted between January 1, 2010 and July 1, 2020 was performed using the MEDLINE and Embase databases utilizing keywords encompassing both the population (patients with VWD) and data of interest. Obtained studies were screened for eligibility by 2 independent reviewers using predefined criteria. Additional pragmatic searches were performed (Google, Google Scholar, Cochrane Library, abstracts from related conferences) and reference lists of 
retained publications were screened. Case reports and interventional clinical trials were excluded. For studies with multiple publications, only the most recent was retained. Sources were evaluated and data from relevant publications were extracted.

Results: Within the 122 sources retained, 18 distinct studies described bleeding events. The proportion of patients with bleeding events varied according to bleeding type (Table 1) and VWD severity, with bleeding located primarily in mucocutaneous sites. Eight studies assessing QoL in patients with VWD reported a variety of measures of general health, bodily pain, and physical or social functions. Patients with VWD had poorer QoL scores than reference/ general populations in 4 of these 8 studies. One of the 8 studies reporting on HCRU showed approximately 2 -fold increases in the rates of outpatient visits and hospitalizations, and longer hospital stays in patients with VWD than in age- and sex-matched controls.

TABLE 1 Proportions of patients with bleeding events reported in observational studies (January 2010 to July 2020)

\begin{tabular}{|c|c|c|c|c|c|c|c|c|}
\hline Bleeding type & $\begin{array}{l}\text { Studies, } \\
\mathrm{n}\end{array}$ & Regions & $\begin{array}{l}\text { Targeted age } \\
\text { (number of } \\
\text { studies) }\end{array}$ & $\begin{array}{l}\text { Patients with } \\
\text { VWD, } \mathrm{n}^{*}\end{array}$ & $\begin{array}{l}\text { Range for } \% \\
\text { of patients } \\
\text { with bleeding } \\
\text { events (overall) }\end{array}$ & $\begin{array}{l}\text { Range for } \\
\text { events by } \\
\text { Type } 1\end{array}$ & $\begin{array}{l}\text { of patients } \\
\text { WD type } \\
\text { Type } 2\end{array}$ & $\begin{array}{l}\text { eeding } \\
\text { Type } 3\end{array}$ \\
\hline Epistaxis & 13 & $\begin{array}{l}\text { Europe, North America, } \\
\text { Asia-Pacific, Middle } \\
\text { East, North Africa, } \\
\text { Latin America }\end{array}$ & $\begin{array}{l}\text { All ages }(8) \\
\leq 19 \text { years }(5)\end{array}$ & 2,295 & $21.8-86.0$ & $\begin{array}{r}38.8- \\
52.9\end{array}$ & $43.8-56.6$ & $36.8-77.3$ \\
\hline $\begin{array}{l}\text { Menorrhagia } \\
\text { among female patients }\end{array}$ & 11 & $\begin{array}{l}\text { Europe, North America, } \\
\text { Asia-Pacific, Middle } \\
\text { East }\end{array}$ & $\begin{array}{l}\text { All ages }(7) \\
\leq 18 \text { years }(3) \\
\geq 16 \text { years }(1)\end{array}$ & 1,573 & $48.3-100$ & $\begin{array}{r}79.9- \\
83.3\end{array}$ & $66.7-75.6$ & $64.7-69.2$ \\
\hline $\begin{array}{l}\text { Wound related } \\
\text { (including minor } \\
\text { wounds/cuts) }\end{array}$ & 7 & $\begin{array}{c}\text { Europe, Asia-Pacific, } \\
\text { Middle East }\end{array}$ & $\begin{array}{l}\text { All }(3) \\
\geq 16 \text { years }(2) \\
\leq 19 \text { years }(2)\end{array}$ & 2,796 & $38.0-78.9$ & $\begin{array}{l}35.0- \\
51.0\end{array}$ & $39.1-43.8$ & 54.5 \\
\hline Gastrointestinal & 13 & $\begin{array}{l}\text { Europe, North America, } \\
\text { Asia-Pacific, Middle } \\
\text { East, North Africa, } \\
\text { Latin America }\end{array}$ & $\begin{array}{l}\text { All (8) } \\
\geq 16 \text { years }(1) \\
\geq 18 \text { years }(1) \\
<18 \text { years }(3)\end{array}$ & $19,625^{\dagger}$ & $1.2-24.0$ & $4.0-14.3$ & $10.3-33.3$ & $4.6-19.2$ \\
\hline Joint & 9 & $\begin{array}{l}\text { Europe, Asia-Pacific, } \\
\text { Middle East, Latin } \\
\text { America }\end{array}$ & $\begin{array}{l}\text { All }(7) \\
\geq 16 \text { years }(1) \\
<18 \text { years }(1)\end{array}$ & 3,475 & $3.8-38.0$ & $1.9-22.0$ & $8.5-20.6$ & $36.6-47.8$ \\
\hline $\begin{array}{l}\text { Central nervous } \\
\text { system }\end{array}$ & 6 & Europe, Middle East & $\begin{array}{l}\text { All }(2) \\
<16 \text { years }(1) \\
\geq 16 \text { years }(2) \\
<18 \text { years }(1)\end{array}$ & 3,223 & $1.0-8.8$ & 0.6 & 0.8 & $1.8-9.1$ \\
\hline $\begin{array}{l}\text { Spontaneous oral and } \\
\text { dental }\end{array}$ & 12 & $\begin{array}{l}\text { Europe, North America, } \\
\text { Asia-Pacific, Middle } \\
\text { East, North Africa, } \\
\text { Latin America }\end{array}$ & $\begin{array}{l}\text { All }(7) \\
\geq 16 \text { years }(1) \\
\leq 18 \text { years }(4)\end{array}$ & 2,750 & $\begin{array}{l}\text { Bleeding }{ }^{\ddagger}: \\
3.6-100\end{array}$ & $27.0-49.0$ & $35.7-56.3$ & $57.6-69.9$ \\
\hline Post-dental extraction & 8 & $\begin{array}{l}\text { Europe, Asia-Pacific, } \\
\text { Middle East, North } \\
\text { Africa, Latin America }\end{array}$ & $\begin{array}{l}\text { All (5) } \\
\geq 16 \text { years }(1) \\
\leq 19 \text { years }(2)\end{array}$ & 2,197 & $6.1-84.6$ & $\begin{array}{l}42.0- \\
53.2\end{array}$ & $40.9-60.0$ & 40.9 \\
\hline
\end{tabular}

${ }^{*}$ Patients included in analyses across all studies. ${ }^{\dagger}$ Includes 16,640 admissions from 1 study.

${ }^{\ddagger}$ Includes oral cavity, gum, and oropharyngeal bleeding.

Conclusions: This literature review highlights the high rates of bleeding-related complications, decreased QoL, and increased HCRU in patients with VWD. Large variations in bleeding rates reflect the variability in study populations and methodologies of the retrieved publications. 
PB0925 | p.G2752S Severely Affects Dimerization of von Willebrand Factor: An Analysis of Type 3 von Willebrand Disease

S. Okamoto ${ }^{1}$; S. Tamura ${ }^{1}$; N. Suzuki ${ }^{2}$; K. Odaira ${ }^{1}$; Y. Hayakawa ${ }^{1}$;

A. Suzuki ${ }^{2}$; T. Kanematsu ${ }^{2}$; F. Hayakawa ${ }^{1}$; H. Kiyoi ${ }^{1}$; T. Kojima ${ }^{3}$;

T. Matsushita ${ }^{2}$

${ }^{1}$ Nagoya University Graduate School of Medicine, Nagoya, Japan;

${ }^{2}$ Nagoya University Hospital, Nagoya, Japan; ${ }^{3}$ Aichi Health Promotion

Foundation, Nagoya, Japan

Background: Intrachain or interchain disulfide bonds in CK domain are essential for C-termina dimerization of VWF. Previous studies reported that missense mutation of cysteine residue in $\mathrm{CK}$ domain impaired intrachain disulfide bond formation and caused type 3 von Willebrand disease (VWD), the most severe type of VWD. However it is not reported type 3 VWD cases with missense mutation in $\mathrm{CK}$ domain other than cysteine residues.

Aims: To examine the molecular pathogenesis of VWF c.8254 G > A (p.G2752S), the missense mutation in CK domain with type3 VWD.

Methods: The Patient was 65-year Japanese male with p.G2752S as homozygotes. We draw his blood samples to observe endothelial colony forming cell (ECFC) and multimer structure. Subsequently, we transiently expressed recombinant VWF (rVWF) of wild type (WT) and p.G2752S in COS-7 cells to examine intracellular localization, extracellular secretion and multimer structure of them.

Results: A tiny amount of VWF was identified in patient derived ECFC and plasma VWF of patient was mainly consisted of dimer and monomer. In the analysis of rVWF, most of rVWF-G2752S was impaired to transport from endoplasmic reticulum (ER) to Golgi apparatus and intracellularly retained. Co-transfection experiments of WT and p.G2752S indicated the dominant negative effect of p.G2752S. Conclusions: In type 3 VWD, VWF c.8254 G > A (p. G2752S) is a novel missense mutation in $\mathrm{CK}$ domain other than cysteine residues and it produces multimerization failure and reduction of extracellular secretion. Furthermore, p.G2752S possibly affects intrachain disulfide bonds formation of CK domain and cause type3 VWD.

PB0926 | Don't Let Bleeding Go Unnoticed - A Global Initiative to Increase Awareness of von Willebrand Disease

F.F. Corrales-Medina ${ }^{1,2}$; E. Berntorp ${ }^{3}$

${ }^{1}$ Division of Pediatric Hematology-Oncology, University of Miami-Miller School of Medicine, Miami, United States; ${ }^{2}$ University of Miami-

Hemophilia Treatment Center, Miami, United States; ${ }^{3}$ Lund University, Faculty of Medicine, Lund, Sweden

Background: Paradoxically, the most common rare bleeding disorder, von Willebrand disease (VWD), is also the most underdiagnosed. An estimated $1 \%$ of the population carries mutations of the von Willebrand factor gene that affect coagulation, but only $1 \%$ of this estimated population have been diagnosed with VWD. Even allowing for a large fraction of asymptomatic mutation carriers, this discrepancy suggests a substantial lack of awareness of VWD among healthcare professionals and the general population, as well as reflecting the complexity of VWD diagnosis.

Aims: To develop a global initiative designed to increase awareness and education around VWD and support diagnosis of the condition. Methods: As part of the initiative, we launched www.vwdtest.com. This multilingual online resource includes a simple self-assessment tool to identify people with potentially abnormal bleeding symptoms and provides diagnostic guidance and expert advice if a bleeding disorder is suspected. We report on engagement with this platform to date, in particular following the launch of a Spanish version of the website in Latin America in September 2020.

Results: Between late September 2020 and the end of January 2021, over 26000 unique users from 47 countries accessed the website. Over $80 \%$ of visitors were from Latin America, predominantly Argentina, reflecting a successful launch of the website and highlighting a potential demand for such a resource in this region. During this period, over 600 visitors completed the online bleeding assessment test.

Conclusions: Our interim experience suggests a high demand for increased education on bleeding disorders, in particular VWD. The high engagement rate from Latin America reflects the need for a global initiative. We aim to further expand this worldwide support platform and initiative, focusing especially on those regions with limited access to diagnostic and educational tools.

PB0927 | Characteristics and Treatment of Patients with von Willebrand Disease (VWD) in General Practice Settings in the United Kingdom

P. Du ${ }^{1}$; K. Wilcox Hagberg ${ }^{2}$; S. Tzivelekis ${ }^{3}$; F. Truong

Berthoz $^{4}$; G. Özen ${ }^{5}$; S. Jick ${ }^{2,6}$

${ }^{1}$ Millennium Pharmaceuticals, Inc., a Takeda Company, Cambridge, United States; ${ }^{2}$ Boston Collaborative Drug Surveillance Program, Lexington, United States; ${ }^{3}$ Shire Plc, a Takeda Company, Boston, United States; ${ }^{4}$ Baxalta GmbH, a Takeda Company, Zürich, Switzerland; ${ }^{5}$ Baxalta US Inc., a Takeda Company, Cambridge, United States; ${ }^{6}$ Boston University School of Public Health, Boston, United States

Background: Previous research has focused mainly on patients with moderate or severe von Willebrand disease (VWD) attending specialist centers. Limited data exist for VWD managed in general practice settings. Aims: To describe the characteristics and management of patients with VWD in UK general practice.

Methods: We conducted a retrospective cohort study of patients with VWD using patient data from the UK Clinical Practice Research Datalink GOLD and Hospital Episode Statistics databases. A random sample of patients with VWD was selected and a paper questionnaire sent to their general practitioner (GP) requesting additional anonymized clinical details, including laboratory results at VWD diagnosis, VWD severity and type (as assessed by the GP), and VWD treatments. 
Results: Results are based on questionnaires completed for 235 patients with confirmed VWD; disease severity or VWD type was reclassified for 53 patients on the basis of GP-provided laboratory values. Female patients accounted for $65.1 \%$ of the study population. Mean (SD) age at first VWD diagnosis was 24.2 (18.1) years. The majority of patients had mild disease ( $n=171 ; 72.8 \%$ ), which was predominantly type 1 ( $n=90,52.6 \%$ ) or unknown type ( $n=57,33.3 \%)$. The most common comorbidities were depression, anemia, and cardiovascular disease, regardless of VWD severity. Heavy menstrual bleeding and hysterectomy were recorded most often in women with moderatesevere disease. Bleeding was recorded in 108 (46.0\%) patients (Table 1); the most frequent bleed types were epistaxis and gastrointestinal bleeding. Tranexamic acid and desmopressin were treatments most commonly reported by GPs, and factor replacement was only reported in $8.1 \%$ of patients since VWD diagnosis (Table 2).

TABLE 1 Bleed site by severity of VWD*

\begin{tabular}{lllll} 
& & & & \\
\cline { 3 - 4 } Bleed site & Total $^{\dagger}(\mathbf{N}=108)$ & Mild $^{\dagger}(\boldsymbol{n}=76)$ & Moderate-severe $^{\dagger, \ddagger}(\boldsymbol{n}=18)$ & Unknown \\
Nose/epistaxis & $53(49.1)$ & $38(50.0)$ & $9(50.0)$ & $6(42.9)$ \\
Gastrointestinal & $37(34.3)$ & $24(31.6)$ & $7(38.9)$ & $6(42.9)$ \\
Kidney & $15(13.9)$ & $10(13.2)$ & NR & NR \\
Skin & $11(10.2)$ & $6(7.9)$ & NR & NR \\
Joint & $9(8.3)$ & $7(9.2)$ & NR & NR \\
\hline
\end{tabular}

Values are no. of patients (\%).

${ }^{*} n=108$ patients with a completed questionnaire and record of bleeding

${ }^{\dagger}$ Not mutually exclusive - patients counted once per bleed site.

${ }^{\ddagger}$ Counts $<5$ not reported.

NR, not reportable (counts $<5$ ).

TABLE 2 Reported VWD treatments and reason for use

\begin{tabular}{|c|c|c|c|c|c|}
\hline Treatment & Overall $(N=235)$ & \multicolumn{4}{|c|}{ Reason for use* } \\
\hline Factor replacement $^{\dagger}$ & $19(8.1)$ & NR & $10(4.3)$ & $8(3.4)$ & $N R$ \\
\hline Desmopressin & $58(24.7)$ & $21(8.9)$ & $29(12.3)$ & $7(3.0)$ & $12(5.1)$ \\
\hline Antifibrinolytics ${ }^{\ddagger}$ & $85(36.2)$ & $23(9.8)$ & $33(14.0)$ & $27(11.5)$ & 27 (11.5) \\
\hline Tranexamic acid & $68(28.9)$ & - & - & - & - \\
\hline
\end{tabular}

Values are no. of patients (\%).

*Not mutually exclusive: GPs circled all that applied and some GPs did not provide a reason.

${ }^{\dagger}$ Factor replacement included VWF concentrate (unspecified), VONCENTO ${ }^{\circledR}$ (human FVIII/human VWF; CSL Behring GmbH, Marburg, Germany), FVIII (unspecified), HUMATE-P ${ }^{\circledR}$ (antihemophilic factor/VWF complex [human]; CSL Behring GmbH, Marburg, Germany), ALPHANATE ${ }^{\circledR}$ (human FVIII/human VWF; Instituto Grifols, Barcelona, Spain), WILATE ${ }^{\circledR}$ (human FVIII/human VWF; Octapharma, Manchester, UK); antihemophilic factor (unspecified).

${ }^{\ddagger}$ Antifibrinolytics included tranexamic acid and aminocaproic acid.

${ }^{\S}$ Females only, $n=153$; included oral, intrauterine device, and other (patch, injectable, implant, progesterone only, and multiple types).

"Reason for use = heavy menstrual bleeding.

FVIII, factor VIII; NR, not reportable (counts <5).

Conclusions: This study captures previously undescribed patients with VWD - those managed by GPs in the UK. Many of these patients experienced bleeding events, despite a mild disease status.
Increasing access to treatments and care of patients with mild VWD may improve outcomes for a previously under-studied VWD population. 
PB0928 | Results of a Survey Regarding the Diagnosis, Investigation and Management of Unclassified Bleeding Disorders amongst United Kingdom Haemophilia Centre Doctors' Organisation (UKHCDO) Members

W. Thomas ${ }^{1}$; K. Downes ${ }^{1}$; G. Evans ${ }^{2}$; G. Gidley ${ }^{3}$; G. Lowe ${ }^{4}$;

S. MacDonald ${ }^{1}$; S. Obaji ${ }^{5}$; J. O'Donnell ${ }^{6}$; F. Pinto ${ }^{7}$; M. Desborough ${ }^{8,9}$ ${ }^{1}$ Cambridge University Hospitals NHS Foundation Trust, Cambridge, United Kingdom; ${ }^{2}$ East Kent Hospitals Trust, Canterbury, United Kingdom; ${ }^{3}$ Leeds Teaching Hospitals NHS Trust, Leeds, United Kingdom; ${ }^{4}$ University Hospitals Birmingham, Birmingham, United Kingdom;

${ }^{5}$ University Hospital of Wales, Cardiff, United Kingdom; ${ }^{6}$ Royal College of Surgeons in Ireland, Dublin, Ireland; ${ }^{7}$ Royal Hospital for Children, Glasgow, United Kingdom; ${ }^{8}$ Guy's \& St Thomas' Hopsital, London, United Kingdom; ${ }^{9}$ Oxford University Hospitals NHS Foundation Trust, Oxford, United Kingdom

Background: Around $50 \%$ of patients investigated for a mildmoderate bleeding tendency will not have a conventional haemostatic defect found. Where patients have a clear bleeding tendency with normal haemostatic tests then these have been previously termed 'unclassified bleeding disorder' (UBD).

Aims: To investigate current clinical practice in the United Kingdom regarding UBD patient practice.

Methods: A survey was circulated in January/February 2021 to United Kingdom Haemophilia Centre Doctors' Organisation centres and one response per centre was requested to gain information on current practice regarding UBD. This study had approval from the Health Research Authority (IRAS: 275873).

Results:

TABLE 1 Responses from haemophilia centres $(n=52)$ regarding the management of a 36 year old female with an ISTH-BAT score of 8 , with normal haemostatic tests, undergoing various haemostatic challenges. ${ }^{*}$ centres could choose more than 1 option

First-line haemostatic precautions to cover a dental extraction, $n$ (\%)

Tranexamic acid

Tranexamic acid + desmopressin

Desmopressin

First-line haemostatic precautions to cover a 2(2) major surgical procedure, $n(\%)^{*} \quad 49(54)$

Manage expectantly 26 (28)

Tranexamic acid 1 (1)

Desmopressin 3 (3)

Prothrombin complex concentrate 6 (7)

Fresh frozen plasma 3 (3)

Platelet infusion

Recombinant factor VIla
Would prophylactic low-molecular heparin $26(50)$ be advised if the patient was at risk of venous $26(50)$ thromboembolism (VTE) on the local risk assessment tool after major surgery, $n$ (\%)

Yes

No

In a $3^{\text {rd }}$ pregnancy would fetal precautions be ad$26(50)$ vised (no Ventouse/rotational forceps/fetal scalp $26(50)$ blood sampling/electrodes and oral vitamin $\mathrm{K}$ ), $n$ (\%)

Yes

No

In a $3^{\text {rd }}$ pregnancy would centres recommend $43(82)$ avoiding spinal anaesthesia, $n$ (\%) 9 (8) Yes

No

In a $3^{\text {rd }}$ pregnancy would centres recommend

$47(90)$ avoiding spinal anaesthesia, $n$ (\%)

Yes

No

$52 / 67(78 \%)$ of centres responded; $50 \%$ of respondents were haemophilia treatment centres and $50 \%$ were comprehensive care centres. 35 (67\%) respondents register patients with a convincing bleeding history and normal laboratory tests as UBD; 28 call these patients UBD, where-as 4 use the term undiagnosed bleeding disorder and 3 bleeding of unknown cause. Centres were asked about management of a 36 year old female patient with an ISTH-BAT of 8 (previous post-partum haemorrhage in 2 pregnancies (regional anaesthesia not needed for either), bruising, epistaxis \& heavy menstrual bleeding) undergoing various haemostatic challenges and the results of this are shown in table 1. Of the 35 centres that register UBD cases, $31 / 35$ (89\%) would register this patient as having a UBD. For management of heavy menstrual bleeding in UBD, in addition to gynaecological treatments, all centres would recommend tranexamic acid and $24 / 52$ (46\%) would also recommend desmopressin.

Conclusions: The management of UBD remains uncertain due to lack of clinical trials and knowledge of the natural history. This study gives an insight into current United Kingdom practice in this area, and variability in practice which is potentially due to a lack of clear evidence and guidance within this area of practice. Further research into this patient group is required. 
PB0929 | Prevalence and Predictors of Iron Deficiency Anemia in Women with von Willebrand Disease: An NIS Study

A. Amouzegar ${ }^{1}$; K. Jeong ${ }^{2}$; J.G. Yabes ${ }^{2}$; M.V. Ragni ${ }^{3}$

${ }^{1}$ Department of Medicine, University of Pittsburgh Medical Center, Pittsburgh, United States; ${ }^{2}$ Center for Research on Health Care Data Center, Department of Medicine, University of Pittsburgh, Pittsburgh, United States; ${ }^{3}$ Department of Medicine, Division of Hematology/ Oncology, University of Pittsburgh Medical Center, Pittsburgh, United States

Background: Von Willebrand disease (VWD) is estimated to affect $1 \%$ of the general population. The morbidity associated with bleeding in females with VWD is high and associated with iron deficiency (IDA). However, the prevalence and factors associated with IDA in females with VWD have not been clearly identified.

Aims: To assess prevalence of and factors associated with IDA in VWD in a national discharge database.

Methods: Retrospective analysis of discharge data from the National Inpatient Sample (NIS) between 2005 and 2014 was performed. Adult females with and without VWD, with or without IDA, were identified by International Disease Classification (ICD) codes. Prevalence estimates were weighted using NIS-provided dischargelevel weights to reflect national estimates. Categorical variables were analyzed by Rao-Scott chi square test, and continuous variables by weighted simple linear regression. Covariates associated with IDA were identified by weighted multivariable logistic regression.

Results: A total of 19,774 female admissions with diagnosis of VWD were identified, among whom IDA prevalence was $6.3 \%$. VWD discharges with IDA were more likely to have upper GI bleeding (GIB) (14.9\% vs. $2.6 \%$ ), lower GIB (8.1\% vs. $1.3 \%$ ), chronic kidney disease (7.3\% vs. $3.7 \%$ ), thrombocytopenia ( $5.7 \%$ vs. $2.8 \%$ ), angiodysplasia with hemorrhage (3.6\% vs. $0.3 \%)$, and heavy menstrual bleeding (HMB) $(3.5 \%$ vs. $0.8 \%$ ) compared to VWD without IDA, all $P<0.001$. Compared with non-VWD with IDA, VWD with IDA had 3-fold more post-procedure bleeding $(P<0.001)$. Among patients with VWD, the odds of IDA is higher among those with (versus without) lower $\mathrm{GIB}(\mathrm{OR}=6.84[\mathrm{Cl} 4.71$, 9.93]); with upper $\mathrm{GIB}(\mathrm{OR}=6.01[\mathrm{Cl} 4.80,7.52])$; with $\mathrm{HMB}(\mathrm{OR}=5.29$ $[\mathrm{Cl} 3.61,7.75])$; with epistaxis $(\mathrm{OR}=2.41[\mathrm{Cl} 1.55,3.75])$; or with postpartum hemorrhage (OR $=2.03[\mathrm{Cl} 1.19,3.47])$.

Conclusions: GIB and HMB were significantly associated with IDA among women with VWD, but the low IDA prevalence suggests testing is infrequent.
PB0930 | Von Willebrand Factor (VWF) Collagen IV Binding Defect - A Potentially Overlooked Cause for Excessive Bleeding Phenotype

M. Escobar ${ }^{1}$; N. Montanez ${ }^{1}$; J. Lemons ${ }^{1} ;$ K. Friedman ${ }^{2}$

${ }^{1}$ University of Texas Health and Science Center of Houston, McGovern Medical School, Gulf States Hemophilia and Thrombophilia Center, Houston, United States; ${ }^{2}$ Diagnostic Laboratories, Versiti (Blood Center of Wisconsin), Milwaukee, United States

Background: Interaction of VWF with collagen at sites of vascular injury supports initial platelet tethering. Defective collagen-binding may increase the bleeding risk in type $1 \mathrm{VWD}$ and is a mechanism of type 2M VWD. The VWF A3 domain interacts with collagens I and III while the A1 interacts with collagens IV and VI.

Aims: Describe a single Center's experience in diagnosis of VWF collagen IV binding defect.

Methods: Retrospective record review of a 56 y/o Caucasian female with presumptive clinical diagnosis of "Ehlers Danlos Syndrome Classic type" with history of excessive bruising, heavy menstrual bleeding, and post-operative bleeding that required RBC transfusion, suggesting additional bleeding risk factors.

Results: Testing of primary, secondary and fibrinolytic system were without evidence of abnormalities. VWF antigen, platelet-binding and multimers, as well as Factor I - XIII, platelet aggregation studies, and flow cytometry for platelets and thromboelastography (ROTEM) were within normal limits. Comprehensive bleeding disorder genetic analysis (51 genes) identified heterozygosity VWF c.4196G>A (p. Arg1399His). Subsequent VWF collagen binding profile showed decreased VWF collagen IV binding activity, with reduction of the collagen IV binding activity to VWF antigen ratio (0.58, reference interval 0.77-1.27). VWF collagen III binding, ratio of collagen III binding to VWF antigen and VWF multimers were all normal. VWF c.4196G>A (p. Arg1399His) has been shown through expression studies to have impaired binding to collagen type IV and association with a hemostatic defect in a mouse model (Slobodianuk 2019), potentially increasing bleeding score in patients with type 1 VWD (Flood 2016).

Conclusions: The interaction of VWF with collagen is physiologically important, but is uncommonly assessed in the evaluation of patients with excessive bleeding phenotype. Different VWF domains interact with collagen I/III versus collagen IV/VI. Although isolated collagen-binding defects are rare, such evaluation should be considered in patients with unexplained bleeding, as such defects may contribute to bleeding phenotype.

\section{PB0931 | Vascular Abnormalities in Patients with Von} Willebrand Disease: A Scoping Review

N. Chornenki ${ }^{1}$; M. Shanjer ${ }^{2}$; P. James ${ }^{1}$

${ }^{1}$ Queens University, Kingston, Canada; ${ }^{2}$ McMaster University, Hamilton, Canada

Background: Qualitative or quantitative defects of Von Willebrand Factor (VWF) such as in Von Willebrand Disease (VWD) are associated with vascular abnormalities, especially in the gastrointestinal (GI) tract. 
However, the locations, extent, and natural history of these abnormalities in patients with VWD is not well understood.

Aims: In order to summarise the existing literature on the topic we conducted a scoping review of vascular abnormalities in patients with VWD.

Methods: We searched MEDLINE and EMBASE from inception to September $1^{\text {st }}, 2020$ for studies clinically describing vascular malformations in VWD patients. Screening and data extraction was completed independently and in duplicate. If more than one site of malformation was reported in a patient, each was documented individually.

Results: After screening, 54 studies reported patient-level data comprising 146 patients were included. Patient information is detailed in Table 1. Type 2A (39\%) and Type $3(14.4 \%)$ were the most common VWD subtypes. The most common site of vascular malformation was the $\mathrm{Gl}$ tract, occurring in 124 patients (84.9\%), while $18(12.3 \%)$ had non-GI vascular abnormalities and $4(2.7 \%)$ had both $\mathrm{GI}$ and non-GI vascular abnormalities. Fifteen patients also had a diagnosis of Hereditary Hemorrhagic Telangiectasia. Angiodysplasia was most commonly diagnosed with endoscopy (71 conventional, 37 video capsule, 11 push endoscopy). Treatment for bleeding angiodysplasia was varied and included statins (19 patients), tamoxifen (5 patients), thalidomide (10 patients), surgery (18 patients), and endoscopic interventions (42 patients). With respect to outcomes 3 patients (2\%) died, $53(36.2 \%)$ had bleeding resolve, and 9 (6.2\%) had ongoing bleeding. Outcomes were not specified in the majority (55.5\%) of patients.

TABLE 1 Demographics and disease characteristics of included patients

$$
N=146 \text { patients }
$$

$\begin{array}{ll}\text { Gender } & 71(48.6 \%) \\ \text { Male } & 56(38.4 \%) \\ \text { Female } & 19(13 \%) \\ \text { Unspecified } & \\ & \\ \text { VWD Type } & 17(11.6 \%) \\ \text { Type 1 } & 57(39 \%) \\ \text { Type 2A } & 18(12.3 \%) \\ \text { Type 2B } & 11(7.5 \%) \\ \text { Type 2M } & 1(0.7 \%) \\ \text { Type 2N } & 2(1.4 \%) \\ \text { Type 2 Unspecified } & 21(14.4 \%) \\ \text { Type 3 } & 19(13 \%) \\ \text { Unspecified } & \end{array}$

Conclusions: Vascular abnormalities in patients with VWD occur predominantly in the gastrointestinal tract and in patients with Type 2 or Type 3 VWD. The clinical treatment and natural history of these abnormalities remain understudied and further research is needed.
PB0932 | Patient-reported Outcomes in Autosomal Inherited Bleeding Disorders: A Systematic Literature Review

W. Al Arashi ${ }^{1}$; M.E. Houwing ${ }^{1}$; E.S. van Hoorn ${ }^{2}$; F.W. Leebeek ${ }^{3}$; J.A. Hazelzet ${ }^{2}$; S.C. Gouw ${ }^{4}$; R.E. Schutgens ${ }^{5}$; S.E. Schols ${ }^{6}$; H.F. Lingsma ${ }^{2}$; M.H. Cnossen ${ }^{1}$; The SYMPHONY consortium ${ }^{1}$ Department of Paediatric Haematology, Erasmus MC - Sophia Children's Hospital, University Medical Center Rotterdam, Rotterdam, Netherlands; ${ }^{2}$ Department of Public Health, Erasmus MC, University Medical Center Rotterdam, Rotterdam, Netherlands; ${ }^{3}$ Department of Haematology, Erasmus University Medical Centre Rotterdam, Rotterdam, Netherlands; ${ }^{4}$ Department of Paediatric Haematology, Amsterdam University Medical Centre - Emma Children's Hospital, Amsterdam, The Netherlands, Rotterdam, Netherlands; ${ }^{5}$ Central Diagnostic Laboratory - Research, Utrecht University, Utrecht, Netherlands; ${ }^{6}$ Radboud University Medical Center, Nijmegen, Netherlands

Background: Currently, it is unknown which patient-reported outcomes are important for patients with autosomal inherited bleeding disorders.

Aims: The purpose of this study is to systematically review the available literature assessing patient-reported outcomes and their measurement methods in autosomal inherited bleeding disorders.

Methods: The Embase, Medline ALL, Web of Science Core Collection, Cochrane Central Register of Controlled Trails and Google Scholar databases were searched from inception until August $1^{\text {st }} 2020$ using a combination of registered and non-registered terms. Studies on patient-reported outcomes in von Willebrand disease, inherited platelet function disorders and rare factor deficiencies were included.

Results: The systematic literature search yielded a total of 1959 nonduplicate references, of which 21 articles met the inclusion criteria. Figure 1 shows the risk of bias assessment of the included studies. Three studies were assessed as having poor quality and therefore a high risk of bias. The remaining articles had a fair quality rating. The majority of included studies focused on patients with von Willebrand disease. Patients with von Willebrand disease were reported to have lower health-related quality of life compared to the general population. Overall, this trend was especially visible in the following domains: vitality, physical and social functioning and pain. Women with inherited bleeding disorders generally scored lower on health-related quality of life compared to men, especially those with heavy menstrual bleeding. Health-related quality of life was associated with bleeding assessment tool scores in most studies. Patients with joint bleeds or heavy menstrual bleeding experienced an increased level of pain. 


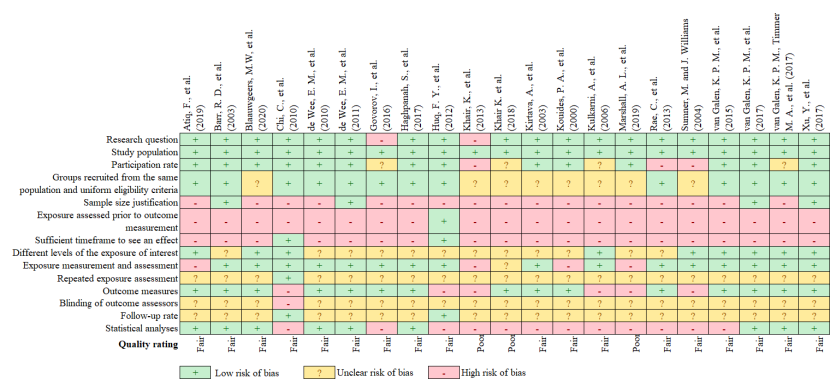

FIGURE 1 Risk of bias assessment using the quality assessment tool for observational cohort and cross-sectional studies from the National Heart, Lung, and Blood Institute

Conclusions: Our systematic review highlights the urgent need for studies using established, standardised measurement methods to analyse patient-reported outcomes in patients with autosomal inherited bleeding disorders, in order to effectively capture all aspects of disease. Future studies should especially focus on women and the association between bleeding phenotype and patient-reported outcomes.

PB0933 | A Quantitative Evaluation of vWF Multimeric Structure in Patients with Different Types of vWD and aVWS

A. Poletaev ${ }^{1}$; E. Seregina ${ }^{1,2}$; N. Karamyan ${ }^{1}$; P. Zharkov ${ }^{1}$

${ }^{1}$ Dmitry Rogachev National Medical Research Center of Pediatric

Hematology, Oncology and Immunology, Moscow, Russian Federation;

${ }^{2}$ Center for Theoretical Problems of Physicochemical Pharmacology,

Moscow, Russian Federation

Background: Multimeric assay is an important diagnostic tool in typing von Willebrand disease. However, this method allows only a visual assessment of the multimeric profile.

Aims: To develop an algorithm for the quantitative evaluation of von Willebrand factor multimers.

Methods: The semi-automatic method for evaluating multimers HYDRAGEL 5 vov Willebrand Multimers (Sebia, France) was used. The molecular ladder 1 Kb DNA Ladder M12 10000bp and Thermo Scientific FastRuler DNA Radder 100-5000 were used to assess the throughput of the gel, followed by staining with ethidium bromide. The gel of patients with established diagnosis of VWD and aVWS was evaluated, and PNP was used as a normal control. For a quantitative assessment of the photograph of the gel, the Imagej program was used, where the brightness of the bands was estimated by fractions of HF multimers - low(LMWM), medium(IMWM), high molecular weight(HMWM), and total amount.

Results: On gel with molecular ladder, 8 heavy and 2 medium ladder marks were visualized. The $8000 \mathrm{bp}$ label according to a molecular weight of $5200 \mathrm{kDa}$, which allows the separation of high and medium molecular weight forms, the border runs between 3 and 4 bands on the gel. The $4000 \mathrm{bp}$ label according to $2600 \mathrm{kDa}$, separating the medium and low molecular forms between bands 1 and
2. Accordingly, the first band on gel - LMWM, 2,3 bands - IMWM, 4 and further - HMWM. There was a good correlation(Pearson) between VWF:Ag and the overall brightness of the bands for all patients-0.95( $P<0,01)$. Correlation between the brightness of HMWM and VWF:Rco in patients with VWD-0.99( $P<0,01)$, in patients with aVWS - 0.54( $P<0,34)$.

Conclusions: Evaluation of throughput capacity of the gel allowed to define the limits of the various forms multimers, which in fact differ from published data. The quantification algorithm has shown excellent correlations with vWF:Rco and vWF:Ag, and needs more research.

PB0934 | Efficacy of hFVIII/VWF Concentrate in Pediatric Patients with von Willebrand Disease (VWD): The French Experience

A. Harroche ${ }^{1}$; L. Rugeri ${ }^{2}$; R. D'Oiron ${ }^{3}$; A. Hassoun ${ }^{4}$; Y. Repesse ${ }^{5}$; B. Frotscher ${ }^{6}$; A. Fournel'; D. Bracquart ${ }^{8}$; C. Martin ${ }^{8}$; M. Trossaert ${ }^{9}$; S. Meunier ${ }^{2}$

${ }^{1}$ Department of Hematology, CHU Necker, Paris, France; ${ }^{2}$ Clinical Hemostasis Unit, CHU Lyon, Lyon, France; ${ }^{3} \mathrm{CRC}$ (Regional Hemophilia Treatment Center), CHU Bicêtre, AP-HP, Le Kremlin Bicêtre, France; ${ }^{4}$ Haemophilia Treatment Center, Hospital of Simone Veil, Eaubonne, Montmorency, France; ${ }^{5} \mathrm{CHU}$ de Caen, Hematology Laboratory, Caen, France; ${ }^{6} \mathrm{CHRU}$ Nancy, Vandoeuvre Les Nancy, France; ${ }^{7} \mathrm{CRC}$ (Regional Hemophilia Treatment Center), CHU Besançon, Besançon, France; ${ }^{8}$ CSL Behring, Paris, France; ${ }^{9}$ Laboratory of Hematology, Haemophilia Center, University Hospital, Nantes, France

Background: This French national observational study (OPALE) evaluated the use of human factor VIII (hFVIII)/von Willebrand factor (VWF) concentrate to prevent and treat bleeding episodes in patients with inherited von Willebrand disease (VWD) in real-life settings.

Aims: To determine the efficacy of hFVIII/VWF concentrate in a French pediatric population.

Methods: Data were collected May 2016 to May 2020 from patients treated with hFVIII/VWF concentrate for prophylaxis, treatment of hemorrhage or surgical bleeding.

Results: Among 116 patients enrolled in the OPALE study by 17 French centers, 19 were pediatric patients ( $<6$ years, $n=5$ and $\geq 6-12$ years, $n=14)$, including $5(26.3 \%)$ females. In this pediatric cohort, patients were diagnosed with type $1(26.7 \%)$, type $2 \mathrm{~A}(17.2 \%)$, type $2 \mathrm{M}(13.8 \%)$, type $2 \mathrm{~B}(12.9 \%)$, type $2 \mathrm{~N}(5.2 \%)$, type $2 \mathrm{~B} / 2 \mathrm{~N}(9.5 \%)$, or type $3(14.7 \%)$ VWD. Seven patients received prophylaxis, including four patients previously receiving prophylaxis with another VWF concentrate and two previously treated episodically; one received hFVIII/VWF concentrate as first treatment. Patients received prophylaxis $1 \times /$ week $(n=2), 2 \times /$ week $(n=4)$ or $3 \times /$ week $(n=1)$; mean (range) dose per infusion was 85 (62-109) IU/kg, 53 (45-100) $\mathrm{IU} / \mathrm{kg}$ and $31 \mathrm{IU} / \mathrm{kg}$, respectively. Bleeding events $(n=23)$ were reported in six patients outside of any prophylactic period; events 
included epistaxis $(n=9)$ in three patients and hemarthrosis $(n=2)$ in one patient. In addition, 9 patients received hFVIII/VWF concentrate during 10 surgical procedures including two tonsillectomies, four adenoidectomies, one tooth extraction, one postectomy, one umbilical hernia repair and one esogastroscopy. Hemostatic efficacy was rated as "excellent" or "good" by investigators for all treatment regimens (prophylaxis, episodic and surgery) in both patients $<6$ years and $\geq 6-12$ years. No safety concerns have been reported in this study to date.

Conclusions: This study demonstrates that hFVIII/VWF concentrate is effective in the prevention and treatment of bleeding in this pediatric French cohort.

\section{PB0935 | Congenital Bleeding Disorders in Adolescent Females} Evaluated for Menorrhagia and Iron Deficiency Anemia

A. Trillo' ${ }^{1}$ R. Leeman ${ }^{1}$; J. Davis ${ }^{2}$; F.F. Corrales-Medina ${ }^{2}$

${ }^{1}$ Jackson Memorial Hospital/Holtz Children's Hospital, Miami, United

States; ${ }^{2}$ University of Miami-Miller School of Medicine, Miami, United States

Background: In post-menarcheal females, congenital bleeding disorders (CBD) often present as menorrhagia and iron-deficiencyanemia (IDA). Bleeding symptoms including epistaxis, gingival bleeding, easy bruising, and other bleeding manifestations, including significant post-operative bleeding, joint/muscle bleeding and postpartum hemorrhage, may also occur. Delayed diagnosis of CBD in females may cause significant clinical ramifications for which early recognition and diagnosis is crucial.

Aims: To examine the prevalence of CBD among post-menarcheal adolescent females referred to a hemophilia treatment center for evaluation of menorrhagia or IDA.

Methods: A retrospective chart review was performed after IRB approval. Included patients were post-menarcheal females younger than 22-years-of-age referred for evaluation of menorrhagia or IDA between January 2015 and November 2020 to the University of Miami-Hemophilia Treatment Center. Medical records were identified by ICD 10 code. Excluded patients were those with an established bleeding disorder diagnosis.

Results: Eighty-one patients met inclusion criteria. Thirty-three patients (40.7\%) were referred for IDA, 23 (28.4\%) for menorrhagia, and 25 (30.9\%) had both diagnoses. Thirty-three patients $(40.7 \%)$ were ultimately diagnosed with a CBD. The most prevalent diagnoses were type 1 von Willebrand Disease (VWD) ( $n=18,54.6 \%$ ), factor VII (FVII) deficiency ( $n=10,30.3 \%$ ) and platelet function disorders ( $n=2,6.1 \%)$. Factor XI deficiency $(n=1,3 \%)$, hemophilia A carrier $(n=1,3 \%)$, factor VIII deficiency $(n=1,3 \%)$ and combined FVII deficiency and VWD ( $n=1,3 \%$ ) were also identified.

Increased bleeding tendency was common in patients with CBD. Reported symptoms included epistaxis (27\%), gingival bleeding (27\%), and other bleeding manifestations (88\%). Presence of bleeding manifestations in the family was significantly associated with a
CBD diagnosis $(25 / 33,75.8 \%$ vs. $16 / 48,33.3 \%, P<0.05)$, although only $30.8 \%$ ( $n=8 / 33$ ) of these patients had a family member diagnosed with $\mathrm{CBD}$.

Conclusions: $\mathrm{CBD}$ are highly prevalent among post-menarcheal adolescent females presenting with menorrhagia and IDA. Personal or family history of bleeding manifestations should warrant clinical investigation to rule-out underlying $C B D$.

PB0936 | High Plasma von Willebrand Factor Level is Associated with Increased Risk of upper Gastrointestinal Bleeding in Patients with Chronic Coronary Syndromes Receiving Long-term Antiplatelet Therapy

V. Korobkova ${ }^{1}$; A. Komarov ${ }^{1}$; O. Shakhmatova ${ }^{1}$; A. Dobrovolsky ${ }^{1}$; E. Novikova ${ }^{1}$; E. Guskova ${ }^{1}$; E. Titaeva ${ }^{1}$; E. Yarovaya ${ }^{2}$; A. Shuleshova ${ }^{1}$; E. Panchenko ${ }^{1}$

${ }^{1}$ Federal State Budget Educational Institution National Medical Research Centre of Cardiology, Moscow, Russian Federation; ${ }^{2}$ Federal State Budget Educational Institution of Higher Education M.V. Lomonosov Moscow State University, Faculty of Mechanics and Mathematics, Moscow, Russian Federation

Background: Plasma von Willebrand factor (VWF) levels have been proposed as a marker of atherosclerotic burden and as a risk factor for cardiovascular events. Numerous clinical and experimental reports suggest that high VWF levels reflect damage to the endothelium or endothelial dysfunction. It is unclear whether variations in VWF levels may determine the rate of bleeding complications in pts with atherosclerosis receiving antithrombotic therapy.

Aims: To assess the predictive value of VWF levels for upper gastrointestinal bleeding (UGIB) in patients with chronic coronary syndromes (CCS) receiving long-term antithrombotic therapy.

Methods: Single center prospective Registry of Long-term AnTithrombotic TherApy (REGATTA-1 NCT04347200) included 934 pts with CCS (78.6\% males, age $61 \pm 10.7 \mathrm{yrs}, 76 \%$ after elective $\mathrm{PCI})$. The UGIB annual incidence was 1.9 events per 100 patient-years. VWF was determined in baseline blood samples from 28 pts with UGIB and 141 controls, matched for age, sex and main clinical risk factors.

Results: The median for VWF was 139[interquartile range 107168] \%. Frequency of UGIB was higher in the Q2-Q5 (> 105\%) compared to the lower quintile of VWF distribution (20.8\% vs $2.6 \%$, $P=0.008)$. VWF showed acceptable discriminatory ability for UGIB, $A U C=0.67,95 \% \mathrm{Cl}=0.59-0.74, P=0.0014$ (figure 1). High VWF remained significant after adjustment for anatomical and clinical variables in regression model taking into account ESC panel's UGIB risk factors (OR 14.02, 95\% Cl 1.41-139.42; $P=0.023$ ). 


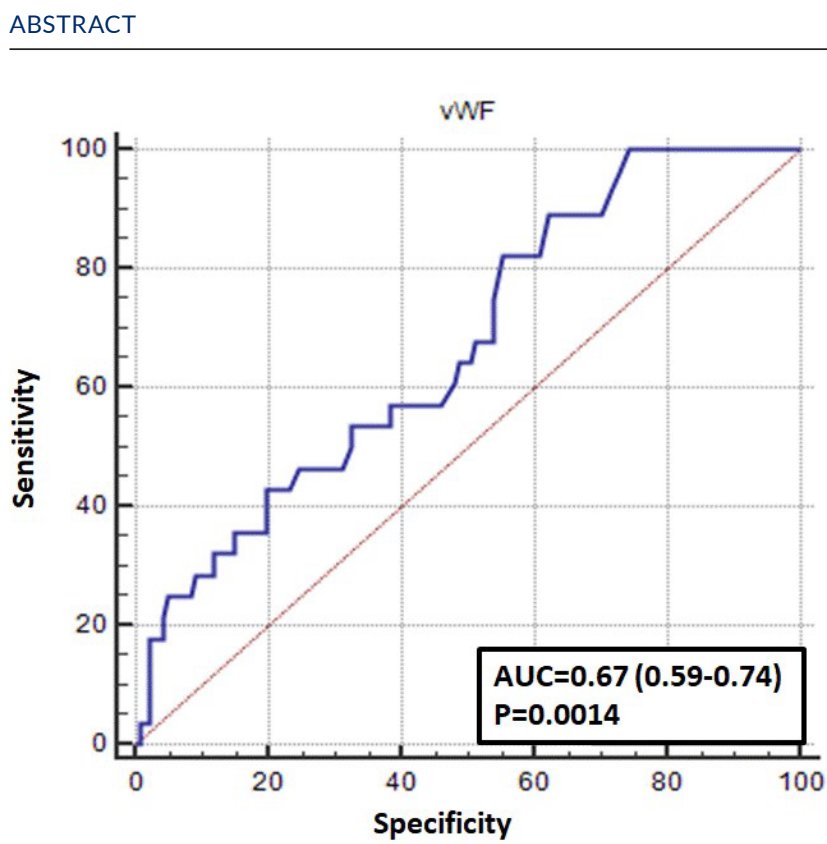

FIGURE 1 VWF as prognostic biomarker for upper gastrointestinal bleeding in patients with chronic coronary syndromes (ROC curve analysis)

Conclusions: VWF should be considered as a valuable prognostic biomarker to improve the prediction of UGIB in addition to wellknown scoring systems in CCS patients receiving long-term antithrombotic therapy.

PB0937 | Diagnosis of von Willebrand Disease-intricacies and Challenges: An Experience from a Tertiary Care Centre in Southern India

R. Kar; K. Balakrishnan; A. Logaiyappan; J. Jayachandan; D. Basu Jawaharlal Institute of Postgraduate Medical Education and Research, Puducherry, India

Background: The diagnosis of von Willebrand Disease (VWD) is an intricate process. The basic diagnostic panel includes von Willebrand factor antigen assay (VWF:Ag), VWF ristocetin cofactor activity (VWF:RCo), and Factor VIII:C.

Aims: To analyze the spectrum and coagulation profile of VWD cases diagnosed based on a simplified algorithm (Figure 1).

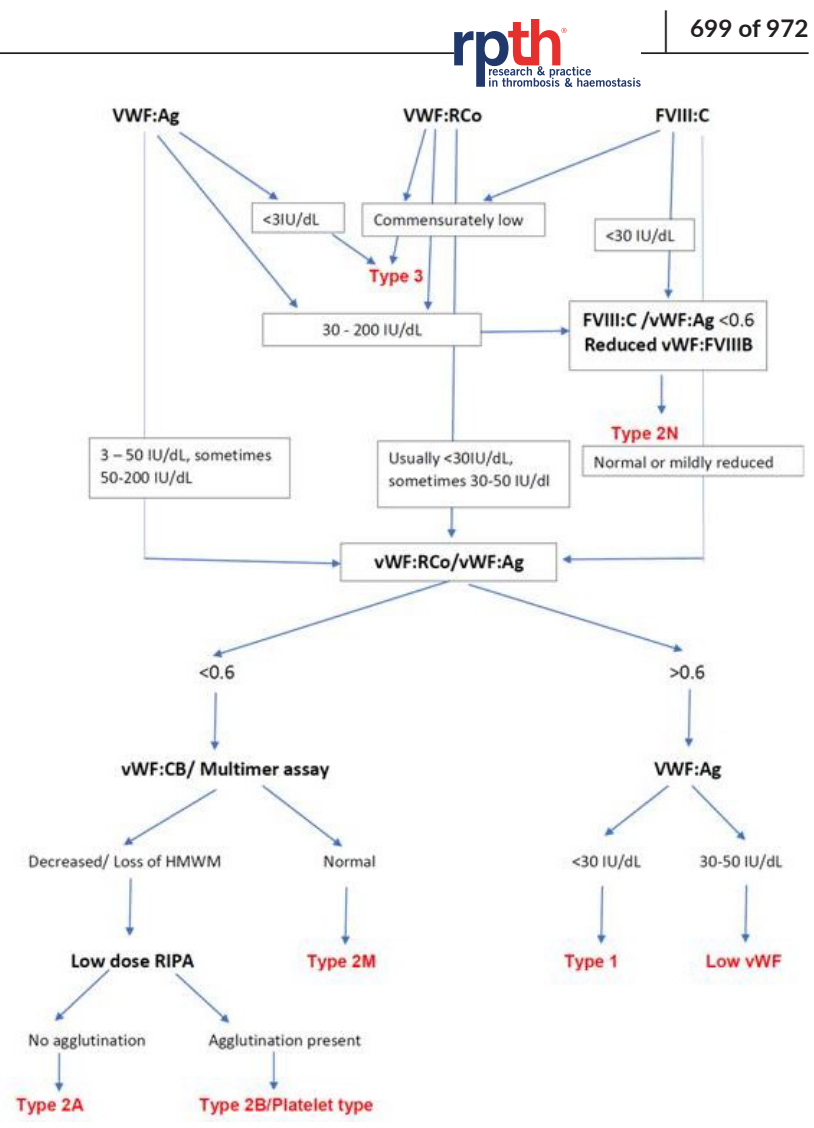

FIGURE 1 A simplified algorithm for diagnosis and subtyping of VWD

Methods: This was a cross-sectional study over five and half years. Cases with normal screening coagulogram, or isolated activated partial thromboplastin time prolongation, or with prolonged bleeding time where platelet function defect was excluded, VWF:Ag assay by either ELISA [Raybiotech Life, Georgia, United States] or automated coagulometer [STA compact CT, Diagnostica Stago, Asnièressur-Seine, France], VWF: RCo [490-2D, Chronolog Corporation, Havertown, PA, USA] and FactorVIII:C [automated] were done. Ratios of function to antigen parameters which included VWF:RCo/ VWF:Ag and FVIII:C /VWF:Ag were derived. Multimer assay [Hydragel 5 von Willebrand Multimers kits, Sebia, Lisses, France] was done in a few cases.

Results: Forty-two patients had some form of VWD/ defect of VWF as follows: Type 3 in 13, Type $2 \mathrm{~N}$ in 7, Type 2N/3 (incomplete work-up) in 2, Type 2 (not further categorized) in 9, Low VWF in 10, and one patient of Waldenstrom Macroglobulinemia with acquired VWD. The mean age of presentation was either in the second or third decade with a female predominance with common bleeding patterns of epistaxis, bleeding gums, easy bruising, and menorrhagia. The hemostasis parameters of the various categories are summarized in Table 1. 
TABLE 1 Clinical and hemostatic parameters of the various subtypes of VWD

\begin{tabular}{|c|c|c|c|c|}
\hline Parameters/ Diagnosis (n) & VWD Type 3 (13) & VWD Type 2N (7) & VWD Type2 (9) & Low VWF (10) \\
\hline Age in years, Mean (SD) & $29.4(15.9)$ & $18.8(12.4)$ & $12.7(7.7)$ & 15.7(10.3) \\
\hline Gender, Male\%: Female\% & $30.8: 69.2$ & 28.6: 71.4 & 44.4: 55.6 & $30: 70$ \\
\hline BT in min, Median (Range) & $>15(2->15)$ & $4: 30(3-10: 30)$ & $3: 45(1: 30->15)$ & $3(1: 30-5)$ \\
\hline aPTT in sec, Mean (SD) & $58.1(13.7)$ & $53.5(4.7)$ & $40.1(6.8)$ & $30.9(2.5)$ \\
\hline FVIII level in \%, Median (Range) & $5.6(1-24)$ & $4(1-17)$ & $35(31-44)$ & Not available (NA) \\
\hline VWF:RCo in \%, Median (Range) & $0(0-4)$ & $72(56.2-128)$ & $13(0-45)$ & 31 (NA) \\
\hline $\begin{array}{l}\text { VWF:Ag in \% or ng/ml, Median } \\
\text { (Range) }\end{array}$ & $1(0-3)$ & $118.1(50-183)$ & $56(18-95)$ & $47(37-49.6)$ \\
\hline $\begin{array}{l}\text { Ristocetin aggregation \%, } \\
\quad \text { Median (Range) }\end{array}$ & $7.5(0-16)$ & $42(19-85)$ & $26(3-95)$ & $55(17-79)$ \\
\hline $\begin{array}{l}\text { Multimer assay (Total done/ } \\
\text { Pattern) }\end{array}$ & 7 , Absent in all & 2, Normal pattern & 2, Lack of HMWM (type 2A) & NA \\
\hline
\end{tabular}

Conclusions: The combination of VWF: Ag assay, VWF:RiCo, and FactorVIII:C forms the tripod for diagnosis and classification of major VWD types. Further subtyping can be done by multimer analysis. A greater proportion of severe types of VWD were observed in our study. However, this would not be representative of the population prevalence of various types since patients with more severe bleeding phenotypes are likely to have a hospital referral.

PB0938 | Acquired von Willebrand Disease: The Diagnosis and Management of an Underdiagnosed Coagulopathy

A. Ferretti ${ }^{1}$; E. Baldacci ${ }^{1}$; S. Lancellotti ${ }^{2}$; M. Basso ${ }^{2}$; F. Barone ${ }^{2}$;

A. Pallotta ${ }^{3}$; G. Lapietra ${ }^{1}$; M. Sacco ${ }^{2}$; A. Chistolini ${ }^{1}$; E. De Candia ${ }^{2}$; C. Santoro ${ }^{1}$

${ }^{1}$ Unit of Hematology, Department of Translational and Precision Medicine, Sapienza- University of Rome, Rome, Italy; ${ }^{2}$ Fondazione Policlinico Gemelli IRCCS, Università Cattolica Sacro Cuore, Rome, Italy; ${ }^{3}$ Unit of Hematology, Department of Translational and Precision Medicine, Rome, Italy

Background: Acquired Von Willebrand Syndrome (AVWS) is an acquired coagulopathy, often associated to an underlying disorder. The diagnosis is not easy and relies on a negative familial and personal clinical hemorrhagic history and a late onset in life of bleeding symptoms, associated with a laboratory pattern for Von Willebrand Disease (VWD).

Aims: Aim of this study is to describe the experience on diagnosis and management of AVWS patients (pts) in two Italian centers.

Methods: Between 2004-2020 we have diagnosed and managed 14 pts [8F, 6M; median age 62.45 years (45.4-85.9)] affected by AVWS Determination of coagulation parameters, including FVIII, were carried out on an automatic coagulometer (ACL Top 700, Werfen). VWF:antigen (VWF:Ag) and VWF:activity (VWF:RCo) were measured by chemiluminescence assays (HemosIL AcuStar, Werfen). VWF propeptide (VWFpp) level was measured by ELISA immunoassay
(Sanquin, Amsterdam, NL). The screening for VWF:RCo inhibitor was made utilizing mixing studies

Results: At diagnosis, for all pts, we observed the results showed in table 1. VWFpp and multimers were studied just in 9 pts. Except VWFpp median level, all other VWF-related median parameters were significantly reduced. VWFpp/VWF:Ag ratio was significantly higher in all 9 studied pts; multimers were absent in 6 and normal in 2 ; in 1 patient just LMW multimers were present. All the pts did not have either a family or past personal history of bleeding symptoms. Thirteen 14 cases showed a concomitant disorder, one case was idiopathic. In table 2, therapies either for underlying disorders if present or AVWS, and outcomes are shown.

TABLE 1 Clinical and laboratory characteristics of patients

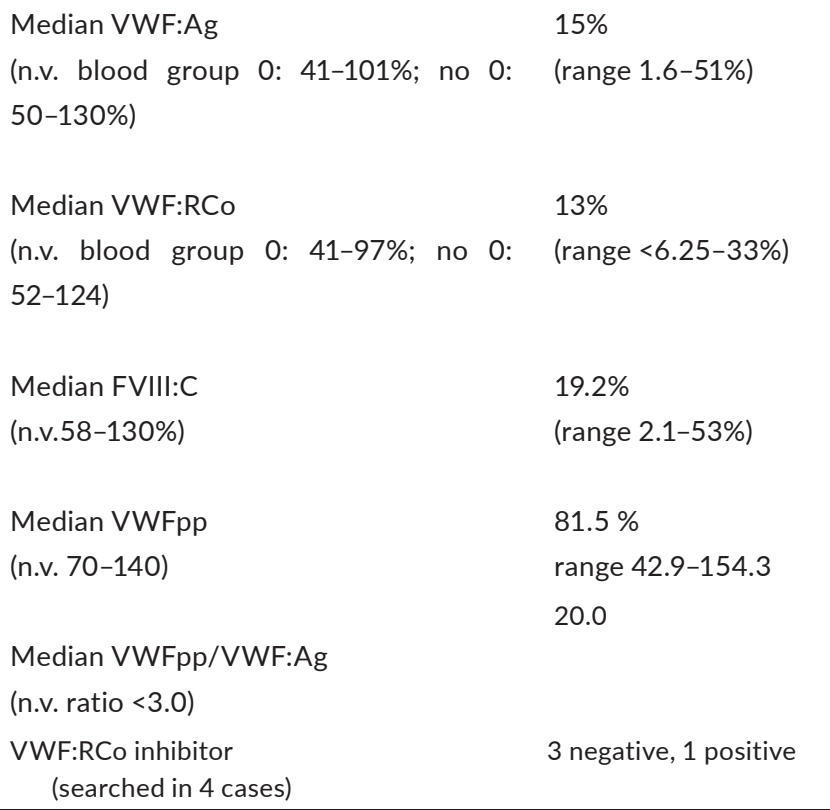


TABLE 2 Management of the patients with lymphoproliferative disorders

\begin{tabular}{|c|c|c|}
\hline Type & Treatment & Response \\
\hline $\begin{array}{l}\text { Gastric B cell MALT Iymphoma } \\
1 \text { patient }\end{array}$ & Rituximab & Complete remission of lymphoma and AVWS \\
\hline Waldenstrom Disease & $\mathrm{R}-\mathrm{CVP}$ & $\begin{array}{l}\text { After } 2^{\text {nd }} \text { line, stable lymphoproliferative disease } \\
\text { and persistent AVWS }\end{array}$ \\
\hline $1^{\text {st }}$ patient & Ibrutinib & \\
\hline Waldenstrom Disease & Rituximab & Partial response \\
\hline $2^{\text {nd }}$ patient & $\begin{array}{l}\text { After two years for disease progression: } \\
\text { Rituximab+-Bendamustine }\end{array}$ & $\begin{array}{l}\text { Persistent AVWS and partial response of } \\
\text { lymphoma }\end{array}$ \\
\hline $\begin{array}{l}\text { Indolent B cell lymphoma } \\
1 \text { patient }\end{array}$ & $\begin{array}{l}\text { Watch and wait strategy } \\
\text { Rituximab }\end{array}$ & Complete remission of lymphoma and AVWS \\
\hline \multicolumn{3}{|c|}{ Strategies to manage AVWS in all patients } \\
\hline Type of disorder & Treatment & Response \\
\hline $\begin{array}{l}\text { MGUS } \\
1 \text { patient }\end{array}$ & $\begin{array}{l}\text { Prednisone + cyclophosphamide and then high } \\
\text { dose immunoglobulins }\end{array}$ & No response to either therapies \\
\hline $\begin{array}{l}\text { MGUS } \\
3 \text { patients }\end{array}$ & $\begin{array}{l}\text { Infusion of high dose immunoglobulins } \\
\text { ( } 2 \text { cases are under chronic treatment with Iv Ig } \\
\text { every } 6-8 \text { weeks) }\end{array}$ & $\begin{array}{l}\text { Transient } C R \text { as regard VWD laboratory } \\
\text { parameters }\end{array}$ \\
\hline $\begin{array}{l}\text { MGUS } \\
4 \text { patients }\end{array}$ & 4 patients & \\
\hline & Prednisone & Response to bleeding's symptoms \\
\hline \multicolumn{3}{|l|}{ Breast cancer } \\
\hline 1 patient & & \\
\hline Idiopathic AVWS & Prednisone & CR on VWD laboratory parameters \\
\hline 1 patients & At relapse Prednisone + cyclophosphamide & CR on VWD laboratory parameters \\
\hline
\end{tabular}

Conclusions: AVWS is a rare syndrome, probably underdiagnosed. AVWS must be suspected whenever a patient presents with a late onset in life of bleeding symptoms associated to laboratory characteristics compatible with VWD. The VWFpp/VWF:Ag ratio could be a valuable biomarker to be considered to help the diagnosis.

Table 1: Clinical and laboratory characteristics of patients

Table 2: Management of the patients with lymphoproliferative disorders
PB0939 | Plasma-derived VWF/FVIII Concentrate (wilate ${ }^{\circledR}$ ) for Haemostasis in Women with VWD during Childbirth

A. Iorio ${ }^{1}$; A. Srivastava ${ }^{2}$; P. James ${ }^{3}$; A. Ma ${ }^{4}$

${ }^{1}$ Department of Health Research Methods, Evidence and Impact, and Department of Medicine, McMaster University, Hamilton, Canada;

${ }^{2}$ Department of Haematology, Christian Medical College, Vellore, India; ${ }^{3}$ Department of Medicine, Queen's University, Kingston, Canada; ${ }^{4}$ Department of Medicine, University of North Carolina School of Medicine, Chapel Hill, United States

Background: Women with von Willebrand disease (VWD) are at risk of excessive bleeding during and after childbirth. Peripartum haemostatic management of women with VWD who have not normalised VWF levels in pregnancy with von Willebrand factor (VWF) concentrate reduces this risk.

Aims: To evaluate the usage, efficacy and safety of wilate ${ }^{\circledR}$, a plasma-derived VWF/FVIII concentrate with a 1:1 VWF:FVIII ratio, 
for prevention of excessive bleeding during childbirth in women with any type of VWD.

Methods: Data on women with VWD who received wilate ${ }^{\circledR}$ for haemostatic coverage for childbirth were pooled from a prospective Phase 3 clinical trial of wilate ${ }^{\circledR}$ in surgery (the WONDERS Study) and a prospective Phase 4 study of wilate ${ }^{\circledR}$ in real-world clinical practice (WIL-20).

Results: Ten women with VWD were treated with wilate ${ }^{\circledR}$ to prevent excessive bleeding for childbirth. The mean (SD) age at enrolment was 29.6 (4.2) years. Two women had Type 1 (VWF:Rco < 65\% and VWF:Ag <25\% at study enrolment), four had Type 2 (two 2A, one $2 \mathrm{M}$ and one unspecified) and four had Type 3 VWD. Of the 10 deliveries, 5 were caesarean sections. All patients received a loading dose of wilate ${ }^{\circledR}$ prior to delivery. Patients received a mean (SD) of $9.5(2.2)$ infusions of wilate ${ }^{\circledR}$ over 6.8 (1.9) exposure days, with a total dose of 234 (126) IU/kg wilate ${ }^{\circledR}$. No excessive bleeding was noted, and efficacy was rated as "excellent" or "good" for all 10 deliveries. Two patients experienced eight possible or probable treatment-related AEs; all were mild or moderate and resolved. No thromboembolic events were observed.

Conclusions: Peripartum doses of wilate ${ }^{\circledR}$ aiming to increase VWF and FVIII levels in VWD patients can prevent excessive bleeding associated with childbirth. These results suggest that wilate ${ }^{\circledR}$ may be an effective treatment for management of bleeding in women with any type of VWD when giving birth.

PB0941 | Perioperative Management of Patients with von Willebrand Disease Undergoing Surgical Interventions

R. Toenges ${ }^{1}$; L. Haack ${ }^{2}$; B. Krammer-Steiner ${ }^{2}$

${ }^{1}$ Department of Medicine, Hematology/Oncology/Hemostaseology, Goethe University, Frankfurt, Germany; ${ }^{2} 3$ rd Department of Internal Medicine, City Hospital Rostock, Rostock, Germany

Background: Patients with von Willebrand disease (vWD) are at increased risk of bleeding following surgical interventions. Both the type and severity of VWD as well as the intervention-associated risk of bleeding need to be considered to ensure individualized management aiming to prevent bleeding complications.

Aims: To analyse a single German hemophilia center experience of vWD patients undergoing surgery.

Methods: Data were collected over a 5-year period for all vWD patients undergoing surgical interventions. All interventions were included without restrictions related to indication and type of surgery. Results: In total, 42 vWD patients (18 to 78 years of age; 34 females and 8 males) with $69 \%$ type 1 vWD underwent 83 surgical interventions. The intervention-associated risk of bleeding was rated as high or moderate in $71 \%$ and $29 \%$ of the interventions, respectively. The total mean dose of von Willebrand factor (VWF)/factor VIII (FVIII) concentrate administered perioperatively and over the days following surgery was $102 \mathrm{IU} / \mathrm{kg}$ and $73.5 \mathrm{IU} / \mathrm{kg} \mathrm{FVIII}$ for surgeries with a high and moderate risk of bleeding, respectively. Treatment was continued for a median of five days postoperatively, regardless of the type of surgery. No postoperative bleeding was reported in $95 \%$ of all interventions and there was no postoperative bleeding in $99 \%$ of all interventions with a high risk of bleeding. Three minor bleeding events were recorded as were four non bleeding-related, minor, postsurgical complications.

Conclusions: vWF/FVIII concentrate can be used to successfully manage the perioperatively increased bleeding risk in vWD patients undergoing surgical interventions. The intervention-associated bleeding risk in combination with the VWD type and severity should guide the individualized replacement approach.

PB0942 | Molecular Heterogeneity in von Willebrand Disease in North Indian Patients

R. Sharma ${ }^{1}$; M. Jamwal ${ }^{1}$; N. Kumar ${ }^{1}$; H.K. Senee ${ }^{1}$; C. Hans ${ }^{1}$;

D. Bansal ${ }^{1}$; A. Trehan; P. Malhotra; R. Das; J. Ahluwalia

PGIMER, Chandigarh, India

Background: von Willebrand Disease (VWD) is the commonest inherited bleeding disorder that occurs due to quantitative (type 1 and 3 ) and qualitative (type 2 subgroups $2 \mathrm{~A}, 2 \mathrm{~B}, 2 \mathrm{M}$, and $2 \mathrm{~N}$ ) deficiency of glycoprotein VWF. Type 1 is the most common whereas type 3 is rare and most severe form of VWD. Molecular testing is mandatory in type 2 and 3 VWD. There is a paucity of information on the genetic basis of VWD in north Indians.

Aims: To study the molecular spectrum of subtypes of von Willebrand Disease in north Indian patients.

Methods: Patients with history of bleeding and decreased levels of VWF antigen, VWF GPIbR and/or an abnormal RIPA test were subcategorized. Family history and informed consent was taken. Thirty-five cases were subjected to targeted resequencing using Ampliseq for Illumina custom panel for library preparation and sequencing was done using MiSeq. The output files (.fastq files) were analyzed using Local run manager software and BaseSpace Variant Interpreter (Illumina). Pathogenicity of variants was predicted using in silico tools. Sanger validation of pathogenic variants was done in the index cases and family members.

Results: Type 3 subtype was most common (16/30 = 53.3\%) followed by type $2(11 / 30=36.6 \%)$ and type $1(2 / 30=6.6 \%)$. Pathogenic variants were found in 30 cases (85.7\%) including 14 missense (45\%), 9 nonsense (29\%), 5 splice site (16\%), 3 indels (9.7\%) of which 13 were novel. Family history and consanguinity were positive in 14 and 4 cases respectively. Most of the mutations were in exon 28.

Conclusions: The molecular spectrum of VWD in the north Indian population is varied and major subcategories of VWD are represented. In this largely non- consanguineous cohort, most variants are non-recurring and exon 28 is a hotspot. The data from this study will help in designing strategies for prenatal diagnosis, predictive testing, and genetic counseling for the affected families. 
PB0943 | Atypical Presentation of VWD Leading to Discovery of Novel VWF Mutation

T. van de Berg ${ }^{1}$; A.M Todaro ${ }^{1} ;$ J. van Beers ${ }^{2} ; \mathrm{K}$.

Wichapong $^{1}$; F. Heubel-Moenen ${ }^{3}$; E. Castoldi ${ }^{1}$; Y. Henskens ${ }^{2}$; E.

Beckers $^{3}$

${ }^{1}$ Cardiovascular Research Institute Maastricht, Department of

Biochemistry, Maastricht, Netherlands; ${ }^{2}$ Central Diagnostic Laboratory,

MUMC+, Maastricht, Netherlands; ${ }^{3}$ Department of Hematology,

MUMC+, Maastricht, Netherlands

Background: Von Willebrand Factor (VWF) is a multimeric protein largely involved in both primary and secondary hemostasis. The diagnosis and classification of von Willebrand Disease (VWD) patients can be challenging. Atypical presentations of VWD might benefit from additional genetic analysis.

Aims: Characterization of a VWD patient with a disproportionately severe bleeding phenotype.

Methods: Routine analysis for VWD was performed. Genetic screening was performed by exome sequencing of hemostasis related genes. VWF mRNA analysis was carried out by RT-PCR and Sanger sequencing.

Results: Routine analysis showed PFA-ADP and PFA EPI $>300 \mathrm{sec}$ onds, VWF:ACT of $37 \%$ with a VWF:AG of $36 \%$. Collagen binding and FVIII-binding were $46 \%$ and $28 \%$ respectively.

Genetic analysis of the VWF gene disclosed 2 heterozygous variants of unknown significance (VUS): c.2771 G>A (exon 21, p.Arg924.

GIn) has a 1-2.5\% population prevalence and has been previously described in type 1 and 2N VWD. The other VUS (c.2278 C>A; exon 17 ) is a novel mutation predicting a major amino acid substitution (p.Arg760Ser) in the D2-domain of VWF. Sequencing of exons 17 and 21 in the patient's VWF mRNA revealed homozygosity for the mutated allele at both mutation sites, indicating that the two variants are in cis and that the 'normal' allele is not expressed at mRNA level. Moreover, an aberrantly spliced mRNA was identified which lacks exon 17, leading to a frameshift and a premature stop codon in exon 18. Structural analysis showed that the Arg760Ser mutation may decrease the affinity of furin to the VWF pro-peptide cleavage site. Conclusions: The patient carried two VUS on the only VWF allele that was expressed at the mRNA level. The Arg760Ser mutation possibly interferes pro-peptide cleavage by furin. The cause of the silencing of the 'normal' allele, the phenotypic impact of the exon 17 variant and the functional impact of the mutations are currently under investigation.
PB0944 | The von Willebrand Disease-Woman (VWD-Woman) Trial: A Pilot Study Comparing Recombinant von Willebrand Factor (rVWF) plus Tranexamic Acid (TA) vs. rVWF Alone in the Prevention of Postpartum Hemorrhage in Women with von Willebrand Disease

N. Machin ${ }^{1}$; S. Caritis ${ }^{1,2}$; C. Seaman ${ }^{1,3} ;$ M. Brooks ${ }^{1}$; D. Vehec ${ }^{1,3}$; M. Rode ${ }^{1,3}$; D. Ivanco ${ }^{1,3}$; D. Fischer ${ }^{2}$; D. Zowacki ${ }^{2}$; M. Ragni ${ }^{1,3}$ ${ }^{1}$ University of Pittsburgh, Pittsburgh, United States; ${ }^{2}$ UPMC MageeWomens Hospital, Pittsburgh, United States; ${ }^{3}$ Hemophilia Center of Western Pennsylvania, Pittsburgh, United States

Background: Von Willebrand disease (VWD) is a quantitative or qualitative deficiency of von Willebrand factor (VWF) that is associated with a 1.5 -fold increased odds of postpartum hemorrhage (PPH). This risk persists despite VWF replacement and may be lifethreatening, lead to hysterectomy, and require blood transfusion with its attendant risk. We hypothesize the increased bleeding risk is due to physiologic postpartum fibrinolysis in the setting of VWF deficiency. We propose that the use of the antifibrinolytic agent tranexamic acid (TA), given IV within the first 3 hours of delivery, will reduce $\mathrm{PPH}$ to a greater degree than $\mathrm{rVWF}$ alone.

Aims: We designed a single-center pilot study to establish the safety and feasibility of enrollment, drug delivery, and lab collection to conduct a future multicenter randomized trial comparing rVWF + TA vs. rVWF alone.

Methods: This is a phase III, randomized, open-label pilot trial of rVWF $80 \mathrm{IU} / \mathrm{kg}$ at delivery and day 1 and 2 postpartum with or without TA 1 gm IV administered within 3 hours of delivery in 20 women with VWD to prevent PPH (Fig 1). The primary endpoint is a reduction in $\mathrm{PPH}$ determined by quantitative blood loss at delivery. Secondary endpoints include patient-reported pictorial blood loss estimate, safety, tolerability, and coagulation studies. Statistical comparisons are descriptive. Informed consent will be obtained under University of Pittsburgh Institutional Review Board approval, PRO20030186, prior to trial activities. This trial is registered at clinicaltrials.gov NCT04344860.

\section{Primary Endpoint}
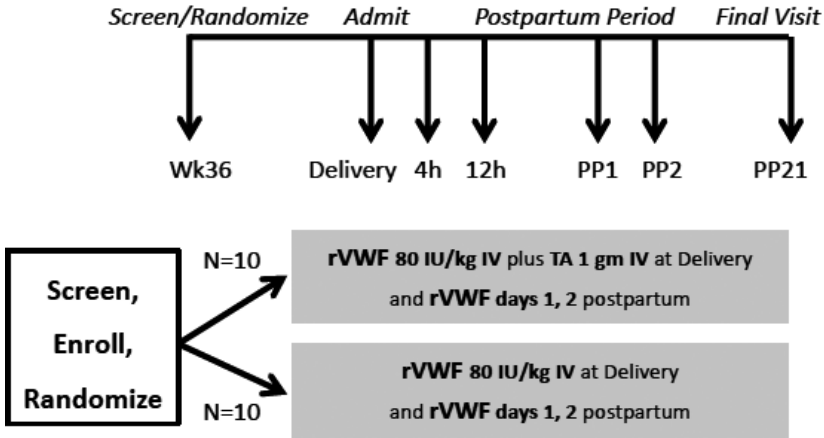

FIGURE 1 Schema of VWD-Woman Trial 
Results: In collaboration with obstetric colleagues, we have completed IRB, DSMB, and regulatory requirements, established a redcap database, a drug-dose calculator, reporting forms, and operations manual.

Conclusions: Women with VWD experience PPH despite VWF replacement. In this pilot phase III trial comparing rVWF +TA to rVWF alone, in women with VWD is proven feasible, we will initiate a multicenter phase III randomized trial.

PB0945 | Finding the Balance between Bleeding Risk and Thromboembolic Risk: A Case Report

B. Merchan Muñoz; S. Herrero Martín; M.I. Nuevo López; M. Mora Argumánez

Hospital Universitario Guadalajara, Guadalajara, Spain

Background: Type 2B Von Willebrand Disease (VWD) is an inherited bleeding disorder caused by changes in Von Willebrand Factor (VWF) that enhances binding of VWF to GPIb on platelets. Patients with this subtype have a more severe bleeding phenotype compared with other type 2 .

Aims: To describe the challenge of managing a patient with VWD and multiple thromboembolic risk factors.

Methods: We report the case of a 74-year-old male with type 2B VWD (variant found in exon 28), responsible for thrombocytopenia and high bleeding risk. He presented high thrombotic risk due to atrial fibrillation, arterial hypertension, critical left renal artery stenosis, parietal thrombosis in the left common iliac artery, as well as positive for anticardiolipin antibodies. Patient data was collected from medical records.

Results: After an evaluation by a multidisciplinary team, percutaneous closure of the left atrial appendage was carried out in order to avoid anticoagulation therapy and started treatment with antiplatelet agent. During the past years, major and frequent gastrointestinal bleeding episodes occurred; one of them was life-threatening. After discussing the risk and benefits of different treatment options for the condition presented, prophylaxis with VWF/FVIII concentrated (1:1 balance of VWF and FVIII) was started: $1500 \mathrm{IU} /$ twice a week to maintain FVIII:C concentrations under $150 \mathrm{IU} / \mathrm{dL}$ in order to reduce the thromboembolic risk. Therapy with VWF/FVIII concentrate significantly reduced the frequency and severity of bleeding episodes with stabilized hemoglobin levels, and bleeding risk was reassessed throughout the course of treatment. Thrombotic episodes or other side effects were not observed following the infusion of the treatment. To reduce the thromboembolic risk we valued the benefits of switching to high-purity VWF concentrate.

Conclusions: This case illustrates the challenging nature of VWD and multiple prothrombotic risk factors. Prophylaxis with VWF/ FVIII showed a reduction in transfusion dependence and significant improvement in quality of life.
PB0946 | Reclassification of von Willebrand Disease Classification and Effects of Vascular Dysfunction on von Willebrand Factor

H. Watson ${ }^{1}$; Y.E. $\mathrm{Ng}^{2}$

${ }^{1}$ Aberdeen Royal Infirmary, Aberdeen, United Kingdom; ${ }^{2}$ University of Aberdeen, Aberdeen, United Kingdom

Background: von Willebrand Disease (vWD) is a bleeding disorder caused by a lack of, or a functional abnormality of von Willebrand Factor (vWF). The United Kingdom Haemophilia Centre Doctors Organisation (UKHCDO) published 2014 guidelines for categorising patients by disease type.

Aims: We aimed to re-evaluate the classification of a cohort of patients, diagnosed with von Willebrand disease in NHS Grampian from 1980 onward, by applying the 2014 guidance to both existing and updated measurements of VWF and Factor VIII. We also assessed changes in vWF levels across time in patients with and without features of degenerative vascular disease.

Methods: Using pre-existing patient data on the UKHCDO registry, patients were reclassified using the 2014 guidelines. In addition, updated factor levels were also collected and reclassification using these updated levels was performed.

Results: 49 patients were included and $57 \%$ of them were reclassified. Most changes in diagnosis were seen in patients who were initially diagnosed with Type $1 \mathrm{vWD}$, and none occurred in patients with Type 3 vWD. 5 patients were reclassified into the category of having 'low vWF level' introduced in 2014. 21 patients were deemed to no longer have vWD.

Most were shown to have increases in VWF antigen (vWF:Ag) and vWF:Ristocetin cofactor activity (vWF:RCo) across time. However, in patients with vascular disease, there was no obvious trend in these factor level changes based on a small number of data.

Conclusions: Using the criteria outlined in the 2014 UKHCDO guidelines resulted in the reclassification of over half of the patients in our study. Reclassification into 'low vWF level' and increasing levels of VWF with age/cardiovascular risk account for most of the changes observed. There has to be clear thinking on the need to change VWD diagnosis based on increases in VWF related to age/cardiovascular disease.

PB0947 | Correlation of VWF:Ab to VWF:Ag Ratio and Abnormal VWF Multimer Pattern

M. Stuart; D. Chen; N. Heikal; R. Pruthi

Mayo Clinic, Rochester, United States

Background: Type 2 variants of congenital von Willebrand disease (VWD) and acquired VW syndrome (AVWSyn) typically have an abnormal VWF multimers (VWFM): decreased or loss of high molecular weight VWF multimers (HMWM)). A VWF activity (VWF:Ab) to VWF antigen (VWF:Ag) ratio of $<0.7$ raises suspicion of congenital 
Type 2 VWD, in acquired AVWSyn the ratio may be higher and the VWF levels are typically normal.

Aims: For patients with normal VWF levels ( $\geq 55 \%$ ), to determine prevalence of abnormal VWFM at a VWF:Ab to VWF:Ag ratio cut off of 0.7 or 0.8 .

Methods: Retrospective analysis of VWD test panels between March and September 2019. ROC analysis of VWF:Ab to VWF:Ag ratio of $<0.8$ vs $<0.7$ for abnormal VWFM was conducted.

Results: Over the study period, of a total 5,340 VWD panels, 1735 were reflexed to VWFM analysis based on a cut off of $<0.8$ of which 1065 had normal VWF levels. Of those with ratio 0.7-0.8 $(n=845)$, 45 (5\%) had abnormal VWF. Of the 212 with ratio <0.7, 97 (46\%) had an abnormal VWFM. Optimal cut-off to diagnostic accuracy assessed with ROC analysis comparing the ratio to qualitative VWFM interpretation. Data set 1, all panels reflexed to VWFM ( $n=1735)$, acheived optimal threshold of 0.620, AUC at 0.834. Data set 2, panels with normal VWF levels ( $n=1065$ ), acheived optimal threshold of 0.620 , AUC at 0.836 .

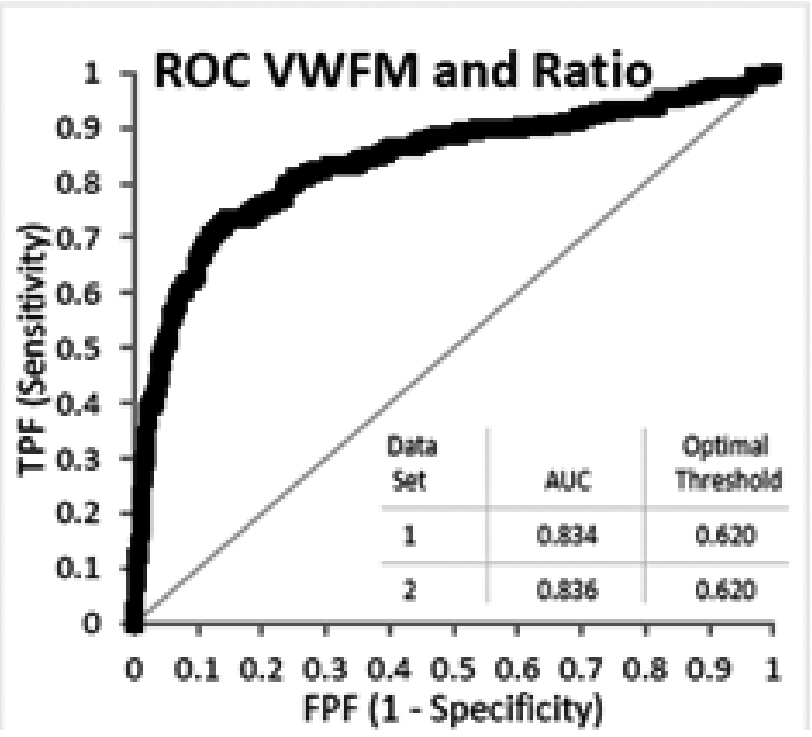

\section{FIGURE 1}

Conclusions: There is a higher prevalence of abnormal VWFM at a VWF:Ab to VWF:Ag ratio cut off of 0.7 and is consistent with recent VWD guidelines for type 2 VWD and AVWSyn. The latter patients with normal VWF levels, may still have abnormal VWFM and thus may need follow up VWFM testing.
PB0948 | Intermittent Prophylaxis for Heavy Menstrual Bleeding in Von Willebrand Disease

P. Laguna $^{1}$; A. Laguna ${ }^{2} ;$ M. Matysiak ${ }^{1}$

${ }^{1}$ Medical University of Warsaw, Warsaw, Poland; ${ }^{2}$ Queen Mary

University, London, United Kingdom

Background: Von Willebrand disease (VWD) is the most common congenital bleeding disorder. Its main symptom is recurrent bleeding from mucosal tissues, which can include heavy menstrual bleeding (HMB), even leading to anaemia and the need for hospitalisation and transfusion with red cell concentrates (RCC).

Aims: To describe case studies illustrating appropriate patient management in $\mathrm{HMB}$.

Methods: Three cases of teenagers with VWD and HMB are presented.

Results: The first patient is a 14.5-year-old girl, who started experiencing heavy menstrual bleeding when she was 13 . Coagulation parameters (APTT, INR, fibrinogen) were always normal. Due to substantial anaemia, the patient often received RCC. Contraceptive treatment was introduced, but was unsuccessful and VWD started being suspected. Laboratory tests revealed FVIII levels of 51\%; VWF:RCo levels of $14.5 \%$ and VWF:Ag of $18.3 \%$. VWD type 2 was diagnosed, which led to end the contraception treatment and start intermittent prophylaxis with pdVWF/FVIII (ratio 2.4:1) for 3 days (40 IU VWF/kg/day). This normalized the menstruation.

The second patient is a 14-year-old girl, whose HMB even required hospitalisation. Contraceptives had no effect, which elicited a suspicion of VWD. Laboratory tests showed FVIII levels of $61.5 \%$, VWF:RCo of $20.8 \%$ and VWF:Ag of $36.3 \%$. After obtaining the results, VWD type 2 was confirmed and pdVWF/FVIII was introduced prophylactically during the first 2 days of the menstrual period ( 40 IU VWF/kg/day). This normalized the menstruation.

The third patient is a 16-year-old girl with VWD type 3. From her first menstrual period, prolonged bleeding was observed, together with the development of substantial anaemia. After introducing pdVWF/FVIII (40 IU VWF/kg/day) the first three days of each period, menstrual bleeding was normalized.

Conclusions: In the case of recurrent HMB despite normal coagulation parameters in the blood test results, one should always suspect VWD. Intermittent prophylaxis with VWF-containing concentrates can be considered in these cases. 
PB0949 | Modern Diagnosis of von Willebrand Disease in Children in Russia: Experience in Centralized Diagnostics

A. Poletaev ${ }^{1}$; E. Seregina ${ }^{1,2}$; D. Fedorova ${ }^{1}$; P. Zharkov ${ }^{1}$

${ }^{1}$ Dmitry Rogachev National Medical Research Center of Pediatric Hematology, Oncology and Immunology, Moscow, Russian Federation; ${ }^{2}$ Center for Theoretical Problems of Physicochemical Pharmacology, Moscow, Russian Federation

Background: The diagnostic capabilities of detecting von Willebrand disease(VWD) in children are limited in many regions of our country, and therefore, in 2019, the Russian Hemophilia Society starts the centralized diagnostics program(Program) that provides for distance diagnosis of VWD in the regions.

Aims: Evaluate the first results of centralized program for VWD diagnostics in children.

Methods: According to clinical state of the patient and to the decision of hematologist the patient was enrolled in Program. Blood samples were collected from peripheral vessel, then freezed and transported to the Laboratory of hemostasis at the Dmitry Rogachev National Medical Research Center Of Pediatric Hematology, Oncology and Immunology. There were 122 frozen samples from 122 children under the age of 18 year, from 21 regions of Russian Federation, received during the period from 04.2019 to 02.2021. The vWF:Ag and VWF:RCo and fVIII activity were evaluated in samples.

Results: Preanalytical errors were detected in 5(4.1\%)samples. The decrease in vWf activity within $30-50 \%$ was detected in $22 \%$ of cases, vWF:RCo less than $30 \%$ in $13.9 \%$ of cases. The vWF:Ag was within $30-50 \%$ in $16.4 \%$ and less than $30 \%$ in $6.5 \%$ of samples respectively. Among patients with vWf:RCo $<30 \%$ type 1 vWD was detected in 4(23.5\%)patients, type 2 - in 12(70.6\%)patients, type 3 - in 1(5.9\%)patient. The decrease in fVIII activity while vWF:Ag and activity were in normal range was observed in 6(4.9\%)cases. The decrease in $\mathrm{fVIII/VWF:Ag} \mathrm{ratio} \mathrm{was} \mathrm{detected} \mathrm{in} 8(6.6 \%)$ children. The centralized diagnostics program allowed to reveal the hemostasis pathology in $36(29.5 \%)$ patients. The $13,9 \%$ of all cases had the typical laboratory pattern of vWD, and $20(16.3 \%)$ patients require the additional diagnostics using an expanded panel of laboratory tests. Conclusions: This program has great potential, especially in sparsely populated regions of the country, where the establishment of regional diagnostics on site may be economically impractical.

PB0950 | Nationwide Screening Programme for Bleeding Disorders Among Adolescent Girls

M. Ross; K. Ilves

North Estonia Medical Centre, Tallinn, Estonia

Background: Estimating the prevalence of Von Willebrand disease (VWD) is a challenging quest due to the considerable number of mild cases in type 1 and the difficulties in making a correct diagnosis between type 1 and 2. Current understanding still reflects that von
Willebrand disease is underdiagnosed and, in many cases, the bleeding disorder is not diagnosed accurately.

Undiagnosed VWD means lost days from school or work due to heavy menstrual bleeding. It is a risk for secondary anaemia and iron deficiency problems and increases the need for iron replacement therapy. An underlying bleeding disorder means higher risk for periand post-operative and peri- and post-partum bleeding, with many women suffering the consequences without appropriate help.

Aims: Estonia has excellent digital health records and e-consultation opportunities among school nurses, GPs and specialty doctors. Based on those advantages, we are establishing a screening programme in addition to our current National Child Health Monitoring Guideline (CHMG).

Methods: According to the CHMG, all children are booked in for a routine GP visit in the 9th grade. Most of the girls at this age (15-17 years old) are already at least one year past menarche, which is a suitable time to screen for bleeding phenotype. During this visit, a standardised pictorial blood loss assessment chart (PBAC) is used for scoring. All girls with a PBAC score $>150$ are booked in through e-consultation for a visit to the haemophilia treatment centre. Girls are encouraged to attend the visit with their mothers. A more accurate and detailed bleeding history and blood samples are taken during the visit.

Results: The main goal of this programme is to find more girls with bleeding disorders and to provide suitable medical attention.

Conclusions: In hereditary conditions, this will also help find boys who are at risk of bleeding problems.

\section{PB0951 | Neurosurgery in a Patient with von Willebrand Disease: A Case-report}

J. Cabral; C. Calaza; M. Calheiros; A. Marques

Immunohemotherapy Service, Hospital de Braga, Braga, Portugal

Background: Von Willebrand disease (vWD) is the most common inherited bleeding disorder.

Optimal surgical management for these patients is dependent on multiple factors (type of surgery, type of vWD, baseline von Willebrand factor (vWF) and factor VIII (FVIII) levels, patient's history of bleeding).

Currently, consensus on the preferred surgical management for all patients is lacking and many of the recommendations regarding the use of replacement therapy are based on expert opinion.

Aims: Describing prophylaxis in a patient with VWD for a neurosurgery.

Methods: Review a clinical case.

Results: A 49-year-old woman with vWD type $2 \mathrm{~N}$ was submitted to a meningioma resection under replenishment with recombinant FVIII concentrate (rcFVIII).

Screening coagulation tests pre-surgery showed: Prothrombin Time (PT) 11.9"; activated Partial Thromboplastin Time (aPTT) 31.3"; Fib. 345 mg/dL; vWF:Ag 209; vWF:Act 224; FVIII 43.8\%. 
Two hours before surgery she received $2000 \mathrm{IU}$ of rCFVIII and tranexamic acid.

Seven hours after administering the rcFVIII we have measured FVIII (67.6\%).

Approximately ten hours after surgery, she did not have hemorrhagic complications. We administered additional 1000 IU of rcFVIII. Twenty hours after surgery, CT scan was performed that showed only small acute hemorrhagic foci.

In D1 post-surgery, she initiated replacement treatment with rcFVIII every 12 hours.

In D3 post-surgery, twelve hours after administering the rcFVIII: FVIII 85.9\%.

After D4 post-surgery, she maintained replacement treatment with rcFVIII 1000 UI each day.

In D7 post-surgery, twenty-four hours after administering the rcFVIII: aPTT 29.3"; vWF:Ag 262; vWF:Act 232; FVIII 59.9\%. An MRI was performed without showing hemorrhages.

In D11 post-surgery, due to maintaining adequate hemostatic values, factor administration was reduced to every other day until D15 post-surgery.

Conclusions: Patients with vWD might have an increased risk of bleeding-related complications, particularly during and after surgery. It is highly recommended that any surgery should be managed by a specialized and experienced multidisciplinary team.

\section{PB0952 | Acquired von Willebrand Disease}

\section{Escobar ${ }^{1}$; K. Chong ${ }^{2}$; N. Montanez ${ }^{1}$}

${ }^{1}$ University of Texas Health and Science Center of Houston, McGovern Medical School, Gulf States Hemophilia and Thrombophilia Center, Houston, United States; ${ }^{2}$ Memorial Hermann Hospital - Texas Medical Center, Medicine and Benign Hematology, Clinical Pharmacist

Specialist, Houston, United States

Background: Acquired von Willebrand syndrome (AVWS) is a rare bleeding disorder characterized by a functional or structural defect of von Willebrand factor (VWF) often secondary to autoimmune or lymphoproliferative disorders. Management concentrates on eradicating the autoantibody and achieving hemostasis. Currently, there are no evidence-based guidelines for management, although effectiveness of intravenous immunoglobulin (IVIG) was demonstrated in an open-label crossover study in patients with AVWS.

Aims: Describe a single Center's experience with management of AVWS.

Methods: Retrospective record review of a 70 y/o Caucasian female diagnosed with AVWS in the setting of Rheumatoid Arthritis (RA) and Parkinson's disease following recurrent Gl bleeding unresponsive to VWF replacement. Coagulation studies suggest severe von Willebrand disease (FVIII 5\%, VWF: Ag 11\%, VWF R:Co <15\%), full gene sequencing of the VWF (exons 1-52) resulted in negative findings, as well as VWF GP1bM and VWF propeptide antigen assay. Suggesting markedly low VWF antigen is due to increased clearance of VWF without inhibition of function secondary to acquired inhibitor, likely resulting from active RA.

Results: IVIG $~ 1000 \mathrm{mg} / \mathrm{kg} /$ dose and high doses of VWF/F8 concentrate achieved normal levels of coagulation proteins for $\sim 1$ week, stopping GI bleed. Maintenance low dose IVIG 1000 mg/kg/dose every 4 weeks has kept patient without bleeding ( 10 months), despite maintaining levels of FVIII between 60-20\%, VWF: Ag 60$20 \%$, VWF R: Co $30-<15 \%$. Eradication of autoantibody remains unachievable with lack of response to management of underlying disease mechanisms (RA) with Rituximab and Methotrexate.

Conclusions: In patients with AVWS, the use of IVIG and high doses of VWF/F8 concentrate can temporarily increase levels of low proteins to normal range. Favorable response to monthly low dose of IVIG can be given "prophylactically" to prevent bleeding but must consider the risks and benefits of this therapy long-term.

P0163 | Systemic Lupus Erythematosus-induced Coagulopathy: A Case Report

\section{G.A. Maia ${ }^{1,2}$}

${ }^{1}$ Clínica Hematológica, Grupo Oncoclínicas, Belo Horizonte, Brazil;

${ }^{2}$ Faculdade de Ciências Médicas de Minas Gerais, Belo Horizonte, Brazil

Background: Systemic lupus erythematous (SLE) presents multiple clinical manifestations associated with laboratory markers. Spontaneous bleeds associated with SLE are uncommon, but they are clinically important.

Aims: Report the diagnosis of SLE in a man with cutaneomucosal bleeding.

Methods: Case report description.

Results: Male, 56 years old, with a history of macroscopic hematuria for about a year and for four months evolving with spontaneous bruises in the upper and lower limbs. There were no reports of abnormal bleeding previously. He was in treatment for bipolar disorder for 36 years. Evaluated by urology, a benign prostatic hyperplasia was identified. In the preoperative period of transurethral resection of the prostate, a change in coagulogram was identified: activated partial thromboplastin time (aPTT) 58 seconds (control 24 seconds), prothrombin activity $32 \%(70-100 \%)$, international normalized ratio 2.18 (1,00-1,30), with normal platelets and fibrinogen. Mixing test was performed on aPTT with the presence of inhibitor. In laboratory tests carried out, a reduction of coagulation factors (XII, VIII, $X I, I X, X I, V)$ and of ristocetin cofactor $22.36 \%$ (40-200\%) were found, but the von Willebrand fator activity were normal 79.2\% (50$150 \%$ ). There were positivity for ANA (homogeneous nuclear standard title 1:640), anticardiolipins IgM and IgG, lupus anticoagulant, coombs test, anti-Ro, Anti-dsDNA, Anti-U1RNP. It was found presence of $0.5 \mathrm{~g} / \mathrm{dL}$ monoclonal serum protein, IgM/Kappa, but myelogram did not have plasmacytosis or dysplasias. In his blood count had normocytic and normochromic anemia, without hemolysis, and leukogram with neutropenia and lymphopenia. On computed tomography, he had mild pericardial effusion, signs of pneumonitis and 
portal hypertension without evidence of thrombosis combined with splenomegaly.

Conclusions: The patient had acquired von Willebrand disease by SLE. Immunosuppressive treatment (prednisone) controled the disease, with improvement of his symptoms and laboratory tests impacting the quality of life.

\section{PLATELETS AND MEGAKARYOCYTES}

\section{MEGAKARYOCYTES AND THROMBOPOIESIS}

\section{PB0953 | Bifunctional Effect of Inflammatory Cytokine TNFa on Human Megakaryopoiesis and Platelet Production}

T. Chu ${ }^{1}$; S. Hu ${ }^{1,2} ;$ J. Qi ${ }^{1,2,3,4} ;$ Y. Han ${ }^{1,2,3,4} ;$ D. Wu ${ }^{1,2,3,4}$

${ }^{1}$ National Clinical Research Center for Hematologic Diseases, Jiangsu Institute of Hematology, The First Affiliated Hospital of Soochow University, Suzhou, China; ${ }^{2}$ Institute of Blood and Marrow Transplantation, Collaborative Innovation Center of Hematology, Soochow University, Suzhou, China; ${ }^{3}$ Key Laboratory of Thrombosis and Hemostasis of Ministry of Health, Suzhou, China; ${ }^{4}$ State Key Laboratory of Radiation Medicine and Protection, Soochow University, Suzhou, China

Background: Platelets are affected by many factors, such as infectious or aseptic inflammation. Tumor necrosis factor a (TNFa) is an important inflammatory cytokine. However, the role of TNFa in thrombopoiesis remains largely elusive.

Aims: This study aims to investigate the effect of TNFa on megakaryopoiesis (MK) and platelet production.

Methods:

Results: Here, we report an increase of TNFa in patients with essential thrombocythemia, which is characterized by megakaryocyte burden. Meanwhile, a significant higher TNFa level is also observed in patients with normal platelet reconstitution after HSCT compared with those with prolonged thrombocytopenia. The ex vivo study demonstrates that escalating concentrations of TNFa differentially modulate human CD34+ cells development toward MK and platelet production. Specifically, a low concentration of TNFa $0.5 \mathrm{ng} / \mathrm{m}$ tends to promote $\mathrm{MK}$ maturation, stress fiber formation, proplatelet formation and platelet production. Otherwise, a high concentration of TNFa $10 \mathrm{ng} / \mathrm{ml}$ or more exhibits a remarkably inhibitory effect on these processes. Of note, the distinct effect of TNFa on MK is mainly dependent on TNFR1 rather than TNFR2. The Transcriptome analysis of cultured MK treated with TNFa shows significant reprogramming of cell adhesion and migration related genes. Further investigations indicate that TNFa $0.5 \mathrm{ng} / \mathrm{ml}$ and $10 \mathrm{ng} / \mathrm{ml}$ also differentially regulate cell cytoskeleton molecules in $\mathrm{MK}$, including RhoA/ ROCK1/Cofilin/MLC2, which have been reported to regulate cell cytoskeleton rearrangement. In addition, both MAPK-ERK1/2 and PI3K-Akt signaling pathways are differentially activated by different concentrations of TNFa. In mice, low $(0.5 \mu \mathrm{g})$ or high doses $(5 \mu \mathrm{g})$ of TNFa administration also differentially affect short-term platelet recovery after bone marrow transplantation. Bone marrow MK number and location with vascular sinusoids in TNFa treated group are also significantly different with control group.

Conclusions: Our data shows a distinct effect of TNFa on regulating MK maturation and thrombopoiesis, and may provide new insights into its treatment implications in platelet abnormality diseases.

PB0954 | Aberrant Expression of Lnc-MEG3 and LncNOTCH1 Predicts Refractory Phenotype in Chronic Idiopathic Thrombocytopenic Purpura

N. El-Khazragy ${ }^{1,2}$; S. Matbouly ${ }^{3}$; H.F. Abdelsamee ${ }^{4}$

${ }^{1}$ Department of Clinical Pathology-Hematology and AinShams Medical Research Institute (MASRI), Cairo, Egypt; ${ }^{2}$ Global Research Labs, Cairo, Egypt; ${ }^{3}$ Department of Pediatrics, Faculty of Medicine, Ain Shams University, Cairo, Egypt; ${ }^{4}$ Internal Medicine-Clinical Hematology Department, Faculty of Medicine, Ain Shams University, Cairo, Egypt

Background: Primary immune thrombocytopenia (ITP) is highly complex, heterogeneous and life threating autoimmune disease. Although, the pathogenesis of ITP is multifactorial, it remains incompletely understood. The main characteristics of ITP etiology is attributed to $T$ and $B$ cell dysfunction, which further lead to auto-immune intolerance and release of auto-immune antibodies. Maternally expressed gene 3 (MEG3), a maternally expressed IncRNA, had closed relationship with autoimmune-related diseases, including ITP. Recently, it was discovered that MEG3 induces immune imbalance of Treg/Th17 in ITP. On the other hand, evidence demonstrated that Notch1 signaling pathway play an important role in the immune system, it regulates the development and differentiation of many other hematopoietic and immune cells. Deregulation of Notch signaling has been linked to multiple human diseases especially T-acute lymphocytic leukemia and recently, it has been related to the development of many autoimmune disorders as ITP.

Aims: The goal of this study is to investigate the expression pattern of Inc-MEG3 and Inc-NOTCH1 genes in patients with chronic ITP, in addition to correlate the expression level with disease phenotype, in order to evaluate its prognostic value.

Methods: A total of 85 cases with chronic ITP and 35 heathy controls were included. The Inc-MEG3 and Inc-NOTCH1 expression level were analyzed in peripheral blood mononuclear cells (PBMCs). Results: 

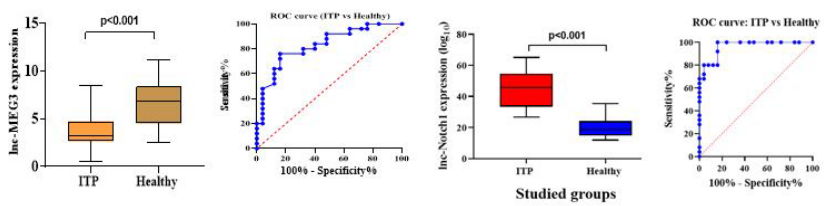

MEG3 was significantly dounregulated in ITP group compared to healthy contro

AUC 0.82 Sensitivity $76 \%$
Specificity $70 \%$

FIGURE 1 The expression level of Inc-MEG3 and Inc-NOTCH1 in PBMCs, and the ROC curves illustrating the potential of the IncRNAs in discriminating ITP patients from healthy controls. Abbreviations: PBMCs: peripheral blood mononuclear cells, ROC: Receiving operating Characteristics, AUC: area under the curve, ITP: Idiopathic thrombocytopenic purpura, MEG3: Maternity expressed gene-3

We demonstrated higher expression level of Notch1 in chronic ITP patients than controls with high statistical significant difference. In addition, higher expression levels of Inc-NOTCH1 is significantly associated with high risk patients. In contrast, Inc-MEG3 was downregulated in chronic ITP patients compared to healthy controls, and lower expression levels were significantly associated with poor prognosis and refractory disease phenotype.

Conclusions: Lnc-MEG3 and Inc-NOTCH1 are independent noninvasive prognostic biomarker in chronic ITP, hence they could be therapeutically targeted in future.

PB0955 | Single-cell RNA Sequencing Reveals Features of Hematopoietic Stem and Progenitor Cells in Immune Thrombocytopenia

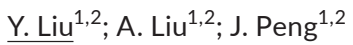

${ }^{1}$ Qilu Hospital of Shandong University, Jinan, China; ${ }^{2}$ Shandong

University, Jinan, China

Background: Primary immune thrombocytopenia (ITP) is an acquired autoimmune disease characterized by isolated thrombocytopenia. A growing body of emerging evidence indicates that abnormalities during any stage of thrombopoiesis and megakaryocytopoiesis can influence platelet production.

Aims: The aim of our study is to explore the cellular heterogeneity, lineage and functional states of the hematopoietic stem and progenitor cells (HSPCs) in ITP patients.

Methods: CD34 ${ }^{+}$HSPCs were isolated from BM of four newly diagnosed ITP patients and four healthy adults as controls by fluorescence-activated cell sorter (FACS), and Single-cell RNA sequencing (scRNA-seq) data was collected using the recommended protocol for the 3' scRNA-seq 10X genomics platform.

Fig1. ScRNA-seq analysis of the BM HSPCs from ITP patients and controls

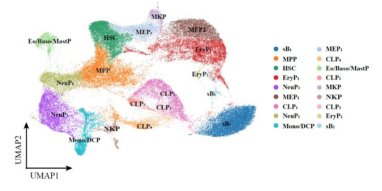

A. UMAP illustration of clustered cells

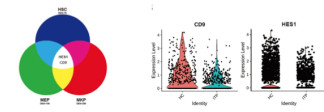

C. HES1 and CD9 are down-regulated in megakaryopoiesis

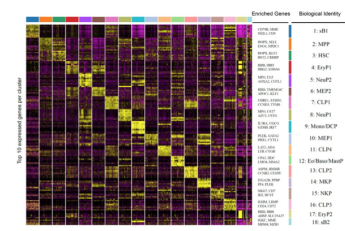

B. Heatmap showing the expression of enriched genes in each cluster

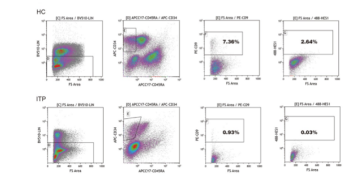

D. Representative flow cytometric analysis of HSPCs showing the down-regulation of HES1 and CD9
FIGURE 1 ScRNA-seq analysis of the BM HSPCs from ITP patients and controls

Conclusions: Using scRNA-seq, we revealed a hierarchicallystructured transcriptional landscape of hematopoietic differentiation of BM CD34+ HSPCs. We observed a significantly descreased expression of HES1 and CD9 in newly diagnosed ITP patients, which might relate with the generation of abnormal MKs and be a biomarker potentially using in diagnosis.

PB0956 | The Role of Matrix-metalloproteinase 9 in Bone Marrow Remodeling after Total Body Irradiation and Hematopoietic Stem Cell Transplantation

K. Mott; D. Semeniak; H. Schulze

Institute of Experimental Biomedicine, University Hospital, Würzburg, Germany

Background: Megakaryocytes (MKs) in the bone marrow (BM) are exposed to extracellular matrix (ECM) proteins to prevent premature platelet release. Total body irradiation (TBI), which is widely used as a conditioning regimen for hematopoietic stem cell transplantation (HSCT), leads to ECM-remodeling by matrix-metalloproteinase MMP9, preceding a massive vasodilation, reduction in MK numbers and thrombocytopenia. Prolonged thrombocytopenia is a frequent complication after HSCT, which is associated with poor prognosis and increased mortality. The underlying mechanisms of long-lasting thrombocytopenia after HSCT are still unknown.

Aims: This study aims to analyze the role of MMP9 in BM remodeling after irradiation and MK engraftment after HSCT.

Methods: Mouse femur sections were stained and subjected to confocal immunofluorescence microscopy to map BM sinusoids, MKs, and ECM proteins. MMP expression and activity was assessed by immunoblot analysis, gelatin-zymography, in situ zymography, and live-cell zymography. Studies were performed using MMP9 $^{-/-}$mice and littermate controls. Ubiquitously dsRed-expressing reporter mice were used as BM donors in HSCT to assess reconstitution of the vasculature and $\mathrm{MK}$ engraftment. 
Results: Collagen IV is selectively degraded at BM sinusoids after sublethal TBI, while we found specific upregulation of MMP9 activity. This appeared not to drive reduction of MK numbers or platelet counts after TBI. MMP9 ${ }^{-/}$mice, however, displayed a delayed recovery of irradiation-induced vasodilation indicating a role of MMP9 in vascular remodeling. $\mathrm{MMP9}^{--}$mice and wildtype controls showed a similar engraftment capacity with donor-derived MKs and platelets being detectable as early as d4 after HSCT. On d7 vasodilation was still increased in $\mathrm{MMP9}^{-/-}$animals. In the transplanted BM we detected remaining gelatinolytic activity in MMP9-deficient mice, suggesting compensatory upregulation of other MMP family members besides MMP2.

Conclusions: The findings presented here show that MMP9 plays a minor role for early MK engraftment, but is a modulator of the vasculature, as reflected by a prolonged vasodilation after HSCT.

PB0958 | Pharmacological Characterization of eNOS-based Megakaryocyte Subpopulations and its Implications for their Platelet Progeny

\section{A. Asgari ${ }^{1}$; P. Jurasz ${ }^{1,2}$}

${ }^{1}$ University of Alberta/Faculty of Pharmacy and Pharmaceutical Sciences, Edmonton, Canada; ${ }^{2}$ University of Alberta/Department of Pharmacology, Edmonton, Canada

Background: Platelets play a key role in atherothrombosis and its associated inflammation. Recently, we identified platelet and megakaryocyte/blast subpopulations based on endothelial nitric oxide synthase (eNOS) and the ability to produce nitric oxide (NO). We showed that eNOS-negative (eNOS ${ }^{\text {neg }}$ ) platelets initiate aggregate formation, while eNOS-positive (eNOS ${ }^{\text {pos }}$ ) platelets inhibit aggregation by generating NO. However, it's unknown how these platelet and megakaryocyte subpopulations arise and the impact of antiatherothrombotic drugs on their formation.

Aims: Therefore, we aimed to

(1) investigate whether pro-inflammatory cytokines known to downregulate eNOS expression decrease the ratio of eNOS ${ }^{\text {pos }}$ to eNOS neg megakaryocytes/blasts and resulting platelets; and

(2) whether anti-atherothrombotic drugs, with known antiinflammatory properties would counteract the effects of proinflammatory cytokines on eNOS expression and eNOS-based megakaryocyte and platelet subpopulation formation.

Methods: The human megakaryoblastic cell line Meg-01 was utilized as a megakaryocyte model. Meg-01 were cultured in the presence of thrombopoietin and pro-inflammatory cytokines (IL-1 $\beta$, IL-6, TNF$a, I F N-\gamma$ ) and anti-atherothrombotic drugs acetylsalicylic acid (ASA 10-30 mM) and atorvastatin (0.03-1 mM). The levels of eNOS neg and eNOS ${ }^{\text {pos }} \mathrm{Meg}-\mathrm{O} 1$ and platelet-like particles was measured using flow cytometry.

Results: Compared to control the cytokine cocktail significantly increased the percentage of eNOS neg Meg-01 and this effect was inhibited by $30 \mathrm{mM} \mathrm{ASA}$ and $0.1 \mathrm{mM}$ atorvastatin (Control $7.4 \pm 0.9 \%$ vs cytokines $11.6 \pm 1.2 \%$ vs cytokines \& ASA and Control $9.4 \pm 1.1 \%$ vs cytokines $8.0 \pm 0.6 \%$ vs cytokines \& atorvastatin). Similarly, although fewer in their relative number compared to their parent Meg-01, platelet-like particles released from eNOS ${ }^{\text {pos }}$ Meg-01 cells decreased in response to inflammatory cytokines and this effect was reversed by ASA and atorvastatin.

Conclusions: The generation of eNOS ${ }^{\text {neg }}$ and eNOS ${ }^{\text {pos }}$ megakaryocytes and platelets may be counter-regulated by inflammatory status. Conversely, anti-atherothrombotic drugs ASA and atorvastatin may promote an anti-thrombotic phenotype, in part, by increasing the formation of eNOS ${ }^{\text {pos }}$ megakaryocytes and platelets.

\section{PB0959 | Medium Plate Volume as a Predictor of Plate Engraftment Time in Patients with Bone Marrow Transplant}

J.A. Avila Rueda; C. Duboscq; J. Ceresetto; S. Palmer; K. Oliveros; S. Rivarola; A.M. Cantillo; M. Carreras; L. Zapata; M.C. Sernaque; N. Flegler; O.R. Oscar Rabinovich; C. Shanley; M. Quarchioni; E. Bullorsky; G. Stemmelin Hospital Britanico de Buenos Aires, Buenos Aires, Argentina

Background: Increase in mean platelet volume (MPV) was related as an indicator of spinal recovery in post-chemotherapy patients, however the behavior of this parameter in patients undergoing bone marrow transplantation (BMT) is unknowed.

Aims: To evaluate if there is an association between platelet volume mean and estimated time of platelet engraftment in patients undergoing BMT.

Methods: A retrospective cohort study was carried out in 40 patients transplanted in our hospital between November 2019 and December 2020. it was determined MPV and platelet count using a counter by impedance for each patient, starting the day transplant (DO) and then every 72 hours until engraftment platelet, defined as count $>20,000 / \mathrm{ml}$ and transfusion independence by 7 days. In the statistical analysis t test and Spearman's coefficient was used.

Results: $80 \%$ of the patients received autologous transplantation and $20 \%$ allogeneic transplantation; $52 \%$ were men. The overall mean age was 51 years (range: 23 to 75). Pathologies that required transplantation were: Multiple myeloma $43 \%$, lymphomas $37 \%$ and acute lymphoblastic and myeloid leukemia $20 \%$. A significant difference was observed ( $p<0.0001$, Student's $t$ ) between the average MPV of D0 and the day of engraftment $(9.6+0.9$ vs $11.2+1.2$, mean and SD). In 70\% (95\% Cl 0.55-0.84), MPV was maximum during or in the 72 hours prior to engraftment. A correlation was observed between the difference obtained from VPM to engraftment vs D0 (DVPM), with the period time elapsed from DO of transplantation to platelet engraftment, taking into account the following DVPM values: 1.07 (6-8 days), 1.27 (9-11 days), 1.59 (12-14 days), 2.6 (15-17 days) and 3.2 (18-20 days), all with statistical significance $(p<0.01$, Spearman). 
Conclusions: The results found in our sample, reported that there is a relationship between the MPV and platelet engrafment in post BMT patients.

\section{PLATELET FUNCTION AND INTERACTIONS}

\section{LPB0033 | The Role of RAP1 Signaling in Platelet-coagulation Interplay}

A. Ballard $^{1}$; D. Adalsteinsson ${ }^{2}$; E. O'Shaughnessy ${ }^{3}$; D. Paul ${ }^{1}$; R. Lee ${ }^{1}$; W. Bergmeier ${ }^{1}$

${ }^{1}$ University of North Carolina at Chapel Hill Department of

Biochemistry and Biophysics, Chapel Hill, United States; ${ }^{2}$ University of North Carolina at Chapel Hill Department of Mathematics, Chapel Hill, United States; ${ }^{3}$ University of North Carolina at Chapel Hill Department of Pharmacology, Chapel Hill, United States

Background: The small GTPase Rap1 is a central regulator of platelet function and hemostatic plug formation. Rap1 is best known for its crucial role in integrin inside-out activation and cellular adhesion. Previous studies also suggest a role for Rap1 signaling in phosphatidylserine (PS) exposure and platelet procoagulant response.

Aims: To determine if and how Rap1 contributes to platelet procoagulant response in vitro and in vivo.

Methods: PS exposure response in vitro was determined by flow cytometry (annexin V). In vivo, PS exposure, platelet adhesion and fibrin formation were monitored in hemostatic plugs by spinning disk confocal microscopy. Three-dimensional stacks of hemostatic plugs were obtained every 10 seconds and analyzed with ImageTank software.

Results: In vitro, deficiency in both Rap1 isoforms in the megakaryocyte lineage (Rap1 ${ }^{\mathrm{mKO}}$ ) resulted in markedly decreased platelet PS exposure following cellular stimulation. The defect in PS exposure was partially compensated by concomitant inhibition of RhoA/ ROCK signaling. In vivo, the importance of procoagulant platelets for fibrin formation was demonstrated in mice lacking cyclophilinD (CypD) in platelets only, generated by adoptive transfer of CypD ${ }^{-1}$ platelets into thrombocytopenic mice. To determine the procoagulant function of Rap1 ${ }^{\mathrm{mKO}}$ platelets in vivo, a mixture of WT/ $\mathrm{CypD}^{-/-}$or Rap1 ${ }^{\mathrm{mKO}} / \mathrm{CypD}^{-/-}$platelets was transfused into thrombocytopenic mice. Co-transfusion with $\mathrm{CypD}^{-/}$platelets was required to prevent prolonged bleeding and to create a situation where only WT or Rap1 ${ }^{\mathrm{mKO}}$ platelets could provide a procoagulant platelet surface. Compared to $\mathrm{WT} / \mathrm{CypD}^{-/-}$mice, fibrin formation was reduced in hemostatic plugs of $\operatorname{Rap}^{\mathrm{mKO}} / \mathrm{CypD}^{-/-}$mice.

Conclusions: In summary, we provide the first evidence for a Rap1RhoA-PS connection in platelets, and proof that platelet Rap1 signaling affects hemostatic plug formation independent of its key role in integrin-mediated adhesion processes.
LPB0034 | Effects of Depression and Antidepressant Use on Platelet Reactivity Traits

J. Grech ${ }^{1} ;$ M. Chan ${ }^{1,2} ;$ A. Lachapelle ${ }^{1} ;$ F. Thibord ${ }^{1} ;$ Z. Schneider ${ }^{1}$; P. Armstrong ${ }^{2}$; C. Wallace de Melendez ${ }^{1}$; T. Warner ${ }^{2}$; M.-H. Chen ${ }^{1}$; A. Johnson ${ }^{1}$

${ }^{1}$ National Heart, Lung, and Blood Institute (NHLBI), Framingham, United States; ${ }^{2}$ The Blizard Institute, Barts and The London School of Medicine \& Dentistry, London, United Kingdom

Background: Depression is a risk factor of cardiovascular disease (CVD) and linked to worsened CVD outcomes. Serotonin is a key neurotransmitter in depressive pathology, contained in platelet dense granules, and a weak activator of platelets.

Aims: Our study assessed the link between platelet reactivity traits, depression and antidepressant (AD)-use in a large population sample. Methods: This study was conducted in the Framingham Heart Study $(N=3,140)$. AD-use $(N=563)$ and aspirin-use $(N=681)$ at the time of platelet assays was noted. Depression was measured using the Center for Epidemiological Studies-Depression (CES-D) survey. Platelet reactivity traits were measured across multiple agonists using light transmission aggregometry (LTA), Multiplate whole blood impedance aggregometry (MP), Total-Thrombus Formation Assay System (T-TAS), Optimul 96-well plate assay, and flow cytometry (FC). We utilized a linear mixed effects model to test associations between platelet reactivity traits and depression, adjusting for age, sex and aspirin-use. Similarly, we analyzed trait associations with any $A D$-use, serotonin-affecting $A D s$, and norepinephrine-affecting ADs, respectively.

Results: There were strong associations with reduced platelet function and $A D$-use, particularly with serotonin-affecting medications. This included decreased Optimul epinephrine $(P<4.49 \mathrm{E}-14)$ and U46619 ( $P<8.56 \mathrm{E}-11)$ maximal aggregation, decreased LTA ADP final aggregation $(P<1.26 \mathrm{E}-07)$, higher LTA ADP disaggregation $(P<2.14 \mathrm{E}-07)$, and decreased ADP activation by FC, among others. Similar associations with increased CES-D symptomology (thresholds $>16$ or $>21$ ) were largely attenuated after adjusting for current $A D$-use. Observations were also significant within the aspirin strata. Conclusions: In the largest study yet of AD-use on platelet function we show that antidepressants, particularly serotonin-affecting drugs, inhibit platelet reactivity. Serotonin potentiates platelet reactivity to weak agonists such as ADP and epinephrine. Thus, our results are consistent with $A D$-use leading to platelet serotonin depletions, decreased stability of platelet aggregates, and overall decreased aggregation to multiple agonists, which may be a mechanism by with ADs increase risk for adverse bleeding events. 


\section{LPB0035 | Desialylation Primes Platelets for Apoptosis: A New Role for ß1 Integrin}

R. Grozovsky ${ }^{1}$; H. Roweth ${ }^{2}$; C. Fraser ${ }^{3}$; K. Sarosiek ${ }^{3}$; E. Battinelli ${ }^{2}$

${ }^{1}$ Sylvester Comprehensive Cancer Center, Miami, United States;

${ }^{2}$ Brigham and Women's Hospital, Boston, United States; ${ }^{3}$ Harvard T.H Chan School of Public Health, Boston, United States

Background: Platelets are short-lived anucleate cells that play an essential role in primary hemostasis. The fast pace of platelet lifespan emphasizes the need for a tight regulatory mechanism to avoid spontaneous bleeding or arterial occlusion.

Aims: Here, we investigate the functional relationship between the two well-established mechanisms that regulate platelet survival: desialylation and apoptosis.

Methods: Biotinylated old and young platelet subpopulations were obtained 60 hs after biotin injection of WT mice followed by immunomagnetic separation. Platelets from the old $\left(\right.$ biotin $^{+}$) fraction were highly desialylated, with increased levels of phosphatidylserine and Neu1 (sialidase) surface exposure compared to the young (biotin) platelets. These data suggest platelets become desialylated and subsequently undergo apoptosis in circulation.

Results: Next, we performed $\mathrm{BH} 3$ profiling to measure mitochondrial readiness to undergo apoptosis on two different models of desialylated platelets (Asgr $2^{-/-}$and St3gal $4^{-/-}$mice) and compared to WT mice treated with the sialidase inhibitor, DANA. We found that both models of desialylated platelets were more primed to undergo apoptosis compared to WT, which was consistent with increased levels of apoptosis detected with Annexin $\mathrm{V}$ and blotting for cleaved caspase 3. Notably, DANA-treated platelets were less primed than WT, indicating that sialidase inhibition suppresses platelet apoptosis. Platelet dependence on the pro-survival protein BCL- $X_{L}$, which has been previously shown to be vital for platelet survival, was also reduced by DANA treatment. This has implications for the use of $B C L-X_{L}$ inhibitors for cancer therapy, which are known to cause ontarget thrombocytopenia.

Conclusions: We also tested if apoptosis alone could induce desiaIylation. Neither in vivo nor in vitro treatment of WT platelets with the BCL-2/BCL- $X_{L}$ inhibitor ABT-737 affected platelet desialylation. Lastly, using galactose-binding lectin chromatography, we identified ß1 integrin as the major glycoprotein highly desialylated in whole cell lysates from biotinylated old (biotin ${ }^{+}$) WT platelets populations, as well as in desialylated platelets derived from $\mathrm{Asgr}^{-/-}$and St3gal $4^{-/-}$mice.
LPB0036 | Elevated Platelet-derived sGPVI Is a Biomarker of Venous In-stent Stenosis in Patients with Post-thrombotic Syndrome

A.M. Gwozdz ${ }^{1}$; S.A. Black ${ }^{1}$; R. Morris ${ }^{1}$; S. Messiha ${ }^{1}$; M. Ikram¹; A.P. Bye ${ }^{2}$; J.M. Gibbins ${ }^{2}$; M.L. Rand ${ }^{3}$; A.S. Patel ${ }^{1}$; B. Modarai ${ }^{1}$; A. Smith ${ }^{1}$; P. Saha ${ }^{1}$

${ }^{1}$ Academic Department of Vascular Surgery, Section of Vascular Risk and Surgery, School of Cardiovascular Medicine and Science, King's College London, London, United Kingdom; ${ }^{2}$ Institute for Cardiovascular and Metabolic Research, School of Biological Sciences, University of Reading, Reading, United Kingdom; ${ }^{3}$ Division of Hematology/Oncology, The Hospital for Sick Children, Toronto, Canada

Background: In-stent stenosis following intervention for postthrombotic syndrome (PTS) occurs in $\sim 30 \%$ of cases, despite therapeutic anticoagulation.

Aims: The aim of this study was to investigate whether platelets have a role in this process.

Methods: Case-matched patients undergoing venous stenting were prospectively recruited. Venous in-stent thrombus specimens were excised and immunohistochemical analysis was performed to detect collagen I, collagen III, CD68 and CD41. Blood samples were taken before and after venous stent placement, and platelet activation markers (P-selectin and phosphatidylserine) and reactivity were determined by flow cytometry and plate-based aggregation, respectively. Soluble glycoprotein VI (sGPVI), shed during activation, was measured in plasma. Patients with in-stent stenosis requiring reintervention ( $>50 \%$ diameter reduction) were compared with those who did not during follow-up.

Results: Forty-five patients were recruited (median age: 43yrs, range: $33-55 y r s ; 65 \%$ female). Re-intervention was required in 19/45 (42\%; median time: $3 \mathrm{wks}$, range: 1 day-3mths). Immunohistochemical analysis of in-stent thrombosis demonstrated a rich network of platelets, both early (collagen III) and late (collagen I) forms of collagen, and inflammatory cell infiltrates. There was no significant difference in platelet activation or reactivity after stenting, but Pselectin exposure pre-stent was significantly higher in patients who developed in-stent stenosis ( $2.7 \% \pm 0.4$ vs $1.7 \% \pm 0.2 ; P<0.05)$. sGPVI levels before stent insertion were increased in patients who developed in-stent stenosis $(18.9 \pm 3.6 \mathrm{ng} / \mathrm{mL}$ vs. $7.4 \pm 0.9 \mathrm{ng} / \mathrm{mL} ; P<0.01)$. Platelet reactivity to collagen-related peptide, a GPVI-specific platelet agonist, was reduced in patients who developed in-stent stenosis $\left(\log \mathrm{EC}_{50}=-6.5 \mathrm{M} \pm 0.3\right.$ vs $\left.-7.2 \mathrm{M} \pm 0.2 ; P<0.01 ; n=33\right)$.

Conclusions: Venous stenting does not activate platelets or alter platelet function, but patients who developed in-stent stenosis exhibited greater levels of pre-stent platelet activation, greater loss of platelet surface GPVI in the form of SGPVI and consequent reduction in reactivity to GPVI activation. SGPVI may have potential to risk-stratify patients undergoing deep venous reconstruction and predict who requires closer surveillance. 
LPB0129 | Platelet-specific Deletion of Acetyl-CoA Carboxylase 1 Decreases Phospholipid Content and Impairs Platelet Functions

M. Octave ${ }^{1}$; L. Pirotton ${ }^{1} ;$ A. Ginion ${ }^{1}$; V. Robaux ${ }^{1}$; S. Lepropre ${ }^{1}$; S. Kautbally ${ }^{1}$; Y. Senis ${ }^{2}$; Z. nagy ${ }^{3}$; J. Ambroise ${ }^{4}$; B. Guigas ${ }^{5}$; M. Giera ${ }^{5}$; L. Bertrand ${ }^{1}$; C. Beauloye ${ }^{1}$; S. Horman ${ }^{1}$

${ }^{1}$ Université catholique de Louvain, Institut de Recherche Expérimentale et Clinique, Pôle de Recherche Cardiovasculaire, Brussels, Belgium; ${ }^{2}$ Université de Strasbourg, Institut National de la Santé et de la Recherche Médicale (INSERM), Etablissement Français du Sang Grand Est, Unité Mixte de Recherche (UMR)-S 1255, Fédération de Médecine Translationnelle de Strasbourg, Strasbourg, France; ${ }^{3}$ University of Würzburg, Institute of Experimental Biomedicine, University Hospital and Rudolf Virchow Center, Würzburg, Germany; ${ }^{4}$ Université catholique de Louvain, Institut de Recherche Expérimentale et Clinique, Centre de Technologies Moléculaires Appliquées, Brussels, Belgium; ${ }^{5}$ Leiden University Medical Center, Leiden, Netherlands

Background: Acetyl-CoA carboxylase (ACC), the first enzyme regulating lipid synthesis, promotes platelet activation and thrombus formation by increasing platelet phospholipid content and thromboxane $\mathrm{A} 2$ generation.

Aims: Our study sought to evaluate whether ACC1 platelet-specific deletion may affect platelet functions by decreasing phospholipid content.

Methods: We generated a new Cre transgenic mouse strain that allows megakaryocyte/platelet specific ACC1 deletion (GplbCre+/- $x$ ACC1 flx/flx mouse). In vitro, platelet functions were assessed by aggregometry and flow cytometry. In vivo, hemostasis was assessed via the measurement of bleeding time. Lipidomics analysis was carried out on the commercial Lipidyzer platform. Thromboxane A2 secretion was evaluated by ELISA.

Results: As expected, ACC1 deletion was restricted to the megakaryocytic lineage. Hematological parameters in platelet-specific ACC1 knockout mice showed a decrease in platelet count by $30 \%$ and an increase in platelet volume by $31 \%$, compared to ACC1 flx/flx platelets. In vitro, platelets from platelet-specific ACC1 knockout mice displayed a decrease in thrombin and CRP-induced platelet aggregation, associated with impaired dense granules secretion. In contrast, ADP-induced platelet aggregation was higher in the absence of ACC1. In vivo, platelet-specific ACC1 knockout mice showed a normal bleeding time. In agreement with our hypothesis, lipidomics analyses showed that ACC1 deletion in platelets was associated with a significant decrease in arachidonic acid contaning phosphotidylethanolamine plasmalogen, and subsequently with a reduced production of thromboxane A2 upon thrombin or CRP stimulation.

Conclusions: Platelet-specific ACC1 deletion led to a decrease in phospholipid content which, in turn, decreased platelet thromboxane $A 2$ generation, dense granules secretion and aggregation upon thrombin and CRP, but not ADP stimulation. Further studies are needed to elucidate the impact of ADP on platelet functions.
LPB0130 | A Multiscale Framework for Patient-specific Predictions of Thrombus Growth

K. Shankar; T. Sinno; S. Diamond

University of Pennsylvania, Philadelphia, United States

Background: While computational modeling of thrombosis has been extensively studied, these studies have been restricted to predictions in idealized geometries, lack individual platelet resolution of agonist stimulation, and/or do not account for patient-to-patient variability in platelet function.

Aims: To extend our previous 2D multiscale model of thrombosis in a microfluidic channel to develop a fully spatially resolved 3D framework with capabilities to simulate patient-specific platelet phenotypes and blood vessel geometries.

Methods: The multiscale framework is composed of four modules. A patient-specific neural network module computes the timedependent degree of platelet activation as indicated by intra-platelet calcium concentration, given the agonist-exposure history for each platelet. A lattice kinetic Monte Carlo module is used to track platelet positions and perform platelet bonding and detachment events. A lattice Boltzmann module is used to update the blood flow field which evolves due to platelet deposition. Soluble platelet agonist concentrations are tracked using the finite-volume method solution to the convection-diffusion equation.

Results:

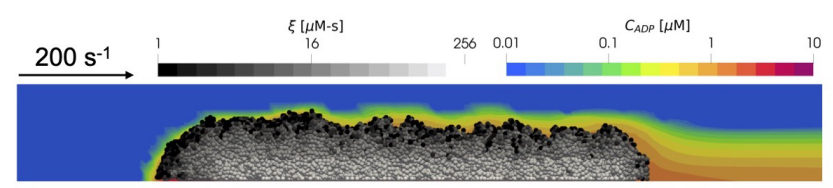

FIGURE 1 Platelet activation (black indicates inactivated and white, fully activated) and deposition in the presence of released ADP and TXA2. Flow: left to right. Inlet of the microfluidic device maintained at constant flow rate that corresponded to an a wall shear rate of $200 \mathrm{~s}^{-1}$.

The model was used to simulate thrombus growth in a microfluidic channel under venous conditions, as shown in Figure 1. Thrombus growth dynamics and morphology predicted by the model agree well with experiments. Furthermore, the model can easily be applied to fully resolved simulations of thrombus growth over a range of shear rates in arbitrary geometries, such as stenoses and bifurcations, as depicted in Figure 2. Moreover, the model can predict thrombus dynamics under different pharmacological conditions corresponding to antiplatelet therapy, and under blood disorders such as von Willebrand disease (Fig.2). 
Enhanced platelet deposition at high shear rate due to vWF stretching

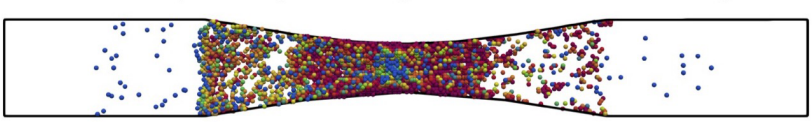

Reduced platelet deposition at high shear rate (von Willebrand disease)

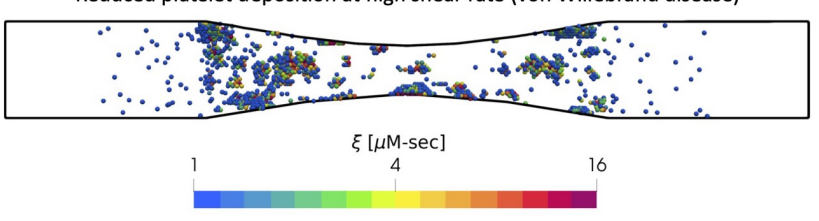

FIGURE 2 Platelet activation (blue indicates inactivated and red, fully activated) and deposition in the presence and absence of vWF stretching. Flow: left to right. Inlet and outlet of the stenosis maintained at constant pressure drop that corresponded to an initial inlet wall shear rate of $1000 \mathrm{~s}^{-1}$

Conclusions: The multiscale framework we have developed allows for the prediction of thrombus growth under flow in the presence of agonist species. To our knowledge, our model is the first of its kind that accounts for patient-specific platelet phenotypes to perform robust 3D simulations of thrombosis in geometries of clinical relevance.

\section{LPB0131 | Inhibition of Surface-induced Platelet Activation on Hydrogel Films}

G. Apte; A. Lindenbauer; J. Schemberg; H. Rothe; T.-H. Nguyen Institut für Bioprozess- und Analysenmesstechnik e.V. iba, Heilbad Heiligenstadt, Germany

Background: The activation of platelets is caused by their immediate contact with any non-physiological surfaces. Understanding the mechanism and repercussions of such interaction is crucial in the context of biomedical research. Selection of a platelet congenial surface is of utmost importance in manufacturing platelet storage bags or even in fabricating an anti-thrombogenic implant surface. Platelet-surface activation can be inhibited by modulating the surface properties.

Aims: We developed agarose and gelatin-based hydrogel films and their nanocomposites to inhibit platelet-surface adhesion.

Methods: The fabricated hydrogel films were characterized by contact angle measurements using the captive bubble technique while the swelling behavior and water retention properties of the hydrogels and their nanocomposite films were investigated to determine the stability of the films. The stiffness of the films was measured by using AFM based indentation technique. The de-adhesion forces between platelets and surfaces were measured in real-time by using platelet force spectroscopy using FluidFM. Platelet morphologies on the gels were obtained by confocal laser microscopy and scanning electron microscopy.

Results: Agarose films exhibit higher surface wettability, better controlled-swelling properties, and greater stiffness compared to gelatin, resulting in a strong reduction of platelet adhesion. The mechanical property and surface wettability of the hydrogel films was varied by adding magnetite $\left(\mathrm{Fe}_{3} \mathrm{O}_{4}\right)$ nanoparticles, however, degree of platelet adhesion did not change.

Conclusions: Agarose and agarose nanocomposite materials strongly reduce platelet adhesion and spread. As several types of nanoparticles carry anti-bacterial properties, agarose nanocomposite can be a promising candidate in the fabrication of platelet storage bags that contains anti-bacterial functions.

PB0960 | Identification of Marginal Zone B Cells as Key Players in a Mouse Model of Platelet Alloimmunization

A. Couvidou; C. Angenieux; L. Ruch; C. Gachet; B. Maitre Université de Strasbourg, INSERM, EFS Grand Est, BPPS UMR-S 1255, FMTS, Strasbourg, France

Background: Platelet alloimmunization remains a potentially serious adverse transfusion event. Alloantibodies produced by the recipient, mainly directed against HLA I donor antigens, can compromise the therapeutic efficiency of upcoming transfusion and may lead to refractoriness. The cellular mechanisms regulating this process are not yet identified. We hypothesize that the cell types involved could be found in the splenic environment where alloimmune response takes place. Within the spleen, the marginal zone B cells (MZB) represent attractive candidates as they are known to rapidly react to bloodborne antigens.

Aims: To assess the role of the MZB in platelet alloimmune response and the associated refractory state.

Methods: Balb/C $\left(\mathrm{H}^{2}{ }^{\mathrm{d}}\right)$ mice were transfused weekly with platelets isolated from $\mathrm{C} 57 \mathrm{BI} / \mathrm{J}$ mice $\left(\mathrm{H} 2^{\mathrm{b}}\right)$ during 3 weeks. Alloantibody production (anti- $\mathrm{H}^{\mathrm{b}}$ ) was evaluated by flow cytometry. To evaluate the role of $M Z B$, mice were either treated with a combination of depleting anti-CD11a/CD49d antibodies, or with FTY720, a S1P1-receptor antagonist that prevents the proper localization of MZB in the marginal zone.

Results: $\mathrm{H} 2^{\mathrm{b}}$-platelets transfused weekly in $\mathrm{Balb} / \mathrm{C}$ mice led to anti$\mathrm{H} 2^{b}$ alloantibody production from the second week. When Balb/C mice were depleted in MZB or treated with FTY720, alloantibody production was significantly reduced in both cases as compared to control mice. To determine whether this alloantibody production could lead to a refractory state, alloimmunized mice were transfused with ex vivo CFDA-SE/oregon green labelled $\mathrm{H} 2^{\mathrm{b}}$-platelets. After 2 hours, transfused platelets were all eliminated from the circulation of the recipient. Interestingly, following MZB-immunodepletion or FTY720-treatment, transfused platelets were still found in the circulation after $24 \mathrm{~h}$, suggesting that the reduction of alloantibody production may prevent the associated refractory state.

Conclusions: This study suggests that targeting MZB cells could be a new therapeutic strategy to minimize platelet alloimmunization and prevent the associated refractory state. 
PB0961 | a-Synuclein: A VAMP Chaperone in the Platelet

Release Reaction

A. Smith; S. Joshi; S. Whiteheart

University of Kentucky, Lexington, United States

Background: Platelets use SNARE-mediated exocytosis to maintain hemostasis and thrombosis. These processes are maintained by the exocytosis of platelet releasate from the three types of granules in platelets: dense, alpha, and lysosomal. Understanding how the process of exocytosis is regulated in secretion, we look for potential SNARE regulators and found the protein a-synuclein. $a$-Synuclein appears to be the only member of the synuclein family present in platelets and is very abundant.

Aims: To address the role of a-synuclein in platelet exocytosis.

Methods: We examined the phenotype of platelets from asynuclein $^{-/-}$mice. Secretion from each granule population was measured and hemostasis was evaluated using a tail-bleeding time assay. Western blotting was used to assess the levels of the platelet secretory machinery.

Results: Secretion kinetic and dose-response assays showed that platelets from $a$-synuclein ${ }^{-/-}$mice have defective release from the dense granules and less so from lysosomal granules (Figure 1). Tail bleeding times for $a$-synuclein ${ }^{-/-}$mice were increase compared to the wild-type mice. To understand the mechanism of this defect, we asked whether $a$-synuclein is acting as a VAMP-chaperone in platelets and thus modulates secretion by controlling V-SNARE levels. The two dominant T-SNARES SNAP-23 and Syntaxin-11 were not altered in a-synuclein ${ }^{-/}$mice. However, the dominant V-SNARE VAMP-8 was reduced. Other V-SNARES VAMP-2, VAMP-3, and VAMP-7 were normal. Additional western blotting experiments demonstrated the presence of the $a$-synuclein-interactor Cysteine String Protein, added a new element to the known platelet secretory machinery (Figure 2).
Figure 1.
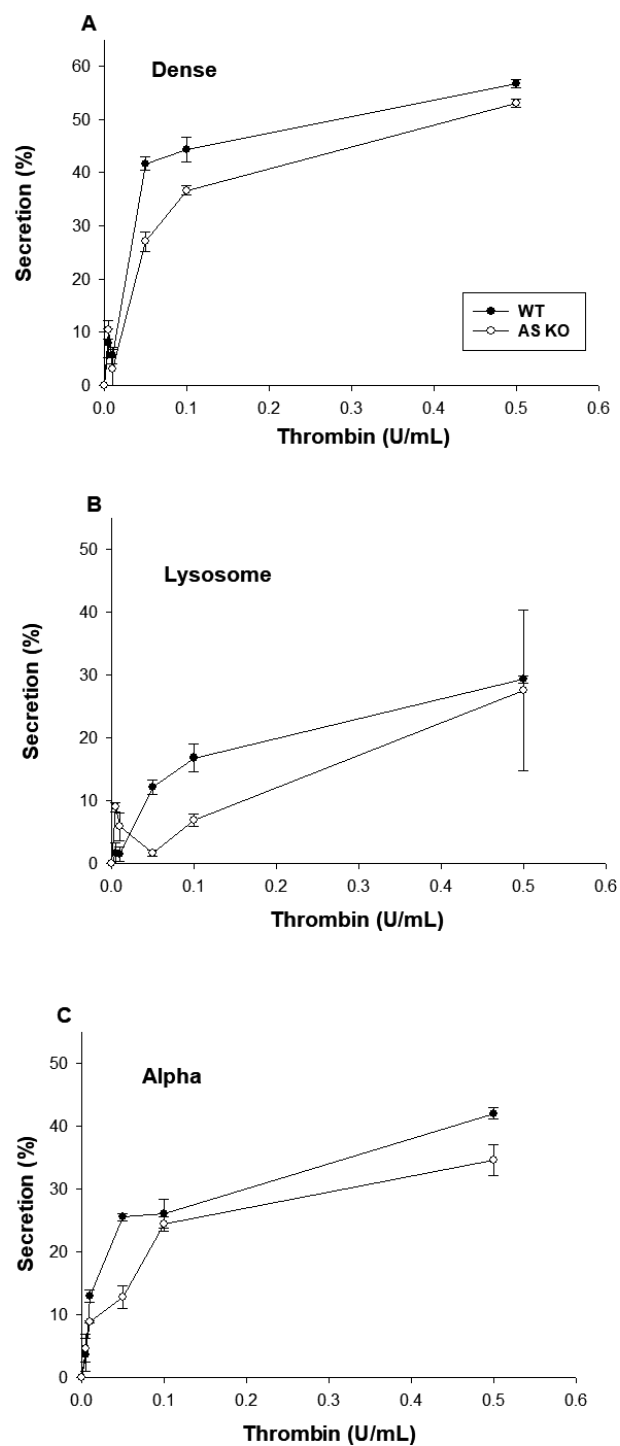

FIGURE 1 a-Synuclein-/- platelets have a mild secretion defect. 


\section{asis}

\section{Figure 2.}

AS

KO

AS

KO

WT WT WT

Alpha Synuclein

CSP

Syntaxin-11

SNAP-23

Actin

Munc18-b

VMAT

VAMP-7

VAMP -3

VAMP_2

VAMP-8

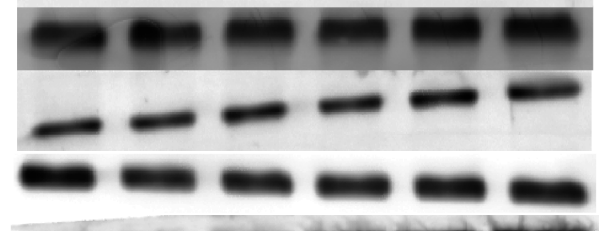

FIGURE 2 Western blots examining protein levels in the various transgenic mice

Conclusions: These experiments demonstrate a role for a-synuclein in platelet exocytosis and hemostasis and will further fill a gap in our knowledge on a-synuclein's physiological function and understanding how the process of platelet exocytosis is regulated.

This work is supported by grants from the NIH, NHLBI, VA Merit Award, and an NSF KY-WV LSAMP BD Fellowship (HL56652, HL138179, HL150818, NSF HRD 2004710) to S.W.W. and A.N.S.

PB0963 | Stimulation of Calpain Activity, Facilitation of Talin Degradation and Shedding of PMPs Exist as the Modalities of Prion-mediated Pathological Effects in Human Platelets

\section{Prakash Gaire ${ }^{1}$; R. Lala Mallick ${ }^{1}$; S. Kumar Karn'ㄹ R. Thapa ${ }^{3}$}

${ }^{1}$ Kathmandu University/Birat Medical College \& Teaching Hospital, Biratnagar, Nepal; ${ }^{2}$ Damak Hospital \& Research Center, Damak, Nepal; ${ }^{3}$ Nirnayak Reference Lab Damak, Damak, Nepal

Background: The amino acid sequence 106-126 of prion proteins i.e., $\operatorname{PrP}(106-126)$ is highly amyloidogenic. It leads to prion-mediated pathologies. As PrP(106-126) is known to be expressed in blood following leakage from brain tissue in prion diseases, we aimed to investigate the modalities of its pathological effects in human platelets.

Aims: We aimed to investigate:

1. Calpain activity

2. Facilitation of talin degradation

3. Shedding of Platelet-derived microparticles (PMPs).

Methods: 1 . Isolation of human blood platelets in resting state by differential centrifugation

2. Platelet aggregation / agglutination and dense granule secretion
3. Flow cytometric based analyses

4. Spectrofluorometric measurement of intracellular free calcium

5. Confocal microscopy for calcium imaging

6. Calpain activity assay

7. Western blot analyses.

Results: 1. $\operatorname{PrP}(106-126)$ induces platelet activation

2. $\operatorname{Pr} P(106-126)$ induces rise in intracellular free calcium in platelets 3. $\operatorname{PrP}(106-126)$-induced rise in intracellular free calcium in platelets was found to involve transient receptor potential channel (TRPC) proteins

4. $\operatorname{PrP}(106-126)$ stimulates calpain activity in human platelets

5. $\operatorname{PrP}(106-126)$ facilitates talin degradation to release the plateletderived microparticles

6. $\operatorname{PrP}(106-126)$ induces shedding of platelet-derived microparticles (PMPs).

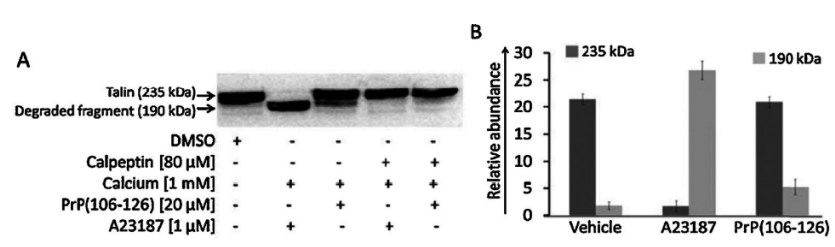

FIGURE 1 Prion-induced partial proteolysis of cytoskeleton protein 'talin'
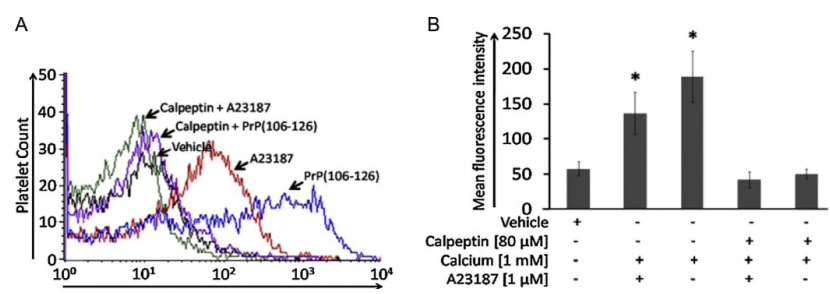

FIGURE 2 Histogram representation of Phycoerrythtrin (PE)-labeled annexin- $\mathrm{V}$ binding to PMPs

Conclusions: Our findings suggested that $\operatorname{Pr} P(106-126)$ induced 30fold rise in intracellular rise in calcium. It was attributable to influx from extracellular fluid. Calcium mobilization was associate with 8-10 fold stimulation in activity of thiol protease that laid to partial cleavage of cytoskeleton-associated protein talin and extensive shedding of platelet-derived microparticles. Both proteolysis of talin and microparticle release were precluded by calpeptin i.e., a specific inhibitor of calpain. As microparticles are endowed with phoshpatidylserine (PS)-enriched surface and so are procoagulant in nature, exposure to prion favors thrombogenic state. 
PB0964 | Genetic and Non-genetic Regulators of Platelet Function in Healthy Tanzanian Individuals

V. Kullaya $^{1}$; G. Temba ${ }^{2}$; N. Fadaq ${ }^{3}$; C. Boahen ${ }^{3} ;$ T. Pecht ${ }^{4} ;$ M. Netea ${ }^{3}$; B. Mmbaga ${ }^{1}$; A. van der Ven ${ }^{3}$; Q. de Mast $^{3}$

${ }^{1}$ Kilimanjaro Clinical Research Institute, Moshi, Tanzania, United Republic of; ${ }^{2}$ Kilimanjaro Christian Medical University College, Moshi, Tanzania, United Republic of; ${ }^{3}$ Radboud University Medical Center, Nijmegen, Netherlands; ${ }^{4}$ University of Bonn, Bonn, Germany

Background: The incidence of cardiovascular diseases (CVD) is rising in Sub-Saharan Africa (SSA) due to life style changes associated with emergent urbanization. Platelets are key cells in thrombosis, hemostasis and inflammation and as such play an important role in the development of ischemic CVD such as myocardial infarction and ischemic stroke. To date, data on determinants of platelet activation and reactivity in populations in SSA is scarce.

Aims: To explore genetic and non-genetic host factors, and environmental determinants of platelet reactivity in healthy Tanzanians.

Methods: We explored genetic, non-genetic and environmental determinants of platelet reactivity in a cohort of 319 healthy Tanzanian adults. We also studied the associations of platelet parameters with circulating inflammatory markers.

Results: Genome wide association study (GWAS) showed unique set of genes associated with platelet activation/reactivity in Tanzania with little overlap with Caucasian populations. We identified 2 novel SNPs, rs903650 and rs4789332, that were associated with platelet function at the genome-wide significance threshold of $P$-value $\leq 5 \times 10-{ }^{8}$, and confirmed polymorphisms in the PAR4 genes previously reported to increase PAR4-dependent reactivity in African descendants. Associations with inflammatory markers yielded positive correlations with plasma levels of alpha-1-antitrypsin (AAT) and negative correlations with interleukin (IL)-1 $\beta$ and IL-18 levels. While environmental factors had little effect on platelet reactivity, interesting associations were observed with food-derived metabolites especially with lipids. Among others, Triglyceride, Dehydrophytosphingosine and 3-methyl-4-(sulfooxy) but-2-enoic acid showed strong positive associations while 4",5,6-Trimethylscutellarein 7-glucoside, Methyl cellulose and Mangostenone $B$ showed strong negative associations. Clustering of subjects based on food metabolites showed significant differences in frequency of food consumption and levels of platelet activation/ reactivity.

Conclusions: Platelet function in a Tanzanian population is driven by unique sets of genetic and non-genetic determinants whereby diet, through food metabolites, pose an important determinant of platelet reactivity. These differences provide novel insights in the possible health consequences of lifestyle changes in SSA.
PB0965 | Expression and Localization of Rab GTPase Proteins in Platelets

N. Nguyen $^{1}$; A. Melrose ${ }^{2}$; E. Fellin ${ }^{3}$; I. Parra-Izquierdo¹; J. Pang ${ }^{1}$; O. McCarty ${ }^{1} ;$ J. Aslan ${ }^{2}$

${ }^{1}$ Oregon Health \& Science University, Department of Biomedical

Engineering, Portland, United States; ${ }^{2}$ Oregon Health \& Science

University, Knight Cardiovascular Institute, Portland, United States;

${ }^{3}$ Oregon Health \& Science University, School of Medicine, Portland, United States

Background: Rab GTPases serve as master regulators of vesicle biogenesis, traffic and fusion in eukaryotic cells. Platelets express over 40 different Rab proteins with potential roles in secretion, receptor trafficking and other cell physiological process critical to platelet function; however, roles for Rab proteins in platelet physiology remain minimally described.

Aims: We aim to determine the localization, distribution and regulation of Rab GTPase system proteins in platelets.

Methods: Through a combination of immunofluorescence microscopy and cell physiology methods, we determine the localization and regulation of $>10$ different Rab GTPases in resting platelets, as well as platelets stimulated with the platelet collagen receptor GPVI/ ITAM agonist crosslinked collagen-related peptide (CRP-XL).

Results: Washed platelets were prepared from blood of healthy human donors for in vitro agonist stimulation. Conventional and super-resolution immunofluorescence microscopy found different Rab proteins localize to distinct vesicle populations in resting and activated human platelets. Rab7 and Rab10 show staining patterns consistent maturing endosomes/lysosomes; whereas Rab 8 and Rab10 appear to localize to multivesicular bodies. Other Rab proteins, including Rab9, Rab13, Rab30, Rab32 and Rab37 also exhibit distinct intracellular staining patterns that redistribute in platelets following activation with CRP-XL and other agonists.

Conclusions: Our work begins to systematically characterize Rab GTPase expression, localization, regulation and function in human platelets to better understand how Rab GTPase activities regulate platelet cellular homeostasis, as well as activation programs in health and disease. 
PB0966 | MAS9 - A Novel Small Molecule Inhibitor of the CLEC2-Podoplanin Interaction

M.A. Sowa ${ }^{1}$; J. van Groningen ${ }^{2} ;$ Y. Di ${ }^{3}$; J.M. Gibbins ${ }^{1}$; Á. García ${ }^{4}$; A.Y. Pollitt ${ }^{1}$

${ }^{1}$ Institute for Cardiovascular and Metabolic Research (ICMR), School of Biological Sciences, University of Reading, Reading, United Kingdom; ${ }^{2}$ Pivot Park Screening Centre, Oss, Netherlands; ${ }^{3}$ Institute of Cardiovascular Sciences, College of Medical and Dental Sciences, University of Birmingham, Birmingham, United Kingdom; ${ }^{4}$ Platelet Proteomics Group, Center for Research in Molecular Medicine and Chronic Diseases (CIMUS), Universidad de Santiago de Compostela, and Instituto de Investigación Sanitaria (IDIS), Santiago de Compostela, Spain

Background: The C-type lectin-like receptor-2 (CLEC-2) is a platelet receptor for the endogenous ligand Podoplanin. This interaction contributes to a number of pathophysiological roles, such as lymphangiogenesis, preservation of blood and lymphatic vessel integrity, organ development and tumour metastasis. Activation of CLEC-2 leads to the phosphorylation of its cytoplasmic hemITAM domain and initiates a signalling cascade involving Syk and PLC $\gamma 2$.

Aims: The aim of this study was to identify a small-molecule inhibitor of the CLEC-2-Podoplanin interaction and to characterise their effect on human platelet activation.

Methods: AlphaScreen-based high-throughput screening identified a small-molecule inhibitor of the CLEC-2-Podopolanin interaction. Light transmission aggregometry, platelet spreading and phosphorylation assays were used to evaluate the effect of the small molecule on CLEC-2 mediated platelet activation.

Results: 18,476 small molecules were screened resulting in 14 candidates. Following the secondary screening, one small molecule (MAS9) was taken forward for further characterisation. $20 \mu \mathrm{M}$ of MAS9 inhibited platelet aggregation in response to the CLEC-2 agonist Rhodocytin. MAS9 dose-dependently inhibited platelet spreading and adhesion on immobilized Podoplanin and Rhodocytin. 30 $\mu \mathrm{M}$ MAS9 inhibited the phosphorylation of Syk, PLC $\gamma 2$ and Src in platelets activated by Rhodocytin. Partial inhibition of GPVI mediated aggregation and spreading was observed but MAS9 did not impact GPVI mediated phosphorylation.

Conclusions: MAS9 potently inhibits CLEC-2-mediated aggregation, platelet spreading and phosphorylation, showing selectivity on CLEC-2 inhibition over GPVI. Further pharmacological and functional experiments will be carried out to establish the potential of MAS9 as a lead compound to identify a novel anti-platelet drug with therapeutic effects in thrombosis and cancer.
PB0967 | Machine Learning-guided Analysis of Adult and Cord Platelet Adhesion Dynamics

J. Sheriff; P. Wang; P. Zhang; Z. Zhang; W. Bahou; Y. Deng; D. Bluestein

Stony Brook University, Stony Brook, United States

Background: Shear-mediated platelet adhesion is essential to initiating clot formation in vascular diseases and prosthetic cardiovascular devices. However, sparse and noisy raw in vitro image data continues to hamper validation of predictive computational models of platelet adhesion under flow.

Aims: To determine if adhesion dynamics is age-specific and intracellular $\mathrm{Ca}^{2+}$-dependent, by applying a novel machine learning (ML)guided approach for accurate image analysis of flowing adult and cord platelets.

Methods: Gel-filtered platelets, prepared from blood drawn from consenting healthy adult volunteers or cord blood obtained from neonates delivered via Caesarean sections under Stony Brook University IRB-approved protocols, were diluted to $150,000 / \mu \mathrm{l}$ and perfused at wall shear stress of $30 \mathrm{dyne} / \mathrm{cm}^{2}$ through $100 \mathrm{\mu g} / \mathrm{ml}$ vWF-coated microchannels, with adhesion events captured at 1000 fps. Platelets were also pre-treated with $20 \mu \mathrm{M}$ BAPTA-AM to evaluate intraceullar $\mathrm{Ca}^{2+}$ dependence. A semi-unsupervised learning system (SULS) classified platelet morphology from DIC microscope images, from which geometric parameters and rolling direction were calculated. Rotational angles and velocities fit to a modified Jeffery orbit model were compared across the age and $\mathrm{Ca}^{2+}$ treatment groups using two-sample t-tests.

Results: SULS accurately predicted moving platelet boundaries (Fig. $1 \mathrm{~A})$, with false prediction area of $0.728 \mu \mathrm{m} 2$. For both adult and cord platelets, we observed distinct periods characterizing longer lift-off from and shorter reattachment to the vWF surface $(P<0.05$, Fig. 2B-C). Cord platelets ( $n=21$ ) flip non-significantly faster than adult platelets ( $n=70, P<0.05$, Fig. 2D). Intracellular Ca2+-depleted cord platelets $(n=3)$ show a 1.16 -fold increase in peak rotational speed compared to untreated cord platelets ( $P<0.05$, Fig. 2D).

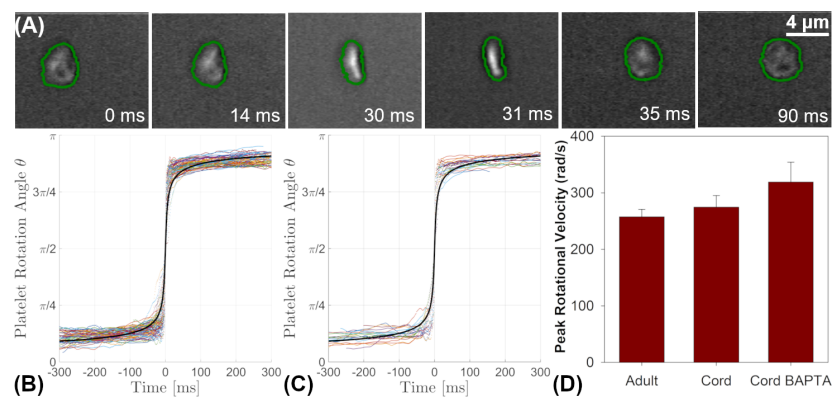

FIGURE 1 (A) Platelet adhesion image sequences are presented with SULS-predicted boundaries. (B) Adult and (C) cord platelet rotation during adhesion. (D) Peak rotational velocities for adult, cord, and 20 $\mu$ M BAPTA-AM-treated cord platelets 
Conclusions: Our integrated ML-microscopy approach allows accurate segmentation of flipping platelets, showing heterogeneity of platelet motion during adhesion and possible dependence on age and intracellular $\mathrm{Ca}^{2+}$ availability. This framework bridges sparse in vitro data and multiscale computational models, which may predict physiologically significant platelet dynamics beyond the capabilities of current imaging technology.

\section{PB0968 | The Role of Septins in Platelet Structure and Function}

\section{O.V. Kim ${ }^{1}$; O. Vagin ${ }^{2}$; R.I. Litvinov ${ }^{1}$; J.W. Weisel ${ }^{1}$}

${ }^{1}$ Department of Cell and Developmental Biology, Perelman School of Medicine, University of Pennsylvania, Philadelphia, United States; ${ }^{2}$ Department of Physiology, Geffen School of Medicine at UCLA, Los Angeles, United States

Background: Platelets are blood cells playing an important role in hemostasis and thrombosis. Activated platelets change shape due to cytoskeleton remodeling. Septins, a family of GTP-binding cytoskeletal proteins, are implicated in cytokinesis, membrane remodeling and intracellular trafficking of nucleated cells, but their contribution to platelet biology is largely unknown.

Aims: To examine septins in resting and activated human platelets and their role in platelet structure and functions.

Methods: Confocal microscopy, flow cytometry, biomechanical and biochemical assays were used to examine structural and functional changes in human platelets. Septins 2 and 9 , and microtubules were stained in resting and activated platelets as well as in platelets spread on a fibrinogen-coated surface.

Results: In resting platelets, septin 2 concentrated the cell periphery, while septin 9 was distributed as small patches over the cell volume, often with a peripheral localization. Both septins colocalized with a microtubule marginal band. In thrombin-activated platelets, septins formed intense fluorescent clusters. Activation with thrombin resulted in a 2-fold increase of septin intensity and decrease in colocalization between septins and a-tubulin. Inhibition of septin assembly with forchlorfenuron (FCF) resulted in disruption and thickening of septin 2 ring, elongation of septin structures, reduction of colocalization between septins and a-tubulin, a decrease of platelet roundness and surface curvature. In FCF-pretreated platelets activated with TRAP, expression of activated integrin allb $\beta 3$ was significantly suppressed. FCF impeded clot contraction with a 6-fold increase of the lag-time and up to a 3-fold decrease of the extent of contraction. Inhibition of septin assembly abrogated platelet spreading by $50 \%$ and accelerated thrombin-induced platelet fragmentation.

Conclusions: Septins are important for stabilizing platelet shape and supporting platelet integrity; septins are involved in platelet surface markers expression and biomechanical functions, such as contractility and adhesion.

Funding: Work supported by AHA grant 17SDG33680177.
PB0969 | Rapid and Complete Clearance of HPA-1a Mismatched Platelets in a Human Model of Fetal and Neonatal Alloimmune Thrombocytopenia by a Hyperimmune Plasma Derived Polyclonal Anti HPA-1a Antibody

C. Geisen ${ }^{1}$; E. Fleck ${ }^{1}$; S.M.G. Schäfer ${ }^{2}$; C. Walter ${ }^{2}$; S. Braeuninger ${ }^{1}$; K. Olsen ${ }^{3}$; Z. Bhagwagar ${ }^{4}$; A. Mortberg ${ }^{5}$; A. Wikman ${ }^{5,6}$; M. Kjaer ${ }^{7,8}$; J. Kjeldsen-Kragh ${ }^{9,10}$; F. Behrens ${ }^{2}$; E. Seifried ${ }^{1}$; M. Köhm² ${ }^{1}$ Institute of Transfusion Medicine and Immunohaematology, German Red Cross Blood Transfusion Service Baden-Württemberg-Hessen gGmbH, Frankfurt am Main, Germany; ${ }^{2}$ Fraunhofer Institute for Translation Medicine and Pharmacology ITMP, Frankfurt am Main, Germany; ${ }^{3}$ Larix A/S, Herlev, Denmark; ${ }^{4}$ Rallybio, New Haven, United States; ${ }^{5}$ Karolinska University Hospital, Stockholm, Sweden; ${ }^{6}$ CLINTEC, Karolinska Institutet, Stockholm, Sweden; ${ }^{7}$ UiT - the Arctic University of Norway, Tromsø, Norway; ${ }^{8}$ Finnmark Hospital Trust, Hammerfest, Norway; ${ }^{9}$ Department of Clinical Immunology and Transfusion Medicine, University and Regional Laboratories, Lund, Sweden; ${ }^{10}$ Department of Laboratory Medicine, University Hospital of North Norway, Tromsø, Norway

Background: RLYB211 is an intravenously administered, investigational, plasma-derived polyclonal anti-Human-Platelet-Antigen-1a (HPA-1a) hyperimmune IgG being developed for the prevention of Fetal and Neonatal Alloimmune Thrombocytopenia (FNAIT). FNAIT is a rare condition in which the mother's immune system attacks the platelets of her fetus, leading to potentially catastrophic fetal and neonatal morbidity and mortality. Fetal-maternal incompatibility in the HPA-1 system is the most common (85-90\%) cause of FNAIT. Treatment with RLYB211 is designed to rapidly eliminate fetal HPA-1a positive platelets from a mother's circulation and prevent maternal alloimmunization, eliminating the risk of FNAIT in the fetus. There are no currently approved treatments for the prevention of FNAIT.

Aims: To determine whether a dose of 1000 IU of anti-HPA-1a antibodies would accelerate the clearance of HPA-1a positive platelets transfused to HPA-1bb (i.e., HPA-1a negative) healthy male volunteers.

Methods: Following approval by the Paul Ehrlich Institut, Germany and the Ethics Committee, University Hospital Frankfurt, informed consent was obtained from 8 healthy male subjects (HPA-1bb and HLA-A2 negative). Subjects were administered either RLYB211 $(n=6)$ or placebo $(n=2) 60$ minutes after administration of $10 \times 10^{9}$ HPA-1ab and HLA-A2 positive platelets. The proportion of normalized HLA-A2 positive platelets in circulation following administration of RLYB211 or placebo was determined by flow cytometry.

Results: RLYB211 showed acceptable safety and tolerability with no serious adverse events and minimal adverse events observed. Administration of RLYB211 markedly accelerated the clearance of HPA-1ab positive platelets compared with placebo (half-life of mismatched platelets 0.32 hrs. vs. 65.29 hrs. respectively; $p$ value $<0.001)$. 


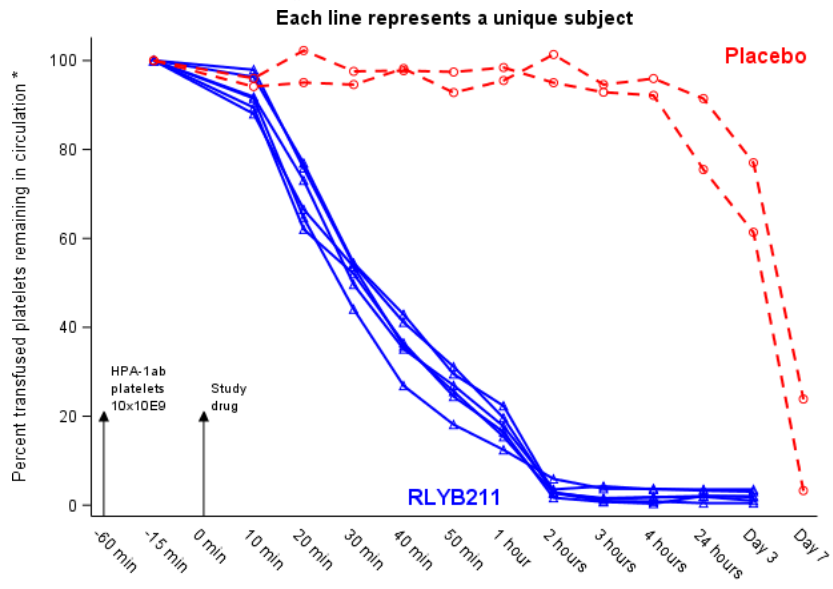

*Data normalized at $100 \%$ for sample collected 15 minutes prior to study drug at 0 mins.

FIGURE 1 Rapid and complete clearance of HPA-1ab positive platelets by anti-HPA-1a antibodies compared with placebo in HPA-1bb healthy male subjects

Conclusions: RLYB211 provides proof of concept of the ability of anti HPA-1a antibodies to rapidly and completely clear mismatched HPA-1a positive platelets in HPA-1bb individuals. Administration of anti-HPA-1a antibodies could therefore be a viable treatment for the prevention of FNAIT in mothers at high risk.

PB0970 | Highly Reactive Juvenile Platelets Express Higher Levels of GPVI in a Size-related Manner

A. Veninga $^{1}$; S. Handtke ${ }^{2}$; B.M.E. Tullemans ${ }^{1}$;

S.L.N. Brouns ${ }^{1}$; A. Greinacher ${ }^{2}$; J.W.M. Heemskerk ${ }^{1}$; P.E.J. van der Meijden $^{1,3} ;$ T. Thiele ${ }^{2}$

${ }^{1}$ Department of Biochemistry, Cardiovascular Research Institute Maastricht (CARIM), Maastricht University, Maastricht, Netherlands; ${ }^{2}$ Institute for Immunology and Transfusion Medicine, University Medicine Greifswald, Greifswald, Germany; ${ }^{3}$ Thrombosis Expertise Center, Heart and Vascular Center, Maastricht University Medical Center, Maastricht, Netherlands

Background: Platelets within one individual display heterogeneity in reactivity, size, age, and expression of surface receptors. We and others have shown that larger platelets show increased responsiveness to activating stimuli compared to smaller platelets. Next to that, it is considered that the RNA content in juvenile platelets is associated with higher reactivity.

Aims: To investigate the combined intra-individual contribution of platelet size, platelet age, and receptor expression levels on the reactivity of platelets.

Methods: Fractions of large and small platelets from healthy donors were separated by differential centrifugation. Multicolour flow cytometry with subsequent automated high-dimensional clustering analysis (FlowSOM) was used to identify and phenotype platelet subpopulations formed in response to different doses of CRP-XL, TRAP6, and ADP. Platelet age correlations were assessed by co-staining of labelled oligo-dA/T with either anti-glycoprotein (GP) VI or anti-HLA-I antibodies. Whole blood reconstituted with sizeseparated platelet fractions were perfused over a collagen-coated surface to assess thrombus formation.

Results: Clustering analysis of the flow cytometric data showed that highly reactive platelet populations are characterised by highest GPVI and HLA-I (marker for juvenile platelets) expression (Figure 1A). Platelets with high expression of GPVI also show a higher RNA content (Figure 1B). Highly reactive juvenile platelets were found to be enriched in the large platelet fraction (Figure 1C). Even when adjusted to the same platelet mass, the larger platelet fraction resulted in faster adhesion to collagen under flow and the formation of larger thrombi, compared to the small platelet fraction.

Conclusions: High GPVI expression is a feature of highly reactive juvenile platelets, which are predominantly found among large platelets and likely promote thrombus formation.
A

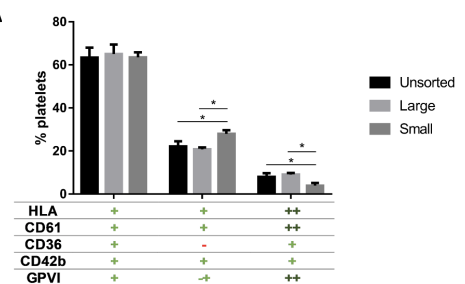

C

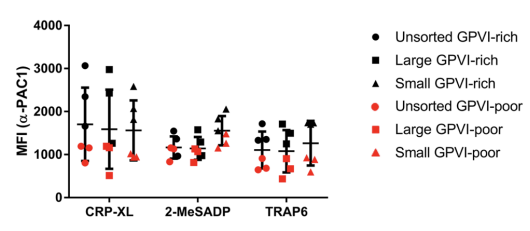

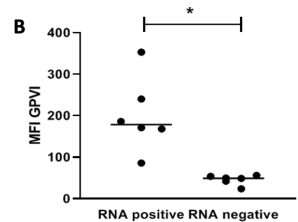

FIGURE 1 (A) Platelet populations in the size-separated platelet fractions resulting from clustering analysis of multicolour flow cytometric data. The $\mathrm{X}$-axis presents the expression characteristics for each population (ranging from low (-) to high (++) expression levels); (B) Expression levels of GPVI in RNA positive/negative platelet fractions distinguished by labelled oligo-dA/T staining; $(C)$ Active integrin allbß3 (PAC1) levels in size-separated platelet fractions gated on GPVI-rich in samples after suboptimal stimulation CRP-XL, 2-MeSADP, or TRAP6

Funded by Landsteiner Foundation for Blood Transfusion Research. 
PB0971 | A Novel Therapeutic Strategy for Hepatocellular Carcinoma Based on the Tumor-platelet Interaction

H. Tanaka ${ }^{1}$; K. Horioka ${ }^{2}$

${ }^{1}$ Division of Tumor Pathology, Department of Pathology, Asahikawa Medical University, Asahikawa, Japan; ${ }^{2}$ Department of Legal Medicine, International University of Health and Welfare, Narita, Japan

Background: We previously reported that platelets markedly accumulated in hepatocellular carcinoma ( $\mathrm{HCC}$ ) and they were activated during tumor progression. Platelets are able to internalize extracellular substances, which are then released upon activation.

Aims: We hypothesized that autologous platelets could be a potential drug carrier for cancer therapy. We propose a unique HCC therapy using autologous platelets as a drug carrier.

Methods: We induced HCC in rats according to the Solt \& Farber protocol. Then, we collected the blood from the tail vein of the tumor-bearing rats. Platelets were isolated and incubated with sorafenib in vitro. The rats were divided into 4 experimental groups: saline, sorafenib, platelets, and platelets incubated with sorafenib (Sora-PLT); saline, sorafenib, platelets and Sora-Plt were injected via the tail vein of the host rats.

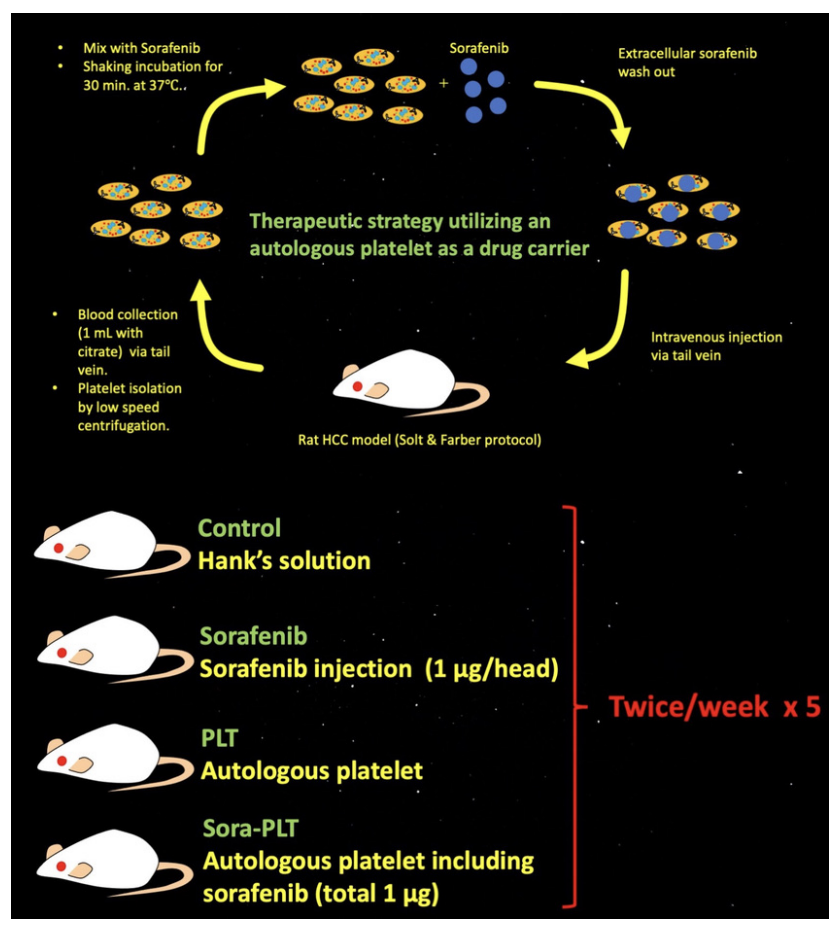

FIGURE 1 Graphical image of our methods

Results: Although platelets did not exert any effects, Sora-PIt treatment induced significant regression of the tumors. The tumorsuppressing effect of Sora-PIt was superior to that induced by sorafenib.

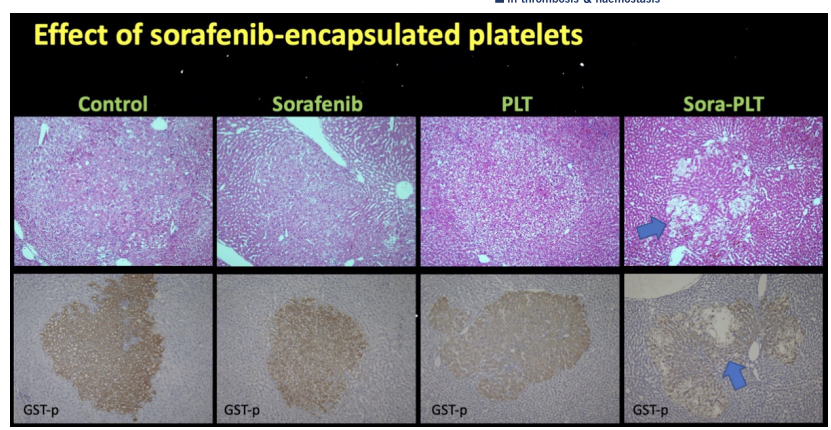

FIGURE 2 Histopathological analysis

Conclusions: Our results indicate that the use of autologous platelets containing anti-cancer drugs could be a novel therapeutic strategy for HCC.

PB0972 | Serglycin, an Intragranular Proteoglycan with Many Effects on Platelet Function

H. Chanzu $^{1}$; J. Lykins ${ }^{1}$; S. Joshi ${ }^{1,2} ;$ M. Chow ${ }^{1}$; I. Pokrovskaya ${ }^{3}$; B. Storrie ${ }^{3}$; G. Pejler ${ }^{4}$; S.W. Whiteheart ${ }^{1,2}$

${ }^{1}$ University of Kentucky, Lexington, United States; ${ }^{2}$ Lexington VA Medical Center, Lexington, United States; ${ }^{3}$ University of Arkansas for Medical Sciences, Little Rock, United States; ${ }^{4}$ Uppsala University, Uppsala, Sweden

Background: Upon vascular injury, platelets are activated and release molecules that affect the vascular microenvironment, promoting coagulation, wound healing, and clot architecture. Previously, we have shown that serglycin (SRGN), an intra-granular proteoglycan, plays multiple roles during platelet granule cargo packaging, retention and release, and in the extracellular environment by affecting receptor shedding.

Aims: To investigate SRGN's function in platelet cargo packaging and release and granule-plasma membrane pore dynamics upon platelet activation. Second, to define SRGN's role in platelet surface proteins shedding and downstream signaling.

Methods: Anti-SRGN nanobodies were produced in alpacas using recombinant, unglycosylated SRGN. These were used in pulldown assays to study the interaction of SRGN with platelet releasate proteins, to identify interacting partners. Serial block face EM was used to study how SRGN affects granule-plasma membrane pore dynamics and release kinetics upon activation. Multiplex, western blotting, and proteomics were used to determine how SRGN affects membrane protein shedding and downstream signaling.

Results: 
A

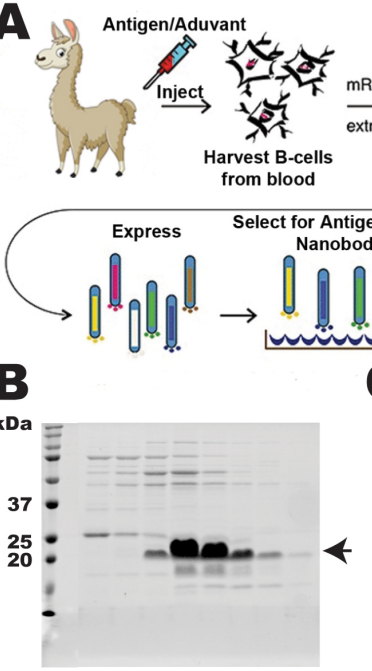

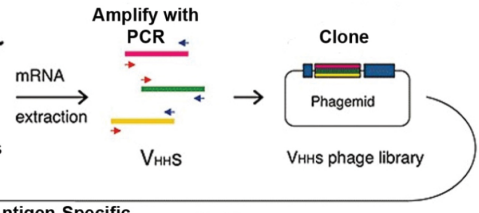

Purify

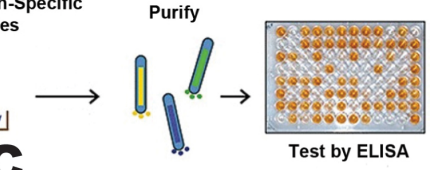

c

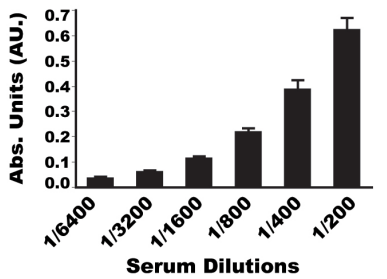

FIGURE 1 Anti-Serglycin Nanobody Production. (A). Nanobody production in Alpaca. (B) Recombinant, unglycosylated SRGN protein (black arrow). C) ELISA measure of anti-SRGN response using sera from immunized alpaca

Platelets from $\mathrm{SRGN}^{-/}$showed reduced a-granule decondensation and swelling upon stimulation. We have generated platelets from $\mathrm{SRGN}^{-1-}$ and wild-type control mice to examine fusion pore expansion by 3D EM analysis. Recombinant SRGN protein and its $\mathrm{N}$ - and $\mathrm{C}$-terminal domains have been produced and used as antigens and for screening our cDNA library. Sera from immunized alpaca was screened by ELISA to confirm the immune response. The initial panning of the libraries shows promising clones that recognize the full-length SRGN. GPVI shedding increased in $\mathrm{SRGN}^{-/-}$platelets after convulxin treatment, but GP1b was unaffected compared to $\mathrm{SRGN}^{+/+}$controls suggesting different roles of SRGN in receptor shedding and downstream signaling.

Conclusions: SRGN regulates a-granule decondensation and swelling after stimulation, affects convulxin-induced GPVI shedding in platelets, and influences cargo packaging and release in both megakaryocytes and platelets.

PB0973 | Utilizing CRISPR-CAS9 Gene Editing Technology in Human Pluripotent Stem Cells to Study Platelet Integrin allb $\beta 3$ Function

K.P.Y. Fong ${ }^{1}$; C. Jobaliya ${ }^{2}$; J.A. Maguire ${ }^{2}$; M. Poncz ${ }^{2}$; D.L. French ${ }^{2}$; J.S. Bennett ${ }^{1}$

${ }^{1}$ University of Pennsylvania, Philadelphia, United States; ${ }^{2}$ Children's Hosptial of Philadelphia, Philadelphia, United States

Background: For platelet integrins such as allb $\beta 3$, the functional consequences of site-directed mutations have previously been studied in epithelial cell lines, such as Chinese hamster ovary ( $\mathrm{CHO})$ cells. Only recently has technology advanced sufficiently to enable study of platelet integrins in their normal cellular environment, the

platelet-producing megakaryocyte. The advent of human induced pluripotent stem cells (iPSC), coupled with CRISPR-CAS9 technology, has provided a way to study allb $\beta 3$ mutants in megakaryocytes. Following platelet stimulation, allb $\beta 3$ undergoes a global rearrangement in which a clasp composed of its extracellular stalk, transmembrane, and membrane-proximal cytoplasmic domains is disrupted causing the allb $\beta 3$ headpiece to open exposing a ligand binding site. Using computational methods, we previously predicted mutations that would destabilize the allb $\beta 3$ stalk, causing allb $\beta 3$ activation.

Aims: To translate these findings to iPSC-derived megakaryocytes, we studied a V760A missense mutation located in the allb stalk that is highly activating in $\mathrm{CHO}$ cells.

Methods: Using an established iPSC line designated CHOPWT14, we created heterozygous and homozygous V760A missense mutations using a CRISPR-CAS9 protocol.

Results: Cell lines were differentiated into megakaryocytes and binding of the activation-dependent monoclonal antibody PAC-1 was used to measure constitutive and agonist-induced allb $\beta 3 \mathrm{li}$ gand binding activity. PAC-1 bound constitutively and specifically to $23.4 \%$ and $26.0 \%$ of megakaryocytes expressing heterozygous and homozygous V760A mutations, respectively, compared to $9.04 \%$ of control megakaryocytes. In addition, thrombin stimulation increased PAC-1 binding to $>65 \%$ in all lines, indicating normal overall allb $\beta 3$ function.

Conclusions: These data show that 1) structure-function studies of computationally identified mutations confirmed in $\mathrm{CHO}$ cells can be analyzed using human iPSC-derived megakaryocytes, 2) mutations shown to be highly active in $\mathrm{CHO}$ cells appear to be constrained or less constitutively active in human megakaryocytes, and 3) more indepth analyses of platelet integrin structure-function relationships will be possible using human megakaryocytes.

PB0974 | Modification of Platelet Characteristics and Hemostasis in Children with Inflammatory Bowel Diseases under Hyperbaric Oxygenation

A. Popovicheva; A. Martusevich; E. Fedulova; I. Neshchetkina Federal State Budgetary Institution "Privolzhsky Federal Research Medical Centre" of the Ministry of Health of the Russian Federation, Nizhny Novgorod, Russian Federation

Background: Inflammatory bowel diseases (IBD) are associated with a violation of the platelets properties and the hemostatic system. The effect of hyperbaric oxygenation (HBO) on these abnormalities in pediatric IBD has not been previously studied.

Aims: To evaluate platelet aggregation and the state of the hemostatic system in children with IBD and the effect of $\mathrm{HBO}$ on these parameters.

Methods: The study involved 27 patients aged 6 to 17 years. The study was approved by the local ethics committee of the PRMU, as well as informed consent to participate in the study. The platelet aggregation was studied in artificial shear flow on a device designed 
according to the principle of Schmid-Schönbein et al. (1975) modified by Levin et al. (2006). The content of soluble fibrin-monomer complexes (SFMC) was determined by the orthophenanthroline method. Similar indicators were studied after a course of HBO performed of 5-8 daily sessions in the mode of 1.3 ata for 40 minutes. The data is presented as the mean \pm standard error. The results of the study were compared with similar indicators of 35 healthy children and processed by the Mann-Whitney test and Wilcoxon pair comparisons. The differences were considered statistically significant at $P<0.05$ and $P<0.001$.

Results: In children with IBD a significant increase of platelet aggregation was revealed (table 1). The number of SFMC in these patients also significantly exceeded the control values (table 2). After a course of HBO platelet aggregation decreases to the control values (table 1). There was a tendency to reduce the level of SFMC in children with IBD after the course of HBO (table 2).

Conclusions: IBD in children is characterized by a significant increase of the platelet aggregation and SFMC. The use of HBO in the treatment of IBD contributes to a pronounced and significant decrease in these indicators.

\section{PB0975 | Effects of Different Concentrations of Apixaban} Combined with Antiplatelet Therapies on Platelet Hemostatic and Thrombogenic Functions. Ex-vivo Exploratory Study in Patients under Various Antiplatelet Regimens

J. Martinez-Sanchez ${ }^{1,2,3}$; L. Castrillo ${ }^{4}$; D. Jerez ${ }^{2}$; S. TorramadeMoix $^{2}$; M. Palomo ${ }^{1,2,3}$; G. Mendieta ${ }^{4}$; M. Diaz-Ricart ${ }^{2,3}$; M. Roque ${ }^{4}$; G. Escolar 2,3

${ }^{1}$ Josep Carreras Leukaemia Research Institute, Barcelona, Spain; ${ }^{2}$ Hematopathology, Department of Pathology, Centre de Diagnostic Biomedic (CDB), Hospital Clinic de Barcelona, Institut d'Investigacions Biomediques August Pi i Sunyer (IDIBAPS), Universitat de Barcelona, Barcelona, Spain; ${ }^{3}$ Barcelona Endothelium Team, Barcelona, Spain; ${ }^{4}$ Department of Cardiology, Institut d'Investigacions Biomediques August Pi i Sunyer (IDIBAPS), Hospital Clinic de Barcelona, Universitat de Barcelona, Barcelona, Spain

Background: Balancing the prevention of thrombotic events vs. the risk of bleeding associated with anticoagulant and antiplatelet therapies in patients with coexisting atrial fibrillation and coronary artery disease is controversial. Recent availability of direct oral anticoagulants and more potent antiplatelet agents has further increased the complexity of this challenge.

Aims: To evaluate the effects of apixaban (Apix), added at different concentrations to blood samples from patients under antiplatelet therapies on the hemostatic and prothrombotic functions of platelets.
Methods: Blood samples were obtained from: control healthy donors ( $n=24)$, and patients treated with aspirin (ASA, $n=21$ ), ASA and clopidogrel (ASA+CLOPI, $n=8$ ), or ASA and ticagrelor (ASA+TICA, $n=20$ ). Samples were incubated with Apix at 0,40 , and $160 \mathrm{ng} / \mathrm{mL}$. We applied:

i) Thrombin generation assay, to analyze the contribution of platelets to thrombin generation;

ii) Rotational thromboelastometry (ROTEM), to evaluate clot formation;

iii) Microfluidic studies in perfusion devices to evaluate platelet and fibrin interactions with a collagen + tissue factor substrate.

Results: Thrombin generation decreased progressively with Apix concentrations, especially for Apix $160 \mathrm{ng} / \mathrm{mL}$ with ASA+TICA $(P<0.01)$. Apix caused dose-dependent alterations of the viscoelastic parameters in ROTEM ( $P<0.01$ at Apix $160 \mathrm{ng} / \mathrm{mL}$ for all groups). Microfluidic studies showed a reduction in the surface covered by platelets, platelet aggregates volumes and fibrin deposition for both Apix concentrations in all groups. Apix $40 \mathrm{ng} / \mathrm{mL}$ showed moderate inhibitory antithrombotic actions on all the tests. At this concentration, Apix preserved better the hemostatic function of platelets with all antiplatelet regimes explored.

Conclusions: Combined presence of Apix with antiplatelet strategies showed inhibitory action on all parameters evaluated. The suppressed action on thrombin generation, fibrin and platelet aggregate formation was more evident at the highest Apix concentration. Apix $40 \mathrm{ng} / \mathrm{mL}$ demonstrated a consistent antithrombotic action, but proved more respectful at preserving hemostatic parameters with all antiplatelet regimens.

Grants: BMS ERISTA, FIS PI19/00888.

\section{PB0976 | Ponatinib Inhibits Collagen Induced Platelet} Aggregation and Coated-platelet Formation in Human Platelets

I. Beke Debreceni ${ }^{1}$; G. Mezei ${ }^{2}$; P. Batár ${ }^{2}$; L. Kozma ${ }^{3}$; J. Kappelmayer ${ }^{1}$ ${ }^{1}$ Department of Laboratory Medicine, Faculty of Medicine, University of Debrecen, Debrecen, Hungary; ${ }^{2}$ Department of Internal Medicine, Division of Hematology, Faculty of Medicine, University of Debrecen, Debrecen, Hungary; ${ }^{3}$ Department of Laboratory Medicine, Faculty of Medicine, University of Debrecen, Debrecn, Hungary

Background: BCR-ABL tyrosine kinase inhibitors (TKI) are effective for the therapy of chronic myeloid leukemia (CML), nevertheless, they impose problems in several patients since both thrombotic events as well as bleeding may complicate TKI treatment. In previous publications, ponatinib was associated with platelet dysfunction or with a prothrombotic state.

Aims: Because of these contradictory data, we aimed to investigate the effect of ponatinib on aggregating and procoagulant platelets in a purified system and in ponatinib treated CML patients. 
Methods: Platelet rich plasmas (PRP) or gel filtered platelets of healthy volunteers were pretreated with different concentration of ponatinib then the quantity of phosphatidylserine (PS), PAR1 and GPVI agonist induced activation of allb $\beta$ IIla integrin and coatedplatelets were measured by flow cytometry. In addition, platelet dependent thrombin generation was measured by fluorimetry and collagen and ADP elicited platelet aggregation response was examined by light transmission aggregometry. These studies were also performed in samples of five CML patients taking ponatinib.

Results: In PRPs of healthy volunteers, ponatinib dose-dependently impaired collagen induced platelet aggregation but did not affect the ADP elicited aggregation response. In addition, ponatinib reduced the formation of coated-platelets, already at $150 \mathrm{nM}(P<0.05)$. It also dose-dependently inhibited the formation of PAR1 and GPVI agonists induced allb $\beta 3$ integrin activation as detected by PAC1 binding. On the other hand ponatinib exerted a significant potentiating effect on PS exposure of platelets $(P<0.05)$ at supra-therapeutic concentration (1000 nM), although this PS elevation was not associated with changes of parameters of thrombin generation. Platelets, isolated from ponatinib treated patients showed impaired collagen and ADP elicited aggregation response and reduced coated-platelet formation in some cases but no systematic effect of ponatinib could be observed in other tests.

Conclusions: Ponatinib itself may activate platelets, but more importantly it impairs activation processes elicited by platelet agonists.

PB0977 | Coagulation and Anticoagulation Factors Affecting Platelet Responses Independently of Thrombin and Fibrin

I. De Simone ${ }^{1,2}$; J.M. Gibbins ${ }^{2}$; H. ten Cate ${ }^{1,3}$; J.W. Heemskerk ${ }^{1}$; C.I. Jones ${ }^{2}$; P.E. van der Meijden ${ }^{1,3}$

${ }^{1}$ Department of Biochemistry, Cardiovascular Research Institute Maastricht (CARIM), Maastricht University, Maastricht, Netherlands;

${ }^{2}$ Institute for Cardiovascular and Metabolic Research, School of Biological Sciences, University of Reading, Reading, United Kingdom; ${ }^{3}$ Thrombosis Expertise Center, Heart and Vascular Center, Maastricht University Medical Center, Maastricht, Netherlands

Background: Platelet and coagulation activation are highly reciprocal processes. Activated platelets secrete several coagulation factors and expose phosphatidylserine. On the other hand, the coagulation cascade generates ligands for platelet receptors, such as thrombin and fibrin. However, the contribution of coagulation processes beyond thrombin and fibrin to platelet functions is an active area of research. Coagulation-related factors known to bind platelets include amongst others coagulation factor (F)XIIla and activated protein C (APC), but their role in platelet functions is unclear.

Aims: To investigate thrombin-independent effects of coagulated plasma (factors) on platelet responses.

Methods: Modulating effects of hirudin-treated, coagulated (fibrindepleted) plasma on platelets were evaluated via well plate-based aggregation and flow cytometry (PAC-1 binding and anti-P-selectin).
Fura-2 loaded platelets were activated with CRP-XL concentrations that induced submaximal platelet activation, in combination with FXa, FXIIla or APC, and changes in cytosolic $\left[\mathrm{Ca}^{2+}\right]_{i}$ were assessed. Spreading assays were performed on surfaces coated with FXIII(a), (A)PC, thrombin and collagen and were assessed by fluorescence microscopy

Results: Platelets exposed to hirudin-treated coagulated plasma showed increased aggregation and surface activation markers, compared to resting plasma. Purified FXa, FXIIla and APC each enhanced CRP-XL-induced rises in platelet cytosolic $\left[\mathrm{Ca}^{2+}\right]_{i}$. Markedly, the potentiating effect of FXa was completely abolished by addition of different thrombin inhibitors. While FXIIla and APC alone did not induce platelet activation in solution, coated FXIIla or APC caused spreading. Interestingly, PAR-1 inhibition diminished spreading on APC, but not on FXIIla. Our ongoing work is further exploring platelet receptors and their pathways involved in platelet spreading on FXIIIa.

Conclusions: Coagulated plasma promotes agonist-induced platelet activation. Measurements of calcium rises and spreading point to a role of FXIIla and APC, but not of FXa herein, partly through thrombin-independent PAR-1 activation.

PB0978 | Platelet Activity and Platelet-induced Endothelial Inflammatory Pathways in Treated HIV Is Lowered by Clopidogrel: A Randomized Control Trial

T. Schwartz; E. Maracantoni; N. Allen; M. Cambria; R. Dann; M. Garshick; J.S. Berger New York University School of Medicine, New York, United States

Background: Patients with Human Immunodeficiency Virus (HIV) exhibit an activated platelet phenotype and an increased risk of Cardiovascular Disease (CVD).

Aims: We conducted a randomized controlled trial to investigate the efficacy of aspirin and clopidogrel (two anti-platelet medications commonly used to prevent CVD) to reduce platelet activation and platelet effector cell properties in HIV patients.

Methods: Fifty five HIV positive patients (mean age $53.5 \pm 7.8$ years, $42.6 \%$ female, mean CD4+ T-cell count 665.6 cells $/ \mathrm{m}^{3}$ ), were enrolled to receive clopidogrel $(n=22,75 \mathrm{mg} / \mathrm{d}$ ), aspirin $(n=22,81$ $\mathrm{mg} / \mathrm{d})$, or no-treatment $(n=11)$ for 14 -days. Platelet aggregation and platelet receptor expression of p-selectin and pac-1 was assessed at baseline and day 14 . To assess the impact of platelet inhibition on the endothelium (in vitro), platelets isolated from 6 patients (2/ group) at baseline and follow-up were incubated in HUVECs and proinflammatory HUVEC gene expression was assessed (Nanostring, 594 transcripts).

Results: Aspirin treatment significantly reduced platelet aggregation to arachidonic acid (AA) (84\% to $31 \%, P<0.01)$ while clopidogrel reduced platelet aggregation to adenosine diphosphate (ADP) (85\% to $41 \%, P<0.001)$, confirming study drug compliance. Clopidogrel treatment decreased platelet $\mathrm{p}$-selectin $(-5.9 \%, P=0.04)$, p-selectin 
plus thrombin (-40.8\%, $P=0.03)$, pac-1 expression $(-8 \%, P=0.02)$, and pac-1 plus ADP $(-24.0 \%, P=0.03)$ and AA $(-24.0 \%, P<0.01)$. In contrast, aspirin did not affect $p$-selectin or pac-1 expression. When compared to no-treatment, HIV patients on clopidogrel exhibited a reduction in the composite pro-inflammatory transcript expression of platelet treated HUVECS ( $\log 2$ Fold $\Delta-0.07 \pm 0.58$ vs. $-0.12 \pm 0.53, P<0.001)$ while aspirin treated platelets upregulated HUVEC transcript expression (Log2 Fold $\Delta-0.07 \pm 0.58$ vs. $0.19 \pm 0.59, P<0.001)$.

Conclusions: Clopidogrel, but not aspirin, reduced platelet activation and HUVEC pro-inflammatory gene expression. Our results suggest that clopidogrel as opposed to aspirin may be preferential to reduce $\mathrm{CV}$ risk in HIV; however, larger clinical trials are needed to expand upon these findings.

PB0979 | Sex Differences in Thrombosis through Platelet Nitric Oxide Production

M. Godwin ${ }^{1,1}$; Z. Hilt ${ }^{2}$; A. Aggarwal ${ }^{1}$; S. Shah ${ }^{2}$; J. Gorski ${ }^{2}$;

S. Cameron ${ }^{1}$

${ }^{1}$ Cleveland Clinic, Cleveland, United States; ${ }^{2}$ University of Rochester, Rochester, United States

Background: A plant-based diet that increases nitrate intake has several benefits, including the normalization of metabolic abnormalities, reduction in blood pressure, and regression of atherosclerosis. The exact mechanism behind these beneficial effects is unclear. We hypothesized that ingesting nitrate augments nitric oxide (NO), which may suppress platelet reactivity.

Aims: To understand the effect of oral nitrate uptake on nitric oxide (NO) production and platelet reactivity and whether this differs in men and women.

Methods: We evaluated, in a randomized, double-blinded, placebocontrolled manner, whether capsules containing nitrate $(2 \times 490 \mathrm{mg}$ potassium nitrate tabs) alter platelet $\mathrm{NO}$ production and receptor agonist sensitivity. $\mathrm{NO}$ and nitrite concentration in saliva and plasma was analyzed using a saliva test strip and the Griess reaction respectively. Confocal microscopy was used to visualize P-selectin and NO in isolated platelets from the placebo and nitrate groups. Platelet activation was assessed by changes in surface P-selectin by Flow Cytometry. Finally, Western blotting was done for PKG and pVASP using Tubulin and VASP as loading controls respectively. MannWhitney $U$ test and t-test were done to determine $P$-value using GraphPad Prism.

Results: Females treated with placebo showed markedly increased platelet reactivity by several orders of magnitude compared to males. Ingestion of a capsular nitrate load augmented saliva and plasma nitrite/nitrate concentration by 4.8 -fold and 5.8 -fold, respectively, coincident with 2 -fold and 10 -fold platelet NO production in males and females respectively (Fig. 1). Our study showed that an oral nitrate load leads to a slight inhibition of platelet reactivity in females, and leads to an augmentation of platelet reactivity in males (Fig. 2).

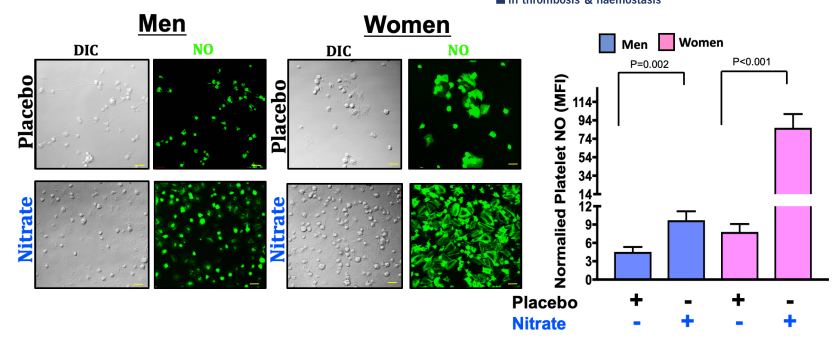

Fig 1. Platelet Nitric Oxide after an Oral Nitrate Load. Healthy subjects were randomized to placebo or nitrate capsule in a double-blinded manner. After 90 minutes, platelets were isolated, stained with a fluorescent probe, 4 -
amino-5-methylamino-2', 7'-difluorofluorescein diacetate (DAF-FM), visualized by confocal microscopy on amino-5-methylamino-2, 7 -diffluorofluorescein diacetate (DAF-FM), visualized by confocal microscopy on
fibrinogen matrix (representative images, left). In a separate experiment, platelet DAF-FM fluorescence was quantified in quadruplicate by FACS as mean $\pm S E M, n=12$ men (blue) and $n=10$ women (pink). Level of significance is noted, Mann-Whitney U test. Yellow bar $=5 \mu \mathrm{m}$. MFI = mean fluorescence intensity.
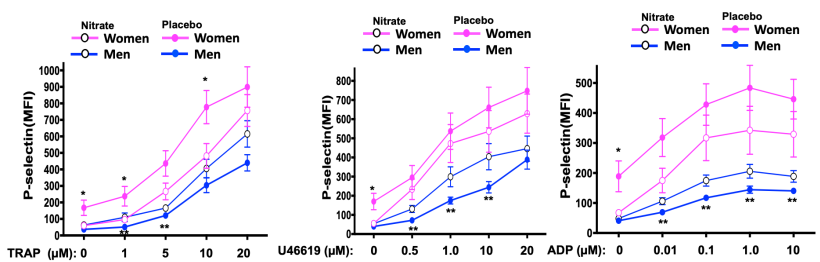

Fig. 2. Platelet Receptor Sensitivity after an Oral Nitrate Load decreases in females and increases in males. Healthy subjects were randomized to placebo or nitrate capsule in a double-blinded manner, each subject serving
as their own control. After 90 minutes, platelets were isolated and stimulated with TRAP (PAR1 agonist). U46619 (thromboxane receptor agonist), and ADP (P2Y 12 receptor agonist); then stained with a PE-tagged p-selectin antibody and quantified in quadruplicate by FACS as mean \pm SEM, $n=12$ men (blue) and $n=10$ women (pink). * $P$ $<0.05$ between female placebo groups vs. female nitrate groups and $* *<0.05$ for male placebo groups vs. male nitrate groups at the concentration shown by the Kruskal-Wallis test followed by Dunn's post test correction. MFI= mean fluorescence intensity.

FIGURE 1 Platelet Nitric Oxide after an Oral Nitrate Load

FIGURE 2 Platelet Receptor Sensitivity after an Oral Nitrate Load decreases in females and increases in males

Conclusions: The effects of an oral nitrate load differ in men and women. Women are typically under-represented in cardiovascular investigations. We suggest that platelet reactivity and thrombosis in those subscribing to a plant-based diet should be evaluated prospectively in men and women.

PB0981 | Role of Platelet's Heme Oxygenase-1 in Obese Patients with and without Type 2 Diabetes Mellitus

R. Liani ${ }^{1}$; P.G. Simeone ${ }^{1}$; R. Tripaldi ${ }^{1}$; S. Ciotti ${ }^{1}$; P. Lanuti ${ }^{1}$; G. Bologna ${ }^{1}$; A. Boccatonda ${ }^{1}$; D. D'Ardes ${ }^{1}$; V. Luca ${ }^{2}$; F. Santilli ${ }^{1}$ ${ }^{1}$ Department of Medicine and Aging, and Center for Advanced Studies and Technology (CAST), 'G. d'Annunzio' University of Chieti-Pescara, Chieti, Italy; ${ }^{2}$ Department of Drug Sciences, University of Catania, Catania, Italy

Background: Oxidative stress plays a pivotal role in the development of microvascular and cardiovascular complications. Obesity is accompanied by a state of chronic, low-grade, systemic inflammation that increases risk for cardiovascular disease (CVD) exacerbating the vascular inflammatory response. High levels of reactive oxygen species (ROS) are linked to obesity and to type 2 diabetes mellitus (T2DM). Enhanced heme turnover catalyzed by heme oxygenase 
(HO)-1, an inducible enzyme, induce anti-inflammatory, antioxidant, and anti-apoptotic functions.

Aims: To determine in a pathological context with a high oxidative stress and prothrombotic risk, as obesity and T2DM, the presence and role of HO-1 in platelet and its release in extracellular compartment.

Methods: Fifty-one obese (OB) (31 male, median age 67 years) and sixty-six non-OB patients (40 male, median age 69 years), with or without T2DM, were enrolled. Blood samples were collected at 8 am after an overnight fasting. PLT's HO-1 was assayed with ELISA kit (EnzoLife). Microvesicles (MVs) were analyzed on fresh sample with CFM. Intima-media thickness (IMT) was obtained with Doppler system (GE Healthcare). Each subject signed written informed consent, and protocol was approved (GR-2011-02350450).

Results: PLT HO-1 levels were significantly higher in non-OB vs. OB patients (all, $P=0.0089$; noT2DM, $P=0.015$ Fig. 1). PLT's MVs were significantly lower in non-OB patients (all, $P=0.017$; noT2DM, $P=0.023$, Fig. 1) and correlated inversely with $\mathrm{HO}-1$ in non-OB patients (all, rho $=-0.486, P=0.025$; non-OB NOT2DM rho $=-0.857$, $P=0.001$ Fig.1).

Indeed, HO-1 correlated inversely with IMT in non-OB patients (sx: all, rho $=-0.428, P=0.003$, noT2DM, rho $=-0.491, P=0.009 ; \mathrm{dx}$, all, rho $=-0-342 P=0.021$, noT2DM, rho $=-0.418, P=0.030$, Fig. 2).

\section{Figure 1}
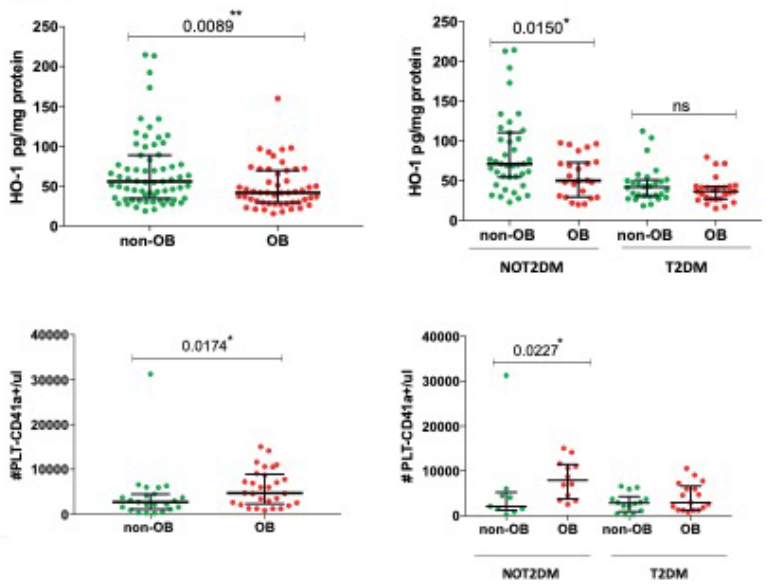

non-OB

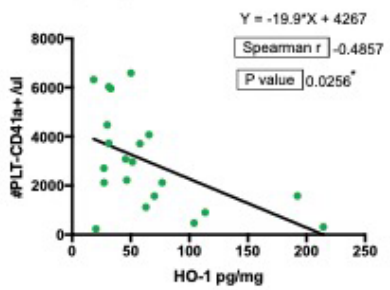

non-OB NOT2DM

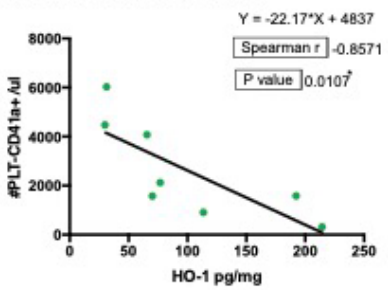

Figure 2
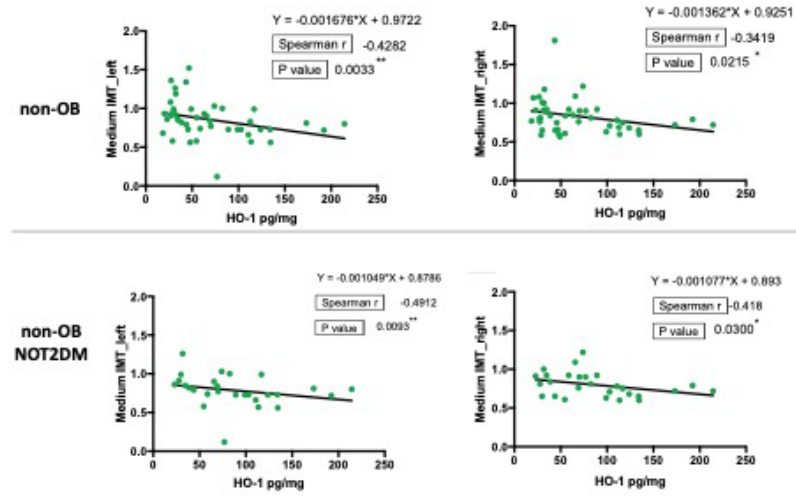

FIGURE 2 PLT's HO-1 and medium_IMT

Conclusions: PLT levels of $\mathrm{HO}-1$ appear to be closely related to the number of MVs of platelet origin, suggesting a role of $M V s$ in its extra-PLT transport. Higher PLT levels of HO-1 may reflect a lower atherothrombotic risk, to further underline its protective role in cardiovascular diseases.

PB0982 | Role of Platelet HIF-2 Alpha to Indorse Thrombogenicity through Synthesis of PAI-1 during Hypoxia

R.L. Mallick ${ }^{1} ;$ S.N. Chaurasia ${ }^{2}$; D. Dash ${ }^{2} ;$ P. Mallick ${ }^{3}$

${ }^{1}$ Kathmandu University / Birat Medical College \& Teaching Hospital, Biratnagar, Nepal; ${ }^{2}$ Banaras Hindu University / Institute of Medical Sciences, Varanasi, India; ${ }^{3}$ Nilkantha Diagnostic Laboratory \& Research Center Pvt. Ltd., Biratnagar, Nepal

Background: High altitude residents and pathologic existence of COPD exist as certain of the oxygen-compromised environments. These surroundings are associated with platelet hyperactivity. Platelets have restricted access to oxygen if they are confined within a core of aggregate. However, to sustain thrombus, they continue to perform some energy-intensive procoagulant activities. So, studying the platelet signaling in relation to mechanistic basis of thrombus stability under hypoxia is critical.

Aims: We aim to:

1. Investigate role of hypoxia-inducible factor-2 alpha (HIF-2a) in hpoxia and

2. Rule out whether hypoxia will induce synthesis of PAI-1 and shedding of extracellular vesicles.

Methods: 1. Platelet preparation and western-blot analysis,

2. Hypoxic stimulation of isolated human platelets,

3. Isolation \& analysis of platelet-derived extracellular vesicles,

4. Measurement of intracellular free calcium,

5. Analysis of platelets from COPD and individuals residing at high altitude.

Results: 1. Human platelets express HIF-2a. The exposure to either hypoxic stress of physiological agonists had augmented the expression of HIF-2a in human platelets. 

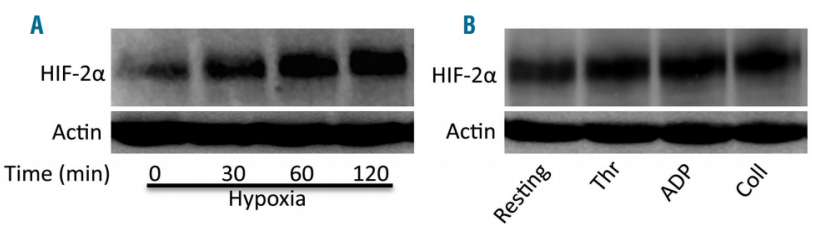

FIGURE 1 Pattern of Expression of HIF-2a in human platelets

2. Hypoxia and hypoxia-mimetics induced the shedding of extracellular vesicles and synthesis of PAI-1 in human platelets.

3. Hypoxia-mimetics induce shedding of extracellular vesicles and a rise in intracellular free calcium in human platelets.

4. Platelets from patients with COPD have higher expression of HIF-2a and PAI-1 than those from healthy counterparts.

5. Platelets from high-altitude residents have higher expression of HIF-2a and PAI-1 than those from lowlander counterparts.

Conclusions: Hypoxia stress stimulates platelets to synthesize PAI-1 and shed extracellular vesicles. Both of these contribute to prothrombotic phenotype associated with hypoxia. The hypoxia mimetics had laid to stabilization of HIF-2a and accelerates thrombus formation. In agreement of these findings, platelets from COPD and high-altitude-residents exhibited thrombotic attributes with abundant expression of HIF-2a and PAI-1. So, the approach to target hypoxia-signaling can be an effective anti-thrombotic strategy.

PB0984 | A comparison of Five Platelet Reactivity Tests in Over 3,000 Participants of the Framingham Heart Study

M.V. Chan ${ }^{1}$; M.-H. Chen ${ }^{1}$; F. Thibord ${ }^{1}$; A. Lachapelle ${ }^{1}$; J. Grech ${ }^{1}$; P.C.J. Armstrong ${ }^{2}$; T.D. Warner ${ }^{2}$; A.D. Johnson ${ }^{1}$

${ }^{1}$ National Heart, Lung and Blood Institute, The Framingham Heart Study, Framingham, United States; ${ }^{2}$ The Blizard Institute, Barts and The London School of Medicine \& Dentistry, Queen Mary University of London, London, United Kingdom

Background: In-depth platelet reactivity testing requires dedicated equipment, personnel and time. Therefore, large studies are rarely conducted and there is a paucity of studies comparing platelet assays.
Aims: In the most recent Framingham Heart Study exam, standard measures of platelet function were collected with an aim to identify correlations among these assays.

Methods: Informed consent was obtained, and the study was approved by the Boston University Medical Center institutional review board. Fasting blood was drawn from participants (self-declared European ancestry, $N=3140,46.4 \%$ male, $54.5 \pm 9.0$ years) into sodium citrate or hirudin anti-coagulant. Citrated blood was centrifuged to obtain platelet-rich plasma (PRP). Five platelet reactivity assays (Table) were performed in whole blood or PRP. Aspirin use was defined as arachidonic acid (AA) final aggregation $<40 \%$ in LTA. Correlation matrices were constructed for the platelet assays. In addition, platelet responses were ranked into quintiles and Cohen's Kappa (к) test was performed to assess the correspondence between the lowest and highest responders for each assay.

Results: Aspirin was associated with a high correlation between AA-mediated responses in LTA and Multiplate. When aspirin takers $(N=681)$ were removed, this correlation was significantly reduced. Strong intra-assay correlation was seen in all assays, particularly in Multiplate (ADP vs TRAP area under the curve [AUC], Pearson's $r=0.619)$. Moderate inter-assay correlation was observed between epinephrine AUC responses in LTA and Optimul ( $r=0.418$ ). Furthermore, female sex increased platelet reactivity in nearly all traits (e.g. Multiplate ADP AUC; $r=0.281$ ). Finally, we showed that highest $20 \%$ of responders to ristocetin were also high responders to TRAP-6 amide (LTA AUC; $\mathrm{k}=0.653$ ) and lowest responders to these agonists were also correlated $(K=0.583)$. 
TABLE 1 Platelet reactivity testing assays in the Framingham Heart Study

\begin{tabular}{|c|c|c|c|c|c|}
\hline & $\begin{array}{l}\text { Multiplate } \\
\text { impedance } \\
\text { aggregometry }\end{array}$ & $\begin{array}{l}\text { Total Thrombus } \\
\text { formation } \\
\text { Analysis System } \\
\text { (T-TAS) }\end{array}$ & Flow cytometry & $\begin{array}{l}\text { Light transmission } \\
\text { aggregometry (LTA) }\end{array}$ & Optimul aggregometry \\
\hline $\begin{array}{l}\text { Arachidonic acid } \\
\qquad \text { (AA) }\end{array}$ & $0.5 \mathrm{mM}$ & & & $1.6 \mathrm{mM}$ & $\begin{array}{c}0.03 \mathrm{mM}, 0.06 \mathrm{mM}, 0.11 \mathrm{mM} \\
0.19 \mathrm{mM}, 0.33 \mathrm{mM} \\
0.57 \mathrm{mM}, 1 \mathrm{mM}\end{array}$ \\
\hline Collagen & $0.061 \mathrm{mg} / \mathrm{mL}$ & Type I & & $0.19 \mathrm{mg} / \mathrm{mL}$ & $\begin{array}{l}0.01 \mu \mathrm{g} / \mathrm{ml}, 0.04 \mu \mathrm{g} / \mathrm{ml} \\
0.16 \mu \mathrm{g} / \mathrm{ml}, 0.62 \mu \mathrm{g} / \\
\mathrm{ml}, 2.5 \mu \mathrm{g} / \mathrm{ml}, 10 \mu \mathrm{g} / \mathrm{ml} \\
40 \mu \mathrm{g} / \mathrm{ml}\end{array}$ \\
\hline Ristocetin & $1.15 \mathrm{mg} / \mathrm{mL}$ & & & $1.5 \mathrm{mg} / \mathrm{mL}$ & $\begin{array}{l}0.14 \mathrm{mg} / \mathrm{ml}, 0.24 \mathrm{mg} / \mathrm{ml} \\
0.43 \mathrm{mg} / \mathrm{ml}, 0.75 \mathrm{mg} / \mathrm{ml} \\
1.31 \mathrm{mg} / \mathrm{ml}, 2.29 \mathrm{mg} / \mathrm{ml} \\
4 \mathrm{mg} / \mathrm{ml}\end{array}$ \\
\hline TRAP- 6 amide & $4.48 \mu \mathrm{M}$ & & & $15 \mu \mathrm{M}$ & $\begin{array}{c}0.03 \mu \mathrm{M}, 0.11 \mu \mathrm{M}, 0.36 \mu \mathrm{M}, \\
1.1 \mu \mathrm{M}, 3.79 \mu \mathrm{M}, \\
12.3 \mu \mathrm{M}, 40 \mu \mathrm{M}\end{array}$ \\
\hline U46619 & & & & & $\begin{array}{l}0.005 \mu \mathrm{M}, 0.02 \mu \mathrm{M}, \\
0.10 \mu \mathrm{M}, 0.44 \mu \mathrm{M}, \\
1.98 \mu \mathrm{M}, 8.89 \mu \mathrm{M}, \\
40 \mu \mathrm{M}\end{array}$ \\
\hline
\end{tabular}

Conclusions: Caution must be taken in extrapolating responses between assay types, even for the same agonist. The dynamics of each assay must be considered when choosing or interpreting the results of a platelet assay.

PB0985 | New Strategies for Minimization of Surface-induced Platelet Activation

T.H. Nguyen; G. Apte; L.-Y. Chen; A. Lindenbauer Institute for Bioprocessing and Analytical Measurement Techniques, Heilbad Heiligenstadt, Germany

Background: Platelets have a strong tendency to be activated when they contact non-physiological and artificial surfaces. Minimization of surface-induced platelet activation is important for many biomedical applications such as in vivo-performance, platelet storage, and acceptance of an implant. However, inhibition of platelet-surface activation is challenging, and to date, controversies and open questions in this field still remain.

Aims: To minimize surface-induced platelet activation by i) modifying contact surface with bio-polymers, and ii) nanopatterning the underneath surface before seeding platelets.
Methods: Nanopatterns were fabricated using electron-beam lithography and FluidFM based atomic force microscopy (AFM). Characteristics of the surfaces were investigated using contact angle measurements while the stiffness of the gel was determined by AFM nanoindentation. Adhesion forces between single platelets and fabricated surfaces were determined by single-platelet force spectroscopy-based AFM. Platelet morphologies on surfaces were obtained by confocal laser microscopy and scanning electron microscopy (SEM). The geometry of nanogroove patterns was imaged with AFM and SEM. Platelet aggregometry was used to determine the effect of polymers on platelet aggregation.

Results: Both laminin and collagen-G gels formed on the glass surface reduced platelet activation. However, laminin showed a slower activation rate than collagen-G. The formation of stable and inert agarose hydrogel films and a mixture of agarose with nanoparticles effectively minimized surface-induced platelet activation even after a long time of storage. Nanopatterns together with laminin coating also strongly reduced platelet-surface adhesion and activation. Particularly, laminin-coated $100 \mathrm{~nm}$ groove patterns inhibited platelet activation better than the $500 \mathrm{~nm}$ size. The adhesion force between single platelets and these surfaces reduced strongly as compared with non-coated and non-patterned surfaces. The alteration of factors including adhesion force, topography, wettability, 
stiffness, swelling, and surface chemistry directly influence platelet morphology.

Conclusions: Surface-induced platelet activation can be minimized by seeding platelets on i) agarose hydrogel films, and ii) laminincoated nanopatterns.

\section{PB0986 | Role of Platelet GARP in TGF $\beta$ Activation}

J. Bodart $^{1}$; C. Dufeys ${ }^{1}$; Y. Senis ${ }^{2}$; Z. Nagy ${ }^{3}$; L. Bertrand ${ }^{1}$;

C. Beauloye ${ }^{1,4}$; S. Lucas ${ }^{5}$; S. Horman ${ }^{1}$

${ }^{1}$ Université Catholique de Louvain (UCLouvain), Institut de Recherche

Expérimentale et Clinique (IREC), Pôle de Recherche Cardiovasculaire

(CARD), Brussels, Belgium; ${ }^{2}$ Université de Strasbourg, Institut National de la Santé et de la Recherche Médicale (INSERM), Etablissement Français du Sang Grand Est, Unité Mixte de Recherche (UMR)-S 1255, Strasbourg, France; ${ }^{3}$ University of Würzburg, Institute of Experimental Biomedicine, University Hospital and Rudolf Virchow Center, Würzburg, Germany; ${ }^{4}$ Cliniques Universitaires Saint-Luc, Division of Cardiology, Brussels, Belgium; ${ }^{5}$ Université Catholique de Louvain (UCLouvain), Institut de Duve, Pôle de Recherche Génétique et Cellulaire (GECE), Brussels, Belgium

Background: Transforming growth factor (TGF) $\beta$ is known to be a central player in the control of cardiac fibroblast properties and fibrosis. However, cellular and molecular mechanisms that trigger its activation remain poorly understood. Platelets are considered as a major source of TGF $\beta$ and recent evidence suggest that they are involved in TGF $\beta$ activation via Glycoprotein A Repetitions Predominant (GARP) present on their surface.

Aims: The present study sought to evaluate the role of platelet GARP in TGF $\beta$ activation using platelet specific GARP knockout mice.

Methods: We generated a new Cre transgenic mouse strain that allowed Megakaryocyte/platelet specific invalidation of GARP (GplbaCre $\times \mathrm{GARPfl} / \mathrm{fl}$ ). The impact of GARP deficiency on platelet function was measured in vitro by flow cytometry using thrombin and CRP. Serum production of total and active TGF $\beta$ was assessed by ELISA.

Results: Platelet count and other hematological parameters were normal in platelet specific GARP knockout mice, except platelet volume, which was increased by $10.3 \%$, as compared to wild-type platelets. Stimulation by thrombin and CRP increased GARP exposure at platelet surface. However, platelets without GARP displayed normal agonist induced activation, as reflected by CD62P and allb $\beta 3$ exposure. Interestingly, the generation of active TGF $\beta$ was drastically impaired in the serum of platelet specific GARP knockout mice, while the amount of total TGF $\beta$ was not affected.

Conclusions: We provided evidence that platelet GARP is a crucia contributor to the systemic activation of TGFß. Future work will aim to determine its role in cardiac fibroblast myodifferentiation and fibrosis.
PB0987 | PI4P and PI(4,5)P2 Immunofluorescence Staining Optimization in Human Platelets

A. Bura; I. Đurić; S. Čabrijan; A. Jurak Begonja

Department of Biotechnology, University of Rijeka, Rijeka, Croatia

Background: Phosphatidylinositol-4-phosphate (PI4P) is a phosphoinositide found mostly at the Golgi apparatus and partially at the plasma membrane (PM). At the PM, PI4P can contribute to the polyanionic lipid pool or can serve for the formation of phosphatidylinositol-4,5bisphosphate $\left[\mathrm{PI}(4,5) \mathrm{P}_{2}\right] . \mathrm{PI}(4,5) \mathrm{P}_{2}$ is primarily localized at the $\mathrm{PM}$ where it regulates actin reorganization. The staining method utilizing antibodies for visualizing these phosphoinositides on the PM has been well established in HeLa cells (Hammond et al 2009. Biochem J) but not in blood cells such as human platelets (hPLTs). When using the established protocol, we observed that the majority of activated hPLTs did not stain while the resting hPLTs and control HEK293T cells displayed phosphoinositide staining.

Aims: We tested if changes in the percentage of the permeabilizing agent, the time of the permeabilization, or different permeabilizing agents would improve the success rate of the phosphoinositide staining in hPLTs.

Methods: hPLTs were fixed as rested or spread on glass, permeabilized with different permeabilizing agents (triton, tween, digitonin, saponin) for different times (5, 30, 45 minutes, and one hour) and immunostained for $\mathrm{PI} 4 \mathrm{P}$ or $\mathrm{PI}(4,5) \mathrm{P}_{2}$. HEK293T cells were used as controls.

Results: hPLTs showed the best staining results for PI4P and $\mathrm{PI}(4,5)$ $P_{2}$ when permeabilized for a shorter period (5 min) with $0,5 \%$ saponin while unpermeabilized cells did not exhibit any staining. In contrast, HEK293T cells were stained in all tested conditions. PI4P and $\mathrm{PI}(4,5) \mathrm{P}_{2}$ were confined to the $\mathrm{PM}$, and the phosphoinositide signals could be modulated with specific inhibitors of PI4 kinase and $\mathrm{PI}(4,5)$ $\mathrm{P}_{2}$ phosphatase.

Conclusions: Our data suggest that PI4P and $\mathrm{PI}(4,5) \mathrm{P}_{2}$ at the $\mathrm{PM}$ of hPLTs are more sensitive to extraction by permeabilizing agents than in HEK293T cells when PLTs undergo spreading, but not in their resting state. Further studies are underway to investigate the exact role of these lipids in platelet activation.

PB0988 | Prototyping of Physiological Stenosis with 2-photon Lithography for Thrombosis Studies

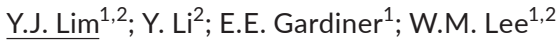

${ }^{1}$ The Australian National University, ACRF Department of Cancer Biology and Therapeutics, Canberra, Australia; ${ }^{2}$ The Australian National University, ACRF INCITe Centre - ANU Node, Canberra, Australia

Background: The rheology within a stenotic vessel contributes to the geometry and stability of a thrombus after vascular injury. Microfluidics provide an experimental platform to simulate this 
microenvironment in vitro, but standard chamber fabrication methods (soft lithography, 3D-printing) require multiple steps or do not achieve submicron resolution. Multiphoton lithography can create stenosis of variable geometries within microchannels, while simultaneously enabling volumetric imaging of thrombus formation at the seconds time scale. We can assess how non-uniform surface shear impacts on localized thrombus growth and stability.

Aims: Using 2-photon lithography we create stenotic geometries and evaluate how they modulate thrombosis kinetics.

Methods: 2-Photon lithography was used to generate stenosis from NOA81 optical adhesive within $200 \mu m$-diameter circular glass capillary tubes. Tubes were coated with $100 \mu \mathrm{g} / \mathrm{mL}$ collagen, then citrated whole blood with AlexaFluor 594-conjugated anti-CD42a antibody to label platelets was flowed at $1800 \mathrm{~s}^{-1} / 10 \mathrm{~min}$, followed by phosphate-buffered saline for 10 min using a syringe pump. Using a custom-built 2-photon microscope to image at 20 frames/volume/ $\mathrm{sec}$, total thrombus volume was quantified based on anti-CD42a fluorescence. The geometry of each stenosis and final thrombus volume were verified by digital holographic microscopy (DHM).

Results: DHM imaging of capillaries demonstrated that 2-photon lithography produced 3D stenosis within capillaries of $40 \times 30 \times 40 \mu \mathrm{m}$, $(\mathrm{L} \times \mathrm{W} \times \mathrm{H}, \mathrm{Fig} 1 \mathrm{~A})$. Preliminary results demonstrate thrombus volume increased by 17 -fold and peaked at $9 \mathrm{~min}$ in the presence of $20 \%$ stenosis compared to 7 -fold at $7 \mathrm{~min}$ without stenosis. However, flow-through of PBS resulted in similar loss of thrombus volume in the absence of stenosis ( $40 \%$ loss) compared to the stenotic microchannel (42\%, Fig2). The mechanisms underlying volume loss/gain are being actively explored.

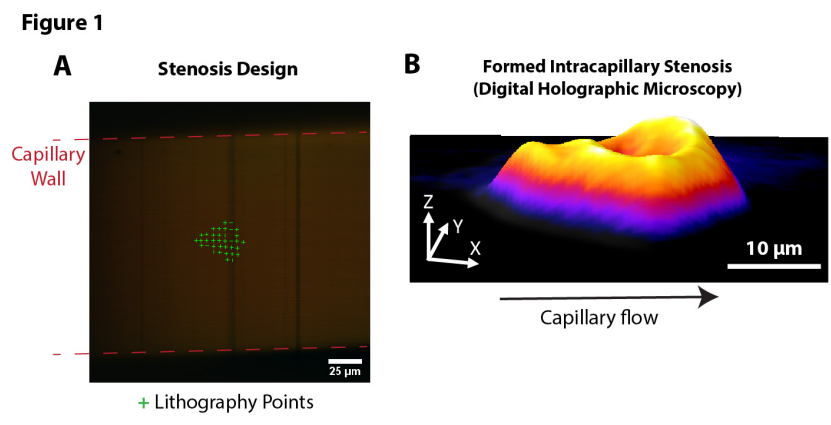

FIGURE 1 Design and intracapillary fabrication of stenosis by 2-photon lithography

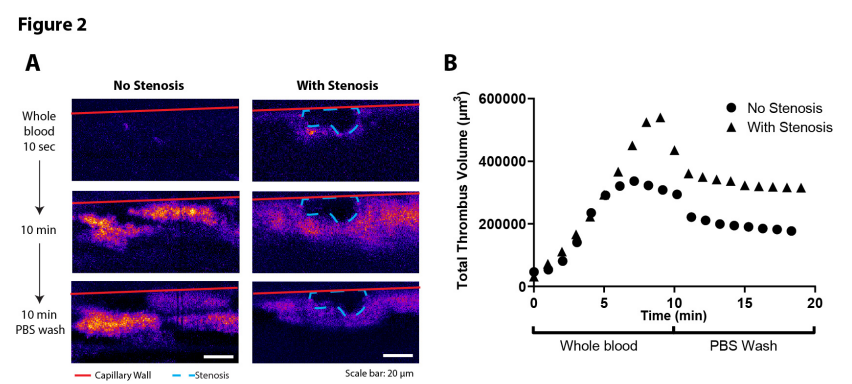

FIGURE 2 2-photon volumetric imaging and quantification of thrombus growth and loss in a capillary with or without stenosis

Conclusions: 2-photon lithography can be paired with multiphoton volumetric imaging to build and assess the impact of stenosis within microchannels on thrombus growth and stability. Stenosis increases duration and volume of thrombus growth.

PB0989 | Assessment of the Effects of Syk and BTK Inhibitors on GPVI-mediated Platelet Signaling and Function

T. Zheng ${ }^{1}$; E. Lofurno ${ }^{2}$; A. Melrose ${ }^{1}$; H. Lakshmanan ${ }^{1}$; J. Pang ${ }^{1}$; K. Phillips ${ }^{3}$; M. Fallon ${ }^{1}$; T. Kohs ${ }^{1}$; A. Ngo ${ }^{1}$; J. Shatzel ${ }^{1}$; M. Hinds ${ }^{1}$; O. McCarty ${ }^{1}$; J. Aslan ${ }^{1}$

${ }^{1}$ Oregon Health \& Science University, Portland, United States; ${ }^{2}$ Oregon State University, Corvallis, United States; ${ }^{3}$ Convergent Genomics, South San Francisco, United States

Background: Spleen tyrosine kinase (Syk) and Bruton's tyrosine kinase (BTK) play critical roles in platelet physiology, facilitating GPVImediated platelet signaling. Small molecule tyrosine kinase inhibitors (TKIs) targeting Syk and BTK have been developed as anti-neoplastic and anti-inflammatory therapeutics, and have also gained interest as anti-platelet agents. However, no studies to date have systematically examined multiple Syk and BTK inhibitors simultaneously to uncover and compare the mechanisms by which these agents affect platelet signaling and function.

Aims: Determine the effects of 12 TKIs (Bay 61-3606, R406/fostamatinib, entospletinib, TAK-659, ibrutinib, acalabrutinib, ONO4059/tirabrutinib, AVL-292/spebrutinib, CG-806, BMS-935177, BMS-986195, fenebrutinib) on platelet functional responses.

Methods: Platelets were isolated from human donors' whole blood and treated with the selected TKIs. Platelets were stimulated and assessed for P-selectin exposure, PAC-1 binding, ATP secretion, adhesion on collagen and fibrinogen surfaces under static and physiological flow conditions, protein phosphorylation levels, and PI3K/ tubulin colocalization to evaluate the effect of each inhibitor on platelet function.

Results: The selected TKIs significantly reduced GPVI-mediated platelet adhesion on collagen (27-70\% reduction) and integrinmediated platelet spreading on fibrinogen (7-45\% reduction in average surface area). Under physiological flow, platelet aggregate volume was reduced by $32-51 \%$ on collagen surfaces when treated 
with selected TKIs. Downstream of GPVI-mediated activation, selected TKIs reduced platelet ATP secretion (53-67\% reduction), the percentage of platelets exposing P-selectin (to 1.37-6.64\%), and percentage of platelets binding PAC-1 (to 0.91-9.09\%) in response to GPVI agonist, cross-linked collagen-related peptide (CRP-XL) activation. All TKIs inhibited GPVI-mediated phosphorylation of PLC $\gamma 2, P K C \delta$ and Akt, but differentially affected the phosphorylation of PI3K p85 regulatory subunit. Fluorescence imaging of PI3K found co-localization with tubulin when untreated, but is disrupted in platelets treated with the selected TKIs.

Conclusions: Clinically relevant TKIs targeting Syk and BTK inhibit platelet functional responses, but may differentially alter PI3K signaling and organization in an inhibitor class specific manner.

\section{PB0990 | Lyophilized Human Platelets Interact with Fresh Platelets to Promote Hemostasis Under Shear in vitro}

B. Ishler $^{1}$; A. Lee ${ }^{1}$; S. Torramadé-Moix² ; S. Samambar ${ }^{2}$; M. DiazRicart $^{2}$; G. Escolar ${ }^{2}$; K. Moskowitz ${ }^{1}$

${ }^{1}$ Cellphire, Inc., Rockville, United States; ${ }^{2}$ Hematopathology, Pathology Department, CDB, Hospital Clinic Barcelona, IDIBAPS, University of Barcelona, Barcelona, Spain

Background: Lyophilized human platelets (LHP) are a stabilized platelet derived hemostatic agent under clinical development as Thrombosomes ${ }^{\circledR}$ for treatment of bleeding secondary to thrombocytopenia.

Aims: To evaluate mechanisms of LHP under shear and the potential role of interactions with fresh platelets during thrombus formation.

Methods: Fibrinogen binding to LHP GPIIb/IIla was assessed by flow cytometry. LHP thrombus formation under shear was quantified with the Total Thrombus-formation Analysis System (T-TAS) using microcapillary channels coated with collagen and tissue factor. Thrombus formation was assessed in plasma before and after the addition of LHP $\left(375 \times 10^{3} / \mu \mathrm{L}\right)$. Whole LMWH-anticoagulated blood at $75 \times 10^{3}$ platelets $/ \mu \mathrm{L}$ was perfused over collagen and tissue factor coated microfluidic channels with and without LHP. Triple-color confocal microscopy imaging was applied to differentiate platelets from LHP and assess adherent cells and fibrin interactions. Association of LHP and fresh platelets was assessed by aggregometry, and fluorescent microscopy was performed on mixed samples with(out) GPIIb/ Illa antagonism.

Results: LHP had a 37-fold increase in binding of the anti-fibrinogen antibody 9F9 relative to fresh resting platelets. Citrated plasma alone did not occlude on T-TAS. When LHP were added to plasma at a $375 \times 10^{3}$ particles $/ \mu \mathrm{L}$, the system reached total occlusion at approximately 12 minutes. Confocal microscopy showed minimal platelet or fibrin deposition under mild thrombocytopenic conditions. Addition of LHP promoted total LHP, platelet, and enhanced fibrin deposition approaching that of native whole blood. LHP and fresh platelets formed mixed aggregates as measured by aggregometry. Treatment of the fresh platelets with the GPIIb/IIla inhibiting peptide RGDS reduced aggregates by $>50 \%$.

Conclusions: LHP adhere to collagen and interact with fresh platelets under shear, likely mediated by LHP surface fibrinogen. LHP further enables hemostasis in thrombocytopenic blood. LHP may thus function as a hemostatic agent by adhering to sites of injury leading to secondary interactions with circulating platelets and final fibrin formation.

PB0991 | Iron Repletion Decreases Platelet Count in Iron Deficient Premenopausal Women

B. Elstrott ${ }^{1}$; H.H.S. Lakshmanan ${ }^{2}$; J. Aslan²; J.J. Shatzel ${ }^{2,3}$

${ }^{1}$ Oregon Health \& Science University, Portland, United States;

${ }^{2}$ Department of Biomedical Engineering, OHSU, Portland, United

States; ${ }^{3}$ Department of Hematology-Oncology, Knight Cancer Institute, OHSU, Portland, United States

Background: Iron deficiency is associated with anemia, and somewhat paradoxically, thrombocytosis. Prior studies have demonstrated platelet count reduction in patients treated with oral iron and patients with chronic kidney disease treated with intravenous (IV) iron, but characterization in premenopausal women receiving IV iron replacement (the most prevalent iron deficient population) is lacking.

Aims: To investigate the frequency of thrombocytosis in iron deficient premenopausal women, and whether correction of iron deficiency alters platelet count and other blood indices.

Methods: A multicenter retrospective cohort study was performed for 231 women between 18 and 40 years of age receiving intravenous ferumoxytol or low-molecular weight iron dextran for iron deficiency (ferritin $<20 \mu \mathrm{g} / \mathrm{L}$ ). Only women who achieved a ferritin $\geq 50 \mu \mathrm{g} / \mathrm{L}$ at follow up were included. Pregnant patients and those with platelet disorders, hematologic malignancy, or receiving antineoplastic chemotherapy were excluded. Thrombocytosis was defined as platelet count $>400 \mathrm{~K} / \mathrm{mm} 3$. Pre- and post-infusion platelet counts were compared by two-sided paired t-test.

Results: Heavy menstrual bleeding was the most prevalent documented cause of iron deficiency in the cohort (50.2\%). The mean ferritin pre and post-infusion was $11.1 \mathrm{ng} / \mathrm{ml}$ (range: $2-19$ ) and $133.2 \mathrm{ng} /$ $\mathrm{ml}$ (52-531) respectively. The mean platelet count was $288( \pm 97) \mathrm{K} /$ mm3 pre-infusion and $261( \pm 84) \mathrm{K} / \mathrm{mm} 3$ post-infusion (Figure 1). Thrombocytosis was present in 18 (7.8\%) patients at pre-infusion and $7(3.0 \%)$ patients at post-infusion. The mean change in platelet count from pre-infusion to post-infusion was $-26.5 \mathrm{~K} / \mathrm{mm} 3(95 \%$ $\mathrm{Cl}$ - $32.1,-20.9, P<0.001)$. While $\mathrm{Hgb}$, Hct, and red cell count were significantly different post-infusion, no changes in white blood cell count or mean platelet volume were observed. 
Platelet Count Before and After IV Iron Therapy

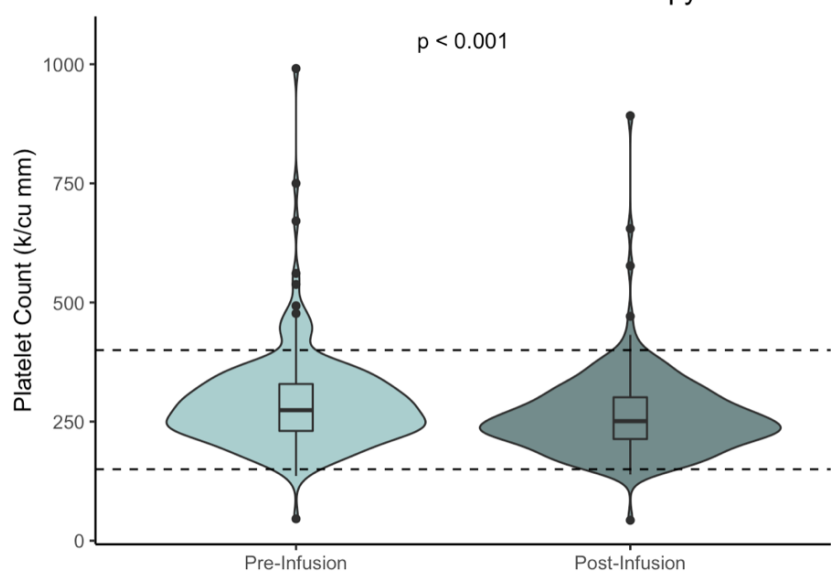

FIGURE 1

Conclusions: Thrombocytosis was present but uncommon in premenopausal women with iron deficiency and generally corrected with iron repletion. A small but significant decrease in platelet counts occurred after IV iron in this population, however changes in platelet volume and white cell counts were not observed.

PB0992 | Desipramine Reduces the Exposure of Phosphatidylserine on the Surface of Human Platelets, but Does Not Inhibit Platelet TF-induced Procoagulant Activity

O. Panes; M.F. Becerra; D. Mezzano

P. Universidad Católica de Chile, School of Medicine, Department of Hematology-Oncology, Santiago, Chile

Background: A recent report (J Wang et al. Blood 2019; 134:645) showed that sphingomyelin (SM), an abundant phospholipid on the outer leaflet of cell membranes, inhibits the activation of TF in mouse monocytes. In fact, blockade of acidic sphingomyelinase (AsMase) by desipramine and imipramine attenuated the LPS-induced procoagulant activity of TF without affecting de novo synthesis of the protein. We have previously shown that human platelets synthesize and contain functional TF, which releases its activity after ristocetininduced VWF(VWF-R)-GPIba binding.

Aims: Now, we examine the effect of desipramine on human platelet procoagulant activity induced by VWR-R-GPIba activation.

Methods: Control PRP platelets and PRP pre-incubated (30 min, $37^{\circ} \mathrm{C}$ ) with $10 \mu \mathrm{M}$ desipramine were stimulated with Ristocetin or TRAP. We measured light transmission platelet aggregation, serotonin secretion and serotonin content (HPLC), P-selectin secretion and Annexin $\mathrm{V}$ binding (FC). The PCA was assessed with Factor Xa generation (fluorometric tenase assay) and thrombin generation in PRP without including TF in the reaction solutions (Thrombinoscope).

Results: Desipramine induced a significant decrease in phosphatidylserine (PS) expression (Annexin V binding) in VWF-R-activated platelets (mean \pm SD of $7.5 \pm 4.7$ to $4.7 \pm 3.3 \%$ of labeled platelets, $P<0.03)$, but not in TRAP-stimulated platelets $(5.4 \pm 5.5$ to $4.8 \pm 3.7$
$\%$ of labeled platelets, p:NS); however, this change was not accompanied with concomitant reductions of FXa and thrombin generation (Endogenous Thrombin Potential and Velocity Index). Similarly, desipramine induced no significant changes in platelet aggregation, secretion of serotonin and P-selectin and platelet serotonin content. Conclusions: Desipramine, an inhibitor of ASMase, decreases PS exposure without affecting GPIba-TF-FVIla clotting pathway in human platelets, contrasting with the observations in mice macrophages. These initial findings suggest that PS translocation would not be determinant in triggering platelet TF-dependent PCA.

PB0993 | Effect of Antiplatelet Therapy on Monocyte-Platelet Aggregates

C.C. Rolling ${ }^{1}$; K. Myndzarr ${ }^{1}$; H. El Bannoudi ${ }^{1}$; T. Schwartz ${ }^{1}$; T.J. Barrett ${ }^{1}$; J.S. Berger ${ }^{1,2}$

${ }^{1}$ Department of Medicine, New York University Grossman School of Medicine, New York City, United States; ${ }^{2}$ Department of Surgery, New York University Langone Health, New York City, United States

Background: In addition to their role in thrombosis and hemostasis, platelets are key mediators of inflammation and altered immunity. Circulating monocyte-platelet aggregates (MPA) represent the crossroads between thrombosis and inflammation and may represent a therapeutic target. While antiplatelet therapy (APT) reduces platelet activity and thrombosis, its effect on MPA is uncertain.

Aims: To analyze the effect of APT on MPA in vitro.

Methods: The effect of different platelet-activating agonists (thromboxane analog U-46619, ADP, PAR4, collagen, and epinephrine) on MPA formation in whole blood (WB) was measured via flow cytometry. Agonist-stimulated WB was incubated in the presence of inhibitors against P-selectin, PSGL-1, PAR1 (ML161), P2Y12 (AZD1283), GPIIb/IIla (eptifibatide), acetyl salicylic acid (ASA), and dipyridamole and assessed for MPA formation. RNA-Seq data sets of monocytes incubated with healthy platelet releasates (PR) were used to identify platelet-induced upregulation of monocyte transcripts and were validated by RT-qPCR in monocyte-PR co-incubation assays in the presence of APT.

Results: Circulating MPA are increased in prothrombotic and inflammatory diseases including the most recent COVID-19. Monocytes aggregated to platelets have more CD40 and tissue factor expression than monocytes not aggregated to platelets $(P<0.05$ for each comparison). As expected, targeting P-selectin (85.4\% reduction) and PSGL-1 (88.2\% reduction) had the greatest attenuation of MPA. Among platelet inhibitors, $\mathrm{P} 2 \mathrm{Y} 12$ inhibition was most effective in lowering MPA formation (30.7\% reduction) (figure 1). 


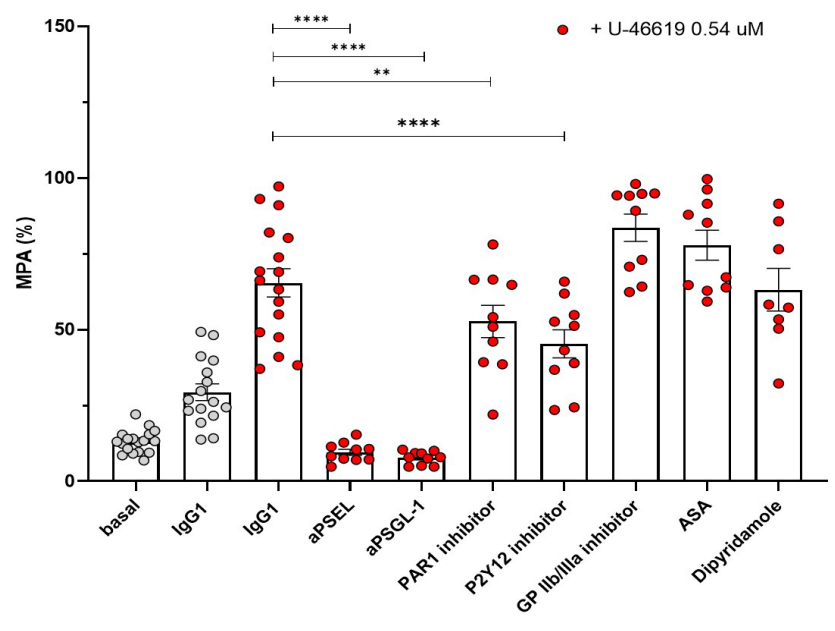

FIGURE 1 APT effect on MPA formation in whole blood

Flow cytometry analysis of MPA.

Incubation of monocytes with platelet releasate induced upregulation of inflammatory mRNA transcripts suppressor of cytokine signaling 3 (SOCS3) and oncostatin $m$ (OSM). Following pretreatment of platelets with APT, both GPIIb/IIla and P2Y12 inhibition was associated with lower expression of SOCS3 and OSM (figure 2).
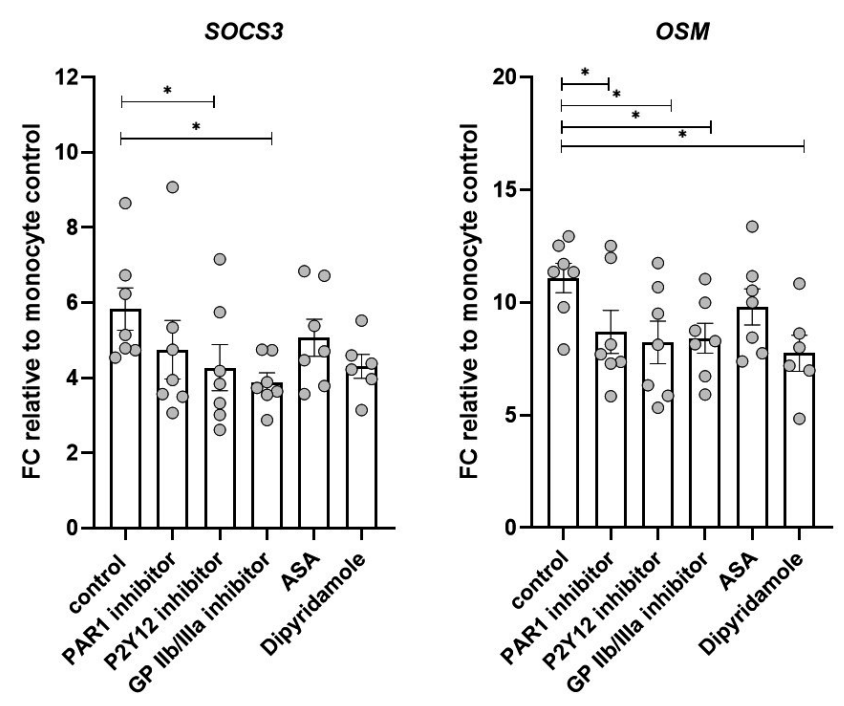

FIGURE 2 Effect of APT on PR-induced inflammatory transcripts in monocytes

SOCS3 and OSM in monocytes incubated with APT-treated PR.

Conclusions: Circulating MPA represent a crossroad of platelet and monocyte activation. We show that APT is associated with both reduced MPA formation and platelet-induced monocyte activation.
PB0994 | Stripping a Platelet "Sugar Coat" by Shear: Shearmediated Platelet Desialylation Promotes Reduction in Platelet Count and Increased Microvesiculation

Y. Roka-Moiia $^{1}$; S. Miller-Gutierrez ${ }^{1}$; J.E. Italiano ${ }^{2}$; M.J. Slepian ${ }^{1}$

${ }^{1}$ Sarver Heart Center, University of Arizona, Tucson, United States;

${ }^{2}$ Brigham and Woman's Hospital, Harvard Medical School, Boston, United States

Background: Mechanical circulatory support (MCS) is vital for patients with advanced heart failure. Yet, long-term MCS is associated with bleeding coagulopathy, felt to be driven by more than anticoagulant excess. Due to undefined etiology, device-related bleeding lacks efficient therapeutic management. We showed that MCS-generated hypershear causes platelet dysfunction via downregulation of adhesion receptors, impairing aggregation, promoting pro-apoptosis, and microvesiculation - all contributors to bleeding. As recently recognized, glycosylation of platelet surface receptors, i.e. platelet "sugar coat," plays a major role in regulation of platelet function and lifespan.

Aims: We tested the hypothesis that hypershear stress promotes platelet desialylation thus facilitating microvesiculation and platelet count reduction.

Methods: Gel-filtered human platelets were exposed to neuraminidase-1, continuous hypershear (70 dynes $/ \mathrm{cm}^{2}, 10$ '), or both; optionally platelets were pre-treated with oseltamivir (neuraminidase inhibitor) prior to shear exposure. Platelets were co-stained with fluorophore-conjugated anti-CD42a, SNA and MAL lectins binding 2,6- and 2,3-linked sialic acids; flow cytometry was performed to quantify platelet surface sialylation, platelets, and microparticles. Fluorescent nanobeads SPHERO ${ }^{\mathrm{TM}}$ were used as a size standard.

Results: Platelet exposure to hypershear stress and sialidase induced platelet surface desialylation as indicated by significant decrease of SNA and MAL lectins' binding (Fig.1A-B); MAL binding indicating 2,3-linked sialic acids was mainly affected by both shear stress and neuraminidase-1. Platelet desialylation induced by shear stress and neuraminidase was associated with a substantial decrease in platelet count and increase in microparticles (Fig.1C-D). Neuraminidase reinforced shear-mediated microvesiculation, but not platelet count drop. Oseltamivir slightly inhibited shear-mediated desialylation, preserved platelet count (30\% increase), and decreased microparticle generation (29\% decrease) (Fig.1A-D). 
A

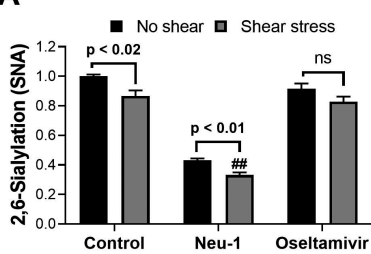

C

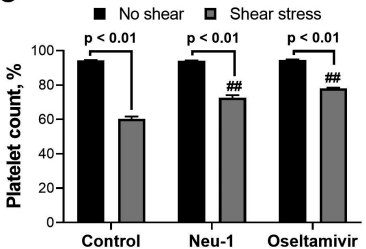

B

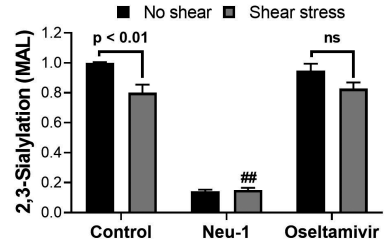

D

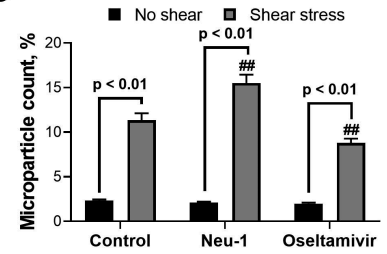

FIGURE 1 Exposure to hypershear stress and neuraminidase-1 (0.1 $\mathrm{mU} / \mathrm{mL}$ ) promotes platelet desialylation. Platelet desialylation is associated with the decrease of platelet count and intensification of platelet microvesiculation. Neuraminidase inhibition by oseltamivir partially preserves platelet sialylation, prevents platelet count drop, and decreases microvesiculation mediated by hypershear. A, $B$ - platelet surface sialylation as indicated by SNA and MAL lectins, binding to 2,6- and 2,3-linked sialic acid moieties respectively; C, D - the number of platelets and platelet-derived microparticles. $N=6$, Mean \pm SEM, one-way ANOVA followed by Sidak's multiple comparisons test: ${ }^{*}$, \#\# - $P<0.01$ as compared with non-sheared and sheared control, respectively

Conclusions: Shear stress induces platelet surface desialylation with associated microvesiculation and reduction in platelet count. Neuraminidase inhibition restores platelet count and decreases microparticle generation caused by hypershear. Developing therapeutic strategies for preservation of platelet sialylation offers significant translational potential for pharmacologic management of MCS-related platelet dysfunction, coagulopathy, and bleeding complications.

PB0995 | Inhibition of ADP and Thromboxane A2 Production Results in Decreased Global Platelet Contraction, but Thromboxane A2 Inhibition Plays a Greater Role in Limiting Local Platelet Contraction

\section{K. Trigani; S. Diamond}

University of Pennsylvania, Philadelphia, United States

Background: Platelet contractility plays a crucial role in clot contraction to provide rigidity and stability to thrombi. Clot contraction has been studied extensively in static conditions, but there are fewer studies that evaluate how shear flow can affect platelet contraction. In particular, there have been limited studies evaluating the role of secondary platelet aggregation on platelet contraction under flow.

Aims: Here, we wanted to evaluate how inhibition ADP and thromboxane A2 (TXA2) would affect clot contraction.

Methods: We ran PPACK-treated whole blood \pm acetylsalicylic acid (ASA), 2-MethylthioAMP (2-MeSAMP), and/or MRS-2179 over collagen (100 s-1) for 7.5 minutes, then stopped flow to observe contraction for 7.5 minutes. We developed two automated methods of quantifying platelet contraction: (1) a one dimensional, "global" measurement of clot length in the direction of flow, and (2) a two dimensional, "local" measurement of the change in area coverage of platelets in a clot.

Results:
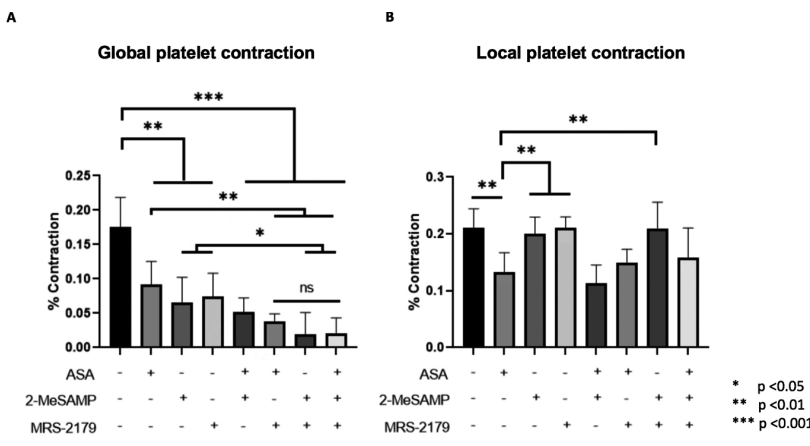

Figure 1: Global platelet contraction is reduced by both ADP and TXA2 inhibition, while local platelet contraction is only reduced by TXA2 inhibition. (A) Global platelet contraction was significantly reduced by inhibition of ADP or TXA2, and was further reduced by the combinations of ADP and TXA2 inhibitors. (B) Local platelet contraction was significantly reduced by inhibition of TXA2, while there were no significant differences in local platelet contraction between the control and conditions with only ADP inhibition.

FIGURE 1 Global platelet contraction is reduced by both ADP and TXA2 inhibition, while local platelet contraction is only reduced by TXA2 inhibition

We found that platelet fluorescence intensity (FI) decreased and global platelet contraction was inhibited when ASA, 2-MeSAMP, or MRS-2179 were added to inhibit TXA2 or ADP production. We observed a correlation between platelet $\mathrm{FI}$ and global platelet contraction $(R 2=0.72)$. Unlike global platelet contraction, local platelet contraction was more pronounced across all conditions; however, we observed that in conditions with ASA, there was significantly reduced local platelet contraction relative to conditions without ASA. We also evaluated P-selectin FI to determine how highly activated platelets were affected by ADP and TXA2 inhibition. P-selectin FI was significantly reduced by ADP and TXA2 inhibition. There was limited global and local contraction in P-selectin+ platelets across all conditions.

Conclusions: Our results demonstrate that global platelet contraction is inhibited by ASA, 2-MeSAMP, and MRS-2179, while ASA has a more pronounced inhibitory effect on local platelet contraction. These results are significant in understanding how different platelet antagonists affect clot contraction and ultimately clot resolution. 
PB0996 | The Proteasome Inhibitor, Bortezomib Induces Apoptosis and Activation in Gel Filtered Human Platelets

$\underline{\text { H. Ghansah }}^{1}$; I. Beke Debreceni ${ }^{2}$; G. Szabó ${ }^{2}$; J. Kappelmayer ${ }^{2}$

${ }^{1}$ Department of Laboratory Medicine, Faculty of Medicine, University of Debrecen,, Debrecen, Hungary; ${ }^{2}$ Department of Laboratory Medicine, Faculty of Medicine, University of Debrecen, Debrecen, Hungary

Background: Bortezomib has been approved for clinical use as a first-line treatment for newly diagnosed multiple myeloma, and for treating relapsed/refractory cases. Thrombocytopenia is a common adverse effect of bortezomib and is mostly thought to be associated with the inhibition of proplatelet formation of megakaryocytes.

Aims: We investigated the effect of bortezomib on platelet apoptotic processes, activation, and subsequent thrombin generation.

Methods: In human gel filtered platelets (GFP), mitochondrial inner membrane potential depolarization and platelet phosphatidylserine(PS) expression were determined by flow cytometry using DiOC6(3) and annexin V-FITC respectively. In both series of experiments, platelets were preincubated with bortezomib, or thrombin and DMSO as positive and negative controls respectively. Thrombin generation was initiated with $1 \mathrm{pM}$ recombinant tissue factor after resuspension of the GFPin autologous platelet poor plasma.

Results: In each of the bortezomib treated groups ( $26 \mathrm{nM}, 260 \mathrm{nM}$ and $2.6 \mu \mathrm{M}$ ) there was a significant increase in the percentage depolarized cells at 15 and 60 minutes. PS expression was also significantly increased in each of the bortezomib treated groups at both time points from $6 \%$ to $40 \%(P=0.0002)$. In the thrombin generation assay, Peak thrombin and Velocity index increased significantly $(P<0.01$ for both parameters) whereas Time to peak and Start Tail values were decreased $(P<0.01)$ in all the bortezomib pretreated groups. We observed no PS expression after bortezomib treatment when platelet rich plasma was used instead of GFP. To demonstrate whether the effect of bortezomib on platelets is neutralized by plasma proteins in vitro, GFP were incubated with $40 \mathrm{mg} / \mathrm{mL}$ human serum albumin, which completely abolished the bortezomib effect on PS expression.

Conclusions: Bortezomib induces platelet apoptotic processes, activation, and subsequent thrombin generation, however these effects can only be studied on gel filtered platelets.
PB0998 | Antiplatelet Activity Produced by Chloroacilhidroquinones via Inhibition of the Mitochondrial Bioenergy

E. Fuentes $^{1}$; D. Méndez ${ }^{1}$; I. Palomo ${ }^{1}$; M. Alarcón ${ }^{1}$; F.A. Urra ${ }^{2}$; A. Trostchansky ${ }^{3}$; J.P. Millas-Vargas ${ }^{4} ;$ R. Araya-Maturana ${ }^{4}$ ${ }^{1}$ Thrombosis Research Center, Universidad de Talca, Talca, Chile; ${ }^{2}$ Molecular and Clinical Pharmacology Program, Institute of Biomedical Sciences, Faculty of Medicine, University of Chile, Santiago, Chile; ${ }^{3}$ Departamento de Bioquimica and Center for Free Radical and Biomedical Research, Facultad de Medicina, Universidad de la República, Montevideo, Uruguay; ${ }^{4}$ Instituto de Química de Recursos Naturales, Programa de Investigación Asociativa en Cáncer Gástrico (PIA-CG), Universidad de Talca, Talca, Chile

Background: Cardiovascular diseases are the leading cause of death in the world. Platelets play a major role in cardiovascular events, binding to the damaged endothelium, activating and forming thrombi.

Aims: Evaluate the antiplatelet effect of hydroquinone derivatives targeting to mitochondria, in human platelets.

Methods: We carry out cytotoxicity studies and platelet aggregation screening to a series of compounds. The compound with the highest activity was compound JP-I (Figure 1). To evaluate the mechanism of action of compound JP-I on platelets, we performed techniques of: Flow cytometry for markers of platelet activation, ROS, mitochondrial membrane potential and intracellular calcium levels; platelet spreading by fluorescence microscopy; Intracellular ATP and ATP secretion by luminescence; and NADH in real time by Fluorimetry; AMPK-ACC signaling western blot, in vitro clot retraction and occlusion time with PFA-200 System. Concentrations close to the IC50 obtained in platelet aggregation were used.

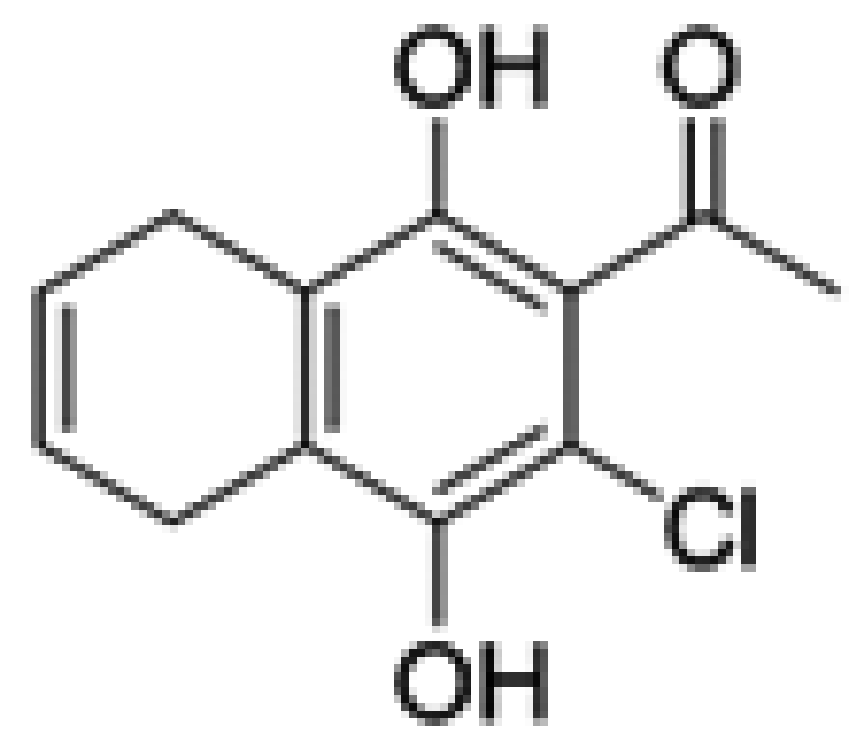

FIGURE 1 JP-I compound chemical structure 
Results: Compound JP-I does not present cytotoxicity and inhibits platelet aggregation, with IC50 values: $3.99 \pm 1.43 \mu \mathrm{M}$ (Thrombin Receptor Activator Peptide-6) and $7.16 \pm 0.9 \mu \mathrm{M}$ (Phorbol 12-myristate13-acetate). Decreases the expression of P-selectin, CD63 and PAC-1, and reduces platelet spreading. It is an inhibitor of mitochondrial NADH oxidation and electron transport in cellular respiration, it depolarizes the mitochondrial membrane, increases ROS levels, increases intracellular calcium levels and produces a decrease in ATP secretion and its intracellular levels. Western blot assays show that the antiplatelet activity induced by compound JP-I is mediated by AMPK-ACC signaling, in response to the decrease in ATP levels. Furthermore, it does not significantly alter the clot retraction (generated by Thrombin) and does not prolong the occlusion time in matrices of Collagen/Epinephrine and Collagen/ADP.

Conclusions: Our data indicate that compound JP-I decreases platelet activation by inhibiting mitochondrial bioenergetics, which activates AMPK-ACC signaling; and has a low risk of bleeding (in vitro). Acknowledgment: This research was funded by ANID/CONICYT, FONDECYT grant No1180427 (EF), and 1180069 (RAM)

\section{PB0999 | An Oxidative Stress-induced Platelet-associated} Tissue Factor Expression Characterizes Patients with Migraine and Patent Foramen Ovale

P. Canzano ${ }^{1}$; D. Trabattoni ${ }^{1}$; M. Brambilla ${ }^{1}$; B. Porro ${ }^{1}$; A. Becchetti ${ }^{1}$; S. Fiorelli ${ }^{1}$; E. Tremoli ${ }^{1}$; M. Camera ${ }^{1,2}$

${ }^{1}$ Centro Cardiologico Monzino, Milan, Italy; ${ }^{2}$ Università degli Studi di Milano, Milan, Italy

Background: Migraine is a complex, debilitating, multifactorial neurovascular disorder. Association with patent foramen ovale (PFO) has been reported with symptom regression after PFO closure. Increased platelet aggregation as well as changes in reduced/oxidized gluthatione (GSH/GSSG) ratio, as marker of oxidative stress, have been documented in patients with migraine.

No data are available on migraineurs with PFO.

Aims: 1) To assess the oxidative stress status in PFO patients with migraine on $100 \mathrm{mg} /$ die aspirin before and after PFO closure and 2) to investigate whether oxidative stress influences platelet activation using an in vitro approach.

Methods: Platelet activation markers (Pselectin, activated glycoprotein Ilbllla [aGPIIbllla] and Tissue Factor [TF]) were evaluated by whole blood (WB) flow cytometry in 62 patients before (TO) and six-months after (T1) PFO closure. Oxidative stress was assessed by mass spectrometry as GSH/GSSG ratio. Eight healthy subjects (HS) treated with aspirin for 15 days were enrolled as controls. The in vitro effect of GSSG on Pselectin and TF expression was assessed on WB from HS pre-incubated with aspirin.

Results: Pselectin and aGPIIbIlla expression was comparable in PFO patients before and after PFO closure and in HS. Conversely, TF expression was significantly higher in PFO patients at TO, returning to HS levels only after PFO closure $(3.3 \% \pm 1.9$ vs $1.7 \% \pm 1$, respectively;
$P<0.0001)$. The GSH/GSSG ratio was significantly lower at T0, returning to HS levels only after PFO closure. In in vitro studies, GSSG concentration-dependently induced platelet-associated TF and Pselectin expression; pre-incubation with aspirin blunted Pselectin expression, while TF expression remained unchanged, thus recapitulating what observed in vivo.

Conclusions: PFO patients are characterized by an oxidative stress status that leads to platelet-associated TF expression, which is not fully controlled by aspirin. After PFO correction, the oxidative imbalance reverted to physiological levels, leading also to the reduction of platelet TF expression.

PB1000 | Reticulated Platelets in Healthy Volunteers. Relationships with Platelet Size Indexes and Platelet Activity

A. Mazurov; V. Bodrova; O. Shustova; S. Khaspekova

National Medical Research Center of Cardiology, Moscow, Russian

Federation

Background: Reticulated platelets (RP) are young, functionally active platelet forms which are detected by RNA staining. Their content in circulation reflects the intensity of thrombocytopoesis in the bone marrow.

Aims: In this study we assessed the relationships of RP content with platelet size and activity in healthy volunteers.

Methods: RP were evaluated by thiazole orange staining using flow cytometry, platelet size indexes included mean platelet volume (MPV), platelet large cell ratio (P-LCR) measured in a Coulter type analyzer and forward scattering (FSC) measured in a flow cytometer. Platelet activity was evaluated by expression of activated glycoprotein (GP) Ilb-IIla (PAC-1 antibody binding) and CD62P using flow cytometry. Platelets were activated by thrombin receptor activating peptide (TRAP) (10 and $1 \mu \mathrm{M})$ and ADP (20 and $2.5 \mu \mathrm{M})$.

Results: The percentage of RP in healthy volunteers varied from $2.9 \%$ to $23.8 \%$ (mean \pm SD $-11.7 \pm 4.7 \%, n=99$ ) and correlated with all platelet size indexes, MPV, P-LCR and FCS ( $r$ from 0.452 to 0.529 , $P<0.001, n=87-99)$. RP were distributed at a ratio 9:1 between $50 \%$ subpopulations of large and small platelets according to FSC index. Expression of activated GP IIb-IIla correlated with RP percentage and platelet size indexes when platelets were activated by both doses of TRAP and ADP ( $r$ from 0.309 to 0.560 , p from 0.014 to $<0.001, n=50-62)$. CD62P expression correlated with RP percentage and platelet size indexes when platelets were activated by $10 \mu \mathrm{M}$ TRAP inducing maximal CD62P expression, ( $r$ from 0.332 to 0.556 , $\mathrm{p}$ from 0.008 to $<0.001, n=65$ ), but not by weaker agonists, $1 \mu \mathrm{M}$ TRAP and both ADP doses ( $r<0.3, n=54-66)$.

Conclusions: High RP content in healthy volunteers is associated with the increase of platelet size and activity in the whole platelet population 
PB1001 | Standardization of Platelet-rich Plasma Preparation with Optimal Platelet Function for the Use in Veterinary Regenerative Medicine

S. Kim; P.K. Chaudhary; S. Kim

Chungbuk National University, Cheongju, Korea, Republic of

Background: Platelet-rich plasma (PRP) has recently been used in human and veterinary regenerative medicine due to its abundant content of growth factors (GFs) and cytokines that can affect tissue regeneration, wound healing, angiogenesis, and cell proliferation. However, no standardized method of PRP preparation has been established. Moreover, there have been no studies on the effects of the currently used methods on platelet function which further affects the optimal use of PRP in the regenerative field.

Aims: Therefore, we determined to optimize the PRP preparation to achieve the highest recovery of platelets with the characterization of relevant platelet function and GF release using canine platelets.

Methods: Whole blood from healthy dogs was centrifuged at different run conditions $(800,1200,1500,2000$, and $3600 \mathrm{rpm})$ for $10 \mathrm{~min}$ single-spin or 5 min dual-spin to obtain PRP. Commercial PRP preparation kit was used to compare its efficiency. The platelet recovery rate of each PRP was determined by $\mathrm{CBC}$. Agonist-induced platelet aggregation and Platelet-Derived Growth Factor-BB (PDGF-BB) release were measured to determine the function of platelets prepared from each PRP.

Results: The PRP obtained by dual-spin centrifugation at $1500 \mathrm{rpm}$ for 5-5 min showed the highest platelet recovery rate with no other white blood cell contamination. Consistently, platelets obtained from this PRP showed the maximum platelet aggregation in response to 2-MeSADP and thrombin, suggesting that optimizing the centrifugal speed and time is indispensable for the utmost collection of PRP with optimal platelet function. Interestingly, PDGF-BB release was dramatically increased time-dependently in PRP adjusted at $1 \times 10^{9}$ platelets/ml indicating the importance of platelet concentration in PRP for maximum GF release.

Conclusions: In conclusion, we standardized the PRP preparation with the optimal platelet function and GF release that will help to achieve the effectiveness and efficacy of the application of PRP in veterinary regenerative medicine.

PB1002 | Pandrial Platelet Reactivity in Aspirin-treated Individuals: A Comparison between Fasting and Non Fasting Ontreatment Platelet Responses

B. Nkambule; A.D. Johnson

NHLBI Population Sciences Branch, Framingham, United States

Background: Platelet hyperreactivity in metabolic disease is associated with an increased risk of major adverse cardiac events. Variable cardiovascular outcomes in patients living with obesity and dyslipidemia are associated with altered platelet function. However, the clinical relevance of monitoring platelet responses in metabolic disease remains controversial. Since platelets uptake triglycerides and oxidized low-density lipoproteins. The lack of consensus on the ideal testing conditions (fasting vs non-fasting) for platelet function testing in metabolic disease may account for inconsistencies across various studies. In this preliminary analysis, we assessed postprandial platelet responses in aspirin-treated individuals.

Aims: In this preliminary analysis, we assessed postprandial platelet responses in aspirin-treated individuals.

Methods: A total of 1865 participants from the Framingham Heart Study (FHS), were retrospectively categorized into four major subgroups and these comprised of a:

(i) fasted aspirin-naïve group ( $n=1456$ );

(ii) nonfasted aspirin-naïve group $(n=68)$;

(iii) nonfasted aspirin treated group ( $n=308)$; and

(iv) fasted aspirin-treated group ( $n=33$ ).

Platelet reactivity to $20 \mathrm{mM}$ of ADP was assessed using a whole blood flow cytometry assay.

Results: In asprin-naïve fasted individuals, the differences in the levels of platelet reactivity were higher in comparison to fasted individuals; PAC-1 (MD: 2.05 [95\%Cl:2.05;1.93]) and CD63 (MD: 0.31 [95\%Cl:-0.39;1.00]. Similarily, in the asprin group the levels of PAC-1 (MD:0.94[95\%Cl:-4.73;6.60]; CD63[MD:0.0.34[95\%:Cl:-0.75;1.439] were higher in fasted individuals compared to non fasted comparators. Notably, P-selectin levels were slightly reduced in fasted compared to nonfasted individuals (MD: $-0.29[95 \% \mathrm{Cl}:-2.42 ; 1.08]$.

Conclusions: Platelet responses to endogenous agonists may be modified by postpandrial factors including transient changes in post-meal lipid profiles. These preliminary findings suggest that the fasting state of indivduals could be a crucial testing variable in flow cytometry-based platelet assays.

PB1003 | Evaluation of Platelet Dense Granules by Transmission Electron Microscopy (PTEM) in Pediatric Patients and Healthy Pediatric Donors

J. Greenmyer; D. Warad; K. Howell; K. Fischer; L. Stromback; T. Gibbons; J. Charlesworth; R. Barness; S. Gossman; J. Gamez; R. Pruthi; D. Chen

Mayo Clinic, Rochester, United States

Background: PTEM whole mount (WM) is a standard method for evaluating platelet dense granules (pDG). However, a pediatric/adolescent $\mathrm{pDG}$ reference range (RR) and prevalence of pDG deficiency in symptomatic patients has not been well-established.

Aims: This study aimed to establish a pediatric/adolescent RR for mean pDG and determine the prevalence of pDG deficiency in a cohort of patients who had clinical suspicion for platelet disorders (PD). Methods: First, WM-PTEM was performed on healthy donors. A mean pDG RR was calculated by averaging DG of 100 plt per patient as previously described (PMID: 29863946). Next, patients undergoing laboratory evaluation of suspected PD were recruited to this 
study. In addition to routine laboratory testing, their ISTH BAT score (normal <3; abnormal $\geq 3$ ) was correlated with mean pDG.

Results: Healthy donors ( $n=77,41.6 \%$ female), ages $3-18$ years, had a mean pDG 2.7 (+/- 0.5) ranging from 1.9 to 3.8. The mean pDG did not correlate with age or gender. The tentative RR was calculated to be 1.9 to $3.8 \mathrm{DG} /$ platelet. Nineteen patients over the age of three $(25.3 \%, n=19 / 75)$ had $<1.9$ mean pDG. Of the 75 symptomatic patients (age 3-18 years, 69.3\% female), 42 and 33 patients had BAT scores $\geq 3$ and $<3$ (range $0-11$ ), respectively. DG/plt in pt with bleeding scores $\geq 3$ (mean $=2.3+/-0.83, n=42$ ) vs. those with bleeding scores $<3($ mean $=2.3+/-0.66, n=32)(P=.206)$ were similar. There was no difference in the number of patients with normal or abnormal bleeding scores in groups with normal vs decreased mean DG/ plt $(P=.595)$

Conclusions: In this study, we established a tentative pediatric RR for platelet DG at 1.9-3.8 DG/plt. Approximately 25\% of patients were found to have DG deficiency. However, pDG did not not correlate with the ISTH BAT.

PB1004 | The Effects of Iron Deficiency on Platelet Function, Indices, and Clinically Reported Hemostasis: A Prospective Clinical Study of Iron Deficient Women Receiving IV Iron

B. Elstrott ${ }^{1}$; M. West ${ }^{2}$; H.H.S. Lakshmanan ${ }^{3}$; J. Aslan ${ }^{3}$; J.J. Shatzel ${ }^{2,3}$

${ }^{1}$ Oregon Health \& Science University, Portland, United States;

${ }^{2}$ Department of Hematology-Oncology, Knight Cancer Institute, OHSU, Portland, United States; ${ }^{3}$ Department of Biomedical Engineering, OHSU, Portland, United States

Background: Iron deficiency, heavy menstrual bleeding, and anemia are highly prevalent issues in women's health impacting an estimated 310 million premenopausal women worldwide. It is unknown how iron deficiency affects platelet function and signaling, or how this may contribute to defects in hemostasis.

Aims: To define the role of iron in platelet physiology including activity, function, and clinically reported hemostasis in women.

Methods: This is a prospective single center study aiming to enrol 50 women with iron deficiency (ferritin $<50 \mathrm{mcg} / \mathrm{L}$ ) assigned to receive high dose iron repletion (low molecular weight iron dextran $1000 \mathrm{mg} \times 1$ ). Pre-infusion and 6-12-week post infusion blood samples and clinical assessment is undertaken.

Results: Enrollment is ongoing, and currently 8 women are enrolled on study. Median age 40 years, with a mean pre and post-infusion ferritin of $9.8 \mathrm{ng} / \mathrm{ml}$ and $135 \mathrm{ng} / \mathrm{ml}$ respectively. Platelet count decreased post infusion by an average of $25 \mathrm{~K} / \mathrm{cu} \mathrm{mm}$. Preliminary platelet function analysis for activated integrin GPIIbIlla (i.e., PAC1FITC), granule secretion (i.e., CD62P), platelet-leukocyte interactions, and phosphatidylserine (PS) exposure (i.e., Annexin V) by flow cytometry is underway. The effects of iron repletion on patient reported hemostasis is likewise ongoing.
Conclusions: Iron deficiency has a well described effect on elevating platelet count. However, while extremely prevalent in premenopausal women, the effects of iron deficiency on platelet function and hemostasis remain poorly understood. Our preliminary data reaffirms that iron repletion lowers platelet count and suggests that iron deficiency may result in alterations of platelet function that can be reversed with iron repletion. Continued enrollment will provide the opportunity for expanded analysis and assessment of clinically reported hemostasis.

PB1005 | Case Report: Skin Necrosis in setting of Heparin and Warfarin Exposure

D.Z.S. Eng; C.P. Coorey; D. Hsu; S. Mallik

Liverpool Hospital, Sydney, Australia

Background: Heparin-induced thrombocytopenia and thrombosis (HIT) is caused by an immune reaction, most frequently lgG, in which antibodies form against heparin and platelet factor 4 complex. These antibodies activate platelets leading to thrombotic complications, including skin necrosis.

Aims: We describe a 81-year-old patient who underwent an elective left upper lobectomy for a pulmonary mass at Liverpool Hospital in Sydney, Australia, and was found to have abdominal skin necrosis eight days following local subcutaneous unfractionated heparin injections for venous thromboembolism prophylaxis, in the absence relative thrombocytopenia from baseline. Baseline thrombocytopenia is related to cirrhosis and associated splenomegaly. This was present since at least 2008 , with a count typically $60-80 \times 109 / \mathrm{L}$ in the preceding 12 months, and 55×109/L on the day of admission. He received warfarin two days prior as well for newly diagnosed paroxysmal atrial fibrillation. The 4T score widely used to assess the pre-test probability of HIT and thereby guiding laboratory testing was low at 3 , as the platelet count was unchanged from baseline. Nevertheless, heparin was immediately ceased and warfarin stopped soon after. A therapeutic dose of fondaparinux was commenced on day 13 once the INR had fallen to below 2.0.

Methods: Case Report.

Results: The HemosIL AcuStar HIT-IgG immunoassay demonstrated strongly positive results, and the serotonin release assay confirmed the presence of heparin-induced antibodies with a result of $104 \%$ with low dose $0.1 \mathrm{U} / \mathrm{mL}$ heparin.

Conclusions: Skin necrosis in the setting heparin and warfarin use are important clinical entities to recognise as they require prompt and accurate diagnosis to prevent serious complications. In our patient where both medications were administered, confirmation of HIT by laboratory methods was crucial in establishing the offending agent, and preserved warfarin as a future therapeutic option. The absence of thrombocytopenia in this case emphasises the need to apply clinical reasoning to all cases that have an atypical presentation. 
PB1006 | Interaction of Blood Platelets with Engineered Fibrinogen Scaffolds of Different Topographies

M. Kenny ${ }^{1}$; S. Stamboroski ${ }^{2,3}$; R. Taher ${ }^{1}$; D. Brüggemann ${ }^{2}$; I. Schoen ${ }^{1}$

${ }^{1}$ Laboratory of MechanoVascular Biology, School of Pharmacy and Biomolecular Sciences, Irish Centre for Vascular Biology, RCSI, Dublin, Ireland; ${ }^{2}$ Institute for Biophysics, University of Bremen, Bremen, Germany; ${ }^{3}$ Fraunhofer Institute for Manufacturing Technology and Advanced Materials, Bremen, Germany

Background: Biomaterial-blood interactions are critical for the successful tissue integration of implants and might be used to guide wound healing. Fibrin(ogen) mediates platelet aggregation and thrombus formation at vascular injury sites. Studies have shown that the local adhesion protein density and substrate stiffness inform platelet responses, yet the influence of fibrin(ogen) topography is poorly understood.

Aims: To investigate platelet adhesion, secretion, and procoagulant activity upon interaction with fibrinogen scaffolds of different nanotopographies.

Methods: Platelet-rich plasma (PRP) or washed platelets were incubated on planar or nanofibrous fibrinogen scaffolds prepared by salt-induced self-assembly (Stapelfeldt et al., Biofabrication 2019; Stapelfeldt et al., Nanolett 2019), as well as on control fibrinogencoated coverslips. Samples were fixed and stained for confocal microscopy to evaluate platelet activation in terms of cytoskeleton adhesion morphology (F-actin organization), a-granule secretion ( $\mathrm{P}$ selectin surface expression), and procoagulant activity (phosphatidylserine exposure).

Results: Platelets in PRP remained round on adsorbed or planar fibrinogen but spread and formed small clots on nanofiber scaffolds. In contrast, washed platelets adhered and spread on all substrates and developed similar adhesion morphologies. Most platelets on all substrates were P-selectin positive but showed heterogeneous variation in phosphatidylserine exposure, independent of the presence of plasma proteins.

Conclusions: These preliminary results suggest that platelets interact more strongly with 3D fibrinogen scaffolds than 2D fibrinogen coatings. Further investigation is required to elucidate the molecular mechanisms underpinning platelet behaviour on 3D substrates.
PB1007 | Thrombogenic Potential in Patients Undergoing Hemodialysis Using Central Venous Catheter and Arteriovenous Fistula

M. Dumont Cunha; M. Henrique Fernandes Ottoni; M. Araújo

Figueiredo; T. Coaglio Lucas

Universidade Federal dos Vales do Jequitinhonha e Mucuri - UFVJM, Diamantina, Brazil

Background: Non-maturation of the arteriovenous fistula (AVF) leads to prolonged use of the central venous catheter (CVC) and can promote the thrombus development.

Aims: To evaluate the thrombogenic potential in patients undergoing hemodialysis with prolonged use of hemodialysis CVC and AVF.

Methods: This cross-sectional study included 68 adult participants with chronic renal failure. Participants were distributed: I: clinical and laboratorial healthy individuals matched by sex and age (controls); II: patients after one month of insertion of the catheter and AVF and III: patients after 4 months of insertion of the catheter and AVF. To the activation of platelets was investigated by GPIIb/IIla and p-selectin expressions using flow cytometry. Thrombomodulin Elisa kit, and all tests were performed as recommended by the respective manufacturers and were run in duplicates. Statistical analysis was performed using the Student $t$, Mann-Whitney and ANOVA as appropriate. Differences statistically significant $(p \leq 0.05)$. The participants signed the Informed Consent Term approved by the Ethics Committee, number 2,740,297.

Results: A significant difference was found by the expression of p-selectin comparing control group $(32.84 \pm 4.805)$ and the percentage obtained from peripheral vein at a time of 1 month $(41.28 \pm 5.849)$ and 4 months $(50.08 \pm 7.415)$ after CVC insertion ( $p<0.0001$ ) (Figure 1).The median values for GPIIb/III expression were 10422(10033-10670),13933(13508-15509) and 19858 (17517-20712) after CVC insertion ( $p<0.0001)$, for groups I, II and III, respectively. To the AVF was not showed significant $(P<0,05)$ changes in the platelet activation among groups I, II and III. The median values for Elisa test were 3547(3222-5057) for the patients after 4 months of the catheter and 2144(1754-3421) for the patients after 4 months of AVF. 


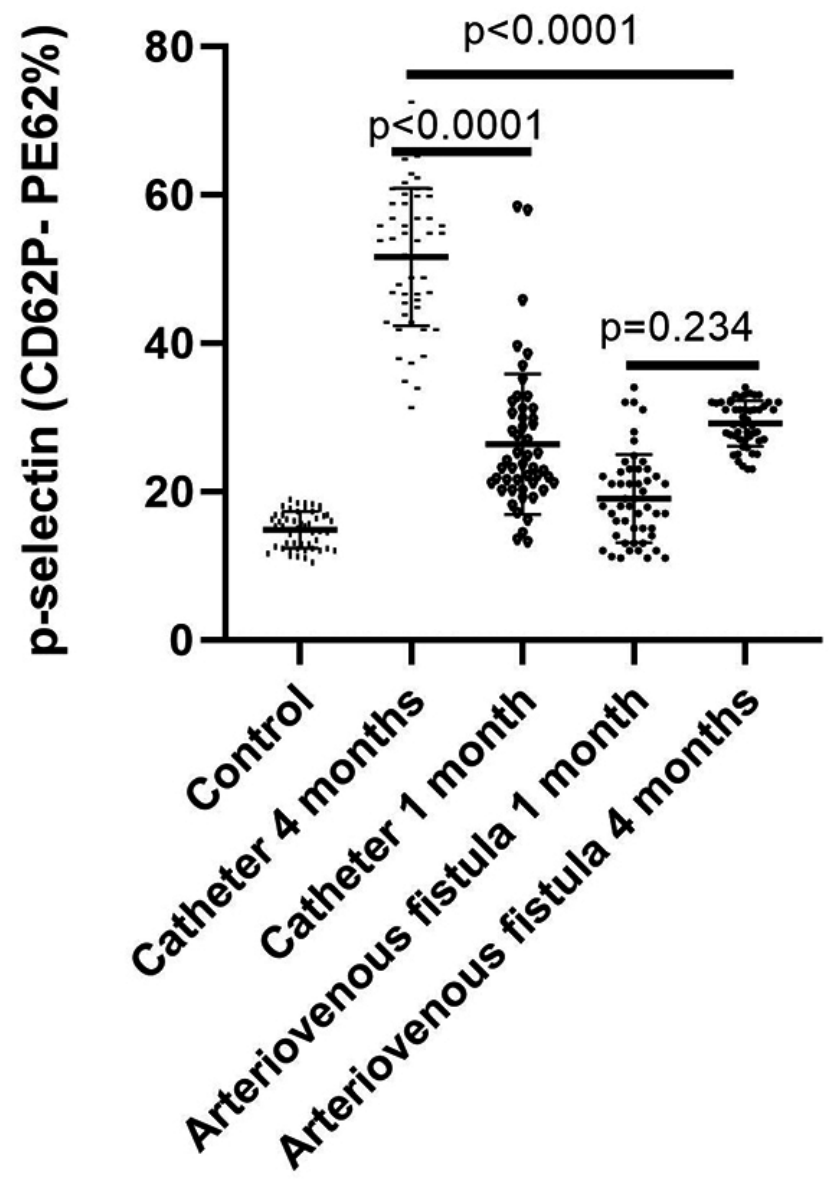

FIGURE 1 Analysis of platelet activation by flow citometry. P-selectin analysis in percentages.Student's test

Conclusions: The current study demonstrated that the patients who use catheters for hemodialysis have a greater tendency to develop thrombi as adverse events when compared to those use AVF.

PB1009 | Importance of Consideration to Differetial Diagnosis of ITP

N. Mirbehbahani

Cancer Research Center of Golestan University of Medical Sciences, Gorgan, Iran, Islamic Republic of

Background: A 4 Years -old boy was admitted in hospital due to gum bleeding in 2010. Laboratory studies performed including CBC, PT, PTT, INR and BMA. There was no hepatosplenomegaly and lymphadenopathy and fever in examination. PT, PTT, INR were normal. Aims: The patient diagnosed as idiopathic thrombocytopenic purpura. He received IVIG and discharged in good condition with $\mathrm{plt}=40000 / \mathrm{mm} 3$. In addition, he had multiple admissions (20times) due to petechiae, epistaxis, gum bleeding and low pIT count until 2017.
Methods: He received in these admissions IVIG 8 times, steroid 3 periods (1-3 weeks), anti-D for 4 weeks and rituximab for

4 weeks. He responded to treatments as platelet count increase from $30000 / \mathrm{mm} 3$ to $80000 / \mathrm{mm} 3$. But after few weeks or months platelets drop again below $10000 / \mathrm{mm} 3$.

On 2018 the patient referred and admitted in pediatric surgery ward of our hospital for splenectomy with diagnosis chronic refractory ITP. Before surgery, our surgeon requested pediatric hematology consultation.

There were 4 important points in review of patient's history:

- cousin marriage in his parents.

- Presence of low PIT in patients cousin

- History of repeated bruising from infancy

- Lack of favorable response to ITP treatment

These clues help me think about hereditary thrombocytopenia.

Results: Since BSS is the second most recognized inherited sever PLT disorder among hereditary thrombocytopenia and considering that in 9 patients registered in our center with BSS 6 cases had been treated and followed-up as ITP for a long time, I though bout BSS in this patient.

Therefore I requested peripheral blood smear and PLT aggregometry test for the patient that disclosed the diagnosis of BSS.

Conclusions: Inherited thrombocytopenia especially BSS has very close similarities to ITP, therefore BSS should be considered in differential diagnosis of persistent and refractory ITP to avoid inappropriate therapies and it's cost and complications.

PB1010 | Pathogen-reduced Platelet Concentrates: Novel Concepts to Elucidate the Impact of Different Technologies on Platelet Function

M. Visser; W. Schwarz; A. Rausch-Mueller; S. Volpe; A. Hilger;

U. Salge-Bartels

Paul-Ehrlich-Institut, Langen, Germany

Background: The safety of platelet concentrates (PC) is of central importance in transfusion medicine, and is mainly guaranteed by the selection of suitable donors, the testing of known infectious agents, and a limitation of the storage time as part of the manufacturing process. Given the emergence and spread of novel transmissible infectious agents, the importance of pathogen reduction procedures of PC has increased significantly in recent years.

Aims: To dissect alterations in platelet function and integrity in pathogen-reduced PC prepared by three different procedures.

Methods: The study design is based on a pool-and-split approach. Platelet products treated with Amotosalen/UVA, Riboflavin/UV, and UVC as well as untreated platelet products are prepared from pooled plasma-reduced PC and stored under standard conditions. Platelet products are tested for functional parameters including platelet aggregation, and P-selectin- and phosphatidylserine expression. 
Mitochondrial membrane potential as a marker of apoptosis is assessed by TMRE (tetramethylrhodamine, ethyl ester) sequestration. Moreover, alterations in adhesive properties and platelet stability are investigated by the application of wall shear stress in collagencoated micro-fluidic channels and after cold treatment at $4^{\circ} \mathrm{C}$.

Results: Our study protocol is designed to examine platelet function after application of three common pathogen reduction procedures compared with untreated controls. The pool-and-split method enables alterations in platelet function to be estimated with little impact of the individual donor-associated variations. Furthermore, our test concept provides for a shear stress challenge on platelets that mimics the physiological situation in the vascular system and could reveal latent defects.

Conclusions: In view of the increasing demands on the infection safety of platelet concentrates, parallel testing and application of challenging stress conditions is a valuable approach for the evaluation of novel manufacturing procedures and their effect on platelet functionality.

\section{PB1011 | Immune Thrombocytopenic Purpura: A Case Report}

\section{A. Berisha; S. Sadiku}

University of Prishtina, Prishtina, Albania

Background: Immune thrombocytopenic purpura (ITP) is an autoimmune disorder characterized by a low platelet count and mucocutaneous bleeding. Immune thrombocytopenic purpura is classified as primary or as secondary to an underlying disorder and as acute (of six months or less in duration) or chronic. The diagnosis of immune thrombocytopenic purpura remains one of exclusion. Prednisone (1 mg per kilogram of body weight) is the conventional initial therapy for patients who have more severe thrombocytopenia.

Aims: The aim of this case report is to better understand ITP, including the clinical features and treatment outcome.

Methods: The data was taken from the patient files in the Hematology clinic of the clinical university center of Kosovo. The patient included in the report was diagnosed with ITP with the exclusion of other secondary causes of ITP including autoimmune disease and hematological disease. The patient was treated with the standard therapy for ITP.

Results: The patient D.H, female presented with mucocutaneous bleeding and a thrombocyte count of $36 \times 10^{3} / \mathrm{mm}^{3}$. The immunological profile including ANA, anti-dsDNA and other markers were all negative. The viral antibody titers were negative for lgM accept for CMV IgG which was positive. The blood smear, bone marrow aspiration and biopsy no hematological disease. The patient was started on corticosteroids but the treatment was not satisfactory.

Conclusions: The patient was initially treated with prednisone for 3 weeks but the thrombocyte count did not increase. For this reason the patient was treated with Rituximab once a week. Even after taking 3 doses of Rituximab improvement. After that she was started with Azathioprine $100 \mathrm{mg}$ once a day.

\section{PLATELET PROTEOMICS AND GENOMICS}

\section{LPB0083 | Temporal Assessment of Platelet Proteome and fFnction during ex vivo Anticoagulation}

S. Tassi Yunga; A. Gowers; A. Melrose; M. Fitzgerald; A. Rajendran; A. Reddy; P. Wilmarth; L. David; O. McCarty; J. Aslan Oregon Health \& Sciences University, Portland, United States

Background: Despite the importance of ex vivo studies of platelets in health and disease, the progressive impact of pre-analytical blood processing conditions on platelet function and composition remains minimally characterized.

Aims: To determine the effects of commonly utilized blood draw anticoagulants, as well as processing time, on ex vivo platelet proteome and function.

Methods: Whole blood was collected from four healthy human donors into four different anticoagulants, including sodium citrate, acid citrate dextrose (ACD), EDTA, and heparin. Platelet function was tracked in anticoagulated blood over 120 hours with biochemical and cellular assays. Whole proteome profiles of platelets purified at 1 hour and 24 hours after blood draw, were determined through a peptide tandem mass tag (TMT) labeling and multiplex mass spectrometry approach.

Results: Collection of blood into heparin, and to a lesser extent sodium citrate, significantly increased platelet-platelet and plateletmonocyte aggregate formation within 1 hour of blood draw. A rapid release of platelet-derived extracellular vesicles was also observed for heparinized blood, whereas a distinct increase in platelet surface P-selectin exposure was noted for platelets in EDTA blood 5 hours after collection. Multiplex TMT proteomics identified 3,357 proteins spanning a dynamic range of 5 orders of magnitude in all platelet samples, where, the duration of blood storage before platelet purification was a strong driver of whole platelet proteome changes associated with metabolism and exocytosis. Compared to platelets from ACD-anticoagulated blood, EDTA platelets showed increased levels of complement $\mathrm{C} 1 \mathrm{r}$ and ficolin 3 proteins. Platelets from heparinized blood contained high levels of histone proteins and neutrophilrelated enzymes. The Association of NET products and platelets was confirmed by flow cytometry and immunofluorescence staining. Conclusions: This study establishes time-dependent and anticoagulant-associated ex vivo effects on the platelet proteosequestrome that may confound characterizations of platelet function in health and disease. 
PB1012 | Single-cell Transcriptomics of Young and Mature Thrombocytes in Zebrafish

W. Fallatah; D. Burks; R. Azad; P. Jagadeeswaran

University of North Texas, Denton, United States

Background: Zebrafish have two populations, young and mature thrombocytes. The mechanism of maturation of young to mature thrombocytes is not fully understood. We believe studying thrombocyte maturation might shed light on megakaryocyte maturation without the interference of polyploidy.

Aims: To perform single-cell RNA sequencing of young and mature thrombocytes in zebrafish.

Methods: Young (RFP+) and mature (GFP+) thrombocytes from GloFli fish were sorted separately in a $5 \mathrm{ml}$ culture tube using BD FACSCanto flow cytometer. The instrument was set at $4^{\circ} \mathrm{C}$ with a nozzle size of 100 . We set $75 \%$ to $90 \%$ cell viability with at least 10,000 cells for optimum analysis. The sorted cell samples were kept on ice and sent immediately to the Next Generation Sequencing core. RNA from these cells was prepared according to $10 x$ Genomics protocols and sequencing was performed after successful library preparation and quality control. Results: We used 2,176 RFP+ young thrombocytes and 1,541 GFP+ mature thrombocytes that survived. The total number of genes detected for GFP+ cells is 8,746 and for RFP+ cells, it is 6,990 genes. RNA-seq analysis of this data showed 6593 genes are expressed in both young and mature thrombocytes. Whereas mature thrombocyte uniquely expresses 2153 genes, mature thrombocyte expressed about 397 genes exclusively. About $80 \%$ of total genes in both GFP+ and RFP+ thrombocytes had human orthologous. The heatmap showed patterns that are consistent with the results mentioned above. We also analyzed the RNA-seq data by PANTHER program and found the gene-specific transcriptional regulators in the young and mature thrombocytes to be 14 and 140 genes, respectively. We also noted other differences.

Conclusions: RNA-seq analysis of young and mature thrombocytes identified differences in expression genes in various categories, including transcriptional regulators. This data should be useful in genomewide knockdown screening to understand thrombocyte maturation.

PB1013 | Common Cardiovascular Disease Polygenic Risk Scores for Arterial and Venous Disease Influence Different Platelet Reactivity Tests

J. Grech ${ }^{1} ;$ M. Chan ${ }^{1,2} ;$ A. Lachapelle ${ }^{1} ;$ F. Thibord ${ }^{1} ;$ Z. Schneider ${ }^{1}$;

P. Armstrong ${ }^{2}$; C. Wallace de Melendez ${ }^{1}$; T. Warner ${ }^{2}$; M.-H. Chen ${ }^{1}$; A. Johnson ${ }^{1}$

${ }^{1}$ National Heart, Lung, and Blood Institute (NHLBI), Framingham, United States; ${ }^{2}$ The Blizard Institute, Barts and The London School of Medicine \& Dentistry, London, United Kingdom

Background: Summary statistics from large genome-wide association studies (GWAS) are used to derive polygenic risk scores (PRS) which combine multiple associated DNA variants' (SNPs) effects to assess individuals' genetic predisposition to diseases. Platelets are contributors to CVD pathogenesis and are effective drug targets. Thus, an individual's genetic propensity to developing CVD may be mediated through effects on platelets.

Aims: We analyzed the association of PRS for multiple CVDs and platelet reactivity traits via five distinct assays.

Methods: PRS for coronary artery disease (CAD), stroke, atrial fibrillation (AF), and venous thromboembolism (VTE) were derived from the largest GWAS. Platelet reactivity traits were measured across multiple agonists in the Framingham Heart Study $(N=3,065)$ using light transmission aggregometry (LTA), Multiplate whole blood impedence aggregometry (MP), Total-Thrombus Formation Assay System (T-TAS), Optimul 96-well plate assay, and flow cytometry (FC). We used linear mixed effects models to test association between each PRS and platelet traits, after adjustment for age, sex and aspirin use.

Results: The strongest positive associations were found between VTE PRS and T-TAS:AUC ( $P=8.2 \mathrm{E}-05)$, MP:collagen AUC ( $P=1.8 \mathrm{E}-03)$, MP:ristocetin AUC ( $P=1.8 \mathrm{E}-03)$, and LTA:ristocetin slope ( $P=4.5 \mathrm{E}-07)$, respectively. A majority of VTE PRS SNPs had congruent effects for these traits with alleles increasing platelet reactivity also increasing VTE risk. Multiple loci were implicated including $A B O, G P 6, F 5, F 11$ and 4 others. CAD, AF, and stroke PRS had positive associations with $\mathrm{ADP}$-induced platelet activation in whole blood and PRP FC assays (PAC-1, CD63, CD62P).

Conclusions: ADP activation associating with arterial disease PRS is consistent with ADP inhibition being efficacious in CVD prevention. Strong associations with different platelet assays and VTE PRS suggests distinct platelet-contributing genes in this disorder. Given an expanding set of anti-platelet drugs (e.g., GP6, GP1b, PAR4 inhibitors) in development, targeting to specific CVD indications might be improved based on the platelet pathways implicated by our results.

PB1014 | Long-term Severe Platelet Dysfunction as a Form of Presentation of Familial Platelet Disorder with Predisposition to Acute Myeloid Leukemia (FPD/AML), due to a RUNX1 Variant

M. Sousa-Pimenta ${ }^{1,2}$; M. Pereira ${ }^{1,3}$; C. Lau ${ }^{4,3} ;$ A. Gonçalves ${ }^{5,3}$; M. E. Oliveira ${ }^{5,3}$; E. Cruz ${ }^{1}$; R. Santos ${ }^{5,3}$; M. Lima ${ }^{4,3}$; S. Morais ${ }^{1,3}$

${ }^{1}$ Unidade de Trombose e Hemostase e Centro de Coagulopatias Congénitas, Serviço de Hematologia Clínica, Centro Hospitalar Universitário do Porto (CHUP), Porto, Portugal; ${ }^{2}$ Serviço de Hemato-Oncologia do Instituto Português de Oncologia do Porto, Porto, Portugal; ${ }^{3}$ Unidade Multidisciplinar de Investigação Biomédica, Instituto de Ciências Biomédicas Abel Salazar, Universidade do Porto (UMIB/ICBAS/UP), Porto, Portugal; ${ }^{4}$ Laboratório de Citometria, Unidade de Diagnóstico Hematológico, Serviço de Hematologia Clínica, Centro Hospitalar Universitário do Porto (CHUP), Porto, Portugal; ${ }^{5}$ Unidade de Genética Molecular, Centro de Genética Médica Doutor Jacinto Magalhães (CGMJM), Centro Hospitalar Universitário do Porto (CHUP), Porto, Portugal

Background: Inherited platelet function disorders (IPFD) are qualitative platelet diseases with mild to severe bleeding, whose diagnosis 
can be a challenge. Familial platelet disorder with predisposition to acute myelogenous leukemia (FPD/AML) is an IPFD caused by germline mutations in the gene encoding the transcription factor RUNX1. Dysregulated expression of RUNX1-targets in platelets, including downregulation of the a 2 subunit (GPla) of collagen receptor $\alpha 2 \beta 1$, has been proposed to guide diagnosis.

Aims: We present a case of a woman with mild thrombocytopenia and severe bleeding whose laboratory study led to the diagnosis of FPD/AML.

Methods: Platelets were evaluated by morphometric, functional (PFA-200, lumiaggregometry), flow cytometry (glycoprotein expression), and genetic (NGS) studies.

Results: The patient, a 32 years-old-woman, bleeding score (ISTHBAT) of 10 , was first evaluated at 10 -years-old because of mucocutaneous bleeding and severe hemorrhage after tonsillectomy requiring red blood cell transfusion; moderate thrombocytopenia with storage-pool deficiency was assumed. There was no family history of hemorrhagic diseases or hematological neoplasms. About twenty years later, during pregnancy, she underwent a new evaluation. Laboratory studies showed moderate thrombocytopenia (87x109/L), extended PFA-200 EPI and ADP closure times (>300s), impaired platelet aggregations, absent in response to $\mathrm{COL}(1 \mu \mathrm{g} /$ $\mathrm{mL}$ ) and decreased in response to ADP $(10 \mu \mathrm{M})$, EPI $(10 \mu \mathrm{M})$, and AA $(1 \mathrm{mM})$, but normal in response to TRAP-6 $(25 \mu \mathrm{M})$; reduced ristocetin $(1 \mathrm{mg} / \mathrm{mL})$ induced agglutination. ATP release was absent with ADP, COL, EPI, and AA, and decreased with TRAP-6. Platelet glycoprotein levels were normal except for CD49b/Gpla (28\%). A likely pathogenic frameshift variant in RUNX1 (c.358del; p.Ala120Profs*2) was identified by NGS.

Conclusions: In this case, a bleeding tendency more severe than expected to the degree of thrombocytopenia, together with a storage pool platelet dysfunction and low platelet Gpla expression, warned for a possible FPD/AML. NGS assisted the diagnosis, by revealing a likely pathogenic RUNX1 variant.

\section{PLATELET RECEPTORS}

\section{LPB0084 | GPVI, a New Binding Partner for pro-coagulant} Factor VIII on Platelets

R. Sekar ${ }^{1}$; V. Proulle ${ }^{1,2}$; S. Loyau ${ }^{3}$; Y. Boulaftali ${ }^{3}$; A. Mimoun ${ }^{1}$; M. Jandrot-Perrus ${ }^{3}$; S. Lacroix-Desmazes ${ }^{1}$

${ }^{1}$ Centre de Recherche des Cordeliers, INSERM UMRS 1138, Sorbonne University, Paris, France; ${ }^{2}$ Service Hématologie Biologique et Hémostase Clinique, Hôpital Cochin, AP-HP Centre - Université de Paris, Paris, France; ${ }^{3}$ INSERM U 1148, Hôpital Bichat, Paris, France

Background: Factor VIII (FVIII) is an essential actor in the intrinsic coagulation pathway, where it acts as a cofactor for activated FIX to form the tenase complex that anchors at the surface of activated platelets. Binding partners for FVIII on platelets include phosphatidylserine and platelet-bound fibrin. GPVI is a platelet-specific glycoprotein, the cross-linking of which by collagen, activates platelets. Upon screening for FVIII interactions with platelet-expressed glycoproteins, we observed FVIII binding to GPVI.

Aims: To characterize the FVIII/GPVI interaction at the molecular level and decipher its potential biological relevance on platelet functions.

Methods: Binding of human full-length and B domain-deleted (BDD) FVIII (Advate, Novoeight, Factane, FVIII ${ }^{\mathrm{HSQ}}$ ) was investigated on immobilized human recombinant GPVI (R\&D) or home-made Fc-fused GPVI. FVIII was pre-incubated alone or with varying amounts of von Willebrand factor (VWF, Wilfactin), or human monovalent monoclonal anti-A2 (BOIIB2), anti-C1 (KM33) or anti-C2 (BO2C11) IgG.

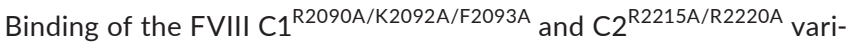
ants was also investigated. Bound FVIII was revealed using SAF8C, a FVIII-specific polyclonal sheep IgG.

Results: Full-length and BDD FVIII, but not plasma-derived FVIII, bound in a dose-dependent manner to recombinant GPVI and GPVI-Fc. VWF inhibited FVIII binding to GPVI. Interestingly, C1 and C2-specific, but not A2-specific, monovalent IgG also inhibited the interaction. Accordingly, FVIII variants mutated in the target epitopes of BO2C11 or KM33 lost binding capacity to GPVI.

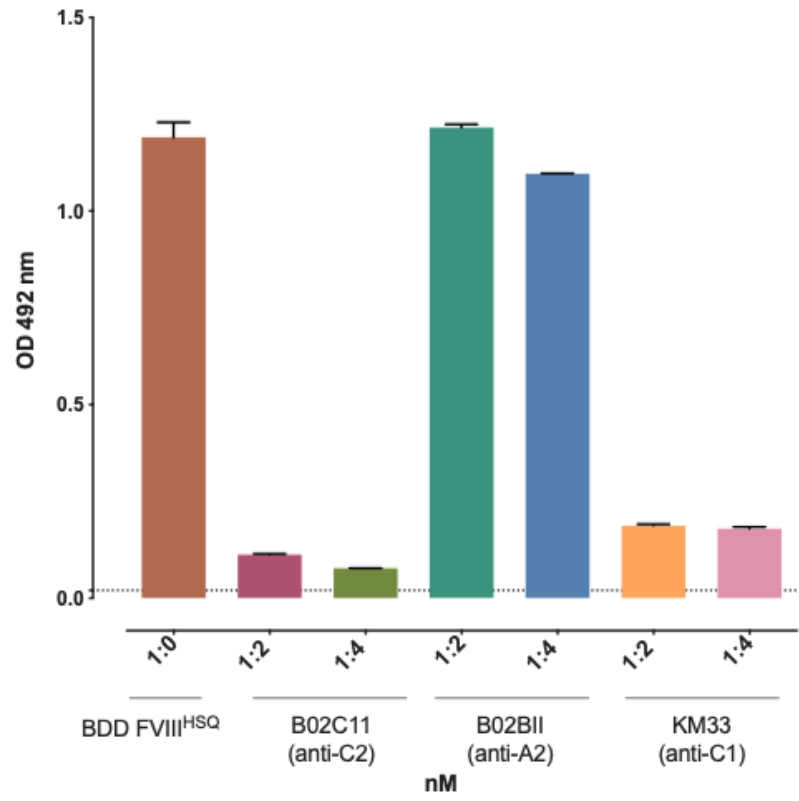

Figure 1: The binding of FVIII to GPVI-Fc in the absence or presence of antiFVIII monovalent IgG against FVIII domains C1, C2 and A2.

$B$ domain deleted $\mathrm{FVIII}$ (BDD FVIII ${ }^{\mathrm{HSQ}}$ ) at $55.5 \mathrm{nM}$ alone or in the presence of corresponding molar excess of anti-FVIII monovalent IgG B02C11 (anti-C2), B02BII (anti-A2) and KM33 (anti-C1) were incubated with GPVI-Fc immobilized wells. Bound FVIII was revealed with biotinylated SAF8C, a polyclonal anti-FVIII sheep IgG by ELISA. Representative of two independent experiments in triplicates (mean $\pm S D$ ).

FIGURE 1 The binding of FVIII to GPVI-Fc in the absence or presence of anti-FVIII monovalent IgG against FVIII domains C1, C2 and A2 
Conclusions: Phosphatidylserine and $a_{11 b} B_{3}$ integrin-bound fibrin have been identified as binding sites on platelets for the light chain of FVIII. The present findings identify GPVI as a novel binding partner for FVIII light chain on platelets; the interaction is however prevented in the presence of VWF. Future work will decipher the domains of GPVI implicated in FVIII binding and the potential biological significance of the interaction.

PB1015 | Salt Bridge Formation Between A1 Domain of von Willebrand Factor and Platelet Glycoprotein (GP) Iba by Molecular Dynamics Simulations

M. Nakayama; S. Goto; S. Goto

Tokai University School of Medicine/Department of Medicine, Iseharashi, Japan

Background: Platelet membrane glycoprotein (GP) Iba binding with von Willebrand factor (VWF) exclusively mediates initial platelet adhesion at site of endothelial damage under blood flow conditions. Single amino-acid mutations, such as G233 in GPIba were shown to lead to clinical phenotype by changing the adhesion characteristics of platelets.

Aims: To clarify salt bridge formation between VWF and GPIba in various mutant at G233 Platelet GPIba.

Methods: All atoms and water molecules constructing the $\mathrm{N}$ terminus GPIba domain containing leucine rich repeat (residues $\mathrm{HSE}^{1}-\mathrm{PRO}^{265}$ ), A1 domain of VWF (residues ASP ${ }^{506}-\mathrm{PRO}^{703}$ ) were targeted for Molecular Dynamics (MD) calculation. The mutations are prepared with G233A (equal function), G233V (gain of function), and G233D (loss of function), already calculated in previous study Salt bridges formed within $4 \AA$ between VWF and GPIba were calculated using the NAMD energy plugin implemented in VMD 1.9.4. The parameter file was using Chemistry at Harvard Macromolecular Mechanics (CHARMM)-36 force field.

Results: Salt bridges formed within $4 \AA$ between VWF and GPIba with various mutations were changed (Fig.1).

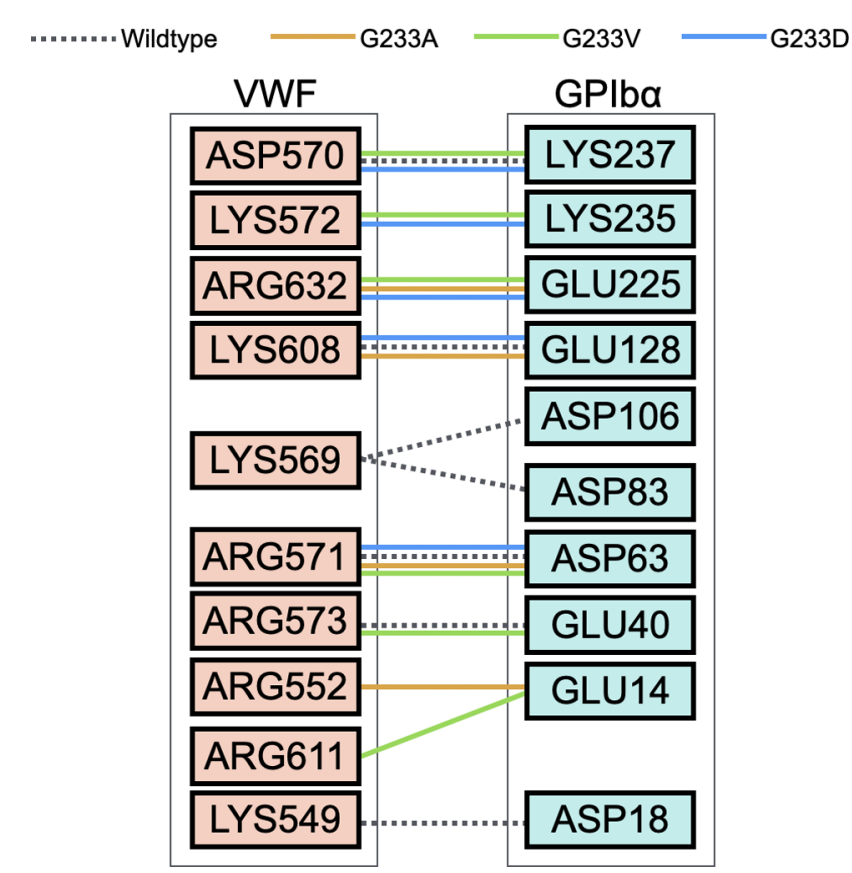

FIGURE 1 The list of salt bridges between VWF and GPIba interaction. Salt bridges within $4 \AA$ A were shown in the list

Non-bond potential energies were shown $-1056.2 \mathrm{kcal} / \mathrm{mol}$ in wild type, $-978.7 \mathrm{kcal} / \mathrm{mol}$ in G233A, $-908.4 \mathrm{kcal} / \mathrm{mol}$ in G233V, and $-903.1 \mathrm{kcal} / \mathrm{mol}$ in $\mathrm{G} 233$. The wild type was shown most stable energetic structure and G233D was less stable compared with others. The pair of amino acids consist of salt bridges of wild type and G233D are shown in Fig.2. In wild type, the salt bridges concentrated in $\mathrm{N}$-terminus side. In G233D, salt bridges were sparse compared with wild type.
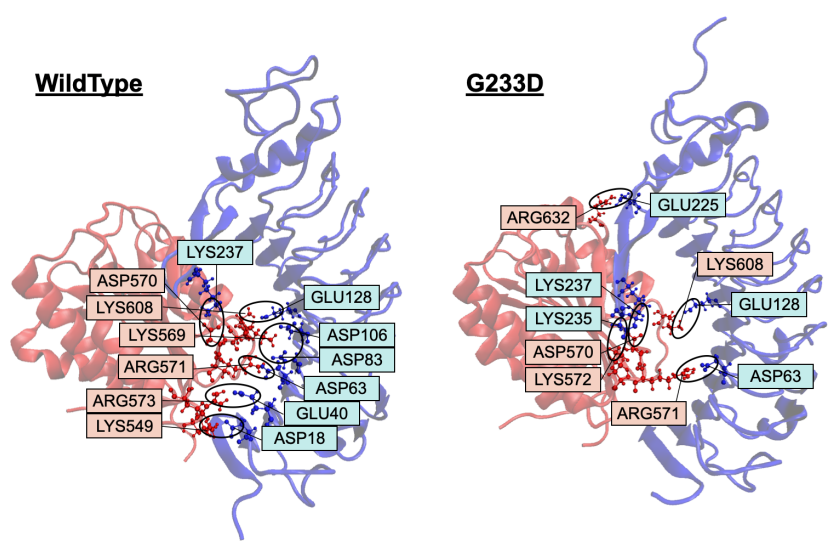

FIGURE 2 The pair of amino acids consist of salt bridges of wild type and G233D. The circle showed the interactions of two or three amino-acid in salt bridge

Conclusions: Mutation at G233 influence biological function of GPIba by changed salt bridge formation between VWF. 
PB1016 | Defining the Molecular Features of Inverse Agonism: Insights from the P2Y12 Receptor and the Antiplatelet Drug Ticagrelor

S. Bancroft; J. Khalil; S. Mundell

University of Bristol, Bristol, United Kingdom

Background: Although many G-protein-coupled receptors (GPCRs) show varying degrees of constitutive activity, a detailed molecular understanding of this phenomena is lacking. Recent studies have revealed that the platelet expressed $P 2 \mathrm{Y}_{12}$ receptor $\left(\mathrm{P} 2 \mathrm{Y}_{12} \mathrm{R}\right)$ displays a high degree of constitutive activity and that ticagrelor, a clinical antiplatelet drug, is an inverse agonist at this receptor (Aungraheeta et al., 2016).

Aims: Use of molecular dynamic simulations (MDs) alongside bioluminescence resonance energy transfer (BRET) assays to further our understanding of the molecular determinants underlying GPCR constitutive activity.

Methods: 1 $\mathrm{ss}$ MDs of several P2Y12-ligand receptor complexes, employing the ff14SB forcefield. Residues believed to be important for regulating activity were mutated and transiently transfected into HEK293 cells where receptor/G protein coupling was assessed by BRET.

Results: MDs revealed that ticagrelor binds to a region of the receptor similar to that of AZD1283 and 2MeSADP, but not ADP. Ticagrelor interacts with transmembrane domains (TM) 3 and 5-7. ADP sits in an alternative region contacting TM1-3, TM5 and TM7. Principal component analysis reveals that ticagrelor induces movements in TM5 resulting in a shift at the intracellular end, towards TM3. Experimental mutation of C194 to an alanine produced a $64 \%$ decrease in ticagrelor inverse agonism.

Conclusions: The orthosteric cavity of the $\mathrm{P} 2 \mathrm{Y}_{12} \mathrm{R}$ can be divided into two pockets with 2MeS-ADP, AZD1283 and ticagrelor binding in a distinct pocket to ADP. Ticagrelor induces a distinct conformation in TM5 bringing it into closer proximity with TM3. This likely occludes G-protein binding and in part defines the ability of ticagrelor to act as an inverse agonist.

Aungraheeta, R., Conibear, A., Butler, M., Kelly, E., Nylander, S., Mumford, A. and Mundell, S. (2016). Inverse agonism at the P2Y12 receptor and ENT1 transporter blockade contribute to platelet inhibition by ticagrelor. Blood, 128(23), pp.2717-2728.

PB1017 | Study of the Affinity of Thrombin towards its Receptors on Platelets

A. Macwan ${ }^{1} ;$ T. Hallström ${ }^{2} ;$ T. Lindahl ${ }^{1}$

${ }^{1}$ Linköping University, Linköping, Sweden; ${ }^{2}$ NanoTemper Technologies, Munich, Germany

Background: Thrombin is the key enzyme for platelet activation and coagulation. Thrombin interacts with platelets through proteaseactivated receptors (PARs) 1 and 4 and von Willebrand factor binding glycoprotein $1 \mathrm{~b}$. Many studies reported high affinity, and low-affinity thrombin binding sites with an estimated number of sites per platelet, however, all these studies considered PAR receptors as a single site, and the exact number of each receptor was not known. Receptor number for PAR4 was reported only recently by $\mathrm{Li}$ et al. in 2020.

Aims: To analyze the interaction of thrombin with platelets using Microscale thermophoresis (MST).

Methods: Microscale thermophoresis (MST) is a technology that can analyze the interactions between biomolecules and is used to measure the affinity between two biomolecules. In this study, we are pioneers in using MST to analyze the interaction of thrombin to platelets. The exact number of each receptor per platelet was used for calculations. We used fluorescently labeled thrombin and washed platelets in the presence of various inhibitors of thrombin exosites, GP1b, PAR1, or PAR4 to study the affinity of thrombin towards its receptors.

Results: GP1b was found to be the low-affinity binding site as blocking of GP1b by its antibody did not affect the thrombin affinity significantly. Blockage of exosite 1 affected thrombin affinity the most as the PAR1 receptor is the high-affinity site and also the PAR1 receptor number is larger than that of PAR4. PAR-specific inhibitors vorapaxar or BMS-986120 did not affect thrombin binding to the platelets.

Conclusions: The affinity of thrombin towards its receptors on platelets is in the order of PAR1>PAR4>GP1b. MST is a useful and non-harmful technique that can be used to study the interaction of biomolecules with platelets.

PB1018 | Structural Characterisation of GPVI in Complex with Nanobody 2 Generates a Domain-swapped GPVI Dimer: Could this Represent a Biologically Active Conformation?

A. Slater $^{1}$; Y. Di ${ }^{1} ;$ J. Clark ${ }^{1,2} ;$ N. Jooss ${ }^{1,3} ;$ E. Martin ${ }^{1} ;$ F. Alenazy $^{1}$; M. Thomas ${ }^{1}$; R. Ariëns ${ }^{4}$; A. Herr ${ }^{5}$; N. Poulter ${ }^{1,2}$; J. Emsley ${ }^{6,2}$; S. Watson ${ }^{1,2}$

${ }^{1}$ Institute of Cardiovascular Sciences, University of Birmingham, Birmingham, United Kingdom; ${ }^{2}$ Centre of Membrane Proteins and Receptors, Birmingham and Nottingham, United Kingdom; ${ }^{3}$ Cardiovascular Research Institute, Maastricht University, Maastricht, Netherlands; ${ }^{4}$ Leeds Institute of Cardiovascular and Metabolic Medicine, University of Leeds, Leeds, United Kingdom; ${ }^{5}$ Division of Immunobiology and Division of Infectious Diseases, Cincinnati Children's hospital, Cincinnati, United States; ${ }^{6}$ Biodiscovery Institute, University of Nottingham, Nottingham, United Kingdom

Background: Glycoprotein VI (GPVI) is the major signalling receptor for collagen on platelets and is a promising anti-thrombotic target. Dimerisation of this receptor is believed to have roles in both ligand binding and signalling, but the mechanisms of GPVI dimerisation remain poorly understood. We have previously raised a series 
of nanobodies against GPVI as novel probes to further study GPVI structure and function.

Aims: We aim to map the binding sites of the nanobodies on GPVI by crystallography and competition assays, and relate to function.

Methods: The ability of the nanobodies to inhibit GPVI in response to collagen was assessed using NFAT activation reporter assays, thrombus formation of whole blood under flow, and binding of recombinant GPVI to a collagen-coated surface. The most potent nanobody was co-crystallised with recombinant GPVI. NFAT reporter assays on a truncated GPVI mutant were performed to validate the novel GPVI dimer conformation.

Results: We show that three of the nanobodies inhibited collageninduced GPVI signalling by $>90 \%$ and significantly reduced thrombus formation in whole blood in response to collagen. This inhibition was due to direct displacement of collagen binding. Solving the crystal structure of the most potent nanobody with GPVI revealed a binding epitope close to the collagen related peptide (CRP) binding site. In addition, GPVI adopted a novel domain-swapped dimer conformation, and through site directed mutagenesis, we demonstrate that the critical domain-swapped hinge region is required for GPVI signalling.

Conclusions: The solved nanobody bound crystal-structure reveals a domain-swapped GPVI dimer, which may represent a biologically active conformation. The inhibitory nanobodies provide new therapeutic frameworks for treating thrombosis.

PB1019 | Charactering the Role of TLT-1 in Inflammatory Pathogenesis of Obesity and Nonalcoholic Fatty Liver Disease (NAFLD)

S. Branfield ${ }^{1}$; B. Nieves Lopez ${ }^{2}$; M. Poynter ${ }^{3}$; A.V. Washington ${ }^{1}$ ${ }^{1}$ Oakland University, Rochester, United States; ${ }^{2}$ University of Puerto Rico, San Juan, Puerto Rico; ${ }^{3}$ University of Vermont, Burlington, United States

Background: Obesity, a nationwide health issue, has related medical costs ranging between \$147-210 billion per year in United States and is associated with a 3.5-fold increased risk of developing NAFLD. In obesity, platelets work in a pleotropic manner with vascular and immune cells to amplify the chronic inflammatory process. The emerging role of activated platelets during obesity induced inflammation introduces the novel concept of platelet targeted therapeutic interventions.

TREM-Like Transcript-1 (TLT-1) is a platelet specific receptor found in the a-granules of platelets and released to the surface upon platelet activation. TLT-1 facilitates platelet aggregation and regulates immune mediated inflammatory bleeding. When placed on a western diet, treml1-/- mice are more prone to weight gain and hyperlipidemia.

Aims: Evaluate the effects of western diet on obesity and NAFLD in the treml1-/- mouse model.
Methods: TLT-1 (treml1 ${ }^{-/}$) and Apolipoprotein E (apoe ${ }^{-/-}$) double null (AT-DKO; $n=11)$ mice and control apoe ${ }^{+/-} /$treml $^{+/-}$littermate controls (AT-Hets; $n=20$ ) were fed western diet for 20 weeks and plasma samples were evaluated for obesity related parameters.

Results: Overall AT-DKO mice gained more weight compared to AT-Hets $(12.94 \pm 8.34$ vs $8.51 \pm 1.573$ grams $P=0.02)$. Plasma analysis demonstrates that the AT-DKO have higher levels of TNF- $a$ (0.54 \pm 0.599 vs $0.118 \pm 0.192 \mathrm{pg} / \mathrm{ml} P=0.03)$, and IL10 $(2.49 \pm 1.422$ vs $1.51 \pm 2.23 \mathrm{pg} / \mathrm{ml} P=0.004)$ compared to littermate controls. Histological analysis of livers illustrates increased lipid vacuoles and inflammatory foci in the AT-DKO mice as compared to controls, while preliminary data is not significant for these differences, liver damage in the AT-DKO was significantly greater as demonstrated by increased AST levels (178.57 \pm 89.48 vs $102 \pm 68.10 \mathrm{U} / \mathrm{L} P=0.02)$.

Conclusions: Mutant AT-DKO mice are more prone to obesity and NAFLD compared to littermate controls, suggesting that TLT-1, a platelet gene, plays a surprising role in metabolism and may be an intervention target. The current state of this project will be reported here.

\section{PB1020 | The Role of Platelet CLEC-2 and GPVI in} Rhabdomyolysis-induced Acute Kidney Injury

S. Oishi ${ }^{1}$; N. Tsukiji ${ }^{2}$; S. Otake ${ }^{2} ;$ N. Oishi ${ }^{3} ;$ T. Sasaki $^{2}$; T. Shirai ${ }^{2}$

Y. Yoshikawa ${ }^{2}$; K. Takano ${ }^{2}$; H. Shinmori ${ }^{4}$; T. Inukai ${ }^{5}$; T. Kondo ${ }^{3}$;

K. Suzuki-Inoue ${ }^{2}$

${ }^{1}$ Yamanashi University, Department of Clinical and Laboratory

Medicine, Faculty of Medicine, Chuo, Japan; ${ }^{2}$ Department of Clinical and Laboratory Medicine, Faculty of Medicine, Yamanashi University, Chuo, Japan; ${ }^{3}$ Department of Pathology, Faculty of Medicine, Yamanashi University, Chuo, Japan; ${ }^{4}$ Faculty of Life and Environmental Science, University of Yamanashi, Kofu, Japan; ${ }^{5}$ Department of Pediatrics, Faculty of Medicine, University of Yamanashi, Chuo, Japan

Background: Rhabdomyolysis is a syndrome due to the breakdown of skeletal muscle caused by various reasons. The most serious complication is acute kidney injury (AKI), which is sometimes fatal, however, no effective treatment has been established. According to a recent study, heme (hemin) released from damaged muscles activates platelets, and the activated platelets induce macrophage extracellular traps (METs), resulting in exacerbation of AKI. We have reported in the last ISTH that hemin binds directly to CLEC-2 and GPVI and activates platelets via the SFK-SYK-PLC 22 pathway, and in rhabdomyolysis mouse model, deletion of CLEC-2 and GPVI reduces renal dysfunction and METs-like structures in the kidney.

Aims: To elucidate the detailed mechanisms of 1) binding of hemin to CLEC-2/GPVI and 2) METs induction by hemin-activated platelets.

Methods: 1)-1 We performed hemin-induced platelet aggregation assay with Co-HP/anti-GPVI antibody, which inhibit the binding known ligands to CLEC-2/GPVI respectively. 
1)-2 Using CLEC-2/GPVI-expressing cell lines, we performed the competitive binding assay by flow cytometry to investigate whether hemin competes with known ligands.

2) To examine whether hemin-activated platelets promote MET formation via platelet SFK pathway, we co-cultured hemin-activated platelets and macrophages with or without SFK inhibitor, and evaluated MET formation.

Results: 1)-1 Co-HP or anti-GPVI antibody inhibited hemin-induced platelet aggregation in FCR $/$ GPVI deficient mice or CLEC-2 depleted mice, respectively.

1)-2 Hemin inhibited binding of podoplanin or CRP to CLEC-2 or GPVI respectively.

2) METs induced by heme-activated platelets were suppressed by the addition of SFK inhibitor to platelets.

\section{Competitive binding assay}

CLEC-2+

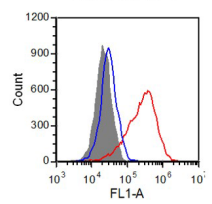

GPVI+
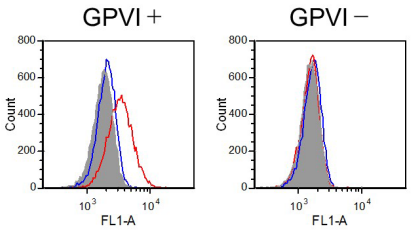

- $\operatorname{ctrl}(1 \% \mathrm{DMSO} / \mathrm{PBS})+\mathrm{hPod}-\mathrm{hFc}$

- Hemin + hPod-hFc

$\operatorname{ctrl}(1 \% \mathrm{DMSO} / \mathrm{PBS})+\operatorname{ctrl}(\mathrm{PBS})$

- $\operatorname{ctrl}(1 \% \mathrm{DMSO} / \mathrm{PBS})+\mathrm{CRP}-\mathrm{AF} 488$

- Hemin + CRP-AF488 $\operatorname{ctrl}(1 \% \mathrm{DMSO} / \mathrm{PBS})+\mathrm{hFc}$

FIGURE 1 Hemin inhibits the binding of podoplanin to CLEC-2 and CRP to GPVI

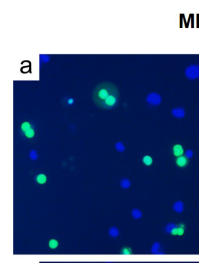

METs induction by hemin-activated platelets in vitro
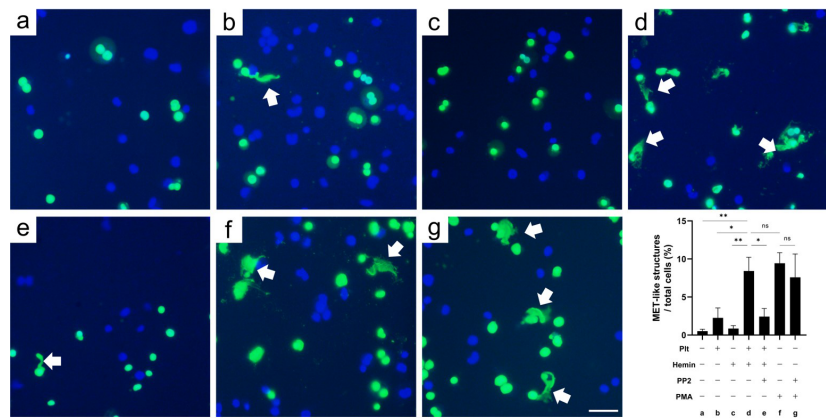

FIGURE 2 MET formation by hemin-activated platelets requires activation of the SFK pathway in platelets

Conclusions: These results suggest that 1) hemin shares the binding sites with known ligands for CLEC-2 and GPVI, and 2) the SFK pathway in platelet, which exists downstream of CLEC-2/GPVI, is important for the induction of METs by hemin-activated platelets.
PB1021 | Impact of the Common GPVI Polymorphism Is Limited to Intracellular Calcium Response upon Activation

M. Stepanyan ${ }^{1,2}$; A. Martyanov ${ }^{1,3}$; A. Boldova ${ }^{1}$; A. Filkova ${ }^{1,3}$;

A.K. Garzon Dasgupta ${ }^{1,2}$; E. Beresneva ${ }^{1}$; A. Balatsky ${ }^{4}$;

E. Ponomarenko ${ }^{3}$; A. Ignatova ${ }^{1,3} ;$ M. Panteleev ${ }^{1,2,3}$;

A. Sveshnikova ${ }^{1,2,3}$

${ }^{1}$ CTP PCP RAS, Moscow, Russian Federation; ${ }^{2} \mathrm{MSU}$, Faculty of

Physics, Moscow, Russian Federation; ${ }^{3} \mathrm{NMRC}$ PHOI, Moscow, Russian

Federation; ${ }^{4} \mathrm{MSU}$, Faculty of Medicine, Moscow, Russian Federation

Background: Glycoprotein-VI (GPVI) is the major platelet collagen receptor. Two haplotypes in GPVI encoding gene (GP6) were identified previously: allele "a" - SKTQH and allele "b" - PEALN. Allele "b» carriers are more likely to suffer from cardiovascular events. These are in contradiction with Cole et al. JTH 2003 and Snoep et al. JTH 2009, who didn't find a link between GPVI polymorphism and development of pathology. The effect of polymorphisms on platelet function (platelet aggregation, $\mathrm{P}$-selectin exposure) is also controversial. Aims: To assess the impact of GPVI polymorphisms on platelet signaling and functional responses.

Methods: 77 saliva samples were collected from healthy donors for polymorphism sequencing. Blood of the selected donors (five "aa", five "ab", two "bb" haplotypes) was collected by venipuncture and assessed by means of endpoint and continuous flow cytometry, fluorescent microscopy of thrombus growth, aggregometry. Computational model of platelet activation by GPVI covering platelet GPVI ligation, SFK and Syk activation, LAT-signalosome formation and calcium release from cytosolic calcium stores was constructed for data interpretation. System of ODEs was integrated using LSODA in Python 3.8.

Results: Platelet functional parameters (fibrinogen binding, alphagranules, dense granules, procoagulant activity, thrombus coverage area, aggregation velocity, etc) were independent from polymorphism presence. Intracellular calcium level was significantly different between carriers and non-carriers of " $b$ " allele $(81 \pm 32 \mathrm{nM}$ for "aa", 33 \pm 22 nM for "ab"+ "bb") upon platelet stimulation by $5 \mu \mathrm{g} / \mathrm{ml}$ of CRP. Computational model predicted these to be dependent on the variations in SFK activity, which was altered in the presence of polymorphism, according to the literature.

Conclusions: The polymorphisms of the platelet GPVI affect platelet calcium response upon stimulation, while platelet functional responses remain unaltered.

The study was supported by Russian Science Foundation (Grant 21-74-20087). 
PB1022 | Soluble Triggering Receptor Expressed on Myeloid

Cells Like Transcript-1 (sTLT-1) as a Biomarker for Stable Cardiovascular Diseases

Z. Bayron ${ }^{1}$; S. Branfield ${ }^{2}$; J. Menendez ${ }^{3}$; B. Nieves ${ }^{1}$; L. Ospina ${ }^{1}$; G. Maldonado ${ }^{1}$; R. Hunter ${ }^{4}$; A. Valance Washington ${ }^{2}$; L.M Melendez ${ }^{5}$; Y.M Cantres ${ }^{6}$

${ }^{1}$ University of Puerto Rico Rio Piedras Campus, San Juan, Puerto Rico; ${ }^{2}$ University of Oakland, Michigan, United States; ${ }^{3}$ University of Puerto Rico, San Juan, Puerto Rico; ${ }^{4}$ Universidad Central del Caribe, Bayamon, Puerto Rico; ${ }^{5}$ Department of Microbiology and Medical Zoology, University of Puerto Rico, Medical Sciences Campus, Translational Proteomics Center, Comprehensive Cancer Center, San Juan, Puerto Rico; ${ }^{6}$ Translational Proteomics Center, Comprehensive Cancer Center, University of Puerto Rico, Medical Sciences Campus, San Juan, Puerto Rico

Background: Cardiovascular diseases are the leading cause of death worldwide, taking an estimated of 17.9 million lives each year, mak ing their study pivotal in the medical field. Platelets are very important in thrombosis, hemorrhage, and inflammation and play a critical role in cardiovascular diseases. TREM-like transcript 1 (TLT-1), a prothrombotic membrane protein exclusive to platelets, has been linked to irregularities in clot formation especially in atherosclerosis and sepsis. Previous work demonstrated that activated platelets release a soluble form of TLT-1 (sTLT-1) found in serum but not in the plasma of healthy individuals.

Aims: 1) Evaluate if ADAMS17 is the main protease that releases TLT-1;

2) Determine if sTLT-1 can be used as marker for disease severity in patients with Cardiovascular Diseases.

Methods: We used western blotting and protease inhibitors to evaluate STLT-1 release. Soluble TLT-1 was measured retrospectively by ELISA in plasma samples from patients that are in the Preventing Events of Angiotensin Converting Enzyme (PEACE) study. We used the program "R" and SPSS to evaluate the statistical relevance of the work.

Results: Figure 1 demonstrates that ADAMS 10 and 17 are only partly responsible for sTLT-1 release. Table 1 shows the analysis of sTLT-1 levels at baseline (day 1 ) samples. The analysis revealed several significant associations. Including increased STLT-1 levels in patients that experienced arrythmia $(P=0.007)$, however, lower levels of sTLT-1 were associated with those patients that underwent percutaneous intervention ( $P=0.03$ ), had their left ventricular function qualitatively abnormal (QUALABS; $P=0.008$ ), or experienced unstable angina $(P=0.043)$. There was not, however, any association with the primary outcomes of $C V$ death $(P=0.21)$ or other death $(P=0.81)$. Interestingly, those patients that used cigarettes had on average significantly lower levels of sTLT-1 $(P=0.00012)$. Multivariant analysis revealed that QUALABS accounted for the raised levels of STLT-1.

Conclusions: Surprisingly, TLT-1 /STLT-1 may be a protective for QUALABS

\section{PLATELET SIGNALING}

LPB0085 | The Predominant Role of Arrestin3 in General GPCR Desensitization in Platelets

P.K. Chaudhary; S. Kim; S. Kim

Chungbuk National University, Cheongju, Korea, Republic of

Background: Arrestin3 and arrestin2 function in G protein-coupled receptor (GPCR) desensitization and its signaling in concert with the GPCR kinases (GRKs) in various cells. Despite the importance of GPCR-mediated signaling in platelets, little is known regarding the mechanism of GPCR desensitization by arrestins in platelets.

Aims: Therefore, we determined the functional differences of arrestin3 versus arrestin2 in the regulation of GPCR signaling and the mechanism of GPCR desensitization by arrestin3 in platelets.

Methods: We used mice lacking arrestin 3 and arrestin2 to evaluate their functional role in platelet activation.

Results: Platelet aggregation and dense-granule secretion induced by 2-MeSADP, thrombin, and AYPGKF were significantly potentiated in arrestin3-deficient platelets compared to wild-type (WT) platelets. However, platelet aggregation and secretion induced by AYPGKF and thrombin were significantly inhibited while 2-MeSADP was minimally affected in arrestin2-deficient platelets compared to WT platelets. Moreover, deficiency of arrestin2 and arrestin3 showed no effect on CRP-induced platelet aggregation and secretion. Recently, we have shown that GRK6 plays a role in platelet function through selective GPCR desensitization as it was not involved in the regulation of serotonin- and epinephrine-induced platelet aggregation. Surprisingly, in contrast to GRK6, platelet aggregation induced by co-stimulation of serotonin and epinephrine was significantly potentiated in arrestin3-deficient platelets suggesting the central role of arrestin3 in general GPCR desensitization in platelets. In addition, the second challenge of ADP and AYPGKF restored platelet aggregation in arrestin3-deficient platelets but failed to do so in WT and arrestin2-deficient platelets, confirming that arrestin3 contributes to GPCR desensitization. Furthermore, 2-MeSADP- and AYPGKFinduced Akt and ERK phosphorylation were significantly increased in arrestin3-deficient platelets. Finally, arrestin3-deficient mice were not protected against $\mathrm{FeCl}_{3}$-induced in vivo thrombosis model, indicating that arrestin3 is critical for thrombus formation in vivo.

Conclusions: In conclusion, arrestin3, not arrestin2, plays a central role in the regulation of platelet functional responses and thrombus formation through general GPCR desensitization. 
LPB0132 | Chronic Edible Dosing of $\Delta$ 9-tetrahydrocannabinol (THC) in Non-human Primates Reduces Systemic Platelet Activity and Function

S.E. Reitsma $^{1}$; J. Johnson ${ }^{1}$; J. Pang ${ }^{1}$; I. Parra-Izquierdo ${ }^{1,2}$; H. Hara Sudhan Lakshmanan ${ }^{1}$; A.R. Melrose ${ }^{2,1}$; M. T. Hinds ${ }^{1}$; J.E. Aslan ${ }^{2,1}$; O.J. McCarty ${ }^{1}$; J.O. Lo ${ }^{3}$

${ }^{1}$ Oregen Health and Science University, Portland, United States;

${ }^{2}$ Knight Cardiovascular Institute, Portland, United States; ${ }^{3}$ Department of Obstetrics and Gynecology, Portland, United States

Background: Medical cannabis is administered for chronic pain treatment based on the premise that the endocannabinoid system signals desensitize pain sensor neurons and produce anti-inflammatory effects. The major psychoactive ingredient of cannabis is $\Delta 9$ tetrahydrocannabinol (THC) which signals through cannabinoid receptor-1 ( $\mathrm{CBr}$ ); beyond neurons, $\mathrm{CBr}$ is expressed in tissues ranging from skin to blood cells including platelets. In vitro, $\mathrm{CBr}$-mediated signaling acutely inhibit platelet activation downstream of the immunotyrosine activation motif (ITAM) platelet collagen receptor GPVI. The systemic effects of chronic THC administration on platelet activity and function is unknown.

Aims: Determine the effects of chronic THC administration on platelet function in non-human primates (NHPs).

Methods: Seven female rhesus macaques (Macaca mulatta) were fed THC edibles daily, titrated up to $2.5 \mathrm{mg} / 7 \mathrm{~kg} /$ day, equivalent to a heavy medical dose in humans, over 3 months. Blood was collected every 3 weeks and platelet function was analyzed by flow cytometry and aggregometry in response to the platelet agonists collagen-related peptide (CRP-XL; GPVI/ITAM agonist), TRAP-6 (GPCR protease-activated receptor-1 agonist), ADP (GPCR P2Y12 agonist) and the Toll-like receptor 2, Pam2CSK4. In parallel, human washed platelets were pretreated with a $\mathrm{CBr}$ agonist followed by CRP-XL stimulation; phosphorylation was analyzed by Western blot. Results: Chronic THC administration in NHPs decreased platelet aggregation in a dose-dependent manner in response to CRP-XL and ADP. Platelet thromboxane production was reduced by $\geq 70 \%$ in THC-treated animals. Granule secretion as measured by $\mathrm{P}$ selectin expression was reduced in a THC dose-dependent manner compared to untreated animals in response to CRP-XL, TRAP-6, and ADP. Platelet activation induced by Pam2CSK4 remained unchanged. In vitro, a CBr agonist inhibited GPVI-mediated phosphorylation of Akt and MAPK substrates while increasing PKA-substrate phosphorylation.

Conclusions: Chronic administration of THC edibles desensitized platelet activity and function in response to ITAM- and GPCR-based activation by interfering with primary and secondary feedback signaling pathways.
PB1023 | Discovery of WNT/Planar Cell Polarity Membrane Receptors in Platelets

S.P Comer $^{1,2}$; N. Alkazemi ${ }^{1,2}$; D. Hamilton ${ }^{1,2} ;$ T. O'Neill $^{3}$;

P. Maguire ${ }^{1,2,4}$

${ }^{1}$ Conway SPHERE Research Group, Conway Institute, University

College Dublin, Dublin, Ireland; ${ }^{2}$ School of Biomolecular and Biomedical

Science, University College Dublin, Dublin, Ireland; ${ }^{3}$ Conway Institute

Imaging Facility, University College Dublin, Dublin, Ireland; ${ }^{4}$ UCD

Institute for Discovery, University College Dublin, Dublin, Ireland

Background: Platelet activity is regulated by a myriad of biochemical signalling pathways which are closely intertwined in function and outcome. We have previously shown canonical WNT signalling effectors in platelets, however, WNT/planar cell polarity (PCP) signalling has not yet been attributed to platelets. WNT/PCP regulates cell polarity and cell movement during key developmental processes such as gastrulation and neural tube closure, through the upstream regulation of small GTPases including RhoA, Rac1 and Cdc42 (Fig. 1).

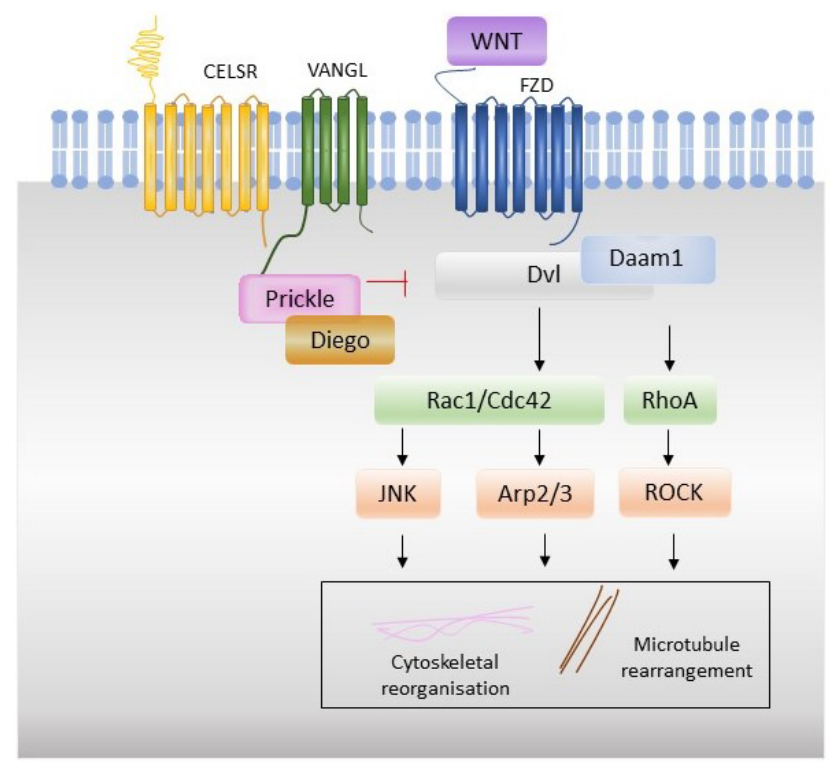

FIGURE 1 The WNT/PCP signalling pathway coordinating planar polarisation

Aims: To investigate the expression, localisation, and interaction of key WNT/PCP membrane receptors Cadherin EGF LAG Seven-pass G-type Receptor (CELSR), Frizzled (FZD), and Van Gogh-Like (Vangl), in both resting and activated platelets.

Methods: Blood was collected from healthy donors who gave consent under the Declaration of Helsinki. SDS-PAGE/Western blotting, immuno-fluorescence confocal microscopy, immuno-gold labelling electron microscopy, and co-immunoprecipitation were utilised to investigate core WNT/PCP membrane receptors in platelets under resting and varied stages of activation. 
Results: We demonstrate that the three key WNT/PCP membrane receptors CELSR, FZD and Vangl, and their downstream signalling effectors, are expressed in both human and murine platelets. In resting platelets, CELSR and Vangl were found to co-localise and co-immunoprecipitate, analogous to WNT/PCP processes regulating cell polarity during morphogenesis. Platelet activation revealed a strong association of Vangl with platelet alpha-granules, translocation of CELSR to the platelet membrane and subsequent release of CELSR on platelet microparticles.

Conclusions: WNT/PCP coordination of morphogenesis is well documented and the discovery of WNT/PCP membrane effectors in platelets adds another layer of complexity to the understanding of platelet signalling processes during activation. The physiologic/ pathologic implications of the discovery of these important cell polarity pathway membrane receptors in platelets remains to be fully elucidated.

PB1024 | Integrin allb33 Regulates Platelet Procoagulant Activity in the Lung

T. Brzoska; T.W. Kaminski; R. Vats; E. Tutuncuoglu; M.T. Gladwin; P. Sundd

University of Pittsburgh, Pittsburgh, United States

Background: Pulmonary thrombosis is a major complication associated with high morbidity. Despite advances in diagnosis and treatment, the pathophysiology of pulmonary thrombosis remains incompletely understood. New clinical evidence suggests that in situ platelet activation resulting in enhanced procoagulant activity may promote pulmonary thrombosis. Improved understanding of the etiological mechanism would enable the development of new therapies for pulmonary thrombosis.

Aims: To elucidate the contribution of allb $\beta 3$-dependent platelet procoagulant activity to the pathophysiology of pulmonary thrombosis.

Methods: Collagen and thromboplastin (TF) were administered intravascularly (IV) to C57BL/6 (WT) mice and the pulmonary microcirculation was visualized using quantitative fluorescence intravital fluorescence lung microscopy (qFILM). Fluorochrome-conjugated anti-mouse CD49b Ab and dextran was administered IV for in vivo staining of circulating platelets and visualization of blood vessels, respectively. Pulmonary thrombosis was defined as occlusion of blood vessels with platelet aggregates leading to pulmonary ischemia. Additionally, quantitative microfluidic fluorescence microscopy (qMFM) was used to study the effect of platelet allb $\beta 3$ inhibition on platelet procoagulant activity in human blood under vascular mimetic flow conditions.

Results: Collagen and TF triggered dose-dependent pulmonary thrombosis in mice in vivo, which involved development of plateletrich thrombi in the pulmonary arteriolar bottlenecks (junction of pulmonary arteriole and capillaries), resulting in a transient ischemia in the arteriole and the down-stream capillary tree. The pulmonary arteriole thrombosis triggered by IV collagen or TF was protracted, lethal and completely abrogated following IV administration of allb $\beta 3$ receptor inhibitor (eptifibatide). Inhibition of platelet allb $\beta 3$ also significantly reduced platelet procoagulant activity, fibrin formation and thrombus formation in human blood flowing through microfluidic channels ex vivo.

Conclusions: Our current findings suggest that allb $\beta 3$-dependent platelet procoagulant activity promotes pulmonary thrombosis. Both our models have potential application in investigating the molecular determinants of pulmonary thrombosis in diverse pulmonary disorders as well as evaluating efficacy of new antithrombotic drugs.

\section{PB1025 | A rRle for the JAK/STAT5 Axis in GPVI-mediated Platelet Function}

I. Parra Izquierdo ${ }^{1}$; A. R. Melrose ${ }^{1}$; J. Pang ${ }^{1}$; S.H. Vasilipalli ${ }^{1}$; S. E. Reistma ${ }^{1}$; H.H. Sudhan Lakshmanan ${ }^{1}$; M. K. Larson ${ }^{2}$; O. J.T. McCarty ${ }^{1}$; J. E. Aslan ${ }^{1}$

${ }^{1}$ Oregon Health \& Science University, Portland, United States;

${ }^{2}$ Augustana University, Sioux Falls, United States

Background: As hematopoietic cells, platelets express Janus kinase (JAK) and signal transducer and activator of transcription (STAT) proteins; however, roles for JAK/STAT and associated innate immunity signaling pathways in platelet function remain unclear. Recent phosphoproteomics studies from our group found activation of a JAK/STAT5 pathway in platelets following stimulation with collagenrelated peptide (CRP)-XL, which signals through the glycoprotein $\mathrm{VI}$ (GPVI) receptor, suggesting a role for JAK/STAT5 in GPVI-mediated platelet function.

Aims: To determine roles for the JAK/STAT5 axis in platelet function induced by GPVI activation in vitro.

Methods: Washed platelets prepared from healthy human volunteers were pretreated with five therapeutic JAK inhibitors, or "jakinibs" (ruxolitinib, oclacitinib, upadacitinib, baricitinib, tofacitinib) before stimulation with CRP-XL. Platelet functional responses were analyzed with biochemical, microscopy, flow cytometry and aggregometry assays.

Results: Ruxolitinib and baricitinib significantly reduced GPVImediated platelet aggregation, adhesion, and a-granule secretion. JAK inhibitors also inhibited platelet cytoskeletal changes, as shown by a reduction in F-actin formation and decreased spreading on fibrinogen. In contrast, platelet responses to the $\mathrm{G}$ protein-coupled receptor agonist thrombin were unaffected by jakinibs. Western blot analyses of platelet lysates probed with phosphorylation sitespecific antisera found that platelet JAK2 and STAT5 were activated in response CRP-XL and inhibited by preincubation with JAK inhibitors. In addition, all the inhibitors impaired Akt pathways activation downstream of GPVI and demonstrated specific effects on downstream mediators such as dual adaptor of phosphotyrosine and 3-phosphoinositides 1 (DAPP1) and p21 activated kinase 1 (PAK1). Pretreatment of platelets with a STAT5 inhibitor also demonstrated a 
reduction in integrin activation and a-granule secretion in response to $C R P-X L$ as well as spreading on collagen and fibrinogen.

Conclusions: The JAK inhibitors ruxolitinib and baricitinib and a STAT5 inhibitor impaired GPVI-mediated platelet adhesion, secretion, and aggregation, suggesting a role for JAK/STAT innate immunity signaling pathways in platelet hemostatic responses.

\section{PB1026 | Acetyl-CoA Carboxylase Inhibition Alters Tubulin} Acetylation and Aggregation in Thrombin-stimulated Platelets

M. Octave ${ }^{1}$; L. Pirotton ${ }^{1} ;$ A. Ginion ${ }^{1}$; V. Robaux ${ }^{1}$; S. Lepropre ${ }^{1}$; S. Kautbally ${ }^{1}$; V. Darley-Usmar ${ }^{2}$; J. Ambroise ${ }^{3}$; B. Guigas ${ }^{4}$; M. Giera $^{4}$; M. Foretz ${ }^{5}$; L. Bertrand ${ }^{1}$; C. Beauloye ${ }^{1}$; S. Horman ${ }^{1}$ ${ }^{1}$ Université Catholique de Louvain, Institut de Recherche Expérimentale et Clinique, Pôle de Recherche Cardiovasculaire, Brussels, Belgium; ${ }^{2}$ University of Alabama at Birmingham, Center for Free Radical Biology, Department of Pathology, UAB Mitochondrial Medicine Laboratory, Birmingham, United States; ${ }^{3}$ Université Catholique de Louvain, Institut de Recherche Expérimentale et Clinique, Centre de Technologies Moléculaires Appliquées, Brussels, Belgium; ${ }^{4}$ Leiden University Medical Center, Leiden, Netherlands; ${ }^{5}$ Université Paris Descartes, Institut Cochin, INSERM, U1016-CNRS UMR8104, Paris, France

Background: Acetyl-CoA carboxylase (ACC), the first enzyme regulating lipid synthesis, promotes thrombus formation by increasing platelet phospholipid content. Inhibition of its activity decreases lipogenesis and concomitantly increases the content in acetyl-CoA which can serve as a substrate for protein acetylation. This posttranslational modification plays a key role in the regulation of platelet aggregation, via tubulin acetylation.

Aims: To demonstrate that ACC inhibition may affect platelet functions via an alteration of lipid content and/or tubulin acetylation.

Methods: Platelets were treated 2 hours with CP640.186, a pharmacological ACC inhibitor, prior to thrombin stimulation. Platelet functions were assessed by aggregometry and flow cytometry. Lipogenesis was measured via ${ }^{14} \mathrm{C}$-acetate incorporation into fatty acids. Lipidomics analysis was carried out on the commercial Lipidyzer platform. Protein phosphorylation and acetylation were evaluated by western blot.

Results: Treatment with CP640.186 drastically decreased platelet lipogenesis. However, the quantitative lipidomics analyses showed that 2 hours preincubation with the compound did not affect significantly global platelet lipid content. Interestingly, this short-term ACC inhibition was sufficient to increase tubulin acetylation level, at basal state and after thrombin stimulation. It was associated with an impaired platelet aggregation, in response to low thrombin concentration, while granules secretion was not affected. Mechanistically, we highlighted a decrease in the small GTPase Rac1 activity, associated with a reduced phosphorylation of its downstream effector PAK2. Surprisingly, actin cytoskeleton was not impacted but we evidenced a significant decrease in ROS production which could result from a decreased NOX2 activity.
Conclusions: Pharmacological ACC inhibition decreases platelet aggregation upon thrombin stimulation. The mechanism depends on increased tubulin acetylation, with subsequent alteration of the Rac1/PAK2/NOX2 signaling pathway

\section{PB1027 | Quantitative Assessment of Heterogeneity of Single Platelet Calcium Responses to Activation}

F. Balabin $^{1,2}$; S. Galkina ${ }^{3}$; I. Zhizhaikina ${ }^{4}$; M. Panteleev ${ }^{1,3,2}$; A. Sveshnikova ${ }^{1,2,4,3}$

${ }^{1}$ Center for Theoretical Problems of Physico-Chemical Pharmacology, Russian Academy of Sciences, Moscow, Russian Federation; ${ }^{2}$ National Medical Research Center of Pediatric Hematology, Oncology and Immunology named after Dmitry Rogachev, Moscow, Russian Federation; ${ }^{3}$ Faculty of Physics, Lomonosov Moscow State University, Moscow, Russian Federation; ${ }^{4}$ Department of Normal Physiology, Sechenov First Moscow State Medical University, Moscow, Russian Federation

Background: Platelet activation relies on calcium signaling, however, platelets display heterogeneity in their calcium dynamics and functional responses upon stimulation. We analyze the heterogeneity of single platelet calcium responses to collagen, ADP and thrombin in healthy donors and hematological patients.

Aims: We aim to develop a diagnostic method for abnormal platelet activation detection relying on classification of single-platelet calcium profiles.

Methods: Whole hirudinated blood of 9 Wiskott- Aldrich Syndrome, 3 immune thrombocytopenia and 7 MYH9-related thrombocytopenia patients and 18 healthy donors was collected in compliance with the Declaration of Helsinki. Fluorescence microscopy was used for characterization of platelet calcium responses.

Results: We distinguished four types of platelet calcium response: "no response" (i) with rare spiking, "spiking" (ii) with calcium oscillations with period $\mathrm{T}=3-10 \mathrm{~s}$, "clusters" (iii) with several clusters of jammed together spikes, and "sustained high" (iiii) with a sustained high calcium level. The distribution of platelets of healthy donors shifted to response types that indicated higher calcium levels upon activation. Using bootstrap method, we obtained confidence intervals for mean fractions of healthy donors' platelet populations within the groups. We provide a tool that tells if a patient has a normal platelet activation pattern by checking if fractions of his or her platelet population fit into these confidence intervals. Platelet calcium response profile of a patient with Wiskott-Aldrich syndrome has demonstrated weaker response to activation on neutral coating, while on collagen it demonstrated strong responses with $4 \%, 20 \%$, $20 \%$ and $56 \%$ fractions of platelet population in groups (i), (ii), (iii), (iiii), respectively.

Conclusions: The distribution of single platelets between response groups could be utilized as a diagnostic technique to determine the fractions of refractory platelets or hyper-active platelets within the 
platelet population. The reported study was funded by RFBR and the Royal Society of London (RS), project number 21-51-10005

PB1028 | Curcumin Inhibits Platelets by Activation of Adenosine A2 Receptor and cAMP/PKA Pathway

N. Rukoyatkina ${ }^{1}$; N. Al Arawe ${ }^{2}$; S. Gambaryan ${ }^{1}$; V. Shpakova ${ }^{1}$

${ }^{1}$ Sechenov Institute of Evolutionary Physiology and Biochemistry of

Russian Academy of Sciences, Saint Petersbug, Russian Federation;

${ }^{2}$ Saint Petersburg State University, Saint Petersburg, Russian Federation

Background: Curcumin is a natural bioactive component derived from Curcuma longa which possesses a range of beneficial activities on human cells. Curcumin inhibits platelet aggregation and activation, however molecular mechanisms of curcumin inhibitory effect are not fully defined.

Aims: Cyclic nucleotides are known as a major inhibitory system in platelets and we tested whether curcumin activated PKA or PKG in these cells.

Methods: Washed platelets were prepared by centrifugation from the whole blood of healthy donors. Activation of PKA or PKG was monitored by phosphorylation of vasodilator-stimulated phosphoprotein (VASP) and calcium and diacylglycerol-regulated guanine nucleotide exchange factor I (CalDAG-GEFI) by Western blotting. Adenylate cyclase (AC) activity was inhibited by SQ22536 (60 $\mu \mathrm{M}), \mathrm{ODQ}(10 \mu \mathrm{M})$ was used as inhibitor of soluble guanylate $c y-$ clase (sGC). For analysis of $A C$ activation adenosine receptor $A 2 A$ (ZM241385) and prostanoid receptors including DP1, IP3, EP4 (BW A868C, CAY 10441, L161.982 respectively) inhibitors were used.

Results: Curcumin at concentration 10 and $50 \mu \mathrm{M}$ significantly increased VASP and CaIDAG-GEFI phosphorylation during $10 \mathrm{~min}$ and 60 min of application. SQ22536, but not ODQ, prevented curcumininduced VASP and CaIDAG-GEFI phosphorylation indicating that curcumin activated cAMP/PKA, not cGMP/PKG system. Next we tested which GPCR was responsible for curcumin-induced $A C$ activation. VASP and CaIDAG-GEFI phosphorylation were prevented only by A2A adenosine receptor inhibitor (ZM241385) and did not change after application of inhibitors of prostanoid receptors.

Conclusions: Platelet inhibition by curcumin, at least partly, is mediated by A2A adenosine receptor and PKA activation. The study was funded by RFBR (19-315-90102).

PB1029 | Annexin V Binding Detects Platelet Procoagulant Commitment but Is Not Sensitive to Reductions in the Level of Platelet PS Exposure

S. Millington-Burgess; M. Harper

University of Cambridge, Cambridge, United Kingdom

Background: Procoagulant platelets expose phosphatidylserine (PS) on their surface, where it supports thrombin generation, through activation of a scramblase protein (TMEM16F). Inhibiting platelet PS exposure could be a novel anti-thrombotic approach, though currently there are no known selective inhibitors of platelet PS exposure. Platelet PS exposure is commonly quantified by the \% of platelets that bind annexin $\mathrm{V}$ ( $\mathrm{VV} \%+\mathrm{ve}$ ). This detection and anlaysis method, though convenient, may not be the most sensitive assay for screening novel inhibitors of platelet PS exposure.

Aims: Characterise the sensitivities of different PS exposure assays. Methods: Washed human platelets were incubated with R5421 or DMSO. Scramblase and flippase activity were measured by flow cytometry using NBD-PS. PS expsoure following stimulation with $10 \mu \mathrm{M}$ A23187 was measured using multiple assays: a plate-based luminesence $A V$-binding assay, end-point and real-time flow cytometry assays using AV-FITC, lactadherin-FITC, or FRET pair AV-eGFP/ AV-Alexa594. Liposomes containing different \%PS were detected using each assay.

Results: Liposomes containing different \%PS demonstrated that end-point AV binding by flow cytometry was the least sensitive measure of membrane PS composition. Decreased PS exposure following treatment with R5421 was not detectable using single colour AV \%+ve analysis but could be detected using NBD-PS-, lactadherin, AV FRET- and luminesence-based assays. Alternative analysis of single colour AV binding could convey the inhibition of PS exposure by R5421.

Conclusions: Anlaysis of platelet PS exposure by AV \%+ve measures the commitment of platelets to becoming procoagulant but is not sensitive to differences in the extent of PS exposure.

\section{PB1030 | Resveratrol Prevents Platelet Activation by Inhibition of ROS Formation}

N. Al Arawe ${ }^{1}$; V. Shpakova ${ }^{2}$; N. Rukoyatkina ${ }^{2}$; S. Gambaryan ${ }^{2,1}$

${ }^{1}$ Department of Cytology and Histology, Saint Petersburg State University, Saint Petersburg, Russian Federation; ${ }^{2}$ Sechenov Institute of Evolutionary Physiology and Biochemistry, Russian Academy of Sciences, Saint Petersburg, Russian Federation

Background: Many natural compounds, including polyphenols, isolated from different plants are characterized by anti-inflammatory and antithrombotic properties and they are often used to reduce the risk of cardiovascular disease. Resveratrol is a natural polyphenol that exhibits many therapeutic effects including inhibition of platelet activation; however the molecular mechanism of its action on platelets is not fully defined.

Aims: Because cyclic nucleotides (cAMP, cGMP) and corresponding protein kinases (PKA, PKG) are the main intracellular platelet inhibitory mechanisms, we tested whether platelet inhibition by resveratrol is mediated by activation of these pathways, or by other mechanisms.

Methods: Platelets were activated by thrombin and collagen-related peptide (CRP). Flow cytometry was used to assess platelet activation (Fibrinogen-Alexa-647), platelet viability (Calcein-AM), and reactive 
oxygen species (ROS) formation (DCF-DA). Activity of kinases involved in platelet stimulatory pathways PKB ERK1,2 were analyzed by Western blotting. Caspase 3 activation was used as a marker of apoptosis. Phosphorylation of the Vasodilator-stimulated protein (VASP) was used as a marker of these kinases activation.

Results: Resveratrol showed a dose-dependent inhibition of washed platelets activation induced by thrombin or CRP followed by diminished phosphorylation of PKB and Erk. Resveratrol, even at high concertation $(100 \mu \mathrm{M})$ did not induce VASP phosphorylation, indicating that its inhibitory effect is not mediated by PKA/ PKG activation. Platelet inhibition also could be connected with initiation of apoptosis, or reduction of platelet viability. Therefore, we tested whether resveratrol activates caspase-dependent apoptosis or induces platelet death. Resveratrol in all tested concentrations (1 $-100 \mu \mathrm{M})$ had no effect on caspase 3 activation and platelet viability. ROS are involved in platelet activatory pathways and CRP and thrombin strongly initiated ROS production in platelets. Resveratrol concentration-dependently inhibited ROS formation induced by CRP and thrombin.

Conclusions: All our data indicated that inhibitory effects of resveratrol in platelet activation are mainly mediated by the prevention of ROS formation.

PB1031 | Combined Antiplatelet Therapy Reduces the Proinflammatory Properties of Activated Platelets

A.C. Heinzmann; D.M Coenen; T. Vajen; J.M. Cosemans; R.R Koenen

Maastricht University, CARIM - School for Cardiovascular Diseases, Maastricht, Netherlands

Background: The cause of atherothrombosis is rupture or erosion of atherosclerotic lesions, leading to myocardial infarction or stroke. Here, platelet activation plays a major role, leading to the release of bioactive molecules, e.g. chemokines and coagulation factors, and to platelet clot formation. Several antiplatelet therapies have been developed for secondary prevention of cardiovascular events, in which anticoagulant drugs are often combined. Besides playing a role in haemostasis, platelets are also involved in inflammation. However, it is unclear whether current antiplatelet therapy also affects platelet immune functions.

Aims: In this study, the possible anti-inflammatory effects of antiplatelet medications were investigated on chemokine release.

Methods: Platelets were treated with various clinically applied antiplatelet drugs (acetyl-salicylic acid, cilostazol, P2Y $_{12}$ antagonists) and chemokine release was measured using ELISA. Chemotaxis of THP-1 cells towards platelet releasates was measured using Boyden chambers.

Results: We found that antiplatelet medication acetylsalicylic acid (ASA) led to reduced Chemokine (C-C motif) ligand 5 (CCL5) and chemokine ( $\mathrm{C}-\mathrm{X}-\mathrm{C}$ motif) ligand 4 ( $\mathrm{CXCL4}$ ) release from platelets, while leukocyte chemotaxis was not affected. Depending on the agonist, $a_{11 b} \beta_{3}$ - and P2Y 12 -inhibitors also affected CCL5 or CXCL4 release. The combination of acetyl-salicylic acid with a $P 2 Y_{12}$ inhibitor or a phosphodiesterase inhibitor did not provide an additive reduction on CCL5 or CXCL4 release. Interestingly, these combinations did reduce leukocyte chemotaxis.

Conclusions: This study provides evidence that combined therapy of ASA and a $\mathrm{P}_{2} \mathrm{Y}_{12}$ or PDE3 inhibitor can decrease the inflammatory leukocyte recruiting potential of the releasate of activated platelets.

PB1032 | Targeted Proteasomal Degradation of Platelet BTK and TEC as a Novel Approach in Thrombosis Prevention

A. Munkacsi; K. Sledz; I. Hers

University of Bristol, Bristol, United Kingdom

Background: Bruton's tyrosine kinase (BTK) plays an important role in platelet signalling downstream of GPVI receptor and has been proposed as a novel target to prevent thrombosis in high-risk patients. Current, clinically used BTK inhibitors (e.g. acalabrutinib, ibrutinib), however, have off target effects and are associated with an increased risk of bleeding.

Aims: Our study is focussed on the targeted chemical degradation of BTK in human platelets by using recently developed heterobifunctional small molecule degraders (PROteolysis TArgeting Chimeras; PROTACs) that utilise the proteasomal system to degrade BTK. We aim to explore the repurposing potential of PROTACs as novel antiplatelet therapy and to see whether these compounds can be used as pharmacological research tools to study platelet function.

Methods: Human platelet rich plasma (PRP) was treated with various concentrations of the PROTACs. Samples were then either lysed and subjected to immunoblotting, or were assessed for integrin $a_{1 \mid b} b_{3}$ activation, $\mathrm{P}$-selectin expression and Annexin $\mathrm{V}$ binding in response to the GPVI and PAR agonists CRP or a-thrombin, respectively.

Results: The multi-tyrosine kinase degrader TL 12-186 and BTK degraders show potent BTK degradation, which can be overcome by addition of proteasomal inhibitors. GPVI-mediated integrin activation, P-selectin expression and PS exposure were significantly impaired, whereas PAR-mediated responses were unaffected. Tandem mass tagging confirmed the high specificity of BTK degradation.

Conclusions: Our data confirm the high susceptibility of human platelets to BTK degraders, therefore PROTACs can be successfully utilised in modulating and studying human platelet function. 
PB1033 | Antiplatelet Effect of Nobiletin Is Mediated by Activation of DP1 Receptor

V. Shpakova ${ }^{1}$; A. Avdeeva ${ }^{2}$; S. Gambaryan ${ }^{1}$; N. Rukoyatkina ${ }^{1}$

${ }^{1}$ Sechenov Institute of Evolutionary Physiology and Biochemistry of Russian Academy of Sciences, Saint Petersbug, Russian Federation;

${ }^{2}$ Saint Petersburg State University, Saint Petersburg, Russian Federation

Background: Nobiletin is a polymethoxylated flavone which possesses a wide range of beneficial effects and a low toxicity. Specifically, inhibition of platelet aggregation and adhesion by Nobiletin was demonstrated, hence, this compound may be used in antiplatelet therapy. However, the exact mechanisms of Nobiletin effects on platelets are not fully defined.

Aims: Here we investigated molecular mechanisms of Nobiletin effects on platelets.

Methods: Washed platelets from healthy donors were incubated with Nobiletin and analyzed by flow cytometry or Western blotting. Phosphatidylserine (PS) surface expression and allb $\beta 3$ integrins activation were assessed by flow cytometry. Vasodilator-stimulated phosphoprotein (VASP) phosphorylation and caspase-3 activation were analyzed by Western blotting.

Results: Nobiletin dose-dependently $(25-75 \mu \mathrm{M})$ inhibited allb $\beta 3$ integrins activation induced by Thrombin or CRP (collagen receptor peptide). Nobiletin did not cause PS exposure or caspase-3 cleavage indicating that inhibitory effect was not related to platelet apoptosis and stipulated by induction of intracellular inhibitory pathways in platelets. The major inhibition mechanisms in platelets are mediated by adenylate (AC) and guanylate cyclases (GC) activation and consequent synthesis of cyclic AMP and GMP which activate protein kinases $A$ and $G$ respectively. Activation of the inhibitory pathways can be monitored by the phosphorylation of the substrate protein VASP. We showed that Nobiletin induces VASP phosphorylation in less than 1 minute after incubation, and the phosphorylation is blocked by AC inhibitor SQ22536 but not GC inhibitor ODQ. We suggested that AC activation in this case is mediated by surface receptors activation. We examined four inhibitors (ZM241385, BWA868C, CAY10441, and L161982) for $A_{2} A$, DP1, IP and $P G E_{2}$ receptors which activate $A C$ in platelets. Only DP1 receptor inhibition blocked Nobiletin-induced VASP phosphorylation.

Conclusions: In conclusion, we showed that inhibitory effect of Nobiletin on platelets is mediated by activation of DP1 receptor. The study was funded by RFBR (19-315-90102).

PB1034 | Investigation of Core and Shell Platelet Mechanics through $\mathrm{P}_{2} \mathrm{Y}_{12}$ Antagonism

M. DeCortin; S. Diamond

University of Pennsylvania, Philadelphia, United States

Background: Hemostatic clots have a P-selectin positive core surrounded by a $\mathrm{P}$-seletctin negative shell where $\mathrm{P} 2 \mathrm{Y}_{12}$ activation is necessary for platelet recruitment.
Aims: To understand the contribution of $\mathrm{P}_{2} \mathrm{Y}_{12}$ signaling to core/ shell mechanics and stability in human thrombi utilizing a 2-step microfluidic assay.

Methods: A 2-stage assay perfused whole blood over a collagen/ tissue factor surface at a wall shear rate of $100 \mathrm{~s}^{-1}$ followed by perfusion of a buffer at $1000 \mathrm{~s}^{-1}$. A P2Y 12 antagonist was utilized to block ADP binding to the integrin. Platelet deposition and P-selectin expression are measured with immunofluorescence.

Results: P2Y 12 antagonism with 2-MeSAMP drastically lowers platelet aggregation and the number of $\mathrm{P}$-selectin positive platelets when blood is perfused over a collagen/tissue factor when compared to the absence of 2-MeSAMP for 20 minutes. Occlusion is not reached with platelets constantly attaching and detaching from the thrombus. Utilizing an extended height 8-channel device, clot growth is immediately stopped when switching to buffer well before clot occlusion. When subjected to increase shear and switch to buffer at $180 \mathrm{~s}, 2$-MeSAMP treated clots are highly unstable, and large platelet masses detach immediately. This is in contrast to control clots which erode at a much slower rate. 2-MeSAMP treated clots erode by almost $60 \%$ at 1200 s compared to $40 \%$ for control clots. $\mathrm{P}_{2} \mathrm{Y}_{12}$ antagonism lowered the stability of P-selectin negative platelets while the P-selectin positive core region is stable under both conditions.

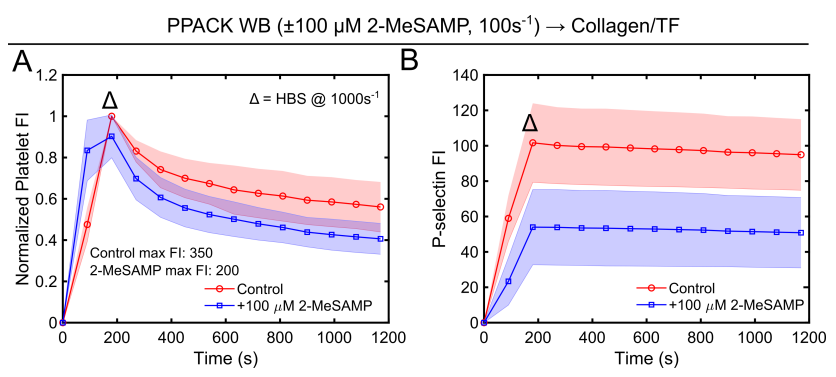

FIGURE 1 P2Y12 antagonism increases platelet erosion (A) and lowers the number of $\mathrm{P}$-selectin positive platelets (B)

Conclusions: The 2-stage microfluidic assay allows for interrogation core/shell mechanics by stopping thrombus growth and then examining platelet-platelet interactions through the use of shear. P2 $\mathrm{Y}_{12}$ antagonism greatly limits the formation of stable shell region while limiting core formation. 


\section{VASCULAR BIOLOGY}

\section{BLOOD CELLS AND VESSEL WALL}

LPB0037 | Neutrophil Extracellular Traps in Thrombosis of Ovine Extracorporeal Membrane Oxygenation Models

Y. Zhang ${ }^{1}$; R. Peng ${ }^{1}$; S. Gao ${ }^{2} ;$ B. Ji ${ }^{2} ;$ Z. Zhou ${ }^{1}$

${ }^{1}$ Department of Laboratory Medicine, State Key Laboratory

of Cardiovascular Disease, Fuwai Hospital, National Center

for Cardiovascular Diseases, Beijing, China; ${ }^{2}$ Department of

Cardiopulmonary Bypass, State Key Laboratory of Cardiovascular

Disease, Fuwai Hospital, National Center for Cardiovascular Diseases,

Beijing, China

Background: The balance between thrombosis and hemostasis is a difficult issue during extracorporeal membrane oxygenation (ECMO) support. The pathogenesis of thrombotic complications under ECMO support, as well as the exploration of predictive markers, are still largely unknown. Neutrophil extracellular traps (NETs) have been reported to participated in thrombosis and related to inflammation. However, the role in thrombotic complications of ECMO has not been studied.

Aims: To explore the role of NETs in thrombosis during ECMO support, and to investigate whether NETs as a predictive biomarker for thrombotic complications with ECMO assistance.

Methods: Ten ovine ECMO models were established, including five venoarterial (VA) and five venovenous (VV) models. Venous blood samples were collected at 1 day before ECMO (baseline), 6 hours (post-ECMO) and $1-5$ days ( $D+1-D+5)$ after the ECMO runs. Markers of NETs were detected in plasma, neutrophils and thrombus specimens on the membrane. The study was approved by medical ethics committee. Statistical significance was assessed by unpaired t test. Results: The levels of neutrophils and the markers of NETs (dsDNA and $\mathrm{CitH} 3$ ) were significantly elevated after $6 \mathrm{~h}$ after the ECMO runs, compared with those at baseline (Figure1 A-B). The levels of dsDNA and $\mathrm{CitH} 3$ at the time points of $6 \mathrm{~h}$ and $\mathrm{D}+1-\mathrm{D}+5$ were significantly higher in the VA than in the VV mode (Figure 1C).

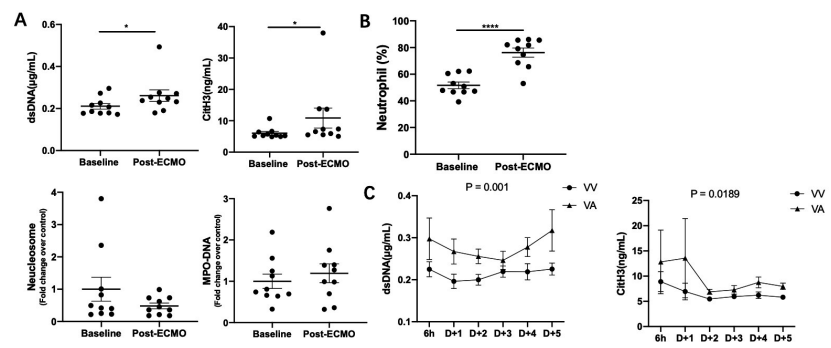

FIGURE 1 The increased levels of neutrophils and markers of NETs at $6 \mathrm{~h}$ after the ECMO runs(A-B). The levels of dsDNA and CitH3 were significantly higher in the $\mathrm{VA}$ than in the $\mathrm{V} V \bmod (\mathrm{C})$
The isolated neutrophils from fresh blood samples at the time of $6 \mathrm{~h}$ could release more NETs (Figure 2A-B). NETs also existed in thrombi on the membrane (Figure $2 \mathrm{C}$ ).
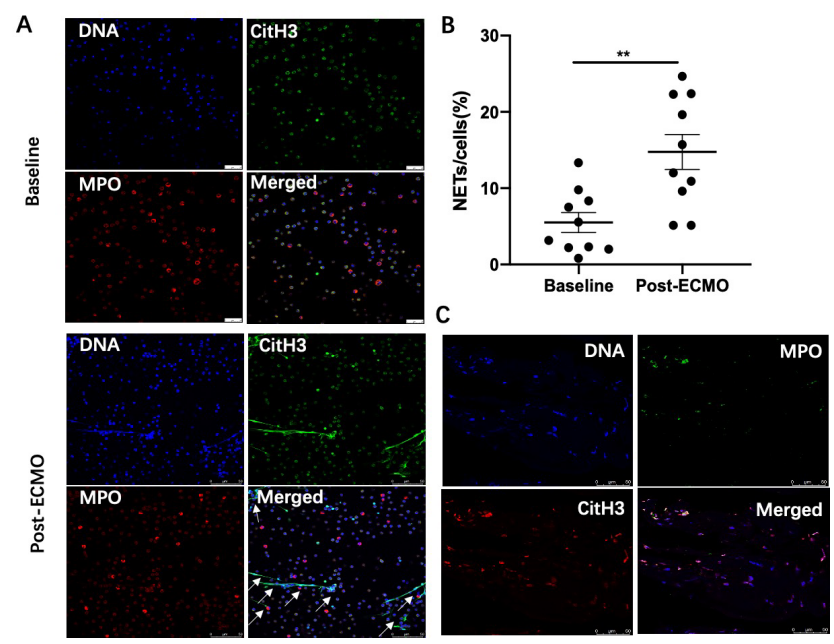

FIGURE 2: The isolated neutrophils at the time of $6 \mathrm{~h}$ could release more NETs (Figure 2A-B). NETs existed in thrombi on the membrane (C)

Conclusions: We are the first to demonstrate that altered NETs levels might play a role in thrombosis during ovine models of ECMO support, especially in the VA mode. These findings provide a new insight into the prevention of thrombotic complications by targeting NETs.

LPB0038 | Neutrophil Binding on E-selectin: a Potential Biomarker of Clinical Course and Response to Therapy in Sickle Cell Disease

$\underline{\text { Y. Man }}{ }^{1}$; E. Kucukal ${ }^{1}$; S. Liü ${ }^{1}$; R. An ${ }^{1}$; U. Goreke ${ }^{1}$; W.J. Wulftange ${ }^{1}$; Z. Sekyonda ${ }^{1}$; K. Cheng ${ }^{1}$; A. Bode ${ }^{1,2}$; A. Hill ${ }^{1,2}$; K. Monchamp ${ }^{1,2}$; J.A. Little ${ }^{3}$; D. Manwani ${ }^{4}$; E.X. Stavrou ${ }^{1,5}$; U.A. Gurkan ${ }^{1}$ ${ }^{1}$ Case Western Reserve University, Cleveland, United States; ${ }^{2}$ University Hospitals Cleveland Medical Center, Cleveland, United States;

${ }^{3}$ University of North Carolina, Chapel Hill, United States; ${ }^{4}$ Albert Einstein College of Medicine, Bronx, United States; ${ }^{5}$ Louis Stokes Cleveland Veterans Administration Medical Center, Cleveland, United States

Background: Neutrophil recruitment to inflamed endothelium significantly contributes to the hypercoagulable state seen in sickle cell disease (SCD). We previously demonstrated that neutrophil binding on E-selectin was significantly enhanced under hypoxia in SCD. However, we observed considerable heterogeneity in adhesion profiles and clinical characteristics of SCD. Here, we interrogated if the differential interaction of neutrophils with E-selectin is mechanistically linked to clinical features and the course of SCD. 
Aims: To investigate if profiles of differential neutrophil binding on E-selectin correlate with clinical characteristics of SCD.

Methods: Venous blood samples were collected from 35 adult patients with homozygous (HbSS) SCD in EDTA vacutainers during a non-crisis clinic visit. Samples were re-calcified with Hank's buffer $(1: 1 \mathrm{v} / \mathrm{v})$ and injected into E-selectin immobilized microchannels at typical shear stress values seen in post-capillary venules. Neutrophils bound on E-selectin under shear were quantified in a 32 $\mathrm{cm}^{2}$ window (Fig 1A-B).

Results: Two groups of patients with distinct lactate dehydrogenase (LDH) levels and absolute reticulocyte counts (ARCs) were identified based on K-means cluttering analysis (Fig. 1C). Group 2 patients $(N=19)$ had significantly higher LDH levels and ARCs as well as lower number of neutrophils bound on E-selectin (Fig. 1D) and fetal hemoglobin (HbF) levels (Fig. 1E) compared to Group 1 patients $(N=16)$. Moreover, 79\% (15/19) of Group 2 patients were transfusion-dependent compared to $31 \%(5 / 16)$ of Group 1 patients. Mechanistically, the degree of neutrophil activation, assessed by L-selectin shedding/blockade, was inversely related to neutrophil binding on E-selectin (Fig. 1F-H).

Conclusions: Our results show that SCD patients with a more severe hemolytic phenotype and greater transfusion dependency have constitutively less neutrophil binding on E-selectin. Further, profiling neutrophil adhesion may help predict response to anti-E-selectin therapy. Future experiments will focus on analyzing neutrophil adhesion on ICAM-1 or endothelial cells for assessing $\alpha_{M} \beta_{2}$ upregulation levels.
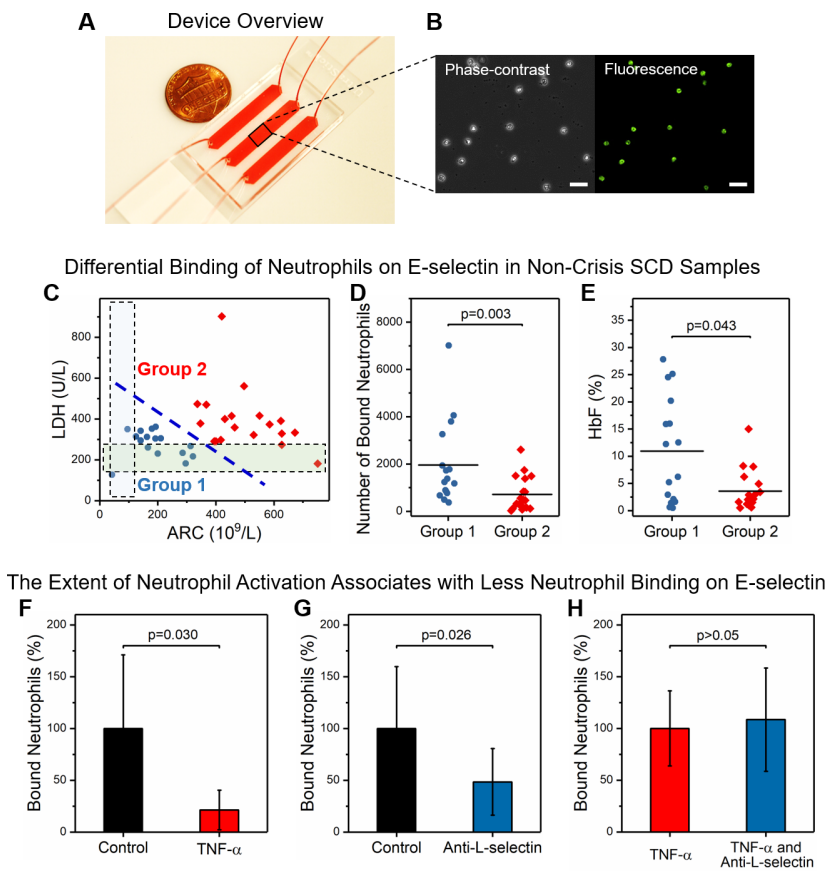

FIGURE 1 Neutrophil binding on E-selectin correlates with clinical variables in SCD. (A) Macroscopic view of the 3-channel microfluidic device whereby 3 identical microchannels with separate inlets and outlets, each 50- $\mu \mathrm{m}$ tall, 4-mm wide, and $25-\mathrm{mm}$ long, are shown.
(B) Adherent cells were permeabilized and stained for neutrophil elastase. Scale: $50 \mu \mathrm{m}$. (C) Two sub-groups of patients with distinct $\mathrm{LDH}$ levels and ARCs were identified via K-means clustering analysis. The dashed rectangular regions represent normal ranges for the given clinical parameters. Group 2 patients $(N=19)$ had significantly lower (D) number of neutrophils bound on E-selectin $(P=0.003$, Mann-Whitney) and (E) HbF levels ( $P=0.043$, Mann-Whitney) compared to Group 1 patients $(N=16)$. (F) Neutrophil activation by $25 \mu \mathrm{g} / \mathrm{mL}$ TNF-a or (G) L-selectin blockade by $10 \mu \mathrm{g} / \mathrm{mL}$ anti-Lselectin antibody led to significantly decreased neutrophils bound on E-selectin compared to the control $(P=0.030$ or $P=0.026$, pared t-test, $N=5$ in each group). Neutrophil adhesion was normalized based on the mean adhesion value of control samples and shown in percentage. (G) L-selectin blockade had no reducing effect on TNF-a stimulated neutrophils binding on E-selectin $(P>0.05, N=4$ in each group). Neutrophil adhesion was normalized based on the mean adhesion value of TNF-a stimulated samples and shown in percentage. LDH: lactate dehydrogenase. ARCs: absolute reticulocyte counts. HbF: fetal hemoglobin. TNF- $a$ : tumor necrosis factor- $a$. Data cross lines represent the mean. Error bars indicate the standard deviation.

LPB0086 | GDP/GTP Exchange Factor MADD Drives Activation and Recruitment of Secretory Rab GTPases to Weibel-Palade Bodies

M. Kat ${ }^{1}$; P. Bürgisser ${ }^{2}$; H. Janssen ${ }^{3}$; I. De Cuyper ${ }^{1}$; I. Conte ${ }^{4}$; A. Hume ${ }^{5}$; T. Carter ${ }^{4}$; J. Voorberg ${ }^{1}$; C. Margadant ${ }^{6}$; R. Bierings ${ }^{2}$ ${ }^{1}$ Sanquin Research and Landsteiner laboratory, Amsterdam, Netherlands; ${ }^{2}$ Erasmus Medical Center, Rotterdam, Netherlands; ${ }^{3}$ Netherlands Cancer Institute, Amsterdam, Netherlands; ${ }^{4} \mathrm{St}$ George's University of London, London, United Kingdom; ${ }^{5}$ University of Nottingham, Nottingham, United Kingdom; ${ }^{6}$ Cancer Center Amsterdam, Amsterdam, Netherlands

Background: Von Willebrand factor (VWF) is an essential hemostatic protein that is synthesized and secreted by endothelial cells and stored in Weibel-Palade bodies (WPBs). The secretory Rab GTPases Rab27A, Rab3B and Rab3D have been linked with WPB trafficking and secretion. How these Rabs are activated and recruited to WPBs remains elusive. Rabs require a guanine exchange factor (GEF) for their activation and recruitment to specific membranes. Previously, MAP-kinase activating death domain (MADD) has been identified as a GEF for Rab27A and Rab3 isoforms in melanocytes and neuroendocrine cells, respectively.

Aims: We investigated if and how MADD is involved in the regulation of VWF secretion.

Methods: We performed shRNA-based knockdown in primary endothelial cells and determined intracellular localization of WPBs and Rabs by immunofluorescence microscopy. Rab activity was assessed using GST-tagged Rab-specific effectors as pulldown bait and readout by Western blotting. VWF secretion was measured by ELISA. 
Results: Rab activity assays revealed a reduction in Rab27A, Rab3D, and Rab3B activation upon MADD silencing (Figure 1). Rab activation was dependent on the DENN domain of MADD. Furthermore, immunofluorescent analysis showed that Rab27A and Rab3D recruitment to WPBs was dramatically decreased upon MADD knockdown, revealing that MADD drives Rab membrane targeting (Figure 2). Artificial mistargeting of MADD using a TOMM70tag abolished Rab27A localization to WPB membranes in a DENN domain-dependent manner, indicating that normal MADD localization in the cytosol is crucial. MADD silencing did not have an impact on functional WPB biogenesis, MADD-depleted cells however exhibited decreased histamine-evoked VWF release, similar to Rab27A-depleted cells. Activation of Rab3B and Rab3D was reduced upon Rab27A silencing, suggesting that activation of these Rabs is enhanced through prior activation of Rab27A by MADD.

Figure 1.

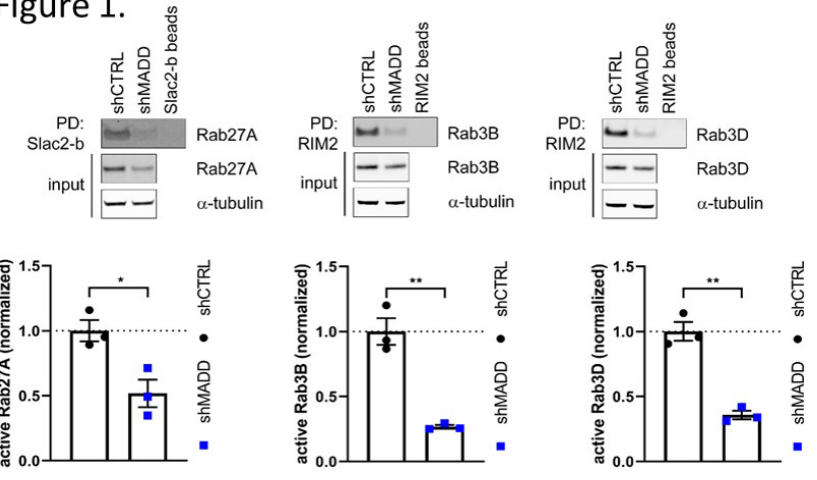

FIGURE 1 MADD is a guanine nucleotide exchange factor for secretory Rabs

Figure 2.
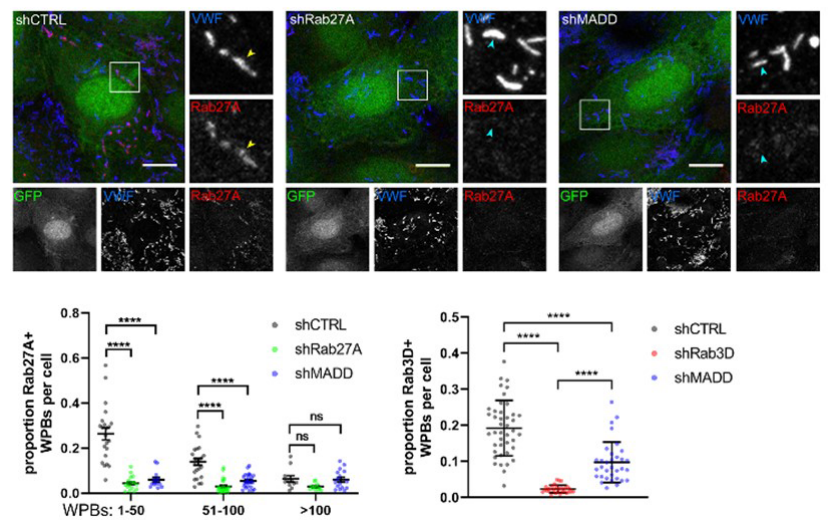

FIGURE 2 Rab recruitment to WPBs is decreased upon MADD silencing

Conclusions: MADD acts as a master regulator in VWF secretion by coordinating the activation and membrane targeting of secretory Rabs to WPBs.
LPB0133 | Endothelial Cell and Neutrophil Interactions Are Required for the Activation of the Blood Coagulation Cascade Leading to Thrombus Formation Following a Laser-induced Injury

E. Carminita $^{1}$; L. Crescence ${ }^{1}$; N. Brouilly ${ }^{2}$; L. Panicot-Dubois ${ }^{1}$;

C. Dubois ${ }^{1}$

${ }^{1}$ C2VN INSERM 1263, Marseille, France; ${ }^{2}$ IBDM, Marseille, France

Background: Platelets are classically described to play an important role in the generation of fibrin at the site of injury by supporting the generation of thrombin. Indeed, in vitro, thrombin and collagen are both needed to induce the expression of negative phospholipids at the surface of (COATED) platelets. However, in the in vivo laserinduced injury model, thrombus formation and fibrin generation are independent of platelet-collagen interactions (Dubois et al., Blood, 2006). Neutrophils were described to be the first cells recruited at the site of injury. The presence and role of procoagulant platelets in this model is to date unknown.

Aims: The goal of our study was to investigate the role of endothelial cells, neutrophils and platelets on the activation of the blood coagulation cascade.

Methods: In vivo, the contribution of endothelial cells and platelets was determined using intravital confocal microscopy. Negative phospholipids signal was detected using fluorescent Annexin-V. In vitro, both static and flow endothelial cell culture (IBIDI system) were studied.

Results: In vivo, following a laser-induced injury, fibrin was colocalized with endothelial cells and neutrophils but not with platelets. Depletion of platelets did not affect the generation of fibrin. The presence of negative phospholipids was detected on the endothelial cells and neutrophils but not on platelets. The interaction of neutrophils with activated endothelial cells is sufficient enough to activate the coagulation cascade. Interestingly, whereas the platelet thrombus reaches a maximal size 80 to $120 \mathrm{sec}$ post-injury, fibrin generation constantly increases for $6 \mathrm{~h}$ following the laser injury surrounding the vessel wall.

Conclusions: We conclude that endothelial cells and neutrophils but not platelets are implicated in the activation of the blood coagulation cascade leading to thrombus formation following a laser induced injury in living mice. Moreover, in vitro experiments confirm that activated endothelial cell express negative phospholipids.

\section{PB1035 | Rapid Internalization and Nuclear Translocation of CCL5 and CXCL4 in Endothelial Cells}

A. Dickhout; M.A. van Zandvoort; R.R Koenen

Maastricht University, CARIM - School for Cardiovascular Diseases, Maastricht, Netherlands

Background: Activated platelets are known to release the chemokines CCL5 and CXCL4, which can be deposited onto the endothelial cells inducing monocyte arrest. 
Aims: In this study, we aimed to elucidate the fate of CCL5 and CXCL4 after endothelial deposition.

Methods: HUVECs and the endothelial cell line EA.hy926 were incubated with CCL5 or CXCL4 for up to 120 minutes and analyzed with light-, confocal- or stimulated emission depletion (STED) microscopy. To quantify internalization, whole cell lysates and organellefractionated cells were analyzed using ELISA. Monocyte arrest was evaluated using laminar flow leukocyte adhesion assays.

Results: Both CCL5 and CXCL4 were rapidly internalized in endothelial cells (<10 min). Whereas CXCL4 remained partly presented on the cell surface, all of the CCL5 was internalized. Endocytosis was dependent on dynamin and clathrin, as internalization was blocked by specific inhibitors. Cell surface proteoglycans, chemokine binding polysaccharides, had a less definite role in the internalization of CCL5 and CXCL4. Combined incubation of CCL5 and CXCL4 with endothelial cells did not influence the internalization or the localization of either of the chemokines. Localization studies by confocal and super-resolution microscopy suggested that both CCL5 and CXCL4 partly have a nuclear localization. This was supported by cell fractionation, which revealed a relatively high nuclear accumulation of both CCL5 and CXCL4. Internalization of chemokines appears less in cells with an inflammatory phenotype, and monocyte-arrest was higher to endothelial cells, when incubated with CCL5 and CXCL4.

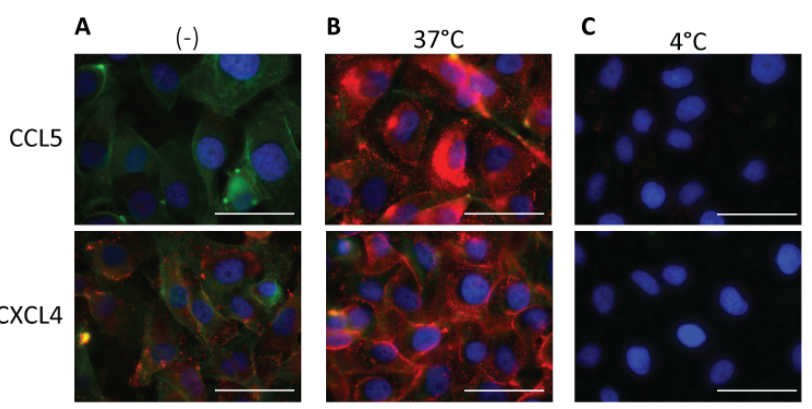

Uptake of chemokines by EA.Hy926 cells after $60 \mathrm{~min}$.

FIGURE 1 Uptake of chemokines (red) by EA.Hy926 cells after 60 min at indicated temperature. Blue: nuclei, green: actin

Conclusions: In summary, endothelial cells rapidly and actively internalize CCL5 and CXCL4 by clathrin and dynamin-dependent endocytosis, where the chemokines appear to be directed to the nucleus. These findings introduce a potential novel, non-canonical role of alpha-granule released chemokines in the cross-talk of activated platelets and endothelial cells, which could have implications for the mechanisms in which leukocytes are attracted to sites of inflammation.
PB1036 | Dasatinib Impairs Vascular Integrity and Promotes Skin Wound Healing in Mice

S. Wichaiyo $^{1}$; S. Svasti ${ }^{2}$; N. Morales ${ }^{3}$

${ }^{1}$ Department of Pharmacology, Faculty of Pharmacy, Mahidol University, Bangkok, Thailand; ${ }^{2}$ Thalassemia Research Center, Institute of Molecular Biosciences, Mahidol University, Nakhon Pathom, Thailand; ${ }^{3}$ Department of Pharmacology, Faculty of Science, Mahidol University, Bangkok, Thailand

Background: A complex interaction between cells and molecules contributes to four phases of wound healing, i.e., hemostasis, inflammation, proliferation, and maturation. Platelet-expressed glycoprotein VI (GPVI) and C-type lectin-like receptor 2 (CLEC-2) function in maintaining vascular integrity to prevent intra-tissue bleeding during skin inflammation. A recent study has shown that lacking both GPVI and CLEC-2 on platelets facilitates wound healing in mice through the impairment of vascular integrity and the associated fibrin/fibrinogen deposition and decreased inflammation. Dasatinib has previously been demonstrated to inhibit Src and Syk, the downstream molecules upon GPVI and CLEC-2 activation, in platelets.

Aims: In this study, we investigated whether dasatinib affects skin wound healing.

Methods: A single full-thickness excisional skin wound (4-mm diameter) was generated on dorsum of 8-week-old C57BL/6 mice. Dasatinib $(5 \mathrm{mg} / \mathrm{kg}$ or $10 \mathrm{mg} / \mathrm{kg}$ ) or DMSO (3\%) vehicle was intraperitoneally injected immediately after biopsy and then daily for up to 3 days post injury (the inflammatory phase). Wound closure was monitored until day 9 post-injury.

Results: The results showed that dasatinib-treated mice had a significant acceleration in wound closure compared to DMSO-treated mice $(n=8)$. However, there was no difference in wound closure between the two dasatinib-treated groups. During the first 3 days after injury, redness at the wound edge was observed in dasatinibinjected animals but not in controls. In addition, macroscopic observation of inner side of the wound revealed bleeding into the wound of dasatinib-injected mice, suggesting a loss of vascular integrity during this period. Moreover, mice treated with dasatinib had increased fibrinogen content in the wound relative to controls $(n=5-6)$ at day 3 post injury. 


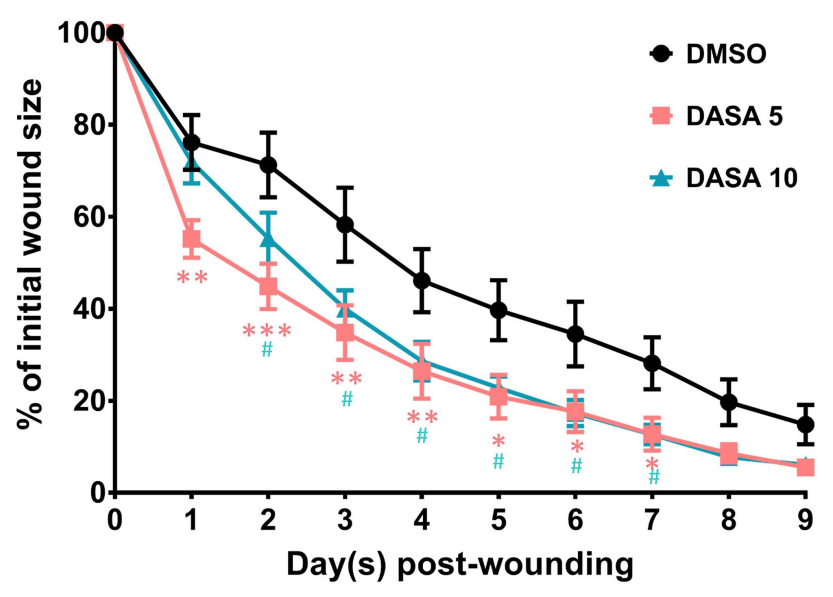

At day 3 post injury

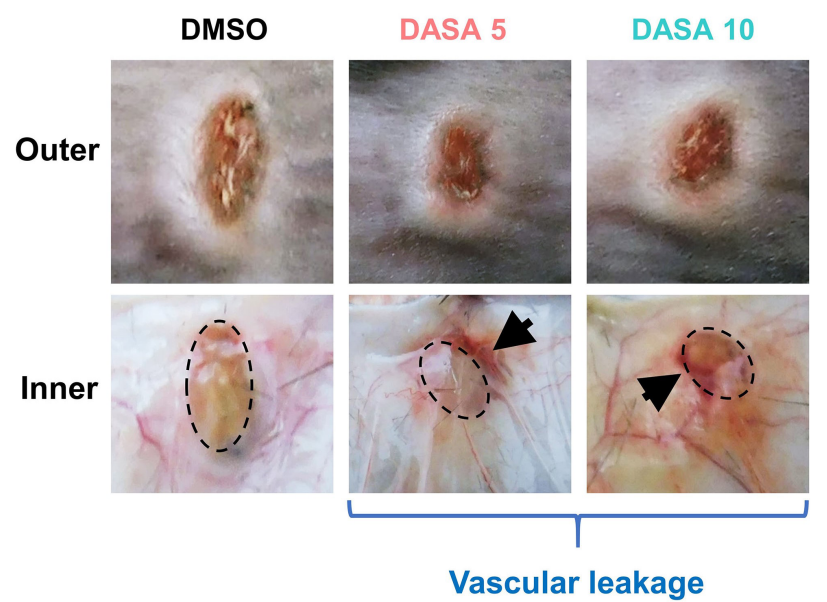

FIGURE 1 Dasatinib enhances skin wound healing by inducing vascular leakage

Conclusions: In conclusion, our results show that dasatinib induces vascular leakage during inflammatory phase of cutaneous wound repair, leading to increased fibrinogen deposition in association with the accelerated rate of wound closure.

\section{PB1037 | Targeting Pathways of Mitochondrial Neutrophil} Extracellular Trap Formation to Inhibit Progression of Abdominal Aortic Aneurysms in Preclinical Models

$\underline{\text { S. Bleichert }}{ }^{1}$; N. Ibrahim ${ }^{1}$; J. Klopf ${ }^{1}$; V. Knöbl ${ }^{1}$; A. Busch ${ }^{2}$; M. Bailey ${ }^{3}$; W. Eilenberg ${ }^{1}$; C. Neumayer ${ }^{1}$; C. Brostjan ${ }^{1}$

${ }^{1}$ Medical University of Vienna, Vienna, Austria; ${ }^{2}$ Technical University

Munich, Munich, Germany; ${ }^{3}$ University of Leeds, Leeds, United

Kingdom

Background: Neutrophil extracellular traps (NETs) have been reported to promote the formation of abdominal aortic aneurysms (AAAs) by propagating an inflammatory response. They are formed by the expulsion of nuclear or mitochondrial DNA which implicates the production of reactive oxygen species (ROS). Moreover, oxidized mitochondrial DNA is known to be involved in chronic inflammatory pathologies like systemic lupus erythematosus (SLE).

Aims: The aim of the study was to test the therapeutic capacity of blocking mitochondrial ROS and mitoNET formation on established AAA disease in murine models. mitoTEMPO, a mitoROS scavenger, was previously shown to diminish NET capacity in a mouse model of SLE. Additionally, metformin, which is an anti-diabetic agent acting in a pleiotropic manner, has been proposed to inhibit nuclear as well as mitochondrial NETs by regulating ROS production.

Methods: AAAs are induced in ApoE KO mice by subcutaneous implantation of osmotic pumps releasing angiotensin II over 28 days. Aneurysms develop by day 8 , when the animals undergo external jugular vein catheterization with an access port for daily intravenous injections with PBS (as control) or anti-NET therapy with mitoTEMPO (3 $\mu \mathrm{g} / \mathrm{g}$ ) or metformin $(0.2 \mu \mathrm{g} / \mathrm{g}$ mouse weight).

Results: Inhibition of AAA progression revealed a significant difference in percent growth of aortic volume at day $28(P=0.0177)$ between the control group treated with daily PBS injections $(n=6$, $337 \%$ growth in aortic volume), and the mitoTEMPO treated group ( $n=7,185 \%$ growth in aortic volume). Moreover, the application of metformin in the same model showed significant inhibition of AAA progression in the treatment group ( $n=7 /$ group, $364 \%$ vs. $199 \%$ growth in aortic volume, $P=0.0133$ ).

Conclusions: Both mitoTEMPO and metformin show inhibition of AAA progression in the ApoE KO mouse model. To document the impact of these inhibitors on mitoNETs, reversal of drug effects by injection of oxidized mitochondrial DNA will be attempted.

\section{PB1038 | Ponatinib Induces Vasculitis with Immune Cells Expressing Coagulation Factors FV \& FVIII}

\section{P. Zeng; A. Merkulova; E. Chan; A.H. Schmaier}

Case Western Reserve University, Cleveland, United States

Background: The tyrosine kinase inhibitor (TKI) ponatinib (poni) is an agent for resistant CML and ALL with the BCR-ABL1 translocation. However, its use is associated with thrombosis in $31 \%$ patients (arterial 26\%, venous 5\%). Poni-treated mice have heightened arterial thrombosis with an aortic vascular infiltrate expressing ROS and apoptosis.

Aims: Characterize the vascular infiltrates and determine how they contribute to thrombosis.

Methods: Mouse model using 20-week-old C57BL/6 mice treated with poni for 2 weeks. Studies include arterial (Rose Bengal) and venous (IVC ligations) thrombosis assay; aortic RNAseq; IPOX and immunofluorescence on aortic sections; and flow cytometry on aortic digests and aortic lymph nodes with data analyzed by FlowJo.

Results: Poni-treated mice also have larger thrombi on the IVC ligation model. Aorta RNA-seq from poni-treated mice on REACTOME show upregulated immune (innate, adaptive, interleukins, and cytokines) and hemostatic (Coagulation, GPVI activation, Platelet Activation) pathways. We determined how these systems interact. 
On IPOX both Ly6G+ and CX3CR1+ immune cells are present. The nitrotyrosine (ROS) and caspase 3 (apoptosis) positive vascular cell infiltrates were identified to be CX3CR1+ immune cells, not Ly6G+ neutrophils. The major CX3CR1+ immune cells were subtyped to be both $C D 3+C D 8 b+$ and CD3-CD19+. RNAseq data also show increased F5 and F8 mRNA (See Figure 1). On IPOX, both FV and FVIII antigen are present in the vascular infiltrate. Immunofluorescent studies show that FV antigen colocalizes with the CD3+CD8b+and CD3-CD19+ immune cells. Aortic Immune cells isolated from ponitreated mice by digests and flow cytometry also have the phenotype of $C D 3+C D 8 \beta+F V+$ and $C D 3-C D 19+F V+$. This phenotype also is seen in aortic lymph nodes, but not peripheral blood.

Conclusions: Ponatinib treatment promotes immune cell vascular inflammation of CX3CR1+CD3+CD8b+ and CX3CR1+CD3-CD19+ cell that express ROS, apoptosis and FV antigen. Immune cell vascular inflammation with FV expression is a novel pathophysiologic mechanism associated with thrombosis in the cancer patient.

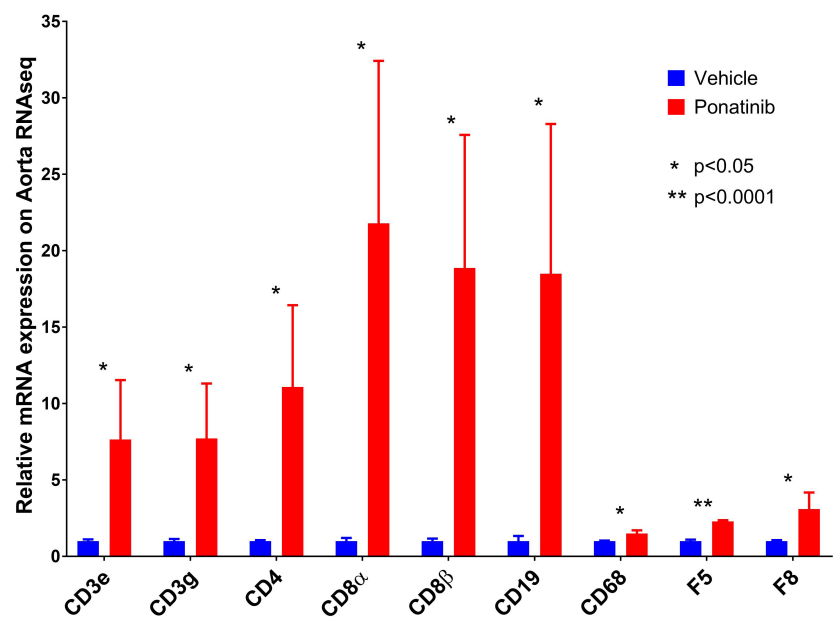

FIGURE 1 Ponatinib-induced upregulation of aortic immune cell markers

PB1039 | The Role of Nitric Oxide and Cationic Groups in Cardiovascular and Respiratory Toxicity of Protamine Sulfate in Zebrafish and Rodent

$\underline{\text { J. Miklosz }}{ }^{1}$; B. Kalaska ${ }^{1}$; P. Podlasz ${ }^{2}$; M. Chmielewska-Krzesinska ${ }^{2}$; M. Zajaczkowski ${ }^{3}$; A. Kosinski ${ }^{3}$; D. Pawlak ${ }^{1}$; A. Mogielnicki ${ }^{1}$ ${ }^{1}$ Medical University of Bialystok, Bialystok, Poland; ${ }^{2}$ University of Warmia and Mazury in Olsztyn, Olsztyn, Poland; ${ }^{3}$ Medical University of Gdansk, Gdansk, Poland

Background: Protamine sulfate (PS) is the only available option to reverse anticoagulant activity of unfractionated heparin (UFH), however it can cause cardiovascular and respiratory complications.
Aims: We explored toxicity of PS and its complexes with UFH in zebrafish and rodents. The involvement of nitric oxide (NO), cationicity of PS and hERG channels in above effects was investigated.

Methods: To study survival and hatching rates, heart rate (HR) and organ toxicity, zebrafish embryos were exposed to the full range of PS concentrations, UFH and L-NAME alone, or together with PS. hERG blockade by PS was measured using the automated patch clamp technique in human embryonic kidney 293 cells. Blood pressure, $\mathrm{HR}$, perfusion of paw vessels, blood oxygen saturation, respiratory rate, and peak exhaled $\mathrm{CO}_{2}$ were registered over 60 minutes after drug administration to rats. Cardiac troponin concentration and heart tissue histopathology were evaluated in mice treated repeatedly for 35 days. All procedures involving animals were approved (No. 2/2018).

Results: We found concentration-dependent lethality, morphological defects, and bradycardia in zebrafish. We also observed hypotension, and cardiovascular and respiratory disturbances more pronounced with increasing dose of PS. We found no effect of PS on hERG channels, or signs of heart damage in mice. The hypotension in rats and bradycardia in zebrafish were partially attenuated by inhibitor of endothelial NO synthase - L-NAME (Figure 1AB). The disturbances in cardiovascular and respiratory parameters were reduced or delayed when cationic groups of PS were neutralized with UFH. Data were analyzed using GraphPadPrism8 with Kruskal-Wallis ANOVA with Dunn's post-hoc test and shown as median with range. Conclusions: Cardiorespiratory toxicity of PS seems to be chargedependent and involves enhanced release of NO. PS administered at appropriate doses and ratios with UFH should not cause permanent damage of heart tissue, although careful monitoring of cardiorespiratory parameters is necessary.

NCN grant number 2016/23/N/NZ7/00442
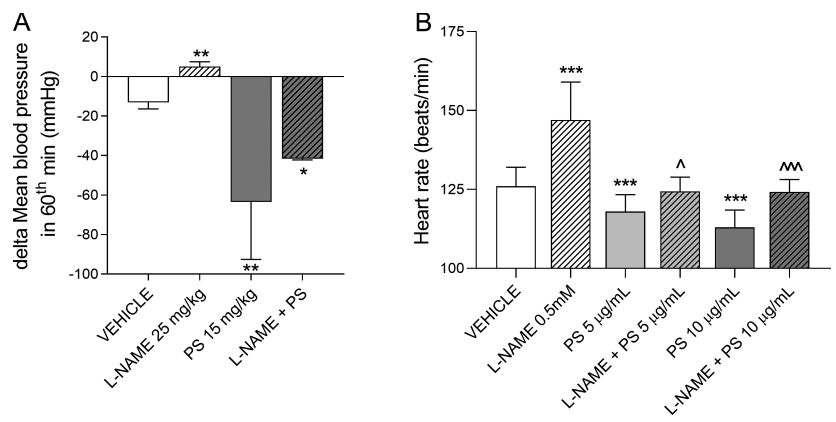

FIGURE 1 The effects of PS alone and together with L-NAME after single administration in rats (A) and zebrafish embryos exposed for 48 hours to drugs (B). ${ }^{*}$ p 
PB1040 | A 3D in vitro Model of Inflammation-associated Bleeding

$\underline{\text { R. Riddle }}{ }^{1} ;$ K. Hansson ${ }^{2}$; K. Jennbacken ${ }^{2}$; M. Harper ${ }^{1}$

${ }^{1}$ University of Cambridge, Cambridge, United Kingdom; ${ }^{2}$ AstraZeneca, Gothenburg, Sweden

Background: Thrombocytopenic patients often experience 'spontaneous' bleeding events, where no obvious cause of bleeding can be found. These events range in severity from petechiae to intracranial haemorrhage. Recently, studies in mice have suggested that this bleeding may be due to inflammation. Upon transmigration, neutrophils create small holes in the endothelium and underlying extracellular matrix (ECM), which platelets would normally plug to prevent red blood cell (RBC) leakage. Although these mouse studies have been very informative, a human in vitro model may have more relevance to thrombocytopenic patients.

Aims: To develop a humanized 3D organ-on-a-chip model of inflammation-associated haemostasis.

Methods: Mimetas Organoplates were used to culture human endothelial vessels against physiologically relevant ECM components. Platelets, neutrophils, and RBCs isolated from human whole blood were perfused through TNF-a-stimulated endothelial vessels and their dynamics studied.

Results: Confluent endothelial vessels formed against Geltrex ECM with a small amount of collagen I incorporated. Stimulation of vessels with TNF- $a$ induced transmigration of neutrophils into the ECM. Red blood cells leaked at sites of neutrophil transmigration in stimulated chips, indicative of inflammation-associated bleeding. Washed platelets prevented this bleeding, consistent with mouse models.

Conclusions: We successfully developed a 3D human endothelial vessel model which supported perfusion of isolated primary human neutrophils, platelets, and red blood cells and recapitulates inflammation-associated bleeding. We will further use this model to investigate how platelets prevent bleeding at inflammatory sites.

PB1041 | Elevated Circulating Endothelial Cells and Success in Endothelial Colony-forming Cells Isolation

L. Silva $^{1}$; A. Justo-Junior ${ }^{2}$; S. Montalvão ${ }^{1}$; R. Medina ${ }^{3}$;

J. Annichino-Bizzacchi ${ }^{1}$

${ }^{1}$ University of Campinas, Campinas, Brazil; ${ }^{2}$ Brazilian Center for

Research in Energy and Materials, Campinas, Brazil; ${ }^{3}$ Queen's

University Belfast, Belfast, United Kingdom

Background: Circulating endothelial cells (CEC) have been associated with vascular injury and are described as potential biomarkers for cardiovascular disease. Besides, current optimized methodologies enable the isolation of a well-characterized subtype of endothelial progenitor known as Endothelial colony-forming cells (ECFCs). Although ECFC isolation methodologies are well described; some discrepancies remain in relation to their isolation efficiency.
Aims: To evaluate the isolation efficacy ECFCs and CEC frequency in human peripheral blood.

Methods: All volunteers signed the informed consent form approved by The Ethics Research Committee of the University of Campinas. CEC enumeration was assessed by flow cytometry in the peripheral blood. $100 \mu \mathrm{L}$ of blood (with a leukocyte concentration between 5 and $10 \times 10^{3} / \mu \mathrm{L}$ ) was incubated with monoclonal anti-human antibodies (CD45 PerCp, CD133 APC, CD31 FITC, and CD146 PE). FACS lysing solution was used for red blood cell removal. 300,000 events or the total volume of the tube were acquired using a FACSCalibur ${ }^{\circledR}$ flow cytometer. To determine the number of cells / $\mu \mathrm{L}$, the percentage of events was multiplied per number of leucocytes and divided per 100. Cell culture from venous blood was used to isolate ECFCs which were confirmed by their cobblestone morphology and immunophenotyping for endothelial markers (CD31, CD146, CD309, and CD144).

Results: ECFC isolation was successful in 9 out of 18 donors (1 male, 8 females; mean age: $35.8 \pm 11.1$ years). CEC frequency was significantly higher $(P=0.004)$ in the group with successful ECFC isolation when compared to the group that did not yield ECFCs $(2.9 \pm 1.2$ $\mathrm{CECs} / \mu \mathrm{L}$ against $1.2 \pm 0,8 \mathrm{CEC} / \mu \mathrm{L})$. This suggests that the success in ECFC isolation might be related to the number of CECs, and therefore ECFC mobilization into circulation may also be linked to vascular injury.

*This study was financed by FAPESP, Finance Code 2018/15618-3.

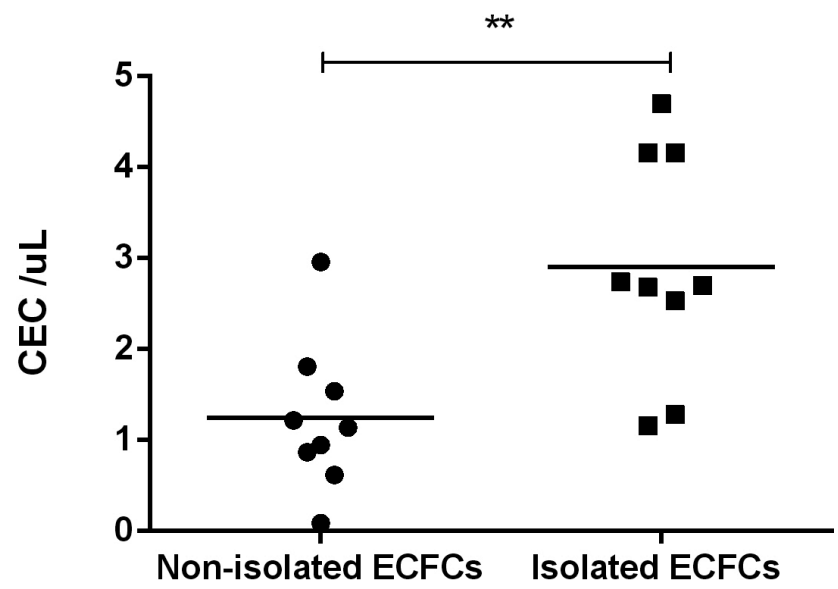

FIGURE 1 Increased number of circulating endothelial cells in peripheral blood, in the group that had success in ECFCs isolation, $n=9$. Unpaired t-test, $P=0.0041$.

Conclusions: Increased number of CECs might be associated with the success of ECFCs isolation. 


\section{PB1042 | Global Coagulation Assays in Chronic Kidney Disease}

B. Lui ${ }^{1}$; D. Barit ${ }^{1}$; M. Sashindranath ${ }^{2}$; C. Selan ${ }^{2}$;

G. Donnan ${ }^{3}$; H. Nandurkar ${ }^{2}$; P. Ho ${ }^{1}$; H.Y. Lim ${ }^{2,1,3}$

${ }^{1}$ Northern Health, Epping, Australia; ${ }^{2}$ Australian Centre for Blood

Diseases, Melbourne, Australia; ${ }^{3}$ University of Melbourne, Melbourne,

Australia

Background: Chronic kidney disease (CKD) is associated with both increased risk of thrombotic and bleeding complications. Currently available coagulation assays are limited in their capacity to predict these outcomes.

Aims: To evaluate the use of global coagulation assays in patients with chronic kidney disease.

Methods: This is a prospective observational study in which patients with estimated glomerular filtration rate (eGFR) $<30 \mathrm{~mL} / \mathrm{min} / 1.73 \mathrm{~m}^{2}$ were recruited $(n=84)$ and compared to healthy controls $(n=153)$. Blood samples were collected for baseline bloods and global coagulation assays, which included whole blood thromboelastography and platelet-poor calibrated automated thrombogram (CAT), overall haemostatic potential (OHP), tissue factor pathway inhibitor (TFPI) and plasminogen activator inhibitor-1 (PAI-1).

Results: Compared to healthy controls (mean age 41.8 years, $67 \% \mathrm{fe}-$ male), CKD subjects (mean age 65.1 years, 38\% female) had increased von Willebrand factor antigen (178 vs 102\%, $P<0.001$ ), factor VIII levels (183 vs $108 \%, P<0.001)$ and prothrombotic thromboelastography parameters with increased maximum amplitude (69.9 vs $60.3 \mathrm{~mm}, P<0.001)$. Fibrin generation parameters were increased (overall coagulation potential 44.5 vs $36.1, P<0.001$ ) with impaired fibrinolytic potential $(65.6$ vs $81.3 \%, P<0.001)$ and peak thrombin was higher in CKD patients despite comparable endogenous thrombin potential. The differences remained with multivariate analysis modelling for age and sex. The global coagulation parameters did not correlate with urea level. D-Dimer (930 vs $430, P<0.001$ ) was increased in the CKD group although it did not correlate with overall haemostatic potential parameters. Comparisons haemodialysis ( $n=45)$ and peritoneal dialysis $(n=15)$ sub-populations demonstrated that peritoneal dialysis patients had higher thrombin with reduced overall fibrinolytic potential.

TABLE 1 Global coagulation assay parameters in CKD patients compared to healthy controls

\begin{tabular}{|c|c|c|c|}
\hline Global coagulation assay parameters & Normal controls $(n=153)$ & CKD eGFR<30 $(n=82)$ & $P$-value* \\
\hline $\begin{array}{l}\text { THROMBOELASTOGRAPHY: Maximum } \\
\text { amplitude (mm), mean (SD) }\end{array}$ & $60.3(6.4)$ & $70.1(6.8)$ & $<0.001$ \\
\hline $\begin{array}{l}\text { CAT: Endogenous thrombin potential (ETP, } \\
\text { nM.min), mean (SD) }\end{array}$ & $1335(258)$ & $1286(228)$ & 0.52 \\
\hline CAT: Peak (nM), mean (SD) & $219.7(67.1)$ & $231.2(65.5)$ & 0.038 \\
\hline CAT: Velocity index (nM/min), median (IQR) & $64.3(43.2,93.5)$ & $68.4(56.3,96.8)$ & 0.005 \\
\hline $\begin{array}{l}\text { OHP: Overall coagulation potential (\%), mean } \\
\text { (SD) }\end{array}$ & $35.5(9.7)$ & $44.7(10.7)$ & $<0.001$ \\
\hline $\begin{array}{l}\text { OHP: Overall haemostatic potential (\%), } \\
\text { median (IQR) }\end{array}$ & $6.4(4.8,9.4)$ & $16.4(10.8,21.6)$ & $<0.001$ \\
\hline $\begin{array}{l}\text { OHP: Overall fibrinolytic potential (\%), median } \\
\text { (IQR) }\end{array}$ & $81.1(77.4,84.1)$ & $64.1(54.3,71.7)$ & $<0.001$ \\
\hline TFPI (ng/mL), median (IQR) & $14.5(6.9,27.4)$ & $38.7(21.4,66.0)$ & $<0.001$ \\
\hline PAI-1(ng/mL), median (IQR) & $8.1(2.8,18.5)$ & $7.4(3.8,19.5)$ & 0.89 \\
\hline
\end{tabular}

Conclusions: CKD appears to confer a prothrombotic state characterised by increased von Willebrand factor antigen, factor VIII levels, clot strength as well as both increased fibrin generation and hypofibrinolysis. The use of global coagulation assays to stratify thrombosis risk warrants further investigation.
PB1043 | The Role of Transforming Growth Factor ß1 (TGFß1) and Fibrinogen in the Induction of Adhesive Properties of Vascular Endothelium (HUVECS) and the Spread of Acute Lymphoblastic Leukemia (ALL)

V. Barilka; V. Matlan; S. Prymak; O. Shalay

SI 'Institute of Blood Pathology and Transfusion Medicine of NAMS of Ukraine', Lviv, Ukraine

Background: TGF $\beta 1$ and fibrinogen $(F)$ are some of the key regulators of endothelial cells (EC) interactions in ALL which can modify the EC properties increasing their adhesiveness to neoplastics cells and their distribution in the body. 
Aims: To determine the attachment of labeled by ${ }^{3} \mathrm{H}$-thymidine cells K562, L1210 to the monolayer of HUVECs in vitro after influence of plasma and conditional mediums (CM) off mononuclear cells from peripheral blood (MNPB) in 21 ALL pts who had not treated before investigation, and to compare obtained data with $\mathrm{F}$ concentration (FC) and endogenous TGF $\beta 1$ (enTGF $\beta 1$ ) level.

Methods: EnTGF $\beta 1$ level was detected by bioassay used CCL64 as a target. The MNPB were obtained after $24 \mathrm{~h}$-incubation in RPMI 1640. The number of blasts in peripheral blood of ALL pts were $58,62 \pm 10,53 \%$. The attachment of K562, L1210 to HUVECs were estimated by the index of adhesion (IA). The FC was estimated in venous citrate blood by simple hemostatic method.

Results: EnTGFB1 concentrations in plasma and CM of MNPB of ALL pts were higher, than in plasma of donors $(2,11 \pm 0,38 \mathrm{ng} / \mathrm{ml} ; P<0,05)$. Elevated FC was found in ALL pts $(7,20 \pm 0,60 \mathrm{mg} / \mathrm{ml})$, than in donors $(4,00 \pm 1,00 ; P<0,02)$. Plasma ALL pts showed comparatively strong

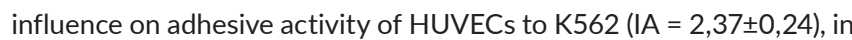
comparison to $L 1210(I A=1,28 \pm 0,8 ; P<0,05)$. CM of MNPB of ALL pts also intensively activated of adhesiveness of HUVECs (IA $=1,99 \pm 0,38$ for K562 and 1,92 $\pm 0,24$ for L1210, respectively). CM secreted higher level of TGF $\beta 1$, than plasma pts $(P<0,05)$.

Conclusions: EnTGFB1 and FC were elevated in plasma and CM of PBMC of ALL pts and may influence on spread of the ALL by stimulating of adhesiveness of endothelial cells. Therefore, the adjustment of enTGFß1 and FC levels in the blood of ALL pts may be an important step in the treatment of $\mathrm{AL}$.

PB1044 | Hemophagocytic Lymphohistiocytosis Complicating Systemic Diseases

I. Chabchoub ${ }^{1}$; R. Ben Salah ${ }^{1}$; F. Abida ${ }^{2}$; F. Frikha ${ }^{1}$;

M. Chaari ${ }^{2}$; Z. Bahloul ${ }^{1}$

${ }^{1}$ Internal Medicine Department, Hedi Chaker Hospital, Sfax, Tunisia;

${ }^{2}$ Hematology Laboratory, Hedi Chaker Hospital, Sfax, Tunisia

Background: Hemophagocytic lymphohistiocytosis (HLH) is a rare and potentially lethal condition. It can be primary or secondary and can complicate other conditions: neoplastic, infectious or autoimmune. Systemic diseases are an uncommon cause of $\mathrm{HLH}$.

Aims: The aim of this work is to study the clinico-biological and etiological characteristics of HLH cases during systemic diseases.

Methods: Retrospective study carried out over a period of 22 years including patients presenting a ( $\mathrm{LHL}$ ) whose diagnosis was retained according to the Histiocyte Society 2004 criteria. Patients presenting a (LHL) related to a systemic disease were studied.

Results: Five patients out of a total of 10 patients with HLH (50\%) were female with an average age of 39.4 years. The reason for hospitalisation was febrile pancytopenia in $60 \%$ of cases, febrile polyarthralgia in $10 \%$ of cases and febrile dyspnoea in $10 \%$ of cases. An alteration in general condition was found in 4 patients, fever in all patients, hepatomegaly in 1 , splenomegaly in 3 , adenopathy in 1 patient and erythematous skin rash in 2 patients. Biology revealed a biological inflammatory syndrome in $60 \%$ cases, cytopenia in $80 \%$ patients: normocytic normochromic anaemia in $80 \%$ cases of inflammatory type in $40 \%$ cases and autoimmune in $40 \%$ cases, leukopenia in $60 \%$ cases, lymphopenia in all cases and thrombocytopenia in $80 \%$ of patients. Hyperferritinemia was objectified in all patients, hypertriglyceridemia in 4 patients and hepatic cytolysis in 3 patients. The myelogram revealed multiple images of haemophagocytosis in $80 \%$ cases. The LHL was secondary to: a severe relapsing SLE in 4 cases and Still's disease in 1 case. The treatment was both symptomatic and etiological using high dose corticosteroid therapy in all case. The evolution was favourable for all our patients.

Conclusions: Systemic diseases are among the aetiologies of (LHL) whose diagnosis can be difficult because of the presence of several clinical and biological signs common to both pathologies.

PB1045 | Global Coagulation Assays and Endothelial Biomarkers in Patients with Diabetes Mellitus

$\underline{\text { H.Y. Lim }}^{1,2,3}$; B. Lui ${ }^{2}$; M. Tacey ${ }^{2}$; A. Kwok ${ }^{2}$; S. Varadarajan ${ }^{2}$; M. Sashindranath ${ }^{3}$; C. Selan ${ }^{3}$; G. Donnan ${ }^{1}$; H. Nandurkar ${ }^{3}$; P. Ho ${ }^{2,1}$

${ }^{1}$ University of Melbourne, Melbourne, Australia; ${ }^{2}$ Northern Health, Epping, Australia; ${ }^{3}$ Australian Centre for Blood Diseases, Melbourne, Australia

Background: Diabetes mellitus (DM) is associated with increased risk of cardiovascular disease although there are no current coagulation studies that predict thrombotic risks.

Aims: To investigate the utility of global coagulation assays and endothelial biomarkers in diabetic patients compared to healthy controls. Methods: Diabetic patients, not on anticoagulation and without active malignancy, were recruited from endocrinology outpatients. Blood samples were collected for baseline tests and global coagulation assays including thromboelastography (TEG), calibrated automated thrombogram (CAT) and overall haemostatic potential (OHP) assay as well as tissue factor pathway inhibitor (TFPI) and plasminogen activator inhibitor-1 (PA1-1). The results were compared to previously recruited healthy controls ( $n=153$ ).

Results: 184 patients consisting of 22 type 1 DM (T1DM), 154 type 2 DM (T2DM) and 8 latent autoimmune diabetes in adults (LADA) were recruited. Compared to healthy controls, diabetic patients demonstrated more hypercoagulable TEG parameters with increased clot strength (maximum amplitude, 68.7 vs $60.5 \mathrm{~mm}, P<0.001$ ). While there was no difference in thrombin generation (CAT), the OHP assay demonstrated significantly higher fibrin generation and lower overall fibrinolytic potential (OFP 73.6 vs $81.1 \%, P<0.001)$. TFPI was significantly increased in diabetic patients ( 36.9 vs $14.5 \mathrm{ng} / \mathrm{mL}, P<0.001)$ while PAI-1 was comparable $(P=0.14)$. On sub-analysis, T2DM patients were more hypercoagulable than T1DM patients on thromboelastography, and fibrin generation with higher PAl-1 (14.8 vs $8.7 \mathrm{ng} / \mathrm{mL}, P=0.017$ ) but comparable for other assays. T1DM patients with known diabetic complications had lower OFP than those without complications while T2DM with known complications had higher thrombin generation parameters with reduced OFP. 
TABLE 1 Global coagulation assay parameters in diabetic patients compared to healthy controls

\begin{tabular}{|c|c|c|c|c|}
\hline $\begin{array}{l}\text { Global coagulation assay } \\
\text { parameters }\end{array}$ & Units & $\begin{array}{l}\text { Normal controls } \\
(n=153)\end{array}$ & All Diabetics ( $n=184)$ & $P$ values* \\
\hline $\begin{array}{l}\text { Thromboelastography: Maximum } \\
\text { amplitude, median (IQR) }\end{array}$ & $\mathrm{mm}$ & $60.5(57.2,63.5)$ & $69.0(66.1,72.6)$ & $<0.001$ \\
\hline $\begin{array}{l}\text { CAT: Endogenous thrombin } \\
\text { potential, median (IQR) }\end{array}$ & $\mathrm{nm} \cdot \min$ & $1320(1164,1510)$ & $1260(1097,1460)$ & 0.061 \\
\hline CAT: Peak height, median (IQR) & $\mathrm{nm}$ & $220.3(173,266.8)$ & $211.3(173.5,256.0)$ & 0.92 \\
\hline $\begin{array}{l}\text { OHP: Overall coagulation } \\
\text { potential, mean (SD) }\end{array}$ & unit & $35.5(9.7)$ & $41.1(10.3)$ & $<0.001$ \\
\hline $\begin{array}{l}\text { OHP: Overall fibrinolytic } \\
\text { potential, median (IQR) }\end{array}$ & $\%$ & $81.1(77.4,84.1)$ & $73.3(65.1,79.5)$ & $<0.001$ \\
\hline Biomarkers: TFPI, median (IQR) & $\mathrm{ng} / \mathrm{mL}$ & $14.5(6.7,27.6)$ & $37.1(2.1,71.4)$ & $<0.001$ \\
\hline Biomarkers: PAI-1, median (IQR) & $\mathrm{ng} / \mathrm{mL}$ & $8.1(2.8,18.5)$ & $9.9(4.5,20.0)$ & 0.14 \\
\hline
\end{tabular}

Conclusions: Our study demonstrates that diabetic patients have a more hypercoagulable profile on global coagulation assays, particularly in T2DM patients as well as patients with known diabetic complications. Further studies with longer term follow-up are ongoing to evaluate the utility of global coagulation assays in predicting patient outcomes.

\section{ENDOTHELIAL CELL SIGNALING}

PB1046 | Collagen Turnover Biomarkers in Atrial Fibrillation and their Use in Clinical Management and the Study of Inflammatory Pathogenesis

K. Wieschhaus; E. Bontekoe; J. Fareed; D. Hoppensteadt;

S. Mushabbar; J. Goldstein

Loyola University Chicago Stritch School of Medicine, Maywood, United States

Background: Atrial fibrillation (AF) is the most diagnosed cardiac arrhythmia in the world. While over 2.7 million Americans have AF, the pathophysiology remains unclear ${ }^{1}$. Current literature reveals that inflammation and atrial remodeling are integral to AF development ${ }^{2,3}$. Aims: This study aims to demonstrate that collagen turnover proteins give a telling narrative of AF progression.

Methods: Baseline citrated blood samples from 91 patients with confirmed diagnosis of AF were collected at Loyola University Medical Center. Normal human plasma (NHP) was obtained from a commercial source (George King Biomedical, Overland Park, KS). Biomarkers of collagen turnover, including MMP-1, MMP-2, TIMP-1, TIMP-2, ICTP, PICP, PINP, PIIICP and PIIINP were measured using commercially available ELISA methods. Results were then statistically analyzed using Graphpad Prism v.8; the results are compiled in Table 1.

Results: Biomarkers including MMP-1 (AF $1.35 \pm 0.19,279 \%$ vs $0.36 \pm 0.096 ; P<0.0001$ ), MMP-2 (AF $263.5 \pm 6.4,38 \%$ vs $191.2 \pm 21.3 ; P<0.02$ ), TIMP-1 (AF $125.9 \pm 9.0,56 \%$ vs $80.8 \pm 21.4$;
$P<0.05$ ), TIMP-2 (AF $137.6 \pm 4.2,43 \%$ vs $96.3 \pm 8.5 ; P<0.004$ ), PIIINP (AF $26.4 \pm 4.3,584 \%$ vs $3.9 \pm 1.2 ; P<0.02$ ), and PINP (AF $422.3 \pm 43.8,51 \%$ vs $280.5 \pm 56.5 ; P<0.02)$ showed significant increase when compared to NHP (Table 1a). Significant correlations were found between TIMP-2 and MMP-1 (0.492; $P<0.0001)$, PIIINP and TIMP-1 (0.313; $P<0.01)$, and PIIINP and PINP $(0.460, P<0.001)$. Conclusions: These results firmly indicate $A F$ upregulates collagen degradation. While collagen synthesis is not as unanimously demonstrated, the increase of PIIINP and PINP, along with PIIINP's strong correlation to TIMP-1, strongly suggest an increase in collagen synthesis, as well. Suggesting that collagen turnover is increased in AF patients. These biomarkers can now be reliably used to correlate against other biomarker findings. Additionally, these collagen turnover biomarkers could be studied to determine their accuracy in scoring fibrosis compared to LGE-MRI.

Table 1: Table 1a: A comparison of the composite parameters for collagen turnover. Parameters AF Samples Controls \% Change P value Average \pm SD SEM (n) Range Average \pm SD SEM (n) Range PICP (ng/ ml) $1.68 \pm 0.370 .048$ (61) 1.2-2.78 $1.71 \pm 0.290 .06$ (21) 1.36-2.19 -0.0170 .408 ICTP $(\mathrm{ng} / \mathrm{ml}) 4.00 \pm 1.080 .13$ (68) 2.23-9.5 $3.39 \pm 0.93$ 0.29 (10) 2.36-5.44 17.8 0.0518 PIIINP (ng/ml) $26.4 \pm 36.14 .25$ (72) $0.77-208.13 .853 \pm 2.891 .18$ (6) $0.55-6.81583 .80 .0156$ PIIICP $(\mathrm{pg} / \mathrm{ml}) 1435 \pm 2461288.0$ (73) 0.00-13625 $1871 \pm 1067477.3$ (5) 0.00-2691 -23.3 0.192 PINP (pg/ml) $422.3 \pm 350.343 .8$ (64) 153.2$1686280.5 \pm 195.956 .5$ (12) 141.8-761.0 50.8 0.0137 MMP-1 (ng/ ml) $1.35 \pm 1.520 .19$ (64) 0.01-10.3 0.36 \pm .350 .096 (13) $0.08-1.12$ 278.9 


\section{INFLAMMATION AND SEPSIS}

LPB0039 | The Extracellular Protease EpiP from S. aureus Triggers Blood Coagulation by Proteolytically Activating Prothrombin and Platelet Protease-Activated Receptor 1

V. De Filippis ${ }^{1}$; I. Artusi ${ }^{1}$; G. Pontarollo²; L. Acquasaliente ${ }^{1}$; A. Pagotto ${ }^{1}$; C.M. Radu ${ }^{3}$; F. Bagnoli ${ }^{4}$; G. Pietrocola ${ }^{5}$; P. Speziale ${ }^{5}$ ${ }^{1}$ Department of Pharmaceutical and Pharmacological Sciences, University of Padua, Padova, Italy; ${ }^{2}$ Center for Thrombosis and Hemostasis (CTH), University Medical Center Mainz, Mainz, Germany; ${ }^{3}$ 2Department o Medicine, University of Padua, Padua, Italy; ${ }^{4}$ GSK Vaccines, Siena, Italy; ${ }^{5}$ Department of Molecular Medicine, Unit of Biochemistry, University of Pavia, Pavia, Italy

Background: Staphylococcus aureus is a Gram-positive bacterium known for its pathogenicity in humans, responsible for both mild and systemic infections, i.e. bacteremia and sepsis. During the last decades, clinical evidence provide new insights for a positive relation between $S$. aureus infections and dramatic thrombotic complications, such as DIC. Among the huge arsenal of virulence factors, extracellular proteases might play a role in triggering thrombotic events in infectious diseases, whereby bacterial proteases could activate the coagulation cascade by proteolytically converting ProT zymogen into thrombin species and stimulating platelet aggregation. Aims: In this work, we investigated if the subtilisin-like protease EpiP from S. aureus is involved in thrombus formation during staphylococcal infections, directly activating Prothrombin (ProT) and platelets.

Methods: Biochemical techniques: limited proteolysis, enzymatic chromogenic assay, mass spectrometry; coagulation assays: fibrin generation and platelet aggregation; fluorescence microscopy.

Results: Staphylococcal EpiP converts ProT into an active species which is able to hydrolyze the thrombin-specific substrate S2238. The time-course analysis of ProT activation allowed to identify the EpiP cleavage sites (Arg155-Ser156, Arg271-Thr272, and Arg320Ile321), identical to those hydrolyzed by factor Xa under physiological conditions. The activation products of ProT by EpiP can induce fibrin clot formation, either from a fibrinogen solution or platelets-free plasma (PFP), and platelets aggregation. Surprisingly, EpiP can proteolyze PAR1(38-60) peptide at the same site of thrombin cleavage (Arg41-Ser42) and electrostatically interact with Gplba(268-282) peptide, as demonstrated by SPR. Ultimately, we directly observed EpiP-mediated platelets agglutination by fluorescence microscopy. Conclusions: The extracellular protease EpiP from S. aureus, can proteolyze the inactive ProT into an active thrombin species which is able to trigger blood coagulation. Moreover, EpiP directly induces platelet aggregation activating PAR1 receptor after binding to Gplba on platelet surface. These results widen our understanding of biochemical mechanism whereby $S$. aureus proteases can initiate coagulation, establishing a direct link between infections and higher thrombotic risk.
LPB0087 | Activated Platelets Transfer miR-223 into Endothelial Cells via Microparticles to Downregulate ICAM-1 Expression among Septic Conditions

B. Szilágyi ${ }^{1}$; Z. Fejes ${ }^{1}$; Á. Rusznyák ${ }^{2}$; F. Fenyvesi ${ }^{2}$; M. Pócsii ${ }^{1}$; S. Halmi ${ }^{3}$; Z. Griger ${ }^{3}$; S.P. Kunapuli ${ }^{4}$; J. Kappelmayer ${ }^{1}$; B. Nagy Jr. ${ }^{1}$

${ }^{1}$ University of Debrecen, Department of Laboratory Medicine, Debrecen, Hungary; ${ }^{2}$ University of Debrecen, Department of Pharmaceutical Technology, Debrecen, Hungary; ${ }^{3}$ University of Debrecen, Institute of Internal Medicine, Debrecen, Hungary; ${ }^{4}$ Temple University School of Medicine, Department of Physiology and Sol Sherry Thrombosis Center, Philadelphia, United States

Background: In sepsis, platelets become activated and shed increased amount of microvesicles containing several bioactive proteins and microRNAs (miRNAs). The latter molecules can be taken up by distinct recipient cells of circulation, thus utilize potent effects to regulate cellular function in different diseases.

Aims: We investigated the release of miR-223 from activated human platelets and transfer via microparticles (PMPs) into endothelial cells to downregulate enhanced intercellular adhesion molecule-1 (ICAM1) expression among septic conditions in vitro.

Methods: To observe whether platelet-derived miR-223 carried by PMPs could enter endothelial cells, human coronary artery endothelial cells (HCAECs) were co-cultured with isolated PMPs from sepsis and normal plasma. Flow cytometry was used for quantification of CD41a/Annexin-V positive PMPs, and immunofluorescence microscopy was performed to detect the internalization of PMPs into endothelial cells. Expression of miR-223-3p and its direct target ICAM1 were quantified by RT-qPCR and ELISA in HCAECs after treatment with TNF-a with or without PMPs.

Results: Leukocyte-depleted platelets (LDPs) isolated from sepsis patients showed decreased expression of intracellular miR-223, while their plasma samples as well as PMPs contained elevated miRNA level compared to healthy samples. Similarly, thrombinreceptor activated LDPs showed reduced miR-223 intracellularly with high level in the supernatants and PMP isolates in vitro. In addition, we found higher PMP count in sepsis plasma compared to controls and increased PMP uptake by HCAECs. TNF-a stimulated HCAECs showed decreased miR-223 with elevated ICAM1, while PMPs caused higher miRNA level that attenuated ICAM1 expression at mRNA and protein levels. Importantly, miR-223 was not transcribed in response to PMPs as pre-miR-223 was not altered by PMPs, while mature miR-223 was still induced in PMP-treated vs. untreated HCAECs after transfection with Dicer1 siRNA.

Conclusions: miR-223 delivery by PMPs from septic platelets can modulate ICAM1 expression in endothelial cells that may be a protective role against sepsis-induced vascular inflammation. 
PB1047 | The Effect of DNase I in Combination with Unfractionated Heparin or Low Molecular Weight Heparin in a Mouse Model of Sepsis

S. Medeiros; N. Sharma; D. Dwivedi; S. Sohrabipour; V. De Sousa;

J. Zhou; P.C Liaw

McMaster University, Hamilton, Canada

Background: Cell-free DNA (CFDNA) has emerged as a prognostic biomarker in patients with sepsis. Circulating CFDNA is hypothesized to be associated with histones in the form of nucleosomes. In vitro, DNA activates coagulation and inhibits fibrinolysis, whereas histones activate platelets and are cytotoxic to endothelial cells. Previous studies have targeted CFDNA or histones in animal models of sepsis using DNasel or heparins, respectively, which improved survival.

Aims: In this study, we explored the possibility that the combination of DNasel and heparins, either unfractionated heparin (UFH) or low-molecular weight heparin (LMWH), may be a better therapeutic approach than monotherapy in a murine model of sepsis.

Methods: Mice $(\mathrm{C} 57 \mathrm{BI} / 6)$ were subjected to either cecal-ligation and puncture (CLP) or sham-surgery. Mice were given either saline, DNasel (40mg/kg/day), UFH (18IU/kg/h), LMWH (140IU/kg/day), or a combination of UFH+DNasel or LMWH+DNasel $(n=18-25)$. Mice were then monitored for $72 \mathrm{~h}$. At study endpoint, organs were harvested for analysis and plasma levels of CFDNA, IL-6, and prothrombin fragment1+2 were measured.

Results: DNasel (70.0\%) or LMWH (68.2\%) monotherapy significantly improved survival compared to CLP saline controls (40.0\%; $P<0.05)$. Although UFH (57.9\%) increased survival compared to saline controls (40.0\%), this was not significant. Combination therapies showed no improvement in survival (40.0\%;44.4\%) compared to saline controls. Furthermore, all CLP mice had elevated levels of CFDNA, IL-6, prothrombin fragment $1+2$, and organ severity scores compared to shams. However, DNasel and LMWH had reduced levels of prothrombin fragment $1+2$ and IL- 6 compared to saline-treated CLP mice.

Conclusions: Compared to saline treatment, administration of either DNasel or LMWH to septic mice significantly improved survival, which may reflect reduced systemic inflammation and thrombosis as evidenced by decreased levels of IL- 6 and prothrombin fragment $1+2$, respectively. Unexpectedly, the combination therapy showed no improvement in survival suggesting that these agents are not synergistic in vivo.

\section{INNATE AND ADAPTIVE IMMUNITY}

\section{LPB0135 | Platelet-rich Neutrophil-platelet Micro-emboli Contribute to Cigarette Smoke-induced Influenza Severity}

T.W. Kaminski; T. Brzoska; X. Li; R. Vats; R. Dubey; K. Nickolich; K. Robinson; T. Nyunoya; P. Sundd University of Pittsburgh, Pittsburgh, United States

Background: Influenza is an acute respiratory disease primarily caused by the influenza-A virus (IAV), which affects over 35million people in the US annually. Epidemiological evidence suggests that prior exposure to cigarette smoke (CS) or habitual smoking increases the risk of IAV-triggered respiratory failure (severe flu). Although emerging evidence supports the role of thrombo-inflammation in the development of CS and IAV-triggered lung injury, the innate immune mechanism contributing to this morbidity remains poorly understood.

Aims: Our aim was to investigate the platelets-neutrophil interplay in lung microcirculation during CS-induced severe flu in mice.

Methods: We have developed a two-hit model of CS-induced severe flu in mice. Mice were exposed to four weeks of room air (air) or CS followed by intranasal administration of A/PR/8/34 (H1N1) IAV. The body weight was measured every day for two weeks after IAV administration followed by assessment of lung injury at days-7 and-14. Lungs were harvested for histological assessment of injury and estimation of viral titer by qPCR. Quantitative fluorescence intravital lung microscopy (qFILM) was conducted 3- and 4-days post-IAV-infection to visualize dynamics of neutrophil and platelet recruitment in the lung of mice IV administered with fluorescent dextran, anti-Ly6G Ab and anti-CD49Abs.

Results: Mice exposed to CS+IAV manifested significantly more weight loss, lung injury, lung congestion, alveolar hemorrhage and hypoxemia compared to mice administered IAV only. QFILM revealed that severity of lung injury was associated with significantly larger area with impaired blood flow and more vascular leakage secondary to vascular occlusion by platelet-rich neutrophil-platelet aggregates in the lung of CS+IAV than IAV administered mice.

Conclusions: These initial results suggest that CS primes innate immune signaling in neutrophils and platelets to promote their recruitment in the lung following flu, leading to severe acute lung injury. Currently, studies are underway to identify innate immune pathways in neutrophils and platelets that drive this hyper thromboinflammatory response. 


\section{NON-CODING RNAS}

LPB0040 | rs2431697 of miR-146a Regulates NETosis

Determining the Thickness of the Carotid Intima-media in Patients with Rheumatoid Arthritis

L. Reguilón Gallego ${ }^{1} ;$ A.M. del los Reyes-García ${ }^{1} ;$ S. Águila ${ }^{1}$; M.P. Fernández-Pérez ${ }^{1}$; N. García Barberá하 L. Zapata-Martínez ${ }^{1}$; I. Ruiz Lorente ${ }^{1}$; M.C. Ábalos-Aguilera²; E. Saiz ${ }^{3}$; M.F. Pina ${ }^{3}$; M.T. Herranz ${ }^{4}$; A. Barceló ${ }^{5}$; I. Hervés ${ }^{5}$; V. Vicente ${ }^{1}$; C. LópezPedrera $^{2}$; C. Martínez ${ }^{1}$; R. González-Conejero ${ }^{1}$

${ }^{1}$ Deparment of Hematology and Medical Oncology, Morales Meseguer University Hospital, Centro Regional de Hemodonación, Universidad de Murcia, IMIB, Murcia, Spain; ${ }^{2}$ Rheumatology Service, Reina Sofia Hospital/Maimonides Institute for Research in Biomedicine of Cordoba (IMIBIC)/University of Cordoba, Córdoba, Spain; ${ }^{3}$ Deparment of Rheumatology, Morales Meseguer University Hospital, Murcia, Spain; ${ }^{4}$ Deparment of Internal Medicine, Morales Meseguer University Hospital, Murcia, Spain; ${ }^{5}$ Deparment of Radiology, Morales Meseguer University Hospital, Murcia, Spain

Background: Rheumatoid arthritis (RA) is a systemic autoimmune disease with cardiovascular complications in which immunothrombosis could take place. Our group has described, in other pathologies, that NET markers in plasma are associated with the rs2431697 of miR-146a whose carriers of the T-allele $(<50 \%$ miR-146a levels) have increased risk of cardiovascular events.

Aims: Our objective is to explore whether rs2431697 is associated with NET markers and to study their relationship with the development of cardiovascular complications in patients with RA.

Methods: We collected clinical variables, plasma and DNA from RA patients $(n=359)$ [mean age $55(28-87)$, women 72\%, $238(66 \%)$ without biological drugs and 121 (34\%) that received them during evolution] and samples from 50 controls. We genotyped rs2431697 using TaqMan probes and evaluated NETs in plasma by quantifying free-DNA (cfDNA) using SYTOXGreen and citrullinated-histone-H3 (citH3)/DNA complexes by ELISA. We evaluated the presence of atheroma plaques and quantified the thickness of the carotid intima (CIMT) using Doppler-ultrasonography.

Results: Frequencies of the rs2431697 genotypes did not show differences between patients and controls. We observed a higher proportion of carriers of T-allele in patients with biological drugs $(P=0.05)$; in this group, citH3/DNA complexes were significantly higher in TT cohort (Fig.1). Doppler-ultrasonography data showed that values of both citH3/DNA (Fig.2A) and cfDNA (Fig.2B) were significantly higher in patients with vascular damage (59 vs 40; $P=0.006 ; 64$ vs 40; $P=0.01$, respectively). Interestingly, the highest levels of citH3/DNA in the pathological CIMT group corresponded to carriers of the T-allele (Fig.2B)
FIGURE 1

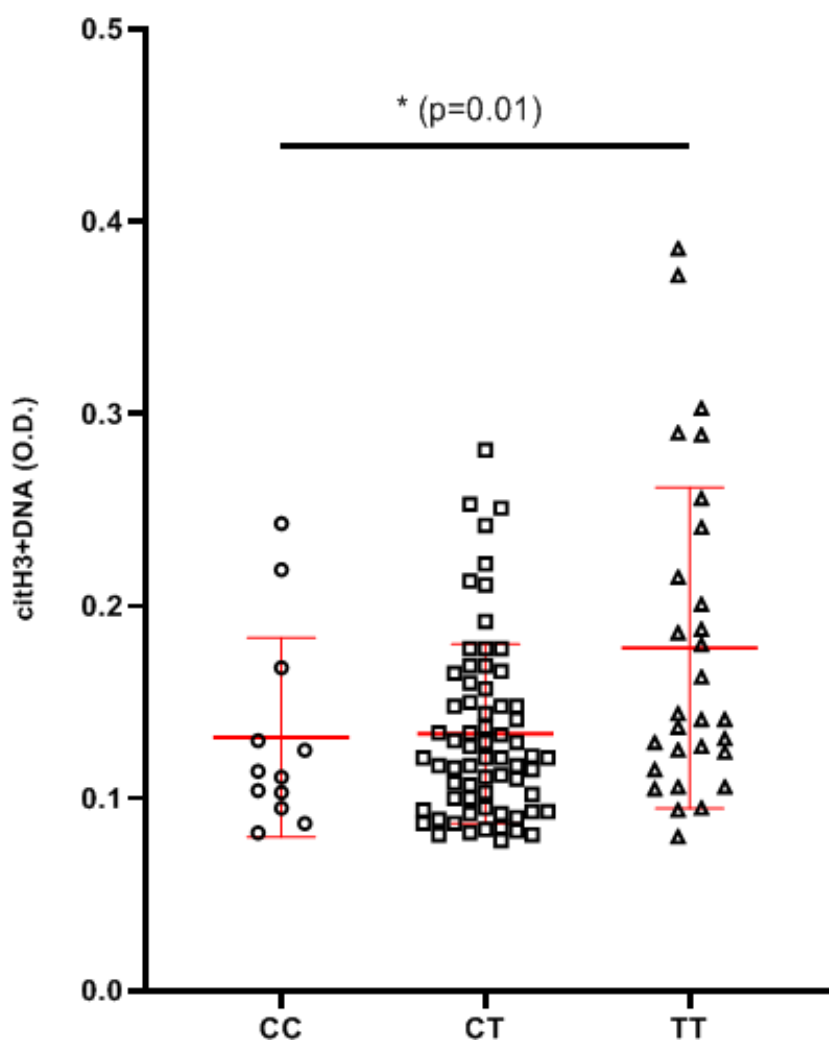

FIGURE 1 citH3+DNA (O.D.) according to genotype in the cohort of patients with biological drugs
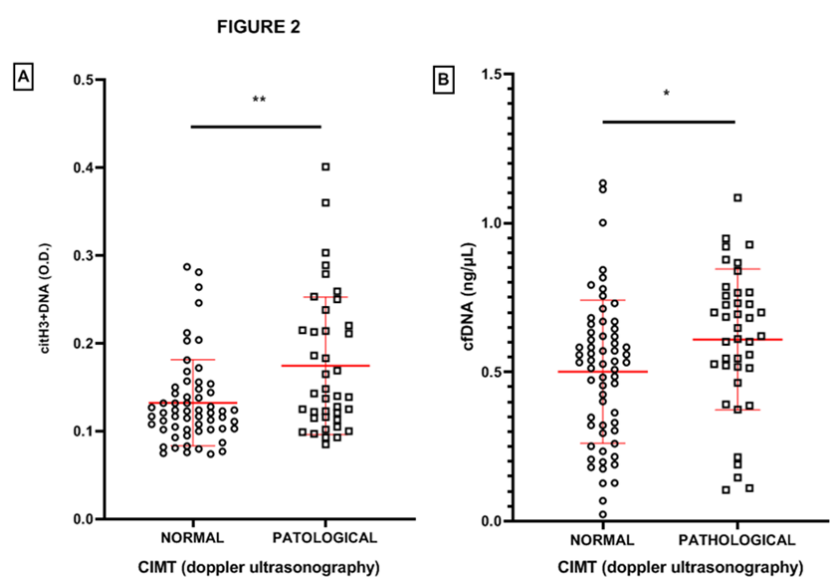

FIGURE 2 A: citH3+DNA (O.D.) according to the result of carotid doppler ultrasonography. B: cfDNA ( $n g / \mu \mathrm{L}$ ) according to the result of carotid doppler ultrasongraphy

Conclusions: We find that miR-146a rs2431697-T genotype is associated with the biological drug requirement and with greater intimal thickness in carriers. This suggests that miR-146a levels could influence both the clinical course and the thrombotic comorbidities associated with the disease. 
LPB0088 | Identification of Small Interfering RNAs for Alleleselective Silencing of Murine von Willebrand Factor

Y.K. Jongejan; R.J. Dirven; A. de Jong; B.J.M. van Vlijmen;

J.C.J. Eikenboom

Leiden University Medical Center, Leiden, Netherlands

Background: High von Willebrand factor (VWF) plasma levels are associated with arterial thrombosis. Current antiplatelet therapy to prevent arterial thrombosis increases bleeding risk and often fails to prevent thrombosis. An alternative therapeutic approach would be to lower VWF by allele-selective silencing of VWF. This approach averts complete knockdown of VWF and thus minimizes bleeding risk.

Aims: The aims of this study are to identify small interfering RNAs (siRNAs) that can distinguish between strain-specific differences in murine $V w f$ to be used in allele-selective knockdown studies in heterozygous mouse models.

Methods: Two commonly used mouse inbred strains, C57BL/6J and $129 \mathrm{~S} 1 / \mathrm{SvlmJ}$, were selected based on genetic differences between their Vwf genes and comparable plasma VWF levels. siRNAs were designed to target one or two of 11 genetic differences between these mouse strains. In silico analysis predicted 14 siRNAs to be active, meaning that they effectively inhibit either of the strain-specific Vwf alleles. All selected siRNAs were identically chemically modified to increase stability, which included 2'O-Methyl and phosphorothioate backbone modifications. Activity and allele/strain-selectivity of these siRNAs were determined, dose-dependently, in HEK293 cells transiently expressing either C57BL/6J or 129S1/SvImJ Vwf or both. Results: 7 out of 14 siRNAs effectively inhibited the targeted allele ( $\geq 80 \%$ at $1 \mathrm{nM}$ siRNA), with minimal inhibition of the untargeted allele. Of the other siRNAs, two showed allele-selective inhibition of approximately $70 \%$, three showed non-selective inhibition of both alleles (at least 60\%), and two showed limited inhibitory effect on both alleles. Two lead candidates were chosen based on strong inhibitory activity (>80\%), strain-selectivity and ability to target one nucleotide difference.

Conclusions: We have identified strain-selective siRNAs that can distinguish between C57BL/6J and 129S1/SvImJ Vwf based on one or two nucleotide(s) difference between their Vwf genes. The selected lead compounds will be tested in F1 hybrids of cross-bred C57BL/6J and 129S1/SvImJ mice.

PB1048 | Platelet microRNA Release in the Context of Agoniststimulation and Thrombosis

T.L. Krammer $^{1}$; S. Zeibig ${ }^{2}$; H.-P. Holthoff ${ }^{2}$; M. Hackl ${ }^{1}$

${ }^{1}$ TAmiRNA GmbH, Vienna, Austria; ${ }^{2}$ advanceCOR GmbH, Martinsried, Germany

Background: Platelets contain a diverse array of miRNA species contributing considerably to the pool of cell-free miRNAs in the circulation. Platelet activation has been linked to the secretion of miRNAs, rendering them promising biomarker candidates.

Aims: We set out to investigate platelet miRNA release upon agonist stimulation and in the context of thrombosis to establish their potential as biomarkers of platelet activation.

Methods: We measured the levels of 11 miRNAs with confirmed associations to platelet function and novel miRNA biomarker candidates using RT-qPCR. Platelets from five donors were stimulated in buffer using two different agonists. Additionally, miRNA release was measured after platelet stimulation in PRP. To increase sensitivity, we separated vesicle- and protein-bound miRNAs applying sizeexclusion chromatography. This panel of miRNAs was also measured in a mouse model of thrombosis (Folts intervention) ( $n=25)$.

Results: Platelet stimulation in buffer led to an unexpectedly high miRNA background in the unstimulated control. Nonetheless, a trend towards elevated miRNA levels upon platelet activation was observed for several miRNAs, including miR-223-3p, miR-24-3p, and the novel biomarker candidate miR-199a-3p. When platelets were stimulated in PRP, several miRNAs showed a significant or near significant increase compared to the control, indicating secretion upon activation. These miRNAs were predominantly found in complex with proteins. In the mouse model of thrombosis, a strong secretion of miRNAs after the intervention was observed. Eight of the standard platelet miRNAs as well as miR-199a-3p showed a significant increase in response to thrombogenesis.

Conclusions: We showed a link between platelet activation and miRNA secretion, however detecting miRNA release in vitro remains challenging. We provide evidence that circulating platelet miRNAs are complexed with proteins and only to a lesser degree vesiclebound. We observed a substantial and significant release of platelet miRNAs upon thrombogenesis in vivo, further corroborating our hypothesis that platelet miRNAs are promising biomarker candidates of platelet reactivity.

\section{PROTEASE ACTIVATED RECEPTORS}

LPB0136 | BMS-986141, a Selective PAR4 Antagonist, Reduces Platelet Deposition in a Microfluidic Thrombosis Assay

J. Chen $^{1}$; C.C. Verni ${ }^{1}$; S.M. Garonzik ${ }^{2}$; V. Perera
J.M. Luettgen

Background: Thrombin activates platelets by hydrolysis of the protease-activated receptors (PAR) PAR1 and PAR4. Small molecule antagonists of PAR1 and PAR4 have demonstrated antithrombotic activity in nonhuman primate models of thrombosis.

Aims: To characterize the pharmacology of BMS-986141, a potent and selective PAR4 antagonist, in platelet calcium mobilization assays and in a microfluidics model of thrombosis. 
Methods: Human blood from healthy donors was spiked with BMS986141. Platelet calcium mobilization assays were performed with agonists including ADP, convulxin, U46619, thrombin, PAR1 and PAR4 agonist peptides. Arterial plaque rupture thrombosis was simulated by flowing blood through microfluidics channels patterned with von Willebrand Factor (vWF) to allow platelet adhesion and lipidated tissue factor (TF) to trigger thrombin generation.

Results: BMS-986141 specifically blocked calcium mobilization by PAR4 agonist peptide (AYPGKF, IC ${ }_{50} \sim 1.3 \mathrm{nM}$ ). The PAR4 antagonist reduced the secondary phase of calcium mobilization in platelets challenged with $200 \mathrm{nM}$ thrombin, without affecting the initial peak calcium, as expected for slower more sustained PAR4 signaling compared to the rapid, short lived signaling of PAR1. For corn trypsin inhibitor (CTI)-treated whole blood perfused over vWF/TF surface under high shear rate $\left(800 \mathrm{~s}^{-1}\right)$ in Figure 1, BMS-986141 reduced platelet deposition by $\sim 20 \%$, but not fibrin deposition ( $N=7$ donors, 27 clots; $P<0.03$ ).

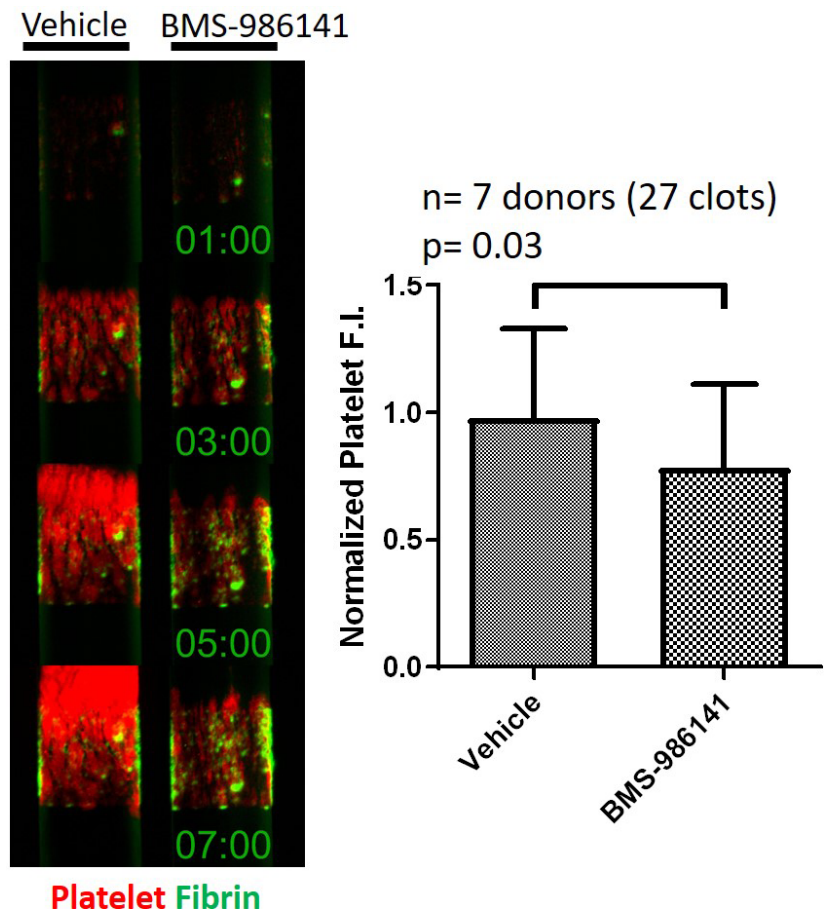

FIGURE 1 PAR4 antagonist, BMS-986141, reduces platelet deposition on a vWF/TF surface at $800 \mathrm{~s}^{-1}$. (F.I. fluorescence intensity)

Conclusions: BMS-986141 is a highly specific antagonist of PAR4. This small molecule reduced platelet deposition in a microfluidic assay of perfused CTI-treated whole blood over patterned surfaces of $\mathrm{VWF} / \mathrm{TF}$, which simulate conditions of arterial thrombosis.

\section{STEM CELLS AND VASCULAR CELL GROWTH}

PB1049 | Impact of ABO Incompatibility on the Outcome of Hematopoeitic Stem Cell Transplantation

M. Borhany; U. Zaidi; M. Abid; S. Zafar; T. Shamsi

National Institute of Blood Disease, Karachi, Pakistan

Background: Allogeneic hematopoietic stem cell transplantation (alIoHSCT) is a curative treatment for a variety of hematological diseases. The impact of $A B O$ incompatibility on the clinical outcome after HSCT has been argued and it has been found to have no negative impact on it.

Aims: To investigate the complications and outcome associated with ABO compatible \& incompatible transplants.

Methods: A retrospective, single-center, cohort study was conducted at our center. Patients were categorized according to ABO compatibility and incompatibility.Laboratory parameters and clinical details were recorded.

Results: A total of 107 patients were recruited, out of which 100 (93.45\%) had allogenic transplants whereas 7 (6.8\%) patients underwent autologous transplants. Among them 68 (63.5\%) were male and 32 (30\%) were female, with a median age of 9.5 years $(\mathrm{IQR}=12.75)$. Full-match related transplants were $69(69 \%)$ as opposed to haploidentical which were 31 (31\%). Comparison of demographics in $\mathrm{ABO}$ compatible and incompatible transplant groups is presented in Table1. Post-transplant outcomes are presented in Table 2. Significant consequences observed were pure red cell aplasia in $4(4 \%)$ i.e. $(P=0.0161)$ patients, whereas gut GVHD in $10(10 \%)$ patients i.e. $(P=0.000)$ in both groups. Mortality was reported in 17 (17.7\%) patients; thus, the overall survival curve of 83 (83\%) patients from the day of transplant to day +100 was found to be insignificant i.e. $(P=0.377)$. Moreover, in the post-transplantation period, the significant mean rank of platelet transfusions was 55.15 in the matched group, while 43.23 mean rank in the mismatched group i.e. $(P=0.045)$. Chimerism between the groups was also not significantly different. 
TABLE 1 Comparison of Demographics in ABO Compatible and Incompatible

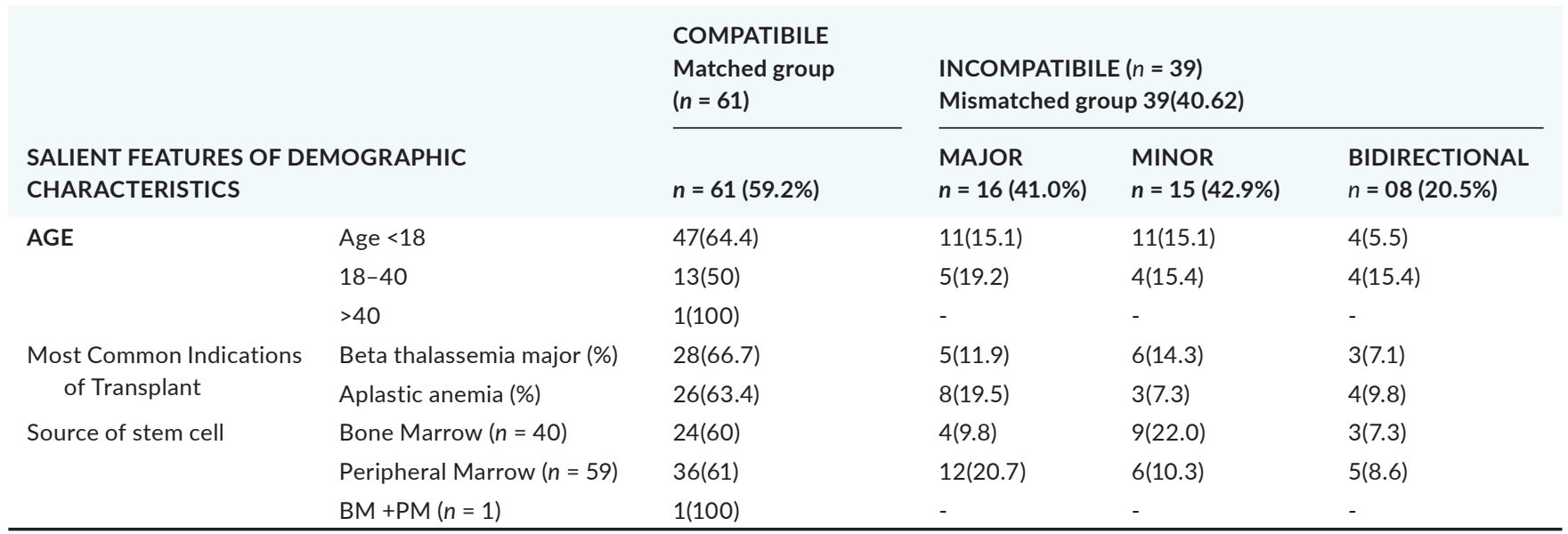

TABLE 2 Post transplant outcomes of Matched and Mismatched group

\begin{tabular}{|c|c|c|c|c|c|c|}
\hline Outcomes & & Matched & Major & Minor & Bidirectional & P-Value \\
\hline \multirow[t]{2}{*}{ Graft Failure } & Primary GF $(n=13)$ & $8(72.7)$ & $3(27.3)$ & - & - & 0.251 \\
\hline & Secondary GF $(n=12)$ & $5(71.4)$ & $1(14.3)$ & $1(14.3)$ & - & 0.861 \\
\hline \multirow{5}{*}{$\begin{array}{l}\text { Acute GVHD (Graft } \\
\quad \text { Versus Host Disease) }\end{array}$} & Skin (\%) & $4(80)$ & $1(20)$ & - & - & 0.922 \\
\hline & Gut (\%) & $8(80)$ & - & $1(10)$ & $1(10)$ & $0.000^{*}$ \\
\hline & Ocular (\%) & $2(100)$ & - & - & - & 0.804 \\
\hline & Liver (\%) & $4(57.1)$ & $1(14.3)$ & $1(14.3)$ & $1(14.3)$ & 0.523 \\
\hline & Oral (\%) & $9(81.8)$ & $1(9.1)$ & - & $1(9.1)$ & 0.291 \\
\hline \multirow[t]{2}{*}{ Complications } & PRCA & - & $2(50)$ & - & $2(50)$ & $0.016^{*}$ \\
\hline & CMV & $13(65)$ & $3(15)$ & $4(20)$ & - & 0.495 \\
\hline
\end{tabular}

Conclusions: $A B O$ incompatibility does not seem to influence the parameters in patients undergoing allogeneic stem cell transplantation. Hence, careful monitoring of patients can help early detection and treat them efficiently by reducing the number of life threatening events.

\section{VENOUS THROMBOEMBOLISM}

\section{ANTIPHOSPHOLIPID SYNDROME}

\section{PB1050 | Could the Adjusted Version of the Global}

Antiphospholipid Syndrome Score (aGAPSS) Be a Useful Tool to Select Patients with Primary Obstetric Antiphospholipid Syndrome (POAPS) for Primary Thromboprophylaxis with Aspirin?

S. Udry ${ }^{1} ;$ F. Aranda ${ }^{1}$; S. Perés Wingeyer ${ }^{1} ;$ S. Stephanie Morales Perez $^{2}$; C. Belizna ${ }^{3}$; J. Alijotas-Reig ${ }^{4}$; E. Esteve-Valverde²; D.S. Fernández Romero ${ }^{5}$; J.O. Latino ${ }^{5}$; G. de Larrañaga ${ }^{1}$ ${ }^{1}$ Hospital of Infectious Diseases 'Dr. Francisco J. Muñiz', Buenos Aires, Argentina; ${ }^{2}$ Althaia Healthcare University Network of Manresa, Barcelona, Spain; ${ }^{3}$ University Hospital Angers, Angers, France; ${ }^{4}$ Valld'Hebron University Hospital, Barcelona, Spain; ${ }^{5}$ Acute Hospital 'Dr. Carlos G. Durand', Buenos Aires, Argentina

Background: POAPS women have an increased risk of thrombosis over time. However, how to know the thrombotic risk profile and prevent it still is a matter of debate.

Aims: To evaluate the clinical utility of aGAPSS to assess the risk of a first thrombotic event on POAPS patients and if stratifying 
according to this score could be helpful to identify those who would be benefited from thromboprophylaxis with low dose aspirin (LDA). Methods: 169 POAPS women were retrospectively evaluated [median age: 32 years; (28-37)], during a follow-up period [median: 7 years (6-12 years)]. Clinical and laboratory variables were evaluated and the aGAPSS was calculated. 69/169 patients received LDA.

Results: 7/169 (4.1\%) presented thrombosis during follow-up. Triple positivity for antiphospholipid antibodies (aPLs) [OR $=10.44$ (95\% Cl: $2.161-50.469), P=0.004]$, an aGAPSS $\geq 7$ [OR $=1.621(95 \% \mathrm{Cl}:$ 1.198-2.193), $P=0.002$ ], arterial hypertension $[O R=16.607(95 \%$ $\mathrm{Cl}: 3.103-88.895), P=0.001]$ and hyperlipidaemia $[\mathrm{OR}=60.00(95 \%$ Cl: 7.756-486.15), $P<0.0001]$, were independent risk factors associated with a first thrombotic event. Remarkably, all of the patients with a first thrombotic event during follow-up had a high aGAPSS $(\geq 7)$. There were significant differences in the incidence of thrombosis between patients with high and low aGAPSS values $(10.44 \%$ vs $0,00 \% ; P=0.002$ ). Other risk factors were not associated with the development of thrombosis in our cohort. No significant differences $(P=0.242)$ in the occurrence of thrombotic events were observed between patients with or without LDA. LDA was marginally associated with a decrease in the risk of thrombosis only in patients with aGAPSS $\geq 7(P=0.048)$.

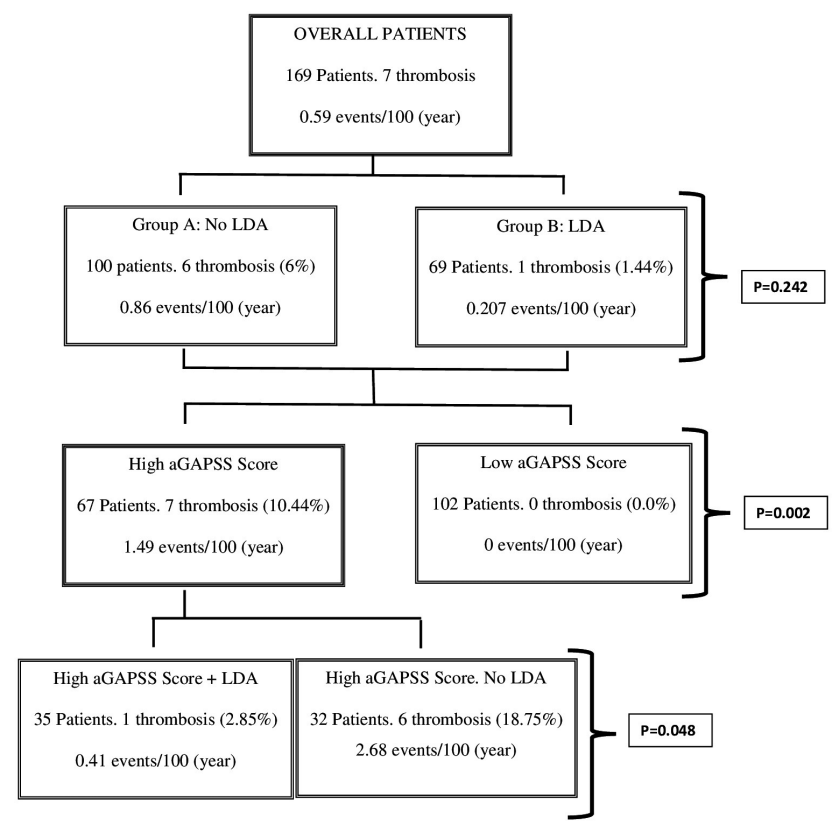

LDA: Low dose aspirin, POAPS: Primary Obstetric Antiphospholipid Syndrome

FIGURE 1 Evaluation of patients according to aGAPSS Score and LDA thromboprophylaxis among POAPS patients

Conclusions: Our findings suggest that the aGAPSS could be a useful tool to predict a first thrombotic event in POAPS patients. In this way, the stratification of patients according to the aGAPSS could be helpful to select patients who would benefit from thromboprophylaxis with LDA as this treatment might significantly decrease the thrombotic risk.
PB1051 | Clinical Course of Thrombotic Antiphospholipid Syndrome with Positive IgA Anticardiolipin or IgA $\beta 2$-glycoprotein I

L. Figueiredo ${ }^{1}$; B. Mazetto ${ }^{2}$; A.P. dos Santos ${ }^{2}$; B. Jacintho ${ }^{2}$; C. Vaz ${ }^{2}$; J.D. Oliveira ${ }^{3}$; G. Mesquita ${ }^{2}$; J. Annichino-Bizzachi ${ }^{2}$; F. Orsi ${ }^{2}$

${ }^{1}$ Pontifícia Universidade Católica de Campinas (PUC-Campinas), Campinas, Brazil; ${ }^{2}$ Faculdade de Ciências Médicas da UNICAMP, Campinas, Brazil; ${ }^{3}$ Faculdade de Ciências Farmacêuticas da UNICAMP, Campinas, Brazil

Background: Although testing for IgA-anticardiolipin $(\mathrm{aCL})$ or IgAanti- $\beta 2$-glycoprotein I (aß2GPI) is not recommended for antiphospholipid syndrome (APS) diagnosis, the role of these IgA isotypes in APS prognosis has not been established.

Aims: To evaluate the association of IgA-aCL or IgA-aß2GPI with the clinical course of APS with thrombosis (t-APS).

Methods: Consecutive patients with confirmed t-APS were tested for IgA-aCL and IgA-aß2GPI by chemiluminescence. The association of IgA-aCL and IgA-aß2GPI and different clinical presentations of the disease was evaluated.

Results: 81 patients with a median follow-up time of 9 years (IQR 714) were included. Women comprised $72 \%$ and primary APS $58 \%$ of the patients (Table1). 24 patients (29.6\%) were positive for $\operatorname{lgA}-\mathrm{aCL}$ or IgA-aß2GPI. $42 \%$ of IgA-positive patients were also triple positive (TP) for antiphospholipids (aPL), while only $12 \%$ of IgA-negative patients were TP $(P=0.001)$. The odds for TP was 6 -fold increased in IgA positive as compared with IgA-negative patients(OR $6.295 \% \mathrm{Cl}$ 1.9-20). Figure 2 shows IgA-aCL and IgA- $\beta 2 \mathrm{GP} 1$ levels by aPL profiles. At baseline, frequency of venous thrombosis was higher in IgApositive (83\%) than in IgA-negative $(63 \%, P=0.06)$ patients; other parameters were similar between groups. During follow-up, $54 \%$ of IgA-positive and $37 \%$ of IgA-negative had recurrent thrombosis $(P=0.24)$. Additionally, $17 \%$ of IgA-positive and no IgA-negative primary APS patients developed systemic lupus erythematosus (SLE) $(P=0.004)$. The median time elapsed from primary APS diagnosis to SLE development was 6.8 years (IQR 4.1-14.1).

TABLE 1 Demographic and clinical characteristics at diagnosis

Thrombotic APS $(n=81)$

Age, median (interquartile range)

$42.7(30.8-54.8)$

Primary APS, $n$ (\%)

$47(58.0)$

Site of the first thrombotic event

Venous, $n$ (\%)

Triple positivity

Cardiovascular risk factors

Hypertension, $n$ (\%)

Dyslipidemia, $n(\%)$

Obesity, $n$ (\%)

$15(18.5)$

Conclusions: IgA-aCL and -aß2GPI were associated with triple aPL positivity and higher risk of developing SLE, which indicates a high risk APS profile. Given that testing for triple positivity may not always 
be feasible, as is the case with anticoagulated patients, testing for IgA antibodies may be useful for improving APS risk assessment.

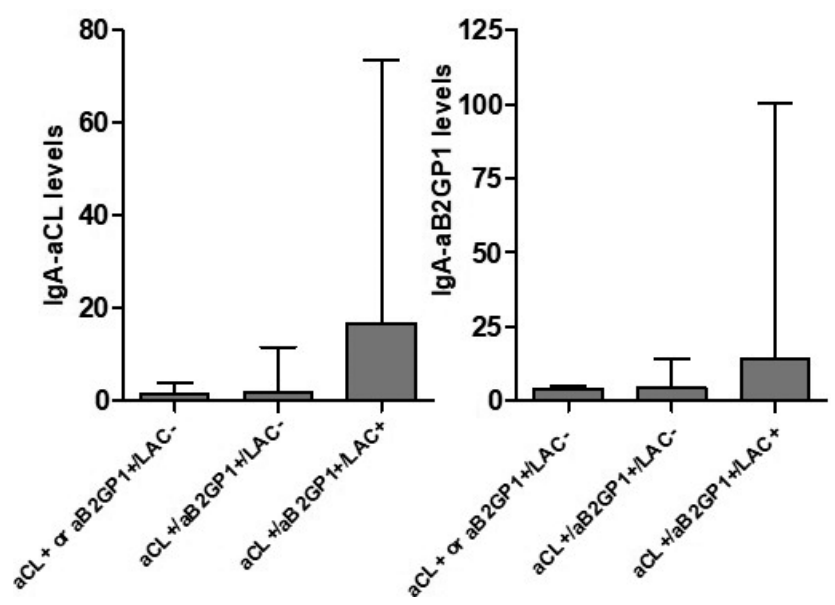

FIGURE 2 Levels (median and 75th quartile) of $\lg \mathrm{A}-\mathrm{aCL}(\mathrm{A})$ and $\lg \mathrm{A}-$ B2GP1 (B) in aPL profiles: single positive $\mathrm{aCL}$ or $\mathrm{a} 32 \mathrm{GP} 1$ without LAC activity ( $\mathrm{aCL}+$ or aß2GP1+/LAC- ; $n=34$ ), double positive $\mathrm{aCL}$ and aß2GP1 without LAC activity (aCL+/aß2GP1+/LAC-; $n=25)$, and triple positive $(\mathrm{aCL}+/ \mathrm{a} 2 \mathrm{GP} 1+/ \mathrm{LAC}+; n=17)$

PB1052 | Risk Factors for Recurrent Thrombosis and Cause of Death in Patients with Antiphospholipid Syndrome; A Swedish Cohort Study

N. Karandyszowska ${ }^{1}$; H. Alagündüz ${ }^{1}$; J. Oesman ${ }^{1} ;$ M. Magnusson ${ }^{2}$; E. Svenungsson ${ }^{3}$; A. Antovic ${ }^{3}$; M. Bruzelius ${ }^{1}$

${ }^{1}$ Department of Medicine Solna, Karolinska Institutet, Stockholm, Sweden; ${ }^{2}$ Clinical Chemistry and Blood Coagulation, MMK, Karolinska Institutet, Stockholm, Sweden; ${ }^{3}$ Karolinska Institutet, Karolinska University Hospital; Division of Rheumatology, Department of Medicine Solna, Stockholm, Sweden

Background: In patients with the antiphospholipid syndrome (APS) recurrent thrombosis is common despite anticoagulation and the mortality rate is high. Identifying high risk patients is challenging, due to the low incidence of APS in the population. Consequently, there are few larger longitudinal studies.

Aims: To estimate the incidence on re-thrombosis and death, evaluate the impact of cardiovascular (CV) risk factors and antiphospholipid antibody ( $\mathrm{aPL}$ ) profiles on re-thrombosis and identify causes of death in a novel APS-cohort.

Methods: This retrospective cohort study comprises all patients identified with APS in the electronic medical records at Karolinska University Hospital, Sweden 2014-2020. Descriptive statistics was presented as median and interquartile range (IQR). Cox proportional hazards regression analyses were used to investigate the effect of risk factors. Ethical approval was obtained from the Swedish Ethical Review Authority (2020-02333).

Results: 217 patients were included in the cohort. Age of APSdiagnosis was 43 years (IQR 31-55) and 66\% were women. During follow-up, 37 re-thrombosis occurred; 23 arterial and 14 venous events, with an incidence of 3.4 per 100 person-years $(95 \% \mathrm{Cl}$ : 2.4-4.7). Significant CV risk factors for re-thrombosis were current smoking; hazard ratio $2.50, P=0.03$ and chronic kidney disease; $3.44, p<0.01$. Twenty-seven (73\%) patients with re-thrombosis were triple positive for aPL compared to 113 (48\%) without any event at follow-up ( $<0.01)$. The cumulative death incidence was $4 \%(n=12)$ with sepsis due to bacterial infection being the most common cause. The median age at death was 63 years (IQR 51-71) and occurred 8 years (IQR 2-10) after diagnosis.

Conclusions: APS-patients suffering re-thrombosis are more likely to have multiple CV risk factors, with smoking and chronic kidney disease being most important. APS-patients are susceptible to sepsis following bacterial infection with high mortality. The treatment of APS-patients should be regularly assessed for concordance to current guidelines.

PB1053 | Effect of Hydroxychloroquine on the Levels of Inflammatory Cytokines and Tissue Factor in Patients with Primary Antiphospholipid Syndrome

B. Mazetto ${ }^{1}$; S. Saraiva ${ }^{2}$; B. Jacintho ${ }^{2}$; G. Vieira-Damiani ${ }^{3,1}$; C. Vaz ${ }^{2}$; J.D. Oliveira ${ }^{2}$; A.P. dos Santos ${ }^{4}$; G. Mesquita ${ }^{4}$; J. AnnichinoBizzacchi $^{2,5}$; F. Orsi ${ }^{5,6}$

${ }^{1}$ School of Medical Sciences, University of Campinas, Campinas, Brazil; ${ }^{2}$ School of Medical Sciences, Department of Clinical Medicine, University of Campinas, Campinas, Brazil; ${ }^{3} \mathrm{Federal}$ Institute of Education Science and Technology of São Paulo, Department of Biology, Capivari, Brazil; ${ }^{4}$ School of Medical Sciences, Department of Medical Sciences, University of Campinas, Campinas, Brazil; ${ }^{5}$ Hematology and Hemotherapy Center, University of Campinas, Campinas, Brazil; 'S School of Medical Sciences, Department of Clinical Pathology, University of Campinas, Campinas, Brazil

Background: Given the high risk of thrombosis in primary antiphospholipid syndrome (PAPS), additional therapies, complementary to anticoagulation, are required.

Aims: To investigate whether hydroxychloroquine (HCQ) affects the inflammatory and coagulation parameters in PAPS with thrombosis (t-PAPS).

Methods: HCQ at $400 \mathrm{mg} /$ day was given to anticoagulated t-PAPS patients for 6 months. After HCQ withdrawal, the same patients were followed for further 12 months. Blood samples were drawn at baseline, 6 months of HCQ use, 6 and 12 months after the end of HCQ use. Levels of tumor necrosis factor-alpha (TNF- $a$ ), interleukin 6 (IL-6), and tissue factor (TF) were quantified by ELISA. 
Results:
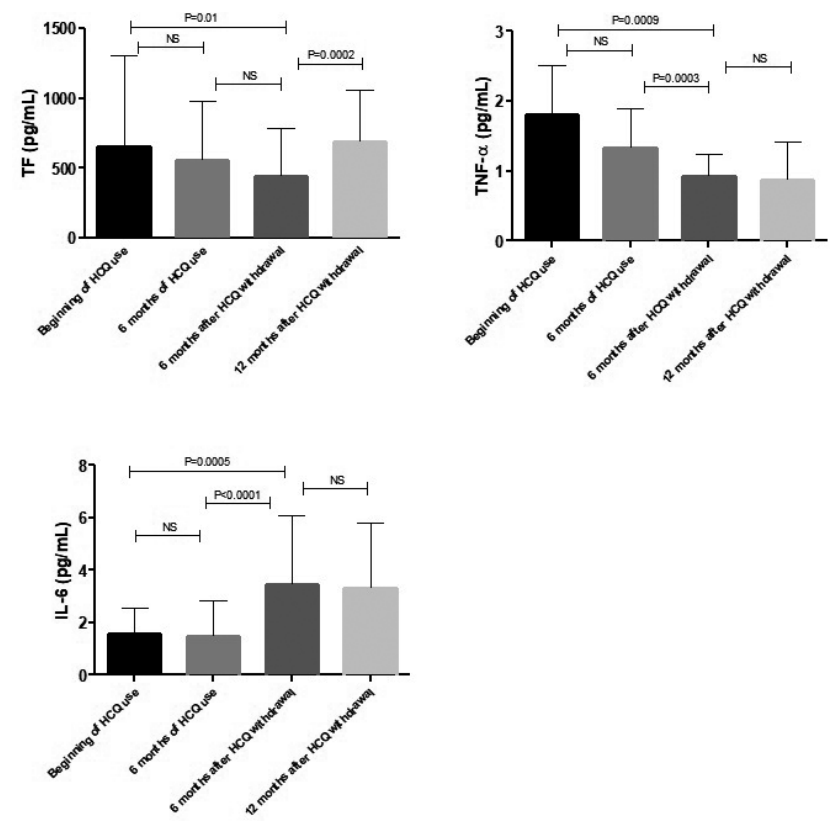

FIGURE 1 The figure illustrates the changes in the levels of TF (mean: $653.5 \mathrm{pg} / \mathrm{mL}$ vs $559.55 \mathrm{pg} / \mathrm{mL}$ vs $442.35 \mathrm{pg} / \mathrm{mL}$ vs $685.65 \mathrm{pg}$ / $\mathrm{mL}$ ), TNF-a (mean: $1.795 \mathrm{pg} / \mathrm{mL}$ vs $1.57 \mathrm{pg} / \mathrm{mL}$ vs $1.14 \mathrm{pg} / \mathrm{mL}$ vs $1.14 \mathrm{pg} / \mathrm{mL}$ ) and IL-6 (mean: $1.55 \mathrm{pg} / \mathrm{mL}$ vs $1.48 \mathrm{pg} / \mathrm{mL}$ vs $3.46 \mathrm{pg} /$ $\mathrm{mL}$ vs $3.30 \mathrm{pg} / \mathrm{mL}$ ) during the study period. Box plots represent means and SD. $P$ value was calculated using paired $t$ test. Legend: $\mathrm{HCQ}=$ Hydroxychloroquine; $P$ = P-value; NS = not significant.

TABLE 1 Demographic and clinical characteristics of the patients at baseline

\begin{tabular}{ll} 
& $\begin{array}{l}\text { Participants } \\
\text { ( } n=27)\end{array}$ \\
Age, years, mean (SD) & $44(12)$ \\
& $10(37)$ \\
Antiphospholipid antibodies profile & \\
Triple positivity, $\mathrm{n}$ (\%) & $8(9)$ \\
Time since the first thrombosis, mean (SD) years & $4(6)$ \\
Time since the last thrombosis, mean (SD) years & $5(33)$ \\
Obstetric complications, $\mathrm{n}(\%)$ & \\
Site of the first thrombotic episode & $22(77)$ \\
$\quad$ Venous thrombosis, $\mathrm{n}(\%)$ & $8(30)$ \\
$\quad$ Arterial thrombosis, $\mathrm{n}(\%)$ & $9(33)$ \\
Recurrent thrombosis, $\mathrm{n}(\%)$ & \\
\hline
\end{tabular}

Twenty-seven patients completed the study. The mean age was 44 years, $78 \%$ were females and $44 \%$ had hypertension or dyslipidemia. Venous thrombosis was the index event in $77 \%$ of patients, $33 \%$ had recurrent thrombosis and $37 \%$ were triple positive for antiphospholipid antibodies (table 1). During HCQ treatment, TF and TNF-a levels decreased by $14.4 \%$ and $12.4 \%$, respectively. FT and TNF- $a$ levels remained decreasing until up to six months after HCQ withdrawal. From the beginning of HCQ use to 6 months after HCQ was interrupted, TF levels decreased by $32.3 \%$ and those of TNF- $a$ by 36.4\% ( $P=0.01$ and 0.0009, respectively). Conversely, IL-6 levels did not change with HCQ use and further increased 6 months after HCQ withdrawal. Twelve months after HCQ with drawal, the levels of IL-6 and TNF- a remained stable, while TF levels significantly increased.

Conclusions: HCQ use reducedTF and TNF-a levels in t-PAPS. This reduction was observed until up to six months after $\mathrm{HCQ}$ with drawal possibly due to a long-term effect of the drug. A possible rebound effect on the levels of TF was also seen 12 months after HCQ withdrawal. These findings support the hypothesis that HCQ may contribute to reduce the thrombotic risk in t-PAPS.

PB1054 | Artificial Intelligence Classifies APS in Anticoagulated Patients Based on Thrombin Generation

R. de Laat-Kremers ${ }^{1}$; D. Wahl ${ }^{2}$; S. Zuily ${ }^{2}$; M. Ninivaggi ${ }^{1}$; W. Chayoua $^{1}$; V. Regnault ${ }^{2}$; J. Musial ${ }^{3}$; P. de Groot ${ }^{1}$; K. Devreese ${ }^{4}$; B. de Laat $^{1}$

${ }^{1}$ Synapse Research Institute, Maastricht, Netherlands; ${ }^{2} \mathrm{CHRU}$ de Nancy, Nancy, France; ${ }^{3}$ Jagiellonian University Medical College, Krakow, Poland; ${ }^{4}$ Ghent University Hospital, Ghent, Belgium

Background: The antiphospholipid syndrome (APS) is characterized by the presence of antiphospholipid antibodies ( $\mathrm{APL}$ ) predominantly directed against $\beta 2$-glycoprotein I. APS is associated with an increased risk of thrombosis and pregnancy morbidity. Diagnosing APS is difficult because most patients are already on anticoagulation when tested for aPL and anticoagulant treatment interferes with aPL assays. Nevertheless, the aPL profile defines patient management, making aPL testing warranted during anticoagulant therapy.

Aims: We developed a neural net (NN) that diagnoses APS in a cohort of anticoagulated patients and controls based on thrombin generation (TG) and thrombin dynamics.

Methods: A NN was developed using TG data obtained with the CAT method in 48 APS patients anticoagulated with vitamin $\mathrm{K}$ antagonists and 64 anticoagulated controls. Input parameters were lag time, peak, ETP, time-to-peak, velocity index, total prothrombin conversion, maximum prothrombin conversion rate, thrombinantithrombin, thrombin- $a_{2}$-macroglobulin, and the thrombomodulin effect. Five NNs were developed and the most accurate NN was selected and clinically validation in the validation cohort of 311 APS patients and controls. The golden standard for APS diagnosis were the ISTH-SSC guidelines.

Results: In the derivation cohort, the NN classifies APS patients under anticoagulant treatment with a sensitivity of $92 \%$ and a specificity of 95\%, $\left(\mathrm{ROC}_{\mathrm{AUC}}=0.9805 ; 0.9542-1.000 ; P<0.0001\right)$. In the validation cohort, the NN was clinically validated in 33 APS patients and 278 controls, including anticoagulated controls $(n=62)$, thrombosis patients ( $n=38$ ), auto-immune disease patients $(n=49$ ), patients visiting the hospital for other indications ( $n=92)$, and normal controls ( $n=37$; Figure 1 ). The sensitivity of the NN was $85 \%$. The 
specificity of the NN was $93 \%$ in the whole validation cohort and ranged from $100 \%$ in normal controls to $76 \%$ in thrombosis controls (Table 1).

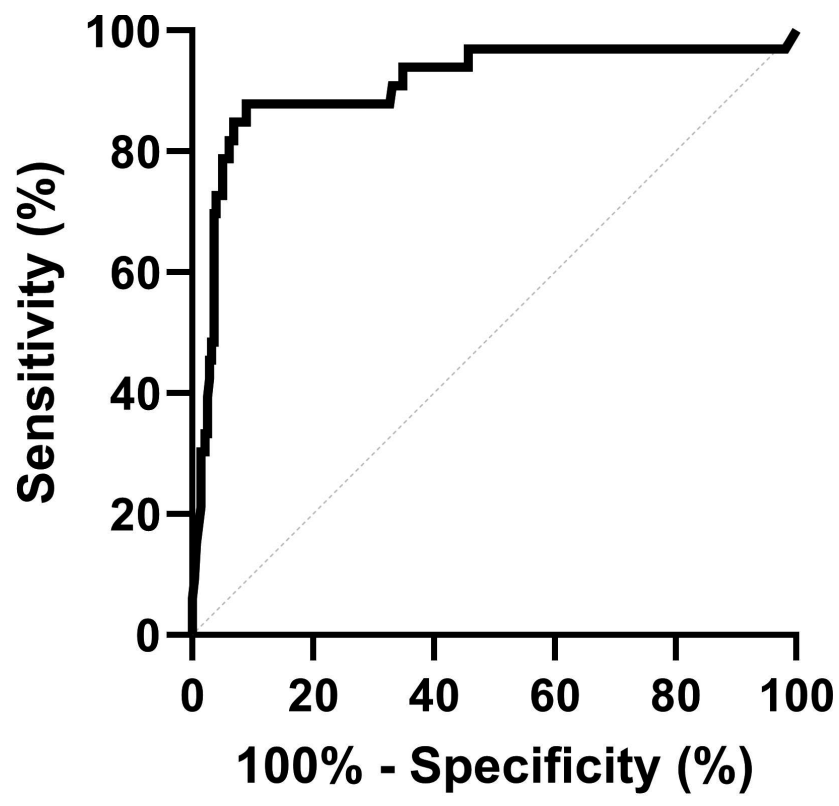

FIGURE 1 ROC curve of the performance of the neural net in the overall APS patient - control cohort. ROC curve of the APS diagnosing neural net in a set of 311 subjects, including 33 APS patients, 49 auto-immune disease patients, 38 thrombosis patients, 92 hospital controls, 62 patients on vitamin $\mathrm{K}$ antagonists, and 37 normal controls. The AUC $=0.9805$ (0.9542-1.000; $p$

Conclusions: We developed a NN that accurately classifies APS under anticoagulant treatment. This NN could be an alternative for the LAC test which is affected by anticoagulation.

PB1055 | A Novel ELISA Assay for the Detection of Antiprothrombin Antibodies in APS Patients at High Risk of Thrombosis

\section{N. Pozzi ${ }^{1} ;$ V. Pengo ${ }^{2}$}

${ }^{1}$ Saint Louis University, St. Louis, United States; ${ }^{2}$ University of Padova, Padova, Italy

Background: Autoantibodies targeting prothrombin bound to phosphatidylserine (aPS/PT) are frequently found in Antiphospholipid Syndrome (APS) patients at high risk of thrombosis. However, their detection has proven difficult to standardize because of the transient nature of the complex, which requires calcium ions, and the variable source/purity of phospholipids and antigen. Furthermore, even though it is assumed that aPS/PT interact with prothrombin, they may react against PS and other plasma proteins capable of interacting with PS.

Aims: Develop an ELISA assay to improve the identification of antiprothrombin antibodies in correlation with thrombosis.

Methods: We engineered a novel prothrombin variant carrying an artificial tag at the $\mathrm{C}$-terminus suitable for site-specific biotinylation (proT-BioT). The structural and functional properties of proT-BioT free and bound to neutravidin were characterized using biochemical and biophysical approaches. Immobilization of proT-BioT was performed using plastic plates coated with neutravidin, using different proT-BioT/neutravidin ratios. Anti-prothrombin antibodies were researched in plasma samples of 32 APS patients and the results compared with commercially available and homemade ELISA assays.

Results: proT-Biot retained identical structural and functional properties of prothrombin wild-type with the advantage that it could be immobilized to neutravidin coated plates at the desired density and with a defined orientation, i.e., pointing the $\mathrm{N}$-terminal fragment-1 toward the solvent (C). Antibodies against proT-Biot (aPT-Bio) were found in 24 out of 32 APS patients (75\%) with triple positivity (LA, $\mathrm{aCL}$, and $\mathrm{a}$ (2GPI). When compared to aPS/PT (A) and aPT-A (autoantibodies targeting prothrombin bound to the plastic plate) (B), aPTBio showed an excellent linear correlation with aPS/PT (R2 = 0.85) (D) but not with aPT-A (R2 $=0.40)(E)$.

Conclusions: Immobilization of proT-Biot to neutravidin-coated plates allows detection of anti-prothrombin antibodies in APS patients at high risk of thrombosis. Since aPT-Bio correlates with aPS/ PT but not with aPT-A, this method may find utility for detecting anti-prothrombin antibodies in correlation with thrombosis.

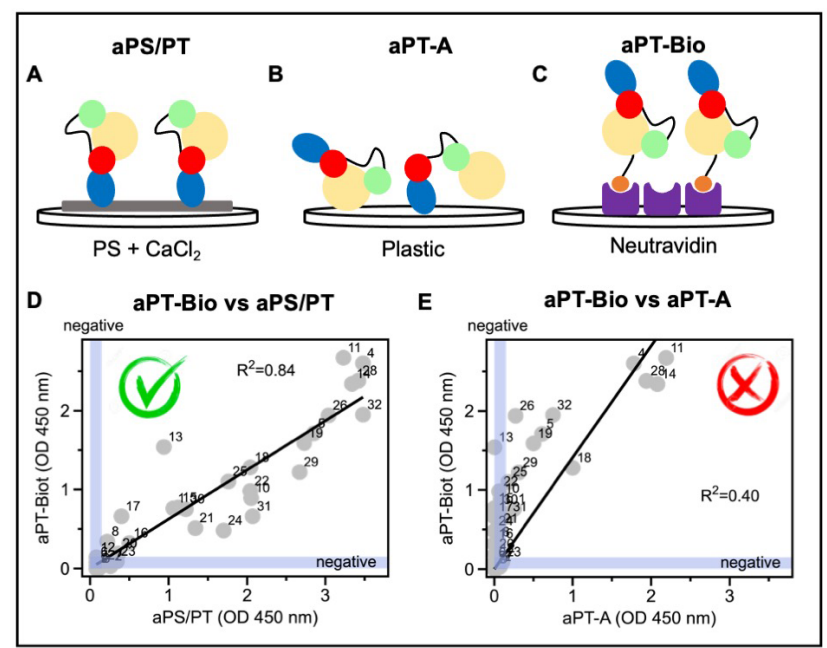

FIGURE 1 Graphical Summary 
PB1056 | The Utility of the Dilute Prothrombin Time Assay in the Diagnosis of Antiphospholipid Syndrome

T. Storozuk; G. Wool

University of Chicago, Chicago, United States

Background: ISTH APS guidelines recommend two lupus anticoagulant (LA) reagent systems: the dilute Russell's Viper Venom Time (DRVVT) and a LA-sensitive aPTT-like assay. Other LA reagents are available, including the dilute prothrombin time (DPT). At UChicago Medicine, we provide a comprehensive APS panel that includes lupus-sensitive aPTT, DRVVT, as well as the DPT LA assay. In combination with the DRVVT, the DPT can serve as an effective screen for confounding anticoagulant medications such as warfarin and $\mathrm{Xa}$ inhibitory DOAC.

Aims: Here we analyze the utility of DPT-based functional LA testing in contributing laboratory evidence of antiphospholipid syndrome as well as in assisting with interpretation of LA panels.

Methods: We retrospectively evaluated all lupus anticoagulant testing in a 4.5 year period (1693 patients, 2015 cases). DPT positivity was defined as a prolonged screening clotting time and a significant shortening of clotting time with high concentration phospholipid, in keeping with ISTH guidelines.

Results: Of the 2015 cases evaluated, DPT was generally positive in concert with other LA studies (Table 1). Only 56 cases showed sole LA positivity in the DPT-based system (2.8\%). In only two cases was repeat LA testing performed and the DPT the only functional system that contributed confirmatory repeat laboratory evidence of antiphospholipid syndrome.

Warfarin and Xa inhibitory DOACs are common causes of interference with DRVVT testing. Prolonged DPT screens with negative confirmatory phase are commonly seen with these anticoagulants. Warfarin interference leads to DPT screen > DRVVT screen while
Xa inhibitory DOAC lead to the opposite pattern (Table 2). These cases receive extra pathologist scrutiny for possible false positive DRVVT.

TABLE 1 Total DPT testing and percent positivity

\begin{tabular}{|c|c|c|c|}
\hline & \multicolumn{3}{|c|}{ Total DPT Testing (2015 total screens) } \\
\hline & Number & $\begin{array}{l}\text { Percent of } \\
\text { total }\end{array}$ & $\begin{array}{l}\text { Percent of } \\
\text { subgroup }\end{array}$ \\
\hline $\begin{array}{l}\text { Prolonged DPT } \\
\text { screen }\end{array}$ & 1154 & $57.3 \%$ & \\
\hline $\begin{array}{l}\text { Abnormal DPT } \\
\text { confirm }\end{array}$ & 213 & $10.6 \%$ & \\
\hline $\begin{array}{l}\text { Abnormal DPT } \\
\text { confirm cases } \\
\text { with other } \\
\text { positive lupus } \\
\text { anticoagulant } \\
\text { studies } \\
\text { (DRVVT, } \\
\text { aPTT) }\end{array}$ & 157 & $7.8 \%$ & $\begin{array}{l}\text { 73.7\% of } \\
\text { abnormal DPT } \\
\text { confirm }\end{array}$ \\
\hline $\begin{array}{l}\text { Abnormal DPT } \\
\text { confirm cases } \\
\text { with no other } \\
\text { positive lupus } \\
\text { anticoagulant } \\
\text { studies }\end{array}$ & 56 & $2.8 \%$ & $\begin{array}{l}\text { 26.3\% of } \\
\text { abnormal DPT } \\
\text { confirm }\end{array}$ \\
\hline $\begin{array}{l}\text { Abnormal DPT } \\
\text { confirm with } \\
\text { no other } \\
\text { positive lupus } \\
\text { anticoagulant } \\
\text { studies and } \\
\text { with clinical } \\
\text { evidence of } \\
\text { APS }\end{array}$ & 27 & $1.3 \%$ & $\begin{array}{l}\text { 50.0\% of } \\
\text { abnormal DPT } \\
\text { confirm cases } \\
\text { with no other } \\
\text { positive lupus } \\
\text { anticoagulant } \\
\text { studies and } \\
\text { available } \\
\text { clinical data }\end{array}$ \\
\hline
\end{tabular}

TABLE 2 Use of DPT to investigate for false positive DRVVT in patients on warfarin or Xa inhibitory DOAC

\begin{tabular}{llll} 
& & Number & $\begin{array}{l}\text { Percent of total DPT testing (2015 } \\
\text { total screens) }\end{array}$ \\
Cases with abnormal DRVVT confirm & & 653 & $32.4 \%$ \\
& - with prolonged DPT screen but & 371 & $18.4 \%$ \\
& negative DPT confirm & & $11.4 \%$ \\
\hline
\end{tabular}

Conclusions: The DPT is only rarely the sole positive LA system.

In combination with the DRVVT, the DPT can serve as an effective screen for anticoagulant interference and contributes to the accuracy of pathologist interpretation of APS panels. 
PB1057 | A Diagnostic Solution for Lupus Anticoagulant Testing in Patients Taking Direct Oral FXa Inhibitors Using DOAC Filter

C. Farkh ${ }^{1}$; S. Ellouze ${ }^{1}$; L. Gounelle ${ }^{1}$; M. Sad-Houari ${ }^{1}$; J. Duchemin ${ }^{1}$; V. Proulle ${ }^{1}$; M. Fontenay ${ }^{1,2} ;$ X. Delavenne ${ }^{3,4} ;$ G. Jourdi $^{1,5,6,7}$

${ }^{1}$ AP-HP, Centre-Université de Paris, Hôpital Cochin, Paris, France;

${ }^{2}$ Institut Cochin, CNRS UMR8104, INSERM U1016, Université De Paris,

Paris, France; ${ }^{3}$ Institut National de la Santé et de la Recherche Médicale U 1059, Dysfonctions Vasculaires et de L'Hémostase, Université de Lyon, Saint-Etienne, France; ${ }^{4}$ Laboratoire de Pharmacologie, Toxicologie, Gaz du Sang, CHU de Saint-Etienne, Saint-Etienne, France; ${ }^{5}$ Université de Paris, Innovative Therapies in Haemostasis, INSERM UMR_S 1140, Paris, France; ${ }^{6}$ Montreal Heart Institute, Montreal, Canada; ${ }^{7}$ Montreal University, Montreal, Canada

Background: Direct oral factor Xa (FXa) inhibitors interfere with Lupus Anticoagulant (LA) assays challenging the diagnosis of antiphospholipid syndrome in treated patients.

Aims: DOAC Filter is a filtration cartridge in which FXa inhibitor compounds are trapped by noncovalent binding while plasma is filtered through a solid phase. We hence evaluated for the first time its potential usefulness for reliable LA testing in real-life clinical practice. Methods: Samples from 147 patients who gave their informed written consent were analyzed before and after filtration: 38 rivaroxaban, 41 apixaban and 68 none. Anticoagulant plasma concentrations were measured using specific anti-Xa assays and HPLC-MS/MS. LA testing was performed using dilute Russell Viper Venom Time (dRVVT) and Silica Clotting Time (SCT).

Results: Baseline median [min-max] concentrations were 64.8 [17.6; 311.4] for rivaroxaban and $92.1 \mathrm{ng} / \mathrm{mL}$ [37.1; 390.7] for apixaban (HPLC-MS/MS). They were significantly correlated with anti-Xa assays results ( $r=0.98$ and $r=0.94$, respectively). dRVVT was positive in $92 \%$ rivaroxaban and $72 \%$ apixaban and SCT in 28 and $41 \%$ of samples, respectively. In post-filtration samples, median $\%$ of neutralization was $100 \%$ with rivaroxaban and apixaban concentrations of respectively $<2$
[<2-2.4] and <2 ng/mL [<2-9.6] using HPLC-MS/MS. No significant effect of DOAC Filter was observed on dRVVT nor SCT screen and confirm assays performed in controls $(n=31)$ and LA positive $(n=37)$ nonanticoagulated samples. dRVVT and SCT remained positive in respectively 16 and $8 \%$ of rivaroxaban and 41 and $18 \%$ of apixaban samples.

Conclusions: As such, DOAC Filter would be an easy-to-use new device allowing FXa inhibitors removal from plasma samples, limiting therefore their interference with LA testing in treated patients.

\section{PB1058 | The Diagnostic Utility of the Taipan Snake Venom} Time in an Era of DOACstop ${ }^{\mathrm{TM}}$

E. Foxton ${ }^{1}$; R. Polgrean ${ }^{1}$; M. Desborough ${ }^{2}$; G. Ling ${ }^{2}$

${ }^{1}$ Viapath Analytics, Diagnostic Haemostasis Laboratory, St Thomas' Hospital, London, United Kingdom; ${ }^{2}$ Guy's and St Thomas' NHS

Foundation Trust, London, United Kingdom

Background: The dilute Russell's viper venom time (dRVVT) and dilute APTT (dAPTT) remain guideline recommended assays for Lupus Anticoagulant (LA) screening. Direct oral anticoagulants (DOAC) can interfere with dRVVT and dAPTT testing. Local LA screening includes Taipan Snake Venom Time (TSVT) for patients on oral anticoagulation. DOAC-Stop ${ }^{\mathrm{TM}}$, an activated charcoal tablet designed to adsorb DOAC to restore normal Xa activity, we assess its use to determine the ongoing need for TSVT.

Aims: To determine LA detection rates pre / post addition of DOACstop ${ }^{\text {TM }}$ to DOAC samples using dRVVT, dAPTT and TSVT.

Methods: Samples from patients on DOACs $(n=70)$ ((Rivaroxaban $(n=39)$, Apixaban $(n=22)$, Edoxaban $(n=9))$ and a control group ( $n=20)$ (VKA / no anticoagulation) were tested using drug specific anti-Xa, dRVVT, dAPTT and TSVT, pre and post addition of DOACStop $^{\mathrm{TM}}$. Data were compared using the paired student t-test.

Results:

TABLE 1 LA test results for neat plasma and post DOAC removal

\begin{tabular}{|c|c|c|c|c|c|c|c|c|}
\hline Anticoagulant & LA Assay & & $n=$ & $\begin{array}{l}\text { Neat Plasma } \\
\text { Median [Range] }\end{array}$ & $n=$ & $\begin{array}{l}\text { DOACstop } \\
\text { Median [Range] }\end{array}$ & $\begin{array}{l}\text { Difference } \\
\text { (\%) }\end{array}$ & $p$-value \\
\hline \multirow[t]{3}{*}{ DOAC } & dRVVT & Screen & 70 & $1.99[0.94-5.00]$ & 70 & 1.01 [0.86-1.95] & -101 & $<0.0001$ \\
\hline & TSVT & TSVT & 70 & $1.06[0.96-1.24]$ & 70 & $1.05[0.92-1.24]$ & -1 & 0.135 \\
\hline & & ET & 17 & 1 [0.93-1.06] & 15 & 0.96 [0.90-1.03] & -4.2 & - \\
\hline \multirow[t]{3}{*}{ VKA/None } & dRVVT & Screen & 20 & 1.39 [0.93-4.07] & 20 & $1.4[0.87-4.17]$ & 0.7 & 0.442 \\
\hline & TSVT & TSVT & 20 & 1.23 [1.06-1.79] & 20 & 1.24 [1.06-1.87] & 0.8 & 0.160 \\
\hline & & ET & 17 & $0.96[0.81-1.14]$ & 18 & 0.99 [0.84-1.13] & 3 & - \\
\hline
\end{tabular}

69/70 (99\%) DOAC anti-Xa levels were reduced from $20-651 \mathrm{ng} / \mathrm{ml}$ to below assay detection limits by use of DOAC-Stop ${ }^{\mathrm{TM}}$, 1 measureable Edoxaban level of $6.6 \mathrm{ng} / \mathrm{ml}$ post DOAC-Stop ${ }^{\mathrm{TM}}$ was reduced from $73.4 \mathrm{ng} / \mathrm{ml}$ (detection limit = $5 \mathrm{ng} / \mathrm{ml}$ ).

Post-treatment results showed normalisation of dRVVT and dAPTT ratio $(p<0.001)$ for patients on DOAC as compared with control samples. No significant change in TSVT results was seen pre/post tablet in either patient group.

Post-DOAC-Stop ${ }^{\mathrm{TM}}$, 17/70 DOAC results were consistent with LA, 15/70 by TSVT and 5/70 by dRVVT/dAPTT, with 3/17 samples positive by both TSVT and dRVVT/dAPTT. 16/20 control results were consistent with LA by TSVT, including 10 positive by both TSVT and DRVVT/ DAPTT (Table 2). 
TABLE 2 LA result interpretation

\begin{tabular}{|c|c|c|c|c|}
\hline Anticoagulant & dRVVT / dAPTT result (post DOACstop ${ }^{\mathrm{TM}}$ ) & TSVT positive & TSVT negative & Total \\
\hline \multirow[t]{3}{*}{ DOAC } & dAPTT +/or dRVVT positive & $3(4.3 \%)$ & $2(2.9 \%)$ & 5 \\
\hline & dAPTT + dRVVT negative & $12(17.1 \%)$ & $53(75.7 \%)$ & 65 \\
\hline & Total & 15 & 55 & 70 \\
\hline \multirow[t]{3}{*}{ VKA / None } & dAPTT +/or dRVVT positive & $10(50 \%)$ & 0 & 10 \\
\hline & dAPTT + dRVVT negative & $7(35 \%)$ & $3(15 \%)$ & 10 \\
\hline & Total & 17 & 3 & 20 \\
\hline
\end{tabular}

Conclusions: DOAC-Stop ${ }^{\mathrm{TM}}$ removed anti-Xa activity, allowing interpretation of dRVVT and dAPTT results, and may reduce the rate of positive LA results seen in our laboratory if TSVT is not used. Further clinical correlation of patients' phenotype with LA results will inform on whether TSVT is still needed given the efficacy of DOAC-Stop.

PB1059 | Evaluation of a Gene Signature Related to Thrombotic Manifestations in Antifospholipid Syndrome

B. Jacintho $^{1}$; B. Mazetto ${ }^{2}$; B. Hounkpe ${ }^{3}$; A.P. Santos ${ }^{4}$; C. Vaz ${ }^{1}$;

G. Vechiatto ${ }^{4}$; J. Oliveira ${ }^{1}$; E. de Paula ${ }^{5,2}$; F. Orsi ${ }^{5,6}$

${ }^{1}$ School of Medical Sciences, Department of Clinical Medicine, University of Campinas, Campinas, Brazil; ${ }^{2}$ School of Medical Sciences, University of Campinas, Campinas, Brazil; ${ }^{3}$ School of Medical Sciences, Department of Medical Physiopathology, Universisity of CampinasUNICAMP, Campinas, Brazil; ${ }^{4}$ School of Medical Sciences, Department of Medical Sciences, University of Campinas,, Campinas, Brazil;

${ }^{5}$ Hematology and Hemotherapy Center, University of Campinas -

UNICAMP, Campinas, Brazil; ' ${ }^{6}$ chool of Medical Sciences, Department of Clinical Pathology, University of Campinas, Campinas, Brazil

Background: Whether different manifestations of thrombotic antiphospholipid syndrome (APS) share pathological mechanisms has not been established. Transcriptome analysis may constitute a new approach to evaluate the mechanisms behind thrombotic manifestations in APS.

Aims: To determine in patients with primary thrombotic APS (tPAPS) the expression of genes already related to venous and arterial thrombosis in the general population.

Methods: mRNA was obtained from total leucocyte and gene expression was measured by qPCR and the results were analyzed by QuantStudio $^{\mathrm{TM}}$ Software.

Results:

TABLE 2 Median fold changes in gene expression according to the subgroups of clinical relevance of t-PAPS. Legend: This table demonstrates that TNFAIP6 mRNA expression is higher in all t-PAPS subgroups in comparison with controls, being particularly elevated in patients who had multiple thrombosis $(P=0,01)$. ANXA3 mRNA expression was slightly higher in t-PAPS than in controls but the difference was not statistically significant. SERPINB2 mRNA expression is lower in all t-PAPS subgroups in comparison with controls, being slightly decreased in patients who had simple thrombosis $(P=0,0025)$. BACH2 mRNA expression was lower in all t-PAPS subgroups in comparison with controls, being slightly decreased in patients who had a single thrombosis $(P=0,002)$ TXK mRNA expression was lower in all t-PAPS subgroups in comparison with control, being slightly decreased in triple positive patients $(P=<0,0001)$.

\begin{tabular}{|c|c|c|c|c|c|c|c|c|}
\hline Genes & \multicolumn{8}{|c|}{ Fold-change in individual studies (FC) } \\
\hline & \multicolumn{8}{|c|}{ Up-regulated genes } \\
\hline TNFAIP6 & 0.93 & 1.38 & 1.49 & 1.27 & 2.17 & 1.33 & 1.85 & Innate immunity \\
\hline SERPINB2 & 1.11 & 0.4 & 0.99 & 0.69 & 1.06 & 0.84 & 0.85 & Hemostasis \\
\hline $\mathrm{BACH} 2$ & 1.62 & 1.01 & 1.19 & 0.95 & 1.15 & 0.98 & 1.27 & Immune regulation \\
\hline TXK & 1.44 & 0.86 & 0.94 & 0.84 & 0.97 & 0.90 & 0.77 & Innate immunity \\
\hline
\end{tabular}


TABLE 1 Clinical and laboratory features of t-PAPS $(n=83)$. Legend: Abbreviations: t-PAPS thrombotic primary antiphospholipid syndrome; IQR interquartile range; aPL antiphospholipid antibodies

\begin{tabular}{ll}
\hline $\begin{array}{l}\text { Age at the diagnosis, median } \\
\text { (IQR) }\end{array}$ & $33(24 ; 48)$ \\
$\begin{array}{l}\text { Time elapsed since the last } \\
\text { thrombotic event in months, } \\
\text { median (IQR) }\end{array}$ & $33.9(11.6 ; 66.9)$ \\
Non-provoked thrombosis, $\mathrm{n}(\%)$ & $48(57.8)$ \\
Site of the first thrombotic event: & \\
Venous thrombosis, $\mathrm{n}(\%)$ & $61(73.5)$ \\
Arterial thrombosis, $\mathrm{n}(\%)$ & $22(26.5)$ \\
Multiple thromboses, $\mathrm{n}(\%)$ & $35(42.2)$ \\
aPL triple positivity, $\mathrm{n}(\%)$ & $14(16.8)$ \\
\hline
\end{tabular}

83 t-PAPS and 85 controls were included. The median age at the enrollment day was 40 years-old (IQR 31-51) in patients and 38 (IQR 29-53) in controls, 66\% of patients and controls were women and cardiovascular risk factors were more prevalent among t-PAPS than in controls (37\% vs $11 \%$ ). The clinical and laboratory features of t-PAPS patients are shown in Table 1. TXK $(P<0.001), B A C H 2$ $(P=0.005)$ and SERPINB2 $(P=0.003)$ mRNA expressions were down-regulated while TNFAIP6 mRNA expression was up-regulated $(P=0.003)$ in t-PAPS when compared to controls. ANXA3 mRNA expression was similar between groups. In a subgroup analysis that considered different manifestations of t-PAPS, such as venous vs. arterial thrombosis, single vs. multiple thrombosis and non-triple positive vs. triple positive, we observed that the increase in TNFAIP6 mRNA expression was more pronounced in t-PAPS with recurrent thrombosis. Table 2 demonstrates the fold changes by t-PAPS subgroups.

Conclusions: In this study, we validated in t-PAPS the expression of genes previously associated with arterial and venous thrombosis in general population. Particularly, the main difference between tPAPS and controls appeared in the expression of genes related to immune regulation. These genes were also associated with disease severity, such as multiple thrombosis and triple positivity. Our findings point towards an association between immune regulation and thrombosis in APS.

Acknowledgments: São Paulo Research Foundation FAPESP (2016/14172-6)
PB1060 | Platelet Activity from Antiphospholipid Syndrome (APS) Patients is Enhanced: Possible Role of the ADP Signaling Pathway

G. Leonardi $^{1}$; C.H. Lescano ${ }^{1}$; A.P.R. Dos Santos ${ }^{2}$; B.C. Jacinto ${ }^{2}$; B.M. Mazetto $^{2}$; F.A. Orsi ${ }^{3,4}$; F.Z. Mónica ${ }^{1}$

${ }^{1}$ Department of Pharmacology, Faculty of Medical Sciences, University of Campinas, Campinas, SP, Brazil; ${ }^{2}$ Faculty of Medical Sciences, University of Campinas, Campinas, SP, Brazil; ${ }^{3}$ Laboratory of Haemostasis, Hematology and Hemotherapy Center, University of Campinas, Campinas, SP, Brazil; ${ }^{4}$ Department of Clinical Pathology, Faculty of Medical Sciences, University of Campinas, Campinas, SP, Brazil

Background: Several studies have evaluated the direct effect of antiphospholipid antibodies in isolated platelets from healthy volunteers, but the literature is scarce about platelet activity obtained from patients with APS.

Aims: To evaluate platelet aggregation from patients with primary APS with thrombosis (t-PAPS) or healthy volunteers with no history of diabetes, hypertension or dyslipidemia.

Methods: Twenty-four patients with t-PAPS $(66.6 \%$ females, mean age: 38 years) and fifty-three healthy volunteers ( $58.5 \%$ females, mean age: 33 years) were included. Firstly, platelet-rich plasma (PRP) was obtained and stimulated with adenosine diphosphate (ADP, 3 or $10 \mu \mathrm{M}$ ), collagen $(1 \mu \mathrm{g} / \mathrm{ml})$ or arachidonic acid (AA, $300 \mu \mathrm{M})$. Next, PRP was pre-incubated with platelets inhibitors, as nitric oxide donor, sodium nitroprusside (SNP, 3 or $10 \mu \mathrm{M}$ ) or the stable analogue of prostacyclin, (iloprost, 3 or $10 \mathrm{nM}$ ) and then stimulated with ADP or collagen.

Results:

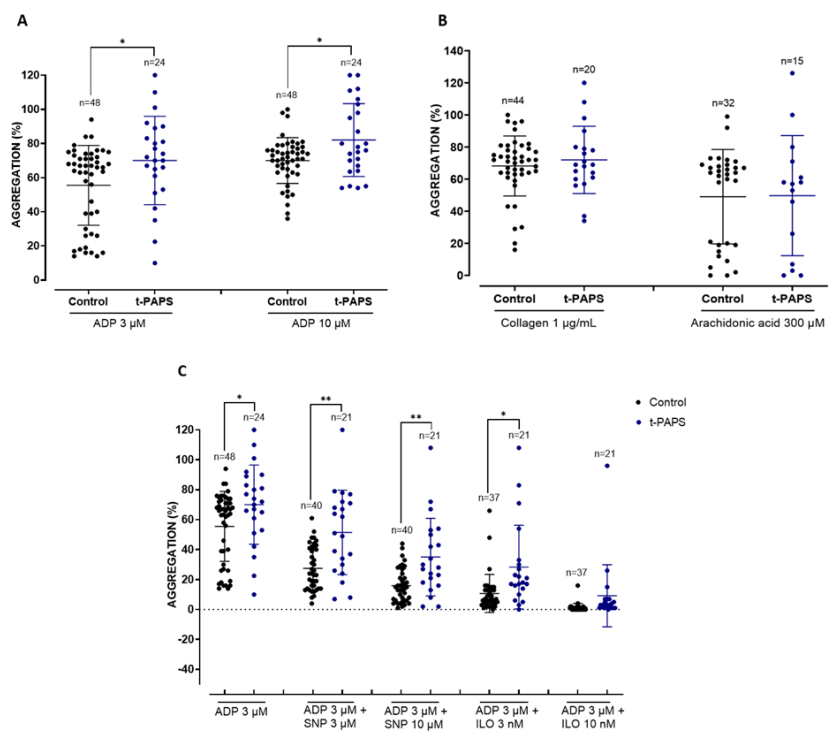

FIGURE 1 Effect of agonists and inhibitors on platelet-rich plasma (PRP). Platelets from patients with thrombotic primary antiphospholipid syndrome (t-PAPS) or healthy volunteers were stimulated with ADP (3 or $10 \mu \mathrm{M})(\mathrm{A})$, collagen $(1 \mu \mathrm{g} / \mathrm{mL})$ or AA (300 $\mu \mathrm{M})(B)$. Panel C: in another set of experiments, PRP from both groups was pre-incubated with SNP (3 or $10 \mu \mathrm{M}, 3$ minutes) or iloprost (3 or $10 \mathrm{nM}, 3$ minutes) 
and then stimulated with $\mathrm{ADP}(3 \mu \mathrm{M})$. Data represent the mean $\pm \mathrm{DP}$, $N=24$ (ADP, 3 or $10 \mu \mathrm{M}$ ), 20 (collagen $1 \mu \mathrm{g} / \mathrm{mL}$ ), 15 (AA, $300 \mu \mathrm{M}$ ) or 21 (ADP $3 \mu \mathrm{M}$ with SNP or iloprost) for t-PAPS and $N=48$ (ADP, 3 or $10 \mu \mathrm{M}$ ), 44 (collagen $1 \mu \mathrm{g} / \mathrm{mL}$ ), 32 (AA, $300 \mu \mathrm{M}$ ), 40 (ADP $3 \mu \mathrm{M}$ with SNP) or 37 (ADP $3 \mu \mathrm{M}$ with iloprost) for control. For statistical analysis, unpaired $t$ test with Welch's correction was performed

ADP-induced platelet aggregation was significantly higher in tPAPS group than in controls $(3 \mu \mathrm{M}$ : $70 \% \pm 26.4 \%$ vs $55.5 \% \pm 23.3 \%$, $P=0.02 ; 10 \mu \mathrm{M}: 82 \% \pm 21.3 \%$ vs $70 \% \pm 13.4 \%, P=0.02)$. No difference in AA- $(49.7 \% \pm 37.3 \%$ vs $49 \% \pm 29.4 \%, P=0.95)$ or collagen$(72 \% \pm 20.9 \%$ vs $68.2 \% \pm 18.6 \%, P=0.51)$-induced aggregation was seen between groups. The aggregation inhibition induced by SNP ( $3 \mu \mathrm{M}: 26.4 \% \pm 40.2 \%$ vs $50 \% \pm 26.1 \%, P=0.001 ; 10 \mu \mathrm{M}$ : $50.5 \% \pm 36.5 \%$ vs $71.1 \% \pm 20.2 \%, P=0.004$ ) or iloprost ( $3 \mathrm{nM}$ : $59.5 \% \pm 39.8 \%$ vs $80.7 \% \pm 22.9 \%, P=0.01$ ) was less prominent in platelets from t-PAPS than in healthy volunteers.

Conclusions: Our results showed that ADP-induced aggregation was increased and the inhibition induced by endothelial mediators was reduced in platelets from t-PAPS patients when compared to controls. Our findings suggest that platelets activity is increased in t-PAPS and point towards a possible role of the ADP signaling pathway in the thrombotic event seen in these patients.
PB1061 | Plasmocytoid Dendritic Cells Activity in Asymptomatic Antiphospholipid Carriers and in Primary and Secondary Antiphospholipid Syndrome with Thrombosis

A.P. Rosa dos Santos ${ }^{1}$; B. Cardoso Jacintho ${ }^{2}$; C. de Oliveira Vaz ${ }^{2}$;

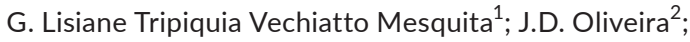
J. Annichino-Bizzacchi ${ }^{3}$; S. Appenzeller ${ }^{4}$; B. Moraes Mazetto ${ }^{5}$; F. A. Orsi ${ }^{6}$

${ }^{1}$ University of Campinas - Unicamp / School of Medical Sciences, Department of Medical Sciences, Campinas, Brazil; ${ }^{2}$ University of Campinas - Unicamp / School of Medical Sciences, Department of Clinical Medicine, Campinas, Brazil; ${ }^{3}$ University of Campinas Unicamp / School of Medical Sciences, Hematology and Hemotherapy Center, Campinas, Brazil; ${ }^{4}$ University of Campinas - Unicamp / School of Medical Sciences, Rheumatology Unit, Department of Clinical Medicine, Campinas, Brazil; ${ }^{5}$ University of Campinas - Unicamp / School of Medical Sciences, Campinas, Brazil; ${ }^{6}$ University of Campinas - Unicamp / School of Medical Sciences, Hematology and Hemotherapy Center, Department of Clinical Pathology, Campinas, Brazil

Background: Plasmacytoid dendritic cells ( $\mathrm{pDCs}$ ) have the ability to produce large amounts of interferon (IFN)-1, a mediator in the immune response. Although pDCs have been associated with systemic autoimmune disorders, whether they play a role in antiphospholipid antibodies (aPL) pathological mechanisms is not determined.

Aims: To determine the frequency and activity of pDCs in different forms of antiphospholipid syndrome (APS).

Methods: We enrolled patients with thrombotic primary and secondary APS (t-PAPS and t-SAPS), asymptomatic aPL carriers and individuals without thrombosis (controls). pDCs and IFN- $a$ expression (with and without oligodeoxynucleotides [CPG] stimulus) were quantified in the peripheral blood by flow cytometry and the results were compared using Kruskal-Wallis test. Ethics Committee approved the study.

Results: 46 t-PAPS patients, 39 t-SAPS, 17 aPL carriers and 50 controls were included. Demographic, clinical and laboratory data are shown in Table 1. Median frequency of $\mathrm{pDC}$ was $0.23 \%$ (interquartile range [IQR] 0.14-0.29) in controls, $0.28 \%$ (IQR 0.14-0.31) in aPL carriers, $0.20 \%$ (IQR 0.11-0.27) in t-PAPS and $0.12 \%$ in t-SAPS (IQR 0.11-0.16; $P<0.001)$. Median IFN- $a$ expression before CPG stimulus was $2.54 \%$ (IQR 2.12-3.21) in controls, $2.33 \%$ (IQR 2.14-2.89) in aPL carriers, 2.63\% (IQR 2.02-3.70) in t-PAPS and 3.08\% (IQR 2.593.75 ) in t-SAPS; $P=0.015$. Median expression of IFN- $\alpha$ after CPG stimulus was 3.17\% (IQR 2.70-3.85) in controls, 3.13\% (IQR 2.803.47 ) in aPL carriers, $3.76 \%$ (IQR 2.69-4.65) in t-PAPS and $4.86 \%$ (IQR 4.36-5.43) in t-SAPS; $P<0.001$.The change in IFN-a expression after CPG stimulus was higher in t-PAPS and t-SAPS, as compared to controls and aPL carriers, as shown in Figure 1. 
TABLE 1 Demographic characterization, clinical and laboratory features of controls, aPL carriers, t-PAPS and t-SAPS patients. Legend: Abbreviations: $n$ = absolute number; SD = standard deviation; aPL antiphospholipid; t-PAPS primary thrombotic antiphospholipid syndrome; t-SAPS thrombotic secondary antiphospholipid syndrome.* hypertension, dyslipidemia or diabetes

\begin{tabular}{|c|c|c|c|c|}
\hline & & aPL carriers & t-PAPS & \\
\hline Demographic characterization & Controls $n=50$ & $n=17$ & $n=46$ & $\mathrm{t}-\mathrm{SAPS} n=39$ \\
\hline Age, mean (SD) & $40.58(13.29)$ & $41(13.60)$ & $40.62(13.21)$ & $40.67(13.35)$ \\
\hline Female, n (\%) & $32(64)$ & $14(82.35)$ & $28(60.86)$ & $34(87.17)$ \\
\hline Venous thrombosis, n (\%) & 0 & 0 & $32(69.56)$ & $29(74.35)$ \\
\hline Multiple thrombosis, n (\%) & 0 & 0 & $19(41.30)$ & $23(58.97)$ \\
\hline \multicolumn{5}{|l|}{ Antiphospholipid profile } \\
\hline Lupus anticoagulant. n (\%) & 0 & $11(64.70)$ & 39 (84.78) & $22(56.41)$ \\
\hline Anticardiolipin antibody IgM or lgG. n (\%) & 0 & $15(88.23)$ & $26(56.52)$ & 31 (79.48) \\
\hline Anti- $\beta 2$-glycoprotein I antibody IgM or IgG. n (\%) & 0 & $20(117.64)$ & $32(69.56)$ & $47(120.51)$ \\
\hline Triple positivity for aPL. $\mathrm{n}(\%)$ & 0 & $5(29.41)$ & $7(15.21)$ & $3(7.69)$ \\
\hline Cardiovascular Risk Factors n (\%)* & $13(26)$ & $7(41.17)$ & $36(78.26)$ & $44(112.82)$ \\
\hline
\end{tabular}

Conclusions: pDC activity is increased in t-PAPS and t-SAPS when compared to controls and aPL carriers. These results suggest that cellular immunity is deranged not only in SAPS but also in PAPS and is not associated with the presence of aPL alone.
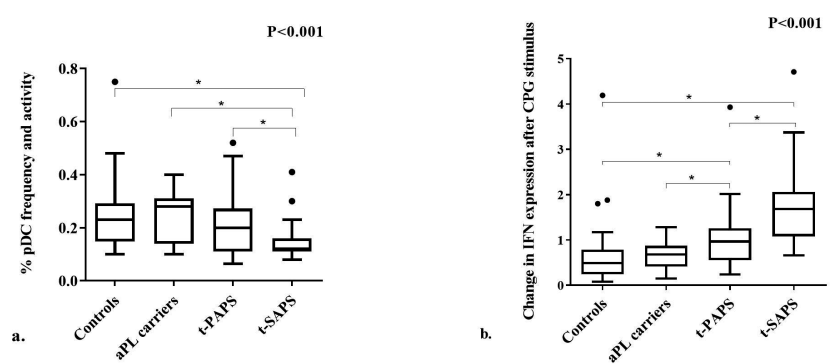

FIGURE 1 pDC frequency and change in intracellular IFN- $a$ expression after stimulus (pDC activity). ${ }^{*} \mathrm{P}$ value $<0.05$. Legend: (a) pDC count in peripheral blood of controls (median $0.23 \%$, interquartile range [IQR] 0.14-0.29), aPL carriers (0.28\%; IQR 0.14-0.31), t-PAPS (0.20\%; IQR 0.11-0.27) and t-SAPS (0.12\%; IQR 0.11-0.16). (b) Change in IFN- $a$ expression after CPG stimulus in controls $(0.48 \%$; IQR 0.24-0.78), aPL carriers (0.68\%; IQR 0.41-0.87), t-PAPS $(0.96 \%$, IQR 0.55-1.25) and t-SAPS (1.68\%; IQR 1.08-2.06).

PB1062 | Rivaroxaban versus Vitamin K Antagonist for the Secondary Prevention of Recurrent Thrombosis in Antiphospholipid Syndrome: A Systematic Review and Metaanalysis

R.D. Agustin; D.L. Villanueva; E. Alajar; M.T. Abola

University of the Philippines - Philippine General Hospital, Manila, Philippines

Background: Vitamin $\mathrm{K}$ antagonists (VKA) such as Warfarin are the mainstay of therapy in Antiphospholipid Syndrome (APS) but this entails frequent INR monitoring, increased bleeding and multiple drug and food interactions. Direct oral anticoagulants such as Rivaroxaban present an alternative and novel therapeutic approach to APS management.

Aims: This study aimed to assess the effect of Rivaroxaban compared with VKA on recurrent thrombosis and major bleeding among patients with APS.

Methods: We included studies satisfying the following criteria:

1) Randomized controlled trials (RCT);

2) APS;

3) recurrent thrombosis andmajor bleeding.

We searched for eligible studies in PUBMED, Clinical Key, Science Direct, Cochrane, Google Scholar, and ClinicalTrials.gov from November 1 to December 31, 2019 and assessed their quality using the Cochrane Risk of Bias Tool. Our primary outcomes of interest included the effect of Rivaroxaban compared to VKA on

1) recurrent thrombosis and

2) major bleeding.

We used Review Manager(RevMan 5.3) for analysis and reported risk ratios (RR) and $95 \%$ confidence intervals $(\mathrm{Cl})$.

Results: Three RCTs with 424 patients were included. There is no significant difference between Rivaroxaban and VKA in terms of overall recurrent thrombosis, but Rivaroxaban use shows a trend towards harm (RR 4.08, Cl 0.48-34.79, $P=0.13, I^{2}=57 \%$ ). Subgroup analysis revealed that there is increased risk of stroke with Rivaroxaban compared to VKA (RR 14.13, Cl 1.87-106.81, P 0.01, $\mathrm{I}^{2}=0 \%$ ). There is no significant difference between Rivaroxaban and VKA in terms of venous thrombosis.In terms of the primary safety outcome of major bleeding, there is no significant difference between Rivaroxaban and VKA (RR 1.10, Cl 0.45-2.68, $P=0.83, I^{2}=0 \%$ ). 


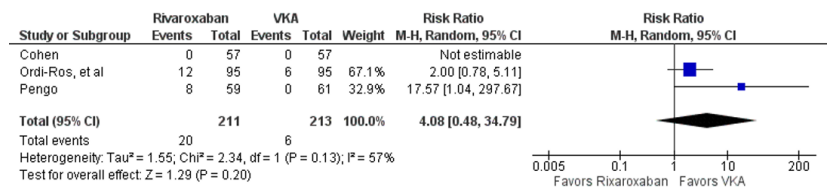

FIGURE 1 Overall Effect of Rivaroxaban versus VKA on Risk of Recurrent Combined Arterial and Venous Thrombosis

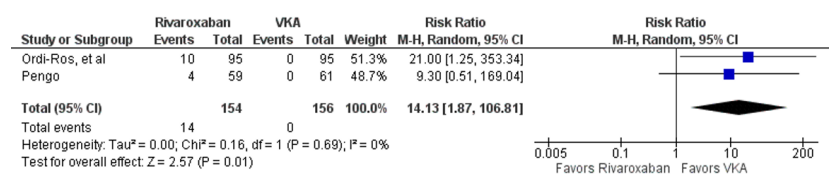

FIGURE 2 Effect of Rivaroxaban versus VKA on Risk of Stroke

Conclusions: There is no conclusive evidence that Rivaroxaban is efficacious in preventing recurrent thrombosis among patients with APS. There is a signal towards harm in terms of stroke and evidence is equivocal in terms of venous thrombosis and major bleeding. Rivaroxaban cannot be recommended as an alternative toVKA at this time.

PB1063 | Clinico-pathologic Characterization of Patients Tested for Antiphospholipid Syndrome at a Tertiary Care Hospital

K. Sajeeve; D. K.V; V. V; A. Catherine Jacob; D. Sidharth; D.

Vidyadharan

Amrita Institute Of Medical Sciences, Eranakulam, India

Background: In the clinical setting, APS can cause protean clinical manifestations apart from the classical symptoms. As a result, patients are referred for testing in a wide variety of scenarios. We conducted a study to identify the common clinical causes for which lupus testing is requested and also to assess the percentage positivity in different conditions.

Aims: To study the clinical pathological characterization of Anti phospholipid syndrome at a tertiary care hospital.

Methods: This study was carried out retrospectively in 130 patients referred for APS workup to the laboratory. LA was evaluated on the basis of either prolonged lupus sensitive APTT or dilute Russell Viper Venom Test (dRVVT). aCL and anti $\beta 2-\mathrm{GPI}$ antibodies were measured with sandwich ELISA. The patient records were retrieved from the Hospital Information System.

Results: Out of the 130 cases, the indications for testing in decreasing order were patients with ITP, SLE, AIHA, thrombosis, pregnancy loss and other autoimmune diseases. 35 out of 130 patients were positive for LA with an M:F ratio of 2:5.

Conclusions: APS testing is done in several clinical scenarios. In our small retrospective analysis, we identified ITP as the most common cause for LA positivity. Further, this small study also highlights the lacunae in testing for other antibodies namely anti- $\beta 2 \mathrm{GPI}$ and $\mathrm{aCL}$.

\section{TABLE 1}

$\begin{array}{ll}\text { Indication for testing } & \text { Number of cases (\%) } \\ \text { Immune thrombocytopenia (ITP) } & 10(28.5 \%) \\ \text { Systemic lupus erythematosus (SLE) } & 6(17 \%) \\ \text { Autoimmune hemolytic anemia (AIHA) } & 4(11.4 \%) \\ \text { Thrombosis } & 3(8.5 \%) \\ \text { Pregnancy loss } & 2(5.7 \%) \\ \text { Adult onset Stills' disease } & 1(2.8 \%) \\ \text { Sjogren's syndrome } & 1(2.8 \%) \\ \text { Autoimmune hepatitis } & 1(2.8 \%) \\ \text { Avascular necrosis } & 1(2.8 \%) \\ \text { Thrombotic microangiopathy } & 3(8.5 \%) \\ \text { Chronic liver disease } & 1(2.8 \%) \\ \text { Autoimmune thyroiditis } & 2(5.7 \%)\end{array}$

PB1064 | Automated Methods for the Enhancement of Lupus Anticoagulant Mixing Study Sensitivities

Z. Cao; R. Bottenus; C. Kung

Instrumentation Laboratory, Orangeburg, United States

Background: Mixing studies in lupus anticoagulant (LA) testing are used to discriminate between LA and factor deficiencies or the presence of other inhibitors. Anticoagulants have been identified with capabilities to enhance LA mixing study sensitivities.

Aims: This study investigates automated LA mixing study solutions with enhanced sensitivities to avoid misclassification of weak LA samples.

Methods: Anticoagulants that greatly enhance LA positive sample test ratio but with minimum impact on LA negative sample test ratio were identified and added to platelet poor normal plasma (PPP-AC). dRVVT screen and confirm tests were modified to include a mixing step of LA samples with PPP-AC.

Results: As shown in Figure 1, for mixing studies that do not require incubation at $37^{\circ} \mathrm{C}, \mathrm{d}$-phenylalanyl-1-prolyl-1-arginine-chloromethyl ketone in PPP (PPP-PPACK) significantly increased the dRVVT screen/confirm (S/C) ratio of an LA positive sample while slightly decreasing the $\mathrm{S} / \mathrm{C}$ ratio of an LA negative sample, leading to an increase of test ratio from 1.38 to 1.74 in the 1:1 mixing study for an LA positive sample with a test ratio of 1.67 . 
A 140
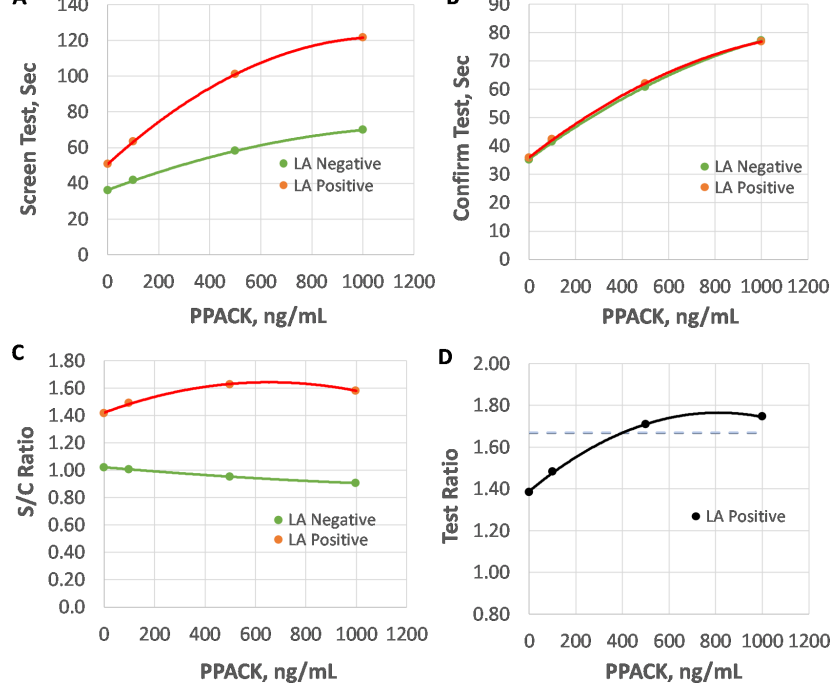

FIGURE 1 Dose Response of PPACK on dRVVT Screen and Confirm Tests in a Mixing Study. Panel A: PPACK significantly increased dRVVT screen time of LA positive samples. Panel B: PPACK equally affected dRVVT confirm time of LA positive and LA negative samples. Panel C: PPACK had an inverse impact on dRVVT S/C ratio for LA positive versus LA negative samples. Panel D: PPACK enhanced the test ratio of an LA positive sample in the mixing study (the dashed line represents the dRVVT test ratio)

In the presence of inhibitors that require incubation at $37^{\circ} \mathrm{C}$ as shown in Figure 2, the impact of hirudin in PPP (PPP-Hirudin) on dRVVT S/C ratio was considerably stronger for a LA positive sample than for a LA negative sample. Thus, the test ratio was greatly improved. A 1:1 mixture of LA negative sample with PPP-Hirudin may be used to normalize the test ratio.
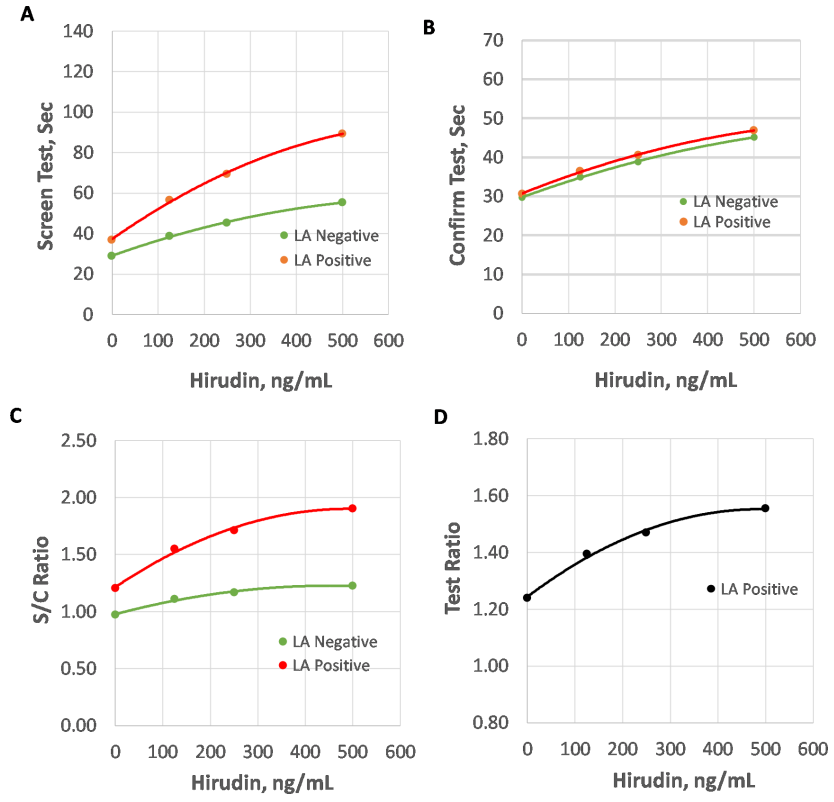

FIGURE 2 Dose Response of Hirudin on dRVVT Screen and Confirm Tests in a Mixing Study. Panel A: Hirudin significantly increased
dRVVT screen time of LA positive samples. Panel B: Hirudin equally affected dRVVT confirm time of LA positive and LA negative samples. Panel C: The impact of hirudin on dRVVT S/C ratio was greater for LA positive than for LA negative samples. Panel D: Hirudin enhanced the test ratio of an LA positive sample in the mixing study

Conclusions: Particular anticoagulants have demonstrated unique impact on dRVVT results. Specifically, PPP-PPACK provides a simple automated mixing study solution with enhanced sensitivity to LA positive samples when incubation at $37^{\circ} \mathrm{C}$ is not needed. PPPHirudin, on the other hand, is useful for mixing studies that require incubation.

\section{PB1065 | Successfully Treated Pediatric Catastrophic} Antiphospholipid Syndrome with Combination of Thrombolytic Agent, Rituximab and Sirolimus

P. Pongphitcha $^{1}$; N. Sirachainan ${ }^{1}$; Y. Okuno ${ }^{2}$; W. Sasanakul ${ }^{1}$; P. Kadegasem ${ }^{1}$; D. Songdej ${ }^{1}$; A. Chuansumrit ${ }^{1}$; S. Vilaiyuk ${ }^{1}$

${ }^{1}$ Faculty of Medicine Ramathibodi Hospital, Mahidol University, Bangkok, Thailand; ${ }^{2}$ Medical Genomics Center, Nagoya University Hospital, Nagoya, Japan

Background: Catastrophic antiphospholipid syndrome (CAPS) is a life-threatening disease which characterized by multi-organ thrombosis and presence of antiphospholipid antibodies. Therapeutic approach reported from the International CAPS Registry were a combination of anticoagulants, glucocorticoids, plasma exchange and/or intravenous immunoglobulins which was able to reduce the mortality rate from $53 \%$ to $33 \%$. However, in severe cases, the other treatment such as rituximab, cyclophosphamide, and eculizumab may be required.

Aims: To demonstrate treatments in two pediatric patients with catastrophic antiphospholipid syndrome.

Methods: After inform consent, two CAPS patients were enrolled. The data including clinical information, etiology, DNAs study, and outcome were obtained.

Results: Patient 1 (P1), a 14-year-old girl, presented with extensive cerebral venous sinus thrombosis, intracardiac thrombus, pulmonary embolism (PE), splenic thrombus and extensive deep vein thrombosis (DVT), while Patient 2 (P2), a 5-year-old-girl, presented with massive PE, extensive DVT and acute arterial occlusion. Both had no underlying diseases. Thrombophilia investigation showed heterozygous PROC gene mutation c.565G>T (p.R189W) in both patients. They initially received standard heparin, pulse methylprednisolone, and plasma exchange. During treatment, the intravascular clot progressed, therefore, both required 2 doses of thrombolytic agent, rituximab, and sirolimus. After that, intravascular clot was controlled. At present, at 2.5 and 0.5 years after initial diagnosis respectively, they have been well. There was the resolution of clot by imaging study but was still present. They still 
receive sirolimus, hydroxychloroquine, and anticoagulant as a maintenance treatment.

Conclusions: The triple therapy with thrombolytic agent, rituximab and sirolimus in refractory CAPS should be considered in uncontrolled patients who had extensive clot despite pulse methylprednisolone, plasma exchange and anticoagulant.

PB1066 | Evaluation of the Determination of Dabigatran, Rivaroxaban, and Apixaban at Lupus Anticoagulans Positive Patients

L. Slavik ${ }^{1}$; J. Jacova ${ }^{2}$; D. Friedecky²; J. Ulehlova ${ }^{1}$; P. Bradacova ${ }^{1}$; A. Skoumalova ${ }^{3}$; J. Prochazkova ${ }^{1}$; A. Hlusi ${ }^{1}$; M. Palova ${ }^{1}$;

A. Kvasnička²; E. Ivanovova ${ }^{2}$; B. Pisklakova ${ }^{2}$

${ }^{1}$ Department of Hematology-Oncology, Faculty of Medicine and Dentistry, Palacky University Olomouc; University Hospital Olomouc, Olomouc, Czech Republic; ${ }^{2}$ Department of Clinical Biochemistry, Faculty of Medicine and Dentistry, Palacky University Olomouc; University Hospital Olomouc, Czech Republic, Olomouc, Czech Republic; ${ }^{3}$ Department of Internal Medicine III-Nephrology, Rheumatology and Endocrinology, Faculty of Medicine and Dentistry, Palacky University Olomouc; University Hospital Olomouc, Czech Republic, Olomouc, Czech Republic

Background: The effect of direct oral anticoagulants (DOAC) on laboratory tests dependent on the production of their targets, factor Ila and factor Xa (FXa), is a well-known problem and can cause both false positive and negative results. In particular, the situation in patients who develop lupus anticoagulant (LA) antibodies is very complex.

Aims: For evaluating the effectiveness of DOAC therapy at lupus positive patiens, 35 samples were enrolled. All patient samples were spiked by free types of DOACs (dabigatran etixilate, rivaroxaban and apixaban) in concentration that significantly influenced screening test fo lupus anticoagulans and thus so can mask the presence of LA. Subsequently, the DOAC was always unbound by the DOAC-STOP procedure. DOAC levels before and after binding were determined by functional assays followed by HPLC MS / MS.

Methods: Determination of DOAC levels was performed by specific functional tests - dabigatran - direct thrombin assay and xabans - determination of anti Xa activity with specific calibration. Our in house LC-MS/MS method enables simultaneous determination of apixaban, dabigatran and rivaroxaban.

Results: The results of LA positive samples show significant differences between functional tests and HPLC MS / MS method both before and after DOAC binding.

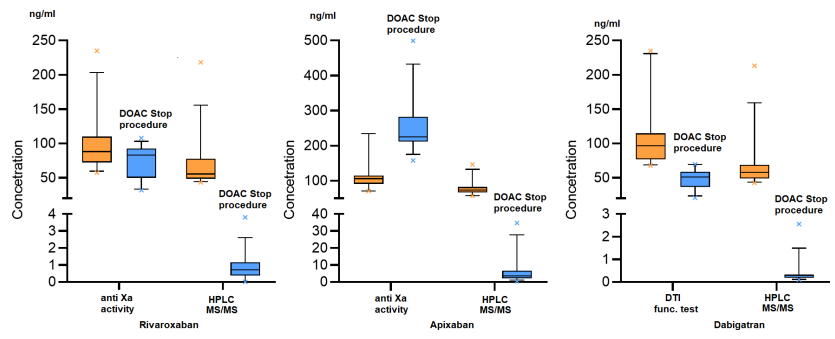

FIGURE 1 The comparison results of function test and HPLC MS/ MS methods for allDOACs at lupus anticoagulans positive sapmles

Conclusions: The severe finding of the presence of LA-type antibodies fundamentally affects the determination of DOAC by functional tests, and in this case it is necessary to use HPLC MS / MS analysis to determine the true value.

If patients treated with DOAC develop LA of medium and higher titers, we do not recommend checking with functional tests.

Supported by grant LF-2021-001 and MH CZ - DRO (FNOI, 00098892)

PB1067 | Laboratory Testing Platform Discrepancy between Current Singleplex-assay and New Multiplex-assay for Detecting and Quantifying IgM-anti-Cardiolipin-antibodies and other AntiPhospholipid (APL)-antibodies: The Potential for Mis-diagnosis of the APL-syndrome

M. Escobar $^{1}$; T.E. Howard ${ }^{2}$; N. Montanez ${ }^{1}$

${ }^{1}$ University of Texas Health and Science Center of Houston, McGovern Medical School, Gulf States Hemophilia and Thrombophilia Center, Houston, United States; ${ }^{2}$ University of Texas Health Rio Grande Valley, Department of Human Genetics, School of Medicine, Brownsville, United States

Background: Anti-phospholipid (APL)-syndrome (APLS) is characterized by presence of both: specific clinical events that include vascular-thromboemboli and/or adverse-pregnancy-outcomes; and persistent abnormal clinical laboratory (i) serologic-tests which include elevated serum levels of one or more anti-phospholipid (APL)-antibodies such as IgM-/IgG-anti-cardiolipin ( $\mathrm{ACL}$ )-antibodies and IgM-/IgG-anti- $\beta 2$-glycoprotein-I (aß2GPI)-antibodies, and/or (ii) coagulation-assays for lupus anticoagulant (LA) [1]. Specificity and sensitivity of laboratory assays for detecting APL-antibodies should be sufficiently high to render accurate diagnoses and optimal medical-outcomes. The most frequently used platform is presently the enzyme-linked-immunosorbent-assay (ELISA), which detects/ quantifies antibodies against a single antigen, novel tests like the addressable-laser-bead-immunoassay (ALBIA), which measures antibodies against multiple antigens, are increasingly being performed. Aims: Describe a single Center's experience with laboratory platform discrepancy between standard and novel technology for $\mathrm{aCL}$ IgM antibody. 
Methods: Retrospective record review of two unrelated females33-y/o with a first-trimester pregnancy loss \& 42-y/o with left renal artery thrombosis-were found to have isolated markedly \& moderately elevated IgM-ACL-antibody titers, respectively, by ELISA (QUANTA Lite), but within-reference-range titers by ALBIA (BioPlex ${ }^{\mathrm{TM}}$ 2200). The tests for other APL-antibodies and LAs were within-reference-range.

\section{TABLE 1 aCL Titer Comparison}

$\begin{array}{lll}\text { Case } & \text { IgM ACL (ELISA) } & \text { IgM ACL (ALBIA) } \\ 33 \text { y/o Female } & 105 \mathrm{MPL} & 2.2 \mathrm{MPL} \\ & 102 \mathrm{MPL} & 2.4 \mathrm{MPL} \\ 42 \text { y/o Female } & 82 \mathrm{MPL} & 4.2 \mathrm{MPL} \\ & 53 \mathrm{MPL} & 3.7 \mathrm{MPL}\end{array}$

Value Interpretation

>40-80 MPL Medium (Diagnostic)

$>80$ MPL High Titer (Diagnostic)

Results: APLS-diagnosis requires repeat APL-antibody testing which different assay-platforms are now available. As decisions concerning antithrombotic-therapy and/or prophylactic-anticoagulation are based on accurate diagnosis, clinical-practice-issues arise with discrepant-laboratory results. As described for these 2 cases, isolated IgM-ACL-antibody elevations were noted with the widely-used singleplex-ELISA but not with the recently introduced multiplexALBIA-immunoassay. Unclear if : 1) elevated ELISA-titers are due to inadequate specificity with this platform (i.e., non-specific ACLantibody binding to immobilized-cardiolipin capture-molecules); or 2) within-reference-range ALBIA-titers are due to an inadequate sensitivity, i.e. due to distinct assay-design feature(s).

Conclusions: Since diagnosis of the APLS requires persistentpositivity of at least one of the specific-laboratory-tests for APLantibodies, it is clear from the current patients with isolated elevated IgM-ACL-antibodies that further study is needed to determine which of these assay-platforms is biologically-accurate.

\section{PB1068 | A Prolonged Actived Partial Thromboplastin Time Revealing a Childhood Systemic Lupus erythematosus}

I. Krichen ${ }^{1}$; S. Samet ${ }^{1}$; F. Megdich ${ }^{1}$; M. Hsairi ${ }^{2}$; L. Gargouri ${ }^{2}$;

A. Mahfoudh ${ }^{2}$; C. Kallel ${ }^{1}$

${ }^{1}$ Laboratory of Hematology, Hospital University Habib Bourguiba, Sfax, Tunisia; ${ }^{2}$ Department of Pediatrics, Hospital University Hedi Chaker, Sfax, Tunisia

Background: Childhood-onset systemic lupus erythematosus (cSLE) is a rare multisystem autoimmune disease. The diagnosis can be ambiguous due to the heterogeneity of clinical manifestations.The occurrence of cSLE is extremely rare before the age of 5 years.

Aims: The purpose of this observation is to highlight the importance of hemostasis tests in the diagnosis of cSLE.
Methods: We report a cSLE's diagnosis of a 4 year-old girl that was established due to abnormal haemostasis tests.

Results: A 4 year-old girl was admitted in the department of pediatrics due to persistent fever $\left(T=38,5^{\circ} \mathrm{C}\right)$ and asthenia. The laboratory investigation revealed an inflammatory syndrome. In fact, the erythrocyte sedimentation rate (ESR) was more than $120 \mathrm{~mm}$ at the first hour and the C-reactive protein (CRP) was positive. The complete blood count showed thrombocytopenia and anemia.

Furthermore, the screening for infectious as well as neoplasic diseases was negative.

Fever persisted despite the prescription of antibiotics and antipyretics.

The hemostasis tests showed an isolated prolonged activated partial thomboplasin time $(54,4 \mathrm{sec}$, ratio $=1,81)$ that was not corrected by mixing test. The Rosner index was $37 \%$.

The levels of factors of coagulation were within the normal ranges $(\mathrm{FVIII}=125 \% ; \mathrm{FIX}=116 \% ; \mathrm{FXI}=107 \% ; \mathrm{FXII}=89 \%)$. Nevertheless, screening for lupus anticoagulant (LA) was positive using dilute Russell's viper venom time (dRVVT).

The diagnosis of cSLE was suspected because its frequent association with the antiphospholipid antibodies. The serologic tests showed positive antinuclear antibody and Anti-double stranded DNA. Based on the clinical findings and the laboratory results, the diagnosis of cSLE was confirmed refering to the American College of rheumatology (ACR) criteria.

The patient received oral hydroxychloroquin.

Conclusions: Infantile SLE is extremely rare and its abnormal appearance in this age group is frequently responsible of a major diagnostic delay. The presence of LA was useful to guarantee an earlier diagnosis and to ensure a better therapeutic managment.

\section{P0164 | Real World Experience Use of DOAC in APLS Patients}

Y.Y. Yap ${ }^{1}$; R.b. Ramli ${ }^{1}$; J. Suriar ${ }^{2}$; J. Sathar ${ }^{1}$

${ }^{1}$ Ministry of Health, Ampang Jaya, Malaysia; ${ }^{2}$ Gleneagles Hospital

Kuala Lumpur, Kuala Lumpur, Malaysia

Background: Antiphospholipid syndrome is associated with high risk of thromboembolic disease in which an effective anticoagulation is essential. The data with direct oral anticoagulant in Malaysia is very limited.

Aims: To study on the effectiveness of DOAC in APLS patients.

Methods: All 16 patients with APLS were identified retrospectively for direct oral anticoagulation (DOAC) Rivaroxaban.

Results: Out of the 16 patients with APLS, 11 were on rivaroxaban $20 \mathrm{mg}$ daily and 5 were on rivaroxaban $15 \mathrm{mg}$ BD. 9 were females and 7 were males. The reason for switching from oral warfarin or low molecular weight heparin to oral rivaroxaban, namely difficult INR monitoring in 7 patients, patient's request in 6 patients, noncompliance in 3 patients. 14 patients were diagnosed primarily with Venous Thromboembolism (VTE), one was diagnosed primarily with ischemic stroke and one was diagnosed primarily with thrombosis 
in unusual sites. Apart from VTE, there were two patients had arterial events and two patients had thrombosis in unusual sites. Six of them were double positive for antiphospholipid antibodies (APLA) and one was triple positive for APLA.

Treatment Outcome: This cohort was followed-up as outpatient for at least 3 years. Three patients were switched back to warfarin. Among the three, first one had developed recurrent VTE and was complicated by Chronic Thromboembolic Pulmonary Arterial Hypertension (CTEPH); second one had recurrent DVT and third one was non-compliant to rivaroxaban. One had an episode of transient ischemic attack due to missing his medication for 2 weeks but was resumed rivaroxaban as patient's preference. Three patients defaulted the treatment and one had migrated to New Zealand. One passed away due to Acute Coronary Syndrome. In the end, only 8 of them remained uneventful while on oral rivaroxaban.

Conclusions: The real world experience illustrates DOAC can be a viable option for long term anticoagulation in patient with uncomplicated APLS.

P0168 | Unusual Laboratory Presentation of Lupus Anticoagulant: Prolonged Prothrombin Time (PT) and Activated Partial Thromboplastin Time (APTT) without Severe Factor Deficiency

C. Guedes ${ }^{1}$; L. Barbosa ${ }^{2}$; D. Marques ${ }^{1} ;$ J. Silva ${ }^{1} ;$ T. Luís ${ }^{1} ;$ R. Salvado ${ }^{1}$; J. Tomaz ${ }^{1}$

${ }^{1}$ Coimbra's Hospital and University Center, Coimbra, Portugal;

${ }^{2}$ Portuguese Intitute of Oncology - Coimbra, Coimbra, Portugal

Background: Lupus Anticoagulant (LA) is a heterogeneous immunoglobulin that prolongs phospholipid-dependent coagulation tests, especially APTT-based. Prolonged PT is a less frequent presentation. The strong presence of LA is likely to give erroneous results in coagulation tests and factor measurements, that can be misleadingly interpreted as a coagulopathy. For bleeding risk assessment it is necessary to exclude congenital or acquired factor deficiencies. Despite of these laboratory findings, LA is associated with hypercoagulability and thrombosis.

Aims: To report a case of a 71-year-old patient referenced to our hospital with a significant prolonged TP and APTT, for bleeding risk assessment pre-colonoscopy. The procedure, scheduled to investigate patient significant weight loss and anorexia, was postponed for intimidation regarding hemorrhage due to laboratory findings. The patient was clinically asymptomatic and stable, and no personal bleeding history was reported. Other clinical findings reported on Fig.1.

Methods: Laboratory investigation included Werfen ${ }^{\circledR}$ LA integrated tests (dRVVT and SCT), one-stage and chromogenic factor assays SIEMENS ${ }^{\circledR}$ and ROTEM Sigma ${ }^{\circledR}$ complete test.

Results: Preliminary laboratory investigation revealed a strong LA and factor deficiencies (Table1a). Most factor deficiencies were not confirmed when assayed at higher plasma dilutions (Table1b), with only FVII, FII and FXI slightly decreased (not justifying screening results prolongation). ROTEM Sigma ${ }^{\circledR}$ showed EXTEM and INTEM slight prolonged CT, probably in relation to LA, without any other abnormality in clot formation.

Conclusions: This case illustrates how the presence of a potent LA can interfere with factor assays interpretations, besides APTT. Significantly prolonged screening tests in a patient without bleeding symptoms, can make us suspect of LA and guide our investigation for a quicker response, mostly in situations when invasive procedure is needed and urgent.

In this context, instead of PT and APTT, viscoelastic tests may be a good strategy since it reflects more accurately and quickly patient coagulation status.

\section{ATRIAL FIBRILLATION}

\section{PB1069 | Association of Retest Interval with INR Control in Warfarin Patients with Markedly Out of Range INRs}

B. Haymart ${ }^{1}$; X. Kong ${ }^{1}$; G.D Barnes ${ }^{1}$; A. Purohit ${ }^{1}$; M. Ali ${ }^{2}$; E. KlineRogers $^{1}$; J.H Kozlowski ${ }^{3}$; G. Krol ${ }^{4}$; V. Shah ${ }^{4}$; J.B Froehlich ${ }^{1}$; S. Kaatz ${ }^{4}$ ${ }^{1}$ University of Michigan-Samuel and Jean Frankel Cardiovascular Center, Ann Arbor, United States; ${ }^{2}$ William Beaumont Hospital-Royal Oak, Royal Oak, United States; ${ }^{3}$ DMC Huron Valley Sinai Hospital, Commerce Charter Twp, United States; ${ }^{4}$ Henry Ford Hospital, Detroit, United States

Background: The ideal INR retest interval following warfarin dose changes for markedly out of range INRs is not clear. Guidelines from the International Society on Thrombosis and Haemostasis suggest retesting VTE patients with INRs $\geq 4.0$ or $\leq 1.5$ within 7 days based on a prior study showing higher time in therapeutic range in centers with shorter retest intervals.

Aims: To determine if prompt retesting ( $\leq 7$ days) results in better INR control across a broad cohort of patients at the patient-INR level.

Methods: INRs $\geq 4.0$ or $\leq 1.5$ from the Michigan Anticoagulation Quality Improvement Initiative $\left(\mathrm{MAQI}^{2}\right)$ registry were identified. INRs from patients with target INR ranges of 2-3 were included, except those within 30 days of warfarin initiation or without a follow-up test. Based on the number of days between warfarin dose adjustment and the date of the next INR, INRs were categorized as promptly ( $\leq 7$ days) or non-promptly retested. INR control was defined by whether or not the retest INR ( $1^{\text {st }}$ follow-up INR) or the $2^{\text {nd }}$ follow-up INR were in range. Comparisons were made using Chi square tests. A two-sided $\mathrm{P}<0.05$ was considered statistically significant.

Results: A total of 36,822 eligible INRs were identified (22,399 $\leq 1.5$; $14,423 \geq 4.0$ ). Prompt retesting occurred in 21,455 (58.3\%). The median retest intervals were 5 days and 12 days for promptly and nonpromptly retested INRs, respectively. Prompt retesting was inferior for the retest INR being in-range ( $34.7 \%$ vs. $42.3 \%, P<0.001)$ as well as the second follow-up INR being in range (42.8\% vs $43.8 \%$, $P=0.049$ ). 
TABLE 1 Prompt vs Non-Promptly Retested INRs

\begin{tabular}{|c|c|c|c|}
\hline & Promptly retested ( $\leq 7$ days) & Non-promptly retested (>7days) & P-value \\
\hline All INRs -n(\%) & $21,455(58.3)$ & $15,367(41.7)$ & - \\
\hline Retest INR in-range $-\mathrm{n}(\%)$ & $7,454(34.7)$ & $6,496(42.3)$ & $<0.001$ \\
\hline $2^{\text {nd }}$ INR in range $-n(\%)$ & $9,179(42.8)$ & $6,733(43.8)$ & 0.049 \\
\hline INRs $\leq 1.5-n(\%)$ & $12,310(55.0)$ & $10,089(45.0)$ & - \\
\hline Retest INR in-range $-n(\%)$ & $3,795(30.8)$ & $4,033(40)$ & $<0.001$ \\
\hline $2^{\text {nd }}$ INR in range $-n(\%)$ & $5,178(42.1)$ & 4,227 (41.9) & 0.72 \\
\hline INRs $\geq 4.0-n(\%)$ & $9,145(63.4)$ & $5,278(36.6)$ & - \\
\hline Retest INR in-range -n(\%) & $3,659(40.0)$ & $2,463(46.7)$ & $<0.001$ \\
\hline $2^{\text {nd }}$ INR in range $-n(\%)$ & $3,992(43.7)$ & $2,506(47.5)$ & $<0.001$ \\
\hline
\end{tabular}

Conclusions: In this $\mathrm{MAQI}^{2}$ cohort of patients, retesting markedly out of range INRs within 7 days appears to provide worse INR control over the short-term follow-up period. Further analysis of this data is needed to account for confounding patient factors that may affect INR control and prescribed retest intervals.

PB1070 | Switching of Oral Anticoagulants in People with Atrial Fibrillation: Insight from Australian General Practices

A. Kefale; G. Peterson; W. Bezabhe; L. Bereznicki

University of Tasmania, School of Pharmacy and Pharmacology,

Hobart, Australia

Background: Oral anticoagulants (OACs) are effective in reducing the risk of ischaemic stroke in people with atrial fibrillation (AF). The availability of the direct-acting OACs (DOACs) changed the landscape of stroke prevention, including the possibility for switching between agents. Switching may affect clinical outcomes and healthcare costs, although it has received little research attention.

Aims: To investigate the pattern of switching after OAC initiation and to identify predictors of OAC switching, using national data from Australian general practices.

Methods: The study analysed general practice data obtained from the NPS MedicineWise dataset, Medicinelnsight. This dataset represents the Australian population in terms of age and gender. We included AF patients who newly-started on an OAC between January 1, 2013, and September 30, 2017. Switching was considered when an OAC other than the index OAC was prescribed within 60 days of the end of the last prescription supply of the index agent. Predictors were assessed using logistic regression.

Results: 15,020 patients (47.3\% female) were included (commenced on: apixaban: 4,274 (28.4\%); dabigatran: 1,541 (10.3\%); rivaroxaban: 4,830 (32.2\%); warfarin: 4,375 (29.1\%)). At 1 year of follow-up, $2.6 \%$ (95\% Cl, 2.2-2.6\%), 8.9\% (95\% Cl, 7.5-10.4\%), 4.0\% (95\% Cl; 3.54.6\%), 9.4\% (95\% Cl, 8.6-10.4\%), and 5.7\% (95\% Cl, 5.3-6.1\%) of apixaban, dabigatran, rivaroxaban, warfarin, and all OAC users had been switched to another OAC, respectively. Half of the switches from warfarin were to rivaroxaban, while the majority $(71.1 \%)$ of switches from DOACs were to another DOAC. Renal impairment was the strongest predictor of switching from DOACs. Conversely, it markedly reduced the likelihood of switching from warfarin.

Conclusions: Overall, switching between OACs was relatively low in Australian general practices. Patients initiated on apixaban and warfarin had the lowest and highest switching rate, respectively. The findings support the relative safety, effectiveness, and patient acceptability of each OAC in real-world practice.

\section{PB1071 | Evaluation of the Effect of Educational Intervention Adapted for Patients on Warfarin with Poor Anticoagulation Control: A Controlled Clinical Trial}

J.M. Costa'; M.S. Marcolino ${ }^{2}$; M.F.S. Praxedes ${ }^{3}$; H.C. Torres ${ }^{4}$; R.P. Souza ${ }^{5}$; D.V. Nascimento ${ }^{1}$; C.B. Ferreira ${ }^{1}$; N.S. Antunes ${ }^{6}$; R.C. Viana ${ }^{1}$; J.A. Araújo ${ }^{1}$; L.A. Fernandes ${ }^{1}$; L.d.F.M. Braga ${ }^{1}$; A.C.R. Silva ${ }^{1}$; H.C. Barbosa ${ }^{2}$; D.D. Ribeiro ${ }^{6}$; M.A.P. Martins $s^{1,2,6,7}$ ${ }^{1}$ Faculdade de Farmácia, Universidade Federal de Minas Gerais, Belo Horizonte, Brazil; ${ }^{2}$ Faculdade de Medicina, Universidade Federal de Minas Gerais, Belo Horizonte, Brazil; ${ }^{3}$ Centro Ciências da Saúde, Universidade Federal do Recôncavo da Bahia, Santo Antônio de Jesus, Brazil; ${ }^{4}$ Escola de Enfermagem, Universidade Federal de Minas Gerais, Belo Horizonte, Brazil; 5 Instituto de Ciências Biológicas, Universidade Federal de Minas Gerais, Belo Horizonte, Brazil; ${ }^{6} \mathrm{Hospital}$ das Clínicas, Universidade Federal de Minas Gerais, Belo Horizonte, Brazil; ${ }^{7}$ Hosptial Risoleta Tolentino Neves, Belo Horizonte, Brazil

Background: Warfarin therapy requires intense patient participation to achieve treatment goals and its promotion is challenging in healthcare settings.

Aims: To evaluate the effect of an educational intervention adapted for patients on warfarin with poor anticoagulation control at two anticoagulation clinics in Brazil.

Methods: This is a controlled clinical trial with participants recruited at two public hospitals in Belo Horizonte, Southeast Brazil. Inclusion criteria were age $\geq 18$ anos, both sexes, AF diagnosis under warfarin therapy, outpatient follow-up for at least six months, poor quality of anticoagulation control. The intervention was based on 
Paulo Freire's theory and comprised four meetings offered over a four-month period. Sociodemographic and clinical data, as well as adherence information, were collected from medical records and patient interviews. The primary outcome was the time in therapeutic range (TTR), and the secondary outcomes were patient knowledge on oral anticoagulation, and self-report adherence to warfarin therapy. These variables were measured in both groups in the pre- and post-intervention phases. TTR $<60 \%$ was considered to classify poor anticoagulation control. Patient knowledge was assessed by using the Oral Anticoagulation Test (OAK Test). Trial registration: Registro Brasileiro de Ensaios Clínicos (ReBEC) RBR- 9cy6py; UTN U11111217-0151 (March, 2019).

Results: Overall, 168 participants were enrolled with 83 allocated to the intervention group and 85 to the control group. The mean age was $65 \pm 12.6$ years with a predominance of women (56.6\%). Most patients presented non-valvar AF $(101 ; 60.1 \%)$ as indication for oral anticoagulation. There was no statistical difference between groups regarding TTR and adherence. The intervention was associated with an increase of three points in the knowledge score $(P<0.001)$.

Conclusions: Our findings showed the contribution of an educational intervention to patient knowledge on warfarin therapy. Further studies are needed to investigate strategies to improve the quality of care provided to warfarin patients with poor anticoagulation control.

PB1072 | A Comparative Cohort Study of Serum Rivaroxaban Levels in Singaporean (SG) and British (UK) Non-valvular Atrial Fibrillation (NVAF) Patients

E.X. Yong ${ }^{1}$; Y.Y. Lin ${ }^{2}$; D. Tan ${ }^{1}$; E. Chan ${ }^{1}$; V. Speed ${ }^{3}$; J. Patel ${ }^{3}$; E.S. Yap ${ }^{4}$; G. Chang ${ }^{2}$; P. Gallagher ${ }^{1}$

${ }^{1}$ National University of Singapore, Singapore, Singapore; ${ }^{2}$ Khoo Teck

Puat Hospital, Singapore, Singapore; ${ }^{3}$ King's College London, London,

United Kingdom; ${ }^{4}$ National University Hospital, Singapore, Singapore

Background: Studies suggest that Asians may require lower doses of antithrombotics as compared to Caucasians. It is worthwhile to explore if serum rivaroxaban levels differ between SG and UK.

Aims: To compare steady-state trough rivaroxaban levels between SG and UK patients and identify factors affecting rivaroxaban plasma levels.

Methods: A total of 104 SG and 173 UK subjects were included in the study. Comparison of the median $\mathrm{Cmin}$,ss between both cohorts was performed among appropriately dosed patients using the MannWhitney $\mathrm{U}$ test (non-normal). Measured $\mathrm{Cmin}$,ss were classified as "higher than range" or "lower than range" with respect to the published reference range to determine factors affecting rivaroxaban levels. Covariates with a $p$ value $<0.1$ from the univariate analysis were included in the multivariate regression to determine factors driving out-of-range $\mathrm{Cmin}$,ss.

Results: Median trough levels for 20mg dose-group $(n=189)$ did not differ between SG and UK groups which were predominantly
Asian and Caucasian respectively. In the $15 \mathrm{mg}$ dose-group (14 SG vs 29 UK), trough rivaroxaban levels were significantly lower for the SG cohort (46.04ng/mL vs 70.08ng $/ \mathrm{mL}, P=0.041$ ). After controlling for body mass index, appropriate dose and time from last dose to blood sampling, "UK cohort" (OR $=6.022,95 \% \mathrm{Cl}=1.293-28.043$, $P=0.022)$ and "poorer renal function" (OR $=0.972,95 \% \mathrm{Cl}=0.948-$ $0.996, P=0.022)$ remained significant factors driving "higher than range" trough levels. A SG subgroup analysis revealed a $3 \%$ and 14.1\% thrombo-embolic (TE) and bleeding risk respectively.

Conclusions: Caucasians are more likely to experience "higher than range" trough rivaroxaban levels as compared to Asians. TE rates in the SG cohort, suggest that the dose requirement could be higher for Asians without causing too many adverse drug reactions. Larger studies should be commissioned to determine all factors affecting TE and bleeding outcomes.

PB1073 | A Comparison of the Incidence of Traumatic Brain Injury in Anticoagulated and Non-anticoagulated Head Injured Patients

F. Al-Haimus ${ }^{1}$; N. Clayton ${ }^{1,2}$; J. Klyn ${ }^{2}$; K. de Wit ${ }^{3,1}$

${ }^{1}$ McMaster University, Hamilton, Canada; ${ }^{2}$ Hamilton Health Sciences, Hamilton, Canada; ${ }^{3}$ Queens University, Kingston, Canada

Background: People who take anticoagulation are assumed to have a higher risk of traumatic brain injury (TBI) after a head injury than those who are not anticoagulated. The aim of this study was to compare the incidence of TBI among anticoagulated head-injured patients to head-injured patients who do not take anticoagulation.

Aims: The aim of this study was to compare the incidence of TBI among anticoagulated head-injured patients to head-injured patients who do not take anticoagulation.

Methods: This was a two-hospital health records review which enrolled head-injured emergency patients with a Glasgow Coma Score (GCS) 13-15 between May and December 2019. Patients were classified as anticoagulant users or non-anticoagulant users. We defined anticoagulant users as those who took warfarin, apixaban, rivaroxaban, edoxaban, dabigatran, low molecular weight heparin, fondaparinux or unfractionated heparin for at least 3 days prior to emergency department (ED) presentation. We defined our outcome, TBI, as the presence of intracranial blood within any intracranial compartment, diffuse axonal injury or isolated skull fracture. We reported TBI diagnosed at the index ED visit, and delayed TBI diagnosed within 30 days. We compare these incidences between anticoagulant users and non-anticoagulant users.

Results: We enrolled 368 patients who were anticoagulant users and 1650 non-anticoagulant users. The mean age of anticoagulant users was $80,49 \%$ were male, non-anticoagulant users was $61,54 \%$ were male. $23 / 368$ (6.3\%, 4.2 to $9.3 \%)$ anticoagulant users were diagnosed with TBI on initial ED visit and no patient (0.0\%, 0.0 to $1.4 \%$ ) was diagnosed with delayed TBI in the following 30 days. 125/1650 (7.6\%, 6.4 to $9.0 \%$ ) non-anticoagulant users were diagnosed with TBI 
on initial ED visit and 4 patients (0.3\%, 0.1 to $0.7 \%)$ were diagnosed with delayed TBI.

Conclusions: This study did not identify a difference in the rate of TBI between anticoagulated and non-anticoagulated head-injured patients.

PB1074 | Efficacy and Safety of Direct Oral Anticoagulants in Patients of Southern Italy with Atrial Fibrillation: Real-world Data from the EGINA Registry

N. Ciavarella ${ }^{1} ;$ A. Ciampa ${ }^{2} ;$ S. Bradamante ${ }^{3} ;$ A. Colucci $^{4}$; G. Dirienzo ${ }^{5}$; A.M. Iannone ${ }^{6}$ G. Polimeno7; G. Rescigno ${ }^{8}$; L. Ria 9 R. Scarafile ${ }^{10}$; . Ciavarella ${ }^{11}$; C. Custodero ${ }^{12}$; C. Sabbà ${ }^{12}$

${ }^{1}$ A.P.T.E.A., Bari, Italy; ${ }^{2}$ Hemostasis and Thrombosis Center, Avellino, Italy; ${ }^{3}$ Thrombosis and Blood Transfusion Center, Taranto, Italy; ${ }^{4}$ Thrombosis and Blood Transfusion, "Di Venere" Hospital, Bari, Italy;

${ }^{5}$ Thrombosis Center, Department of Clinical Pathology, Altamura, Italy; ${ }^{6}$ Thrombosis and Blood Transfusion Center, Molfetta, Italy; ${ }^{7}$ Department of Hematology, Acquaviva delle Fonti, Italy; ${ }^{8}$ Hemostasis and Thrombosis Center, Nocera Inferiore-Pagani-Scafati, Italy; ${ }^{9}$ Department of Internal Medicine, Gallipoli, Italy; ${ }^{10}$ Hemostasis and Blood Transfusion Center, "San Paolo" Hospital, Bari, Italy; ${ }^{11}$ Fondazione IRCCS Ca' Granda Ospedale Maggiore Policlinico, A. Bianchi Bonomi Hemophilia and Thrombosis Center, Milan, Italy; ${ }^{12}$ Department of Interdisciplinary Medicine, University of Bari, Bari, Italy

Background: Oral anticoagulant therapy has been historically managed in Italy in patients with atrial fibrillation (AF) by a network of Anticoagulation Centers (ACs). Patients taking direct oral anticoagulants (DOACs) no longer required periodical blood withdrawal for the modification of the drug dosage and therefore their follow-up might be less strict than before. From 2018 onwards, 19 ACs of southern Italy have been using a clinical model, named EGINA (Excellence model for the Integrated Management of New Anticoagulants), designed to improve the management of such patients.

Aims: To evaluate the incidence of ischemic and hemorrhagic events in patients taking DOACs, followed according to the EGINA model. Methods: This multi-center study included patients with AF who started a DOAC from Jan 2018 to Feb 2020. Data were collected retrospectively by 9 ACs of southern Italy. The observational period lasted a maximum of 12 months from the date of initiation of therapy with a DOAC. Diagnosis of major and minor bleeding was made according to the International Society on Thrombosis and Haemostasis (ISTH).

Results: Overall 395 patients with AF has been assessed. Mean age was 75.76 years ( $S D=9.48$, ranging from 31 to 100 years old) and 170 patients were female (43\%). On average, at baseline assessment CHA2DS2-VASc score was $3.49(\mathrm{SD}=1.3)$ and HAS-BLED 1.79 $(S D=0.95)$. The $33.2 \%$ of patients were naïve for anticoagulation. Apixaban was the most prescribed DOACs (35.4\%), followed by edoxaban (32.4\%), rivaroxaban (17.0\%) and dabigatran (15.2\%). Dose reduction was recommended in $30.9 \%$ of patients. Collectively, we observed a low incidence of major $(0.8 \%)$ and minor bleedings (1.3\%), with no significant difference according the DOACs dosage (standard or reduced). Only one ischemic event was observed.

Conclusions: Our real-world data show a low incidence of bleeding events, demonstrating that the accurate follow-up of patients anticoagulated with DOACs may guarantee good clinical outcomes.

\section{PB1075 | Appropriate dose of Dabigatran in Asian Population}

N. Hantrakun; W. Wongcharoen; K. Thiankhaw; L. Norasetthada; A. Tantiworawit; E. Rattaritamrong; T. Rattanathammethee; S. Huntrakool; P. Piriyakhuntorn; C. Chai-Adisaksopha Chiang Mai University, Chiang Mai, Thailand

Background: Dabigatran, a direct thrombin inhibitor, is commonly used for the prevention of stroke or systemic embolism in patients with atrial fibrillation (AF) and the treatment of venous thromboembolism (VTE). Either dabigatran $110 \mathrm{mg}$ or $150 \mathrm{mg}$ is recommended in AF patients. However, there were limited data on dabigatran levels in Asian patients using two different doses.

Aims: This study aimed to investigate the plasma levels of dabigatran $110 \mathrm{mg}$ (D110) or $150 \mathrm{mg}$ (D150) twice daily in Thai patients who had AF or VTE.

Methods: This was a single center, cross-sectional study. We included all adult patients (age $\geq 18$ years) who were diagnosed with AF or VTE and who were prescribed either dabigatran $110 \mathrm{mg}$ or $150 \mathrm{mg}$ twice daily. We collected data regarding to age, sex, comorbidities, concomitant medications and coagulation tests. Peak and trough levels of dabigatran were measured by diluted thrombin time. Results: There were 80 patients included in the study (39 in D110 and 41 in D150 group). D150 group had lower mean age (64.39 vs 75.85 years) and higher mean creatinine clearance $(\mathrm{CrCl})$ (69.83 vs $47.20 \mathrm{ml} / \mathrm{min}$ ). Comparing between two groups, there was no significant difference in trough and peak plasma dabigatran levels in patients with $\mathrm{CrCl} \geq 50 \mathrm{ml} / \mathrm{min}$ (figure 1). In patients with $\mathrm{CrCl}<50$ $\mathrm{ml} / \mathrm{min}$, there was significant higher mean peak plasma dabigatran level in D150 group compared to D110 group (383.69 ng/mL vs $207.67 \mathrm{ng} / \mathrm{mL}, P=0.01$, figure 1). Patients who had $\mathrm{CrCl}<50 \mathrm{ml} / \mathrm{min}$ were more likely to have over expected range of dabigatran (figure 2). After adjusting for age and $\mathrm{CrCl}, \mathrm{D} 150$ was associated with over expected plasma level (odds ratio, 1.12; 95\% confidence interval, $1.01-1.25 ; P=0.037)$. 
Figure 1. Trough and peak level of dabigatran in patient who received $110 \mathrm{mg}$ compared with $150 \mathrm{mg}$ of dabigatran according to creatinine clearance
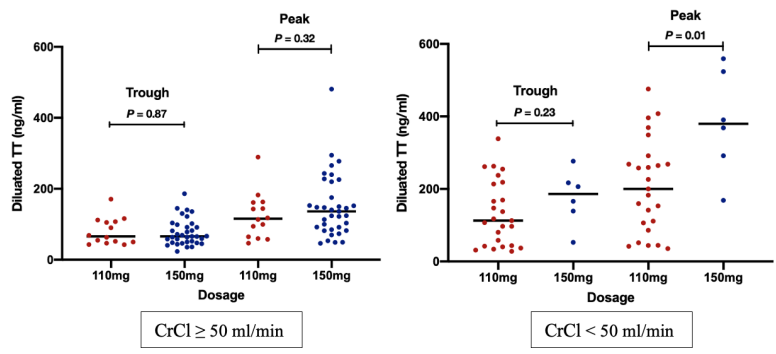

FIGURE 1 Trough and peak level of dabigatran in patient who received $110 \mathrm{mg}$ compared with $150 \mathrm{mg}$ of dabigatran according to creatinine clearance

Figure 2. Peak level of dabigatran categorized by expected range of peak plasma level for standard dose Figure 2. Peak level of dabigatran categorized by exp
(reference from Eur Heart J. 2018;39(16):1330-1393)

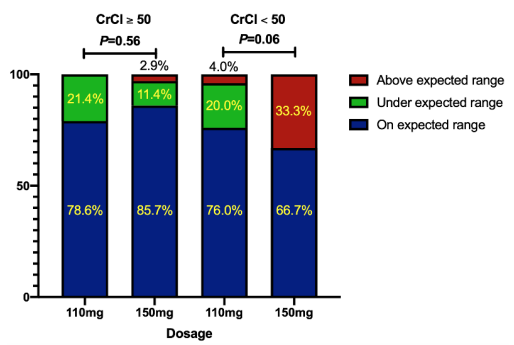

FIGURE 2 Peak level of dabigatran categorized by expected range of peak plasma level for standard dose

Conclusions: In Asian patients, dabigatran 150 mg twice daily was associated with higher plasma dabigatran levels, especially in those with impaired renal function.

PB1076 | Burden and Costs of Stroke/Systemic Embolism and Major Bleeding among Elderly Non-valvular Atrial Fibrillation Patients Prescribed Oral Anticoagulants

A. Dhamane ${ }^{1}$; A. Keshishian ${ }^{2}$; M. Preib ${ }^{2} ;$ T. Bello ${ }^{2}$; C. Russ ${ }^{3}$; M. Di Fusco ${ }^{3}$; M. Ferri ${ }^{1}$

${ }^{1}$ Bristol-Myers Squibb Company, Lawrenceville, United States;

${ }^{2}$ STATinMED Research, Ann Arbor, United States; ${ }^{3}$ Pfizer Inc, New York, United States

Background: Non-Valvular Atrial Fibrillation (NVAF) increases with age and results in a significant financial burden. Stroke/Systemic Embolism (SE) and Major Bleeding (MB) are considered key clinical events in NVAF patient management.
Aims: Examine the rate of Stroke/SE (Ischemic, Hemorrhagic, Other) and Major Bleeding ( $\mathrm{ICH}, \mathrm{GI}$, other site) events and associated medical costs among NVAF patients prescribed oral anticoagulants (OACs).

Methods: Elderly patients with a NVAF diagnosis and OAC prescription (received January 1, 2013 - December 31, 2017) were identified in the fee-for-service Medicare claims database. Patients were followed from OAC initiation to discontinuation, switch, disenrollment, death, or study end. Stroke/SE and MB related hospitalizations and associated costs were identified using ICD-9 and 10 primary diagnosis codes.

Results: 738,283 patients with NVAF were included (apixaban: $34.0 \%$, dabigatran: $5.6 \%$, rivaroxaban: $26.7 \%$, warfarin: $33.6 \%$ ). Patients average age was 78 years with mean $\mathrm{CHA}_{2} \mathrm{DS}_{2}$-VASc score of 4.5 and HAS-BLED score of 3.4. Mean follow-up time was 300.5 days. $3.7 \%$ of patients had a $\mathrm{MB}(\mathrm{GI}: 1.9 \%, \mathrm{ICH}$ : $0.6 \%$, Other: $1.5 \%)$. Among patients with $\mathrm{MB}, \mathrm{MB}$-related average total medical costs were $\$ 19,505$ and the PPPM cost among all patients was $\$ 171$. GI bleed had the lowest total medical costs $(\$ 17,964)$ compared to other MB types, however due to the high event frequency, is the largest cost contributor (Figure). 1.0\% of patients had stroke/SE; among patients with stroke/SE events, stroke/SE-related total costs were $\$ 20,269$ and PPPM cost among all patients was \$59. Ischemic strokes had the highest medical costs PPPM compared to other stroke/SE types (Figure).

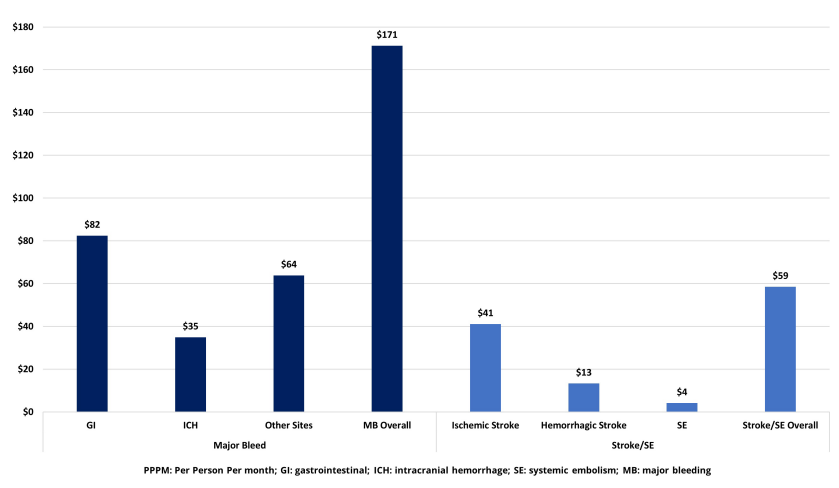

FIGURE 1 Major Bleeding-related and Stroke/SE-related Total Medical Costs PPPM among All Patients

Conclusions: In this study of NVAF patients treated with OACs, major $\mathrm{GI}$ bleed occurred more frequently than $\mathrm{ICH}$, any other bleed, and any stroke/SE event. Managing GI bleeds is important in the NVAF patient population as they contribute to a significant clinical and economic burden. 
TABLE 1 Incidence Rate and Proportions of Patients with a Bleeding and Stroke/SE: Overall and By Site

\begin{tabular}{|c|c|c|c|c|}
\hline & \multicolumn{4}{|l|}{ Major Bleeding } \\
\hline & Major Bleeding Overall & GI & $\mathrm{ICH}$ & Other Site \\
\hline Patients with a Major Bleed, $\mathrm{n}(\%)$ & $27,560(3.7 \%)$ & $14,224(1.9 \%)$ & $4,107(0.6 \%)$ & $10,945(1.5 \%)$ \\
\hline \multirow{3}{*}{$\begin{array}{l}\text { Incidence Rate of Major Bleeding, per } 100 \\
\text { person-years }\end{array}$} & 5.08 & 2.61 & 0.78 & 2.03 \\
\hline & \multicolumn{4}{|l|}{ Stroke/SE } \\
\hline & Stoke/SE Overall & Ischemic & Hemorrhagic & Other \\
\hline Number of Patients with a Stroke/SE, n (\%) & 7,708 (1.0\%) & $5,894(0.8 \%)$ & $1,433(0.2 \%)$ & $381(0.1 \%)$ \\
\hline $\begin{array}{l}\text { Incidence Rate of Stroke/SE, per } 100 \\
\text { Person-years }\end{array}$ & 1.37 & 1.05 & 0.25 & 0.07 \\
\hline
\end{tabular}

$\mathrm{ICH}$ : intracranial hemorrhage; GI: gastrointestinal; SE: systemic embolism.

\section{PB1077 | Quality of INR Control during the COVID-19 Pandemic}

S. Kaatz ${ }^{1}$; X. Kong ${ }^{2}$; B. Haymart ${ }^{2}$; M. Ali ${ }^{3}$; E. Kline-Rogers ${ }^{2}$; G. Krol ${ }^{1}$; V. Shah ${ }^{1} ;$ J. Kozlowski ${ }^{4}$; J.B Froehlich ${ }^{2}$; G.D Barnes ${ }^{2}$

${ }^{1}$ Henry Ford Hospital, Detroit, United States; ${ }^{2}$ University of Michigan-

Samuel and Jean Frankel Cardiovascular Center, Ann Arbor, United States;

${ }^{3}$ William Beaumont Hospital-Royal Oak, Royal Oak, United States; ${ }^{4}$ DMC

Huron Valley Sinai Hospital, Commerce Charter Twp, United States

Background: The COVID-19 pandemic disrupted anticoagulation clinics with staff re-deployment and patient fears of exposure when getting INR testing. The impact on quality metrics of INR control, timeliness of INR testing, and strategies to mitigate barriers are not well described.

Aims: To compare time in therapeutic range (TTR), prescribed interval for next INR, proportion of late INRs, use of extended INR testing $>5$ weeks, switch from warfarin to a direct oral anticoagulant
(DOAC) and use of home INR testing before and after the beginning of the pandemic in March 2020.

Methods: Pre/post comparison of patients in the ongoing MAQI2 quality collaborative registry of 6 anticoagulation clinics in the USA funded by an insurance provider, Blue Cross/Blue Shield of Michigan. Patients initiating warfarin are retrospectively reviewed by trained nurse abstractors. Continuous data was compared with student $\mathrm{t}$-test and categorical with chi square.

Results: The number of patients post pandemic did not change and data in the table reflects delays in abstraction secondary to nurse redeployment. There was no change in TTR, and the proportion of patients with late INRs ( $>1$ day overdue) increased by $1 \%$. There was no change in timeliness contacting patients with INR results, and prescribed next INR increased by 1 day. Use of extending testing intervals increased by $1 \%$, switching from warfarin to DOAC decreased by $2 \%$ and there was no change in the proportion of patients using home INR testing.

TABLE 1 Anticoagulation Management Pre and Post COVID-19

Pre-pandemic (September 2019-February 2020)

Number of patients

Number of follow-ups

Time in therapeutic range (\%)

INRs retested $>1$ day late $(\%)$

Time from INR result to patient contact (mean days \pm SD)

Prescribed INR retest intervals (mean days \pm SD)

Use of extended testing interval in patients (\%)

Pts switched to a DOAC (\#/100 pts followed)

Home testers (\#/100 pts followed)
2,527

23,196

$62.9 \%$

$7,717(33.3 \%)$

$0.78 \pm 1.58$

$14.2 \pm 12.3$

$1,087(4.7 \%)$

108 (4.3\%)

$340(13.5 \%)$
Pandemic (March

2020-August 2020)

1,716

12,453

$62.8 \%$

4,275 (34.3\%)

$0.78 \pm 1.68$

$15.4 \pm 13.7$

708 (5.7\%)

41 (2.4\%)

234 (13.6\%)
$P$-value

0.56

0.04

0.69

$<0.001$

$<0.001$

0.001

0.86

Conclusions: We saw no change in INR control or timeliness of patient contact and only slight delays in patient follow up. There was minimal uptake in suggested strategies to mitigate the need for INR testing. Our anticoagulation clinics performed admirably during the pandemic. 
PB1078 | The Impact of Strong Inducers on Direct Oral Anticoagulant Plasma Levels: A Retrospective Study

A.-L. Sennesael $^{1}$; A.-S. Larock $^{1}$; P. Hainaut ${ }^{2}$; S. Lessire ${ }^{3}$; M. Hardy ${ }^{3,4}$; J. Douxfils ${ }^{5}$ A. Spinewine ${ }^{1,6} ;$ F. Mullier $^{4}$

${ }^{1}$ Université Catholique de Louvain, CHU UCL Namur, NTHC, NARILIS, Department of Pharmacy, Yvoir, Belgium; ${ }^{2}$ Université Catholique de Louvain, Cliniques Universitaires Saint-Luc, Department of Internal Medicine, Brussels, Belgium; ${ }^{3}$ Université Catholique de Louvain, CHU UCL Namur, NTHC, NARILIS, Department of Anesthesiology, Yvoir, Belgium; ${ }^{4}$ Université Catholique de Louvain, CHU UCL Namur, NTHC, NARILIS, Hematology Laboratory, Yvoir, Belgium; ${ }^{5}$ Pharmacy Department, NTHC, NARILIS, Université de Namur, Namur, Belgium; ${ }^{6}$ Clinical Pharmacy Research Group, Louvain Drug Research Institute, Université Catholique de Louvain, Brussels, Belgium

Background: All direct oral anticoagulants (DOAC) are transported by $\mathrm{P}$-glycoprotein (P-gp), and some of them are metabolized by CYP3A4. Therefore, concomitant use of DOAC and strong CYP3A4/ $\mathrm{P}$-gp inducers leads to a potential risk of reduced DOAC levels and therapeutic failure. However, data are scarce in clinical practice.

Aims: To describe DOAC plasma concentrations in patients receiving strong CYP3A4/P-gp inducers, in relation to risk factors for either drug accumulation or loss of efficacy.

Methods: We retrospectively analyzed DOAC measurements performed in clinical practice at the CHU UCL Namur between 2016 and 2021. We included patients receiving simultaneously a DOAC and carbamazepine, phenobarbital, phenytoin, rifampicin or St John's Wort. Socio-demographic, clinical and medication data were collected. DOAC peak and/or trough levels were estimated at steady-state using specific chromogenic assays. They were compared to on-therapy ranges observed in the pivotal trials. Expected ranges were divided into quartiles, from Q1 (lower) to Q4 (upper). For each patient, risk factors for high or low DOAC levels were identified.

Results: We included 16 patients (median age: 75 years), mainly receiving apixaban (8/16) along with carbamazepine (8/16). Five patients (31\%) had peak and/or trough level below the expected range. Among the remaining 11 patients, 8 had at least one measurement in the lower quartile of the range (Q1). The median number of risk factors for drug accumulation was 0 in patients with DOAC levels below the range, compared to 2 in patients with DOAC levels within the range (Figure 1). All DOAC patients aged $\geq 75$ years with renal impairment had plasma concentrations within the range.

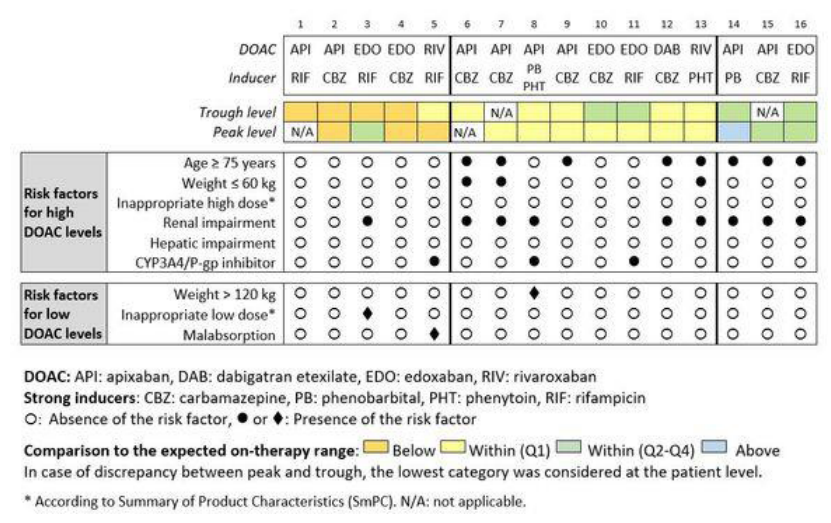

FIGURE 1 Individual risk factors for high or low DOAC plasma levels

Conclusions: Our data suggest a significant risk of reduced DOAC levels in patients taking strong CYP3A4/P-gp inducers, especially in patients without risk factors for drug accumulation. In clinical practice, DOAC measurement could help manage the concomitant use of DOAC and strong inducers.

PB1079 | Effectiveness and Safety of Direct Oral Anticoagulants in Thai Patients with Atrial Fibrillation

S. Srikajornlarp; K. Boonyawat; P. Angchaisuksiri;

K. Likittanasombat; M. Amnueypol; P. Numthavaj; P. Vathesatogkit Ramathibodi Hospital, Mahidol University, Bangkok, Thailand

Background: Direct Oral Anticoagulants (DOACs) Have Been Widely Used in Atrial Fibrillation (AF) Patients for the Prevention of Systemic Thromboembolism. The Study of its Effectiveness and Safety Has Not Been Entirely Elucidated in Thai Patients.

Aims: We aimed to study the effectiveness and safety of DOACs and warfarin among Thai patients with AF.

Methods: A retrospective cohort study of AF patients was conducted at Ramathibodi Hospital during 2013-2018. The data was reviewed from electronic medical records. Patients with confirmed AF receiving warfarin, dabigatran, rivaroxaban or apixaban were included in the study. Patients' baseline characteristics and risk factors were recorded. Primary outcome was a composite of major bleeding, ischemic stroke, and systemic thromboembolism. Secondary outcome were all-cause mortality and disease-specific mortality. All patients were followed for at least 1 year during the study period.

Results: A total of 1,680 AF patients treated with anticoagulants were included into the study (warfarin 1,193, apixaban 140, dabigatran 193, rivaroxaban 114). The baseline characteristics were presented in Table 1. Using inverse probability treatment weighting with regression adjustment method, the estimated incidence of primary outcome was $16 \%$ [95\% confidence interval (CI) 14.0-18.0\%] in the warfarin group, and $12.4 \%(95 \% \mathrm{Cl} 9.4-15.3 \%)$ in the DOACs group ( $p$ value $=0.03)$. Number needed to treat (NTT) was $27.1(95 \%$ $\mathrm{Cl}$ 2.3-51.9). Ischemic stroke occurred in 116 patients (9.7\%) in the warfarin group and 30 patients (67\%) in the DOACs group. For the 
safety outcome, major bleeding occurred in 118 patients (9.9\%) in the warfarin group and 25 patients $(5.6 \%)$ in the DOACs group. Allcause mortality was 95/1,193 (8\%) in the warfarin group, and 22/447 $(4.9 \%)$ in the DOACs group. Other outcomes are shown in Table 2.

TABLE 1 Baseline characteristics between warfarin, combined and separated DOACs groups

\begin{tabular}{|c|c|c|c|c|c|c|}
\hline Characteristics & $\begin{array}{c}\text { Overall } \\
(\mathrm{n}=1640)\end{array}$ & $\begin{array}{l}\text { Warfarin } \\
(\mathrm{n}=1193)\end{array}$ & $\begin{array}{c}\text { Combined } \\
\text { DOACs } \\
(\mathrm{n}=447)\end{array}$ & $\begin{array}{c}\text { Apixaban } \\
(\mathrm{n}=140)\end{array}$ & $\begin{array}{l}\text { Dabigatran } \\
(\mathrm{n}=193)\end{array}$ & $\begin{array}{c}\text { Rivaroxabar } \\
(\mathrm{n}=114)\end{array}$ \\
\hline $\begin{array}{l}\text { Mean age, years }{ }^{*} \\
\text { (min-max) }\end{array}$ & $70.4(15-98)$ & $69.9(15-96)$ & $71.8(31-98)$ & $71.8(45-91)$ & $71.2(34-97)$ & $73(31-98)$ \\
\hline \multicolumn{7}{|l|}{ Gender, n (\%) } \\
\hline Male & $844(51.5)$ & $619(51.9)$ & $225(50.3)$ & $67(47.9)$ & $101(52.3)$ & $57(50)$ \\
\hline \multicolumn{7}{|l|}{ Underlying diseases, $\mathbf{n}$} \\
\hline Hypertension & $1330(81.1)$ & $974(81.6)$ & $356(79.6)$ & $115(82.1)$ & 154 (79.8) & $87(76.3)$ \\
\hline Diabetes* & $614(37,4)$ & $474(39.7)$ & $140(31.3)$ & $48(34.3)$ & $60(31.1)$ & $32(28.1)$ \\
\hline Congestive heart failure & $469(28.6)$ & $354(29.7)$ & $115(25.7)$ & $40(28.6)$ & $50(25.9)$ & $25(21.9)$ \\
\hline History of stroke & $372(22.7)$ & $279(23.4)$ & $93(20.8)$ & $44(31.4)$ & $35(18.1)$ & $14(12.3)$ \\
\hline History of TIA & $78(4.8)$ & $56(4.7)$ & $22(4.9)$ & $9(6.4)$ & $11(5.7)$ & $2(1.8)$ \\
\hline History of VTE & $45(2.7)$ & $36(3)$ & $9(2)$ & $2(1.4)$ & $4(2.1)$ & $3(2.6)$ \\
\hline History of bleeding & $571(34.8)$ & $428(35.9)$ & $143(32)$ & $55(39.3)$ & $64(33.2)$ & $24(21.1)$ \\
\hline Peripheral vascular disease & $593(36.2)$ & $438(36.7)$ & $155(34.7)$ & \begin{tabular}{|l|}
$41(29.3)$ \\
\end{tabular} & $68(35.2)$ & $46(40.4)$ \\
\hline Abnormal renal function* & $83(5.1)$ & $73(6.1)$ & $10(2.2)$ & $3(2.1)$ & $2(1)$ & $5(4.4)$ \\
\hline Abnormal liver function & $95(5.8)$ & $71(6)$ & $24(5.4)$ & $12(8.6)$ & $7(3.6)$ & $5(4.4)$ \\
\hline Antiplatelet or NSAID & $1118(68.2)$ & $806(67.6)$ & $312(69.8)$ & $103(73.6)$ & $133(68.9)$ & $76(66.7)$ \\
\hline $\begin{array}{l}\text { CHA }_{2} \text { DS } 2 \text { VASc Score, } \\
\text { median (QR) }\end{array}$ & $\begin{array}{l}4.0 \\
(3.0,5.0)\end{array}$ & $\begin{array}{l}4.0 \\
(3.0,5.0) \\
\end{array}$ & \begin{tabular}{|l|}
4.0 \\
$(3.0,5.0)$ \\
\end{tabular} & $\begin{array}{l}4.0 \\
(3.0,5.0) \\
\end{array}$ & $\begin{array}{l}4.0 \\
(3.0,5.0) \\
\end{array}$ & $\begin{array}{l}4.0 \\
(2.0,5.0) \\
\end{array}$ \\
\hline $\begin{array}{l}\text { HAS-BLED Score, } \\
\text { median (IQR) }\end{array}$ & $\begin{array}{l}3.0 \\
(2.0,4.0)\end{array}$ & $\begin{array}{l}3.0 \\
(2.0,4.0)\end{array}$ & $\begin{array}{l}3.0 \\
(2.0,4.0)\end{array}$ & $\begin{array}{l}4.0 \\
(2.0,5.0) \\
\end{array}$ & $\begin{array}{l}3.0 \\
(2.0,4.0)\end{array}$ & $\begin{array}{l}.0 \\
(2.0,4.0)\end{array}$ \\
\hline
\end{tabular}

* Significant difference between the warfarin group and the combined DOACs group

TABLE 2 Outcomes between warfarin, combined and separated DOACs groups

Table 2. Outcomes between warfarin, combined and separated DOACs groups

\begin{tabular}{|l|l|l|l|l|l|l|}
\hline Incidence, $\mathbf{n}(\%)$ & $\begin{array}{c}\text { Overall } \\
(\mathrm{n}=1640)\end{array}$ & $\begin{array}{c}\text { Warfarin } \\
(\mathrm{n}=1193)\end{array}$ & $\begin{array}{c}\text { Combined } \\
\text { DOACs } \\
(\mathrm{n}=447)\end{array}$ & $\begin{array}{c}\text { Apixaban } \\
(\mathrm{n}=140)\end{array}$ & $\begin{array}{c}\text { Dabigatran } \\
(\mathbf{n}=193)\end{array}$ & $\begin{array}{c}\text { Rivaroxaban } \\
(\mathbf{n}=114)\end{array}$ \\
\hline Composite & $246(15)$ & $197(16.5)$ & $49(11)$ & $27(19.3)$ & $18(9.3)$ & $4(3.5)$ \\
\hline Major bleeding & $143(8.7)$ & $118(9.9)$ & $25(5.6)$ & $14(10)$ & $8(4.1)$ & $3(2.6)$ \\
\hline Ischemic stroke & $146(8.9)$ & $116(9.7)$ & $30(6.7)$ & $19(13.6)$ & $11(5.7)$ & 0 \\
\hline $\begin{array}{l}\text { Systemic } \\
\text { thromboembolism }\end{array}$ & $28(1.7)$ & $24(2)$ & $4(0.9)$ & $1(0.7)$ & $1(0.5)$ & $2(1.8)$ \\
\hline Mortality, n (\%) \\
\begin{tabular}{l} 
All-cause \\
\hline Specific
\end{tabular} & $117(7.1)$ & $95(8)$ & $22(4.9)$ & $12(8.6)$ & $8(4.1)$ & $2(1.8)$ \\
\hline Combined & $14(0.9)$ & $12(1)$ & $2(0.4)$ & $2(1.4)$ & 0 & 0 \\
\hline Bleeding & $4(0.2)$ & $2(0.2)$ & $2(0.4)$ & $2(1.4)$ & 0 & 0 \\
\hline Stroke & $8(0.5)$ & $8(0.7)$ & 0 & 0 & 0 & 0 \\
\hline $\begin{array}{c}\text { Systemic } \\
\text { thromboembolism }\end{array}$ & $2(0.1)$ & $2(7)$ & 0 & 0 & 0 & 0 \\
\hline Other causes & $103(6.3)$ & $83(7)$ & $20(4.5)$ & $10(7.1)$ & $8(4.1)$ & $2(1.8)$ \\
\hline
\end{tabular}

Conclusions: Thai AF patients receiving DOACs were associated with a lower risk of composite outcome compared to warfarin. Allcause mortality was also lower in the DOACs group.

PB1080 | Use of Dabigatran Assessed By Thrombin Generation Assay (TGA): Paradoxical Results

R. Duarte ${ }^{1}$; C. Ferreira ${ }^{2}$; E. Figueiredo ${ }^{3}$; G. Lopes $^{4}$; L. Paes ${ }^{5}$; D. Rios ${ }^{6}$; M. Carvalho ${ }^{1}$

${ }^{1}$ Faculty of Pharmacy-Federal University of Minas Gerais, Belo Horizonte, Brazil; ${ }^{2}$ Technical College - Federal University of Minas Gerais, Belo Horizonte, Brazil; ${ }^{3}$ Hospital Life Center, Belo Horizonte, Brazil; ${ }^{4}$ Institute of Biological Sciences - Federal University of Minas Gerais, Belo Horizonte, Brazil; ${ }^{5}$ Faculty of Pharmacy-Federal University of Minas Gerais - Brazil, Belo Horizonte, Brazil; ${ }^{6}$ Campus Centro Oeste - Federal University of São João del-Rei, Divinopolis, Brazil

Background: Dabigatran etexilate, a thrombin inhibitor, is widely used for prevention of stroke in patients with atrial fibrillation (AF). Thrombin Generation Assay (TGA) has been widely used for research purposes. Paradoxically elevated thrombin generation has been observed in users of dabigatran, indicating a hypercoagulable profile.

Aims: In view of this paradoxical and curious finding, our objective was to evaluate the performance of TGA in plasma of patients with AF using dabigatran, in comparison with healthy individuals (controls). Methods: Citrated blood samples were collected from patients with AF using a standard dose of dabigatran (110 mg twice a day; $n=5)$ and from controls ( $n=17$ ). TGA was performed using CAT method, with subsequent analysis of Lagtime (min), Endogen Thrombin Potential (ETP, nM.min) and Peak (nM). Data were presented as median and interquartile range and analyzed by Mann-Whitney test ( $p<0.005)$. Results: Patients using dabigatran showed an increase in ETP and Peak values, confirming previous studies. However, Lagtime was prolonged in these patients (Figure 1). Our preliminary data are in line with previous studies (Connolly et al., 2009; Helin et al., 2015; Zhang et al., 2018; Bloemen et al., 2018), who reported similar results. The increase in ETP and Peak values in patients using Dabigatran may occur due to in vitro interference with the TGA technique. According to Bloemen et al. (2018), this finding may be explained due to the inhibition of the calibrator (alpha2M-Thrombin), a TGA reagent, by Dabigratan. If so, an improvement in TGA is necessary to overcome this inconvenience, which can lead to misinterpretations of the results.

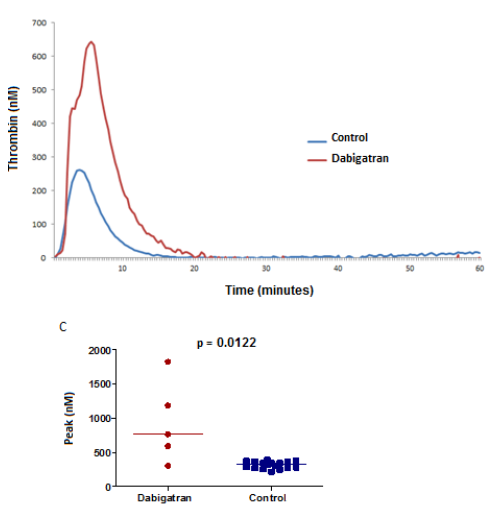

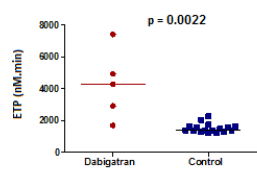

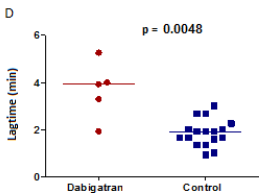


FIGURE 1 Thrombin Generation Profile (A) of patients using dabigatran and the control group. Comparison between the parameters ETP (B), Peak (C) and Lagtime (D)

Conclusions: TGT (CAT method), in its current conditions, cannot be used to assess Dabigatran use. Based on our results, only Lagtime reflected the use of Dabigatran, that is, compatible with the use of an anticoagulant drug.

Support: FAPEMIG/CNPQ.

PB1081 | Accounting of Symptoms and Ejection Fraction May Improve Prediction of Left Atrial Thrombus in Patients with Nonvalvular Atrial Fibrillation before Catheter Ablation or Cardioversion

I. Zaigraev; I. Yavelov; O. Drapkina; E. Bazaeva

National Medical Research Center for Therapy and Preventive Medicine of the Ministry of Health of the Russian Federation, Moscow, Russian

Federation

Background: Optimal approach for prediction of left atrial thrombus (LAT) in patients with nonvalvular atrial fibrillation (NAF) is not established yet.

Aims: To evaluate possibilities for prediction of LAT before catheter ablation or cardioversion in patients with NAF.

Methods: In a retrospective single-center study medical records of 1994 patients with NAF underwent transesophageal echocardiography before catheter ablation or cardioversion were analyzed. LAT was identified in $33(1.6 \%)$ of them. For the control group, 167 patients without LAT were randomly selected from this database. Logistic regression analysis and calculation of C-statistics were used for evaluation of diagnostic values for different risk factors including $\mathrm{CHADS}_{2}, \mathrm{R} 2 \mathrm{CHADS}_{2}, \mathrm{CHA}_{2} \mathrm{DS}_{2}-\mathrm{VASc}, \mathrm{R}-\mathrm{CHA}_{2} \mathrm{DS}_{2}-$ VASc, R2CHA ${ }_{2} \mathrm{DS}_{2}$-VASc, $\mathrm{CHA}_{2} \mathrm{DS}_{2}$-VASc-RAF, mCHA2DS2-VASc, $\mathrm{CHA}_{2} \mathrm{DS}_{2}$-VASc-AFR and ATRIA scores.

Results: The mean age of studied patients was $60.3 \pm 10.9$ years, 110 (55\%) of them were males. Mean $\mathrm{CHA}_{2} \mathrm{DS}_{2}$-VASc score was 2.54 \pm 1.79. In multivariate analysis EHRA score 3-4 (OR 18.7; 95\% Cl 3.8-92.1; $P<0.0001)$, left ventricular ejection fraction [EF] (OR 0.89; $95 \% \mathrm{Cl}$ 0.81-0.98; $P=0.017$ ) and $\mathrm{CHA}_{2} \mathrm{DS}_{2}$-VASc-RAF score (OR 1.24; 95\% $\mathrm{Cl} 1.04-1.50, P=0.017)$ were associated with LAT. Addition of EHRA score 3-4 (+11 points) and EF $<48 \%$ (+6 points) to $\mathrm{CHA}_{2} \mathrm{DS}_{2}-\mathrm{VASc}$ RAF score increased C-statistics from $0.83(95 \% \mathrm{Cl} 0.76-0.91)$ to 0.87 (95\% Cl 0.80-0.94). Optimal cut-off for modified $\mathrm{CHA}_{2} \mathrm{DS}_{2}$-VAScRAF score was $>8$ points (OR 25.8; 95\% Cl 5.9-112.3, $P<0.0001$ ). Sensitivity, specificity, positive and negative predictive values of $\mathrm{CHA}_{2} \mathrm{DS}_{2}$-VASc-RAF and modified $\mathrm{CHA}_{2} \mathrm{DS}_{2}$-VASc-RAF scores are presented in the table 1

Conclusions: Accounting of severity of symptoms and reduced EF may slightly increase predictive value of $\mathrm{CHA}_{2} \mathrm{DS}_{2}$-VASc-RAF score for left atrial thrombus in patients with non-valvular AF before catheter ablation or cardioversion.

TABLE 1 Predictive values of CHA2DS2-VASc-RAF and modified CHA2DS2-VASc-RAF scores for LAT in patients with NAF before catheter ablation or cardioversion

\begin{tabular}{|c|c|c|c|c|}
\hline High values of risk scores & Sensitivity & Specificity & PPV & NPV \\
\hline $\mathrm{CHA}_{2} \mathrm{DS}_{2}$-VASc-RAF $>3$ points & $90,6 \%$ & $57,1 \%$ & $30,2 \%$ & $96,7 \%$ \\
\hline Modified CHA2DS2-VASc-RAF $>8$ points & $93,5 \%$ & $64,0 \%$ & $34,9 \%$ & $97,9 \%$ \\
\hline
\end{tabular}

PPV - positive predictive value; NPV - negative predictive value.

PB1082 | Inappropriate Direct Oral Anticoagulant Dosing in a Spanish Cohort with Atrial Fibrillation

B. Navarro Almenzar ${ }^{1}$; J.J. Cerezo Manchado ${ }^{2}$; F. García Candel ${ }^{3}$

${ }^{1}$ Hospital General Universitario Rafael Méndez, Murcia, Spain;

${ }^{2}$ Hospital General Universitario Santa Lucía, Murcia, Spain; ${ }^{3} \mathrm{Hospital}$

Clínico Universitario Virgen de la Arrixaca, Murcia, Spain

Background: Atrial fibrillation (AF) is the most prevalent arrhythmia worldwide, being the main cause of anticoagulation. DOACS are used often for the stroke prevention in these patients. Each DOAC has two presentations, the standard dose and the reduced dose.

Aims: To assess the frequency, effectiveness and safety of inappropriate DOAC dosing in patients with AF.
Methods: Retrospective study that included patients with AF who started a DOAC (Rivaroxaban, Apixaban, Dabigatran or Edoxaban) from January 1, 2013 to December 31, 2016, in three Spanish hospitals (Hospital Clínico Universitario Virgen de la Arrixaca, Hospital Comarcal del Noroeste and Hospital Vega Baja). Inappropriate dosing was analysed according to labeling recommendations. Thromboembolic, hemorrhagic complications and mortality were recorded. Mean follow-up was 1,6 years. Statistical analysis was performed using SPSS ${ }^{\circledR}$ Statistics program v25 (SPSS Inc., Chicago, Illinois, USA).

Results: A total of 2218 patients were included, of which, 506 patients (23\%) were receiving an inappropriate dose. Among these patients, inappropriate reduced dose (underdosing) predominated (87 \%). Table 1 shows the main characteristics of the cohort. Rivaroxaban was the drug incorrectly prescribed most often. 
TABLE 1 Cohort characteristics $(N=2218)$

$\begin{array}{ll}\text { Age (years) } & 76 \pm 9 \\ \text { Sex (female) } & 1116(53 \%) \\ \text { Smoking } & 142(6 \%) \\ \text { Alcoholism } & 49(2 \%) \\ \mathrm{CrCl}(\mathrm{ml} / \mathrm{min}) & 74 \pm 35 \\ \text { Hypertension } & 1943(88 \%) \\ \text { Diabetes mellitus } & 734(33 \%)\end{array}$

\begin{tabular}{ll} 
Heart failure & $416(19 \%)$ \\
$\mathrm{CHA}_{2} \mathrm{DS}_{2}$-VASc & $4.0 \pm 1.6$ \\
HAS-BLED & $2.4 \pm 0.9$ \\
\hline
\end{tabular}

Compared to the correctly dosing group, a higher rate of mortality was observed in the underdosing group (13,6 vs $8,5 \%, P<0,001)$. However, regarding ischemic stroke and major bleeding, we could not find differences between groups (table 2).

TABLE 2 Events according to appropriate dosing of DOAC

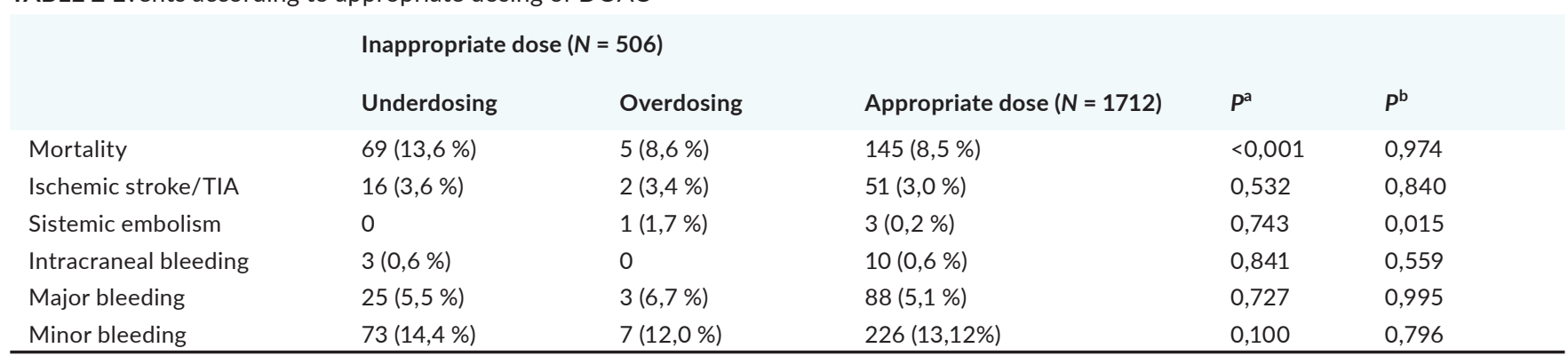

$P^{\mathrm{a}}$ underdosing vs appropriate dosing. $P^{\mathrm{b}}$ :underusing vs appropriate dosing.

Multivariate analysis revealed that underdosing was associated to elderly, prior major bleeding and the drug rivaroxaban, while overdosing was associated to elderly, higher CHA2DS2-VASc score and the drug dabigatran.

Conclusions: Almost a quarter of the population was receiving an offlabel dose. This is traduced in a higher rate of mortality in patients undersing. A higher rate of ischemic events in the underdosing group or a higher rate of bleeding events in the overdosing group were not shown.

Figure 2: Events according to appropriate or innapropriate dosing of DOAC

PB1083 | Comparative Effectiveness of Alternative Bridging Therapies for Subtherapeutic INR in Patients with Left Ventricular Assist Devices

G. Chung ${ }^{1}$; E. Salem ${ }^{2}$; E. Sippola ${ }^{3}$; S. Shore ${ }^{3}$; L. Baumann Kreuziger ${ }^{2}$; G. Barnes ${ }^{3}$

${ }^{1}$ University of Michigan School of Public Health, Ann Arbor, United States; ${ }^{2}$ Medical College of Wisconsin, Milwaukee, United States;

${ }^{3}$ University of Michigan Medical Center, Ann Arbor, United States

Background: Patients with left ventricular assist device (LVAD) often require bridging anticoagulation for low INRs. There is currently a lack of comparative data between unfractionated heparin (UFH) and low-molecular-weight heparin (LMWH) bridging in this population.
Aims: To predict LMWH vs. UFH use and compare rates of ISTH major bleeding or thromboembolism within 30 days of stopping bridging.

Methods: We conducted a retrospective cohort study of adult patients with LVAD implantation between January 1, 2014 and December 31, 2018 from two academic medical centers. Data were collected for each subtherapeutic anticoagulation episode for which either UFH or LMWH was used and followed for 30-days after bridging was discontinued. We performed logistic regression, adjusting for LVAD type and clustering at the site and patient levels.

Results: Data were collected from 282 patients and 1976 bridging episodes (Table 1). Age (OR 0.84 per decade $95 \% \mathrm{Cl} 0.74-0.94$ ), an additional bridging episode within 30 days (OR $0.54,95 \% \mathrm{Cl} 0.43-0.69$ ), and aspirin dosage of $>100 \mathrm{mg}$ daily (OR $2.62,95 \% \mathrm{Cl} 1.79-3.82$ ) are predictors of LMWH use. Compared to HeartMate 3, having a HeartMate II (OR 0.34, 95\% Cl 0.19-0.61) or Heartware HVAD (OR 0.34, 95\% Cl $0.22-0.52$ ) is associated with lower odds of LMWH use. There was no significant difference in the unadjusted rates of major bleeding or thromboembolism between LMWH and UFH (40/1410 [2.8\%] vs. $15 / 566$ [2.7\%], respectively, $P=0.91$ ). In adjusted analysis, the combined risk of major bleeding or thromboembolism was not significantly elevated for LMWH versus UFH (OR: 1.75, 95\% Cl 0.86-3.55) (Table 2).

TABLE 1 Patient-level and bridging episode-level characteristics

Patient-Level Characteristics $(n=282)$

Study Site

University of Michigan

Medical College of Wisconsin

Age at time of LVAD implantation
Bridging Episode-Level Characteristics ( $n=1,976)$

Bridging Type

$N(\%)$

215 (76.2)

UFH

$566(28.6)$

$67(23.8)$

LMWH

$1,410(71.4)$

Duration of bridging (in days) 
Patient-Level Characteristics $(n=282)$

Study Site

Median (IQR)

Type of LVAD

HeartMate 3

HeartMate II

Heartware HVAD

Additional course of bridging administered in 30-day period

No

$82(29.1)$

Yes
Bridging Episode-Level Characteristics $(n=1,976)$

Bridging Type

$N(\%)$

Median (IQR)

$5(3-9)$

Aspirin dosage

$\leq 100 \mathrm{mg}$ daily

$371(19.6)$

$>100 \mathrm{mg}$ daily
TABLE 2 Results of two-level multivariable logistic regression analysis examining the association between LMWH use and 30-day major bleeding or thromboembolism

\begin{tabular}{lll} 
& $\begin{array}{l}\text { Major Bleeding or } \\
\text { Thromboembolism }\end{array}$ & \\
Characteristics & OR $(95 \% \mathrm{Cl})$ & p-value \\
LMWH use & $1.75(0.86-3.55)$ & 0.123 \\
Type of LVAD & & \\
Heartmate 3 & Ref. & \\
Heartmate II & $6.43(2.09-19.79)$ & 0.001 \\
Heartware HVAD & $1.52(0.58-4.02)$ & 0.397 \\
\hline
\end{tabular}

Conclusions: Patients receiving LMWH had a non-significantly elevated risk of major adverse events as compared to UFH bridging for subtherapeutic INRs. A well powered, prospective comparison of these two management strategies is needed given significant healthcare cost and resource implications.

PB1084 | Procedural Success of Left Atrial Appendage Occlusion Device in Patients with Chronic Kidney Disease: Metaanalysis of Real-world Data

A. Singh ${ }^{1}$; S. Hussain ${ }^{2}$; B. Antony ${ }^{1}$

${ }^{1}$ Menzies Institute for Medical Research, University of Tasmania, Hobart Australia, Hobart, Australia; ${ }^{2}$ Institute of Biostatistics and Analyses, Faculty of Medicine, Masaryk University, Brno, Czech Republic

Background: Percutaneous left atrial appendage occlusion (LAAO) devices are a therapeutic alternative used for the prevention of stroke in patients with atrial fibrillation (AF). Population with chronic kidney disease (CKD) are at high risk of procedural complications after transcatheter intervention; however, there is no meta-analysis on procedural success in CKD vs. non-CKD population receiving LAAO interventions.

Aims: To perform a systematic review and meta-analysis of the realworld evidence (RWE) data on the procedure success of LAAO in CKD population compared to the non-CKD population.
Methods: PubMed, EMBASE, and Cochrane database were searched, from inception to January 2021, for published RWE studies reporting the procedural success rate for LAAO device implantation in patients with or without CKD. Two researchers independently screened search results and extracted data for study details (design, follow-up, assessment etc.), outcome results (procedure success rate), and conclusion. The quality of the included studies was assessed using Newcastle-Ottawa Scale (NOS) and random-effect model was used for meta-analysis.

Results: Of 65 articles identified, four studies (2 from Germany, 1 from China, and 1 multinational) comprising 678 CKD and 978 non-CKD patents receiving LAAO fulfilled the inclusion criteria. The majority $(n=3)$ of the studies were of high quality. CKD was defined as an eGFR $<60 \mathrm{ml} / \mathrm{min}$ per $1.73 \mathrm{~m} 2$ across studies. Three studies reported procedural success rate as successful implantation and absence of significant residual leak ( $\geq 5 \mathrm{~mm}$ ). The mean age was above 70 years and Aamplatzer cardiac plug was used in most of the LAAO procedures $(68 \%, n=1122)$, across studies. The metaanalysis showed a similar procedural success rate for LAAO procedure $(C K D=98.4$ and non-CKD $=96.9)$ with a pooled risk difference of 0.01 [(95\%Cl: $0.00-0.03), P=0.07]$.

\begin{tabular}{|c|c|c|c|c|c|c|c|c|}
\hline Study & Country & Follow-up & CKD assessment & $\begin{array}{l}\text { Gender, }(\mathrm{m} / f) \\
C K D / \text { ron- } K K D\end{array}$ & $\begin{array}{l}\text { Age, mean (SD) } \\
C K D / \text { nan- } C K D\end{array}$ & & $\begin{array}{l}\text { Ulation } \\
\text { Non-CKD }\end{array}$ & LAAO device \\
\hline 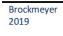 & Germany & 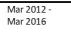 & 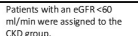 & $\mid(242 / 39) /(42 / 23)$ & $78.2(7.3 / 3 / 74.4(7.1)$ & 81 & 65 & 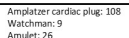 \\
\hline Xue 2018 & Germany & 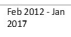 & 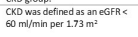 & $(92 / 59) /(111 / 38)$ & $77.0(7.2) / 73.217 .88$ & 151 & 149 & $\begin{array}{l}\text { Whuthese } \\
\text { Watchman: } 3000\end{array}$ \\
\hline So 2018 & China & $\begin{array}{l}\text { Jun 2009- } \\
\text { Aug } 2017\end{array}$ & 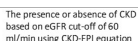 & Deverall: 128/168 & Overall: 72 (8) & 71 & 125 & NR \\
\hline xeter 2016 & Multinational & $\begin{array}{l}\text { Dece } 2008 \text { - } \\
\text { Nov } 2013\end{array}$ & 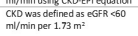 & $(205 / 169) /(425 / 2125)$ & $77.9(7.3 / 1 / 73.18(8.4)$ & 375 & 639 & Amplatzer cardibac plus: 1014 \\
\hline
\end{tabular}

FIGURE 1 Study characteristics

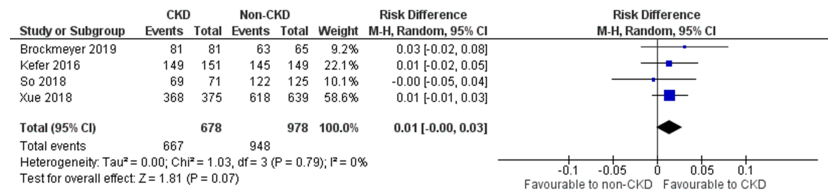

FIGURE 2 Meta-analysis of the LAAO procedure success in CKD vs non-CKD population 
Conclusions: The procedural success rate of LAAO did not differ between the CKD and non-CKD patients. LAAO demonstrated similar real-word procedural safety for patients with and without CKD.

PB1085 | Self-report Instruments for Assessing Adherence to Oral Anticoagulation with Warfarin: A Systematic Review

M.S. Vianna ${ }^{1}$; M.F.S. Praxedes²; V.E. Araújo ${ }^{3}$; C.B. Ferreira ${ }^{2}$; W.J.F.N. Sousa ${ }^{4}$; C.C. Viana ${ }^{1}$; M.A.P. Martins ${ }^{4,1}$

${ }^{1}$ Faculdade de Farmácia, Universidade Federal de Minas Gerais, Belo Horizonte, Brazil; ${ }^{2}$ Centro Ciências da Saúde, Universidade Federal do Recôncavo da Bahia, Santo Antônio de Jesus, Brazil; ${ }^{3}$ Faculdade de Odontologia, Pontifícia Universidade Católica de Minas Gerais, Belo Horizonte, Brazil; ${ }^{4}$ Faculdade de Medicina, Universidade Federal de Minas Gerais, Belo Horizonte, Brazil

Background: The analysis of adherence to warfarin therapy is important in clinical practice to identify patients who need closer monitoring and educational interventions.

Aims: We sought to identify validated self-report instruments to assess adherence to warfarin therapy.

Methods: This systematic review searched for articles on MEDLINE (Pubmed), LILACS, EMBASE and Cochrane Library. Search strategies used indexed terms from Medical Subject Heading (MeSH), with no date limit for publication andno language restriction. Inclusion criteria: age $\geq 18$ years, chronic warfarin use for any indication of oral anticoagulation, employment of validated self-report instruments to assess adherence to warfarin therapy. Exclusion criteria: duplicates, narrative or systematic review and meta-analysis, case reports/series and studies involving the determination of drug concentrations in plasma. A data extraction form was used for data collection from selected articles. The risk of bias was minimized by independent screening of articles and data extraction, performed by two reviewers. Disagreements were resolved by a third reviewer. The quality assessment was performed using the Agency for Research and Health Quality Methodology Checklist, Newcastle-Ottawa Scale and Cochrane Collaboration's ROBINS-I tool.

Results: Nineteen studies were selected for qualitative synthesis. Study designs encompassed seventeen cross-sectional studies, a cohort study and a quasi-experimental study, with publication timeframe 2009-2019. Four validated instruments were identified, including: the Morisky Medication Adherence Scale (MMAS), Morisky Medication Adherence Scale (MMAS-8), Treatment Adherence Measurement (MTA) and Brief Medication Questionnaire (BMQ). All instruments focused exclusively on the isolated use of the medication.

Conclusions: Four validated self-report instruments focused on adherence to warfarin therapy were identified in this review. None of them were adapted and validated to address the broad complexity of warfarin therapy, including diet, laboratory monitoring, and caution while taking concomitant medications.. Further studies are needed to develop and validate more comprehensive instruments as an attempt to assess adherence to warfarin therapy.
PB1086 | Prescribing Patterns, Efficacy and Safety of Direct Oral Anticoagulants (DOAC) Usage in a Tertiary Hospital

K.Y. Yong ${ }^{1}$; S. Leow ${ }^{2}$; E.S. Yap ${ }^{1}$; P.S. Ong ${ }^{2}$; S.C. Tan ${ }^{1}$; P.W. Goh ${ }^{1}$

${ }^{1}$ National University Hospital Singapore, Singapore, Singapore;

${ }^{2}$ National University Singapore, Singapore, Singapore

Background: The use of Direct oral anticoagulants (DOACs) have been increasing due to its convenience and equivalent efficacy with warfarin. We would like to evaluate prescribing patterns of apixaban and rivaroxaban and to compare the efficacy and safety of these DOACs in our hospital.

Aims: To evaluate prescribing patterns, safety and efficacy of DOACs.

Methods: Retrospective analysis of patients newly initiated with DOACs and data was collected from the electronic database of the hospital. Prescribing patterns were evaluated for appropriateness based on product-insert leaflet (PIL) and FDA-approved recommendations. Primary efficacy outcomes were ischaemic stroke and any systemic embolism that occurred during the 1-year follow-up. Primary safety outcomes were any major or clinically relevant non-major bleeding.

Results: A total of 395 patients initiated with DOACs were identified with a median age of 72 (range 44-91 years old). 81 (20.5\%) of them were switched from warfarin to DOAC and the choice of DOACs are Apixaban ( $n=303, n=76.7 \%$ ) followed by Rivaroxaban ( $n=92$, 23.3\%). Most of the indications of anticoagulations are atrial fibrillation ( $n=350,88.4 \%$ ) followed by venous thromboembolism ( $n=35$, $8.8 \%)$ and cardiovascular event $(n=9,2.3 \%)$ respectively. Most patients were male $(n=218)$ with gender ratio of $1.2: 1$. $45.5 \%$ have normal BMI. Appropriate prescribing was identified in 289 patients (73.2\%) of the patients and inappropriate prescribing was mainly attributed to under-dosing (16.5\%). 93.6\% of them were compliant to the treatment. Primary efficacy and safety outcomes were not significantly different between apixaban and rivaroxaban (Hazard ratio [HR] 0.756; 95\% Cl, 0.156-3.679; $P=0.729$ for efficacy outcomes; Hazard ratio $[\mathrm{HR}], 0.828 ; 95 \% \mathrm{Cl}, 0.505-1.359 ; P=0.456$ for safety outcomes). 8 (2\%) and 88 (22\%) of them developed major bleeding and clinically relevant non-major bleeding, respectively.

Conclusions: Majority of the prescriptions for DOACs were in line with recommendations. Apixaban and rivaroxaban appeared to have similar efficacy and safety outcomes.

PB1087 | Should We Check the Anticoagulant Activity Of Edoxaban, at Least at the Beginning of the Treatment?

N. García-León; M.T. Calderon-López; A. Matilla-García Hospital Central de la Defensa, Gómez Ulla, Madrid, Spain

Background: Edoxaban is a direct oral anticoagulant and does not require monitoring, however it could be useful in certain situations. Aims: To check the anticoagulant activity of edoxaban, measured with the Low Molecular Weight Heparin (LMWH) calibrated anti-Xa assay. 
Methods: Samples were obtained one month after the beginning of the treatment, at the through (median time of 19h). We do not have the edoxaban specific calibrator, as we work with the ACL TOP 500 (Werfen ${ }^{\circledR}$ ), so we used the LMWH calibrated anti-Xa assay. We used the cut-off $<0.10 \mathrm{UI} / \mathrm{mL}$ for excluding the presence of clinically relevant concentration of anti-Xa inhibitor.

Results: 36 patients were studied from 2018 to 2020. 32 were anticoagulated because of atrial fibrillation and 2 for venous thrombosis. 18 were on $60 \mathrm{mg}$ and $18 \mathrm{on} 30 \mathrm{mg}$. 7 had the $30 \mathrm{mg}$ dose, because of low weight, with a median weight of $55 \mathrm{~kg}(40-63)$ and 10 because of creatinine clearance $(\mathrm{CrCl})<50 \mathrm{~mL} / \mathrm{min}$, with a median $\mathrm{CrCl}$ of 41 $\mathrm{mL} / \mathrm{min}$ (21-41). Only 1 patient fulfilled both criteria.

Median age of patients on $60 \mathrm{mg}$ was 78 (57-93), 66,6\% were women (12) and $33,3 \%$ (6) were men. Median age in the group of $30 \mathrm{mg}$, was 81 ( 50-92), 72\% were women (13) and 28\% (5) were men.

3 patients had an anti-Xa activity $<0.10 \mathrm{IU} / \mathrm{mL}$, confirmed in two other different times, all of them were on $60 \mathrm{mg}$. 1 out of 3 had a $\mathrm{CrCl}>95 \mathrm{~mL} / \mathrm{min}$ and the other $2 \mathrm{a} \mathrm{CrCl}>88 \mathrm{~mL} / \mathrm{min}$. None of them had any drug interaction or a cause that justified it.

Conclusions: We found 3 patients taking edoxaban $60 \mathrm{mg}$ with no clinically relevant anticoagulant activity and only one had an obvious cause, a $\mathrm{CrCl}>95 \mathrm{~mL} / \mathrm{min}$. Hence, it could be useful to check the anticoagulant activity of edoxaban, in the first months of treatment in order to confirm the patient is correctly anticoagulated.

\section{CANCER ASSOCIATED THROMBOSIS}

\section{LPB0041 | Improved Patient-reported Treatment Satisfaction} with Rivaroxaban as Compared to Low Molecular Weight Heparins for Cancer Patients with Acute Venous Thromboembolism Results from the CONKO-011 Trial

$\underline{\text { H. Riess }}{ }^{1}$; M. Sinn ${ }^{2}$; A. Lohneis ${ }^{3} ;$ M. Hellmann ${ }^{4}$; J. Striefler ${ }^{1}$; T. Südhoff ${ }^{5}$; U. Pelzer ${ }^{1}$; M. Stahl' ${ }^{6}$; A. Schlenska-Lange ${ }^{7}$; A. Krziwanie ${ }^{8}$; R. Trappe ${ }^{9}$; S. Rutzner ${ }^{10} ;$ J. Heinz ${ }^{11}$; K.-D. Wernecke ${ }^{12}$

${ }^{1}$ Charité, University Medicine Berlin, Berlin, Germany; ${ }^{2}$ Universtity Hospital Eppendorf, Hamburg, Germany; ${ }^{3}$ University Hospital Köln, Köln, Germany; ${ }^{4} \mathrm{PIOH}$, Frechen, Germany; ${ }^{5}$ Klinikum Passau, Passau, Germany; ${ }^{6}$ Ev. Kliniken Essen, Essen, Germany; ${ }^{7}$ Krankenhaus Barmherzige Brüder Regensburg, Regensburg, Germany; ${ }^{8}$ Krankenhaus Halle, Halle, Germany; ${ }^{9}$ Diako, Bremen, Germany; ${ }^{10}$ Universiti Hospital Erlangen, Erlangen, Germany; ${ }^{11}$ University Hospital Freiburg, Freiburg, Germany; ${ }^{12}$ Sostana GmbH, Berlin, Germany

Background: Guidelines recommend low molecular weight heparins (LMWHs) or factor-Xa-inhibitors for $\geq 3$ months in patients with cancer-associated venous thromboembolism (CAT).

Aims: We studied patient satisfaction with anticoagulant treatment, which may be essential for the choice of drug and anticoagulation adherence.
Methods: CONKO-011, is an open-label, prospective study approved by ethics committees in patients with symptomatic CAT randomized after informed consent to center-specific LMWHs or rivaroxaban. Patient satisfaction with anticoagulant treatment was measured by the Anti-Clot Treatment Scale (ACTS). The 12-item ACTS Burdens scale (primary endpoint after 4 weeks) and the 3-item ACTS Benefits scale were analysed at 4,8 and 12 weeks; clinical outcome parameters for up to week 24.

Results: 247 patients were randomized. Characteristics were well balanced (Table 1). At 4 weeks the relative range of ACTS Burdens and Benefits Scores with rivaroxaban were $88 \%$ (53/60) and $77 \%$ (12/15), respectively. Mean ACTS Burdens scores after 4 weeks were 52.8 versus 51.2 in favour of rivaroxaban $(P=0.006)$ with mean score differences ranging from 3.3 (week $8 ; P=0.001$ ) to 2.4 (week 12; $P=0.006$ ). As result from multivariate longitudinal variance analysis, treatment effect of ACTS burden was consistent over treatment time $(P<0.001)$. The ACTS Benefits scores were in favor of rivaroxaban at $4(P=0.042)$ and $8(P=0.055)$ weeks, but not at 12 $(P=0.546)$ weeks. More patients on LMWH requested to stop study treatment preterm (19.4\% versus 11.1\%). There were 8 and $15 \mathrm{SAE}$ $\geq 4^{\circ}$ in the rivaroxaban and LMWH groups, respectively. Venous and arterial thromboembolic as well as major bleeding events did not differ between groups (Table 2).

TABLE 1 Patient's characteristics

\begin{tabular}{lll} 
& LMWH & Rivaroxaban \\
$n$ & 124 & 123 \\
Age (mean \pm SD) / male (n) & $64.47 \pm 10.91 / 58$ & $62.94 \pm 11.35$ \\
& & $/ 64$ \\
Weight $[\mathrm{kg}]$ (mean $\pm \mathrm{SD})$ & $75.71 \pm 18.20$ & $78.43 \pm 16.95$ \\
Index-VTE Distal DVT & $29.6 \%$ & $29.0 \%$ \\
$\quad$ Proximal DVT & $9.6 \%$ & $10.5 \%$ \\
$\quad$ Pulmonary embolism & $37.6 \%$ & $35.5 \%$ \\
Cancer Loco-regional & $31.2 \%$ & $29.8 \%$ \\
$\quad$ Metastasized & $68.8 \%$ & $70.2 \%$ \\
Anti-cancer therapy & $87.2 \%$ & $86.3 \%$ \\
\hline
\end{tabular}

TABLE 2 Study outcomes at 24 weeks

\begin{tabular}{|c|c|c|}
\hline & LMWH & Rivaroxaban \\
\hline $\begin{array}{l}\text { Preterm stop of study } \\
\text { medication }\end{array}$ & $39.5 \%$ & $43.1 \%$ \\
\hline "Patient's request" & $19.4 \%$ & $11.1 \%$ \\
\hline Cancer related death & $8.9 \%$ & $12.2 \%$ \\
\hline Major bleeding & $0.8 \%$ & $0.0 \%$ \\
\hline $\begin{array}{l}\text { Severe adverse events } \\
\quad \geq 3^{\circ}(\mathrm{SAE} ; \mathrm{n})\end{array}$ & 59 & 50 \\
\hline Recurrent VTE [rVTE] (n) & 5 & 3 \\
\hline $\begin{array}{l}\text { rVTE + Myocardial } \\
\quad \text { infarction + Stroke (n) }\end{array}$ & 4 & 5 \\
\hline Major bleeding (n) & 5 & 5 \\
\hline $\begin{array}{c}\text { Clinically relevant non- } \\
\text { major bleeding (n) }\end{array}$ & 5 & 13 \\
\hline
\end{tabular}


Conclusions: The CONKO-011 trial comparing real-life use of different LMWHs to rivaroxaban in CAT patients supports (i) an improved patient reported treatment satisfaction with rivaroxaban, particularly in reducing anticoagulation-related burden, resulting in less patient requested treatment stops and (ii) a balanced risk-benefit relation for rivaroxaban compared with $\mathrm{LMWH}$.

\section{LPB0042 | Characterization of Thrombotic Risk in Patients with}

\section{Cancer}

J. Trujillo Santos ${ }^{1}$; M. Salgado ${ }^{2}$; I. García Escobar ${ }^{3} ;$ R. Molina ${ }^{4}$; A. Araújo ${ }^{5}$; E. Horváth ${ }^{6}$; R. Porta ; J.C. Benítez ${ }^{8}$; M. Lobo $;$ E. Martí ${ }^{10}$; F. Garicano ${ }^{11}$; M. Malheiro ${ }^{12}$; A.L. Costa ${ }^{13}$; A.C. Albuquerque ${ }^{14}$; M.J. Ribeiro ${ }^{15}$; B. Campos Balea ${ }^{16}$; A. Cañada Martínez ${ }^{17}$; E. Brozos ${ }^{18}$; M. Masvidal ${ }^{19}$; P. Cerezuela ${ }^{20}$; CARTAGO study group ${ }^{1}$ Hospital General Universitario Santa Lucía, Cartagena, Spain; ${ }^{2}$ Complexo Hospitalario Universitario de Ourense, Ourense, Spain; ${ }^{3}$ Hospital Universitario Virgen de las Nieves, Granada, Spain; ${ }^{4} \mathrm{Hospital}$ Príncipe de Asturias, Alcalá de Henares, Spain; ${ }^{5}$ Centro Hospitalar Universitário do Porto, Porto, Portugal; ${ }^{6}$ Hospital Universitari Son Espases, Palma de Mallorca, Spain; ${ }^{7}$ Institut Català d'Oncologia, Girona, Spain; ${ }^{8}$ Hospital Universitari MútuaTerrassa, Terrassa, Spain; ${ }^{9}$ General University Hospital of Valencia, Valencia, Spain; ${ }^{10}$ Hospital Clínic Universitari de Valencia, Valencia, Spain; ${ }^{11}$ Hospital Universitario Galdakao, Galdakao, Spain; ${ }^{12}$ Hospital de São Francisco Xavier, Lisboa, Portugal; ${ }^{13}$ Hospital de Sta. Maria (CHLN), Lisboa, Portugal; ${ }^{14}$ Hospital de Setúbal, Setúbal, Portugal; ${ }^{15}$ Hospital São João, Porto, Portugal; ${ }^{16}$ Hospital Universitario Lucus Augusti, Lugo, Spain; ${ }^{17}$ Instituto de Investigación Sanitaria La Fe, Valencia, Spain; ${ }^{18}$ Complejo Hospitalario Universitario de Santiago, Santiago de Compostela, Spain; ${ }^{19}$ Hospital Universitari Sant Joan de Reus, Reus, Spain; ${ }^{20}$ Hospital Clínico Universitario Virgen de la Arrixaca, Murcia, Spain

Background: Venous thromboembolism (VTE) is a frequent complication of cancer. Most VTE events occur outside the hospital and the validated tool to predict them is the Khorana score. Despite being the leading tool to predict VTE in ambulatory cancer patients, given its limitations, it is unlikely to be incorporated into daily use.

Aims: To develop a new risk assessment model for VTE in patients undergoing anticancer treatment.

Methods: Ambulatory cancer patients from Spain and Portugal were included between February 2018 and December 2019 in a prospective, observational study. Patients with breast cancer on adjuvant chemotherapy; metastatic breast cancer treated only with hormone therapy; non-metastatic and metastatic hormone-sensitive prostate cancer, and patients receiving pharmacological VTE prophylaxis were excluded. We also explored the Khorana risk model in this population. Results: We present the 6-month descriptive data from 1781 patients. The main cancers were colorectal (31.6\%), lung (24.4\%), pancreatic (8.5\%), gastric (8.2\%), gynecological (3.7\%), and urothelial (3.1\%). The extension of cancer at the time of inclusion were localized (17.4\%), locally advanced (29.6\%), and metastatic (53.0\%).
The incidence of VTE and major or clinically relevant bleeding was 129 (7.2\%) and 38 (2.1\%) respectively. The most thrombogenic tumors were hepato-biliary (14.8\%), pancreatic (14.5\%), or urothelial (11.3\%). The most hemorrhagic tumors were gastric (3.5\%) and pancreatic (2.6\%). VTE was more common in patients with 1) metastatic or locally advanced, solid cancers ( $8.3 \%$ vs $3.8 \% ; P=0.007) ; 2$ ) venous compression by mass ( $14.8 \%$ vs $7.4 \%$; $P=0,038$ ); 3 ) reduced mobilization (14.7\% vs $1.9 \%$; $p<0.001)$; 4 ) disease progression (12.2\% vs $7.0 \%$; $P=0.01)$, and 5$)$ ECOG $\geq 1$ (9.9\% vs $4.3 \% P<0.001)$. Khorana score revealed scant capacity to predict VTE events (AUC 0.55).

Conclusions: VTE was frequent in this sample. Hemorrhagic risk was relevant in upper digestive tumors. Khorana score was not predictive of thrombosis in the Iberian population with cancer.

\section{LPB0089 | Outcome of Cancer-associated Venous} Thromboembolism Is More Favourable among Patients with Hematologic Malignancies than with Solid Tumors

R. Lecumberri ${ }^{1}$; P. Ruiz-Artacho ${ }^{2}$; I. Tzoran ${ }^{3}$; B. Brenner ${ }^{3}$; D. FargeBancel $^{4}$; V. Rosa ${ }^{5}$; I. Francisco ${ }^{6}$; L. Hernández-Blasco $;$; J. TrujilloSantos $^{8}$; M. Monreal ${ }^{9}$; RIETE Investigators ${ }^{1}$ University Clinic of Navarra. IdISNA. CIBERCV, Pamplona, Spain; ${ }^{2}$ University Clinic of Navarra. CIBERES, Madrid, Spain; ${ }^{3}$ Rambam - Health Care Campus, Haifa, Israel; ${ }^{4}$ Hôpital Saint-Louis, AP-HP, Université de Paris, Paris, France; ${ }^{5}$ Hospital Universitario Virgen de Arrixaca, Murcia, Spain; ${ }^{6}$ Hospital Universitari de Girona Dr. Josep Trueta, Girona, Spain; ${ }^{7}$ Hospital General Universitario de Alicante. ISABIAL, Alicante, Spain; ${ }^{8}$ Hospital General Universitario Santa Lucía. Universidad Católica de Murcia, Murcia, Spain; ${ }^{9}$ Hospital Germans Trias i Pujol. CIBERES, Badalona, Spain

Background: The incidence and prognosis of cancer-associated venous thrombosis (CAT) varies between different types of cancer. Current knowledge of specific outcomes among patients with hematologic malignancies (HM) is scarce, since these patients were poorly represented in pivotal clinical trials.

Aims: To evaluate the rates of recurrent venous thromboembolism ( $\mathrm{VVTE}$ ), major bleeding (MB) and death during anticoagulant therapy in patients with VTE associated to an HM vs solid tumors (ST).

Methods: Consecutive patients with an active cancer registered in the RIETE Registry until December 2020 were included. Baseline characteristics, treatments and outcomes were recorded. Univariate and multivariate competing-risk analysis were performed.

Results: 16,694 patients with CAT were included. 1,062 (6.4\%) suffered a HM. Hematologic patients presented less often with pulmonary embolism (48\% vs 63\%) and more frequently with upper-limb deep vein thrombosis (25 vs 18\%). Concomitant chemotherapy at the time of index VTE was more frequent among hematologic patients (67 vs $41 \%$ ), as were the proportion of patients with anaemia (66\% vs $61 \%)$ or with thrombocytopenia ( $<50 \times 10 \mathrm{e} 9 / \mathrm{L})(5.6 \%$ vs $0.7 \%)$. Low-molecular weight heparin was used as initial and longterm therapy in most patients, for a median duration of 150 and 127 
days, respectively. Compared to patients with ST, the rate ratios of events per 100 patient-years were: $0.73(95 \% \mathrm{Cl}, 0.56-0.95)$ for rVTE; $0.72(95 \% \mathrm{Cl}, 0.53-0.98)$ for $\mathrm{MB}$, and $0.49(95 \% \mathrm{Cl}, 0.41-0.57)$ for mortality (Table 1). All outcomes were particularly better in patients diagnosed with multiple myeloma. The multivariate analysis confirmed a lower incidence of rVTE or MB (HR 0.78; $95 \% \mathrm{Cl}, 0.64-$ 0.96 ), and a lower mortality (HR 0.53; $95 \% \mathrm{Cl}, 0.43-0.66$ ) among patients with HM.

TABLE 1 Outcomes during the course of anticoagulation

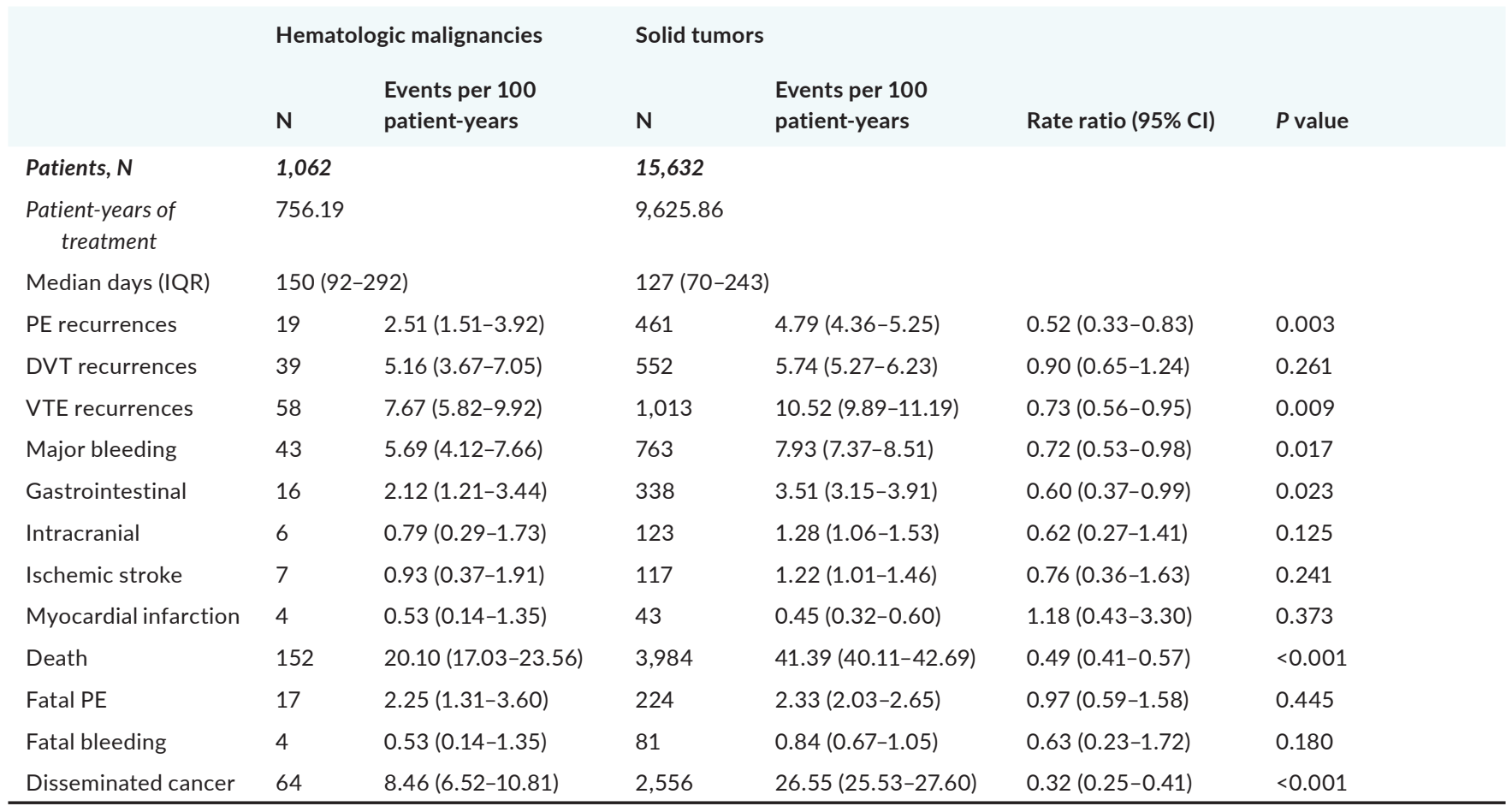

Conclusions: Patients with VTE associated with a HM, particularly multiple myeloma, have lower rates of rVTE, MB and death than patients with ST. This finding can be relevant for the interpretation of previous clinical trials and the design of future studies.

LPB0090 | Active Cancer and Venous Thromboembolism: Safety and Effectiveness of Edoxaban in Patients from the Noninterventional Global ETNA-VTE Program

A. Cohen ${ }^{1}$; M. Nakamura ${ }^{2}$; K.-M. Chiu ${ }^{3,4}$; W.-I. Choi ${ }^{5}$; P.-E. Reimitz ${ }^{6}$; W. Jiang ${ }^{7}$; C. Chen ${ }^{7}$; M. Unverdorben ${ }^{7}$; G. Agnelli ${ }^{8}$ ${ }^{1}$ Guy's and St Thomas' NHS Foundation Trust, King's College London, London, United Kingdom; ${ }^{2}$ Nakamura Medical Clinic, Department of Internal Medicine, Pediatrics and Cardiology, Kuwana, Japan; ${ }^{3} \mathrm{Far}$ Eastern Memorial Hospital, Cardiovascular Center, New Taipei City, Taiwan, Province of China; ${ }^{4}$ Yuan Ze University, Electrical Engineering, Taoyuan City, Taiwan, Province of China; ${ }^{5}$ Hanyang University Myongji Hospital, Department of Internal Medicine, Goyang-si, Korea, Republic of; ${ }^{6}$ Daiichi Sankyo Europe $\mathrm{GmbH}$, Clinical Operations and Biostatistics and Data Operations, Munich, Germany; ${ }^{7}$ Daiichi Sankyo, Inc., Basking Ridge, United States; ${ }^{8}$ University of Perugia, Internal and Cardiovascular Medicine-Stroke Unit, Perugia, Italy

Background: Active cancer is a major risk factor for recurrent venous thromboembolism (VTE) and major bleeding (MB). The direct oral anticoagulant edoxaban was noninferior to dalteparin for the composite endpoint of recurrent VTE or MB in the randomized Hokusai VTE Cancer trial. Data from real-world practice setting complement clinical trial results and can be of particular clinical relevance.

Aims: To investigate the safety and effectiveness of edoxaban in patients with active cancer and VTE in real world clinical practice Methods: The prospective, noninterventional global ETNA-VTE program enrolled from European and Asian countries unselected patients with acute, symptomatic VTE who were treated with edoxaban. Written informed consent and ethics committee approvals were obtained. Baseline and clinical event data were collected over a period of up to 12-months. Patients with active cancer were identified according to medical history and included in this analysis.

Results: Of 4,595 patients enrolled, 539 (11.7\%) had active cancer, most of whom (77\%) were from Japan. Baseline characteristics of patients with and without active cancer were similar, with a few exceptions: patients with active cancer had lower body weight, lower percentage of VTE history, higher percentage of bleeding history, and higher VTE-BLEED score (Table 1). The vast majority (89\%) of patients with active cancer received the recommended edoxaban dose according to prescribing information. At 1-year follow up, the annualized clinical event rate was $6.3 \%$ for recurrent VTE, $8.2 \%$ for ISTH MB (intracranial hemorrhage $0.6 \%$, major gastrointestinal bleeding 2.5\%). Malignancy-related deaths accounted for the majority of all-cause mortality (Table 2 ). 
TABLE 1 Baseline characteristics and medical history

$\begin{array}{llll} & \begin{array}{l}\text { All Patients } \\ (N=4,595)\end{array} & \begin{array}{l}\text { Patients with active cancer } \\ (n=539)\end{array} & \begin{array}{l}\text { Patients with no active cancer } \\ (n=4,056)\end{array} \\ \text { Age - yr, Mean (SD) } & 64.9(15.5) & 66.9(11.9) & 64.6(15.9) \\ \text { Male gender, } \mathrm{n}(\%) & 2,222(48.4) & 233(43.2) & 1,989(49.0) \\ \text { Weight - kg, Mean (SD) } & 72.8(19.2) & 61.8(15.1) & 74.3(19.2) \\ \text { Creatinine Clearance - mL/min, Mean (SD) } & 87.9(40.6) & 82.0(36.2) & 88.8(41.1) \\ \text { VTE-BLEED score, mean (SD) } & 1.8(1.5) & 3.9(1.3) & 1.6(1.3) \\ \text { HAS-BLED score*, mean (SD) } & 1.7(1.2) & 1.6(1.2) & 1.7(1.2) \\ \text { History of VTE } & 793(17.3) & 40(7.4) & 753(18.6) \\ \text { History of bleeding } & 207(4.5) & 47(8.7) & 160(3.9) \\ \text { History of major bleeding } & 96(2.1) & 18(3.3) & 78(1.9)\end{array}$

* Modified HAS-BLED score excluding labile INR

TABLE 2 Annualized rates of clinical events

Data shown as \%/year, [95\%
Recurrent VTE
PE with or without DVT
DVT only
Major bleeding (ISTH)
Intracranial hemorrhage
Major GI bleeding
All-cause death
Malignancy death
Cardiovascular death

Conclusions: In the real-world global ETNA-VTE program, patients with active cancer had higher VTE and bleeding event rates than those without. Edoxaban demonstrated a safety and effectiveness profile in patients with VTE and active cancer that is consistent with the findings from previous randomized controlled trial.

LPB0137 | Validation of the HULL Clinical Prognostic Rule (CPR) for Incidental Pulmonary Embolism in Ambulatory Cancer Patients

F. Haque ${ }^{1,2}$; G. Bozas ${ }^{1}$; C. Huang ${ }^{2}$; A. Pillai ${ }^{1}$; S. Mirza ${ }^{1}$; J. Ryde ${ }^{1}$; S. Sethi ${ }^{1}$; M. Kolodziej ${ }^{1}$; A. Stephens ${ }^{1}$; S. Raper ${ }^{1}$; E. Gollins ${ }^{1}$; G. Avery ${ }^{1,2} ;$ A. Maraveyas ${ }^{1,2}$

${ }^{1}$ Hull University Teaching Hospital NHS Trust, Hull, United Kingdom;

${ }^{2}$ Hull York Medical School, Hull, United Kingdom

Background: Risk stratification clinical prognostic rules (CPRs) commonly used to predict adverse outcomes of suspected pulmonary embolism (PE) have limitations in discriminating outcomes for ambulatory cancer patients with incidental PE (IPE). The HULL score, derived from the prospective cohort of 234 ambulatory cancer patients from 2010-2014, uses a 5-point scoring system incorporating performance status and self-reported new or recently evolving

$\begin{array}{ll}\begin{array}{l}\text { Patients with active cancer } \\ (n=539)\end{array} & \begin{array}{l}\text { Patients with no active cancer } \\ (n=4,056)\end{array} \\ 6.33[3.87 ; 9.77] & 2.79[2.26 ; 3.41] \\ 2.81[1.29 ; 5.34] & 1.04[0.73 ; 1.44] \\ 4.39[2.40 ; 7.36] & 1.76[1.35 ; 2.27] \\ 8.23[5.37 ; 12.06] & 1.91[1.48 ; 2.43] \\ 0.62[0.08 ; 2.24] & 0.58[0.35 ; 0.89] \\ 2.48[1.07 ; 4.90] & 0.49[0.28 ; 0.78] \\ 31.89[26.03 ; 38.67] & 2.67[2.15 ; 3.27] \\ 25.08[19.92 ; 31.17] & 0.52[0.31 ; 0.82] \\ 2.79[1.27 ; 5.29] & 0.92[0.63 ; 1.30]\end{array}$

symptoms at IPE diagnosis (1). It stratifies patients into low, intermediate and high risk for adverse outcomes at 30,90 and 180 days. Aims: To validate the HULL CPR in a prospective cohort of ambulatory cancer patients with IPE derived from the same clinical setting. Methods: 284 consecutive patients managed under the IPE-acute oncology service in HUTH NHS trust from January 2015 to March 2020 were included. Primary outcome measures were 30-day, 90day, 180-day mortality and overall survival (OS) for the three risk categories. Other parameters studied were hospitalization within 30-days post IPE diagnosis, recurrent venous thromboembolism (VTE) and major bleeding. 
Results:

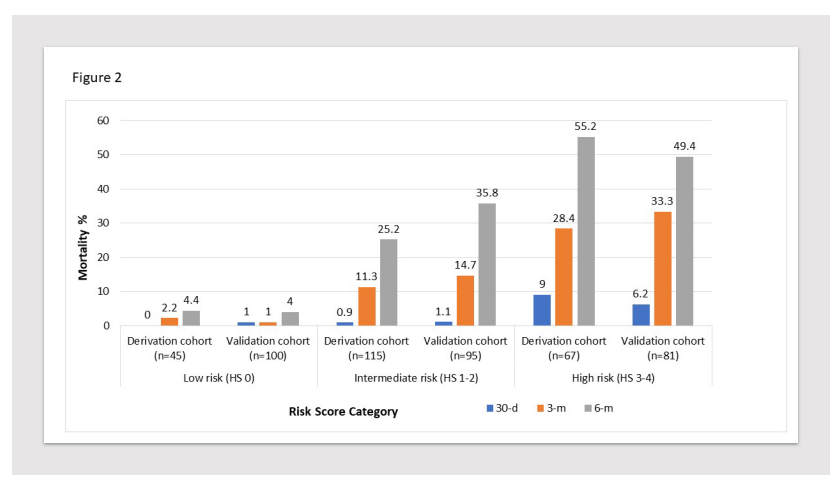

FIGURE 1 Survival curves for the Hull score groups for the first 12 months of follow-up for validation cohort. Line separators for the 30-day, 90-day, 180-day cut-offs and the median for survival are included

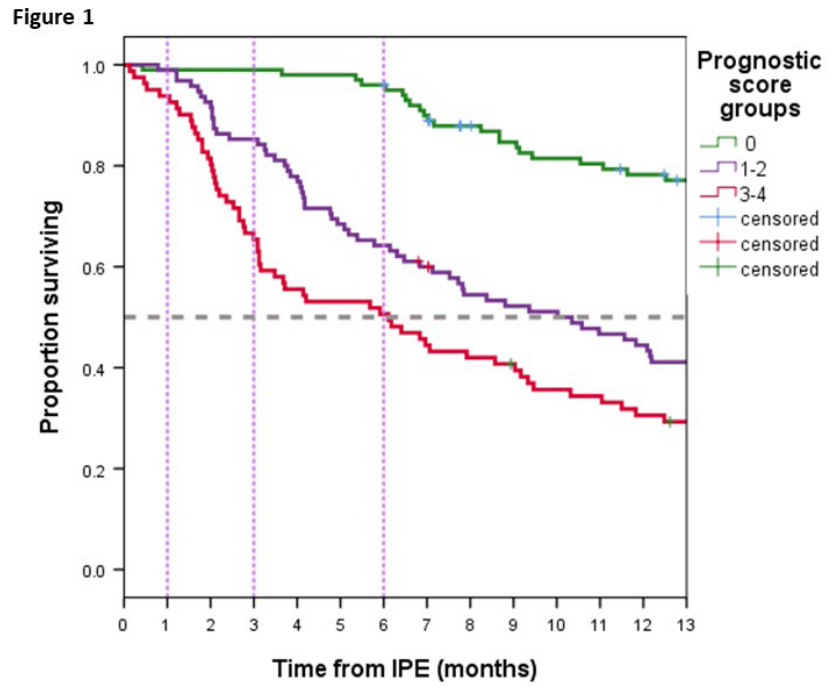

FIGURE 2 Mortality by HULL CPR score in derivation (2010-2014) and validation (2015-2020) cohorts

30-day, 90-day and 180-day mortality for the whole cohort was $3.4 \%$ $(n=7), 21.1 \%(n=43)$ and $39.2 \%(n=80)$ respectively. Recurrence occurred in $10.6 \%$ (30), major bleeding in $3.9 \%$ (11); $12.8 \%$ (36) hospitalized within 30 days post IPE. The HULL score stratified them into low 35.5\% (100), intermediate 33.7\% (95) and high $28.7 \%$ (81) risk groups (Figure 1). Correlation of the risk categories with 30-day, 90-day,180-day mortality and OS was consistent with the derivation cohort (area under curve [AUC] 0.72 [95\% Cl 0.52, 0.91], AUC 0.77 [95\% Cl 0.70, 0.84], AUC 0.75 [95\% Cl 0.69, 0.81] respectively, $p<0.001$ for OS). (Figure 2).

Conclusions: This study validates the capacity of the HULL score to stratify mortality risk in ambulatory cancer patients with IPE. It is composed of practical oncological parameters and can guide the outpatient management of IPE in an acute oncology setting.
LPB0138 | Results of a Prospective, Observational, Multicenter Cohort Study of Venous Thromboembolism Outcomes in Thrombocytopenic Cancer Patients (TROVE Study)

B. Carney ${ }^{1}$; T.-F. Wang ${ }^{2}$; S. Ren ${ }^{3}$; G. George ${ }^{4}$; A. Al Homssi ${ }^{5}$; M. Gaddh ${ }^{6}$; G. Connolly ; V. Shah ${ }^{8}$; T. Bogue ${ }^{1}$; A. Bartosic ${ }^{9}$; D. Neuberg ${ }^{3}$; L. Baumann Kreuziger ${ }^{10}$; J. Zwicker ${ }^{1}$ ${ }^{1}$ Beth Israel Deaconess Medical Center, Boston, United States; ${ }^{2}$ University of Ottawa at The Ottawa Hospital and Ottawa Hospital Research Institute, Ottawa, Canada; ${ }^{3}$ Dana-Farber Cancer Institute, Boston, United States; ${ }^{4}$ University of Colorado, Aurora, United States; ${ }^{5}$ Medical College of Wisconsin, Milwaukee, United States; ${ }^{6}$ Emory University School of Medicine, Atlanta, United States; ${ }^{7}$ Lipson Cancer Institute and Rochester Regional Health, Rochester, United States; ${ }^{8}$ Henry Ford Health System, Detroit, United States; ${ }^{9}$ The Ohio State University Wexner Medical Center, Columbus, United States; ${ }^{10}$ Versiti Blood Research Institute, Milwaukee, United States

Background: Venous thromboembolism (VTE) is a frequent complication of cancer. Thrombocytopenia is common in cancer, either due to the disease itself or a side effect of therapy. Retrospective studies have evaluated either full-dose anticoagulation with transfusion support or dose-modified anticoagulation. We performed a prospective multicenter observational study to assess bleeding and thrombosis outcomes in cancer patients with thrombocytopenia diagnosed with acute VTE.

Aims: To determine the cumulative incidence of recurrent VTE and hemorrhage in patients with cancer who developed acute VTE in the setting of thrombocytopenia according to anticoagulation regimen administered (full-dose with platelet transfusion support versus dose-modified anticoagulation). Enoxaparin was the only low molecular weight heparin used, and full dose was defined as $\geq 1.5$ $\mathrm{mg} / \mathrm{kg} /$ day.

Methods: A prospective, multi-center observational cohort study was performed in patients with active malignancy with acute VTE and concurrent thrombocytopenia (platelet count $<100,000 / \mathrm{mcL}$ ). Patients were monitored for 60 days for the development of recurrent VTE and hemorrhage. Major hemorrhage was defined by ISTH criteria. All clinical outcomes were adjudicated by two physicians. Results:

TABLE 1 Baseline Demographics

\begin{tabular}{|l|c|c|c|}
\hline Characteristic & $\begin{array}{c}\text { Full Dose } \\
\mathbf{n}=75\end{array}$ & $\begin{array}{c}\text { Modified Dose } \\
\mathbf{n}=33\end{array}$ & $\begin{array}{c}\text { None } \\
\mathbf{n}=13\end{array}$ \\
\hline Male, $\mathbf{n}(\%)$ & $39(52)$ & $16(48)$ & $10(77)$ \\
\hline Mean age at enrollment (range) & $59(23-88)$ & $60(26-78)$ & $65(42-78)$ \\
\hline Inpatient, $\mathbf{n}(\%)$ & $70(93)$ & $30(91)$ & $11(85)$ \\
\hline Cancer diagnosis, $\mathbf{n}$ (\%) & & & \\
\hline Hematologic malignancy & $43(57)$ & $31(94)$ & $11(85)$ \\
\hline Solid tumor & $32(43)$ & $2(6)$ & $2(15)$ \\
\hline Index VTE Event & & & \\
\hline Lower extremity DVT & 27 & 11 & 4 \\
\hline Upper extremity DVT & 23 & 17 & 8 \\
\hline Pulmonary embolism & 36 & 7 & 2 \\
\hline Other thrombus & 4 & 0 & 0 \\
\hline Anticoagulant & & & 0 \\
\hline Low molecular weight heparin & 40 & 26 & 0 \\
\hline Unfractionated heparin & 23 & 3 & 0 \\
\hline Direct oral anticoagulant & 12 & 4 & \\
\hline
\end{tabular}


A total of 121 patients were enrolled (Table). Baseline characteristics differed in some aspects, e.g. cancer diagnosis. In patients who initially received therapeutic anticoagulation, the cumulative incidence of major hemorrhage at 30 days was $6.7 \%(95 \% \mathrm{Cl}, 0.01-12.4 \%)$ and $0 \%$ in the dose-modified anticoagulation group (sHR $2.18,95 \% \mathrm{Cl}$ 1.21-3.93). The cumulative incidence of recurrent VTE at 30 days in patients who initially received full-dose anticoagulation was $4.1 \%$ $(95 \% \mathrm{Cl}, 0 \mathrm{8.8 \%})$ and $0 \%$ in patients who initially received dosemodified anticoagulation.

Conclusions: In patients with cancer who develop VTE in the setting of thrombocytopenia, dose-modified anticoagulation was associated with a lower rate of major hemorrhage than full-dose anticoagulation. Dose-modified anticoagulation was not associated with an increased rate of recurrent VTE.

PB1089 | The Role of Primary Thromboprophylaxis in People with Cancer: A Systematic Review and Meta-analysis

F.A. Dewar ${ }^{1}$; K.M. Musgrave ${ }^{1}$; J. Simpson ${ }^{1}$; J. Hanley ${ }^{2}$

${ }^{1}$ Translational and Clinical Research Institute, Newcastle University, Newcastle upon Tyne, United Kingdom; ${ }^{2}$ Newcastle Upon Tyne Hospitals NHS Trust, Newcastle upon Tyne, United Kingdom

Background: Patients with cancer receiving chemotherapy are at an increased risk of venous thromboembolism (VTE)(Khorana,2005). In the United Kingdom, the use of primary thromboprophylaxis is not recommended in ambulatory patients with cancer (NICE,2018). Recent data evaluating the directly acting oral anticoagulants for primary prophylaxis of cancer associated thrombosis (CAT) has emerged. There is a requirement to include this within a review.

Aims: This systematic review and meta-analysis examined the efficacy and safety of primary thromboprophylaxis in ambulatory patients with cancer receiving chemotherapy. Hypothesising that primary thromboprophylaxis is associated with a reduction in VTE occurrence and does not increase rates of major bleeding.

\section{Methods:}

Ovid's Medline (R), Embase, Clinicaltrials.gov, Cochrane's CENTRAL database and the ISCRTN registry were searched using relevant terms, and without restrictions, up to the 2 nd June 2020. Randomised control trials meeting pre-defined inclusion criteria were selected (see figure 1). VTE and major bleeding outcome data were extracted alongside methodological and participant characteristics.

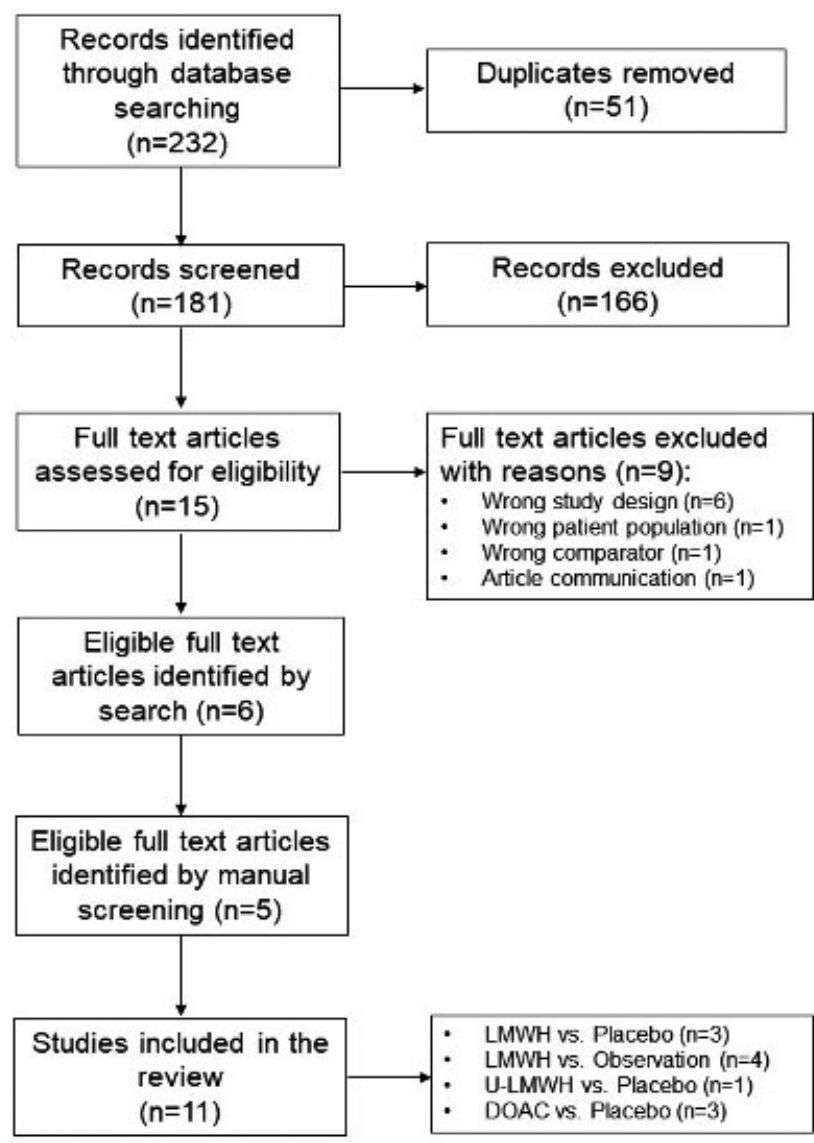

FIGURE 1 A PRSIMA flow diagram detailing the study selection process with reasons for the exclusion of studies given at each stage. Meeting one exclusion criterion excluded a study and the first criterion met was recorded for the reason for exclusion

Results: Eleven randomised control trials (7375 participants) were included. VTE occurred in $1.94 \%$ of participants receiving thromboprophylaxis and $5.71 \%$ of controls [OR 0.39 95\% Cl 0.27, 0.56 I2 41\% $P=<0.00001$ ] (see figure 2), NNT $=27$. The enrolment of high risk participants was not associated with an increased treatment effect. Major bleeding events occurred in $1.89 \%$ of participants receiving thromboprophylaxis and $1.39 \%$ of controls [OR $1.3995 \% \mathrm{Cl} 0.96$, $2.04120 \% P=0.08$ ] (see figure 2). There was no significant alteration in the probability of major bleeding when studies were grouped by degree of bias, anticoagulant agent or risk score.
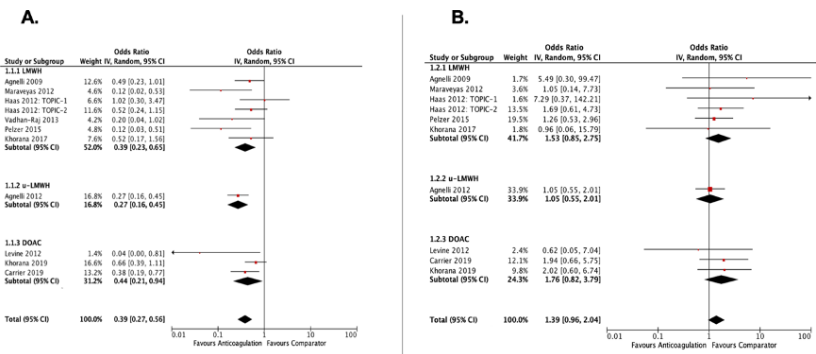
Figure 2 Forest plots for the two major outcomes of the review. Inset (a) represents the primary efficacy outcome of VTE occurrence, the overall effect estimate shows a reduction in the odds of VTE for those participants who received thromboprophylaxis (OR 0.39 [95\% $\mathrm{Cl} 0.27,0.56])$. Inset (b) represents the primary safety outcome of major bleeding, the overall effect estimate shows a non-significant increase in the odds of major bleeding with confidence intervals spanning one (OR 1.39 [95\% Cl 0.96, 2.04])

Conclusions: Primary thromboprophylaxis decreased venous thromboembolic events in patients with cancer receiving chemotherapy, and was not significantly associated with an increase in major bleeding. The inclusion of DOAC data within the review lowered the number needed to treat (Di Nisio,2016), indicating these agents are a viable option for CAT prophylaxis.

PB1090 | Plasma Biomarkers for Predicting Risk of Venous Thromboembolism (VTE) in Ambulatory Cancer Patients Receiving Chemotherapy

D. Roy ${ }^{1}$; T.F. Wang ${ }^{2,3}$; M. Carrier ${ }^{2,3}$; E. Mollanji ${ }^{2,3}$; P. Liu ${ }^{4}$; P. Wells ${ }^{2,3}$

${ }^{1}$ School of Epidemiology and Public Health, University of Ottawa,

Ottawa, Canada; ${ }^{2}$ Department of Medicine, University of Ottawa,

Ottawa, Canada; ${ }^{3}$ Ottawa Hospital Research Institute, Ottawa,

Canada; ${ }^{4}$ University of Ottawa Heart Institute, Ottawa, Canada
Background: Growth Differentiation Factor 15 (GDF-15), N-terminal pro-B-type natriuretic peptide (NT-proBNP) and high-sensitivity cardiac Troponin T (hs-TnT) are associated with an increased risk of VTE in non-cancer patients, however, the performance of these biomarkers in cancer patients is unknown.

Aims: To assess the performance of GDF-15, NT-proBNP and hs-TnT in predicting VTE in patients with cancer.

Methods: A post-hoc analysis using 1-month plasma samples from patients enrolled in the AVERT trial (a randomized, placebo-controlled, double-blind trial to assess the efficacy and safety of apixaban as primary thromboprophylaxis in ambulatory cancer patients with intermediate to high risk for VTE) to determine if levels of GDF-15, NT-proBNP and hs-TnT are associated with VTE. Logistic regression analysis was used to calculate adjusted odds ratios (OR) across tertiles of these biomarkers. A first model was constructed for all cancer patients, a second excluding patients with brain cancer (justified by lower GDF-15 and NT-proBNP, and the potential influence of the blood-brain barrier), a third for gynecologic cancer. All models were adjusted for age, sex, treatment group, and concomitant antiplatelet therapy [Table 1].

Results: 477 patients were analysed. In Model 1, 2 and 3, participants with highest tertile GDF-levels had adjusted ORs for VTE of 1.66 ( $P=0.199), 3.16(P=0.002)$, and 4.27( $P=0.010)$ versus the lowest tertile, respectively [Table 2]. For NT-proBNP, levels in the moderate tertiles had a significant association with VTE for patients with all cancers and non-brain cancers [adjusted ORs: $1.84(P=0.011)$ and 3.28( $P=0.033)$, respectively]. Hs-TnT levels in the moderate tertiles was associated with a decreased risk of VTE compared to the highest tertile as reference in the all cancer and non-brain cancer models.

TABLE 1 Descriptive Statistics According to Venous Thromboembolism Events

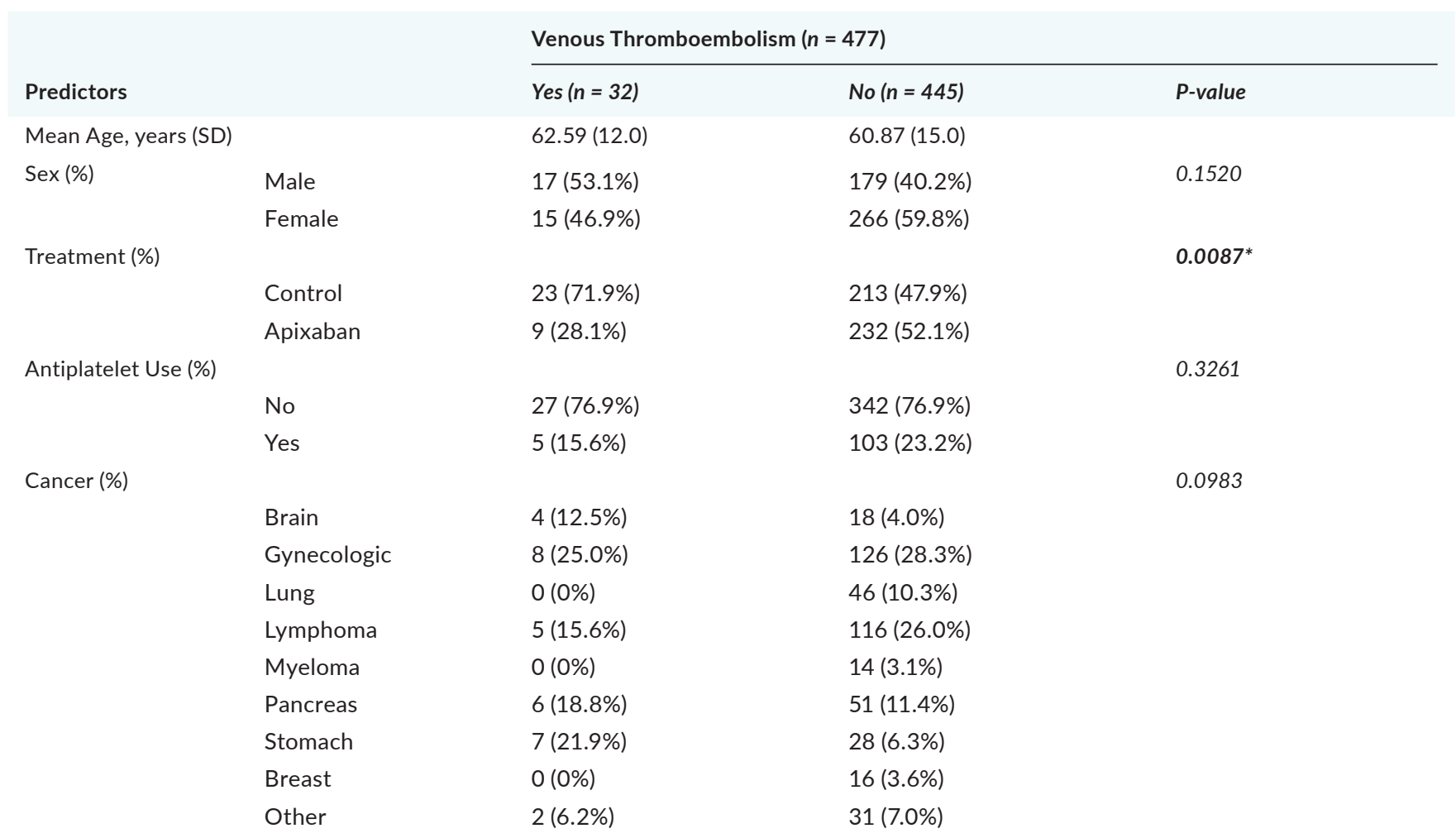


Venous Thromboembolism $(n=477)$

Predictors

Median GDF-15, pg/mL (IQR)

Median NT-ProBNP, pg/mL (IQR)

Median hs-TnT, pg/mL (IQR)

\begin{tabular}{lll}
\hline Yes $(n=32)$ & No $(n=445)$ & $P$-value \\
$2352(2726)$ & $1910(2064)$ & 0.2036 \\
$175(179)$ & $111.80(182.33)$ & 0.1045 \\
$8.54(13.08)$ & $6.10(7.28)$ & 0.0661
\end{tabular}

TABLE 2 Logistic Regression Models Looking at Associations between Biomarkers and VTE

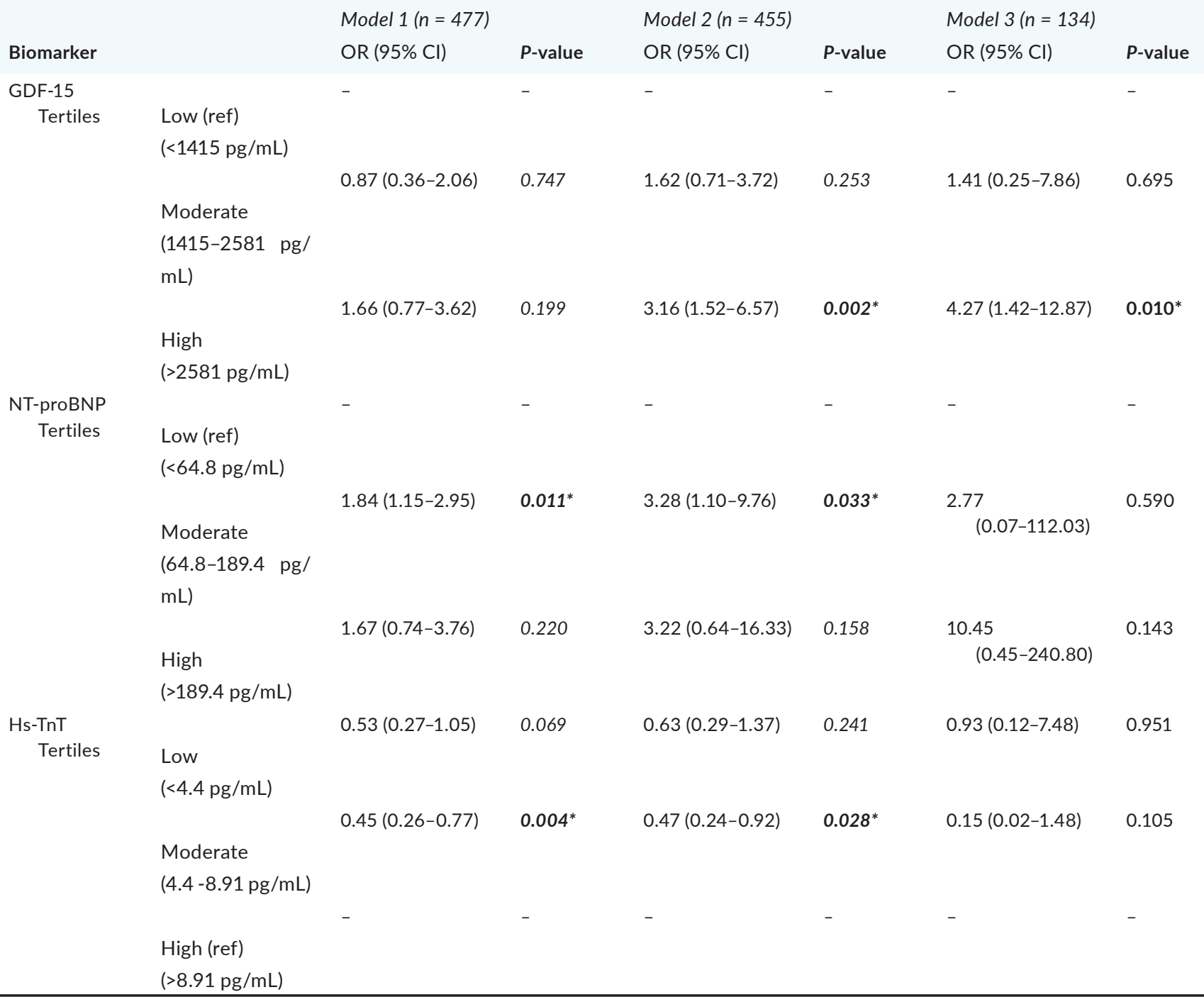

Conclusions: In this first study to evaluate the predictive performance of GDF-15, proBNP and hs-TnT for VTE in patients with cancer, higher tertile GDF-15 and NT-proBNP predicted increased VTE risk whereas higher hs-TnT predicted decreased VTE risk. 
PB1091 | Characteristics and Outcomes of Patients on Concurrent Direct Oral Anticoagulants and Targeted Anticancer Therapies - TacDOAC Registry

T.-F. Wang ${ }^{1}$; L. Baumann Kreuziger ${ }^{2}$; A. Leader ${ }^{3,4}$; G. Spectre ${ }^{3,4}$; M. Lim5; A. Gahagan'; R. Gangaraju'; K. Sanfilippo7;

R. Mallick ${ }^{8}$; J. Zwicker ${ }^{9}$; M. Carrier ${ }^{1}$

${ }^{1}$ University of Ottawa at The Ottawa Hospital and Ottawa Hospital Research Institute, Ottawa, Canada; ${ }^{2}$ Blood Research Institute, Versiti, Department of Medicine Medical College of Wisconsin, Milwaukee, United States; ${ }^{3}$ Institute of Hematology, Davidoff Cancer Center, Rabin Medical Center, Petah Tikva, Israel; ${ }^{4}$ Sackler School of Medicine, Tel Aviv University, Tel Aviv, Israel; ${ }^{5}$ Division of Hematology and Hematologic Malignancies, Department of Internal Medicine, University of Utah, Salt Lake City, United States; ${ }^{6}$ Division of Hematology/Oncology, Department of Medicine, University of Alabama at Birmingham, Birmingham, United States; ${ }^{7}$ Division of Hematology, Washington University School of Medicine, Saint Louis, United States; ${ }^{8}$ Clinical Epidemiology Program, Ottawa Hospital Research Institute, Ottawa, Canada; ${ }^{9}$ Division of Hemostasis and Thrombosis, Beth Israel Deaconess Medical Center and Harvard Medical School, Boston, United States

Background: Cancer patients are increasingly prescribed direct oral anticoagulants (DOACs) and targeted anticancer therapies, but limited data are available on the outcomes during concurrent use.
Aims: We conducted an international registry through the SSC of ISTH to evaluate bleeding and thrombotic outcomes in patients receiving concurrent DOACs and targeted anticancer therapies.

Methods: Patients receiving concurrent DOACs and selected targeted anticancer therapies were included (mostly retrospectively) and followed for 6 months after the start of concurrent use. Data including patient and cancer characteristics, major bleeding, nonmajor bleeding events, venous or arterial thromboses were collected and analyzed. The primary outcome was major bleeding by ISTH criteria. Analyses considered death as a competing risk. Local ethics committee approved the study.

Results: 202 patients were included from six institutions in the United States and Israel: 134 with venous thromboembolism (VTE) and 73 with atrial fibrillation. The most common malignancies were hematologic ( $N=57,28.2 \%)$, followed by breast $(N=50,24.8 \%)$ and lung $(N=44,21.8 \%)$. The most common anticancer therapies were EGFR and ALK inhibitors ( $N=43,21.3 \%$ ), followed by BTK inhibitors ( $N=42,20.8 \%)$ and palbociclib $(N=42,20.8 \%)$. During the 6-month follow up, there were 9 major bleeding and 12 non-major bleeding events, corresponding to cumulative incidences of $4 \%$ (95\% confidence interval [Cl]: $2-8 \%)$ and $6 \%(95 \% \mathrm{Cl}: 3-10 \%)$, respectively. The cumulative incidence of major bleeding events was highest in BTK inhibitor users (10\%), followed by VEGF inhibitor users (7\%) (Table and Figure). There were 3 VTE and 2 arterial thromboses, corresponding to cumulative incidences of $1.5 \%$ (95\% Cl: $0.4-4.0 \%)$ and 1.0\% (95\% Cl: 0.2-3.3\%), respectively.

TABLE 1 Outcomes by anticancer therapies

\begin{tabular}{|c|c|c|c|c|c|c|c|c|c|}
\hline \multirow{2}{*}{$\begin{array}{l}\text { Anticancer therapies (one } \\
\text { patient was on palbociclib } \\
\text { and everolimus at different } \\
\text { time points) }\end{array}$} & \multirow[b]{2}{*}{$N=202(\%)$} & \multicolumn{2}{|c|}{ Major bleeding } & \multicolumn{2}{|c|}{ Non-major bleeding } & \multicolumn{2}{|c|}{ Venous thromboembolism } & \multicolumn{2}{|c|}{ Arterial thrombosis } \\
\hline & & $N(\%)$ & $\begin{array}{l}\text { Cumulative } \\
\text { incidence } \\
\%,(95 \% \mathrm{Cl})\end{array}$ & $N(\%)$ & $\begin{array}{l}\text { Cumulative } \\
\text { incidence } \\
\%,(95 \% \mathrm{Cl})\end{array}$ & $N(\%)$ & $\begin{array}{l}\text { Cumulative } \\
\text { incidence \%, } \\
(95 \% \mathrm{Cl})\end{array}$ & $N(\%)$ & $\begin{array}{l}\text { Cumulative } \\
\text { incidence \%, } \\
(95 \% \mathrm{Cl})\end{array}$ \\
\hline $\begin{array}{l}\text { EGFR and ALK inhibitors } \\
\text { (osimertinib, } \\
\text { alectinib) }\end{array}$ & $43(21.3 \%)$ & $1 / 43(2.3 \%)$ & $2(0.2-11)$ & $1 / 43(2.3 \%)$ & $2(0.2-11)$ & 0 & & $\begin{array}{l}1 / 43 \\
(2.3 \%)\end{array}$ & $2(0.2-11)$ \\
\hline & $42(20.8 \%)$ & $4 / 42(9.5 \%)$ & $10(3-21)$ & $6 / 42(14.3 \%)$ & $14(6-27)$ & 0 & & 0 & \\
\hline $\begin{array}{l}\text { BTK inhibitors (ibrutinib, } \\
\text { acalbrutinib) }\end{array}$ & & & & & & & & & \\
\hline $\begin{array}{l}\text { Cyclin-dependent kinase } \\
\text { inhibitor (palbociclib) }\end{array}$ & $42(20.8 \%)$ & $2 / 42(4.8 \%)$ & $5(1-14)$ & $3 / 42(7.1 \%)$ & $7(2-18)$ & 0 & & 0 & \\
\hline $\begin{array}{l}\text { VEGF inhibitors (sunitinib, } \\
\text { cabozantinib) }\end{array}$ & $28(13.9 \%)$ & $2 / 28(7.1 \%)$ & $7(1-21)$ & $1 / 28(3.6 \%)$ & $4(0.2-16)$ & $2 / 28(7.1 \%)$ & $7(1-21)$ & 0 & \\
\hline $\begin{array}{l}\text { BCR-ABL inhibitors (imatinib, } \\
\text { nilotinib) }\end{array}$ & 22 (10.9\%) & 0 & & 0 & & 0 & & $1 / 22(4.6 \%)$ & $5(0.3-19)$ \\
\hline mTOR inhibitor (everolimus) & $18(8.9 \%)$ & 0 & & $1 / 18(5.6 \%)$ & $6(0.3-23)$ & 0 & & 0 & \\
\hline HER2 inhibitors (lapatinib) & $4(2.0 \%)$ & 0 & & 0 & & 0 & & 0 & \\
\hline $\begin{array}{l}\text { BRAF inhibitors (dabrafenib, } \\
\text { vemurafenib) }\end{array}$ & $4(2.0 \%)$ & 0 & & 0 & & $1 / 4(25 \%)$ & $25(0.3-71)$ & 0 & \\
\hline
\end{tabular}


Major Bleed and Therapy Type

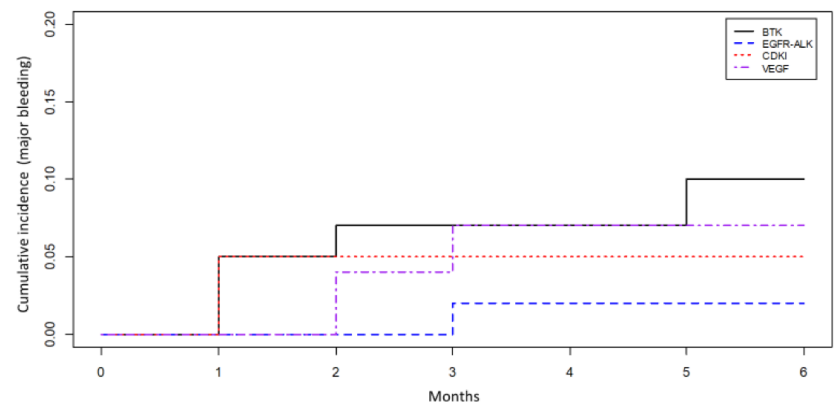

FIGURE 1 Cumulative incidence of major bleeding events by types of anticancer therapies

Conclusions: In this cohort receiving concurrent DOACs and targeted anticancer therapies, the incidence of bleeding is higher compared to thrombosis, particularly with selective anticancer therapies such as BTK inhibitors. Future larger prospective studies are needed for better assessment to improve care for these patients.

PB1092 | Management of Cancer Associated Thrombosis in France: A National Survey in Vascular Disease and Supportive Care Specialists

I. Mahé ${ }^{1}$; C. Chapelle ${ }^{2}$; S. Laporte ${ }^{3}$; L. Bertoletti ${ }^{4}$; P. Mismetti ${ }^{4}$; G. Meyer ${ }^{5}$; D. Mayeur ${ }^{6}$; G. Mahé7; F. Couturaud ${ }^{8}$

${ }^{1}$ Université de Paris, APHP, Inserm UMR_S1140, F-CRIN INNOVTE, Colombes, France; ${ }^{2}$ SAINBIOSE INSERM U1059, Université Jean Monnet, Université de Lyon, Unité de Recherche Clinique, Innovation, Pharmacologie, Saint Etienne, France; ${ }^{3}$ SAINBIOSE INSERM U1059, Université Jean Monnet, Université de Lyon, Unité de Recherche Clinique, Innovation, Pharmacologie, F-CRIN INNOVTE, Saint-Etienne, France; ${ }^{4}$ Service de Médecine Vasculaire et Thérapeutique, $\mathrm{CHU}$ de St-Etienne, INSERM, UMR1059, Université Jean-Monnet, ; INSERM, CIC-1408, F-CRIN INNOVTE, Saint-Etienne, France; ${ }^{5}$ Université de Paris, APHP, F-CRIN INNOVTE, Paris, France; ${ }^{6}$ AFSOS (Association Francophone des Soins Oncologiques de Support), Oncologie médicale, Centre Georges Francois Leclerc, Dijon, France; ${ }^{7}$ SFMV (Société Française de Médecine Vasculaire), Unité de Médecine Vasculaire, CHU de Rennes, Rennes, France; ${ }^{8}$ F-CRIN INNOVTE, Hôpital de la Cavale Blanche CHRU de Brest, CIC INSERM 1412; EA 3878 (GETBO), CHU de Brest, Brest, France

Background: For many years, low molecular weight heparins (LMWHs) were recommended by international guidelines for at least 3 to 6 months in patients with cancer associated thromboembolism (CAT). Following clinical trials assessing direct oral anticoagulants (DOACs), DOACs have been proposed as alternative to low-molecular-weight heparins (LMWHs) by many guidelines. In clinical practice, specialists in charge of CAT management, have to decide which anticoagulant to prescribe.

Aims: To describe the therapeutic management proposed for patients diagnosed with CAT according to medical specialties in France.
Methods: An electronic survey tool including vignettes and questions about management of patients with CAT was sent to members of the Société Française de Médecine Vasculaire (SFMV), Association Francophone pour les Soins Oncologiques de Support (AFSOS) and INNOVTE network.

Results: Among the 414 specialists who answered the survey, 376 were in charge of patients with CAT : 40 cancer-related specialists (10.6\%) and 336 vascular-related specialists (89.4\%).LMWHs were indicated as the first choice by most of the specialists for the initial treatment of symptomatic CAT. The prescription of DOACs within the first 3 weeks of CAT diagnosis was highly dependent of cancer's site : $5.9 \%, 18.6 \%$ and $24.5 \%$ in patients with locally advanced colorectal, lung and breast cancer respectively; $76.9 \%$ chose the same therapeutic option regardless of primary tumor's site. The determinants for the choice of treatment were mostly related to cancer (site and stage or evolution) and to anticancer treatments (Table). For $61 \%$ of physicians, some anticancer treatments were contra-indications to DOACs, mainly due to hemorrhagic risk. However, almost $90 \%$ of physicians consider switching to DOAC after a median 3-months of LMWHs therapy.

\begin{tabular}{|c|c|c|c|}
\hline × & $\begin{array}{l}\text { Cancer } \\
\text { specialists } \\
(\mathrm{N}=-40) \mathrm{d}\end{array}$ & $\begin{array}{l}\text { Vascular- } \\
\text { specialists } \\
(\mathrm{N}==-336) \not\end{array}$ & $\begin{array}{c}\text { All } \\
(\mathrm{N}==376) \mathrm{x}\end{array}$ \\
\hline 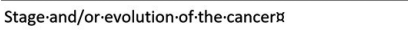 & $19 \cdot(47.5 \%) \not$ & $130 \cdot(38.7 \%)$ म & $149 \cdot(39.6 \%) \not$ \\
\hline Site of cancery & $13 \cdot(32.5 \%) \not \mathbf{~}$ & $126 \cdot(37.5 \%)$ घ & $139 \cdot(37.0 \%) \not$ \\
\hline Patients'comorbidities/additional-risk·factors $\not$ & $15 \cdot(37.5 \%)$ म & $109 \cdot(32.4 \%)$ ) & $124 \cdot(33.0 \%) \bar{x}$ \\
\hline Risk.of.drug.interaction & $16 \cdot(40.0 \%) \not 8$ & $103 \cdot(30.7 \%) \not$ & $119 \cdot(31.6 \%)$ \\
\hline Anticancer-treatment. $\$$ & $8 \cdot(20.0 \%) \bar{R}$ & $56 \cdot(16.7 \%) \times$ & $64 \cdot(17.0 \%) \square$ \\
\hline Index event.typex & $2 \cdot(5.0 \%)$ ㅁ & $55 \cdot(16.4 \%) x$ & $57 \cdot(15.2 \%)$ घ \\
\hline Patient-preference & $0 \cdot(0.0 \%) \times$ & $40 \cdot(11.9 \%) \times$ & $40 \cdot(10.6 \%)$ \\
\hline $\begin{array}{l}\text { Some-anticancer-treatment-are-contra-indications.to- } \\
\text { DOACs } x\end{array}$ & $26 \cdot(65.0 \%)$ 口 & $204 \cdot(60.7 \%)$ 叫 & $230 \cdot(61.2 \%) \times$ \\
\hline Main-reasons & a & x & $x$ \\
\hline Hemorrhagic.riskar & $21 \cdot(80.8 \%) \not$ & $120 \cdot(58.8 \%)$ म & $141 \cdot(61.3 \%) x$ \\
\hline Thromboembolic.riskra & $0 .(0.0 \%) \mathrm{x}$ & $53 \cdot(26.0 \%)$ म & $53 .(23.0 \%)$ म \\
\hline $\begin{array}{l}\text { Toxicity.of.antitumor.treatmenta } \\
\text { O } \\
\text { Ineffectiveness. of } \cdot \text { antitumor.treatmentr }\end{array}$ & $\begin{array}{l}2 \cdot(7.7 \%) \ltimes \\
3 \cdot(11.5 \%) \ltimes\end{array}$ & $\begin{array}{c}23 \cdot(11.3 \%) \not x \\
8 \cdot(3.9 \%) \not x\end{array}$ & $\begin{array}{l}25 \cdot(10.9 \%) \not x \\
11 \cdot(4.8 \%) \times\end{array}$ \\
\hline
\end{tabular}

FIGURE 1 Determinants of the decision for initial anticoagulant treatment of CAT according to the medical specialty

Conclusions: LMWHs and DOACs are now considered by specialists of CAT, both in vascular and oncologic diseases, depending of the site of cancer. The role of anticancer treatments in the decision remains to be further investigated. 
PB1093 | Prevalence, Treatment and Prognosis of Tumor Thrombi in Renal Cell Carcinoma

F.H.J. Kaptein ${ }^{1}$; S.J.E. Braken ${ }^{1}$; E.M.E. du Chatinier ${ }^{1}$;

M.C. Burgmans ${ }^{2}$; J.T. Buijs ${ }^{1}$; S.C. Cannegieter ${ }^{1,3}$; E.J. van Gennep ${ }^{4}$;

R.C.M. Pelger ${ }^{4}$; E.L. van Persijn van Meerten ${ }^{2}$; H.H. Versteeg ${ }^{1}$;

M.V. Huisman ${ }^{1}$; T. van der Hulle ${ }^{5}$; F.A. Klok ${ }^{1}$

${ }^{1}$ Department of Thrombosis and Hemostasis, Leiden University

Medical Center, Leiden, Netherlands; ${ }^{2}$ Department of Radiology,

Leiden University Medical Center, Leiden, Netherlands; ${ }^{3}$ Department

of Clinical Epidemiology, Leiden University Medical Center, Leiden,

Netherlands; ${ }^{4}$ Department of Urology, Leiden University Medical

Center, Leiden, Netherlands; ${ }^{5}$ Department of Oncology, Leiden

University Medical Center, Leiden, Netherlands

Background: Renal cell carcinoma (RCC) can be complicated by a venous tumor thrombus (TT), of which the optimal management is unknown.

Aims: To assess the prevalence of TT in RCC, its management and its association with venous thromboembolism (VTE), major bleeding (MB) and mortality in daily practice.

Methods: 649 patients diagnosed with RCC between 2010-2019 in our hospital were included and followed from diagnosis until last known follow-up visit or death. The study was approved by the local Institutional Review Board and oral informed consent was obtained. All endpoints were adjudicated. Cumulative incidences were estimated using Kaplan-Meier and cumulative incidence competing risk methods. Outcome predictors were determined with multivariable (time-dependent) Cox regression models.

Results: The median follow-up was 25 months (IQR 7.0-57): 86 patients had TT at RCC diagnosis (13\%), 200 patients died (31\%), and during follow-up 57 were diagnosed with VTE (8.8\%) and 55 with MB (8.5\%). Of the TTs, 71 (83\%) were limited to the renal vein or inferior vena cava below the diaphragm (limited TT), and 15 extended above the diaphragm (17\%; extensive TT): 26 patients (30\%) started therapeutic anticoagulation and 45 (52\%) underwent thrombectomy with/without anticoagulant therapy. Patients with TT were more often diagnosed with VTE (aHR 4.9, 95\% Cl 2.5-9.6) and faced a higher mortality (aHR $1.5,95 \% \mathrm{Cl} 1.1-2.2$ ) than patients without TT. Relative to limited TT, extensive TT was associated with higher VTE incidence (aHR 6.2, 95\% Cl 2.2-17; Table). The adjusted 2-year cumulative VTE incidence in TT patients who did not receive anticoagulation was $17 \%(95 \% \mathrm{Cl} 8.3-29)$. Anticoagulation use in TT (vs. non-TT) patients was associated with a higher MB incidence (HR 3.3, 95\% Cl 0.94-11).

TABLE 1 Adverse outcomes in the total cohort and in tumor thrombi patients

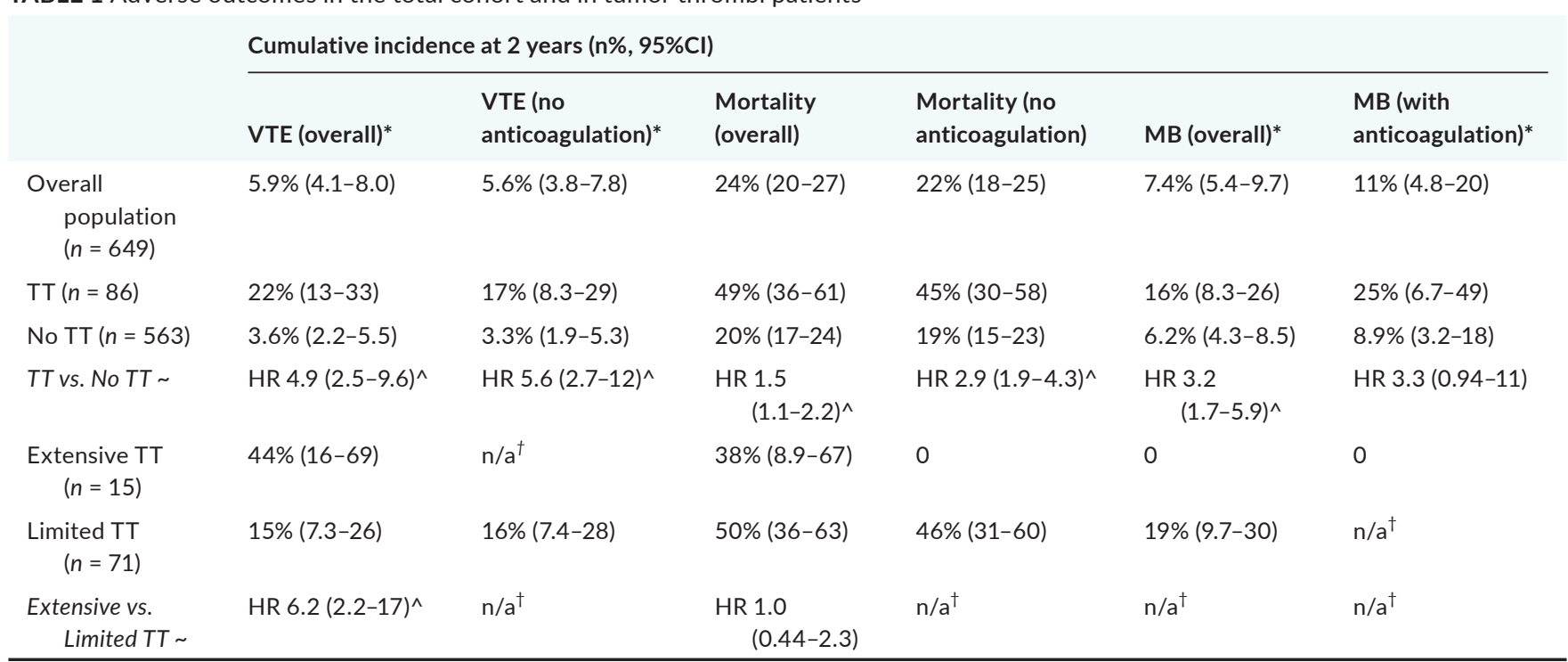

Note: $\mathrm{VTE}=$ venous thromboembolism, $\mathrm{MB}=$ major bleeding, $\mathrm{TT}=$ tumor thrombus, $\mathrm{Cl}$ = confidence interval, $\mathrm{n} / \mathrm{a}=$ not available, $\mathrm{Limited} \mathrm{TT}=\mathrm{TT}$ in renal vein or inferior vena cava below the diaphragm, Extensive TT $=$ TT extending above the diaphragm, $\mathrm{HR}=$ hazard ratio.

*Adjusted for competing risk of death.

'Significant at the $\mathrm{P}<0.05$ level.

Cox regression analysis with total follow-up time.

${ }^{\dagger}$ Analysis not possible or not reliable, due to very small numbers.

Conclusions: Patients with RCC-associated TT were at high risk of VTE and had a poorer overall survival than RCC patients without TT. Future trials should establish the optimal anticoagulation management of RCC-associated TT. 
PB1094 | Thromboembolic and Bleeding Complications fFowing Surgery among Patients with Ovarian Cancer - A Single Centre Retrospective Cohort Study

H.M. Wiegers ${ }^{1}$; M. Schaafsma ${ }^{1}$; H. Zelisse ${ }^{1}$; N. van Es ${ }^{1}$;

S. Middeldorp ${ }^{2}$; C.H. Mom ${ }^{1}$

${ }^{1}$ Amsterdam UMC, Amsterdam, Netherlands; ${ }^{2}$ Radboud UMC, Nijmegen, Netherlands

Background: Venous thromboembolism (VTE) is a major complication after gynaecological oncology surgery. International guidelines recommend extended thromboprophylaxis up to 28 days following surgery in ovarian cancer patients. More data are needed to guide decisions about the optimal dose and duration of thromboprophylaxis. Aims: To assess the incidence of thromboembolic and bleeding events 30 days following surgery in ovarian cancer patients and to evaluate the association between the duration of thromboprophylaxis and the incidence of VTE.

Methods: We performed a single-centre, retrospective cohort study in women surgically treated for ovarian cancer FIGO stages I-IV between January 2015 and December 2020. We estimated the risks of VTE, arterial thromboembolism (ATE), major bleeding, and mortality occurring in the 30-day postoperative period using Kaplan-Meier method. Cox regression analysis was performed to identify factors associated with VTE and major bleeding, and to evaluate the association between the duration of thromboprophylaxis and the incidence of VTE.

Results: A total of 601 patients with a median age of 65 years were included. Of these patients, 466 and 77 patients received thromboprophylaxis for 7 days and 28 days, respectively. Fifty-six patients were treated with therapeutic anticoagulants. During 30-day follow-up, 22 patients developed VTE (cumulative incidence $3.6 \%$; $95 \% \mathrm{Cl}: 2.0-5.2$ ) and five ATE (cumulative incidence $0.9 \% ; 95 \% \mathrm{Cl}$ 0.1-1.6). During surgery or in the following 30 days, 104 patients experienced major bleeding (cumulative incidence 15.3\%; $95 \% \mathrm{Cl}$ 12.2-18.3) and six patients died (cumulative incidence 1.1\%; 95\% $\mathrm{Cl}$ : 0.2-1.9). Extended duration of thromboprophylaxis for 28 days was not significantly associated with the incidence of VTE (HR 1.13 95\% Cl: 0.33-3.88) and the incidence of major bleeding (HR 1.39; 95\% Cl: 0.79- 2.43).

Conclusions: The cumulative incidence of VTE in ovarian cancer patients in the 30 days following cancer surgery was 3.6\%. Extended thromboprophylaxis for 28 days was not associated with a lower risk of VTE in this observational study.
PB1095 | Risk of Venous Thromboembolism and Major Bleeding in the Clinical Course of Osteosarcoma and Ewing Sarcoma Patients

M.A.M. Stals ${ }^{1}$; F.HJ Kaptein ${ }^{1}$; R.E Evenhuis ${ }^{2}$; AJ Gelderblom ${ }^{3}$; M.V Huisman ${ }^{1}$; DSA Karis ${ }^{2}$; RWD Noten ${ }^{1}$; SC Cannegieter ${ }^{1,4}$; $\mathrm{HH}$ Versteeg ${ }^{1}$; MAJ van de Sande ${ }^{2}$; FA Klok ${ }^{1}$ ${ }^{1}$ Department of Thrombosis and Hemostasis, Leiden University Medical Center, Leiden, Netherlands; ${ }^{2}$ Department of Orthopedic Surgery, Leiden University Medical Center, Leiden, Netherlands; ${ }^{3}$ Department of Medical Oncology, Leiden University Medical Center, Leiden, Netherlands; ${ }^{4}$ Department of Clinical Epidemiology, Leiden University Medical Center, Leiden, Netherlands

Background: Patients with osteosarcoma and Ewing sarcoma are considered to have a high risk of venous thromboembolism (VTE), although the exact incidence is understudied.

Aims: To study the incidence of VTE and major bleeding (MB) in these patients.

Methods: We performed a chart review in patients treated for osteosarcoma (OS) or Ewing sarcoma (ES) in our hospital between 1980 and 2018. The study was approved by the Institutional Review Board. Patient charts were scrutinized for patient and tumor characteristics, and outcome events, the latter adjudicated by an independent expert. Cumulative incidences were estimated using Kaplan-Meier and cumulative incidence competing risk (CICR) method. Multivariable time-dependent Cox models were used to determine the association between outcome events and mortality.

Results: Baseline characteristics of 520 osteosarcoma and 165 Ewing sarcoma patients are shown in Table 1. VTE occurred in 65 OS patients (13\%) and $11 \mathrm{ES}$ patients (6.7\%), MB in 31 OS patients (6.0\%) and $9 \mathrm{ES}$ patients (5.5\%). Adjusted cumulative incidences for VTE and MB at 3, 6, 9, 12 and 24 months are presented in Table 2. The most frequent VTE presentation was arm vein thrombosis $(32 \%$ and $45 \%$ in OS and ES, respectively), mostly related to central venous catheters (CVC; in 18/21 OS patients and 5/5 ES patients). From the $31 \mathrm{MB}$ complications in osteosarcoma patients, 13 patients were known to use prophylactic or therapeutic anticoagulants (42\%). The majority of MBs ( $84 \%$ in OS, $78 \%$ in ES) were bleedings within metastases or rebleeds after surgery. In osteosarcoma, VTE and MB were both associated with all-cause mortality (adjusted HRs $1.9(95 \% \mathrm{Cl} 1.3-2.7)$ and $3.3(95 \% \mathrm{Cl} 2.1-5.1)$ respectively), whereas this association was only present for $\mathrm{MB}$ in Ewing sarcoma (adjusted HR 2.9 (95\%Cl 1.0-8.1)). 
TABLE 1 Baseline characteristics of osteosarcoma and Ewing sarcoma patients

\begin{tabular}{|c|c|c|}
\hline Baseline characteristics & Osteosarcoma & Ewing sarcoma \\
\hline Number of patients & 520 & 165 \\
\hline Age at diagnosis - years & $32(19)$ & $23(13)$ \\
\hline Mean (+/- SD) & $3.5-84$ & $1.2-67$ \\
\hline \multicolumn{3}{|l|}{ Range } \\
\hline Gender - no (\%) & $288(55)$ & $113(69)$ \\
\hline Male & $232(45)$ & $52(32)$ \\
\hline \multicolumn{3}{|l|}{ Female } \\
\hline $\begin{array}{l}\text { Presence of distant metastases at time of } \\
\text { diagnosis - no (\%) }\end{array}$ & $107(21)$ & $50(30)$ \\
\hline Tumor grade - no (\%) & $453(87)$ & NA \\
\hline High grade sarcoma & $40(7.7)$ & NA \\
\hline Low grade sarcoma & $10(1.9)$ & NA \\
\hline Intermediate grade & $17(3.3)$ & NA \\
\hline \multicolumn{3}{|l|}{ Undefined } \\
\hline Presence of CVC - no (\%) & $394(76)$ & $152(92)$ \\
\hline Yes & $91(18)$ & $3(2)$ \\
\hline No & $35(6.7)$ & $10(6.1)$ \\
\hline \multicolumn{3}{|l|}{ Unknown } \\
\hline Patients died - no (\%) & $257(49)$ & $88(53)$ \\
\hline Median follow-up time in months - IQR & $60(22-142)$ & $42(19-124)$ \\
\hline
\end{tabular}

SD: standard deviation; no: number; CVC: central venous catheter; IQR: interquartile range; NA: not available

TABLE 2 Adjusted cumulative incidences of venous thrombotic complications and major bleeding events in osteosarcoma patients and Ewing sarcoma patients

\begin{tabular}{|c|c|c|c|c|}
\hline $\begin{array}{l}\text { Index date: date of } \\
\text { histological diagnosis of } \\
\text { OS or ES }\end{array}$ & $\begin{array}{l}\text { Osteosarcoma } \\
\text { VTE } \\
\%(95 \% \mathrm{Cl})\end{array}$ & $\begin{array}{l}\text { Ewing sarcoma } \\
\text { VTE } \\
\%(95 \% \mathrm{Cl})\end{array}$ & $\begin{array}{l}\text { Osteosarcoma } \\
\text { MB } \\
\%(95 \% \mathrm{Cl})\end{array}$ & $\begin{array}{l}\text { Ewing sarcoma } \\
\text { MB } \\
\%(95 \% \mathrm{Cl})\end{array}$ \\
\hline 3 months & $5.2(3.5-7.3)$ & $1.8(0.50-4.8)$ & $1.2(0.48-2.4)$ & 0 \\
\hline 6 months & $6.7(4.8-9.1)$ & $1.8(0.50-4.8)$ & $2.3(1.3-3.9)$ & $0.61(0.06-3.1)$ \\
\hline 9 months & $7.5(5.4-10)$ & $4.2(1.9-8.1)$ & $2.5(1.4-4.1)$ & $0.61(0.06-3.1)$ \\
\hline 12 months & $8.3(6.1-11)$ & $6.1(3.1-10)$ & $3.3(2.0-5.1)$ & $1.2(0.24-4.0)$ \\
\hline 24 months & $8.8(6.6-11)$ & $6.7(3.5-11)$ & $4.0(2.6-6.0)$ & $2.5(0.81-5.8)$ \\
\hline
\end{tabular}

OS: osteosarcoma; ES: Ewing sarcoma; VTE: venous thromboembolism; MB: major bleeding; Cl: confidence interval

Adjusted cumulative incidence: cumulative incidence adjusted for competing risk of death (following the CICR method).

Conclusions: Our findings provide accurate estimations of the incidence and prognostic relevance of VTE and MB in patients with osteosarcoma and Ewing sarcoma. 


\section{PB1096 | Factor VIIIc Predicts Venous Thromboembolism in Gynecological Cancer Patients}

E. Ibrahim ${ }^{1,2} ;$ S. O'Toole ${ }^{1,3,4}$; M. Ward ${ }^{4,3}$; F. Abu Saadeh ${ }^{1,2}$; L. Norris $^{1,3}$

${ }^{1}$ Department of Obstetrics and Gynecology, Trinity College Dublin, Dublin, Ireland; ${ }^{2}$ Division of Gynae-oncology, Trinity St. James's Cancer Institute, Dublin, Ireland; ${ }^{3}$ Trinity St. James's Cancer Institute, Dublin, Ireland; ${ }^{4}$ Dept of Histopathology, Trinity College Dublin, Dublin, Ireland

Background: Gynecological cancers patients are at high risk of venous thromboembolism(VTE) particularly following surgery and during chemotherapy. We have shown that the thrombin generation is increased in these patients and can be used to predict VTE in gynecological cancers (10.1002/rth2.12342), however the key pathways involved are not understood. Our group has shown that Factor VIIIC(FVIIIC), free Protein S(PS) and Factor V(FV) are significant determinants of thrombin generation in cancer patients (10.1016/ S0049-3848(16)30157-8) and may be useful as predictors of VTE.

Aims: The aim of this study was to compared pre-operative FVIIIc, PS and FV levels in gynecological cancer patients who developed VTE post surgery compared with those who remained thrombosis free. A secondary aim was to determine the effects of FVIIIc, Protein $S$ and Factor $V$ on overall(OS) and progression free survival(PFS). Methods: 51 gynecological cancer patients who developed VTE during the one year follow-up were matched with 154 gynecological cancer patients who remained thrombosis free. All patients gave full informed written consent and the study was approved by the hospital ethics committee. FVIIlc was measured using a chromogenic substrate assay (Hyphen Biomed), FV (Abcam) PS (Hyphen) were determined by ELISA, 24-48 hours before surgery.

Results: FVIIIc levels were significantly higher in the VTE group $(P<0.004)$ compared to matched controls. There was no significant difference in FV and PS levels between the groups. Factor VIIIc levels above the 75th centile were associated with increased risk of VTE (HR = 1.97 (95\% Cl1.089-3.583) after adjustment for chemotherapy treatment during follow up (HR = $1.863(95 \%$ $\mathrm{Cl} 1.087-3.38)$, the association was still significant. There was no association between FV, FVIIIc or PS and OS or PFS.

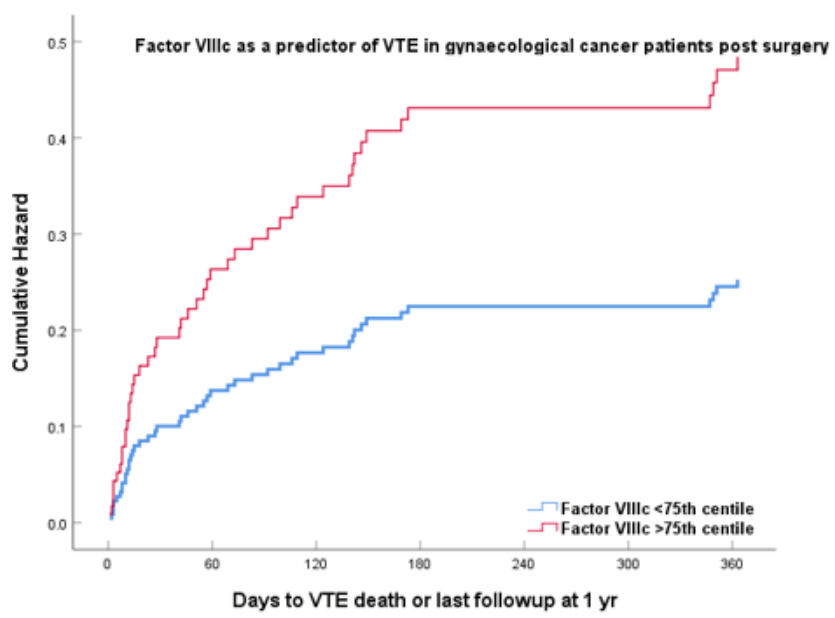

FIGURE 1 Factor VIIIc $>$ 75th percentile (Factor VIIIc >166\%) predicts VTE in gynaecological cancer patients post surgery $(N=206)$

Conclusions: FVIIIc is increased in gynecological cancer patients prior to the development of VTE. Larger prospective studies are underway in our center to determine the utility of FVIIIc as a predictive tool for VTE in gynecological cancer.

\section{PB1097 | Could Thromboprophylaxis Influence the Clinical Course of Patients with Active Cancer? Preliminary Results from the ACT4CAT Study}

N. Tsoukalas; A. Christopoulou; E. Timotheadou; A. Koumarianou; I. Athanasiadis; S. Peroukidis; G. Samelis; A. Psyrri; N. Kapodistrias; C. Kalofonos; C. Andreadis; A. Ardavanis; E. Samantas; A. Bokas; V. Barbounis; N. Kentepozidis; D. Mavroudis; A. Athanasiadis; P. Papakotoulas; I. Boukovinas

On behalf of the Hellenic Society of Medical Oncology (HeSMO, http://www.hesmo.green), Athens, Greece.

Background: The cross talk between malignancy and thrombosis imposes serious consequences for patients with active cancer. Cancer Associated Thrombosis (CAT) is the $1^{\text {st }}$ cause of death in ambulatory patients undergoing chemotherapy. Tumor type, treatment, and patient related factors contribute to High Thrombotic Burden (HTB) in ambulatory patients with active cancer.

Aims: The study ACT4CAT aims to record clinical practice for CAT management regarding anticoagulation in patients with active cancer. 
Methods: A prospective observational clinical study (ACT4CAT) conducted by HeSMO across Greece involved ambulatory, active cancer patients who received thromboprophylaxis. Patients enrolled after informed consent form signing.

Results: Preliminary results regarding 431 patients from 18 oncology departments are presented; $65.4 \%$ of them have completed the study. Tumor types were: $39.8 \%$ gastrointestinal, $28.8 \%$ lung, $7.0 \%$ gynecological, $7.0 \%$ urological, $4.4 \%$ breast and $20 \%$ others; $88.2 \%$ of patients treated with High-Risk for Thrombosis Chemotherapy Agents (HRTCAs) including: $55.9 \%$ platinum, $44.7 \%$ antimetabolites and $12.6 \%$ immunotherapy. Concerning clinical setting: $62.1 \% 1^{\text {st }}$ line, $18.4 \% 2^{\text {nd }}$ line, $8.9 \%$ adjuvant and $2.4 \%$ neoadjuvant. Analysis depicted in Table 1.

TABLE 1 Analysis

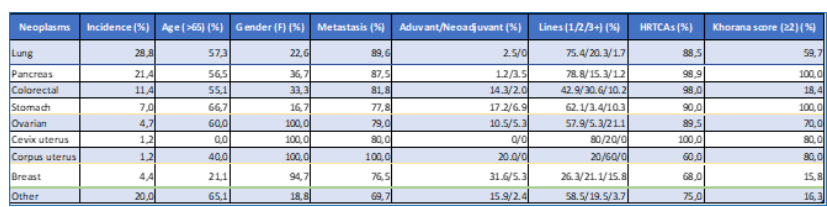

Average thromboprophylaxis duration was $5.3 \pm 3.6$ months. Duration per tumor type depicted in Figure. Anticoagulants administered: tinzaparin $90.8 \%$, fondaparinux $5.5 \%$, bemiparin $1.5 \%$, enoxaparin $1.2 \%$, apixaban $0.5 \%$ and rivaroxaban $0.5 \%$. Intermediate doses received $70.9 \%$ of patients regardless clinical setting $\left(1^{\text {st }}\right.$ or $2^{\text {nd }}$ line, adjuvant, neoadjuvant: $70.2 \%, 79.2 \%, 51.3 \%, 70.0 \%$ respectively, $P=0.0254)$, although intermediate dose used more in metastatic stages (OR: $2.495 \% \mathrm{Cl}: 1.4-4.2, P=0.0028$ ).

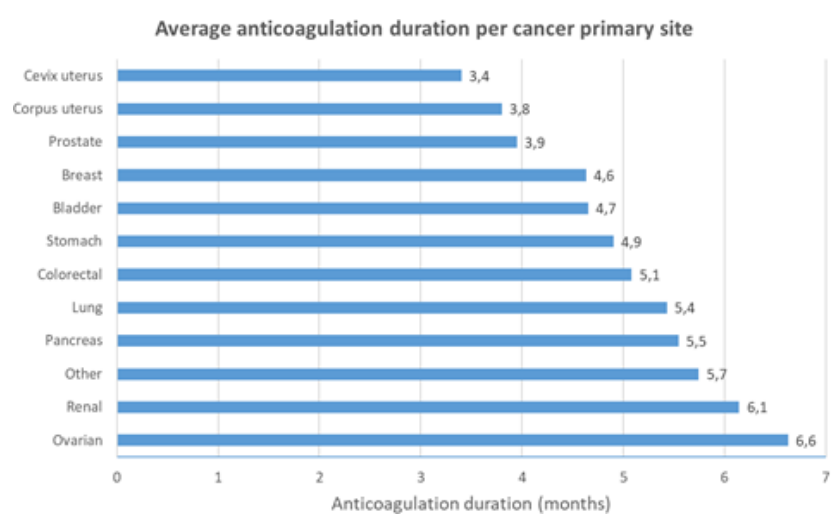

FIGURE 1 Anticoagulation duration (months)

About efficacy: 9 thrombotic events reported $(2.1 \%, 95 \% \mathrm{Cl}$ : $1.1-$ $3.9 \%)$, with a trend towards prophylactic doses. Eleven minor bleedings reported $(2.6 \%, 95 \% \mathrm{Cl}: 1.4-4.5 \%)$, regardless clinical setting or dose used.

Conclusions: Thromboprophylaxis in patients with active cancer is safe and effective. Besides Khorana score, factors such as metastases, HRTCAs and drug-drug interactions influence the clinical decision of thromboprophylaxis in cancer patients mainly with LMWHs and frequently on intermediate doses regardless clinical setting. CAT can be preventable.

\section{PB1098 | Association of KRAS Mutation with Arterial} Thromboembolism in Advanced Lung and Gastrointestinal Cancer

S. Maharaj; S. Bhandari; X. Wu; S. Rai; V. Sharma

University of Louisville, Louisville, United States

Background: Patients with cancer are at increased relative risk of arterial thromboembolic events (ATE) and these increase morbidity and mortality. In advanced GI and NSCLC, molecular subtyping has increased use of next-generation sequencing (NGS).

Aims: To investigate the association between tumor mutation profile and ATE risk.

Methods: We conducted a retrospective cohort study of consecutive GI/NSCLC patients from 2014-2019 with NGS and follow-up at Brown Cancer Center. The NGS platform detected substitutions, indels, copy number alterations and select rearrangements in 324 genes. Patients with thrombophilia, prior anticoagulant use or $>1$ malignancy were excluded. ATE was defined as any arterial thromboembolic event including arterial stroke, myocardial infarction, peripheral arterial thrombosis and visceral arterial thromboses; within 6 months prior to diagnosis or any time after. For statistical analysis SAS 9.5 was used with significance at alpha $=0.05$. Multinomial logistic regression was performed, in which the log odds of ATE was modeled as a linear combination of the genes. Odds ratios and $95 \%$ confidence intervals for ATE were generated.

TABLE 1 Demographics and characteristics of the study population

\begin{tabular}{|c|c|c|c|c|}
\hline & & All patients & No ATE & ATE \\
\hline 1 & Number of patients, $n(\%)$ & $326(100)$ & $298(91.4)$ & $28(8.6)$ \\
\hline \multirow[t]{3}{*}{2} & Gender, n (\%) & & & \\
\hline & Female & $152(46.6)$ & $139(42.6)$ & $13(4.0)$ \\
\hline & Male & $174(53.4)$ & $159(48.8)$ & $15(4.6)$ \\
\hline \multirow[t]{3}{*}{3} & Smoking history, $\mathrm{n}(\%)$ & & & \\
\hline & Current/ former smoker & $249(76.4)$ & $230(70.5)$ & $19(5.8)$ \\
\hline & Never smoker & $77(23.6)$ & $68(20.9)$ & $9(2.8)$ \\
\hline 4 & Age, Mean (95\% Cl) & $59.2(57.9-60.5)$ & $59.1(57.8-60.5)$ & $59.6(55.5-63.6)$ \\
\hline 5 & $\begin{array}{l}\text { Brain metastases at } \\
\text { diagnosis }\end{array}$ & $38(11.7)$ & $33(10.1)$ & $5(15.3)$ \\
\hline \multirow[t]{4}{*}{6} & Type of cancer & & & \\
\hline & Colorectal & $108(33.1)$ & $93(28.5)$ & $15(4.6)$ \\
\hline & Gastrointestinal & $30(9.2)$ & $28(8.6)$ & $2(0.6)$ \\
\hline & Lung (NSCLC) & $188(57.7)$ & $177(54.3)$ & $11(3.4)$ \\
\hline
\end{tabular}


TABLE 2 Correlation between tumor mutations and ATE in patients with advanced NSCLC and GI malignancy

\begin{tabular}{lll} 
Gene & $\begin{array}{l}\text { Odds Ratio for } \\
\text { ATE }\end{array}$ & 95\% Confidence Limits \\
BRAF & 1.846 & $(0.548,6.218)$ \\
FGF6 & 10.061 & $(0.673,150.344)$ \\
FGF23 & 1.142 & $(0.093,13.968)$ \\
KRAS & 2.456 & $(1.077,5.602)$ \\
MPL & 2.519 & $(0.362,17.519)$ \\
PIK3CA & 2.172 & $(0.709,6.656)$ \\
PTCH1 & 0.313 & $(0.016,6.146)$ \\
SMAD4 & 0.585 & $(0.121,2.831)$ \\
\hline
\end{tabular}

Results: A total of 364 patients were reviewed; after exclusions 326 patients were included comprising Stage III/IV NSCLC (58\%), metastatic colorectal (33\%) and other metastatic GI cancers - gastric, duodenal, esophageal, pancreatic and cholangiocarcinoma (9\%). Approximately half (53\%) were males with mean age of $59.1 \mathrm{yrs}$ and 76.4\% current/former smokers (Table 1). There was a low level of microsatellite instability (0.9\%). ATE occurred in 28 patients (8.6\%). Statistical analysis showed KRAS mutation significantly increased odds of ATE (Table 2).

Conclusions: Patients with KRAS mutations had significantly higher ATE risk. This tumor mutation and the associated pathways deserve further investigation in patients with cancer.

PB1099 | Laboratory Monitoring of Coagulation State in Children with Acute Lymphoblastic Leukemia

E. Seregina $^{1,2}$; L. Zharikova ${ }^{1}$; N. Trubina ${ }^{1}$; M. Korsantiya ${ }^{1}$; M. Gracheva ${ }^{1}$; A. Poletaev ${ }^{1}$; T. Vuimo ${ }^{1,2}$; F. Ataullakhanov ${ }^{1,2,3,4}$; U. Rumyantseva ${ }^{1}$; A. Karachunskiy ${ }^{1}$

${ }^{1}$ Dmitry Rogachev National Medical Research Center for Pediatric Hematology, Oncology and Immunology, Moscow, Russian Federation; ${ }^{2}$ Center for Theoretical Problems of Physicochemical Pharmacology RAS, Moscow, Russian Federation; ${ }^{3}$ Department of Physics, Moscow State University, Moscow, Russian Federation; ${ }^{4}$ The Faculty of Biological and Medical Physics, Moscow Institute of Physics and Technology, Dolgoprudniy, Russian Federation

Background: Thrombosis in children with Acute Lymphoblastic Leukemia(ALL) present in nearly $40 \%$ of patients due to CVSs and chemotherapy including L-asparaginase.

Aims: The aim was to develope the laboratory panel to evaluate the group of patients with the high risk of thrombosis.

Methods: 103 patients ( 60 boys, 43 girls, 1-17 yr, median 5 yr) with ALL were enrolled in this study. APTT, TT, PR, fibrinogen, ATIII, Ddimer levels, Thromboelastography(TEG), Thrombodynamics(TD), thrombomodulin(TM) and endothelin-1(ET-1) levels were used for coagulation assessment in patients before and during the treatment with L-asparaginase (10 points of blood collection during consolidation phase of ALL-MB-2015 protocol).

Results: TEG parameters and standard clotting tests were normal(nearly $60 \%$ ) or in hypocoagulation(nearly $40 \%$ ) area during the treatment due to L-asparaginase induced coagulopathy and decrease of platelets count. Fibrinogen and ATIII were both decreased during the treatment in nearly $55 \%$ of points respectively. Thrombosis was visualized with ultrasound in 57 patients(55\%). TD revealed hypercoagulation in $82 \%$ of points. There were increased levels of TM and ET-1 levels only in patients with thrombosis. We've devided patients in two groups: the group with high and normal Ddimer levels. If there were hypercoagulation in TD in there were $42 \%$ of thrombosis in group with normal D-dimer levels compared to group with high $\mathrm{D}$-dimer levels: there were only $11 \%$ of thrombosis. There was no thrombosis in points with normal TD.

Conclusions: The dysfunction in lysis system of hemostasis confirmed by high TM levels, normal D-dimer levels during hypercoagulation by TD is probably the cause of high thrombosis risks in ALL. TD, TM and D-dimer level are the possible group of assays to predict thrombotic complication in children with ALL.

PB1100 | Incidence and Impact of Venous Thromboembolism and Major Bleeding in Patients with Glioblastoma

F.H.J. Kaptein ${ }^{1}$; M.A.M. Stals ${ }^{1}$; E. Klaase ${ }^{1}$; M.Y. Kapteijn ${ }^{1}$; R. van Eijk $^{2}$; S.C. Cannegieter ${ }^{1,3}$; S.G. van Duinen ${ }^{2}$; M.J.B. Taphoorn ${ }^{4,5}$; L. Dirven ${ }^{4,5}$; H.H. Versteeg ${ }^{1}$; J.T. Buijs ${ }^{1}$; M.V. Huisman ${ }^{1}$;

J.A.F. Koekkoek ${ }^{4,5}$; F.A. Klok ${ }^{1}$

${ }^{1}$ Department of Thrombosis and Hemostasis, Leiden University Medical Center, Leiden, Netherlands; ${ }^{2}$ Department of Pathology, Leiden University Medical Center, Leiden, Netherlands; ${ }^{3}$ Department of Clinical Epidemiology, Leiden University Medical Center, Leiden, Netherlands; ${ }^{4}$ Department of Neurology, Leiden University Medical Center, Leiden, Netherlands; ${ }^{5}$ Department of Neurology, Haaglanden Medical Center, Den Haag, Netherlands

Background: Patients with glioblastoma are considered to be at high risk of venous thromboembolism (VTE) and major bleeding (MB), although reliable incidence estimates are lacking.

Aims: To assess the cumulative incidence, risk factors and prognostic impact of VTE and MB in a large cohort of glioblastoma patients. Methods: Patients diagnosed with glioblastoma between 20042020 from the Leiden University Medical Center and Haaglanden Medical Center were included and followed from 1 year before date of histopathological glioblastoma diagnosis up to two years after, or until an outcome of interest (VTE and MB) or death occurred. Cumulative incidences were estimated using Kaplan-Meier and cumulative incidence competing risk methods. Outcome predictors were determined with multivariable (time-dependent) Cox models. The study was approved by the local Institutional Review Board and informed consent was waived. 
Results: Of the 973 included patients, 615 died, 103 were diagnosed with VTE and 134 with MB during a median follow-up of 20 months (IQR 15-28). Ambulant patients did not routinely receive pharmacological thromboprophylaxis. The adjusted 3-year-cumulative incidence of VTE was $12 \%(95 \% \mathrm{Cl} 10-15)$ and of MB $15 \%(95 \% \mathrm{Cl} 12-17)$ (Figures 1\&2). One year increase in age (HR 1.03, 95\% Cl 1.01-1.05), tumor resection (vs. biopsy: $\mathrm{HR} 0.55,95 \% \mathrm{Cl} 0.34-0.88$ ) and performance status (ECOG $>1$ vs $\leq 1$ : HR $1.8,95 \% \mathrm{Cl} 1.2-2.8$ ) were independent predictors of VTE (a biopsy rather than resection was performed in patients with the worst prognosis). The majority of MBs occurred within 6 weeks post-surgery (57\%) and intracranially (93\%). MB and VTE both predicted all-cause mortality (adjusted HR $1.6,95 \% \mathrm{Cl} 1.3-2.0$ and $1.3,95 \% \mathrm{Cl} 0.99-1.7$ respectively).

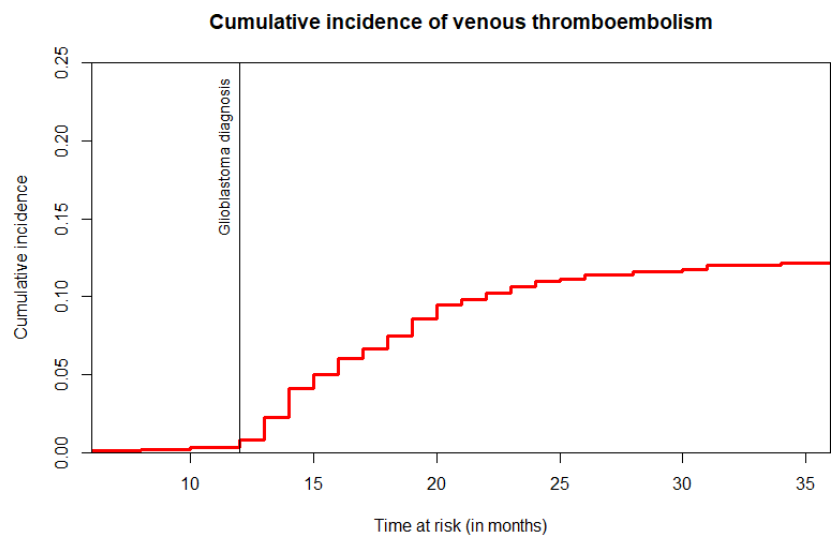

Note: The cumulative incidence (adjusted for the comping risk of death) of venous thromboembolism, during the total follow-up period (i.e. 1 year before until 2 years after histopathological glioblastoma diagnosis)

FIGURE 1 Cumulative incidence of venous thromboembolism

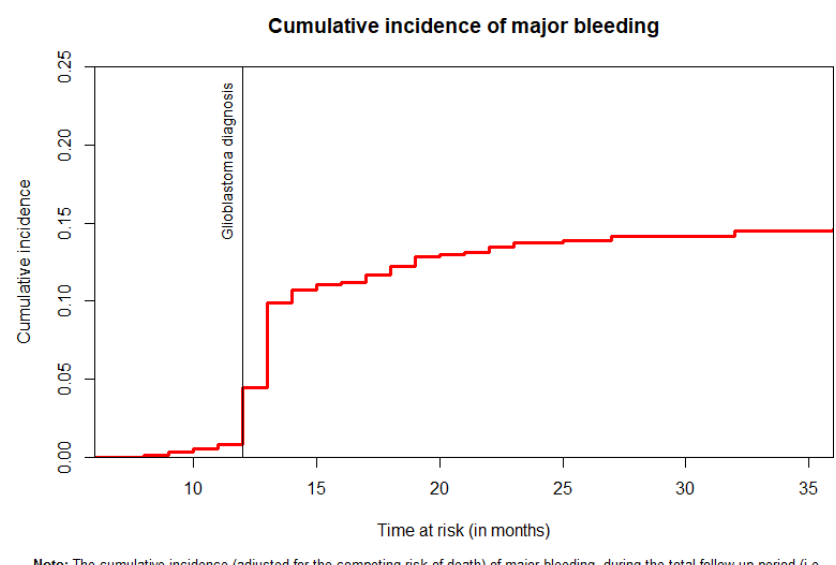
1 year before until 2 years after histopathological glioblastoma diagnosis)

FIGURE 2 Cumulative incidence of major bleeding

Conclusions: The incidences of VTE and MB in patients with glioblastoma are high, with both complications associated with a poor prognosis. Our observations emphasize the need for prospective studies to determine optimal thromboprophylaxis and VTE treatment strategy in these patients.
PB1101 | Association between Cancer and Anatomical Site of Venous Thromboembolic Disease

D. Froehling ${ }^{1}$; W. Wysokinski ${ }^{1}$; R. McBane ${ }^{1}$; D. Houghton ${ }^{1}$;

D. Vlazny ${ }^{1}$; D. Hodge ${ }^{2}$; L. Peterson ${ }^{1}$; A. Casanegra ${ }^{1}$

${ }^{1}$ Mayo Clinic, Rochester, United States; ${ }^{2}$ Mayo Clinic, Jacksonville, United States

Background: There is limited published data on the association between malignancy and the location of venous thromboembolism (VTE) in the body.

Aims: Assess the location of VTE in the body in patients with active cancer and compare these results in patients without malignancy.

Methods: Consecutive patients enrolled in the Mayo Clinic VTE Registry between March 1, 2013 and November 30, 2019 for acute VTE were followed prospectively. Anatomical site of thrombosis and malignancy status were recorded. Patient outcomes were assessed in person, by mailed questionnaire, or by a scripted phone interview. Active cancer was defined as treatment for malignancy within the last six months or not yet in remission.

Results: During the study period there were 2,798 patients with acute VTE (1256 with and 1542 without active cancer). Pulmonary emboli were more common in patients with active cancer compared to patients without cancer $(49.5 \%$ vs. $39.7 \%, P<0.001)$. Upper extremity deep vein thrombosis ( $11.4 \%$ vs. $7.7 \%, P<0.001)$, renal vein thrombi $(1.4 \%$ vs. $0.2 \%, P<0.001)$ and splanchnic vein thrombi $(9.3 \%$ vs. $6.0 \%, P=0.001$ ) were all more common in patients with active cancer compared to patients without cancer.

Conclusions: Compared to those without malignancy, patients with active cancer were more likely to have pulmonary emboli, upper extremity deep vein thrombosis, renal vein thrombi, and splanchnic vein thrombi.

PB1102 | Risk Factors for Symptomatic Venous

\section{Thromboembolism in Children and Adolescents with Lymphomas}

\section{Evstratov; P. Zharkov; N. Myakova}

Dmitry Rogachev National Medical Research Center of Pediatric Hematology, Oncology and Immunology, Moscow, Russian Federation

Background: Lymphoma is the third most common cancer in children and adolescents. Venous thromboembolism (VTE) is a frequent complication in pediatric patients with lymphomas, but there is lack of data about risk factors for symptomatic VTE (sVTE) in this cohort of patients.

Aims: To evaluate risk factors for VTE in children and adolescents with lymphomas.

Methods: Our study is a monocentric retrospective analysis of 262 patients aged $<18$ years with lymphoma that were treated in our Center since 2013 to 2019 year. The epidemiological characteristics of patients are presented in table 1. 
TABLE 1 Epidemiological characteristics of children and adolescents with lymphomas

\begin{tabular}{|c|c|c|c|}
\hline Characteristic & Patients without sVTE (\%) & Patients with sVTE (\%) & $P$, Chi-square \\
\hline ABO - Group O & $84(34.9 \%)$ & $3(14.3 \%)$ & $P=0.057$ \\
\hline ABO - Group "Non-O" & $157(65.1 \%)$ & $18(85.7 \%)$ & \\
\hline Hodgkin lymphoma & 75 (31.1\%) & $9(42.9 \%)$ & $P=0.27$ \\
\hline Non-Hodgkin lymphoma & $166(68.9 \%)$ & $12(57.1 \%)$ & \\
\hline Mediastinal involvement - & $159(66 \%)$ & $6(28.6 \%)$ & $P=0.001$ \\
\hline Mediastinal involvement + & $82(34 \%)$ & $15(71.4 \%)$ & \\
\hline $\begin{array}{l}\text { Intensive care unit treatment during the first } \\
30 \text { days of hospitalization - }\end{array}$ & $200(83 \%)$ & $14(66.7 \%)$ & $P=0.064$ \\
\hline $\begin{array}{l}\text { Intensive care unit treatment during the first } \\
30 \text { days of hospitalization }+\end{array}$ & 41 (17\%) & $7(33.3 \%)$ & \\
\hline Male & $165(68.5 \%)$ & 7 (33.3\%) & $P=0.87$ \\
\hline Female & $76(31.5 \%)$ & $14(66.7 \%)$ & \\
\hline
\end{tabular}

We took only sVTE as the event, data of asymptomatic VTE was censored. Patients were followed since the start of treatment to sVTE, relapse, death, +100 day after allogenic hematopoietic stem cell transplantation or loss to follow-up, whatever came. VTE diagnosed before the start of the treatment was recorded as the time 0 . The median time of follow up was 2,18 years (IQR 0,71-3,85 years). Risk factors were analyzed by univariate and multivariate analysis with logistic regression. ROC-analysis was used for the determination of optimal cutoff. P-value $<0,05$ was considered as significant.

Results: Among all patients sVTE was diagnosed in $21(8 \%, 95 \% \mathrm{Cl}$ : 4,73-11,3). Median time to diagnosis was 22 (IQR 17-36,5) days. The optimal cutoff for duration of treatment in the intensive care unit during the first 30 days was $>2$ days. The optimal cutoff for the volume of mediastinal lymphadenopathy was $250 \mathrm{ml}$. The optimal cutoff for age was $\geq 12$ years. Results of the univariate and multivariate analysis are presented in the table 2 .

TABLE 2 Univariate and multivariate analysis of risk factors for sVTE in children and adolescents with lymphomas

\begin{tabular}{|c|c|c|c|c|}
\hline & Univariate analysis & & Multivariate analysis & \\
\hline Characteristic & Odds ratio $(95 \% \mathrm{Cl})$ & $P$-value & Odds ratio $(95 \% \mathrm{Cl})$ & $P$-value \\
\hline ABO group "Non-O" & $3.2(0.925-11.28)$ & 0.066 & $4.86(1.26-18.65)$ & 0.02 \\
\hline $\begin{array}{l}\text { Volume of mediastinal } \\
\qquad \text { lymphadenopathy } \geq 250 \mathrm{ml}\end{array}$ & $6.3(2.49-16.1)$ & 0.0001 & $4.38(1.59-12.1)$ & 0.004 \\
\hline ICU hospitalization $\geq 2$ days & $3.6(1.4-9.8)$ & 0.01 & $2.92(0.98-8.68)$ & 0.053 \\
\hline
\end{tabular}

Conclusions: Blood group "Non-O", volume of mediastinal lymphadenopathy $\geq 250 \mathrm{ml}$ and age $\geq 12$ years are independent risk factors for SVTE in children and adolescents with lymphomas. These factors could be used for further studies of primary antithrombotic prophylaxis in this cohort of patients.

PB1103 | Identification of Tumor-expressed MicroRNAs Associated with Venous Thrombosis in Colorectal Cancer

R.J.S. Anijs; E.H. Laghmani; B. Ünlü; S.M. Kielbasa; H. Mei;

S.C. Cannegieter; F.A. Klok; P.J.K. Kuppen; H.H. Versteeg; J.T. Buijs Leids Universitair Medisch Centrum, Leiden, Netherlands

Background: Colorectal cancer (CRC) patients have an increased risk of developing venous thromboembolism (VTE), resulting in increased morbidity and mortality. Available risk prediction tools for identifying patients at high risk of VTE show poor clinical performance. MicroRNAs (miRNAs) are small RNAs, which regulate a variety of cellular processes, are relatively stable and are detectable in body fluids. We hypothesize that miRNAs can be used to improve VTE prediction in CRC patients.

Aims: The aim of this study is to identify novel tumor-expressed miRNAs associated with VTE.

Methods: In a cohort of 418 CRC patients diagnosed between 20012015 at the Leiden University Medical Center (LUMC), 23 patients developed VTE 1 year before or after cancer diagnosis. Based on availability of frozen tumor material, age, gender and tumor stage, 17 patients with VTE and 18 patients without VTE were selected. Tumor cells were isolated using laser capture microdissection and samples were subsequently analyzed on the Illumina sequencing platform NovaSeq600 using a 150 bp paired-end sequencing. The 
paired-end raw reads were processed using the BioWDL small-RNA pipeline version 1.2.0 developed at LUMC. Differential miRNA expression was analysed using edgeR; the Benjamini-Hochberg method was used to adjust $p$-values for false discovery.

Results:

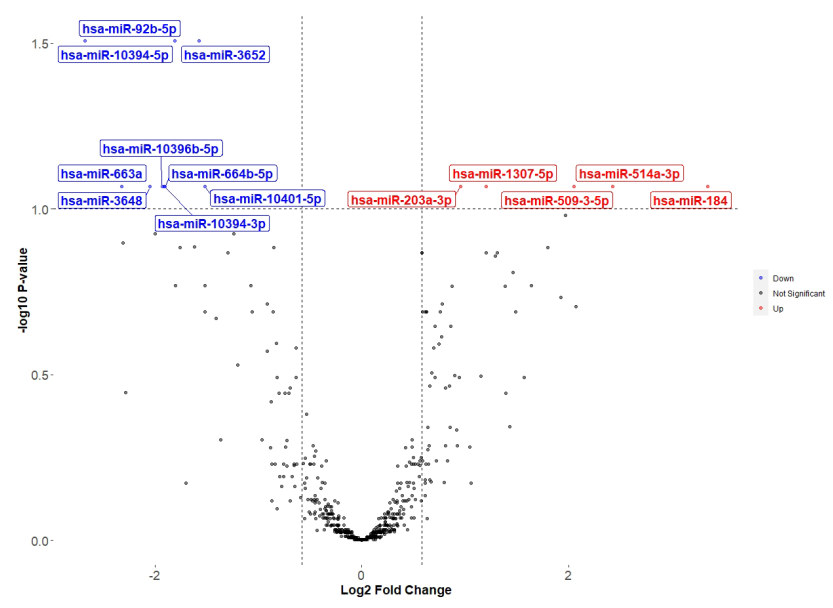

FIGURE 1 Volcano plot illustrating the top 14 differentially expressed miRNAs in tumor tissue from CRC patients with VTE compared to those without VTE. miRNAs in red indicate significantly upregulated and miRNAs in blue indicates significantly downregulated. CRC = colorectal cancer, VTE = venous thromboembolism

A total of 548 miRNAs were detected. Applying a minimum 1.5 fold change (FC) difference and a FDR value of $<0.1,14$ miRNAs were differentially regulated in CRC patients with VTE, compared to without VTE (table 1 \& figure 1). In a sub-analysis, we assessed miRNAs associated with VTE in the early disease course and not affected by cancer treatment (1 year before cancer diagnosis). Seven significant miRNAs were identified (downregulated; hsa-miR-10394-3p, hsamiR-10394-5p, hsa-miR-483-5p, hsa-miR-182-5p, hsa-miR-3654; upregulated; hsa-miR-223-3p, hsa-miR-363-3p).

\begin{tabular}{|c|c|c|c|c|c|}
\hline mirNa & Regulation & $\begin{array}{l}\text { Average loge? Fold } \\
\text { Change }\end{array}$ & $\begin{array}{l}\text { P.value } \\
\text { nat }\end{array}$ & Adjusted P-value & Predicted target genes \\
\hline hsa-mir-3652 & Down & $-1,57$ & 0.000136 & 0.031095 & 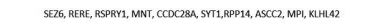 \\
\hline hss-mir-92b-5p & Down & $-1,81$ & 0,000164 & 0,031095 & 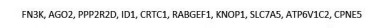 \\
\hline hss-mir-103945p & Down & $-2,68$ & 0,000171 & 0,031095 & 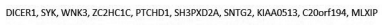 \\
\hline Hes-mile-184 & up & 3,34 & 0,000956 & 0,085692 & 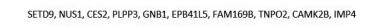 \\
\hline hso-mir-3648 & Down & $-2,05$ & 0,000981 & 0,085692 & 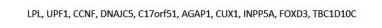 \\
\hline hasa-mir-10399-3p & Down & $-1,91$ & 0,001249 & 0.085692 & PREK2, TRAF3, PEAK3, CLEGG, EVSL, HDAC10, APBA1, RALGAPB, MYYODI, WWA1 \\
\hline hso-mir-10401-5p & Down & $-1,52$ & 0,001513 & 0.085692 & FOXX2, BMPQ, KLF7, ZNFF80, RELN, CHD2, MPIGGB, GRRP2, GNAT1, TRAAG1 \\
\hline hssomir. $5093.5 \mathrm{p}$ & up & 2,05 & 0,001644 & 0.085692 & 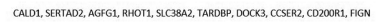 \\
\hline hsam mil-1307-5p & up & 1,20 & 0,001847 & 0,085692 & IYPLAR, LRRCA5, IPH3, SBF1, KRT13 \\
\hline hsa-mik-103966-5p & Down & $-1,93$ & 0,001867 & 0,085692 & ABO, ESPN, ACS13, M12, SCRT1, TNFASF, NFFX, SLC29A3, SRRT, AP521 \\
\hline hso- mir-663a & Down & $-2,32$ & 0,001991 & 0,085692 & 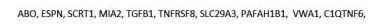 \\
\hline iss-mil-664b-5p & Down & $-1,90$ & 0,002003 & 0,085692 & 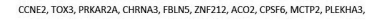 \\
\hline hsom mil:5 540 -3p & up & 2,42 & 0,002043 & 0,085692 & 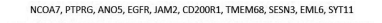 \\
\hline hss-mil-203a-3p & up & 0,95 & 0,002193 & 0,0856992 & 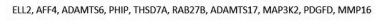 \\
\hline
\end{tabular}

FIGURE 2 Differentially tumor-expressed miRNAs in CRC patients with VTE compared to those without VTE. CRC = colorectal cancer, $\mathrm{VTE}=$ venous thromboembolism
Conclusions: We identified 19 tumor-expressed miRNAs significantly expressed in cancer-associated VTE, which may have the potential to serve as novel, non-invasive predictive biomarkers for VTE in CRC.

PB1104 | The Role of Thrombophilia in Asparaginase Related Venous Thromboembolism in Pediatric and Young Adult Patients Affected by Acute Lymphoblastic Leukemia

A. Serrao $^{1}$; G.M. Assanto ${ }^{1}$; C. Santoro ${ }^{1}$; S. Bianchi ${ }^{1}$; S. Olivieri ${ }^{2}$;

M. Canichella ${ }^{1}$; A.M. Testi ${ }^{1}$; A. Chistolini ${ }^{1}$

${ }^{1}$ Sapienza University of Rome, Rome, Italy; ${ }^{2}$ Policlinico Umberto I, Rome, Italy

Background: Venous thromboembolism (VTE) is one serious complication in patients with acute lymphoblastic leukemia (ALL) with an incidence of 1-14\% in thromboembolic symptomatic patients and up to $37 \%$ in the asymptomatic. In these patients, VTE is a multifactorial event: leukemia, central venous catheter (CVC), chemotherapy, in particular, asparaginase (ASP), and steroids. ASP is an effective chemotherapeutic agent that catalyzes the hydrolysis of asparagine. Treatment with ASP increases the risk for VTE. Among the causes that can determine an increased thromboembolic risk, the presence of congenital or acquired thrombophilia can play a crucial role.

Aims: The aim of our study was to evaluate the role of thrombophilia on VTE events in pediatric patients (age $<18$ years) and in adolescents and young adults (AYA) $<40$ years old with ALL during ASP chemotherapy regimens.

Methods: We defined ASP-associated thrombosis when the event occurred within 1 month from the drug administration. All patients performed the inherited and acquired thrombophilia assays at disease diagnosis before chemotherapy start.

Results:

TABLE 1 Patients' characteristics

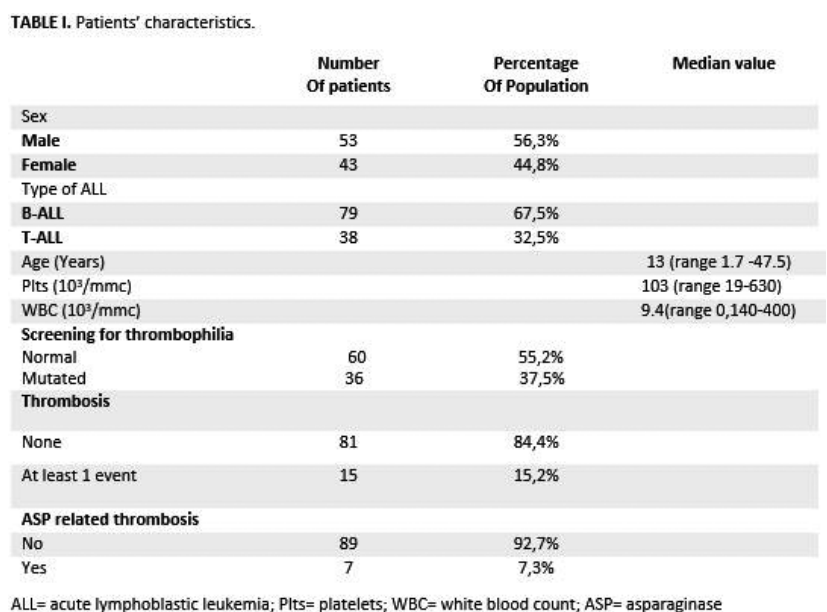


We studied 96 ALL patients and their characteristics are summarized in table 1. Thrombotic complications occurred in 15/96 (15.6\%) patients. Overall, 6/15 (40\%) patients with the thrombotic complication presented thrombophilia $(P=0.265)$. Of the 15 thrombotic events, 7/15 (47\%) were associated with the use of ASP. Notably, $5 / 7$ presented thrombophilia $(P=0.008)$. In the multivariate analysis, among the predictive factors for ASP-related thrombosis considered (sex, age, thrombophilia, and T-ALL), the only significant factor for thrombotic events development was thrombophilia $(P=0.0029$, IC 0.025-0.823; HR 0.143).

Conclusions: Although thrombophilia is a well-known risk factor for VTE in ALL, our study focused on its link with ASP-related VTE. We suggests that screening for thrombophilia should be performed in ALL patients before ASP administration, since thrombophilic ALL patients may benefit from VTE prophylaxis.

PB1105 | Cumulative Incidence of Recurrent Venous Thromboembolism by Cancer and Provoking Status - A Population-based Study

K. Ding ${ }^{1}$; L. Ford ${ }^{1} ;$ A. Wendelboe ${ }^{1} ;$ A. Adamski² ; N. Reyes ${ }^{2}$; K. Abe ${ }^{2}$; G. Raskob ${ }^{1}$

${ }^{1}$ University of Oklahoma Health Sciences Center, Oklahoma City, United States; ${ }^{2}$ Centers for Disease Control and Prevention, Atlanta, United States

Background: The incidence of recurrent venous thromboembolism (VTE) stratified by patient's cancer and provoking status has been understudied in racially diverse populations.

Aims: To measure the incidence of first recurrent VTE among adult patients, stratified by cancer and provoking status.

Methods: A prospective population-based cohort study of VTE in Oklahoma County, OK, with racial distribution similar to the United States, during April 1, 2012-March 31, 2014. VTE was diagnosed using imaging studies. Cancer status at the first identified VTE diagnosis was categorized as either active cancer (defined as metastatic or diagnosed within 6 months previously), a history of cancer $\geq 6$ months previously, or no history of cancer, classified further by provoking status using ISTH criteria.

Results: We identified 3,231 incident VTE events among adults, of whom 304 (9.4\%) had active cancer, 424 (13.1\%) had a history of cancer $\geq 6$ months previously, and 2,503 (77.5\%) had no history of cancer; 4 patients $(0.1 \%)$ had insufficient data for analysis. Among patients without a history of cancer, 58 (2.3\%) were classified as persistent provoked, 1,182 (47.2\%) as transient provoked and 1,263 (50.5\%) as unprovoked. Recurrent VTE occurred in 38 patients (12.5\%) with active cancer, 34 (8\%) with a history of cancer $\geq$ 6 months previously, and among those without cancer, in 10 patients (17.2\%) classified as persistent provoked, 115 patients (9.7\%) as transient provoked, and 122 patients (9.7\%) as unprovoked. The Figure shows timing of recurrent VTE.

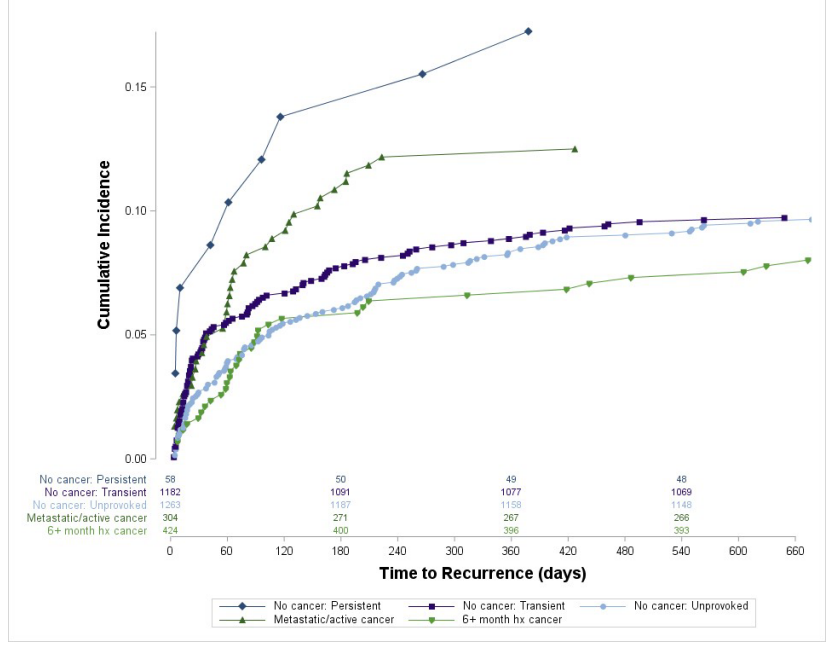

FIGURE 1 Cumulative incidence of first recurrent venous thromboembolism

Conclusions: The cumulative incidence of recurrent VTE is higher in patients with active cancer and in those without cancer with persistent provoking factors, and was similar (8-9.7\%) among the other provoking status categories. Further clinical trials of extended anticoagulation seem warranted for patients with VTE associated with transient provoking factors or a history of cancer $\geq 6$ months previously.

PB1106 | Bleeding and Venous Thromboembolism Recurrence in Patients with Gastrointestinal and Genitourinary Malignancies Treated with Anticoagulation

D. Attia ${ }^{1}$; X. Jia ${ }^{2}$; M. Wilks ${ }^{3}$; B. Tripp ${ }^{3}$; C. D’Andrea ${ }^{1}$; K.R. McCrae ${ }^{1}$; D.E. Angelini ${ }^{4}$; A.A. Khorana ${ }^{4}$

${ }^{1}$ Cleveland Clinic Foundation-Taussig Cancer Institute, Cleveland, United States; ${ }^{2}$ Department of Quantitative Health Sciences, Cleveland Clinic, Cleveland, United States; ${ }^{3}$ Cleveland Clinic Foundation, Cleveland, United States; ${ }^{4}$ Taussig Cancer Institute, Cleveland Clinic Foundation, Cleveland, United States

Background: Direct oral anticoagulants (DOACs) are a new treatment option for cancer associated venous thromboembolism (VTE). Randomized trials comparing DOACs to low molecular weight heparins (LMWHs) showed treatment with DOACs conferred less risk of recurrent VTE,but potentially higher rates of bleeding, especially in patients with gastrointestinal (GI) and genitourinary (GU) cancers.

Aims: To report rates of bleeding and recurrent VTE of cancer patients(pts)treated with anticoagulation in a centralized cancer associated thrombosis (CAT) Clinic.

Methods: A prospective cohort of pts referred to our CAT clinic from 8/2014-05/2020 ( $N=1,651)$. Pts with primary GI and GU malignancies treated with LMWH or DOACs for acute VTE were included. Bleeding was defined using the ISTH criteria for major and clinically relevant non-major bleeding (CRNMB). The comparison of bleeding 
rates among treatment groups were examined using Fisher's exact test.Rates of recurrent VTE were analyzed using a competing model in which death was treated as a competing risk.

Results: Of506 pts with acute VTE,49 (9.7\%) primary GI cancer and $54(10.7 \%)$ GU cancers.48.1\% males, median age 64 years (19-95) and $17.1 \%$ had a prior VTE. Overall, pts with GI cancers were less likely to be treated with DOACs compared to other sites (4\% upper GI, 4.8\% lower GI, 11.5\% GU, $12.4 \%$ others, P0.05). Overall,the 6-month,1-year, and 2-year VTE recurrence rate among GI cancer pts was $6 \%, 8 \%, 6 \%$, respectively with similar recurrence rates between DOAC and LMWH groups (HR0.76,95\% Cl 0.17-3.32, p0.72) VS. 4\%, 4\%, 4\% among GU cancer pts with no difference between LMWH vs DOAC groups (HR 0.76,95\% Cl 0.05-11.19, p0.83). Of 506 pts for whom 6-month follow-up data was available, 80 (14.3\%) had bleeding events, of which 26 (5.1\%) major bleeding and 54 (10.7\%) CRNMB with no difference across treatment groups ( $p$ 0.072). No difference in bleeding rate in pts with $\mathrm{GI}$ malignancies while pts with GU cancers tend to experience more CRNMB (table 1).

Table 1 6-month Bleeding Rates in GI and GU malignancies

\begin{tabular}{|c|c|c|c|c|c|c|}
\hline \multirow[b]{2}{*}{ Bleeding rates } & \multicolumn{3}{|c|}{ GI Cancer } & \multicolumn{3}{|c|}{ GU Cancer } \\
\hline & $\mathrm{N}$ & $\%$ & $\mathbf{P}$ & $\mathrm{N}$ & $\%$ & $\mathbf{P}$ \\
\hline Major & 7 & $15.2 \%$ & & 5 & $8.1 \%$ & \\
\hline CRNMB & 4 & $8.7 \%$ & & 8 & $12.9 \%$ & \\
\hline DOAC & 21 & $16.8 \%$ & & 19 & $11.5 \%$ & \\
\hline Others & 3 & $2.4 \%$ & & 6 & $3.6 \%$ & \\
\hline Warfarin & 1 & $0.8 \%$ & & 2 & $1.2 \%$ & \\
\hline
\end{tabular}

Conclusions: In our CAT clinic,There was no difference in the rate of recurrent VTE or bleeding among pts with GI and GU cancers treated with LMWH or DOACs.In both cohorts,bleeding rates were high within 6 months of starting anticoagulation.

\section{PB1107 | Role of Tissue Factor in the Procoagulant Shift} of Endothelial Cells upon Exposure to Cancer Cell-derived Microparticles

$\underline{\text { R. Djedidi-Amrane }}^{1,2}$; P. Vandreden ${ }^{2}$; G. Gerotziafas ${ }^{1,3,4}$

${ }^{1}$ Research Group "Cancer-Angiogenesis-Haemostasis" INSERM 4938

Faculty of Medicine, Sorbonne University, Paris, France; ${ }^{2}$ Clinical Research Department, Diagnostica Stago, Gennevilliers, France; ${ }^{3}$ Clinical Hemostasis and Thrombosis, Department of Hematology and Cell Therapy, Saint Antoine Hospital, AP-HP.6, Paris, France; ${ }^{4}$ Department of Biological Haematology, Tenon University Hospital, APHP.6, Paris, France

Background: Endothelium activation is essential in pathogenesis of cancer associated thrombosis (CAT). Endothelial cell (EC) is a potential target of cancer cell derived microparticles (CaCe-dMPs). We recently showed that EC exposed to $\mathrm{CaCe}-\mathrm{dMPs}$ acquire a procoagulant phenotype characterized by an enhancement of thrombin generation (TG) transferable to daughter cells.

Aims: We investigated the implication of tissue factor (TF) in the new procoagulant profile acquired by EC exposed to CaCe-dMPs and if TF alone is capable of inducing this change.
Methods: Microparticles released in conditioned medium from pancreas adenocarcinoma cells (BXPC3) were isolated with differential centrifugation. Human umbilical vein endothelial cells (HUVEC) were cultured for $72 \mathrm{~h}$ according to 5 experimental conditions: in presence of (a) BXPC3-dMPs (b) BXPC3 conditioned medium depleted in MPs (c) MP-Reagent (no TF and 4uM of phospholipids) (d) PPP-Reagent High (5pM TF; 4uM phospholipids) (e) PPP-Reagent Low (1pM TF; 4uM phospholipids) or (f) Dade Innovin (5nM TF, phospholipids, calcium). Capacity of exposed-EC to enhance TG in PPP was assessed with CAT assay (Thrombinoscope, Diagnostica Stago, France). TF concentration was determined by using the Zymutest Tissue Factor kit (Hyphen, France). 
Results:

TABLE 1 Thrombogram parameters in normal PPP of HUVEC cells exposed or not (control) to respectively BXPC3 derived vesicles (BXPC3-dEVs), BXPC3 conditioned medium depleted in vesicles (BXPC3-MC), human recombinant TF Dade ${ }^{\circledR}$ Innovin ${ }^{\circledR}$ (5nM TF, phospholipids and calcium), PPPReagent High (5pM TF and $4 \mu \mathrm{M}$ phospholipids), PPP-Reagent Low (1pM TF and $4 \mu \mathrm{M}$ phospholipids) or MP-Reagent (no TF and $4 \mu \mathrm{M}$ of phospholipids). Values are mean \pm sd of 3 experiments

\begin{tabular}{|c|c|c|c|c|c|c|c|}
\hline & $\begin{array}{l}\text { HUVEC } \\
\text { control }\end{array}$ & $\begin{array}{l}\text { HUVEC + } \\
\text { BXPC3- } \\
\text { dEVs }\end{array}$ & $\begin{array}{l}\text { HUVEC + } \\
\text { BXPC3-MC }\end{array}$ & $\begin{array}{c}\text { HUVEC + } \\
\text { Innovin }\end{array}$ & $\begin{array}{c}\text { HUVEC + } \\
\text { PPP-R } \\
\text { High }\end{array}$ & $\begin{array}{c}\text { HUVEC + } \\
\text { PPP-R } \\
\text { LoW }\end{array}$ & $\begin{array}{c}\text { HUVEC + } \\
\text { MP-R }\end{array}$ \\
\hline Lagtime (min) & $\begin{array}{l}10,92 \pm \\
0,83\end{array}$ & $\begin{array}{l}3,50 \pm \\
0,24\end{array}$ & $9,59 \pm 2,01$ & $\begin{array}{l}7,92 \pm \\
0,59\end{array}$ & $\begin{array}{l}9,75 \pm \\
1,06\end{array}$ & $\begin{array}{l}10,25 \pm \\
2,72 \\
\end{array}$ & $\begin{array}{l}11,09 \pm \\
0,59\end{array}$ \\
\hline ETP (nM.min) & $\begin{array}{l}800,98 \pm \\
48,03\end{array}$ & $\begin{array}{l}698,07 \pm \\
80,49\end{array}$ & $\begin{array}{l}627,62 \pm \\
183,48\end{array}$ & \begin{tabular}{|l|}
$743,73 \pm$ \\
107,75
\end{tabular} & $\begin{array}{l}621,16 \pm \\
44,80\end{array}$ & \begin{tabular}{|l|}
$841,77 \pm$ \\
38,64 \\
\end{tabular} & $\begin{array}{l}762,31 \pm \\
18,17\end{array}$ \\
\hline Peak (nM) & $\begin{array}{l}56,26 \pm \\
15,94\end{array}$ & $\begin{array}{l}43,12 \pm \\
2,85\end{array}$ & $\begin{array}{l}52,05 \pm \\
9,69\end{array}$ & $\begin{array}{l}40,87 \pm \\
4,14\end{array}$ & $\begin{array}{l}32,91 \pm \\
1,87\end{array}$ & $\begin{array}{l}64,27 \pm \\
5,90\end{array}$ & $\begin{array}{l}54,76 \pm \\
1,14\end{array}$ \\
\hline ttPeak (min) & $\begin{array}{l}17,92 \pm \\
1,77\end{array}$ & $\begin{array}{l}12,42 \pm \\
0,12\end{array}$ & $\begin{array}{l}16,94 \pm \\
2,01\end{array}$ & $\begin{array}{l}16,34 \pm \\
0,47\end{array}$ & $\begin{array}{l}18,33 \\
0,71\end{array}$ & $\begin{array}{l}16,84 \pm \\
2,60 \\
\end{array}$ & $\begin{array}{l}18,50 \pm \\
0,71\end{array}$ \\
\hline MRI (nM/min) & $\begin{array}{l}8,26 \pm \\
3,38\end{array}$ & $\begin{array}{l}3,50 \pm \\
0,24\end{array}$ & $7,08 \pm 1,32$ & $\begin{array}{l}4,85 \pm \\
0,43\end{array}$ & $\begin{array}{l}3,83 \pm \\
0,06\end{array}$ & $\begin{array}{l}9,75 \pm \\
0,72\end{array}$ & $\begin{array}{l}7,39 \pm \\
0,84\end{array}$ \\
\hline
\end{tabular}

Table I. Thrombogram parameters in normal PPP of HUVEC cells exposed or not (control) to respectively $\mathrm{BXPC} 3$ derived vesicles (BXPC3-dEVs), BXPC3 conditioned medium depleted in vesicles (BXPC3-MC), human recombinant TF Dade® Innovin $(5 \mathrm{nM} \mathrm{TF}$, phospholipids and calcium), PPPReagent High ( $5 \mathrm{pM}$ TF and $4 \mu \mathrm{M}$ phospholipids), PPP-Reagent Low (1pM TF and $4 \mu \mathrm{M}$ phospholipids) or MP-Reagent (no TF and $4 \mu \mathrm{M}$ of phospholipids). Values are mean \pm sd of 3 experiments.

TABLE 2 Tissue factor concentration of HUVEC cells exposed or not (control) to respectively BXPC3 derived vesicles (BXPC3-dEVs), BXPC3 conditioned medium depleted in vesicles (BXPC3-MC), human recombinant TF Dade ${ }^{\circledR}$ Innovin ${ }^{\circledR}$, PPP-Reagent High, PPPReagent Low or MP-Reagent. Values are mean \pm sd of 3 experiments

\begin{tabular}{|l|l|l|l|l|l|l|l|l|}
\hline & $\begin{array}{c}\text { HUVEC } \\
\text { control }\end{array}$ & $\begin{array}{c}\text { HUVEC+ } \\
\text { BXPC3- } \\
\text { dEVs }\end{array}$ & BXPC3-MC & $\begin{array}{c}\text { HUVEC } \\
+ \\
\text { BXPC3- } \\
\text { MC }\end{array}$ & $\begin{array}{c}\text { HUVEC } \\
+ \\
\text { Innovin }\end{array}$ & $\begin{array}{c}\text { HUVEC } \\
\text { + PPP-R } \\
\text { High }\end{array}$ & $\begin{array}{c}\text { HUVEC } \\
\text { + PPP-R } \\
\text { Low }\end{array}$ & $\begin{array}{c}\text { HUVEC } \\
\text { + MP-R }\end{array}$ \\
\hline $\begin{array}{l}\text { TF } \\
(\mathbf{p g} / \mathbf{m l})\end{array}$ & 0,00 & $\begin{array}{l}563,84 \pm \\
47,47\end{array}$ & $\begin{array}{l}361,1 \pm \\
47,49\end{array}$ & 0,00 & 0,00 & 0,00 & 0,00 & 0,00 \\
\hline
\end{tabular}

Table II. Tissue factor concentration of HUVEC cells exposed or not (control) to respectively BXPC 3 derived vesicles (BXPC3-dEVs), BXPC3 conditioned medium depleted in vesicles (BXPC3-MC), human recombinant TF Dade $®$ Innovin $₫$, PPP-Reagent High, PPP-Reagent Low or MP-Reagent Values are mean \pm sd of 3 experiments.

HUVEC exposed to BXPC3-dMPs acquired a procoagulant profile with a significant enhancement of TG as compared to control experiment (non-exposed HUVEC). However, HUVEC exposed to BXPC3 conditioned medium, Innovin, MP-R, PPP-R high or low are not capable to enhance TG and display thrombogram parameters similar to the control (Table I). Furthermore, only HUVEC exposed to BXPC3dMPs display a high amount of TF $(563,84 \pm 47,47 \mathrm{pg} / \mathrm{ml})$ (Table II). Conclusions: According to the histological type of cancer, $\mathrm{CaCe}$ dMPs induce a procoagulant shift of EC that present high TF activity. However, exposition to soluble TF and thus, also with high concentration, can't induce this procoagulant shift. Indeed, only TF+ MPs can induce this change and represent a potent activator of EC.
PB1108 | Impact of a Smartphrase Venous Thromboembolism Risk Assessment Tool on Prophylaxis Prescribing Rates in a Gynecologic Oncology Clinic

M. Duco; J. MacDonald; B. Orr; E. Weeda; N. Bohm

Medical University of South Carolina, Charleston, United States

Background: Gynecologic cancer confers a high risk for developing venous thromboembolism (VTE). Current guidelines recommend VTE prophylaxis for cancer patients with a Khorana score $\geq 2$. One report found that $<10 \%$ of oncology practitioners use a risk assessment tool, leading to low prescribing rates of VTE prophylaxis.

Aims: The primary objective was to assess the utilization of VTE prophylaxis in gynecologic oncology patients before and after implementation of a Khorana score-based smartphrase tool.

Methods: A smartphrase tool for VTE risk assessment was implemented in a gynecologic oncology clinic in October 2020. Adult patients initiating chemotherapy for newly diagnosed or recurrent disease between January 2014 through January 2020 were included in a historical cohort. Patients initiating chemotherapy between October 2020 and December 2020 were included in a prospective cohort. Data relating to VTE was collected for up to 6 months after treatment initiation.

Results: Of 110 patients included in the historical cohort, 48 (43.6\%) had a Khorana score $\geq 2$, compared to 6 of 16 (37.5\%) in the prospective cohort. None of the historical patients received prophylaxis, compared to 3 of $6(50 \%)$ in the prospective cohort $(P<0.001)$. Thrombosis occurred in 10 historical patients (9.1\%) compared to 2 (12.5\%) in the prospective cohort, all in patients not receiving VTE prophylaxis.

Conclusions: Implementation of a Khorana score screening tool significantly increased utilization of VTE prophylaxis in a gynecologic oncology clinic; however, thrombosis rates remained similar. Additional risk factors may need to be incorporated, and ongoing assessment of the intervention impact is necessary. Similar tools should be considered to improve prophylaxis prescribing rates in clinics with low uptake.

\section{PB1109 | The RIETE Score: A Useful Screening Tool for Occult Malignancy in VTE and STP?}

D. McBriar ${ }^{1}$; B. Jacob ${ }^{2}$; G. Benson ${ }^{1}$

${ }^{1}$ Belfast City Hospital, Belfast, United Kingdom; ${ }^{2}$ NHS Fife, Edinburgh, United Kingdom

Background: Venous thromboembolism (VTE) and superficial thrombophlebitis (STP) have long been associated with cancer. Investigation of patients with VTE or STP may allow early diagnosis of an occult malignancy, however the benefit of extensive screening remains controversial. The Registro Informatizado de Pacientes con Enfermedad TromboEmbólica (RIETE) score aims to identify patients 
at higher risk of occult malignancy. It is calculated using only patient information and a full blood count.

Aims: To evaluate the efficacy of the RIETE score at identifying occult malignancy in patents diagnosed with VTE or STP.

Methods: We analyzed all patients managed through our outpatient VTE service in 2019. At present patients are reviewed in clinic, along with a panel of bloods including tumor markers and a myeloma screen. The RIETE score was retrospectively applied and we followed patients for a minimum of one year to determine if they were subsequently diagnosed with a malignancy.

Results: After exclusions, 390 patients were included in the study, of which 28 (7\%) were identified as having a malignancy during this time.

The RIETE score was applied using a cut off score of 3 or greater. This returned a sensitivity for cancer of 0.54 ( $95 \%$ confidence interval 0.34-0.72), a positive predictive value of $0.17(0.12-0.27)$ and a negative predictive value was $0.96(0.93-0.98)$. Looking specifically at patients with PE the positive predictive value was 0.23 (0.050.54), with DVT 0.18 (0.09-0.31) and with STP 0.11 (0.02-0.29).

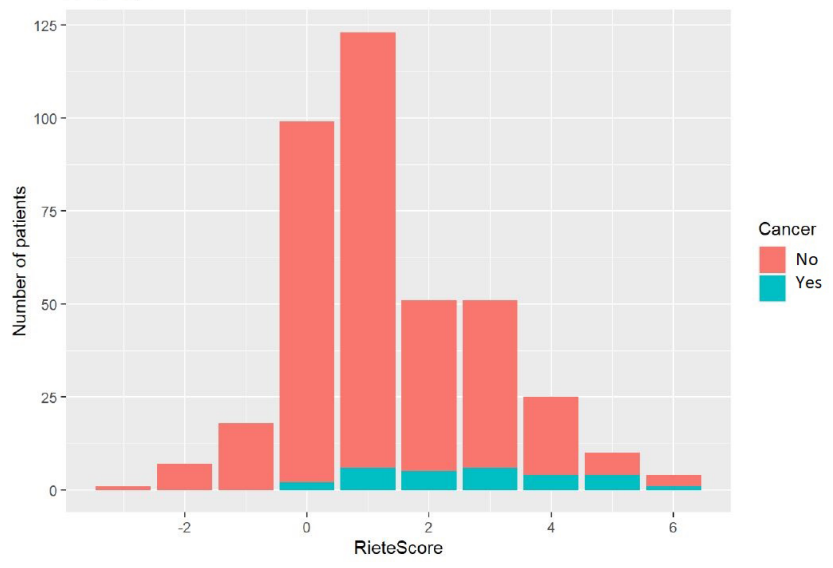

FIGURE 1 Bar chart to show the distribution of patients by RIETE score and the outcome of cancer screening

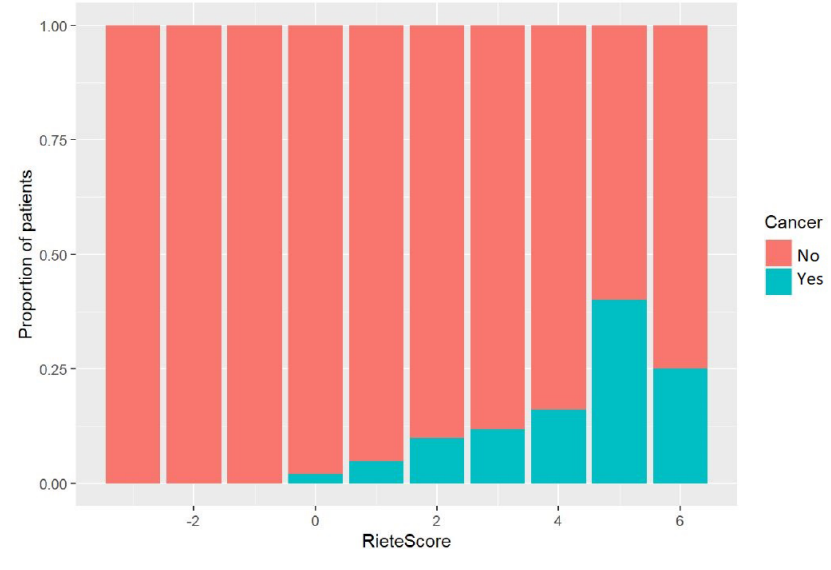

FIGURE 2 Bar chart to show the proportion of patients at each RIETE score who went on to be diagnosed with malignancy

Conclusions: This retrospective analysis demonstrates acceptable positive and negative predictive values for patients with PE, DVT and STP. Wider use could assist the diagnosis of occult malignancy in these patients, as well as reducing unnecessary investigation and consequent patient anxiety. Following this study, we will use the RIETE score to discriminate which patients undergo further investigation.

PB1110 | Differential Associations of Aromatase Inhibitors and Tamoxifen with Thrombin Generation and Sensitivity to Thrombomodulin: A Prospective Cohort Study

M. Blondon; L. Thouvenin; T. Lecompte; M. Righini; P. Fontana;

A. Bodmer; A. Casini

Geneva University Hospitals, Geneva, Switzerland

Background: Endocrine treatments with aromatase inhibitors (AI) or tamoxifen reduce the mortality of estrogen-positive breast cancer. While tamoxifen increases the risk of venous thromboembolism (VTE), whether Al influence hemostasis and the risk of VTE remains unclear. This information is critical when assessing women with breast cancer at high risk of VTE.

Aims: Among women with breast cancer, to evaluate the associations of $\mathrm{Al}$ or tamoxifen with changes on thrombin generation and sensitivity to thrombomodulin (TM)

Methods: We included 107 women with localized breast cancer, $\geq 4$ weeks after breast surgery. Exclusion criteria were a planned chemotherapy, a personal history of VTE and a recent use of exogenous hormones. We measured the laboratory hemostatic profile before and 10-16 weeks after initiation of Al or tamoxifen. Thrombin generation (calibrated automated thrombography) was determined in platelet-poor plasma using $5 \mathrm{pM}$ of tissue factor, $4 \mu \mathrm{M}$ of phospholipids, and with/without $2 \mathrm{nM}$ of TM. Variables of thrombin generation and of endogenous thrombin potential-based normalized TM sensitivity ratios (nTMsr) were compared using paired T-tests. All women provided informed consent. 
Results: Compared with women using AI ( $n=65)$, women using tamoxifen ( $n=42)$ were younger (49.5y (SD $=8.9)$ vs. 65.5y (SD = 9.4)) Previous cardiovascular disease was rare (1.9\%). Most common breast cancer stages were IA (51.4\%), IIA (19.6\%) and IIB (10.3\%). Compared with baseline, the ETP and thrombin peak height were increased with tamoxifen therapy $(+174 n \mathrm{Mxmin}, 95 \% \mathrm{Cl} 34-142$ and $+33 \mathrm{nM}, 95 \% \mathrm{C} \mathrm{I} 21-44)$ but not with $\mathrm{Al}$ (+46nMxmin, $95 \% \mathrm{Cl}-4$ to 95 and $+8 \mathrm{nM}, 95 \% \mathrm{Cl}-2$ to 17$)$. NTMsr were increased with tamoxifen $(+0.26,95 \% \mathrm{Cl} 0.19-.033)$ but not with $\mathrm{Al}(+0.03,95 \% \mathrm{Cl}-0.02$ to 0.08 ).

Conclusions: Tamoxifen is associated with an in vitro hypercoagulable state that is not found in users of Al. This analysis provides some evidence supporting the use of $\mathrm{Al}$ in women with breast cancer at high risk of VTE.

PB1111 | Direct Oral Anticoagulants vs. Low-molecular-Weight Heparin for the Treatment of Acute Venous Thromboembolism Associated with Gastrointestinal Cancer: A Systematic Review and Meta-analysis

T. Rungjirajittranon; W. Owattanapanich; Y. Chinthammitr;

T. Ruchutrakool; B. Suwanawiboon

Division of Hematology, Department of Medicine, Faculty of Medicine Siriraj Hospital, Mahidol University, Bangkok, Thailand

Background: The association of gastrointestinal (GI) cancer and a high incidence of venous thromboembolism (VTE) is well-known. Prior randomized studies demonstrated that direct oral anticoagulants (DOACs) effectively treated cancer-associated VTE (CAT). However, some DOACs appeared to increase the risk of bleeding, particularly in patients with GI malignancies. Therefore, the current systematic review and meta-analysis were conducted to evaluate the safety and efficacy of DOACs in GI cancer-associated thrombosis.

Aims: To study the efficacy and safety of DOACs vs. low-molecularweight heparin (LMWH) for the treatment of acute VTE in patients with GI cancer.

Methods: All relevant studies that compared DOACs and LMWH in GI cancer-associated thrombosis published before December 2020 were individually searched for in two databases (MEDLINE and EMBASE) by two investigators. The effect estimates and $95 \%$ confidence intervals $(\mathrm{Cl})$ from each eligible study were combined using the Mantel-Haenszel method.

Results: A total of 7 eligible studies were included in this metaanalysis. Major bleeding rate was similar in both groups (OR 1.71, $95 \% \mathrm{Cl}, 0.93-3.14, P=0.08,12=46 \%)$. However, the clinically relevant non-major bleeding (CRNMB) rate was significantly higher in the DOACs group (OR 2.28, 95\% Cl: $1.45-3.59, P=0.0004,12=0 \%$ ). The risk of recurrent VTE was not statistically different in both groups (OR 0.72, 95\% Cl: 0.40-1.29, $P=0.27,12=0 \%$ ).

Conclusions: Current data suggest that treatment of CAT with DOACs increased the risk of CRNMB but not major bleeding in patients with GI malignancies. The efficacy of DOACs for preventing recurrent VTE in GI cancer was similar to that of $\mathrm{LMWH}$.

PB1113 | Risk of Vascular Occlusive Events with PARPis in Cancer: A Systematic Review and Meta-analysis

H. Haguet $^{1}$; L. Ronvaux ${ }^{1}$; J. Douxfils ${ }^{1,2}$

${ }^{1}$ UNamur, Namur, Belgium; ${ }^{2}$ QUALIblood s.a., Namur, Belgium

Background: Poly(ADP-ribose) polymerase inhibitors (PARPis) are anticancer drugs that blocked PARP-1 auto-PARylation. As PARP-1 possesses pro-inflammatory functions involved in the thrombotic process (e.g. expression of adhesion molecules, production of proinflammatory cytokines), we hypothesized that PARPis could prevent the development of vascular occlusive events (VOEs).

Aims: To investigate the risk of VOE in patients with cancers treated with PARPis in randomized clinical trials (RCTs).

Methods: The literature search (data lock point: April 17, 2020) was conducted according to a registered protocol (PROSPERO CRD42020179676). All RCTs comparing a PARPi versus placebo or standard of care (SoC) for cancer treatment were included. Two independent investigators were responsible of the screening, the review and the data extraction.

The meta-analysis was performed using a random (REM) and a fixed (FEM) effect model according to the characteristics of the included studies. ORs with $95 \% \mathrm{Cls}$ were computed using the Peto method for the analysis of VOE and the Mantel-Haenszel method for progression-free survival (PFS) and overall survival (OS). Publication bias was assessed by funnel plots.

Results: Among the 2424 abstracts identified, 13 RCTs fulfilled established criteria. Overall, $2.78 \%$ (84/3028) of patients developed a VOE with a PARPi compared with 1.94\% (30/1549) in the control group (FEM OR ${ }_{\text {PETO }} 1.40 ; 95 \% \mathrm{Cl}, 0.94-2.09$ ). This result is consistent whatever the comparator (i.e. placebo and SoC). PARPis significantly improve PFS (REM OR $\left.{ }_{M-H} 1.70 ; 95 \% \mathrm{Cl}, 1.10-2.61\right)$. This difference is non-significant when PARPis are compared to SoC $\left(R E M \mathrm{OR}_{\mathrm{M}-\mathrm{H}}\right.$ 0.86 ; $95 \% \mathrm{Cl}, 0.64-1.14)$. OS was not significantly improved with the use of PARPis compared to controls (REM OR $_{\mathrm{M}-\mathrm{H}} 1.13 ; 95 \% \mathrm{Cl}, 0.98-$ 1.31). Funnel plots demonstrate no evidences of publication bias. 


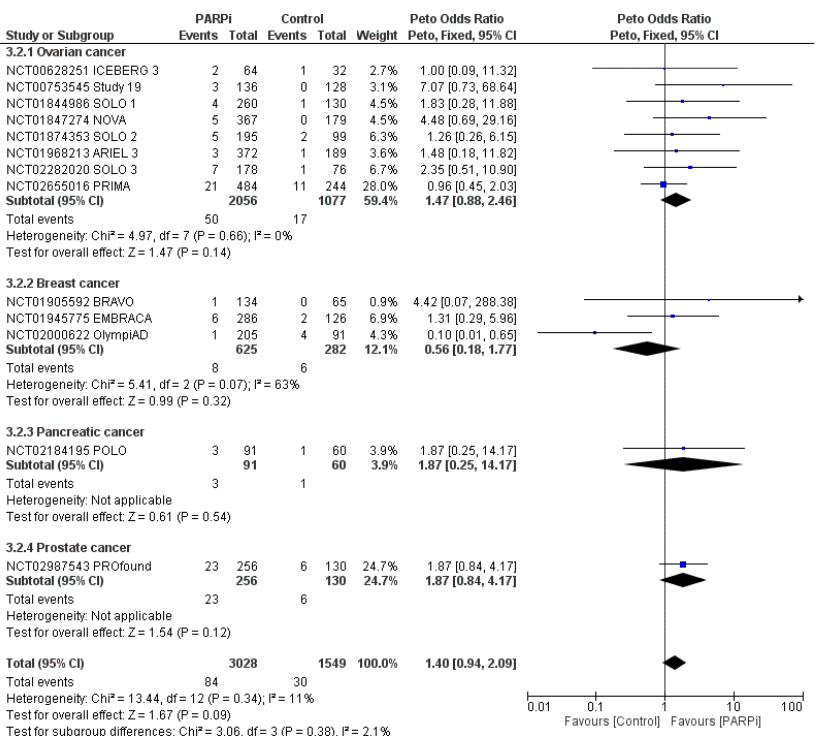

FIGURE 1 Forest plot of vascular occlusive events comparing PARPis to SoC or placebo stratified by cancer type

Conclusions: Our meta-analysis indicates a tendency toward an increased risk of VOE with PARPis compared to SoC or placebo, requiring careful pharmacovigilance activities of these treatments.

PB1114 | Clinical Risk Factors for Venous Thromboembolism in Hematologic Malignancies

A. López Sacerio ${ }^{1} ;$ N. Alvarez Basulto ${ }^{2} ;$ M. Acosta Alvarez ${ }^{1}$;

M.C. Tejeda Ramón ${ }^{1}$

${ }^{1}$ Arnaldo Milián University Hospital, Santa Clara, Cuba; ${ }^{2}$ Amalia Simoni University Hospital, Camagüey, Cuba

Background: During the last year, 1558 deaths were caused by Hematologic Malignancies (HM) in Cuba. Patients with Hematologic Malignancies (HM) are at high risk of venous thromboembolism (VTE). Some clinical risk factors have been related to thrombotic events, but prophylaxis in hospitalized patients is still inconsistent and not risk adjusted in our country.

Aims: To identify clinical risk factors associated to VTE and to determine overall survival (OS) in patients with $\mathrm{HM}$.

Methods: A case-control study was carried out at Arnaldo Milián University Hospital in Santa Clara, Cuba from January 2018 to January 2021. The study population was 222 patients ( 66 with VTE, 156 without VTE), hospitalized with a diagnosis of HM. For the univariate analysis chi-square test, Odds Ratio (OR) with 95\% confidence interval and Cramer's $\mathrm{V}$ were applied. The logistic regression was performed to identify potential predictors of VTE in the multivariate analysis. OS was determined by the Kaplan Meier method. Informed consent was obtained and the study was approved by a medical ethics committee according to the Declaration of Helsinki.
Results:

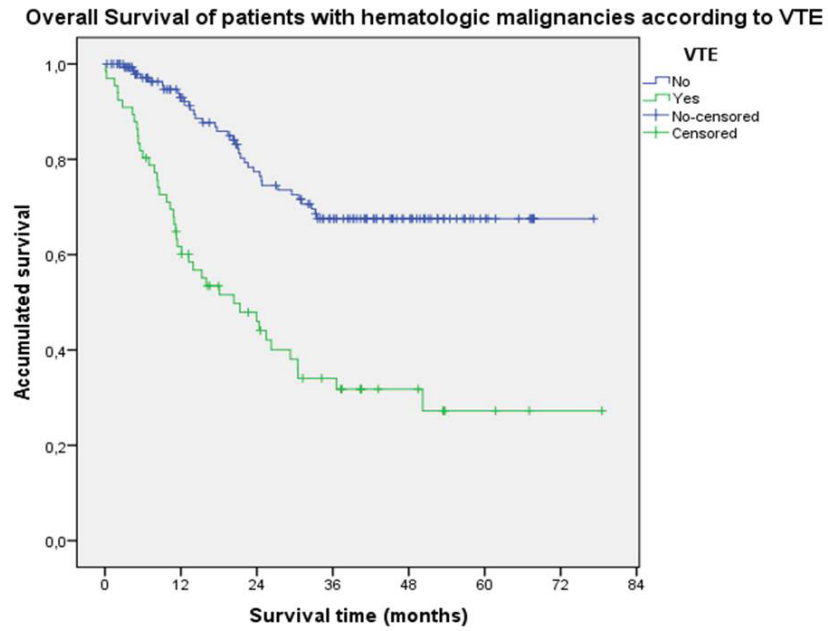

FIGURE 1 Overall survival of patients with Hematologic malignancies according to VTE

On univariate analysis, variables associated with an increased VTE risk were: bedding $(O R=5.73, p 0.000)$, diabetes mellitus $(O R=4.54$, p 0,000), hypercholesterolemia ( $O R=5.48, p$ 0.000), tumoral activity $(O R=7.22, p 0.000$ ), obesity (OR $=2.50$, p 0.012), history of prior thrombosis(OR $=3.52, \mathrm{p}$ 0.002) and use of thrombogenic drugs ( OR $=1.95, \mathrm{p} 0.030$ ), mainly steroids and hormonal therapy. By logistic regression bedding, tumoral activity, diabetes mellitus, hypercholesterolemia and the use of thrombogenic drugs were identified as predictive factors. At 36 months, the OS of patients without VTE were $67.5 \%$ vs $31.8 \%$ in the group with VTE (figure 1 ).

Conclusions: Several risk factors are associated to the occurrence of VTE in patients with HM. VTE has an important role in the diminished OS of this patients.

PB1115 | Treatment of Venous Thromboembolism (VTE) Events with Cancer as a Risk Factor in a Teaching Hospital: 2015-2020

H. Rowswell; T. Nokes

University Hospitals Plymouth NHS Trust, Plymouth, United Kingdom

Background: Cancer is a well-known risk factor for VTE. Data is presented on various metrics, for patients with VTE in the context of underlying Cancers and treatment.

Aims: To identify all VTE events diagnosed between 2015 and 2020, associated with active cancer as a risk factor. Assess treatment management over the study period looking at anticoagulation use and any changes to this over the study period.

Methods: All VTE events identified from targeted radiological data, comprising CT pulmonary angiograms. SPECT-CT and Doppler ultrasounds of upper and lower limbs, were reviewed for evidence of active cancer as a risk factor. Cancer type and degree of metastasis was also assessed, together with anticoagulant used. 
Results:

TABLE 1 Cancer Associated VTE events 2015-2020

\begin{tabular}{|c|c|c|c|c|c|}
\hline YEAR & Total VTE & Number Cancer RF & DVT (Met) & PE (Met) & Total \\
\hline 2016 & 861 & 188 & $75(17)$ & $113(18)$ & 188 \\
\hline 2018 & 822 & 179 & $67(15)$ & $112(28)$ & 179 \\
\hline 2019 & 769 & 167 & $60(10)$ & 107 (19) & 167 \\
\hline Total & 4881 & 1102 & $396(36 \%)$ & $706(64 \%)$ & 1102 \\
\hline
\end{tabular}

A total of 4881 VTE events occurred over six years. 1102 (23\%) of these had active cancer as a risk factor. Of the 3800 communityacquired (CAT) events, 721 (19\%) were cancer-associated, compared to 381 of the 1081 hospital acquired (HAT) events (35\%).There is a significant association of PE over DVT for cancer associated thrombosis. Overall 214/1102 (19\%) demonstrated metastatic cancer. The most prevalent cancers were lung, prostate, breast and bowel, comprising $54 \%$ of all cancers associated with VTE. Low molecular weight heparin (LMWH) was most widely prescribed anticoagulation in $66 \%$ of cases, then Rivaroxaban at $23 \%$ and Apixaban at $6 \%$. The use of direct oral anticoagulants generally, has increased significantly over the last two years.

Conclusions: Cancer is a major risk factor for VTE. Using the UK Department of Health, VTE risk assessment tool, only age (>60 years) demonstrated a larger risk within our VTE outcome data. The most common cancers identified are not those known to have the highest VTE risk, but are the more prevalent cancers, therefore explaining this outcome. With trials now published around this topic further use of direct oral anticoagulants would be expected to be seen.

PB1116 | Treatment of Cancer-associated Thrombosis with Six Months of Prophylactic-dose Enoxaparin after Initial Full-dose Anticoagulation: A Pilot Study

J. Popov' ${ }^{1}$; S. Coelho ${ }^{2}$; M. Carrier ${ }^{3}$; C. Sperlich ${ }^{4}$; S. Solymoss ${ }^{5,6}$; N. Routhier ${ }^{7,8}$; S. Shivakumar ${ }^{9,10}$; W. Aibibula ${ }^{2}$; S. Kahn ${ }^{1,2}$; V. Tagalakis ${ }^{1,2}$

${ }^{1}$ McGill University, Montreal, Canada; ${ }^{2}$ Jewish General Hospital/Lady Davis Institute, Montreal, Canada; ${ }^{3}$ Ottawa Hospital Research Institute at the University of Ottawa, Ottawa, Canada; ${ }^{4}$ CSSS Charles-Le Moyne, Greenfield Park, Canada; ${ }^{5}$ cGill University Health Centre, Montreal, Canada; ${ }^{6}$ St. Mary's Hospital, Montreal, Canada; ${ }^{7}$ Université de Montréal, Montréal, Canada; ${ }^{8}$ Hôpital Sacré Coeur, Montréal, Canada; ${ }^{9}$ Dalhousie University, Halifax, Canada; ${ }^{10}$ Queen Elizabeth II Health Sciences Centre, Halifax, Canada

Background: Patients with cancer-associated thrombosis (CAT) are treated with full-dose anticoagulation for at least three months. Optimal dosing thereafter is unknown.
Aims: We explored efficacy and safety of reduced-dose low molecular weight heparin (LMWH) following a minimum of three months of full-dose LMWH.

Methods: We conducted a multicenter prospective pilot study of patients with CAT who completed at least three months of weightadjusted LMWH. Patients received six months of prophylactic-dose (40 mg) subcutaneous enoxaparin. The primary outcome was recurrence of deep vein thrombosis (DVT) or pulmonary embolism (PE) and secondary outcomes included major, clinically relevant nonmajor (CRNM), and minor bleeding. The research ethics boards of participating centers approved the study, and informed consent was obtained from all participants.

Results: From August 2016 to May 2019, 52 patients with a mean age of $64.1 \pm 9.7$ years were included. The study was stopped early due to poor recruitment following approval of direct oral anticoagulants for CAT. Breast (23.1\%) and colon (11.5\%) were the most common cancers; $61.0 \%$ had stage IV malignancy. CAT consisted of DVT alone in $57.7 \%$ of patients and PE with or without DVT in $42.3 \%$. Patients received a mean of $7.6 \pm 7.4$ months of weight-adjusted LMWH prior to enrollment. During a mean follow-up of $5.6 \pm 1.4$ months, 1 patient was diagnosed with incidental PE. There were no major bleeding events, and 1 CRNM and 1 minor bleeding event. Eight (15.4\%) patients died: 6 due to cancer and 2 due to respiratory disease unrelated to PE.

Conclusions: Among patients with CAT treated with at least three months of full-dose anticoagulation, we observed one incidental PE and two non-major bleeding events during six months of prophylactic-dose LMWH. Our results provide support for clinical trials of reduced-dose anticoagulation for secondary prevention of CAT. 
PB1117 | Venous Thromboembolism in Children with Acute Lymphoblastic Leukemia in China: A Multi-center Clinical Study

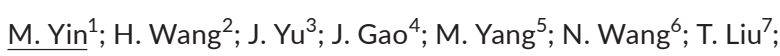
S. Shen ${ }^{8}$; A.W. Leung ${ }^{9}$; F. Zhou ${ }^{10} ;$ X. Wu ${ }^{11}$; J. Huang ${ }^{12} ;$ H. Lii $^{13}$; S. $\mathrm{Hu}^{14} ;$ X. Tian ${ }^{15} ;$ H. Jiang ${ }^{16}$; X. Zhai ${ }^{2}$; J. Tang ${ }^{8}$; Q. Hu ${ }^{1}$

${ }^{1}$ Department of Pediatrics, Tongji Hospital of Tongji Medical College, Huazhong University of Science and Technology, Wuhan, China; ${ }^{2}$ Department of Hematology/Oncology, Children's Hospital of Fudan University, Shanghai, China; ${ }^{3}$ Department of Hematology/Oncology, Chongaing Medical University Affiliated Children's Hospital, Chongqing, China; ${ }^{4}$ Department of Pediatrics, Key Laboratory of Birth Defects and Related Disease of Women and Children, Ministry of Education, West China Second University Hospital, Sichuan University, Chengdu, China; ${ }^{5}$ Department of Pediatrics, Xiangya Hospital Central South University, Changsha, China; ${ }^{6}$ Department of Pediatrics, Anhui Medical University Second Affiliated Hospital, Anhui, China; ${ }^{7}$ State Key Laboratory of Experimental Hematology and Division of Pediatric Blood Diseases Center, Institute of Hematology and Blood Diseases Hospital, Peking Union Medical College, Chinese Academy of Medical Sciences, Tianjin, China; ${ }^{8}$ Department of Hematology/Oncology, Shanghai Children's Medical Center, School of Medicine, Shanghai Jiao Tong University, Key Laboratory of Pediatric Hematology \& Oncology of China Ministry of Health, Shanghai, China; ${ }^{9}$ Department of Pediatrics, Hong Kong Children's Hospital, The Chinese University of Hong Kong, Hong Kong, China; ${ }^{10}$ Department of Pediatrics, Union Hospital of Tongji Medical College, Huazhong University of Science and Technology, Wuhan, China; ${ }^{11}$ Department of Pediatrics, Nanfang Hospital, Southern Medical University, Guangzhou, China; ${ }^{12}$ Department of Hematology/Oncology, Children's Hospital of Nanjing Medical University, Nanjing, China;

${ }^{13}$ Department of Hematology/Oncology, Shanghai Children's Hospital, Shanghai Jiao Tong University, Shanghai, China; ${ }^{14}$ Department of Hematology/Oncology, Children's Hospital of Soochow University, Suzhou, China; ${ }^{15}$ Department of Hematology/Oncology, Kunming Children's Hospital, Kunming, China; ${ }^{16}$ Department of Hematology/ Oncology, Guangzhou Women and Children's Medical Center, Guangzhou, China

Background: Venous thromboembolism (VTE) is a severe complication in children with acute lymphoblastic leukemia (ALL).

Aims: Investigating the incidence of VTE, clinical features and factors associated with VTE in ALL children.

Methods: We conducted a multi-institutional clinical study in 7640 consecutive newly diagnosed ALL patients treated on China Children's Cancer Group ALL-2015 protocol in 20 hospitals between January 2015 and December 2019.We summarized the clinical characteristics of ALL patients with VTE. Data were compared using the Chi-square or Fisher's exact test.

Results: A total of 159 (2.08\%, 159/7640) patients with a first VTE occurred during the median time follow-up period of 3.3 years, and overall survival rate was $96.23 \%$ (153/159). The incidence rate of VTE was 62.1 per 10000 person-years, and 56.6\% (90/159) occurred during induction therapy. Of the 159 VET patients, 108
(67.92\%, 108/159) patients with an initial VTE in upper extremities, $21(13.21 \%, 21 / 159)$ in lower extremities, 28 (17.61\%,28/159) in cerebral veins, $1(0.63 \%, 1 / 159)$ in right atrium, and $1(0.63 \%, 1 / 159)$ with pulmonary embolism. Clinical factors associated with VTE included T-ALL $(P=0.015)$, mediastinal mass $(P=0.032), B C R / A B L 1$ fusion ( $P=0.049$ ), hepatosplenomegaly $(P=0.04), \mathrm{WBC} \geq 50 \times 10^{9} / \mathrm{L}$ at diagnosis $(P=0.04)$, age between $12-18$ years $(P=0.027)$, and developed sepsis $(P=0.003)$. The abnormal of some biomarkers such as serum albumin $(P=0.005)$, PLT $(P<0.001)$, D-2 polymer $(P<0.001)$ and so on may predict the occurrence of VTE. No association between VTE occurrence and prognoses of ALL children $(P=0.294)$. Thromboprophylaxis cannot reduce VTE incidence $(P=0.107)$.

Conclusions: The incidence rate of VTE in ALL children was $2.08 \%$, and thrombosis most occurred in induction therapy and upper extremities. T-ALL, mediastinal mass, BCR/ABL1 fusion, hepatosplenomegaly, $W B C \geq 50 \times 109 / L$ at diagnosis, older age, and developed sepsis were associated with VTE occurrence. VTE had no association with ALL prognoses.

Correspondence: Xiaowen Zhai\&Jingyan Tang\&Qun Hu

This work was supported by VIVA-China Children's Cancer Foundation.

PB1118 | Anticoagulant Therapy and Related Bleeding Complications in Internal Medicine Wards

M.T. Sartori; F. Boscaro; A. Bozzolin; C. Zurlo; F. Fabris Department of Medicine, University Hospital of Padova, Padova, Italy

Background: Anticoagulant therapy (ACT) is widely used; however, there is little data on bleeding complications associated with ACT use in patients admitted to Internal Medicine wards - often elderly and/or with multiple comorbidities requiring a thorough assessment of its risk-benefit ratio.

Aims: To evaluate the prevalence of ACT use and related bleeding complications in patients admitted to Internal Medicine wards. To identify potential predictors of bleeding in ACT patients.

Methods: We conducted an observational, retrospective study using medical records of all consecutive patients discharged between January-June 2020. We subdivided ACT patients into bleeders and non-bleeders; we collected length of stay, mortality and clinical findings to identify predictors of bleeding. Patients taking prophylactic anticoagulation were excluded.

Results: We enrolled 511 patients (age $74.6 \pm 14.6$ years), 193 (37.8\%) undergoing ACT. Hospitalization for ACT-related bleeding was $6.2 \%$. Bleeders $(32 / 193,16.6 \%)$ had longer hospital stays $(16.0 \pm 0.6$ days vs. $9.9 \pm 7.4$ days, p 0.01 ), higher mortality rate ( $15.6 \%$ vs. $5.6 \%$, p 0.04) vs. non-bleeders. Most bleeders initiated ACT within 90 days (OR 2.22, 95\% Cl 1.02-4.79, p 0.04), had higher CRP values (76.2 \pm 75.6 vs. $45.9 \pm 76.3 \mathrm{mg} / \mathrm{L}, \mathrm{p} 0.001$ ) and active cancer (OR 3.79, $\mathrm{Cl}$ 1.72-8.82, $\mathrm{p}$ 0.002). LMWH associated with higher bleeding risk vs. DOACs and VKAs [OR 7.50 (2.86-19.68; p 0.0001) and OR 6.36 (2.13-19.03; p 0.001), respectively]. At multivariate analysis, 
concomitant LMWH and antiplatelet therapy were independent risk factors for ACT-related bleeding. Major bleedings occurred in $47 \%$ of patients, clinically relevant non-major bleedings in $34 \%$. Bleeding prompted hospitalization in $65 \%$ of cases.

Conclusions: In Internal Medicine wards ACT use and ACT-related bleeding complications were significant. LMWH was a risk factor for bleeding; unclear whether it stems from comorbidities or the drug itself.

PB1119 | Circulating Extracellular Vesicles in Patients with Cancer and Venous Thromboembolism: A Systematic Review and Meta-analysis

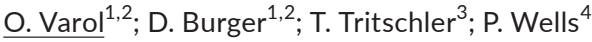

${ }^{1}$ Department of Cellular and Molecular Medicine, University of Ottawa, Ottawa, Canada; ${ }^{2}$ Kidney Research Center, Ottawa Hospital Research Institute, Ottawa, Canada; ${ }^{3}$ Department of General Internal Medicine, Inselspital, Bern University Hospital, University of Bern, Bern, Switzerland; ${ }^{4}$ Department of Medicine, University of Ottawa, and Hospital Research Institute, Ottawa, Canada

Background: Venous thromboembolism (VTE), defined as pulmonary embolism or deep vein thrombosis, is the second leading cause of mortality in cancer patients, second only to cancer itself. A number of reports suggest that circulating extracellular vesicles (EVs) may be increased in cancer patients with VTE.

Aims: The aim of this study was to determine if circulating EVs are higher in cancer patients with VTE compared to those without VTE. Methods: A comprehensive literature search was performed systematically in the databases Embase and MEDLINE from inception until April 20th, 2020. Study selection was performed by 2 authors independently. The pooled standardized mean difference (SMD) for EVs of any phenotype were compared between patients with and without VTE, and the summary odds ratio (OR) for VTE was estimated using random-effects meta-analysis.

Results: A total of 21 studies met the inclusion criteria and consisted of 9 cohort studies, 8 case-control studies, 3 cross-sectional studies, and 1 randomized control trial. The levels of Tissue Factor (TF)bearing EVs were higher in cancer patients presenting with VTE than in cancer patients that did not have VTE (pooled SMD 1.27; 95\% confidence interval [Cl], 0.27-2.27; $\left.P<0.001 ; I^{2}=95 \%\right)$. The SMD in case-control studies was $1.43\left(95 \% \mathrm{Cl},-0.02-2.87 ; \mathrm{I}^{2}=96 \%\right)$ and that in cohort studies was 1.09 (95\% Cl: $\left.0.27-1.91, \mathrm{I}^{2}=88 \%\right)$. The overall OR for VTE was $2.68\left(95 \% \mathrm{Cl}, 1.69-4.25 ; \mathrm{I}^{2}=59 \%\right)$. Similar results were found for $\mathrm{EV}$ analysis detection methods. Analysis for publication bias testing and sensitivity and subgroup analysis is ongoing.

Conclusions: Our meta-analysis demonstrates that EV levels are increased in cancer patients with VTE. These data suggest that EVs may be a biomarker of VTE in individuals with cancer.
PB1120 | Venous Thromboembolisms are Significantly Associated with Central Venous Catheter Loss in Pediatric Cancer Patients

Z. Forbrigger ${ }^{1}$; P. Moorehead ${ }^{2}$; K. Kulkarni ${ }^{3}$

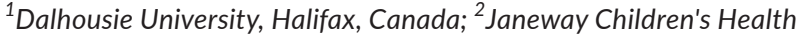
and Rehabilitation Centre, St. John's, Canada; ${ }^{3}$ WWK Health Centre, Halifax, Canada

Background: Central venous catheters (CVC's) are necessary for the treatment of pediatric cancer patients (PCPs). Loss of CVC during treatment requires immediate replacement. Venous thromboembolism (VTE) is a common side effect in PCPs, with a high proportion of VTE associated with CVC's. Data on CVC loss in relation to VTE is limited.

Aims: To determine the association between VTE and CVC loss.

Methods: This retrospective population-based study includes PCPs from Atlantic Canada (Prince Edward Island, New Brunswick, Nova Scotia, and Newfoundland). Patient demographics, diagnosis, CVC and thrombosis information was collected from 2000-2019. Patients without a CVC were excluded. The proportion of lost CVC's was determined by patients having greater than 1 CVCs.

Results:

TABLE 1 Demographic and clinical information of 1098 pediatric oncology patients treated between 2000 to 2019 in Atlantic Canada. Abbreviations: SD: standard deviation; VTE: venous thromboembolism; CVC: central venous catheter

$\begin{array}{lll} & \text { VTE }(N=92) & \text { No-VTE (N =1006) } \\ & N(\%) & N(\%) \\ \text { Sex (Male) } & 55(60) & 552(55) \\ \text { Diagnosis } & & \\ \text { Leukemia } & 35(38) & 366(36) \\ \text { Lymphoma } & 18(20) & 151(15) \\ \text { Sarcoma } & 17(18) & 133(13) \\ \text { Other } & 22(24) & 356(35) \\ \text { CVC mean (SD) } & 2.3(1.6) & 1.6(0.98) \\ 1 \text { CVC } & 41(45) & 647(64) \\ >1 \text { CVC } & 51(55) & 358(36)\end{array}$

A total of 1098 patients were included in the study. The M:F ratio was $55: 45 \%$. The age breakdown was $0-5$ years (47\%), 6-10 years (21\%), $11-15$ years (22\%), and $16+$ years (10\%). Diagnosis were leukemia (37\%), lymphoma (15\%), sarcoma (14\%), and other (34\%). Out of the 1098 patients, 92 developed a VTE (8.4\%). Seventy-four (80\%) had one VTE and $18(20 \%)$ had $>1$ for a total of 111 VTE's. Among patients with VTE, 68 (61\%) were CVC-associated. Sixty three percent of VTE were associated with a Port-a-Cath, $20 \%$ with a PICC/other CVC, and 17\% with a broviac/hickman, 
Patients with VTE had a mean of 2.3 CVCs while non-VTE patients had $1.6(P<0.001)$. Fifty-five percent of VTE patients experienced CVC loss compared to $36 \%$ of non-VTE patients $(P<0.001)$.

Conclusions: We demonstrate that PCPs with a VTE require significantly more CVCs during treatment and that VTE patients have a higher percent loss of CVCs in comparison to patients without a VTE. Future studies to reduce VTE and consequent CVC loss are prudent.

PB1121 | A Population-Based Descriptive Study of Long-term Anticoagulation in the Treatment and Secondary Prophylaxis of Venous Thromboembolism in Men with Prostate Cancer in Sweden

Y. Balabanova ${ }^{1}$; B. Farahmand ${ }^{2}$; P. Stattin ${ }^{3}$; H. Garmo ${ }^{3}$; G. Brobert ${ }^{2}$ ${ }^{1}$ Bayer AG, Berlin, Germany; ${ }^{2}$ Bayer AB, Stockholm, Sweden; ${ }^{3}$ Uppsala University, Uppsala, Sweden

Background: For most people with cancer-associated thrombosis, current guidelines recommend anticoagulation, usually with lowmolecular weight heparin $(\mathrm{LMWH})$ or a non-vitamin $\mathrm{K}$ oral anticoagulant (NOAC); however, the recommended duration varies. Observational data describing the anticoagulation received by men with prostate cancer in routine clinical practice are limited.

Aims: We aimed to evaluate the type and duration of long-term anticoagulant therapy prescribed for the treatment and secondary prophylaxis of venous thromboembolism (VTE) in men with prostate cancer in Sweden.

Methods: This population-based record-linkage cohort study used data from the National Prostate Cancer Register, the Prescribed Drug Registry and the Patient Registry in Sweden. We identified men with a first VTE between 2013 and 2017 and described the type and duration of outpatient anticoagulation they received.

Results: Among 1413 included men, 96\% started outpatient anticoagulant therapy within four weeks after VTE diagnosis. Almost two-thirds (64\%) were prescribed parenteral anticoagulation, 31\% a NOAC and $20 \%$ a VKA (men could receive $>1$ type of anticoagulant). Over the study period, use of parental anticoagulation declined from $83 \%$ to $53 \%$, VKA use declined from $45 \%$ to $4 \%$, and NOAC use increased from $5 \%$ to $51 \%$. Men received their first prescription within 1-6 days after their recorded VTE (median of 2 days). The median duration of use was 7 months (interquartile range [IQR] 3-13). A fifth of men were dispensed $\leq 3$ months anticoagulation, over half $(57 \%)$ $>6$ months' anticoagulation, and a quarter $>1$ year of anticoagulation. Duration was longer for pulmonary embolism (median 8 months, IQR 5-15) than for deep vein thrombosis (median 6 months, IQR 3-9).

Conclusions: Men with prostate cancer and VTE in Sweden were dispensed an average of 7 months' anticoagulation; however, a wide range of treatment durations were observed reflecting the individualised approach to treating VTE in these heterogeneous patients.
PB1122 | Janus Kinase 2 (JAK2V617F) Mutations in Patients with Splanchnic Vein Thrombosis, a Single Centre Experience

S. Chong; N.L. Mohd Ramli; E.S. Zulkefli; Z. Juhari; C.L. Phan;

R. Hamzah; V. Selvaratnam; S.M. Tan

Hospital Ampang, Ampang, Malaysia

Background: The JAK2V617F mutation is recurrent in polycythaemia vera and essential thrombocythaemia, which are myeloproliferative neoplasms frequently associated with arterial and venous thromboembolism. It has also been reported as a marker for occult Myeloproliferative Neoplasm (MPN) in patients with splanchnic venous thrombosis. However, compared to JAK2V617F mutationpositive, patients with an CALR mutation-positive present with less risk of thrombosis.

Aims: We evaluate the frequency of JAK2V617F and CALR mutations in patients presenting with splanchnic vein thrombosis in samples sent to clinical Referral Haematology Laboratory, Hospital Ampang, which is the tertiary referral laboratory, the only laboratory offered JAK2V617F and CALR mutational analysis for all hospitals within the Ministry of Health in Malaysia.

Methods: We retrospectively investigated all the samples sent for JAK2V617F mutations from year 2016-2020 with presenting history of splanchnic vein thrombosis which include: portal, splenic, hepatic and superior mesenteric vein thrombosis which are confirmed either by ultrasound or Computed tomography (CT) imaging. JAK2V617F mutation analysis was done by Amplification Refractory Mutation System (ARMS) PCR and CALR mutation analysis by High Resolution Melting (HRM).

Results: Preliminary result of a total of 53 cases (29men and 24 women) with presenting history of splanchnic vein thrombosis was sent for screening of JAK2V617F in our database during 2016 and 2017. 13 patients were found to have the JAK2V617 mutation, and the remaining of 40 patients were found to be negative for both JAK2V617F and CALR mutation.

Conclusions: Despite thrombosis being a common manifestation of Myeloproliferative Neoplasm(MPN), the role of screening the JAK2V617F mutations in all patients presenting with splanchnic vein thrombosis without other features of MPN is unclear. However, the significance of screening for this mutation provides an accurate diagnosis and further understanding the role of JAK2V617F mutation in thrombosis is a great potential for the future development of therapeutics for disease intervention.

\section{PB1123 | The Use of DOACS in Catheter Related Thrombosis in Cancer}

U. Faruqi; G. Bahra; P.-L. Luo; Z. Mahir; A. Danaee

Guys and St Thomas' NHS Foundation Trust, London, United Kingdom

Background: Currently, there is no consensus on the optimal management of catheter related upper extremity deep vein thrombosis 
(UEDVT) in cancer patients. There is increasing evidence for DOACs in cancer associated thrombosis (CAT) but there is a paucity of data for the use of these agents in UEDVT.

Aims: To describe a single centre experience on the use of DOAC in cancer patients with catheter related UEDVT.

Methods: Retrospective cohort study on the management of UEDVT in cancer patients between 2019 and 2020 at Guys and St Thomas Hospital London. Data was collected by reviewing electronic case notes and thrombosis confirmed on ultrasound doppler reports.

Results: There were 53 patients with UEDVT: 48 (90.5\%) PICC, 3 (5.6\%) portacath and 2 (3.7\%) cannula associated thrombosis. The PICC thromboses were sub-analysed (Table 1 ).

PICC lines were in situ for a mean of 48.8 days (inter quartile range 41.7 days) until thrombosis development. All patients were initiated on low molecular weight heparin (LMWH) until review in CAT clinic when anticoagulation was converted to DOAC in 20 patients $(43.7 \%)$ after risk assessment.

Two patients $(9.5 \%)$ reported bleeding on DOACs. One minor bleeding and one clinically relevant non-major bleeding event with epistaxis requiring attendance to hospital. Bleeding was defined as per the ISTH criteria.

There were no recurrent line thromboses on anticoagulation with DOACs or LMWH. Three patients developed recurrent thromboses on DOACS (one superficial vein, two PE) and two patients on LMWH (one superficial vein, one PE). 13 (27\%) patients continued DOAC prophylaxis after three months treatment dose with no recurrent line thrombosis.

TABLE 1 Baseline demographics of patients presenting with PICC associated UEDVT

\begin{tabular}{|c|c|c|}
\hline & & $\begin{array}{l}\text { Number of patients } \\
\mathrm{N}(\%)\end{array}$ \\
\hline \multirow[t]{2}{*}{ Gender } & Male & $13(27.0)$ \\
\hline & Female & $35(72.9)$ \\
\hline \multirow[t]{8}{*}{ Cancer } & $\begin{array}{l}\text { Gastrointestinal } \\
\text { tract }\end{array}$ & $15(31.2)$ \\
\hline & Breast & $10(20.8)$ \\
\hline & Oesophageal & $6(12.5)$ \\
\hline & Gynaecological & $5(10.4)$ \\
\hline & Haematological & $4(8.3)$ \\
\hline & Lung & $3(6.2)$ \\
\hline & Pancreas & $2(4.1)$ \\
\hline & Other & $3(6.2)$ \\
\hline Initial anticoagulation & LMWH & $48(100)$ \\
\hline \multirow{3}{*}{$\begin{array}{c}\text { Anticoagulation } \\
\text { converted }\end{array}$} & Apixaban & $6(12.5)$ \\
\hline & Rivaroxaban & $4(8.3)$ \\
\hline & Edoxaban & $10(20.8)$ \\
\hline \multirow{2}{*}{$\begin{array}{l}\text { Secondary } \\
\text { prophylaxis }\end{array}$} & LMWH & $2(4.1)$ \\
\hline & DOAC & $13(27.0)$ \\
\hline
\end{tabular}

Conclusions: Although this is a small study, there were no recurrent line thromboses or major bleeding events in patients treated with DOAC which suggests that DOAC may be a safe and efficacious alternative to LMWH. Studies including randomised controlled trials are required for further assessment.

\section{PB1124 | Factor VIII and Prothrombin Time during Induction Therapy Correlate with Response to Treatment in Transplant- eligible Newly Diagnosed Multiple Myeloma Patients}

J. Vieira; A. Vagos Mata; I. Paulos Mesquita; S. Durão; H. Martins;

A. Rodrigues; A. Garção; Á. Beleza; J. Raposo; G. Esteves Hospital Santa Maria, Centro Hospitalar Universitário Lisboa Norte, Lisbon, Portugal

Background: Several hemostatic abnormalities have been reported in patients with multiple myeloma (MM). Multiple contributing factors have been proposed, including type of treatment and disease stage.

Aims: Assess hemostatic abnormalities in MM patients during induction therapy and its correlation with depth of response to treatment, before and after autologous stem cell transplant (ASCT).

Methods: Single centre retrospective study, including newly diagnosed transplant-eligible patients, treated between January 2015 and December 2019. Prothrombin time (PT), activated partial thromboplastin time (APTT), factor VIII, plasma fibrinogen and von Willebrand factor were assessed during induction therapy, at the time of peripheral blood stem cell collection. Response evaluation according to the International Myeloma Working Group (IMWG) criteria.

Results: 95 patients were included, $57.9 \%$ male. Median age of 59 years-old (IQR 53-65). ISS stage was I, II and III in 41.6\%, $25.8 \%$ and $32.6 \%$, respectively. Patients were treated with a bortezomibbased regimen, $50.6 \%$ in association with an immunomodulatory drug and $49.4 \%$ with cyclophosphamide. Median follow up time of 45 months. Factor VIII, plasma fibrinogen and von Willebrand factor were above the reference range in $69.5 \%, 40 \%$ and $92.1 \%$ of patients, respectively. PT and APTT were above the reference range in $13.7 \%$ and $4.2 \%$ of patients, respectively. Patients who achieved $\geq$ very good partial response (VGPR) before ASCT had significantly lower levels of factor VIII at the time of apheresis, compared to those who achieved $\leq \mathrm{PR}$ (mean 172, SD \pm 45.7 vs. mean $212, \mathrm{SD} \pm$ $53, P<0.001$ ). Factor VIII levels did not correlate with response to treatment at day+100 after ASCT. Higher values of TP at the time of apheresis were significantly associated with $\leq \mathrm{PR}$ at day+100 after ASCT (HR 1.989, 95\% Cl 1.1-3.5, $P=0.02$ ).

Conclusions: Factor VIII levels correlate with response to treatment before ASCT. TP levels correlate with response to treatment at day+100 after ASCT. 
PB1125 | Prediction of Venous Thromboembolism among Ambulatory Cancer Patients with Gastrointestinal Malignancies - A Retrospective Analysis

K. Pavithran; H. Jayamohanan; S. Soman

Amrita Institute of Medical Sciences and Research Center, Kochi, India

Background: Patients with cancer are at high risk of venous thromboembolic (VTE) events. Cancer-associated thrombosis (CAT) is an important risk factor for increased morbidity and mortality. There are several risk prediction scores for VTE, but these scores' clinical implication among different populations is not very clear. The occurrence of VTE is high in gastrointestinal (GI) malignancies.

Aims: To study the performance of Khorana and CONKO scores risk prediction scores to predict VTE development in GI malignancies.

Methods: Ambulatory cancer patients with GI malignancies undergoing treatment at AIMS were included in this retrospective analysis. The clinical data were collected from the hospital's electronic medical records during the period 2017-2019. The study's primary outcome was the occurrence of any venous thromboembolic event - either deep vein thrombosis (DVT) or pulmonary embolism (PE) during or after treatment.

Results: 250 patients with GI malignancies were included in this analysis. The median age was 62 years. $58.4 \%$ of the study population were males. The common sites of cancer were colon (33.6\%), rectum (26.4\%), stomach (17.6\%), and pancreas (15.6\%). $96 \%$ of the cases were adenocarcinoma. Out of 9 VTE events, half of the events 5 (55.6\%) occurred in patients with stage IV; stage II and III patients had two events each. According to the Khorana risk score, $83.2 \%$ of the patients were at high risk of VTE, and $16.8 \%$ at intermediate risk. Only 9 (3.6\%) patients had one episode of VTE during treatment ( pulmnary embolism and and 8 deep vein thrombsosi). According to the CONKO score, $63.6 \%$ belonged to the low-risk category and $36.4 \%$ in the high-risk group. As per the CONKO scoring, 7 events occurred in the low-risk and 2 events in the high-risk group.

Conclusions: The present finding does not support either Khorana or CONKO risk scores for risk stratification of VTE in patients with GI malignancies.

PB1126 | The Association among von Willebrand Factor, ADAMTS-13, D-dimer, and Soluble P-selectin with Venous Thromboembolism Incidence in Cancer Patients: Systematic Review and Meta-analysis

B.P. Putra ${ }^{1}$; F.N. Putra ${ }^{2}$

${ }^{1}$ Medical Doctor, Blitar, Indonesia; ${ }^{2}$ Faculty of Medicine Universitas Airlangga, Surabaya, Indonesia

Background: Cancer patients tend to have hypercoagulable state with increased venous thromboembolism (VTE) incidence that raises morbidity and mortality. Previous limited number of studies suggest any inconclusive association among von Willebrand factor (vWF), a disintegrin-like and metalloproteinase with thrombospondin type 1 motif, member 13 (ADAMTS-13), D-dimer, and soluble P-selectin (sP-selectin) with the VTE incidence among cancer patients.

Aims: This study aims to measure the association among vWF, ADAMTS-13, D-dimer, and sP-selectin with VTE incidence in cancer patients.

Methods: We did both comprehensive searching and hand-searching in online databases of Pubmed, EMBASE, ScienceDirect, and The Cochrane Library, to include all relevant literature from 2000 until 2021 then followed the PRISMA guideline. We included all observational studies that access the association among vWF, ADAMTS-13, $\mathrm{D}$-dimer, and SP-selectin with VTE incidence in all cancer patients. Bias risk was accessed by using The Newcastle-Ottawa Scale. Analysis was performed to provide pooled risk ratio (RR) with $95 \%$ confidence interval $(\mathrm{Cl})$ using random-effect heterogeneity test.

Results: We included 1 case-control and 2 cohort studies fulfilled our inclusion criteria. Increased vWF is significantly associated with VTE incidence among cancer patients (pooled $\mathrm{RR}=2.00,95 \% \mathrm{Cl} 1.28$ $3.13, P=0.002, I^{2}=28 \%$ ). The ADAMTS-13 (pooled RR $=1.13,95 \%$ Cl $0.88-1.44, P=0.35, I^{2}=68 \%$ ), D-dimer (pooled RR $=1.20,95 \%$ $\mathrm{Cl} 0.78-1.85, P=0.40, I^{2}=71 \%$ ), and sP-selectin (pooled RR = 1.13, $95 \% \mathrm{Cl} 0.64-2.00, P=0.67, I^{2}=21 \%$ ) are associated with higher VTE risk among cancer patients although not statistically significant.

Conclusions: The von Willebrand factor, ADAMTS-13, D-dimer, and sP-selectin can be potential predictors of VTE incidence among cancer patients. However, further studies are needed to establish the associations.

PB1127 | Endothelial Dysfunction in Patients with Polycythemia Vera on Cytoreductive and / or Antiplatelet Therapy

N. Silina; N. Korsakova; O. Golovina; M. Fominykh; E. Efremova; S. Voloshin; L. Papayan

Russian Research Institute of hematology and Transfusiology, Saint Petersburg, Russian Federation

Background: Polycythemia vera (PV) is a clonal disease that belongs to the group of myeloproliferative neoplasms. PV is characterized by thrombotic complications. Currently there is not general diagnostic laboratory method for the development of thrombosis. Activation of endothelium that can lead to endothelial dysfunction is one of the factors that contribute to the progress of thrombosis. Prevention of thrombotic complications is a major issue in the management of these patients.

Aims: Aim to compare the effect of cytoreductive and / or antiplatelet therapy on endothelium activation in patients with PV.

Methods: 38 patients were examined. 18 people were treated with acetylsalicylic acid in prophylactic doses (group 1) and 20 patients were on cytoreductive therapy in combination with antiplatelet agents (group 2). The control group consisted of 43 healthy individuals. Ristocetin cofactor activity and von Willebrand factor level (vWF R:Co and Ag vWF respectively) were determined. Microsoft 
office Excel and STATISTICA 12.0 was used. The results are given as median (Me) with 50\% confidence intervals ( $\mathrm{Cl}$ ). Mann-Whitney test was used to compare the studied groups. $P<0.05$ was considered statistically significant.

\section{TABLE 1}

Parameters

Group $1(n=18)$

Group $2(n=20)$

138.00* **

$107.25-167.30$

$143.75^{*}$

$125.15-160.20$

\section{vWF R:Co, \%}

Ag $\vee W F, \%$
80.00

$74.00-110.00$
$91.90-143.30$
Results: The data are shown in the table. Patients on cytoreductive and antiplatelet therapy had a significant increasing of vWF R:Co and Ag vWF. Endothelial activation was more pronounced in the second group that is the sign of endothelial dysfunction in that patients and it is a potential risk factor of thrombotic complications.

${ }^{*}-P<0.05$ compare to controls

**- $P<0.05$ differences between Group 1 and Group 2

Conclusions: Patients with PV on cytoreductive and antiplatelet therapy have a higher damage to the vascular wall than that in patients on acetylsalicylic acid only. Endothelial dysfunction due to its activation is a generally recognized risk factor for thrombotic complications.

\section{PB1128 | Breast Cancer, Doxorubicin and Cardiotoxicity: Can Thrombin Generation Test Be Useful?}

R.M.C. Pestana ${ }^{1}$; R.C.F. Duarte ${ }^{1}$; A.d.P. Sabino ${ }^{1}$; L.M. Silva ${ }^{2}$;

R. Simões ${ }^{1}$; K.B. Gomes ${ }^{1}$; M.d.G. Carvalho ${ }^{1}$

${ }^{1}$ Federal University of Minas Gerais, Belo Horizonte, Brazil; ${ }^{2}$ Ezequiel

Dias Foundation, Belo Horizonte, Brazil

Background: Anthracyclines, especially doxorubicin (DOXO), are widely used in the treatment of patients with breast cancer. However, secondary cardiotoxicity is a limiting outcome to its use. Cancer patients commonly present hemostatic abnormalities correlating with the worsening of the disease. Thrombin Generation Test (TGT) may be a useful tool for detecting such abnormalities in patients with breast cancer using DOXO, particularly those who develop cardiotoxicity.

Aims: To evaluate the performance of TGT in patients with breast cancer before and after treatment with DOXO, with or without treatment-related cardiotoxicity.

Methods: Blood samples were collected from 80 women with breast cancer at different times, before (T0), shortly after (T1) and one year after the use of DOXO (T2) (median dose was $380 \mathrm{mg} / \mathrm{m}^{2}$ ). Invasive carcinoma was observed in 72 participants (90.0\%) followed by other less frequent types. TGT was performed using the CAT method. The study received approval from the local Ethics Committee and is in accordance with the Declaration of Helsinsk.

Results: Of the total participants, 27 (33.75\%) had cardiotoxicity, while 53 (66.25\%) did not. Only at T0, an increased value for the Peak was observed in those who developed cardiotoxicity
$(P=0.030)$, suggesting that hypercoagulability, a priori, may predispose to this complication. However, the use of DOXO increased Peak and Endogenous Thrombin Potential (ETP) values in relation to their baseline levels (T0 x T1 and T0 x T2; $p<0.001$ for both), only in those who did not develop cardiotoxicity, that is, in those who were less hypercoagulable in TO.

Conclusions: This finding suggests that the use of DOXO increases the hypercoagulable status. Moreover, evaluation by TGT before treatment with DOXO may be a useful predictor of cardiotoxicity in women with breast cancer who will be treated with this drug.

PB1129 | Risk Factors of Catheter-related Thrombosis in Patients with Multiple Myeloma Undergoing Autologous Stem Cell Transplantation

A. Hoppe; J. Rupa-Matysek; B. Malecki; D. Dytfeld; L. Gil

Department of Hematology and Bone Marrow Transplantation, Poznan University of Medical Sciences, Poznan, Poland

Background: Despite the novel targeted therapies in multiple myeloma, high dose melphalan treatment supported by autologous stem cell transplantation (ASCT) remains the standard of care in transplant-eligible patients. In the majority of patients short term non-tunneled central venous catheter (CVC) is inserted during the procedure. CVC implantation carries the risk of catheter-related thrombosis (CRT).

Aims: The aim of the study was to identify risk factors of CRT in myeloma patients during ASCT.

Methods: We retrospectively analyzed 276 patients undergoing autologous stem cell transplantation in 2009-2019 in the Department of Hematology and Bone Marrow Transplantation. Each patient had CVC insertion before the ASCT procedure. Clinical symptoms of CRT were confirmed with a Doppler ultrasound examination. We examined the impact of patient-related, disease-related, catheterrelated and treatment-related factors on CRT development (Table 1). 
TABLE 1 Factors analyzed in the study Abbreviations: BMI - Body mass index, HCT-Cl - Hematopoietic Cell Transplantation-specific Comorbidity Index, Charlson Cl - Charlson Comorbidity Index, MM - Multiple myeloma, M protein - monoclonal protein, D-S - Durie Salmon staging system, ISS - International Staging System, R-ISS - Revised International Staging System, ASCT - Autologous stem cell transplantation, CVC - Central Venous Catheter, CVS - Cardiovascular system, GI - Gastrointestinal

\section{Group}

Patient-related

Disease-related

Catheter-related

Treatment-related

\section{Parameters}

Age

Gender

BMI

HCT-Cl

Charlson $\mathrm{Cl}$

Previous thrombotic history

Number of thromboembolic events

Thrombotic complications on initial therapy of MM

M protein type

Stage D-S, ISS, R-ISS

Bone disease

Kidney insufficiency before ASCT

Location

Time from placement to removal

Conditioning type (myeloablative/non-myeloablative)

Complications:

Infectious/Non-infectious

CVC infection

CVS complications/GI complications
Results: Clinically symptomatic CRT was present in $2.5 \%$ (7/276) of patients. Univariate analysis showed an increased risk of CRT in patients with a catheter-related infection (OR 2.4; 95\% Cl 1.11-6.53, $P=0.022)$, previous thrombotic episode during initial $M M$ treatment (OR 2.75; 95\%Cl 1.15-5.39, $P=0.021$ ), any previous thrombotic episode before ASCT (OR 2.49; 95\% Cl 1.15-5.39, $P=0.021$ ) and non-thrombotic/non-infectious complications (OR $2.60 ; 95 \% \mathrm{Cl}$ 1.10-6.15, $P=0.029$ ), especially in patients with gastrointestinal complications such as vomiting and diarrhea (OR $3.87 ; 95 \% \mathrm{Cl}$ 1.57-9.53, $P=0.003$ ). In multivariate analysis, catheter-related infection (OR 2.78; 95\% $\mathrm{Cl} 1.21-6.39, P=0.016$ ), previous thrombotic episode before ASCT (OR 2.88; 95\% Cl 1.24-6.70, $P=0.014$ ) and non-thrombotic/non-infectious complications (OR $2.75 ; 95 \% \mathrm{Cl}$ 1.09-6.91, $P=0.031$ ) were all associated with higher CRT incidence. Conclusions: The incidence of symptomatic CRT in ASCT in MM is lower than in other CVC implantation studies. Previous thrombotic events, especially during induction of $M M$ treatment increase the CRT risk during ASCT. Dehydration following gastrointestinal complications is associated with higher CRT incidence.
PB1130 | Risk Factors for Reccurence of Catheter Related upper Extremity Deep Venous Thrombosis in Cancer Patients

R. Hakem; S. Soudet; M.A. Sevestre

CHU Amiens Picardie, Amiens, France

Background: Patients with cancer have a substantially increased risk of venous thromboembolism (VTE) compared with patients without cancer. Long term indwelling central venous catheters (CVC) use has considerably increased to secure vascular access that improve the quality of life. Catheter related upper extremity deep venous thrombosis (CVC-RT) is a common complication. It occurs in approximately $2-6 \%$ of cancer patients with a CVC. Studies are needed to define the optimal management of patient with CVC-RT and the risk of recurrence of VTE should be better known.

Aims: To determine the influence of anticoagulation duration on the incidence of recurrent VTE in cancer patients.

Methods: We conducted a retrospective, monocentric observational study at the Amiens-Picardie University Hospital. All consecutive patients with upper extremity thrombosis were included between January 2015 and July 2020. The incidence of thrombosis was estimated using Gray's method for competitive risk with death as the competitive event. Thrombosis risk factor analysis was performed 
with the Fine \& Gray univariate model for competitive risk with death as the competitive event.

Results:

Mean age was 56 years. $22 \%$ had a previous history of VTE. $80 \%$ had metastatic status. Mean follow-up was 24 months. 25,5\% of patients had reccurence (figure 1), clinical characteristics are detailed in table 1. At reccurence, 41 patients $80,4 \%$ still had anticoagulation treatment. In multivariate analysis, previous VTE and maintain of CVC were significant reccurence risk factors. Without CVC, anticoagulant effect had HR = $1.05[1.01 ; 1.09](P=0.0290)$. With CVC effect was $\mathrm{HR}=1.02[0.99 ; 1.04](P=0.1890)$. Anticoagulant treatment effect trends to decrease when CVC is maintained but not significantly $(P=0.22)$.

TABLE 1 Clinical characteristics of Recurrent VTE

\begin{tabular}{ll} 
Variable & $N(\%)$ \\
Recurrent VTE, $n$ & $51(25,5 \%)$ \\
During anticoagulation treatment & \\
EP, $\mathrm{n}(\%)$ & $41(80,4)$ \\
DVT of lower limb, $\mathrm{n}(\%)$ & $20(10 \%)$ \\
CVC-RT, $\mathrm{n}(\%)$ & $8(4 \%)$ \\
CVC status & $30(15 \%)$ \\
Removed, $\mathrm{n}(\%)$ & $5(9.8 \%)$ \\
Maintained, $\mathrm{n}(\%)$ & $46(90.2 \%)$ \\
\hline
\end{tabular}

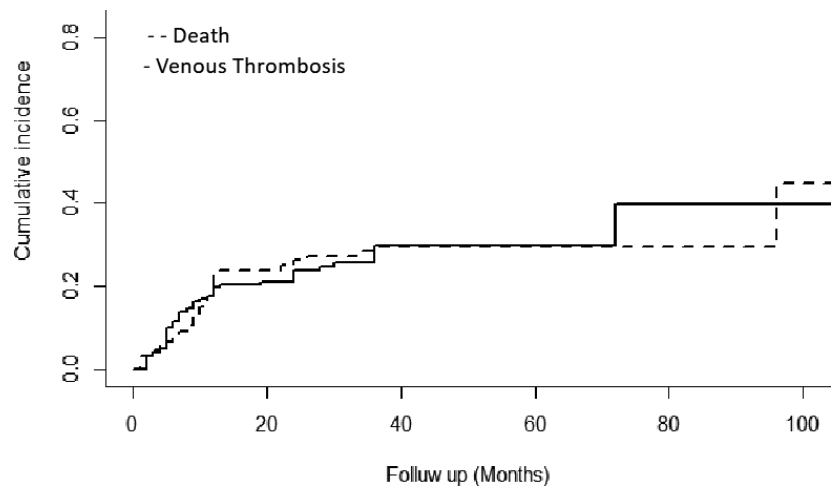

FIGURE 1 Reccurence of venous thrombosis and death

Conclusions: Reccurence of venous thrombosis is frequent after CVC-RT, risk factors are previous VTE and maintain of CVC. Presence of CVC did not influence significantly the risk of reccurence.
PB1131 | Effect of Therapy on Thrombin Generation in Patients with Polycythemia Vera

N. Silina; N. Korsakova; O. Golovina; O. Matvienko; M. Fominykh;

E. Efremova; S. Voloshin; L. Papayan

Russian Research Institute of Hematology and Transfusiology, Saint

Petersburg, Russian Federation

Background: Polycythemia vera (PV) is a clonal disease that belongs to the group of myeloproliferative neoplasms. The most patients have a JAK2V617F mutation that plays a common role in the etiology and pathogenesis of disease and it is a risk factor of thrombosis. Prevention of thrombotic complications is a major issue in the management of these patients.

Aims: Aim to compare the effect of cytoreductive and / or antiplatelet therapy on thrombin generation in patients with PV.

Methods: 38 patients were examined. 18 people were treated with acetylsalicylic acid in prophylactic doses (Group 1) and 20 patients were on cytoreductive therapy in combination with antiplatelet agents (Group 2). The control group consisted of 43 healthy individuals. Thrombin generation was assessed by calibrated automated thrombinography (CAT) according to Hemker et al. with or without thrombomodulin (TM). The following parameters were evaluated: endogenous thrombin potential (ETP, nM*min) and peak thrombin (Peak thrombin, nM). Sensitivity ETP and Peak thrombin for TM were calculated as percent of decreasing of these parameters after adding to assay of TM (Sensitivity ETP, \% and Sensitivity Peak thrombin, \% respectively). Decreasing of these parameters indicates disability of anticoagulant protein $\mathrm{C}$ system and it is a potential risk factor of thrombotic complications. Microsoft office Excel and STATISTICA 12.0 was used. The results are given as median (Me) with $50 \%$ confidence intervals ( $\mathrm{Cl}$ ). Mann-Whitney test was used to compare the studied groups. $P<0.05$ was considered statistically significant. 
Results:

\section{TABLE 1}

\begin{tabular}{|c|c|c|c|}
\hline Parameters of CAT & Group $1(n=18)$ & Group $2(n=20)$ & Healthy controls $(n=43)$ \\
\hline \multirow[t]{2}{*}{ ETP, $\mathrm{nM} \times \mathrm{min}$} & 1702.25 & $1342.67^{*}$ & 1624.00 \\
\hline & $1249.48-1884.90$ & $1288.42-1664.39$ & 1489.88-1777.00 \\
\hline \multicolumn{4}{|l|}{ Sensitivity ETP, \% } \\
\hline & $43.80^{*}$ & $29.57^{*}, * *$ & 52.90 \\
\hline & $31.02-48.83$ & $25.38-36.13$ & $47.83-57.67$ \\
\hline \multicolumn{4}{|l|}{ Peak thrombin, $\mathrm{nM}$} \\
\hline & $206.82^{*}$ & $211.84^{*}$ & 285.60 \\
\hline & $164.79-236.84$ & $170.24-252.50$ & $265.50-311.81$ \\
\hline \multicolumn{4}{|c|}{ Sensitivity Peak thrombin, \% } \\
\hline & $20.70^{*}$ & $14.29^{*},{ }^{* *}$ & 42.13 \\
\hline & $17.18-31.88$ & $10.35-17.82$ & $36.20-47.00$ \\
\hline
\end{tabular}

\footnotetext{
*-P $<0.05$ compare to controls

${ }^{* *}-P<0.05$ differences between Group 1 and Group 2
}

The data are shown in the table. Sensitivity to TM was significantly reduced in patients of both groups. The parameters of sensitivity to $\mathrm{TM}$ in patients of the second group were significantly lower than those in the first group.

Conclusions: The treating with the combination therapy has a more pronounced risk of thrombotic complications than that in patients on the antiplatelet therapy.

PB1132 | Concurrent Atypical Thrombotic Complications of Acute Promyelocytic Leukemia (APL) in an Anticoagulated Patient: A Rare Case Report

A. Ashwath; A. Cordova Sanchez; S. Rao; R. Denley SUNY Upstate Medical University, Syracuse, United States

Background: Coagulopathy in APL incorporates disseminated intravascular coagulation and primary hyperfibrinolysis. Thrombotic events in APL are known to occur, but hemorrhagic complications predominate the literature as they are the leading cause of mortality. Therapeutic all-trans retinoic acid (ATRA) has dramatically improved survival rates in APL, but its utilization may alter hemostatic balance increasing hypercoagulability and risk of atypical thromboses.

Aims: Describe rare concurrent thrombotic events in an APL patient undergoing treatment.

Methods: A 28-year-old female was diagnosed with pulmonary embolism in the setting of leukopenia. A bone marrow biopsy revealed APL with t(15;17). She was initiated on ATRA and arsenic trioxide for low-risk disease, and therapeutic systemic anticoagulation for her PE.
During induction, she developed headache, diplopia, and papilledema. An MRI/MRV of the brain showed a transverse sinus thrombosis requiring urgent mechanical thrombectomy. Days later, she complained of chest pain prompting a CT angiogram. A new intraventricular thrombus was seen affixed to the right ventricular wall and was confirmed by echocardiogram and cardiac MRI. Due to poor candidacy for thrombolysis or mechanical retrieval, she continued anticoagulation as primary management.

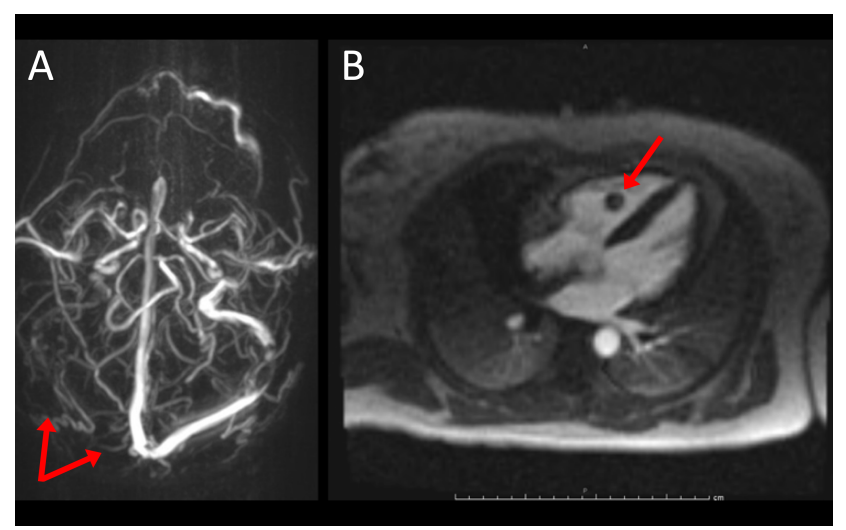

FIGURE 1 Lack of contrast within the right cerebral transverse sinus consistent with thrombus in a brain MRV $(A)$ and right intraventricular filling defect by cardiac MRI (B)

Results: Cerebral venous sinus thrombosis (CVST) and intraventricular thrombus (IVT) in APL are sparsely reported in the medical literature. We believe to be reporting the first case of their coexistence. 
TABLE 1 Published cases of cerebral venous sinus thrombosis (CVST) or intraventricular thrombosis (IVT) in patients with APL

\begin{tabular}{lllll} 
Author (Year) & Patient & Site of Thrombus & Timing of Thrombus & Treatment Regimen \\
Hazani A, et al. (1988) & $11 \mathrm{M}$ & CVST & Diagnosis & N/A \\
Torromeo C, et al. (2001) & $50 \mathrm{M}$ & IVT & Induction & ATRA + idarubicin \\
Torromeo C, et al. (2001) & $32 \mathrm{~F}$ & IVT & Induction & ATRA + idarubicin \\
Dally N, et al. (2005) & -- & CVST & Induction & ATRA + daunorubicin \\
Dally N, et al. (2005) & -- & CVST & Induction & ATRA + daunorubicin \\
Breccia M, et al. (2007) & $32 \mathrm{~F}$ & IVT & Induction & ATRA + idarubicin \\
Breccia M, et al. (2007) & $50 \mathrm{M}$ & IVT & Induction & ATRA + idarubicin \\
Ciccone M, et al. (2008) & $35 \mathrm{~F}$ & CVST & Remission & ATRA + idarubicin \\
Beslow LA, et al. (2009) & $12 \mathrm{M}$ & CVST & Diagnosis & N/A \\
Kayal S, et al. (2011) & $3 \mathrm{~F}$ & IVT & Induction & ATRA + daunomycin \\
Lee KR, et al. (2014) & $22 \mathrm{~F}$ & CVST & Induction & ATRA + idarubicin \\
Song LX, et al. (2014) & $28 \mathrm{~F}$ & CVST & Induction & ATRA \\
Ashwath, et al. (2021) & $28 \mathrm{~F}$ & CVST + IVT & ATRA + arsenic trioxide \\
\hline
\end{tabular}

Conclusions: Thrombotic events in APL occur in $2-15 \%$ of patients and are almost exclusively myocardial infarctions, strokes, or DVT/ PE. Sixty percent of these events occur following ATRA therapy. This may be explained by ATRA mediated IL-1B, CD2, and CD15 expression leading to leukocytosis, leukoagglutination, tissue damage by microvascular occlusion, and finally thrombosis. The onset of our patient's symptoms suggest her rare thrombi occurred while anticoagulated for her pulmonary embolism underscoring the potent thrombogenic potential of APL. Patients presenting with acute symptoms during or following ATRA treatment should additionally be evaluated for atypical sites of thrombosis.

PB1133 | Safety and Effectiveness of an Institutional Recommendation on the Management of Cancer Associated Venous Thrombosis: Results of a Real World Study with One-year Follow-up

C. Rothschild; A.A.G.S. Brandão; A. Duran; A.E. Zerati; G.F. Saes; C.S. Bittar; I.B.S.d.S. Costa; M.H.H.d.S. Rehder; S.M.R. Fonseca; M.D.P.E. Diz; L.B.d.O. Alves; J. Pereira; V. Rocha Instituto do Cancer do Estado de Sao Paulo, Hospital das Clinicas HCFMUSP, Faculdade de Medicina, Universidade de Sao Paulo, São Paulo, Brazil

Background: Management of cancer associated venous thrombosis (CAT) is challenging due to coexistent risk of bleeding and thrombosis (VTE) recurrence, that are 6 and approximately 4 fold higher compared to non-cancer patients, respectively. Anticoagulant choice needs to be careful and can be guided by a recommendation to avoid those complications.

Aims: To evaluate effectiveness and safety of a public cancer institution recommendation on CAT management.

Methods: Retrospective cohort study carried out from October 2018 to January 2020. The recommendation on CAT management included defined criteria to select the proper anticoagulant for each patient. A 3-month ambulatory was scheduled to evaluate eligibility of outpatients on enoxaparin to switch to rivaroxaban (new drug at the institution), based on the recommendation. All consecutive adult oncological outpatients on enoxaparin in the institution were considered eligible for the study. Data on cancer, comorbidities, bleeding, VTE recurrence and death during 1 year since the first visit were captured. Cumulative incidence of bleeding and VTE recurrence were calculated by Kaplan-Meier method and log-rank test.

Results: 326 patients were referred to the ambulatory and 275 were included in the analyses, with a median age of 63 years (IQR 53-71). $61 \%$ were female. $86 \%$ had solid tumours ( $53 \%$ metastatic). The main sites were gastrointestinal (27\%), breast (12\%) and pulmonary (10\%). $45 \%$ of the patients switched to rivaroxaban and $5 \%$ were able to stop anticoagulation. The main reason for not switching was drugdrug interaction (27\%). One-year cumulative incidence of VTE recurrence was $11.2 \%$ (95\% Cl 7.7-16.2) and of overall bleeding was $13.4 \%$ (95\% Cl 9.4-18.7). Major bleeding occurred in 8 patients. Death rate was $32 \%$, with no one due to bleeding. No significant difference of outcomes was observed between patients who had switched or not. Conclusions: Recommendation-based decision on CAT management showed similar effectiveness and safety independently of the chosen anticoagulant. 
PB1134 | Development of a Multi-language Mobile App to Implement the 2019 ITAC Clinical Practice Guidelines for the Treatment and Prophylaxis of Venous Thromboembolism in Cancer Patients

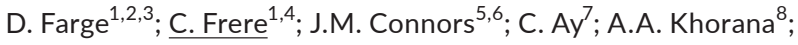
A. Munoz ${ }^{9,10}$; B. Brenner ${ }^{11}$; A. Kakkar ${ }^{12,13}$; H. Rafii ${ }^{1}$;

S. Solymoss (in memoriam) ${ }^{3}$; D. Brilhante ${ }^{14}$; M. Monreal ${ }^{15,16}$; H. Bounameaux ${ }^{17}$; I. Pabinger ${ }^{7}$; J. Douketis ${ }^{18}$

${ }^{1}$ Assistance Publique Hôpitaux de Paris, Paris, France; ${ }^{2}$ Université de Paris, Paris, France; ${ }^{3}$ McGill University, Montreal, Canada; ${ }^{4}$ Sorbonne Université, Paris, France; ${ }^{5}$ Brigham and Women's Hospital, Boston, United States; ${ }^{6}$ Harvard Medical School, Boston, United States; ${ }^{7}$ Medical University of Vienna, Vienna, Austria; ${ }^{8}$ Taussig Cancer Institute, Cleveland Clinic Foundation, Cleveland, United States; ${ }^{9}$ Hospital General Universitario Gregorio Maranon, Madrid, Spain; ${ }^{10}$ Universidad Complutense, Madrid, Spain; ${ }^{11}$ Rambam Health Care Campus, Haifa, Israel; ${ }^{12}$ Thrombosis Research Institute, London, United Kingdom; ${ }^{13}$ University College London, London, United Kingdom; ${ }^{14}$ Francisco Gentil Portuguese Institute of Oncology, Lisbon, Portugal;

${ }^{15}$ Hospital Germans Trias i Pujol, Barcelona, Spain; ${ }^{16}$ Universidad Católica de Murcia, Barcelona, Spain; ${ }^{17}$ University Hospitals of Geneva, Geneva, Switzerland; ${ }^{18}$ McMaster University, Hamilton, Canada

Background: The International Initiative on Thrombosis and Cancer (ITAC) has recently published updated evidence-based clinical practice guidelines (CPGs) for the management of venous thromboembolism (VTE) in patients with cancer. However, implementation of these CPGs in daily oncology practice appears as particularly challenging in these complex patients.

Aims: To describe the process development and contents of a clinical decision support tool to implement the ITAC-CPGs for the treatment and prophylaxis of VTE in patients with cancer.

Methods: We constructed a multilayer framework to translate the ITAC-CPGs into executable knowledge. We followed an iterative process of CPGs executive summary, algorithms elaboration, app development using the Objective- $\mathrm{C}$ and Java programming languages, and testing, with revision through each step, to support the design of 3 validated modules (prophylaxis of VTE; treatment of non-catheterrelated VTE; treatment of catheter-related VTE).

Results: The resulting workable mobile app integrates a large amount of information and recommended processes into a single handheld tool. In 2021, the ITAC app is available in English, French, Spanish, Portuguese and Russian in any iOS (iTunes App Store) or Android (Google Play) devices for free. This ITAC app constitutes a practical, step-by-step guide for the prophylaxis and treatment of VTE in cancer patients, based on simple decision-tree algorithms. By answering a series of questions, health care users can quickly determine a recommended course of action for any given patient based on his clinical scenario.

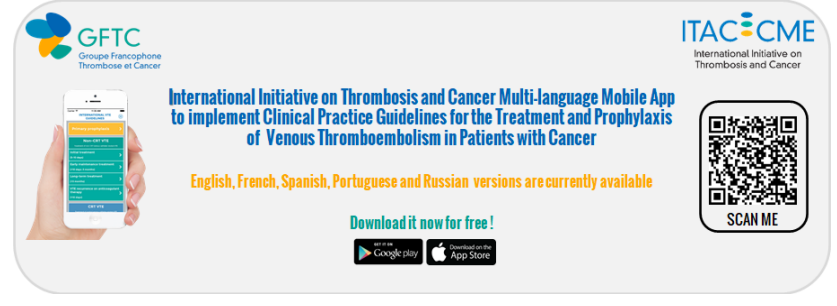

FIGURE 1 Multi-language mobile App to implement the International Initiative on Thrombosis and Cancer Clinical Practice Guidelines for the Treatment and Prophylaxis of venous Thromboembolism in Patients with Cancer

Conclusions: By translating written ITAC-CPGs into timely and effective decision-making, the multi-language ITAC app shall allow physicians and other healthcare professionals to overcome cancerassociated management challenges worldwide.

PB1135 | Thrombotic and Hemorrhagic Complications in Patients Undergoing Allogeneic Hematopoietic Stem Cell Transplantation

E. Gonzalez Gomez; N. Fernandez Mosteirin; M. Moreno Carbonell; S.F. Pinzon Mariño; A. Garcia Ortego; A. Gomez Martinez; C.F. Hernandez Mata; S. Martin Consuegra; M. Civeira Marin; A. Lopez Peña; C. Rodriguez Lefler; M.S. Ordas Miguelez; M.d.M. Herrero Gutierrez; P.E. Lopez Gomez; M.P. Delgado Beltran; J.M. Calvo Villas Hospital Universitario Miguel Servet, Zaragoza, Spain

Background: Allogeneic hematopoietic stem cell transplantation (AHSCT) is an established therapy in the treatment of hematological malignancies. In recent years, different studies have reported thromboembolic complications with increased morbidity and bleeding complications with higher mortality in patients undergoing AHSCT.

Aims: To analyze the thrombotic and hemorrhagic complications in patients undergoing AHSCT.

Methods: All patients who underwent a related donor AHSCT between 2010 and 2019 at the Miguel Servet University Hospital (Zaragoza, Spain) were included. Data related to the patient, the hematological disease, AHSCT and follow-up were collected through clinical and laboratory data. The results are expressed in percentages for qualitative variables, and in means, standard deviation (SD) for continuous variables. IBM SPSS Statistics ${ }^{\circledR} 20$ program was used for their analysis.

Results: 87 patients, 53 men, were included for the study. The mean age at AHSCT was 48.5 years (SD 12.8). The average follow-up was 30 months (SD 31.1). 15 patients had a thrombosis after AHSCT, 12 were deep vein thrombosis (DVT), 6 of which were associated with a central venous catheter (CVC). The mean time from AHSCT to diagnosis of thrombosis was 18 months (SD 20.2). The most commonly used treatment for thrombosis was anticoagulation with low molecular weight heparin $(n=10)$. Post-AHSCT bleeding complications 
were detected in 41 patients, mainly gastrointestinal $(n=14)$ and genitourinary $(n=13) .7$ patients had graft-versus-host disease, 6 had $B K$ virus cystitis, and 6 had thrombocytopenia at the time of bleeding. The mean time from AHSCT to detection of bleeding was 9 months (SD 16.9).

Conclusions: The incidence of thrombotic and hemorrhagic complications is similar to that previously published. Most were diagnosed months after AHSCT. The main thrombotic complications were DVT, mostly in patients with a CVC. The hemorrhagic complications were more frequent, located mostly at the digestive and genitourinary level.

PB1136 | Pancreatic-miRaCan-VTE: A R Shiny App to Aid in the Diagnosis of Pancreatic Cancer-associated VTE

J. Oto ${ }^{1}$; A.C Larsen ${ }^{2}$; E. Plana ${ }^{3,1}$; R. Herranz ${ }^{1}$; F. Cana ${ }^{1}$; D. Hervás ${ }^{4}$; A. Cañada ${ }^{4}$; Á. Fernández-Pardo ${ }^{1}$; S.R Kristensen ${ }^{5,6}$; O. ThorlaciusUssing $^{2,6}$; P. Medina ${ }^{1}$

${ }^{1}$ Haemostasis, Thrombosis, Atherosclerosis and Vascular Biology Research Group, Medical Research Institute Hospital La Fe, Valencia, Spain; ${ }^{2}$ Department of Gastrointestinal Surgery and Center of Clinical Cancer Research, Aalborg University Hospital, Aalborg, Denmark; ${ }^{3}$ Angiology and Vascular Surgery Service, La Fe University and Polytechnic Hospital, Valencia, Spain; ${ }^{4}$ Data Science, Biostatistics and Bioinformatics Unit, Medical Research Institute Hospital La Fe, Valencia, Spain; ${ }^{5}$ Department of Clinical Biochemistry, Aalborg University Hospital, Aalborg, Denmark; ${ }^{6}$ Department of Clinical Medicine, Aalborg University, Aalborg, Denmark

Background: Cancer-associated venous thromboembolism (VTE) increases mortality and morbidity. However, limited tools are available to identify high risk patients. microRNAs (miRNAs) are small non-coding RNAs that regulate protein expression. miRNAs seem to regulate cancer progression and VTE. We have previously reported a profile of 7 plasma miRNAs able of estimate the risk of future VTE at diagnosis in biliopancreatic cancer patients (AUC $=0.95 ; 95 \%$ Confidence Interval $[0.987,1])(1)$

Aims: To develop an open online app to rapidly calculate the risk of future VTE events in biliopancreatic cancer patients using a plasma profile of 7 miRNAs.

Methods: The interface was created with using R (v4.0.2) and Shiny (v1.4). We created a short introduction followed by a friendly menu where any researcher can upload the expression level of 7 miRNAs in their patients and obtain a VTE prediction value.

Results: In this tool, any researcher worldwide can introduce the miRNA expression data from their biliopancreatic cancer patients and rapidly obtain a prediction of future VTE events. The steps in the process are:

1) Quantify by RT-qPCR the expression levels of 7 miRNAs (miR486-5p, miR-106b-5p, let-7i-5p, let-7g-5p, miR-144-3p, miR-19a-3p and miR-103a-3p) and the normalizer (miR-93-5p).

2) Upload raw Ct values in a user-friendly interface.
3) Download a file with the predictions for each study subject. Conclusions: We have developed the Shiny App PancreaticmiRaCan-VTE, a free available online tool to easy and rapidly estimate the risk of future VTE events in biliopancreatic cancer patients. The use of Pancreatic-miRaCan-VTE could support the personalized management of biliopancreatic cancer patients in whom to tailor thromboprophylaxis to prevent adverse thrombotic complications. ISCIII-FEDER (PI17/00495, PI20/00075), GVA (ACIF/2017/138), Sociedad Española de Trombosis y Hemostasia.

1. Oto J et al. Int J Mol Sci 2020 Jan 28;21(3):840. https://doi. org/10.3390/ijms21030840.

PB1137 | Biomarkers Of Thrombosis As Predictors Of Venous Thromboembolism In Cancer Patients: Preliminary Results From Fiit Project

S. Lopes ${ }^{1}$; J. Marinho ${ }^{2}$; F. Azevedo ${ }^{1}$; L. Vieira ${ }^{1}$; I. Leão ${ }^{2}$; E. Neto ${ }^{2}$; I. Legath Alonso ${ }^{1}$; E. Silva ${ }^{3}$; H. Coelho ${ }^{4}$; E. Mesquita ${ }^{5}$ T. Costa ${ }^{2}$; D. Ferreira ${ }^{1}$

${ }^{1}$ Centro Hospitalar Vila Nova de Gaia/Espinho, Immunohemotherapy Department, Vila Nova de Gaia, Portugal; ${ }^{2}$ Centro Hospitalar Vila Nova de Gaia/Espinho, Medical Oncology Department, Vila Nova de Gaia, Portugal; ${ }^{3}$ Centro Hospitalar Vila Nova de Gaia/Espinho, Multidisciplinary Thoracic Tumor Unit, Vila Nova de Gaia, Portugal; ${ }^{4}$ Centro Hospitalar Vila Nova de Gaia/Espinho, Hematology Department, Vila Nova de Gaia, Portugal; ${ }^{5}$ Instituto de Saúde Pública da Universidade do Porto, ISUP, Porto, Portugal

Background: Venous thromboembolism (VTE) is an important cause of morbimortality in cancer patients. Comprehensive management of VTE, includes not only its effective its treatment, but also identification of patients who will benefit from thromboprophylaxis. Khorana score is the main validated tool for VTE risk-stratification.

Aims: Develop a predictive model of VTE in ambulatory cancer patients, combining thrombosis biomarkers ( $D$-dimers and thrombin generation potential) with Khorana model.

Methods: This is a prospective observational study that Includes patients with a cancer diagnosis, proposed for anti-tumour treatments (chemotherapy, immunotherapy or targeted therapies). Patients with major bleeding in the last 3 months, major surgery in the last 28 days, on anticoagulation/antithrombotic therapy were excluded. Patients' disease characteristics, blood count values and thrombosis biomarkers were collected at baseline. The primary endpoint is the occurrence of symptomatic or incidental VTE at 6 and 12 months. The study was approved by the local ethics committee. Informed consent was obtained prior to study inclusion.

Results: From April-December 2019, 211 patients were enrolled, 171 analysed (40 were excluded). Median age was 56 [21-81], $53 \%$ were female. The majority had breast $(22 \%, n=38)$, colorectal $(20 \%, n=34)$ and gastric/gastroesophagic $(14 \%, n=24)$ malignancies, $68 \%$ had advanced disease (stage III-IV). The majority initiated chemotherapy+/-targeted therapies $(95 \%, n=163)$. At 6 months, 9 
patients (5.3\%) were diagnosed with VTE (4 pulmonary embolisms, 3 catheter-related and 2 deep venous thrombosis), 56\% $(n=5)$ were incidental findings on exams. VTE was more frequent in metastatic or locally advanced solid cancers $(78 \%, n=7)$, and in gastric/gastroesophagic/biliary $(44 \%, n=4)$ and coloretal $(22 \%, n=2)$. At 12 months VTE was present in $8.8 \%(n=15)$.

Conclusions: Recruitment achieved $74 \%$ by December 2020 , lower than expected due to COVID-19 pandemics. This study is ongoing and will result in a comprehensive risk model adapted to new realities taking into account the diversity of anti-tumour treatments available.

P0165 | A Rare Case of Bi-atrial Thrombi in a Stage IV Pancreatic Cancer Patient with Atrial Fibrillation on Warfarin

K.Y. Kim ${ }^{1}$; N.H. Kim ${ }^{1} ;$ T. Alrifai ${ }^{2}$

${ }^{1}$ AMITA Health St Joseph Hospital Chicago, Chicago, United States;

${ }^{2}$ Rush University Medical Center, Chicago, United States

Background: Atrial fibrillation (AF)-related systemic thromboembolic events are the leading cause of mortality and morbidity in AF patients, necessitating anticoagulation in high risk patients. Pancreatic cancer is associated with a hypercoagulable state, and the choice of an ideal anticoagulant in patients with concomitant AF and cancer remains a controversy.

Aims: We present an unusual case of a 78-year old male with Stage IV pancreatic cancer and underlying AF who was found to have biatrial thrombi while on warfarin therapy.

Methods: Case report with information extracted from patient chart per CARE (CAse REport) guidelines.

Results: CT abdomen and pelvis for surveillance revealed smal thrombi within the right atrium and left atrial appendage, which was new compared to CT imaging performed 3 months prior. At the time of diagnosis, the patient was noted to have supra-therapeutic prothrombin time-international normalized ratio(PT-INR) of 3.3. The patient was admitted and initiated on unfractionated heparin infusion. He declined transitioning to low molecular weight heparin as this required daily subcutaneous injections. He was instead discharged on apixaban.

Conclusions: The development of thrombi despite being on anticoagulation in this patient implores clinicians to consider thromboembolism in cancer patients regardless of their anticoagulation usage. Traditionally, warfarin has been the mainstay of anticoagulation therapy for stroke and systemic thromboembolism prevention in patients with AF. However, our case of failed anticoagulation with warfarin suggests that this agent may be a less ideal choice in patients with underlying malignancy, particularly in the setting of hepatic dysfunction. Recent clinical evidence suggests that direct oral anticoagulants (DOACs) can effectively prevent thrombotic events with a safety index in cancer patients comparable to conventional therapy. Prudent consideration of patient factors (thrombotic and bleeding risk, drug-drug interactions, renal and hepatic function, nutritional status, patient preferences) is encouraged in order to deliver individualized, safe, and effective anticoagulation therapy.

P0171 | Cerebral Thrombosis during Treatment of Childhood Acute Lymphoblastic Leukemia

A. Canbolat Ayhan

Istanbul Medeniyet University Pediatric Hematology-Oncology

Department, Istanbul, Turkey

Background: Therapy for childhood acute lymphoblastic leukaemia (ALL) is performed using intense chemotherapy protocols with a high risk of therapy-related complications such as thrombosis. Chemotherapy agents, infections are some of the factors which contribute increased risk of thrombosis.

Aims: The aim of this study is to evaluate the frequency of cerebral thrombosis in children who were treated for ALL.

Methods: For this purpose the clinical records of 153 patients diagnosed with ALL and treated with ALL-Berlin-FrankfurtMünster (BFM) treatment protocol in the Department of Pediatric Hematology of Istanbul Medeniyet University Prof Dr Suleyman Yalcın Training Hospital between August 2009 and January 2021 were analyzed retrospectively.

Results: Of 153 patients 62 (40.5\%) were female, 91(59.5\%) were male, aged between 7 months-17.8 years, mean age 9.3 years. Of these 18 patients were diagnosed with T-cell ALL, 135 with precursor B-cell ALL. We observed cerebral thrombosis in two (1.3\%) patients. The characteristics of these patients:

Case1: A 5.8 year-old-girl diagnosed with common-ALL; in standard risk group; developed superior sagital sinüs thrombosis on the 43rd day of remission induction chemotherapy.

Case 2: A 8.5 yer-old-boy diagnosed with common-ALL; in median risk group; developed superior sagittal sinüs thrombosis on the 29th day of remission induction chemotherapy.

Inherited thrombophilia screening tests were negative for both of the patients and thrombosis was likely due to L-asparaginase. They were treated with low molecular weight heparin successfully. Anticoagulant treatment was continued until the end of the chemotherapy. They continued to receive L-asparaginase according to the treatment protocol and they did not have recurrence.

Conclusions: Sinus venous thrombosis represents one of the serious chemotherapy-related complications in childhood ALL. Especially L-asparaginase is significantly related with development of thrombosis. We need more studies to be done to establish the optimal duration for treatment of cerebral thrombosis in childeren. 
P0177 | Coagulation Abnormalities At Presentation of Childhood Acute Lymphoblastic Leukemia

\section{A. Canbolat Ayhan}

Istanbul Medeniyet University Pediatric Hematology-Oncology

Department, Istanbul, Turkey

Background: Acute lymphoblastic leukemia (ALL) is the most common malignancy in childhood. Most of the patients have trombocytopenia at presentation of the disease which may lead to bleedings but coagulation abnormalities are not frequent at initial diagnosis of childhood ALL.

Aims: The aim of this study is to evaluate the coagulation profile on the onset of ALL before the start of chemotherapy in children.

Methods: For this purpose the medical records of patients diagnosed with ALL were analyzed retrospectively. Eighty patients were included in the study. Laboratory results for prothrombin time (PT), activated partial thromboplastin time (APTT) and fibrinogen which were obtained at their first admission to hospital were recorded.

Results: Of 80 patients 34 (42.5\%) were female, 46 (57.5\%) were male, they were aged between 7 months and 17.8 years. Seven (8.8\%) patients were diagnosed with T-cell, 73 (91.2\%) patients were diagnosed with precursor B-cell ALL.

Fibrinogen levels ranged between 55 and $658 \mathrm{mg} / \mathrm{dl}$. In 44 (55\%) patients fibrinogen levels were in normal $(200-400 \mathrm{mg} / \mathrm{dl})$ range, in 29 (36.3\%) patients, fibrinogen was high $(400-658 \mathrm{mg} / \mathrm{dl})$, in $3(3.7 \%)$ patients it was between $100-200 \mathrm{mg} / \mathrm{dl}$ and in $4(5 \%)$ patients it was between $55-100 \mathrm{mg} / \mathrm{dl}$.

PT levels ranged between $11.5 \mathrm{~s}$ and 21.9 s. In 65 (81.2\%) patients PT was in normal (11.5-15.5 s) limits . In 15 (18.8\%) patients PT was prolonged (15.5-21.9 s).

APTT levels ranged between $25.6 \mathrm{~s}$ and $35.2 \mathrm{~s}$. In 48 (60\%) patients APTT was in normal (25.6-35.2 s) levels. In six (7.5\%) patients APTT was prolonged ( $>35.2 \mathrm{~s}$ ). In $16(20 \%)$ patients APTT shortened ( $<25.6 \mathrm{~s}$ ).

Conclusions: Childhood ALL may be associated with alterations in coagulation tests which can be predictor to bleeding or thrombotic events. Recognition of these abnormalities is important in management of these complications.
P0179 | Management of Venous Thrombosis in Non-Hodgkin Lymphoma

V. Musteata

State University of Medicine and Pharmacy 'N. Testemitanu', Institute of Oncology, Chisinau, Moldova

Background: Thrombotic complications frequently develop during the evolution of hematological malignancies, significantly influencing the rates of morbidity and mortality.

Aims: The study objectives were to identify the features and evaluate the results of treatment of venous thrombosis in non-Hodgkin lymphoma (NHL).

Methods: We performed a clinical-analytical, descriptive study of one case with stage IIA diffuse large B-cell NHL, which was treated and followed up at the Institute of Oncology between 2018-2020. The patient was a female of 46 years old, with concomitant pathology: Essential hypertension, gr. II, moderate additional risk. Hypertensive heart disease. Grade I obesity. The diagnosis was proved according to the Revised 2017 WHO Classification of Tumors of Hematopoietic and Lymphoid Tissues by the histopathological and immunohistochemical examinations of the biopsied lymph node and standard staging procedures, including CT scan.

Results: Three adjusted cycles of R-CHOP chemotherapy were performed for the remission induction on the background of cardiotropic and antiplatelet medication, with positive clinical-imaging dynamics and without side effects. Taking into account the persistence of residual tumor lymphadenopathy, the locoregional radiotherapy (LRT) was initiated at the ilioinguinal lymph nodes. The LRT was temporarily stopped at a dosage of $14 \mathrm{~Gy}$ due to the appearance of the clinical signs of venous thrombosis of the right femur and calf. Duplex sonography of the legs veins proved the diagnosis of the acute bilateral grade II phlebothrombosis. The patient responded with the tendency of venous recanalization to the daily oral antiplatelet and anticoagulant therapy under the control of INR, prothrombin index and the angiosurgeon follow-up (Figure 2). The patient accomplished the LRT, achieved the complete remission and underwent the maintenance Bleo-COP cycles. 


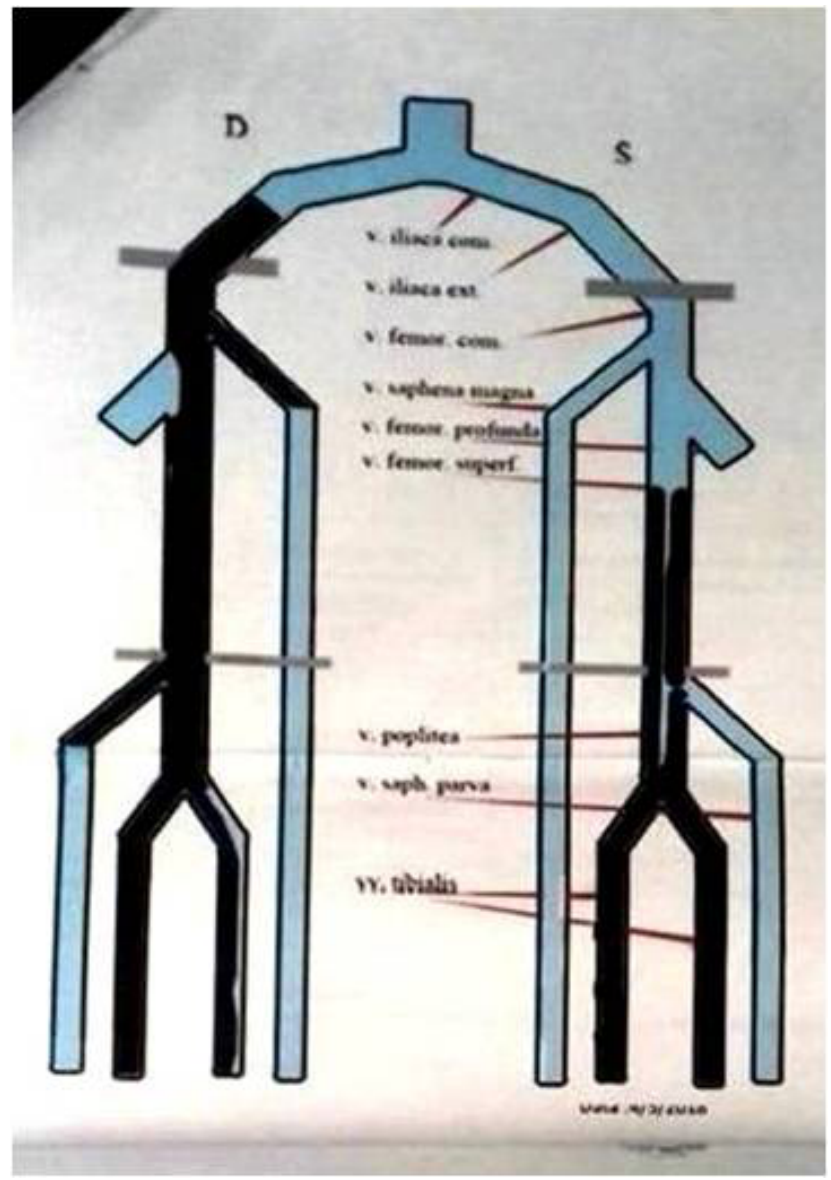

FIGURE 1 Duplex sonography of the inferior limbs veins: phlebothrombosis before the treatment

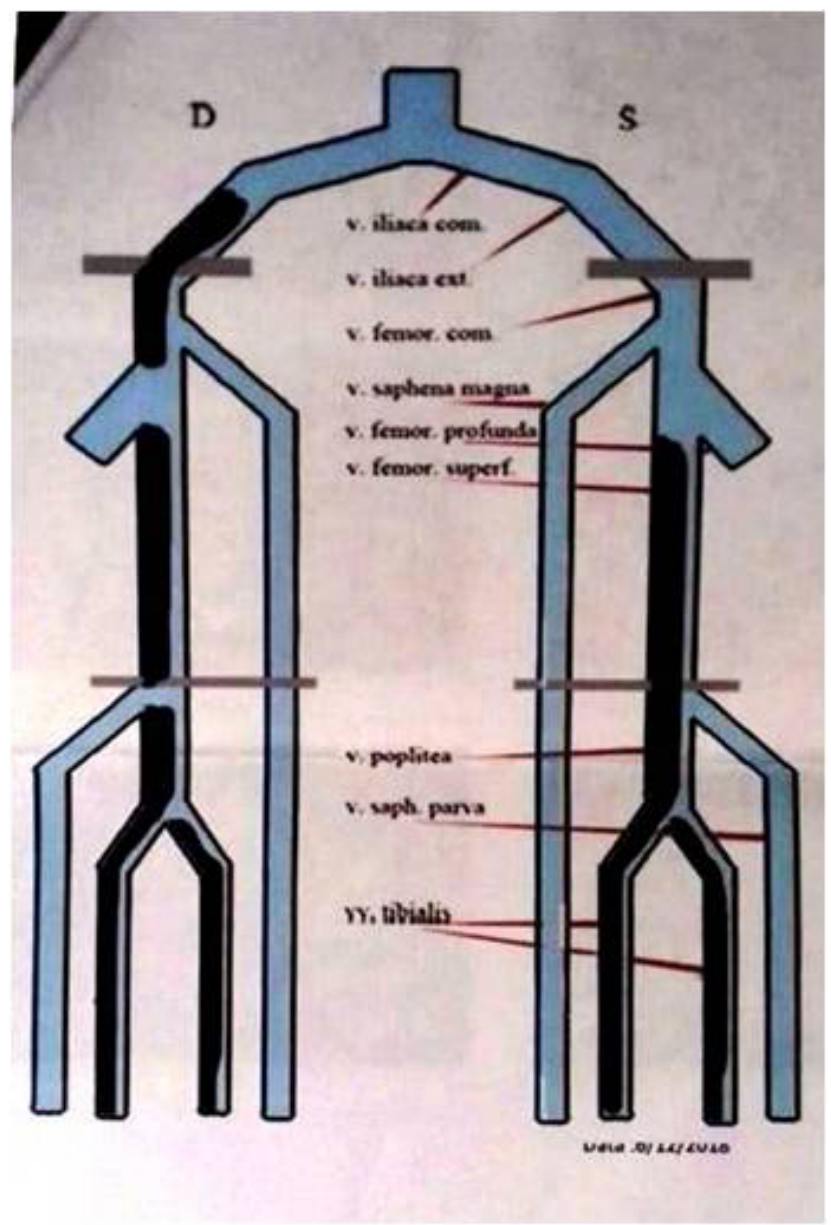

FIGURE 2 Duplex sonography of the inferior limbs veins under the treatment: tendency to recanalization

Conclusions: NHL in association with obesity may be complicated with phlebothrombosis during the LRT. The phlebothrombosis regression occurred after the complete remission and under the oral antiplatelet and anticoagulant therapy.

P0182 | Venous Thromboembolism as the First Presentation of Multiple Myeloma

$\underline{\mathrm{X} . \mathrm{Ye}}$

Second Affiliated Hospital of Guangzhou Medical University, Guangzhou, China

Background: Multiple myeloma (MM) is a kind of malignant plasma cell disease which can lead to disturbance in coagulation system. Cases of coagulopathy caused by MM and treatment-associated thrombosis have been reported. But cases of venous thrombosis as the first presentation of MM had rarely been reported.

Aims: To report two cases presented with venous thrombosis or pulmonary embolism (PE) before they were diagnosed with $\mathrm{MM}$.

Methods: Case 1 was a 45-year-old male patient who was diagnosed intracranial venal sinus thrombosis with cranial MR contrast imaging. 
Anticoagulation therapy partially relieved his condition. 4 months later, he was diagnosed IgG-type MM. Case 2 was a 28-year-old female who was found to have portal vein, splenic vein thrombosis and superior mesenteric vein thrombosis and received interventional therapy and anticoagulation therapy. Four months later, she was diagnoses MM. She had received splenectomy and cholecystectomy a year before due to epigastric pain and splenomegaly as a result of $\beta$-thalassemia and chronic hepatitis B. She had high platelet count and positive lupus anticoagulant.

Results: In case 1, With anticoagulation and chemotherapy, the intracranial sinus thrombosis was cleared 2 months later. The thrombosis did not recur for four years now and his $M M$ was in partial remission. In case 2, anticoagulation and chemotherapy for $\mathrm{MM}$ had to be suspended constantly due to recurrent upper digestive bleeding. Her MM was in remission after courses of chemotherapy. But, unfortunately, she died of delayed treatment for PE after she discontinued anticoagulants and had diarrhea.

The report here has got informed consent from the patient and their relatives.

Conclusions: DVT can be the first presentation of MM and should be paid attention to and serum Ig concentration should be checked. Successful treatment of MM together with anticoagulation therapy is helpful for the clearance of thrombosis.

P0188 | "Heparin Failure" in Seminoma-related Dramatic Hypercoagulable Melieu and Extended Vein Thrombosis: Is it BEP Protocol Ongoing Responsible?

A.M. Fioretti $^{1}$; T. Leopizzi ${ }^{1}$; L. Palermo ${ }^{2}$; V. Lorusso ${ }^{2}$; S. Oliva ${ }^{1}$

${ }^{1}$ Cardio-Oncology Unit, IRCCS Istituto Tumori "Giovanni Paolo II", Bari, Italy; ${ }^{2}$ Medical Oncology Unit, IRCCS Istituto Tumori "Giovanni Paolo II", Bari, Italy

Background: TEV is a common cancer complication with $20 \%$ incidence.

Aims: LMWH is the standard therapy for efficacy, safety and ease of use. However, some scenarios are deeply challenging for intercurrent prothrombotic anticancer drugs.
Methods:

\section{esaote}

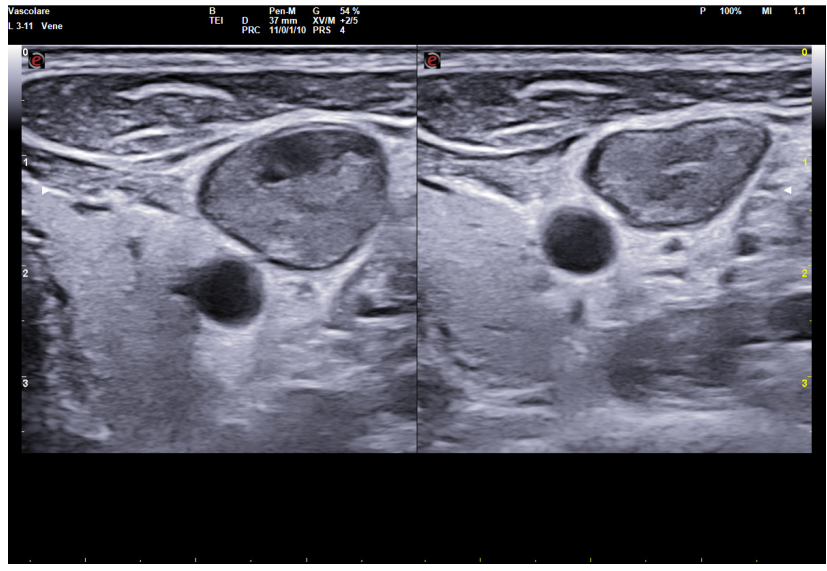

FIGURE 1 Left internal giugular vein thrombosis

A 35 y-o man reported dysphagia, EGDS: esophagus ulcers, thyroid echography: thoracic mass compressing proximal borders. Vascular ultrasound: thrombosis of left internal giugular, subclavian, axillary and brachial veins; he began enoxaparin $4000 \mathrm{IU} \times 2 /$ die. CT: solid anterior-superior mediastinum vascularized mass $(16 \times 13$ $\mathrm{cm}$ ) incorporating great thoracic vessels with $20 \mathrm{~cm}$ cranio-caudal longitudinal extension with trachea dislocation. PET-CT: massive superior-anterior mediastinum pathological ${ }^{18} \mathrm{~F}$-FDG accumulation suggestive for malignancy. Lung perfusion scan: absence of left lung perfusion. Angio-CT: showed compression of pulmonary artery trunk and of branches. He presented marked asthenia, sweating and presyncope. D-dimer: $6026 \mu \mathrm{g} / \mathrm{L}$, NT-proBNP: $1417 \mathrm{pg} / \mathrm{mL}$. Mediastinum biopsy exhibited seminoma (ki67+: 65\%), he started BEP Protocol (etoposide, cisplatin, bleomycin), till now. 
Results:

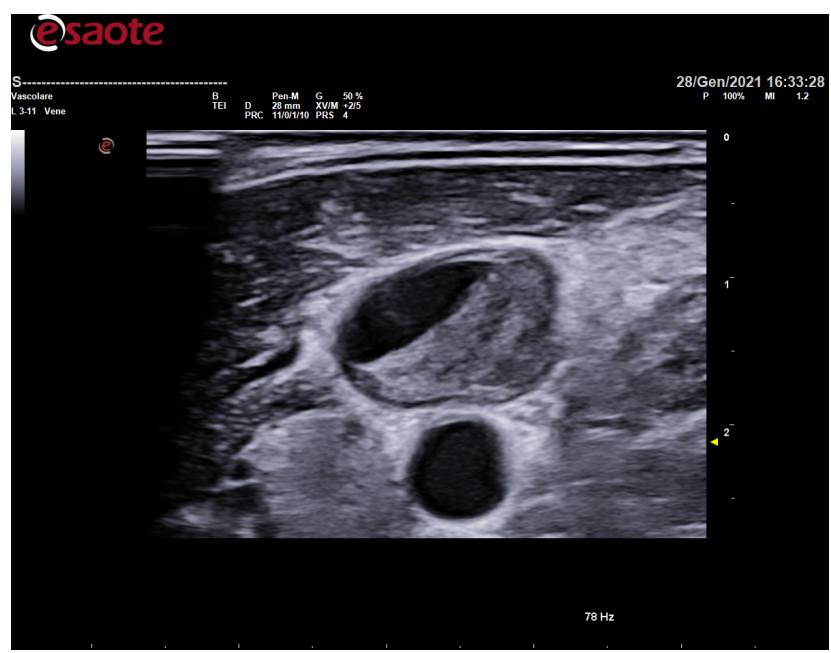

FIGURE 2 Partial recalization of left internal giugular vein thrombosis

TTE: periaortic cuff from mediastinum mass which ab-extrinseco compressed pulmonary artery trunk and branches with occlusion of left one, right chambers dilatation, sovra-epatic veins and inferior vena cava $(21 \mathrm{~mm}$ ) ectasia, decreased inspiratory collapse, pulmonary hypertension (SPAP: 52 mmHg), EF: 55\%. After 2 months enoxaparin, vein ultrasound: persistent DVT and positive CUS (Figure 1). So, we replaced enoxaparin with edoxaban $60 \mathrm{mg} / \mathrm{die}$. After 1 month edoxaban, vein ultrasound showed initial recanalization of left internal giugular vein (Figure 2); D-dimer: $1554 \mu \mathrm{g} / \mathrm{L}$. After 2 months BEP Protocol, CT: decrease mediastinum mass $(6 \times 12 \mathrm{~cm})$ dimensions. Conclusions: Cancer associated thrombosis is complex complication, worsening mortality, morbidity and decision making. Cancer stage and drugs favour development of severe thrombosis, not solvable with $\mathrm{LMWH}$, the cornerstone anticoagulant therapy in cancer-related thrombosis. DOACs appear as new and successful therapeutical option, especially in most challenging cases of highly thrombotic profile after "heparin failure".

\section{GENETIC RISK FACTORS OF THROMBOSIS}

PB1138 | Secondary Prophylaxis of Venous Thromboembolism with Direct Oral Anticoagulants in Patients with Major Congenital Thrombophilia vs Non-thrombofilic Patients

\section{A. Serrao ${ }^{1}$; G.M. Assanto ${ }^{1}$; C. Santoro ${ }^{1}$; A. Pallotta ${ }^{2}$; A. Chistolini ${ }^{1}$ \\ ${ }^{1}$ Sapienza University of Rome, Rome, Italy; ${ }^{2}$ Policlinico Umberto I, \\ Roma, Italy}

Background: Direct oral anticoagulants (DOACs) are widely used for the treatment and secondary prophylaxis of venous thromboembolism (VTE). Congenital thrombophilia is a condition that predisposes to a higher incidence of VTE, more frequent VTE recurrences, some also in atypical sites, and often require long-term anticoagulation for secondary prevention. It is less clear the efficacy of DOACs s in patients with major thrombophilia.

Aims: The aim of our study was to evaluate the efficacy, in terms of VTE prevention, and safety, in terms of absence of bleeding complications, in patients with major thrombophilia compared to nonthrombophilic patients candidate to long-term anticoagulation for recurrent VTE.

Methods: We evaluated consecutive patients who required longterm anticoagulation for recurrent VTE, treated with DOACs, and compared the outcomes between patients affected by major thrombophilia and non-thrombophilic patients. All patients presented at least 2 thrombotic events. Major thrombophilia was defined as the presence of physiologic inhibitors deficiency (protein C, protein S and antithrombin; homozygous Factor $V$ Leiden, homozygous Factor II G20210A, combined heterozygosity of these defects.

Results:

TABLE 1 Population's characteristics

\begin{tabular}{|c|c|c|c|}
\hline $\begin{array}{l}\text { Patients under DOAC } \\
\qquad 167\end{array}$ & $\begin{array}{l}\text { Patients with } \\
\text { thrombophilia }\end{array}$ & $\begin{array}{l}\text { Control } \\
\text { Patients }\end{array}$ & p value* \\
\hline Number of patients & 72 & 94 & \\
\hline Sex (Male/Female) & $39 / 33$ & $62 / 32$ & 0.12 \\
\hline Body Mass Index (median) & 24,6 (range $16.8-45$ ) & 27 (range 20-39) & 0.007 \\
\hline $\begin{array}{l}\text { Age at start of therapy } \\
\text { (median years) }\end{array}$ & 42,4 (range 18-91) & 55,9 (range 18-94) & $<0,0001$ \\
\hline $\begin{array}{l}\text { Platelets at start of therapy } \\
\text { (Median10 } 10^{3} / \mathrm{mmc} \text { ) }\end{array}$ & 228 (range 148-380) & 234 (range 130-600) & 0.24 \\
\hline $\begin{array}{l}\text { Creatinine at start of therapy } \\
\text { (median } \mathrm{mg} / \mathrm{dl} \text { ) }\end{array}$ & 0,8 (range $0.2-1.50$ ) & 0.93 (range 0.2-1.53) & 0.005 \\
\hline \multicolumn{4}{|l|}{ DOAC administered } \\
\hline Rivaroxaban & $29(40.33)$ & $26(27.7 \%)$ & \\
\hline Edoxaban & $7(9.796)$ & $20(21.3 \%)$ & \\
\hline Apixaban & 28 (38.996) & $43(45.7 \%)$ & \\
\hline Dabigatran & $8(11.1 \%)$ & $5(5.3 \%)$ & \\
\hline \multicolumn{4}{|l|}{ Thrombophilia } \\
\hline Protein C Deficiency & $7(9.69)$ & & \\
\hline Protein S Deficiency & $20(27,436)$ & & \\
\hline Antithrombin Deficiency & $12(16.436)$ & & \\
\hline $\begin{array}{l}\text { FV Leiden Homozyzous } \\
\text { Mutated }\end{array}$ & $18(24.796)$ & & \\
\hline $\begin{array}{l}\text { FII G20210A Homozygous } \\
\text { Mutated }\end{array}$ & $5(6.8 \%)$ & & \\
\hline $\begin{array}{l}\text { Double Heterozygous } \\
\text { mutation }\end{array}$ & $10(13.7 \%)$ & & \\
\hline
\end{tabular}

The examined patients were 167: 72 (43.4\%) with major thrombophilia and 94 (66.6\%) non-thrombophilic. All patients' characteristics are specified in table 1 . The median time of DOACS therapy was 32 months (range 6-90) in the group with major thrombophilia; 20 months (range 6-80) in the other group. No significative difference was observed in the 2 groups in the incidence of thrombotico events ( $p$ 0.4) and for the onset of hemorrhagic complications ( $p$ 0.14).

Conclusions: Although major thrombophilia predisposes to a higher incidence of VTE, our data suggest that in this setting of patients, 
DOACs are effective and safe, with comparable results to nonthrombophilic patients.

PB1139 | Is Hematopoietic Clonality of Indetermined Potential a Risk Factor for Pulmonary Embolism?

S. Soudet; G. Jedraszak; O. Evrard; L. Garcon; J.P. Marolleau; M.A. Sevestre

CHU Amiens Picardie, Amiens, France

Background: Idiopathic pulmonary embolism (iPE) is a severe and frequent condition accounting for half of pulmonary embolisms. Characterization of new risk factors is mandatory in order to identify patients that would benefit from a long-term treatment.

Aims: To evaluate if idiopathic pulmonary embolism is associated with the presence of clonal hematopoiesis of indetermined significance (CHIP).
Methods: We conducted a pilot retrospective monocentric observational study. Patients with iPE between 18 to 65 years old, after a first documented episode of PE were included. PE was considered non provoked, when no transient nor persistant risk factor was present and when extensive thrombophilia testing was negative. Patients with documented atherosclerosis, previous VTE, familial history of VTE, presence of cytopenias, presence of any driven mutation of myeloproliferative neoplasms were excluded. CHIP proportion in iPE patients were analyzed using next generation sequencing of the coding sequence of a custom panel composed by DNMT3A, ASXL1, SF3B1, TET2 and TP 53. Presence of CHIP was considered with a variation allelic fraction higher than $1 \%$.

Results: Upon 61 patients with iPE consecutively included, a total of 19 somatic mutations were found in 12 patients (20\%). 15 mutations were found in DNMT3A gene, 3 in ASXL1 and one in TET2. No mutation in SF3B1 nor TP53 genes were identified. There was no difference in terms of age, PE location, DVT presence and risk stratification in CHIP carriers and non carriers. Median follow-up was 2 years.

TABLE 1 Comparison between CHIP carriers and non carriers

$\begin{array}{ll} & \text { CHIP carriers } \\ \text { Median age, IQR, y } & 59.5[56.25-65] \\ \text { Sex ratio, \% } & 16 \\ \text { PE location (proximal), \% } & 33 \\ \text { DVT proportion, \% } & 42 \\ \text { Median hematocrit, IQR, \% } & 0.42[39.5-43.5] \\ \text { Median platelet numeration, IQR, } & 214[175-246] \\ 10^{9} / \mathrm{L} & \\ \text { Median WBC, IQR, 10\% /L } & 6.5[5.7-8]\end{array}$

\begin{tabular}{ll} 
CHIP non carriers & $P$ \\
$54[46-58]$ & 0.08 \\
12 & 0.64 \\
45 & 0.54 \\
47 & 0.66 \\
$0.43[38-44.3]$ & 0.61 \\
$207[176-298]$ & 0.55 \\
5 [3.5-7] & \\
\hline
\end{tabular}

Conclusions: We report, for the first time, an association between idiopathic pulmonary embolism and CHIP, that may become a new risk factor of VTE. CHIP-induced inflammation of vascular endothelium, well documented for TET2 mutation, leading to atherosclerosis and potentially clinical iPE, may represent the missing link between arterial and venous thrombosis. These results need to be confirmed in a prospective study including.

PB1140 | Tumor Somatic Mutations as Predictors of CDK4/6 Inhibitor Associated Thromboembolism in Women with Metastatic Breast Cancer

M. West; R. Thawani; J. Shatzel

Oregon Health \& Sciences University, Portland, United States

Background: CDK4/6 inhibitors (CDKi) are integral therapy for metastatic hormone receptor positive Her2 negative breast cancer, although venous thromboembolism occurred in up to $5 \%$ of patients in clinical trials. Real-world studies describe rates of thrombosis up to $10 \%$ at one year, of which a third were arterial events, however traditional risk assessment models fail to predict which patients are at high risk for thrombosis. Increasingly, tumor somatic mutations appear to be independent risk factors for thrombosis. Breast cancer somatic mutations associated thrombosis have yet to be identified.

Aims: To identify and describe the thrombotic risk associated with tumor somatic mutations in metastatic breast cancer patients receiving CDKi.

Methods: A retrospective multi-institutional review of 65 women with metastatic breast cancer treated with CDKi who received tumor next generation sequencing analysis. The presence of thrombosis during or up to 30 days of discontinuation of CDKi was collected from chart review. The analysis was exploratory and thus unpowered. Descriptive statistics and fisher's exact test were performed to define association between tumor mutational status and thrombosis.

Results: Thrombotic events occurred in 6 of the 65 total patients while on CDKi (9.2\%). In the 6 patients who developed thrombosis, 46 total somatic mutations were identified. The most prevalent mutations in those with thrombosis were in PIK3CA (4), followed by TP53 (3), CCND1 (2), MAP2K4 (2), FGF4 (2), FGF3 (2), FGF19 (2), CKND2A (1). The strongest association with thrombosis was seen 
in mutations of the fibroblast growth factor/FGF receptor pathway ( FGF19 $P=0.056)$ and PIK3CA $(P=0.072)$ Table 1 . No arterial events occurred. .

Table 1: Association between tumor somatic mutations and thrombosis

\begin{tabular}{llll}
$\begin{array}{l}\text { Somatic } \\
\text { Mutation }\end{array}$ & Thrombosis & No thrombosis & $P$-value \\
FGF4 & $28.6 \%$ & $6.9 \%$ & 0.122 \\
FGF19 & $27.2 \%$ & $5.6 \%$ & 0.056 \\
FGF3 & $25.0 \%$ & $7.0 \%$ & 0.155 \\
MAP2K4 & $25.0 \%$ & $7.0 \%$ & 0.155 \\
CCND1 & $20.0 \%$ & $7.3 \%$ & 0.228 \\
PIK3CA & $16.0 \%$ & $2.6 \%$ & 0.072 \\
TP53 & $10.5 \%$ & $8.7 \%$ & 0.571 \\
CDKN2A & $8.3 \%$ & $9.4 \%$ & 0.695 \\
\hline
\end{tabular}

Conclusions: CDKi associated thrombotic events may be associated with somatic tumor mutations in FGF-FGFR pathway, MAP2K4, CCND1, PIK3CA and CDKN2A. Larger studies evaluating tumor somatic mutations and thrombosis are needed to identify high risk patients who may benefit from thromboprophylaxis.

PB1141 | ABO Blood Groups as a Risk Factor for the Development of Deep Vein Thrombosis of the Lower Extremities in Elderly and Senile Patients

A. Tomchenko; A. Chechulova; V. Soroka; S. Nokhrin; I. Khomchuk Saint Petersburg I.I. Dzhanelidze Research Institute of Emergency Medicine, Saint Petersburg, Russian Federation

Background: It is believed that non-ABO blood groups increase the likelihood of deep vein thrombosis (DVT), however, their effect on the risk of VTE in the elderly and senile has not been sufficiently studied.

Aims: To evaluate the effect of different $A B O$ blood groups on the risk of deep vein thrombosis of the lower extremities in elderly and senile patients.

Methods: 55 patients with DVT were examined and their ABO blood groups were determined. There were 33 males (60\%) and 22 females (40\%). The median age was $74.2( \pm 8.7)$ years. The control group was represented by patients without a history of DVT $(n=49)$. All subjects were divided into two groups: elderly (60-74 years) and senile (75-90 years).

Results: In the general group of DVT B blood group was the most common, which was almost three times more than in patients without a history of DVT. Group B in half of the cases occurred in women with DVT, which was twice as much as in men with DVT (50.7\% vs. $24.2 \%$; OR $=3.1 ; P=0.05)$ and seven times as much as in women of the control group $(50.7 \%$ vs. $7.1 \%$; OR $=13.0 ; 95 \% \mathrm{Cl}$ : 2.5-68.6; $P<0.001)$. O blood group was detected almost three times less frequently among patients with DVT than in the control group. Non-O blood groups were found in the elderly in $90 \%$ of cases, which was 1.6 times more common than in the control group of the same age category (90\% vs. 55.6\%; OR = 7.2; 95\% Cl:1.8-28.9; $P=0.003$ ) and almost 1.5 times more common than in patients with DVT older than 75 years ( $90 \%$ vs. $66.7 \%$; OR $=4.5 ; 95 \% \mathrm{Cl}: 1.1-19.9$; $P=0.038$ ).

Conclusions: Non-O blood groups can be considered as a risk factor for the development of DVT in elderly patients. Women with B blood type have the highest exposure to DVT.

PB1142 | Hemostatic Genetic Risk Factors of Aseptic Cerebral Venous Thrombosis

K. Stepanchenko; O. Tsymbal; A. Zhydkova

Kharkiv Medical Academy of Postgraduate Education, Kharkiv, Ukraine

Background: Aseptic cerebral venous thrombosis (ACVT) can develop in patients with hereditary and acquired thrombophilic conditions, which are diagnosed in $34 \%$ of cases. Common hereditary factors include polymorphisms in the Leiden $\mathrm{V}$ and prothrombin genes, deficiencies in proteins $\mathrm{C}$ and $\mathrm{S}$, and antithrombin III.

Aims: To study the frequency of hemostatic gene polymorphisms in patients with ACVT.

Methods: Two groups:

Group 1-47 patients (age 62,7 77,7 years) with ACVT; Group 2 (control) - 40 patients $(59,7 \pm 10,2$ years) without ACVT and VTE were formed in non-randomized retrospective-prospective study.

Brain MRI and CT-/MR-venography were performed. DNA diagnostics of thrombophilic genetic polymorphisms of the plasminogen activator inhibitor PAI-1 (5G6754G); prothrombin gene FII (G20210A); fibrinogen beta FGB (G455A); platelet fibrinogen receptor ITGB3 (T1565C); coagulation factor V (G1691A); activated factor XIII (fibrinase) gene FXIIIA1 (G103T); integrin alpha (Gp1a glycoprotein) gene ITGA2 (C807T); coagulation factor VII (G10976A) - were carried out using PCR.

Results: Hemostatic gene polymorphisms were found in $42(89,4 \%)$ of 47 patients (table 1 ). 
Table 1: The most common genotypes among patients examined for hemostatic gene polymorphisms The presence of polymorphisms in the genes of the hemostasis system

\begin{tabular}{lllllll}
$\begin{array}{l}\text { Number of patients } \\
(\%)\end{array}$ & & PAI-1 & F13A1 & ITGA2 & FVII & FGB \\
$8(17 \%)$ & M:F & 5G6754G & G103T & C807T & G10976A & G455A \\
$5(10,6 \%)$ & $3: 4$ & $\mathrm{P}$ & $\mathrm{N}$ & $\mathrm{N}$ & $\mathrm{N}$ & $\mathrm{N}$ \\
$4(8,5 \%)$ & $1: 3$ & $\mathrm{P}$ & $\mathrm{P}$ & $\mathrm{N}$ & $\mathrm{N}$ & $\mathrm{N}$ \\
$3(6,4 \%)$ & $1: 3$ & $\mathrm{P}$ & $\mathrm{N}$ & $\mathrm{P}$ & $\mathrm{N}$ & $\mathrm{N}$ \\
$3(6,4 \%)$ & $1: 2$ & $\mathrm{P}$ & $\mathrm{N}$ & $\mathrm{N}$ & $\mathrm{P}$ & $\mathrm{N}$ \\
\hline
\end{tabular}

$\mathrm{N}$ - normal genotype

$\mathrm{P}$ - homozygous or heterozygous gene polymorphism

Polymorphism of one gene was present in $9(19,1 \%)$ patients, two genes - in 15 (31,9\%) patients, three genes - in 18 (38,3\%) patients, and four genes - in $5(10,6 \%)$ patients. Among patients with hemostatic gene polymorphisms, $15(35,7 \%)$ had a homozygous state, and $38(90,5 \%)$ - a heterozygous state. Most often homozygous gene polymorphism in 9 cases $(19,1 \%)$, as well as heterozygous gene polymorphisms in 29 (61,7\%) patients were found in the gene of the plasminogen activator inhibitor PAI-1 5G6754G.

Conclusions: Hemostatic gene polymorphisms in ACVT were detected in $89,4 \%$ of cases. Gene polymorphism of the plasminogen activator inhibitor PAI-1 5G6754G was the most common. The polymorphism of this gene leads to an increase in the functional activity of the plasminogen activator inhibitor protein and thus risk of thrombosis.

PB1143 | VKORC1 -1639AA Genotype Is a Possible Protective Factor for Venous Thromboembolism Development in Patients with FII G20210A Mutation from North-Western Russia

S. Kapustin ${ }^{1}$; A. Chechulova ${ }^{2}$; S. Svitina ${ }^{1}$; J. Sidorova ${ }^{1}$; V. Burakov ${ }^{1}$; V. Soroka ${ }^{2}$ ․ V. Soldatenkov ${ }^{1}$; L. Papayan ${ }^{1}$

${ }^{1}$ Russian Research Institute of Hematology and Transfusiology, Saint Petersburg, Russian Federation; ${ }^{2}$ Dzhanelidze Research Institute of Emergency Medicine, Saint Petersburg, Russian Federation

Background: Vitamin $\mathrm{K}$ plays a crucial role in hemostasis by activating both procoagulant (FII, VII, IX, X) and anticoagulant (proteins C, S, Z) factors. Vitamin K-epoxide reductase 1 (VKORC1) G-1639A gene polymorphism is known to affect an enzyme activity and bioavailability of vitamin $\mathrm{K}$. To date, there is a little data on the role of VKORC1 G-1639A variation in venous thromboembolism (VTE) development, in particular, in patients with inherited thrombophilia. Aims: To assess effect of the VKORC1 G-1639A gene polymorphism on the risk of VTE development in patients from North-Western Russia.

Methods: We included 600 VTE patients (294 men and 306 women, mean age $43.6 \pm 15.3$ years) originated from the North-Western region of Russia in the study. The control group (CG) consisted of 200 healthy individuals of the same origin. Genotyping for the VKORC1 G-1639A, FII G20210A and FV G1691A variations was performed by PCR-RFLP. Intergroup differences in genotype frequencies were assessed by Fisher's exact test. The study was approved by the local ethical committee.

Results: Distribution of the VKORC1 G-1639A gene variants was similar in both VTE patients and controls. Frequency of the VKORC1 -1639AA genotype in patients with the FV Leiden was 4-fold higher than in those having the FII G20210A mutation (19.6\% vs. $4.4 \%$, respectively; $O R=5.2 ; 95 \% \mathrm{Cl}: 1.2-23.6 ; P=0.021)$. In the group of patients without FII and FV gene mutations, the frequency of the VKORC1 -1639AA genotype was almost equal to that in CG $(17.1 \%$ vs. $16.5 \%$, respectively). When compared to CG, the VKORC1 -1639AA variant was significantly underrepresented in VTE patients with the FII G20210A gene mutation (4.4\% vs. 16.5\%, respectively; $\mathrm{OR}=0.2 ; 95 \% \mathrm{Cl}: 0.1-1.0 ; P=0.035)$.

Conclusions: We suggest that VKORC1 -1639AA genotype could have protective effect on VTE development in patients with FII G20210A mutation from North-Western Russia. Further studies are needed to confirm this finding.

PB1144 | FACTOR XII 46 C/T Gene Polymorphism as a Possible Risk Factor for Late-onset Venous Thromboembolism in Patients from the North-Western Russia

A. Chechulova $^{1}$; S. Kapustin ${ }^{2}$; V. Soroka ${ }^{1}$; V. Soldatenkov ${ }^{2}$;

L. Papayan ${ }^{2} ;$ M. Galchenko ${ }^{3}$

${ }^{1}$ Dzhanelidze Research Institute of Emergency Medicine, St. Petersburg, Russian Federation; ${ }^{2}$ Russian Research Institute of Hematology and Transfusiology, St. Petersburg, Russian Federation; ${ }^{3}$ Saint Petersburg State Agrarian University, St. Petersburg, Russian Federation

Background: Factor XII (FXII, Hageman factor) is involved in initiation of internal blood coagulation pathway, regulation of fibrinolysis and kallikrein-kinin system. The FXII $46 \mathrm{C} / \mathrm{T}$ gene polymorphism is associated with decrease of both level and activity of this factor. Role of the FXII $46 \mathrm{C} / \mathrm{T}$ polymorphism in venous thromboembolism (VTE) development is still not clear. 
Aims: To evaluate the role of the FXII $46 \mathrm{C} / \mathrm{T}$ gene polymorphism in VTE development in patients from the North-Western Russia.

Methods: We examined 600 patients (294 men and 306 women, mean age $-43.6 \pm 15.3$ years) with VTE. In 400 patients, the first episode of VTE was diagnosed at young age (45 years or less). Other 200 patients composed the group with late-onset VTE. The control group (CG) consisted of 200 age- and sex-matched healthy persons. All individuals originated from the North-Western Russia and gave informed consent for participation in the study. Genotyping for the FXII 46 C/T polymorphism was performed by PCR-RFLP. The differences in genotypes distribution between the groups were estimated by Fisher's exact test.

Results: Distribution of the FXII $46 \mathrm{C} / \mathrm{T}$ variants was similar between VTE patients and CG. Frequencies for the CC, CT and TT genotypes were $48.2 \%, 43.0 \%, 8.8 \%$ in patients, and $48.0 \%, 45.5 \%, 6.5 \%$ in controls, respectively. The $46 \mathrm{~T}$ allele was more frequently present in patients with late-onset VTE $(58.0 \%$ vs. $48.8 \%$ in young patients; $\mathrm{OR}=1.5 ; P=0.038)$. Homozygosity for the $46 \mathrm{~T}$ allele was found in $24(12.0 \%)$ patients with late-onset VTE and 29 (7.3\%) young patients $(O R=1.7 ; P=0.066)$. When compared to $C G$, the frequency of 46TT genotype was almost 2-fold increased in patients with VTE manifested after 45 years old $(12.0 \%$ vs. $6.5 \%$, respectively; $\mathrm{OR}=2.0 ; P=0.083$ ).

Conclusions: Our data suggest that the FXII $46 \mathrm{C} / \mathrm{T}$ gene polymorphism could be a possible risk factor for late-onset VTE development in patients from the North-Western Russia.

\section{PB1145 | The Evaluation of the Children with Intracranial} Thrombosis

A. Meral Güneș ${ }^{1}$; S. Güler ${ }^{1}$; Ö. Tüfekçi ${ }^{2}$; M. Sezgin Evim ${ }^{1}$; B. Baytan ${ }^{1}$; Ş. Yılmaz ${ }^{2}$; H. Ören ${ }^{2}$

${ }^{1}$ Bursa Uludag University, Bursa, Turkey; ${ }^{2}$ Dokuz Eylul University, Izmir, Turkey

Background: Intracranial thrombosis (ICT) is rare in children. Early diagnosis is important for the outcome.

Aims: The aim of this study was to evaluate the etiology, clinical findings and imaging, and prognosis in children with ICT.

Methods: We, retrospectively analyzed the ICT diagnosed within the last 10 years excluding neonates and children with malignancy.

Results: A total of 54 patients (M / F: 27/27) were included. The median age was 62.2 (2-193)months. The mean follow-up period was 53 (0-186) months. The majority of cases had arterial thrombosis (59\%) whereas venous thrombosis was detected in $41 \%$ of children. The most frequently involved artery and veins were arteria cerebri media (33\%) and transverse sinus (28\%). Family history was found positive in $15 \%$ of the patients. Mutation analysis and Protein C, S levels were available $87 \%$ and $70 \%$ of cases, respectively. Homozygous MTHFR (32\%), FVL (19\%) homozygous PAI (4\%) and prothrombin mutations (4\%) were detected. Protein S and C deficiencies were found in 13\% (n: $5 / 38)$ and $3 \%(n=1 / 38)$ of cases, respectively. The most common acquired risk factors were infection (28\%), surgery (9\%) and trauma (7\%). No acquired risk factors were identified in 22 (41\%) children. Seizures (28\%), vomiting (22\%), fever (19\%) and headache (19\%) were the most common symptoms. Hemiplegia/hemiparesis (35\%), papillary edema (11\%), and facial paralysis (5.5\%) were frequent. LMWH and ASA were used for treatment. Prophylaxis was applied to $70 \%$ of children. None of the children died of thrombosis. Although $59 \%$ of the children recovered, chronic sequelae occurred in $43 \%$. The thrombosis recurred only in one child.

Conclusions: It is important to reach an accurate and rapid diagnosis of ICT. Majority of the patients had a genetic risk factor (94\%; $n=44 / 54)$. Therefore, we strongly suggest that all children with ICT should be searched for thrombophilic mutations. Children should be closely followed for chronic sequelaes.

PB1146 | Pulmonary Embolism in the Onset of Hematogenous Thrombophilia

V. Voitsekhovsky; T. Zabolotskikh; I. Sayapina; O. Prikhodko; T. Luchnikova; E. Romantsova; N. Grigoriev

Amur State Medical Academy, Blagoveshchensk, Russian Federation

Background: The term "thrombophilia" means a tendency to thrombosis with an early age-related onset, family history, severity of thrombosis, disproportionate to the known causative factor and episodes of recurrent thrombosis.

Aims: To investigate young patients with pulmonary embolism (PE), in the absence of an obvious causative agent, for markers of hematogenous thrombophilia.

Methods: The study involved 81 patients between the ages of 17 and 45 years, with various thrombotic complications. Somatic diseases, surgical interventions in which secondary thrombophilia may occur were excluded. In 32 patients (38\%), the disease debuted with $\mathrm{PE}$; in 11 (14\%) - ischemic stroke; in 3 (5\%) - myocardial infarction; in 35 (43\%) - pathology of the veins of the lower extremities.

Results: The following thrombophilia markers were found in 32 patients with PE (20 men, 12 women): F5 Leiden mutations - in 15, prothrombin F2 G20210A - in 5, MTHFR - in 10, antithrombin III deficiency - in 2 and protein C in - 4, hyperhomocysteinemia - in 15, antiphospholipid syndrome - in 4 patients. In four patients, a mutation of one gene took place, in the remaining cases a combined form of thrombophilia was diagnosed. In 25 cases, heredity was diagnosed for pathological thrombus formation. In 8 women, the provoking factor was the intake of hormonal drugs. The age composition of the surveyed: 4 patients - at the age of 17-20 years, 10-21-30, 12-31-40, 6-41-45 years. At the time of diagnosis of thrombophilia, 6 had recurrent PE. For the secondary prevention of thrombosis, direct oral anticoagulants were used. Replacement therapy was used in the congenital deficiency of protein $\mathrm{C}$ and antithrombin III.

Conclusions: PE is often the primary clinical manifestation of hematogenous thrombophilia (38\%). All cases of PE at a young age should be examined for markers of thrombophilia. Appointment 
of adequate anti-thrombotic therapy contributes to a relapse-free course of the disease.

PB1147 | Evaluation of Fibrinogen Level In non-Diabetic Hypertensive Patients at the University of Nigeria Teaching Hospital Ituku-Ozalla

E. Ezigbo $^{1} ;$ O. Achunine ${ }^{2} ;$ T. Nwagha ${ }^{3}$

${ }^{1}$ Thrombosis \& Haemostasis Unit, Department of Medical Laboratory

Sciences, University of Nigeria, Enugu Campus, Enugu, Nigeria;

${ }^{2}$ Department of Medical Laboratory Sciences, University of Nigeria

Enugu Campus, Enugu, Nigeria; ${ }^{3}$ Department of Haematology,

University of Nigeria Nsukka/University of Nigeria Teaching Hospital, Ituku Ozalla, Enugu, Nigeria

Background: The presence of platelet microparticles in hypertension has been reported, and these are believed to play a role in mediating target organ injury in severe hypertension.

Aims: This project aimed to assess the coagulation profile of nondiabetic hypertensive patients.

Methods: Fifty (50) non-diabetic hypertensive patients at the medical outpatient (MOP) Department of University of Nigeria Teaching Hospital, Ituku Ozalla, Enugu, Nigeria were investigated, they comprised of 25 males and 25 females. Besides, fifty age and sexmatched healthy subjects served as the control group. The University of Nigeria Research Ethics Committee approved the study. The coagulation profile and biochemical analysis carried out include: prothrombin Time, (PT) Activated partial Thromboplastin Time (APTT), Fibrinogen Assay, platelet count, Fasting Blood Glucose, sodium ion, Potassium ion, Chloride ion, Bicarbonate ion $\left(\mathrm{HCO}_{3}{ }^{-}\right)$, Urea, Creatinine, Alkaline phosphatase, Total Bilirubin (TB), Conjugated Bilirubin, SGOT and SGPT.

Results: The Mean age (years) and range for the hypertensive patients was 58(40-78) and for the control subjects 59(48-75). The following variables show significant differences between the hypertensive group and the controls; PT $13.76 \pm 0.20$ and $13.18 \pm 0.15$ p-value (0.0248). Fibrinogen Assay $5.45 \pm 0.27$ and $3.16 \pm 0.10(<0.0001)$; SGOT $14.82 \pm 0.19$ and $13.64 \pm 0.18(<0.0001)$; D.B $0.56 \pm 0.01$ and $0.51 \pm 0.01$ (0.0316); $\mathrm{HCO}_{3} 21.46 \pm 0.31$ and 26.16 \pm 0.69 (<0.0001); Urea $34.74 \pm 0.9371$ and $28.68 \pm 0.91 \quad(<0.0001)$; Creatinine $0.93 \pm 0.02$ and $1.00 \pm 0.01$ (0.0008) respectively.

Conclusions: The increased PT and fibrinogen in the non-diabetic hypertensive subjects may indicate a hypercoagulable state of patients recruited for this study.

\section{POST-THROMBOTIC SYNDROME}

LPB0091 | Predictors of the Development of the Postthrombotic Syndrome: A Sub-analysis of the ATTRACT Trial

F. Rinfret $^{1,2}$; C.-S. Gu ${ }^{3}$; S. Vedantham ${ }^{4}$; S. Kahn ${ }^{2,1}$

${ }^{1}$ McGill University, Montreal, Canada; ${ }^{2}$ Jewish General Hospital, Montreal, Canada; ${ }^{3}$ Centre for Regulatory Excellence, Statistics and Trials, Ottawa, Canada; ${ }^{4}$ Washington University School of Medicine, St. Louis, United States

Background: The post-thrombotic syndrome (PTS) occurs in 30$40 \%$ of patients following deep vein thrombosis (DVT). Factors such as sex, older age, elevated body mass index (BMI) and ipsilateral prior DVT have previously been identified as strong predictors of PTS.

Aims: To investigate known and novel predictors of the development of PTS in participants of the ATTRACT Trial, which evaluated pharmacomechanical catheter-directed thrombolysis plus anticoagulation vs. anticoagulation alone to treat proximal DVT.

Methods: Using the ATTRACT Trial database, we used multivariate logistic regression to identify baseline and post-baseline factors that were predictive of the development of PTS during study follow-up, as defined by a Villalta score $\geq 5$ from 6-24 months after enrolment. Results: Among 691 study participants, median age was 53 years and $62 \%$ were male. In the multivariate analysis, age (OR 1.03 per year increase [95\% Cl 1.02 to 1.04$]$ ) and BMI (OR 1.05 per $1 \mathrm{~kg} / \mathrm{m}^{2}$ [95\% $\mathrm{Cl} 1.02$ to 1.07]) were independent predictors of PTS. Additional predictors identified were Villalta score at baseline (OR 1.09 per 1unit increase [95\% $\mathrm{Cl} 1.05$ to 1.13$]$ ), leg pain severity at day 10 (OR 1.28 [95\% $\mathrm{Cl} 1.13$ to 1.45$]$ per 1 point increase in a 7-point scale) and employment status (unemployed due to disability OR 3.31 [95\% Cl 1.72 to 6.35 ] vs. employed $>35$ hours per week). Use of rivaroxaban on day 10 appeared to be protective (OR 0.53 [95\% Cl 0.33 to 0.86]) when compared to warfarin.

Conclusions: We confirmed that age and BMI were predictors of PTS in the ATTRACT population. We also identified that baseline Villalta score, leg pain severity at 10 days, and unemployed due to disability are independent predictors of PTS. Our findings also suggest that the initial choice of anticoagulant to treat DVT may have an impact on the development of PTS. 
PB1148 | Statins for Venous Event Reduction in Patients with Venous Thromboembolism: A Multicenter Randomized Controlled Pilot Trial with a Focus on Prevention of Post-thrombotic Syndrome

A. Delluc ${ }^{1}$; W. Ghanima ${ }^{2}$; M. Kovacs ${ }^{3}$; S. Shivakumar ${ }^{4}$; S. Kahn ${ }^{5}$; P.M. Sandset ${ }^{6}$; C. Kearon ${ }^{7} ;$ M. Rodger ${ }^{5}$

${ }^{1}$ Ottawa Hospital Research Institute, Ottawa, Canada; ${ }^{2}$ Ostfold Hospital, Ostfold, Norway; ${ }^{3}$ University of Western Ontario, London, Canada; ${ }^{4}$ Dalhousie University, Halifax, Canada; ${ }^{5}$ McGill University, Montreal, Canada; ${ }^{6}$ University of Oslo, Oslo, Norway; ${ }^{7}$ McMaster University, Hamilton, Canada

Background: Post-thrombotic syndrome (PTS) is one of the most frequent complication of venous thromboembolism (VTE). It has been postulated that rosuvastatin could prevent PTS via inhibiting expression of thrombus-associated mediators of inflammation.

Aims: To explore in a multicenter randomized controlled trial if generic rosuvastatin can prevent PTS.

Methods: 312 patients receiving standard anticoagulation for a newly diagnosed VTE were randomly allocated to adjuvant rosuvastatin $20 \mathrm{mg}$ once daily for 180 days ( $n=155$ ) or no rosuvastatin ( $n=157)$. At the end of the trial, an independent observer who was blinded to study treatment performed a PTS assessment on each patient using the Villalta scale. The primary clinical outcomes were mean Villalta score and presence of PTS defined by Villalta score $>4$ at Day 180.

Results: At Day 180, the Villalta score was $3.5 \pm 0.3$ in the rosuvastatin arm vs. $3.3 \pm 0.3$ in the control arm $(P=0.59)$, and presence of PTS was in $29.7 \%$ the rosuvastatin arm vs. $25.5 \%$ in the control arm $(P=0.41)$. Secondary analyses showed no difference between trial arms for presence of severe PTS (Villalta score $\geq 15$ ) at Day 180 $(2.0 \%$ vs. $2.7 \%, P=1)$ and for changes in Villalta score between baseline and Day $180(-3.7 \pm 4.4$ vs. $-4.0 \pm 5.0, P=0.59)$.

Conclusions: This randomized controlled trial did not demonstrate efficacy of short-term use of rosuvastatin to prevent PTS. Future studies with longer rosuvastatin treatment are needed to exclude any benefit in preventing PTS. (NCT02679664)

PB1150 | Role of Individual Venous Symptoms and Signs at Baseline in Predicting Future Development of Post-thrombotic Syndrome: Sub-analysis of the ATTRACT Trial

F. Rinfret $^{1,2}$; C.-S. Gu ${ }^{3}$; S. Vedantham ${ }^{4}$; S. Kahn ${ }^{2,1}$

${ }^{1}$ McGill University, Montreal, Canada; ${ }^{2}$ Jewish General Hospital, Montreal, Canada; ${ }^{3}$ Centre for Regulatory Excellence, Statistics and Trials, Ottawa, Canada; ${ }^{4}$ Washington University School of Medicine, St. Louis, United States

Background: The post-thrombotic syndrome (PTS) occurs in 30$40 \%$ of patients following deep vein thrombosis (DVT). The Villalta Scale score at baseline (total score, maximum 33 , based on severity of 5 venous symptoms [maximum score 15] and 6 signs [maximum score 18]) has been shown to predict development of PTS.

Aims: To describe the prevalence of individual venous symptoms and signs components of the Villalta Scale at baseline, and to assess if these predict the development of PTS in participants of the ATTRACT trial, which evaluated pharmacomechanical catheterdirected thrombolysis plus anticoagulation vs. anticoagulation alone to treat proximal DVT that extended above the popliteal vein.

Methods: Using the ATTRACT Trial database, we calculated the prevalence of individual components of the Villalta Scale at baseline and used logistic regression to assess if they predicted development of PTS, defined by a Villalta score $\geq 5$ from 6-24 months after enrolment. Results: Among 691 study participants, mean (SD) Villalta total score at baseline was 9.7 (5.4). In univariate analysis, all 5 symptoms similarly predicted development of PTS (ORs ranged from 1.5 to 2.0 per unit increment in score, $\mathrm{p}$ values $\leq 0.0003$ ). Among 6 signs, only hyperpigmentation (OR $=2.09$ [95\% Cl: 1.42 to 3.06]), venous ectasia $(\mathrm{OR}=2.00$ [1.50 to 2.67]) and pretibial edema (OR = 1.96 [1.28 to 3.01]) were predictive of PTS. In multivariate analysis, total Villalta symptoms score and total Villalta signs score similarly predicted risk of developing PTS (OR = 1.07 [1.01 to 1.13] and 1.11 [1.04 to 1.18] per unit increment in score, respectively).

Conclusions: Several individual symptoms and signs predicted development of PTS. In multivariable analysis, total symptoms score and total signs score were similarly predictive of risk of developing PTS, suggesting that either alone could be used when assessing future risk of PTS.

PB1151 | Development of a New Disease-specific Healthrelated Quality of Life Questionnaire after Deep Vein Thrombosis: Qualitative Phase of a Mixed Method Development

E. Asady $^{1,2}$; W. Ghanima ${ }^{2,1,3}$; L.-P. Jelsness-Jorgensen ${ }^{2,4}$; F. Klok ${ }^{5}$;

G.J. Boon ${ }^{5}$; H. Skuterud Wik ${ }^{3}$

${ }^{1}$ University of Oslo, Oslo, Norway; ${ }^{2} \varnothing$ stfold Hospital Trust, Grålum, Norway; ${ }^{3}$ Oslo University Hospital, Oslo, Norway; ${ }^{4} \varnothing$ stfold University College, Halden, Norway; ${ }^{5}$ Leiden University Medical Center, Leiden, Netherlands

Background: Several Health-related quality of life (HRQoL) questionnaires for deep vein thrombosis (DVT) are available. None of these have however been developed in full accordance with current standards for questionnaire development.

Aims: To develop a new disease-specific HRQoL questionnaire for DVT in accordance with state-of-the-art standards, as defined by current guidelines.

Methods: The questionnaire development was setup as a phase I-IV study, of which results from phase II are presented in this abstract. Phase II consisted of item generation by conducting interviews with the target population. Accordingly, we conducted seven semistructured group interviews with 40 DVT patients, at least 12 weeks after the acute event, in Norway and The Netherlands. Thematic 
analysis based on Braun \& Clarke's method was used to analyze the transcripts from the interviews, in which relevant issues were identified and grouped into themes.

Results: Four main themes concerning issues that affected patients most in their daily life were identified from the interview material. Theme 1) Leg pain; Theme 2) Discomfort; consisting of the following issues: heavy legs; cramps; swelling; itching; fatigue; discomfort due to use of elastic compression stockings; Theme 3) Limitations; consisting of limitations in daily activity; social life and work; Theme 4) Psychological influence; consisting of worries about cause of thrombosis and risk of recurrent DVT or bleeding; bad mood; frustration; feeling of helplessness; concentration problems; altered choice of clothing; and feeling mortal.

Conclusions: During the semi-structured group interviews, we identified several issues in the chronic phase after DVT. These issues will form the basis for further evaluation and testing in phase III and IV in the questionnaire development.

\section{THROMBOPHILIA}

\section{PB1152 | Pathologically Stiff Erythrocytes Can Promote Thrombosis by Impeding Contraction of Blood Clots}

V. Tutwiler ${ }^{1,2}$; R.I. Litvinov ${ }^{1,3}$; A. Protopopova ${ }^{1}$; C. Nagaswami ${ }^{1}$; C. Villa ${ }^{1}$; O. Abdulmalik ${ }^{4}$; D. Siegel ${ }^{1}$; J.E. Russell ${ }^{1}$; V. Muzykantov ${ }^{1}$; W. Lam ${ }^{5}$; D. Myers ${ }^{5}$; J.W. Weisel ${ }^{1}$

${ }^{1}$ University of Pennsylvania, Philadelphia, United States; ${ }^{2}$ Rutgers

University, Piscataway, United States; ${ }^{3}$ Kazan Federal Research University, Kazan, Russian Federation; ${ }^{4}$ Children's Hospital of Philadelphia, Philadelphia, United States; ${ }^{5}$ Emory University, Atlanta, United States

Background: Blood clot contraction, volume shrinkage of the clot is driven by platelet contraction and accompanied by compaction of the erythrocytes and their gradual shape change from biconcave to polyhedral, with the resulting cells named polyhedrocytes.

Aims: Here, we examined the role of erythrocyte rigidity on clot contraction and erythrocyte shape transformation.

Methods: We used an optical tracking methodology that allowed us to quantify changes in contracting clot size over time.

Results: Erythrocyte rigidity has been shown to be increased in sickle cell disease (SCD), and in our experiments erythrocytes from SCD patients were 4-fold stiffer than those from healthy subjects. On average, the final extent of clot contraction was reduced by $53 \%$ in the clots from the blood of patients with SCD compared to healthy individuals, and there was significantly less polyhedrocyte formation. To test if this reduction in clot contraction was due to the increase in erythrocyte rigidity, we used stiffening of erythrocytes via chemical cross-linking (glutaraldehyde), rigidifying Wrightbantibodies (Wrb) and naturally more rigid llama ovalocytes. Results revealed that stiffening erythrocytes results in impaired clot contraction and fewer polyhedrocytes.

Conclusions: These results demonstrate the role of erythrocyte rigidity in the contraction of blood clots and suggest that the impaired clot contraction/shrinkage in SCD is due to the reduced erythrocyte deformability, which may be an underappreciated mechanism that aggravates obstructiveness of erythrocyte-rich (micro)thrombi in SCD.

PB1153 | Abnormal Thrombin Generation in Children with Thrombosis

S. Kuldanek; D. Thornhill; L. Jacobson; C. Baird; A. Chan; J. Smith; R. Shearer; M. Manco-Johnson

University of Colorado Anschutz Medical Campus, Aurora, United States

Background: Abdominal thrombosis in children is rare and understudied.

Aims: To apply the calibrated automated thrombogram (CAT) assay of thrombin generation with and without thrombomodulin to investigate thrombophilia in children with a history of abdominal thrombosis.

Methods: This investigation was conducted on biobank samples and clinical data from a consented prospective cohort study. Samples were collected at least 3 months following thrombus detection, on no interfering anticoagulants. Thrombophilia evaluations included antithrombin (AT), protein C (PC), protein S (PS), factor V Leiden (FVL), prothrombin 20210 mutation (PTM), factor VIII activity (FVIII), lipoprotein (a) (Lp(a)) and the antiphospholipid antibodies (APA), lupus anticoagulant (LA), anticardiolipin antibody (ACA) and anti- $\beta 2$ GP1 antibodies. Fibrinogen evaluations were performed as indicated. The calibrated automated thrombogram (CAT) assay of thrombin generation was performed with and without thrombomodulin (TM) using manufacturers procedures (CAT, CAT-TM, Diagnostica Stago). CAT detects increased thrombin generation. CAT-TM detects a failure to down-regulate thrombin generation by activated protein $\mathrm{C}$. Results were stratified by participant age under 2 years, or 2 and greater to separate out presumed perinatal events.

Results: Table 1 shows thrombophilia results in 44 children. Samples were available for CAT assays in 21 participants and the CAT-TM in 19. Table 2 shows CAT and CAT-TM relative to individual assayed traits. CAT thrombin generation was increased in 3 children with consistent thrombophilia traits and 2 with fibrinogen abnormalities. CAT was negative in 16 children, none with defects expected in CAT. CAT-TM showed decreased thrombin suppression in 12 children, 2 with no expected traits. CAT-TM failed to detect 4 expected abnormalities.

TABLE 1 Genetic Thrombophilia \& APA Detected in Children with Abdominal Thrombosis

$\begin{array}{ll}\text { Number of Traits } & \text { Number of Participants } \\ 0 & 15(34 \%) \\ 1 & 14(32 \%) \\ 2 & 10(23 \%) \\ 3 & 5(11)\end{array}$

Traits detected: AT (3), PC (3), PS (2), FVL heter (5), FVL homo (1), PTM (1), fibrinogen

Abnormalities (5), elevated Lp(a) (7), elevated FVIII (7), APA (14) 
TABLE 2 Results of CAT and CAT-TM in Children with Abdominal Thrombosis

Group
$<2$ years, CAT +
$<2$ years, CAT -
$\geq 2$ years, CAT +
$\geq 2$ years, CAT -
$<2$ years, CAT TM +
$<2$ years, CAT TM-
$\geq 2$ years, CAT TM +
$\geq 2$ years, CAT TM -

Conclusions: Children with abdominal thromboses showed a high rate of abnormal thrombin generation in their steady state. Most abnormal CATs were corroborated with known consistent thrombophilia traits, but some unexplained positives were determined. Future work will evaluate the usefulness of the CAT and CAT-TM as a screening tool for hypercoagulability.

PB1154 | Additional Risk Factors of Hereditary Thrombophilia in the North-Western Region of Russia

V. Soldatenkov; O. Soldatenkova; N. Mineeva; S. Kapustin;

L. Papayan; N. Silina; A. Chechetkin; K. Komissarov

Russian Scientific Research Institute of Hematology and Transfusiology, Saint-Petersburg, Russian Federation

Background: The role of the most important prothrombotic genetic risk factors, such as FV Leiden mutation or Prothrombine G20210A mutation, is obvious. Nowadays it is necessary to proceed searching for additional factors of hereditary thrombophilia.

Aims: To study laboratory characteristics of patients with verified thrombosis and different forms of hereditary thrombophilia and to evaluate some additional prothrombotic markers.

Methods: 101 case-records of patients, who underwent treatment in Russian Scientific Research Institute of Hematology and Transfusiology in 2017-2021, were studied. Inclusion criteria: verified arterial or venous thrombosis and confirmed hereditary thrombophilia. Exclusion criteria: JAK2, CALR, MPL mutations. The following groups of patients were formed on the base of the molecular genetics and coagulation tests: I - isolated FV Leiden mutation ( $n=16$ ), II - isolated mutation in Prothrombine G20210A ( $n=8)$, III - isolated antithrombine deficiency $(n=2), I V$ - isolated Protein C deficiency $(n=1), \mathrm{V}$ - isolated FVIII elevation ( $n=10), \mathrm{VI}$ - isolated hyperhomocysteinemia (MTHFR, MTRR mutations, confirmed phenotypically) ( $n=8)$, VII - isolated primary antiphospholipid syndrome ( $n=4)$, VIII - combination of three and more thrombophilia markers $(n=3)$, IX - combination of two strong thrombophilia markers $(n=4), X$ - strong and moderate thrombophilia markers combination $(n=45)$. Clinical and laboratory data of those groups were analyzed.
Thrombophilia Traits Identified that Can be Detected in CAT or CAT-TM

AT (2)

No traits associated with increased thrombin generation

PTM (1), [dysfibrinogenemia (2)]

No traits associated with increased thrombin generation

PC (1), FVL hetero (1)

FVL homo \& ACA (1)

FVL hetero (1), Lp(a) (2), APA (3), Lp(a) \& APA (2), Unknown (2)

FVL hetero (1), APA (1), Lp(a) (1)

Results: 1. 158 strong or moderate thrombophilia markers were found. The most common was hyperhomocysteinemia (30.4\%).

2. Combined thrombophilias were found in $51.48 \%$. Combination of Leiden mutation with hyperhomocysteinemia was the most common combined thrombophilia (34.6\%).

3. Venous thrombosis was found in $74.2 \%$, arterial - in $15.8 \%$, combined - in $10 \%$ of patients.

4. Mutation of FI gene was found in $36,6 \%$ of patients. $54 \%$ of them had confirmed hyperfibrinogenemia.

5. Incidence of B (III) blood group was 2,56 times higher than in average population.

6. Incidence of Rh-negative blood type was 1,3 times higher.

Conclusions: Mutation in Fl gene, blood types B (III) and Rh-negative may be considered as an additional prothrombotic markers.

\section{PB1155 | A Potential Risk of Venous Thrombosis Induced by} Nanomaterials and the Protection Role of Nrf2

Y. $\operatorname{Bian}^{1,2} ;$ J. $\mathrm{Pi}^{1}$

${ }^{1}$ China Medical University, Shenyang, China; ${ }^{2}$ Seoul National University, Seoul, Korea

Background: Nanomaterials are widely used in food packaging, coatings, aerospace and medical fields owing to their various good properties such as bactericidal, photocatalytic properties and etc. However, its potential health risks still lack systematic research. Venous thrombosis, as a contributor to the largest proportion of deaths worldwide, seriously threatens human life and health. Erythrocytes (also called RBCs), as one of the most important target cells when nanomaterials enter the body, alterations of procoagulant activity of which are closely related to venous thrombosis. Aims: However, whether and how nanomaterials trigger venous thrombosis by affecting the procoagulant activity of erythrocytes are still unclear. In this study, we aim to elucidate the effects of nano silver (AgNPs) and nano titanium dioxide ( $\mathrm{TiO}_{2} \mathrm{NPs}$ ) mainly on erythrocytes-associated coagulation pathways, eventually leading to venous thrombosis. 
Methods: Here, venous thrombosis animal model are performed for observation of venous thrombosis in vivo, in addition, freshly isolated human erythrocytes are applied to evaluate pro-coagulant activity as well as other related indicators. Nuclear factor E2-related factor 2 (Nrf2; also known as NFE2L2) belongs to a family of CNCbZIP proteins and is recognized as a master regulator of the cellular adaptive response to various stresses, in particular oxidative insults. To further investigate the underlying mechanism, global Nrf2 gene knockout animal models are used, in the presence or absence of Nrf2 regulator.

Results: Upon these experiments, we aim to clarify the cell-specific roles and related molecular mechanisms of adaptive antioxidant responses with Nrf2 as the core regulatory factor in venous thrombosis induced by nanomaterials.

Conclusions: Of note, our study attempts to figure it out that the Nrf2 signaling pathway serves as a pathophysiological basis for key molecular targets for prevention and treatment, providing an experimental basis for the risk assessment and scientific prevention of venous thrombosis caused by nanomaterials exposure.

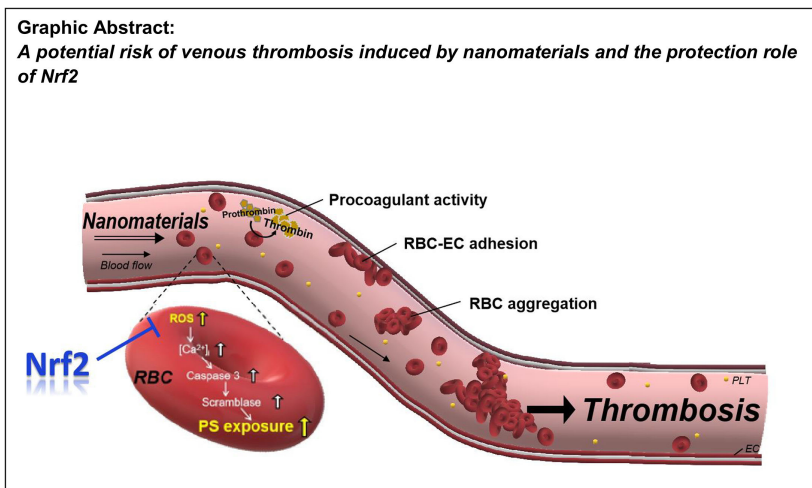

FIGURE 1 Graphic Abstract: A potential risk of venous thrombosis induced by nanomaterials and the protection role of Nrf2

PB1156 | Impact of Thrombophilia Testing in Splanchnic Vein Thrombosis Management

$\underline{\text { R. Rouached }}^{1,2} ;$ H. Baccouche ${ }^{1,2} ;$ A. Laabidi ${ }^{3,2} ;$ H. Kechir ${ }^{4,2}$;

J. Boubaker ${ }^{3,2}$; N. Laamari ${ }^{4,2}$; N. Ben Romdhane ${ }^{1,2}$

${ }^{1}$ Hematology Department La Rabta Hospital, Tunis, Tunisia; ${ }^{2}$ Faculty of Medecine of Tunis, Tunis, Tunisia; ${ }^{3}$ Gastroenterology and Hepatology Department (A) La Rabta Hospital, Tunis, Tunisia; ${ }^{4}$ Gastroenterology and Hepatology Department (B) La Rabta Hospital, Tunis, Tunisia

Background: Splanchnic vein thrombosis (SVT) is an unusual site of venous thromboembolism. Screening for inherited thrombophilias, as part of the etiological assessment of these thrombotic events, as well as its impact on anticoagulant therapy duration are nuanced and controversial.
Aims: This study aimed to evaluate the impact of thrombophilia testing in both clinical and therapeutic management of SVT.

Methods: This was a retrospective study, including 90 patients followed for SVT from January 2008 to December 2020. Thrombosis associated with an hepatocellular carcinoma were not included. Clinical characteristics of patients, data on thrombotic events and results from thrombophilia screening were recorded.

Results: The mean age was 50 years old. 54 patients were women (60\%). Portal vein was the most frequent localization $(84,5 \%)$. Underlying diseases were cirrhosis in 42 patients $(46,7 \%)$, myeloproliferative neoplasms in 9 patients (10\%), inflammatory bowel disease in 5 patients $(5,56 \%)$ and other aetiologies in 34 patients $(37,74 \%)$. A thrombophilia testing was performed in 61 patients (67.8\%). The decision to screen for thrombophilia was significantly associated to young age (75,51\% vs $24,49 \%)$. Combined deficiencies were found in 34 patients due to liver failure $(n=20)$ or antivitamin $K$ treatment $(n=9)$. A heriditary deficiency of coagulation inhibitors was documented in 5 patients. Thrombophilia abnormalities had no significant impact on the decision to start or not anticoagulation. However, other factors such as complete obstruction $(89,19 \%$ vs $10,81 \%)$, recent thrombosis $(86,84 \%$ vs $13,16 \%)$ and low bleeding risk $(82,19 \%$ vs $17,8 \%$ ) were significantly associated to the decision to start anticoagulation. Besides, both the absence of underlying liver disease ( $75 \%$ vs $25 \%$ ) and the absence of vein recanalization $(85,71 \%$ vs $14,29 \%)$ were significantly related to an indefinite treatment.

Conclusions: Thrombophilia testing was often performed in young patients with SVT but the results did not influence therapeutic management. Duration of anticoagulation therapy was rather associated to underlying disease and thrombus characteristics.

\section{PB1157 | Thrombin Generation in Newly Diagnosed Multiple} Myeloma

D. Velasco-Rodríguez ${ }^{1}$; I. Martínez-Alfonzo ${ }^{1}$; A. VelascoValdazo $^{2}$; R. Vidal Laso ${ }^{1}$; C. Díaz ${ }^{1}$; N. Revilla ${ }^{3}$; A. García-Raso ${ }^{1}$; J.M. Alonso-Domínguez ${ }^{1}$; S. Martín-Herrero'; E. Askari'; E. Prieto; M.J. Blanchard ${ }^{3}$; F.J. López-Jiménez ${ }^{3}$; P. Llamas-Sillero ${ }^{1,2}$

${ }^{1}$ Hospital Universitario Fundación Jiménez Díaz, Madrid, Spain; ${ }^{2}$ Hospital Universitario Rey Juan Carlos, Móstoles, Spain; ${ }^{3}$ Hospital Universitario Ramón y Cajal, Madrid, Spain

Background: Venous thromboembolism (VTE) is a common cause of morbidity and mortality in Multiple Myeloma (MM) patients. However, the risk factors for VTE in these patients are not well defined. Thrombin generation (TG) assessment has proved to identify and quantify hypercoagulability.

Aims: To determine whether TG assessment could reveal a hypercoagulable status in MM patients prior to initial treatment compared to healthy individuals.

Methods: This prospective and longitudinal observational study included 19 newly diagnosed MM patients from three centers in Madrid (Hospital Universitario Fundación Jiménez Díaz, Hospital Universitario 
Rey Juan Carlos and Hospital Universitario Ramón y Cajal). Blood samples were collected before the start of treatment. Congenital thrombophilia study was negative in all of them. Calibrated automated TG was measured in PPP using STG-ThromboScreen ${ }^{\circledR}$ reagent system, with and without thrombomodulin (+TM/-TM) in ST-Genesia ${ }^{\circledR}$ analyzer (Diagnostica Stago). TG-derived assessed parameters were: lag time, peak height $(\mathrm{PH})$, time-to-peak, endogenous thrombin potential (ETP), velocity index (Vel.index), and ETP\%inh (percentage of ETP inhibition after adding TM). MM cases were compared to 35 presumably healthy subjects. A comparison between MM cases with (higher $\mathrm{PH}$ and/or higher ETP and/or lower ETP\%inh) and without hypercoagulability was also performed. All statistical analyses were performed using SPSS version 19.0 for Windows. P values $<0.05$ were considered to be statistically significant.

Results: Characteristics of MM cases and control group are summarized in Table 1. Six MM patients (31.57\%) showed hypercoagulability. Prolonged lag time (3.11 min vs. $2.40 \mathrm{~min}, P=0.01$ ) and higher Vel.index (127.96 $\mathrm{nM} / \mathrm{min}$ vs. 82.79, $P=0.03$ ) were found in $\mathrm{MM}$ patients (Table 2). No significant differences were found in $\mathrm{PH}$, timeto-peak, ETP and \%ETPinh.

\begin{tabular}{|c|c|c|}
\hline & MULTIPLE MYELOMA (N=19) & CONTROL GROUP ( $\mathrm{N}=35$ ) \\
\hline Age (years), median (IQR) & $70(59-80)$ & $44(30-55)$ \\
\hline \multicolumn{3}{|l|}{ Gender, n (\%) } \\
\hline Male & $8(42.1)$ & $11(31.4)$ \\
\hline Female & $11(57.9)$ & $24(68.6)$ \\
\hline \multicolumn{3}{|l|}{ ECOG, n (\%) } \\
\hline 0 & $9(47.4)$ & \\
\hline 1 & $7(36.8)$ & \\
\hline 2 & $2(10.5)$ & \\
\hline 3 & $1(5.3)$ & \\
\hline BMI (kg/m²), median (IQR) & $25.43(23.01-31.09)$ & \\
\hline Obesity, n (\%) & $7(36.8)$ & \\
\hline \multicolumn{3}{|l|}{ Diagnosis, $n$ (\%) } \\
\hline Multiple Myeloma & $14(73.7)$ & \\
\hline Bence Jones & $3(15.8)$ & \\
\hline Plasmocytomas & $2(10.5)$ & \\
\hline \multicolumn{3}{|l|}{ ISS-R, n (\%) } \\
\hline 1 & $5(29.4)$ & \\
\hline 2 & $7(36.8)$ & \\
\hline 3 & $5(26.3)$ & \\
\hline Serum monoclonal protein (g/dL), median (IQR) & $2.38(0.62-3.11)$ & \\
\hline Kappa free light chains (mg/dL), median (IQR) & $277.20(8.78-689.49)$ & \\
\hline Lambda free light chains (mg/dL), median (IQR) & $13.85(6.99-942.69)$ & \\
\hline Hemoglobin, median (IOR) & $10.2(9.5-11.6)$ & \\
\hline Creatinine clearance ( $\mathrm{ml} / \mathrm{min})$, median (IQR) & $72.64(38-90.77)$ & \\
\hline High risk cytogenetic abnormalities, $n$ (\%) & $6(31.6)$ & \\
\hline Bone fractures, $n(\%)$ & $8(44.4)$ & \\
\hline Plasma cells by cytology (\%), median (IQR) & $30(12-50)$ & \\
\hline Plasma cells by flow cytometry (\%), median (IQR) & $11.6(4.37-19.50)$ & \\
\hline PT (seg), median (IQR) & $11.7(11.2-12.3)$ & $11.2(11-11.7)$ \\
\hline INR, median (IQR) & $1.04(0.9-1.07)$ & $0.99(0.97-1.05)$ \\
\hline APTT (seg), median (IQR) & $28.3(27.7-29.6)$ & $31(28.7-32.8)$ \\
\hline APTT ratio, median (IQR) & $0.91(0.88-0.94)$ & $0.98(0.91-1.03)$ \\
\hline Fibrinogen ( $\mathrm{mg} / \mathrm{dL}$ ), median (IQR) & $459(364-515)$ & $369(321-452)$ \\
\hline D-Dimer (ng/mL), median (IQR) & $838(378-3332)$ & \\
\hline
\end{tabular}

APTT= activated partial thromboplastin time; BMI= body mass index; INR= international normalized ratio; IQR= interquartile range; $\mathrm{PT}=$ prothrombin time

FIGURE 1 Characteristics of MM patients and control group
Table 2. Comparison of TG parameters of MM subjects and control group.

\begin{tabular}{|l|c|c|c|}
\hline & $\begin{array}{c}\text { Myeloma group } \\
\mathbf{n = 1 9}\end{array}$ & $\begin{array}{c}\text { Control group } \\
\mathbf{n = 3 5}\end{array}$ & $\boldsymbol{p}$ \\
\hline Lag time (min) & 3.11 & 2.40 & 0.010 \\
\hline Lag time (ratio) & 1.53 & 1.22 & 0.026 \\
\hline Peak Height (nM) & 209.29 & 180.60 & 0.179 \\
\hline Peak Height (\%) & 81.65 & 67.22 & 0.83 \\
\hline Time to peak (min) & 5.66 & 5.52 & 0.713 \\
\hline Time to peak (ratio) & 1.36 & 1.38 & 0.852 \\
\hline ETP (nM.min) & 1164.15 & 1164.81 & 0.993 \\
\hline ETP (\%) & 82.15 & 84.66 & 0.665 \\
\hline Vel index (nM/min) & 127.96 & 82.79 & 0.030 \\
\hline Vel index (\%) & 84.49 & 50.97 & 0.012 \\
\hline \%ETPinh (\%) & 57.27 & 57.72 & 0.925 \\
\hline
\end{tabular}

FIGURE 2 Comparison of TG parameters of MM patients and control group

Conclusions: Both a significant delay and higher velocity in the onset of thrombin formation can be observed in newly diagnosed $\mathrm{MM}$ patients. However, no differences were observed in TG parameters of hypercoagulability.

\section{PB1158 | Clinical and Molecular Characterization of Chinese Patients with Protein S deficiency}

X. Zhang ${ }^{1}$; D. Zhang ${ }^{2}$; R. Yang ${ }^{2}$; F. Zhou ${ }^{1}$

${ }^{1}$ Hematology Department of Zhongnan Hospital of Wuhan University, Wuhan, China; ${ }^{2}$ State Key Laboratory of Experimental Hematology, National Clinical Research Center for Hematological Disorders, Institute of Hematology and Blood Diseases Hospital, Chinese Academy of Medical Sciences and Peking Union Medical College, Tianjin, China

Background: Hereditary Protein S deficiency is a rare disease characterized by reduced activity of protein $\mathrm{S}$, a plasma serine protease that has a complex role in blood coagulation, inflammation and apoptosis.

Aims: To analyze the mutations of PROS1 gene and identify the potential correlation between genotype and phenotype.

Methods: We collected clinical data of 17 Protein S deficiency patients, analyzed mutations of PROS1 gene at the genomic DNA by the next generation sequencing (NGS), and further determined the potential correlation between genotype and phenotype.

Results: Of these 17 probands, 52.9\% (9/17) experienced multi-site and/or recurrent thrombotic episodes, mainly manifested as deep venous thrombosis. Additional risk factors of VTE were observed in $41 \%(7 / 17)$ probands who exhibited a significantly higher rate of recurrent VTE compared with those not, in which 3 probands were complicated by anti-phospholipid syndrome. Most patients and family members exhibited quantitative Protein $\mathrm{S}$ deficiency with impairment of both activated protein $\mathrm{C}$ and tissue factor pathway inhibitor cofactor activities. A total of 15 unique mutations identified, including 8 novel mutations. Most mutations (11/15, 73\%) were missense 
or nonsense mutations, whereas two frameshift mutations ( p.S194fs and p.N583fs) were located in exons 6 and 14 respectively, and one splcing mutation, c.1493-17T>C, was located in Intron 12.

Conclusions: PROS1 gene analysis can make a definite diagnosis of Protein S deficiency and identify mutation carriers, and this research provides a framework for correlating the clinical pathogenesis of Protein S deficiency to genetic backgrounds in the Chinese population.

PB1159 | Role of Inherited Thrombophilia in the Occurrence of Arterial Thrombosis after Venous Thromboembolism

A. Ciavarella ${ }^{1,2}$; M. Abbattista ${ }^{1} ;$ F. Gianniello $^{1} ;$ M. Capecchi ${ }^{1,2}$

A. Artoni ${ }^{1}$; I. Martinelli ${ }^{1}$; F. Peyvandi ${ }^{1,3}$

${ }^{1}$ Fondazione IRCCS Ca' Granda Ospedale Maggiore Policlinico, A. Bianchi Bonomi Hemophilia and Thrombosis Center, Milan, Italy; ${ }^{2}$ Department of Biomedical Sciences for Health, Università degli Studi di Milano, Milan, Italy; ${ }^{3}$ Department of Pathophysiology and Transplantation, Università degli Studi di Milano, Milan, Italy

Background: Patients with venous thromboembolism (VTE) have a high risk of subsequent arterial thrombosis. This may be explained by sharing some acquired risk factors, but the role of inherited thrombophilia in the occurrence of arterial thrombosis after VTE is still unknown.

Aims: To evaluate the incidence of arterial thrombosis after VTE in patients with or without inherited thrombophilia.

Methods: This single-center retrospective cohort study included patients referred to our center from Jan 2009 to Dec 2018 for a thrombophilia work-up after an episode of VTE (deep vein thrombosis of the lower limbs and/or pulmonary embolism). Patients with arterial thrombosis before VTE, on antiplatelets therapy or with antiphospolipid antibodies were excluded. The observational period lasted a maximum of 5 years from the date of anticoagulation withdrawal to the date of arterial thrombosis, recurrent VTE, or last visit. Such arterial thrombosis as myocardial infarction, ischemic stroke, transient ischemic attack, arterial thrombosis of the lower limbs, and acute mesenteric ischemia were considered.

Results: This preliminary report evaluated 563 patients, of whom 237 met the inclusion criteria (91 with and 146 without thrombophilia abnormalities). Baseline characteristics are shown in Table1. Arterial thrombosis was observed in 14 patients, for an incidence rate of 2.3\% (95\% Cl 1.3-3.8\%) patient-year. Patients with thrombophilia had a higher risk of arterial thrombosis after VTE than those without (IR 4.3\%, 95\% Cl 2.2-7.5\% vs $1.1 \%$, 95\% Cl 0.4-2.6\% patientyear, HR 3.59, 95\% Cl 1.19-11.53).

TABLE 1 Baseline characteristics of patients included in the study

$\begin{array}{llll}\text { Characteristics } & \text { VTE patients } N=237 & \text { Characteristics } & \text { VTE patients } N=237 \\ \text { Male, } n \text { (\%) } & 110(46) & \text { Cardiovascular risk factors, } n(\%) & 58(25) \\ \text { Age at VTE, mean (SD) } & 43(15) & \text { Smoking } & 150(44) \\ \text { BMl class } & & \text { Hypercholesterolemia } & 4(2) \\ \text { Normal weight } & 120(51) & \text { Diabetes } & 38(16) \\ \text { Overweight } & 78(33) & \text { Hypertension } & 146(62) \\ \text { Obesity } & 39(16) & \text { Inherited thrombophilia*, } n(\%) & 21(9) \\ & & \text { Any } & 70(30)\end{array}$

* severe thrombophilia included antithrombin, protein C and protein S deficiency, homozygous factor V Leiden and prothrombin G20210A mutations and double heterozygosis; mild thrombophilia included heterozygous of factor V Leiden and prothrombin G20210A mutations and high factor VIII plasma levels. 


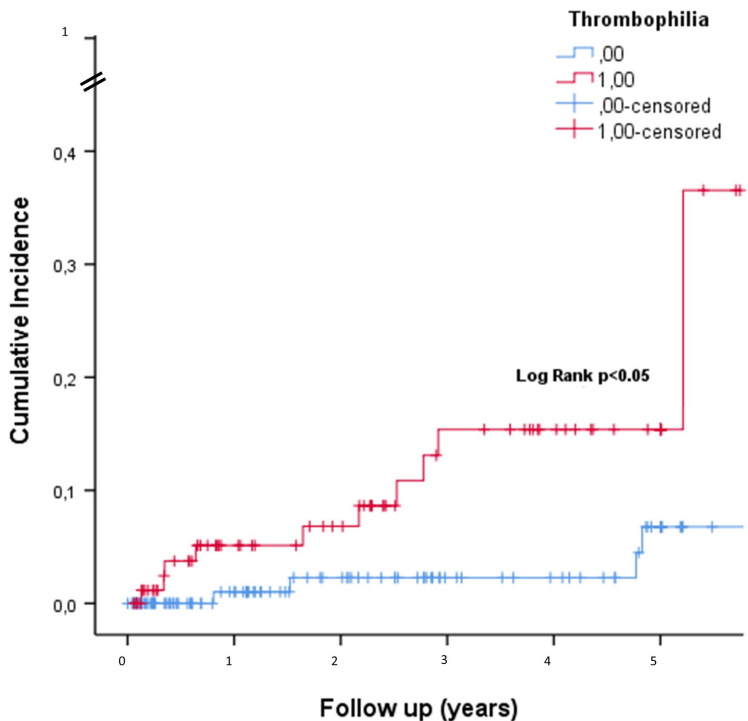

Patients at risk

$\begin{array}{lllllll}\text { THR - } & 146 & 95 & 73 & 55 & 50 & 37\end{array}$

$\begin{array}{lllllll}\mathrm{THR}+ & 91 & 61 & 52 & 39 & 30 & 22\end{array}$

FIGURE 2 Kaplan-Meier curves for the risk of arterial thrombosis after VTE

Conclusions: Patients with inherited thrombophilia have a higher risk to develop arterial thrombosis after VTE. Thrombophilia screening could be useful to identify patients with a higher risk of arterial thrombosis after VTE.

PB1160 | Reversible Factor Xa Inhibitors Specifically Inhibit, in Clot Growth, via the Intrinsic Tenase Complex: A Tool to Determine Specifically the In- and Extrinsic Contribution to Clot Growth

\section{Kluft; S. Naji}

Good Biomarker Sciences, Sassenheim, Netherlands

Background: Reversible, direct factor Xa inhibitors (Xa-DOACs) are used frequently in clinical practice, and concern Rivaroxaban, Apixaban, and Edoxaban.

Aims: We studied the effects in the thrombodynamics method on clot growth, and on thrombin generation.

Methods: Thrombodynamics with spiked plasma samples, recording layer thickness and thrombin formation.

Results: In clot growth the Xa-DOACSs, all similarly, inhibited stationary growth rate partially (50-45\%) in normal plasma and not at all in factor IX and VIII deficient plasma up to high concentrations of $4 \mu \mathrm{M}$.

The thrombin generation close to the surface-bound tissue factor in the thrombodynamics, showed an inhibition with IC-50 $2.5 \mu \mathrm{M}$. The thrombin generation further from the surface (intrinsic TG), depending on the factor XI-VIII-IX route was dose-dependently inhibited to a maximum of $60 \%$ inhibition.
We can take advantage of this specific inhibition to determine the contribution of extrinsic and intrinsic factor Xa formation in individuals.

In 10 apparently normal, young individuals we determined both portions of factor Xa activation and arrived for the fibrin layer thickness after 5000 secs at total layer thickness $2896 \mu \mathrm{m}$ (SD 20\%) and for the apixaban sensitive portion at $1666 \mu \mathrm{m}$ (SD 17\%) and the inhibited portion at $1230 \mu \mathrm{m}$ (SD 44\%). The extrinsic portion shows less interindividual variability (CV 17\%) compared to the intrinsic portion (CV 44\%). The variable intrinsic portion integrates contributions of factors XI, IX and VIII.

Conclusions: In view of the relationships between elevations of factors XI, IX and VIII in epidemiological studies, we consider the specific, integrated testing of the intrinsic portion relevant for thrombosis. On the other hand, the relation of decreases in those factors in relation to bleeding also renders this test relevant in this respect.

PB1161 | Activated Charcoal to Remove DOAC from Patients'

Plasma. Potential Usefulness for Routine Coagulation and Thrombophilia Testing in DOAC Treated Patients

M. Vannini ${ }^{1}$; B. Dahmani ${ }^{2}$; A. Appert-Flory ${ }^{1}$; F. Fischer ${ }^{1}$;

D. Jambou ${ }^{1} ;$ P. Toulon ${ }^{1}$

${ }^{1}$ Cote d'Azur University, Pasteur University Hospital, Hematology, Nice, France; ${ }^{2}$ Centre Hopitalier Princesse Grace, Hematology, Monaco,

Monaco

Background: Direct (anti-Ila and anti-Xa) oral anticoagulants (DOACs) are known to induce changes in various coagulation parameters. Different devices able to neutralize or abolish their effect in vitro enable their reliable measurement.

Aims: To evaluate the performance of the activated charcoal DOACRemove (5-Diagnostic, Basel, Switzerland) in extracting DOACs from plasma samples.

Methods: We evaluated left-over plasmas from untreated patients and patients treated with dabigatran rivaroxaban, or apixaban obtained in the laboratory workload. Tests were performed before and after a $x$ min-incubation of $0.8 \mathrm{~mL}$ plasma sample with 1 tablet of the charcoal. This included DOAC were measured using a specific direct thrombin inhibitor or an anti-Xa assay, routine coagulation testes (PT, aPTT, and fibrinogen), and thrombophilia panel including antithrombin, protein $\mathrm{C}$ (PC, chromogenic and clotting assays), and PS (free PS antigen and clotting assays). All assays were performed using reagents from Werfen on the ACL TOP 700 analyzer.

Results: In untreated patients, the DOAC-Remove had no significant effect on the test results ( $n>30$ for each parameter). In treated patients, the DOAC-Remove completely eliminated any of the three DOACs, with levels below the detection limit of the techniques $(n>30$ for each DOAC), with a dramatic correction of the DOAC-induced prolongations of PT and APTT. The same applied to elevated PC and PS anticoagulant activities (clotting assays) found in plasmas from 
patients treated with any of the three DOACs, and for antithrombin activity in the plasma from patients on rivaroxaban and apixaban. The DOAC-Remove had no impact on PC activity evaluated using a chromogenic assay, and on free PS antigen concentration, which were not affected with any of the 3 DOACs.

Conclusions: The DOAC-Remove effectively removed all 3 tested DOACs from the plasma of treated patients. Accordingly, routine coagulation and thrombophilia assays can validly be performed in treated patients without withholding the treatment..

PB1162 | Appropriateness of Thrombophilia Evaluation and Anticoagulation Management: A Multi-specialty Survey of Practitioners at Tower Health Reading Hospital

T. Johnson; D. Forman

Reading Hospital Tower Health, West Reading, United States

Background: The management of anticoagulation initiation, maintenance and completion is contextual in practice. Various factors can influence the appropriate anticoagulant, as well as the overall duration. When these factors are not truly appreciated, practitioners may provide unnecessary treatment which exposes patients to undue risk. Further, the implication of incorrectly interpreted thrombophilia testing may unnecessarily place patients on extended anticoagulation.

Aims: This study aims to determine if there was an identifiable knowledge gap between practitioners of varying degrees, specialties, or experience of practice, as it relates to the appropriateness of thrombophilia evaluation and anticoagulation management.

Methods: A total of 438 physicians and advanced practice providers (APPs) were surveyed with a questionnaire composed of both subjective and objective items related to their knowledge and practice experience with anticoagulation and thrombophilia. 404 of the 438 surveys were fully completed and analyzed using a combination of chi-square, Kruskal-Wallis with post-Hoc Dunn test, unpaired T-test and test item analysis where appropriate.

Results: The overall median physician score of $61.3 \%$ was significantly higher compared to APPs score of $52.4 \%(P=<0.001)$ Between specialties for the physicians, the Hospitalist and cardiology sub-groups had significantly higher median score compared to various medical specialties (table 1). The knowledge differential between physician and APP was not significant at $0-3$ years of experience but was present at $\geq 4$ years of experience $(P=<0.05)$.

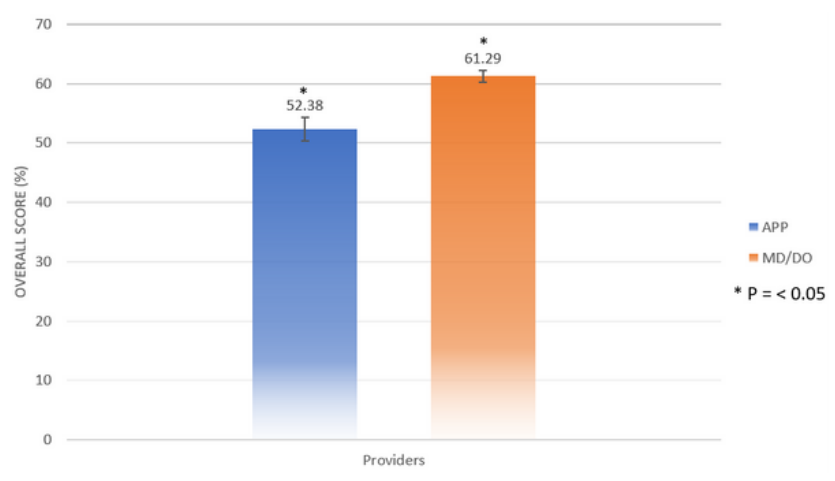

FIGURE 1 Physician Vs APP Total Score

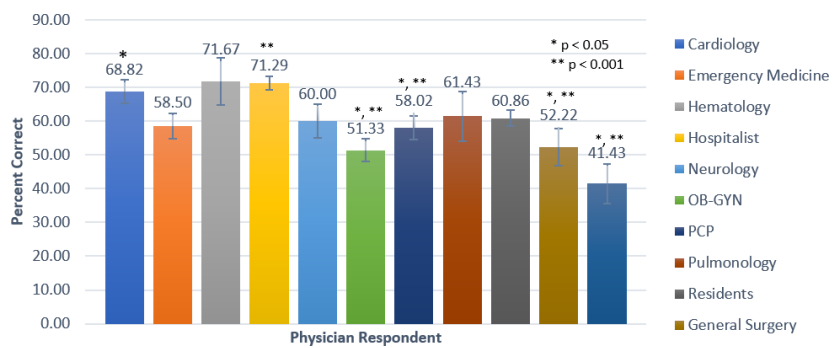

FIGURE 2 Scores of Physician Respondents based on Specialty

Conclusions: Our study shows a significant knowledge gap between providers of varying degrees and providers within different subspecialties. This may allow for a specific target audience for increased education regarding aspects of anticoagulation management and thrombophilia evaluation in the future.

PB1163 | C20209T Prothrombin Gene Mutation in a Caucasian Woman with a History of Venous Thrombosis

D. Jambou ${ }^{1}$; N. Saut ${ }^{2}$; V. Queyrel-Moranne ${ }^{3}$; A. Appert-Flory ${ }^{1}$; F. Fischer ${ }^{1}$; P. Suchon ${ }^{2}$; P. Toulon ${ }^{1}$

${ }^{1}$ Cote d'Azur University, Pasteur University Hospital, Hematology, Nice, France; ${ }^{2}$ Assistance Publique Hopitaux de Marseille, Hematology, Marseille, France; ${ }^{3}$ Cote d'Azur University, Pasteur University Hospital, Rheumatology, Nice, France

Background: Whereas the G20210A (c.*97G>A) prothrombin gene variant has been associated with an increased risk of venous thromboembolism (VTE), other rare mutations in F2 gene have been reported, with unknown frequency and potential association with VTE.

Aims: To report a case with a rare F2 mutation.

Methods: A 69 years old Caucasian woman presented with an unprovoked deep venous thrombosis of the leg. The thrombophilia workup included standard coagulation tests, antithrombin, protein C, protein S, anticardiolipin and antibeta2GP1 antibodies, (isotypes G and $\mathrm{M}$ ), lupus anticoagulant (LA) and F2 and F5 gene polymorphisms 
determination by melting curve analysis after real-time PCR on a capillary-based Lightcycler ${ }^{\mathrm{TM}}$. Finally, sequencing of the $3^{\prime} U T R$ region encompassing the 20210 position of the F2 gene was studied using an ABI 3500XL.

Results: Antithrombin, proteins $\mathrm{C} / \mathrm{S}$, anticardiolipin and antibeta2GP1B were within their normal ranges. LA was negative and F5 genotyping for G1691A transition revealed a wild-type genotype. By contrast, melting curve analysis for $\mathrm{G} 20210 \mathrm{~A}$ polymorphism showed an unusual curve progression (figure 1), with $\operatorname{Tm}\left(57.5^{\circ} \mathrm{C}\right.$ and $63.5^{\circ} \mathrm{C}$ ), whereas the melting points ${ }^{\mathrm{TM}}$ corresponding to the wild-type and the $20210 \mathrm{~A}$ allele were defined by the manufacturer to be between $61-67^{\circ} \mathrm{C}$ and $51-56^{\circ} \mathrm{C}$, respectively. Sequencing revealed a $\mathrm{C}>\mathrm{T}$ heterozygous transition at position 20209. The retrospective evaluation of test results obtained in our two hospitals between 2007 and 2018, revealed 21 heterozygous carriers of the C20209T mutation among 26.000 determinations (incidence: $0.08 \%$ ).

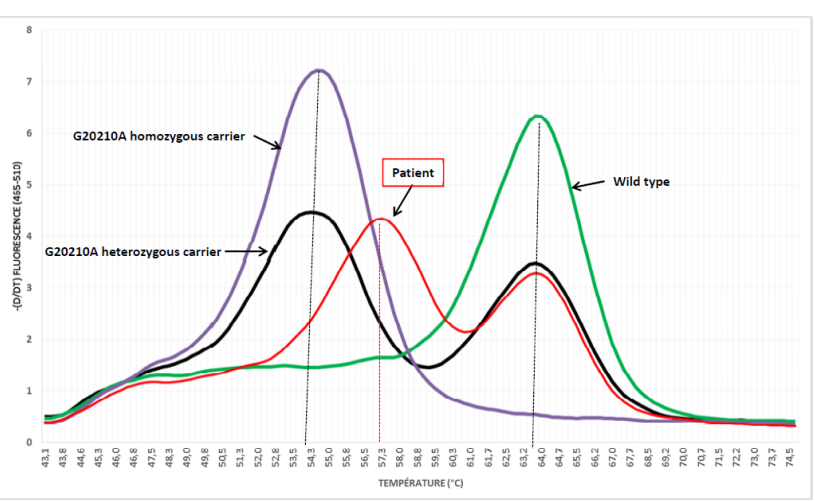

\section{FIGURE 1}

Conclusions: The C20209T mutation detected in our patient is rare among Caucasians, and is mainly found in non-Caucasian patients particularly Africans, African-Americans and Caribbeans. Its role as VTE risk factor is still unknown and the frequency misjudged. One possible reason could rely on the widely used specific assays for the G20210A mutation such as the classical RFLP, in contrast with the Lightcycler $^{\mathrm{TM}}$ used in the present study.

PB1164 | Heterozygosity for factor V Leiden with a Catastrophic Presentation

R. Pombal; L. Vieira; S. Lopes; R. Neto; H. Gomes; M. Figueiredo Centre of Thrombosis and Hemostasis and Department of Transfusion Medicine, Centro Hospitalar Vila Nova de Gaia/Espinho, E.P.E., Vila Nova de Gaia, Portugal

Background: Factor $\mathrm{V}$ Leiden (FVL) results from a mutation in the F5 gene, which encodes the coagulation factor $V$ protein, increasing the risk of venous thromboembolism (VTE). Only 5-10\% of FVL heterozygotes will experience VTE during their lifetime. The reasons for the highly variable phenotype are incompletely understood.
Aims: Description of a heterozygote FVL with a catastrophic clinical presentation.

Methods: Collection of clinical data in SClínico ${ }$ application.

Results: Woman, 26 years old, with overweight and estrogencontaining oral contraceptive as medical history, developed on august 2020, mild dyspnoea, with sudden worsening at the $5^{\text {th }}$ day of symptoms, culminating in cardiopulmonary arrest. Prehospital resuscitation was successfully performed. At the emergency room admission, the electrocardiogram and echocardiogram showed S1;T3 and right ventricle dilatation. Pulmonary embolism with obstructive shock was suspected and alteplase thrombolysis performed. Computed tomography angiography confirmed the diagnosis: extensive bilateral acute pulmonary embolism, with left and right lobar arteries thrombosis. She was admitted to the Intensive Care Unit (ICU) and hypocoagulant treatment with unfractionated heparin was iniciated. The patient presented a favourable evolution and was discharged from ICU 4 days later. She remained hospitalized for 4 more days. Deep vein thrombosis was excluded and age-appropriate cancer screening was negative. There was a progressive clinical improvement under hypocoagulant treatment, first with low molecular weight heparin and later with apixaban, which she maintained after discharge.

Following the acute event, thrombophilia study was performed and a heterozygosity for FVL diagnosed with genetic testing. Nowadays, she maintains follow-up, hypocoagulated with apixaban, without haemorrhagic events, with non-estrogen-containing contraceptive and with a complete recovery of the cardiopulmonary function.

Conclusions: A patient with heterozygosity for FVL, without a personal and family history of thrombosis, presents as an initial manifestation of a multifactorial disease a life-threatening event. With this case, we intend to highlight that, although rare, these severe events can unfortunately occur.

PB1165 | The Role of Thrombophilia in Deep Vein Thrombosis of Unusual Sites

I. Chabchoub ${ }^{1}$; R. Ben Salah ${ }^{1}$; F. Megdiche ${ }^{2}$; C. Kallel ${ }^{2}$; Z. Bahloul ${ }^{1}$

${ }^{1}$ Internal Medicine Departement, Hedi Chaker Hospital, Sfax, Tunisia;

${ }^{2}$ Hematology Laboratory, Habib Bourguiba Hospital, Sfax, Tunisia

Background: Deep vein thrombosis of unusual location refers to deep vein thrombosis of location other than the lower limbs. They are infrequent and, unlike thrombosis of the lower limbs, they most often occur in an underlying anomaly.

Aims: The aim of our work is to determine the etiological profile of deep vein thromboses of unusual location.

Methods: A retrospective study of 146 files of patients with thromboembolic venous disease over a 5-year period (2013-2017).

Results: Twenty-one cases (14.3\%)had unusual sites venous thrombosis: 9 men and 12 women with an average age of 37.1years. DVT of the abdominal veins was dominant observed in 8patients with 1case of portal thrombosis, 1case of splenic venous thrombosis, 4cases of 
mesenteric venous thrombosis, 1case of the renal vein, and 4cases of the ovarian vein. DVT of the inferior vena cava was observed in 6patients, that of the cerebral veins in 2cases and that of the upper limbs in 1case. Risk factors for abdominal venous thrombosis were dominated by intra-abdominal surgery (4cases), cirrhosis with portal hypertension (2cases), inflammatory bole disease (2cases) and a history of thrombosis (4cases). The other venous thrombosis of unusual site had occurred following bed rest (7 cases), surgery (6cases), post partum (5 cases) and in the context of Behçet's disease in 1case. An etiological assessment, carried out in all cases had shown that constitutional thrombophilia was observed in 10patients (47.6\%), dominated by resistance to activated protein C (APCR). (APCR) was isolated in 8cases, associated with protein S deficiency in 1case and antiphospholipid syndrome in 1 case. Unusual venous thromboses associated with RPCA were located in the abdominal veins in 4cases, the inferior vena cava in 3 cases and the ovarian veins in 3 cases. Acquired thrombophilia (antiphospholipid syndrome) was observed in 4 patients (19\%).

Conclusions: Thrombophlebitis with unusual localisation is a serious condition that requires exploration and adequate symptomatic and etiological management.
PB1166 | Incidence of Thrombosis in Patients with MTHFR C677T Homozygosity and Hyperhomocysteinemia

G.M. Nicolò $^{1}$; G. Sottilotta ${ }^{2}$; F. Luise ${ }^{3}$; V. Oriana ${ }^{2}$; A. Piromalli ${ }^{3}$

${ }^{1}$ Clinical Pathology and Clinical Biochemistry, University of Catania, Catania, Italy; ${ }^{2}$ Hemophilia Centre - Thrombosis and Hemostasis Service, Great Metropolitan Hospital, Reggio Calabria, Italy; ${ }^{3}$ Analysis Laboratory, Great Metropolitan Hospital, Reggio Calabria, Italy

Background: Methylene tetrahydrofolate reductase (MTHFR) is a key enzyme in homocysteine (HCY) metabolism. Previous studies have demonstrated that homozygosis for the MTHFR C677T mutation is associated with an increased risk of thrombosis, even in the absence of hyperhomocysteinemia (hHCY). However, this relationship remains controversial.

Aims: To determine the incidence of thrombosis in patients homozygous for C677T MTHFR mutation, with or without hHCY, compared to healthy individuals with the same characteristic; we divided the patients into two groups: those with normal HCY levels and those with $\mathrm{hHCY}$, assessing the incidence of thrombosis in both groups. The data obtained from the two groups were analyzed using chisquare test

Methods: We retrospectively analysed the clinical data of 570 subjects with homozygosity for the C677T MTHFR mutation followed by our centre in the last 10 years: 149 males, 421 females; average age: 39.7 (4-88). 382 had normal HCY and 188 had hHCY. All subjects with other congenital or acquired thrombophilia states were excluded from the study. hHCY has been defined if greater than 15 micromol/L.

Results:

Table 1 Incidence of thrombosis in homozygous C677T MTHFR patients with and without hyperhomocysteinemia.

\begin{tabular}{lll} 
& $\begin{array}{l}\text { Homozygous C677T MTHFR patients } \\
\text { with thrombosis }\end{array}$ & $\begin{array}{l}\text { Homozygous C677T MTHFR patients } \\
\text { without thrombosis }\end{array}$ \\
$\begin{array}{l}\text { Normal Homocysteine ( } \leq 15 \mathrm{micromol} / \mathrm{L}) \text { and } \% \\
(n=382)\end{array}$ & $57(14.9 \%)$ & $325(85.1 \%)$ \\
$\begin{array}{l}\text { High Homocysteine (>15.1 micromol/L) and \% } \\
(n=188)\end{array}$ & $29(15.4 \%)$ & $159(84.6 \%)$ \\
Total: 570 & $86(15.1 \%)$ & $484(84.9 \%)$ \\
\hline
\end{tabular}

We found very similar incidence of thrombosis in homozygous subjects with hHCY, 29/188 (15.4\%), compared to those with normal homocysteine, 57/382 (14.9\%). The results showed statistical significance by Chi-square test: X2 $(1, N=570)=0.025, P=.874398$. Data are summarized in table 1.

Conclusions: Unlike other authors, our data did not confirm the importance of $\mathrm{hHCY}$ as an independent thrombotic risk factor; the incidence of thrombosis in C677T MTHFR homozygotes also appears to be lower than that shown in the literature. Prospective and randomized studies, especially in comparison to subjects without MTHFR mutations, are necessary to understand better the real prothrombotic role of C677T MTHFR and hHCY.

\section{PB1167 | Deep Vein Thrombosis in Young Woman Reveals a Novel Mutation on SERPINC1 Gene}

F. Bargado; F. Rincón; A. Ribeiro; A. Mascarenhas; M.C. Romeiras; T. Araújo

Centro Hospitalar Universitário Lisboa Central, EPE - Serviço de Imunohemoterapia, Lisboa, Portugal

Background: Antithrombin deficiency is associated with an increased risk of thromboembolism. It can be congenital, due to gene variation, or acquired, as consequence of certain clinical conditions or therapeutics. Congenital antithrombin deficiency is one of the most severe thrombophilia affecting $0,02-0,2 \%$ of the general 
population and exerts a dominant inheritance with incomplete penetrance and variable expression. SERPINC1 is the gene that codes for antithrombin. So far, more than $\mathbf{3 5 0}$ mutations in this gene are known to cause disease.

Aims: Report a new mutation in the SERPINC1 gene responsible for congenital antithrombin deficiency.

Methods: Collection of data in hospital clinical software.

Results: A 36-years-old woman presented with lower extremity deep vein thrombosis without apparent trigger factor. The patient reported low levels of antithrombin in previous isolated blood tests, after a DVT family study. The study we carried out after the acute phase of the disease confirmed deficiency of antithrombin, presenting low antithrombin activity values (20-30\%). SERPINC1 gene mutation search was requested and identified a novel heterozygous mutation variant c.332C $>$ T, p.(Ser111Leu). The patient underwent therapeutic anticoagulation with $\mathrm{LMWH}$ and fully recovered from the event after 6 months of therapeutics. Based on the results we choose to maintain prophylaxis anticoagulation indefinitely with rivaroxaban $10 \mathrm{mg}$.

Conclusions: Congenital antithrombin deficiency presents with clinical heterogeneity. Genetic sequencing makes it possible to identify mutations already known or novel mutations, allowing a fully characterization of the disease that may have an impact on its management. In our case we provide genetic counseling to the patient and are currently studying her family.

PB1168 | Duration of Thromboprophylaxis with Low Molecular Weight Heparins after Cesarean Delivery in Carriers of Leiden Mutation, F5g1691a Genotype

M. Nikolaeva ${ }^{1,2} ;$ K. Shchekleina ${ }^{1}$

${ }^{1}$ FSBEl of Higher Education Altai State Medical University, Barnaul, Russian Federation; ${ }^{2}$ FSBI "National Medical Research Center of Hematology" Altai Branch Office, Barnaul, Russian Federation

Background: Pregnancy is the proved risk factor of venous thromboembolism (VTE). The peak of thrombotic events falls on the postnatal period, increasing their number at 5-15 times. A carriage of prothrombotic Leiden mutation during pregnancy is an additional risk factor of VTE and assumes carrying out thromboprophylaxis with low molecular weight heparins (LMWHs) in the antenatal and postnatal period. Thus, duration of therapy with LMWHs after cesarean delivery is a subject of a scientific discussions.

Aims: to study efficiency prolonged thromboprophylaxis after cesarean delivery in carriers of Leiden mutation, F5G1691A genotype, based on studying of a laboratory phenotype.
Methods:

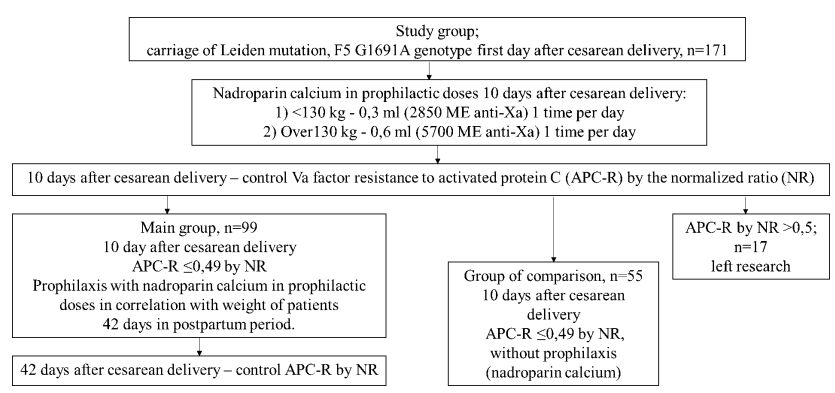

FIGURE 1 Design of research prolonged thromboprophylaxis after cesarean delivery in carriers of Leiden mutation, F5 G1691A genotype

A single-center randomized controlled study, the period of supervision was 2008-2020 years. The design of research is presented in figure 1.Efficiency of appointment nadroparin calcium was estimated on number of cases of VTE registered in the main group in relation to group of comparison.

Results: Statistical processing of the received results has shown the lack of episodes of VTE in the main group (RR $=0.037 ; 95 \% \mathrm{Cl}$ 0.02-0.64; $P=0.0235$ ). 7 cases of VTE are registered in group of comparison: 4 supervision $(16,18,21$ and 37 days of the postnatal period) - thrombosis of deep veins of femur, one of which has become complicated with pulmonary embolism; 3 cases - thrombosis of deep veins of crus (19, 21 and 27 days). Using the declared method it is necessary to treat 8 patients (NNT $=7.7$; $95 \% \mathrm{Cl}$ : $5.05-16.76$; $\mathrm{P}=0.0223$ ) to avoid 1 case of development of VTE in patients with Leiden mutation, F5G1691A genotype.

Conclusions: at carriage cases of Leiden mutation, F5G1691A genotype, with the shown laboratory phenotype in the form of APC-R $\leq 0,49$ on NR, after cesarean delivery it is reasonably to use prolonged thromboprophylaxis with LMWHs up to 42 days in prophilactic doses.

PB1169 | Discrepancy between Genotype and Phenotype for Factor V Leiden Mutation in Recipients of Liver and Stem Cell Transplantation

\section{J. Rigano}

Alfred Health, Melbourne, Australia

Background: Activated protein C resistance (APCR) is the most common hereditary risk factor for venous thromboembolism (VTE) in Caucasian population. Approximately $90-95 \%$ of cases, the coagulation disorder results from factor $V$ Leiden (FVL) mutation (R506Q) in the factor $\mathrm{V}(\mathrm{FV})$ gene causing activated $\mathrm{FV}$ to be resistant to cleavage by APC. It has been established that liver transplantation (LTX) and stem cell transplantation (SCTX) recipients are at risk of VTE. 
Recipients of these transplants can acquire or lose APCR and FVL mutation.

Aims: To investigate genotype and phenotype discrepancies for FVL mutation in recipients of LTX and SCTX both associated with deep vein thrombosis (DVT).

Methods: First case was 68-year-old male who presented with a DVT nine months post LTX for liver cirrhosis. Second case was 42-year-old female who presented with a CRT four months post SCTX for AML. Both patients reported no history of thrombosis prior to transplantation. Thrombophilia assays were performed using HemosIL $®$ reagents on the ACL TOP CTS 500 and AcuStar analysers (Instrumentation Laboratory; Werfen). Molecular thrombophilia assays for FVL and prothrombin gene (G20210A) mutations were performed using the Qiagen Rotor-Gene by PCR and HRM analysis. Results: APCR was detected in both patients with ratios of 1.75 and 1.64 for case one and two respectively (normal APCR ratio 2.2-3.3). All other thrombophilia assays were negative. In case one, APCR was acquired and detected in donor liver and the recipient's leucocyte DNA lacked FVL mutation. In case two, APCR was inherited and detected in the recipient's liver and the peripheral leucocyte DNA of the donor lacked the FVL mutation. Since both patients reported no history of thrombosis, thrombophilia testing was never previously indicated.

Conclusions: Consideration should be given to thrombophilia testing of LTX and SCTX donors and recipients which may indicate anticoagulation to prevent additional morbidity.
PB1170 | Inpatient Thrombophilia Testing - Adding Unnecessary Cost to the Clot?

S. Bandikatla ${ }^{1}$; A. Dadlani ${ }^{1}$; A. Pinter ${ }^{1}$; S. Maharaj ${ }^{2}$; A. Rojan ${ }^{2}$

${ }^{1}$ University of Louisville, Department of Internal Medicine, Louisville, United States; ${ }^{2}$ University of Louisville, Division of Medical Oncology/ Hematology, Louisville, United States

Background: Thrombophilia testing (TT) generally has a limited role in the management of patients with thrombosis in the inpatient setting. Inaccuracies with testing following acute thromboses or anticoagulation can lead to patient anxiety, inappropriate prolongation of anticoagulation, or false reassurance. Indiscriminate TT also adds to cost of healthcare for thrombosis.

Aims: To analyze the prevalence and cost of inpatient TT and investigate whether the results of these tests changed management.

Methods: This was a retrospective analysis at the University of Louisville Hospital from 7/1/2020 to $12 / 30 / 2020$. Patients were included if they were admitted with thrombosis (arterial and/or venous) and underwent inpatient TT (any test as listed in Table 1). Chart review was done to study demographics, details of TT, and any subsequent change in management. Based on available evidence and guidelines [Baglin 2010, Van Cott 2002, Pengo 2009, Nicolaides 2005], the rate of inappropriate TT was assessed. Cost data were obtained from Clinical Laboratory Fee Schedule.

Results: Over the 6-month period, TT included 156 tests in 38 patients (average 4.1 tests/patient). The majority was female (63\%) with a mean age of 48.3 years [range: $19-75]$. The reasons for TT are detailed in Table 2; recurrent VTE was the most common. Twothirds of TT (67\%) were classed as inappropriate. Overall, 6 tests were positive (3.8\%); none of the positive tests changed management. The total cost of TT was estimated at $\$ 38,944$; inappropriate TT was estimated at $\$ 28,165$.

TABLE 1 Inpatient thrombophilia tests studied - frequency and yield of testing

\begin{tabular}{|c|c|c|c|c|c|}
\hline Test name & Number ordered & $\begin{array}{l}\text { Number resulting } \\
\text { positive }\end{array}$ & Test name contd. & Number ordered & $\begin{array}{l}\text { Number resulting } \\
\text { positive }\end{array}$ \\
\hline Protein $\mathrm{C}$ activity & 6 & 1 & JAK2 mutation & 8 & 0 \\
\hline $\begin{array}{l}\text { Antithrombin III } \\
\text { activity }\end{array}$ & 10 & 1 & Lupus anticoagulant & 17 & 0 \\
\hline Factor $\vee$ activity & 7 & 0 & $\begin{array}{l}\text { Beta-2 glycoprotein } 1 \\
\text { Antibody (IgA/ IgG/ IgM) }\end{array}$ & 17 & 1 \\
\hline $\begin{array}{r}\text { Prothrombin } \\
\text { mutation }\end{array}$ & 16 & 0 & $\begin{array}{l}\text { Anti-cardiolipin Antibody } \\
\text { (IgA/ IgG/ IgM) }\end{array}$ & 18 & 1 \\
\hline MTHFR mutation & 7 & 0 & & & \\
\hline
\end{tabular}


Table 2 Charted reasons for inpatient thrombophilia testing across 6 months in an inpatient setting

$\begin{array}{ll}\text { Charted reason } & \text { Number (\%) } \\ \text { Recurrent Venous Thromboembolism (VTE) } & 21(55 \%) \\ \text { VTE in an unusual location (cerebral, splanchnic) } & 11(29 \%) \\ \text { Stroke at a young age or recurrent cryptogenic stroke/arterial thrombosis } & 3(7 \%) \\ \text { Massive Pulmonary embolism } & 1(2 \%) \\ \text { Pulmonary embolism in pregnancy } & 3(7 \%)\end{array}$

Conclusions: Inpatient TT at an urban tertiary hospital was expensive (average estimate $\$ 1,024$ per patient) with a high rate of inappropriate TT, low positive result rate (3.8\%), and no change in management. We plan to implement interventions to improve this. The data suggest a role for systems-based hematology in the inpatient setting to improve the quality and value of care to patients admitted with thrombosis.

PB1171 | Frequency of Hereditary Thrombophilia in Venous Thromboembolic Disease

\section{K. Mendi}

Laboratoire Central et CTS, Hopital Bachir Mentouri - EPH de Kouba, Algiers, Algeria

Background: Venous thromboembolic (VTE) disease is a multifactorial pathology. It's a disorder that includes deep vein thrombosis (DVT) and pulmonary embolism (PE). Hereditary thrombophilia plays a major role in the development of this disease because it's predispose to thrombosis. The most common inherited thrombophilias are factor V Leiden, prothrombin G20210A; deficits in protein C, S and antithrombin.

Aims: Our objective was to determine the frequency of deficits in physiological coagulation inhibitors and activated protein $C$ resistance in patients with VTE, and to analyze their epidemiological and clinical characteristics.

Methods: This retrospective study involved 379 patients with proven venous thrombosis, authenticated with medical imaging. These patients were selected according to the recommendations of GEHT. The thrombophilia assessment included the functional assay of physiological coagulation inhibitors and the search for activated protein $\mathrm{C}$ resistance.

Results: 379 patients were included in the study : 112 men and 267 women, a sex ratio M / F of 0.42 . The mean age was 35 years. An hereditary thrombophilia was found in 42 patients ( $11,1 \%$ of the cases) : we found 01 case $(0,2 \%)$ of antithrombin deficiency, 04 cases $(1,1 \%)$ of protein $\mathrm{C}$ deficiency, 14 cases $(3,7 \%)$ of protein $\mathrm{S}$ deficiency and 23 cases $(6,1 \%)$ of activated protein $C$ resistance. This is 13 men and 29 women, a sex ratio of 0,44 . The mean age was 37 years. We found 31 cases of DVT, 10 cases of cerebral venous thrombosis and 01 case of PE. 14 patients presented also acquired risk factors and 20 patients had thrombosis's antecedents. The family investigation revealed 59 asymptomatic patients.
Conclusions: Hereditary thrombophilia plays a key role in the development of venous thromboembolism, hence the value of research.

P0166 | Thrombophilia and Thromboembolic Venous Disease in Southern Tunisia

I. Chabchoub ${ }^{1}$; R. Ben Salah ${ }^{1} ;$ F. Megdiche ${ }^{2}$; C. Kallel ${ }^{2}$; Z. Bahloul ${ }^{1}$

${ }_{1}^{1}$ Internal Medicine Department, Hedi Chaker Hospital, Sfax,

Tunisia, ${ }^{2}$ Hematology Laboratory, Habib Bourguiba Hospital, Sfax, Tunisia

Background: Thromboembolic venous disease (TVD) is a multifactorial pathology. Thrombophilia, which is a state of hypercoagulability linked to constitutional and/or acquired haemostasis abnormalities, is one of the main etiological factors of TVD.

Aims: The aim of our work is to study the thrombophilia profile in a series of patients hospitalised for TVD.

Methods: A monocentric retrospective study over a period of 5 years (2013-2017). All the records of patients hospitalised for VTE and for whom an etiological assessment of thrombophilia was carried out were pooled.

Results: There were 146 patients: 69men (47.3\%) and 77women $(52.7 \%)$ with a sex ratio (M/F) of 0.89 . The average age of our patients was 42.5 years. 62patients $(42.46 \%)$ had a thrombophilic anomaly: 46 cases (31.5\%) of isolated constitutional thrombophilia, 13 cases $(8.9 \%)$ of isolated acquired thrombophilia, 3cases $(2.05 \%)$ of mixed thrombophilia.

During constitutional thrombophilia, antithrombin III deficiency was found in 1 case $(0.68 \%)$, Protein S deficiency in 5 cases $(3.4 \%)$ and Protein $\mathrm{C}$ deficiency in 2cases (1.36\%). Resistance to activated protein C was observed in $45 / 146$ patients (30.82\%). Acquired thrombophilia was diagnosed in 16 patients (11\%). The associated etiologies were SLE (1case), Behçet's disease (1case) and cancer and/ or its treatment (1case).

Out of a total of 124cases of deep vein thrombosis of the lower limbs, hereditary thrombophilia was noted in 42cases (33.8\%), acquired thrombophilia in 12cases(9.6\%) and mixed thrombophilia in 3 cases(2.4\%). PRCa was the most common constitutional abnormality observed during lower limb DVT, noted in 36patients(27\%). During the unusual venous thromboses represented by 21 cases, hereditary thrombophilia was noted in 9cases(42.8\%), acquired thrombophilia in 3cases(14.2\%) and mixed thrombophilia in 1case(4.7\%). 
The most frequent hereditary abnormality was CPRa. It was isolated in 8patients.

Conclusions: Thrombophilia is one of the major causes of VTE. The indication of the thrombophilia assessment must be known by the clinician given its clinical involvement and its therapeutic consequences in a patient suffering from VTE.

P0169 | Investigation of Hereditary Thrombophilia in Women with Recurrent Fetal Loss in LUMHS Hyderabad

K. Ilyas; I. Ujjan; B. Bhatti; A. Naz; S. Abbasi

Liaquat University of Medical and Health Sciences Jamshoro, Hyderabad, Pakistan

Background: Thrombophilia is a condition of hypercoagulability and has been associated with adverse pregnancy outcomes. It may be hereditary or acquired. Five to $20 \%$ of women experience one or more pregnancy loss during their reproductive age. Thrombophilia has been suggested to play a key role in recurrent pregnancy losses, yet controversy exists as to whether thrombophilia is a cause or an association. Internationally, the prevalence of hereditary thrombophilia differs among races, geographical areas, and communities.

Aims: To evaluate the role of Antithrombin, Protein C, Protein S, and Factor $\mathrm{V}$ Leiden in recurrent pregnancy loss and to compare the cause of hereditary thrombophilia among women with recurrent fetal loss.

Methods:

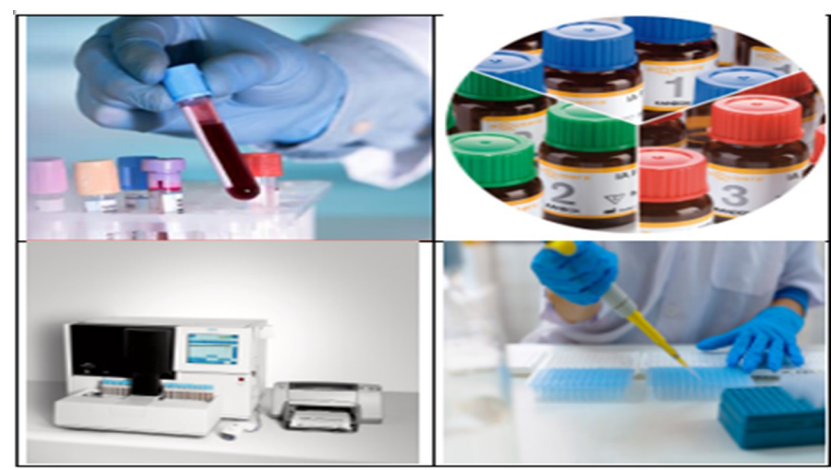

\section{FIGURE 1}

Patients with two or more pregnancy loss having ages 17-45 years included in the descriptive cross-sectional study. While patients with liver disease, uterine fibroids. Cardiac disease, congenital abnormalities associated with pregnancy loss were excluded. A total of six $m$ of blood was taken and distributed in an EDTA tube and in sodium citrate. $C B C$ was performed on automated CBC analyzer Sysmex CA 1000. PT, APTT were performed on Sysmex CA 600. Antithrombin screening was measured by INNOVANCE Antithrombin Kit. Protein $C$ activity was performed by using Berichrome Protein $C$ Kit. Protein
S screened by Siemens Protein S Ac kit. Factor V Leiden was measured by using a Pro $\mathrm{C}$ global kit with factor $\mathrm{V}$ deficient plasma. Quality control and standard curves were performed as per kits instructions Results:

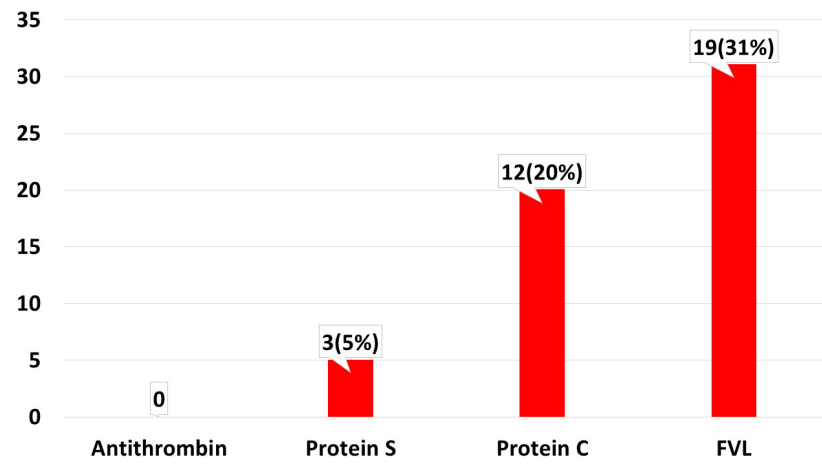

FIGURE 2

In our study of sixty women with recurrent fetal loss, the mean ages were 28.1 \pm 3.7. Among these 19 (31\%) patients had Factor $V$ Leiden. Protein C was found in 12 (20\%). Whereas, Protein S was found in 3 (5\%) patients. Antithrombin was not detected in any patients.

Conclusions: Our research-based on the results concludes that Factor V Leiden has a significant association with recurrent fetal loss.

P0170 | How to Use the Data about Risk Factors for Thrombosis in Practice

L. Stanciakova ${ }^{1}$; K. Chromekova ${ }^{1}$; K. Vajdova ${ }^{1}$; M. Dobrotova ${ }^{1}$; P. Holly'; M. Brunclikova ${ }^{1}$; T. Bolek ${ }^{2}$; M. Samos ${ }^{2}$; P. Kubisz ${ }^{1}$; J. Stasko ${ }^{1}$

${ }^{1}$ National Center of Hemostasis and Thrombosis, Department of Hematology and Transfusion Medicine, Comenius University in Bratislava, Jessenius Faculty of Medicine in Martin, Martin University Hospital, Martin, Slovakia; ' Department of the Internal Medicine I, Comenius University in Bratislava, Jessenius Faculty of Medicine in Martin, Martin University Hospital, Martin, Slovakia

Background: Development of venous thromboembolism (VTE), as well as arterial thrombosis is associated with the presence of inherited and acquired risk factors. Despite the current advances in research, unfortunately, a lot of questions remain unanswered. For instance, despite the coincidence of several risk factors for VTE, in some patients the thrombotic episode will not be developed. On the other hand, in some individuals, despite the absence of risk factors, thromboembolic event is diagnosed repeatedly.

Aims: The assessment of the influence of inherited and acquired risk factors on the development of arterial and venous thrombosis.

Methods: The authors analyzed risk factors and circumstances of the development of thromboembolism in patients followed-up for 
the management of thrombosis in the National Center of Hemostasis and Thrombosis.

Results: In this complex study, the authors confirmed the influence of acquired risk factors, such as polytrauma, surgical intervention, renal failure, immobilisation, pregnancy, inflammation, travelling and malignancy on the higher risk of arterial and venous thrombosis. In selected groups of patients, they evaluated the influence of acquired risk factors including increase in factor VIII activity, protein S and antithrombin deficiency on the development of thromboembolic complications. The most common form of arterial thrombosis was ischemic stroke present in $7.4 \%$ of the patients, the commonest form of venous thromboembolism ws deep venous thrombosis of lower extremities developed in $42.6 \%$ of the cases. Pregnancy complications occurred in $7.4 \%$.

Conclusions: The results of laboratory parameters may contribute to individualization of primary or secondary thromboprophylaxis and increase the quality of life of affected persons.

Acknowledgements: Authors thank the support of the projects of the Scientific Grant Agency (Vega) 1/0549/19 and Agency for the Support of Research and Development APVV-16-0020.

\section{P0183 | Misdiagnosing Protein C and Protein S Deficiency}

C. Peixoto; Á. Beleza; M. Galvão; A. Freixo; T. Quaresma; J. Amorim; M.M. Deveza

Hospital Santa Maria, Lisboa, Portugal

Background: Protein C (PC) and protein S (PS) are two vitamin Kdependent plasma proteins that act together as a natural anticoagulant system. PC and PS deficiencies are two clinical conditions that predispose to thromboembolic events and can be inherited or acquired. Multiple factors can interfere with PC and PS plasma concentrations, such as age, gender, inflammatory state, and therapy with vitamin $\mathrm{K}$ antagonists (VKA).

Aims: To draw attention to the relevance of VKA treatment as a possible cause of acquired PC and/or PS deficiencies.

Methods: A 59-year-old female was on VKA (warfarin) anticoagulation therapy since an idiopathic venous thrombosis (left lower limb at the age of 44). Thrombophilia screening presented a decreased activity of PC and free PS: 55\% (reference 70-130\%) and 23\% (reference 53-109\%), respectively. A major inherited thrombophilia due to conjugated PC and PS deficiencies was assumed and indefinite anticoagulation recommended.

Recently, this patient had an invasive lobular carcinoma and was sent to our anticoagulation clinic for having high INR levels induced by dicumarinic intoxication. Anticoagulation strategy was switched to a low-molecular-weight heparin (LMWH) due to fewer pharmacologic interactions.

Results: Since the combination of genetically determined inherited PC and PS deficiencies is very rare, particularly with no family history of thrombophilia or thromboembolism, the diagnosis of inherited thrombophilia was questioned. After 24 hours of LMWH suspension, she was retested for PC and free PS activity levels, and the results demonstrated normal levels of both $(127 \%$ and $59 \%$, respectively).

Conclusions: VKA are a major cause of acquired PC and/or PS deficiencies. Despite this, as well as other disadvantages, VKA is still widely used as anticoagulant treatment. The present case report also raises awareness to the timing for inherited thrombophilia testing, interference of anticoagulants and the need to repeat abnormal test results for confirmation, especially in the absence of a family history.

\section{VISCERAL VEIN THROMBOSIS}

LPB0043 | Annexin A2-mediated Surface Fibrinolysis Is Impaired in Non-alcoholic Steatohepatitis (NASH) Cirrhosis and May Contribute to Portal Vein Thrombosis

H.I Lim ${ }^{1}$; B. Fortune ${ }^{2}$; M.T DeSancho'; K.A Hajjar ${ }^{3,4}$

${ }^{1}$ Weill Cornell Medicine, Division of Hematology and Oncology, Department of Medicine, New York, United States; ${ }^{2}$ Weill Cornell Medicine, Division of Gastroenterology and Hepatology, Department of Medicine, New York, United States; ${ }^{3}$ Weill Cornell Medicine, Division of Hematology and Oncology, Department of Pediatrics, New York, United States; ${ }^{4}$ Weill Cornell Medicine, Department of Cell and Developmental Biology, New York, United States

Background: Annexin A2 (A2) is a cell surface fibrinolytic receptor for tissue plasminogen activator (tPA) and plasminogen (PLG) that stimulates efficient plasmin generation on the cell surface. Non-alcoholic steatohepatitis (NASH) cirrhosis is a leading cause of chronic liver disease, and is associated with venous thrombosis, especially portal vein thrombosis (PVT).

Aims: Our objective was to determine the potential role of $A 2$ in NASH cirrhosis and NASH thrombogenesis.

Methods: Peripheral blood mononuclear cell (PBMC) lysates from obese controls, and NASH with or without cirrhosis, were analyzed for A2 expression by immunoblot. The effects of patient plateletpoor plasma (PPP) on A2 expression in human umbilical vein endothelial cells (HUVECs) were tested. Formalin-fixed human liver tissue samples with normal, steatosis, and NASH cirrhosis pathologies were analyzed by immunofluorescence for A2 expression. A2dependent PBMC surface fibrinolysis was assessed using a plasmin generation assay. Systemic fibrinolysis was assessed via plasminanti-antiplasmin (PAP) complex and D-dimer ELISAs.

Results: Total A2 expression did not differ in PBMC lysates among subjects with varying degrees of NASH cirrhosis versus controls (Figure 1A). Plasma from subjects with varying disease severity had no effect on total A2 expression in cultured HUVECs (Figure 1B). 


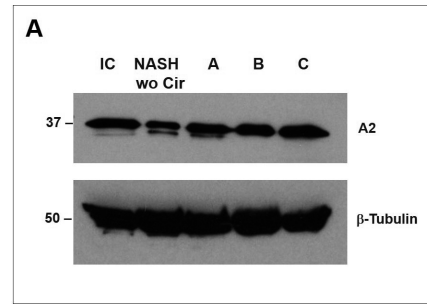

B

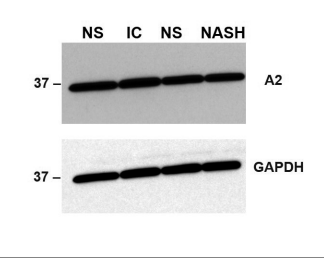

FIGURE 1 Annexin A2 expression in PBMCs from NASH patients and in HUVECs incubated with NASH plasma. (A) Immunoblot analysis of PBMC lysates from internal control (IC), NASH without cirrhosis (NASH wo Cir), and varying degrees of NASH cirrhosis (Child-Turcott-Pugh A (A), B (B), and C (C)). Levels in all cohorts compared to the beta-tubulin control were similar. (B) Representative immunoblot analysis of lysates from HUVECs incubated with human plasma and probed with anti-A2 IgG. There was no difference in A2 expression upon incubation without plasma (NS), or with plasma from an internal control (IC) or NASH cirrhosis patient (NASH). GAPDH was used as loading control

However, patients with NASH cirrhosis who developed PVT had decreased $A 2$ to vessel lumen ratios by quantitative immunofluorescence (Figure 2A), and PBMC surface plasmin generation decreased as disease severity worsened (Figure 2B). At the same time, systemic fibrinolysis increased in patients with cirrhosis, especially as their disease worsened (Figures 2C, 2D).
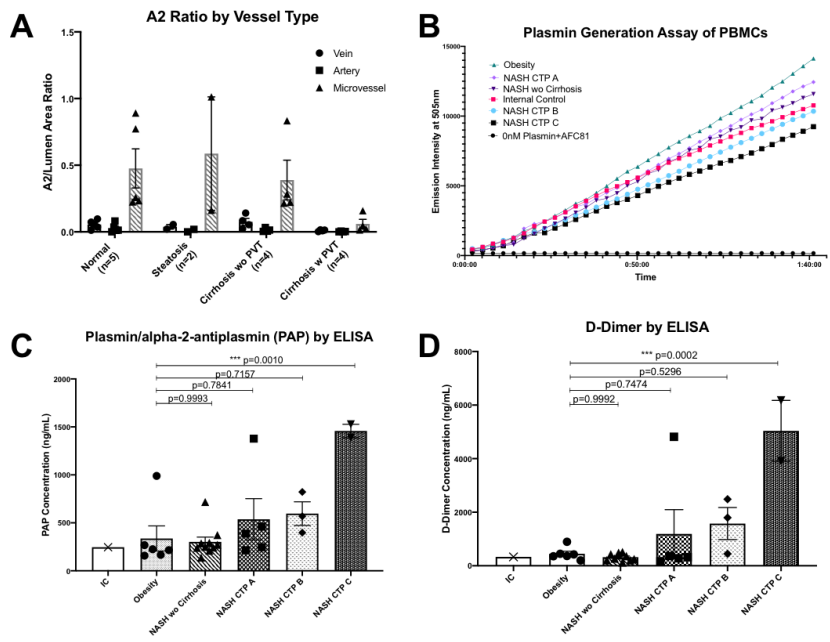

FIGURE 2 Fibrinolytic function in NASH. (A) Immunofluorescence analysis of human liver tissue. A2 area to vessel lumen area ratio was assessed by quantitative analysis. Vein, artery, and branching vessels (microvessels) within portal triads were analyzed. The NASH cirrhosis with portal vein thrombosis (Cirrhosis w PVT) cohort showed decreased A2/lumen area ratio compared to normal, steatosis, and NASH cirrhosis without portal vein thrombosis (Cirrhosis wo PVT) groups in microvessels. (B) PBMC surface plasmin generation assay showed the highest rate of plasmin generation among obese controls, with decreasing rates as NASH cirrhosis progressed. Rates below that of the internal control (IC) were seen in decompensated cirrhosis patients (NASH CTP B, NASH CTP C). OnM of plasmin with fluorescent plasmin substrate (AFC81) was used as a negative control. (C) Plasmin/alpha-2-antiplasmin (PAP) levels by ELISA in platelet-poor plasma (PPP) of subgroups. Cohorts with the most severe cirrhosis (NASH CTP C) had significantly higher levels of PAP compared to obese control (Obesity). (D) D-dimer levels by ELISA in PPP of subgroups. D-dimer levels were significantly elevated in severe, decompensated cirrhosis (NASH CTP C) compared to obese controls (Obesity)

Conclusions: Together, these data suggest that, despite preserved total A2 expression and more activated systemic fibrinolysis in NASH cirrhosis, A2-mediate surface fibrinolysis decreased as NASH cirrhosis worsened. In addition, the A2 expression ratio in hepatic vasculature decreased in subjects with PVT. This is the first evidence that impairment in cell-surface A2 activity and cell surface fibrinolysis may contribute to PVT in NASH cirrhosis.

PB1172 | Non-malignant Portal Vein Thrombi in Patients with Cirrhosis Consist of Intimal Fibrosis with or without a Fibrin-rich Thrombus

E.G. Driever ${ }^{1}$; F.A. von Meijenfeldt ${ }^{1}$; J. Adelmeijer ${ }^{1}$; R.J. de Haas $^{2}$; M.C. van den Heuvel ${ }^{3}$; C. Nagasami ${ }^{4}$; J.W. Weisel ${ }^{4}$; R.J. Porte ${ }^{5}$; A. Blasi ${ }^{6}$; N. Heaton ${ }^{7}$; S. Gregory ${ }^{8}$; P. Kane ${ }^{8}$; W. Bernal ${ }^{7}$; Y. Zen ${ }^{7}$; T. Lisman ${ }^{1,5}$.

${ }^{1}$ University Medical Center Groningen, Department of Surgery, Surgical Research Laboratory, Groningen, Netherlands; ${ }^{2}$ University Medical Center Groningen, Department of Radiology, Groningen, Netherlands; ${ }^{3}$ University Medical Center Groningen, Department of Pathology and Medical Biology, Division of Pathology, Groningen, Netherlands; ${ }^{4}$ University of Pennsylvania School of Medicine, Philadelphia, Pennsylvania, United States; ${ }^{5}$ University Medical Center Groningen, Department of Surgery, Section of Hepatobiliary Surgery and Liver Transplantation, Groningen, Netherlands; ${ }^{6} \mathrm{Hospital}$ Clinic-IDIBAPS, Department of Anesthesiology, Barcelona, Spain; ${ }^{7}$ King's College Hospital NHS FT, Institute of Liver Studies, London, United Kingdom; ${ }^{8}$ King's College Hospital NHS FT, London, United Kingdom

Background: Portal vein thrombosis (PVT) is a common complication of cirrhosis. The exact pathophysiology remains largely unknown and treatment with anticoagulants only lead to recanalization of the portal vein in a proportion of patients. A better insight in the structure and composition of portal vein thrombi may assist in developing a more rational treatment approach.

Aims: The aim of this study was to define the structure and composition of portal vein thrombi in patients with cirrhosis at the time of liver transplantation.

Methods: Eight prospectively and 63 retrospectively collected non-malignant portal vein thrombi from cirrhotic patients who underwent liver transplantation were included. Histology, 
immunohistochemistry and scanning electron microscopy were used to assess structure and composition of the thrombi. Most recent CT scans were reanalysed for thrombus characteristics. Clinical characteristics were related to histological and radiological findings. Results: All prospective and retrospective samples showed a thickened, fibrotic tunica intima. Fibrin-rich thrombi were present on top of the fibrotic intima in 4/8 prospective cases and in $21 / 63$ retrospective cases. A minority of the fibrotic areas stained focally positive for fibrin(ogen) (fg, 16\% of the cases), Von Willebrand Factor (VWF, 10\%) and CD61 (platelets, 21\%), while most of the fibrin-rich areas stained positive for those markers (fg, 100\%; VWF, 77\%; CD61, $100 \%)$. No associations were found between clinical characteristics including estimated thrombus age and presence of fibrin thrombi.

Conclusions: Here we demonstrated that PVT in cirrhotic patients consists of intimal fibrosis with an additional fibrin-rich thrombus in only a third of the cases. These results suggest that the majority of portal vein thrombi in cirrhotic patients are unlikely to recanalise by anticoagulant therapy.

PB1173 | Subacute Mesenteric Venous Thrombosis Secondary to COVID-19: A Late Thrombotic Complication in a Non-severe Patient

\section{Cano Cevallos ${ }^{1}$; W. Alemán ${ }^{2}$}

${ }^{1}$ Universidad Católica de Santiago de Guayaquil, Guayaquil, Ecuador; ${ }^{2}$ Universidad Espíritu Santo, Samborondón, Ecuador

Background: Subacute mesenteric venous thrombosis (SMVT) is a vascular complication commonly associated with hypercoagulability, resulting in abdominal pain and ischemia of the intestines.

Aims: This case exemplifies the heterogeneous presentation of late thrombotic complications in COVID-19 and the relevance of prophylactic measures against hypercoagulability.

Methods: We carried a full investigation of the patient to gather all the information needed to conclude the origin of his thrombotic episode.
Results:

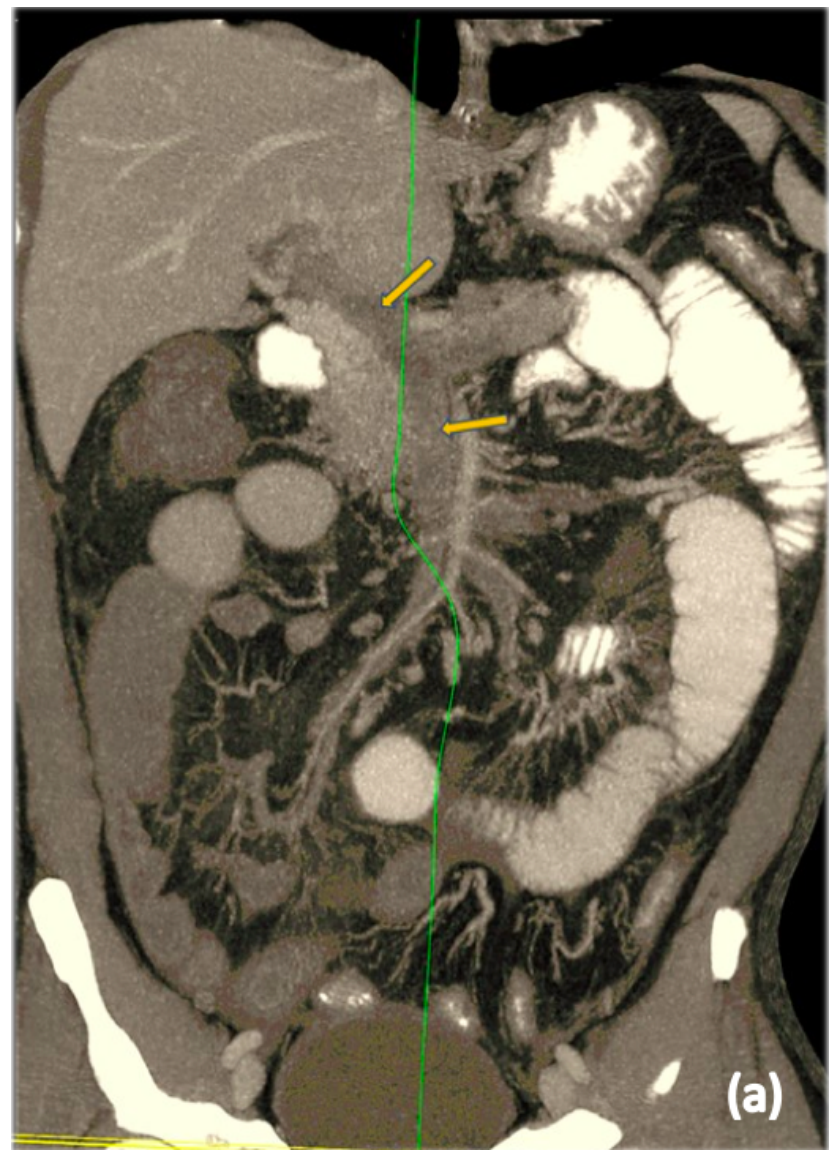

FIGURE 1 Computed tomography

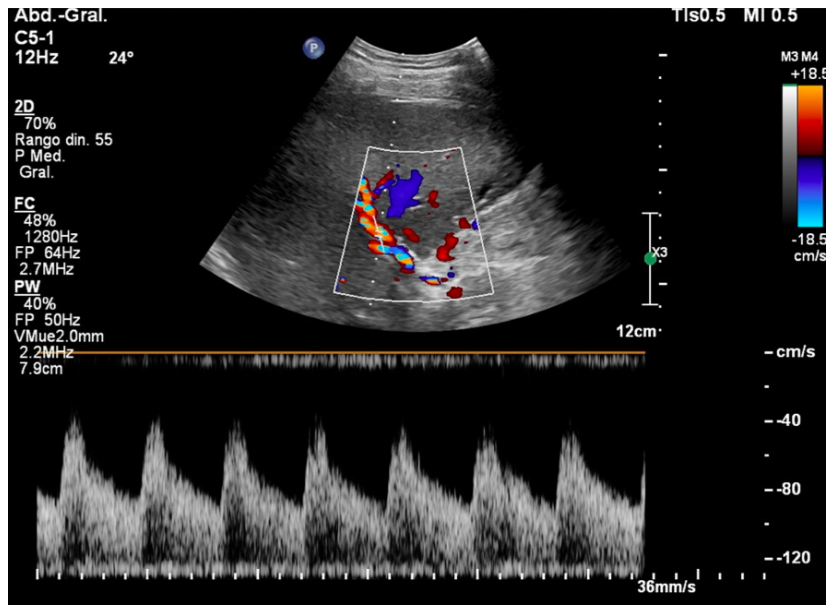

FIGURE 2 Doppler ultrasound

We report a 44 y/o male without relevant history and COVID-19 disease who developed abdominal pain after the onset of respiratory symptoms. The initial differential diagnosis from surgical evaluation for the patient's abdominal pain included mesenteric ischemia, 
bowel obstruction, and pancreatitis. Imaging studies by computed tomography (Fig.1) demonstrated defined hypodensities in the portal vein, venous filling defects, vein enlargement, and engorgement (yellow arrows). Doppler ultrasound (Fig.2) showed abnormal flow consistent with thrombosis of mesenteric veins. He was successfully treated with anticoagulation therapy.

Conclusions: Reports on coagulopathy are on the rise yet the question remains on how to predict these complications. It is relevant to consider prophylactic measures for avoiding hypercoagulability. Progressive diffuse abdominal pain with no significant alterations on coagulation profile or other risk factors should raise the awareness for mesenteric thrombosis. Actually, few cases of intestinal thrombosis exist in the literature considering our patient one of the first cases of subacute mesenteric venous thrombosis in a non-severe COVID-19 patient. More case reports and descriptive data are needed in the literature to increase the index of suspicion for these types of complications.

PB1174 | Does Anticoagulation Affect Outcome of Splenic Vein Thrombosis in Acute Pancreatitis?

L. Vieira; S. Lopes; R. Pombal; R. Neto; A. Magalhães; M. Figueiredo Immunohemotherapy Service, Vila Nova de Gaia/Espinho Hospital Centre, Vila Nova de Gaia, Portugal

Background: Splanchnic venous thrombosis (SVT) is a wellestablished complication of acute pancreatitis (AP) and may affect splenic, portal and superior mesenteric veins, either isolated or in combination. Its pathogenesis is closely associated to inflammation, leading to cellular infiltration, formation of pancreatic/peripancreatic collections that contribute to venous stasis and systemic activation of haemostasis.

Aims: Description of a case of SVT AP-associated.

Methods: Collection of clinical data in SClínico ${ }^{\circledR}$ application.

Results: A 47-year-old female patient, with antecedents of previous AP secondary to hypertriglyceridemia, was admitted to emergency department with pain in upper quadrants of abdomen, radiating towards the back, with nausea and vomiting, over the past few hours. Through clinical, analytical and imaging evaluation, the diagnosis of AP secondary to hypertriglyceridemia was established. The patient was hospitalized and, 4 days later, due to clinical worsening, a computed tomography (CT) was performed, revealing splenic vein thrombosis and pancreatic necrosis. Enoxaparin in therapeutic dose was initiated. The patient remained hospitalized for 18 days and enoxaparin was replaced by rivaroxaban $20 \mathrm{mg}$ once daily at discharge. Three months later, CT showed persistence of thrombosis, with perigastric/perisplenic collateral circulation. Considering this extensive collateral circulation, complete recanalization was no longer expected. Anticoagulation was maintained for a total period of 6 months.

Conclusions: Management of thrombosis in AP remains challenging. There's no consensus on anticoagulation in this setting, with some studies concluding that there's no difference in collateral formation, recanalization and mortality, whether anticoagulation had been prescribed or not. These findings emphasize the predominant role of inflammation, increasing uncertainty of risk/benefit ratio of anticoagulation. When portal and superior mesenteric veins are affected, anticoagulation seems a reasonable attitude, considering the risk of hepatic decompensation and bowel ischemia. More studies are needed to consolidate this evidence and to establish well-defined recommendations in other situations (e.g., isolated thrombosis of splenic vein, as in this case).

\section{VTE DIAGNOSIS}

\section{PB1175 | Detection of Right Ventricular Dysfunction in Acute Pulmonary Embolism by CT Scan: A Systematic Review and Meta- analysis}

N. Chornenki ${ }^{1}$; K. Poorzargar ${ }^{2}$; M. Shanjer ${ }^{2}$; L. Mbuagbaw ${ }^{2}$; M. Crowther ${ }^{2}$; A. Delluc ${ }^{3}$; D. Siegal ${ }^{3}$

${ }^{1}$ Queens University, Kingston, Canada; ${ }^{2}$ McMaster University, Hamilton, Canada; ${ }^{3}$ University of Ottawa, Ottawa, Canada

Background: Right ventricular (RV) dysfunction predicts worse outcomes in acute pulmonary embolism (PE). CT pulmonary angiography visualizes cardiac structures and may be a potential method for assessing RV without the need for transthoracic echocardiography. Aims: To conduct a systematic review and meta-analysis to assessing the diagnostic accuracy of CT scan findings for detecting RV dysfunction compared to echocardiography.

Methods: We searched MEDLINE and EMBASE from inception to April 2020 for studies comparing RV dysfunction on CT scan to echocardiography standard. Study quality was assessed with the QUADAS-2 risk of bias tool. Meta-analysis was performed using a bivariate mixed-effects regression framework.

Results: Overall, 26 studies (3,508 patients) were included. Indicators of RV dysfunction included reflux of contrast into the inferior vena cava (IVC reflux;4 studies; 445 patients), increased RV/ LV ratio (21 studies; 3,111 patients), and septal deviation (5 studies; 459 patients). Septal deviation had the highest specificity of 0.98 $(95 \% \mathrm{Cl}=0.90-1.00)$ a positive likelihood ratio of $13.6(95 \% \mathrm{Cl}=3.1-$ 60.4), and a positive predictive value of 0.92 (0.87-0.98) for RV dysfunction (Table 1). For combinations of measurements, any of septal deviation, IVC reflux, or RV/LV > 1.0 had $95 \%$ sensitivity and $88 \%$ specificity for RV dysfunction in one study $(n=59)$. Overall, most studies were judged to be at high risk of bias by the QUADAS-2 tool (Figure 1).

Conclusions: CT scanning is performed in most patients with suspected PE. RV dysfunction can be detected by CT but the diagnostic accuracy compared to echocardiography varies depending on the specific findings. The presence of septal bowing appears to be highly specific for RV dysfunction suggesting that echocardiography is not necessary when it is present. Although preliminary, our 
findings suggest that findings on CT suggestive of RV dysfunction may negate the need for echocardiography - access to which may be delayed or not available. Further studies are warranted.

\section{PB1176 | ISTH Definition of Pulmonary Embolism-related Death and Classification of the Cause of Death in Venous Thromboembolism Studies: An Autopsy Study}

$\underline{\text { T. Tritschler }}^{1}$; S.P. Salvatore ${ }^{2}$; S.R. Kahn ${ }^{3,4}$; D. Garcia ${ }^{5}$; A. Delluc ${ }^{6}$; N. Kraaijpoel ${ }^{7}$; N. Langlois ${ }^{6}$; P. Girard ${ }^{8}$; G. Le Gal ${ }^{6}$

${ }^{1}$ Department of General Internal Medicine, Inselspital, Bern University Hospital, University of Bern, Bern, Switzerland; ${ }^{2}$ Department of Pathology and Laboratory Medicine, Weill Cornell Medical College/ NewYork-Presbyterian Hospital, New York, United States; ${ }^{3}$ Department of Medicine, McGill University, Montreal, Canada; ${ }^{4}$ Divisions of Internal Medicine and Clinical Epidemiology, Jewish General Hospital/ Lady Davis Institute, Montreal, Canada; ${ }^{5}$ Division of Hematology, Department of Medicine, University of Washington, Seattle, United States; ${ }^{6}$ Department of Medicine, Ottawa Hospital Research Institute, University of Ottawa, Ottawa, Canada; ${ }^{7}$ Department of Vascular Medicine, Amsterdam UMC, University of Amsterdam, Amsterdam, Netherlands; ${ }^{8}$ Institut du Thorax Curie-Montsouris, Institut Mutualiste Montsouris, Paris, France

Background: The ISTH's SSC recently proposed a definition of pulmonary embolism (PE)-related death, aiming to increase the reproducibility of adjudication of death events in venous thromboembolism studies.

Aims: To evaluate the accuracy and interrater reliability of the ISTH definition of PE-related death.

Methods: We used data from an autopsy study that was performed at the NewYork-Presbyterian-Hospital between 01/2010 and $07 / 2019$. Included in this study were all patients with autopsyconfirmed PE-related death (cases) during that time frame, combined with patients who died in 2018 from a cause other than PE (controls). Based on clinical summaries which were retrospectively collected from the electronical health records and autopsy reports, two adjudicators independently, blinded to case-to-control ratio and autopsy results, determined the cause of death in each patient using the ISTH definition and classification (Figure). Patients with conflicting adjudications for cause of death were independently assessed by a third adjudicator. The primary outcome was autopsy-confirmed PE-related death. We determined the sensitivity and specificity of the ISTH definition for autopsy-confirmed PE-related death, and its interrater reliability using the percentage agreement and Cohen's kappa.
RECOMMENDATIONS AND GUIDELINES

Definition of pulmonary embolism-related death and classification of the cause of death in venous thromboembolism studies: Communication from the SSC of the ISTH

Tobias Tritschler $^{1,2} \odot$ | Noémie Kraaijpoel ${ }^{3}$ | Philippe Girard ${ }^{4,5}$ | Harry R. Büller ${ }^{3}$ | Nicole Langlois ${ }^{1}$ | Marc Righini ${ }^{6}$ | Sam Schulman ${ }^{7,8}$ | Annelise Segers ${ }^{9}$ | Grégoire Le Gal ${ }^{1} \odot$ | for the Subcommittee on Predictive and Diagnostic Variables in Thrombotic Disease

A. Pulmonary embolism (PE)-related death

A1. Autopsy-confirmed PE in the absence of another more likely cause of death

A2. Objectively confirmed PE before death in the absence of another more likely cause of death

Definition of objectively confirmed PE includes $\geq 1$ of the following situations in the last 48 hours* before death:

- PE diagnosed by imaging

- Objectively confirmed proximal deep vein thrombosis of the lower extremity in patients with clinical signs and symptoms of PE

A3. PE is not objectively confirmed, but is most likely the main cause of death

B. Undetermined cause of death

B1. Cause of death is undetermined, despite available information

B2. Insufficient clinical information available to determine the cause of death

C. Cause of death other than PE

*Longer time period may apply on a case-by-case basis.

FIGURE 1 ISTH definition for PE-related death and classification of the cause of death in venous thromboembolism studies

Results: A total of 126 deaths (median age, 68 years [range, 21-94], 60 [48\%] women) were adjudicated, of which 29 were autopsyconfirmed PE-related deaths. The ISTH definition's sensitivity and specificity for autopsy-confirmed PE-related death were $45 \%(95 \% \mathrm{Cl}$, 26-64\%) and 99\% (95\% Cl, 94-100\%), respectively. Interrater reliability for PE-related death was substantial (percentage agreement, 94\%; kappa, 0.73; 95\% Cl, 0.50-0.91; Table). When deaths classified in category $\mathrm{B}$ were also considered to be PE-related, sensitivity and specificity for autopsy-confirmed PE-related death were $83 \%$ (95\% $\mathrm{Cl}, 64-94 \%)$ and $74 \%(95 \% \mathrm{Cl}, 64-83 \%)$, respectively, and the interrater agreement was moderate (percentage agreement, 71\%; kappa, 0.41; 95\% Cl, 0.24-0.57). 
TABLE 2 Classification of the cause of death by adjudicator 1 (columns) and adjudicator 2 (rows)

\begin{tabular}{llllll} 
Category & A2 & A3 & B1 & B2 & C \\
$\begin{array}{c}\text { A2. Objectively } \\
\text { confirmed PE }\end{array}$ & 5 & - & - & - & - \\
$\begin{array}{c}\text { A3. PE most likely } \\
\text { the main cause } \\
\text { of death }\end{array}$ & 3 & 3 & 5 & - & - \\
$\begin{array}{c}\text { B1. Undetermined } \\
\text { despite } \\
\text { information }\end{array}$ & - & 1 & 19 & - & 13 \\
$\begin{array}{c}\text { B2. Insufficient } \\
\text { information }\end{array}$ & 1 & - & 9 & - & 4 \\
$\begin{array}{c}\text { C. Cause of death } \\
\text { other than PE }\end{array}$ & - & - & 20 & - & 43 \\
\hline
\end{tabular}

Abbreviation: PE, pulmonary embolism.

Subcategory A1 is not displayed, because the study design did not allow us to classify death events as autopsy-confirmed PE.

Conclusions: Adjudication of the cause of death using the ISTH definition results in very high specificity, moderate sensitivity and good interrater reliability for PE-related death.

\section{PB1177 | Comparing "Clinical Hunch" against Clinical Decision Scores (PERC Rule, Wells Score, Revised Geneva Score, YEARS Criteria) in Acute Pulmonary Embolism Diagnostics}

$\underline{\text { K. Medson }}{ }^{1}$; J. Yu ${ }^{2}$; L. Liwenborg ${ }^{1}$; E. Westerlund ${ }^{1}$; P. Lindholm ${ }^{1}$

${ }^{1}$ Karolinska Institutet, Stockholm, Sweden; ${ }^{2}$ Karolinska University

Hospital, Stockholm, Sweden

Background: Pulmonary embolism (PE) is a common and potentially life-threatening condition. Since it is considered a "do not miss" diagnosis, PE tends to be over-investigated beyond

the evidence-based clinical decision support systems (CDSS), which in turn exposes patients to unnecessary radiation and contrast agent exposure with no apparent benefits in terms of outcome.

Aims: Using computed tomography pulmonary angiogram (CTPA) as the gold standard for diagnosis of acute pulmonary embolism (PE), we evaluated the predictive performance of clinical hunch (gestalt) and four CDSS; PERC Rule, Wells score, revised Geneva score, and Years criteria.

Methods: A review was conducted on the Electronic Medical Records (EMR) of 1655 patients from the Emergency Department in a tertiary teaching hospital who underwent CTPA from 1 Jan 2018 to 31 Dec of 2019. Based on the data from EMR, the scores for the four CDSS was calculated retrospectively. The patients were divided into five groups: "clinical hunch", PERC rule, Wells score, revised Geneva score and YEARS criteria. We considered a CTPA ordered purely on a clinical hunch when there was no mention of CDSS in the EMR and no D-dimer.
Results: Of the total 1655 patients who underwent CTPA, 279 were positive for PE. The five groups' positive predictive value (PPV) were as follows - clinical hunch: $15 \%$, PERC rule: $18 \%$, Wells score: $21 \%$, revised Geneva score: 26\% [EW1] and YEARS criteria: 27\%. The negative predictive value (NPV) can be calculated for the CDS and were as follows: revised Geneva: 92\%, PERC rule: $93 \%$, Wells score: $93 \%$, and YEARS criteria: $94 \%$.

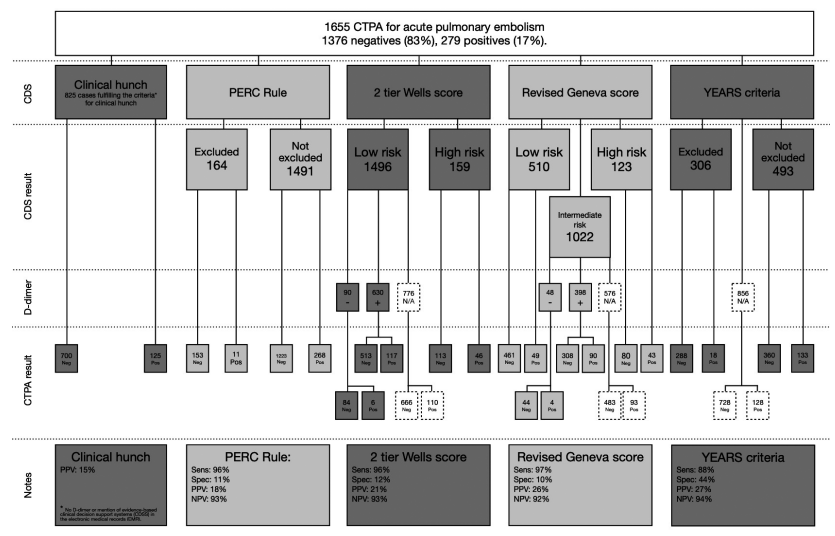

FIGURE 1 Flowchart evidence-based clinical decision support systems (CDSS)

Conclusions: Clinicians should trust the evidence-based clinical decision support systems in line with the international guidelines to diagnose pulmonary embolism. This due to the clearly higher PPV of CDSS compared to clinical hunch.

PB1178 | Pulmonary Embolism Diagnosis: Let's Practice what we Preach. A Quality Improvement Study to Increase Adherence to Evidence-based PE Diagnosis in the Emergency Department

F. Germini; F. Al-haimus; Y. Hu; S. Niaz; N. Clayton; S. Mondoux; Q. Ibrahim; L. Thabane; K. de Wit McMaster University, Hamilton, Canada

Background: We found that emergency physicians do not always use D-dimer for pulmonary embolism (PE) testing. We implemented $\mathrm{PE}$ testing pathway which required D-dimer testing for all patients suspected of having PE.

Aims: To evaluate the adherence to and clinical impact of the new PE testing pathway.

Methods: We enrolled consecutive adult patients tested for PE between January 2018 and January 2021 in two Canadian emergency departments. In November 2019, we implemented a new PE testing pathway. Outcomes before and after the intervention were compared using two-level generalized linear model, adjusting for patient age and gender, time of the day (day vs night-time), days of week (weekdays vs weekend), physician gender and years of experience 
and study site. Adjusted odds ratio (aOR) are presented with the relative $95 \%$ confidence intervals.

Results: 5085/70,911 (7.2\%) eligible patients were tested for PE before the intervention, 3854/36,530 (10.6\%) after, with an aOR of $1.42(1.35,1.50)$. The aOR for following the protocol was $3.10(2.53$, $3.80)$, for use of imaging $1.01(0.92,1.11)$, imaging positive yield 0.97 $(0.79,1.19)$, use of imaging in patients with a negative $D$-dimer 0.28 $(0.23,0.35)$, PE diagnosis among tested patients $0.98(0.81,1.19)$, central PE (segmental or more proximal) among all PEs 1.44 (0.80, 2.58). The false negative rate for $P E$ testing was $4 / 5085$ (0.08\%) before and $1 / 3854(0.03 \%)$ after intervention [percentage difference of $-0.05(-0.15,0.04)]$.

Conclusions: Implementation of a new PE testing pathway was associated with an increase in PE testing, improved adherence to Ddimer use and no change in imaging use or PE testing yield.

PB1179 | Agreement between Physician and Nurse Derived Wells Score as Part of the Assessment of Deep Vein Thrombosis in a Rapid Access Ambulatory Clinic

C. Martínez ${ }^{1,2}$; B. Rossignol ${ }^{3}$; M. Zwetkow ${ }^{3}$; V. Tagalakis ${ }^{1,4}$

${ }^{1}$ McGill University, Montreal, Canada; ${ }^{2}$ The Rosalind and Morris Goodman Cancer Research Centre, Montreal, Canada; ${ }^{3}$ Jewish General Hospital, Montreal, Canada; ${ }^{4}$ Centre for Excellence in Thrombosis and Anticoagulation, Division of General Internal Medicine, Jewish General Hospital, Montreal, Canada

Background: The Jewish General Hospital Clinical Access Service (CAS) is a rapid referral clinic for the diagnosis of deep vein thrombosis (DVT) in Montreal, Canada. Ambulatory patients with suspected DVT are referred with a physician completed Wells score. A modified Wells score $<2$ suggests DVT is unlikely and $\geq 2$ suggests DVT is likely. Depending on the score, patients may undergo d-dimer testing with or without venous doppler ultrasound (DUS) or DUS alone. The CAS registered nurses were recently trained to use the Wells score.

Aims: To determine correlation and agreement between physician (MD) and registered nurses (RN) assigned Wells scores in patients with suspected DVT in an outpatient setting.

Methods: RNs assessed the Wells score of 141 patients with suspected DVT between April 15thand December 28th, 2020. RNs were blinded to physician scores. Physician and registered nurses scores agreement was determined by Pearson Correlation. Proportions were used to describe concordance of unlikely $(<2)$ and likely $(\geq 2)$ scores.

Results: RNs assessed the Wells score of 141 patients with suspected DVT between April 15thand December 28th, 2020. RNs were blinded to physician scores. Physician and registered nurses scores agreement was determined by Pearson Correlation. Proportions were used to describe concordance of unlikely $(<2)$ and likely $(\geq 2)$ scores.
Table 1 Physician and registered nurse Wells score assignment and agreement

\begin{tabular}{|c|c|c|c|}
\hline & Physician scores & \multicolumn{2}{|c|}{ Registered nurse scores } \\
\hline $\begin{array}{l}\text { Modified } \\
\text { Wells } \\
\text { score }<2\end{array}$ & $63(44.7)$ & \multicolumn{2}{|l|}{$97(68.8)$} \\
\hline $\begin{array}{l}\text { Modified } \\
\quad \text { Wells } \\
\quad \text { score } \geq 2\end{array}$ & $78(55.3)$ & \multicolumn{2}{|l|}{$44(31.2)$} \\
\hline Total (n) & \multicolumn{3}{|l|}{141} \\
\hline \multicolumn{4}{|c|}{$\begin{array}{l}\text { Agreement between physician and registered nurse Wells scores } \\
\qquad(\mathrm{n}, \%)\end{array}$} \\
\hline & Concordance & Discordance & Total \\
\hline $\begin{array}{l}\text { Physician and } \\
\text { registered } \\
\text { n nurse } \\
\text { Wells } \\
\text { score < } 2\end{array}$ & $61(96.8)$ & $2(3.2)$ & $63(44.6)$ \\
\hline $\begin{array}{l}\text { Physician and } \\
\text { registered } \\
\text { n nurse } \\
\text { Wells } \\
\text { score } \geq 2\end{array}$ & $42(53.8)$ & $36(46.2)$ & 78 (55.3) \\
\hline
\end{tabular}

Conclusions: We observed moderate interobserver agreement between physician and RN for the use of Wells scores as part of a DVT assessment algorithm in a fast-track clinic. Discordance was more frequent among patients assessed with a high DVT risk. Future studies are needed to better understand the use of the Wells score in a fast-track outpatient clinic according to assessor.

PB1181 | Echocardiographic Parameters Predicted Short-term Outcomes in Acute Pulmonary Embolism

\section{Nadejda; T. Cuzor}

Institute of Cardiology, Chisinau, Moldova

Background: The role of transthoracic echocardiography (TTE) in acute pulmonary embolism (PE) remains incompletely defined. TTE is often considered for risk stratification of patients with acute pulmonary embolism (PE).

Aims: To determine the role of early TTE (within 24 hours of PE diagnosis) and the association between TTE findings for predicting adverse outcome in 7 days and during the first 3 months.

Methods: This was a prospective analysis of a single academic center, within the national research project 2020-2023, focused on patients with acute VTE.

Results: In this prospective study we included 84 of patients ( 59.3 +12.5 years $(62,9 \%$ men) with acute PE , of whom $80 \%$ underwent early TTE (during the first 24 hours). Of these $25 \%$ met the primary endpoint within 7 days, including 5,8\% deaths, 14\% systemic thrombolysis, $12 \%$ requiring vasopressor due to systemic hypotension, 
a patient develop $\mathrm{MI}$ of RV. In statistical analyses, right atrial (RA) enlargement (OR: 3.74; 95\% Cl, 3.50-6.0), right ventricular (RV) hypokinesis $32 \%$ (OR 1.94; 95\% Cl, 1.50-4.0) and right heart thrombi 9,2\% (OR 2.14; 95\% Cl, 1.50-3.0), high PASP (>50 mm Hg- 64\%) (OR $3.54 ; 95 \% \mathrm{Cl}, 2.50-6.3)$ were associated with adverse outcome during the first 14 days and the first 3 months of observation. In addition was included a new parameter as combined echocardiographic ratio of TAPSE divided by PASP (OR: 0.038, 95\% Cl 0.025-0,055, $P<0,0001$ ), which was significantly better than either TAPSE or PASP alone.

Conclusions: The association between 4 echocardiographic variables (RA enlargement $>3,75 \mathrm{~cm}$, RV hypokinesis, presence of right heart thrombi, decreased ratio TAPSE/PASP $<0,4$ ) constitute significant after adjustment for NYHA class and 6 min walk test distance. We hypothesized that these parameters may improve risk stratification and identification of the patients that will suffer short-term deterioration after high and intermediate-risk PE

PB1182 | A Simplified Decision Rule to Rule out Deep Vein Thrombosis Using Clinical Assessment and D-dimer

K. Xu' ${ }^{1}$ K. de Wit ${ }^{2,3}$; G.-J. Geersing ${ }^{4} ;$ T. Takada ${ }^{4} ;$ R. Schutgens ${ }^{4}$; J. Elf ${ }^{5}$; C. Kearon ${ }^{2,3} ;$ S. Parpia ${ }^{6,7}$.

${ }^{1}$ Department of Statistics and Actuarial Science, University of Waterloo, Kitchener, Canada; ${ }^{2}$ Department of Medicine, McMaster University, Hamilton, Canada; ${ }^{3}$ Thrombosis and Atherosclerosis Research Institute, McMaster University, Hamilton, Canada; ${ }^{4}$ Julius Centre for Health Sciences and Primary Care, University Medical Centre Utrecht, Utrecht, Netherlands; ${ }^{5}$ Department of Emergency Medicine, Lund University, Lund University Hospital, Lund, Sweden; ${ }^{6}$ Department of Oncology, McMaster University, Hamilton, Canada; ${ }^{7}$ Department of Health Research Methods, Evidence, and Impact, McMaster University, Hamilton, Canada

Background: Current clinical decision rules to exclude deep vein thrombosis (DVT) are under-utilized partly because of their complexity.

Aims: To develop a new simple clinical decision rule to safely exclude DVT using D-dimer and Wells-rule items.

Methods: We used individual patient data from four prospective outpatient diagnostic DVT studies. A bootstrap approach with logistic regression was used to select Wells-rule items that would form the new clinical decision rule. Risk groups were then defined based on combinations of the selected items. D-dimer was applied with predetermined thresholds of $<500 \mathrm{ng} / \mathrm{mL}$; 500 to $999 \mathrm{ng} / \mathrm{mL}$; and $\geq 1,000 \mathrm{ng} / \mathrm{mL}$ to rule out DVT. DVT was considered safely excluded if the upper $95 \%$ confidence interval (CI) of DVT prevalence was < $2 \%$.

Results: Four studies and 3368 patients were included in the analysis. Overall prevalence of DVT was $17 \%$. In addition to D-dimer, 'calf swelling $>3 \mathrm{~cm}$ ' and 'DVT as the most likely diagnosis' were included in the new rule. Based on these two variables, two clinical pre-test probability (CPTP) groups were defined; low (none of the two items present) and high (at least one of the items present). DVT can be safely excluded in patients with low CPTP with a D-dimer < $500 \mathrm{ng} / \mathrm{mL}$ (prevalence $=0.1 \%$; $95 \% \mathrm{Cl}$ : 0.0-0.8\%), low CPTP with a D-dimer between $500 \mathrm{ng} / \mathrm{mL}$ and $999 \mathrm{ng} / \mathrm{mL}$ (prevalence $=0.3 \%$; 95\% Cl: 0.0-1.7\%), and high CPTP with D-dimer $<500 \mathrm{ng} / \mathrm{mL}$ (prevalence $=0.3 \%$; $95 \% \mathrm{Cl}: 0.0-1.0 \%$ ).

Conclusions: We derived a simple clinical decision rule with 3 items as shown in Figure 1. The results suggest that the rule can safely exclude DVT. Prospective validation is required.

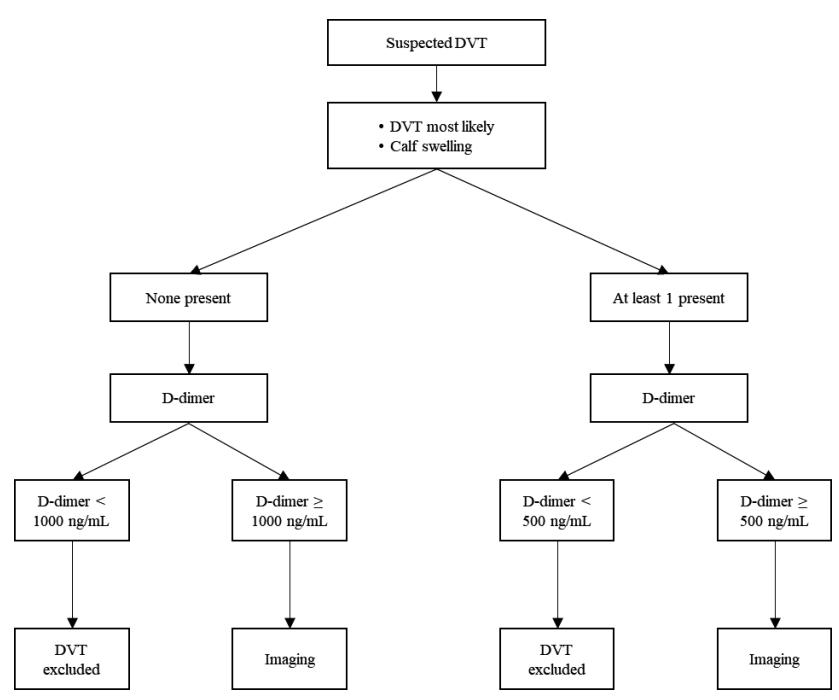

FIGURE 1 Diagnostic work-up for suspected deep vein thrombosis (DVT) using the new rule

PB1183 | D-dimer to Rule out Venous Thromboembolism during Pregnancy: A Systematic Review and Meta-analysis

M. Bellesini ${ }^{1,2}$; H. Robert-Ebadi ${ }^{1}$; C. Combescure ${ }^{3}$; C. Dedionigi ${ }^{2}$; G. Le Gal ${ }^{4}$; M. Righini ${ }^{1}$

${ }^{1}$ Division of Angiology and Hemostasis, Geneva University Hospitals and Faculty of Medicine, Geneva, Switzerland; ${ }^{2}$ Department of Medicine and Surgery, Research Center on Thromboembolic Disorders and Antithrombotic Therapies, University of Insubria, Varese and Como, Italy; ${ }^{3}$ Division of Clinical Epidemiology, Department of Health and Community Medicine, Geneva University Hospitals, Geneva, Switzerland; ${ }^{4}$ Department of Medicine, University of Ottawa, Ottawa Hospital Research Institute, Ottawa, Canada

Background: The usefulness of D-dimer measurement to rule out venous thromboembolism (VTE) during pregnancy is debated.

Aims: We performed a systematic review and meta-analysis to investigate the safety of D-dimer to rule out acute VTE in pregnant women with suspected pulmonary embolism (PE) and/or deep vein thrombosis (DVT). 
Methods: Two reviewers independently identified studies through Pubmed and Embase until February 2020, Week 1. We supplemented our search by manually reviewing reference lists of all retrieved articles, clinicalTrials.gov and reference literature. Prospective or retrospective studies in which a formal diagnostic algorithm was used to evaluate the ability of D-dimer to rule out VTE during pregnancy were eligible.

Results: We identified 576 references through systematic database and additional search strategies; 41 studies were retrieved in full, of which four were included, after applying exclusion criteria. Three studies were prospective, and one had a retrospective design. The three-month thromboembolic rate in pregnant women left untreated after a negative D-dimer was $1 / 312$ (0.32\%; $95 \% \mathrm{Cl}, 0.06-$ 1.83). The pooled estimate values were $99.5 \%$ for sensitivity $(95 \%$ $\left.\mathrm{Cl}, 95.0-100.0 ; \mathrm{I}^{2}, 0 \%\right)$ and $100 \%$ for negative predictive value $(95 \%$ $\left.\mathrm{Cl}, 99.19-100.0 ; \mathrm{I}^{2}, 0 \%\right)$. The prevalence of VTE and the yield of Ddimer were $7.4 \%\left(95 \% \mathrm{Cl}, 3.8-12 ; \mathrm{I}^{2}, 83 \%\right)$ and $34.2 \%(95 \% \mathrm{Cl}, 15.9$ $\left.55.23 ; I^{2}, 89 \%\right)$ respectively.

Conclusions: Our results suggest that D-dimer allows to safely rule out VTE in pregnant women with suspected VTE at low-intermediate pretest probability. However, data are limited, and further trials are needed to refine the optimal management strategy in this setting.

PB1184 | Diagnostic Accuracy of D-dimer for Splanchnic Vein Thrombosis: A Systematic Review and Meta-analysis

L.M. Attard ${ }^{1} ;$ K. Vella ${ }^{1} ;$ N. Riva ${ }^{1}$; J. Calleja Agius ${ }^{1} ;$ A. Gatt ${ }^{1}$;

A. Squizzato ${ }^{2}$

${ }^{1}$ University of Malta, Msida, Malta; ${ }^{2}$ University of Insubria Department of Medicine and Surgery, Varese/Como, Italy

Background: D-dimer is included in the diagnostic algorithm for deep vein thrombosis of the lower limbs and pulmonary embolism. However, its role for the diagnosis of splanchnic vein thrombosis (SVT) is still debated, especially considering that several conditions predisposing to SVT are associated with increased D-dimer (malignancy, abdominal surgery, liver cirrhosis).

Aims: To evaluate the accuracy of D-dimer in the diagnosis of SVT by performing a systematic review of the literature and meta-analysis. Methods: The protocol of this systematic review was registered a priori in PROSPERO (CRD42020184300). We performed a bibliographic search within the electronic databases MEDLINE, EMBASE, and CENTRAL up to July 2020, in order to identify all studies which evaluated D-dimer accuracy in patients with suspected SVT. The index test was any D-dimer assay. The reference standard was radiological imaging (abdominal ultrasound, computed tomography, magnetic resonance, angiography) or abdominal surgery. The QUADAS-2 checklist was used for the risk of bias assessment. We calculated pooled weighted mean specificity/sensitivity and positive/negative likelihood ratios.

Results: Among the 11 included studies, 9 enrolled only cirrhotic patients ( 5 of them post-surgery). Risk of bias was high in 9 studies.
Only 4 studies tested D-dimer within 24 hours from the reference standard, while 3 studies did not use the D-dimer manufacturers' cut-off.

Overall, mean prevalence of SVT was $34.6 \%(95 \% \mathrm{Cl}, 23.0-47.2 \%$, $\left.\mathrm{I}^{2}=95.1 \%\right)$. D-dimer sensitivity was $95.3 \%$ (95\% Cl, 70.0-99.4\%), specificity was $41.6 \%(95 \% \mathrm{Cl}, 15.4-73.6 \%)$. Positive likelihood ratio was 1.63 (95\% Cl, 0.99-2.68), negative likelihood ratio was 0.11 (95\% Cl, 0.02-0.54). The area under the ROC curve was 0.81 (95\% $\mathrm{Cl}, 0.78-0.84)$.

Conclusions: This systematic review showed high risk of bias and high heterogeneity in the published literature on this topic. Although D-dimer seems to have high sensitivity also for the diagnosis of SVT, there is strong need for more robust evidence.

\section{PB1185 | Clinical Implications of V/Q SPECT Scans Being Inconclusive in $20 \%$ of Patients Referred on Suspicion of Pulmonary Embolism}

K. Medson ${ }^{1}$; A. Hassler ${ }^{2}$; R. Taeri ${ }^{2}$; M. Bagge ${ }^{3}$; P. Lindholm ${ }^{1}$; E. Westerlund ${ }^{1}$

${ }^{1}$ Karolinska Institutet, Stockholm, Sweden; ${ }^{2}$ Karolinska University Hospital, Stockholm, Sweden; ${ }^{3}$ Danderyds Sjukhus, Stockholm, Sweden

Background: Pulmonary embolism (PE) is a common and potentially life-threatening condition. V/Q SPECT is one of the diagnostic procedures, but inconclusive results are causing problems in the clinical setting.

Aims: To analyze the further diagnostic workup and the initiation or change of anticoagulation treatment after an inconclusive V/Q SPECT in patients with suspected pulmonary embolism (PE).

Methods: A retrospective review of electronic medical records was made for all patients with suspected PE who underwent V/Q SPECT 2014-2019 at two teaching hospitals. The frequency of additional imaging with computed tomography pulmonary angiography (CTPA) and the resulting anticoagulation therapy (ACT) of patients was analyzed.

Results: A total of 865 patients with suspected PE were included, 574 acute and 291 chronic PE. 502 female and 363 male, age 19-99 years (mean 65, SD 19) for both groups. There were 131 (15\%) positive and 568 (66\%) negative cases for PE of V/Q SPECT. The remaining 166 cases (19\%) had an inconclusive result. 20\% $(n=112)$ in the acute and 19\% $(n=54)$ in the chronic PE group.

In the group with suspected acute PE and inconclusive V/Q SPECT result, only $9 \%(n=10)$ underwent further diagnostics with CTPA, which were all negative.

Of the remaining 102 patients, $10 \%(n=10)$ were already on a longterm therapeutic dose of ACT. $14 \%(n=14)$ were started on ACT and $76 \%(n=78)$ did not receive any ACT.

See the figure for the chronic PE group. 


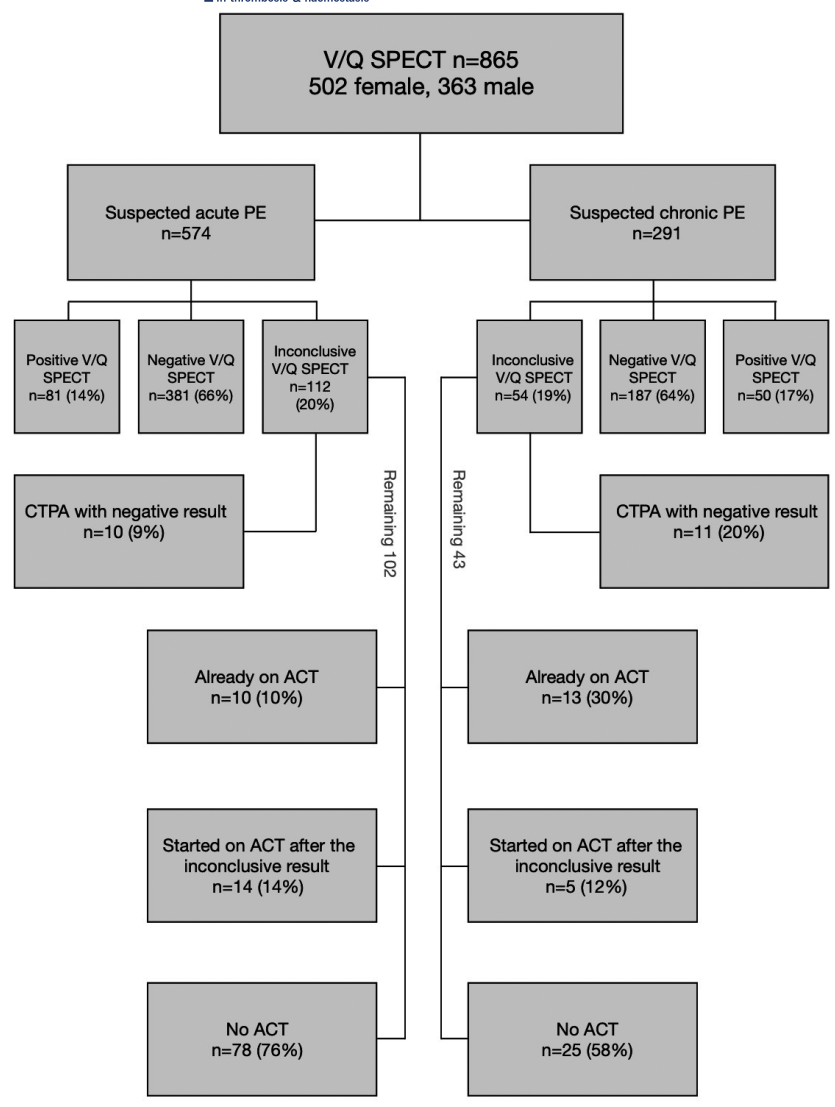

FIGURE 1 V/Q SPECT flow chart

Conclusions: Our study shows that $20 \%$ of patients with suspected PE receive an inconclusive V/Q SPECT result. The clinical consequences for this group were that most (76\%) were not treated with ACT. Considering the high mortality of pulmonary embolism, the need for a more accurate diagnostic method in patients with contraindication for CTPA is urgent.

\section{PB1186 | Predictors for Prognosis in Patients with Nonfatal Pulmonary Embolism in COVID-19 Pandemic}

N. Diaconu; T. Cuzor; D. Lupu; A. Grosu; L. Caldare Institut of Cardiology, Chisinau, Moldova

Background: Pulmonary embolism (PE) is a devastating clinical problem with the high mortality rate, including mortality due to recurrent PE.

Aims: The objective of this study was to determine predictors for short- and middle-term prognosis of patients with pulmonary embolism (PE) and the clinico-instrumental predictors of poor outcome.

Methods: This was a single-center prospective study of inpatients admitted in Institut of Cardiology of RM, with first-time PE (during 2020). Clinical data were collected from patients with objectively confirmed PE, and a 1-year follow up was conducted.

Results: Eighty-four patients with $\mathrm{PE}$, on age $59.3+12.5$ years $(62,9 \%$ men), were selected in the study. Pulmonary embolism was confirmed by CT angiography in all the patients, while DVT was confirmed by ultrasound in 34 patients. Study population was followed up for 9.7 months. Multivariate regression analysis was done where right ventricular (RV) diameter (mean $3.74 \mathrm{~cm}$ ), mean PASP (66 \pm 23 $\mathrm{mm} \mathrm{Hg}$ ), RV hypokinesis, presence of RV thrombi, decreased ratio TAPSE/PASP $<0,4(0.038,95 \% \mathrm{Cl}, 0.025-0,055, P<0,0001)$ measured by echoCG, d-dimer level at baseline $3615.5 \pm 420.3 \mathrm{ng} / \mathrm{mL}$ and number of comorbidities ( $3.4 \pm 0.7)$ entered the model. D-dimer level was revealed as a predictor for the length of hospitalization $(\beta=10,97, P=0.05)$ and active cancer $(\mathrm{OR}=6.142,95 \% \mathrm{Cl} 1.233-$ 30.587) and COVID history (OR-4,1, 95\% Cl, 4,3-80) were associated with a poor prognosis for acute $\mathrm{PE}$ in the short term. Cox regression analysis showed that elevated PASP( $\geqslant 55 \mathrm{mmHg})(\mathrm{HR}=6.240,95 \%$ $\mathrm{Cl}, 2.307-37.013)$ and active cancer with $\mathrm{PE}(\mathrm{HR}=3.700,95 \% \mathrm{Cl}$, 1.010-13.562) were associated with an increased risk of mid-term mortality after a follow-up period of 1 years.

Conclusions: Our results show that the baseline measurement of these parameters independently influence both the short-term and middle-term prognosis of patients with nonfatal PE.

\section{PB1187 | Elevated D-dimer Levels and DVT Following Neurosurgery}

M.T. Sartori ${ }^{1}$; A. Bozzolin ${ }^{1}$; G. Camporese ${ }^{2}$; P. Ciccarino ${ }^{3}$; F. Boscaro'; A. Rizzoli ${ }^{3} ;$ G. Avruscio ${ }^{2}$; F. Fabris ${ }^{1}$

${ }^{1}$ Clinical Medicine 1, Department of Medicine, University Hospital of Padova, Padova, Italy; ${ }^{2}$ Unit of Angiology, Department of Cardiac, Thoracic and Vascular Sciences, University Hospital of Padova, Padova, Italy; ${ }^{3}$ Neurosurgery, Department of Neuroscience, University Hospital of Padova, Padova, Italy

Background: Despite thromboprophylaxis, neurosurgical patients carry a high post-operative thromboembolic risk, due to various factors including paresis of lower extremity, immobilization, and hypercoagulability linked to neoplasia, trauma, steroids use. However, clinical signs and symptoms of DVT are often absent in these patients due to consciousness and/or neurological impairment.

Aims: To investigate whether elevated D-dimer levels may help diagnose post-surgery DVT in neurosurgical patients.

Methods: We conducted a retrospective observational study on all patients who underwent venous US for suspected DVT from elevated $D$-dimer levels after neurosurgery between January and August 2020. Patients were subdivided into 2 groups, DVT and non-DVT, and we compared the following parameters: clinical and demographic characteristics, type and duration of the surgical procedure, and D-dimer levels. Logistic regression analysis was used to ascertain any association between elevated $D$-dimer levels and risk of developing DVT in patients investigated between the $3^{\text {rd }}-15^{\text {th }}$ day after surgery. The ROC curve identified the cutoffs values for suspected DVT.

Results: Among 556 neurosurgical patients, 73 underwent postsurgery venous US for increased D-dimer levels and 35 had a DVT; 
between the $3^{\text {rd }}-15^{\text {th }}$ post-operative day, DVT occurred in 20/43 (46.5\%) patients. We found no association between DVT and all variables considered, except D-dimer levels, which were higher in patients who developed DVT. Logistic regression analysis revealed OR 1.98 (Cl 1.04-3.78, p 0.03) for each $1000 \mu \mathrm{g} / \mathrm{L}$ D-dimer increase. The ROC curve identified an optimal D-dimer cutoff $1334 \mu \mathrm{g} / \mathrm{L}$ (SEN

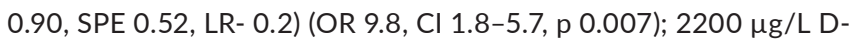
dimer levels were associated with the best positive predictive value (SPE 0.87, PPV 77\%, LR+ 3.8).

Conclusions: Elevated D-dimer levels were associated with increased risk of developing DVT after neurosurgery.

PB1188 | Diagnosing Deep Venous Thrombosis using Artificial Neural Networks and Metabolomics by ${ }^{1} \mathrm{H}-\mathrm{NMR}$ Data

T.D. Martins ${ }^{1}$; M. Quintero ${ }^{2,3}$; L. Tasic ${ }^{3}$; T.B.B.C. Costa ${ }^{3}$;

D. Stanisic ${ }^{3}$; S.A.L. Montalvão²; S.C. Huber²; E.V. de Paula²;

R.M. Filho ${ }^{4}$; J.M. Annichino-Bizzacchi ${ }^{2}$

${ }^{1}$ Universidade Federal de São Paulo, Diadema, Brazil; ${ }^{2}$ Hemostasis

and Thrombosis Laboratory, Hematology and Hemotherapy Center,

University of Campinas, Campinas, Brazil; ${ }^{3}$ Chemical Biology

Laboratory, Department of Organic Chemistry, Institute of Chemistry,

University of Campinas, Campinas, Brazil; ${ }^{4}$ School of Chemical

Engineering, University of Campinas, Campinas, Brazil

Background: The metabolic profile of a patient can changes when Deep Venous Thrombosis (DVT) takes place. It can be used on the disease's diagnosis and to develop of an artificial intelligence predictive model.

Aims: To obtain a neural network (ANN) to classify patients who have DVT (or not) based on their metabolic profile, obtained by Magnetic Resonance spectroscopy (NMR), and clinical data.

Methods: Table 1 summarizes the clinical patient characteristics. These patients presented a unique confirmed previous DVT of lower limbs or cerebral area and up to three years after the acute episode. Data were collected between 2015 and 2018. The inputs were: sex, age, body mass index, first and second-grade family history of a previous episode, diabetes, arterial hypertension, plus the considered metabolites (signal intensity at the respective chemical shift) lipids (unsaturated fatty acids, triacylglycerides, and glycerides), isoleucine, leucine, valine, lactate, alanine, and glucose. These metabolites were previously determined as important biomarkers using the liquid-state ${ }^{1} \mathrm{H}$-NMR data acquisition combined with chemometrics analysis. The ANNs had 56 inputs and the output was the DVT outcome. The best ANN was defined by a 3-fold cross-validation scheme. ANNs with one and two hidden layers were tested. The number of neurons ranged from 5 to 35 .
Table 1 Clinical summary of the patients.

\begin{tabular}{lll} 
& Thrombosis & Control \\
Number of Patients & 40 & 50 \\
Age (years) & $37.5(18-78)$ & $40(23-66)$ \\
Male (\%) & 20 & 26 \\
Average Body Mass & $25(19-60)$ & $24(18-36)$ \\
$\quad$ Index $\left(\mathrm{kg} / \mathrm{m}^{2}\right)$ & 23 & 0 \\
Family History of VTE & 9 & 8 \\
Obesity & 2 & 0 \\
Surgery & 3 & 0 \\
Gestacional/postpartum & 19 & 0 \\
Hormonal contraceptive & 19 & \\
\hline
\end{tabular}

Results: The best result was obtained with a two hidden layer ANN, containing 20 and 5 neurons in the first and second hidden layers, respectively. All the statistical parameters found for the best model are shown in Table 2. The model presented high ACC, AUC, TPR, and TNR. Also, it presented a low probability to indicate false results, which was confirmed by the LR+ and LR- values.

Table 2 Summary results obtained for the validation dataset

$\begin{array}{ll}\text { Performance Metric } & \text { Value } \\ \text { Accuracy (ACC) } & 93.3 \% \\ \text { AUC } & 0.982 \\ \text { Positive Predicted Value (PPV) } & 0.860 \\ \text { Negative Predicted Value (NPV) } & 1.000 \\ \text { True Positive Rate (TPR) } & 1.000 \\ \text { False Positive Rate (FPR) } & 0.110 \\ \text { True Negative Rate (TNR) } & 0.890 \\ \text { Positive Likelihood Ratio (LR+) } & 9.000 \\ \text { Negative Likelihood Ratio (LR-) } & 0.000\end{array}$

Conclusions: ANNs have potential to contribute to the diagnosis of DVT. If this model can be validated in another group of patients they can be used by clinicians on daily basis.

\section{PB1317 | Gender-specific Risk Prediction of Venous} Thromboembolism for Patients Admitted to Intensive Care Units (ICU)

I. Labbene $^{1,2}$; N. Mezghani ${ }^{3}$; W. Sellami ${ }^{1,2} ;$ A. Ghorbel ${ }^{4}$; F. Kadri ${ }^{4}$; N. Nouri ${ }^{4} ;$ M. Ferjani ${ }^{1,2}$

${ }^{1}$ Department of Anesthesiology and Critical care Medicine, Tunis, Tunisia; ${ }^{2}$ Research Laboratory LR12DN01, Faculté de Médecine de Tunis, Tunis, Tunisia; ${ }^{3}$ Laboratoire de Recherche en Imagerie et Orthopédie, TELUQ University, Montreal, Canada; ${ }^{4}$ Medilsys, Tunis, Tunisia

Background: Venous thromboembolism (VTE) is the third major cause of mortality in the world after heart attack and stroke. Most 
of patients admitted to ICUs are at high risk of VTE, especially with sedation, immobilization and mechanical ventilation. A number of studies have pointed out that woman present higher risk of VTE compared to men. That is why the prediction factors of such a risk should be analyzed seperatly according genders.

Aims: The purpose of this study is to develop a gender-specific computer-aided system to predict the risk of VTE complications, one hour early, for critically ill patients admitted to ICU.

Methods: All patients admitted to a 20 beds ICU between July 2019 and December 2020 were involved in the study. Exclusion criteria include patients with thromboembolic events prior to admission and patients having had other than a VTE-related event. Collected data included demographic characteristics, vital signs and laboratory measurements evaluated during the patient ICU stay. For each gender, the developed computer-aided system is based on logistic regression model to detect VTE complications risk one hour early.

Results: The performance of the developed computer-aided systems has been evaluated on a data set of 443 patients, 140 females (mean $63.8+/-17.3$ years) and 303 males (56.36 +/- 19.8 years). Results show that the retained discriminant features are different for male and female. The AUC is higher for female than for male (85\% against $71 \%)$. Moreover, the ability of the developed model to correctly predict a thromboembolic events reach $88 \%$ for females against $61 \%$ for males. The false negative prediction is higher for male (39\% against $12 \%$ for females).

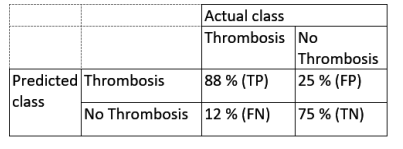

Performance of the female prediction model

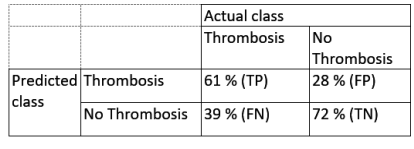

Performance of the male prediction model
FIGURE 1 The performances of the developed computer-aided systems

Conclusions: These results demonstrate

(1) the performances of the computer-aided system to predict one hour early the risk of VTE complications which could be useful to provide adequate therapeutic strategy and

(2) the predictions model is gender-specific.

P0184 | Thrombosis in Elderly: Watch out Antipsychotic Agents!

M. Mrouki; H. Hamitou; P. Tissot; I. Lusamvuku; S. Maillard;

C. Bonhoure

Centre Hospitalier Perpignan - Geriatric Department, Perpignan, France

Background: Venous thrombo-embolism (VTE) is a frequent condition especially in elderly. It's mainly caused by neoplastic disease and immobilization in these patients but other etiologies may occur. Often, VTE is caused by multifactorial causes. The iatrogenic etiology, such as antipsychotic agents, is not rare.
Aims: The aim of this study is to describe and explain the mechanism of VTE secondary to neuroleptics through a case-series.

Methods: This was a retrospective, longitudinal and descriptive study involving patients over 75 years old hospitalized in the geriatric department and having VTE secondary to a neuroleptic treatment between January 2020 and December 2020.

Results: Six patients were included. The average age was 85.3 (7597). All patients were women. It was an isolated deep vein thrombosis (DVT) in one case, a pulmonary embolism (PE) in 3 cases and both DVT and PE in one case. An isolated tachycardia was noted in 2 cases. Hypoxemia was noted in 2 cases. No electric signs of PE in ECG in any case. Three patients were having Risperidone, Loxapine, Haldol and Tiapride respectively were prescribed in one case each. No other etiology was found in any patient. The VTE seemed to be idiopathic in every case.

Conclusions: VTE secondary to antipsychotic medication was largely described but still controversial. The mechanism has not been fully understood. It can be direct since some antipsychotic agents are known to possibly raise the risk factors of recurrent VTE events or secondary to the sedative effect of these medications causing immobilization of the patient.

P0189 | Association of Complete Blood Parameters, D-dimer and Lupus Anticoagulant with VTE Risk Score in Hospitalized Patients

E. Ezigbo $^{1}$; I. Isiwu ${ }^{2}$; L. Nnamani ${ }^{2} ;$ T. Nwagha ${ }^{3}$

${ }^{1}$ Thrombosis \& Haemostasis Unit, Department of Medical Laboratory Sciences, University of Nigeria, Enugu Campus, Enugu, Nigeria; ${ }^{2}$ Department of Medical Laboratory Sciences, University of Nigeria Enugu Campus, Enugu, Nigeria; ${ }^{3}$ Department of Haematology, University of Nigeria Nsukka/University of Nigeria Teaching Hospital, Ituku Ozalla, Enugu, Nigeria

Background: A dose-related increased risk of VTE with higher monocyte count and red cell distribution width (RDW)has been reported in patients with thrombosis. The most appealing maker, the D-dimer assay is not without a high incidence of negative scans. Also, in a limited-resource setting as ours, screening methods emphasizing cost-effectiveness are needed.

Aims: To investigate the association of Complete Blood Count (CBC), D-dimer and Lupus Anticoagulant (LA) with VTE risk score in hospitalized patients at risk of VTE in Enugu State Nigeria.

Methods: The study populations include medical patients, surgical patients and healthy control subjects. The Caprini risk assessment model (RAM) was used for the study (figure 1). The University of Nigeria Research Ethics Committee approved the study. 
Results:

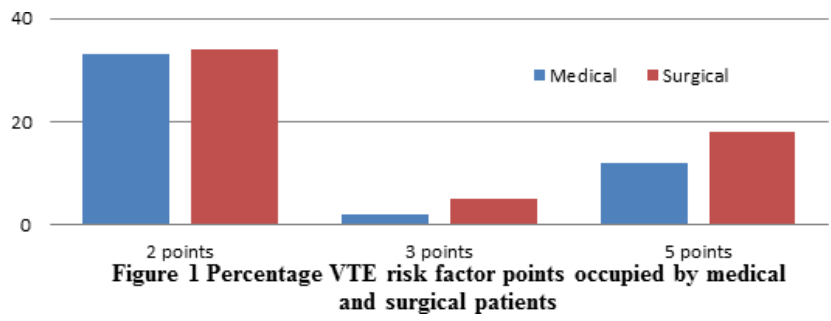

FIGURE 1

In the surgical patients, it was observed that all the assayed parameters gave no significant univariate association $(P<0.05)$ with a high risk of VTE, except $\mathrm{MCHC}$ (OR: 0.54, 95\% Cl: 0.30-0.98, $P=0.044$ ). After adjustment for sex, age, $\mathrm{BMI}$ and $\mathrm{ABO}$ blood group (multivariate model), the association still persist between $\mathrm{MCHC}$ and high risk of VTE (OR: $0.39,95 \% \mathrm{Cl}$ : 0.18-0.86, $P=0.020$ ), together with lymphocyte count (OR: $0.36,95 \% \mathrm{Cl}: 0.30-0.98, P=0.037$ ). The univariate associations between blood count parameters and high risk of VTE in medical patients gave no significant association $(P<0.05)$ in all the parameters.

Conclusions: This study showed an association between $\mathrm{MCHC}$ and VTE risk score, but more data and a follow-up study are needed to determine the endpoint development of VTE event in these patients.

Keywords: venous thromboembolism; complete blood count parameters; VTE Risk.

\section{VTE EPIDEMIOLOGY}

LPB0092 | Alteration of the Circulating Plasma Proteome by FXa Inhibition in Venous Thromboembolism and the Relationship with Clinical Outcome

A. Pallares Robles $^{1}$; V. ten Cate ${ }^{1,2} ;$ A. Schulz ${ }^{2} ;$ J.H Prochaska ${ }^{1,2,3}$; S. Rapp ${ }^{2}$; T. Köck ${ }^{2,3}$; S. Heitmeier ${ }^{4}$; S. Schwers ${ }^{4}$; K. Leineweber ${ }^{4}$; H.A. Ghofrani ${ }^{5}$; F.J. Meyer ${ }^{6}$; C. Espinola-Klein $;$; K.J. Lackner ${ }^{8,3}$; T. Münzel ${ }^{7,3,9} ;$ S.V. Konstantinides ${ }^{9,10}$; P.S. Wild ${ }^{1,2,3}$

${ }^{1}$ Clinical Epidemiology and Systems Medicine, Center for Thrombosis and Hemostasis (CTH), Mainz, Germany; ${ }^{2}$ Preventive Cardiology and Preventive Medicine, Department of Cardiology, University Medical Center of the Johannes Gutenberg University Mainz, Mainz, Germany; ${ }^{3}$ German Center for Cardiovascular Research (DZHK), Partner Site Rhine Main, University Medical Center of the Johannes Gutenberg University Mainz, Mainz, Germany; ${ }^{4}$ Bayer AG, Wuppertal, Germany; ${ }^{5}$ University Hospital Gießen and Marburg, Ambulance for Pulmonary Hypertension, Gießen, Germany; ${ }^{6}$ Lung Center Munich, München Klinik Bogenhausen, Department of Pneumology and Pneumological Oncology, München, Germany; ${ }^{7}$ Department of Cardiology - Cardiology I, University Medical Center of the Johannes Gutenberg University Mainz, Mainz, Germany; ${ }^{8}$ Institute of Clinical Chemistry and Laboratory Medicine, University Medical Center of the Johannes Gutenberg University Mainz, Mainz, Germany; ${ }^{9}$ Center for Thrombosis and Hemostasis (CTH), Mainz, Germany; ${ }^{10}$ Department of Cardiology, Democritus University of Thrace, Thrace, Greece

Background: Diverse studies have demonstrated non-haemostatic effects of factor $\mathrm{Xa}$ (FXa) inhibition.

Aims: To evaluate whether use of FXa inhibitors alters the concentration of circulating plasma proteins in patients with venous thromboembolism (VTE) in the acute phase and after 12 months of follow-up, compared to individuals not treated with anticoagulants before blood sampling.

Methods: Circulating levels of 444 proteins were measured by proximity extension assay in the acute setting of VTE (baseline) in 147 individuals treated with $\mathrm{FXa}$ inhibitors and in 89 individuals not receiving anticoagulants recruited in the GMP-VTE project, a multi-center, prospective cohort study on VTE. At the 12-month follow-up evaluation, plasma samples of 103 individuals treated with FXa inhibitors and 59 individuals not treated with anticoagulants were analyzed. LASSO-regularized logistic regression was used to identify plasma proteins altered by FXa inhibitors at both time points. Multivariable linear regression was used to assess the association of identified proteins with coagulation tests, and age and sexadjusted proportional hazards Cox regression was performed to test their associations with clinical outcome over 2 years of follow-up.

Results: At baseline, 19 proteins were identified as altered by FXa inhibition. At the 12-month follow-up examination, 6 proteins with altered levels were identified. The candidate proteins showed moderate procoagulant or anticoagulant effects as assessed with coagulation tests. Fibroblast growth factor-19 (Hazard ratio [HR]:0.56, 95\% Confidence Interval [CI]: 0.36-0.87), Brother of CDO (HR:1.76, 
95\% Cl:1.10-2.81) and granulysin (HR:1.75, 95\%Cl:1.26-2.45) concentrations were age and sex-independently associated with thromboembolic events or death. For clinically relevant bleeding, an age and sex independent association was found for tumour necrosis factor receptor-associated factor family member-associated NF-kB activator (HR:1.59, 95\% Cl: 1.05-2.41) only.

Conclusions: This investigation has identified candidate proteins related to FXa inhibition that are not directly related to coagulation, providing new insights into non-haemostatic mechanisms of action of FXa inhibitors in the setting of VTE.

LPB0139 | Association of FXI Activity with Thromboinflammation, Extracellular Matrix Interactions, Lipid Metabolism and Apoptosis in Venous Thromboembolism

A. Pallares Robles ${ }^{1}$; V. ten Cate ${ }^{1,2}$; A. Schulz ${ }^{2}$; J.H. Prochaska ${ }^{1,2,3}$; S. Rapp ${ }^{2}$; T. Köck ${ }^{2,3}$; M. Panova Noeva ${ }^{1,2}$; S. Heitmeier ${ }^{4}$;

S. Schwers ${ }^{4}$; K. Leineweber ${ }^{4}$; H.-J. Seyfarth ${ }^{5}$; C.F. Opitz ${ }^{6}$;

H. Spronk ${ }^{7}$; C. Espinola-Klein ${ }^{8}$; K.J. Lackner ${ }^{9,3}$; T. Münzel ${ }^{8,3,10}$; M.A. Andrade-Navarro ${ }^{11}$; S.V. Konstantinides ${ }^{10,12}$; H. ten Cate; P.S. Wild ${ }^{1,2,3}$

${ }^{1}$ Clinical Epidemiology and Systems Medicine, Center for Thrombosis and Hemostasis (CTH), Mainz, Germany; ${ }^{2}$ Preventive Cardiology and Preventive Medicine, Department of Cardiology, University Medical Center of the Johannes Gutenberg University Mainz, Mainz, Germany; ${ }^{3}$ German Center for Cardiovascular Research (DZHK), Partner Site Rhine Main, University Medical Center of the Johannes Gutenberg University Mainz, Mainz, Germany; ${ }^{4}$ Bayer AG, Wuppertal, Germany; ${ }^{5}$ Department of Pneumology, University of Leipzig, Leipzig, Germany; ${ }^{6}$ Department of Cardiology, DRK-Kliniken Westend, Berlin, Germany; ${ }^{7}$ Cardiovascular Research Institute Maastricht (CARIM), Maastricht University Medical Center, Maastricht, Netherlands; ${ }^{8}$ Department of Cardiology - Cardiology I, University Medical Center of the Johannes Gu-tenberg University Mainz, Mainz, Germany; ${ }^{9}$ Institute of Clinical Chemistry and Laboratory Medicine, University Medical Center of the Johannes Gutenberg University Mainz, Mainz, Germany; ${ }^{10}$ Center for Thrombosis and Hemostasis (CTH), University Medical Center of the Johannes Gutenberg University Mainz, Mainz, Germany; ${ }^{11}$ Institute of Organismic and Molecular Evolution, Johannes Gutenberg University Mainz, Mainz, Germany; ${ }^{12}$ Department of Cardiology, Democritus University of Thrace, Thrace, Greece

Background: Animal experiments and early phase human trials suggest that inhibition of factor Xla ( $\mathrm{FXla}$ ) safely prevents venous thromboembolism (VTE), and specific murine models of sepsis have shown potential efficacy in alleviating cytokine storm. These latter findings support the relevant role of FXI beyond coagulation.

Aims: To discover associations between FXI activity (FXI:c) and the plasma protein profile of patients with VTE that illustrate the role FXI beyond coagulation.

Methods: FXI activity was measured with a modified activated partial prothrombin time (aPTT) clotting time assay that used recombinant human thromboplastin by a coagulometric method. Proximity extension assay-based protein profiling was performed on plasma collected from subjects from the Genotyping and Molecular Phenotyping of Venous Thromboembolism (GMP-VTE) Project collected during an acute VTE event $(n=557)$ and 12 -months after the acute event $(n=194)$. Machine learning and bioinformatic methods were applied for the analysis of the resulting data.

Results: Mean \pm SD FXI:c levels were $199.5 \pm 35.1 \%$ in the acute VTE event and $82.3 \pm 35.2 \%$ at the 12 -month follow-up examination, reflecting its involvement in the thrombotic event. C-reactive protein (CRP) concentrations and platelet count positively correlated with FXI:c. Among the 444 proteins investigated, respectively 46 and 87 were associated with $\mathrm{FXI}: \mathrm{c}$ during the acute VTE event and at the 12-months follow-up examination. 17 proteins were identified as FXI:C-associated at both time points. The FXI:c-related proteins were enriched in immune pathways involved in thrombo-inflammation (IL-1 signaling, NF-KB signaling, TLR4 signaling, cytokine signaling and $B$ cell receptor signaling), extracellular matrix interactions, lipid metabolism and apoptosis.

Conclusions: This is an exploratory analysis evaluating the complex interactions of $\mathrm{FXI}: \mathrm{C}$ and the plasma protein profile in humans, illustrating its crosstalk with various systems. The results of this study offer important new avenues for future research into the multiple properties of FXIa, which are of high clinical interest given the current development of FXIa inhibitors.

LPB0140 | Long-term Survival after Venous Thromboembolism: A Prospective Cohort Study

H. Nilius ${ }^{1}$; T. Mertins ${ }^{1}$; R. Boss ${ }^{1} ;$ M. Knuchel ${ }^{1}$; E. Blozik ${ }^{2}$; J.A. Kremer Hovinga ${ }^{3}$; S. Eichinger ${ }^{4}$; M. Nagler ${ }^{1,3}$

${ }^{1}$ University of Bern / University Institute of Clinical Chemistry, Bern, Switzerland; ${ }^{2}$ Helsana Group/Department of Health Sciences, Zürich, Switzerland; ${ }^{3}$ Inselspital Bern University Hospital/Department of Hematology and Central Hematology Laboratory, Bern, Switzerland; ${ }^{4}$ Medical University of Vienna / Department of Medicine 1, Wien, Austria

Background: Little is known about long-term survival after the initial treatment of venous thromboembolism (VTE).

Aims: In a prospective cohort study, we aimed to assess the longterm mortality and key predictor variables relating to disease severity, treatment intensity, and comorbidities.

Methods: Between 1988 and 2018, 6'243 consecutive patients with previous VTE from a University outpatient unit were prospectively included and followed until December 2019; clinical characteristics, measures of disease severity, and treatment details were recorded. Dates of death were retrieved from the Swiss Central Compensation Office. Standardized mortality ratios (SMR) were computed using data from the Swiss Federal Statistics Office. Univariate and multivariate Cox proportional-hazard models were fitted to the data. 
Results: Two-hundred and fifty-four deaths occurred over an observation period of 57'212 patient-years. Compared to the Swiss population, the SMR was 1.30 (95\% confidence interval [CI] 1.14, 1.47; overall mortality rate: 4.44 per 1'000 patient-years). The following predictors were associated with increased mortality: Unprovoked VTE (hazard ratio [HR]: 5.06; 95\% Cl: 3.29, 7.77), transient triggering risk factors (HR: 3.46; $95 \% \mathrm{Cl}: 2.18,5.48$ ), previous VTE (HR 2.05; $95 \% \mathrm{Cl}: 1.60,2.62$ ), pulmonary embolism (HR: 1.45 , 95\% Cl: 1.10 , 1.89), permanent anticoagulant treatment (HR 3.14; $95 \% \mathrm{Cl}: 2.40$, 4.12), prolonged anticoagulant treatment (7-24 months; HR 1.70; $95 \% \mathrm{Cl}: 1.16,2.48)$, and cardiovascular comorbidities. Unprovoked VTE, previous VTE, permanent and prolonged anticoagulation remain independent risk factors after adjustment for age, sex, and comorbidities.

Conclusions: Survival after VTE was significantly reduced compared to the Swiss general population, especially in patients with more severe disease, cardiovascular comorbidities, and longer anticoagulant treatment. Close monitoring and reduced-intensity treatment schemes are potential targets to improve long-term care in patients with severe VTE.

PB1190 | Thromboembolism is Prevalent and Adversely Affects Overall Survival in Adults with Hemophagocytic Lymphohistiocytosis

J. Croden ${ }^{1}$; L. Bilston ${ }^{2}$; J. Grossman ${ }^{3}$; H. Sun ${ }^{4}$

${ }^{1}$ Department of Medicine, University of Alberta, Edmonton, Canada;

${ }^{2}$ Department of Medicine, University of Calgary, Calgary, Canada;

${ }^{3}$ Division of Hematology and Bone Marrow Transplantation, Department of Medicine, University of Calgary, Calgary, Canada; ${ }^{4}$ Division of Hematology, Department of Medicine, University of Alberta, Edmonton, Canada

Background: Hemophagocytic lymphohistiocytosis (HLH) is a rare syndrome characterized by uncontrolled immune activation that results in multi-organ dysfunction and a high risk of death. Minimal data exists on the prevalence of thromboembolism (TE) in HLH.

Aims: We aimed to report the cumulative incidence of TE in adults admitted with $\mathrm{HLH}$ and determine the impact of TE on survival.

Methods: We performed a multicentre retrospective cohort study, which included adults admitted with HLH (1999-2009) in Alberta, Canada. Potential cases were ascertained upon chart review by two independent reviewers. Overall survival (OS) was calculated using Kaplan-Meier estimates. The impact of TE on OS was assessed using the Cox proportional-hazards model.

Results: We identified 96 adults with HLH (median age 45 years, $67 \%$ male). The cumulative incidence of TE was $14 \%$, at a median of 9 (IQR 6-25) days from admission, including 3 (3\%) arterial and 10 (10\%) venous thromboembolism (VTE). The cumulative incidence of bleeding events was $41 \%$. Patients with VTE had significantly higher rates of major bleeding than those without ( $70 \%$ vs $28 \%$, $P=0.08$ ), which may be attributed to worse thrombocytopenia and coagulopathy (Table 1). In addition to hospitalization and immobility, all VTE patients had $\geq 1$ additional risk factor for developing VTE. Only 2 (20\%) and 4 (40\%) VTE patients received pharmacologic and mechanical thromboprophylaxis, respectively. Median OS was 18.0 months, with significantly worse OS in patients with vs without VTE (median 0.7 vs 30.8 months, log-rank $P=0.007$; Figure 1). VTE was independently associated with an increased hazard of death on multivariable Cox proportional-hazards regression (adjusted hazard ratio 2.35 , 95\% confidence interval 1.01-5.46).

Table 1 Demographics and disease characteristics of patients with or without VTE

\begin{tabular}{|c|c|c|c|}
\hline & VTE $(n=10)$ & No VTE $(n=86)$ & P-value \\
\hline Median age (IQR) & $61(50-71)$ & $43(32-61)$ & 0.036 \\
\hline Male sex, n (\%) & $6(60)$ & $58(67)$ & 0.73 \\
\hline HLH trigger & $3(30)$ & $28(33)$ & 0.89 \\
\hline - Malignancy & $5(50)$ & $31(36)$ & \\
\hline - Infection & $2(20)$ & $19(22)$ & \\
\hline $\begin{array}{l}\text { - Autoimmune } \\
\text { - Idiopathic }\end{array}$ & $0(0)$ & $8(9)$ & \\
\hline $\begin{array}{l}\text { Major bleeding } \\
\text { events, n (\%) }\end{array}$ & $7(70)$ & $24(28)$ & 0.012 \\
\hline Coagulation pro- & $1.5(0.4)$ & $1.3(0.5)$ & 0.23 \\
\hline file, mean (SD) & $43.4(10.3)$ & $36.7(9.5)$ & 0.074 \\
\hline - INR & $1.3(1.1)$ & $1.7(1.3)$ & 0.31 \\
\hline - aPTT & $20(23)$ & $42(72)$ & 0.041 \\
\hline $\begin{array}{l}\text { - Fibrinogen } \\
\text { - Nadir platelets } \\
\text { - D-dimer }\end{array}$ & $10.3(n=4)$ & $7.1(n=35)$ & 0.42 \\
\hline $\begin{array}{l}\text { ICU admission, } \\
\mathrm{n}(\%)\end{array}$ & $7(70)$ & $47(55)$ & 0.51 \\
\hline $\begin{array}{l}\text { Central venous } \\
\text { catheter, } \mathrm{n}(\%)\end{array}$ & $7(70)$ & $38(44)$ & 0.18 \\
\hline $\begin{array}{l}\text { Recent surgery, } \\
\text { n (\%) }\end{array}$ & 0 & $6(7)$ & 1.0 \\
\hline IVIG use, n (\%) & $2(20)$ & $30(35)$ & 0.49 \\
\hline OCP use, n (\%) & 0 & $1(1)$ & 1.0 \\
\hline
\end{tabular}

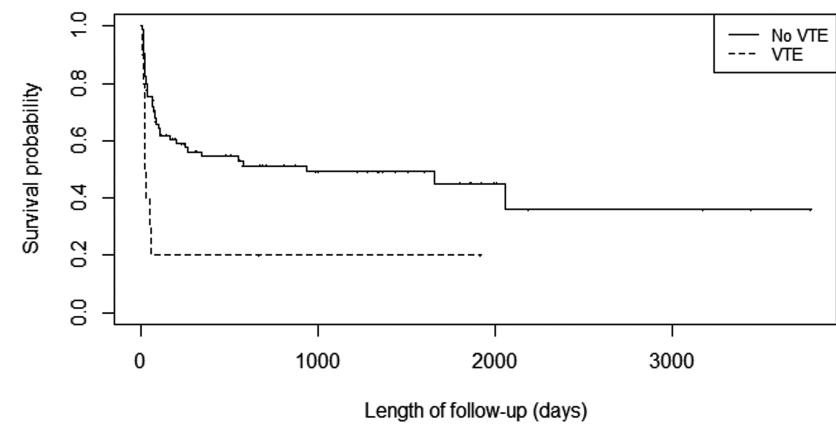

FIGURE 1 Overall survival of patients with or without VTE 
Conclusions: In adult patients with HLH, TE may be more common than previously described and is an independent predictor of increased mortality. Prevention and treatment of VTE are complicated by high bleeding rates in this patient population.

PB1191 | Erythrocytosis and Thromboembolic Events in Transgender Individuals Undergoing Masculinizing Therapy with Testosterone

M. Oakes; A. Arastu; C. Kato; J. Somers; H. Holly; G. Dy; T. Kohs; O. McCarty; V. Raghunathan; J. Shatzel Oregon Health \& Science University, Portland, United States

Background: Gender-affirming hormonal therapy, often including testosterone, is integral to transgender care. While secondary erythrocytosis is a well-recognized consequence of testosterone, the prevalence of erythrocytosis and its contribution to thrombotic risk in this population remain poorly defined.

Aims: To evaluate the incidence, sequelae, and management of erythrocytosis in transgender and non-binary (TGNB) individuals receiving testosterone.

Methods: A retrospective observational study of TGNB individuals $>18$ years receiving testosterone was undertaken from June 2017 to June 2020 at a university hospital in Portland, Oregon. We then performed an analysis of 14,294,784 hospitalizations from the 2016-17 US National Inpatient Sample (NIS).

Results: 923 transgender individuals receiving testosterone were identified in the university sample (mean age 29 years), with 519 having documented pre- and post-testosterone hemoglobin and hematocrit $(\mathrm{Hgb} / \mathrm{Hct})$. Testosterone cypionate was the most common formulation administered in $837 / 923$ patients (90.7\%). The mean baseline and peak Hgb was 13.5 and $15.7 \mathrm{~g} / \mathrm{dL}$, while the mean baseline and peak Hct were $40.2 \%$ and $47.0 \%$, respectively. The mean time-to-peak Hgb/Hct was 31.2 months. 41/519 patients (7.8\%) developed a hemoglobin $>17.5 \mathrm{~g} / \mathrm{dL}$, whereas 104/519 (20\%) developed a hematocrit of $>50 \%$. Ferritin values were available in 120 individuals with a mean of $50 \mathrm{ng} / \mathrm{mL}$. Dose reduction occurred in $42 \%$ of patients with erythrocytosis and $4.8 \%$ underwent phlebotomy Thromboembolic events occurred in $5 / 519$ patients $(0.9 \%)$, of which $4 / 5$ (80\%) had developed erythrocytosis, including two superficial vein thrombosis, two calf vein thrombosis, and one ischemic stroke. Analysis of the NIS identified 4141 admission involving transgender individuals. Of those, only seven were noted to have erythrocytosis, of which one had a concurrent venous thromboembolic event.

Conclusions: Hematocrit $>50 \%$ occurs in as many as 1 in 5 TGNB individuals undergoing masculinizing therapy with testosterone. Despite the high incidence of erythrocytosis, thromboembolic events and hospitalizations involving erythrocytosis were uncommon.
PB1192 | Venous Thromboembolism in Patients Hospitalized with COVID-19: Results from the Brazilian COVID-19 Registry

W.C. Silveira ${ }^{1}$; S.M. Rezende ${ }^{1}$; M.A.P. Martins ${ }^{1}$; M.C. Pires ${ }^{1}$; R.T. Silva ${ }^{1}$; L.E.F. Ramos ${ }^{1}$; M.V.R.S. Silva ${ }^{1}$; T.L.S. Sales ${ }^{2}$; C.C.R. Cimini ${ }^{3}$; M.H. Guimarães Júnior ${ }^{4}$; F.G. Aranha ${ }^{5}$; M.C.A. Nogueira ${ }^{6}$; L.B. Moreira ${ }^{7}$; T.O. Fereguetti ${ }^{8}$; P.L. Assaf ${ }^{9}$; M. Carneiro ${ }^{10}$; M.S. Marcolino ${ }^{1}$; S.F. Araújo ${ }^{11}$; A.V. Schwarbold ${ }^{12}$; G.A.N. Bastos ${ }^{13}$

${ }^{1}$ Federal University of Minas Gerais, Belo Horizonte, Brazil; ${ }^{2}$ Federal University of São João del-Rei, Divinópolis, Brazil; ${ }^{3}$ Hospital Santa Rosália, Teófilo Otoni, Brazil; ${ }^{4}$ Hospital Márcio Cunha, Ipatinga, Brazil; ${ }^{5}$ Hospital SOS Cárdio, Florianópolis, Brazil; ${ }^{6}$ Hospital Rede Mater Dei, Belo Horizonte, Brazil; ${ }^{7}$ Hospital das Clínicas de Porto Alegre, Porto Alegre, Brazil; ${ }^{8}$ Hospital Eduardo de Menezes, Belo Horizonte, Brazil; ${ }^{9}$ Hospital Metropolitano Doutor Célio de Castro, Belo Horizonte, Brazil; ${ }^{10}$ Hospital Santa Cruz, Santa Cruz do Sul, Brazil; ${ }^{11}$ Hospital Semper, Belo Horizonte, Brazil; ${ }^{12}$ Hospital Universitário de Santa Maria, Santa Maria, Brazil; ${ }^{13}$ Hospital Moinhos de Vento, Porto Alegre, Brazil

Background: COVID-19 patients are at increased risk of venous thromboembolism (VTE), and this complication leads to a worse prognosis. However, to diagnose VTE on COVID-19 patients is a challenge to physicians, as the symptoms of pulmonary embolism can often be mistaken for the overlapped viral pneumonia. Herein, there is still little information on VTE incidence and associated risk factors specifically for this population.

Aims: To assess the incidence and associated risk factors for VTE in hospitalized COVID-19 patients in Brazilian hospitals.

Methods: Retrospective multicenter cohort in 15 Brazilian hospitals. Consecutive adult patients ( $\geq 18$ years-old) with laboratoryconfirmed COVID-19 between March and September 2020 were included. Study data were collected from medical records using Research Electronic Data Capture (REDCap) tools. The study was approved by the National Research Ethics Commission waiving off the application of informed consent.

Results: Of 4,021 patients included, 234 (5.8\%) had VTE. When comparing VTE and non-VTE groups (Tables 1 and 2), there was no statistical difference in terms of sex and age between groups. The median age was 63 years-old (IQR 51-72 years-old) in VTE group. The most common comorbidities for both groups were hypertension and diabetes. Obesity, chronic pulmonary obstructive disease, previous VTE and recent surgery were more frequent in VTE group. D-dimer, C-reactive protein, lactate dehydrogenase levels and lymphocyte count were higher in the VTE group. Admission to intensive care units (37.6\% vs $69.7 \% ; P<0,001)$ and in-hospital mortality $(19.0 \%$ vs $28.3 \% ; P<0,01)$ were significantly higher in those who had VTE. 
Table 1 Main demographic features of the COVID-19 hospitalized patients cohort

\begin{tabular}{lllll} 
Characteristic & Total Number & no-VTE $(n=3,787)$ & VTE $(n=234)$ & $P$-value \\
Age (years) & 4,021 & $61.0(48.0,72.0)$ & $63.0(51.0,72.0)$ & 0.161 \\
$\begin{array}{l}\text { Male sex at birth } \\
\text { Comorbidities }\end{array}$ & 4,020 & $2,098(55.4 \%)$ & $126(53.8 \%)$ & 0.689 \\
$\quad$ Hypertension & & & \\
COPD & 4,021 & $2,059(54.4 \%)$ & $129(55.1 \%)$ & 0.874 \\
Diabetes mellitus & 4,021 & $228(6.0 \%)$ & $24(10.3 \%)$ & 0.014 \\
Obesity (BMI $\left.{ }^{3} 30 \mathrm{~kg} / \mathrm{m}^{2}\right)$ & 4,021 & $1,068(28.2 \%)$ & $74(31.6 \%)$ & 0.293 \\
Previous VTE & 4,021 & $683(18.0 \%)$ & $55(23.5 \%)$ & 0.044 \\
Surgery up to 90 days & 4,021 & $25(0.7 \%)$ & $5(2.1 \%)$ & 0.028 \\
\hline
\end{tabular}

Table 2 Main clinical characteristics, laboratory-values and outcomes of the COVID-19 hospitalized patients cohort

\begin{tabular}{|c|c|c|c|c|}
\hline Characteristic & Total No & no-VTE $(n=3,787)$ & VTE $(n=234)$ & $P$-value \\
\hline \multicolumn{5}{|l|}{ Laboratory parameters } \\
\hline D-dimer & 3,275 & $2.0(1.1,7.8)$ & $4.1(1.5,27.5)$ & $<0.001$ \\
\hline $\mathrm{C}$ reactive protein $(\mathrm{mg} / \mathrm{L})$ & 3,602 & $73.0(33.7,131.0)$ & $92.3(55.0,172.1)$ & $<0.001$ \\
\hline Lactate dehydrogenase & 2,565 & $376.0(273.0,514.0)$ & $407.0(328.0,603.0)$ & $<0.001$ \\
\hline Leukocytes count & 3,940 & $6.9(5.1,9.5)$ & $8.7(5.9,12.4)$ & $<0.001$ \\
\hline \multicolumn{5}{|l|}{ Outcomes } \\
\hline In hospital mortality & 4,013 & 719 (19.0\%) & $66(28.3 \%)$ & $<0.001$ \\
\hline Intensive care unit & 4,017 & 1,424 (37.6\%) & 163 (69.7\%) & $<0.001$ \\
\hline
\end{tabular}

Conclusions: Overall, $5.8 \%$ of COVID-19 hospitalized patients had VTE. Elevated laboratory values were associated with increased risk of this condition. VTE was associated with higher rates of intensive care admission and in-hospital mortality.

PB1193 | Association between Cardiovascular Risk Factors and Venous Thrombosis in the Elderly

$\underline{\text { H. Wang }}{ }^{1}$; M. Cushman ${ }^{2}$; F.R Rosendaal ${ }^{1}$; A. van Hylckama Vlieg ${ }^{1}$

${ }^{1}$ Department of Clinical Epidemiology, Leiden University Medical Center, Leiden, Netherlands; ${ }^{2}$ Department of Medicine, Larner College of Medicine at the University of Vermont, Vermont, United States

Background: Several cardiovascular risk factors have been associated with the risk of venous thrombosis (VT) in young and middleaged populations.

Aims: To investigate whether cardiovascular risk factors (BMI, smoking and alcohol intake) are associated with the risk of VT in elderly and to assess the combined effect between genetic risk factors for VT (factor V Leiden (FVL)/prothrombin 20210A (PT20210), positive family history of VT and non-O blood group) and cardiovascular risk factors.

Methods: Analyses were performed in the AT-AGE study, a multicenter case-control study performed in Vermont, USA and Leiden, $\mathrm{NL}$, comprising of 401 cases with a first VT and 431 control subjects, all aged $\geq 70$ years. To assess the risk of VT, odds ratios (OR) with 95\% confidence intervals( $\mathrm{Cl})$ were calculated, after adjustment for potential confounders. All participants provided written informed consent. The study was approved by the Medical Ethical Committee of the Leiden University Medical Center and by the Committee of Human Research of the University of Vermont.

Results: BMI was positively associated with the risk of VT:OR 1.2 (95\%Cl: 0.8-1.9) for BMl>30 kg/m² compared with $\mathrm{BMI}<25 \mathrm{~kg} / \mathrm{m}^{2}$. This risk was more pronounced for DVT rather than PE and for unprovoked VT (OR: 1.6-1.7). No association with VT was observed for smoking and alcohol intake (Table 1 ). In the presence of a genetic predisposition, individuals with a $\mathrm{BMI}<30 \mathrm{~kg} / \mathrm{m}^{2}$ had a similar risk of VT compared with individuals with a BMI>30 kg/m². Similarly, for smoking and alcohol intake, in the presence of a genetic risk factor, the cardiovascular risk factor didn't further affect the risk of VT (Table 2). 
Table 1 The risk of venous thrombosis associated with cardiovascular risk factors.

\begin{tabular}{|c|c|c|c|c|c|c|}
\hline Risk factor & $\begin{array}{l}\text { OR crude } \\
(95 \% \mathrm{Cl})\end{array}$ & $\begin{array}{l}\text { OR overall* } \\
(95 \% \mathrm{Cl})\end{array}$ & $\begin{array}{l}\text { OR DVT* } \\
(95 \% \mathrm{Cl})\end{array}$ & $\begin{array}{l}\text { OR PE } \pm D V T^{*} \\
(95 \% \mathrm{Cl})\end{array}$ & $\begin{array}{l}\text { OR provoked* } \\
(95 \% \mathrm{Cl})\end{array}$ & $\begin{array}{l}\text { OR unprovoked* } \\
(95 \% \mathrm{Cl})\end{array}$ \\
\hline \multicolumn{7}{|l|}{$\mathrm{BMI}\left(\mathrm{kg} / \mathrm{m}^{2}\right)^{\#}$} \\
\hline Healthy & 1 (ref) & 1 (ref) & 1 (ref) & 1 (ref) & 1 (ref) & 1 (ref) \\
\hline Obese & $1.1(0.8-1.7)$ & $1.2(0.8-1.9)$ & $1.7(0.9-3.1)$ & $0.9(0.6-1.6)$ & $0.9(0.5-1.6)$ & $1.6(0.9-2.8)$ \\
\hline \multicolumn{7}{|l|}{ Smoking } \\
\hline Never+former & 1 (ref) & 1 (ref) & 1 (ref) & 1 (ref) & 1 (ref) & 1 (ref) \\
\hline Current & $0.8(0.5-1.3)$ & $0.7(0.5-1.2)$ & $1.0(0.5-1.8)$ & $0.6(0.3-1.0)$ & $0.5(0.3-1.0)$ & $1.0(0.6-1.8)$ \\
\hline \multicolumn{7}{|l|}{ Alcohol intake } \\
\hline No & 1 (ref) & 1 (ref) & 1 (ref) & 1 (ref) & 1 (ref) & 1 (ref) \\
\hline Yes & $0.9(0.7-1.2)$ & $1.1(0.8-1.5)$ & $1.4(0.9-2.3)$ & $1.0(0.7-1.4)$ & $0.8(0.5-1.2)$ & $1.5(1.0-2.3)$ \\
\hline
\end{tabular}

Table 2 Combined effect of cardiovascular risk factors and a genetic predisposition (combined) on the risk of venous thrombosis.

\begin{tabular}{lllll}
$\begin{array}{l}\text { Genetic } \\
\text { predisposition(combined) }\end{array}$ & Cardiovascular risk factors & $\mathrm{OR}_{\text {obesity }}{ }^{* \#}(95 \% \mathrm{Cl})$ & $\mathrm{OR}_{\text {smoking }}{ }^{*}(95 \% \mathrm{Cl})$ & $\mathrm{OR}_{\text {alcohol }}{ }^{*}(95 \% \mathrm{Cl})$ \\
No & no & 1 (ref) & $1(\mathrm{ref})$ & $1(\mathrm{ref})$ \\
No & yes & $2.0(0.8-4.8)$ & $0.6(0.3-1.3)$ & $0.7(0.4-1.3)$ \\
Yes & no & $2.2(1.2-4.1)$ & $1.7(1.1-2.5)$ & $1.3(0.7-2.2)$ \\
Yes & yes & $2.5(1.4-4.3)$ & $1.5(0.8-2.8)$ & $1.6(1.0-2.8)$ \\
\hline
\end{tabular}

Conclusions: In the elderly, BMI was associated with the risk of VT, while smoking and alcohol intake were not. In the presence of genetic predisposition, cardiovascular risk factors did not affect the risk of VT.

PB1194 | Premature Mortality Burden Related to Pulmonary Embolism in Cuba

K. Valdés-Díaz; H. Hernández-Negrín

Arnaldo Milian Hospital, Santa Clara, Cuba

Background: Despite improvements in recent decades, mortality related to pulmonary embolism remains high and may be exhibiting an increasing trend in some low- and middle-income countries. However, little research has been done on the premature mortality burden related to pulmonary embolism in Cuba.

Aims: To identify the premature mortality burden related to pulmonary embolism in Cuba.
Methods: Data were obtained from the mortality database of the Ministry of Public Health of Cuba (International Classification of Diseases-10, codes: 126.x, 180.x, 182.2, 182.4, 182.6, 182.8, 182.9, O22.2, O22.3, O22.9, O87.0, O87.1, 087.9 and O88.2, as underlying cause of death) between 2015-2018. Years of life lost (YLL) were calculated using the standard life expectancy of the WHO's global health estimates. Age- and sex-specific YLL rates were calculated using the Cuban mid-year population estimates and the agestandardized YLL rate (ASYR) was estimated by the direct method using the WHO's standard population.

Results: A total of 2292 pulmonary embolism-related deaths were identified, 1131 (49.3\%) male and 1161 (50.7\%) female. The median age at death in men was 75 years (interquartile range[IR]): 63 to 83) and in women 77 years (IR: 66 to 86). A total of 40568 YLL were accumulated, with an average of 17.7 YLL per death. The absolute YLL count and crude YLL rate according to age, sex and causes of death are shown in Figure 1 and Table 1. ASYR between 2015 and 2018 increased by $90.6 \%$ in men (43 vs 82 YLL per 100000 inhabitants) and by $52 \%$ in women (48 vs 73 YLL per 100000 inhabitants).

TABLE 1 YLL number and crude YLL rate due to premature mortality related to pulmonary embolism by cause of death. Cuba, 2015-2018

\begin{tabular}{|c|c|c|c|c|c|c|c|}
\hline & & & & & & & \\
\hline \multirow[b]{2}{*}{ Cause of death } & \multirow[b]{2}{*}{ ICD-10 codes } & \multicolumn{2}{|l|}{ Male } & \multicolumn{2}{|l|}{ Female } & \multicolumn{2}{|l|}{ Total } \\
\hline & & $\overline{Y L L}$ & crudeYLL rate & $\overline{Y L L}$ & crudeYLL rate & $\overline{Y L L}$ & crudeYLL rate \\
\hline \multicolumn{8}{|l|}{ Pulmonary embolism } \\
\hline Pulmonary embolism & $126 . x$ & 14138 & 0.63 & 11990 & 0.53 & 26128 & 0.58 \\
\hline $\begin{array}{l}\text { Acute embolism and } \\
\text { thrombosis of specified } \\
\text { or unspecified veins }\end{array}$ & $\begin{array}{l}182.2,182.4 \\
182.6 \\
182.8,182.9\end{array}$ & 1400 & 0.06 & 1321 & 0.06 & 2721 & 0.06 \\
\hline
\end{tabular}




\begin{tabular}{|c|c|c|c|c|c|c|c|c|}
\hline ABSTRACT & & & & & & & & 877 of 972 \\
\hline $\begin{array}{l}\text { Obstetric } \\
\text { thromboembolism }\end{array}$ & 088.2 & - & - & 467 & 0.02 & 467 & 0.02 & \\
\hline Limb vein thrombosis & & & & & & & & \\
\hline $\begin{array}{l}\text { Deep vein thrombosis in } \\
\text { pregnancy }\end{array}$ & O22.3 & - & - & 181 & 0.01 & 181 & 0.01 & \\
\hline $\begin{array}{l}\text { Phlebitis and } \\
\text { thrombophlebitis }\end{array}$ & $180 . x$ & 5493 & 0.24 & 5439 & 0.24 & 10932 & 0.24 & \\
\hline
\end{tabular}

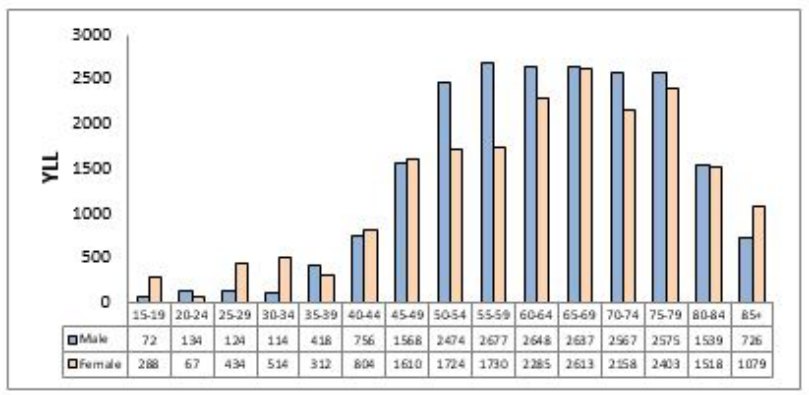

a-) YLL number.

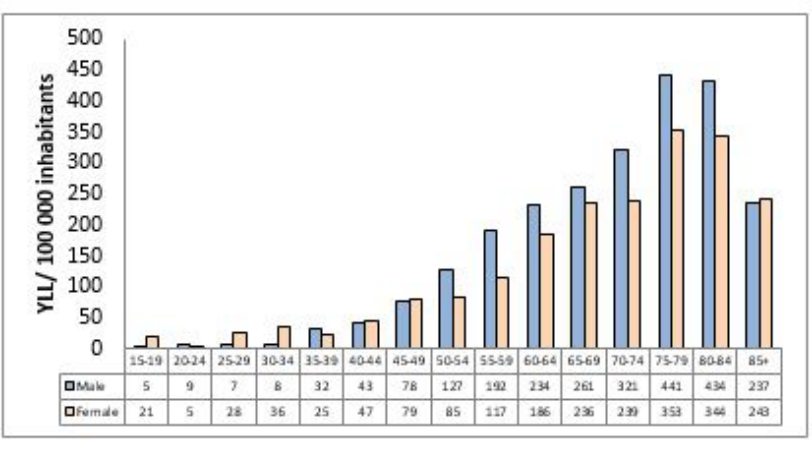

b-) Crude YLL rate.

FIGURE 1 Premature mortality related to pulmonary embolism by age and sex. Cuba, 2015-2018

Conclusions: Premature mortality burden related to pulmonary embolism in Cuba is high and appears to be increasing, affecting more males and the elderly. Further epidemiological research is needed to improve our understanding of this condition and its impactin our setting.
PB1195 | Location and Burden of Extremity Vein Thrombosis in Patients with Solitary versus Multiple Subsegmental Pulmonary Emboli

R. Meverden ${ }^{1}$; Y. Hirao-Try ${ }^{1}$; D. Vlazny ${ }^{1}$; A. Casanegra ${ }^{1}$;

D. Houghton ${ }^{1}$; D. Hodge ${ }^{2}$; L. Peterson ${ }^{1}$; R. McBane ${ }^{1}$;

W. Wysokinski ${ }^{1}$

${ }^{1}$ Mayo Clinic, Rochester, United States; ${ }^{2}$ Mayo Clinic, Jacksonville, United States

Background: Subsegmental pulmonary embolism (SSPE) is relatively benign and can be managed with surveillance if bleeding risk is high and there are no other thromboses. SSPE may involve one or more subsegmental branches. It remains unexplored if solitary versus multiple SSPE have significant coexistence with upper and lower extremity deep vein thrombosis (DVT).

Aims: The aim of the study was to compare the location and burden of DVT in upper and lower extremities in solitary versus multiple SSPE.

Methods: Consecutive patients with SSPE anticoagulated at Mayo Clinic Thrombophilia Clinic (03/01/2013-12/31/2020) were followed prospectively.

Results: Out of 1542 patients with PE, 1317 patients had proximal PE and 225 (14.6\%) SSPE, either solitary $(n=139)$ or multiple ( $n=86 ; 47$ with bilateral and 39 unilateral emboli). Of the proximal PE, 670 (50.9\%) had coexisting DVT compared to 68 (30.2\%) with SSPE $(P<0.001)$. Proportion of patients with upper extremity DVT was not significantly different in solitary SSPE group compared to multiple SSPE group (21.6\% vs 6.9\%, $P=0.166)$. Proportion of patients with lower extremity DVT was not significantly different in multiple SSPE group compared to solitary SSPE group $(96.6 \%$ vs $83.8 \%, P=0.124)$. No DVT in the axial iliac-common femoral venous segment was found (one DVT in internal iliac vein) in solitary SSPE group versus 4 in multiple SSPE group $(P=0.033)$. Thrombus burden assessed by a specially designed scoring system (Table 2 ) revealed more extensive DVT burden in multiple SSPE compared to solitary SSPE group (5.7 vs 2.4, $P=0.008$ ).

VTE recurrence, major bleeding, clinically relevant non-major bleeding, and mortality was not different in SSPE with DVT compare to those without DVT (data not shown). 
Table 1 Extremity deep vein thrombosis (DVT) location and burden in patients with solitary versus multiple subsegmental pulmonary emboli (SSPE).

\begin{tabular}{|c|c|c|c|c|}
\hline DVT Location & $\begin{array}{l}\text { Solitary } \\
N=37\end{array}$ & $\begin{array}{l}\text { Multiple } \\
N=29\end{array}$ & $\begin{array}{l}\text { Total } \\
N=66\end{array}$ & $P$-value ${ }^{a}$ \\
\hline Iliac or Common Femoral Veins, n (\%) & $1(2.7 \%)$ & $5(17.2 \%)$ & $6(9.1 \%)$ & 0.079 \\
\hline $\begin{array}{l}\text { Common Iliac, External Iliac, or } \\
\text { Common Femoral Veins, } \mathrm{n}(\%)\end{array}$ & $0(0.0 \%)$ & $4(13.8 \%)$ & $4(6.1 \%)$ & 0.033 \\
\hline Femoral or Popliteal Veins, n (\%) & $13(35.1 \%)$ & $12(41.4 \%)$ & $25(37.9 \%)$ & 0.620 \\
\hline Calf Veins, $\mathrm{n}(\%)$ & $25(67.6 \%)$ & $22(75.9 \%)$ & $47(71.2 \%)$ & 0.586 \\
\hline Lower Extremity Veins, n (\%) & $31(83.8 \%)$ & $28(96.6 \%)$ & $59(89.4 \%)$ & 0.124 \\
\hline Upper Extremity Veins, n (\%) & $8(21.6 \%)$ & $2(6.9 \%)$ & $10(15.2 \%)$ & 0.166 \\
\hline Upper or Lower Extremity Veins, n (\%) & $37(100 \%)$ & $29(100 \%)$ & $66(100 \%)$ & 0.170 \\
\hline \multirow{2}{*}{$\begin{array}{l}\text { Extremity DVT burden score: Mean } \\
\quad(\mathrm{SD})^{\text {b }} \text {, Range }\end{array}$} & $2.4(2.0)$ & $5.7(6.6)$ & $3.8(4.9)$ & \multirow[t]{2}{*}{0.008} \\
\hline & $0.5-11.0$ & $0.5-30.0$ & $0.5-30.0$ & \\
\hline
\end{tabular}

${ }^{a} \mathrm{P}$-values result from analysis of variance for continuous variables and Chi square test for categorical variables

${ }^{\mathrm{b}} \mathrm{SD}=$ standard deviation

Table 2 Extremity deep vein thrombosis (DVT) burden scoring system.

\begin{tabular}{|c|c|c|c|}
\hline DVT Location & $100 \%$ Vein Involvement & $50-99 \%$ Involvement & $<50 \%$ Involvement \\
\hline Iliac (Common, External, Internal) & 5 & 3 & 2 \\
\hline Innominate, Subclavian, Common Femoral, Popliteal & 3 & 2 & 1 \\
\hline \multirow[t]{2}{*}{ Femoral divided into Proximal, Middle, Distal } & Each segment $=3$ & & \\
\hline & $100 \%$ Vein Involvement & $<100 \%$ Involvement & \\
\hline $\begin{array}{l}\text { Axillary, Brachial, Internal Jugular, Tibial (Anterior, } \\
\text { Posterior), Peroneal, Soleal, Gastrocnemius }{ }^{c}\end{array}$ & 1 & 0.5 & \\
\hline \multirow[t]{2}{*}{ Superficial Vein Thrombus Extending to Deep Vein Location } & Sapheno-femoral junction = 2 & & \\
\hline & Sapheno-Popliteal junction = 1 & & \\
\hline
\end{tabular}

Conclusions: Extremity thrombus burden is significantly higher in multiple SSPE compared to solitary SSPE. DVT involving the axial iliac-common femoral segment is associated with multiple SSPE. Upper and lower extremity DVT were evenly distributed between groups.
PB1196 | The Roles of Individual and Neighborhood Income on Regional and Racial Differences in Risk of Venous Thromboembolism in the United States: The REasons for Geographic and Racial Differences in Stroke Study (REGARDS)

J. Munger ${ }^{1}$; K. Wilkinson ${ }^{2}$; I. Koh ${ }^{2}$; L. Long ${ }^{3}$; G. Lovasi ${ }^{4}$; L. McClure ${ }^{4}$; J. Brown ${ }^{4}$; G. Howard ${ }^{3}$; V. Howard ${ }^{3}$; T. Plante ${ }^{1,5}$; M. Cushman ${ }^{2,5}$; N. Zakai ${ }^{2,5}$

${ }^{1}$ University of Vermont, Larner College of Medicine, Burlington, United States; ${ }^{2}$ Department of Pathology \& Laboratory Medicine, Larner College of Medicine at the University of Vermont, Burlington, United States;

${ }^{3}$ School of Public Health at the University of Alabama at Birmingham, Birmingham, United States; ${ }^{4}$ Dornsife School of Public health at Drexel University, Philadelphia, United States; ${ }^{5}$ Department of Medicine, Larner College of Medicine at the University of Vermont, Burlington, United States

Background: In the United States (U.S.), Black individuals have higher venous thromboembolism (VTE) risk than White individuals, with the highest disparity in the Southeast.

Aims: To determine whether individual income (I-income) and neighborhood income ( $\mathrm{N}$-income) explain the race and region differences VTE risk. 
Methods: From 2003-2007, REGARDS, a U.S. national cohort study, enrolled 30,239 Black and White participants aged 45+ years and followed them for incident VTE through 2011. N-income was estimated using census records and I-income was self-reported in categories. The race-specific hazard ratio (HR) of VTE by region was estimated with cox proportional hazards models adjusting for age, sex, race, region, body mass index, C-reactive protein and physical activity. To estimate the excess VTE risk by race and region, we developed a ratio of ratios (RoR) which is the Black-White HR for VTE in the Southeast divided by the corresponding Black-White HR for the rest of the U.S.
Results: The median follow-up was $\sim 5$ years with 92 and 153 incident VTE among Black and White participants. In the Southeast there was a higher risk of VTE in Black than White participants relative to the rest of the country (RoR: 1.84; p for race*region interaction $=0.02$ ). When stratified by above and below median $\mathrm{N}$-income, in above median $\mathrm{N}$-Income areas, southeast-residing Black participants had 2.64-times excess risk of VTE compared to White participants (RoR: 2.64; p for interaction $=0.03$ ) and equal risk (RoR: 1.03: $\mathrm{p}$ for interaction $=0.95$ ) for below median $\mathrm{N}$-income. No significant differences were observed when stratified by I-income.

Table 1 Impact of Neighborhood and Individual Income on the Association of Race and Region with VTE Risk in the United States

\begin{tabular}{|c|c|c|c|c|c|}
\hline & \multicolumn{5}{|c|}{ Black-White Hazard Ratio (95\% Cl) } \\
\hline & Southeast & Rest of Country & Ratio of Ratios* & $P$ for interaction & VTE events \\
\hline \multicolumn{6}{|c|}{ Neighborhood Income } \\
\hline Above Median & $1.40(0.71,2.75)$ & $0.53(0.30,0.95)$ & 2.64 & 0.03 & 127 \\
\hline Higher Income & $1.41(0.85,2.34)$ & $0.79(0.46,1.36)$ & 1.68 & 0.12 & 136 \\
\hline Lower Income & $1.20(0.84,1.72)$ & $0.65(0.44,0.96)$ & 1.85 & 0.08 & 134 \\
\hline
\end{tabular}

Conclusions: These data suggest that after adjustment for VTE riskfactors, black-white differences in VTE risk are obfuscated by lower $\mathrm{N}$-income and enhanced by higher $\mathrm{N}$-income. Further research will be needed to study the mechanisms for how income, a known factor in health disparities, affects the black-white health disparity in VTE risk.

PB1197 | Coagulation Factors Levels during and after Anabolic Androgenic Steroid Use: Data from the HAARLEM Study

E. Camilleri ${ }^{1}$; D.L. Smit ${ }^{2}$; N. van Rein ${ }^{1,3}$; S. Le Cessie ${ }^{1}$; O. de Hon ${ }^{4}$; M. den Heijer ${ }^{5}$; S.C. Cannegieter ${ }^{1,6}$; W. de Ronde ${ }^{2}$

${ }^{1}$ Department of Clinical Epidemiology, Leiden University Medical

Center, Leiden, Netherlands; ${ }^{2}$ Department of Internal Medicine, Spaarne Gasthuis, Haarlem, Netherlands; ${ }^{3}$ Department of Pharmacy, Amsterdam University Medical Centers - Location AMC, University of Amsterdam, Amsterdam, Netherlands; ${ }^{4}$ Doping Authority Netherlands, Capelle aan den IJssel, Netherlands; ${ }^{5}$ Department of Internal Medicine, Section of Endocrinology, Amsterdam UMC, Amsterdam, Netherlands; ${ }^{6}$ Department of Internal Medicine, Division of Thrombosis and Hemostasis, Leiden University Medical Center, Leiden, Netherlands

Background: Anabolic androgenic steroids (AAS) are frequently used by amateur strength athletes to enhance muscle mass. An increased risk of cardiovascular events and venous thrombosis has been reported after AAS use, but the mechanism behind this association is unclear and the level of evidence is low. Also, no studies describe the extent of recovery of coagulation factor levels after AAS are withdrawn.

Aims: To assess the change of coagulation factor levels during AAS use and their recovery after.

Methods: The HAARLEM study enrolled 100 men intending to start a self-initiated AAS cycle between 2015-2018. Coagulation factors (factor[F]II, FVIII, FIX, von Willebrand factor [vWF], protein S [PS], $D$-Dimer $[D D]$ ) were measured before AAS use $\left(T_{0}\right)$, at the end of the cycle $\left(T_{1}\right)$ and 3 months after discontinuation $\left(T_{2}\right)$. Multivariable linear regression, adjusted for possible confounders, was used to assess the association between weekly AAS dose and cycle duration and changes in coagulation factors between $T_{0}$ and $T_{1}$, and between $\mathrm{T}_{0}$ and $\mathrm{T}_{2}$ to assess recovery.

Results: Subject performed an AAS cycle with a median duration of 13 weeks (range 2-52) and median dose of $900 \mathrm{mg}$ (range 88-3721). Mean levels of procoagulant factors FII, FIX and DD increased at T1 compared to TO (Table 1), whereas FVIII were unchanged and VWF levels decreased. Levels of the natural anticoagulant PS increased the most $(22 \%, 95 \% \mathrm{Cl} 15-29)$. An increase of weekly AAS dose and a shorter cycle duration were associated with an adjusted increase in PS between T1 and TO. Coagulation factor levels returned to baseline at T2; neither weekly dose or cycle length were associated with the recovery of coagulation parameters. 
Table 1 Mean changes in coagulation parameters between the last week (T1) and the start (TO) of the cycle
T0
T1
$\Delta \mathrm{T} 1=\mathrm{T} 1-\mathrm{T} 0^{*}$

Mean $\Delta \mathrm{T} 1$ increase by $100 \mathrm{mg}$ increase of

AAS weekly dose

Mean Mean Mean difference Mean $\triangle \mathrm{T} 1$ increase

(SD) (SD)

(95\% Cl)

$98.2 \quad 112.0$

FII

[\%]

FVIII

[\%]

FIX

[\%]

vWF

[\%] (42.1) (39.9)

PS $\quad 113.4 \quad 134.8$

[\%] (30.5) (35.7)

DD $\quad 285.9 \quad 404.5$

$[\mathrm{ng} / \mathrm{mL}] \quad$ (212.8) (580.5)
(95\%Cl)

0.7

(0.1 to 1.4 )

0.3

$(-0.5$ to 1.1$)$

1.0

(0.02 to 2.0)

0.4

(-0.9 to 1.6$)$

2.1

(0.9 to 3.3 )

$-5.6$

$(-24.7$ to 13.4$)$
Adjusted Mean $\Delta \mathrm{T} 1$

increase $t$

(95\% Cl)

0.2

$(-0.6$ to 0.9$)$

$-0.2$

$(-1.1$ to 0.7$)$

0.4

(-0.8 to 1.5$)$

$-0.3$

(-1.9 to 1.2$)$

2.9

(1.4 to 4.3 )

$-10.5$

$(-32.1$ to 11.1$)$
Mean $\Delta \mathrm{T} 1$ increase by 1 week increase of cycle length

Mean $\Delta \mathrm{T} 1$ increase

$(95 \% \mathrm{Cl})$

Adjusted Mean $\Delta \mathrm{T} 1$

increase $t$

(95\% Cl)

$-0.4$

(-0.7 to 0.03$)$

$-0.7$

$(-0.7$ to 0.3$)$

$-0.4$

(-1.0 to 0.2$)$

$-0.4$

$(-1.2$ to 0.4$)$

$-0.06$

(-0.8 to 0.7$)$

$-2.8$

$(-14.1$ to 8.5$)$

${ }^{*} \mathrm{TO}=$ before the start of the cycle, T1 = in the last week of the cycle, T2 $=3$ months after the cycle

t adjusted for number of different agents used, the use of AAS at time of T1, the use of other performance and image-enhancing drugs (e.g. growth hormone, anti-estrogenic, aromatase inhibitors), recreational drugs use, previous AAS use, age and weight

Table 2 Mean changes in coagulation parameters between 3 months after (T2) and the start (TO) of the cycle

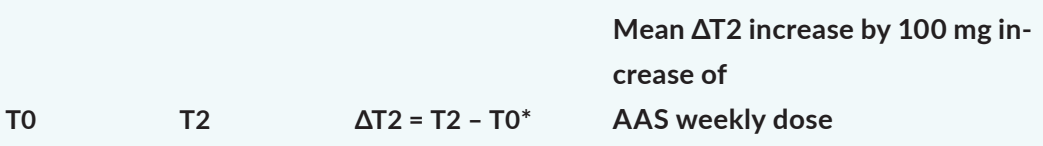

Mean Mean

(SD)

$\begin{array}{ll}\text { FII } & 98.2 \\ \text { [\%] } & (11.2 \\ \text { FVIII } & 121.0\end{array}$

[\%] (25.7)

(SD)

96.8

(12.0)

123.3

(28.2)

[\%]

(19.5)

(17.9)

138.6

(41.6)

[\%] (42.1)

PS

113.4

[\%]

(30.5)
111.6

(28.1)
Mean difference

(95\% Cl)

$-1.2$

(-3.3 to 0.9$)$

2.6

$(-1.5$ to 6.7$)$

3.0

(-0.7 to 6.7)

0.8

crease of

$\begin{array}{lllll}\text { Mean } \Delta \mathrm{T} 2 & \text { Adjusted Mean } & \text { Mean } & \Delta \mathrm{T} 2 & \text { Adjusted } \\ \text { increase } & \Delta \mathrm{T} 2 \text { increase }^{\mathrm{x}} & \text { increase } & \Delta \mathrm{T} 2 \text { increase }^{\mathrm{x}} \\ (95 \% \mathrm{Cl}) & (95 \% \mathrm{Cl}) & (95 \% \mathrm{Cl}) & (95 \% \mathrm{Cl})\end{array}$

0.1

0.07

(-0.4 to 0.5$)$

0.2

(-0.6 to 1.0$)$

$-0.3$

$(-1.2$ to 0.6$)$

0.3

$-0.08$

(-0.4 to 1.0$)$

(-0.9 to 0.7$)$

0.3

$(-0.7$ to 1.3$)$

$-0.3$

(-4.6 to 6.3$)$

$-0.8$

(-5.9 to 4.3$)$

Mean $\Delta T 2$ increase by 1 week increase of cycle length

$(-0.3$ to 0.09$)$

$-0.3$

-0.5 to 0.01$)$

0.2

$-0.4$

$(-0.7$ to 0.2$)$

$(-1.0$ to 0.1$)$

$-0.2$

$-0.5$

(-0.6 to 0.1$)$

$(-1.0$ to -0.03$)$

$-0.5$

$-0.6$

(-1.1 to 0.04$) \quad$ (-1.4 to 0.1$)$

$0.2 \quad 0.7$

0.09

0.5

(-0.8 to 1.2$) \quad$ (-0.5 to 1.8$)$

(-0.4 to 0.6$)$

(-0.2 to 1.2$)$ 


$\begin{array}{lllllllll}\mathrm{DD} & 285.9 & 351 & 56.9 & -7.7 & -11.6 & -3.9 & -5.4 \\ {[\mathrm{ng} / \mathrm{mL}]} & (212.8) & (643.2) & (-54.7 \text { to } 168.5) & \begin{array}{l}(-30.2 \\ 14.9)\end{array}\end{array}$

${ }^{*} \mathrm{T0}=$ before the start of the cycle, $\mathrm{T} 1$ = in the last week of the cycle, $\mathrm{T} 2=3$ months after the cycle

${ }^{x}$ adjusted for number of different agents used, the use of post-cycle therapy (e.g. anti-estrogen therapy), the use of other performance and imageenhancing drugs during the cycle, recreational drugs use, previous AAS use, age and weight

Conclusions: AAS use was associated with increased levels of both procoagulant and anticoagulant factors. A higher weekly AAS dose and shorter cycle durations were associated with a stronger increase in PS.

PB1198 | Acute VTE in HIV versus Non-HIV population Nationwide Analysis of Mortality, Morbidity, Demographics and Healthcare Utilization

M.J. Tariq ${ }^{1}$; M.U. Almani ${ }^{1}$; J. Tufail ${ }^{2} ;$ M.A. Elsebaie ${ }^{1}$; B. Baral ${ }^{1}$;

M. Usman ${ }^{1}$; S. Gupta ${ }^{1}$

${ }^{1}$ John H Stroger Hospital of Cook County, Chicago, United States; ${ }^{2} \mathrm{Al}$

Nafees Medical College and Hospital, Islamabad, Pakistan

Background: HIV infection is considered a prothrombotic condition associated with a 2- to 10-fold increase in VTE in HIV-infected patients compared to general population.

Aims: We aim to compare outcomes of patients admitted with acute VTE with HIV (VTE-HIV) and without HIV (VTE-no-HIV).
Methods: US Healthcare Cost and Utilization Project National Inpatient Sample (HCUP-NIS) was queried to identify HIV and non-HIV acute VTE admissions between 2016-2018. We studied socio-demographic differences, medical comorbidities, healthcare utilization, all-cause mortality and secondary outcomes listed in Table-1. Statistics were performed using t-test and univariate and multinomial logistic regression.

Results: We identified 3050 VTE-HIV and 866,745 VTE-no-HIV admissions. VTE-HIV patients were significantly younger (mean age 51.6 vs 62.8 years), male (73\% vs 48\%), African American (AA) $(59 \%$ vs $19 \%$ ), admitted to teaching hospitals ( $81 \%$ vs $67 \%$ ), on Medicaid (34\% vs $12 \%$ ), all $P<0.001$. Rates of CKD, hemodialysis, liver disease and protein energy malnutrition were significantly higher in HIV-VTE while dyslipidemia, hypertension, obesity and smoking were significantly higher in VTE-no-HIV, all $P<0.05$. VTE-HIV group had lower adjusted inpatient mortality (aOR 0.25, Cl:0.13-0.48, $P<0.001$ ) while mean length of stay (LOS) (5.6 vs 4.4 days, $P<0.01$ ) and mean total hospital charges (THC) $(54,961$ vs $47,007, P<0.01)$ were higher than VTE-no-HIV. Rates of thrombolysis, thrombectomy, cardiac arrest were similar while VTE-HIV was associated with lower rates of ICU admissions $(P<0.05)$. Table-1.

Table 1 Clinical outcomes of patients admitted to hospital with acute VTE with and without HIV in the US from 2016 through 2018 , analysis of National Inpatient Sample

\begin{tabular}{|c|c|c|c|c|}
\hline Outcome & Without HIV & With HIV & aOR $(95 \% \mathrm{Cl})$ & $\mathrm{p}$-value \\
\hline In-hospital mortality & $2.3 \%$ & $1.6 \%$ & $0.25(0.13-0.48)$ & $0.00^{*}$ \\
\hline \multicolumn{5}{|l|}{ Secondary outcomes } \\
\hline \multirow[t]{2}{*}{$\begin{array}{l}\text { Length of Stay } \\
\text { (mean) (days) }\end{array}$} & $4.4(4.3-4.4)$ & $5.6(4.7-6.5)$ & $-1.2(-1.6 \text { to }-0.9)^{\#}$ & $0.00^{*}$ \\
\hline & & & & $0.00^{*}$ \\
\hline Total hospital charges & 47,007 & 54,961 & -7954 & \\
\hline (mean) \$ & $(46,350-47,665)$ & $(48,136-61,786)$ & $(-13307 \text { to }-2601)^{\#}$ & \\
\hline tPA administration & $4.8 \%$ & $3 \%$ & $1.1(0.65-1.8)$ & 0.7 \\
\hline Thrombectomy & $2.9 \%$ & $2.8 \%$ & $1.3(0.76-2.3)$ & 0.3 \\
\hline Intracranial Hemorrhage & $0.25 \%$ & $0.16 \%$ & $0.08(0.01-0.58)$ & $0.01^{*}$ \\
\hline Cardiac Arrest & $1.1 \%$ & $0.66 \%$ & $0.51(0.18-0.14)$ & 0.19 \\
\hline ICU admission & $2.4 \%$ & $2.1 \%$ & $0.52(0.29-0.9)$ & $0.026^{*}$ \\
\hline
\end{tabular}

Abbreviations: ${ }^{*}=$ statistically significant, ${ }^{\#}=$ unadjusted mean difference, aOR $=$ adjusted odds ratio, $\mathrm{Cl}=\mathrm{confidence}$ interval, $\mathrm{tPA}=\mathrm{tissue}$ plasminogen activator, ICU = Intensive Care Unit.

Adjusting factors: age, race, gender, hospital location and teaching status, hospital bed size and region, insurance, dyslipidemia, coronary artery disease, hypertension, diabetes mellitus, obesity, heart failure, chronic kidney disease, smoking, liver disease, hemodialysis, proteinenergy malnutrition.

Conclusions: VTE-HIV patients are younger, AA, men with significantly lower mortality and ICU-admissions compared to HIV-no-VTE but higher LOS and THC amounting to over \$24 million in 3 years. While higher prevalence of CKD and hemodialysis may partly 
explain the increased THC in HIV-VTE patients, these discrepancies between outcome and healthcare utilization need to be studied further to save costs.

PB1199 | Recurrence and Mortality Following First Venous Thromboembolism among Saudi Population: Single-centre Cohort Study

F. Aleidan; R. Almanea; N. Shalash; N. Alrajhi; A. Almoneef;

S. Almousa; K. Abuelgasim

King Abdulaziz Medical City, Riyadh, Saudi Arabia

Background: Little is written about recurrence and mortality rates after a first episode of venous thromboembolism (VTE) among Saudi population.

Aims: To estimate the incidence rates and assess the risk factors associated with recurrence and mortality after a first VTE events.

Methods: We retrospectively followed up 1124 patients aged $\geq 18$ years with a symptomatic VTE confirmed by imaging test. In this single-centre Saudi cohort study, the incidence of VTE recurrence and mortality were assessed. The association between characteristics of patients and VTE recurrence and mortality were explored by estimating hazard ratio (HR) and $95 \%$ confidence interval $(\mathrm{Cl})$ using univariate and multivariate cox regression. Furthermore, we also explored the difference between cancer-related VTE, provoked and unprovoked VTE in term of recurrence and mortality using KaplanMeier curves and compared groups using the log-rank test.

Results: Of the 1124 patients with primary VTE, 214 patients developed recurrent VTE and 192 patients died with an overall incidence rates of 15.8 per 100 patient-year $(95 \% \mathrm{Cl}, 13.8-18.0)$ and 10.0 per 100 patient-year $(95 \% \mathrm{Cl}, 8.7-11.5)$, respectively. Presence of active cancer and Pulmonary embolism (PE) with or without deep vein thrombosis (DVT) were found independent risk factors associated with recurrence (HR, 2.87; 95\% Cl, 2.02-4.08; $P<.001$ and HR 2.10; 95\% Cl 1.57-2.82; $P<.001$, respectively). VTE recurrence predicted significantly higher mortality rate $(\mathrm{HR}, 7.0 ; 95 \% \mathrm{Cl}, 5.00-$ 9.81; $P<.001)$. Using Kaplan-Meier, the estimate recurrence mean time (months) was significantly lower in cancer-related VTE (18.7) than provoked (29.0) and unprovoked VTE $(28.4, P<.001$ by the log-rank test). The estimate survival mean time (months) was significantly lower in cancer-related VTE (21.8) than in provoked (30.5) and unprovoked VTE (29.8, $P<.001$ by the log-rank test).

Conclusions: The presence of active cancer and PE with or without DVT were a statistically significant risk factor for recurrence. Patients who developed recurrent VTE had 7-fold higher mortality rate than patients with no recurrences.
PB1200 | Development of a Computable Phenotype for Venous Thrombosis Present on Admission: The Medical Inpatient Thrombosis and Hemostasis (MITH) Study

R. Thomas ${ }^{1}$; I. Koh ${ }^{1}$; K. Wilkinson ${ }^{1} ;$ A. Li ${ }^{2}$; N. Roetker ${ }^{3}$; N. Smith ${ }^{4}$; A. Repp ${ }^{1}$; C. Holmes ${ }^{1}$; T. Plante ${ }^{1}$; M. Cushman ${ }^{1}$; N. Zakai ${ }^{1}$ ${ }^{1}$ University of Vermont Medical Center, Burlington, United States; ${ }^{2}$ Baylor College of Medicine, Houston, United States; ${ }^{3}$ Chronic Disease Research Group, Minneapolis, United States; ${ }^{4}$ University of Washington, Seattle, United States

Background: Venous thromboembolisms (VTEs) are largely preventable and currently there is not a computable phenotype to quickly and accurately identify VTE using electronic health record (EHR) data. Computable phenotypes make it possible to rapidly identify a condition without manual chart abstraction.

Aims: We sought to develop and validate an accurate and reproducible computable phenotype for newly diagnosed VTE that is present at admission (POA). Our goal is to differentiate VTE POA from VTE that is hospital acquired, previously diagnosed/treated, or miscoded. Methods: We captured all admissions to the medical services between 2010-19 at the University of Vermont Medical Center. A computable phenotype for VTE was developed using International Classification of Diseases (ICD) 9 or 10 discharge codes with the POA billing flag, current procedure terminology (CPT) codes for VTE-directed imaging studies, and anticoagulant medication administration. The algorithm that was created was compared with the gold standard for VTE POA - physician chart abstraction. 120 charts were abstracted from five different categories and the sensitivity and specificity of the computable phenotype vs. gold standard was assessed using survey weighting methodology.

Results: For the 120 charts that were abstracted for the computable phenotype, 71 charts were marked as POA VTE by the computable phenotype and 63 of these were confirmed as POA VTE with manual abstraction. Using survey weighting methodology to recreate the source population, the VTE case definition had a specificity of $95.9 \%$ and a sensitivity of $99.6 \%$ (Table 1 ).

TABLE 1 Weighted POA VTE data comparing physician chart abstraction and the computable phenotype

\begin{tabular}{|c|c|c|}
\hline & \multicolumn{2}{|c|}{ Chart Review VTE (Gold Standard) } \\
\hline EHR VTE Computable Phenotype & Yes & No \\
\hline Yes & 2717 & 262 \\
\hline No & 117 & 59984 \\
\hline & & \\
& Sensitivity (95\% Cl) - & Specificity (95\% Cl) - \\
& $95.9 \%(95.1-96.6 \%)$ & $99.6 \%(99.5-99.6 \%)$ \\
\hline
\end{tabular}

Conclusions: We developed a computable phenotype to identify POA VTE with excellent sensitivity and specificity. This can be used to further define risk factors for VTE using EHR data and to differentiate VTE POA from hospital-acquired VTE. 
PB1201 | National Survey of Hospital-associated Venous Thromboembolism Prevention in NHS England: Findings from the GIRFT Thrombosis Survey

L.N. Roberts ${ }^{1}$; M. De Caro ${ }^{2}$; A.-M. Ridgeon ${ }^{3}$; C. Moroy ${ }^{2}$; T. Briggs ${ }^{4,3}$; B.J Hunt ${ }^{5}$; R. Arya ${ }^{1}$

${ }^{1}$ King's College Hospital NHS Foundation Trust, London, United Kingdom; ${ }^{2}$ Edge Health, London, United Kingdom; ${ }^{3}$ Getting It Right First Time, London, United Kingdom; ${ }^{4}$ Royal National Orthopaedic Hospital NHS Trust, London, United Kingdom; ${ }^{5}$ Guy's and St Thomas' Hospital NHS Foundation Trust, London, United Kingdom

Background: In 2010, a national Venous Thromboembolism (VTE) Prevention Programme was introduced in England to prevent hospital associated VTE (HA-VTE). Mandatory documented risk assessment for VTE on hospital admission underpinned implementation, supported by NICE guidance on thromboprophylaxis. VTE risk assessment rates remain high nationally, but there is little data on thromboprophylaxis use and the impact on HA-VTE. A national survey of VTE prevention practice and rates of HA-VTE was undertaken through Getting It Right First Time (GIRFT); a national quality improvement initiative aimed at reducing unwarranted variation in clinical practice.

Aims:

1. To survey appropriate use of thromboprophylaxis and supply of patient information on VTE during hospitalisation.

2. To estimate rates of HA-VTE and proportion that were potentially preventable events.

Methods: Three thromboprophylaxis surveys were distributed to all NHS England hospitals with data required from October 2019 to March 2020 \& collected centrally. They assessed 1. organisational resource, 2. Rates of thromboprophylaxis and information provision to patients at high risk of VTE ( $n=20$ per site/month), and 3. Details of HA-VTE.

Results: 98 hospitals ( $68 \%$ of 144 invited) participated in $\geq 1$ survey. 98 hospitals contributed data regarding 9553 patients to survey 2 . Written patient information was provided to 2859 (31\%; variation 0100\%). Anticoagulant thromboprophylaxis was prescribed to 6544 (88\%, of 7399 with an indication) with significant inter-hospital variation (40-100\%). Missed doses were common (8.1\%; variation $0-55 \%)$

84 hospitals submitted 4595 HA-VTE cases, of which 595 (13\%, variation $0-100 \%$ ) were considered potentially preventable. The median rate of HA-VTE was 1.89 per 1000 admissions (IQR 0.61-3.17) with variation by admission type; medical 4.0 (1.6-7.9), surgical 1.4 (0.672.3) and obstetric 0.84 (0.53-1.3) per 1000 admissions.

Conclusions: There remains significant variation in VTE prevention care throughout NHS England. Provision of patient information and ensuring anticoagulant prophylaxis is given are key areas for improvement.
PB1202 | How Common Are Uterine Venous Plexus Thrombi in Women Attending the Gynaecology Clinic?

T. Amin ${ }^{1} ; \underline{\text { H. Cohen }}{ }^{2} ;$ M. Wong ${ }^{2}$; D. Jurkovic ${ }^{2}$

${ }^{1}$ Guy's and St Thomas's NHS Foundation Trust, London, United

Kingdom; ${ }^{2}$ University College London Hospitals NHS Foundations Trust, London, United Kingdom

Background: Venous thromboembolism (VTE) has been a leading cause of direct maternal deaths in the U.K. for over two decades. Venous thrombosis (VT) commonly affects the deep veins of the lower limbs but can occur in unusual sites such as the vena cava, abdomen/pelvis and upper limbs. With improved gynaecological imaging, pelvic thrombi can also be detected on transvaginal ultrasound (TVS).

Aims: To determine the prevalence of uterine venous plexus thrombosis (UVPT) in women attending gynaecology clinic and identify potential risk factors.

Methods: A prospective observational, cross-sectional study was conducted within a gynaecology clinic setting at a university teaching NHS hospital in London, U.K. over a 16-month period. Women presented with a variety of symptoms and were recruited by a single operator. The diagnosis of UVPT was based on established criteria for thrombi. The exclusion criteria included: age <18 years, unable to undergo TVS, previous hysterectomy. Women diagnosed with UVPT were managed in conjunction with the haematologists and had thrombophilia screening and lower limb venous duplex imaging.

Results:

Table 1 Demographic data

\begin{tabular}{lll} 
Demographic information & UVPT $(N=39)$ & $\begin{array}{l}\text { No UVPT } \\
(N=1259)\end{array}$ \\
Age & $44 \cdot 7 \pm 10 \cdot 1$ & $42 \cdot 8 \pm 12 \cdot 5$ \\
Parity 0 & $8(20 \cdot 5 \%)$ & $573(45 \cdot 5 \%)$ \\
Parity 1 & $7(18 \cdot 0 \%)$ & $231(18 \cdot 4 \%)$ \\
Parity 2 & $10(25 \cdot 6 \%)$ & $245(19 \cdot 5 \%)$ \\
Parity $>3$ & $14(35 \cdot 9 \%)$ & $210(16 \cdot 7 \%)$ \\
Pre-menopausal & $33(84 \cdot 6 \%)$ & $977(77 \cdot 6 \%)$ \\
Post-menopausal & $6(15 \cdot 4 \%)$ & $282(22 \cdot 4 \%)$ \\
History of VTE & $1(2 \cdot 6 \%)$ & $21(1.7 \%)$ \\
\hline
\end{tabular}

We screened 1,298 women and 39 women were diagnosed with UVPT, giving a prevalence of 3.0\% (95\% Cl, 2.1-4.1\%). Demographic data are shown in Table 1. Multivariate analysis for UVPT risk factors showed significant associations between multiparity, premenopausal status, recent surgery, presence of leg varicose veins and a family history of venous thromboembolism. Thrombophilia was detected in $12.8 \%$ women with UVPT.

Conclusions: The prevalence of UVPT in a general gynaecological population was 3.0\%. Several demographic and clinical factors were found to be associated with UVPT, which could help to identify 
women at risk of this condition and facilitate its early detection. This would provide a basis to assess the natural history and clinical significance of this novel clinical finding, and the development of an optimal management approach.

PB1203 | Machine Learning and Algorithmic Diagnosis Identification of PatientsTreated by Direct Oral Anticoagulants Using Medico-administrative Databases

J. Emmerich ${ }^{1}$; A. Chekroun-Martinot ${ }^{2}$; C. Petri ${ }^{3}$; R. Sigogne ${ }^{2}$;

L. Perray ${ }^{2}$; M. Maravic ${ }^{4}$

${ }^{1}$ University of Paris, Groupe Hospitalier Paris Saint-Joseph and INSERM

CRESS 1153, Paris, France; ${ }^{2}$ IQVIA, La Défense, France; ${ }^{3} \mathrm{NHLI}$

Centre for Cardiac Engineering, Faculty of Medicine, Imperial College

London Guy, London, United Kingdom; ${ }^{4}$ Hôpital Lariboisière - AP-HP

(Rheumatology) and IQVIA, Real World Solutions, La Défense, France

Background: New oral anticoagulants (DOAC) are indicated in atrial fibrillation (AF), thromboembolic events (VTE), and prevention of VTE after hip or knee prosthesis (PVTE).

Aims: The objective was to differentiate these indications using medico-administrative databases.

Methods: Two sources of data were used, LPD and LRx, including data of near 2.5 and 40 million patients, respectively. LPD, a medicalized database, included 56,665 patients treated by DOAC in 2019 followed-up by 1,800 general practitioners and/or specialists who participating in a permanent longitudinal observatory of prescription in ambulatory medicine. LRx, contained all anonymized medication dispenses prescribed in outpatient care from a representative panel of $45 \%$ of all French retail pharmacies. After derivation on LPD, the best gradient boosting model was selected in order to identify AF and/or VTE (accuracy of $91.5 \%$ and $90.5 \%$ for AF, and VTE, respectively). The model was then implemented in LRx in order to obtain AF and VTE patients in 2019. In order to identify PVTE patients on LRx, rules-based algorithm was defined. We obtained a raw number of DOAC patients and performed demographic characteristics. We calculated the extrapolated number of DOAC patients.

Results: Over the 944,892 DOAC patients identified in LRx, 73\% were classified as having AF, 20\% VTE, 4\% AF/VTE, 3\% PVTE, and $0.3 \%$ unclassified. The percentage of female and mean age (SF) were $45 \%$ and 79 (10), 54\% and 65 (15), 57\% and 76 (9), 51\% and 77 (11), 93\% and 88 (9), for AF, VTE, AF/VTE, PVTE, unclassified, respectively. The extrapolated number of DOAC patients in France in 2019 was 1,8 million.

Conclusions: A combined approach using machine learning and rules-based algorithm could be used in order to distinguish the different indications for which DOAC could be prescribed. According to demographics characteristics in France of each medical condition our results correspond to patients seen in clinical practice and literature.
PB1204 | Risk Factors of Venous Thromboembolism at Patients with Endoscopic Urological Interventions

D. Shorikova; E. Shorikov; P. Shorikov

Bukovinian State Medical University, Chernivtsy, Ukraine

Background: Venous thromboembolism is accompanied with superficial thrombophlebitis in $25 \%$ of patients. Lethality in 3-6 months after deep vein thrombosis, according to different authors, is $7-15 \%$. The role of preoperative risk factors for venous thromboembolism in endoscopic urological interventions have to be analized.

Aims: To examine the clinical, gender and coagulologic parameters of preoperating risk for venous thromboembolic complications factors before endoscopic urological interventions.

Methods: 1918 patients were examined, including 414 patients with venous thromboembolic postoperative complications (223 male, 191 female, average age $48.55 \pm 13.77$ years). Cumulative incidence $(\mathrm{Cl})$, cumulative incidence (UI) reduction, relative risk (RR) and odds ratio (OR) were analyzed.

Results: After prolonged prospective observation among 1918 patients in preoperating period it was set, that preceding chronic venous insufficiency could be the main basic risk factor of venous thrombosis during surgical interventions $(P<0,05)$. It was proven the linear dependence between the class of chronic venous insufficiency and incident of peripheral thrombotic complications $(P<0,05)$, with the highest risk for $C 3$ and $C 4$ classes $(P<0,05)$.

It was estimated that women sex $(P<0,05)$ reliably promotes the risk of venous thromboembolism with valid relative $\operatorname{risk}(1,53[1,11-2,12])$ and odds ratio (1,59 [1,13-2,27]) before surgical intervetions.

It was set that coagulogram indexes as the level of fibrinogen, prothrombin time and activated partial thromboplastine time should be analized in pre-operating period, but could not be the reliable markers of venous thromboembolism incidence before surgical intervetions $(P<0,05)$.

Conclusions: Preexisting chronic venous insufficiency is the main risk factor for venous thromboembolism in urological interventions $(P<0.05)$ with significant effect of C3-C4 $(P<0.05)$. In female $(P<0.05)$ was set significantly increases risk of venous thromboembolism after endoscopic urological interventions.

PB1205 | Hypoalbuminemia, Not Proteinuria, Is Associated with Increased Venous Thromboembolism Risk among Primary Glomerulonephritis Patients with Nephrotic Syndrome: A Metaanalysis of Cohort Studies

B.P. Putra ${ }^{1}$; F.N. Putra ${ }^{2}$

${ }^{1}$ Medical Doctor, Blitar, Indonesia; ${ }^{2}$ Faculty of Medicine Universitas Airlangga, Surabaya, Indonesia

Background: Venous thromboembolism (VTE) spectrum recently has been considered as second life-threatening cause after infection among primary glomerulonephritis with nephrotic syndrome (NS) 
patients. Based on the previous studies, there is inconclusive results whether proteinuria and hypoalbuminemia, which are hallmarks of NS patients, are associated with VTE incidence.

Aims: This study aims to measure the association between proteinuria and hypoalbuminemia with the VTE incidence among NS patients. Methods: We searched comprehensively in online databases of Pubmed, ScienceDirect, EMBASE, and The Cochrane Library, to include all relevant studies from 2000 until 2021. We followed the PRISMA guideline for conducting this study. We included all cohort studies that access the association between proteinuria and hypoalbuminemia with the VTE incidence among primary glomerulopathies NS patients. Bias risk were accessed by using The Newcastle-Ottawa Scale for cohort study. Analysis was performed to provide pooled risk ratio (RR) with $95 \%$ confidence interval $(\mathrm{Cl})$ using random-effect heterogeneity test.

Results: We included 10 cohort studies matched our inclusion criteria. Patients with proteinuria above $3.5 \mathrm{~g} /$ day is associated with increased VTE risk although statistically not significant. (pooled $\left.\mathrm{RR}=1.11,95 \% \mathrm{Cl} 0.99-1.25, P=0.06, \mathrm{I}^{2}=76 \%\right)$. Otherwise, NS patients whose hypoalbuminemia below $3 \mathrm{~g} / \mathrm{dL}$ are associated with significant incremental VTE incidence risk (pooled RR $=1.41,95 \% \mathrm{Cl}$ 1.13-1.74, $\left.P=0.002, I^{2}=78 \%\right)$.

Conclusions: Hypoalbuminemia, not proteinuria, is associated with increased VTE incidence among primary glomerulonephritis with nephrotic syndrome patients. However, further studies are needed to establish the association and causality.

PB1206 | Incidence of Venous Thromboembolic Disease during Primary Hyperparathyroidism: A 20-year Single Center Study

M. Orlova; Y.J. Zin; M.L. Posadas Martínez; M.F. Grande Ratti; D.H. Giunta; M. Burgos; F.J. Vázquez

Hospital Italiano de Buenos Aires, Buenos Aires, Argentina

Background: Primary Hyperparathyroidism (HPTP) has been associated with cardiovascular events and inflammatory and prothrombotic markers. There are no studies that explore the occurrence of Venous ThromboEmbolism (VTE).

Aims: To estimate the incidence of VTE in patients with HPTP.

Methods: Retrospective cohort which included adult patients affiliated to an institutional prepaid-insurance (Hospital Italiano de Buenos Aires) between 1999-2019. HPTP diagnosis was defined as hypercalcemia (calcium value $>10.5 \mathrm{mg} / \mathrm{dL}$ or ionic calcium value $>1,35 \mathrm{mmol} / \mathrm{L}$ ) and PTH measurement $>77 \mathrm{pg} / \mathrm{dL}$, each case was individually confirmed by an expert review. Patients were followed up until the occurrence of VTE (deep vein thrombosis -DVT- and/ or pulmonary thromboembolism -PE-), surgery, death or administrative censored. VTE events were captured from secondary databases (Institutional Registry of Thromboembolic Disease). No sample size calculation was performed because we included all eligible patients. Cumulative Incidence was estimated using Kaplan-Meier analysis.
We reported different times ( 6 months and from 1 to 5 years) with their respective $95 \% \mathrm{Cl}$.

Results: From a total of 1,354 potentially eligible patients, 653 were confirmed with HPTP by a specialist. The overall median follow-up of the cohort was 7.23 years with time at risk of 1,812,313 persons/ days. A total of 28 VTE events occurred, 22 DVT and 6 PE, with a median of 1211 days (3.3 years). The incidence of VTE was: $0.15 \%$ $(95 \% \mathrm{Cl} 0.02-1.08)$ at 6 months and 1-year, $1.07 \%(95 \% \mathrm{Cl} 0.51-2.24)$ at 2 -year, $2.03 \%(95 \% \mathrm{Cl} 1.82-3.47)$ at 3 -year, $2.87 \%(95 \% \mathrm{Cl} 1.82-$ $4.53)$ at 4 -year, and $3.24 \%(95 \% \mathrm{Cl} 2.10-4.98)$ at 5 -year period. The median of PTH levels in patients who had VTE was 138 (IQR 101.7) and 157.5 (IQR 102.4) in patients without VTE. The mean of calcium levels in patients who had VTE was 11,72 (SD 0.32) and 11.93 (SD 0.75 ) in patients without VTE.

\section{Conclusions:}

HPTP patients had an increased occurrence of VTE compared to the general population.

\section{PB1207 | Incidence and Treatment Pattern of Venous Thromboembolism from 2014 to 2018: The 3rd Korean Nationwide Study}

H.-G. Hwang ${ }^{1}$; J.H. Lee ${ }^{2}$; S.-A Kim²; Y.-K. Kim ${ }^{3}$; H.-Y. Yhim ${ }^{4}$; J. Hong ${ }^{5}$; S.-M. Bang ${ }^{2}$

${ }^{1}$ Soonchunhyang University Gumi Hospital, Gumisi, Korea, Republic of; ${ }^{2}$ Department of Internal Medicine, Seoul National University College of Medicine, Seoul National University Bundang Hospital, Seongnam, Korea, Republic of; ${ }^{3}$ Respiratory Division, Department of Internal Medicine Soonchunhyang University, School of Medicine, Seoul Hospital, Seoul, Korea, Republic of; ${ }^{4}$ Department of Internal Medicine, Chonbuk National University Medical School, Jeonju, Korea, Republic of; ${ }^{5}$ Department of Internal Medicine, Seoul National University College of Medicine, Seoul National University Hospital, Seoul, Korea

Background: The incidence of venous thromboembolism (VTE) gradually increased in Korean population even though it is still low compared to western population.

Aims: The aim of the $3 \mathrm{rd}$ nationwide study is to evaluate the annual age- and sex-adjusted incidence rates (ASR) of VTE and to describe the trend of anticoagulation over years since direct oral anticoagulants (DOAC) from 2014 to 2018.

Methods: Using the Korean Health Insurance Review and Assessment Service (HIRA) database, VTE patients from 2014 to 2018 were retrospectively identified by both diagnostic codes and medication codes of anticoagulants used within 6 months of initial index event. DOAC-based regimen was defined as DOAC only or DOACs combined with UFH and/or LMWH except warfarin; LMWH and/or UFH regimen; warfarin-based regimen as warfarin only, or $\mathrm{LMWH}$ /warfarin; mixed anticoagulation regimen as anticoagulants including both warfarin and DOACs.

Results: We identified 95,205 individuals with VTE (54,085 female, $56.8 \%$ ). The ASR of VTE, deep vein thrombosis (DVT) only, and 
pulmonary embolism with or without DVT (PE) per 100,000 individuals continued to increase from $32.83,13.82$ and 19.01 cases in 2014 to $53.66,22.79$ and 30.87 in 2018, respectively. The incidence rate for VTE was significantly higher in 2018 than in 2014 (relative risk [RR] of 1.63 ; $95 \% \mathrm{Cl}, 1.6$ to $1.67 ; p$-value< 0.0001 ); among patients aged 80 or older than 30-39 age group (RR $25.4095 \% \mathrm{Cl}$, 24.63 to 26.18; $p$-value<0.0001); in female than male (RR 1.29; $95 \%$ $\mathrm{Cl}, 1.28$ to $1.31 ; p$-value<0.0001). Among anticoagulants, the portion of DOACs-based prescription increased from $40.5 \%$ to $72.8 \%$; UFH and/or LMWH decreased from 24.3 to $18.4 \%$; warfarin-based prescription, from $27 \%$ to $5.6 \%$ / mixed anticoagulation, from $8.2 \%$ to $3.2 \%$ in 2014 and 2018 , respectively.

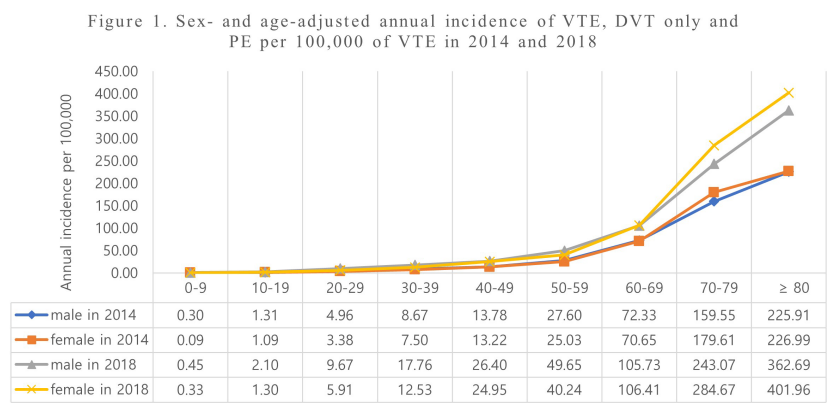

Figure 1 Sex- and age-adjusted annual incidence of VTE, DVT only and PE per 100,000 of VTE in 2014 and 2018

Figure 2. Trend of anticoagulants for VTE from 2014 to 2018

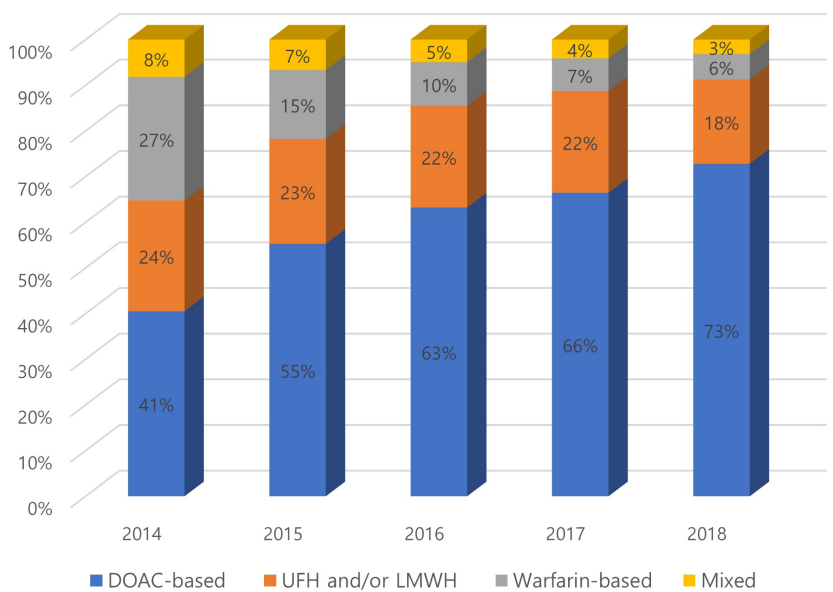

Figure 2 Trend of anticoagulants for VTE from 2014 to 2018

Conclusions: The ASR of VTE in Korea still continuously increased from 2014 to 2018. DOACs became main option for anticoagulation in two-third of VTE patients.
PB1208 | Solitary versus Multiple Subsegmental Pulmonary Emboli: Clinical Characteristics and Outcomes

Y. Hirao-try ${ }^{1}$; D. Vlazny ${ }^{1}$; D. Houghton ${ }^{1}$; A. Casanegra ${ }^{1}$;

R. Meverden ${ }^{1}$; D. Hodge ${ }^{2}$; L. Peterson ${ }^{1}$; R. McBane ${ }^{1}$;

W. Wysokinski ${ }^{1}$

${ }^{1}$ Mayo Clinic, Rochester, United States; ${ }^{2}$ Mayo Clinic, Jacksonville, United States

Background: Current guidelines prefer clinical surveillance over anticoagulation for isolated subsegmental pulmonary embolism (ISSPE) depending on coexisting bleeding risks. The numeric frequency of ISSPE is not considered in the decision process whereby single (solitary) or multiple subsegmental pulmonary embolism are treated the same way.

Aims: To assess the clinical relevance of numeric frequency of ISSPE, the demographics and clinical outcomes of solitary and multiple ISSPE were compared.

Methods: Patients receiving anticoagulation for ISSPE at Mayo Thrombophilia Clinic between 03/01/2013 and 12/31/2020 were followed prospectively. Demographic, clinical characteristics and clinical outcomes including venous thromboembolism (VTE) recurrence, major bleeding, clinically relevant non major bleeding (CRNMB), and mortality were compared for solitary and multiple ISSPE.

Results: Among 3878 patients with VTE, 1542 (40\%) had pulmonary embolism including 225 (6\% with ISSPE either solitary $(n=139)$ or multiple ( $n=86$; 47 with bilateral and 39 unilateral emboli)) ISSPE. A lower proportion of symptomatic events and coexisting proximal leg deep vein thrombosis was observed in solitary compared to multiple ISSPE (Table1). One patient with solitary and two with multiple ISSPE experienced recurrent VTE (1.14 vs 3.76 per 100 personyears, $P=0.26$ ). The solitary group experienced 2 episodes of major bleeding (2.36 per 100 person-years) while the multiple group had no major bleeding. Seven patients in each group had CRNMB events (8.20 vs 14.09 per 100 person-years for solitary and multiple patients respectively, $P=0.25)$. Patients with solitary ISSPE had a higher death rate compared to multiple (43.07 vs 22.96 per 100 person-years, $P=0.04$ ) but it was no longer statistically different after adjusting for cancer $(P=0.41$, see Table 2$)$. 
TABLE 1 Demographic and clinical variables of patients with solitary versus multiple isolated subsegmental pulmonary emboli

\begin{tabular}{llll} 
& Solitary & Multiple & \\
Variables & $n=139$ & $n=86$ & $p$-value \\
Age, mean (SD) & $61.8(14.4)$ & $61.7(13.6)$ & 0.450 \\
Female, $\mathrm{n}(\%)$ & $66(47.5 \%)$ & $35(40.7 \%)$ & 0.320 \\
Symptomatic, $\mathrm{n}(\%)$ & $46(33.6 \%)$ & $42(49.4 \%)$ & 0.019 \\
Previous VTE, $\mathrm{n}(\%)$ & $14(10.4 \%)$ & $16(19.8 \%)$ & 0.056 \\
Leg, DVT only, $\mathrm{n}(\%)$ & $31(22.3 \%)$ & $30(34.9 \%)$ & 0.039 \\
Provoked PE, $\mathrm{n}(\%)$ & $117(85.4 \%)$ & $67(77.9 \%)$ & 0.152 \\
Active cancer, $\mathrm{n}(\%)$ & $87(62.6 \%)$ & $44(51.2 \%)$ & 0.091 \\
\hline
\end{tabular}

TABLE 2 Venous thromboembolism (VTE) recurrence, major bleeding, clinically relevant non-major bleeding (CRNMB), and death in patients anticoagulated for solitary versus multiple isolated subsegmental pulmonary emboli without concomitant proximal deep vein thrombosis.

\begin{tabular}{|c|c|c|c|}
\hline \multirow[b]{2}{*}{ Outcome } & \multicolumn{2}{|c|}{$\begin{array}{l}\text { Sub-segmental } \\
\text { pulmonary embolism }\end{array}$} & \multirow[b]{2}{*}{$P$-value } \\
\hline & $\begin{array}{l}\text { Solitary } \\
N=124\end{array}$ & $\begin{array}{l}\text { Multiple } \\
n=74\end{array}$ & \\
\hline VTE recurrence (n) & 1 & 2 & 0.26 \\
\hline Total follow up person-years & 87.70 & 53.17 & \\
\hline $\begin{array}{l}\text { Major bleeding rate } \\
100 \text { person-years }\end{array}$ & 1.14 & 3.76 & \\
\hline Major bleeding (n) & 2 & 0 & $\mathrm{~N} / \mathrm{A}$ \\
\hline Total follow up person-years & 84.60 & 56.61 & \\
\hline $\begin{array}{l}\text { Major bleeding rate } \\
100 \text { person-years }\end{array}$ & 2.36 & $\mathrm{~N} / \mathrm{A}$ & \\
\hline CRNMB (n) & 7 & 7 & 0.25 \\
\hline Total follow up person-years & 85.39 & 49.66 & \\
\hline $\begin{array}{l}\text { CRNMB rate } \\
100 \text { person-years }\end{array}$ & 8.20 & 14.09 & \\
\hline Death (n) & 38 & 13 & 0.04 \\
\hline Total follow up person-years & 88.23 & 56.61 & \\
\hline $\begin{array}{l}\text { Death rate } \\
\text { per } 100 \text { person-years }\end{array}$ & 43.07 & 22.96 & \\
\hline
\end{tabular}

Conclusions: There were more solitary than multiple ISSPE and more bilateral than unilateral in the latter group. The rates of VTE recurrence, major bleeding, CRNMB, and death do not appear to be influenced by the numeric frequency of ISSPE.
PB1209 | ISTH Membership and Research Output: A Comparative Study of Sub-Saharan Africa and Europe

E. Olayemi $^{1}$; D. Fiawoo ${ }^{2}$

${ }^{1}$ University of Ghana Medical School, Accra, Ghana; ${ }^{2}$ Korle Bu Teaching Hospital, Accra, Ghana

Background: The International Society on Thrombosis and Haemostasis (ISTH) was created in 1969 to advance the understanding, prevention, diagnosis and treatment of conditions related to thrombosis and haemostasis. ISTH currently has over 5000 members in 100 countries.

The vast majority of ISTH members are either citizens or residents of High and Middle-Income Countries; similarly, most of the research in the area of Thrombosis and Haemostasis originate in these countries. Previously, it was wrongly assumed that the prevalence of disorders of the coagulation system was low, in other parts of the world and this presumably explained the low representation of health workers and scientists from low-income countries in the activities of the society. To address this imbalance, the ISTH has instituted several programs including the Reach-the-World program.

Aims: This study was designed to compare the membership of ISTH and research output from sub-Saharan Africa (SSA) and Europe.

Methods: We collected and compared data on the number of ISTH members from Europe and SSA as at September 2020, using the ISTH membership directory. We also compared the number of accepted abstracts (oral and poster), to the ISTH 2020 Virtual Congress from both parts of the world.

Results: As shown in table 1, Europe with a population far lower than SSA has 20 times more ISTH members and almost 40 times more abstracts were from Europe compared to SSA. The top 3 countries in terms of membership and abstracts from both regions is shown in table 2 .

Table 1 ISTH members and number of accepted abstracts from selected regions of the world

\begin{tabular}{|c|c|c|}
\hline & Europe & $\begin{array}{c}\text { Sub-Saharan } \\
\text { Africa }\end{array}$ \\
\hline ISTH Members & 1645 & 82 \\
\hline Number of Accepted Abstracts & 1383 & 37 \\
\hline 2020 Population & 747 Million & 1.1 Billion \\
\hline
\end{tabular}

Table 2 Top 3 Three Countries from both Regions

\begin{tabular}{ccc} 
& Europe & Sub-Saharan Africa \\
Top 3 Countries & United Kingdom (332), & South Africa (36), \\
(Membership) & Netherlands (188), & Nigeria (28), \\
& Germany (175) & Kenya (6) \\
Top 3 Countries & Italy (206), United & Nigeria (17), South \\
(Abstracts) & Kingdom (186), & Africa (7), \\
& Germany (140) & Senegal (3). \\
\hline
\end{tabular}

Conclusions: Although, ISTH has instituted several programs to encourage participation by health workers and scientists from under represented parts of the world in its activities. More needs to be 
done in promoting the society in underserved and under reached region as well as providing educational support to scientists and health workers from these regions.

PB1210 | Prospective Cohort: A 10 Years Experience with Hospitalized Patients with Venous Thromboembolic Disease from a Single-center Institutional Registry, Argentina

F.J. Vázquez; M.F. Grande Ratti; M.A. Burgos; M.E. Zapiola;

D.H. Giunta; M.L. Posadas-Martinez

Hospital Italiano de Buenos Aires, Buenos Aires, Argentina

Background: Venous thromboembolic embolism (VTE) is the most frequent cause of preventable mortality in hospitalized patients and the third leading cause of mortality due to vascular diseases.

Aims: We aim to describe patients with VTE who required hospitalization, their clinical characteristics, management, and evolution after discharge.

Methods: Prospective cohort which included patients with acute and symptomatic VTE who required hospitalization in a single tertiary center for their management in a 10 years' period (between 2006 and 2016). Patients were included at the time of VTE diagnosis; we collected baseline characteristics, risk stratification and initial therapeutic approach. Then, they were periodically, prospectively, and systematically followed up to evaluate clinical outcomes: recurrence, bleeding and/or death. Time-to-event analysis was performed for these outcomes related to evolution after discharge.

Results: We included 3,457 VTE confirmed episodes for the analysis (1,985 deep vein thrombosis -DVT-, 930 pulmonary embolism -PE- and $542 \mathrm{DVT}+\mathrm{PE}$ ), corresponding to 3,157 patients. Most were elderly, the median age was 68 years (SD 16) with a predominance of women $57 \%(1,955)$. Most frequent predisposing factors were immobility (45\%), active cancer (35\%), recent surgery (33\%) Onefourth of PE had a Pulmonary Embolism Severity Index (PESI) with 0 points. More than $91.7 \%$ received some kind of treatment: $86 \%$ lowmolecular-weight heparin, of which $33 \%$ were prescribed in combination with a vitamin $\mathrm{K}$ antagonist since the beginning. Recurrence, major bleeding and mortality rates within 30 days were $10 \%, 5 \%$ and $14 \%$ respectively., obesity (26\%), recent hospitalization (22\%) and previous VTE (21\%).
Table 1. VTE: characteristics of hospitalization and initial therapy management

\begin{tabular}{|c|c|c|c|c|}
\hline & VTE & DVT only & PE only & PE with DVT \\
\hline $\begin{array}{l}\text { Hospital stay, in } \\
\text { days * }\end{array}$ & $10(5.6-21)$ & $12(6-26)$ & $8.60(5-16)$ & $7.37(5-14)$ \\
\hline $\begin{array}{l}\text { Respiratory } \\
\text { Assistance }\end{array}$ & $3.50 \%(121)$ & $4.28 \%(85)$ & $2.80 \%(26)$ & $1.85 \%(10)$ \\
\hline $\begin{array}{l}\text { Need for Intensive } \\
\text { care unit }\end{array}$ & $47.73 \%(1650)$ & $44.43 \%(882)$ & $53.76 \%(500)$ & $49.45 \%(268)$ \\
\hline Initial therapy & $91.73 \%(3171)$ & $89.62 \%(1779)$ & $93.55 \%(870)$ & $96.31 \%(522)$ \\
\hline \multicolumn{5}{|l|}{ Type of therapy } \\
\hline $\begin{array}{l}\text { Only low molecular } \\
\text { weight (LMWH) }\end{array}$ & $52.85 \%(1676)$ & $55.31 \%(984)$ & $50.80 \%(442)$ & $47.89 \%(250)$ \\
\hline $\begin{array}{l}\text { LMWH + vitamin K } \\
\text { antagonist }\end{array}$ & $33.55 \%(1064)$ & $29.96 \%(533)$ & $37.93 \%(330)$ & $38.50 \%(201)$ \\
\hline $\begin{array}{l}\text { Unfractionated } \\
\text { heparin }\end{array}$ & $5.92 \%(188)$ & $7.25 \%(129)$ & $3.79 \%(33)$ & $4.98 \%(26)$ \\
\hline $\begin{array}{l}\text { Inferior vena cava } \\
\text { filter }\end{array}$ & $1.51 \%(48)$ & $1.79 \%(32)$ & $0.68 \%(6)$ & $1.91 \%(10)$ \\
\hline $\begin{array}{l}\text { Direct oral } \\
\text { anticoagulants }\end{array}$ & $0.75 \%(24)$ & $0.73 \%(13)$ & $0.49 \%(4)$ & $1.34 \%(7)$ \\
\hline $\begin{array}{l}\text { Thrombectomy } \\
\text { and/or thrombolytic }\end{array}$ & $0.47 \%(15)$ & $0.39 \%(7)$ & $0.57 \%(5)$ & $0.60 \%(3)$ \\
\hline Anti platelets & $0.12 \%(4)$ & $0.22 \%(4)$ & - & - \\
\hline Missing data & $4.83 \%(152)$ & $4.35 \%(77)$ & $5.74 \%(50)$ & $4.78 \%(25)$ \\
\hline
\end{tabular}

FIGURE 1 VTE episodes between 2006 and 2016

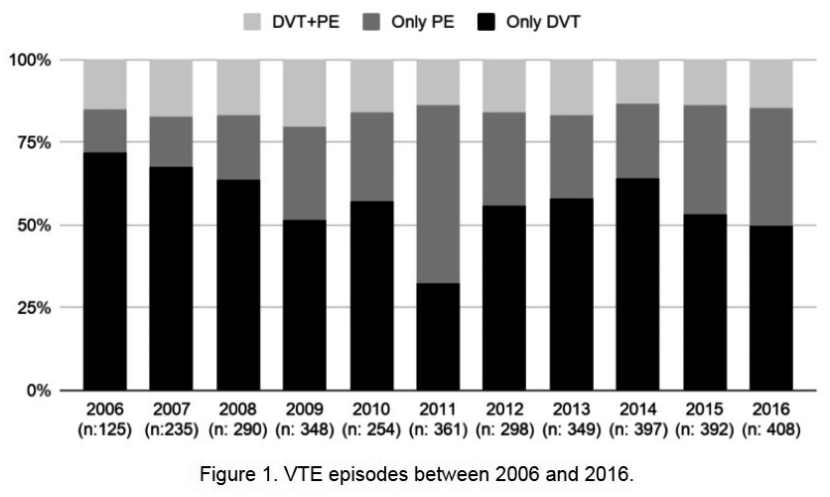

FIGURE 2 VTE: characteristics of hospitalization and initial therapy management

Conclusions: A high proportion of VTE requires hospitalization for their management, even PE with low sPESI. Mortality and complication remain elevated. We encourage the development and maintenance of more VTE registries in Latin America, to fully understand the local characteristics of this disease.

PB1211 | Differences of Profile and Management of Cancerassociated Thrombosis in a RIETE's Hospital in Brazil

J. de Abreu Couto Vieira; R. Santos de Oliveira Júnior;

A.T. Cavalcanti Rocha

Escola Bahiana de Medicina e Saúde Pública, Salvador, Brazil

Background: Clinical management and treatment of cancerassociated thrombosis (CAT) are important challenges for physicians that deal with venous thromboembolism (VTE). 
Aims: To evaluate the profile and management of patients with CAT in a private hospital in Salvador, participating in the RIETE registry. Methods: Consecutive patients from 5/2018 to 12/2019 were evaluated and differences between CAT patients and other-VTE patients were compared.

Results: Of the 95 patients, $67.4 \%$ were women, the mean age 53.29 years $( \pm 18.99)$ and, higher for CAT, $65.82( \pm 15.63)$ than for otherVTE, 49.52 ( \pm 18.36$), P<0.001)$. There were 64.2\% PE, 17.9\% DVT/ PE and $17.9 \%$ DVT, with high rates of PE between CAT and otherVTE and there was no incidental PE. The primary cancer site was breast $27.3 \%$, prostate $18.2 \%$ and lung $13.6 \%$. Among the risk factors for CAT, $13.6 \%$ used hormones, $18.2 \%$ history of VTE, $22.7 \%$ had obesity, 13.6\% immobility >4 days, 9.1\% smoking, 4.5\% recent travel $>6$ hours, none had family history of VTE; hypertension was more frequent in CAT than other-VTE: $63.6 \%$ vs. $39.7 \%, P=0.05$. The mean length-of-stay was $9,13 \pm 5.99$ days, being shorter for CAT (7.77 \pm 6.52 days). The initial choice of anticoagulant for all cancer patients was enoxaparin $100 \%$; the choice for maintenance therapy after discharge for this group was rivaroxaban $41.2 \%$ and apixaban $41.2 \%$. There were some differences between the choice of anticoagulants for CAT and other VTE, however, both enoxaparin and rivaroxaban were the most used in both groups and in both situations, respectively.

TABLE 1 Anticoagulation Therapy for Maintenance after Discharge

\begin{tabular}{|c|c|c|c|c|}
\hline & Total & No cancer & Cancer & \\
\hline \multicolumn{5}{|c|}{ Anticoagulant of maintenance } \\
\hline Enoxaparin & $3(3.9)$ & $2(3.3)$ & $1(5.9)$ & 0.33 \\
\hline Warfarin & $25(19.5)$ & $14(23.3)$ & $1(5.9)$ & \\
\hline Rivaroxaban & $36(46.8)$ & $29(48.3)$ & $7(41.2)$ & \\
\hline Edoxaban & $3(3.9)$ & $2(3.3)$ & $1(5.9)$ & \\
\hline Total & $95(100)$ & $73(76.8)$ & $22(23.2)$ & - \\
\hline
\end{tabular}

TABLE 2 Primary Cancer Sites among Patients with Diagnosis of Thromboembolism (VTE)

\begin{tabular}{ll} 
Primary site of cancer & Total $=\mathbf{2 2}-\mathbf{n}(\%)$ \\
Breast & $6(27.3)$ \\
Prostate & $4(18.2)$ \\
Lung & $3(13.6)$ \\
Colorectal & $2(9.1)$ \\
Kidney & $2(9.1)$ \\
Pancreas & $2(9.1)$ \\
Hematological & $2(9.1)$ \\
Others & $1(4.5)$ \\
\hline
\end{tabular}

PB1212 | Trombophilia and Other Risk Factors of Cerebral Venous Sinus Thrombosis in 255 Patients

A. Gwozdowska ${ }^{1}$; E. Odnoczko²; B. Baran ${ }^{2}$; J. Windyga ${ }^{1,2}$; A. Gwozdowska; E. Odnoczko; B. Baran; 1;2 J. Windyga

${ }^{1}$ Department of Haemostasis Disorders and Internal Medicine, Institute of Hematology and Transfusion Medicine (IHTM), Warsaw, Poland;

${ }^{2}$ Department of Haemostasis and Metabolic Disorders, Institute of Hematology and Transfusion Medicine (IHTM), Warsaw, Poland

Background: Cerebral venous sinus thrombosis (CVST) is a rare manifestation of venous thromboembolism (VTE). The estimated CVST incidence in adults is $2-5$ per million cases. Among potential risk factors for CVST are congenital and acquired thrombophilia.

Aims: Evaluation of the prevalence of congenital thrombophilia, antiphospholipid syndrome (APS) and other risk factors for CVST in a large cohort of patients.

Methods: Our study comprised 255 patients with definitive diagnosis of CVST: 188 women and 67 men, mean age 39 (14-83) years. Medical interview included family history of VTE, age and presence of environmental thrombosis risk factors (pregnancy, delivery, oral contraceptives and hormone replacement therapy (OCPs/HRT), infection, neoplasm) at CVST diagnosis. Laboratory tests comprised thrombophilic defects: deficiency of antithrombin, protein $C$ and protein $\mathrm{S}$ as well as factor $\mathrm{V}$ Leiden and prothrombin gene mutation G20210A and antiphospholipid antibodies. 
Results: Forty-two (16.5\%) patients had congenital thrombophilia and fourteen (5.5\%) were diagnosed with APS. In 11 (4.3\%) patients myeloproliferative neoplasms (MPN) was investigated due to complete blood count aberrations and in 9 of them $(3.5 \%$ of all patients) JAK-2 V617F positive MPN was confirmed (Essential Thrombocytemia (ET) in 5, Policythemia Vera (PV) in 1, ET/Primary Myelofibrosis (PMF) in 1, PMF in 1 and MF post PV in 1). One patient had triple-negative ET and one - Myelodysplastic Syndrome/MPN Two patients $(0.8 \%)$ had congenital thrombophilia coexistent with MPN. At the time of CVST diagnosis 86/188 (45.7\%) women were taking OCPs/HRT, while in twenty-nine (15.4\%) females CVST occurred during pregnancy or at delivery. Thirty one patients (12.1\%) reported a positive family history of VTE.

Conclusions: In the study patients the main risk factors for CVST were oral contraceptives and hormone replacement therapy as well as congenital thrombophilia, pregnancy and delivery.

PB1213 | Prevalence and Incidence of Deep Vein Thrombosis in Medical Critically III Patients: A Prospective Analysis in a Single Tertiary Care Centre in Thailand

S. Arunothai ${ }^{1}$; T. Panpikoon ${ }^{2}$; K. Boonyawat ${ }^{3}$; Y. Sutherasan ${ }^{4}$; P. Theerawit ${ }^{4}$

${ }^{1}$ Department of Medicine, Faculty of Medicine Ramathibodi Hospital, Mahidol University, Bangkok, Thailand; ${ }^{2}$ Division of Body Intervention, Department of Radiology, Faculty of Medicine Ramathibodi Hospital, Bangkok, Thailand; ${ }^{3}$ Division of Hematology, Department of Medicine, Faculty of Medicine Ramathibodi Hospital, Bangkok, Thailand; ${ }^{4}$ Division of Pulmonary and Critical Care Medicine, Department of Medicine, Faculty of Medicine Ramathibodi Hospital, Bangkok, Thailand

Background: Critically ill patients are at high risk for developing venous thromboembolism. Currently, the prevalence and incidence of deep vein thrombosis (DVT) in Thai medical intensive care unit (ICU) is unknown.

Aims: We aimed to evaluate prevalence and incidence of DVT in patients who admitted to critical care units and associated risk factors of DVT.

Methods: A single-center, prospective cohort study of critically medically ill patients was conducted at Ramathibodi Hospital during 2019-2020. Patients were screened for proximal DVT by Doppler ultrasound on admission, day 5 , day 7 , and then weekly until discharge from ICU. Patients who have a prior history of venous thromboembolism, femoral venous catheter insertion, end stage renal disease (ESRD) requiring hemodialysis, require platelet transfusion were defined as a high-risk group.

Results: A total of 200 patients were enrolled. Mean age (standard deviation, SD) was 66.5 (16.4) years. Mean APACHE II score was 27. The prevalence of DVT was $8.5 \%$ and the incidence of DVT was 7\%. No pulmonary embolism occurred in incident DVT patients. During admission, 2 (1\%) patients had received pharmacologic thromboprophylaxis and 12 (6\%) received therapeutic anticoagulant. There was no significant difference in DVT between high risk and low risk group (8.9 \% vs. $6.3 \%$, respectively). There was no significant difference in mortality in those with and without DVT. No risk factor was associated with the development of DVT.

Conclusions: Despite low rate of thromboprophylaxis, we found a low incidence of DVT. Pharmacological thromboprophylaxis in our critical care unit should be discussed individually.

PB1214 | Low Awareness of Venous Thromboembolism among the General Population: A Call for Increased Public Enlightenment Programs

H. Okoye ${ }^{1}$; T. Nwagha ${ }^{1}$; E. Ezigbo ${ }^{2}$; O. Nnachi ${ }^{3} ;$ O. Obodo ${ }^{4}$; O. Nnachi ${ }^{3} ;$ N. Amu ${ }^{4}$; I. Anigbogu ${ }^{1}$

${ }^{1}$ Department of Haematology and Immunology, College of Medicine, University of Nigeria, Enugu, Nigeria; ${ }^{2}$ Thrombosis and Haemostasis Unit, Department of Medical Laboratory Sciences, University of Nigeria, Enugu Campus, Enugu, Nigeria; ${ }^{3}$ Department of Haematology and Blood Transfusion, Alex Ekwueme Federal University Teaching Hospital Abakaliki Ebonyi, Abakaliki, Nigeria; ${ }^{4}$ Department of Haematology and Blood Transfusion. University of Nigeria Teaching Hospital, Enugu, Nigeria

Background: Venous thromboembolism (VTE) is a notable but often ignored cause of disability and death. Improved public awareness of the symptoms and risks associated with VTE reduces morbidity and nortality from the disease.

Aims: We aimed to determine the awareness of VTE among the general population.

Methods: We conducted a population-based study using a pretested pre-validated Ipsos-Reid questionnaire between October 2019 to March 2020. The questionnaire was distributed to consenting adults in the capital cities of Enugu and Ebonyi states of SouthEastern Nigeria to determine their level of awareness and knowledge of VTE: deep vein thrombosis (DVT) and pulmonary embolism (PE). We also sought to determine their knowledge of the symptoms and risk factors of VTE.

Results: A total of 284 adults participated with a mean age of $32.73 \pm 10.33$ years and majority $(70.8 \%)$ had a post-secondary level of education. While the majority of them were aware of other medical conditions like a heart attack (96.1\%), stroke (97.2\%), diabetes (98.2\%), HIV/AIDS (98.6\%), cancer (97.2\%) and malaria (98.2), just a few of the subjects were aware of thrombosis (41.5\%) and DVT (33.8\%). Less than half (42.4\%) correctly described DVT as a blood clot in the vein and $13.7 \%$ of the respondents knew what PE feels like. A minority of them knew the risk factors of VTE included hospital stay (19.0\%), surgery (37.2\%), cancer (31.6\%), pregnancy (31.6\%) and old age (29.6\%). Age and gender showed no statistically significant association with awareness of VTE ( $p$ value: 0.491 and 0.287 respectively. 
TABLE 1 Awareness of Risk factors of Blood Clot

\begin{tabular}{lcc}
$\begin{array}{l}\text { Table 1: Awareness of Risk factors of Blood Clot } \\
\text { Variable }\end{array}$ & Frequency & Per cent \\
\hline Risk factors (multiple response, $\boldsymbol{n}=\mathbf{9 5 7}$ ) & 48 & 19.0 \\
Hospital stay & 94 & 37.2 \\
Surgery & 80 & 31.6 \\
Cancer & 118 & 46.6 \\
Immobility & 80 & 31.6 \\
Pregnancy or just giving birth & 65 & 25.7 \\
Use of oral contraception pills or hormone & & \\
replacement therapy & 86 & 34.0 \\
A family history of clot & 75 & 29.6 \\
Older age (65years plus) & 32 & 12.6 \\
Too much exercise & 99 & 39.1 \\
High blood cholesterol & 34 & 13.4 \\
Donating blood & 69 & 27.3 \\
High blood pressure & 5 & 2.0 \\
Other factors & 3 & 1.2 \\
None & 69 & 27.3 \\
Not sure of any & & \\
\hline
\end{tabular}

Conclusions: The awareness of VTE in the general population is low. The creation of awareness programs should be a public health priority in order to reduce morbidity and mortality associated with VTE.

\section{PB1215 | Venous Thromboembolism Profile and Management} Differences between Ages in a RIETE's Hospital of Brazil

R. Santos de Oliveira Júnior; J. Abreu Couto Vieira;

\section{A.T. Cavalcanti Rocha}

Escola Bahiana de Medicina e Saúde Pública, Salvador, Brazil

Background: Although mortality rate of venous thromboembolism (VTE) is lower in younger patients, its incidence remains high even with various modifiable risk factors, principally in Brazil, where VTE management data is scarce.

Aims: Evaluate the management of patients with deep venous thromboembolism (DVT) and/or pulmonary embolism (PE) in private hospital in Salvador, participating in the RIETE registry.

Methods: Prospective cohort which evaluated consecutive patients from 5/2018-9/2019 and compared differences between ages, classic inferential descriptive analyzes were used.

Results: Of the 95 patients, $67.4 \%$ were women, the mean age 53,3 years ( \pm 19 ) and $32.8 \%$ of women, vs. $9.6 \%$ of men were $\leq 40$ years $(P=0.01)$. Among risk factors for VTE, $64.2 \%$ were overweight $31.6 \%$ had obesity, $23.2 \%$ cancer, $20 \%$ history of VTE, $28.1 \%$ of women used hormones, $17.9 \%$ surgery $\leq 2$ months. Thus, only cancer and hormone therapy differed significantly between ages. There were $64.2 \% \mathrm{PE}, 17.9 \%$ DVT and 17.9\% DVT/PE, with more patients $>40$ years-old having PE $(64.8 \%$ vs $62.5 \%)$ and more patients $\leq 40$ having combined DVT/PE (20.8\% vs $16.9 \%)$. Diagnostic tool more utilized was angiotomography, used in $85.3 \%$ of patients; $32.6 \%$ were admitted to intensive care unit; mean length-of-stay was longer for patients $>40$ (10.2 \pm 14 vs $5.8 \pm 3.7$ days). The initial choice of anticoagulant was enoxaparin in $88.4 \%$, rivaroxaban $6.3 \%$, warfarin $2.1 \%, 3.2 \%$ others DOACS. There was more indication of anticoagulation after discharge in patients $\leq 40$ (91.7\% vs $78.9 \%)$; the choice for maintenance was rivaroxaban $37.9 \%$, apixaban $21.1 \%$, $16.8 \%$ warfarin, $4.2 \%$ edoxaban and $3.2 \%$ enoxaparin.

Table 1 Risk factors of patients with diagnosis of venous tromboembolism (VTE)

\begin{tabular}{|c|c|c|c|c|}
\hline $\begin{array}{l}\text { Risk factors } \\
\text { for VTE }\end{array}$ & $\begin{array}{l}\text { Total } \\
N(\%)\end{array}$ & $\begin{array}{l}\leq 40 \\
\text { years } \\
N(\%)\end{array}$ & $\begin{array}{l}>40 \\
\text { years } \\
N(\%)\end{array}$ & $P$ value \\
\hline Overweight & $61(64.7)$ & $13(54.2)$ & $48(67.6)$ & 0.23 \\
\hline Obesity & 30 (31.6) & $7(29.2)$ & $23(32.4)$ & 0.76 \\
\hline Smoking & $7(7.4)$ & $1(4.2)$ & $6(8.5)$ & 0.48 \\
\hline Cancer & $22(23.2)$ & $1(4.2)$ & $21(29.6)$ & 0.01 \\
\hline $\begin{array}{c}\text { Immobility > } 4 \\
\text { days }\end{array}$ & $9(9.5)$ & $0(0)$ & 9 (12.7) & 0.06 \\
\hline History of VTE & $19(20)$ & $6(25)$ & $13(18.3)$ & 0.47 \\
\hline $\begin{array}{l}\text { Hormone } \\
\text { therapy }\end{array}$ & $18(18.9)$ & $10(41.7)$ & $8(11.3)$ & 0.001 \\
\hline $\begin{array}{l}\text { Surgery, past } 2 \\
\text { months }\end{array}$ & $17(17.9)$ & $4(16.7)$ & 13 (18.3) & 0.85 \\
\hline
\end{tabular}

Table 2 Anticoagulation therapy for maintenance after discharge

\begin{tabular}{lllll} 
& TotalN & $\leq 40$ years & $>40$ years & $P$ \\
Anticoagulant & $(\%)$ & $\mathbf{N}(\%)$ & $\mathbf{N}(\%)$ & value \\
$\begin{array}{c}\text { Indication for } \\
\quad \text { maintenance }\end{array}$ & $78(82.1)$ & $22(91.7)$ & $56(78.9)$ & 0.15 \\
Rivaroxaban & $36(37.9)$ & $11(45.8)$ & $25(35.2)$ & 0.35 \\
Apixaban & $20(21.1)$ & $5(20.8)$ & $15(21.1)$ & 0.97 \\
Edoxaban & $4(4.2)$ & $0(0)$ & $4(5.6)$ & 0.12 \\
Warfarin & $16(16.8)$ & $5(20.8)$ & $11(15.5)$ & 0.54 \\
Enoxaparin & $3(3.2)$ & $1(4.2)$ & $2(2.8)$ & 0.74 \\
Total & $95(100)$ & $24(100)$ & $71(100)$ & \\
\hline
\end{tabular}

Conclusions: This cohort had more women with VTE, particularlly the group of patients $\leq 40$ years-old; high rates of $\mathrm{PE}$ and strong risk factors such as cancer, hormone use and obesity. Enoxaparin was still the main choice for initial treatment; younger population had more indication of anticoagulation after discharge and DOACS were more common in the maintenance of the coagulation.

PB1216 | Clinical Observations in Patients with Cerebral Venous Sinus Thrombosis

T.W Htut ${ }^{1}$; H.G Watson ${ }^{1}$; G. Mackay ${ }^{2}$; M.M Khan ${ }^{1}$

${ }^{1}$ Department of Haematology, Aberdeen Royal Infirmary, Aberdeen, United Kingdom; ${ }^{2}$ Department of Neurology, Aberdeen Royal Infirmary, Aberdeen, United Kingdom

Background: Cerebral venous sinus thrombosis (CVST) is a rare form of cerebrovascular disease, accounting for $0.5-1 \%$ of all strokes. 
Aims: To review associations with the development of CVST including patient behaviours and characteristics. To review outcomes of anticoagulant therapy.

Methods: Retrospective single centre observational study over 4 years practice. Patients diagnosed with CVST between December 2016 and December 2020 were included. A diagnosis of CVST was confirmed by either CT +/- angio/ venography or MRI +/- angio/ venography. We reviewed clinical records for patient characteristics and provoking factors for venous thromboembolism (VTE) and evidence of thrombotic and haemorrhagic events whilst on anticoagulation.

Results: Among total 17 patients included, the mean age was 42 years (range 21-88) and the majority of patients were female (70.6\%). None of the patients had a previous history of VTE.

11 patients had one or more risk factors for thrombosis at the time of diagnosis. This included active use of COCP in 7 out of 12 female patients (58.3\%), local and systemic infections in 4 patients (23.5\%) antiphospholipid syndrome (APS) in 5 patients (33\%), and antithrombin deficiency in 1 patient (6.6\%) out of 15 patients tested for APS and thrombophilia.

There was no reported recurrent thrombotic event during a median duration of 20 months follow up (range 3-47). No major bleeding occurred and only one patient reported CRNMB due to vaginal bleeding related to her underlying cervical cancer.

Conclusions: In our cohort the majority of patients affected were female and were strongly associated with APS and use of the COCP both of which may induce a state of activated protein $\mathrm{C}$ resistance. No recurrent CVST or other form of VTE was reported during follow up.

\section{VTE PROPHYLAXIS}

PB1217 | Implementation of a Multidisciplinary Surveillance Team to Improve Inferior Vena Cava Filter Retrieval and Outcomes

$\underline{\text { S. Chao }}{ }^{1}$; H. Bortz ${ }^{2}$; H. Stevens ${ }^{3,4,5}$; J. McFadyen ${ }^{3,4,5} ;$ H. Tran ${ }^{3,4}$

${ }^{1}$ Monash University, Melbourne, Australia; ${ }^{2}$ Pharmacy Department, Alfred Health, Melbourne, Australia; ${ }^{3}$ Clinical Haematology, Alfred Health, Melbourne, Australia; ${ }^{4}$ Australian Centre for Blood Diseases, Monash University, Melbourne, Australia; ${ }^{5}$ Atherothrombosis and Vascular Biology Program, Baker Institute, Melbourne, Australia

Background: Inferior vena cava filters (IVCF) are commonly used to prevent pulmonary embolism in high risk patients deemed unsuitable for venous thromboembolism (VTE) chemoprophylaxis, or as adjunct therapy in patients with established VTE who are unable to receive anticoagulation due to high bleeding risk. However, insertion of IVCF may result in significant complications such as IVC occlusion or filter embolisation. Therefore, timely filter retrieval is critical.

Aims: To evaluate whether the introduction of a multidisciplinary surveillance team (MDST) impacts IVCF retrieval rate, time-to-retrieval, filter-related complications, and rate of VTE after IVCF insertion.
Methods: Retrospective study at a tertiary institution evaluating all patients with IVCF insertion between January 2015 and December 2018. A MDST, involving haematology and pharmacy, was introduced in July 2017. The team had oversight of all patients with IVCF inserted and established systematic follow up and planning for filter retrieval. All patients were followed up to 12 months after IVCF insertion. Outcomes were compared between the groups before (pre-MDST) and after (post-MDST) introducing the MDST.

Results: Four hundred and sixty-six patients had retrievable IVCF inserted, with 280 patients in the pre-MDST group and 186 patients in the post-MDST group. Overall, 208 (44.6\%) patients received an IVCF due to acute VTE, and 258 (55.4\%) patients received the filter as VTE prophylaxis. After the introduction of a MDST, the rate of successful IVCF retrieval was significantly increased from $51.1 \%$ to 71.5\% $(P<0.0001)$, and patients lost-to-follow-up decreased from $18.9 \%$ to $3.8 \%(P<0.0001)$ (Table 1$)$. No significant differences between the pre-MDST and post-MDST groups were found in time-toretrieval, filter complications, or VTE after IVCF insertion.

TABLE 1 Summary of results

\begin{tabular}{|lccc|}
\hline & $\begin{array}{c}\text { Pre MDST } \\
(\mathbf{n = 2 8 0})\end{array}$ & $\begin{array}{c}\text { Post MDST } \\
(\mathbf{n = 1 8 6 )}\end{array}$ & P value \\
\hline Successful IVCF retrieval, $n$ (\%) & $143(51.1 \%)$ & $133(71.5 \%)$ & $<0.0001$ \\
\hline Lost-to-follow-up, $n$ (\%) & $53(18.9 \%)$ & $7(3.8 \%)$ & $<0.0001$ \\
\hline $\begin{array}{l}\text { Median time to retrieval (days) } \\
\text { Patients with IVCF } \\
\text { complications, } n(\%)\end{array}$ & 126 & 140 & NS \\
\hline VTE post IVCF insertion, $n(\%)$ & $29(10.4 \%)$ & $20(10.8 \%)$ & NS \\
\hline
\end{tabular}

Conclusions: The establishment of an MDST for IVCF resulted in a significant improvement in filter retrieval rates and notable reduction in patients lost-to-follow-up. These findings support the implementation of a supervisory team to improve IVCF monitoring and retrieval to improve patient-centred care.

PB1218 | Comparison of 0.9\% Normal Saline (NS) Flush and Unfractionated Heparin (UFH) Flush for Maintenance of Central Venous Catheter Line Patency

A.N. Landmesser ${ }^{1,2}$; R.J. Roberts ${ }^{2}$; R.P. Rosovsky ${ }^{3,4}$; M.E. Barra ${ }^{2}$ ${ }^{1}$ Northeastern Univeristy, Boston, United States; ${ }^{2}$ Massachusetts General Hospital, Department of Pharmacy, Boston, United States; ${ }^{3}$ Massachusetts General Hospital, Department of Medicine, Division of Hematology, Boston, United States; ${ }^{4}$ Harvard School of Medicine, Boston, United States

Background: Central venous catheters (CVCs), an important tool often utilized in the care of critically ill patients, provides access for medication and fluid administration as well as hemodynamic monitoring and diagnostics. To maintain line patency, CVCs are often flushed with unfractionated heparin (UFH) or $0.9 \%$ normal saline (NS). However, 
conclusive evidence surrounding the optimal CVC maintenance strategy is unknown. During a critical heparin shortage in $2019,0.9 \%$ NS became the only CVC flush option to maintain line patency at our institution.

Aims: To compare safety and effectiveness of $0.9 \%$ NS versus UFH flush in the maintenance of CVC.

Methods: We conducted a retrospective analysis at a tertiary academic medical center of critically ill adult patients with documented CVC placed for at least 72 hours, between February 2019 and February 2020. The primary efficacy outcome was a composite of CVC occlusion, CVC removal due to thrombosis, or intracatheter alteplase administration. The secondary safety outcomes included major and clinically relevant non-major bleeding (CRNMB). Data was extracted from the electronic health record, and the results were analyzed using JMP ${ }^{\circledR}$ Pro 15 (SAS Institute Inc.) software.

Results: Five hundred consecutive patients were analyzed consisting of 250 patients in each cohort. Among the entire cohort 275 patients were male, median age was 62.2 years [51.0-72.6], median length of stay in the intensive care unit was 11 days [6.0-21.0], and the median duration of CVC was 8.3 days [5.4-13.8]. Twenty-nine patients (11.6\%) in the UFH group experienced the primary outcome versus 22 patients (8.8\%) in the NS group. Combined rates of major bleeding and CRNMB were similar between the two groups ( $6 \%$ in NS and $8 \%$ in UFH).

\section{TABLE 1 Population Baseline Characteristics}

\section{Table 1}

Population Baseline Characteristics

\begin{tabular}{|l|c|c|}
\hline Patient Characteristics & $\mathbf{0 . 9} \%$ NS ( $\mathbf{n = 2 5 0 )}$ & UFH (n=250) \\
\hline Age (years) & $62.8[49.8-74.2]$ & $61.7[51.9-70.2]$ \\
\hline Sex (female) & $117(46.8)$ & $108(43.2)$ \\
\hline Body Mass Index $\left(\mathrm{kg} / \mathrm{m}^{2}\right)$ & $27.7[23.6-33.3]$ & $28.3[23.6-32.3]$ \\
\hline Coronary Artery Disease & $84(33.6)$ & $83(33.2)$ \\
\hline Heart Failure & $77(30.8)$ & $76(30.4)$ \\
\hline Diabetes Mellitus & $85(34.0)$ & $88(35.2)$ \\
\hline Atrial Fibrillation & $62(24.8)$ & $68(27.2)$ \\
\hline Thrombotic Event & $110(44.0)$ & $111(44.4)$ \\
\hline Malignancy & $75(30.0)$ & $66(26.4)$ \\
\hline
\end{tabular}

Malignancy

$75(30.0)$

Abbreviations: NS, normal saline; UFH, unfractionated hepari

'All data presented as $n(\%)$ or median [interquartile range]

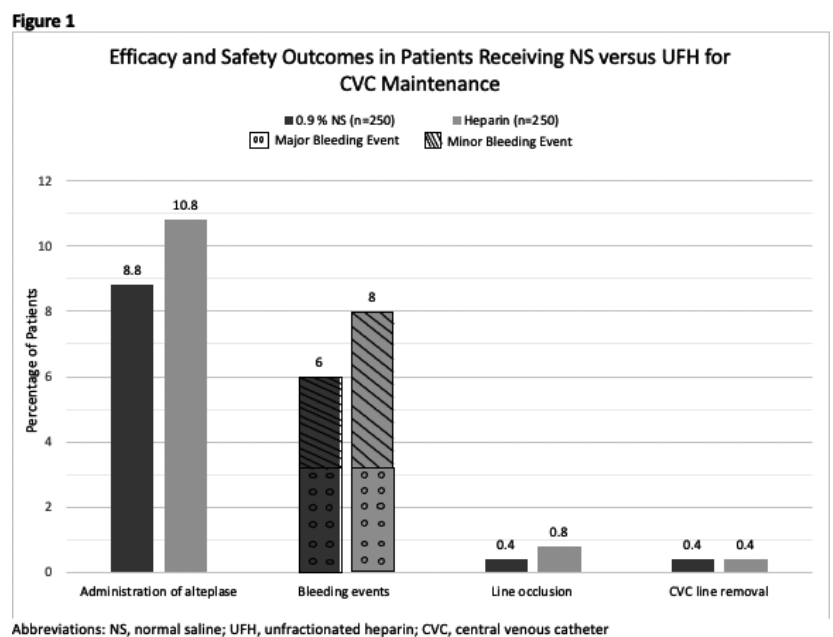

Conclusions: In this large retrospective analysis, there was no difference in CVC patency or bleeding events between patients who received NS versus UFH flush when used for maintenance of CVC.

PB1219 | Can Combining a Risk Score Derived from Ubiquitous Biomarkers with a Clinical Risk Assessment Model Better Predict 90-day Post-discharge Hospital-associated Venous Thromboembolism among Medical Patients?

S. Woller ${ }^{1,2} ;$ S. Stevens ${ }^{1,2} ;$ J. Bledsoe ${ }^{1,3} ;$ J. Lloyd $^{1} ;$ G. Snow $^{1}$;

M. Fazili ${ }^{1}$; L. Venner ${ }^{1}$; J. Christensen ${ }^{1}$; B. Horne ${ }^{1,3}$

${ }^{1}$ Intermountain Medical Center, Murray, United States; ${ }^{2}$ University of Utah School of Medicine, Salt Lake City, United States; ${ }^{3}$ Stanford University, Stanford, United States

Background: Some discharged medical patients are at risk for hospital-associated venous thromboembolism (HA-VTE) and identifying at-risk patients can inform extended-duration thromboprophylaxis decision-making. We reported the derivation and validation of a thrombosis risk estimation tool derived from the RDW, BUN, age, glucose, WBC, platelet count, RBC, and sodium that is predictive of post-discharge 90-day VTE with an area under the received operating characteristic curve $=0.6$ (The Hospital-Associated Venous ThromboEmbolism InterMountain Risk Score (HA-VTE IMRSCHEST2020 Late Breaking Abstract). From our original study's validation set of 15,224 patients, 297 (2.0\%) experienced 90-day post-discharge VTE. Two clinical risk assessment models (RAMs) for the outcome of VTE derived with weighted clinical characteristics, the UTAH score and the Kucher score have been described.

Aims: We assess if the addition of a clinical RAM, the UTAH score, or the Kucher score, might improve on the predictiveness of the HAVTE IMRS.

Methods: We performed 2 Cox regression analyses. Table 1 (yellow-top) reports the incremental predictiveness for each additional 1 point for the HA-VTE IMRS and each clinical RAM. Table 1 (blue-bottom) reports each risk assessment tool's predictiveness at the pre-specified high-risk cut point (HA-VTE IMRS $\geq 7$, UTAH Score $\geq$ 1 , Kucher Score $\geq 4$ ) formerly identified as predictive of 90 -day HAVTE. Table 2 demonstrates the AUC for the risk prediction scores. Results: For the outcome of 90-day HA-VTE the combination of the UTAH score with the HA-VTE IMRS yielded the most favorable cstatistic of 0.686 . The HA-VTE IMRS alone may be calculated without clinical history. If clinical history is readily available the clinical history added to the HA-VTE IMRS is useful. 
Table 1 Cox Regression for 90-day Post-Discharge Venous Thromboembolism

Hazard ratios and $95 \%$ confidence intervals are per +1 point for each score. Ranges of scores were: HA-VTE IMRS: 0-16; UTAH score: 0-4; Kucher score: 0-14. N = 15,224 (297 VTE, 14,927 event-free).

\begin{tabular}{|c|c|c|c|}
\hline & Univariable & $\begin{array}{l}\text { IMRS + UTAH } \\
\text { Score }\end{array}$ & $\begin{array}{l}\text { IMRS + } \\
\text { Kucher } \\
\text { Score }\end{array}$ \\
\hline HA-VTE IMRS & $\begin{array}{c}1.12(1.08 \\
1.15)^{*}\end{array}$ & $\begin{array}{c}1.07(1.04 \\
1.11)^{*}\end{array}$ & $\begin{array}{c}1.08(1.04, \\
1.12)^{*}\end{array}$ \\
\hline UTAH Score & $\begin{array}{c}1.91(1.70 \\
2.13)^{*}\end{array}$ & $\begin{array}{c}1.79(1.60 \\
2.02)^{*}\end{array}$ & \\
\hline Kucher Score & $\begin{array}{c}1.21(1.16 \\
1.26)^{*}\end{array}$ & & $\begin{array}{c}1.18(1.14, \\
1.23)^{*}\end{array}$ \\
\hline \multicolumn{4}{|c|}{$\begin{array}{l}\text { Hazard ratios and } 95 \% \text { confidence intervals for dichotomous } \\
\text { comparisons }\end{array}$} \\
\hline $\begin{array}{l}\text { HA-VTE } \\
\qquad \begin{array}{l}\text { IMRS } \geq 7 \\
\text { ( } n=5,242) \\
\text { vs. }<7 \\
(n=9,982)\end{array}\end{array}$ & $\begin{array}{c}1.69(1.35 \\
2.13)^{*}\end{array}$ & $\begin{array}{c}1.39(1.11 \\
1.76) \dagger\end{array}$ & $\begin{array}{c}1.43(1.14 \\
1.81) \ddagger\end{array}$ \\
\hline $\begin{array}{l}\text { UTAH Score } \geq 1 \\
\qquad \begin{array}{l}(n=5,884) \\
\text { vs. } 0 \\
(n=9,340)\end{array}\end{array}$ & $\begin{array}{l}3.25(2.55 \\
4.13)^{*}\end{array}$ & $\begin{array}{c}3.07(2.41 \\
3.92)^{*}\end{array}$ & \\
\hline $\begin{array}{l}\text { Kucher Score } \geq \\
\begin{array}{l}4(n=4,120) \\
\text { vs. }<4 \\
(n=11,104)\end{array}\end{array}$ & $\begin{array}{c}2.62(2.09, \\
3.30)^{*}\end{array}$ & & $\begin{array}{c}2.45 \text { (1.94, } \\
3.09)^{*}\end{array}$ \\
\hline
\end{tabular}

${ }^{*} P<0.001, \dagger P=0.005, \ddagger P=0.002$

UTAH Score: prior VTE, cancer, central venous catheter, order for bedrest; 1 point each; a score $\geq 1$ confers high risk for thrombosis

Table 2 Predictiveness of each risk assessment method for the outcome of 90-day hospital-associated VTE among medical patients following discharge

$\begin{array}{ll}\text { Risk Assessment } & \text { AUC }(95 \% \mathrm{Cl}) \\ \text { HA-VTE IMRS } & 0.600(0.596-0.631) \\ \text { UTAH Score } & 0.658(0.625-0.690) \\ \text { HA-VTE IMRS + UTAH Score } & 0.686(0.657-0.715) \\ \text { Kucher Score } & 0.636(0.602-0.669) \\ \text { HA-VTE IMRS + Kucher Score } & 0.663(0.633-0.693)\end{array}$

AUC: area under receiver operating characteristic curve.

Conclusions: Once programmed, the HA-VTE IMRS calculation may be automated and presented effortlessly in the EMR from laboratory results ubiquitous in routine care. If clinical history that populates a RAM is readily available, this added to the HA-VTE IMRS appears useful.
PB1220 | VTE Prophylaxis in Foot and Ankle Surgery: A Worldwide Survey

R. Zambelli ${ }^{1,2} ;$ S. Frolke ${ }^{3}$; B. Nemeth ${ }^{4} ;$ D. Baumfeld ${ }^{2}$; C. Nery ${ }^{5}$; C. Ortiz ${ }^{6}$; S. Cannegieter ${ }^{4}$; S. Rezende ${ }^{2}$

${ }^{1}$ Mater Dei Healthcare Network, Belo Horizonte, Brazil; ${ }^{2}$ Federal University of Minas Gerais, Belo Horizonte, Brazil; ${ }^{3}$ Amsterdam University Medical Centre, Amsterdam, Netherlands; ${ }^{4}$ Leiden University Medical Center, Leiden, Netherlands; ${ }^{5}$ Albert Eistein Hospital, São

Paulo, Brazil; ${ }^{6}$ Universidad de los Andes, Santiago, Chile

Background: Current recommendations on thromboprophylaxis for foot and ankle (FA) surgery are often inconsistent and generally based on weak evidence.

Aims: The aim of this international survey study was to evaluate the current practice and rationale among orthopaedic surgeons regarding VTE prophylaxis following FA surgery.

Methods: From February 2019 to March 2020, a 12-item online questionnaire was sent by e-mail to orthopaedic societies across the world. The questionnaire was hosted by the ISTH RedCAP platform. Topics of interest were VTE rate, prescription, duration and type of thromboprophylaxis, bleeding complications, VTE risk factors, guidelines, risk assessment models and screening of VTE.

Results: A total of 693 FA orthopaedic surgeons from all continents completed the survey of whom 392 (57\%) performed $>200$ FA procedures per year. More than half of respondents (403, 58\%) reported that 1-5 patients experienced a symptomatic VTE in the postoperative year. In case thromboprophylaxis was prescribed, aspirin (325, 47\%), LMWH (291, 42\%) and DOACs (216, 31\%) were the preferred choice. The use of aspirin was more common in North America compared to the rest of the world (Figure 1). In Europe, LMWH was predominantly prescribed (Figure 1). Half of surgeons prescribed thromboprophylaxis for the duration of immobilization. Previous DVT, immobility, obesity and inherited thrombophilia were considered the main risk factors indicative of thromboprophylaxis.

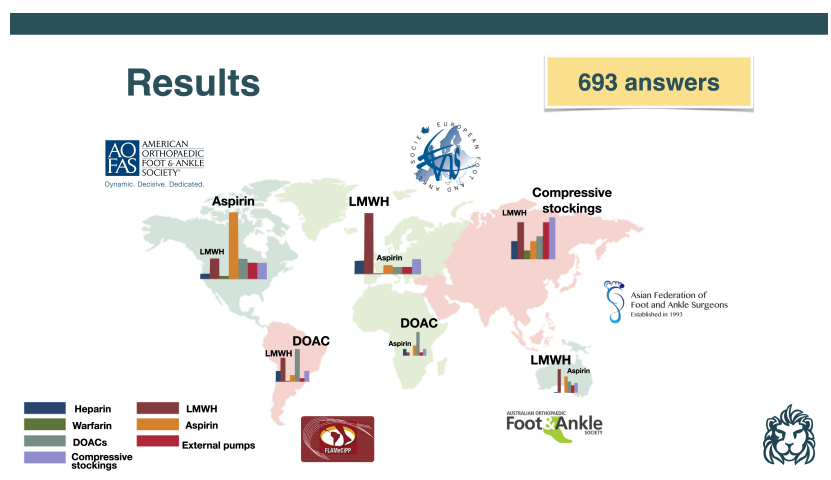

FIGURE 1 Distribution of thromboprophylaxis prescription worldwide 
Conclusions: Administration, duration and type of thromboprophylaxis differs greatly between FA surgeons and there is a strong intercontinental discrepancy. Although not recommended by the guidelines, orthopaedic surgeons are more likely to use thromboprophylaxis in FA surgery in case of previous DVT, immobility, obesity and inherited thrombophilia. Uniform guidelines are needed to optimize prophylactic strategies in FA procedures.

PB1221 | Safety and Efficacy of Low-Molecular Weight Heparin Regimes for Venous Thromboembolism Prophylaxis in Bariatric Surgery

A. Li ${ }^{1}$; A. Eshaghpour ${ }^{2}$; G. Lee ${ }^{3} ;$ J. Deng ${ }^{4}$; R. Ikesaka ${ }^{5}$; M. Carrier ${ }^{6}$; M. Crowther ${ }^{5}$

${ }^{1}$ University of Ottawa, Faculty of Medicine, Ottawa, Canada;

${ }^{2}$ McMaster University, Michael G. DeGroote School of Medicine, Hamilton, Canada; ${ }^{3}$ University of Toronto, Temerty Faculty of Medicine, Toronto, Canada; ${ }^{4}$ McMaster University, Faculty of Health Sciences, Hamilton, Canada; ${ }^{5}$ McMaster University, Department of Medicine, Hamilton, Canada; ${ }^{6}$ University of Ottawa, Division of Hematology, Department of Medicine, Ottawa Hospital Research Institute, General Campus, Ottawa, Canada

Background: Patients undergoing bariatric surgery are at an increased risk of venous thromboembolic events (VTE). The optimal low-molecular weight heparin (LMWH) thromboprophylactic dosing is debated. Different dosing regimens have reported bleeding and VTE rates ranging between $0 \%$ to $6.4 \%$ and $0 \%$ to $6.7 \%$, respectively. Aims: We sought to analyze the safety and efficacy of different thromboprophylactic regimens using LMWH following bariatric surgery.

Methods: We conducted a search of Medline and Embase from inception until November 2020. Screening and data abstraction were independently done by two authors (GL and JD). Observational studies or randomized controlled trials that assessed thromboprophylaxis using LMWH following bariatric surgery were included. LMWH regimens group by duration ( $<10$-days or $\geq 10$-days of anticoagulation and were classified as either weight-adjusted or non-weight adjusted, with non-weight adjusted doses grouped using the ASH classification of anticoagulant regimens (Table 1). The primary efficacy and safety outcomes were bleeding and VTE, respectively, and analyzed with the help of a biostatistician using the Comprehensive Meta-Analysis Software.

TABLE 1 Classification of LMWH regimens, adapted from ASH Classification of Anticoagulant Regimens by Intensity

Prophylactic Regimen

Bemiparin $3500 \mathrm{U}$, SC OD

Dalteparin 5000 U, SC OD

Enoxaparin $30 \mathrm{mg}$ (3000 U), SC OD

(for GFR 15-30)

Enoxaparin $30 \mathrm{mg}$ (3000 U), SC BID

(for $\mathrm{BMI} \geq 40 \mathrm{~kg} / \mathrm{m} 2$ )

Enoxaparin $40 \mathrm{mg}$ (4000 U), SC OD

Enoxaparin $40 \mathrm{mg}$ (4000 U), SC BID

(for $\mathrm{BMI} \geq 40 \mathrm{~kg} / \mathrm{m} 2$ )

Unfractionated heparin $5000 \mathrm{U}, \mathrm{SC}$ BID

Unfractionated heparin $5000 \mathrm{U}, \mathrm{SC}$ TID

Unfractionated heparin $7500 \mathrm{U}, \mathrm{SC}$ $\mathrm{BID}$ (for $\mathrm{BMI} \geq 40 \mathrm{~kg} / \mathrm{m} 2$ )

Nadroparin 2850 U, SC q24h (postop general surgery)

Nadroparin $5700 \mathrm{U}, \mathrm{SC}$ q24h (highrisk medical patients $>70 \mathrm{~kg}$ )

Nadroparin $3800 \mathrm{U}, \mathrm{SC}$ q24h (highrisk medical patients $\leq 70 \mathrm{~kg}$ or postop hip replacement surgery

Tinzaparin $3500 \mathrm{U}$, SC OD

Tinzaparin $4500 \mathrm{U}$, SC OD

Tinzaparin $75 \mathrm{U} / \mathrm{kg}$, SC OD

Results: Out of the 2692 studies identified by our search, a total of 41 articles were included. The majority of studies were observational cohort studies with enoxaparin being the most common LMWH. The rates of bleeding and VTE are reported in Figure 2. The rate of VTE ranged from $0.5 \%$ to $2 \%$ for $<10$-day regimens and $0.6 \%$ to $1.1 \%$ for $\geq 10$-day regimens. The rate of bleeding ranged from $1.5 \%$ to $3.1 \%$ for $<10$-day regimens and $2.4 \%$ to $3 \%$ for $\geq 10$-day regimens. The overall degree of heterogeneity was moderate for VTE and high for bleeding.

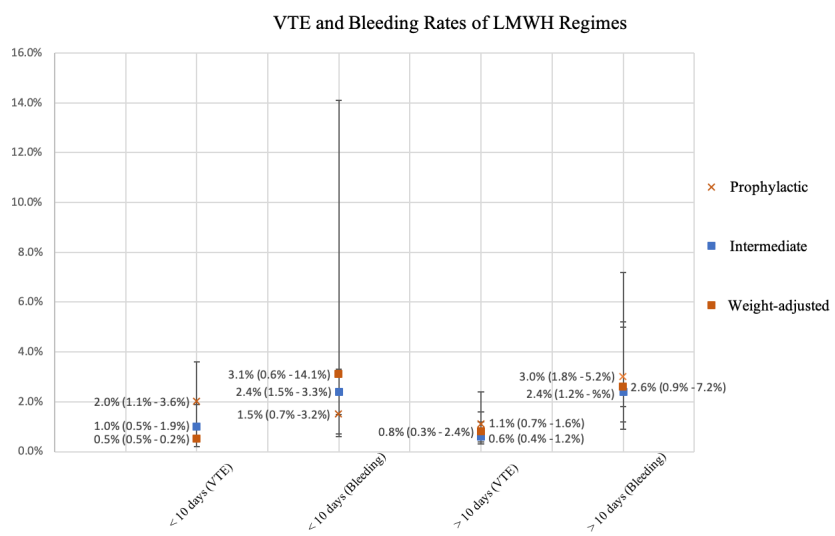

FIGURE 1 Bleeding and VTE rates of regimens with 95\% confidence intervals 
Conclusions: There was no statistically significant difference in terms of the VTE and bleeding rate between the different regimes of $\mathrm{LMWH}$. The heterogeneity of the data calls for prospective studies using standardized measurements of VTE and bleeding for further clarification.

\section{PB1222 | Comparing Adherence of Low Molecular Weight} Heparin versus Unfractionated Heparin for Thromboprophylaxis of Medical Inpatients

M. Trybula ${ }^{1} ;$ Z. Ahart ${ }^{1}$; E. Stacy ${ }^{2}$

${ }^{1}$ University of Cincinnati, Cincinnati, United States; ${ }^{2}$ UC Health University of Cincinnati Medical Center, Cincinnati, United States

Background: Both low molecular weight heparin (LMWH) administered once daily and unfractionated heparin (UFH) given thrice daily are routinely used for venous thromboembolism (VTE) prophylaxis in the inpatient setting. There is little data regarding a difference in adherence between these regimens.

Aims: The primary outcome was comparison of adherence to LMWH and UFH doses ordered for VTE prophylaxis of medical inpatients. Secondary outcomes included adherence rate among subgroup populations, incidence of VTE, and adherence rates of greater than $80 \%$ and $90 \%$ of doses ordered.

Methods: This is a retrospective study of 1444 adult patients admitted to a primary medicine team and receiving VTE prophylaxis in a 726-bed tertiary care center from January $1^{\text {st }}$ to October $1^{\text {st }}$, 2020. Patients with body mass index $(\mathrm{BMI})>40 \mathrm{~kg} / \mathrm{m}^{2}$, creatinine clearance $<30 \mathrm{~mL} / \mathrm{min}$, and COVID positive status were excluded. Adherence was defined as the percentage of ordered doses documented as administered within the electronic medical record.

Results: 456 patients received $\mathrm{LMWH}$ and 998 received UFH. Compared to UFH, LMWH had a significantly higher adherence with a median of $100 \%$ [IQR 66.7-100] vs $83.3 \%$ [IQR 50.0-97.9] $(P<0.001)$ and mean of $75.7 \%$ vs $68.4 \%(P<0.001)$. There was a statistically significant increase in adherence among various subgroups including: males, females, age $>50$ years old, and BMI 18.540. Patients in the LMWH group were more likely to have adherence rates of greater than $80 \%(62.9 \%$ vs $52.0 \%, P<0.001)$ and $90 \%$ (57.0\% vs $37.0 \%, P<0.001)$ when compared to UFH. There was no statistically significant difference in new VTE events between the LMWH and UFH groups.

Conclusions: Guidelines equally recommend LMWH and UFH for thromboprophylaxis in hospitalized medicine patients. This study demonstrates that LMWH has a higher adherence rate than UFH in the clinical setting, and providers should take this into consideration when making choices about VTE prophylaxis for hospitalized patients.
PB1223 | A Systematic Review and Meta-analysis on the Use of Chemoprophylaxis in the Prevention of Venous Thromboembolism in Colorectal Cancer Surgery in Asian Population

S.J.J. Tan ${ }^{1}$; A.Y. Chok ${ }^{1}$; Y.Y. Ng${ }^{1}$; I. Seow-En ${ }^{1}$; R. Sultana ${ }^{2}$; J.C. Allen ${ }^{2}$; E.K.W. Tan ${ }^{1}$; R. Mathew ${ }^{1}$.

${ }^{1}$ Singapore General Hospital, Singapore, Singapore; ${ }^{2}$ Duke-NUS

Medical School, Singapore, Singapore

Background: Venous thromboembolism (VTE) is associated with a significant risk of morbidity and potentially preventable death in surgical patients. However, it is widely believed that the incidence of VTE in Asian population is less frequently observed than the Western population. Therefore, routine chemoprophylaxis may not be necessary.

Aims: This systematic review aims to evaluate the incidence of venous thromboembolism (VTE) in colorectal cancer (CRC) surgery patients in the Asian population with and without the use of chemoprophylaxis. The secondary aim is to assess potential bleeding complications associated with it.

Methods: Studies published up to June 302020 were searched from PubMed, Embass, Cochrane Database of Systematic Reviews, Cochrane Central Register of Controlled trials and google scholar. Articles were found using the keywords: Asia, Asian, venous thromboembolism, deep vein thrombosis, pulmonary embolism, colorectal surgery with the inclusion criteria:

1) all randomized controlled trials (RCT), case-control and cohort studies,

2) population studied was primarily Asian or the study was conducted in an Asian country,

3) patients must undergo primary resection for the colorectal cancer, 4) studies reported incidence of DVT/PE.

Results: 12 studies (3 RCTs and 9 observational cohort studies) encompassing 8107 patients were included. The distribution of the population was in the following numbers: Korean ( $n=4162,51.3 \%)$, Japanese ( $n=2327,28.7 \%$ ), Chinese ( $n=1519,18.7 \%$ ) and Indian $(n=99,1.2 \%)$. Overall incidence of VTE was $1.9 \%$. Addition of chemoprophylaxis did not result in a significant decreased in incidence of VTE $(0.9 \%$ versus $3.2 \%, P=0.19)$, but associated with an increased in incidence of bleeding complications ( $0.8 \%$ versus $0.6 \%, P=0.004$.) Conclusions: There may not be additional benefit in the use of chemoprophylaxis for VTE prevention for colorectal cancer surgery in Asian population. The decision to introduce chemoprophylaxis should be individualized and also take into account the significant bleeding risks. 
PB1224 | Pharmacologic Profiles of Direct Oral Anticoagulants in Patients Receiving Rituximab-CHOP Chemotherapy

T. Punnachet $^{1}$; T. R. Cressey ${ }^{1}$; P. Apiwatnakorn ${ }^{2}$; A. Koonarat ${ }^{3}$;

L. Norasetthada ${ }^{1}$; A. Tantiworawit ${ }^{1}$; E. Rattaritamrong ${ }^{1}$;

T. Rattanathammethee ${ }^{1}$; S. Huntrakool ${ }^{1}$; P. Piriyakhuntorn ${ }^{1}$;

C. Chai-Adisaksopha ${ }^{1}$

${ }^{1}$ Chiang Mai University, Chiang Mai, Thailand; ${ }^{2}$ Lamphun Hospital,

Chiang Mai, Thailand; ${ }^{3}$ Nakornping Hospital, Chiang Mai, Thailand

Background: Rivaroxaban and dabigatran have been approved for prophylaxis and treatment of thromboembolic diseases in patients with active cancer. However, drug-drug interaction between chemotherapy and direct oral anticoagulant (DOAC) is unknown.

Aims: To evaluate the potential drug-drug interaction between rivaroxaban/dabigatran and $\mathrm{R}-\mathrm{CHOP}$ regimen.

Methods: This study was an open-label, pharmacokinetic study. Eligible subjects were adults diagnosed with non-Hodgkin lymphoma, diffuse large B-cell subtype, who were planned to receive $\mathrm{R}$-CHOP chemotherapy regimen. Enrolled patients were given rivaroxaban $10 \mathrm{mg}$ once daily or dabigatran $110 \mathrm{mg}$ twice daily. Each patient was tested for plasma DOAC levels 11 samples before and 11 samples after R-CHOP administration. Plasma rivaroxaban and dabigatran levels were measured using anti-factor Xa for rivaroxaban and diluted thrombin time, respectively.

Results: There were 17 patients ( 8 in rivaroxaban group 9 in dabigatran group with a median age of 66 years (range 59-70). The median creatinine clearance was $67 \mathrm{~mL} / \mathrm{min}$ (range 50-79). The plot of plasma rivaroxaban and dabigatran level by the time were shown in Figure $1 \mathrm{~A}$ and $1 \mathrm{~B}$. In rivaroxaban group, there was no statistically significant difference between mean area under the curve (AUC) of rivaroxaban before and concurrent with $\mathrm{R}-\mathrm{CHOP}$ administration, mean difference $36.50 \mathrm{ng} / \mathrm{mL}$ (95\% confidence interval $(\mathrm{Cl})-47.53$ to $120.50 \mathrm{ng} / \mathrm{mL}, P=0.34$ ), Figure $2 \mathrm{~A}$. In dabigatran group, there was no statistically significant difference between mean AUC of dabigatran before and concurrent with R-CHOP administration, mean difference $-39.53(95 \% \mathrm{Cl}-139.1$ to $60.0 \mathrm{ng} / \mathrm{mL}, P=0.38)$, Figure $2 \mathrm{~B}$. There was no adverse event in both groups.

Conclusions: Concomitant administration of R-CHOP chemotherapy do not significantly alter plasma levels of rivaroxaban and dabigatran.
Figure 1A/1B. Mean anti-FXa rivaroxaban and dTT $( \pm 95 \% \mathrm{CI})$ versus time curves before and concurrent with R-CHOP administration
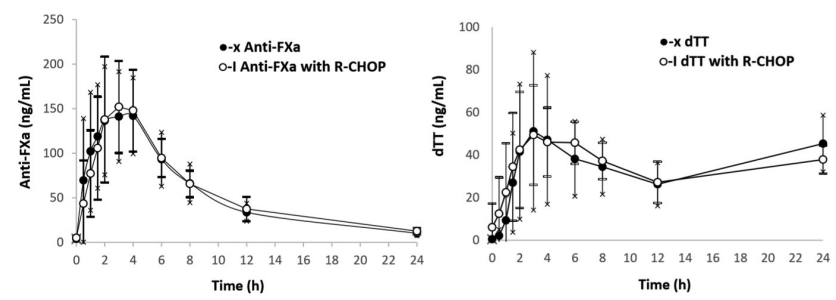

FIGURE 1 (A, B) Mean anti-FXa rivaroxaban and dTT ( $\pm 95 \% \mathrm{Cl}$ ) versus time curves before and concurrent with R-CHOP administration

Figure 2A/2B. Mean area under the curve (AUC) of plasma rivaroxaban and dabigatran before and concurrent with R-CHOP administration
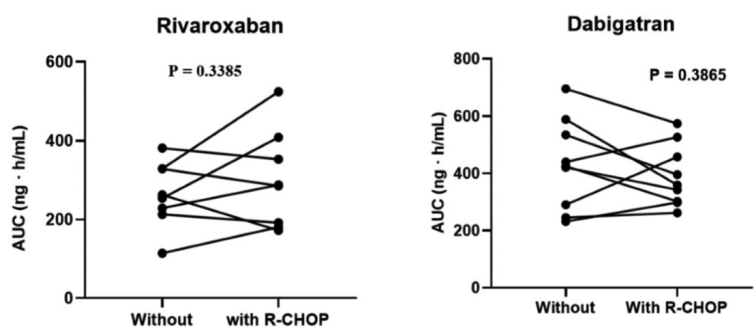

FIGURE 2 ( $\mathrm{A}, \mathrm{B})$ Mean area under the curve (AUC) of plasma rivaroxaban and dabigatran before and concurrent with $\mathrm{R}-\mathrm{CHOP}$ administration

\section{PB1225 | Venous Thromboembolism Risk Profiles and}

Prophylaxis in Medical Oncology Inpatients: a Subgroup Analysis of the Observational DissolVE-2 Study

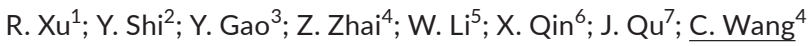

${ }^{1}$ Sun Yat-sen University Cancer Center, State Key Laboratory of Oncology in South China, Collaborative Innovation Center for Cancer Medicine, Department of Medical Oncology, Guangdong, China; ${ }^{2}$ National Cancer Center, Chinese Academy of Medical Sciences and Peking Union Medical College, Beijing Key Laboratory of Clinical Study on Anticancer Molecular Targeted Drugs, Department of Medical Oncology, Beijing, China; ${ }^{3}$ The First Affiliated Hospital of Zhengzhou University, Department of Pharmacy, Henan, China; ${ }^{4}$ Center of Respiratory Medicine, China-Japan Friendship Hospital, Institute of Respiratory Medicine, Chinese Academy of Medical Sciences, National Clinical Research Center for Respiratory Diseases, Department of Pulmonary and Critical Care Medicine, Beijing, China; ${ }^{5}$ West China Hospital, Sichuan University, Department of Respiratory and Critical Care Medicine, Department of Pulmonary and Critical Care Medicine, Sichuan, China; ${ }^{6}$ Zhongshan Hospital, Fudan University, Department of General Surgery, Shanghai, China; ${ }^{7}$ Rui jin Hospital, School of Medicine, Shanghai Jiao Tong University, Department of Respiratory Medicine,

Shanghai, China

Background: • Appropriate thromboprophylaxis for patients at-risk can reduce the incid ence of venous thromboembolism (VTE). 
- In recent years, the incidence of VTE has been increasing in China. This suggests that despite the American College of Chest Physicians (ACCP) guideline's recommendations, utilization of anticoagulants is suboptimal.

- Active cancer is one of the risk factors of VTE. Hence, improvement of awareness and practice of VTE prophylaxis in the medical oncology inpatients is urgently needed.

Aims: To investigate the risk profile of VTE and evaluate VTE prophylaxis implementation in patients with cancer in China.

Methods: DissolVE-2 was a retrospective, multicenter, observational, cross-sectional study enrolling 14000 patients in China. Here we report the results from the subgroup analysis in patients with cancer.

Results: A total of 1535 patients with cancer were included. According to the Padua score, 826 (53.8\%) patients were at low risk of VTE, while 709 (46.2\%) patients were at high VTE risk. Among patients with high VTE risk, 93.9\% did not receive any VTE prophylaxis, and only 11 (1.6\%) patients received appropriate VTE prophylaxis as per ACCP guidelines. VTE events occurred in 9 (0.6\%; 4 and 5 patients in the low and high VTE risk group respectively) patients during hospitalization.Among high risk patients who received VTE prevention, no VTE events were observed.(Table 1) A total of 55 (3.6\%) patients (24 patients with low risk of bleeding, 31 patients with higher risk of blood) occurred bleeding events. (Table 2) 53 patients were identified as unrelated to VTE therapy and 2 patients could not be determined.

TABLE 1 VTE events in medical patients with cancer during hospitalization

Table1. VTE events in medical patients with cancer during hospitalization

\begin{tabular}{lcc}
\hline \multicolumn{1}{c}{ VTE events occurred } & Patients number $\%$ & $95 \% \mathrm{CI}$ \\
\hline Total patients & $9 / 1535(0.6 \%)$ & $(0.3 \%, 1.1 \%)$ \\
Patients with low VTE risk & $4 / 826(0.5 \%)$ & $(0.1 \%, 1.2 \%)$ \\
Patients with high VTE risk & $5 / 709(0.7 \%)$ & $(0.2 \%, 1.6 \%)$ \\
Patients received any VTE prophylaxis & $0 / 43(0.0 \%)$ & $(-,-)$ \\
Patients received no VTE prophylaxis & $5 / 666(0.8 \%)$ & $(0.2 \%, 1.7 \%)$ \\
Patients received appropriate VTE methods* & $0 / 28(0.0 \%)$ & $(-,-)$ \\
Patients received appropriate VTE prophylaxis* & $0 / 11(0.0 \%)$ & $(-,-)$ \\
\hline
\end{tabular}

*Appropriate prophylaxis means full dose and full course pharmacological prophylaxis following the recommendations of the ACCP guidelines.

*VTE Methods include pharmacological and mechanical prophylaxis.
TABLE 2 Bleeding events in medical patients with cancer

Table2. Bleeding events in medical patients with cancer

\begin{tabular}{lcc}
\hline \multicolumn{1}{c}{ Bleeding events occurred } & Patients number \% & $95 \%$ CI \\
\hline & & \\
Total patients & $55 / 1535(3.6 \%)$ & $(2.71 \%, 4.64 \%)$ \\
Patients with low bleeding risk & $24 / 1209(2.0 \%)$ & $(1.28 \%, 2.94 \%)$ \\
Received any VTE prophylaxis & $2 / 56(3.6 \%)$ & $(0.44 \%, 12.31 \%)$ \\
Received no VTE prophylaxis & $22 / 1153(1.9 \%)$ & $(1.20 \%, 2.87 \%)$ \\
Patients with high bleeding risk & $31 / 326(9.5 \%)$ & $(6.55 \%, 13.23 \%)$ \\
Received any VTE prophylaxis & $4 / 19(21.1 \%)$ & $(6.05 \%, 45.57 \%)$ \\
Received no VTE prophylaxis & $27 / 307(8.8 \%)$ & $(5.88 \%, 12.54 \%)$ \\
\hline
\end{tabular}

Conclusions: Nearly half of the in-patients with cancer were at high risk of VTE, but most of them did not receive VTE prophylaxis. This real-world data highlights an urgent need to increase awareness and appropriate VTE prophylaxis in China to improve the management of VTE risk in oncology inpatients.

\section{PB1226 | Using the Caprini Risk Score to Increase VTE} Awareness in the Community. The Know Your Score Project

P. Shetye ${ }^{1}$; S. Gavankar ${ }^{1}$; H. Saadaldin ${ }^{2,3} ;$ A. Tafur ${ }^{4,5}$; J. Caprini ${ }^{4,5}$ ${ }^{1}$ GTF Group, Suwanee, United States; ${ }^{2}$ October 6 University; 6th of October City, Egypt; ${ }^{3}$ American Venous Forum, East Dundee, United States; ${ }^{4}$ NorthShore University HealthSystem, Skokie, United States; ${ }^{5}$ The University of Chicago Pritzker School of Medicine, Chicago, United States

Background: Venous Thromboembolism (VTE) is a leading preventable cause of death following surgery or hospitalization. Risk-stratified thromboprophylaxis is the key to reducing VTE rates, including pulmonary emboli. However, globally, VTE risk awareness in the community is low.

Aims: We aimed to pilot an educational program intended to empower the community to understand VTE risk assessment.

Methods: The Caprini Risk Score (CRS) is a comprehensive list of 40 thrombosis risk factors validated in more than 5 million patients. To empower patients to participate in informed decision-making, we disseminated the validated patient-friendly CRS to the community. Electronically, the CRS and a letter with epidemiological facts were distributed to high school students by two classmates (PS, SG) with instructions to share among relatives as a snowball distribution. The data was collected for one month.

Results: A total of 1,219 responses were received from friends, family members, members of the Global Thrombosis Forum from residents in Florida, Georgia, and Missouri. Family history of blood clots was reported in 268/1219 (22.0\%) of respondents. 59.4\% were $\leq 41$ years of age, $28.5 \%$ had a $\mathrm{BMI}>25,10.4 \%$ were hospitalized for infection, and $10.7 \%$ reported swollen legs during the past month. The substantial incidence of certain co-morbidities in the group (almost $60 \%$ of whom 
were young) emphasizes the importance of individual risk assessment. The majority of patients had a baseline score in the high-risk category.

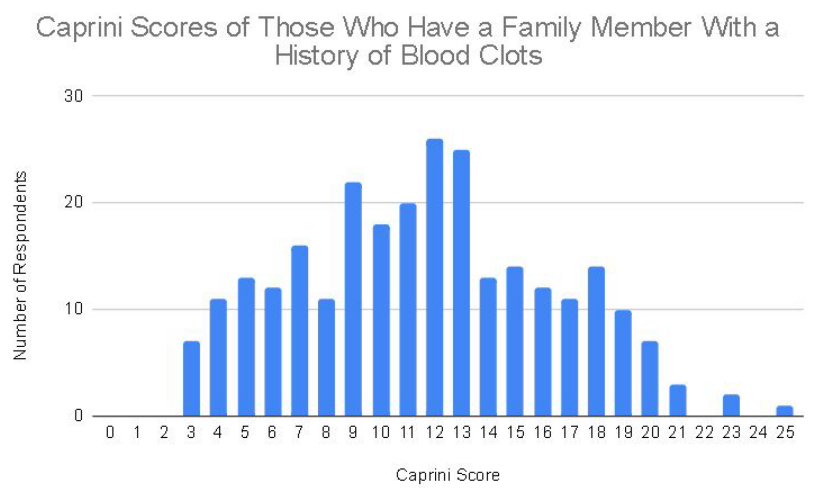

FIGURE 1 Caprini Score of those who have a family member with history of blood clots

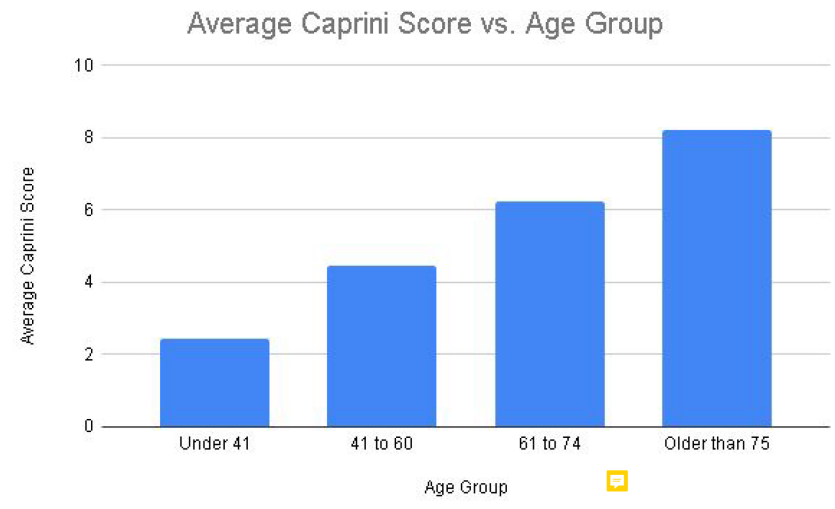

FIGURE 2 Average Caprini Score vs. Age Group

Conclusions: Using community-based strategies utilizing school endorsement and word of mouth snowball marketing can empower the community to participate in shared decision-making about their VTE risk. The next step of the "Know Your Score project" is to measure the effectiveness of educational methods in the community and integrate the CRS into the respondent's medical record.

PB1227 | Assessing Use of Surrogate Outcome Measures in Randomized Controlled Trials Investigating Venous Thromboembolism Prophylaxis

\section{A. Eshaghpour ${ }^{1} ;$ A. Li ${ }^{2}$; J. Park ${ }^{3} ;$ T. Cho ${ }^{3} ;$ M. Crowther ${ }^{4}$}

${ }^{1}$ Michael G. DeGroote School of Medicine, McMaster University, Hamilton, Canada; ${ }^{2}$ Faculty of Medicine, University of Ottawa, Ottawa, Canada; ${ }^{3}$ Faculty of Health Sciences, McMaster University, Hamilton, Canada; ${ }^{4}$ Department of Medicine, McMaster University, Hamilton, Canada

Background: Outcome measures in randomized controlled trials (RCTs) can be broken down into patient-important and surrogate outcomes. Venous thromboembolism (VTE) prophylaxis trials often use surrogate outcome measures given the low incidence of VTE. These outcomes often consist of radiographic asymptomatic VTE or a composite of asymptomatic and symptomatic VTE, as opposed to solely symptomatic VTE. The clinical relevance of such outcomes is controversial, especially when comparing VTE vs bleeding risk.

Aims: To establish the prevalence of surrogate outcome measures in VTE prophylaxis trials.

Methods: A comprehensive search of MEDLINE, Embase and Cochrane (CENTRAL) databases from January 2000 to October 2020 was conducted. Any English-language RCT examining VTE prophylaxis of any kind with at least 150 participants was included. Baseline characteristics, type of outcome measure, acknowledgement of surrogate outcome limitations, and presence of VTE vs bleeding risk discussions were recorded. Binary logistic regression analysis was done to assess the relationship of impact factor, citation count, and sample size with use of a surrogate outcome measure Results: 209 studies were included in the analysis. 81\% (170/209) of studies used a surrogate outcome measure. Of these studies, 20\% (34/170) acknowledged this as a potential limitation and $89 \%(152 / 170)$ discussed bleeding vs thrombosis risk. There was no statistically significant relationship between citation count $(\beta 1 O R=0.999 ; 95 \% \mathrm{Cl}=0.998-1.001 ; P=0.248)$ or sample size $(\beta 10 R=0.999,95 \% \mathrm{Cl}=0.999-1.000, P=0.320)$ and use of a surrogate outcome measure. There was a statistically significant relationship between impact factor and use of a surrogate outcome measure ( $\beta 10 \mathrm{R}=0.981,95 \% \mathrm{Cl}=0.969-0.994, P=0.003)$

Conclusions: Surrogate outcome measures are highly prevalent in VTE prophylaxis literature and are used more frequently in journals of lower impact factor. While using patient-important outcome measures may not always be feasible, we recommended clinicians acknowledge the potential limitation of surrogate outcomes, especially when discussing bleeding vs thrombosis risk.

\section{PB1228 | Venous Thromboembolism Prophylaxis in Patients} with Severe Burns in a Large Trauma Hospital

R. Brazilek; H. Cleland

Alfred Health, Melbourne, Australia

Background: Burns patients have significantly increased risk for Venous Thromboembolism (VTE) development due to concurrent dehydration and global inflammation inducing a hypercoagulable response. VTE events correlate with increased morbidity and mortality and longer inpatient stays. It is therefore critical to elucidate factors surrounding VTE prophylaxis prescription that may reduce this likelihood.

Aims: This study aimed to demonstrate key factors relating to VTE prescription in patients with severe burns, including temporal factors affecting VTE development and mode of thromboprophylaxis. Methods: 226 records of patients who had sustained a $>20 \%$ total body surface burn between 2009-2019 were reviewed. 20 
incidences of VTE were identified, and a retrospective cohort study was performed to identify diagnostic modalities, thromboprophylaxis timing and identify key risk factors for VTE development.

Results: The most common thromboprophylaxis was enoxaparin ( $85 \%, n=192)$, followed by heparin $(13.71 \%, n=31$ ), then sequential compression devices $(0.88 \%, n=2)$. Patients who developed a VTE vs. those who did not had a mean difference in time from admission to thromboprophylaxis initiation of 1.90 days $(95 \% \mathrm{Cl}=[-1.50$ to 4.92], $P<0.05, n=20$ ), and 10.51 days in those who developed a PE (95\% Cl $=$ [3.73 to 17.3], $P=0.0006, n=6$ ). By a 4 day threshold, $96 \%$ of patients who did not develop a VTE had been initiated on thromboprophylaxis compared with $32 \%$ of those who developed a PE.

Conclusions: This review of a large cohort of patients with severe burn injury demonstrates a clear increase in risk of PE development in those who have delayed VTE prophylaxis prescription. While multiple factors complicate adequate and timely dosing of burns patients, this study provides support for early institution of thromboprophylaxis post injury, with special consideration given to high risk populations, such as the obese.

\section{PB1229 | International Survey of Secondary} Thromboprophylaxis Practice Patterns in Pediatrics

H.P. Wilson ${ }^{1}$; R. Capio ${ }^{1}$; I. Aban ${ }^{1}$; N. Goldenberg ${ }^{2}$

${ }^{1}$ University of Alabama at Birmingham, Birmingham, United States;

${ }^{2}$ Johns Hopkins All Children's Hospital, St. Petersburg, United States

Background: Despite the increase in clinicians identifying venous thromboembolism (VTE), we lack robust research to support clinical care guidelines. Although most children are at low risk for recurrence, some have higher risks due to persistent prothrombotic risk factors. However, pediatric-specific guidance and expert consensus to recommend long-term anticoagulation in children is lacking.

Aims: Understanding provider practices is a vital first step to addressing current limitations in pediatric guidelines for secondary thromboprophylaxis among such high-risk children.

Methods: We performed an international survey of pediatric thrombosis experts to approximate the number of children with persistent prothrombotic risk factors and characterize the contemporary prac tice patterns for the use of secondary thromboprophylaxis. Potential participants were identified using the membership registries of three of the primary pediatric thrombosis networks: Duration of Therapy for Thrombosis in children investigators, Children's Hospital Acquired Thrombosis consortium and the VENUS pediatric thrombosis subgroup. Surveys were disseminated December 2020 through January 2021 using Qualtrics (Provo, UT).

Results: The survey was distributed electronically to 124 potential participants with 80 respondents. After exclusions, 61 complete surveys were evaluable. The majority of responders were from the United States, practicing more than 10 years at freestanding, academic pediatric hospitals with dedicated pediatric thrombosis programs. Providers were more likely to prescribe secondary thromboprophylaxis to adolescents and teenage patients as compared to infants and children. Respondents reported they were most likely to initiate chronic secondary thromboprophylaxis (therapeutic or low dose) in children with a potent thrombophilia, recurrent unprovoked VTE and in those with underlying inflammatory disorders and/or chronic central venous catheters.

Conclusions: Our results show variation in practice patterns but less so in areas where we have more definitive guidelines. These findings highlight the need for a multicenter prospective cohort study to determine the impact of various risk factors on recurrence rates to ultimately inform the design of eventual interventional studies.

\section{PB1230 | A Multi-disciplinary Approach to Improving Temporary IVC Filter Retrieval Rates}

J. Kamp; T. Ammar; J. Wilkins; L. Roberts

King's College Hospital, London, United Kingdom

Background: Poor inferior vena cava (IVC) filter retrieval rates is an ongoing global problem with an increasing awareness of the need for timely retrieval. The national UK guidelines updated in 2020 reaffirms the recommendation for IVC filter retrieval as soon as anticoagulation can be re-established. Historically low retrieval rates prompted the collaboration between our haematology and interventional radiology departments to improve our retrieval service. Measures taken included automatic IVC filter retrieval scheduling at the time of insertion, haematology review of all patients with filters and the development of a database to track filter outcomes.

Aims: To assess the effect of new protocols in achieving high IVC filter retrieval rates.

Methods: All patients who received an IVC filter from December 2018 to December 2019 were retrospectively followed up for a minimum period of 12 months. Patient lists were generated from procedural codes pulled from the local computerised radiology information system (CRIS) and matched against the existing IVC database. Filter retrieval outcome was then independently verified.

Results: A total of 112 filter insertions were identified. 62/63 (98\%) patients that required local retrieval had a median time to attempt of 66 days (IQR 33-110). A single patient refused retrieval, and two attempts were appropriately abandoned intra-operatively due to thrombus in the filter and subsequently made permanent. Of the remaining 60 procedures, there was one failure secondary to filter tilt, resulting in a $98 \%(59 / 60)$ retrieval success rate. There were no procedural complications. Of the remaining 49/112 patients, 31 died, 4 filters were declared permanent, and 14 were discharged to their base hospital. No patients were lost to follow-up, and all patients discharged to base hospitals had written correspondence to the responsible clinician instructing retrieval.

Conclusions: Collaborative work at our institution has led to excellent local retrieval rates and robust follow-up culminating in an exemplary IVC retrieval service. 
PB1231 | Incidence of Thrombohaemorrhagic Complications in the Periprocedural of Oral Anticoagulant Treatment with Vitamin K Antagonists

M. Argüello Marina; I. Gutiérrez Jomarrón; L. Castilla García; E. Flores Ballester; I. Sánchez Prieto; S. Valenciano Martínez; J.M. Aspa Cilleruelo; C. Martínez Vázquez; G. López de Hontanar Torres; P.A. Rodríguez Barquero; M. López Rubio; J. García Suárez Hospiral Universitario Príncipe de Asturias, Alcalá de Henares, Spain

Background: Patients with oral anticoagulation therapy (OAT) with vitamin $\mathrm{K}$ antagonists (VKA) undergo invasive procedures. Deciding wether to withdraw or maintain the OAT, as well as the needing to implement low molecular weight heparin (LMWH) as bridge therapy (BT), will be determined by the patient's thrombotic risk (TR) and surgery's haemorrhagic risk (HR).

Aims: To decrease bleeding events related to surgery by limiting the use of LMWH with patients having high TR.

Methods: Prospective, unicentric, observational study including 200 patients with OAT undergoing surgery (December 2019-February 2021), with 4 weeks of follow-up. The median age is 73 (33-93). Most patients are low TR on CHADs-VASc score. OAT periprocedural management was in accordance with the approved hospital protocol which classifies patients according to TR (Table 1 ) and surgeries according to HR . VKA is suspended 3 days before surgery and resumed 24 hours after. LMWH as BT is reserved for patients with high TR or suspected active cancer (therapeutic dose), starting with OAT suspension and reintroduced along with it 24 hours after surgery for 3 days (if no bleeding complications).

TABLE 1 Thrombotic Risk Stratification

\begin{tabular}{|c|c|c|c|}
\hline \multirow[t]{2}{*}{ Risk } & \multicolumn{3}{|c|}{ Anticoagulation reason } \\
\hline & $\begin{array}{l}\text { Prosthetic heart } \\
\text { valves }\end{array}$ & Atrial fibrillation & $\begin{array}{c}\text { Venous } \\
\text { Thromboembolism }\end{array}$ \\
\hline High & $\begin{array}{c}\text { Mitral position } \\
\text { Tricuspid position } \\
\text { (includying biological } \\
\text { valves) } \\
\text { Aortic position } \\
\begin{array}{c}\text { Stroke/TIA }<6 \\
\text { months }\end{array}\end{array}$ & $\begin{array}{c}\text { CHA2DS2-VASc 7-9 } \\
\text { Stroke/TIA }<3 \text { months } \\
\text { Rheumatic } \\
\text { valvulopathy mitral }\end{array}$ & $\begin{array}{c}\text { VTE }<3 \text { months } \\
\\
\text { Severe thrombophilia } \\
\text { (Leyden's factor V in } \\
\text { homozygosis, } 20210 \\
\text { prothrombin, protein C, } \\
\text { Sor antithrombin III } \\
\text { deficiency, múltiples } \\
\text { deficiencys, } \\
\text { Antiphospholipid } \\
\text { Syndrome) }\end{array}$ \\
\hline Moderate & $\begin{array}{c}\text { Aortic position } \\
+1 \text { Risk factor (AF, } \\
\text { history of Stroke/TIA } \\
>6 \text { months, DM, } \\
\text { congestive heart } \\
\text { failure, Age }>75 \\
\text { years) }\end{array}$ & $\begin{array}{l}\text { CHA2DS2-VASC 5-6 } \\
\text { Stroke/TIA > } 3 \text { months }\end{array}$ & $\begin{array}{c}\text { VTE 3-12 months } \\
\text { Not Severe } \\
\text { thrombophilia (Leyden's } \\
\text { factor } V \text { in heterozygosis } \\
\text { or } 20210 \mathrm{~A} \text { prothrombin } \\
\text { mutation) } \\
\text { Recurrent VTE } \\
\text { VTE + active cancer }\end{array}$ \\
\hline Low & $\begin{array}{c}\text { Aortic position } \\
\text { With no risk factors }\end{array}$ & $\begin{array}{l}\text { CHA2DS2-VASc 1-4 } \\
\text { with no previous } \\
\text { Stroke/TIA }\end{array}$ & VTE $>12$ months \\
\hline
\end{tabular}

TIA: Transient Ischemic Attack; AF: Atrial fibrillation; DM: Diabetes Mellitus; VTE: Venous thromboembolism. CHADSVASC: 1 point Congestive Heart Failure, 1 point High blood pressure, 1 point Age above 65 (two points if above 75), 1 point Diabetes, 1 point Previous stroke or Clot, 1 point Vascular disease, 1 point if woman.
Results: Interventions and clinical events during the follow-up are shown in Table 2 .

Eleven post-surgical haemorrhagic events $(5,5 \%)$ are described[GJ1]

. Three (two hemoperitoneum and one haematoma of anterior rectus abdominis muscle) required hospitalisation for management, being solved with conservative treatment. Eight were mild events, handled outpatiently. No thrombotic events were recorded. Generally, OAT was re-induced the day after surgery in patients undergoing minimal and low HR interventions, being the percentage lower in patients undergoing high $\mathrm{HR}$ interventions.

TABLE 2 Incidence of haemorrhagic and thrombotic complications according to the TR of the patient and the HR of the intervention

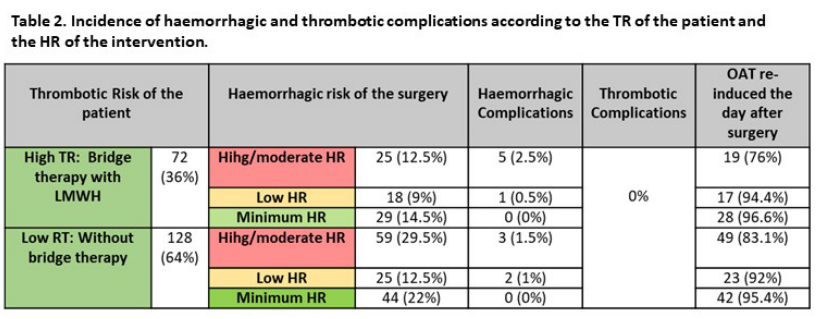

$T R$ : thrombotic risk: $H R$ : haemorrhagic risk; LMWH: low molecular weight heparin.

Conclusions: The standardization of periprocedural management protocol for OAT adjusting BT with LMWH according to individual risk factors for each patient resulted in a reduction in the incidence of haemorrhagic complications without secondary increase of thromboembolic events. This study demonstrates the importance of a central, unified periprocedural management protocol.

PB1232 | Final Results (765 Answers) of the Brazilian National Survey in Thromboprophylaxis for Varicose Vein Surgery

A.J. Ribeiro $^{1}$; M.A. Marques ${ }^{2}$; F.L. Erzinger ${ }^{3}$; A. Ribeiro ${ }^{4}$. ${ }^{1}$ Clínica de Veias, Brasilia, Brazil; ${ }^{2}$ UERJ e UNIRIO, Rio de Janeiro, Brazil; Instituto da Circulação, Curitiba, Brazil; ${ }^{4}$ Clinica Villas Boas, Brasilia, Brazil

Background: Venous thromboembolism (VTE) is not common following varicose vein surgery. Further, there is a lack of specific evidence-based clinical guidelines regarding thromboprophylaxis in this type of surgery.

Aims: We have conducted a national survey to investigate the current practice among Brazilian vascular surgeons regarding thromboprophylaxis for varices surgery.

Methods: An anonymous online questionnaire was emailed to all 3.766 members of the Brazilian Society of Angiology and Vascular Surgery (SBACV) and a message was sent to approximately 1.500 members of the Vascular Forum WhatsApp $\AA$ group in Brazil.

Results: We received 765 responses. With regard to VTE prevention, $48.3 \%$ surgeons stratify the patients pre-operatively, $10.6 \%$ 
never do it and $7.4 \%$ rarely do so. $30.6 \%$ surgeons always prescribe pharmacological prophylaxis, $21.9 \%$ rarely do it and $18.4 \%$ never do so. The agents are prescribed for just one day in $44.2 \%$ of the answers, $5.9 \%$ for two days, $7.3 \%$ for three days and $23.2 \%$ for seven days. The enoxaparin is prescribed in $84 \%$ of the cases, and $44.2 \%$ use a single dose. The DOACS have been used off label by $13 \%$ of the surgeons and rivaroxaban was the chosen in $93 \%$ of the answers. If the patients are taking contraceptive pills, $61 \%$ of the respondents continue to use them. Routine post procedure duplex scan is carried out by $18 \%$. Moreover, $73 \%$ of respondents claim to know their post-operative VTE rate which varies from 0\% (26\%), 1\% (50\%) 2 to $5 \%$ (11\%). The post op VTE occurred on the day 3 to 10 in $60.3 \%$ of the answers. $5.8 \%$ of the surgeons reported death cases after the varicose vein surgery.

\section{Do you stratify your patients regarding the risks of thrombolembolism in venous surgery?}

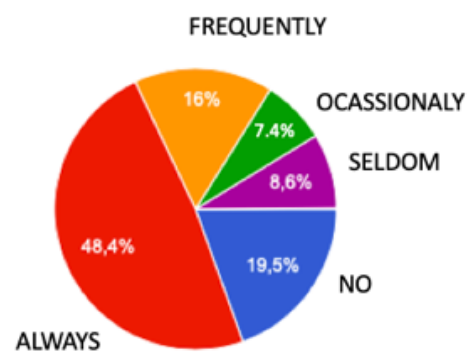

FIGURE 1 Patients stratification

\section{For how many days do you prescribe the prophylaxy?}

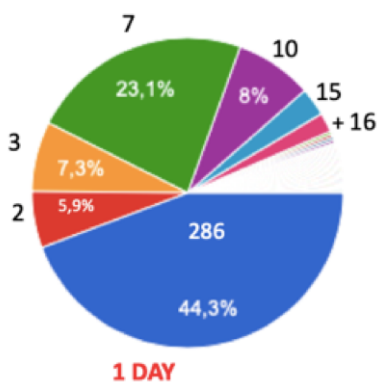

$57.5 \%=1$ to 3 DAYS

FIGURE 2 Days of pharmacoprophylaxi

Conclusions: The majority of respondents use peri-procedure pharmacoprophylaxis for varicose vein surgery, mainly enoxaparin in a single dose. DOACS have been used off label in $13 \%$ of the cases. VTE rates were low. However, the true incidence may be higher.

PB1233 | Success of Online CME at Improving Knowledge and Confidence around Guideline-directed Management of Cancerassociated Thrombosis

M. Harris ${ }^{1}$; J. Spyropoulos ${ }^{1}$; M. LaCouture ${ }^{1}$; C. Padbury ${ }^{1}$;

M. Zec Bonomo ${ }^{2}$; C. Capparelli; MBA; $\mathrm{CHCP}^{1}$

${ }^{1}$ Medscape Education, New York, United States; ${ }^{2}$ University of Chicago

Pritzker School of Medicine, Center for Continuing Medical Education, Chicago, United States

Background: Recent guidance statements recommend the use of direct oral anticoagulants (DOACs) as primary thromboprophylaxis in ambulatory patients with cancer who are starting chemotherapy and in patients with cancer-associated thrombosis (CAT) at low risk of bleeding and no drug-drug interactions. Yet, many clinicians lack knowledge and confidence with integrating DOACs into management strategies for patients with cancer in accordance to guideline recommendations.

Aims: We sought to determine if online continuing medical education (CME) could improve the knowledge and confidence of hematologists/oncologists regarding guideline-directed use of DOACs in the management of CAT.

Methods: This CME intervention comprised of a 30-minute online video-based roundtable discussion among experts in the field of CAT management. Responses to 3 multiple-choice, knowledge questions and 1 self-efficacy, 5-point Likert scale confidence question were analyzed using a repeated pairs pre-/post-assessment study design. A chi-square test ( $P<.05$ is considered significant) assessed pre- to post-activity change. Data were collected December 2019 through February 2020.

Results: In total, 71 hematologists/oncologists were included in this study. Overall, there were knowledge and confidence improvements (reported as relative) seen among participants from pre- to post-assessment:

- $27 \%(P<.01)$ improved at identifying appropriate guidelinerecommended DOAC therapy for thromboprophylaxis in cancer patients.

- $27 \%(P<.01)$ improved at selecting guideline-appropriate treatment options for CAT.

- $44 \%$ had an increase in confidence in managing CAT.

Continued educational gaps:

- $25 \%$ failed to select guideline-recommended DOAC therapy for thromboprophylaxis in cancer patients.

- $45 \%$ failed to select guideline recommended DOAC therapy for treatment of CAT. 
- $66 \%$ still remain at only a rating of 1 to 3 on a scale of 1 to 5 in their confidence managing CAT.

Conclusions: This study demonstrates the success of online, CMEaccredited, video-based roundtable discussion on significantly improving knowledge and confidence of hematologists/oncologists related to the guideline-recommended use of DOACs in the management of CAT. Continued gaps were identified for future educational targets.

\section{PB1234 | VTE Risk at Discharge}

K. Patel $^{1}$; R. Fulton ${ }^{1}$; J. Knapp ${ }^{1} ;$ T. Knox ${ }^{1}$; H. Lombardo' ${ }^{1}$; M. Lóser ${ }^{1}$; A. Hallam ${ }^{2}$; A. Macchiavelli ${ }^{1,2}$

${ }^{1}$ Geisinger Commonwealth School of Medicine, Scranton, United States; ${ }^{2}$ AtlantiCare Health System, Atlantic City, United States

Background: Venous thromboembolism (VTE) poses a significant risk to patients in the hospital setting, and studies have shown that VTE prophylaxis should be incorporated into the management of high-risk patients during their hospital stay. However, many of these patients are still high-risk for VTE at the time of discharge and for up to three months after discharge. In fact, $50 \%$ of VTE events occur approximately 30 days after hospital discharge, with patients often discharged home without any form of VTE prophylaxis.

Aims: To identify a need for change in hospital discharge protocols in order to reduce the incidence of VTE events among non-ICU, medically ill patients hospitalized at our regional medical centers by focusing on extended prophylaxis for patients discharged from our institution.

Methods: This retrospective study utilized data that was collected from 100 patients admitted to our medical service in 2019. Patients who expired during their hospitalization were excluded. The patients were evaluated using three different VTE risk scales to determine their risk for VTE at time of discharge: IMPROVE, Padua, and Geneva.

Results: At the time of hospital discharge, $56 \%$ of patients were at high-risk for VTE, indicated by a Padua Score $\geq 4$. 78\% of patients were at high-risk for VTE based on a Geneva Score $\geq 3$ at discharge. Additionally, 24\% of patients were at high-risk for VTE at time of discharge with an IMPROVE Score $\geq 3$.

\section{Conclusions:}

There is a need for continued prophylaxis after discharge, as $24-78 \%$ of the patients included in our study were still at high-risk for VTE at the time of discharge, which is an indication for VTE prophylaxis. Based on these results and other recent studies, our recommendation is to implement a new protocol at our institution that requires patients with a high-risk score for VTE to receive extended prophylaxis upon discharge, either with Rivaroxaban or Betrixaban.

\section{PB1235 | D-Dimer Test and Postoperative Thromboprophylaxis}

T. Makarovska Bojadjieva ${ }^{1}$; E. Velkova ${ }^{1}$; V. Dejanova llijevska ${ }^{1}$; E. Petkovic ${ }^{1}$; E. Ristovska ${ }^{1}$; S. Useini ${ }^{1}$; R. Grubovic ${ }^{1}$; M. Tashkovska ${ }^{2}$; B. Todorovski ${ }^{3}$

${ }^{1}$ Institute for Transfusion Medicine, Skopje, North Macedonia, The Republic of; ${ }^{2}$ City General Hospital 8th September, Skopje, North Macedonia, The Republic of; ${ }^{3}$ Institute for Transfusion medicine, Skopje, North Macedonia, The Republic of

Background: Postoperative pharmacological thromboprophylaxis is well established practice. Dosing and duration depends on the type of surgery, body mass as well as patient-related risk factors. A growing tendency towards routine monitoring of D-dimers in order to discontinue postoperative thromboprophylaxis is being observed in our clinical practice.

Aims: To investigate the reasons for routine ordering D-dimer test and extended thromboprophylaxis with low molecular with heparin (LMWH) after hospitalization.

Methods: We investigated 57 adult outpatients with recent abdominal surgery. They received LMWH during and after hospitalization. D-dimer test was ordered at the end of hospitalization and every 7 days depending on the $D$-dimer (normal level $<500 \mathrm{ng} / \mathrm{mL}$ ).

Results: The mean age of patients was 54 years and none of them had previous thromboembolic event, nor additional risk for venous thromboembolism. The mean time of hospitalization was 5 days. No postoperative complications were observed. At discharge, D-dimers were in the range from 1900 to $3800 \mathrm{ng} / \mathrm{mL}$. Thromboprophylaxis with enoxaparin was prescribed for 7 days. Subsequent D-dimers ranged from 1400 to $3100 \mathrm{ng} / \mathrm{mL}$ in 47 patients who came for the second laboratory check and from 800 to $1700 \mathrm{ng} / \mathrm{mL}$ in 32 patients who came for the third check. LMWH was continued in all of the patients for another 7 days, so they received LMWH at least two to three weeks post discharge. The criteria for ordering the D-dimer test and the targeted D-dimer level to stop the thromboprophylaxis remained uncertain.

Conclusions: It is well known that elevated levels of D-dimer may be seen in conditions such as surgery, trauma, inflammation, infection, etc. Therefore, there is no evidence-based need for routine ordering of the D-dimer test, nor for the extended LMWH thromoprophylaxis on regular basis after surgery in outpatient setting. It is imperative that every hospital should develop a cost-effective thromboprophylactic strategy according to the international and local guidelines.

\section{P0172 | Delays of VTE Prophylaxis}

K. Patel $^{1}$; H. Lombardo ${ }^{1}$; R. Fulton ${ }^{1} ;$ J. Knapp $^{1} ;$ T. Knox ${ }^{1}$; M. Lóser ${ }^{1}$; A. Hallam ${ }^{2}$; A. Macchiavelli ${ }^{1,2}$

${ }^{1}$ Geisinger Commonwealth School of Medicine, Scranton, United States; ${ }^{2}$ AtlantiCare Health System, Atlantic City, United States

Background: Venous thromboembolism (VTE) is a significant cause of morbidity and mortality for hospitalized patients, and VTE 
are considered preventable events with appropriate prophylaxis. However, prophylaxis is frequently delayed on hospital admission. We investigated the incidence of VTE prophylaxis delays for highrisk patients admitted to our institution.

Aims: To reduce VTE prophylaxis delays by $75 \%$ in the next year for all high-risk patients admitted to the medical floor.

Methods: We performed a retrospective chart review of 100 medically ill patients admitted to our institution from the Emergency Department from December 2018 to March 2019. Data collection consisted of patient demographics, length of stay, timing of prophylaxis for VTE, type of VTE prophylaxis, and Padua Prediction Score. Results: The first dose of prophylaxis was given within 24 hours of arrival to $75 \%$ of patients, with only $25 \%$ of patients receiving their first dose within 8 hours. Of all 100 patients, 13 patients did not receive prophylaxis at all. The length of time between prophylaxis order and first dose administration showed that $74 \%$ of patients received prophylaxis within 12 hours; only $36 \%$ of patients received prophylaxis in under 4 hours from the time the order was placed. In addition, $62 \%$ of patients had a Padua score $\geq 4$ at the time of admission, suggesting significant risk for VTE.

Conclusions: Our investigation revealed a disparity in length between admission time and first dose of prophylaxis. The study also showed a $2 \%$ mortality rate, with $3 \%$ of all patients developing a VTE. These results would require further study to demonstrate a relationship between delays in VTE prophylaxis and adverse outcomes in the medically ill population. We recommend implementation of a standard STAT order for patients at high risk for VTE according to the Padua Prediction score to receive prophylaxis by the admitting team and then a routine order to follow.

P0173 | Venous Thromboembolism Prophylaxis during Hospitalization: Evidence-based Recommendation (by an Internal Medicine Team)

\section{F.J. Vázquez; M.F. Grande Ratti; M.G. Vallone; P.G. Paula}

Guadalupe Gutierrez; M.B. Bonella; D. Andresik; A. Freixas; M.L. Peroni; D.S. Thomas; C.M.U. Ungaro; M. Burgos; D.H. Giunta; H.M. Recchioni; M.M. Serra; B. Martinez; H. Michelangelo;

\section{J.A. Pollán; B.R. Boietti; V.A. Peuchot}

Hospital Italiano de Buenos Aires, Buenos Aires, Argentina

Background: Hospitalization is an important opportunity for applying prevention efforts. International clinical practice guidelines are for individual healthcare practitioners, who should use them in their work in conjunction with their own judgment and discussion. That's why in 2019, a committee with physicians from Internal Medicine at Hospital Italiano de Buenos Aires, initiated an effort to develop evidence-based recommendations related to Venous Thromboembolism prophylaxis during clinical hospitalization, that meet the highest standards of development, rigor, and trustworthiness.
Aims: To develop our own recommendation/s based on the best available evidence, and to describe the process (search, update and check the quality).

Methods: A project-manager provided administrative support, planning and scheduling the work, and arranging meetings. The specialist team identified relevant questions and only one was selected due to feasibility, by using the Hanlon Method. The committee made a literature search to answer it, created databases to manage the search results and kept a log of search results and strategies (using Pubmed and Rayyan). The evidence review team identified, reviewed and summarised the evidence.

Results: The selected PICO question was: what is the efficacy and safety of thromboprophylaxis with low molecular weight heparin versus unfractionated heparin in patients hospitalized for nonsurgical reasons?

The original search found 498 articles, reviewed by title/abstract, leaving 30 for review by text. Quality tools (PRISMA and AMSTAR2) were applied to a total of 18 papers, with 7 systematic reviews and meta-analyses finally selected.

The reviewers critically appraised this evidence, distilled it into a Summary of Findings Table, and wrote brief summaries.

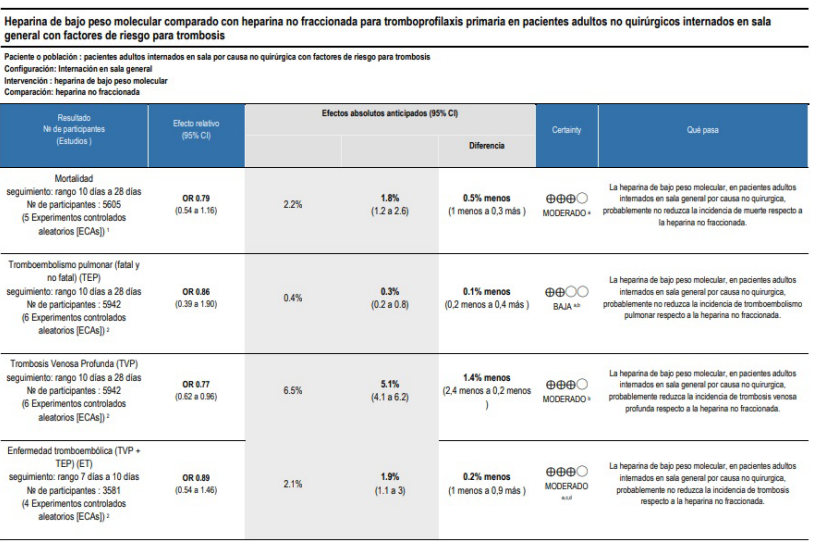

FIGURE 1 Summary of findings table (Part I)

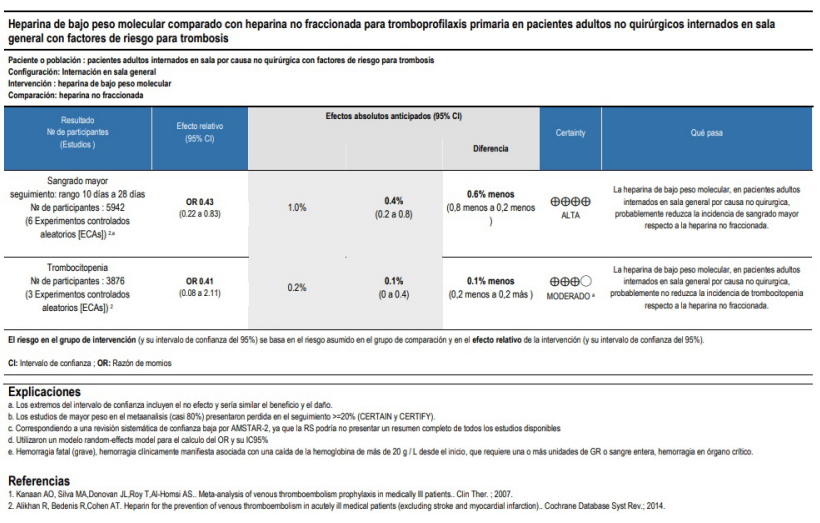

FIGURE 2 Summary of findings table (Part II) 
Conclusions: The consolidation of a working group, the construction of an academic space -in order to learn, disseminate the GRADE methodology and critical reading of evidence-, the training and participation of the potential users of the final recommendations, could improve health care outcomes. It is pending to measure postimplementation adherence.

P0174 | Pharmacological Venous Thromboembolism Prophylaxis in an Internal Medicine Ward: Cross-sectional Observational Study

G. Durao-Carvalho; A. Canoso; J. Louro; R. Amorim

Department of Internal Medicine | Centro Hospitalar do Oeste Unidade de Caldas da Rainha, Caldas da Rainha, Portugal

Background: Half of the venous thromboembolism (VTE) cases occur in inpatients.

Aims: Use of pharmacological VTE prophylaxis in medical inpatients. Methods: Observational, cross-sectional study with inpatients ( $\geq$ 24h since admission) at the Internal Medicine ward, on a random weekday. IMPROVE VTE and IMPROVE bleeding risk (BR) scores were calculated, with stratification into 3 risk cohorts.

Results: 38 patients, $44,7 \%$ males, median age of 80 years old. $60,5 \%$ had a low-molecular-weight heparin (LMWH) prescribed (15 patients - prophylactic dose; 8 - therapeutical dose). No other anticoagulation drug was found.

- VTE low-risk cohort: 6 patients were anticoagulated. 2 of them had clinical indication related to the hospitalization diagnosis (pulmonary embolism [PE] and acute coronary syndrome). The other 4 were doing prophylactic LMWH for no clinical reason.

- VTE moderate-risk cohort ( $n=16$ ), 11 had LMWH - 5 patients on a therapeutical dose due to the main diagnosis and/or because of other comorbidities and the remaining 6 on a prophylactic dose. Among these 6 patients, 5 had a low BR $(<7)$ and the researchers agreed with the prescription. The remaining patient had an IMPROVE BR score $\geq 7$, which gave a major BR of $4,1 \%$ (global BR 7,9\%). Because VTE risk was lower (1,5\%), we think that this prophylactic LMWH was inappropriate. In the subgroup without LMWH $(n=5)$, all patients had a low BR (major BR 0,4\%; any hemorrhage risk $1,5 \%)$.

- VTE high-risk cohort $(n=8), 6$ patients had LMWH (5 - prophylactic dose; 1 - therapeutical dose). Among the 5 patients with prophylactic dose, 2 patients had high BR. The patient with LMWH therapeutical dose had a PE and a low BR. In the VTE high-risk level, 2 pts were not doing LMWH (1- high BR; 1- low BR).

Conclusions: $13,2 \%$ of patients were inadequately prescribed prophylactic LMWH. Among those with formal indication to prophylactic $\mathrm{LMWH}, 15,8 \%$ were not doing it.
P0185 | Analysis of Risk Factors for Hemorrhagic Complications of Anticoagulant Therapy

N. Vorobyeva $^{1,2}$; A. Shchapkov ${ }^{2}$; E. Melnichuk ${ }^{2}$

${ }^{1}$ National Medical Research Center for Hematology (Northern Branch) Ministry of Health of Russia, Arckhangelsk, Russian Federation;

${ }^{2}$ Northern State Medical University Ministry of Health of Russia, Arckhangelsk, Russian Federation

Background: Anticoagulant therapy (ACT) with vitamin $\mathrm{K}$ antagonists (AVC) and direct oral anticoagulants (DOAC) requires an assessment of such risk factors as concomitant pathology and its therapy. Aims: The aim is to analyze the factors contributing to the development of hemorrhagic complications on the background of ACT.

Methods: The analysis of the case histories of 50 patients admitted to the First State Clinical Hospital named after E. E. Volosevich in the period 2014-2020 was made. The presence of causes, outcomes of complications, their frequency, concomitant pathology, the level of INR (international normalized ratio) and blood pressure (BP) during hospitalization were studied.

Results: 50 patients ( 23 women and 22 men) aged 46 to 83 years ( $\mathrm{lu}=67)$ who received the ACT were hospitalized with a diagnosis of "hemorrhagic stroke/intracranial hemorrhage", confirmed clinically and on CT. Fatal outcome in $40 \%(n=20)$ of patients. 37 patients (74\%) took Warfarin, 13 patients (26\%) - DOAC.

6 patients received Omeprazole (12\%), 5 - Digoxin (10\%), 1 Rosuvastatin (2\%), 17 - Atorvastatin (34\%). Taking these drugs together with Warfarin requires monitoring the degree of hypocoagulation.

At the admission of 16 patients (32\%) with blood pressure within: 160 / 100-179 / 109 and 20- (40\%) with a blood pressure of 180/110 or higher. Uncontrolled blood pressure can increase the risk of hemorrhagic complications.

$40 \%(n=20)$ of patients had impaired renal function, liver $-20 \%$ ( $n=10)$, thyroid $-12 \%(n=3)$. The INR value in admission was more than 3 in $50 \%(n=25)$ of patients taking AVC-excessive hypocoagulation.

Conclusions: BP, impaired kidney and liver function, their therapy can increase the rate of fatal bleeding. It is necessary to correct the concomitant pathology, to assess the pharmacokinetics of the drugs and the patient's adherence to treatment.

\section{P0186 | Efficacy of Preoperative Prevention in Venous} Thromboembolism at Endoscopic Urological Interventions

E. Shorikov; P. Shorikov; D. Shorikova

Bukovinian State Medical University, Chernivtsy, Ukraine

Background: Different studies indicate a frequency of postoperative thrombosis from 20 to $59 \%$. More than $70 \%$ of venous thrombosis after endoscopic interventions are asymptomatic and undiagnosed (Cushman M., 2007). However, in 3.9\% of cases they be accompanied 
by severe thromboembolic complications (Decousus H., 2010, Gillet JL, 2015; Avram J., 2010).

Aims: To analize the efficacy of venous thromboembolism prevention with different prophylactic schemes in preoperative period of endoscopic urological interventions.

Methods: It was researched 559 clinical cases after urological endoscopic interventions: 177 patients with preoperative prevention by unfractionated heparin, 136 patients with prophylaxis with low molecular heparin, 127 patients with preoperative cava-filters implantation, 119 patients with rivaroxaban prophylaxis were included. Results: It is proved that antithrombotic preoperative prophylaxis in abdominal surgery is effective and mandatory in patients with high risk of venous thromboembolism. The highest efficiency of preoperative prophylaxis is proved by using cava-filters $(P=0,069)$. It was not shown the reliable differences between unfractionated and low molecular weight heparins in the incidence of venous thromboembolism $\left(x^{2}=0,165 ; p_{1}=0,685 ; p_{2}=0,983\right)$ after usage of these schemes in preoperative period at surgical patients. It has been established that combined prevention schemes and usage of new oral anticoagulants (direct inhibitors of Xa factor) is more efficiency, than therapy with heparin for postoperative thrombosis prophylaxis $\left(x^{2}=12,382 ; p_{1}=0,002 ; p_{2}=0,006\right)$.

Conclusions: After long-term prospective observation it has been proven, that therapy with new oral anticoagulants (rivaroxaban) in postoperative period is necessary step for thromboembolism prevention, which contributes to the clott regression, such clinical as ultrasound.

\section{VTE TREATMENT}

PB1236 | Outcomes of Non-bleeding Patients on Warfarin with an INR > 10, who Received Vitamin K or Conservative Therapy

A. Jones $^{1}$; S. Vazquez ${ }^{2}$; G. Barnes ${ }^{3}$; C. Anderson ${ }^{4}$; S. Woller ${ }^{4}$; S. Stevens ${ }^{4}$; N. Clark ${ }^{5}$; T. Delate ${ }^{6}$; M. Crowthrm ${ }^{7}$; D. Witt ${ }^{8}$

${ }^{1}$ University of Utah School of Medicine, Salt Lake City, United States;

${ }^{2}$ University of Utah Health, Murray, United States; ${ }^{3}$ University of Michigan Health System, Ann Arbor, United States; ${ }^{4}$ Intermountain Healthcare, Murray, United States; ${ }^{5}$ Kaiser Permanente Colorado, Aurora, United States; ' Kaiser Permanente National Pharmacy, Aurora, United States; ${ }^{7}$ McMaster University, Hamilton, Canada; ${ }^{8}$ University of Utah College of Pharmacy, Salt Lake City, United States

Background: Warfarin frequently causes increased healthcare utilization for major bleeding. Warfarin's anticoagulant effect is measured by the international normalized ratio (INR). Elevated INRs are associated with an increased risk of bleeding. Currently, consensus guidelines based on low-quality evidence suggest treating patients who are not bleeding and have an INR $\geq 10$ with oral vitamin $\mathrm{K}$. In contrast, recommendations for patients with elevated INRs from 4.5-10 are to simply hold warfarin.

Aims: Assess the association between temporary discontinuation of warfarin with or without any over-the-counter or dietary vitamin $\mathrm{K}$ (conservative therapy) versus prescription vitamin $\mathrm{K}$ (vitamin $\mathrm{K}$ ) and bleeding (ISTH definitions for major and clinically relevant nonmajor), any arterial or venous thromboembolism (TE), and all-cause mortality at 30 days after initial INR $\geq 10$, and time to INR $<4.0$.

Methods: This was a multi-center observational cohort study. Data were pooled using multivariable random-effects modeling for outcome analysis.

Results: Across four sites, 563 and 705 patients comprised the conservative and vitamin $\mathrm{K}$ groups, respectively (Table). There were 53 (9.4\%) and 52 (7.4\%) bleeding events in the conservative and vitamin $\mathrm{K}$ groups, respectively. TE events occurred in $4(0.8 \%)$ and 11 (1.6\%) of conservative and vitamin $\mathrm{K}$ group patients, respectively. Unadjusted odds ratios (with 95\% confidence intervals) comparing conservative therapy to vitamin $\mathrm{K}$ at 30 days were (Figure): all bleeding (OR: 1.22 [0.77-1.92]), major bleeding (OR: 1.07 [0.55-2.09]), TE (OR: 0.45, [0.14-1.45]), and all-cause mortality (0.67 [0.46-0.97]). The mean difference in time to INR $<4.0$ was 0.77 days [ $95 \% \mathrm{Cl} 0.03-$ $1.52, P=0.04]$ favoring vitamin $\mathrm{K}$. 
TABLE 1 Characteristics of Non-bleeding Patients with INRs $>10$ at each site stratified by treatment (Vitamin K versus Conservative Therapy)

\begin{tabular}{|c|c|c|c|c|c|c|c|c|}
\hline & \multicolumn{2}{|c|}{$\begin{array}{l}\text { University of Utah } \\
(n=121)\end{array}$} & \multicolumn{2}{|c|}{$\begin{array}{l}\text { University of Michigan } \\
(n=71)\end{array}$} & \multicolumn{2}{|c|}{$\begin{array}{l}\text { Intermountain } \\
\text { Healthcare }(n=272)\end{array}$} & \multicolumn{2}{|c|}{$\begin{array}{l}\text { Kaiser Permanente } \\
\text { Colorado }(n=809)\end{array}$} \\
\hline & VitK & CT & VitK & CT & Vit $K$ & CT & & CT \\
\hline & $n=101$ & $n=14$ & $n=48$ & $n=21$ & $n=224$ & $n=48$ & VitK $n=332$ & $n=477$ \\
\hline Mean Age, years (SD) & $62(16)$ & $56(12)$ & $58(17)$ & $53(15)$ & $74(13)$ & $70(14)$ & $72(15)$ & $72(15)$ \\
\hline Male & $45 \%$ & $47 \%$ & $52 \%$ & $48 \%$ & $45 \%$ & $44 \%$ & $63 \%$ & $39 \%$ \\
\hline$\geq 1$ bleeding RF & $74 \%$ & $71 \%$ & $80 \%$ & $66 \%$ & $64 \%$ & $44 \%$ & $\mathrm{n} / \mathrm{a}$ & $\mathrm{n} / \mathrm{a}$ \\
\hline $\begin{array}{l}\text { Time to INR < } 4.0 \text { mean days, } \\
\text { (SD) }\end{array}$ & $2.5(2.6)$ & $\begin{array}{l}3.4 \\
(2.3)\end{array}$ & $4.5(3.8)$ & $3.4(2.7)$ & $3.2(4.1)$ & 4.7 (3.1) & $2.2(1.8)$ & $2.4(2.3)$ \\
\hline
\end{tabular}

Vitk = Vitamin $\mathrm{K}, \mathrm{CT}=$ Conservative Therapy, $\mathrm{RF}=$ risk factor, $\mathrm{n} / \mathrm{a}=$ not available

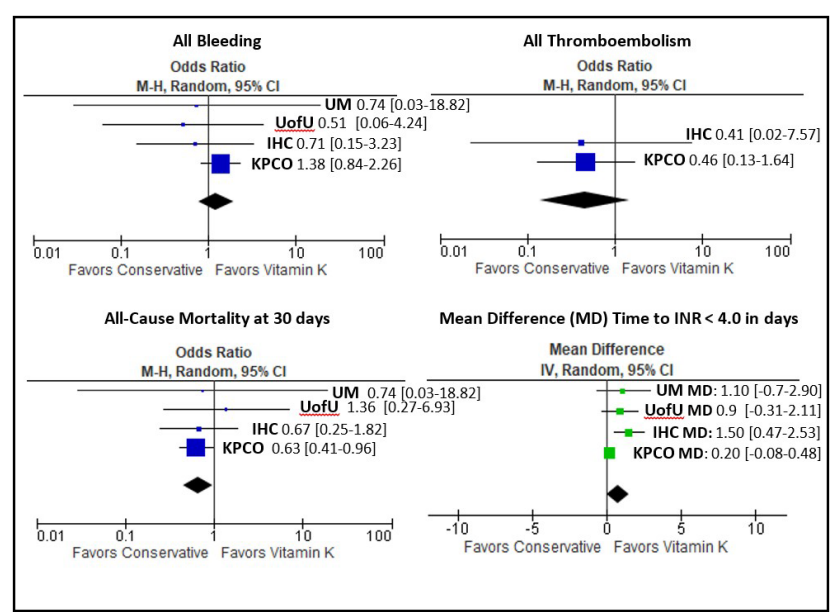

FIGURE 1 Forest Plots for All Bleeding, Venous Thromboembolism and Mortality at 30 days, and Mean Difference to INR

Conclusions: Compared to vitamin K, conservative therapy is associated with lower mortality and no differences in bleeding and TE and is thus a reasonable strategy for asymptomatic patients presenting with INRs $\geq 10$. The difference in time to achieve an INR $<4.0$ was statistically but not clinically different between groups.
PB1237 | Long-term Risk of Recurrent Venous

Thromboembolism after a First Contraceptive-related Event: Data from REVERSE Cohort Study

D. Aziz ${ }^{1,2}$; L. Skeith ${ }^{3,4}$; M. Rodger ${ }^{5,6}$; E. Sabri ${ }^{1}$; M. Righini ${ }^{7}$; M. Kovacs ${ }^{8}$; M. Carrier ${ }^{1,2}$; S. Kahn ${ }^{5,9}$; P. Wells ${ }^{1,2}$; D. Anderson ${ }^{10}$; I. Chagnon ${ }^{11}$; S. Solymoss ${ }^{5}$; M. Crowther ${ }^{12}$; R. White ${ }^{13}$; G. Le Gal ${ }^{1,2}$ ${ }^{1}$ The Ottawa Hospital, Ottawa, Canada; ${ }^{2}$ University of Ottawa, Ottawa, Canada; ${ }^{3}$ University of Calgary, Calgary, Canada; ${ }^{4}$ Foothills Medical Centre, Calgary, Canada; ${ }^{5}$ McGill, Montreal, Canada; ${ }^{6}$ McGill University Health Centre, Montreal, Canada; ${ }^{7}$ Geneva University Hospital, Geneva, Switzerland; ${ }^{\circ}$ Lawson Health Research Institute,

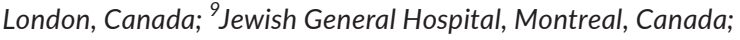

${ }^{10}$ Dalhousie University, Halifax, Canada; ${ }^{11}$ Hôpital du Sacré-Cœurde-Montréal, Montreal, Canada; ${ }^{12}$ McMaster University, Hamilton, Canada; ${ }^{13}$ University of California, Davis, United States

Background: The reported risk of recurrent venous thromboembolism (VTE) after a combined oral contraceptive (COC) associated VTE is heterogeneous.

Aims: We assessed the long-term risk of recurrent VTE in women on $\mathrm{COC}$ at the time of a first VTE, in comparison to women without $\mathrm{COC}$ use. Our secondary aim assessed the impact of $\mathrm{COC}$ use on the recurrent VTE risk in high risk and low risk HERDOO2 subgroups.

Methods: The REVERSE cohort study derived the HERDOO2 clinical decision rule to predict recurrent VTE in patients who discontinued anticoagulation after 5-7 months for a first unprovoked VTE. Incidence rates of recurrent VTE among women with and without COC exposure were calculated as the number of recurrent VTE over the number of person-years of follow-up, and Cox proportional hazards model was used to compare risks between groups. 
Results: The risk of recurrent VTE among COC users was 1.1\% (95\% $\mathrm{Cl}$ 0.3-2.9) per patient-year as compared with $3.2 \%$ per patientyear (95\% Cl 2.4-4.3) among non-users ( $\mathrm{HR}, 0.37,95 \% \mathrm{Cl}, 0.1-1.0$ ). Women who were $\mathrm{COC}$ users and high risk by HERDOO2 score had a recurrence rate of $3.5 \%(95 \% \mathrm{Cl} 0.4-12.5)$ as compared to $6.1 \%$ (95\% $\mathrm{Cl} 4.3-8.5)$ among women who were non-COC users and at high risk by HERDOO2 score (HR 0.6, 95\% Cl, 0.1-2.5).

Conclusions: Women who were $\mathrm{COC}$ users at the time of an otherwise unprovoked VTE event had a lower VTE recurrence rate during long-term follow-up, compared to non-users. The use of HERDOO2 rule may help identify higher risk women with COC use.

PB1238 | Results of DOAC Dipstick Test in Outpatients with Venous Thromboembolic Disease Are Comparable to Plasma Levels of Direct Oral Inhibitors - An Efficient Assessment Tool

L. Papageorgiou $^{1,2}$; J. Harenberg ${ }^{3}$; S. Auge ${ }^{4}$; L. Tredler ${ }^{5}$; I. Elalamy ${ }^{1,2} ;$ G. Gerotziafas ${ }^{1,2}$

${ }^{1}$ Service d'Hématologie Biologique, Hôpital Tenon, Paris, France; ${ }^{2}$ Cancer Biology and Therapeutics, INSERM U938, Paris, France; ${ }^{3}$ DOASENSE GmbH, Heidelberg, Germany; ${ }^{4}$ Service de Médecine Interne et Hématologie, Hôpital Tenon, Paris, France; ${ }^{5}$ Service de Médecine Interne et Hématologie, Paris, France

Background: The efficacy and safety of direct oral anticoagulants (DOACs) in outpatients is closely related to patient's adherence to therapy. Objective documentation of drug intake may be a useful tool for patients' education and improvement of adherence to treatment.

Aims: We aim to analyze the accuracy of DOAC Dipstick ${ }^{\mathrm{TM}}$-patient device compared to plasma concentration for evaluation of the presence of DOACs in outpatients' urine samples treated with DOACs for venous thromboembolism.

Methods: A prospective observational ongoing cohort study is performed including outpatients on treatment with DOACs. All participants are routinely assessed for DOACs' plasma concentration by specific chromogenic assays and for renal function by CockroftGault equation. The dipstick test is performed from patients' urine samples and trained staff evaluated colors of factor-Xa (FXA) and thrombin inhibitors (THR) pads visually according the instructions for use.

Results: Interim analysis was performed after enrolment of 72 patients (female $n=40$, age $56 \pm 16$ years, mean $\pm S D$ ). standard deviation). 47 received rivaroxaban, 23 apixaban, and 2 dabigatran. All patients had normal renal function. Plasma anti Xa levels were $151.41 \pm 114.79 \mathrm{ng} / \mathrm{mL}$ (mean, SD) and anti-Ila levels 191.66 \pm 110.34 $\mathrm{ng} / \mathrm{mL}$. The color of the FXA pad was judged as positive in $69 / 70$ patients (correct positive for DXI: 98.5\%) and of THR pad as negative in all cases treated with DXI (correct negative for DTI: 100\%), respectively. The pads of the 2 DTI treated patients were judged correctly as positive (THR pad) and as correctly negative (FXA pad).
Conclusions: The results show a high agreement of therapeutic plasma concentrations of DXI treated patients with presence of DXI and absence of DTI in urine samples by DOAC Dipstick test. Given the encouraging results despite the limited number of patients treated with dabigatran, the ongoing study should allow the device's validation as an accurate, easy-to-use assessment tool

\section{PB1239 | Differences in the Clot Based and Amidolytic Anti-Xa Assays for the Neutralization Profile of Apixaban, Betrixaban, Edoxaban and Rivaroxaban}

F. Siddiqui $^{1}$; A. Tafur ${ }^{2}$; D. Hoppensteadt ${ }^{1}$; E. Bontekoe ${ }^{1}$; W. Jeske ${ }^{1}$; B. Lewis ${ }^{1}$; O. Iqbal ${ }^{1}$; J. Fareed ${ }^{1}$

${ }^{1}$ Loyola University Medical Center, Maywood, United States; ${ }^{2}$ North Shore University Health Systems, Evanston, United States

Background: A specific antidote namely andexanet alfa (AA) is approved for the management of bleeding complications with apixaban and rivaroxaban. The dosing regimen of andexanet alfa ranges from 400-800 $\mathrm{mg}$ bolus followed by $4-8 \mathrm{mg} / \mathrm{min}$ for up to two hours. This dosing regimen is projected to result in a circulating concentration of $75-150 \mathrm{ug} / \mathrm{ml}$.

Aims: This study is designed to compare the neutralization efficacy of andexanet alfa for various factor Xa inhibitors in clot-based and anti-Xa assays.

Methods: Stock solutions of each of the agents were prepared at $1 \mathrm{mg} / \mathrm{ml}$ and working concentrations at 100 and $10 \mathrm{ug} / \mathrm{ml}$. Each of the factor Xa inhibitor was supplemented in plasma in the concentration range of $0-1 \mathrm{ug} / \mathrm{ml}$. The neutralization profile of AA was studied at $100 \mathrm{ug} / \mathrm{ml}$ and $200 \mathrm{ug} / \mathrm{ml}$ and saline was used as a control. The $I C_{50}$ for the Xa inhibitory activity were calculated for the control drugs and after supplementation with AA.

Results: Apixaban and edoxaban exhibited similar inhibitory profiles in the anti-Xa assays, where as rivaroxaban and betrixaban exhibited much higher $\mathrm{IC}_{50 \text { s. }}$. AA effectively neutralized the anticoagulant effects of all agents at both the 100 and $200 \mathrm{ug} / \mathrm{ml}$, however the neutralization profile in the clot based was not proportional to the anti-Xa effects. At $200 \mathrm{ug} / \mathrm{ml}$ only betrixaban was completely neutralized whereas all the other agents were partially neutralized. The order of neutralization at 200ug/ml was betrixaban > rivaroxaban > apixaban > edoxaban.

Conclusions: These studies show that the neutralization profiles of various Xa agents by AA exhibit differential characteristic response for each of the individual agent. These results indicate that individual dosing based on clinical endpoints for each agent rather than the neutralization of anti-Xa activity measurement may be more relevant to the clinical outcome. 
PB1240 | Association of Adding Antiplatelet Therapy to Warfarin for Management of Venous Thromboembolism with Bleeding and Other Adverse Events

M. Song ${ }^{1}$; B. Haymart ${ }^{1}$; X. Kong ${ }^{1}$; M. Ali ${ }^{2}$; J. Kozlowski ${ }^{3}$; G. Krol ${ }^{4}$; S. Kaatz ${ }^{4}$; J. Froehlich ${ }^{1}$; G. Barnes ${ }^{1}$

${ }^{1}$ University of Michigan, Ann Arbor, United States; ${ }^{2}$ Beaumont Hospital, Royal Oak, United States; ${ }^{3}$ Detroit Medical Center, Detroit, United States; ${ }^{4}$ Henry Ford Hospital, Detroit, United States

Background: Historically, guidelines regarding anticoagulation and antiplatelet treatment regimens have focused on patients with coronary artery disease (CAD) and atrial fibrillation. Few studies have examined the use of anticoagulation and antiplatelet therapy in patients with venous thromboembolism (VTE) and other comorbidities, including CAD.

Aims: To evaluate the frequency and outcomes of antiplatelet therapy in addition to warfarin for patients with VTE.

Methods: Using a registry-based cohort study of adults enrolled at six anticoagulation clinics in Michigan, USA from 2009 to 2020, we evaluated patients started on warfarin for VTE without comorbid atrial fibrillation/flutter, antiphospholipid syndrome, or history of valve replacement. Adverse event rates were calculated via Kaplan-Meier survival analysis. Adjusted associations between number of antiplatelets and outcomes of thrombosis and bleeding were assessed using Cox proportional hazards. Results: Of the study cohort of 2918 patients (1333 men [45.7\%]; mean [SD] age, 61.0 [16.4] years), 820 patients (28.1\%) received warfarin plus one or more antiplatelet medication. Incidence of CAD was greater among those on combination warfarin and antiplatelet therapy versus warfarin alone $(74.6 \%$ vs. $13.4 \%, P<0.001)$

Incidence of bleeding events was also greater in patients on combination warfarin and antiplatelet therapy versus warfarin alone (28.8\% vs. $21.9 \%, P<0.001)$. In unadjusted analysis, an increase in number of antiplatelet medications was associated with increased rates of bleeding. After controlling for age, gender, race, provoked status of VTE, and RIETE score for risk of major bleeding, use of one antiplatelet medication (hazard ratio, 1.21; 95\% Cl, 1.02-1.43) and use of two antiplatelet medications (hazard ratio, 2.37; $95 \% \mathrm{Cl}, 1.65-$ 3.39) remained significantly associated with increased bleeding.

TABLE 1 Rates of Adverse Events by Treatment Type

\begin{tabular}{clll} 
& $\begin{array}{l}\text { Warfarin } \\
\text { only } \\
(n=2098)\end{array}$ & $\begin{array}{l}\text { Warfarin and } \\
1 \text { antiplatelet } \\
(n=730)\end{array}$ & $\begin{array}{l}\text { Warfarin } \\
\text { and } 2 \\
\text { antiplatelets } \\
(n=90)\end{array}$ \\
$\begin{array}{lll}\text { Rate of first } \\
\text { thrombotic } \\
\text { event per 100 }\end{array}$ & 3.3 & 2.7 & 2.2 \\
$\begin{array}{l}\text { patient-years } \\
\text { Rate of first bleeding } \\
\text { event per 100 } \\
\text { patient-years }\end{array}$ & 20.0 & & \\
\hline
\end{tabular}

\begin{tabular}{llll} 
& $\begin{array}{l}\text { Warfarin } \\
\text { only } \\
(n=2098)\end{array}$ & $\begin{array}{l}\text { Warfarin and } \\
1 \text { antiplatelet } \\
(n=730)\end{array}$ & $\begin{array}{l}\text { Warfarin } \\
\text { and } 2 \\
\text { antiplatelets } \\
(n=90)\end{array}$ \\
$\begin{array}{l}\text { Rate of first ER visit } \\
\text { for bleeding }\end{array}$ & 8.8 & $14.2^{* * *}$ & $34.1^{* * *}$ \\
$\begin{array}{l}\text { Rate of first admission } \\
\text { for bleeding }\end{array}$ & 5.1 & $8.4^{* *}$ & $14.6^{* *}$ \\
$\begin{array}{l}\text { Rate of first bleed } \\
\text { that required } \\
\text { treatment (e.g., } \\
\text { RBC transfusion, } \\
\text { vitamin K, or FFP) }\end{array}$ & 8.7 & 9.9 & $19.6^{* *}$ \\
\hline
\end{tabular}

Note: Poisson test was used to calculate $\mathrm{p}$-values, in comparison to warfarin only reference group. ${ }^{*}$ denotes $P<0.05,{ }^{* *}$ denotes $P<0.01$, *** denotes $P<0.001$
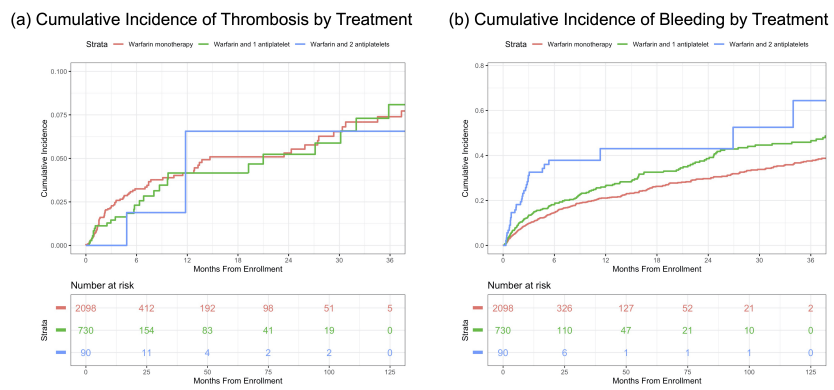

FIGURE 1 Cumulative Incidence of Thrombosis or Bleeding by Treatment Type

Conclusions: Among patients with VTE, combination warfarin and antiplatelet therapy was associated with increased bleeding and similar rates of thrombosis, compared to warfarin monotherapy. Further research is needed to evaluate bleeding risk among patients with VTE compared to similarly matched patients with atrial fibrillation.

\section{PB1241 | Development of a Modified Thrombin Generation} Assay with Improved Sensitivity to Factor Xla Inhibition by the Novel Small Molecule Milvexian (BMS-986177/JNJ-70033093)

M. Bunce ${ }^{1} ;$ F. Bonilla ${ }^{1} ;$ J. Cardenas ${ }^{2}$; J. Kotha ${ }^{2}$; L. Jennings ${ }^{2}$;

M. Chintala ${ }^{1}$

${ }^{1}$ Janssen Research \& Development, Spring House, United States; ${ }^{2}$ MLM Medical Labs, Memphis, United States

Background: Milvexian (formerly known as BMS-986177/JNJ70033093) is a novel small molecule FXla inhibitor currently in Phase II clinical trials with the potential for reduced bleeding risk compared to currently approved direct oral anticoagulants (DOACs). Since FXla inhibition represents a new mechanism of antithrombotic therapy, establishing assays sensitive to milvexian to facilitate measuring its 
anticoagulant activity is desirable. The thrombin generation assay (TGA) has been used clinically with currently approved DOACs, but its use of tissue factor (TF) to generate thrombin via the extrinsic pathway limits sensitivity toward the intrinsic pathway.

Aims: Develop a modified TGA sensitive to FXla inhibition by milvexian using a specific activator of the intrinsic pathway.

Methods: TGAs were performed in platelet-poor plasma (PPP) using the Thrombinoscope method (Stago). Thrombin generation (TG) was initiated with TF reagent or dilute aPTT reagents. After establishing preliminary assay conditions, a validation study was performed to assess reproducibility of TGA parameters from 0.1-10 $\mu \mathrm{M}$ milvexian spiked into plasma from 20 healthy donors.

Results: TGAs initiated with dilute aPTT reagents clearly differentiated normal from FXI-deficient plasmas, versus a modest difference in reactions initiated with TF. Primary TGA endpoints (Lag Time, Peak Thrombin, Time to Peak, and Endogenous Thrombin Potential (ETP)) correlated dose-dependently with FXI plasma concentration or with a small molecule FXla inhibitor. A validation study with milvexian confirmed reproducible dose response in plasma from 20 healthy donors. Intraassay, inter-assay, inter-operator, and aPTT reagent lot-to-lot precision were within acceptable ranges $(<20 \% \mathrm{CV})$.

Normalized Peak Thrombin

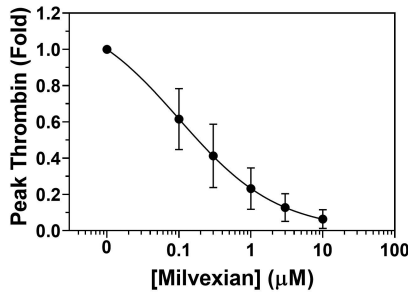

FIGURE 1 Thrombin Generation Initiated with Tissue Factor or Kaolin aPTT Reagent in Normal and FXI-Deficient Plasma. PNP pooled normal plasma; FXI-ID, FXI-immunodepleted plasma; TF, tissue factor
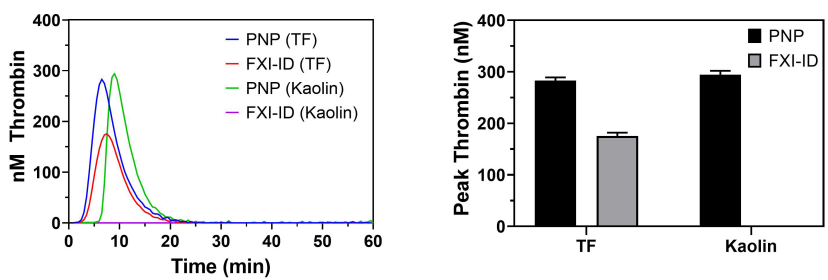

FIGURE 2 Inhibition of Thrombin Generation with Milvexian. ETP, endogenous thrombin potential
Conclusions: Initiating TGAs with dilute aPTT reagent enables sensitive measurement of changes in $\mathrm{FXI}(\mathrm{a})$ activity resulting from differences in FXI antigen or FXla inhibitors. A validation study confirmed the ability to sensitively measure $\geq 100 \mathrm{nM}$ milvexian in PPP. The modified TGA has consequently been included as an exploratory pharmacodynamic assay in the AXIOMATIC TKR trial (NCT03891524).

PB1242 | Recurrent Thromboembolic Risk in Paroxysmal Nocturnal Hemoglobinuria Patients not on Anticoagulation Treated with Terminal Complement Inhibition

G. Gerber; A. DeZern; S. Chaturvedi; R. Brodsky

Johns Hopkins University, Baltimore, United States

Background: Before therapeutic C5 inhibition, thromboembolism accounted for $40-67 \%$ of deaths in paroxysmal nocturnal hemoglobinuria (PNH). Anticoagulation alone is ineffective in preventing thromboembolism. Further, bleeding risk is significant due to cooccurrence of marrow failure and hepatic dysfunction. C5 inhibition decreases recurrent thromboembolism, however many patients remain on anticoagulation. There is limited data whether anticoagulation in PNH patients with history of thromboembolism can be safely discontinued.

Aims: Compare the risk of recurrent thromboembolism in $\mathrm{PNH}$ patients with and without anticoagulation on C5 inhibition.

Methods: We reviewed the electronic medical records of patients at Johns Hopkins Hospital between 1/2005-10/2020 with documented PNH clones treated with eculizumab or ravulizumab for $>6$ months. Patients with history of thromboembolism by imaging or high clinical suspicion were selected. The period on C5 inhibition included thromboembolic events from treatment initiation through last follow-up or bone marrow transplant, as long as therapy was continued with <1-week interruption. Thromboembolic rates for the period pre-C5 inhibition and during $\mathrm{C} 5$ inhibition were calculated as the total events divided by the time in years on a per patient basis and compared using the Fisher exact test. This study was approved by the Johns Hopkins IRB.

Results: Of 21 patients with history of thromboembolism, 11 discontinued anticoagulation, 6 never received or could not tolerate anticoagulation, and 4 continued anticoagulation after initiation of C5 inhibition (Figure 1, Table 2). Thrombosis rate pre-C5 inhibition was 26.3 events/100 patient-years compared with 1.5 events/100 patient-years on anti-C5 monotherapy $(P<0.001)$ and 5.4 events/100 patient-years on combined anticoagulation and $C 5$ inhibition $(P=0.016)$. Two thromboembolic events on anti- $C 5$ monotherapy were provoked and treated with 3-6 months of anticoagulation. Thrombosis rates between the anti-C5 monotherapy and C5 inhibitor plus anticoagulation groups were not significantly different $(P=0.4)$. 


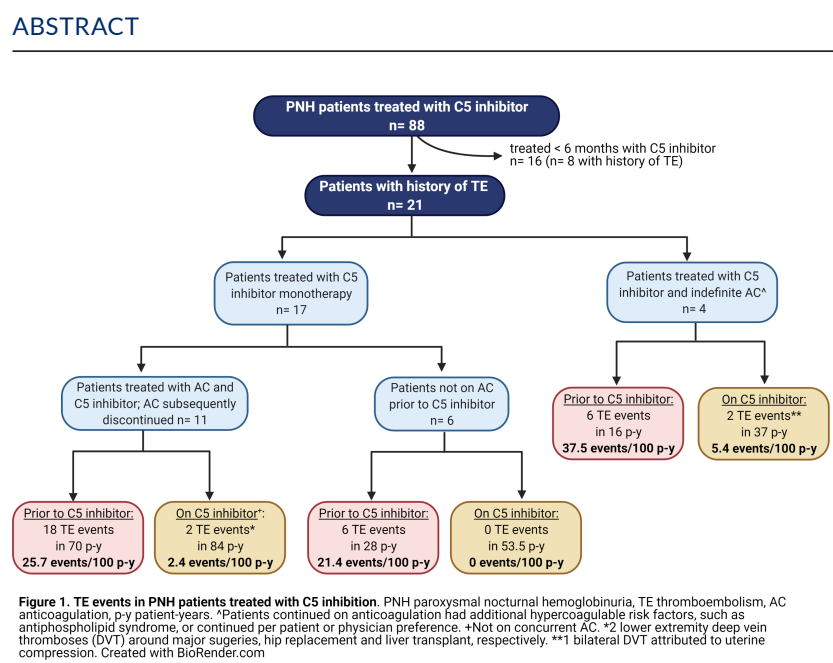

FIGURE 1 Thromboembolic events in $\mathrm{PNH}$ patients treated with $\mathrm{C} 5$ inhibition

TABLE 1 Baseline patient characteristics and thromboembolic (TE) events

\begin{tabular}{|c|c|c|}
\hline Patient Group & $\begin{array}{l}\text { Patients treated } \\
\text { with anti-C5 } \\
\text { monotherapy } \\
(n=17)\end{array}$ & $\begin{array}{l}\text { Patients treated } \\
\text { with C5 inhibitor } \\
\text { and indefinite } \\
\text { anticoagulation } \\
(n=4)\end{array}$ \\
\hline Diagnosis & 10 & 4 \\
\hline Classical PNH & 7 & - \\
\hline \multicolumn{3}{|l|}{$\mathrm{PNH} / \mathrm{AA}$} \\
\hline Sex & 10 & 1 \\
\hline Male & 7 & 3 \\
\hline \multicolumn{3}{|l|}{ Female } \\
\hline $\begin{array}{l}\text { Median age of diagnosis } \\
\text { (range) }\end{array}$ & 24 years $(10-59)$ & 41 years $(36-61)$ \\
\hline $\begin{array}{l}\text { Median granulocyte clone } \\
\text { (range) }\end{array}$ & $97 \%(73-100)$ & $87 \%(78-99)$ \\
\hline $\begin{array}{l}\text { Median time prior to } \\
\text { anti-C5 treatment } \\
\text { (range) }\end{array}$ & 5 years (1-14) & 3.5 years $(1-8)$ \\
\hline $\begin{array}{l}\text { Median time on anti-C5 } \\
\text { treatment (range) }\end{array}$ & $\begin{array}{l}10 \text { years } \\
\quad(0.5-14)\end{array}$ & 9.5 years $(5-13)$ \\
\hline $\begin{array}{l}\text { Median time on } \\
\text { anticoagulation } \\
\text { (range) }\end{array}$ & $\begin{array}{c}1 \text { month (0-11 } \\
\text { years) }\end{array}$ & 9 years $(5-19)$ \\
\hline
\end{tabular}

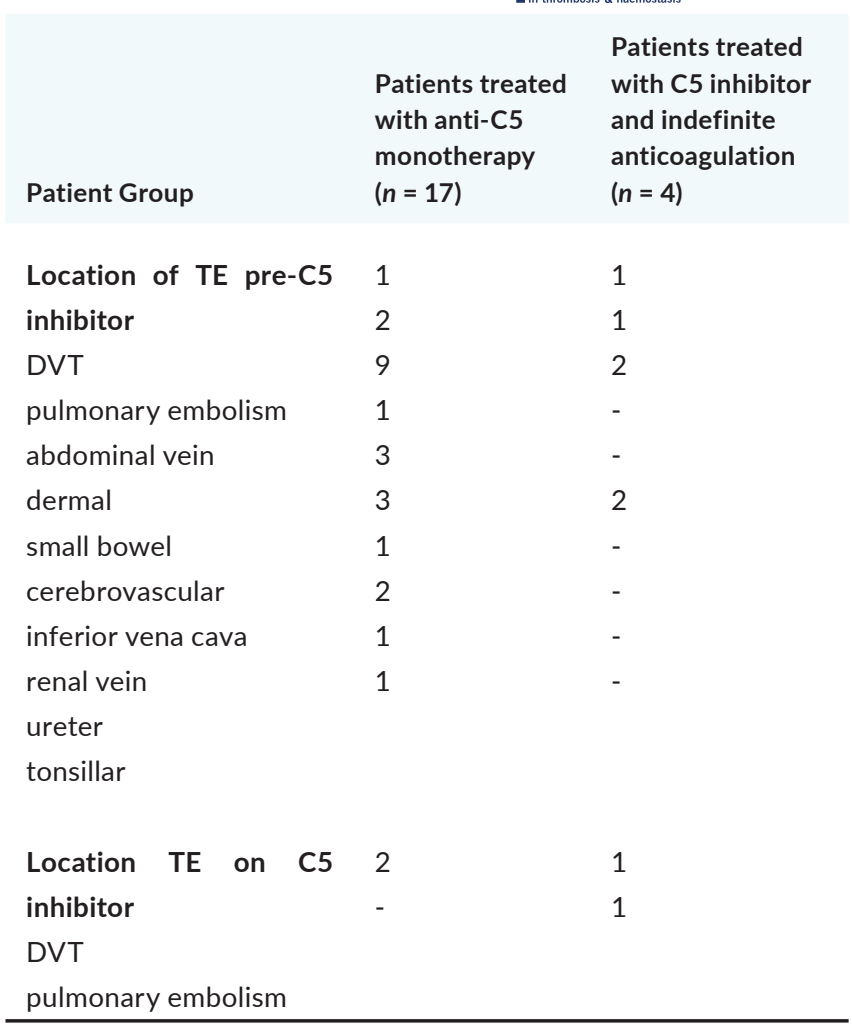

Conclusions: Discontinuation of anticoagulation for secondary prevention of thromboembolism in $\mathrm{PNH}$ patients well-controlled on terminal complement inhibition appears safe.

PB1243 | DASH Score for Prediction of Recurrent Venous Thromboembolism: Updated Long-term Outcomes from a Singlecentre

A. Banerjee ${ }^{1}$; M. Berks ${ }^{1}$; M. Hu ${ }^{1}$; R. Umeria ${ }^{1}$; Y. Zhou ${ }^{1}$; W. Thomas ${ }^{2}$. ${ }^{1}$ University of Cambridge School of Clinical Medicine, Cambridge, United Kingdom; ${ }^{2}$ Cambridge University Hospitals NHS Foundation Trust, Cambridge, United Kingdom

Background: Management of venous thromboembolism (VTE) after the initial 3 months anticoagulation remains controversial. Determining which patients may benefit from indefinite anticoagulation remains a key question; risk prediction tools (e.g. DASH score, Vienna score and HERDOO2) have been used to help determine recurrence risk. The DASH score comprises the D- dimer 1 month after stopping anticoagulation ( +2 if positive), age $\leq 50(+1)$, sex ( +1 if male) and use of hormonal therapy ( -2$)$. A score $\leq 1$ predicts a relatively low recurrence risk (3.1\% annually; $95 \%$ confidence interval (CI) 2.3 - 3.9) and has been used to stop anticoagulation where otherwise there is clinical equipoise.

Aims: To provide long-term data on patients treated with the DASH score with unprovoked VTE (proximal deep vein thrombosis (DVT) \& pulmonary embolism (PE)), who had a score $\leq 1$ and that didn't have long-term anticoagulation. 
Methods: Single- centre retrospective service evaluation of patients seen in the thrombophilia clinic between 1.1.2013-31.12.2016. The project was registered with the hospital audit department. The outcome of these patients was determined. The census date was 31.12.20, recurrent VTE or death (whichever was soonest).

Results: 145 patients were included. Mean age at index VTE was 62 years (standard deviation (SD) 15 ) and $52.4 \%$ patients were male. 1 patient had a previous history of provoked VTE. 5.5\% patients continued low dose aspirin after anticoagulation was stopped. 10.3\% patients had hormone provoked VTE. Mean weight (available for 118 patients) was $86.9 \mathrm{~kg}$ (SD 20.2). Median follow-up 4.7 years. In 635 patient years follow-up there were 39 recurrences; 6.1/100 patient years. 15 recurrences were as DVT, 22 as PE and 2 were DVT/PE.

Conclusions: A DASH score $\leq 1$ was insufficient to determine a threshold at which anticoagulation could be stopped; this may be due to other risk factors (e.g. weight and post-thrombotic syndrome) not accounted for in the tool.

PB1244 | Anticoagulation Strategies during Conditioninginduced Thrombocytopenia in Hematopoietic Cell Transplant Patients with Venous Thromboembolism

$\mathrm{K}$ Martens $^{1}$; C. Amos ${ }^{2,3}$; C. Rojas Hernandez ${ }^{4}$; P. Kebriaei ${ }^{5}$; R. Basom ${ }^{6}$; C. Davis ${ }^{6}$; M. Kesten ${ }^{6}$; M. Carrier ${ }^{7}$; D. Garcia ${ }^{8}$; S. Lee ${ }^{6,9}$; A. $\mathrm{Li}^{6,10}$

${ }^{1}$ Department of Medicine, University of Washington School of Medicine, Seattle, United States; ${ }^{2}$ Division of Epidemiology and Population Science, Baylor College of Medicine, Houston, United States; ${ }^{3}$ Institute of Clinical and Translational Medicine, Baylor College of Medicine, Houston, United States; ${ }^{4}$ Section of Benign Hematology, The University of Texas MD Anderson Cancer Center, Houston, United States; ${ }^{5}$ Department of Stem Cell Transplantation and Cellular Therapy, The University of Texas MD Anderson Cancer Center, Houston, United States; ${ }^{6}$ Clinical Research Division, Fred Hutchinson Cancer Research Center, Seattle, United States; ${ }^{7}$ Department of Medicine, Ottawa Hospital Research Institute, University of Ottawa, Ottawa, Canada; ${ }^{8}$ Division of Hematology, University of Washington, Seattle, United States; 'Division of Oncology, University of Washington, Seattle, United States; ${ }^{10}$ Section of Hematology-Oncology, Baylor College of Medicine, Houston, United States

Background: History of venous thromboembolism (VTE) is common in patients undergoing hematopoietic cell transplantation (HCT). Management of VTE and anticoagulation during conditioninginduced thrombocytopenia remains challenging due to concerns about increased risks for bleeding.

Aims: Assess impact of continuing versus temporarily withholding anticoagulation during thrombocytopenia on short-term VTE recurrence and bleeding. Methods: Patients undergoing first autologous or allogenic HCT 2006-2015 were selected from our institutional database. Patients included in this retrospective study had confirmed VTE prior to HCT and were receiving therapeutic anticoagulation upon arrival for HCT. Patients were allocated to two cohorts based on anticoagulation strategy at the onset of thrombocytopenia, defined as platelets $\leq 50 \times 10^{9} / \mathrm{L}$ or first platelet transfusion after conditioning. Those who continued on therapeutic anticoagulation received platelet transfusions to maintain threshold of $50 \times 10^{9} / \mathrm{L}$ for $\geq 3$ days, while those who temporarily held did so until platelet recovery. Inverse probability of weighting (IPW) using propensity score was used to account for potential confounders and estimate the causal effect associated with differential management. Primary outcomes included VTE recurrence, PE/DVT recurrence, major bleeding (WHO grade 3-4), and overall bleeding (WHO grade 1-4) at 30 days after HCT.

Results: Of 3,722 HCT patients over 10 years, 340 patients met inclusion criteria, of which 227 continued anticoagulation and 113 temporarily withheld (Figure 1). Median duration of thrombocytopenia was 14 days. Adequate balance with standardized difference $<0.10$ was achieved on all covariates after IPW. In IPW-weighted analysis, continuing versus holding anticoagulation was not significantly associated with decreased risk of VTE recurrence within the first 30 days (3\% vs $4 \%)$, however trended toward higher risk of both major bleeding $(7 \%$ vs $5 \%$ ) and overall bleeding (41\% vs $32 \%$ ) (Table 1 ).

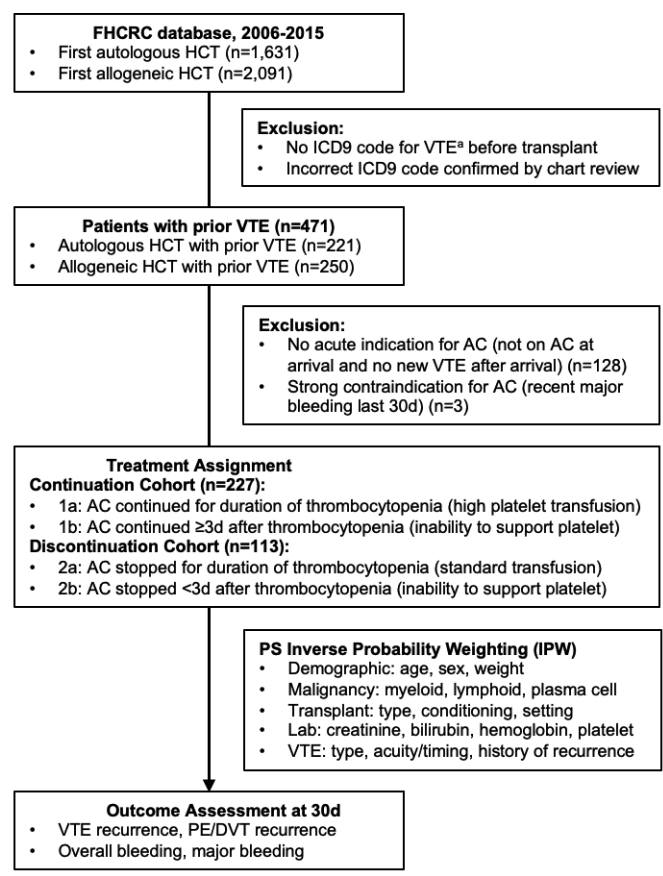

Abbreviations: FHCRC, Fred Hutchinson Cancer Research Center; HCT, hematopoietic cell transplantation; ICD, International Classification of Diseases; VTE, venous thromboembolism; AC, anticoagulation; PS, propensity score; PE, pulmonary embolism; $\mathrm{DVT}$, deep vein thrombosis

a Patients identified by ICD9 codes 12.51, 58.61, 415, 451, and 453 from January 1, 2006-Dec 31, 2015

FIGURE 1 Study design and cohort selection 
TABLE 1 Thrombotic and bleeding outcomes 30 days after transplant. Results are shown before and after IPW weighting

\begin{tabular}{|c|c|c|c|c|c|c|c|}
\hline Outcome at $30 \mathrm{~d}$ & \multicolumn{3}{|c|}{ Pre-IPW Weighting $(n=340)$} & \multicolumn{4}{|c|}{ Post-IPW Weighting $(n=340)$} \\
\hline All VTE recurrence & $3 \%$ & $2 \%$ & 1.00 & $3 \%$ & $4 \%$ & $-1.5 \%$ & 0.61 \\
\hline All bleeding & $42 \%$ & $35 \%$ & 0.20 & $41 \%$ & $32 \%$ & $+9.9 \%$ & 0.08 \\
\hline Major bleeding & $7 \%$ & $4 \%$ & 0.47 & $7 \%$ & $5 \%$ & $+2.2 \%$ & 0.42 \\
\hline
\end{tabular}

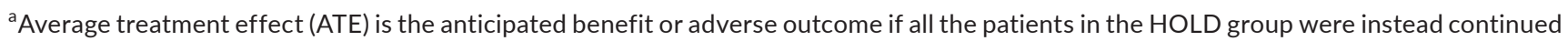
on anticoagulation.

Abbreviations: IPW, inverse-probability weighting; ATE = average treatment effect; VTE, venous thromboembolism; PE, pulmonary embolism; DVT, deep venous thrombosis

Conclusions: In patients taking anticoagulant medication for prior VTE, temporarily withholding anticoagulant therapy may reduce bleeding without significantly increasing risk of recurrent VTE within the first 30 days following HCT.

PB1245 | Effectiveness and Safety of DOACs for the Prevention of Recurrent VTE: A Prospective Cohort Study

A. Vinci ${ }^{1}$; M.C. Vedovati ${ }^{1}$; M.G. De Natale ${ }^{1}$; L. Pierpaoli ${ }^{2}$;

F. Di Filippo²; G. Agnelli ${ }^{1}$; C. Becattini ${ }^{1}$

${ }^{1}$ University of Perugia, Perugia, Italy; ${ }^{2}$ S. Maria delle Croci Hospital,

Ravenna, Italy

Background: In the direct oral anticoagulants (DOACs) era, extended anticoagulation after 6-12 months of treatment is an attractive strategy in patients with venous thromboembolism (VTE). Real-life data on the clinical benefit of DOAC over time is lacking.

Aims: The aim of this study is to assess the effectiveness and safety of DOACs in patients with acute VTE treated for variable periods.

Methods: Data on patients with an objective diagnosis of acute VTE treated with DOACs were included in prospective cohort study. Study outcomes were recurrent VTE and major bleeding (ISTH definition).

Results: Overall, 934 patients were included (mean age 67.0 \pm 16.0 , male gender $51.4 \%$ ). Three-hundred and forty-six patients had a deep vein thrombosis (37.0\%), 98 (10.5\%) had isolated pulmonary embolism and 490 (52.5\%) had both. One-hundred and sixty-nine patients (18.1\%) had an active cancer, 59 (6.3\%) a history of cancer and 365 patients (39.1\%) an unprovoked VTE. During DOAC treatment (mean 21.6 months), 7 recurrent VTEs and 25 major bleedings occurred. In 546 and in 98 patients, DOAC was continued with full and reduced doses, respectively. In 290 patients (43.8\% unprovoked, $13.8 \%$ active cancer, $42.4 \%$ associated with non-cancer risk factor), anticoagulants were withdrawn (average treatment duration 8.8 months) and 22 recurrent VTEs occurred over a followup off-treatment period of 31.9 months. In these patients, 2 episodes of major bleeding were observed. Overall, 201 patients died; fatal PE occurred in 4 and fatal bleeding in 1 patient. Time course for recurrent VTE according to 2019 ESC risk for recurrence is reported in the Figure.

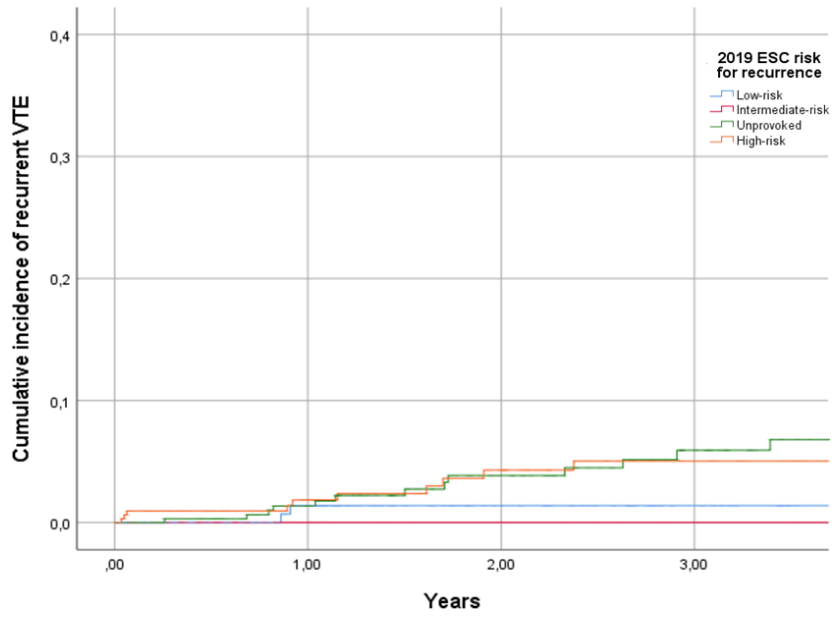

FIGURE 1 Cumulative incidence of recurrent VTE

Conclusions: In this cohort study, DOACs showed a good risk to benefit profile in the extended phase after an acute VTE event. 
PB1246 | Antithrombotic Management of Patients with Deep Vein Thrombosis and Venous Stents: An International Registry

A. Cervi $^{1}$; D. Applegate ${ }^{2}$; S.M. Stevens ${ }^{2,3}$; S.C. Woller ${ }^{2,3}$;

L. Baumann Kreuziger ${ }^{4}$; K. Puchhalapalli ${ }^{4}$; T.-F. Wang ${ }^{5}$;

R. Lecumberri' ${ }^{6}$ S. Schulman ${ }^{7,8}$; G. Foster ${ }^{9,10}$; J. Douketis ${ }^{1}$

${ }^{1}$ Department of Medicine, McMaster University, Hamilton, Canada;

${ }^{2}$ Intermountain Healthcare, Murray, United States; ${ }^{3}$ School of Medicine,

University of Utah, Salt Lake City, United States; ${ }^{4}$ Department of

Medicine, Blood Research Institute, Versiti, Medical College of Wisconsin,

Milwaukee, United States; ${ }^{5}$ Department of Medicine, University of Ottawa at The Ottawa Hospital and Ottawa Hospital Research Institute, Ottawa, Canada; ${ }^{6}$ Hematology Service, University Clinic of Navarra, Pamplona, Spain; ${ }^{7}$ Department of Medicine, Thrombosis and Atherosclerosis Research Institute, McMaster University, Hamilton, Canada; ${ }^{8}$ Department of Obstetrics and Gynecology, The First I.M. Sechenov Moscow State Medical University, Moscow, Russian Federation; ${ }^{9}$ Department of Health Research Methods, Evidence and Impact, McMaster University, Hamilton, Canada;

${ }^{10}$ Biostatistics Unit, St. Joseph's Healthcare Hamilton, Hamilton, Canada

Background: There is no standard approach to antithrombotic management for acute deep vein thrombosis (DVT) treated with catheter-based strategies and adjunct venous stents.

Aims: To create an international registry of patients with leg DVT who received venous stents as part of their acute management.

Methods: We enrolled patients across 5 clinical centres through the ISTH with venous stents inserted from 2005-2019. We collected data on baseline clinical characteristics and pre-and post-venous stent antithrombotic therapy.

Results: We recruited 173 patients with venous stents; 100 (58\%) were under 50 years of age, 106 (61\%) were female, and 126 (74\%) had thrombosis risk factors. DVT was iliofemoral in 95 (55\%) patients and catheter-based treatment was administered within 7 days of diagnosis in $92(53 \%)$ patients. Among the $30 \%(N=52)$ of patients that received anticoagulation preceding stent insertion, $17 \%(N=30)$ received low molecular weight heparin and $13 \%(N=22)$ received unfractionated heparin. After stent insertion, patients were most likely to receive a single anticoagulant [109 (63\%)], often a direct oral anticoagulant (DOAC) [50 (29\%)] for 3-6 months [26 (15\%)] or indefinitely [21 (12\%)]. Fiftynine patients (34\%) received combined anticoagulant-antiplatelet therapy for variable durations; rivaroxaban and clopidogrel was the most common regimen [(14 (8\%)]. Determinants of combined anticoagulantantiplatelet use, as compared to anticoagulant only use were: increasing number of antecedent percutaneous interventions such as thrombolysis, thrombectomy, and aspiration (odds ratio $[\mathrm{OR}]=5.11,95 \%$ confidence interval $[\mathrm{Cl}]: 1.45,18.05)$; use of balloon angioplasty $(\mathrm{OR}=2.62$, $95 \% \mathrm{Cl}: 1.2,5.76)$; and complications of stent insertion, such as stent restenosis (OR $=7.2,95 \% \mathrm{Cl}: 1.45,35.89)$. Repeat pharmacomechanical catheter-based interventions were needed in 20 (11.5\%) patients. Conclusions: DOAC therapy appears to be the most common anticoagulant strategy for adjunct venous stents after acute DVT. Further prospective research is warranted to determine outcomes of venous stents in relation to antithrombotic practices.
PB1247 | Safety Profile of Rivaroxaban in First-time Users Treated for Deep Vein Thrombosis and Pulmonary Embolism without a Recent History of Cancer

A. Ruigomez ${ }^{1}$; T. Schink ${ }^{2}$; A. Voss ${ }^{2}$; R.MC Herings ${ }^{3}$; E. Smits ${ }^{3}$; K. Swart-Polinder ${ }^{3}$; Y. Balabanova ${ }^{4}$; G. Brobert ${ }^{5}$; L.A. García Rodríguez ${ }^{1}$

${ }^{1}$ CEIFE - Centro Español de Investigación Farmacoepidemiológica, Madrid, Spain; ${ }^{2}$ Leibniz Institute for Prevention Research and Epidemiology - BIPS GmbH, Bremen, Germany; ${ }^{3}$ The PHARMO Institute, Utrecht, Netherlands; ${ }^{4}$ Bayer AG, Berlin, Germany; ${ }^{5}$ Bayer AB, Solna, Sweden

Background: A post-authorisation study of rivaroxaban use was conducted between 2011-2020.

Aims: To study major bleeding and its risk factors among first-time users of rivaroxaban (RVX) or vitamin $\mathrm{K}$ antagonists (VKA) for venous thromboembolism treatment in routine clinical practice in four countries.

Methods: This cohort study used data from four European countries: IQVIA Medical Research Data-UK in the UK, the German Pharmacoepidemiological Research Database, the Dutch PHARMO Database Network and Swedish national health registries. Patients $\geq 2$ years with a first prescription of RVX or VKA from December 2011-December 2017 temporally close to diagnostic codes for VTE-T, without codes for atrial fibrillation and no recent cancer history, were followed until the date of each safety outcome (intracranial, gastrointestinal, urogenital or other bleeding leading to hospitalization), death, or study end (December 2017 or December 2018, country-dependent). Crude incidence rates (IR; [95\% CI]) of safety outcomes per 100 personyears were calculated for RVX and VKA users; nested case-control analyses examined risk factors for each safety outcome per database.

Results:

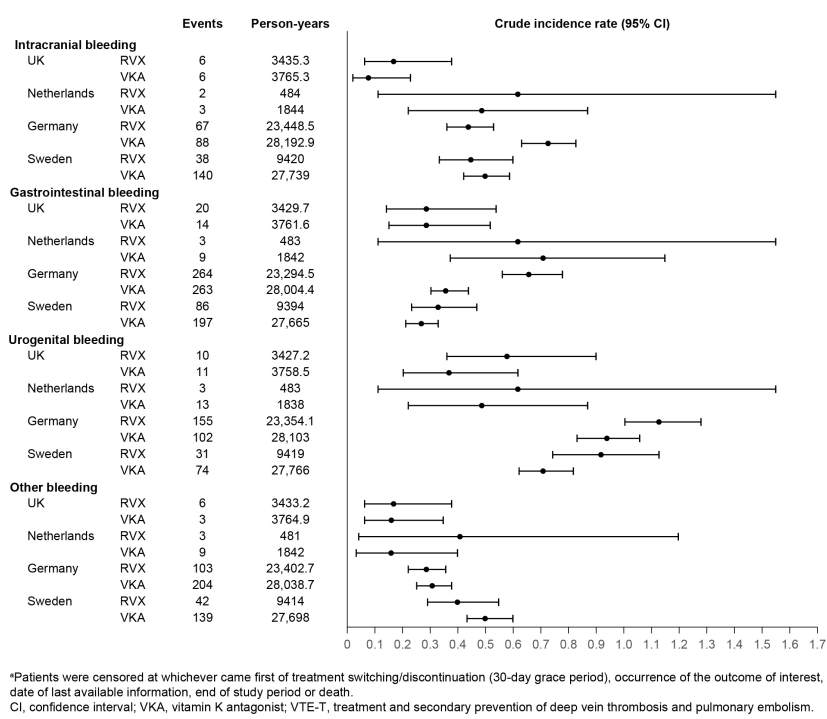

FIGURE 1 Incidence rate of safety outcomes associated with first use of rivaroxaban and VKA (first episode of treatment a) - VTE-T without recent history of cancer sub-cohort 
Overall, 44,737 (RVX) and 45,842 (VKA) first-time users were identified. Crude IR estimates were similar between RVX and VKA users for intracranial and urogenital bleeding, with minor variations between studies and somewhat higher for RVX than VKA for gastrointestinal bleeding, in line with the findings from the pivotal clinical trial (Figure). Nested case-control analyses will also be presented in detail.

Conclusions: Bleeding incidence rate estimates broadly agreed with pivotal clinical trial findings. Observed variations in estimates may reflect differences between data sources and healthcare systems, as well as in the methods followed for capturing events prior to the start date.

PB1248 | Usefulness of Measuring Anti-Factor Xa Activity to Guide the Treatment with Low-molecular-weight Heparins in Patients with Acute Pulmonary Embolism

J. Bonorino; J. Bilbao; H. Fernandez; N. Torres; M. Iwanowski;

P. Alarcon; R. Melchiori; J. Santucci; M.E. Aris Cancela; P. Colimodio; C. Rosa; M.C. Ferrario; P. Rubio; S. Marquez; G. Garcia; M. Bivort; A. Sanchez; N. Perez Caceres; S. Baratta; A. Hita

Hospital Universitario Austral, Pilar, Argentina

Background: Anticoagulation with enoxaparin is recommended in acute pulmonary embolism (PE) and active cancer, and in patients who will receive vitamin $\mathrm{K}$ antagonists, who must overlap both drugs for several days. Pretreatment with enoxaparin during 5-10 days is needed in patients who will be treated with dabigatran or edoxaban. Usefulness of measuring Anti-Factor Xa activity (Anti-Xa) is uncertain since it's unclear whether excessive or insufficient activity could be associated with higher bleeding or thrombotic risks, respectively.

Aims: Determine the existence of prognostic variables that could alter Anti-Xa in patients with PE anticoagulated with enoxaparin.

Methods: Single-center observational cohort registry. A total of 268 patients were hospitalized with PE between 2008-2018, and were eligible for this study those anticoagulated with enoxaparin in whom Anti-Xa was measured. The following prognostic factors were considered to establish variations in Anti-Xa: High-thrombus burden $(\mathrm{H}-\mathrm{ThB})$, right ventricular dysfunction, creatinine clearance $<50 \mathrm{ml} /$ min; obesity; active cancer and elderly.

Results: We included 126 patients; $59 \pm 16$ years (51\% female). Enoxaparin dose modifications ocurred in $39 \%$.

Patients with $\mathrm{H}-\mathrm{ThB}$ required more frequently dose modifications of enoxaparin (38\% vs 17\%; p:0.02) and were older ( $64 \pm 15$ vs $56 \pm 16$; p:0.01).

In patients who required dose modifications, $58 \%$ increased doses. No differences were observed between those who required dose modifications of enoxaparin vs those who didn't, with regard to bleeding events (4.4 vs 3.8\%), in-hospital mortality ( 2.1 vs $2.9 \%$ ) and 30 -day mortality (2.4 vs 5.3\%), respectively. After multivariate analysis, only $\mathrm{H}-\mathrm{ThB}$ was associated with dose modifications of enoxaparin (OR 2.9, 95\%Cl,1.03-8.71; p:0.04).
Conclusions: Measurement of Anti-Xa was useful in patients with PE anticoagulated with enoxaparin and $\mathrm{H}-\mathrm{ThB}$, since more than one third required modifications of the initial dose and of them, more than half increased the dose with similar bleeding rates. Likewise, thrombus burden quantification may be useful to guide treatment with enoxaparin.

\section{PB1249 | A Retrospective Evaluation of the Management of Isolated Distal Deep Vein Thrombosis in Australia}

B. Lui ${ }^{1}$; J. Lai ${ }^{2}$; Z. Khattak ${ }^{1}$; A. Kwok ${ }^{2}$; P. Ho ${ }^{1}$; H.Y. Lim ${ }^{1,2}$

${ }^{1}$ Northern Health, Melbourne, Australia; ${ }^{2}$ The University of Melbourne, Melbourne, Australia

Background: Isolated distal deep vein thrombosis (IDDVT) is often viewed to be of less clinical significance than major venous thromboembolism (VTE). However, studies report variable recurrence rate (2-19\%) with significant heterogeneity in the IDDVT management.

Aims: To evaluate the characteristics of IDDVT in our study population.

Methods: Retrospective evaluation of IDDVT events managed at Northern Health, Melbourne, Australia from January 2012 to June 2019 (median follow-up 5.7 years). Analysis included demographics, associated factors, management and outcomes.

Results: 429 patients (median age 63 years (range 18-102), 56\% females) presented with 438 cases of IDDVT in this time period. The majority ( 297 cases, 68\%) were provoked, most commonly due to injury/immobility ( $n=142,33 \%$ ) followed by surgery ( $n=116,26 \%)$. Prior VTE history was present in $82(19 \%)$ cases. Twenty-nine patients (7\%) had active malignancy at time of diagnosis. The median duration of anticoagulation was 3 months for provoked events compared to 4 months for unprovoked events $(P=0.015)$. Warfarin was the most common anticoagulant used (189 cases, 43\%), followed by direct oral anticoagulants (DOACs) (152, $35 \%)$. Of note, DOACs were only listed by Pharmaceutical Benefits Scheme for use in Australia in 2013. There were 53 (12\%) patients with recurrent VTE (including 18 (34\%) as major VTE) and 9 (2\%) patients with clinically significant major bleeding. An analysis of the overall database demonstrated that IDDVT patients had comparable VTE recurrence rate to those with major VTE (12\% vs $11 \%, P=0.44$ ) but lower major bleeding rates ( $2 \%$ vs $4 \%, P=0.036)$. There were four bleeding-related deaths (all on warfarin/enoxaparin), with no thrombosis-related deaths. Fourteen cases (3\%) were diagnosed with subsequent malignancy.

Conclusions: The majority of IDDVT were provoked although the risk of recurrent thrombosis was comparable to major VTE despite a lower major bleeding rate. These data suggest that IDDVT is not always as benign as assumed. 
PB1250 | Comparative Effectiveness of Oral Anticoagulants in Venous Thromboembolism: On-treatment Analysis in GARFIELD-VTE

S. Haas $^{1}$; H. Bounameaux ${ }^{2}$; A.E. Farjat ${ }^{3}$; W. Ageno ${ }^{4}$; J.I. Weitz ${ }^{5}$; S.Z. Goldhaber ${ }^{6}$; A.G.G. Turpie ${ }^{7}$; S. Goto ${ }^{8}$; P. Angchaisuksiri ${ }^{9}$; P. MacCallum ${ }^{3,10}$; H. Ten Cate ${ }^{11}$; E. Panchenko ${ }^{12}$; M. Carrier ${ }^{13}$; C.J. Sanchez Dias ${ }^{14,15}$; H. Gibbs ${ }^{16}$; P. Jansky ${ }^{17}$; G. Kayani ${ }^{3}$; S. Schellong ${ }^{18}$; P. Prandoni ${ }^{19}$; A.K. Kakkar ${ }^{3,20}$; on behalf of the GARFIELD-VTE investigators

${ }^{1}$ Formerly Technical University of Munich, Munich, Germany; ${ }^{2}$ Faculty of Medicine, University of Geneva, Geneva, Switzerland; ${ }^{3}$ Thrombosis Research Institute, London, United Kingdom; ${ }^{4}$ Department of Medicine and Surgery, University of Insubria, Varese, Italy; ${ }^{5}$ McMaster University and the Thrombosis and Atherosclerosis Research Institute, Hamilton, Ontario, Canada; ${ }^{6}$ Brigham and Women's Hospital and Harvard Medical School, Boston, United States; ${ }^{7}$ McMaster University, Hamilton, Ontario, Canada; ${ }^{8}$ Department of Medicine (Cardiology), Tokai University School of Medicine, Tokyo, Japan; ${ }^{9}$, Department of Medicine, Ramathibodi Hospital, Mahidol University, Bangkok, Thailand; ${ }^{10}$ United Kingdom; Queen Mary University of London, London, United Kingdom; ${ }^{11}$ Department of Vascular Medicine and Internal Medicine, Maastricht University Medical Center (MUMC+), Maastricht, Netherlands; ${ }^{12}$ National Medical Research Center of Cardiology of Ministry of Health of the Russian Federation, Moscow, Russian Federation; ${ }^{13}$ Department of Medicine, The Ottawa Hospital, Ottowa, Canada; ${ }^{14}$ Escuela de Medicina y Ciencias de la Salud. Tecnológico de Monterrey, Monterrey, Mexico; ${ }^{15}$ Instituto de Cardiología y Medicina Vascular, TecSalud, Monterrey, Mexico; ${ }^{16}$ Department of General Medicine, Alfred Health, Melbourne, Australia; ${ }^{17}$ Motol University Hospital, Department of Cardiovascular Surgery, Prague, Czech Republic; ${ }^{18}$ Medical Department 2, Municipal Hospital Dresden, Dresden, Germany; ${ }^{19}$ Arianna Foundation on Anticoagulation, Bologna, Italy; ${ }^{20}$ University College London, London, United Kingdom

Background: Direct oral anticoagulants (DOACs) provide a safe and effective alternative to vitamin $\mathrm{K}$ antagonists (VKAs) for treatment of venous thromboembolism (VTE), as shown in a previous intentionto-treat comparative effectiveness analysis. However, on-treatment analysis is imperative in observational studies because the duration and choice of anticoagulation is at the investigators' discretion.

Aims: Compare the effectiveness of DOACs and VKAs on 12-month outcomes in VTE patients using on-treatment analysis.

Methods: GARFIELD-VTE (ClinicalTrials.gov: NCT02155491) is a global, prospective, non-interventional study of real-world treatment practices. This on-treatment analysis included 8,034 patients treated with either VKA ( $n=3,043,37.9 \%)$ or DOAC ( $n=4,991$, $62.1 \%$ ), with or without parenteral anticoagulation bridging. The causal treatment effect was estimated using marginal structural models with inverse probability weights to account for the timevarying nature of exposure and confounders, and to correct for the effect of major bleeding on dropout.

Results: After adjustment for baseline characteristics, all-cause mortality was significantly lower with DOACs than with VKAs (hazard ratio [HR]: 0.58; 95\% confidence interval $[\mathrm{Cl}] 0.42-0.79, P=0.001$ ) (Figure 1). Patients receiving VKAs were more likely than those receiving DOACs to die of VTE complications (4.9\% vs. $2.2 \%$ ) or from bleeding (4.9\% vs. $0.0 \%)$. There was no significant difference in recurrent VTE (HR: 0.74, 95\% Cl 0.55-1.01), major bleeding (HR: 0.76, 95\% $\mathrm{Cl} 0.47-1.24)$, or overall bleeding (HR: $0.87,95 \% \mathrm{Cl} 0.72-1.05)$ with DOACs or VKAs (Table 1). VTE patients with active cancer were more likely to die if they received a VKA than a DOAC (52.51 [37.33-73.86] vs 26.52 [19.37-36.29] per 100 person-years, respectively). This was also true for VTE patients with concomitant renal insufficiency (9.97 [7.51-13.23] vs 4.70 [3.25-6.81] per 100 person-years, respectively). Conclusions: With similar rates of recurrent VTE and major bleeding, DOACs were associated with reduced rates of all-cause mortality and a lower likelihood to die from VTE or fatal bleeding compared to VKAs.
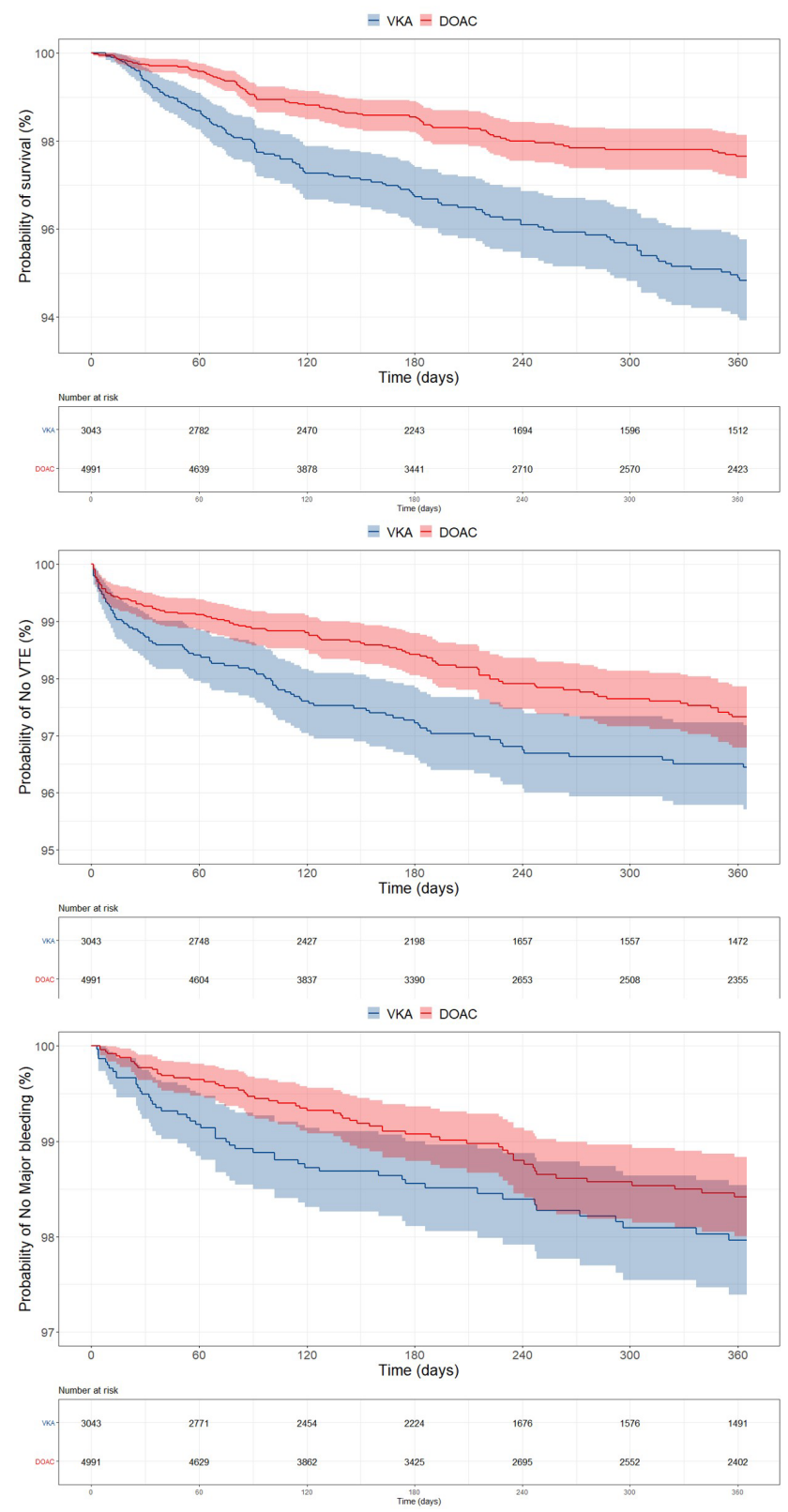

FIGURE 1 Kaplan-Meier curves for all-cause mortality, recurrent VTE and major bleeding in VTE patients receiving oral anticoagulants. Abbreviations; DOAC: direct oral anticoagulant, VKA: vitamin $\mathrm{K}$ antagonist, VTE: venous thromboembolism 
TABLE 1 Incidence rates (per 100 person-years) and adjusted hazard ratios for VTE patients receiving oral anticoagulants. Event rates are shown per 100 person-years. HR values

\begin{tabular}{|c|c|c|c|c|c|c|c|}
\hline Outcome & \multicolumn{2}{|l|}{ VKA } & \multicolumn{2}{|l|}{ DOAC } & Adjusted* HR & $95 \% \mathrm{Cl}$ & $P$-value \\
\hline All-cause mortality & 5.69 & $4.76-6.79$ & 2.61 & $2.12-3.20$ & 0.58 & $0.42-0.79$ & 0.001 \\
\hline Recurrent VTE & 4.32 & $3.52-5.30$ & 2.97 & $2.44-3.61$ & 0.74 & $0.55-1.01$ & 0.058 \\
\hline Major bleeding & 2.35 & $1.78-3.10$ & 1.69 & $1.30-2.18$ & 0.76 & $0.47-1.24$ & 0.27 \\
\hline Any bleeding & 12.65 & $11.20-14.29$ & 12.02 & $10.89-13.26$ & 0.87 & $0.72-1.05$ & 0.151 \\
\hline Stroke/TIA & 0.37 & $0.19-0.75$ & 0.61 & $0.40-0.93$ & 1.57 & $0.53-4.66$ & 0.416 \\
\hline
\end{tabular}

PB1251 | Using an App to Educate Patients on the Pros and Cons of Extended Anticoagulation after Venous Thromboembolism: A Randomized Controlled Trial

M.A. de Winter ${ }^{1}$; T. Timmers ${ }^{2,3}$; M.M.C. Hovens ${ }^{4}$;

A. Iglesias del Sol ${ }^{5}$; A.T.A. Mairuhu' ${ }^{6}$; H.A.H. Kaasjager ${ }^{1}$;

M. Nijkeuter ${ }^{1}$

${ }^{1}$ Department of Acute Internal Medicine, University Medical Center Utrecht, Utrecht, Netherlands; ${ }^{2}$ Interactive Studios, Rosmalen, Netherlands; ${ }^{3}$ Radboud University Medical Center, Radboud Institute for Health Sciences, IQ Healthcare, Nijmegen, Netherlands; ${ }^{4}$ Department of Internal Medicine, Rijnstate Hospital, Arnhem, Netherlands; ${ }^{5}$ Department of Internal Medicine, Alrijne Hospital, Leiderdorp, Netherlands; ${ }^{6}$ Department of Internal Medicine, Haga Teaching Hospital, Den Haag, Netherlands

Background: Adequate patient education is essential to enable patients to engage in shared decision-making (SDM) when deciding to stop or continue anticoagulation after 3 months of anticoagulation for venous thromboembolism (VTE).

Aims: To evaluate the effect of an interactive, educational app on patients' level of satisfaction with information, perceived level of knowledge, decisional conflict and SDM when deciding on treatment duration after VTE.

Methods: This randomized controlled trial in 1 academic and 3 general hospitals in The Netherlands included adult patients with VTE without malignancy or other indication for anticoagulation. Patients were randomized in a 1:1 ratio to receive the app (intervention group) in addition to the standard of care. The app contains information on VTE and anticoagulation on an interactive timeline, created for this study. In the week preceding the consultation when treatment duration is decided, patients were provided with daily videos using push notifications. Outcomes were assessed through online, self-reported questionnaires at baseline, 1-2 days before and 1 day after the consultation. Data were analyzed using t-tests and linear mixed models for repeated measurements.

Results: The trial was terminated early as the inclusion rates dropped due to the COVID-19 pandemic. Data of 56 patients were analyzed (mean age $57 \pm 13 ; 27 \%$ female). Satisfaction with received information was very heterogeneous and generally higher after the consultation (Figure 1). On a numeric rating scale from 0 to 10, patients who received the app were 0.86 points $(95 \% \mathrm{Cl} 0.04$ to $1.68 ; \mathrm{p}$ 0.04 ) more satisfied with the provided information (Table 2). Patients who received the app experienced significantly less decisional conflict. No significant differences in satisfaction with knowledge, perceived knowledge and physician-reported SDM were observed.

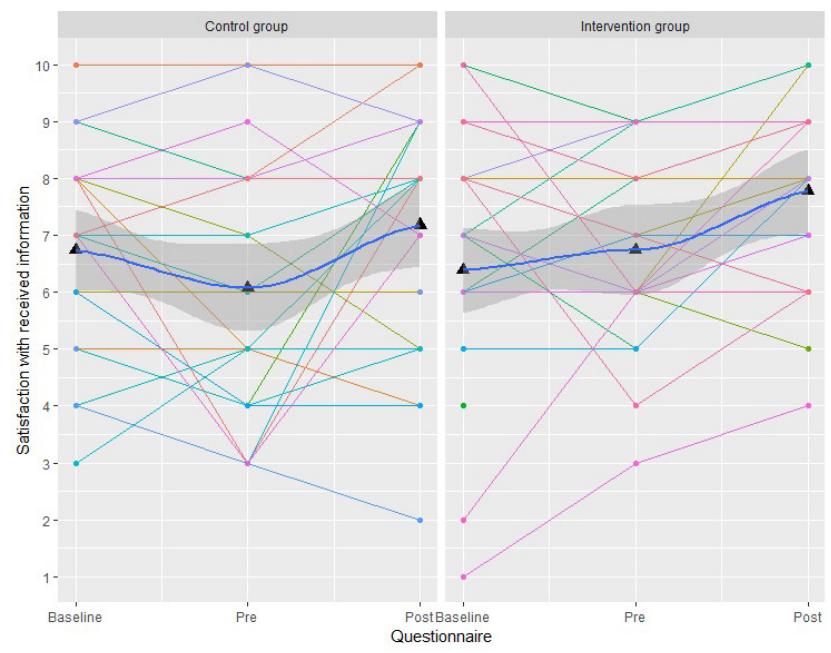

FIGURE 1 Change of satisfaction with received information over time stratified by intervention group. Measured at baseline, 1-2 days prior to consultation ("pre") and 1 day after consultation ("post"). Colored lines indicate individual patients; blue thick line and confidence band indicate mean and 95\% confidence interval 
TABLE 1 Intention to treat analysis of primary and secondary outcomes in 26 patients receiving the app (intervention) and 30 patients receiving standard of care only (control). All data are shown as mean \pm SD. All scores are positively associated with the outcome (e.g. higher decisional conflict scale means more decisional conflict). Abbreviation: $\mathrm{Cl}$ confidence interval. ${ }^{*}$ indicates p-value

\begin{tabular}{|c|c|c|c|c|c|c|}
\hline & \multicolumn{2}{|c|}{ Before consultation } & \multicolumn{2}{|c|}{ After consultation } & \multirow{2}{*}{$\begin{array}{l}\text { Model estimated } \\
\text { difference (intervention } \\
\text { vs. control) }(95 \% \mathrm{Cl})\end{array}$} & \multirow[b]{2}{*}{$P$-value } \\
\hline & Intervention & Control & Intervention & Control & & \\
\hline $\begin{array}{l}\text { Satisfaction with received information } \\
\text { (range 1-10) }\end{array}$ & $6.7 \pm 1.7$ & $5.9 \pm 2.4$ & $7.8 \pm 1.5$ & $7.1 \pm 2.0$ & 0.9 (0.0 to 1.7$)$ & 0.04 \\
\hline Satisfaction with knowledge (range 1-10) & $6.7 \pm 2.2$ & $6.2 \pm 2.4$ & $8.0 \pm 1.3$ & $7.2 \pm 2.0$ & $0.7(-0.3$ to 1.5$)$ & 0.11 \\
\hline Perceived level of knowledge (range 5-25) & $13.8 \pm 3.0$ & $13.1 \pm 3.8$ & $17.1 \pm 3.3$ & $15.2 \pm 4.3$ & $1.0(-0.6$ to 2.6$)$ & 0.22 \\
\hline Decisional Conflict Scale (range 0-100) & n.a. & n.a. & $29.8 \pm 15.2$ & $46.2 \pm 22.5$ & n.a. & 0.01 \\
\hline $\begin{array}{l}\text { Shared decision-making (physician) (range } \\
0-45 \text { ) }\end{array}$ & n.a. & n.a. & $31.8 \pm 9.5$ & $31.6 \pm 9.0$ & n.a. & 0.94 \\
\hline
\end{tabular}

Conclusions: An educational app about VTE and anticoagulation increases patients' satisfaction and reduces decisional conflict when deciding to stop or continue anticoagulation after initial treatment for VTE.

PB1252 | Real-world Data on the Use of Oral Anticoagulants for the Treatment of Venous Thromboembolism in Sweden: Oral Anticoagulant Use and Baseline Characteristics from the Venous Thromboembolism Treatment Study

A. Gottsäter ${ }^{1}$; H. Pohjantähti-Maaroos ${ }^{2}$; K. Bokenberger ${ }^{3}$;

S. Graham ${ }^{4}$; A. Jenkins ${ }^{5}$; D. Lambrelli ${ }^{4}$; K. Mercaldi ${ }^{6}$; M. Raluy ${ }^{3}$; W. Ghanima ${ }^{7}$

${ }^{1}$ Lund University, Malmö, Sweden; ${ }^{2}$ Kuopio University Hospital, Kuopio,

Finland; ${ }^{3}$ Evidera, Stockholm, Sweden; ${ }^{4}$ Evidera, London, United

Kingdom; ${ }^{5}$ Pfizer, Tadworth, United Kingdom; ${ }^{6}$ Evidera, Waltham, United Kingdom; ${ }^{7}$ University of Oslo, Oslo, Norway

Background: Non-vitamin K oral anticoagulants (NOACs) are recommended as a first-line treatment for venous thromboembolism (VTE). Observational data from nationwide registries allow for the investigation of how clinical recommendations have impacted practice.

Aims: The Venous Thromboembolism Treatment (VOLT) study describes the characteristics, treatment patterns, healthcare resource utilisation, comparative effectiveness, and safety of OACs in treating VTE in Sweden, Finland, and Norway. This abstract presents the initial descriptive data from Sweden.
Methods: A cohort study using prospectively collected administrative data identified treatment-naïve, newly diagnosed adult VTE patients dispensed apixaban, dabigatran, edoxaban, rivaroxaban, or warfarin between 1 January 2013 and 30 September 2018 using Swedish nationwide registries. Patient demographics, clinical characteristics, and subgroups (deep vein thrombosis [DVT] or pulmonary embolism [PE]; diagnostic setting and etiology) are described per treatment cohort. This study was approved by the Swedish Ethical Review Authority (2019-04722).

Results: A total of 33,979 patients from Sweden were included, with the vast majority receiving rivaroxaban (38.8\%), warfarin $(33.2 \%)$ or apixaban (26.8\%); few were treated with dabigatran (1.1\%) or edoxaban (0.1\%). There was a marked increase in NOAC use and decrease in warfarin use during the follow-up period (Figure 1). Almost half (47.2\%) of all patients were diagnosed with PEs (with or without a DVT). Over half (62.2\%) of VTE was unprovoked. The mean age for both the total and apixaban cohorts was 64.6 years; the warfarin cohort was slightly older (65.9 years) and the rivaroxaban cohort was slightly younger (63.4 years) (Table 1). Comorbidities were more frequent in the warfarin cohort and less common in the rivaroxaban cohort. The apixaban cohort had a higher prevalence of prior bleeding. 
TABLE 1 Age and key subgroups for patients with VTE in Sweden from 1 January 2013 to 30 September 2018 (overall and for each OAC cohort at the time of OAC treatment initiation). Abbreviations: DVT = deep vein thrombosis; $\mathrm{PE}=$ pulmonary embolism; SD = standard deviation; $\mathrm{VTE}=$ venous thromboembolism; ${ }^{*}=$ data suppressed to maintain confidentiality, includes a cell with $<5$ patients.

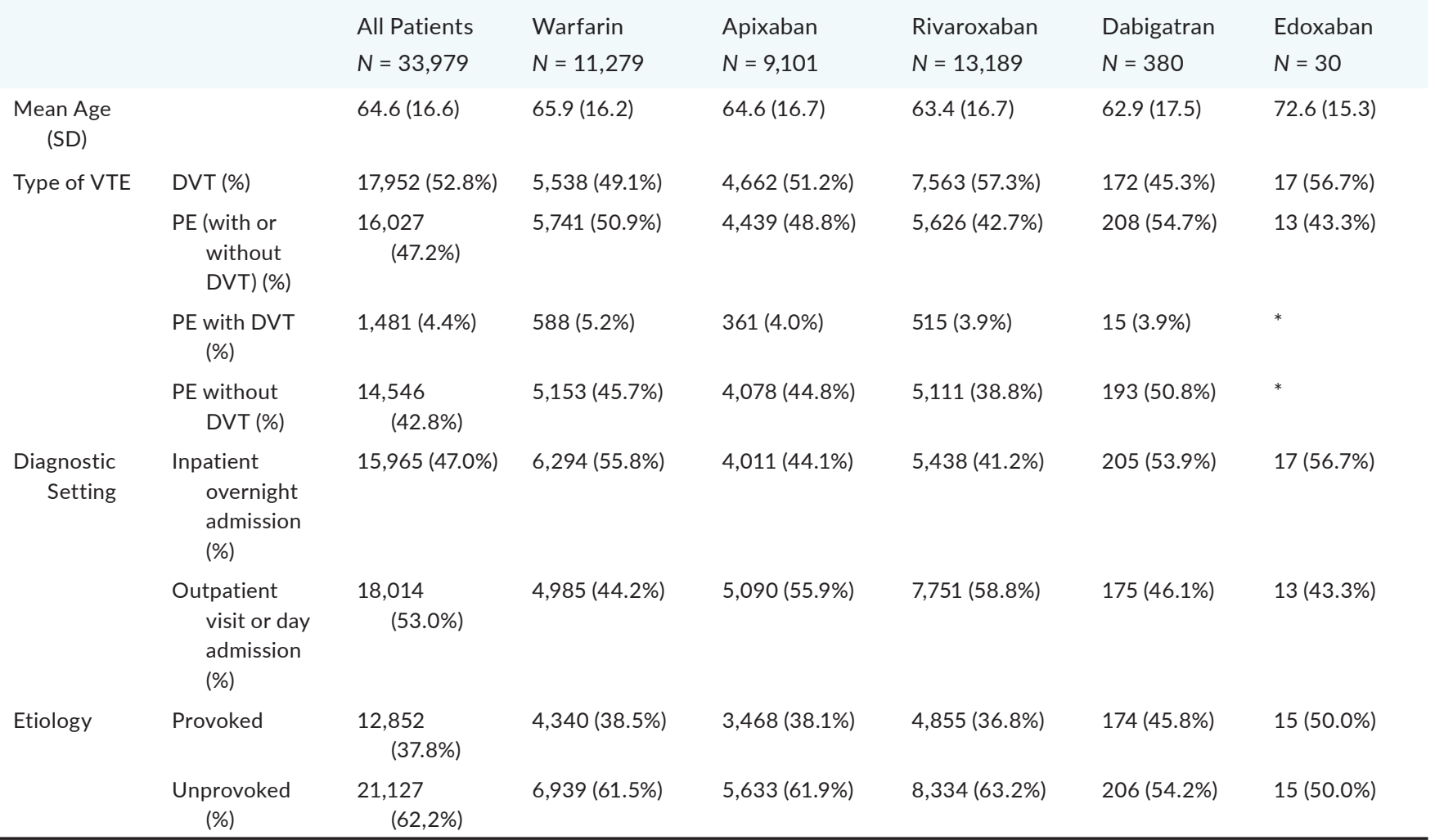

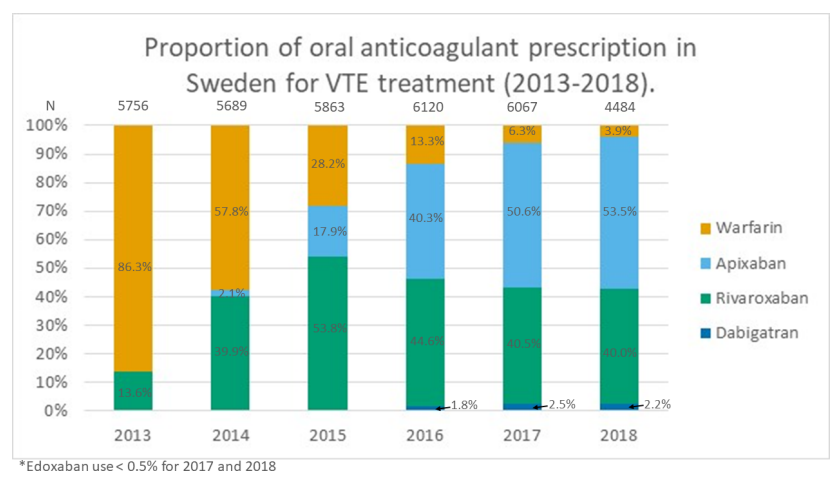

FIGURE 1 Proportion of oral anticoagulant prescription in Sweden for VTE treatment (2013-2018)

Conclusions: From 2013 to 2018, there was a marked increase in NOAC use for VTE treatment, particularly for rivaroxaban and apixaban, which coincides with the publication of clinical guidelines recommending their use.

\section{PB1253 | Neutralization of the Oral and Parenteral Anti-Xa Agents by Andexanet Alfa}

F. Siddiqui $^{1}$; D. Hoppensteadt ${ }^{1}$; J. Walenga ${ }^{1}$; W. Jeske ${ }^{1}$; A. Tafur ${ }^{2}$; E. Ramacciotti ${ }^{1}$; J. Fareed ${ }^{1}$

${ }^{1}$ Loyola University Medical Center, Maywood, United States; ${ }^{2}$ North Shore University Health Systems, Evanston, United States

Background: Beside the oral anti-Xa agents, parenteral forms of the inhibitors of factor Xa such as otamixaban (Sanofi Aventis, Paris, France) and DX9065a (Mitsubishi Pharmaceuticals, Tokyo, Japan) have also been developed. Andexanet alfa is a broad-spectrum neutralizing agent for anti-Xa drugs.

Aims: This study is designed to compare the neutralization profile of andexanet alfa for apixaban and rivaroxaban with otamixaban and DX9065a in various laboratory assays. 
Methods: Anti-Xa agents were supplemented in plasma in the concentration range of $0.0-1.0 \mathrm{ug} / \mathrm{ml}$. Individual aliquots of samples were supplemented with either saline or andexanet alfa at a final concentration of $100 \mathrm{ug} / \mathrm{ml}$. Factor Xa activity was measured by using an amidolytic method. APTT, and thrombin generation inhibition studies were also carried out. The inhibitory effects of each of these agents towards factor Xa were calculated and their reversal by andexanet alfa was determined. Results were compiled as mean SD of multiple determination. Results: Both the oral and parenteral anti-Xa agents produced a concentration dependant inhibition of factor-Xa with the IC50 values ranging from 0.17-1.1 ug/ml in control group. Supplementation of andexanet alfa at $100 \mathrm{ug} / \mathrm{ml}$ resulted in the neutralization of the anti-Xa activities of these agents with the IC50 values ranging from $0.22-1.1 \mathrm{ug} / \mathrm{ml}$. Andexanet alfa at $100 \mathrm{ug} / \mathrm{ml}$ effectively neutralized the anticoagulant effects of otamixaban in comparison to Apixaban and rivaroxaban.

Conclusions: Our results suggest that andexanet alfa is capable of neutralizing the effects of potent parenteral anti-Xa agents such as otamixaban. These results also underscore that the in-vitro anti-Xa potency of both the oral and parenteral anti-Xa agents does not fully reflect their inhibitory effects on the overall coagulation process. Nevertheless, andexanet alfa may be a useful agent in the neutralization of parenteral anti-Xa agents.
PB1254 | Oral Anticoagulant Use in Patients with Morbid Obesity: A Systemic Review and Meta-analysis

T.-F. Wang ${ }^{1} ;$ M. Carrier ${ }^{1}$; K. Fournier ${ }^{2}$; D. Siegal ${ }^{1}$; G. Le Gal ${ }^{1}$; A. Delluc ${ }^{1}$

${ }^{1}$ University of Ottawa at The Ottawa Hospital and Ottawa Hospital Research Institute, Ottawa, Canada; ${ }^{2}$ Library, University of Ottawa, Ottawa, Canada

Background: Obesity is associated with increased risks of venous thromboembolism (VTE) and atrial fibrillation (AF) for which anticoagulation is commonly used.

Aims: We conducted a systemic review and meta-analysis to evaluate the efficacy and safety of direct oral anticoagulants (DOACs) or vitamin $\mathrm{K}$ antagonists (VKA) in the treatment of VTE or AF in patients with morbid obesity.

Methods: We searched the electronic databases including MEDLINE, Embase, Scopus, and Cochrane Central Register of Controlled Trials from inception. We included randomized controlled trials (RCTs) and observation studies which reported outcomes of interest in adult patients with weight $\geq 120 \mathrm{~kg}, \mathrm{BMI} \geq 40 \mathrm{~kg} / \mathrm{m}^{2}$, or classified as morbid obesity by ICD codes who received DOACs or VKA for VTE or AF. The primary efficacy outcome was VTE recurrence in VTE population and stroke or systemic embolism in AF population, and the primary safety outcome was major bleeding. We calculated the pooled annual incidence rates of outcomes and compared DOAC with VKA by incidence rate ratio using $\mathrm{R}$ software (version 4.0.3). The quality of studies was assessed by ROBINS-I and Cochrane RoB 2 tools.

Results: Fifteen studies (3 RCTs and 12 observational studies) with 68,250 morbidly obese patients were included for meta-analysis. Nine studies involved VTE population and 10 involved AF. Table 1 summarized the incidence rates of outcomes. VKA was associated with a numerically higher rate of recurrent VTE compared to DOAC in VTE population. In both populations, DOAC was associated with significantly lower risks of major bleeding compared to VKA. However, all observational studies had moderate to serious risks of bias. Patients prescribed VKA were commonly reported to have more co-morbidities.

TABLE 1 The incidence rates and incidence rate ratios of thrombotic and bleeding outcomes

\begin{tabular}{|c|c|c|c|c|c|}
\hline \multirow[b]{2}{*}{ Population } & \multirow[b]{2}{*}{ Anticoagulant } & \multicolumn{2}{|c|}{$\begin{array}{l}\text { Recurrent venous thromboembolism or } \\
\text { stroke/systemic thrombosis }\end{array}$} & \multicolumn{2}{|l|}{ Major bleeding } \\
\hline & & $\begin{array}{l}\text { Incidence rate per } \\
100 \text { person-years } \\
(95 \% \mathrm{Cl})\end{array}$ & $\begin{array}{l}\text { Incidence rate } \\
\text { ratio }(95 \% \mathrm{Cl})\end{array}$ & $\begin{array}{l}\text { Incidence rate per } \\
100 \text { person-years } \\
(95 \% \mathrm{Cl})\end{array}$ & $\begin{array}{l}\text { Incidence rate ratio } \\
(95 \% \mathrm{Cl})\end{array}$ \\
\hline $\begin{array}{l}\text { Venous } \\
\text { thromboembolism }\end{array}$ & Direct oral anticoagulants & $3.83(3.08-4.76)$ & $0.78(0.48-1.27)$ & $1.63(1.17-2.28)$ & $0.72(0.54-0.96)$ \\
\hline \multirow[t]{2}{*}{ Atrial fibrillation } & Direct oral anticoagulants & $1.16(0.86-1.59)$ & $0.96(0.78-1.16)$ & $2.74(1.68-4.48)$ & $0.75(0.59-0.96)$ \\
\hline & Vitamin $\mathrm{K}$ antagonists & $1.18(0.67-2.08)$ & & $3.61(1.78-7.31)$ & \\
\hline
\end{tabular}

Conclusions: Patients with morbid obesity on fixed-dose DOAC did not appear to have worse outcomes compared to VKA. However, the strength of evidence remained low given that results were mostly observational with high risk of confounding. 
PB1255 | Statins for Venous Event Reduction in Patients with Venous Thromboembolism: A Randomized Controlled Pilot Trial Assessing Feasibility

A. Delluc ${ }^{1}$; W. Ghanima ${ }^{2}$; M. Kovacs ${ }^{3}$; S. Shivakumar ${ }^{4}$; S. Kahn ${ }^{5}$; P.M. Sandset ${ }^{6}$; C. Kearon 7 M. Rodger ${ }^{5}$

${ }^{1}$ Ottawa Hospital Research Institute, Ottawa, Canada; ${ }^{2}$ Ostfold Hospital, Ostfold, Norway; ${ }^{3}$ University of Western Ontario, London, Canada; ${ }^{4}$ Dalhousie University, Halifax, Canada; ${ }^{5}$ McGill University, Montreal, Canada; ${ }^{6}$ University of Oslo, Oslo, Norway; ${ }^{7}$ McMaster University, Hamilton, Canada

Background: Statins may reduce the risk for recurrent venous thromboembolism (VTE), however, no randomized trials have explored this hypothesis.

Aims: To determine feasibility of recruitment of a larger trial of secondary VTE prevention with rosuvastatin.

Methods: Patients with a newly diagnosed symptomatic proximal deep vein thrombosis and/or pulmonary embolism, receiving standard anticoagulation, were randomly allocated to adjuvant rosuvastatin $20 \mathrm{mg}$ once daily for 180 days or no rosuvastatin for 6 months.

Results: Between November 2016 and December 2019, 3391 patients were assessed for eligibility in 6 centres. Of these patients, 1347 (39.7\%) were eligible and approached for participation in the trial and 312 (23.1\%) were randomized. The mean rate of randomization was $8.2 \pm 4.3$ patients per month. During follow-up, 5 recurrent VTE events were observed, $3(1.9 \%)$ in the rosuvastatin group ( 2 pulmonary embolism, 1 deep vein thrombosis) and 2 (1.3\%) in the control group (2 pulmonary embolism) $(P=0.68)$. One major arterial event occurred in the rosuvastatin arm and none in the control arm $(0.6 \%$ vs. $0 \%, P=0.50)$. Efficacy and safety clinical outcomes are summarized in Table 1.

TABLE 1 Efficacy and safety clinical outcomes

\begin{tabular}{|c|c|c|c|}
\hline Outcomes & $\begin{array}{l}\text { Rosuvastatin } \\
(n=155)\end{array}$ & $\begin{array}{l}\text { Control } \\
(n=157)\end{array}$ & $P$ value \\
\hline \multicolumn{4}{|l|}{ Thrombotic events, n (\%) } \\
\hline $\begin{array}{l}\text { Recurrent major VTE } \\
\text { (total) }\end{array}$ & $3(1.9)$ & $2(1.3)$ & 0.68 \\
\hline Recurrent DVT & $1(0.6)$ & 0 & 0.50 \\
\hline Recurrent PE & $2(1.3)$ & $2(1.3)$ & 1 \\
\hline $\begin{array}{l}\text { Recurrent non-major } \\
\quad \text { VTE }\end{array}$ & $1(0.6)$ & 0 & 0.50 \\
\hline Arterial events (total) & $1(0.6)$ & 0 & 0.50 \\
\hline Myocardial infarction & 0 & 0 & 1 \\
\hline Stroke/TIA & $1(0.6)$ & 0 & 0.50 \\
\hline Acute limb ischemia & 0 & 0 & 1 \\
\hline $\begin{array}{l}\text { Death from any cause, } \\
\text { n (\%) }\end{array}$ & 0 & $1(0.6)$ & 1 \\
\hline Major bleed, n (\%) & 0 & $1(0.6)$ & 1 \\
\hline
\end{tabular}

\begin{tabular}{llll} 
Outcomes & $\begin{array}{l}\text { Rosuvastatin } \\
(n=155)\end{array}$ & $\begin{array}{l}\text { Control } \\
(n=157)\end{array}$ & P value \\
$\begin{array}{c}\text { Clinically relevant } \\
\text { nonmajor bleeding, } \\
\mathrm{n}(\%)\end{array}$ & $2(1.3)$ & $1(0.6)$ & 1 \\
$\begin{array}{c}\text { Major muscle toxicity } \\
(\text { CK }>10 \mathrm{D} \text { (10), } \mathrm{n}(\%)\end{array}$ & $1(0.6)$ & 0 & 1 \\
$\begin{array}{c}\text { Muscle-related adverse } \\
\text { events, } \mathrm{n}(\%)\end{array}$ & $11(7.1)$ & $1(0.6)$ & 0.01 \\
\hline
\end{tabular}

Conclusions: In conclusion, this pilot trial established feasibility of a larger scale randomized controlled trial to determine the efficacy of adjuvant rosuvastatin for secondary prevention of VTE. (NCT02679664).

PB1256 | A Real-world Experience of Venous Thromboembolism (VTE) Management in Australia

B. Lui ${ }^{1}$; A. Kwok ${ }^{2}$; J. Lai ${ }^{2}$; Z. Khattak ${ }^{1}$; P. Ho ${ }^{1}$; H.Y. Lim ${ }^{1,2}$

${ }^{1}$ Northern Health, Melbourne, Australia; ${ }^{2}$ The University of Melbourne, Melbourne, Australia

Background: Venous thromboembolism (VTE), including deep vein thrombosis (DVT) and pulmonary embolism (PE), is increasingly recognised as a chronic disease with significant recurrence rates and resulting morbidity or mortality. The availability of direct oral anticoagulants (DOACs), listed by Pharmaceutical Benefits Scheme in 2013 in Australia, has changed the landscape of VTE management.

Aims: To characterise the real-world experience of VTE management in our study population.

Methods: Retrospective evaluation of VTE events managed at Northern Health, Melbourne, Australia from January 2012 to June 2019 (median follow-up 5.6 years). The analysis included patient demographics, associated risk factors, management and outcomes.

Results: There were 2055 VTE events analysed involving 1932 individuals (median age 65 years (range 16-102); 53\% females). These events included 1450 (71\%) DVTs, 965 (47\%) PEs and 360 (18\%) with concurrent DVT/PE. 337 (16\%) patients had active malignancy. 60\% events ( $n=1233$ ) were provoked with the most common provoking factor being injury/immobility ( $n=486,24 \%$ ) followed by surgery ( $n=344,17 \%) .280$ (14\%) events occurred despite being on some form of anticoagulation. The median duration of anticoagulation was 6 months. 872 (42\%) cases were managed with warfarin, 365 (18\%) with enoxaparin and 673 (33\%) with a DOAC. 221 (11\%) patients experienced recurrent VTE while 75 patients (4\%) experienced clinically significant major bleeding $(P<0.001)$. Patients on warfarin and/ or enoxaparin had higher rates of clinically significant major bleeding compared to DOACs (31/872 on warfarin (4\%) vs $30 / 365$ on enoxaparin (8\%) vs $12 / 673$ on DOAC ( $2 \%), P<0.001)$. Thrombosis and bleeding-related mortalities were comparable (30 (1.6\%) vs 22 (1.1\%), $P=0.26) .68$ patients (4\%) were diagnosed with subsequent malignancies. 
Conclusions: The recurrent thrombosis rate was $11 \%$ with a $4 \%$ rate of clinically significant major bleeding in this study. Bleeding rates were lower in patients treated with DOAC supporting the use of DOACs as first-line therapy in appropriately selected patients.

PB1257 | VTE Treatment Planning: A Mixed-method Analysis of Clinical Challenges, Knowledge, and Confidence Gaps in Selecting Evidence-based Treatment

A. Spyropoulos ${ }^{1}$; P. Lazure ${ }^{2}$; S. Kaatz ${ }^{3}$; A. Khorana ${ }^{4}$; S. Zahabi ${ }^{2}$; K. McCrae ${ }^{4}$; C. Pollack ${ }^{5}$; S. Péloquin ${ }^{2}$

${ }^{1}$ Northwell Health at Lenox Hill Hospital, New York, United States; ${ }^{2}$ AXDEV Group Inc., Brossard, Canada; ${ }^{3}$ Henry Ford Hospital, Detroit, United States; ${ }^{4}$ Cleveland Clinic, Cleveland, United States; ${ }^{5}$ University of Mississippi Medical Center, Jackson, United States

Background: Studies have revealed underutilization of direct oral anticoagulants (DOACs) in the treatment of venous thromboembolism (VTE) despite being addressed in guidelines, including those specific to patients with cancer. Evidence identifies complexity of patient profiles as a potential barrier to the uptake of optimal VTE treatment.

Aims: This study aimed to identify barriers, practice gaps, and causalities related to secondary prevention of VTE in patients with a first occurrence, as well prevention, treatment, and management of risks associated with VTE in cancer. Findings on underutilization of DOACs are presented here.

Methods: A mixed-methods study (semi-structured interviews and quantitative survey) was conducted with physicians from 5 specialties in U.S. community practice settings. Qualitative data was thematically analyzed and survey data was analyzed using Chi-squares and Kruskal Wallis $\mathrm{H}$ tests.

Results:

TABLE 1 Percentages of participants reporting sub-optimal levels of knowledge by profession/specialty groups

\begin{tabular}{|c|c|c|c|c|c|c|c|}
\hline \multirow[b]{2}{*}{ Sub-optimal knowledge of: } & \multicolumn{5}{|c|}{ Profession/specialty groups } & \multirow[b]{2}{*}{$\begin{array}{l}\text { Participants } \\
\text { with sub- } \\
\text { optimal } \\
\text { knowledge } \\
\text { (of total } \\
n=241 \text { ) }\end{array}$} & \multirow[b]{2}{*}{$\begin{array}{l}\text { Significance } \\
\text { (differences } \\
\text { between } \\
\text { profession/ } \\
\text { specialty } \\
\text { groups)* }^{*}\end{array}$} \\
\hline & $\begin{array}{l}\text { Primary } \\
\text { care } \\
\text { physicians }\end{array}$ & $\begin{array}{l}\text { Hematologists- } \\
\text { oncologists }\end{array}$ & $\begin{array}{l}\text { Community } \\
\text { oncologists }\end{array}$ & $\begin{array}{l}\text { Emergency } \\
\text { department } \\
\text { physicians }\end{array}$ & $\begin{array}{l}\text { VTE specialists } \\
\text { (e.g., hematologists, } \\
\text { cardiologists, } \\
\text { pulmonologists, } \\
\text { vascular medicine } \\
\text { specialists) }\end{array}$ & & \\
\hline $\begin{array}{l}\text { Latest randomized control } \\
\text { trials on VTE treatments }\end{array}$ & $75 \%(39)$ & $48 \%(23)$ & $66 \%(29)$ & $90 \%(43)$ & $49 \%(24)$ & $65.6 \%(158)$ & $\mathrm{p}<.000$ \\
\hline $\begin{array}{l}\text { Guidelines specific to acute } \\
\text { treatment for VTE } \\
\text { patients }\end{array}$ & $46 \%(24)$ & $40 \%(19)$ & $34 \%(15)$ & $54 \%(26)$ & $27 \%(35)$ & $40.4 \%(97)$ & $P=.067$ \\
\hline $\begin{array}{l}\text { Recently FDA-approved } \\
\text { treatments for VTE }\end{array}$ & $52 \%(27)$ & $40 \%(19)$ & $48 \%(21)$ & $60 \%(29)$ & $40.8 \%(20)$ & $48.1 \%(116)$ & $P=.226$ \\
\hline
\end{tabular}

Chi-square

TABLE 2 Mean (SD) confidence levels (visual analogue scale; 0 = not at all confident to $100=$ highly confident) by profession/specialty groups

Profession/specialty groups

Primary care physicians

Hematologists-oncologists

Community oncologists

Emergency department physicians
Deciding which type of VTE treatment to use according to specific patient profile

71.9 (21.1)

73.4 (19.0)

$67.4(22.6)$

$60.3(21.8)$
Using DOACs with cancer patients

$63.3(24.0)$

$75.1(20.2)$

$70.2(21.5)$

$57.4(22.4)$ 


\begin{tabular}{|c|c|c|}
\hline TRACT & & 923 of 972 \\
\hline $\begin{array}{l}\text { VTE specialists (e.g., hematologists, } \\
\text { cardiologists, pulmonologists, } \\
\text { vascular medicine specialists) }\end{array}$ & $73.5(14.7)$ & $67.7(22.4)$ \\
\hline $\begin{array}{l}\text { Asymptotic Significance (differences } \\
\text { between profession/ specialty } \\
\text { groups)* }\end{array}$ & $P=.006$ & $P=.002$ \\
\hline Representative quote & $\begin{array}{l}\text { "With cancer patients often times their comorbidities } \\
\text { make decision-making more challenging. They may have } \\
\text { metastatic disease that puts them at high risk for bleed- } \\
\text { ing or, if they do bleed, at high risk for morbidity related } \\
\text { to a bleed, particularly if they have intracranial metasta- } \\
\text { ses or spinal metastases. [...] Some of these patients are } \\
\text { very advanced, and by the time they're diagnosed with } \\
\text { VTE it's pretty much the end of the road. So, discus- } \\
\text { sions about what their goals of care are and end-of-life } \\
\text { decision-making-we're not always equipped to do that } \\
\text { very well." } \\
\text { - ED Physician }\end{array}$ & $\begin{array}{l}\text { "NOACs, those new anticoagulants, are } \\
\text { still not approved in the cancer treat- } \\
\text { ment patients, so technically we still } \\
\text { need to use Coumadin if you want to } \\
\text { be going by the book. [...] so it's a chal- } \\
\text { lenge, because Coumadin is an old drug } \\
\text { and it's hard to monitor. Because of } \\
\text { their cancer, a patient may have prob- } \\
\text { lems with their monitoring parameters, } \\
\text { which could be falsely elevated or } \\
\text { decreased." } \\
\text { - Community Oncologist }\end{array}$ \\
\hline
\end{tabular}

* Kruskal Wallis $\mathrm{H}$

A total of 262 healthcare providers (HCPs) participated in the study, 21 completed interviews and 241 completed surveys. Findings showed a conservative approach to treatment (avoiding DOACs), driven by lack of knowledge and perceived uncertainty of optimal management from guidelines among non-VTE specialists. Qualitative data clarified low knowledge and confidence levels, revealing HCPs' underlying doubts regarding: DOACs safety, clarity of antithrombotic guidelines, and applicability to cancer. HCPs lacked confidence using patient profile information to guide treatment decisions. Emergency Department (ED) physicians, community oncologists, and primary care providers lacked knowledge about new treatments compared with professionals specialized in VTE and weighed risks of complication heavily. HCPs' conservative approach regarding DOACs was prevalent when caring for patients with comorbidities or in ED settings.

Conclusions: This study demonstrates the need for education supporting HCPs' decision-making using DOACs for treating VTE in cancer patients and in acute settings. While HCPs generally understand the benefits of DOACs, they lack confidence in guidelines and can overweight the risks of complication in more constrained and potentially riskier scenarios.

\section{PB1258 | Symptomatic versus Incidentally Noted Isolated Subsegmental Pulmonary Embolus Outcomes}

D.T Vlazny ${ }^{1}$; Y. Hirao-Try ${ }^{1}$; D.O Hodge ${ }^{2}$; A.I Casanegra ${ }^{1}$;

D.E Houghton ${ }^{1}$; R.A Meverden ${ }^{1}$; L.G Peterson ${ }^{1}$; R.D McBane ${ }^{1}$;

W.E Wysokinski ${ }^{1}$

${ }^{1}$ Mayo Clinic, Rochester, United States; ${ }^{2}$ Mayo Clinic, Jacksonville, United States

Background: Many isolated subsegmental pulmonary emboli (ISSPE) are found incidentally. Whether the clinical outcomes of incidental ISSPE differ from symptomatic events is unclear.

Aims: To determine if demographics or clinical outcomes differ between incidentally noted versus symptomatic ISSPE.

Methods: Consecutive patients with ISSPE enrolled in the Mayo Clinic VTE registry (March 1, 2013, through December 31, 2020) were followed prospectively. ISSPE was defined based on radiologic criterion with clot isolated to subsegmental pulmonary vasculature without involvement of more proximal vasculature or evidence of right ventricular strain. The primary efficacy outcome was venous thromboembolism (VTE) recurrence with death as the secondary outcome. The primary safety outcome was major bleeding.

Results: There were 225 patients with ISSPE of which $60 \%$ were incidentally noted. Patients with incidental ISSPE were older with lower body weight than symptomatic patients. A higher percentage of malignancy was found in incidental ISSPE $(72.6 \% \vee 36.7 \%$, $P<0.0001$, Table 1). Incidentally noted ISSPE had a delay of anticoagulation initiation by a mean of 0.6 days $(P=0.0027)$. Outcomes, including death, were similar between the groups (Table 2). However, there were no major bleeding events in the incidental group. 
TABLE 1 Demographic and clinical variables of patients with symptomatic versus incidental, isolated subsegmental pulmonary embolism

$\begin{array}{llll}\text { Variables } & \text { Symptomatic }(\boldsymbol{n}=90) & \text { Incidental }(\mathbf{n}=135) & P \text {-value } \\ \text { Age, years, mean (SD) } & 57.8(16.5) & 63.0(12.6) & 0.040 \\ \text { Weight, kilograms, mean (SD) } & 90.8(23.2) & 79.7(20.6) & <0.001 \\ \text { Female, } \mathrm{n}(\%) & 39(43.3) & 62(45.9) & 0.702 \\ \text { Provoked pulmonary emboli, } \mathrm{n}(\%) & 67(76.1) & 117(86.7) & 0.043 \\ \text { Active cancer, } \mathrm{n}(\%) & 33(36.7) & 98(72.6) & <0.001 \\ \text { Chemotherapy, } \mathrm{n}(\%) & 22(71.0) & 6(66.0) & 0.607 \\ \text { Immobility, } \mathrm{n}(\%) & 18(20.0) & 11(8.1) & 0.009 \\ \text { Other, } \mathrm{n}(\%) & 17(18.9) & 14(10.4) & 0.062 \\ \text { Time to anticoagulation start for diagnosis, } & 0.4(1.5) & 1.0(2.2) & 0.003\end{array}$

TABLE 2 Venous thromboembolism (VTE) recurrence, major bleeding, clinically relevant non-major bleeding, and death in patients anticoagulated for symptomatic versus incidental, isolated subsegmental pulmonary embolism without concurrent proximal DVT

\begin{tabular}{|c|c|c|c|}
\hline Outcomes & \multicolumn{3}{|c|}{ Isolated Subsegmental Pulmonary Embolism } \\
\hline VTE $^{\mathrm{b}}$ recurrence $(\mathrm{n})$ & 1 & 2 & 0.89 \\
\hline Total follow up person-years & 48.79 & 91.82 & \\
\hline $\mathrm{VTE}^{\mathrm{b}}$ recurrence rate & 2.05 & 2.18 & \\
\hline \multicolumn{4}{|l|}{100 person-years } \\
\hline Major bleeding (n) & 2 & 0 & $\mathrm{~N} / \mathrm{A}$ \\
\hline Total follow up person-years & 45.87 & 95.07 & \\
\hline Major bleeding rate & 4.36 & $N / A$ & \\
\hline \multicolumn{4}{|l|}{100 person-years } \\
\hline \multicolumn{4}{|l|}{100 person-years } \\
\hline Death (n) & 20 & 31 & 0.45 \\
\hline Total follow up person-years & 49.50 & 95.07 & \\
\hline Death rate & 40.40 & 32.60 & \\
\hline per 100 person-years & & & \\
\hline
\end{tabular}

Conclusions: ISSPE is often found incidentally, particularly in cancer patients. Compared to those presenting with symptoms, VTE recurrence, major bleeding, CRNMB and death occur with similar frequency. Delayed anticoagulation initiation is a common feature of incidental ISSPE. These results suggest that incidentally noted ISSPE carries similar gravity as those identified in symptomatic patients.
PB1259 | Neutralization of the Anticoagulant Effects of

\section{Sulodexide by Protamine Sulfate}

B. Daravath; O. Iqbal; D. Hoppensteadt; W. Jeske; J. Fareed Loyola University Medical Center, Maywood, United States

Background: In view of the current shortage of heparin there is a need to develop a suitable alternative for this anticoagulant. Sulodexide is a glycosaminoglycan-derived drug, composed of fastmoving heparin $(80 \%)$ and dermatan sulfate $(20 \%)$, representing a suitable substitute to heparin. 
Aims: The purpose of this study is to compare the anticoagulant effects of Sulodexide and its protamine neutralization profiles in the activated clotting time (ACT).

Methods: In order to study the neutralization a saline control was also performed. The blood was drawn up to $2 \mathrm{ml}$ mark in each of the syringes to obtain a final concentration of Sulodexide at 50, 25, 12.5, 6.2 and $0 \mathrm{ug} / \mathrm{ml}$. In order to study neutralization by protamine sulfate, 200ul of Sulodexide (1056) at a final concentration of 50,25,10 $\mu \mathrm{g} / \mathrm{ml}$, together with $200 \mathrm{ul}$ of protamine sulfate at final concentration of $25 \mathrm{ug} / \mathrm{ml}$ was placed in a separate set of labeled syringes. After gently mixing the contents of the syringes, ACT was immediately performed and the clotting time recorded in seconds.

Results: All 6 different Sulodexide batches showed a concentrationdependent anticoagulant response. At a final concentration of $25 \mu \mathrm{g} /$ $\mathrm{ml}$, Sulodexide-1056 (331 \pm 22 seconds), sulodexide-1285(303 \pm 21 sec), sulodexide-2516(335 $\pm 24 \mathrm{sec})$, sulodexide-2604(276 $\pm 27 \mathrm{sec}$ ), sulodexide-3274 (309 $\pm 21 \mathrm{sec})$, sulodexide- 4190 (291 $\pm 18 \mathrm{sec}$ ), compared to a saline control value of $145 \pm 14$ seconds. The anticoagulant effects of one specific batch-1056 Sulodexide at final concentrations of 50,25 and $10 \mu \mathrm{g} / \mathrm{ml}$ was differentially neutralized by protamine sulfate at $25 \mathrm{ug} / \mathrm{ml}$ with ACT values of $332 \pm 33 \mathrm{sec}-$ onds,171 $\pm 14 \mathrm{sec}, 148 \pm 11 \mathrm{sec}$, respectively, when compared to protamine and saline ACT value of $152 \pm 12$ seconds.

Conclusions: Sulodexide at concentrations of $6.25-50 \mu \mathrm{g} / \mathrm{ml}(0.62-$ $5.0 \mathrm{USP} / \mathrm{ml}$ ) produced comparable anticoagulant effects to heparin which were neutralized by protamine sulfate.

\section{PB1260 | AntiXa Heparin Dosing Protocol Evaluation at a Large} Academic Medical Center

T. Ahuja $^{1}$; M. Williams ${ }^{1}$; S. Arnouk ${ }^{1}$; D. Lum ${ }^{1}$; J. Papadopoulos ${ }^{1}$; V. Raco ${ }^{2}$; D. Green ${ }^{1}$

${ }^{1}$ NYU Langone Health, New York, United States; ${ }^{2}$ NYU Langone

Hospital- Brooklyn, Brooklyn, United States

Background: The activated partial thromboplastin time (aPTT) and anti-factor Xa (anti-Xa) test are often used as surrogate markers of heparin's effects, though anti-Xa may be preferred due to less variability and closer relation to heparin's activity in the body. The antithrombotic and hemostatic therapy oversight group at NYU Langone Health (NYULH) implemented a nurse-titrated protocol, utilizing anti-Xa to titrate and monitor time in therapeutic range with heparin.

Aims: To evaluate adherence to the nurse-driven continuous infusion unfractionated heparin protocol and assess time to therapeutic anti-Xa, median time in therapeutic range, and clinical outcomes including bleeding and thrombotic events.

Methods: This was a retrospective chart review. Adult patients ( $>18$ years old) who received heparin based on nurse-driven titration protocol between March 2019 and June 2019 at NYULH were included. Patients that received heparin for less than 24 hours, had an interruption in heparin for more than 12 hours, or received a direct oral anticoagulant (DOAC) prior to heparin initiation were excluded. Data collection included baseline characteristics, relevant concomitant medications, heparin administration including bolus dose, infusion rate, length of therapy, time within therapeutic range, bleeding events, and thrombotic events. The primary outcome was adherence to the protocol. Secondary outcomes included time within therapeutic range, thrombotic or bleeding events.

Results: Adherence to the protocol, defined as deviation of not more than $25 \%$ of protocol elements, including but not limited to adequate bolus dose, correct body weight used, time to first anti-Xa, and correct adjustment (units/kg) in heparin dose based on anti-Xa was observed $85 \%$ of the time, with the majority of dose titrations occurring by nursing, per protocol in $82 \%$ of patients. Therapeutic anti-Xa levels were achieved at a median of 30 hours into IV UFH therapy.

Conclusions: It remains a challenge to achieve a stable goal anti-Xa level within 24 hours of heparin initiation.

PB1261 | Tailored Anticoagulant Treatment after a First Venous Thromboembolism: Protocol of the Leiden Thrombosis Recurrence Risk Prevention (L-TRRiP) Study, a Cohort Based Randomized Controlled Trial

J.L.I. Burggraaf ${ }^{1}$; N. van Rein ${ }^{1}$; R.H.H. Bemelmans ${ }^{2}$; S. Benimadho ${ }^{3}$; J.W.K. van den Berg ${ }^{4}$; M. Coppens ${ }^{5}$; A. Dees ${ }^{6}$; L.M. Faber ${ }^{7}$; W.K. de Jong ${ }^{8}$; T. Koster ${ }^{9}$; A.T.A. Mairuhu ${ }^{10}$; K. Meijer ${ }^{11}$; M. Nijkeuter ${ }^{12}$; M.E. van den Akker - van Marle ${ }^{13}$; S. le Cessie ${ }^{13,1}$; M.V. Huisman ${ }^{14}$; F.A. Klok ${ }^{14}$; S.C. Cannegieter ${ }^{1,14}$; L-TRRiP study investigators

${ }^{1}$ Department of Clinical Epidemiology, Leiden University Medical Center, Leiden, Netherlands; ${ }^{2}$ Department of Internal Medicine, Ziekenhuis Gelderse Vallei, Ede, Netherlands; ${ }^{3}$ Department of Internal Medicine, Sionsberg, Dokkum, Netherlands; ${ }^{4}$ Department of Pulmonology, Isala Hospital, Zwolle, Netherlands; ${ }^{5}$ Department of Vascular Medicine, Amsterdam University Medical Centers, University of Amsterdam, Amsterdam, Netherlands; ${ }^{6}$ Department of Internal Medicine, Ikazia Ziekenhuis, Rotterdam, Netherlands; ${ }^{7}$ Department of Internal Medicine, Red Cross Hospital, Beverwijk, Netherlands; ${ }^{8}$ Department of Pulmonology, Ziekenhuis Gelderse Vallei, Ede, Netherlands; ${ }^{9}$ Department of Internal Medicine, Groene Hart Hospital, Gouda, Netherlands; ${ }^{10}$ Department of Internal Medicine, Haga Teaching Hospital, The Hague, Netherlands; ${ }^{11}$ Department of Hematology, University Medical Center Groningen, Groningen, Netherlands; ${ }^{12}$ Department of Internal Medicine, University Medical Center Utrecht, Utrecht, Netherlands; ${ }^{13}$ Department of Biomedical Data Sciences, Leiden University Medical Center, Leiden, Netherlands;

${ }^{14}$ Department of Medicine - Thrombosis and Hemostasis, Leiden University Medical Center, Leiden, Netherlands

Background: Patients with a first venous thromboembolism (VTE) are at risk of recurrence (rVTE). A rVTE can be prevented by prolonged anticoagulant therapy, but this may come at the cost of major bleeding (MB). The L-TRRiP and VTE-BLEED prediction scores have been developed to classify the risk of rVTE (low, intermediate, high) 
and MB (low vs high), respectively (table). However, their combined use in finding the optimal balance to minimize both long-term risks is unclear.

TABLE 1 Factors in the L-TRRiP and VTE-BLEED models

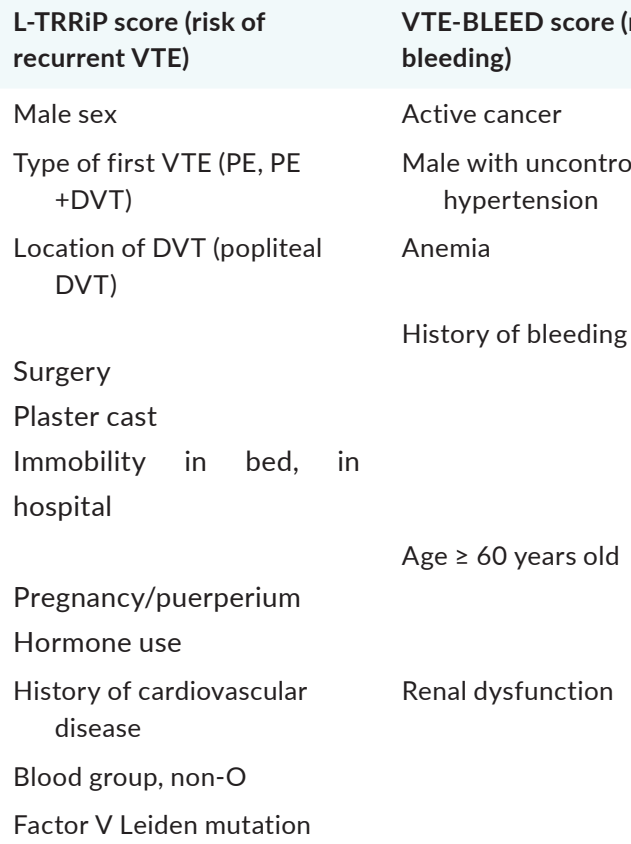

Aims: To evaluate tailored duration of long-term anticoagulant treatment based on individualized risk assessments of rVTE and MB risks. Methods: The L-TRRiP study is a multicenter, open-label, cohort based randomized controlled trial including 1600 participants with a first VTE. After the initial three months of anticoagulant treatment, each patient's individual rVTE and MB risk will be determined using the L-TRRiP and VTE-BLEED prediction scores, respectively. Patients with a low rVTE risk will discontinue anticoagulants, whereas patients with a high rVTE risk and low MB risk will continue. The other groups, with unclear benefit of prolonged treatment, will be randomized to continue or discontinue anticoagulants (Figure 1). The primary outcome is the incidence of rVTE and MB in the randomized group after 2 years follow-up. Secondary outcomes are quality of life, cost-effectiveness and functional outcomes in all groups and incidence of rVTE and MB in the non-randomized groups. The study has been approved by the Medical Ethics Committee Leiden Den Haag Delft. All participants will provide informed consent. The L-TRRiP study is funded by ZonMw, the Netherlands; grant number: 848017007.

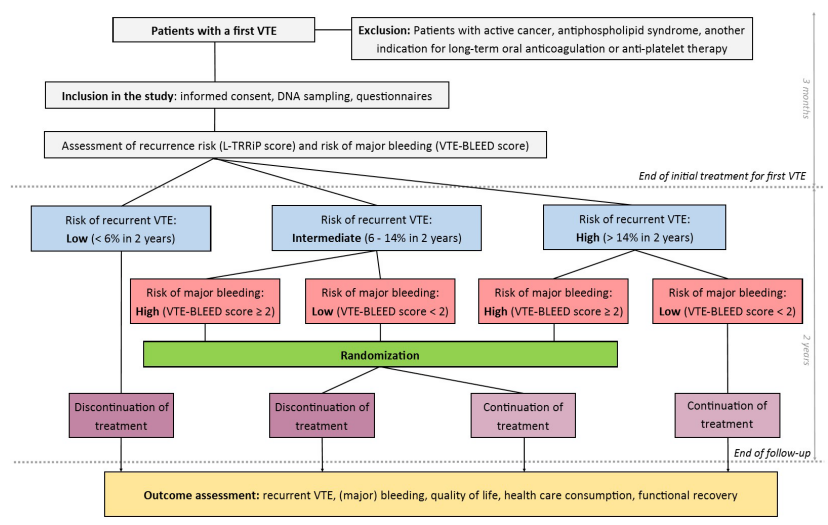

FIGURE 1 Design of the L-TRRiP study

Results: Results are expected in 2025.

Conclusions: The L-TRRiP study will assess whether a tailored strategy, based on classification of both rVTE risk and MB risk, leads to minimized risks of both complications.

PB1262 | Duration and Treatment Outcomes between Idiopathic and Provoked Non-catheter-Related Axillary and Subclavian Vein Thromboses

A. Sliskovic ${ }^{1}$; M. Vrkic Kirhmajer ${ }^{1}$; M. Narancic ${ }^{2}$; I. Sabol ${ }^{3}$;

M. Vodanovic ${ }^{1}$; A. Boban ${ }^{1}$; D. Pulanic ${ }^{1}$

${ }^{1}$ University Hospital Centre Zagreb, Zagreb, Croatia; ${ }^{2}$ General Hospital Zadar, Zadar, Croatia; ${ }^{3}$ Institute Ruder Boskovic, Zagreb, Croatia

Background: Axillary and subclavian vein thrombosis (ASVT) is a rare condition, and the lack of randomized trials results in insufficiently standardized treatment, especially regarding idiopathic type of ASVT.

Aims: This study aimed to investigate differences in treatment and outcomes of patients with idiopathic and other non-catheter-related ASVT.

Methods: In this single-center retrospective study, we analyzed the characteristics and outcomes of patients with non-catheter-related ASVT treated at University Hospital Center Zagreb from December 2008 to September 2020.

Results: 54 patients were analyzed with a median age of 38 years (18-82), 59\% were male. Fourteen patients (26\%) had idiopathic ASVT. They were treated with: warfarin 7, rivaroxaban 4, apixaban 2 , dabigatran 1. Forty patients (74\%) had apparent etiological factors of ASVT: 14 on-effort thrombosis without thoracic outlet syndrome (TOS), 7 TOS, 5 malignant disease, 6 antiphospholipid syndrome, 6 thrombophilia, and 2 other. They were treated with: warfarin (16), rivaroxaban (10), apixaban (5), LMWH (3), and 6 patients switched anticoagulant therapy (ACT). There was no statistical significant difference in ACT duration between idiopathic and provoked ASVT (8 vs 12 months, $P=0,7108$ ).

Seven patients (13\%) had a relapse of venous thrombosis, 3 with idiopathic ASVT, and 3 relapses occurred during the ACT. There was 
no statistical significant difference in the relapse rate between idiopathic and provoked ASVT $(P=0,5264)$ and between different anticoagulant drugs $(P=0,088)$.

Conclusions: Results of our study showed similar duration and treatment outcome between idiopathic and other non-catheter-related ASVT. This finding indicates need for further research in prospective design with longer follow-up

PB1263 | Comparison of Real-world Effectiveness and Safety of Enoxaparin versus Tinzaparin or Dalteparin in Cancer Patients with Venous Thromboembolism. The RIETECAT Cohort Study

M. Monreal $^{1}$; J. Trujillo-Santos ${ }^{2}$; D. Farge-Bancel ${ }^{3}$; J.M. Pedrajas ${ }^{4}$;

C. Gómez-Cuervo ${ }^{5}$; A. Ballaz ${ }^{6}$; A. Braester ${ }^{7}$; I. Mahé ${ }^{8}$

${ }^{1}$ Hospital Universitari Germans Trias i Pujol, Badalona, Spain;

${ }^{2}$ Universidad Católica de Murcia, Murcia, Spain; ${ }^{3}$ Université de Paris, Paris, France; ${ }^{4}$ Hospital Clinico San Carlos, Madrid, Spain; ${ }^{5}$ Hospital Universitario 12 de Octubre, Madrid, Spain; ${ }^{6} \mathrm{Hospital}$ de Galdakao, Galdakao, Spain; ${ }^{7}$ Bar-Ilan University, Safed, Israel; ${ }^{8}$ Hôpital Louis Mourier, Colombes, France

Background: Venous thromboembolism (VTE) is a frequent complication in patients with active cancer, and a leading cause of morbidity and death.

Aims: To compare the long-term effectiveness and safety of enoxaparin versus tinzaparin or dalteparin for the secondary prevention of VTE in adults with cancer.

Methods: RIETECAT was an observational cohort study performed using data from the RIETE (Registro Informatizado Enfermedad ThromboEmbolica) registry. Patients initiating with full dose treatment of enoxaparin, tinzaparin or dalteparin between January 2009 to June 2018 and within 48h from the date of diagnosis of their primary episode of VTE were included. VTE recurrences and major bleeding events were assessed in the 6 months following treatment initiation.

Results: Overall, 4,451 cancer patients with VTE were included (enoxaparin [ $N=3526]$; tinzaparin [ $=754]$; dalteparin [ $N=171]$ ). Recurrent VTE occurred in 70 patients $(2.0 \%)$ in the enoxaparin subgroup and 23 patients (2.5\%) in tinzaparin/dalteparin subgroup (odds ratio [OR]: $0.79,[95 \% \mathrm{Cl}: 0.49-1.28], P=0.343,[P=0.004$ for non-inferiority]). There were no differences between subgroups in the rate of recurrences of deep vein thrombosis, pulmonary embolism or fatal pulmonary embolism (Table1). Major bleeding occurred in 111 patients (3.1\%) in the enoxaparin subgroup and 18 patients (1.9\%) in tinzaparin/dalteparin subgroup (OR: 1.64; 95\%Cl: $0.99-$ 2.71, $P=0.052$ ). Incidence of all-cause death was similar between treatment subgroups (18.9\% vs $17 \%$; OR: 1.14 ; $95 \% \mathrm{Cl}$ : $0.94-1.38$; $P=0.182)$. After propensity score matching analysis, there were no differences between subgroups for risk of VTE recurrences (adjusted hazard ratios [aHR]: $0.81 ; 95 \% \mathrm{Cl}: 0.48-1.38)$, major bleeding (aHR: $1.41 ; 95 \% \mathrm{Cl}: 0.80-2.46 ; P=0.235$ ) or all-cause death (aHR: 1.07; 95\%Cl: 0.88-1.30; $P=0.476)$.
Conclusions: In real-life clinical practice, enoxaparin had a comparable effectiveness and safety profile to tinzaparin/dalteparin in cancer patients with VTE.

Figure 1: Outcome Enoxaparin ( $N=3526$ ) Tinzaparin or Dalteparin $(\mathrm{N}=925)$ P-value OR $(95 \% \mathrm{Cl})$ Primary outcome Recurrence of VTE 70 (2.0\%) 23 (2.5\%) 0.34280 .79 (0.49-1.28) Secondary outcomes Symptomatic DVT PE (fatal or non-fatal) Fatal PE 36 (1.0\%) 35 (0.99\%) 33 (0.94\%) 11 (1.2\%) 12 (1.3\%) 6 (0.65\%) 0.65600 .41970 .4041 0.86 (0.43-1.69) $0.76(0.39-1.48) 1.45(0.60-3.46)$ Safety outcomes Major bleeding (fatal or non-fatal) Site of bleeding Gastrointestinal Cerebral Haematoma Retroperitoneal 111 (3.1\%) 44 (1.2\%) 15 (0.43\%) $10(0.28 \%) 11$ (0.31\%) 18 (1.9\%) 6 (0.65\%) 6 (0.65\%) 2 (0.22\%) 0 (0.00\%) 0.05240 .12380 .37790 .72500 .08901 .64 (0.99-2.71) 1.94 (0.82-4.56) 0.65 (0.25-1.69) 1.31 (0.29-6.00) - Fatal major bleeding $15(0.43 \%) 5(0.54 \%) 0.64120 .79(0.28-2.17)$ Non-major bleeding of clinical significance 87 (2.5\%) 24 (2.6\%) 0.82520 .95 (0.60-1.50) Composite safety outcome\# 191 (5.4\%) 41 (4.4\%) 0.23061 .23 (0.871.74) All-cause death 666 (18.9\%) 157 (17.0\%) 0.18171 .14 (0.94-1.38) Fatal PE or fatal bleeding related death 48 (1.4\%) 11 (1.2\%) 0.68371 .15 (0.59-2.22) Cl: confidence intervals; DVT: deep vein thrombosis; OR: odds ratio; PE: pulmonary embolism; VTE: venous thromboembolism * Composite efficacy outcome $=$ symptomatic DVT and fatal or non-fatal PE. \# Composite safety outcome = major bleeding events (fatal or nonfatal) and non-major bleeds of clinical significance.

PB1264 | Direct Oral Factor-Xa-inhibitors for the Treatment of VTE in the Obese: Do Body Weight and Body Composition Matter? A Prospective Pilot Study

K. Guetl ${ }^{1}$; D.R. Leitner ${ }^{2}$; A. Beck ${ }^{2}$; J. Rabensteiner ${ }^{3} ;$ T. Gary $^{1}$; R.B. Raggam ${ }^{1} ;$ H. Toplak $^{2}$

${ }^{1}$ Medical University of Graz, Department of Internal Medicine, Division of Vascular Medicine, Graz, Austria; ${ }^{2}$ Medical University of Graz, Lipid Clinic, Graz, Austria; ${ }^{3}$ Medical University of Graz, Clinical Institute od Medical \& Chemical Diagnostics, Graz, Austria

Background: Even after a decade of direct oral anticoagulants' (DOACs) use in clinical routine, uncertainty about safety and efficacy in the obese still remains.

Aims: The aim of the BIARIVA prospective pilot study was to investigate the association of body composition parameters with specific blood coagulation parameters in patients on anticoagulant treatment with oral factor Xa inhibitors rivaroxaban or edoxaban.

Methods: Specific blood coagulation parameters including antifactor Xa activity levels and direct plasma concentration levels at peak and trough were explored for their association with body composition parameters by correlation analysis. Plasma concentrations levels were directly measured for rivaroxaban and calculated by formula for edoxaban. Body composition measurement comprised determination of fat-mass and fat-free mass by use of two different scaling systems, the bipolar BIACorpus RX 4000 scale and the tetrapolar Seca mBCA 500 scale. All patients provided written 
informed consent. The study protocol was approved by the local ethics committee.

Results: A total of 39 patients in the edoxaban group and 41 patients in the rivaroxaban group were considered for the final analysis. Median body mass index (BMI) was 33.60 [23.30-36.20] in edoxaban patients and 31.90 [24.50-37.75] kg/m² in rivaroxaban patients. Among edoxaban patients, a significant but only modest positive correlation was found for absolute fat-free mass with the absolute increase and also with peak levels of both anti-Xa activity and plasma concentration for both scale measures. No significant correlation was found for absolute fat mass with coagulation parameters. Results for percentage fat-mass did not present a conclusive trend. In the rivaroxaban group, correlations of blood coagulation parameters with body composition parameters did not reach a level of significance anyway.

TABLE 1 The association of blood coagulation parameters with body composition parameters in patients receiving anticoagulant fulldose treatment with edoxaban or rivaroxaban

\begin{tabular}{|c|c|c|c|c|c|c|c|c|c|c|c|c|c|c|}
\hline & \multicolumn{7}{|c|}{ edoxaban } & \multicolumn{7}{|c|}{ rivaroxaban } \\
\hline & & \multicolumn{3}{|c|}{ Seca mBCA 500} & \multicolumn{3}{|c|}{ BLACorpus RX 4000} & & \multicolumn{3}{|c|}{ Seca mBCA 500} & \multicolumn{3}{|c|}{ BLACorpus RX 4000} \\
\hline & & 量司 & 商㝴 & 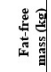 & 啚㦴 & 骨: & 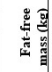 & & 量或 & : & 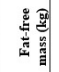 & 佥国 & 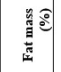 & 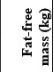 \\
\hline \multirow{2}{*}{$\begin{array}{c}\text { Anti-factor Xa } \\
\text { (trongh; } \mathrm{U} / \mathbf{m l})\end{array}$} & corr & $-0,006$ & 0,117 & $-0,088$ & $-0,129$ & $-0,084$ & $-0,007$ & cocr & $-0,131$ & $-0,041$ & $-0,196$ & $-0,181$ & $-0,123$ & $-0,179$ \\
\hline & sig & 0,970 & 0,486 & 0,598 & 0,442 & 0,616 & 0,968 & is & 0,421 & 0,003 & 0,225 & 0,265 & 0,49 & 0,270 \\
\hline \multirow{2}{*}{$\begin{array}{l}\text { Anti-factor Xa } \\
\text { (peak; } \mathbf{U} / \mathbf{m I})\end{array}$} & corr & $-0,080$ & 0,283 & $-457 *$ & $-0,049$ & 0,310 & $-.444^{*}$ & ocr & $-0,089$ & $-0,027$ & $-0,110$ & $-0,130$ & $-0,127$ & $-0,118$ \\
\hline & aig & 0,633 & 0,085 & 0,004 & 0,771 & 0,058 & 0,005 & aig & 0,585 & 0.871 & 0,501 & 0,425 & 0,434 & 0,470 \\
\hline \multirow{2}{*}{$\begin{array}{l}\text { Anti-factor } \mathbf{X a} \\
\text { (abs.diff;; } \\
\text { U/ml) }\end{array}$} & corr & $-0,072$ & 0,289 & $-.461^{*}$ & $-0,027$ & $333^{2}$ & $-456^{*}$ & corr & $-0,118$ & $-0,056$ & $-0,119$ & $-0,144$ & $-0,130$ & $-0,143$ \\
\hline & aig & 0,666 & 0,078 & 0,004 & 0,874 & 0,041 & 0,004 & aig & 0,470 & 0,733 & 0,464 & 0,374 & 0,424 & 0,379 \\
\hline \multirow{2}{*}{ 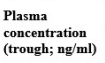 } & corr & $-0,006$ & 0.117 & -0.088 & $-0,129$ & -0.084 & $-0,007$ & $\mathrm{cor}$ & -0.154 & $-0,055$ & -0.221 & -0.204 & -0.122 & $\mid-0.192$ \\
\hline & sig & 0.970 & 0.486 & 0.598 & 0.42 & 0.516 & 0.968 & sig & 0.342 & 0.736 & 0.171 & 0.206 & 0.453 & 0.235 \\
\hline \multirow{2}{*}{$\begin{array}{l}\text { Plasma } \\
\text { concentration } \\
\text { (peak; ng } \mathbf{g} \text { /ml) }\end{array}$} & corr & $-0,072$ & 0,289 & $.461^{*}$ & $-0,027$ & $333^{\circ}$ & $-450^{\circ}$ & $\mathrm{corr}$ & $-0,071$ & $-0,010$ & $-0,134$ & $-0,109$ & $-0,099$ & $-0,130$ \\
\hline & sig & 0,060 & 0,078 & 0,004 & 0,874 & 0,041 & 0,004 & sig & 0,068 & 0,952 & 0,415 & 0,511 & 0,548 & 0,431 \\
\hline \multirow{2}{*}{$\begin{array}{l}\text { Plasma } \\
\text { concentration } \\
\text { (abs.diff:; } \\
\text { ng/ml) }\end{array}$} & corr & $-0,051$ & 0,291 & . $.439^{*}$ & 0,007 & $360^{*}$ & $-.438^{*}$ & corr & $-0,045$ & $-0,025$ & $-0,066$ & $-0,072$ & $-0,093$ & \begin{tabular}{|l|l|}
$-0,079$ \\
\end{tabular} \\
\hline & aig & 0,762 & 0,077 & 0,006 & 0,968 & 0,026 & 0,006 & aig & 0,785 & 0,881 & 0,692 & 0,662 & 0,575 & 0,631 \\
\hline
\end{tabular}

Conclusions: The BIARIVA prospective pilot study did not unveil a definite trend towards blood coagulation parameters dependency on body composition indicating safety and efficacy of edoxaban and rivaroxaban even in the obese.

PB1265 | Patient Satisfaction with Quality of Care from a New Multidisciplinary Thrombosis Service

$\underline{\text { S. Young }}^{1,2}$; K.O. Bonsu ${ }^{1}$; T. Lee ${ }^{1,2}$; H.V Nguyen ${ }^{1}$; R. Chitsike ${ }^{3,4}$

${ }^{1}$ Memorial University School of Pharmacy, St. John's, Canada; ${ }^{2}$ Eastern

Regional Health Authority Pharmacy Department, St. John's, Canada;

${ }^{3}$ Eastern Regional Health Authority Division of Hematology, St. John's, Canada; ${ }^{4}$ Memorial University Faculty of Medicine (Hematology), St. John's, Canada

Background: We opened a multidisciplinary Adult Outpatient Thrombosis Service (TS) in 2017 in a Canadian health region. The TS is a comprehensive thrombosis and anticoagulation management program with unique, interrelated clinics. The TS is staffed by Pharmacists, a Thrombosis Physician/Hematologist, and Clerical staff.

Aims: This study assessed patient satisfaction with the TS.

Methods: We conducted a cross-sectional mailed survey of eligible patients $(n=1058$ ) attending the TS between 2017 and 2019 . Patient satisfaction was measured using the validated, seven-item Short Assessment of Patient Satisfaction (SAPS). The SAPS assesses core satisfaction domains of treatment satisfaction, explanation of treatment results, clinician care, participation in decision-making, respect by the clinician, time with the clinician, and satisfaction with clinic care, and utilizes 5-point responses (0-4), with the range from 0 (extremely dissatisfied) to 28 (extremely satisfied).

Results: Of the 563 surveys returned, nine were excluded for missing SAPS items. Most respondents (87\%) were 50 or more years, with $51 \%$ male. Two-thirds (67\%) had post-secondary education. About half $(54 \%)$ were taking a direct oral anticoagulant, 19\% taking warfarin, and $19 \%$ no anticoagulant. Almost half (47\%) had taken the anticoagulant for one to five years, $28 \%$ less than one year, and $25 \%$ greater than five years. The mean SAPS score was 22.1 (SD 4.1, range 8 to 28). Multivariate analysis showed patients with post-secondary education were more satisfied with the TS ( $\beta=16.153, P=0.024)$, and patients taking warfarin were less satisfied $(\beta=-15.832, P=0.039$ ). The SAPS tool demonstrated high internal consistency (Composite reliability tests 0.86 ). Of the $40 \%$ that provided written responses to one open-ended question, most described positive experiences and perceptions of the TS. Content analysis revealed four major themes: service organization and coordination of care, patient-provider relationship, pharmacist-led medication therapy management, and patient-centered delivery and communication of health information. Conclusions: The majority of respondents were satisfied with the multidisciplinary TS.

PB1266 | Using the Functional Resonance Analysis Method to Explore how Elastic Compression Therapy Is Organized and Could Be Improved

$\underline{\text { R.H. Schreurs }}^{1,2,3} ;$ M.A. Joore ${ }^{4} ;$ H. Ten Cate Ca, $^{1,2,3}$

A.J. Ten Cate-Hoek ${ }^{1,2}$

${ }^{1}$ Thrombosis Expert Centre Maastricht and Laboratory for

Clinical Thrombosis and Haemostasis, Maastricht, Netherlands;

${ }^{2}$ Cardiovascular Research Institute Maastricht (CARIM), Maastricht, Netherlands; ${ }^{3}$ Department of Internal Medicine, Maastricht University Medical Centre, Maastricht, Netherlands; ${ }^{4}$ Department of Clinical Epidemiology and Medical Technology Assessment, Maastricht, Netherlands

Background: Elastic compression stocking (ECS) therapy is an important treatment for patients with deep venous thrombosis (DVT) and chronic venous insufficiency (CVI). Practically, the impression exists that there is a substantial variation in how ECS therapy is implemented. Aims: This study aimed to provide insight into the structure and variability of the elastic compression stocking therapy process, its 
effects on outcomes, and to elicit improvement themes from a multiple stakeholder perspective.

Methods: This study was conducted in two regions in The Netherlands. Thirty semi-structured interviews with professionals and patients were performed. The study was approved by the medical ethical committee of MUMC+ and all participants gave verbal informed consent. The essential functions for the process of elastic compression stocking therapy were extracted to create two work-as-done models using the Functional Resonance Analysis Method (FRAM). These findings were used to guide discussion between stakeholders to identify improvement themes. The study was funded by a grant from ZonMw, the Netherlands Organisation for Health Research and Development.

Results: Two FRAM models were created (one for each region). The variability of the functions and their effect on outcomes, as well as interdependencies between functions were identified. These were presented in stakeholder meetings to identify the structure of the process and designated variable and uniform parts of the process and its outcomes. Ultimately six improvement themes were identified: dissemination of knowledge of the entire process; optimizing and standardizing initial compression therapy; optimizing timing to contact the medical stocking supplier (when edema has disappeared); improving the implementation of assistive devices; harmonizing follow-up duration for CVI-patients; and personalizing follow-up and treatment duration in DVT-patients.

Conclusions: This study provides a detailed understanding of how elastic compression stocking therapy is delivered in daily practice by describing major functions and variability in performances and elicited six improvement themes from a multi-stakeholder perspective.

\section{PB1267 | International Multicentric Study: Laboratory in} Patients Treated with Apixaban or Rivaroxaban in Latin America (Larila): Final Results

E. Cortina $^{1,2}$; D. Garcia ${ }^{3,2}$; G. Conte ${ }^{4,2}$; M.C. Guillermo ${ }^{5,2}$; M. Cúneo ${ }^{4,2}$; P. Turcatti ${ }^{5,2}$; R. Izaguirre I,2 $^{1,2}$

${ }^{1}$ Instituto Nacional de Cardiologia 'Ignacio Chavez', Mexico, Mexico;

${ }^{2}$ Grupo Cooperativo Latinoamericano de Hemostasis y Trombosis, Mexico, Mexico; ${ }^{3}$ Clínica 25 de Mayo, Mar del Plata, Argentina; ${ }^{4}$ Clinical Hospital University of Chile, Santiago, Chile; ${ }^{5}$ Hospital de Clínicas, Facultad de Medicina, Montevideo, Uruguay

Background: A Latin American Group (Argentina, Chile, Mexico, Uruguay) for Laboratory Study of Direct Oral Anticoagulants (DOACs), LARILA, was created in January 2019. Analytical, prospective, Ethics Committees approved study.

Aims: To standardize the laboratory control of Rivaroxaban and Apixaban in Latin America, to correlate anti Xa activity and coagulation, to register adverse events.

Methods: Patients > 18 yo. Non-valvular atrial fibrillation (NVAF) and/or Venous Thromboembolic Disease (VTE) on Rivaroxaban 20 or $10 \mathrm{mg}$ po/day or Apixaban 5 o 2.5 mg /12 hr between January 2019 and January 2020. Anti-Xa activity: STA Compact Max coagulometer, STA $®$-Liquid Anti-Xa, Stago calibrator, control for Rivaroxaban and Apixaban, PT, APTT, TT, Fibrinogen.

Results: Patients:199, W:M:1.07:1 (53\% W, 46.7\% M), median age 70 years (21-101). Mexico 44.7\% (89/199), Argentina 38.7\% (77/199), Chile 10.6\% (21/199), Uruguay 6.0\% (12/1993). NVAF 53.3\% (106/199), VTE 33.7\% (67/199), PE 8.0\% (16/199), STROKE 2.0\% (4/199), SVT 3.0\% (6/199). Apixaban 63.8\% (127/199), Rivaroxaban 36.2\% (72/199). Doses: (138/199) 69.3\% full doses, (61/199) $30.7 \%$ half doses. Bleedings 5.52\% (11/199), 0.5\% major (1/199), 5.02\% minor (10/199). Non significant differences in Anti Xa between bleeding and non bleeding groups. Recurrence: 1.0\% (2/199).

Anti Xa U/ml: Mean +/- SD: Visit 1 APIXABAN ( $N=127)$ trough 87.6 +/- 58.2; peak $185.4 \pm 82.9$; Visit $2(N=70)$; trough $102.2 \pm 75.2$; peak $196.6 \pm 98.5$

RIVAROXABAN Visit 1 ( $N=72$ ): trough $34.7 \pm 17.6$, peak $185.4 \pm 82.9$, Visit $2(N=30)$ : trough $34.0 \pm 17.5$, peak $224.0 \pm 77.8$. Rivaroxaban higher anti-Xa at peak in Visit 2, $P=0.035$.

Between Argentina and Mexico, Apixaban trough at $5 \mathrm{mg} / 12 \mathrm{hrs}$ showed difference $(P=0.023)$. Not other statistically significant differences between countries were found.

Conclusions: Anti Xa values obteined were in accordance with data has been published $(1,2)$. We observed an interinvidual variation in the second Rivaroxaban peak. This is the first Latin American cooperative study focused on the analysis of the population treated with DOACs.

\section{PB1268 | Nationwide Children's Hospital Pediatric and Adult Comprehensive Anticoagulation Program: A Report on its Anticoagulation Management and Clinical Outcomes}

V. Rodriguez; J. Stanek; J. Giver; A. Dunn; A. Sankar; K. Monda; J. Canini; B. Kerlin

Nationwide Children's Hospital/The Ohio State University, Columbus, United States

Background: Dedicated anticoagulation programs have demonstrated improvement in patients' anticoagulation management and outcomes. Our anticoagulation program, established in 2014, is unique as it provides comprehensive care to pediatric and adult patients expanding diverse geographical areas in the state of Ohio.

Aims: (1) Compare the impact of an anticoagulation program preand post-implementation, on the quality of anticoagulation as measured by \% time in therapeutic range (\%TTR) and compliance.

(2) To assess clinical outcomes (bleeding and thrombosis complications) prior and following anticoagulation program implementation. Methods: Medical records were retrospectively reviewed for the years 2014-2019. Patient demographics, indications and type of anticoagulants, INR range, days on anticoagulation, TTR, \% TTR and compliance were obtained. Percentage TTR was calculated by Rosendaal linear interpolation method. Bleeding complications were defined according to the ISTH-SSC for non-surgical patients. New 
thrombotic events were reviewed. Comparisons were made using non-parametric analyses.

Results:

\section{TABLE 1}

Long-term warfarin patients

$N=308$

Male sex

$180(58.4)$

Median age, years (range)

24 (2-69)

Age group

$91(29.5)$

Pediatrics (<18 y)

$217(70.5)$

Adults

Warfarin indication

$161(52.3)$

Mechanical valve

55 (17.9)

Fontan

$45(14.6)$

DVT/PE

$31(10.1)$

$16(5.2)$

Other (heart failure, pulm. HTN, etc.)

Home INR

Aspirin

$155(50.3)$

Bleeds pre-clinic

Major

$3(1.0)$

Non-major/minor

Bleeds while followed by clinic

Major

Non-major/minor

Venous thromboembolic events

VTE pre-clinic

VTE while followed by clinic

Non-warfarin long-term or short-term warfarin

$N=114$

patients

$16(0-45)$

Median age at VTE, years (range)

$98(86.0)$

Age Group

$16(14.0)$

Pediatrics $(<18 y)$

Adults

Major/Minor bleeds

9 (7.9)

VTE events while on anticoagulation

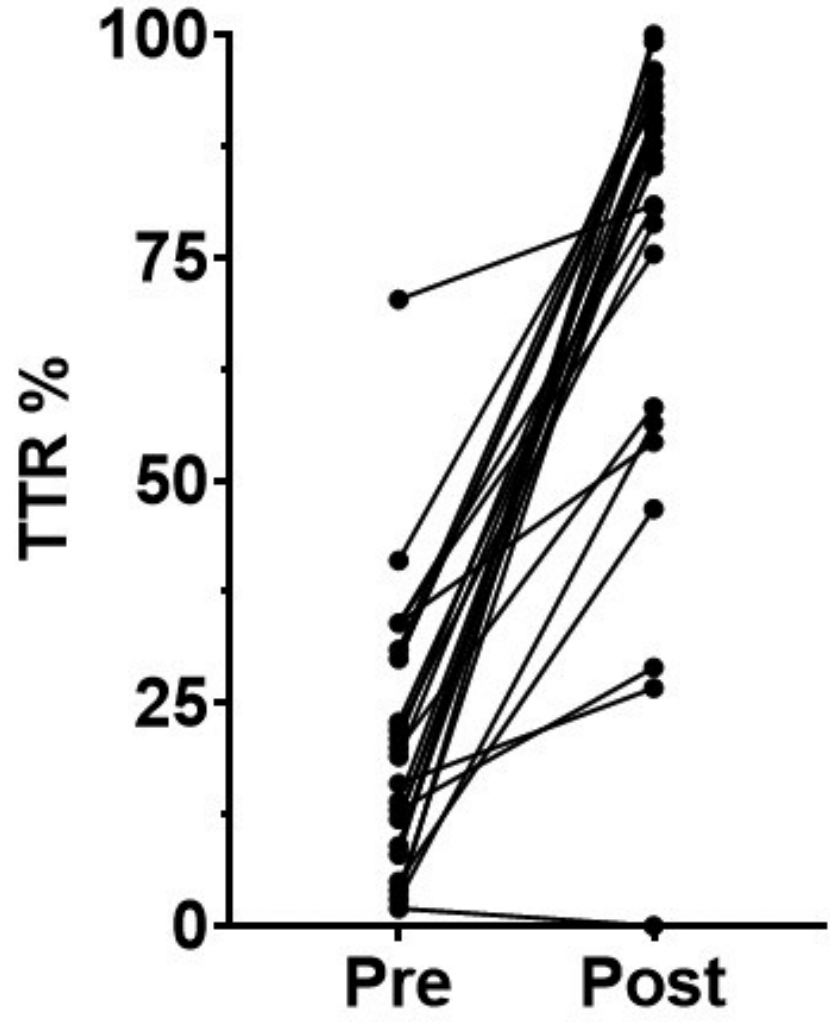

FIGURE 1 Median TTR \% pre-clinic was 17.5\%, vs the median TTR $\%$ post-clinic was $87 \%$; patients increased their TTR $\%$ by $63 \%$ on average $P$

Table 1 summarizes demographic data. Long-term warfarin therapy group included 308 patients with $87 \%$ of these being cardiac related indications. Median age 24 y (range: 2-69 y). The second group $(N=114)$ comprised short-term and non-warfarin long-term anticoagulation (e.g. LMWH, DOAC) [median age 16 (range: 0-45 y)].Median \%TTR pre-anticoagulation clinic for 26 patients was $17.5 \%$ versus median TTR post-clinic of $87 \%$ (Fig 1A). Median \%TTR 81.2 (range: 77.7-85.4) for the years 2014-2019. Similarly, compliance increased by an average of $28.6 \%$. Thrombosis events while on anticoagulation was no different pre- and post-clinic (Table 1; $P=0.59)$. Bleeding events were higher post-clinic $[N=17$; mean age 35 y (range: $22-69$ y)] versus pre-clinic [ $N=7$; mean age 25.8 (range: 2-69 y)].

Conclusions: Our anticoagulation program has significantly improved and sustained \%TTR and compliance. A greater proportion of major bleeding events were documented post-clinic implementation perhaps related to the increased age and complexity of our patient population. 
PB1269 | Enhancement of Thrombin Generation in Lymphoma Cohort by Andexanet Alfa

F. Siddiqui ${ }^{1}$; E. Bontekoe ${ }^{1}$; D. Antic ${ }^{1}$; D. Hoppensteadt ${ }^{1}$;

G. Gerotziafas ${ }^{2}$; I. Elalamy ${ }^{2}$; J. Fareed ${ }^{1}$

${ }^{1}$ Loyola University Medical Center, Maywood, United States; ${ }^{2} T e n o n$ University Hospital, Paris, France

Background: The prevalence of thrombosis in lymphoma patients is reportedly high and ranges from $3-10 \%$, and further increased at advanced stages of the disease especially in hgNHL. The thrombin generation potential in these patients is decreased.

Aims: This study was designed to compare effect of andexanet alfa (AA) on the thrombin generation potential and its relevance to the generation of thrombin.

Methods: Citrated blood samples from 78 patients with confirmed diagnosis of non-Hodgkin lymphoma (NHL), Hodgkin lymphoma (HL) and Chronic lymphocytic leukemia/Small lymphocytic lymphoma (CLL/SLL) were collected from the Clinic of Hematology Unit, University of Belgrade, Belgrade, Serbia. 50 samples of normal human plasma (NHP) was obtained from George King Biomedical (Overland park, KS). NHP was prepared for referencing purposes. Individual samples were supplemented with andexanet alfa at 100 $\mathrm{ug} / \mathrm{ml}$. Thrombin generation studies were carried out using a commercially available a kinetic fluorogenic substrate method (calibrated automated thrombogram; CAT).

Results: On a cumulative basis, lymphoma patients showed an increase in LT (2.9 1.15) in comparison to NHP (2.06 0.2) which decreases with AA (1.94 0.1). The PT levels were decreased (120.34 44.3) in comparison to NHP (159.20 4.8) and increases with AA (153.70 37.2). The AUC was also decreased (668.85 284.3) in comparison to NHP (756.46 34.5) which increases with AA (758.32 226.4). Table 1 shows the data on the sub-group analysis on the effect of andexanet on thrombin generation in lymphoma patients.

\section{TABLE 1}

\begin{tabular}{|c|c|c|c|c|c|c|}
\hline \multicolumn{6}{|c|}{ Table 1: Inhibition of thrombin generation potential in lymphoma cohort by andexanet alfa } \\
\hline \multirow{2}{*}{ Groups } & \multicolumn{2}{|c|}{ Peak Thrombin } & \multicolumn{2}{c|}{ AUC } & \multicolumn{2}{c|}{ Lag Time } \\
\cline { 2 - 7 } & Pre Andexanet & Post Andexanet & Pre Andexanet & Post Andexanet & Pre Andexanet & Post Andexanet \\
\hline Control & $159.20 \pm 4.8$ & $188.15 \pm 7.4$ & $756.46 \pm 34.5$ & $829.14 \pm 50.2$ & $2.06 \pm 0.2$ & $1.94 \pm 0.1$ \\
\hline HL & $128.16 \pm 68.2$ & $180.75 \pm 35.4$ & $781.91 \pm 343.8$ & $938.31 \pm 220.2$ & $3.67 \pm 1.3$ & $3.38 \pm 1.0$ \\
\hline NHL & $113.99 \pm 45.4$ & $154.16 \pm 36.8$ & $627.81 \pm 221.0$ & $744.52 \pm 222.6$ & $3.07 \pm 1.2$ & $2.50 \pm 0.6$ \\
\hline CLL & $129.77 \pm 31.9$ & $142.46 \pm 33.8$ & $738.18 \pm 402.5$ & $725.26 \pm 224.2$ & $2.23 \pm 0.6$ & $2.32 \pm 0.5$ \\
\hline Lymphoma & $120.34 \pm 44.3$ & $153.70 \pm 37.2$ & $668.85 \pm 284.3$ & $758.32 \pm 226.4$ & $2.87 \pm 1.2$ & $2.52 \pm 0.7$ \\
\hline
\end{tabular}

Conclusions: The observed decrease in thrombin generation potential in these patients may be due to the consumption of prothrombin and other coagulation factors. Increase in thrombin generation potential in post andexanet samples indicate that andexa promotes thrombogenesis and should be given cautiously in predisposing conditions. Wide variations among these lymphoma groups are noted.
PB1270 | A Survey of Current Anticoagulation Patient Education Practices and Development

A. Jones $^{1}$; J. Saunders ${ }^{2}$; S. Vazquez ${ }^{3}$; A. Fagerlin ${ }^{1}$; D. Witt ${ }^{2}$

${ }^{1}$ University of Utah School of Medicine, Salt Lake City, United States;

${ }^{2}$ University of Utah College of Pharmacy, Salt Lake City, United States;

${ }^{3}$ University of Utah Health, Murray, United States

Background: Most patient education is misremembered or forgotten. Anticoagulants are high-risk medications widely used to prevent and treat thrombotic events, resulting in a need for adequate patient education to minimize harm. While anticoagulant patient education interventions can improve knowledge and surrogate outcomes, they may not represent current practice.

Aims: Determine provider perceptions of anticoagulation therapy patient education at their institution.

Methods: A quantitative survey was distributed through a national professional organization and across several health systems. Questions included educational delivery methods, perceived effectiveness, evaluation of patient knowledge, development of patient education, and demographics.

Results: The 61 survey respondents were $79.0 \%$ female, an average age of $43,86.2 \%$ identified as white, and $80.3 \%$ were pharmacists. Most respondents (95\%) complete patient education interventions in one session averaging 35 minutes for warfarin and 22.5 minutes for direct oral anticoagulants. Providers reviewed educational topics in $37 \%$ of daily patient interactions. $91.9 \%$ of respondents perform either a formal or informal knowledge check during patient education activities. 59\% of respondents reported reasons for not performing patient knowledge checks included no formal process requiring knowledge checks, lack of time, and forgetting. The majority (93.4\%) reported their patient education process was somewhat or very effective. The main reason underlying perceived less effective patient education was too much information in one session. Thirty-four respondents had some knowledge of patient education development at their institution. Most of these (82.9\%) indicated their educational process's development relied on expert opinion. In comparison, $22.9 \%$ indicated utilizing user-based design, and 10 (28.9\%) respondents used learning theories.

TABLE 1 Completion of knowledge checks by anticoagulation providers
Format of knowledge checks, $n=61$

Both Formal and Informal Checks

Formal Check Only

Informal Check Only

No check

Types of knowledge check, $n=19^{1}$
$N, \%$

$20,27.9 \%$

$3,4.9 \%$

$35,57.4 \%$

$6,9.8 \%$ 
Format of knowledge checks, $n=61$

$N, \%$

Multiple types of tests ${ }^{2}$

6, $31.6 \%$

Written Test

$1,5.2 \%$

Teach Back

$11,57.9 \%$

Verbal Test

$1,5.2 \%$

When do you use knowledge checks, $n=57^{1}$

Formal part of education process

$12,20.7 \%$

If I think patient does not understand

$45,77.6 \%$

Patient behavior demonstrates deficit

$52,89.7 \%$

of knowledge

Why are knowledge checks not used, $n=36^{1}$

Take too much time

$11,30.6 \%$

Not useful

2, $5.6 \%$

I forget to use them

7, $19.4 \%$

Not part of our formal process

$18,50 \%$

I never learned to use them

$1,2.8 \%$

Other

7, $19.4 \%$

${ }^{1}$ Respondents could give more than one response

${ }^{2}$ Types of tests included any combination of written, teach-back method, the Indian Health Services model, and verbal tests

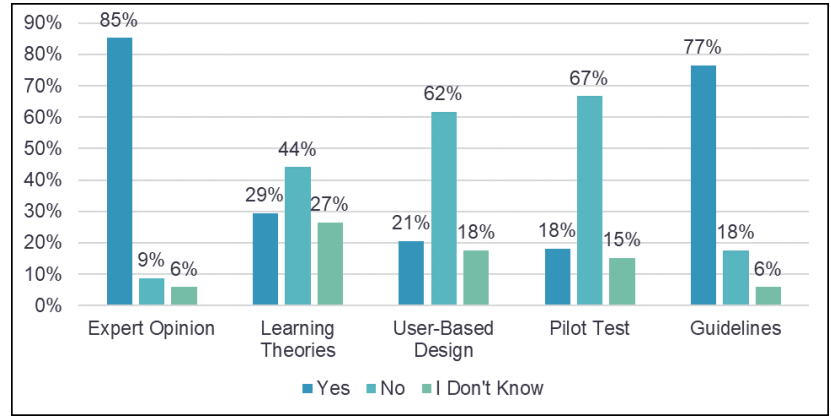

FIGURE 1 Different methods of patient education development that are used by providers involved in creating anticoagulation patient education $(n=34)$

Conclusions: While most providers felt patient education was at least somewhat effective, they reported reviewing education with patients daily, indicating a lack of understanding. The lack of formal knowledge checks and best practices in developing patient education tools are significant gaps to address.
PB1271 | Statin Use and Recurrence of Venous Thromboembolic Event

H.J. Peroni; P.R. Saunero; T. Politi; F. Holguin; J. Wisnivesky;

M. Burgos; M.F. Grande Ratti; F.J. Vázquez

Hospital Italiano de Buenos Aires, Buenos Aires, Argentina

\section{Background:}

Prevalence of Recurrence of Venous Thromboembolism (RVTE) is high.

Aims: To assess the association of statin use with RVTE.

Methods: A retrospective cohort study with consecutive adults patients with a confirmed first episode VTE, who were included in the Thromboembolic Disease Institutional Registry, between 06/2006 and $12 / 2016$. All patients were classified according to the exposure variable (Statin Use -SU-, defined as the initial questionnaire at first VTE episode) and were followed until the occurrence of RVTE, death or date of last contact. We used time to event analyses, the KaplanMeier method and multivariate Cox proportional hazards model.

Results:

A total of 3,157 patients with a first episode of VTE were included, of which 638 were SU and 2,518 NSU. The most frequent statin was atorvastatin (55.24\%).

Cumulative incidence of RVTE were similar between groups as shown Figura 1 (at on-year of follow up: 5.4\% in SU and 6.7\% in NSU), and in Figure 2 with log rank test ( $p$-value $=0.275$ ).

The univariate HR of SU for RETV was $0.81(95 \% \mathrm{Cl} 0.56-1.17$; $P=0.277$ ). After adjustement by confounder variables (Age, Sex, NSAIDs, Corticosteroids, Immobility, Anticoagulant treatment, Hypertension, Diabetes mellitus, Obesity, Coronary heart disease, Stroke, Chronic heart failure and Oncological disease) adjusted-HR was 0.95 (95\% Cl 0.62-1.45; $P=0.839)$.

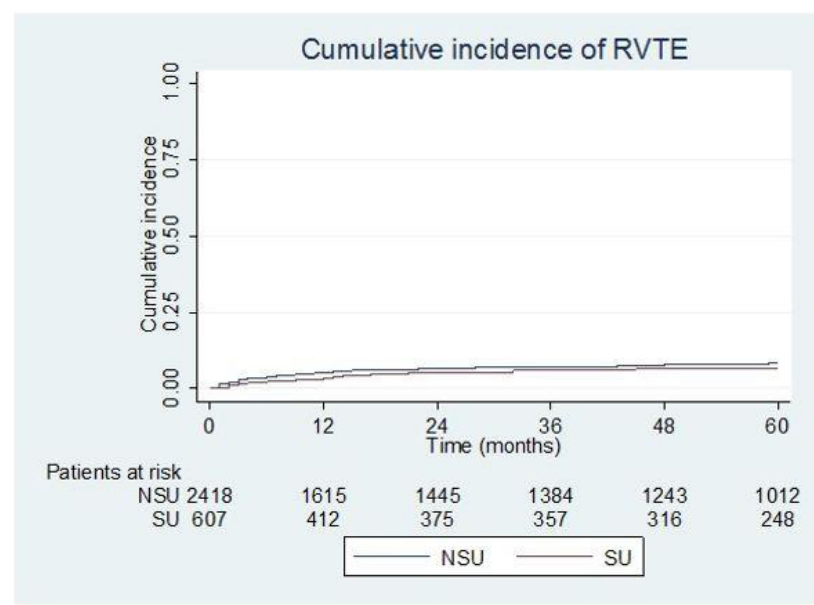

FIGURE 1 Cumulative incidence of RTVE 


\begin{tabular}{|c|c|c|}
\hline $\begin{array}{c}\text { Cumulative incidence } \\
\text { of RVTE } \\
\text { (months) }\end{array}$ & $\begin{array}{c}\text { Statin Use (SU) } \\
\text { (\% con IC95\%) }\end{array}$ & $\begin{array}{c}\text { No Statin Use (NSU) } \\
\text { (\% con IC95\%) }\end{array}$ \\
\hline 12 & $3.2(1.9-5.2)$ & $5.4(4.5-6.4)$ \\
\hline 24 & $5.4(3.7-7.9)$ & $6.7(5.7-7.9)$ \\
\hline 36 & $6.2(4.3-8.9)$ & $7.1(6.1-8.4)$ \\
\hline 48 & $6.5(4.5-9.2)$ & $7.7(6.6-9.0)$ \\
\hline 60 & $6.5(4.5-9.2)$ & $8.2(7.0-9.6)$ \\
\hline
\end{tabular}

FIGURE 2 Cumulative incidences at different times (in months)

Conclusions: Statin use was not associated RVTE. Probably because of the low recurrence rate, the small sample size of $\mathrm{SU}$, and the short follow-up period. Future studies will need to explore the pleiotropic and antiinflammatory effect of statins.

PB1272 | A Realist Evaluation on Therapeutic Elastic Compression Therapy Pathways for DVT- and CVD Patients to Develop Evidence-informed Improvement Strategies

R.H. Schreurs $^{1,2,3}$; M.A. Joore ${ }^{4}$; D. De Bruijn-Geraets ${ }^{4}$; H. Ten Cate ${ }^{1,2,3}$; A.J. Ten Cate-Hoek ${ }^{1,2}$

${ }^{1}$ Thrombosis Expert Centre Maastricht and Laboratory for Clinical Thrombosis and Haemostasis, Maastricht, Netherlands; ${ }^{2}$ Cardiovascular Research Institute Maastricht (CARIM), Maastricht, Netherlands; ${ }^{3}$ Department of Internal Medicine, Maastricht University Medical Centre, Maastricht, Netherlands; ${ }^{4}$ Department of Clinical Epidemiology and Medical Technology Assessment, Maastricht, Netherlands

Background: Elastic compression stocking (ECS) therapy is well established in clinical practice for patients with deep venous thrombosis (DVT) and chronic venous disease (CVD). However, considerable variations exist for its implementation in clinical practice, which may impact patient outcomes. We previously identified six improvement themes for optimization of ECS therapy using the Functional Resonance Analysis Method (FRAM).

Aims: This study aims to expand theoretical understanding using a realist evaluation to refine the initial improvement theory and thereby improve chances for implementation.

Methods: The realist evaluation included a mixed-method design consisting of semi-structured interviews with patients $(n=5)$ and involved health care professionals $(n=25)$, stakeholder meetings ( $n=2)$, and surveys $(n=114)$. The study was approved by the recognized medical ethical committee of MUMC+ and all participants gave verbal informed consent. Both quantitative and qualitative data were extracted to create the content of context-mechanism-outcomeconfigurations (CMOCs) important in ECS therapy. The study was funded by a grant from ZonMw, the Netherlands Organisation for Health Research and Development.
Results: We identified seven CMOcs that should be used to inform implementation strategies to support the implementation and optimization of ECS therapy. These CMOcs targeted four optimal outcomes: type of initial compression therapy with maintenance of patient's self-reliance, selection of ECS material, maintenance of patient's self-reliance using assistive devices, follow-up (for DVTand CVD-patients), and duration of ECS therapy (for DVT-patients). Furthermore, the main clinical care pathways for ECS therapy and associated patient volumes were identified, providing insight into the potential impact of various strategies.

Conclusions: This study provides a detailed insight into what needs to be in place to optimize ECS-therapy and to inform implementation strategies.

\section{PB1273 | Recurrent Thrombosis Rescued by Fondaparinux in High-risk Patients: Case Series and Review of Literature}

M. Tanguay ${ }^{1}$; C. Séguin ${ }^{2}$

${ }^{1}$ McGill University, Department of Medicine, Montreal, Canada; ${ }^{2} \mathrm{McGill}$ University, Department of Medicine, Division of Hematology, Montreal, Canada

Background: Thrombotic disease treatment options are limited when anticoagulation with dose escalation of low-molecular-weight heparin (LMWH) or unfractionated heparin (UFH) fail. Fondaparinux is a pure, synthetic pentasaccharide that consists of heparin's essential five sugar chain that binds antithrombin to inactivate factor Xa. There is scarce data regarding fondaparinux's use in recurrent thrombosis.

Aims: To highlight fondaparinux's potential role in anticoagulantrefractory thrombosis.

Methods: Six patients received fondaparinux after thrombosis progression in our center between 2016 and 2021. Their clinical data was retrieved and analysed.

Results: Patient characteristics and evolution on treatment are included in table 1 . Of those six patients, two were previously diagnosed with a high-risk thrombophilia (triple positive antiphospholipid syndrome (APS) and homozygous factor V Leiden) and four had an underlying malignancy. Half of the patients were female, and their median age was 52 (range 28-71). Four had thrombosis recurrence on increased $\mathrm{LMWH}$ weight-adjusted doses (mean increase of $27.5 \%$, range $15-40 \%$ ) and two had thrombosis recurrence on therapeutic UFH. While on LMWH or UFH: the APS patient developed a valvular thrombus with arterial microemboli, one patient had progressive upper extremity catheter-related deep vein thrombosis (DVT), another patient developed lower extremity DVT and pulmonary emboli and three others experienced worsening lower extremity DVTs. Heparin-induced thrombocytopenia was considered and ruled out in every case. Hence, all patients were treated with fondaparinux for a duration ranging from 26 days to 16 months. All patients had a normal renal function. No progression of thrombus was observed in $100 \%$ of patients. Reduction of valvular thrombus 
was demonstrated in the APS patient and DVT recanalization either partial or complete was shown on venous duplex in the other five patients. No bleeding complications were documented while on fondaparinux.
Conclusions: In our case series, fondaparinux was a limb-saving and potential life-saving option when other standard methods of anticoagulation failed.

TABLE 1 Patient characteristics and evolution on treatment

\begin{tabular}{|c|c|c|c|}
\hline $\begin{array}{l}\text { Patient (sex, } \\
\text { age, weight) }\end{array}$ & $\begin{array}{l}\text { High thrombotic risk } \\
\text { condition }\end{array}$ & Thrombotic disease and treatment & Evolution \\
\hline $\begin{array}{l}\text { Female, } \\
28 \mathrm{y}, \\
64 \mathrm{~kg}\end{array}$ & $\begin{array}{l}\text { Triple positive APS } \\
\text { Ischemic toe at } 25 y \text {, } \\
\text { PE (on Warfarin) at } \\
26 y, 1^{\text {st }} \text { pregnancy at } \\
27 y \text { (on LMWH): pla- } \\
\text { cental abruption at } \\
28 \text { weeks and HELLP } \\
\text { syndrome }\end{array}$ & $\begin{array}{l}\text { At } 33 \text { weeks of } 2^{\text {nd }} \text { pregnancy: developed a TIA while on LMWH } \\
\text { - LMWH dose increased } 20 \% \\
\text { Elective delivery at } 36 \text { weeks } \\
2 \text { days post-partum: developed several cerebral microinfarcts } \\
\text { - another } 20 \% \text { LMWH increase (total } 40 \% \text { ) } \\
4 \text { days post-partum: found to have a large mitral valve thrombus ( } 21 \times 9 \\
\mathrm{~mm} \text { ) and worsening neurological symptoms } \\
\text { - fondaparinux } 10 \mathrm{mg} \times 1 \text {, then } 7.5 \mathrm{mg} \text { die } \times 25 \text { days (with Warfarin bridging) }\end{array}$ & $\begin{array}{l}1 \text { month post } \\
\text { fondaparinux } \\
\text { initiation: } \\
\text { reduction in } \\
\text { mass size to } 3.4 \\
\text { mm, no further } \\
\text { embolic events }\end{array}$ \\
\hline $\begin{array}{l}\text { Female, } \\
30 y, \\
92 \mathrm{~kg}\end{array}$ & $\begin{array}{l}\text { Homozygous factor } \\
\mathbf{V} \text { Leiden and May- } \\
\text { Thurner syndrome } \\
\text { diagnosed after } \\
\text { post-partum DVT: } \\
\text { left common iliac vein } \\
\text { stent was inserted }\end{array}$ & $\begin{array}{l}\text { In-stent left common iliac vein thrombosis on Warfarin ( } 4 \text { months post } \\
\text { insertion) } \\
\text { - switched to LMWH } \\
30 \text { days later: Left leg DVT progression } \\
\text { - LMWH increased } 25 \% \\
7 \text { days later: Right common femoral DVT } \\
\text { - fondaparinux } 10 \text { mg die } \times 14 \text { months, followed by Warfarin }\end{array}$ & $\begin{array}{l}\text { Duplex } 5 \text { months } \\
\text { post: patent } \\
\text { right leg veins, } \\
\text { partial DVT } \\
\text { regression on } \\
\text { the left }\end{array}$ \\
\hline $\begin{array}{l}\text { Male, } \\
54 \mathrm{y}, \\
65 \mathrm{~kg}\end{array}$ & $\begin{array}{l}\text { Testicular metastatic } \\
\text { germ cell tumor }\end{array}$ & $\begin{array}{l}\text { Bilateral above-knee DVTs } \\
\text { - started on LMWH 15\% above weight-adjusted dose } \\
7 \text { months later: acute bilateral above-knee DVTs } \\
\text { - fondaparinux } 7.5 \mathrm{mg} \text { die } \times 5 \text { months, followed by Apixaban }\end{array}$ & $\begin{array}{l}\text { Duplex } 5 \text { months } \\
\text { post: bilateral } \\
\text { partial } \\
\text { recanalization }\end{array}$ \\
\hline $\begin{array}{l}\text { Male, } \\
50 y, \\
57 \mathrm{~kg}\end{array}$ & $\begin{array}{l}\text { Stage IV Pancreatic } \\
\text { cancer }\end{array}$ & $\begin{array}{l}\text { Right above-knee DVT - started on LMWH } \\
3 \text { weeks later: Acute left above-knee DVT } \\
\text { - LMWH increased } 30 \% \\
2 \text { months later: Bilateral PEs } \\
\text { - fondaparinux } 7.5 \mathrm{mg} \text { die } \text { x } 40 \text { days (then died from cancer) }\end{array}$ & $\begin{array}{l}\text { Duplex } 1 \text { month } \\
\text { post: partial } \\
\text { recanalization of } \\
\text { bilateral DVTs }\end{array}$ \\
\hline $\begin{array}{l}\text { Female, } \\
61 \mathrm{y}, \\
63 \mathrm{~kg}\end{array}$ & Hodgkin's lymphoma & $\begin{array}{l}\text { Catheter-related subclavian DVT } \\
\text { - started on UFH (about } 40000 \text { units/24h for therapeutic PTT) } \\
\text { After } 3 \text { days of UFH: DVT extension in axillary vein } \\
\text { - fondaparinux } 7.5 \text { mg die } x 8 \text { months }\end{array}$ & $\begin{array}{l}\text { Duplex } 2 \text { months } \\
\text { later: partial } \\
\text { recanalization }\end{array}$ \\
\hline $\begin{array}{l}\text { Male, } \\
71 \mathrm{y}, \\
75 \mathrm{~kg}\end{array}$ & Multiple myeloma & $\begin{array}{l}\text { Right above-knee DVT and PEs - started on LMWH } \\
10 \text { months later: spontaneous thigh hematoma requiring transfusion, du- } \\
\text { plex showed acute right above-knee DVT } \\
\text { - IVC filter insertion and stopped anticoagulation } \\
2 \text { weeks later: Left above-knee DVT } \\
\text { - started on UFH (about } 28000 \text { units/24h for therapeutic PTT) } \\
\text { After } 4 \text { days of UFH: Worsening bilateral limb-threatening DVTs } \\
\text { - fondaparinux } 7.5 \mathrm{mg} \text { die } x 16 \text { months, followed by Apixaban }\end{array}$ & $\begin{array}{l}1 \text { week post: } \\
\text { near resolution of } \\
\text { D-dimers } \\
\text { Duplex } 3 \text { months } \\
\text { post: bilateral par- } \\
\text { tial recanalization }\end{array}$ \\
\hline
\end{tabular}

APS: antiphospholipid syndrome; PE: pulmonary emboli; LMWH: low-molecular-weight heparin; HELLP: hemolysis, elevated liver enzymes, and low platelets; TIA: transient ischemic attack; DVT: deep vein thrombosis; UFH: unfractionated heparin; PTT: partial thromboplastin time; IVC: inferior vena cava 
PB1274 | May-Thurner Syndrome-associated Deep Vein Thrombosis: Is Oral Anticoagulation the Novel Approach?

F.A. Lo

Tan Tock Seng Hospital, Singapore, Singapore

Background: May-Thurner Syndrome (MTS) has been reported in 1-2 out of five patients presenting with left ilio-femoral deep vein thrombosis (DVT). Anticoagulation, catheter-directed thrombolysis (CDT) with stenting, thrombectomy, and bypass surgery are among the treatment options.

Aims: To follow-up the clinical outcomes of patients with MTSassociated DVT who received different treatment options in a span of four years.

Methods: Inclusion criteria were adult patients followed-up at our institution's Vascular Medicine Clinic with MTS detected by computed tomography (CT) scan, and followed-up for at least two years with or without repeat CT scan. We excluded patients who were followed-up in a non-Vascular Medicine specialty clinics, and those with interior vena cava (IVC) filters. Three treatment groups were identified: (A) anticoagulation (warfarin or direct oral anticoagulation), (B) CDT with stenting, or (C) graft bypass.

Results: Fifteen (15) patients were identified. One patient was excluded due to presence of IVC filter, and one patient died while on the second year of follow-up. Group A had eight patients, three in Group B, and two in Group C ( $n=13)$.

After four years of follow-up, repeat CT scans in Group A showed documented clearance of DVT in 6 patients, Group B had stable stents with no evidence of DVT in the three cases, while one patient in Group C showed graft patency. Post-thrombotic syndrome was seen in 2 patients in Group A, and none in Groups B and C. No bleeding complications were seen in all treatment groups.

Conclusions: As catheter-directed therapy appears to be a more suitable interventional approach compared to graft bypass surgery; oral anticoagulation therapy for MTS-associated DVT may be offered to patients as an alternative. This is especially suitable for patients who have high perioperative risk, or for those who decide not to undergo interventional or surgical therapy.
PB1275 | Heparin Therapeutic Range for Five aPTT Reagents in Plasma and One Point of Care

E. Cortina-de la Rosa; K.G. Cortés-Cortés; M.O. Romero-

Arroyo; F.A. Grimaldo-Gómez; M.M. Salcedo-Hernández;

Á.A. Arrieta-Alvarado; A. Ramírez-Hernández; S. Vázquez-Olvera;

R. Izaguirre-Ávila

National Institute of Cardiology Ignacio Chávez, Mexico City, Mexico

Background: Due unfractionated heparin (UFH) has an unstable pharmacokinetics, it requires close monitoring that means a challenge for medical attention. The most used assay to monitoring the UFHs therapy has been the activated Partial Thromboplastin Time (aPTT). It has been suggested different ways to establish heparin therapeutic ranges (HTR) for the best use of aPTT to monitoring the UFH therapy.

Aims: To get the HTR for five different aPTT reagents and from a point of care (POC) CoaguchekPro-II, for the evaluation of UFH as anticoagulation or prophylaxis therapy.

Methods: There were taken a venous blood sample from 83 patients (some of them more than once) to get 125 determinations of aPTT and anti-Xa activity. At the same time, we took a capilar blood sample to get the aPTT in the Coaguckek-Pro-II using the aPTT test strip CoaguChek aPTT Test. There were used three platforms (BCS$X P ® S i e m e n s$, STA-Compact Max $®$ Stago and Cobas-t $411 \circledR$ Roche) with five different aPTT reagents: Pathromtin-SL $®$ Siemens (Path-SL), CK-Prest ${ }^{\circledR S t a g o}$ (CK-Prest),Cephascreen ${ }^{\circledR S t a g o}$ (Cephas), LowS $₫$ Roche (LowS) and MediumS $®$ Roche (MedS).

It was calculated the linear regression between the APTT and UFH values to use the intercept and slope results and calculate the aPTT value for each UFH anticoagulation interval: $<0.3 \mathrm{UI} / \mathrm{mL}$ (sub-anticoagulation),0.3-0.7 $\mathrm{UI} / \mathrm{mL}$ (anticoagulation),0.7-1.1UI/ $\mathrm{mL}$ (over-anticoagulation low-risk) and >1.1 (over-anticoagulation high-risk).

Results:

TABLE 1 aPTT median and intervals (5-95p) for the five aPTT plasma measurements and for the POC

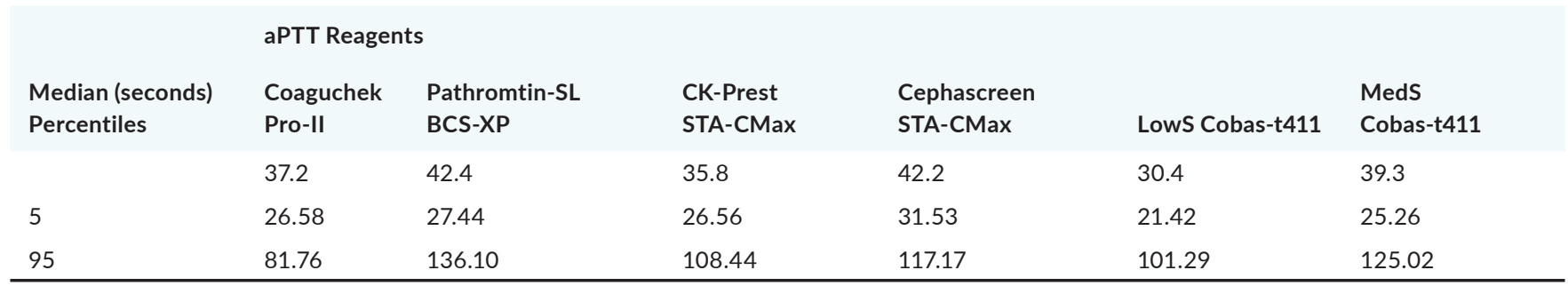


TABLE 2 Lineal regression elements (slope and intercep), aPTT results for each UFH values and their correspondent kappa

\begin{tabular}{|c|c|c|c|c|c|c|}
\hline & \multicolumn{6}{|c|}{ aPTT (seconds) } \\
\hline intercept & 32.36 & 28.95 & 30.49 & 25.41 & 19.30 & 26.30 \\
\hline$r$ & 0.723 & 0.870 & 0.928 & 0.914 & 0.900 & 0.854 \\
\hline UFH 0.3-0.7* & $48.6-70.1$ & $73.8-133.6$ & $63.7-108.0$ & $55.4-95.4$ & $53.1-98.2$ & $74.2-138.0$ \\
\hline UFH 0.7-1.1* & $70.1-91.7$ & $133.6-193.4$ & $108.0-152.3$ & $95.4-135.3$ & $98.2-143.3$ & 138.0-201.8 \\
\hline UFH >1.1* & $>91.7$ & $>193.4$ & $>152.3$ & $>135.3$ & $>143.3$ & $>201.8$ \\
\hline Kappa & 0.521 & 0.763 & 0.639 & 0.724 & 0.673 & 0.757 \\
\hline
\end{tabular}

${ }^{*} \mathrm{UI} / \mathrm{mL}$

Conclusions: We consider that the kappa value that we got for the capilar aPTT (CoaguChek Pro-II),let us to use it in an intensive care unit as a first anticoagulation approach. The kappa values for all of the aPPT reagents can be taken as considerable, and may be used with confidence to know the anticoagulation degree of the patient. Of course it is important to note,at least that there are patients with a high inflammation state that will present shorten APTT values despite the UFH anti-Xa activity.

PB1276 | Bleeding Complications in Patients on New Oral Anticoagulants for Venous Thromboembolism in Kenya

\section{A. Obayo}

Aga Khan University Hospital, Nairobi, Kenya

Background: The incidence of bleeding complications in patients with venous thromboembolism (VTE) on new oral anticoagulants (NOACs) has not been widely studied in contemporary practice in Africa.

Aims: To determine the rates of bleeding associated with NOAC use in daily clinical practice.

Methods: A retrospective review of adult patients diagnosed with venous thromboembolism and treated with NOACs at Aga Khan University Hospital from January 2014 to December 2019 was carried out. Clinical and outcome data were collected from medical records and the hospital mortality database. They were followed up from the time of commencement of oral anticoagulation to completion of therapy or to the time of the first clinically relevant non-major bleed (CRNM) or major bleed. Data on minor bleeding was also obtained. Data on bleeding was obtained from the hospital data base and through telephone interviews. Unadjusted rates of first major bleeding event or clinically relevant non-major bleeding (CRNM) was calculated as the number of bleeding events per 100 person-years Results: 243 patients with VTE were recruited. 222(91.4\%) were initiated on rivaroxaban, 12(4.9\%) on dabigatran, 9(3.7\%) on apixaban with a median follow-up of $213(119,477)$ days. The median age of the patients was $57(45,71)$ years. A total of 64 bleeding events were identified in 41(16.9\%) patients. Of all events, $18.8 \%$ were major, $17.2 \%$ were clinically relevant non-major (CRNM), and $64.1 \%$ were minor. Overall, the incidence rate for bleeding events was 22.1 per 100 patient-years. Approximately $4 \%$ of all patients had a major bleeding event. Gastrointestinal bleeding was the most common major bleeding site. There were more females with bleeding events (70.7\%) compared to males.

Conclusions: Most of these bleedings are minor with the GIT being the most common source of major bleeding and menorrhagia being the commonest cause of bleeding. Females had more major and clinically relevant non-major bleeds than men.

PB1277 | Predictors of Recurrence of Venous Thromboembolic Disease after Suspension of Anticoagulation

M.L. Posadas-Martinez ${ }^{1}$; F. Torres Gómez ${ }^{1}$; C. Juana ${ }^{2}$; M.F. Grande Ratti ${ }^{1}$; M. Burgos ${ }^{1}$; D. Mezzarobba ${ }^{1}$; N. Schutz ${ }^{1}$;

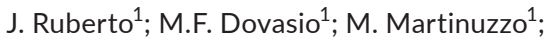

F. Gonzalez Bernaldo De Quiros ${ }^{1}$; F.J. Vazquez ${ }^{1}$; D.H. Giunta ${ }^{1}$ ${ }^{1}$ Hospital Italiano de Buenos Aires, Buenos Aires, Argentina; ${ }^{2}$ Instituto Universitario Hospital Italiano, Buenos Aires, Argentina

Background: The risk of recurrent venous thromboembolism (VTE) is high.

Aims: To evaluate associated factors with recurrence intra-treatment and one-year after stopping anticoagulation.

Methods: Prospective cohort which included all consecutive patients with a first episode of deep vein thrombosis, included in the Institutional Registry from $1 / 6 / 2015$ to $30 / 3 / 2019$, who started anticoagulant treatment. We excluded patients with cancer or having a permanent IVF implantation or informed consent refusal. All patients were evaluated at 30 days pre-suspension of anticoagulation for d-dimer (DD) and ultrasound; at one-year after stopping anticoagulant with ultrasound and thrombophilia evaluation (if they were under 55 years or had no known risk factors for VTE). Outcomes 
were defined as recurrence (progression or new symptomatic event considered only during anticoagulation and once anticoagulation was suspended), bleeding (major and minor) and death.

Results: We included 304 patients, 73\% were female and the median age was 80 years. The highest comorbidities were hypertension (61\%) and dyslipidemia (35\%). Risk factors for VTE: thrombophilia (5\%), immobilization (3\%) and trauma/surgery (2\%). All patients received anticoagulation at the time of the event. The most frequent treatments were acenocoumarol and enoxaparin. The recurrence rate was $5 \%(n=16,95 \% \mathrm{Cl} 3-8 \%)$ during anticoagulation and $4 \%$ ( $n=11,95 \% \mathrm{Cl} 2-6 \%$ ) after suspension. The overall bleeding rate was $13 \%(n=39,95 \% \mathrm{Cl} 9-17 \%)$, and major bleeding 5\%. Regarding associated factors with VTE recurrence, these patients presented higher baseline DD (age adjusted ratio), neutrophils and monocytes, more echographic whole leg affectation and shorter anticoagulation duration, without statistical significance.

Conclusions: In non oncology-patients, even when some parameters were elevated in the patients with VTE recurrence, these differences were not significant, probably because of the small sample size, the low recurrence rate, and the short follow-up period after anticoagulation suspension.

PB1278 | The Accelerated Regimen of Low Dose Recombinant Tissue-type Plasminogen for the Treatment of Acute Pulmonary Embolism: A Case Series from Vietnam

B.H. Hoang ${ }^{1}$; G.P. Do ${ }^{2}$; D.L. Le ${ }^{3}$; T.H.T. Bui ${ }^{4}$; N.T. Bui ${ }^{5}$; M.Q. Nguyen ${ }^{3}$; D.A. Nguyen ${ }^{4}$; M.M Dinh ${ }^{6}$; L.H. Nguyen ${ }^{1}$

${ }^{1}$ Hanoi Medical University Hospital \& Hanoi Medical University, Hanoi, Vietnam; ${ }^{2}$ Hanoi Medical University Hospital, Hanoi, Vietnam; ${ }^{3}$ Thu Duc District Hospital, Ho Chi Minh City, Vietnam; ${ }^{4}$ Hanoi Medical University, Hanoi, Vietnam; ${ }^{5}$ Thu Duc District Hospital - Pham Ngoc Thach Medical School, Ho Chi Minh City, Vietnam; ${ }^{6}$ Royal Prince Alfred Hospital- The University of Sydney, Sydney Medical School, Sydney, Australia

Background: Pulmonary reperfusion in acute pulmonary embolism (PE) by using a fixed full dose regimen of $100 \mathrm{mg}$ of recombinant tissue-type plasminogen activator (rt-PA) over 2 hours or an accelerated low-dose rt-PAregimen has not only been controversial in Vietnam.

Aims: To describe the outcomes of an accelerated low-dose rt-PA regimen for the treatment of high to intermediate mortality risk $\mathrm{PE}$ in Vietnamese patients.

Methods: This was a case series study, the PE patients of high to intermediate mortality risk were enrolled in ninedifferent hospitals across Vietnam. The selected PE patients received an injection alteplase with the dosage of $0.6 \mathrm{mg}$ per kilogram of body weight (maximum of $50 \mathrm{mg}$ ) over 15 minutes. The main outcome of study was the survival rate at hospital discharge and at three months post discharge; in-hospital haemorrhage.
Results: A total of 80 patients were enrolled in this study. There were 48 patients (60\%) with high-risk mortality PE and 32 patients (40\%) classified as intermediate risk mortality PE. Seven patients (8.75\%) had died at the time of hospital discharge and all of these were classified in the high-risk mortality group. All 73 patients who were alive at discharge were alive after 3 months follow up. There were 1 patient $(1.25 \%)$ of major bleeding and $7(8.75 \%)$ of minor bleeding.

Conclusions: Accelerated regimen with $0.6 \mathrm{mg}$ per kilogram of body weight over 15 minutes of alteplase was initiallyeffective and safe on Vietnamese patients with acute pulmonary embolism after 3 months follow up.

Table 1: The characteristic of dead case Patient Sex Age LOS Cardiac arrest due to PE Detail No 1. Female 548 Yes Comatose with multiorgan failure following cardiac arrest No 2. Female 9418 No Initially improved but develiped ventilator associated pneumonia and septic shock at day 3. No 3. Male 691 No Hemodynamic were not improved. Patients delegate did not agree to thrombectomy (patient had pre-existing colon cancer). No 4. Female 752 Yes Comatose after cardiac arrest in spite of returning to spontaneous circulation No 5. Male 591 Yes Refractory shock No 6. Female 631 Yes Cardiac arrest after diagnosis with no ROSC No 7. Male 741 No Lung cancer was found 1 day after using alteplase. Hemodynamic were not improved and treatment withdrawn

Table 2: In-hospital adverse events High- risk mortality PE group $n=48$ Intermediate-high risk PE group $n=32$ Total $N=80$ Probability value Age $60.6 \pm 18.8463 .1 \pm 18.7161 .6 \pm 18.710 .42$ (sign test) Length of stay $9.7 \pm 6.767 .9 \pm 4.918 .9 \pm 5.990 .25$ (sign test) Inhospital bleeding complications Major intracranial bleeding 00 Major bleeding or needing a blood transfusion 01 (blood loss due to menstrual bleeding) Minor bleeding (bleeding due to urethral/stomach catheter placement, bleeding tooth) 2 (4.2\%) 5 (15.6\%) 0.086 (Fisher's exact test) Furthermore, there were 2/80 (2.5\%) patients who developed chronic thromboembolic pulmonary hypertension at three months follow up.

PB1279 | Efficacy and Safety Comparison of DOACs versus Enoxaparin or Heparin with Concomitant Warfarin in Patients with Acute Venous Thromboembolism - A Systematic Review and Meta-analysis

I. Rinaldi ${ }^{1}$; K. Winston ${ }^{2}$; J. Leoni ${ }^{2}$; Y. Samudera ${ }^{2}$

${ }^{1}$ Division of Hematology and Medical Oncology, Department of Internal Medicine, Cipto Mangunkusumo National General Hospital, Faculty of Medicine, Universitas Indonesia, Jakarta, Indonesia; ${ }^{2}$ Research Assistant, Faculty of Medicine, Universitas Indonesia, Jakarta, Indonesia

Background: Venous thromboembolism (VTE) is one of the leading causes of mortality worldwide. The standard treatment and prevention for VTE are often enoxaparin or heparin with concomitant warfarin. Recently DOACs are introduced as potential alternative. Additionally, DOACs have several advantages such as fixed dosing, 
oral route, rapid action, and lack of drug interaction. However, the efficacy and safety of DOACs need to be confirmed versus standard therapy in acute venous thromboembolism patients.

Aims: We aim to conduct a systematic review and meta-analysis to compare the efficacy and safety of DOACs versus enoxaparin or heparin with concomitant warfarin in acute VTE.

Methods: We conducted literature search on PubMed, Scopus, EBSCOhost, and JSTOR for RCTs that compare efficacy and safety of DOACs versus enoxaparin or heparin with concomitant warfarin in acute VTE patients. The measured outcomes were VTE recurrences and major bleeding which were displayed as risk ratio (RR) with $95 \%$ confidence intervals $(\mathrm{Cl})$. Heterogeneity tests were presented in $1^{2}$ value. Meta-statistics were conducted using Review Manager software version 5.4 with random-effects model.

Results: Five RCTs with a total of 13852 patients were identified. Meta-analysis showed that there was no statistically significant difference between DOACs and enoxaparin or heparin with concomitant warfarin in VTE recurrence (RR: 0.87; 95\% Cl: 0.70-1.08; p: $0.21 ; I^{2}: 0 \%$ ) (Figure 1). Major bleeding risk was observed to be lower in DOACs group (RR: 0.46; 95\% Cl: 0.31-0.67; p: <0.0001; $\mathrm{I}^{2}$ : 20\%). All RCTs were assessed to have low risk of bias.

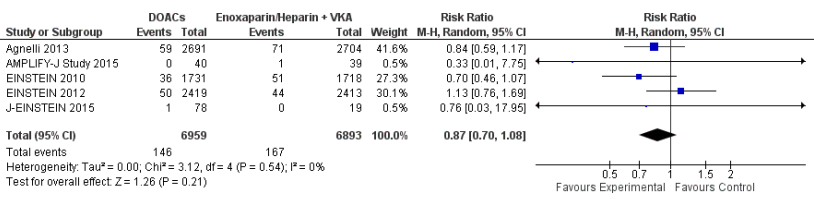

FIGURE 1 Forest plot showing risk ratio of VTE recurrences

Conclusions: There was no difference in efficacy of DOACs versus standard treatment of enoxaparin or heparin with concomitant warfarin in preventing VTE recurrence for acute VTE patients. However, there was a lower risk of major bleeding from the use of DOACs. Hence, DOACs may be considered as an alternative treatment of choice for acute VTE patients.

\section{PB1280 | Experience with Direct Oral Anticoagulants (Doacs)} for the Treatment of Cancer Associated Thrombosis

C. Aguilar ${ }^{1}$; M. Valles ${ }^{2}$; D. Condori ${ }^{2}$; A.B Dueñas ${ }^{1}$; f. Sevil ${ }^{1}$;

C. Dominguez ${ }^{1}$

${ }^{1}$ Department of Haematology, Hospital General Santa Bárbara, Soria, Spain; ${ }^{2}$ Department of Oncology, Hospital General Santa Bárbara, Soria, Spain

Background: The growing use of DOACs for the treatment of cancer associated thrombosis (CAT) as recommended by most international guidelines is gradually changing the treatment paradigm in this setting.
Aims: We report some real-world experience on the efficacy and safety of DOACs for the treatment of CAT in a community hospital in Spain.

Methods: Twenty two patients with CAT were referred from the Oncology to the Haematology Department for management of anticoagulant treatment and agreed to start on a DOAC. All patients had active cancer and underwent regular outpatient follow-up in order to evaluate any episodes of recurrent VTE or bleeding. DOACs are licensed but not reimbursed in Spain for the treatment of venous thromboembolism (VTE).

Results:

TABLE 1 Baseline features of patients assessed

\begin{tabular}{llll} 
Characteristic & $\mathrm{N}(\%)$ & Characteristic & $\mathrm{N}(\%)$ \\
Cancer type & & Khorana score \\
Lung & $7(31.8 \%)$ & 0 & $5(22.7 \%)$ \\
Breast & $1(4.5 \%)$ & 1 & $10(45.5 \%)$ \\
Lymphoma & $2(9 \%)$ & 2 & $6(27.3 \%)$ \\
Colon & $1(4.5 \%)$ & 3 & $1(4.5 \%)$ \\
Gynaecologic & $3(13.5 \%)$ & Type VTE event & \\
Renal & $1(4.5 \%)$ & DVT & $6(27.3 \%)$ \\
Neurinoma & $1(4.5 \%)$ & PE & $9(41 \%)$ \\
Head/neck & $1(4.5 \%)$ & DVT+PE & $3(13.5 \%)$ \\
Prostate & $2(9 \%)$ & Catheter & $3(13.5 \%)$ \\
Urine bladder & $1(4.5 \%)$ & Portal/mesenteric & $1(4.5 \%)$ \\
Brain & $1(4.5 \%)$ & Incidental & $13(59 \%)$ \\
Liver & $1(4.5 \%)$ & DOAC prescribed & \\
Metastatic & $13(59 \%)$ & Rivaroxaban & $20(91 \%)$ \\
& & Edoxaban & $2(9 \%)$ \\
& & Previous VTE & $2(9 \%)$ \\
& & Chemotherapy & $14(63.6 \%)$ \\
\hline
\end{tabular}

Table 1 shows patient characteristics. Male/female ratio was 1. Median age and duration of DOAC therapy were 69 years (range 4987) and 112 days (range 29-740) respectively; 41\% were treated for over 6 months. Twenty one patients had been prescribed a low molecular weight heparin for a median of 3 months (range $0-15$ ) before switching to a DOAC. Two patients $(9 \%)$ showed thrombotic recurrence during follow-up in association with recurrent and/or locally advanced tumor progression.

No major bleeding episodes were reported. Four patients (18.1\%) presented with clinically relevant non-major bleeding (3 cases of haematuria, 1 case of haemoptisis; incidence 1.3 per 100 patientsyear) at a median of 2 months (range 1-4) from DOAC start. Nine patients died during follow-up (41\%) after a median of 88 days of DOAC therapy (range 54-740); in all cases cancer was the cause of death.

Conclusions: Our real-world experience in an unfavourable profile cancer patient setting confirms that DOACs are an efficient and safe for the treatment of CAT even when prescribed for longer periods than those reported in clinical trials. 


\section{PB1281 | Practical Considerations for Treatment of Left} Ventricular Thrombus with Direct-acting Oral Anticoagulants

R. Brazilek; H. Bortz; C. Corallo; J. McFadyen

Alfred Health, Melbourne, Australia

Background: Left ventricular thrombi (LVT) arise when the myocardium demonstrates dyskinesis, often in conjunction with hypercoagulable states. Warfarin is the mainstay of therapy but requires frequent monitoring and has common drug and dietary interactions. Direct-acting Oral Anticoagulants (DOACs) may offer an alternative. There is a paucity of guidance regarding practical elements of DOAC prescription in LVT treatment.

Aims: This study aimed to assess clinician rationale and considerations for off-label DOAC prescription in treatment of LVT, and report on modalities used for follow-up and treatment outcomes, including time until thrombus resolution.

Methods: A retrospective cohort study was conducted of patients with echocardiograph-diagnosed LVT prescribed a DOAC. Collected characteristics included DOAC application justification, follow-up modality, and mean follow-up time.

Results: This study presents 15 off-label applications for 13 individual patients for DOACs to treat LVT. Twelve applications $(80.0 \%)$ were for compliance concerns. One patient was lost to follow-up three were followed-up at external health services, one patient received clinic follow-up only, and one patient was awaiting results at time of writing. No bleeding events were recorded. Of the 7 patients with follow-up imaging, LVT resolution occurred in 6 patients at a mean of 5.6 months ( $95 \% \mathrm{Cl}:-0.5$ to $10.6, n=6$ ).

Conclusions: Patient factors such as compliance concerns due to access to testing facilities, rurality or intravenous drug use history may be appropriate clinical justifications for DOAC use in LVT treatment. Small case numbers and the retrospective nature of the study limit interpretation, but these results support published data that suggest DOACs may have efficacy in LVT treatment in those with compliance concerns. The study highlights the need for a consistent approach to monitoring and patient follow up.

PB1282 | Standardized Direct Oral Anticoagulants Prescription for the Treatment of Acute Deep Vein Thrombosis in the Emergency Department: A Quality Improvement Initiative

C. Simard ${ }^{1}$; L. Poirier-Blanchette ${ }^{1}$; T. Cafaro ${ }^{2}$; R. Kerzner ${ }^{3}$; H. Mantzanis ${ }^{3}$; M. Koolian ${ }^{2}$

${ }^{1}$ McGill University, Faculty of Medicine, Montreal, Canada; ${ }^{2}$ McGill University, Jewish General Hospital, Division of General Internal Medicine, Department of Medicine, Montreal, Canada; ${ }^{3}$ Jewish General Hospital, Department of Pharmacy, Montreal, Canada

Background: Acute deep vein thrombosis (DVT) is a frequently encountered pathology and current guidelines support the use of direct oral anticoagulants (DOAC) as the mainstay of therapy. Studies have demonstrated significant rates of inappropriate DOAC prescriptions in this and other populations. Standardized prescriptions have shown to decrease the incidence of prescription errors in different contexts.

Aims: To determine the impact of a standardized prescription on inappropriate DOAC prescriptions in patients with acute DVT discharged from the Emergency Department (ED).

Methods: We conducted a retrospective study of patients with acute DVT discharged with a DOAC from the ED between December $27^{\text {th }}, 2019$ and December $27^{\text {th }}, 2020$ following the implementation of a standardized DOAC prescription. The impact of the prescription was measured using the absolute risk reduction (ARR) in the proportion of inappropriate prescriptions between pre- and postimplementation periods. Results from a previous audit of DOAC prescription appropriateness were used for the pre-implementation period. Appropriateness was determined using the Medication Appropriateness Index (MAI) with categories: A for appropriate, $B$ for inappropriate with limited clinical significance, and $C$ for inappropriate.

Results: A total of 28 patients discharged from the ED with acute DVT were prescribed a DOAC. Among the prescriptions, 3/28 (10.7\%) were categorized as B compared to $24 / 44$ (54.0\%) preimplementation (ARR 43.3\%), 1/28 (3.6\%) met at least one B and one $\mathrm{C}$ compared to $5 / 44$ (11.3\%) pre-implementation (ARR 7.7\%) and $1 / 28$ (3.6\%) was categorized as C compared to $10 / 44$ (22.7\%) preimplementation (ARR 19.1\%). The proportion of prescriptions that used the standardized prescription was $8 / 28(28.6 \%)$ and increased with time.

Conclusions: A standardized prescription reduces inappropriate DOAC prescriptions in patients with acute DVT. While a secular decrease is expected as clinicians become more familiar with this class of drugs, a standardized prescription likely enhances this effect and offers a promising avenue to improve patient safety outcomes.

\section{PB1283 | Evaluation of the Efficacy and Safety of Enoxaparin} Biosimilar Treatment in Our Center

M.A. Garcia Ruiz ${ }^{1}$; P. Romero Garcia ${ }^{2}$; E. Morente Constantín ${ }^{1}$; M. Jurado Chacon ${ }^{1}$

${ }^{1}$ Hospital Universitario Virgen de las Nieves, Granada, Spain; ${ }^{2}$ Complejo Asistencial de Soria, Soria, Spain

Background: A biosimilar is a medicine of biotechnological origin, produced according to specific requirements established by the European Medicines Agency (EMA) regarding quality, efficacy and safety.

Aims: The main objective is to evaluate the efficacy and safety of using enoxaparin sodium biosimilar (Inhixa $($ ) ) available in our center, for the prophylaxis of venous thromboembolic disease and the treatment of deep vein thrombosis, as well as hemorrhagic or thrombotic complications.

Methods: We evaluated two groups of patients:

1. Patients anticoagulated who were suspended oral anticoagulation. 
- 25 patients. Weight adapted doses/24h, initially, and then, according to anti-Xa levels.

- 50 and 88 years old.

- Indication: 5 arrhythmia, 18 VTE and 2 prosthetic valves.

- Most of the cases presented several associated diagnoses.

2. Anticoagulated patients with VKA who have required bridging therapy at full doses.

- 98 patients. $1.5 \mathrm{mg} / \mathrm{kg} / 24$ h. Prophylactic dose 4,000 IU 12h before the procedure and 6-12 hours after it, depending on the risk of thrombosis/bleeding.

- 21 to 87 years old.

- Indication: 10 arrhythmias, 58 VTE and 30 prosthetic valves.

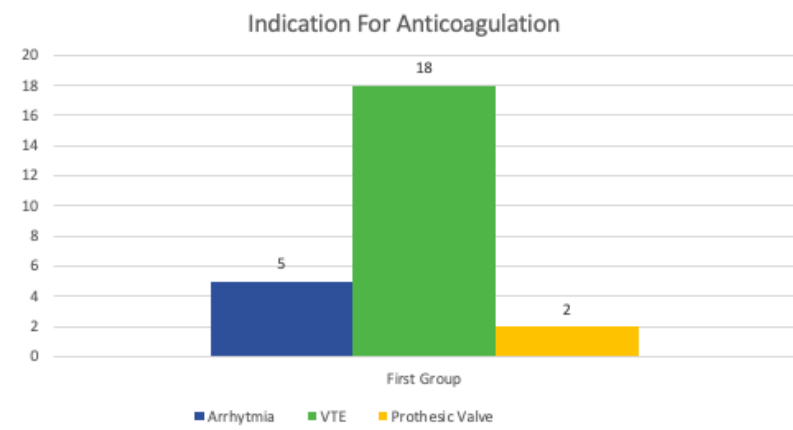

Indication For Anticoagulation

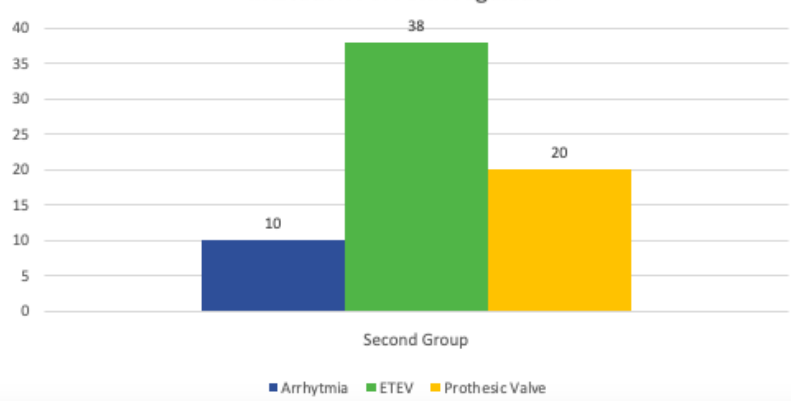

FIGURE 1 First and second group indication for anticoagulation

Results: • In patients who had secondary bleeding to VKA, it totally disappeared with heparin.

- 3 patients had an allergic reaction to heparin.

- No bleeding or thrombotic phenomena.

- 6 hematomas at the puncture sites.

Conclusions: Although these are few cases, the results obtained confirm the efficacy, safety and cost-effectiveness of the continuous use of enoxaparin sodium biosimilar in the prevention of VTE in patients requiring anticoagulation. Biosimilar enoxaparin sodium administered at therapeutic doses in the perioperative period, according to the scheme described, is associated with a low incidence of VTE recurrence and bleeding. Treatment should be personalized according to each patient.

Further studies will provide more confirmatory data. Biosimilar drugs have the potential to offer the National Health System cost savings and expand access to therapeutic innovations.
PB1284 | Thromboembolic disease (VTE) and Inmune Trombocytopenia (ITP): A Case Report

M. R. Lopez; E. Lopez Ansoar; A. Nieto Vazquez; C. Albo Lopez Hospital Alvaro Cunqueiro, Vigo, Spain

Background: Although ITP is a hemorrhagic disease, scientific evidence has increased regarding its role as a thrombophilic risk factor. The thrombopoietin (TPO) agonists were associated with incidence of VTE of 6\%, confirmed in extension studies. Therefore, the absence of International Clinical Guidelines for proper management of VTE in this context stands out,; some articles and National Guidelines guides treatments. Finally, Bemiparin sodium (Rovi ${ }^{\circledR}$ ) subcutaneous once a day in treatment of VTE has shown in some study non-inferiority vs Enoxaparin sodium (every 12 hours), which can facilitate adherence to treatment

Aims: Explain our management of VTE and concomitant ITP with trombocytopenia (grade $4 \mathrm{WHO}$ ).

Methods: Woman, 69 years, admitted from Urgencies with suspicion of ITP (petechiae in lower extremities, oral bleeding and Platelets $4000 / \mathrm{mm}^{3}$ ). She started Dexamethasone 4 days, according to Spanish Guideline (2011), with complete response (CR) on day 9 but after new relapse and fail to respond to Dexamethasone and poliespecific Inmunoglobulins,we started Romiplostim ${ }^{\circledR}$ (3 micrograms / Kg / week) after exclude central thrombocytopenia (Bone Marrow Biopsy). Results: At day 9 of Romiplostim, platelet count of $8000 / \mathrm{mm}^{3}$, she reported pain in left calf;ECOdoppler found "distal popliteal DVT, with floating thrombus in common femoral". Treatment sequence: First, Lower Vein Cava Filter was inserted. When Platelet > 50,000 / $\mathrm{mm}^{3}$, she started Hibor ${ }^{\circledR} 115 \mathrm{IU} / \mathrm{Kg} /$ day, 3 months. Finally, prophylaxis with Hibor ${ }^{\circledR} 3500$ IU / day, 3 months (during treatment with Romiplostim). After CR, prophylactic antiplatelet therapy.

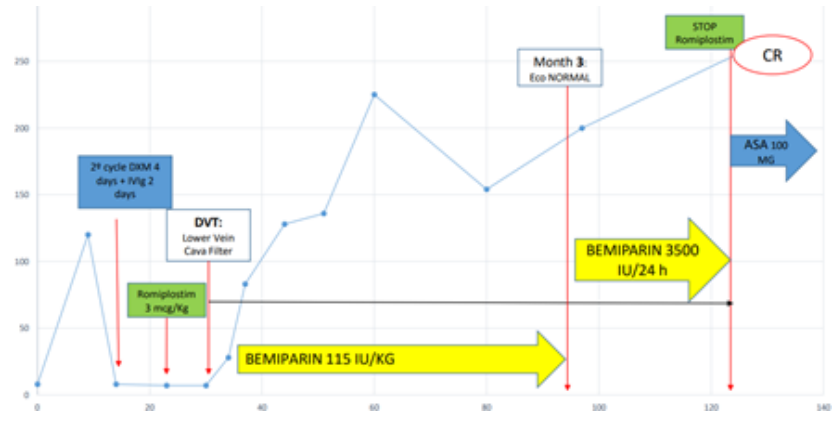

FIGURE 1

Conclusions: Despite being considered a bleeding disorder, there is growing interest in its prothrombotic nature, conditioned in part by the use of treatments with potential intrinsic risk of VTE in patients with risk factors for it; More studies are required to know the real impact of the problems and thus develop specific guidelines for the management of VTE in this context 
P0176 | Efficacy and Safety of Direct Oral Anticoagulants in Morbidly Obese Patients with Acute Venous Thromboembolism: A Meta-analysis

\section{B. Borea; M. Righini}

Geneva University Hospitals, Geneva, Switzerland

Background: Direct oral anticoagulants (DOACs) are approved in most countries for the treatment of venous thromboembolic disease (VTE) and for non-valvular atrial fibrillation (AF). Limited data on anticoagulation dose in patients with morbid obesity are available. The guidelines of the International Society on Thrombosis and Haemostasis (ISTH), recommend the use of DOACs at the usual dose in patients with BMI less or equal than $40 \mathrm{~kg} / \mathrm{m} 2$ and a weight less than $120 \mathrm{~kg}$. Because of the lack of clinical data in patients with a BMI $\geq 40 \mathrm{~kg} / \mathrm{m} 2$ and or a weight above $120 \mathrm{~kg}$ DOACs are not recommended in this population. Aims: We aimed to perform a systematic review and meta-analysis of the use of DOACS in morbid obese patients or with a weight above $120 \mathrm{~kg}$.

Methods: We performed a systematic literature search of papers published between 01.01.2007 and 01.02.2020. All studies including patients aged 18 years and over with a weight $>120 \mathrm{~kg}$ and/or a $\mathrm{BMI} \geq 40 \mathrm{~kg} / \mathrm{m} 2$ requiring therapeutic anticoagulation for VTE or for VTE and/or AF were included. Efficacy outcome was recurrent VTE event and the safety outcome was major and non major but clinically relevant bleeding according to the ISTH definition.

Results: Ten studies were published between 01.01.2016 (date of release of the ISTH guidelines) and 01.02.2020 met the preestablished criteria, for a total of 9,711 patients, of which 5,271 under traditional treatment and 4,440 under DOACs.

Conclusions: In regards of outcomes of efficacy and safety compared to traditional treatment ( $\mathrm{LMWH} / \mathrm{AVK}$ ), the results are reassuring and suggest that DOACs in this population maintain good efficacy with a good safety profile. For the future, randomized controlled studies targeted to this population are necessary to confirm our data.

\section{P0178 | An Insilco Approach to Venous Thromboembolism} (VTE) Using Network Based Medicine with Direct Oral Anticoagulants (DOACs)

A. Khan ${ }^{1,2} ;$ M.J. Khan ${ }^{1}$

${ }^{1}$ Comsats University Islambad, Islamabad, Pakistan; ${ }^{2}$ Shifa College of Medicine Islamabad, Islamabad, Pakistan

Background: Thromboembolism is characterized by aberrant activation of coagulation pathways often results in thrombus or clot formation in the large blood vessels followed by dislodgement from the primary site of formation into the distal site. Thromboembolism is of two types; 1. arterial thromboembolism (ATE) and

2. venous thromboembolism (VTE).

Direct oral anticoagulants (DOACs) are the greatest innovation in the field of anticoagulation therapy. It has several advantages when compared to traditional vitamin antagonists (VKA).
COVID Patients are at greater risk of thromboembolic complications. So, Direct oral anticoagulants such as Rivaroxaban and Apixaban are the two choices for clinicians firstly because it has greater advantages over Vitamin $\mathrm{K}$ antagonists (VKA) as they have broader therapeutic window and secondly because they don't require monitoring hence there is less patients visits for blood sample withdrawal thus minimizing the spread of infection.

Aims: The aim of our study was to decipher the underlying molecular mechanisms in venous thromboembolism (VTE) and establishing the connection of certain miRNAs and treatment with DOACs.

To overcome the potential bleeding episodes in patients who are prone to bleeding epistaxis and intracranial hemorrhages $(\mathrm{ICH})$.

Methods: We obtained a raw datasets of Differentially expressed genes (DEG's) from NCBI in patients with who are diagnosed with Venous Thromboembolisms (VTE) which were then further subjected to statistical analysis based on $\mathrm{p}$-value and Log fold changes. Results: The table 1 shows the molecular docking results of $p$ glycoprotein and the anticoagulant Rivaroxaban(Anti-Xa).

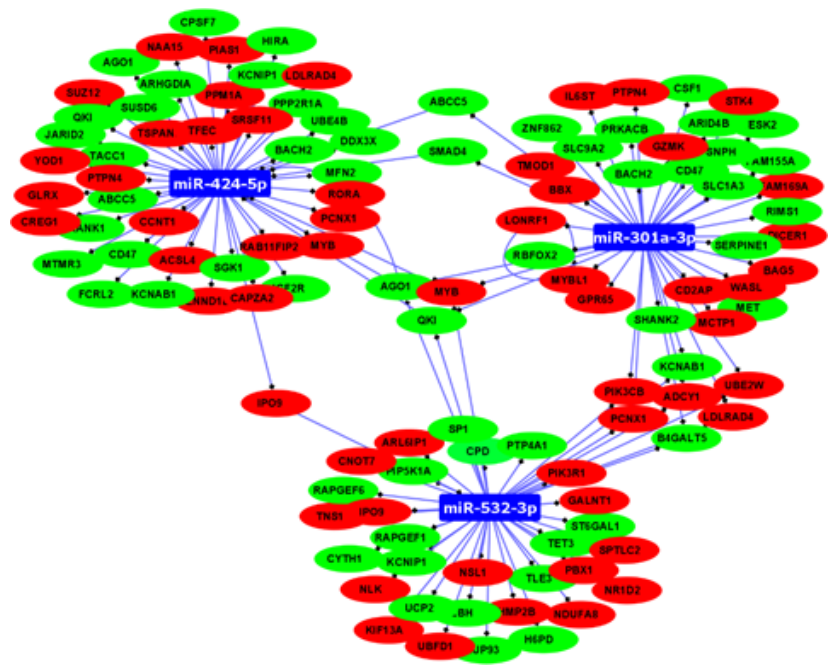

FIGURE 1 The network illustration shows the targeted by each of the 03 miRNAs

Conclusions: Metabolism of the dug in the human body is a complex mechanism that are govern by several mechanisms including dietary intake \& concomitant administration of drugs. There are number of enzymes involved in the biotransformation of a drug into active compounds capable of exerting therapeutic responses. 
P0180 | Management of Anticoagulation Therapy in Patients with Idiopathic Mondor's Disease

G. Sottilotta ${ }^{1}$; M.G. Fava ${ }^{2}$; L. Turano ${ }^{3}$; F. Luise ${ }^{4}$; G.M. Nicolo ${ }^{15}$; V. Oriana ${ }^{1}$; A. Piromalli ${ }^{4}$

${ }^{1}$ Hemophilia Centre - Thrombosis and Hemostasis Service, Great Metropolitan Hospital, Reggio Calabria, Italy; ${ }^{2}$ Unit of Surgery, Great Metropolitan Hospital, Reggio Calabria, Italy; ${ }^{3}$ Unit of Radiology, Great Metropolitan Hospital, Reggio Calabria, Italy; ${ }^{4}$ Analysis Laboratory, Great Metropolitan Hospital, Reggio Calabria, Italy; ${ }^{5}$ Clinical Pathology and Clinical Biochemistry, University of Catania, Catania, Italy

Background: Mondor's disease (MD) in women is characterized by thrombophlebitis of the breast or anterior chest wall. Hormone therapy,breast cancer,thrombophilic conditions,surgery or traumas, have been identified as etiological factors, but mostly it is idiopathic.Anti-inflammatory and anticoagulant drugs are used in the treatment.

Aims: The aim of our study was to analyze the MD cases followed by our Center to underline any etiological correlation with congenital or acquired thrombophilia and to verify the efficacy of the anticoagulant treatment.

Methods: We examined the medical records of two women (52 and 44 aged), with MD, followed by our center because under anticoagulation. They underwent research of thrombophilia, breast cancer and autoimmune diseases.

Results:
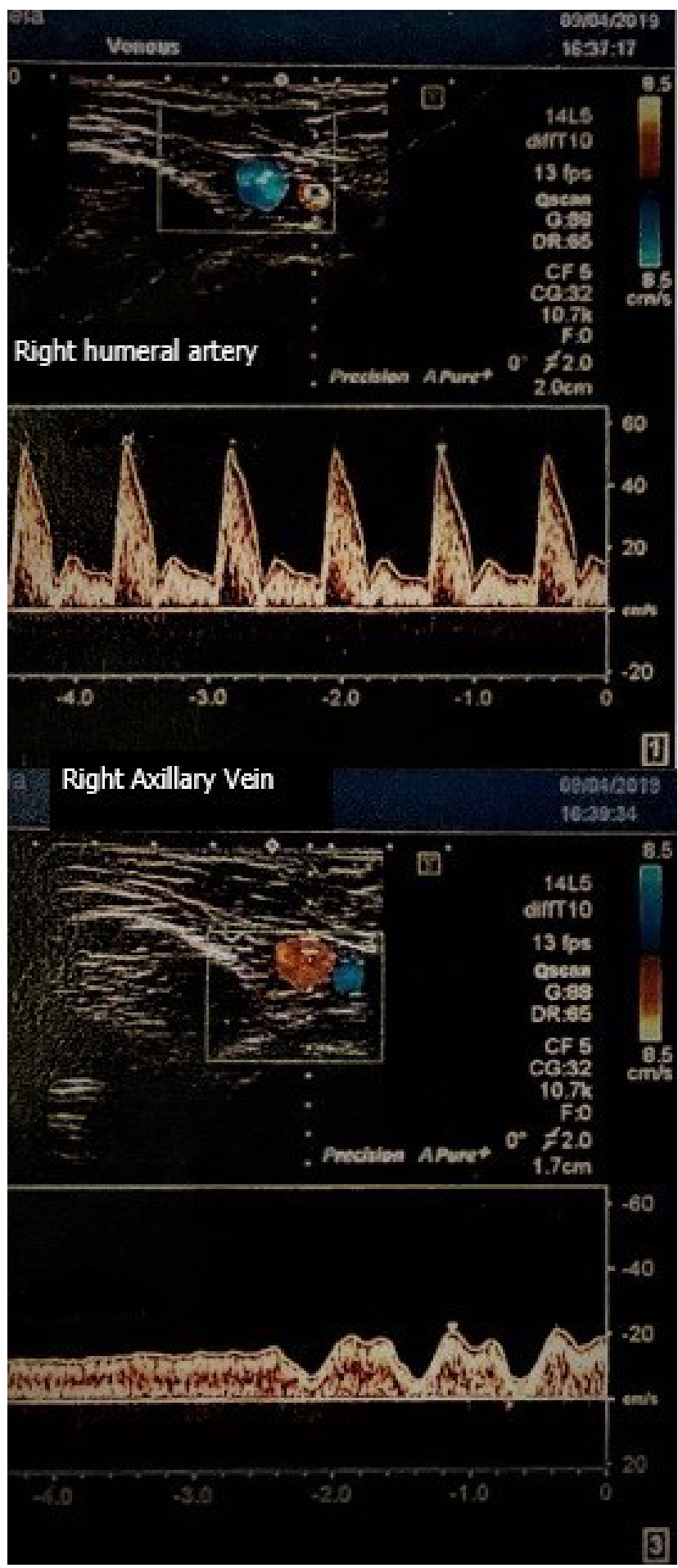

FIGURE 1 Echocolordoppler of right axillary vascular district shows good reperfusion under anticoagulant therapy in the first patient

Patients were subjected to specialist and radiological examinations of breast and were found negative for breast cancer.The first,with a thoraco-mammary right thrombophlebitis,had fibromyalgia and had undergone thyroidectomy for thyroiditis several years before. No thrombophilia was found.She was treated with warfarin for six months,but after 8 months direct oral anticoagulants were resumed for lower limb thrombophlebitis.The second patient had an axillary 
left vein thrombophlebitis;she reported recurrent unexplained abortions and a benign breast fibroadenoma.The research of thrombophilia showed heterozygosis for Factor $\mathrm{V}$ Leiden.She was treated first with enoxaparin for a month,then with sulodexide twice daily for 2 weeks until the symptomatology remitted; now she is on sulodexide daily to prevent thrombosis recurrence.

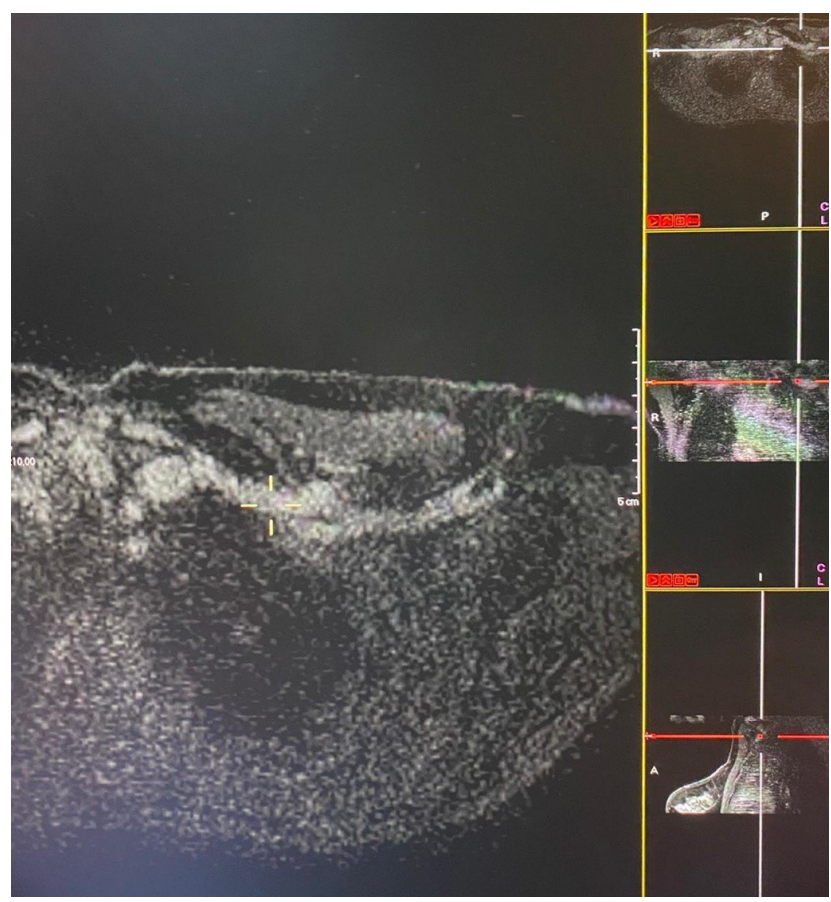

FIGURE 2 Left axillary vein reconstruction in breast Magnetic Resonance Imaging with contrast evidences the stop sign due to thrombosis (second patient)

Conclusions: Our experience, although limited to only two cases,seems to confirm the well known variability of the causes associated with the onset of MD, as well as symptoms and treatments. Although we found a thrombophilic condition in only one patient, in our opinion, the presence of congenital or acquired prothrombotic defects should be always investigated in MD patients for a better choice and duration of the anticoagulant treatment. In any case, periodic follow-up checks with Haemostasis and Breast Specialists are needed for a safe and effective MD management.

P0181 | Long Term Recurrence of Venous Thromboembolism: A Retrospective Case-control Study

G. Turatti; L. Spiezia; M. Marobin; A. Poretto; E. Borella; C. Simion; P. Simioni

University of Padua, Padua, Italy

Background: Venous thromboembolism, (VTE) including both deep vein thrombosis (DVT) and pulmonary embolism (PE), is reported to be the third most frequent cardiovascular disease. People affected need anticoagulant therapy for at least 3 months but often "unprovoked" events tend to remain treated life-long, with a relevant bleeding risk.

\section{Aims: To assess}

i) the risk of recurrence in the long term period (beyond 5 years), and ii) the influence of other factors (presence/discontinuation of therapy, sex, age).

\section{Methods:}

In this retrospective study we collected data from outpatients during follow-up visits at our centre. We compared the risk of recurrence after at least 5 years from the diagnosis of VTE between provoked vs unprovoked events and the Odds Ratio were calculated.

Results: Among 1124 events, 440 (39.1\%) were unprovoked and 684 (60.9\%) were provoked. Recurrence occurred in 57 (\%) patients with an unprovoked event and in 78 (\%) patients with a provoked event with global rate of recurrence in our population of $12.0 \%$ (Odds Ratio (OR) 1.16 (95\% confidence interval 0.8-1.66; $P=0.43$ ). We observed no significant difference in patients with or without extended therapy neither in the overall population (OR 2.19, 95\% confidence interval 0.99-4.83; $P=0.052$ ) nor in the group with an unprovoked event (OR 1.17, 95\% confidence interval 0.47-2.91; $P=0.73$ ).

Conclusions: In our study we found no statistical significance between the risk of long-term recurrence, independently from the etiology of the first event or the presence of a "long-term" therapy.

\section{P0187 | Rare Complications of DOAC Treatment}

M. Hulikova ${ }^{1}$; S. Hulik ${ }^{2}$; J. Hulikova ${ }^{1}$

${ }^{1}$ Center of Hemostasis and Thrombosis, Unilabs Slovakia, Kosice, Slovakia; ${ }^{2}$ University Hospital of L.Pasteur, Kosice, Slovakia

Background: DOACs are effective in preventing and treating VTE. However, in clinical practice, treatment failure (recurrent VTE, postthrombotic syndrome) and unexpected changes in coagulation tests occur. Aims: We present rare complications of DOAC treatment (rivaroxaban, dabigatran, apixaban) in adequately anticoagulated patients: recurrent VTE, post-thrombotic syndrome, thrombocytopenia, coagulation factor deficiency, FVIII inhibitor.

Methods: 18 patients with proximal lower limb thrombosis, pulmonary embolism, adequately anticoagulated; laboratory tests: platelets, PT, APTT, antithrombin, coagulation factors, FVIII inhibitor, endogenous thrombin potential, DOAC concentration.

Results: Recurrence of VTE was observed in two patients, postthrombotic changes were observed in one patient after 6 months of anticoagulant treatment, and recurrent VTE was observed 4 to 32 weeks after cessation of treatment in three patients. In 9 patients we found out a false coagulation factor deficiency, a false FVIII inhibitor, during DOAC treatment, and in three patients, we found DOAC-induced thrombocytopenia.

Conclusions: Therapeutic strategies in such situations are limited. Optimal management of DOAC treatment failure is not clear and the options include the following: dose escalation, switching over to an 
alternative anticoagulant, adding an antiplatelet agents. In clinical practice, it is important to decide whether treatment failure is due to drug (DOAC) or underlying disease. It is important to correctly diagnose recurrence of VTE, to distinguish recurrence from the residual thrombosis, and to correctly interpret the laboratory test results.

\section{WOMEN'S HEALTH}
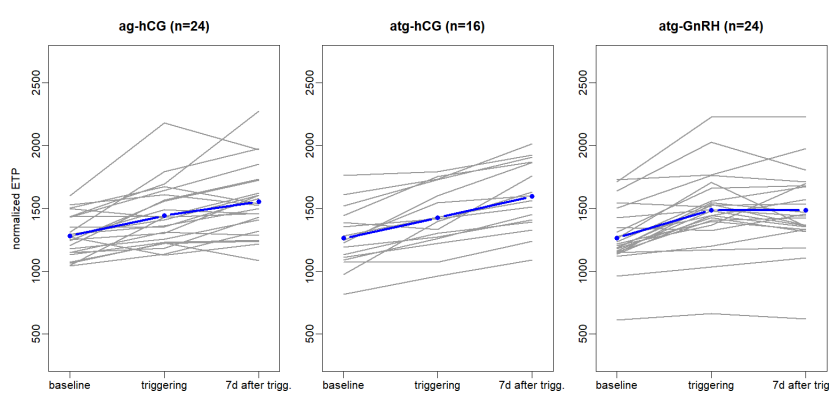

\section{ESTROGENS AND PROGESTINICS}

FIGURE 1 Normalized ETP evolution over time in 3 different ART protocols

Conclusions: The hypercoagulable state was higher and persistent LPB0044 | Prothrombotic Biomarkers during Controlled Ovarian Stimulation for Assisted Reproductive Techniques

J. Hugon-Rodin ${ }^{1}$; A. Casini ${ }^{2}$; J. Bénard ${ }^{2}$; A. Poncet ${ }^{2}$; P. Fontana ${ }^{2}$; N. Vulliemoz ${ }^{3}$; I. Streuli ${ }^{2}$

${ }^{1}$ Hospital Saint Joseph, Paris, France; ${ }^{2}$ University Hospitals of Geneva, Geneva, Switzerland; ${ }^{3}$ University Hospitals of Lausanne, Lausanne, Switzerland

Background: Controlled ovarian stimulation (COS) for assisted reproductive techniques (ART) is associated with a hypercoagulable state and an increased risk of venous thrombosis. The impact of the different ART protocols on coagulation biomarkers is unknown.

Aims: To assess the evolution of coagulation biomarkers throughout and after the ovarian stimulation comparing 3 different ART protocols. Methods: In this observational multicentre cohort study, infertile women undergoing COS for ART in 2017-2019 were included. Written informed consent was obtained and the study was approved by the ethics committees. Our primary outcome was endogenous thrombin potential (ETP) assessed by calibrated automated thrombinography (using $5 \mathrm{pM}$ of tissue factor). ETP was measured before stimulation (baseline), on the day of ovulation triggering (triggering) and seven days after triggering. Three protocols were prescribed according to the standards used: agonist protocol with hCG trigger (ag-hCG); antagonist protocol with hCG trigger (atg-hCG) or GnRH agonist trigger (atg$\mathrm{GnRH}$ ); evolution of ETP was estimated and compared among groups using mixed effects linear regression model.

Results: A total of 64 women, mean aged (SD) 37.8 (2.8), participated to the study: 24 received ag-hCG, 16 atg-hCG, and 24 atg-GnRH. The mean serum estradiol levels in atg-GnRH were statistically higher at trigerring and lower 7days after. Overall, ETP evolution over time was statistically different between groups $(P=0.013)$. If values were similar at baseline and increased at triggering in each group, the greatest difference occurred between triggering and 7days after triggering: while ETP continued to increase in ag-hCG $(+110)$ and atg-hCG (+170), it remained stable in atg-GnRH (+0). Protein $\mathrm{C}$ and protein $\mathrm{S}$ levels were stable, while $\mathrm{D}$-dimers, fibrinogen and factor VIII increased at triggering and 7days after in all groups.

after stimulation in the ag-hCG and atg-hCG groups groups compared to the atg- $\mathrm{GnRH}$ group.

LPB0141 | Platelet Activation and Platelet Indices as Markers for Disease Progression in Women with Breast Cancer

Y. Tera ${ }^{1,2}$; H. Azzam ${ }^{1}$; N. Abousamra ${ }^{1}$; M. Zaki ${ }^{3} ;$ A. Eltantawy ${ }^{4}$; M. Awad ${ }^{4}$; H. Ghoneim¹; M. Othman ${ }^{2}$

${ }^{1}$ Clinical Pathology Department, Faculty of Medicine, Mansoura, Egypt;

${ }^{2}$ Biomedical and Molecular Sciences, Faculty of Medicine, Queen's University, Kingston, Ontario, Canada; ${ }^{3}$ Pathology Department, Faculty of Medicine, Mansoura, Egypt; ${ }^{4}$ Oncology Department, Faculty of Medicine, Mansoura, Egypt

Background: Ex-vivo activation of platelet was reported in women with breast cancer (BC). Mixed evidence from literature exists that mean platelet volume (MPV) is higher in cancer vs benign breast tumors and maybe associated with larger tumors, higher stage and poorer prognosis. Predictive markers are required to identify highrisk patients who may develop metastasis.

Aims: To systematically evaluate platelet activation and platelet volume indices in women with BC in chemo-naïve locally invasive and metastatic disease.

Methods: Patients were recruited from oncology center at our local hospital between 2019 to 2020 following ethics approval. Patient groups included: 80 locally invasive BC patients (stages I,II,III), 20 metastatic (stage IV) and 100 age-matched controls. Platelet activation in response to ADP was assessed by light-transmission aggregometry. Platelet P-selectin (CD62P) expression with and without ADP stimulation was assessed by flowcytometry. Comprehensive analysis of platelet count and all platelet volume indices (PVIs) (MPV, $\mathrm{PDW}, \mathrm{MPV} / \mathrm{P}$ and PDW/P). Data were analyzed in relation to tumor pathology, hormone receptors (ER, PR, HER-2) and proliferation index $\mathrm{Ki}-67$. Regression analyses were conducted for prediction of poor prognosis, tumor aggression and metastatic potential.

Results: There was significant increase in platelet aggregation (MA), CD62P expression, CD62P+ADP, MPV, PDW, MPV/P and PDW/P in the metastatic group compared to the locally invasive group (Table1). Tumor size and grade were significantly correlated with 
ADP-MA, CD62P, CD62P+ADP, MPV, PDW, MPV/P and PDW/P. The area under curve showed $(0.98,0.9,0.97,0.93,0.66,0.7,0.8$, 0.73) for ADP MA, CD-62, CD-62P+ADP, CD-62P Diff., MPV, PDW,
MPV/P, PDW/P) respectively. Univariate regression analysis showed significance for MA, CD62P, CD62P+ADP, CD-62P Diff., PLTs count, MPV, PDW, MPV/P, PDW/P (Table2).

TABLE 1 Comparison between control group and BC patient group in relation to platelet volume indices (PVIs), aggregation and flowcytometry work-up. MPV: mean platelet volume, PDW: platelet distribution width, MPV/P: MPV divided by platelet count. ADP-MA: maximum platelet aggregation with ADP, CD-62P and CD-62P+ADP: P-selectin expression at basal level and with ADP. CD-62P Diff: difference between basal and activated CD-62P

\begin{tabular}{|c|c|c|c|c|}
\hline Parameters / Groups & Control group & Locally invasive $B C$ group & Metastatic BC group & $P$ value \\
\hline Platelets $10^{9} / \mathrm{L}$ Mean \pm SD & $234 \pm 43$ & $11.8 \pm 1.6$ & $10.5 \pm 1.4$ & $\leq 0.001$ \\
\hline MPV fL Mean \pm SD & $10.9 \pm 0.9$ & $14.6 \pm 1.5$ & $16.7 \pm 1.1$ & 0.02 \\
\hline PDW fL Mean \pm SD & $13.9 \pm 2.17$ & $16.1 \pm 3.9$ & $18.4 \pm 5.2$ & 0.044 \\
\hline $\mathrm{MPV} / \mathrm{P} \mathrm{fL} / 10^{9} / \mathrm{L}$ Mean $\pm \mathrm{SD}$ & $0.048 \pm 0.01$ & $1.3 \pm 0.25$ & $1.6 \pm 0.16$ & $\leq 0.001$ \\
\hline $\mathrm{PDW} / \mathrm{P} \mathrm{fL} / 10^{9} / \mathrm{L}$ Mean $\pm \mathrm{SD}$ & $0.067 \pm 0.01$ & $1.43 \pm 0.03$ & $1.77 \pm 0.2$ & 0.044 \\
\hline APD MA $\%$ Mean $\pm S D$ & $54.7 \pm 12.1$ & $73.7 \pm 19$ & $115 \pm 5$ & $\leq 0.001$ \\
\hline $\mathrm{CD}-62 \mathrm{P} \%$ Mean $\pm \mathrm{SD}$ & $2.7 \pm 1.3$ & $3.5 \pm 1.4$ & $6.9 \pm 1.9$ & 0.02 \\
\hline $\mathrm{CD}-62 \mathrm{P}+\mathrm{ADP} \%$ Mean $\pm \mathrm{SD}$ & $23.4 \pm 10$ & $46.8 \pm 17.7$ & $81.5 \pm 5$ & $\leq 0.001$ \\
\hline CD-62P Diff. Mean $\pm S D$ & $20.5 \pm 10$ & $43.5 \pm 18.5$ & $74.5 \pm 5.5$ & 0.001 \\
\hline
\end{tabular}

TABLE 2 Univariate regression analysis for prediction of tumor aggression and metastatic potential of BC. OR: odds ratio, Cl: confidence interval, MPV: mean platelet volume, PDW: platelet distribution width, MPV/P: MPV divided by platelet count. ADP-MA: maximum platelet aggregation with ADP, CD-62P and CD-62P+ADP: P-selectin expression at basal level and with ADP. CD-62P Diff: difference between basal and activated CD-62P

\begin{tabular}{lllll} 
Parameters & P value & OR & Lower $95 \% \mathrm{Cl}$ & $\mathrm{Higher} 95 \% \mathrm{Cl}$ \\
APD MA & $<0.001$ & 1.29 & 1.127 & 1.479 \\
CD-62P & 0.022 & 2.027 & 1.5 & 2.7 \\
CD-62P + ADP & 0.001 & 1.3 & 1.126 & 1.502 \\
CD-62P Diff. & 0.01 & 1.205 & 1.097 & 1.324 \\
Platelets & 0.002 & 0.56 & 0.386 & 0.812 \\
MPV & 0.044 & 1.409 & 0.995 & 1.996 \\
PDW & 0.011 & 0.978 & 0.967 & 0.989 \\
MPV/P & 0.03 & 143.5 & 9.8 & 210.1 \\
PDW/P & 0.003 & 0.04 & 0.01 & 0.095 \\
\hline
\end{tabular}

Conclusions: PVIs (MPV/P and PDW/P) can be used as simple, easily available and low cost predictors for cancer progression and poor prognosis. Also, MA and CD62P+ADP can be regarded significant predictors for metastatic $B C$.

LPB0142 | Association between Non-O Blood Group and the Risk of Venous Thromboembolism due to Oral Contraceptive Therapy

A. Poretto; L. Spiezia; G. Turatti; M. Marobin; E. Borella;

E. Campello; D. Tormene; P. Simioni

University of Padua, Padua, Italy

Background: Several studies report the association between non-O blood group and the risk to develop a venous thromboembolic event (VTE). Nevertheless, the role of non-O blood type and its interactions with inherited thrombophilia (i.e. antithrombin, protein $\mathrm{S}$ and $\mathrm{C}$ deficiencies, factor $\mathrm{V}$ Leiden and prothrombin variant) as possible risk factors for venous thrombosis in women undergoing oral contraceptive therapy (OCT) remain unclear.

Aims: We aimed to retrospectively evaluate the prevalence of non-O blood type and inherited thrombophilia in a large group of women with a venous thromboembolic event due to OCT.

Methods: A group of n.223 women with a personal history of venous thrombosis and/or pulmonary embolism due to OCT assumption, referred to the Medical Department of Padova University Hospital between December 2009 and December 2019, were enrolled. Women under $18 \mathrm{yrs}$ and those with acquired thrombophilia were excluded. Two hundred and twenty-three healthy women underwent OCT for at least six months acted as controls. The Odds Ratios (ORs) and 95\% confidence intervals (Cls) were calculated as an estimate of VTE developing. 
Results: Out of n. 223 enrolled women, n. 168 (75\%) had non-O blood group. The prevalence of non-O blood group was significantly higher in cases than controls $(p<0.001)$. Inherited thrombophilia was observed in n. 86 (39\%) patients. The prevalence of thrombophilia was significantly associated with the risk to develop OCT related VTE ( $p<0.01$ respectively). Moreover, the combination of non-O blood type and inherited thrombophilia was associated with a 4.4fold (CI 95\% 1.98-9.78) increased risk to develop OCT related VTE.

Conclusions: Either non-O blood group or inherited thrombophilia are associated with an increased risk to develop OCT related VTE. The combination of these two conditions further increase this thrombotic risk. Larger studies are needed to confirm our results and to clarify the possible clinical meaning of our findings.

\section{PB1285 | Hormonal Contraception (HC) and Thrombosis:} Identifying the Gaps in Knowledge among Young Woman

$\underline{\text { Y. Deng }}{ }^{1,2}$; S. Tierney ${ }^{2}$; A. Geauvreau ${ }^{1} ;$ M. Velez ${ }^{3}$; M. Othman ${ }^{1,2}$

${ }^{1}$ St Lawrence College, School of Baccalaureate Nursing, Kingston, Canada; ${ }^{2}$ Queen's University, Department of Biomedical and Molecular Sciences, Kingston, Canada; ${ }^{3}$ Queen's University, Department of Obstetrics and Gynecology, Kingston, Canada

Background: Estrogen-based medication is among the risk factors for thrombosis. This research is centred around the knowledge of potential risks in young women of venous thromboembolism (VTE) related to $\mathrm{HC}$. This can be significantly reduced with a proactive knowledge translation intervention. Global health initiatives focus on raising awareness of contraception options and improving understating of side effects, risks and benefits.

Aims: To identify the gaps in knowledge of $\mathrm{HC}$ among young women particularly around thrombosis, to better inform about the risks/ various choices available, and to support prevention programs.

Methods: 681 females aged $18-30$ years in various academic programs in two post-secondary instructions completed an anonymous online survey exploring three sections: demographics, use/type/duration (UD) of HC, and knowledge of risks and benefits associated with $\mathrm{HC}$ including thrombosis. Mann Whitney $\mathrm{U}$ test and Spearman Correlation were used to determine differences in knowledge level about contraceptives across age groups, education levels (EL), and use/type/duration of HC.

Results: Of the 681 participants, 476 were users of HC (264>1 year) and 199 non-HC users. 45\% have post-secondary diplomas or degrees. The knowledge level of $\mathrm{HC}$ risks was associated with duration of use and overall knowledge level of thrombosis and HC. Knowledge level of thrombosis was positively correlated with UD, EL and age. Participants with higher EL had an increased knowledge surrounding thrombosis. Those who have been using $\mathrm{HC}$ for a duration of 5 years or longer had a significantly higher level of knowledge of thrombosis and risks of HC than users with a shorter UD. Finally, the knowledge level of $\mathrm{HC}$ risks is correlated with one another.

TABLE 1 Spearman correlation of knowledge level of HC across age, EL, methods of contraception, UD, use type

\begin{tabular}{|c|c|c|c|c|c|c|c|c|c|}
\hline Variables & $n$ & 8 & 7 & 6 & 5 & 4 & 3 & 2 & 1 \\
\hline 1.Age & 679 & & & & & & & & - \\
\hline 2.Education level & 679 & & & & & & & - & $.00^{* *}$ \\
\hline $\begin{array}{l}\text { 3.Method of } \\
\text { contraception }\end{array}$ & 674 & & & & & & - & .65 & .51 \\
\hline 4.Use duration & 299 & & & & & - & .65 & .09 & $.00^{* *}$ \\
\hline 5.Use type & 267 & & & & - & .99 & .51 & .63 & $.00^{* *}$ \\
\hline $\begin{array}{l}\text { 6.Subscore of knowledge } \\
\text { level }\end{array}$ & 610 & & & - & .13 & $.00^{* *}$ & .31 & $.00^{* *}$ & $.00^{* *}$ \\
\hline $\begin{array}{l}\text { 7.Subscore of Knowledge } \\
\text { level of } \mathrm{HC} \text { risks }\end{array}$ & 602 & & - & $.00^{* *}$ & .25 & $.04^{*}$ & .68 & .44 & .81 \\
\hline 8.Total scores & 622 & - & $.00^{* *}$ & $.00^{* *}$ & .34 & .06 & .27 & .59 & .53 \\
\hline
\end{tabular}

Conclusions: Misconceptions remain among young women concerning the benefits and risks of $\mathrm{HC}$ and targeted educational intervention can address this to better inform young women and to support prevention programs.
PB1286 | A New Pharmacodynamic Test to Evaluate Effects of Oral Contraceptives on Coagulation

P. de Kruijf ${ }^{1}$; S. Naji ${ }^{1}$; C. Krijnen ${ }^{1}$; J. Jespersen ${ }^{2}$; C. Kluft ${ }^{1}$

${ }^{1}$ Good Biomarker Sciences, Leiden, Netherlands; ${ }^{2}$ University of Southern Denmark, Esbjerg, Denmark

Background: The use of combined oral contraceptives has been associated with an increased risk of thromboembolic disease. Current in vitro tests are focusing on one or some sets of multiple factors but are not really pharmacodynamic tests. In this study we present thrombodynamics, 
which is an elegant in vitro analysis that resembles in vivo coagulation starting from a prothrombotic surface and gives insight in the spatial dynamics of the formation of a fibrin clot and thrombin generation.

Aims: Investigate the effect of 6 month treatment of three different types combined oral contraceptives belonging to either the $2^{\text {nd }}$ generation or a 3rd generation COC (Ethinylestradiol $30 \mu \mathrm{g}-$ Desogestrel $150 \mu \mathrm{g}$; or Ethinylestradiol $20 \mu \mathrm{g}$ - Desogestrel 150 $\mu \mathrm{g})$ in healthy woman on fibrin clot growth and thrombin generation. Methods: The coagulation state of blood plasma samples was quantitatively evaluated using a Thrombodynamics Analyzer System T-2 (Hemacore). This system records the fibrin clot light scattering changes in a time-lapse video microscopy mode and records the fluorescence signal during cleavage of synthetic AMC-based substrate by thrombin. Next, numerical parameters are calculated describing the coagulation process. Results: Data show that fibrin clot formation and thrombin generation are increased after 6 months treatment of combined oral contraceptives. Figure 1 shows the size of the clot before and after 6 month treatment; Figure 2 shows the individual changes in thrombin formation in the clot.
A

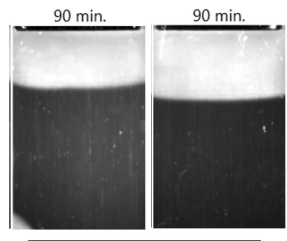

2nd generation (30EELNG)
B

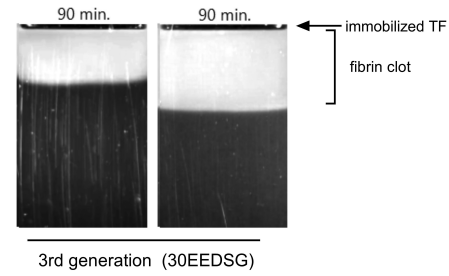

FIGURE 1 Example of images of fibrin clot formed from inmmobilized tissue-factor into thin layer of plasma sample before (left) and after (right) 6 months 2nd (A) or 3rd generation (B) COC treatment

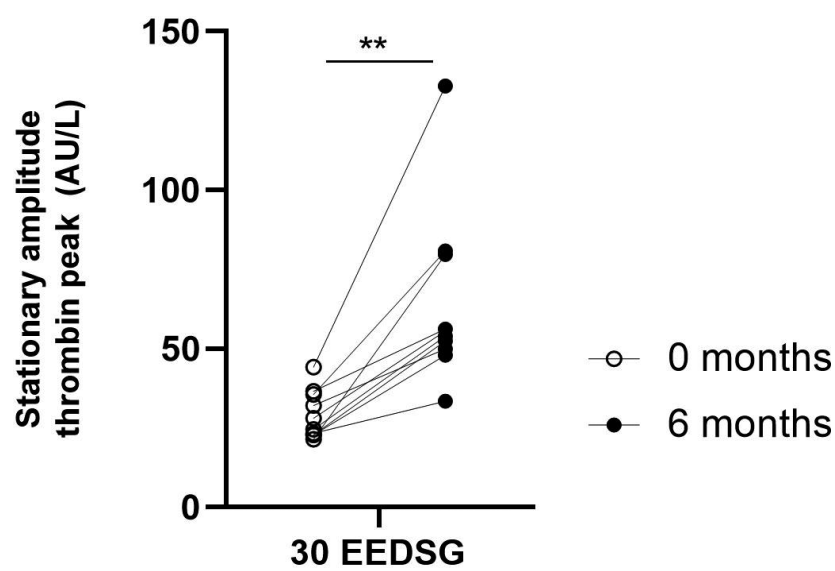

FIGURE 2 The stationary amplitude of thrombin within the clot before and after 6 months COC treatment of 10 subjects

Conclusions: The use of three types of COC (30EELNG, 30EEDSG and 20EEDSG) is involved in the increased risk of thromboembolic disease. Both the fibrin clot size and thrombin generation increased after 6 months treatment of COC. The read outs are consequences of multiple factors unlike reductionistic approaches of other tests.
Furthermore, with thrombodynamics the 'high hemostatic responders' can be identified who may have the highest risk to develop thrombogenic effects while using COCs.

PB1287 | A Major Knowledge Gap in Women's Health: The Combined Effect of Antifibrinolytics and Estrogen on Risk of Thromboembolism - A Scoping Review

D. Meschino ${ }^{1,2}$; D. Lindsay ${ }^{2,3}$; G. Tang ${ }^{2,1,4}$; P. James ${ }^{5,6}$; M. Sholzberg 2,1,4

${ }^{1}$ University of Toronto, Toronto, Canada; ${ }^{2}$ St. Michael's Hospital, Toronto, Canada; ${ }^{3}$ University of Waterloo, Waterloo, Canada; ${ }^{4} \mathrm{Li} \mathrm{Ka}$ Shing Knowledge Institute, Toronto, Canada; ${ }^{5}$ Queen's University, Kingston, Canada; ${ }^{6}$ Kingston Health Sciences Centre, Kingston, Canada

Background: Up to $30 \%$ of reproductive-age women experience heavy menstrual bleeding, negatively affecting quality of life. Estrogen-containing contraceptives and antifibrinolytics are firstline treatments. Despite proven efficacy and safety of these agents, plus the use of antifibrinolytics in other high-estrogen states such as post-partum, prescribers are frequently uncomfortable prescribing them in combination due to theoretical thrombotic risk.

Aims: To systematically evaluate the literature exploring the combined effect of pharmacologic or high physiologic estrogen and antifibrinolytics on thromboembolism risk in reproductive-age women when used for heavy menstrual or post-partum bleeding.

Methods: Medline, EMBASE, CINAHL, and Scopus were searched from inception to July 2020. Included articles' references were screened and a grey literature search was conducted. English language studies exploring the risk of thromboembolism in reproductive-age women prescribed antifibrinolytics alongside estrogen-containing contraceptives or with physiologic high-estrogen states were included. Screening and data abstraction were performed by two independent reviewers (DM, DL) and conflicts were adjudicated by a third reviewer (MS).

Results: A total of 2389 title and abstracts were identified. 33 studies with 27933 participants were extracted for full-text review. 31 studies investigated antifibrinolytic use for post-partum bleeding: 22 randomized controlled trials, 3 retrospective cohort studies, 1 combined prospective-retrospective cohort study, 2 case series, and 3 case reports. Almost all (29/31) studies found no increased thromboembolism risk/rate. However, only 4 studies were powered to make this assessment. The 2 studies describing thromboembolism with post-partum antifibrinolytic use were a case report and case series $(N=18)$, respectively. Only 2 case reports described thromboembolism with concomitant estrogen-containing contraceptives and antifibrinolytic use, both of which reported thromboembolism in women using both agents for $<3$ months.

Conclusions: We found no evidence that antifibrinolytic use in high physiologic or pharmacologic estrogenic states results in higher rates of thromboembolism. Prospective studies are warranted to provide an accurate assessment of risk and inform prescribing practices. 
PB1288 | Heavy Menstrual Bleeding: Knowledge and Practice Assessment among Tunisian Primary Care Physicians

E. Hammami; I. Helali

Medical School of Tunis, Tunis, Tunisia

Background: Heavy Menstrual Bleeding (HMB) is a public health issue. Women with HMB have a reduced quality of life. Nevertheless, it's still an underdiagnosed women's health issue. Hence, physicians' knowledge in this area still needs to be evaluated and improved. Therewithal, Primary care physicians (PMP) play a key role in bleeding disorders' diagnosis including HMB. Yet, little data have been published regarding PMP knowledge about HMB.

Aims: To assess HMB practices and knowledge among Tunisian PCP. Methods: We developed a self-administrated online survey. The latter was composed of nine questions regarding demographics, knowledge, and practices related to the management of HMB. The survey was designed using Survey Monkey. Tunisian PCP were targeted and the survey was distributed using social media. Data were analyzed using the 25 th version of SPSS.

Results: Twenty-one family physicians answered the survey. Among them, $52.38 \%(n=11)$ were family residents while $19.05 \%(n=4)$ were private PCP. Seventeen out of 21 were women and $90.48 \%$ $(n=19)$ had between 25 and 34 years. Less than a half (42.86\%) considered that they had satisfactory knowledge regarding HMB. Less than a half (42.86\%) chose the right answer when asked about the normal duration of menstruations. Only five PCP evaluated HMB using specific charts. Ten out of 21 chose estrogens as a potential treatment for HMB while 10 PCP chose anti-fibrinolytic agents such as tranexamic acid. Eighteen PCP agreed that HMB was a public health issue.

Conclusions: Survey results were partially satisfactory. However, misdiagnosis and therapeutic delays may increase the burden of disease in women with $\mathrm{HMB}$. We advocate the implementation of a training curriculum for PCP that focuses on the management of bleeding disorders including HMB.

\section{PB1289 | Sex-specific Association of Endogenous PCSK9 with} Memory Function in Elderly Subjects at High CV Risk

P. Simeone $^{1}$; F. Vadini ${ }^{2}$; R. Tripaldi ${ }^{1}$; R. Liani ${ }^{1}$; S. Ciotti ${ }^{1}$;

A. Di Castelnuovo ${ }^{3}$; F. Cipollone ${ }^{1} ;$ F. Santilli $^{1}$

${ }^{1}$ Department of Medicine and Aging, and Center for Advanced

Studies and Technology (CAST), Via Luigi Polacchi, Chieti, Italy;

${ }^{2}$ Psychoinfectivology Service, Pescara General Hospital, Pescara, Italy;

${ }^{3}$ Mediterranea Cardiocentro, Naples, Italy

Background: Growing evidence indicates that cognitive decline and cardiovascular diseases (CVDs) share common vascular risk factors. Protease proprotein convertase subtilisin/kexin type 9 (PCSK9) is associated with CV disease risk and has been also involved in neuronal differentiation.
Aims: Evaluate whether in patients at high CV risk cognitive function is related to PCSK9 levels.

Methods: One hundred sixty-six patients ( 67 female) were enrolled. A detailed neuropsychological (NP) assessment was performed PCSK9 levels were measured with ELISA.

Results: Men had significantly higher short-term memory, executive function and praxic and mental representation skills, as reflected by Forward Digit Span (FDS) $(P=0.005)$, Trail Making Test-A (TMT-A) $(P=0.047)$, Clock Drawing Test (0.016). Endogenous PCSK9 levels were higher in female $(P=0.005)$. On linear regression analysis PCSK9 predicts short term memory only in females (Beta $=0.408$, $P=0.001$ ), with an interaction between PCSK9 and gender ( $P=0.004$ for interaction PCSK9 by sex). The association of PCSK9 with FDS in female was partially mediated by waist circumference (mediation effect 8.5\%).

Conclusions: In patients at high CV risk short term memory was directly related to PCSK9 levels only in women, revealing the relevance of sex in this relationship. The association of PCSK9 with memory function may be mediated, at least in part, by waist circumference.

\section{PB1290 | Transferability of the ETP-based APC Resistance Assay on the ST Genesia System}

L. Morimont $^{1,2}$; M. Didembourg ${ }^{1}$; J.-M. Dogné ${ }^{1}$; A. Carlo ${ }^{3}$;

J. Douxfils ${ }^{1,2}$

${ }^{1}$ University of Namur, Department of Pharmacy, Namur Thrombosis and Hemostasis Center (NTHC), Namur Research Institute for Life Sciences (NARILIS), Namur, Belgium; ${ }^{2}$ QUALIblood S.A., Namur, Belgium; ${ }^{3}$ Diagnostica Stago, Asnières-sur-Seine, France

Background: Regulatory bodies recommend assessing the endogenous thrombin potential (ETP)-based activated protein C (APC) resistance assay during the development of combined oral contraceptives (COC) in women. In 2019, this assay was validated on the Calibrated Automated Thrombogram (CAT) device using commercially available reagent to ensure batch-to-batch traceability, and reproducibility of the method over time. However, in view of its screening potential, its implementation in clinical routine is essential. Aims: This study aimed at implementing the ETP-based APC resistance assay on the automated ST Genesia system (Stago, France), using reagent STG-ThromboScreen -TM (TS -TM) (Stago, France) with exogenous APC added (Stago, France).

Methods: Healthy pooled plasma (HPP) constituted of 20 healthy individuals ( 10 males and 10 women not using COC, not carrier of FV Leiden or G20210A mutation) was used to define APC concentration leading to $90 \%$ of inhibition of the ETP. Dose-response curves were performed on two different batches of TS -TM. The intra- $(N=5)$ and inter-run $(N=10)$ variability were assessed on three samples: STGRefPlasma TS, STG-QualiTest Norm TS and STG-QualiTest High TS. STG-QualiTest Low TS was not assessed as thrombin generation was completely inhibited in presence of APC. Twenty samples from healthy individuals were analyzed to define reference ranges. 
Results: The APC concentration [IC95\%] leading to $90 \%$ of inhibition of the ETP, on both batches of TS -TM was defined at $652 \mathrm{mU} / \mathrm{mL}$ [534-805 mU/mL]. Intra- and inter-run variability showed standard deviations (SD) values below $2.5 \%$ and $4.0 \%$ respectively, regardless of the tested samples. The mean inhibition percentage $[ \pm \mathrm{SD}]$ of the 20 healthy subjects was $90.6 \%[ \pm 7.4 \%]$

Conclusions: The ETP-based APC resistance assay has been successfully transferred onto the ST Genesia, a routine clinical device for evaluating thrombin generation. Confirmation of promising results on thrombogenicity identification for all COC users is still needed before this test can become commercially available.

\section{PB1291 | Thrombin Generation Test and Use of Combined Oral Contraceptive}

M. Sousa; R.C. Figueiredo Duarte; M.d.G. Carvalho; I.d.F. Oliveira Silva; D. Serenini; L. Dusse

Faculty of Pharmacy-Federal University of Minas Gerais, Brazil, Belo Horizonte, Brazil

Background: The use of combined oral contraceptives (COC) has been associated with hypercoagulability. Through the analysis of the parameters of the thrombin generation test (TGT) it is possible to gain a better understanding of the relationship between use of hormones and hemostatic disorders.

Aims: To evaluate the profile of thrombin generation in women using or not combined oral contraceptives.

Methods: The study included young, healthy, university Brazilian women aged between 18 and 30 years using combined oral contraceptives (COC, $n=20$ ) or not (non-COC, $n=20$ ), of whom a sample of blood was collected. TGT was performed according to the CAT method, with low (LTF) and High (HTF) Tissue Factor, with subsequent analysis of the parameters Lagtime, Time to peak, Peak, Endogen Thrombin Potential (ETP).

Results: An increase in thrombin generation was observed in women using COC compared to women not using this drug, with greater discrimination between the two groups when the TGT was performed with LTF, as follows: Peak (COC x non- COC: $456 \pm 141 \times 279 \pm 122$ $\mathrm{nM} ; \mathrm{P}=0.001$ ), and ETP (COC $\times$ non-COC: $2006.0 \pm 431.0 \times$ $1443.0 \pm 350.3$ nM.min; $p<0.001$ ) and Lagtime [COC $x$ non-COC: 2.3 (1.1) x 3.5 (3.0) $\mathrm{min}$.; $P=0.019$ ] and Time to peak [COC $\times$ nonCOC: 4.3 (1.2) x 6.5 (6.0) min; $P=0.022$ ]. Confirming previous findings, this increase in thrombin generation is related to the use of $\mathrm{OC}$ that triggers an increase in procoagulants (factors VII, VIII, X and fibrinogen) and a decrease in natural anticoagulants (AT, PC, PS and TFPI).

Conclusions: Our results reinforce the importance of seeking the standardization and validation of TGT, a tool with great potential for application to assess hypercoagulable states in women using COC, among numerous diseases and other conditions related to hemostatic disorders.
PB1292 | The Influence of Thrombophilic Anamnesis and Thrombotic Complications Heredity

N. Vorobyeva $^{1,2} ;$ D. Gamyrkina ${ }^{2}$

${ }^{1}$ National Medical Research Center for Hematology (Northern Branch) Ministry of Health of Russia, Arckhangelsk, Russian Federation;

${ }^{2}$ Northern State Medical University Ministry of Health of Russia, Arckhangelsk, Russian Federation

Background: Antithrombin III (AT III) provides at least $75-80 \%$ of the anticoagulant activity of plasma. AT III deficiency is the serious risk factor of the progression of venous and, much less frequently, arterial thrombosis. Combined oral contraceptives (COC) is one of the most effective and commonly used methods of preventing unwanted pregnancies in the world.

Aims: To study the influence of thrombophilic history and thrombotic complications heredity on the AT III level in COC recipients.

Methods: A cross-clinical laboratory study was carried out among 100 women at the average age of 29.0 (23.3-35.0) years. Depending on the COC reception mode, women were divided into two groups $(n=50$ in each group). The progressive activity of AT III was discovered and a questionnaire was conducted to determine the presence of a thrombophilic history and thrombotic complications heredity. The method of linear regression was used to identify independent factors, having impact on the level of AT III.

Results: Both analyzes, onefactor and multifactor, showed a statistically significant effect of aggravated anamnesis and thrombotic complications heredity ( $P=0.022$ and $P=0.027$, respectively) on the decrease in AT III activity. The results indicate the importance of collecting a thrombophilic history before prescribing COCs.

According to the results of this study taking $\mathrm{COC}$ does not have a significant effect on the level of AT III.

Conclusions: This conclusion can be explained, firstly, by the fact that almost all women participating in the study were prescribed COCs by a gynecologist, and secondly, all participants in our study used COCs with a maximum dose of EE of $30 \mu \mathrm{g}$.

\section{P0194 | Venous Thromboembolism in a 30 Year Old Female:} Synergic Effect of Oral Contraceptive and Elevated Factor VIII?

F. Laouan Brem ${ }^{1}$; F. Mouedder ${ }^{1}$; C. Toutai ${ }^{1} ;$ N. Ismaili ${ }^{1,2}$; N. El Ouafi ${ }^{1,2}$

${ }^{1}$ Department of Cardiology, Mohammed VI University Hospital, Faculty of Medicine and Pharmacy of Oujda, Mohammed First University, Oujda, Morocco; ${ }^{2}$ Epidemiological Laboratory of Clinical Research and Public Health, Faculty of Medicine and Pharmacy of Oujda, Mohammed First University, Oujda, MAR, OUJDA, Morocco

Background: Birth pills control, used for contraceptive or noncontraceptive benefits, are associated with a high risk of venous thromboembolism generally higher with those containing desogestrel or gestodene rather than levonorgestrel, and also during the first year of use. 
Aims: We report the case of a 30 -year-old woman previously healthy who used oral contraception discontinuously for 9 years and diagnosed with pulmonary embolism and the right hypogastric vein thrombosis. The investigation found a high level of factor VIII.

Methods: A 30-year-old woman with an active lifestyle presented to the emergency department for progressive chest pain and dyspnea that had begun four days earlier. The physical examination revealed no abnormalities. The electrocardiogram (EKG) was normal as well as the laboratory findings except a high D-dimer level at $6500 \mathrm{ng} / \mathrm{mL}$. The transthoracic echocardiography (TTE) showed a dilated right ventricular with a normal systolic function. The computed tomography showed bilateral pulmonary embolism and right hypogastric vein thrombosis. She reported using an oral contraceptive discontinuously, for thirteen years as the treatment of polycystic ovary syndrome. The thrombophilia study showing normal results except a high level of FVIII. The patient underwent anticoagulation therapy with Acenocoumarol.

Results: Many previous studies have described several alterations in circulating coagulation blood factors such as Factor VIII in OCP users. Furthermore, an elevated thrombotic risk due to OCP use is reported in women with an elevated factor VIII and both factors have synergistic effects. However, there is no evidence that the interaction between a high factor VIII and the use of birth pills control will lead to an excess of thrombotic events.

Conclusions: With regards to the possible joint risk of VTE in patients with inherited or acquired thrombophilia under $\mathrm{OCP}$, we suggest that physicians should identify patients at a high risk of thrombophilia and tailor the laboratory testing, before starting on OCP.

\section{PREGNANCY AND PREGNANCY COMPLICATIONS}

LPB0045 | Principles of Care for Women and Girls with Inherited Bleeding Disorders

K. van Galen ${ }^{1}$; M. Lavin ${ }^{2}$; N. Skouw-Rasmussen ${ }^{3}$; D. Noone ${ }^{3}$; D. Pollard ${ }^{4}$; K. Khair ${ }^{5}$; K. Gomez ${ }^{6}$; K. Fischer ${ }^{1}$; E. van Loon; C. Bagot ${ }^{8}$; P. Elfvinge ${ }^{9}$; R. D'Oiron ${ }^{10} ;$ R. Kadir ${ }^{11}$

${ }^{1}$ Van Creveldkliniek, University Medical Center Utrecht, Utrecht University, Utrecht, Netherlands; ${ }^{2}$ Irish Centre for Vascular Biology, School of Pharmacy and Biomedical Sciences, RCSI, Dublin, Ireland; ${ }^{3}$ European Haemophilia Consortium, Brussels, Belgium; ${ }^{4}$ Dormandy Haemophilia \& Thrombosis Centre, Royal Free London NHS Foundation Trust, London, United Kingdom; ${ }^{5}$ Heamnet Foundation, London, United Kingdom; ${ }^{6}$ Haemophilia Centre and Thrombosis Unit Haematologist, Royal Free London, London, United Kingdom; ${ }^{7}$ UZ Leuven, Leuven, Belgium; ${ }^{8}$ Glasgow Royal Infirmary, Glasgow, United Kingdom; ${ }^{9}$ Karolinska University, Stockholm, Netherlands; ${ }^{10}$ APHP Paris Saclay, Hôpital Bicêtre, Inserm U 1176, Le Kremlin Bicêtre, Paris, France; ${ }^{11}$ Royal Free Foundation Hospital and Institute for Women's Health, University College London, London, United Kingdom

Background: Despite increasing awareness of the issues faced by women and girls with inherited bleeding disorders (WGBD) standards of care are lacking, with disparities in access to diagnosis and treatment for WGBD across the world.

Aims: To define generalizable principles of care $(\mathrm{PoC})$ in an iterative process, involving relevant stakeholders, with the eventual goal to optimize diagnosis and management for WGBD.

Methods: Supported by the European Association for Haemophilia and Allied Disorders and following four multidisciplinary meetings of health care providers (HCPs) experienced in WGBD care and European Haemophilia Consortium representatives, combined with broad patient and HCP consultations in the European haemophilia community, practical PoC were formulated to promote standardization of care for WGBD within Haemophilia Treatment/Comprehensive Care Centres (HTCs).

Results: Ten PoC for WGBD were developed (Table 1) which stress the importance and benefits of a centralized, multidisciplinary, comprehensive, family-centred approach to support and manage WGBD at all life stages. These principles emphasise the right to equitable access and quality of care for all people with bleeding disorders, irrespective of gender.

TABLE 110 Principles of Care for Women and Girls with inherited bleeding disorders

1 Equitable access and quality of care for all individuals with bleeding disorders, irrespective of gender

2 Timely and accurate diagnosis of bleeding disorders in women and girls

3 Awareness of the additional challenges faced by WGBD throughout life

4 Provision of comprehensive care in a family centred approach 
5 Inclusion of a dedicated obstetrician and gynaecologist in the multidisciplinary team

6 Education of WGBD and their families regarding the menstrual cycle and management

7 Early recognition and optimal management of heavy menstrual bleeding

8 Provision of pre-conception counselling and access to prenatal diagnostics

9 Provision of a patient-centred comprehensive management plan throughout pregnancy and the post-partum period

10

Involvement of WGBD in registries, clinical research and innovation

Conclusions: Ten PoC for WGBD were defined after an iterative process among appropriate stakeholders in Europe. They can serve as a benchmark for diagnosis and comprehensive multidisciplinary management of WGBD, and improve awareness of their unique challenges. They provide a framework to guide HTCs in providing equitable care for all WGBD, both in their own services and other healthcare settings. Implementation of and adherence to these principles is expected to positively impact the health, wellbeing and quality of life for WGBD.

LPB0046 | The Impact of the ABO Blood Group on Postpartum Haemorrhage Risk among Women with Thrombocytopenia

S. Arcudi ${ }^{1}$; A. Ronchi ${ }^{2}$; M. Capecchi ${ }^{3}$; M.W. Ossola ${ }^{2}$; I. Mancini ${ }^{1}$; A.M. Marconi ${ }^{4}$; G. Podda ${ }^{5}$; A. Artoni ${ }^{6}$

${ }^{1}$ Fondazione IRCCS Ca' Granda Ospedale Maggiore Policlinico, Angelo Bianchi Bonomi Hemophilia and Thrombosis Center, Università degli Studi di Milano, Department of Pathophysiology and Transplantation, Milan, Italy; ${ }^{2}$ Department of Obstetrics and Gynecology, Fondazione IRCCS Ca' Granda Ospedale Maggiore Policlinico, Milan, Italy;
${ }^{3}$ Fondazione IRCCS Ca' Granda Ospedale Maggiore Policlinico, Angelo Bianchi Bonomi Hemophilia and Thrombosis Center, Università degli Studi di Milano, Department of Biomedical Sciences for Health, Milan, Italy; ${ }^{4}$ Department of Obstetrics and Gynecology, ASST Santi Paolo e Carlo, Università degli Studi di Milano, Milan, Italy; ${ }^{5}$ Medicina III, ASST Santi Paolo e Carlo, Dipartimento di Scienze della Salute, Università degli Studi di Milano, Milan, Italy; ${ }^{6}$ Fondazione IRCCS Ca' Granda Ospedale Maggiore Policlinico, Angelo Bianchi Bonomi Hemophilia and Thrombosis Center, Milan, Italy

Background: Previous literature investigating the effect of the blood group on the development of postpartum haemorrhage (PPH) is controversial. It is known that $\mathrm{O}$ blood group subjects carry lower levels of Von Willebrand Factor (VWF) and consequently lower levels of factor VIII, but its effect on the risk of PPH in case of thrombocytopenia during delivery is not known.

Aims: To define whether blood group $O$ may strengthen the risk of $\mathrm{PPH}$ in thrombocytopenic women.

Methods: We performed a multicentre retrospective study. We enrolled consecutive women undergoing vaginal delivery or caesarean section with moderate/severe thrombocytopenia, without prophylactic platelet transfusions and without congenital thrombocytopenia or an already known immune thrombocytopenia (ITP). Exclusion criteria were the presence of a congenital bleeding disorder, ongoing anticoagulant therapy or the presence of cancer. Women with more than $150.000 / \mu \mathrm{L}$ platelets at delivery were selected as controls and matched for age, type of birth and ethnicity. Blood group was determined in each participant. Odds ratios (ORs) with their $95 \%$ confidence intervals $(95 \% \mathrm{Cl})$ were calculated as risk estimates. The analyses were repeated after stratifying for the O/non-O blood group.

Results:

TABLE 1 Demographic, obstetrical and laboratory characteristics of the study population. Comparison between thrombocytopenic and nonthrombocytopenic women is shown. Dichotomous variables are expressed as numbers and percentages; continuous variables as median and interquartile range (IQR). Statistical analysis has been performed using chi-square test for dichotomous variables and Student's t-test for continuous variables. PLT, platelet count; ITP, immune thrombocytopenia; HELLP syndrome, Hemolysis elevated liver enzymes and low platelets count syndrome; $\mathrm{PPH}$, postpartum haemorrhage; $P, P$-value

\begin{tabular}{|c|c|c|c|c|}
\hline & & Platelets $<100.000 / \mu \mathrm{L}$ & Platelets $>150.000 / \mu \mathrm{L}$ & $P$ \\
\hline \multirow[t]{10}{*}{ Blood group } & $\mathrm{O}$ & $44(47 \%)$ & $39(40 \%)$ & $P=0.46$ \\
\hline & non-O & $50(53 \%)$ & $55(60 \%)$ & \\
\hline & Platelets $/ \mu \mathrm{L}$ & 90000 (79000-97000) & $229000(198000-260000)$ & $<0.01$ \\
\hline & Haemoglobin g/dl & $11.4(10.1-12.3)$ & $11.4(10.8-12.1)$ & $P=0.15$ \\
\hline & Fibrinogen mg/dl & $429(374-479)$ & $463(402-524)$ & $<0.01$ \\
\hline & Blood loss (ml) & $500(300-1000)$ & $300(200-500)$ & $<0.01$ \\
\hline & $\begin{array}{l}\text { Red Blood Cell } \\
\text { Transfusions }\end{array}$ & $15(16 \%)$ & $1(1 \%)$ & $<0.01$ \\
\hline & Peripartum hysterectomy & $1(1 \%)$ & 0 & - \\
\hline & Deaths & 0 & 0 & - \\
\hline & PPH (number, percentage) & $37(37 \%)$ & $10(10 \%)$ & $<0.01$ \\
\hline
\end{tabular}


Ninety-four thrombocytopenic women and 94 controls were enrolled in the study. The rate of PPH was significantly higher in thrombocytopenic women than in controls ( $37 \%$ vs $10 \%, P<0.001$ ); a higher risk of PPH was observed in the thrombocytopenic group when compared to the control group (OR $5.47 ; 95 \% \mathrm{Cl} 2.4-12.4$ ).
When we stratified the patients into $\mathrm{O}$ and non-O blood groups carriers, we found that carrying blood group $\mathrm{O}$ confers a higher risk of developing PPH in thrombocytopenic women (OR 12.7; 95\% Cl 2.955.3) than in healthy controls (OR 3.2; 95\% Cl 1.1-9.5).

\section{Conclusions:}

TABLE 2 Analyses of postpartum haemorrhage risk expressed in the entire cohort of patients and then stratified for O/non-O blood group. Crude OR, crude Odds Ratio; OR adj 1, crude OR adjusted for matching factors and confounders (age, ethnicity, mode of delivery); OR adj 2 , crude OR adjusted for age, ethnicity, mode of delivery and other risk factors for PPH (nulliparity, placental disorders, labour induction, gestational age < 32 weeks, fetal macrosomia); Ref, reference; Ter, tertile; p, p-value; PLT, platelets; PPH, postpartum haemorrhage

\begin{tabular}{|c|c|c|c|c|c|c|c|c|}
\hline & PPH & no PPH & $\begin{array}{l}\text { crude } \\
\text { OR( } 95 \% \mathrm{Cl})\end{array}$ & $P$-value & $\begin{array}{l}\text { ORadj } 1 \\
(95 \% \mathrm{Cl})\end{array}$ & $P$-value & $\begin{array}{l}\text { ORadj } 2 \\
(95 \% \mathrm{Cl})\end{array}$ & $P$ value \\
\hline Thrombocytopenic & 35 & 59 & & & & & & \\
\hline Healthy controls & 10 & 84 & $\begin{array}{l}4.98 \\
\quad(2.3-10.8)\end{array}$ & $<0.01$ & $5.47(2.4-12.4)$ & $<0.01$ & $4.5(1.9-10.8)$ & $<0.01$ \\
\hline Thrombocytopenic $O$ & 20 & 24 & & & & & & \\
\hline non-O & 15 & 35 & $7.3(2.2-24.0)$ & $<0.01$ & $12.7(2.9-55.3)$ & $<0.01$ & $13.3(2.2-82.2)$ & $<0.01$ \\
\hline Healthy controls $\mathrm{O}$ & 4 & 35 & & & & & & \\
\hline non-O & 6 & 49 & $3.5(1.2-9.9)$ & 0.01 & $3.2(1.1-9.5)$ & 0.03 & $2.7(0.8-8.7)$ & 0.09 \\
\hline Total & 94 & 94 & & & & & & \\
\hline
\end{tabular}

Our study shows that the blood group O phenotype is a strong risk factor for PPH if associated with a platelet count below 100.000/ $\mu \mathrm{L}$ at delivery.

LPB0047 | Management of Peripartum Anticoagulation in Women with Venous Thromboembolism: An International Survey of Clinical Practice

C. Simard ${ }^{1}$; I. Malhamé ${ }^{2}$ E. Rey ${ }^{3}$; M.P. Carson ${ }^{4}$; V. Tagalakis ${ }^{5}$ ${ }^{1}$ McGill University, Faculty of Medicine, Montreal, Canada; ${ }^{2}$ McGill University, McGill University Healthcare Center, Division of General Internal Medicine, Department of Medicine, Research Institute of the McGill University Health Center, Montreal, Canada; ${ }^{3}$ Université de Montreal, Departments of Medicine and Obstetrics and Gynaecology, Montreal, Canada; ${ }^{4}$ Jersey Shore University Medical Center and Seton Hall Hackensack-Meridian School of Medicine, Nutley, United States; ${ }^{5}$ McGill University, Centre for Clinical Epidemiology of the Lady Davis Institute for Medical Research, Jewish General Hospital, Division of General Internal Medicine, Department of Medicine, Montreal, Canada

Background: Pregnancy associated venous thromboembolism (VTE) is an important cause of maternal morbidity and mortality. In most cases, treatment consists of weight adjusted low-molecular-weightheparin (WA LMWH). While guidelines exist regarding the antepartum management of VTE, guidance is lacking with regards to the management of anticoagulation at the time of delivery.

Aims: To describe physician practices in the peripartum management of anticoagulation in women with VTE.

Methods: An electronic survey, active between September $15^{\text {th }}$ and December $15^{\text {th }}, 2020$, was created and validated by content experts. The target sample was members of organizations including the Canadian Venous Thromboembolism and Outcomes Research Network (CanVECTOR), Thrombosis Canada, the North American Society of Obstetric Medicine (NASOM), the International Society of Obstetric Medicine (ISOM), and the Canadian Society of Internal Medicine (CSIM). Descriptive analyses were performed.

Results: Survey respondents were General Internists (54/96, 56.3\%), Hematologists (21/96, 21.9\%), Obstetricians (6/96, 6.3\%) and other specialists $(15 / 96,15.6 \%)$. For the management of a VTE in the first trimester, physicians opted to: continue WA LMWH until planned induction and omit the dose the day prior (46/96, 47.9\%), switch to twice daily WA LMWH dosing at 36 weeks and omit the dose the evening prior 42/96, 43.8\%), continue once daily WA LMWH and bridge with intravenous heparin $(4 / 96,4.2 \%)$ or had other management strategies (4/96, 4.2\%). In the management of a VTE in the third trimester, physicians opted to: continue once daily WA LMWH and omit one dose prior to planned induction (29/96, 30.2\%), switch to twice daily dosing at 36 weeks $(37 / 96,38.5 \%)$ or bridge with intravenous heparin (30/96, 31.3\%).

Conclusions: The survey highlights a wide variability of practice in the management of peripartum anticoagulation. Larger scale studies are needed to evaluate bleeding and thrombotic risks associated with different anticoagulation strategies at the time of delivery. 
LPB0093 | An Overall Evaluation of the Adjusted Version of Global Antiphospholipid Syndrome Score (aGAPSS) on Primary Obstetric Antiphospholipid Syndrome (POAPS) Patients

G. de Larrañaga ${ }^{1}$; F. Aranda ${ }^{1}$; S. Perés Wingeyer ${ }^{1} ;$ S. Udry ${ }^{1,2}$; S. Morales Perez ${ }^{3}$; C. Belizna ${ }^{4}$; J. Alijotas-Reig ${ }^{5}$; E. EsteveValverde $^{3}$; D.S. Fernández Romero ${ }^{2}$; J. Latino ${ }^{2}$ ${ }^{1}$ Hospital of Infectious Diseases F. J. Muñiz, CABA, Argentina; ${ }^{2}$ Acute Hospital Dr. Carlos G. Durand, CABA, Argentina; ${ }^{3}$ Althaia Healthcare University Network of Manresa, Barcelona, Spain; ${ }^{4}$ University Hospital Angers, Angers, France; ${ }^{5}$ Valld'Hebron University Hospital, Barcelona, Spain

Background: About 20\% of POAPS patients, even under conventional treatment (CT) during pregnancy, still are at a high risk of suffering from obstetric morbidity.

Aims: To evaluate the clinical utility of aGAPSS to assess the risk of a new obstetric event on POAPS patients under $\mathrm{CT}$ during pregnancy. Methods: 107 pregnancies from women with POAPS treated with CT were retrospectively evaluated [median age: 31 years; (28-36)].
CT included low dose aspirin and a prophylactic low molecular weight heparin/day. At the time of diagnosis, clinical and laboratory variables were evaluated and the aGAPSS was calculated. Treatment failure was defined as any of the following obstetric outcomes: early or late miscarriage, fetal loss, early severe pre-eclampsia and/or fetal growth restriction.

Results: Among the 107 pregnancies that were followed up, in 91 (85.1\%) live births were achieved while 16 (14.9\%) resulted in pregnancy loss: 12 (11.2\%) miscarriages and 4 (3.7\%) fetal losses. Fetal growth restriction in 7 (6.5\%) and early severe pre-eclampsia in 4 (3.7\%) pregnancies. Thus, CT failed in 23 pregnancies (21.5\%).

The presence of triple positivity for antiphospholipid antibodies (aPL) $[\mathrm{OR}=8.410(95 \% \mathrm{Cl}: 2.732-26.210), P<0.001]$, and an aGAPSS $\geq 7$ [OR $=3.664$ (95\% Cl: 1.407-9.541), $P=0.008$ ] were associated with a higher chance of treatment failure. However, after multivariate analysis, only the triple positivity for aPL $[O R=8.462(95 \% \mathrm{Cl}$ : 2.732-26.210); $P<0.0001]$ was found to be a strong risk factor independently associated with treatment failure.

TABLE 1 Pregnancy outcomes on POAPS patients with CT according to the aGAPSS and other risk factors

\begin{tabular}{llll} 
APS Risk Factors & Conventional treatment failure & & $P$ \\
& No $(\boldsymbol{n}=84)$ & Yes $(\boldsymbol{n}=23)$ & $8.7( \pm 4.37)$ \\
aGAPSS value & $5.8( \pm 2.99)$ & $43.5 \%(10 / 23)$ & $P<0.001$ \\
Triple positivity of aPL & $8.3 \%(7 / 84)$ & $56.6 \%(13 / 23)$ & $P=0.01$ \\
aGAPSS $\geq 7$ & $26.2 \%(22 / 84)$ & & $N S$ \\
Cardiovascular Risk Factors: & & $4.3 \%(1 / 23)$ & $N S$ \\
$\quad$ Hyperlipidemia & $4.8 \%(4 / 84)$ & $13.0 \%(3 / 23)$ & $N S$ \\
$\quad$ Arterial hypertension & $8.3 \%(7 / 84)$ & $17.4 \%(4 / 23)$ & NS \\
$\quad$ Smoking habit & $7.1 \%(6 / 84)$ & $13.0 \%(3 / 23)$ & \\
$\quad$ Obesity & $8.3 \%(7 / 84)$ & & \\
\hline
\end{tabular}

Conclusions: A high aGAPSS ( $\geq 7$ ) would not be an independent risk factor for CT failure. In fact, the triple positivity of aPL would actually be an indicator of poorer response to $\mathrm{CT}$ and worse prognosis. Arterial hypertension and hyperlipidemia might have a significantly lesser contribution than triple positivity of aPL to the aGAPSS thus decreasing its potential as a prognostic marker.
LPB0095 | Bleeding Complications in Women with Venous Thromboembolism during Pregnancy: A Systematic Review of the Literature

\footnotetext{
C. Simard ${ }^{1}$; I. Malhamé ${ }^{2}$; A. Douros ${ }^{3}$; K.B. Filion ${ }^{3}$; V. Tagalakis ${ }^{4}$

${ }^{1}$ McGill University, Faculty of Medicine, Montreal, Canada; ${ }^{2}$ McGill University, McGill University Healthcare Center, Division of General Internal Medicine, Department of Medicine, Research Institute of the McGill University Health Center, Montreal, Canada; ${ }^{3}$ McGill University, Centre for Clinical Epidemiology, Lady Davis Institute, Montreal, Canada; ${ }^{4}$ McGill University, Centre for Clinical Epidemiology of the Lady Davis Institute for Medical Research, Jewish General Hospital, Division of General Internal Medicine, Department of Medicine, Montreal, Canada
}

Background: Pregnant women are at higher risk of venous thromboembolism (VTE), which represents an important cause of maternal 
morbidity and mortality. Estimates of bleeding associated with anticoagulation in patients with VTE during pregnancy are not well described.

Aims: To describe the frequency of major bleeding and postpartum hemorrhage in women receiving therapeutic anticoagulation for pregnancy associated VTE by means of a systematic review of the literature.

Methods: An electronic search was conducted from database inception to January 21, 2021 using Medline, Embase, Scopus and Web of Science. Key words related to anticoagulation including "heparin", "low molecular weight heparin" and key words related to bleeding including "postpartum", "antepartum" or "peripartum" and "hemorrhage" were used. There was no language or geographic location restriction. Included studies (1) described women treated for an acute pregnancy associated VTE, (2) receiving therapeutic anticoagulation and (3) a defined bleeding outcome was reported. Two independent reviewers extracted the data using predefined criteria, and clinical bleeding outcomes were collected.

Results: Of 1636 deduplicated references identified, seven studies including a total of 2338 women receiving therapeutic anticoagulation for VTE were included. Four studies were retrospective. Bleeding definitions varied between studies. Frequency of bleeding ranged between $1.41 \%$ and $8.40 \%$ and postpartum hemorrhage between $1.90 \%$ and $30.0 \%$. Three of the eight studies included control groups, two of which found no significant difference in the risk of bleeding between groups, while the third found a significantly increased bleeding risk.

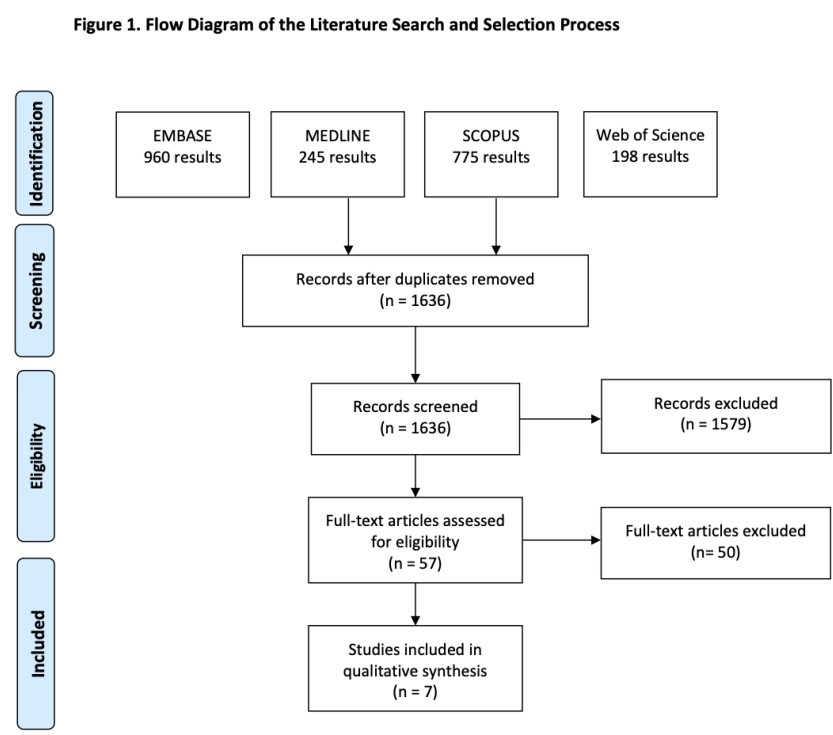

From: Moher D, Liberati A, Tetzlaff J, Altman DG, The PRISMA Group (2009). Preferred Reporting Hems for Systematic Reviews and MetaFor more information, visit www.prisma-statement.org.

FIGURE 1 Flow Diagram of the Literature Search and Selection Process

TABLE 1 Basic Characteristics and Bleeding Results of Included Studies

\begin{tabular}{|c|c|c|c|c|}
\hline Study & Study Design & $\begin{array}{l}\text { Population } \\
\text { Size (n) }\end{array}$ & $\begin{array}{l}\text { Control } \\
\text { Group } \\
\text { (n) }\end{array}$ & Bleeding Definition \\
\hline $\begin{array}{l}\text { Blanco- } \\
\text { Molina } \\
\text { et al. }\end{array}$ & $\begin{array}{c}\text { Prospective } \\
\text { cohort }\end{array}$ & 136 & NA & $\begin{array}{l}\text { Major bleeding: overt and associate } \\
\text { with decrease in hemoglobin } \\
>20 \mathrm{~g} / \mathrm{L},>2 \text { units of blood or } \\
\text { retroperitoneal/intracranial } \\
\text { bleeds. }\end{array}$ \\
\hline Chan et al. & $\begin{array}{l}\text { Retrospective } \\
\text { cohort }\end{array}$ & 60 & NA & ISTH definition of major bleeding*. \\
\hline Chen et al. & $\begin{array}{l}\text { Systematic } \\
\text { review and } \\
\text { meta- } \\
\text { analysis }\end{array}$ & 746 & $3296^{* *}$ & Variable. \\
\hline $\begin{array}{l}\text { Côté- } \\
\text { Poirier } \\
\text { et al. }\end{array}$ & $\begin{array}{l}\text { Retrospective } \\
\text { cohort }\end{array}$ & 232 & NA & $\begin{array}{l}\text { Major hemorrhagic complication: } \\
\text { requiring surgery, hospital } \\
\text { readmission, admission to } \\
\text { ICU, red blood cell transfusion } \\
\text { or fluid resuscitation of }>1 \mathrm{~L} \\
\text { or crystalloids (for bleeding } \\
\text { concern). }\end{array}$ \\
\hline
\end{tabular}

Bleeding Result

4/136 (2.9\%) had major bleeding 


\begin{tabular}{|c|c|c|c|c|c|}
\hline \multirow[b]{2}{*}{ Study } & \multirow[b]{2}{*}{ Study Design } & \multirow[b]{2}{*}{$\begin{array}{l}\text { Population } \\
\text { Size (n) }\end{array}$} & \multirow[b]{2}{*}{$\begin{array}{l}\text { Control } \\
\text { Group } \\
\text { (n) }\end{array}$} & \multirow[b]{2}{*}{ Bleeding Definition } & \multirow[b]{2}{*}{ Bleeding Result } \\
\hline & & & & & \\
\hline Knol et al. & $\begin{array}{c}\text { Retrospective } \\
\text { cohort }\end{array}$ & 88 & 352 & $\begin{array}{l}\text { VD: } \mathrm{PPH}>500 \mathrm{~mL} \text {, severe PPH } \\
>1000 \mathrm{~mL} \\
\text { CS: } \mathrm{PPH}>1000 \mathrm{~mL}\end{array}$ & $\begin{array}{l}\text { In VD, } \mathrm{PPH} \text { occurred in } 30 \% \text { vs. } 18 \% \text { in } \\
\text { treatment vs. control group (OR } 1.19, \\
95 \% \mathrm{Cl} 1.1-3.5, \mathrm{P}=0.029 \text { ) and risk of } \\
\text { severe } \mathrm{PPH} 5.6 \% \text { vs. } 5.0 \% \text { (OR } 1.1,95 \% \\
\mathrm{Cl} 0.4-3.6) . \text { In CS, risk of PPH was } 12 \% \\
\text { vs. } 4 \% \text { (OR } 2.9,95 \mathrm{Cl} 0.5-19.4 \text { ). }\end{array}$ \\
\hline $\begin{array}{l}\text { Romualdi } \\
\text { et al. }\end{array}$ & $\begin{array}{l}\text { Systematic } \\
\text { review and } \\
\text { meta- } \\
\text { analysis }\end{array}$ & 981 & NA & Variable. & $\begin{array}{l}\text { The weight mean incidence of major } \\
\text { bleeding was } 1.41 \%(95 \% \mathrm{Cl} 0.60- \\
2.41 \%) \text { antenatally and } 1.90 \%(95 \% \mathrm{Cl} \\
0.80-3.60 \%) \text { during the first } 24 \mathrm{~h} \text { after } \\
\text { delivery. }\end{array}$ \\
\hline $\begin{array}{r}\text { Roshani } \\
\text { et al. }\end{array}$ & $\begin{array}{l}\text { Retrospective } \\
\text { cohort }\end{array}$ & 95 & 524 & $\begin{array}{l}\mathrm{PPH}>500 \mathrm{~mL} \text {, severe } \mathrm{PPH}>1000 \\
\quad \mathrm{~mL}\end{array}$ & $\begin{array}{l}\text { PPH occurred in } 18 \% \text { in treatment group } \\
\text { vs. } 22 \% \text { in controls (RR for PPH } 0.8 \text {, } \\
95 \% \mathrm{Cl} 0.5-1.4 \text { ). Incidence of severe } \\
\text { PPH was the same in both group (6\%) } \\
\text { (RR } 1.295 \% \mathrm{Cl} 0.5-2.9 \text { ). }\end{array}$ \\
\hline
\end{tabular}

*Major bleeding ISTH definition: fatal, in critical area (intracranial, intra-spinal, retroperitoneal), bleeding causing drop > $20 \mathrm{~g} / \mathrm{L}$ in hemoglobin level or transfusion of 2 or more units of blood.

**The control group received prophylactic anticoagulation.

Abbreviations: $\mathrm{NA}=$ non-available, ISTH = International Society on Thrombosis and Haemostasis, CRNMB = clinically relevant non-major bleeding, $\mathrm{Cl}=$ confidence interval, $\mathrm{OR}=$ odds ratio, $\mathrm{ICU}=$ intensive care unit, $\mathrm{CS}=$ cesarian delivery, $\mathrm{VD}=$ vaginal delivery, $\mathrm{PPH}=$ postpartum hemorrhage, $\mathrm{RR}=$ relative risk.

Conclusions: Among pregnant women anticoagulated for VTE, reported bleeding frequency is variable. The ability to make inference is limited by the observational nature of studies, small number of patients and heterogeneity of bleeding definitions. Large scale prospective studies with standard bleeding definitions are needed to provide accurate bleeding estimates and anticipate the healthcare needs of pregnant women with VTE.

LPB0143 | Is standard dose thromboprophylaxis (TP) as recommended by the Royal College of Obstetricians and Gynaecologists (RCOG) adequate in pregnancy for women with prior venous thrombo-embolism (VTE)?

E. Treharne ${ }^{1}$; A. Alexander²; E. Jackson²; B. Myers ${ }^{3}$

${ }^{1}$ University of Leicester Medical School, Leicester, United Kingdom;

${ }^{2}$ The University of Leicester Medical School, Leicester, United Kingdom;

${ }^{3}$ University Hospitals of Leicester, Leicester, United Kingdom

Background: Pregnancy-associated VTE is associated with a high morbidity rate, with pulmonary embolism (PE) a leading cause of maternal deaths. RCOG guidelines on prevention of VTE were updated in the guideline document "Reducing the Risk of Venous Thromboembolism during Pregnancy and the Puerperium" in 2015 Aims: Our aim, in this retrospective study, was to evaluate the utility of these guidelines in preventing recurrence of VTE in pregnancy. Methods: We identified 290 pregnancies from our local data-base, 2015-2019, in whom a past VTE was recorded in 190. For each record, we documented women's characteristics and assessed whether appropriate thromboprophylaxis (TP) was used as recommended and if this prevented recurrence

Results: Appropriate weight-related thromboprophylaxis with Dalteparin had been used in all cases. Four cases had breakthrough VTEs (2.11\%). Of these, one case had very poor compliance, a second had severe thrombotic tendency having antiphospholipid syndrome and Factor $V$ Leiden heterozygosity. The third had a recurrence early in her 10th pregnancy at the time of confirmed pregnancy; therefore before TP was commenced. She later had post-partum thrombophlebitis despite recommended TP, although her compliance was poor. The final case had no extra risks to explain thromboprophylactic failure. Excluding cases 1 and 3, as these did not constitute TP failure, the frequency of recurrence was low (1\%). Conclusions: Frequency of VTE recurrence was low, in keeping with the New Zealand study, Cox et al, who reported $1.2 \%$ recurrence rate on TP with Enoxaparin. The Netherland study, van Lennep et al, using Nadroparin had 5.5\% recurrence rate, but all cases were in 'high-risk' women, as in our 2nd case. Although we use low molecular weight heparins interchangeably there are differences in their half-lives and other characteristics which could also impact on recurrence risk. We conclude that for the vast majority of cases standard weight-adjusted TP is adequate for prevention of PA-VTE 
LPB0144 | Thrombocytopenia in Pregnancy: Identification and Management at a Reference Centre in Pakistan

M. Borhany; M. Abid; S. Zafar; T. Shamsi

National Institute of Blood Diseases, Karachi, Pakistan

Background: Thrombocytopenia is an important finding encountered during pregnancy, which is second only to anemia. It is diagnosed in approximately $5-6 \%$ of pregnancies. Increased platelet turn-over or dilutional effect of increased blood volume during pregnancy may be responsible for thrombocytopenia.

Aims: The study aimed to evaluate the causes of thrombocytopenia in pregnancy and its management along with the outcome.

Methods: The study aimed to evaluate the causes of thrombocytopenia in pregnancy and its management along with the outcome.

Results: A total of 130 pregnant women with thrombocytopenia were enrolled, with the mean age being $27.3 \pm 4.64$ years. Mean platelet counts at baseline were $48.0 \pm 24$. Main clinical manifestations at baseline included: anemia $65.9 \%$, bruises $23.25 \%$, and edema $9.3 \%$. Causes of thrombocytopenia were: gestational thrombocytopenia (GT) 65 (50\%), acute fatty liver 4 (3.1\%), pre-eclampsia in 10 (7.7\%) and eclampsia 6 (4.6\%). Causes not specific to pregnancy include 24 (18.4\%) cases of ITP, hepatitis $C$ and nutritional deficiency was reported in 8 (6.1\%) patients each. 17 (70.8\%) ITP patients received treatment with steroids with complete response in $64.4 \%$ of patients as presented in Table 1. Maternal outcomes in patients of thrombocytopenia as displayed in Table 2. Overall, 35 (27\%) women with bleeding symptoms and platelet counts $<50 \times 10^{9} / \mathrm{L}$ received platelet transfusions.

TABLE 1 Response to treatment in ITP patients

\begin{tabular}{llll} 
& COMPLETE & PARTIAL & NO. \\
TREATMENT & RESPONSE & RESPONSE & RESPONSE \\
Prednisolone (oral) & $5(29.4 \%)$ & $2(11.7 \%)$ & $1(5.8 \%)$ \\
Methylprednisolone (IV) & $2(11.7 \%)$ & $1(5.8 \%)$ & 0 \\
Dexamethasone & $1(5.8 \%)$ & 0 & 0 \\
IVIG & $1(5.8 \%)$ & 0 & 0 \\
Prednisolone + IVIG & $2(11.7 \%)$ & $1(5.8 \%)$ & $1(5.8 \%)$ \\
\hline
\end{tabular}

TABLE 2 Maternal outcomes in patients of thrombocytopenia

\begin{tabular}{|c|c|c|}
\hline OUTCOMES & $\begin{array}{l}\text { SPECIFIC TO } \\
\text { PREGNANCY }\end{array}$ & $\begin{array}{l}\text { NOT SPECIFIC } \\
\text { TO PREGNANCY }\end{array}$ \\
\hline Antepartum bleeding & $3(2.3 \%)$ & $5(3.8 \%)$ \\
\hline Postpartum bleeding & $10(7.7 \%)$ & $4(3 \%)$ \\
\hline Normal delivery & $56(43 \%)$ & $12(9.2 \%)$ \\
\hline C-Section & $49(37.6 \%)$ & $10(7.7 \%)$ \\
\hline Maternal death & 0 & 0 \\
\hline Abortion & 2 (1.5\%) & $1(0.7 \%)$ \\
\hline $\begin{array}{l}\text { Neonatal } \\
\quad \text { thrombocytopenia }\end{array}$ & 0 & $2(1.5 \%)$ \\
\hline Pre term & $3(2.3 \%)$ & $1(0.7 \%)$ \\
\hline Fetal death & 0 & 0 \\
\hline
\end{tabular}

Conclusions: The study shows that pre-eclampsia and eclampsia are serious conditions with high risk for complications, while GT is a benign and the most common cause of thrombocytopenia which requires no active treatment.The other causes are in between and require individualized management.

\section{PB1293 | Thromboprophylaxis in High-risk Obese Pregnant Women: How Much Is Enough?}

A. Rodríguez Alén ${ }^{1}$; M. De la Torre De la Paz ${ }^{1}$; N. Rollón Simón ${ }^{1}$; S. Daza Pozo ${ }^{1}$; M.O Abío Calvete ${ }^{1}$; L. Parrilla Navamuel ${ }^{1}$; G. Figaredo García-Mina; M. Jiménez Sánchez ${ }^{2}$; O. Rodríguez Gómez²; A. García López²; S. Moreno Ramírez; K.G. Albiño Salazar ${ }^{1}$; J. Cuesta Tovar ${ }^{1}$ ${ }^{1}$ Hospital Universitario de Toledo, Servicio de Hematología y Hemoterapia, Toledo, Spain; ${ }^{2}$ Hospital Universitario de Toledo, Servicio de Obstetricia y Ginecología, Toledo, Spain

Background: Venous thromboembolism (VTE) is a major cause of death and morbidity in pregnant women. Obesity is a wellrecognized risk factor in this setting, but data about which of these women should receive thromboprophylaxis, and the optimal low molecular weight heparin (LMWH) dosage are scarce.

Aims: To evaluate the optimal thromboprophylaxis regimen and pregnancy outcomes in high-risk obese women.

Methods: We performed a retrospective analysis of all obese pregnant women $\left(\mathrm{BMI}>30 \mathrm{~kg} / \mathrm{m}^{2}\right)$ referred to our hematology dedicated clinic for thromboprophylaxis assessment between 01/05/2015 and 01/05/2020. Demographics, risk factors, antithrombotic treatment, bleeding and thrombotic events and pregnancy outcomes were collected from the electronic patient record.

Results: 71 pregnancies (66 women) were included. Mean age was 35 years (18-47) and weight was 94 kg on average (62-155). Risk factors are shown in table 1.

TABLE 1 Risk factors (other than obesity)

$\begin{array}{ll}\text { Risk factors } & n(\%) \\ \text { Age>35 } & 33(46.5) \\ \text { Thrombophilia (hereditary or acquired) } & 28(39.4) \\ \text { Smoking } & 21(29.6) \\ \text { Previous VTE } & 17(23.9) \\ \text { Medical comorbidities } & 18(25.4) \\ \text { IVF/ART } & 14(19.7) \\ \text { Multiple pregnancy } & 8(11.3) \\ \text { Family history of VTE } & 7(9.8) \\ \text { Parity } \geq 3 & 4(5.6)\end{array}$

Anti-Xa levels were performed at least once every quarter in all but two pregnancies, and LMWH (enoxaparin) was prescribed to reach an anti-Xa peak level of $0.3-0.4 \mathrm{IU} / \mathrm{mL}$. Enoxaparin mean dose was $80 \mathrm{mg}$ once daily. 55\% were also treated with antiplatelet agents. Bleeding 
was reported in 3 patients, only one severe, needing transfusion. Two patients suffered from superficial venous thrombosis (one of them before thromboprophylaxis was started). Cesarean section was performed in $45.6 \%$ of the deliveries. $92.6 \%$ of the women received neuraxial analgesia (all of them uneventful). Postpartum prophylaxis was prescribed for most women, with a mean length of 27 days (Table 2).

TABLE 2 Pregnancy complications and outcomes

\begin{tabular}{ll} 
Pregnancy complications & $\mathbf{n}(\%)$ \\
Miscarriages (first trimester) & $3(4.4 \%)$ \\
Pre-eclampsia & $8(11.8 \%)$ \\
Preterm birth (<37 weeks) & $6(8.8 \%)$ \\
Intrauterine growth retardation & $3(4.4 \%)$ \\
Thrombosis (superficials) & $2(2.9 \%)$ \\
Bleeding & \\
$\quad$ Minor & $2(2.9 \%)$ \\
$\quad$ Major & $1(1.5 \%)$ \\
Local allergic reactions & $2(2.9 \%)$ \\
Cesarean section & $31(45.6 \%)$ \\
Neuraxial analgesia & $63(92.6 \%)$ \\
\hline
\end{tabular}

\section{Conclusions:}

- Individualized thromboprophylaxis in high-risk obese pregnant women is safe and effective.

- In our series, most deliveries could be performed under neuraxial analgesia without complications.

- In our experience, anti-Xa levels can help in finding the dose of LMWH with the best risk-benefit ratio.

- Collaboration between hematologists and obstetricians is mandatory in these high-risk patients.

PB1294 | Antepartum Anemia and Risk of Postpartum Hemorrhage: A Comprehensive Review of the Literature

H. Glonnegger ${ }^{1}$; L. Lancaster ${ }^{2}$; R. Barnes ${ }^{2}$; A. von Drygalski ${ }^{2,3}$

${ }^{1}$ University of Freiburg, Division of Pediatric Hematology and Oncology, Freiburg, Germany; ${ }^{2}$ University of California San Diego, Division of Hematology/Oncology, San Diego, United States; ${ }^{3}$ The Scripps Research Institute, Department of Molecular Medicine, La Jolla, United States

Background: Postpartum hemorrhage (PPH) is responsible for 30$50 \%$ of maternal deaths worldwide. Anemia appears to facilitate $\mathrm{PPH}$. However, evidence is conflicting, derived mostly from a few studies applying strict World Health Organisation (WHO) definitions of PPH and/or anemia.
Aims: To study anemia and $\mathrm{PPH}$ risk by considering studies with conformant and non-conformant WHO definitions.

Methods: A MEDLINE database search was conducted applying the terms anemia OR hemoglobin AND postpartum hemorrhage. Study titles and abstracts were screened. Exclusion criteria were: not peerreviewed, non-English, uterotonic studies, case reports, in-vitro/ animal reviews.

Data extraction included: study type, sample size, PPH/anemia definition, anemia severity and information regarding associations of anemia and PPH. We used a random-effects meta-analysis model to estimate an overall odds ratio.

Results: Of 1060 articles, 1021 were excluded, leaving 39 studies originating from Africa $(n=14)$, Asia $(n=12)$, Europe $(n=7)$, America/ Oceania $(n=2)$, South America $(n=1)$ or multi-country $(n=1)$. Only $46 \%$ and $15 \%$ of studies used the WHO definition for PPH and performed objective blood loss (OBL) measurements. The WHO definition of antepartum anemia ( $\mathrm{Hgb}<11 \mathrm{~g} / \mathrm{dL}$ ) was used in $76 \%$ of studies, with WHO sub-categorization by anemia severity in only $31 \%$. The overall prevalence of anemia across studies ranged from 1-88\%. Of 39 studies 32 reported positive associations between anemia and $\mathrm{PPH}(n=25)$ or adverse outcomes of anemia in PPH $(n=7)$ (Figure 1 ).

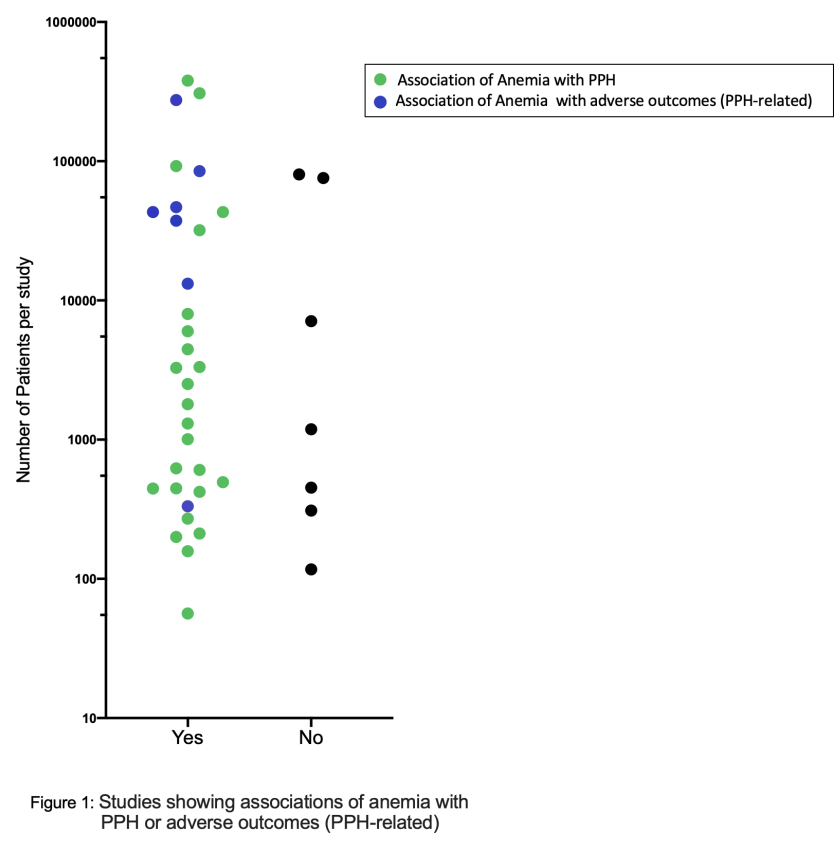

FIGURE 1 Studies showing associations of anemia with PPH or adverse outcomes (PPH-related)

A meta-analysis of studies providing crude ORs $(n=12)$ showed a greater risk of $\mathrm{PPH}$ in patients with anemia (OR 1.47; $95 \% \mathrm{Cl}: 1.25$, 2.33) (Figure 2). 


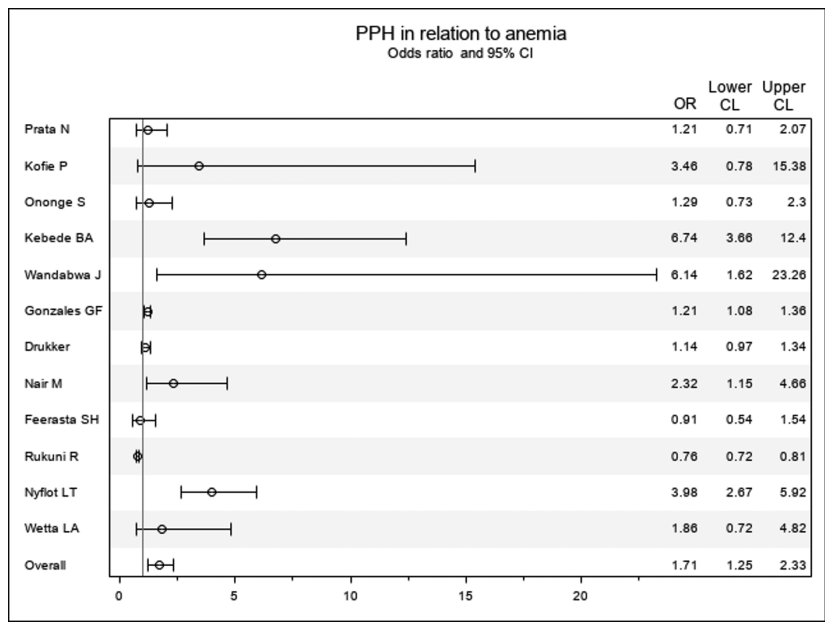

FIGURE 2 Forest-plot of odds of PPH in antepartum anemia

Conclusions: Antepartum anemia appears to influence the risk of $\mathrm{PPH}$ and/or adverse maternal outcomes. However, this analysis revealed a lack of uniform definitions of PPH and anemia, OBL measurements and large geographic variations in anemia prevalence, altogether highlighting the need for harmonized prospective studies to inform future intervention strategies.

PB1295 | Diagnostic Significance of Definitions Soluble Fibrin Monomer Complexes In Patients with Preeclampsia

\section{B. Kurbanov}

Tashkent Pediatric Medical Institute, Tashkent, Uzbekistan

Background: Despite the close attention of researchers, the problem of preeclampsia is far from being solved. With preeclampsia, almost all endothelial functions are impaired: regulation of hemostasis, vascular tone, immune processes, vasopermeability.

Aims: The purpose of our work was to study the diagnostic role of soluble fibrin-monomer complexes (SFMC) in the development of preeclampsia.

Methods: We studied the state of the hemostasis system in 100 pregnant women with preeclampsia and in 30 women with physiological pregnancy in the third trimester. The study of the state of platelet, procoagulant and fibrinolytic links of the hemostasis system and their assessment were carried out in the dynamics of observation. The level of soluble fibrin monomer complexes (SFMC) was determined by the ortho-phenanthroline method using standard test systems "RFMK-test" from Tekhnologiya - Standart, Russia. Results: The indices of the hemostasis system in pregnant women with preeclampsia in the third trimester differ significantly from those in women with the physiological course of pregnancy.

In pregnant women with preeclampsia in the third trimester was revealed, a statistically significant shortening of activated recalification time $-42.7 \pm 0.8 \mathrm{sec}$ compared to the control $-51.4 \pm 0.4 \mathrm{sec}$ $(\mathrm{P}<0.001)$.
When studying markers of blood activation, we found a significant increase in SFMC - $6.9 \pm 0.7 \mathrm{mg} / 100 \mathrm{ml}$ in the main group, in contrast to the control group $-2.89 \pm 0.11 \mathrm{mg} / 100 \mathrm{ml}(\mathrm{P}<0.001)$. The presence of markers of intravascular blood coagulation in pregnant women with severe preeclampsia indicate the activation of intravascular thrombus formation as a chronic form of DIC.

Conclusions: Analysis of observations of patients indicates the staging of the development of platelet hyperaggregation followed by hypercoagulation. An increase in the frequency of positive tests for the presence of SFMC was evidence of the intensification of intravascular blood coagulation.

PB1296 | Utility of Royal College of Obstetricians and Gynaecologists (RCOG) Guidelines in Preventing Pregnancy Related Venous Thrombo-embolism (PA-VTE)

E. Jackson ${ }^{1}$; E. Treharne ${ }^{1}$; A. Alexander ${ }^{1}$; B. Myers ${ }^{2}$

${ }^{1}$ The University of Leicester Medical School, Leicester, United Kingdom;

${ }^{2}$ University Hospitals of Leicester, Leicester, United Kingdom

Background: Guidelines for pregnancy and post-partum thromboprophylaxis are largely expert-based, and differ widely from country to country. We performed a retrospective analysis of a local database with respect to RCOG guidelines to assess the utility in preventing pregnancy-related VTE.

Aims: The aim of the study was to assess the utility in preventing pregnancy-related VTE (PA-VTE).

Methods: A local database was used to identify women who had suffered a PA-VTE, and the notes reviewed to assess their RCOG score. 290 previously pregnant women were identified, and of these 225 had no previous history of VTE. The notes were obtained and a review of risk factors as listed in RCOG were available for 216 cases.

TABLE 1 RCOG risk factor score and Specific risk factors for VTE

\begin{tabular}{lll}
$\begin{array}{l}\text { RCOG Risk Factor } \\
\text { Score }\end{array}$ & $\begin{array}{l}\text { No of women with } \\
\text { no VTE }\end{array}$ & $\begin{array}{l}\text { No of women with } \\
\text { VTE }\end{array}$ \\
0 & $17(61 \%)$ & $11(39 \%)$ \\
1 & $45(66 \%)$ & $23(34 \%)$ \\
2 & $46(79 \%)$ & $21(21 \%)$ \\
3 & $39(32 \%)$ & $83(68 \%)$ \\
Specific Risk Factor & & \\
$\quad$ BMI >30 & $80(77 \%)$ & $24(23 \%)$ \\
$\quad$ Age over 35 & $47(76 \%)$ & $15(24 \%)$ \\
\hline
\end{tabular}

Results: Those with 4 or more risk factors were analysed in a separate submission; table 1 shows the results of number of risk factors and number of women who did or did not have a PA-VTE. For 3 risk factors, those women who suffered a clot did so prior to 28 weeks. A further stratification focused on the RCOG risk factors "BMI >30", which showed $23 \%$ of cases experienced a PA-VTE, and the RCOG 
risk factor "Age over 35", which showed $24 \%$ of cases of women aged 35 experienced a PA-VTE.

Conclusions: There was no clear predictive value of the RCOG guidance from this small study, in particular with the factors of maternal age and BMI not demonstrating a clear positive correlation of adverse outcomes in VTE, either antenatally or postnatally.

\section{PB1297 | Interactions of Anemia with Race and Peripartum Transfusion in Three Large US Registries}

\section{E. Davis $^{1}$; R. Amdur ${ }^{2} ;$ H. Ahmadzia ${ }^{3}$}

${ }^{1}$ George Washington University School of Medicine and Health Sciences, Washington, DC, United States; ${ }^{2}$ Department of Surgery, George Washington University, Washington, DC, United States; ${ }^{3}$ Division of Maternal-Fetal Medicine, Department of Obstetrics and Gynecology, George Washington University, Washington, DC, United States

Background: Postpartum hemorrhage (PPH) is a prevalent cause of maternal morbidity and mortality in the United States. Non-Hispanic Black patients experience higher rates of $\mathrm{PPH}$, as well as greater prevalence of anemia in pregnancy (AIP), a known risk factor for $\mathrm{PPH}$.

Aims: To describe the racial distribution of AIP across three nationwide datasets, and to determine the peripartum transfusion rate and characteristics of transfusion recipients, to better understand disparities in hemostatic outcomes.

Methods: Using Eunice Kennedy Shriver National Institute of Child Health and Human Development (NICHD) Maternal Fetal Medicine Units Network Cesarean Registry (CR), NICHD Consortium on Safe Labor Registry (CSL), and a cohort of deliveries at Universal Health Services (UHS) hospitals, univariable associations were described between race, anemia and transfusion. Multivariable logistic regressions examined associations of race with transfusion, independent of anemia, and of anemia with transfusion, independent of race. Multivariable models included covariates: age, parity, smoking during pregnancy, BMI, and delivery mode. The anemia by race interaction was also investigated.

Results: We included $n=56,964$ from CR, 140,324 from CSL, 87,465 from UHS (with 28\%, 24\%, 12\% Black respectively). Prevalence of anemia was $8 \%$ in CR, $13 \%$ in CSL, $7 \%$ in UHS. Anemia was more common in Black patients (CR OR 2.52 [95\%Cl 2.37-2.68], CSL OR 1.48 [95\% Cl 1.43-1.53], UHS OR 2.61 [95\% Cl 2.45-2.79]) and was universally associated with transfusion (OR 6.46 [5.78-7.22], OR 1.27 [1.18-1.37], OR 5.79 [4.58-7.32], respectively). After adjusting for covariates, Black patients were more likely to receive blood transfusions in CR (aOR 1.32 [1.16-1.50, $P<.0001]$ ), but not in UHS or CSL (aORs 1.19 [0.89-1.59, $P=.24$ ], 0.40 [0.36-0.44, $P<.0001]$ respectively).

Conclusions: Although anemia was more common among Black patients, the relationship between race and transfusion differed between databases, suggesting other unexplored factors are involved.
PB1298 | Pregnancy and Heparin: Peripartum Management. Experience of Two Centers in Argentina

$\underline{\text { S. Molnar }}{ }^{1}$; C. Gumpel ${ }^{2}$

${ }^{1}$ Clinica Universitaria Reina Fabiola, Cordoba, Argentina; ${ }^{2}$ Laboratorio de Hemostasia y Trombosis y Centro de Especialidades Medicas,

Rosario, Argentina

Background: Low molecular weight heparin (LMWH) is the most frequently used drug in pregnancy for prophylaxis or treatment of thromboembolic disease or obstetric complications. Delivery timing is challenging both preventing bleeding and also thrombosis. Intermediate or high doses could be associated with an increased risk of peripartum bleeding. Expert recommendations range from education, programmed suspension, dose reduction, or induction of labor.

Aims: To describe the heparin peripartum management and its association with hemorrhagic and/ or thrombotic complications in our population.

Methods: Retrospective multicenter study to analyze the peripartum management of $\mathrm{LMWH}$-treatment pregnant women between 2004 to 2020. Data were analyzed by Chi (2).

Results: 499 pregnancies in 448 women were included (Table 1). Median age: 35 years (19-50).

28/ 499 programmed labor induction, and 22/ 28 (79\%) were successful.

Prepartum hemorrhage was presented in $14 / 499$ pregnancies $(2.8 \%)$, but there was no significant (NS) correlation with the dose of heparin No patients presented spinal hematoma.

19/ 499 (3,8 \%) presented postpartum hemorrhage. 6/19 had major bleeding according ISTH SSC.

There was NS association between bleeding and last heparin dose: urgent cesarean section ( $<12 \mathrm{hrs}$ last heparin dose), patient education group (12-24 hrs last heparin dose) or programmed suspension ( $>24$ hrs to $\geq 1$ week).

Also there was NS association between heparin dose (prophylactic, intermediate or therapeutic) and bleeding.

There was only one event of thrombotic complication (superficial venous thrombosis).

TABLE 1 Clinical characteristics

Pregnancies n 499 $N(\%)$

LMWH indication

499

Obstetric

344 (68)

Infertility

98 (19.6)

Hematologic

55 (11.02)

Hematologic + Obstetric 
Pregnancies n 499

$N(\%)$

$\begin{array}{ll}\text { LMWH doses } & 499 \\ \text { Prophylactic* } & 338(67.7) \\ \text { Intermediate } & 147(29.4) \\ \text { Therapeutic** } & 14(2.8) \\ & \\ \text { LMWH antepartum suspension } & 499 \\ <12 \text { hours } & 10(2) \\ \text { 12-24 hours } & 395(79.15) \\ >24 \text { hours }-6 \text { days } & 77(15.43) \\ \geq 1 \text { week } & 17(3.4) \\ & \\ \text { Delivery Mode and Anesthesia } & 499 \\ \text { Vaginal delivery } & 129(25.85) \\ \text { Labor epidural analgesia } & 107 / 129(83) \\ \text { Caesarean section } & 370(74.15) \\ \text { Neuroaxial anesthesia } & 367 / 370(99.2) \\ \text { General anesthesia } & 3 / 370(0.8) \\ & \end{array}$

Conclusions: We did not find any significant association between heparin dose or the time from last dose to delivery and bleeding or thrombotic complications.

There was a high rate of elective caesarean sections.

The education of the pregnant woman for the optimal time to hold heparin prior to delivery is a safe method without significantly in creasing the risk of hemorrhagic or thrombotic complications.

PB1299 | New Criteria for Assessing Hemostasis Disorders in Pregnant Women with Chronic Kidney Disease

I. Vasilenko ${ }^{1,2}$; I. Nikolskaya ${ }^{3}$; E. Shestero ${ }^{3}$; V. Metelin ${ }^{1,2}$; D. Kassina ${ }^{1}$

${ }^{1}$ M.F. Vladimirsky Moscow Regional Clinical and Research Institute (MONIKI), Moscow, Russian Federation; ${ }^{2}$ A.N. Kosygin Russian State University, Moscow, Russian Federation; ${ }^{3}$ Moscow Regional Research Institute of Obstetrics and Gynecology (MONIIAG), Moscow, Russian

Federation

Background: The pregnancy of women with kidney disease is followed by increased frequency of gestational complications development such as endotheliosis, inflammation, oxidative stress, and hemostatic impairments. In this connection, a particular relevance is the search for objective and informative criteria for blood clotting disorders in pregnant women.

Aims: The aim - to make easier the forecasting of possible complications in mother and a child, evaluation of their condition severity, and therapy efficacy.
Methods: A group of 48 women with physiologic course of pregnancy was observed (mean age $27.3 \pm 4.1$ years) as well as a group of 44 pregnant women with chronic kidney disease (CKD) (mean age $29.1 \pm 3.6$ years) in the third trimester of gestation. Investigations were carried out using laboratory diagnostic test "Thrombodynamic register T-2" which allowed to characterize the process of spatiotemporal blood clot formation at a new level. In parallel, In parallel, routine hemostasis assessment tests were used.

Results: It was established, that in a majority of female patients (88.9\%) with CKD, some indicators were decreased (APTT by $14.8 \%$, and INA by $21.7 \%$ ) but fibrinogen concentration in blood increased by $15.8 \%$, i.e. there were hypercoagulable changes. The thrombodynamic data showed that the speed of fibrin clot growth increased by $31.7 \%$; initial speed of clot growth increased by $17.5 \%$, and stationary speed of clot growth increased by $9.5 \%$, and the size of clot grew by $15.9 \%$ as compared with indicators in women with physiologic pregnancy.

Conclusions: The results obtained in the conducted study indicate the significant increase of blood coagulation potential in the pregnant women with CKD. In our opinion, the global Thrombodynamic test is an objective method of investigation allowing clear differentiation among hyper-, hypo-, and isocoagulation, and identification of patterns of hemostatic disorders in pregnant women with CKD eventually determining the outcome of pregnancy and the health of a newborn child.

\section{PB1300 | Preeclampsia and Inflammatory Lipid Mediators: A} Longitudinal Study

L. Perucci ${ }^{1}$; K. Pinto ${ }^{1}$; S. Silva ${ }^{1}$; E. Lage ${ }^{2}$; P. Teixeira ${ }^{2}$; A. Barbosa ${ }^{2}$; P. Alpoim²; L. Sousa ${ }^{2}$; L. Dusse ${ }^{2} ;$ A. Talvani ${ }^{1}$

${ }^{1}$ Federal University of Ouro Preto, Ouro Preto, Brazil; ${ }^{2}$ Federal

University of Minas Gerais, Belo Horizonte, Brazil

Background: The levels of pro-inflammatory (e.g. leukotriene B4-LTB4) and pro-resolving (e.g. lipoxin A4-LXA4, and resolvin D1-RvD1) lipid mediators have been recently investigated in preeclampsia (PE). However, the production of these mediators throughout gestation in both healthy and diseased contexts remains unclear. Aims: Investigate LTB4, LXA4, and RvD1 levels throughout gestation in pregnant women with risk factors for PE who either developed $(N=11)$ or did not develop $(N=7)$ the disease.

Methods: The ethics committee of the Federal University of Minas Gerais (\#0618.0.203.000-10) approved the study protocol and all participants provided written informed consent. LTB4, LXA4 and RvD1 plasma levels were measured by immunoassays at three timepoints: 12-19, 20-29, and 30-34 weeks of gestation in both groups. Results: Table 1 shows patients' clinical characteristics. 
TABLE 1 Clinical characteristics of the studied pregnant women

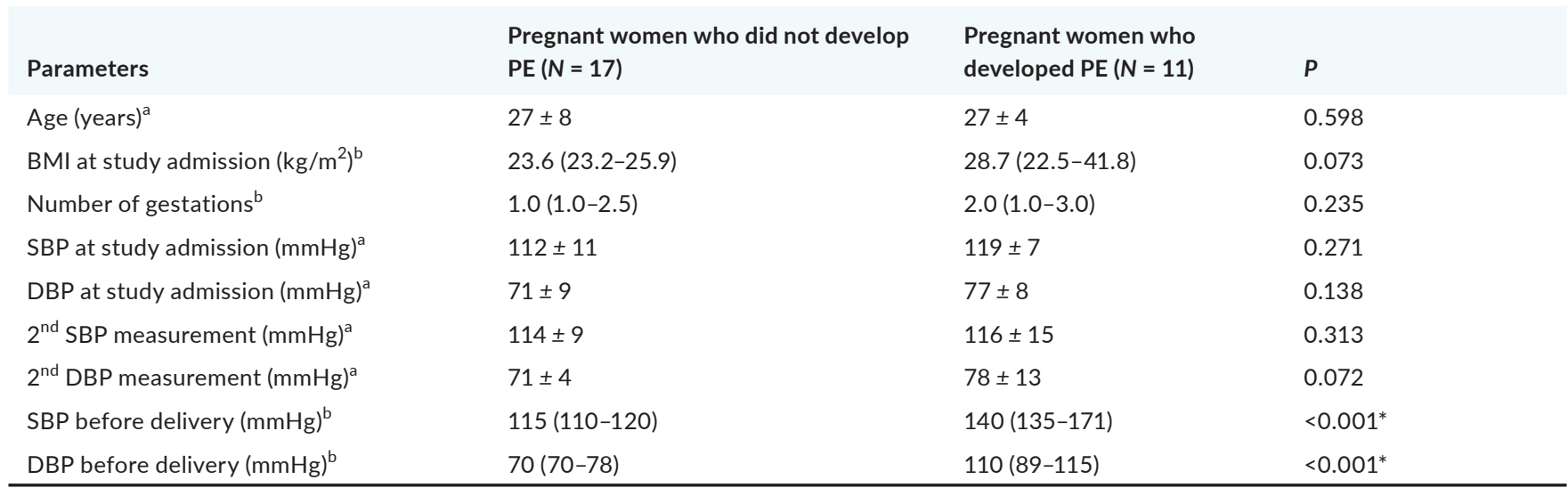

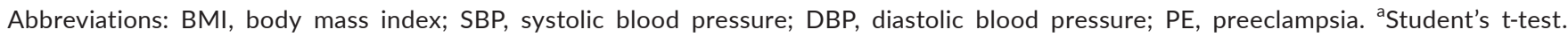
Parametric data are presented as mean \pm standard deviation (SD). ${ }^{b}$ Mann-Whitney U-test. Non-parametric data are presented as median (25 ${ }^{\text {th }}$ - $75^{\text {th }}$ percentiles). ${ }^{*} P<0.05=$ statistically significant.

There was a trend of upregulated LTB4 levels throughout gestation in women who developed PE, but this difference was significant only at 30-34 weeks (Fig.1A). LXA4 levels showed a similar pattern with no difference between groups in any gestational age (Fig. 1B). Pregnant women who developed PE had lower RvD1 levels (Fig. 1C) and a decreased RvD1/LTB4 ratio (Fig. 1E) at 30-34 weeks compared to normotensive pregnant women (Norm). Contrarily, RvD1 levels were decreased in normotensive pregnant women at 12-19 weeks (Fig. 1C). LXA4 and RvD1 levels were higher at 30-34 weeks compared to 20-29 weeks in both groups (Fig. 1B and $1 \mathrm{C}$, respectively). The LXA4/LTB4 ratio did not differ between groups in any gestational age evaluated, but it was higher at 30-34 weeks compared to 20-29 and 12-19 weeks of gestation in both groups (Fig. 1D). There was an interaction between the gestational outcome (preeclamptic vs. normotensive pregnancy) and the gestational age only for RvD1.
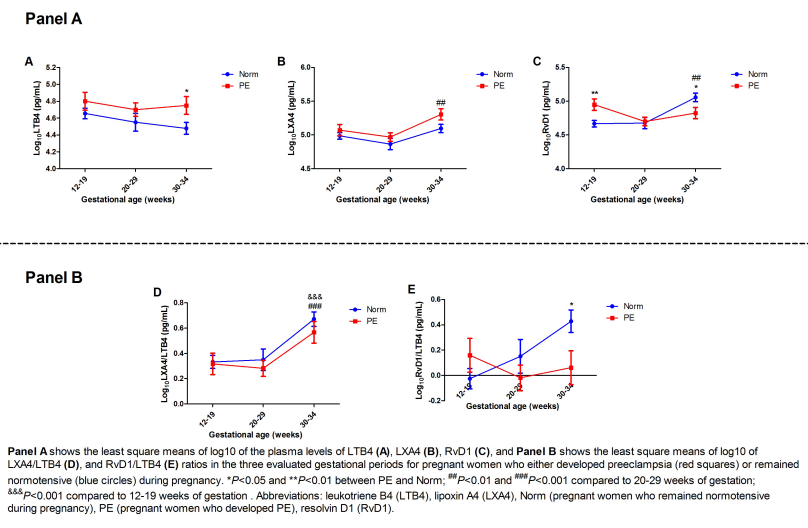

FIGURE 1 Plasma levels of inflammatory lipid mediators, and the ratios between pro-resolving and pro-inflammatory lipid mediators throughout preeclamptic and normotensive pregnancies.
Conclusions: Imbalanced levels of LTB4, LXA4, and RvD1 may be associated with the excessive systemic inflammation that underlies PE pathogenesis.

Financial support: CNPq, FAPEMIG, CAPES and UFOP/PROPP.

PB1302 | Impact of Tissue Factor Pathway Inhibitor Gene Polymorphisms (33T/C and 264V/M) on Plasma TFPI Levels and their Influence on Risk of Recurrent Pregnancy Loss in India

K. Kishor $^{1}$; A. Sharma ${ }^{1}$; S. Maharana ${ }^{2}$; R. Ranjan ${ }^{1}$; R. Kumar ${ }^{1}$; S. Tyagi ${ }^{1}$; R. Saxena ${ }^{3}$; M. Mahapatra ${ }^{1}$

${ }^{1}$ All India Institute of Medical Sciences, New Delhi, India; ${ }^{2}$ Central University of Tamil Nadu, Thiruvarur, India; ${ }^{3}$ Medanta, the Medcity, Gurugram, India

Background: Recurrent pregnancy loss (RPL) is a complex, multifactorial disease, with a frequency of $0.5-3 \%$ in all couples trying to conceive. Etiology of approximately $40-50 \%$ of all RPL remain unexplained. Low TFPI level increased the risk of RPL in the West. However, association of TFPI levels with its polymorphisms and their role in Indian RPL patients is not yet studied.

Aims: To find out the distribution of TFPI 33T/C and 264V/M polymorphisms, their effects on TFPI levels and risk of RPL in India.

Methods: RPL patients with at least 3 consecutive pregnancy losses before 20 weeks of gestational age and equal number of healthy women with, at least one naturally conceived pregnancy studied. Plasma TFPI levels were determined by ELISA and its normal range determined (Mean \pm 2 SD of TFPI levels in controls). TFPI polymorphisms, 33T/C and 264V/M were detected by PCR-RFLP.

Results: 80 RPL patients, median age 33 years range (21-44 years) were recruited. Mean TFPI level was significantly lower in patients $(37.32 \pm 13.92 \mathrm{ng} / \mathrm{ml})$ than controls $(48.15 \pm 13.35 \mathrm{ng} / \mathrm{ml}, P=0.001)$. Moreover, 11 patients had low TFPI $(<21.45 \mathrm{ng} / \mathrm{ml})$, whereas no control had low TFPI. CT and TT genotypes of 33T/C polymorphism 
was significantly lower 31 (38.75\%) in patients than 44 (55\%) controls $(P=0.039)$. The distribution of heterozygous genotype (VM) of $264 \mathrm{~V} / \mathrm{M}$ polymorphism was similar $5(6.25 \%)$ in patients and $3(3.75 \%)$ controls $(P=0.719)$ however, no homozygous mutant genotype (MM) was observed in study subjects. Relation between the TFPI levels and their genotypes and relative risk of RPL are shown in table.

TABLE 1 TFPI levels according to genotypes of TFPI polymorphisms and relative risk of RPL

$\begin{array}{llll}\text { TFPI polymorphisms } & \text { TFPI Levels ng/ml (Mean } \pm \text { SD) } & \text { OR }(95 \% \mathrm{Cl}) & \text { OR }^{\mathrm{A}}(95 \% \mathrm{Cl}) \\ \text { 33T/C TT } & 34.02 \pm 11.51 & 1.0 & 1.0 \\ \text { TC } & 45.60 \pm 11.72 & 0.536(0.279-0.927) & 0.441(0.22-0.85) \\ \text { CC } & 70.96 \pm 13.78 & 0.327(0.112-1.062) & 0.202(0.07-0.822) \\ & 0.001^{*}, 0.001^{\$}, 0.001^{\#} & & 1.0 \\ 264 V / M V V & 48.46 \pm 13.76 & 1.0 & 2.85(0.91-13.07) \\ \text { VM } & 42.88 \pm 12.65 & 2.260(0.609-9.78) & -\end{array}$

P-value <0.05 is statistically significant: *Overall p value, \# Wild Vs Heterozygous, \$Wild Vs Homozygous, ORA: adjusted for BMI, DM-Type-II, hypertension.

Conclusions: As in the West, low TFPI level predisposes to RPL. 33T/C polymorphism associated with high plasma TFPI level and has a protective role against RPL. 264V/M polymorphism neither associated with TFPI level nor with risk of RPL. A study with large sample size is required to confirm these findings.

PB1303 | Warfarin Anticoagulation and Fetal Central Nervous System Abnormalities: A Case Report

R. Monaheng; E. Schapkaitz; H. Rhemtula; M. Louw; A. Gerber University of the Witwatersrand, Medical School, Johannesburg, South Africa

Background: Anticoagulation of pregnant woman with mechanical prosthetic heart valves is associated with significant maternal and fetal risks. In order to reduce the risks of embryopathy associated with first trimester vitamin $\mathrm{K}$ antagonists such as warfarin, sequential therapy with dose adjusted therapeutic low molecular weight heparin and warfarin has been recommended. Nonetheless, this does not obviate the risks of central nervous system (CNS) abnormalities, miscarriages and stillbirths, which have been described following warfarin exposure during any trimester.

Aims: To describe the CNS abnormalities of a fetus of a pregnant woman with a mechanical valve replacement on lifelong warfarin therapy.

Methods: A 30-year-old African female, presented at 11 weeks' gestation. She was receiving warfarin (Aspen Pharmacare) $7.5 \mathrm{mg}$ daily for a second generation mechanical mitral valve replacement for rheumatic heart disease. This was associated with a time in therapeutic range of $88.8 \%$. Past obstetric history included recurrent first trimester and one second trimester miscarriages associated with anticoagulation. On presentation, the international normalized ratio (INR) was 6.78 with no associated signs of bleeding. $A$ termination of pregnancy was performed. Written informed consent was obtained and the research was approved by the institutional Ethics Committee (M-200547).

Results: Post-mortem examination of the male fetus showed a small flattened skull. No cerebellar or cerebral tissue was present in the skull confirming anencephaly (Figure 1 ).

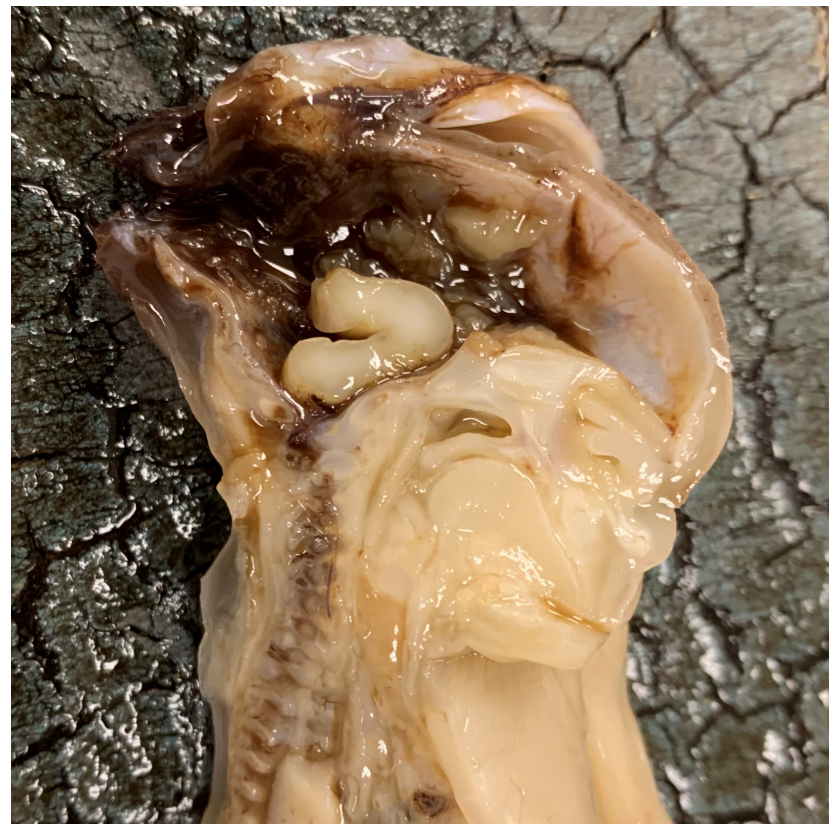

FIGURE 1 Transverse section through the skull, confirming presence of brain stem without cerebellum or cerebrum

Histological assessment confirmed a meningocoele with hemorrhage (Figure 2). 


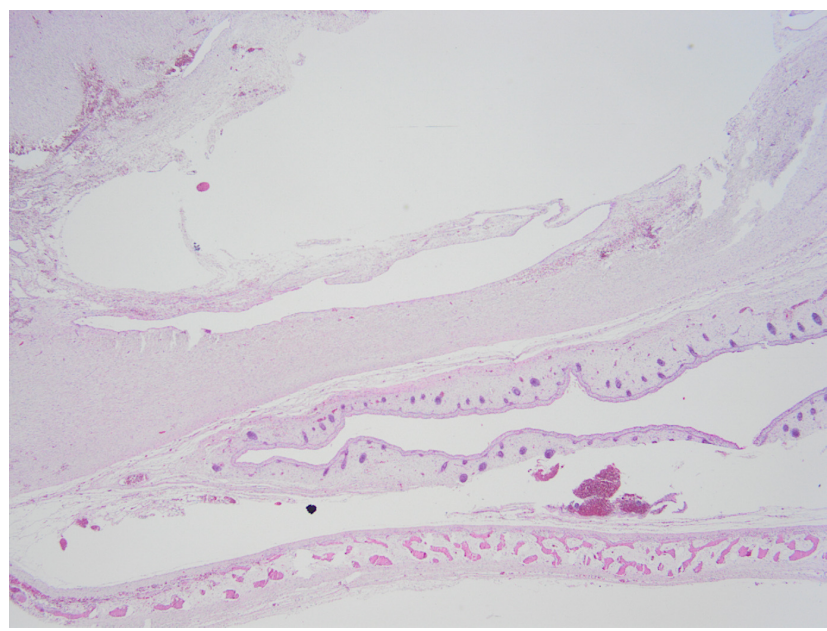

FIGURE 2 Meningocele

Conclusions: The findings of this case report, add to the few previously described cases of anencephaly and a meningocele. These CNS abnormalities are caused by intrauterine hemorrhage. In order to improve fetal outcomes, a multidisciplinary approach in pregnant women with mechanical prosthetic heart valves is essential. This includes contraception advice at anticoagulation clinics, prepregnancy planning and early presentation or replacement of warfarin with heparin prior to conception.

\section{PB1304 | Evaluation of the Effectiveness of Multidisciplinary} Care Plans for the Perinatal Management of Women with Bleeding Disorders

N. O'Neill' ${ }^{1} ;$ F. Meffe ${ }^{2,3} ;$ J. Baker ${ }^{4,5,6} ;$ R. Martin ${ }^{7,8}$; M. Sholzberg $9,10,11,12,13$

${ }^{1}$ Temerty Faculty of Medicine, University of Toronto, Toronto, Canada;

${ }^{2}$ Department of Obstetrics and Gynecology, University of Toronto, Toronto, Canada; ${ }^{3}$ Department of Obstetrics and Gynecology, St. Michael's Hospital, Toronto, Canada; ${ }^{4}$ Department of Paediatrics, University of Toronto, Toronto, Canada; ${ }^{5}$ Department of Pediatrics,
St. Michael's Hospital, Toronto, Canada; ${ }^{6}$ Department of Paediatrics, Division of Haematology-Oncology, The Hospital for Sick Children, Toronto, Canada; ${ }^{7}$ Anesthesiology and Pain Medicine, University of Toronto, Toronto, Canada; ${ }^{8}$ Department of Anesthesia, St. Michael's Hospital, Toronto, Canada; ${ }^{9}$ Department of Medicine, University of Toronto, Toronto, Canada; ${ }^{10}$ Department of Laboratory Medicine and Pathobiology, University of Toronto, Toronto, Canada; ${ }^{11}$ Department of Medicine, St. Michael's Hospital, Toronto, Canada; ${ }^{12}$ Department of Laboratory Medicine and Pathobiology, St. Michael's Hospital, Toronto, Canada; ${ }^{13} \mathrm{Li}$ Ka Shing Knowledge Institute, Toronto, Canada

Background: The perinatal period poses hemostatic challenges to both mother and baby. The challenges are particularly heightened if the mother has an underlying bleeding disorder. Bleeding risk at labour and delivery, risk of postpartum hemorrhage, anesthesia management, and the possibility of a bleeding disorder in the neonate all require careful consideration. Our Multidisciplinary Clinic for Women with Bleeding Disorders creates personalized care plans to promote optimal perinatal management.

Aims: The primary objective was to determine the clinical effectiveness of the care plan perceived by women with bleeding disorders and their healthcare team.

Methods: This study involved women with bleeding disorders and healthcare providers who had experience using the multidisciplinary care plan. Participants completed an online survey to determine the perceived value of and satisfaction with the care plan, and healthcare provider adherence to the care plan. Research ethics board approval was obtained.

Results: Twenty-seven women with bleeding disorders completed the online survey (Table 1). The majority (93\%) agreed or strongly agreed that the care plan was useful and $88 \%$ of women thought that their medical team followed the care plan. Eighteen healthcare providers completed the survey (Table 2). Healthcare provided participants included 4 anesthesiologists, 2 hematologists, 1 nurse practitioner, 4 obstetricians/gynecologists and 7 pediatricians. The majority (89\%) agreed or strongly agreed that the care plan was useful, and all agreed or strongly agreed that it met their needs as a healthcare professional.

TABLE 1 Survey answers from women with bleeding disorders who had been provided a multidisciplinary care plan

\begin{tabular}{|c|c|c|c|c|c|c|}
\hline & $\begin{array}{l}\text { The care } \\
\text { plan was } \\
\text { useful. }\end{array}$ & $\begin{array}{l}\text { The care plan } \\
\text { made me } \\
\text { more aware of } \\
\text { my bleeding } \\
\text { disorder. }\end{array}$ & $\begin{array}{l}\text { The care plan } \\
\text { helped me feel } \\
\text { safer during } \\
\text { my labour and } \\
\text { delivery. }\end{array}$ & $\begin{array}{l}\text { The care plan } \\
\text { helped make } \\
\text { me feel more in } \\
\text { control of my } \\
\text { bleeding disorder. }\end{array}$ & $\begin{array}{l}\text { The care plan } \\
\text { met my needs as } \\
\text { a woman with a } \\
\text { bleeding disorder. }\end{array}$ & $\begin{array}{l}\text { I had a good } \\
\text { experience with } \\
\text { the care plan. }\end{array}$ \\
\hline Strongly agree & 20 (74\%) & $16(59 \%)$ & 21 (78\%) & 18 (67\%) & 18 (67\%) & $15(56 \%)$ \\
\hline Agree & $5(19 \%)$ & $7(26 \%)$ & 4 (15\%) & 4 (15\%) & $6(22 \%)$ & $8(30 \%)$ \\
\hline Disagree & $0(0 \%)$ & $1(4 \%)$ & $0(0 \%)$ & $0(0 \%)$ & $1(4 \%)$ & $1(4 \%)$ \\
\hline Strongly disagree & $0(0 \%)$ & $0(0 \%)$ & $0(0 \%)$ & $0(0 \%)$ & $0(0 \%)$ & $0(0 \%)$ \\
\hline
\end{tabular}


TABLE 2 Survey answers from healthcare providers who had experience using a multidisciplinary care plan

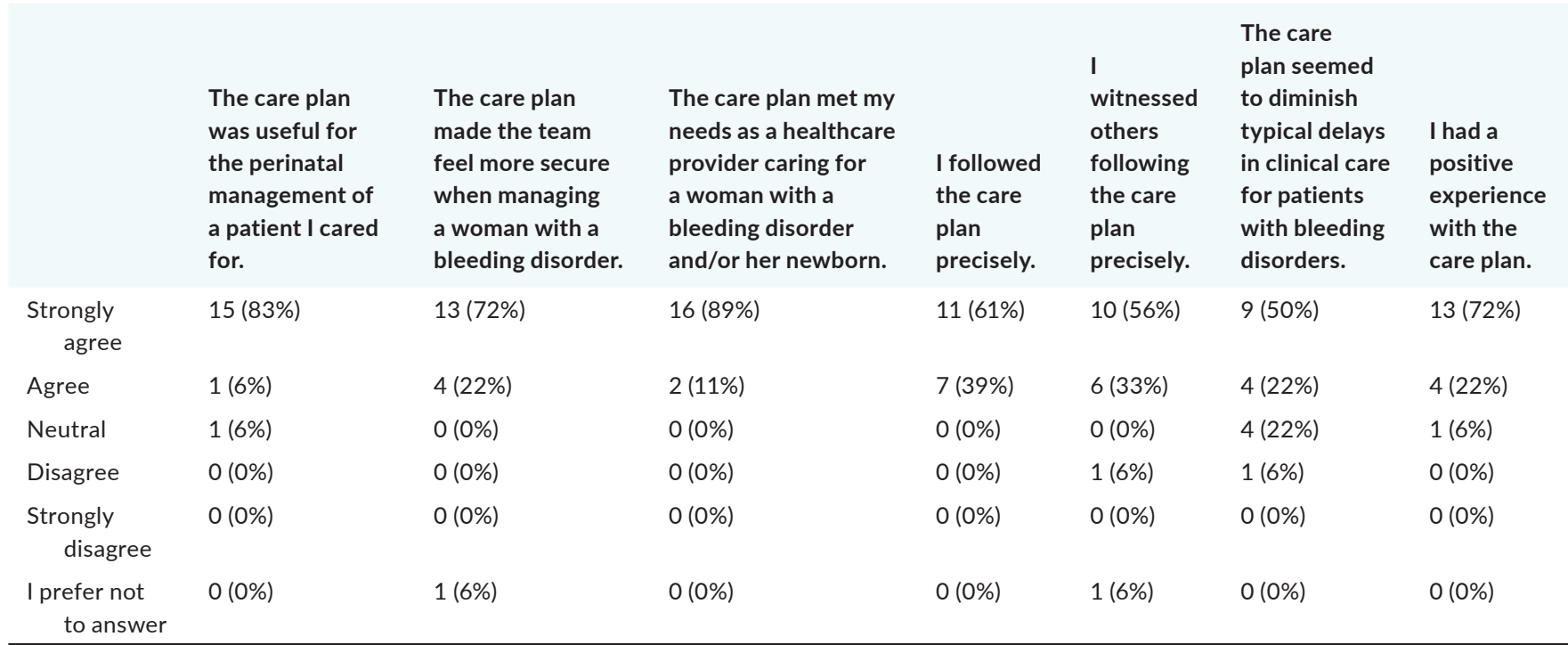

Conclusions: We found that women with bleeding disorders and their potentially affected neonates can experience a satisfactory delivery and postpartum period with a multidisciplinary labour and delivery management plan. These care plans appear to empower healthcare providers and patients alike to facilitate optimal perinatal care. We have the opportunity to improve the care plan and extend its use externally to further enhance the care of this vulnerable patient population.

\section{PB1305 | Deconstructing the Obstetric Antiphopholipid Syndrome (APS)}

\section{B. Grand; L.S. Voto}

Hospital "Juan A. Fernández", Department of Maternal and Fetal Medicine. School of Medicine. University of Buenos Aires, Caba, Argentina

Background: Deconstruction, form of philosophical and literary analysis, derived mainly from work begun in the 1960s by the French philosopher Jacques Derrida. To "deconstruct" an opposition is to explore the tensions and contradictions between the hierarchical ordering assumed (and sometimes explicitly asserted) in the text and other aspects of the text's meaning. The point of the deconstructive analysis is to restructure, or "displace," the opposition, not simply to reverse it. Deconstruction's influence widened to include a variety of other disciplines, such as medical practice.

Aims: To deconstruct the explicit representative model of APS (updated in Sydney).

Methods: This work is only referred to the called non-thrombotic obstetric APS and do not is applicable to other clear well established autoimmune diseases (LES, Rheumatoid Arthritis etc.). Material: The International Classification Criteria propose to define the APS and the use in the daily medical practice of the antiphospholipid antibodies (aPL). Method: In $1^{\text {st }}$ column we describe the traditional "scientific perspective of the classical APS"; in the $2^{\text {nd }}$ column the social widespread and real-world use of $\mathrm{APL}$ and the $3^{\text {rd }}$ we propose what is needed to be deconstructed before new/future classifications of APS.

Results: Deconstruction of traditional obstetric APS is needed. See Table.

\section{TABLE 1}

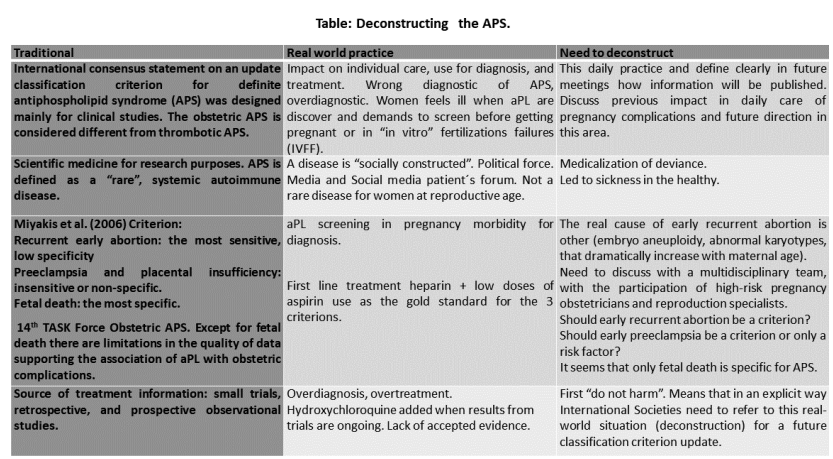

Conclusions: APS has become a familiar concept and the truth for daily practice in women with pregnancies complications. We propose an effort to open a new way of looking the APS at health care. We need to give a correct answer to women at reproductive age and not create false expectative when detecting aPL. The scientific consensus of APS classification criteria contrasts with the real daily practice. After deconstruction of the APS a new reconstruction of this syndrome can open a clearer way for the diagnosis and treatment pregnancies complications. 
PB1306 | Novel Point of Care Rapid Coagulation Analyzer for Obstetrics - Quantra (SEER Sonorheometry)

A. Karuppiah; B.S. Kodali

University of Maryland, Baltimore, United States

Background: Hemorrhage continues to be a prominent contributor to obstetric morbidity and mortality around the world. A rapid coagulation test would be a significant improvement for the diagnosis of coagulopathy and the management of obstetric hemorrhage. Conventional tests are time consuming, and while viscoelastic tests provide rapid results, but they require training and manipulation of samples that prevent widespread adoption. The Quantra ${ }^{\circledR}$ Hemostasis analyzer is a novel, rapid coagulation analyzer that utilizes ultrasound to induce resonance and measure clot stiffness over time.

Aims: We evaluated the sensitivity of the Quantra compared to conventional coagulation tests $(C L)$ to varying levels of fibrinogen, platelets and clotting factors in blood samples from pregnant women.

Methods: Blood was collected from healthy pregnant subjects at term into citrated tubes. Each subject's samples were pooled, and aliquots were analyzed or diluted and reconstituted with plasma from pregnant or non-pregnant women to mimic fluid resuscitation (Figure 1). Neat or reconstituted samples were run in parallel on the Quantra and CL. Data was analyzed using regression analysis to correlate the Quantra parameters against CL.

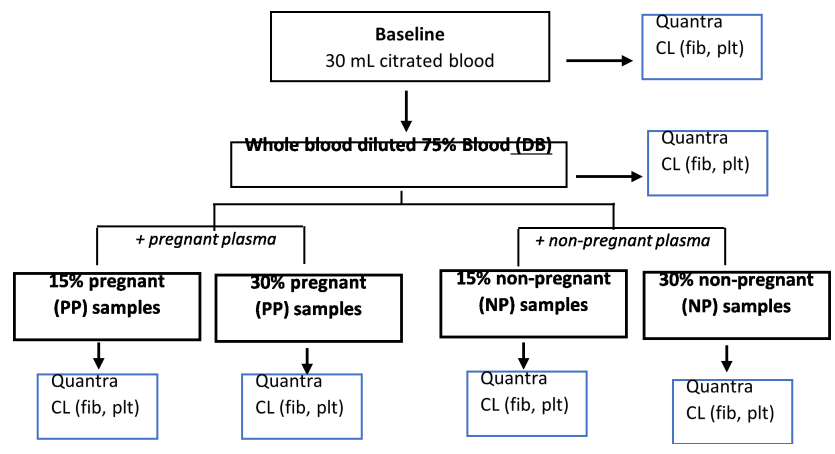

FIGURE 1 Schematic of blood dilutions, reconstitutions and data analysis for each subject

Results: Eighty-nine samples from 13 parturients at term were analyzed. Figure 2 provides the mean (SD) of key Quantra and CL parameters at term gestation. There was a very high linear correlation between Fibrinogen and CS $(R=0.93, P<0.001)$, Fibrinogen and FCS $(R=0.77, P<0.001)$, and Platelet and PCS $(R=0.90)$. FCS and CS strongly predicted a fibrinogen $<200 \mathrm{mg} / \mathrm{dL}$ based on the area under the ROC curves of $0.931(95 \% \mathrm{Cl} 0.881-0.980)$ and $0.943(95 \% \mathrm{Cl}$ 0.868-1.0), respectively. The optimal threshold for FCS to detect fibrinogen $<200 \mathrm{mg} / \mathrm{dL}$ in this patient population was $2.45 \mathrm{hPa}(95 \%$ $\mathrm{Cl}$ 2.35-3.05).

\begin{tabular}{|c|c|c|c|c|c|c|c|}
\hline & $\mathrm{CT}(\mathrm{s})$ & $\operatorname{CS}(\mathrm{hPa})$ & PCS (hPa) & FCS (hPa) & APTT & \begin{tabular}{|l|}
$\begin{array}{l}\text { Fibrinogen } \\
\mathrm{mg} / \mathrm{dL}\end{array}$ \\
\end{tabular} & \begin{tabular}{|l}
$\begin{array}{l}\text { Platelet } \\
\times 10^{9} / \mathrm{L}\end{array}$ \\
\end{tabular} \\
\hline Baseline & $127(18)$ & $32.8(9.8)$ & $7.9(7.7)$ & $4.9(2.5)$ & $27.0(1.5)$ & $494(103)$ & $243(57)$ \\
\hline Diluted $75 \%$ & $183(14)^{*} t$ & $6.4(2.2)^{*} \dagger$ & $5.6(1.5)^{*} t$ & $1.1(0.4)^{*}+$ & $75.6(12))^{*} t$ & $105(31)^{*}+$ & $55(11)$ \\
\hline $15 \% \mathrm{NP}$ & $157(14)^{*}+t$ & $8.5(1.6)^{*}$ & \begin{tabular}{|l|l|l}
$6.4(1.3)^{*}$ \\
\end{tabular} & \begin{tabular}{|l|}
$1.9(0.5)^{*}$ \\
\end{tabular} & $51.7(3.4) * t$ & \begin{tabular}{|l|}
$137(23)^{*}$ \\
\end{tabular} & ND \\
\hline $15 \% \mathrm{pp}$ & $160(17)^{\circ}$ & $10.9(3.2)^{*}$ & $8.4(2.5)^{*}$ & $2.5(0.9)^{-}$ & $49.7(10)^{*}$ & $162(38)^{*}$ & ND \\
\hline $30 \% \mathrm{NP}$ & $156(11)^{*}$ & $8.8(2.1)^{*}$ & $6.4(1.7)^{*}$ & $2.4(0.8)^{*}$ & $42.5(3.2)^{*}$ & $162(25)^{*}$ & ND \\
\hline $30 \% \mathrm{pp}$ & $152(14)^{*}$ & $14.9(4.5) * \tilde{T}$ & $11.2(3.6) * \widetilde{T}$ & \begin{tabular}{|l|}
$3.8(1.6)$ \\
\end{tabular} & $38(2.3)^{*}$ & $214(46)^{*}$ & ND \\
\hline Resuscitated & $128(18) \gamma$ & $18.9(4.5)^{\circ}$ & $15.3(3.8)^{*}$ & $3.9(1.5)$ & $33.1(1.6)$ & $279(38) *{ }^{*}$ & $107(41)$ \\
\hline
\end{tabular}

Values represent mean (standard deviation). Quantra parameters include: Clot time (CT), Clot Stiffness (CS), Platelet contribution (PCS), fibrinogen contribution (FCS).

$*(P<0.001)$ significant different from baseline $+(P<0.001), \forall(P<0.01), \breve{P}(P<0.05)$ significant different between the current value and the previous value on the trend. ND, not determined.

Figure 2 Effects of dilution and reconstitution on Quantra and conventional coagulation test parameters

Conclusions: The Quantra is a user-friendly cartridge-based coagulation monitoring system that rapidly provides a measure of a patient's coagulation status which correlates well with conventional fibrinogen and platelet measurements.

PB1307 | Outcomes of Treatment of Thromboembolic Disease during Pregnancy and Postpartum - Single-center Experience

Š. Stražišar; M. Kozak

University Medical Centre Ljubljana, Medical Faculty of Ljubljana, Ljubljana, Slovenia

Background: Pregnancy and the postpartum period are risk factors for developing venous thromboembolic disease, which is an important cause of morbidity in this population. Since peroral medications are contraindicated due to possible fetal complications, the drug of choice is low molecular weight heparin (LMWH). There are some differences according to different guidelines in LMWH dosing especially around the delivery when the suggestions are not so clear. There is also scarce data about the long-term outcome.

Aims: We wanted to assess the efficacy and safety of our VTE treatment scheme during pregnancy and postpartum and asses also the long-term outcome in patients with deep venous thrombosis (DVT). Methods: All pregnant patients on treatment due to previous or VTE in current pregnancy treated in our clinics from 2010 to 2019 were included. An initial dose of LMWH was adjusted according to the body weight and increased when body weight went up. Some adjustments were done also according to anti-Xa testing. Treatment was stopped the day before delivery when the patient got only half dose and started when vaginal delivery after 12 , and after cesarean section after 8 hours, then therapeutic dose every 24 hours till the end of the 6th week after delivery. Recurrent VTEs and all bleedings were looked for and long-term efficacy in patients with deep venous thrombosis (DVT) was assessed by Villalta score and ultrasound investigation 5-12 months after delivery.

Results: We included 116 women. The outcomes - recurrent VTEs, bleedings, and incidence of a post-thrombotic syndrome are shown in table 1. 
TABLE 1 Outcomes in treated patients

\begin{tabular}{ll} 
Recurrent VTE on treatment & $N(\%)$ \\
DVT & $1(0.9)$ \\
PE & 0 \\
All & $1(0.9)$ \\
Bleedings on treatment & \\
Minor during pregnancy & $6(5.2)$ \\
Minor clinically important during pregnancy & $3(2.6)$ \\
Major peripartum+* & $1(0.9)$ \\
Major postpartum* & $1(0.9)$ \\
All & $11(9.6)$ \\
Postthrombotic syndrome** in 95 patients with DVT & $6(6.3)$ \\
\hline
\end{tabular}

* Gynecological cause by a gynecologist, ${ }^{* *}$ Villalta score $>5$.

Conclusions: In our study the incidence of recurrent VTEs and bleedings was low. Only 6 women reported the development of the postthrombotic syndrome. We believe that our therapeutic regime is effective and safe.

PB1308 | Bernard Soulier Syndrome in Pregnancy: Management of Two Pregnancies in a Patient with Variant Bernard Soulier Syndrome

B. Madan ${ }^{1}$; G. Gray²; S. Araujo ${ }^{1}$; D. Holloway ${ }^{2}$

${ }^{1}$ Centre for Haemostasis and Thrombosis, St Thomas' Hospital, London, United Kingdom; ${ }^{2}$ Department of Women's Health, St Thomas' Hospital, London, United Kingdom

Background: Bernard Soulier Syndrome is a rare inherited platelet disorder with mainly autosomal- recessive inheritance. The frequency is 1 in one-million people. It is characterised by macro-thrombocytopenia, reduced expression of platelet-glycoprotein $\mathrm{Ib}-\mathrm{IX}-\mathrm{V}$ and genetic mutations. Literature on obstetric-management is limited in this rare disorder. We report on two pregnancies in a single patient.

Aims: This patient had a life-long history of menorrhagia, gastrointestinal and urinary-tract bleeding. She required a multidisciplinaryapproach to the management of her pregnancies and deliveries. The aim of this report is to shed light on challenges of obstetric management-strategies that are required.

Methods: The patient received counselling regarding increased risk of bleeding associated with pregnancy. However, she made an informed decision to proceed with a planned first pregnancy. The second pregnancy occurred due to failure of contraception and she decided to proceed with this. Due to maternal co-morbidities, an elective caesarean section was planned for both the pregnancies Close liaison with the National Blood Service was essential as she had anti-D antibodies and for monitoring of HPA and HLA platelet antibodies.
Results: The antepartum-course in both pregnancies was complicated by episodes of gastrointestinal-bleeding necessitating hospital admissions. During the first delivery by caesarean section the blood loss was $600 \mathrm{ml}$. However, it was complicated postoperatively by an infected pelvic haematoma which required laparotomy and drainage. The second delivery by elective caesarean section was complicated by 1.5 litre post-partum haemorrhage. In both deliveries, intrapartum and post-partum haemorrhage was managed with blood product support requiring HLA matched platelets, red cell transfusions, recombinant VIla and tranexamic acid. The two pregnancies resulted in delivery of two healthy female infants.

Conclusions: Pregnancy, especially labour and delivery are complicated by a significantly increased risk in bleeding in Bernard Soulier Syndrome. Individualised treatment-plans and close liaison is required between obstetricians, haematologists, gynaecologists and blood transfusion service to manage these complex pregnancies.

PB1309 | Assessment of the Utility of the Pregnancy-adapted YEARS Algorithm in Predicting Presence of Pulmonary Embolism (PE) in Pregnant Women (PA-PE)

A. Alexander $^{1}$; E. Treharne ${ }^{1}$; E. jackson ${ }^{1}$; B. Myers ${ }^{2}$

${ }^{1}$ The University of Leicester Medical School, Leicester, United Kingdom;

${ }^{2}$ University Hospitals of Leicester, Leicester, United Kingdom

Background: PA-PE is a leading cause of maternal morbidity and mortality in the UK. The current assessment of PEs in pregnancy is difficult and unreliable, leading to the need for better diagnostic strategies. There is debate regarding whether an algorithm such as the pregnancy- adapted YEARS score (figure 1) can be helpful in order to simplify this diagnostic process; the algorithm has been subject to conflicting reports on its efficacy, especially considering the conclusions of the DiPEP biomarker study (2018) which showed no correlation between D-dimer and VTE in pregnancy.

Aims: The study aims to assess the utility of the YEARS algorithm in relation to PA-PE.

Methods: From a local data-base, women who had suffered a PA-PE and who had a D-dimer performed in pregnancy were identified and notes obtained. There were full sets of data available for 36 women. The pregnancy-adapted YEARS algorithm was applied retrospectively to assess its utility. The criteria are summarised in figure 1. 


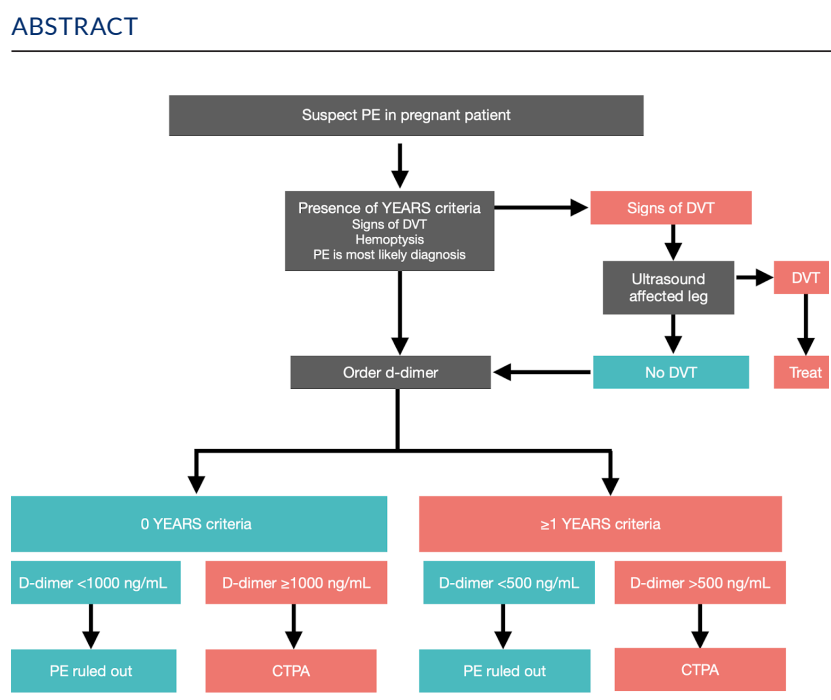

FIGURE 1 The YEARS algorithm

Results: A total of 36 cases of PA-PE were identified, of which 23 were antenatal and 13 were postnatal events. The YEARS score was 1-3 in all cases. D-dimer was raised above the threshold in all but 2 cases (520-9,530ng/ml). CTPA or V/Q scans were performed and PE confirmed in 30 cases and no PE in 4 cases. Two women had d dimer below the threshold and on CTPA did not have a PE. (Table 1)

TABLE 1 Summary of YEARS algorithm results

\begin{tabular}{lll} 
YEARS-predicted & Proven PE & PE not found \\
36 & $30(89 \%)$ & $4(11 \%)$ \\
PA-VTE & AN & PN \\
& $23(64 \%)$ & $13(36 \%)$ \\
CTPA/VQ & Presence of PE & No PE present \\
& $30(89 \%)$ & $4(11 \%)$ \\
\hline
\end{tabular}

Conclusions: No PEs were missed but the score predicted presence of PE in $11 \%$ of cases which had negative scans. These were antenatally suspected VTEs in all four cases. It is reassuring that no VTEs were missed but the specificity of the algorithm is low as dependent on the experience of the assessor as to whether PE is 'the most likely diagnosis'.

PB1310 | Perinatal Outcome of Normal Pregnant and Pregnant Women Hospitalized for Hypertension (PIH: Pregnancy Induced Hypertension) and Studied for Antiphospholipid Antibodies (aPL) at Time of PIH Onset

\section{B. Grand; L.S. Voto}

Hospital "Juan A. Fernández", Department of Maternal and Fetal Medicine. School of Medicine. University of Buenos Aires., Caba, Argentina

Background: The association between $\mathrm{PPL}$ and $\mathrm{PIH}$ remains unclear and controversial. There is no general agreement on which aPL profile confers the greatest obstetric risk and its relationship with rothicher

perinatal outcome in comparison with patients at same gestational age without aPL. Current treatment fails in $20-30 \%$ of pregnancies.

Aims: To assess prospectively the impact of aPL on perinatal outcome in control normal pregnant women studied after 20 weeks and patients hospitalized for $\mathrm{PIH}$ and studied at time of its diagnosis.

Methods: Normal pregnant women (NP) $(n=40)$ : $>20$ weeks of gestation, without infection, hypertension, autoimmune disease, antithrombotic drugs, thromboembolic and/or pregnancy complications, delivering a newborn (NB) at term with adequate birthweight (bw) for gestational age (GA). Patients: Hospitalized for PIH $(n=100)$. Laboratory tests: Blood samples were taken at the time of PIH onset and after $20 \mathrm{w}$ of gestation. Lupus anticoagulant (LA) according to the ISTH recommendations; ELISA tests aPL Ig G and IgM (Louisville) anti b2 Glycoprotein I antibodies IgG and IgM (ab2GPI). GA and bw were documented, and statistical analysis results are showed in table.

Results: Results: LA was positive in $24 \%$ of PIH and in $5 \%$ of NP (p <0.01). Only Ig M ab2GPI was significantly higher: 5, $57 \pm 3,21 \mathrm{UI} /$ $\mathrm{ml}$ vs $7,77 \pm 4,57 \mathrm{UI} / \mathrm{ml}(P<0.01)$. GA and BW between NP and PIH. See Table: Perinatal results of $\mathrm{aPL}$ in $\mathrm{PIH}$ and normal pregnant women

TABLE 1 Perinatal results of aPL in PIH and normal pregnant women

\begin{tabular}{|l|c|c|c|c|c|c|}
\hline & $\begin{array}{c}\text { NB WEIGHT } \\
\mathrm{mg}\end{array}$ & $\begin{array}{c}\text { Std D } \\
\pm\end{array}$ & $P$ & GA w & $\begin{array}{c}\text { Std D } \\
\pm\end{array}$ & $P$ \\
\hline NP & 3379,25 & 428,422 & 0,001 & 39,20 & 1,324 & 0,001 \\
\hline PIH & 2193,67 & 1044,945 & & 34,21 & 4,289 & \\
\hline PIH aPL (-) & 2615,23 & 1035,287 & NS & 35,93 & 4,044 & NS \\
\hline PHI aPL (+) & 2252,60 & 1112,730 & & 34,48 & 5,332 & \\
\hline NP aPL: LA & $4 \%$ & & 0,001 & & & \\
\hline PIH > 34 w aPL: LA & $9 \%$ & & & & & \\
\hline PIH < 34 w aPL: LA & $18 \%$ & & & & & \\
\hline
\end{tabular}

Conclusions: Gestational age is an indicator of perinatal morbimortality. In our work LA was strongly associated with early PIH, but we did not found differences between the gestational age in women with and without $\mathrm{aPL}$ and with $\mathrm{PIH}$.

\section{PB1311 | Review of Intrapartum Management of ITP in Pregnancy in John Radcliffe Hospital}

J. Dhanapal; S. Pavord

Oxford University Hospital, Oxford, United Kingdom

Background: ITP which is the most common cause of thrombocytopenia in early pregnancy can be challenging due to limited treatment options in pregnancy and at times an unexpected fall in the platelet count on arrival in labour.

Aims: We reviewed the adherence to the intrapartum care plans provided by the Obstetric Haematology team using the 2019 International Consensus Guidelines as standard.

Methods: 20 patients selected at random with diagnosis of ITP in pregnancy. Characteristics were:median age 31(age 21 to 43),10 
prima gravid patients, 9 patients with history of ITP while 11 patients had newly diagnosed ITP in pregnancy.

Results: 4 patients in their third trimester with platelet count between 40 to 60 were started on Prednisolone $20 \mathrm{mg}$ daily with good response. 1 patient dropped platelet count to 7 in second trimester. She responded well to $20 \mathrm{mg}$ prednisolone and had uneventful delivery. 8 patients with platelet count above 70 had neuraxial anaesthesia without complications. 1 patient had splenectomy prior to pregnancy and did not have a relapse during pregnancy.13 patients had spontaneous vaginal delivery and 4 had caesarean section for obstetric reasons. None of the patients required emergency treatment at delivery. 5 of 20 babies were born with neonatal thrombocytopenia. One baby with a sibling affected with neonatal thrombocytopenia had platelet count of 89 at birth which further dropped to 39 a week later and recovered spontaneously 3 days later.None of the women reported any significant bleeding in the post partum period. There were no deviations from the recommendations specified in the intrapartum care plan in any of the cases.

Conclusions: In conclusion, we note that our patients responded well to prednisolone $20 \mathrm{mg}$ without complications. Neonatal thrombocytopenia was higher than expected at $25 \%$, however was managed as advised with no issues.Our study reassured us of the value of the documented intrapartum care plan and how advice was reliably followed.

PB1312 | Prophylactic Radiological Interventions to Reduce Postpartum Haemorrhage in Patients with Placenta Accreta Spectrum Disorders

$\underline{\text { L. Bonsen }}^{1,2}$; V. Harskamp ${ }^{3}$; S. Feddouli ${ }^{1,3}$; J. Duvekot ${ }^{4}$; A. Pors ${ }^{3}$; K. Bloemenkamp ${ }^{5}$; J. van Roosmalen ${ }^{1,6}$; M. van Kraaij ${ }^{7}$; J. Zwart ${ }^{8}$; J. van Lith ${ }^{1}$; T. van den Akker $^{1,9}$; D. Henriquez ${ }^{1,2,3}$; J. van der Bom ${ }^{2,3}$; TeMpOH-3 study group

${ }^{1}$ Department of Obstetrics and Gynaecology, Leiden University Medical Centre, Leiden, Netherlands; ${ }^{2}$ Department of Clinical Epidemiology, Leiden University Medical Centre, Leiden, Netherlands; ${ }^{3}$ Jon J. van Rood Centre for Clinical Transfusion Research, Sanquin Research, Leiden, Netherlands; ${ }^{4}$ Department of Obstetrics and Gynaecology, Erasmus University, Rotterdam, Netherlands; ${ }^{5}$ Department of Obstetrics, Birth Center, Wilhelmina's Children Hospital, Division Woman and Baby, University Medical Center Utrecht, Utrecht, Netherlands; ${ }^{6}$ Athena Institute, VU University, Amsterdam, Netherlands; ${ }^{7}$ Department of Internal Medicine, Radboud University Nijmegen Medical Center, Nijmegen, Netherlands; ${ }^{8}$ Department of Obstetrics and Gynecology, Deventer Hospital, Deventer, Netherlands; ${ }^{9}$ National Perinatal Epidemiology Unit, University of Oxford, Oxford, United Kingdom

Background: Placenta accreta spectrum disorder (PAS) has a high maternal morbidity and mortality rate caused by severe postpartum haemorrhage. The role of prophylactic endovascular interventions to reduce blood loss in these patients is controversial.
Aims: To quantify the association of prophylactic placement of balIoon catheters or transcatheter internal iliac arterial sheaths with total blood loss in women at high risk of PAS.

Methods: We included women suspected to have PAS based on their medical history or on radiological imaging who had undergone a planned cesarean section. We selected these women from databases of two national registries and birth registries of 69 participating hospitals in The Netherlands. We determined the effect of the intervention on total blood loss from a linear regression model. Women without balloon catheters were the reference group. Results were adjusted for patient and PAS characteristics.

Results: We included 351 women with suspected PAS: 290 based on history of whom 21 had the intervention and 61 based on imaging of whom 22 had the intervention.

Women with PAS based on history without intervention had median blood loss of $750 \mathrm{~mL}$ (interquartile range, IQR, 500-1500) vs 1000 $\mathrm{mL}(\mathrm{IQR}, 550-1750)$ in women with intervention; adjusted blood loss by intervention: $+2 \mathrm{~mL},(95 \% \mathrm{Cl},-251-377), P=0.99$.

Women with PAS based on imaging without intervention had median blood loss of $2500 \mathrm{~mL}$ (IQR, 1200-5000) vs $2000 \mathrm{~mL}(\mathrm{IQR}, 850-$ 4000); adjusted blood loss reduction by intervention: $-590 \mathrm{~mL}$, $(95 \%$ $\mathrm{Cl},-1018-130), P=0.09$.

In women with confirmed PAS disorder the adjusted blood loss reduction by intervention was $-872 \mathrm{~mL},(95 \% \mathrm{Cl},-1411-84), P=0.07$.

Conclusions: Preoperative placement of balloon catheters in women with PAS might be associated with reduced blood loss. As the results of this study did not reach statistical significance and several previous studies have proven inconclusive as well, we will perform a meta-analysis.

\section{PB1313 | Not Yet to Be Born: A Clinical Case of} Pseudothrombocytopenia in a Pregnant Woman

A.C.B. Marques; A.V.d. Barros; JS. Matias; M. Manaças; F. Carriço;

A. Miranda

Clinical Pathology Department, Hospital de Santa Maria, Centro Hospitalar Universitário Lisboa Norte, Lisboa, Portugal

Background: Pseudothrombocytopenia is a platelet count (PC) erroneously below the reference value, due to platelet aggregation or satellitism, when autoantibodies bind to glycoprotein IIb/IIla in vitro, in the presence of EDTA.

Aims: The authors present a clinical case of pseudothrombocytopenia. Methods: A 32-year-old pregnant woman, gesta 5, para 4, at 36 weeks and 2 days was admitted to the Obstetrics Emergency Room with complains of low back pain, cramps, chills, vomiting and nausea with 1 day evolution. Obstetrics past history of 2 preterm labours. At the inicial observation, she presented no significant clinical changes. Analytically, only revealed thrombocytopenia $\left(126 \times 10^{9} / \mathrm{L}\right)$ and C-RP: $3,93 \mathrm{mg} / \mathrm{dL}$. Urine dipstick test confirmed leukocyturia. SARS-CoV-2 RT-PCR test was positive. Cardiotocography (CTG) showed a normal pattern. The patient was hospitalized with CTG monitoring and 
started cefuroxime for acute pyelonephritis. Prophylactic enoxaparin was started on the $2^{\text {nd }}$ day. The patient remained clinically stable, analytically highlighting a progressive decrease in PC from the $2^{\text {nd }}$ day on. On the $4^{\text {th }}$ day of hospitalization with thrombocytopenia of $11 \times 10^{9} / \mathrm{L}$ and sFIt-1/PIGF ratio ongoing, a variety of different diagnosis were raised: pre-eclampsia, HELLP syndrome, heparin induced thrombocytopenia (HIT) or COVID-19-induced thrombocytopenia. Results:

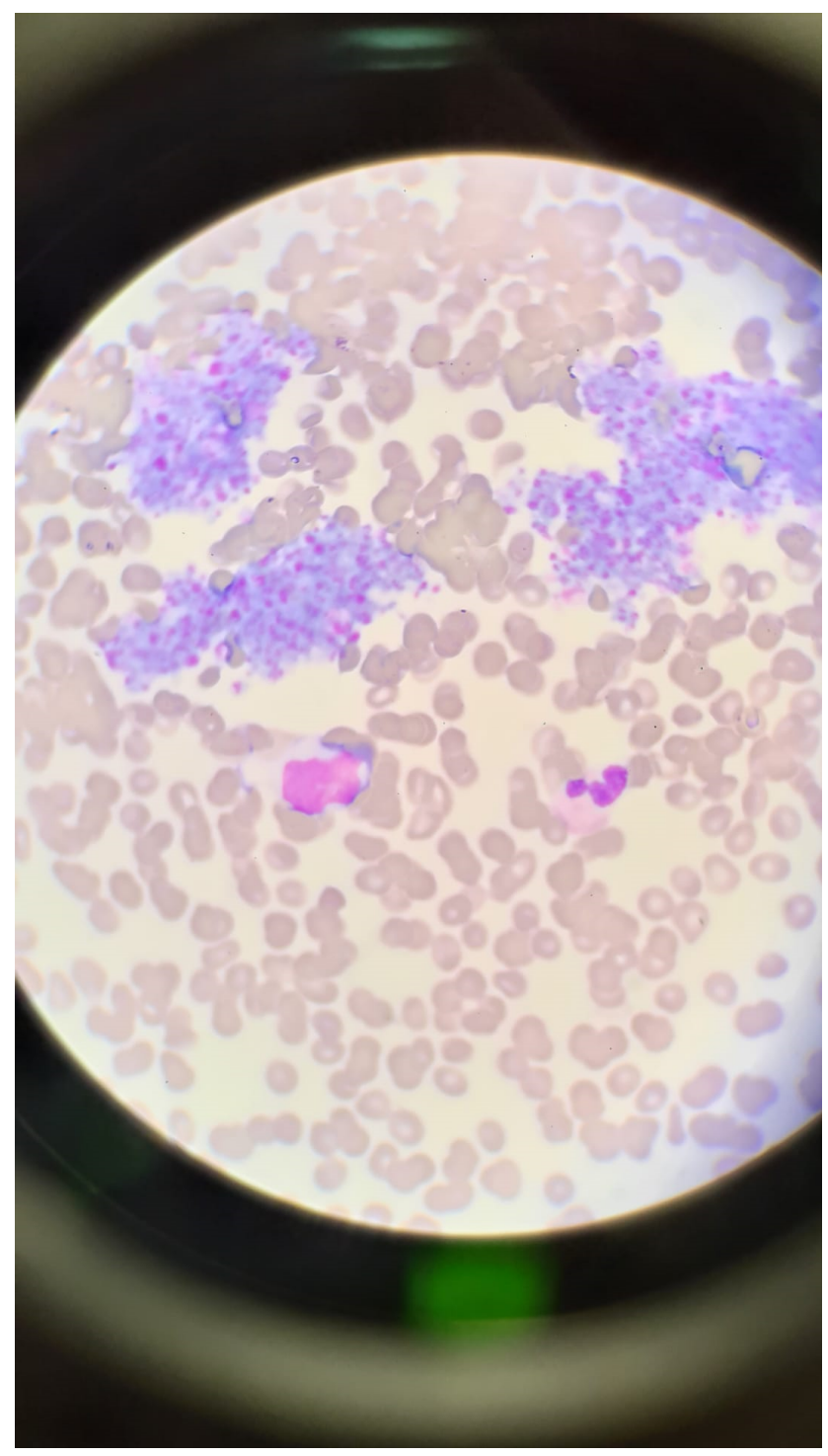

\section{FIGURE 1}

HIT test was negative and the blood smear revealed many platelet aggregations. New blood collections in trisodium citrate solution and in $\mathrm{Mg}^{2+}$ compound collection tubes revealed a $\mathrm{PC}$ of $31 \times 10^{9} / \mathrm{L}$ and $123 \times 10^{9} / \mathrm{L}$, respectively. A significant difference in the PC remained after COVID-19 cure when measured in an EDTA and in
$\mathrm{Mg}^{2+}$ compound collection tubes. On the $5^{\text {th }}$ day she was discharged. The delivery happened later, at 39 weeks of gestation.

Conclusions: The identification of a pseudothrombocytopenia avoided an iatrogenic preterm labour. When confronted with a patient with no signs or symptoms of bleeding or haemorrhagic dyscrasia and no past history of thrombocytopenia, pseudothrombocytopenia should be considered. Its early detection may avoid extensive investigations and unnecessary therapeutic procedures.

PB1314 | The Effect of Prothrombin Complex Concentrate and Cryoprecipitate on the Frequency and Severity of Multiple Organ Dysfunction Syndrome in Massive Obstetric Haemorrhage

V. Sedinkin; O. Klygunenko; O. Volkov

Dnipropetrovsk Medical Academy of Health Ministry of Ukraine,

Dnipro, Ukraine

Background: Effective treatment of massive obstetric haemorrhage is important; however, the optimal therapy is still not known.

Aims: We aimed to compare the efficacy of first-line therapy using cryoprecipitate (CP) or prothrombin complex concentrate (PCC) for the reversal of induced coagulopathy, and consequently the development of multiple organ dysfunction syndrome (MODS).

Methods: Having agreed with the local Ethics Committee and obtained the informed consents, 75 women (whose delivery or early postpartum period was complicated by severe hemorrhage with blood volume (BV) deficit of $40-52 \%$ ) were examined. Patients were randomized into 2 groups depending on the characteristics of the BV replenishment. Both groups were similar in relation to age, height, gestation term, blood loss volume $(2570 \pm 510 \mathrm{ml})$. In 1 group $(n=42)$ of first-line prevention of progression severe coagulopathy using CP (5-8 $U)$. In group 2 ( $n=33$ ), the first-line drug was used for $1500 \mathrm{IU}(20 \mathrm{mg}$ / kg) PCC. Clinical signs of MODS are accounted for myocardial insufficiency (frequency and duration of vasopressor support); respiratory dysfunction (frequency and duration of mechanical ventilation); acute kidney injury (frequency and duration of use of diuretics), gastrointestinal dysfunction (frequency and duration of the enteroparesis).

Results: Analysis showed that use of PCC reduced total infusiontransfusion volume by $24.5 \%$ (1300 ml). Patients 2 group had decreased frequency and duration of VS, frequency and duration of $\mathrm{MV}$, had reduced the number of patients requiring RRT, and it duration. The frequency GIT dysfunction reduced more than twice. This provided a significant reduction in the duration of stay in the ICU from $10.7 \pm 1.5$ ( 1 group) to $7.8 \pm 2.1$ days (2 group).

Conclusions: Our results underline the importance of early and effective factor concentrates supplementation for severe coagulopathy in massive obstetric haemorrhage. The available sample size in our study appears sufficient to make some conclusions that first-line PCC is superior to CP. 
PB1315 | Hereditary Thrombophilia in Macedonian Women with in vitro Fertilization Failure

E. Petkovikj; R. Grubovic Rastvorceva; T. Makarovska Bojadzieva;

E. Velkova; V. Dejanova; E. Ristovska; S. Useini

Institute for Transfusion Medicine, Skopje, North Macedonia, The

Republic of

Background: Many factors contribute for in vitro fertilization (IVF) failure, and hereditary thrombophilia as hypercoagulable condition has been mentioned as one of them. There are limited data on the association between thrombophilia with IVF failure in Macedonian population.

Aims: The aim of the study was to examine the representation of thrombophilic mutations in Macedonian women with IVF failure and healthy controls.

Methods: In this case-control study we evaluated 70 women, divided in two groups. The case group included 34 women with history of women with three or more failed cycles of IVF. The control group included 36 women, age matched, who gave birth to at least one healthy baby without obstetric complications. All women included in the study have signed the informed consent approved by the Ethical Committee of the Medical faculty in Skopje. Presence of gene mutations for prothrombin G20210A, factor V Leiden G1691A (FVL) and methylentetrahydrofolate reductase C677T (MTHFR) was examined in both groups.

Results: Prothrombin G20210A heterozygous was found in $5.9 \%$ of the case group vs. $5.5 \%$ in the control group; FVL heterozygous was found in $20.6 \%$ of the case group vs. $2.8 \%$ in the control group with a significant statistical difference $(P=0.0194)$; MTHFR homozygous was found in $20.6 \%$ of the case group vs. $5.5 \%$ in the control group. Combined thrombophilic mutations were present in $17.6 \%$ in the case group and $5.6 \%$ in the control group.

Conclusions: Hereditary thrombophilia is more prevalent in women with IVF failure than the control group. The presence of at least one thrombophilic mutation especially FVL heterozygous and MTHFR homozygous may have significant role in IVF failure.

\section{PB1316 | Bombay Phenotype and Twin Pregnancy: Case Report} and Literature Review

\section{Krigstein; N. Cromer}

Royal North Shore Hospital, St Leonards, Australia

Background: Bombay phenotype is rare and case reports of antenatal care in these patients are scarce. We present an even rarer case of a Bombay female pregnant with twins and detail her multidisciplinary management and outcome.

Aims: In conjunction with a literature review of all published cases, we hope this assists other clinicians with their decision making in the antenatal management of this uniquely challenging scenario.
Methods: We describe a case report of a Bombay female pregnant with dichorionic diamniotic twins. We summarise the literature to date to better inform decision making and patient blood management in the antenatal care of Bombay patients.

Results: Haematinics were optimised to ensure an optimise haemoglobin. Anti-H titres were tracked throughout pregnancy and were 1:256 at both 28 weeks and 36 weeks gestation. Regular middle cerebral artery dopplers were performed to assess for fetal anaemia. There was constant communication with obstetrics and anaesthetics throughout the antenatal period. Both autologous frozen and directly donated fresh red cells were available as part of a clear detailed transfusion plan for the patient (Figure 1). Transfusion was not required and neither child was affected by haemolytic disease of the foetus and newborn. The neonates were blood group O, DAT negative, and blood group A, DAT positive. Maternal anti-A was detected in the neonatal eluate.

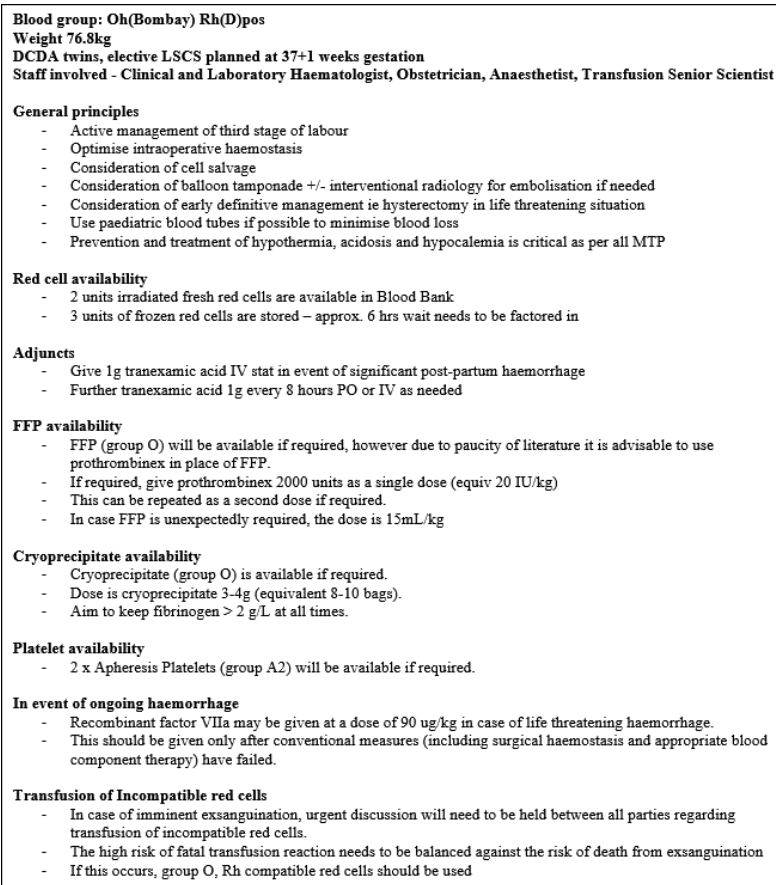

FIGURE 1 Detailed Haemostasis / Transfusion Plan for our twin delivery with Bombay blood group

Conclusions: Bombay phenotype poses unique challenges during pregnancy, particularly when postpartum haemorrhage risk is increased such as twin pregnancy. Through employing patient blood management strategies, engaging a collaborative multidisciplinary approach involving anaesthetics and high risk obstetrics, and a clear detailed delivery plan, these challenges can be surmounted. 


\section{P0190 | Successful Infertility Treatment and Pregnancy} Outcome in a Woman with Severe Treatment-refractory ITP

B. Krastev; P. Arabadjikova; I. Sarbianova; G. Grigorov; M. Eneva;

G. Stamenov

MHAT Hospital for Women Health Nadezhda, Sofia, Bulgaria

Background: A proportion of patients with idiopathic thrombocytopenic purpura (ITP) are refractory to treatment and in young women this poses risk to pregnancy and delivery.

Aims:

Methods:

Results:

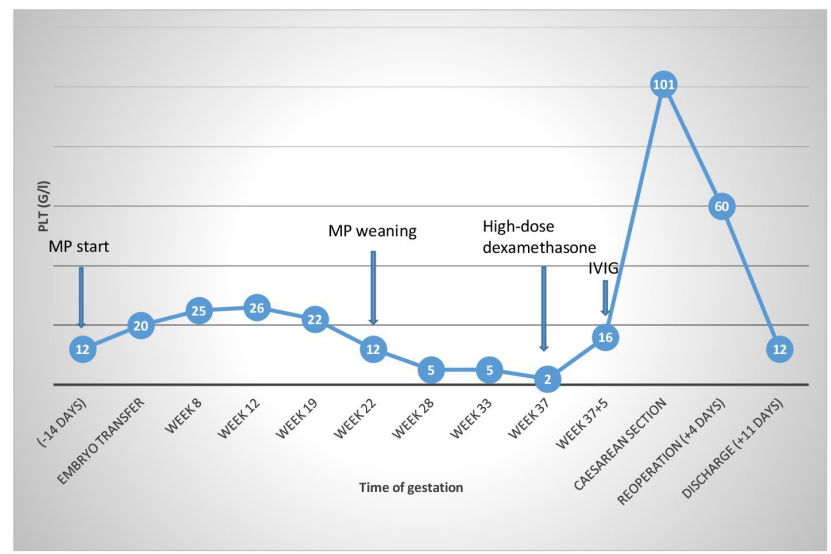

FIGURE 1 PLT dynamics during pregnancy

A 37-year-old woman presented with ITP since adolescence. She had already tried different therapeutic options, including glucocorticosteroids, splenectomy, cytostatics, thrombopoetin agonists and intravenous immunoglobulins (IVIG). All treatment had led to modest and transient effect and at that moment she received no therapy. Despite usual platelet counts (PLT) of around $10 \mathrm{G} / \mathrm{l}$, the patient had no history of significant bleeding.For the last eight years, the woman had been struggling to conceive, including two unsuccessful in vitro ferilization (IVF) attempts. We planned another IVF procedure and 14 days before it, oral methylprednisolone (MP) was started. A single embryo was transfered at PLT $20 \mathrm{G} / \mathrm{l}$ and three weeks later pregnancy was confirmed. MP was continued throughout pregnancy, sustaining PLT slightly above $20 \mathrm{G} / \mathrm{l}$, until gestational week 22 when the effect was lost and therapy was weaned.At week 37 , the patient was hospitalized for planned caesarean section with PLT $2 \mathrm{G} /$ l. High-dose dexamethasone was tried but PLT increased insignificantly. At that point, we applied a single infusion of IVIG $1 \mathrm{~g} / \mathrm{kg}$ and 48 hours later PLT were 101 $\mathrm{G} / \mathrm{I}$. The caesarean section was performed with normal intraopearitve blood loss. However, on day 4 after delivery, the woman had to be reoperated due to subfascial hematoma of the abdominal wall. This necessitated additional hematological support and after a week of recovery, the woman was discharged. Her newborn was clinically well but with severe neonatal thrombocytopenia, having PLT $6 \mathrm{G} / \mathrm{l}$ at birth.
Conclusions: Pregnancy should not be discouraged in women with refractory ITP. High-dose IVIG could rescue delivery and mitigate postpartum maternal bleeding but neonates are still at risk of severe thrombocytopenia.

\section{P0191 | The Case of Obstetric APS - A Therapeutic Challenge}

\section{J. Teliga-Czajkowska ${ }^{1}$; K. Czajkowski²; A. Sikorska ${ }^{3}$}

${ }^{1}$ Medical University of Warsaw, Department of Obstetrics and Gynecology Didactics, Warsaw, Poland; ${ }^{2}$ Medical University of Warsaw; 2nd Department and Clinic of Obstetrics and Gynecology,, Warsaw, Poland; ${ }^{3}$ Institute of Hematology and Transfusion Medicine, Department of Disorders of Hemostasis and Internal Medicine,, Warsaw, Poland

Background: Antiphospholipid syndrome - APS - is a systemic autoimmune disorder characterized by thrombotic venous or arterial circulation in the presence of antiphospholipid antibodies -aPL: lupus anticoagulant -LA, anticardiolipin antibodies, and antibeta2glycoprotein-I antibodies - anti-beta GPI. APS can be either primary or secondary when it occurs in the presence of an underlying autoimmune disorder. Pathophysiologic mechanism underlying thrombosis and pregnancy loss in APS include aPL induced cellular activation, inhibition of both natural anticoagulant and fibrinolytic system and complement activation. There is a high rate of pregnancy complications and thrombotic events especially in triple positive patients. Aspirin with low molecular weight or unfractionated heparin may reduce the incidence of pregnancy loss in obstetric APS and are therefore a standard treatment.

Aims: To present a case of obstetric APS which presented a therapeutic challenge.

Methods: The 34 years old primigravida with APS was admitted to the Clinic in 21st gestational week with epigastric pain, hypertension, elevated liver enzymes and low platelets. there was a positive culture of E.coli in urine and uterine cervix which were accordingly treated with antibiotics. The fetus was symmetrically hypothrophic. Due to the further decrease in platelets count till 10000 with no hemorrhagic diathesis, we investigated the presence of EBV and ADAMTS-13 level. Only heparin-dependent antiplatelet antibodies against heparin/PF4 were detected. LMWH was stopped and steroid treatment was initiated. In 23rd gestational week a stillbirth was discovered. Before induction of abortion, the patient received platelets transfusion. The post-surgery course was uneventful but the heparin dependent antiplatelet antibodies were still detected 6 months later.

Results: 
Lab-tests results

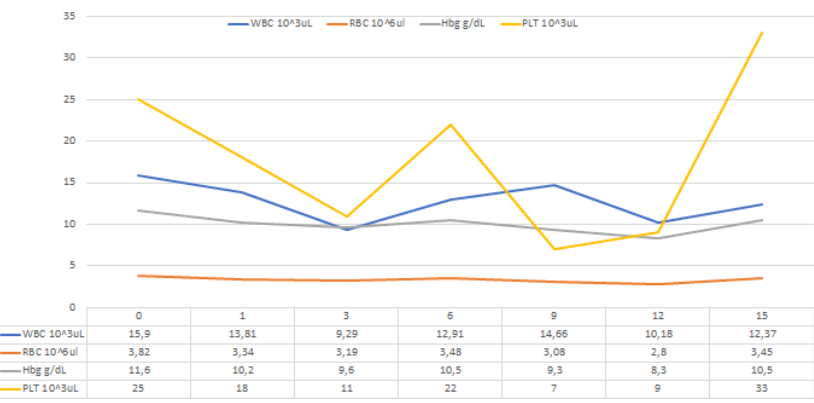

FIGURE 1 Coagulation lab-tests results

\section{Coagulation lab-tests results}

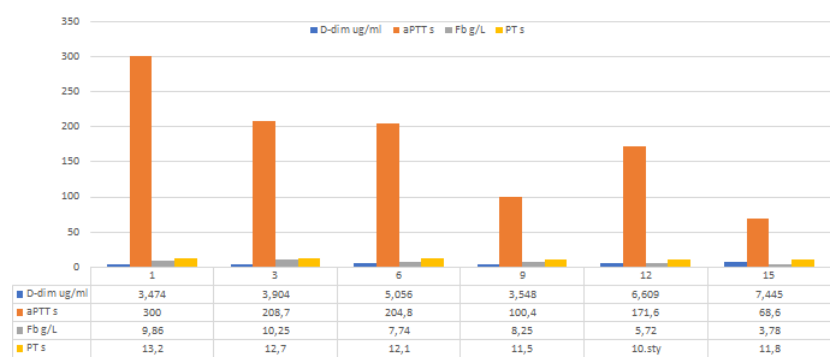

FIGURE 2 Lab-tests results 2

Conclusions: Pregnancy complications that are observed in APS may be overlapped by some other conditions. The standard treatment may not be sufficient. Recent insights into the pathogenesis of APS have led to the identification of new potential therapeutic interventions, including anti-inflammatory and immunomodulatory therapies.

P0192 | The Role of Hydroxychloroquine in a Secondary

Prevention of Recurrent Thrombosis in Patient with Thrombophilia and Antiphospholipid Syndrome. A Case Report

A. Movsisyan ${ }^{1}$; I. Karapetyan ${ }^{2}$; A. Harutyunyan ${ }^{2}$; H. Grigoryan ${ }^{1}$;

L. Hambardzumyan ${ }^{2}$; J. Simonyan ${ }^{2}$; N. Sargsyan ${ }^{1,2}$; G. Tamamyan ${ }^{1,2}$;

S. Danelyan ${ }^{1}$; L. Sahakyan ${ }^{1}$; H. Khachatryan ${ }^{1,2}$

${ }^{1}$ Hematology Center after Prof. R.H. Yeolyan, Yerevan, Armenia;

${ }^{2}$ Yerevan State Medical University, Yerevan, Armenia

Background: Antiphospholipid syndrome (APS) is characterized by a high incidence of recurrent venous or arterial thrombosis and obstetric morbidity. Conventional anticoagulation therapy may be ineffective in antithrombotic prophylaxis. It remains an issue in the management of APS. Hydroxychloroquine (Plaquenil) is reported to reduce antiphospholipid antibody titers in patients with antiphospholipid syndrome and to improve pregnancy outcomes. Several data suggest the role of Hydroxychloroquine in lowering incidence of thromboembolic events in patients with persistently elevated levels of antiphospholipid antibodies.

Aims: To decrease events of recurrent thrombosis, to show the efficacy of Hydroxychloroquine in prophylaxis of recurrent thrombosis in patients with antiphospholipid syndrome.

Methods: The patient is a 22-year-old primigravida, at the gestational age of 10 weeks, with a history of thrombotic episodes, homocysteinemia, and antiphospholipid syndrome.

She was diagnosed with deep vein thrombosis of the legs a few years ago. Anticoagulation treatment was initiated with Low-molecularweight heparin (LMWH) and Rivaroxaban. Within a year after the first thrombotic episode re-thrombosis occurred, which was followed by the identification of several mutations, including FII/prothrombin G20210A mutation high-risk heterozygous, FITGL, ITGB, PAI low-risk heterozygous, MTR homozygous, MTRR, MTHFR heterozygous mutations. Additionally, she has persistently tested positive for antiphospholipid antibodies (anti- $\beta 2$-glycoprotein 1 IgAMG, anti-cardiolipin IgAMG). Anticoagulant agents were replaced by Warfarin and Hydroxychloroquine, the result was beneficial. After pregnancy diagnosis, the former was changed to LMWH. Plaquenil $200 \mathrm{mg}$ daily administration during the entire pregnancy is recommended, except of bleeding episodes and hematoma

Results: The patient experienced recurrent venous thromboembolism while being treated with conventional anticoagulant therapy. The change of the treatment plan with Hydroxychloroquine inclusion was associated with the improvement of clinical presentation and imaging tests' results.

Conclusions: Experience showed that Hydroxychloroquine administration allows to prevent recurrent thrombosis, safe pregnancy, prevents the risk of miscarriages. 